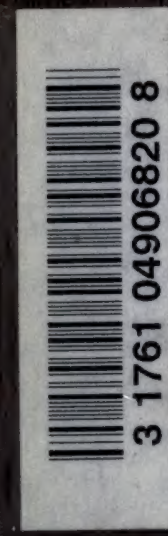

. 




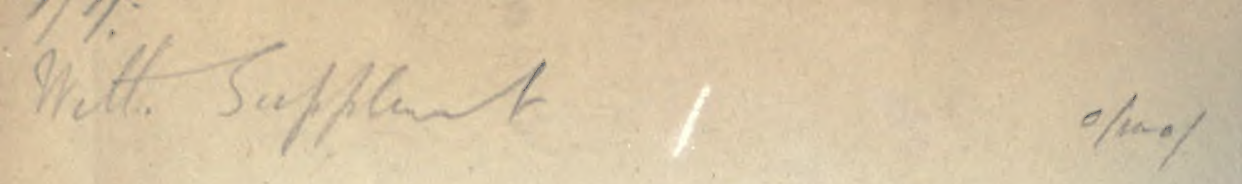
Glat. 
Digitized by the Internet Archive in 2007 with funding from Microsoft Corporation 



\section{DICTIONARY}

OF

ARTS, MANUFACTURES, AND MINES.

VOL. I. 

S.D

W $753 \mathrm{~d}$

\section{URE'S DICTIONARY}

OF

\section{ARTS, MANUFACTURES, AND MINES}

costante

A CLEAR EXPOSITION OF THEIR PRINCIPLES AND PRACTICE

BT

\section{ROBERT HUNT, F.R.S.}

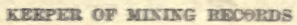

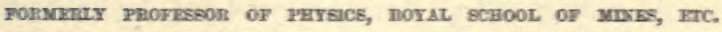

ACTHOR OP 'REEEARCHES OS UGKT' 'THE POETEY OF SCHESCE' ITC.

ascisted by

F. W. RUDLER, F.G.S.

and by aumerous Contributors eminemt in Science asd fomitiar cailh Monvfactures

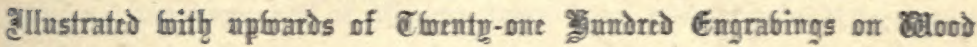

\section{SEVENTH EDITION}

COMPLETELY REVISED AND GREATLY ENLARGED

IN THREE VOLUMES

VOL. I.

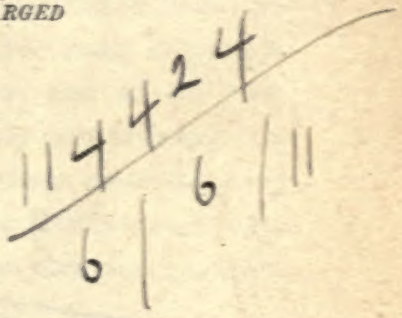

LONDON

LONGMANS, GREEN, AND CO. 
जरामा घh,

Pan

पintueit?

$$
\begin{gathered}
\text { SEEN BY } \\
\text { PRESERVATION } \\
\text { SERVICES } \\
\text { DATE99 }
\end{gathered}
$$




\section{PREFACE}

To

\section{THE SEVENTH EDITION.}

This Dictionary of Arts, Manufactures, and Mines passed through four Editions during the lifetime of Dr. ANDREW URE; and this is the third Edition which has been required by the public, since, in 1858 , it was committed to my care.

These volumes have received the same unrelaxing attention, to every detail, which was bestowed upon the previous Editions, and which secured for them the confidence of the manufacturer, the miner, the metallurgist, and the general public.

Every division of the Arts; each special process of Manufacture, and all the branches of Mining, have been most cautiously examined, and such improvements, as have been proved to be of real utility, have been recorded in all necessary detail. This has led to an increase in the size of the volumes, to the rejection of many articles which had, with the progress of advancing knowledge, become obsolete, and. to the curtailing of others which were of less importance than the new ones which it was necessary to introduce. The more important articles have been, for the most part, rewritten, and all of them subjected to a critical revision, while many entirely new articles have been introduced. It is needless to particularise these, since the most hasty comparison between the volumes of the last Edition, and those of the present one, will at once render the new and the amended articles sufficiently obvious.

The type for the whole of the work has been entirely reset, and nearly two hundred woodcut illustrations have been added. 
The list of contributors will show to whom we are indebted for the techuical articles which distinguish this Dictionary; and the articles themselves will give evidence of their having been treated, in all cases, by men who are thoroughly experienced in the processes which they have described. When the initial letters at the end of the articles do not indicate the authors of them, I am directly responsible for them. In a work treating of the useful applications of Science, it became necessary to introduce such portions of those sciences, which have been economically useful to man, as would render the processes described sufficiently intelligible to all readers, and show the aid which has been rendered by scientific enquiry. Therefore, several divisions of Physics, Chemistry, Geology, and Mineralogy, are succinctly dealt with. Of Chemistry, it should be remarked, that the very rapid advances of discovery in that science, have involved a revolution in the mode of viewing the constitution of bodies, and necessitated the construction of a new mode of expressing that constitution. During the transition period, there naturally arises much difficulty in determining with exactness the formulæ which shall correctly express the composition of a compound. This natural difficulty has been aggravated by the unfortunate introduction of hypothetical views respecting the constitution of compounds, and the creation of systems of notation which are constantly liable to somewhat capricious alteration. As this Dictionary is for the use of a public which cannot be expected to be acquainted with each change in the views entertained by the different schools of Chemistry, it has been thought desirable to retain the formulø with which they have been long familiar, and to give in another (black) type the formulw which have been adopted by most modern chemists.

It will be observed that in some cases the Imports and Exports of productions used in the Arts or Manufactures, which were given in the former Editions, do not appear in the present one. This arises from the impossibility of obtaining them: the Custom House authorities now entering a large number of articles under the head of 'Unenumerated,' which were formerly given in detail. This is much to be regretted, since it removes the power of tracing the progress of the special use, or industry, to which the article in question belongs.

To all those gentlemen who have favoured me with contributions my best thanks are due. I have, however, to express my obligations more especially - to Mr. Higarn, whose article on 'Calico Printing' gives a more satisfactory description of that industry than any other to be 
found in any language; to Mr. Hilary Bauerman, who has brought the articles 'Iron' and 'Steel' up to the most recent date, and to a condition of great completeness; and to Mr. John Darlingron, who has submitted to a complete revision all the articles connected with Mining. Of the latter it may be safely said, that they, taken collectively, form a description of the modes of obtaining and of preparing the useful minerals for the market such as is not to be found in any other work in the English language.

My obligations to Mr. F. W. RUdLER, who has assisted me in my heavy labours of producing these volnmes, are great. During a period, when the disturbed condition of my health rendered it even dangerous for me to give any prolonged fixed attention to the Dictionary, that gentleman, with almost enthusiastic zeal, devoted his best energies to the tedious details of the work, and gave me all the advantages of his general and exact knowledge.

With the aid that has been received, and the attention which has been bestowed on this work, I feel that it may with confidence be commited to the public, believing that it will be found a useful guide in all those industries which are connected with the Arts, Manufactures, and Mines.

ROBERT HUNT, F.R.S.

April 27, 1875. 



\title{
LIST OF THE CONTRIBUTORS.
}

\author{
General Editor-ROBERT HUNT, F.R.S. \\ KEEPER OF MINING RECORDS, ETC. \\ Assistant Editor-F. W. RUDLER, F.G.S.
}

W. C. AITKIN, EsQ.

Birmingham.

GEORGE F. ANSELL, Esq.

EMERSON BAINBRIDGE, Esq. Mining Fingineer, Sheffield.

H. K. BAMBER, Esa., F.C.S., åc.

H. BAUERMAN, Ese., F.G.S. Author of 'A Treatise on the Metallurgy of Iron."

E. W. BINNEY, Esa., F.G.S., \&c. Manchester.

(The late) H. W. BONE, Ese. Enamellex.

HENRY W. BRISTOW, Ese., F.R.S. F.G.S. Author of "Glossary of Mineralogy." Director of the Geological Survey of England and Wales.

R. J. COURTNEY, EsQ.

JAMES DAFFORNE, EsQ. Assistant Editor of the 'Art Journal.'

JOHN DARLINGTON, Esa. Mining Kngineer. Author' of 'Miner's Hand. book."

M. DARTON, Ese. Bookbinder, Loudon.

(The late) F. W. FAIRHOLT, Esq, F.R.A.S, Anthor of 'Costume in England,' 'Dictionary of Terms in Art,' \&e.

E. FRANKLAND, EsQ., Pн.D., F.R.S., F.C.S. Profeseor of Chemistry, Royal School of Mines.

ALFRED FRYER, Ese. Sugar Reflner, Manchester.

(The late) T. H. HENRY, Esa., F.R.S., F.C.S.
E. HELM, Ese.

Manchester. Author of papers on 'Cotton Manufacture.'

(The late) W. HERAPATH, Ese, M.D., \&c.

R. HERRING, EsQ. Author of 'History of Paper Manufacture.'

JAMES HIGGIN, Ese., F.C.S. Manchester.

SAMUEL HOCKING, Esq., C.E. Camborne, Cornwall.

G. MANLEY HOPWOOD, EsQ. Chemist, Manchester.

GEORGE HUNT, EsQ. Brewer, Oldham.

T. B. JORDAN, EsQ. Engineer; Inventor of Wood-carving Machinery.

JANES B. JORDAN, Ese.

Assistant in Mining Record Office, Museum of Practical Geology.

WILLIAM LINTON, Ese. Artist. Author of "Ancient and Modern Colonrs.'

(The late) JAMES Mc ADAM, Jun., EsQ. Late Secretary of the Royal Society for the Cnltiration of Flax in. Ireland.

H. Mc CALL, EsQ.

Lisburn, Ireland. Author of Essays on 'Linen and Flax,'

(The late) HERBERT MACKWURTH, EsQ., C.E., F.G.S. One of H.M.'s Inspectors of Coal Mines.

HENRY MARLES, EsQ., L.R.C.P.

(The late) DAVTD MORRIS, Ese.

Manchester. Author of 'Cottonopolis,' dec. 
D. NAPIER, Esq., C.E., \&c.

JAMES NAPIER, EsQ, F.C.S. Author of 'Manual of Dyoing, 'ElectroMetallurgy,' 'Ancient Works in Metal,' \&c.

HENRY M.NOAD, Esq., Ph.D., F.R.S. Author of 'A Mannal of Electriclty,' \&c.

(Thelate) A. NoRMANDY, EsQ., M.D. F.C.8. Author of 'Handbook of Commercial Chomistry."

AUGST.B. NORTHCOTE, EsQ.,F.C.S. Chemist, Univeraity of Oxford.

ROBERT OXLAND, EsQ., F.C.S. One of the Authors of ' Metals and their Alloys."

THOMAS JOHN PEARSALL, EsQ., F.C.S., \&c., \&c.

JOHN ARTHUR PHILIPS, Eso. Mem. Inst. C.F., F.G.S., F.C.S. Graduate of the Imperial School of Mines, Paris. Anthor of 'Elements of Metallurgy,' and 'The Mining Metallurgy of Gold and Silver.

SEPTLMUS PIESSE, Ese., F.C.S. Author of 'Treatise on Art of Perfumery,' \&c.

ANDREW CROMBIE RAMSAY, Ese, LL.D., F.R.S., F.G.S. Professor of Geology, Royal School of Mines; Director-General of the Greological Survey of the United Kingdom.

T. A. READWIN, Esq., F.G.S. Manchester.

(Thelate) EBENEZER ROGERS, C.E., F.G.S.

CHARLES SANDERSON, Esq. Sheffield. Anthor of Papers on "Steel and Iron.

E. SCHUNK, Esq., PH.D., F.R.S. F.C.S.

R. ANGUS SMITH, Esq.,PH.D.,F.R.S. Author of various Papers on 'Air and Water,' 'Life of Dalton,' and 'History of Atomic 'Theory,' \&c.

RICHARD SMITH, EsQ.

Saperintendent of Metallurgical Laboratory, Royal School of Mines.
WARINGTON W. SMYTH, EsQ., M.A. F.R.8., F.G.S. Professor of Mining and Mineralogy, Royal School of Mines, and Inspector of Crown Mines.

THOMAS SOPWITH, EsQ., M.A., F.R.S., F.G.S. Author of 'Isometrical DruW ing,' '\&o.

JOHN SPILLER, EsQ., F.C.S.

President of the Photographic Socioty.

ANDREW TAYLOR, Esq., F.C.S. Chemist, Edinburgh.

(The late) ROBERT DUNDAS THOISON, EsQ., M.D., F.R.S. Professor of Chemis. try in St. Thomas's Hospital College.

JOHN W. TURNER, ESR.

Bradford. Author of Papers on 'Wool anil Woollen Mannfacture.

ALFRED TYLOR, Es\&., F.G.S. Anthor of 'Treatise on Metal Work.'

A. VOELCKER, Esa., Pн.D., F.R.S., F.C.S. Consulting Chemist to the Royal Agricaltural Society of England.

CHARLES V. WALKER, EsQ., F.R.S. F.R.A.S. Engineer of Telegraphs and Time to the South-Eastern Railway Company. Author of 'Mlectrotype Manipulation'; Translator of 'Kæemtz's Meteorology,' 'De la Rive's Electricity;' \&c.

W. J. WARD, Esa., Metallurgical Laboratory, School of Minos.

C. GREVILLE WILLIAMS, Ese., F.R.S. Author of "A Handbook of Chemical Manipulation,' \&ce.

WM. MATTIEU WILLIAMS, Ese., F.C.S.

(The late) HENRY M. WITT, EsQ. F.C.S. Assistant Chemist, Government School of Mines.

With special assistance and information from the late Sir WM. REID, C.B.; Sir Wy. Armstrone, C.B., \&c.; Robert Maldet, Esq., F.R.S., dc. ; Captain Drayson, R.A.; Georen W. Lenox, Esq.; and many oihers. 


\section{Omissions.}

Page 157 insert AmruAmras. See Watts's 'Dietionary of Chemistry.'

" 203 " ARACEs. A natural order of monocotyledonous plants, to which the genus Arum gives the name.

" 1008 " Cow TrRz. The milk tree of Demerara,

". " CRAB OII. An oil obtained in South America, from the seed of the Carapa Guinanensis. 



\title{
A DICTIONARY
}

\author{
OP
}

\section{ARTS, MANUFACTURES, AND MINES.}

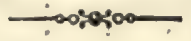

A

Aaz. A red dye used in Central India for imparting a permanent colour to the native cotton cloth. It is yielded by the roots of the Morinda citrifolia, a small tree belonging to the Rubiacea, or madder order. Professor T. Anderson has obtained from the aal root a pale yellow crystalline substance which he calls morindin, and this whon subjected to distillation yields a crystalline sublimate termed morindone. It has been found that this morindone is identical with alizarine, one of the colouring principles of madder; and it is conjectured that the morindin may correspond with ruberythric acid.

ABA. A woollen stuff manufactured in Turkey.

ABACA. A species of fibre obtained in the Philippine Islands in abundance. Some authorities refer those fibres to the palm-tree known as the Abaca, or Anisa textilis. Thero seem, indeed, to be several well-known varieties of fibre included under this name, some so fine that they are used in the most delicate and costly textures, mixed with fibres of the pine-apple, forming Pina muslins and textures equal to the best muslins of Bengal. Of the coarser fibres, mats, cordage, and sail-cloth are made. M. Duchesne states that the well-known fibrous manufactures of Manilla have led to the manufacture of the fibres themselves, at Paris, into many articles of furniture and dress. Their brilliancy and strength give remarkable fitness for bonnets, tapestry, carpets, network, hammocks, \&c. The only manufactured articles exported from the Philippine Islands, enumerated by Thomas de Comyn, Madrid, 1820 (transl. by Walton), besides a few tanned buffalo hides and skins, are 8,000 to 12,000 pieces of light sail-cloth, and $200,000 \mathrm{lbs}$. of assorted abaca cordage.

ABICErT2. An arsenate of copper found occasionally in the copper mines of Cornwall and of the Hartz. It usually consists of 54 per cent, of protoxide of copper and 30 per cent. of arsenic acid, with water.

ArIIs (in Botany). The fir; a genus of trees which belong to the coniforous order. These trees are well known from their ornamental character, and for the valuablo timber which they produce. They yield several resins or gum-resins, which are nseful in the arts.

Abizs Barsames. The Balm of Gilead fir. It is a native of Canada and Nova Scotia; it produces the Canada Balsam. This elegant tree grows most abundantly in the colder regions of North America. (See Canada Batsam).

Abies Crorus (Cedrus Iibani,. The Cedar. It is a nativo of Mount Lebanon and the range of Mount Taurus. Cedar wood is said to be very indestructible, and its wood is used in the manufacture of ormamental boxes, on account of its odour. See Crdar Woom.

Arias Excruss of De Candolle (Pinus abies of Linnæus). The Norway Spruce fir, or Dantric Deal, a native of Germany, Russia, Norway, and other parts of Northern Europe. It yields-a resinous exudation known as Frankincense or Thus, while the wood forms the 'White Deal' of the carpenter. The well-known Burgundy Pitch is

Vot, I. 
propared from the resin obtnined from this tree. See Burgundy Pitcr; FrankIXCEXSE.

Amiss Larix (Larix europera). The Common Larch, producing the Venice Turpen. tine, and a manna called Briançon Manna which exudes from the leaves. Seo Vesice TURPENTINE.

Ariss Nigra. The Black Spruce fir, indigenous to the most inclement regions of North America. A considerable quantity of the essence of spruce is extracted from the young branches of this tree; it is, however, also obtained from other varieties of the spruce fir. See Sprucu Essneck.

Aburs Prcra of Linnæeus (Abies pectinata of De Candolle). The Silver fir, producing the true Strasburg turpentine. Seo Turpentine.

Of the woods of these trees the following quantities were imported in 1871 :-

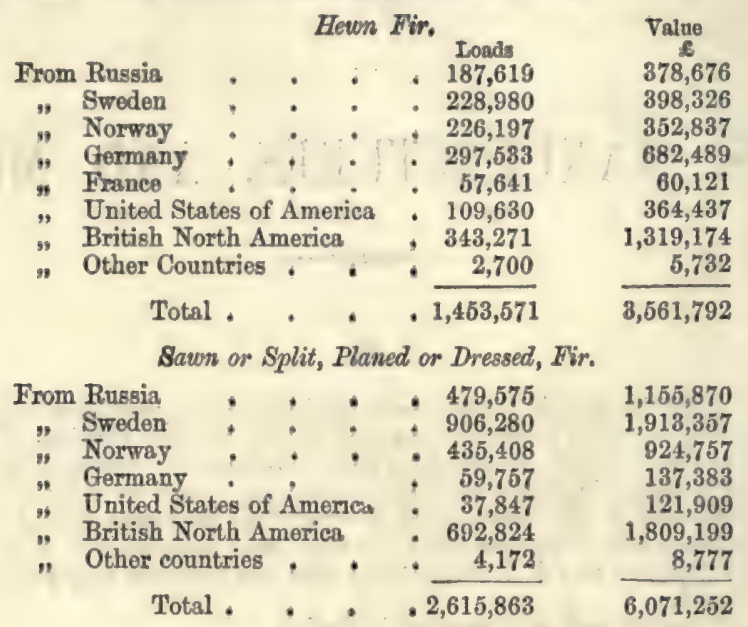

ABIDTsNz. This hydrocarbon is the product of distillation of the terebinthinate exudation of a coniferous tree indigenous to California, viz., the Pinus sabiniana, a tree met with in the dry sides of the foot hills of the Sierra Nevada mountains, and locally known as the nut-pine or digger-pine, on account of the edible quality of its fruit. A gum resin, or rather balsam, is obtained from this treo by incisions made in its wood, and the balsam is submitted to distillation almost immediately after it has been collected, owing to the great volatility of the hydrocarbon (or essential oil, because abietene really stands in the same relation to the balsam alluded to, as oil of turpentine stands to the exudation derived from other species of Pinus). The crudeoil, as usually met with for sale at San Francisco, is a colourless limpid fluid, requiring only to be redistilled to obtain it quite pure. The commercial article is used under different names, abietene, exasine, theoline, \&c., for the removal of grease and paint from clothing and woven fabries, and likowise as an efficient substitute for petroleum-benzine. Pure abietene is a colourless fluid, possessing a strungly penetrating odour, bearing some resemblance to oil of oranges; sp. gr. at $165^{\circ}=0.694$ : it is very volatile, highly combustible, burning with a brilliant white smokeless tlame, almost insoluble in water, and soluble in 5 parts of alcohol at 95 per cent. Abietene is not acted upon by dry hydrochloric acid gas nor by nitric acid (sp. $\mathrm{gr}_{\mathrm{s}}=1 \cdot 43$ ) in the cold, but heat being applied, a slight reaction takes place; neither concentrated sulphuric acid nor potassium acts upon this hydrocarbon; when treatod with chlorine, abietene is converted into fluid of the consistency of glycerine, insoluble in water, colourless, soluble in warm alcohol, and having a sp. gr. $=1 \cdot 666$. Abietone readily dissolves iodine and bromine, and is a powerful solvent for fixed and volatile oils, castor oil excepted, and also Peruvian balsam and Canada balsam; castor oil is absolutely insoluble in abietene, while, curiously enough, the last-named substance is dissolved by castor oil to some extent. When burned in an ordinary spirit-lamp with not too large a flame, a brilliant white light is obtained without smoke: the vapour of abicteno is a powerful angesthetic when inhaled, and it has been used with success as an insecticido against moths, \&ce, whon sprinkled in closed receptacles.

A BIExrar. A pale yollow, transparent, viseid exudation from the Abies picea. It contains 35 per cent. of a volatile oil of an agroeablo smell, combined with a 
resin, and a small quantity of the acid of amber, as well as the peculiar body called abictin.

A BIETTE, or ABIZ, is a name given to several species of fish, but particularly to the Bleak, the scales of which are employed for making the pearl essence which is used in the manufacture of artificial pearls. See Pearls, Artificial.

ABrasrow. The figuration of materials by wearing down the surface. In general, the abrasive tool or grinder is exactly a counterpart of the form to be produced; thus, for plane surfaces a flat grinder is employed, and for concave surfaces a convex grinder.

ABRAUM SAITS ((Abraumsalze, Ger., 'Salts to be removed'). Towards tho upper part of the great salt deposits now extensively worked at Stassfurt, in Prussia, the chloride of sodium becomes largely mixed with certain salts of potassium and magnesium, representing the more soluble compounds which remained dissolved in the mother liquor after the greater part of the chloride of sodium had been separated by crystallisation. These mixed salts were formerly regarded as worthless, and were hence termed Abraumsalze. Indeed the workings at Stassfurt having been originally undertaken with the view of procuring common rock-salt, it was naturally considered unprofitable to bore through the thick beds of impure salt before reaching the underlying deposit of purer chloride of sodium. The high commercial value of these so-called Abraumsalze is now, however, fully recognised; and at the present time they are employed on a very large seale for the production of chloride of potassium. In addition to the rock salt, the chief constituents of the Abraumsalze are the minerals known as Carnallite, a double chloride of potassium and magnesium; Sylvine, or chloride of potassium; and Kieserite, or sulphate of magnesia. Similar doposits

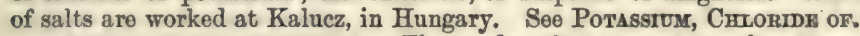

AzRUs PRECATORIA. The seeds, often strung together as rosaries and necklacos, are well known as 'Prayer Beads.' They are of a brilliant scarlet colour, with a black spot on one side, and are hence termed 'Crabs' Eyes.' In India, they are used by druggists and jewellers as weights, the seeds weighing uniformly about one grain each. The Abrus belongs to the Leguminose, or Pea-order.

A BsINTE. A liquor flavoured with wormwood (Artemesia absinthium, Natural Order Composite) and other species containing the bitter principle termed absinthine. To prepare absinth the leaves and flower-heads of the wormwood are steeped in spirit somewhat above 'proof' for several days, with other aromatic and stimulant herbs-such as angelica root, Calamus aromaticus, aniseed, dittany leaves and wild marjoram. Tho liquid is then distilled, and the green essence thus obtained is mixed with certain aromatic extracts. A brilliant tint is obtained by the use of indigo and other vegetable colouring matters: sulphato of copper is said to have been employed for this purpose; and it is also asserted that the liquor is occasionally adulterated with chloride of antimony in order to produce a characteristic milkiness.

Absinth is largely prepared in Switzerland, especially in the canton of Neufehatel ; and indeed the strongest liquor is often known in trade as 'Swiss absinth,' though it may not have been prepared in Switzerland. Of late years, it has been drunk immoderately in France, and large quantities are also consumed in America. Certain French physiologists have alleged that the essential oils in absinth act as an energetic poison, especially affecting the nervous system; other authorities attribute the injurious effects of absinth-drinking to the Calamus aromaticus said to be used in its proparation; whilst others again maintain that the effects are merely those which follow the long-continued use of any strong alcoholic liquor-excepting, of course, the poisonous action of any copper-salts which may be present in adulterated absinth.

ABYSSINIAN GOID. A yellow metal of a fine colour if properly prepared. It is an alloy of 90.74 parts of copper and 8.33 of zinc. The ingot is plated on one side with a thin plate of gold, and it is then rolled out into sheets, from which articles of jewellery are formed in the usual way. The gold on the articles as sold varies from 0.03 to 1.03 per cent. This is also known as Talmi gold. The term Abyssinian Gold is sometimes applied in trade to Aluminium Bronze. See Aruminiom Bronzz.

ACACIA. (Lat. acacia, a thorn; Gr. ách, a point). The acacia is a very extensive genus of trees, or shrubby plants belonging to the Leguminose or Pea-order. The acacias inhabit the tropical regions generally, but extend in some instances into the temperate zone; being found, for example, in Australia and the neighbouring islands. Botanists are acquainted with nearly 300 species of the acacia, some of them yielding gum arabic and other gums known in commerce; while others give a large quantity of tannin, especially a species which grows in Van Diemen's Land or Tasmania. The Acacia vera is a native of Arabia and of Africa from Senegal to Egypt; the A. Arabica is found in the samo countries and in India; the A. Karoo belongs to the Cape of Good Hope, producing the Cape gum; A. gummifera is chiefly found in Africe near Mogador A. Scyal in Senegambia; A. tortilis grows in the 
Desert, as does also A. Ehrewbergii, their gums being collected by the Bedouin Arabs; and $A$. Senegal grows in Arabia and Afriea, from Senegal to the Cape of Good Hope. Seo Amaric, Gom; Gux.

ACACIA CATzCrE. The catechu acacia (Mimosa catechu of Linnæus) is a tree with a moderately high and stout stem, growing in mountainous places in various parts of Indis. Its unripe pods and wood, by decoction, yield the catechu. This kind of catechu is known under the name of Kutch or Cutch, and must not be confounded with the official catechu (Catechu pallidum). This catechu is imported in large masses of $1 \mathrm{cwt}$ and upwards. It is used in the preparation of some leathers and by dyers. See CaTrchu.

ACACIN. A name for common Ǵum Arabic.

ACAJOU (Bors D'). The French name for mahogany. See Marogary.

ACARord RISIN. A resin sometimes called Botany Bay resin, prodnced by a liliaceous tree growing in New Holland. It contains benzoic and cinnamic acids, which give it, espocially when burnt, a grateful odour.

ACrsCrsNT. Substances which have a tendency to pass into an acid state; as an infusion of malt, \&ce.

ACETAX. $\mathrm{C}^{12} \mathrm{H}^{14} \mathrm{O}^{4}$. $\left(\mathbf{C}^{6} \mathbf{I}^{14} \mathbf{O}^{2}\right)$. One of the products of the oxidation of alcohol under the influence of oxygen condensed in platinum-black. Pieces of wellcleaned pumice-stone are moistened with nearly absolute alcohol, and placed at the bottom of a wide-mouthed flask, which is then filled with capsules containing platinum-black, and exposed to a temperature of $20^{\circ}$ Cent. $\left(67^{\circ} \mathrm{Fahr}\right.$.), till the whole of the alcohol is acidified. It is a colourless, mobile, ethereal liquid, boiling at $221^{\circ} \mathrm{F}$. Its density in the fluid state is 0.821 at $72^{\circ}$. The specific gravity of its vapour 4.138 Stas, (mean of three experiments): calculation gires $4 \cdot 0.83$ for four volumes of vapour. The researches of Wurtz render it evident that the construction 0 . acetal is quite different from what has generally been supposed, and that it is in fact glycole in which two atoms of hydrogen are replaced by two of ethyle. -C. G. W.

АсгтAтв. (Acétate, Fr.; Essigsäure, Ger.) Any saline compound in which the acid constituent is acetic acid. All acetates are soluble in water; the least soluble being the acetates of tungsten, molybdenum, silver, and mercury. The ncetates, especially those of lead and alumina, are of great importance in the arts. The acetates are all described under their respective bases;-a rulo which will be adopted with all the acids: See Acm.

ACITrC ACID. (Acide acétique, Fr.; Essigsäure, Ger.; Acidum aceticum, Iat.; Eisel, Sax.) The word 'acetic' is derived from the Latin acetum, applied to vinegar; probably the earliest known body possessing the sour taste and other properties which characterize acids; hence the term AcID, now become generic; both the Latin word, and also the Saxon acid, being from the root acies (Greek arin), an edge or point, in reference to the sharpmess of the taste.

Vinegar must have been known from the most remote periods of antiquity. It is mentioned by Moses.' Hippocrates employed it in medieine under the namo okjus." Hannibal, in his passage over the Alps, is said to have softened the rocks by fire and vinegar. ${ }^{3}$ It was known to the alchemists in the more concentrated state in which it is obtained by the distillation of acetate of copper (verdigris); being mentioned both by Geber" and Stahl.

Crystallised acetic acid was first obtained by Westendorff' and Lowitz.

Acetic acid exists in nature only in the organised kingdoms, or as a product of the oxidation of organic bodies. According to Vauquelin and Morin it is found in the juices of certain plants, and it probably exists in certain animal fluids.

Gmelin and Geiger state that it has been found in mineral waters, which is quite possible, having been derived from the decay of organic matter originally present.

Acetic acid is produced either by the oxidation, or the destructive distillation of organic bodies containing its elements-carbon, hydrogen, and oxygen.

The oxidation of organic bodies, in order to convert them into acetic acid, may be effected either-1, by exposing them in a finely divided state to the action of air or oxygen gas; 2, by submitting them to the action of ferments in the presence of a free supply of atmospheric air ; or, 3 , by the action of chemical oxidising agents.

When acetic acid is procured by the axidation of organic bodies, it is generally nleohol that is employed; but by whatever process alcohol is transformed into acetic acid, it is always first converted into an intermediate compound, aldehyde; and this

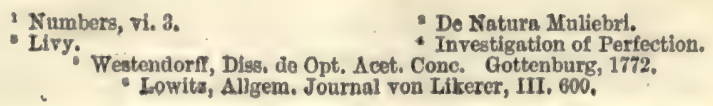


boing a very volatile body, it is desirable always to effect the oxidation as completely and rapidly as possible, to avoid the loss of alcohol by the evaporation of this aldehyde,

$\begin{array}{ll}\text { Alcohol contains } & \mathrm{C}^{4} \mathrm{H}^{4} \mathrm{O}^{2}\left(\mathbf{C}^{2} \mathbf{m}^{8} \mathbf{0}\right) \\ \text { Aldehyde } " & \mathrm{C}^{4} \mathrm{H}^{4} \mathrm{O}^{2}\left(\mathbf{C}^{2} \mathbf{m}^{4} \mathbf{0}\right) \\ \text { Acetic agid " } & \mathrm{C}^{4} \mathrm{H}^{4} \mathrm{O}^{4}\left(\mathbf{C}^{2} \mathbf{m}^{4} \mathbf{O}^{2}\right)\end{array}$

The process, therefore, consists first in the removal of two atoms of hydrogen from alcohol, which are converted into water-aldehyde being produced-and then the further union of this aldehyde with two atoms of oxygen to convert it into acetic acid. Seo ALDEHYDE,

By the oxidation of alcohol, pure acetic acid is obtained : but the vinegars of commerce are mixtures of the pure acetic acid with water; with saccharine, gummy, and colouring matters; with certain ethers (especially the acetic ether), upon which their agreeable aromatic flavour depends; with empyreumatic oils, \&c.

The pure acetic acid (free from water and other impurities) may be obtained most advantageously, according to Melsens, ${ }^{1}$ by distilling pure acotate of potash with an excess of acetic acid (which has been obtained by the redistillation of ordinary acetic acid, procured either by oxidising alcohol, or by the destructive distillation of wood): the acid which first passes over contains water; but finally it is obtained free.

Properties of pure Acetic Acid.-When absolutely pure, acetic acid is a colourless. liquid of specific gravity 1.064 , which at temperatures below $62^{\circ} \mathrm{F}$. (17 $\mathrm{C}$.) solidifies into a colourless crystalline mass. It has strongly acid properties, being as powerfully corrosive as many mineral acids, causing vesication when applied to the skin ; and it possesses a peculiarly pungent, though not a disagreeable smell.

The vapour of the boiling acid is highly combustible, and burns with a blue flame. Hydrated acetic acid dissolves camphor, gliadine, resins, the fibrine of blood, and several organic compounds. When its vapour is conducted through a slightly ignited porcelain tube, it is converted entirely into carbonic acid and acetone, an atom of the acid being resolved into an atom of each of the resultants. At a white heat the acid vapour is converted into carbonic acid, carburetted hydrogen, and water.

It attracts water with great avidity, mixing with it in all proportions. Its solution in water increases in density with the increase of acetic acid up to a certain point; but beyond this point its density again diminishes. Its maximum density being 1.073 , and corresponding to an acid containing $\mathrm{C}^{4} \mathrm{H}^{4} \mathrm{O}^{4}+2 \mathrm{Aq}$, which may be extemporaneously produced by mixing $77 \cdot 2$ parts of crystallised acetic acid with 22.8 parts of water. This hydrate boils at $104^{\circ} \mathrm{C} .\left(219^{\circ} \mathrm{F}\right.$ ), whilst the crystallised acid boils only at $120^{\circ} \mathrm{C}$. $\left(248^{\circ} \mathrm{F}\right.$.)

The proportion of acetic acid in aqueous mixtures may therefore be ascertained, within certain limits, by determination of the specific gravity. See AoETIMETRY.

The following Table, by Mohr, indicates the per-centage of acetic acid in mixtures of different specific gxavities; but of course this is only applicable in cases where no Bugar of other bodies are present which increase the specific gravity :-

\section{Abstract of Mohr's Table of the Speoific Gravity of Mixtures of Acetio Aoid and Water.}

\begin{tabular}{|c|c||c|c|}
\hline $\begin{array}{c}\text { Yer-centage of Acetic } \\
\text { Acid, } \mathrm{C}^{*} \mathrm{H}^{*} \mathrm{O}^{4}\end{array}$ & Density & $\begin{array}{c}\text { Per-centage of Acetic } \\
\text { Acid, } \mathrm{C}^{*} \mathrm{H}^{4} \mathrm{O}^{*}\end{array}$ & Density \\
\hline 100 & 1.0635 & 45 & 1.055 \\
95 & 1.070 & 40 & 1.051 \\
90 & 1.073 & 35 & 1.046 \\
85 & 1.073 & 30 & 1.040 \\
80 & 1.0735 & 25 & 1.034 \\
75 & 1.072 & 20 & 1.027 \\
70 & 1.070 & 15 & 1.022 \\
65 & 1.068 & 10 & 1.015 \\
60 & 1.067 & 5 & 1.0067 \\
55 & 1.064 & 1 & 1.001 \\
60 & 1.060 & & \\
\hline
\end{tabular}

Comptes Rendus, xix. 611.

s Mohr, Ann, der Ohem, und Phar, xxxi, 227. 
Which numbers closely agree with those obtained by Dr. Ure .-

\begin{tabular}{|c|c|c|c|c|c|}
\hline Aold & $\mathrm{Sp}$. Gr. & scid & Sp. Gr. & Acld & sp. Gr. \\
\hline 100 & $1 \cdot 0620$ & 76 & 1.0743 & 52 & 1.0617 \\
\hline 98 & 1.0650 & 74 & 1.0740 & 50 & 1.0603 \\
\hline 96 & 1.0680 & 72 & 1.0733 & 45 & 1.0558 \\
\hline 94 & 1.0700 & 70 & 1.0725 & 40 & 1.0512 \\
\hline 92 & 10716 & 68 & 1.0716 & 35 & 1.0459 \\
\hline 90 & 1.0728 & 66 & 1.0712 & 30 & $1 \cdot 0405$ \\
\hline 88 & 1.0730 & 64 & 1.0701 & 25 & 1.0342 \\
\hline 86 & 1.0735 & 62 & 1.0687 & 20 & 1.0282 \\
\hline 84 & 1.0738 & 60 & 1.0675 & 15 & 1.0213 \\
\hline 82 & 1.0740 & 68 & 1.0665 & 10 & 1.0147 \\
\hline 80 & $1 \cdot 0750$ & 56 & 1.0647 & 6 & 1.0075 \\
\hline 78 & 1.0748 & 54 & 1.0634 & & \\
\hline
\end{tabular}

Acetic acid was formerly (and is still by some chemists) viewed as the hydrated teroxide of a radical, Acotyl. $\left(\mathrm{C}^{4} \mathrm{H}^{3}\right) \mathrm{O}^{3}, \mathrm{HO}$

Acetyl.

And therefore an anhydrous acetic acid, $\mathrm{C}^{4} \mathrm{H}^{3} \mathrm{O}^{3}$, was supposed to exist. Many attempts have been made to isolate this anhydrous acetic acid $\mathrm{O}^{4} \mathrm{H}^{3} \mathrm{O}^{3}$; and a body which has received this name has been obtained by Gerhardt, ${ }^{1}$ by the double decomposition of chloride of acetyl and an alkaline acetate, thus-

$$
\underbrace{\mathrm{C}^{4} \mathrm{H}^{8}\left(\mathrm{O}^{2} \mathrm{Cl}\right)}_{\begin{array}{c}
\text { Chlortde of } \\
\text { acetyl, }
\end{array}}+\underbrace{\mathrm{KO} \cdot \mathrm{C}^{4} \mathrm{H}^{3} \mathrm{O}^{3}}_{\begin{array}{c}
\text { Acotate of } \\
\text { potaih. }
\end{array}}=\underbrace{\mathrm{C}^{8} \mathrm{H}^{\circ} \mathrm{O}^{8}}_{\begin{array}{c}
\text { (So-colled) } \\
\text { Anhydrous } \\
\text { acetic acid. }
\end{array}}+\underbrace{\mathrm{K} \mathrm{Cl}}_{\begin{array}{c}
\text { Chloride of } \\
\text { potassium. }
\end{array}}
$$

$\mathbf{C}^{2} \mathbf{H}^{3} \mathrm{OCI}+\mathrm{ZCC}^{2} \mathrm{H}^{3} \mathrm{O}^{2}=\mathrm{C}^{4} \mathbf{H}^{6} \mathrm{O}^{3}+\mathrm{ZCCl}$

This body Gerhardt describes as a colourless liquid having a strong smell of acetic acid, but associated with the flavour of hawthorn blossom, having a specific gravity of 1.073 , and boiling at $137^{\circ} \mathrm{C} .\left(278^{\circ} \mathrm{F}\right.$.); falling in water in the form of oily drops, only dissolving on gently heating that fluid. It is, however, not anhydrous acetic acid, but a compound isomeric with the hypothetical anhydrous acetic acid $\mathrm{C}^{4} \mathrm{H}^{3} \mathrm{O}^{3}$, containing, in fact, double the amount of matter, its formula being $\mathrm{C}^{8} \mathrm{H}^{6} \mathrm{O}^{6}$. See ISOMERISM.

The impure varieties of acetic acid known as vinegar, pyroligneous acid, \&c., are the products met with in commerce, and therefore those require more minute deseription in this work.

Before describing the manufacture of these commercial articles, it may be interesting to allude to a method of oxidising alcohol by means of spongy platinum; which may yet meet with extensive practical application. It is a well-known fact that spongy platinum (e.g. platinum black), from its minute state of division, condenses the oxygen of the air within its pores; consequently, when the vapour of alcohol comes in contact with this body, a supply of oxygen in a concentrated state is presented to it, and the platinum, without losing any of its properties, effects the combination between the oxygen and the alcohol, converting the latter into acetic acid.

This may be illustrated by a very simple experiment. Place recently ignited spongy platinum, loosely distributed on a platinum-gauze, at a short distance over a saucer containing warm alcohol, the whole standing under a bell-glass supported by wodges on a glass dish, so that on removing the stopper from the bell-glass a slow current of air circulates through the apparatus; the spongy platinum soon begins to glow, in consequence of the combustion going on upon its surface, and acetic acid vapours are abundantly produced, which condense and run down the sides of the glass. The simultaneous formation of aldehyde is, at the same time, abundantly proved by its peculiar odour.

In Germany this method has been actually carried out on the large scale, and, if it wore not for the high price of platinum and the heavy duty on alcohol, it might be extensively employed in this country on account of its elegance and extreme simplicity. See Acras. 


\section{ACETIC ACID}

The processes employed for the manufacture of Acetic Acid, which is principally used in the arts, and those for the production of Vinegar, which is chiefly for domestic use, are widely different and carried on in separate works ; they will, therefore, be best described under thoir respective hoads.

\section{Masuracturs of Acric Acm (Pyroligarmods Acm). \\ A.-By destructive distillation of Wood.}

The genersl nature of the process of destructive distillation will be found detailed under the head of Distillatron, Destructrve; as well as a list of products of the rearrangement of the molecules of organic bodies under the influence of heat in closed vessels. We shall, therefore, at once proceed to the details of the process as specially applied in the manufacture of acetic acid from wood.

The forms of apparatus very generally employed on the Continent for obtaining at the same time crude acetic acid, charcoal, and tar, are those of Schwartz and Reichenbach ; but in France the process is carried out with special reference to the production of acetic acid alone.

The following is a deseription of that in use at Nuits and Rouen:-

Into large cylindrical vessels (fig. 1) made of rivetted sheet iron, and having at their top and side a small sheet-iron cylinder, the wood intended for making charcoal is introduced. To the upper part of this vessel a cover of sheet iron, B, is adapted, which is fixed with bolts. This vessel, thus closed, represents, as we see, a vast retort. When it is prepared, as we have said, it is lifted by means of a swing

1

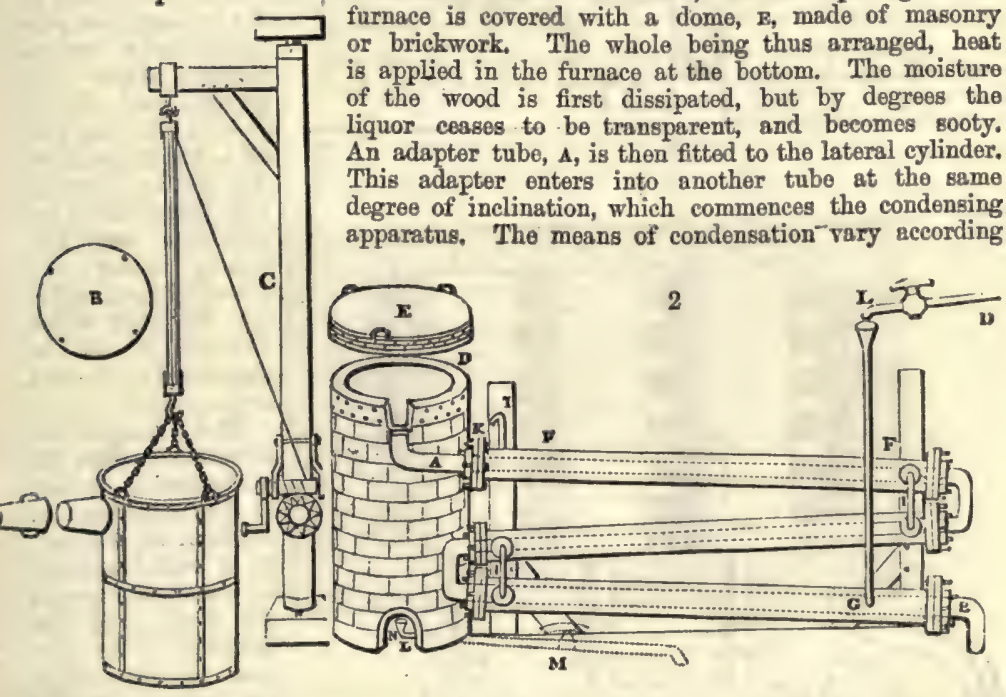

to the localitios. In certain works they cool by means of air, by making the vapour pass through a long series of cylinders, or sometimes, even, through a series of casks connected together; but most nsually water is used for condensing, when it can be easily procured in abundance. The most simple apparatus employed for this purpose consists of two cylinders, FF (fig. 2), the one within the other, and which leave between them a sufficient space to allow a considerable body of water to circulate along and cool the rapours. This double cylinder is adapted to the distilling vessel, and placed at a certain inclination. To the first double tube, F F, a second, and sometimes a third, entirely similar, are connected, which, to save space, return upon themselves in a zigzag fashion. The water is set in circulation by an ingenious means now adopted in many different manufactories. From the lower extremity, $G$, of the system of condensers, a perpendicular tube rises, whose length should be a little more than the most elevated point of the system. The water, furnished by a reservoir $\mathrm{L}$, enters by means of the perpendicular tube through the lower part of the system, and fills the whole space between the double cylinders. When the apparatus is in action, the vapours, as they condense, raise the temporature. 
of the water, which, by the column in $\mathrm{L} G$, is pressed to tse upper part of the cylinders, and runs over by the spout $\mathrm{K}$. To this point a very short tube is attachod, which is bent towards the ground, and serves as an overflow.

The condensing apparatus is terminated by a conduit in bricks covered and sunk In the ground. At the extremity of this species of gutter is a bent tube, $\mathrm{x}$, which discharges the liquid product into the first cistorn. When it is full, it empties itself, by means of an overflow pipe, into a great reservoir; the tube which terminates the gutter plunges into the liquid, and thus intercepts communication with the inside of the apparatus. The disengaged gas is brought back, by means of pipes $M I$, from

3

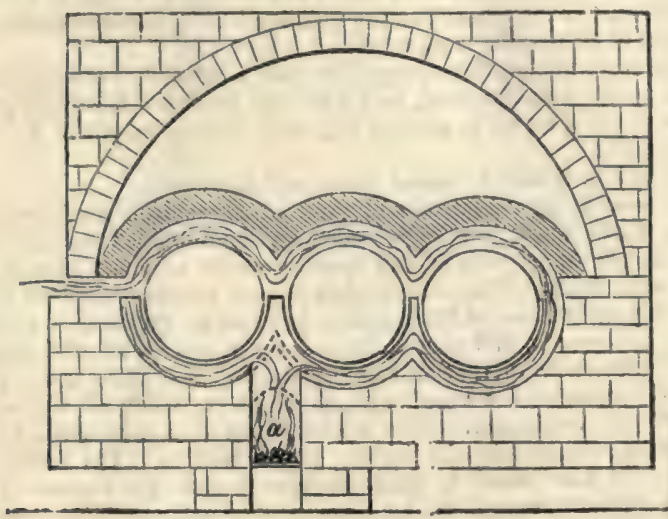

4

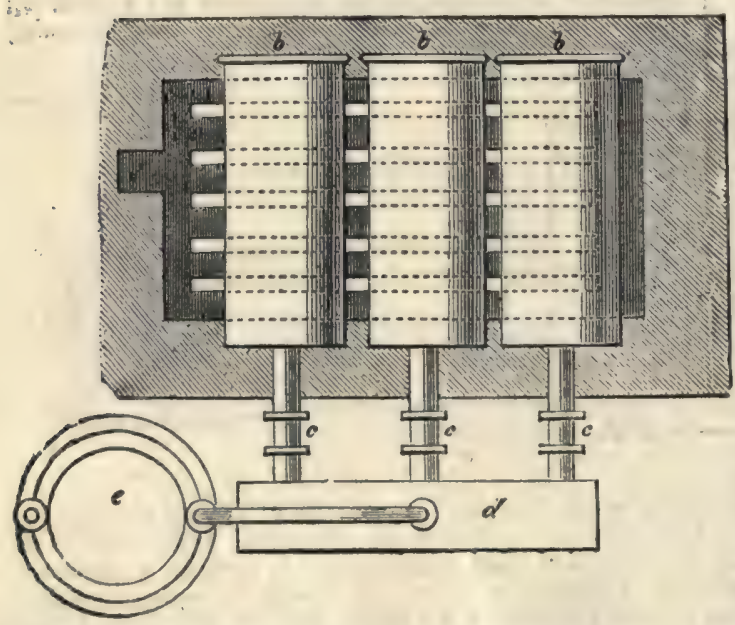
one of the sides of the conduit to the under part of the ashpit of the furnace. These pipes are furnished with stopeocks, $\mathbf{x}$, at some distance in front of the furnace, for the purpose of regulating the jet of the gas, and interrupting, at pleasure, communication with the inside of the apparatus. The part of the pipes which torminates in the furnace rises perpendicularly several inches above the ground, and is expanded like the rose of a watering-ean, $x$. The gas, by means of this disposition, can distribute itsolf uniformly under the vessel, without suffering the pipe which conducts it to bo obstrueted by the fuel or the ashes.

The temperature necessary to effect the carbonisation is not at first considerable: however, at the last, it is raised so high as to make the vessels red hot; and the duration of the process is necessarily proportional to the quantity of wood car. bonised. For a vessel which shall contain about 5 meters cube (nearly 6 cubic yards), 8 hours of fire is sufficient. It is known that the carbonisation is complete by the colour of the flame of the gas : it is first of a yellowish red ; it becomes afterwards blue, when more carbonic oxide than carburetted hydrogen is evolved; and towards the end it becomes entirely white, - a circumstance owing, probably, to the furnace being more heated at this period, and the combustion therefore more complete. There is still another means of knowing the state of the process, to which recourse is more frequently had : that is the cooling of the first tubes, which are not surrounded with water: a fow drops of this fluid are thrown upon their surface, and if they evaporate quietly, it is judged that the calcination is sufficient. The adapter tube is then unluted, and is slid into its junction pipe; the orifices are immediately stopped with plates of iron and plaster loam. The brick cover, $\mathrm{z}$, of the furnace is first remored 
by means of the swing crane, then the cylinder itself is lifted out and replaced immediately by another one previously charged. When the cylinder which has been

s taken out of the furnace is entirely cooled, its cover is removed, and the chareoal is emptied. Five cubic meters of wood furnish about 7 chaldrons and a half of chareoal.

The carbonisers of Reichenbach and Schwartz are usually employed with special reference to the manufacture of wood-charcoal, the condensation of the volatile products being only a secondary consideration.

In England the distillation of wood, with especial reference to the manufacture of pyroligneous acid, is generally carried out in large iron retorts, placed horizontally in the furnace; the process, in fact, elosely resembling the distillation of coal in the manufacture of coal gas, excepting that the retorts are generally larger, being sometimes 4 feet in diameter, and 6 or 8 feet long. Generally two, or even three, are placed in each furnace, as shown in fig. 3 , so that the fire of the single furnace, $a$, plays all round them. The doors for charging the retorts are at one end, $b$ (fig. 4), and the pipe for carrying off the volatile products at the other, $c$, by which they are conducted, first to the tar-condenser, $d$, and finally through a worm in a large tub, $e$, where the crude acetic acid is collected.

Of course, in differont localities an endless variety of modifications of the process are employed.

In the Forest of Dean, instead of cylindrical retorts, square sheet-iron boxes are used, $4 \mathrm{ft} .6 \mathrm{in}$. by $2 \mathrm{ft} .9 \mathrm{in}$., which are heated in large square ovens.

Dr. Ure gives the following description of special works in Glasgow :-

The cylinders here employed are 6 feet long, and both ends project a little beyond the briekwork. One end has a disk, or round plate of cast-iron, well fitted and

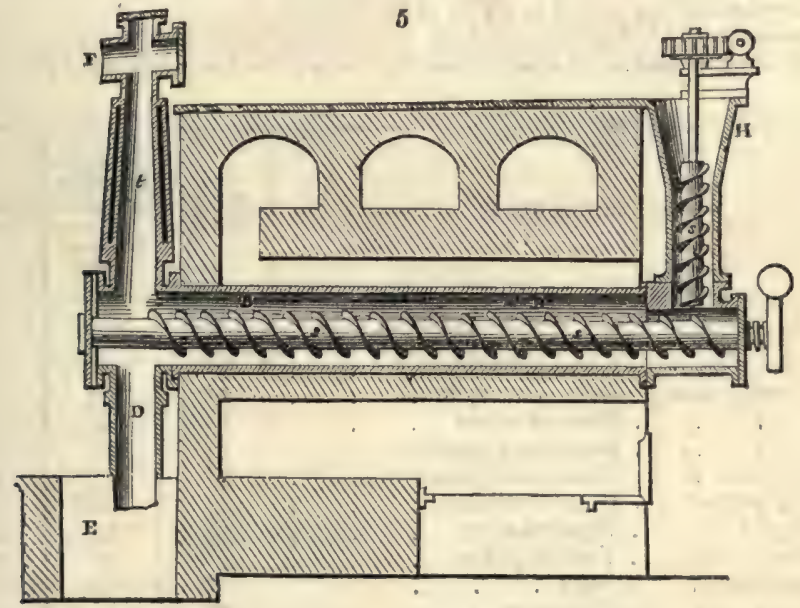

firmly bolted to it, from the centre of which an iron tube, about 6 inches in diameter, proceeds, and enters at a right angle the main tube of refrigeration. The diameter of this tube may be from 9 to 14 inches, according to the number of cylinders. The other end of the cylinder is called the mouth of the retort; this is closed by a dise of iron, smeared round its edge with clay-lute, and secured in its place by iron wedges. The charge of wood for such a cylinder is about $8 \mathrm{cwt}$. The hard woods-oak, ash, birch, and beech-are alone used in this manufactory - fir not being found to answer. The heat is kept up during the day, and the furnace allowed to cool during the night. Next morning the door is opened, the charcoal removed, and a new charge of wood introduced. The average product of crude vinegar is 35 gallons. It is much contaminated with tar, is of a deep brown colour, and has a specific gravity of $1 \cdot 025$. Its total weight is therefore about $300 \mathrm{lbs}$.; but the residuary charcoal is found to weigh no more than one-fifth of the wood employed; hence nearly onehalf of the ponderable matter of the wood is dissipated in incondensable gases.

With regard to the relative advantages of eylindrical retorts or square boxes, it should be remarked, that the cylinders are more adapted for the distillation of the large billets of Gloucestershire and the refuse ship timber of Glasgow, Newcastle, 
and Liverpool ; but, on the other hand, where light wood is used, such as that generally carbonised in the Welsh factories, the square ovens answer better.

An ingenious improvement in the manufacture of pyroligneous acid was patonted some years ago by the late Mr. A. G. Halliday, of Manchester, and adopted by several large manufacturers. The process consists in effecting the destructive distillation of waste materials, such as sawdust and spent dyewoods, by causing them to pass in continuous motion through heated retorts. For this purpose the materials, which are almost in a state of powder, are intruduced into a hopper. $\mathbf{H}$ (fig. 5), whence they descend into the retort, B, being kept all the while in constant agitation, and at the same time moved forward to the other end of the retort by means of an endless screw, 8 . By the time they arrive there the charge has been completely carbonised, and all the pyroligneous acid evolved at the exit tabe, $t$. The residuary charcoal falls through the pipe $D$ into a vessel of water, $k$, whilst the volatile products escape at $\mathbf{F}$, and are condensed in the usual way.

Several of these retorts are generally set in a furnace side by side; the retorts are only 14 inches in diameter, and eight of these retorts produce in 24 hours as much acid as 16 retorts 3 feet in diameter upon the old system. In the manufacturing districts of Lancashire and Yorkshire, where such immense quantities of spent dyewoods aceumulate, and have proved a source of annoyance and expense for their remoral, this process has afforded a most important means of economically converting them into valuable products-charcoal and acetic acid.

Mention should also be made of Messrs. Solomons and Azulay's patent for employing superheated steam to effect the carbonisation of the wood, which is passed directly into the mass of materials. Since the steam accompanies the volatile products, it necessarily dilutes the acid; but this is in a great degree compensated fir by employing these vapours to concentrate the distilled products, by causing them to traverse a coil of tubing placed in a pan of the distillates.

As regards the yield of acetic acid from the different kinds of wood, some valuable facts have boen collected and tabulated by Stolze, in his work on Pyroligneous Acid:-

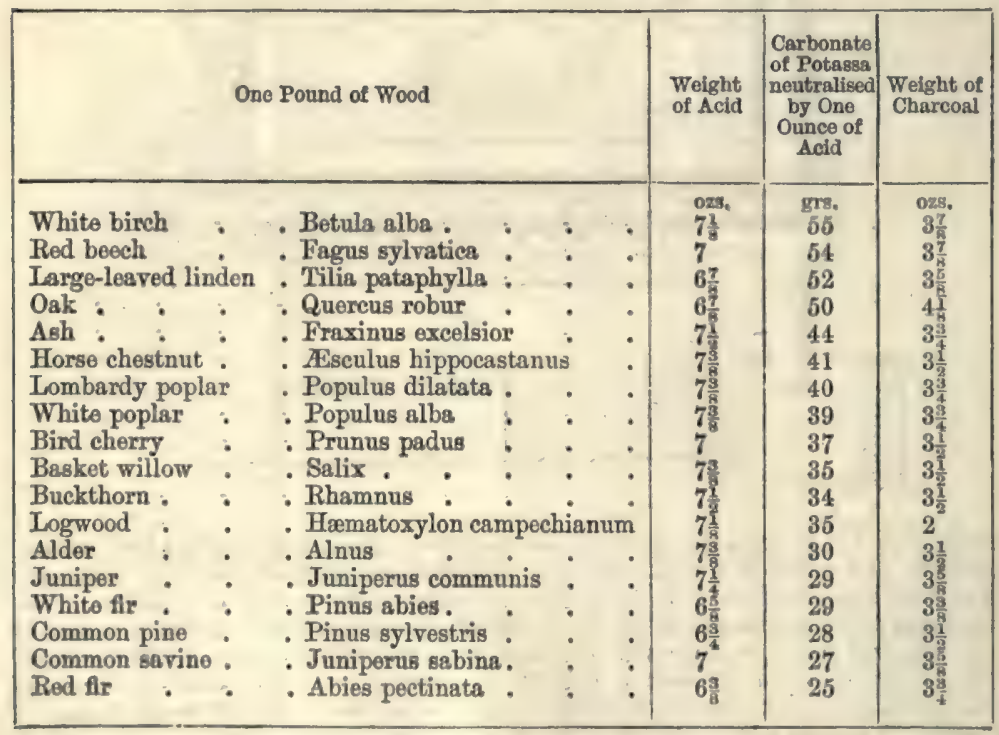

\section{Properties of the crude Pyroligneous Acid.}

The crude pyroligneous acid possesses the properties of acetic acid, combined with those of the pyrogenous bodies with which it is associated. As first obtained, it is black, from the large quantity of tar which it holds in solution; and although certain resins are removed by redistillation, yet it is impossible to remove some of the empyreumatic oils by this process, and a special purification is necessary.

In consequence of the presence of creosote, and other antiseptic hydrocarbons, in the crude pyroligneous acid, it possesses, in a very eminent degree, anti-putrescent. 
properties. Flesh steeped in it for a few hours may be afterwards dried in the air without corrupting; but it becomes hard, and somewhat leather-like; so that this mode of preservation does not answer well for butcher's meat. Fish are sometimes cured with it.

\section{Purification of Pyroligneous Acid.}

This is effected either, 1st, by converting it into an acetate-acetate of lime or soda-and then, after the purification of these salts by exposure to heat sufficient to destroy the tar, and repeated recrystallisation, liberating the acid again by distilling with a stronger acid, e.g. sulphuric.

Or, 2ndly, by destroying the pyrogenous imfurities by oxidising agents, such as binoxide of manganese in the presence of sulphuric acid, \&c.

The former is the method generally adopted.

After the naphths has been expelled, the acid liquor is run off into tanks to deposit part of its impurities; it is then syphoned off into another vessel, in which is either milk of lime, quicklime, or chalk; the mixture is boiled for a short time, and then allowed to stand for 24 hours to deposit the excess of lime with any impurities which the latter will carry down with it. The supernatant liquor is then pumped into the ovaporating pans.

The evaporation is effected either by the heat of a fire applied beneath the evaporating pans, or more frequently by a coil of pipe in the liquor through which stenm is passed-the liquor being kept constantly stirred, and the impurities which rise to the surface during the process carefully skimmed off.

From time to time, as the evaporation advances, the acetate of lime which separates is removed by ladles, and placed in baskets to drain; and the residual mother-liquor is evaporated to dryness. This mass, by ignition, is converted into carbonate of lime and acetone.

If the acetate of lime have been procured by directly saturating the crude acid, it is called brown acetate; if from the acid once purified by redistillation, it is called grey acetate.

From this grey acetate of lime acetate of sods is now prepared, by adding sulphate of soda to the filtered solution of the acetate of lime. In performing this operation, it is highly important to remember that, for every equivalent of acetate of lime, it is necessary to add two equivalents of sulphate of soda, on account of the formation of a double sulphate of soda and lime. The equation representing the change being :-

$$
\begin{aligned}
& \underbrace{\mathrm{CaO}, \mathrm{C}^{4} \mathrm{H}^{3} \mathrm{O}^{3}}_{\text {Acetate of lime. }}+\underbrace{2\left(\mathrm{NaO}, \mathrm{SO}^{3}\right)}_{\text {Sulphate of soda. }}=\underbrace{\mathrm{NaO}, \mathrm{C}^{4} \mathrm{H}^{3} \mathrm{O}^{3}}_{\text {Acetate of soda. }}+\underbrace{\mathrm{CaO}, \mathrm{SO}^{3} \cdot \mathrm{NaO}, \mathrm{SO}^{3}}_{\text {Double salt. }} \\
& \mathrm{Ca}\left(\mathrm{C}^{2} \mathrm{H}^{3} \mathrm{O}^{2}\right)^{2}+\mathrm{ANa}^{2} \mathrm{SO}^{4}=\mathrm{ZNaC}^{2} \mathbf{H}^{3} \mathrm{O}^{2}+\mathrm{CaSO}^{4} \cdot \mathrm{Na}^{2} \mathrm{SO}^{4} \text {. }
\end{aligned}
$$

Or, if sulphuric acid be considered as a bibasic acid, which this rery reaction so strongly justifies -

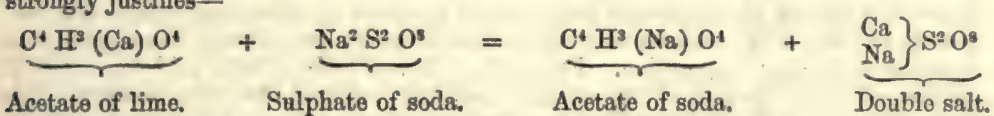

If this point be neglected, and only one equivalent of sulphate of soda be used, onehalf of the acetate of lime may escape decomposition, and thus be lost.

After the separation of the double salt, the solution of acetate of soda is drawn off, any impurities allowed to subside, and then concentrated by evaporation until it has a density of $4 \cdot 3$ - when the acetate of soda crystallises out, and may be further purified, if requisite, by another re-solution and re-crystallisation. The contents of the mother liquors are converted into acetone and carbonate of soda, as before.

The crystallised acetate of soda is now fused in an iron pot, at a temperature of about $400^{\circ}$, to drive off the water of crystallisation, the mass being kept constantly stirred. A stronger heat must not be applied, or we should effect the docomposition of the salt.

For the production of the acetic acid from this salt, a quantity of it is put into a stout copper still, and a deep cavity made in the contre of the mass, into which sulphuric acid of specific gravity 1.84 is poured in the proportion of 35 per cent. of the weight of the salt; the walls of the eavity are thrown in upon the acid, and the whole briskly agitated with a wooden spatula, The head of the still is then luted, and connected with the condensing worm, and the distillation carried on at a very gentlo heat. The worm should be of silver or porcelain, as also the still-head; and even silver solder should be used to connect the joinings in the body of the still. The still is now generally heated by a steam 'jacket.' Soo Dismu.uAtion. 
The acid which passes over is nearly colourless, and has a specific gravity of 1.05 . That which collects at the latter part of the operation is liable to be somewhat om. pyreumatic, and therofore before this point is reached the receiver should be changed; and throughout the ontire operation, care should be taken to avoid applying too high a temporature, as the flarour and purity of the acid will invariably suffer.

Any trace of empyreuma may bo removed from the acid by digestion with animal charcoal and redistillation.

A considerable portion of this acid erystallises at a temperature of from $40^{\circ}$ to $50^{\circ}$ F., constituting what is called glacial acetic aoid, which is the compound $\mathrm{C}^{4} \mathrm{H}^{4} \mathrm{O}^{4}$, or $\mathrm{C}^{4} \mathrm{H}^{3} \mathrm{O}^{3}, \mathrm{HO}\left(\mathbf{C}^{2} \mathbf{E R}^{4} \mathrm{O}^{2}\right)$.

For culinary purposes, pickling, \&c., the acid of specific gravity 1.05 is diluted with five times its weight.of water, which renders it of the seme strength as Revenue proof vinegar.

Several modifications and improvements of this process have recently been introduced, which require to be noticed.

The following process depends upon the difficult solubility of sulphate of sods in strong acetic acids :-100 lbs. of the pulverised salt being put into a hard glazed stoneware receiver, or deep pan, from 35 to $36 \mathrm{lbs}$, of concentrated sulphuric acid are poured in one stream upon the powder, so as to flow under it. The mixture of the salt and acid is to be made very slowly, in order to moderate the action and the heat generated, as much as possible. After the materials have been in intimate contact for a fow hours, the decomposition is effected; sulphate of soda in crystalline grains will occupy the bottom of the vessel, and acetic acid the upper portion, partly liquid and partly in crystals. A small portion of pure acetate of lime addod to the acid will freo it from any remainder of sulphate of soda, leaving only a little acetate in its place; and though a small portion of sulphate of soda may still remain, it is unimportant, whereas the presence of any free sulphuric acid would be very injurious. This is easily detected by evaporating a little of the liquid, at a moderate heat, to dryness, when that mineral acid can be distinguished from the neutral soda sulphate. This plan of superseding a troublesome distillation, which is due to M. Mollerat, is one of the greatest improvements in this process, and depends upon the insolubility of the sulphate of soda in acetic acid. The sulphate of soda thus recovered, and well drained, serves anew to decompose acetate of lime; so that nothing but this cheap earth is consumed in carrying on the manufacture. To obtain absolutely pure acetic acid, the above acid has to be distilled in a glass retort.

Völckel recommends the use of hydrochloric instead of sulphuric acid for decomposing the acotate.

The following is his description of the details of the process:-

- The crude acetate of lime is separated from the tarry bodies which are deposited on neutralisation, and evaporated to abont one-half its bulk in an iron pan. Hydrochloric acid is then added until a distinctly acid reaction is produced on cooling; by this means the resinous bodies are separated, and come to the surface of the boiling liquid in a melted state, whence they can be removed by skimming, while the compounds of lime, with creosote, and other volatile bodies, are likewise decomposed, and expelled on further evaporation. From 4 to $6 \mathrm{lbs}$. of hydrochloric acid for every 33 gallons of wood vinegar is the average quantity required for this purpose. The acetate having been dried at a high temperature on iron plates, to char and drive off the remainder of the tar and resinous bodies, is then decomposed, by hydrochloric acid, in a still with a copper head and leaden condensing tube. To every $100 \mathrm{lbs}$. of salt about 90 to $95 \mathrm{lbs}$. of hydrochloric acid of specific gravity $1 \cdot 16$ are required. The acid comes over at a temperature of from $100^{\circ}$ to $120^{\circ} \mathrm{C}$. $\left(212^{\circ}\right.$ to $248^{\circ} \mathrm{F}$.), and is very slightly impregnated with empyreumatic products, while a mere cloud is produced in it by nitrate of silver. The specific gravity of the product varies from 1.058 to 1.061 , and contains more than 40 per cent. of real acid; but as it is seldom required of this strength, it is well to dilute the 90 parts of hydrochloric acid with 25 parts of water. These proportions then yield from 95 to 100 parts of acetic acid of specifle gravity 1.015.

This process is recommended on the score of economy and greater purity of product. The volatile empyreumatic bodies are said to be more easily separated by the use of hydrochloric than sulphuric acid; moreover, the chloride of calcium being a more easily fusible salt than the sulphate of lime, or even than the double sulphate of lime and soda, the acetic acid is more freely evolved from the mixture. The resinous bodies also decompose sulphuric acid towards the end of the operation, giving rise to sulphurous acid, sulphuretted hydrogen, \&c., which contaminate the product.

The decomposition of acetate of lime or lead by means of sulphuric acid has many inconveniences, and there is danger of the product being contaminated with sulphuric 
acid. Christl ${ }^{1}$ was therefore induced to employ hydrochloric acid as a decomposing agent, and has found that when this acid is not used in excess, the distillate contains scarcely an appreciable trace of chlorine. A mixture of $100 \mathrm{lbs}$. of raw acetate of lime, obtained from the distillation of wood, and containing 90 per cent. of neutral acetate, with $120 \mathrm{lbs}$. of hydrochloric acid $\left(20^{\circ}\right.$ Baumé) is allowed to stand during a night, and then distilled in a copper vessel. The application of heat requires to be gradual, in order to prevent the somewhat thick liquor from running over. The product of acetic acid amounts to about $100 \mathrm{lbs}$. of $8^{\circ} \mathrm{B}$; it has a faint yellow colour and empyreumatic odour, which may be perfectly removed by treatment with wood charcoal and subsequent rectification.

In order to obtain the acetate of lime sufficiently pure, Völckel ${ }^{2}$ adopts the following process:- The raw pyroligneous acid is saturated with lime without previous distillation. A part of the resinous substances dissolved in the acid are thus separated in combination with lime. The solution of impure acetate of lime is allowed to stand until it becomes clear, or it is filtered, then evaporated in an iron pan to about onehalf, and hydrochloric acid added until a drop of the cooled liquid distinctly reddens litmus-paper. A part is sometimes distilled off in a copper still, in order to obtain wood-spirit. The addition of acid serves to separate a great part of the resin still held in solution, which collects together in the boiling liquid, and may be skimmed off, and likewise decomposes the compounds of lime with creosote, and some other imperfectlyknown volatile substances which are driven off by further evaporation. As these volatile substances have little or no action upon litmus-paper, its being reddened by the liquor is a sign, that not only are the lime compounds of these substances decomposed, but also a small quantity of acetate of lime. The quantity of acid necessary for this purpose varies, and depends upon the nature of the pyroligneous acid, which is again dependent upon the quantity of the water in the wood from which it is obtained. Three hundred pints of wood-liquor will require from 4 to $6 \mathrm{lbs}$. of hydrochloric acid.

The solution of acetate of lime is evaporated to dryness, and a tolerably strong heat applied at last, in order to remove all volatile substances. Both operations may be performed in the same iron pans; but when the quantity of salt is large, the latter may be more advantageously effected upon cast-iron plates. The drying of the salt requires very great care, for the empyreumatic substances adhere very strongly to the acetate of lime, as well as to the compound of resin and acetic acid mixed with it, and when not perfectly separated, pass over with the acetic acid in the subsequent distillation with an acid, communicating to it a disagreeable odour. The drying must therefore be continued until, upon cooling, the acetate does not smell at all, or but very slightly. It then has a dirty brown colour. The acetic acid is obtained by distillation with hydrochloric acid, in a still with a copper head and leaden condenser; and when proper precautions are taken, the acetic acid does not contain a trace of either metal. The quantity of hydrochloric acid required cannot be exactly stated, because the acetate of lime is mixed with resin, and already formed chloride of calcium. In most instances 90 or 95 parts by weight of acid, 1.16 specific gravity, are sufficient to decompose completely 100 parts of the salt, without introducing much hydrochloric acid into the distillate.

The distilled acetic acid possesses only a very faint empyreumatic odour, very different from that of the raw pyroligneous acid; it is perfectly colourless, and should only become slightly turbid on the addition of nitrate of silver. If the acid has a yellowish colour, this is owing to resin having been spirted over in the distillation. It is therefore advisable to remove the resin, - which is separated on the addition of hydrochloric acid, and floats upon the surface of the liquid,-either by skimming or filtration through a linen eloth. The distilled acid has a specific gravity ranging between 1.058 and.1.061, containing upwards of 40 per cent, of anhydrous acetic acid. It is rarely that acid of this strength is required; and as the distillation is easier when the mixture is less concentrated, water may be added before or towards the ond of the distillation. Völckel recommends as convenient proportions-

\section{0 parts of acetate of lime,}

90 to 95 hydrochloric acid,

25 parts water,

which yield from 905 to 100 parts of acetic acid of $1 \cdot 105$ specific gravity; 150 litres of raw pyroligneous acid yield about $50 \mathrm{lbs}$, of acetic acid of the above specific gravity.

The acid prepared in this way thay be still further purifled by adding a small 
quantity of carbonate of soda and redistilling; it is thus rondered quite freo from chlorine, and any remaining trace of colour is likewiso removed. The slight empyreumatic smell may be remored by distilling the acid with about 2 or 3 per cent. of acid chromate of potash. Oxide of manganese is less efficacious as a purifying agent.

Although pure acetic acid may be procured by the distillation of vinegar, the whole of the acid cannot be obtained except by distilling to dryness, by which means the extractive substances are burnt, and the distillate rendered impure. In order to obviato this difficulty, Stoin ${ }^{2}$ proposes to add $30 \mathrm{lbs}$. of salt to every $100 \mathrm{lbs}$. of vinegar; the boiling point is thus raised, and the acid passes over completely.

\section{B.-Manufacture of Acetic Acid from Acetate of Copper.}

Before thy process for pyroligneous acid, or wood vinegar, was known, there was only one method of obtaining strong vinegar practisod by chemists; and it is still followed by some operators, to prepare what is called radical or aromatic vinegar. This consists in decomposing, by heat alone, the crystallised binacetate of copper, commonly, but improperly, called distilled verdigris. With this view, we take a stoneware retort (fig. 6), of a size suited to the quantity we wish to operate upon, and coat it with a mixture of fireclay and horsedung, to make it stand the heat better. When this coating is dry, we introduce into the retort the crystallised acetate slightly bruised, but very dry; we fill it as far as it will hold without spilling when tho beak is considerably inclined. We then set it in a proper furnace. Wo attach to its neek an adapter pipe, and two or three globes with opposite tubulures, and a last globe with a vertical tubulure. The apparatus is terminated by a Welter's tube, with a double branch; the shorter issues from the last globe, and the other dips into a flask filled with distilled vinegar. Everything being thus arranged, we lute the joinings with a putty made of pipeclay and linseod oil, and cover them with glue paper.

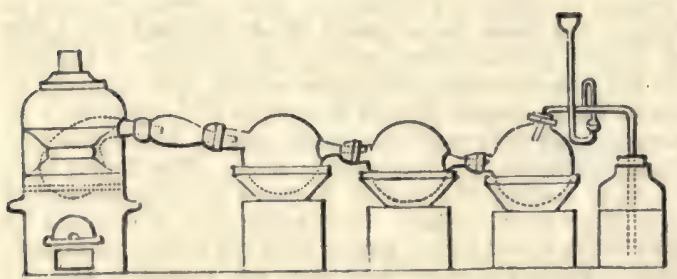

Each globe is placed in i separate basin of cold water, or the whole may be put into an oblong trough, through which a constant stream of cold water is made to flow. The tubes must be allowed a day to dry. Next day we proceed to the distillation, tempering the heat very nicely at the beginning, and increasing it by very slow degrees till we see the drops follow each other pretty rapidly from the neck of the retort, or the end of the adapter tube. The vapours which pass over are very hot, whence a series of globes are necessary to condense them. We should renew, from time to time, the water of the besins, and keep moist pieces of cloth upon the globes; but this demands great care, especially if the firo be a little too brisk, for the ressels become, in that case, so hot, that they wotld infallibly be brokon if touched suddenly with cold water. It is always easy for us to regulate this operation according to the emission of gas from the extremity of the apparatus. Whon the air-bubbles succeed each other with great rapidity, we must damp the fire.

The liquor which passes in the first half hour is weakest; it proceeds, in some mensure, from a little water sometimes left in the crystals, which, when well made, however, ought to be anhydrous. A period arrives towards the middle of the process when we see the extremity of the beak of the retort, and of the adapter, covered with crystals of a lamellar or needle shape, and of a pale green tint. By degrees these crystals are carried into the condensed liquid by the acid vapours, and give a colour to the product. These crystals are merely some of the cupreous salt forced over by the heat. As the process approaches its conclusion, we find more difficulty in raising the vapours; and we must then augment the intensity of the heat, in order to continue their disengagement. Finally, we judge that the process is altogether finished, when the globes become cold, notwithstanding the furnace is at the hottest, and when no more vapours are erolved. The fire may then be allowed to go out, and the retort to cool.

As tho acid thus obtained is slightly tinged with copper, it must be rectified before bringing it into the market. For this purpose we may make use of the same apparatus, only substituting for the stoneware retort a glass one, placed in a sand-bath. 
All the globes ought to be perfectly clean and dry. The distillation is to be conductod in the usual way. If we divide the product into thirds, the first yields tho feeblest acid, and the third the strongest. We could not push the process quite to dryness, because there remain in the last portions certain impurities which would injure the flavour of the acid.

The total acid thus obtained forms nearly one-half of the weight of the acetate employed, and the residuum forms three-tenths; so that about two-tenths of the acid have been decomposed by the heat, and are lost,-Ure.

Other metallic acetates may be used instead of the acetate of copper, but with variable results as to the amount of acetic acid which they yield. Acetates which have easily reducible oxides-as those of copper, silver, mercnry, lead, \&c.-afford a larger proportion of acetic acid; but acetone and marsh gas, as well as carbonic oxide and carbonic acid, invariably accompany it. The acetate of silver gives no acetone; whilst those of the alkaline earths yield chiefly acetone or marsh gas, and are converted into carbonates. See AcETONE.

Anhydrous Acetic Acid, as made by Gerhardt, is obtained by mixing perfectly dry fused acetate of potash with about half its weight of chloride of benzoyle, and applying a gentle heat; when a liquid distils over, which, after being rectified, has a constant boiling point of $279^{\circ} \mathrm{F}$., and is heavier than water, with which it does not mix until after it has been agitated with it for some time. It dissolves at once in hot water, forming acetic acid.

\section{Uses of Acetic Acid.}

Acetic acid is extensively employed in the arts, in the manufacture of the various acetates, especially those of alumina and iron, so extensively employed in calicoprinting as mordants, sugar of lead, \&c. It is likewise used in the preparation of varnishes, for dissolving gums and albuminous bodies; in the culinary arts, especially in the manufacture of pickles and other condiments; in medicine, externally, as a local irritant, and internally, to allay fever, \&c.

For the treatment in cases of poisoning, we refer to Taylor, Pereira, and other medical authorities.

For the Manufacture of Vinegar, see Vinggar.

ACखTIC 5THFRs. (Acetate of Lthyl. Essigäther. Essignaphtha. Essigsäures Ethyloxyd.) These are compounds of acetic acid with the alcohol radicals. See Radicats, Azcohol, and Radicals, Chemicat,

ACETIMETIR. An apparatus used in the processes for determining the strength of vinegar. Consult WATTs's Dictionary of Chemistry.

ACETrmeTrx. Determination of the Strength of Vinegar.-If in vinegars, we were dealing with mixtures of pure acetic acid and water, the determination of the density might to a certain extent afford a criterion of the strength of the solution: but vinegar, especially that obtained from wine and malt, invariably contains gluten, saccharine, and mucilaginous matters, which increase its density and render this method altogether fallacious.

An accurate means of determining the strength of vinegar is by ascertaining the quantity of carbonate of soda or potash neutralised by a given weight of the vinegar under examination. This is performed by adding to the vinegar a standard solution of the alkaline carbonate of known strength from a burette, until, after boiling to expel the carbonic acid, a solution of litmus previously introduced into the liquid is rondered distinctly blue.

The details of this process, which is equally applicable to mineral and other organic acids, will be found fully described under the head of AcipncETRY.

Roughly, it may be stated that every 53 grains of the pure anhydrous carbonate of soda, or every 69 grains of carbonate of potassa (i.e. one equivalent), correspond to 60 grains of acetic acid $\left(\mathrm{C}^{4} \mathrm{H}^{4} \mathrm{O}^{4}\right)$.

It is obvious that preliminary examinations should be made to ascertain if sulphuric, hydrochloric, or other mineral acids are present; and, if so, their amount determined; otherwise they will be reckoned as acetic acid.

The British malt vinegar is stated in the 'London Pharmacopoia' to require a drachm (60 grains) of crystallised carbonate of soda (which contains 10 equiralents of water of erystallisation), for saturating a fluid ounce, or $4 \times 46$ grains; it contains, in fact, from $4 \cdot 6$ to 5 per cent. of real acotic acid.

The same authorities consider that the purified pyroligneous acid should require 87 grains of carbonate of soda for saturating 100 grains of the acid.

Dr. Ure suggests the use of the bicarbonate of potash. Its atomic weight, referred to hydrogen as unity, is 100.584 , while the atomic weight of acetic acid is 51.563 ; if we estimate 2 grains of the bicarbonate as equivalent to 1 of the real acid, we shall 
commit no appreciable error. Hence a solution of the carbonate containing 200 grains in 100 measures will form an acetimeter of the most perfect and convenient kind; for the measures of test liquid expended in saturating any measure-for instance, an ounce or 1,000 grains of acid-will indicate the number of grains of real acetic acid in that quantity. Thus 1,000 grains of the above proof would require 50 measures of the acetimetrical alkaline solution, showing that it contains 50 grains of real acetic acid in 1,000 , or 5 per cont.

Although the bicarbonate of potash of the shops is not absolutely constant in composition, yot tho method is no doubt accurato enough for all practical purposes.

The acetimetrical method employed by the Fixcise is that recommonded by Messrs. J. and P. Taylor, and consists in estimating the strength of the acid by the specific gravity which it acquires when saturated by hydrate of lime. Acid which contains 5 per cent. of real acid is equal in strength to the best malt vinegar, called by the makers No. 24, and is assumed as the standard of vinegar strength, under the deno. mination of 'proof vinegar.' Acid which contains 40 per cent. of real acetic acid, is therefore, in the language of the Revenue, 35 per cent. over proof; it is the strongest acid on which duty is charged by the acetimeter. In the case of vinegars which have not been distilled, an allowance is made for the increase of weight due to the mucilage present; hence, in the acetimeter sold by Bate, a weight, marked $\mathbf{M}$, is provided, and is used in trying such vinegars. As the hydrate of lime employed causes the precipitation of part of the mucilaginous matter in the vinegar, it serves to remove this difficulty to a certain extent. (Pereira.)

As the colour of malt vinegar or impure acetic acid sometimes obscures the exact termination of the reaction, when a standard solution of carbonate of soda is used, with litmus as an indicator, it is better to use the ammoniacal solution of copper recommended by Kiefer. This is made by dissolving sulphate of copper in water and adding solution of ammonia till the precipitate of basic salt, which forms at first, just redissolves. The strength of the copper solution is then ascertained by means of a standard solution of sulphuric acid. To use it a certain quantity of the vinegar to be tested is measured with a pipette and placed in a beaker or other suitable ressel and the copper solution gradually run into it from a burette. The bluish green precipitate formed disappears on stirring as long as any free acid remains, but as soon as it is completely neutralised a permanent turbidity is produced. A sheet of darkcoloured paper placed under the beaker enables the end of the experiment to be distinguished with greater facility. It is necessary that the acid should be so dilute that the precipitate, which is seen on adding the first drop of copper solution, only disappears on agitating the mixture; it is then of a suitable strength.

An excellent method, equally applicable to every description of acotic acid, has been proposed by Mohr. Pure precipitated carbonate of lime or baryta is added in excess to a known quantity of the acid to be tested. When the effervescence has ceased the mixture is heated, to complete the saturation of the acid and to expel the carbonic acid gas. The excess of the earthy carbonate employed is then filtered off, washed with hot water, and its amount ascertained by means of a standard acid and an alkaline solution, as deseribed in the article on alkalimetry, The result thus obtained is subtracted from the weight of the carbonate added, and gires the quantity which has been consumed in saturating the acid. 100 parts of carbonate of lime dissolved, represent 102 of acetic acid, viewed as anhydrous, or 120 of the hydrated acid.

ACrToNm. A volatile spirit obtained by the distillation of the acetates of the alkaline earths. It may also be propared by the destructive distillation of citric acid, or by distilling starch, sugar, or gum with quicklime. The formula of acetone is $\mathrm{C}^{6} \mathrm{H}^{6} \mathrm{O}^{2}\left(\mathbf{C}^{3} \mathbf{H}^{8} \mathbf{0}\right)$. See Prrodchitic Spirtx.

ACrTYI. Some chemists (following Berzelius, who denied the existence of oxidised radicals) regard acetyl as a radical, the teroxide of which constituted acetic acid. The followers of Gerhardt, on the other hand, consider acetic acid to contain a radical of the formula $\mathrm{C}^{4} \mathrm{H}^{4} \mathrm{O}^{2}\left(\mathbf{C}^{2} \mathbf{H}^{3} \mathbf{O}\right)$. The latter is generally known as acotyl. Dr. Williamson proposes to eall it othyl.

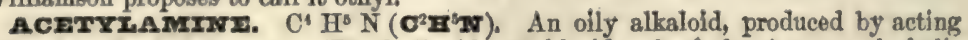
with the oil of olefiant gas (Dutch liquid, or chloride of ethylene) on an alcoholic solution of ammonit.

ACETrImx工. A hydrocarbon containing $\mathrm{C}_{4} \mathrm{H}_{2}\left(\mathbf{C}_{2} \mathbf{Z}_{2}\right)$. By passing a roltaic current through carbon points in an atmosphere of hydrogen, acetylene is formed by the direct union of its elements. It may also be produced by the incomplete combustion of certain hydrocarbons, and indeed by the imperfect oxidation of most organic compounds. Acetylene is a colourless gas, possessing a peculiar odour, and burning with an intensely luminous flame. A highly characteristic reaction of acetylene, by which its presence may readily bo determined, is the formation of a 
rod precipitate when brought in contact with an ammoniacal solution of subchloride of copper. This red compound explodes either by percussion, or on being suddenly heated to a temperature a little above that of boiling water. It has been suggested that this is probably the cause of certain gas explosions which have occurred in unserewing the brass fittings of gas-meters. Acetylene is one of the constituents of coal gas, and the red explosive compound is liable to be formed by contact of the gas. with the brass-work.

A.CHROMA TIC, destitute of colour. White light consists, as is shown by its de. composition by a prism, of several coloured rays, having different degrees of refrangibility. When, therefore, whito light passes through any transparent body, such as a lens, it is liable to this decomposition to a greater or a less extent, and hence colour is produced. This is termed chromatic aberration. Many, especially oldfashioned, telescopes exhibit objects surrounded by beautifully coloured fringes. Now the means which have been devised to prevent this are termed achromatic, signifying the deprivation of colour. See LitHT.

ACHronATIC rzNs. Hale, in 1733, constructed lenses which did not produce chromatic dispersion. In 1757 Dollond arrived, by a perfectly independent examination, at the same discovery, and published it.

$A$ lens may be regarded as a number of prisms united round a centre; therefore a ray of light falling on a lenticular glass is decomposed, and the rays being of unequal refrangibility, they have on its axis as many foci as there are colours. The images, therefore, of objects which are produced at these points are superimposed, more or less, and the edges fringed with indistinct colours. The least refrangible rays unite at foci further away than the more refrangible; and the object sought for, and attained, by both Hale and Dollond, was the means of uniting these rays at one focal point. They combined flint-glass with crown-glass, and found that, by a suitable curvature given to the object-glasses, the images seen through them were distinct, and free from these adventitious colours.

Telescopes, microscopes, \&ce, fittod up with such combinations of lenses as those described, are called achromatic telescopes.

ACrCuIrxz. A name applied to Aikenito (a native sulphide of bismuth, copper and lead), in allusion to its occurrence in acicular, or needle-like crystals. See

\section{ATKRNTTE,}

ACrD. (Acidus, sour, L.) The term acid was formerly applied to bodres which were sour to the taste, and in popular language the word is still so used. It is to be regretted that the necessities of science have led to the extension of this word to any bodies combining with bases to form salts, whether such combining body is sour or otherwise. Had not the term acid been established in language as expressing a sour body, there would have been no objection to its use; but chemists now apply the term to substances which are not sour, and which do not change blue vegetable colours; and consequently they fail to convey a correct ides to the popular mind.

Hobbes, in his 'Computation or Logic,' says, 'A name is a word taken at pleasure to serve for a mark which may raise in our mind a thought like to some thought we had before, and which, being pronounced to others, may be to them a sign of what thought the speaker had, or had not, before in his mind.' This philosopher thus truly expresses the purpose of a name; and this purpose is not fulfilled by the term acid, as now employed.

Mr. John Stuart Mill, in his 'System of Logic,' thus, as it appears not very happily, endeavours to show that the word acid, as a scientific term, is not inappropriate or incorrect.

'Sciontific definitions, whether they are definitions of scientifle terms, or of common terms used in a scientific sense, are almost always of the kind last spoken of : their main purpose is to serve as the landmarks of scientific classification. And, since the classifications in eny science are continually modified as scientific knowledge advances, the definitions in the sciences are also constantly varying. A striking instance is afforded by the words acid and alkali, especially the former. As experimental discovery advanced, the substances classed with acids have been constantly multiplying; and, by a natural consequence, the attributes connoted by the word have receded and become fewer. At first it connoted the attributes of combining with an alkali to form a neutral substance (called a salt), being compounded of a base and oxygen, causticity to the taste and touch, fluidity, \&ce. The true analysis of muriatic acid into chlorine and hydrogen caused the second property, composition from a base and oxygen, to be excluded from the connotation. The same discovery fixed the attention of chemists upon hydrogen as an important element in acids; and more recent discoveries having led to the recognition of its presence in sulphurie, nitric, and many other acids, where its existonce was not previously suspected, there is now a tendency to include the presence of this element in the connotation of the word. But carbonic acid, silica,

Yor. I. 
and sulphurous acid, have no hydrogen in their composition; that property cannot, therefore, be connoted by the term, upless those substances are no longer to be considered acids. Causticity and fluidity have long since been excluded from the charactoristics of the class by the inclusion of silica and many other substances in it; and the formation of neutral bodies by combination with alkalis, together with such electro-chemical peculiarities as this is supposed to imply, are now the only differentia which form the fixed connotation of the word acid as a term of chemical science,'

The term Alkatr, though it is included by Mr. J. 8. Mill in connection with acid in his romarks, does not stand, oven as a scientific term, in the objectional position in which we find acid. Alkali is not, strictly speaking, a common name to which any definito idea is attached. Acid, on the contrary, is a word commonly employed to signify sour. The highest chemical authorities, following Gerhardt, now define AciDs to be Salts of Hydrogen, or compounds in which the hydrogen may be readily replaced by a metal so as to form an ordinary salt.

An acid must now be defined to be a body which has the power of destroying more or less completely the characteristic properties of alkalis-at the same time losing its own distinguishing peculiarities. Seo Alkatr ; ANHYDRrdes.

In this Dietionary all the acids named will bo found under their respective heads, as Acurtic, Nitric, Sutphuric Acids, \&c.

ACIDIFInR. Any body whose presence appears to be necessary for the production of an acid.

ACIDIMETDR. An instrument for measuring the strength or quantity of real acid contained in a free state in liquids. The construction of that instrument is founded on the principle that the quantity of real acid present in any samplo is proportional to the quantity of alkali which a given weight of it can neutralise. The instrument, like the alkalimeter (see ALKALIMETRR), is made to contain 1,000 grains in weight of pure distilled water, and is divided accurately into 100 divisions, each of which therefore represents 10 grains of pure distilled water; but as the specific gravity of the liquids which it serves to measure may be heavier or lighter than pure water, 100 divisions of such liquids are often called 1,000 grains' measure, irrespectively of their weight (specific gravity), and accordingly 10-20 \&c. divisions of the acidimeter are spoken of as 100-200 \&c. grains' measure; that is to say, as a quantity or measure which, if filled with pure water, would have weighed that number of grains.

ACIDIMFTRY. Acidimetry is the name of a chemical process of analysis by means of which the strength of acids-that is to say, the quantity of pure free acid contained in a liquid - can be ascertained or estimated. The principle of the method is based upon Dalton's law of chemical combinations; or, in other words, upon the fact that, in order to produce a complete reaction, a certain definite weight of reagent is required.

If, for example, we take 1 equivalent, or 49 parts in weight, of pure oil of vitriol of specific gravity 1.8485 , dilute it (of course within limits) with no matter what quantity of water, and add thereto either soda, potash, magnesia, ammonia, or their carbonates, or in fact any other base, until the acid is neutralised-that is to say, until blue litmus-paper is no longer, or only very faintly, reddened when moistened with a drop of the acid liquid under examination - it will be found that the respective weights of each base required to produce that effect will greatly differ, and that with respect to the bases just mentioned these weights will bo as follows:-

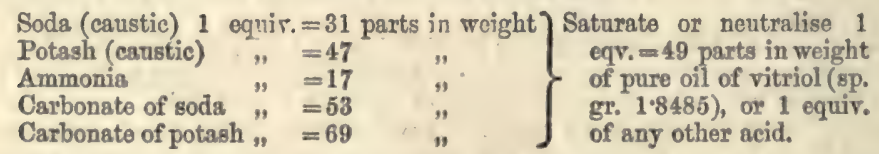

This being the case, it is evident that if we wish to ascertain by such a method the quantity of sulphuric acid or of any other acid contained in a liquid, it will be necessary, on the one hand, to weigh or measure aecurately a given quantity of that liquid to be examined, and, on the other hand, to dissolve in a known volume of water the weight above mentioned of any one of the bases just alluded to, and to pour that solution gradually into that of the acid until neutralisation is obtained; the number of volumes of the basic solution which will have been required for the purpose will evidently indicate the amount in weight of acid which existed in the liquid under examination. Acidimetry is therefore exactly the reverse of alkalimetry, since in principle it depends on the number of volumes of a solution of a base diluted with water to a definite strength, which are required to neutralise a known weight or measure of the different samples of acids. 
The solution containing the known weight of base, and capable therefore of saturating a known weight of acid, is called a 'test-liquor;' and an aqueous solution of ammonia, of a standard strength, as first proposed by Dr. Ure, affords a most exact and convenient means of effecting the purpose, when gradually poured from a graduated dropping-tube or acidimeter into the sample of acid to bo examined.

The strength of the water of ammonia used for the experiment should be so adjusted that 1,000 grains' measure of it (that is, 100 divisions of the alkalimeter) really contain one equivalent (17 grains) of ammonia, and consequently neutralise one equivalent of any one real acid. The specific gravity of the pure water of ammonia employed as a test for that purpose should be exactly 0.992 , and when so adjusted, 1,000 grains' measure (100 divisions of the acidimeter) will then neutralise exactly

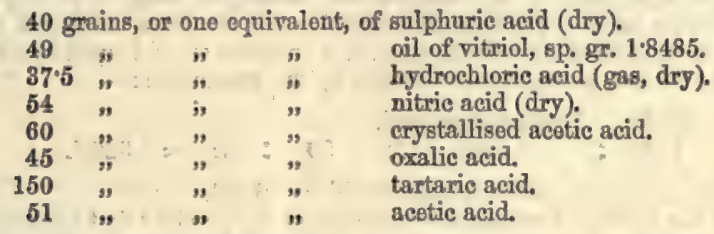

And so forth with the other acids.

A standard liquor of ammonia of that strength becomes, therefore, a universal acidimeter, since the number of measures or divisions used to effect the neutralisation of 10 or of 100 grains of any one acid, being multiplied by the atomic weight or equivalent number of the acid under examination, the product, divided by 10 or by 100 , will indicate the per-centage of real acid contained in the sample; the proportion of free acid being thus determined with precision, even to $\frac{x}{50}$ th of a grain, in the course of five minutes, as will be shown presently.

The most convenient method of preparing the standard liquor of ammonia of that specific gravity is by means of a glass bead, not but that specific-gravity bottles and hydrometers may, of course, be employed; but Dr. Ure remarks, with reason, that they furnish incomparably more tedious and less delicate means of adjustment. The glass bead, of the gravity which the test-liquor of ammonia should have, floats, of course, in the middle of such a liquor at the temperatiure of $60^{\circ} \mathrm{F}$; ; but if the strength of the liquor becomes attenuated by evaporation, or its temperature increased, the attention of the operator is immediately called to the fact, since the difference of a single degree of heat, or the loss of a single hundredth part of a grain of ammonia per cent., will cause the bead to sink to the bottom-a degree of precision which no hydrometer can rival, and which could not otherwise be obtained, except by the troublesome operation of accurate weighing. Whether the solution remains uniform in strength is best ascertained by introducing into tho bottle containing the ammonia test-liquor two glass beads, so adjusted that one, being very slightly heavier than the liquid, may remain at the bottom; whilst the other, being very slightly lighter, reaches the top, and remains just under the surface as long as the liquor is in the normal state; but when, by the evaporation of some ammonia, the liquor becomes weaker, and consequently its specific gravity greater, the bead at the bottom rises towards the surface, in which case a few drops of strong ammonia should be added to restore the balance.

An aqueous solution of ammonia, of the above strength and gravity, being pre. pared, the acidimetrical process is in every way similar to that practised in alkalimetry; that is to say, a known weight, for example, 10 or 100 grains of the sample of acid to be examined, are poured into a sufficiently large glass vessel, and diluted, if need be, with water, and a little tincture of litmus is poured into it, in order to impart a distinct red colour to it; 100 divisions, or 1,000 grains' measure, of the standard ammonia test-liquor above alluded to, are then poured into an alkalimeter (which, in the present case, is used as an acidimeter), and the operator proceeds to pour the ammonia test-liquor from the alkalimeter into the vessel containing the acid under examination, in the same manner, and with the same precautions used in alkalimetry (see Alkatmortry), until the change of colour, from red to blue, of the acid liquor in the ressel indicates that the neutralisation is complete and the operation finished.

Let us suppose that 100 grains in weight of a sample of sulphuric acid, for example, have required 61 divisions (610 water-grains' measure) of the acidimeter for their completo neutralisation, since 100 divisions (that is to say, a whole acidimeter full) of the test-liquor of ammonia is capable of neutralising exactly 49 grains-one equivalent - of oil of vitriol, of specific gravity 1.8485 , it is clear that the 61 divisions employed will have neutralised 29.89 of that acid, and, consequently, the sample of 
sulphuric acid examined contained that quantity per cent. of pure oil of vitriol, xopresenting $24^{\cdot 4}$ per cont. of pure anhydrous sulphuric acid: thus-

$$
\begin{aligned}
& \text { Divisions, Oil of Vitriol } \\
& 100 \quad: \quad{ }_{49}:: 61: t=29.89 \text {. } \\
& \text { Anhydrous Acid. } 61 \quad: \quad \approx=24 \cdot 4 \text {. }
\end{aligned}
$$

The specific gravity of an acid of that strength is 1.2178.

In the same manner, suppose that 100 grains in weight of hydrochloric acid have required 90 divisions ( 900 grains' measure) of the acidimeter for their completo neutralisation, the equivalent of dry hydrochloric acid gas being $36^{\cdot 5}$, it is clear that since 90 divisions only of the ammonia test-liquor have been employed, the sample operated upon must have contained per cont. a quantity of acid equal to 33.30 of dry hydrochloric acid gas, in solution, as shown by the proportion :-

Divis. Hydrochloric acid.

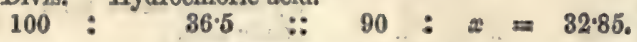

The specific gravity of such a sample would be 1'1646.

Instead of the ammonia test-liquor just alluded to, it is clear that a solution containing one equivalent of any other base-such as, for example, carbonate of soda, or carbonate of potash, caustic lime, \&c.-may be used for the purpose of neutralising the acid under examination. The quantity of these salts required for saturation will of course inaicate the quantity of real acid, and, by calculation, the per-centage thereof in the sample, thus:-The equivalent of pure carbonate of sods being 53, and that of carbonate of potash 69, either of these weights will represent one equivalent, and consequently 49 grains of pure oil of vitriol, $36^{\circ} 5$ of dry hydrochloric acid, 60 of crystallised, or 51 of anhydrous acotic acid, and so on. The acidimetrical assay is performed as follows:-

If with carbonate of soda, take 530 grains of pure and dry carbonate of soda, obtained by igniting the bicarbonate of that base (see AtKATIMETRY), and dissolve them in 10,000 water-grains' measure (1,000 acidimetrical divisions) of distilled water. It is evident that each acidimeter full (100 divisions) of such a solntion will then correspond to one equivalent of any acid, and accordingly if the test-liquor of carbonate of soda be poured from the acidimeter into an weighed quantity of any acid, with the same precautions as before, until the neutralisation is complete, the number of divisions employed in the operation will, by a simple rule of proportion, indicate the quantity of acid present in the sample as before. Pure carbonate of soda is easily obtained by recrystallising once or twice the crystals of carbonate of soda of commerce, and carefully washing them. By heating them gradually they melt, and at a very low red heat entirely lose their water of crystallisation and become converted into pulverulent anhydrous neutral carbonato of soda, which should be kept in wellclosed bottles.

When carbonate of potash is used, then, since the equivalent of carbonate of potash is 69 , the operator should dissolve 690 grains of it in the 10,000 grains of puro distilled water, and the acidimeter being now filled with this test-liquor, the assay is carried on again precisely in the same manner as before. Neutral carbonate of potash for acidimetrical use is prepared by heating the bicarbonate of that base to redness, in order to expel one equivalent of its carbonic acid; the residue left is pure neutral carbonate of potash; and in order to prevent its absorbing moisture, it should be put, whilst still hot, on a slab placed over concentrated sulphuric acid, or chloride of calcium, under a glass bell, and, whon sufficiently cool to be handled, transferred to bottles carefully closed.

To adapt the above methods to the French weights and measures, now used also generally by the German chemist, we need only substitute 100 decigrammes for 100 grains, and procoed with the graduations as already described.

A solution of caustic lime in cane sugar has likewise been proposed by M. Peligot for acidimetrical purposes. To prepare such a solution, take pure caustic lime, ob. tained by heating Carrara marble with charcoal in a furnace; when sufficiently roasted to convert it into quicklime, slake it with water, and pour upon the slaked lime as much water as is necessary to produce a milky liquor; put this milky liquor in a bottle, and add thereto, in the cold, a certain quantity of pulverised sugar-candy; close the bottle with a good cork, and shake the whole mass well. After a certain time it will be observed that the milky liquid has become very much clearer, and perhaps quito limpid; filter it, and the filtrate will be found to contain about 50 parts of lime for every 100 of sugar employed. The liquor should not be heated, because saccharate of lime is much more soluble in cold than in hot water, and if heat were. 


\section{AOIDIMETRY}

applied it would become turbid or thick, though on cooling it would bocome clear again. ${ }^{i}$

A concentrated solution of lime in sugar being thus obtained, it should now be diluted to such a degree that 1,000 water-grains' measure of it may be capablo of saturating exactly one equivalent of any acid, which is done as follows:-Take 100 grains of hydrochloric acid of specific gravity $1 \cdot 1812$, that weight of acid contains exactly one equivalent $=36.5$ of pure hydrochloric acid gas ; on the other hand, fill the acidimeter up to 0 (zero) with the solution of caustic lime in sugar prepared as abovesaid, and pour the contents into the acid until exact neutralisation is obtained, which is known by testing with litmus-paper in the usual manner already described. If the whole of the 100 divisions of the acidimeter had been required exactly to neutralise the 100 grains' weight of hydrochloric acid of the specific gravity mentioned, it would have been a proof that it was of the right strength; but suppose, on the contrary, that only 50 divisions of the lime solution in the acidimeter have been sufficient for the purpose, it is evident that it is half too strong, or, in other words, one equivalent of lime $(=28)$ is contained in those 50 divisions instead of in 100. Pour, therefore, at once, 50 divisions or measures of that lime-liquor into a glass cylinder accurately divided into 100 divisions, and fill up the remaining 50 divisions with water; stir the whole well, and 100 divisions of the lime-liquor will, of course, now contain as much lime as was contained before in the 50 ; or, in other words, 100 acidimetrical divisions will now contain 1 equivalent of lime, and therefore will be crpable of exactly neutralising 1 equivalent of any acid.

When, however, saccharate of lime is used for the determination of sulphuric acid, it is necessary to dilute it considerably, for otherwise a precipitate of sulphate of lime would be produced. This reagent, moreover, is evidently applicable only to the determination of such acids the lime salts of which are soluble in water.

Instead of a solution of caustic lime in sugar, a clean dry piece of white Carrara marble may be used. Suppose, for example, that the acid to be assayed is acetic acid, the instructions given by Brande are as follows:-A clean dry piece of marble is selected and accurately weighed; it is then suspended by a silk thread in a known quantity of the vinegar or acetic acid to be examined, and which is cautiously stirred with a glass rod, so as to mix its parts, but without detaching any splinters from the weighed marble, till the whole of the acid is saturated, and no further action on the marble is observed. The marble is then taken out, washed with distilled water, and weighed; the loss in weight which it has sustained may be considered as equal to the quantity of acetic acid present, since the atomic weight of carbonate of lime $(=50)$ is very nearly the same as that of acetic acid $(=51)$. Such a process, however, is obviously less exact than those already described.

But, whichever base is employed to prepare the test-liquor, it is clear that the acid tested with it must be so far pure as not to contain any other free acid than that for which it is tested, for in that case the results arrived at would bo porfectly fallacious. Unless, therefore, the operator has reason to know that the acid, the strength of which has to be examined by that process, is genuine of its kind, he must make a qualitative analysis to satisfy himself that it is so; for in the contrary case the acid would not be in a fit state to be submitted to an acidimetrical assay.

The strength of acids may also be ascertained by determining either the volumes or the weight of carbonic acid gas disengaged from pure bicarbonate of soda by a given weight of any acid.

For measuring exactly the volumes of carbonic acid thus expelled, Dr. Uro's apparatus, represented in fig. 7, may be used. As it is absolutely requisite, for the success of the experiment, that the whole of the acid taken for examination should be completely saturated, the operator must accordingly take care to use a little more bicarbonate than is necessary for the purpose.

Now the equivalent number of bicarbonate of soda is 75 , and the carbonic acid contained therein $=44$; that of oil of vitriol is 49 ; wherefore by mixing together 75 grains of pure bicarbonate of soda with 49 grains of pure oil of vitriol, 44 grains of carbonic acid gas will be expelled, equal in bulk or volume to 2,381 acidimetrical divisions (23,810 water-grains' measure). These proportions, however, would be inconvenient, the more especially as the acidimeter in question should contajn exactly 10,000 water-grains' measure, marked in series of 10 divisions from 0 (zero) at the top down to 100 , such an arrangement at onco enabling the operator to read off the amount of real acid per cent.; and accordingly a weight, or proportion of acid capablo of disengaging exactly 10,000 water-grains' measure of carbonic acid from a quantity of

3 The directions given by M. Violette for the preparation of Saccharate of Lime are as follow:Digest in the cold 50 grammes of slaked caustic lime in 1 utre of water containing 100 grammes of sugar. 
bicarbonato more than suffleient to supersaturate it is used. That woight or portion varies, of course, with each kind of acid, thus :-

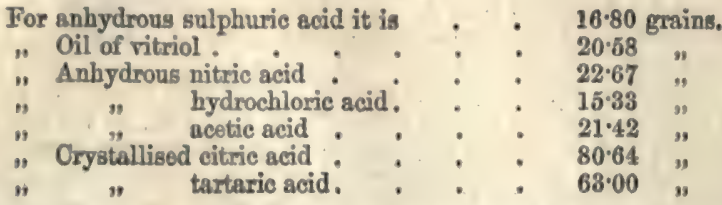

Therefore by taking, of any sample of acid to be examined, the exact number of grains corresponding to each of the above-mentioned acids, we shall obtain a volume of car-

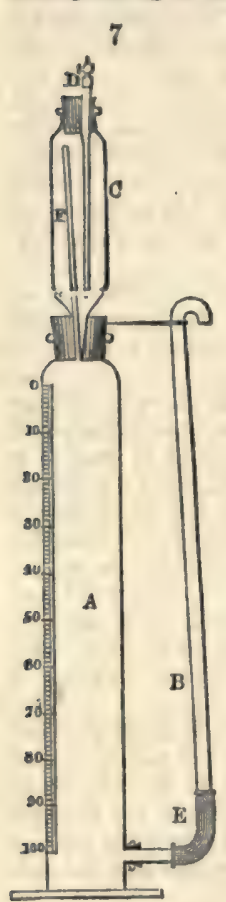
bonic acid gas proportioned to the strength and purity of the sample of each of them respectively. The modus operandi is as follows:-Charge the glass cylinder $\mathrm{A}$ with water, and pour upon the surface of the latter a layer of olive oil, about 1 inch in thickness, so that the level corresponds exactly to the 0 (zero) of the graduated scale etched on the glass cylinder. Through the cork in the mouth of the cylinder, push the taper tail of the flask c, air-tight; introduce into this flask c about 50 grains of bicarbonate of soda, in powder, and pour upon them a little more water than is sufficient to cover the powder; and if, for example, the object is to determine the amount of pure oil of vitriol contained in a given sample of that acid, weigh now accurately 20.58 grains of that sample, dilute it with water, and suck it up into the taper dropping glass tube, D ; shut the stopeock, introduce the dropping-tube, pushing it air-tight through the perforated cork uutil its extromity plunges into the mixture of bicarbonate of sods and water in the flask, c. On opening now slightly the stopeock of the dropping-tube, the acid contained therein coming in contact with the bicarbonate will cause the evolution of a volume of carbonic acid proportioned to its strength. Supposing the same sample of sulphuric acid which was found by the acidimetrical process first described to contain 29.89 of oil of vitriol, or 24.4 of anhydrous sulphuric acid, por cent., to be now examined by the present method, it will be found that the 20.58 grains of that acid taken for the experiment have disengaged a rolume of carbonic acid gas corresponding nearly to the number $\mathbf{3 0}$ of the graduated scale of the glass cylinder, thereby indicating nearly 30 per cent, of pure oil of vitriol in the sample under consideration.

In the same manner the sample of hydrochloric acid, which by the former process was found to contain 32.85 per cent. of pure hydrochloric acid, would now disengage a volume of carbonic acid gas which would depress the lovel of the water in the glass cylinder nearly to the point marked 33 , and therefore the operator wonld at once know that the quantity of pure hydrochloric acid gas contained in the sample was a little less than 33 per cent., a degree of accuracy quite sufficient for all commercial purposes, and which might besides be rendered still more accurate by lengthening the glass cylinder and diminishing its bore, so that the divisions may be sufficiently distant as to admit of being subdivided into fractions.

The principal objection to this form of acidimeter, howevor, is its expense, and also the difficulty or trouble of introducing into it the whole of the accurately weighed quantity of acid, a circumstance which renders it less applicable to acidimetry than to alkalimetry. By suppressing, however, tho top flask, c, and using instoad of it a common Florence flask, connected with the cylinder, the cost is considorably reduced, and the operator is at once enabled to secure the complete reaction of the whole of the accurately weighed acid upon the bicarbonate of soda. The arrangement has, bosides, several other advantages, which the simple inspection of fig. 8 renders apparent. It consists of a 10,000 water-grains'-measuro glass cylinder, $\Delta$, graduatod in the same mannor, and provided with a discharge-tube, B, as before; but the mouth of the cylinder need not be larger than that of an ordinary winebottle, which allows of its being corked air-tight with greater ease and certainty. This cork is perforated, and provided with a tubo passing air-tight through it, and connected - by a length of vulcanised india-rubber, $\mathrm{c}$ - with the disengagement tube of an ordinary Florence flask, into which the bicarbonate of soda and a certain quantity 


\section{ACIDIMETRY}

of water has been previously introdnced, and likewise a small tost-tubo, $\mathrm{s}$, containing the exactly weighed quantity of acid to be examined. All the joints being perfectly air-tight, if the Florence flask be now carefully tilted on one side, a portion of the acid in the test-tube will, of course, flow down upon the bicarbonate of soda, and a corresponding quantity of carbonic acid gas being evolved will depress the water in the glass cylinder, causing an overflow from the tube $\mathrm{B}$, which should be held over a basin, and progressively lowered so as to keep the discharging aperture on a lovel with the descending water in the cylinder. The operation is terminated when, all the acid in the test-tube having been completely upset and all effervescence being entirely at an end, the level of the water in the cylinder $\Delta$ remains stationary; the number of divisions of the scale corresponding to that level are then read off; they indicate the per-centage strength of the sample.

The bicarbonate of soda of commerce frequently contains some neutral carbonate of soda which should be removed before using it for that and for the following process; this is easily done by washing it with a moderate quantity of cold water, which dissolves the neutral carbonate, but leaves the greater portion of the bicarbonate in an undissolved state; it should then be dried spontaneously by spreading it in the air, and then kept in stoppered bottles; for though bicarbonate of soda does not undergo decomposition by exposure to dry air, a moist atmosphere converts a portion of it into a neutral carbonate, with 5 equivalonts of water $\left(\mathrm{NaO}, \mathrm{CO}^{2} .6 \mathrm{HO}\right)$.

8

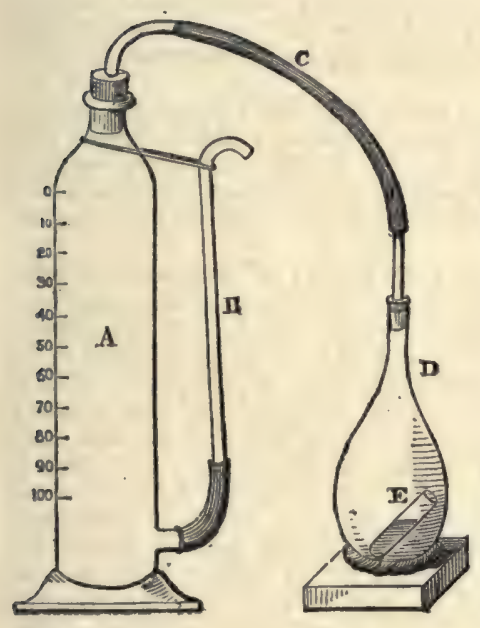

B

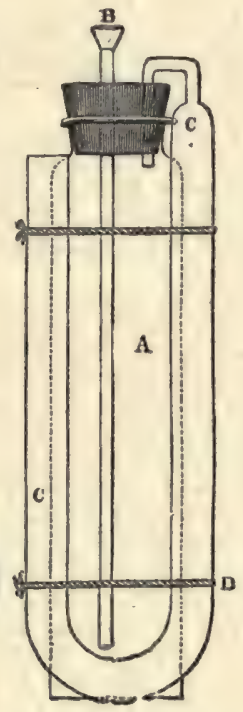

10

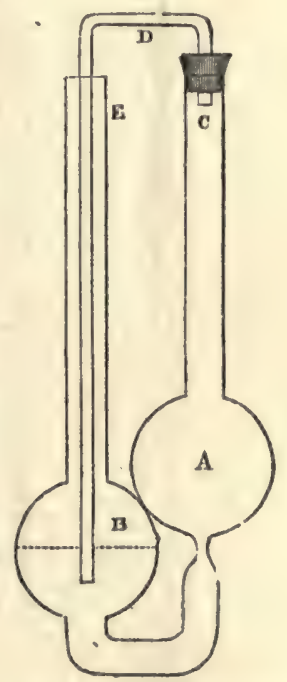

Acidimetrical operations may likewise be performed by determining the weight instead of the volumes of the carbonic acid expelled from bicarbonate of potash, or of soda, by a given quantity of acid. For this purpose either of the apparatus contrived by Dr. Ure, and represented above, may be used. The details of their construction are given in ALKALIMETRX, to which the reader is referred.

Since 1 equivalent of any acid will disengage 2 equivalents $(=44)$ of carbonic acid from 1 equivalent $(=75)$ of bicarbonate of soda, it is evident that by determining what quantity of any pure acid is capable of disongaging or expelling 10 grains of carbonic acid gas, then taking that quantity of the acid to be examined, and causing it to react upon a mass of bicarbonate of soda more than sufficient to saturate or neutralise it (in order to make sure that the acid has produced all its effeet), the loss sustained after the operation from the carbonic gas expelled, multiplied by ten, will at once indicate the exact per-centage of real acid contained in the sample examined. Of course the weight of acid capable of disengaging exactly 10 grains of carbonic acid gas varies with each kind of acid; and that weight is found by dividing 10 times the atomic weight of the acid, whatever it may be, by 44 ; that is to say, by the atomic weight of the two equivalents of carbonic acid gas contained in the bicarbonate of sods. 
For eulphurie acid, for example, the proportion would be as follows:-

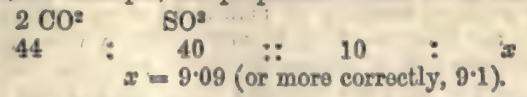

Applying this rule, the weights to bo taken are as follows, in roference to-

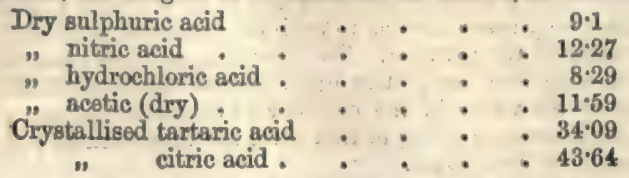

Each of these quantities of real acid, with 25 or 26 grains of bicarbonate of soda, will give off 10 grains of carbonic acid gas; and hence, by adding a eypher, that is, multiplying by ten, whatever weight the apparatus loses denotes the per-centage of acid in the sample under trial, without the necessity of any arithmetical reduction. Let us suppose, for example, that the apparatus, being charged with 9.1 grains of a sample of sulphuric acid, is found, after the experiment, to have lost $7 \cdot 5$ grains; this multiplied by $10=75.0$; therefore the sample contained 75 per cent, of dry sulphuric acid. If the apparatus had lost $2 \cdot 44$ grains thus, it would have indicated $24 \cdot 4$ per cent. of dry or anhydrous acid. Persons accustomed to the Freneh metrical system may use

11

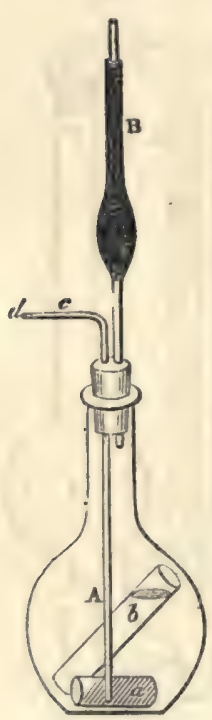
decigrammes instead of grains, and they will arrive at the same per-centage results.

Another apparatus for ascertaining the weight of carbonic expelled for the purposes of either acidimetry or alkalimetry, and which the operator himself may readily construct, is reprosented in fig. 11 .

$\Delta$ is a small matrass, with a somewhat wide mouth, capable, however, of being hermetically closed by a cork perforated with two holes, through one of which a bulbed tube, $\mathrm{B}$, passes filled with fragments of chloride of calcium; through the other hole a tube, $c$, is introduced, suffciently long to reach the bottom of the matrass $\boldsymbol{\Lambda}$.

A certain quantity (say 25 grains) of bicarbonate of soda, greater than is required for saturation, is then introduced into the matrass $\mathbf{A}$, and likewise enough water to cover it. $A$ small glass test-tube is next charged with the proper quantity of the acid to be examined, namely, 9.1 if for sulphuric acid, 12.27 if for nitric acid, \&c. \&cc., as before mentioned, and it is carefully introduced into the matrass $\mathbf{A}$, taking care that the acid - does not come in contact with the biearbonate of soda, which is easily avoided by lowering the tube containing the acid into the matrass with a thread, or by carefully sliding it down, and keeping it nearly in an upright position, leaning against the sides of the matrass, as shown by the letter $b$. The matrass is then to be closed with the cork provided with its trabes, as above directed, and the whole is accurately weighed. This done, the apparatus is gently jerked, or tilted, on one side, so as to cause a portion of the acid in the tube $b$ to flow among the bicarbonate of soda on which it is resting. A disengagement of carbonic acid gas immediately takes place from the decomposition of the carbonate of soda by the acid. When the violent effervescence has subsided, a fresh quantity of acid is again jerked, or spilled, out of the tube, until the whole of the acid is emptiod, the tube occupying now a horizontal position, as represented by letter $a$. The water, which is mechanically carried off by the carbonic acid, is arrested by the chloride of calcium of the bulbed tube $\mathbf{B}$. When all disengagement of carbonic acid gas has ceased, even after shaking the apparatus, tho residuary gas is sucked up through the bulbed tube $\mathrm{B}$, while the atmospheric air enters at the oriflee, $d$, of the bent tube, $c$, to replace it. If the apparatus has become warm during the reaction, it should be allowed to cool complotely, and it is then weighed again accurately. The difference between the first and second weighing, the loss, represents, of course, the weight of the carbonic acid gas expelled, and consequently the per-contage of real acid contained in the sample.

Instead of the preceding arrangement, the apparatus contrived by Drs. Fresenius and Will may be used. The annexed figure at once renders the construction of that apparatus intelligible, and as a full description of it is given in the article on ALKatiMETEX; the reader is accordingly referred thereto. When that contrivance is 
used for acidimatrical purposes, proceed as follows :-Fill bottle $A$ with ordinary oil of vitriol to about one-half of its capacity, and pour into bottlo B the accurately weighed quantity of acid to be examined, namely, $9 \cdot 1$ grains for sulphuric acid, $12 \cdot 27$ for nitric acid, \&ce \&c., according to the rulo and table given (page 24), and dilute it with water, so that bottle B may be one-third full. Put now into a testtube a quantity of bicarbonate of soda sufficient to saturate the weight of acid contained in bottle $\mathrm{B}$, and suspend it into that bottle by means of a thread, kept tight by the pressure of the cork. Weigh now the whole apparatus accurately; this done, carefully loosen the thread, so that the testtube charged with bicarbonate of soda may fall into the acid, and the cork being instantly adjusted air-tight, the whole of the carbonic acid gas disengaged is led by tube $c$ into the concentrated sulphuric acid of bottle $\mathrm{A}$, which absorbs all its moisture before it finally escapes through the tube $a$. When all effervescence has ceased, the operator, by applying his lips to that tube $a$, sucks out all the residuary carbonic acid gas contained in the apparatus, and replaces it by atmospheric air, which enters at $d$. The apparatus,

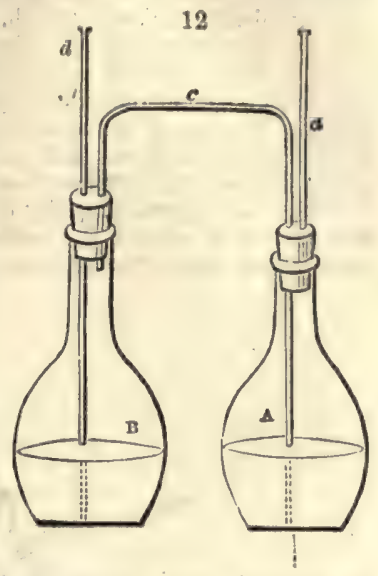
if it have become warm, should be allowed to cool completely, and on weighing it again the loss indicates the per-centage of real acid present in the sample.

The balance used in these methods should, of course, be sufficiently delicate to indicate small weights when heavily laden.

We shall terminate this article by a description of Liebig's acidimetrical method of determining the amount of prussic acid contained in solutions; for example, in medicinal prussic acid, in laurel and bitter-almond water, essence of bitter almonds, and cyanide of potassium. The process is based upon the following reaction :- When an excess of caustic potash is poured into a solution which contains prussic acid, cyanido of potassium is, of course, formed; and if nitrate of silver be then poured into such a liquor, a precipitate of cyanide of silver is produced, but it is immediately redissolved by shaking, because a double cyanide of silver and of potassium ( $\mathrm{Ag} \mathrm{Cy}+$ $\mathrm{K} \mathrm{Cy}$ ) is formed, which dissolves, without alteration, in the excess of potash employed. The addition of a fresh quantity of nitrate of silver produces again a precipitate which agitation causes to disappear as before; and this reaction goes on until half the amount of prussic acid present in the liquor has been taken up to produce cyanide of silver, the other half being engaged with the potassium in the formation of a double cyanide of silver and of potassium, as just said. As soon, however, as this point is reached, any new quantity of nitrate of silver poured in the -liquor causes the cyanide of potassium to react upon the silver of the nitrate, to produce a permanent precipitate of cyanide of silver, which indicates that the reaction is complete, and that the assay is terminated. The presence of chlorides, far from interfering, is desirable, and a certain quantity of common salt is accordingly added, the reaction of chloride of silver being analogous to that of the cyanide of the same metal.

To determine the strength of prussic acid according to the above process, a test or normal solution should be first prepared, which is as follows :-

Since 1 equivalent of nitrate of silver $(=170)$ represents, as we have seen, 2 equivalents of prussic acid $(=54)$, dissolve, therefore, 170 grains of pure fused nitrate of silver in 10,000 water-grains' measure of pure water; 1,000 water-grains' measure (1 acidimeter full) of such solution will therefore contain 17 grains of nitrate of silver, and will therefore represent 5.4 grains of prussic acid; and consequently each acidimetrical division 0.054 grain of puro prussic acid.

ACIP Farsmr. A genus of cartilaginous ganoid fishes, to which the Sturgeon belongs, and from which isinglass is obtained. The roe of the sturgeon yields caviaire. There are at least eight species ; four, however, appear to yield the Isinglass of commerce. The Beluga or large Sturgeon, Acipenser Huso. The Osseter, A. Güldenstadtii. The Sterlet, $A$. Ruthenus, and the Sewraga, A. Stellatus. These inhabit the Black and Caspian Sea, and the great rivers flowing into them. Soe

Caviatras ; Istrgiass.

ACONITINE. $\mathrm{C}^{100} \mathrm{H}^{47} \mathrm{NO}^{14}\left(\mathbf{C}^{30} \mathrm{ZZ}^{47} \mathrm{2rO}^{7}\right)$. A poisonous alkaloid constituting the active principle of the Monkshood (Aconitum Napellus) and other species of Aconite. ACorrirua. (akóvitov.) The Greek name for the Hemlock. See Coniux.

Acosuruar is now the namo of a genus of plants belonging to the Ranunculacea, 
nearly all the species being remarkable for their poisonous properties. A. Napellus is the Monkshood or Wolf's-bane, commonly cultivatod in gardens as a showy flower, but tho learos and root are highly poisonous, and doath has rosulted from eating the root by mistake for horse-radish. The Bikh, Bish, or Nabeo poison, used by the hilltribes of Northern India for poisoning arrows, is obtained from $A$. ferox, which is said to be a moro powerful poison than either of the other species; the quantity of the poisonous alkaloid Aconitine depending on the temperature in which the plant has grown. The root of the Aconite or Monkshood having been very frequently mistakon for the horse-radish root, and sereral deaths having boen produced by eating it, a fow of the distinctions between thom aro given. The aconite root, as shown in fig. 13, is conical and tapering rapidly to a point. The horse-radish is slightly conical at the crown, then of almost the same thickness for several inches. Aconite is colourod more or less brown, the horse-radish is externally white. The odour of the aconite is

13

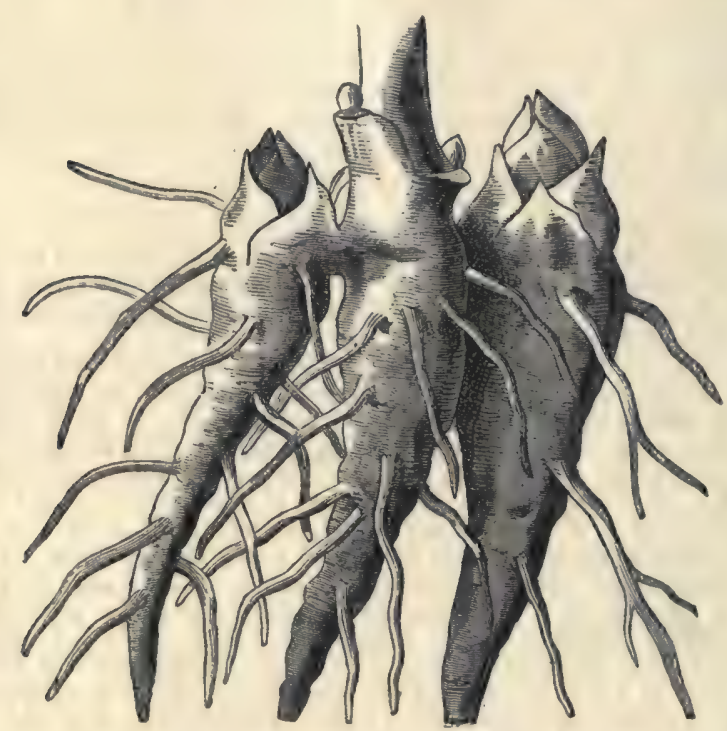

merely earthy, that of horse-radish pungent and irritating. Aconite root is the most virulent in the winter months and early spring, when the leaves are absent.

Acorws. The fruit of the oak (Quercus). These possess some of the properties of the bark, but in a very diluted degree. Acorhs are now rarely used. Pigs are sometimes fed upon them. The acorn-cups of Quercus LFgilops are used in tanning and dyeing, and are imported under the name of Valonia. See VALONIA.

Acorus caramus. The common sweet flag. This plant is a native of England, growing abundantly in the rivers of Norfolk, from which-county the London market is chiefly supplied. The radix calami aromatica of the shops occurs in flattened pieces about one inch wide and four or five inches long. It is employed medicinally as an aromatic, and it is said to be used by some distillers to flavour gin. The essential oil (oleum acori calami) of the sweet flag is used by snuff-makers for scenting snuff, and it sometimes enters as one of the aromatic ingrodients of aromatic vinegar. Tho Acorus belongs to the Aracece or arum-order. See Arace

ACrosprnz. (Plumule, Fr.; Blattkeim, Ger.). The sprout at the end of seeds when they begin to germinate. Maltsters use the name to express the growing of the barley. 'The first leaves that appear when corn sprouts.'-Lindley.

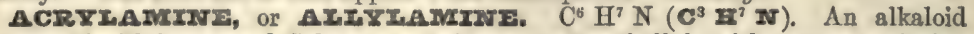
obtained by Hofmann and Cahours, by boiling cyanate of allyle with a strong solution of potash. It boils at about $365^{\circ}$. C. G. W.

ACTrorssm. (From àktiv, a ray; signifying merely the power of a ray, without defining what character of ray is intended.)

As early as 1812, M. Berard (in a communication to the Academy of Sciences, on some observations made by him of the phenomona of solar action) drew attention to the 
fact, that three very distinct sets of physical phenomena were manifested : Light, Heat, and Chemical action. Chaptal, Berthollet, and Biot reported on this paper by M. Berard; and, as showing the extent to which this very important inquiry had proceeded in the hands of this philosopher, the following quotation is given from their report:

- M. Berard found that the chemical intensity was greatest at the violet end of the spectrum, and that it extended, as Ritter and Wollaston had observed, a little beyond that extremity. When he left substances exposed for a certain time to the action of each ray, he observed sensible effects, though with an intensity continually decreasing, in the indigo and blue rays. Hence we must consider it as extremely probable, that if he had been able to employ reactions still more sensible, he would have observed analogous effects, but still more feeble, even in the other rays. To show clearly the great disproportion which exists in this respect between the energies of different rays, $\mathbf{M}$. Berard concentrated, by means of a lens, all that part of the spectrum which extends from the green to the extreme violet, and he concentrated, by another lens, all that portion which extends from the green to the extremity of the red ray. This last pencil formed a white point, so brilliant that the eyes were scarcely able to endure it, yet the muriate of silver remained exposed more than two hours to this brilliant point of light, without undergoing any sensible alteration. On the other hand, when exposed to the other pencil, which was much less bright, and less hot, it was blackened in less than six minutes. . . . . If we wish to consider solar light as composed of three distinct substances, one which oceasions light, another heat, and the third chemical combinations, it will follow that each of those substances is separable by the prism into an infinity of different modifications, like Light itself; since wo find, by experiment, that each of the three properties, chemical, calorific, and colourific, is spread, though unequally, over a certain extent of the spectrum. Hence we must suppose, on

14

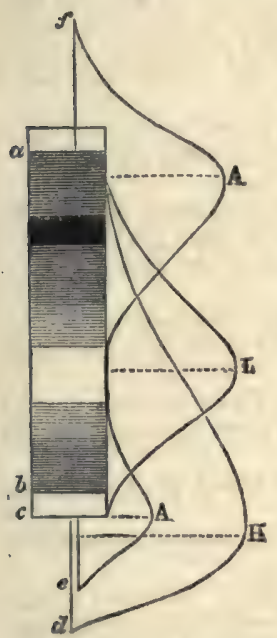
that hypothesis, that there exists three spectrums, one above another; namely, a calorific, a colourific, and a chemical spectrum.'

This was the earliest indication of the probable existence of a physical influence, in the solar rays, distinct from Light and Heat, A large number of philosophers still hold to the ides that the chemical changes produced by the sunbeam are due to light, and this idea is confirmed in the public mind by the universal adoption of the torm photography (lightdrawings) to indicate the production of pictures by the agency of the sunbeam. See PHOTOGRAPHY.

The actual conditions of the sunbeam will be understood by reference to the annexed woodcut, fig. 14, and attention to the following doscription: $a b$ represents the prismatic spectrum -as obtained by the decomposition of white light by the prism-or Nevtonian luminous spectrum, consisting of certain bands of colour. Newton determined those rays to be seven in number; red, orange, yellow, green, blue, indigo, and violet; recent researches, by Sir John Herschel and others, have proved the existence

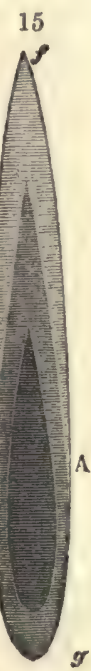

I.

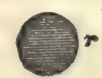

crimson ray $c$, found at the least refrangible end of the spectrum, the other occurring at the most frangible end, or beyond the violet rays, which is a lavender or grey ray. Beyond this point up to $f$, Professor Stokes has discovered a new set of rays, which are only brought into view when the light is received upon the surfaces of bodies which possess the property of altering the refrangibility of the rays. Those rays have been called the fluorescent rays, from the circumstance that some of the varieties of Fluor Spar exhibit this phenomenon in a remarkable manner. (Seo Fluorescence.) The curved line I from $a$ to $c$ indicates the full extent of the luminous spectrum, the point marked $x$ showing the maximum of illuminating power, which exists in the yellow ray.

Sir William Herschel and Sir Henry Englefield determined, in the first instance, the maximum point for the calorific rays, and Sir John Herschel subsequently confirmed their results, proving that the greatest heat was found below the red ray, and that it gradually diminished in power with the increase of refrangibility in the rays, ceasing entirely in the violet ray. Heat rays have been detected down to the point $d$, and the curved line $\mathrm{I}$ indicates the extent of thoir action, 
Now, if any substance capable of undergoing chomical change be exposed to this spectrum, the result will be found to be such as is represonted in the accompanying drawing, fig. 15. Over the space upon which the greatest amount of light falls, i.e.,

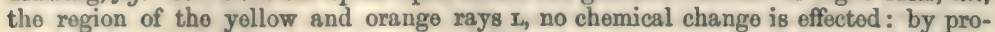
longed action a slight change is brought about where the red ray falls, $r$, but from the mean green ray $g$ up to the point $f, a$ certain amount of chomical action is maintainod; the maximum of action being in the blue and violet rays $\Delta$. Thus the curved line (fig. 14) from $e$ to $f$ represents the extent and degree of chemical power as manjested in the solar spectrum. Two maxima aro markod $\mathbf{A} \mathbf{A}$, differing widely, however, in their degreo.

Here, as in Berard's experiments, we see that where the light is the strongest, there is no chemical action, and that as the luminous power diminishes the chemical force is more decidedly manifested.

Again, we find that if we take a piece of yellow glass, stained with oxide of silver, we have a medium which ontirely prevents the permeation of the chemical rays, though it obstructs no Light. But, if a very dark blue glass is taken, we find that rinety per cent. of the luminous rays are obstructed, while the chemical rays permeate it most freely. Numerous experiments of an analogous charactor appear to prove that the chemical and luminous powers of the sunbeam are balanced against each other (see Hunt's 'Researches on Light'), that they are indeed antagonistic principles or powers. That there are three very distinct sets of phenomena, every one admits.

LigHT (luminous power), to which belongs the phonomena of vision and the production of colour.

HEAT (calorific power), the function of which appears to be the determining the physical condition of all matter, as regards its solid, fluid, or gaseous condition.

Actinism (chemical power), to which all the phenomena of photography are due, and many of the more remarkable changes observed in the vegetable kingdom.

ActrNo-chemrstre was a torm first applied by Sir John Herschel, and has beon generally adopted to indicate the phenomena of chemical change by the action of the solar rays. Actinism was first proposed to express the chemical principle of the sunbeam by the Editor of this Dietionary at the meting of the British Association at York.

ACTrNoGrapr. A name given to an instrument for recording the variations in the chemical (actinic) power of the solar beams. The name signifies ray writer.

ACTrivoIIT2. A variety of Hornblendo Seo HorkBLENDE.

ACXINOMITrR. (Ray measurer.) The name of various forms of instuments, the objects of which are to measure the direct heat radiations from the sun. The torm has also been applied to instruments employed to measure the varying intensities of Light.

ADAMANTINE SPar. An old name for Corundum. See CorUNDUM.

ADANRTE. A native hydrous arsenate of zinc, occurring in the silver-mines of Chañarcillo in Chili, and in the Dép. du Var, France.

ADAM'S NEIDIE, A name commonly given to the Yucca gloriosa, a plant belonging to the Liliacece or Lily-order, the fibres of which have been used in the manufacture of paper.

Adarsonia Digrmata. The Baobab tree, a native of Western Africa. It yiolds a fibre which has lately been used in paper-making.

ADDITIOxs. Such articles as are added to the fermenting wash of the distiller, were of old distinguished by this trivial name.

ADrzsrow (sticking together). The union of two surfaces. With the phonomena which are dependent upon bringing two surfaces so closely together that the influence of cohesion is exerted, we have not to deal. In arts and manufactures, adhesion is effected by interposing between the surfaces to be united some body possessing peculiar properties, such as gum, plaster, resin, marine or ordinary glue, and various kinds of cement. Adhesion should be restricted to mean, sticking together by means of some interposed substance; cohesion, the state of union effected by natural attraction.

Not only is adhesion exhibited in works of art or manufacture; we find it very strikingly displayed in nature. Fragments of rocks which have been shattered by convulsion are found to be cemented together by silica, lime, oxide of iron, and the like; and broken parts of mineral lodes are frequently reunited by the earthy minerals.

ADIPIC ACID. $\mathrm{C}^{12} \mathrm{H}^{10} \mathrm{O}^{3} .\left(\mathbf{C}^{6} \mathbf{H}^{10} \mathbf{O}^{4}\right.$. $)$ One of the fixed fatty acids produced by the action of nitric acid on oleic acid, suet, spermaceti, and other fatty bodies, See Watts's 'Dictionary of Chemistry.'

АDIPoCIRz. From adeps, fat; cera, wax. (Adipocire, Fr.; Fettwachs, Ger.) The fatty matter supposed to be generated in dead bodies buried under peculiax circumstances. It is chiefly margarate of ammonia, In 1786 and 1787, when the 
churchyard of the Innocents, at Paris, was cleaned out, and the bones transported to the Catacombs, it was discovered that not a few of the cadavres were converted into a saponaceous white substance, more especially many of those which had boen interred for fifteen years in one pit, to the amount of 1,500 , in coffins closely packed together. These bodies were flattened in consequence of their mutual pressure; and though they generally retained their shape, there was deposited round the bones of several of them a greyish white, somewhat soft, flexible substance. Fourcroy presented to the Academy of Sciences, in 1789, a memoir which appeared to prove that the fatty body was an ammoniacal soap containing phosphate of lime; that the fat was similar to spermaceti, as it assumed, on slow cooling, a foliated crystalline structure; as also to wax, as, when rapidly cooled, it became granular; hence he called it adipocire. Its molting point was $52.5^{\circ} \mathrm{C}$. $\left(126.5^{\circ} \mathrm{F}\right.$. $)$

This substance was again examined by Chevreul, in 1812, and was found by him to contain margaric acid, oleic acid, combined with a yellow colouring odorous matter, besides ammonia, a little lime; potash, oxide of iron, salts of lactic acid, an azotised substance; and was therefore considered as a combination of margaric and oleic acids, in variable proportions. These fat acids are obviously generated by the reaction of the ammonia upon the margarine and oleïne, though they eventually lose the greater part of that volatile alkali. It is sometimes confounded with chlorestine. Bog butter is said to be a similar substance. See Fat and Fattr Bodies.

ADIPOSE פUBSTANCE or ADIPOsz TrSsur. (Tissu graisseux, Fr.) An animal oil, resembling in its essential properties the regetable oils. During life, it appears to exist in a fluid or semi-fluid state; but, in the dead animal, it is frequently found in a solid form, constituting suet, which, when divested of the membrane in which it is contained, is called tallow. See TALLOW, Ors, \&c.

ADIT or ADIT IFVבI. The horizontal entrance to a mine; a passage or level driven into the hill-side. The accompanying section gives, for the purpose of distinctness, an exaggerated section of a portion of the subterranean workings of a metalliferous mine. It should be understood that $d$ represents a mineral-lode, upon which the shaft, $a$, has been sunk. At a certain depth from the surface of the hill the miners would be inconvenienced by water, consequently a level is driven in from the side of the hill, $b$, through which the water flows off, and through which also the miner can bring ont the broken rock, or any

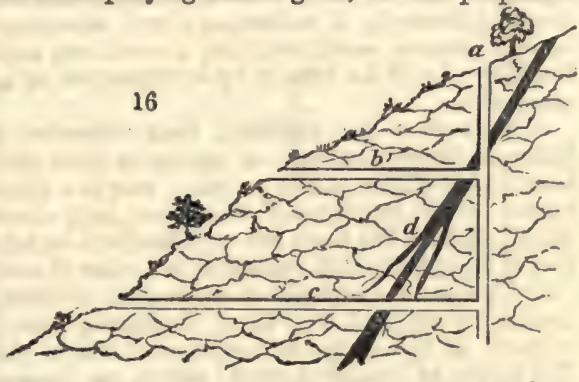
ores which he may obtain. Proceeding still deeper, supposing the workings to have commenced, as is commonly the case, at a certain elevation above the sea-level, similar conditions to those described again arising, another level is driven so as to intersect the shaft or shafts, as shown at $c$. In this case, $b$ would bo called the shallow, and $c$ the deep adit. The economy of such works as these is great, saving the cost of expensive pumping machinery, and also of considerable labour in the removal of ores or other matter from the mine.

The great Gwennap Adit, in Cornwall, with its branches, was cut through the solid rock for nearly 30 miles; through it, numerous mines are drained to a certain depth, and the water pumped from greater depths discharged. The Nentforce Level, or Adit, in Alston Moor, has been wrought under the course of the River Nent, and it extends about $3 \frac{1}{4}$ miles into that important mining district, serving to drain a considerable number of the Nenthead mines. Many of the mines in Cumberland and in Derbyshire are worked by the Adit called a Day-level only; the adit, as at $c$, being carried into the hill until it reaches the lode. The ore is obtained by working up into the hill. It falls into the level, and is carried out in tram-wagons. Soe Mintra.

Adurarra. A variety of orthoclase. See Frispar.

ADUXTFRATION. The practice of debasing any product of manufacture by the introduction of cheap and often injurious materials. The extent to which the adulteration of almost every useful article is carried, is at once a disgrace to the trading community, and a standing reflection on an age and country which boasts of its high moral character and its devotion to Christianity.

ADzE. A cutting instrument; differing from the axe by the edge being placed at nearly right angles to the handle, and being slightly curved up or inflected towards it. The instrument is held in both hands, whilst the operator stands upon his work in a 
stooping position; the handle boing from twonty-four to thirty inches long, and the woight of the blado from two to four pounds. The adzo is swung in a circular path almost of the same curvature as the blade, the shoulder joint being the centre of motion, and the entiro arm and tool forming, as it were, one inflexible radius; the tool, therefore, makes a succession of small ares, and in each blow the arm of the workman is brought in contact with the thigh, which serves as a stop to prevent accident. In coarse preparatory works, the workman directs his adzo through the space between his two feet; he thus surprises us by the quantity of wood removed; in fine works ho frequently places his toes over the spot to be wrought, and the adze penetrates two or three inches beneath the sole of the shoo; and he thus surprises us by the apparent danger, yet perfect working of the instrument, which, in the hands of a shipwright in particular, almost rivals the joiner's plane; it is with him the nearly universal paring instrument, and is used upon works in all positions.-Holtzapffel.

ENOIAN zARP. A musical instrument; the invention of Kircher; although it was probably indicated by Hero of Alexandria. The musical sounds aro produced by the action of a current of air upon strings placed above a long box of thin deal. The wires of the electric telegraph on the sides of our railroads are frequently set in such a state of vibration by the wind, that thoy bocome gigantic Acolian harps.

AIRATID WATPR. Tho common commercial name of water artificially impregnated with carbonic acid or oxygen.

Arroxriss. Meteoric stones. It has long been woll established that masses of solid matter have fallen from the atmosphere upon this earth. Various hypotheses have been proposed to account for them; amongst others the following may be named:-

1. That they are aggregations of solid matter which take place in the higher regions of the air. It is known, however, that our atmosphere does not contain the chemical elements of meteorites; and, moreover, the large size of many of these meteoric masses-some weighing several tons each-renders it extremely improbable that thoy should be formed by condensation or aggregation in a highly attenuated atmosphere.

2. That they are projected from volcanoes in the moon. The researches of Nasmyth, Smyth, and others appear to show that our satellite, whatever may have been her condition at one period, is now in a state of comparative, if not of perfect, repose. Some astronomers think they have observed changes in some parts of the moon's surface, but there are no indications sufficiently clear to warrant the assumption of there being any voleanoes in a state of activity.

3. That belts composed of fragments of matter circulate in certain fixed orbits around the sun, and that these fragments, sometimes entering our atmosphere, are involved in the earth's influences, and fall in obedience to the law of gravitation. The flights of 'shooting stars' which are observed at particular periods appear to favour this viow.

It has not been proved, however, that meteorites move in circumsolar orbits ; and indeed evidence may be adduced tending to show that they have probably come from regions of space beyond the limits of the solar system.

It is usual to distinguish between aerolites, or meteoric stones, and siderites, or masses of meteoric iron; but the two classes pass into each other through certain meteorites, termed siderolites, which are partly metallic and partly stony. An aerolite, or meteoric stone, is composed of a number of crystalline minerals, usually loosely aggregated, and presenting a peculiar spherular structure. The surface of the stone is invariably coated with an incrustation, in most cases lustrous and of a black colour. This crust seems to be the result of suporficial fusion consequent upon the great development of heat due to the resistance which the stone suddenly encounters on entering the earth's atmosphere.

Among the minerals found in aerolites may be noticed-olivine and angite (two silicates of magnesia), several alloys of iron and nickel, troilite (sulphido of iron), schreibersite (phosphide of iron and nickel), graphite, and certain hydrocarbons similar to what are commonly regarded as organic compounds. The following average per-centage composition of an aorolite has been calculated, by Reichonbach, from a very large number of trustworthy analyses: silica, 40; iron, 25; magnesia, 20 ; alumina, 2 ; sulphur, 2 ; nickel, 1.5 ; lime, 1.5 ; chromium, 0.5 ; manganese, 0.33 ; sodium, 0.33 ; other elements, 1.34 ; oxygen, hydrogen, and loss, $5 \cdot 5$.

Some interesting experiments on the artificial formation of meteorites have been carried out, within the last fow years, by M. Daubrée. This chemist has been successful in producing, on a small scalo, cortain products strongly resembling meteorites, both in structure and composition. The artificial aerolites were produced by fusing a rock called Lherzolite, the fusion being effected either alone or in the 
presence of cortain reducing agents. Other experiments have been made by heating iron, silicon, and magnesium, in an imperfectly oxidising atmosphore.

It is perhaps unnecessary in this place to do moro than refer to Dr. Mayer's theory, which seeks to explain the source of solar heat by the impact of meteorites falling into the mass of the sun - a subject on which Mr. Waterston and Sir W. Thomson have also written. It has oven been suggested that the zodiacal light may be a luminous crowd of meteoric stones showered down upon the sun. This hypothesis has not, however, received the support it claimed, and the whole question remains in a state of considerable uncertainty.

AmROSTATION; AmRONADTCS. The ascent into the atmosphere by means of balloons, which are either filled with hot air-FIrs-BaILooss, or a light gas-AIr-BatLoons.

The Montgolfier balloon is a bag filled with air, which is rarefied by the action of fire, which is kept burning under the mouth of the bag; and thus the whole mass is rendered specifically lighter than the surrounding medium.

The investigations of Cavendish led to the use of hydrogen gas - the lightest of known bodies-to inflate silken bags; and since his time our balloons have been inflated with either pure hydrogen, or with common coal gas-carburetted hydrogen.

Notwithstanding the numerous attempts which have been made to navigate the air, nothing has yet been done to enable the aeronaut to steer his balloon. In whatever current of air he may be, with that current he moves; and, until this difficulty is overcome, we cannot expect any satisfactory results from aeronautics. The great use of balloons during the siege of Paris led to considerable improvements in the art of aerostation. They were largely employed during the siege of Paris in enabling the besieged to communicate outside the city. A balloon-post (Poste aérienne) was thus established, and no fewer than 54 ascents were made between October 1870 and January 1871. M. Dupuy de Lôme has constructed balloons characterized by remarkable stability of the car, and furnished with screws and rudder, whereby the speed and direction of the balloon are brought, to a certain extent, under the control of the aeronaut; nevertheless, the great problem of aerostation yet remains unsolved. Some interesting and useful experiments have been made by using captive balloons, by which we have arrived at some facts connected with the upper regions of the air, which could not be obtained by any other means. By means of balloons, valuable meteorological observations have been made at great altitudes in the atmosphere by Mr. Glaisher and other scientific aeronauts.

स्R UGO. (Verdigris. Acetate and carbonate of copper.) The name formerly given to the bright green rust, produced by the oxygen of the air and carbonic acid, upon copper, and its alloys, bronze and brass. The Romans gave this name; they considered that the cerugo added much to the beauty of their statues; and adjusted the composition of their alloys with the view of producing the finest green colour. This was frequently effectod artificially; and to distinguish the real from the artificial they used for the former the term errego nobilis. This is the patina of the Italians; it is a form of verdigris. See Verdigris; Copper, Acetate and Carbonate.

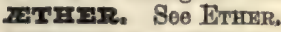

2mTITOPS MINIRAI. The black sulphide of mercury prepared by rubbing mercury and sulphur together. The term Fithiops was applied by the old pharmacentical chemists to several mineral preparations of a black or dark colour.

ETHops ANTTMONiALIs was a sulphide of antimony and mercury.

\#imops Martiatis. Black oxide of iron.

सETHIOPS NARCotrcus. Sulphide of metcury obtained by precipitation. air.

ETHLOPS PER SE. The grey powder obtained by exposing impure mereury to the

Arrivrsy. The term used by chemists to denote the peculiar attractive force which produces the combination of dissimilar substances - as an acid with an alkali, or of sulphur with a metal. See CHesmcar, AmFintry.

AIRICAN HIMP. A fibre prepared from the leaves of Sansevieria Zeylanica, a member of the lily order extensively distributed through tropical Africa and India.

Arrtcars rear. A taluable wood for ship-building, the produce of Oldfieldia Africana, Bth., a tree belonging to the spurge order. This wood is to be carefully distinguished from the trite teak. See Trak.

AGarmatorrse. The 'Figure-stone, or Pagodite, of China; a soft mineral in which carvings are commonly executed by the Chinese. Under the common name of agalmatolite, are included several minerals similar in physical characters, but essentially distinct in chemical composition. The true agalmatolite is a hydrous silicate of alumina and potash, closely allied to pinito. Professor Brush has shown that certain specimens of so-called agalmatolito are really a compact form of 
pyrophyllito (hydrous silicate of alumina), whilst others aro silicates of magnesia, either hydrous or anhydrous.

AGAR-AGAR. A seaweed forming a large article of pommerce in the East. It is frequently called Bengal Isinglass, from the fact of its being found largely in the Bongal market, It is used for making jellies and for stiffening purposes. Seo Axas.

AGArrcus, A genus of the class Fungi, so numerous that 4,000 species have been enumerated. The mushrooms are of this order. The Agaricus campestris is the one commonly used in this country as food, and from which the sance called ketchup is made. In Italy this species is considered poisonous, while many species used there and in France are unused here. The truffle is a mushroom, Tuber ciharium, and its commercial value is so great that in Rome the yearly average of taxed mushrooms from 1837 to 1847 was between 60,000 and 30,000 lbs. weight. The Agaricus muscaria is a poisonous species, though used by the natives of Kamtschatka and Kores to produce intoxication; the Russian name is monchomore, and an infusion of this taken with some liquor produces raving delirium and not unfrequently a desire to commit suicide or assassination. Another variety is beautifully phosphorescent. The Agarici grow in decaying animal or vegetable matter; they are cellular plants, with a rounded thallus on a stalk; the spores or seeds occur underneath the cup in the gills or hymenium. Their growth is remarkably rapid, and there is often great difficulty in distinguishing between the edible and poisonous verieties.

All the fungi of this genus contain a larger amount of nitrogen than either peas or beans. The following are a few of the analyses given in Watts's 'Chemical Dictionary:'

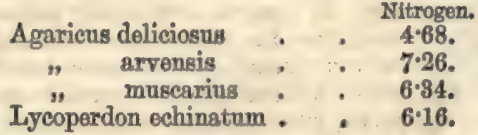

Upon this depends their nutritive properties.

ACAT2. (Agate, Fr.; Achat, Ger.) The term Agate is not employed to denote any distinct mineral of uniform composition, but is applied rather to certain mixtures of siliceous minerals, consisting of different varieties of chalcedony usually associated with jasper, quartz, amethyst, and other natural forms of silica. The agate is the ax $\chi$ 'ं $\eta s$ of the Greeks, by whom it was so called after the river in Sicily of that name (now the Drillo), whence, according to Theophrastus, agates were first procured. Bochart, with much probability, deduces the name from the Punic and Hebrew, nakad; spotted.

In some agates, as in certain varieties from Saxony and Bohemia, the chalcedony and other component minorals have been deposited in fissures, thus forming true veins; but in by far the greater number of cases the materials of the agate have been formed, layer after layer, in the cavities of a vesicular rock. When the formation of the agate has proceeded with regularity, a transverse section of the stone exhibits a number of concentric lines representing the edges of tho successive deposits-these deposits differing one from another in colour, density, and other physical characters, and thus producing the variegated patterns exhibited by most agates. As the component minerals are formed in regular sequence, the suceessive layers being deposited from without inwards, it follows that the innermost portion of an agate must always be the most recents.

Agates are usually found either embedded in a rock called melaphyre, or in the form of free nodules, liberated by decomposition of the matrix. Although the term melaphyre has been somewhat loosely applied, it is now generally used to designate a fine-grained eruptive rock, composed mainly of a felspar-either oligoclase or labradorite-and augite, with more or less magnetic iron-ore: it is chiefly associated with stratified rocks of palrozoic age. When fresh, the melaphyro, as its name implies, ( $\mu$ é $\lambda$ as, black) is usually of a black or very dark colour, but on weathering it often becomes green or brown; it is the altered varieties of melaphyre that most commonly contain agates. Some varieties are porphyritic, and were formerly called augitic porphyry.

Unwilling to admit the igneous origin of the agate-bearing melaphyres, some authorities, as Bischof, have maintained that the cavities now oceupied by siliceous minerals have been formed by the removal of crystals in the porphyritic rock-theso cavities having been enlarged and their angles rounded off by subsequent solution. Although such an explanation may be admissible in certain cases, it seems much more probable that in by far the larger number of rocks the cavities were 
originally formed by the disengagement of gas or steam at a time when the melaphyre was in a molten or partially molten state. The formation of such vesicles in a plastic mass may be well illustrated by the spongy texture of a loaf of bread. Originally the form of the bubbles in the viscid rock would be more or less globular, but by movement of the pasty mass the hollows might become elongated, or, as often happens, pointed at one extremity; if the mass were slowly moving upwards the point would be directed downwards. In many cases the cavities have been much extended and laterally compressed; and hence the agates now occupying such hollows are elongated, flattened and pointed, thus resembling almonds, and the rock containing them is consequently termed amygdaloidal melaphyre. Evidence of the movement of the viscous rock-mass is further afforded by the parallelism often observable in the amygdaloidal agates as they lie in the rock - the longer axes of these agates being all arranged in one direction. The smaller agates are often perfectly amygdaloidal, but the larger specimens are usually distorted. It is likely that the cavities of the large amygdaloids may have been produced by the coalescence of several smaller vesicles.

On the cooling of the igneous mass, water charged with carbonic acid would percolate through the rock, and effect the decomposition of some of the mineral constituents - the chemical changes being perhaps aided by the heat still lingering in the rock. The products of this decomposition might be carried into the vesicular cavities, and thrown down as a lining on their inner walls. Among the first formed of such products are the minerals called delessite, or ferruginous chlorite, and the somewhat similar substance termed 'green earth ;' many vesicular cavities exhibit nothing more than a layer of such green minérals, derived probably from the decomposition of the augite in the rock, whilst most agates-especially the smaller ones-when freed from their matrix present a green external coating of a like nature. In other cases a hydrous peroxide of iron appears to have been formed, perhaps by further alteration of the green earth; and hence many large agates present a rusty exterior or exhibit a pitted surface due to the former presence of a coating of this mineral. The smaller cavities in amygdaloidal rocks are commonly filled with carbonate of lime, and the solid nodules removed from such rocks consequently appear as small green-coated masses of calcito. But in most cases silica has been separated from the silicates decomposed in the rock, and has been thrown down in a gelatinous state on the walls of the cavity as a coating of chalcedony. This coating may be nothing more than a thin rind, thus forming a hollow agate or geode. It generally happens that crystalline silica is deposited on the inner surface of the chalcedonic layer, which thus bears a crop of erystals of quartz-the free pointed ends of these crystals being all directed towards the centre of the geode. Fine amethysts are often found seated in such situations. In other cases the cavity may contain stalactitic deposits of chalcedony, or erystals of calcite, or of various hydrated silicates called zeolites, and rarely of certain metallic minerals-as iron-glance, copper-pyrites, native copper, \&c.

By continued deposition of chalcedony, or of alternate layers of chalcedony and crystalline quartz, the cavity may become completely filled, and a solid agate thus formed.

Many agates exhibit, on section, tubular orifices, which are commonly supposed to have served as inlets through which the agate-forming materials have been introduced by infiltration. In some specimens these inlets have become choked up at an early period in the history of the stone, and the introduction of fresh matter was thus prevented; in other cases the tubes have remained open until the cavity was completely filled, and connexion has thus been maintained between the very heart of the agate and the exterior. This 'infiltration theory' has been staunchly supported by Von Buch and Nöggerath. On the other hand, Haidinger supposed that the silica, instead of being introduced through special apertures, exuded through the general walls of the cavity. After a lining had been laid down uniformly over the interior, more silica in solution might pass from the exterior through this layer by a kind of osmosis, and thus gain access to the interior. But in describing the modern method of colouring agates, it will be shown that certain layers of chalcedony are quite impermeable to fluids, at least under ordinary conditions of temperature and pressure. As soon, then, as a dense impervious layer was deposited, it would seem at first sight that all action must cease; but it has been suggested that siliceous solutions might still find their way to the interior through the cracks with which agates are almost invariably rent.

A different theory of agate formation has been advanced by Reusch. He supposes that warm siliceous solutions were from time to time introduced into the cavities by the action of intermittent thermal springs, the cavities being thus alternately filled and emptied. The intermission of the action may account for the definite succession of the deposits, and the sharp lines of separation between the several strata. This theory has been ingeniously extended by Lange, who seeks to explain the formation of Vor. I. 
both concentric and horizontal layers in the same stone, an association often observed in tho South American agatos, and always difficult of explanation. Lango maintains that whon the golatinous silica, depositod in a warm state, had choked up tho entrance, the tension of the steam confined in this closed cavity would cause the siliceous jelly to be pressed equally in all directions against the inner surface of the cavity, thus forming a continuous lining, until the steam finally burst tho coating at its weakest point, and so effected its egress : the so-callodinlots of infiltration may thoreforo be really canals of eruption. If the cavity were largo, the elasticity of the vapour might be insufficient to press the golntinous matter against the sides of the hollow, and the contents would then be precipitated, in obedience to the force of gravity, in horizontal strata on the floor of the cavity : hence the co-existence of concentric and horizontal layers in a single agate.

According to the varying conditions under which the succossive strata of an agate have been formed, different varieties of the mineral are produced, and many of theso are sufficiently well characterized to receive special names. Thus, when the cavity in which the stone has been formed presents angular contours, the layers naturally adapt themselves to these angles, and the cut stone thus exhibits a zigzag pattern, whence it is termed fortification agate. If the deposits form concentric rings the stono is called eye agate. Such trivial names as ribbon agate, landscape agate, clonded agate, and the like, sufficiently explain thomselves. A beautiful brecciated agate, in which angular fragments of a banded variety are comented together, chiefly by amethyst, is well known from Schlottwitz in Saxony.

The colours of agate are arranged in parallel or concentric bands, or assume the form of clouds or spots, or arborescent and moss-like stains. These colours are due to the presence of metallic oxides. When black and white strata alternate, the stone is ealled an onyx; when the layers are brown, or red and white, it becomes a sardonyx. If the white stratum of an onyx be so thin as to appear bluish white, the stone is termed by jewellers a nicolo. The mocha stone is a variety containing dendritic markings due to the presence of oxide of manganese, or of iron, whilst the moss agate is a chalcedony, containing green moss-like markings, referable to includod inorganic colouring-matter disposed in these patterns.

The chief localities which yield agates to any extent are the melaphyre rocks in the Galgenberg and elsewhere in the neighbourhood of Oberstein in Rhenish Bavaria, and the beds of the Rio Pardo, the Taquarie, and other rivers in Uruguay, which yield the nodules commonly called 'Brazilian agates.' Fine Oriental agates are imported from India, chiefly from Cambay, whero they are largely cut and polished. The well-known 'Scotch pebbles' are true agates found in various localities in Scotland, especially in the amygdaloidal rocks of Kinnoul Hill in Perthshire; near Montrose in Forfarshire; at Dunbar; at Dunglass in Haddingtonshire, and elsewhere. The pebbles found on the south coast of England are not true agates, but are simply flints derived from the upper chalk, and often exhibit patterns due to the presence of choanites, ventriculites, and other organic remains. At the same time large numbers of agates are also sold, at low prices, at most English watering-places, but these, so far from being local 'pebbles,' are generally South American agates, cut and polished in Germany, whence they are largely imported. Fine pebbles of agate and other siliceous stones are found in the Vaal River in South Africa, and also in the Nile. Mr. Daintree has rocently described a local eruption of molaphyre, parts of which contain fine agates, at Agate Creok, a tributary of the Gilbert River in Queensland. Some beautiful polished examples of Queensland agates were exhibited in the International Exhibition of 1872 .

Agates are used in the arts for a variety of purposes, such as knife-edges of delicate balances, small mortars for chemical purposes, burnishers for gold and silver, styles for writing, seal-handles, brooches, bracelets, beads, and an endless variety of small ornamental objects.

These hard stones are cut and polished almost exclusively in a small district at the foot of the Southern Hundsriick in Western Germany. The works are chiefly situated along the Valley of the Idar, a small stream which flows into the Nahe. At a distance of about $\mathbf{4 0}$ miles from Bingen, where the Nahe empties itself into the Rhine, the small town of Oberstein is situated. Oberstein and Idar-about two miles distantare the chief centres of the agate trade. Although it is truo that there are many other localities where agate-working is carried on to a limited extent, it is only in this district-at least in Western countries-that the trado is systematically pursued, and forms the staple industry. From the low value of labour, the agates are there cut and polished at incredibly low prices, and vast quantities of the cut stones are sent thence to all the continental fairs and watering-places. A large trade is also carried on with London, Birmingham, Paris, New York, and other distant localities ; considerable quantities, too, are exported to the interior of Africa. 
Tho localisation of the agate industry in the neighbourhood of Oberstein and Idar, at a very early date - certainly more than 400 years ago-may be traced to the plentiful occurrence of agates in the surrounding hills, especially in the hill called the Galgenberg or Steinkaulonberg. These hills consist of a melaphyre, moro or less amygdaloidal, which has burst through the sandstones of the Saarbrück coal-field. Adits were formerly driven into the bill-side, and the agate-boaring rocks were systematically quarried. The workings in these quarries have for several years past been almost, if not entirely, abandoned, chiefly through the large importations constantly being received from South America.

In 1827 some Idar polishers who had emigrated to Uruguay accidentally diseovored some fine agates, used at that time as paving-stones. Large quantities of the amygdaloidal nodules were easily collected, as loose pebbles, from the bed of the River Taquarie, and were despatehed to Oberstein by way of Hamburg. The South American agates are evidently derived from decomposed melaphyre rocks, for although nerer found actually embedded in the matrix, portions of the mother-stone are occasionally seen adhering to the pebbles. As the agate-nodules are obtained without the expense of quarrying, the cast is confined to that of collecting them from the surface of the ground, or separating them from the superficial detritus. The stones are brought duwn to the coast on mules, or in waggons drawn by oxen, and are received at Porto Alegre, or at Salto, whence they are taken to Monte Video and Buenos Ayres, and shipped from these ports to Europe. Formerly they left the country free of duty, and were brought over as ballast, but an export duty has now to be paid, amounting in Uruguay to six per cent. of their value, and in Brazil to ten per cent. Arrived at Hamburg, Antwerp, or Havre, the agates are conveyed by rail to Oberstein-the rough stones travelling in open trucks, while the choicer carnelians are packed in cases. The total cost of transport by sea and land amounts to from $3 s$. to $6 s$. per cwt. Iarge pareels of the agates are displayed in the courtyards of the inns, and after due advertisement are sold by public auction. Prior to the sale the polishers inspect the lots, and break off samples of the stones, which are taken home and tested as to their power of taking colour by methods to be presently described. From forty to fifty auctions take place annually, and though the prices of the stones vary greatly according to their quality, it may be said that ordinary agates may be bought on an average for about 15s. per cwt. In 1867, the auction sales for the year realised a gross sum of nearly 16,000 .

The agates are first roughly dressed with chisel and hammer. From the texture of the stone the experienced workman is able to judge in what directions the stone will most readily split, and hence by a fow skilfully-directed blows he manages to trim the agate rudely into the shape which it is intended to assume. The more valuable stones are, however, sawn into shape with emery and water. The grinding is effected on large red grindstones, mounted on a horizontal axis, and rotating in a vertical plane. Each axle carries from three to five stones, and communicates on the outside with a water-wheel. These wheols vary from 10 to 18 feet in diameter, and are usually undershot. Most of the mills are situated on the Idar, a stream which rises in one of the highest parts of the Hochwald. At the village of Idar it is about 1,012 feet above the sea-level, and at Oberstein, where it debouches into the Nahe, it is 905 feet high. It is in the Idar valley, between these two villages, that most of the agate-mills are situated. In consequence of the want of water during a dry season, the mills often stand idle, and hence in a few of the larger works steam-power has of late years been introduced. The grindstones, to which the water-wheel or steamengine gives motion, are made of new red sandstone, quarried at the Kaiserslautern near Mannheim, and are about 5 feet in diameter and one foot in width. They usually make three revolutions per minute. When the stones have been badly selected, or used too soon after having left the quarry, they have been known to fly to pieces with great violence, and in this way several fatal accidents formerly occurred. The wheels revolve in a well, the horizontal shaft being nearly on a level with the floor, below which the lower half of the wheel is concealed. A small stream of water is introduced by a launder above, and by constantly trickling over the stones keops them moist. Two workmen are generally employed, side by side, at each stone. The workman lies in an almost horizontal position, resting his chest and stomach on a bare wooden grinding-stool, adapted to the shape of his body, whilo he presses his feet against a block of wood fastened to the floor: the reaction of this fixed block enables him to apply any object with great force to the moving grindstone. The specimen to be ground is either held directly in the hand or applied to the stone by means of a short pioce of soft wood. The form is given to the object by holding it in cortain channels cut on the circumference of the stone. During grinding, the friction causes the agate to glow with a beautiful reddish phosphorescent light, visible oven in the daytime, and quite distinct from sparks elicited by friction. After having been 
ground, the stones are polished with tripoli on a cylinder of hard wood, or on a plate of tin or lead.

An important branch of the agate trade is that of artificially colouring the stonesan art which has attained great perfection in rocent times, but is far from being a modern invention. Pliny states (bk. xxxvir. cap. 75) that in Arabia agates are found which aro purified and prepared for the cutter by being heated in honoy for seven days and seven nights. Evidently the mere absorption of this saccharine matter into the pores of the stones would be insufficient to materially alter the appearance of the agate; but if the absorbod honey could have been carbonized by the action of sulphuric acid-as at present practised-the dark colour of the brownish layers would have been considerably heightened, and the stones improved for the cameoworker. Believing that this was really the process deseribed by Pliny, though ho was ignorant of the entire secret, some authorities have argued in favour of the knowledge of oil of vitriol by the ancient Romans, while others have suggested that the nativo sulphuric acid from volcanic emanations was probably employed; others, again, have maintained that the honey was meroly charred by exposure to heat, as is said to be still practised in tho East. Be that as it may, it appears that the secret of artificially producing a black colour in agates was for ages handed down traditionally by the Italian cameo-workers. From time to time, Italian travellers visited Idar, and purchased stonos, which were taken to Rome and there coloured. At length the secret of this art oozed out under peculiar circumstances, and, being once known in Idar, rapidly spread, and has not only been the means of greatly developing the agateindustry, but has, to a large extent, caused the removal of the seat of stone-engraving from Italy to Germany.

All artificial colouration of these hard stones depends for its success on variations in texture and density presented by the different layers. Sections of agate under the microscope often exhibit distinct pores, and the unequal texture of the several layers is well seen by the action of hydrofluoric acid, which readily attracts certain strata while it is resisted by others, thus producing an uneven surface, from which an impression of the agate may be naturally printed-a process which has beon beautifully carried out by Dr. Leydolt. Not only do the component layers of an agate absorb liquids with different degrees of facility, but the stones as a whole exhibit like differences; some stones absorbing the colour rapidly, others requiring months to do so, and others, again, entirely refusing to take colour. The South American agates, as a rule, lend themselves with peculiar facility to this artificial colouration, and are much more porous than the true German agates.

Black or dark brown colours are those commonly developed in agates-these dark strata, when alternating with dense white layers, forming beautiful onyses well fitted for cameo-work. To produce the dark colour, the stones, having been well washed and dried, are placed in honey, thinned with water, and are exposed in a warm place for several days, in some cases as long as three woeks. The vessel containing them is heated by being placed in hot ashes or on a stove, but the syrup is never allowed to boil. After having lain in the warm honey for a sufficient time, depending on the texture of the stone, they are removed, well washed, and placed in a vessel with sufficient commercial oil of vitriol to cover them; the vessel boing covered with a slate, and exposed to a moderate temperature. The sulphuric acid carbonises the saccharine matter previously absorbed by the porous layers of the agate, and produces a black or a deep brown colour according as the action is more or less intense. Olive oil is used by the Italians instead of honey, but the chemical reactions are, of course, essentially the same in the two cases. Some stones blacken in a few hours, others require several days, while bad stones never take colour. When sufficiently tinted the stones are removed from the acid, washed, dried, and polished; they are then generally laid in oil to improve the lustre, and are finally dried in bran. Whilst the charring of the syrup or oil gives a dark colour to the porous layers, it is said that the white colour of the denser strata is also heightened; but this is probably merely the effect of contrast. If the darker parts of the stone should be too deep, the colour can be 'drawn,' or made lighter by the action of nitric acid.

The art of agate-colouration has reached so advanced a state that stones can now be tinted to almost any desired hue; some of the processes are, however, still kept secret by the polishers. In 1845 the method of colouring agate blue was first introduced. This is now effected in various ways. One of the best modes is to submit the stone successively to the action of solutions of yellow prussiate of potash (ferrocyanide of potassium), and of a per-salt of iron, thus causing a precipitate of Prussian blue to be thrown down in the pores of the stone. Or, red prussiate of potash and common green vitriol may be used; or an ammoniacal sulphate of copper may be formed, by placing the stone in a solution of blue vitriol and then in ammonia. A green colour was formerly produced by nitrate of nickel, but it is now obtained by using chromic acid. 
The greon stones are largely used as artificial chrysoprase, and the colour is more intonse and said to be more durable than that of the natural chrysoprase. A yellow tint is obtained by prolonged digestion in warm hydrochloric acid, the acid acting on the oxide of iron normally present in the stone, and thus forming a yellow perchloride. One of the commonest and at the same time most easily developed colours is the red tint of the carnelian. It was long ago observed that yellow and grey chalcedony might be caused to assume \& bright red colour by mere exposure to sunshine, the heat being sufficient to expel the water more or less completely from the hydrated peroxide of iron normally present in the stone. In India it has long been the practice to convert yellowish chalcedony into red carnelian by solar heat. The Idar workers generally expose the agates to the heat of an oven, gradually raised until all hygroscopic water is expelled. The stones are then moistened with sulphuric acid, and raised to a red heat, whereby an anhydrous peroxide of iron, of fine red colour, is developed. Or a pernitrate of iron may be formed by throwing a handful of old nails into half-a-pint of aquafortis mixed with a pint of water, and the stones having been soaked in this solution are heated so as to decompose the absorbed salt, and cause a precipitate of ferric oxide in the pores of the agate. Several organic colours have been introduced by the agate-worker, but they are generally fugitive: mauve and magonta agates, and other equally unnatural stones, are common in the market. The ingenuity of the Idar workmen also enables them to imitate successfully the dendritic markings in mocha-stones.

Another important branch of industry connected with the agate-trade is that of mounting the finished objects. Though dignified by the name of Goldschmiede, the mounters usually work in gilt tomback; some of the better kinds of agate-ware are now, however, set in silver. Drilling, engraving, cameo-cutting, and etching with hydrofluoric acid, are also extensively practised in Idar; and, in addition to agates, large quantities of Oriental blood-stones and other semi-precious stones, and even pastes, are largely cut and mounted in this district.

In 1867 there wero in Birkenfeld and the neighbourhood 724 grindstones working in 153 mills. The number of grinders and polishers in Birkenfeld was 1,129, but in addition to this number about 300 workmen dwelt beyond the limits of the Principality. There were also about 258 persons engaged in drilling and boring the agates, and about 700 so-called goldsmiths. Ineluding those employed in the sale of the stones, in making paper cases, and otherwise connected with the trade, it may be said that upwards of 3,000 persons depend for their support on the agate-industry in the neighbourhood of Idar and Oberstein.

[For further information on the subject of this Article the reader may consult the following works:-Billing's 'The Science of Gems' 1867, p. 48 et seq.; Kluge, 'Edelsteinkunde,' 1860, p. 401; Lange, 'Die Halbedelsteine aus der Familie der Quarze und die Geschichte der Achatindustrie,' 1868; Nöggerath, 'Die Kunst, Onyxe, Carneole, Chalcedone u, andere verwandte Steinarten zu färben,' Karsten's 'Archiv: xxii.' 1848, p. 262, and 'Edin. Now Phil. Journ.', xlviii. 1850, p. 166 ; Nöggerath, 'Ueber die Achatmandeln in den Melaphyren,' Haidinger's 'Naturwissentschaftliche Abhandlungen,' iii. 1850, p. 93; Kenngott, 'Ueber die Achatmandeln in den Melaphyren, namentlich über die von Thesis in 'Tirol,' Op. cit. iv. 1851, p. 72 ; Haidinger's 'Bexichte,' vi. 1850, p. 62 ; Bischof, 'Lehrbuch d. Chem. u. Phys. Geologie,' ed. 2 , ii. p. 853 ; iii. p. 457, 464, 623 et seq. ; Reusch, 'Ueber den Agat'; Poggendorf's 'Annalen,' exxiii. 1864, p.94].-F. W. R.

ACATz. An instrument used by gold-wire drawers, so called from the agato fixed in the middle of it.

AxCA METAX. An alloy patented in 1860 by Johann Aich, and recommended for use in ship-building and sheathing. In composition it approaches close to Koir's metal. The following are found to be the best proportions: copper, $60 \mathrm{lbs}$; zinc, 38 lbs. 2 ozs.; and iron, 1 lb. 8 ozs. The proportion of zinc may, however, be increased to 40 per cent., and that of iron may vary between 0.5 and 3 per cent.

Arrmerrz. A native sulphide of bismuth, lead, and copper, crystallising in needle-shaped crystals belonging to the prismatic or orthorhombic system. It is found at Bersow in the Urals, and is believed to occur in Georgia and North Carolina in the Xnited States.

Arr. The gaseous onvelopo which surrounds this earth is emphatically so called; it consists of the gases nitrogen and oxygen.

About 79 measures of nitrogen, or azote, and 21 of oxygen, with $\frac{1}{100}$ th of carbonic acid, constitute the air we breathe. The torm air is applied to any permanently gasoous body. And we express different conditions of the air, as good air, bad air, foul air, \&c. See AtMrosphers.

AIR-zRrcis. A brick of the ordinary sizo and kind, but perforated so as to admit the pessage of air through the openings when built into the walls. 
ArR, Comprassrad. For its employment in some mining operations, seo Minive.

ArR-IN crNE. The considerable expansibility of air by heat naturally suggested its use as a motive power long before theoretical investigation demonstrated its actual value. The great advanco made during the last fow years in our knowledge of the mechavical action of heat has enabled us to determine with certainty the practical result which may be obtained by the use of any contrivance for employing heat as a primo mover of machinery. We are indebted to Sir Wm. Thomson for tho fundamental theorem which decides the economy of any thermo-dynamic engine. It is-that in any perfectly constructed engine the fraction of heat converted into work is equal to the range of temperature from the highest to the lowest point, divided by the lighost temperature reckoned from the zoro of absolute temperature. Thus, if wo havo a perfect engine in which the highest temperature is $280^{\circ}$ and the lowest $80^{\circ}$

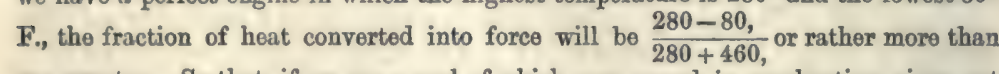
ono quartor. So that, if we use a coal of which one pound in combustion gives ont heat equivalont to $10,380,000$ foot-pounds, such an engine as we have just described would produce work equal to $2,805,405$ foot-pounds for each pound of coal consumed in the furnaco. From the above formula of Sir Wm. Thomson, it will appear that the economy of any perfect thermo-dynamic engine deponds upon the range of temperature we can obtain in it. And as the lowest temperature is generally nearly constant, being ruled by the temperature of the surface of the earth, it follows that the higher we can raise the highost temperature, the more economical will be the engine. The question is thus reduced to this:-In what class of ongine can we practically use the highest temperature? In the steam-engine workod with saturated vapour, the limit is obviously determined by the amount of pressure which can be safely employed. In the steam-engine worked with super-heated vapour-i.e. in which the vapour, after passing from the boilor, receives an additional charge of heat without being allowed to take up more water-and also in tho air-ongine, the limit will depend upon the temperature at which steam or air acts chemically upon the metals employed, as well as upon the power of the metals themselves to resist the destructive action of heat. It thus appears that the steam-ongine workod with superheated steam possesses most of the economical advantages of the air engine. But when wo consider that an air-engine may be made available where a plentiful supply of water cannot be readily obtained, the importance of this kind of thermo-dynamic engine is incontestable. The merit of first constructing a practical air-engine belongs to Mr. Stirling. Mr. Eriesson has subsequently introduced various refinements, such as the respirator-a reticulated mass of metal, which, by its extensive conducting surface, is able, almost instantaneously, to give its own temperature to the air which passes through it. But various practical difficulties attend these refinements, which, at best, only apply to enginos worked between particular temperatures. The least complex engine, and that which would probably prove most offectual in practico, is that described in the 'Philosophical Transactions,' 1852, Part I. It consists of a pump, which comprosses air into a receiver, in which it receives an additional charge of heat; and a cylinder, the piston of which is worked by the heated air as it escapes. The difference betwoen the work produced by the cylinder and that absorbod by the pump constitutes the force of the engine; which, being compared with the heat communicated to the receiver, gives results exactly conformable with the law of Sir Wm. Thomson above described.-J.P.J.

Dr. Jonle has proposed various ongines to be worked at temperatures below redness, which, if no loss occurred by friction or radiation, would realise about one-half the work due to the heat of combustion, or about four times the economical duty which has, as yet, been attained by the most perfect steam-engine.

A detailed account of Ericsson's Calorific Engine may be useful, especially as a certain amount of success has attended his efforts in applying the expansive power of heat to move machinery. Ericsson's engines have been for some years at work in the foundry of Messirs. Hogg and Delamater, in Now York; one engine being of five and another of sixty horse-power. The latter has four cylinders. 'Two, of seventy-two inches diamoter, stand side by side. Over each of these is placed one much smaller. Within these are pistons exactly fitting their respective cylinders, and so connected, that those within tho lower and upper cylinders move together. Under the bottom of each of the lower cylinders a fire is applied, no other furnaces being employed. Neither boilers nor water aro used. The lower is called the working cylinder; the upper, the supply cylinder. As the piston in the supply cylinder moves down, valves placed in its top open, and it bocomes filled with cold air. As the piston rises within it, those valves close, and the air within, unablo to oscape as it came, passes through another sot of valves into a 
receiver, from whence it has to pass into the working cylinder, to force up the working piston within it. As it leaves the receiver to perform this duty, it passes through what is called the regenerator, where it becomes heated to about $450^{\circ}$; and upon entering the working cylinder, it is further heated by the supply underneath. For the sake of illustration, merely, let us suppose that the working cylinder contains double the area of the supply cylinder; the cold air which entered the upper cylinder will, therefore, but only half fill the lower one. In the course of its passage to the latter, however, it passes through the regenerator; and as it enters the working cylindor, wo will suppose that it has become heated to about $480^{\circ}$, by which it is expanded to double its volume, and with this increased capacity it enters the working cylinder. We will further suppose the area of the piston within this cylinder to contain 1,000 square inches, and the area of the piston in the supply cylinder above to contain but 500 . The air presses upon this with a mean force, we will suppose, of about eleven pounds to each square inch; or, in other words, with a weight of 5,500 pounds. Upon the surface of the lower piston the heated air is, however, pressing upwards with a like force upon each of its 1,000 square inches; or, in other words, with a force which, after overcoming the weight above, leaves a surplus of 5,500 pounds, if we make no allowance for friction. This surplus furnishes the working power of the engine. It will be seen that after one stroke of its pistons is made, it will continue to work with this force so long as sufficient heat is supplied to expand the air in the working cylinder to the extent stated; for, so long as the area of the lower piston is greater than that of the upper, and a like pressure is upon every square inch of each, so long will the greater piston push forward the smaller, as a two-pound weight upon one end of a balance will be sure to bear down a one-pound weight placed upon the other. We need hardly say, that after the air in the working cylinder has forced up the piston within it, a valve opens; and as it passes out, the pistons, by the force of gravity, descend, and cold air again rushes into and fills the supply cylinder. In this manner the two cylinders are alternately supplied and discharged, causing the pistons in each to play up and down substantially as they do in the steam-engine.

The regenerator must now bo described. It has been stated that atmospherie air is first drawn into the supply cylinder, and that it passes through the regenerator into the working cylinder. The regenerator is composed of wire net, like that used in the manufacture of sieves, placed side by side, until the series attains a thickness of about 12 inches. Through the almost innumerable cells formed by the intersections of the wire, the air must pass on its way to the working cylinder. In passing through these it is so minutely divided that all parts are brought into contact with the wires. Supposing the side of the regenerator nearest the working cylinder is heated to a sigh temperature, the air, in passing through it, takes up, as we have said, about $450^{\circ}$ of the $480^{\circ}$ of heat required to double the volume of the air; the additional $30^{\circ}$ are communicated by the fire beneath the cylinder.

The air has thus become expanded, it forces the piston upwards; it has done its work-valves open, and the imprisoned air, heated at $480^{\circ}$, passes from the cylinder and again enters the regenerator, through which it must pass before leaving the machine. It has been said that the side of this instrument nearest the cylinder is kept hot; the other side is kept cool by the action upon it of the air entering in the opposite direction at each up-stroke of the pistons; consequently, as the air from the working cylinder passes out, the wires absorb the heat so effectually, that when it leaves the regenerator it has been robbed of it all, except about $30^{\circ}$.

The regenerator in the 60 -horse engine measures 26 inches in height and width internally. Each dise of wire composing it contains 676 superficial inches, and the net has ten meshes to the inch. Each superficial inch, therefore, contains 100 meshes, which, multiplied by 676 , gives 67,600 meshes in each disc; and, as 200 dises are employed, it follows that the regenerator contains $13,520,000$ meshes ; and consequently as there are as many spaces between the dises as there are meshes, we find that the air within it is distributed in about $27,000,000$ minute cells. Thence every particle of air, in passing through the regenerator, is brought into very close contact with a surface of metal which heats and cools it alternately. Upon this action of the regenerato:, Ericsson's Calorific Engine depends. In its application on the large seale, contemplated in the great Atlantic steamer called 'The Eriesson,' the result was not satisfactory. Wo may, however, notwithstanding this result, safely predicate, from the investigation of Messrs. Thomson and Joule, that the expansion of air by heat will eventually, under some conditions, take the place of steam as a motive power.

Arr-Grarravc. A kind of air-brick built into walls to admit air under the floors or into close places. Air-gratings are often made of iron.

AIR-GUN. This is a weapon in which the elastic force of air is made use of to project the ball. It is so arranged, that in a cavity in the stock of tho gun, air can be, 
by means of a piston, powerfully condensed. Here is a reserved force, which, upon its being relieved from pressure, is at once exerted. Whon air has been condensed to about $\frac{1}{10}$ th of its bulk, it exorts a forco which is still very inferior to that of gunpowdor. In many other respects the air-gun is but an imperfect weapon, consequently it is rarely employed.

Arr-Forms. Tho cavities in a motal casting-produced by the escape of air through the liquid metal.

Arn-PUMP. A machine by which the air can be exhausted from any vessel containing it. It is employed in sciontific investigations for exhibiting many very interesting phenomena in connoction with the pressure of air, and its presenco or absence; and it is connected with, and forms an important part of, the improved modern steam-engine. Similar machines are also used for condensing atmospheric air; these have been employed on a large scale in some civil engineering purposes. For a description of the Sprengel pump, see Aspirator.

ATR-SEArT. In Mining, a shaft devoted to the purpose of maintaining the circulation of air in a colliory or metalliferous mine. (Seo Mining, Ventration.)

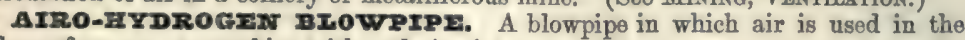
place of oxygen, to combine with and give intonsity of heat to a hydrogen flame for the purposes of soldering. See Aurogreos Soldmenve.

AJUrACE. A tube through which water is discharged-as in a fountain.

Aтаваsтrz. Gypsum (Albâtre, Fr.; Alabaster, Ger.), a sulphate of lime. (See Atabaster, Ordantrax.) When massive, it is called indifferently alabaster or gypsum ; and when in distinct and separate crystals, it is termed selenite. Massive alabaster ocenrs in Britain in the new red or keuper marl: in Glamorganshire, on the Bristol Channel ; in Leicestershire, at Syston; at Tutbury and near Burton-on-Trent, in Staffordshire; at Chellaston, in Derbyshire; near Droitwich it is associated in the marl with rock salt, in strata respectively 40 and 75 feet in thicknoss; and at Northwich and elsewhere the red marl is intorsected with frequent veins of gypsum. At Tutbury it is quarried in the open air, and at Chellaston in caverns, where it is blasted by gunpowder: at both places it is burned in kilns, and otherwise prepared for the market. It lies in irregular beds in the marl, that at Chellaston being about 30 feet thick. There is, however, reason to suppose that it was not originally deposited along with the marl as sulphate of lime, but rather that calcareous strata, by the access of sulphuric acid and water, have beon converted into sulphate of limea circumstance quite consistent with the bulging of the beds of marl with which the gypsum is associated, the lime, as a sulphate, occupying more space thau it did in its original state as a carbonate. At Tutbury, and elsewhere, though it, lies on a given general horizon, yet it can scarcely be said to be traly bedded, but ramifies among the beds and joints of the marl in numerous films, veins, and layers of fibrous gypsum. A thick bed of white crystalline alabaster, or gypsum, has been discovered during the Sub-Wealden Exploration in Sussex. It occurs in beds which are probably of Purbeck ago.

A snow-white alabaster occurs at Volterra, in Tuscany, much used in works of art in Florence and Loghorn. In the Paris basin it occurs as a granular crystalline rock, in the lower Tertiary rocks, known to geologists as the upper part of the Middle Eocene freshwater strata. It is associated with beds of white and green marls; but in the Thüringerwald there is a great mass of sulphate of lime in the Permian strata. It has been sunk through to a depth of 70 feet, and is believed to be metamorphosed magnesian limestone or Zechstein. In the United States this calcareous salt occurs in numerous lenticular masses in marly and sand strata of that part of the Upper Silurian strata known as the Onondaga salt group. It is excavated for agricultural purposes. For mineralogical character, \&c., see GYPsuM.-A. C. R.

The fineness of the grain of alabaster, the uniformity of its texture, the beauty of its polished surface, and its semi-transparency, are the qualities which render it valuable to the seulptor and to the manufacturer of ornamental articles.

The alabaster is worked with the same tools as marble; and as it is many degrees softer, it is so much the more essily cut; but it is more difficult to polish, from its little solidity. After it has been fashioned into the desired form, and smoothed down with pumice-stone, it is poliahnd with a pap-like mixture of chalk, soap, and milk; and, last of all, finished by friction with flannel. It is apt to acquire a yellowish tinge.

Besides the harder kinds, employed for the sculpture of large figures, there is a softer alabaster, pure white and semi-transparent, from which small ornamental objects are made, such as boxes, vases, lamps, stands of timepieces, \&c. This branch of business is much prosecuted in Florence, Leghorn, Milan, \&c., and employs a great many turning-lathos. Of all the alabasters, the Florentine merits the preference, on account of its beauty and uniformity. Othor sorts of gypsum, such as that 


\section{ALBERTITE}

of Salzburg and Austria, contain sand veins and hard nodules, and require to be quarried by cleaving and blasting operations, which are apt to erack it and render it unfit for all delicate objects of sculpture. It is, besides, of a grey shade, and often stained with darker colours.

The alabaster best adapted for the fine arts is white when newly broken, and becomes yet whiter on the surface by drying. It may be easily cut with the knife or chisel, and formed into many pleasing shapes by suitable steel tools. It is worked either by the hand alone, or with the aid of a turning-lathe. The turning tools should not be too thin or sharp-edged; but such as are employed for ivory and brass are most suitable for alabaster, and are chiefly used to shave and to scratch the surface. The objects which cannot be turned may be fashioned by the rasping tools, or with minute files. Fine chisels and graving tools are also used for the better pieces of statuary.

For polishing such works, a peculiar process is required: pumice-stone, in fine powder, serves to smooth down the surfaces very well, but it soils the whiteness of the alabaster. To take away the unevenness and roughness, dried shave-grass (equisetum) answers best. Friction with this plant and water polishes down the asperities left by the chisel: the fine streaks left by the grass may be removed by rubbing the pieces with slaked lime, finely pulverised, sifted, and made into a paste; or with putty-powder (oxide of tin) and water. The polish and satin-lustre of the surface are communicated by friction, first with soap-water and lime, and finally with powdered and elutriated tale or French chalk.

Such articles as consist of several pieces are joined by a cement composed of quicklime and white of egg, or of well-calcined and well-sifted Paris plaster, mixed with the least possible quantity of water.

Alabaster objects are liable to become yellow by keeping, and are especially injured by smoke, dust, \&c. They may be in some measure restored by washing with soap and water, then with clear water, and again polished with shave-grass. Grease-spots may be removed either by rubbing with talc powder, or with oil of turpentine.

The surface of alabaster may be etched by covering over the parts that are not to be touched with a solution of wax in oil of turpentine, thickened with white lead, and immersing the articles in pure water afior the varnish has set. The action of the water is continued from 20 to 50 hours, more or less, according to the depth to which the etching is to be cut. After removing the varnish with oil of turpentine, the etched places, which are necessarily deprived of their polish, should be rubbed with a brush dipped in finely-powdered gypsum, which gives a kind of opacity, contrasting well with the rest of the surface.

Alabaster may be stained either with metallic solutions, with spirituons tinctures of dyeing plants, or with coloured oils, in the same way as marbles.

AIABASTrR, ORIENTAI. Oriental alabaster is a form of stalagmitic or stalactitic carbonate of lime, an Egyptian variety of which is highly esteemed. It is also procured from the Pyrenees, from Chili, and from parts of the United States of America. Ancient quarries are still in existence in the province of Oran, in Algeria. The well-known 'Gibraltar stone' and the Californian marble are similar stalagmitic forms of carbonate of lime. This Oriental alabaster, or alabaster of the ancients, is to be carefully distinguished from the mineral now commonly known as alabaster; the former is a carbonate, the latter a sulphate of lime. See ONYx, ALGRRIAN.

AIBAN. A white resinous substance extracted from gutta-percha by either alcohol or ether. See GuTTA-PERCHA.

AIBANI STONg. (Lapis albanus). The Peperino of modern geologists. A dark volcanic tufa found in Italy, much used at Rome before building with marble became common. The Italian name peperino is derived from pepe, pepper, which it somewhat resembles.

AIBATA PIATE, a name given to one of the varieties of white metal now so commonly employed. See Coppere and Attoxs.

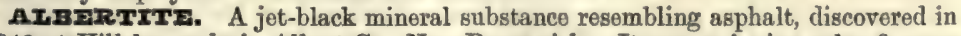
1849 at Hillsborough, in Albert Co., New Brunswick. It occurs in irregular fissures in Lower Carboniferous rocks, and is now usually regarded as the residue left on the drying-up of a great body of petroleum. "The deposit of the Albert mine would thus be a vein or fissure constituting an ancient reservoir of petroleum, which, by the loss of its more volatile parts and partial oxidation, has been hardened into a coaly substance.' (Dawson). Albertite has been largely used in the United States for the distillation of oil and coke. The yiold per ton is said to be 100 (erude) gallons of oil, and 14,500 cubic feet of illuminating gas, while a residue of good coke remains in the retorts. 
AIBIT2. A soda felspar. Seo Fmspar.

AInorrrr. A coment prepared by calcining native carbonate of magnesia (magnesite) and mixing the magnesia thus obtained with silica.

AInum crsecum. The whita frees of dogs, hyrenas, \&c. After the hair has been removed from skins, this is used to preserve the softness of them, and prepare them for the tan-pit. Fowls' dung is considered by practical tanners as superior to the dung of dogs, and this is obtained as largely as possible. These excrota may be said to be essentially phosphate of lime and mucus. We are informed that various artificial compounds which represent, chemically, the conditions of those natural ones, havo been tried without producing the same good results. It is a reflection on our science, if this is really the case. Album Grocum is frequently found fossilised in bone-cavorns which have been used as hyæna dens.

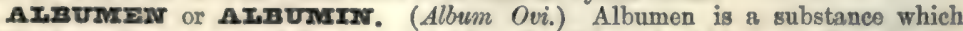
forms a constituont part of the animal fluids and solids, and which is also found in the vegetable kingdom. It exists nearly pure in the white of egg. Albumen 'according to sevoral analyses consists of :-

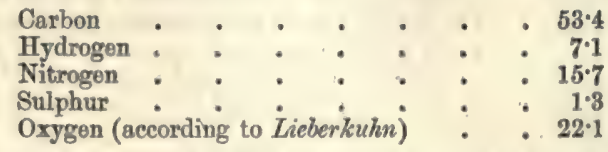

The coagulation of albumen by heat is illustrated in the boiling of an egg. The salts of tin, bismuth, lead, silver, and mercury form with albumen white insolublo precipitates; therefore, in cases of poisoning by corrosivo sublimate, nitrate of silver, or sugar of lead, the white of egg is the best antidote which can be administered.

Albumen is employed for clarifying vinous and syrupy liquids; when boiled with them it coagulates and entangles the colouring mattor, either falling to the bottom or floating on the surface of the fluid, according to its specific gravity. In this way it is employed, especially as it is found in the serum of blood, in sugar-refining. It is also used in fixing the colours in calico-printing. It is employed in photography, and mixed with recently slaked lime it forms a very useful cement, becoming in a short time as hard as stone.

Vegrtabus Albuman is identical in composition with that of the white of egg. It is abundant in nearly all the roots used for food, as the potato, turnip, carrot, \&re. It is found in large quantity in wheat-flour, and in most oleaginous seeds. See Watts's 'Dictionary of Chemistry."

AImUaMzNISzD PAPra. A paper prepared with the white of egg for photographic purposes. Seo ProtograpuY.

ArBUmInTorDs. A term applied to componnds which play an important part in the economy of both animal and vegetable life. The more important are ArBuman, FuBRT, and CASEn:.

AICARAzmas. Porous earthenware vessels mado in Spain from a sandy marl, and but slightly fired. They are used for cooling liquors. Those vessols are made in France under the name of hydrocérames; similar kinds of earthenware are also manufactured in Staffordshire and Derbyshize.

AxcID2:. A family of sea-birds, to which the Guillomots and Penguins belong. The Patagonian Penguin is larger than a goose, slate-coloured on the back, and white with a black mark on the breast, encircled by a citron-yellow cravat. The plumage is very close, and the breast is used as tippets and for trimming ladies' dresses. The black Guillemot ( Uria grylle) also yields its feathers to meet the requirements of fashion.

Axcozox. (Alcool, Fr.; Alkohol, or Weingeist, Ger.) The word alcohol is derived from the Hebrew word 'kohol,' hry to paint. The Oriental females were and are still in the habit of painting the eyebrows with varions pigments ; the one generally employed was a preparation of antimony, and to this the term was generally applied. It became, however, gradually extended to all substances used for the purpose, and ultimately to strong spirits, which were employed, probably, as solvents for certain colouring principles. The term was subsequently exclusively used to designate ardent spirits, and ultimately the radical or principle upon which their strength depends.

As chemistry advanced, alcohol was found to be a member only of a class of bodies agreeing with it in general characters; and hence the term is now generic, and we speak of the various alcohols. Of these, common or vinous alcohol is the best known; sad, in common life, by 'alcoholic liquors,' we invariably mean those containing the original or rinous alcohol. 
When the characters of ordinary alcohol have beon stated, allusion will be mado to the class of bodies of which this is the type.

Fermented liquors were known in the most remote ages of antiquity. We read (Genesis ix.) that after the flood 'Noah planted a vineyard, and he drank of the wine and was drunken.' . Homer, who certainly lived 900 years before the Christian era, also frequently mentions wine, and notices its effects on tho body and mind (Odyssey IX. and XXI.); and Herodotus tolls us that the Egyptians drank a liquor fermented from barley. The period when fermented liquors were submitted to distillation, so as to obtain 'ardent spirits,' is shrouded in much obscurity. Raymond Lully' was acquainted with 'spirits of wine,' which he called aqua ardens. The separation of absolute alcohol would appear to have been first offected about this period (1300), by Arnauld de Villeneuve, a colebrated physician residing in Montpollier (Gerhardt), and its analysis was fixst performod by Th. de Saussure. ${ }^{2}$

The preparation of alcohol may be divided into three stages :-

1. The production of a fermented vinous liquor-the Fermentation.

2. The preparation from this of an ardent spirit-the Distillation.

3. The separation from this ardent spirit of the last traces of water-the Rectification.

1. Fermentation. The term 'fermentation' is now applied to those mysterious changes which vegetable (and animal) substances undergo when exposed, at a certain temperature, to contact with organic or even organised bodies in a state of change.

There are several bodies which suffer these metamorphoses, and under the influence of a great number of different excrting substances, which are tormed the 'ferments ;' moreover, the resulting products depend greatly upon the temperature at which the change takes place.

The earliest known and best studied of these processes is the one commonly rocognised as the vinous or alcoholic fermentation.

In this process solutions containing sugar-either the juice of the grape (seo WINE) or an infusion of germinated barley, malt (see Bere)-are mixed with a suitable quantity of a ferment; beer or wine yeast is usually employed (see YrasT), and the whole maintained at a temperature of between $70^{\circ}$ and $80^{\circ} \mathrm{F}$. $\left(21^{\circ}\right.$ to $26^{\circ} \mathrm{C}$.)

Other bodies in a state of putrefactive decomposition will effect the same result as the yeast, such as putrid blood, white of egg, \&c.

The liquid swolls up, a considerable quantity of froth collects on the surface, and an abundance of gas is disengaged, which is ordinary carbonic acid $\left(\mathrm{CO}^{2}\right)$. The composition of (pure) alcohol is expressed by the formula $\mathrm{C}^{4} \mathrm{H}^{6} \mathrm{O}^{2}\left(\mathbf{C}^{2} \mathbf{x}^{6} \mathbf{0}\right)$, and it is produced in this process by the breaking up of grape sugar, $\mathrm{C}^{12} \mathrm{H}^{12} \mathrm{O}^{12} .2 \mathrm{HO}$ $\left(\mathbf{C}^{6} \mathbf{E}^{12} \mathbf{0}^{6} \cdot \mathbf{X}^{2} \mathbf{O}\right)$ into alcohol, carbonic acid, and water, thus :-

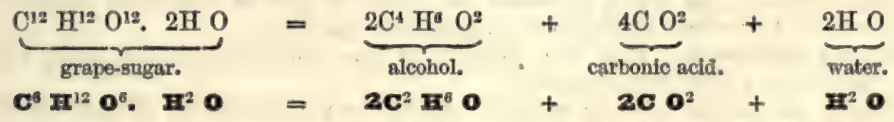

It is invariably the grape sugar which undorgoes this change; if the solution contains cane sugar, the cano sugar is first converted into grape sugar undor the influenco of the ferment. See Suarar.

Much diversity of opinion exists with respect to the office which the ferment performs in this process, since it does not itself yield any of the products. See FirMENTATION.

The liquid obtained by the vinous fermentation has received different names, according to the source whence the saccharine solution was dorived. When procured from the expressed juice of fruits-such as grapes, currants, gooseberries, \&c. - the product is denominated wine; from a decoction of malt, ale or beer; from a mixture of honey and water, mead; from apples, cider; from the leaves and small branches of spruce-fir (Abies excelsa, \&c.), together with sugar or troacle, spruce; from rice, rice beer (which yields the spirit arrack); from cocon-nut juice, palm wine.

It is an interesting fact that alcohol is produced in very considerable quantities (in the aggregate) during the raising of broad. The carbonic acid which is generated in the dough, and which during its expulsion raises the bread, is one of the products of the fermentation of the sugar in the flour, under the influence of the yeast addod; and of course at the same time the complementary product, alcohol, is gonerated. As

Thomeor's History of Chemistry, 1. 41. (1880).

- Annales de Chimie, xlll. 225. 
Messrs. Ronalds and Richardson remark:" 'the onormous amount of bread that is baked in large towns-in London, for instanco, 8.8 millions of cwts. yearly-would render the small amount of alcohol contained in it of sufficient importance to be worth collecting, provided this could be done sufficiently cheaply.' In London it has been estimated that in this way about 300,000 gallons of spirit are annually lost; but the cost of collecting it would far exceed its value.

2. Distillation. By the process of distillation, ardent spirits are obtained, which have likewise received different names according to the sources whence the formented liquor has been derived: viz. that produced by the distillation of wine boing callod brandy, and in France cognac, or eau de vie; that produced by the distillation of the fermontod liquor from sugar and molasses, rum. There are several varieties of spirits made from the fermented liquor produced from the cereals (and especially barley), known according to their peculiar mothods of manufacture, flavour, \&cc. - as whisky, gin, Hollands - the various compounds and liqueurs. In India, the spirit obtained from a fermented infusion of rice is called arrack.

3. Rectification; preparation of absolute alcohol. It is impossible by distillation alone to deprive spirit of the whole of the water and other impuritios-to obtain, in fact, pure or absolute alcohol.

This is effected by mixing with the liquid obtained after one or two distillations, certain bodies which have a powerful attraction for water. The agents commonly employed for this purpose are quicklime, carbonate of potash, anhydrous sulphato of copper, or chloride of calcium. Perhaps the best adapted for the purpose, especially where large quantities are required, is quicklime; it is powdered, mixed in the retort with the spirit (previously twice distilled), and the neck of the retort being securely closed, the whole is left for 24 hours, with occasional shaking; during this period the lime combines with the water, and then on carefully distilling, avoiding to continue the process until the last portions come over, an alcohol is obtained which is free from water. If not quite free, the same process may be again repeated.

In experiments on a small scale, an ordinary glass retort may be employed, heated by a water-bath, and fitted to a liebig's condonser cooled by ice-water, which passes lastly into a glass receiver, similarly cooled.

Although alcohol of sufficient purity for most practical purposes can be readily obtained, yet the task of procuring absolute alcohol entirely free from a trace of water, is by no means an easy one.

Mr. Drinkwater ${ }^{2}$ effected this by digesting ordinary alcohol of specific gravity 850 at $60^{\circ} \mathrm{F}$. for 24 hours with carbonate of potash previously exposed to a red heat; the alcohol was then carefully poured off and mixed in a retort with as much fresh-burnt quicklime as was sufficient to absorb the whole of the alcohol; after digesting for 48 hours, it was slowly distilled in a water-bath at a temperature of about $180^{\circ} \mathrm{F}$. This alcohol was carefully redistilled, and its specific gravity at $60^{\circ} \mathrm{F}$. found to be 7947 , which closely agrees with that given by Gay-Lussac as the specific gravity of absolute alcohol. He found, moreover, that recontly ignited anhydrous sulphate of coppor was a less efficient dehydrating agent than quicklime.

Graham recommends that the quantity of lime employed should never exceed three times the weight of the alcohol.

Chloride of calcium is not so well adapted for the purification of alcohol, since the alcohol forms a compound with this salt.

Many other processes have been suggested for depriving alcohol of its water.

A curious process was proposed many years ago by Soemmering, ${ }^{3}$ which is dependent upon the peculiar fact, that whilst water moistens animal tissues, alcohol does not, but tends rath bo enclosed in an ox bladder, the water gradually traverses the membrane and evapo. rates, whilst the alcohol does not, and consequently by the loss of water the spirituous solution becomes concentrated.

This process, though an interesting illustration of exosmose, is not practically ap. plicable to the production of anhydrous alcohol; it is, however, an economical method, and well suited for obtaining alcohol for the preparation of varnishes. Smugglers, who bring spirits into France in bladders hid about their persons, have long known, that although the liquor decreased in bulk, yet it increased in strength; hence the people preforred tho article conveyed clandestinely. Professor Graham ingeniously proposed to concentrate alcohol as follows:-

'A large shallow basin is covered, to a small depth, with recently burnt quicklime, in coarse powder, and a smaller basin, containing three or four ounces of com.

' Chemical Technology, by Dr. F. Knapp : edited by Messrs. Ronalds and Richardson. Vol. ii. 199,

- On the Preparation of Absolute Alcohol, and the Composition of Proof Spirit. See Memoirs of the Ohemical Soclety, vol. ili. p. 447.

- Soemmering: 'Denkschriften d. k. Akad. d. Wlssenschaften zu Munchen,' 1711 to 1824. 
mercial alcohol, is made to rest upon the lime; the whole is placed under the low receiver of an air-pump, and the exhaustion continued till the alcohol evinces signs of ebullition. Of the mingled vapours of alcohol and water which now fill the receiver, the quicklime is capable of uniting with the aqueous only, which is thorefore rapidly withdrawn, while the alcohol vapour is unaffected; and as water cannot remain in the alcohol as long as the superincumbent atmosphere is devoid of moisture, more aqueous vapour rises, which is likewise abstracted by the lime, and thus the process goes on till the whole of the water in the alcohol is removed. Several days aro always required for this purpose.'

\section{Properties of Alcohol (Absolute).}

In the state of purity, alcohol is a colourless liquid, highly inflammable, burning with a pale blue flame, very volatile, and having a density of 0.792 at $155^{\circ} \mathrm{C}$. (60 F.) (Drinkwater). It boils at $78.4^{\circ} \mathrm{C} .\left(173^{\circ} \mathrm{F}\right.$.) It has never yet been solidified, and the density of its vapour is 1.6133 .

Anhydrous alcohol is composed by weight of 52.18 carbon, 13.04 hydrogen, and $34 \cdot 78$ of oxygen. It has for its formula $\mathrm{C}^{4} \mathrm{H}^{6} \mathrm{O}^{2}=\mathrm{C}^{4} \mathrm{H}^{5} \mathrm{O}+\mathrm{HO}$, or hydrated oxide of ethyle. It has a powerful affinity for water, removing the water from moist substances with which it is brought in contact. In consequence of this property, it attracts water from the air, and rapidly becomes weaker, unless kept in very wellstopped vessels. In virtue of its attraction for water, alcohol is very valuable for the preservation of organic substances, and especially of anatomical preparations, in consequence of its causing the coagulation of albuminous substances; and for the same reason it causes death whon injected into the veins.

When mixed with water a considerable amount of heat is evolved, and a remarkable contraction of volume is observed, these effects being greatest with 54 per cent. of alcohol and 46 of water, and thence decreasing with a greater proportion of water. For alcohol which contains 90 per cent. of water, this condensation amounts to 1.94 per cent. of the volume; for 80 per cent., $2 \cdot 87$; for 70 per cent., 3.44 ; for 60 per cent., 3.73 ; for 40 per cent., 3.44 ; for 30 per cont., $2 \cdot 72$; for 20 per cent., $1 \cdot 72$; for 10 per cont., $0 \cdot 72$.

Alcohol is prepared absolute for certain purposes, but the mixtures of alcohol and water commonly met with in commeree are of an inferior strength. Those commonly sold are 'Rectified Spirit' and 'Proof Spirit.'

'Proof Spirit' is defined by Act of Parliament, 58 Geo. III. c. 28, to be 'such as shall, at the temperature of fifty-one degrees of Fahrenheit's thermometer, weigh exactly twelve-thirteonth parts of an equal measure of distilled water.' And by very careful experiment, Mr. Drinkwater has determined that this proof spirit has the following composition:-

\begin{tabular}{|c|c|c|c|c|}
\hline \multicolumn{3}{|c|}{ Alcohol and Water } & \multirow{2}{*}{$\begin{array}{l}\text { Speciflc Gravity } \\
\text { at } 60^{\circ} \mathrm{F} \text {. }\end{array}$} & \multirow{2}{*}{$\begin{array}{l}\text { Balk of the mixture } \\
\text { of } 100 \text { measures of } \\
\text { Alcohol, and } 81 \cdot 82 \\
\text { of Water }\end{array}$} \\
\hline By weight & By mes & & & \\
\hline 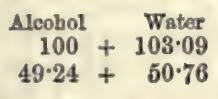 & $\begin{array}{l}\text { Alcohol } \\
100+\end{array}$ & $\begin{array}{l}\text { Water } \\
81 \cdot 82\end{array}$ & •919 & $175 \cdot 25$ \\
\hline
\end{tabular}

Spirit which is weaker is called ' under proof;' and that stronger, 'above proof.' The origin of these terms is as follows:-Formerly a very rude mode of ascertaining the strength of spirits was practised, called the proof; the spirit was poured upon gunpowder and inflamed. If, at the end of the combustion, the gunpowder took fire, the spirit was said to be above or over proof. But if the spirit contained much water, the powder was rendered so moist that it did not take fire: in which case the spirit was said to be under or below proof.

Rectified spirit contains from 54 to 64 per cent. of absolute alcohol ; and its specific gravity is fixed by the London and Edinburgh Colleges of Physicians at 0.838, whilst the Dublin College fixes it at 0.840.

In commerce the strength of mixtures of alcohol and water are stated at so many degrees, according to Sykes's hydrometer, above or below proof. This instrument will be explained under the head of AxconогомгтRт.

As will have been understood by the preceding remarks, the specific gravity or density of mixtures of alcohol and water rises with the diminution of the quantity of 
alcohol present; or, in other words, with the amount of water. And since the strongth of spirits is detorminod by ascortaining their density, it bocomes highly im. portant to determine the precise ratio of this increase. This incroase in donsity with the amount of water, or diminution with the quantity of alcohol, is, however, not directly proportional, in consequence of the contraption of volume which mixtures of alcohol and water suffer,

It therefore became necessary to determine the density of mixtures of known composition, propared artificially. This has been dope with great care by Mr. Drinkwater ; ${ }^{2}$ and the following Table by him is recommended as one of the most acourate :-

Table of the Quantity of Aloohol, BY wHIGHT, contained in Mistures of Aloohol and Water of the following Specifio Gravities:-

\begin{tabular}{|c|c|c|c|c|c|c|c|c|c|}
\hline $\begin{array}{l}\text { Bpeolfie } \\
\text { Gravity at } \\
60^{\circ} \mathrm{F} \text {. }\end{array}$ & $\begin{array}{l}\text { Alcohol } \\
\text { per cent. } \\
\text { by } \\
\text { weight. }\end{array}$ & $\begin{array}{l}\text { Speoific } \\
\text { Gravity } \\
\text { at } 60^{\circ} \mathrm{F} \text {. }\end{array}$ & $\begin{array}{c}\text { Alcohol } \\
\text { per cent. } \\
\text { by } \\
\text { weight. }\end{array}$ & $\begin{array}{l}\text { Specific } \\
\text { Gravity } \\
\text { at } 60^{\circ} \mathrm{F} \text {. }\end{array}$ & $\begin{array}{c}\text { Alcohol } \\
\text { per cent. } \\
\text { by } \\
\text { weight. }\end{array}$ & $\begin{array}{l}\text { Speciflo } \\
\text { Gravity } \\
\text { at } 60^{\circ} \mathrm{F} .\end{array}$ & $\begin{array}{c}\text { Alcohol } \\
\text { per cent. } \\
\text { by } \\
\text { weight. }\end{array}$ & $\begin{array}{l}\text { Specific } \\
\text { Gravity } \\
\text { at } 60^{\circ} \text { F. }\end{array}$ & $\begin{array}{l}\text { Alcohol } \\
\text { per cent. } \\
\text { by } \\
\text { weight. }\end{array}$ \\
\hline 1.0000 & 0.00 & •9967 & $1 \cdot 78$ & .9934 & $3 \cdot 67$ & $\cdot 9901$ & $5 \cdot 70$ & $\cdot 9869$ & $7 \cdot 85$ \\
\hline$\cdot 9999$ & 0.05 & .9966 & 1.83 & .9933 & $3 \cdot 73$ & .9900 & $5 \cdot 77$ & .9868 & $7 \cdot 92$ \\
\hline •9998 & 0.11 & •9965 & 1.89 & ·9932 & $3 \cdot 78$ & ·9899 & 5.83 & .9867 & $7 \cdot 99$ \\
\hline$\cdot 9997$ & $0 \cdot 16$ & -9964 & 1.94 & •9931 & $3 \cdot 84$ & .9898 & 5.89 & .9866 & 8.06 \\
\hline •9996 & $0 \cdot 21$ & .9963 & 1.99 & .9930 & $3 \cdot 90$ & ·9897 & $5 \cdot 96$ & .9865 & $8 \cdot 13$ \\
\hline •9995 & $0 \cdot 26$ & .9962 & $2 \cdot 05$ & .9929 & $3 \cdot 96$ & •9896 & 6.02 & •9864 & $8 \cdot 20$ \\
\hline •9994 & 0.32 & .9961 & $2 \cdot 11$ & .9928 & 4.02 & .9895 & 6.09 & .9863 & $8 \cdot 27$ \\
\hline .9993 & $0 \cdot 37$ & .9960 & $2 \cdot 17$ & .9927 & 4.08 & .9894 & $6 \cdot 15$ & .9862 & $8 \cdot 34$ \\
\hline .9992 & 0.42 & .9959 & $2 \cdot 22$ & .9926 & $4 \cdot 14$ & .9893 & 6.22 & 9861 & 8.41 \\
\hline •9991 & 0.47 & •9958 & $2 \cdot 28$ & .9925 & $4 \cdot 20$ & .9892 & $6 \cdot 29$ & .9860 & $8 \cdot 48$ \\
\hline .9990 & 0.53 & .9957 & $2 \cdot 34$ & .9924 & $4 \cdot 27$ & .9891 & 6.35 & .9859 & $8 \cdot 55$ \\
\hline •9989 & 0.58 & •9956 & $2 \cdot 39$ & .9923 & $4 \cdot 33$ & $\cdot 9890$ & $6 \cdot 42$ & .9858 & $8 \cdot 62$ \\
\hline .9988 & 0.64 & .9955 & $2 \cdot 45$ & .9922 & $4 \cdot 39$ & .9889 & 6.49 & .9857 & 8.70 \\
\hline$\cdot 9987$ & $0 \cdot 69$ & $\cdot 9954$ & $2 \cdot 51$ & •9921 & $4 \cdot 45$ & ·9888 & 6.55 & .9856 & $8 \cdot 77$ \\
\hline .9986 & 0.74 & .9953 & $2 \cdot 57$ & .9920 & 4.51 & .9887 & $6 \cdot 62$ & .9855 & 8.84 \\
\hline$\cdot 9985$ & 0.80 & .9952 & $2 \cdot 62$ & .9919 & 4.57 & .9886 & $6 \cdot 69$ & .9854 & 8.91 \\
\hline .9984 & 0.85 & .9951 & $2 \cdot 68$ & .9918 & 4.64 & .9885 & 6.75 & .9853 & 8.98 \\
\hline$\cdot 9983$ & 0.91 & $\cdot 9950$ & $2 \cdot 74$ & .9917 & 4.70 & $\cdot 9884$ & 6.82 & -9852 & 9.05 \\
\hline .9982 & 0.96 & .9949 & $2 \cdot 79$ & .9916 & $4 \cdot 76$ & .9883 & 6.89 & .9851 & $9 \cdot 12$ \\
\hline .9981 & 1.02 & .9948 & $2 \cdot 85$ & .9915 & 4.82 & •9882 & $6 \cdot 95$ & .9850 & $9 \cdot 20$ \\
\hline .9980 & 1.07 & .9947 & $2 \cdot 91$ & .9914 & 4.88 & .9881 & $7 \cdot 02$ & $\begin{array}{r}.9849 \\
\end{array}$ & $9 \cdot 27$ \\
\hline •9979 & $1 \cdot 12$ & .9946 & $2 \cdot 97$ & .9913 & 4.94 & .9880 & $7 \cdot 09$ & .9848 & $9 \cdot 34$ \\
\hline •9978 & $1 \cdot 18$ & $\cdot 9945$ & 3.02 & •9912 & 5.01 & 9879 & $7 \cdot 16$ & $\cdot 9847$ & $9 \cdot 41$ \\
\hline$\cdot 9977$ & 1.23 & .9944 & 3.08 & .9911 & 5.07 & .9878 & $7 \cdot 23$ & $\cdot 9846$ & $9 \cdot 49$ \\
\hline$\cdot 9976$ & 1.29 & $\cdot 9943$ & $3 \cdot 14$ & .9910 & $5 \cdot 13$ & .9877 & $7 \cdot 30$ & $\cdot 9845$ & $9 \cdot 56$ \\
\hline .9975 & 1.34 & .9942 & $3 \cdot 20$ & .9909 & $5 \cdot 20$ & $\cdot 9876$ & $7 \cdot 37$ & .9844 & $9 \cdot 63$ \\
\hline$\cdot 9974$ & $1 \cdot 40$ & •9941 & $3 \cdot 26$ & $\cdot 9908$ & $5 \cdot 26$ & .9875 & $7 \cdot 43$ & .9843 & $9 \cdot 70$ \\
\hline$\cdot 9973$ & 1.45 & .9940 & $3 \cdot 32$ & .9907 & $5 \cdot 32$ & .9874 & $7 \cdot 50$ & .9842 & $9 \cdot 78$ \\
\hline$\cdot 9972$ & 1.51 & $\cdot 9939$ & $3 \cdot 37$ & 9906 & 5.39 & $\cdot 9873$ & $7 \cdot 57$ & $\cdot 9841$ & $9 \cdot 85$ \\
\hline .9971 & 1.56 & .9938 & $3 \cdot 43$ & .9905 & 5.45 & •9872 & $7 \cdot 64$ & .9840 & $9 \cdot 92$ \\
\hline .9970 & 1.61 & $\cdot 9937$ & $3 \cdot 49$ & $\cdot 9904$ & $5 \cdot 51$ & .9871 & $7 \cdot 71$ & •9839 & 9.99 \\
\hline •9969 & 1.67 & .9936 & $3 \cdot 55$ & $\cdot 9903$ & 5.58 & .9870 & $7 \cdot 78$ & -9838 & 10.07 \\
\hline •9988 & 1.73 & $\cdot 9935$ & $3 ॰ 61$ & $\cdot 9902$ & $5 \cdot 64$ & & & & \\
\hline
\end{tabular}

The preceding Table, though very accurate as far as it goes, is not sufficiently extensive for practical purposes, only going, in fact, from 6 to 10 per cent. of alcohol; the Table of 'Tralle's (page 50) extends to 50 per cent. of absolute alcohol.

Moreover, Drinkwater's Table has the (practical) disadvantage (though scientifically more correct and useful) of stating the per-centage by weight; whereas in Tralle's Table it is given by volume. And since liquors are vended by measure, and not by weight, the centesimal amount by volume is usually preferred. But as the bulk of liquids generally, and particularly that of alcohol, is increased by heat, it is necessury that the statement of the density in a certain volume should have reference to some normal temperature. In the construction of Tralle's Table the tomperature of the

Memoirs of the Chemical Society, vol, iil. p. 454. 
liquids was $60^{\circ} \mathrm{F}$.; and of course, in using it, it is necessary that the density should be observed at that temperature.

In order to convert the statement of the composition by volume into the content by weight, it is only necessary to multiply the per-centage of alcohol by volume by the specific gravity of absolute alcohol, and then divide by the spoeific gravity of the liquid.

It has been thought desirable to retain the following remarks by $\mathrm{Dr}$. Ure, and to give Mr. Gilpin's Tables in addition to the others.

The importance of extreme accuracy in determining the density of alcoholic mixtures in the United Kingdom, on account of the great revenue derived from thom to the State, and their consequent high price in commerce, induced the Lords of the Treasury a few years ago to request the Royal Society to examine the construction and mode of applying the instrument now in use for ascertaining and charging the duty on spirits. This instrument, which is known and described in the law as Sikes's hydrometer, possesses, in many respects, decided advantages over those formerly in use. The committes of the Royal Society state, that a definite mixture of alcohol and water is as invariable in its value as absolute alcohol can be; and can be more readily, and with equal accuracy, identified by that only quality or condition to which rocourse can bo had in practico, namoly, specific gravity. The committeo further proposed, that the standard spirit be that which, consisting of alcohol and water alone, shall have a specifie gravity of 0.92 at the temperature of $62^{\circ} \mathrm{F}$., water being unity at the same temperature; or, in other words, that it shall at $62^{\circ}$ weigh $\frac{92}{100}$ ths or $\frac{25}{25}$ ths of an equal bulk of water at the same temperature.

This standard is rather stronger than the old proof, which was $\frac{12}{13}$ ths or 0.923 ; or in the proportion of nearly 1.1 gallon of the present proof spirit per cent. The proposed standard will contain nearly one-half by weight of absolute alcohol. The hydrometer ought to be so graduated as to give the indication of strength; not upon an arbitrary scale, but in terms of specific gravity at the temperature of $62^{\circ}$.

The committeo recommend the construction of an equation table, which shall indicate the same strength of spirit at every temperature. Thus in standard spirit at $62^{\circ}$ the hydrometer would indicate 920 , which in this table would give proof spirit. If that same spirit were cooled to $40^{\circ}$, the hydrometer would indicate some higher number; but which, being combined in the table with the temperature as indicated by the thermometer, should still give proof or standard spirit as the result.

It is considered advisable, in this and the other tables, not to express the quality of the spirit by any number over or under proof, but to indicate at once the number of gallons of standard spirit contained in, or equivalent to, 100 gallons of the spirit under examination. Thus, instead of saying 23 over proof, it is proposed to insert 123 ; and in place of 35.4 under proof, to insert its difference to 100 , or $64 \cdot 6$.

It has been considerod expedient to recommend a second table to be constructed, so as to show the bulk of spirit of any strength at any tomperature, relative to a standard bulk of 100 gallons at $62^{\circ}$. In this table a spirit which had diminished in volume, at any given temperature, 0.7 per cent., for example, would be expressed by 99.3 ; and a spirit which had increased at any given temperature 0.7 per cent., by 100.7 .

When a sample of spirit, therefore, has been examined by the hydrometer and thermometer, these tables will give first the proportion of standard spirit at the observed temperature, and next the change of bulk of such spirit from what it would be at the standard temperature. Thus at the temperature of $51^{\circ}$, and with an indication (specific gravity) of $8,240,100$ gallons of the spirit under examination would be shown by the first table to be equal to 164.8 gallons of standard spirit of that temperature; and by the second table it would appear that 99.3 gallons of the same spirit would become 100 at $62^{\circ}$, or in reality contain the 164.8 gallons of spirit in that state only in which it is to be taxed.

But as it is considered that neither of these tables can alone be used for charging the duty (for neither can express the actual quantity of spirit of a speciflc gravity of 0.92 at $62^{\circ}$ in 100 gallons of stronger or weaker spirit at temperatures above or below $62^{\circ}$ ), it is considered essential to have a third table, combining the two former, and expressing this relation directly, so that upon mere inspection it shall indicate the proportion of standard spirit in 100 gallons of that under examination in its then present state. In this table the quantities should be set down in the actual number of gallons of standard spirit at $62^{\circ}$, equivalont to 100 of the spirit under examination; and the column of quantities may be expressed by the term value, as it in reality ox. presses the proportion of the only valuable substance present.

The following specimen Table has been given by the committeo :- 


\begin{tabular}{|c|c|c|c|c|c|}
\hline \multicolumn{3}{|c|}{ Temperature $45^{\circ}$} & \multicolumn{3}{|c|}{ Temparature $75^{\circ}$} \\
\hline Indication ${ }^{2}$ & Btrength & Value & Indication & Strength & Value \\
\hline 9074 & $114 \cdot 5$ & & 8941 & $114 \cdot 5$ & \\
\hline 7 & $114^{\circ} 3$ & . & 4 & $114 \cdot 3$ & 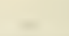 \\
\hline 9 & $114 \cdot 2$ & & 5 & $114 \cdot 2$ & \\
\hline 81 & 114.0 & & 8 & 114.0 & \\
\hline 3 & $113 \cdot 9$ & & 9 & $113 \cdot 9$ & \\
\hline 5 & $118 \cdot 7$ & & 52 & $113 \cdot 7$ & \\
\hline 6 & 113.6 & & 8 & 113.6 & \\
\hline 9 & $113 \cdot 4$ & & 6 & $113 \cdot 4$ & 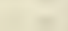 \\
\hline 90 & 113.3 & & 7 & $113 \cdot 3$ & \\
\hline 3 & $113 \cdot 1$ & & 9 & $113 \cdot 1$ & \\
\hline
\end{tabular}

The mixture of alcohol and water, taken as spirit in Mr. Gilpin's Tables, is that of which the specific gravity is 0.825 at $60^{\circ} \mathrm{F}$, water boing unity at the samo temperature. The specific gravity of water at $60^{\circ}$ being 1,000 , at $62^{\circ}$ it is 99,981 . Hence, in order to compare the specifle gravities given by Mr. Gilpin with those which would result when the specific gravity of water at $62^{\circ}$ is talken at unity, all the former numbers must be divided by 99,981 .

Table of the Specific Gravities of different Mixtures, BY WEIGHT, of Alcohol and Wator, at different Temperatures; constructed by Mr. Gilpin, for the use of the British Revenue on Spirits.

\begin{tabular}{|c|c|c|c|c|c|c|c|c|c|c|c|}
\hline 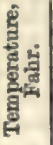 & $\begin{array}{c}\text { Pure } \\
\text { Alcohol }\end{array}$ & $\begin{array}{c}100 \\
\text { Alcohol } \\
5 \\
\text { Water }\end{array}$ & $\begin{array}{c}100 \\
\text { Aloobol } \\
10 \\
\text { Water }\end{array}$ & $\begin{array}{c}100 \\
\text { Alcohol } \\
15 \\
\text { Water }\end{array}$ & $\begin{array}{c}100 \\
\text { Alcohol } \\
20 \\
\text { Water }\end{array}$ & \begin{tabular}{|c|}
100 \\
Alcohol \\
25 \\
Water
\end{tabular} & $\begin{array}{c}100 \\
\text { Alcohol } \\
30 \\
\text { Water }\end{array}$ & $\begin{array}{c}100 \\
\text { Aloohol } \\
35 \\
\text { Water }\end{array}$ & $\begin{array}{c}100 \\
\text { Alcohol } \\
40 \\
\text { Water }\end{array}$ & $\begin{array}{c}100 \\
\text { Alcohol } \\
45 \\
\text { Water }\end{array}$ & $\left\{\begin{array}{c}100 \\
\text { Alcohol } \\
50 \\
\text { Water }\end{array}\right.$ \\
\hline $\begin{array}{c}\text { Deg. } \\
30 \\
35 \\
40 \\
45 \\
50 \\
55 \\
60 \\
65 \\
70 \\
75 \\
80 \\
85 \\
90 \\
95 \\
100\end{array}$ & $\begin{array}{r}0.83896 \\
\cdot 83672 \\
.83445 \\
\cdot 83214 \\
.82977 \\
.82736 \\
.82500 \\
.82262 \\
.82028 \\
.81780 \\
\cdot 81530 \\
.81291 \\
.81044 \\
.80794 \\
.80848\end{array}$ & $\begin{array}{c}0.84995 \\
.84769 \\
.84539 \\
.84310 \\
.84076 \\
.83834 \\
.83599 \\
.88362 \\
.83124 \\
.82878 \\
.82631 \\
.82396 \\
.82150 \\
.81900 \\
.81657\end{array}$ & $\begin{array}{l}0.85957 \\
.85729 \\
.85507 \\
.85277 \\
.85042 \\
.84802 \\
.84568 \\
.84334 \\
\cdot 84092 \\
.83851 \\
.83603 \\
.83371 \\
.83126 \\
.82877 \\
-82689\end{array}$ & $\begin{array}{r}0.86825 \\
.86587 \\
.86361 \\
-86131 \\
.85902 \\
.85664 \\
.85430 \\
.85193 \\
.84951 \\
.84710 \\
-84467 \\
.84243 \\
.84001 \\
-83758 \\
-83513\end{array}$ & \begin{tabular}{|}
0.87585 \\
.87357 \\
.87184 \\
.86905 \\
.86676 \\
.86441 \\
.86208 \\
.85976 \\
.85756 \\
.85496 \\
.85248 \\
.85036 \\
.84797 \\
.84550 \\
.84038
\end{tabular} & $\begin{array}{c}0.88282 \\
.88059 \\
.87838 \\
.87613 \\
.87984 \\
.87150 \\
.86918 \\
.86686 \\
.86451 \\
.86212 \\
.85966 \\
.85757 \\
.85518 \\
.85272 \\
.85031\end{array}$ & $\begin{array}{r}0.88921 \\
.88701 \\
.88481 \\
.88255 \\
.88030 \\
.87796 \\
.87569 \\
.87337 \\
.87105 \\
.86864 \\
.86622 \\
.86411 \\
.86172 \\
.85928 \\
.85688\end{array}$ & $\begin{array}{r}0.89511 \\
.89294 \\
.89073 \\
.88849 \\
.88626 \\
.88393 \\
.88169 \\
.87938 \\
.87705 \\
.87466 \\
.87228 \\
.87021 \\
.86787 \\
.86542 \\
.86302\end{array}$ & $\begin{array}{r}0 \cdot 90054 \\
.89839 \\
-89617 \\
.89396 \\
.89174 \\
.88945 \\
.88720 \\
.88490 \\
-88254 \\
.88018 \\
.87776 \\
.87590 \\
.87360 \\
-87114 \\
\cdot 86879\end{array}$ & \begin{tabular}{|}
0.90558 \\
.90345 \\
.90127 \\
.89909 \\
.89684 \\
.89458 \\
.89232 \\
.89006 \\
.88773 \\
.88538 \\
.88301 \\
.88120 \\
.87889 \\
.87654 \\
$\cdot 87421$
\end{tabular} & $\begin{array}{r}0.91023 \\
-90811 \\
.90596 \\
-90380 \\
.90160 \\
.89933 \\
.89707 \\
.89479 \\
\cdot 89252 \\
.89018 \\
-88781 \\
.88609 \\
.88376 \\
.88146 \\
\cdot 87915\end{array}$ \\
\hline Tem & $\begin{array}{l}\text { perature, } \\
\text { cahr. }\end{array}$ & $\begin{array}{c}100 \\
\text { Aloohol } \\
55 \\
\text { Water }\end{array}$ & $\begin{array}{c}100 \\
\text { Alcohol } \\
60 \\
\text { Water }\end{array}$ & $\begin{array}{c}100 \\
\text { Alcohol } \\
65 \\
\text { Water }\end{array}$ & $\begin{array}{c}100 \\
\text { Alcohol } \\
70 \\
\text { Water }\end{array}$ & $\begin{array}{c}100 \\
\text { Alcohol } \\
75 \\
\text { Water }\end{array}$ & $\begin{array}{c}100 \\
\text { Alcohol } \\
80 \\
\text { Water }\end{array}$ & $\begin{array}{c}100 \\
\text { Alcohol } \\
85 \\
\text { Water }\end{array}$ & $\begin{array}{c}100 \\
\text { Alcohol } \\
90 \\
\text { Water }\end{array}$ & $\begin{array}{c}100 \\
\text { Alcohol } \\
95 \\
\text { Water }\end{array}$ & $\begin{array}{c}100 \\
\text { Alcolol } \\
100 \\
\text { Water }\end{array}$ \\
\hline & $\begin{array}{c}\text { Deg. } \\
30 \\
35 \\
40 \\
45 \\
50 \\
55 \\
60 \\
65 \\
70 \\
75 \\
80 \\
85 \\
40 \\
95 \\
100\end{array}$ & $\begin{array}{r}0 \cdot 91449 \\
\cdot 91241 \\
\cdot 91026 \\
\cdot 90812 \\
\cdot 90596 \\
\cdot 90367 \\
\cdot 90144 \\
\cdot 89920 \\
\cdot 89695 \\
\cdot 89464 \\
\cdot 89225 \\
\cdot 89043 \\
\cdot 88817 \\
\cdot 88588 \\
\cdot 883571\end{array}$ & $\begin{array}{r}0 \cdot 91847 \\
\cdot 91640 \\
\cdot 91428 \\
\cdot 91211 \\
\cdot 90997 \\
\cdot 90768 \\
\cdot 90549 \\
\cdot 90328 \\
\cdot 90104 \\
\cdot 89872 \\
\cdot 89689 \\
\cdot 89460 \\
\cdot 89230 \\
\cdot 89003 \\
\cdot 88769\end{array}$ & $\begin{array}{r}0 \cdot 92217 \\
\cdot 92009 \\
\cdot 91799 \\
\cdot 91584 \\
\cdot 91370 \\
\cdot 91144 \\
\cdot 90927 \\
\cdot 90707 \\
\cdot 90484 \\
\cdot 90252 \\
\cdot 90021 \\
\cdot 89843 \\
\cdot 89617 \\
\cdot 89890 \\
\cdot 89158\end{array}$ & $\begin{array}{c}0 \cdot 92563 \\
.92355 \\
.92151 \\
.91937 \\
.91723 \\
.91502 \\
.91287 \\
.91066 \\
.90847 \\
.90617 \\
.90385 \\
.90209 \\
.89988 \\
.89763 \\
.89536\end{array}$ & $\begin{array}{r}0 \cdot 92889 \\
-92680 \\
.92476 \\
.92264 \\
\cdot 92051 \\
\cdot 91837 \\
\cdot 91622 \\
-91400 \\
\cdot 91181 \\
\cdot 90952 \\
\cdot 90723 \\
\cdot 90558 \\
\cdot 90342 \\
\cdot 90119 \\
\cdot 99889\end{array}$ & $\begin{array}{c}0 \cdot 98191 \\
\cdot 92986 \\
\cdot 92783 \\
\cdot 92570 \\
\cdot 92858 \\
\cdot 92145 \\
\cdot 91933 \\
\cdot 91715 \\
\cdot 91498 \\
\cdot 91270 \\
\cdot 91046 \\
\cdot 90882 \\
\cdot 90688 \\
\cdot 90443 \\
\cdot 90215\end{array}$ & $\begin{array}{r}0.98474 \\
.93274 \\
\cdot 93072 \\
\cdot 92859 \\
.92947 \\
.92436 \\
.92225 \\
.92010 \\
\cdot 91798 \\
.91569 \\
.91340 \\
.91186 \\
.90967 \\
.90747 \\
.90522\end{array}$ & $\begin{array}{c}0 \cdot 93741 \\
.93541 \\
.93341 \\
.99181 \\
.92919 \\
.92707 \\
.92499 \\
.92283 \\
.92069 \\
.91849 \\
.91622 \\
.91465 \\
.91248 \\
.91029 \\
.90805\end{array}$ & $\begin{array}{r}0.93991 \\
.93790 \\
.93592 \\
.99382 \\
.98177 \\
.92963 \\
.92758 \\
.92546 \\
.92338 \\
.92111 \\
.91891 \\
.91729 \\
.91511 \\
.91290 \\
.91066\end{array}$ & $\begin{array}{r}0 \cdot 94222 \\
\cdot 94025 \\
\cdot 93827 \\
\cdot 93621 \\
\cdot 93419 \\
\cdot 93208 \\
\cdot 93002 \\
\cdot 92794 \\
\cdot 92580 \\
\cdot 92364 \\
\cdot 92142 \\
\cdot 91969 \\
\cdot 91751 \\
\cdot 91531 \\
\cdot 91310\end{array}$ \\
\hline
\end{tabular}


Table of the Spectfo Gravities of different Mixtures, \&c. (continued.)

\begin{tabular}{|c|c|c|c|c|c|c|c|c|c|c|}
\hline 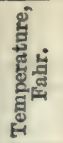 & $\begin{array}{c}95 \\
\text { Alcohol } \\
100 \\
\text { Water }\end{array}$ & $\begin{array}{c}80 \\
\text { Alcobol } \\
100 \\
\text { Water }\end{array}$ & $\begin{array}{c}85 \\
\text { Aloohol } \\
100 \\
\text { Water }\end{array}$ & $\begin{array}{c}80 \\
\text { Alcohol } \\
100 \\
\text { Water }\end{array}$ & $\begin{array}{c}75 \\
\text { Alcohol } \\
100 \\
\text { Water }\end{array}$ & $\begin{array}{c}70 \\
\text { Alcohol } \\
100 \\
\text { Water }\end{array}$ & $\begin{array}{c}65 \\
\text { Alcohol } \\
100 \\
\text { Water }\end{array}$ & $\begin{array}{c}60 \\
\text { Alcohol } \\
100 \\
\text { Water }\end{array}$ & $\begin{array}{c}58 \\
\text { Alcohol } \\
100 \\
\text { Water }\end{array}$ & $\begin{array}{c}50 \\
\text { Alcohol } \\
100 \\
\text { Water }\end{array}$ \\
\hline $\begin{array}{c}\text { Deg. } \\
30 \\
35 \\
40 \\
45 \\
50 \\
55 \\
60 \\
65 \\
70 \\
75 \\
80\end{array}$ & $\begin{array}{l}0.94447 \\
.94249 \\
.94058 \\
.93860 \\
.93658 \\
.93452 \\
.93247 \\
.93040 \\
.92828 \\
.92819 \\
.92993\end{array}$ & $\begin{array}{r}0.94675 \\
.94484 \\
.94295 \\
.94096 \\
.93897 \\
.98696 \\
.93498 \\
.93495 \\
.93076 \\
.92865 \\
.92646\end{array}$ & $\begin{array}{r}0.94920 \\
\cdot 94734 \\
\cdot 94547 \\
\cdot 94348 \\
\cdot 94149 \\
\cdot 93948 \\
\cdot 93749 \\
\cdot 93546 \\
\cdot 93337 \\
\cdot 93132 \\
\cdot 92917\end{array}$ & $\begin{array}{r}0 \cdot 95173 \\
.94988 \\
.94802 \\
.94605 \\
.94414 \\
.94213 \\
.94018 \\
.93822 \\
.93616 \\
.03413 \\
\cdot 93201\end{array}$ & $\begin{array}{r}0.95429 \\
.95246 \\
.95060 \\
.94871 \\
.94683 \\
.94486 \\
.94296 \\
.94099 \\
.93898 \\
.93695 \\
.93488\end{array}$ & $\begin{array}{l}0.95681 \\
\cdot 95502 \\
\cdot 95328 \\
\cdot 95143 \\
\cdot 94958 \\
.94767 \\
.94579 \\
\cdot 94388 \\
.94198 \\
.93989 \\
\cdot 93785\end{array}$ & $\begin{array}{r}0.95944 \\
.95772 \\
.95602 \\
.95423 \\
.95243 \\
.95057 \\
.94876 \\
.94689 \\
.94500 \\
.94301 \\
.94102\end{array}$ & $\begin{array}{r}0.96209 \\
.96048 \\
.95879 \\
.95703 \\
.95534 \\
.95357 \\
.95181 \\
.95000 \\
.94813 \\
.94628 \\
.94431\end{array}$ & $\begin{array}{r}0.96470 \\
.96315 \\
.96159 \\
.95993 \\
.95831 \\
.95662 \\
.95493 \\
.85818 \\
.95139 \\
.94957 \\
.94768\end{array}$ & $\begin{array}{r}0.96719 \\
.96579 \\
\cdot 96434 \\
\cdot 96280 \\
\cdot 96126 \\
.95966 \\
.95804 \\
.95635 \\
.95469 \\
\cdot 95292 \\
\cdot 95111\end{array}$ \\
\hline Temp & $\begin{array}{l}\text { serature, } \\
\text { ahr. }\end{array}$ & $\begin{array}{c}45 \\
\text { Alcohol } \\
100 \\
\text { Water }\end{array}$ & $\begin{array}{c}40 \\
\text { Alcohol } \\
100 \\
\text { Water }\end{array}$ & $\begin{array}{c}25 \\
\text { Alcohol } \\
100 \\
\text { Water }\end{array}$ & $\begin{array}{c}80 \\
\text { Alcohol } \\
100 \\
\text { Water }\end{array}$ & $\begin{array}{c}25 \\
\text { Alcohol } \\
100 \\
\text { Water }\end{array}$ & $\begin{array}{c}20 \\
\text { Alcohol } \\
100 \\
\text { Water }\end{array}$ & $\begin{array}{c}15 \\
\text { Alcohol } \\
100 \\
\text { Water }\end{array}$ & $\begin{array}{c}10 \\
\text { Alcohol } \\
100 \\
\text { Water }\end{array}$ & $\begin{array}{c}5 \\
\text { Aleohol } \\
100 \\
\text { Water }\end{array}$ \\
\hline 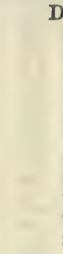 & $\begin{array}{l}45 \\
50 \\
65 \\
60 \\
65 \\
70 \\
75 \\
80\end{array}$ & $\begin{array}{r}0.96967 \\
.96840 \\
.96706 \\
.96568 \\
.96420 \\
.96272 \\
.96122 \\
.95962 \\
.05802 \\
.95638 \\
.95467\end{array}$ & $\begin{array}{r}0 \cdot 97200 \\
\cdot 97086 \\
\cdot 96967 \\
\cdot 96840 \\
\cdot 96708 \\
\cdot 96575 \\
\cdot 96437 \\
\cdot 96288 \\
\cdot 96148 \\
\cdot 95987 \\
\cdot 95826\end{array}$ & $\begin{array}{r}0.97418 \\
\cdot 97319 \\
.97220 \\
\cdot 97110 \\
.96995 \\
\cdot 96877 \\
.96752 \\
\cdot 96620 \\
\cdot 96484 \\
.96344 \\
.96192\end{array}$ & $\begin{array}{r}0.97635 \\
.97556 \\
.97472 \\
.97384 \\
.97284 \\
.97181 \\
.97074 \\
.96959 \\
.96836 \\
.96708 \\
.96568\end{array}$ & $\begin{array}{r}0.97860 \\
\cdot 97801 \\
\cdot 97737 \\
\cdot 97666 \\
.97589 \\
\cdot 97500 \\
.97410 \\
.97309 \\
.97203 \\
.97086 \\
.96968\end{array}$ & $\begin{array}{r}0.98108 \\
.98076 \\
.98033 \\
.97980 \\
.97920 \\
.97847 \\
.97771 \\
.97688 \\
.97596 \\
.97495 \\
.97385\end{array}$ & $\begin{array}{r}0.98412 \\
.98397 \\
.98378 \\
.98338 \\
.98293 \\
.98239 \\
.98176 \\
.98106 \\
.98028 \\
.97943 \\
.97845\end{array}$ & $\begin{array}{r}0.98804 \\
.98804 \\
.98795 \\
.98774 \\
.98745 \\
.98702 \\
.98654 \\
.98494 \\
.98527 \\
.98454 \\
.98367\end{array}$ & $\begin{array}{r}0.99334 \\
-99344 \\
-99345 \\
.99338 \\
.99316 \\
.99284 \\
.99244 \\
.99194 \\
.99134 \\
.99066 \\
.98991\end{array}$ \\
\hline
\end{tabular}

Fxperiments were made, by direction of the committee, to verify Gilpin's Tables, which showed that the error introduced in ascertaining the strength of spirits by Tables founded on Gilpin's numbers must be quite insensible in the practice of the Revenue. The discrepancies thus detected, on a mixture of a given strength, did not amount in any ope instance to unity in the fourth place of decimals. From a careful inspection of such documents the committeo are of opinion that Gilpin's Tables possess a degree of accuracy far surpassing what could be expected, and sufficiently perfect for all practical or scientific purposes.

The following Table is given by Mr. Lubbock, for converting the apparent specific gravity, or indication, into true specific gravity:-

\begin{tabular}{|c|c|c|c|c|c|c|c|c|c|c|c|c|c|}
\hline 5 & & & 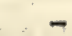 & & & Temr & are & & & + & & & 묭 \\
\hline 름 & $80^{\circ}$ & $82^{\circ}$ & $87^{\circ}$ & $12^{\circ}$ & $\mathbf{4} 7^{\circ}$ & $52^{\circ}$ & $57^{\circ}$ & $62^{\circ}$ & $67^{\circ}$ & $72^{\circ}$ & $77^{\circ}$ & $80^{\circ}$ & 5 \\
\hline$\cdot 82$ & $\cdot 00083$ & $\cdot 00078$ & .00065 & .00052 & $\cdot 00089$ & .00025 & .00012 & & .00011 & $\cdot 00024$ & $\cdot 00085$ & $\cdot 00042$ & $\cdot 82$ \\
\hline .83 & .00084 & .00079 & .00066 & .00052 & .00039 & .00026 & .00012 & & .00012 & .00024 & 00036 & .00042 & $\cdot 88$ \\
\hline$\cdot 84$ & .00085 & .00080 & .00066 & .00053 & .00039 & .00026 & $\cdot 00013$ & & 0012 & .00024 & .00036 & .00043 & $\cdot 84$ \\
\hline .85 & $\cdot 00086$ & $\cdot 00081$ & .00067 & .00054 & $\cdot 00040$ & .00026 & $\cdot 00013$ & & 12 & .00025 & 0037 & 3 & .85 \\
\hline$\cdot 86$ & $\cdot 00087$ & .00082 & .00068 & $\cdot 00054$ & .00040 & .000 & 3 & & 12 & 5 & 037 & 14 & $\cdot 86$ \\
\hline$\cdot 87$ & $\cdot 00088$ & .00083 & .00069 & $\cdot 00055$ & .00041 & $\cdot 0$ & $\cdot 0$ & & 00 & .00 & .00037 & 00 & .87 \\
\hline$\cdot 88$ & .00089 & .00084 & .00070 & .00055 & $\cdot 00041$ & .00027 & .000 & & .00 & .00 & .00038 & .00 & .88 \\
\hline$\cdot 89$ & .00090 & .00085 & .00070 & .00055 & .00042 & .00028 & .000 & & .000 & .000 & .00038 & .000 & .89 \\
\hline .90 & .00091 & .00085 & 00071 & .00056 & .00042 & .00028 & $\cdot 00014$ & & .00 & .00026 & $\cdot 00089$ & 00046 & -90 \\
\hline .91 & .00092 & $\cdot 00086$ & $\cdot 00072$ & $\cdot 00057$ & .00043 & $\cdot 00028$ & .00014 & & .000 & .00026 & $\cdot 00039$ & .000 & $\cdot 91$ \\
\hline •92 & .00093 & $\cdot 00087$ & $\cdot 00073$ & $\cdot 00058$ & $\cdot 00043$ & .00029 & $\cdot 00014$ & & .000 & .00027 & .00040 & .006 & $\cdot 92$ \\
\hline$\cdot 93$ & .00094 & $\cdot 00088$ & $\cdot 00073$ & $\cdot 00059$ & .00044 & .00029 & .00014 & & $\cdot 00$ & .00027 & $\cdot 00040$ & .00 & $\bullet 98$ \\
\hline$\cdot 94$ & $\cdot 00095$ & .00089 & .00074 & $\cdot 00059$ & .00044 & .00029 & .000 & & .00 & .00027 & .00040 & .00048 & $\cdot 94$ \\
\hline .95 & .00096 & .00090 & .00075 & $\cdot 00060$ & .00045 & $\cdot 00029$ & .00014 & & .00 & .000 & .00041 & .00048 & .95 \\
\hline •96 & $\cdot 00097$ & $\bullet 00091$ & .00076 & .00060 & .00045 & .00030 & .00014 & & .00013 & .00028 & .00041 & 00049 & $\bullet 96$ \\
\hline$\cdot 97$ & .00098 & $\cdot 00092$ & .00077 & -00061 & .00046 & .00030 & .00015 & & .00014 & .00028 & .00042 & $\cdot 00049$ & $\cdot 97$ \\
\hline •98 & .00099 & .00083 & .00077 & $\cdot 00062$ & .00046 & .00030 & .00015 & & .00014 & .00028 & .00042 & .00050 & -98 \\
\hline -99 & .00100 & $\cdot 00094$ & .00078 & .00062 & .00047 & $\cdot 00031$ & .00015 & & .00014 & .00029 & $\cdot 00048$ & .00050 & $\bullet 99$ \\
\hline 100 & $\cdot 00101$ & $\cdot 00095$ & $\bullet 00079$ & $\cdot 00088$ & .00047 & $\cdot 00031$ & $\cdot 00015$ & & & & & & 100 \\
\hline
\end{tabular}

VoL. I. 
Tralle's Table of the Composition BY voLuan of Mixtures of Alcohol and Water of different Densities.

\begin{tabular}{|c|c|c|c|c|c|c|c|c|}
\hline $\begin{array}{c}\text { Per- } \\
\text { centage } \\
\text { of } \\
\text { Alcohol } \\
\text { by } \\
\text { volume }\end{array}$ & $\begin{array}{c}\text { Speoifio } \\
\text { Gravity at } \\
60^{\circ} \mathrm{F} \text {. }\end{array}$ & $\begin{array}{l}\text { Differ. } \\
\text { ence of } \\
\text { the spe- } \\
\text { cific gra- } \\
\text { vities }\end{array}$ & $\begin{array}{c}\text { Per. } \\
\text { centage } \\
\text { of } \\
\text { Alcohol } \\
\text { by } \\
\text { volume }\end{array}$ & $\begin{array}{l}\text { Specific } \\
\text { Gravity at } \\
60^{\circ} \mathrm{Y} .\end{array}$ & $\begin{array}{l}\text { Differ- } \\
\text { ence of } \\
\text { the spe- } \\
\text { cific gra- } \\
\text { vitles }\end{array}$ & $\begin{array}{c}\text { Per- } \\
\text { centage } \\
\text { of } \\
\text { Alcohol } \\
\text { by } \\
\text { volume }\end{array}$ & $\begin{array}{l}\text { Specific } \\
\text { Gravity at } \\
60^{\circ} \mathrm{F} \text {. }\end{array}$ & $\begin{array}{l}\text { Differ- } \\
\text { ence of } \\
\text { the spe- } \\
\text { cific gra- } \\
\text { vities }\end{array}$ \\
\hline 0 & $0 \cdot 9991$ & & 84 & 0.9596 & 13 & 68 & 0.8941 & 24 \\
\hline 1 & $0 \cdot 9976$ & 15 & 35 & 0.9583 & 13 & 69 & 0.8917 & 24 \\
\hline 2 & 0.9961 & 15 & 36 & 0.9570 & 13 & 70 & 0.8892 & 25 \\
\hline 3 & 0.9947 & 14 & 37 & 0.9556 & 14 & 71 & 0.8867 & 25 \\
\hline 4. & 0.9933 & 14 & 38 & 0.9541 & 15 & 72 & 0.8842 & 25 \\
\hline 5 & 0.9919 & 14 & 39 & 0.9526 & 15 & 73 & 0.8817 & 25 \\
\hline 6 & 0.9906 & 18 & 40 & 0.9510 & 16 & 74 & $0 \cdot 8791$ & 26 \\
\hline 7 & 0.9893 & 13 & 41 & 0.9494 & 16 & 75 & 0.8765 & 26 \\
\hline 8 & $0 \cdot 9881$ & 12 & 42 & 0.9478 & 16 & 76 & 0.8739 & 26 \\
\hline 9 & 0.9869 & 12 & 48 & 0.9461 & 17 & 77 & 0.8712 & 27 \\
\hline 10 & 0.9857 & 12 & 44 & 0.9444 & 17 & 78 & 0.8685 & 27 \\
\hline 11 & 0.9845 & 12 & 45 & $0 \cdot 9427$ & 17 & 79 & 0.8658 & 27 \\
\hline 12 & 0.9834 & 11 & 46 & 0.9409 & 18 & 80 & 0.8631 & 27 \\
\hline 13 & 0.9823 & 11 & 47 & 0.9391 & 18 & 81 & 0.8603 & 28 \\
\hline 14 & 0.8912 & 11 & 48 & 0.9373 & 18 & 82 & 0.8575 & 28 \\
\hline 15 & 0.9802 & 10 & 49 & 0.9354 & 19 & 83 & 0.8547 & 28 \\
\hline 16 & 0.9791 & 11 & 50 & 0.9335 & 19 & 84 & $0 \cdot 8518$ & 29 \\
\hline 17 & 0.9781 & 10 & 51 & 0.9315 & 20 & 85 & 0.8488 & 30 \\
\hline 18 & 0.9771 & 10 & 52 & 0.9295 & 20 & 86 & 0.8458 & 30 \\
\hline 19 & 0.9761 & 10 & 58 & 0.9275 & 20 & 87 & 0.8428 & 30 \\
\hline 20 & 0.9751 & 10 & 54 & 0.9254 & 21 & 88 & 0.8397 & 81 \\
\hline 21 & 0.9741 & 10 & 55 & 0.9234 & 20 & 89 & 0.8365 & 32 \\
\hline 22 & 0.9731 & 10 & 56 & 0.9213 & 21 & 90 & 0.8332 & 33 \\
\hline 23 & 0.9720 & 11 & 57 & 0.9192 & 21 & 91 & 0.8299 & 33 \\
\hline 24 & 0.9710 & 10 & 58 & 0.9170 & 22 & 92 & 0.8265 & 34 \\
\hline 25 & 0.9700 & 10 & 59 & 0.9148 & 22 & 93 & 0.8230 & 35 \\
\hline 26 & 0.9689 & 11 & 60 & 0.9126 & 22 & 94 & 0.8194 & 36 \\
\hline 27 & 0.9679 & 10 & 61 & 0.9104 & 22 & 95 & 0.8157 & 37 \\
\hline 28 & 0.9668 & 11 & 62 & 0.9082 & 22 & 96 & 0.8118 & 39 \\
\hline 29 & 0.9657 & 11 & 63 & 0.9059 & 23 & 97 & 0.8077 & 41 \\
\hline 30 & 0.9646 & 11 & 64 & 0.9036 & 23 & 98 & 0.8034 & 43 \\
\hline 31 & 0.9634 & 12 & 65 & 0.9013 & 23 & 99 & 0.7988 & 46 \\
\hline 32 & 0.9622 & 12 & 66 & 0.8989 & 24 & 100 & 0.7939 & 49 \\
\hline 33 & 0.9609 & 18 & 67 & 0.8965 & 24 & & & \\
\hline
\end{tabular}

In order, however, to employ this Table for ascertaining the strength of mixtures of alcohol and water of different densitios (which is the practical use of such Tables), it is absolutely necessary that the determination of the density should be performed at an invariable temperature, - viz. $60^{\circ} \mathrm{F}$. The methods of determining the density will be hereafter described; but it is obvious that practically the experiment cannot be conveniently made at any fixed temperature, but must be performed at that of the atmosphere.

M. Gay-Lussac has constructed a most valuable Table, of which the following is an abstract, which is supplied with his 'Alcoometre.' (See AxcoHoLomotru.) It enables one to ascertain, from the observed density at any given temperature, the density at the normal temperature $15 \cdot 5^{\circ} \mathrm{C} .\left(60^{\circ} \mathrm{F}\right.$.), and hence the strength; or, vice versa, from the observed density at $60^{\circ} \mathrm{F}$. to find the density at any other tomperature.

The first vertical column of this Table contains the temperatures, from $0^{\circ}$ to $30^{\circ} \mathrm{C}$. ; and the first horizontal line the indications of the alcoometre. In the same Table he has most ingeniously inserted a corroction of the volume of the spirits when the temperature differs from $15.6^{\circ} \mathrm{C} .\left(60^{\circ} \mathrm{F}\right.$.). All the numbers printed in small characters, under each real strength, i.e. per-centage of absolute slcohol, indicate the volume which 1,000 litres (the litre being 1.760773 pints) of a spirituous liquor would have when moasured at the temperature at which its apperent strength is given. 
Alcoömetrical Table of real Strength, by M. Gay-Lussac.

\begin{tabular}{|c|c|c|c|c|c|c|c|c|c|c|}
\hline $\begin{array}{c}\text { Temp. } \\
\text { O. }\end{array}$ & 810 & 820 & 830 & 840 & 850 & $36 \mathrm{c}$ & $37 \mathrm{c}$ & 880 & 890 & 400 \\
\hline $\begin{array}{c}\text { Deg. } \\
10\end{array}$ & $\begin{array}{l}33.0 \\
1002 \\
\end{array}$ & $\begin{array}{c}34 \\
1002 \\
\end{array}$ & $\begin{array}{c}85 \\
1003 \\
\end{array}$ & $\begin{array}{c}36 \\
1003 \\
\end{array}$ & $\begin{array}{c}37 \\
1008 \\
\end{array}$ & $\begin{array}{c}38 \\
1008 \\
\end{array}$ & $\begin{array}{r}39 \\
1009 \\
\end{array}$ & $\begin{array}{c}40 \\
1008 \\
\end{array}$ & $\begin{array}{c}41 \\
1008 \\
\end{array}$ & $\begin{array}{c}42 \\
1003 \\
\end{array}$ \\
\hline 11 & $\begin{array}{l}32 \cdot 6 \\
1002 \\
\end{array}$ & $\begin{array}{l}33 \cdot 6 \\
1002 \\
\end{array}$ & $\begin{array}{l}34 \cdot 6 \\
1002 \\
\end{array}$ & $\begin{array}{l}35 \cdot 6 \\
1002 \\
\end{array}$ & $\begin{array}{l}36 \cdot 6 \\
1002 \\
\end{array}$ & $\begin{array}{l}37 \cdot 6 \\
1002 \\
\end{array}$ & $\begin{array}{l}38.6 \\
1002 \\
\end{array}$ & $\begin{array}{l}39 \cdot 6 \\
1002 \\
\end{array}$ & $\begin{array}{l}40.6 \\
1003 \\
\end{array}$ & $\begin{array}{l}41 \cdot 6 \\
1003\end{array}$ \\
\hline 12 & $\begin{array}{l}32 \cdot 2 \\
1001 \\
\end{array}$ & $\begin{array}{l}33 \cdot 2 \\
1001 \\
\end{array}$ & $\begin{array}{l}34 \cdot 2 \\
1002 \\
\end{array}$ & $\begin{array}{l}35 \cdot 2 \\
1002 \\
\end{array}$ & $\begin{array}{l}36 \cdot 2 \\
1002 \\
\end{array}$ & $\begin{array}{l}37 \cdot 2 \\
1002 \\
\end{array}$ & $\begin{array}{l}38 \cdot 2 \\
1002 \\
\end{array}$ & $\begin{array}{l}39 \cdot 2 \\
1002 \\
\end{array}$ & $\begin{array}{l}40.2 \\
1002 \\
\end{array}$ & $\begin{array}{l}41 \cdot 2 \\
1002\end{array}$ \\
\hline 13 & $\begin{array}{l}31 \cdot 8 \\
1001\end{array}$ & $\begin{array}{l}32 \cdot 8 \\
1001 \\
\end{array}$ & $\begin{array}{l}33 \cdot 8 \\
1001\end{array}$ & $\begin{array}{l}34 \cdot 8 \\
1001 \\
\end{array}$ & $\begin{array}{l}35 \cdot 8 \\
1001 \\
\end{array}$ & $\begin{array}{l}36.8 \\
1001 \\
\end{array}$ & $\begin{array}{l}37 \cdot 8 \\
1001 \\
\end{array}$ & $\begin{array}{l}38.8 \\
1001 \\
\end{array}$ & $\begin{array}{l}39 \cdot 8 \\
1001 \\
\end{array}$ & $\begin{array}{l}40.8 \\
1001 \\
\end{array}$ \\
\hline 14 & $\begin{array}{l}1 \cdot 4 \\
1001 \\
\end{array}$ & $\begin{array}{l}32 \cdot 4 \\
1001 \\
\end{array}$ & $\begin{array}{l}33.4 \\
1001 \\
\end{array}$ & $\begin{array}{l}34 \cdot 4 \\
1001 \\
\end{array}$ & $\begin{array}{l}35^{\circ} 4 \\
1001 \\
\end{array}$ & $\begin{array}{l}36.4 \\
1001 \\
\end{array}$ & $\begin{array}{l}37 \cdot 4 \\
1001 \\
\end{array}$ & $\begin{array}{l}38.4 \\
1001 \\
\end{array}$ & $\begin{array}{l}39 \cdot 4 \\
1001 \\
\end{array}$ & $\begin{array}{l}40.4 \\
1001 \\
\end{array}$ \\
\hline 15 & $\begin{array}{c}31 \\
1000\end{array}$ & $\begin{array}{c}32 \\
1000 \\
\end{array}$ & $\begin{array}{c}33 \\
1000\end{array}$ & $\begin{array}{c}34 \\
1000\end{array}$ & $\begin{array}{c}35 \\
1000 \\
\end{array}$ & $\begin{array}{c}36 \\
1000 \\
\end{array}$ & $\begin{array}{c}37 \\
1000 \\
\end{array}$ & $\begin{array}{c}38 \\
1000 \\
\end{array}$ & $\begin{array}{c}39 \\
1000 \\
\end{array}$ & $\begin{array}{c}40 \\
1000 \\
\end{array}$ \\
\hline 16 & $\begin{array}{l}30 \cdot 6 \\
1000 \\
\end{array}$ & $\begin{array}{l}31 \cdot 6 \\
1000 \\
\end{array}$ & $\begin{array}{r}32.5 \\
999 \\
\end{array}$ & $\begin{array}{c}33 \cdot 5 \\
899 \\
\end{array}$ & $\begin{array}{l}34 \cdot 5 \\
999 \\
\end{array}$ & $\begin{array}{c}35.5 \\
999 \\
\end{array}$ & $\begin{array}{c}36 \cdot 5 \\
999 \\
\end{array}$ & $\begin{array}{c}37 \cdot 5 \\
999 \\
\end{array}$ & $\begin{array}{c}38.5 \\
999\end{array}$ & $\begin{array}{c}39 \cdot 5 \\
999\end{array}$ \\
\hline 17 & $\begin{array}{c}30 \cdot 2 \\
999 \\
\end{array}$ & $\begin{array}{c}31 \cdot 2 \\
999 \\
\end{array}$ & $\begin{array}{c}32 \cdot 1 \\
999 \\
\end{array}$ & $\begin{array}{c}33 \cdot 1 \\
999 \\
\end{array}$ & $\begin{array}{c}34 \cdot 1 \\
999 \\
\end{array}$ & $\begin{array}{c}35 \cdot 1 \\
999 \\
\end{array}$ & $\begin{array}{c}36 \cdot 1 \\
999 \\
\end{array}$ & $\begin{array}{c}37 \cdot 1 \\
899 \\
\end{array}$ & $\begin{array}{c}38 \cdot 1 \\
999 \\
\end{array}$ & $\begin{array}{c}39 \cdot 1 \\
999 \\
\end{array}$ \\
\hline 18 & $\begin{array}{c}29 \cdot 8 \\
999\end{array}$ & $\begin{array}{c}30.8 \\
999\end{array}$ & $\begin{array}{l}31 \cdot 7 \\
998\end{array}$ & $\begin{array}{c}32 \cdot 7 \\
998\end{array}$ & $\begin{array}{c}33: 7 \\
998\end{array}$ & $\begin{array}{c}34: 7 \\
998\end{array}$ & $\begin{array}{c}35 \cdot 7 \\
998\end{array}$ & $\begin{array}{c}36 \cdot 7 \\
998\end{array}$ & $\begin{array}{c}37 \cdot 7 \\
998\end{array}$ & $\begin{array}{c}38 \cdot 7 \\
998\end{array}$ \\
\hline 19 & $\begin{array}{c}29 \cdot 4 \\
998 \\
\end{array}$ & $\begin{array}{c}30^{\circ} 4 \\
998\end{array}$ & $\begin{array}{l}31.3 \\
998 \\
\end{array}$ & $\begin{array}{c}32 \cdot 3 \\
088 \\
\end{array}$ & $\begin{array}{c}33 \cdot 3 \\
998 \\
\end{array}$ & $\begin{array}{c}34 \cdot 3 \\
998 \\
\end{array}$ & $\begin{array}{c}35 \cdot 3 \\
998 \\
\end{array}$ & $\begin{array}{c}36 \cdot 3 \\
998 \\
\end{array}$ & $\begin{array}{c}37 \cdot 3 \\
997 \\
\end{array}$ & $\begin{array}{c}38 \cdot 3 \\
897 \\
\end{array}$ \\
\hline 20 & $\begin{array}{l}29 \\
998 \\
\end{array}$ & $\begin{array}{l}30 \\
998 \\
\end{array}$ & $\begin{array}{c}30 \cdot 9 \\
997 \\
\end{array}$ & $\begin{array}{c}81 \cdot 9 \\
997 \\
\end{array}$ & $\begin{array}{c}32 \cdot 9 \\
997 \\
\end{array}$ & $\begin{array}{c}33 \cdot 9 \\
997 \\
\end{array}$ & $\begin{array}{c}34 \cdot 9 \\
997 \\
\end{array}$ & $\begin{array}{c}35 \cdot 9 \\
997 \\
\end{array}$ & $\begin{array}{c}36 \cdot 9 \\
997 \\
\end{array}$ & $\begin{array}{l}37 \cdot 9 \\
997 \\
\end{array}$ \\
\hline 21 & $\begin{array}{c}28 \cdot 6 \\
997 \\
\end{array}$ & $\begin{array}{c}29.6 \\
997 \\
\end{array}$ & $\begin{array}{c}30.5 \\
997 \\
\end{array}$ & $\begin{array}{l}31 \cdot 5 \\
997 \\
\end{array}$ & $\begin{array}{c}32 \cdot 5 \\
997 \\
\end{array}$ & $\begin{array}{c}33.5 \\
997 \\
\end{array}$ & $\begin{array}{l}34.5 \\
997 \\
\end{array}$ & $\begin{array}{c}35 \cdot 5 \\
996 \\
\end{array}$ & $\begin{array}{c}36 \cdot 5 \\
996 \\
\end{array}$ & $\begin{array}{l}37 \cdot 5 \\
996\end{array}$ \\
\hline 22 & $\begin{array}{l}28 \cdot 2 \\
997\end{array}$ & $\begin{array}{c}29 \cdot 2 \\
997\end{array}$ & $\begin{array}{c}30 \cdot 1 \\
996\end{array}$ & $\begin{array}{c}31 \cdot 1 \\
996\end{array}$ & $\begin{array}{l}32 \cdot 1 \\
996\end{array}$ & $\begin{array}{c}33 \cdot 1 \\
996\end{array}$ & $\begin{array}{c}34 \cdot 1 \\
996 \\
\end{array}$ & $\begin{array}{c}35 \cdot 1 \\
996 \\
\end{array}$ & $\begin{array}{c}36 \cdot 1 \\
996\end{array}$ & $\begin{array}{c}37 \cdot 1 \\
996\end{array}$ \\
\hline 28 & $\begin{array}{c}27 \cdot 8 \\
996 \\
\end{array}$ & $\begin{array}{c}28 \cdot 8 \\
996 \\
\end{array}$ & $\begin{array}{r}29 \cdot 7 \\
996 \\
\end{array}$ & $\begin{array}{c}30 \cdot 7 \\
996 \\
\end{array}$ & $\begin{array}{c}31 \cdot 7 \\
996 \\
\end{array}$ & $\begin{array}{c}32 \cdot 7 \\
996 \\
\end{array}$ & $\begin{array}{c}33 \cdot 7 \\
896 \\
\end{array}$ & $\begin{array}{c}34 \cdot 7 \\
995 \\
\end{array}$ & $\begin{array}{c}35 \cdot 7 \\
995 \\
\end{array}$ & $\begin{array}{c}36 \cdot 7 \\
995 \\
\end{array}$ \\
\hline 24 & $\begin{array}{c}27 \cdot 4 \\
996\end{array}$ & $\begin{array}{c}28 \cdot 4 \\
996 \\
\end{array}$ & $\begin{array}{c}29 \cdot 3 \\
995 \\
\end{array}$ & $\begin{array}{l}30.3 \\
995 \\
\end{array}$ & $\begin{array}{l}31 \cdot 3 \\
995\end{array}$ & $\begin{array}{c}32 \cdot 3 \\
995 \\
\end{array}$ & \begin{tabular}{|l}
$33 \cdot 3$ \\
995 \\
\end{tabular} & $\begin{array}{l}34 \cdot 3 \\
995 \\
\end{array}$ & $\begin{array}{c}35 \cdot 3 \\
995 \\
\end{array}$ & $\begin{array}{l}36 \cdot 3 \\
994\end{array}$ \\
\hline 25 & $\begin{array}{r}27 \\
995\end{array}$ & $\begin{array}{r}28 \\
995\end{array}$ & $\begin{array}{c}28 \cdot 9 \\
995\end{array}$ & $\begin{array}{c}29 \cdot 9 \\
995\end{array}$ & $\begin{array}{c}30.9 \\
995\end{array}$ & $\begin{array}{c}31 \cdot 9 \\
994\end{array}$ & $\begin{array}{c}32 \cdot 9 \\
994\end{array}$ & $\begin{array}{c}33 \cdot 9 \\
994\end{array}$ & $\begin{array}{c}34 \cdot 9 \\
994\end{array}$ & $\begin{array}{l}85 \cdot 9 \\
994\end{array}$ \\
\hline $\begin{array}{c}\text { Temp. } \\
\text { C. }\end{array}$ & 410 & 420 & $48 \mathrm{c}$ & $\$ 4 c$ & 450 & $\$ 60$ & 470 & 480 & $49 c$ & 800 \\
\hline $\begin{array}{c}\text { Deg. } \\
10\end{array}$ & $\begin{array}{c}43 \\
1003\end{array}$ & $\begin{array}{c}44 \\
1004\end{array}$ & $\begin{array}{c}45 \\
1004\end{array}$ & $\begin{array}{c}46 \\
1004\end{array}$ & $\begin{array}{l}46 \cdot 9 \\
1004\end{array}$ & $\begin{array}{l}47 \cdot 9 \\
1004\end{array}$ & $\begin{array}{l}48.9 \\
1004\end{array}$ & $\begin{array}{l}49 \cdot 9 \\
1004\end{array}$ & $\begin{array}{l}50 \cdot 9 \\
1004\end{array}$ & $\begin{array}{l}51 \cdot 8 \\
1004\end{array}$ \\
\hline 11 & $\begin{array}{l}42 \cdot 6 \\
1003 \\
\end{array}$ & $\begin{array}{l}43 \cdot 6 \\
1003 \\
\end{array}$ & $\begin{array}{l}44^{\prime} 6 \\
1003 \\
\end{array}$ & $\begin{array}{l}45 \cdot 6 \\
1003 \\
\end{array}$ & $\begin{array}{l}46 \cdot 6 \\
1003 \\
\end{array}$ & $\begin{array}{l}47 \cdot 6 \\
1003 \\
\end{array}$ & $\begin{array}{l}48 \cdot 6 \\
1003 \\
\end{array}$ & $\begin{array}{l}49.5 \\
1008 \\
\end{array}$ & $\begin{array}{l}50 \cdot 5 \\
1008 \\
\end{array}$ & $\begin{array}{l}51 \cdot 5 \\
1003 \\
\end{array}$ \\
\hline 12 & $\begin{array}{l}42 \cdot 2 \\
1002 \\
\end{array}$ & $\begin{array}{l}43.2 \\
1002 \\
\end{array}$ & $\begin{array}{l}4 \cdot 2 \\
1002 \\
\end{array}$ & $\begin{array}{l}45 \cdot 2 \\
1002 \\
\end{array}$ & $\begin{array}{l}46 \cdot 2 \\
1002 \\
\end{array}$ & $\begin{array}{l}47 \cdot 2 \\
1002 \\
\end{array}$ & $\begin{array}{l}48 \cdot 2 \\
1002 \\
\end{array}$ & $\begin{array}{l}49 \cdot 2 \\
1002 \\
\end{array}$ & $\begin{array}{l}50 \cdot 2 \\
1002 \\
\end{array}$ & $\begin{array}{l}51 \cdot 1 \\
1002 \\
\end{array}$ \\
\hline 18 & $\begin{array}{l}41 \cdot 8 \\
1001 \\
\end{array}$ & $\begin{array}{l}42 \cdot 8 \\
1001 \\
\end{array}$ & $\begin{array}{l}43 \cdot 8 \\
1001 \\
\end{array}$ & $\begin{array}{l}44.8 \\
1002 \\
\end{array}$ & $\begin{array}{l}45 \cdot 8 \\
1002 \\
\end{array}$ & $\begin{array}{l}46 \cdot 8 \\
1002 \\
\end{array}$ & $\begin{array}{l}47 \cdot 8 \\
1002 \\
\end{array}$ & $\begin{array}{l}48 \cdot 8 \\
1002 \\
\end{array}$ & $\begin{array}{l}49 \cdot 8 \\
1002 \\
\end{array}$ & $\begin{array}{l}50.8 \\
1002 \\
\end{array}$ \\
\hline 14 & $\begin{array}{l}41 \cdot 4 \\
1001 \\
\end{array}$ & $\begin{array}{l}42 \cdot 4 \\
1001 \\
\end{array}$ & $\begin{array}{l}43 \cdot 4 \\
1001 \\
\end{array}$ & $\begin{array}{l}44 \cdot 4 \\
1001 \\
\end{array}$ & $\begin{array}{l}45 \cdot 4 \\
1001 \\
\end{array}$ & $\begin{array}{l}46.4 \\
1001 \\
\end{array}$ & $\begin{array}{l}7 \cdot 4 \\
1001 \\
\end{array}$ & $\begin{array}{l}48 \cdot 4 \\
1001 \\
\end{array}$ & $\begin{array}{l}49 \cdot 4 \\
1001 \\
\end{array}$ & $\begin{array}{l}50.4 \\
1000 \\
\end{array}$ \\
\hline 15 & $\begin{array}{r}41 \\
1000 \\
\end{array}$ & $\begin{array}{r}42 \\
1000 \\
\end{array}$ & $\begin{array}{r}43 \\
1000 \\
\end{array}$ & $\begin{array}{r}44 \\
1000 \\
\end{array}$ & $\begin{array}{r}45 \\
1000 \\
\end{array}$ & $\begin{array}{r}46 \\
1000 \\
\end{array}$ & $\begin{array}{r}47 \\
1000 \\
\end{array}$ & $\begin{array}{r}48 \\
1000 \\
\end{array}$ & $\begin{array}{c}49 \\
1000 \\
\end{array}$ & $\begin{array}{r}50 \\
1000 \\
\end{array}$ \\
\hline 16 & $\begin{array}{c}40^{\circ} 6 \\
999 \\
\end{array}$ & $\begin{array}{c}41 \cdot 6 \\
999 \\
\end{array}$ & $\begin{array}{c}42^{\circ} 6 \\
999 \\
\end{array}$ & $\begin{array}{c}48 \cdot 6 \\
999 \\
\end{array}$ & $\begin{array}{c}44 \cdot 6 \\
999 \\
\end{array}$ & $\begin{array}{c}45 \cdot 6 \\
999 \\
\end{array}$ & $\begin{array}{c}46 \cdot 6 \\
999 \\
\end{array}$ & $\begin{array}{c}47^{\circ} \cdot 6 \\
999 \\
\end{array}$ & $\begin{array}{c}48.6 \\
999 \\
\end{array}$ & $\begin{array}{r}49 \cdot 6 \\
999 \\
\end{array}$ \\
\hline 17 & $\begin{array}{c}40.2 \\
999 \\
\end{array}$ & $\begin{array}{c}41 \cdot 2 \\
999 \\
\end{array}$ & $\begin{array}{c}42 \cdot 2 \\
999 \\
\end{array}$ & $\begin{array}{c}43 \cdot 2 \\
998 \\
\end{array}$ & $\begin{array}{c}44 \cdot 2 \\
298 \\
\end{array}$ & $\begin{array}{c}45 \cdot 2 \\
998 \\
\end{array}$ & $\begin{array}{c}46 \cdot 2 \\
998 \\
\end{array}$ & $\begin{array}{c}47 \cdot 2 \\
998 \\
\end{array}$ & $\begin{array}{c}48 \cdot 2 \\
998 \\
\end{array}$ & $\begin{array}{c}49 \cdot 2 \\
998 \\
\end{array}$ \\
\hline 18 & $\begin{array}{c}39 \cdot 8 \\
998 \\
\end{array}$ & $\begin{array}{c}40.8 \\
998 \\
\end{array}$ & $\begin{array}{c}41.8 \\
998 \\
\end{array}$ & $\begin{array}{c}42 \cdot 8 \\
998 \\
\end{array}$ & $\begin{array}{c}43.8 \\
998 \\
\end{array}$ & $\begin{array}{c}44 \cdot 9 \\
998 \\
\end{array}$ & $\begin{array}{c}45 \cdot 9 \\
998 \\
\end{array}$ & $\begin{array}{c}460^{\circ} \\
998 \\
\end{array}$ & $\begin{array}{r}47 \cdot 9 \\
998 \\
\end{array}$ & $\begin{array}{c}48 \cdot 9 \\
998 \\
\end{array}$ \\
\hline 19 & $\begin{array}{c}39 \cdot 4 \\
997 \\
\end{array}$ & $\begin{array}{c}40.4 \\
997\end{array}$ & $\begin{array}{l}41 \cdot 4 \\
997\end{array}$ & $\begin{array}{l}42.5 \\
997 \\
\end{array}$ & $\begin{array}{l}43.5 \\
997 \\
\end{array}$ & $\begin{array}{c}44 \cdot 5 \\
997 \\
\end{array}$ & $\begin{array}{l}45 \cdot 5 \\
997 \\
\end{array}$ & $\begin{array}{c}46.5 \\
997 \\
\end{array}$ & $\begin{array}{c}47 \cdot 5 \\
997 \\
\end{array}$ & $\begin{array}{c}48 \cdot 5 \\
997 \\
\end{array}$ \\
\hline 20 & $\begin{array}{l}39 \\
997 \\
\end{array}$ & $\begin{array}{l}40 \\
997 \\
\end{array}$ & $\begin{array}{l}41 \\
997 \\
\end{array}$ & $\begin{array}{l}42 \cdot 1 \\
997\end{array}$ & $\begin{array}{c}43 \cdot 1 \\
996 \\
\end{array}$ & $\begin{array}{c}44 \cdot 1 \\
996 \\
\end{array}$ & $\begin{array}{c}45 \cdot 1 \\
996 \\
\end{array}$ & $\begin{array}{c}46 \cdot 1 \\
896 \\
\end{array}$ & $\begin{array}{c}47^{\circ} \cdot 2 \\
996 \\
\end{array}$ & $\begin{array}{c}48 \cdot 2 \\
996 \\
\end{array}$ \\
\hline 21 & $\begin{array}{c}38 \cdot 6 \\
996\end{array}$ & $\begin{array}{l}39 \cdot 6 \\
996\end{array}$ & $\begin{array}{l}40^{\circ} 6 \\
996\end{array}$ & $\begin{array}{l}41 \cdot 7 \\
906\end{array}$ & $\begin{array}{l}42 \cdot 7 \\
996\end{array}$ & $\begin{array}{c}43 \cdot 7 \\
996\end{array}$ & $\begin{array}{l}44 \cdot 8 \\
928\end{array}$ & $\begin{array}{c}45 \cdot 8 \\
996\end{array}$ & $\begin{array}{l}46 \cdot 8 \\
295\end{array}$ & $\begin{array}{l}47 \cdot 8 \\
995\end{array}$ \\
\hline
\end{tabular}


Alcoometrical Table of real Strength, by M. Gay-Inssao (continuod).

\begin{tabular}{|c|c|c|c|c|c|c|c|c|c|c|}
\hline $\begin{array}{l}\text { Temp. } \\
\text { C. }\end{array}$ & 410 & 420 & 480 & $44 c$ & 450 & 460 & 470 & 480 & 490 & 800 \\
\hline $\begin{array}{c}\text { Deg. } \\
22\end{array}$ & $\begin{array}{c}38 \cdot 2 \\
996 \\
\end{array}$ & $\begin{array}{c}89 \cdot 2 \\
998\end{array}$ & $\begin{array}{c}40.2 \\
385\end{array}$ & $\begin{array}{c}41.3 \\
895 \\
\end{array}$ & $\begin{array}{c}42 \cdot 3 \\
908 \\
\end{array}$ & $\begin{array}{c}43 \cdot 9 \\
995 \\
\end{array}$ & $\begin{array}{c}44 \cdot 3 \\
995 \\
\end{array}$ & $\begin{array}{c}45{ }^{\circ} 3 \\
995 \\
\end{array}$ & $\begin{array}{c}46.4 \\
095 \\
\end{array}$ & $\begin{array}{c}47 \cdot 4 \\
995\end{array}$ \\
\hline 28 & $\begin{array}{l}37 \cdot 8 \\
995\end{array}$ & $\begin{array}{c}38 \cdot 8 \\
993\end{array}$ & $\begin{array}{c}39.8 \\
995\end{array}$ & $\begin{array}{l}40 \cdot 9 \\
984\end{array}$ & $\begin{array}{l}41 \cdot 9 \\
994\end{array}$ & $\begin{array}{l}42 \cdot 9 \\
894\end{array}$ & $\begin{array}{l}43.9 \\
994\end{array}$ & $\begin{array}{l}44.9 \\
994\end{array}$ & $\begin{array}{l}46 \\
894\end{array}$ & $\begin{array}{l}47 \\
904\end{array}$ \\
\hline 24 & $\begin{array}{l}87 \cdot 4 \\
994\end{array}$ & $\begin{array}{c}38 \cdot 4 \\
994\end{array}$ & $\begin{array}{c}39 \cdot 4 \\
394 \\
\end{array}$ & $\begin{array}{c}40.5 \\
994 \\
\end{array}$ & $\begin{array}{l}41.5 \\
994 \\
\end{array}$ & $\begin{array}{c}42 \cdot 5 \\
994 \\
\end{array}$ & $\begin{array}{c}43 \cdot 6 \\
994 \\
\end{array}$ & $\begin{array}{c}44.6 \\
994 \\
\end{array}$ & $\begin{array}{r}45 \cdot 6 \\
998 \\
\end{array}$ & $\begin{array}{r}46.6 \\
993 \\
\end{array}$ \\
\hline 25 & $\begin{array}{l}37 \\
994\end{array}$ & $\begin{array}{l}38 \\
994\end{array}$ & $\begin{array}{l}89 \\
993\end{array}$ & $\begin{array}{c}40 \cdot 1 \\
998\end{array}$ & $\begin{array}{c}42 \cdot 1 \\
998\end{array}$ & $\begin{array}{c}42 \cdot 2 \\
993\end{array}$ & $\begin{array}{c}43 \cdot 2 \\
993\end{array}$ & $\begin{array}{c}44 \cdot 2 \\
993\end{array}$ & $\begin{array}{c}45 \cdot 2 \\
998\end{array}$ & $\begin{array}{c}46 \cdot 3 \\
993\end{array}$ \\
\hline $\begin{array}{l}\text { Temp. } \\
\text { C. }\end{array}$ & 510 & 520 & 830 & 540 & 650 & 560 & 570 & 580 & $69 \mathrm{c}$ & $60 c$ \\
\hline $\begin{array}{c}\text { Deg. } \\
10\end{array}$ & $\begin{array}{l}52 \cdot 9 \\
1004 \\
\end{array}$ & $\begin{array}{l}53 \cdot 8 \\
1004 \\
\end{array}$ & $\begin{array}{l}54.8 \\
1004 \\
\end{array}$ & $\begin{array}{l}55 \cdot 8 \\
1004 \\
\end{array}$ & $\begin{array}{l}56 \cdot 8 \\
1004 \\
\end{array}$ & $\begin{array}{l}57 \cdot 8 \\
1004 \\
\end{array}$ & $\begin{array}{l}58 \cdot 8 \\
1004 \\
\end{array}$ & $\begin{array}{l}59 \cdot 7 \\
1004 \\
\end{array}$ & $\begin{array}{l}60.7 \\
1004 \\
\end{array}$ & $\begin{array}{l}61 \cdot 7 \\
1004 \\
\end{array}$ \\
\hline 11 & $\begin{array}{l}52 \cdot 5 \\
1008 \\
\end{array}$ & $\begin{array}{l}53.5 \\
1008 \\
\end{array}$ & $\begin{array}{l}54.4 \\
1003 \\
\end{array}$ & $\begin{array}{l}55.4 \\
1008 \\
\end{array}$ & $\begin{array}{l}56.4 \\
1003 \\
\end{array}$ & $\begin{array}{l}57 \cdot 4 \\
1003 \\
\end{array}$ & $\begin{array}{l}58 \cdot 4 \\
1003 \\
\end{array}$ & $\begin{array}{l}59 \cdot 4 \\
1003 \\
\end{array}$ & $\begin{array}{l}60.4 \\
1008 \\
\end{array}$ & $\begin{array}{l}61 \cdot 4 \\
1003 \\
\end{array}$ \\
\hline 12 & $\begin{array}{l}52 \cdot 1 \\
1002 \\
\end{array}$ & $\begin{array}{l}53 \cdot 1 \\
1002 \\
\end{array}$ & $\begin{array}{l}54 \cdot 1 \\
1002 \\
\end{array}$ & $\begin{array}{c}55 \\
1002 \\
\end{array}$ & $\begin{array}{c}56 \\
1002 \\
\end{array}$ & $\begin{array}{c}57 \\
1002 \\
\end{array}$ & $\begin{array}{c}68 \\
1002 \\
\end{array}$ & $\begin{array}{c}59 \\
1002 \\
\end{array}$ & $\begin{array}{c}60 \\
1002 \\
\end{array}$ & $\begin{array}{c}61 \\
1002 \\
\end{array}$ \\
\hline 13 & $\begin{array}{l}51 \cdot 8 \\
1002 \\
\end{array}$ & $\begin{array}{l}52 \cdot 7 \\
1002 \\
\end{array}$ & $\begin{array}{l}58 \cdot 7 \\
1002 \\
\end{array}$ & $\begin{array}{l}54 \cdot 7 \\
1002 \\
\end{array}$ & $\begin{array}{l}55 \cdot 7 \\
1002 \\
\end{array}$ & $\begin{array}{l}56 \cdot 7 \\
1002 \\
\end{array}$ & $\begin{array}{l}57 \cdot 7 \\
1002 \\
\end{array}$ & $\begin{array}{l}58 \cdot 7 \\
1002 \\
\end{array}$ & $\begin{array}{l}59 \cdot 7 \\
1002 \\
\end{array}$ & $\begin{array}{l}60 \cdot 7 \\
1002 \\
\end{array}$ \\
\hline 14 & $\begin{array}{l}51 \cdot 4 \\
1001 \\
\end{array}$ & $\begin{array}{l}52 \cdot 3 \\
1001 \\
\end{array}$ & $\begin{array}{l}53 \cdot 3 \\
1001 \\
\end{array}$ & $\begin{array}{l}54 \cdot 3 \\
1001 \\
\end{array}$ & $\begin{array}{l}55 \cdot 3 \\
1001 \\
\end{array}$ & $\begin{array}{l}56 \cdot 3 \\
1001 \\
\end{array}$ & $\begin{array}{l}57 \cdot 3 \\
1001 \\
\end{array}$ & $\begin{array}{l}58 \cdot 3 \\
1001 \\
\end{array}$ & $\begin{array}{l}59 \cdot 3 \\
1001 \\
\end{array}$ & $\begin{array}{l}60.3 \\
1001 \\
\end{array}$ \\
\hline 15 & $\begin{array}{c}51 \\
1000 \\
\end{array}$ & $\begin{array}{r}52 \\
1000 \\
\end{array}$ & $\begin{array}{c}53 \\
1000 \\
\end{array}$ & $\begin{array}{c}54 \\
1000 \\
\end{array}$ & $\begin{array}{c}55 \\
1000 \\
\end{array}$ & $\begin{array}{c}56 \\
1000 \\
\end{array}$ & $\begin{array}{c}57 \\
1000 \\
\end{array}$ & $\begin{array}{c}58 \\
1000 \\
\end{array}$ & $\begin{array}{c}59 \\
1000 \\
\end{array}$ & $\begin{array}{c}60 \\
1000 \\
\end{array}$ \\
\hline 16 & $\begin{array}{c}50 \cdot 6 \\
999 \\
\end{array}$ & $\begin{array}{c}51 \cdot 6 \\
999 \\
\end{array}$ & $\begin{array}{c}52 \cdot 6 \\
999 \\
\end{array}$ & $\begin{array}{c}53 \cdot 6 \\
999 \\
\end{array}$ & $\begin{array}{c}54 \cdot 6 \\
999 \\
\end{array}$ & $\begin{array}{c}55 \cdot 6 \\
999 \\
\end{array}$ & $\begin{array}{l}56 \cdot 6 \\
999 \\
\end{array}$ & $\begin{array}{c}57^{\circ} 6 \\
899 \\
\end{array}$ & $\begin{array}{c}58.6 \\
899 \\
\end{array}$ & $\begin{array}{c}59.6 \\
999 \\
\end{array}$ \\
\hline 17 & $\begin{array}{c}50 \cdot 3 \\
998 \\
\end{array}$ & $\begin{array}{c}51 \cdot 3 \\
998 \\
\end{array}$ & $\begin{array}{c}52 \cdot 3 \\
998 \\
\end{array}$ & $\begin{array}{c}53 \cdot 3 \\
998 \\
\end{array}$ & $\begin{array}{c}54 \cdot 3 \\
988 \\
\end{array}$ & $\begin{array}{c}55 \cdot 3 \\
998 \\
\end{array}$ & $\begin{array}{l}56 \cdot 3 \\
998 \\
\end{array}$ & $\begin{array}{c}57 \cdot 3 \\
998 \\
\end{array}$ & $\begin{array}{c}58 \cdot 3 \\
998 \\
\end{array}$ & $\begin{array}{c}59 \cdot 3 \\
998 \\
\end{array}$ \\
\hline 18 & $\begin{array}{c}49 \cdot 9 \\
998 \\
\end{array}$ & $\begin{array}{c}50 \cdot 9 \\
998 \\
\end{array}$ & $\begin{array}{c}51 \cdot 9 \\
998 \\
\end{array}$ & $\begin{array}{c}52 \cdot 9 \\
998 \\
\end{array}$ & $\begin{array}{c}53 \cdot 9 \\
998 \\
\end{array}$ & $\begin{array}{c}54 \cdot 9 \\
998 \\
\end{array}$ & $\begin{array}{c}55 \cdot 9 \\
998 \\
\end{array}$ & $\begin{array}{c}56 \cdot 9 \\
997 \\
\end{array}$ & $\begin{array}{c}57 \cdot 9 \\
997 \\
\end{array}$ & $\begin{array}{c}58 \cdot 9 \\
997 \\
\end{array}$ \\
\hline 19 & $\begin{array}{c}49 \cdot 5 \\
997 \\
\end{array}$ & $\begin{array}{l}50 \cdot 6 \\
997 \\
\end{array}$ & $\begin{array}{c}51 \cdot 6 \\
897 \\
\end{array}$ & $\begin{array}{l}52 \cdot 6 \\
997 \\
\end{array}$ & $\begin{array}{c}53 \cdot 6 \\
897 \\
\end{array}$ & $\begin{array}{c}54 \cdot 6 \\
-997 \\
\end{array}$ & $\begin{array}{c}55 \cdot 6 \\
998 \\
\end{array}$ & $\begin{array}{c}56.6 \\
897 \\
\end{array}$ & $\begin{array}{c}57 \cdot 6 \\
997 \\
\end{array}$ & $\begin{array}{c}58 \cdot 6 \\
997 \\
\end{array}$ \\
\hline 20 & $\begin{array}{c}49 \cdot 2 \\
996 \\
\end{array}$ & $\begin{array}{l}50 \cdot 2 \\
996 \\
\end{array}$ & $\begin{array}{c}51 \cdot 2 \\
996 \\
\end{array}$ & $\begin{array}{c}52 \cdot 2 \\
996 \\
\end{array}$ & $\begin{array}{c}53 \cdot 2 \\
996 \\
\end{array}$ & $\begin{array}{l}54 \cdot 2 \\
996\end{array}$ & $\begin{array}{c}55 \cdot 2 \\
996 \\
\end{array}$ & $\begin{array}{c}56 \cdot 2 \\
996 \\
\end{array}$ & $\begin{array}{c}57 \cdot 2 \\
996 \\
\end{array}$ & $\begin{array}{c}58 \cdot 2 \\
996\end{array}$ \\
\hline 21 & $\begin{array}{c}48 \cdot 8 \\
995 \\
\end{array}$ & $\begin{array}{c}49 \cdot 8 \\
995 \\
\end{array}$ & $\begin{array}{c}50 \cdot 8 \\
995 \\
\end{array}$ & $\begin{array}{c}51.8 \\
995 \\
\end{array}$ & $\begin{array}{c}52 \cdot 9 \\
995 \\
\end{array}$ & $\begin{array}{c}53 \cdot 9 \\
995 \\
\end{array}$ & $\begin{array}{c}54 \cdot 9 \\
995 \\
\end{array}$ & $\begin{array}{c}55 \cdot 9 \\
995 \\
\end{array}$ & $\begin{array}{c}56.9 \\
995 \\
\end{array}$ & $\begin{array}{c}57 \cdot 9 \\
993 \\
\end{array}$ \\
\hline 22 & $\begin{array}{l}48^{\circ} 4 \\
995 \\
\end{array}$ & $\begin{array}{c}49 \cdot 4 \\
995 \\
\end{array}$ & $\begin{array}{l}50 \cdot 4 \\
995 \\
\end{array}$ & $\begin{array}{c}51 \cdot 4 \\
994 \\
\end{array}$ & $\begin{array}{c}52 \cdot 5 \\
994 \\
\end{array}$ & $\begin{array}{c}53 \cdot 5 \\
994 \\
\end{array}$ & $\begin{array}{l}54 \cdot 5 \\
994 \\
\end{array}$ & $\begin{array}{c}55.5 \\
994 \\
\end{array}$ & $\begin{array}{c}56 \cdot 5 \\
894 \\
\end{array}$ & $\begin{array}{c}57 \cdot 5 \\
995 \\
\end{array}$ \\
\hline 23 & $\begin{array}{r}48 \\
994 \\
\end{array}$ & $\begin{array}{r}49 \cdot 1 \\
994 \\
\end{array}$ & $\begin{array}{r}50.1 \\
-994 \\
\end{array}$ & $\begin{array}{c}51 \cdot 1 \\
994 \\
\end{array}$ & $\begin{array}{c}52 \cdot 1 \\
994 \\
\end{array}$ & $\begin{array}{c}53 \cdot 1 \\
994 \\
\end{array}$ & $\begin{array}{c}54 \cdot 1 \\
994 \\
\end{array}$ & $\begin{array}{c}55 \cdot 1 \\
998 \\
\end{array}$ & $\begin{array}{c}56 \cdot 1 \\
998 \\
\end{array}$ & $\begin{array}{c}57 \cdot 1 \\
993 \\
\end{array}$ \\
\hline 24 & $\begin{array}{c}47 \cdot 6 \\
998 \\
\end{array}$ & $\begin{array}{r}48 \cdot 7 \\
998 \\
\end{array}$ & $\begin{array}{r}49 \cdot 7 \\
998 \\
\end{array}$ & $\begin{array}{r}50.7 \\
998 \\
\end{array}$ & $\begin{array}{r}51 \cdot 8 \\
998 \\
\end{array}$ & $\begin{array}{c}52.8 \\
998 \\
\end{array}$ & $\begin{array}{c}53 \cdot 8 \\
993 \\
\end{array}$ & $\begin{array}{c}54.8 \\
998 \\
\end{array}$ & $\begin{array}{c}55^{\circ} 8 \\
993 \\
\end{array}$ & $\begin{array}{c}56.8 \\
992 \\
\end{array}$ \\
\hline 25 & $\begin{array}{c}47 \cdot 3 \\
998\end{array}$ & $\begin{array}{c}48 \cdot 3 \\
998\end{array}$ & $\begin{array}{c}49 \cdot 3 \\
993\end{array}$ & $\begin{array}{l}50 * 3 \\
992\end{array}$ & $\begin{array}{l}51 \cdot 4 \\
992\end{array}$ & $\begin{array}{l}52 \cdot 4 \\
898\end{array}$ & $\begin{array}{l}53 \cdot 4 \\
992\end{array}$ & $\begin{array}{c}54 \cdot 4 \\
992\end{array}$ & $\begin{array}{c}55 \cdot 5 \\
992\end{array}$ & $\begin{array}{r}56 \cdot 6 \\
992\end{array}$ \\
\hline $\begin{array}{c}\text { Temp. } \\
\text { C. }\end{array}$ & 61c & $62 c$ & $63 \mathrm{c}$ & 640 & $65 c$ & 660 & $67 \mathrm{c}$ & 680 & $69 c$ & 700 \\
\hline $\begin{array}{c}\text { Deg. } \\
10\end{array}$ & $\begin{array}{l}62 \cdot 7 \\
1004 \\
\end{array}$ & $\begin{array}{l}63 \cdot 7 \\
1004 \\
\end{array}$ & $\begin{array}{l}64 \cdot 7 \\
1004 \\
\end{array}$ & $\begin{array}{l}65 \cdot 7 \\
1004 \\
\end{array}$ & $\begin{array}{l}66 \cdot 7 \\
1004 \\
\end{array}$ & $\begin{array}{l}67^{\circ} 6 \\
1004 \\
\end{array}$ & $\begin{array}{l}68^{\circ} 6 \\
1004 \\
\end{array}$ & $\begin{array}{l}69.6 \\
1004 \\
\end{array}$ & $\begin{array}{l}70.6 \\
1004 \\
\end{array}$ & $\begin{array}{l}71 \cdot 6 \\
1004 \\
\end{array}$ \\
\hline 11 & $\begin{array}{l}62 \cdot 4 \\
1008 \\
\end{array}$ & $\begin{array}{l}63 \cdot 4 \\
1003 \\
\end{array}$ & $\begin{array}{l}64.4 \\
1008 \\
\end{array}$ & $\begin{array}{l}65 \cdot 4 \\
1008 \\
\end{array}$ & $\begin{array}{l}66 \cdot 4 \\
1008 \\
\end{array}$ & $\begin{array}{l}67 \cdot 3 \\
1003 \\
\end{array}$ & $\begin{array}{l}68 \cdot 3 \\
1008 \\
\end{array}$ & $\begin{array}{l}69 \cdot 3 \\
1004 \\
\end{array}$ & $\begin{array}{l}70.3 \\
1004 \\
\end{array}$ & $\begin{array}{l}71 \cdot 3 \\
1004 \\
\end{array}$ \\
\hline 12 & $\begin{array}{r}62 \\
1002 \\
\end{array}$ & $\begin{array}{r}63 \\
1002 \\
\end{array}$ & $\begin{array}{c}64 \\
1002 \\
\end{array}$ & $\begin{array}{c}65 \\
1002 \\
\end{array}$ & $\begin{array}{c}66 \\
1002 \\
\end{array}$ & $\begin{array}{c}67 \\
1002 \\
\end{array}$ & $\begin{array}{r}68 \\
-1008 \\
\end{array}$ & $\begin{array}{r}69 \\
1008 \\
\end{array}$ & $\begin{array}{c}70 \\
1008 \\
\end{array}$ & $\begin{array}{c}71 \\
1008 \\
\end{array}$ \\
\hline 13 & $\begin{array}{l}61 \cdot 7 \\
1002 \\
\end{array}$ & $\begin{array}{l}62 \cdot 7 \\
1002 \\
\end{array}$ & $\begin{array}{l}63 \cdot 7 \\
1002 \\
\end{array}$ & $\begin{array}{l}64 \cdot 7 \\
1002 \\
\end{array}$ & $\begin{array}{l}65 \cdot 7 \\
1002 \\
\end{array}$ & $\begin{array}{l}66 \cdot 7 \\
1002 \\
\end{array}$ & $\begin{array}{l}67 \cdot 7 \\
1002 \\
\end{array}$ & $\begin{array}{l}68.7 \\
1002 \\
\end{array}$ & $\begin{array}{l}69 \cdot 6 \\
1002 \\
\end{array}$ & $\begin{array}{l}70.6 \\
1002 \\
\end{array}$ \\
\hline 14 & $\begin{array}{l}61 \cdot 3 \\
1001 \\
\end{array}$ & $\begin{array}{l}62 \cdot 3 \\
1001 \\
\end{array}$ & $\begin{array}{l}63 \cdot 3 \\
1001 \\
\end{array}$ & $\begin{array}{l}64 \cdot 3 \\
1001 \\
\end{array}$ & $\begin{array}{l}65 \cdot 3 \\
1001 \\
\end{array}$ & $\begin{array}{l}66.3 \\
1001 \\
\end{array}$ & $\begin{array}{l}67 \cdot 3 \\
1001 \\
\end{array}$ & $\begin{array}{l}68 \cdot 3 \\
1001 \\
\end{array}$ & $\begin{array}{l}69.3 \\
1001 \\
\end{array}$ & $\begin{array}{l}70 \cdot 3 \\
1001 \\
\end{array}$ \\
\hline 15 & $\begin{array}{c}61 \\
1000\end{array}$ & $\begin{array}{r}62 \\
1000\end{array}$ & $\begin{array}{c}63 \\
2099\end{array}$ & $\begin{array}{c}64 \\
1000\end{array}$ & $\begin{array}{c}65 \\
1000\end{array}$ & $\begin{array}{c}66 \\
1000\end{array}$ & $\begin{array}{c}67 \\
1000\end{array}$ & $\begin{array}{c}68 \\
1000\end{array}$ & $\begin{array}{c}60 \\
1000\end{array}$ & $\begin{array}{r}70 \\
1000\end{array}$ \\
\hline
\end{tabular}


Alcöometrical Table of real Strength, by M. Gay-Lussac (continued).

\begin{tabular}{|c|c|c|c|c|c|c|c|c|c|c|}
\hline $\begin{array}{l}\text { Temp. } \\
\text { C. }\end{array}$ & .610 & $62 \mathrm{c}$ & $68 \mathrm{c}$ & 640 & 650 & 660 & $67 \mathrm{c}$ & $68 \mathrm{c}$ & $69 \mathrm{c}$ & $70 \mathrm{c}$ \\
\hline $\begin{array}{c}\text { Deg. } \\
16\end{array}$ & $\begin{array}{c}60.6 \\
999 \\
\end{array}$ & $\begin{array}{c}61 \cdot 7 \\
999 \\
\end{array}$ & $\begin{array}{r}62.7 \\
999 \\
\end{array}$ & $\begin{array}{r}63 \cdot 7 \\
999 \\
\end{array}$ & $\begin{array}{r}64.7 \\
999 \\
\end{array}$ & $\begin{array}{c}65 \cdot 7 \\
999 \\
\end{array}$ & $\begin{array}{c}66^{\circ} 7 \\
999 \\
\end{array}$ & $\begin{array}{c}67 \cdot 7 \\
999 \\
\end{array}$ & $\begin{array}{r}68 \cdot 7 \\
-990 \\
\end{array}$ & $\begin{array}{c}69 \cdot 7 \\
999\end{array}$ \\
\hline 17 & $\begin{array}{c}60 \cdot 3 \\
998 \\
\end{array}$ & $\begin{array}{c}61 \cdot 3 \\
998 \\
\end{array}$ & $\begin{array}{c}62.3 \\
388 \\
\end{array}$ & $\begin{array}{c}63 \cdot 3 \\
998 \\
\end{array}$ & $\begin{array}{c}64: 3 \\
998 \\
\end{array}$ & $\begin{array}{c}65^{\circ} 3 \\
998 \\
\end{array}$ & $\begin{array}{c}66^{\circ} 3 \\
998 \\
\end{array}$ & $\begin{array}{c}67 \cdot 3 \\
998 \\
\end{array}$ & $\begin{array}{c}68 \cdot 3 \\
998 \\
\end{array}$ & $\begin{array}{c}69.3 \\
998 \\
\end{array}$ \\
\hline 18 & $\begin{array}{c}59.9 \\
997 \\
\end{array}$ & $\begin{array}{r}61 \\
997 \\
\end{array}$ & $\begin{array}{r}62 \\
997 \\
\end{array}$ & $\begin{array}{r}63 \\
997 \\
\end{array}$ & $\begin{array}{r}64 \\
997 \\
\end{array}$ & $\begin{array}{r}65 \\
997 \\
\end{array}$ & $\begin{array}{r}66 \\
997 \\
\end{array}$ & $\begin{array}{r}67 \\
997 \\
\end{array}$ & $\begin{array}{r}68 \\
997 \\
\end{array}$ & $\begin{array}{r}69 \\
997\end{array}$ \\
\hline 19 & $\begin{array}{c}59 \cdot 6 \\
997\end{array}$ & $\begin{array}{c}60.6 \\
997\end{array}$ & $\begin{array}{c}61^{\circ} 6 \\
997 \\
\end{array}$ & $\begin{array}{c}62 \cdot 7 \\
997 \\
\end{array}$ & $\begin{array}{c}63.7 \\
997 \\
\end{array}$ & $\begin{array}{c}64 \cdot 7 \\
997 \\
\end{array}$ & $\begin{array}{c}65^{\circ} 7 \\
997\end{array}$ & $\begin{array}{c}66.7 \\
897 \\
\end{array}$ & $\begin{array}{c}67 \cdot 7 \\
996 \\
\end{array}$ & $\begin{array}{c}68 \cdot 7 \\
996\end{array}$ \\
\hline 20 & $\begin{array}{c}59.2 \\
996 \\
\end{array}$ & $\begin{array}{c}60 \cdot 3 \\
996 \\
\end{array}$ & $\begin{array}{c}61 \cdot 3 \\
996 \\
\end{array}$ & $\begin{array}{c}62 \cdot 3 \\
996 \\
\end{array}$ & $\begin{array}{c}63 \cdot 3 \\
996 \\
\end{array}$ & $\begin{array}{c}64 \cdot 3 \\
996 \\
\end{array}$ & $\begin{array}{c}65 \cdot 4 \\
996 \\
\end{array}$ & $\begin{array}{c}66 \cdot 4 \\
996 \\
\end{array}$ & $\begin{array}{c}67 \cdot 4 \\
996 \\
\end{array}$ & $\begin{array}{c}68 \cdot 4 \\
996 \\
\end{array}$ \\
\hline 21 & $\begin{array}{c}58 \cdot 9 \\
995\end{array}$ & $\begin{array}{c}59.9 \\
995\end{array}$ & $\begin{array}{c}61 \\
995 \\
\end{array}$ & $\begin{array}{r}62 \\
895 \\
\end{array}$ & $\begin{array}{l}63 \\
895 \\
\end{array}$ & $\begin{array}{r}64 \\
995 \\
\end{array}$ & $\begin{array}{c}65 \\
995 \\
\end{array}$ & $\begin{array}{l}66 \\
995 \\
\end{array}$ & $\begin{array}{l}67 \\
995 \\
\end{array}$ & $\begin{array}{r}68 \cdot 1 \\
895\end{array}$ \\
\hline 22 & $\begin{array}{c}58 \cdot 5 \\
994\end{array}$ & $\begin{array}{c}59 \cdot 5 \\
994 \\
\end{array}$ & $\begin{array}{c}60.6 \\
924 \\
\end{array}$ & $\begin{array}{c}61 \cdot 6 \\
994 \\
\end{array}$ & $\begin{array}{c}62 \cdot 7 \\
994 \\
\end{array}$ & $\begin{array}{c}63 \cdot 7 \\
994\end{array}$ & $\begin{array}{c}64 \cdot 7 \\
994 \\
\end{array}$ & $\begin{array}{c}65 \cdot 7 \\
994 \\
\end{array}$ & $\begin{array}{c}66 \cdot 7 \\
994 \\
\end{array}$ & $\begin{array}{c}67 \cdot 8 \\
994 \\
\end{array}$ \\
\hline 23 & $\begin{array}{c}58 \cdot 1 \\
993 \\
\end{array}$ & $\begin{array}{c}59 \cdot 2 \\
993 \\
\end{array}$ & $\begin{array}{c}60.2 \\
993 \\
\end{array}$ & $\begin{array}{l}61 \cdot 3 \\
998 \\
\end{array}$ & $\begin{array}{l}62.3 \\
993 \\
\end{array}$ & $\begin{array}{c}63 \cdot 3 \\
993 \\
\end{array}$ & $\begin{array}{c}64.3 \\
993 \\
\end{array}$ & $\begin{array}{c}65 \bullet 4 \\
998 \\
\end{array}$ & $\begin{array}{c}66 \cdot 3 \\
998 \\
\end{array}$ & $\begin{array}{c}67 \cdot 4 \\
.993 \\
\end{array}$ \\
\hline 24 & $\begin{array}{l}57 \cdot 8 \\
992\end{array}$ & $\begin{array}{c}58 \cdot 9 \\
992\end{array}$ & $\begin{array}{c}59 \cdot 9 \\
992\end{array}$ & $\begin{array}{c}61 \\
992\end{array}$ & $\begin{array}{l}62 \\
992\end{array}$ & $\begin{array}{r}63 \\
992\end{array}$ & $\begin{array}{r}64 \\
992\end{array}$ & $\begin{array}{r}65 \\
992\end{array}$ & $\begin{array}{c}66 \\
992\end{array}$ & $\begin{array}{c}67 \cdot 1 \\
992\end{array}$ \\
\hline 25 & $\begin{array}{c}57 \cdot 5 \\
992\end{array}$ & $\begin{array}{c}58 \cdot 5 \\
992\end{array}$ & $\begin{array}{c}59 \cdot 5 \\
992\end{array}$ & $\begin{array}{c}60.6 \\
991\end{array}$ & $\begin{array}{l}61 \cdot 6 \\
991\end{array}$ & $\begin{array}{c}62 \cdot 6 \\
291\end{array}$ & $\begin{array}{l}63 \cdot 7 \\
991\end{array}$ & $\begin{array}{c}64 \cdot 7 \\
991\end{array}$ & $\begin{array}{c}65 \cdot 7 \\
991 .\end{array}$ & $\begin{array}{c}66.7 \\
991\end{array}$ \\
\hline $\begin{array}{l}\text { Temp. } \\
\text { C. }\end{array}$ & 710 & $72 c$ & $73 \mathrm{c}$ & $74 \mathrm{c}$ & 750 & 760 & $77 \mathrm{c}$ & $78 \mathrm{c}$ & $79 \mathrm{c}$ & $80 c$ \\
\hline $\begin{array}{c}\text { Deg. } \\
10\end{array}$ & $\begin{array}{l}72 \cdot 6 \\
1004\end{array}$ & $\begin{array}{l}73.5 \\
1004 \\
\end{array}$ & $\begin{array}{l}74.5 \\
1005 \\
\end{array}$ & $\begin{array}{l}75.5 \\
1005 \\
\end{array}$ & $\begin{array}{l}76.5 \\
1005 \\
\end{array}$ & $\begin{array}{l}77.5 \\
1005 \\
\end{array}$ & $\begin{array}{l}78.5 \\
1005 \\
\end{array}$ & $\begin{array}{l}79 \cdot 5 \\
1005 \\
\end{array}$ & $\begin{array}{l}80.5 \\
1005 \\
\end{array}$ & $\begin{array}{l}81 \cdot 5 \\
1005 \\
\end{array}$ \\
\hline 11 & $\begin{array}{r}72 \cdot 3 \\
1004 \\
\end{array}$ & $\begin{array}{l}73 \cdot 2 \\
1004 \\
\end{array}$ & $\begin{array}{l}74 \cdot 2 \\
1004 \\
\end{array}$ & $\begin{array}{l}75 \cdot 2 \\
1004 \\
\end{array}$ & $\begin{array}{l}76.2 \\
1004 \\
\end{array}$ & $\begin{array}{l}77 \cdot 2 \\
1004 \\
\end{array}$ & $\begin{array}{l}78 \cdot 2 \\
1004 \\
\end{array}$ & $\begin{array}{l}79 \cdot 2 \\
1004 \\
\end{array}$ & $\begin{array}{l}80.2 \\
1004 \\
\end{array}$ & $\begin{array}{l}81 \cdot 2 \\
1004 \\
\end{array}$ \\
\hline 12 & $\begin{array}{r}72 \\
1003 \\
\end{array}$ & $\begin{array}{l}72 \cdot 9 \\
1008 \\
\end{array}$ & $\begin{array}{l}73 \cdot 9 \\
1003 \\
\end{array}$ & $\begin{array}{l}74.9 \\
1003 \\
\end{array}$ & $\begin{array}{l}75 \cdot 9 \\
1003 \\
\end{array}$ & $\begin{array}{l}76 \cdot 9 \\
1008 \\
\end{array}$ & $\begin{array}{l}77 \cdot 9 \\
1003 \\
\end{array}$ & $\begin{array}{l}78 \cdot 9 \\
1008 \\
\end{array}$ & $\begin{array}{l}79.9 \\
1003 \\
\end{array}$ & $\begin{array}{l}80 \cdot 9 \\
1003 \\
\end{array}$ \\
\hline 13 & $\begin{array}{l}71 \cdot 6 \\
1002 \\
\end{array}$ & $\begin{array}{l}72 \cdot 6 \\
1002 \\
\end{array}$ & $\begin{array}{l}73 \cdot 6 \\
1002 \\
\end{array}$ & $\begin{array}{l}74 \cdot 6 \\
1002 \\
\end{array}$ & $\begin{array}{l}75 \cdot 6 \\
1002 \\
\end{array}$ & $\begin{array}{l}76 \cdot 6 \\
1002 \\
\end{array}$ & $\begin{array}{l}77 \cdot 6 \\
1002 \\
\end{array}$ & $\begin{array}{l}78 \cdot 6 \\
1002 \\
\end{array}$ & $\begin{array}{l}79 \cdot 6 \\
1002 \\
\end{array}$ & $\begin{array}{l}80.6 \\
1002 \\
\end{array}$ \\
\hline 14 & $\begin{array}{l}11 \cdot 3 \\
1001 \\
\end{array}$ & $\begin{array}{l}72 \cdot 3 \\
1001 \\
\end{array}$ & $\begin{array}{l}73 \cdot 3 \\
1001 \\
\end{array}$ & $\begin{array}{l}74: 3 \\
1001 \\
\end{array}$ & $\begin{array}{l}75 \cdot 3 \\
1001 \\
\end{array}$ & $\begin{array}{l}76 \cdot 3 \\
1001 \\
\end{array}$ & $\begin{array}{l}77: 3 \\
1001 \\
\end{array}$ & $\begin{array}{l}78 \cdot 3 \\
1001 \\
\end{array}$ & $\begin{array}{l}79.3 \\
1001 \\
\end{array}$ & $\begin{array}{l}80 \cdot 3 \\
1001 \\
\end{array}$ \\
\hline 15 & $\begin{array}{c}71 \\
1000 \\
\end{array}$ & $\begin{array}{c}72 \\
1000 \\
\end{array}$ & $\begin{array}{c}73 \\
1000 \\
\end{array}$ & $\begin{array}{c}74 \\
1000 \\
\end{array}$ & $\begin{array}{c}75 \\
1000 \\
\end{array}$ & $\begin{array}{c}76 \\
1000 \\
\end{array}$ & $\begin{array}{c}77 \\
1000 \\
\end{array}$ & $\begin{array}{c}78 \\
1000 \\
\end{array}$ & $\begin{array}{r}79 \\
1000 \\
\end{array}$ & $\begin{array}{c}80 \\
1000 \\
\end{array}$ \\
\hline 16 & $\begin{array}{r}70 \cdot 7 \\
999 \\
\end{array}$ & $\begin{array}{r}71 \cdot 7 \\
999 \\
\end{array}$ & $\begin{array}{r}72 \cdot 7 \\
999 \\
\end{array}$ & $\begin{array}{r}73 \cdot 7 \\
999 \\
\end{array}$ & $\begin{array}{r}74 \cdot 7 \\
999 \\
\end{array}$ & $\begin{array}{r}75 \cdot 7 \\
999 \\
\end{array}$ & $\begin{array}{r}76.7 \\
999 \\
\end{array}$ & $\begin{array}{r}77 \cdot 7 \\
999 \\
\end{array}$ & $\begin{array}{r}78 \cdot 7 \\
999 \\
\end{array}$ & $\begin{array}{r}79 \cdot 7 \\
999 \\
\end{array}$ \\
\hline 17 & $\begin{array}{c}70 \cdot 3 \\
998 \\
\end{array}$ & $\begin{array}{c}71.3 \\
998 \\
\end{array}$ & $\begin{array}{r}72 \cdot 3 \\
998 \\
\end{array}$ & $\begin{array}{r}73 \cdot 3 \\
998 \\
\end{array}$ & $\begin{array}{r}74 \cdot 3 \\
998 \\
\end{array}$ & $\begin{array}{r}75.4 \\
998 \\
\end{array}$ & $\begin{array}{r}76.4 \\
998 \\
\end{array}$ & $\begin{array}{r}77 \cdot 4 \\
998 \\
\end{array}$ & $\begin{array}{c}78 \cdot 4 \\
998 \\
\end{array}$ & $\begin{array}{r}79.4 \\
998 \\
\end{array}$ \\
\hline 18 & $\begin{array}{c}70 \\
997 \\
\end{array}$ & $\begin{array}{l}71 \\
997 \\
\end{array}$ & $\begin{array}{c}72 \\
997 \\
\end{array}$ & $\begin{array}{r}73 \\
897 \\
\end{array}$ & $\begin{array}{r}74 \\
997 \\
\end{array}$ & $\begin{array}{c}75 \cdot 1 \\
997 \\
\end{array}$ & $\begin{array}{c}76 \cdot 1 \\
997 \\
\end{array}$ & $\begin{array}{c}77 \cdot 1 \\
997 \\
\end{array}$ & $\begin{array}{c}78.1 \\
997 \\
\end{array}$ & $\begin{array}{c}79 \cdot 1 \\
997 \\
\end{array}$ \\
\hline 19 & $\begin{array}{r}69 \cdot 7 \\
996 \\
\end{array}$ & $\begin{array}{c}70 \cdot 7 \\
996 \\
\end{array}$ & $\begin{array}{c}71.7 \\
996 \\
\end{array}$ & $\begin{array}{c}72 \cdot 7 \\
996 \\
\end{array}$ & $\begin{array}{c}73 \cdot 7 \\
996 \\
\end{array}$ & $\begin{array}{c}74 \cdot 7 \\
996\end{array}$ & $\begin{array}{c}75 \cdot 8 \\
996 \\
\end{array}$ & $\begin{array}{l}76.8 \\
996 \\
\end{array}$ & $\begin{array}{c}77.8 \\
996 \\
\end{array}$ & $\begin{array}{l}78 \cdot 8 \\
996 \\
\end{array}$ \\
\hline 20 & $\begin{array}{c}69 \cdot 4 \\
996 \\
\end{array}$ & $\begin{array}{c}70 \cdot 4 \\
996 \\
\end{array}$ & $\begin{array}{l}71 \cdot 4 \\
995 \\
\end{array}$ & $\begin{array}{c}72 \cdot 4 \\
995 \\
\end{array}$ & $\begin{array}{r}73 \cdot 4 \\
995 \\
\end{array}$ & $\begin{array}{c}74.4 \\
995 \\
\end{array}$ & $\begin{array}{c}75^{\circ} 5 \\
995 \\
\end{array}$ & $\begin{array}{c}76.5 \\
895 \\
\end{array}$ & $\begin{array}{r}77 \cdot 5 \\
995 \\
\end{array}$ & $\begin{array}{c}78 \cdot 5 \\
995 \\
\end{array}$ \\
\hline 21 & $\begin{array}{c}69 \cdot 1 \\
995 \\
\end{array}$ & $\begin{array}{c}70 \cdot 1 \\
995 \\
\end{array}$ & $\begin{array}{c}71 \cdot 1 \\
995 \\
\end{array}$ & $\begin{array}{c}72 \cdot 1 \\
994 \\
\end{array}$ & $\begin{array}{c}73 \cdot 1 \\
994 \\
\end{array}$ & $\begin{array}{c}74 \cdot 1 \\
994 \\
\end{array}$ & $\begin{array}{c}75 \cdot 2 \\
994 \\
\end{array}$ & $\begin{array}{c}76 \cdot 2 \\
994 \\
\end{array}$ & $\begin{array}{c}77 \cdot 2 \\
994 \\
\end{array}$ & $\begin{array}{c}78.2 \\
894 \\
\end{array}$ \\
\hline 22 & $\begin{array}{c}68 \cdot 8 \\
994 \\
\end{array}$ & $\begin{array}{c}69.8 \\
994 \\
\end{array}$ & $\begin{array}{c}70^{\circ} 8 \\
994 \\
\end{array}$ & $\begin{array}{c}71 \cdot 8 \\
994\end{array}$ & $\begin{array}{c}72 \cdot 8 \\
993 \\
\end{array}$ & $\begin{array}{c}73 \cdot 8 \\
993 \\
\end{array}$ & $\begin{array}{c}74.8 \\
993 \\
\end{array}$ & $\begin{array}{c}75 \cdot 9 \\
993 \\
\end{array}$ & $\begin{array}{c}76 \cdot 9 \\
993 \\
\end{array}$ & $\begin{array}{c}77 \cdot 9 \\
983 \\
\end{array}$ \\
\hline 23 & $\begin{array}{c}68 \cdot 4 \\
993 \\
\end{array}$ & $\begin{array}{c}69 \cdot 4 \\
993 \\
\end{array}$ & $\begin{array}{l}70.5 \\
993 \\
\end{array}$ & $\begin{array}{c}71.5 \\
998\end{array}$ & $\begin{array}{c}72.5 \\
992 \\
\end{array}$ & $\begin{array}{c}73.5 \\
892 \\
\end{array}$ & $\begin{array}{c}74.5 \\
392 \\
\end{array}$ & $\begin{array}{c}75.5 \\
992 \\
\end{array}$ & $\begin{array}{c}76.6 \\
992 \\
\end{array}$ & $\begin{array}{c}77 \cdot 6 \\
992 \\
\end{array}$ \\
\hline 24 & $\begin{array}{c}68 \cdot 1 \\
992 \\
\end{array}$ & $\begin{array}{c}69.1 \\
992 \\
\end{array}$ & $\begin{array}{c}70 \cdot 1 \\
992 \\
\end{array}$ & $\begin{array}{c}71 \cdot 2 \\
992 \\
\end{array}$ & $\begin{array}{c}72 \cdot 2 \\
992 \\
\end{array}$ & $\begin{array}{c}73 \cdot 2 \\
992 \\
\end{array}$ & $\begin{array}{c}74 \cdot 2 \\
992 \\
\end{array}$ & $\begin{array}{r}75 \cdot 2 \\
991 \\
\end{array}$ & $\begin{array}{c}76.3 \\
991 \\
\end{array}$ & $\begin{array}{c}77 \cdot 3 \\
991 \\
\end{array}$ \\
\hline 25 & $\begin{array}{c}67 \cdot 8 \\
991\end{array}$ & $\begin{array}{c}68 \cdot 8 \\
991\end{array}$ & $\begin{array}{c}69 \cdot 8 \\
901\end{array}$ & $\begin{array}{c}70 \cdot 8 \\
991\end{array}$ & $\begin{array}{l}71 \cdot 8 \\
991\end{array}$ & $\begin{array}{l}72 \cdot 8 \\
991\end{array}$ & $\begin{array}{l}73 \cdot 9 \\
991\end{array}$ & $\begin{array}{l}74 \cdot 9 \\
991\end{array}$ & $\begin{array}{r}76 \\
991\end{array}$ & $\begin{array}{r}77 \\
991\end{array}$ \\
\hline
\end{tabular}


Alcoömetrical Table of real Strength, by M. Gay-Lussac (continued).

\begin{tabular}{|c|c|c|c|c|c|c|c|c|c|c|}
\hline $\begin{array}{c}\text { Trmp. } \\
\text { C. }\end{array}$ & $81 \mathrm{c}$ & 820 & 880 & 840 & 850 & 860 & 870 & 880 & 890 & 900 \\
\hline $\begin{array}{c}\text { Deg. } \\
10\end{array}$ & $\begin{array}{l}82 \cdot 4 \\
1005\end{array}$ & $\begin{array}{l}83 \cdot 4 \\
1005\end{array}$ & $\begin{array}{l}84 \cdot 4 \\
1005\end{array}$ & $\begin{array}{l}85 \cdot 4 \\
1005\end{array}$ & $\begin{array}{l}86.4 \\
1005\end{array}$ & $\begin{array}{l}87 \cdot 4 \\
1005\end{array}$ & $\begin{array}{l}88 \cdot 3 \\
1005\end{array}$ & $\begin{array}{l}89 \cdot 3 \\
1005\end{array}$ & $\begin{array}{l}90 \cdot 2 \\
1005\end{array}$ & $\begin{array}{l}91 \cdot 2 \\
1005\end{array}$ \\
\hline 11 & $\begin{array}{l}82 \cdot 2 \\
1004\end{array}$ & $\begin{array}{l}83 \cdot 1 \\
1004 \\
\end{array}$ & $\begin{array}{l}84 \cdot 1 \\
1004 \\
\end{array}$ & $\begin{array}{l}85 \cdot 1 \\
1004\end{array}$ & $\begin{array}{l}86 \cdot 1 \\
1004 \\
\end{array}$ & $\begin{array}{l}87 \cdot 1 \\
1004 \\
\end{array}$ & $\begin{array}{c}88 \\
1004\end{array}$ & $\begin{array}{r}89 \\
1004 \\
\end{array}$ & $\begin{array}{c}90 \\
1004 \\
\end{array}$ & $\begin{array}{c}91 \\
1004 \\
\end{array}$ \\
\hline 12 & $\begin{array}{l}81 \cdot 9 \\
1003 \\
\end{array}$ & $\begin{array}{l}82 \cdot 9 \\
1003\end{array}$ & $\begin{array}{l}83 \cdot 9 \\
1003 \\
\end{array}$ & $\begin{array}{l}84 \cdot 8 \\
1003\end{array}$ & $\begin{array}{l}85 \cdot 8 \\
1003 \\
\end{array}$ & $\begin{array}{l}86 \cdot 8 \\
1003 \\
\end{array}$ & $\begin{array}{l}87 \cdot 8 \\
1003 \\
\end{array}$ & $\begin{array}{l}88 \cdot 7 \\
1003\end{array}$ & $\begin{array}{l}89 \cdot 7 \\
1003\end{array}$ & $\begin{array}{l}90 \cdot 7 \\
1003\end{array}$ \\
\hline 13 & $\begin{array}{l}81 \cdot 6 \\
1002 \\
\end{array}$ & $\begin{array}{l}82 \cdot 6 \\
1002 \\
\end{array}$ & $\begin{array}{l}83 \cdot 6 \\
1002 \\
\end{array}$ & $\begin{array}{l}84 \cdot 6 \\
1002\end{array}$ & $\begin{array}{l}85.5 \\
1002 \\
\end{array}$ & $\begin{array}{l}86 \cdot 5 \\
1002 \\
\end{array}$ & $\begin{array}{l}87 \cdot 5 \\
1002 \\
\end{array}$ & $\begin{array}{l}88 \cdot 5 \\
1002 \\
\end{array}$ & $\begin{array}{l}89.5 \\
1002 \\
\end{array}$ & $\begin{array}{l}90.5 \\
1002 \\
\end{array}$ \\
\hline 14 & $\begin{array}{l}81 \cdot 3 \\
1001 \\
\end{array}$ & $\begin{array}{l}82 \cdot 3 \\
1001 \\
\end{array}$ & $\begin{array}{l}83 \cdot 3 \\
1001 \\
\end{array}$ & $\begin{array}{l}84^{\circ 3} \\
1001 \\
\end{array}$ & $\begin{array}{l}85 \cdot 3 \\
1001 . \\
\end{array}$ & $\begin{array}{l}86 \cdot 3 \\
1001 \\
\end{array}$ & $\begin{array}{l}87 \cdot 3 \\
1001 \\
\end{array}$ & $\begin{array}{l}88 \cdot 2 \\
1001 \\
\end{array}$ & $\begin{array}{l}89 \cdot 2 \\
1001 \\
\end{array}$ & $\begin{array}{l}90 \cdot 2 \\
1001 \\
\end{array}$ \\
\hline 15 & $\begin{array}{c}81 \\
1000 \\
\end{array}$ & $\begin{array}{c}82 \\
1000 \\
\end{array}$ & $\begin{array}{c}83 \\
1000 \\
\end{array}$ & $\begin{array}{c}84 \\
1000 \\
\end{array}$ & $\begin{array}{c}86 \\
1000 \\
\end{array}$ & $\begin{array}{c}86 \\
1000 \\
\end{array}$ & $\begin{array}{c}87 \\
1000\end{array}$ & $\begin{array}{c}88 \\
1000 \\
\end{array}$ & $\begin{array}{c}89 \\
1000\end{array}$ & $\begin{array}{c}90 \\
1000 \\
\end{array}$ \\
\hline 16 & $\begin{array}{c}80 \cdot 7 \\
999 \\
\end{array}$ & $\begin{array}{l}81 \cdot 7 \\
999\end{array}$ & $\begin{array}{c}82 \cdot 7 \\
999 \\
\end{array}$ & $\begin{array}{c}83 \cdot 7 \\
999 \\
\end{array}$ & $\begin{array}{c}84 \cdot 7 \\
999 \\
\end{array}$ & $\begin{array}{c}85 \cdot 7 \\
999 \\
\end{array}$ & $\begin{array}{c}86 \cdot 7 \\
999\end{array}$ & $\begin{array}{c}87 \cdot 7 \\
999\end{array}$ & $\begin{array}{c}88 \cdot 7 \\
989\end{array}$ & $\begin{array}{c}89 \cdot 7 \\
999\end{array}$ \\
\hline 17 & $\begin{array}{c}80 \circ 4 \\
998\end{array}$ & $\begin{array}{l}81 \cdot 4 \\
998\end{array}$ & $\begin{array}{c}82 \cdot 4 \\
998\end{array}$ & $\begin{array}{c}83.4 \\
998\end{array}$ & $\begin{array}{c}84 \cdot 4 \\
998 \\
\end{array}$ & $\begin{array}{c}85.4 \\
998\end{array}$ & $\begin{array}{c}86.4 \\
998\end{array}$ & $\begin{array}{c}87 \cdot 4 \\
998\end{array}$ & $\begin{array}{c}88 \circ 4 \\
998\end{array}$ & $\begin{array}{c}89 \cdot 5 \\
998\end{array}$ \\
\hline 18 & $\begin{array}{l}80 \cdot 1 \\
997 \\
\end{array}$ & $\begin{array}{l}81 \cdot 1 \\
997 \\
\end{array}$ & $\begin{array}{c}82 \cdot 1 \\
997 \\
\end{array}$ & $\begin{array}{c}83 \cdot 1 \\
997 \\
\end{array}$ & $\begin{array}{c}84 \cdot 1 \\
997 \\
\end{array}$ & $\begin{array}{c}85 \cdot 2 \\
997 \\
\end{array}$ & $\begin{array}{l}86.2 \\
997 \\
\end{array}$ & $\begin{array}{c}87 \cdot 2 \\
997 \\
\end{array}$ & $\begin{array}{l}88 \cdot 2 \\
997\end{array}$ & $\begin{array}{c}89 \cdot 2 \\
997 \\
\end{array}$ \\
\hline 19 & $\begin{array}{r}79 \cdot 8 \\
996 \\
\end{array}$ & $\begin{array}{c}80.8 \\
996 \\
\end{array}$ & $\begin{array}{c}81 \cdot 9 \\
996 \\
\end{array}$ & $\begin{array}{c}82 \cdot 9 \\
996 \\
\end{array}$ & $\begin{array}{c}83 \cdot 9 \\
896 \\
\end{array}$ & $\begin{array}{c}84 \cdot 9 \\
996 \\
\end{array}$ & $\begin{array}{c}85 \cdot 9 \\
996 \\
\end{array}$ & $\begin{array}{c}86 \cdot 9 \\
996 \\
\end{array}$ & $\begin{array}{l}87 \cdot 9 \\
906 \\
\end{array}$ & $\begin{array}{c}88 \cdot 9 \\
996 \\
\end{array}$ \\
\hline 20 & $\begin{array}{l}79.5 \\
995 \\
\end{array}$ & $\begin{array}{l}80.5 \\
995\end{array}$ & $\begin{array}{c}81 \cdot 6 \\
995 \\
\end{array}$ & $\begin{array}{c}82 \cdot 6 \\
995 \\
\end{array}$ & $\begin{array}{c}83 \cdot 6 \\
995 \\
\end{array}$ & $\begin{array}{c}84^{\circ} 6 \\
995\end{array}$ & $\begin{array}{c}85 \cdot 6 \\
995 \\
\end{array}$ & $\begin{array}{c}86^{\circ} 6 \\
-995 \\
\end{array}$ & $\begin{array}{c}87 \cdot 7 \\
995 \\
\end{array}$ & $\begin{array}{c}88 \cdot 7 \\
995 \\
\end{array}$ \\
\hline 21 & $\begin{array}{l}79 \cdot 2 \\
994 \\
\end{array}$ & $\begin{array}{l}80 \cdot 2 \\
994 \\
\end{array}$ & $\begin{array}{c}81 \cdot 3 \\
994\end{array}$ & $\begin{array}{c}82 \cdot 3 \\
994 \\
\end{array}$ & $\begin{array}{c}83 \cdot 3 \\
994 \\
\end{array}$ & $\begin{array}{c}84 \cdot 3 \\
994 \\
\end{array}$ & $\begin{array}{c}85 \cdot 3 \\
994 \\
\end{array}$ & $\begin{array}{c}86 \cdot 4 \\
994 \\
\end{array}$ & $\begin{array}{c}87 \cdot 4 \\
994 \\
\end{array}$ & $\begin{array}{c}88 \cdot 4 \\
994 \\
\end{array}$ \\
\hline 22 & $\begin{array}{c}78 \cdot 9 \\
993 \\
\end{array}$ & $\begin{array}{c}79 \cdot 9 \\
998 \\
\end{array}$ & $\begin{array}{c}81 \\
993 \\
\end{array}$ & $\begin{array}{r}82 \\
998 \\
\end{array}$ & $\begin{array}{c}83 \\
993 \\
\end{array}$ & \begin{tabular}{|c|}
84 \\
998 \\
\end{tabular} & $\begin{array}{l}85 \\
993 \\
\end{array}$ & $\begin{array}{c}86 \cdot 1 \\
993 \\
\end{array}$ & $\begin{array}{c}87 \cdot 1 \\
983 \\
\end{array}$ & $\begin{array}{c}88 \cdot 2 \\
998 \\
\end{array}$ \\
\hline 23 & $\begin{array}{c}78 \cdot 6 \\
992 \\
\end{array}$ & $\begin{array}{c}79 \cdot 6 \\
992 \\
\end{array}$ & $\begin{array}{c}80 \cdot 7 \\
992 \\
\end{array}$ & $\begin{array}{c}81 \cdot 7 \\
992 \\
\end{array}$ & $\begin{array}{c}82 \cdot 7 \\
992 \\
\end{array}$ & $\begin{array}{c}83 \cdot 8 \\
982 \\
\end{array}$ & $\begin{array}{c}84 \cdot 8 \\
992 \\
\end{array}$ & $\begin{array}{c}85 \cdot 8 \\
992 \\
\end{array}$ & $\begin{array}{c}86.8 \\
992 \\
\end{array}$ & $\begin{array}{c}87 \cdot 9 \\
992 \\
\end{array}$ \\
\hline 24 & $\begin{array}{l}78: 3 \\
991\end{array}$ & $\begin{array}{c}79 \cdot 3 \\
991\end{array}$ & $\begin{array}{l}80 * 4 \\
991\end{array}$ & $\begin{array}{c}81 \cdot 4 \\
991\end{array}$ & $\begin{array}{c}82 \cdot 4 \\
991\end{array}$ & $\begin{array}{c}83.5 \\
991\end{array}$ & $\begin{array}{c}84 \cdot \overline{5} \\
991\end{array}$ & $\begin{array}{c}85.5 \\
991\end{array}$ & $\begin{array}{l}86.5 \\
991\end{array}$ & $\begin{array}{c}87 \cdot 6 \\
991 \\
\end{array}$ \\
\hline 25 & $\begin{array}{l}78 \\
991\end{array}$ & $\begin{array}{l}79 \\
991\end{array}$ & $\begin{array}{c}80 \cdot 1 \\
990\end{array}$ & $\begin{array}{l}81 \cdot 1 \\
990\end{array}$ & $\begin{array}{c}82 \cdot 1 \\
990\end{array}$ & $\begin{array}{l}83 \cdot 2 \\
990\end{array}$ & $\begin{array}{l}84 \cdot 2 \\
990\end{array}$ & $\begin{array}{c}85 \cdot 2 \\
990\end{array}$ & $\begin{array}{l}86 \cdot 3 \\
990\end{array}$ & $\begin{array}{c}87 \cdot 4 \\
990\end{array}$ \\
\hline
\end{tabular}

The boiling point of mixtures of alcohol and water likewise differs with the strength of such mixtures.

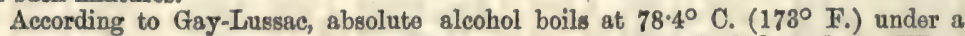
pressure of 760 millimetres (the millimetre being 0.03937 English inches). When mixed with water, of course its boiling point rises in proportion to the quantity of water present, as is the case in general with mixtures of two fluids of greater and less volatility. A mixture of alcohol and water, however, presents this anomaly, according to Soemmering: when the mixture contains less than six per cent. of alcohol, those portions which first pass off are saturated with water, and the alcoholic solution in the retort becomes richer, till absolute alcohol passes over; but when the mixture contains more than six per cent. of water the boiling point rises, and the quantity of alcohol in the distillate steadily diminishes as the distillation procoeds.

\begin{tabular}{|c|c|c||c|c|c|}
\hline Temperature & $\begin{array}{c}\text { Alcoholic con- } \\
\text { tent of the } \\
\text { vapour }\end{array}$ & $\begin{array}{c}\text { Alcoholic con- } \\
\text { tent of the boil- } \\
\text { ing liquid }\end{array}$ & Temperature & $\begin{array}{c}\text { Alcoholic con- } \\
\text { tent of the } \\
\text { vapour }\end{array}$ & $\begin{array}{c}\text { Alcoholic con- } \\
\text { tent of the boil- } \\
\text { ing lquid }\end{array}$ \\
\hline Fahr. $170 \cdot 0$ & 93 & 92 & Fahr. $189 \cdot 8$ & 71 & 20 \\
$171 \cdot 8$ & 92 & 90 & $192 \cdot 0$ & 68 & 18 \\
172 & 91 & 85 & 164 & 66 & 15 \\
$172 \cdot 8$ & 901 & 80 & $196 \cdot 4$ & 61 & 12 \\
174 & 90 & 70 & $198 \cdot 6$ & 55 & 10 \\
$174 \cdot 6$ & 89 & 70 & 201 & 50 & 7 \\
176 & 87 & 65 & 203 & 42 & 5 \\
$178 \cdot 3$ & 85 & 50 & $205 \cdot 4$ & 36 & 3 \\
$180 \cdot 8$ & 82 & 40 & $207 \cdot 7$ & 28 & 2 \\
183 & 80 & 35 & 210 & 13 & 1 \\
186 & 78 & 30 & 212 & 0 & 0 \\
$187 \cdot 4$ & 76 & 25 & & & \\
\hline
\end{tabular}


According to Gröning's researches, the precoding temperatures of the alcoholic vapours correspond to the accompanying contonts of alcohol in per-centage of volume which are disengaged in the boiling of the spirituous liquid.

Gröning undertook this investigation in order to employ the thermometer as an alcoholometer in the distillation of spirits; for which purpose he thrust the bulb of the thermometer through a cork inserted into a tube fixed in the capital of the still. The state of the barometer ought also to be considered in making comparative oxperiments of this kind. Since, by this method, the alcoholic content may be compared with the temperature of the vapour that passes over at any time, so also the contents of the whole distillation may be found approximately; and the method serves as a convenient means of making continual observations on the progress of the distillation.

From the mean of a great many experiments, Dr. Ure drew up the following Table, which shows the boiling point of alcohol of various specific gravities :-

\begin{tabular}{|c|c|c|c|}
\hline Boiling Point & Specific Gravity & Boiling Point & Speciflc Cravity \\
\hline $\begin{array}{l}178.5 \quad \mathrm{~F} . \\
179^{\circ} 75 \\
180^{\circ} 4 ", \\
182^{\circ} 01, " \\
183^{\circ} 40 "\end{array}$ & $\begin{array}{l}0.9200 \\
0.9321 \\
0.9420 \\
0.9516 \\
0.960\end{array}$ & $\begin{array}{l}185.6 \mathrm{~F} . \\
189.0 \% " \\
191.8 \% " \\
196.4 \% \\
202.0 "\end{array}$ & $\begin{array}{l}0.9665 \\
0.9729 \\
0.9786 \\
0.9850 \\
0.992\end{array}$ \\
\hline
\end{tabular}

Density of the Vapour.-One volume of alcohol yields 488.3 volumes of vapour at $212^{\circ} \mathrm{F}$. The specific gravity of the vapour, taking air as unity, was found by Gay-Lussac to be 1.6133. [Its vapour-density, referred to hydrogen as unity, is $13 \cdot 3605$ ? ]

Spirituous vapour passed through an ignited tube of glass or porcelain is converted into carbonic oxide, water, hydrogen, carburetted hydrogen, olefiant gas, naphthaline, empyreumatic oil, and carbon; according to the degree of heat and nature of the tube, these products vary. Anhydrous alcohol is a non-conductor of electricity, but is decomposed by a powerful voltaic battery. Alcohol burns in the air with a blue flame into carbonic acid and water; the water being heavier than the spirit, because 46 parts of alcohol contain 6 of hydrogen, which form 54 of water. In oxygen the combustion is accompanied with great heat, and this flame, directed through a small tube, powerfully ignites bodies exposed to it.

Platinum in a finely divided state has the property of determining the combination of alcohol with the oxygen of the air in a remarkable manner. A ball of spongy platinum, placed slightly above the wick of a lamp fed by spirit and communicating with the wick by a platinum wire, when once heated, keeps at a red heat, gradually burning the spirit. This has been applied in the construction of the socalled 'philosophical pastilles;' eau-de-cologne or other perfumed spirit being thus mado to diffuse itself in a room.

Mr. Gill has also practically applied this in the construction of an alcohol lamp without flame.

A coil of platinum wire, of about the one-hundredth part of an inch in thickness, is coiled partly round the cotton wick of a spirit-lamp, and partly above it, and the lamp lighted to heat the wire to redness; on the flame being extinguished, the alcohol vapour keeps the wire red hot for any length of time, so as to be in constant readiness to ignite a match, for example. This lamp affords sufficient light to show the hour by a watch in the night, with a very small consumption of spirit.

This property of condensing oxygen, and thus causing the union of it with combustible bodies, is not confined to platinum, but is possessed, though in a less degree, by other porous bodies. If wo moisten sand in a capsule with absolute alcohol, and cover it with previously heated nickel powder, protoxide of nickel, cobalt powder. protoxide of cobalt, protoxide of uranium, or oxide of tin (these six bodies being procured by ignition of their oxalates in a crucible), or finely powdered peroxide of manganese, combustion takes place, and continues so long as the spirituous vapour lasts.

Solvent Power.-One of the properties of alcohol most valuable in the arts is its solvent power.

It dissolves gases to a very considerable oxtent, which gases, if they do not enter into combination with the alcohol, or act chomically upon it, are expelled again on boiling the alcohol.

Soveral salte, especinlly the deliquoscent, ure dissolved by it, and some of them 
give a colour to its flame; thus the solutions of the salts of strontia in alcohol burn with a crimson flame, thoso of copper and borax with a green one, lime a reddish, and baryta with a yellow flame.

This solvent power is, however, most remarkable in its action upon resins, ethers, essential oils, fatty bodies, alkaloids, as well as many organic acids. In a similar way it dissolves iodine, bromino, and in small quantities sulphur and phosphorus. In general it may be said to be an excellent solvent for most hydrogenised organic substances.

Uses.-In consequence of this property it is most extensively used in the chemical arts: e.g. for the solution of gum-resins, \&c., in the manufacture of varnishes; in pharmacy, for the separating of the active principles of plants, in the preparation of tinctures. It is also omployed in the formation of chloroform, ether, spirits of nitre, \&c.

The great use of alcohol, in its various states of mixture, is, and has been from time immemorial, as a beverage. There cannot be a doubt that alcoholic liquors are beneficial to most healthy persons when moderately enjoyed; and the man who advocates their rational use cannot be held answerable for their abuse.

Absolute alcohol (or strong spirits) acts locally as an irritant, contracting the tissues; but its effects on the organism, when taken internally, arises from its action, by the nerves, on the brain. Dr. Pereira has graphically described three stages of their effects :-
1. First or mildest degree
2. Second degreo .
Fixcitement.
3. Third degree : : Coma, or true apoplexy.

These effects are tolorably familiar, and for a more minute description of them we must refer to Dr. Pereira ' and other medical anthors.

The important applications of alcohol in the arts, as a solvent for resins, \&ce, have been before alluded to. To the chemist it is a most valuable agent of separation. By its means he is enabled, in complicated organic mixtures, to separate those substances which are soluble from those which are insoluble in this menstruum. It may likewise be employed for separating certain salts - e.g. the chloride of strontium from that of barium, \&c. \&ce.

From it are also manufactured ether, chloroform, chloral, and, indirectly, acetic acid; and in pharmacy, sweet spirits of nitre, the various tinctures, \&c. \&c.

The Spirits imported and retained for Home Consumption were as follows:-

\begin{tabular}{|c|c|c|c|c|c|c|}
\hline \multirow{3}{*}{ Spirita } & \multicolumn{4}{|c|}{ ImPORTs } & \multicolumn{2}{|c|}{$=2$} \\
\hline & \multicolumn{2}{|c|}{1870} & \multicolumn{2}{|c|}{1871} & \multicolumn{2}{|c|}{1872} \\
\hline & Quantity & . Value & Quantity & Value & Quantity & Value \\
\hline $\begin{array}{l}\text { Rum, } \\
\text { Brandy : } \\
\text { Other Sorts }\end{array}$ & \begin{tabular}{|l|} 
Proof gallons \\
$6,915,117$ \\
$7,942,965$ \\
$2,332,049$
\end{tabular} & $\begin{array}{r}808,809 \\
2,153,699 \\
184,869\end{array}$ & $\left|\begin{array}{l}\text { Proof gallons } \\
\mathbf{7 , 6 5 7 , 4 2 2} \\
\mathbf{5 , 3 7 3 , 4 8 6} \\
1,877,390\end{array}\right|$ & $\begin{array}{r}771^{\ell}, 598 \\
1,905,276 \\
186,826\end{array}$ & \begin{tabular}{|l|} 
Proof gallons \\
$6,586,257$ \\
$3,519,413$ \\
$1,558,166$
\end{tabular} & $\begin{array}{r}675,820 \\
1,329,644 \\
187,160\end{array}$ \\
\hline \multicolumn{7}{|c|}{$\begin{array}{l}\text { These Spirits were chiefly imported:-Rum, from British West India Islands, } \\
\text { British Guiana, Mauritius, Spanish West India Islands; and Brandy, from France, } \\
\text { Holland, Germany. }\end{array}$} \\
\hline & \multicolumn{5}{|c|}{ The quantities retained for Hosm Cossumprios were:- } & 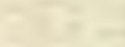 \\
\hline $\begin{array}{l}\text { Rum } \\
\text { Brandy: } \\
\text { Other Sorts }\end{array}$ & $\left|\begin{array}{l}3,851,863 \\
3,526,132 \\
1,027,857\end{array}\right|$ & . & $\left|\begin{array}{l}4,168,905 \\
3,715,675 \\
1,010,929\end{array}\right|$ & & $\begin{array}{r}4,405,192 \\
3,944,725 \\
680,918\end{array}$ & 2 \\
\hline
\end{tabular}

Pereira, Materia Medica, vol, H. p. 1948. 
Spirits Exported.

\begin{tabular}{|c|c|c|c|c|c|c|}
\hline Spirits & 1867 & 1868 & 1869 & 1870 & 1871 & 1872 \\
\hline & Gallons & Gallons & Gallons & Gallons & Gallons & Gallons \\
\hline British and Irish & $1,286,598$ & $1,364,155$ & $1,673,773$ & $1,457,265$ & $1,607,061$ & $1,795,868$ \\
\hline Foreign: & & & & & & \\
\hline Brandy . & 365,316 & 459,857 & 415,546 & 847,492 & 420,324 & \\
\hline Rum . & $2,468,478$ & $2,507,175$ & $1,396,157$ & $1,334,358$ & $1,680,289$ & \\
\hline Unenumerated . & 883,050 & 999,705 & $1,743,469$ & $1,762,894$ & $1,832,455$ & \\
\hline
\end{tabular}

Total quantities of Spirits distilled and charged with Excise Duty for consumption in England and Wales, Scotland and Ireland, in each of the years ending 31 st of March, 1866, 1867, 1868.

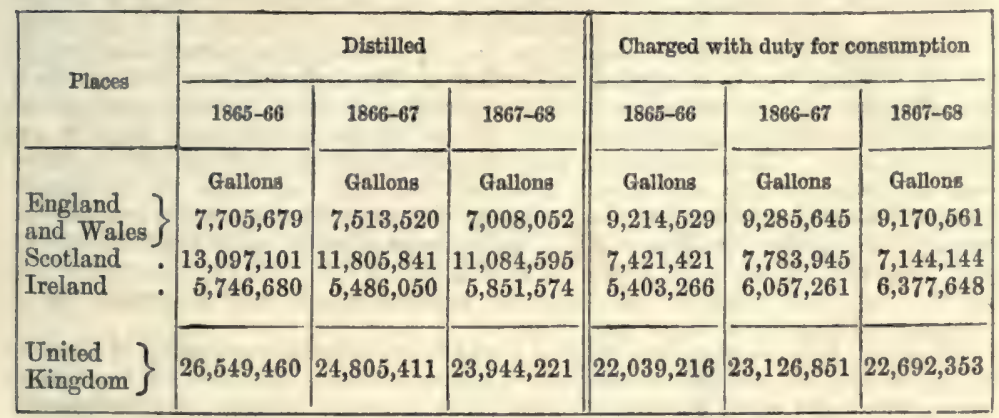

The following are the quantities of spirits charged with duties of excise in each of the years named :-

Gallons.

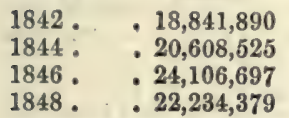

Gallons.

$$
\begin{aligned}
& 1850 \cdot \quad \cdot 23,919,432 \\
& 1852 \cdot-25,270,262 \\
& 1854 . \quad \cdot 26,148,511 \\
& 1856 . \quad \cdot 23,922,453
\end{aligned}
$$

Gellons.

$$
\begin{aligned}
& 1858 \text {. } 23,686,751 \\
& 1860 \text {. : 21,873,369 } \\
& 1862 \text {. } 19,700,250 \\
& 1864 \text {. } 21,039,582
\end{aligned}
$$

Every English distiller has now to pay a licence-duty of ten guineas before he can lawfully conduct operations, and afterwards a duty of $78.10 d$. per imperial gallon of spirits, proof strength, which he produces.

The Scoteh and Irish distillers had to pay the same licence-fee as the English; and in addition to this, the Scotch distiller paid a duty of $4 s .8 d$. per imporial gallon of proof strength, and the Irish a duty of 3s. $4 d_{0}$; but the duties are now equalized.

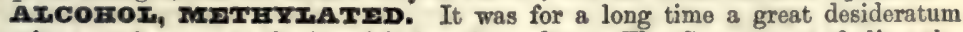
for the manufacturer to obtain spirit free from duty. The Government, feeling the necessity for this, have sanctioned the sale of spirit which has been flavoured with mothyl-alcohol, so as to render it unpalatable, free of duty, under the name of ' methylated spirit.' This methylated spirit can now be obtained, in large quantities by giving suitable security to the Board of Inland Revenue of its employment for manufacturing purposes only, and must prove of great value to those manufacturers who are large consumers.

Professors Graham, Hofmann, and Redwood, in their 'Report on the Supply of Spirit of Wine, free of duty, for use in the Arts and Manufactures,' addressed to the Chairman of the Board of Inland Revenue, came to the following conclusions :-

- From the results of this inquiry it has appeared that means exist by which spirit of wine, produced in the usual way, may be rendered unfit for human consumption, as a beverage, without materially impairing it for the greater number of the more valuable purposes in the arts to which spirit is usually applied. To spirit of wine, of not less strength than corresponds to density 0.830 , it is proposed to make an addition of 10 per cont. of purified wood-naphtha (wood or methylic spirit), and to issue this mixed spirit for consumption, duty-free, under the name of Methylated Spirit. It has been shown that methylated spirit resists any process for its purification; the 
removal of the substance addod to the spirit of wine being not only difficult, but, to all appoarance, impossiblo; and further, that no danger is to bo apprehended of the mothylated spirit being ever compounded so as to make it palatable. . . . . It may be found safe to reduce eventually the proportion of the mixing ingredient to 5 per cent., or even a smaller proportion, although it has been recommended to begin with the larger proportion of 10 per cent."

And further, the authors justly remark:- The command of alcohol at a low prico is sure to suggest a multitudo of improved procosses, and of novol applications, which can scarcely be anticipated at the present moment. It will be felt far beyond the limited runge of the trades now more immediately concerned in the consumption of spirits; like the repeal of the duty on salt, it will at once most vitally affect the chemical arts, and cannot fail, ultimately, to exert a beneficial influence upon many branches of industry.'

And in additional observations, added subsequently to their original Report, the chemists above named recommend the following restriction upon the sale of the methylated spirit:- That the methylated spirit should be issued, by agents duly authorised by the Board of Inland Revenue, to none but manufacturers, who should themselves consume it: and that application should always be made for it according to a recognised form, in which, besides the quantity wanted, the applicant should state the use to which it is to be applied, and undertake that it should be applied for that purpose only. The manufacturer might be permitted to retail varnishes and other products containing the methylated spirit, but not the methylated spirit itself in an unaltered state.' They recommend that the methylated spirit should not be mado with the ordinary crude, very impure wood-naphtha, since this could not be advantageously used as a solvent for resins by hatters and varnish-makers, as the less volatilo parts of the naphtha would be retained by the resins after the spirit had evaporated, and the quality of the resin would be thus impaired. If, however, the methylated spirit be originally prepared with the crude wood-naphtha, it may be purified by a simple distillation from 10 per cent. of potash.

It appears that the boon thus afforded to the manufacturing community of obtaining spirit duty free has been acknowledged and appreciated; and now for most purposes, where the small quantity of wood-spirit does not interfere, the methylated spirit is generally used.

It appears that even ether and chloroform, which one would expect to derive an unpleasant flavour from the wood-spirit, are now made of a quality quite unobjectionable from the methylated spirit; but care should be taken, especially in the preparntion of medicinal compounds, not to extend the employment of the methylated spirit beyond its justifiable limits, lest so useful an article should got into disroputo. ${ }^{1}$ Methylated spirit can be procured also in small quantities from tho wholesale dealers, containing in solution 1oz. to the gallon of shellac, under the name of 'finish.'

AxcomoxATrs, or AxCoATrs. Graham has shown that alcohol forms crys. tallisable compounds with several salts. These bodies, which he called 'Alcoholates,' are in general rather unstable combinations, and almost always decomposed by water. Among the best known are the following:-
Alcoholate of chloride of calcium . $2 \mathrm{C}^{4} \mathrm{H}^{6} \mathrm{O}^{2}, \mathrm{Ca} \mathrm{Cl}$, " $"$ of rine $\mathrm{C}^{4} \mathrm{H}^{6} \mathrm{O}^{2}, \mathrm{Zn} \mathrm{Cl}$, " bichloride of tin . : $\mathrm{C}^{4} \mathrm{H}^{6} \mathrm{O}^{2}, \mathrm{Sn} \mathrm{Cl}^{2}$,
" nitrate of magnesia $\quad$ - $3 \mathrm{C}^{4} \mathrm{H}^{6} \mathrm{O}^{2}, \mathrm{MgO}^{2} \mathrm{~N} \mathrm{O}^{5}$,

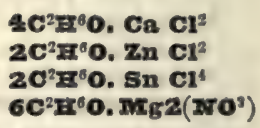

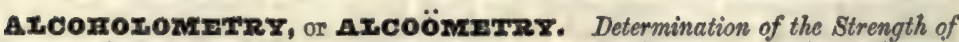
Mixtures of Alcohol and Water. Since the commercial value of the alcoholic liquor's, commonly called 'spirits,' is determined by the amount of pure or absolute alcohol present in thom, it is evident that a ready and accurate means of determining this point is of the highest importance to all persons ongaged in trade in such articles.

If the mixture contain nothing but alcohol and water, it is only necessary to deter. mine the density or specific gravity of such a mixture; if, however, it contain saccharine matters, colouring principles, \&c., as is the case with wine, beor, \&ce, other processes become necessary, which will be fully discussed hereafter.

The determination of the specific gravity of spirit, as of most other liquids, may be effectod, with porhaps greater accuracy than by any other process, by means of a stoppered specific-gravity bottle. If the bottle be of such a size as exactly to hold 1,000 grains of distilled water at $60^{\circ} \mathrm{F}$, it is only necessary to weigh it full of the spirit at the same temperatume, when (the weight of the bottle being known) the specific gravity is obtained by a very simple calculation. See Specric Gravity.

2 Some difference of opinion appears to exist whether Chloroform can be obtained pure from methylated spirit. Bee Mistry. 


\section{ALCOHOLOMETRY}

This process, though very accurate, is somewhat troublesome, espocially to persons unaceustomed to accurate chemical experiments, and it involves the possession of a delicate balance. The necessity for this is, however, obviated by the employment of one of the many modifications of the common hydrometer. This is a floating instrument, the use of which depends upon the principle, that a solid body immersed into a fluid is buoyed upwards with a force equal to the weight of the fluid which it displaces, i.e. to its own bulk of the fluid; consequently, the denser the spirituous mixture, or the less alcohol it contains, the higher will the instrument stand in the liquid; and the less dense, or the more spirit it contajns, the lower will the apparatus sink into it.

There are two classes of hydrometers. 1st. Those which are always immersed in the fluid to the same depth, and to which weights are added to adjust the instrument to the density of any particular fluid. Of this kind are Fahrenheit's, Nicholson's, and Guyton de Morvean's hydrometers.

2nd. Those which are always used with the same weight, but which sink into the liquids to be tried, to different depths, according to the density of the fluid. Of this class are most of the common glass hydrometers, such as Baumé's, Curteis's, Gay-Lussac's, 'Twaddle's, \&c.

Sykes's and Dicas's combine both principles. See HronoMCETERS.

Sykes's hydrometer, or alcoholometer, is the one employed by the Board of Excise, and therefore the one most extensively used in this country.

This instrument does not immediately indicate the density or the per-centage of absolute alcohol, but the degree above or below proof-the meaning of which has been before detailed (p. 45.)

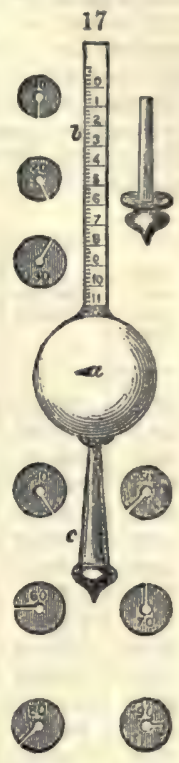

It consists of a spherical ball or float, $a$, with an upper and lower stem of brass, $b$ and $c$. The upper stem is graduated into ten principal divisions, which are each subdivided into five parts. The lower stem, $c$, is made conical, and has a loaded bulb at its extremity. There are nine moveable weights, numbered respectively by tens from 10 to 90 . Each of these circular weights has a slit in it, so that it can be placed on the conical stem, $c$. The instrument is adjusted so that it floats with the surface of the fluid coincident with zero on the scale, in a spirit of specific gravity .825 at $60^{\circ} \mathrm{F}$., this being accounted by the Excise as 'standard alcohol.' In weaker spirit, which has therefore a greater density, the hydrometer will not sink so low; and if the density be much greater, it will be necessary to add one of the weights to cause the entire immersion of the bulb of the instrument. Each weight represents so many principal divisions of the stem, as its number indicates; thus, the heaviest weight, marked 90 , is equivalent to 90 divisions of the stem, and the instrument, with the weight added, floats at 0 in distilled water. As each principal division on the stem is divided into five subdivisions, the instrument has a range of 500 degrees between the standard alcohol (specific gravity 825 ) and water. There is a line on one of the side faces of the stem $b$, near division 1 of the drawing, at which line the instrument with the weight 60 attached to it floats in spirit exactly of the strength of proof, at a tomperature of $51^{\circ} \mathrm{F}$.

In using this instrument, it is immersed in the spirit, and pressed down by the hand until the whole of the graduated portion of the upper stem is wet. The force of the hand required to sink it will be a guide to the selection of the proper woight. Having taken one of the circular weights necessary for the purpose, it is slipped on to the lower conical stem. The instrument is again immersed, and pressed down as before to 0 , and then allowed to rise and settle at any point. The eye is then brought to the level of the surface of the spirit, and the part of the stem cut by the surface, as seen from below, is marked. The number thus indicated by the stem is added to the number of the weight, and the sum of these, together with the temperature of the spirit, observed at tho same time by means of a thermometer, enables the operator, by reference to a Table which is sold to accompany the instrument, to find the strength of the spirit tested.

These Tables are far too voluminous to be quoted here; and this is unnecessary, since the instrument is never sold without them.

A modification of Sykes's hydrometer has been adopted for testing alcoholic liquors which is perhaps more convenient, as the necessity for the loading weights is done away with, the stem being sufficisntly long not to require them. It is 
constructed of glass, and is in the shape of a common hydrometor, the stem being divided into degrees; it carries a small spirit thermometer in the bulb, to which a Bcale is fixed, ranging from $30^{\circ}$ to $82^{\circ} \mathrm{F}$. $\left(0\right.$ to $12^{\circ} \mathrm{O}$.) There are Tables supplied with the hydrometer, which are headed by the degrees and half degrees of the thermometric scale; and the corresponding content of spirit, over or under proof at the respective degree of the Table, is placed opposite each degree of the hydrometer.

By means of either of these instruments, and by the use of the Tables accompanying them, we learn the strength, in degrees, above or below proof; and the following Table by Dr. Ure will be found most useful in converting these numbers into specific gravities :-

\begin{tabular}{|c|c|c|c|c|c|c|c|c|c|}
\hline $\begin{array}{l}\text { Per } \\
\text { cent. } \\
\text { over } \\
\text { Proof }\end{array}$ & $\begin{array}{l}\text { Bpecific } \\
\text { Gravity }\end{array}$ & $\begin{array}{l}\text { Per } \\
\text { cent. } \\
\text { over } \\
\text { Proof }\end{array}$ & $\begin{array}{l}\text { Specific } \\
\text { Gravity }\end{array}$ & $\begin{array}{c}\text { Per } \\
\text { cent. } \\
\text { over } \\
\text { Proof }\end{array}$ & $\begin{array}{l}\text { 8pecific } \\
\text { Gravity }\end{array}$ & $\begin{array}{l}\text { Per } \\
\text { cent. } \\
\text { over } \\
\text { Proof }\end{array}$ & $\begin{array}{l}\text { Specific } \\
\text { Gravity }\end{array}$ & $\begin{array}{c}\text { Per } \\
\text { cent. } \\
\text { over } \\
\text { Proof }\end{array}$ & $\begin{array}{l}\text { Specific } \\
\text { Gravity }\end{array}$ \\
\hline $67 \cdot 0$ & 8156 & $43 \cdot 1$ & 8597 & $19 \cdot 3$ & 0.8948 & $7 \cdot 0$ & 0.9282 & $53 \cdot 3$ & 0.9698 \\
\hline 66.5 & 0.8166 & $42 \cdot 6$ & 604 & $19 \cdot 1$ & & $8 \cdot 0$ & 0.9295 & 54.8 & 0.9701 \\
\hline $66 \cdot 1$ & 0.8174 & $42 \cdot 0$ & 8615 & $18^{\circ} 6$ & 0.8959 & $9 \cdot 0$ & 0.9306 & $56 \cdot 2$ & 0.9709 \\
\hline $65 \%$ & 0.8188 & 41.5 & 8622 & 18.0 & 0.8966 & $10 \cdot 0$ & 0.9318 & $57 \cdot 6$ & 0.9718 \\
\hline $65^{\circ} 0$ & 0.8199 & $41 \cdot 1$ & 8629 & $17 \cdot 5$ & 0.8974 & $11 \cdot 0$ & 0.9329 & $58 \cdot 3$ & 0.9722 \\
\hline $64 \cdot 5$ & 0.8210 & $40 \cdot 6$ & 8636 & 16.9 & 0.8981 & $12 \cdot 1$ & 0.9341 & $59 \cdot 0$ & 0.9726 \\
\hline 64.0 & 0.8221 & 40 & $\cdot 8646$ & 16.4 & 0.8989 & $13 \cdot 1$ & 0.9353 & $60 \cdot 4$ & 0.9734 \\
\hline 63.6 & 0.8227 & 39. & 3 & $15 \cdot 9$ & 0.8996 & $14 \cdot 2$ & 0.9364 & $61 \cdot 1$ & 0.9738 \\
\hline $63 \cdot 1$ & 0.8238 & $39^{\circ}$ & 0.8660 & $15 \cdot 6$ & 0.9000 & $15 \cdot 3$ & 0.9376 & $61 \cdot 8$ & 0.9742 \\
\hline $62 \cdot 5$ & 0.8249 & & & $15 \cdot 0$ & 8 & 16.0 & & & 0.9750 \\
\hline $62 \cdot 0$ & 0.8259 & & 0.8678 & 14.5 & 0.9015 & $17 \cdot 1$ & 0.9396 & 63.9 & 0.9754 \\
\hline $61^{\circ}$ & 0.8266 & 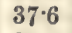 & & $1:$ & 3 & 18.2 & 9407 & $65 \cdot 3$ & 0.9762 \\
\hline $61 \cdot 1$ & 0.8277 & $37 \cdot 1$ & 0.8692 & & 0.9030 & 19.3 & 0.9419 & 66.0 & 0.9766 \\
\hline 60 & 0.828 & 3 & & & 4 & 20.0 & & $67 \cdot 4$ & 0.9774 \\
\hline 60.0 & 0.8298 & $?$ & 0.8 & 12.5 & 0.9041 & $21 \cdot 2$ & 37 & $68 \cdot 0$ & 0.9778 \\
\hline $59 \cdot 6$ & 0.83 & & & & & 22 & 8 & $69 \cdot 4$ & 0.9786 \\
\hline $59 \cdot 1$ & 0.83 & & 0.87 & $\cdot 4$ & 0.8 & $23 \cdot 1$ & 0.9 & $70 \cdot 1$ & 0.9790 \\
\hline 58.6 & 0.83 & & & & & $23 \cdot 9$ & 34 & 71 & 0.9798 \\
\hline 58.0 & 0.8336 & & 0 & 6 & 7 & $24: 3$ & 9468 & $72 \cdot 1$ & 0.9802 \\
\hline $57 \cdot 5$ & 0.83 & & & & & $25 \cdot 1$ & & $73 \cdot 5$ & 0.9810 \\
\hline $57 \cdot 1$ & 0.83 & & & & & $26 \cdot 3$ & & $74 \cdot 1$ & 0.9814 \\
\hline $56 \cdot 6$ & & & & & & $27 \cdot 1$ & & $75 \cdot 4$ & 0.9822 \\
\hline 56.0 & 0. & & & & & & & & 0.9826 \\
\hline $55^{\circ}$ & & & & & 0 & $29 \cdot 2$ & & $77 \cdot 3$ & 0.9834 \\
\hline 55 & & & & & & & & & \\
\hline 54. & & & & & & & & $79 \cdot 2$ & 0.9846 \\
\hline $64^{\circ}$ & & & & & & & & & \\
\hline 53 & & & & & & $3 \cdot 2$ & & $81 \cdot 1$ & 0.9858 \\
\hline $53 \cdot]$ & & & & & & & & & 0.9866 \\
\hline 52 & & & & & 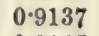 & 1 & & $83 \cdot 5$ & 0.9874 \\
\hline 52. & & & & 4 & & & & & \\
\hline 51 & & & & & & 2 & & 8 & 0.9886 \\
\hline 51 & & & & & & $38 \cdot 1$ & & $86 \cdot 3$ & 0.9894 \\
\hline 50 & & & & & & 3 & & 4 & 0.9902 \\
\hline 50 & & & & & & $40 \cdot 1$ & & 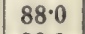 & 906 \\
\hline 49 & & & & & & & & 0.1 & 0.9914 \\
\hline 49 & & & & & & $42 \cdot 2$ & & 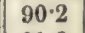 & 0.3922 \\
\hline $48^{\circ}$ & & & & & & & & & \\
\hline 48 & & & & & & & & $92 \cdot 3$ & 0.9938 \\
\hline $47 \cdot 6$ & & & & & & & & & \\
\hline 47 & & & & & & $46 \cdot 1$ & 0.9646 & $94: 3$ & 0.9954 \\
\hline $46 \cdot 6$ & & & & & & & & & \\
\hline 46 & & & & & & & 0.9 & 96.4 & 0.9970 \\
\hline $45^{\circ} \mathrm{C}$ & & & & 3 & & & & & 0.9978 \\
\hline 45 & & & & & & & & $98 \cdot 2$ & 0.9986 \\
\hline $44 \cdot 6$ & 0.857 & $20 \cdot 4$ & & 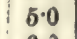 & 0.92 & 51.0 & 0.9677 & $99 \cdot 1$ & 0.9993 \\
\hline 43. & $0.8=0$ & $19 \cdot 9$ & 0.8940 & $6 \cdot$ & 0.9270 & $52 \cdot 2$ & 0.9685 & 100.0 & 1.0000 \\
\hline & & & & & & & & & \\
\hline
\end{tabular}




\section{ALCOHOLOMETRY}

And now, by reference either to Drinkwater's, Tralle's, or Gay-Lussac's Tables, the operator will be enabled to find, by the knowledge of the density or specific gravity, at the temperature at which the operation was performed, the per-centage of real alcohol, either by weight or by rolume.

In France, Gay-Lussac's alcoölometre is usually employed. It is a common glass hydrometer, with the scale on the stem divided into 100 parts or degrees. The lowest division, marked 0 , denotes the specific gravity of pure water; and 100, that of absolute alcohol, both at $15^{\circ} \mathrm{C} .\left(59^{\circ} \mathrm{F}\right.$.) The intermediate degrees, of course, show the per-centage of absolute alcohol by volume at $15^{\circ} \mathrm{C}$.; and the instrument is accompanied by the Tables already given for ascertaining the per-centage at any other tomperature.

\section{Alcoholometry of Liquids containing besides Alcohol, Saccharine Matters, Colouring Principles, go., such as Wines, Beer, Liqueurs, gc.}

In order to determine the proportion of absolute alcohol contained in wines, or other mixtures of alcohol and water with saccharine and other non-volatile substances, the most accurate method consists in submitting a known volume of the liquid to distillation (in a glass retort, for instance); then, by determining the specific gravity of the distilled product, to ascertain the per-centage of alcohol in this distillate, which may be regarded as essentially a mixtnre of pure alcohol and water. The distillation is carried on until the last portions have the gravity of distilled water; by then ascertaining the total volume of the distillate, and with the knowledge of its per-centage of alcohol and the volume of the original liquor used, the method of calculating the quantity of alcohol present in the wine, or other liquor, is sufficiently obvious.

In carrying out these distillations care must be taken to prevent the evaporation of the spirit from the distillate, by keeping the condenser cool. And Professor Mulder recommends the use of a refrigerator, consisting of a glass tube fixed in the centre of a jar, so that it may be kept filled with cold water. The tube must be bent at a right angle, and terminate in a cylindrical graduated measure-glass, shaped like a bottlo.'

It is well to continue the distillation until about two-thirds of the liquid has passed over.

This process, though the most accurate for the estimation of the strength of alcoholic liquors, is still liable to error. The volatile acids and ethers pass over with the alcohol into the distillate, and, to a slight extent, affect the specific gravity. This orror may be, to a great extent, overcome by mixing a little chalk with the wine, or other liquor, previous to distillation.

By this method Professor Brande made, some years ago, determinations of the strength of the following wines, and other liquors: ${ }^{2}-$

\section{Proportion of Spirit per Cent. by Measure.}

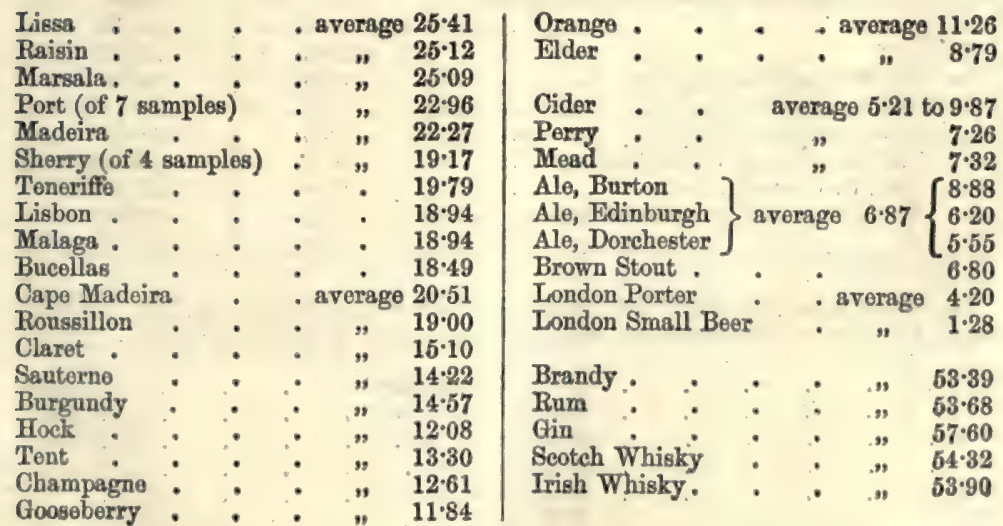

1. The Chemistry of Wine, by G. J. Mulder, edited by H. Bence Jones, M.D.

- Brande"s Marnal of Ohemistry ; slso Philosophical Trans. 1811. 
The following results wore obtained by the same chemist more recontly by this process (1854):-

\section{Prr-centage of Aloohol by Volume.}

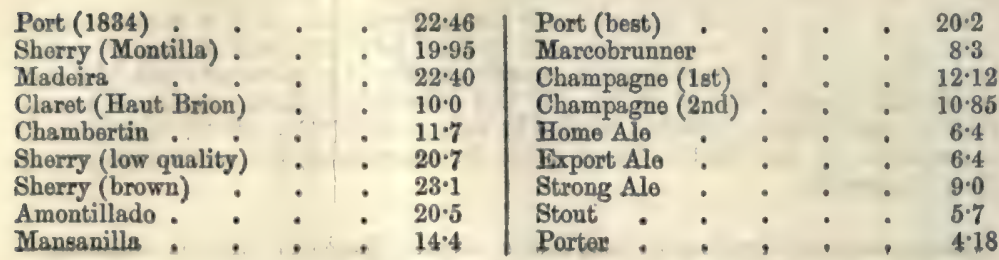

Dr. Chrtstison determined the Aloohalic Strength of Wines as follows :-

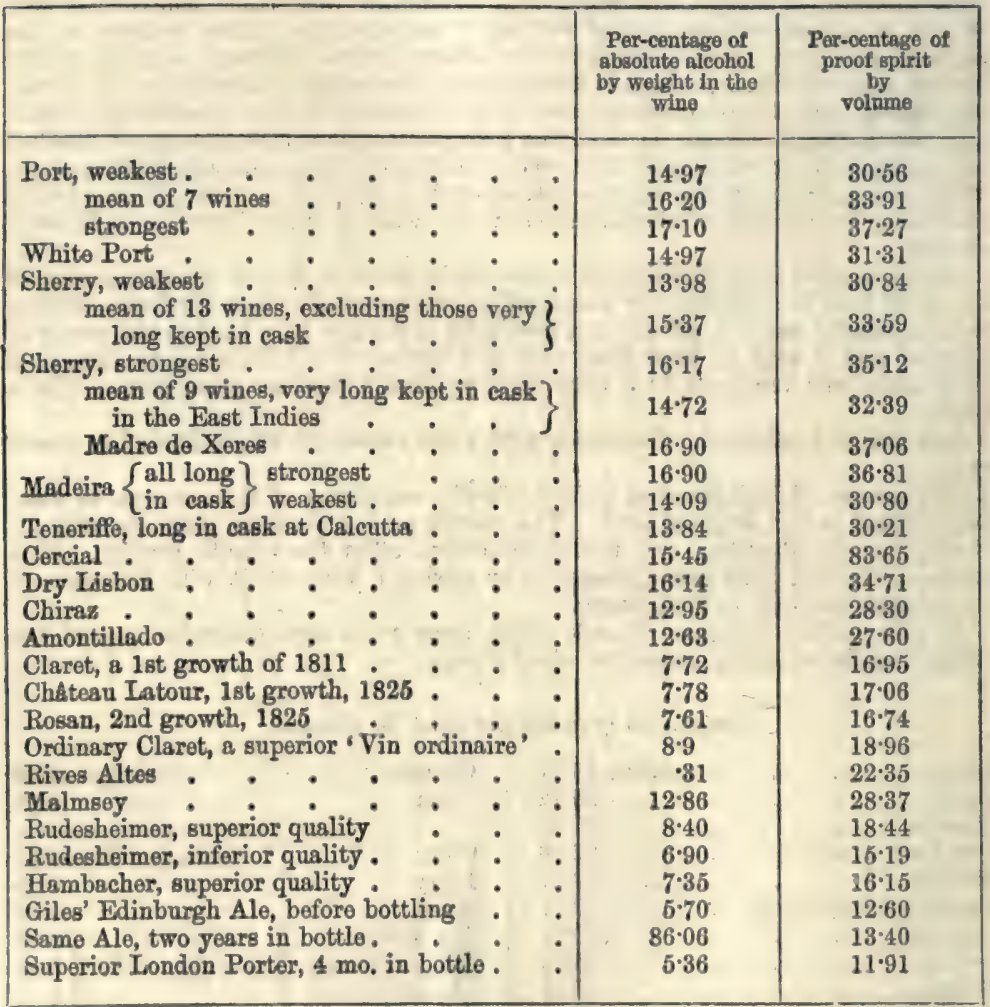

Dr. Bence Jones states that the different fermented liquids which he has examined might, in regard to their strength, or stimulating power, be arranged thus:-

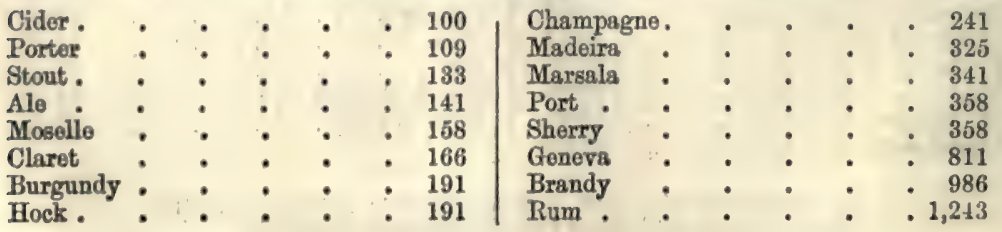


Thus, ten glasses of cider or porter, six glasses of elaret, five glasses of Burgundy, four glasses of champagne, three glasses of pert, sherry, or Marsala, are equivalent to one glass of brandy.

M. l'Abbé Brossard-Vidal, of Toulon,' has proposed to estimate the strength of alcoholic liquors by determining their boiling point. Since water boils at $100^{\circ} \mathrm{C}$. $\left(212^{\circ}\right.$ F.), and absolute alcohol at $78.4^{\circ}\left(173^{\circ} \mathrm{F}\right.$.), it is evident that a mixture of water and alcohol will have a higher boiling point the larger the quantity of water present in it. This method is even applicable to mixtures containing other bodies in solution besides spirit and water, since it has been shown that sugar and salts, when present (in moderate quantities), have only a very trifling effect in raising the boiling point, and the process has the great advantage of facility and rapidity of execution, though of course not comparable to the method by distillation, for accuracy.

Mr. Field's patent (1847) alcoholometer is likewiso founded upon the same principle. The instrument was subsequently improved by Dr. Ure.

The apparatus consists simply of a spirit-lamp placed under a little boiler containing the alcoholic liquor, into which fits a thermometer of very fine bore.

When the liquor is stronger than proof spirit, the variation in the boiling point is so small that an accurate result cannot possibly be obtained; and, in fact, spirit approaching this strength should be diluted with an equal volume of water before submitting it to ebullition, and then the result doubled.

Another source of error is the elevation of the boiling point, when the liquor is kept heated for any length of time; it is, however, nearly obviated by the addition of common salt to the solution in the boiler of the apparatus, in the proportion of $\mathbf{3 5}$ or 40 grains. In order to correct the difference arising from higher or lower pressure of the atmosphere, the scale on which the thermometric and other divisions are marked is made moveable up and down the thermometer tube; and every time, before commencing \& set of experiments, a preliminary experiment is made of boiling some pure distilled water in the apparatus, and the zero point on the scale (which indicates the boiling point of water) is adjusted at the level of the surface of the mercury. On p. 55 will be found a Table showing the boiling point of alcohol of difforent specific gravities.

But even when performed with the utmost care, this process is still liable to very considerable errors, for it is extremely difficult to observe the boiling point to within a degree; and after all, the fixed ingredients present undoubtedly do seriously raise the boiling point of the mixture-in fact, to the extent of from half to a whole degree, according to the amount present.

Silbermann's Method. - M. Silbermann 2 has proposed another method of estimating the strength of alcoholic liquors based upon their expansion by heat. It is well known that, between zero and $100^{\circ} \mathrm{C} .\left(212^{\circ} \mathrm{F}\right.$.), the dilatation of alcohol is triple that of water, and this difference of expansion is even greater between $25^{\circ} \mathrm{C}$. $\left(77^{\circ} \mathrm{F}\right.$.) and $50^{\circ} \mathrm{C}$. $\left(122^{\circ} \mathrm{F}\right.$.); it is evident, therefore, that the expansion between these two temperatures becomes a measure of the amount of alcohol present in any mixture. The presence of salts and organic substances, such as sugar, colouring, and extractive matters, in solution or suspension in the liquid, is said not materially to affect the accuracy of the result; and M. Silbermann has devised an apparatus for applying this principle, in a ready and expeditious manner, to the estimation of the strength of alcoholic liquors. The instrument may be obtained of the philosophical instrument makers of London and of Liverpool.

It consists of a brass plate, on which are fixed-1st, an ordinary mercurial thermometer graduated from $22^{\circ}$ to $50^{\circ} \mathrm{C}$. $\left(77^{\circ}\right.$ to $122^{\circ}$ F.), these being the working temperatures of the dilatatometer; and 2ndly, the dilatatometer itself, which consists of a glass pipette, open at both ends, and of the shape shown in the figure. A valve of cork or india-rubber closes the tapering end $\Delta$, which valve is attached to a rod, $b b$, fastened to the supporting plate, and connected with a spring, $n$, by which the lower orifice of the pipette can be opened or closed at will. The pipette is filled, exactly up to the zero point, with the mixture to be examined-this being accomplished by the

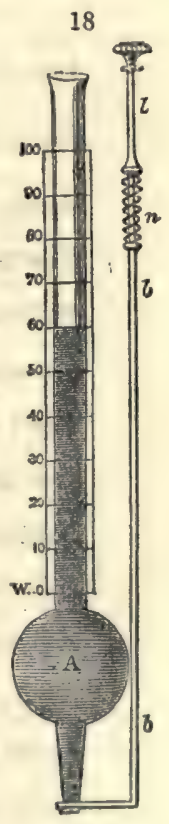
aid of a piston working tightly in the long and wide limb of the pipette; the action of which serves also another valuablo purpose-viz. that of drawing any 
bubbles of air out of the liquid. By now observing the dilatation of the column of liquid when the temperature of the whole apparatus is raised, by immersion in a water-bath, from $25^{\circ}$ to $50^{\circ}$, the co-efficient of expansion of the liquid is obtained, and hence the proportion of alcohol - the instrument being, in fact, 80 graduated, by experiments previously made upon mixtures of known composition, as to give at once the per-centage of alcohol.

Another alcoholometer, which, like the former, is more remarkable for the great facility and expodition with which approximative results can be obtained than for a high degree of accuracy, was invented by $\mathbf{M}$. Geisler, of Bonn, and depends upon the measurement of the tension of the vapour of the liguid, as indicated by the height to which it raises a column of mercury.

Geisler's Alcoholometer. It consists of a closed vessel in which the alcoholic mix19 ture is raised to the boiling point, and the tension of the rapour ob19 indication being rendered more manifest by the elevation of the other end
of the column.

The wine or other liquor of which it is desired to ascortain the strength, is put into the little flask, $\mathrm{F}$, which, when completely filled, is serewed on to the glass which contains mercury, and is closed by a stnpeock at 8. The entire apparatus, which at present is in an inverted position, is now stood erect, the flask and lower extremity of the tube being immersed in a water-bath. The vinous liquid is thus heated to the boiling point, and its vapour forces the mercury up into the long limb of the tube. The instrument having been graduatod, once for all, by actual experiment, the per-centage of alcohol is read off at once on the stem by the height to which the mercurial column rises.

To show how nearly the results obtained by this instrument agree with those obtained by the distillation process, comparative experiments were made on the same wines by Dr. Bence Jones.'

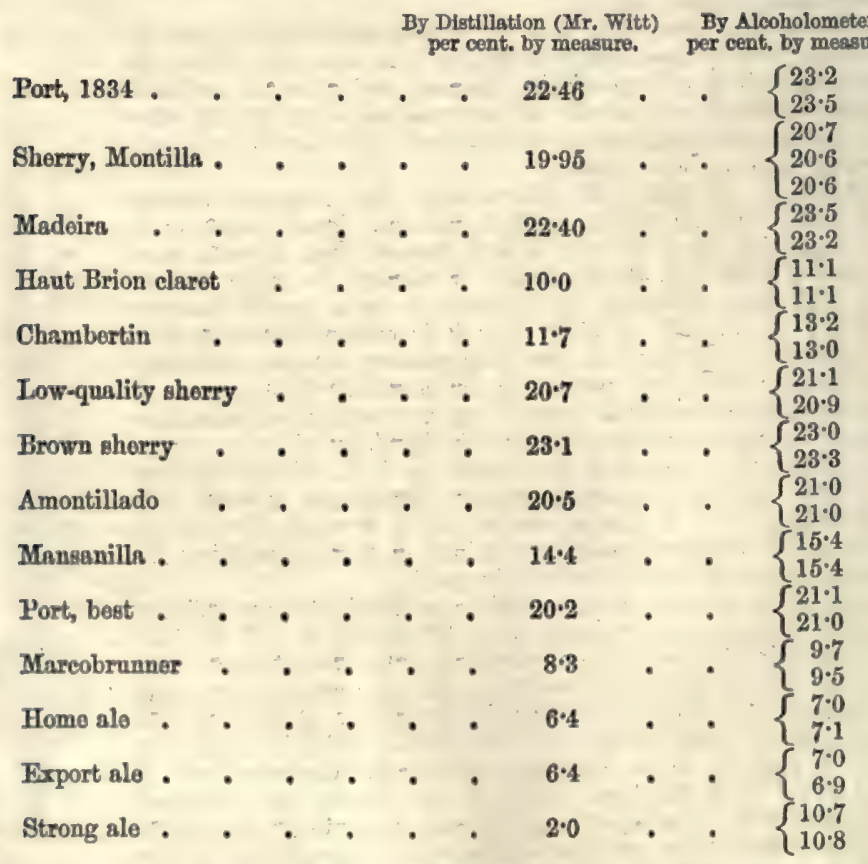

Tabaril's Method.-There is another method of determining the alcoholic contents of mixtures, which especially recommends itself on account of its simplicity. The

'On the Acidity, Sweetness, and Strength of different Wines, by H. Bence Jones, M.D., F.R.S, Proceedings of the Boyal Institution, Pebruary, 1854. 
specific gravity of the liquor is first determined, half its volume is next evaporated in the open air, sufficient water is then added to the remainder to restore its original volume, and the specitic gravity again ascertained. By deducting the specific gravity before the expulsion of the alcohol from that obtained afterwards, the difference gives a specifie gravity indicating the per-centage of alcohol, which may be found by referring to Gay-Lussac's or one of the other Tables. Tabarié has constructed a peculiar instrument for determining these specific gravities, which he calls an enometer; but they may be performed either by a specific-gravity bottle or by a hydrometer in the usual way.

Of course this method cannot be absolutely accurate; nevertheless, Prof. Mulder's experience with it has led him to prefer it to any of the methods before described, especially where a large number of samples have to be examined. He states that the results are almost as accurate as those obtained by distillation. The evaporation of the solution may be accolerated by conducting hot steam through it.

Adulterations,-Absolute alcohol should be entirely free from water. This may be recognised by digesting the spirit with pure anhydrous sulphate of copper. If the spirit contain any water, the white salt becomes tinged blue, from the formation of the blue hydrated sulphate of copper.

Rectified spirit, proof spirit, and the other mixtures of pure alcohol and water, should be colourless, free from odour and taste. If containing methylic or amylic alcohols, they are immediately recognised by one or other of these simple tests.

Dr. Ure states that if wood-spirit be contained in alcohol, it may be detected to the greatest minuteness by the test of caustic potash, a little of which, in powder, causing wood-spirit to become speedily yellow and brown, while it gives no tint to alcohol. Thus 1 per cent. of wood-spirit may be discovered in any sample of spirits of wine.

The admixture with a larger proportion than the due amount of water is of course determined by estimating the per-centage of absolute alcohol by one or other of the several methods just described in detail.

The adulterations and sophistications to which the various spirits known as rum, brandy, whisky, gin, \&c. are subjected, will be best described under these respective heads, since these liquors are themselves mixtures of alcohol and water with sugar, colouring matters, flavouring ethers, \&c.

AIDERYDI. Aldehyde was first obtained by Döbereiner, who named it Iight Oxygen Ether. The name is an abbreviation of Alcohol dehydrogenatum. It is the fluid obtained from alcohol by the removal of two atoms of hydrogen. Thus, alcohol being represented by the formula $\mathrm{C}^{4} \mathrm{H}^{6} \mathrm{O}^{2}\left(\mathbf{C}^{2} \mathbf{m}^{6} \mathbf{0}\right)$, aldehyde becomes $\mathrm{C}^{4} \mathrm{H}^{4} \mathrm{O}^{2}$ $\left(\mathbf{C}^{2} \mathbf{T}^{4} \mathbf{0}\right)$. Seo LAMTPIC ACID.

Preparation.-Aldehyde is prepared by various processes of oxidation. Liebig has published several methods, of which the following is perhsps the best. Three parts of peroxide of manganese, three of sulphuric acid, two of water, and two of alcohol of 80 per cent., are well mixed and carefully distilled in a spacious retort. The extreme volatility of aldehyde renders good condensation absolutely necessary. The contents of the retort are to be distilled over a gentle and manageable fire until frothing commences, or the distillate becomes acid. This generally takes place when about one-third has passed over. The fluid in the receiver is to have about its own weight of chloride of calcium added, and, after slight digestion, is to be carefully distilled on the water-bath. The distillate is again to be treated in the same way. By these processes a fluid will be obtained entirely free from water, but containing several impurities. To obtain the aldehyde in a state of purity it is necessary, in the first place, to obtain aldehyde-ammonia ; this may be accomplished in the following manner:-The last distillate is to be mixed in a flask with twice its volume of ether, and, the flask being placed in a vessel surrounded by a freezing mixture, dry ammoniacal gas is passed in until the fluid is saturated. In a short time crystals of the compound sought separate in considerable quantity. The aldehyde-ammonia, boing collected on a filter, or in the neck of a funnel, is to be washed with ether, and dried by pressure between folds of filtering paper, followed by exposure to the air. It now becomes necessary to obtain the pure aldehyde from the compound with-ammonia. For this purpose two parts are to be dissolved in an equal quantity of water, and three parts of sulphuric acid, mired with four of water, are to be added. The whole is to be distilled on the water-bath, the temperature, at first, being very low, and the operation being stopped as soon as the water boils. The distillate is to be placed in a retort connected with a good condensing apparatus, and, as soon as all the joints are known to be tight, chloride of calcium, in fragments, is to be added. The heat arising from the hydration of the chloride causes the distillation to commence, but it is carried on by a water-bath. The dis. tillate, after one more rectification over chloride of calcium, at a temperature not

Vot. I. 
excoeding $80^{\circ}$ F., will consist of pure aldehyde. Aldohyde is a colourless, very volatile, and mobile fluid, having the density 0.800 at $32^{\circ}$. It boils, under ordinary atmospheric pressure, at $70^{\circ} \mathrm{F}$. Its vapour density is 1.532 . Its formula corrosponds to four volumes of vapour; we consequently obtain the theoretical vapour density by multiplying its atomic weight $=44$ by half tho donsity of hydrogen, or 0346. The number thus found is 1.5224 , corresponding as noarly as could be desired to the experimental result.

Aldehyde is produced in a great number of processes, particularly during the destructive distillation of various organic matters, and in processes of oxidation. From alcohol aldehydo may be procured by oxidation with platinum black, nitric acid, chromic acid, chlorine (in presence of wator), or, as we have seen, a mixture of peroxide of manganese and sulphuric acid. Certain oils, by destructive distillation, yield it. Wood vinegar in the crude state contains aldehydo as well as wood-spirit. Lactic acid, when in combination with weak bases, yiolds it on destructive distillation. Various animal and vegotable products afford aldehydo by distillation with oxidizing agents, such as sulphuric acid and peroxide of manganese, or bichromate of potash.

The word aldehyde, like that of alcohol, is gradually becoming used in a much more extended sense than it was formerly. By the term is now understood any organic substance which, by assimilating two atoms of hydrogen, yields a substanco having the properties of an alcohol, or, by taking up two atoms of oxygen, yields an acid. It is this lattor property which has induced cortain chomists to say that there is the same relation between an aldehyde and its acid as between inorganic acids onding in ous and ic. Several very interosting and important substances are now known to belong to the class of aldehydes. The essential oils are, in several instances, composed principally of bodies having the properties of aldehydes. Among the most prominent may be mentioned the oils of bitter almonds, cumin, cinnamon, rue, \&ze. Now that tho character of the aldehydes is becoming better understood, the chances of artificially producing the essential oils above alluded to in the commercial scale become greatly increased. A substitute for one of them has been for some years known under the very incorrect name of artificial oil of bitter almonds. See Nimrobanzorr, - C. G. W.

AIDEEYDI GRarI, Aniline Green, or Emeraldine. This dye was discovorod in 1863 by M. Cherpin, of Saint Ouen, and is employed for dyeing silk; the colour is especially brilliant by candle-light. It may be conveniently prepared by adding one-half part of aldehyde to a cold solution of one part of magenta in throe of strong sulphuric acid, and one of water. This mixture, when heated, yields an unstablo blue substance known as aldehyde blue. By pouring this into a large bulk of boiling water containing about three or four times as nuch hyposulphite of soda as magenta originally employed, and then boiling and filtering the product, the aldehyde green will be obtained in the filtrate. See Avmrns.

AxDEr. (Aune, Fr. ; Erle, Ger.; Alnus glutinosa, Lin.) A tree, different species of which are indigenous to Europe, Asia, and America. The common alder seldom grows to a height of more than 40 feet. The wood is stated to be very durable under: water. The piles at Venice, and those of Old London Bridge, are stated to have been of alder; and it was much used for pipes, pumps, and sluices. The charcoal of this wood is used for gunpowder.

AIE. The fermented infusion of pale malted barley, combined with infusion of

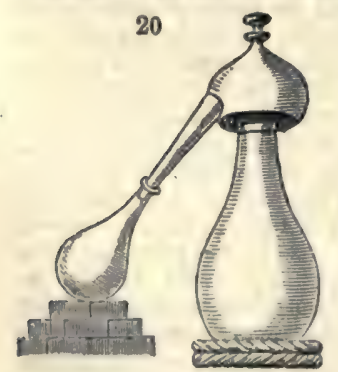
hops. See Berr.

Axzmezc. A Still. See Disminatiox. The term is, however, applied to a still of peculiax construction, in which the head, or capital, is a separato piece, fitted and ground to the neck of the boiler, or cucurbit, or otherwise carefully united with a luto. The elembic has this advantage over the common retort, that the residue of distillation may be easily cleared out of the body. It is likewise capable, when skilfully managed, of distilling a much larger quantity of liquor in a given time than a retort of equal capacity. In France the term alembic, or rather alambic, is used to designate a glass still.

AIFMrgotr, SAxT OF. (Sal Alembroth.)

The salt of wisdom of the alchemists; a compound of bichloride of mercury and asl ammoniac. If two atoms of bichloride of mercury are mixed with one atom of sal ammoniac and eight atoms of water, at $140^{\circ}$ this mixturo is fluid, but the salt of alembroth crystallises on cooling. 
Arnercon raca. A French lece, of which it is one of the richest, finest, strongest, and most expensive. It has a six-sided mosh of two threads wove with pure hand-spun linen thread. See LAcz.

AIFXANDRITr. A variety of Chrysoberyl occurring in large twin crystals, which are usually arranged in six-rayed star-shapod groups. The mineral presents an emerald-green colour, due probably to the presence of chromium; but by transmitted light the colour is columbine-red. It is found in the emerald-mines of Takowaja, 180 versts east of Katerinburg, in the Ural Mountains; but is not suffciently clear and free from tlaws to be used as a precious stone.

AIG 2 . (Varech, Fr. ; Seegras, or Alge, Ger.) An order of cryptogamous planta, including the seaweeds (fucus) and the lavors (ulva) growing in salt wator, and the freshwater confervas. Wo havo only to deal with those seavoeds which are of any commercial value.

Dr. Pereira gives the following list of esculent seeweeds :

\begin{tabular}{l|l} 
Rhodomenia palmata (or Dulso) & Iridea edulis. \\
Rhodomenia ciliata. & Alaria esculenta. \\
Laminaria saccharina. & Ulva latissima.
\end{tabular}

Rhodomenia palmata passes under a variety of names, dulse, dylish, or dellish, and amongst the Highlanders it is called dulling, or waterleaf. It is omployed as food by the poor of many nations; when well washed, it is chewed by the peasantry of Ireland without being dressed. It is nutritious, but sudorific, has the smell of violets, imparts a mucilaginous feel to the mouth, leaving a slightly acrid taste. In Iceland the dulse is thoroughly washed in fresh water and dried in the air. When thus treated it becomes covered with a white powdery substanee, which is sweet and palatable; this is mannite (80e MANNA), which Dr. Stenhouse proposes to obtain from seaweeds. 'In the dried state it is used in Iceland with fish and butter, or else, by the higher classes, boiled in milk, with the addition of rye flour. It is preserved packed in close casks: a fermented liquor is produced in Kamtschatka from this seaweed, and in the North of Europe and in the Grecian Archipelago cattlo are fed upon it.' - Stenhouse.

Laminaria saccharina yields $12 \cdot 15$ per cent, of mannite, while the Rhodomenia palmata contains not more than 2 or 3 per cent.

Iridce edulis. - The fronds of this woed are of a dull purple colour, flat, and succulent. It is employed as food by fishermen, either raw or pinched between hot irons, and its taste is then said to resemble roasted oysters.

Alaria esculenta.-Mr. Drummond informs us that on the coast of Antrim, 'it is often gathered for eating, but the part used is the leaflets, and not the midrib, as is commonly stated. These have a very pleasant taste and flavour, but soon cover the mouth with a tenacious greenish crust, which causes a sensation somewhat like that of the fat of a heart or kidney.'

Ulva latissima (Broad green laver).-This is rarely used, being considered inferior to the Porphyra laciniata (Laciniated purple laver). This alga is abundant on all our shores. It is picklod with salt, and sold in England as laver, in Ireland as sloke, and in Scotland as slaak. The London shops are mostly supplied with lavor from the coasts of Devonshire. When sterred, it is brought to the table and eaten with pepper, butter or oil, and lemon-juice or vinegar. Some persons stew it with loeks and onions. The pepper dulse (Laurencia pinnatifida), distinguished for its pungent taste, is often used as a condiment when other seaweeds are eaten. "Tanglo" (Laminaria digitata), so called in Scotland, is tormed 'red-ware' in the Orkneys, 'soa-wand ' in the Highlands, and 'sea-girdles ' in England. The flat leathery fronds of this weod, when young, are employed as food. Mr. Simmonds tells us, "There was a time when the cry of "Buy dulse and tangle" was as common in the streets of Edinburgh and Glasgow, as is that of "water-cresses" now in our metropolis.'Society of Arts' Journal.

Laminaria potatorum.- - The large sea tangle is used abundantly by the inhabitants of the Straits of Magellan and by the Fuegians. Under the name of 'Bull Kelp' it is used as food in New Zealand and Van Diomen's land. It is stated to be excoedingly nutritive and fattening.

Chondrus crispus (chondrus, from $\chi$ évôos, cartilage).--Carrageen, Irish, or pearl moss. For purposes of diet and for medicinal uses, this alga is collected on the west coast of Ireland, washed, bleached by exposure to the sun, and dried. It is not unfrequently used in Ireland by painters and plasterers as a substitute for size. It has also beon successfully applied, instead of isinglass, in making blanc-mango and jellies; and in addition to its use in medicine, for which purpose it was introduced by Dr, Todlunter, of Dublin, sbout 1831, a thick mucilage of carrageen, scented with 
somo propared spirit, is sold as bandoline, fixature, or clysphitique, and it is omployed for stiffening silks. According to Dr. Davy, carrageen consists of

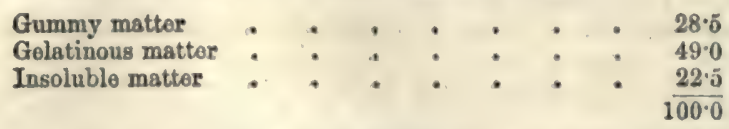

The following results, obtained by Dr. Apjohn and Dr. Davy, show, in a satisfao. tory manner, the value of the algø. The amount of wator is less than that which belongs to the alga when fresh from the soa, all these having undergono a partial drying in the progress of carriage from the coast:-

Specimens supplied by Dr. Davy, and dried at $212^{\circ}-$

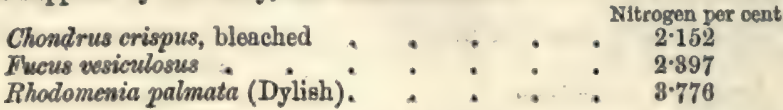

\begin{tabular}{|c|c|c|c|c|}
\hline Kinds of Algw & Weter & $\begin{array}{c}\text { Dry } \\
\text { Matter }\end{array}$ & $\begin{array}{l}\text { Per Cent. } \\
\text { of Nitrogen } \\
\text { in Dry } \\
\text { Matter }\end{array}$ & $\begin{array}{l}\text { Protein } \\
\text { contained } \\
\text { In Dry } \\
\text { Matter }\end{array}$ \\
\hline $\begin{array}{l}\text { Chondrus crispus, bleached } \\
\text { Chondrus crispus, unbleached, Bally- }\end{array}$ & $17 \cdot 92$ & $82 \cdot 08$ & 1.534 & $9 \cdot 587$ \\
\hline Gigartina mammillosa, Ballyesstle : & $21 \cdot 47$ & $78 \cdot 53$ & $2 \cdot 142$ & $13 \cdot 387$ \\
\hline Chondrus crispus, bleached, (2nd expe- & 2100 & 7840 & $2 \cdot 1$ & $13 \cdot 737$ \\
\hline $\begin{array}{l}\text { riment) } \\
\text { Chondrus crispus, unbleached, Bally: }\end{array}$ & $19 \cdot 79$ & $80 \cdot 21$ & $1 \cdot 485$ & $9 \cdot 281$ \\
\hline $\begin{array}{l}\text { castle, (2nd experiment) } \\
\text { Laminaria digitata, or Dulse tangle, }\end{array}$ & $19 \cdot 96$ & 80.04 & $2 \cdot 510$ & $15 \cdot 687$ \\
\hline $\begin{array}{l}\text { Ballycastle } \\
\text { Laminaria digitata, or Black tangle, }\end{array}$ & $21 \cdot 38$ & $78 \cdot 62$ & 1.588 & $9 \cdot 925$ \\
\hline $\begin{array}{l}\text { Ballycastle } \\
\text { Rhodomenia palmata, or Dylish, Bally- }\end{array}$ & $31 \cdot 05$ & 68.95 & 1.396 & $8 \cdot 725$ \\
\hline castle & $16 \cdot 56$ & $83 \cdot 44$ & $3 \cdot 465$ & $21 \cdot 656$ \\
\hline Porphyra laciniata, Ballycastle . & 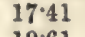 & 82.59 & 0 & $29 \cdot 062$ \\
\hline $\begin{array}{l}\text { Iridea edulis, Ballycastlo } \\
\text { Alaria esculenta, or Murlins, Ballycastle }\end{array}$ & $\begin{array}{l}19 \cdot 61 \\
17 \cdot 91\end{array}$ & $\begin{array}{l}80 \cdot 39 \\
82 \cdot 09\end{array}$ & $\begin{array}{l}3 \cdot 088 \\
2 \cdot 424\end{array}$ & $\begin{array}{l}19 \cdot 300 \\
12 \cdot 150\end{array}$ \\
\hline Mean composition of theso Algro & $20 \cdot 42$ & $79 \cdot 58$ & $2 \cdot 407$ & $15 \cdot 0405$ \\
\hline
\end{tabular}

The quantity of nitrogen contained in some of these plants is remarkably large, and will, of course, with the protcinaceous substances detected in all the Algre, account for the high nutritive value ascribed to them.

Plocaria candida.-Coylon moss; Edible moss. This moss is exported from the islands of the Indian Archipelago, forming a portion of the cargoes of nearly all the junks. It is stated by Mr. Crawford, in his 'History of the Indian Archi. pelago,' that on the spots where it is collected, the prices seldom exceed from 5s. $8 d$. to 78. 6d. per ewt. The Chinese use it in the form of a jelly with sugar, as a sweetmeat, and apply it in the arts as an excollent paste. The gummy matter which they employ for covering lanterns, varnishing papcr, \&cc., is mado chiefly from this moss.

As ordinarily sold in Ceylon, it appears to consist of several varietios of marine productions, with the Plocaria intermixed.

The Agar-Agar of Malacea belongs to this variety; and probably seaweods of this character are used by the Salangana or esculent swallow in constructing their nests, which aro esteemed so great a delicacy by the Chinese. The plant is found on the rocks of Pulo Ticoos and on the shores of the neighbouring islands. It is blanched in the sun for two days, or until it is quite white. It is obtained on submerged banks in tho noighbourhood of Macassar, Celebes, by the Bajow-lout, or sea-gipsios, who sond it to China. It is also collected on the reefs and rocky submerged lodges in the 
neighbourhood of Singapore. Mr. Montgomery Martin informs us that Agar-Agar produces in China from six to eight dollars por pecul in its dry and bulky state. From 6,000 to 12,000 peculs are produced annually, the pecul being equal to 100 catties of $1.333 \mathrm{lbs}$, each.

Similar to this, perhaps the same in character, is the Agal-Agal, another species of seaweed. It dissolves into a glutinous substance, and its principal use is for gumming silks and paper, as nothing equals it for paste, nor is it liable to be eaten by insects. The Chinese make a beautiful kind of lantern formed of netted thread washed over with this gum, and which is extremely light and transparent,

Dr. Macgowan, of Ningpo, forwarded to the Society of Arts, through Sir John Bowring, the following algæ, which he thus names and describes:-

Tan-shwin grass, so named from the place, on the coast of Formosa, whence it is procured. It is used for making yang-trai (ocean-regetable).

Nin-mau (ox-hair) grass. Mado into an iced jelly, and sold in the streets, in hot weather, sugared.

Hâi-tâi (sea-tape). Sent into the interior, wherever fossil coal is used. It is considered corrective of the deleterious exhalations of that fuel. It is usually boiled with pork. This kind comes from Shantung province.

Tsz-tsai (purple vegetable). Often oaton as it is, to give a relish to rice, or cooked.

Fah-tsai (hair vegetable). Boiled, either with animal or. vegetable articles, forms a broth. Also the gills eaten with sugar.

Ki-tsai (hen-foot vegetable). Cooked with soy or vinegar. Used by women to make the hair glossy, and to strengthen it. A kind of Bandoline.

Sea-tape, from Japan. It is preferred to the former.

Within the last few years considerable improvements have been effected ir the economic applications of algæ or seaweeds. Mr. E. C. C. Stanford's method of utilising the marine algæ is carried out at the works of the British Seaweed Company in the Hebrides. The seaweed is collected during the winter, and the Company is thus enabled to employ a large number of hands at a time when they would otherwise be unoccupied. The dried and compressed weed is distilled in retorts at a low red heat; a larger quantity of iodine is thus obtained than would be yielded by burning the weed for kelp in the ordinary way, whilst the alkaline salts are obtained more easily and economically. Further, a number of volatile organic products - such as tar, ammonia, and acetic acid-are collocted from the distillation, and an illuminating gas is also obtained : indeed, the Company's works are lighted by seaweed gas. Finally, the carbonaceous residue in the retort, known as seaweed charcoal, is recommended for use as a valuable deodorizer instead of earth in the dry-closet system. A colloction of products obtained from seaweed by Stanford's process was exhibited in the International Exhibition of 1871. See Krep; Ionink.

\section{arigaroba. Seo Ataravitu.}

AIGAROTE, POWDER OF. Powder of Algarotti,-English Powder. This salt was discovered by Algarotti, a physician of Verona. Chloride of antimony is formed by boiling black sulphide of antimony with hydrochloric acid : on pouring the solution into water, a white flocky precipitate falls, which is an oxychloride of antimony. If the water be hot, the precipitate is distinctly erystalline; this is the powder of algaroth. This oxychloride is used to furnish oxide of antimony in the preparation of tartar emetic.

AICAROVInIA. This substance is called by the Spaniards Algaroba, from the resemblance it bears to the fruit of the Carob (Ceratonia siliqua), which is a native of Europe, in the southern countries of Spain and Portugal. It is the fruit of a tree which grows in Chili, of which the botanical name is Prosopis pallida, according to Captain Bagnald, R.N., who first brought a sample of it to this country in the year 1832. It consists of pods bruised and agglutinated more or less with the extractive exudation of the seeds and husks. According to a more recent determinstion; algarorilla is said to be the product of the tree Juga Marthre of Santa Martha, a province of New Carthagena.

It is an astringent substance repleto with tannin, capable, by its infusion in water, of tanning leather, for which purpose it possesses more than four times the power of good oak-bark. Its active matter is very soluble in water at a boiling temperature. The soeds are merely nutritive and demulcent, but contain no astringent property. This resides in the husks. The seeds in the entire pod constitute about one-fifth of the weight, and they are three or four in number in each oblong pod. Alcohol of 80 per cent. over proof dissolves 64 parts in 100 of this substance. The solution consists chiofly of tannin, with a very little resinous matter. Water dissolvos somewhat moro of it, and affords a very styptic-tastod solution, which precipitates solution 
of isinglass very copiously, like infusion of galls and catechu. Its solution forms with sulphate of irom a black procipitate.

AIcrinIAN ONYX, or ONYX MARnIs. A stalagmitic carbonate of lime, resembling the alabaster of the ancients. The chief quarries are at Ain-Tekbalet in the province of Oran, in Algeria. The deposit there forms regular beds, nearly horizontal, presenting a thickness of from 6 to 10 mètres, and extending over an area of more than 100 hectares. Originally the quarries were worked by the Romans, and subsequently by the Moors of Tlemcen. A few years ago they were re-discovered, and are now actively worked; the marble being highly prized as an ornamental stone. A similar material has been found in the Caucasus, and is worked at Tiflis.

AIIMnsirT. (Alimentum, from alo, to feed). The food necessary for the human body, and capable of maintaining it in a state of health.

1. Nitrogenous substances are required to deposit, from the blood, the organised tissue and solid muscle.

2. And carbonaceous, non-nitrogenous bodies, to aid in the processes of respiration, and in the supply of carbonaceons elements, as fat, \&c., for the due support of animal heat.

For information on these substances, consult Liebig's 'Animal Chemistry,' tho investigations of Dr. Lyon Playfair, and Dr. Robert Dundas Thompson's 'Experimental Researches on Food,' 1846. See Foon.

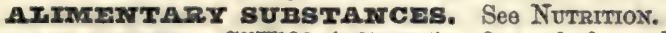

AITZARIJE. $\mathrm{O}^{28} \mathrm{H}^{8} \mathrm{O}^{8}\left(\mathbf{C}^{1 / \mathbf{H}^{8}} \mathbf{O}^{4}\right)$. One of the red colouring principles of Madder. See Maddrar.

In 1869 Messrs. Graebe and Liebermann made the important discovery that alizarine might be produced artificially from anthracene, one of the heavy products of coal-tar distillation. Both scientifically and commercially, the discovery was one of unusual interest: it furnished the first instance of the synthetical formation of an organic colouring matter, and at the same time oponed up a new branch of industry.

According to Messrs. Graebe and Liebermann's original process, the anthracene was first subjected to the action of certain oxidising agents, and thus converted into a compound called anthraquinone or oxanthracene. This conversion may be effected by heating one part of anthracene with two parts of bichromate of potash and sulphuric acid, or by heating one part of anthracene with two parts of bichromate of potash together with one part of glacial acetic acid. The anthraquinone thus obtained is then converted into a bibromide by being heated with bromine. Finally, this dibromanthraquine is heated to about $356^{\circ} \mathrm{F}$. with caustic potash or soda, and thus yields a blue product, which when cold is treated with water ; an excess of acid is then added to the filtered solution, and the yellow precipitate thus thrown down is washed and dried at a gentle heat. This precipitate is identical in chemical composition and in its properties with the alizarine of the madder-root.

Valuable as these researches were in a scientific point of view, it remained for $\mathrm{Mr}$. Perkin to render the process economically available to the manufacturer by introducing important modifications, whereby the use of an expensive agent like bromine was dispensed with. Mr. Perkin showed that when anthraquinone is strongly heated with sulphuric acid of specific gravity $1: 84$, it is converted into disulphoanthroquinonic acid, and that this acid, when heated with hydrate of potash to a temperature of $356^{\circ} \mathrm{F}$, ultimately yields sulphite and alizarate of potash, from which the alizarine may be thrown down, as a bright yellow precipitate, by addition of hydrochloric acid.

AIXAIr. A term derived from the Arabians, and introduced into Europo when the Mahometan conquerors pushed their conquests westward. Al, el, or ul, as an Arabic noun, denotes 'God-Heaven-Divine.' As an Arabic particle, it is prefixed to words to give them a more emphatic signification, much the same as our particle the; ss in Alcoran, the Koran, alchymist, the ehemist.

Kali was the old name for the plant producing potash (tho glasswort, so called from its use in the manufacture of glass), and alkali signified no more than the kali plant. Potash and soda were for some time confounded together, and were hence called alkalis. Ammonia, which much resembles them when dissolved in water, was also called an alkali. Ammonia was subsequently distinguished as the volatile alkali, potash and soda being called fixed alkalis. Ammonia was also called the animal alkali; soda was tho mineral alkali, being derived from rock-salt, or from the ocean; and potash received the name of vegetable alkali, from its source being the ashes of plants growing upon the land. Alkalis are characterized by being very soluble in water, by neutralising the strongest acids, by turning to brown the vegetable yellows, and to green the vegetable reds and blues.

Some chemists classify all salifiable bases under this name.

In commercial language, the torm has been hitherto applied to an impure soda, but now it is understood to comprehend both soda and potash. The imports and exports of the alkalis are given on the opposite page. See Potasi and SoDA. 
Of Alkali manufactured in the United Kingdom the following quantities were EXPORTED :-

\begin{tabular}{|c|c|c|c|c|c|c|c|}
\hline & & \multicolumn{2}{|c|}{1870} & \multicolumn{2}{|c|}{1871} & \multicolumn{2}{|c|}{1872} \\
\hline & & Quantity & Value & Quantity & Value & Quantity & Value \\
\hline $\begin{array}{l}\text { To Russia : } \\
\text { "Germany : } \\
\text { " Holland : France : } \\
\text { " United States : } \\
\text { " Other Countries: }\end{array}$ & $: \quad:$ & $\begin{array}{c}\text { cwts } \\
256,210 \\
485,454 \\
217,892 \\
146,027 \\
1,903,640 \\
844,170\end{array}$ & $\begin{array}{r}\qquad 5 \\
129,427 \\
155,258 \\
57,212 \\
53,657 \\
755,838 \\
334,653\end{array}$ & $\begin{array}{r}\text { cwts } \\
241,692 \\
682,627 \\
266,846 \\
195,777 \\
1,924,510 \\
865,216\end{array}$ & 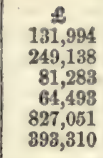 & $\begin{array}{r}\text { c17ts } \\
264,129 \\
677,594 \\
260,085 \\
88,924 \\
2,190,559 \\
976,754\end{array}$ & $\begin{array}{r}\mathbf{8} \\
177,017 \\
332,829 \\
104,244 \\
\mathbf{4 4 , 8 7 8} \\
1,250,591 \\
\mathbf{5 7 9 , 8 0 4}\end{array}$ \\
\hline Total & - . & $8,858,393$ & $1,486,045$ & $4,176,667$ & $1,747,269$ & $4,458,045$ & $2,480,368$ \\
\hline
\end{tabular}

The Isronts of alkali were as follows:-

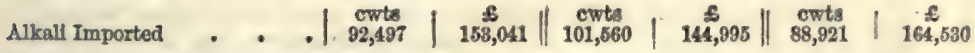

These quantities were from Germany, Spain, the United States, and other parts of America, and small quantities from a few other places.

AIXAII, ORGANIC, or AIKAIOID. During the last few years the organic alkaloids have so greatly increased in number, that a considerable volume might be devoted to their history. In Watts's 'Chemical Dictionary' will be found a long list of the most important nitrogen-alkaloids, natural and artificial, with a statement of the sources from which they are derived, or a description of their mode of formation. There are, however, only a few which have become articles of commerce. The principal sources from whence they are obtained are the following: -1. The animal kingdom. 2. The vegetable kingdom. 3. Destructive distillation. 4. The action of potash on the cyanic and cyanuric ethers. 5. The action of ammonia on the iodides, \&c. of the alcohol radicals. 6. The action of reducing agents on nitro-compounds. The principal bases existing in the animal kingdom are creatine and sarcosine. The vegetable kingdom is much richer in them, and yields a great number of organic alkalis, of which several are of extreme value in medicine. Modern chemists regard all organic alkalis as derived from the types ammonia or oxide of ammonium. Their study has led to results of the most startling character. It has been found that not only may the hydrogen in ammonia and oxide of ammonium be replaced by metals and compound radicals without destruction of the alkaline character, but even the nitrogen may be replaced by phosphorus or arsenic, and yet the resulting compounds remain powerfully basic. In studying the organic bases, chemists have constantly had in view the artificial production of the bases of cinchona bark. It is true that this result has not as yet been attained; but, on the other hand, bodies have been formed having so many analogies, both in constitution and properties, with the substances sought, that it cannot be doubted the question is merely one of time. The part performed by the bases existing in the juice of flesh has not been ascertained, and no special remedial virtues have been detected in them; but this is not the case with those found in vegetables; it is, in fact, among them that the most potent of all modicines are found-such, for example, as quinine and morphia. It is, moreover, among vegetable alkaloids that we find the substances most inimical to life, for aconitine, atropine, brucine, coniine, curarine, nicotine, solanine, strychnine, \&c. \&c., are among their number. It must not be forgotten, however, that, used with proper precaution, even the most virulent are valuable medicines. The fearfully poisonous nature of some of the organic bases, together with an ides that they are difficult to detect, has unhappily led to their use by the poisoner; strychnine, especially, has acquired a painful notoriety, in consequence of its employment by a medical man to destroy persons whose lives he had insured. Fortunately for society, the skill of the analyst has more than kept pace with that of the poisoner; and without regarding the extravagant assertions made by some chemists as to the minute quantities of regetable poisons they are able to detect, it may safely be asserted that it would be very difficult to administer a fatal dose of any ordinary vegetable poison without its being discovered. Another check upon the poisoner is found in the fact that those most difficult of isolation from complex mixtures are those which cause such distinct symptoms of poisoning in the victim, that the medical attendant, if moderutely observant, can scarcely fail to have his suspicions aroused.

Under the heads of the various alkaloids will be found (where deemed of sufficient 
importance), not merely the mode of preparation, but also the easiest mothod of detection.-C. G. W.

Axrexrmargin. There are various kinds of alkalimeters, but it will be more convenient to explain their construction and use in the article on Aukarmotry, to which the reader is referred.

AITAIIMITRX. 1. The object of alkalimetry is to determine the quantity of caustic alkali or of carbonato of alkali contained in the potash or soda of commerce. The principle of the method is, as in acidimetry, based upon Dalton's law of chemical combining ratios - that is, on the fact that in order to produce a complete reaction a certain definite weight of reagent is required, or, in other words, in order to saturate or completely neutralise, for example, one equivalent of a base, exactly one equivalent of acid must be employed, and vice versâ. This having been thoroughly explained in the article on AcmNETRY, the reader is referred thereto.

2. The composition of the potash and of the soda met with in commerce presents very great variations; and the value of these substances being, of course, in proportion to the quantity of real alkali which they contain, an easy

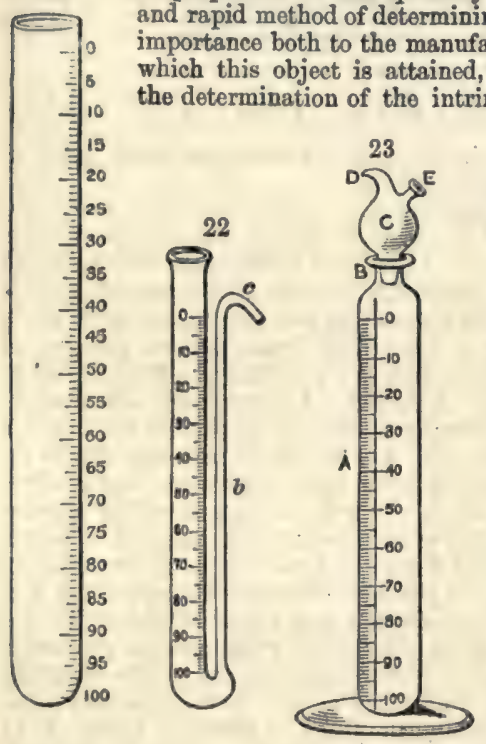
(he greatest ufacturer and to the buyer. The process by which this object is attained, though originally contrived exclusively for the determination of the intrinsic value of these two alkalies (whence its name Alkalimetry), has since been extonded to that of ammonis and of earthy bases and their carbonates, as will bo shown presently.

3. Before, however, entering into a description of the process itself, we will give that of the instrument employed in this method of analysis, which instrument is called an alkalimeter.

4. The common alkalimeter is a tube closed at one end (see fig. 21), of about $\frac{3}{4}$ ths of an inch internal diameter, about $9 \frac{3}{2}$ inches long, and is thus capable of containing 1,000 grains of pure distilled water. The space occupied by the water is divided accurately into 100 divisions, numbering from above downwards, each of which, therefore, represents 10 grains of distilled water.

5. When this alkalimeter is used, the operator must carefully pour the acid from it by closing the tube with his thumb, so as to allow the acid to trickle in drops as occasion may require; and it is well also to emear the edge of the tube with tallow, in order to prevent any portion of the test acid from being wasted by running over the outside after pouring, which accident would, of course, render the analysis altogether inaccurate and worthless; and, for the same reason, after having once begun to pour the acid from the alkalimeter by allowing it to trickle between the thumb and the edge of the tube, as above mentioned, the thumb must not be removed from the tube till the end of the experiment, for otherwise the portion of acid which adheres to it would, of course, be wasted and vitiate the result. This uncomfortable precaution is obviated in the other forms of alkalimeter now to be described.

6. That represented in fig. 22 is Gay-Lussac's alkalimeter; it is a glass tube about 14 inches high, and $\frac{1}{2}$ an inch in diameter, capable of holding more than 1,000 grains of distilled water; it is accurately graduated from the top downwards into 100 divisions in such a way that each division may contain exactly 10 grains of water. It has a small tubo, $b$, communicating with the larger one, which small tube is bent and bevelled at the top, $c$. This very ingenious instrument, known also under the names of 'burette' and 'pouret,' was contrived by Gay-Lussac, and is by far more convenient than the common alkalimeter, as by it the test acid can be unerringly poured drop by drop, as wanted. The only drawback is the fragility of the small side-tube, $b$, on which account the common alkalimeter, represented in fig. 21, was generally used, especially by workmen, because, as it has no side-tube, it is less liable to be broken; but it gives less accurate results, \& portion of the acid being wasted in various ways, and it is besides less manageable. Gay-Lussac's 'burette' is therefore preferable; and if melted wax be run between the space of the large and of the small tube, the 
instrument is rendered much less liable to injury; it is generally sold with a soparate wooden foot or socket, in which it may stand vertically.

7. The preceding form of alkalimeter (fig. 23), which I contrived several jears ago, will, I think, be found equally delicate, but more convenient still than that of Gay-Lussac. It consists of a glass trbe $\mathrm{A}$, of the same dimensions and graduated in the same manner as that of Gay-Lussac; but it is provided with a glass foot, and the upper part, $\mathrm{B}$, is shaped like the neck of an ordinary glass bottle; $\mathrm{c}$ is a bulb blown from a glass tube, one end of which is ground to fit the neck, $\mathbf{B}$, of tho alkalimeter, like an ordinary glass stopper. This bulb is drawn to a capillary point at $\mathrm{D}$, and has a somewhat large opening at $\mathbf{s}$. With this instrument the acid is porfectly under the control of the operator, for the globular joint at the top enables him to see the liquor before it actually begins to drop out, and he can then regulate the pouring to the greatest nicety, whilst its more substantial form renders it much less liable to accidents than that of Gay-Lussac; the glass foot is extremely convenient, and is at the same time a great additional security. The manner of using it will be described further on.

8. Another alkalimeter of the same form as that which I have just described,

24

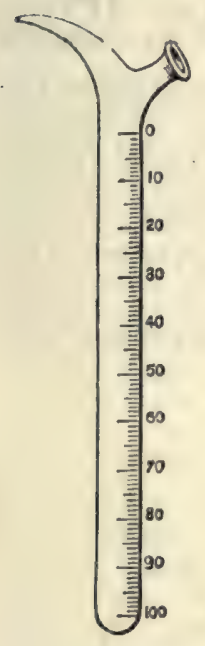

25

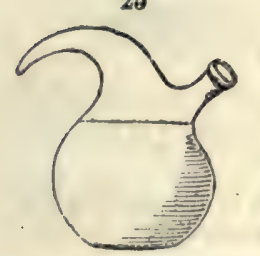

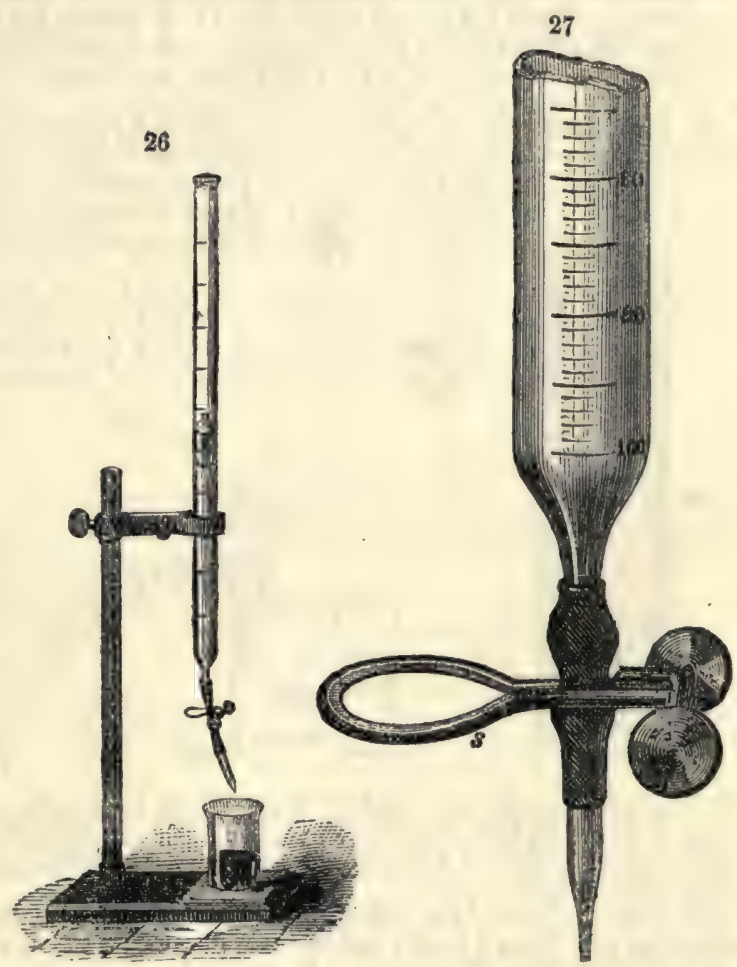

except that it is all in one piece, and has no globular enlargement, is represented in fig. 24. Its construction is otherwise the same, and the results obtained are equally delicate; but it is less under perfect control, and the test acid is very liable to run down the tube outside : this defect might be easily remedied by drawing the tube into a finer and more delicate point, instead of in a thick blunted projection, from which the last drop cannot be detached, or only with difficulty and imperfectly. A glass foot would moreover be an improvement.

9. With Schuister's alkalimeter (represented in fig. 25), the strength of alkalis is determined by the weight, not by the measure, of the acid employed to neutralise the alkali : it is, as may be seen, a small bottle of thin glass, having the form of the head of the alkalimeter represented in fig. 23. We shall describe further on the process of analysis with this alkalimeter.

10. The alkalimeter most used now is that known as Mohr's (fig. 26). It consists of a straight graduated tubo, having its lowor extremity contractod and drawn out so as 
to form a short narrow tube, over which is secured a piece of caoutchone tnbing. This is torminated by a fine glass jet mado by drawing out a piece of quill tubing, 80 as to deliver the solution contained in the alkalimeter in a fine stream, or by single drops, as required. A spring clamp 8 (fig. 27) is used to regulate the flow of the liquid. A serew clamp, with one end attached to the support of the burette, is still better, as it enables the operator to deliver the test liquor at any desired rate, and leaves both hands free. Instead of a clamp a short piece of glass rod, of a diameter slightly greater than the bore of the caoutchouc tube, may be introduced between the end of the alkalimeter and the jet. By slightly pinching the rod between the thumb and forefinger, the caoutchoue tube is expanded laterally, and allows the liquid to escape betweon the glass rod and its inner surface, effectually closing again tho moment the pressure is relieved. A float (fig. 28) may be used with advantage with these burettes. It consists of a short glass tube, closed at both ends, and weighted with mercury, so as to float upright and rise but little out of the liquid. A line is etched round the middle of the float, and from this all readings off are made, withont regard to the upper surface of the fluid. The float should fit pretty closely, but easily, and the line round it should be perfectly horizontal, or inaccuracies will be introduced into the readings. There are several other forms of alkalimeter, but whichever of them is employed the process is the same-namely, pouring carefully an acid of a known strength into a known weight of the alkali under examination, until the neutralising point is obtained, as will be fully explained presently.

11. Blue litmus-paper being immediately reddened by acids is the reagent used for ascertaining the exact point of the neutralisation of the alkali to be tested. It is prepared

28

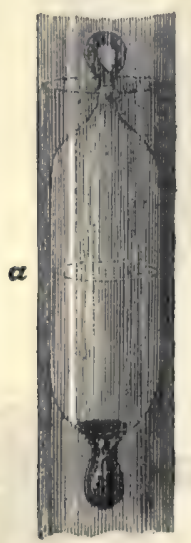
by pulverising one part of commercial litmus, and digesting it in six parts of cold water, filtering, and dividing the blue liquid into two equal portions, adding carefully to one of the portions, and one drop at a time, as much very dilute sulphuric acid as is sufficient to impart to it a slight red colour, and pouring the portion so treated into the second portion, which is intensely blue, and stirring the whole together. The mixture so obtained is neutral, and by immersing slips of white

29

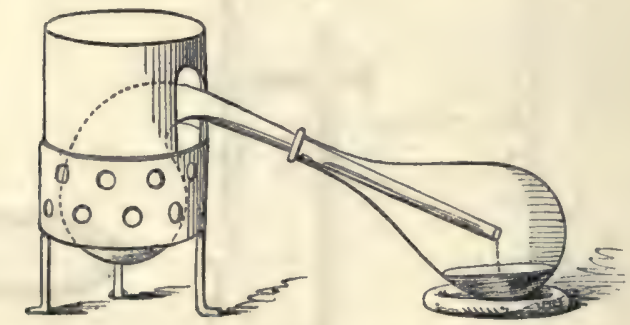

blotting-paper into it, and carefully drying them by hanging them on a stretched piece of thread, an exceedingly sensitive test paper of a light blue colour is obtained, which should be kept in a wide-mouth glass-stoppered bottle, and sheltered from the air and light.

12. Since the principle on which alkalimetry is based consists in determining the amount of acid which a known woight of alkali can saturate or noutralise, it is clear that any acid having this power can bo employed.

18. The test acid, however, generally preferred for the purpose, is sulphuric acid, bocause the normal solution of that acid is more easily prepared, is less liablo to change its strength by keoping, and has a stronger reaction on litmus-paper than any other acid. It is true that other acids-tartaric acid, for examplo - can be procured of greater purity, and that as it is dry and not caustic, the quantities required can bo more comfortably and accurately weighed off; and on this account some chemists, after Buchner, recommended its use, but the facility with which its aqueous solution becomes mouldy is so serious a drawback, that it is hardly ever resorted to for that object.

14. When sulphuric acid is employed, the pure acid in the maximum state of concentration, or, as it is called by chemists, the pure hydrate of sulphuric acid, specific gravity 1.8485 , is preferable. Such an acid, however, is never met with in commerce, for the ordinary English oil of vitriol is seldom pure, and never to the maximum state of concentration; the operator, howover, may prepare it by distilling ordinary oil of vitriol, but as the specific heat of the vapour of sulphuric acid is very small, the 
distillation is a somewhat hazardous operation, unless peculiar procaution be taken. The following apparatus, however, allows of the acid being distilled in a perfectly safo and convenient manner; it consists of a plain glass retort, charged with oil of vitriol : a little protosulphate of iron is added, for the purpose of destroying any nitrous products which the acid may evolve, and it is then placed into a cylinder of iron, the bottom of which is perforated with holes about threc-quarters of an inch in diameter, except in the middle, where a large hole is cut of a suitable size for the retort to rest upon; the sides of the cylinder are likewise perforated, as represented in fig. 29. Ignited charcoal is then placed all round the retort, the bottom of which protruding out of the influence of the heat, allows the ebullition to proceed from the sides only. It is well to put into the retort a few fragments of quartz or a few lengths of platinum wire, the effect of which is to render the ebullition more regular.

15. In order to prevent the acid fumes from condensing in the neck of the retort, it should be covered with a cover of sheet iron, as represented in fig. 29.

16. The first fourth part which distils over should be rejected, because it is too weak; the next two-fourths are kept, and the operation is then stopped, leaving the last fourth part of the acid in the retort. The neck of the retort should be about four feet long and about one and a half inches in the bore, and be connected with a large receiver; and as the necks of retorts are generally much too short for the purpose, an adapter tube should be adjusted to it and to the receiver, but very loosely ; this precaution is absolutely necessary, for otherwise the hot acid falling on the sides of the receiver would crack it; things, in fact, should bo so arranged that the hot drops of the distilling acid may fall into the acid which has alroady distilled over. Do not surround the receiver with cold water, for the hot acid dropping on the refrigerated surface would also certainly crack it. The acid so obtained is puro oil of vitriol, or monohydrated sulphuric acid, $\mathrm{SO}^{3}, \mathrm{HO}$, and it should bo kept in a well-stoppered and dry flask.

17. For commercial assays, however, and, indeed, for every purpose, the ordinary concentrated sulphuric acid answers very well : when used for the determination of the value of potashes, it is made of such a strength that each division (or 10 watergrains' measure) of the alkalimeter saturates exactly one grain of pure potesh; an acid of that particular strength is prepared as follows:-

18. Take 112.76 grains of pure neutral and anhydrous carbonate of soda, and dissolve them in about 5 fluid ounces of hot water. ${ }^{1}$ This quantity, namely, 112.76 grains, of neutral carbonate of soda will exactly saturate the same quantity of pure sulphuric acid $\left(\mathrm{SO}^{3}\right)$ that 100 grains of pure potash would. It is advisable, however, to prepare at once a larger quantity of test solution of carbonate of soda, which is of coturse easily done, as will be shown presently.

19. Mix, now, 1 part, by measure, of concentrated sulphuric acid with 10 parts of water, or rather-as it is advisable, where alkalimetrical assays have frequently to be mude, to keep a stock of test acid-mix 1,000 water-grains' measure of concentrated sulphuric acid with 10,000 grains of water, or any other larger proportions of concentrated sulphuric acid and water, in the above respective proportions; stir the whole well, and allow it to cool. The mixture of the acid with the water should be made by first putting a certain quantity of the water into a glass beaker or matrass of a suitable size, then pouring the concentrated acid slowly therein, while a gyratory motion is imparted to the liquid. The vessel containing the acid is then rinsed with the water, end both the rinsing and the rest of the water are then added to the whole mass. When quite cold, fill the graduated alkalimeter with a portion of it up to the point maxked $0^{\circ}$, taking the under line of the liquid as the true level; and whilst stiming briskly with a glass rod the aqueous solution of the 112.76 grains of neutral carbonate of soda above alluded to, drop the test acid from the alkalimeter into the vortex produced by stirring, until, by testing the alkaline solution with a strip of reddened litmus-paper after every addition of acid, it is found that it no longer shows an alkaline reaction (which is known by the slip of reddened litmus-paper not being rendered blue), but, on the contrary, indicates that a very slight excess of acid is present (which is known by testing with a slip of blue litmus-paper, which will then turn slightly red).

20. If, after having exhausted the whole of the 100 divisions (1,000 water-grains' measure) of the diluted acid in the alkalimeter, the neutralisation is found to be exactly attained, it is a proof that the test acid is right.

21. But suppose, on the contrary (and this is a much more probable case)-suppose that only 80 divisions of the acid in the alkalimeter have been required to neutralise the alkaline solution, it is then a proof that the test acid is too strong, and accord-

- Anhydrous, or dry, nentral carbonate of soda may be obtained by keeping a certain quantity of pure bicarbonate of soda for a short time at a dull red heat, in a platinam cruclble: the bicarbonate is converted into fts neutral carbonate, of course free from water. 
ingly it must be further dilutod with water, to bring it to the standard strength; and this may at once be done, in the present instance, by adding 20 measures of water to every 80 measures of the acid. This is best accomplished by pouring the whole of the acid into a large glass cylinder, divided into 100 equal parts, until it reaches the mark or scratch corresponding to 80 measures; the rest of the glass, up to 100 , is thon filled up with water, so that the same quantity of real acid will now be in the 100 measures as was contained before in 80 messures.

22. The acid adjusted as just mentioned should be labelled ' Test Sulphuric Acid for Potash,' and kept in well-stoppered bottles, otherwise evaporation taking place would render the remaining bulk more concontrated, consequently richer in acid than it should be, and it would thus, of course, become valueless as a test acid until roadjusted. Each degree or division of the alkalimeter of such an acid ropresonts 1 grain of pure potash.

23. The alkalimetrical assay of soda is also made with sulphuric acid, in preference to other acids, but it must bo so adjusted that 100 alkalimetrical divisions $(1,000$ water-grains' measure) of acid will exactly neutralise 170.98 of pure anhydrous carbonate of soda, that quantity containing 100 grains of pure sode.

24. Dissolve, therefore, 171 grains of pare anhydrous nentral carbonate of soda, obtained as indicated before, in five or six ounces of hot water, and prepare in the meantime the test sulphuric acid, by mixing 1 part, by measure, of ordinary concentrated sulphuric acid with about 9 parts by measure of water, exactly as described before; stir the whole thoroughly, let the mixture stand until it has become quite cold, then pour 1,000 water-grains' measure of the dilute acid so prepared into an alkalimeter-that is to say, fill that instrument up to $0^{\circ}$, taking the under line as the true level, and then, whilst stirring briskly the aqueous solution of the 171 grains of carbonate of soda with a glass rod, pour the acid, with increased precaution as the saturating point is approaching, into the vortex produced, until by testing the liquor alternately with reddened and with blue litmus-paper, or with grey litmus-paper, as before mentioned, the exsctly neutralised point is hit.

25. If the whole of the 100 alkalimetrical divisions ( 1,000 water-graing' measure) have been required to effect the neutralisation, it is a proof that the acid is of the right strength, but if this be not the case, it must be adjusted as described beforethat is to say:-

26. Suppose, for example, that only 75 alkalimetrical divisions or measures of the acid in the alkalimeter have been required to neutralise the 171 grains of nentral carbonate of soda operated upon, then 75 measures of the acid should be poured at once into a glass cylinder accurately divided into 100 parts; the remaining 25

30

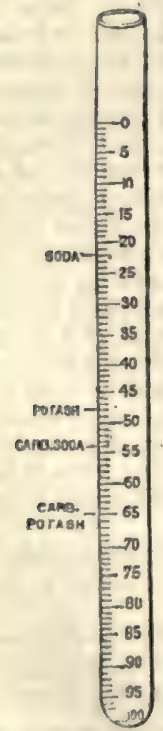
divisions should then be filled with water, and the whole being now stirred up, 100 parts of the liquor will of course contain as much real acid as 75 parts contained before, and accordingly the acid may now be used as a test acid for the alkalimetrical assay of soda, each degree or division of the alkalimeter representing one grain of pure soda.

27. The stock of test acid should be kept in well-stoppered flasks, that it may not vary in strength by evaporation, and bo labelled 'Test Sulphurio Acid for Soda.'

28. Instead, however, of keeping two kinds of 'test sulphuric acid,' of different saturating powers as described, the one for potash, the other for soda, one kind only may bo propared so as to serve for both alkalis, by constructing, as is very often done, an alkalimeter adjusted so as to indicate the quantities of the acid of a given strength required for the saturation or neutralisation of both potash or soda, or of their respective carbonates; and this, in fact, is the alkalimeter most in use in the factory.

It should be in shapo similar to that of Gay-Lussac's (see fig. 22), or that described in figs. 23 and 24 ; but, like that represented by fig. 21 , it generally consists of a tube closed at one end, about threefourths of an inch internal diameter and about $9 \frac{1}{2}$ inches in length; it is gradusted into 100 equal parts, and every division is numbered from above downwards (see fig. 80 ).

The following directions for their construction are given by Professor Faraday. 'Let the tube represented in the margin have 1000 grains of water weighed into it; then let the space it occupies be graduated into 100 equal parts, and every ten divisions numbered from above downwards. At $22 \cdot 1$ parts, or 77.99 parts from the bottom, make an extra line, a little on one side or even on the opposite vide of the graduation, and write at it with a scratching diamond, soda; lower dowa 
at 48.62 parts, make another line, and write potash ; still lower, at 54.43 parts, a third line marked carb. soda, and at 65 parts a fourth, marked $c a r b$. potash. It will be observed that portions are measured off beneath these marks in the inverse order of the equivalent number of these substances, and consequently directly proportionate to the quantities of any particular acid which will neutralise equal weights of the alkalis and their carbonates. As these points are of great importance, it will be proper to verify them by weighing into the tubes first 350 , then $513 \cdot 8$, and lastly $779 \cdot 9$ grains of water, which will correspond with the marks if they are correct, or the graduation may be laid down from the surface of the four portions of fluid when weighed in, without reference to where they fall upon the general scale. The tube is now completed, except that it should be observed whether the aperture can be perfectly and securely covered by the thumb of the left hand, and if not, or if there be reason to think it not ultimately secure, then it should be heatod and contractod until sufficiontly small.'

29. The test acid for this alkalimeter should have a specific gravity of $1 \cdot 1268$; and such an acid may be prepared by mixing one part, by weight, of sulphuric acid, specific gravity 1.82, with four parts of water, and allowing the mixture to cool. In the meantime, 100 grains of pure anhydrous carbonate of soda, obtained as indicated before, should be dissolved in water, and the test sulphuric acid, of spocific gravity $1 \cdot 1268$, prepared as above said, having become quite cold, is poured into the alkalimeter up to the point marked carbonate of soda ; the remaining divisions are filled up with water, and the whole should be well mixed by shaking.

30. If the whole of the sulphuric acid, adjusted as was said, being poured carefully into the solution of the 100 grains of the neutral carbonate of soda, neutralise them exactly-which is ascertained, as usual, by testing the solution with litmus-paper, which should not be either reddened or rendered bluer by it-it is of course a sign that the test is as it should be-that is to say, is of the proper strength; in the contrary case it must be finally adjusted in the manner already indicated, and which need not be repeated. See $\$ \$ 20,21$.

31. The best and most convenient process for the analyst, however, consists in preparing a test acid of such a strength that it may serve not only for all alkalis, but indeed for every base; that is to say, by adjusting the test acid so that 100 alkalimetrical divisions of it (1,000 water-grains' measure) may exactly saturato or neutralise one equivalent of every base. This method, which was first proposed by Dr. Ure, is exceedingly convenient, and the possession of two reciprocal test liquids, namely, the ammonia test liquor of a standard strength, of which we gave a description in the article on Acidimetry, and the standard test acid of which we are now speaking, affords, as Dr. Ure observes, ready and rigid means of verification. For microscopic analysis of alkaline and of acid matter, a graduated tube of a small bore, mounted in a frame, with a valve apparatus at top, so as to let fall drops of any size and at any interval, is desirable; and such an instrument Dr. Ure employed for many years; but instead of a tube with a valve apparatus at top, the operator may use a graduated tube of a small bore, terminated by a small length of vulcanised india-rubber tube pinched in a clamp, which may be relaxed in such a way as to permit also the escape of drops of any size at any interval of time, the little apparatus being under perfect command.

32. The test sulphuric acid, of such a strength that 100 alkalimetrical divisions of it can saturate one equivalent of every base, should have a specific gravity of 1.032 , and is prepared as follows:-

Take 53 grains (one equivalent) of pure anhydrous neutral carbonate of soda, obtained in the manner indicated before (seo $\$ 18$ ), and dissolve them in about one fluid ounce of water. Prepare in the meantime the test sulphuric acid by mixing one part, by measure, of concentrated sulphuric acid with about 11 or 12 parts of water, and stir the whole well. The mixture having become quite cold, fill the alkalimeter with the cold diluted acid up to the point marked $0^{\circ}$, taking the under line of the liquid as the true level, and whilst stirring briskly the aqueous solution of the 53 grains of carbonate of soda above alluded to, pour the acid carefully from the alkalimeter into the vortex produced by stirring, until, by testing the liquor alternatoly with reddened and with blue litmus-paper, or, more conveniently still, with grey litmus-paper, the neutralising point is exactly hit.

33. If the whole of the 100 divisions of the alkalimeter had been required to neutralise exactly the 53 grains of pure anhydrous carbonate of soda, it would be a proof that the acid is of the right strength; but if this is not the case, it must be adjusted in the manner described before, that is to say :-

34. Let us suppose, for example, that only 50 measures in the alkalimeter have been required to saturate or neutralise the 53 grains of carbonate of soda, then 60 moasures should be poured at once into a glass cylinder accurately divided into 100 
parts, the remaining 60 divisions should be fillod up with water, and the whole being well stirrod, 100 parts of tho acid liquor will now contain as much real acid as was eontained before in the 60 parts.

35. The acid may now be labelled simply, 'Test or Normal Sulpharic Aoid.' Each one hundred alkalimetrical divisions, or 1,000 water-grains' moasure of it, contain one equivalent, or 40 grains of real sulphuric acid; and, consoquontly, each 100 alkalimetrical divisions of it will neutralise one equivalent, or 31 grains of soda, 47 of potash, 17 of ammonia, 28 of lime, and so forth, with respect to any othor base.

36. The stock of test or normal sulphuric acid should, as usual, be kept in wollstoppered bottles, in order to prevent concentration by evaporation. By keoping in the flask containing it a glass bead, exactly adjusted to the specific gravity of 1.032 , the operator may always ascertain, at a glance, whether the acid requires readjusting.

37. With a Schüster's alkalimeter, it is convenient to prepare the test acid of such a strength that, according as it has been adjusted for potash or for soda, 10 grains of it will exactly saturate one grain of one or the other of these bases in a pure state. It is considered that the alkalimeter may be charged with a known weight of any of the other sulphuric test acids of a known strength. Suppose, for example, that the test sulphuric acid taken have a specific gravity of 1.032 , we know, as we have just shown, that 1.032 grains weight of that acid contains exactly one equivalent of pure sulphuric acid $=40$, and is capable, therefore, of neutralising one equivalent of any base; and, consequently, by taking a certain weight of this acid before beginning the assay, and weighing what is left of it after the assay, it is very easy to calculate, from the quantity of acid consumed in the experiment, what quantity of base has been neutralised. Thus a loss of $21.96,60 \cdot 70,33.29$ grains' weight of this test acid represents one grain of potash, of ammonis, of sode respectively, and so on with the other bases.

38. The operator being thus provided with an appropriate test acid, we shall now describe how he should proceed with each of them in making an alkalimetrical assay with potash.

In order to obtain a reliable result, a fair average sample must be operated upon, To secure this the sample should be taken from various parts of the mass and at once put in a wide-mouth bottle, and woll corked up until wanted; when the assay has to be made, the contents of the bottle must be reduced to powder, so as to obtain a fair mixture of the whole; of this weigh out 1,000 grains exactly-or less, if that quantity cannot be spared-and dissolve them in a porcelain capsule, in about 8 fluid ounces of distilled hot water, or in that proportion; and if there be left anything like an insoluble residue, filter, in order to separate it, and wash it on the filter with small quantities of distilled water, and pour the whole solution, with the washings and rinsings, into a measure divided into 10,000 water-grains' measure. If the water used for washing the insoluble residue on the filter has increased the bulk of the solution beyond 10,000 water-grains' measure, it must be reduced by evaporation to that quantity; if, on the contrary, the solution poured in the measure stands below the

31

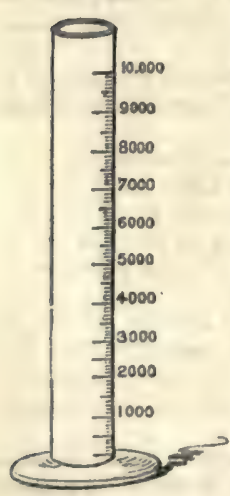
mark 10,000 water-grains' measure, then as much water must be added thereto as will bring the whole mass exactly to that point. In order to do this correctly, the cylindrical measure should stand well on a table, and the under or lower line formed by the liquid, as it reaches the scratch 10,000 , is taken as the true level.

39. This being done, 1,000 grains' measure of the filtrate, that is to say, one-tenth part of the whole solution, is transferred to a glass beaker, in which the saturation or neutralisation is to be effected, which is best done by means of a pipette capable of containing exactly that quantity when fillod up to the scratch, $a$. In order to fill such a pipette it is sufficiont to dip it into the alkaline solution and to suck up the liquor a little above the scratch, $a$; the upper orifice should then be stopped with the first finger, and by momentarily lifting it up, the liquor is allowed slowly to fall from the pipetto back again into the 10,000 grains' measure until its level reaches exactly the scratch, $a$. The last drop which remains hanging from the point of the pipette

32

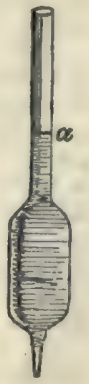

may be readily detached by touching the sides of the glass measure with it. The 1,000 grains being thus rigorously measured in the pipette should then be transferred to the glass beaker, in which the noutralisation is to take place, by removing the finger altogether, blowing into it to detach the last drop, and rinsing it with a little water. 
10. Or, instead of the pipette just described, the operator may measuro 1,000 grains by taking an alkalimeter full of the alkaline solution, and emptying it into the glass beaker in which the neutralisation is to take place, rinsing it with a little water, and of course adding the rinsing to the mass in the said glass beaker.

41. Whichever way is adopted, a slight blue colour should be imparted to the 1,000 grains' measure of the alkaline solution, by pouring into it a small quantity of tincture of litmus. The glass beaker should then be placed upon a sheet of white paper, or a slab of white porcelain, in order that the change of colour produced by the gradual addition of the test acid may be better observed.

42. This being done, if the operator have decided upon using the test sulphuric acid for potash (\$\$ 17-22), he should take one of the alkslimeters represented in figs. 21,22, 23 , or 24, and fill it up to $0^{\circ}$ (taking the under line of the liquid as the true level); then taking the alkalimeter thus charged in his right hand, and in his left the glass beaker containing the alkaline solution coloured blue by tincture of litmus, he should gradually and carefully pour the acid liquor into the alkaline solution in the glass beaker, to which a circular motion should be given whilst pouring the acid, or which should be briskly stirred, in order to insure the rapid and thorough mixing of the two liquors, and therefore their complete reaction; moreover, in order at once to detect any change of colour from blue to red, the glass beaker should be kept over the white sheet of paper or the white porcelain slab, as before stated.

43. At first no effervescence is produced, because the carbonic acid expelled, in. stead of escaping, combines with the portion of the alkaline carbonate as yet undecomposed, which it converts into bicarbonate of potash, and accordingly no sensible change of colour is perceived; but as soon as a little more than half the quantity of the potash present is saturated, the liquor begins to effervesce, and the blue colour of the solution is changed into one of a vinous, that is, of a purple or bluish-red hue, which is due to the action of the carbonic acid upon the blue colour of the litmus. More acid should be still added, but from this moment with very great care and with increased caution, gradually as the point of neutralisation is approached, which is ascertained by drawing the glass rod used for stirring the liquor across a slip of blue litmus-paper. If the paper remains blue, or if a red or reddish streak is thereby produced which disappears on drying the paper and leaves the latter blue, it is a proof that the neutralisation is not.yet complete, and that the reddish streak was due only to the action of the carbonic acid; more acid must accordingly be poured from the alkalimeter, but one drop only at a time, stirring after each addition, until at last the liquor assumes a distinct red or pink colour, which happens as soon as it contains an extremely slight excess of acid; the streaks made now upon the litmus-paper will remain permanently red, even after drying, and this indicates that the reaction is complete and that the assay is finishod.

44. If the potash under examination were perfectly caustic, the solution would suddenly change from blue to pink, because there would be no evolution of carbonic acid at all, and consequently no vinous or purple colour produced; if, on the other hand, the potash was altogether in the state of bicarbonate, the first drops of test acid would at once decompose part of it and liberate carbonic acid, and impart a vinous colour to the solution at the very outset, which vinous colour would persist as long as any portion of the bicarbonate would remain undecomposed.

45. The neutralising point being attained, the operator allows the sides of the alkalimeter to drain, and he then reads off the number of divisions which have beon employed. If, for example, 50 divisions have been used, then the potash examined contained 50 per cent. of real potash. See observ., $\$ 48-49$.

46. Yet it is advisable to repeat the assay a second time, and to look upon this first determination only as an approximation which enables the operator, now that he knows about where the point of neutralisation lies, to arrive, if need be, by increased caution as he reaches that point, at a much greater degree of precision. He should accordingly take again an alkalimeter full (1,000 water-grains measure)-that is to say, another tenth part of the liquor left in the 10,000 grains' measure-and add thereto at once 48 or 49 alkalimetrical divisions of the test acid, and after having thoroughly agitated the mixture, proceed to pour the acid carefully, two drops only at a time, stirring after such addition, and touching a strip of litmus-paper with the end of the glass rod usod for stirring; and so he should go on adding two drops, stirring, and making a streak on the litmus-paper until tho liquor assumes suddenly a pink or onion-red colour, and the streak made on the litmus-paper is red also. The alkalimeter is then allowed to drain as before, and the operator reads off the number of divisions employed, from which number 2 drops (or $\frac{2}{10}$ ths of a division) should bo deducted; Gry-Lussac having shown that, in alkalimetrical assays, tho sulphates of alkalis produced retard the manifestation of the red colour in that proportion. One alkalimetrical division generally consists of 10 drops, but as this is not always the 
case, the operator should determine for himself how many drops are necessary to make up one division, and take account of them in the assay according to the ratio thus found. In the example given before, and supposing 10 drops to form one alkalimetrical division, then the per-centage value of the sample of potash under examination would probably be as follows :-

$$
\begin{aligned}
& \begin{array}{l}
\text { Number of divisions of acid omployed } \\
-2 \text { drops acid in excess . }
\end{array} \\
& \text { Real por-centage of potash . } \quad . \quad, \frac{50.0}{49.8}
\end{aligned}
$$

47. When the alkalimeter described in fig. 23 is employed, the test acid may, at the beginning of the experiment, be poured from the larger opening, $\mathrm{E}$; but towards the end-that is, when the neutralising point is approaching-the acid should be carefully poured from the point, $\mathbf{D}$, in single drops, or only two drops at a time, until the saturating point is hit, as we have just said. If the operator wishes to pour only one drop, he should close the larger opening, $x$, of the bulb with the thumb, and then fill the bulb with the test acid by inclining the alkalimeter; putting now the alkalimeter in an upright position, and removing the thumb, a certain quantity of acid will bo retained in the capillary point, $\mathbf{D}$; end if the thumb be now pressed somewhat forcibly against the opening, $x$, the acid contained in the capillary point will be forced out and form one drop, which will then fall into the alkaline solution if it be held over it. If the saturation be complete, the operator, without removing the bulb stopper, may, by applying his lips to the large opening, $\mathrm{E}$, suck the acid engaged in the capillary point back into the alkalimeter.

48. If there should be in the mind of the operator any doubt as to what is meant by the onion-red colour which the liquor tinged blue with tincture of litmus acquires when slightly supersaturated, he may pour into a glass beaker a quantity of pure water equal to, or even larger than, the alkaline solution operated upon, and tinge it blue with a little tincture of litmus, to about the same degree of intensity as the alkaline liquor uuder examination. If he now pour into the pure water coloured blue with litmus, one single drop of the test acid, it will acquire at once, by stirring, the onionred colour alluded to, and which he may now use as a standard of comparison.

49. Considering the rapidity with which these alkalimetrical operations can be performed, the operator, unless he have acquired sufficient practice, or unless a great degree of accuracy be not required, should repeat the assay two or three times, looking upon the first determination only as an approximation, and as a sort of guide as to the quantity of acid which will bo required in the subsequent experiments, whereby he will now be enabled to proceed with increased caution as he approaches the point of saturation; but, at any rate, if he will not take the little extra trouble of a repetition, he should, before he begins to pour the acid, take a little of the filtered alkaline solution out of the glass beaker, as a corps de réserve, which he adds to the rest after the saturating point has been approximated, and from that moment he may proceed, but with great care, to complete the neutralisation of the whole.

50. Do not forget that as the test sulphuric acid must always be added in slight excess to obtain a distinct red streak on the litmus-paper, a correction is absolutely necessary; that is to say, the excess of sulphuric acid employed must be deducted if a strictly accurate result is sought.

51. If, instead of the special alkalimeter for potash above described, the operator prefers using that prepared of such a strength that 100 divisions of the alkalimeter (1,000 water-grains' measure) contain exactly one equivalent of each alkali or base, which test sulphuric acid, as we have seen, has a specifle gravity of 1.032 (see $\$ \S 31-36)$, he should proceed exactly as indicated in $\$ 38$ and following; and the alkalimeter being filled with that test acid, of specific gravity 1.032 , up to $0^{\circ}$, it (the acid) should bo poured carefully into the aqueous solution of the alkali tinged blue with litmus, until exact neutralisation is attained, precisely in the same manner as in $\$ 38$ and following.

52. The neutralising point being hit, let us suppose that the whole of the contents of the alkalimeter have been employed, that the aqueous solution tinged blue with litmus is not yot saturated, and that, after having refilled the alkalimeter, the 4 divisions more (altogether 104 divisions) have been required to neutralise the alkali in the aqueous solution; then, since 100 divisions (1,000 water-grains' measure) of the test acid now employed saturate exactly one equivalent, that is, 47 of potash, the question is now, What quantity of potash will have been saturated by the 104 divisions of acid employed? The answer is found, by a simple rule of proportion, tc b. nearly 49.

$100: 47:: 104: x=48 \cdot 88$. 
The sample of potash oxaminod contained, therefore, nearly 49 per cent. of puro potash.

53. If instead of the special test sulphuric acid for potash (\$17), or of the test sulphuric acid for potash, soda, and other bases $(\$ 28)$, the operator uses the potash and soda alkalimeter (\$\$ 31-36), the method to bo followed is exactly similar to that described in $\$ 42$ and following. Some of the test sulphuric acid, of specific gravity $1 \cdot 1268$, is to be poured into the alkalimeter until it reaches the point marked 'potash' (that is to say, 48.62 divisions of the alkalimeter), taking the under line of the liquid as the true level, and the remaining divisions up to $0^{\circ}$ are carefully filled with water. The operator then closes the aperture of the alkalimeter with the thumb of his left hand, and the whole is violently shaken so as to obtain a perfect mixture.

54. The acid so mixed must now be carefully poured from the alkalimeter into the alkaline solution of the potash under examination until neutralisation is attained, precisely as described in $\$ 42$ and following.

55. The neutralising point being hit, the operator allows the sides of the alkalimotor to drain, and he then reads off the number of divisions employed in the experiment, which number indicates the per-centage of real potash contained in the sample.

Had the operator wished to estimate the quantity of potash as carbonate of potash, he should have poured the test acid into the alkalimeter up to the point marked ' carbonate of potash,' filled the remaining divisions of the alkalimeter up to $0^{\circ}$ with water, and proceeding exactly as just mentioned, the number of divisions of acid employed would indicate the per-centage of potash contained in the sample as carbonate of potash.

56. The most accurate and expeditious method of determining the value of a sample of an alkali is by means of a standard acid and a solution of a caustic alkali of corresponding strength contained in two burettes (fig. 26). The solution of soda ash, for instance, having been prepared as directed in $\$ 38-40$, is tinted with litmus solution, and a quantity of the standard acid more than sufficient to saturate the whole of the alkali is run into it, and the mixture boiled till the carbonic acid is entirely expelled from it and the clear red of the litmus solution is seen. The number of divisions of acid added is then noted and the neutral tint restored by the careful addition of the solution of caustic alkali. From the absence of carbonic acid the reaction is very sharp and decided, and even if the neutral point be overshot, the addition of a few drops of acid followed by the more cautious use of the alkaline solution will enable a correct result to be obtained. If the two standard solutions are of exactly equal strength, it is only necessary to subtract the number of divisions of the alkaline solution used, from that of the acid one, to give at once the number of those of the latter required for the neutralisation of the substance tested, and hence by a simple calculation the per-centage of real alkali.

57. If a Schiuster's alkalimeter (fig. 25) be used, and supposing, for example, that the acid to be employed therewith is so adjusted that 10 grains weight of it neutralise exactly 1 grain in weight of potash, proceed as follows :-Take 100 grains in weight of a fair average of the sample, previously reduced to powder, dissolve them in water, filter with the precautions which have already been described before ( $\$ 38$ and following), and pour this solution into a glass cylinder graduated into 100 parts, and capablo of containing 10,000 water-grains ; fill it up with water exactly as described before; of this take now 100 alkalimetrical divisions, that is to say, $\frac{1}{10}$ th of the whole solution, and pour it in a glass beaker. On the other hand, charge the Schüster's alkalimeter with a cortain quantity of the test acid, and weigh it, along with the alkalimeter itself, in a good balance. This done, proceed with the neutralisation of the solution in the glass beaker, by pouring the acid from the alkalimeter in the usual way, and with the usual precautions, until the saturation is completed. Replace the alkalimeter, with the quantity of unconsumed acid, in the scale of the balance, weigh accurately, and since every grain of acid represents $\frac{1}{10}$ th of a grain of potash, the number of grains of acid used in the experiment indicates at once the per-centage of real potash present in the sample.

58. When, however, potash is mixed with soda, as is frequently the case with the potash of commerce, either accidentally or for fraudulent purposes, the determination of the amount of the cheaper alkali could not, until a comparatively recent period, bo estimated, except by the expensive and tedious process of a regular chemical analysis. In 1844, however, M. Edmund Pesier, professor of Chemistry at Valenciennes, published an easy and commercial method for the estimation of the quantity of soda which potash may contain, by means of an areometer of a peculiar construction, to which the name of 'Natrometer' has been given by the talented professor.

59. The rationale of the method is grounded upon the increase of specifie gravity which sulphate of soda produces in a solution saturated with pure sulphate of potash, and is deduced from the fact that a solution saturated with neutral sulphate of potash possesses a uniform and constant density when the saturation is made at the same temperature, and that the density of such a solntion increases progressively in propor.

VoI. I. 
tion to the quantity of sulphate of soda present; an increase of density so much ths more readily observable, that the solubility of the sulphate of potash is greatly augmented by the presence of sulphate of soda. It had at first been thought that, in order to obtain anything like accuracy, it would be necossary to combine all the potash with one same acid, preferably sulphuric acid; and, consequently, that as the potash of commorco always contains a littlo, and sometimes a rather considerable quantity, of chloride of potassium, the lattor salt should first be docomposed. Further exporiments, however, establishod the fact that in dissolving chloride of potassium in a saturated solution of sulphate of potash, the specific gravity of the liquor is not materially increased, since the introduction of as much as 50 per cent. of chloride of potassium does not increase that density more than 3 per cent. of soda would do whon examined by the natrometer-a degree of accuracy quite sufficient for commercial purposes. When soda is added to a saturated solution of sulphate of potash, the further addition of chloride of potassium thereto renders the specific gravity of the liquor less than it would have been without that addition - an apparent anomaly duo to the fact that chlorine, in presence of sulphuric acid, of potash, and of soda, combines with the latter base to form chloride of sodium; and it is this salt which increases the solubility of sulphato of potash, though in a somewhat less dogree than sulphate of soda. Thus, if to a saturated solution of sulphate of potash 0.14 of soda be added along with 0.20 of chloride of potassium, the natrometer indicates only 0.125 of soda. Seoing, therefore, that in such an exceptional case the error does not amount to more than 0.015 of errur, it will probably be found unnecessary in most cases to decompose the chloride contained in the potashes of commerce, that quantity being too small to materially affect the result. Yet, as the accurate determination of soda in potash was a great desideratum, M. Pesier contrived two processes, one of which, in the hands of the practised chemist, is as perfect as, but much more rapid than, those ordinarily resorted to ; the other, which is a simplification of the first, yields results of sufficient accuracy for all commercial purposes.

60. First process.-Take 500 grains of a fair average sample of tho potash to be examined, dissolve them in as littlo water as possiblo, filter, and wash tho filter until the washings are no longer alkaline. This filtering, however, may bo dispensed with when the potash is of good quality and leaves but a smoll residue, or when an extrome degree of accuracy is not roquired.

61. The potash boing thus dissolved, a slight excess of sulphuric acid is added thereto; the excess is necessary to decompose the chlorides and expol the muriatic acid. The liquor so treated is then evaporated in a porcelain capsule, about six inches in diameter; and when it begins to thicken, it should be stirred with a glass rod, in order to avoid projections. When dry, the fire must be urged until the residue fuses, and it is then kept in a stato of tranquil fusion for a fow minutes. The capsule should then be placed upon, and surrounded with, hot sand, and allowed to cool down slowly, to prevent its cracking, which would happen without this precaution.

62. The fused mass in the capsule having become quite cold should now be treatod with as little hot water as possible, that is to say, with less than 3,000 grains of hot water; and this is best done by treating it with successive portions of fresh water. All the liquors thus successively obtained should then bo poured into a flask capable of holding about 10,000 grains of water, and the excess of sulphuric acid must be accurately neutralised by a concentratod solution of pure carbonate of potash-that is to say, until tho colour of litmus-paper is no longer affected by the liquor, just as in ordinary alkalimetrical or acidimetrical assays. During this operation, a pretty considerable precipitate of sulphate of potash is, of course, produced.

63. The noutralising point being exactly hit, a saturated solution of sulphate of potash is prepared, and brought to the atmosphoric temperature; a condition which is expedited by plunging the vessel which contains the solution into a basin full of cold water, and stirring it until the thermometer plunged in the liquor indicates that the temperature of the latter is about the same as, and proferably less than, that of the air, because in the latter case it may be quite correctly adjusted by grasping the vessel with a warm hand. In order, however, to secure exactly the proper temperature, the whole should be left at rest for a few minutes aftor having withdrawn the vessel from the basin of cold water used for refrigerating it, taking care simply to stir it from time to time, and to ascertain that the thermometer remains at the same degroe of temperature. This done, the liquor is filtered into a glass cylinder, c, on which a scratch, $\mathbf{B}-\mathbf{I}$, has been made, corresponding to 3,000 water-grains' measure. If the directions given have been exactly followed, it will be found that the filtrate is not sufficient to fill it up to that mark; the necessary volume, however, should be completed by washing the deposit of sulphate of potash in the filter, B, with a saturated solution of the same salt (sulphato of potash) previously prepared. It is advisable to use a saturated solution of sulphate of potash which has been kept for some 


\section{ALKALIMETRY}

time, and not one immediately prepared for the purpose, because sulphate of potash in dissolving produces a cortain amount of cold, which would creato delay, since it would be necessary to wait until the temperature of the mass had become tho same as that of the air.

64. The liquor occupying 3,000 water-grains' measure in the cylinder should be next rondered homogeneous by stirring it well, aftor which the natromotor may be immersed in it. The natrometer is simply an areometer of a peculiar construction, provided with two scales: the one of a pink colour shows the degrees of temperature, and indicates, for each degree of the Centigrade thermometer, the level at which a solution saturated with pure sulphate of potash would stand; on the other scale, each degree represents 1 per cent. of sode (oxide of sodium), as represented in fig. 34 .

65. The $0^{\circ}$ of the two scales coincide with each other. If the experiment take place at the temperature of $0^{\circ}$, the quantity of soda will be directly determined by observing the number of degroes on the soda scale; but if the experiment be performed at $25^{\circ}$, for example, it will be seen that the point at which the instrument would sink in a liquor saturated with pure

\section{3}

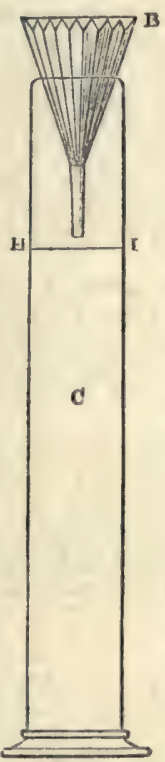
sulphate of potash corresponds to $\frac{8}{100}$ th of soda, and, in this case, it is from this point that the $0^{\circ}$ of the soda scale should be supposed to begin, which is easily accomplished by a simple subtraction, as will be seen presently.

66. Experiment having shown that the degrees of soda cannot bo equidistant, but that, on the contrary, they become smaller and smaller as the quantity of soda incresses, the number of degrees of soda axe obtained as follows:From the number of degrees of temperature now indicated on the pank scale of the natrometer, subtract the number of degrees of temperature indicated by an ordinary thermometer at starting; then look at the soda scale for the number of soda degrees which correspond to the number of degrees of temperature left after subtraction, and each of the soda degrees, beginning from the $0^{\circ}$ of the natrometer, represents 1 per cent.

67. For example:- Suppose the experiment to have been made at starting, and as indicated by an ordinary thermometer, at $+20^{\circ}$

3.)

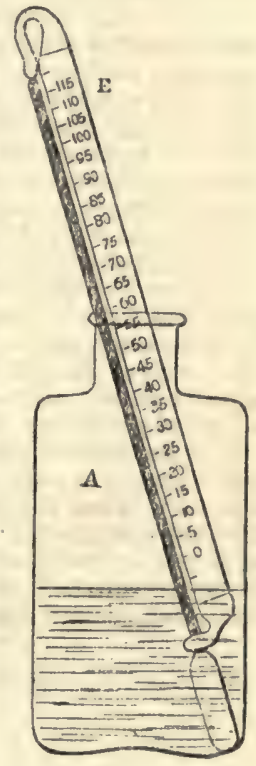

Centigrade, and that the level of the solution is now found to stand at $59^{\circ}$ on tho pink scale of temperature of the natrometer, then by deducting 20 (the original temperature) from 59 (number of degrees indicated by the floating point on the pink scale of temperatures of the natrometer), there remains, of course, 39 . Drew the instrument out, and looking now on the said pink scale for $39^{\circ}$, there will be found exactly opposite, on the soda scale, the number 13, which number signffies that the potash under examination contains 13 per cent. of soda (oxide of sodium).

68. As the deposit of sulphate of potash separated by filtering might retain some sulphate of soda, it is advisable, in order to avoid all chance of emor, to wash it with a saturated solution of sulphate of potash, adding as much of it as is necessary to bring the whole mass of the liquor up to the mark 3,000 water-grains' measure, in which the natrometer being again immersed, the minute quantity of soda indicated should be added to the per-centage found by the first operation.

69. If a great degreo of accuracy is required, the fractions of degree of the instrument must be taken account of; otherwise they may be neglected without the result being materially affected, since 3 degrees of the scale of temperature correspond only to about 1 per cent. of soda.

70. For commercial purposes, the process may be slightly varied, as follows :Take 500 grains of a fair average sample of the potash to be examined, proviously reduced to powder, and throw them into a flask, $\mathbf{A}$ (fig. 35), capable of containing 
about 6,000 grains of water; pour upon them about 2,000 grains of wator, and shako until dissolved. Add now sulphuric acid thereto; this will produce a smart offorvescence, and in all probability a deposit of sulphate of potash. Wo say in all probability, because it is clear that if the potash in question is largely aduiterated with soda, or was altogrothor nothing elso than carbonato of soda, as has oceasionally happened, it is evident that no deposit of sulphate of potash would take placo; and yet, as it is necessary to the success of the operation that the liquor should contain an excess of this lattor salt, a certain quantity of it previously reducod to fine powdor must in that.case be purposely added to the solution.

71. After the disengagement of gas has ceased, it is necessary to pour the dilute acid cautiously, and only drop by drop, until the neutralising point is correctly hit, which will be known as usual by testing with litmus-paper. But if, by accident, too much acid havo been used, which is known by the reddening of the litmus-papor, the slight overdose may be neutralised by adding a small quantity of weak solution of potash.

72. As this reaction produces hoat, it is necessary to lower the liquor down to the tomperature of the atmosphere, decant in a filter placed over the glass cylinder, and fill it up to the scratch 3,000 , by washing the residue on the filter with a saturated solution of sulphato of potash, exactly as described in $\$ 63$.

73. The glass cylinder being properly filled up to the scratch, remove the funnel, close the orifice of the glass cylinder with the palm of the hand, and shake the whole violently; holding the natrometer, which should be perfectly clean, by its upper extremity, slowly immerse it in the solution. If the potash under examination bo pure, the pink scale will indicate the degree of temperature at which the experiment has been made, taking the under line as the true level of the liquid; but if, on the contrary, it contains soda, the pink scale of temperatures will indicate a fow degrees more than the real temperature, and this surplus number of degrees, being compared with those of the soda scale contiguous to it, on the opposite side, will express the per-centage of soda present in the sample.

74. For example :- Suppose the experiment to have been mado at $+12^{\circ}$ Centigrade, and to have given a solution marking $25^{\circ}$ on the pink scale of temperatures of the natrometer, that is $13^{\circ}$ more than the real temperature;-looking, thorefore, at number 13 on the pink scale of temperature, it will be seen that the number exactly opposite on the soda scale, and corresponding to it, is 4 , which indicates that the sample of potash examined contains 4 per cent. of soda.

It is important to bear in mind that all commercial potashes contain naturally a small quantity of soda, which quantity, in certain varieties, may even be considerable; it is only when the proportion of soda is more considerable than that which is naturally contained in the species of potash submitted to analysis, that it should be considered as fraudulently added. The following Table, published by M. Pesier, shows the average composition of the principal varieties of potash found in commerce, when in an unadulterated stato:-

Average Composition of Potashes.

\begin{tabular}{|c|c|c|c|c|c|c|c|c|c|c|}
\hline & & & 3 & & है & $\begin{array}{l}\text { Potsuhe } \\
\text { is the I } \\
\text { by e }\end{array}$ & $\begin{array}{l}\text { E obtained } \\
\text { Laboratory } \\
\text { es leiniag }\end{array}$ & से है & 1851 & 1855 \\
\hline & हैँ & 递 & c & $\frac{\text { aี }}{\mathrm{g}}$ & 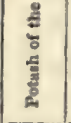 & 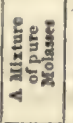 & 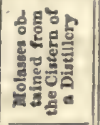 & 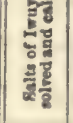 & 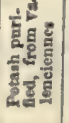 & 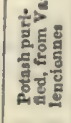 \\
\hline Sulphate of potash . . & $18 \cdot 47$ & $14 \cdot 11$ & $15 \cdot 32$ & $14 \cdot 38$ & $38 \cdot 84$ & $4 \cdot 27$ & $2 \cdot 98$ & $16 \cdot 19$ & 1.50 & 0.70 \\
\hline Cbloride of potassium. & 0.95 & $2 \cdot 09$ & $8 \cdot 15$ & $3 \cdot 64$ & $9 \cdot 16$ & $18 \cdot 17$ & $19 \cdot 69$ & 38.89 & $1 \cdot 60$ & $1 \cdot 70$ \\
\hline Carbonate of potash & $74 \cdot 10$ & $69 \cdot 61$ & $68.07^{3}$ & $71 \cdot 38$ & $38 \cdot 63$ & $51 \cdot 83$ & $53 \cdot 90$ & $26 \cdot 64$ & $89 \cdot 95$ & 95.24 \\
\hline Carbonate of soda (dry) . & $3 \cdot 01$ & $3 \cdot 09$ & $5 \cdot 85$ & $2 \cdot 31$ & $4 \cdot 17$ & $24: 17$ & $23 \cdot 17$ & $19 \cdot 60$ & $5 \cdot 12$ & $2 \cdot 12$ \\
\hline Insoluble resid ne & 0.65 & $1 \cdot 21$ & $3 \cdot 35$ & 0.44 & $2 \cdot 66$ & & & & & \\
\hline Moisture $\bullet$ & $7 \cdot 28$ & $8 \cdot 82$ & $\begin{array}{l}\text { unde- } \\
\text { ter- } \\
\text { mined }\end{array}$ & $4 \cdot 56$ & $5 \cdot 34$ & - $\cdot$ & - $\bullet$ & - • & 0.50 & \\
\hline $\begin{array}{l}\text { Phosphoric acld, llme, sillica, } \\
\text { Ec. }\end{array}$ & 0.54 & $1 \cdot 07$ & ditto & $3 \cdot 29$ & $1 \cdot 20$ & $1 \cdot 56$ & 0.26 & 3.68 & $1 \cdot 38$ & 0.24 \\
\hline . & $100^{\circ} 00^{\prime}$ & $100^{\circ} 00$ & & $100 \cdot 00$ & $100 \cdot 00$ & $100 \cdot 00$ & $100 \cdot 00$ & $100 \cdot 00$ & $100 \cdot 00$ & $100 \cdot 00$ \\
\hline Alkalimetrie degrees & 56 & $58 \cdot 1$ & 65 & $84 \cdot 4$ & $31 \cdot 6$ & 60 & 59.7 & $86 \cdot 5$ & $68 \cdot 6$ & $69 \cdot 5$ \\
\hline
\end{tabular}

- In the impossibility of estimating exactly the loss by calcination, and the quantity of oxide of potassium in the caustic state (hydrate of potash), we have reduced the potash to the state of car. bonate, to make comparison more easy. 
75. The alkalimetrical assay of soda is performed exactly in the same manner as that of potash - that is to say: From a fair average sample of the soda to be examined, take 1,000 grains' weight (or less if that quantity cannot be spared), and boil it five or six minutes in about eight fluid ounces of water, filter in order to separate the insoluble portion, and wash the residue on the filter with boiling water until it no longer drops from the filter with an alkaline reaction, and the bulk of tho filtered liquid and the washings received in a graduated glass cylinder form 10,000 grains' measure. Should the water which may have been required to wash the residue have increased the bulk of the solution beyond that quartity, it should be evaporated to reduce it to the bulk mentioned.

76. This being done, 1,000 water-grains' measure-that is to say, $\frac{1}{10}$ th part of the aqueous solution of the soda ash above mentioned ( $\$ 75)$-are transferred to the glass beaker or vessel in which the saturation is intended to take place: it is tinged distinctly blue with tincture of litmus, and the operation is performed in the same manner and with the same precautions as for potash, the glass beaker containing the blue alkaline solution being placed upon a sheet of white paper, or a slab of white porcelain, the better to observe the change of colour which takes place when the saturating point is approaching.

77. Having put into a glass beaker the 1,000 grains' measure of the aqueous solution of soda ash to be examined ( $\$ 75)$, and if the test sulphuric acid for soda, described before ( $\$ \S 23-27$ ), the alkalimeter, fig. 22,23 , or 24 , should be filled with that test acid up to the point marked $0^{\circ}$ (taking the under line of the liquid as the true level), and poured therefrom with the precaution already indicated, stirring briskly, at the same time, the liquid in the beaker. As is the case with the alkalimetrical assay of potash, the carbonic acid expelled by the test acid reacting upon the as yet undecomposed portion of the soda ash, converts it into bicarbonate of soda, so that at first no effervescence is produced; but as soon as half the quantity of tho soda in the solution is saturated, a brisk effervescence takes place. At first, therefore, the operator may pour at once, without fear, a pretty large quantity of the test acid into the alkaline solution, but as soon as this effervescence makes its appearance he should proceed with increased precaution gradually as the saturating point is approached. The modus operandi is, in fact, precisely as already detailed for the assay of potash, precisely the same kind and amount of care is requisite, and the assay is known to be terminated when the streaks made upon the litmus-paper with the stirring rod remain distinctly and permanently of a pink colour.

78. After saturation, and after having allowed the sides of the alkalimeter to drain, the number of divisions at which the test acid stands in the alkalimeter indicate at once the per-centage of the soda assayed, since, as we said, each division of this particular test acid represents one grain of pure soda. If, therefore, the test acid stands at 52 in the alkalimeter, then the soda assayed contained 52 per cent. of real soda. See, besides, the observations of $\$ 48$ and following, and also $\$ 81$.

79. If, instead of the special test acid for soda just alluded to, the operator employs that which has a specific gravity of 1.032 , and 100 alkalimetrical divisions of which saturate one equivalent of each base, the modus operandi is the same-that is to say, the alkalimeter is filled with it up to $0^{\circ}$, and it is poured therefrom carefully into the alkaline solution; but as the equivalent of soda is 31 , and 100 alkalimetrical divisions of the test sulphuric acid now employed are capable of saturating only that quantity of soda, it is clear that with the soda ash taken as an example in the preceding case, and containing 52 per cent. of real soda, the operator will have to refill his alkalimeter with the same test acid, and that a certain number of divisions of this second filling will have to be employed to perfect the saturation. In this instance the operator will find that nearly 68 divisions more, altogether 168 divisions (correctly, $167^{\circ} \cdot 74$ ) have been required to effect the saturation.

80. If, instead of the special test sulphuric acid for soda (\$\$23-27), or the test sulphuric acid for potash, soda, and other bases (\$8 31-34), the operator uses the potash and sods alkalimeter ( $\$ 828-35$ ), the method is always the same $(\$ \S 74,75)-$ that is to say, the aqueous solution of the soda ash is poured into the glass beaker, the difference being merely, that instead of the alkalimeter being quite filled up with the test sulphuric acid, which, in the present instance, has a specific gravity of $1.268(\$ 29)$, the said test acid is poured into the alkalimeter only up to the point marked 'soda' (taking the under-line of the liquid as the true level), and the remaining divisions of the alkalimeter are carefully filled up with water. The mouth of the tube should then be thoroughly closed with the thumb of the left hand, and the whole violently shaken until perfectly mixed, taking great care, of course, not to squirt any of the acid out of the tube, which evidently would cause an amount of error proportionate to the quantity of the test acid which would have thus been lost. The acid should then be poured from the alkalimeter with the usual precaution 
(8 76) into the glass beaker containing the aqueous solution of the soda ash unctcr examination, until complete neutralisation is attainod, stirring briskly all the time, or after ench addition of tho test acid. The neutralisation point being hit, the sides of the alkalimeter are allowed to drain, and the operator then reads off the number of divisions employed, which numbor indicates the per-centage of real soda contained in tho samplo assayed. Thus, if the sample operated upon be the same as that allucled to before, the number of divisions omployed being 52 would indicato 52 per cont. of real soda.

81. If the operator wishes to estimate the amount of soda in the sample as car. bonate of soda, he should fill the alkalimeter with the test acid in question (specific gravity 1.268 ) up to the point marked carbonate of soda, and fill the remaining divisions with water, shake the whole well, and proceed with tho neutralisation of tho aqueous solution of the sample in the glass beaker as just described. Supposing, as before, that the sample in question contains 52 per cent. of real soda, it will now be found that the number of divisions employed altogether to saturato the sample complotoly aro vory nearly 89 , for 52 of caustic soda correspond to 88.90 of tho carbonate of that alkali.

82. If the soda ash is very poor, instead of operating upon 1,000 water-grains' measure, or one-tenth part of the whole solution ( $=100$ grains' weight of the soda ash, $\$ 876-77)$, it is advisable to take three or four thousand water-grains' measure of the alkaline solution, and to divide, by three or four, the result obtained by saturation. Suppose, for example, that the quantity of real soda found is 46 ; this, if only 1,000 grains' measure had been taken, would, of course, indicate 46 per cent.; but as 4,000 water-grains' measure of solution have been taken instead, that number 46 must, accordingly, be divided by 4 , which gives $11 \frac{1}{2}$ per cent. only of real soda contained in the sample under examination.

83. The soda ash of commerce contains generally a per-centage of insoluble substances, which are removod by filtering, as wo said, and a greater or less quantity of chloride of sodium (common salt) and of sulphato of soda, which, howerer, do not in the slightest degree interfere with the accuracy of the result. But there is a source of error resulting from the presence in the soda ash of sulphide of calcium, of sulphite, and sometimes also, though more rarely, of hyposulphite, of soda. When sulphuret of calcium is present in the ash, on heating the latter by hot water, a double decomposition takes place: the sulphuret of calcium, reacting upon the carbonate of soda, forms sulphuret of sodium and carbonate of lime. Now snlphuret of sodium saturates the test acid just as carbonate of soda ; but as it has no commercial value, it is clear that if the ash contains a quantity of the useless sulphuret at all considerable, a very serious damage may be sustained by the purchaser if the per-centage of that substance present in the ash be taken account of as being soda. Sulphite of soda is produced from the oxidation of this sulphuret of sodium, and is objectionable inasmuch that when the test acid is added slowly to the nquoous solution of the ash, the effect is to convert the sulphite into bisulphite of soda, before any evolution of sulphurous acid, and consequently before the pink reaction on litmus-paper is produced.

84. In ordor to obviate the inaccuracies resulting from the neutralisation of a portion of the test acid by these substances, it is necossary to convert them into sulphates of soda, which is easily done by calcining a quantity of tho sample with five or six per cont. of chlorate of potash, as recommended by Gay-Lussac and Weltor. The operator, therefore, should intimately mix 50 or 60 grains' weight of pulverised chlorate of potash with 1,000 grains of the pulverised sample, and fuse the mixturo in a platinum crucible, for which purpose a blowpipe gas-furnace will be found excoedingly convenient. The fused mass should be washed, and the filtrate being received into a 10,000 water-grains' measure, and mado up with water to occupy that bulk, may then be assayed in every respecb as described beforc with one or other of the test acids mentioned.

85. When, however, the soda ash contains some hyposulphite of soda-which fortunately is seldom the case, for this salt is very difficultly produced in presence of a very large excess of alkali-it should not be calcined with chlorato of potash, because in that case one equivalent of hyposul phite becomes transformed, not into one equivalent of sulphate, but, reacting upon one equivalent of carbonato of soda, expels its carbonic acid, and forms with the soda of tho decomposed carbonato a second equivalent of sulphate of soda, cach equivalent of hyposulphito becoming thus converted into two equivalents of sulphate, and therefore creating an error proportionate to the quantity of the hyposulphite present, each equivalent of which would thus destroy one equivalent of real and available alkali, and thus render the estimation of the sample inaccurate, and possibly to a very considerable extent.

86. When this is the case, it is therofore advisable, according to Messrs. Fordos and Golis, to change the condition of tho sulphurets, sulphites, and hyposulphites, by add. 
ing a little neutral cliromate of potash to the alkaline solution, whence results sulphate of chromium, water, and a separation of sulphur, which will not affect the accu. racy of the alkalimetrical process.

87. Whether the sample to be analysed contains any sulphuret, sulphite, or hyposulphite, is easily ascertained as follows:- If, on pouring sulphuric acid upon a portion of the sample of soda ash under examination, an odour of sulphuretted hydrogen - that is, an odour of rotten eggs-is evolved, or if a portion of the soda ash, being dissolved in water, and then filtered, produces a black precipitate (sulphuret of lead) when solution of acetate of lead is poured into it, then the sample contains a sulphuret.

88. And if, after adding to some dilute sulphuric acid as much bichromate of potash as is necessary to impart to it a distinct roddish-yellow tinge, and a certain quantity of the solution of the soda ash under examination being poured into it, but not in sufficient quantity to neutralise the acid, tho reddish-yellow colour becomes green, it is a proof that the sample contains either sulphite or hyposulphite of soda, the green tinge being due to the transformation of the chromic acid into sesquioxide of chromium.

89. And if, muriatic acid being poured into the clear solution of the soda ash, a turbidness supervenes aftcr some time if left at rest, or at once if heat is applied, it is due to a doposit of sulphur, an odour of sulphurous acid being evolved, and hyposulphite of soda is probably present. We say ' probably', because if sulphurets and sulphites are present, the action of muriatic acid would decompose both, and liberate sulphuretted hydrogen and stlphurous acid; but as these two gases decompose each other, a turbidnoss due to a separation of sulphur is also formed; thus $2 \mathrm{HS}+\mathrm{SO}^{2}$ $=2 \mathrm{HO}+2 \mathrm{~S}$.

90. As wo havo already had occasion to romark, the soda ash of commerce frequently contains some, and occasionally a large quantity of caustic soda, the proportion of which it is at times important to determine. This may be done, according to Mr. Barreswill, by adding a solution of chloride of barium to the aqueous solution of the soda ash, by which the carbonate of soda is converted into carbonate of baryta, whilst the caustic soda, reacting upon the chloride of barium, liberates a quantity of caustic baryta proportionate to that of the caustic soda. in the soda ash. After this addition of chloride of barium, the liquor is filtered in order to separate the precipitated carbonate of baryta produced, and which remains on the filter, on which it should be washed with pure water. A few lumps of chalk are then put into \% Florence flask, $a$, and some muriatic acid being poured upon it, an effervescence due to a disengagement of carbonic acid is produced; the flask is then closed with a good cork, provided with a bont tube, $b$, reaching to the bottom of the vessel $c$, and the stream of carbonic acid produced is then passed through the liquor $c$, filtered from the carbonate of baryta above mentioned. The stream of carbonic acid produces a precipitate of carbonate of baryta, which should be also collected on a separate filter, washed, dried, and weighed. Each grain of this second precipitate of carbonate of baryta corresponds to 0.3157 of caustic soda.

91. As the soda ash of commerce almost invariably contains earthy carbonates, the sample operated upon should always be dissolved in hot water, and filtered in order to separate the carbonate of lime which otherwise would saturate a proportionate quantity of the tost acid, and thus render the analysis worthless.

92. The quantity of water contained in either potash or soda ash is ascertained by heating a weighed quantity of the sample to redness in a covered platinum capsule or crucible. The loss after ignition indicates the proportion of water. If any caustic alkali is present, 1 equivalent $=9$ of water, is retained, which cannot be thus eliminated, but which may, of course, be determined by calculation after the proportion of caustic soda has been found, as shown before, each 31 grains of caustic soda containing 9 grains of water.

93. Besides the alkalimetrical processes which have been explained in the preceding pages, the proportion of availablo alkali contained in the samplo may be estimatod from the amount of carbonic acid which can bo expelled by supersaturating the alkali with an acid. The determination of the valuo of alkalis from the quantity of card bonic acid thus evolved by the supersaturation of the carbonate actod upon has long been known. Dr. Uro, in tho 'Annals of Philosophy,' for October, 1817, and thon in his 'Dictionary of Chemistry,' 1821, and more recently in his pamphlet 'Chemistry Simplified,' described sereral instrumonts for analysing earthy and alkaline 
carbonates, and for a description of which the reader is referred to the article on AcDimetry. The ingenious little apparatus of Drs. Fresenius and Will for the same purpose, and to which we have already alluded in the same article, gives accurato results; but it should be observed that when the potash or soda of commerce contains any caustic alkali, or bicarbonate, or earthy carbonates, or sulphuret of alkali-which, as we have seen, is frequently, and, indeed, almost invariably, the case, the process is no longer applicable without first submitting the sample to soveral operations-which render this process troublesome and unsuited to unpractised hands. Thus, if caustic potash is present, the sample must be first mixed and triturated with its own weight of pure quartzose sand and about one-third of its weight of carbonate of ammonia. The mass is thon moistened with aqueous ammonia, and then put into a small iron capsule

37

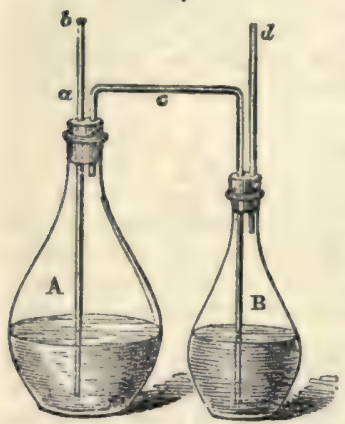
and evaporated to dryness, so as to expel completely the ammonia and carbonate of ammonia. The mass is then treated by water, filtered, washed, and concentrated to a proper bulk by evaporation, transferred to the apparatus, and treated as will be seen presently. If the sample contains caustic sods, instead of onethird, at least half of its weight of carbonate of ammonia should be employed. But for the estimation of pure carbonates, Drs. Fresensius and Will's method is both accurate and easy. The apparatus consists of two flasks, $\Delta$ and $B$; the first should have a capacity of from two to two ounces and a half; the second, or flask B, should be of a somewhat smaller size, and hold about one and a half or two ounces. Both should be provided with perfectly sound corks, each perforated with two holes, through which the tubes $a, c, d$ are passing. The lower extremity of the tube $a$ must be adjusted so as to reach nearly to the bottom of the flask $\Delta$, and its upper extremity is closed by means of a small pellet of wax, $b$; $c$ is a tube bent twice at right angles, one end of which merely protrudes through the cork into the flask $\mathbf{A}$, but the other end reaches nearly to the bottom of the flask B. The tube $d$ of the flask B merely protrudes through the cork into the flask.

94. The apparatus being so constructed, a certain quantity-100 grains, for example - of the potash or soda ash under examination (and which may have been previously dried) is weighed and introduced into the flask $\mathbf{A}$, and water is next pourod into this flask to about one-third of its capacity. Into the other flask, or flask B, concentrated ordinary sulphuric acid is poured, and the corks are firmly put in the flasks, which thus become connected, so as to form a twin-apparatus, which is then carried to a delicate balance, and accurately woighed. This done, the operator removes the apparatus from the balance, and applying his lips to the extremity of the tube $d$, sucks out a few air-bubbles, which, as the other tube, $a$, is closed by the wax pellet, rarefies the air in the flask $\Delta$, and consequently causes the sulphuric acid of flask $B$ to ascend a certain height (after the suction) into the tube $c$; and if, after a short time, the column of sulphuric acid maintains its height in the tube $c$, it is a proof that the apparatus is air-tight, and therefore as it should be. This boing ascertained, suction is again applied to the extremity of the tube $d$, so that a portion of the sulphuric acid of the flask $B$ ascends into the tube $c$, and presently falls into the flask $A$, the quantity which thus passes over being, of course, proportionate to the vacuum produced by the suction. As soon as the acid thus falls in the water containing the alkaline carbonate in the flask $\mathbf{A}$, an effervescenco is immediately produced, and as the carbonic acid disengaged must, in order to escape, pass, by the tube $c$, through the concentrated sulphuric acid of the flask $\mathrm{B}$, it is thereby completely dried before it can finally make its exit through the tube $d$. The effervescence having subsided, suction is again applied to the tube $d$, in order to cause a fresh quantity of sulphuric acid to flow over into the flask $\Delta$, as before; and so on, till the last portion of sulphuric acid sucked over produces no efforvescence, which indicates, of course, that all the carbonate is decomposed, and that, consequently, the operation is at an end, A powerful suction is now applied to the tube $d$, in order to cause a tolerably large quantity of sulphuric acid, but not all, to flow into the flask $\Delta$, which thus becomes very hot, from the combination of the concentrated acid with the water, so that the carbonic acid is thereby thoroughly expelled from the solution. The little wax pellet which served as a stopper is now removed from the tube $d$, and suction applied for some time, in order to sweep the flasks with atmospheric air, and thus displace all tho carbonic acid in the apparatus, which is allowed to become quite cold, and weighed again, together with the wax pellet, tho difference between the first and the second weighings - that is to say, the loss-indicating the quantity of carbonic acid which was contained in the 
carbonate, which has escaped, and from which, of course, the quantity of the carbonated alkali acted upon may be calculated. Suppose in effect, that the loss is 19 grains: taking the

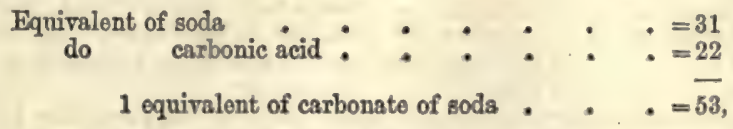

it is clear that the 19 grains of carbonic acid which have been expelled represent 45.77 grains of carbonate of soda, or, in other words, 100 grains of soda ash operated upon contained $45 \cdot 77$ of real carbonate of soda, thus :-

$$
\begin{array}{cl}
\mathrm{CO}^{2} & \mathrm{NaO} . \mathrm{CO}^{2} \mathrm{CO}^{2} \\
22: 53:: 19:= & \mathrm{NaO} \cdot \mathrm{CO}^{2}
\end{array}
$$

95. As the soda ash of commerce always contains earthy carbonates, and very froquently sulphurets, sulphites, and occasionally hyposulphites, instead of putting the 100 grains to be operated upon directly into the flask $\mathrm{A}$, it is absolutely necessary first to dissolve them in boiling water, to filter the solution, and to wash the precipitate which may be left on the filter with boiling water. The solution and the washings being mixed together, should then be reduced by evaporation to a proper volume for introduction into the flask $\mathrm{A}$, and the process is then carried on as described. If sulphurets, sulphites, or hyposulphites are present, the ash should be treated exactly as mentioned in $\$ \$ 83-91$, previous to pouring the solution into the flask $\mathrm{A}$, since otherwise the sulphuretted hydrogen and sulphurous acid, which would be disengaged along with the carbonic acid, would apparently augment the proportion of the latter, and render the result quite erroneous.

96. The balance used for this mode of analysis should be capable of indicating small weights when heavily laden.

AIRAIINI EARTHS-BARYTA, Lrme, and Strontu. These earths are so called to distinguish them from the earths Magnesra and Auvmiva. They are solublo in water, but to a much less extent than the alkalies. Their solutions impart a brown colour to turmeric paper, and neutralise acids. They are, however, distinguishod from the alkalies, by their combination with carbonic acid being nearly insoluble in water.

AITAII WASTz. A by-product obtained in the manufacture of soda-ash. By heating sulphate of soda with chalk and carbonaceous matter, a mixture of carbonate of soda and sulphide of calcium is obtained. The former salt is dissolved out on lixiviation, whilst the latter remains as an insoluble residue. It is this residue which constitutes 'alkali waste.' 'The accumulation of this material is aften a source of great annoyance to the manufacturer, especially by the evolution of sulphuretted hydrogen. Several methods have, of late years, been introduced for the utilisation of this product, and the recovery of the sulphur which it contains. For a description of these processes see SoDA.

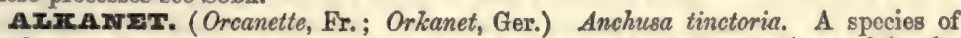
bugloss, or boragewort, cultivated in the neighbourhood of Montpellier and in the Levant. It is sometimes called the bugloss of Languedoc, or the dyer's bugloss. The anchusa is a rough plant, with downy and spear-shaped leaves, and clusters of small purple or reddish flowers, the stamens of which are shorter than the corolla. It affords a fine red colour to alcohol and oils, but a dirty red to water. Its prineipal use is for colouring ointments, oils, and pomades. The spirituous tincture gives to white marble a beautiful deep stain; but, usually, wax is coloured with the anchusa, and then applied to the surface of warm marble. It stains it flesh-colour, and the stain sinks deep into the stone. Oil coloured by alkanet is used for staining wood in imitation of rosewood.

Alkanet root was analysed by Dr. John, who found the constituents to be a peculiar colouring matter (pseudo-alkanium), 5.50; extraclive, 1.00; gum, 6.25; mattors extracted by caustic potash, $65 \cdot 00$; woody fibre, $18 \cdot 00$.

The colouring matter resides in the cortical part of the root, and was regarded by Pelletior as a kind of fatty acid (anchusic acid); but it is now usually considered to be a resinoid (anchusine), whose composition is $\mathrm{C}^{33} \mathrm{H}^{20} \mathrm{O}^{3}\left(\mathbf{C}^{35} \mathbf{z}^{40} \mathbf{O}^{8}\right)$. This root is sometimes termed the spurious alkanet root (radix alkannce spuries), to distinguish it from the Al-kenna.

Ax-KENNA, or AX-Hewra, is the name of the roots and leaves of Lawsonia inermis, which have been long employed in the East to dye the nails, teeth, hair, garments, \&c. The leaves, ground and mixed with a little limewater, servo for dyeing the, tails of horses in Persia and Turkey. 
It is the same as the herb Henna froguently referred to by the Oriontal poots. Tho powder of the leaves, boing wet, forms a pasto, which is bound on the nails for a night, and the colour thus given will last for several wooks.

This plant is clistinguished as the truo alkanot root (radix alkanne vera).

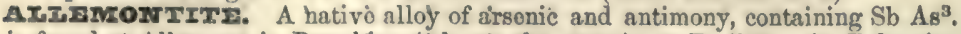
It is found at Allomont, in Dauphiny (whonco tho namo); at Przibram, in Bohemia ; and at Andreasberg, in the Hartz.

AIIIGATION. An arithmetical formula, useful on many occasions for ascertaining the proportion of constituents in a mixture, when thoy have undergone no change of volume by chomical action, or for finding the price or value of compounds consisting of ingredients of different values. Thus, if a quantity of sugar worth $8 d$. tho pound, and another quantity worth $10 d$., aro mixed, the question to be solved by alligation is, what is the value of the mixture by the pound? Alligation is of two kinds-medial, and alternate; medial, whon the rato of mixture is sought from the rates and quantities of tho simples; alternate, when the quantities of the simples aro sought from the rates of the simples and the rate of the mixture. - Webster.

AxzIOIב. Alliole is obtained by distilling crudo naphtha, and collecting all that leaves tho still in the first distillation before the boiling temperature reaches $194^{\circ} \mathrm{F}$.; and on the second distillation, all below $176^{\circ} \mathrm{F}$. It boils, when nearly free from benzole, at a tomperature of from $140^{\circ}$ to $158^{\circ} \mathrm{F}$., and possesses an alliaceous odour somewhat resembling that of bi-sulphide of carbon.

AIIIUIN. A genus of plants belonging to the Liliacee or lily-order. The bulbs of many species are esculont. Allium Cepa is the onion; A. sativum, garlic; A. pormu, the look; A. Ascalonicum, the shallot; A. Schonoprasum, the chive. The so-callod. 'Spanish onions,' imported from Spain, Portugal, and Egypt, aro mexely the largo bulbs of varieties of the common onion, which, cultivated in warm dry countries, lose much of their pungency.

AIIOCIASz. I mineral found in the Bannat-once regarded as a Cobaltglance. It appears to be of a very complex and variable composition. Essentially it is a compound of sulphur, arsenic, iron, ginc, and cobalt.

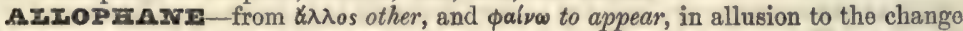
of appearance which this mineral undergoes before the blowpipe flame. This mineral, which is a hydrated silicate of alumina, consists essentially of silica $24 \cdot 22$, alumina $40 \cdot 39$, and water $35 \cdot 39$. It is generally found lining small cavities, and in veins in marl or chalk. Allophanes havo been found containing from 14 to 19 per cent of oxide of copper, which give them a green colour.

AIxorroory. Allotropic Condition. A name introduced by Berzolius to signify

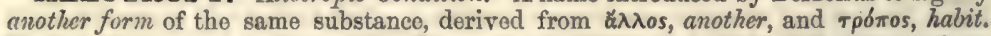
Carbon, for example, exists as the diamond, a brilliant gem, with difficulty combustible; as graphite, a dark, opaque mass, often crystalline, also of great infusibility; and as charcoal, a dark porous body, which burns with facility.

Sulphur, when melted, is at $230^{\circ} \mathrm{F}$. perfectly liquid. Being heated to $430^{\circ} \mathrm{F}$., it becomes thick and so tenacious that it can scarcely be poured out of the vessel in which it is melted. When heated to $480^{\circ}$ it again becomes liquid, and continues so until it boils. These examples are sufficient to explain tho meaning of this term. An extensive series of bodies appears to assume similar allotropic modifications. The probability is that, with the advance of physical and chemical science, many of the substances now supposed to be elementary will be proved to be but allotropic states of some one form of matter. See Isomkrism.

AxтоY. (Alliage, Fr.; Legirung, Ger.) From the French allier, to unite or mix ; or the Latin alligo, to bind. This term formerly signified mixing some baser metal with gold and silvor, and this meaning is still preserved in reforence to coinage; but, in chemistry, it now means any compound of any two or more metals whatever. Thus, bronze is an alloy of copper and tin; brass, an alloy of copper and zinc; and typo metal, an alloy of lead and antimony. All the alloys possess metallic lustre, oven when cut or broken to pieces; they are opaque; are excellent conductors of hoat and electricity; are frequently susceptible of erystallising; are more or less ductile, malleable, elastic, and sonorous. An alloy which consists of motals differently fusible is usually malleable when cold, and brittle when hot, as is exemplified with brass and gong metal.

Many alloys consist of definite or atomic proportions of the simple component metals, though some alloys seem to form in any proportion, like combinations of salt or sugar with water. It is probable that peculiar properties belong to the atomic ratio, as is oxemplified in the superior quality of brass made in that proportion.

The experimonts of Crookewitt upon amalgams appear to provo that the combination of metals in alloys oboys some laws of a similar charactor to those which prevail between combining bodies in solution; $i, e$, that a true combining proportion existed. 
By amalgamation and straining through chamois leather, he obtained erystalline motallic compounds of gold, bismuth, lead, and cadmium, with mercury, which appeared to exist in true definite proportions. With potassium he obtained two amalgams, $\mathrm{KHg}^{20}$ and $\mathrm{KHg}^{23}$. With silver, by bringing mercury in contact with a solution of nitrate of silver, according to the quantity of mercury employed, he obtained such amalgams as $\mathrm{Ag}^{5} \mathrm{Hg}^{16}, \mathrm{AgH}^{2}, \mathrm{AgH}^{3}, \mathrm{AgHg}^{4}$.

Beyond those there are many experiments which appear to prove that alloys are true chemical compounds; but, at the same time, it is highly probable that the true chemical alloy is very often dissolved (mechanically disseminated) in that metal which is largely in excess. In some cases, however, the alloy appears to be nothing more than a mechanical misture of the component metals.

Some years since, the Editor, at the request of Sir Henry De la Beche, and guided by the advice of Professor Graham, carried out a series of experiments in the laboratory of the Museum of Practical Geology, with the view of obtaining a good alloy for soldiers' medals, and the results confirmed the views respecting the laws of definite proportional combination among the metals. Many of those alloys were struck at the Mint, and yielded beautiful impressions; but there were many objections urged against the use of any alloy for a medal of honour.

One metal does not alloy indifferently with every other metal, but it is governed in this respect by poculiar affinities; thus, silver will hardly unito with iron, but it combines readily with gold, copper, and lead. In comparing the alloys with their constituent metals, the following differences may be noted. In general, the ductility of the alloy is less than that of the separate metals, and sometimes in a very remarkablo degree; on the contrary, the alloy is usually harder than the mean hardness of its constituents. The mercurial alloys or amalgams are, perhaps, exceptions to this rule.

The specific gravity is rarely the mean between that of each of its constituents, but is sometimes greater and sometimes less; indicating, in the former case, a closer cohesion, and, in the latter, a recedure, of the particles from each other in the act of their union. The alloys of the following metals have been examined by Crookewitt, and he has given their specific gravities as in the following Table; the specific gravity of the unalloyed metals being-

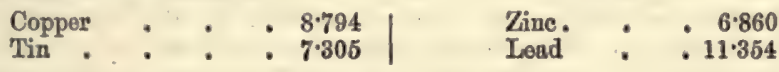

That of the alloys was-

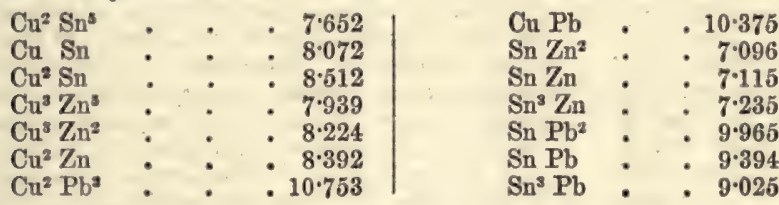

The following Tables of binary alloys exhibit this circumstance in experimental detail :-

\section{Alloys having a density greater than the mean of their constituents.}

Gold and zine

Gold and tin

Gold and bismuth

Gold and antimony

Gold and cobalt

Silver and rine

Silver and lead

Silver and tin

Silver and bismuth

Silver and antimony

Copper and rine

Copper and tin

Copper and palladium

Copper and bismuth

Iead and antimony

Platinum and molybdenum

Palladium and bismuth
Alloys having a density less than the mean of their constituents.

Gold and silver

Gold and iron

Gold and lead

Gold and copper

Gold and iridium

Gold and nickel

Silver and copper

Iron and bismuth

Iron and antimony

Iron and lead

Tin and lead

Tin and palladium

Tin and antimony

Nickel and arsenic

Zine and antimony 
There are many points of great physical as well as chemical interest in connection with alloys, which require a closer study than they have yet received. There are some striking facts, brought forward by M. Wertheim, deduced from experimonts earried on upon fifty-four binary alloys and nine ternary alloys of simple and known composition, which will be found in the 'Journal of the French Institute,' to which the reader desiring information on this point is reforred.

It is hardly possible to infer the melting point of an alloy from that of each of its constituent metals; but, in general, the fusibility is increased by mutual affinity in their state of combination. Of this a remarkable instance is afforded in the fusible metal consisting of 8 parts of bismuth, 5 of lead, and 3 of tin, which melts at the heat of boiling water, or $212^{\circ} \mathrm{F}$., though the melting point deduced from the mean of its components should be $514^{\circ} \mathrm{F}$. This alloy may be rendered still more fusible by adding a little mercury to it, when it forms an excellent material for anatomical injections. See Fusubize Mistat.

\section{On the Melting Point of Certain Alloys.}

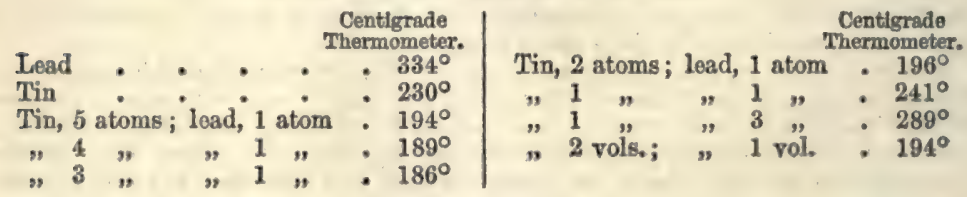

In these experiments of M. Kupffer, the temperatures were determined with thermometers of great delicacy, and the weighings were carefully carried out.-Ann. de Chimie, xl. 285-302; Brewster's Edin. Jour. Sci. i. N.S. p. 299.

The colours of alloys do not depend in any considerable dogree upon those of the separate metals; thus, the colour of copper, instead of being rendered paler by a large addition of zinc, is thereby converted into a rich-looking yellow metal, brass.

By menns of alloys, we multiply, as it were, the number of useful metals, and sometimes give usefulness to such as are separately of little value. Since these compounds can be formed only by fusion, and that many metals are apt to oxidise readily at their melting temperature, proper precautions must be taken in making alloys to prevent this occurrence. Thus, in combining tin and lead, resin or grease is usually put on the surface of the melting metals, the carbon produced by the decomposition of which protects them, in most cases, sufficiently from oxidation. When wo wish to combine tin and iron, as in the tinning of cast-iron tea-kettles, we rub sal-ammoniac upon the surfaces of tho hot metals in contact with each other, and thus exclude the atmospheric oxygen by means of its fumes. When there is a notable difference in the specific gravities of the metals which we wish to combine, we often find great difficulties in obtaining homogeneous alloys; for each metal may tend to assume the level due to its density, as is remarkably exemplified in alloys of gold and silver made without adequate stirring of the melting metals. If the mass be large and slow of cooling, after it is cast in an upright cylindrical form, the metals sometimes separate, to a certain degree, in the order of their densities. Thus, in casting large bells and cannon with copper alloys, the bottom of the casting is apt to contain too much copper and the top too much tin, unless very dexterous manipulation in mixing the fused materials has been employed immediately before the pouring out of the melted mass. When such inequalities are observed, the objects are broken and re-melted, after which they form a much more homogeneous alloy. This artifice of a double melting is often had recourse to, and especially in casting the alloys for the specula of telescopes.

When we wish to alloy three or more metals, we often experience difficulties, either because ane of the metals is more oxidisable, or denser, or more fusible, than the others, or because there is no direct affinity between two of the metals. In the latter predicament, we shall succeed better by combining the three motals first in pairs, for example, and then melting the two pairs together. Thus, it is difficult to unite iron with bronze directly; but if, instead of iron, we use tin plate, we shall immediately succeed, and the bronze, in this manner, acquires valuable qualities from the iron. Thus, also, to render brass better adapted for some purposes, a small quantity of lead is sometimes added to it, but this cannot be done directly with advantage; it is better to melt the lead first along with the zine, and then to add this alloy to the melting copper, or the copper to that alloy, and fuse them together.

One of the alloys most useful to the arts is brass; it is more ductile and less easily oxidised than even its copper constituent, notwithstanding the opposite nature of the zinc. (See Brass.) This alloy may exist in many different proportions, under which 
it has different names, as tombac, similor, pinchbeck, \&c. Copper and tin form compounds of remarkable utility, known under the name of hard brass, for the bushes, steps, and bearings of the axles, arbours, and spindles in machinery; and of bronze, bell-metal, \&c. (See Bronzs, \&c.) Gold and silver, in their puro state, aro too soft and flexible to form either vessels or coins of sufficient strength and durability ; but when alloyed with a littlo copper, they aequire the requisite hardness and stiffness for these and other purposes. Aluminium has been found by Dr. Porcy to possess the same hardening property. See Axuminium Bronze.

When we have occasion to unite several pieces of the same or of different metals, we employ the process called soldering, which consists in fixing together the surfaces by means of an interposed alloy, which must be necessarily more fusiblo than the metal or metals to be joined. That alloy must also consist of metals which possess a strong affinity for the substances to be soldered together. Hence each metal would seem to require a particular kind of solder, which is, to a certain extent, true. Thus, the solder for gold trinkets and plate is an alloy of gold and silver, or gold and copper; that for silver trinkets is an alloy of silver and copper; that for copper is either fine tin, for pieces that must not be exposed to the fire, or a brass alloy called hard solder, of which the zine forms a considerable proportion. The solder of lead and tin plate is an alloy of lead and tin, and that of tin is the same alloy with a little bismuth. Tinning, gilding, and silvering may also be reckoned as species of alloys, since the tin, gold, and silver are superficially united in these cases to other metals.

Metallic alloys possess usually more tenacity than could be inferred from their constituents; thus, an alloy of 12 parts of lead with 1 of zine has a tenacity double that of zinc.

The cohesive force of alloys is well shown in the annexed Table (p. 94), in which the results are mostly those obtained by Muschenbroek. ${ }^{1}$

Metallic alloys are generally much more easily oxidised than the separate metals, a phenomenon which may be ascribed to the increased affinity for oxygen which results from the tendency of the one of the oxides to combine with the other. An alloy of tin and lead heated to redness takes fire, and continues to burn for some time like a pioce of bad turf.

Every alloy is, in reference to the arts and manufactures, a new metal, on account of its chemical and physical properties. A vast field here remains to bo explored. Not above 60 alloys have been studied by the chemists out of many hundreds which may be made; and of these but few have been yet practically employed. Very slight modifications often constitute valuable improvements upon metallic bodies. Thus, the brass most esteemed by turners at the lathe contains from 2 to 3 per cent. of lead; but such brass does not work well under the hammer; and, reciprocally, the brass which is best under the hammer is too tough for turning.

M. Chaudet has made some experiments on the means of detecting the metals of alloys by the cupelling furnace, and they promise useful applications. The testing depends upon the appearance exhibited by the metals and their alloys when heated on a cupel. The following were Chaudet's results:-

Metals.-Pure tin, when heated this way, fuses, becomes of a greyish-black colour, fumes a little, exhibits incandescent points on its surface, and leaves an oxide which, when withdrawn from the fire, is at first lemon-yellow, but, when cold, white. Antimony melts, preserves its brilliancy, fumes, and leaves the vessel coloured lemonyellow when hot, but colourless when cold, except a few spots of a rose tint. Zine burns brilliantly, forming a cone of oxide; and the oxide, much increased in volume, is, when hot, greenish, but, when cold, perfectly white. Bismuth fumes, becomes covered with a coat of melted oxide, part of which sublimes, and the rest enters the pores of the cupel; when cold, the cupel is of a fine yellow colour, with spots of a greenish hue. Lead resembles bismuth very much; the cold cupel is of a lemonyellow colour. Copper melts, and becomes covered with a coat of black oxide; sometimes spots of a rose tint remain on the cupel.

Alloys. - Tin 75, antimony 25, melt, become covered with a caat of black oxide, have very fow incandescent points; when cold, the oxide is nearly black, in consequence of the action of antimony; $a \frac{1}{400}$ th part of antimony may be ascertained, in this way, in the alloy. An alloy of antimony containing tin leaves oxide of tin in the cupel; a so th part of tin may be thus detected. An alloy of tin and zine gives an oxide which, whilst hot, is of a green tint, and resembles philosopher's wool in appearance. An alloy containing 99 tin 1 zinc did not present the incandescent points of pure tin, and gave an oxide of greenish tint when cold. Tin 95 , bismuth 5 parts, gave an oxide of a grey colour. Tin and lead give an oxide of a rusty brown colour. An alloy of lead and tin, containing only 1 per cent. of the latter metal, when 


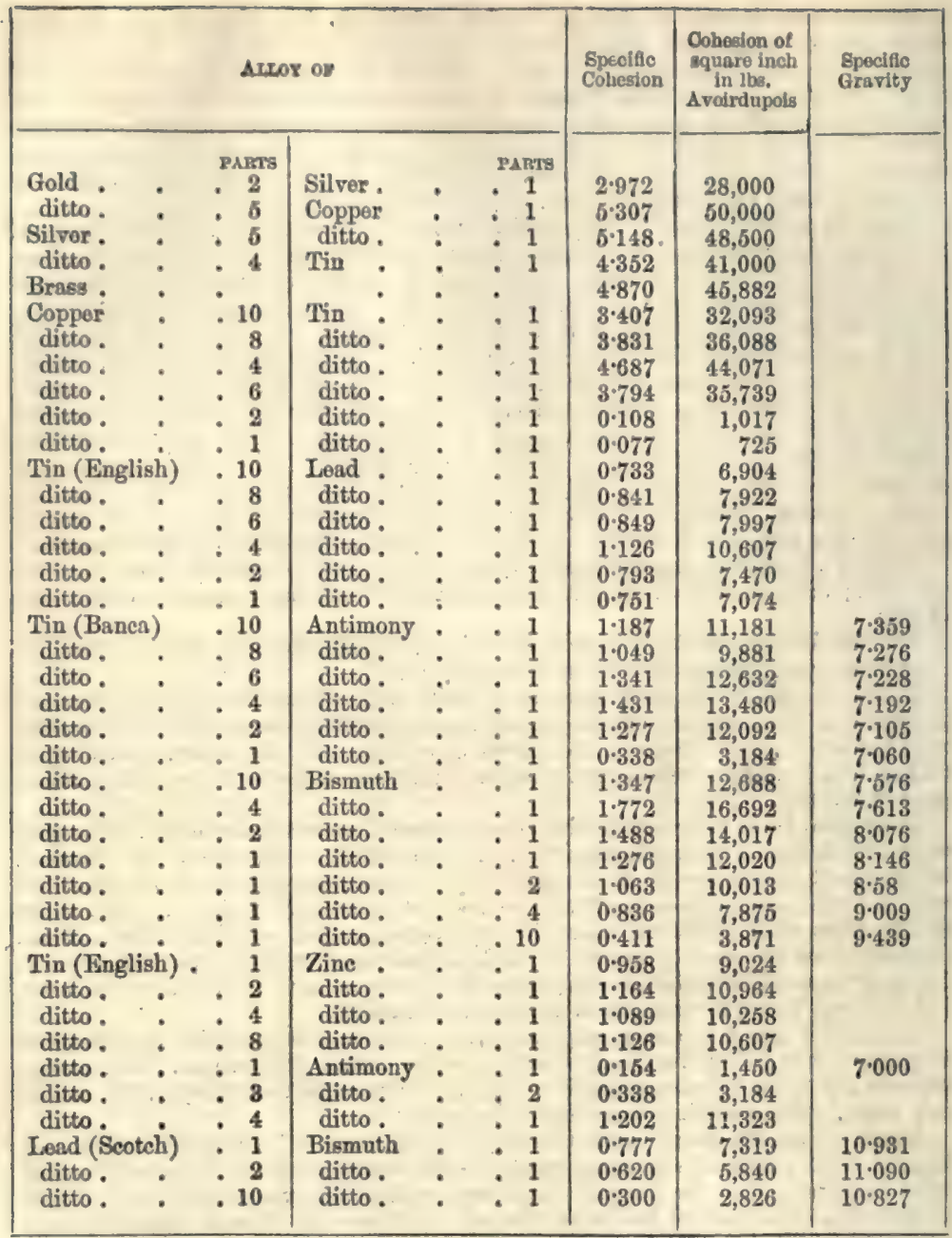

heated, does not expose a clean surface, like lead, but is covered at times with oxide of tin. Tin 75 and copper 25 gave a black oxide: if the heat be much elevated, the underpart of the oxide is white, which is oxide of tin; the upper part is black, being the oxide of copper, and the cupel becomes of a rose colour. If the tin be impure from iron, the oxide produced by it is marked with spots of a rust colour.

The degree of affinity between metals may be in some measure estimated by the greater or less facility with which, when of different degrees of fusibility or volatility, they unite, or with which they can, after union, be separated by heat. The greater or less tendency to separate into differently proportioned alloys, by long-continued fusion, may also give some information upon this subject. Mr. Hatchett remarked, in his elaborate researches on metallic alloys, that gold made standard with the usual precautions, by silver, copper, lead, antimony, \&c., and then cast, after long fusion, into vertical bars, was by no means an uniform compound; but that the top of the bar, corresponding to the metal at the bottom of the crucible, contained the larger proportion of gold. Hence, for a more thorough combination, two red-hot crucibles should be employed, and the liquefied metals should be alternately poured from the one into the other. To prevent unnecessary oxidisation from tho air, the crucibles should contain, besides the metal, a mixture of common salt and pounded charcoal. The metallic alloy should also be occasionally stirrod up with a rod of carthenware. 
When there is a strong affinity between the two metals, their alloy is generally. denser than the mean, and vice versâ. This is exemplified, as previously shown, in the alloys of copper with zine and tin, on the one hand, and with copper and lead on the other. When one of the metals, however, is added in excess, there result an atomic compound and an indefinite combination, as would appear from Muschenbroek's exporiments. Thus :-

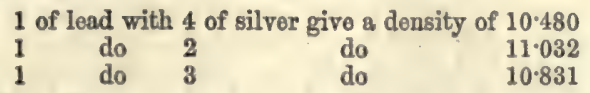

The proportion of the constituents is on this principle estimated in France by the test of the ball applied to powtor; in which the weight of the alloyed ball is compared with that of a ball of pure tin or standard pewter cast in the same mould. Alloys possess the elasticity belonging to the mean of their constituents, and also the specific heat.

According to M. Rudberg, while lead solidifies at $325^{\circ} \mathrm{C}$, and tin at $228^{\circ}$, their atomic alloy solidifles at $187^{\circ}$, which he calls the fixed point, for a compound $\mathrm{Pb} \mathrm{Sn}^{3}$.

An alloy too slowly cooled is often apt to favour the crystallisation of one or moro of its components, and thus to render it brittle; and hence an iron mould is preferable to one of sand when there is danger of such a result.

It is not a matter of indifference in what order the metals are melted together in making an alloy. Thus, if we combine 90 parts of tin and 10 of copper, and to this alloy add 10 of antimony; or if we combine 10 parts of antimony with 10 of copper, and add to that alloy 90 parts of tin, we shall have two alloys chemically the same; and still it will be easy to discover that, in other respects - fusibility, tenacity, \&c.-they totally differ. Whence this result? Obviously from the nature of their combination, dependent upon the order pursued in the preparation, and which continues after the mixture. In the alloys of lead and antimony also, if the heat be raised in combining the two metals together much above their fusing points, the alloy becomes harsh and brittle; probably because some alloy formed at that high temperature is not soluble in the mass.

In common eases the specifie gravity affords a good criterion whereby to judge of the proportion of two metals in an alloy. But a very fallacious rule has been given in some respectable works for computing the specific gravity that should result from the alloying of given quantities of two metals of known densitios, supposing no chomical condensation or expansion of volume to take place. Thus, it has been taught, that if gold and copper be united in equal woights, the computed specific gravity is merely the arithmetical mean between the numbers denoting the two specific gravities. Whereas, the specific gravity of any alloy must be computed by dividing the sum of the two weights by the sum of the two volumes, compared, for convenience sake, to wator reckoned unity. Or, in another form, the rule may be stated thus :-Multiply the sum of the weights into the products of the two specific-gravity numbers for a numerator; and multiply each specific-gravity number into the weight of the other body, and add the two products together for a denominator. The quotient obtained by dividing the said numerator by the denominator is the truly computed mean specific gravity of the alloy. On comparing with that density the density found by experiment, we shall see whether expansion or condensation of volume has attended the metallic combination. Gold having a spocific gravity of $19 \cdot 36$, and eopper of $8 \cdot 87$, when they are alloyed in equal weights, give, by the fallacious rule of the arithmetical mean of the densities $\frac{19 \cdot 36+8 \cdot 87}{2}=14 \cdot 11$; whereas the rightly computed density is

only $12 \cdot 16$. It is evident that, on comparing the first result with exporiment, we should be led to infer that there had been a prodigious condensation of volume, though expansion has actually taken place. Let $\mathrm{W}$, w be the two weights; $\mathrm{P}, \mathrm{p}$ the two specific gravities, then M, the mean specific gravity, is given by the formula

$$
\mathrm{M}=\frac{(\mathrm{W}+\mathrm{w}) \mathrm{Pp}}{\mathrm{Pw}+\mathrm{pW}} \quad \therefore 2 \Delta=-\frac{(\mathrm{P}-\mathrm{p})^{2}}{\mathrm{P}+\mathrm{p}}
$$

$=$ twice the error of the arithmetical mean; which is therefore always in excess.

Alloys of a somewhat complex character aro made by Mr. Alexander Parkos, of Birmingham, of a white or pale colour, by melting together 33! lbs. of foreign zine, 64 of tin, $1 \frac{1}{4}$ of iron, and 3 of copper; or 50 zinc, 48 tin, 1 iron, and 3 copper ; or any intermediate proportion of zine and copper may be used. The iron and copper are first melted together in a crucible, the tin is next introduced, in such quantities at a time as not to solidify the iron and copper; the zinc is added lastly, and the whole mixed by stirring. The flux rocommended for this alloy is, 1 part of lime, 1 part of Cumberland iron ore, and 3 parts of al-ammoniac. 
Another of his alloys is composed of $66 \mathrm{lbs}$. of foreign zinc, $33 \frac{1}{\mathrm{l}}$ tin, $3 \frac{1}{4}$ antimony ; or $70 \frac{3}{4}$ zine, $191 \mathrm{~s}$ tin, and $2 \frac{3}{4}$ antimony ; or any intermediato proportions, and with or without arsenic. He uses black flux. When to be applied to the sheathing of ships, from 8 to $16 \mathrm{oz}$. of metallic arsenic are added to every $100 \mathrm{lbs}$. of alloy. $A$ third class of alloys consists of equal parts of iron and nickel; the coppor is next added, and lastly the zine, or the copper and zine may bo added as an alloy. $100 \mathrm{lbs}$. may consist of $45 \mathrm{lbs}$. of iron and nickel (partes equales), and $10 \mathrm{l}$ lbs. of foreign zinc; or $30 \%$ lbs. of alloy of iron and nickel $(p, a$. $), 46$ copper, and $26 \frac{1}{2}$ zine; or any intermediate proportions of zine and copper. He uses also an alloy of $60 \mathrm{Ibs}$. of copper, 20 of zinc, and 20 of silver; or 60 copper, 10 nickel, 10 silver, and 20 zine; the coppor and nickel being first fused together. His fifth alloy is called by him a non-conductor of heat! It is made of 25 nickel, 25 iron, and 50 copper; or 15 nickel, 25 iron, and 60 coppor; the last being added after the fusion of the others.

It may prove convenient to give a general statement of the more striking peculiarities of the important alloys. More dotailed information will be found under the heads of the respective metals.

Gold AND Silver AlloYs.-The British standard for gold coin is 22 parts puro gold and 2 parts alloy, and for silver, 222 parts pure silver to 18 parts of alloy.

The alloy for the gold is an indefinite proportion of silver and copper: some coin has a dark rod colour from the alloy being chiefly copper; the lighter the colour a larger portion of silver is indicated, sometimes even (when no copper is present) it approaches to a greenish tinge, but the proportion of pure gold is the same in either case.

The alloy for silver coinage is always copper; and a very pure quality of this motal is used for alloying, both for the gold and silver coinage, as almost any other metal being present, even in very small quantities, would make the motals unfit for coinage, from rendering the gold, silver, and copper brittle, or not sufficiently malleable.

The standard for plate (silver) is the same as the coin, and requires the same quantity of copper, and carefully melting with two or three bits of charcoal on the surface while in fusion, to prevent the oxidation of the copper by heat and exposure to the atmosphere.

The gold standard for plate and jewellery varies, by an Act of Parliament, from the 22 carats pure, to 18,12 , and 9 : the alloys are gold and silver, in various proportions according to the taste of the workmen; the colour of the articles manufactured depending, as with the coin, on the proportions; if no copper is used in qualities under 22 carats fine gold, the colour varies from a soft green to a greonish white, but a proportion of copper may be used so as to bring the colour to nearly that of 22 fine, 1 silver, and 1 copper.

Wire of either gold or silver may be drawn of any quality, but the ordinary wire for fine purposes, such as lace, contains from 5 to 9 pennyweights of copper in the pound of 240 pennyweights, to render it not so soft as it would be with pure silver.

Gold, silver, and copper may be mixed in any proportions without injury to the ductility, but no reliable scale of tenacity appears to have been constructed, although gold and silver in almost any proportions may be drawn to the very finest wire.

The alloys of silver and palladium may be made in any proportions; it has been found that even 3 per cent. of palladium prevents silver tarnishing so soon as without it; 10 per cent. very considerably protects the silver, and 30 per cent. of palladium will prevent the silver being affected by fumes of sulphuretted hydrogen unless very long exposed: the latter alloy has been found useful for dental purposes, and the alloy with less proportions - say 10 to 15 per cent.-has been usepd for graduated scales of mathematical instruments.

The alloy of platinum and silver is made for the same purposes as those of palladium, and, by proper care in fusion, are nearly equally useful, but the platinum doos not seem to so perfectly combine with the silver as the palladium. Any proportion of palladium with gold injures the colour, and even 1 per cent. may be detectod by sight, and 5 per cent. renders it a silver colour, while about 10 per cent. destroys it; but the ductility of the alloy is not much injured.

Gold leaf for gilding contains from 3 to 12 grains of alloy to the ounce. The gold used by respectable dentists is nearly pure, but necessarily contains about 6 grains of copper to the ounee troy, or $\frac{1}{80}$ th part.

Antimony in the proportion of $\frac{1}{1920}$ quite destroys the ductility of gold.

Gold and platinwm alloy forms a somewhat elastic metal. Hermstadt's imitation of gold consists of 16 parts of platinum, 7 parts of copper, and 1 of zinc, put in a crucible, covered with charcool powder, and molted into a mass:-P. J. 
Dentist's Alloy. For the ordinary purposes of mounting artificial teeth \& peculiar metal is required. It must be sufficiently hard and tough, and it must not be liable to corrosion by either acid or acrid fluids. Experience has shown that an alloy of gold, silver, and copper most nearly meets all the required conditions. Dentists use 16-carat gold, which is $\frac{2}{3}$ fine gold and $\frac{1}{3}$ alloy, the alloy boing always nearly equal portions of silver and copper, which is not, for these purposes, in tho slightest dogree injurious, - See AxrazgaM.

Copper Allors.-Copper alloyed with zinc forms Brass, and with tin we have Bronzz. (See those articles.) The best Kingston's Metal is an alloy of copper, tin, and mercury; it is much used for bearings. (See Knngston's Metal.) The alloys of the ancients were usually either brasses or bronzes. The following analyses of ancient coins, \&rc., by Mr. John Arthur Phillips, are of great ralue:-. .

\begin{tabular}{|c|c|c|c|c|c|c|c|c|c|c|c|}
\hline • & & & & & & & & & & & \\
\hline & B.C. & A.D. & Copper & Tin & Lead & Iros & Zlne & sulver & Sujph. & Niekel & Coonit \\
\hline Ws & 500 & 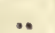 & $69 \cdot 69$ & $7 \cdot 16$ & $21 \cdot 82$ & -47 & ** & .. & trace & trace & • \\
\hline Bemis : & 500 & $\cdots$ & $62 \cdot 04$ & $7 \cdot 66$ & $29 \cdot 32$ & 18 & :. & $\because$ & trace & $\cdot 19$ & 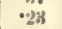 \\
\hline Quadrans : & 500 & $\therefore$ & $72 \cdot 22$ & $7 \cdot 17$ & $19 \cdot 56$ & -40 & ... & $\because$ & trace & 20 & $\because 9$ \\
\hline Hiero I. & 470 & $\therefore$ & $94 \cdot 15$ & $5 \cdot 49$ & .. & .32 & . &. & & & \\
\hline Alexander the Great : & 885 & 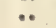 & $86 \cdot 77$ & $12 \cdot 99$ & .. & .. & $\cdots$ & .. & .06 & & \\
\hline Philippus III. . & 323 & $\therefore$ & $90^{-27}$ & $9 \cdot 43$ & & & & & & & \\
\hline Phillippus V. & 200 & .. & $85 \cdot 15$ & $-11 \cdot 12$ & $2 \cdot 85$ & $\cdot 42$ & .. & .. & trace & & \\
\hline Copper coin of Athens & $\frac{7}{70}$ & $\because$ & $88: 34$ & 9.95 & $\cdot 63$ & -26 & .. & . & .. & trace & trace \\
\hline Egyptian, Ptolemy IX. & $\begin{array}{l}70 \\
53\end{array}$ & 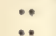 & $84 \cdot 21$ & $15 \cdot 64$ & 药 & trace & .. & $\cdots$ & trace & .. & truce \\
\hline $\begin{array}{l}\text { Pompey. First Brass } \\
\text { Chin of the Atilia }\end{array}$ & & .. & $74 \cdot 17$ & $8 \cdot 47$ & & & & & & & \\
\hline Family & 45 & .. & $68 \cdot 69$ & $4 \cdot 86$ & $25 \cdot 48$ & $\cdot 11$ & . & .. & & trace & trace \\
\hline Julius and Angustus . & 42 & .. & $79 \cdot 13$ & $8: 00$ & $12 \cdot 81$ & trace & 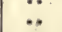 & $\ldots$ & trace & & \\
\hline Augustus and Agrippa & 80 & *. & 78.45 & $12 \cdot 96$ & $8 \cdot 62$ & trace & .. & $\cdots$ & trace & & \\
\hline $\begin{array}{l}\text { Large Brass of the } \\
\text { Cassia Family. }\end{array}$ & 20 & & & & & & $17 \cdot 81$ & & & & \\
\hline Sword-blade. : : & .. & $\because$ & $\begin{array}{l}82 \cdot 26 \\
89 \cdot 69\end{array} \mid$ & $9: 58$ & $\because$ & $\begin{array}{l}45 \\
.33\end{array}$ & $\begin{array}{c}17 \cdot 01 \\
. .\end{array}$ & $\ddot{. .}$ & $\begin{array}{l}\text { trace } \\
\text { trace }\end{array}$ & & \\
\hline Broken sword-blade & .. & .. & $85 \cdot 62$ & $10^{\circ} 02$ & .. & .44 & 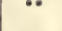 & & & & \\
\hline $\begin{array}{l}\text { Fragment of a sword. } \\
\text { blade. }\end{array}$ & & & $91 \cdot 79$ & & & & & & & & \\
\hline Broken spear-head & $\ddot{.}$ & .: & $99 \cdot 71$ & $8 \cdot 17$ & $\because$ & trace & $\because$ & $\because$ & trace & & \\
\hline Celt . & .. & .. & $90 \cdot 68$ & $\because 43$ & $\because \cdot 28$ & trace & $\because$. & $\because$ & trace & & \\
\hline Celt & .. & .. & $90 \cdot 18$ & $9 \cdot 81$ &.. & trace & & & & & \\
\hline Celt . . & .. & $\therefore$ & 33 & $9 \cdot 19$ & & .83 & .. & .. & 24 & & \\
\hline Celt ${ }^{\circ}$ - & $\because$ & $\ddot{60}$ & $83 \cdot 61$ & $10^{\circ} 79$ & $3 \cdot 20$ & $\cdot 58$ & 10.87 & *. & . & trace & • 34 \\
\hline $\begin{array}{l}\text { Large Brass of Nero } \\
\text { Titus }\end{array}$ & $\because$ & $\begin{array}{l}60 \\
79\end{array}$ & $\begin{array}{l}81 \cdot 07 \\
83 \cdot 04\end{array}$ & $1 \cdot 05$ & $\cdots$ & $\because 0$ & $\begin{array}{l}17.81 \\
15.84\end{array}$ & & & & \\
\hline $\begin{array}{l}\text { Titus } \\
\text { Hadrian }\end{array}$ & $\because$ & 120 & $\left|\begin{array}{l}83 \cdot 04 \\
85 * 67\end{array}\right|$ & $\ddot{1}_{14}$ & $\ddot{1 * 73}$ & .74 & 10.85 & & & & \\
\hline & :. & 165 & $79 \cdot 14$ & 4.97 & $9 \cdot 18$ & $\cdot 23$ & $6 \cdot 27$ & & & & \\
\hline $\begin{array}{l}\text { Greek Imperial Samo- } \\
\text { sata }\end{array}$ & & 212 & $70 \cdot 91$ & $6 \cdot 75$ & 21.96 & race & & & & & \\
\hline Victorinus, Sen. (No. 1) & $\because$. & 262 & $\begin{array}{l}10 \cdot 91 \\
95 \cdot 37\end{array}$ & $\begin{array}{r}099 \\
\cdot 99\end{array}$ & ace & trace & & $1 \cdot 60$ & & & \\
\hline Victorinus, Sen. (No. 2) & 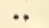 & 262 & 3 & $\cdot 10$ & trace & 1.01 & * & $1 \cdot 76$ & & & \\
\hline Tetrius, Sen. (No. 1). & . & 267 & 98.50 & $\cdot 37$ & trace & .46 & .. & $\cdot 76$ & & & \\
\hline Tetrins, Sen. (No. 2) : & $\because$ & 268 & $98 \cdot 00$ & -51 & 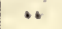 & -05 & $*$ & $1 \cdot 15$ & & & \\
\hline $\begin{array}{c}\text { Claudins Gothicus, } \\
(N 0.1)\end{array}$ & & & $81 \cdot 60$ & $7 \cdot 41$ & $8 \cdot 11$ & $*$ & & 1.86 & & & \\
\hline "Gothicus," & & 268 & & & & $* 0$ & 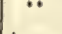 & & & & \\
\hline (No.2) , : & $\cdots$ & & $84 \cdot 70$ & 8.01 & $2 \cdot 67$ & 31 & trace & $7 \cdot 98$ & & & \\
\hline Tacitus (No. 1) . & .. & 275 & $86 \cdot 08$ & "ّ̈'63 & $4 \cdot 87$ & $\ddot{3}$ & .. & 4.42 & & & \\
\hline $\begin{array}{l}\text { Tacitus (No. 2) } \\
\text { Probus (No. 1) }\end{array}$ & $\because$ & & $\begin{array}{l}91 \cdot 46 \\
90 \cdot 68\end{array}$ & 200 & $2 \cdot 38$ & $\begin{array}{r}2.81 \\
\cdot 61\end{array}$ & $\ddot{1} \cdot 39$ & $\begin{array}{l}5 \cdot 92 \\
2 \cdot 24\end{array}$ & & 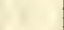 & \\
\hline Probus (No.2) : & $\because$ & 275 & 94.65 & 45 & 45 & .80 & $\ldots$ & $3 \cdot 22$ & & & \\
\hline
\end{tabular}

Copper, when united with half its weight of lead, forms an inferior alloy, resembling gun-metal in colour, but it is softer and cheaper. This alloy is called pot-metal and cock-metal, because it is used for large measures and in the manufacture of taps and cocks of all deseriptions.

Sometimes a small quantity of zinc is added to pot-metal; but when this is considerable the copper seizes the zinc to form brass, and leaves the lead at liberty, a largo portion of which separates on cooling. Zine and lead are not disposed to unito; but a little arsenic occasions them to combine.

It is not a little curious to find that some of the coins of high antiquity contain zinc: this must have been introduced by the use of calamino, since it does not appear that zinc was known as a metal before 1280 A.D., when Albertus Magnus speaks of it as a semi-metal, and calls the alloy of copper and zinc golden marcasite; or rather, perhaps, he means to apply that name to zine, from its power of imparting a golden colour to copper. The probability is that calamine was known from the earliest times as a peculiar earth, although it was not thought to be an ore of zine or of any otber metal. - See 'Watson's Chemical Essays.'

VOL. I. 
Lead Arzors. - Of the alloys of copper and lead, Mr. Holtzapfel gives the following deseription:-Two ounces lead to one pound copper produces a red-colourod and ductile alloy.

Four ounces lead to 1 pound copper gives an alloy less red and ductile. Neither of these is so much used as those following (p. 100), as the object is to employ as much lead as possible.

Tabular Statement of the Physical Pecnliarities of the Principal Alloys, adopted, with some alterations, from the 'Encyclopédie Technologique.'

\begin{tabular}{|c|c|c|}
\hline \multicolumn{3}{|c|}{ BRITTLE METALS } \\
\hline Ansentc & AstivosY & Bismutr \\
\hline $\begin{array}{l}\text { With Zisc, rendering it } \\
\text { brittle, }\end{array}$ & This alloy is very brittle. & Unknown. \\
\hline $\begin{array}{l}\text { With Inos and STREL, } \\
\text { hardening, whitening, } \\
\text { and rondering those me- } \\
\text { tals susceptible of a fine } \\
\text { polish : much used for } \\
\text { steel chains and other } \\
\text { ornaments. }\end{array}$ & $\begin{array}{l}30 \text { of iron and } 70 \text { of anti- } \\
\text { mony are fusible; very } \\
\text { hard, and white. An } \\
\text { alloy of two of iron and } \\
\text { one of antimony is vory } \\
\text { hard and brilliant. }\end{array}$ & Doubtful. : : \\
\hline $\begin{array}{l}\text { With Goud, a grey metal, } \\
\text { very brittle. }\end{array}$ & $\begin{array}{l}\text { Forms readily a pale yel- } \\
\text { low alloy, breaking with } \\
\text { a fracture like porcelain. }\end{array}$ & $\begin{array}{l}\text { Similar to antimony ; of a } \\
\text { yellow-green colour. }\end{array}$ \\
\hline $\begin{array}{l}\text { With Copper. Composed } \\
\text { of } 62 \text { parts of copper and } \\
32 \text { argenic, a grey, bril: } \\
\text { liant, brittle metal, In- } \\
\text { ereasing the quantity of } \\
\text { copper, the alloy be- } \\
\text { comes white and slightly } \\
\text { ductile: used in the ma- } \\
\text { nufacture of buttons un- } \\
\text { der the name of white } \\
\text { copper, or ToмBAc. }\end{array}$ & $\begin{array}{l}\text { Alloys readily: the alloys } \\
\text { are brittle. Those formed } \\
\text { with equal parts of the } \\
\text { two metals are of a fine } \\
\text { violet colour. } \\
\text {.. }\end{array}$ & $\begin{array}{cc}\text { Pale-red brittle metal. } \\
\vdots \\
\vdots \\
\vdots\end{array}$ \\
\hline $\begin{array}{l}\text { With Snvgr. } 23 \text { of silver } \\
\text { and } 14 \text { arsenic form a } \\
\text { greyish - white brittle } \\
\text { metal, }\end{array}$ & $\begin{array}{l}\text { These have } a \text { strong af- } \\
\text { fnity; their alloys are } \\
\text { álways brittle. }\end{array}$ & $\begin{array}{l}\text { Alloys hrittle and lamel- } \\
\text { lated. }\end{array}$ \\
\hline $\begin{array}{l}\text { With Leap. Arsenic ren- } \\
\text { ders lead brittle. The } \\
\text { combination is very in- } \\
\text { timate; not decomposed } \\
\text { by heat. }\end{array}$ & $\begin{array}{l}\text { Antimony gives hardness } \\
\text { to lead. } 24 \text { parts of an- } \\
\text { timony and } 76 \text { of lead, } \\
\text { corresponding to } \mathrm{Pb} \mathrm{S}^{2} \mathrm{Sb} \text {, } \\
\text { appear the point of satu- } \\
\text { ration of the two metals. }\end{array}$ & $\begin{array}{l}\text { The alloys of bismuth and } \\
\text { lead are less brittle and } \\
\text { more ductile than those } \\
\text { with antimony; but the } \\
\text { alloy of } 3 \text { parts of lead } \\
\text { and } 2 \text { of bismuth is } \\
\text { harder than lead. These } \\
\text { alloys are very fusible. }\end{array}$ \\
\hline $\begin{array}{l}\text { With Tis. Brittle, grey } \\
\text { lamellated; less fusible } \\
\text { than tin. }\end{array}$ & $\begin{array}{l}\text { The alloys of antimony } \\
\text { and tin are rery white. } \\
\text { They become brittle } \\
\text { when the arsenic is in } \\
\text { large quantity. }\end{array}$ & $\begin{array}{l}\text { Tin and bismuth unite in } \\
\text { all proportions by fusion. } \\
\text { All the alloys are more } \\
\text { fusible than tin. }\end{array}$ \\
\hline $\begin{array}{l}\text { With Mercury. Without } \\
\text { interest. }\end{array}$ & A gritty white alloy. & $\begin{array}{l}\text { Mercury dissolres a large } \\
\text { quantity of bismuth with- } \\
\text { out losing its fluidity; } \\
\text { but drops of the alloy } \\
\text { elongate, and form a tail. }\end{array}$ \\
\hline
\end{tabular}




\begin{tabular}{|c|c|c|c|}
\hline \multicolumn{4}{|c|}{ DUCTILE METALS. } \\
\hline Inos & Gotw & Coppren & Snver ' \\
\hline $\begin{array}{l}\text { With Zixc. See } \\
\text { G A I v A N I \& nD } \\
\text { Irox. }\end{array}$ & $\begin{array}{l}\text { A greonish-yellow } \\
\text { alloy, which will } \\
\text { take a fne polish. }\end{array}$ & See Brass. & $\begin{array}{l}\text { Silver and zinc com- } \\
\text { bine easily, form- } \\
\text { ing a somewhat } \\
\text { brittle alloy. }\end{array}$ \\
\hline $\begin{array}{l}\text { With Iros or } \\
\text { STEkL. }\end{array}$ & $\begin{array}{l}\text { Gold and iron alloy } \\
\text { with ease, and } \\
\text { form yellowish al- } \\
\text { loys, varying in } \\
\text { colour with the } \\
\text { proportions of the } \\
\text { metals. Three or } \\
\text { four parts of iron } \\
\text { united with one of } \\
\text { gold is very hard, } \\
\text { and is used in the } \\
\text { manufacture of } \\
\text { cutting instru- } \\
\text { ments. }\end{array}$ & $\begin{array}{l}\text { Iron and copper do } \\
\text { not form trie al- } \\
\text { loys. When fused } \\
\text { together, the iron, } \\
\text { however, retains a } \\
\text { little copper.-Se- } \\
\text { veral methods for } \\
\text { coating iron with } \\
\text { copper and brass } \\
\text { will be described. }\end{array}$ & $\begin{array}{l}\text { When } 1 \text { of silver } \\
\text { and } 500 \text { of isteel } \\
\text { are fused, a very } \\
\text { perfeet button is } \\
\text { formed. Stodart } \\
\text { and Faraday. } \\
\qquad \therefore\end{array}$ \\
\hline 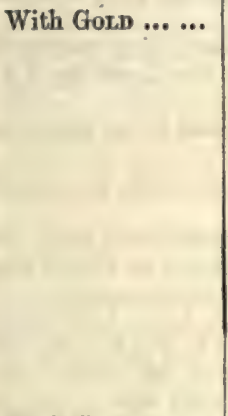 & $\begin{array}{lll}\cdots & \ldots & \ldots\end{array}$ & $\begin{array}{l}\text { Copper and gold al- } \\
\text { loy in all propor- } \\
\text { tions, the copper } \\
\text { giving hardness to } \\
\text { the gold. This al- } \\
\text { loy is much used } \\
\text { in coin and in the } \\
\text { metal employed in } \\
\text { the manufacture } \\
\text { of jewellery. }\end{array}$ & $\begin{array}{l}\text { Gold and silver mix } \\
\text { easily together; } \\
\text { but they do not } \\
\text { appear to form a } \\
\text { true combination. } \\
\text { Jewellers often } \\
\text { employ } \text { or vert, } \\
\text { which is composed } \\
\text { of } 70 \text { parts of gold } \\
\text { and } 30 \text { of silver, } \\
\text { which corresponds } \\
\text { very nearly to the } \\
\text { alloy possessing } \\
\text { the maximum } \\
\text { hardness. }\end{array}$ \\
\hline With Copprin & $\cdots \quad \cdots \quad \cdots$ & $\cdots \quad \cdots \quad \cdots$ & $\begin{array}{l}\text { Silver and copper } \\
\text { alloy in all propor- } \\
\text { tions. These al- } \\
\text { loys aro much used } \\
\text { in the arts. The } \\
\text { maximum hard- } \\
\text { ness appears to be } \\
\text { produced when } \\
\text { the alloy contains } \\
\text { a fifth of copper. }\end{array}$ \\
\hline $\begin{array}{l}\text { With InAD, does } \\
\text { not appear to } \\
\text { form any alloy. }\end{array}$ & $\begin{array}{l}\text { A very brittle alloy. } \\
\text { A thousandth pt. } \\
\text { of lead is sufficient } \\
\text { to alter the duc- } \\
\text { tility of gold. }\end{array}$ & $\begin{array}{l}\text { Do not appear to } \\
\text { form a truo alloy. }\end{array}$ & $\begin{array}{l}\text { Unite in all propor- } \\
\text { tions; but a very } \\
\text { small quantity of } \\
\text { lead will greatly } \\
\text { diminish the duc- } \\
\text { tility of silver. }\end{array}$ \\
\hline $\begin{array}{l}\text { With Tr. A } \\
\text { rery little iron } \\
\text { diminishes the } \\
\text { malleability of } \\
\text { tin, and gives it } \\
\text { hardness. }\end{array}$ & $\begin{array}{l}\text { The alloys of gold } \\
\text { and tin are brit- } \\
\text { tle; they preserve, } \\
\text { however, some } \\
\text { ductility when the } \\
\text { proportion of tin } \\
\text { does not exceed } \frac{1}{2} \text {. }\end{array}$ & $\begin{array}{l}\text { Of greatimportance. } \\
\text { Soe Browze. }\end{array}$ & $\begin{array}{l}\text { Alloys readily. A } \\
\text { very small quan- } \\
\text { tity of tin destroys } \\
\text { the ductility of } \\
\text { silvor. }\end{array}$ \\
\hline $\begin{array}{l}\text { With Mrecuny. } \\
\text { Mercury has no } \\
\text { action on iron. }\end{array}$ & $\begin{array}{l}\text { Mercury has a most } \\
\text { powerful action on } \\
\text { gold. See AMaL- } \\
\text { GAX. }\end{array}$ & $\begin{array}{l}\text { An amalgam which } \\
\text { is formed with dif- } \\
\text { ficulty, and with- } \\
\text { out interest. }\end{array}$ & $\begin{array}{l}\text { The amalgamation } \\
\text { of these two me- } \\
\text { tals is a little less } \\
\text { energetic than be- } \\
\text { tween mercury } \\
\text { and gold. See } \\
\text { AMAIAAMATUS. }\end{array}$ \\
\hline
\end{tabular}


Six ounces lesd to one pound copper is the ordinary pot-metal, called dry pot-metal, as this quantity of lead will be taken up without soparating on cooling; this alloy is brittle when warmed.

Seven ounces lead to one pound copper forms an alloy which is rather short, or disposed to break.

Eight ounces lead to one pound copper is an inferior pot-metal, called wet potmetal, as the load partly oozes out in cooling, especially when the new motals are mixed; it is therefore always usual to fill the crucible in part with old metal, and to add new for the remainder. This alloy is very brittle when slightly warmed. Moro lead can scarcely be used as it separates on cooling.

Antimony twenty parts and lead eighty parts form the printing-type of France; and lead and antimony are united in various proportions to form the type-metal of our printers. See TrPE-Mratak.

Mr. James Nasmyth, in a letter to the 'Athenæum' (No. 1176, p. 511), directed attention to the employment of lead, and its fitness as a substitute for all works of art hitherto executed in bronze or merble. He says the addition of about 5 per cent. of antimony to the lead will give it, not only great hardness, but enhance its capablity to run into the most delicate details of the work.

Baron Wetterstedt's patent sheathing for ships consists of lead, with 2 to 8 per cent. of antimony; about 3 per cent. is the usual quantity. The alloy is rolled out into sheets. We are not aware that this alloy has ever been employed.

Emery wheels and grinding tools for the lapidary are formed of an alloy of antimony and lead.

Organ pipes are sometimes made of lead and tin, the latter metal being employed to harden the lead. The pipes, however, of the great organ in the Town Hall a Birmingham are principally made of sheet zinc.

Lead and arsenic form shot-metal. The usual proportions are, said to be $401 \mathrm{lbs}$. of motallic arsenic to one ton of lead.

In addition to these, the alloys of iron appear of sufficient importance to require some further notice.

Iros and Mavganese. Mr. Mushet concludes, from his experiments, that the maximum combinations of manganese and iron is 40 of the former to 100 of the latter. The alloy $71^{\cdot 4}$ of tin and $28^{\circ} 6$ of manganese is indifferent to the magnet.

Irox and SuvEr; STrke and Silver.-Various experiments have been made upon alloys of iron and steel with other metals. The only alloys to which sufficient importance has been given are those of iron and silver and steel and silver. M. Guyton states, in the 'Annales de Chimie,' that he found iron to alloy with silver in greater quantity than the silver with the iron. 'Iron can,' he says, 'therefore no longer be said to refuse to mix with silver; it must, on the contrary, be acknowledged that those two metals, brought into perfect fusion, contract an actual ehemical union; that, whilst cooling, the heaviest metal separates, for the greatest part; that notwithstanding each of the two metals retains a portion of the other, as is the case in every liquation, that the part that remains is not simply mixed or interluid, but chemically united; lastly, that the alloy in these proportions possesses peculiar properties, particularly a degree of hardness that may render it extremely useful for various purposess'

The experiments of Faraday and Stodart on the alloys of iron and steel are of great value; the most interesting being the alloy with silver. The words of these experimentalists are quoted:-

'In making the silver alloys, the proportion first tried was 1 silver to 160 steel; the resulting buttons were uniformly steel and silver in fibres, the silver being likewise given out in globules during solidifying, and adhering to the surface of the fused button; some of these, when forged, gave out more globules of silver. In this stato of mechanical mixture the little bars, when exposed to a damp atmosphere, evidently produced voltaic action; and to this we are disposed to attribute the rapid destruction of the metal by oxidation, no such destructive action taking place when the two metals are chemically combined. These results indicated the necessity of diminishing the quantity of silver, and 1 silver to 200 steel was tried. Here, again, were fibres and globules in abundance; with 1 to 300 the fibres diminished, but still were present; they were detected eren when 1 to 400 was used. The successful experiment remains to be named. When 1 of silver to 500 steel were properly fused, a very perfect button was produced; no silver appeared on its surface; when forged and dissected by an acid, no fibres were seen, although examined by a high magnifying power. The specimen forged remarkably well, although very hard; it had in every respect the most favourable appearance. By n delicate test every part of the bar gave silver. This alloy is decidedly superior to the very best steel; and this excellence is unquestionably owing to a combination with a minute quantity of silver. It has been 
repeatedly made, and always with equal success. Various cutting tools have been made from it of the best quality. This alloy is, perhaps, only inferior to that of steol and rhodium, and it may be procured at small expense; the value of silver, where the proportion is so small, is not worth naming; it will probably be applied to many important purposes in the arts.'

Messrs. Faraday and Stodart show from their researches that not only silver, but platinum, rhodium, gold, nickel, copper, and oven tin, have an affinity for steel suffciently strong to make them combine chemieally.

Iros and Nrcker anito in all proportions, producing soft and tonacious alloys. Some few years since, Mr. Nasmyth drew attention to the combination of silicon with steel. Fresh interest has been excited in this direction by the investigations of a French chemist, M. St. Claire Deville, who has examined many of the alloys of silicon. For other alloys of iron, soe Irox.

Irov and Surcos combine to form an alloy, which is a sort of fusible steel in which carbon is replaced by silicon. The siliciurets-aro all of them quite homogeneous, and are not capable of being separated by liquation.

COPPsR and SmICON united in various proportions, according to the same chemist. A very hard, brittle, and white alloy, containing 12 per cent. of silicon, is obtained by melting together three parts silico-fluoride of potassium, one part sodium, and one part of copper, at such a temperature that the fused mass remains covered with a very liquid scoria. The copper takes up the whole of the silicon, and remains as a white substance less fusible than silicon, which may serve as a base for other alloys. An alloy with 5 per cent. silicon has a beautiful bronze colour, and vill probably receive important applications.

Mr. Oxland and Mr. Truran have given, in 'Metals and their Alloys,' the following useful tabular view of the composition of the alloys of copper. In addition to those given, the alloy of copper and aluminium is now most important. See AuvMInTUX BRoNze.

The principal alloys of copper with other metals are as follows:-

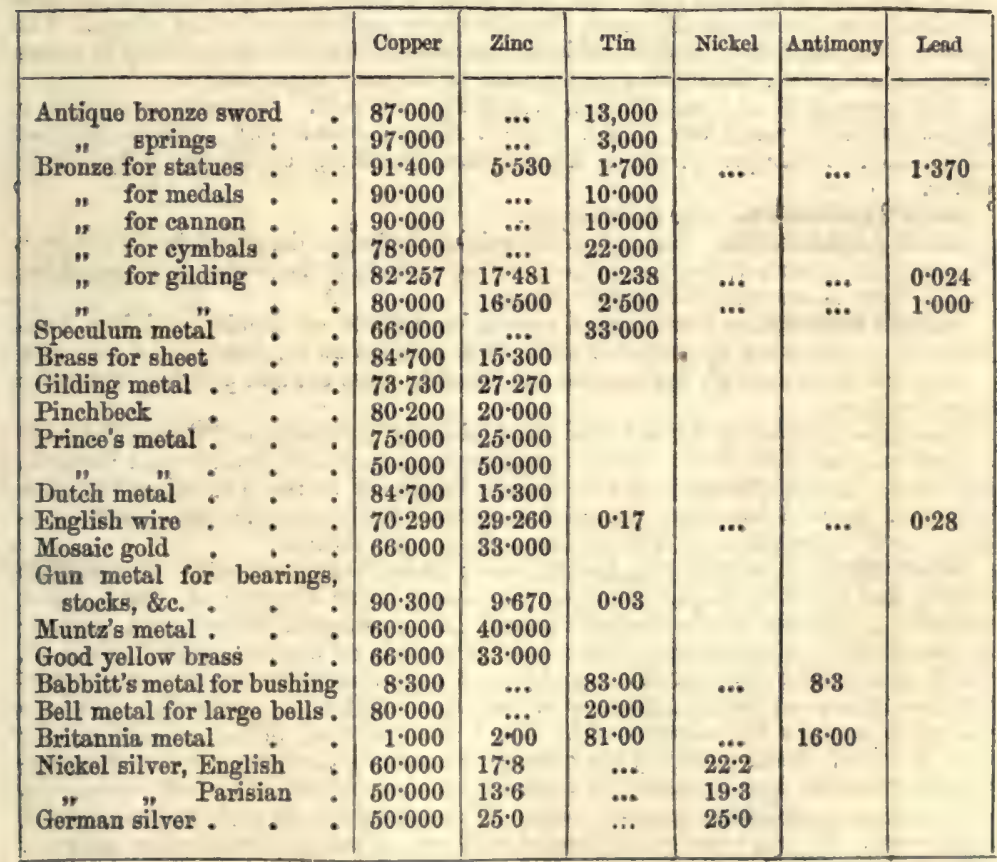

Some valuable researches on the nature of alloys were undertaken by the late Dr. Matthiessen, the results of which are embodied in his 'Report on the Chomical Nature of Alloys' (Report of the British Aseociation for the Advancement of Science, 1863, p. 37), and in a discourse 'On Alloys' delivered before the Chemical Socioty (Journal of the Chem. Boc., 1867, p. 201). 
Axzox, NATrve. Osmium and Iridium, in the proportions of 72.9 of the former and 24.5 of the latter. See Osmivs, Inubur.

ArIspICz. Pimento, or Jamaica pepper, Bo called because its flavour is thought to comprehend the flavour of cinnamon, cloves, and nutmegs. The tree produeing this spice (Eugenia pimenta) is cultivated in Jamaica in what are called Pimento walks. It is importod in bags, almost entirely from Jamaica. Mr. Montgomery Martin informs us that pimento was exported in one year (1837) from the different districts of Jamaica as follows. (See Pmonito, Prppera.)

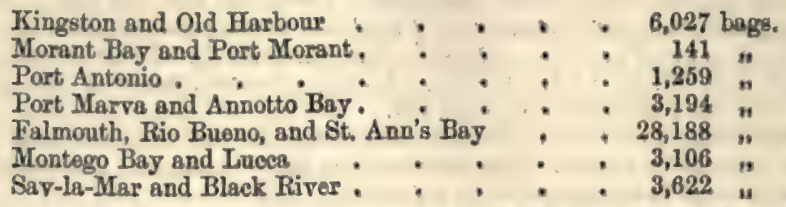

Axxuvruax. (Alluo, to wash upon; or alluvio, an inundation.) Earth, sand, gravel, stones, and other transported matter which has boen washed away, and thrown down-by rivers, floods, or other causes-upon land not permanently submerged beneath the wators of lakes or seas-Lyell.

AIIYI. $\mathrm{C}^{12} \mathrm{H}^{10}\left(\mathbf{C}^{6} \mathbf{z}^{10}\right)$. This radical exists in the oils of mustard and garlic, but is usually obtained by the decomposition of the iodide of allyl, which is obtained by acting on glycerine with iodine and phosphorus.

AIIXI, SUIPHIDE OF. This compound is contained in the essential oils produced by distilling with water the leaves and soeds of various plants of the liliaceous and cruciferous orders. It forms the principal constituent of the oil obtained from the bulbs of garlic (Allium Cepa). It is also found with oil of mustard in the leaves and seed of Thlaspi arvesse. The Alliaria officinalis distilled yields 90 per cent. of oil of mustard and 10 per cent. of oil of garlic; small quantities are also obtained from the Shepherd's purse, Capsella Bursa pastoris, and other plants. The power of the sulphide of Allyl to precipitate some of the metals appears likely to render it of use in the arts. The following are some of the more important:-

Gold precipitate, a beautiful yollow, and films of gold. Platisum precipitate, a yollowish-brown precipitate, which forms a Kermes brown with hydrosulphide of ammonium. Silver precipitate, a dark brown, becoming eventually sulphide of silver.

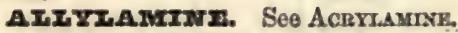

AxMAGrFrIx. An anhydrous sulphate of zinc, describod by Broithaupt. It oceurs in crystals belonging to the rhombic system, at the Barranca Jarosa Mino, in the Sierra Almagrera, in Southern Spain.

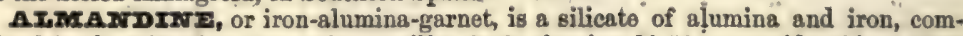
bined in the following proportions: silica $36^{\circ} 3$, alumina $20^{\circ} 56$, protoxide of iron $43 \cdot 2$.

It occurs in Groenland, Ceylon, and the Brazils; when cut and pqlished, it forms a beautiful gem.

The name is probably derived from the Alabandic carbuncles of Piliny, which were cut and polished at Alabanda. Several localities for garnets in Devonshire and Cornwall are given by Mr. Collins in his excellent 'Handbook to the Mineralogy' of these counties; but it is doubtful if the specimens found in Cornwall are true almandinotherofore those localities are given under Garnet. See GARNET,

AxmowD. (Amande, Fr.; Mandel, Ger.; Amygdalus communis.) De Candollo admits five varieties of this species. A. amara, bittor almond; $A$. dulcis, sweet almond; A. fragilis, tender-shelled almond; $A$. macrocarpa, large-fruited almond; A. persicoides, peach almond. There are two kinds of almond usually employed, which do not diffor in chemical composition, only that the bitter, by a curious chemieal reaction of its constituents, generates in the act of distillation a quantity of rolutile oil which contains hydrocyanic acid. Vogel obtained from bittor almonds 8.5 per cent. of husks. After pounding the kernels, and heating thom to coagulate the albumen, he procured, by expression, 28 parts of an unctuous oil, which did not contain the smallest particle of hydrocyanic acid. The whole of the oil could not be extracted in this way. The expressed mass, treated with boiling water, afforded sugar and gum, and, in consequence of the heat, some of that acid. The sugar constitutes 6.5 per cent. and the gum 3. The vegetable albumen extracted, by means of caustic potash, amounted to 30 parts : the vegetable fibre to only 5 . The poisonous aromatic eil, according to Robiquet and Boutron-Charlard, does not exist ready-formed in the bitter almond, but seems to be produced under the influence of ebullition with water. These chomists havo shown- 


\section{ALMOND OIL}

1st. That neithor bitter almonds nor their residuary cake yield any volatile oil by pressure.

2nd. They yield no oil when digested in alcohol or in ether, though the volatile oil is soluble in both these liquids.

3rd. Alcohol extracts from bitter-almond cake, sugar, resin, and amygdalin; when the latter substance has been removed, the cake is no longer capable of furnishing the volatile oil by distillation.

4th. Ether extracts no amygdalin, and the cake left, after digestion in ether, yields tho rolatilo oil by distillation with water; but alcohol dissolves out a peculiar whito crystallino body, without smell, of a sweetish taste at first, and afterwards bitter, to which they gave the name of amygdalin. This substance does not seem convertible into volatilo oil.-Pereira. See Watts's 'Dictionary of Chemistry.'

Sweet almonds, by the analysis of Boullay, consist of 54 parts of the bland almond oil, 6 of uncrystallisable sugar, 3 of gum, 24 of vegetable albumen, 24 of woody fibre, 5 of husks, 3.5 of water, 0.5 of acetic acid, including loss. We thus see that sweet almonds contain nearly twice as much oil as bitter almonds do.

Three varieties are known in commerce.

1. Jordan Almonds, which are the finest, come from Malaga. Of these there are two kinds : the one above an inch in length, flat, with a clear brown cuticle, sweet, mucilaginous, and rather tough; the other more plump and pointed at one end, brittle, but equally sweet with the former.

2. Valentia almonds are about three-eighths of an inch broad, not quite an inch long, round at one end, and obtusely pointed at the other, flat, of a dingy brown colour and dusty cuticle.

3. Barbary and Italian almonds resemble the latter, but are generally smaller and less flattened,-Brande, Dietionary of Pharmacy.

Our importation and exportation of AlmoNDs in 187.1 were as follows:-

Isronts, : 1871.

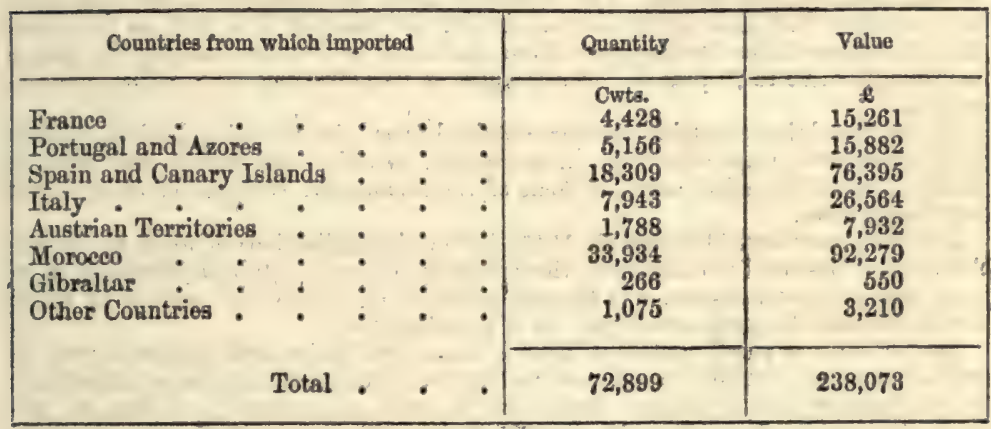

Exponts.

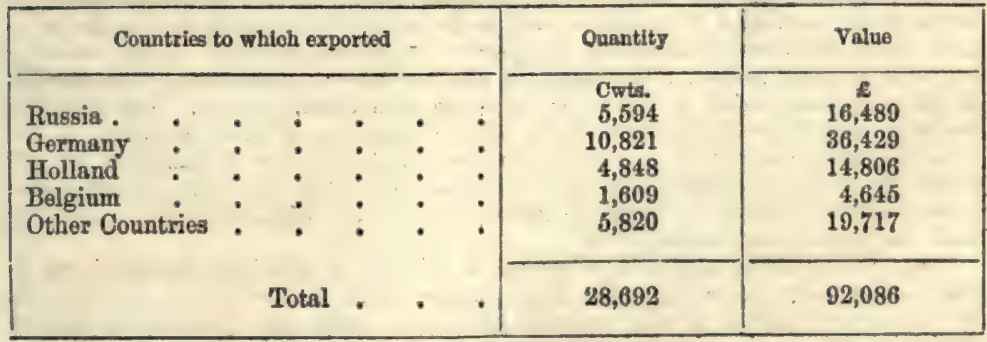

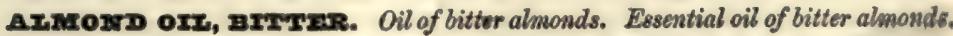
(Oleum Amygdale Amare.)

After the fixed oil has been expressed from the bitter almonds, the residual cake is mixed with water and distilled. A volatile oil comes over. It has been convincingly proved that this fine-flavoured essential oil is produced during the process, by some decomposition of the amygdalin and the emulsion of the soeds. It is highly 
poisonous, owing to the presence of hydrooyanic aeid. Seo Berzorc Acrd, Benzole, \&c.

ArmoxD oxn, SwDEx. A bland fixed oil, obtained by expression from bitter or sweet almonds-usually from the former, on account of their cheapness as well as the greater value of the residual cake. The average produce is from 48 to $62 \mathrm{lbs}$. from $1 \mathrm{cwt}$. of almonds.

This is commonly known as Oil of sweet almonds, the Oleum Amygdala of the Pharmacopcia. When first obtained it is opaque, and of a yellow colour; but it speedily becomes quite transparent, and is bleached by exposure to light. This oil has a bland taste, and does not congeal at a temperature which solidifies olive-oil. It is often sold for nut-oil; which is supposed to possess this property of remaining fluid in an eminent degree.

ATMOND POWDER (farina amygdale) is the ground almond cake after the oil has been expressed; it is employed for washing the hands, and it is used by chemists as a lute to connect the parts of their distillatory apparatus together.

AIOE. (Aloès, Fr.; Aloe, Ger.) In botany a genus of the order Litiacee. There are many species, all natives of warm climates.

In Africa the leaves of the Guinea aloe are made into dumble ropes. Of one species are made lines, bowstrings, stockings, and hammocks : the leaves of another spocies are used to hold rain-water. A series of trials has boon made, within a fow years, in Paris, to ascertain the comparative strength of cables made of hemp and of the aloe from Algiers; and they are said to have all turned to the advantage of the aloe. Of cables of equal size, that made of aloe raised a woight of 2,000 kilogrammes (2 tons nearly), that made of hemp a weight of only 400 kilogrammes. The fibre of the aloe 18 used extensively in Belgium for the ropes used for winding the coal from the very deep coal-pits of Charleroi. The Belgium engineers state that they could not raise the eoal with equal facility and safety with any other kind of rope.

The following varietios of the inspissated juices of the Aloe-called Arozs, and often Brrtrk Arors - are known in commerce: Socotrine, Hepatic, Barbadoes, Cape, Mocha, Catalline, and Indian. The Socotrine Aloes are regarded as the best kind, but that from Barbadoes is the most abundant, and much of it is sold as Socotrine. The Barbadoes Aloes are importéd from Barbadoes or Jamaica, usually in gourds woighing from 60 to 70 pounds, but sometimes in boxes holding about half a hundredweight.

It is believed that Socotrine aloes are obtained from Aloe Socotrina, Barbadoes aloes from $A$. vulgaris, Cape aloes from $A$. spicata and its allies, and Indian aloes from $A$. Indica; but the botanical source of some of the commercial varieties of aloes is not definitely known.

A patent was taken (January 27,1847 ) for certain applications of aloes to dyeing. Although it has not beon employed, the colouring mattor so obtainod promising to be very permanent and intense, it is thought advisable to describe the process by which it was proposed to prepare the dye. It is as follows:-

Into a boiler or vessel capable of holding about 100 gallons, the patentee puts 10 gallons of water, and $132 \mathrm{lbs}$. of aloes, and heats the same until the aloes are dissolved; he then adds $80 \mathrm{lbs}$. of nitric or nitrous acid in small proportions at a time, to prevent the disengagement of such a quantity of nitrous gas as would throw part of the contents out of the boiler. When the whole of the acid has been introduced, and the disengagement of gas has ceased, $10 \mathrm{lbs}$. of liquid caustic soda, or potash of commeree, of about $30^{\circ}$, are added to neutralise any undecomposed acid romaining in the mixture, and to facilitate the use of the mixture in dyeing and printing. If the colouring matter is required to be in a dry state, the mixture may bo incorporated with $100 \mathrm{lbs}$. of china-clay, and dried in stoves, or by means of a current of air. The colouring matter is used in dyeing by dissolving a sufficient quantity of water, according to the shade required, and adding as much hydrochloric acid or tartar of commerce as will neutralise the alkali contaned in the mixture, and leave the dye-bath slightly acidulated. The articles to be dyed are introducod into the bath, which is kept boiling until the desired shade is obtained.

When the colouring matter is to be used in printing, a sufficient quantity is to be dissolved in water, according to the shade required to be produced; this solution is to bo thickened with gum, or other common thickening agent, and hydrochloric acid, or tartar of commerce, or any other suitable supersalt, is to be added thereto. After the fabrics have been printed with the colouring matter, they should be subjected to the ordinary process of steaming, to fix the colour.-Napier.

Aloktrc ACID. The colouring matter of the aloes depends on this acid, which has been examined by Schunck and Mulder. Aloetic acid is deposited from nitric acid, which has been heated with aloes, as a yellow powder: it dissolves in ammonia with a violet colour; when treated with protochloride of tin it forms a dark-violet heavy 
powder; and this again, when treated with potash, ovolves ammonia, and assumes a violet-blue colour. This solution of aloetic acid in ammonia is violet.

AIPACA. (Alpaga, Fr.) An animal of Peru, of the Llama specios; also the name given to a woollen fabric woven from the wool of this animal, or a mixture of silky goat's hair with the harsher fibre of sheep's wool. See Liuau.

AxsToNrrz. A double carbonate of lime and baryta, crystallising in tho prismatic or orthorhombic system. It occurs at Fallowfield, near Hoxham, in Northumborland; and at Bromley Hill, near Alston, in Cumborland.

AIUDEI. The aludels of the earlier chemists were a series of pear-shaped pots, generally made of earthenware, but sometimes of glass, open at both ends. Each aludel had a short neck at top and bottom, so that a series of them could be fitted togother, by means of the neck, in succession. The earthenware pear-8haped vessels in which the mercurial vapours are condensed, at Almaden, in Spain, are also known as aludels. See Mercury.

Arow. (Alun, Fr.; Alaun, Ger.) A saline body or salt, consisting of alumina, or the peculiar earth of clay, united with sulphuric acid, and these again united with sulphate of potash or of ammonia. In other words, it is a double salt, consisting of sulphato of alumina and sulphate of potash, or sulphato of alumina and sulphate of ammonia. The common alum crystallises in octahedrons, but there is a kind which takes the form of cubes. It has a sour or rathor subacid taste, and is peculiarly astringent. It reddens the blue colour of litmus or red cabbage, and acts like an acid on many substances. Other alkalis may take the place of the ammonia or potash, and other metals that of the aluminium.

Alum was known to the ancients, who used it in medicine, as it is now used, and also as a mordant in dyeing and calico-printing, as at the present day. Old historians do not describe correctly, either the mode of obtaining it or its exact characteristics, so that it is confounded with sulphate of iron, with which it seems generally to have been mixed. But that some qualities were made with very little iron in it, is clear from the fact that it was employed when white for dyeing bright colours. (Pliny, $\mathbf{x x r}$. 15.) It is said by Pliny that the purchasers tosted it with tannin (pomegranate juice), in order to see if it blackened. He says that the white kind blackened as well as the black; but in all probability this was a test applied by the dyers to see which blackened least, so as to obtain a good mordant for reds. Pliny's description, although confused, leaves this fact perfectly clear-that there were men in whose minds the knowledge was much clearer than in his, or a manufacture of such magnitudo could not have existod. There is mention of some boing made from stone, and crystallising in fine hairs, but the characteristics given do not enable us to decide that this was either alum or the peculiar sulphate of alumina which takes that form. The alum was sometimes boiled down to dryness, and heated till it was spongy or like pumice-stone. It was used as burnt alum.

The ancients. used it also for preventing the combustibility of wood and wooden buildings. But although the knowledge of it was very accurate, their writers always imagine that sulphate of iron was a kind of alum, because it is said that tho black alum was used for dyeing dark colours. They used iron as a mordant, and found its charactor by galls or by pomegranate juice, which contains tannin. Their alum was chiefly a natural production, and they removed the fine efflorescing crystals which first appeared, or which gradually are raised above the rest, as the finest kind. 'It was produced in Spain, Egypt, Armenia, Macedonia, Pontus, and Africa; the islands Sardinia, Molos, Lipari, and Stromboli. The best was got in Egypt, the next in Melos.' The word is probably Egyptian, as it was best and most abundantly obtained in Egypt. It is not probable that it was the double salt in all eases, but simply a sulphato of alumina. Pliny, indeed, says that a substance called in Greek íypa, or watery, probably from its very soluble nature, and which was milk-white, was used for dyoing wool of bright colours. This may have been the mountain butter of the German mineralogists, which is a native sulphate of alumina, iron, \&c., of a soft texture, waxy lustre, and unctuous to the touch. The stypteria of Dioscorides and the alumen of Pliny comprehended, no doubt, a variety of saline substances besides sulphate of iron and alum.

It secms to have come to Europe in later times as alum of Pocca, the name of Edessa, or that place where the Italians first learnt the art; but it is not impossible that this namo was an Italian prefix, which has romajned to this day undor tho form of Rock alum, Allume di rocca. The East has always had some manufactures of it, and Phocis, Lesbos, and other places, wero ablo to supply the Turks with alum for their magnificent Turkey red. It was also made at Foya Nova, near Smyrna, and at Constantinople. The Genoese and other trading people of Italy imported alum into Western Europe for the use of the dyers of red cloth.

A Genoese merchant, Bartholomew Perdix, who had boen in Syria, obsorved a 
stone suitable for alum in the island Ischia ; ho burnt it, and obtained a good result, being the flrst who introduced the manufacture into Europe. This was in the yoar 1459; about the samo timo John di Castro learnt tho method at Constantinoplo, and manufucturod alum at Tolfa. This discovery of the mineral noar Civita Vecehin was considered 80 important by John di Castro, that ho announced it to the Popo as a great victory over the Turks, who annually took from the Christians 300,000 pieces of gold for their dyed wool. A statue was erected to tho 'Discoverer of Alum.'Beckman.

The manufacture of alum was then made a monopoly of the Papal Powors, and instead of buying it as before from the East, it was considered Christian to obtain it only from the States of the Church, and, as such, was mado compulsory in the Wost. The manufacture then went to Spain, to a spot near Carthagena. Germany legan so early as 1554 to mako alum, although Basil Valentine seems to havo known of its existence there somowhat sooner. The first establishment known was at Oberkaufungen in Hesse-Cassel, where it still exists. It was not introduced as a manufacture into England until the year 1600, when Sir Thomas Chaloner, the son of Quoen Filizabeth's minister of that name, found that his own estate of Guisborough, in Yorkshire, contained alum. This ho is said first to haro observed from tho vegetation, which had a very weak green. Di Castro had flust been led to it by the appearance of the holly, but neither can be said to bo decisive tests of its presence, nor are the geological features of Tolfa and Guisborough at nll aliko. The violont denunciations of the Pope did not prevent tho manufacture from growing to unexpected magnitude in England. The mines of the samo district have over since sent out alum, which is now known as Whitby alum, and oven those at Guisborough itself are now at work, although for seventy years of the period since their diseovery they wore disused. The manufacture was begun at Hurlet, in Scotland, by Nicholson and Lightbody, in 1766, abandoned, and resumed by Macintosh and Wilson in 1797.

The chemical composition of alum varies with the nature of the bases present, but all alums are constructed on a common type, expressed by the general formula MO. $\mathrm{SO}^{3} \cdot \mathrm{M}^{2} \mathrm{O}^{3} \cdot 3 \mathrm{SO}^{3}+24 \mathrm{HO}$. They are, therefore, double sulphates, containing both s protoxide and a sesquioxide, combined with a constant number of molecules of water. The protoxide is genorally an alkali-usually potash or ammonia-but other alums are known, though at present of no importance in the arts, in which the metal of tho protoxide is silver, thallium, ceesium, or rubidium. On the other hand, the sesquioxide, though generally alumina, may take the form of sesquioxide of iron, of manganese, or of chromium. It is desirable to exhibit this relation between some of the more important alums; and, as the formule representing these double salts are somewhat complex, it may be useful to compare their symbolic expressions constructed with both the old and the now atomic woights :-

\begin{tabular}{|c|c|c|c|}
\hline & & Old formules & Wew formulve \\
\hline Potash alum . & & $\mathrm{KO} . \mathrm{SO}^{3} \mathrm{Al}^{2} \mathrm{O}^{3} \cdot 3 \mathrm{SO}^{3}+24 \mathrm{HO}$ & IRAI2(SO $\left.0^{4}\right)+12 I^{2} O$ \\
\hline Soda alum & & $\mathrm{NaO} \cdot \mathrm{SO}^{3} \cdot \mathrm{Al}^{2} \mathrm{O}^{3} \cdot 3 \mathrm{SO}^{3}+24 \mathrm{HO}$ & $\operatorname{TaA12}\left(80^{\prime}\right)+12 \pi^{2} 0$ \\
\hline Ammonia alum & & $\mathrm{NH}^{4} \mathrm{O} \cdot \mathrm{SO}^{3} \cdot \mathrm{Al}^{2} \mathrm{O}^{3} \cdot 3 \mathrm{SO}^{3}+24 \mathrm{HO}$ & $\mathrm{NBR} \cdot \mathrm{Al2}\left(\mathrm{SO}^{\prime}\right)+12 \mathrm{E}^{2} \mathrm{O}$ \\
\hline Iron alum . & 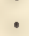 & $\mathrm{KO} . \mathrm{SO}^{3} \cdot \mathrm{Fe}^{2} \mathrm{O}^{3} .3 \mathrm{SO}^{3}+24 \mathrm{HO}$ & $\operatorname{Irez}\left(\mathrm{SO}^{1}\right)+12 \mathrm{Ex}^{2} \mathrm{O}$ \\
\hline Chromo alum . & - & $\mathrm{KO} . \mathrm{SO}^{3} \cdot \mathrm{Cr}^{2} \mathrm{O}^{3} \cdot 3 \mathrm{SO}^{3}+24 \mathrm{HO}$ & $\operatorname{ICr} 2\left(\mathrm{SO}^{4}\right)+12 \mathrm{E}^{2} \mathrm{O}$ \\
\hline Manganese alum & . & KO.SO $. \mathrm{Mn}^{2} \mathrm{O}^{3} \cdot 3 \mathrm{SO}^{2}+24 \mathrm{HO}$ & $\operatorname{ISMn} 2\left(50^{\prime}\right)+12 E^{8} 0$ \\
\hline
\end{tabular}

The composition of pure potashalum may be represented centesimally and atomically as follows :-

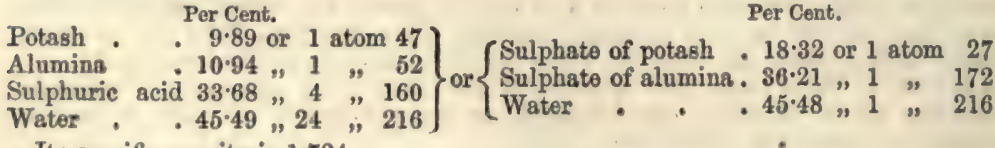

Its spocific gravity is $1 \cdot 724$.

100 parts of water dissolve, at 32 degrees Fahrenheit,

$3 \cdot 29$ alum

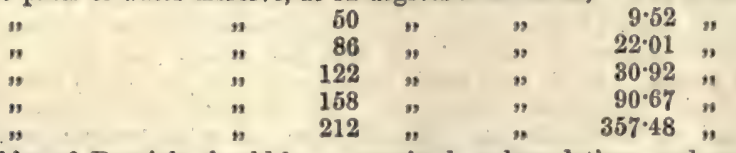

These Tubles of Poggiale should be ro-examined, and gradations made more usoful for this country. 


\section{ALUM}

One part of crystallised potash alum is solublo-

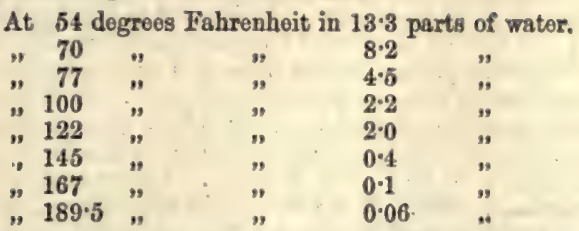

A solution saturated at $46^{\circ}$ is 1.045 specific gravity. This difference in the rate of solubility in hot and cold water renders it easily separated from many other salts. The erystals are permanent in the air, or nearly 80, unless the air be very dry; if kept at $180^{\circ}$ they lose 18 atoms of water, but alum deprived of its water, and exposed to the air of summer, took up 18 atoms in 47 days. It melts at a low temperature in its water of crystallisation. At $356^{\circ}$ it loses 43.5 per cent. of water, or 23 atoms; the last atom is only lost when approaching red heat. At a red heat the sulphate of alumina loses its acid, and the alumina seems then able to remove some acid from the potash, losing it again by heat. Alum, when heated with common salt, acts liko sulphurie acid, and gives off muriatic acid; the same with chlorides of potassium and ammonium. If boiled with a saturatod solution of chloride of potassium, hydrochloric acid is formed and a subsulphate of alumina falls down; this oceurs only to a small extont with chloride of sodium, and still less with sal-ammoniac.

Ammonia-Ahum, which is now very extensively prepared, contains:-

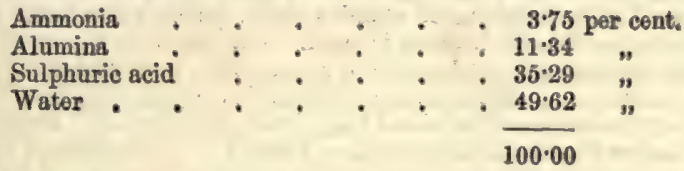

This salt also occurs in octahedrons, and can only be known from potash-alum by trial. The addition of caustic lime, soda, or potash gives out the ammonia, easily distinguished by the smell. Ammonia-alum readily loses all its ammonia when heated, and the sulphuric acid may be driven off from the renaining sulphate of alumina, so that the pure earth, alumina, will remain.

The greater proportion of the alum at present used in this country is ammonia-alum -an abundant and convenient source of ammonia boing furnished by the ammoniacal liquor obtained in the manufacture of coal-gas. In commerce ammonia and potashalums are sometimes found mixed.

Soda-alum is not an article of commerce, nor is it used in the arts. Nevertheless, the great commercial value of compounds of potash or of ammonia renders it obviously desirable to replace them, if possible, by the cheaper compounds of soda. The cost of sulphate of soda, for example, is trifling compared with that of sulphate of potash or of ammonia- the latter salts being especially in demand by the agriculturist as fertilising agents. Some experiments on the formation and erystallisation of sodaalum were undertaken a fow years ago by Mr. J. Carter Bell. Up to the present time, however, there appear to have been great difficultios in the manufacture of this kind of alum, especially in respect to its crystallisation, but these difficulties may not be altogether insuperablo. Mr. J. Berger Spence, who has studied the preparation of soda-alum, remarks that 'it may ultimately, now that the practicability of producing soda-alum on the commercial scale has been demonstrated, even with all the difficulty of crystallisation, be a more economical way of producing this double salt.' '

For the composition of potash-, soda- and ammonia-8lums found ready formed in nature, see ALUM, NATIVE.

Applications of Alum.-Alum is an astringent. Its immediate effoct on man is to corrugate the fibres and contract the small vessels. It precipitates albuminous liquids and combines with gelatine. It causes dryness of the mouth and throat, and checks the secrotions of the alimentary canal, producing constipation-in large quantities, nausea, vomiting, and purging. It is given in lead colic, to convert the lead into sulphate of lead, and is used externally. Its principal use is in dyeing; calico-printers print it as a mordant; the eloth is then put into the dye, and the printed parts absorb the colour.

It is largely employed by the calico-printer in the preparation of acotate of alumina

' On the Phenomena of the Crystallisation of a Double Salt, by J. Berger Spenoo, F.C.S.-'Chern. ienl Nows, " vol, 2xili, 1870, p. 181. 
by procipitating a solution of alum with sugar of lead. Papor-makers use alum in their size, and bookbinders in thoir paste. It is used in tanning leather, and sometimes, both in Asia and Europe, it is used for precipitating rapidly the impurities of wator. This is a dangerous process, unless there be a great amount of alkalino salts, such as carbonate of lime or soda, to neutralise the acid. It is extensively used in correcting the baking qualities of bad flour, for which the experience of many has decided that it is a valuablo remedy; unfortunately, it is also used to make excellent flour whiter, when there is no need of its presence. Liebig says that lime is equally good, and of course much safer. It is also used in the adulteration of beer. From time immemorial it has been used to provent the combustibility of wood and cloth. Milner's fire-proof safes are said to be lined with a mixture of alum and sulphate of lime.

Alum heated with charcoul or carbonacoous substances forms Homberg's phosphorus, which inflames spontanoously. It is composed of alumina, sulphide of potassium, and charcoal.

Burnt Alum, or dried alum, is mado by gently heating alum till the water is drivon off. The alum first melts in its water of crystallisation, and is then driod. It has a stronger action than the hydrated crystals, and is a mild escharotic. It reabsorbs water.

Neutral Alum is a name sometimes given erroneously to alum which has had some of its acid noutralised by an alkali. It is in fact a basic salt of alumina, which may also be made by dissolving alumina in ordinary alum. It deposits a basic salt more readily than ordinary alum, and may be of service in some cases of printing. Properly speaking, the common alum is the neutral salt.

Testing of Alum.-Alum being generally in large crystals, any impurity is more readily seen; this is said to be the roason for keeping up the practice of making this substance instead of the sulphate of alumina alone, which is less bulky and fitted for nearly overy purpose for which alum is used. But probably the ancient accidental discovery of the potash form has determined its use to the present day. Iron is readily found in it, by adding to a dilute solution forrocyanide of potassjum or yellow prussiato of potash, which throws down Prussian-blue. A very delicate test is sulphide of ammonium, which throws down both the alumina and iron, but the blacking of the precipitate depends on the amount of iron. The total amount of iron is got by adding pure caustic potash or soda till the solution is strongly alkaline, washing and filtering off the oxide. To look for lime, precipitate the alumina and iron by ammonia, boil and filter-the lime and magnesia are in the solution-add oxalate of ammonia; add tartaric acid to keep up the iron and alumina, make alkaline by ammonia, then precipitate the lime by oxalate of ammonia, filter, and precipitate the magnesia by a phosphate. Silica and insoluble basic sulphates are obtained by simply dissolving the alum in water and filtering. If silica, it is insoluble in acids; if a basic sulphate, it will dissolve in sulphuric acid, and the addition of sulphate of potash or ammonia wilt convert it into potash-or ammonia-alum.

Pure alum gives a white precipitate with ammonia, no precipitate with sulphurotted hydrogen gas, and no precipitato with oxalato of ammonia and ammonia, if tartaric acid be previously added.

The addition of ammonia to a solution of alum, or the addition of any other alkali, in insufficiont quantity, causes a precipitate, not of pure alumina, as one might suppose, but of a subsulphate of alumins. Even an excess of alkali will not remove all the sulphuric acid without heat being applied; an excess, on the other hand, is apt to dissolve some of the alumina, especially if fow salts are present, and the solution not much boiled. Sulphide of ammonium precipitates it thoroughly.

In a saturated solution of tersulphate of alumina, the crystals of alum are almost insoluble.

If we dissolve alum in 20 parts of water, and drop this solution slowly into water of caustic ammonia till this be nearly, but not entirely, saturated, a bulky whito precipitate will fall down, which, when properly washed with water, is pure aluminous earth or hydrate of alumina; and, dried, forms 10.94 per cent. of tho weight of the alum. If this earth, while still moist, be dissolved in dilute sulphuric acid, it will constitute, when as neutral as possible, simple sulphate of alumina, which requires only two parts of cold water for its solution. If we now decompose this solution, by pouring into it water of ammonia, there appears an insoluble white powder, which is subsulphate of alumina, or basic alum, and contains throe times as much earth as exists in the neutral sulphate. If, however, we pour into the solution of the neutral sulphate of alumina a solution of sulphate of potash, a white powder will fall if the solutions bo coneentrated, which is true alum; if the solutions be dilute, by evaporating their mixture, and cooling it, crystals of alum will be obtained.

When newly precipitated alumina is boiled in a solution of alum, a portion of the earth onters into combination with tho ealt, constituting an insoluble compound which fulls in the form of a whito powder. The same combination takes place, if we decom- 
pose a boiling hot solution of alum with a solution of potash, till the mixture appears nearly neutral by litmus-paper. This insoluble or basic alum exists native in the alum-stoni of Tolfa, near Cívita Vecchis. (See below.)

Ores or Raw Material. - The chief difficulty in manufacturing alum has been the solution of the alumina. This substance is generally combined with silica in such a strong combination, that even powerful acids cannot remove it without assistance. The older methods, however, took no notice of these difficulties, and obtrined the alum more or less directly from nature. The method now practised at the Solfatara. di Pozzuoli and in the island Vulcano is simply to take the offloresconce and the earth containing it, wash it with water, and concentrate. But it very seldom contains a sufficient amount of potash to form alum. A salt of potash is then added, chiefly a carbonate. To transform this into a sulphate, a portion of the sulphate of alumina is decomposed. The use of a carbonate is a wasteful method of modern times; the ancients would have felt no difficulty, but boiled all down, and so obtained the whole alumina there. Their product, therefore, would have been basic sulphate of alumina, which it evidently was when this practice was resorted to. When they merely concentrated and then crystallised, they got pure alum; but they lost a great deal of their alumina.

Alum occurs ready formed in nature in the alum-stones of Italy, \&c., as an efflo. rescence on stones, and in certain mineral waters in the East Indies. (See AvuM, Native.) The alum of European commerce is manufactured artificially, eithor from the alum-schists or stones, or from clay. The mode of manufacture differs according to the nature of these earthy compounds. Some of them, such as the alum-stone, contain all the elements of the salt, but mixed with other matters, from which it must be freed. The schists contain only the elements of two of the constituents, namely, clay and sulphur, which are convertible into sulphate of alumina, and this may be then made into alum by adding the alkaline ingredient. To this class belong the alum-slates, and other analogons schists, containing brown coal. Alum has of late years been rery extensively prepared by Spence's process, in which the raw material is a carbonaceous shale from the coal-measures. Quito recently a new method of alum manufacture has been introduced, in which the raw material is a siliceous phosphate of alumina and iron from Redonda in the West Indies. Each of these methods of manufacturing alum will now be separatoly described.

I. Manufacture of Alum from the Alum-Stone.-The alum-stone or alunite is a mineral of limited occurrence, being found in moderate quantity at Tolfa (near Civita Vecchia, in the Roman States), and in larger quantity in Hungary, at Beregszaz and Muszay, where it forms entire beds in a hard substance, partly characterized by numerous cavities, containing drusy crystallisations of pure alum-stone or basic alum. It is also found in the Isle of Milo and elsewhere in the Grecian Archipelago. The alum-stone appears to be confined to volcanic districts, where it is formed by the action of sulphurous acid gas and steam on trachytic and other felspathic rocks. The ordinary alum-stone is a massive rock, often cellular in texture, and sometimes sufficiently hard to be employed as a millstone.

The composition of ordinary alum-stone is fairly represented by the following solection of analyses :-

\begin{tabular}{|c|c|c|c|c|c|c|}
\hline \multirow{2}{*}{\multicolumn{2}{|c|}{ 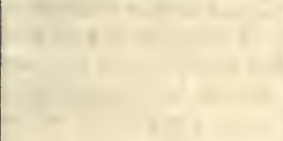 }} & Klaproth & Rammelsberg & Klaproth & Descotils & Condier \\
\hline & & $\begin{array}{l}\text { Tolfa, } \\
\text { Italy }\end{array}$ & $\begin{array}{l}\text { Tolfa, } \\
\text { Italy }\end{array}$ & $\begin{array}{l}\text { Beregazan, } \\
\text { Hungary }\end{array}$ & $\begin{array}{l}\text { Montione, } \\
\text { Tuscany }\end{array}$ & $\begin{array}{l}\text { MCont d'Or, } \\
\text { France }\end{array}$ \\
\hline Silica & & 56.5 & $1 \cdot 94$ & $62 \cdot 3$ & . & $28 \cdot 4$ \\
\hline Alumina & . & $19 \cdot 0$ & $34 \cdot 02$ & $17 \cdot 5$ & 40.0 & $31 \cdot 8$ \\
\hline Sulphuric acid . & . & $16 \cdot 5$ & $36 \cdot 94$ & $12 \cdot 5$ & $35 \cdot 6$ & $27 \cdot 0$ \\
\hline Potash & - & $4 \cdot 0$ & $10 \cdot 38$ & 1.0 & $13 \cdot 8$ & $5 \cdot 8$ \\
\hline Water & . & 30 & $16 \cdot 72$ & $5 \cdot 0$ & 10.0 & $3 \cdot 7$ \\
\hline Peroxide of iron & $\bullet$ & $\cdots$ & ... & $\ldots$ & ...' & $1 \cdot 4$ \\
\hline
\end{tabular}

The older analysts examined the rock as a whole, including all impurities, and hence the proportion of silica in their detorminations appears much higher than in the more recent analyses, which relate to the alunito alone, separated as far as possible from mechanically-associated quartzose matter.

The purest specimens of alunite, which exhibit the mineral in rhombohedral crystals, consist of a basic sulphate of alumina with sulphate of potash, referable to

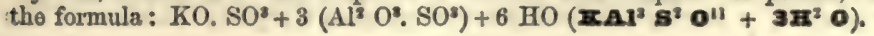


In preparing alum from the alum-stone, the ore is first sorted. The larger lumps contain more or fower flints disseminated through them, and are, according to their quality, either pickod out to make alum, or thrown away. The sorted pieces aro ronstod or calcined, by which operation apparently the hydrate of alumina, associated with the sulphato of alumina, loses its water and its affinity for alum. It becomes, therofore, froe; and during the subsequent exposure to the weather the stone gets disintegrated, and the alum becomes soluble in water.

The calcination is performed in common limekilns in the ordinary way. In the regulation of the fire it is requisite, here, as with gypsum, to prevent any fusion or running together of the stones, or even any disengagement of sulphuric or sulphurous acids, which would cause a corresponding diminution in the produce of alum. For this reason the contact of the ignited stones with carbonaceous matter ought to be avoided.

The calcinod alum-stones, piled in heaps from 2 to 3 feet high, are to be exposed to the weather, and meanwhile they must be continually kept moist by sprinkling them with water. As the water combines with the alum the stones crumblo down, and fall, eventually, into a pasty mass, which must be lixiviated with warm wator, and allowed to settle in a large cistern. The clear supernatant liquor, being drawn off, is to be evaporated, and then crystallised. A second crystallisation finishes the process, and furnishes a marketable alum. Thus the Roman alum is made, which is covered with a fine red film of peroxide of iron.

Roman Alum crystallises-partly in octahedrons, like other alums, partly in cubes. If these cubes are dissolved in water of about $110^{\circ} \mathrm{F}$, the evaporated liquid gives crystals of common or octahedral alum. It was said that on heating, it depositod subsulphate of alumina; but Loewel says that such crystals were impure, and he finds no real difference of composition. All that seems to be known with cortainty is, that it is formed when thore is a salt of alumina in solution with the alum containing more alumina than the neutral or common alum. This can very readily occur in the Roman alum, where there is a great excess of alumina in the alum-stone. The Roman alum is prized for its great freedom from iron; it was said by MM. Thenard and Roard to contain only $\frac{2}{2200}$ th of sulphate of iron, whilst the ordinary alum contained 1000th.

II. Alum Manufacture from Alum-Schist.-The greater portion of the alum found in British commerce was until recently made from alum-slate and analogous substances, This slate contains more or less iron pyrites, mixed with coaly or bituminous matter, which is occasionally so alundant as to render the schist somewhat combustible. In the strata of brown coal and bituminous wood, where the upper layers lie immediately under clay beds, they consist of the enaly substance rendered impure with clay and pyrites. This triple mixture constitutes the essence of all good alum-schists, and it operates spontaneously towards the production of sulphate of alumina. The coal, besides burning, serves to make the texture open, and to allow the air and moisture to penotrate freely, so as to change the sulphur and iron present into acid and oxide. When these schists are exposed to a high temperature in contact with air, the pyrites loses ono-half of its sulphur, in the form of sublimed sulphur of of sulphurous acid, and becomes a black sulphide of iron, which speodily attracts oxygen, and changes to sulphate of iron, or green vitriol. The brown-coal schists contain, commonly, some green vitriol crystals spontaneously formed in them. The sulphate of iron transfors its acid to the clay, progressively, as the iron, by the action of the air with a little elevation of temperature, becomes peroxidised; whereby sulphate of alumina is produced. A portion of the green vitriol remains, however, undecomposed, and so much tho more as there may happen to be less of other salifiable bases present in the clay-slate. Should a little magnesia or lime be present, tho vitriol gets more completely decomposed, and a portion of Epsom salt and gypsum is produced.

The production of alum from alum-stone, in which the whole ingredients have been found, has been far from enough for the supply of the world, and recourse has been had to substances very different in composition,-alum-shale, or schist, and clay. Until within a few years the only supply of alum in Britain has been from the lias shales of Whitby, and the lower coal-measures of Campsie and Hurlet, near Glasgow, and they are still the only places where it is manufactured from the 'ore,' as it is called.

The manufacture of alum from alum-schists may be described under the six following heads:-1. The preparation of the alum-shale. 2. The lixiviation of the shale. 3. The evaporation of the lixivium. 4. The addition of the saline ingredients, or the precipitation of the alum. 5. The washing of the aluminous salts; and, 6. The crystallisation.

1. Preparation of the Alum-Shale.-Some alum-shales are of such a nature that, being piled in heaps in the open air, and moistened from time to time, they get spontaneously hot, and by dogrees fall into a pulverulent mass, ready to be lixiviated. Tho 
groator part, however, require the process of ustulation, from which they derive many advantages. The cohesion of the dense shale is thereby so much impaired that its decomposition becomes more rapid; the decomposition of the pyrites is quickened by tho expulsion of a partion of the sulphur; and the ready-formed greon ritriol is partly docomposed by the heat, with a transference of its sulphuric acid to tho clay, and tho production of sulphato of alumina.

Such alum-shales as contain too little bitumen or coal for the roasting process must be interstratified with layers of small coal or brushwood over an extensive surface. At Whitby tho alum-rock, broken into small pieces, is laid upon a horizontal bed of fuel, composed of lrushwood; but at Hurlet small coal is chiefly used for the lower bod. When about four feet of rock is piled on, fire is set to the bottom in rarions parts; and whonever the mass is fairly kindled; more rock is placed ovor the top. At Whitby this piling process is continued till the calciming heap is raised to the height of 90 or 100 feet. 'The horizontal area is also' augmented at the same time till it forms a groat bed nearly 200 feet square, having therefore about 100,000 yards of solid measurement. The rapidity of the combustion is tempered by plastering up the crevices with small schist moistened. Whon surch an immense mass is inflamod, the heat is sure to rise too high, and an immense waste of sulphur and sulphuric acid must ensue. This evil has been noticed at the Whitby works. At Hurlet the height to which the heap is piled is only a few feet, while the horizontal area is expanded: which is a much more judicious arrangement. At Whitby 130 tons of calcined schist produces on an average 1 ton of alum. In this humid climato it would be ad. visable to pile up on the top of the horizontal strata of brushwood or coal and schist, a pyramidal mass of schist, which, having its surface plastered smooth, with only a few air-holes, will protect the mass from the rains, and at the same time prevent the combustion from becoming too vehement. Should heavy rains supervene, a gutter must be scooped out round the pile for recoiving the aluminous lixivium, and conducting it into the reservoir.

It may be observed, that certain alum-schists contain abundance of combustible matter, to keep up a suitable calcining heat after the fire is once kindled; and therefore nothing is needed but the first layer of brushwood, which, in this case, may be laid over the first bed of the bituminous schist.

A continual but very slow heat, with a smothered fire, is most beneficial for the ustulation of alum-slate. When the fire is too brisk, the sulphide of iron may mun with the earthy matters into a species of slag, or the sulphur will be dissipated in rapour, by both of which accidents the product of alum will be impaired. Those lituminous alum-schists which have been used as fuel under steam boilers have suffered such a violent combustion that their ashes yield almost no alum. Even the best regulated calcining pipes are apt to burn too briskly in high winds, and shonld havo their draught-holes carefully stopped under such circumstances. It may be laid down a.s a general rule, that the slower the combustion the richer the roasted ore will be in sulphate of alumina. When the calcination is completo, the heap diminishes to one-half its original bulk; it is covered with a light reddish ash, and is open and porous in the interior, so that the air can circulate freely throughout the mass. To favour this access of air, the masses should not be too lofty; and in dry weather a Jittle water should be occasionally sprinkled on them, which, by dissolving away some of the saline matter, will make the interior more open to the atmosphere.

The following analyses of shales are by G. Kersten :-

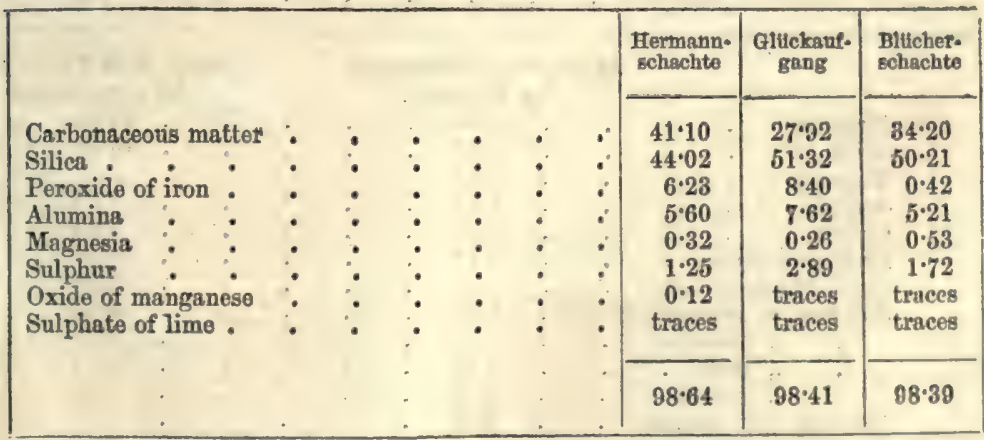

Messrs. Richardson and Ronalds have given some very detailed analyses of the Whitby and Campsie shiales :- 


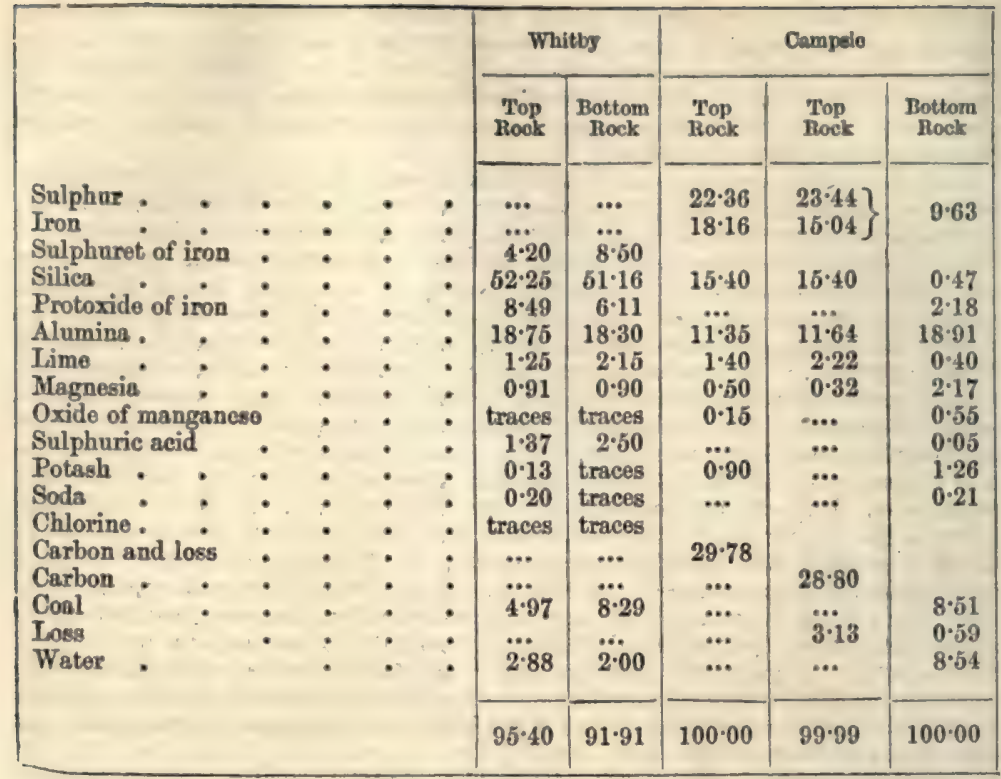

As the Top rock contains a larger excess of iron pyrites than the Bottom, they are mixed so as to diffuse the sulphuric acid equally.

Erdmann has thus analysed his German specimens:-

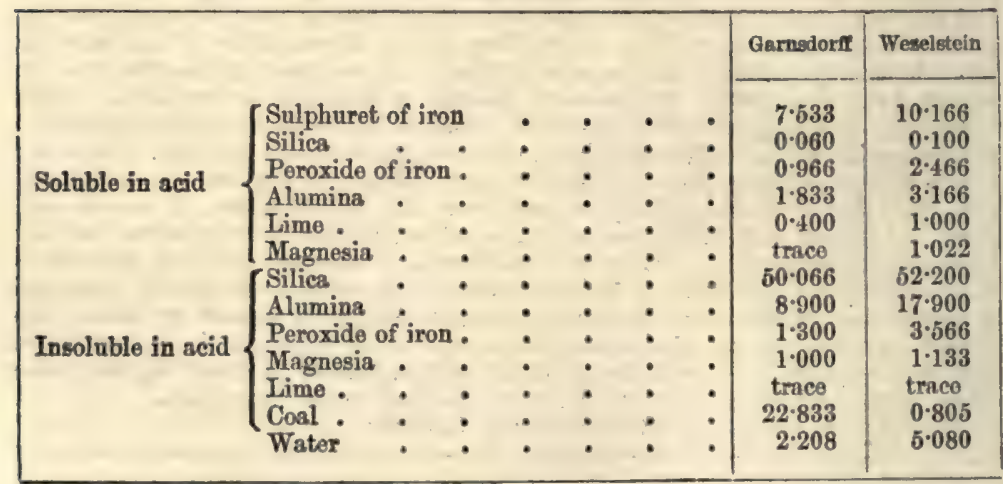

Shales from Freienwalde, by Klaproth.

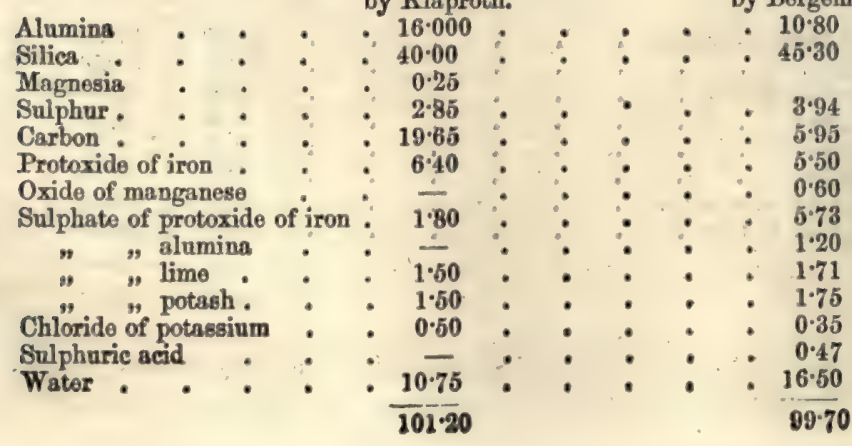

Shales from Piizberg, by Bergemann.

$$
\begin{array}{r}
10.80 \\
45.30 \\
\\
3.94 \\
5.95 \\
5.50 \\
0.60 \\
5.73 \\
1.20 \\
1.71 \\
1.75 \\
0.35 \\
0.47 \\
16.50 \\
99.70
\end{array}
$$




\section{ALUM}

Here the sulphur has evidently oxisted in combination with iron, which has boen united to oxygen by the analysts. The amount of sulphate shows a partial disintegration and other changes.

Lampadius gives another analysis with much more sulphur:Alum-Shale from Siehda.

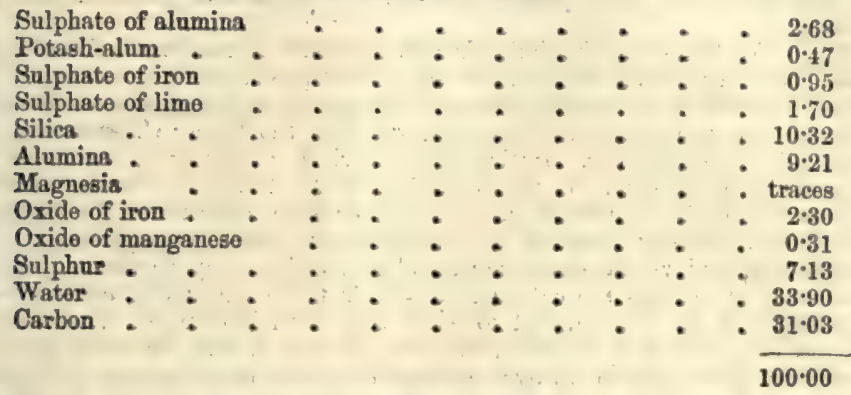

When alum is made of such shale, the object is first of all to oxidise the sulphur, forming sulphuric acid. This acid then dissolves the slumina. The result may be accomplished by allowing the shale to disintegrate spontanoously in the air, the sulphur oxidising and dissolving the alumina. But in general, as at Whitby and Campsie, combustion must be resorted to. This can be accomplished without the use of coal, further than is needful simply to set fire to that portion which exists in the shale itself. Indeed, the Campsie shale, having more coal than is desirable for slow combustion, is mixed with some spent material, in order to diminish the force of the heat.

The sulphur is united with the iron, forming a bisulphide, each atom of which must, therefore, take up seven atoms of oxygen, $\mathrm{FeS}^{2}+70=\mathrm{FeO}^{2} \mathrm{SO}^{3}+\mathrm{SO}^{3}$. When combustion takes place, the sulphur oxidises: if rapid combustion is permitted, sulphurous acid gas escapes; if slow combustion, the sulphurous acid penetrates the mass slowly, receives another atom of oxygen, unites to a base, and a sulphate is the consequence. Sulphate of iron is formed, and free sulphuric acid. In the process it is probable that the oxidation is completed by means of the iron. Protoxide of iron readily becomes peroxide; the sulphurous acid-readily decompeses peroxide, forming sulphurie acid and protoxide of iron. This protoxide of iron is again convorted into peroxide, and if not dissolved is rendered, to a great extent, difficult to dissolve, by reason of the heat of the mass. For this reason partly, there is less sulphate of iron in the alum than might be expected: To effect these changes it is desirablo to burn very slowly, so as to allow no loss - of sulphurous acid, and, in washing, to allow the water to stand a long time on the burnt-ore. Another method, by which the sulphuric acid is transferred to the alumina, is the peroxidation of the protoxide in the sulphate of iron; acid - is by this means set free and begins to act on the alumina.

The protosulphate of iron being formed, it is removed by boiling down the liquor until the protosulphate of iron crystallises out, at the same time the solution becoming saturated with the aluminous salt. The sulphate of iron is soluble in 0.3 of hot water, the alum in 0.06 . The liquid around the erystals on the remaining mothorliquor contains iron also; this is washed off by adding pure liquors.

The presence of lime or magnesia in the ores is, of course, a means of abstracting acid, preventing the alumina being dissolved, and even precipitating it when dissolved.

Knapp says that at Salzweiler, near Duttweiler, in Rhenish Prussia, the roasting of the ore takes place in the pit or mine. The stratum of brown coal which lies under it, having been accidentally set fire to in 1660 , has smouldered till the present time without intermission.

When the ores are rossted, one-half of the sulphur is freed and sent into the mass, or escapes as sulphurous acid; and the remaining protosulphide of iron is afterwards converted into green vitriol.

When the calcined mineral becomes thoroughly cold, we may proceed to the lixiviation. But as, from the first construction of the piles or beds till their complete calcination, many weeks, or even months, may elapse, care ought to be taken to provide a sufficient number or extent of them, so as to have an adequate supply of material for carrying on the lixiviating and crystallising processes during the

Vor. I. 
course of the year, or at least during the severity of the winter season, when the ealcination may be suspended, and the lixiviation becomes unsatisfactory. The beds are known to be sufficiently decomposed by the effloreseence of the salt which appears npon the stones, from the strong aluminous taste of the ashes, and from the appropriate chemical test of lixiviating an aliquot average portion of the mass, and seeing how much alum it will yield with solution of sulphate of potash or chloride of potassium.

2. The Lixiviation.-The lixiviation is best performed in stone-built cisterns; those of wood, howover strong at first, are soon decomposed, and noed repairs. They ought to be erected in the neighbourhood of the calcining hoaps, to save the labour of transport, and so arranged that the solutions from the higher cisterns may spontanoously flow into the lower. In this point of view, a sloping terrace is the best situation for an alum work. In the lowest part of this terrace, and in the neighbourhood of the boiling-house, there ought to be two or more large tanks, for holding the crude lixivium, and they should be protected from the rain by a proper shed. Upon a somewhat higher level the cisterns, of the clear lixivium may. be placed. Into the highost range of cisterns the caleined mineral is to be put, taking care to lay the largest lumps at tho bottom, and to cover thom with lighter ashes. A sufficient quantity of water is now to be run over it, and allowed to rest for some time. The lixivium may then be drawn off, by a stopcock connected with a pipe at the bottom of the cistern, and run into another cistern at a somewhat lower level. Fresh water must now be poured on the partly exhausted schist, and allowed to remain for a sufficient time. This lixivium, being weak, should be run off into a separate tank. In some cases a third addition of fresh water may be requísite, and the weak lixivium which is drawn off may be reserved for a fresh portion of calcined mineral. In order to save evaporation, it is always requisite to strengthen weak leys by employing them instead of water for fresh portions of calcined schist. Upon the ingenious disposition and form of these lixivisting cisterns, much of the economy and success of an alum work depends. The hydrometer should be always used to determine the degree of concentration which the solutions acquire.

The lixiviated stone, being thus exhausted of its soluble ingredients, is to be removed from the cisterns, and piled up in a heap in any convenient place, where it may be left, either spontaneously to decompose, or, after drying, subjected to another calcination.

After calcining and washing the Campsie ores, the residue had the following com. position:-

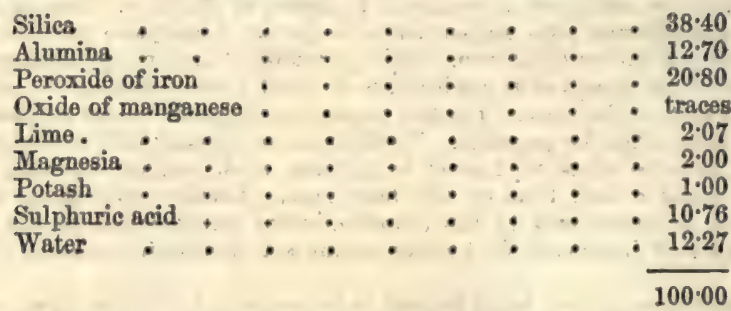

It is, therefore, very far from being a complete process; but it is not considered profitable to remove the whole of the alumina. In some places the exhausted ore is burnt a second time with frosh ore, as at Campsie, but we are not told the estimated exhaustion.

The density of the solution may be brought, upon an average, up to the specific gravity of from $1 \cdot 09$ to $1 \cdot 15$. The latter density may always be obtained by pumping up the weaker solutions upon fresh calcined mine. This strong liquor is then drawn off, when the sulphate of lime, the oxide of iron, and the earths are deposited. It is of advantage to leave the liquor exposed to air for some time, whereby the green vitriol may pass into a persulphate of iron with the deposition of some oxide, when the acid will act better on the clay present, so as to increase the quantity of sulphate of alumins. The manufacture of alum is the more imperfect, as the quantity of sulphate of iron left undecomposed is greater; and therefore every expedient ought to be tried to convert the sulphate of iron into sulphate of alumina.

3. The Evaporation of the Schist Lixivium.-As the aluminous liquors, however well settled at first, are apt, on the great scale, to deposit earthy matters in the course of their concentration by heat, they are best evaporated by a surface fire, such as that employed at Hurlet and Campsie. A water-tight stone cistern must be built, having a layer of well-rammed clay behind the flags or tiles which line its bottom and sides. 
The cistorn may be 4 or 6 feet wide, 2 or 3 foet deep, and 30 or 40 feet long, and it is covered in by an arch of stone or brickwork. At one extremity of this tunnel, or covered canal, a fire-grate is set, and at the other a lofty chimney is erected. The cistern being filled to the brim with the alum ley, a strong fire is kindled in the reverberatory grate, and the flame and hot air are forced to sweep along the surface of the liquor, so as to keep it in constant ebullition, and to carry off the aqueous parts in vapour. The soot which is condensed in the process falls to the bottom and leaves the body of the liquor clear. As the concentration goes an, more of the rough lixivium is run in from the settling cistern, placed on a somewhat higher level, till the whole gots charged with a clear liquor of a spocific gravity sufficiently high for transferring into the proper load boilers.

At Whitby, the lead pans are 10 feet long, 4 feet 9 inches wide, 2 feet 2 inches deep at the one end, and 2 feet 8 inches deep at the other. This increase of depth and corresponding slope facilitates the decantation of the concentrated lixivium by means of a syphon applied at the lower end. The bottom of the pan is supported by a series of parallel iron bars placed very near each other. In these lead pans the liquor is concentrated, at a brisk boiling heat, by means of the flame of a flue beneath them. Every morning the pans are emptied into a settling cistern of stone or lead. The specific gravity of the liquor should be about 1.4 or 1.5 , being a saturated solution of the saline matters present. The proper degree of density must varys however, with different kinds of lixivia, and according to the different views of the manufacturer. For a liquor which consists of two parts of sulphate of alumina, and one part of sulphate of iron, a specific gravity of 1.25 may be sufficient; but for a solution which contains two parts of sulphate of iron to one of sulphate of alumina, so that the green vitriol must bo withdrawn first of all by crystallisation, a specific gravity of i.4 may be requisite.

The construction of an evaporating furnace well adapted to the concentration of aluminous and other crude lixivia is described under SoDA. The liquor basin may be made of tiles or flags puddled in clay, and secured at the seams with a good hydraulic coment. A mortar made of quicklime mixed with the exhausted schist in powder, and iron turnings, is said to answer well for this purpose. Sometimes over. the reverberatory furnace a flat pan is laid, instead of the arched top, into which the crude liquor is put for neutralisation and partial concentration. In Germany, such a pan is made of copper, because iron would waste too fast, and lead would be apt to melt. From this preparation-basin the under evaporating trough is gradually supplied with hot liquor. At one side of this lower trough, there is sometimes a door, through which the sediment may be raked out as it accumulates upon the bottom. Such a contrivance is convenient for this mode of evaporation, and it permits, also, any repairs to be readily made; but, indeed, an apparatus of this kind, well mountod at first, will serve for many years.

In the course of the final concentration of the liquors, it is customary to add some of the mother-waters of a former process, the quantity of which must be regulated by a proper analysis and knowledge of their contents. If these mother-waters contain much free sulphuric acid, they may prove useful in dissolving a portion of the alumina of the-sediment which is always present in greater or less quantity.

4. The Precipitation of the Alum by adding Alkaline Salts.-As a general rule, it is most advantageous to separate, first of all, from the concentrated clear liquors, the alum in the state of powder or small crystals, by addition of the proper alkaline matters, and to leave the mingled foreign salts, such as the sulphate of iron or magnesia, in solution, instead of trying to abstract those saits by a previous crystallisation. In this way we not only simplify and accolerate the manufacture of alum, and leave the mother-waters to be worked up at any convenient season, but we also avoid the risk of withdrawing any of the sulphate of alumina with the sulphate of iron or magnesia. On this account the concentration of the liquor ought not to be pushed so far as that, when it gets cold, it should throw out crystals, but merely to the verge of this point. This density may be determined by suitable experiments. The powder of alum is also called flour.

The clear liquor should now be run off into the precipitation eistern, and have the sulphate of potash or chloride of potassium, or impure sulphato or carbonato of ammonia, added to it. The sulphate of potash, which is the most direct, forms 18.34 parts out of 100 of crystallised alum; and therefore that quantity, or an equivalent in chloride of potassium, or other potash, or ammoniacal salts, must be introducod into the aluminous liquor. Since sulphate of potash takes 10 parts of cold water to dissolve it, but is much more soluble in boiling water, and since the precipitation of alum is more abundant the more concentrated the mingled solutions are, it would be prudent to add the sulphate solution as hot as may be convenient: but, as chloride of potassium is fully three times more soluble in cold water, it is to be prot 
ferred as a precipitant, when it can be procured at a cheap rato. It has, also, the advantage of decomposing the sulphate of iron present into a chloride, a salt very difficult of crystallisation, and, theref ore, less apt to contaminate the crystals of alum. Of late years chloride of potassium has been largely obtained from carnallite, and sulphate of potash has been procured from kainite, two minerals found in great abuadance in the salt-mines of Stassfurt, in Prussia. The quantity of alkaline salts requisite to precipitate the alum, in a granular powder, from the lixivium, depends on their richness in potash or ammonia, on the one hand, and on the richness of the liquors in sulphate of alumina on the other; and this must be ascertained, for each large quantity of product, by a preliminary experiment in a precipitation glass. Here, an aliquot measure of the aluminous liquor being taken, the liquid precipitant must be added in successive portions, as long as it causes any cloud, when the quantity added will be indicated by the graduation of the vessel. A very exact approximation is not practicable upon the great scale; but, as the mother-waters are afterwards mixed together in one cistern, any excess of the precipitant at one time is corrected by excess of aluminous sulphate at another, and the resulting alum-meal is collectod at the bottom. When the precipitated saline powder is thoroughly settled and cooled, the supernatant mother-water must be drawn off by a pump, or rather a syphon or stopeock, into a lower cistern. The more completely this drainage is effected, the more easily and completely will the alum be purified.

100 parts of alum are formed from the sulphate of alumina liquor,

$$
\begin{aligned}
& \text { by } 18.32 \text { of sulphate of potash, } \\
& \text { or } 13.86 \text { of sulphate of ammonia, } \\
& \text { or } 15.69 \text { of chloride of potassium. }
\end{aligned}
$$

Sulphate of ammonia is soluble in 1 of hot and 2 of cold water; sulphate of potash in nearly 10 , and chloride of potassium in 3 , of water of ordinary temperature; alum, in 13 parts of water. A portion of the alum formed will remain in solution; this will depend on the quantity of liquid; the rest fulls as a powder.

This mother-liquor has generally a specific gravity of 1.4 at a medium tempersture of the atmosphere, and consists of a saturated solution of sulphate or muriate of black and red oxide of iron with sulphate of magnesia, in certain localities, and chlorido of sodium, when kelp salts have been used as a precipitant, as also a saturated solution of sulphate of alumina. By adding some of it, from time to time, to the fresh lixivia, a portion of that sulphate is converted into alum; but, eventually, the mother-water must be evaporated, so as to obtain from it a crop of ferruginous erystals; after which it becomes capable, once more, of giving up its alum to the alkaline precipitants.

When the aluminous lixiria contain a groat deal of sulphate of iron, it may be good policy to withdraw a portion of it by erystallisation before precipitating the alum. With this view the liquors must be eraporated to the density of $1 \cdot 4$, and then run off into crystallising stone cisterns. After the green vitriol has crystallised, the liquor should be pumped back into the evaporating pan, and again brought to the density of 1.4. On adding to it, now, the alkalino-saline precipitants, the alum will fall down from this concentrated solution, in a very minute crystalline powder, easy to wash and purify. But this method requires more ressels and manipulation than the proceding, and should only be had recourse to from necessity; sinee it compels us to carry on the manufacture of both the valuable alum and the lower-priced salts at the same time; moreover, the copperns extracted at first from the schist liquors carries with it, as we have said, a portion of the sulphate of alumina, and acquires thereby a dull aspect; whereas the copperas obtained after the separation of the alum is of a brilliant appearance.

5. The Washing, or Edulcoration, of the Alum Powder.-This crystalline pulverulent matter has a brownish colour, from the admixture of the ferruginous liquors ; but it may be freed from it by washing with very cold water, which dissolves not more than one-eighteenth of its weight of alum. After stirring the powder and the water well together, the former must be allowed to settle, and then the washing must be drawn off. A second washing will render the alum nearly pure. The less water is employed and the more effectually it is drained off, the more complete is the process. The second water may be used in the first washing of another portion of alum powder, in the place of pure water. These washings may be added to the schist lixivis. This powder is now extensively sold without further manipulation.

6. The Crystallisation.-The washed alum is put into a lead pan, with just enough water to dissolve it at a boiling heat; fire is applied, and the solution is promotod by stirring. Whenever it is dissolved in a saturated state, it is run off into the crystallising vessels, which are called roching casks. These casks are about five 
foet high; three foet wide at the top, and somewhat wider at the botfom; they are made of very strong staves, nicely fitted to each other, and held together by strong. iron hoops, which are driven on pro tempore, so that they may be easily knocked off again, in order to take the staves asunder. The concentrated solution, during its slow cooling in these close vessels, forms large regular crystals, which hang down from the top, and project from the sides, while a thick layer or cake lines the whole interior of the cask. At the end of eight or ten days, more or less according to the weather, the hoops and staves are removed, when a cask of apparently solid alum is disclosed to view. The workman now pierces this mass with a pickaxe at the side near the bottom, and allows the mother-water of the interior to run off on tho sloping stone floor into a proper cistern, whence it is taken and added to another quantity of washed powder to be crystallised with it. The alum is next broken into lumps, exposed in a proper place to dry, and is then put into the finished bing for market. There is sometimes a little insoluble basic alum (sub-sulphate) left at the bottom of the cask. This, being mixed with the former mother-liquors, gets sulphuric acid from them; or, being mixed with a little sulphuric acid, it is equally conrerted into alum.

Alum Liquors. - In the alum works on the Yorkshire coast, eight different liquors are met with:

1st. 'Raw Liquor.' The calcined alum-shale is steeped in water till the liquor has acquired a specific gravity of 9 or 10 pennyweights, according to the language of the alum-maker.

2nd. 'Clarified Liquor.' The raw liquor is brought to the boiling point in lead pans, and suffered to stand in a cistern till it has cleared; it is then called clarified liquor. Its gravity is raised to 10 or 11 pennyweights.

3rd. 'Concentrated Liquor.' Clarified liquor is boiled down to about 20 pennywoights. This is kept merely as a test of the comparative value of the potash salts used by the alum-maker.

4th. 'Alum Mother-Liquor.' The alum pans are fed with clarified liquor, which is boiled down to about 25 or 30 pennyweights, when a proper quantity of potash salt in solution is mixed with it, and the whole run into coolers to crystallise. The liquor pumped from these rough crystals is called 'alum mothers.'

5th. 'Salts Mothors,' The alum mothers are boiled down to a crystallising point, and afford a crop of 'Rough Epsom,' which is a sulphate of magnesia and protoxido of iron.

6th and 7th. 'Alum Washings.' The rough crystals of alum (No. 4) are washed twice in water, the first washing being about 4 pennyweights, the second about $2 \frac{2}{2}$, the difference in gravity being due to mother-liquor clinging to the crystals.

8th. 'Tun Liquor.' The washed crystals are now dissolved in boiling water, and run into the 'roching tuns' (wood vessels lined with lead) to crystallise. The mother-liquor of the 'roch alum' is called 'tun liquor:' it is, of course, not quite so pure as a solution of roch alum in water.

The alum-maker's specific-gravity bottle holds 80 pennyweights of water, and by 10 pennyweights he means 10 more than water, or 90.

'The numbers on 'Twaddle's hydrometer, divided by $2 \cdot 5$, give alum-makers' pennyweights.

The alum-maker tests his samples of potash salts comparatively by dissolving equal weights of the different samples in equal measures of alum liquor at 20 pennyweights, heated up to the boiling-point, and weighing the quantity of alum crystals produced on cooling.

For the above information wo are indebted to Mr. Maurice Scanlan, who superintended for some time the Mulgrave Alum Works.

According to him $6 \frac{1}{2}$ tons of the alum rock at the Mulgrave Works, to the north of Whitby, yield, after calcination, \&c., one ton of alum.

The true value of the Whitby alums consists in the amount of soluble alumina which they contain, and for calico-printing also in their freedom from iron.

The alum-shales not being very generally found over the country, and nature heving interposed certain limits to the amount manufactured and the speed of the process, many attempts have been made to obtain alum and sulphato of alumina from other sources.

A number of these processes will be afterwards described in chronological order, but the following are those only which are at present largely applied in this country. One of the great advantages of the modern processes is the rapidity with which the alum can be manufactured; thus, an order can now be executod in three 
woeks which formerly would havo occupied many months. For cortain purposes, however, the old-fashioned Whitby alum is still preforred.

III. Alum Manufacture from Coal-Measure Shales.-The manufacture of alum has of late years taken an entirely new shape, and the two procosses of Mr. Sponce and Mr. Pochin have absorbed the whole of the manufacture in the north-west.

Mr. Spence, who has a manufactory of ammonia-alum at Newton Heath, near Manchester, called the Pendleton Alum Works, has now become the largest maker of this substance in the world, as his regular production amounts to upwards of 150 tons per week. His method of manufacture is also largely carried on at Goole, in Yorkshire. In Spence's process, which he patented fifteen years ago, he uses for the production of his sulphate-of-alumina solution the carbonacoous shale of the coalmeasures. ${ }^{1}$ This substance contains from 5 to 10 per cent. of carbonaceous matter, and, when ignited by a small quantity of burning coal, the combustion continues of itself. The raw shale is piled in heaps about 20 feet long, 4 or 5 feet high, and 2 or 3 feot broad. To supply air during the calcination of tho shale, a brick drain is constructed under each heap. The calcination occupies about ten days, and must be conducted below a red heat; at higher temperatures, the material has a tendency to vitrify, and the alumina thus becomes insoluble in sulphuric acid. After calcination, the shale is removed to the pans, in which it is treated with oil of vitriol. These pans are each about 40 feet long, 10 feet wide, and 3 feet deep. They are constructed of sheets of cast iron, screwed together, and lined with lead, and the bottom of each pan before use is covered with earthen tiles about 9 inches square. A charge of 20 tons of calcined shale is introduced into each pan, and digested with about 10 tons of oil of vitriol, of specific gravity $1 \cdot 25$, which dissolves out the alumina of the shale. The digestion is conducted at a temperature of $220^{\circ} \mathrm{F}$., this temperature being maintained partly by fires under the pans, and partly by the introduction of hot gaseous ammonia evolved from the gas-liquor evaporated in boilers. A double sulphate of alumina and ammonia, or ammonia-alum, is thus formed. Aftor boing heated for four or five days, the liquor is drawn off into the coolers, which are large shallow rectangular vessels of lead, each about 29 feet long, 17 feet wide, and $1 \frac{3}{4}$ feet deop. In these ressels the liquid is kept in constant agitation by means of a long wooden arm, worked by steam. The formation of large crystals is thus prevented; but, after the lapse of about fourteen hours, the small crystals, which then form a bed several inches thick at the bottom of the vessel, are removed, and thrown into a large square box, lined with lead, in which they are washed with the mother-liquor, and having drained are thrown upon an iron gxating formed of bars set about half-an-inch apart. The masses of crystals are thus broken, and the mother-liquor washed out. The crystals are then transferred to a cylindrical ressel, two or three feet high, and about 2 feet in diameter. This vessel has two divisions, one open and the other closed. The alum is placed in the open compartment, and dissolved by means of steam, introduced at a pressure of about 20 lbs. per square inch, through holes in a coil of leaden pipe at the bottom of this division of the vessel. A pipe leads from the top of the cylinder to a wooden vessel, called the dissolving box, which receives the solution of alum before it is drawn off into the roching pans. This box is 3 feet deep, 14 feet long, and 8 feet broad; it is covered with boards, the joints between which are closely packed with cotton waste. After the solution has remained for some hours in this vessel, a quantity of sizo-about 4 quarts - is poured in, and the susponded impurities are thus caused to settle. The clear solution is tapped off from the dissolving box into the crystallising tubs; each tub is about 6 feet high, and wider below than abovo. At the bottom of each tub is a round flagstone, upon which the staves of the tub, lined with lead, are built up and kept in place by strong iron hoops screwed together. The hot solution is run into these tubs, and protected by a wooden cover. After standing for about forty days, a sufficient thickness of alum will have crystallised to allow the sides of the tub to be taken down. The block is allowed to stand for about a fortnight longer, and a hole is then made in the lower part of the block, through which the mother-liquor runs out, and is received in tanks. On breaking open the block, the sides of the internal cavity are found to be studded with fine octahedral crystals, presenting a slightly violet tinge. These blocks weigh about 4 tons each.

IV. Alum Manufacture from Mineral Phosphates.-In 1870 Mr. Peter Spence patented a novel process for manufacturing alum, using as his raw material a phos: phate of alumina and iron obtained from the Island of Redonda, near Antigua, in the West Indies; other mineral phosphates containing alumina may, however, be employed.2 The process has special value, since not only is the alumina of the

- This description of Spence's process is taken from a paper on 'The Past and Present Tistory, of AIum,' by J. Carter Bell, Esq. F.C.S., Associate of the Royal School of Mines, 'Chemical News,' vol. If. 1865 , pp. 221, 284.

Specification of Patents, A.D. 1870, No. 1676. 
mineral dissolved out by sulphuric acid, and used in the manufacture of alum, but the phosphoric acid set free forms a highly valuable by-product.

The following analysis may be cited as that of a good sample of the Redonda phosphate :-

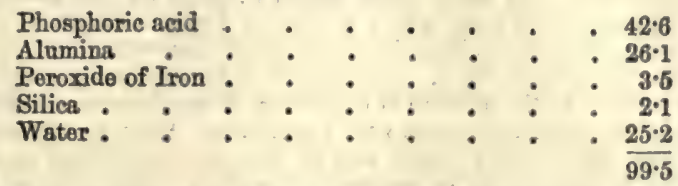

The mineral is first calcined at a red heat, whereby the water is expelled, and the material rendered porous, so as to be more freely acted on by sulphuric acid. If desirable, the calcination may be dispensed with, and the raw material merely prepared by grinding. In either case the phosphate is digested in leaden vessels with sulphuric acid of specifie gravity $\mathbf{1}^{\cdot 6}$.

The proportion of sulphuric acid varies with the richness of the mineral in alumins ; thus, if it contain 20 per cent. of alumina, an equal weight of acid will be used; if only 12 per cent. of alumina, about three-fifths its weight of acid; and so forth. To facilitate solution of the mineral by the acid, heat is applied-most conveniently by blowing steam into the vessel. As solution proceeds, the density of the liquid rises, and the strength of the liquor is then reduced by adding water, or weak liquors from subsequent stages of the process, and the liquid is boiled until its specific gravity ultimately reaches 1.45 , or $90^{\circ}$ Twaddle. This liquid is then transferred to a closed leaden vessel, and ammoniacal vapour from gas-liquor is distillod into it. The sulphate of ammonia thus formed combines with the sulphate of alumina to form ammonia-alum. From 600 to 900 gallons of gas-liquor are used to each ton of phosphate. After introduction of ammonia, the liquor is allowed to settle, and the clear solution, which has now a specific gravity of 1.4 , or $80^{\circ}$ Twaddle, is run into leaden coolers, where the alum crystallises. When the phosphate contains 20 per cent. of alumina, it yields about a ton-and-a-half of alum per ton. One of the great difficultios in this process consists in completely freeing the alum from the associated phosphoric acid.

Whilst the alumina of the phosphate is thus utilised for the production of alum, the phosphoric acid, which is at the same time eliminated, forms a product of high value to the agxiculturist. Indeed the mother-liquor from which the alum is deposited consists chiefly of a solution of phosphoric acid, with a small proportion of sulphate of alumina, iron, and sulphato or phosphate of ammonia. This liquid may be used $a^{\prime}$ a fertilising agent either directly or made into an artificial manure by being absorbed in sawdust and dried at a low heat.

We believe that Mr. Spence's new process is now carried out on a large scale at the Pendleton Alum Worke.

Other Methods of Alum Manufacture.-In addition to the four principal processes of manufacture already described, numerous other methods have from time to time been proposed, and in some cases worked to a limited extent. The more important of these suggested improrements are included in the following notices, which are arranged in chronological order.

In 1743, Ambrose Newton wished to economise the manufacture by boiling the scum of the alum works, the muddy deposit in YYorkshire, and adding to the concentrated solution of 45 pennyweights, stale urine, which is ammonia, until the solution became 27 pennyweights. The liquor stands "for four days, and strikes out into small allom, and afterwards melted and roached into casks, which stand 14 days, and are taken down and the allom is finished.'

Another patent in 1765 , by Holme, Cropper, and two Nicholsons, uses stale urine and kelp liquor. They seem to use, by a mistake in names, iron pyrites only for their alum, but no doubt it contained both iron and alumina. They took advantage of the potash, and perhaps also of the soda, of the kelp.

In 1780, Matthew Sanderson patented a plan for making alum by burning the metallic sulphurets, obtaining the sulphuric acid, and uniting it with aluminous earth -a far-soeing plan, not till long after adopted.

In 1794 Lord Dundonald patented a process for 'washing aluminous, vitriolic, or pyritous schist or materials with sea-water or solutions of salts containing muriate of soda, or mixing muriate of soda with aluminous or vitriolated salts or pyritous substances. He also proposed the use of muriatic acid. It is probable, then, that both a soda- and an ammonja-alum have boen manufactured when the whole mothod was not very clearly understood. 
Macques, Foureroy, and Vauquelin having discovered the component parts of alum, Chaptal made it from its elements, using clay. Ho says, 'Pure clay upon which the sulphuric acid is digested is dissolved with difficulty.' He then says, 'I calcine my elays, and reduce them into small pieces, which I spread on the floor of my leaden chambers. The sulphuric acid, which is formed by combustion of a mixture of sulphur and saltpotre, expands, itself in the cavity of these chambers, and exists for a certain time in the vaporous form. In this form it has a stronger action than when it has been weakened by the mixture of a quantity of water more or less considerable, 80 that it seizes the earths, combines with them, causes them to increase in bulk by the effervescence which takes place, and at the end of several days the whole surface exposed to the vapour is converted into alum. Caro is taken to stir these earths from time to time, that they may successively present all thoir surfaces to the action of the acid.' 'But whatever process may be used to combine the acid with clay, it is necessary to expose the aluminised earths to the air during a greater or less space of time, in order that the combination may be more accurate, and the saturation more complete.' This is, in fact, the mode of making the sulphate of alumina. It was then dissolved in water, drawn off clear, to free it from the silica and undissolved matter, mixed with sulphate of potash, evaporated, and erystallised.

The manufacture of the alum from clay seems to have been a good deal used in France. Their method at present, according to Regnault, is as follows:- 'They choose clays, such as kaolins, which contain little iron. The clays are then calcined at a low red heat in a furnace; they are ground to powder in a mill, and mixed with half their weight of sulphuric acid of 1.45 specific gravity. The mixture is then heated in another furnace until the sulphuric acid begins to evaporate. It is then taken out, and left to stand for several days.' After some time the combination becomes intimate, and the usual method of removing the sulphate of alumina from the insoluble matter is resorted to, and the potash, or ammonia-salt, is added, to convert it into alum.

The most usual method has been to allow it to stand some weeks, or months, until the combination has been effected. This has partially arisen from a supposition of the necessity of giving it as much time as is needful with the shales, as it was not known until lately how completely the acid may decompose the clays.

A patent was obtained in November, 1839, by Mr. William Wiesmann, of Duesburg, for improvements in the manufacture of alum. He subjects potters' clay to a moderate red heat, grinds it, and subjects the powder, in leaden pans, to the action of concentrated sulphuric acid $\left(66^{\circ}\right.$ B. $)$, taking care to use excess of clay and a moderate heat. The mixture is to be stirred till it is dry, then treated with boiling water, in order to dissolve the sulphate of alumina formed. So far the process is old and well known. The novelty consists in freeing the saline solution from iron by ferrocyanide of potassium (prussiate of potash). Whon the iron has been all thrown down in the form of Prussian-blue, the liquor is allowed to settle, the supernatant pure sulphate is drawn off, and evaporated till it forms, on cooling, a concrete mass, which may be moulded into the shape of bricks, \&zc., for the convenience of packing. This was manufactured at Lee-Moor, near Plympton, on a small scale; but it is no longer made in this country. Dr. Muspratt's analysis made it a basic sulphate $=2 \mathrm{Al}^{2} \mathrm{O}^{3}$. $5 \mathrm{SO}^{3}+33 \mathrm{Aq}$; and he adds that manufacturers objected to it because it was impossible to judgo of its purity by its merely physical appearance. Mohr's analysis gave-

Alumina
Sulphuric acid : $: \quad \vdots:$
Water
Sulphate of potash

By having an excess of clay, Wiesmann intended to have all his acid saturated. He found that he could not dissolve all the alumine by using only its equivalent of acid; he preferred, therefore, to lose some of the alumina, as in the other processes.

Hervey's patent of 1839. - Clay is dried, ground, and sieved; it is then mixed with sulphuric acid of from $10^{\circ}$ to $80^{\circ} \mathrm{Twad}$, and from to an equal quantity of clay, used aceording to its quality. The mixture is then woll stirred; a great obullition consues, and aftor ebullition it is again stirred. This is the formation of the sulphate of alumina, which is washed out, and made into alum in the ordinary way.

- In $1842 \mathrm{Mr}$. Turner patented a method, said to be originally Sprengel's proposal, of - extracting the alumina and potash from felspar to make alum. The felspar is heated with sulphate of potash to melting, then carbonate of potash is added. This gives a soluble,glass, which, in boiling water, takes up two-thirds of the silica and as much potash as wass added to the felspar. This being heated with carbonic acid, gives a golatinous mass of silica. When dried, the carbonate of potash may be washed out. 
The insoluble portion of the glass contains the original felspar, minus two-thirds of its silica-a light, porous substance, similar in composition to elreolite. This is boiled with sulphuric acid of 1.2 specific gravity. The intense heat needed has prevented the success of this process.

In 1842 Kagenbusch proposed to cover the schist over with a plastering of clay, or mud, for several months, and wash with water; thon to have it burnt in kilns fittod with air-holes. In this process turf is used, on which the schist rests. The air-holes regulato the combustion, which lasts three days. He uses kelp to obtain the alkali.

In $1850 \mathrm{~J}$. T. Wilson proposed a method of collecting the ammonia from smoke, and using it in making ammonia-alum. What is wanting, ho supplements by potash salts, cansing a mixed potash- and ammonia-alum to bo manufactured.

In 1854 Richardson adds iron pyrites, to increase the amount of sulphur, and, consequently, of sulphuric acid, in the shale; but it does not seem to have been used.

In $1855 \mathrm{Dr}$. Frankland precipitated the subsulphate of alumina, and added sulphuric acid, thus obtaining the base by a small expenditure of precipitant.

In $1856 \mathrm{~J}$. Metcalf makes a cake similar to the alum-cake described at p. 122 ; but he uses coanse clay.

In 1856 Henry Pease and Thomas Richardson mix clay with chloride of potassium or with common salt; they convert both into sulphates; the muriatic acid set freo dissolves the alumina, and the chloride of aluminium formed is used as alum.

In 1856 Spilsburg's patent purposed to mako alum from cryolito.

The Boghead Cannel-coal ash contains about 30 per cent. of alumina, which it has been proposed to dissolve for making alum; but it has not hitherto been found a convenient material.

Among the modern methods adopted for manufacturing alum on the Continent, some of the more interesting are those in which eryolite is the raw material (see Cryolite). According to Thomson's method the cryolite is ignited with carbonate of lime, whereby aluminate of soda and fluoride of calcium are produced; the former being soluble and the latter insoluble in water. The aluminate is, therefore, dissolved out, and a current of carbonic acid gas transmitted through tho solution. A gelatinous precipitate of alumina is thus thrown down, while carbonate of soda remains in solution. Or bicarbonate of soda may be added to the solution of the aluminate, and the alumina thus precipitated in a compact form. The alumina obtained by either of these methods is dissolved in dilute sulphuric acid, and the sulphate of alumina thus formed is converted into alum.

In another process, introduced by Sauerwein, the cryolite having been powdered, is boiled with water and limo; and, as before, aluminate of soda and fluoride of calcium are obtained. The solution of aluminate is mixed with an excess of finely-powdered cryolite, whereby alumina and fluoride of sodium are produced. The alumina is then converted into sulphate, and this into alum, as previously described.

Sulphate of alumina may also be obtained from cryolite by treating it directly with sulphuric acid.

Alum has likewise been prepared from the mineral called Bauxite, which, on ignition with sulphate of soda and charcoal, yields sulphate of alumina. Again, blastfurnace slag may be employed as the raw matorial; the slag, treated with hydrochloric acid, yields chloride of aluminium, from which alumina may readily be precipitated.

Alum has been made extensively in England and France from an artificial sulphate of alumina, prepsred from clays which are. chosen as free as possible from carbonate of lime and oxide of iron. They are calcined in a reverberatory furnace, in order to expel the water, to peroxidise the iron, and to render the alumina more easily acted on by the acid. The expulsion of the water renders the clay porous and capable of absorbing the sulphuric ncid by capillary nttraction. The peroxidation of the iron renders it less soluble in the sulphuric acid; and the silica of the clay, by rencting on the alumina, impairs its aggregation, and makes it more readily attracted by the acid. The clay should therefore be moderately calcined; but not so as to indurato it like pottery-ware, for certain combinations would then be effected which would make it resist the action of acids. The clay is usually calcined in a reverberatory furnace, the flame of which serves afterwards to heat two evaporating pans and a basin for containing a mixture of the calcined clay and sulphuric acid. As soon as the clay has become friable in the furnace it is takon out, reduced to powder, and passed through a fine siove. With 100 parts of the pulverised elay, 45 parts of sulphurie acid, of specific gravity 1.45 , are well mixed, in a stone basin, arched over with brickwork. The flame and hot air of the reverboratory furnace are made to play along the mixture, in the same way as described for eraporating alum-schist liquors. (Seo Sons.) The mixture, boing stirred from timo to timo, is at the end of a fow days to be raked out, and to be sot aride in a warm place, for the acid to work on the clay, 
during six or eight weeks. At the end of this time, it must be washed, to extract the snlphate of alumina. With this view, it may be treated like the roasted alum ores previously described. If potash-alum is to be formed, this sulphate of alumina is evaporated to the specific gravity of 1.38 ; but if ammonia-alum, to the specific gravity of only 1.24 ; because the sulphate of ammonia, being soluble in twice its woight of water, will cause a precipitation of pulverulent alum from a weaker solution of sulphate of alumins than the less soluble sulphate of potash could do.

In preparing alum from clay or shale, it is of infinite importance that so much and no more heat be applied to the clay or shale, in the first instance, as will just expel the wator of combination without inducing contraction. A tomperature of $600^{\circ} \mathrm{F}$. is well adapted to effect this object, provided it be maintained for a sufficient period. When this has been carefully done, the silicate of alumina remaining is easily enough acted upon by sulphuric acid, oither slightly diluted or of the ordinary commercial strength. The best form of apparatus is a leaden boiler, divided into two parts by a perforatod soptum or partition, also in lead; though on a very large scale, brickwork set in clay might be employed. Into one of the compartments the roasted clay or shale should be put, and diluted sulphuric acid being added, the bottom of the other compurtment may be exposed to the action of a well-regulated fire, or, what is better, heated by means of steam through the agency of a coil of leaden pipe. In this way a circulation of the fluid takes place throughout the mass of shale; and, as the alumina dissolves, the dense fluid it produces, falling continually towards the bottom of the boiler, is replaced by dilute acid, which, becoming in its turn saturated, falls like the first; and so on in succession, until either the whole of the alumina is taken up, or the acid in great part neutralised. The solution of sulphate of alumina thus obtained is sometimes evaporated to dryness, and sold under the name of 'concen. trated alum;' but more generally it is boiled down until of the specific gravity of about 1.35 ; then one or other of the carbonates or sulphates of potash or ammonia, or chloride of either base, or a mixture of these, is added to the boiling fluid, and as soon as the solution is complete, the whole is run out into a cooler to crystallise. The rough alum thus made is sometimes purified by a subsequent recrystallisation, after which it is 'roched' for the market-a process intended merely to give it the ordinary commercial aspect, but of no real value in a chemical point of view.

Alum Cake.-This substance owes its value to the amount of sulphate of alumina it contains, and is in fact another means of making soluble alumina accessible. We have already seen the many attempts to obtain alumina from clay, and the tedious nature of the operation of solution in acid, as well as the long after-processes of lixiviation and conversion into sulphate of alumina, or into alum, by reboiling or crystallising. Mr. Pochin, of Manchester, has found a method of removing all the difficulties, both of the first and after processes. He uses very fine china clay, free from iron, heats it in a furnace, mixes it thoroughly with acid, and finds that, when the process is managed carefully, tho combination of the alumina and sulphuric acid is not only complete, but so violent that he is obliged to dilute his acid considerably, in order to calm the action. When mixed, it is passed into cisterns with moveable sides, where, in a fow minutes, it heats violently and boils. The thick liquid gradually becomes thicker, until it is converted into a solid porous mass - the pores being made by the bubbles of steam which rise in the mass, which is not fluid enough to contract to its original volume. The porons mass is perfectly dry, although retaining a large amount of combined water. It retains, of course, all the silica of the original clay, but this is in such fine division that every particle appears homogeneous. The silica gives it a dryness to the touch not easily gained by the sulphate only.

When pure sulphate of alumina is wanted in solution, the silica is allowed to precipitate before using it, but, in many cases, the fine silica is no hindrance; then the solution is made use of at once.

Some quantity of alum has been exported from China, chiefly to India, within a short period. The Chinese use alum very largely in their cements.

The alum mines are in the neighbourhood of Peh-kwan harbour, $2^{\circ} 9^{\prime} 10^{\prime \prime} \mathrm{N}$., $12^{\circ} 32^{\prime} 6^{\prime \prime} \mathrm{E}$. Ton alum-making establishments appear to exist there, and the process, as described in the North China Mail, is similar to that employed where, in this country, the alum-shale is used.

AIUM IARTE. A variety of aluminous schist found associated with the lignites of the tertiary beds, especially on the Rhine.

AIUM, РЕАтrER. A hydrated sulphate of protoxide and sesquioxide of iron, called by Dans Halotrichite. The name 'feather alum' is also sometimes applied to 's hydrated sulphate of alumina, known also as Alunogen. The latter is found crystallised in a close mass of fine, white, flexible needles, of a feather or hair form, and has been, like a fow other substances, called hair-salt. It is also found with 
various degrees of impurity, sometimes with a smaller amount of water. Knapp has collected the list of analyses shown in the Table :-

Analyses of Natural Sulphate of Alumina or Feather Alum.

\begin{tabular}{|c|c|c|c|c|c|c|c|c|c|c|c|}
\hline \multirow{2}{*}{ 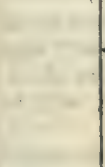 } & \multirow{2}{*}{ 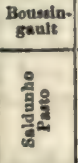 } & \multirow{2}{*}{ Bartwell } & \multicolumn{4}{|c|}{ I. Rose } & \multirow{2}{*}{ Gobel } & \multirow{2}{*}{ 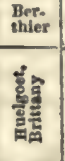 } & \multicolumn{2}{|c|}{ Th, Themeos } & \multirow{2}{*}{ 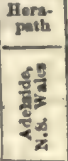 } \\
\hline & & & 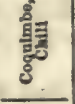 & $\begin{array}{l}\frac{6}{8} g \\
\frac{8}{2} \\
\frac{2}{2}\end{array}$ & 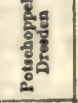 & है & & & gัँ & $\frac{\frac{6}{8}}{\frac{1}{8}}$ & \\
\hline \multirow{3}{*}{$\begin{array}{l}\text { Sulphurie } \\
\text { acid } \\
\text { Alnmina } \\
\text { Peroxide } \\
\text { of iron. }\end{array}$} & $36 \bullet 400$ & $40 \cdot 31$ & $86 \cdot 97$ & $87 \cdot 380$ & $85 \cdot 710$ & $38 \div 637$ & $58 \cdot 58$ & $12 \cdot 9$ & $85 \cdot 872$ & $40: 425$ & \multirow{4}{*}{$\begin{array}{c}25 \cdot 63 \\
17^{\circ} 09 \\
0.04 \\
\text { Oxide } \\
\text { ofCop. } \\
\text { per }\end{array}$} \\
\hline & $16 \cdot 000$ & $14 \cdot 98$ & $14 \cdot 63$ & $14 \cdot 867$ & $12 \cdot 778$ & $11 \cdot 227$ & $88 \cdot 75$ & $41 \cdot 5$ & $14 \cdot 645$ & 10.485 & \\
\hline & 0.004 & . & $2 \cdot 58$ & $\bullet$ & $\because$ & $\cdots$ & $\cdots$ & $\cdots$ & $0 \cdot 300$ & $8 \cdot 580$ & \\
\hline $\begin{array}{l}\text { Protoxide } \\
\text { of iron. }\end{array}$ & $\because$ & 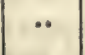 & $\cdots$ & $2 \cdot 168$ & $0 \cdot 667$ & 0.718 & $+80^{2} 2 \cdot 78$ & $\cdots$ & $\cdots$ & & \\
\hline \multirow{7}{*}{$\begin{array}{l}\text { Protoxide } \\
\text { Man- } \\
\text { ganese } \\
\text { Potagh : } \\
\text { Boda : } \\
\text { Lime.: } \\
\text { Magneala } \\
\text { Muriatic } \\
\text { acld. : } \\
\text { Sillca. } \\
\text { Water : }\end{array}$} & . & $\ddot{0} 26$ & $\because$ & $\ddot{0} 215$ & $\begin{array}{l}1.018 \\
0.824\end{array}$ & $\begin{array}{l}6 \cdot 807 \\
0.480\end{array}$ & & & & & p p \\
\hline & $\therefore$ & $1 \cdot 18$ & $\because$ & $\because 10$ & 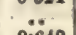 & $\because$ & 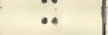 & $\because$ & $\ddot{2} \ddot{262}$ & 1072 & \\
\hline & $\begin{array}{l}0^{\circ} 002 \\
0^{\circ} 004\end{array}$ & $\ddot{0} \cdot 85$ & Oi1s & $\begin{array}{c}0.149 \\
\ldots *\end{array}$ & $\begin{array}{l}0.640 \\
0.273\end{array}$ & $\begin{array}{l}0 \circ 449 \\
1 \cdot 012\end{array}$ & & & & & \\
\hline & $\cdots$ & 0.10 & & & & & & & & & \\
\hline & 19.00 & $\begin{array}{r}1 \cdot 13 \\
40 \cdot 94\end{array}$ & $1 \cdot 37$ & $4 \pi \cdot 164$ & $47 \cdot 092$ & $0 \cdot 430$ & ・ & $3 \cdot 5$ & $0 \cdot 100$ & 38.095 & 0.50 \\
\hline & 20000 & & & & 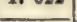 & & & & & & \\
\hline & 99.010 & $100 \cdot 00$ & $100 \cdot 33$ & $100 \cdot 238$ & $98 \cdot 432$ & $100 \cdot 000$ & $100 \cdot 11$ & $100 \cdot 0$ & $99 \cdot 754$ & $96 \cdot 904$ & $98 \cdot 96$ \\
\hline
\end{tabular}

AIUM, NATrVI. This term includes several compounds of sulphate of alumina with the sulphate of some other base, as magnesia, potash, soda, the protoxides of iron, manganese, \&ce. They occur generally as efflorescences, or in fibrous masses ; when crystallised they assume octahedral forms ; they are soluble in water, and have an astringent taste. The following species are the chief native alums, but it should be understood that the proportion of water of erystallisation is not the same in all, and hence in some cases they cannot be brought within the general formula given under the aricle Axus.

Kalinite, or Potash Alum, found as an efflorescence on alum-slate at Whitby, Hurlet and Campsio.

Mendozite, or Soda-alum, found in South America. The following analyses may be citod:-

Soda-ahum from Peru, by T. Thomson.

Sulphato of soda.

$\therefore 6.50$

Alumins : $: 22.55$

Sulphuric acid : : : $32 \cdot 95$

Water $: 39 \cdot 20$

$\overline{101 \cdot 20}$
Soda-alum from the Andes.

Sulphuric acid . . $\quad$. $36 \cdot 199$

Alumina . ., 11.511

Soda $: 7.259$

Water. $: 48819$

Silica . . . 0.180

Lime . . . 0.255

Peroxide of iron . . : 0:199

Protoxide of iron: . . 0.760

$100 \cdot 162$

Trchermigite, or Ammonia-alum, found at Tschermig in Bohomia. Analyses have been made of specimons from Tschermig, by Stromeyer :-

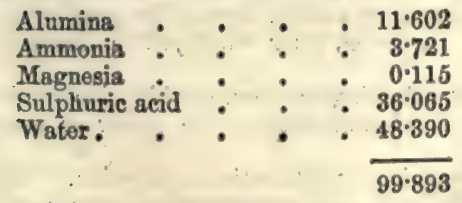

Sulphate of alumina . $\quad 38 \cdot 688$

Sulphate of ammonia . $12 \cdot 478$

Sulphate of magnesia : $\quad 0.337$

Water. . 48.390

$99 \cdot 893$

Pickeringite, or Magnesia-alum, found near Iquique in Pern, and in Nova Scotia, Apjohnite, or Manganese-alum, from Lagos Bay in South Africa. 
Bosjomanite, a Manganese-alum, found near the Bosjeman River in South Africa, and at Alum Point, near the Salt Lake, Utah.

AIUx, Romax (Alumen Romanum. Allwmo di rocca, It.), called sometimes Roch or Rock Alum. Seo Acurc.

AxUm ROOT. The root of the Geranium maculatum, a North American plant. It contains a largo proportion of tannin, and is used as a powerful astringent.

AIUAM-SEATE. The chief natural source from which the alum of commerce is derived in this country. It occurs in a remarkable manner near Whitby, in Yorkshire, and at Hurlet and Campsie, near Glasgow. A full description of the alumshale, and of the processes by which the crystallisable alum is separated, will bo found under ALUs.

AIUM-SIATr. A clay-slate containing bitumen and sulphide of iron, found in the North of England, in Scotland, in Seandinavia, and other places. The common variety effloresces and acquires the taste of alum. The glossy Alum-slate is of a bluish-black colour, with a semi-metallic lustre. It swells upon exposure to the air, owing to the saline efflorescence formed between the foliations, and is eventually disintegrated.

AIUM-STOxz of AIUNrTz. (Alun, Fr.; Alaunstein, Ger.)-This mineral, in its purest form, is composed of alumina $37 \cdot 13$, sulphuric acid $38 \cdot 53$, potash $11 \cdot 34$, water 13.00. Silica is also frequently present as an impurity, sometimes to the extent of 60 per cent. It is a white, greyish, or reddish mineral; affording a white streak, and an uneven, flat, conchoidal fracture, which is splintery in the massive varieties. It is transparent or subtranslucent.

Alum-stone is one of the sources of the alum of commerce, which is obtained from it in crystals after frequent roasting, and lixiviation in wator. See Axvx.

Alum-stone is found at Tolfa, near Civita Vecchia, in the Roman States (sometimes in crystals); at Muszaly in Hungary; at Pie de Sancy, in France, and in the Grecian Archipelagu. The compact varieties from Hungary are so hard as to be used for millstones.

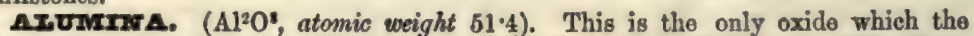
metal aluminium forms, and it is assumed to be a sesquioxide on account of its isomorphism with sesquioxide of iron.

Alumina occurs in a native state, forming the gems known as the sapphire and the ruby, and in a less pure form as the minerals corundum and emery. In a stato of hydrate, or in chemical combination with water, alumina constitutes the species known as diaspore, gibbsite, and hydrargyllite. Such of these minerals as are of economic value will be noticed under their respective heads.

Alumi a is obtained in the state of hydrate from common alum (KO, $\mathrm{SO}^{3} ; \mathrm{Al}^{2} \mathrm{O}^{3}$, $3 \mathrm{SO}^{3}+24 \mathrm{HO}$ ) by adding a solution of ammonia (or better, carbonate of ammonia) to the latter salt and boiling. The precipitate is white, and gelntinous in a high degree, and retains the salts, in the presence of which it has been formed, with remarkable pertinacity, so that it is very difficult to wash.

By drying and igniting this hydrate, the anhydrous alumins is produced; but it may be obtained more readily by heating ammonia-alum $\left(\mathrm{NH}^{4} \mathrm{O}, \mathrm{SO}^{3} ; \mathrm{Al}^{2} \mathrm{O}^{3} 3 \mathrm{SO}^{3}+\right.$ 24HO). All the constituents of this salt are volatile, with the exception of the alumina.

Alumina. is insoluble in. water, but soluble both in acids and alkalis. Towards the former it plays the part of a.base, producing the ordinary alumina salts; whilst, with the latter, it also enters into combination, but in this case it is an acid, forming a series of compounds which may be callod aluminates.

The important application of alumina and its compounds in the arts of dyeing and calico-printing depends upon a peculiar attraction which it possesses for organic bodies. This affinity. is so strong, that when digested in solutions of vegetablo colouring matters the alumina combines with and carries down the colouring matter, removing it entirely from the solution. Pigments thus obtained, which are combinations of alumina with the vegetable colouring matters, aro called 'lakes.'

Alumina has not only an affinity for the colouring matters, but at the same time also for the vegetable fibres, cotton, silk, wool, \&c.; and hence, if alumina be precipitated upon cloth in the presence of a colouring matter, a most jintimate union is effected between the cloth and the colour. Alumina, whę employed in this way, is called a 'mordint.'

Other bodies have a similar attraction for colouring mattere, e.g. binoxide of tin and sesquioxide of iron: each of these gives its peculiar shade to the çolour or combination, alumina changing it least.

Mr. Walter Crum' has discovered a peculiar soluble modification of alumina. The binacetate of alumina has been found by Mr. Crum to possess tho very curious property

- Obemical Bociety's Quarterly Journal, vi. 216. 
of parting with its acetic acid until the whole is expelled, by the long-continued application of heat to $a$ solution of this salt; the alumina remains in the solution, in a soluble allotropic condition, forming what has boen called metalumina. Its coagulum with dyewoods is translucent, and entirely different from the opaque cakes formed by ordinary alumins; hence this solution cannot act as a mordant. But this solution of alumina, which is perfoctly colourless and transparent, has the alumina separated from it by the slightest causes. A minute quantity of either an acid, an alkali, even of a neutral salt, or of a vegetable colouring matter, effects the change. The precipitated alumina is insoluble in acids, even boiling sulphuric; this shows another allotropic condition. But it is dissolved by caustic alkalis, by which it is restored to its common state.

AIUMIXA, ACzTATz Or. The acotates of alumina aro extensively used in the arts on account of the property which they possess of being readily decomposed with deposition of their alumina on the fibre of eloth; hence they are used as mordants, in the mannor deseribed under Calrco-Printing; and sometimes in dyeing they are mixed with the solution of a colouring matter; in this the textile fabrie is immersed, whilst, on heating, the alumina is precipitated upon the fabric, which, in consequence of its affinities beforo alluded to, carries down the colouring mattor with it, and fixes it on the cloth.

The acetate of alumina thus employed is obtained by treating sulphate of alumina with neutral acetate of lead, and filtering off the solution from the precipitato of sulphate of lead. Acetate of lime is also used; but the sulphate in this case does not leave the solution so cloar or so rapidly.

According to Mr. Walter Crum, ' the solution resulting from the decomposition of sulphate of alumina $\mathrm{Al}^{2} \mathrm{O}^{2}, 3 \mathrm{SO}^{\prime}\left(\mathbf{A l}^{2} \mathbf{3 8 0 ^ { 4 } )}\right.$ by monobasic acetate of lead contains the salt $\mathrm{Al}^{2} \mathrm{O}^{2}, 2\left(\mathrm{C}^{4} \mathrm{H}^{3} \mathrm{O}^{3}\right.$. $\left.\mathrm{HO}\right)\left[\mathrm{Al}^{2} \mathrm{O}^{2} .4 \mathrm{C}^{2} \mathrm{E}^{4} \mathrm{O}^{2}\right]$ (binacetate of alumina), together with one equivalent of free acetic acid, the compound $\mathrm{Al}^{2} \mathrm{O}^{3}, 3 \mathrm{C}^{4} \mathrm{H}^{3} \mathrm{O}^{3}\left(\mathbf{A l}^{2} \mathbf{C C}^{2} \mathbf{H}^{3} \mathrm{O}^{2}\right)$ not appearing to exist. By evaporating this solution at low temperatures, e.g. in a very thin layer of fluid below $38^{\circ} \mathrm{C}$. $\left(100^{\circ} \mathrm{F}\right.$.), Crum obtained a fixed residuo completely soluble in water, the composition of which, in the dry state, approached $\mathrm{Al}^{2} \mathrm{O}^{3}, 2 \mathrm{C}^{4} \mathrm{H}^{2} \mathrm{O}^{2}+4 \mathrm{HO}\left(\mathbf{A l}^{2} \mathrm{O}^{3} . \mathbf{C}^{2} \mathbf{B}^{4} \mathbf{O}^{3}+\mathbf{2} \mathrm{H}^{2} \mathrm{O}\right)$

AIUMmMA, SIIICATHS Or. Silicate of alumina is the chief constituent of common clay; it occurs also associated with the silicates of iron, magnesia, lime, and the alkalis in a great variety of minerals, which will be found described elsewhero. The most interesting of these are the felspars and the zeolites. Sec ClaY, Frzspar, ZEOLITS.

Of course, being present in clay, silicate of alumina is the essential constituent of porcelain and earthenware. See Eirthrarware and Porcetals.

AIUMTrA, SUIPHATE Or. The neutral sulphate of alumina, $\mathrm{Al}^{2} \mathrm{O}$ ?,

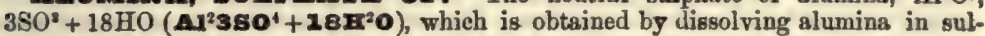
phuric acid, crystallises in noedles and plates; but sulphuric acid and alumina combine in other proportions, e.g. a salt of the formula $\mathrm{Al}^{2} \mathrm{O}^{3}, 3 \mathrm{SO}^{3}+\mathrm{Al}^{2} \mathrm{O}^{3}\left(\mathbf{A l}^{2} \mathbf{3 s O}^{4}+\mathbf{A l}^{2} \mathrm{O}^{2}\right.$ ) was obtained by Mons, and the solution of this salt, when largely diluted with water, splits into the neutral sulphate and an insoluble powder containing $\mathrm{Al}^{2} \mathrm{O}^{2}, 3 \mathrm{SO}^{2}$ $+2 A I^{2} O^{3}+9 H O\left(A^{2} 35 O^{2}+2 \mathbf{A l}^{2} O^{3}+9 \mathbf{H}^{2} O\right)$. This subsalt forms the mineral aluminite, or Websterite, found near Newhaven, and was found by Humboldt in the schists of the Andes.

The sulphate of alumina is now extensively used in the arts instead of alum, under the name of ' concentrated alum' (see p. 122). For most of the purposes for which alum is employed, the sulphate of potash is an unnecessary constituent, being only added in order to facilitate the purification of the compound from iron; for in consequence of the ready crystallisability of alum, this salt is easily purified. Nevertheless, Wiesmann has succeeded in removing the iron from the crude solution of sulphate of alumina obtained by treating clay with sulphuric acid, by adding ferrocyanide of potassium, which throws down the iron as Prussian-blue; the solution, when evaporated to drynese, is found to consist of sulphate of alumina, containing about 7 per cent. of potash-alum. About 1750 tons of this article were produced at Newcastle-onType alone in the year 1872. See also Arvar.

: ATUMINAT2 OP SODA. $3 \mathrm{NaO} \cdot \mathrm{Al}^{2} \mathrm{O}^{3}\left(3 \mathrm{Na}^{2} \mathrm{O}, \mathbf{A l}^{2} \mathrm{O}^{2}\right)$. This salt is how manufactured on a large scale for use as a mordant in dyeing and calico-printing; in the preparation of lakes for pigments; in the manufacture of certain forms of artificisl stone; and in candle and soap manufacture. The salt is generally obtained by heating Bauxite with carbonato of soda in a reverberatory furnace. The alumina present in the Bauxite combines with the soda, while carbonic acid is expelled. On lixiviating the product, the aluminate of soda is dissolved out; the 
solution is then filtered, and the flltrato ovaporated to dryness. The salt is thus obtained as a whitish powder. See Bauxrrs.

AI UMTNITZ. See WEBsTrRTTR.

ArUmrurum. (Sym. Al., at. wt. 13.7.) The name Aluminium is derived from the Latin alumen, for alum, of which salt this metal is the notable constituent.

Aluminium, though never found in the free state, occurs extensively diffused in nature in alumina and certain of its salts, especially the silicates.

The native varietios of anhydrous alumina are, the sapphire, ruby, and corundum, whilst the hydrate occurs in nature in the minersls, diaspore a:d gibbsito. But the chief quantity of aluminium is found in the endless varieties of the mineral silicates of alumina with other bases, such as the felspars, micas, many kinds of clay, the zeolites, \&e.

Alumina was first decomposed by Davy, who discovered the metal soon after decomposing the other earths and alkalis; but he never seems to have obtained it without some mixture of potassium. It is evident, however, that the earth was completely reduced to the metallic state by him.

Wöhler obtained aluminium pure in $1827^{\prime}$ by the reduction of the chloride of aluminium in the form of a grey powder. Later $(1845), 2$ he succeeded by the same process in obtaining it in globules, which he describes as tin whito, tolerably malleable and ductile, not materially oxidised by exposure to the sir, of a specific gravity of $2 \cdot 5$, but, when hammered, of $2 \cdot 67$; unacted upon by water at the common temperature, but slowly disengaging hydrogen from water at the boiling point.

In $1854^{3}$ Deville's first experiments on the preparation and properties of aluminium were published. The method he adopted for the liberation of the metal was essentially the same as that originally employed by Wöhler. But, by dint of improvements in the details of the process, he succeeded in procuring the metal in Iarger globules, which were silver-white, having a fusing point nearly approaching that of silver, which were unoxidised when exposed to the air, even in a fused state, and remaining bright even in boiling water, unattacked by either dilute or concentrated nitric or sulphuric acid in the cold; but dissolved by hydrochloric acid with evolution of hydrogen.

Oersted was undoubtedly the first to prepare the ehloride of aluminium, ${ }^{4}$ and it is even stated that he also procured the metal by the following method:- 'Pure alumina, intimately mixed with powdered charcoal, was introduced into a porcelain tube; through this, when strongly heated, a stream of chlorine was directed, and the chloride of aluminium formed was collected in a separate vessel. By mixing this compound with an amalgam of potassium, containing a large proportion of the latter body, and immediately heating the mixture, chloride of potassium was found, and the aluminium combined with the mereury. This, on being distilled out of contact with the air, gave off the mercury, whilst aluminium remained in the form of a metallie button, closely resembling tin." "s

Deville's researches raised the hope that the metal might be cbtained in sufficient quantity to become of high technical importance, since it was probable that the chlorido of aluminium might be decomposed by cheaper metals at a higher temperature; and he obtained a grant from the late Emperor of the Fronch for the purpose of prosecuting his investigations on a sufficient scale.

Bunsen also showed in $1854^{\circ}$ that aluminium could be obtained in reguline masses by submitting the double chloride of aluminium and sodium or potassium to electrolysis: in a fused state.

By fusing the chloride of aluminium (obtained by the process which will be found deseribed under the head of the chloride) with an equal equivalent of common salt, he obtained a double chloride, which fused below $200^{\circ} \mathrm{C}$. (360 F.), and from which the metal is readily reduced by the same electrolytic process previously employed by Bunsen in the case of magnesium. Seo Magnesiuxr.

Bunsen. pointed out that the discrepancy existing in the properties of the metal in the two states, as obtained respectively by Wöhler and Deville, arose from its physical condition; for Bunsen found that it was only the massive metal which possessed the properties ascribed to it by Deville, that in fact the pulverulent modification does decompose water at $100^{\circ} \mathrm{C}$., as stated by Wöhler.

Almost at the same time Devillo published the results of his experiments upon the production of aluminium on a larger scale.? He quite gave up the hope of succoeding

Poggendorrt's Annalen, xl. 146. Ann, Ch, et. Pharm, lill, 422.

- Ferussal, Bul. des. Sc. Mathemat. \&c. 1826, 275:

- Record of MLining aud Metallurgy, Phillips and Darlington.

- Pogg. Ann, xoll, 618, "Comptes Rendus, xuxix. 321. 


\section{ALUMTNIUM}

in effecting the reduction of the chlorides by any of the common metals. Ho adhered to Wöhler's and Bunsen's methods, carrying them out on a largor scale, with modifications and improvements in the details, which onabled him to obtain the metal in such quantities, and thus to study its properties with so much success, as to suggest numerous applications, the probability of which never entered the minds of the original discoverers. Very great eredit is therefore due to M. Devilio, although it is the practice amongst the German chemists to detract from, or even deny, his merit.

The following is the method described by M. Devillo for the preparation of this interesting metal:- -

Having obtained the chloride of aluminium, he introduces into a wide glass (or porcelain) tube 200 or 300 grammes of this salt betwoen two plugs of asbestos (or in a boat of porcelain or even copper), allows a current of hydrogen to pass from the generator through a desiccating bottle containing sulphuric acid and tubes containing chloride of calcium, and finally through the tube containing the chloride; at the same time applying a gentle heat to the chloride, to drive off any free hydrochloric acid which might be formed by the action of the air upon it. He now introduces at the other extremity of the tube a porcelain boat containing sodium; and when the sodium is fused the chloride of aluminium is heated, until its vapour comes in contact with the fused sodium. A powerful reaction ensues, considerable heat is evolved, and by continuing to pass the vapour of the chloride over the sodium until the latter is all consumed, a mass is obtained in the boat of the double chloride of aluminium and sodium $\mathrm{NaCl}, \mathrm{Al}^{2} \mathrm{Cl}^{3}\left(\mathbf{2 N a C l} . \mathbf{A l}^{2} \mathbf{C l}^{\mathrm{s}}\right)$, in which globules of the newly-reduced motal are suspended. It is allowed to cool in the hydrogen, and then the mass is treated with water, in which the double chloride is soluble, the globules of metal boing unacted upon.

These small globules are finally fused together in a porcelain crucible, by heating them strongly under the fused double chloride of aluminium and sodium, or even under common salt.

This process, which succeeds without much difficulty on a small scale, is performod far more successfully as a manufacturing operation. Two cast-iron cylinders are now employed instead of the glass or porcelain tube, the anterior one of which contains the chloride of aluminium, whilst in the posterior one is placed the sodium in a tray, about 10 lbs. being omployed in a single operation. A smaller iron cylinder intsrmediate between the two former is filled with seraps of iron, which serve to separate iron from the vapour of chloride of aluminium, by converting the perchloride of iron into the much less volatile protochloride. Thoy also separate free hydrochloric acid and chloride of sulphur.

During the progress of the operation the connecting tube is kept at a temperature of about $400^{\circ}$ to $600^{\circ} \mathrm{F}$; but both the cylinders are but very gently heated, since the chloride of aluminium is volatile at a comparatively low temperature, and the reaction between it and the sodium when once commenced generatos so much heat that frequently no external aid is required.

Preparation of Aluminium by Electrolysis.-Mr. Gore has succeeded in obtaining plates of copper coated with aluminium by the electrolysis of solutions of chloride of aluminium, acetate of alumina, and even common alum; ${ }^{1}$ but the unalloyed metal cannot be obtained by the electrolysis of solutions. Deville, however, produced it in considerable quantities by the method originally suggested by Bunsen, viz. by the electrolysis of the fused double chloride of aluminium and sodium $\mathrm{Na} \mathrm{Cl}_{4} \mathrm{Al}^{2} \mathrm{Cl}^{3}$ $\left(\mathbf{2 v a C l} . \mathbf{A l}^{2} \mathbf{C l}^{\circ}\right)$; but since this process is far more troublesome and expensive than its reduction by sodium, it has beon altogether superseded.

Preparation of Aluminium from Cryolite. So early as March 30, 1855, a specimen of aluminium was exhibited at one of the Friday-evening meetings of the Royal Institution, which had been obtained in Dr. Percy's laboratory by Mr. Allan Dick, by a process entirely different from that of Deville, which promised, on account of its great simplicity, to supersede all others. ${ }^{2}$ It consisted in heating small pieces of sodium, placed in alternate layers with powdered cryolite, a mineral now found in considerable abundance in Greenland, which is a double fluoride of aluminium and sodium, analogous to the double chloride of aluminium and sodium, jts formula being $3 \mathrm{Na} \mathrm{F}$, $\mathrm{Al}^{2} \mathrm{~F}^{3}$ (62ra $\left.\mathbf{F} \cdot \mathbf{A l}^{2} \mathbf{F}^{6}\right)$. The process has the advantage that one of the materials is furnished ready formed by nature.

The experiment was only performed on a small scale by Mr. Dick in a platinum crucible lined with magnesia ; the small globules of metal, which were obtained at the bottom of the mass of fused salt, being subsequently fused together under chloride of potassium or common salt.

Before the description of these experiments was published, M. Rose, of Berlin, 
-published a papor in September, 1855, on the same subject.' In Rose's experiments he employed cast-iron crucibles, in which was heated ten parts of a mixture of equal weights of cryolite and chloride of potassium with 2 parts of sodium. The aluminium was obtained in small globules, which were fused together under chloride of potassium, as in Mr. Dick's exporiments.

Rose experiencod a slight loss of aluminium by fusion under chloride of potassium, and found it more advantageous to perform this fusion under a stratum of the double chloride of aluminium and sodium, as Doville had done,

He never succeeded in extracting the whole quantity of aluminium present in the cryolite (13 per cent.), chiefly on account of the ready oxidisability of the metal when existing in a very finely divided state, as some of it invariably does.

It does not appear that any attempt has since been made to obtain aluminium on the large scale from cryolite, probably from the supply of the mineral not proving so abundant as was at one time anticipated.

In all the processes which have been found practicable on any considerable scale, for the manufacture of aluminium, the powerful affinities of sodium are employed for the purpose of eliminating it from its compounds. The problem of the diminution of the price of aluminium therefore resolves itself into the improvement of the methods for procuring sodium, so as to diminish the cost of the latter metal. M. Devillo's attention was therefore directed, in the early steps of the inquiry, to this point; and very considerable improvements have been made by him, which will be found fully. described under the head of Soprur..

Deville ${ }^{3}$ has since suggested the employment at once of the double salt of chloride of aluminium and chloride of sodium ( $\left.\mathrm{NaCl}, \mathrm{Al}^{2} \mathrm{Cl}^{3}\right)$, instead of the simple chloride of aluminium, so as to obtain the metal by means of sodium. Ho uses 400 parts of this double salt, 200 of common salt, 200 of fluor spar, and 75 to 80 of socium. The above-mentioned salts are dried, powdered, and mixed together; then with these the sodium, in small pieces, is mixed, and the whole heated in a crucible under a layer of common salt. After the reaction is complete, the heat is raised so as to promote the separation of the aluminium in the form of a button. It was found, however, that eryolite was, with advantage, substituted for the fluor spar.

C. Brunner ${ }^{3}$ employs artificially propared fluoride of aluminium; but this method cannot offer any advantage over the employment of the chloride, which is cheaper, or the cryolite, which nature affords.

The following remarks on the manufacture of aluminium are from the pen of Mr. Isaac Lowthian Bell of Newcastle, at whose works at Washington this metal was, until lately, produced:-

- Upon the introduction of its manufacture at Washington, the source of the alumina was the ordinary ammonia-alum of commerce - a nearly pure sulphate of alumina and ammonia. Exposure to heat drove off the water, sulphuric acid, and ammonia, leaving the alumina behind. This was converted into the double chloride of aluminium and sodium, by the process described by the French chemist, and practised in France, and the double chloride was subsequently deeomposed by fusion with sodium. Faint, however, as the traces might be of impurity in the alum itself, they to a great extent, if not entirely (being of a fixed character when exposed to heat), were to be found in the alumina. From the alumina, by the action of chlorine on a heated mixture consisting of this earth, common salt, and charcoal, these impurities, or a large proportion thereof, found their way into the sublimed double chloride; and once there, it is unnecessary to say that, under the influence of the sodium in the process of reduction, any silica, iron, or phosphorus found their way into the aluminium sought to be obtained. Now, it happens that the presence of foreign matters, in a degree so small as almost to be infinitesimal, interferes so largely with the colour as well as with the malleability of the aluminium, that the use of any substance containing them is of a fatal character. Nor is this all, for the nature of that compound which hitherto has constituted the most important application of this metal -aluminium-bronze -is so completely changed by using aluminium containing the impurities referred to, that it ceases to possess any of those properties which render it valuable. As an example of the amount of interference exercised by very minute quantities of impurity, it is perhaps worthy of notice that very fow varieties of copper have boen found susceptible of being employed for the manu. facturo of aluminium-bronze; and hitherto wo have not at Washington, nor have they in France, been able to establish in what the difference consists between copper fit for the production of aluminium-bronze, and that which is usually unsuitable for the purpose. These considerations have led us, both hero and in France, to adopt tho use of another raw material for the production of aluminium, which either does

- Poggendorrt' Annalen, and Phil. Mag. x. 283. Chemical Gasette, $1856,838$.

Ann. de Chim, et Phys, xlvi. 415. 
not contain the impurities roferrod to as so projudicial, or contains them in such a form as to admit of their easy soparation. This matcrial is Baurite, so called from the name of the locality where it is found in France. The Bauxite is ground and mixed with the ordinary soda-ash of commerce, and then heated in a furnaco. The soda combines with the alumina, and the aluminate of soda so formed is soparated from the insoluble portions-viz., peroxide of iron, silico-aluminato of soda, \&c. - by lixiviation. Muriatic acid or carbonic acid is then added to the solution, which throws down pure alumina. The remainder of the process is precisely that which is described by Mons. St. Claire Deville. The alumina is mixed with common salt and charcoal, made into balls the size of an orange, and dried. These balls are placed in vertical earthen retorts, kept at a red heat, and through the heated contents chlorino gas is passed. The elements of the earth, under the joint influence of carbon and chlorine at that temperature, are separated, the carbon taking the oxygen and the chlorine the aluminium. The latter substance accompanied by chloride of sodium (common salt) sublimes over, and is collected as a double chlorido of aluminium and sodium. Sodium,- - being required to effect the decomposition of this compound, - is thus prepared. In small iron retorts kept at as high a temperature as iron can bear, a mixture of soda (carbonate of soda) and carbonaceous matter with a little ground chalk is placed. The metallic base of the alkali distils over, and is collected in coal oil. A portion of the double chloride of aluminium and sodium, and the metallic sodium, along with fluxes, is exposed to a full red heat in a reverberating furnace. The sodium seizes the chlorine combined with the aluminium, and thus liberates the latter metal, which falls to the bottom of the fixed mass. Aluminium is used in sufficiont quantity to keop the only work in England-viz., that at Washingtorpretty actively omployed. As a substanco for works of art, whon whitened by means of hydrofluoric and phosphoric acid, it appears well adapted, as it runs into the most complicated patterns, and has the advantage of preserving its colour, from the absence of all tendency to unite with sulphur, or to become affected by sulphuretted hydrogen, as happons with silver.' Aluminium has been used in the manufacture of woights and scale-beams for chemists, and for opera- and field-glasses, for which purposes it is exceedingly valuable, by reason of its lightness and its not being liable to tarnish. A greatly increased activity has been given to the manufacture of aluminium by its use in the manufacture of alumininm-bronze-a compound of exceoding beauty, so much like gold that it can scarcely be distinguished from that precious motal.

Properties. - The metal is white, but with a bluish tinge, and even when pure has a lustre far inferior to silver.

Specific gravity, $2 \cdot 56$, and when hammered, $2 \cdot 67$.

Conducts electricity eight times better than iron, and is feebly magnetic. It is highly sonorous.

Its fusing-point is between the melting-points of zinc and silver.

By electrolysis it is obtained in forms which Deville belioves to be regular octahedra; but Rose, who has also occasionally obtained aluminium in a crystalline state (from cryolite), denies that they belong to the regular system.

When pure, it is unoxidised even in moist air; but most of the commercial specimens (probably from impurities present in the metal) become covered with a bluishgrey tarnish.

It is unaffected by cold or boiling water; oven stoam at a rod heat is but slowly decomposed by it.

It is not acted upon by cold nitric acid, and only very slowly dissolved even by the boiling acid; scarcoly attacked by dilute sulphuric acid, but readily dissolved by hydrochloric acid, with evolution of hydrogen.

Sulphuretted hydrogen and sulphides havo no action upon it; and it is not oven attacked by fused hydrated alkalis. Professor Wheatstone' ${ }^{\prime}$ has shown that in the voltaic series, aluminium, although having so small an atomic numbor, and so low a specific gravity, is moro electro-negative than zinc; but it is positive to cadmium, tin, lead, iron, copper, and platinum.

Impurities in Aluminium.-Many of the diserepancies in tho properties of aluminium, as obtained by different experimenters, are due to the impurities which are present in it.

If the naphtha be not carefully removed from the sodium, the aluminium is liable to contain carbon.

Frequently, in preparing aluminium, by the action of the chloride on sodium, by Devillo's original process, copper boats havo been used for holding the sodium; in this case the metal becomes contaminated, not only with copper, but also with any 
other metals which may bo prosent in the copper-e.g. Salm-Horstmar' found copper in the aluminium sold in Paris, and Erdmann dotected zine; ${ }^{2}$ and in every caso the metal is very liable to become mixed with silicon, either from the earthonware tubes, boats, or crucibles; hence Salvétat found, even in the aluminium prepared by Deville himself, 2.87 per cent. of silicon, 2.40 of iron, 6.38 of copper, and traces of lead.

The following analyses of commercial aluminium were communicated to the British Association, at its meoting in 1857 , by Profossor Mallet :-

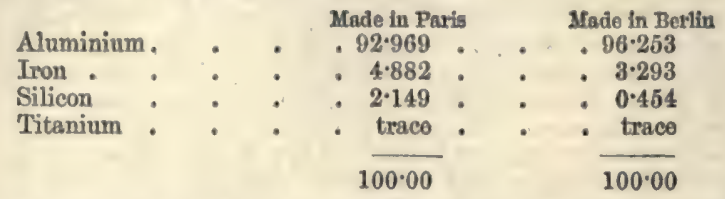

Alloys of Aluminium. - Very small quantities of other metals suffice to destroy the malleability and ductility of aluminium. An alloy containing only $\frac{1}{20}$ th of iron or copper cannot be worked, and the presence of $\frac{1}{10}$ th copper renders it as brittle as glass. Silver and gold produce brittleness in a less dogree. An alloy of 5 parts of silver with 100 of aluminium is capable of being worked like the pure metal, but it is harder, and therefore susceptible of a finer polish; whilst the alloy, containing 10 per cent, of gold, is softer, but, nevertheless, not so malleable as the pure motal. The presence of even $\frac{1}{1000}$ th part of bismuth renders aluminium brittle in a high degree.

These statements by Tissier, ${ }^{3}$ however, require confirmation; for Debray states that aluminium remains malleable and tough when containing as much as 8 per cont. of iron, or 10 per cent. of copper, but that a larger quantity of either of these metals renders it brittle.

It is curious that only 3 per cent. of silver are sufficient to give aluminium the brilliance and colour of pure silver, over which the alloy has the great advantage of not being blackened by sulphuretted hydrogen.

On the other hand, small quantities of aluminium combined with other metals change their properties in a remarkable manner. Thus copper alloyed with only $\frac{1}{10}$ th of its weight of aluminium has the colour and brilliance of gold, and is still very malleable (Tissier); and when the aluminium amounts only to $\frac{1}{5}$ th (i.e. 20 per cont.) the alloy is quite white (Debray). See Aluninium Bronze.

An alloy of 90 parts of copper and 10 of aluminium is harder than common bronze, and is capable of being worked at high temperatures easier than the best varieties of iron. Larger quantities of aluminium render the metal harder and brittle.-Debray."

An alloy of 100 parts of silver with 5 of aluminium is a's hard as the alloy employed in the silver coinage, although the other properties of the silver remain unchanged (Tissier). Similar alloys have likewise been prepared by Dr. Percy."

Messrs Calvert and Johnson describe ${ }^{6}$ an alloy of 25 parts aluminium to 75 of iron, which has the valuable property of not oxidising by exposure to moist air.

Uses of Aluminium.-No very important application of aluminium has yet been made: although, at the time M. Deville's experiments were commenced, sanguine hopes were entertained that aluminium might be produced at a price sufficiently low to admit of its practical application on a large scale, these anticipations have not been realised; and as yet, on account chiefly of its high price, the applications which have been made of this interesting metal are but few.

Its low specific gravity, combined with sufficient tenacity, recommends it for many interesting uses. The fractional weights used by chemists, which are made of platinum, are so extromely small that they are constantly being lost; their much greater volume in aluminium renders this metal peculiarly suitable. In the construction of the beams of balances, strength combined with lightness are desiderata; and M. Deville has had very beautiful balance-beams made of this motal; but at present its high price has prevented their extensive adoption.

These same qualities render this metal suitable for the construction of holmets and other armour; but at present these are but curiosities, and are likely to remain so,

1 Journal pr. Chem. Ixvil. 493.

C. and J. Tissier, Comptes Rendus, xilit. 885.

- Proceedings of the Royal Institution, March 14, 1856.
- Journal pr. Chem. Ixvil. 494. - Comptes Rendus, xliii. 925. 
unless some cheaper method of eliminating the metal than by the agency of sodium be discovered.

Mr. Gordon has shown that the amount of tension which aluminium wire was capable of resisting will be found to be between that of the best iron and the best steel wire.

Probably one of the most interesting of the applications of aluminium (at least in a scientific point of view) that has been made, is that by Deville and Wöhler, of employing it in the production of erystalline allotropic modifications of certain other elements previously unknown in that state-e.g. boron, silicon, and titanium. It deponds upon the fact that these elements, in the amorphous state, dissolve in fused aluminium, and on cooling the molten solution, thoy slowly separate from the aluminium in the crystalline state.

Aluminium may be gilded by being dipped into a solution of the hyposulphite of gold after it has been well cleaned by the successive use of potash, nitric acid, and water. Aluminium is soldered with difficulty. The most successful method is to coat the aluminium with copper by the electrotype process, after which soldering can be effected in the usual way.

ArUMrnIUM-Bronzz. This alloy was a discovery of Dr. John Percy, F.R.S., and it appears to be a true chemical compound. Mr. I. I. Bell, who manufactured this beautiful metallic compound on a large scale, thus describes its manufacture:- Copper is melted in a plumbago crucible, and, after being removed from the furnace, the solid aluminium is added. The union of the two metals is attended with such an increase of temperature, that the whole becomes white hot, and uniess the crucible containing the mixture is of a refractory material, it is fused by the intensity of the heat. A vessel which has resisted a heat sufficient to effect the fusion of copper has been melted when the aluminium has been added.'

The value of aluminium-bronze will be gathered from the following statement of results obtained by Lieut.-Colonel A. Strange, F.R.A.S., and communicated by him to the Astronomical Society. Lieut.-Colonel Strange recommended this metal as a valuable material for the construction of astronomical and other philosophical instruments. Regarding the most important qualities as-1. Tensile strength; 2. Resistance to compression; 3. Malleability; 4. Transverse strength or rigidity; 5. Expansive ratio; 6. Founding qualities; 7. Behaviour under files, cutting tools, \&c,; 8. Resistance to atmospheric influences; 9. Fitness to receive graduation; 10. Flasticity ; 11. Fitness for being made into tubes; 12. Specific gravity-experiments were made to determine each of those coriditions.

Tensile strength. - The result of trials by Mr. Anderson of Woolwich was- the average tenacity of this metal proved to be 22 tons $12 \mathrm{cwt}$. (50,624 lbs.) breaking weight per square inch. Elongations did not take place until 4,300 lbs. in the one case, and $3,600 \mathrm{lbs}$. in the other had been applied, when a permanent elongation was noticed of 009 of an inch in the first specimen and 034 of an inch in the last.

Resistance to compression.-The ultimate amount of compression applied was 59 tons 2 cwt. 1 qr. 4 lbs. $(132,416$ lbs.), under which the specimen became much distorted. "The specimen subjected to this enormous pressure, distorted though it is, does not exhibit the trace of a fissure. The cohesion of its particles is inviolate.' Compression was not perceptible until 9 tons 2 cwt. per square inch $(20,384$ lbs.) was applied, when the specimen gave way to the extent of 006 of an inch; and on the weight being removed an elasticity of $\cdot 001$ was observed, which gives the first permanent compression as 005 of an inch.

Malleability. - The quality of this metal for forging purposes would appear to be excellent. There were specimens in the International Exhibition of 1862, showing that the alloy could be drawn out under the hammor almost to a needle-point.

Transverse strength. - These experiments were made by Messrs. Simms. The same weight applied to these bars altered the index of the instrument as under :- -

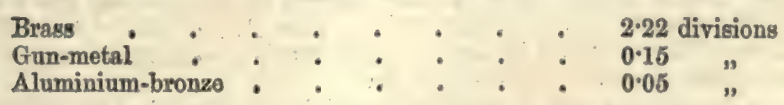

Hence aluminium-bronte is 3 times more rigid than gun-metal, and 44 times more rigid than brass.

Expansive ratio.-Aluminium-bronze is affected by change of temperature a little less than gun-motal, and much less than brass.

Founding qualities. - The alloy produces admirable castings of any size.

Behaviour under files, cutting tools, \&c. - In this rospect it leaves nothing to bo 
desired. It does not clog the file; and in the lathe and planing-machino the tool removes long elastic sharings, loaving a fine, bright, smooth surface.'

Resistance to atmospheric influcnces. - This alloy tarnishes much less readily than any motal usually omployod-riz. gun-motal, brass, silver, cast-iron, or stocl.

Fitness to receive graduation. - The lines aro remarkably puro and equable, and very distinct under the microscope, notwithstanding the yellow colour of the motal.

Elasticity.-No wires tried for the suspension of Foucault's pendulum for jllustrating the rotation of the earth were so durable - not oven those of steel-under that severe ordeal, as wires of aluminium-bronzo.

Fitness for being made into tubes.-It admits of every procoss necessary for this purpose.

Spocific Gravity.-The specific gravities of alloys of aluminium and copper aro:-

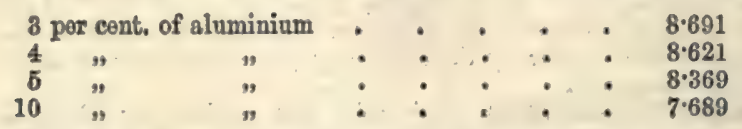

At the Elswick Works Captain Noble, R.A., confirmed previous experiments on the capability of aluminium-bronze to resist longitudinal and transverse fracture; and in addition to this he ascertained that its position to withstand compression stood halfway between that of the finest steel and the best iron.

The bronze, containing 10 parts of aluminium and 90 of copper, affords an alloy endowed with the greatest strength, malleability, and ductility. 'The colour of the copper is affected by a very trifling addition of the other constituent, and the alloy gradually improves in theso valuable qualities just mentionod, until the proportions given above are reached. After this-i.e. when more than 10 per cent. of aluminium enters into the composition of the bronze-tho alloy gradually becomes weaker and less malleable, and at length so brittle that it is easily pounded in a mortar.

AIUMINTUM, CETORIDE Or. $\mathrm{Al}^{2} \mathrm{Cl}^{8}=133^{\circ} 9\left(\mathbf{A l}^{2} \mathbf{C l}^{8}\right)$. Preparation.Chloride of aluminium cannot be prepared by treating alumina with hydrochloric acid, as in the case of most chlorides; for on evaporating the solution to dryness, hydrochloric acid is ovolved and alumina alone remains.

The method at present used is, in principle, the same as that originally suggested by Oersted, which has since found numerous other applications. It is impossiblo to convert alumina into the chloride by the direct action of chlorine alone; at any temperature the chlorine is as incapable of displacing the oxygen from the alumina as it would from lime. But if the attraction of the chlorine for the metal be supported by the affinity of carbon for the oxygen, then the compound is, as it were, torn asundercarbonic acid or carbonic oxide resulting on the one hand, and the chloride of aluminium on the other.

On the large seale the chlorine is passed over a previously ignited mixture of clay and coal-tar, contained in retorts like those used in the manufacture of conl-gas, which are heated in a furnace; the chloride, which on account of its volatility is carried off, being condensed in a chamber lined with plates of earthenware, where it is deposited in a crystalline mass.

Properties. - It is a yellowish crystalline solid, readily decomposed by the moisture of the air into hydrochloric acid and alumina, and volatile at a dull red heat. It is very soluble in water, but cannot be recovered by evaporating the solution.-H.M.W.

Chloride of aluminium has been brought into use as a disinfectant under the name of Chloralum.

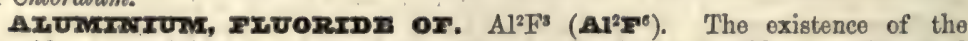
fluoride of aluminium in nature, in the form of the double fluoride of sodium and aluminium, namely, $3 \mathrm{Na} F, \mathrm{Al}^{2} \mathrm{~F}^{3}\left(\boldsymbol{\sigma} \mathbf{N} \mathbf{a r}, \mathbf{A l}^{2} \mathbf{T}^{0}\right)$, as cryolite, and the use of this mineral in the manufacture of aluminium, have been already alluded to. The fluorido of aluminium likewise exists in several other minerals, namely, the topar, pyenite, fluellite, chiolite, pachnolite, gearksutite, \&re.

The pure fluoride can only be obtained artificially by dissolving pure aluminium in hydrofluoric acid. It has a great tendency to form double salts with the fluorides of potassium and sodium.-H. M. W.

ArUmocaxcrTz. A milk-white mineral, from Fibenstock, in Saxony. It appears to be an impure form of opal, containing-in addition to the hydrous silicaa notable proportion of lime and alumina.

azva or Axra. See Espatio Grass.

Axvrrz, A Norwegian mineral, described by Mr. David Forbes and Mr. T. 
Dahll. It is a hydrous silicate of complex constitution, containing yttria, thoria, zirconis, glucina, alumine, and sesquioxide of iron.

AMADOU. (Amadou, Fr.; Zunderschwamm, Ger.) The namo of a spongy com. bustible substance, prepared from a species of fungus, the Boletus igniarius, which grows on the trunks of cherry-trees, sshes, boeches, \&ce.; it is sometimes known as spunk, and as touchwood, but commonly in this country it is called German tinder. It must be plucked in the months of August and September. This plant grows horizontally on the several trees on which it is indigenous; when it makes its first appearance it is a little round wart-like body, not larger than a pea; it gradually increases in size and hardness till it becomes of a darkish brown, and is as large as an apple. It afterwards takes a horizontal direction, forms a border, and bocomes covered with numerous closely-packed tubes on its under surface. The Boletus fomentarius is another indigenous fungus, found on tho oak and birch. This is also used in preparing Amadou. It was formerly used in surgery, and has hence been called Surgeon's Agaric. Amadou is propared by removing the outer bark with a knife, and separating carefully the spongy substance- of a yollow brown colour, which lies within it-from the ligneous matter below. This substance is cut into thin slices, and beaten with a mallet to soften it, till it can be easily pulled asunder between the fingers. In this state the boletus is a valuable substance for stopping oozing hremorrhages, and some other surgical purposes. To convert it into tinder it must receive a finishing preparation, which consists in boiling it in a strong solution of nitre, drying it, beating it anew, and putting it a second time into the solution. Sometimes, indeed, to render it very inflammable, it is imbued with gunpowder, whence the distinction of black and brown amadou.

All the puff-balls of the lycopodium genus of plants, which have a fleshy or flamentous structure, yield a tinder by soaking in gunpowder-water. The Hindoos employ a leguminous plant, which they call solu, for the same purpose. Its thick spongy stem, being reduced to charcoal, takes fire like amadou. See Agaric.

AMAICAM. When mercury is alloyed with any metal, the compound is called an amalgam of that metal; as, for example, an amalgam of tin, bismuth, \&c.

Some amalgams are solids and others fluids; the former aro often crystalline, and the latter may be probably regarded as the solid amalgam dissolved in mereury.

Silver Amalgam may be formed by mixing finely-divided silver with mereury. The best process is to precipitate silver from its solution by copper, when wo obtain it in a state of fine powder, and then to mix it with the mereury.

A native amalgam of mercury and silver occurs in fine crystals in the minos of Moschellandsberg, in the Palatinate : it is said to be found where the veins of copper and silver intersect each other. Its existence is also recorded in Hungary and Sweden; at Allemont, in Dauphiné ; Almaden, in Spain ; Kongsberg, in Norway ; and in Chili ; and the following analyses have been quoted :-

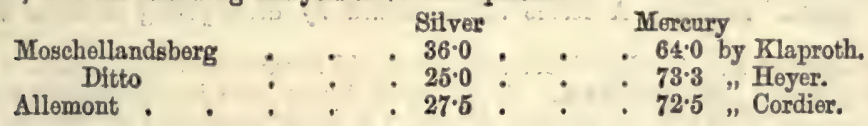

If six parts of a saturatod solution of nitrate of silver with two parts of a saturated solution of the protonitrate of mercury are mixed with an amalgam of silver one part and mercury seven, the solution is speedily filled with beautiful arborescent crystals - the Arbor Diane, the tree of Diana, - or the silver tree.

Gold Amalgam is made by heating together mercury with grains of gold, or goldfoil; when the amalgam of gold is heated, the mercury is volatilised and the gold left. This amalgam is employed in the process known as that of fire-gilding, although, since electro-gilding has been introduced, it is not so frequently omployed. A gold amalgam is obtained from the platinum region of Columbia ; and it has been reported from California, especially from near Mariposa. Schneider gives its composition, mercury, $57 \cdot 40$; gold, $38 \cdot 89$; silver, $5 \cdot 0$.

Tin Amalgam.-By bringing tinfoil and mercury together, this amalgam is formed, and is used for silvering looking-glasses. If melted tin and mercury are brought together in the proportion of three parts mercury and one part tin, the tin amalgam. is obtained in cubio crystals. See StuvernNa Grass.

Electric Machine Amalgam.-Melt equal parts of tin and eine together, and add three parts of mereury; the mass must be shaken until it is cold, and rubbed down with some lard to give it the proper consistence.

Sodium Amalgam, used for separating gold from its ores. See Gord, SruvER.

Copper Amalgam.-The French dentists have long made uso of this for stopping tooth. It is sold in small rolls of about a drachm and a half in weight; it is ooverod with a groyish tarnish, has a hardnoss much greater than that of bone, and its cohesion 
and solidity are considerable. When heatod nearly to the point of boiling water this amalgam swells up, drops of moreury oxuding, which disappoar again on tho cooling. of the substance. If a piece, thus heated, be rubbed up in a mortar, a plastic mouldable mass, like poor clay, is obtained, the consistence of which may, by continued kneading, bo incroased to that of fat elay. If the moulded mass be left for ton or twolve hours, it hardens, acquiring again its former proporties, without altering its specifie gravity. Hence the stopping, after it has hardened, remains tightly fixed in tho hollow of the tooth. The softening and hardening may be repeated many times with the same sample. Pettenkofer ascribes these phonomona to a state of amorphism, into which the amalgam passes from the crystalline condition in the process of softoning. All copper amalgams containing between 0.25 to 0.30 of copper exhibit the same behaviour. The above ehemist recommends, as the best modo of preparing this amalgam, that a crystalline paste of sulphato of suboxide of moreury (prepared by dissolving mercury in hydrated sulphuric acid at a gentlo heat) be saturated under water at a tomperature of from $60^{\circ}$ to $70^{\circ}$, with finely divided reguline copper (prepared by precipitation from sulphate of copper with iron). One portion of the coppor precipitates the mercury, with formation of sulphate of copper ; the other portion yields with mercury an amalgam : 100 parts of dissolved mereury require the copper precipitated, by iron, from 232.5 parts of sulphate of copper. As in dissolving the mercury the protoxide is easily formed instead of the suboxide, particularly if too high a tomperature be maintained, it is advisable, in order to avoid an excess of mercury in the amalgam, to take 223 parts of sulphate of copper, and to add to the washed amalgam, which is kept stirred, a quantity of mereury in minute portions, corresponding to the amount of suboxide contained in the mercury salt, until the whole has become sufficiently plastic. This amalgam may be obtained by moistening finely-divided copper with a fow drops of a solution of nitrate of suboxide of morcury, and then triturating the metal with mercury in a warmed mortar. The rubbing may be continued for some time, and may be carried on under hot water, mercury being added until the required consistence is attained.

A remarkable depression of temperature during the combination of amalgams has been observed by several chemists.

Döbereiner states that when 816 grains of amalgam of lead (404 mercury and 412 lead) were mixed, at a temperature of $68^{\circ}$, with 688 grains of the amalgam of bismuth (404 mercury and 284 bismuth) the tomperature suddenly fell to $30^{\circ}$, and by the addition of 808 grains of mercury (also at $68^{\circ}$ ), it became as low as $17^{\circ}$; the total depression amounting to $51^{\circ}$.

In certain proportions of mixture of the constituents of fusible metal (tin, lead, and bismuth) with mercury, Döbereiner formed surprising depressions of temperaturo; the temporature, he records of one experiment, sank instantly from $65^{\circ}$ to $14^{\circ}$.

AmaIgamatrow. See Gold and Sirver.

AMAIIC ACID. A feoble acid obtained by decomposing caffeine with chlorine.

AMANDINa. An albuminous compound, said to exist in eweet almonds.

AMANTTA IMUSCARIA. A poisonous fungus used in Kamtschatka and Siboria as a narcotic and intoxicating agent. The specific name has roference to its use, when stooped in milk, as a fly-poison. An organic base, called amanitine, has been separated from this fungus. By some authoritios the poisonous properties are reforred to the presence of a peculiar acid called muscaric acid.

AMarrwz, or Benzoline. $\mathrm{C}^{42} \mathbf{H}^{19} \mathrm{~N}^{2}\left(\mathbf{C}^{21} \mathbf{E}^{18} \mathbf{N}^{2}\right)$. An organic base obtained by boiling hydrobenzamide with solution of caustic potash. The hydrobenzamide is prepared by the action of ammonia on pure oil of bitter almonds.

AMAzox sTONz. A bluish-green variety of Folspar(Orthoclase). It is found at Lake Ilmen in Russia, of a verdigris-green variety. That from Baikal in Siberia is composed of silvery spangles in a green base, of which small vases and other ornaments are made. See Fraspar.

AMBAR, IIQUTD. (Ambre Liquide, Fr.) In former editions of this Dictionary, the liquid-ambar, as it was called, was confounded with liquid storax or styrax. It is obtained from the Liquidambar styraciflua of Linnæus, growing in Louisiana and Mexico, whereas the storax is procured chiofly-it is now entirely-from Trieste; storax was originally extracted from the Styrax officinalis, which grows in various parts of Greece, but this resin is lost to commerce, and the present commercial liquid storax appears to be obtained from Liquidambar orientale (Muiller). Pereira, quoting Buchner's 'Repertorium,' informs us that the storax is known in the East as buchurijag. Liquid-ambar is rarely used in any art or manufacture. It is brownish ashgrey, of the consistence of turpentine, dries up readily, smells agreeably, like benzoin, has a bitterish, sharp, burning tasto; is solublo in 4 parts of alcohol, and contains only 1.4 per cent, of benzoic acid. 
Amrnen. (Succin, Fr.; Bernstein, Ger.) The Electron of the Greoks, to whom this substance appears to have been woll known. From its peculiar property of manifesting electrical phenomena, we have derived our word electricity. It appears to have been known to the Romans under tho names of lynourium (a name also applied by Theophrastus to a gem-stone, perhaps either tourmaline or zircon), and, because of its supposed vegetable origin, succinum. The ancients also gave the name electrum to a yellow metal containing gold and silver. See Euctrum.

Amber is a mineral solid, of a yellow colour of various shades, which burns entirely away with flame, and consists of carbon, hydrogen, and oxygen, in nearly the same proportions, and in the same state of combination, as in vegetable resin. The chomical composition of amber, according to Schrötter, is-

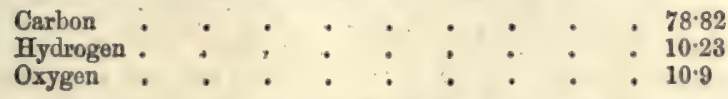

Its specific gravity varies, by Dr. Uro's trials, from 1.080 to 1.085 . It becomes negatively and powerfully electrical by friction. When applied to a lighted candle it takes fire, swells considerably, and exhales a white smoke of a pungent odour; but does not run into drops. Copal, which resembles it in several respects, differs in boing softer, and in melting into drops at the flame; and mellite, or honoy-stone, which is a mineral of a similar colour, becomes white when laid on a red-hot coal.

The texture of amber is resino-vitreous, its fracture conchoidal, and lustro glassy. It is perfectly homogeneous; sufficiently hard to scratch gypsum, and to take a fino polish. It is, however, scratched by calcareous spar. When amber is distilled in a retort, crystalline needles of succinic acid sublime into the dome, and oil of amber drops from the beak into the receiver. Fossil resins, such as that of Highgate, found in the Iondon-clay formation, do not afford succinic acid by heat; nor does copal.

It is now admitted that amber is not a simple resin. For the most part it consists of a peculiar resin, which is said to resist the action of all known solvents; and it is to this substance that Dana has applied the term succinite, as a definite mineral spocies. But in addition to this rosin-which forms from 85 to 90 per cont. of amber-there are two other resins soluble in alcohol and ether, together with the oil and succinic acid above mentioned. It would appear that several distinet resinous substances, occurring in a fossil state, have been classed together under the common name of amber, while in commerce copal and gum anime are occasionally sold for true amber.

When amber is found embedded in its original position, it is usually in beds of the brown-coal formation of lower tertiary age; but fossil resins, apparently identical with amber, also occur in upper cretaceous rocks, and in strata of even greater ago. As a rule, the amber is found almost uniformly in separate nodules, disseminated in the sand, clay, or fragments of lignite of the plastic-clay formation. The size of these nodules varies from that of a nut to a man's head; but this magnitude is very rare in true amber. It does not occur either in continuous beds, like the chalk-flints, or in veins; but it lies at one time in the earthy or friable strata which accompany or include the lignites; at another entangled in the lignites themselves. The pieces of amber found in the sands, and other formations evidently alluvial, those mot with on the sea-coasts of certain countries, and especially Pomerania, come undoubtedly from the above geological formation; for the organic matters found still adhering to the amber leave no doubt as to its primitive place.

The vegetable origin of amber is satisfactorily determined by its chemical composition, its optical properties, - as shown by Sir David Brewster, - and by the condition in which insects and the remains of insects are found in this resin, along with fragments of leaves and stalks. Certain families of insects occur more abundantly than others. Thus the hymenoptera, or insects with four marked membranacoous wings, as the bee and wasp, are not abundant. The diptera, or insects with two wings, as gnats and flies, are more numerous. Then come the spider tribe, some coleoptcra (insects with crustaceous shells or elytra, which shut together and form a longitudinal suture down the back), or beetles-principally those which live on trees, as the elaterides, or lespers, and the chrysomelida. The insects appear evidently to have struggled after being entangled in the then viscous fluid, and occasionally a log or a wing is found at some distance from the body, which had been detached in the efforts of the insect to escape from the resin. Göppert has named the tree supposed to have yielded most of the amber, Pinites succinifer, but he has shown that several other coniferous trees, of older tertiary age, havo also yielded this product. 
Germar and Schwreiger state that the insects enveloped in ambor are in general such as sit on the trunks of trees, or live in the fissures of their bark. Theso naturalists have not been ablo to rofer thom to any living species; but it has been observed that they resemble more the insects of hot climates than those of the temperate zones. The Rev. F. W. Hope, F.R.S., in his paper on the 'Succinic Insects,' states them to be altogether extra-Europosn. D. T. 'Tessler sent to the Exhibition, in 1851 , a piece of amber containing the leg of a toad.

Amber is found abundantly on the Prussian coast of the Baltic, occurring from Dantzic to Memol, especially between Pillau and Dorfo Gross-Hubnicken. It is also found in many of the lignite workings opened in the great plain of North Germany. A rich and unexpected locality was discovered a fow years ago in Kurland. It oceurs also on the coast of Denmark and Sweden; in Gallicia, near Lemberg; and at Missan, in Poland; in Moravia, at Boskowitz; in the Uralian Mountains, Russia; near Christiania, Norway; in Switzerland, near Basle, and other places. Small quantitios are occasionally found in the clay of the Paris and the London basins. - Amber is occasionally met with in the gravel-pits near London, and I havo soon specimens which were found in Hyde Park. At Aldborough after a raking tido it is thrown on the beach in considerable quantities, along with masses of jet.'Rev. F. W. Hope, Trans. Ent. Soc. On tho Sicilian coast amber is sometimes found having a peculiar blue tinge. Large discoveries of amber havo been reported from Australia.

Amber is collected on the coast of Prussis in soveral ways. It is found in the bods of streams; in the sand-banks of rivers; in pieces thrown up by the sea and rounded by the waves; it is sought for in the cliffs, and in some places mining operations for it are carried on.

The amber-fishers, clothed in leather dresses, wade into the sea, and seok to discover the amber floating on its surface, which they secure with bag-nets hung at the ends of long poles. They conclude that much amber has been detached from its bed, when they discover many pieces of lignite floating about. Mining is carried on by sinking through the sand and superficial strata to the beds containing the amber and lignite; many of these pits are sunk to the dopth of 130 feet. The faces of the precipitous cliffs are explored in boats, and masses of loose earth or rock supposed to contain the object of search aro detached with long poles having iron hooks at thoir onds.

The most extensive use of amber is for the construction of mouth-pieces to pipes ; these form an essential constituent of the genuine meerschaum and the Turkish pipe. There is a curront belief in Turkey that amber is incapable of transmitting infoction, and as it is a great mark of politeness to offer the pipe to a stranger, this supposed negative property of amber accounts in some measure for the estimation in which it is held. Amber necklaces aro not uncommon: the Russian peasant girls adorn themselves with double and treble rows of amber beads, but it not unfrequently happens that copal is substituted for the genuine article.

The Prussian Government is said to draw a considerable annual revenue from amber. A good piece of a pound weight fetches 50 dollars. A mass weighing 13 pounds has been found, the value of which at Constantinople was said to bo not less than 30,000 dollars.

When amber is to be worked into trinkets, it is first split on a leaden plate at a lathe, and then smoothed into shape.on a Swedish whetstone. It is polished on the lathe with chalk and water, or vegetable oil, and finished by friction with flannel.

Amber, after having been filed, may bo polished with Trent sand, or scraped Flanders brick on flannel with water, or with rotten-stone with oil on flannel, or the same material dry on the hand. Turned works are, however, generally polished first with glass-paper and then with rotten-stone and oil. Necklaces and other ornaments in amber are frequently cut into facets by the gold-cutters, those artisans who cut and polish facottod works.-Holtzapffel.

From the electrical character of amber, it frequently during the process of polishing becomes so excited as to crack and fly to pieces. The workmen, therefore, take several pieces, and work them each for a short time and in regular order. These men are said to be seized with nervous tremors in their wrists and arms from the electricity thus developed.

Pieces of amber may be neatly joined by smearing their edges with linseed oil and pressing them strongly together whilo they are hold over a chareoal fire.

AMBER, ACID OF. See Succivic ACD.

AMrzme, orr or. (Oleum succini). This is obtained by distilling amber, for which purpose chippings of amber and inferior pieces are used. When it is distilled with charcoal, the first product is the rectified oil of amber. The oil of amber has a composition of $\mathrm{C}^{20} \mathrm{H}^{16}\left(\mathbf{C}^{10} \mathbf{E}^{16}\right)$. When 1 part of rectified oil of amber is dissolved in 
24 parts of alcohol of 830 , and 96 of caustic ammonie of 916 , eave de lucs is formed. Eau de luce was a celebrated old perfume, but it is now rarely mado.

If nitrie acid is poured into eau de luce a viscid resinous mass is formod, which has the smell of musk, and is known as artificial musk. Formerly this proparation, dissolved in alcohol, was considered as a specifle in whooping-cough, and it was froquently administered in spasmodio diseases.

AMAER VARNrsII. A strong and durablo varnish is mado by dissolving amber in drying linseed oil. The amber is, however, previously heated in an iron pot, over a clear red fire, till it softens and assumos a somi-fluid form. The oil, which has been also made hot, is to be poured on the meltod amber, and the mixturo diligently stirred.

The following proportions are stated to be the best:-16 ounces of amber and 10 ounces of linseed oil. When these are, by the above method, thoroughly incorporated, and the liquid is somewhat cooled, a pound of oil of turpentine must be added.

Black coachmaker's varnish is prepared by melting 16 ounces of amber and adding thereto about half a pint of boiling-hot drying linseed oil, 3 ounces of asphaltum, and the same quantity of resin. After these have been thoroughly mixed over tho firo, the vessel containing the varnish is removed, and, after cooling, a pint of warm oil of turpentine is added.

Amber is composed of a mixture of resins, two of which are solublo in alcohol ether, and in certain hydrocarbons; whilst the third, which forms by far tho greater part of the amber, is insoluble in all known solvents. Varnishes are propared from the soluble portions, and sold undor the name of amber spirit varnishes; but these are frequently composed of either copal or mastic. They have been much used for varnishing collodion pictures.

Ammarars. (Ambregris, Fr.; Ambra, Ger.) A morbid secretion from the liver and intestines of the spermaceti whale (Physeter macrocephalus). It is found usually swimming on the sea upon the coasts of Coromandel, Japan, the Moluccas, and Madagasear, and also on various parts of the east coast of Africa. Ambergris has not been found in any whales but such as have been dead or sick; its production has therefore been attributed to disease. As portions of the food of the whale are invariably found in any large pieces of ambergris, there is little doubt that it originates in the intestines of that animal.

The best ambergris is ash-coloured, with yellow or blackish veins or spots, scarcely any taste, and very little smell unless heatod or much handled, whon it yields an agreeable odour. Exposed in a silver spoon it melts without bubblo or scum, and on whe heated point of a knifo it vaporisos completely away.

The Chinese try the genuineness of ambergris by scraping it fine upon boiling tea; it should dissolve and diffuse itself generally. Black or whito is bad; tho smooth and uniform is gonerally factitious. It has often a black streak, or is marbled yellow and black; has a fatty taste, is lighter than water, melts at $60^{\circ} \mathrm{C} .\left(140^{\circ} \mathrm{F}\right.$.), dissolves readily in absolute alcohol, in ether, and in both fat and volatile oils.

The chemical composition of ambergris is represented by the following formula, $\mathrm{C}^{93} \mathrm{H}^{92} \mathrm{O}\left(\mathbf{C}^{3 s} \mathbf{z}^{84} \mathbf{O}\right)$. True ambergris is very rarely met with, by far the largest proportion of that which is sold as ambergris being a preparation scented with civet or musk. The alcoholic tincture of ambergris is highly fluorescent in sunlight, exhibiting a characteristic yellow green rim on the surface of the solution. This is a test by which genuine ambergris may be distinguished from such as is spurious.

Capt. Alex. Hamilton, in his 'Thirty Years' Experience,' says, 'Sometimes, in the south-west monsoons, they find ambergrease floating on the sea. I saw a piece in Adda Rajah's possession as big as a bushel ; and he valued it at 10,000 rupees, or 1,250l. sterling.' This was at the Laccadive Islands, 170 miles from the Malabar Coast. - New Account of the East Indies, 1688 to 1730.

In France the duty upon ambergris is 62 francs per kilogrammo when imported in French vessels, and 67 franes when imported in foreign vessels.

Mr. Temple, of British Honduras, speaks of an odorous substance thrown off by the alligator, which appoars to resemble ambergris.

AmBorsa WOOD. A beautiful wood much used for inlaid work. Several varictios are imported, but probably all are producod by ono species-the Pterospermum Indicum, a treo belonging to the Byttneriacee, or chocolate order.

AMBREIns. The fragrant substance of amborgris, which may be obtained by digesting amborgris in hot alcohol, from which, on cooling, it is dopositod in a crystalline form. It is composed of $\mathrm{C} 88 \cdot 37$, H $3 \cdot 32,0$ 3.31.

AMrarme. A fossil resin occurring in large massos in Now Zealand, and much rosembling the common resin of the Dammara Australis, with which it is often exported.

AMEnosme. A resinous mineral found in tho phosphatic bods near Charleston, South Carolina, U.S. 
AMrstryst. (Amethyste occidentale, Fr.; Amethyst, Ger.) One of the vitreous varioties of quartz, of a cloar purple or bluish-violet tint; but the colour is frequently irregularly diffused, and gradually fades into white. The colour is supposed to bo due to the presence of a small per-centage- of manganese, but Hointz attributes it to a compound of iron and soda. The amothyst, from the beauty of its colour, has always been estoemed and used in jowellery. It was one of the stones called by the ancionts àkévotos, a name which they conferred on it from its supposed power of preserving the wearer from intoxication. Tho most beautiful specimens are procured from India, Ceylon, and Persia, where they occur in geodes and pebbles: it is also found at Oberstoin, in the Palatinate; in Transylvania; near Cork, and in the Island of May, in Iroland,-H. W. B.

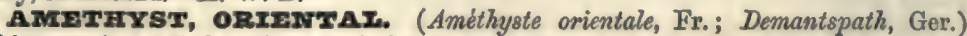
This torm is applied to those varietios of corundum which aro of a violet colour. See Corundor, - H. W. B.

Amravreres is the name given to the whiter and more delicate varieties of asbestos, which possess a satin-like lustre, in consequence of the greater separation of the fibres of which they are composed. A variety of amianthus (the amianthoide of Haüy) is found at Oisens, in France, the fibres of which are in some degree elastic. The word amianthus (from a alayros, undefilod) is expressive of the easy manner by which, when soiled, it may be cleansed and restored to its original purity, by being heated to redness in a fire. See AsBrstos.

AMIDE. This torm and amidogen are applied to a class of substances which contain ammonia deprived of an atom of hydrogen.

AMrDIrrz. A name given to the soluble portion of starch.

AMrDON. The name for starch on the Continent.

AMrrv Es. Chemical substances resembling Amides, but containing basic radicals. Soo Watts's 'Dictionary of Chemistry.'

Ammonra. NH',at. wt. 17. (Ammoniaque, Fr.; Ammoniak, Ger.) The name given by Bergman in 1782 to the gas prepared by treating sal-ammoniac with lime or a caustic alkali. It was first isolated by Dr. Black in 1756, and distinguishod by him from its carbonate, with which it had been previously confounded. The aqueous solution had been long known, and is mentioned by Raymond Lully in the thirteenth century. Ammonia being a product of the putrefactive decay, as well as of the destructive distillation of organic substances containing nitrogen, is widely diffused in nature, but, from the very circumstances of its formation, it is rarely, if ever, evolved in a free or uncombined state. It exists in the atmosphere, though the relative quantity is small; according to Liebig, if all the ammonia were collected at the level of the sea and had a density corresponding to the atmospheric pressure there, it would form a stratum less than a quarter of an inch in depth; yet ho belioves that the nitrogen of plants is derived entirely from this source. The opinions of chemists are, however, divided upon this point; Liebig's view is supported by Boussingault and opposed by Mulder and Ville. The ammonia present in the air is carriod down by rain, sometimes partly in the form of nitrite or nitrate; the maximum amount of combined nitrogen found in numerous analyses by Lawes, and Gilbert, and Way, being 0.032 part in 100,000 parts of rain-water. Rocent experiments by Dr. R. Angus Smith have shown that the proportion of ready-formed ammonia varies with the locality; thus, at Valentia, on the west coast of Ireland, it was as low as 0.018 , whilst from the burning of coals and other causes, and the diminished area of absorbent soil and vegetation, it rises in large towns, London giving 0.345 and Glasgow even 0.910 in 100,000 . In addition there are certain nitrogenous bodies capable of yielding ammonia, designated by $\mathrm{Dr}$. Smith ' albumonoid ammonia,' since it is formed whon these bodies are treated with the same chemical reagents which ovolve ammonia from albumon.

Ammonia is found in many mineral and brine springs, some kinds of rock salt, in deep well-water, river-, and sea-water. In voleanic districts its salts are at times exhaled in such quantity as to form an article of commerce. The eruption of Vesuvius in 1794 produced so much sal-ammoniac that the peasants collected it by hundredweights ; in an eruption of Hecla in 1845 a similar phenomenon was observed; also at Etna it is sometimes found in sufficient abundance to create a profitable trade. Dr. Daubeny is of opinion that the volcanic ammonia is produced by the action of water upon mineral nitrides (perhaps the nitrides of silicon), similar in properties to the nitrides of titanium and boron, which have been recently more carefully examined by M. St. Claire Deville. The suffioni of Tuscany yield, besides boracic acid and several different salts, sulphate of ammonia as an important by-product. All cultivable soils, espocially those of ferruginous or argillaceous naturo, contain an appreciable quantity of ammonia, and a considerable evolution of its salts has been observed recently on meadow-land being overflowed by a stream of lava. The 
chloride has also beon found as a sublimate arising from the combustion of coal strata. Salts of ammonia exist in plants, but to a much greater extent in the liquid and solid excroments of some animals. As a urate it forms the chiof constituent of the excrement of the boa, as well as that of many birds : hence the large quantity of ammoniacal salts in guano. Seo Guavo.

In the guano deposits of South America large quantities of bicarbonate have boen met with and exported to Europe. In several manufacturing processes ammonia is generated as in the purification of caustic soda, by heating it with nitrate of soda, and possibly in this process in sufficient quantity to pay for condensation; but of all sources of supply the so-called 'ammoniacal liquor" of the gas-works is the most important. This is produced during the dry distillation of coal for the manufacture of illuminating gas, and consists mainly of an aqueous solution of sesquicarbonate of ammonia with some sulphide and sulphocyanide of ammonium, \&c.

Formation of Ammonia.-No process has yet been devised for inducing the direct combination of nitrogen and hydrogen to produce ammonia; but under the disposing influence of the production of other compounds, in the presence of these olements, as well as when these gases are presented to each other in the nascent state, their union is effected.

Thus, when electric sparks are passed through a mixture of nitrogen and oxygen in the presence of hydrogen and aqueous vapour, nitrate of ammonia is generated. If, while zine is being dissolved in sulphuric acid, nitric acid be added, much ammonia is formed (Nesbit); so again, if hydrogen and binoxide of nitrogen be passed over spongy platinum, torrents of ammonia are produced, the hydrogen converting the oxygen of the binoxide into water, when the nitrogen, at the moment of its liberation, combines with the hydrogen to form ammonia.

It has even been proposed to carry out this last mothod on a manufacturing scale.

Messrs. Crane and Jullien, in their patent of January 18, 1848, describe a mothod of manufacturing ammonia in the state of carbonate, hydrocyanate, or free ammonia, by passing any of the oxygen compounds of nitrogen, together with any compound of hydrogen and carbon, or any mixture of hydrogen with a compound of carbon or oven freo hydrogen, through a tube or pipe containing any catalytic or contact substance, as follows:-Oxides of nitrogen (such, for instance, as the gases liberated in the manufacture of oxalic acid), however procured, are to be mixed in such proportion with any compound of carbon and hydrogen, or such mixture of hydrogen and carbonic oxide or acid as results from the contact of the vapour of water with ignited carbonaceous matters, and the hydrogen compound or mixture containing hydrogen may be in slight excess, so as to ensure the conversion of the whole of the nitrogen contained in the oxide so employed into either ammonia or hydrocyanic acid, which may be known by the absence of the characteristic red fumes on allowing some of the gaseous matter to come in contact with atmospheric air. The catalytic substance which Messrs. Crane and Jullien prefer is platinum, which may be in the stato of sponge, or it may be asbestos coated with platinum. This catalytic substance is to be placed in a tube, and heated to about $600^{\circ} \mathrm{F}$., so as to increase the temporature of the product, and at the same time prevent the deposition of carbonate of ammonia, which passes onwards into a vessel of the description well known and employed for the purpose of condensing carbonate of ammonia. The condenser for this purpose must be furnished with a safety pipe, to allow of the escape of uncondensed mattor, and made to dip into a solution of any substance capablo of combining with hydrocyanic acid or ammonia where they would be condensed. A solution of salt of iron is preferable for this purpose."

Chemical Characters. - The gaseous ammonia liberated from its salts by lime (in a manner to be afterwards described) is a colourless gas of a peculiar pungent odour. It is composed, by weight, of 1 atom of nitrogen and 3 of hydrogen; or, by volume, of 2 measures of nitrogen and 6 of hydrogen, condensed to four; and may be resolved into these constituent gases by passing over spongy platinum heated to redness, or by a current of electric sparks. By a pressure of 6.5 atmosphores at $50^{\circ} \mathrm{F}$., it is condensed into a colourless liquid. It is combustible, but less so than hydrogen on account of the incombustible nitrogen which it contains; but its inflammability may be readily seon by passing it into an argand gas flame reduced to a minimum.

Ammonia is very soluble in water, water at $32^{\circ} \mathrm{F}$. absorbing no less than 1,149 times its volume of this gas, and at $68^{\circ} 681.8$ times its volume; and the solution has a less density and a lower boiling point than pure water. The following Table of the density of solutions of ammonia in water, of different strengths, is by Dr. Uro:-

Pharm, Journ, xiil, 114. 


\begin{tabular}{|c|c|c|c|c|c|}
\hline$\underset{\text { in } 100}{\text { Amonis }}$ & Water in 100 & $\begin{array}{l}\text { Spectlo Gravity } \\
\text { by Risperiment }\end{array}$ & $\begin{array}{c}\text { Ammonia } \\
\text { in } 100\end{array}$ & Water in 100 & $\begin{array}{l}\text { Specific Gravity } \\
\text { by Experiment }\end{array}$ \\
\hline $26 \cdot 500$ & 73.500 & 0.9000 & $13 \cdot 250$ & $86 \cdot 750$ & 0.9455 \\
\hline $25 \cdot 175$ & $74 \cdot 825$ & 0.9045 & $11 \cdot 325$ & 88.075 & 0.9510 \\
\hline $23 \cdot 850$ & $76 \cdot 150$ & 0.9090 & $10 \cdot 600$ & $19 \cdot 400$ & 0.9564 \\
\hline 22.525 & $77^{\circ} 475$ & 0.9133 & $9 \cdot 275$ & $90 \cdot 725$ & 0.9614 \\
\hline $21 \cdot 200$ & 78.800 & 0.9177 & $7 \cdot 950$ & 92.050 & 0.9662 \\
\hline 19.875 & $80 \cdot 125$ & 0.9227 & 6.625 & $93 \cdot 375$ & 0.9716 \\
\hline 18.550 & $81 \cdot 450$ & 0.9275 & $5 \cdot 300$ & $94 \cdot 700$ & 0.9768 \\
\hline $17 \cdot 225$ & 82.775 & 0.9320 & 3.975 & 96.025 & 0.9828 \\
\hline 15.900 & $84 \cdot 100$ & 0.9363 & $2 \cdot 650$ & $97 \cdot 350$ & 0.9887 \\
\hline 14.575 & 85.425 & 0.9410 & 1.325 & 98.675 & 0.9945 \\
\hline
\end{tabular}

Upon this variation in density of solutions of ammonia in proportion to their strength, Mr. J. J. Griffin has constructed a useful instrument called an Ammonia-meter. It is founded upon the following facts:-That mixtures of liquid ammonia with water possess a specitic gravity which is the mean of the specific gravities of their components; that in all solutions of ammonia, a quantity of anhydrous ammonia, weigh. ing $212 \frac{1}{2}$ grains, which he calls a test-atom, displaces 300 grains of water, and reduces the specific gravity of the solution to the extont of $\cdot 00125$; and, finally, that the strongest solution of ammonia which it is possible to prepare at the temperature of $62^{\circ} \mathrm{F}$. contains in an imperial gallon of solution 100 test-atoms of ammonja.

We extract the following paragraph from Mr. Griffin's paper in the 'Transactions of the Chemical Society, explanatory of the accompanying Table :-

"The first column shows the specific gravity of the solutions; the second column the weight of an imperial gallon in pounds and ounces; the third column the per-centage of ammonia by weight; the fourth column the degree of the solution, as indicated by the instrument, corresponding with the number of test-atoms of ammonia presont in a gallon of the liquor; the fifth column shows the number of grains of ammonia contained in a gallon; and the sixth column the atomic volume of the solution, or that measure of it which contains one test-atom of ammonia. For instance, one gallon of liquid ammonia, specific gravity 880, weighs $8 \mathrm{lbs}$. or $128 \mathrm{oz}$. avoirdupois; its percentage of ammonia, by weight, is 33.117 ; it contains 96 test-atoms of ammonia in one gallon, and $20400^{\circ} 0$ grains of ammonia in one gallon; and, lastly, 104.16 septems containing one test-atom of ammonia. Although no hydrometer, however accurately constructed, is at all equal to the Centigrade mode of chemical testing, yet the Ammonia-metor, and the Table accompanying it, will be found very useful to the manufacturer, enabling him not only to determine the actual strength of any given liquor, but the precise amount of dilution necessary to convert it into a liquor of any other desired strength, whilst the direct quotation of the number of grains of real ammonia contained in a gallon of solution of any specific gravity will enable him to judge at a glance of the money-value of any given sample of ammonia.'

\section{Table of Liquid Ammonia (Griffin).}

One Test-Atom of Anhydrous Ammonia $=\mathrm{NH}^{3}$ weighs 212.5 grains.

Specific Gravity of Water $=1 \cdot 00000$. One Gallon of Water weighs $10 \mathrm{lbs}$., and contains 10,000 Septems. Temperature $62^{\circ} \mathrm{F}$.

\begin{tabular}{|c|c|c|c|c|c|c|}
\hline $\begin{array}{l}\text { Specific Gravity } \\
\text { of the Liquid } \\
\text { Ammonia }\end{array}$ & $\begin{array}{r}\text { Weigh } \\
\text { Imperial } \\
\text { Avoirdu } \\
\text { and }\end{array}$ & $\begin{array}{l}\text { ht of an } \\
\text { Gallon in } \\
\text { upois lbs. } \\
\text { d ozs. }\end{array}$ & $\begin{array}{c}\text { Per-centage of } \\
\text { Ammonia by } \\
\text { Weight }\end{array}$ & $\begin{array}{c}\text { Test-atoms } \\
\text { of Am- } \\
\text { monis in } \\
\text { one Gallon }\end{array}$ & $\begin{array}{c}\text { Grains of } \\
\text { Ammonis in one } \\
\text { Gallon }\end{array}$ & $\begin{array}{l}\text { Septems } \\
\text { containing one } \\
\text { Test-atom of } \\
\text { Ammonis }\end{array}$ \\
\hline .87500 & $\begin{array}{l}\text { lba } \\
8\end{array}$ & $\begin{array}{r}\text { ozs. } \\
12.0\end{array}$ & $34 \cdot 694$ & 100 & $21250 \cdot 9$ & $100 \cdot 00$ \\
\hline$\cdot 87625$ & 8 & $12 \cdot 2$ & $34 \cdot 298$ & 99 & $21037 \cdot 5$ & $101 \cdot 01$ \\
\hline$\cdot 87750$ & 8 & $12 \cdot 4$ & $33 \cdot 903$ & 98 & $20825 \cdot 0$ & $102 \cdot 04$ \\
\hline$\cdot 87875$ & 8 & $12 \cdot 6$ & $33 \cdot 509$ & 97. & $20612 \cdot 5$ - & 103.09 \\
\hline .88000 & 8 & $12 \cdot 8$ & $33 \cdot 117$ & 96 & 0.0 & $104 \cdot 16$ \\
\hline .88125 & 8 & $13 \cdot 0$ & $32 \cdot 725$ & 95 & $20187 \cdot 5$ & $105 \cdot 26$ \\
\hline$\cdot 88250$ & 8 & $13 \cdot 2$ & $32 \cdot 335$ & 94 & $19975^{\circ} 0$ & $106 \cdot 38$ \\
\hline$\cdot 88375$ & 8 & $13 \cdot 4$ & $31 \cdot 946$ & 93 & $19762 \cdot 5$ & $107 \cdot 53$ \\
\hline$\cdot 88500$ & 8 & $13 \cdot 6$ & $\therefore 31.558 \ldots$ & 92 & $19550 \cdot 0$ & $108 \cdot 70$ \\
\hline$\cdot 88625$ & 8 & $13 \cdot 8$ & $31 \cdot 172$ & 91 & $19337 \cdot 5$ & $109 \cdot 89$ \\
\hline$\cdot 88750$ & 8 & 14.0 & 30.785 & $90-$ & 19125.0 & $111 \cdot 11$ \\
\hline
\end{tabular}


Table of Liquid Ammonia (continued).

\begin{tabular}{|c|c|c|c|c|c|c|}
\hline $\begin{array}{l}\text { Specific Gravity } \\
\text { of the Liquid } \\
\text { Ammonia }\end{array}$ & $\begin{array}{r}\text { Welgh } \\
\text { Imperial } \\
\boldsymbol{\Lambda} \text { voirdu } \\
\text { and }\end{array}$ & $\begin{array}{l}\text { ht of an } \\
1 \text { Gallon in } \\
\text { upois lbs. } \\
\text { d ozs. }\end{array}$ & $\begin{array}{c}\text { Per-centage of } \\
\text { Ammonia by } \\
\text { Weight }\end{array}$ & $\begin{array}{c}\text { Test-atoms } \\
\text { of Am- } \\
\text { monia in } \\
\text { one Gallon }\end{array}$ & $\begin{array}{c}\text { Grains of } \\
\text { Ammonia in one } \\
\text { Gallon }\end{array}$ & $\begin{array}{l}\text { Septems } \\
\text { containing one } \\
\text { Test-ntom of } \\
\text { Ammonia }\end{array}$ \\
\hline 88875 & $\begin{array}{l}\text { lhos. } \\
8\end{array}$ & $\begin{array}{c}\text { oss. } \\
14 \cdot 2\end{array}$ & 30.400 & 89 & $18912 \cdot \bar{b}$ & $112 \cdot 36$ \\
\hline .89000 & 8 & $14 \cdot 4$ & 30.016 & 88 & 18700.0 & $113 \cdot 64$ \\
\hline$\bullet 89125$ & 8 & $14 \cdot 6$ & $29 \cdot 633$ & 87 & $18487 \cdot 5$ & $114 \cdot 94$ \\
\hline$\cdot 89250$ & 8 & $14 \cdot 8$ & $29 \cdot 252$ & 86 & $18275^{\circ} 0$ & $116 \cdot 28$ \\
\hline$\cdot 89375$ & 8 & 15.0 & $28 \cdot 871$ & 85 & $18062 \cdot 5$ & $117 \cdot 65$ \\
\hline$\cdot 89500$ & 8 & $15 \cdot 2$ & $28 \cdot 492$ & 84 & 17850.0 & 119.05 \\
\hline$\cdot 89625$ & 8 & $15 \cdot 4$ & $28 \cdot 113$ & 83 & $17637 \cdot 5$ & $120 \cdot 48$ \\
\hline$\cdot 89750$ & 8 & $15^{\circ} 6$ & $27 \cdot 736$ & 82 & $17425^{\circ} 0$ & $121 \cdot 95$ \\
\hline •89875 & 8 & $15 \cdot 8$ & $27 \cdot 359$ & 81 & $17212 \cdot 5$ & $123 \cdot 46$ \\
\hline$\cdot 90000$ & 9 & 0.0 & $26 \cdot 984$ & 80 & 17000.0 & $125 \cdot 00$ \\
\hline .90125 & 9 & 0.2 & $26 \cdot 610$ & 79 & $16787 \cdot 5$ & $126 \cdot 58$ \\
\hline$\cdot 90250$ & 9 & 0.4 & $26 \cdot 237$ & 78 & $16575 \cdot 0$ & $128 \cdot 21$ \\
\hline .90375 & 9 & 0.6 & $25 \cdot 865$ & 77 & $16362 \cdot 5$ & $129 \cdot 87$ \\
\hline$\cdot 90500$ & 9 & 0.8 & $25 \cdot 493$ & 76 & $16150 \cdot 0$ & $131 \cdot 58$ \\
\hline$\cdot 90625$ & 9 & 1.0 & $25 \cdot 123$ & 75 & $15937 \cdot 5$ & $133 \cdot 33$ \\
\hline .90750 & 9 & $1 \cdot 2$ & $24 \cdot 754$ & 74 & $15725^{\circ} 0$ & $135 \cdot 13$ \\
\hline$\cdot 90875$ & 9 & $1 \cdot 4$ & $24: 386$ & 73 & $15512 \cdot 5$ & $136 \cdot 98$ \\
\hline$\cdot 91000$ & 9 & $1 \cdot 6$ & $24 \cdot 019$ & 72 & 15300.0 & $138 \cdot 99$ \\
\hline$\cdot 91125$ & 9 & $1 \cdot 8$ & $23 \cdot 653$ & 71 & $15087 \cdot 5$ & $140 \cdot 85$ \\
\hline$\cdot 91250$ & 9 & $2 \cdot 0$ & $23 \cdot 288$ & 70 & $14875^{\circ} 0$ & $142 \cdot 86$ \\
\hline .91375 & 9 & $2 \cdot 2$ & $22 \cdot 924$ & 69 & $14662 \cdot 5$ & $144 \cdot 93$ \\
\hline .91500 & 9 & $2 \cdot 4$ & 22.561 & 68 & $14450^{\circ} 0$ & $147 \cdot 06$ \\
\hline$\cdot 91625$ & 9 & $2 \cdot 6$ & $22 \cdot 198$ & 67 & $14237 \cdot 5$ & $149 \cdot 25$ \\
\hline$\cdot 91750$ & 9 & $2 \cdot 8$ & $21 \cdot 837$ & 66 & $14025^{\circ} 0$ & $151 \cdot 51$ \\
\hline$\cdot 91875$ & 9 & $3 \cdot 0$ & $21 \cdot 477$ & 65 & $13812 \cdot 5$ & $153 \cdot 85$ \\
\hline .92000 & 9 & $3 \cdot 2$ & $21 \cdot 118$ & 64 & 13600.0 & $156 \cdot 25$ \\
\hline$\cdot 92125$ & 9 & $3 \cdot 4$ & $20 \cdot 760$ & 63 & $13387 \cdot 5$ & $158 \cdot 73$ \\
\hline .92250 & 9 & $3 \cdot 6$ & $20 \cdot 403$ & 62 & $13175^{\circ} 0$ & $161 \cdot 29$ \\
\hline$\cdot 92875$ & 9 & $3 \cdot 8$ & 20.046 & 61 & 12962.5 & $163 \cdot 93$ \\
\hline .92500 & 9 & $4: 0$ & $19 \cdot 691$ & 60 & 12750.0 & $166 \cdot 67$ \\
\hline .92625 & 9 & $4 \cdot 2$ & $19 \cdot 337$ & 59 & $12537^{\circ} 5$ & $169 \cdot 49$ \\
\hline .92750 & 9 & $4 \cdot 4$ & $18 \cdot 983$ & 58 & $12325^{\circ} 0$ & $172 \cdot 41$ \\
\hline$\cdot 92875$ & 9 & $4: 6$ & 18.631 & 57 & $12112 \cdot 5$ & $175 \cdot 44$ \\
\hline .93000 & 9 & $4 \cdot 8$ & $18 \cdot 280$ & 56 & $11900 \cdot 0$ & 178.57 \\
\hline$\cdot 93125$ & 9 & $5 \cdot 0$ & $17 \cdot 929$ & 55 & $11687 \cdot 5$ & $181 \cdot 82$ \\
\hline .93250 & 9 & $5 \cdot 2$ & $17 \cdot 579$ & 54 & $11475^{\circ} 0$ & $185 \cdot 18$ \\
\hline .93375 & 9 & $5 \cdot 4$ & $17 \cdot 231$ & 53 & 11262.5 & $188 \cdot 68$ \\
\hline .93500 & $\theta$ & $5 \cdot 6$ & $16 \cdot 883$ & 52 & $11050^{\circ} 0$ & $192 \cdot 31$ \\
\hline$\cdot 93625$ & 9 & $5 \cdot 8$ & $16 \cdot 536$ & 51 & $10837 \cdot 5$ & 196.08 \\
\hline$\cdot 93750$ & 9 & 6.0 & $16 \cdot 190$ & 50 & 10625.0 & 200.00 \\
\hline -93875 & 9 & $6 \cdot 2$ & $15 \cdot 846$ & 49 & $10412 \cdot 5$ & $204 \cdot 08$ \\
\hline$\cdot 94000$ & 9 & $6 \cdot 4$ & $15 \cdot 502$ & 48 & 10200.0 & $208 \cdot 33$ \\
\hline .94125 & 9 & $6 \cdot 6$ & $15 \cdot 158$ & 47 & $9987 \cdot 5$ & $212 \cdot 77$ \\
\hline .94250 & 9 & $6 \cdot 8$ & $14 \cdot 816$ & 46 & $9775 \cdot 0$ & $217 \cdot 39$ \\
\hline$\cdot 94375$ & 9 & $7 \cdot 0$ & $14 \cdot 475$ & 45 & $9562 \cdot 5$ & $222 \cdot 22$ \\
\hline .94500 & 9 & $7 \cdot 2$ & $14 \cdot 135$ & 44 & 9350.0 & $227 \cdot 27$ \\
\hline$\cdot 94625$ & 9 & $7 \cdot 4$ & 13.795 & 43 & $9137 \cdot 5$ & $232 \cdot 56$ \\
\hline$\cdot 94750$ & 9 & $7 \cdot 6$ & $13 \cdot 456$ & 42 & $8925^{\circ} 0$ & 238.09 \\
\hline$\cdot 94875$ & 9 & $7 \cdot 8$ & $13 \cdot 119$ & 41. & $8712 \cdot 5$ & $243 \cdot 90$ \\
\hline$\cdot 95000$ & 9 . & 8.0 & $12 \cdot 782$ & 40 & $8500^{\circ} 0$ & 250.00 \\
\hline .95125 & 9 & $8 \cdot 2$ & $12 \cdot 446$ & 39 & $8287 \cdot 5$ & $256 \cdot 41$ \\
\hline$\cdot 95250$ & 9 & $8 \cdot 4$ & $12 \cdot 111$ & 38 & $8075^{\circ} 0$ & $263 \cdot 16$ \\
\hline -94375 & 9 & $8: 6$ & $11 \cdot 777$ & 37 & 7862.5 & 270.27 \\
\hline .95500 & 9. & $8 \cdot 8$ & $11 \cdot 444$ & 36 & 7650.0 & $277 \cdot 78$ \\
\hline •95025 & 9 & $9 \cdot 0$ & $11 \cdot 111$ & 35 & $7437 \cdot 5$ & $285 \cdot 71$ \\
\hline .95750 & 9 & $9 \cdot 2$ & $10 \cdot 780$ & 34 & $7225^{\circ} 0$ & $294 \cdot 12$ \\
\hline$\cdot 95875$ & 9 & 9.4 & $10 \cdot 4490$ & 33 & $7012 \cdot 5$ & 303.03 \\
\hline .96000 & 9 & $9 \cdot 6$ & $10 \cdot 1190$ & 32 & $6800 \cdot 0$ & $312 \cdot 50$ \\
\hline$\cdot 96125$ & 9 & $9 \cdot 8$ & $9 \cdot 7901$ & 81 & $6587 \cdot 5$ & $322 \cdot 58$ \\
\hline
\end{tabular}


Table of Liquid Ammonia (continued).

\begin{tabular}{|c|c|c|c|c|c|c|}
\hline $\begin{array}{l}\text { Speciflc Gravity } \\
\text { of the Liquid } \\
\text { Ammonis }\end{array}$ & $\begin{array}{l}\text { Weight } \\
\text { Imperial } \\
\text { Avoirdur } \\
\text { and }\end{array}$ & $\begin{array}{l}\text { t of an } \\
\text { Gallon in } \\
\text { pois lbs. } \\
\text { ozs. }\end{array}$ & $\begin{array}{c}\text { Per-centage of } \\
\text { Ammonia by } \\
\text { Weight }\end{array}$ & $\begin{array}{c}\text { Test-atoms } \\
\text { of Am- } \\
\text { monia in } \\
\text { one Gallon }\end{array}$ & $\begin{array}{c}\text { Grains of } \\
\text { Ammonia in one } \\
\text { Gallon }\end{array}$ & $\begin{array}{l}\text { Beptems } \\
\text { containing one } \\
\text { Test-atom of } \\
\text { Ammonia }\end{array}$ \\
\hline$\cdot 96250$ & $\begin{array}{l}\text { llber: } \\
9\end{array}$ & $\begin{array}{r}\text { ovas. } \\
10.0\end{array}$ & 9.4620 & 30 & $6375 \cdot 0$ & $883 \cdot 83$ \\
\hline$\cdot 96375$ & 0 & $10 \cdot 2$ & $9 \cdot 1347$ & 29 & $6162 \cdot 5$ & $844 \cdot 83$ \\
\hline$\cdot 96500$ & 9 & 10.4 & $8 \cdot 8083$ & 28 & 5950.0 & $857 \cdot 14$ \\
\hline$• 96625$ & 9 & $10 \cdot 6$ & $8 \cdot 4827$ & 27 & $5737 \cdot 6$ & $370 \cdot 37$ \\
\hline •96750 & $\theta$ & $10 \cdot 8$ & $8 \cdot 1580$ & 26 & $5525 \cdot 0$ & $884^{\circ} 62$ \\
\hline$• 96875$ & 9 & 11.0 & $7 \cdot 8341$ & 25 & $5312 \cdot 5$ & $400 \cdot 00$ \\
\hline$\cdot 97000$ & 9 & $11 \cdot 2$ & $7 \cdot 5111$ & 24 & 5100.0 & $416 \cdot 67$ \\
\hline$\cdot 97125$ & 9 & $11 \cdot 4$ & $7 \cdot 1888$ & 23 & $4887 \cdot 5$ & $434 \cdot 78$ \\
\hline$\cdot 97250$ & 9 & $11 \cdot 6$ & 6.8674 & 22 & $4675 \cdot 0$ & $454 \cdot 54$ \\
\hline$\cdot 97375$ & 9 & $11 \cdot 8$ & 6.5469 & 21 & 4462.5 & $476 \cdot 19$ \\
\hline .97500 & 9 & $12 \cdot 0$ & $6 \cdot 2271$ & 20 & 4250.0 & 500.00 \\
\hline$\cdot 97625$ & 9 & $12 \cdot 2$ & $5 \cdot 9082$ & 19 & $4037 \cdot 5$ & $526 \cdot 32$ \\
\hline$\cdot 97750$ & 9 & $12 \cdot 4$ & $5 \cdot 5901$ & 18 & $3825 \cdot 0$ & $555 \cdot 56$ \\
\hline$\cdot 97875$ & 9 & $12 \cdot 6$ & $5 \cdot 2728$ & 17 & 3612.5 & $588 \cdot 24$ \\
\hline -98000 & 9 & $12 \cdot 8$ & $4 \cdot 9563$ & 16 & $3400^{\circ} 0$ & $625 \cdot 00$ \\
\hline •98125 & 9 & $13 \cdot 0$ & $4^{\circ} 6406$ & 15 & $3187 \cdot 5$ & $666 \cdot 67$ \\
\hline$\cdot 98250$ & 9 & $13 \cdot 2$ & $4 \cdot 3255$ & 14 & 2975.0 & $714 \cdot 29$ \\
\hline •98375 & 9 . & $13 \cdot 4$ & $4 \cdot 0111$ & 13 & 2762.5 & $769 \cdot 23$ \\
\hline$\cdot 98500$ & 9 & $13 \cdot 6$ & 3.6983 & 12 & 2550.0 & $833 \cdot 33$ \\
\hline •98625 & 9 & $13 \cdot 8$ & $3 \cdot 3858$ & 11 & $2337 \cdot 5$ & $909 \cdot 09$ \\
\hline$\cdot 98750$ & 9 & $14 \cdot 0$ & 3.0741 & 10 & $2125 \cdot 0$ & $1000 \cdot 00$ \\
\hline$\cdot 98875$ & 9 & $14 \cdot 2$ & $2 \cdot 7632$ & 9 & $1912 \cdot 5$ & $1111 \cdot 10$ \\
\hline .99000 & 9 & $14 \cdot 4$ & $2 \cdot 4531$ & 8 & 1700.0 & $1250 \cdot 00$ \\
\hline$\cdot 99125$ & 9 & $14 \cdot 6$ & $2 \cdot 1438$ & 7 & $1487^{\circ}$ & $1428 \cdot 60$ \\
\hline 99250 & 9 & $14 \cdot 8$ & $1 \cdot 8352$ & 6 & $1275^{\circ} 0$ & $1666 \cdot 70$ \\
\hline$\cdot 99875$ & 9 & $15^{\circ} 0$ & $1 \cdot 5274$ & 5 & $1062 \cdot 5$ & $2000 \cdot 00$ \\
\hline$\cdot 99500$ & 9 & $15 \cdot 2$ & $1 \cdot 2204$ & 4 & 850.0 & $2500 \cdot 00$ \\
\hline •99625 & 9 & $15^{\circ} 4$ & 0.9141 & 3 & $637 \cdot 5$ & 3333:30 \\
\hline •99750 & 9 & $15 \cdot 6$ & 0.6087 & 2 & $425^{\circ} 0$ & $5000 \cdot 00$ \\
\hline$\cdot 99875$ & 9 & $15 \cdot 8$ & 0.3040 & 1 & $212 \cdot 5$ & 10000.00 \\
\hline $1 \cdot 0000$ & $10 \mathrm{lbs}$. & Water & & 0 & & \\
\hline
\end{tabular}

Ammoniacal gas combines directly with hydrated acids, forming a series of salts, tho constitution of which is peculiar, and must be here briefiy discussed, that the formulæ hereafter employed in describing them may be understood.

These compounds may be viewed as direct combinations of the ammonia with the hydrated acids; thus, the compound with

Hydrochloric acid as the Hydrochlorate ( $\mathrm{NH}^{3}, \mathrm{HCl}$ ) (2ran ${ }^{3} . \mathbf{m C 1}$ ).

Hydrosulphuric acid ", Hydrosulphate $\left(\mathrm{NH}^{3}, \mathrm{HS}\right)\left(\mathbf{2 N r \mathbf { Z } ^ { 3 }} . \mathbf{m}^{2} \mathbf{S}\right)$.

Sulphuric acid " Hydrated sulphato $\left(\mathrm{NH}^{3} ; \mathrm{HO}^{\left.-\mathrm{SO}^{3}\right)}\left(\mathbf{2} \mathbf{2 r} \mathbf{m}^{3} . \mathbf{m}^{2} \mathbf{S O}^{4}\right)\right.$ :

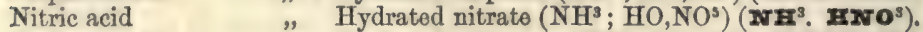

Carbonic acid $\quad "$ Hydrated carbonate $\left(\mathrm{NH}^{3} ; \mathrm{HO}, \mathrm{CO}^{2}\right),\left(\mathbf{2} \mathbf{N r}_{\mathbf{2}}{ }^{2}, \mathbf{r r}^{2} \mathbf{0}, \mathbf{C O}^{2}\right)$.

But the close analogy of these compounds, in all their properties, to the corresponding salts of potash and soda has led chemists to the assumption of the existonce of a group of elemonts possessing the characters of a metal, of a basyl or hypothetical metallic radical, called ammonium $\left(\mathrm{NH}^{4}\right)$, in these salts; which theory of their constitution brings out the resemblance to the potash and soda salts more clearly, thus :-

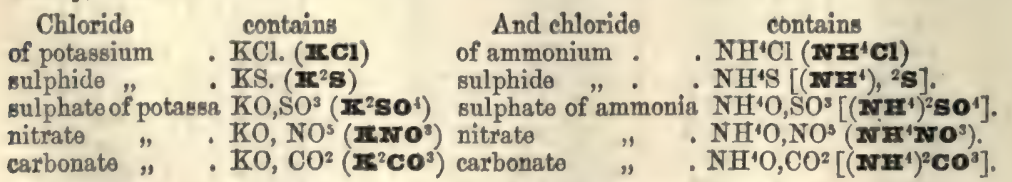

Although it may be objected to this view that the metal ammonium is not known, yet a curious metallic compound of this metal with mercury has boen obtained; and, 
after all, it is by no means necessary that the motal should be isolated, for the existence of numerous basic radicals has been assumed in organic chemistry which have never been isolated.

It is true, also, that the oxide of ammonium is unknown, but substitution-products of it have been produced, which are solid bodies, soluble in water, exhibiting all the characters of potash solution, being as powerfully caustic and alkaline. In fact, ammonia is in reality but the type of a vast number of compounds. It is capable of having its hydrogen replaced by metals (as copper, mercury, calcium, \&c.), as well as by metallic or basic compound radicals, producing the endless number of artificial organic bases, which are primary, secondary, or tertiary nitrides, according as one, two, or three equivalents of the ammonia is replaced. When the substitution of the hydrogen in ammonia is effected by acid radicals, the compounds are called amides.

Preparation of Ammonia.-Ammonia is obtained by the decomposition of one of the salts of ammonia, either the chloride of ammonium, $\mathrm{NH}^{+} \mathrm{Cl}$ (sal-ammoniac), or the sulphate, by a metallic oxide, e.g. lime.

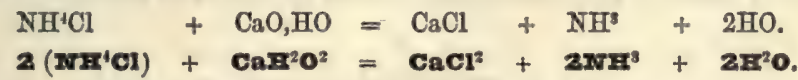

On the small scale in the laboratory the powdered ammoniacal salt is mixed with slaked lime, in a Florence flask or a small iron retort, and gently heated; the ammoniacal gas being dried by passing it through a bottle containing lime. Chloride of calcium must not be employed in the desiccation of ammonia, since the ammonia is absorbod by this salt, producing a curious compound, the chloride of caliammonium.

It has been proposed by Knab to make use of the property of chloride of calcium to absorb ammonia and give it up again when heated, for the purpose of storing and transporting it. Solution of ammonia of the usual commercial strength, specific gravity 0.880 , contains only 33.12 per cent., whilst the chloride of calcium compound is said to retain 50 per cont.

The gaseous ammonia must be collected over mercury, on account of its solubility in water.

This operation is carried out on the large scale for the purpose of making the aqueous solution of ammonia (liquor ammonice, or spirits of hartshorn).

\section{Solution of Ammonia.}

Preparation.-In preparing the aqueous solution, the gas is passed into water contained in Woolfe's bottles, which on the small scale are of glass, whilst on the large scale they are made of earthenware.

A sufficiently capacious still or retort of iron or lead should be employed, which is provided with a moveable neck; and it is desirable to pass the gas through a worm, to cool it, before it enters the first Woolfe's bottle. Each of the series of Woolfe's bottles should be furnished with a safety-funnel in the third neck, to avoid accidents by absorption. The whole of the condensing arrangements should be kept cool by ice or cold water.

Properties.-In the London and in the Edinburgh 'Pharmacopoia' two solutions of ammonia are directed to be prepared, the stronger having the specific gravity 0.882 , and containing about 32.5 per cent. of ammonia ; the weaker of specific gravity 0.260 , containing, therefore, about 10 per cent. of the gas.

Sometimes the commercial solution of ammonia is made by treating impure ammoniacal salts with lime, and it then contains empyreumatic oils; in fact, the various volatile products of the distillation of coal which are soluble in or miscible with water.

Pyrrol may be detected in ammonia by the purple colour which it strikes with an excess of nitric or sulphuric acid. If the residue of its distillation bo mixed with potash, Picoline is detected by its peculiar odour. Naphthaline is discovered not only by its odour, but may also be soparated by sublimation or heating, after converting the ammonia in the solution into a salt by sulphuric or hydrochloric acid.Dr. Maclogan.

Wo imported into England of sulphate and liquor of ammonia as follows :-

$$
\begin{aligned}
& \text { Ammionia, sulphate of . . } 1856 \text {. . 1bs. 23,904 }
\end{aligned}
$$

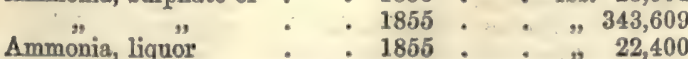

Since, for the purpose of purification on the large scale, ammonia is invariably 
converted into chloride or sulphate, the dotails of the manufacture of the ammoniacal salts will be givon under those heads. For the determination of ammonia, see Nitroger.-H. M. W.

AMmONIA, CARBONATE Or. [The sesquicarbonale of commorce,

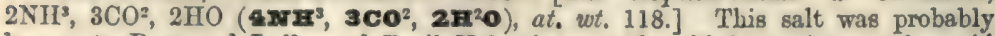
known to Raymond Lully and Basil Valontine, as the chiof constituent of putrid urine. The roal distinction between ammonia and its carbonate was pointed out by Dr. Black.

Carbonate of ammonia is formed during the putrefaction of animal substances, and by their destructive distillation. Its presence in rain-water has been bofore alluded to.

The carbonate of ammonia of commorce is obtained by submitting to sublimation a mixture either of sal-ammoniac or sulphate of ammonia with chalk.

This is generally carried out in cast-iron retorts, similar in size and shape to those used in the manufacture of coal gas. The retorts are charged through a door at one end, and at the other they communicate with large square leaden chambers, supported by a wooden frame, in which the sublimed salt is condensed. Fig. 38.

38

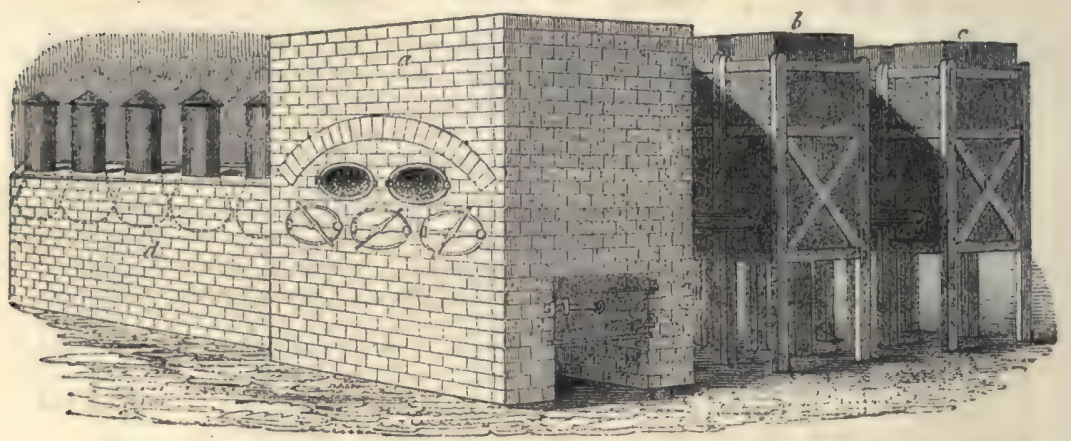

The product of this first process is impure, being especially discoloured by the presence of carbonaceous matter, and has to be submitted to resublimation. This is carried out in iron pots surmounted by moveablo leaden caps. These pots are either set in brickwork, and heated by the flue of the retort furnace, or are placed in a waterbeth, as shown in fig. 39 . In fact, a temperature not exceeding $150^{\circ} \mathrm{F}$. is found oufficiont.

39

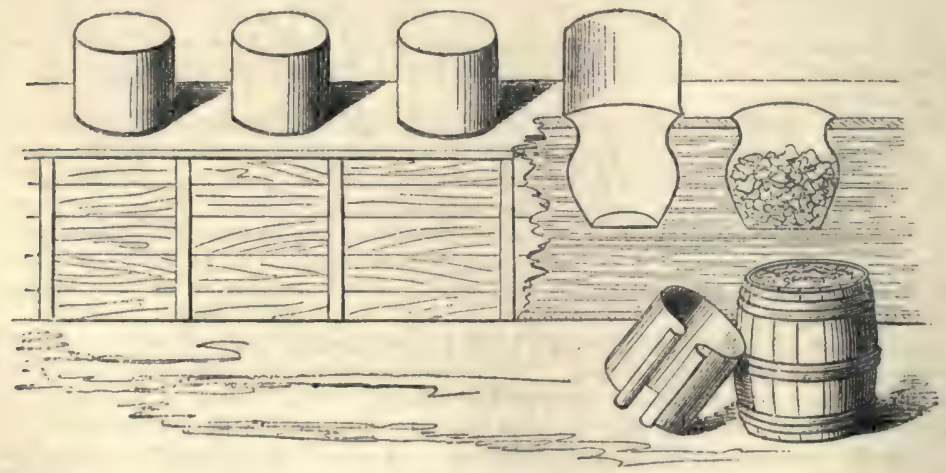

The charge of a retort consists usualiy of about $65 \mathrm{lbs}$. of sulphate of ammonia (or an equivalent quantity of the chloride) to $100 \mathrm{lbs}$. of chalk, which yield about $40 \mathrm{lbs}$. of crude carbonate of ammonis.

Modifications of the Process.-Mr. Taming has suggestod to bring ammonia and carbonic acid gases into mutual contact in a loaden chamber having at the lower part a lajer of water, and then to crystallise the salt by evaporating this aqueous solution. 
He also proposes to prepare carbonate of ammonia from the sulphide of ammonium of gas liquors, by passing carbonic acid gas into the liquor, which enrbonic gas is generated by heating a mixture of oxide of copper and charcoal, in the proportion of twelve parts of the former to one of the latter.

Mr. Hills has described his mode of obtaining sesquicarbonate of ammonia from guano. To effect this, the guano is first mixed with charcoal or powilered coko; the mixture is then heated, and the sesquicarbonate of ammonia obtained by sublimation. The process does not appear to be much employed.

Manufacture of Ammonia from Peat and Shale.-Mr. Hills, in his patent of August 11th, 1846, specified the following method of obtaining ammonia from peat. The peat is placed in an npright furnace and ignited; the air passes through the bars as usual, and the ammonia is collected by passing the products of combustion through a suitable arrangement of apparatus to effect its condensation. This plan of obtaining ammonia from peat appears to be precisely similar to that patented by Mr. Rees Reece (January 23rd, 1849), and made to form an important feature in the operations of the British and Irish Peat Company. The first part of Mr. Reece's patent is for an invention for causing peat to be burned in a furnace by the aid of a blast, 80 as to obtain inflammable gases and tarry and other products from peat. For this purpose, a blast furnace with suitable condensing apparatus is used. The gases, on their exit from the condensing apparatus, may be collected for use as fuel or otherwise; and the tarry and other products pass into a sultable receiver. The tarry products may be employed to obtain paraffine and oils for lubricating machinery, \&c. ; and the other products may be made available for evolving ammonia, wood-spirit, and other matters by any of the existing processes. Dr. Hodges, of Belfast, states that in his experiments ho obtained nearly $22 \frac{3}{4} 1 \mathrm{bs}$. of sulphate of ammonia from a ton of peat. Sir Robert Kane, who was employed by Government to institute a series of experimental researches on the products obtainable from peat, states that he obtained sulphate of ammonia at the rate of $24 \frac{8}{10} \mathrm{lbs}$. per ton of peat. Messrs. Drew and Stocken patented, in 1846, the obtaining ammonia from peat by distillation in close vessels, as practised in the carbonisation of wood. It will thus be seen that the peat is a source of ammonia, but whether this source is a profitable or economical one, in a commercial point of view, is a problem which has not yet received solution.

Ammonia from Schist.-Another source of ammonia is bituminous schist, which, when submitted to destructive distillation, gives off an ammoniacal liquor which may be employed in the manufacture of ammonincal salts by any of the usual processes. The obtaining of ammonia from schist forms part of a patent granted to Count do Hompesch, Soptomber 4, 1841.

Chemical Composition and Constitution.-The true neutral carbonate of ammonia, $\mathrm{NH}^{4} \mathrm{O}, \mathrm{CO}^{2}\left[\left(\mathbf{M H}^{\prime}\right)^{2} \mathbf{C O}^{8}\right]$, does not appear to exist. The sesquicarbonate of ammonia of the shops was found by Rose to have the composition assigned to it by Mr. Philips, as given at the head of this article; and it may be viowed as a compound of the true bicarbonate (i.e. the double carbonate of ammonia and water), $\mathrm{NH}^{\circ} \mathrm{O}, \mathrm{CO}^{\prime \prime}$; $\mathrm{HO}, \mathrm{CO}^{2}\left[\left(\mathbf{N} \mathbf{H}^{4}\right)^{2} \mathbf{C O}^{3} ; \mathbf{H}^{2} \mathbf{C O}^{3}\right]$; with a peculiar compound of anhydrous carbonic acid with ammonia itself, $\mathrm{NH}^{3}, \mathrm{CO}^{2}\left[\left(\mathbf{M r a}^{3}\right)^{2} \mathbf{C O}^{2}\right]$.

It is invariably found that a certain quantity of water and ammonia are liberated during the distillation, and hence the anomalous character of the compound. In fact, in operating upon 3 equivalents of the sulphate or chloride of the 3 equiralents of the true carbonate of ammonia $\left(\mathrm{NH}^{4} \mathrm{O}, \mathrm{CO}^{2}\right)$ which may be supposed to be generated, two are decomposed, one losing an equivalent of ammonia, the other an equivalent of water; of course, the ammonia thus liberated, amounting to 14 parts for each 100 of earbonate of ammonia obtained, is not lost; it is passed into water to be saturated with acid, and thus again converted into sulphate or chloride.

Properties. - Sesquicarbonate of ammonia (as it is commonly called) is met with in commeree in the form of fibrous white translucent cakes, about two inches thick.

When exposed to the air the constituents of the less stable compound $\mathrm{NH}^{3}, \mathrm{CO}^{2}$ are volatilised, and a white opaque mass of the true bicarbonate remains. Hence the odour of ammonia always emitted by the commercial carbonate. Mr. Scanlan has also shown that by treatment with a small quantity of water, the carbonate is dissolved, leaving the bicarbonate. It is soluble in four times its woight of cold water, but boiling water decomposes it.

Impurities.-The commercial salt is sometimes contaminated with empyreumatic oil, which is recognised by its yielding a brownish coloured solution on treatment with water.

It may contain sulphate and chloride of ammonium. For the recognition of the presence of these acids, see SUI.PHURIC and HrDrochloric acids.

Sulphide and hyposulphite of ammonia are sometimes present, and likewiso lead, from the chambers into which the salt has been sublimed.

Vox. I. 
Other Carbonatcs of Ammoniu.-Besides the nontral or monocarbonato of ammonia beforo alludod to, the truo bicarbonato $\mathrm{NH}^{4} \mathrm{O}, \mathrm{CO}^{2} ; \mathrm{IIO}, \mathrm{CO}^{2}\left[\left(\mathbf{N} \mathbf{x}^{4}\right)^{2} \mathbf{C O}^{3} ; \mathbf{F}^{2} \mathbf{C O}^{3}\right]$ and the sesquicarbonato of the shops, Rose has described about a dozen other dofinito compounds; but, for their doseription, wo must refor to Watts's 'Dictionary of Chomistry.'

Ammoniacum, Gom: Gum-rosin. (Gomme Ammoniaque, Fr.; Ammoniak, Ger.) This is the inspissated juice of an umbelliferous plant (the Dorema ammoniacum), the gum-bearing heracleum, which grows in Persia, tho East Indies, and Afrien. In the Fronch colony of Algiers this plant grows naturally, and it appears likely to become an object of cultivation. It comes to us either in small white tears clustered together, or in brownish lumps, containing many impurities. It possesses a poculiar smoll, somewhat like that of assafœtida, and a bitterish tasto. It is employed in medicine. Its only use in the arts is for forming a cement to join brokon pieces of china and glass, which may bo prepared as follows: "Take isinglass 1 ounce, distilled water 6 ounces, boil together down to 3 ounces, and add $1 \frac{1}{2}$ ounce of strong spirit of wine;-boil this mixture for a minute or two; strain it; add while hot; first, half an ounce of milky emulsion of gum ammoniac, and then 5 drachms of an alcoholic solution of resin mastic.'

Ammorra, Nrmants or.-This salt is not mado on an extensive scale; but as it has a certain consumption for making the protoxide of nitrogen (laughing gas), now largoly used by dentists as an anosthetic, a fow remarks respecting it may not be ont of place here.

It is obtained by saturating solution of ammonia, or the carbonate, with nitric acid, and then evaporating the solution till crystallisation takes place. It ought to be perfectly free from chloxide of ammonium.

This salt crystallises in six-sided prisms, boing isomorphous with nitrate of potash.

Its composition is $\mathrm{NH}^{4} \mathrm{O}, \mathrm{NO}^{5}\left[\left(\mathbf{M r}^{4}\right) \mathbf{N O}^{3}.\right]$ It is incapable of existing without tho presence of an equivalent of water, in addition to $\mathrm{NH}^{3}$ and $\mathrm{NO}^{5}$. If heat be applied, the salt is entirely docomposed into protoxide of nitrogen and water; thus-

$$
\begin{aligned}
& \mathrm{NH}^{4} \mathrm{O}, \mathrm{NO}^{s}=2 \mathrm{NO}+4 \mathrm{HO} \text {. } \\
& \left(\mathrm{MH}^{4}\right) \mathrm{NTO}^{3}=2 \mathrm{Tr} \mathrm{O}+2 \mathrm{Ir}^{2} \mathrm{O}
\end{aligned}
$$

Besides its use in the laboratory for making protoxide of nitrogen, it is a constituent of frigorific mixtures, on account of the cold which it produces on dissolving in water.

Iastly, it is very convenient for promoting the deflagration of organic bodies, both its constituents being volatile on heating.

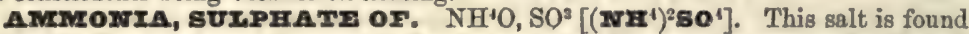
native in fissures noar volcanoes, under the name of Mascagnin, associated with salammoniac. It also forms in ignited coal-beds-as at Bradley, in Staffordshire-with chloride of ammonium.

This salt is prepared by saturating the solution of ammonia, obtained by any of the processes before described (either from animal refuse, from coal, in the manufacture of coal gas, from guano, or from any other source), with sulphuric acid, and then evaporating the solution till the salt crystallises out.

Frequently, instead of adding the acid to the ammoniacal liquor, the crude ammoniacal liquor is distilled in a boiler, either alone or with lime, and the erolved ammonia is passod into the sulphuric acid, containod in a largo tun or in a series of Woolfe's bottles; or a modification of Coffey's still may bo usod with advantage, as in the case of the saturation of hydrochloric acid by ammonia.

If Coffey's still be employed, a considerable concentration of the liquor is offected during the process of saturation, which is subsequently completed generally in iron pans; but great care has to be taken not to carry the evaporation too far, to avoid decomposition of tho sulphato by the organic matter invariably presont, which reduces it to the state of sulphite, hyposulphito, and even to sulphide, of ammonium.

The salt obtained by this first crystallisation is much purer than the chloride produced under similar circumstances, and ono or two recrystallisations effect its purification sufficiently for all commercial purposes.

It is on account of the greater facility of purification which the sulphate affords by crystallisation than the chloride of ammonium, that the former is often produced as a preliminary stage in the manufacture of the latter compound, the purified sulphato being then converted into sal-ammoniac by sublimation with common salt. The acid mother-liquor left in the first crystallisation is returnod to be again treated, togethor with some. additional acid, with a fresh quantity of ammonia:

Preparation. Modifications in details and patents.- Since it is in the production of 
the sulphate of ammonia that the modification of Coffey's still, called the ammonia still, is generally employed, it may be well to introduce here a detailed account of its arrangement.

This apparatus is an upright vessel, divided by horizontal diaphragms or partitions into a number of chambers. It is proposed to construct the vessel of wood, lined with lead, and tho diaphragms of sheot iron. Each diaphragm is perforated with many small holes, so regulated, both with regard to number and size, as to afford, under some pressure, passage for the elastic vapours which ascend, during the use of the apparatus, to make their exit by a pipe opening from tho uppor chambor. Fitted to each diaphragm are several small valves, so weighted as to riso whonover elastic vapours accumulato under them in such quantity as to exort more than a certain amount of pressure on the diaphragm. A pipe also is attached to each diaphragm, passing from about an inch above its upper surface to near the bottom of a cup or small reservoir, fixed to the upper surface of the diaphragms next underneath. This pipe is sufficiently large to transmit freely downwards the whole of the liquid which enters for distillation at the upper part of the upright vessel; and the cup or reservoir into which the pipe dips forms, when full of liquid, a trap by which the upward passage of elastic vapours by the pipe is prevented. The vessel may rest on a clase cistern, contrived to receive the descending liquid as it leaves the lowest chamber, and from this eistern it may be run off, by a valve or cock, whenever expedient. The cistorn, or in its absence the lowest chamber, contains the orifice of a pipe which supplies the steam for working the apparatus. The exact number of chambers into which the upright vessel is divided is not of essential importance; but the quantity of liquid and the surface of each diaphragm being given, the distillation, within cortain limits, will be more complete the greater the number of chambers used in the process. The liquid undergoing distillation in this apparatus necessarily covers the upper surface of each diaphragm to the depth of about an inch, being prevented from passing downward through the small porforations by the upward pressure of the rising steam and other elastic vapours; and, on the other hand, the steam being prevented, by the traps, from passing upwards by the pipes, is forced to ascend by the perforations in the diaphragms; so that the liquid lying on them becomes heated, and in consequence gives off its volatile matters. When the ammonircal liquid accumulates on one of the diaphragms to the depth of an inch, it flows over one of the short pipes into the trap below, and overflows into the next diaphragm, and so on. Seo Distritation.

The management of the apparatus varies in some measure with the form in which it is desirable to obtain the ammonia. When the ammonia is required to lesve the upper chamber in the form of gas, either pure or impure, it is necessary that the steam which ascends and the current of ammoniacal liquid which descends, should be in such relative proportions that the latter remains at or near the atmospheric tempersture during its passage through some of the upper chambers, becoming progressively hotter as it descends, until it reaches the boiling temperature; in which state it passes through the lower chambers, either to make its escape, or to enter a cistern provided to receive it, and in which it may for some time be maintained at a boiling heat. On the contrary, if the ammonia, either pure or impure, bo required to leave the uppor chamber in combination with tho vapour of water, the supply of steam entering below must bear such proportion to that of the ammoniacal liquid supplied above, that the latter may be at a boiling temperature in the upper part of the apparatus. ${ }^{1}$

The use of this apparatus was patented in the name of Mr. W. E. Newton, Nov. 9, 1841.

Mr. Hills' process, patented Oct. 19, 1848, for concentrating ammoniacal solutions by causing them to descend through a tower of coke through which steam is ascending, is, in fact, nothing more than a rough mode of carrying out the same principle, which is more effectually and elegantly performed by the modification of Coffey's still above described. The concentrated ammonia liquor is then treated with acid and evaporated in the usual way.

Mr. Wilson patented, Dec. 7,1850 , another method of saturating the ammonia with the acid by passing the crude ammonia rapour, obtained by heating the ammoniacal liquor of the gas-works, in at the bottom of a high tower fillod with coke, whilst the sulphuric acid descends in a continuous current from the top; in this manner the acid and ammonia are exposed to each other over a greatly extended surface.

Dr. Richardson (patent, Jan. 26, 1850) mixes the crude ammonia liquors with sulphato of magnesia, thon evaporates the solution, and submits the doublo sulphnto 
of magnesia and ammonia, which separates, to sublimation; but it would not appear that any great advantage is derived from proceoding in this way, eithor pecuniary or otherwise.

Mr. Laming passes sulphurous acid through the gas liquor, and finally oxidises the sulphite thus obtained to the state of sulphate, by exposure to the air. (Patont, Aug. 12, 1852.)

Michiel's mode of obtaining sulphato of ammonia, patented A pril 30, 1850, is as follows:-The ammoniacal liquors of the gas-works are combined with sulphate and oxide of lead, which is obtained and prepared in the following way:-Sulphuret of lead in its natural state is taken and reduced to small fragments by any convenient crushing apparatus. It is then submitted to a roasting.process, in a suitably arranged reverberatory furnace of the following construction:- The furnace is formed of two sholves, or rather the bottom of the furnace, and one shelf, and there is a communication from the lower to the upper. The galena or sulphuret of lead, previously ground, is then spread over the surface of the upper shelf, to a thickness of about 2 or $2 \frac{1}{2}$ inches, and there it is submitted to the heat of the furnace. It remains thus for about two hours, at which time it is drawn off the upper shelf, and spread over the lower shelf or bottom of the furnace, where it is exposed to a greater heat for a certain time, during which it is well stirred, for the purpose of exposing all the parts equally to the action of the heat, and at the same time the fusion of any portion of it is prevented. By this process the sulphuret of lead becomes converted partly into sulphate and partly into oxide of load. This product of sulphate and oxide of lead is to be crushed by any ordinary means, and reduced to about the same degree of fineness as coarse sand. It is now to be combined with the ammoniacal liquors, when sulphate of ammonis and sulphuret and carbonate of lead will bo producod.

The sulphate of ammonia is separated by treatment with water, and the residuary mixture of sulphide and carbonate of lead is used for the manufacture of lead compounds.

Properties.-The sulphate of ammonia obtained by either of the methods above described is a colourless salt, containing, according to Mitscherlich, one atom of water of crystallisation. It is isomorphous with sulphate of potash.

It deliquesces by exposure to the air; 1 part dissolves in 2 parts of cold water, and 1 of boiling water. It fuses at $140^{\circ} \mathrm{C} .\left(284^{\circ} \mathrm{F}\right.$.), but at $280^{\circ} \mathrm{C}$. $\left(536^{\circ} \mathrm{F}\right.$. $)$ it is decomposed, being volatilised in the form of freo ammonia, sulphite, water, and nitrogen.

For the other sulphates, the sulphites, and those salts which are but little used in the arts and manufactures-we refer to Watts's 'Dictionary of Chemistry.'

Uses.-The chief consumption of ammoniacal salts in the arts is in the form of sal-ammoniac, the sulphate of ammonia being principally used as a material for the manufacture of the chloride of ammonium. It may, however, be employed directly in making ammonia-alum, or in the production of free ammonia by treatment with lime.

Ammoxrum. (NH') The radical supposed to exist in the various salts of ammonia. Thus $\mathrm{NH}^{4} \mathrm{O}\left[\left(\mathbf{v m}^{4}\right)^{2} \mathbf{O}\right]$ is the oxide, $\mathrm{NH}^{4} \mathrm{Cl}$ the chloride, of ammonium, Ammonium constitutes one of the best established chemical types, See Formor.s, Chemicas.

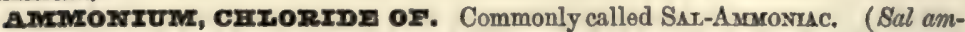
moniac, Fr.; Salmiak, Ger.) The early history of this salt is involved in much

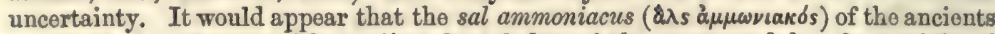
was, in fact, rock salt. The earliest knowledge of the compound has been claimed both for the Arabians and the Egyptians; but the late Dr. Royle remarked, that " the salt must have been familiar to the Hindoos ever since they have burnt bricks, as they now do, with the manure of animals, for somo may usually be found crystallised at the unburnt extremity of the kiln.'

This salt is formed in the solid state by bringing in contact its two gaseous constituents, hydrochloric acid and ammonia. The gases combine with such force as to generate, not only heat, but sometimes even light. It may also be prepared by mixing the aqueous solutions of these gases, and evaporating till crystallisation takes place.

When ammoniacal gas is brought into contact with dry chlorine, a violent reaction ensues, attended by the evolution of heat and even light. The chlorine combines with the hydrogen to produce hydrochloric acid, which unites with the remainder of the ammonia, forming chloride of ammonium, the nitrogen being liberated. The same reaction takes place on passing chlorine gas into the saturated aqueous solution of ammonia.

Manufacture of Chloride of Ammonium from Camels' Dung.-In Egypt-which un. doubtedly was the great seat of the manufacture of this salt from the beginning of 
the thirteenth to the middle of the seventeenth century, and whence all the European markets were supplied-the following is the process by which it is obtained:-

The original source was the urine and dung of the camel, which are dried by plastering them upon the walls, and burning, other fuel being very scarce in that country. A fire of this material evolves a thick smoke, charged with chloride of ammonium, part of which is condensed with the soot.

In every part of Egypt, but especially in the Delta, peasants are seen driving asses loaded with bags of that soot, on their way to the sal-ammoniac works.

Here it is extracted in the following manner:-Glass globes, coated with loam, are filled with the soot, pressed down by wooden rammers, a space of only two or three inches being loft vacant, near their mouths. These globes are set in round orifices formed in the ridge of a long vault or large horizontal furnace flue. Heat is gradually applied by a fire of dry camels' dung, and it is eventually increased till tho globes become obscurely red. As the chloride of ammonium is volatile at a temperature much below ignition, it rises out of the soot in vapour, and gets condonsed into a cake upon the inner surface of the top of the globe. A considerable portion, however, escapes into the air; and another portion concretes in the mouth, which must be cleared from time to time by an iron rod. Towards the end, the obstruction becomes very troublesome and must be most carefully attended to and obviated, otherwise the globes would explode by the uncondensed vapours. In all cases when the subliming process approaches to a conclusion, the globes crack or split; and when they come to be removed, after the heat has subsided, they usually fall to pieces. The upper portion of the mass is separated, because to it the white salt adheres; and, on detaching the pieces of glass with a hatchet, it is ready for the market. At the bottom of each balloon a nucleus of salt remains, surrounded with fixed pulverulent matter. This is reserved, and, after being bruised, is put in along with the charge of Boot in a fresh operation.

The sal-ammoniac obtained by this process is dull, spongy, and of a greyish hue; but nothing better was for a long period known in commerce. Fifty years ago, it fetched 2s. $6 d$. a pound; whereas now, perfectly pure sal-ammoniac may bo had at one-fifth of that price,

Manufacture of Sal-Ammoniac from Bones and other Animal Matter.-Various nnimal offals develop, during their spontaneous putrefactive fermentation, or their decomposition by heat, a large quantity of free or carbonated ammonia among their volatile products. Upon this principle many sal-ammoniac works have been established.-Watts's ' Dictionary of Chemistry.'

The first attempts made in France to obtain sal-ammoniac profitably in this manner failed. A very extensive factory of the kind, which experienced the same fate, was under the superintendence of the celebrated Baumé. It was established at Gravelle, near Charenton, and caused a loss to the shareholders in the speculation of upwards of 400,000 francs, which result closed the concern in 1787 . For 10 years after that event, all the sal-ammoniac consumed in France was imported from foreign countries. Since then the two works of MM. Payen and Pluvinet were mounted, and seem to have been tolerably successful. Coal soot was, prior to the introduction of the gasworks, a good deal used in Great Britain for obtaining sal-ammoniac.

In France, bones and other animal matters are distilled in large iron retorts for the manufacture of both animal charcoal and sal-ammoniac.

'The annexed numbers show the produce of a French manufactory of ammonia and its salts, from the distillation of bones and other matters.

'The matorials were-

and the produce was-

46,754 tons of bones of various kinds.

$30 " \quad$ silk waste and old leather.
$11 \frac{1}{2} " \quad$ sulphuric acid.
$80, \quad$ chloride of sodium.
$2 \frac{3}{4} "$ sulphate of lime.

$$
\begin{aligned}
2,400 & \text { tons of animal charcosi. } \\
44 & \text { " chloride of ammonium. } \\
100 & \text { sulphate of soda. } \\
4 & \text { " liquor ammonia. } \\
\text { and } 25 & \text { " sulphate of ammonia.' }
\end{aligned}
$$

- Muspratt.

These rotorts are iron cylinders, two or three foet in diameter and six foet long. Figs, 40 and 41 show the form of the furnace, and the manner in which the cylinders are arranged, the first being a longitudinal, the second a transverse section of it. $\mathbf{\Delta}$, the ashpits under the grates; $B$, tho fire-placos, arched orer at top; $c$, the vault of 
bench of fire-bricks, perforated inside with oight flues for distributing tho flame; $\mathbf{D}, \mathbf{a}$ great arch, with a triple voissoir $\mathrm{D}, d^{\prime \prime}, d^{\prime \prime \prime}$, undor which the rotorts are sct. The first arch, $\mathbf{D}$, is perforated with twenty vent-holes, the socond with four vont-holes, through

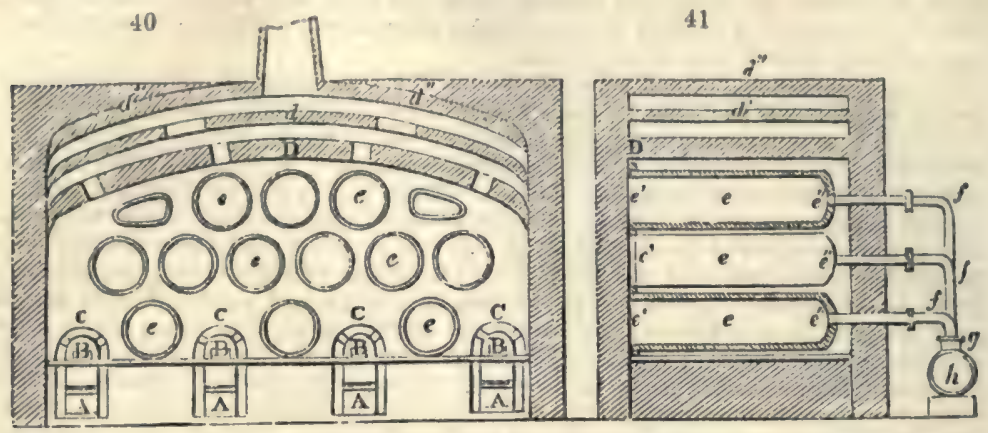

which the flame passes to the third arch, and thence to the common chimney-stalks. The retorts are shut by the door $e^{\prime}(f g .41)$, luted, and made fast with screw-bolts. Their other ends $e^{\prime \prime}$, terminate in tubes, $f, f, f$, which all enter the main pipe $h$. The condensing pipe procoeds slantingly downwards from the further ond of $h$, and dips into a large sloping iron cylinder immersed in cold water.

The filters used in the large sal-ammoniac works in France are ropresented in fig. 42. The apparatus consists - 1 , of a woodon chost, $a$, linod with lead, and which is

42

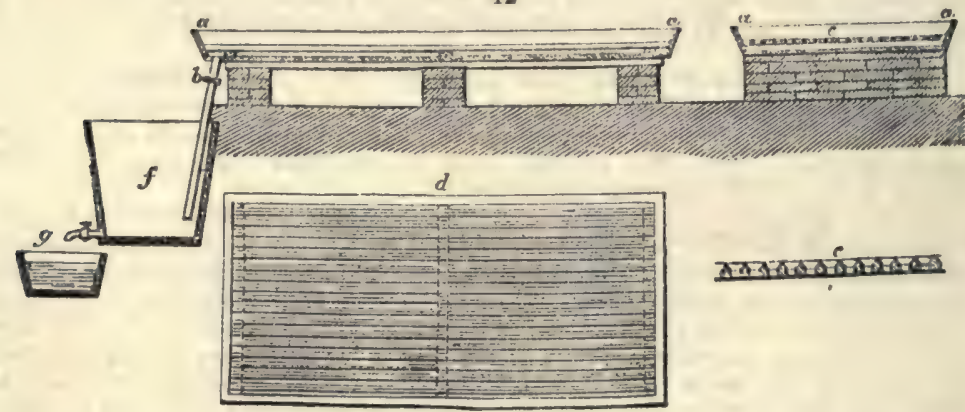

turned over at the edges; a socket of lead, $b$, soldered into the lowest part of the bottom serves to discharge the liquid; 2 , of a wooden crib or grating, formed of rounded rods, as shown in the section $c, c$, and the plan $d$; this grating is supported one inch at least above the bottom, and set truly horizontal, by a series of wedges; 3 , of an open fabric of canvas or strong calico, laid on the grating, and secured over the edges so as to keep it tense. A large wooden reservoir, $f$, lined with lead, furnished with a cover, is placed under each of the filters; a pump throws back once or twice upon the filters what has already passed through. A common reservoir, $g$, below the others, may be made to communicate at pleasure with one of them by means of intermediato stopeocks.

The two boilers for eraporating and decomposing are made of lead, about one - quarter of an inch thick, set upon a fire-brick vault, to protect thom from the direct action of the flame. Through the whole extent of their bottoms above the vault, horizontal cast-iron plates, supported by ledges and brick compartments, compel the flame and burned air, as they issue from tho arch, to take a sinuous course before thoy pass up the chimncy. This floor of cast-iron is intended to support the bottom of the boiler, and to diffuse the heat more equably. The leaden boilers are surrounded with brickwork, and supported at their edges with a wooden framo. They may be omptied at pleasure into lower receivers, called crystallisers, by means of leadon syphons and long-necked funnels.

The crystallisers aro wooden chests lined with Jead, 15 inches deep, 3 or 4 feot broad, and from 6 to 8 feet long, and may be inclinod to ono side at pleasure. A 
round cistern receives the drainings of the mother-waters. The pump is made of lead hardened with antimony and tin.

The subliming furnace is shown in figs. 43 and 44 , by a transwerso and longitudinal section; $a$ is the ashpit; $b$, the grate and fixeplace; $c$, the arch above them. This arch, destined to protect the bottles from the direct action of tho fire, is perfornted with vent-holes, to give a passage to the products of combustion between the subliming ressels; $d, d$, are bars of iron, upon which the bottom of the bottles rests; $e$, stonoware bottles, protected by a coating of loam from the flamo.

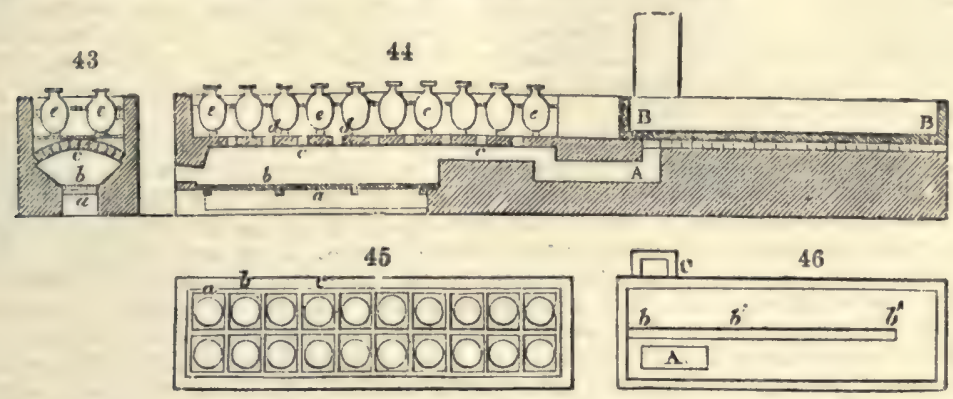

Fig. 45 shows the cast-iron plates, $a, b, c$, which, placed above the vaults, receive each two bottles in a double circular opening.

At the extremity of the above furnace, a second one, called the drier, receives the products of the combustion of the first at $\mathbf{\Delta}$, under horizontal cast-iron plates, and upon which the bottom of a rather shallow boiler, 3, rests. After passing twice under these plates, round a longitudinal brick partition, $b, b^{\prime}, b^{\prime \prime}$, the products of combustion entor the smoke chimney, c. See plan, fig. 46.

The boiler set over this furnace should have no soldered joints. It may be $3 \frac{1}{2}$ feet broad, 9 or 10 feet long, and 1 foot deep. The concrete sal-ammoniac may be crushed under a pair of edge millstones, when it is to be sold in powder.

Bones, blood, flesh, horns, hoofs, woollen rags, silk, hair, scrapings of hides and leather, \&c., may be distilled for procuring ammonia. When bonos are used, the residuum in the retort is bone-black. The charcoal from the other substances will servo for the manufacture of Prussian blue. The bones should undergo a degree of calcination beyond what the ammoniacal process requires, in order to convert them into the best bone-black; but the other animal matters should not be calcined up to that point, otherwise they are of littlo use in the Prussian blue works. If the bones bo calcined, however, so highly as to become glazed, their decolorising power on syrups is nearly destroyed. The other substances should not be charred beyond a red-brown heat.

The condensed vapours from the cylinder-retorts afford a compound liquor holding carbonate of ammonia in solution, mixed with a large quantity of empyreumatic oil, which floats at top. Lest incrustations of salt should at any time tend to obstruct the tulos, a pipe should be inserted within them, and connected with a steam-boiler, so as to blow steam through them occasionally.

The whole liquors mixod have usually a density of $8^{\circ}$ or $9^{\circ}$ Baumé $(1 \cdot 060)$. The simplest process for converting their carbonate of ammonia into the chloride of ammonium is to saturato them with hydrochloric acid, to evaporate the solution in a loaden boiler till a pellicle appears, to run it off into crystallisers, and to drain the crystals. Another process is, to decompose the carbonate of ammonia, by passing its crude liquor through a layer of sulphate of lime, 3 or 4 inches thick, spread upon the filters, fig. 42. The liquor may be laid on with a pump; it should never stand higher than 1 or 2 inches above the surface of the bruised gypsum, and it should be closely covered with boards, to prevent the dissipation of the volatile alkali in the air. When the liquor has passed through the first filter, it must be pumped up on to the second; or the filters being placed in a terrace-form, the liquor from the first may flow down upon the second, and thus in succession. The last filter should be formed of nearly fresh gypsum, so as to insuro the thorough conversion of the carbonate into sulphate. The resulting layers of carbonate of lime should be washed with a little water, to extract the sulphate of ammonia interposed among its particlos. The ammoniacal liquor thus obtained must be complotely saturated, by adding the requisite quantity of sulphuric acid; even a slight excoss of acid can do no harm. It is then to be eraporated, and the oil must bo skimmed off in the course of tho concentration. 
Whon the liquid sulphate has acquired the density of about $1 \cdot 160$, sea-salt should be added, with constant stirxing, till the whole quantity equivalent to the double decomposition is introduced into the lead boiler.

The fluid part must now be drawn off by a syphon into a somewhat deop reservoir, whero the impurities are allowed to subside; it is then evaporatod by boiling till the sulphate of soda falls down in graniular crystals, as the result of the mutual reaction of the sulphate of ammonia and chloride of sodium; while the more soluble chloride of ammonium romains in the liquor. During this precipitation, the whole must be occasionally agitated with wooden paddles; the procipitato being in the intervals removed to tho cooler portion of the pan, in order to be taken out by coppor rakes and shovels, and thrown into draining-hoppers, placed near the edges of the pan. The drained sulphate of soda must bo afterwards washed with cold water, to extract all the adhering sal-ammoniac.

The liquor thus freed from the greater part of the sulphate, when sufficiontly concentrated, is to be drawn off by a lead syphon into the crystallisers, where, at tho end of 20 or 30 hours, it affords an abundant crop of crystals of sal-ammoniac. The mother-water may then be run off, the crystallisers set aslope to drain the salt, and the salt itself must be washed, frst by a weak solution of sal-ammoniac, and lastly with water. It must bo next clesiccated, by tho apparatus fig. 46 , into a perfectly dry powder, then put into the subliming stoneware balloons, by means of a funnol, and well rammed down. The mouth of the bottle is to be closed with a plate or inverted pot of any kind. The fire must be nicoly regulated, so as to effect the sublimation of the pure salt from the under part of the bottle, with due regularity, into a white cake in the upper part. The neck of the bottle should be cleared from time to time with a long steel skewer, to prevent the risk of ehoking, and consoquont bursting; but in spito of every prectution, several of the bottles crack almost in erery operation.-Ure.

The pots are of variable dimensions, but those most frequently employed are about 18 inches in height in the body, and the cups about 10 or 12 inches, with a breadth of 16 inches at the widest part.

In Scotland a process somewhat similar is pursued, the salt being sublimod in castiron pots lined with fire tiles; the condensation being effected in globular heads of green glass, with which each of the iron pots is capped.

Manufacture of Sal-Ammoniac from Gas-Liquor.-By far the largest quantity of the ammoniacal salts now met with in commerce is prepared from 'gas-liquor,' the quantity of which annually produced in the metropolis alone is quite extraordinaryone of the London gas-works producing in one year 224,800 gallons of gas-liquor, by the distillation of 51,100 tons of coal; and the total consumption of coal in London for gas-making is estimatod at about 840,000 tons.

The principle of the conversion of the nitrogen of coal into ammonia by destructive distillation, as in the manufacture of coal gas, will be found described in connection with the processes of gas manufacture and the products produced by the destructive distillation of cosl.

In the purification of the coal gas, the bodies soluble in water are all contained in the 'gas-liquor' (see COAL GAS), together with a certain quantity of tarry matter. The ammonia is chiefly present in the form of carbonate, together with certain quantities of chloride, sulphide, cyanide, and sulphocyanide of ammonium, as well as the salts of the compound ammonias.

For the purpose of preparing the chloride, if hydrochloric acid be not too costly, the liquor is saturated with hydrochloric acid-the solution evaporated to cause the salt to crystallise, and then, finally, the crude sal-ammoniac is purified by sublimation.

Before treatment with the acid, the liquor is frequently distilled.

This is generally effected in a wrought-iron boiler, the liquors passing into a modification of the Coffey's still, by which the solution of ammonia is obtained freor from tar and more concentrated.

The Saturation of the Ammoniacal Liguor with the acid is generally effected by allowing the acid to flow, from a large leaden vessel in which it is held, into an underground tank (fig. 47) containing the liquor, which is furnished with an exit tube, passing into a chimney, to carry off the sulphuretted hydrogen and other offensive gases which are disengaged.

Or, in other works, tho gas-liquor is put into large tuns, and the acid lifted in guttapercha carboys by cranes, thrown into the liquor and stirred with it by means of an agitator; tho offonsiro gases being in this case made to traverse the fire of the steam. engine.

Sometimes the vapours produced in the distillation of the crude gas-liquor are passed in at the lower extremity of a column filled with coko, down which the acid trickles. 
The Evaporation of the crude Saline Solution is generally performed in square or rectangular cast-iron vats, capable of holding from 800 to 1,500 gallons. They are

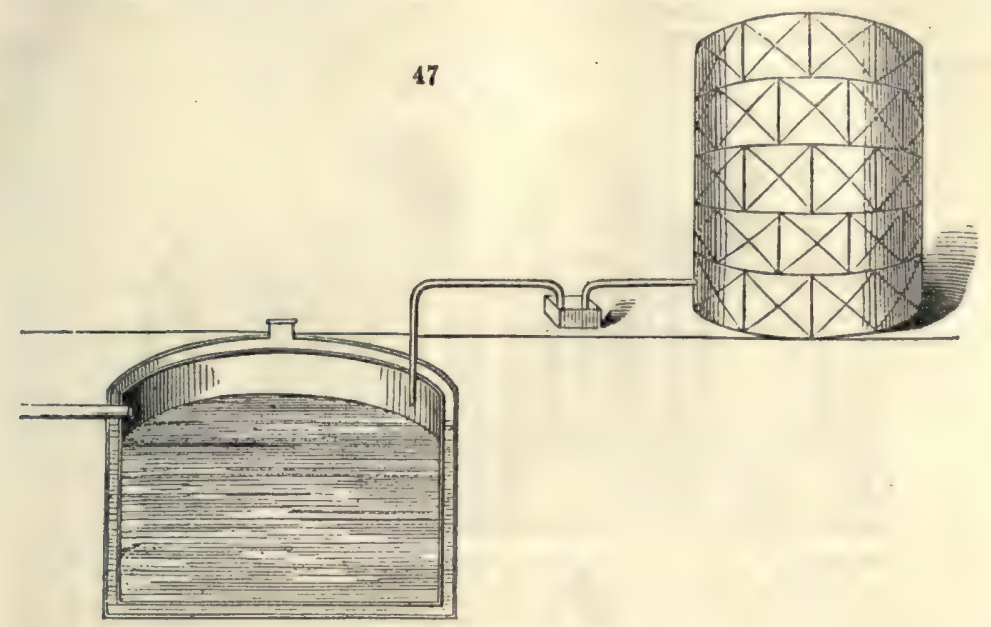

encased in brickwork, the heat being applied by a firo, the flne of which takes a sinuous course beneath the lining of brickwork on which the pan rests, as shown in fig. 48.

When the liquor is evaporated to a specific gravity of 1.25 , it is transferred to the erystallising pans; but during the process of concentration a considerable quantity of

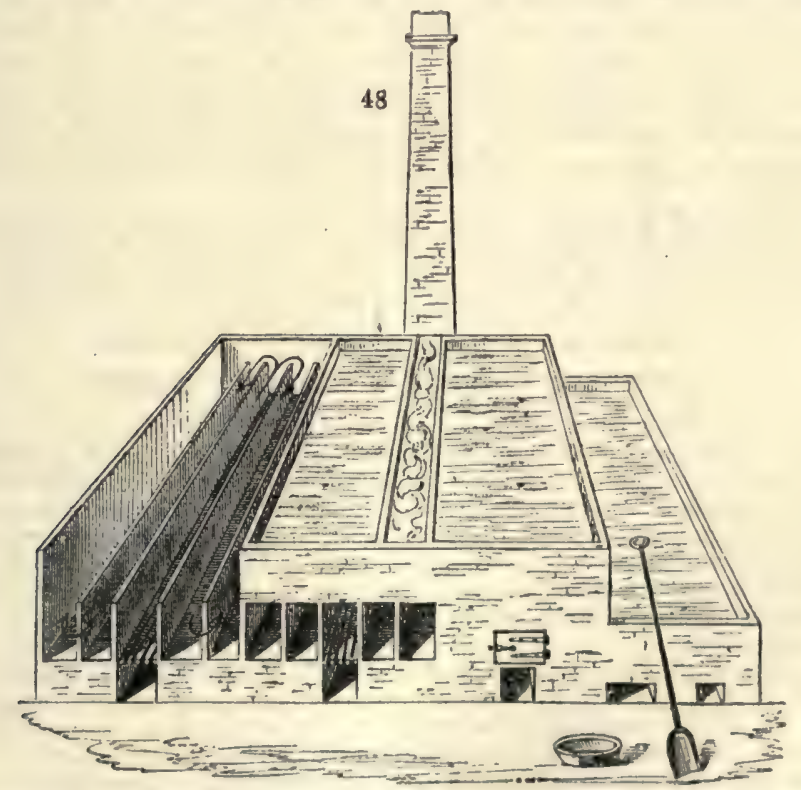

tur separates on the surface, which must be remored, from time to time, by skimming, since it seriously impedes eraporation.

The crystallisation, which takes place on cooling, is performed in circular tubs, from 7 to 8 feet wide, and 2 to 3 doep, which are generally embedded cntirely or partially in the ground. To prevent the formation of large crystals, which would be 
inconvouiont in the subsequent process of sublimation, the liquor is agitated from time to time. The crudo mass obtained, which is contaminated with tarry matter, free acid, and water, is next dried, by gently lioating it on a cast-iron plato under a domo. The groyish-whito mass remaining is now ready to be transforred to tho sublimers.

Tho mothod of sublimation generally adopted in this country consists in beating down into the metal pots, shown in fig. 49 , tho chargo of dry coarsely crystallised sal-

49

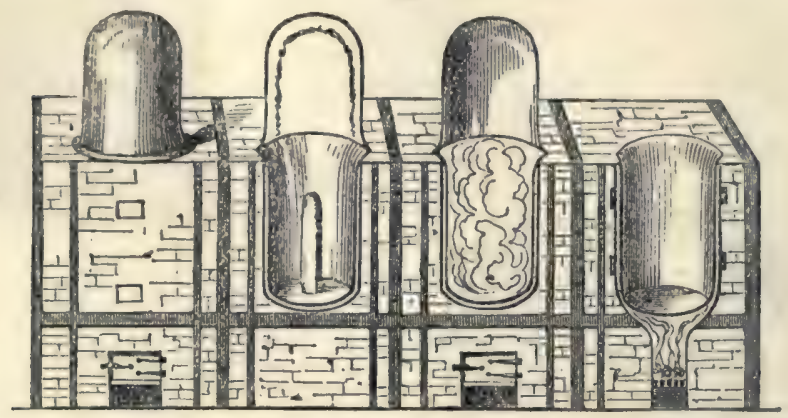

ammoniac. These pots are heated from bolow and by flues round the sides. The body of the subliming vessel is of cast-iron, and the lid usually of lead, or, less frequently, jron. Thero is a small hole at the top, to permit the escape of steam, sometimes loosely closed by a plug of sal-ammoniac, which is removed from time to time to observe the progress of the sublimation; great attention is requisite in the management of the heat, for if it be applied too rapidly a large quantity of sal-ammoniac is carried off with the steam, or even the whole apparatus may be blown up; whilst, if the temperature bo too low, the cake of sul-ammoniac is apt to be soft and yellow.

The sublimation is never continued until the whole of the salt has been rolatilised, since the heat required would decomposo the carbonacoous impurities, and they, emitting volatile oily hydrocarbons, diminish tho purity of the product. In consequence of this incomplete sublimation, a conical mass (shown in the fig. 49) is left behind, called the 'yolk.' After cooling, the dome of the pot is taken off and the attached cake carefully removod. This cake, which is from 3 to 5 inches thick, is nearly pure, only requiring a littie scraping, where it was in contact with the dome, to fit it for the market.

Modifications of the Process.-If, as is often the case, sulphuric acid is cheaper or more accessible than hydrochloric, the gas liquor is neutralised with sulphuric acid, and then the sulphate of ammonia thus obtained is sublimed with common salt (chloride of sodium), and thus converted into sal-ammoniac.

$$
\begin{aligned}
& \mathrm{NH}^{4} \mathrm{O} \cdot \mathrm{SO}^{3}+\mathrm{NaCl}=\mathrm{NH}^{4} \mathrm{Cl}+\mathrm{NaO}_{2} \mathrm{SO}^{3}
\end{aligned}
$$

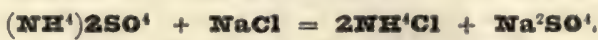

Mr. Croll has taken out a patent for converting crude ammonia into the chloride, by passing the vapours evolved in the first distillation through the crude chloride of manganese, obtained, as a by-product in the preparation of chlorine, for the manufacture of chloride of lime: crude chloride of iron may be used in the same way.

Mr. Laming patented in July, 1843, the substitution of a solution of chloride of calcium for treating the crudo gas-liquor, instead of the mineral acids. Mr. Hills, August, 1846, proposed chloride of magnesium for use in the same way; and sevcral other patents have been taken out by both those gentlemen, for the use of various salts in this way.

Manufacture of Sal-Ammoniac from Guano.-Mr. Young took ont a patent, November 11 th, 1841, in which ho describes his method of obtaining ammonia and its salts from guano. He fills a retort, placod vertically, with a mixture of two parts by weight of gunno, and one part by weight of hydrate of lime. These substances are thoroughly mixed by giving a reciprocating motion to the agitator placed in the retort; a modorato degroe of heat is then appliod, which is gradually increased until tho bottom of the retort becomes red-hot. The ammoniacal gas thus given off is absorbed by water in a condenser, whilst other gases, which are giren off at the same time, being insoluble in water, pass off. Solutions of carbonate, bicarbonato, or sesquicarbonato of ammonia aro produced, by filling the condenser with a solution of ammonia, and passing car- 
bonic acid through it. A solution of chloride of ammonium or sulphato of ammonia is obtained by filling the condenser with diluted hydrochloric or sulphuric acid, and passing the ammonia through it as it issues from the retort.

Dr. Wilton Turner obtained a patent, March 11th, 1844, for obtaining salts of ammonia from guano. The following is his method of obtaining chloride of ammonium in conjunction with cyanogen compounds:-The guano is subjected to destructive distillation in close vessels, at a low red heat during the greater part of the oporstion; but this temperature is increased towards the end. The products of distillation are collected in a series of Woolfe's bottles, by means of which the gases evolved during the operation may be mado to pass two or three times through water, before escaping into the air. These products consist of carbonate of ammonia, hydrocyanic acid, and carburetted hydrogen, the first two of which are rapidly absorbed by the water, with the formation of a strong solution of cyanide of ammonium and carbonato of ammonia. After the ammoniacal solution has been remored from the Woolfo's apparatus, a solution of protochloride of iron is added to it, in such quantities as will yield sufficient iron to convert the latter into Prussian blue, which is formed on the addition of hydrochloric acid in sufficient quantity to neutralise the free ammonia; the precipitate thus formed is now allowed to subside, and is carefully separated from the solution, and by being boiled with a solution of potash or soda, will yield the alkaline ferrocyanide, which is obtained by crystallising in the usual way. The solution (after the removal of the precipitate) should be freed from any excess of iron it may contain, by the careful addition of a fresh portion of the ammoniacal liquor, by which means the oxide of iron will be precipitated, and a neutral solution of ammonia obtained. When the precipitated oxide and cyanide of iron have subsided, the solution of chloride of ammonium is drawn off by a syphon, and the sal-ammoniac obtrined from it by the usual processes; the oxide of iron is added to the ammoniacal solution next operated upon.

If sulphate of iron and sulphuric acid are used, sulphate of ammonia is the ammoniacal salt produced, the chemical changes and operations being similar to the above.

Since the greater part of the nitrogen present in guano exists in the state of ammoniacal salts, which are decomposod at a red heat, nearly the whole of the ammonia which it is capable of yielding is obtained by this method; still there cannot be a doubt that the conversion of the urea, uric acid, and other nitrogenised organic bodies into ammonia, is greatly facilitated by mixing the guano with lime before heating it, as in Mr. Young's process.

Manufacture of Sal-Ammoniac from Urinc.-The urea in the urine of man and other animals is extremely liable to undergo a fermentative decomposition in the presence of the putrefiable nitrogenous matters always presont in this excrement, by which it is converted into carbonate of ammonia.

By treating stale urine with hydrochloric acid, sal-ammoniac separates on evaporation.

Properties.-Chloride of ammonium (or sal-ammoniac) usually occurs in commerce, in fibrous masses of the form of large hemispherical cakes, with a round hole in the centre, having, in fact, the shape of the domes in which it has beon sublimed. By slowly evaporating its aqueous solution, the salt may occasionally be obtained in cakes nearly an inch in height; but it generally forms feathery crystals, which are composed of rows of minute octahedra, attached by their extremities. Its specific gravity is 1.45 , and by heating it sublimes without undergoing fusion. It has a sharp and acrid taste, and one part dissolves in 2.72 parts of water at $6 \tilde{5}^{\circ} \mathrm{F}$, or in an equal weight of water at $212^{\circ} \mathrm{F}$.

It is recognised by its being completely rolatile on heating, giving a whito curdy precipitate of chloride of silver on the addition of nitrate of silver to its aqueous solution, and by the copious evolution of ammonia on mixing it with lime, as well as the production of the yellow precipitate of the double chloride of ammonium and platinum $\mathrm{NH}^{4} \mathrm{Cl}, \mathrm{PtCl}^{2}\left(\mathbf{2 N \mathbf { H } ^ { 4 }}{ }^{4} \mathbf{C l}\right.$. PtCl$\left.{ }^{4}\right)$ on the addition of bichloride of platinum.

Impurities.-In the manufacture of chloride of ammonium, if the purification of the liquor be not effected before crystallising the salt, some traces of protochloride of iron are generally present, and frequently a considerable proportion. Even whon the salt is sublimed, the chloride of iron is volatilised togother with the ehloride of ammonium, and appears to exist in the salt in the form of a double compound (probably of $\mathrm{FeCl}, \mathrm{NH}^{4} \mathrm{Cl}$, analogous to the compound which chloride of ammonium forms with zinc and tin); and this not only in the brown seams of the cake, but likewise in the colourless portion. This accounts for the observation so often made in the laboratory, that a solution of sal-ammoniac, which, when recontly prepared, was perfectly transparent and colourless, bccomes gradually red from the peroxidation of the iron and its precipitation in the form of sesquioxide.

It is in consequence of the existence of the iron in the state of this double salt, that Wurtz found that chloride of ammonium containing iron in this form gnve no incli. 
cations of its presence by the usual reagents until after the addition of nitric acid; and it is curious that there likewise exists a red compound of this class in which the iron exists in the stato of perchloride similarly marked, in fact as $\mathrm{NH}^{4} \mathrm{Cl}, \mathrm{F}^{2} \mathrm{Cl}^{3}$.

A very simple method of removing the iron, suggested by $\mathrm{Mr}$. Brewer, consists in passing a fow bubbles of chlorine gas through the hot concentrated solution of the salt, by which the protochloride of iron is converted into the perchloride.

$$
2 \mathrm{Fe} \mathrm{Cl}^{0}+\mathrm{Cl}=\mathrm{Fe}^{2} \mathrm{Cl}^{3} \text {. }
$$

$\mathbf{2} \mathbf{P C l}^{2}+\mathbf{C l}^{2}=\mathbf{F e}^{2} \mathbf{C l}^{8}$.

The free ammonia always present in the solution decomposes this perchloride with precipitation of sesquioxide, and formation of an additional quantity of sal-ammoniac.

$$
\begin{aligned}
& \mathrm{Fe}^{2} \mathrm{Cl}^{3}+3 \mathrm{NH}^{4} \mathrm{O}=\mathrm{Fe}^{2} \mathrm{O}^{8}+3 \mathrm{NH}^{4} \mathrm{Cl} . \\
& \mathbf{F} \mathbf{e}^{2} \mathbf{C l}^{8}+3\left(\mathbf{M} \mathbf{R}^{4}\right)^{2} \mathbf{O}=\mathbf{F e}^{2} \mathbf{O}^{3}+6 \mathbf{N B}^{4} \mathbf{C l} .
\end{aligned}
$$

The sesquioxide of iron, which is of course present in the form of a brown hydrate, is filtered off or separated by decantation, and a perfectly pure solution is obtained.

The only precaution necessary is to avoid passing more chlorine than is requisito to peroxidise the iron, since the ammonia salt itself will be decomposed with evolution of nitrogon, and the dangerously explosive body, chloride of nitrogen, may result from the union of the liberated nitrogen with chlorine.

Uses.-The most important use of sal-ammoniac in the arts is in joining iron and other metals, in tinning, \&c. It is also extensively used in the manufacture of ammonia-alum, which is now largely employed in the manufacture of mordants instead of potash-alum. A considerable quantity is also consumed in pharmacy.

Sal-ammoniac is one of those salts which possesses, in a high degree, the property of producing cold whilst dissolving in water; it is, therefore, a common constituent of frigorific mixtures. See Frementa Mrxturks.

AMMONIUM, SUIPAIDES OF. When sulphuretted hydrogen gas is passed into a solution of ammonia in excess, it is converted into tho doublo sulpliclo of ammonium and hydrogen - or, as it is frequently called, the hydrosulphate of sulphide of ammonium.- NH'S, HS ( $\mathbf{z r}^{4}$, $\mathbf{z s}$ ).

This solution is extensively employed as a re-agent in the chemical laboratory, for the separation of those metals the sulphides of which are soluble in acids-viz. nickel, cobalt, manganese, zinc, and iron, which are precipitated by this reagent in alkaline solutions.

By exposure to the air, the hydrosulphuric acid which it contains is decomposed, the hydrogen being oxidised and converted into water, whilst the liberatod sulphur is dissolved by the sulphide of ammonium, forming the bisulphide, or even higher sulphide.

This solution of the polysulphide of ammonium is a valuable reagent for dissolving the sulphides of certain metals, such as tin, antimony, and arsenic, the sulphides of which play the part of acids and form salts with the sulphide of ammonium.

By this deportment with sulphide of ammonium, these metals are separated, both on the small scale in the laboratory, and also on the large seale, from the sulphides of those metals - such as lead, copper, mercury, \&ce-the sulphides of which are insoluble in sulphide of ammonium.

The higher sulphides, viz. the tersulphide and the pentasulphide, are bodies of purely scientific interest. They are obtained by distilling the corresponding sulphides of potassium with sal-ammoniac.

All the sulphides of ammonium are soluble in water without decomposition.

Ammonia combines with all the inorganic and organic acids, but for an account of these compound bodies we must refer to Watts's 'Dictionary of Chemistry,' as they have but fow applications in the arts and manufactures.

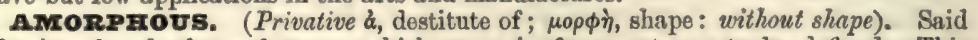
of mineral and other substances which oceur in forms not easy to be defined. This torm may be regarded as the opposito of crystalline. Some elements exist in both the crystalline and the amorphous states, as carbon, which is amorphous in charcoal, but erystalline in the diamond.

The peculiarities which give rise to these conditions-eridently depending upon molecular forces which have not yot been defined-present one of the most fertile fields for study in the range of modern science.

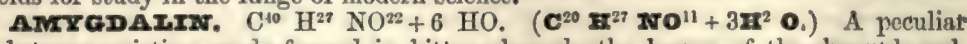
substance, existing ready-formed in bitter almonds, the loaves of the cherry-laurel, the kernels of the plum, cherry, peach, and the leaves and bark of Prunus padus, and in the young sprouts of the $I^{\prime}$. domestica. It is also found in the sprouts of several epecies of Sorbuts, such as S. aucuparia, S. torminalis, and others of the same order. 2o prepare it, the bitter almonds are subjected to strong pressure between hot plates 
of metal. This has the effect of removing the bland oil known in commerce as almondoil. Tho residue, when powdered, forms almond-meal. To obtain amygdalin fromthe meal, the latter is extracted with boiling alcohol of 90 or 95 por cent. The tincture is to be passed through a cloth and the residue prossed, to obtain the fluid mechanically adhorent to it. The liquids will be milky, owing to the presence of some of the oil. On keeping the fluid for a few hours, it may be separated by pouring off, or by means of a funnel, and so obtained clear. The alcohol is now to be removed by distillation, the latter being continued until five-sixths have come over. The fluid in the retort, when cold, is to have the amygdalin precipitated from it by the addition of half its volume of ether. The crystals are to be pressed between folds of filtering paper, and recrystallised from concentrated boiling alcohol. As thus prepared, it forms pearly seales, very soluble in hot alcohol, but sparingly whon cold; it is insoluble in ether, but water dissolves it readily and in large quantity. The erystals contain six atoms of water of erystallisation. Most persons engaged in chemical operations have noticed, when using almond-meal for the purpose of luting, that, before being moistoned with water, it has little odour, and what it has is of an oily kind; but, after moistening, it soon acquires the powerful and pleasant perfume of bitter-almond oil. This arises from a singular reaction taking place between the amygdalin and the vegetable albumen or emulsin. The latter merely acts as a ferment, and its elements in no way enter intr the products formed. The decomposition, in fact, takes place between one atom of amygdalin and four atoms of water; the product being one atom of bitter-almond oil, two atoms of grape-sugar, and one of prussic acid.

In preparing amygdalin, some chemists add water to the residue of the distillation of the tincture, and then yeast, in order to remove the sugar present, by fermentation, previous to precipitating with ether: the process thus becomes much more complex, because it is necessary to filter the fermented liquid, and concontrate it again by evaporation, before precipitating the amygdalin.

The proof that the decomposition which is experienced by the bitter-almond cake, when digested with water, is owing to the presence of the two principles mentioned, rests upon the following considerations. If the mare, or pressed residue of the bitter almond, be treated with boiling water, the emulsin-or vegetable albumen - will become coagulated, and incapable of inducing the decomposition of the amygdalin. It is only the bittor almond which contains amygdalin; the sweet variety is, therefore, incapable of yielding the essence by fermentation. But sweet almonds resemble the bitter in containing emulsin; and it is excoedingly interesting -as illustrating the truth of the explanation given above - that if a little amygdalin be added to an emulsion of sweet almonds, the bitter-almond essence is immediately formed. A temperature of $100^{\circ}$ is the most favourable for the digestion.-C. G. W.

AMYrEx tion of amylic alcohol by sulphuric acid; also by the dry distillation of amyl-sulphate of calcium. It is a colourless thin liquid, with a faint offensive odour. It has been tried as a substitute for chloroform without success.

AMXIUM Maxprocer. Mandioea or Cassava starch. See Cassava and MaNDiocA.

ANACARDTUM IrUT. Dr. Bottyer, in the 'Bayerisches Industrie- und Gewerbe-Blatt,' states that the juice of the Anacardium nut, Anacardium Orientale, contains an oily matter, which, by exposure to the air, assumes a fine black colour, which is quite pormanent against the influences of acids or alkalies, chlorine or cyanide of potassium. It is recommended for use as a marking ink; and if the linen be moistened with a little ammonia, the black is very intense and quite permanent.

AxAIYSIs. In chemistry, a term which is employed to signify the art of resolving a compound substance into its constituont parts. Every manufacturer should so study this art, in the proper treatises and schools of chemistry, as to enable him properly to understand and regulate his business.-Soe Watts's 'Dictionary of Chemistry.' Seo also Spectrom Axalysis,

AmATAsz. An oxide of titanium, of the same composition as Brookite and Rutile. It occurs in Dauphiny with felspar and ilmenite, in Devonshire in chlorite, and in North Wales with Brookite. It is said to be found in the slags from the iron furnaces of Orange County, United States.

AJCHOR. (Ancre, Fr. ; Anker, Ger.) An iron hook, of peculiar construction and of considerable weight and strength, for enabling a ship to lay hold of the ground, and fix itself in a certain situation by means of a rope called the cable. The necessity for securing boats, canoes, or ships in a certain position, has led to the adoption of anchors, of some description, amongst every nation dwelling upon the shores of seas, lakes, or rivers. They were often of the rudest description. We are informed that the Grocks at first used stone anchors, but that they subsequently employed in. 
struments of iron, having one, two, and threo toeth, which were not apparently very different from those wo now employ. The auchors which are used by many of the races inbabiting the shores of the Indian Ocoan are made of tho so-called 'iron-wood,' which is so dense that it sinks in sea-water. The anchor is an instrument of the greatest importance to the navigator, since upon its taking and keoping hold depends his safety upon many occasions, espocially near a leo shore, whero he might be othorwise stranded or shipwrocked. Anchors are gonerally mado of wrought iron, except among nations who cannot work this metal well, and who therofore use copper. The modo in which an anchor oporates will be understood from inspection of fig. 50 , whoro,

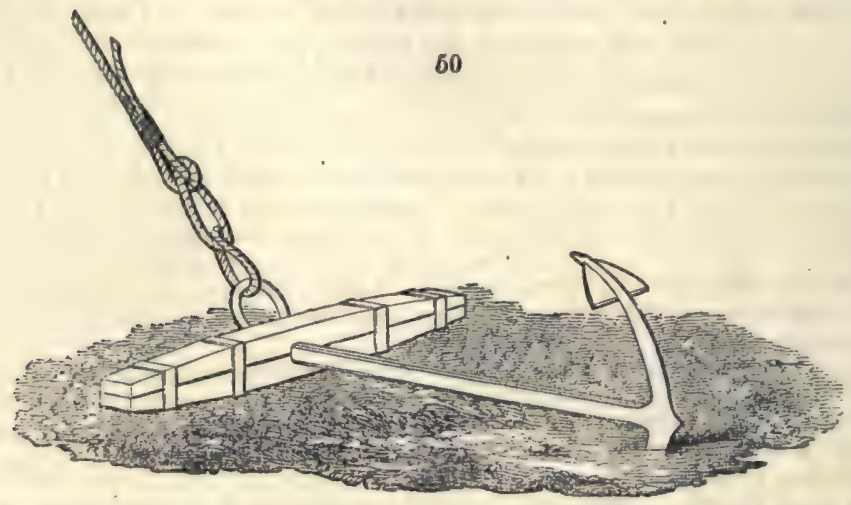

from the direction of the strain, it is obvious that the anchor cannot move without ploughing up the ground in which its hook or fluke is sunk. When this, however, unluckily takes place, from the nature of the ground, from the mode of insertion of the anchor, or from the violence of tho winds or currents, it is called dragging the anchor. When the hold is good, the cable or the buried arm will sooner break than the ship will drive. Anchors are of different sizes, and have difforont names, according to the purposes they serve; thus there are bower, stream, and kedge anchors. Ships of the first class have sevon anchors, and smaller vessels, such as brigs and schooners, throe.

The metal omployed for anchors of wrought iron is known as 'scrap iron,' and for the bost anchors, such as Lonox's, they also use good 'Welsh mine iron.'

It is not practical, without occupying more space than can be afforded, to describe in detail the manufacture of an anchor. It does not, indeed, appear desirable that we should do so, sinco it is so special a form of mechanical industry, that fow will consult this volume for the sake of learning to make anchors. The following will therefore suffice. The anchor-smith's forge consists of a hearth of brickwork, raised about 9 inches above the ground, and generally about 7 foet square. In the centre of this is a cavity for containing the fire. A vertical brick wall is built on one side of the hearth, which supports the dome, and a low chimnoy to carry off the smoke. Behind this wall are placed the bellows, with which the fire is urged; the bellows boing so placed that they blow to the centre of the fire. The anvil and the crane by which the heavy masses of motal aro moved from and to the fire are adjustod near tho hearth. The Hercules, a kind of stamping machine, or the steam hammer, noed not be doscribod in this place.

To make the anchor, bars of good iron are brought togother to be faggoted; the number varying with the size of the anchor. The faggot is kept together by hoops of iron, and tho whole is placed upon the properly arrangod hearth, and covered up by small coals, which are thrown upon a kind of oven mado of cinders. Great caro and good management is required to keop this temporary ovon sound during the combustion;- $-\mathbf{s}$ smith strictly attends to this. When all is arranged, the bellows are set to work, and a blast urged on tho firo; this is continued for about an hour, when a good welding heat is obtained. The mass is now brought from the fire to the anvil, and the iron welded by the hammers. One portion having been welded, the iron is returned to fire, and the operation is repeated until the whole is welded into one mass.

This will be understood by roferring to the annexed figures (fig. 51), in which the bars for tho shanks, $\mathbf{\Delta} \mathbf{\Delta}$, and the arms, $\mathbf{B} \mathbf{B}$, are shown, in plan and sections, as bound together, and thoir shapes after boing welded beforo union; and c c represents the palm. 
The different parts of the anchor being made, the arms are united to the ond of the shank. This must be done with groat care, as the goodness of the anchor depends entirely upon this procoss being effectivoly performed. The arms boing wolded on,

51
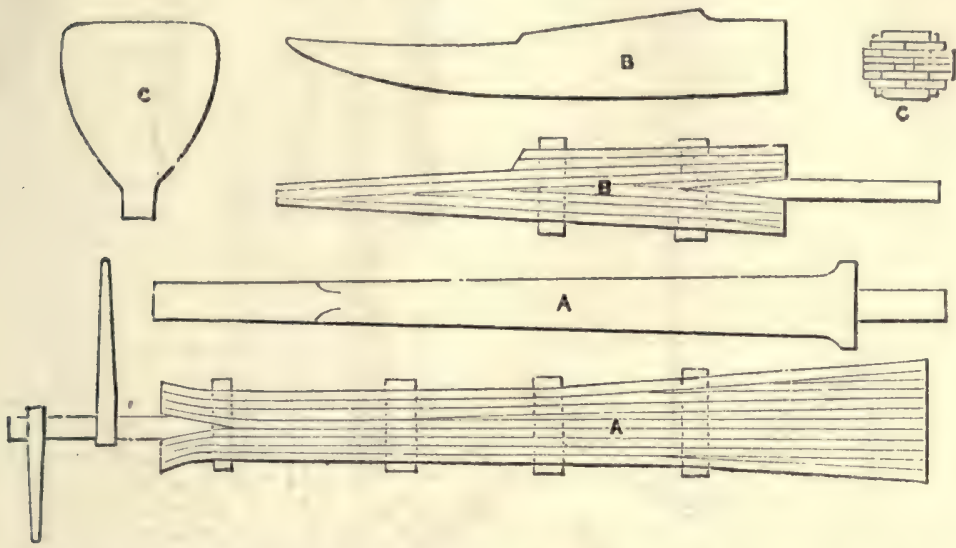

the ring has to be formed and welded. The ring consists of several bars welded together, drawn out into a round rod, passed through a hole in the shank, bent into a circle, and the ends welded together. When all the parts are adjusted, the whole anchor is brought to a red heat, and hammered with lighter hammers than those used for welding, the object being to give a finish and evenness to the surface.

The toughest iron which can be procured should be used in the manufacture of an anchor, upon the strength of which both the security of raluable liros and much property depends.

The manufacture of anchors requires great knowledgo of the structure of iron, and skill in the art of working it. The various parts of an anchor are thus niamed:In fig. $52, \mathrm{~A}$ is the shank; $\mathrm{B}$, the arm or fluke; c, the palm; $\mathrm{D}$, the blade; $\mathrm{B}$, the square; $\mathrm{F}$, the nut; $\mathrm{G}$, the ring; $\mathbf{H}$, the crown,- the proportional weights of the several parts being as follows:-

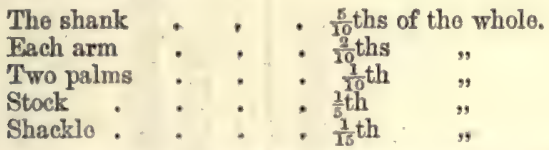

The drawings on next page (fig. 53) show an anchor on the old plan and the dissected parts of which it is composed; and (fig. 54), the patent anchor as invented by Mr. Perring, with its several parts dissected as before.

Perring's improved ancher was a very ingenious one. The bars and half the breadth of the anchor are first welded soparately, and then placed side by side, when the upper half is worked into one mass, while the lower part is left disunited, but it has carrier iron bars, or porters, as the prolongation rods $(3,3$, fig. 53), are commonly called, welded to the extremity of each portion. The lower part is now heated and placed in the clamping machine, which is merely an iron plate firmly bolted to a mass of timber, and bearing upon its surface four iron pins. One end of the crown is placed botween the first of these pins, and passed under an iron strap; the other end is brought between the other pins, and is bent by the leverage power of the elongated rodis or porters.

Thus a part of the arm being formed out of the crown gives much greater security, when a true union of fibres is effected, than when the junction was made merely by a short searf.

The angular opening upon the side opposite is flled with the chock, formed of short iron bars placed upright. When this has been firmly welded, the truss-piece is brought over it. This piece is made of plates similar to the above, except that their 
edges are here horizontal. The truss-piece is half the broadth of the arm; so that, when united to the crown, it constitutes, with the other parts, the total breadth of the arms at those places.

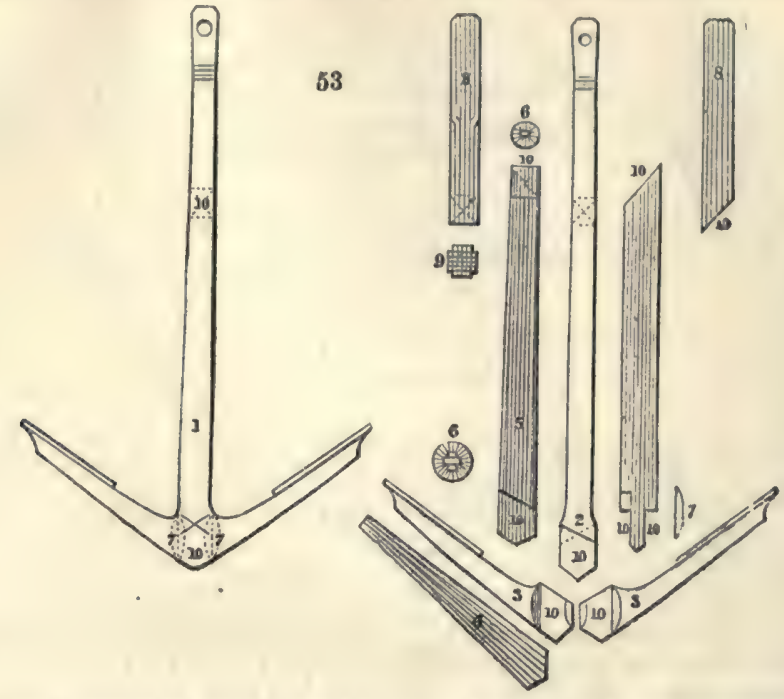

The shank is now shat upon the crown; the square is formed, and the nuts welded to it; the hole is punched out for the ring, and the shank is then fashioned.

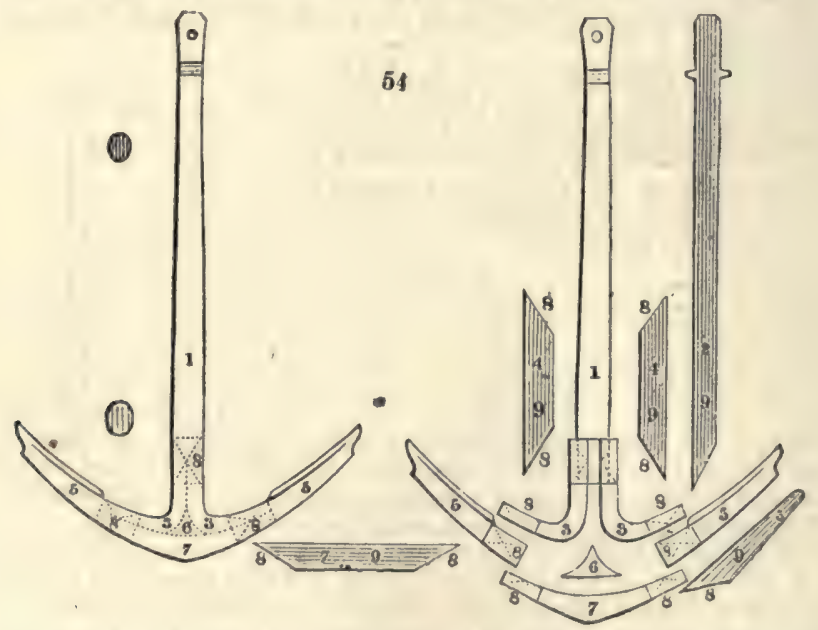

The blade is made much in the way above described. In making the palm, an iron rod is first bent into the approximate form, notching it so that it may more readily take the desired shape. To one end a porter rod is fastened, by which the palm is carried and turned round in the fire during the progress of the fabrication. Iron plates are next laid side by side upon the rod, and the joint at the middle is broken by another plate laid over it. When the mass is worked, its under side is filled up by similar plates, and the whole is completely welded; pieces being added to the sides, if necesgary, to form the angles of the palm. The blade is then shut on to the palm, after which the part of the arm attached to the blade is united to that which constitutes the crown. The smith-work of the anchor is now finished.

The junction - or shutting-on, as the workmen call it-of the several members of an anchor is effected by an instrument callod a Horcules, which is merely a mass of iron 
raised to a certain height, between paralleI uprights, as in the pile-engine or vertical ram, and let fall upon the metal previously brought to a welding heat.

The end of the shank is squared to receive and hold the stock steadily and keep it from turning. To prevent it shifting along, there are two knobs or tenon-like projections. The point of the angle $\mathbf{H}$, betwoen the arms and the shank, is sometimes called the throat. The arm, B C, generally makes an angle of $56^{\circ}$ with the shank A ; it js either round or polygonal, and about half the length of the shank.

The stock of the anchor ( fig. 50) is made of oak. It consists of two beams which embrace the square, and are firmly united by iron bolts and hoops, as shown in the figure. The stock is usually somewhat longer than the shank, has in the middlo a thickness about one-twelfth of its length, but tapers at its under-side to nearly onehalf this thickness at the extromities.

$\because$ An ingenious form of anchor was made the subject of a patent, by Lientenant Rodger, of the Royal Navy, in 1828, and was afterwards modified by him in a second patent, obtained in August, 1829. The whole of the parts of the anchor are to be bound together by means of iron bands or hoops, in place of bolts or pins.

Fig. 55 is a side view of a complete anchor, formed upon his improved construction, and fig. 56 a plan of the same; fig. 57, an ond view of the crown and flukes; or arms ; fig. 56 represents the two prin. cipal iron plates, $a$, of which the shank is constructed, but so as to form parts of the-stump-sirms to which the flukes aro to be connected.

The crown-piece is to be welded to the stump-piece, $\odot c ; f i g .58$, as well as to the end, $l$, of the centre-piece, $h h$, and the gearfs, $m m$, are to be cut to receive the armis or flukes. Previously, however, to uniting the arms or flukes to the stump-arms, the crown and throat of the anchor are to be strengthened by the applieation of the crown slabs, $w$, fig. 58 , which are to be welded. npon each side of the crown, overlapping the end of the pillar, $h$, and the throat or knees of the stump-arms and the crown-piece. The stump-arms are then to be strengthened in $a$ similar manner, by the thin flat pieces, $p p$, which are to be welded upon each side. The palms are united to the flukes in the usual way, and the flukes are also united to the stump-arms by means of the long searfs, $m m$. When the shank of the anchor has been thus formed, and united with the flukes, the anchor-smith's work may be said to be complete.

Another of the improrements in the

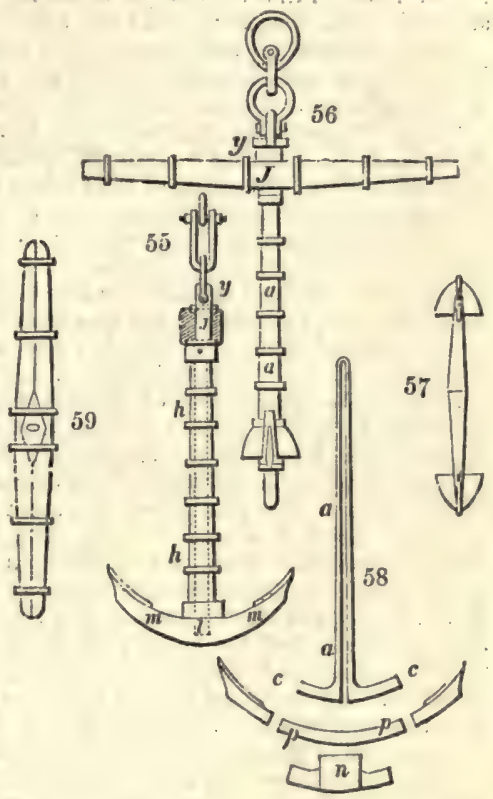
construction of anchors, claimed under

this patent, consists in a new method of affixing the stock upon the shank of the anchor, which is effected in the following manner: in fig. 55 the stock is shown affixed to the anchor; in fig. 58 it is shown detached. It may be made either of one or two pioces of timber, as shall be found most convenient. It is, however, to be observed that the stock is to be completed before fitting on to the shank. After the stock is shaped, a hole is to be made through the middle of it, to fit that part of the shank to which it is to be affixed. Two stock plates are then to be let in, one on each side of the stock, and made fast by counter-sunk nails and straps, or hoops ; other straps or hoops of iron are also to be placed round the stock; as usual.

In place of nuts, formed upon the shank of the anchor, it is proposed to secure the stock by means of a hoop and a key, shown above and below $\mathrm{J}$, in fig. 56 . By this contrivance the stock is prevented from going nearer to the crown of the anchor than it ought to do, and the key prevents it from sliding towards the shackle.

Since fitting the stock to the shank of an anchor by this method prevents tho use of a ring, as in the ordinary manner, the patentee says that he in all cases substitutes a shackle for the ring, and which is all that is required for a chain cable; but when a hempen cable is to be used, he connects a ring to the usual shackle, by means of a joining shackle, as in figs, 55 and 56 . The stock is shown in fig. 69 .

Vot. I. 
Mr. Rodger proposes, under another patent, dated July, 1833, to alter the size and form of the palms; having found from experionce that anchors with small palms will not only hold better than with large ones, but that the arms of the anchor, evon without any palms, hare been found to take more securo hold of tho ground than anchors of the old construction of similar weight and length. Ho has accordingly fixed upon one-fifth of the length of the arm, as a suitable proportion for the length or depth of the palm. He makes the palms, also, broader than they are long or deep:

Previously to the introduction of Lieutenant Rodger's small-palmed anchor, ships wore supplied with heavy, cumbersome contrirances with long shanks and broad palms extending half-way up the flukes. So badly wore they proportioned, that it was no uncommon thing for them to break in falling on the bottom, particularly if the ground was rocky. But, if once firmly imbedded in stiff holding ground, there was considerable difficulty in breaking them out. The introduction of the small palm, therefore, forms an important era in the history of anchors.

The next important introduction was Porter's anchor, with moveable flukes or arms. One grand object sought to be attained here, was the prevention of fouling by the cable. It was considered, also, that as great injury was frequently occasioned by a ship grounding on her anchor, the closed upper arm would remedy the evil. It was found, however, that the anchor would not take the ground properly as at first constructed, and hence the 'shark's fin' upon the outside of each fluke.

Rodger's invention was for some time viewed with distrust; but, from time to time, improvements were introduced, until the patent, which gained the Exhibition prize, was brought out. On this the jurors reported as follows :-

- Many remarkable improvements have been recently mado by Lieutenant Rodger, R.N., insuring a better distribution of the metal in the direction of the greatest strains. The palm of the anchor, instead of being flat, presents two inclined planes, calculated for cutting the sand or mud instead of resisting perpendicularly; and the consequence is, that these new anchors hold much better in the ground. The committee of Lloyd's-so competent to judge of every contrivance likely to preservo ships-have resolved to allow for the anchors of the ships they insure a sixth less weight if made according to the plan of Lieutenant Rodger.'

The original Porter's anchor has also undergone considerable modification; and, under the name of 'Trotman's anchor,' has now a conspicuous place.

Another invention is that of Mitcheson's, which, in form and proportions, strongly resembles Rodger's; but the palm is that adopted in Trotman's, or Porter's anchor. It is a trifle longer in the shank than Rodger's, and has a peculiar stock, which-although original in its form-lacks originality in its design, since Rodger had previously introduced a plan for an iron stock to obviate the weakness caused by making a hole for the stock to pass through. Mr. Lenox was the inventor of an anchor which differed somewhat from the Admiralty's anchor-a modification of Rodger's-in being shorter in the shank and thicker in the flukes, the palms being spade-shaped. Mr. J. Aylen, the Master-Attendant of Sheerness Dockyard, modified the Admiralty's anchor. Instead of the inner part of the fluke, from the crown to the pea, being rounded, as in the Admiralty plan, or squared as in Rodger's and Mitcheson's, it is hollowed. An American anchor, known as Isaac's, has a flat bay of iron from palm to palm, passing the shank elliptically on both sides; and from the end of the stock to the centre of the shank two other bars are fixed to prevent its fouling.

With the anchors thus briefly described the Admiralty ordered trials to be made at Woolwich, and at the Nore. The results of those trials-the particulars of which need not be given here-were, that Mitcheson's, Trotman's, Lenox's, and Rodger's were solected as the best.

A competent authority, writing in the Inited Service Gazette, says:- 'The general opinion deduced from the series of experiments is, that although Mitcheson's lus been so successful, the stock is not at prosent seaworthy. 'Trotman's has come out of the trial very successfully, but the construction is too complicated to render it a good working anchor. Whon once in the ground, its holding properties are very superior; in fact, a glance at its grasp will show that it has the capabilities of an anchor of another construction one-fifth largor. There are, however, drawbacks not easily to be overcome. Its taking the ground is more precarious than with othor anchors; and if a ship should part her cable, it would scarcely be possible to sweop the anchor. It is also an awkward anchor to fish and to stow. Yet there are other merits which render it, upon the whole, a most valuable invention, and no ship should go to sea without one. Of Lenox's it is sufficient to say that it has been found equal to, and that it has gained an advantage over, Rodger's; but so strong is the professional feeling in farour of the latter, that it will over remain a favourite. Our recommendation would be thus:- Lenox and Rodger for bower anchors, Mitcheson for a sheet, and 'Trotman for a spare anchor.' 
The following Table gives at one view the results of the experiments made by the Admiralty upon breaking the trial-anchors, and the time occupied upon each experiment:-

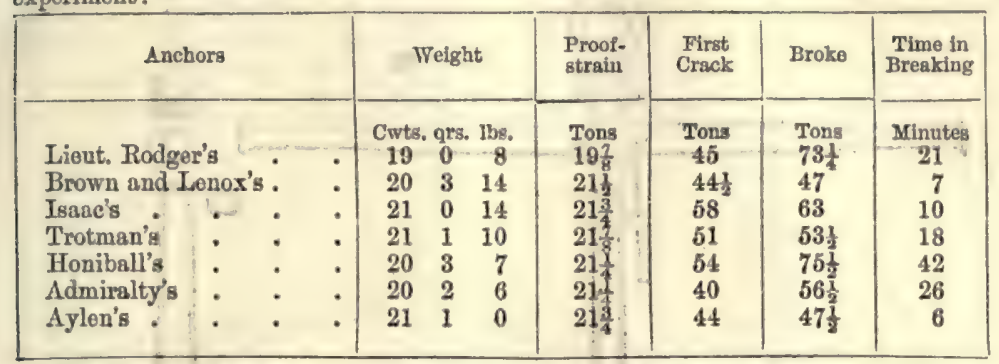

The history of the introduction of Lenox's anchors to the British navy was as follows :

After sundry attempts to induce the Admiralty to give up entirely the use of hempen cable anchors, in consequence of their breaking when applied to chain cables, Mr. Lenox, in 1832, was permitted to alter some of the old anchors to such proportions and shape as would enable them to stand a proof-strain upon the machine in Woolwich Dockyard. It was found, as previously apprehended and asserted, that from the inequality of material in the old anchors, not above one in three was successfully altered, and Mr. Lenox was ordered to supply new anchors, which were proved, and then approved of. This state of things continued until 1838, when Mr. Lenox was requested to reconsider and complete the shape and proportions of anchors for the navy, with a view to a contract being given out for the supply of such anchors to the service. Then was constructed the shape called the 'Admiralty,' or 'Sir William Parker's Anchor' (Sir William being then Store Lord). Mr. Lenox suggested to Sir William the doing away with every sharp edge and line in an anchor, and adopting the smooth long-oval (in the section) for the general shape of shank and arm. This was approved of by Sir William, and he brought it out as his anchor. An entire Table of proportions was furnished; but that it might meet with no opposition from the influence of dockyard authority, it was sent to the officers of Portsmouth Yard for their approval. They returned it after a few months, with some slight alterations in the proportions of some of the sizes, and recommended the construction to be on 'Perring's principle' of the cushioned, or made-up, crown. It was so adopted, and continued to be made by Brown and Lonox for about a year or two, when the great and unnecessary expense incurred by the plan was pointed out. It was contended it was without any good; because, if the crown of the anchor, or any shut or weld, was made sound and perfect, the amalgamation of the grain of the iron would be complete, and assume its full power or strength, whatever way it might be put together; and the strongest form was that which exposed the least surface of iron to the wolding heat, and consequently to injury. About the latter end of 1839 , the subject was again opened. Mr. Lenox renewed his objections, by letter to Sir William Parker, to 'Perring's plan' of shutting-up, and the consequence was - a contract, with specification, \&c. \&c. appeared, and an improved or modified plan of shutting-up (as it is called) was proposed by Mr. Tyler, mastersmith of Portsmouth Yard, which was adopted; and Mr. Lenox's shape and proportions (slightly altored, as before said) came out as 'Sir William

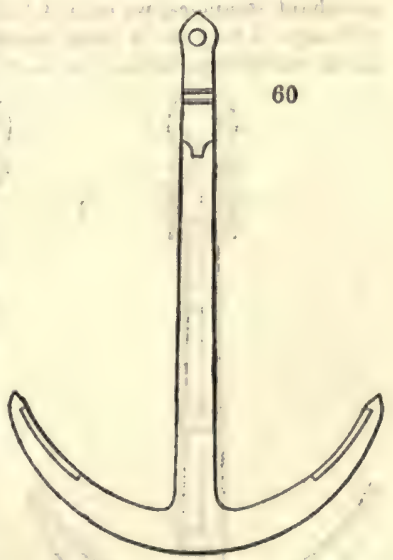
Parker's,' or the 'Admiralty Anchor,' and continued, until after the trials in 1852, with every success in actual service that a good anchor could maintain, and they were made and sold in quantities to all the world.

In tho navy of England, and in nearly all foreign navies, this anchor, of which fig. 60 represents the form, was adopted. They are also largely employed in the merchant service; but theso are not so nicely proportioned as the anchors made for the Government, nor are they so highly finished. Many merchant captains, however, take Rodgor's anchor, and our steamers almost invariably take Portor's or Trotman's anchor. 
Trotman's Anchor is represented in fig. 61, under its various positions. Although for convonionce Trotman's anchor is, as wo have already stated, largely used by the

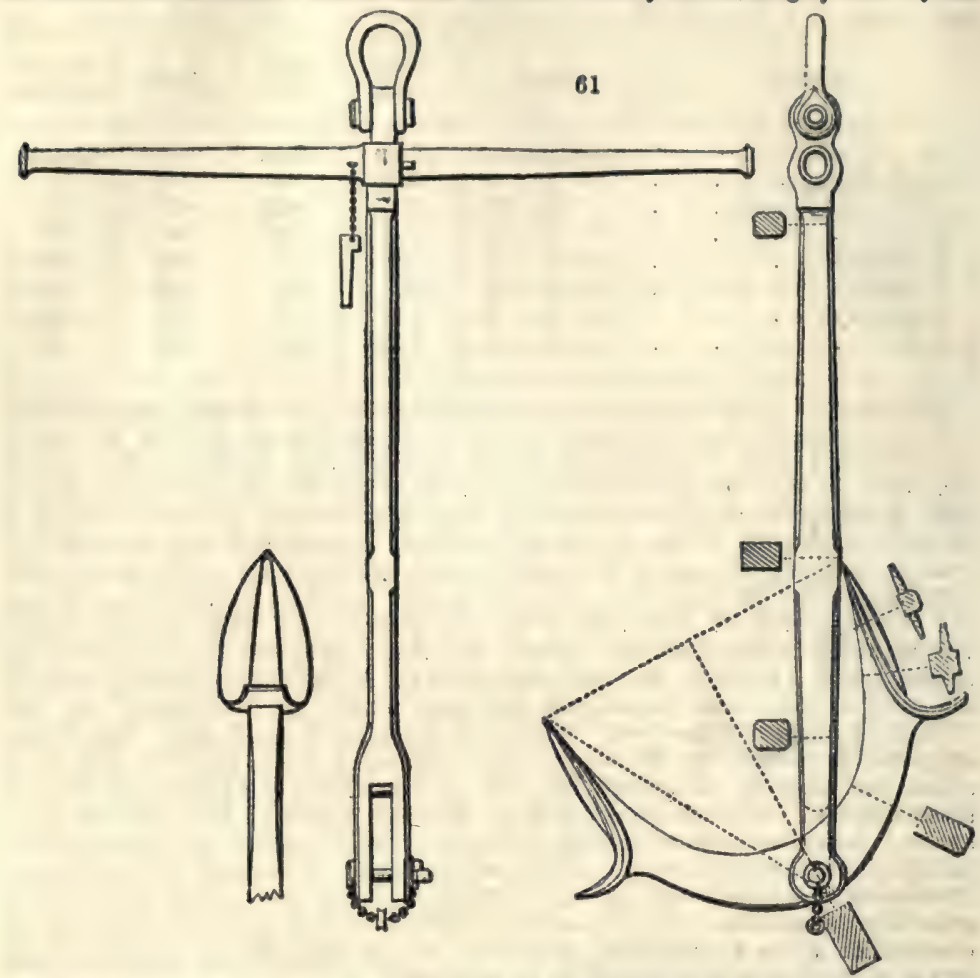

morchant steamers, wo cannot but feel that the soparation of the fluke from the shaft, nlthough it may be in many cases unobjectionable, is attended with the risk that when, in an emergency, the anchor is required, the means of connexion may be at fault.

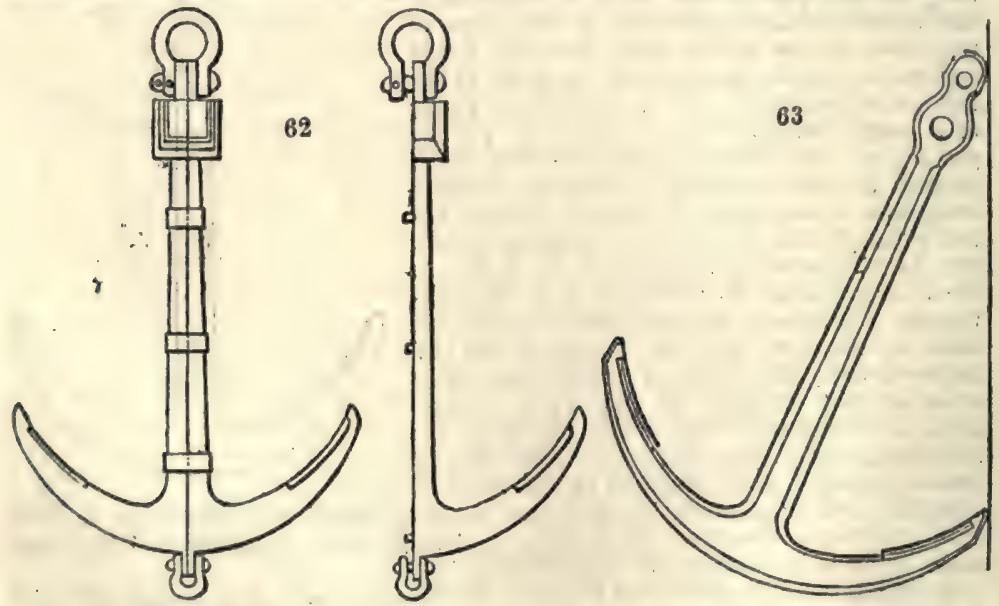

Captain Hall's anchor is a very valuable one, from the circumstance that it is capable of division, as shown in fig. 62 , so that it can bo taken out in boats.

There are various other shapes of anchors; but attention has been confined to those genezalty employed, 


\section{$\triangle N C H O R$}

Wo are not in a position to offer any opinion upon the value of the several anchors which have been named. Having described their peculiarities, there remains but little to be said. The solidity of Lonox's anchors - as shown in fig. 63, and again in their more recent modifications, in plan and section, with the new form of iron stock, fig. 64-has recommended them strongly, and hence thoir general use.
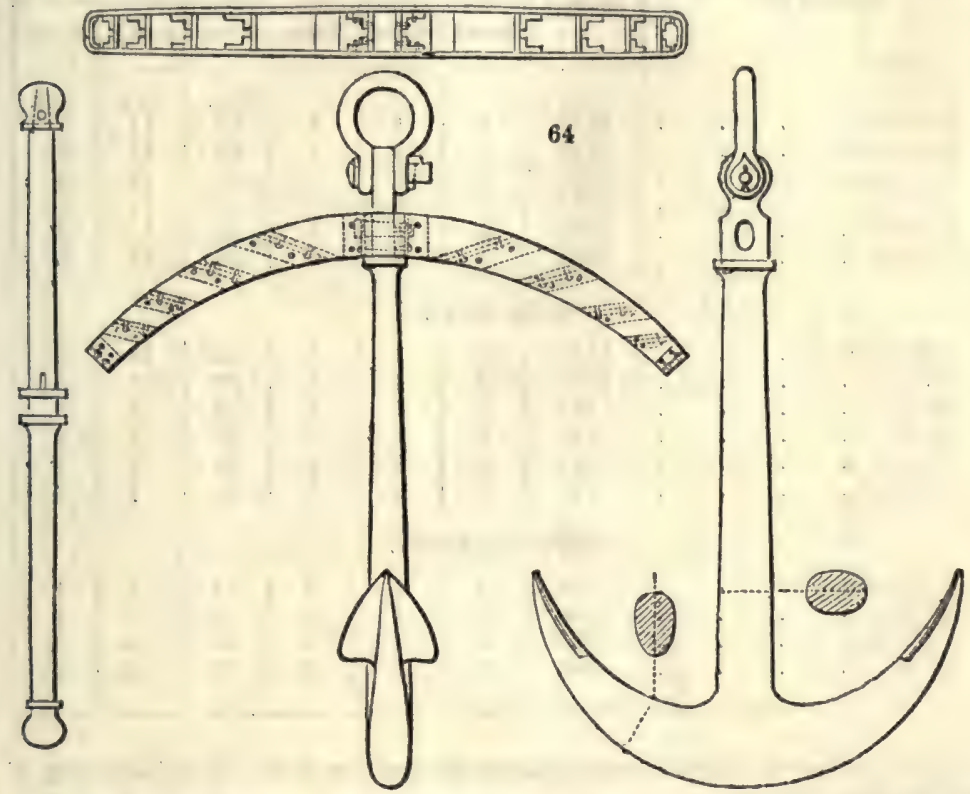

The weight of anchors for different vessels is proportioned to the tonnago. Tho following Tables show the number of anchors now carried, and the weights of ench anchor, by the ships of the Nary, under the Admiralty regulations, and by merchant vessels by the regulation of Lloyd's:-

\section{Admiraxty Regumatioxs.}

Sailing Vessels.

\begin{tabular}{|c|c|c|c|c|c|c|c|c|c|c|}
\hline \multirow{2}{*}{\multicolumn{2}{|c|}{ Name of Ship }} & \multirow{2}{*}{ 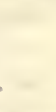 } & & \multirow{2}{*}{ Tonnage } & \multicolumn{3}{|c|}{ Nưmber } & \multicolumn{3}{|c|}{ Weight } \\
\hline & & & & & Bower & Stream & Kedge & Bower & Stream & Kedge \\
\hline $\begin{array}{l}\text { Queen } \\
\text { Camperdown } \\
\text { Albion } \\
\text { Vanguard } \\
\text { Cambridge } \\
\text { Revenge. } \\
\text { Edinburgh } \\
\text { Southampton } \\
\text { Endymion } \\
\text { Stag : } \\
\text { Thalia : } \\
\text { Vestal : } \\
\text { Didö } \\
\text { Volage : } \\
\text { Columbine } \\
\text { Cygnet } \\
\text { Nautilus : } \\
\text { Small brigs } \\
\text { Cutters }\end{array}$ & $\begin{array}{l}: \\
\vdots \\
: \\
: \\
: \\
: 1 \\
: \\
: \\
: \\
: \\
:\end{array}$ & 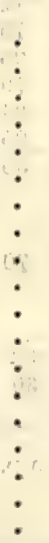 & 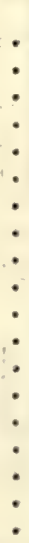 & $\begin{array}{c}\text { Tons } \\
3099 \\
2404 \\
3082 \\
2609 \\
2139 \\
1954 \\
1772 \\
1476 \\
1277 \\
1218 \\
1082 \\
913 \\
731 \\
516 \\
492 \\
350 \\
233 \\
\ldots . \\
\ldots\end{array}$ & $\begin{array}{l}4 \\
4 \\
4 \\
4 \\
4 \\
4 \\
4 \\
4 \\
4 \\
4 \\
4 \\
4 \\
4 \\
4 \\
4 \\
4 \\
4 \\
3 \\
2\end{array}$ & $\begin{array}{l}1 \\
1 \\
1 \\
1 \\
1 \\
1 \\
1 \\
1 \\
1 \\
1 \\
1 \\
1 \\
1 \\
1 \\
1 \\
1 \\
1 \\
1 \\
1\end{array}$ & $\begin{array}{l}2 \\
2 \\
2 \\
2 \\
2 \\
2 \\
2 \\
2 \\
2 \\
2 \\
2 \\
2 \\
2 \\
2 \\
2 \\
2 \\
2 \\
2 \\
1 \\
1\end{array}$. & $\begin{array}{r}\text { Cwit. } \\
99 \\
94 \\
92 \\
85 \\
81 \\
77 \\
73 \\
61 \\
53 \\
50 \\
47 \\
38 \\
31 \\
27 \\
23 \\
18 \\
13 \\
11 \\
0\end{array}$ & $\begin{array}{c}\text { Cwt. } \\
25 \\
23 \\
23 \\
21 \\
20 \\
19 \\
18 \\
15 \\
14 \\
13 \\
12 \\
10 \\
9 \\
8 \\
7 \\
6 \\
5 \\
4 \\
8\end{array}$ & $\begin{array}{l}\text { Cwt. } \\
12 \\
12 \\
12 \\
10 \\
10 \\
9 \\
9 \\
8 \\
7 \\
6 \\
6 \\
5 \\
5 \\
4 \\
4 \\
3 \\
3 \\
2 \\
2\end{array}$ \\
\hline
\end{tabular}


ANCHOŔ

Steam Frigates.

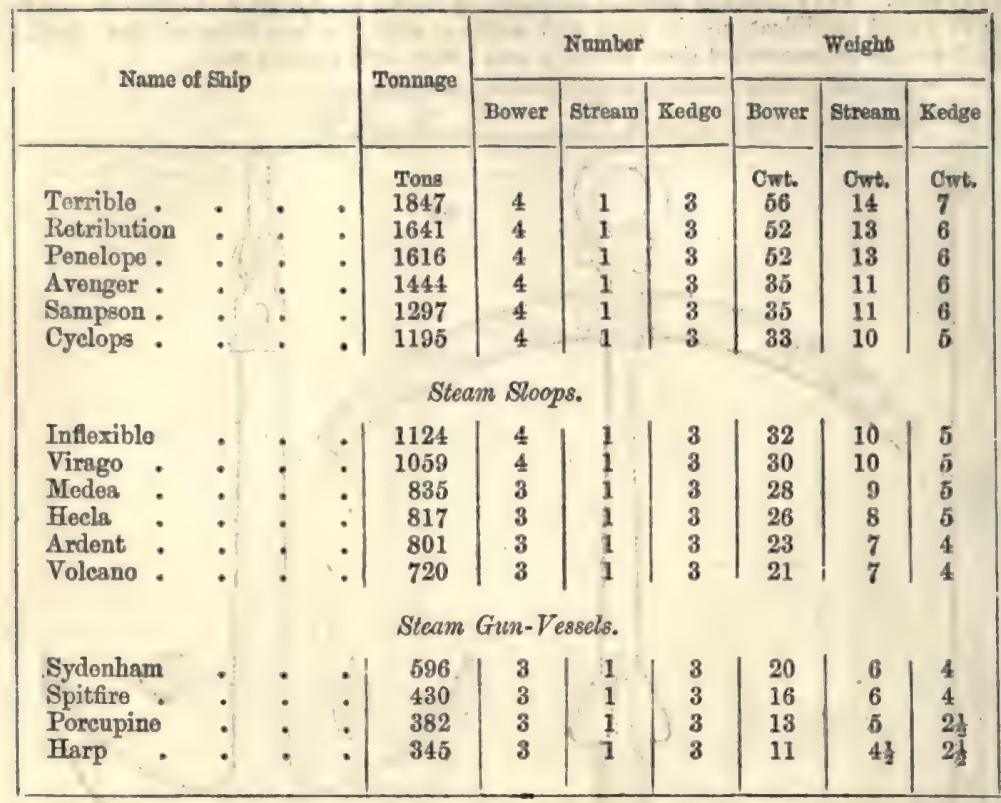

For the following Tables I am indebted to the kindness of the Chief Constructor of her Majesty's Navy :-

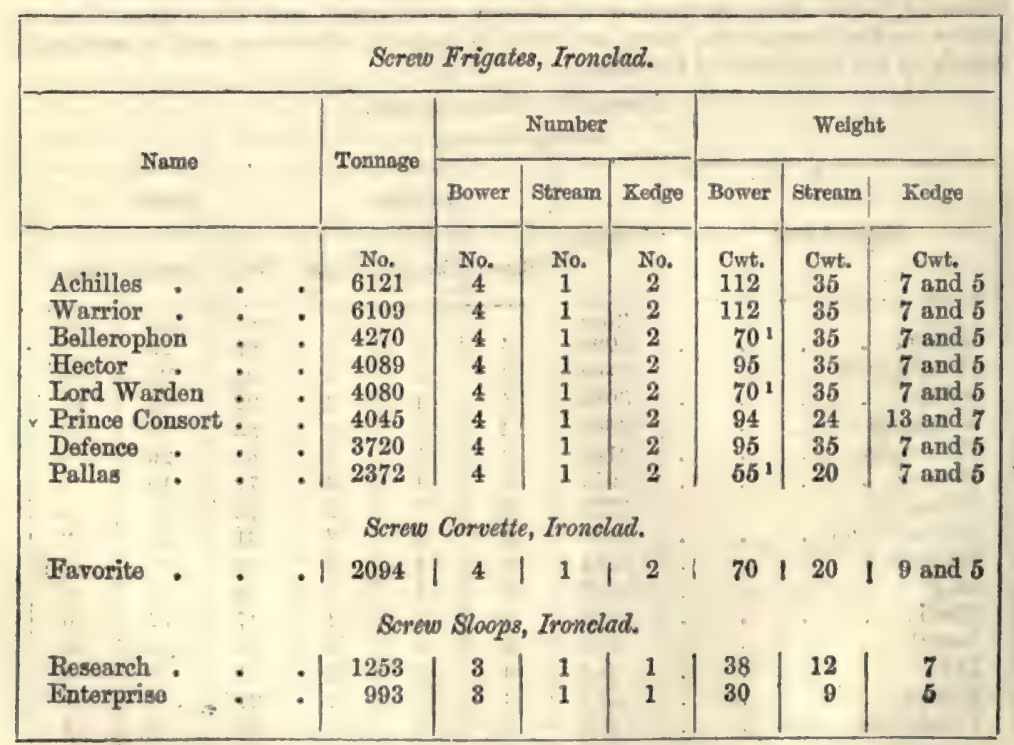

(For Screw Frigates, not Ironclads, see Table on p. 169.)

Rodger'w anchors, 
ANCHOR

\begin{tabular}{|c|c|c|c|}
\hline के & & है & 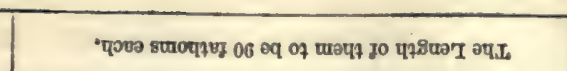 \\
\hline ร5 & 幽 & 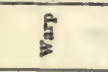 & 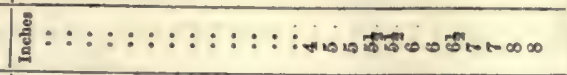 \\
\hline है & 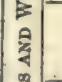 & 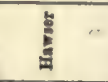 & 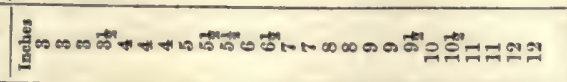 \\
\hline & & $\sum_{\ddot{m}}^{2}$ & 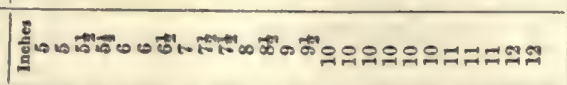 \\
\hline$\frac{8}{2}$ & & है & 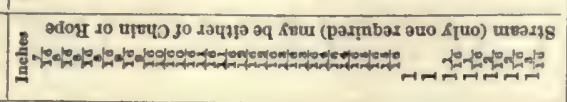 \\
\hline & & & 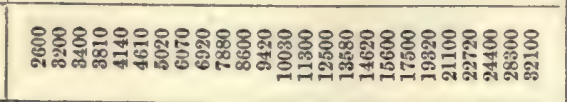 \\
\hline हैำ & & 高嬉 & | \\
\hline & t. & 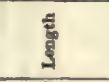 & 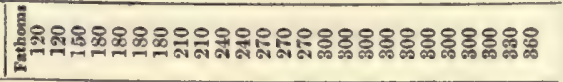 \\
\hline ₹ & 它 & 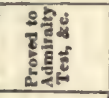 & 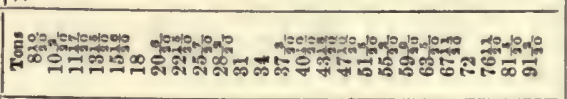 \\
\hline है & 宸 & 产最㩆 & 蒡 \\
\hline$E$ & & 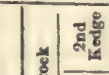 & 竞: : : : : : : - \\
\hline s. & & 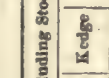 & 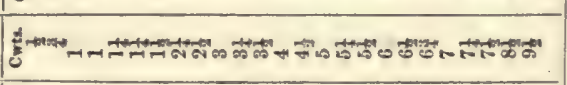 \\
\hline 3 & & $\frac{1}{5}$ & 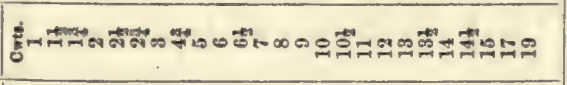 \\
\hline हूँ & 营 & 臂 & : \\
\hline & 要 & 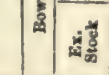 & 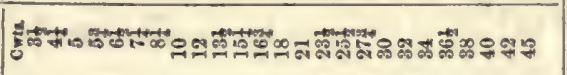 \\
\hline & & है & 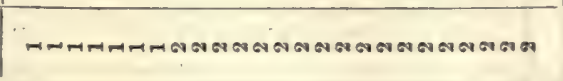 \\
\hline & & 量 & 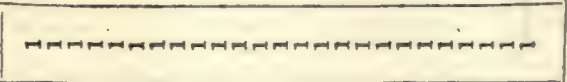 \\
\hline & & 喜 & worase \\
\hline & & 裹 & "ైణ \\
\hline : & & 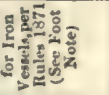 & 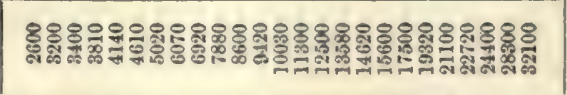 \\
\hline
\end{tabular}

By Section 27 of the Rules, dated February 24, 1870 , for the Building and Classification of Iran Ships, it is provided that 'Their equipment is to he regulated by the Number produced by the sum of the addition of the balf moulded breadth of the vessel amidships, her depth from the upper part of keel to the top of the upper deck beam, and the girth of her half midship section to the same height, multiplied by the vessel's length, for one, two, and three-decked vessels, and for spar-decked steam-vessels.'

For a vessel with an awning-deck, the equipment number to be increased one-aixth beyond that which it would be if ghe were flush decked and withont an awning-deck.

For a vessel with a partial awning-deck, poop, topgallant forecastle, or a raised quarter-deck, the oquipment number to be increased one-tenth beyond that which it would be if she were fiush decked. 


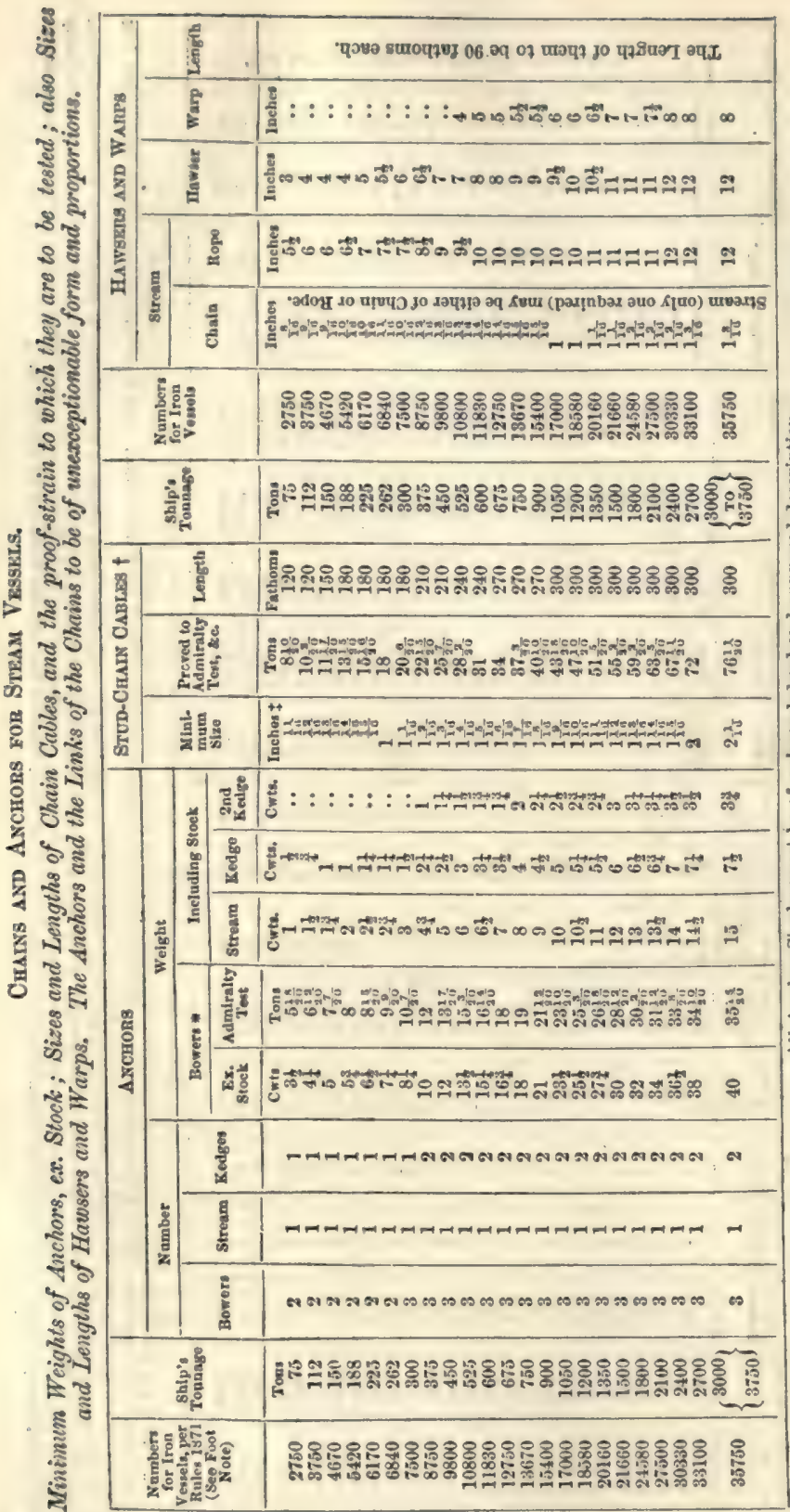

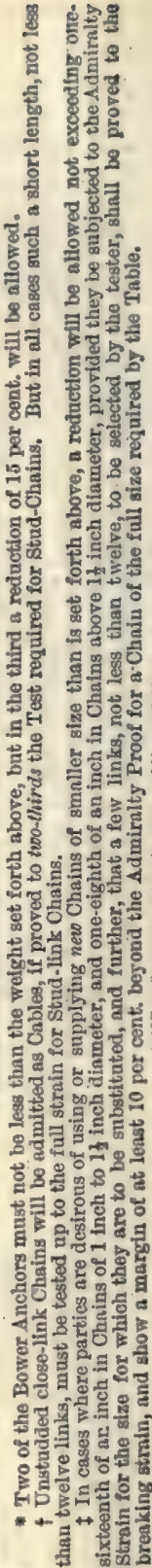

By Section 27 of the Rules dated February 24, 1870, for the Building and Classffication of Iron Ships, it is provided that 'Their equipment is to be regulated by the Number prodnced by the sum of the addition of the half moulded breadth of the vessel amidships, her depth from the upper part of keel to the top of the upper deck beam, and the girth of her half midship section to the same height, multiplled by the vessel's length, for one, two; and three-decked vessels, and for spar-decked steam ressels.'

For a vessel with an awning-deck, the equipment number to be increased one-sixth beyond that which it wonld be if she were flush decked and without an nwning-deck.

For a resael with a partial awning-deck, poop, tongallant forecastle, or a mised quarter-deck, the equipment number to be incrensed one-tenth beyond that which it would be if she were fiush-decked. 


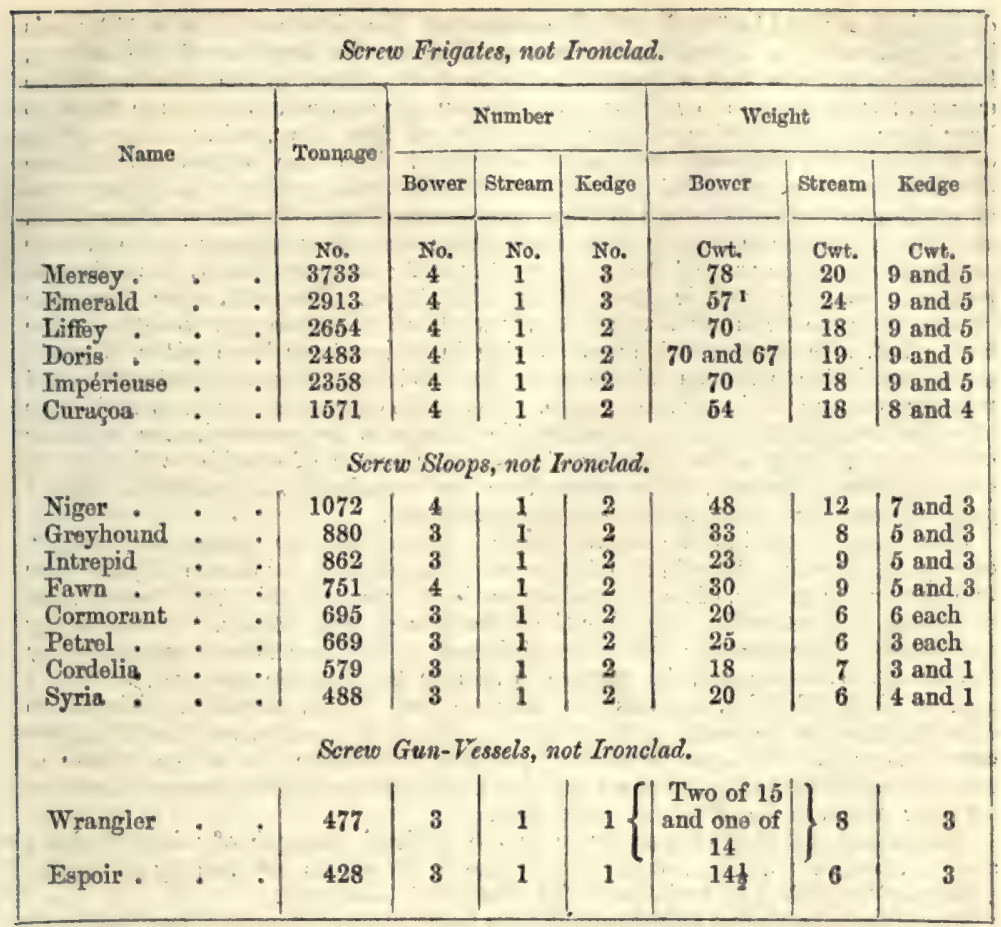

Avcrovx. (Anchois, Fr.; Accinghe, It.; Anschove, Ger.) The Clupea encrasicolns of Linnæeus (Engraulis encrasicolus), a small fish, common in the Moditerranean Sea. Anchovies are preserved as a delicacy, and used in the manufacture of anchovy sauce. The Gorgona anchovy is considered the best. Thoy are abundant off the coasts of Cornwall and Wales, but the fishery is entirely neglected. See SARDINRS.

AxCEUSIC ACID or AxCBusrn. The colouring principle of the Alkanet root (Anchusa tinctoria). Seo Al.

AMDAQUIns wax. A wax produced by a species of bee found on tho banks of the Amazon and Orinoco rivers. It is used as a substitute for ordinary bees'-wax in the manufacture of candles.

ANDIrows, or mAND-rmoxs, also called Firedogs. Before the introduction of raised and close fireplaces, these articles were in general use. Strutt, in 1775, says, "These awndirons are used at this day, and are called "cob-irons;" they stand on the hearth, where they burn wood, to lay it upon; their fronts are usually carved, with a round knob at the top ; some of them are kept polished and bright: anciently many of them were embellished with a variety of ornaments.'

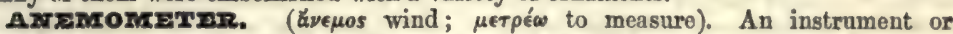
machine to measure the wind, its direction and force. Three descriptions of anemometers are now usually employed-1, Dr. Whewell's; 2, Mr. Follett Osler's; 3, Dr. Robinson's. This is not the place to describe either of those most ingenious instruments, a full account of which will be found in the "Transactions of the British Associatlon' and of the 'Royal Irish Academy.' It is also an instrument designed for measuring the force and relocity of currents of air in mines; and our description of those instruments will be confined to such as aro so employed.

It has not been unusual to determine the rate at which the air travels in the gallery or in the shaft of a mine, by the smoke of gunpowdor, or by floating light bodies, such as thistle-down, in the air. Thero is, however, but little accuracy in those methods. The primitive mode of ascertaining the velocity of currents of air in mines was that of choosing a part of the gallery forming the air-way, having as uniform sectional 
dimensions as conld be found, and after measuring off a distance of 100 to 150 yards in length, taking a lighted candle and walking in the direction of the current, holding the flame in such a position as to be fully exposed to the influence of the current, but taking care to walk at the particular rato required, to cause the flame to burn in an upright position, without being deflected from the vertical, either by the current or by the progress of the person carrying it. The time required to traverse the distance measured off, being noted by a seconds-watch, enabled the averago rate of walking to be determined; and the average rate so found, from three or four trials, was assumed to be the velocity of the air-current; and this, multiplied by the average sectional area of the part of the air-way selected for the experiment, was taken to represent the quantity of air passing in the unit of time. Formerly, when this mode of measuring the air in mines was in use, it would afford a close approximation to the truth; but, with the ventilation now existing in many of our large mines, it would not be practicable to walk as quickly as the currents travel in the principal splits; and running is not a sufficiently stesdy pace. One of the objections to this, as well as to all other methods that require a considerable distance to be traversed, over which to observe the velocity, is the difficulty of obtaining a gallery of equal area throughout over a sufficient distance; but in cases where this is attainable, this method admits of great accuracy for velocities up to 400 feet per minute.

One of the principal of the second modes employed for the measurement of air, consists in observing the velocity of the smoke from an exploded charge of gunpowder in a part of the gallery, of nearly uniform sectional area; and this, until recently, was the means most generally adopted in the coal mines of this country, for ascertaining the velocity of air-currents; and although it has of late been largely superseded by the use of the anemometer, the practice is still in considerable use, and, so far as rogards shaft-velocities, remains the only method. It is, therefore, desirable to ascertain how far the results obtained by this, and similar methods of measuring aircurrents, can be relied upon for accuracy; and to investigate the various sources of error connected with them, with a view of either avoiding or making proper allowances for their effects, so far as may be practicable.

The sudden explosion of gunpowder in the confined passages of mines produces several effects, which tend to cause inaccuracies in the results obtained by noting the passage of the smoke, as an index of the velocity of the current.

Experiments prove (as, indeed, might have been anticipated, considering the small quantities of gunpowder used), that in general neither the increase of bulk due to the introduction into the current of the products of combustion, nor that due to the elevation of temperature, have any appreciable effect on its velocity. But other experiments show that the force of the explosion, when a considerable quantity of gunpowder is used in a feeble current, gives an impulse to the current, and creates a velocity in excess of the normal one. A revolving anemometer was placed in an air-passage traversed by a feoble current, so regulated as to be just strong enough to produce thirty revolutions of the instrument per minute. The explosion of a cubic inch of gunpowder, at a distance of seventy feet, did not in any way affect the instrument; but when the charge of gunpowder was increased to twonty cubic inches, the explosion caused a sudden and violent increase of its rate of revolving, acting as a temporary impulse, the revolutions very quickly decreasing to the original number again. The amount of error arising from this source, and which tends to increase the apparent velocity, depends on the quantity of gunpowder used, the sectional area of the air-way, and the velocity of the current, increasing with the quantity of gunpowder employed.

These errors moy be overcome by using anemometers, or apparatus of various forms; and these may be divided into three classes :- (a) Anemometers having vanes or wands, made to revolve by the current of air impinging upon them, the rate at which they revolve being indicated by pointers on dials forming a part of the instrument-the pointers being made to revolve by means of wheels connecting them with the axis of the vanes or wands. The anemometers of Combes, Biram, Whewell, Osler, and Robinson, are instances of this class of instruments now in use in this country, all of which require a correction for friction. (b) Instruments which are affected by the force or impulse of the wind, without being subjected to any continuous rerolving motion, such as Dr. Lind's, Henaut's, Bongui's, and Dickinson's anemometers. (c) Anemometers of a more complex character, such as Leslie's.

One of the most common forms of anemometer is that derised by Mr. Combes. This consists of a delicately mounted axlo turning with the utmost freedom, upon which are monnted four rectangular plano wings, equally inclined as to a plane perpendicular to the axis. In the middle of the axle is an endless scrow which drives $\mathrm{a}$ wheel with a hundred teeth : this is adjusted so that it advances one tooth for each revolution of the axlo. The first wheel carries a cam, which acts upon the teeth of a 


\section{ANEMOMETER}

second wheel which has fifty teeth. At each rerolution of the first wheel with the hundred teeth, the cam starts the second wheel with fifty teeth by one tooth. The method of using this instrument will be understood from this concise description. The wings and first wheel are adjusted at rero, and kept immovable until the moment of commencing the observation. Then for every complete revolution of the wings the flrst wheel is advanced one tooth, and when this wheel has completed its revolution, or advanced a hundred toeth, one tooth is moved forward on the wheel of fifty teeth. An index-pointer fixed upon light uprights indicates the number of revolutions of the axle of the wings. The manner of using this instrument is easily understood. The limbs aro placed at zero, and the instrument in the axis of the air-tubes, keeping the limbs immovable by means of a catch, which is loosened at the moment of commencing the observations, and fastened at the end of the same. The division of the limbs does not admit of counting over 5,000 turns, which for a velocity of air at 9.84 feet per second, would correspond with a duration of nearly 3 minutes.

The anemometer of $\mathrm{Dr}$. Robinson is constructed on the assumption that the force of impact of the air against hollow hemisphe-
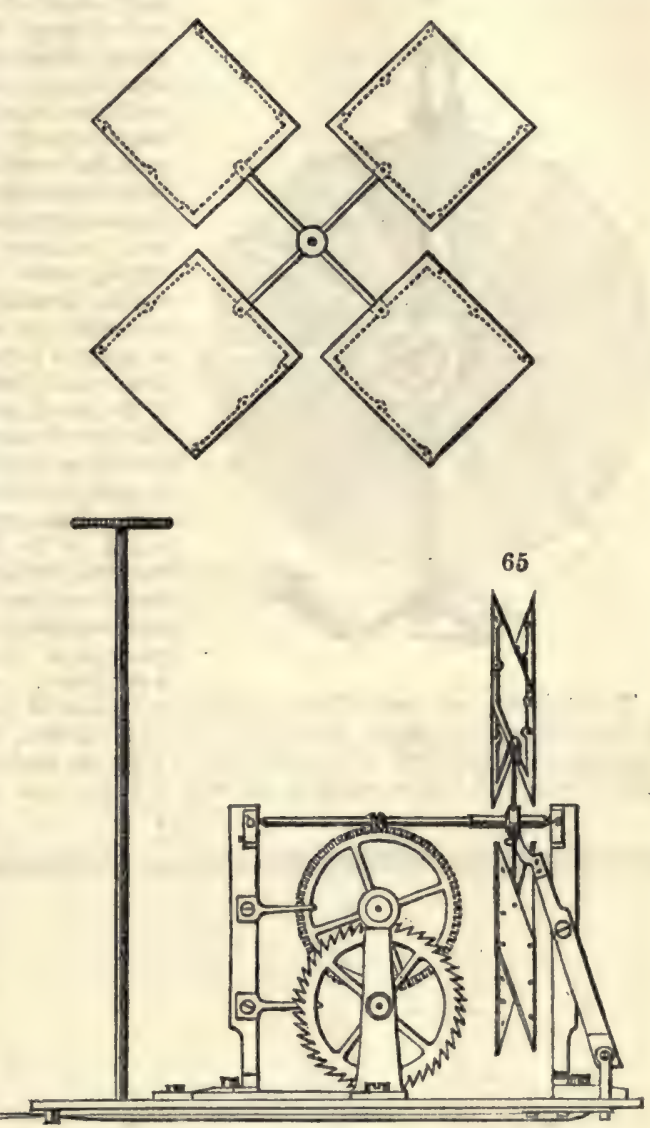

rical cups is twice as great on the concave as on the convex side of the cups, and that the vanes revolve at the rate of one-third the velocity of the current, except in so far as the velocity of revolution is modified by friction.

The mechanism of this instrument is very strong, and allows of the revolutions being recorded throughout the whole day; it would, therefore, be a very euitable anemometer to have near a furnace, or in the principal intake or return of a mine.

Pressure Anemometer. - Perhaps the best known of the pressure anemometers are M. Bongui's, Dr. Lind's, that of Henaut, described by Ponson, and Dickinson's, one of her Mrajesty's Inspectors of Mines. The anemometer of Bongui consists of an apparatus like a spring balance, furnished with a float-board, or plain surface of given area, and the pressure or impulso is indicated by marks on the sliding-rod of the spring; it is figured and described in the 'Edinburgh Encyclopæedia.'

The anemometer most generally used in the coal mines of England is that introduced by the late Mr. Biram. It consists of a series of vanes, which revolve with the action of the air-eurrent-the number of rovolutions, or, rather, numbers proportional to the revolutions, being registered by pointers on the face of a dial forming a part of the instrument itself. It is made of three sizes, 4, 6, and 12 inches; is very portable; and is not, with proper care, liable to get out of order, especially the smaller size. A certain force of current is required to overcome the friction, and put the instrument into motion. Some of these instruments will continue to revolve in a current as low as 30 feet per minute, but with the most of them a velocity of about 50 feet is required. 
Every one who has oceasion to use this anomometer should be aware that it does not fegister the actual velocity of the air, ospecially in feoble air-currents; nor yet the

66

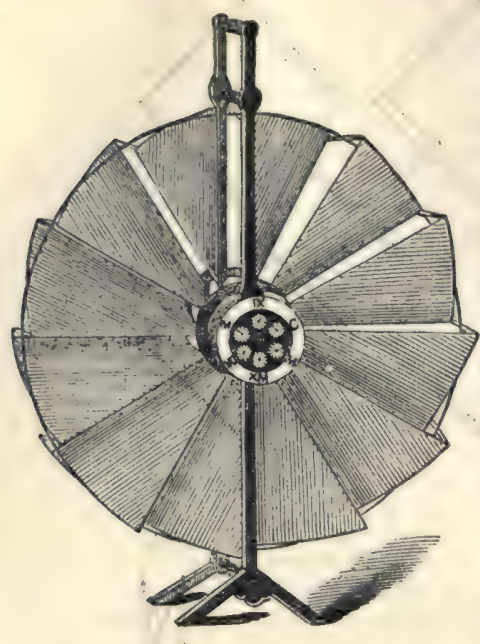
number of revolutions of the wands, but only a number proportional to the latter; and although it is of great value, as indicating an increase or decrease in the velocity from time to time, such as the periodical variations in any particular current, it is of comparatively little value, as generally used, for ascortaining real velocities, such, for instance, as oceur in changing or splitting air-currents, when it is of great importance to know the actual quantities. To obtain, with this instrument, accurato results, available for all purposes, it is necessary, as with Combes anemometer, to apply a formula to its recorded revolutions, or, rather, to the number indicated by the index, in order to ascertain the actual velocity of any current; each particular instrument requiring special experiments to be made with it, in order to determine the value of the constants required to be employed in the formula. These constants, however, remain the same for the same instrument, so long as it remains in the same condition, and are independent of the velocities of the currents of air in which it is employed.

To obtain the constants of this formula, as applicable to any particular instrument, it is absolutely necessary, in making the experiments, to know correctly the true volocity, as a standard of comparison. As before explained, none of the ordinary modes employod for ascertaining the real relocities are reliable; the investigators,

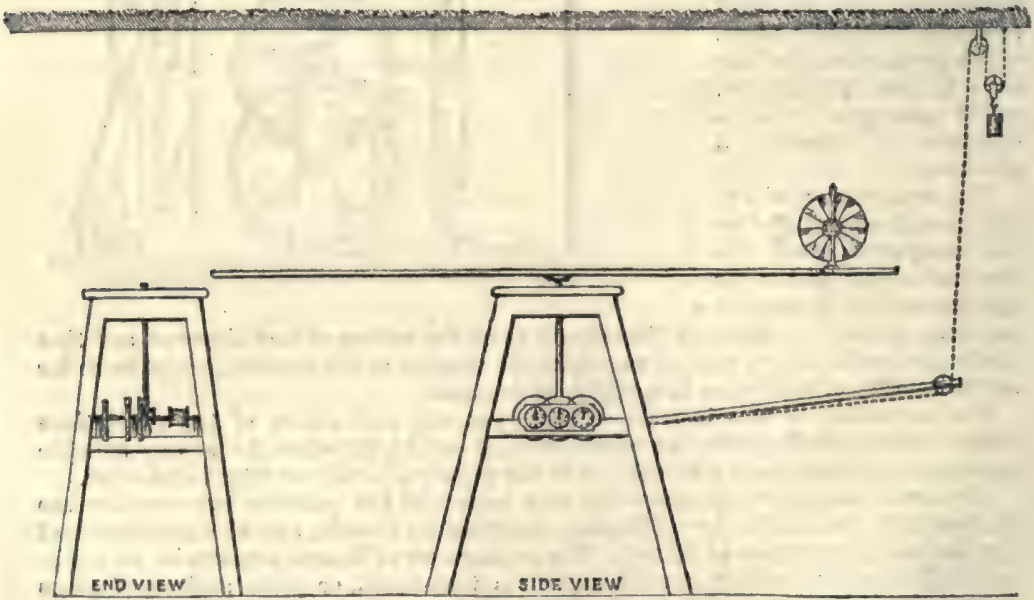

therefore, hand a Whirling Machine constructed, the wand of which, in revolving. describerl a circle of 25 feet in circumference; the number of its rerolutions being indicated by a pointer on a dial.

In the first instance, this Whirling Machine was turned by the hand, but as this did not give a sufficiontly uniform velocity, a small drum, and a rope with a descending weight attached to it, was employed, to give motion to the machine; and worked thus, it gave extremoly accurate results, so far, at least, as regards the uniformity of its own relocity. By fixing the anemometor on the ond of the wand, the velocity with which it passes through the air can bo ascertained and compared with the rovolutions of the anemometer, as indicated on its dial. Fig. 67 ropresents this machine. 
It has been stated by some writers that there is a difference between the force or impulse of air moving upon a body at rest, and the resistance which a body moring through a still atmosphero meots with in its passage, supposing the velocity to be tho samo in each case; and besides this, tho effect of a body moving in a circle, in a still atmosphere, may not be the same as when moving in a straight line. The experiments of Hutton and othors appear, however, to indicate that tho force of impact of a wind against a stationary body is always proportional to the resistanco which a solid, moved through a still atmosphere, meets with at the same volocity.

A valuable series of experimonts were made with this instrument by the late Mr. Atkinson, ono of her Majesty's Coal Inspectors, and Mr. John Daglish, for. which we must refor to the Transactions of the North of England Institute of Mining Enginecrs. The Tables that are givon in connection with the Memoir there published are of the highest possible value.

Water-gauges are sometimos used in determining the rate at which air passes through the shafts or galleries of a mine. They are ordinarily $\mathrm{U}$-shaped tubes with a measured quantity of water, one limb of the tube being bent down, so as to be presented to the current of air.

The anemometer of $\mathrm{Dr}$. Lind resembles the photometer of Pitôt it determines the volocity of tho wind by its action on a small quantity of water in a $U$-shaped tube. As tho same instrument is much used in coal mines as a water-gauge for indicating the difference of pressure between tho down-cast and up-cast air-column, it will not be at all necessary to give a detailed description of it. From numerous experiments, Ds. Lind considered that the pressure of the wind in direct impulse is nearly proportional to the square of its velocity.

Mr. John Daglish, F.G.S. introduced certain improvements in the construction of the water-gauge, which he communicated to the Manchester meeting of the North of England Institute of Mining Engineers, July 14th, 1865.

This communication was made in the following words:-

- Every one who has been much occupied in conducting experiments on the ventilation of mines will have probably felt the inconvemiences attending the use of the ordinary form of water-gange.

- The form of water-gauge introduced by Mr. Daglish, and now extensively in use in the North of England, is constructed with special regard to portability, accuracy, and endurunce.

'As the maximum pressure seldom exceeds three inches of water-column, it is not necessary that the travel of the index-scale should exceed this; the scale is divided simply on either side into inches and tenths, the pressuremarkings on the scale of the ordinary watergauge being not only useless but confusing, and prevent the accurate determination of the difference of the level of the water in each tube. The npper end of one of the tubes is bent over, and open to the external air only by a contracted aperture. This prevents the passage of dust into the tube, which is a fruitful source of annoyance in the ordinary watorgauge whon placed permanently in exposed siturtions in dusty mines. The sealo is moved by a threadod rod working through a female screw attached to the scale: this insures not only the accurato adjustment of the scale in the

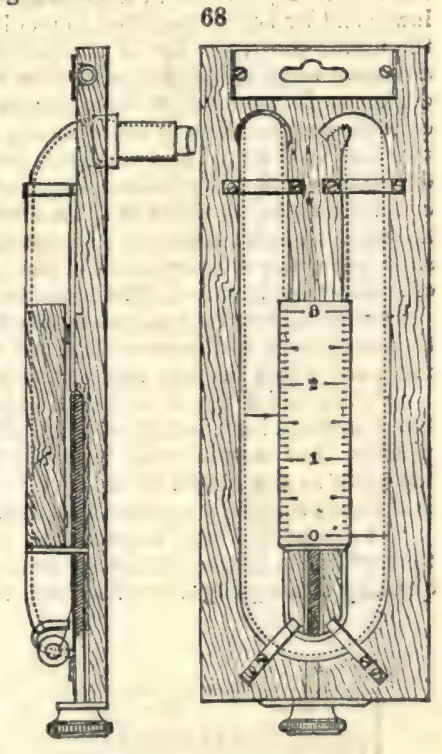
first instance, but its retention in sith, when adjusted. In the ordinary form, if the brass spring-clips, which attach the scale to the tubes, bo too strong, the scale cannot bo accurately adjusted, especially when the water column vibrates much; if, on the other hand, thoy are too weak, the scale will not romain in situ, but falls when released, and this latter is always the tendency after much use; and unless the tubes are perfectly parallel, the scale will be too stiff in ono position, and fall in another. The upper end of the other tube is bent at right angles and fitted up with a short piece of flexible tubing, to the other end of which is attached a short brass tube to be inserted into the aperture to which it is required to attach the wator-gaugo; this short picco of interposed flexible tubing. betweon the rigid brass and glass prerents the liability to frocture of the tubes, in fixing the 
apparatus, which is of such frequent occurrence with the ordinary form of watergauge. The tube is contracted at the bottom bend to provent the oscillations of the wator-column, especially when usod near the ventilating shafts; in the original anomometer of Dr. Lind, which was similar in principle to the water-gauge, this contraction was used.

- The tubes are fitted to the surface of a flat piece of wood, which ontirely prevents liability to fracture, and the apparatus can safely bo carried in tho pocket; a small bulb-tube is fixed to the wood to allow of it being adjusted perfectly lovel when in use, and this is of considerable importance, for any deviation from tho perpendicular is attended with an alteration in the levol of the liquid in each tube. Whon in use, the writer generally mixes a drop of tincture of rosaniline in the water: with this the position of the surface of the water in each tube is clearly distinguished, and the specific gravity of the fluid not appreciably altored.

- Where any great accuracy is required, a vernier, worked by another threaded screw, could readily be attached to the present scale.'

A new application of the water-gauge for ascortaining the pressure of the ventilating column in mines was made by Mr. John Daglish:-

- The ordinary mode of using the water-gauge in mines, for ascertaining tho ventilating pressure, is to place it between the intake and return currents, as near as possiblo to the bottom of the shaft in the mine. The water-gauge, however, placed in this position, does not give the actual difference of pressure due to the differences of tho weight of the downcast and upcast columns of air at their different tomporatures, but only the excess of this amount of pressure over the pressure absorbed by the friction of the curronts in the shafts.

- This loss of pressure from shaft-friction in deep pits, especially where large quantities of air are moving at high velocities in the shafts, reaches a considerable amount, and very sensibly reduces the indications of the water-gaugo as ordinarily employed. By placing in the downeast shaft, however, a range of pipes closed at the lower end by being connected to one leg of a water-gauge, the othor leg being open to the upcast shaft, the loss of pressure due to the friction of the air passing down tho doroncast shaft is avoided, and thus not only is the advantage gained of a greater difference in the level of the water in the two tubes than exists under ordinary circumstances, and thereby enabling the existing state of the ventilating pressure to bo more easily observed and recorded; but inasmuch as the velocity in the downeast shaft diminishes with a diminishod temperature of the upeast shaft, the increased differenco of level referred to above is not a constant quantity, but also varies with tho heat of the upeast shaft; hence, a water-gauge so fixed, not only gives a greater extent of scale under the ordinary state of the ventilation of the mine, but also a greater range of scale under variation of tomperature.

- Another advantage gained by this mods of using a water-gauge is its freedom from the momentary oscillations which are so objectionable in the water-gange as ordinarily used, and which prevent very accurate readings.

- But the chief benefit to be derived from the application of a long range of tubes in the downcast shaft, is that of being able to place the water-gauge on the surfuce in any position that may be most desirable, as its action is not interfored with by extonding the pipes to any length or in any direction, inasmuch as tho column of air in the pipo is dormant and its pressure consequently not reduced by friction.

- At Seaton Colliery, belonging to Earl Vane, the depth of the shaft is 254 fathoms to the furnace in the Hutton-seam; the upcast shaft is 14 feet in diameter, and tho quantity of air going down the downcast shaft is 200,000 cubic feet per minute, and

69

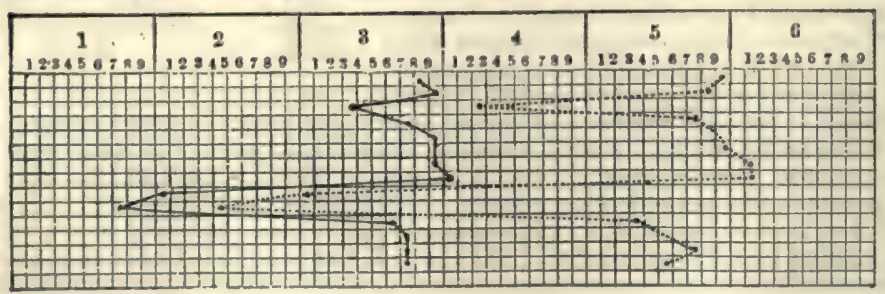

this quantity becomes increased to 300,000 cubic feet per minute in the upcast shaft by expansion due to the high temperature. The ordinary ventilating pressure of the 
mine, as indicated by a water-gange fixed in the mine in the usual way, is 3 inches, and becomes as low as 0.7 inches when the furnaces are out for repairs to the shaft.

- The dotted line on the preceding diagram (fig. 69) exhibits the range of the water-gauge readings, placed in the ordinary position in the mino between the upcast and downeast shafts, taken one each day for a fortnight. The black line exhibits the readings of the water-gauge placed in the colliery office, by means of a pipe from the bottom of the downcast shaft, on the principle previously explained.

'The office is 200 yards distant from the top of the pit, which is 508 yards deop; there is, therefore, 708 yards of pipe (in this case the ordinary half-inch gas-pipe).

'It will be observed that in the customary application of the water-gauge, the height of the water-column due to the ventilating pressure is 3 inches, falling to 0.7 inches when the furnaces are out, being a range of little more than 2 inches; whilst in the new application, the ordinary reading is 5 inches, falling to a minimum of 1.5 inches, having, therefore, a range of $3 \cdot 5$ inches, or nearly double.

' By the addition of a galvanic battery the instrument could be made, if this be considered advisable, to ring a bell when the pressure became reduced below a fixed point."

ATrzRorD BAROMrSTER. This instrument was invented by M. Vidi, of Paris. In its latest form it consists of a cylindrical case, about 4 or 6 inches in diameter, and $2 \frac{1}{4}$ inchos deep, in which lies a thin motal box, near to, and parallel with, the curved boundary of tho case, its two ends being distant about half an inch from each other. From this box the air has been partially exhausted, and the pressure of the external atmosphere on it causes it to alter its form. The accompanying figure (70) shows a section of this box. It is made of thin corrugated plates of metal, so that its elasticity is great. By means of the tube $r$, the air is partially exhausted,

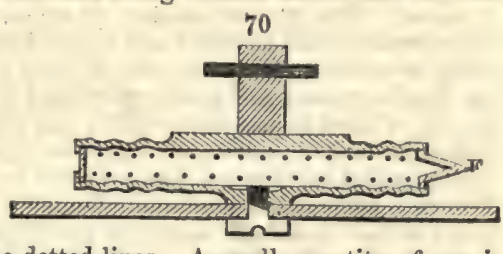
when the box takes the form shown by the dotted lines. A small quantity of gas is introduced after exhaustion, the object of which is to compensate for the varying elasticity of the metal at different temperatures. The pressure of the air on the box in ordinary instruments is between 40 and $50 \mathrm{lbs}$., and it will easily be understood that any variation in this pressure will occasion the distances between the two plates to vary, and consequently the stalk will have a free motion in or out. This is, by an ingonious contrivance, changed from a vertical motion to a motion parallel to the faco of the dial, and this is converted into a rotatory one by the application of a watch-chain to a small cylinder or drum. The original very slight motion is augmented by the aid of levers. This is so effectually done, that when the corrugated surfaces move through only the 250th part of an inch, the index-hand on the face turns over a space of three inches. The extreme portability of this little instrument, and its comparative freedom from risk of injury, render it exceedingly useful to the traveller. Its accuracy is proved by the experiments of Professor Lloyd, who placed one under the receiver of an air-pump, and found that its indications corresponded with those of the mereurial gauge to less than 0.01 of an inch; and within ordinary variations of atmospheric pressure the coincidences are very remarkable.-Lloyd, Nichol, Dreto. Seo BAROMETER.

ANETEUM GRAVIozmss. The common garden Dill. This plant is cultivated in England and imported from the South of Europe. It is used medicinallychiefly on account of its hot and sweetish taste, and for flavouring spirituous cordials.

Axcerrca. (Angélique, Fr.; Angelika, Gor.) The Archangelica officinalis. The dried angelica root is imported from Hamburg in casks. The tender stems, stalks, and the midribs of the leaves, are made, with sugar, into a sweetmeat (candied angelica). The angelica root and seeds are used by rectifiers and compounders in the preparation of gin, and as an aromatic flavouring for 'bitters.' The quantity cultivated in some moist places in this country is sufficient to meet our requirements.

AsraxE-IzON. A piece of iron rolled out in the shape of $L$ to form joints.

ANTGIE-RATIIE or EIP-RATTIR. A piece of timber which runs from the angle of the building to the ridge of the roof, into which it is framed.

AxGIE-STATr. Strips of wood placed upon the vertical angles to protect the plastering.

Ascorzscra. A sulphate of lead found native, generally produced by the decomposition of galena (sulphide of lead). It was first found at Pary's mine in Anglesea, whence the name.

Axroora woox. (Poil de chevron a'Angora, Fr.) Called also angola and angona. The wool of the Angora goat (Capra Angorensis), employed in the manu- 
facture of shawls, camlots, and fine cloth, \&c., is obtained from the long-haired goat of Angora, to which provinee this animal is peculiar. Lieutonant Conolly has given an account of this goat and some other varieties, Capra lanigera, the Cashmere goat, and Changwe or the shawl-goat of Thibet:-

"The country whero it is found was thus deseribed to us-"Tako Angora as a centro, then Kizzil Ermak (or Ilaly's) Chomgero, and from oight to ton hours' march (say thirty miles) beyond; Beybazar, and the same distanco beyond, to near Nalaban? Sevree, Hissar, Yoorrook, Tosiah, Costambool, Geredoh, and Cherkesh, from the whole of which tract the common bristly goat is excluded, and the white-haired goat alone is found." The fleece of the white Angors goat is called tifik (the Turkish for goat's hair), in distinetion to yun, or yapak, sheep's wool. After the goats have com: pleted their first year, they are clipped annually, in April or May, and yield progressively, until they attain full growth, from 150 drachms to $1 \frac{1}{2}$ oke of tiftik (from $1 \mathrm{lb}$. to $4 \mathrm{lbs}$. English). The hair of the tiftik goat is exported from its native districts raw, in yarn, and woven in the delicate stuffs for which Angora has been long celebrated. The last are chiefly consumed in Turkey, while the yarn and raw material are sent to France and England. It appears that the first pareols of Angora wool were shipped from Constantinople for England in 1820, and it was so little appreciated that it fotched only 10d. the pound.

- Within the last two or three years, a new texture made of goats' wool has, how* ever, been introduced both into France and this country, which calls for particular attention. This texture consists of stripes and checks expressly manufactured for ladies' dresses, and having a soft feel and silky appearance. The wool of which this article is made is chiefly the wool of the Angora goat. This wool reaches us through the Mediterranean, and is chiefly shipped at Smyrna and Constantinople. In colour it is the whitest known in the trade, and now more generally used in the manufacture of fine goods than any other. There are, however, other parts of Asiatic Turkey from which limited supplies are received; but in quality not so good as that produced in Angora. After the manufacture of shawls with goats' wool declined in France, this raw material remained neglected for a long while. About two or three years ago, however, the French made another attempt, and brought out a texture for ladies' dresses, in checks and stripes, which they call 'poil de chevre.' The warp is a fine spun silk, coloured, and the wort Angora or Syrian white wool, which was thus thrown on the surface. This article has a soft foel, and looks protty, but in wearing is apt to cut. The price of a dress of French manufacture has been from $2 l$. 10s. to 31. ; but by adopting a cotton warp, the same article is now made in England and sold for 15s.; and it is found that the cotton warp, as a mixture, suits the goats' hoir best.'-Southey on Colonial Sheep and Wools, London, 1852.

The prizcipal manufactures of 'poil de cheure' in Franee are at Paris, Cronyon, Thelle (Oise), Ecrus (Oise), Montataire (Oise), and Ledau. In England, the wool is chiefly spun at Bradford, and partly manufactured there; at Norwich, and also in Scotland; part of the yarn is exported. Mr. Southey informs us, that the quantity of goats' wool imported into the United Kingdom in 1848 was 896,365 lbs.; in 1849 the quantity rose to $2,536,039 \mathrm{lbs}$.

The quantity of goats' wool or hair, in which the alpace wool is included, was :-

\begin{tabular}{|c|c|c|c|c|c|}
\hline & Countries & $\cdots 1$ & & & \\
\hline $\begin{array}{c}\text { From } \\
" \\
" \\
"\end{array}$ & $\begin{array}{l}\text { France } \\
\text { Austrian Territories } \\
\text { Turkey Pritish Possessions in } \\
\text { South Africa } \\
\text { Other Countries }\end{array}$ & $\begin{array}{r}158 \\
253,638 \\
72,357 \\
7,882,359 \\
\quad 235,860 \\
371,150\end{array}$ & $\begin{array}{r}\qquad \\
11,977 \\
6,813 \\
1,005,922 \\
13,322 \\
17,214\end{array}$ & $\begin{array}{l}\text { Ibs. } \\
\overline{-} \\
\overline{-} \\
-\end{array}$ & $\frac{8}{-}$ \\
\hline & Total. & $8,815,364$ & $1,055,248$ & $6,495,482$ & 757,089 \\
\hline
\end{tabular}

In France this article is now applied to the manufacture of a new kind of lace. which in a great measure supersedes the costly fabrics of Valenciennes and Chantilly. The Angors-wool lace is more brilliant than that made from silk, and costing only half the price, it has come into very general wear among the middle classes. The, same material is also manufactured into shawls, which sell from $4 l$. to $16 l$. each. See MoHatr and Cashmrrr. 
AxtryDrides. A name now given by chemists to the so-called oxygen acids, or those which are clearly results of a combination of an element with oxygen.

Thus, the gaseous compound of sulphur and oxygon, commonly called sulphurous acid $\left(\mathrm{SO}^{2}\right)$, is now termed sulphurous anhydride; indeed, in its anhydrous stato, the gas does not exhibit any of the characteristic reactions of an acid, and hence in modern chemistry the term sulphurous acid is restricted to the combination of the anhydride with water $\left(\mathrm{SO}^{2}\right.$. $\mathrm{HO}$, or $\left.\mathbf{F}^{2} \mathbf{S O}^{\mathbf{3}}\right)$.

ANryDrrxe. A mineral consisting of anhydrous sulphate of lime. It occurs in granular masses; in crystals belonging to tho orthorhombic system; in crystalline masses presenting cleavage in three rectangular directions, and hence termed dicespar; and in certain curiously contorted forms known as tripe-stone. Anhydrite is frequently found in beds of rock-salt, where it is often associated with gypsum, or hydrous sulphate of lime; indeed, anhydrite may readily pass into gypsum by combination with water, and in certain localities - as at Box, in Switzerland-extensive beds of anhydrite have been thus hydrated. The compact and granular varieties of anhydrite are often worked into ornamental forms, and the coarser kinds, when occurring in sufficient quantity, have been used for building purposes. The mineral is also useful to the agriculturist, like gypsum, for improving certain soils.

AxrI. The name of the American species of the Indigo plant (Indigofera). It - is a shrubby plant, growing from $2 \frac{1}{2}$ to 3 feot high. From it the name Aniline is derived. Seo Armuks and Irdigo.

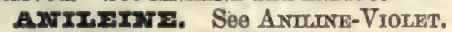

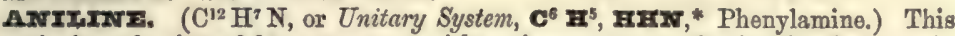
organic base having of late years met with an important application in the arts, in the production of beautiful dye-colours, a description of the methods of preparing it, and of some of its characters, becomes necessary; though for details of its moro interesting relations in scientific chemistry, we must refer to Watts's 'Dictionary of Chemistry.'

Preparation. - There are few bodies which admit of being prepared in a greater variety of ways-all of them interesting in tracing the chemical history of this most curious body; and we proceed to describe Dr. Hofmann's original mode of procuring it from the basic oil of coal-tar, although this method is no longer resorted to for the manufacture of aniline.

The oil is,agitated with hydrochloric acid, which seizes upon the basic oils; after decanting the clear liquor, which contains the hydrochlorates of these oils, it is evaporated over an open fire until it begins to disengage acrid fumes, which indicato a commencement of decomposition, and is then filtered, to separate any adhering noutral compounds. The clear liquor is then decomposed with potash or milk of lime, which liberates the bases themselves in the form of a brown oil, consisting chiefly of a mixture of aniline $\left(\mathrm{C}^{6} \mathrm{H}^{7} \mathrm{~N}\right)$, and leucol or quinoleine $\left(\mathrm{C}^{9} \mathrm{H}^{8} \mathrm{~N}\right)$. This mixture is submitted to distillation, and the aniline is chiefly found in that portion which passes over at or about $360^{\circ} \mathrm{F}$. $\left(182^{\circ} \mathrm{C}\right.$.) : repeated rectification and collection of tho product distilling at this temperature purifies the aniline; but to complete the purification, it is well to treat the partially purified aniline once more with hydrochloric acid, to separato the bases again by an alkali, and then to rectify carefully.

The violet reaction of aniline with solution of bleaching-powder enables the operator to test the distillate from time to time, to ascertain when aniline ceases to pass over, since loucol does not possess this property.-Hofmann.

Aniline may also be obtained from indigo.

When indigo-blue is dissolved by the aid of heat in a strong solution of potash, and the mass, after evaporation to dryness, submitted to destructive distillation, it intumesces considerably, and aniline is liberated, which condenses in the receiver in the form of a brown oil, together with a little water and ammonia disengaged with it. The aniline is purified by rectification, as in the method before described. By this process, the quantity of aniline obtained is about 18 to 20 per cent. of the indigo used.-Fritzsche. (Seo Irvico.)

$\mathrm{By}$ treatment with potash, the indigo-blue $\left(\mathrm{C}^{3} \mathrm{H}^{3} \mathrm{NO}\right)$ is converted into chrysanilic acid and anthranilic acid $\left(\mathrm{C}^{7} \mathrm{H}^{7} \mathrm{NO}^{2}\right)$, and it is this latter body which, by destructive distillation, yields carbonic acid and aniline.

$$
\mathrm{C}^{7} \mathrm{H}^{7} \mathrm{NO}^{2}=\mathrm{C}^{8} \mathrm{H}^{7} \mathrm{~N}+\mathrm{CO}^{2} \text {. }
$$

Nitrobenzol may be converted into aniline, either by the action of sulphuretted hydrogen, or, moro conveniently, as has been shown by M. Béchamp, by the action of a basic acetate of iron.

* The formule employed throughout this article, and in the following articles on aniline colours, are constructed with the modorn atomic welghts, although not printed in black type.

Vor. I. 
For this purpose, the following proportions havo been found convenient by the writer; mix in a retort $\frac{1}{4} \mathrm{lb}$. of iron filings, with about 2 ounces of acetic acid, then add about an equal volume of nitrobenzol. After a few minutes a brisk efforvescenco sets in, and the aniline distils over together with water. The reaction may require to bo aided by the application of a very gentle heat; but it takes place with the greatest ease, and an ordinary condensing arrangemont should be employed. The aniline having so nearly the density of water, does not readily separate on the surface, but the addition of a small quantity of salt, which dissolves in the water, brings it to the surface. It may then be decanted off, dried by standing for a short time over chloride of calcium, and then purified by rectification, as before described.

Properties.-Aniline is one of the organic basic derivatives of ammonia. In fact, it may be viowed as ammonja in which one equivalent of hydrogen is replaced by the compound radical Phenyl $\left(\mathrm{C}^{8} \mathrm{H}^{3}\right)$, thus :-

$$
N\left\{\begin{array}{l}
\mathrm{C}^{6} \mathrm{H}^{3} \\
\mathrm{H}
\end{array}\right.
$$

Just as phenyl is one of a series of homologous radicals, so aniline is the first of a series of homologous bases, in which the one equivalent of hydrogen is replaced by these radicals respectively, thus :-

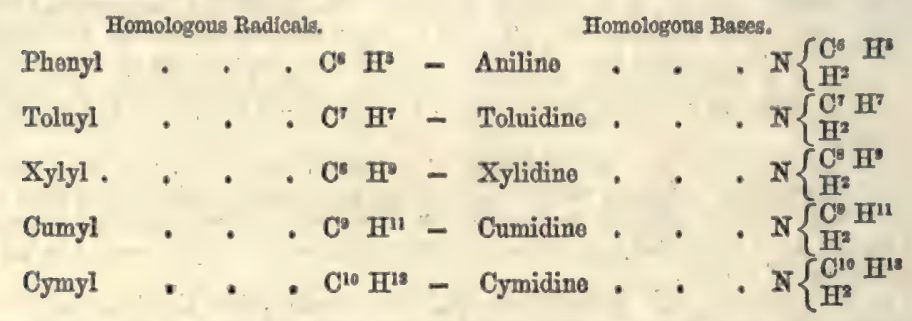

When pure, it is a colourless liquid of a high refractive power, density 1.028 , and of an aromatic odour. It is slightly soluble in water, and mixes in all proportions with alcohol and ether. It boils at $360^{\circ} \mathrm{F}$. $\left(182^{\circ} \mathrm{C}\right.$.). It dissolves sulphur and phosphorus when cold and coagulates albumen. It has no action on litmus-paper, but turns delicate vegetable colours, such as dahlia-petal infusion, blue.

Its basic characters are well developed; thus it precipitates the oxides from the salts of iron, zinc, and alumina, just like ammonia, and yields, with chloride of platinum, a double salt similar to ammonia, the chloro-platinate of aniline $\left(2 \mathrm{C}^{8} \mathrm{H}^{8}\right.$ $\mathrm{NCl}, \mathrm{PtCl}^{4}$ ), which on ignition is entirely decomposed, leaving only a residue of platinum. These characters, together with the beautiful blue colour which it strikes with solution of bleaching-powder, or the alkaline hypochlorites generally, are suffcient for the recognition and distinetion of this body. (See Presy , \&c. \&c.)

Salts of Axilnse-Aniline combines with acids, forming a long series of salts which are in every respect analogous to the corresponding salts of ammonia. They are nearly all soluble and crystallisable, and are decomposed by the mineral alkalies with liberation of aniline. They are generally colourless, but become red by exposure to the air.

Sulphate of Aniline. $\left(\mathrm{C}^{6} \mathrm{H}^{7} \mathrm{~N} ; \mathrm{H}^{2} \mathrm{SO}^{4}\right)$ - This salt is employed in the manufacture of Mr. Perkins' aniline colours. It is prepared by treating aniline with dilute sulphuric acid, and evaporating gently till tho salt separates. It crystallises from boiling alcohol in the form of beautiful colourless plates of a silvery lustre, for the salt is scarcely at all soluble in cold alcohol. It is very soluble in water, but insoluble in ether.

The crystals redden by exposure to the air ; they can be heated to the boiling point of wator without change, but when ignited they are charred with disengagement of aniline and sulphurous acid.

Oxalate of Aniline. $\left(2 \mathrm{C}^{6} \mathrm{H}^{7} \mathrm{~N} ; \mathrm{C}^{2} \mathrm{H}^{2} \mathrm{O}^{4}\right.$.)-This is one of the best defined salts of aniline: it separates as a crystalline mass on treating an alcoholic solution of oxalic acid with aniline. It is very soluble in hot water, much less so in cold, only slightly soluble in alcohol, and insoluble in ether.

A large number of other salts are known: the hydrochlorate, hydrobromate, hydriodate, nitrate, several phosphates, citrate, tartrate, \&c. \&c.; but they aro of purely scientifle interest. The same remark applies to the various products of tho 
decomposition of aniline, which have boon so ably investigated by Fritzsche, Zinin, Hofmanin, Gerhardt, and other chemists.

Since this was written, the aniline dyes have received such an important development, that it is necessary to give a more detailed description of these beantiful colours.

It appears desirable that a Dictionary of the Arts should comprehend a succinet history of the discovery of a body which has performed so important a part in the advancement of a special industry as Aniline has done.

In 1826 Unverdorben, a German chemist, when exposing indigo to destructive distillation, discovered an oily substance which formed crystalline compounds with acids, to which he gave the name of Crystalline. Runge, also a German, subsequently observed in coal-tar oil a substance capable of forming saline compounds, and of striking a violet-blue colour with chloride of lime. To this he gave the name of Kyanol, blue oil. At a later period, Fritzsche, when investigating the action of potash on indigo, obtained a quantity of a basic oil, which he analysed, and to which he gave the name of Aniline. Zinin, about the same time, found that Nitrobenzol, when submitted to the action of sulphuretted hydrogen, was converted into a peculiar and, as he thought, a new substance, to which he gave the name of Benzidam (an ammonia derived from Benzol). Hofmann was the first who submitted crystalline, kyanol, aniline, and benzidam to careful experimental comparison, and proved them to be identically the same substance, which now took its place in chemistry under the name of aniline.

In 1825 Michael Faraday, during an examination of the oily products separated in the compressed oil-gas holders-then largely used-discovered Benzol, which he then described as a bicarburetted hydrogen. On this important discovery, Dr. Hofmann remarks: 'In this investigation, as indeed throughout the whole series of his immortal researches, Faraday's object was the elaboration of truth for its own intrinsic value and beauty; and in the same spirit has the work been continued by those, who, after Faraday, engaged in the further scientific examination of this subject. Nobody in those early days of benzol, when the substance simply existed as a laboratory curiosity, dreamed of the brilliant career looming in the distance for this body, nor of the marvellous transformation it was destined to undergo. But the experience of the last few years in this matter has only corroborated the old axiom, which cannot be too often repeated, that the search after the true, for its own sake, leads on to the discovery of its natural corollaries, the useful and the beautiful. For those, indeed, lie folded up in truth, to be in due time evolved therefrom, even as the great tree unfolds itself from out the little seed.'

Mitscherlich, some years later, found that benzoic acid, distilled with caustic potash, gave \& colourless volatile liquid, identical with the hydrocarbon discovered by Faraday; and hence the name this substance now retains-Benzol. Dr. Hofmann, in 1845, proved the presence of benzol in coal-tar oil, and in 1848 Mansfield showed that an inexhaustible quantity of it could be procured from that souxce. See BENZOL.

Now, the crude tar of the gas-works is subjected to regulated distillation. Thus is obtained separately naphtha or light oil (oily liquid lighter than water), and then dead oil or heavy oil (oily liquid heavier than water), and finally remaining in the retort pitch. From the light eil, benzol is separated by further fractional distillation. Mitscherlich showed, if this benzol is dissolved in fuming nitric acid, and the clear liquid mixed with water, a compound (nitrobenzol) is precipitated as a dense yellow liquid. This is the well-known artificial oil of bitter almonds, which is now prepared easily and economically on a large scale. It was Zinin, already named, who discovered that sulphuretted hydrogen converted this nitrobenzol into aniline, and who, believing the substance thus produced to be a new one, described it under the name of benzidam, or an ammonia derived from benzol. (See Nitrobenzol.)

The successive changes of benzol are thus expressed in chemical symbols.

First change.

Transformation of benzol into nitrobenzol.

$$
\underbrace{\mathrm{C}^{\circ} \mathrm{H}^{3}}_{\text {Benzol. }}+\underbrace{\mathrm{HNO}}_{\begin{array}{c}
\text { Nitric } \\
\text { Acid. }
\end{array}}=\underbrace{\mathrm{C}^{6} \mathrm{H}^{3} \mathrm{NO}^{2}}_{\text {Nitrobenzol. }}+\underbrace{\mathrm{H}^{2} \mathrm{O}}_{\text {Water. }}
$$

Second change.

Transformation of nitrobenzol into aniline.

$$
\underbrace{\mathrm{C}^{6} \mathrm{H}^{3} \mathrm{NO}^{2}}_{\text {Nitro- }}+\underbrace{3 \mathrm{H}^{2} \mathrm{~S}}_{\text {Sulphurettod }}-\underbrace{\mathrm{C}^{6} \mathrm{H}^{2} \mathrm{~N}}_{\text {Aniline. }}+\underbrace{2 \mathrm{H}^{2} \mathrm{O}}_{\text {Water. }}+\underbrace{3 \mathrm{~S}}_{\text {Sulphur }}
$$

benzol. Hydrogen. 
Dr. Hofmann remarks that sulphuretted hydrogen is not by any means the most conveniont reducing agent to effect this conversion, and he has shown that when nitrobenzol is placed in contact with motallic zinc and hydrochloric acid, it is rapidly conferted into aniline. Béchamp submitted nitrobenzol to the action of metallic iron and acetic acid, and thus transformod it into aniline. This process is now almost universally adopted. Equal woights of nitrobonzol, acetic acid, and cast-iron turnings are very gradually mixed in cast-iron vessels, so that tho heat produced by the reaction does not raise the temperature of the mixture too high. The semi-solid mass which is produced consists of acetate of iron and acetate of aniline. This is distilled sometimes alone, or by some manufacturers with the addition of lime; the distillato consisting generally of acetone, aniline, unaltered nitrobonzol, and several other products arising from the impurity of the nitrobenzol. The crude aniline mixture is redistilled, and the aniline obtained sufficiently pure by collecting the products distilling between $175^{\circ} \mathrm{C}$. and $190^{\circ} \mathrm{C}$. Thus produced, aniline is a slightly brownish liquid, a little heavier than water.

AxITINT-BIAck. This colour can scarcely be said to have been satisfactorily produced. The green tints (see ANurive-GRein) are turned black, according to a process devised by Messrs. Wood and Wright, by mixing chlorato of potash with a metallic salt and a salt of aniline; for the metallic salt they prefer ferric salts. Aniline-black may also be produced by treating the colour on the fabric by solutions of bichromate of potash, or of weak bleaching-powdor. Nitrate of copper may be mixed with hydrochlorate of aniline, without the addition of chlorate of potash, and the mixture printed on the fabric, when gradually a black tint is produced.

According to Brandt aniline-blacks are very variable in their composition and properties. Some of them resist the action of light and of reagents much better than others. Some turn of an unpleasant greenish hue if exposed to air charged with acid or sulphurous vapours. The more intense an aniline-black the better it resists reagents. It is true that this intensity depends partly on the degree of concentration of the colour, but other circumstances influence its solidity. A black developed in presence of an excess of aniline is always faster than one of the same degree of concentration which is developed in presence of an excess of acid. In the latter case, beside weakening the tissue, blacks aro obtained which turn green, and cannot stand the application of bleaching-powder. In this case, if the gas used for lighting the premises contains a little sulphur, the fumes cause the folds of every piece in the warehouse to turn greed.

With excess of base a black is produced which shows less disposition to turn green, and bears chlorine better. Such a black must be developed rapidly enough to avoid the volatilisation of the aniline. For this end chlorate of aniline is used instead of chlorate of potash, diminishing the amount of the aniline salt by a corresponding amount. Chlorate of potash, in presence of an excess of aniline, does not decompose very rapidly.

A niline-blacks result from two distinct reactions. There is, firstly, decomposition of the chlorate of aniline; and secondly, oxidation of the other salt of aniline mixed with the chlorate. The decomposition of the former gives rise to chlorinised products derived from aniline. There are probably various stages of substitution-a fact which cxplains the diversity of the results. Besides these, there is formed another product, the result of the oxidation of the salt of aniline.

Aniline-black consists, therefore, of two distinct blacks: the one, formed of the chlorine substitution of aniline, is exceedingly fast, and resists almost all chemicals, but it is not so fine as that produced by a judicious mixture of the two blacks, as it does not acquire its lustre and offect without the aid of the second. This latter is an intense violet-blue, which appears black when concentrated. It is less solid than the former, and turns greenish with the smallest amount of acid. It resists the action of soap very well. The brown-black and blue-black mixed together form a fine anilineblack. The object of the maker is to combine the maximum of beauty with the maximum of solidity. This depends on the due proportion of the chlorate.

Comparative trials have been made with pure aniline on the one hand, and, on the other. with anilines containing toluidine and pseudo-tolxidine. The results werc similar in each case.

AmIxIN2-2aruz. MM. Girard and De Laire, in M. Pelouze's laboratory, discovered the reactions which give rise to the aniline-blues. This reaction consists in heating a salt of rosaniline, or a mixture of substances capable of giving rise to its formation, for several hours with an excess of aniline. The blue colouring-matters thus obtained are the Bleu de Paris and the Bleu de Lyons. The operation on a large scalo is carried out by allowing the mixture of a salt of rosaniline, with an excess of aniline, to digest at a temperature of $150^{\circ}$ or $160^{\circ}$ for a considerable time. If a 
mixture of 2 kilogrammes of dry hydrochlorate of rosaniline and 4 kilogrammes of aniline be employed, the operation is completed in four hours. The crude blue is purified, by treating it successively with boiling water, acidulated with hydrochloric acid, and with pure water, until it presents the purest possible hue. Mr. Nicholson has devised and patented a process of purifying the blue colouring-matter, which he dissolves for this purpose in concentrated sulphuric acid, afterwards digesting the solution for half an hour at a temperature of $150^{\circ} \mathrm{C}$. On adding water to this solution, the blue colouring-matter is precipitated in a modified condition, having, in fact, become soluble in pure water.

Dr. Hofmann has determined the nature of aniline-blue, which he thus describes :'The blue colouring-matters, as might have been expected, are saline compounds of a colourless base, which may be obtained in a state of perfect purity by dissolving one of the salts (the hydrochlorate, for instance) in alcohol, and filtering the solution into alcoholic ammonia. The deop blue immediately disappears, and the slightly reddish solution yields the free base on addition of water, in the form of a white curdy precipitate, which gradually assumes an indistinctly crystalline character. Dried in vacuo, this substance remains colourless, or assumes a slightly bluish tint; at $100^{\circ}$ it cakes, and becomes brown; and is found by analysis to contain-

$$
\mathrm{C}^{98} \mathrm{H}^{93} \mathrm{~N}^{3} \mathrm{O}=\mathrm{C}^{38} \mathrm{H}^{31} \mathrm{~N}^{3}, \mathrm{H}^{2} \mathrm{O} \text {. }
$$

This formula exhibits an extremely simple relation between aniline-blue and anilinered; indeed, aniline-blue is triphenylic rosaniline:-

$$
\begin{aligned}
& \text { Aniline-red } \mathrm{C}^{20} \mathrm{H}^{19} \mathrm{~N}^{3}, \mathrm{H}^{2} \mathrm{O} \text { Rosaniline. } \\
& \text { Aniline-blue } \mathrm{C}^{20}\left[\frac{\mathrm{H}^{18}}{3 \mathrm{C}^{8} \mathrm{H}^{5}}\right] \mathrm{N}^{3}, \mathrm{H}^{2} \mathrm{O}\left\{\begin{array}{l}
\text { Triphenylic } \\
\text { Rosaniline. }
\end{array}\right.
\end{aligned}
$$

The salts of the triphenylic derivative correspond to the rosaniline salts. The composition of the hydrochlorate, which Mr. Nicholson prepared in a state of perfect purity, is analogous to that of the monacid hydrochlorate of rosaniline :-

Hydrochlorate of rosaniline $\mathrm{C}^{20} \mathrm{H}^{19} \mathrm{~N}^{2}, \mathrm{HCl}$.

Hydrochlorate of triphenylic rosaniline $\quad \mathrm{C}^{20}\left[\begin{array}{l}\mathrm{H}^{10} \\ 3 \mathrm{C}^{8} \mathrm{H}^{3}\end{array}\right] \mathrm{N}^{3}, \mathrm{HCl}$.

The genesis of aniline-blue is represented by the following equation :-

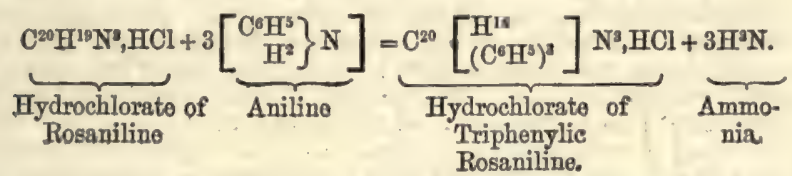

Aniline-blue, when submitted to the action of reducing agents, such as nascent hydrogen or sulphide of ammonium, is converted into a colourless, difficultly crystellisable substance, which in composition corresponds to leucaniline. It contains $\mathrm{C}^{38} \mathrm{H}^{33} \mathrm{~N}^{3}$ ?'

The recognition of the nature of aniline-blue led Dr. Hofmann to some experiments which soon became of great industrial importance. Seeing that the substitution of three atoms of phenyl for three atoms of hydrogen in rosaniline induces a change from red to blue, the idea naturally suggested itself to replace the hydrogen by other radicals, such as methyl, ethyl, and amyl. Experiment furnished results of much interest. Rosaniline is readily attacked by the iodides of these radicals : a series of new substances being produced, obviously the salts of trimethyl, triethyl, and triamyl rosaniline, the blue and violet colours of which are similar to that of the phenylated compound. These new colouring-matters are now manufactured on a largo scale by Messrs. Brooke, Simpson, and Spiller, of London.

Under the title of 'Improvements in preparing Colouring-Matters for Dyeing and Printing,' Dr. Hofmann patented (sealed August 29th, 1863) the following processes :"I take the substance now well known as " rosaniline," being the base obtained from the various salts of rosaniline found in commerce under the names of "roseine," "magenta," and also by other names, and which is usually prepared from aniline and the homologues thereof, and I mix it with the iodides, bromides, or other salts of the "alcohol radicals," such as iodide of ethyl, methyl, or amyl, or bromides of the same. I employ the substances, by preference, in the proportion of one equivalent of rosaniline to three equivalents of the salt of the alcohol radical; I then heat the mixture, either alone or together with methylated spirit, to a temperature betwoen $212^{\circ}$ and $300^{\circ} \mathrm{F}$. 
in a close vessel under pressure; it is convenient to use an iron boiler provided with a safety-valve. I continue the heat until the desirod result is obtained. During the heating the mixture passes through several phases of colouration, being eventually converted into a blue substance. If the process is stopped before the whole is converted jnto the blue, the mixture is then of a violet or purple colour. For the purpose of dyeing and printing the mixture may bo usod in the same mannor as that in which the aniline colours are employed.'

Nicholson's method of making aniline-blue soluble in water was discovered in 1862, but other soluble aniline-blues aro now in the market. Sulphurie acid acting upon aniline-blue can give a series of products varying according to the intensity of the reaction. All are sulphacids of triphenylrosaniline. Bulk has proved the existence of four of these bodies. Sulphuric acid gives, according to circumstances, sulphate of triphenylrosaniline, or its mono- bi-tri- or tetra-sulphuric acid. When we treat hydrochlorate of triphenylrosaniline with strong sulphuric acid and cool the mixture, we obtain a deop red liquor, and hydrochloric acid is liberated. On putting this mixture into water, sulphate of triphenylrosaniline is precipitated unaltered in tho form of a fine blue powder. If, instead of cooling the mixture, it is heated and kept for five or six hours at a temperature of $30^{\circ} \mathrm{C}$., it yields equally, when poured into water, a blue precipitate, insoluble in that liquid, but which differs from the last-mentioned blue precipitate in being soluble in a solution of soda, in which it gives a rod solution. This latter precipitate is the monosulphuric compound of aniline-blue. When recently prepared, it forms deep blue masses, which, when dried in the water-bath, take a fine metallic lustre. It is a monobasic acid, forming with the alkalies salts soluble in water; those of the earths are sparingly soluble. To obtain these salts it is needful to treat the freshly-prepared acids with caustic alkalies. They are scarcely soluble in cold water, and dissolve in hot water with a feeble colouration. The soda salt of this acid is known in commeree as Nicholson's or alkali-blue. It is prepared by digesting th $\theta$ monosulphuric acid of triphenylrosaniline with a solution of caustic soda, not enough to saturate the acid. It is then filtered and evaporated down to dryness at $100^{\circ} \mathrm{C}$. It is an amorphous black-grey mass. It dissolves in hot water with a blue colour. The colour of the aqueous solutions of the salts in question is very feeble, but becomes strong if the acid is set free. If acetic acid is used the colour is unalterable by air in the cold. It is decomposed by hot acetic acid and by cold mineral acids. Wool extracts these salts from their solutions in a colourless state if borax or silicate of soda is added. The salt thus fixed on the wool adheres very firmly, and cannot be removed by washing. When the wool is plunged into an acid bath, the colour appears in its full beauty and intensity. The bisulphuric compound is formed by dissolving the aniline-blue in six times its volume of sulphuric acid, and keeping it for five hours at $60^{\circ} \mathrm{C}$. It is then poured into water. The greater part is precipitated, but a little remains dissolved, forming a blue liquid. The blue precipitate is bisulphuric acid, and the liquid is the trisulphuric. The bisulphuric compound is scarcely soluble in water, but dissolves in alkalis forming salts soluble in cold water. The soda-salt is known in commerce as 'soluble blue.' It is moro soluble than the soda-salt of the former acid. The salts of the heavy metals are mostly insoluble. The trisulphuric acid is prepared by decomposing the blue liquid formed along with the bisulphuric, by means of hydrochloric acid. The precipitate is soluble in water and in alcohol. The highest compound, the tetrasulphuric, is formed by digesting aniline-blue in fuming sulphuric acid at $140^{\circ} \mathrm{C}$. The liquid, when poured into water, forms a blue solution, from which the free sulphuric acid may be withdrawn by means of carbonate of lead. On filtering and evaporating wo obtain a salt of lead saturated with the tetra-acid. It is soluble in water, and precipitable by alcohol. Its alkaline salts are soluble in water and in excoss of alcohol. Those of the heavy metals are soluble in water, but not in alcohol. Silk scarcely withdraws the colour from alkaline and neutral solutions of this acid, but is readily dyod in an acidulated bath. The sulphuric compounds of aniline-violet are analogous, but as their colours are not fine, they are less interesting.

ANIIINZ-GREm. Aniline assumes a beautiful indigo-blue colour by the action of chlorate of potash, to which a quantity of hydrochloric acid has been added, and also under the influence of a solution of chlorous acid. Dr. Crace Calvort and Messrs. Lowe and Clift have produced similar blues, and described them under the name of Azurine. Most of those blues possess the property of acquiring, under the influence of acids, a green tint called Emeraldine. Dr. Calvert obtains this colour directly upon cloth by printing with a mixture of an aniline salt and chlorate of potash, and allowing it to dry. In about twolve hours the green colour is developed. This colour may be converted into blue by being passed through a hot dilute alkaline solution, or through a bath of boiling soap. See Verpres.

In the process of forming the bluer shades of ethylated violet by Hofmann's patent, 
described above, there is always produced simultaneously a certain quantity of a green colouring-matter, which may be purified by taking advantage of the circumstance that it is froely soluble in alkaline solutions. When crystallised or precipitated by suitablo reagents, it is commonly known by the name of 'iodine-green,' and may be regarded chemically as a double compound, resulting from the union of iodide of ethyl with the already ethylated rosaniline. The colour has an affinity for silk and wool, it is remarkably brilliant, and is much used on that account for dyeing bright green, the shades being modified at pleasure by developing with picric acid. This body undergoes a remarkable alteration by heat, which resolves it again into its constituents, the colour changing from green to violet.

The following method of applying the aniline-green dye on straw will be useful. The straw is laid for some time in boiling water and then washed in cold water. It is next bleached in a bath containing 20 grammes of chloride of lime, and 7 to 9 grammes of sulphuric acid. No more water is used than is needful to give sufficient room for the straw to be well covered and turned about. It is then well washed in cold water. A mordant is then made up of sumach, alum, and tartar. In this it is well worked, and allowed to steep for 15 minutes. Half the mordant is then run off, and the vessel is filled up with pure water. The straw having been taken out, anilinegreen, and, if necessary, picric acid, are added, and the straw is worked in this till the right shade is obtained.

ANIIINE-RED. The discovery of this colour is clearly due to Dr. Hofmann. In 1858 this chemist wrote, in the 'Proceedings of the Royal Society' (vol. ix. p. 284) as follows:- 'The aqueous solution yiolds, on addition of potassa, an oily precipitate containing a considerable portion of unchanged aniline; on boiling this precipitate with dilute potassa in a retort, the aniline distils over, whilst a viscid oil remains behind, which generally solidifies with a crystalline structure. Washing with cold alcohol, and two or three crystallisations from boiling alcohol, render this body perfectly whito and pure, a very soluble substance of a magnificent crimson colour remaining in solution. The portion of the black mass, which is insoluble in water, dissolves almost entirely in dilute hydrochloric acid, from which it is reprecipitated by the alkalies in the form of an amorphous pink or dingy precipitate soluble in alcohol, with a rich crimson colour. The greater portion of this body consists of the same colouring principle, which accompanies the white crystalline substance.'

This rod colouration was indeed noticed in 1843 by Dr. Hofmann, while studying the action of fuming nitric acid upon aniline. In 1856 Natanson observed it when examining the action of Dutch liquid upon aniline. The industrial discovery of aniline-red was made in 1859 by Messrs. Verguin and Renard Brothers, of Lyons. Their process for obtaining it was as follows:-A mixture of ten parts of aniline, and from six to seven parts of tetrachloride of tin, either anhydrous or hydrated, is heated to ebullition for fifteen or twenty minutes. The liquid first becomes yellow, and then gradually more and more red, until at last the colour is so intense that the mass appears black. The mixture is allowed to cool, and then treated with a large quantity of boiling water, which acquires a magnificont red colour, and, without any other proparation, forms a splendid dye both for silk and wool. It is found advisable to previously purify the red colouring-matter, for which purpose its insolubility in saline solutions is made available. If the concentrated red liquid be partially saturated with carbonate of soda, and a quantity of common salt added, the aniline-red is precipitated in a solid state, and constitutes the fuchsine of the chemist. This has only to be dissolved in water, alcohol, or acetic acid to prepare the dyebath.

Renard and Franc stated, at the same time, that anhydrous mercuric, ferric, and cupric chlorides might be used in the place of the chloride of tin.

Several other processes for obtaining aniline-red were suggested, and some of thom used. Gerber-Keller treated aniline with the nitrates of mercury. Lauth and Depouilly used nitric acid; and six months before the discovery by these French dyors, two English chomists-Medlock and Nicholson-had separately patented, within a fow days of each other, as the result of their experiments, a similar process.

Medlock and Nicholson, and Girard and De Laire, all, in 1860, patented the use of arsenic acid. This process, being now almost exclusivoly employed, must be described. Arsenic acid is combined with a slight excess of aniline; the crystalline mass is heated by means of a slow fire to about $120^{\circ}$ to $140^{\circ} \mathrm{C}$., care being taken not to exceed $160^{\circ} \mathrm{C}$. The operation, according to the scale on which it is carried on, requires from four to nine hours for completion. A perfectly homogeneous mass, fluid above $100^{\circ} \mathrm{C}$, is thus obtained, which on cooling solidifies to a hard substance, with metallic bronze-coloured lustre. When dissolved in boiling water it produces a 
solution of great richness and purity of colour. If, in the treatment of aniline with arsenic acid, the latter be considerably beyond the proportion of aniline employed, violet and blue dyes may bo formed. The production of such has boen patonted by Girard and De Laire.

Laurent and Casthelaz have obtained aniline-red direct from benzol, without tho proliminary isolation of aniline. Nitrobenzol is treated with $n$ mixture of iron and hydrochloric acid, or with forric chloride. On heating the mixture, the ferric chloride reacts on the aniline contained in the mixture, transforming it into aniline-red. It is doubtful if the colouring-matter obtained by this process is equal in beauty to that procured from the aniline, and the process is no longer employed. The name of erythrobened was given by the discoverers to the colouring-matter thus obtained, but it probably consists principally of rosaniline.

The processes described are amongst the best yet devised for the preparation of crude aniline-red. Numerous other methods have been patented, amongst others the following:-

Messrs. Renard Brothers include in their patent the ebullition of aniline with stannous, stannic, mercurous, and mercuric sulphates, with ferric and uranic nitrates and nitrate of silver, and with stannic and mercuric bromides.

Messrs. John Dale and Caro patont the action of nitrate of lead upon aniline, or hydrochlorate of aniline.

Mr. Smith claims the ebullition of aniline with perchloride of antimony, or the action of antimonic acid, peroxide of bismuth, stannic, ferric, mercuric, and cupric oxides upon hydrochlorate or sulphate of aniline at the temperature of $180^{\circ}$.

M. Gerber-Keller (Heilmann in England) claims the production of aniline red from all the metallic salts of the oxacids of nitrogen, sulphur, chlorine, bromine, iodine, phosphorus, arsenic, and, in fact, almost every compound within the range of chemistry. It may well be said of such patents, that 'scientific men cannot speak otherwise than in terms of reprehension. Their claims are founded on random guesswork, not on the results of patient investigation. They are attempts to pre-occury the whole field, and forestall all the rewards which should be left open to real inventive genius to cultivate and win.'

These processes have reference to the preparation of crude aniline-red. The crude colours contain some undecomposed aniline, mostly in the form of salts. They contain also tarry matters, some insoluble in water and dilute acids; others soluble in bisulphide of carbon, naphtha, or in caustic or carbonated alkalis. If, therefore, the crude red be boiled with an excess of alkali, the undecomposed aniline is expelled, the acid which exists in the product being fixed. On treating the residuo with acidulated boiling water, the red is dissolved, while certain tarry matters romain insoluble. If now the boiling solution be filtered, and then saturated with an alkali, the colouring-matter is precipitated in a tolerable state of purity. By re-dissolving the precipitated red in an acid, not employed in excess, a solution is obtained which frequently crystallises, or from which a pure red may be thrown down by a new addition of chloride of sodium, or other alkaline salt. The hydrnchlorate of aniline. red is employed in dyeing in France, while the acetate is used in England.

To Dr. Hofmann we are principally indebted for the investigation into the nature of aniline-red. In his paper on the 'Formation of Aniline-Red,' he says, he is 'painfully aware of the imperfections of his researches upon this subject: as yet only a corner of the veil which conceals the truth is raised. The genesis and constitution of aniline-red still remain to be investigated, though the chemical nature and composition of the substance itself are no longer doubtful.'

Aniline-reds are salts of a very remarkable compound, which plays the part of a well-defined base, and to which Dr. Hofmann gave the name of rosaniline. This compound, in its anhydrous state, is represented by the formula of $\mathrm{C}^{19} \mathrm{H}^{1}{ }^{2} \mathrm{~N}^{3}$. (See Rosantwirm.)

The formation of aniline-red will be best given in Dr. Hofmann's own words :-

- It had been observed by many manufacturers that some varieties of commercial aniline yield much more rosaniline than others. Samples of aniline, boiling at temperatures much higher than the boiling point of the puro compound, are found to be particularly adapted for the production of the rod. This observation led Dr. Hofmann to examine carefully the deportment of pure aniline under the influence of the several agents by which commercial aniline is converted into aniline-red. To this examination he subjected a sample of puro aniline derived from indigo, and other samples made from pure benzol ; this being derived in some cases from benzoic acid, in other cases from coal-tar. These experiments elicited the remarkable fact, that pure aniline, from whatever source obtained, is incapable of furnishing the red dye-a result fully confirmed by Mr. E. C. Nicholson, who indeed had been long acquaintod with this circumstance. It thus became obvious that commercial aniline must contain 
another base, the presence of which determines the formation of the colouring-matter. The idea very naturally suggested itself that toluidine - which, owing to the difficulty of soparating toluol from betzol, is always present in commorcial aniline-might be the true source of the colouring-matter. Experiments made with pure toluidine showed, however, that this base is not more capable of yielding the red than pure aniline itself. But the red colouring-matter is instantaneously produced when a mixture of pure aniline and pure toluidine is treated with the chlorides of mereury or tin, or with arsenic aoid, plainly showing that the two bases must co-operate in the formation of the red.'

This result, Dr. Hofmann believes, contains the clue by which may be explained not only the genesis of aniline-red, but also that of the tinctorial ammonias generally. It points, moreover, to the necessity of ascertaining how far the formation of tho violet and other colouring-matters, hitherto believed to be exclusively derived from aniline, require the co-operation of toluidine.

Returning to the red derivatives of aniline obtained by the different processes above described, we may state broadly that all these products are composed essentially of rosaniline salts. The red, prepared by the process of Messrs. Renard and Franc, or fuchsine, consists chiefly of hydrochlorate of rosaniline. The azalëine, or anilinered prepared by nitric acid, is principally nitrate of rosaniline.

In the crude red, produced by the action of arsenic acid, we should find the arseniate of rosaniline, which, by the subsequent treatment, for the sake of purification, is converted into hydrochlorate or acotate of rosaniline:

This is the place to record the fact that for some time past a colourless (or at least slightly rose-coloured) paste, with an alkaline reaction, has been delivered into commeree from Mulhouse, in France; which paste has only to be treated with acetic acid, in which it dissolves with the greatest facility, in order to obtain an extremely rich and beautiful carmine solution, capable of being at once employed for printing stuffs. Now that the characters of rosaniline are known, there cannot be the least doubt that this colourless paste, and certain pink powders of similar properties, consist almost ontirely of rosaniline more or less pure, and that they have been prepared by precipitating a solution of a rosaniline salt by an excess of a powerful base, such as soda or lime.

Remarks on the Phenomena observed in the Applications of Aniline-Red.-We are now, moreover, enabled to explain perfectly what takes place when a stuff which has been dyed with aniline-red is acted upon by a powerful acid or alkali. On printing with a powerful acid, the stuff is decolorised with the formation of a yellowish stain, because a rosaniline salt with three equivalents of acid is formed; the triacid salts of this substance being in fact all yellowish, and possessing but little colour. When the material is washed with water, the excess of acid is removed, and the monacid salt reproduced, the red colour being restored.

On printing with a powerful base, for example, with eanstic soda, the red disappears as the red rosaniline salt is decomposed, and rosaniline liberated in a colourless condition. But on washing out the soda with water, the red colour reappears, the rosaniline becoming probably carbonated.

If a powerful volatilo base be employed, as ammonia, the red also disappears, on account of the liberation of the colourless rosaniline; but in proportion as the ammonia evaporates the red colouration returns, particularly on slightly warming-as the rosaniline, which is a fixed base, expels the ammonia, and re-forms the primitive salt of rosaniline with its peculiar colour. If the rosaniline-dyed fabric be left for a considerable length of time (from twelve to twenty hours) in contact with weak ammonia, the colour, as has been pointed out by Mr. W. Crum, scarcely returns on rinsing with water. This is obviously due to the increased solubility of rosaniline in water containing ammonia, which separates the colouring-mattor even from the mordant.

The salts of rosaniline, which are chiefly employed for dyeing silk and wool, are the acetate and the hydrochlorate, and their application is simple in the extreme. The silk is dyed by passing it through a warm aqueous solution of the salt: for the dyeing of wool the solution is heatod to a temperature varying between $50^{\circ}$ and $60^{\circ} \mathrm{C}$.

The force and rapidity with which rosaniline is fixed by silk and wool is the only difficulty to be encountered in this branch of dyeing. In fact, this magnificont colour is precipitated and fixed with such avidity and promptitude by silk and wool, that it is necessary to take particular precautions, and to operate with solutions that are at first comparatively weak, and are only gradually strengthened, to prevent the dyeing being unequal, and the portions first immersed in the bath being more strongly coloured than those afterwards introduced. Cotton is also difficult to dye with rosaniline salts, but for the exactly opposite roason-that it does not present any 
attraction for this colouring-matter. The fixation of the colour on cotton can therefore only be effected by first treating the cotton with some animal mordant, or with tannic acid.

There are two modes of proceeding for this purpose:-

1. The fabric is printed with the thickened organic mordant; for goods intended to be dyed throughout the mordant is uniformly spread on the whole surface of the stuff, and fixed either by drying or steaming beforo tho fabrics are introduced into the dyebath. The colour is only fixed on the mordanted portions, and the shades themselves may be varied according to tho naturo and composition of the mordant.

Among the substances employed as organic mordants for rosaniline are:-Albumen, whether made from white of egg or from blood; prepared gluten, prepared casoin, gelatine, and tannin-this latter being used either in its combinations with the metallic oxides (as antimonic, stannic, or plumbic), or as tannato of gelatino. For some time oily preparations were employed, as, for example, the sulphomargaric and sulpholeic acids.

2. The mordant is thickened, and at the same time some aniline-red is dissolved in it; the stuff is printed therewith, dried, and steamed; the whole is then fixed on the fabric, which then has only to be washed and dried.

This method is principally employed when using albumen, in which acetate of rosaniline is dissolved. Latterly tannin has been employed in preference for fixing aniline-red, particularly in dyeing ordinary articles; for the mordanting of which albumen, although giving the most satisfactory results, is too costly an article.

It is to Mr. Perkin that wo are indebted for the first application of tannin for fixing aniline colours upon cotton.

MM. Kuhlmann and Lightfoot have called attention to the advantages presented by the use of tannate of gelatine; and Mr. Walter Crum has minutely indicated the treatment by which gluten, the cheapest substitute for albumen, may bo rendered available in printing and dyeing with aniline colours.

AnIrIvr-vroxIT. To this colour in its varieties the names of Mauve, Anileine, Indisine, Phenameine, Violine, Rosolane, Tyroline, \&c. have been given. Mr. Perkin's patent dates from 26th August, 1856, and this was the original of all the numerous colorific compounds commercially produced. Aniline-violet was first obtained in the crystalline condition in 1860 by M. Scheurer-Kestner, who used monohydrated acetic acid as a solvent. Dr. Hofmann informs us that in 1862 Kestner obtained 'splendid well-developed prisms of perfectly pure aniline-violet, which that chemist produced by operating on a very large scale.' The chemical composition of this colour has not yet been definitely established, but the aniline-purple prepared by Perkin's process is the sulphate of a base called Mauveine, having the composition $\mathrm{C}^{27} \mathrm{H}^{21} \mathrm{~N}^{4}$. The process of manufacture is as follows :-A cold and dilute solution of sulphate or any other salt of commercial aniline is mixed with a solution, also cold and dilute, of bichromate of potash. The mixture is well stirred, and allowed to stand for ten or twelve hours. A black precipitate is produced, which is collected upon a filter, washed with cold water, and dried. The black matter is digested with light coal-tar oil, when a brownish-black tarry substance is dissolved from the colouring-matter obtained in the precipitate. The insoluble residue is then dried and digested with wood-spirit or alcohol, or indeed with any liquid capablo of dissolving the colouring-matter. The clear solution is separated by filtration or decantation, and distilled in order to recover the alcohol or wood-spirit; the residue left in the retort is Mr. Perkin's aniline-violet.

The black precipitate, after having been washed with cold water, is extracted by a prolonged obullition with large quantities of water (sometimes acidulated with from one to two per cent, of acetic acid), an operation which effects the solution of the colouring-matter. The filtered solutions are concentrated as much as possible, and while boiling are precipitated by the addition of caustic soda. The precipitate is filtered off and washed for some time with an alkaline solution, which facilitates the extraction of the excess of bichromate of potash, and removes a reddish colouringmatter affecting the purity of the aniline-violet. It is then treated with cold water until the alkali is removed, and the washings become coloured. The violet is thus obtained in the form of a paste.

The following processes have also been proposed for the production of aniline-violet:-

1. Oxidation of an aniline salt by a solution of pormanganate of potassium.Williams.

2. Oxidation of an aniline salt by a solution of ferricyanide of potassium, -Smith.

3. Oxidation of a cold and dilute solution of hydrochlorate of aniline by a dilute solution of chloride of lime.-Bolley, Beale, and Kirkman.

4. Oxidation of a salt of aniline in an aqueous solution by peroxide of manganese. -Kay. 
5. Oxidation of a salt of aniline by the peroxide of load under the influence of an acid.-Price.

6. Oxidation of a salt of aniline by free chlorine or free hypochlorous acid.-Smith.

7. Oxidation of a salt of aniline by the double chloride of copper and sodium.Dale and Caro.

The only processes which are employed industrially are those in which bichromate of potash, chloride of lime, and chloride of copper are used. The dyeing of silk or wool by means of aniline-violet is a process of the easiest description.

Dyeing Silk.-An alcoholic solution of the violet purple is diluted with eight times its volume of hot water previously acidulated with tartaric acid. This liquid is poured into the dyeing-bath of cold water slightly acidulated. Through this the silk is passed until the required shade is attained. The tint is rondered bluish by means of indigo-carmine. Sulphuric acid added to the dye-bath causes the violet to assume a greyish shade. This may be increased until a very beautiful grey (gris perle) is obtained. This colour is much used by the silk-dyers of Lyons.

Dyeing Wool. - This is condueted at a temperature of $50^{\circ}$ or $60^{\circ} \mathrm{C}$., the dye-bath consisting simply of a dilute aqueous solution of colouring-matter, without any acid.

Printing upon Silk and Wool.--The paste of aniline-violet is dissolved in about five times its weight of acetic acid (speeific gravity, 1.060), and mixed with enough gumwater to form a printing material. The fabrics when printed are submitted to the action of steam, and then washed.

Dyeing Cotton-Since cotton does not, like silk or wool, present much attraction for the aniline colours, the dyer employs albumen, soluble gluten, or tannin. In some eases the oxides of tin, of aluminium, of lead, or of antimony are used. Sulphuric acid has also the property of fixing the aniline-violet on cotton. The animal or tannin mordants are printed on the cotton, which is steamed so as to fix it, and it is then dyed in an acidulated solution of the colour.

Violet Imperial.-This violet, which is essentially different from the mauve, is formed by modifications of the aniline reds and blues. It is produced thus :-Equal quantities of aniline and dry hydrochlorate of rosaniline are heated to a temperature of $180^{\circ} \mathrm{C}$. for about four hours. MM. Girard and De Laire first patented this colour, and on account of its great beauty it is much used, although not quite so permanent as the mauve. Mr. E. C. Nicholson forms another violet by heating aniline-red in a suitable apparatus to a temperature between $200^{\circ}$ and $215^{\circ} \mathrm{C}$. This mass is exhausted with acetic acid, and the deop-violet solution diluted with enough alcohol to give to the dye a convenient strength.

Aniline-violet resists the action of light to a very considerable extent, although Chevreul has shown that it is inferior in this respect to either madder, cochineal, or indigo.

Avrurmz-ymInOW. In the preparation of aniline-red there arises a great number of secondary products. Amongst others, a yellow colouring-matter has been separated by Mr. Nicholson and examined by Dr. Hofmann. The name of Chrysaniline has been given to this very beautiful yellow colour, which has been proved to be a woll-defined base. The proparation of chrysaniline is simple. The residue from which the rosaniline has been extracted is submitted for some time to a current of steam, when a quantity of the base passes into solution. Addition of nitric acid to this solution precipitates the chrysaniline in the form of a difficultly soluble nitrate.

Hofmann has shown that chrysaniline is intimately related to rosaniline and leucaniline, only differing from the first by two equivalents, and from the second by four equivalents of hydrogen:-

\section{Chrysaniline $\mathrm{C}^{20} \mathrm{H}^{17} \mathrm{~N}^{3}$. \\ Rosaniline $\mathrm{C}^{20} \mathrm{H}^{19} \mathrm{~N}^{3}$. \\ Loucaniline $\mathrm{C}^{20} \mathrm{H}^{21} \mathrm{~N}^{3}$.}

Chrysaniline forms two series of salts, the greater number of which are well crystallised. The most remarkable salt of ehrysaniline is the nitrate, which is so insoluble in water that nitric acid may be precipitated, even from a dilute aqueous solution, by means of the more soluble hydrochlorate or acetate of chrysaniline. These, when poured into a nitric solution, rapidly give rise to the formation of an orange-red chrysaniline precipitate of nitrate of chrysaniline. This chrysaniline and its salts dye silks and wools a splendid golden-yellow colour. Such is the general character of the aniline colours. They have been used as printing-inks, which are very permanent. See 'Watts's Dictionary of Chemistry.'

E. Waller, E.M., in the 'American Chemist,' gives the following list of the names of the various coal-tar colours, both commorcial and chemical, together with their chemical formulre, when those could be found. (It should be observed that the old notation has been employed throughout). 'Many of the compounds hero men- 
tioned are not now in nse as dyes, either because they are too expensive, or because their use is inconvenient, requiring too complicated a trentmont or rare chomicals to fix them upon the goods, or because they have been superseded by dyes of better quality. Several colours, black especially, are usually produced on the fibre of the goods by printing them with various salts of aniline and rosaniline, and then with salts of copper, chlorates, \&c. Most of the dyes called aniline-blacks give, when dissolvod, deep green solutions. Of those to which the formulæ are not given, the chemical formulie are not as yet known, or they have not been given by those works to which I have resorted on the subject. The derivatives of aniline and toluidine are given together, as the two are with difficulty separated from each othor, and in course of manufacture the separation is never attempted.'

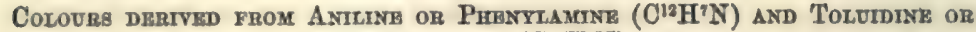 TozUYLAMTras ( $\left.\mathrm{C}^{14} \mathrm{H}^{9} \mathrm{~N}\right)$.}

Reds.

Hydrochlorate of rosaniline$\mathrm{C}^{40} \mathrm{H}^{10} \mathrm{~N}^{3}, \mathrm{HCl}$.

Called also aniline red, now red, magenta, solferino, fuchsine, arileine rougé, roseine, and azaline.

Acetate of rosaniline$\mathrm{C}^{40} \mathrm{H}^{19} \mathrm{~N}^{3}, \mathrm{HO}, \mathrm{C}^{4} \mathrm{H}^{3} \mathrm{O}^{8}$.

Known also by the same names as the above.

Nitrate of rosaniline-

$$
\mathrm{C}^{40} \mathrm{H}^{10} \mathrm{~N}^{3}, \mathrm{HO}, \mathrm{NO}^{5} \text {. }
$$

Known by the same names as the hydrochlorate; also as rubine and rubine imperial.

Dicodhydrate of trimethylehrysanilino$\mathrm{C}^{40} \mathrm{H}^{14}\left(\mathrm{C}^{2} \mathrm{H}^{3}\right)^{3} \mathrm{~N}^{3}, 2$ (HOI).

Called also chrysaniline red.

Nitrosophenyline, $\mathrm{C}^{6} \mathrm{H}^{6} \mathrm{~N}^{2} \mathrm{O}$.

Chomical formula unknown-

Xylidine, tar red, soluble ruby.

\section{Blues and Violets.}

These shade into one another so gradually that they cannot well be separated.

Hydrochlorate of monophenylrosaniline, $\mathrm{C}^{10} \mathrm{H}^{18}\left(\mathrm{C}^{12} \mathrm{H}^{3}\right) \mathrm{N}^{3}, \mathrm{HCl}$.

Called also rosaniline violet, red monophenylrosaniline, and Hofmann's violet.

Hydrochlorate of diphenylrosanilino-

$$
\mathrm{C}^{40} \mathrm{H}^{19}\left(\mathrm{C}^{12} \mathrm{H}^{5}\right)^{2} \mathrm{~N}^{3}, \mathrm{HCl} \text {. }
$$

Also known as rosaniline violot and Hofmann's violet.

Triphenylrosaniline, or triphenyl rosaniline, $\mathrm{C}^{40} \mathrm{H}^{16}\left(\mathrm{C}^{12} \mathrm{H}^{3}\right)^{8}, \mathrm{~N}^{3}$.

Called also aniline blue, rosaniline blue, Hofmann's blue; bleu de Paris, bleu de Lyons, bleu de Mulhouse, bleu de Mexique, bleu de nuit, bleu lumière; bleuine, azurine, and night blue.

Hydrochlorate of triphenylrosaniline$\mathrm{C}^{10} \mathrm{H}^{16}\left(\mathrm{C}^{12} \mathrm{H}^{5}\right)^{3} \mathrm{~N}^{3}, \mathrm{HCl}$.

Known also by the same names as the above.

Acetate of triphenylrosaniline-

$\mathrm{C}^{40} \mathrm{H}^{16}\left(\mathrm{C}^{12} \mathrm{H}^{3}\right)^{3} \mathrm{~N}^{2}, \mathrm{HO}, \mathrm{C}^{4} \mathrm{H}^{3} \mathrm{O}^{8}$.

Known also by the same names as the above.

Bisulphotriphenylrosaniline acid$\mathrm{C}^{40} \mathrm{H}^{18}\left(\mathrm{C}^{12} \mathrm{H}^{3}\right)^{3} \mathrm{~N}^{3}, 4 \mathrm{SO}^{3}$.

Called also Nicholson's blue and soluble blue.
Hydrochlorate of monethylrosaniline$\mathrm{C}^{10} \mathrm{H}^{18}\left(\mathrm{C}^{12} \mathrm{H}^{3}\right) \mathrm{N}^{3}, \mathrm{HCl}$.

Called also Hofmann's red violet.

Hydriodate of ethylrosaniline$\mathrm{C}^{40} \mathrm{H}^{18}\left(\mathrm{C}^{4} \mathrm{H}^{3}\right) \mathrm{N}^{3}, \mathrm{HI}$.

Called also Hofmann's red violet.

Ethyliodate of ethylrosaniline$\mathrm{C}^{10} \mathrm{H}^{18}\left(\mathrm{C}^{4} \mathrm{H}^{3}\right) \mathrm{N}^{3} \mathrm{C}^{4} \mathrm{H}^{3} \mathrm{I}$.

Called also fuchsine with a blue tint, and Hofmann's violet red.

Hydrochlorate of diethylrosaniline$\mathrm{C}^{10} \mathrm{H}^{17}\left(\mathrm{C}^{4} \mathrm{H}^{5}\right)^{2} \mathrm{~N}^{3}, \mathrm{HCl}$.

Called also Hofmann's blue.

Ethyliodate of diethylrosaniline$\mathrm{C}^{40} \mathrm{H}^{17}\left(\mathrm{C}^{4} \mathrm{H}^{5}\right)^{2} \mathrm{~N}^{8}, \mathrm{C}^{4} \mathrm{H}^{5} \mathrm{I}$

Called also Hofmann's red violet and ethylic rosaniline violet.

Hydrochlorate of triethylrosaniline$\mathrm{C}^{40} \mathrm{H}^{16}\left(\mathrm{C}^{4} \mathrm{H}^{5}\right)^{3} \mathrm{~N}^{3}, \mathrm{HCl}$.

Called also Hofmann's blue.

Ethyliodate of triethylrosaniline$\mathrm{C}^{40} \mathrm{H}^{16}\left(\mathrm{C}^{4} \mathrm{H}^{3}\right)^{2} \mathrm{~N}^{3}, \mathrm{C}^{4} \mathrm{H}^{3} \mathrm{I}$.

Called also Hofmann's blue and ethylic rosaniline violet.

Ethylbromate of triethylrosanilino- $\mathrm{C}^{40} \mathrm{H}^{16}\left(\mathrm{C}^{4} \mathrm{H}^{5}\right)^{3} \mathrm{~N}^{3}, \mathrm{C}^{4} \mathrm{H}^{3} \mathrm{Br}$.

Called also brimula.

Hydrochlorate of methylrosaniline$\mathrm{C}^{40} \mathrm{H}^{18}\left(\mathrm{C}^{2} \mathrm{H}^{3}\right) \mathrm{N}^{3}, \mathrm{HCl}$.

Hydriodate of methylrosaniline$\mathrm{C}^{40} \mathrm{H}^{18}\left(\mathrm{C}^{2} \mathrm{H}^{3}\right) \mathrm{N}^{3}, \mathrm{HI}$.

Hydrochlorate of dimethylrosaniline$\mathrm{C}^{40} \mathrm{H}^{17}\left(\mathrm{C}^{2} \mathrm{H}^{3}\right)^{2} \mathrm{~N}^{3}, \mathrm{HCl}$.

Hydrochlorate of trimethylrosaniline$\mathrm{C}^{40} \mathrm{H}^{16}\left(\mathrm{C}^{2} \mathrm{H}^{3}\right)^{3} \mathrm{~N}^{3}, \mathrm{HCl}$

Methylaniline, $\mathrm{C}^{12} \mathrm{H}^{6}\left(\mathrm{C}^{2} \mathrm{H}^{3}\right) \mathrm{N}$.

Called also methylic rosaniline violet and violet de Paris.

Mauraniline, $\mathrm{C}^{38} \mathrm{H}^{17} \mathrm{~N}^{3}$.

Voilanile; $\mathrm{C}^{30} \mathrm{H}^{15} \mathrm{~N}^{3}$.

Mauveine, $\mathrm{C}^{54} \mathrm{H}^{2+} \mathrm{N}^{4}$.

Called also mauve, aniline purple, Per. kin's violet, indisine, analeine harmaline, violine, and mauve rosolane.

Hydrochlorate of mauveino$\mathrm{C}^{34} \mathrm{H}^{24} \mathrm{~N}^{4}, \mathrm{HCl}$.

Known also by the same names as mau: veine.

Hydrochlorate of ethylmaureino$\mathrm{C}^{54} \mathrm{H}^{23}\left(\mathrm{C}^{4} \mathrm{H}^{5}\right) \mathrm{N}^{4}, \mathrm{HCl}$.

Called also dahlia.

Ditolylrosaniline. $\mathrm{C}^{10} \mathrm{H}^{17}\left(\mathrm{C}^{14} \mathrm{H}^{7}\right)^{2} \mathrm{~N}^{3}$. 
Called also toluidine blue.

Tritolylrosaniline, ${ }^{10} \mathrm{H}^{16}\left(\mathrm{C}^{14} \mathrm{H}^{7}\right)^{3} \mathrm{~N}^{3}$. Chemical formulæ unknown-

Regina blue, opal blue, regina purple, bleu de Fayolle, violet de Mulhouse, Britannia violet, geranosine, violet imperial.

\section{Greens.}

$\mathrm{C}^{40} \mathrm{H}^{39}\left(\mathrm{C}^{4} \mathrm{H}^{3}\right) \mathrm{N}^{3}, 2 \mathrm{HO}$.

Known as Aldehyde green, aniline green, viridine, and emeraldine.

$\mathrm{C}^{40} \mathrm{H}^{19}\left(\mathrm{C}^{2} \mathrm{H}^{3}\right)^{6} \mathrm{~N}^{3}, 2 \mathrm{HO}$.

Known as iodine green and iodide of methyl green.

Chemical formulæ unknown-

Iodide of ethyl green, Perkin's green.

\section{Yellows. \\ Chrysaniline, $\mathrm{C}^{10} \mathrm{H}^{17} \mathrm{~N}^{2}$.}

Called also Phosphine, aniline yellow, and yellow fuchsine,

Nitrophenyldiamine, $\mathrm{C}^{20} \mathrm{H}^{6} \mathrm{O}^{8}, \mathrm{HCl}$.

Chrysotoluidine, $\mathrm{C}^{42} \mathrm{H}^{20} \mathrm{~N}^{3}$.

Zinaline, $\mathrm{C}^{10} \mathrm{H}^{19} \mathrm{~N}^{2} \mathrm{O},{ }^{2}(?)$.

Chemical formulæe unknown-

Dinitroaniline, Field's orange (?).

\section{Browns.}

Chemical formulæ unknown.

Havanna brown, Bismarck brown, aniline brown, aniline maroon, Napoleon brown.

\section{Greys.}

Chemical formulæ unknownAniline grey, argentine.

\section{Black.}

Chemical formulæ unknownAniline black.

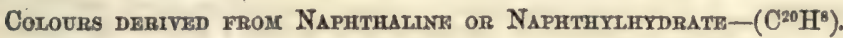

\section{Reds.}

Chloroxynaphthylic acid, $\mathrm{C}^{20} \mathrm{H}^{3} \mathrm{ClO}^{\circ}$.

Called also pseudoalizarine, naphthylic red, and chloronaphthaline acid.

Chemical formulæ unknown-

Roseonaphthaline, carminaphtha.

\section{Yellows. \\ Binitronaphthaline, $\mathrm{C}^{20} \mathrm{H}^{6}\left(\mathrm{NO}^{4}\right)^{2}$.}

Called also binitronaphthal, nephthalamine yellow, naphthylic yellow, golden yellow, and Manchester yellow.

Binitronaphthylic acid-

$$
\mathrm{C}^{20} \mathrm{H}^{5}\left(\mathrm{NO}^{4}\right)^{2} \mathrm{O}, \mathrm{HO} \text {. }
$$

Known also by the same names as the above.

Trinitronaphthylic acid-

$$
\mathrm{C}^{20} \mathrm{H}^{4}\left(\mathrm{NO}^{4}\right)^{3} \mathrm{O}, \mathrm{H} \mathrm{H} \text {. }
$$

\section{Colovrs derived from Carbolic Acto and Phemic Acid, Phenyluydrate} or PuexoI- $\left(\mathrm{C}^{12} \mathrm{H}^{8} \mathrm{O}^{2}\right)$.

\section{Reds.}

Picramic acid, $\mathrm{C}^{12} \mathrm{H}^{5}\left(\mathrm{NO}^{4}\right)^{2} \mathrm{NO}^{2}$.

Called also picramine acid.

Coralline, $\mathrm{C}^{20} \mathrm{H}^{8} \mathrm{O}^{4}$.

Called also peonine.

Coralline amide, $\mathrm{C}^{20} \mathrm{H}^{9} \mathrm{NO}^{2}$.

Called also red coralline.

\section{Blue.}

Isopurpuric acid, $\mathrm{C}^{12} \mathrm{H}^{3}\left(\mathrm{NO}^{4}\right)^{2} \mathrm{NO}^{3}, 2 \mathrm{Cl}$. Called also bicyanide of pieramyl and Grénat.

\section{Greens.}

Chemical formulæ unknownChloropierine.

\section{Yellows.}

Picric acid, $\mathrm{C}^{12} \mathrm{H}^{2}\left(\mathrm{NO}^{4}\right)^{3} \mathrm{O}$, HO.
Called also trinitrophenic acid and carbazotic acid.

Aurine, $\mathrm{C}^{24} \mathrm{H}^{12} \mathrm{O}^{6}$.

Called also rosolic acid.

\section{Brouns.}

Picrate of ammonia$\mathrm{NH}{ }^{4} \mathrm{O}, \mathrm{C}^{\cdot 2} \mathrm{H}^{2}\left(\mathrm{NO}^{4}\right)^{2} \mathrm{O}$.

Isopurpurate of potash. Chemical formulæ unknown.

Phenyl brown, or rotheine, or phénicienne.

Azuline (blue), $\mathrm{C}^{24} \mathrm{H}^{11} \mathrm{NO}^{4}$, and another viridine (formula unknown), are both an aniline and a carbolic acid colour, being produced by the action of carbolic acid on a derivative of aniline.

Cyanine (blue), $\mathrm{C}^{\text {no }} \mathrm{H}^{\text {s3 }} \mathrm{N}^{2} \mathrm{I}$, is deseribed in some works among the coal-tar colours, but is derived from chinchonine.

Among the blues and violets, the names Hofmann's blue or Hofmann's riolet are seen frequently to occur. These are distinguished in commerce by suffixing the letters $\mathrm{B}$ or $\mathbf{R}$ the number of times that either is repeated, showing the relatire blueness or redness of the dye. They range from BBBB to RRRR. The reddish shades are those in which the least substitution has taken place, as in the monethyl- or monophenyl-rosaniline, \&c., while the bluest of these are those which are triethyl- triphenylrosanilines, \&c. The series of names given for triphenylrosaniline and the hydrochlorato of the samo base are frequently interchanged. On the othyliodates and ethylbromates. authorities appear to differ as to whether they really are ethyliodates, \&c., or not. The colours are prepared by the action of iodide of ethyl, \&c. upon rosaniline or some of its salts, and iodine is given off in the operation; but whether one 
eqnimalent of the iodide of ethyl remains bohind or not in a state of chemical combination, doos not appear to be fully establishod. Were the latter supposition the case, the $\mathrm{C}^{4} \mathrm{H}^{3} \mathrm{I}$ of those formulæ would be replaced by $2 \mathrm{HO}$, and the chemical name would necessarily be changed to correspond. The same question might be mised regarding some of the Hofmann violets with their equivalent of $\mathrm{HCl}$ or $\mathrm{HO}, \mathrm{C}^{4}$ $\mathrm{H}^{3} \mathrm{O}^{3}$, but as one of these acids is used in the solution when purifying the colour, the probabilities are that the base unites with it, and the colour goes to market as a salt and not as an isolated base. The terms, direct blues and purified blues, are simply commercial terms indicating the amount of purification which the dyes have received, the first-named being the most impure. Among the greens the terms aniline-green and emeraldine are synonymous terms applied to a colour formed in the fibre of the goods. Viridine is a name applied to a true green, but the term has also been used for a mixture of indigo and picric acid, which cannot properly be called an aniline colour.

ANIMAI BIACK. Refuse animal matters are placed in a retort, and submitted to destructive distillation. The gases, evolved on decomposition, are usually burnt, while water, oily matter, and ammoniacal compounds distil over, and are condensed. There remains in the retort a carbonaceous mass, which, when levigated and ground in a mill, forms ' animal black.' It is in a more finely-divided state than bone-black, and is used in the preparation of blacking and of printing-ink.

ANImax crarcoar. See Bone-Black.

Avxanz. A resin of a pale brown yellow colour, transparent and brittle. It exudes from a large American tree, called by Piso, jetaiba; and by the Indians courbaril. It appears to be a species of Hymenca. It occurs in pieces of various sizes, and it often contains so many insects belonging to living species, as to have merited its name, as being animated. It contains about a fifth of 1 per cent, of a volatile oil, which gives it an agreeable odour. Alcohol does not dissolve the genuine animé, as I have ascertained by careful experiments, nor does caoutchoucine; but a mixture of the two, in equal parts, softens it into a tremulous jelly, though it will not produce a liquid solution. When reduced to this state, the insects can be easily picked out, without injury to their most delicate parts. On the contrary, Dr. R. D. Thomson says, animé resin is distinguished from copal by its ready solubility in alcohol; and that when digested in cold alcohol a portion remains undissolved, which may be dissolved in hot alcohol, from which it erystallises on cooling. Sir $\mathrm{R}$. Kane gives $\mathrm{C}^{40} \mathrm{H}^{\text {s3 }} \mathrm{O}$ ( $\mathbf{C}^{10} \mathbf{Z}^{\text {s8 }} \mathbf{O}$ ), as the composition of this gum-resin. - See 'Watts's Dictionary of Chemistry.'

The specific gravity of the different specimens of animé varies from 1.054 to 1.057 . When exposed to heat, in a glass retort over a spirit-flame, it softens, and, by careful management, it may be brought into liquid fusion without discolouration. It then exhales a white vapour of an ambrosial odour, which being condensed in water, and the liquid being tested, is found to be succinic acid.

It is extensively used by the varnish-makers, who fuse it at a pretty high heat, and in this state combine it with their oils or other varnishes. It is also employed, on account of its agreeable smell when burning, in the mnnufacture of pastilles.

Gum-animé is sometimes mistaken for amber, but the fossil resin can generally be distinguished by its greater hardness. The gum-animé of Zanzibar is a semi-fossil resin, believed to be the produco of a species of Trachylobium.

ANIsFrD. (Anis, Fr.; Anis, Ger.) The fruit or seed of the Pimpinella anisum, largely eultivated in Malta, Spain, and Germany; used in the preparation of the oil of anise (oleum anisi), the spirit of anise (spiritus anisi), and anise-water (aqua anisi). It is also used in cordials. In 1855, 963 cwts. were imported. The oleum badiani, or the oil of star anise (illicium anisatum), has the colour and taste. of the oil of anise; but it proserves its fluidity at $35^{\circ} 6^{\circ} \mathrm{F}$. It is sometimes fraudulently substituted for oloum anisi-Pereira.

AVIrER. A liquid measure of Amsterdam, which contains 32 gallons English. During the war, when communication with Holland was constant, and sailors and soldier's were constantly passing from one country to the other, the anker was as commonly used as a measure in our seaports as in those of Holland. The anker of brandy was frequently smuggled into this country.

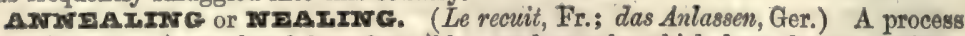
by which glass is renderod less frangible; and metals which have become brittle, either in consequence of fuston or long-continued hammering, are again rendered malleable. When a glass vessel is allowed to cool immediately after being made, it will, if a small splinter of flint, or an angular fragment of quartz, is dropped gently into it, fly to pieces with great violence, sometimes immediately, sometimes after a fow minutes. This extreme fragility is prevented by annealing, or placing the vessels in a hot oven, where they take several hours, or even some days, to cool.

Similar phenomena are exhibitod in a higher degree by glass-tears, or Prince Rupert's drops; produced by letting drops of melted glass fall into cold water. Their 
form resembles that of a pear, rounded at one extremity, and tapering to a very slender tail at the other. If a part of tho tail be broken off, the wholo drop flies to pieces with a loud explosion; and yet the tail of a drop may be cut away by a glasscutter's wheel, or the thick end may be struck smartly with a hammer, without the fear of sustaining any injury. When heated to redness, and permitted to cool gradually in the open air, they lose these peculiarities, and do not differ sensibly from common glass.

The peculiar brittleness of unannealed glass is, by many manufacturers, referred to the following conditions. The exterior surface of the glass cooling quicker than the layers of glass beneath, the two portions of glass are supposed to be in different degrees of tension; as they technically express it, a stretchcd skin of glass is formed; and as the arrangement of the particles is different in this film from their disposition in those parts which have cooled more slowly, there is a constant tendency to fracture, the slightest scratch upon this 'skin' disturbing the entire molecular arrangement.

If any mass of glass or of metal cools rapidly, there will be, according to the thickness of the mass, a greater or less difference between the arrangement of the constituent particles on the outer and inner sections. The process of annealing secures an equal arrangement throughout the mass.

When metals have been extended to a certain degree under the hammer, they become brittle, and incapable of being further extended without cracking. In this case the workman restores their malleability, sometimes by annealing, or, in other cases, by heating them red-hot and allowing them to cool slowly. The rationale of this process seems to be, that the hammering and extension of the metal destroys the kind of arrangement which the particles of the metal had previous to the hammering; and that the annealing, by softening the metal, enables it to recover its original structure.

Of late years a mode has been discovered of rendering cast-iron malleable, without subjecting it to the action of puddling. The process is somewhat similar to that employed in annealing glass. The metal is kept imbedded in ground charcoal, or in powdered hæmatite, for several hours at a high temperature, and then allowed to cool slowly. In this manner vessels are made of cast-iron which can sustain considerable violence without being broken. See Iron, Matlrabrie.

ANTNOTYO or ANATTO. Seo ARNatTo.

AN ORThITz. A lime felspar. Seo FeLspar.

ANTEIOPE EORN is used occasionally for ornamental knife-handles. Seo HORN.

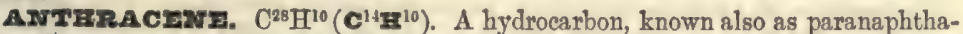
line, discovered by J. Dumas in 1831. It has become of considerable commercial importance since Messrs. Graebe and Liebermann discovered, in 1869, that anthraceno could be converted into a valuable colouring-matter identical with the natural alizarine of the madder-root.

Arthracene is produced in the dry distillation of coal, bituminous shale, or wood; and is found in the heavy semi-fluid portion of the tar which comes over towards the close of the distillation. 'The substance known as 'green grease,' obtained by distilling coal-tar, and used as a common lubricating agent for machinery, contains about 20 per cent. of anthracene associated with naphthaline and other hydrocarbons. From this product, crude anthracene may be obtained by the use of the hydro-extractor, and by submitting the raw product to strong pressure. The crude anthracene is purified by solution in hot coal-tar naphtha and repeated recrystallisation. A yellow tint, due to the presence of chrysogen, may be expelled by exposure to sunlight, and the anthracene is finally purified from any other hydrocarbons by boiling with alcoholic picric acid.

Thus obtained, the pure anthracene appears in small, well-defined, lustrous crystalline laminæ of a clear white colour, and exhibiting, when pure, a beautiful violet fluorescence. The specific gravity of anthracene is $1 \cdot 149$. It melts at about $415^{\circ} \mathrm{F}$, and sublimes at higher temperatures. Anthracene is insoluble in water, but readily soluble in boiling alcohol, in ether, benzole, volatile oils, and bisulphide of carbon. By prolonged exposure to light it passes into an isomeric modification known as paranthracene. Under the influence of oxidising agents it is converted into anthraquinone, from which artificial alizarine may be prepared. Seo Alizarine.

ANTHRACENT-RID. A name for artificial alizarine. See AlizARINE.

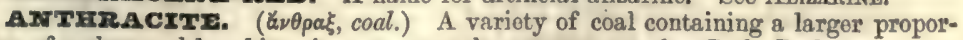
tion of carbon and less bituminous matter than common coal. -De la Beche.

'We see the same series of coal-beds becoming so altered in thoir horizontal range, that a set of beds bituminous in one locality is observed gradually to change into anthracitic in another. Taking the coal-measures of South Wales and Monmonthshire, we have a scries of accumulations in which the coal-beds become not only more anthracitic towards the west, but also exhibit this change in a plane which may 
bo considerod as dipping SSE. at a moderate angle, the amount of which is not yot clearly ascertained, so that in the natural sections afforded wo have bituminous coals in the high grounds and anthracitic coals beneath. This fact is readily observed either in the Neath or Swansea valloys, where we have bituminotis coals on the south and anthracite on the north; and more bituminous coal-beds on the hoights than beneath, some distance up these valloys - those of the Nedd and Tawe. Though the terms bituminous coal and anthracite have been applied to marked differences, the changes are so gradual that there is no sudden modification to be seen. To some of the intermediate kinds the term 'froe-burning' has boen given, and thus three chief differoneos havo been recognised.'-Memoirs of the Geological Survey.

Tho term Culm is applied both to an inferior kind of anthracito only worked for making lime, or for mixing with clay, and to the small pieces of good anthracite obtained in working the true anthracite beds. It is also called Blind Coal, Glance Coal, and Kilkenny Coal.

The term Culm is applied generally to anthracite in our parliamentary returns.

There are three very distinct 'trades' in anthracite. Thero is, first, that where the coal is sold exactly as it is worked, 'through and through,' as it is termed, or Through Culm, which is used entirely for lime-buruing. This coal is not of so puro a kind as that from which the large coal is picked out, and is sometimes called Bastard Stone Coal. The trade in the Neath district is entiroly of this kind. In Swansea and Llanelly it is partly of this kind, and partly of the kind where the large coal is picked out, and sold as Stone Coal for the rarious purposes to which that cosl is applied, leaving the small to be shipped, also for lime-burning purposes, under the name of Stone Coal Culm. In Pembrokeshiro no 'through culm' is shipped. There is one curious lot of 4,000 tons annually shipped in Swansea under the name of Lambskin, which is almost dust; it is sent to one market-Cardiganshire, where it is used entirely for mixing with clay; the mixture, under the name of Fireballs, being usod for household purposes. This mixture, made of the ordinary Stone Coal Culm, is also very commonly used in parts of Pembrokeshire and Caermarthenshire.

Anthracite coal is obtained in the western divisions of the South Wales coal-fiold, at Bideford in Devonshire, at Walsall in Staffordshire, in Ireland, and in Scotland, in Switzerland, Savoy and Italy. Commencing at the top of the Neath valley, and following the north crop of the South Wales coal-field downwards to Kidwelly, from east to west. there are 48 anthracite collieries - and in Pembrokeshire there are 16 of these collieries. It is found abundantly in America. Professor H. D. Roger's 'Transactions of American Geologists' states that in the great Apalachian coal-field, extending 720 miles, with a chief breadth of 180 miles, the coal is bituminous towards the western limit, whore it is level and unbroken, becoming anthracitic towards the south-west, where it is disturbed. Anthracitic coal is also found in the coal-fields of France, especially in the departments of Isère, the High Alps, Gard, Mayenne, and of Sarth; about 42,271,000 kilogrammes (of 2.2046 avoirdupois pounds each) are produced "annually. Anthracite is also raised in Belgium.

The following analyses of bituminous and anthracite coals will sufficiently show the differences:-

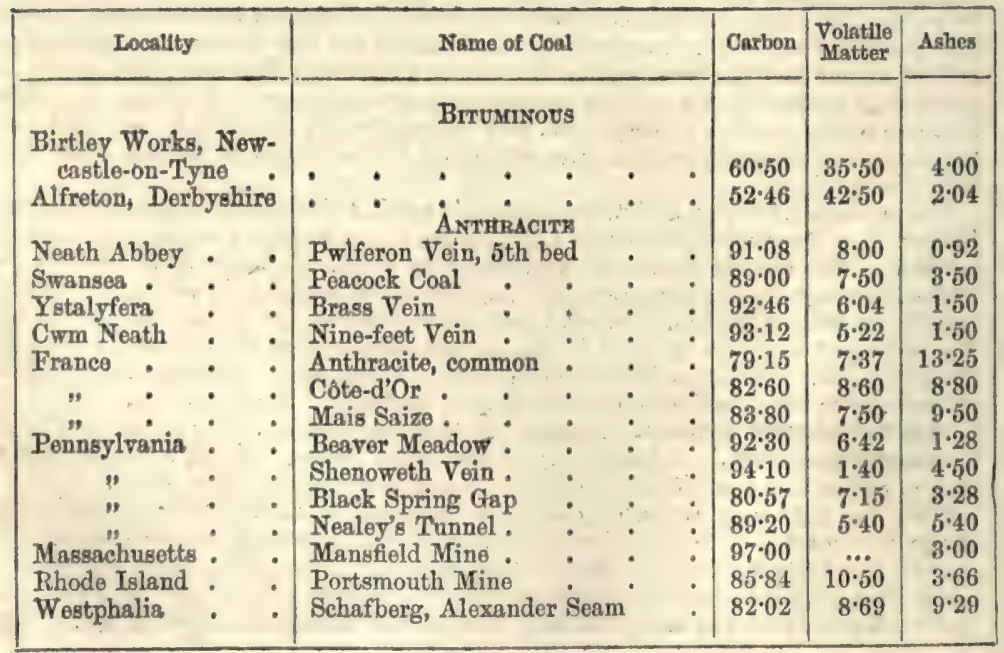


Anthracite is not an original variety of coal, but a modifleation of the same beds which remain bituminous in other parts of the region. Anthracite beds, therefore, aro not separsto deposits in another sea, nor coal-measures in another area, nor interpolations among bituminous coals; but the bituminous beds themselves, altored into a natural coke, from which the volatile bituminous oils and gases have been driven off. $-J$. P. Lesley, on Coal.

Anthracite-now exclusively used for iron-making, steam-engines, and for domestic purposes in the United States-Was some 50 years since regarded as incombustible rofuso, and thrown away.

Principal Localities of Anthracite and Anthracitio Coal, \&c.

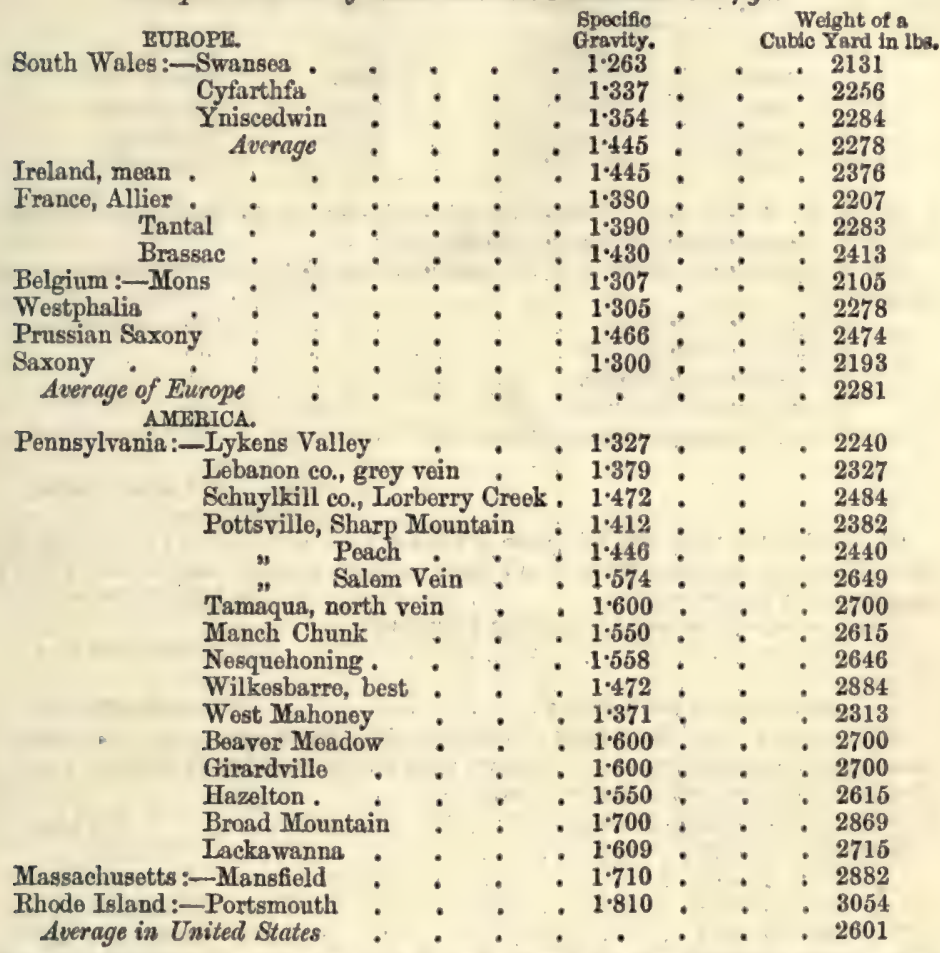

The calorific value of anthracite coal is well shown by the following results from Dr. Fyfe's experiments to compare Scotch and English bituminous coals with anthracito, in regard to their evaporative power, in a high-pressure boiler of a 4 -horso engino laving a grate with 8.15 square feet of surface; also in a waggon-shaped copper boiler, open to the air, surface 18 feet, grate 1.55 .

\begin{tabular}{|c|c|c|c|c|c|c|c|c|c|}
\hline $\begin{array}{l}\text { Kind of Fuel } \\
\text { employed }\end{array}$ & 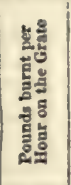 & 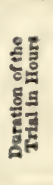 & 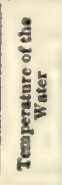 & 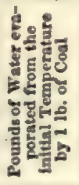 & 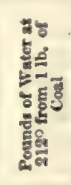 & 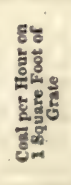 & 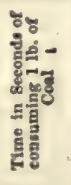 & 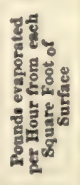 & Remarks \\
\hline Middlerig Scotch & $81 \cdot 83$ & 9 & $45^{\circ}$ & $6 \cdot 66$ & $7 \cdot 74$ & $10^{*} 00$ & $44 \cdot 27$ & $\because$ & \multirow{2}{*}{$\begin{array}{c}\text { Pressure } 17 \mathrm{lbs} . \\
\text { per sq. inch. } \\
\text { Ditto. }\end{array}$} \\
\hline $\begin{array}{l}\text { Scotch coal, dif- } \\
\text { ferent variety } \\
\text { from preceding }\end{array}$ & 108 & 3 & 170 & $6 \cdot 62$ & 6.89 & $13 \cdot 25$ & $83 \cdot 33$ & .. & \\
\hline AYTHRACTTE & $47 \cdot 94$ & 81 & 45 & $8 \cdot 73$ & $10 \cdot 10$ & $5 \cdot 88$ & $75 \cdot 09$ & & \multirow{3}{*}{$\begin{array}{c}\text { Ditto. } \\
\text { Low pressure, } \\
\text { open copper } \\
\text { boller. } \\
\text { Ditto. }\end{array}$} \\
\hline $\begin{array}{l}\text { Scotch coal, from } \\
\text { near Bdinburgh }\end{array}$ & $8 \cdot 24$ & $8 \frac{1}{2}$ & 50 & 8.88 & 6.90 & $8 \cdot 81$ & $436 \cdot 89$ & $8 \cdot 15$ & \\
\hline $\begin{array}{l}\text { English bitumi- } \\
\text { nous conl. }\end{array}$ & 6.07 & $8 \cdot 4$ & 80 & $7 \cdot 84$ & $9 \cdot 07$ & $8 \cdot 91$ & $503 \cdot 08$ & 806 & \\
\hline
\end{tabular}


Spree will not admit of our entering fully into the question of the evaporative power of anthracite ; but its advantages under cortain conditions are fully established.

In this country anthracite coal is used in the manufacture of iron in the following furnaces:-

Ystalyfera in Glamorganshire . . . 11 furnaces in blast in 1871

Yniscedwyn in Brocknockshire : . : 2 -" "

The quantity of anthracite-iron made in 1871 being 34,761 tons.

The following table shows the progress of production in America of anthracite from 1862 to 1871 inclusive, from Schuylkill, Lehigh, and Wyoming:-

\begin{tabular}{|c|c||c|c|}
\hline Years & Tons & Years & Tons \\
\hline 1862 & $7,387,422$ & 1867 & $11,725,588$ \\
1863 & $9,187,588$ & 1868 & $12,912,751$ \\
1864 & $9,657,723$ & 1869 & $12,746,938$ \\
1865 & $8,814,995$ & 1870 & $14,266,190$ \\
1866 & $10,498,970$ & 1871 & $13,855,307$ \\
\hline
\end{tabular}

Mr. P. W. Sheafer, in an address recently delivered to the students of the Pardee Scientifie Departiment in Lafayette College, says :-

'In our estimates of the areas of the anthracite coal-fields of Pennsylvania, we place that of the

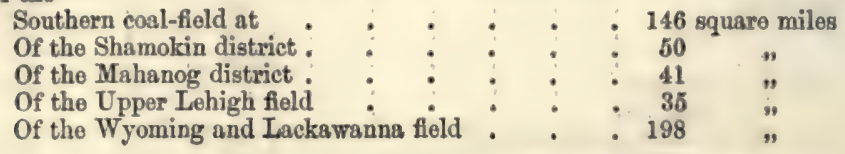

Total. 470 square miles, or 300,800 acres.

- Averaging the total coal thickness of the southern coal-field at 75 feet, and that of the middle and northern fields at 45 feet, we have a total content (one cubic yard equalling one ton) of say . . . . . 26,361,076,000 tons

Deduct one-half for waste in mining, preparing,

and faults

and we have a net result of . . . . . . 13,180,538,000 tons

'The amount mined from 1820 to 1870 , the first fifty years of the anthracite coaltrade, was $206,666,325$ tons ; so that we have yet in store $12,973,878,675$ tons. The progress of our coal-trade is thus shown:-

In 1820 the production was . . . . . . 365 tons

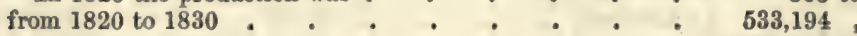

" 1830 to 1840 . . . . . $5,406,711$ "

" 1840 to 1850 . . . . . . . . $15,952,893$ "

" 1850 to 1860 . . . . . . . . 42,088,644 "

", 1860 to 1870 : : : : : 50,337,354 "

'The production of anthracite coal in Pennsylvania in the year 1872 was unprecedently large, as will bo soen by the following Table, illustrating the progress of the extraction year by year during the last thirty years :

\begin{tabular}{|c|c||c|c|}
\hline Year & Tons & Year & \multicolumn{1}{|c|}{ Tons } \\
\hline 1843 & $1,263,598$ & 1858 & $6,839,369$ \\
1844 & $1,630,850$ & 1859 & $7,808,255$ \\
1845 & $2,013,013$ & 1860 & $8,513,123$ \\
1846 & $2,344,005$ & 1861 & $7,954,264$ \\
1847 & $2,882,309$ & 1862 & $7,869,407$ \\
1848 & $3,089,238$ & 1863 & $9,566,006$ \\
1849 & $3,242,966$ & 1864 & $10,177,475$ \\
1850 & $3,358,899$ & 1865 & $9,652,391$ \\
1851 & $4,448,916$ & 1866 & $12,703,882$ \\
1852 & $4,993,471$ & 1867 & $12,988,725$ \\
1853 & $5,195,151$ & 1868 & $13,834,132$ \\
1854 & $6,002,334$ & 1869 & $13,723,030$ \\
1855 & $6,608,567$ & 1870 & $15,849,899$ \\
1856 & $6,927,580$ & 1871 & $15,113,407$ \\
1857 & $6,644,941$ & 1872 & $18,400,000$ \\
\hline
\end{tabular}




\section{ANTTMONY}

AYTirRAPURPURn. A colouring-matter recently obtained by Mr. W. H. Perkin from commercial artificial alizarine. The crude alizarine is dissolved in dilute solution of carbonate of soda, and the product woll agitated with freshlyprecipitated alumina, which combines with the alizarine, leaving the anthrapurpurin in solution. This solution, having been filtered and heated to the boiling point, is acidified with hydrochloric acid, whereby the colouring-matter is precipitated; this may be purified by repented boiling in alcohol.

As a dyeing agent anthrapurpurin greatly resembles alizarine, giving red colours with alumina, and purple and black with iron mordants. The shades of colour, however, are different with tho two materials; the anthrapurpurin reds being much purer and less blue, whilst the purples are bluer and the blacks more intense than those with alizarine. (See 'Journ. Chem. Soc.' May, 1873.)

ANTI-ATIRITION, or, ANTI-PRICTION COMPOSITION. Various preparations have been, from time to time, introduced for the purpose of removing, as much as possible, the friction of machinery. Black lead, or plumbago, mixed with a tenacious grease, has been much employed. Peroxide of iron, finely divided hrematite, \&c., have also been used.

AxTICEI0R2. A term employed by bleachers to the means of obviating the pernicious after-effects of chlorine upon the pulp of paper, or stuffs, which have been bleached therewith. Manufacturers have been in the habit of using sulphite of soda, whose action upon the adhering bleaching salt, which cannot be removed by washing, gives rise to the formation of sulphate and hydrosulphate of soda and chloride of sodium. Chloride of tin has been recommended by some chemists for this purpose.

Hyposulphite of soda is now extensively used as an antichlore, as also are certain salts of lime, as sulphide of calcium.

ANTI-FRICTION METAI. Tin and pewter are often employed as antifriction metals for the bearings of locomotive engines. One-half of each tin and copper is now nsed at some of the large railway works.

Babbet's metal is prepared by taking about fifty parts of tin, five of antimony, and one of copper.

Tin, or pewter, used alone, owing to its softness, spreads out and escapes under the superincumbent weight of the locomotive, or other heavy machinory. It is usual, therefore, to add antimony, for the purpose of giving these metals hardness.

Fenton's anti-friction metal, which is much employed, is a mixture of tin, copper, and spelter. Its adrantages are stated to be cheapness in first cost, low specific gravity, being 20 per cent. lighter than gun-metal; and being of a moro unctuous or Boapy character than gun-metal, less greaso or oil is required.

The softer metal is often supported by brasses cast of the required form, the tin alloy being east upon them. The brasses, or bearings, being properly tinned, and an exact model of the axle having been turned, the parts aro heated, put together in their relative positions, luted with plastic clay, and the fluid anti-friction metal poured in, which then becomes of the required form, and effectually solders the brass.

The following compositions aro recommended to railway engineers as having been employed for several years in Belgium. In those cases where the objects are much exposed to friction, 20 parts of copper, 4 of tin, 0.5 of antimony, and 0.25 of lead. For objects which are intended to resist violent shocks, 20 parts of copper, 6 of zinc, and 1 of tin. For those which are exposed to heat, 17 parts of copper, 1 of zinc, 0.5 of tin, and 0.25 of lead. The coppor is added to the fused mass containing the other metals. See Arror and Knngston's Metal; Astrmosy.

AxTI-GUGGIFR. A small syphon of metal, which is inserted into the mouths of casks, or large bottles called carboys, to admit air over the liquor contained in them, and thus to facilitate their being emptied without agitation or a guggling noise.

Axrrmoxr. (Antimoine, $\mathrm{Fr}_{\mathrm{r}}$; Antimon, Spiessglanz or Spiessglas, Ger.) Symbol Sb. (Stibium, Lat.); Atomic Weight, 122; Specific Gravity, 6.715. The sulphide or sulphuret is the only ore of this metal found in sufficient abundance to be largely smelted, and therefore forms the chief and most common source of the antimony of commerce, and of the greater number of the pharmacontical preparations of that metal.

Antimony Glance, Antimonite, Stibnite, or Grey Antimony Ore, sometimes occurs compact, but usually in very long prismatic or acicular crystals, or in a fibrous form. It is of a lead or steel-grey colour, somotimes with an iridescent lustre, sectile and flexible when in thin laminæ. It may be distinguished from a similar ore of manganese by its perfect diagonal cleavage and easy fusibility. Grey antimony is com. posed of antimony 72 , sulphur 28, corresponding to the formula $\mathrm{SbS}^{3}\left(\mathbf{S b}^{3} \mathbf{g}^{3}\right)$. It fuses readily in the flame of a candle, to which it imparts a greenish tint. On charcoal, in the flame of a blowpipe, it gives ont a strong smell of sulphur, with white fumes, and 
yields a white slag. When pure, it is perfectly soluble in muriatic acid. Its specific gravity is $4 \cdot 5$.

The most celcbrutod localitios of this ore aro Felsübanya, Schomnitz, and Kremnitz, in Hungary, whore it oceurs in diverging prisms severul inches long. It is also found in the Hartz, at Andrensborg; in Cornwall, at Padstow and Tintagel; at Now Cumnick, in Ayrshire; in Victoria and South Australia; and abundantly in Borneo.

This ore was called by the ancients $\pi \lambda a \tau u\langle\phi \theta a \lambda \mu o \nu-\pi \lambda a r \dot{s}$ broad $\phi \theta a \lambda \mu \delta$ s eye -from the use to which it was applied in increasing the apparent siz: of the eye, as is still practised among Oriental nations, by staining the upper and under edges of the eyelids. It was also used as a hair-dye and to colour the eyebrows.

It was the Lupus Metallomem of tho alchemists. 'Crudo antimony' is obtained from it by simple fusion, and from this product the pure motal is extractod.

The other principal ores of antimony are the following:-

Native Antimony is a mineral of a tin-white colour and streak, and of a metallic lustre; it sometimes contains silver, iron, and arsenic, with which last it is commonly associated. It is brittle, and possesses a specific gravity of 6.62 to 6.72 . It is generally lamellar, sometimes botryoidal, or reniform. Before the blowpipe it soon molts, and continues to burn after the heat is removed; but if the heat be continued, it oraporates in white fumes, and is redepositod around the globule.

Native antimony occurs at Sahlberg in Sweden, Andreasberg in the Hartz, Allomont in Dauphiny, in Mexico, in Borneo, \&ze.

Allemontite, or Arsenical Antimony. Seo Ansravic.

Dyscrasite, or Antimonial Silver, is a silver-whito metallic mineral of somewhat rariable composition, containing from 15 to 27 per cent. of antimony, and from 73 to 85 per cent. of silver. It is a rare mineral, oecurring at Andreasberg in the Hartz, at Allemont in Dauphiny, in Bolivia, \&c.

Breithauptite or Antimonial Nickel is a mineral containing 67.5 of antimony, and 32.5 of nickel, found at Andreasberg in the Hartz.

Oxides of Antimony. - Threo mineral spocies consist wholly of native oxides of antimony-the result of the alteration of groy antimony, native antimony, and other ores of that metal. Valentinite, teroxide of antimony, or antimonious oxide, $\mathrm{SbO}^{3}$ $\left(\mathbf{s b}^{2} \mathbf{0}^{3}\right)$, occurs in rectangular plates and acicular prisms belonging to the orthorhombic system. It possesses a shining pearly lustre and a snow-white colour, but is sometimes pinkish, or ash-grey, or brownish. It affords a white streak. It is composed of antimony 84.32 , oxygen 15.68 . Specific gravity $=5 \cdot 56$. It is found in tabular crystals in veins traversing the primary rocks at Prziluram in Bohemia, near Freiberg in Saxony, Allomont in Dauphiny, \&c. Senarmontite.-This also consists sololy of teroxide of antimony $\mathrm{SbO}^{3}\left(\mathbf{S b}^{-} \mathbf{O}^{3}\right)$; but, unlike Valentinite, crystallises in regular octahedrons: hence the native oxide is dimorphous. The crystals are colourless or greyish, with a resinous or subadamantine lustre. Tho per-centage composition is, of course, the samo as that of Valontinite. Senarmontite occurs in the provinee of Constantine in Algeria ; at Pernick in Hungary; at Endellion in Cornwall; and at South Ham in Canada. Cervantite, or Antimony Ochre. - This is an oxide containing $\mathrm{Sb} \mathrm{O}^{4}\left(\mathbf{S b}^{2} \mathbf{O}^{4}\right)$, probably a combination of antimonious and antimonic oxides ( $\mathrm{SbO}^{3}+$ $\mathrm{SbO}^{5}$ ). It occurs as a crust or powder, or in acicular crystals, with a greasy or earthy lustre, and of a pale yellow or nearly white colour. Specific gravity $=4.08$. It is found at Cervantes, in Spain; in Hungary, and tho Auvergno.

Red Antimony (Kermesite) is a compound of oxido of autimony 30.2 , and sulphide of antimony $69 \cdot 8$; or antimony $74 \cdot 45$, oxygen $5 \cdot 29$, and sulphur $20 \cdot 49$. It occurs generally in capillary six-sided prismatic crystals of a cherry-red colour, affording a brownish-red streak. It has a specific gravity of from $4^{\circ}{ }^{\circ}$ to $4^{\circ} 6$. It is feebly translucent, and possesses an adamantine lustre. It occurs at Malaczka in Hungary, Bräunsdorf in Saxony, and at Allemont in Dauphiny.

Antimony also occurs in a large number of othor minerals, which are for the most part double sulphides, such as Jamesonite, Zinekenite, Bournonito, Plumosite, Boulangerite, Wolfsbergite, Panabase, Berthiorite, Miargyrite, Pyrargyrite, \&c.

Assayng of ANTIMony Ores.-The chief mineral or ore which has to be submitted to assay is the sulphide of antimony $\left(\mathrm{SbS}^{s}\right)$, sometimes the oxides of antimony ( $\mathrm{SbO}^{*}$, $\mathrm{SbO}^{4}$, and $\mathrm{SbO}^{5}$ ), and occasionally native antimony.

I. Sulphide of Antimony.

(a.) Estimation of the Sulphide of Antimony when it occurs intermixed with more or less of vein-stuff.-About 2,000 to 7,000 grains of the ore are broken into fragments from about $\frac{1}{2}$ to 1 inch in diameter, so as to produce as littlo dust as possible. Two crucibles are selectod, so that the bottom of the upper one can be inserted into the mouth of the lower one to the extent of about 1 inch; a hole is made in the bottom of the upper crucible. This bole is partially closed by placing over it a small lump 
of the ore or a fragment of charcoal. The ore is then introduced; portions of chareoal about the size of the ore being intermixed during the charging, the fine ore placed on top, a layer of charcoal afterwards added, and the crucible-cover fitted and well luted down. The bottom of the pot thus charged is inserted into the mouth of the lower one. Heat is now applied; the two pots thus arranged being placed so that the lower pot is under the bars of the furnace and the upper one surrounded with hot fuel. The temperature should bo carefully regulated, and the operation completed in about 1 to $1 \frac{1}{2}$ hours. When cold, the liquated regulus or sulphide of antimony will be found in the lower crucible, and from its weight the per-centage of pure ore contained in the sample operated on can be calculated. The regulus should bo well fused, bluish grey in colour, and the fracture bright and fibrous-crystalline. When pure, sulphide of antimony contains $71 \cdot 76$ per cont. of metallic antimony; so that, if desirable, from the weight of the regulus obtained the quantity of metal in the sample opcrated on can readily be calculated. The residual vein-stuff in the upper crucible should be examined, to see that it is practically free from sulphido of antimony.

(b.) Assay of Orc rich in Sulphide of Antimony, or Regulus obtained by Liquation (a) or otherwise, by roasting, $\& c .-100$ to 500 grains of the finoly powdered ore are placed in a crucible or roasting-dish, and roasted at a very low and carefully regulated temperature, with frequent stirring, especially at the first part of the operation, so as to prevent clogging or loss from antimony fume being given off. The roasted product, which is generally grey or greyish white in colour, is then mixed with carbonate of soda and charcoal powder, or tartar, or with black flux, and the assay made as for oxide of antimony.

(c.) By Cyanide of Potassium.-With 100 to 200 grains of the finely powdered ore aro mixed about four times its weight of coarsely powdered cyanide of potassium, the mixture transferred to a crucible, and the whole submitted to a low red heat for about 20 to 30 minutes. The fused contents are poured out into a mould, and when cold the slag detached and the button of metallic antimony weighed. It should be bright, bluish white, brittle, and break with a largely crystalline fracture.

II. Oxides of Antimony.

(a.) By Carbonate of Soda and Carbon. -100 to 500 grains of the finely powdered ore are mixed with from 300 to 500 grains of carbonate of soda, and from 20 to 50 grains of charcoal powder, and the mixture submitted in a crucible to a temperature gradually increasing to a red heat at the end of from 20 to 30 minutes. When the fused contents are tranquil, it is poured out into a mould, and when cold the slag cautiously detached, and the button of antimony cleaned and weighed.

(b.) By Cyanide of Potassium.-100 to 200 grains of the oro are weighed and mixed with about four times its weight of cyanide of potassium, and from 20 to 30 grains of charcoal powder, the whole exposed in a crucible to a low red heat for about 20 to 30 minutes, and the process completed as before described for the assay of sulphide of antimony with cyanide of potassium.

Professor Henry Rose, of Berlin, in a memoir on the natural, not oxidised, combinations of antimony and arsenic, gives the following analyses : '

\begin{tabular}{|c|c|c|c|c|c|c|c|c|c|}
\hline \multicolumn{2}{|c|}{ 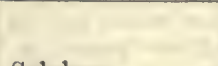 } & 1 & 2 & s & 4 & 5 & 6 & $\tau$ & 8 \\
\hline Sulphur & . $\quad$. & $22 \cdot 08$ & $21 \cdot 05$ & $22 \cdot 15$ & $22 \cdot 53$ & $10 \cdot 72$ & $6 \cdot 42$ & 20.31 & \multirow{2}{*}{$\begin{array}{r}17 \cdot 04 \\
5 \cdot 09\end{array}$} \\
\hline Antimony & . $\quad$. & $44 \cdot 3$ & $30 \cdot 14$ & 3 & $34 \cdot 9$ & & & $26 \cdot 28$ & \\
\hline Lead . & $\ldots$ & $31 \cdot 84$ & $\ldots$ & 40.75 & 36.71 & $46 \cdot 87$ & $\ldots$ & $40 \cdot 84$ & \multirow[b]{2}{*}{$\begin{array}{r}64 \cdot 29 \\
0 \cdot 23\end{array}$} \\
\hline $\begin{array}{l}\text { Silver } \\
\text { Copper }\end{array}$ & - $\quad$ & $\ldots$ & $36 \cdot 40$ & i & $0^{\circ}$ & .. & $\begin{array}{r}68 \cdot 54 \\
0 \cdot 64\end{array}$ & 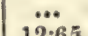 & \\
\hline \multirow{4}{*}{$\begin{array}{l}\text { Copper } \\
\text { Iron . } \\
\text { Lime } \\
\text { Arsenic }\end{array}$} & $\therefore \quad:$ & $\begin{array}{l}0.42 \\
\ldots\end{array}$ & $\begin{array}{l}1.06 \\
0.62\end{array}$ & $\begin{array}{l}0.13 \\
2.30\end{array}$ & $\begin{array}{l}019 \\
2 \cdot 65\end{array}$ & $\dddot{x}_{30}$ & $\begin{array}{l}064 \\
\ldots\end{array}$ & $\begin{array}{l}12 \cdot 65 \\
\ldots .\end{array}$ & 0.06 \\
\hline &.$\quad$. & $\ldots$ & ... & $\ldots$ & $\ldots$ & 0.08 & & & \\
\hline & . & $\ldots$ & ... & ... & $\ldots$ & $\ldots$ & ... & $\ldots$ & $3 \cdot 74$ \\
\hline & (n) & $99 \cdot 23$ & $99 \cdot 17$ & $99 \cdot 73$ & $96 \cdot 17$ & $99 \cdot 01$ & $100 \cdot 28$ & 100.08 & $100 \cdot 15$ \\
\hline
\end{tabular}

1. Zinkenite, from Wolfsberg, in the Eastern Hartz.

2. Miargyrite, from Bräunsdorf, in Saxony.

3, 4. Jamesonite, from Cornwall.

5. Plumose Grey Antimony, from Wolfsberg, in the Eastern Hartz.

6. Brittle Silver Glance, from Schemnitz, Hungary.

7. Bournonite, from the Pfaffenberg mine, Eastern Hartz.

8. Pelybasite, from Mexico. 
Metaluver of Antimony. - In treating cortain ores to obtain the metal, the first object is to separate the gangue, which was formerly done by filling crucibles with the

71

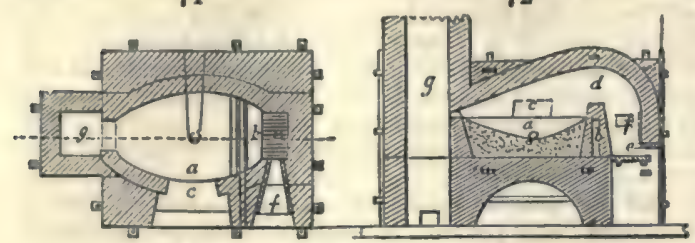

mixed matorials, placing them on the hearth of an oven, and exposing them to a moderate heat. As the sulphide easily melts, it runs ont through a hole in the bottom of the crucible into a pot placod beneath, and out of the reach of the fire. But the great loss from the breakage of the crucibles has caused another method to be adopted. In this, the broken ore, being sorted, is laid on the bottom of a concave reverberatory learth, where it is reduced.

Figs. 71 and 72 represent $a$ wind or flame furnace, for the reduction of antimony. The hearth is formed of sand and clay solidly beat together, and slopes from all sides towards the middle, where it is connected with the orifice a, which is closed with dense coal-ashes; $b$ is the air-channel up through the bridge; $c$, the door for introducing the prepared ore, and running off the slags ; $d$, the bridge; $e$, the grato; $f$, the fire or fuel-door; $g$, the chimncy. With 2 or $3 \mathrm{cwts}$. of ore, the smelting process is completed in from 8 to 10 hours. The metal thus obtained is not pure enough, but must be fused under coal-dust, in portions of 20 or 30 pounds, in crucibles placed upon a reverberatory hearth.

At Malboac, in the department of Ardèche, in France, the separation of the sulphide

73

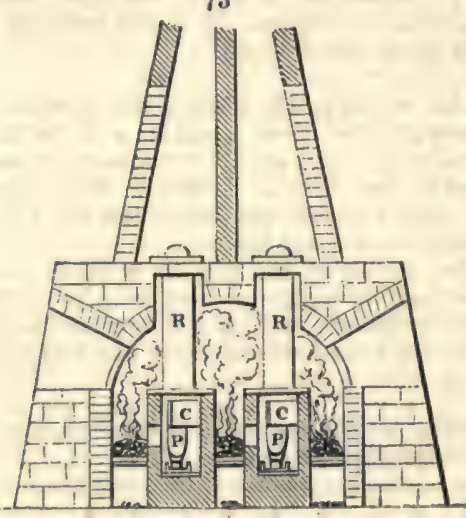
of antimony from its associated gangue is, or was, effected by means of a peculiar apparatus (fig. 73). The mineral is placed in large retorts, $\mathbf{R} \mathbf{R}$, of which four are set in each furnace. An aperture is left at the bottom of each of these cylinders, which corresponds with a similar opening by which they are supported. Boneath these, in the chambers, c c, are placed earthen pots, $\mathbf{P} \mathbf{P}$, in which is received the molted sulphide as it descends through the openings in the cylinders. The fuel consumed on the grate consists of fir-wood; and the sulphide obtained is converted into metallie antimony by roasting in a reverberatory furnsce, and subsequent reduction by a mixture of 20 per cent of powdered chareoal which has been saturated with a strong solution of the carbonate of soda.

Metallic antimony, as obtained by the preceding process, is the antimony of commerce, but is not absolutely pure; containing frequently minute portions of iron, lead, and even arsenic, the detection and separation of which belong to the province of analytical chemistry; but considerable purity may be secured by repeatedly fusing tho metal, mixed with a little of its sulphide and some carbonate of soda, in a crucible. From 100 parts of the impure metal in this way 94 of pure antimony are obtained. The addition of sulphide serves the purpose of making fluid compounds of the sulphides of iron, arsenic, and copper, with the soda. Wöhler purifies antimony completely from arsenic (not from iron and copper) by deflagrating 10 parts of the crude ore with 12 of nitre and 15 of carbonate of soda ; washing away the arsenic salt, and then smelting the residuary antimoniate of potash with black flux. Lead can be soparated only by the humid analysis.

To obtain antimony free from iron, it should be fused with some antimonic oxide in a crucible, whereby the iron is oxidised and separated. The presence of arsenic in antimony is detected by the garlic smoll, emitted by such an alloy when heated at the blowpipe; or, better, by igniting it with nitro in a crucible; in which ease insoluble antimonite and antimonate of potash will be formed along with soluble arsenate. Water digested upon the mixture, filtered, and then tested with nitrate of silver, will afford the brown-red precipitate characteristic of arsenic acid.

According to Berthier, the following materials afford, in smelting, an excellent product of antimony. From 100 parts of sulphide, 60 of protoxide of iron from the shingling or rolling mills (Hammerschlag), 45 to 50 of carbonate of soda, and 10 of 
charcoal powder, from 65 to 70 parts of metallic antimony or regulus should be obtrined. Glauber salts may bo used advantageously instead of soda. Another formula is, 100 parts of sulphide of antimony, 42 of metallic iron, and 10 of dry sulphate of soda. The product thence is said to be from 60 to 64 parts of motal.

In the works where antimonial ores are smelted, by means of tartar (argol, bitartrate of potash), the alkaline scoriæ which cover the. metallic ingots are not rejected as useless, for they hold a certain quantity of antinıonial oxide in combination -a property of the potash flux which is propitious to the purity of the metal. These scoriæ, consisting of sulphide of potassium and antimonate of potash, being treatod with water, undergo a reciprocal decomposition; the elements of the water act on those of the sulphide, and the resulting alkaline hydrosulphide reacts on the antimonial solution so as to form a species of kermes mineral, which precipitates. This is dried, and sold at a low price as a veterinary medicine under the name of kermes, by the dry way.

Metallic antimony is now largely obtained from the native sulphide by the reducing action of iron. A quantity of scrap-iron or of tin-plate clippings is thrust into the molten sulphide, previously separated from the gangue, and the antimony is thus reduced, with formation of a regulus of protosulphide of iron (ferrous sulphide). The process of smelting antimony by means of iron resolves itself into three distinct operations, which may be thus described:-1

1. Singling. - A charge of about 40 lbs. of the ore, broken up into pieces each about half the size of an egg, is introduced into a red-hot crucible, with a quantity of slag obtained in the operation of doubling at a previous smelting. Above this ore and slag is placed a mass of refuse iron, consisting generally of tin-plate in the form. of old kettles and saucepans, and beaten for convenience' sake into the shapo of a cone. Assuming that the ore contains from 50 to 55 per cent. of antimony, about 20 lbs. of iron would be added to the quantity of ore and slag specified above. When the charge has melted, the iron cone is pressed into the molten mass, and the reduction thus effected by action of the metal on the fused sulphide. The crucible is then removed from the fire, and the contents poured into a large conical cast-iron mould. On the cooling of the mass, a button of crude antimony is found below the regulus, from which it is readily separated by a tap with the hammer.

2. Doubling. - The 'singles,' or buttons of metallic antimony from the first melting nre sorted, so that those which contain an excess of sulphur may be melted with those which have an excess of iron. Seventy or eighty pounds of these sorted singles are put into a crucible with a little salt-cake (crude sulphate of soda) and melted. This re-melted antimony is then poured into a cast-iron bowl, where it solidifies, and forms the product known as 'bowl metal.'

3. Refining or Frenching.-A charge of from 60 to 70 lbs. of bowl metal is introduced into a red-hot crucible, with a pound or two of American potash and about 10 lbs, of slag from the previous refining. When melted, the mass is stirred with an iron bar, and the character of the slag adhering to the stirrer enables the workman to judge whether the refining be complete or no. The refined motal is poured into moulds, where it slowly cools and acquires the crystalline structure characteristic of this metal; to favour this crystallisation the metal, while cooling, is covered with slag, and should be left quite undisturbed.

It was shown by the late Dr. Matthiessen that the tendency. of antimony to crystallise is due to the presence of a small proportion of impurity; antimony, in a pure state, cannot be readily caused to crystallise.

Antimony is a brittle metal, of a silvery white colour, with a tinge of blue, a lamellar texture, and crystalline fracture. When heated at the blowpipe, it melts with great readiness, and diffuses white vapours, possessing somewhat of a garlic smell. If thrown in this melted state on a flat sheet of paper, the globule sparkles and bursts into a multitude of small spheroids, which retain their incandescence for a long time, and run about on the paper, leaving traces of the white oxide produced during the combustion. When this oxide is fused with borax, or other vitrifying matter, it imparts a yellow colour to it. Metallic antimony, treated with hot nitric acid in a concentrated state, is converted into a powder, called antimonious acid, which is altogether insoluble in tho ordinary acid menstrua-a property by which the chemist can separate that metal from iron and copper. According to Bergmann, the specific gravity of antimony is 6.86 ; but that of the purest is 6.715 . The alchemists had conceived the most brilliant hopes of this metal; the facility with which it is alloyed with gold, since its fumes aione render this most ductile motal immediately brittle, led them to assign to it a royal lineage, and distinguished it by the title of regulus, or the little king.

'The substance of this description is taken from some 'Notes on Antimony,' in the 'Mining and Smelting Magazine,' vol. Iil. p. 136. 
Its chief employment is in making the alloys called typo motal, stercotypo metal, music plates, and Britannin metal ; the first consisting of 6 of lead and 2 of antimony; the second of 6 of lead and 1 of antimony ; the third of lead, tin, and antimony; and the fourth also of lead, tin, and antimony, with ocersionally a little copper, bismuth, and nickel. Antimony is much used in alloys with tin, tin and load, and in some cases copper, in various proportions, for machinery bearings, instead of gun-metal. In cases of rapid and continuous revolution, as the shafts of screw-steamers, these aro found much better than gun-metal. It is also used by the Ordnance in hardening bullets and shot.-Watts's Dictionary of Chemistry.

Melted with tin, antimony has of lato been used as an antifriction alloy for railway axles, and other bearings ; in motallic rings, or collars, for machinery. As this alloy is not so much heated by friction as the harder metals, less grease is consumed. See Alzoys; Asturatction Metai.

The shipments of Antimony Ore and Regulus from Victora have been as follows:-

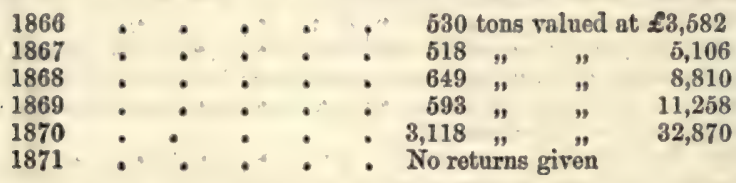

From Borneo the shipments were:-In

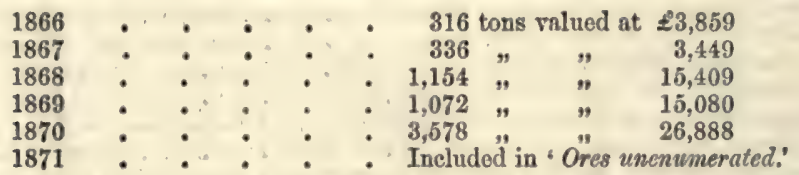

ANTIMONY, CHIORIDEs Or. Two chlorides are known-the terchloride, or antimonious chloride $\left(\mathrm{Sb} \mathrm{Cl}^{3}\right)$, and the pentachloride, or antimonic chloride $\left(\mathrm{Sb} \mathrm{Cl}^{5}\right)$. The former, known to the older chemists as butter of antimony, may be propared by distilling metallic antimony with corrosive sublimatc, or by acting on the tersulphide of antimony with hydrochloric acid, and distilling the product. The pentachloride is obtained by passing chlorine over metallic antimony, and separating the mixed chlorides by distillation. The old Powder of Algaroth was an oxychloride of antimony.

AxTrmosx, Crocus Or. An impure sulphide of antimony and sodium, forming the scoria which is produced in smelting antimony by heating the roasted sulphide with charcoal and carbonate of soda.

ANTrmoNX GIANCD. Native tersulphide of antimony, the principal ore of this metal. See ANTrMonY.

ANTIMONY, GIAss Or. This substance, secording to M. Soubeiran, contained:- Teroxide of antimony, 91.5 ; silica, 4.5 ; peroxide of iron, 3.2 ; sulphuret of antimony, $1 \cdot 9 .=101 \cdot 1$.

A xTrmovr varmxxyow. A rod pigment consisting of artificial tersulphide of antimony, formed by pouring a solution of chloride of antimony in hydrochloric acid into a dilute solution of hyposulphite of lime in excess; when the liquid is hoated, a yellow precipitate falls, and this gradually assumes an orange-red colour.

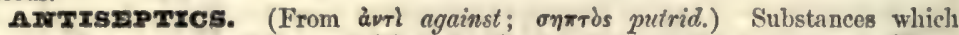
prevent the spontaneous decomposition of animal and vegetable substances. Theso are chiefly the mineral acids, charcoal, chloride of lime, chlorine, culinary salt, nitre, spices, sugar, creosote, and yeast-which operate partly by inducing a change in the animal or vegetable fibres, and partly by combining with and rendering the aqueous constituent unsusceptible of decomposition. Seo Disinfectasts; Food; Provisions, curnze of; and Presereved Mrats.

ANVII. A mass of iron, having a smooth and nearly flat top-surface of stecl, upon which blacksmiths, and rarious other artificers, forge metals with the hammer. The common anvil is usually made of seven pieces: 1 , the core, or body; $2,3,4,5$, the four corner-pieces, which serve to onlarge its base; 6 , the projecting end, which has a square hole for the reception of tho tail, or shank of a chisel, on which iron bars may be cut through; and 7, the beak, or horizontal cone, round which rods or slips of motal may bo turnod into a circular form, as in making rings. These six piecos are welcled separately to the first, or core, and then hammered into a uniform body. In manufacturing large anvils, two hearths are needed, in order to bring each of the 
two pieces to be welded to a proper heat-by itself; and several men are employed in working them together briskly in the welding state, by heavy swing hammers. The steel facing is applied by wolding in the same manner. The anvil is then hardenod, by heating it to a cherry red, and plunging it into cold water-a running stream being preferable to a pool or cistern. The facing should not be too thick a plate; for, when such, it is apt to crack in the hardoning. Tho face of the anvil is now smoothed upon a grindstone, and finally polished with emery and crocus, for all delicate purposes of art.

The blacksmith, in general, sets his anvil loosely upon a wooden block, and in preforence, on the root of an oak treo. The cutlers and file-makers fasten their anvils to a large block of stone, their peculiar work rendering it an advantage to have the anvil fixed as firmly and solidly as possible.

The whitesmith, or brightsmith, when working at the anvil, unless the piece undor the hammer should be very light, is assisted by a striker, who wields a sledge-hammer. In forging round articles, such as bolts, axles, \&c., the smith makes use of swagespieces of steel formed somewhat like hammer-heads-with a groove in one corresponding with a hollow in the other. In forging small spindles, the boss, or lower piece, is permanently fixed upon the anvil. For convenionce in managing heavy articles, a crane is so fixed in the workshops, that the arm traverses between the fire and the anvil.

APATITE. (anatáw to deckive). A name proposed by Werner, in 1786, for the native crystallised phosphate of lime of Saxony, and since extended to all minerals of like chemical composition. The name is suggestire of the deceptive appearances which the mineral often presents, and which naturally onough led the early mineralogists into the error of mistaking many of its varieties for widely-different substances. As apatite, when occurring in sufficient quantity to be adrantageously worked, has always a high economic value, it is desirable to describe the species somewhat in detail.

Apatite crystallises in forms belonging to the hexagonal system, frequently in short six-sided prisms, each terminated either by a pyramid of as many faces, or by a simple flat plane. The horizontal edges at the ends of the crystal are often variously modified, and the lateral faces of the prism frequently exhibit vertical striæ. Cleavage is generally not well marked. In colour the mineral varies from white to various shades of green and blue, passing sometimes into violet and brown; but the 'streak,' readily seen on seratching the mineral, is invariably white. The lustre is generally glassy, but rather inclined to resinous. Some varieties of the mineral are transparent, while others are quite opaque: it is notablo that a bluish opalescence may occasionally be detected in the direction of the vertical axis of the crystal. For a salt of lime, apatite is rather hard, readily scratching glass, but being itself scratched by felspar. Its specific gravity varies from $2 \cdot 92$ to $3 \cdot 25$. In ehemical composition all apatite consists mainly of phosphate of lime (calcium phosphate), but it was shown by Rose in 1827 that this phosphate is almost invariably associated to a greater or less extent with either chloride or fluoride of calcium, or with both. The general composition of apatite may be thus formulated: $3\left(3 \mathrm{CaO}, \mathrm{PO}^{5}\right)+\mathrm{Ca}(\mathrm{Cl}, \mathrm{F})$; $\left[\mathbf{3}\left(\mathbf{C a}^{3} \mathbf{P}^{2} \mathbf{O}^{8}\right)+\mathbf{C a}\left(\mathbf{C l}^{2}, \mathbf{I}^{2}\right)\right]$. The amount of phosphoric acid (phosphoric anhydride) in apatite usually varies from 40 to 45 per cont. Heated before the blowpipe, the mineral fuses, with difficulty, on the edges. Mrany massive varietios emit a phosphorescent glow when heated, and are hence termed phosphorite.

Among the minerals commonly found in association with apatite, may bo speciully mentioned tin-stono, topaz, and tourmaline. It is with such associates that apatite occurs in veins in St. Michael's Mount, and in soveral Cornish tin-mines; and it is also found under similar conditions in the tin-deposits on both the Saxon and the Bohemian sides of the Erzgebirge, especially at Ehrenfriedersdorf, Zinnwald, and Schlaggenwald. A variety in small white crystals, with curved faces, and in crystalline messes, occurring at Huel Franco and Fowey Consols, in Cornwall, has been tormed Francolite. Near Bovey Tracey, in Devonshire, apatite was formerly found in large white crystals, associated with fine specimens of black tourmaline. At Carrock Fells, in Cumberland, it occurs in groen crystals, with smoky quartz, molybdenite and gilbertite. The dark greenish-blue crystals found in Norway and elsewhere have been termed Moroxite. Snarum, in Norway, yields large white crystals, apparently decomposed. A crystalline apatite, flesh-red in colour, and looking much like a felspar, is found at Krageröe in Norway, where it has been largely worked for export to England. The variety termed by Werner Spargelstein ('asparagus stono') occurs in beautifully formed clear yellowish-green crystals, associated with speeular iron-ore, in a peculiar rock, apparently rolcanic, at Jumilla, in the province of MLurcia, Spain. A proposnl was lately made to work the rock containing these crystals. At Logrossan, in the province of Estremadura, there aro rast doposits of massive or con. 
eretionary hard phosphate of lime, known as phosphorite. These doposits were doseribed in $\mathbf{1 8 4 4}$ by the late Dr. Daubeny, and have been worked for many years on a very extensive seale. In the neighbourhood of Staffel, near Limburg, on the Lahn, and elsewhere in Nassau, valuable deposits of phosphorite havo been actively worked. At Staffel the phosphorite is sometimes incrusted with a peculiar mineral, generally in botryoidal or reniform masses, tormed Staffelite. This may have been derived from the alteration of the phosphorite, and is said to contain, in addition to phosphate of lime, upwards of 9 per cent. of carbonate of lime, and is further peculiar in containing traces of jodine. Phosphorite is also worked at Amberg, Diez, and other localities in Bararia. The mineral called Osteolite is said by Professor Church to be an altered impure form of apatite. In the United States there are a large numbor of localities yielding apatite. Crystals of considerable size have been found in St. Lawrence co., in Orange co., in Rossio, and elsewhere in tho State of New York. At Hurdstone, in Sussex co., New Jersey, ' $a$ shaft has been sunk, and the apatite mined; masses brought out weigh occasionally $200 \mathrm{lbs}$., and some cleavage prisms have the planes 3 in. wide' (Dana). Apatite is widely disseminated through many of the erystalline limestones of the Laurentian series in Canada; indeed, in some parts of the limestone it is so abundant as to form a large proportion of the rock. The most remarkable deposits of Laurentian apatite are in the townships of Burgess and Elmsley. 'In North Elmsley it forms an irregular bed parallel to the stratification of the limestone; the breadth of the bed seems to be about 10 feet, of which 3 feet are nearly pure crystalline apatite, sea-green in colour, and with a small admixture of black mica. Masses of this gave an average of 88 per cent. of phosphate of lime' (T. Sterry Hunt).

When apatite or phosphorite occurs in sufficient quantity to be systematically worked, it becomes a mineral of great commercial value. Treated with oil of vitriol, it is converted into superphosphate of lime, and in this soluble form is highly valued by the agriculturist as a fertilising agent for the soil. Apatito has also been used, in the place of bone-ash, as a constituent of certain kinds of soft porcelain. For other mineral substances containing phosphate of lime, see Somrserrite, Coprolites, and Gravo,-F.W.R.

APPIzs. The fruit of the Pyrus malus (apple-tree). Employed in the manufacture of cider. See Cidrr.

APRIE-TREz. (Pyrus malus.) The wood of the apple-tree is much used in the Tunbridge turnery manufacture, and the millwright employs the wood of the crab-tree for the teeth of mortice wheels.

APRIz-Wrra. Cider. Winckler finds that the wine from apples is distinguished from the wine from grapes by the absence of bitartrate of potash and of menanthic acid, by its containing a smaller amount of alcohol and more tannin, but espocially by the presence of a characteristic acid, which he regards as lactic acid, notwithstanding that this opinion is not confirmed by the degree of solubility of its. salts with oxide of zinc, lime, and magnesia. See Cmrr.

AQUArorTrs. Nitric acid, somewhat dilute, was so named by the alchemists on account of its strong solvent and corrosive action upon many mineral, vegetable, and animal substances. It is still employed as the commercial name of nitric acid. See Nitric Acrd.

This acid is usually obtained by distilling either common nitre or cubic nitre with sulphuric acid.

It may, however, be usefully borne in mind, that this term of aquafortis, or strong water of the old chemist, was also applied to solutions which answered their special purposes. Thus Salmon, in 1685, gives the composition of aquafortis from cortain mixtures of acids, not nitric, and salts, and distinctly refers to the Pharmacopoeia for the other kind. This may be of service when applying old recipes for processes in the arts. Aquafortis did not always mean nitric acid.

AQUAMARIN 2 is the name given to those varieties of beryl which are of clear shades of sky-blue, or greenish-blue. It occurs in longitudinally-striatod hexagonal crystals, sometimes a foot long, and is found in the Brazils, Hindostan, and Siberia. See Berxt.

AQUA Rzera. Royal water. The name given by the alchemists to that mix. ture of nitric and muriatic acids which was best fitted to dissolve gold; it is now called nitro-muriatic acid, or nitro-chlorohydric acid, or hypochloro-nitric acid.

Aqua regia, prepared under different conditions, appears to give difforent results. Gay-Lussac observed that aqua regia, when heated in a water-bath, evolves a gaseous body which, dried and exposed to a frigorific mixture, separates into chlorine and a durk lemon-yellow liquid, boiling at $70^{\circ} \mathrm{F}$. This yellow liquid was found to contain $60^{\circ} 4$ per cent. of chlorine, the caleulated quantity for the formula, $\mathrm{NO}^{2} \mathrm{Cl}^{2}$, being $70^{\circ} 2$. Gay-Lussac refutes the assertion of E. Davy and Baudrimont, that the properties of 
aqua regia are due to its containing a compound of chlorine, nitrogen, and oxygen, and confirms the generally received view, that its action depends upon free chlorine. From the vapour evolved in the action of nitric upon hydrochloric acid, a liquid may be condensed which is nearly of the composition $\mathrm{NO}^{2} \mathrm{Cl}^{2}\left(\mathbf{I X C l}^{2}\right)$, containing, however, no free chlorine: this compound, in the gaseous form, is known as chloronitric gas.

The best proportions for forming aqua regia appear to be about one volume of strong nitric to three of hydrochloric acid. Aqua regia is used for dissolving both gold and platinum.

AQUA VITIs. The name given to alcohol when used as an intoxicating beverage. It is derived from the alchemists, who, having obtained-in all probability from the Arabian physicians, since Avicenna uses the term-the product by distillation of saccharine fermentation, al-kohol (alcohol), gave, upon the same principle as guided them in calling the nitro-muriatic acid aqua regia, the namo of aqua vite to several ardent spirits; and it has been retained especially with reference to whisky and brandy.

ARABIC, Gunx. Gum Arabic exudes from several species of Acacia, as A. vera. It is also found in the roots of the mallow, comfrey, and some other plants. Gum Arabic never crystallises, is transparent, and has a vitrenus fracture.

ARABIr. The principal constituent of Gum Arabic. If gum is treated with hydrochloric acid and alcohol, the lime, magnesia, and potash, in combination, are decomposed, and the arabin is separated as a gum, exhibiting the properties of an acid. In the moist state it dissolves in cold water, forming mucilage, from which it is precipitated by alcohol. After drying, it no longer dissolves, but swells into a gelatinous mass. Dried at $212^{\circ} \mathrm{F}$. it has the composition $\mathrm{C}^{12} \mathrm{H}^{11} \mathrm{O}^{11}\left(\mathbf{C}^{12} \mathbf{H}^{22} \mathbf{O}^{11}\right)$. See Acacla, Crrasine, Dextruns, Gum.

ARAGorrrz. So called from Aragon, in Spain, where it was first discovered. A carbonate of lime, crystallised in rhombic prisms, or in forms derived from the samo, Sometimes written Arragonitr, Seo Lims.

ArBor vrTx. Several species of Thuja, found in America and China, are called arbor vite. It is a light, soft, and fine-grained wood, which is used in several kinds of carpentry.

Arcer. As this dictionary is not intended to include articles connected with engineering or with architecture, it would be out of place to deseribe the conditions required to ensure the stability of the arch, which is manifestly one of great importance to the practical builder. (For the theory of the equilibrium of the arch, Gwilt's treatise on the subject should be consulted, or the article Arch, 'Encyclopredia Britannica.') It simply remains to define the arch as a structure of stone or brick, supported by its own curve; or of wood or iron, supported by the mechanical arrangements of the work.

The curvature of an arch may vary very considerably. Where the arch is low, the circle it belongs to becomes very large; and the strength of arches varies greatly with their forms; they may be either segments of a circle, a parabola, an ellipse, an hyperbola, or a catenary.

The arch in architecture is the means of passing from one pillar to another; and we have the circular form, which was succeeded by the pointed arch, and all its modifiod forms of foliation, \&c.

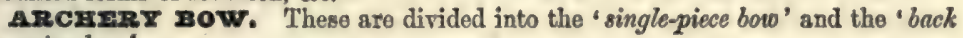
or union bow.'

The single-piece bow is made of one rod of hickory, lance-wood, or yew-tree, which last, if perfectly free from knots, is considered the most suitable wood.

The union bow is made of two or sometimes three pieces glued together. The back' piece, or that furthest from the string, is of rectangular section, and always of lance-wood or hickory; the 'belly, which is nearly of semicircular section, is made of any hard wood that can be obtained straight and clean, as ruby-wood, rose-wood, green-heart, king-wood, snake-wood, \&c. Sometimes the union bow is imitated by one solid piece of straight cocoa-wood of the West Indies (not that of the cocoa-nut palm), in which case the tough fibrous sap is used for the back. The Palmyrea is also used for bows, - Holtzapffel.

Arcrrx. (Orseille, Fr.; Orseille, Ger.; Oricello, Ital.) The namo of archil is given to a colouring-matter obtained, by the simultaneous action of the air, moisture, and an ammoniacal liquor, from many of the lichens, the most esteemed being the Roccella.

It appears in commerce in three forms: 1, as a pasty matter called archil; 2, as a mass of a drier character, named persis; and 3 , as a reddish powder called cudhear.

The lichen from which archil is propared is known also as the canary weed or orchilla weed. It grows in great abundance on some of the islands near the African 
coast, particularly in the Canarios and soveral of tho islands of tho Archipelago. Its colour is sometimes a light and sometimes a dark grey.

There appenrs to bo good evidence for supposing that archil was known to the Romans, and Beckmann is disposed to beliero that tho anciont Greeks wore familiar with this dye. This ingenious and industrious author gives the following account of the modern introduction of the archil.

'Among the oldest and principal Florentine families is that known under the name of Oricellarii or Rucollarii, Ruscellai or Rucellai, several of whom have distinguished themselves as statesmen and men of letters. This family is descended from a German nobleman, named Forro or Frederigo, who livod in the boginning of the 12 th century. One of his descondants, in the year 1300, carried on a great trade in the Levant, by which he acquired considerable riches, and roturning at length to Florence with his fortune, first made known in Europe tho art of dyeing with archil. It is said that a little before his return from the Levant, happening to make water on a rock covered with this lichen, he observed that the plant, which was there called respio or respo, and in Spain orciglia, acquired by the urine a purple colour, or, as others say, a red colour. Ho, therefore, tried several experiments, and when he had brought to perfection the art of dyeing wool with this plant, he made it known at Florence, where he alone practised it for a considerable time, to the great benefit of the state. From this useful inrention the family receired the name of Oricellarii, from which at last was formed Rucollai.'- History of Inventions.

For more than a century Italy possessed the exclusive art of making archil, obtaining the lichens from the islands of the Mediterranean. Teneriffe furnished anuually 500 quintals (of 110 lbs. each) of lichen; the Canary Isles, 400 ; Fucrta Ventura, 300 ; Iancerot, 300 ; Gomera, 300 ; Isle of Ferro, 800 . This business, in the islands of Teneriffe and Canary, belonged to the Crown of Spain, and in 1730 brought in a revenue of 1500 piastres. The farmers paid from 15 to 20 reals for the right to gather each quintal.

Since 1402 the largest quantity of the lichens for the preparation of archil has been obtained in the Canary Islands; a smaller quantity has, however, been procured from the Cape de Verde Islands. It is stated that the archil from the lichens of the latter place dye wool of a deoper colour than the archil from the Canaries, but that the dye is not so rich. The labour of collecting these lichens is very great, and mon aro exposod to the greatest risks, being suspended by cords over the face of stupendous eliffs. Upon the coasts of Spain, Scotland, and Ireland, the peasantry have for a very long period used lichens for the purpose of dyeing red.

The chemical constitution of archil was first investigatod by M. Cocq ('Annales do Chimie,' vol. 1xxxi.); and subsequently, yet more extensively, by Robiquet ('Annales de Chimie,' vol. xlii, 2nd series).

From the Variolaria, Robiquet obtained Orcine, by digesting the lichen in alcohol, evaporating to dryness, dissolving the extract in water, concentrating the solution to the thickness of a syrup, and setting it aside to erystallise. It forms, when quito pure, colourless prisms, of a nauseous sweot taste, which fuse easily, and may be sublimed unaltered. Its formula is $\mathrm{C}^{14} \mathrm{H}^{8} \mathrm{O}^{4}\left(\mathbf{C}^{7} \mathbf{r}^{8} \mathbf{O}^{2}\right)$; when crystallised from its aqueous solution it contains $5 \mathrm{Aq}$.

If orcine be exposed to the combined action of air and ammonia, it is converted into a crimson powder orceine, which is the most important ingredient in the archil of commorce. Orcëine may be obtained by digesting dried archil in strong alcohol, evaporating the solution in a water-bath to dryness, and treating it with ether as long as anything is dissolved; it remains as a dark blood-red powder, being sparingly soluble in water or other, but abundantly in alcohol. Its formula is $\mathrm{C}^{14} \mathrm{H}^{7} \mathrm{NO}^{6}\left(\mathbf{C}^{7} \mathbf{H}^{7} \mathrm{Nr}^{3}\right)$.

Oreëine dissolves in alkaline liquors with a magnificent purple colour; with metallic oxides it forms lakes, also of rich purple of various shades. In contact with deoxidising agents, it combines with hydrogen as indigo does, and forms leuc-orcëino. When bleachod by chlorine, a yellow substance is formed, chlor-orcëine.

Dr. Schunck, by an examination of several species of Lecanora, has proved that, although under the influence of ammonia and of air, they ultimately produce orcëine, these lichens do not contain orcine ready formed, but another body, Lecanorine, which, under the influence of bases, acts as an acid, and is decomposed into oreine and carbonic acid. If lecanoric acid bo dissolved in boiling alcohol, it unites with ether, forming lecanoric ether, which crystallises beautifully in pearly scales. In the Roccella tinctora and the Evernia prunastri erythric acid is found By the oxidation of this acid amarythrine or crythrine bitter is formed. These substances have been carefully examined by Schunck, Stenhouse, and Kane. The chemical history of these and some other compounds is of great interest; but as they do not bear directly upon the manufacture of arehil, or its use in dyeing, further space cannot be devoted to their consideration, 
Kano found archil and litmus of commerce to contain two classes of colouringmatters, as already stated, orcine and orceine, derived from it. Beyond these there were two bodies, one containing nitrogen, azocrythrine, and tho other destitute of nitrogen, erythroleic acid. This latter acid is separated from the other bodies present in archil by means of other, in which it dissolves abundantly, forming a rich crimson solution. It gives with alkalis purplo liquors, and with earthy and metallic salts coloured lakes.

Beyoud those already named there are several other species of lichen which might bo employed in producing an analogous dye, were they prepared, like the precoding, into the substance called archil. Hellot gives the following method for discorering if they possess this property. A little of the plant is to be put into a glass ressel; it is to bo moistened with ammonia and lime-water in equal parts; a little muriate of ammonia (sal-ammoniac) is added, and the small vessel is corked. If the plant be of a nature to afford a red dye, after three or four days the small portion of liquid which will run off on inclining the vessel, now opened, will be tinged of a crimsou red, and the plant itself will have assumed this colour. If tho liquor or the plant does not take this colour, nothing need be hoped for; and it is useless to attempt its proparation on the great scale. Lowis says, however, that he has tested in this way a great many mosses, and that most of them afforded him a yellow or reddish-brown colour; but that he obtained from only a small number a liquor of a deep red, which communi. cated to cloth merely a yellowish-red colour.

Prepared archil gives out its colour very readily to water, ammonia, and alcohol. Its solution in alcohol is used for filling spirit-of-wine thermometers; and when these thermometers are well freed from air, the liquor loses its colour in some years, as Abbe Nollet observed; but the contact of air restores the colour, which is destroyed anew, in vacu, in process of time; but the watery infusion loses its colour, by the privation of air, in a fow days; a singular phenomenon, which merits new researches.

The infusion of archil is of a crimson bordering on violet. As it contains ammonia, which has already modified its natural colour, the fixed alkalis can produce little change on it, only decpening the colour a little, and making it more violet. Alum forms in it a precipitate of a brown red; and the supernatant liquid retains a yellowish-red colour. The solution of tin affords a reddish precipitate, which falls down slowly; the supernatant liquid retains a feeble red colour.

The researches on the lichens, as objects of manufacture, by Westring, of Stockholm, are worthy of attention. He examined 150 species, among which he found several which might be rendered useful. He recommends that the colouring-matter should be extracted in the places where they grow, which would save a vast expense in curing, package, carriage, and waste. He styles the colouring substance itself cudbear, porsio, or turnsole; and distributes the lichens as follows:-1st. Those which, left to themselves, exposed to moderate heat and moisture, may be fixed without a mordant upon wool or silk; such are the Lichenes cinereus, cematonta, ventosus, corallinus, Westringii, saxatilis, conspassus, barbatus, plicatus, vulpinus, \&c.

2. Those which develop a colouring-matter fixable likewise without mordant, but which require boiling and a complicated prepuration; such are the Lichenes subcarneus, dillenii, farinaceus, jubatus, furfuraceus, pulmonareus, comigatus, cocciferus, digitatus, ancialis, aduncus, \&c. Saltpetre or sea-salt is requisite to improve the lustre and fastness of the dye given by this group to silk.

3. Those which require a peculiar process to develop their colour, such as those which become purple through the agency of stale turine or ammonia. Westring employed the following mode of testing:- He put 3 or 4 drachms of the dried and powderod lichen into a flask, moistened it with 3 or 4 measures of cold spring water, put the stuff to be dyed into the mixture, and left the flask in a cool place. Sometimes he added a little salt, saltpetro, quicklime, or sulphate of copper. If no colour appeared, he then moistened the lichen with water containing $\frac{1}{20}$ th of sal-ammoniac and $\frac{1}{i 0}$ th of quicklime, and set the mixture aside in a cool place from 8 to 14 days. There appoarod in most cases a reddish or violet coloured tint. Thus the Lichene cinereus dyed silk a deep carmelite and wool a light carmelite; the $L$. physodes gave a yellowishgrey ; the pustulatus, a rose red; sanguinarius, grey; tartareus, found on the rocks of Norway, Scotland, and England, dyes a crimson-red. Cudbear is made from it in Jutland by grinding the dry lichen, sifting it, then setting it to ferment in a close vessel with ammonia. The lichen must be of the third year's growth to yiold an abundant dye; and that which grows near the sea is the best. It loses half its weight by drying. A single person may gather from 20 to 30 pounds a day in situations whero it abounds. No less than $2,239,685$ pounds were manufactured at Christiansund, Flekkefiord, and Fakrsund, in Norway, in the course of tho six years prior to 1812 . Since more solid dyes of the same shade have been invented, the archil has gone much into disuse. 
To propare arehil, the lichens omployed are ground up with water to a uniform pulp, and this is thon mixed with as much water as will make the whole fluid; ammoniacal liquors from gas or from ivory-black works, or stale urine, are from time to time addod, and the mass frequently stirred so as to promote the action of the air. The oreine or orythrine which exists in the lichen absorbs oxygen and nitrogen, nnd forms orcëine. The roccelline absorbs oxygen and forms erythroleic acid; these being kept in solution by the ammonia, the whole liquid becomes of an intense purple, and constitutes ordinary archil, - Kane.

Archil alone is not used for dyeing silk, unless for lilacs; but silk is frequently passed through a bath of anchil, either before dyeing it in other baths or after it has been dyed, in order to modify different colours or to give thom lustre. It is sufficient here to point out how white silks are passed through the archil bath. The same process is performed with a bath more or loss churged with this colour, for silks already dyed.

Archil, in a quantity proportioned to the colour desired, is to be boiled in a copper. The clear liquid is to be run off quite hot from the archil bath, leaving the sediment at the bottom, into a tub of proper size, in which the silks, newly scoured with soap, are to be turned round on the skein-sticks with much exactness, till they have attained the wished-for shade. After this they must receive one bectling at the river.

Archil is, in general, a very useful ingredient in dyeing; but as it is rich in colour, and communicates an alluring bloom, dyers are often tempted to abuse it, and to exceed the proportions that can add to the beauty without at the same time injuring, in a dangerous manner, the permanence of the colours. Nevertheless, the colour obtained when solution of tin is employed, is less fugitive than without this addition: it is red, approaching to scarlet. Tin appears to be the only ingredient which can increase its durability. The solution of tin may be employed, not only in the dyoing bath, but for the preparation of the silk. In this case, by mixing the archil with other colouring substances, dyes may be obtained which have lustre with sufficient durability.

To dye wool with archil, the quantity of this substance deemed necessary according to the quantity of wool or stuff to be dyed, and according to the shade to which they are to be brought, is to be diffused in a bath of water as soon as it begins to grow warm. The bath is then heated till it be ready to boil, and the wool or stuff is passed through it without any other preparation excopt keeping that longest in which is to have the deepest shade. A fine gridelin, bordering upon violet, is thereby obtained; but this colour has no permanence. Hence archil is rarely employed with any other view than to modify, heighten, and give lustre to the other colours. Hellot says, that having employed archil on wool boiled with tartar and alum, the colour resisted the air no more than what had received no preparation. But he obtained from herb archil ('orseille d'herbe) a much more durable colour, by putting in the bath some golution of tin. The archil thereby loses its natural colour, and assumes one approaching more or less to searlet, according to the quantity of solution of tin employed. This process must be executed in nearly the same manner as that of searlet, except that the dyeing may be performed in a single bath.

Archil is frequently had recourse to for varying the different shades and giving them lustre; hence it is used for violets, lilacs, mallows, and rosemary-flowers. To obtain a deeper tone, as for the deep soupes au vin, sometimes a little alkali or milk of lime is mixed with it. The suites of this browning may also afford agates, rosemary-flowers, and other delicate colours, which cannot be obtained so beautiful by other processes.

The herb archil, just named, called especially orcëille de terre, is found upon the volcanic rocks of the Auvcrgne, on the Alps, and the Pyrenees.

These lichens are gathered by men whose whole time is thus occupied; they scrape them from the rocks with a peculiarly shaped knife. They prefer collecting the orceille in rainy weather, when they are more easily detached from the rocks. They gather about 2 kilogrammes a day, or about $4 \frac{1}{2}$ pounds. When they take their lichens to the makers of archil or litmus for the purpose of selling them, they submit a sample to a test, for the purpose of estimating their quality. To this end they put a little in a glass containing some urine, with a small quantity of lime. As the lichens very rapidly pass into fermentation if kept in a damp state, and thus lose much of their tinctorial power, great care is taken in drying them; when dry they may be preserved without injury for some time.

Archil is perhaps too much used in some cloth factories of England, to the discredit of our dyes. It is said, that by its aid $\frac{1}{3} r d$ of the indigo may be saved in the blue vat; but the colour is so much the more perishable. The fine soft tint induced upon much of the black eloth by means of archil is also deceptive. One half pound of cudbear will dye one pound of woollen cloth. A crimson red is obtained by 
adding to the decoction of archil a little salt of tin (muriate), and passing the cloth through the bath after it has been prepared by a mordant of tin and tartar. It must bo afterwards passed through hot water.

Dyeing with archil with the aid of oil has been patented by Mr. Lightfoot, on the same principle as has been so long used in the Turkey-red cotton dye, who also has recourso to metallic and earthy bases. See Cunbear and Lrrarus.

Under the names of archil carmine and archll purple, or French Purple, two brillinnt dyes wore introduced about a dozen years ago; these contained the colouring principles of the lichens in a very pure form. But since the introduction of the brilliant and stable colours derived from coal-tar, archil and the other dye-stuffes yielded by lichens have greatly fallen into disuse.

ARCHITECTURE. The art of constructing buildings, which involves the consideration of very dissimilar points.

1. UTILITX, - as it regards any specified object, as -

a. Domestic accommodation in a dwelling-house.

b. Acoustic arrangements in all buildings intended for public purposes. This consideration is entirely lost sight of by many modern architects.

c. Ventilation, which is a matter upon which a very large amount of empiricism has been exponded with exceedingly small results.

2. Durabizitr. - If we examine the walls of our ruined abbeys and castles, we shall find that the stones employed still retain the marks of the workman's tool; and that in numerous cases the ornamental work is as sharp as if it had been executed but yesterday. This should prove to us that the selection of stone was of great importance.

ARDENT SPIRITS-called formerly Aqua ardens-are the spirituous products of a considerablo variety of fermentable substances. The term is more strictly applicable to those spirits which, by careful distillation, have boen deprived of a large quantity of the water in combination. Rectified spirit is alcohol with 16 per cent. of water. Proof spirit is 5 pints of rectifled spirit with 3 pints of distilled water.

ARECA. A genus of palms, containing two species-1. The Areca catecha, producing the betel nut, which is so universally chewed in the East Indies. 2. The Areca oleracea, or cabbage-palm; the cabbage is eaten in the West Indies, both raw and boiled; and the trunk, which is often 100 feet long, is used in Jamaica for waterpipes, which are said to become, when buried, almost as hard as iron.

A. catechu is one of the most beautiful palm-trees growing in India. It is chiefly cultivated in Malabar, Ceylon, and Sumatra. Ono tree will produce, according to situation, age, and culture, from 200 to 800 nuts. See Acacia Cateche.

ARzомгтzR. An instrument to measure the densities of liquids. (See AtcoHoLONETRY.) The principle will be well understood by remombering that any solid body will sink further in a light liquid than in a heavy one. The areometer is usually a glass tube, having a small glass bulb loaded with either shot or quicksilver, so as to set the tube upright in any fluid in which it will swim. Within the tube is placed a graduated scale: wo will suppose the tube placed in distilled water, and the line cut by the surface of the fluid to be marked; that it is then removed and placed in strong alcohol-the tube will sink much lower in this, and consequently we shall have two extremities of an arbitrary scale, on which we can mark any intermediate degrees.

The areometer of Baume is used in France, and the following scale is adopted by the French chemists :-

Specific Gravity Numbers corresponding with Baume's Areometric Degrees.

\begin{tabular}{|c|c|c|c|c|c|c|c|c|c|}
\hline \multicolumn{6}{|c|}{ Liquids denser than Water } & \multicolumn{4}{|c|}{ Less dense than Water } \\
\hline $\begin{array}{l}\text { De- } \\
\text { grees }\end{array}$ & $\begin{array}{l}\text { Specifle } \\
\text { Qravity }\end{array}$ & $\begin{array}{l}\text { De- } \\
\text { grees }\end{array}$ & $\begin{array}{l}\text { Specific } \\
\text { Gravity }\end{array}$ & $\begin{array}{c}\text { De- } \\
\text { grees }\end{array}$ & $\begin{array}{l}\text { Specifio } \\
\text { Gravity }\end{array}$ & $\begin{array}{c}\text { De- } \\
\text { grees }\end{array}$ & $\begin{array}{l}\text { Specific } \\
\text { Gravity }\end{array}$ & $\begin{array}{c}\text { De- } \\
\text { grees }\end{array}$ & $\begin{array}{l}\text { Specifle } \\
\text { Gravity }\end{array}$ \\
\hline $\mathbf{0}$ & 1.0000 & 26 & $1 \cdot 2063$ & 52 & $1 \cdot 5200$ & 10 & 1.0000 & 36 & 0.8488 \\
\hline 1 & 1.0066 & 27 & $1 \cdot 2160$ & 53 & 1.5353 & 11 & 0.9932 & 37 & 0.8439 \\
\hline 2 & 1.0133 & 28 & $1 \cdot 2258$ & 54 & $1 \cdot 5510$ & 12 & 0.9865 & 38 & 0.8391 \\
\hline 3 & 1.0201 & 29 & 1.2358 & 55 & 1.5671 & 13 & 0.9799 & 39 & 0.8343 \\
\hline 4 & 1.0270 & 30 & 1.2459 & 56 & 1.5833 & 14 & 0.9733 & 40 & 0.8295 \\
\hline 5 & 1.0340 & 31 & $1 \cdot 2562$ & 57 & $1 \cdot 6000$ & 15 & 0.9669 & 41 & 0.8249 \\
\hline 6 & 1.0411 & 32 & 1.2667 & 58 & 1.6170 & 16 & 0.9605 & 42 & 0.8202 \\
\hline 7 & $1 \cdot 0483$ & 33 & $1 \cdot 2773$ & 69 & $1 \cdot 6344$ & 17 & 0.9542 & 43 & 0.8156 \\
\hline 8 & 1.0556 & 34 & $1 \cdot 2881$ & 60 & $1 \cdot 6522$ & 18 & 0.9480 & 44 & 0.8111 \\
\hline 9 & 1.0630 & 35 & $1 \cdot 2992$ & 61 & 1.6705 & 19 & 0.9420 & 45 & 0.8066 \\
\hline 10 & 1.0704 & 36 & 1.3108 & 62 & 1.6889 & 20 & 0.9359 & 46 & 0.8022 \\
\hline
\end{tabular}




\begin{tabular}{|c|c|c|c|c|c|c|c|c|c|}
\hline \multicolumn{6}{|c|}{ Llquids denser than Water } & \multicolumn{4}{|c|}{ Iess dense than Water } \\
\hline $\begin{array}{l}\text { De- } \\
\text { grees }\end{array}$ & $\begin{array}{l}\text { Specific } \\
\text { Gravity }\end{array}$ & $\begin{array}{l}\text { De- } \\
\text { greess }\end{array}$ & $\begin{array}{l}\text { Spectfle } \\
\text { Gravity }\end{array}$ & $\begin{array}{l}\text { De- } \\
\text { grees }\end{array}$ & $\begin{array}{l}\text { Specific } \\
\text { Gravity }\end{array}$ & $\begin{array}{c}\text { De- } \\
\text { greess }\end{array}$ & $\begin{array}{l}\text { Specifte } \\
\text { Gravity }\end{array}$ & $\begin{array}{c}\text { De- } \\
\text { grees }\end{array}$ & $\begin{array}{l}\text { Specific } \\
\text { Gravity }\end{array}$ \\
\hline 11 & 1.0780 & 37 & $1 \cdot 3217$ & 63 & $1 \cdot 7079$ & 21 & 0.9300 & 47 & 0.7978 \\
\hline 12 & 1.0857 & 38 & $1 \cdot 3333$ & 64 & $1 \cdot 7273$ & 22 & 0.9241 & 48 & 0.7935 \\
\hline 13 & 1.0935 & 39 & $1 \cdot 34551$ & 65 & 1.7471 & 23 & 0.9183 & 49 & 0.7892 \\
\hline 14 & $1 \cdot 1014$ & 40 & 1.3571 & 66 & 1.7674 & 24 & 0.9125 & 50 & 0.7849 \\
\hline 15 & $1 \cdot 1095$ & 41 & $1 \cdot 3694$ & 67 & $1 \cdot 7882$ & 25 & 0.9068 & 51 & 0.7807 \\
\hline 16 & $1 \cdot 1176$ & 42 & $1 \cdot 3818$ & 68 & $1 \cdot 8095$ & 26 & 0.9012 & 52 & 0.7766 \\
\hline 17 & & 43 & $1 \cdot 3945$ & 69 & 1.8313 & 27 & 0.8957 & 53 & 0.7725 \\
\hline 18 & $1 \cdot 1343$ & 44 & 1.4074 & 70 & 1.85037 & 28 & 0.8902 & 54 & 0.7681 \\
\hline 19 & $1 \cdot 1428$ & 45 & 1.4206 & 71 & 1.8760 & 29 & 0.8848 & 55 & $0 \cdot 7643$ \\
\hline 20 & $1 \cdot 15 ๊ 15$ & 46 & 1.4339 & 72 & 1.9000 & 30 & 0.8795 & 56 & 0.7604 \\
\hline 21 & $1 \cdot 1603$ & 47 & & 73 & & 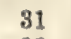 & & 67 & 0.7656 \\
\hline 22 & $1 \cdot 1692$ & 48 & 1.4615 & 74 & & 32 & 0.86 & 58 & 0.7526 \\
\hline 23 & $1 \cdot 1783$ & 49 & 1.4758 & 75 & 1.9740 & 33 & 0.8639 & 69 & $0 \cdot 7487$ \\
\hline 24 & $1 \cdot 1875$ & 50 & $1 \cdot 4902$ & 76 & $2 \cdot 0000$ & 34 & 0.8588 & 60 & 0.7449 \\
\hline 25 & $1 \cdot 1968$ & 61 & $1 \cdot 4951$ & & & 35 & $0 \cdot 8538$ & 61 & 0.7411 \\
\hline
\end{tabular}

Azminaczous. (Arena, sand.) Sandy. Rocks composed of particles of sand, or containing much sand, as the grits and sandstonos, are said to be arenaceons. If they contain lime they are callod arenaceo-calcareous.

ARGAX orx. An oil expressed from the kornels of the Argania Sideroxylon, a shrub growing in Morocco.

ARCrIIACzOUs. Composed of clay, or clayey. This name is applied to all rocks composed of clay. If containing also sand or lime, they are distinguished as argillo-arenaceous or argillo-calcareous. Argillaccous rocks, when breathod on, have a peculiar earthy odour, by which they may be distinguished.

ARGIInACEOUS EARTII. (Argilla, clay, Lat.) The earth of clay, called in chemistry, alumina, because it is obtained in greatest purity from alum. See Alomina, China Cray, Cray, Kaoms.

ARGoI, or ARGaI. (Tartre, Fr.; Wenstein, Ger.) The tartrate of potash is known in commerce as the white and red argol; the white being tho crust let fals by white wines, which is of a pale pinkish colour, and the red the crust deposited from red wines, and of a dark red colour. See Tartar, Crram of Tartar, \&c.

ARICIrz. An alkaloid discovered by Pelletior and Corriol in a cinchona bark from Arica in Poru. It is separated by the same process as quinine.

ARMrNTAN BOTr. See Bolz.

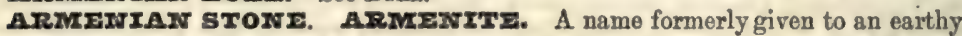
copper ore mixed with limestone, of an azure colour, or to quartz coloured with carbonate of copper. Seo Lapis Lazuli.

ARMoUz-PIATEs. Massive wrought-iron plates used for coating ships of war. The iron for these plates should be as tough and soft as possible; and steel, though possessing high tonsile strength, should not be used. It is an object of the manufacturer to produce a plate of considerable thicknoss, as it is known that the resistance of a single solid plate is greatly superior to that of a number of superimposed plates of the same aggregate thickness, however carefully the several plates may have boen fastened together. It is difficult, however, in manufacturing thick plates, to completely squeeze out the liquid cinder interposed between the several layers of iron which are welded together; and hence armour-plates, when exposed to the impact of heavy shot, often exhibit a tendency to lamination. The large plates are manufactured either by rolling in a mill or by forging under the steam-hammer: it is said that the structure of a hammered plate is more likely to be uniform than that of a rolled plate. 'The desideratum in all armour-plates is that they shall bulge with the least possible amount of cracking. Large radiating fractures at the back indiente brittleness, and should immediately condemn a plate. - (Percy.)

ARNATTO, ARNOTTO, or ANs Ger.) A somewhat dry and hard paste, brown without and red within. It is usually imported in cakes of two or three pounds weight, wrapped up in leaves of large reeds, packed in casks, from America, where it is prepared from the seeds of a certin tree, culled the arnatto tree; it is the Bixa orellana of Linnseus.

The shrub producing the arnatto is originally a native of South America; it is 
now cultivated in Guiana, St. Domingo, and in the East Indies. In the 'Annales de Chimio' we have the following description of the arnatto tree:- 'The tree produces oblong bristled pods, somewhat resembling those of $y$ chestnut. These are at first of a beautiful rose-colour, but, as they ripen, change to a dark brown; and bursting open, display a splendid crimson farina or pulp, in which are contained from thirty to forty seeds, somewhat resembling raisin-stones. As soon as they arrive at maturity, these pods are gathered, divested of their husks, and bruised. Their pulpy substance, which seems to be the only part which constitutes the dye, is then put into a cistern, with just onough water to cover it, and in this situation it remains for seven or eight days, or until the liquor begins to ferment, which, however, may require as many wooks, according to circumstances. It is then strongly agitated with wooden paddles or beaters, to promote the separation of the pulp from the seeds. This operation is continued until these have no longer any of the colouring-matter adhering to them; it is then passed through a sieve, and afterwards boiled, the colouring-matter being thrown to the surface in the form of scum, or, otherwise allowed to subside: in either case, it is boiled in coppers till reduced to a pasto, whon it is made into cakes and dried.'

Instead of this long and painful labour, which occasions diseases by the putrefaction induced, and which affords a spoiled product, Leblond proposed simply to wash the seeds of the bixa till they are entirely deprived of their colour, which lies wholly on their surface; to precipitate the colour by means of vinegar or lemonjuice, and to boil it up in the ordinary manner, or to drain it in bags, as is practised with indigo.

The experiments which Vauquelin made on the seeds of the bixa, imported by Leblond, confirmed the efficacy of the process which he proposed; and the dyers ascertained that the arnatto obtained in this manner was worth at least four times more than that of commerce; that, moreover, it was more easily employed; that it required less solvent; that it gave less trouble in the copper, and furnished a puror colour.

Arnatto dissolves better and more readily in alcohol than in water, when it is introduced into the yellow varnishes for communicating an orange tint.

The decoction of arnatto in water has a strong peculiar odour, and a disagreeable taste. Its colour is yellowish-red, and it remains a little turbid. An alkaline solution renders its orange-yellow clearor and more agreeable, while a small quantity of a whitish substance is separated from it, which remains suspended in the liquid. If arnatto be boiled in water along with an alkali, it dissolves much better than when alone, and the liquid has an orange hue.

The acids form with this liquor an orange-coloured precipitate, soluble in alkalis, which communicate to it a doep orange colour. The supernatant liquor retains only a pale yollow hue.

When arnatto is used as a dye, it is always mixed with alkali, which facilitates its solution, and gives it a colour inclining less to red. The arnatto is cut in pieces, and boiled for some instants in a copper with its own weight of crude pearl-ashes, provided the shade wanted do not require less alkali. The cloths may be afterwards dyed in this bath, either by these ingredients alone, or by adding others to modify the colour; but arnatto is seldom used for woollen, because the colours which it gives are too fugitive, and may be obtained by more permanent dyes. Hellot employed it to dye a stuff prepared with alum and tartar; but the colour acquired had littlo permanence. It is almost solely used for silks.

For silks intended to become aurora and orange, it is sufficient to scour them at the rate of 20 per cent. of soap. When they have been well cleansed, they are immersed in a bath prepared with water, to which is added a quantity of alkaline solution of arnatto more or less considerable, according to the shade that may be wanted. This bath should have a mean temperaturo between that of tepid and boiling water.

When the silk has become uniform, one of the hanks is taken out, washed, and wrung, to see if the colour be sufficiently full; if it be not so, more solution of arnatto is added, and the silk is turned again round the sticks: the solution keops without alteration.

When the desired shade is obtained, nothing remains but to wash the silk, and give it two beetlings at the river, in order to free it from the redundant arpatto, which would injure the lustre of the colour.

When raw silks are to be dyed, those naturally white aro chosen, and dyed in the arnatto bath, which should not be more than tepid, or even cold, in order that the alkali may not attack the gum of the silk, and deprive it of the elasticity which it is desirable for it to preserve.

What has now been said regarls the silks to which the aurora shades are to be given: but to make an orango hue, which contains more red than the aurora, it is

Vot, I. 
requisite, aftor dyeing with arnatto, to rodden the silks with vinegar, alum, or lemonjuice. The acid, by saturating the alkali employed for dissolving the arnatto, destroys the shade of yellow that the alkali had given, and restores it to its natural colour, which inelines a good deal to red.

For the deep shades, the practice at Paris, as Macquer informs us, is to pass the silks through alum; and if the colour be not red onough, they are passed through a faint bath of Brazil wood. At Lyons, the dyers who uso carthamus sometimos omploy old baths of arnatto for dipping the deep oranges.

When the orange hues have been reddened by alum, they must be washed at the. river; but it is not necessary to beetle them, unless the colour turns out too red.

Shades may bo obtained also by a single operation, which retain a reddish tint, employing for the arnatto bath a less proportion of alkali than has been pointed out.

Guhliche recommends to avoid heat in the preparation of arnatto. He direets it to be placed in a glass vessel, or in a glazed earthen one; to cover it with a solution of pure alkali ; to leave the mixture at rest for 24 hours; to decant the liquor, filter it, and add water repeatedly to the residuum, leaving the mixture each time at rest for two or three days, till the water is no longer coloured; to mix all these liquors, and preserve the whole for use in a well-stopped vessel.

He macerates the silk for 12 hours in a solution of alum, at the rate of an eighth of this salt for one part of silk, or in a water rendered acidulous by the aceto-citric acid above described, and he wrings it well on its coming out of this bath.

Silk thus prepared is put into the arnatto bath quite cold. It is kept in agitation there till it has taken the shade sought for; or the liquor may be maintained at a heat far below ebullition. On being taken out of the bath, the silk is to be washed and dried in the shade.

For lighter hues, a liquor less charged with colour is taken; and a little of the acid liquor which has served for the mordant may be added, or the dyed silk may be passed through the acidulous wator.

We have seen the following preparation employed for cotton velvet:-1 part of quicklime, 1 of potash, 2 of soda.

Of these a ley is formed, in which one part of arnatto is dissolved; and the mixture is boiled for an hour and a half. This bath affords the liveliest and most brilliant auroras. The buff (chamois) fugitivo dyo is also obtained with this solution. For this purpose only a littlo is wanted; but we must never forget that the colours arising from arnatto are all fugitive.

Dr. John found in the pulp surrounding the unfermented fresh seeds, which are about the size of little peas, 28 parts of colouring resinous matter, 26.5 of regetable gluton, 20 of ligneous fibre, 20 of colouring extractive matter; 4 formed of matters analogous to vegetable gluten and extractive, and a trace of spicy and acid mattors.

The Gloucestershire cheese is coloured with arnatto, in the proportion of 1 cwt. to an ounce of the dye: butter is sometimes coloured with it.

When used in calico-printing, it is usually mixed with potash or ammonia and starch.

Arnatto was considered to contain two distinct colouring-matters, a yellow and red, till it was shown by M. Preisser that one is the oxide of the other, and that they may be obtained by adding a salt of lead to a solution of arnatto, which precipitates the colouring-matter. The lead is separated by sulphuretted hydrogen; and the substance being filtered and evaporated, the colouring-matter is deposited in small erystals of a yellow-white colour. These crystals consist of bixine; they become yellow by exposure to the air, but if they are dissolved in water they undergo no change. When ammonia is added to bixine, with free contact of air, there is formed a fine deep red colour, like arnatto, and a new substance, called bixeine, is produced, which does not crystallise, but may be obtained as a red powder; this is coloured blue, by sulphuric acid, and combines with alkalis; it is bixine with addition of oxygen. When arnatto, in the form of paste, is mixed from time to time with stale urine, it appears probable that the improvement consists in the formation of bixeine from the bixine by the ammonia of the urine. It has hence been suggested that, to improve the colour of arnatto, it might be mixed with a littlo ammonia, and subsequently exposed to the air, previously to its being used for dyeing.

A solution of arnatto and potash in water is sold under the name of Scott's Nankeen Dye.

Flag arnatto paid a duty of $188.8 d$. per ewt, and the other sorts $5 l .12 s$, previously to 1832 . The duty was subsequently reduced to 18 . per cwt. on the former and 48 . on the latter. It was repealed in 1845 .

AREICA. A genus of plants belonging to the Natural order Composite, and Suborder Corymbifera. The Arnica montana, called Leopard's Bane, or Mountain Tobaceo, is a native of the North of Europe, and of the Alps. The plant contains a 
volatile oil, oil of Amica; and an alkaline bitter principle, Arnicine. It is used oceasionally in medicine.

AROMATrC VINZGAR. (Acetum aromaticum.) This is a compound of strong acetic acid with certain powerful essential oils or aromatic herbs. The 'Edinburgh Pharmacopoia ' orders it to be made with concentrated acetic acid, $1 \frac{1}{2}$ pints ; rosemary and thyme dried, of each 1 oz.; lavender, also dried, $\frac{1}{2}$ oz.; cloves, bruised, $\frac{1}{2}$ drachm. Macerate for seven days, strain, and express strongly, and filter the liquor. Henry's aromatic vinegar is prepared by dissolving oils of cloves, lavender, rosemary, and the like, in concentrated acetic acid. Camphorated acetic acid is sometimes substituted for the acetum aromaticum. These preparations have been in great repute as prophylactics in contagious fevers. The name of 'Le vinaigre des quatre voleurs' has been given to aromatic vinegar in Franco-it is said from the confessions of four thieves who, during the plague at Marseilles, plundered the dead bodies with perfect impunity after protecting themselves with aromatic vinegar.

ARQUIRIT. A silver amalgam from the mines of Arqueros, near Coquimbo. It occurs crystalline. Domeyko finds it to consist of silver $86 \cdot 49$, mercury $13 * 51$.

ARra CE. A spirituous liquor from the East Indies. This term, or its corruption, rack, is applied to any spirituous liquor in the East. The true arrack is said to be distilled from toddy - the fermented juice of the cocoa-nut tree. It is, however, frequently distilled from rice and sugar, fermented with the cocoa-nut juice.

ARROBA. A measure of capacity and weight in general use throughout all those parts of South America which ever belonged to Spain. It is also used in Manilla and the East. According to Spanish standard weight it should be $25.36 \mathrm{lbs}$. English. As a wine measure it is equal to 3.54 imperial gallons; as an oil measure it is but $2 \cdot 78$ imperial gallons.

ARRoræ. Sherry boiled to a syrup; used for colouring other wines.

ARzowroor. (Racine féchière, Fr.; Pfeilwurz, Ger.) The rhizome of the Maranta arundinacea, a plant which grows in the West Indies, and furnishes, by pounding in mortars, and elutriation through sieves, a peculiar species of starch, commonly, but improperly, called arrowroot. It is reckoned more nourishing than the starch of wheat or potatoes, and is generally also freer from peculiar taste or flavour. The fresh root consists, according to Benzon, of 0.07 of volatile oil; 26 of starch (23 of which are obtained in the form of powder, while the other 3 must be extracted from the parenchyma in a paste by boiling water); 1.58 of vegetable albumen; 0.6 of a gummy extract; 0.25 of chloride of calcium; 6 of insoluble fibrine; and 65.5 of water. This plant was brought from the Island of Dominica, by Colonel James Walker to Barbadoes, and there planted. From thence it was sent to Jamaica. The root appears to have been used by the Indians to yield a poison with which to smear their arrows, and henee its name.

This plant has been lately cultivated with great success, and its root manufactured in a superior manner, upon the Hopewell estate, in the Island of St. Vincent. It grows there to the height of about 3 feet, and it sends down its tap roots from 12 to 18 inches into the ground. Its maturity is known by the flagging and falling down of the leaves, which takes place when the plant is from 10 to 12 months old. The roots being dug up with the hoe, are transported to the washing-house, where they are thoroughly freed from all adhering earth, and next taken individually in the hand, and deprived by a knife of every portion of their skins, while every unsound part is cut away. This process must be performed with great nicety, for the cuticle contains a resinous matter which imparts colour and a disagreeable flavour to the fecula which no subsequent treatment can remove. The skinned roots are thrown into a large cistern, with a perforated bottom, and there exposed to the action of a copious cascade of pure water till this runs off quite unaltered. The cleansed roots are next put into the hopper of the mill, and are subjected to the powerful pressure of two pairs of polished rollers of hard brass, the lower pair of rollers being set much eloser together than the upper. (See fig. 74.) The starchy matter is thus ground into a pulp, which falls into the receiver placed beneath, and is thence transferred to large fixed copper cylinders, tinned inside, and perforated at the bottom with numerous minute orifices, like a kitchen drainer. Within these cylinders, wooden paddles are made to revolve with great velocity, by the power of a water-wheel, at the same time that a stream of pure water is admitted from above. The paddle-arms beat out the fecula from the fibres and parenchyma of the pulp, and discharge it in the form of a milk through the perforated bottom of a cylinder. This starchy water runs along pipes, and then through strainers of fine muslin, into large reservoirs, where, after the fecula has subsided, the supernatant liquid is drawn off, and fresh water being let on, the whole is agitated and left again to repose. When the water ceases to remove anything from the arrowroot, all the deposits of fecula are collected into one cistern, covered, and agitated with a fresh charge of water, and 
left until the following morning. The water boing allowed to run off, the surfuce of the deposit is carefully scraped with German-silver palette-knives, to remove any impure or coloured parts, and the lower portions only are dried and prepared for the market. The greatest care is taken in drying; and when dry, tho fecula is packed in tin-cases for exportation.

Fig. 74, plan of arrowroot grinding mill, and two sets of copper cylinder

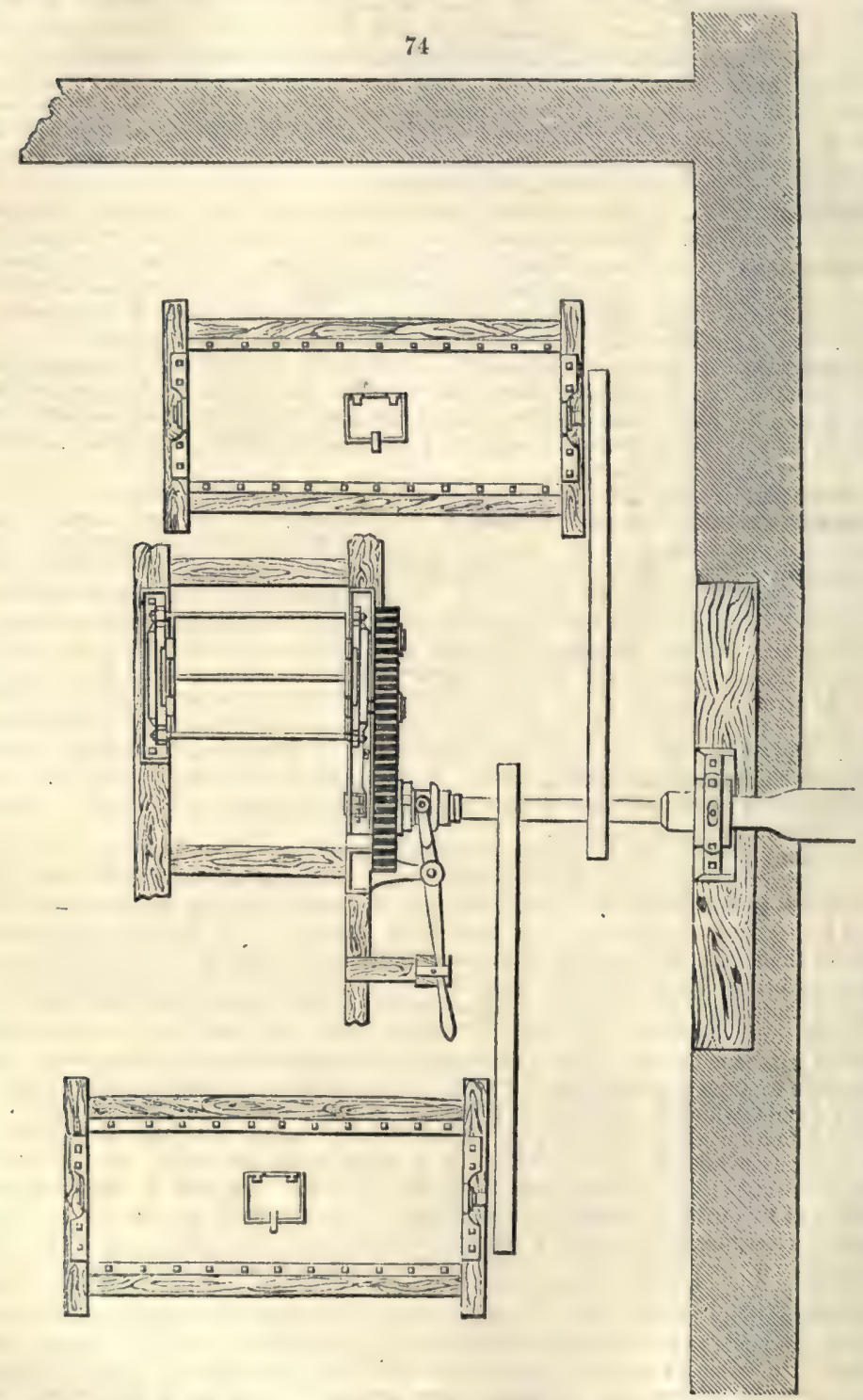

washing-machines, with the connecting machinery for driving them, the washing* agitator being driven from the connecting shaft with leathern belts. Fig. 75, endelevation of copper washing-cylinder, with press framing, \&c. The washing-cylinders are $6 \frac{1}{2}$ feet long and $3 \frac{1}{2}$ in diameter. The mill rollers are 3 feet long and 1 foot in diameter. Fig. 76, end-elevation of arrowroot mill, with wheols and pinions, dist ongaging lever, \&c. 
Arrowroot is brought into the market from Bermuda, St. Vincent, Jamaica, Brazil, the East Indies, Natal, and Sierra Leone. It is subject to a duty of 48 . per cwt. The, Bermuda arrowroot was in 1865 sold wholesale at 1s. $2 d$. the pound, the other sorts varying from $2 \frac{1}{2} d_{0}$ to $6 d$.

The uses of arrowroot are too well known and acknowledged to require recounting here. It is the most elegant and the richest of all the feculas. Liebig places the powers of arrowroot, as a nutriment to man, in a very remarkable point of view, when he states that 15 pounds of flesh contain no more carbon for supplying animal heat by its combustion into carbonic acid in the systom than 4 pounds of starch ; and that if a savage, with one animal and an equal weight of starch, could maintain life and health for a cortain number of days, he would be compelled, if confined to flesh alone, in order to procure the carbon necessary for respiration during the same time, to consume five such animals. All

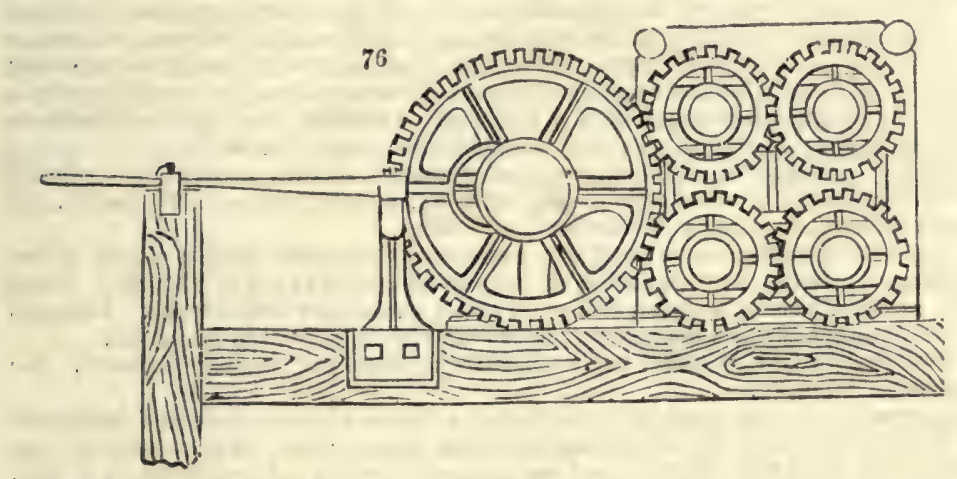

the starches are readily converted into sngar and fat, but they are low in their fleshproducing power.

In commerce, the term arrowroot is frequently used generically to indicate a starch or fecula, as:-East India arrovroot, prepared from the Curcuma angustifolia. Brazilian arrowroot or Cassava, the fecula of Jatropha manihot. English arrowroot, the starch of the potato (Solanum tuberosum). Portland arrowroot, a white amylaceous powder, formerly prepared in the Isle of Portland, from the Amum maculatum, the common Cuckoo-pint, called also Wake-robin and Lords and Ladies. Tahiti arrowroot, the fecula of Tacea oceanica, which has been imported into London and sold as ' arrowroot prepared by the native converts at the missionary stations in the South Sea Islands.'

The presence of potato-starch in arrowroot, with which it is often adulterated, may be discovered by the microscope. Arrowroot consists of regular ovoid particles of nearly equal size, whereas potato-starch consists of particles of an irregular ovoid or truncated form, exceedingly irregular in their dimensions, some being so large as $\frac{1}{300}$ th of an inch, and others only $\frac{1}{2000}$ th. Their surfaces in the arrowroot are smooth and free from the streaks and furrows to be seen in the potato-particles by a good microscope. The arrowroot, moreover, is destitute of that fetid unwholesome oil extractable by alcohol from potato-starch. But the most convenient test is dilute nitric acid of 1.10 (about the strength of single aquafortis), which, when triturated in a mortar with the starch, forms immediately a transparent, very viscid paste or jelly. Flour-starch exhibits a like appearance. Arrowroot, however, forms an opaque paste, and takes a much longer time to become viscid.

ARSINATES. Compounds of arsenic acid with alkaline and metallic bases

ARSINIC, derived from the Greek a $\rho \sigma \in \nu \in k \delta \nu$, masculine, a name applied to orpiment on account of its potent powers. Arsenic occurs native, in veins, in crystalline rocks, and the older schists; it is found in tho stato of oxide, and also combined with sulphur, when it is known under the names of yellow and red arsenic (orpiment and realgar). Arsenic is associated with a great many metallic ores; in this country chiefly with those of tin, but on the Continent arsenical cobalt is the chief source of the compounds of arsenic. 
The following are the principal ores of arsenic:-

Native Arsenic.-The most common form of native arsonic is that of reniform and stalactitic masses, often mammillated, and splitting off in thin successive layers like those of a shell. It possesses a somewhat metallic lustre, and a tin-whito colour and streak, which soon tarnishes to a dark grey. Its specific gravity is 5.93. Before the blowpipe it gives out an alliacoous odour, and volatilises in white fumes. It is found in the silver mines of Freiberg, Annaberg, Marienberg, and Schneeberg in Saxony ; also at Joachimstahl in Bohemia, Andreasberg in the Hartz, Kapnik in Transylvania, Orawitza, in the Bannat, Kongsberg in Norway, Zimeoff in Siberia, in Alsace, in Borneo, and, according to Dana, at Haverhill, and at Jackson, N. H. in the United States.

Arsenical Antimony. - This mineral occurs at Allemont; also at Przibram in Bohemia, where it occurs in metallie veins associated with blende, antimony, and spathic iron; at Schladming in Styria; and Andreasberg in the Hartz. Its composition is :-Arsenic, 63.62 ; antimony, $36 \cdot 38$. When exposed to the action of the blowpipe, this mineral emits fumes of arsenic and antimony; and fuses to a metallic globule, which takes fire and burns away, leaving oxide of antimony on the charcoal.

White Arsenic or Arsenious Acid (Arsenolite) is often formed by the decomposition of other arsenical ores, and is composed of arsenic $75 \cdot 76$, and oxygen $24 \cdot 24$. It occurs either in minute radiating capillary crystals and crusts investing other substances, or in a stalactitic or botryoidal form. Before the blowpipe it volatilises in white fumes: in the inner flame it blackens and gives out an alliacoous odour; its specific gravity is 3.69. It is white, sometimes with a yellowish or reddish tinge, and has a silky or vitreous lustre. It possesses an astringent, sweetish taste.-H. W. B.

Realgar (anciently called Sandaraca), red orpiment, or ruby sulphur, is a sulphide of arsenic, having a composition, sulphur 29.91 , arsenic $70.09=\mathrm{AsS}^{2}\left(\mathbf{A s}^{2} \mathbf{s}^{2}\right)$. It occurs in Hungary, Saxony, Switzerland, and China.

Orpiment (a corruption of its Latin name, auripigmentum - golden paint), yellow sulphide of arsenic: its composition is, sulphur 39, arsenic $61=A_{s S^{3}}\left(\mathbf{A s}^{2} \mathbf{S}^{3}\right.$.) Burns with a blue flame on charcoal, and emits fumes of sulphur and arsenic. Dissolves in nitro-muriatic acid and ammonia. It is found in Hungary, the Hartz, \&c.

Both realgar and orpiment are artificially propared and used as pigments. See OrptMant, Reatcar.

ARsanc is a brittle metal, of an iron-grey colour, with a good deal of brilliancy. It may be prepared by triturating arsonious acid, or the white arsenic of commerce, with black flux (charcoal and carbonate of potash), and subliming in a tube. If arsenical pyrites bo ignited in close tubes, metallic arsenic sublimes, and sulphuret of iron remains. This metal, when exposed in the air, gradually absorbs oxygen, and falls into a grey powder (suboxide). This is sold on the Continent as fly-powder.

To prepare arsenic on a larger scale, mispickel, or the other ores employed, are pounded; some pieces of old iron are mixed with the ore, to retain the combined sulphur, and the mixture placed in retorts between four and five feet in length, to which receivers are adapted. The retorts are moderately heateu by a fire placed beneath them; the ores are decomposed, and metallic arsenic is sublimed and condensed in the receivers. Tho arsenic obtained in this way is purified by a second distillation with a little charcoal. The atomic weight of arsenic is $\mathbf{7 5}$; its symbol As.

Arsenic is used in small quantities in the preparation of several alloys; whilst arsenious acid is employed in the manufacture of opal glass; and is much used in the manufacture of shot, to which the reduced arsenic imparts a certain degree of hardness; and, by preventing the distortion of the falling drops of metal, and thus securing regular globules, the manufacture is greatly facilitated. It is also used in pyrotechny.

ARsEJTIC ACrD. As $\mathrm{O}^{5} .3 \mathrm{HO}\left(\mathbf{H}^{3} \mathbf{A s O}^{4}\right)$. This acid was first produced on a large scale by M. G. Kopp. He employs nitric acid to convert arsenious acid by oxidation into arsenic acid, and by passing the nitrous acid fumes evolved, together with air, over coke moistened with water, he recovers two-thirds or three-fourths of the nitric acid employed. The proportions he adopts are 303 kilogrammes (nearly $2 \frac{1}{4}$ lbs. avoirdupois each) of nitric acid of $1.35 \mathrm{sp}$. gr. to 400 kilogrammes of arsenious acid, and by adding the nitric acid gradually, the oxidising action may be accomplished without the application of heat.

Arsenic acid is now almost universally employed in the manufacture of Rosaniline, and is therefore an article of great consumption. It is also largely used for the white discharge of Turkey red.

M. Kopp has noticed, that without any injury to the general health, a natural tendency to stoutness was produced whilst working with arsenic acid. In the course of ten weeks, while engaged on experiments with arsenic acid, M. Kopp himself inereased in weight considerably more than twenty pounds, which he lost again when the expe- 
riments were concluded. The workpeople engaged in the manufacture of Rosaniline are similarly affected.

Arsenate of Potash is prepared, in the small way, by exposing to a moderate heat, in a crucible, a mixture of equal parts of white arsenic and nitro in powder. After fusion the crucible is to be cooled ; the contents being dissolved in hot water, and the solution filtered, will afford regular crystals on cooling. It is an acid salt, usually called the binarsenate of potash. This article is prepared upon a great scale, in Saxony, by melting nitre and arsenious acid together in a cylinder of cast-iron, A neutral arsenate also is readily formed by saturating the excess of acid in the above salt with potash; it does not crystallise. The acid arsenate is occasionally used in calico-printing, for preventing certain points of the cotton cloth from taking on the mordant; with which view it is mixed up with gum-water and pipe-clay into a paste, which is applied to such places with a block.

Arsenate of Soda.-An acid arsenate of soda, propared by heating white arsenic and nitrate of soda, is now used in calico-printing.

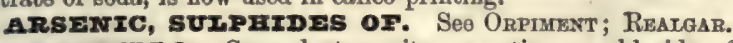

ARszNIII0. Ground atacamite, or native oxychloride of copper, sometimes used in Chili as sand for letters.

ARsmirrous ACID. White Arsenic, Flowers of Arsenic. $\mathrm{AsO}^{3}\left(\mathbf{A s}^{2} \mathbf{O}^{3}\right)$ This is the white arsenic of commerce, usually called Arsenic. It is obtained in this country from the arsenical ores of iron, tin, \&c., and on the Continent from those of cobalt and nickel. It is prepared by heating the ores containing arsenic on the sole of a reverberatory furnace, through which a current of air, after passing through the grate, is allowed to play. The following ores are the more remarkable of this class - the quantity of arsenic in 100 parts is giren in each case.

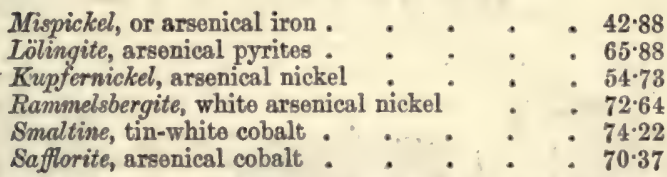

In the roasting of tin ores, a considerable quantity of arsenious acid is collected in the flues leading from the furnaces in which this process is effected.

The extraction of white arsenic from the cobalt ores is performed at Altenberg and at Reichenstein, in Silesia, with an apparatus excellently contrived to protect the health of the smelters from the vapours of this poisonous sublimate.

Figs. 77 to 80 represent the arsenical furnaces at Altenberg. Fig. 77 is a vertical section of the poison tower; fig. 78, a longitudinal section of the subliming furnace $\mathrm{A}$, with the adjoining vault $\mathrm{B}$, and the poison tower in part at $n ;$ fig. 79 , the transverse section of the furnace $\Delta$, of fig. $78 ;$ fig. 80 ground-plan of the furnace $\Delta$, where the left half shows the part above, and the right the part below the muffle or oblong retorts ; $\mathrm{B}^{\prime}$ is the upper view, $\mathrm{B}^{\prime \prime}$ the ground plan of the vault $\mathrm{B}$, of fig. 78 ; $m, n$, the base of the poison tower. In the several figures the same letters denote the same objects; $a$ is the muffle; $b$ is its mouth for turning over the arsenical schlich, or ground ore; $c, c, c$, fire-draughts or flues; $d$, an aperture for charging the muffle with fresh schlich; $e$, the smoke chimney; $f$, two channels or flues for the ascent of the arsenious fumes, which proceed to other two flues $g$, and then terminate both in $h$, which conducts the fumes into the vault $B_{0}$ They issue, by the door $i$, into the conduit $k$, thence by $l$ into the spaces $m, n, o, p, q, r$, of the tower. The incondensable gases escape by the chimney 8 . The cover $t$ is removed after completion of the process, in order to push down the precipitate into the lower compartments.

The arsenious schlichs, to the amount of 9 or $10 \mathrm{cwt}$. for one operation (1 roastpost, or roasting round), are spread 2 or 3 inches thick upon the bottom of the muffle, and heated with a brisk fire to redness, then with a gentler heat, in order to oxidise completely, before subliming, the arsenical ore. With this view the air must have free entrance, and the front aperture of the muffle must be left quite open. After 11 or 12 hours, the calcined materials are raked out by the mouth of the muffle, and fresh ones are introduced by the openings indicated abore, which are closed during the sublimation.

The arsenious acid found in these passages is not marketable till it be resublined in large iron pots, surmounted with a series of sheet-iron drums or cast-iron cylinders, upon the sides of which the arsenic is condensed in its compact glassy form. The top cylinder is furnished with a pipo which terminates in a condensing chamber.

Figs. 81, 82, represent the arsenic-refining furnaces at Roichenstein. Fig. 81 shows at $\Lambda, a$ vertical section of the furnace, the kettle, and the surmounting drums or cylinders; over $\mathrm{B}$ it is seen in elevation; fig. 82 is a ground-plan of tho four firo- 
places. $a$ is the grate; $b$, tho ash-pit; $c$, the openings for firing; $d$, the fire-place; $e$, iron pots or kottlos which are charged with the arsenious powder; $f$, the firo-flues

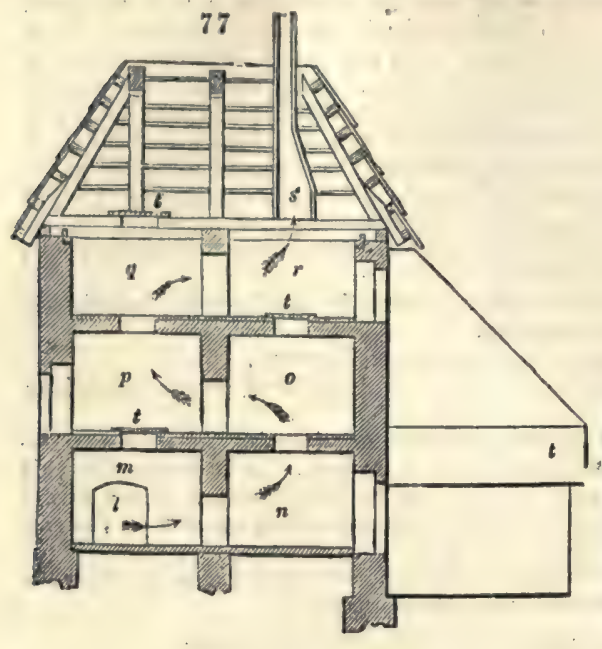

80
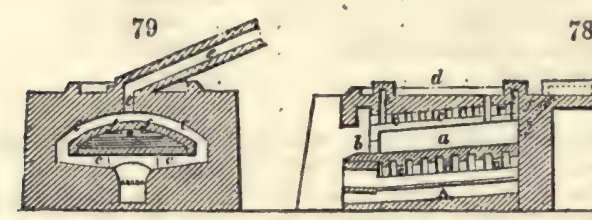

78

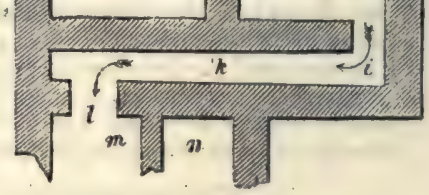

A.
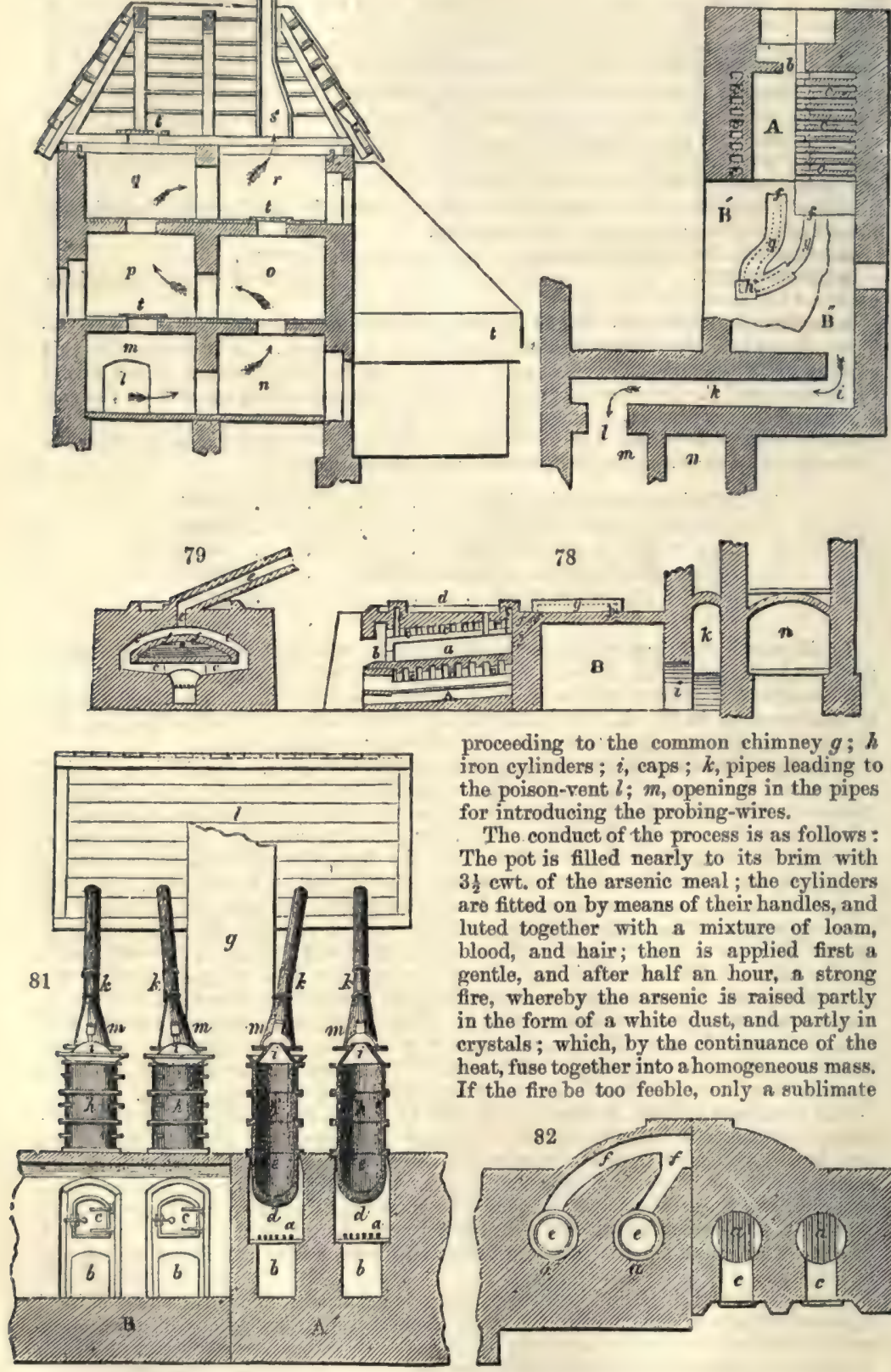

proceeding to the common chimney $g ; h$ iron cylinders ; $i$, caps ; $k$, pipes leading to the poison-vent $l ; m$, openings in the pipes for introduoing the probing-wires.

The conduct of the process is as follows: The pot is filled nearly to its brim with $3 \frac{1}{2}$ ewt. of the arsenic meal; the cylinders are fitted on by means of their handles, and luted together with a mixture of loam, blood, and hair; then is applied first a gentle, and after half an hour, a strong fire, whereby the arsenic is raised partly in the form of a white dust, and partly in crystals; which, by the continuance of the heat, fuse together into a homogeneous mass. If the fixe be too feoble, only a sublimate

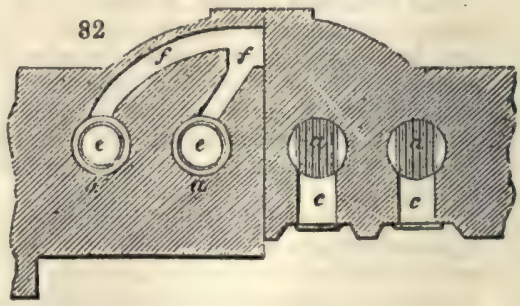

is obtained; but if too violent, much of the arsenic is volatilised into the pipes. The workmen judge by the heat of the cylinders whether the operation be going on well or 
not. After 12 hours the furnace is allowed to cool, prorided the probe-wires show that the sublimation is over. The cylinders are then lifted off, and the arsenious glass is detached from their inner surface. According to the quality of the poisonflour, it yields from $\frac{3}{4}$ ths to $\frac{7}{8}$ ths of its weight of the glass or enamel. Should any dark particles of metallic arsenic be intermixed with the glass, a fresh sublimation must be had recourse to.

In these operations, if any sulphur is present it is converted into sulphurous acid, which escapes through the chimney, while the arsenious acid is condensed in proper chambers, placed in the flues to receive it. Freshly prepared arsenious acid is a perfectly transparent solid mass; but by exposure it becomes transformed into an opaque body resembling porcelain.

White arsenic is extensively used in the preparation of various pigments, as the bisulphide, or realgar, the tersulphide, or orpiment, and also in the mineral greens used by paper-stainers. It has been stated that paper stained with the arsenical greens is injurious to health. Very much has been said on this subject; but the following remarks by Mr. Alfred Fletcher appear to settle the question :- 'Now, it is stated that in a medical work an instance is noted in which injury has been received by those living in rooms decorated with these colours: surely, were the proximity of such materials injurious, it would not be necessary to search in recondite books for the registry of isolated cases. The fact of the large extent to which such materials have always been employed is a sufficient proof that there is no danger attending their use ; moreover, workmen who have been daily employed for many years in manufacturing large quantities of these colours, under the necessity of constantly handling them, are in the regular enjoyment of perfect health, though exposed also to the general influences of a chemical factory. Let blame be laid at the right door, and let the public be assured that it is not the looking at cheerful walls, the fingering of brightly ornamented books, nor the wearing of tastefully coloured clothing, that will hurt them, but the dwelling in ill-ventilated rooms.'

Arsenite of Copper.-Scheele's green is a combination of arsenious acid with oxide of copper, or an arsenite of copper. See Schrexte's Grees.

Arsentc, Poisonivg BY.-This poison is so commonly the cause of death, by accident and by design, that it is important to name an antidote which has been employed with very great success.

This is the hydrated peroxide of iron. This preparation has no action on the system, and it may therefore be administered as largely and as quickly as possible. The following statement will render the action of this hydrated salt intelligible. When hydrated peroxide of iron is mixed in a thin paste with the solution of arsenious acid, this disappears, boing changed into arsenic acid (a far loss active oxide), and the iron into protoxide, $2 \mathrm{Fe}^{2} \mathrm{O}^{3}$ and $\mathrm{As} \mathrm{O}^{3}$, producing $4 \mathrm{Fe} \mathrm{O}+\mathrm{As}^{3}$. The hydrated peroxide of iron may be made in a few minutes by adding carbonate of soda to any salt of the red oxide of iron (perchloride, acetate, \&c.). It noed not be washed, as the liquor contains only a salt of soda, which would be, if not beneficial, certainly not injurious.-Kane.

\section{Detection of Arsenic in Cases of Poisoning.}

Arsenious acid, which is almost always the form in which the arsenic has entered the system, possesses the power of preventing the putrefaction of animal substances; and hence the bodies of persons that have been poisoned by it do not readily putrefy. The arsenious acid combines with the fatty and albuminous tissues to form solid compounds, which are not susceptible of alteration under ordinary circumstances. It hence has frequently occurred that the bodies of porsons poisoned by arsenic have been found, long after death, scarcely at all decomposed; and even where the general mass of the body had completely disappeared, the stomach and intestines had remained preserved by the arsenious acid which had combined with them, and by its detection the crimes committed many yoars before have been brought to light and punished.-Kane.

The presence of arsenic may be determined by one of the following methods :-

1. Portions of the contents of the stomach or bowels being gently heated in a glass tube, open at both ends, the arsenic, if in any quantity, will be sublimed, and collectod as minute brilliant octahedrons of arsenious acid.

2. Or if the ignition is effectod in a tube closed at one end, metallic arsenic sublimes, forming a steel-grey coat, and emitting a strong smoll of garlic.

3. Ammonineal Nitrate of Silver produces a canary-yollow procipitate of arsenite of silver in a solution of arsenious acid. The tribasic phosphate of soda produces a 
yellow precipitato of tribasic phosphate of silvor, which exactly resombles the arsenite. The phosphate is, however, the more soluble in ammonia, and when hoated gires no rolatile product; while the arsenite is decomposed into white arsenic and oxygen, leaving metallic silver behind.

4. Ammoniacal Sulphate of Copper produces a fine apple-green precipitate of arsenite of copper, which is dissolved in an excess of either acid or ammonia. It is, however, uncertain, unless the precipitate bo dried and reduced.

5. The Reduction Test.-Any portion of the suspected matter, being dried, is mixed with equal parts of cyanide of potassium and carbonate of potash, both dry. This mixture is to be jntroduced into a tube terminating in a bulb, to which heat is applied, when metallic arsenic sublimes.

6. Marsh's Test.-This is ono of the most delicate and useful of tests for this poison, and when performed with due care there is little liability to error. The liquid contents of the stomach, or any solution obtained by boiling the contents, is freed as

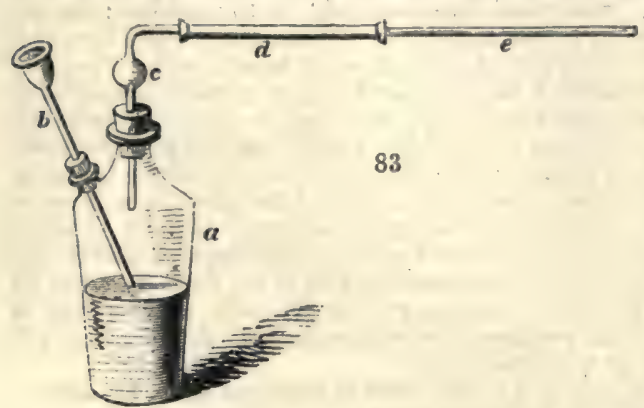
much as possible from animal matter by any of the wellknown methods for doing so. This fluid is then rendered moderately acid by sulphuric acid, and introduced into a bottle properly arranged.

Fig. 83 is the best form for Marsh's apparatus:- $a$ is a bottle capable of holding half, or, at most, a pint. Both necks are fitted with new perforated corks, which must be perfectly tight. Through one of these the funnel-tube $b$ is passed air-tight, and through the other the bent tube $c$, which is expanded at $e$ into a bulb about an inch in diameter. This bulb serves to collect the particles of liquid which are thrown up from the contents of the bottle, and which drop again into the latter from the end of the tube. The other ond of the tube is connected, by means of a cork, with the tube $d$, about six inches long, which is filled with fused chloride of calcium, free from powder, destined to retain the moisture. In the opposite end of the tube $d$ is fixed, air-tight, another tube, $e$, made of glass free from lead, 12 inches long, and, at most, $\frac{1}{12}$ th of an inch in internal diameter. It must be observed that the funnel-tube $d$ is indispensably necessary to introduce the fluid to the pieces of perfectly pure motallic zine already placed in the bottle. Hydrogen gas is at once formed, and if arsenic be prosent, in even the smallest quantity, it combines with the hydrogen, and escapes as arseniuretted hydrogen. If the gas as it issues from the jet is set on fire, no product but water is generated if the hydrogen be pure; and by holding against the flame a cold white porcelain basin, or piece of glass, or of mica, no steam is produced, and a dow is formed upon the cold surface. If arsenic be present, a deposit is obtained, which, according to the part of the flame in which the substance to receive it is placed, will be either a brown stain of metallic arsenic, or a whito one of arsenious acid. If the quantity of arsenic is too small to be detected in this way, it will be well to ignite the horizontal part of the tube. All the arseniuretted hydrogen will, in passing that point, become decomposed, and deposit its arsenic. The heat will drive this forward, and a little beyond the heated portion metallic arsenic will be condensed. Several precautions are necossary to be observed; but for the details of those we must refer to works especially directed to the consideration of this subject. One source of error must, however, be alluded to. A compound of antimony and hydrogen is formed under similar circumstances ; and this gas in many respects resembles the compound of arsenic and hydrogen. If the stain formed by the flame is arsenic, it will dissolve, when heated, in a drop or two of sulpho-hydride of ammonia, and a lemon-yellow spot is left; if antimony is present, it leaves a yellow stain.-Wöhler.

If a drop of bromene is placed on a saucer, and a capsule containing arsemical spots inverted over it, the spots take a very bright lemon-yellow tingo in a short timo. Antimonial spots, under the same circumstances, are acted on much more rapirly (in about five seconds at a temperature of $52^{\circ} \mathrm{F}$.), and assume an orange shade. Both become colourless if exposed to the air, and are again restored if treated with a strong solution of sulphuretted hydrogen. The secondary yellow of the arsenical spots, as observed by Lassaigne, disappears on the addition of ammonia, whilst that of the antimonial spots remains untouched. A concentrated solution of iodate of potash turns 
arsenical spots of a cinnamon-red and dissolves them almost immediately. On antimonial spots it has no visible action within three or four hours. Solutions of the hypochlorites (chlorides) of soda and lime and chlorine-water dissolve arsenical spots instantaneously, leaving those of antimony. A concentrated solution of the chlorate of potash gradually acts upon arsonical spots, but not upon those of antimony. The nitroprusside of potassium, on the other hand, slowly dissolves antimony, producing no perceptible effect upon arsenic. The statement of Bischoff, that arsenical spots were soluble, antimonial insoluble, in a solution of the chloride of sodium, could not be verified, as, after repeated trials, it was found to leave both not perceptibly affected. The chloride of barium, the hypochlorate and the sulphite of ammonia, afforded likewise no distinguishing action. The nitrate of ammonia dissolves arsenical more rapidly than antimonial stains. Of these reactions the most decisive are those of iodate of potash, hypochlorites of soda and $\operatorname{lime}$, and fresh chlorine-water.

It is well known that fluids mixed with glutinous matter are very liable to froth up when hydrogen is disengaged in them, from the mutual action of zinc and a dilute acid; and that the froth obstructs the dne performance of the experiment of Marsh. A committee appointed by the Prussian Government contrived an ingenious modification of Marsh's apparatus, the annexed form (fig. 84) representing a convenient simplification of it by Dr. Ure:- $\boldsymbol{\Lambda}$, is a narrow glass cylinder, open at top, about 10 inches high, and $1 \frac{1}{4}$ or $1 \frac{1}{2}$ inch diameter inside; $\mathbf{B}$ is a glass tube, about 1 inch dia. meter outside, drawn to a point at bottom, and shut with a cork at top. Through the centre of this cork the small tube c passes down air-tight, and is furnished at top with a stopcock, into which the bent small tube of glass (without lead) $\mathrm{s}$ is cemented. The bent tube $\mathbf{F}$ is joined to the end of $\mathbf{x}$ with a collar of caoutchoue, or a perforated cork, which will be found more convenient.

The manner of using this apparatus is as follows:- Introduce a fow oblong slips of ginc, freo from arsenic, into $n$, and then insert its air-tight cork with the attached tubes. Having opened the stopeock, pour into $A$ as much of the suspected liquid, acidulated with dilute hydrochloric or sulphuric acid (each pure) as will rise to the top of the cork, after B is full, and immediately shut the stopcock. The generated

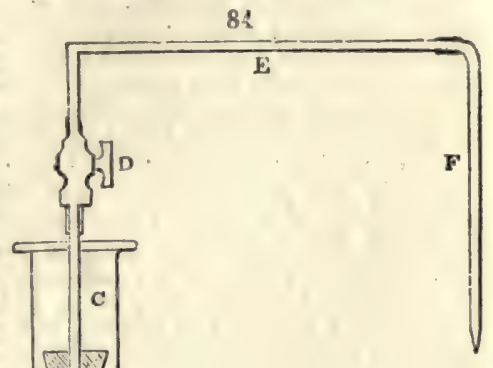
hydrogen will force down the liquid out of the lower orifice of $B$ into $\Delta$, and raise the level of it above the cork. The extremity of the tube $F$ being dipped beneath the surface of a weak solution of nitrate of silver, and a spirit-flame being placed a little to the left of the letter $\mathbf{E}$, the stopeock is then to be slightly opened, so that the gas which now fills the tube $B$ may escape so slowly as to pass off in separate small bubbles through the silver-solution. By this means the whole of the arsenic contained in the arsenjuretted hydrogen will bo deposited either in the metallic state upon the inside of the tube $\mathrm{E}$, or with the silver in the characteristic black powder. The first charge of gas in $B$ being expended, the stopcock is to be shut till the liquid be again expolled from it by a fresh disengagement of hydrogen. The ring of metallic arsenic doposited beyond may be chased onwards by placing a second flame under it, and thereby formed into an oblong brilliant steel-like mirror. It is evident that by the patient use of this apparatus the whole arsenic in any poisonons liquid may be collected, weighed, and subjected to every kind of chemjcal verification. If $\mathrm{F}$ be joined to $\mathrm{x}$ by means of a perforated cork, it may readily bo turned about, and its taper point raised into a position such as when the hydrogen issuing from it is kindled, the flame may be made to play upon a surface of glass or porcelain, in order to produce the arsenical mirror,

7. Reinsch's Test.-Professor Reinsch proposed an entirely different mothod of detecting arsenic, which consists in acidulating any suspected fluid with hydrochloric acid, heating in it a thin plate of bright copper, upon which the arsenic is deposited in the form of a thin motallic crust, and then separating the arsenic from the copper in the stato of oxide by subjecting the copper to a low rod heat in a glass tube. Organic 
fluids and solids suspected to contain arsenic, may be prepared for this purposo by boiling them for half an hour with a littlo hydrochloric acid; solid matters being cut into small shreds, water being added in sufficient quantity to let the ebullition go on quietly, and care being taken to continue the boiling until the solids fore either dissolved, as generally happens, or are reduced to a state of minute division.

The method of Reinsch is exceedingly delicate, for it is adequate to detect a 250,000 th part of arsenic in a fluid. It is also perfeet in another respect : it does not leave any arsenic in the subject of analysis; none, at least, which can be detected by nny other means, even by the most delicate process yet proposed, that of Mr. Marsh.

Cut the coppor on which the arsence is deposited into small chips, so that they may be easily packed in the bottom of a small glass tube, and apply a low red heat. A white crystalline powder sublimes; and if this be examined in the sunshine, or with a candle near it, a magnifier of four or five powers will enable the observer to distinguish the equilateral triangles composing the facets of the octahedral crystals, which are formed by arsenious acid when it sublimes. Sometimes the three equal angles, composing a corner of tho octahedron, may be seen by turning the glass in various directions. If triangular facets cannot be distinguished, owing to the minuteness of the crystals, then shake out the copper chips, close the open end of the tube with the finger, and heat the sublimed powder over a very minute spirit-lamp flame, chasing it up and down the tube till crystals of adequate size are formed. Next boil a little distilled water in the tube over the part where the crystalline powder is collocted; and when the solution is cold, divide it into three parts, to be tested with ammoniacal nitrate of silver, ammoniacal sulphate of copper, and sulphuretted hydrogen, either in the state of gas or dissolved in water.

8. Fleitmann's Test. - If a solution containing arsenic be mixed with a large excess of concentrated solution of potassa, and boiled with fragments of granulated zinc, arseniuretted hydrogen is evolved, and may be easily recognised by allowing it to pass on to a piece of filter paper spotted over with solution of nitrate of silver. These spots assume a purplish-black colour, even when a small quantity of arsonic is present. This experiment may be performed in a small flask, furnished with a perforated cork carrying a piece of glass tube of about $\frac{1}{4}$ inch diameter. It will be obsorved that this test serves to distinguish arsenic from antimony.

The following remarks on the Toxicological Discovery of Arsenic deservo attention:-

'This active and easily administered poison is fortunately one of those most easily and certainly discovered; but the processes require great precaution to prevent mistaken inferences: if due care is taken, arsenic can be found after any lapse of time, as well as after the most complete putrefaction of the animal-romains. The longest time after which it has been discovered by myself is eight years, which was the case of an infant; nothing but the bones of the skeleton remained, the coffin was full of earth, and large roots of a tree had grown through it. The metal was obtainod from the bones, and in the earth immediately below where the stomach had existed. Many cases have occurred in my experience, where one, two, three, four, and five years have elapsed; in one case, after fourteen months, where the body of a boy had been floating in a coffin full of water. The poison is given in one of three states, white arsenious acid, yellow sulphuret ("orpiment"), or " realgar," red sulphuret of arsenic ; and it is worthy of notice that putrefaction will turn either white or rod into yellow, but will never turn yellow into either white or red; this is owing to the hydrosulphuret of ammonia disengaged during decomposition.

'Modern toxicologists have abandoned all the old processes for the detection of this poison, and have adopted one of two, which have beon found more expeditious, as well as more certain. The first was proposed by Marsh, of Woolwich: it is founded upon the principle that nascent hydrogen will absorb and carry off any arsenic which may be present, as arseniuretted hydrogen; but as I prefer the principle first proposed by Reinsch, and have always acted upon it, I shall confine my description to the processes foundod upon it. The principle is this : arsenic mixed or combined with any organic matter will, if boiled with pure hydrochloric acid and metallic coppor, be deposited upon the copper; but as this dopositing property is also possessed by mercury, antimony, bismuth, lead, and tellurium, subsequont operations are required to

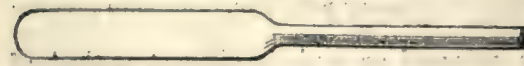
discriminate between the deposits. I take pieces of copper wire, about No. 13 size, and $2 \frac{1}{2}$ inehes long; these I hammor upon a polished plane with a polished hammer, for half their longth ( fig. 85), and having brought the suspected matters to a state of dryness, and boiled the copper blade in tho pure hydrochloric acid, to prove that it contains no metal capable of depositing, I introduce a portion of the suspected matter 
and cuntinue the boiling; if the copper becomes now either steel-grey, blue, or black, I remove it, and wash it free of grease in another vessel in which there is hot dilutod hydrochloric acid; I now dry it, and, with a scraper with a fine edge, take off the deposit with some of the adhering copper, and repeat the boiling, washing and seraping, so as to have four or five specimens on copper; one of these is sealed up hermetically in a tube for future production. I now take a piece of glass tube, and having heated it in the middle, draw it out, as in fig. 86, dividing it at $\mathrm{A}$, each section being about 2 inches long, the wide orifices being about $\frac{3}{10}$ ths of an inch in diamoter, and $\frac{1}{2}$ an inch long, the capillary part $\frac{1}{4}$ th of an inch in diameter and $1 \frac{1}{2}$ inch long; now, by putting one portion of the serapings into one of the tubes at $\mathrm{B}$, and holding it upwards over a very small flame, so that the volatile products may slowly ascend into the narrow portion of the tube, we prove the nature of the deposit: if mereury, it condenses in minute white shining globules; if lead or bismuth, it does not rise, but melts into a yellowish glass, which adheres to the copper; if tellurium, it would fall as a white amorphous powder; if antimony, it would not rise at that low temperature; but arsenious acid condenses as minute octahedral crystals, looking with the microscope like very transparent grains of sand. I make three such sublimates, ono of which is sealed up like the arsenic for future production. I now cut the capillary part of another of the tubes in pieces, and boil it in a few drops (say 10) of distilled water, and when cold drop three or four drops on a plate of white porcelain, and with a glass rod drop one drop of ammoniacal sulphate of eopper in it: and now to make the colours from this and the next test more conspicuous, I keep a chalk-stone, planed and cleaned, in readiness, and placing on it a bit of clean white filtering paper, I conduct the drops of copper-test upon the paper, which permits the excess of copper-solution to pass through into the chalk, but retains the smallest proportion of Scheele's green; the other few drops of the solution are treated the same way with the ammoniacal nitrate of silver. When I get the yellow precipitate of arsenito of silver, the papers, with these two spots, are now dried and sealed up in a tube as before, and that with the silver must bo kept in the dark, or it will become black. I have still one of the tubes with the arsenical sublimate remaining; through this I diroct a stream of hydrosulphuric acid gas for a fow seconds, which converts the sublimate into yellow orpiment. I have now all five tests : the metal, tho acid, arsenite of copper, arsenite of silver, and yellow sulphuret; and the $\frac{1}{100000}$ th of a grain of arsenic is sufficient in adroit hands to produce the whole; but all five must be present, or there is no positive proof, for many matters will cause a darkness of the copper in the absence of arsenic, - sulphurets even from putrefaction;-but there is no sublimate in the second operation, because the sulphur burns into sulphurous acid and passes off upwards. Corn, grasses, and earth slightly darken it from some unknown cause, but produce no sublimate; so, if the solution of suspected arsenious acid is tested with the copper-test while hot, it will produce a greenish deposit of oxide of copper, through the heat dissipating a little ammonia, or if the copper blade has not been deprived of grease by the diluted hydrochloric acid, the sublimed acid from the grease will proeipitate copper from that test; but as much of the sulphuric acid of commeree, and nearly all such hydrochloric acid and some commercial zine contains arsenie, nothing can excuse a toxicologist who attempts to try for arsenic if he has not previously experimented with all his reagents before he introduces the suspected matters. I should also mention that this metal is to be found in all parts of the body, but longest, and in greatest quantity, in the liver, where it is frequently found many days after it has diseppeared from the intestines. $-W$. Herapath.

Arsenious acid of commerce is frequently adulterated with chalk or plaster of Paris. These impurities are very easily detected, and their proportions estimated. Arsenious acid is entirely volatilised by heat, consequently it is sufficient to expose a weighed quantity of the substance to a temperature of about $400^{\circ} \mathrm{F}$. in a capsule or crucible. The whole of the arsenic will pass off in fumes, while the impurities will bo left behind as a fixed residuum, which can, upon cooling, be weighed.

The mines of Cornwall and Devonshire produced in 1871, 4,147 tons, 15 cwt. of arsonic, the value of which was $15,519 l$. 18s.; a considerable quantity is also produced at Swansea, from the roasting of arsenical copper ores.

AxSIN2. A name used by some modern chemists for arseniuretted hydrogen.

ARTESTAN WIII. This is a description of well or borehole in which water is obtained by means of a perforation bored vertically down through impermeable strata to an underlying stratum of a more or less permeable character, such stratum to be charged with water and to exist either in the shape of a basin-shaped depression, or to be so inclined as to reach, at some distance from the point at which the borehole may be made, the surface of the earth. The namo is derived from the 
fact that wells of this description were first known in North-Western Europe, in the provinee of Artois in France, where this method of obtaining water has been practisod from a very early period. Properly speaking, an Artesian well is one in which the water from the lower stratum rises about the surface of the superincumbent impermeable stratum; but by extension tho phrase has been applied of late years to any woll in which the waters of a lower stratum are enabled to rise sufficiently noar to the surface to allow of their being economically used. It will be seen heroafter that in many instances, borings, which woro originally strictly Artesian, havo at a later period lost the characteristic property of yielding waters flowing over the surface.

When the water falls upon the exposed surface of the outerop of the permeable stratum from which the supply for any Artesian well is derived, it passes under the edge of the overlapping impermeable stratum, and over such inferior retentive stratum as it may meet with. Then if it cannot find, or make to itself an outlet, it will follow the surface of the impermeable upholding stratum, in strict accordance with the laws which regulate the flow of water above ground. If, under these circumstances, an opening should be made through the overlying impermeable stratum, the water will rise to a height corresponding to the level at which it passed under such stratum, excepting in so far as it may be affected by friction, or by the existence of any natural overflows, created by interruptions of the containing basin, or by any. disturbance of the lower retentive strata of a nature to place the water-bearing stratum in contact with still lower strata having no communication with the surface.

M. Lofebvre ('Comptes Rendus de l'Acad. des Sciences, 1838,') describes several very ancient Artesian wells, which were discovered by M. Ayme in the Oasis of Thebes. These appear to have been sunk through 80 foet of clay and marls, and then through 300 feet of limestone. M. Ayme states that in the Libyan desert, where there are no rivers or springs, and upon which rain never falls, formerly a large population was supplied with water by Artesian wells, several of which have been cleared out and restored by this French engineer with perfect success, The 'Wells of Solomon, in the plains of Tyre, are supposed to be of this description.

The first Artesian well in London was put down in the year 1794. This description of well has been used for a long period in the East and in Italy,

The term Artesian may really be applied to all wells or borings which may be put down, having for their special purpose the obtaining of water; and the advisability of endeavouring to find water by means of such wells will depend upon several considerations, namely:-on the quantity and quality of water required; on tho physical position of the strata existing in the district where the water-supply is required, and of the surface of the ground where the water-bearing rocks are known to come to the surface, and on the outerop of such rocks being denuded or covered with any description of drift; on the mechanical formation of the rocks to be perforated, with special reforence to their compactness or porosity as the case may be, and on the lithological character and thickness of the water-bearing deposit; lastly, on the application of the processes by means of which the impermeable strata can be passed through, and the water-bearing strata reached.

The first three of these considerations will now be dealt with; the fourth point, which comprises the engineering of the work, will be brought into notice under the head of Bonuvg. Overflowing wells owe their origin, as a rule, to the infiltration of the waters falling upon the surface of the globe, which, percolating through the various pores and fissures of the strata, are passed into, and held by such strata of sand or gravel as will contain water in very large quantities. If the water be carried in this manner from some high point on the surface of the globe to some subterranean point where the surface of the ground is at a lower level than the point at which the source of the water is formed, the hydrostatic pressure is sufficient in case a connection with the surface is formed, either by faults or fissures in the strata, or by a borehole put down from the surface, to cause the water to rise and orerflow in a stream more or less constant.

In the case of all borings used for the purpose of obtaining water, the chief considerations are naturally.as follows, -

1st. To obtain a certain quantity of wator.

2nd. To have such water pure.

.3rd. To have the position of the borehole so fixed as to make a constant supply over a cortain period of time to be depended upon.

The site of a boring for water may of necessity have to be fixed upon within a certain limited space. The strata to be passed through, and the physical character of the surface of the ground adjacont to the sito of the boring, will probably partake of one of the following conditions:-

1st. The ground to be passed through may have a steep inclination extending to 
the bottom of the water-bearing beds. In this case the quantity of water which can be obtained by the borehole is necessarily limited. A large quantity of water may, howerer, be obtained under these conditions (which are illustrated by fig. 87) should the waterbearing strata be very porous, and have a considerable lateral extension.

2nd. On the other hand the inclination of the strata may be very gradual, as shewn in fig. 88, and in this case the area of sur. face receiving the rainfall is much greater. This is due not only to the surface of the water-bearing rocks having a larger superficial area, but also to the fact that near to the surface tho overlying rocks are generally found to be more open than they are at a considerable depth from the surface. Hence, whilst in fig. 87 the rainfall from $\mathrm{z}$ to $\mathrm{x}$ is the

85

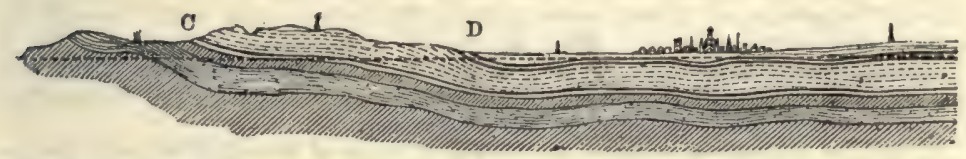

most that can be expected to reach the borehole, in fig. 88 it is probable that most of the rainfall on the area from $\mathbf{C}$ to $\mathbf{D}$ will percolate through to the water-bearing strata. This is the condition under which the largest quantity of water may bo expected to be obtained in the prosecution of Artesian wells.

3rd. Another condition is where a boring has to be made to water-bearing strata through other rocks, which, though compact in their nature, are not impermeable. This condition naturally affects the quantity of water obtainable, and in such a case it is important to obtain increased hydrostatic pressure.

4th. In some cases several qualities of water may be met with in one boring, some of which may be found chemically objectionable. When satisfactory water has been found, the impure water can only be kept back by the insertion of tubes in the borehole. With the third condition mentioned above, the application of tubes will also sometimes be found advantageous. They are always necessary where running sand or very loose strata are met with.

5th. Another case occurs when the water met with by a boring has so little hydrostatic pressure that it will not rise in the borehole to the surface. In this case the water has to be raised, when its lovel is within 30 feet of the surface, by some description of pump. When the level of the water is at a greater distance than this from the top, a plunger-pump has to be used.

In cases 1 and $2 \mathrm{a}$ bed of impermeable rock is assumed to intervene between the surface and the water-bearing strata. It need hardly be mentioned that the quantity of water found in any class of strata does not depend only on the surface of such strata exposed to the rainfall, but is much influenced by the degree of porosity of the strata, which is the test of its saturative capability.

An illustration of this is afforded by the results of the sinkings of many of the deepest coal-mines in Great Britain, where such sinkings hare passed through the Permian beds before reaching the coal-measures. Betweon the magnesian limestone and coal-measures is found in nearly every instance a bed of red sand, varying in thickness from a few inches to 12 feet. Whilst feoders of water of a fow hundred gallons per minute only have been encountered whilst passing through the limestone, the feeders met with on reaching the more porous sand-bed referred to, have frequently been enormous, in several instances amounting to over 4,000 gallons per minuto. In such cases the quantity of water can doubtless be traced to the principles indicated on fig. 88.

Under the conditions referred to in the fifth head, may be mentioned cases where water may be obtained by short holes, bored a fow yards into the ground, the object being to collect the surface-drainage, and the water being obtained by small pumps. 
Where grarol only is found, water eannot genorally be procured by short holos, but where gravel rests on an impervious clay, success is almost certain.

In cases whero there is apparontly a considerable hydrostatic prossure, there is a condition which will prevent any large quantity of water falling upon porous strata, passing to subterranoan depths. Should any river emanato from, or pass through, such strata, the river will probably carry away a large proportion of the water which otherwise would have saturated the permeable rocks.

It will be understood that the geological formations in which Artesian explorations can be made with most prospects of success are those which combine compact and impermoable strata with porous and open rocks. The particular systems of rocks which appear to present as a rule these conditions are those contained by and lying adjacent to the chalk series, embracing the London clay, the chalk, upper greensand, the gault, and the lower greensand. These rocks present the necessary conditions, and in the sites where they are chiefly found, the north of Franee and south-east of England, a large number of wells lave been put down, many of which are producing large volumes of water.

In older formations it is much more difficult to discovor rocks of sufficiont openness to carry large quantities of water, and further than this, as tho older rocks lie very irregularly, and frequently at a heary inclinntion, they aro not so suitable for the purpose of obtaining water by means of boring. Hence it is usual in districts where such rocks prevail to obtain the water-supply, whero river-water either is not usod or is not obtainable, from reservoirs situated at some high level where they can bo arranged to catch the surface-drainage.

The hot springs which burst out of the ground in districts where the so-called primary formations are found, came undoubtedly from a great depth bencath the surface, and derive thoir heat from an exalted subterranean tomperaturo; but it would not be practicable to bore to such extreme depths as would be necessary in theso rocks. A miniature representation of such springs is exhibited in the intermitting fountains of fresh water on the shoulder of Vesuvius.

It will be interesting to record the results of some of the chiof artesian borings which have been mado in this and other countries. The most famous example is that of the boring commenced in $\mathbf{1 8 3 3}$ at Grenelle, a suburb of the SW. of Paris where there was a great scarcity of water. Here the chalk was overlaid by gravels, marls, and clays, which were known to be capable of intercepting tho passago of water. Honce, as it was known that below tho chalk water-bearing sand would be mot with, M. Mulot, the engineer of the well, supported by the authority of MM. Arago and Walferdin, resolved to seek a supply of water by boring through the chalk into the sub-cretaceous strata. At Elbouf tho chalk had been travorsed in this manner, and the water had risen to a height of $\mathbf{8 2}$ feet above the level of the ground, or 109 foet above the level of the sea, and it was considered that as the surface of the ground at Grenello was about 104 feet above the level of the sea, and as the outcrop of the water-bearing strata was nearer to the proposed borehole than at Elbœuf, the water at Grenclle might be expected to flow over the surfaco. This reasoning was found to bo correct, and in February, 1841, aftor eight years' labour, tho rods suddenly desecnded sevural yards, having pierced into the vault of tho subterranean waters so long sought after by the indefatigable ongineer. A fow hours afterwards he was rewarded for all his anxious toils; for the water, rising to the surface, discharged itsclf at the rate of 881,884 gallons in evory twenty-four hours; the temperature of the water being nearly $82^{\circ} \mathrm{F}$. At first it brought up so great a quantity of sand that the tube was several times choked up by it, and even now it is not free from occasional though rare interruptions, but the force of the column of water has always proved sufficient to clear its way after a short interval. The water flows in a clear, continuous struam, and is carried by pipes to a reservoir near the Pantheon, whence it is distributod over the adjacent parts of the city, as woll as along the line of the Boulevards from the abattoir to the Observatory. By means of small pipes, the Ecole Militaire, the Invalides, and two or threo other public establishments, are also supplied with this wator. The surface of the ground at the well is 102 feet above the level of the sea, and the wator is capable of being carried above this to a height of 120 feet. The exposed surface of the water-bearing beds which supply the well of Grenelle is about 117 square miles; the subterranean area in connection with these lines of outcrop may possibly be about 20,000 square miles, and the average thickness of the sand of the grès verts, sorving in their underground range as a reservoir for the water, does not probably exceed thirty or forty feet.-Prestwich on the Water-bearing Strata of London.

After the completion of the Grenelle well others were quickly undertaken. Amongst the most important of these were the borings undertaken in the Rhenish provinees for bringing to the surface the waters of the brine-springs of that district, some of 
which even exceoded the depth of 2,400 feet from the surface. M. Degoussée mentions in his 'Guide de Sondeur' (1847), that he himself had executed no less than sixteen deep borings in the Département de l'Indre et Loire, of which ten are in the town of Tours and six in its neighbourhood, presenting an average depth of about 500 feot. Two of these borings were, however, unsuccessful, and it appears that the conditions under which they occurred with respect to the great watercourses of the district, led to the supposition that the underground course of the waters was interrupted by means of a fault or upheaval.

At Calais the results obtained by the great artesian well there sunk were even more striking than those obtained near Tours; for after having in this place passed through the drift above the chalk, the chalk itself and the whole of the sub-cretaceous strata, the boring was continued in the transition rocks until it had attained a total dopth from the surface of about 1,150 feet. It will be necessary hereafter to refer to this well, and to the abnormal state of the geological formations under this district.

An important Artesian well was also put down at Chichester, being carried through the great Hampshire tertiary basin to the upper greensand, where it was stopped at a depth of 1,054 feet from surface. Very little water was obtained.

At Southampton the upper and lower chalk and chalk-marl were passed through, but at a depth of 1,317 feet no valuable supply of water was obtained. A great number of Atrtesian wells had in the meantime been sunk in the tertiary basins of both London and Hampshire, and the drain thus established upon the subterranean water-courses of those formations was so great that the waters which originally had flowed over the surface of the ground, were no longer able to reach that height; and it became evident that the demand upon those water-bearing strata was rapidly exceeding the supply. Under these circumstances the Hampstead and Highgate Waterworks Company resolved to renew under London the attempt which had been abandoned at Southampton; and their advisers argued that, inasmuch as the outcrop of the sub-cretaceous formations was continuous around the margin of the cretaceous basin surrounding and underlying the London tertiaries, excepting on the eastern border, those sub-cretaceous strata would be found under London just as they had been actually found at Paris.

This reasoning proved to be correct so far as the chalk-marl, the upper greensand, and the gault were concerned; but when those formations had beon traversed (to a depth of $1,113 \frac{1}{2}$ feet), the boring tools, instead of entering upon the lower greensand, which theoretically had been expected, entered upon and traversed, to a total depth of 1,302 feet, a series of marls, clays, and sandstones, which appear in all probability to belong to the new red-sandstone series: all the intermodiate strata being absent.

A boring at Harwich also proved the existence of transition rocks of an early period at a depth of 1,200 feet from the surface; various rocks from the tertiaries to the upper greensand and gault having been passed through.

From the above data the interesting fact will be observed that no borehole in the London basin has as yet succeeded in proving and obtaining water from the lower greensand rocks; the Southampton, Calais, Highgate, and Harwich wells having all proved failures in this respect. It is a question to be proved by experiment whether to the north-west of London the tertiary rocks would not be less likely to give place to rocks of older formations, as they appear to do in the boreholes referred to. It will be understood from the particulars given of the few boreholes, how much uncertainty attends the art of boring; at least, as regards the obtaining of water by this means.

Some particulars may now be given of a number of the chief Artesian boreholes put down in France and England. The following Table shows the depth and cost of several of the French Artesian wells :-

Grenelle,
Calais
Douchery
St. Fargeau
Lille
Crosne
Brou
Ardres
Claye
Chaville

$\begin{array}{cl}\text { Dept. } & \text { Seine } \\ " & \text { Pas de Calais } \\ " & \text { Ardennes : } \\ \text { " } & \text { Yonne } \\ " & \text { Nord : } \\ " & \text { Seine et Oise } \\ " & \text { Marne } \\ " & \text { Nord } \\ \text { " Seine et Marne } \\ \text { Oise. }\end{array}$

1,798 feet
1,138,
$1,215 "$
$666 "$,
$592 "$
$333 "$
$246 "$,
$155 "$
108,
$65 "$

The deep wells of London are all in the chalk. The dopths of some of the most important are given in the following Table, which has been compiled from data given by Mr. W. Whitaker, 'Memoirs of the Geological Survey,' vol. iv. (1872):-

Vor. I. 
Sections of some of the Deop Wells in Isondon and the adjoining Conentry.

\begin{tabular}{|c|c|c|c|c|c|c|c|}
\hline MTDDLEAEX & & 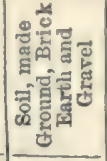 & 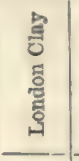 & 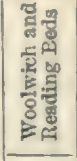 & 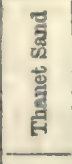 & 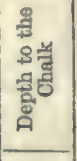 & 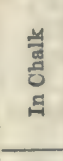 \\
\hline Apothecaries' Hall, Blackfriars. & . & ft. & ft. & $\begin{array}{ll}\text { ftho } \\
48\end{array}$ & ft. & ft. & ft. \\
\hline Bank of England & . & 26 & 111 & $58 \frac{1}{2}$ & 39 & $234 \frac{1}{2}$ & 100 \\
\hline Blackwall, Trinity Wharf. & . & 63 & 68 & 34 & 72 & 237 & 10 \\
\hline Bow . . & . & 19 & 48 & 56 & 51 & 174 & 150 \\
\hline Broad Street, Golden Square & - & $10 \frac{1}{2}$ & 98 & 58 & 25 & $191 \frac{1}{2}$ & 25 \\
\hline Camden Station $\cdot$. & . & $18^{\circ}$ & 144 & 64 & 8 & 234 & 166 \\
\hline Castleboar Hill, near Ealing . & . & $\ldots$ & 300 & 60 & $\ldots$ & 360 & ... \\
\hline Chiswick, Griffin Brewery & - & 40 & 140 & 90 & 29 & 299 & 46 \\
\hline Colney Hatch Lunatic Asylum . & . & $\ldots$ & 137 & 27 & 25 & 189 & 141 \\
\hline Fulham & . & 25 & 135 & 90 & $\cdots$ & 250 & 66 \\
\hline Hackney Road. & . & 14 & $48 \frac{1}{2}$ & 48 & 42 & $152 \frac{1}{2}$ & 259 \\
\hline Haggerstone . & . & 16 & $56 \frac{1}{2}$ & $\underbrace{44 \frac{1}{2}}$ & $47 \frac{1}{2}$ & $164 \frac{\pi}{2}$ & 256 \\
\hline Hampstead, Lower Heath & . & $\ldots$ & 289 & \multicolumn{2}{|c|}{89} & 378 & .72 \\
\hline Hampstead Road . . & . & 23 & 59 & $39 \frac{1}{2}$ & $24 \frac{1}{2}$ & 146 & 37 \\
\hline Hanwell Lunatic Asylum & . & 21 & 194 & 75 & $\ldots$ & 290 & 30 \\
\hline Harrow Waterworks . & . & $\ldots$ & 111 & 48 & $\ldots$ & 159 & 254 \\
\hline Haverstock Hill . & - & ... & 223 & 61 & 28 & 312 & 78 \\
\hline Hayes $\cdot \because$ & - & 14 & 134 & $88 \frac{1}{2}$ & $\ldots$ & 281 & 88 \\
\hline Highbury : • & - & $\ldots$ & 106 & $57 \frac{1}{2}$ & $16 \frac{1}{2}$ & 180 & 134 \\
\hline Holloway City Prison & . & $\because$ & 135 & $69^{\circ}$ & 13 & 217 & 102 \\
\hline Hoxton . . & - & 18 & 69 & 34 & 30 & 151 & $\ldots$ \\
\hline Hyde, The, Edgeware Road & - & $\ldots$ & 66 & $34 \frac{1}{2}$ & & $100 \frac{1}{2}$ & 37 \\
\hline Hyde Park Corner . . . & . & 5 & 229 & $64^{\circ}$ & 21 & $319^{\circ}$ & 18 \\
\hline Isle of Dogs . & . & 39 & $\ldots$ & 43 & $42 \frac{1}{2}$ & $124 \frac{1}{2}$ & 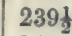 \\
\hline Islington Green & - & 12 & 48 & $100 ?$ & $16^{\circ}$ & $176^{2}$ & $144^{\circ}$ \\
\hline Kensington, Horticultural Socicty & - & 40 & 198 & 54 & 25 & 317 & 84 \\
\hline Konsington Gardons, N. . . & . & 2 & 172 & 56 & 33 & 263 & 58 \\
\hline Kentish Town Waterworks & . & ... & 236 & $61 \frac{1}{2}$ & 27 & $324 \frac{1}{2}$ & $645^{1}$ \\
\hline Leicester Square . . & 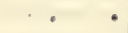 & $5 \frac{1}{2}$ & $148 \frac{1}{2}$ & 60 & 28 & 244 & 101 \\
\hline Limehouse : & 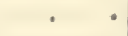 & $29^{\circ}$ & 19 & 47 & $44 \frac{1}{2}$ & $139 \frac{1}{2}$ & \\
\hline Long Acre . & - & 20 & $120 \frac{1}{2}$ & $58 \frac{1}{2}$ & 24 & $222^{2}$ & $258^{2}$ \\
\hline Mile End & $\therefore$ & 13 & 86 & $63-$ & $40 \frac{1}{2}$ & $202 \frac{1}{2}$ & $2^{2}$ \\
\hline Mile End Road (City of London L & Union) & 35 & $60 \frac{1}{2}$ & 41 & $38 \frac{1}{2}$ & 175 & 10 \\
\hline Pentonville Model Prison & . & 17 & 113 & $54 \frac{3}{2}$ & 35 & 2191 & 151 \\
\hline Pimlico, Simpson's Factory, Grosve & renor Road & 26 & 112 & $67 \frac{1}{2}$ & $25 \frac{1}{2}$ & 231 & 100 \\
\hline Pinner $: \quad: \quad$ : & . & $3 \frac{1}{2}$ & 29 & $27 \frac{1}{2}$ & 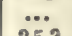 & 60 & 80 \\
\hline Ponder's End. & - & 13 & 15 & $49 \frac{1}{2}$ & 35 ? & $112 \frac{1}{8}$ & $290 \frac{1}{2}$ \\
\hline Ratcliffe. . & . & 14 & 56 & 26 & 54 & $150^{\circ}$ & $102^{2}$ \\
\hline Shoreditch, Truman's Brewery . & 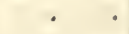 & $22 \frac{1}{2}$ & $80 \frac{1}{2}$ & 53 & 43 & 199 & 331 \\
\hline Shoreditch Workhouso . & . & $18^{\circ}$ & $60^{\circ}$ & 40 & 39 & 157 & 100 \\
\hline Staines . . . & . & 33 & 267 & 79 & $\cdots$ & 369 & 154 \\
\hline Sudbury . . & 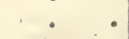 & 2 & 68 & 60 & $\ldots$ & 120 & 80 \\
\hline Tottenham & . & 20 & 52 & 61 & 15 & 148 & $250^{2}$ \\
\hline Tottenliam Court Road (Melux's) & ) & 22 & 64 & $51 \frac{1}{2}$ & 21 & 158 & $207^{\mathrm{s}}$ \\
\hline Towor Hill (Royal Mint) . . & ${ }^{\circ}$ & 24 & 94 & $67^{\circ}$ & $20 \frac{1}{2}$ & $195 \frac{1}{2}$ & 202 \\
\hline Trafalgar Square . . & $\therefore$ & 23 & 142 & 41 & 42 & $248^{-}$ & 147 \\
\hline Twyford, near Eizling & . & $\cdots$ & 211 & 41 & $\cdots$ & $\ddot{0}$ & $\ddot{0}$ \\
\hline Uxbridge Union & . & 12 & 94 & 75 & $\ldots$ & 175 & 38 \\
\hline Westbourne Estate Waterworks & $\therefore$ & $\cdots$ & 217. & 62 & 18 & 297 & 15 \\
\hline West Drayton . . . . & - & 32 & 88 & 66 & $\ldots$ & 186 & 100 \\
\hline Westminster, Elliot's Brewery . & . & 32 & 140 & $67 \frac{1}{2}$ & $31 \frac{1}{2}$ & 271 & 127 \\
\hline Thorne's Brewery & . & $27 \frac{x}{2}$ & 100 & $66 \frac{1}{2}$ & 36 & 230 & 70 \\
\hline W inchmore Hill & - & $\cdots$ & 186 & 44 & $\cdots$ & 230 & $m$ \\
\hline
\end{tabular}

This boring was carried down 332 feet below the bottom of the chalk. 2 Or more.

More since. 


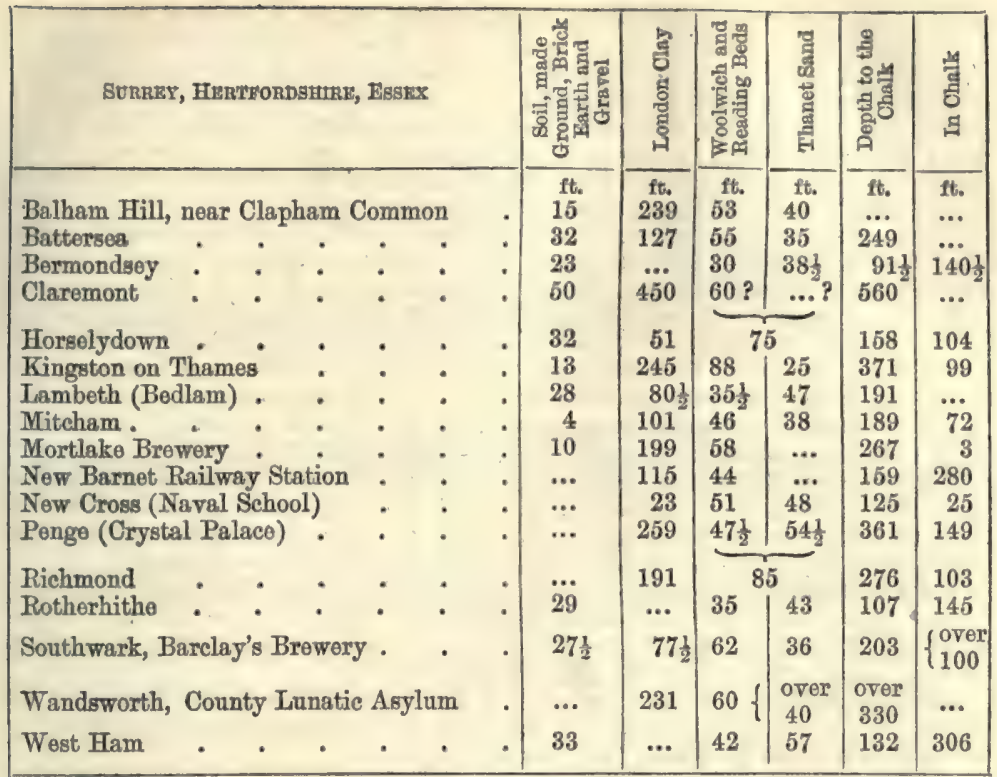

The Artesian wells in Essex which overflow, are of the following depths, according to Dr. Mitchell :-Foulness Island, 450 feet; Mersey, and adjoining islands, 300 feet; Wallis Island, 400 feet; Little Wigborough, 250 feet; Woodham, 350 feet; North Ockenden, 80 ; Fobbing, 100 feet; Bulpham Fen, 70-80.

The difficulty of making any calculation, even in a well-known district, as to the quantity of water which can be expected to drain to any one borehole, is considerable, sinee, though it may be possible to judge of the breadth of surface over which the rainfall may be expected to sink into the water-bearing strata, it is impossible to tell with any degree of exactness the lateral extension of the drainage, except the conditions happen to be those in which the borchole is put down in the centre, or some part of a basin. Naturally the more porous and saturable the water-bearing strata are, the greater the proportion of the drainage which may be expected to be conveyed to any one point.

Some years ago Mr. Prestwich computed the quantity of rain falling over the district surrounding London with a view to estimate the supply of water which a boring through the chalk to the lower greensand formation would furnish. The following Fable exhibits the results of his investigation:-

\begin{tabular}{|c|c|c|c|c|}
\hline & & $\begin{array}{l}\text { Probable } \\
\text { extent of } \\
\text { effective } \\
\text { Area }\end{array}$ & $\begin{array}{l}\text { Quantity of Rainwater } \\
\text { received }\end{array}$ & $\begin{array}{l}\text { Probablo Quantity } \\
\text { absorbed }\end{array}$ \\
\hline $\begin{array}{l}\text { Lower Tertiaries } \\
\text { Upper Greensand. } \\
\text { Lower Greensand }\end{array}$ & $\because \quad$ & $\begin{array}{c}\text { sq. miles } \\
24 \\
70 \\
230\end{array}$ & $\begin{array}{l}\text { Inches Gallons in } \\
\text { annually } 24 \text { hours } \\
25=23,749,656 \\
28=77,660,660 \\
26 \frac{1}{2}=241,500,920\end{array}$ & 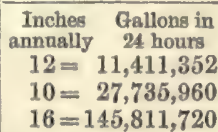 \\
\hline
\end{tabular}

'These calculations, although offered as only very general approximations, give results sufficiently marked and decided, that even admitting the necessity of not inconsiderable corrections, I think they establish strong prima facie evidence in favour of tho upper and lower greensands beneath London containing unusually largo quantities of wator, which may be rendered available for the supply of the metropolis by means of Artesian wolls. What thoir yiold might be could only be determined exactly by actual experiment; but, judging from analogy, if the lower tertiary sands, with dimonsions comparatively so limitod, can novertholess furnish not loss than $3,000,000$ to $4,000,000$ gallons daily (and if, as is probable, they supply much of the 
water found in the upper beds of the chalk beneath London, their yiold may amount to $8,000,000$ or $10,000,000$ ), then, I submit, that there is a reasonable probability, after allowing for tho present over-drainage, of the tertiaries of the upper greensand, with an effective area and a thickness 3 times greater than those of the lower tertiaries, yielding daily, and without diminution, from $6,000,000$ to $10,000,000$, and of the lower groensands, which excoeds by 10 times the lower tertiaries in both respects, of thoir yielding daily and without diminution from $30,000,000$ to $40,000,000$ gallons of water in the twonty-four hours, taken at about surface level.

'Since the beds of the lower greonsand are 200 foet thick, and they occupy an area above and below ground of 4,600 square miles, and since a mass of one mile square and one foot thick will hold more than $60,000,000$ of gallons of water, it is cvidont that a year's consumption of water by the population of London would not occasion a fall of one foot in the water-level over the entire area ; that is, supposing no rain had fallen during the year. Such wells, too, would have the advantage of adding to the adornmont of the metropolis, as if the water of the lowor greensand was liberated by merns of Artesian wolls, fountains would be at once formed, projecting their water from 100 to 150 feet above the level of 'Trinity high-water mark.' '-E. B.

AmTEsian wrIIs, Negative. Borings into the earth which are intended to carry off the waters from the surface. They have been proposed for the purpose of draining large tracts of swampy country. Especial information on this subject will bo found in the 'Society of Arts' Journal' for 1856, and Ansted's 'Geology.'

ARTrCmores. (Cynara Scolymus). $\Lambda$ thistle-like plant, a native of the South of Europe, cultivated for the sake of the fleshy sweet receptacle of its flowers. JERUSATEX ARTICHOKES are the tubers of the Helianthus Tuberosus, and derive their name by $a$ corruption, from the Italian girasole, sunflower.

ARTrCUIтTz. A name proposed by Dr. Wetherill for flexible sandstone, in allusion to the articulated structure of the stone, seen on microscopic examination.

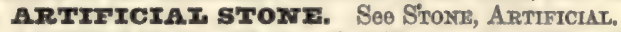

ARTIIIIRY. The earliest European artillery of large size consisted of 'serpentines' and 'bombards,' both being formed of longitudinal bars of wrought-iron, arranged like the staves of a cask, and hooped all over, or nearly so, with wroughtiron rings, shrunk-on hot upon the bars. The serpentine was of small calibre, but of enormous length. A gun of this character, taken by the Swiss from Charles lo Téméraire, at the battle of Granson, in 1476, is described and figured in the Emperor Napoleon's work, 'Passé et l'Avenir d'Artillerie.' This example is preserved in the collection of the Arsenal of Neuville, Canton of Berne; it is only about two inches calibre, but about ten feet in length of chase, formed with wrought-iron, with rings shrunk-on at some inches apart. It is embedded to its horizontal diameter, and for its whole length, in a timber bed.

The bombard was usually a much shorter piece, often of immonse calibre. The great gun of Ghent, known as Dulle Griette, or the Raging Meg, is of this character. Voisin thus describes it:- This enormous cannon, or ancient bombard, is one of the most curious pieces of artillery known, both in dimensions and construction, which is a chef-d'ceuvre of the art of forging. It is 18 feet in length, by 10 feet 6 inches in circumference; the mouth is $2 \frac{3}{4}$ feet in circumference ; it is forged from bars of iron, and weighs $33,606 \mathrm{lbs}$., and throws a stone ball of $600 \mathrm{lbs}$. weight. Its construction appears to date from the early years of the invention of artillery; in all probability it was forged while Philip Van Artevelde, Riswaert of Flanders, was besieging Oudenarde, in 1382. It is certain that the people of Ghent, at war with their Duke, Philippe, used it in 1411, and at the attack of Oudenarde, in 1452.'

In the arsenal of St. Petersburg is a bombard which is 21 feet long; but it only weighs $17,435 \mathrm{lbs}$., and its calibre is only $68 \mathrm{lbs}$.

The Mons Meg of Scotland, which now quietly reposes on the King's Bastion, Edinburgh, is formed of longitudinal stave bars, in one ply only, and of superimposed rings, driven and shrunk-on upon the taper. This will be understood from the accompanying figures $(89,90)$. This gun was made by one $\mathbf{M}$ 'Kin, to whom the people of Kirkeudbright contributed the bars of iron. Mons Meg was used at the siege of Dumbarton, in 1489; at Norham, in 1497; it was used to fire a salute in 1548; and in 1682, when firing a salute in honour of the Duke of York, the iron rings, which are now partly wanting near the breech, were blown away without much disturbing the longitudinal bars. The gun actually discharged balls of Galloway granite against

${ }^{2}$ Consult Prestwich, 'Water-bearing Strata of the Country round London;' 'Mylne's Sections of the London Strate;' M. Garnier's 'Traité sur les Puits Artésiens ;' Swindell, 'Rudimentary Treatise on Well Digging and Boring;' Buckland's 'Bridgewater Treatise on Geology and Mineralogy ;' De la Beche's 'Geological Observer;' Héricart de Thury's 'Considérations sur la Cause du Jallisse. ment des Eaux des Puits Forcés ;' Dégousse and Laurent, 'Guide du Soudeur;' Whitaker, 'Geological Survey Memoirs,' vol. I $v_{0}, 1872$, etc. etc. 
Threave Castle. The woight of a granite ball of $19 \mathrm{z}$ inches diameter is about $330 \mathrm{lbs}$ 。

Colonel Symes, in his 'Embassy to Ava, in 1795, informs us that he found that cannon formed of prismatic bass of wrought hoop-iron hooped together were known

89

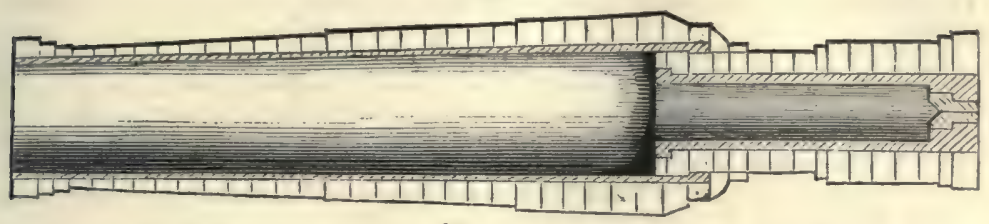

90

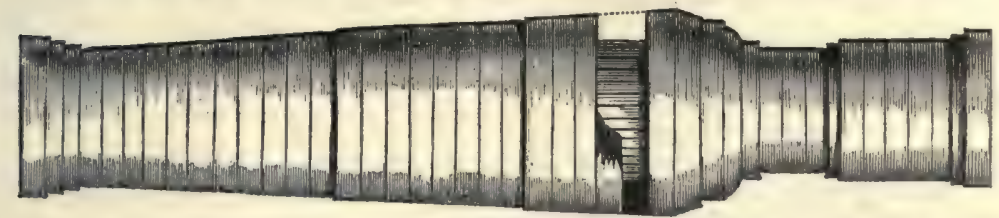

in India from a remote antiquity. In Meyor's 'Historical Manual' will be found a curious history of the progress of wrought-iron cannon, from 1494-when Charles VIII. suppressed wrought-iron bombards, and had no other artillery than that of bronzo--to the present day. In 1856, Daniel Treadwell published a memoir 'on the Practicability of construeting Cannon of Great Calibre, capable of enduring long-continued Use under full Charges.' In this he proposes a very large wrought-iron gun, which should be capable of projecting a shot or shell of a ton weight through the space of six miles. He says, in a note to this paper, 'Betwoen the years 1841 and 1845 , I made upwards of twenty cannon of this material (wrought-iron). They were all made up of rings, or short hollow cylinders, welded together endwise. Each ring was mado of bars wound upon an arbour spirally, like winding a ribbon upon a block, and, being welded and shaped in dies, were joined endwise when in the furnace and at a welding heat, and afterwards pressed together in a mould by a hydrostatic press of 1,000 tons force.' Finding in the early stage of the manufacture that the softness of the wrought-iron was a serious defect, he formed those made afterwards with a lining of steel, the wrought-iron bars being wound upon a previously formed steel ring.

Mr. Nasmyth undertook, in 1854, an enormous wrought-iron gun, of 13 inches calibre; but there was some failure in the forging.

In 1856, Messrs. Horsfall, of Liverpool, completed, and proved with a solid shot of $300 \mathrm{lbs}$, and $45 \mathrm{lbs}$. of powder, a wrought-iron gun, 13 inches calibre, and $13 \frac{1}{2}$ feet length of chase, perhaps the largest and most remarkable forging ever made. Two wrought-iron mortars, of 36 inches calibre, built up of separate pieces, were constructed about the same time for the Government, from the designs of Mr. Mallet. A detailed account of this monster mortar is given at page 235 .

Cast-Iron Guns.-The date of the introduction of cast-iron guns is very uncertain. Blast furnaces for smelting replaced the old Catalan methods about the commencement of the fiftoenth century, were known in the Hartz, in Westphalia, in Flanders, and seem to have come to us thence, and were not uncommon about the middle of the century. There is in the repository at Woolwich an 18-inch Pierrière, captured at Corfu, with the date 1684 upon it, an early example of cast-iron.

In the sixteenth and seventeenth centuries, the average sizes of guns in Fingland were as follow :-

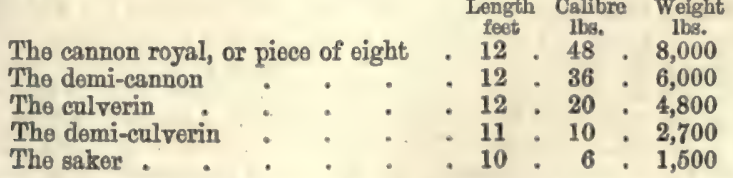

The smaller sizes were called minion, falcon, falconet, rabinet, and base, the last of which only carried a 5-ounce ball of lead.

Cannon of Bronze. - The earliest bronze guns appear to have been cast in Europe about 1370. Between that and 1400 , bombards were cast (after the more ancient models of iron) in bronze with separate and with attached chambers (canons à boite), 
the ancestors of all modern breoch-loading guns; and culverins, which replaced the iron serpentines, and wore of onormous length, 35 to 60 calibres, and great strongth towards tho breoch, but of small calibre. Many examples romain of a later date: one at Dover Castle, another in the Dial Square, Woolwich Arsenal, and the celebrated one of Nancy (1598), above 21 feet in length, carrying about an 18-pound iron ball. In England the earliest bronze guns are said to lavo been cast by one John Owen, in 1535 .

Fow examples are met with of guns formod of metal in strictly atomic proportions; but alloys are found therein presenting every formula, from $7 \mathrm{Cu}+\mathrm{Sn}$ up to $83 \mathrm{Cu}+4 \mathrm{Sn}$. The proportions most approved of in the arsenals of Europe appear to vibrate between 100 by weight of coppor to 9 of tin, up to 100 of copper and 12 of tin. In France, 100 copper +11 tin by weight is the proportion fixed by law, and invariably aimed at. In the Unitod States, 100 coppor +12.5 tin is adopted for certain species of guns.

The proportions of tin and copper used in making bronze guns in tho United States :-

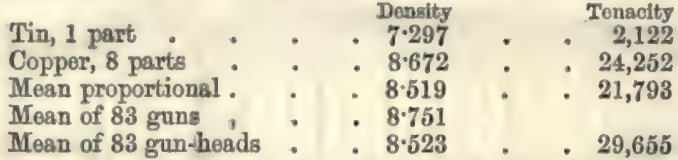

Bronze guns are liable to drop at the muzzle; this is due to the unequal temperature of the inside and of the outside of the gun.

Brass ordnance are made of what is called GUN-METAI, composed of about 10 parts of copper and 1 of tin.

One of the first inquiries of importance in connection with the construction of pieces of artillery is that of the liability to fracture in the metal. Upon this point the researches of Mr. Mallet furnish much important matter. He tells us, as the result of his investigation, that it is a law of the molecular aggregation of crystalline solids, that when their particles consolidate under the influence of heat in motion, their crystals arrange and group themselves with their principal axes in lines perpendicular to the cooling or heating surfaces of the solid: that is, in the lines of the direction of the heat-wave in motion, which is the direction of least pressure within the mass. And this is true, whether in the case of heat passing from a previously fused solid in the act of cooling and crystallising in consolidation, or of a solid not having a crystallino structure, but eapable of assuming one upon its tomperature being sufficiently raised, by heat applied to its external surfaces, and so passing into it.

Cast-iron is one of those erystallising bodies which, in consolidating, obeys, more or less perfectly according to conditions, the above law. In castings of iron the planes of crystallisation group themselves perpendicularly to the surfaces of external contour. Mr. Mallet, after examining the experiments of Mr. Fairbairn-who states ('Trans. Brit. Ass.' 1853) that the grain of the metal and the physical qualitios of the casting improve by some function of the number of meltings; and he fixes on the thirteenth melting as that of greatest strength-shows that the size of crystals, or coarseness of grain in castings of iron, depends, for any given 'make' of iron and given mass of casting, upon the high temperature of the fluid iron above that just necessary to its fusion, which influences the time that the molten mass takes to cool down and assume again the solid state.

The very lowest temperature at which iron remains liquid enough fully to fill every cavity of the mould without risk of defect, is that at which a large casting, such as a heary gun, ought to be ' poured.' Since the cooling of any mass depends upon the thickness of the casting, it is important that suddon changes of form or of dimensions in the parts of cast-iron guns should be avoided. In the sea and land service 13-inch mortars, where, at the chamber, the thickness of metal suddenly approaches twice that of the chase, there is evidently a malconstruction.

The following statements of experiments made to determine the effect produced on the quality of the iron in guns, by slow or rapid cooling of the casting, are from the report of Major W. Wade, of the South Boston Foundry, to Colonel George Bomford, of the Ordnance Department of the United States. Three six-pounder cannon were cast at the same time from the same melting of iron. The moulds were similar and prepared in the usual manner. That in which No. 1 was cast was heated before casting, and kept heatod aftorwards by a fire which surrounded it, so that the flask and mould were nearly red hot at the time of easting; and it was kept up for three days. Nos. 2 and 3 were cast and cooled in the usual way.

At the end of the fourth day the gun No. 1 and flask were withdrawn from the heating eylinder while all parts were yet hot. Nos. 1 and 2 were bored for 6pounders in the usual way; No. 3 for a 12-pounder howitzer, with a 6-pounder 
chamber. The firing of the guns was in every respect the same. Nos, 1 and 2 were fired the samo number of times with similar charges. No. 1 burst at the 27 th fire, and No. 2 at the 25th. It appears from these results, that no matorial effect is produced on the quality of the iron by these different modes of cooling the castings.

A very extensive series of experiments was made, by the order of the Unitod States' Government, on the strength of guns cast solid or hollow. In these it was confirmed that the guns cast hollow endured a much more severe strain than those cast solid. Considerable differences were also observed, whether the casting was cooled from within or without; and lieutenant Rodman's method of cooling from the interior is regarded as tending to provent injurious strains in cooling.

Major Wado informs us that time and repose have a surprising effect in removing strains caused by the unequal coolings of iron castings.

Great advances have been made in improving the quality of iron guns. Guns cast prior to 1841 had a donsity of $7 \cdot 148$, with a tonacity of 23,638 . Guns cast in 1851 had a density of $7 \cdot 289$, with a tenacity of 37,774 .

The following Table gives the results of all the trials made for the United States Government, showing the various qualities of different metals :-

\begin{tabular}{|c|c|c|c|c|c|c|c|c|}
\hline \multirow{2}{*}{ Metals } & & \multirow{2}{*}{ Density } & \multirow{2}{*}{ Tenacity } & \multirow{2}{*}{$\begin{array}{l}\text { Transverse } \\
\text { strength }\end{array}$} & \multicolumn{2}{|c|}{ Torsion } & \multirow{2}{*}{$\begin{array}{l}\text { Com. } \\
\text { pressive } \\
\text { strength }\end{array}$} & \multirow{2}{*}{$\begin{array}{l}\text { Hard- } \\
\text { nees }\end{array}$} \\
\hline & & & & & $\begin{array}{l}\text { At Halt } \\
\text { Degree }\end{array}$ & Ultimate & & \\
\hline \multicolumn{9}{|l|}{ Cast-iron:- } \\
\hline Least & $*$ & $6 \cdot 900$ & 9,000 & 5,000 & 3,861 & 5,605 & 84,592 & 4.57 \\
\hline Greatest . & - & $7 \cdot 400$ & 45,970 & 11,500 & 7,812 & 10,467 & 174,120 & $33 \cdot 51$ \\
\hline \multicolumn{9}{|c|}{ Wrought-iron:- } \\
\hline Least . & • & $7 \cdot 704$ & 38,027 & 6,500 & 3,197 & $\ldots$ & 40,000 & $10 \cdot 45$ \\
\hline $\begin{array}{l}\text { Greatest . } \\
\text { Bronze:- }\end{array}$ & - & $7 \cdot 858$ & 74,592 & ... & 4,298 & 7,700 & 127,720 & $12 \cdot 14$ \\
\hline Least .. & - & $7 \cdot 978$ & 17,698 & $\cdots$ & 2,021 & 5,511 & ... & $4 \cdot 57$ \\
\hline Greatest . & - & $8 \cdot 953$ & 56,786 & $\cdots$ & $\cdots$ & $\cdots$ & $\ldots$ & $5 \cdot 94$ \\
\hline $\begin{array}{l}\text { Cast-steal:- } \\
\text { Iorat }\end{array}$ & • & $7 \cdot 729$ & & & & & 198.944 & \\
\hline Greatest . & . & $7 \cdot 862$ & 128,000 & 23,000 & $\ldots$ & $\cdots$ & 391,985 & \\
\hline
\end{tabular}

The following analyses of the metal of iron guns of three qualities are important:-

Influence of Single Ingredients.

\begin{tabular}{|c|c|c|c|c|c|c|c|c|c|}
\hline \multirow{2}{*}{ Classes } & \multicolumn{2}{|c|}{ Mechanical Testa } & \multicolumn{7}{|c|}{ Chemical Constitnents } \\
\hline & $\begin{array}{l}\text { Specific } \\
\text { Gravity }\end{array}$ & $\begin{array}{l}\text { Tensile } \\
\text { Strength }\end{array}$ & $\begin{array}{c}\text { Combined } \\
\text { Carbon }\end{array}$ & Graphite & Silicium & Slag & $\begin{array}{l}\text { Phos- } \\
\text { phorus }\end{array}$ & Sulphur & $\begin{array}{l}\text { Earthy } \\
\text { Mfetals }\end{array}$ \\
\hline 1 & $7 \cdot 204$ & 28,865 & $\cdot 0977$ & $\cdot 0507$ & $\cdot 0417$ & .0215 & $\cdot 0239$ & $\cdot 0017$ & $\cdot 0117$ \\
\hline 2 & $7 \cdot 140$ & 24,767 & $\cdot 0819$ & .0576 & .0538 & $\cdot 0200$ & $\cdot 0300$ & .0021 & $.009 t$ \\
\hline 3 & $7 \cdot 088$ & 20,176 & .0726 & .0560 & .0531 & .0219 & .0321 & $\cdot 0021$ & .0144 \\
\hline
\end{tabular}

Influence of Two or more Ingredients.

\begin{tabular}{|c|c|c|c|c|c|c|c|c|}
\hline \multirow[b]{2}{*}{ Classes } & \multicolumn{2}{|c|}{ Mechanical Tests } & \multicolumn{6}{|c|}{ Chemical Constituents } \\
\hline & $\begin{array}{l}\text { Speclfic } \\
\text { Gravity }\end{array}$ & $\begin{array}{l}\text { Tensile } \\
\text { Strength }\end{array}$ & $\begin{array}{l}\text { Sullicium } \\
\text { and } \\
\text { Carbon }\end{array}$ & $\begin{array}{l}\text { Silicium } \\
\text { and Slag }\end{array}$ & $\begin{array}{l}\text { Graphite } \\
\text { and Slag }\end{array}$ & $\begin{array}{l}\text { Graphite, } \\
\text { Silicium, } \\
\text { and Slag }\end{array}$ & $\begin{array}{l}\text { Graphite, } \\
\text { Slag, Silicium, } \\
\text { and Phos- } \\
\text { phorus }\end{array}$ & $\begin{array}{c}\text { Total } \\
\text { Carbon }\end{array}$ \\
\hline 1 & $7 \cdot 204$ & 28,865 & •1394 & $\cdot 0632$ & .0722 & $\cdot 1139$ & $\cdot 1378$ & $\cdot 1484$ \\
\hline 2 & $7 \cdot 140$ & 24,767 & $\cdot 1357$ & $\cdot 0738$ & .0776 & $\cdot 1314$ & $\cdot 1614$ & $\cdot 1395$ \\
\hline 3 & $7 \cdot 088$ & 20,176 & $\cdot 1257$ & .0750 & 80 & $\cdot 1311$ & $\cdot 1632$ & $\cdot 1286$ \\
\hline
\end{tabular}


An inspection of the first of the foregoing Tables, representing the average amount of each foreign ingredient in gun-motal deduced from all the analyses, shows a considerable difference in the proportions of those ingredients in esch of the three classes into which guns are divided. It will be observed, that whilo the proportion of combined carbon diminishes from the 1st to the 3rd class, that of silicium sinilariy increases, so that their united amounts are nearly the same. In other words, it appears that silicium can replace the carbon to a cortain extent; but that the quality of the metal is injured where the amount of the silicium approaches that of the aar bon. Karsten made a similar observation in determining the limits betwoen cast-iron and steel, but did not notice the influence of that substitution.

But the differences become more striking by combining the ingredients variousily together, as in the second of those Tables; and especially by comparing the extremes, which are each derived from a larger number of observations than the mean.

After showing the total amount of carbon (both combined and uncombined), silicium and combined carbon are thrown together, which indicates the replacement by silicium of that fortion of carbon set free in the form of graphite. The column 'silicium and slag' shows the general depreciation of the metal as the silicious metal increases.-From the Report of Campbell Morfit and James C. Booth to the Undnawpe Office, United States' Army.

The following analyses (rejecting those substances of which only a mere trace has been discovered), from the same chemists, are selected as showing striking poculiarities :-

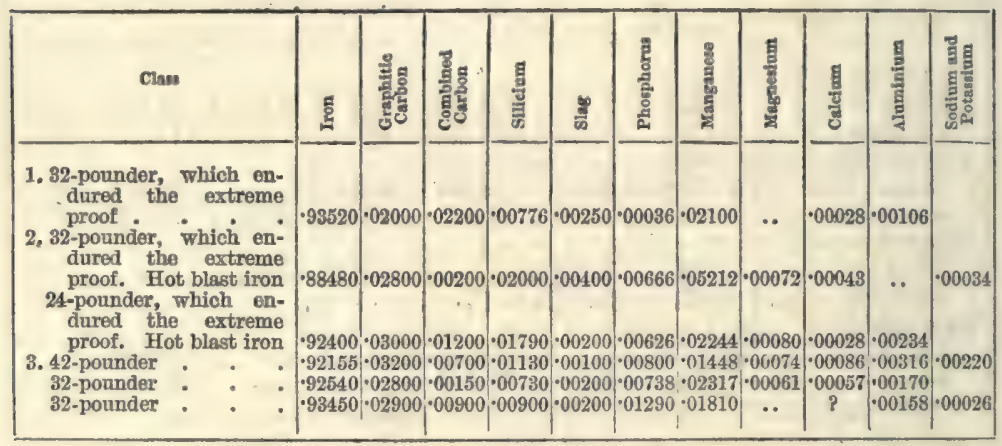

Comparison of Weight, Strongth, Extensibility, and Stiffness; Cast-Iron being unity within practical limits to static forces only.

\begin{tabular}{|c|c|c|c|c|c|c|}
\hline Material & & $\begin{array}{l}\text { Weight for } \\
=\text { Volume }\end{array}$ & Strength & Extensibility & Stiffness & Torsion \\
\hline Cast-ixon & . . & 1.00 & 1.00 & 1.00 & $1 \cdot 00$ & 1.00 \\
\hline Gun-metal. & . . & $1 \cdot 18$ & $0 \cdot 65$ & $1 \cdot 27$ & 0.53 & 0.55 \\
\hline Wrought-iron & . . & 1.07 & $3 \cdot 00$ & 0.45 & $2 \cdot 20$ & $1 \cdot 11$ \\
\hline Stoel . . & - & 1.07 & $4 \cdot 75$ & 0.32 & $3 \cdot 15$ & $2 \cdot 11$ \\
\hline
\end{tabular}

We find that wrought-iron guns are more than fivefold as durable as those of gun-metal, and twenty-two times as durable as those of cast-iron. And taking first cost and durability together, gun-metal cannon are about seventy-seven times, and cast-iron guns about thirty times, as dear as wrought-iron artillery. Again: the cost of horse-labour, or other means of transport for equal strongth (and, of course, therefore, for equal effective artillery power), is about five times as great for gun-metal, and nearly three times as great for cast-iron as for wrought-iron guns. In every respect in which we have submitted them to a comparison, searching and rigid, and that seems to have omitted no important point of inquiry, wrought-iron stand preeminently superior to every other material for the fabrication of ordnance. - United States' Report.

The advantages possessed by rolled bars for the construction of artillery are thus summed up by Mr. Mallet, in his 'Memoir on Artillery :'- 
1. The iron constituting the integrant parts is all in moderate-sized, straight, prismatic pieces, formed of rolled bars only; hence, with its fibres all longitudinal, perfectly uniform, and its extensibility the greatest possible, and in the same direction in which it is to be strained; it is, therefore, a better material than any forged iron can, by possibility, be made.

2. The limitation of manufacture of the iron, thus, to rolling, and the dispensing with all massive forgings, insures absolute soundness and uniformity of properties in the material.

3. The limited size of each integrant part, and the mode of preparation and combination, afford unavoidable tests of soundness and of perfect workmanship, step by stop, for every portion of the whole: unknown or wilfully concealed defects are impossible.

4. Facility of execution by ordinary tools, and under easily obtained conditions, and without the necessity either for peculiarly skilled labour on the part of 'heavy forgemen,' or for steam or other hammers, \&c., of unusual power, and very doubtful utility; and hence very considerable reduction in cost as compared with wrought-iron artillery forged in mass.

5. Facility of transport by reduction of weight, as compared with solid guns of the same or of any other known material.

6. A better material than massive forged iron, rolled bars are much more scientifically and advantageously applied; the same section of iron doing much more resisting work, as applied in the gun built-up in compressed and extended plies, than in any solid gun.

7. The introduction thus into cannon of a principle of elasticity, or rather of elastic range (as in a carriage-spring divided into a number of superimposed leaves), greater than that due to the modulus of elasticity of the material itself; and so acting, by distribution of the maximum effort of the explosion, upon the rings successively recipient of the strain during the time of the ball's trajet through the chase, as materially to relieve its effects upon the gun.

Considerable attention has been given, of late years, to the construction of very powerful pieces of ordnance. Cast-iron cannon are usually employed, but these very soon become useless when exposed to the sudden shocks of rapid firing. Cast-iron is, comparatively speaking, a weak substance for resisting extension, or for withstanding the explosive energy of gunpowder, compared with that of wrought-iron, the proportion being as 1 is to 5 ; consequently, many attempts have been made to substitute wroughs iron cannon for cast.

A gun, exhibited in 1851 by the Belgian Government, made of cast-iron ' prepared. with coke and wood,' was said to have stood 2,116 rounds, and another, $3,6+7$ rounds, without much injury to the touch-hole or vent. Another is said to have been twice 'rebouched,' and has stood 6,002 rounds without injury. As few guns of cast-iron will stand more than 800 rounds without becoming unserviceable, this mode of preparing the iron appears to be a great improvement. At St. Sebastian 2,700 rounds were fired from the English batteries, but, as was observed by an eye-witness, 'you could put your fist into the touch-holes.'-Colonel James, R.E.

In Prussia they have for some time made cannon of 'forged cast-steel.' To get over the difficulty of forging the gun with the trunnions on, the gun has been made without them, and a hollow casting with trunnions afterwards slipped over the breech, and secured in its proper position by screoning in the cascable. The tenacity of this metal must be very great.

CAsTINe of GuNs. - Guns have long been cast in a vertical position, and with a certain amount of 'head of metal' above the topmest part of the gun itself. One object gained by this (of great value) is to afford a gathering-place for all scoria, or other foreign matter; an end that might bo much more effectually accomplished wero the metal always run into the cavity of the mould by ' gaits' leading to the bottom, or lowest point, in place of the metal being thrown in at the top, with a fall, at first, of several feet, as is now the common practice, by which much air and scoria are carried down and mixed with the metal, some of which never rises up again, or escapes as 'air-bubbles.'

The value of the 'head of metal' in casting of guns is shown by the following Table, constructed by Mr. Robert Mallet, after a series of carefully conducted experiments, which he published in a paper entitled ' On the Physical Conditions involved in the Construotion of Artillery':- 
General Clasifioation of the Primcipal Makes of British Cast-Irons as applicable to Artillery. (All docluced from equal Piooes, oast One Inch thick and Wive Inches square.)

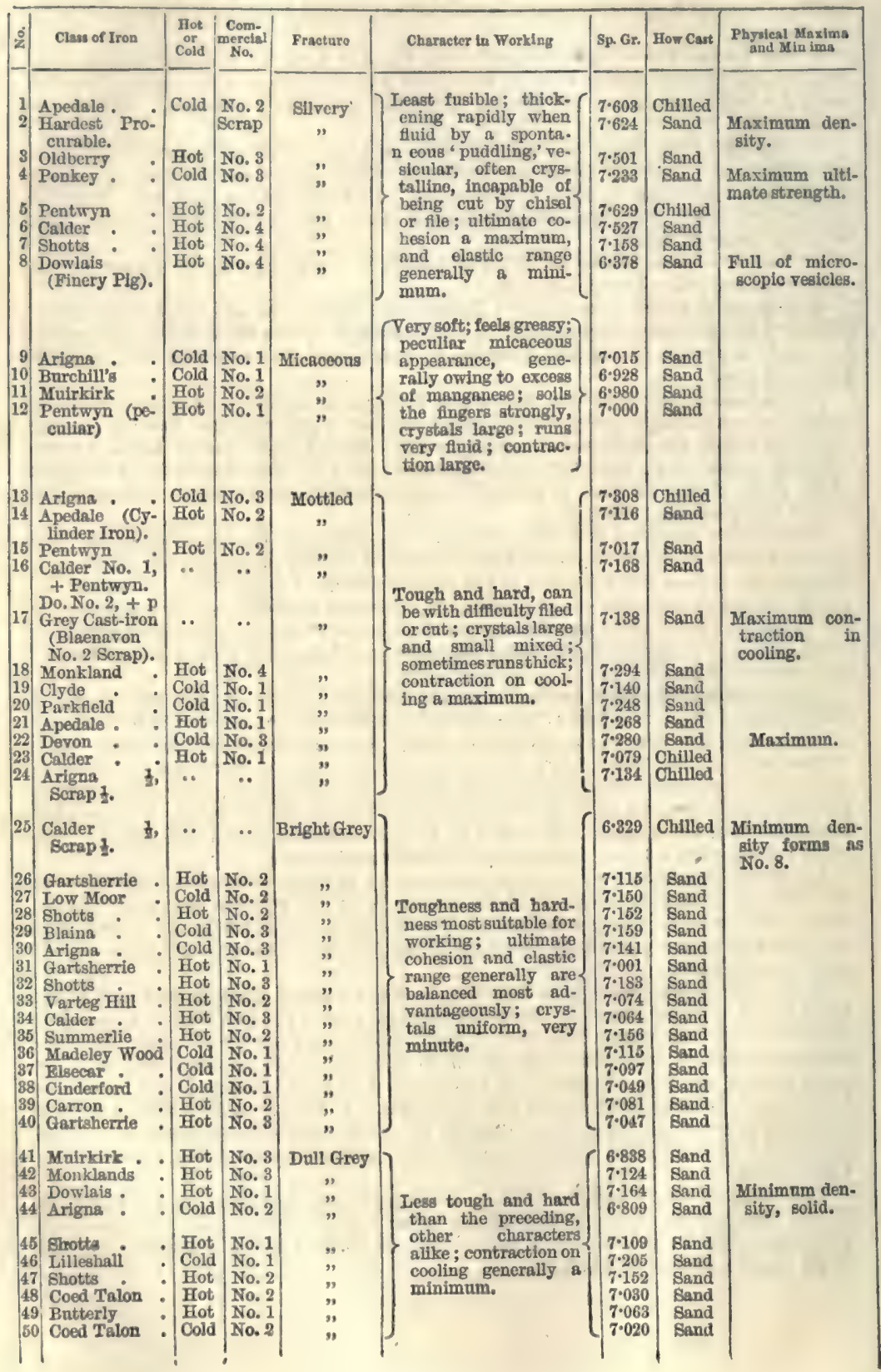




\begin{tabular}{|c|c|c|c|c|c|c|c|c|}
\hline$\frac{5}{4}$ & Clase of Iren & $\begin{array}{l}\text { Hot } \\
\text { or } \\
\text { Cold }\end{array}$ & $\begin{array}{c}\text { Com } \\
\text { mercial } \\
\text { Nio. }\end{array}$ & Fracture & Character in Working & Sp. Gr. & How Cast & $\begin{array}{l}\text { Physical Maxims } \\
\text { and Minina }\end{array}$ \\
\hline $\begin{array}{l}51 \\
52 \\
53 \\
54 \\
55 \\
56 \\
57 \\
58 \\
59\end{array}$ & 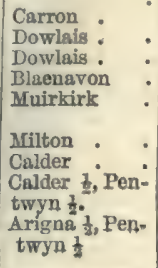 & \begin{tabular}{|c|} 
Cold \\
Cold \\
Cold \\
Cold \\
Cold \\
Hot \\
Hot \\
$\cdots$ \\
$\ldots$
\end{tabular} & $\begin{array}{c}\text { No. } 2 \\
\text { No. } 3 \\
\text { No. } 1 \\
\text { No. 1 } \\
\text { No. 2 } \\
\text { No. } 1 \\
\cdots \\
\cdots\end{array}$ & $\begin{array}{c}\text { Dark Grey } \\
\text { " } \\
" \\
" \\
" \\
\text { " } \\
\text { " } \\
\text { " }\end{array}$ & $\begin{array}{l}\text { Most fusible; remains } \\
\text { long fluid, exudes } \\
\text { graphite in cooling; } \\
\text { soils the fingers; crys- } \\
\text { tals large and lamel- } \\
\text { lar; ultimate cohe- } \\
\text { sion a minimum, and } \\
\text { elastic range gene- } \\
\text { rally a maximum. }\end{array}$ & $\begin{array}{l}7 \cdot 107 \\
7 \cdot 159 \\
7 \cdot 192 \\
7 \cdot 143 \\
7 \cdot 076 \\
7 \cdot 073 \\
7 \cdot 027 \\
6 \cdot 978 \\
7 \cdot 050\end{array}$ & $\begin{array}{l}\text { Sand } \\
\text { Sand } \\
\text { Sand } \\
\text { Sand } \\
\text { Sand } \\
\text { Sand } \\
\text { Sand } \\
\text { Band } \\
\text { Sand }\end{array}$ & $\begin{array}{l}\text { Minimum ulti- } \\
\text { mate strength. }\end{array}$ \\
\hline
\end{tabular}

Table showing the Increase of Density in Castings of large Size, due to their Solidification under a Head of Metal, varying from two to fourteen Feet:-

\begin{tabular}{|c|c|c|c|c|c|c|c|c|c|c|}
\hline \multirow{2}{*}{ 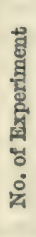 } & \multicolumn{3}{|c|}{$\begin{array}{c}\text { Calder Cast-iron, No. 1, } \\
\text { Hot Blast }\end{array}$} & \multicolumn{3}{|c|}{$\begin{array}{l}\text { Blaenaron, No. 1, } \\
\text { Cold Blast }\end{array}$} & \multicolumn{3}{|c|}{$\begin{array}{l}\text { Apedale, No. } 2 \text {, } \\
\text { Hot Blast }\end{array}$} & \\
\hline & 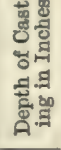 & 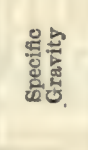 & 䓵总 & 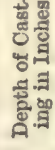 & 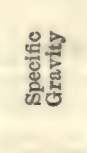 & 递 & 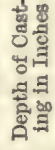 & 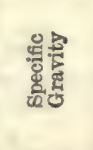 & 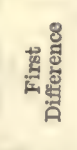 & 运 \\
\hline 1 & 0 & 6.9551 & & 0 & $7 \cdot 0$ & & 0 & $7 \cdot 0328$ & & .0 \\
\hline 2 & 24 & 6.9633 & .0082 & 24 & & .0097 & 24 & 7. & .0089 & 6.4 \\
\hline 3 & 48 & $7 \cdot 0145$ & .0512 & 48 & $7 \cdot 0777$ & .0201 & 48 & $7 \cdot 0558$ & .0141 & $12 \cdot 8$ \\
\hline 4 & 72 & & .03 & 72 & & & 72 & & .0111 & 19. \\
\hline 5 & 96 & $7 \cdot 0642$ & .01 & 96 & $7 \cdot 10$ & .0122 & 96 & $7 \cdot 0$ & .0120 & 25 . \\
\hline 6 & 120 & $7 \cdot c$ & .01 & 120 & & $\cdot 01$ & 120 & & .0 & 32 . \\
\hline 7 & 144 & $7 \cdot 09$ & .01 & 144 & $7 \cdot 1$ & .01 & 144 & $7 \cdot 1046$ & $\cdot 0131$ & 38 . \\
\hline 8 & 168 & $7 \cdot 1035$ & .0128 & 168 & $7 \cdot 1430$ & .0142 & 168 & $7 \cdot 1183$ & .0137 & $44 \cdot 8$ \\
\hline
\end{tabular}

The experiments were made upon cylindrical shafts of cast-iron, cast vertically in dry sand-mould, under heads gradually increasing up to fourteon feet in depth, and all poured from 'gaits' at the bottom.

These experiments show an increase of density due to fourteen feet head, about equal to a pressure of $44.8 \mathrm{lbs}$. per square inch on the casting; from 6.9551 to 7.1035 for Scotch cast-iron.

About the latter end of 1854, the attention of Mr. Robert Mallet, C.E., was directed to the mathematical consideration of the relative powers of shells in proportion to their increase of size or of diameter. His inquiries resulted in a memoir presented by him to Government, in which he investigated the increase of power in shells with increase of diameter, under the heads of:-1. Their penetrative power. 2. Their increased range and greater accuracy of fire. 3. Their explosive power. 4. Their power of demolition, or of levelling earthworks, buildings, \&c. 5. Their fragmentary missile power. 6. and lastly, their moral effect,-in every case viewing the shell, not as a weapon against troops, but as an instrument of destruction to an enemy's works. The result so convinced Mr. Mallet of the rapid rate at which the destructive powers of a shell increase with increase of size, that he was induced to propose to Government the employment of shells of a magnitude never before imagined by any one, namely, of a yard in diameter, and weighing, when in flight, about a ton and a quarter each : and to prepare designs, in sereral respects novel and peculiar, for the construction of mortars capable of projecting theso enormous globes. Such a mortar was made, and on the 19th of October, 1857, the first of those colossal mortars, constructed from Mr. Mallet's design ( fig. 91), was fired on Woolwich Marshes, with charges (of projection) gradually increasing up to $70 \mathrm{lbs}$; and with the latter charge a shell weighing $2,550 \mathrm{lbs}$. was thrown a horizontal range of upwards of a mile and a half, to a height of probably three-quarters of a mile, and falling, penetrated the compact and then hard dry earth of the Woolwich Rango to a depth of more than 18 feet, throwing about cartloads of earth and stones by the mere splash of the fall of the empty shell. 
The drawing of this remarkable pioce of artillery is presented-although, except experimentally, the mortar has never beon used. It certainly is a remarkable example

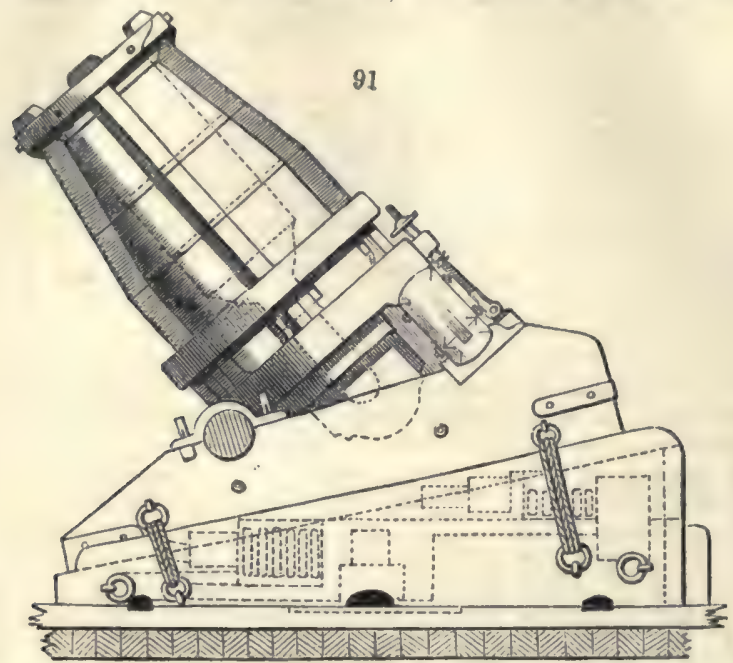

of engineering skill. Mallet's mortar is formed wholly of wrought-iron in concentric rings, and each mortar is separable at pleasure with 13 pieces, the heaviest weighing about 11 tons, the entire mass being 52 tons.

The position attained by Rifled Ordnance manufactured on the principles advocated by Sir William Armstrong is such, that it appears desirable to describe the mode of constructing those guns. Sir William Armstrong himself describes the principles by which he has been guided in the construction of his guns, in his paper communicated to the 'British Association,' and reprinted in 'The Industrial Resources of the Tyne, Wear, and Tees.' With some slight alteration this has been retained as the best possible souree of information.

'In the month of December, 1854, my friend Mr. Rendell, the well-known engineer, submitted to Sir James Graham a communication he had received from me suggesting the expediency of enlarging the ordinary rifle to the standard of a field-gun, and using elongated projectiles of lead instead of balls of cast-iron. This communication was handed by Sir James Graham to the Duke of Newcastle, then Minister of War, with whom I had an interview on the subject in company with Mr. Rendell.

'At this interview I was authorised by his Grace to carry my views into effect, by constructing, upon the plan I had suggested, one or more guns, not exceeding six in number, and to make the necessary exporiments in connection with the subject.

'In acting upon the authority thus received, I deemed it expedient to confine myself, in the first instance, to the production of a single gun, but to make that one gun the test, not only of the principles I had recommended, but also of the feasibility of loading field-pieces at the breech, and applying certain mechenical arrangements to counteract recoil, and facilitate the pointing of the gun.

' The substitution of elongated solid projectiles for spherical bullets is an essential step to the attainment of very extended range in artillery-practice; but the lengthening of a solid projectile involves the necessity of strengthening the gun to onable it to resist the greater intensity of force which becomes necessary to give the required velocity ; and this object can only be effected, consistently with lightness, by constructing the gun of steel or wrought-iron instead of cast-iron or bronze. The tensile strength of these several materials is exhibited in the following Table :-

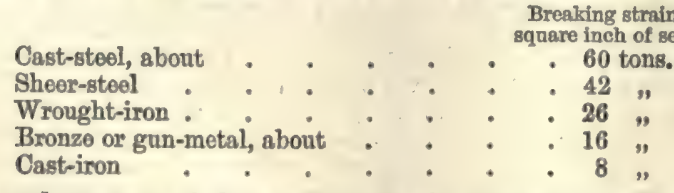

'The first and strongest of these substances, viz. cast-steel, may be set ont of the question, as it appears impracticable, in the present state of manufacture, to produce 
it in masses sufficiently large without the occurrence of flaws, which, in the great majority of cases, would destroy its efficiency. Sheer-steel may be forged, like wrought-iron, into large pieces: but in a gun made from a solid mass of either of these substances, the full strength of the material can never be realised, because the tonacity of wrought-iron or steel is always less in the lateral than in the longitudinal direction : and it is the lateral strength which, in $a$ gin so manufactured, would be chiofly brought into action. There is also much uncertainty in the lateral strength of wrought-iron or steel, because the flaws or imperfections of welding which exist in all thick masses of those materials almost invariably run in the direction of the length, and in general, therofore, only detract from the strength in the transverse direction. It is for these reasons that the barrels of muskets and sporting guns are formed by twisting long slips of iron into spiral tubes, and then welding together the edges, by which means the longitudinal strength of the slip becomes opposed to the explosive foree of the powder, and the weldings being transverse with the bore, have no important influence in lessening the strength of the barrel. It is also to be observed, in reference to the strength of steel or wrought-iron cannon, that the resistance of a cylinder to internal pressure does not increase in the ratio of its thickness. If the cylinder be regarded as made up of a number of concentric layers, each capable of sustaining without injury a degree of extension proportionate to its length, it is obvious, that the greater the circumference of each layer, the less will it be stretched by a given distention of the bore, and, consequently, the less will it contribute to the general strength of the cylinder. The ratio of this decrease is very rapid, being as the square of the circumference, or distance from the centro inversely; and, consequently, when the cylinder is thick, the deficiency of strength from this cause becomes very great.

'Now this defect can only be remedied by giving to the external portion of the cylinder a certain initial tension, gradually decreasing and finally passing into compression towards the centre; and although this condition cannot be effected by any known process of forging or casting, yet where wrought-iron or steel is the material used, it may in a great measure be attained by shrinking an outer cylinder upon an inner one, and in like manner superadding others until the requisite thickness has been acquired.

- The method, however, of forming steel or wrought-iron guns, by simply forging the material into the required form, and boring it in the usual manner, was so much recommended by its facility, that I was induced to make some experiments to test its sufficiency.

- With this view a number of cylinders were forged, each twolve inches long and five inches in the outward diameter. These were bored to an internal diameter of one and three-quarter inches, and tested in the following manner:-Each cylinder was entirely filled with gunpowder, and the open end was pressed by screws against a very thick iron tube bored to the same diameter, and containing a cylindrical shot of lead equal in weight to about three spherical shot of the same diameter and material. Several of the cylinders burst on the first discharge, and those which remained uninjured were afterwards reduced in thickness, and tested a second time. If they still resisted the explosion, the thickness was further diminished; and this mode of proceeding was continued until fracture took place in all of them.

'The results obtained in this manner showed, as had beon apprehended, great uncertainty in the strength of the material, and rendered it impossible to define the thickness necessary to resist a given charge of powder. I felt compelled, therefore, to dismiss this mode of construction, and to adopt another more correct in principle, but more difficult of execution.

'In the above experiment it was found that steel was more subject to defects of welding than iron; but being a harder substance, and therefore more fitted to form the surface of a bore, I determined to apply it as an internal lining, and to obtain the necessary strength by encircling it with twisted cylinders of wrought-iron, tightly contracted upon the steel core by the usual process of cooling after previous expansion by heat. Considerable difficulties were encountered in carrying this plan into practice; but I ultimately succeeded in completing a gun, of which the following is a description.

'The gun, when fired, recoils upon an ascending slide without displacing the carriage, and then returns to its place by gravity. The slide-frame turns upon a pirot, which permits the gun to be pointed to either side without moving the carriage. The gun is elevated and depressed by means of a screw, which is fixed to and moves with the slide, and a similar screw is applied for the traversing or horizontal movement. The arrangement for loading at the breech may be described as follows:-At the back end of the gun a powerful screw is applied, having a hole through the centre, forming a prolongation of the bore, and through which hole the bullet and charge are delivered into the gun. A 'breech-piece' with a mitred face, fitting a similar face 
at the end of the bore, is then dropped into a recess, and by the action of the serew pressed tightly into its seat, so as effectually to close tho bore.

'In order to facilitate the loading, the bullet and cartridgo are placed in a tubo, from which they are thrust into the gun by means of a rammer.

'Tho breech-piece contains a vent, with a cavity for receiving a small quantity of powder to ignite the charge; and as the breech-piece is prepared for firing while the gun is being loaded, no time is lost in subsequent priming.

'Several of those breech-pieces accompany the gun, some being arranged to fire by percussion-caps, and others by friction-tubes or port-fires.

'The bore of the gun is one and three-quarter inches in diameter, and contains oight spiral grooves, having an inclination equal to one turn in twolvo feet. These grooves terminate at a distance of fourteon inches from the breech, and the bore then gradually expands in a longth of three inches, from one and three-quarters inches to one and seven-eighths inches in diameter. The bullet, in the operation of loading, passes freely through this widened space; but its diameter being a littlo in excess of the bore, it lodges in the tapered contraction at the commencement of the grooves.

'The mode in which the gun is made up of separate parts consists in surrounding the steol centre with twisted cylinders of wrought-iron, made in a similar manner to gunbarrels, and being shrunk upon the steel, they are in that state of initial tension which is necessary to bring their entire strength into operation.

'The weight of the gun by itself is about 5 cwts. ; but, including the carriage, its weight is nearly identical with that of a light 6-pounder with its carriage complete. It is probably heavier than necessary, but recoil might be inconveniently increased if the weight were much reduced.

'Having now described the gun and its carriage, I shall proceed to speak of the projectile.

'The resistance which a projectile encounters in passing through the air is mainly dependent upon the area of its cross-section, and the advantage of lengthening a bullet consists in augmenting the weight without increasing this sectional area; but in order to realise this advantage it is essential that the bullet be guided endways in its course, and this can only be effected by causing it to rotate rapidly upon its longer axis, which is accomplished by ffring it from a rifled bore.

'This peculiar influence of rotation, in giving persistency of direction to the axis of a projectile, is entirely distinct from that which it also possesses of correcting the tendency to aberration arising from irregular form or density; and in order to investigate experimentally the nature of this action, I constructed an apparatus by which a cylindrical bullet could be put into extremely rapid rotation, and be then suspended in a manner which left it free to turn in any direction.

' When thus suspended, the rotating bullet exhibited the same remarkable properties as are possessed by the revolving disc in the recently invented instrument called the 'Gyroscope.' When pressure was applied to either end of the axis, the movement which took place was not in the direction of the pressure, but at right angles to it. Thus a vertical pressure deflected the axis horizontally, while lateral pressure deflected it vertically. But the important point elicited was this, that the time required to produce these indirect movements beeame greator as the velocity was increased, and, consequently, that the amount of deflection produced in a given time by a giren pressure, diminished as the rotation was accelerated. Now, all disturbing forces which operate upon a projectile during its flight must necessarily be of very short continuance, and can therefore have but little influence in diverting the axis when thus stiffened by rapid rotation.

'I also found that a cylindrical bullet with tapored extremities was more easily deflected than one of equal weight with flat or merely rounded ends, because the mean diameter of the bullet, and consequently the mean velocity of rotation, were thereby diminished. So far, therefore, as aceuracy of flight depends upon the rigidity of the axis, it would appear that the nearest practicable approach to a plain cylinder is the most desirable form for a projectile, but there are other considerations which modify this conclusion.

'It is also to be observed, that since the rigidity of the axis (relatively to the magnitude of the projectile) depends upon the mean velocity of rotation, the inclination of the spiral grooves in a rifled gun should vary inversely with the diameter of the bore. Thus, if one turn in eight feet be assumed as sufficient for a rifled bore of one inch in diameter, one turn in forty-eight feet should be sufficient for a bore of six inches, provided the same form of projectile be used.

'The forms of bullet which I actually tried with the gun were exceedingly numerous, and the materials used for these bullets was in all cases lead hardened by an intermixture of antimony and tin; and tho weight raried from two to three and a half pounds. 
'In trying these various bullets, a number of each kind was fired against a vortical hank at a distance of 435 yards. The gun was constantly pointed at the same object, and the closeness of the bullet-holes to each other was taken as the criterion of accuracy, while tho drop below the level of the aim furnished an indication of comparative range.

'The conclusions arrived at from these and other experiments may be concisely stated as follows :-

' 1st.-A pointed form at the front end of the bullet is unfavourable to accuracy of flight, unless the cylindrical part of the bullet be of considerable length; but, on the other hand, a pointed or conoidal form behind, has the effect of increasing the accuracy attained. This may be explained upon the very probable supposition that the blast from the mouth of the gun, impinging upon the rear of the bullet, will operate more unfarourably upon a flat or hollow end than upon a rounded or conical one.

' 2 nd. - Increase of length in the cylindrical part of a bullet always increases precision; but, when carried beyond a certain limit, lessens the initial velocity, even where the charge is proportionately augmented.

' $3 x d$. - Both range and accuracy were affected in an important degree by the manner in which the bullet fitted the contraction in the gun. When the fitting part was in front of the bullet, the pressure of the gas operating upon its sides compressed it, and the same effect was produced, though in a less degree, when the conical, or rounded. end at the back, projected too far into the powder-chamber.

'These effects were rendered apparent by inspection of bullets recovered after firing, many of which were found in nearly the precise condition in which they quitted the gun.

- The bullet ultimately selected is a little longer and heavier than those experimented with, and differs from the pointed bullet, in being longer in the cylindrical part and having a coned, instead of a rounded, end behind; and although its drop in a range of 435 yards is considerably more than that of several of the shorter bullets, yet there is little doubt it will excel them in range at higher elerations of the gun ; because I have found that a pointed front only operates in sustaining the flight of the bullet when the range is long; and a high initial velocity, which materially lessens the drop in short distances, does not produce the same effect, in a corresponding degree, when the distance is increased.

'Great improvements were effected in the accuracy of the firing, by modifying the shape of the projectile; but although the experiments were very protracted, I feel that they require to be further prolonged in order to arrive at the greatest attainable perfection in the form of the bullet.

- The ranges at differont elevations were not ascertained with the form of bullet ultimately adopted; but with a three-pound pointed bullet and charges of twelve ounces of powder, they were as follows:-

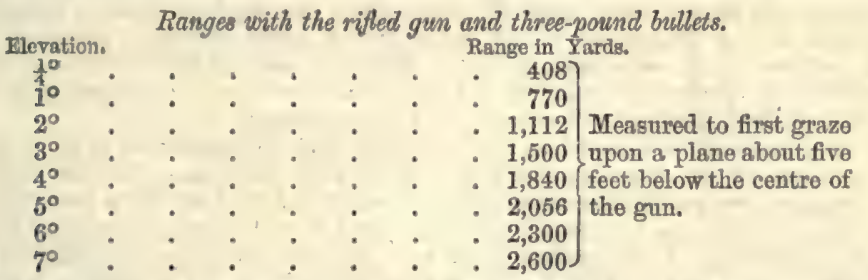

'The powdsr used was a mixture of blasting and 'double-seal' powder in equal proportions. The distances given are in most cases averages of several shots; but in some instances they were only approximately determined. When the gun had more elevation than $7^{\circ}$ the bullets could only be fired out to sea, and the range could not be ascertained.

'By way of comparison with these results, an extract is here given from the last edition of Sir H. Douglas's 'Naval Gunnery,' specifying the ranges obtained with a 68-pounder throwing shot with full charges, which ranges, it will be seen, are, upon the whole, no greater than those of the three-pound bullet fired from the rifled gun:--

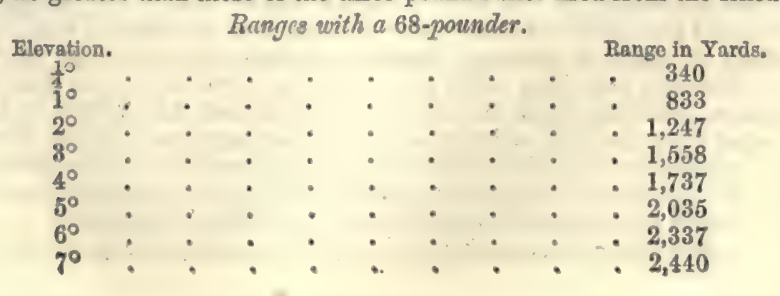


'In trying the initial velocity of the shot by means of a ballistic pendulum, I was enabled to observe its penetrating power. When fired with charges of $13 \mathrm{oz}$. of powder, a 3-lb. bullot passed through 2 feet 2 inches of hard elm timber, and flattened against a cast-iron block forming the back of the pendulum. The initial velocity was similar to that generally obtained with round shot fired with proportionato charges, viz about 1,550 foet per second.

'In the course of the experiments made with the gun, upwards of 500 rounds were fired ; and ample opportunity was thus afforded of judging as to the durability of the parts affected by the loading at the breech. At first the fitting surfaces which closed the bore were of unhardened steel; but these soon failed, being cut away in numerous small channels by the ignited gases. The steel was then hardened; but instead of being rendered more durable, it yielded to the action of the powder more rapidly than before. Conceiving, therefore, that the erosion was not a mechanical action, but a chemical effect of combustion, and that a metal which was a better conductor of heat than steel or iron would be less liable to burn on the surface, I was led to substitute copper as the material of the parts affected, and no further difficulty was experienced. The copper fittings applied for this purpose consist of two annular pieces, one of which is screwed into the breech end of the gun, and the other fixed upon the breechpiece. These fittings can very easily and quickly be repaired, when necessary, by means of a tool provided for that purpose, and can also be removed and replaced by others kept in readiness for use; and there is nothing to prevent these operations being performed by the gunners when on service, if they be previously instructed.

'The advantages of loading at the breech may be stated as follows:-

' 1 st. - It permits of a bullet being used of a larger diameter than the bore, by which means accuracy of fit is secured, and the material of the bullet is forced into the grooves of the bore.

' 2 nd.-Any ignited matter remaining in the gun after firing may with ease and certainty be removed, or, if left in the gun, it will be thrust forward from the part where its presence would be dangerous, by the insertion of the succeeding bullet.

' 3 rd. - In the arrangement which I have adopted, the perishable part of the gun, viz. the vent and its vicinity, is comprised in the moveable breech-piece, which may be easily replaced when worn or otherwise injured:

' 4 th. - A rifled gun loaded at the breech may be more rapidly fired than a rifled gun loaded at the muzzle, because the fouling of the bore presents no impediment to the insertion of the bullet when introduced from behind; but as compared with smooth bored ordnance of the ordinary deseription, there is probably nothing to gain in poir $t$ of quickness of firing.

The gun was remarkably free from tendency to become heated by firing, a fact which can only be explained upon the supposition that the heating of a cannon is occasioned, not by the contact of the flame, but by some molecular action of the metal, produced by the explosion, and more effectually resisted by wrought-iron than by cast-iron or bronze; but possibly the compound structure of this gun may also operate to deaden vibration, and prevent the evil in question.

'It may, perhaps, be objected to this gun, that from the smallness of the bore, it cannot be applied for throwing shells as well as solid projectiles; but the fact is, these two purposes are incompatible with each other, unless both be imperfectly attained, for while the one necessarily requires a large bore, the other demands a small one; and it therefore seems preferable to have separate guns specially adapted for each application. As a civilian, I speak with diffidence upon the advantages which I believe the long range of this description of field-gun will afford in its military application; but I may be permitted to observe, that the incident which chiefly contributed to direct my attention to this subject still appears to furnish a forcible illustration of its importance. I allude to the memorable service rendered at Inkermann, by means of two 18-pounders, laboriously dragged from the batteries, and ultimately directed with great gallantry and success against the Russian artillery, at a distance from which the numerous but lighter guns of the enemy could not effectually reply. Now, these two battery-guns were but a clumsy substitute for light, long-range guns, which would have rendered the same important service with more promptitude and ease, and could have operated at a greater distance from the enemy's fire. It is, perhaps, chiefly as 'guns of position,' commanding important points at great but ascertained distances, that these rifled guns would be valuable, because long range can only be made available where distance can be determined, which it cannot easily be in the rapid operations for which 'field-pieces' are employed. It is, therefore, as adjuncts to, and not as substitutes for, the present description of field ordnance that I propose the adoption of these guns; and when fully brought to perfection, I believe they will furnish a most important addition to the artillery of an army.

'With respect to the construction of heavy ordnance by the process of twisting 
wrought-iron bars into cylinders and combining them in the manner described, there appears to be no great difficulty in so doing, if proper apparatus be provided for the purpose. It would not, however, be adrisable (except in peculiar cases) to apply the principle of loading at the breech except to guns of small dimensions, because in heary ordnance the moveable parts would become too cumbrous to be conveniently handled.'

The essential features of the Armstrong method of construction are :-

1st. The disposal of the fibre of the metal round the bore by coiling, so as to resist the tangential strain, the welds running in the direction of the least strain as regards their separation.

2nd. The employment of a breoch-piece, to support the bottom of the bore, with the fibro running lengthwise so as to resist longitudinal strain.

3rd. The shrinking-on the different portions, so that the exterior of the gun takes a due share of the strain. Mr. Whitworth's method of building-up is as follows :'The tube of the gun is made taper, being in the $5 \frac{1}{3}$-inch-bore gun 1 inch larger in dirmeter at the breech end than at the muzzlo end; then a series of hoops are made, which are screwed together so as to form another tube, that is put on by hydraulie pressure; each layer is put on a little tighter than the succeeding one.'-Evidence Report on Ordnance.

The method of elosing the bore of a built-up gun is an important question. The inner tubes of some of the large M. L. ordnance lately constructed in the Royal Gun Factories, as well as those of some of Blakely's guns, have what are termed closed ends, that is, the tube is not bored through to the bottom; the solid end of the tube in service-guns is supported by a cascable serewed into the breech-piece, and in some of them also by a shoulder in the breech-pioce. Sir W. Armstrong, Mr. Whitworth, and Major Palliser use open tubes and close them by a plug of wrought-iron or copper. The cascable in the Whitworth guns is not, like Armstrong's, cylindrical in form, but is shaped into two or more (screwed) cylinders, their respective diameters increasing from bore to breech.

92

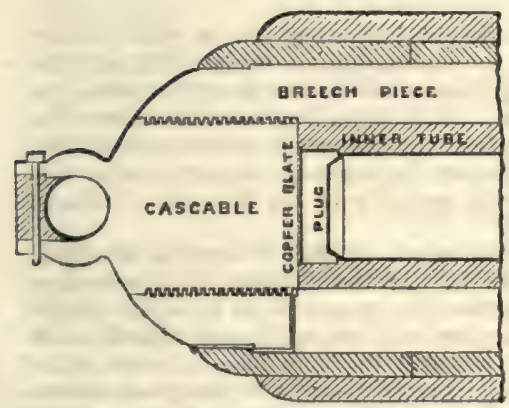

Breech of Armstrong's 10.5-inch gun.
93

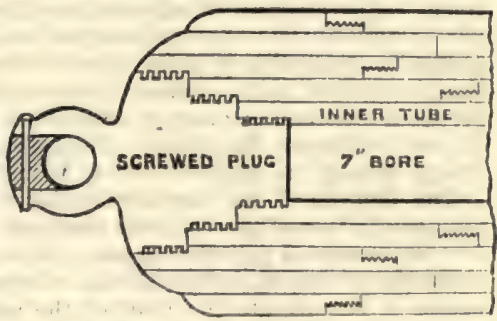

Breech of Whitworth's 7-inch gun.

In closing the bore of a M. I, or B. L. gun, one important principle should not be neglected, viz. that as the gas exerts an equal force in every direction, the thickness of the metal should be as great, or nearly so, behind as over the charge. Inattention to this principle, or its sacrifice to other considerations, is a source of weakness in many B. I. guns. One advantage of an open end is, that the metal of the inner tube is relieved to some extent from longitudinal strain.

The relative cost of large ordnance made in different ways was, when the last edition was published, as follows:-

Cast-iron guns :
Armstrong built-up ditto
Krupp's steel ditto
Gun-metal ditto :

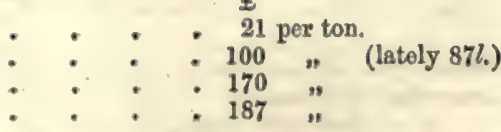

and, excepting the variation due to the price of the metal, it continues relatively about the same.

Bronze guns are vaIuable for recasting. Mr. Frazer has introduced modifications, by which it is said the cost of built-up ordnance will be much reduced, viz, to $40 l$ a ton with a coiled inner tube, and to $55 l$. a ton with a steel tube. Mr. Whitworth told the Committes on Ordnance that his $5 \frac{1}{3}$-inch gun weighing 4 tons, and made of homogeneous metal (soft steel) cost 700l., which is about 175l. per ton. The question of the relative advantages of breech-and muzzle-loading ordnance has been frequently dis-

Vor, I. 
cussol. The subject appenrs vory fiirly put by Major C. H. Owon, Profossor of Artillery, at Woolwich. Ho says:- Various opinions are hold as to the rolative advantages of brech- and muzzle-londing ordnance, but the latter would appoar to bo the best adapted to general service, as they aro stronger for equal woights of metal and simpler in construction. The adrantages of londing cannon at the breech are, that $\Omega$ projectile of larger diameter than tho bore ean be used, and its axis will consequently be stablo; thut the gun can be loadod when run-np, the gunners being therefore less exposed; that the gun can be worked in a smaller space (than $n \mathrm{M}$. L. pieco); tho elearing of tho bore ean be moro readily effected, and any ignited substance left in the bore can be scen and removed; also there is no danger of the shot not being loome. This plan, however, is attended with the following disadvantages, viz; that tho construction is more complicated than that of a muzzle-loading picee; that if the gun bo of largo calibre, the broech-loading apparatus, when sufficiently strong and heavy, will be unwieldy; and that with the same weight of metal, the breech: loading is a weaker and less enduring construction than the muzzle-loading. On the other hand, a muzalo-loading gun has a simpler and stronger construction, but tho gun detachments are more exposed than with a breech-loading gun, and if loaded carelessly, the shot may not be rammed home, in which ease the metal of the gun may be fracturel by the suddenly condensed gas.'

On the Systems of Rifling, Major Owen, R. A., one of our best authorities, writes :- In what does a system of rifling consist? Essentially in the method of giving the rotatory motion to the projectile. This definition will not satisfy some inventors, who wish to claim a particular twist as a part of their system of rifling. It would, however, be quito as reasonable to elaim a particular charge. The rotatory motion, as you all know, is given to prevent the projectile from turning over in flight; and the yelocity of rotition required depends upon the form, length, and weight of the projectile, no matter what the system of rifling may be; in fact, the number of rerolutions made by a shot $=\frac{\text { initial velocity }}{\text { length of twist }}$, and therefore, with the same charge, the same twist must be obviously necessary.'

'A twist, like a charge, may suit a particular rifled gun, but this is quite another thing. A gaining twist is advantageous, for, by employing it, the initial strain upon tho gun is reduced, the rotatory motion not being given whon the shot is set in motion, but gradually acquired as it moves down the bore. It is better to give rather more twist than is required under ordinary circumstances at the expense of a little extri strain; for should the twist be merely sufficient to impart the necessary rotatory motion with the service-charge, the velocity of rotation will probably be too low with a roducod charge to keep the projectile steady in flight.'2

The diameter of the bore has also been often mixed up with the system of rifling, with which it can have nothing whatever to do. As the diameter is decreased, so will the elongated projectile oppose a less surface (in proportion to its weight) to tho resistance of the air, or that of the substance fired at; but, on the other hand, it will expose a less area to the foree of the gas, and will therefore have a lower initial velocity ; it will have less capacity as a shell; its cartridgo must bo olongated, theroby throwing the strain forward; the amount of powder that can. bo usefully employed will be less; and if the length of the bore bo not increased, the expansion of the gas will bo moro limited. This question has been discussed, and should be thoroughly understood. In the Table below the loss of initial velocity by decrease in the size of the bore is elearly shown:-

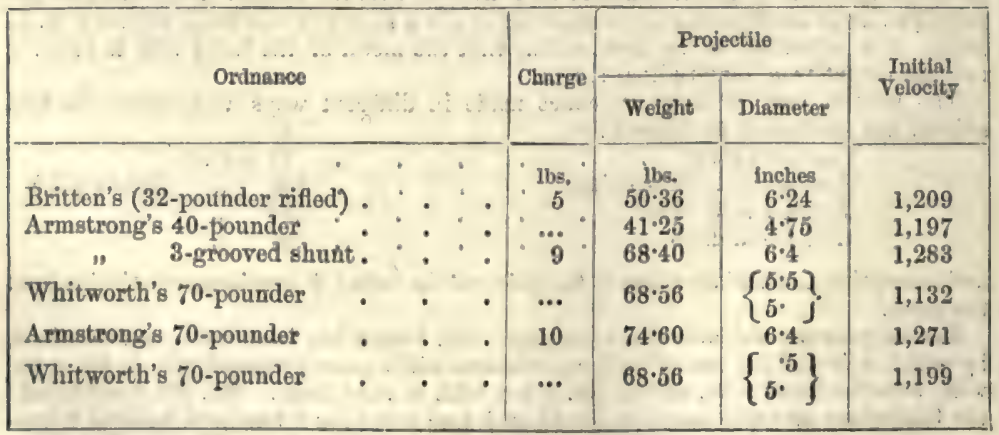

- The Initial Velocity is very little affected by the system of rifling.

This was. particularly shown by the inaccurate practice of the 600 -pounder, when fired with a small charge. 
Mr. Whitworth and his admirers have constantly assertod that his small-bore give 8 a flatter trajectory than the larger bores chosen by Armstrong, Britten, and others: This is, howover, not the case under all circumstances, and arose partly from the fact of the. Whitworth guns first tried being fired with charges of $\frac{3}{\mathrm{a}}$ th of the woight of the projectile, whoreas the greater number of other rifled guns used charges of only $\frac{1}{8}$ th or $\frac{1}{10}$ th. Mr. Whitworth is quite right to use as large a charge. as he can, but it must be taken into account in drawing comparisons. In practico from two rifled guns of different calibres, but firing projectiles of the same weight with equal charges, the large bore will give a lower trajectory; but as the projectile with the smaller diameter is less retarded, its trajectory will gradually become lower, as compared with the other, until beyond a cortain range the small bore will give the lower trajectory. Small bores have found little favour on the Continent, and it is for the authorities to demand either a large or a small bore as circumstances máy require.

The following conditions are requisite in any rifled gun to onsure accuracy of fire:- e rotatory motion must be given to the projectile round an axis parallel to; or coincident with, that of the bore; and the velocity of rotation imparted to the projectile must be sufficient to counteract the pressure of air tending to turn the shot over or render it unsteady in flight:

Great numbers of rifled guns with projectiles to correspond have been proposed, but most of tho systoms of rifling that have been adopted by any service, or tried on the practice-ground, may be divided into the following classes :-

(1.) Muzzle- or breech-loading guns, having projectiles of iron fitting the peculiar form of the bore mechanically.

(2.) Muzzlo-loading guns, with projectiles having soft-metal studs or ribs to fit the

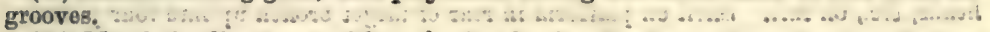

(3.) Muzzlo-loading guns, with projectiles having a soft-metal envelope, coating, or cup, which is expanded by the gas in the bore.

(4.) Breech-loading guns, with projectiles having a soft-metal coating larger in diameter than the bore, but which is compressed by the gas to the form of the bore.

The effects of those guns, and consequently their relative values, will be best shown by extracting from the 'Proceedings of the Royal Artillery Institution ' some of the results as officially stated by the officors in charge of the experiments. The more important of the targets have been selected, as representing the actual conditions of our ships at the time (1866), and of the poyer which can be brought to bear upon the armour-clad ships of an enemy.

\section{'Warrior' Target.}

This target was 10 foet by 12 foet, consisted of three plates, made at the Park Head forge, all $4 \frac{1}{2}$ inches thick, and varying from 12 feet by 3 feet to 12 feet by 3 feet 4 inches.

The Horsfall gun used weighed 24 tons 3 qrs. 2 lbs., diameter of bore $13 \cdot 014$ inches, diameter of shot 12.8 inches. It was first firod at 200 yards' range, with a solid cast-iron shot, weighing $279 \mathrm{lbs}$. and a charge of powder $\mathbf{7 4 . 4 0} \mathrm{lbs}$, which gave an initial velocity of 1,630 feet, reduced at 40 yards to about 1,610 feet per second.

The shot completely pierced the target through and through, making an irregular hole in the armour 2 foet square, and eracking but not buckling it.

From this and other similar experiments it appeared that the "Warrior' ship at 200 yards would be completely pierced by the Horsfall shot. A solid shot of annoaled cast-iron woighing 285 lbs. was fired at the same target with the same charge as before, from a range of 800 yards. This grazed the ground 17 yards short, and struck the target in the junction of two plates, breaking a large hole about 2 feet square through the armour, and burying itself in the timber backing. This proves that at 800 yards the real 'Warrior' would be severely injured, but the skin would not be penetrated by an individual shot.

Mr. Whitworth made a series of experiments to prove the penetration of his projectiles. The shot and shell were fired from a 12-pounder breech-losder, a 70-poundex muzale-loader, and a 120-pounder muzzlo-loader. Most of the shot and shell pierced the turget; the following were the more remarkable results. A target was made in the form of a box, with the object of putting to the test Mr. Whitworth's boast that -he could drivo:a shell through the side of an armour-clad ship, and make it burst between decks. . The shell fired on this occasion, with an initial velocity of 1,275 feet, passed completely through the 4 -inch armour-plate and its oak backing, bnd exploded on the rear side of the box, the plate of which was indented $2 \frac{1}{2}$ inches, bursting the box and blowing all six sides ontwards.

A shell of homogeneous metal, weighing 127 lbs, with a bursting charge of $3 \mathrm{lbs}$. $8 \mathrm{oz}$, and without a fuse, was fired at the same range with a charge of $25 \mathrm{lbs}$. of 
powder, giving a terminal velocity of about 1,263 foet. This shell wont completoly through everything, much to the astonishment of every one present.

Some experiments were made on the 'Warrior' target to prove the effect of steel shell fired from a 13.3" Armstrong M. L. wrought-iron shunt rifled gun at 2,000 yards' range. The initial velocity of the shell, with 51 \& lbs. charge, is about 990 feet, and the striking velocity 940 ',

Mean weight of shell empty
Burstor
Diameter of shell
Length $\left\{\begin{array}{l}\text { over all } \\ \text { of shell }\end{array}\right.$
Mean recoil

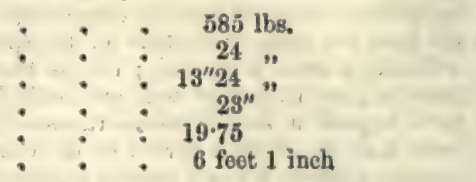

The third round struck about 10 yards short, and ricochetted on to centre of target, striking centro plate $4^{\prime} 2^{\prime \prime}$ from left side; hole in plate $16^{\prime \prime} \times 13 \frac{1}{2}^{\prime \prime}$, centro plate started forward $6 \frac{1}{2}$ " at top and $4 \frac{1}{2}$ " at bottom on right side, and $3^{\prime \prime}$ at top, and $0.5^{\prime \prime}$ at bottom on left side; 11 armour-plate bolts started in centre plate and one in bottom plate. Upper plate blown off and lying at foot of target in front; all the bolts (except three) of this plate broken at nut-the three being drawn bodily out of the plato ; bottom of upper plate, where shell entered, indented $1 \frac{1}{2}$ " in length of 1 foot. At the back ragged holo $4^{\prime} \times 2^{\prime} 3^{\prime \prime}$ between third and fourth ribs from right side; two ribs broken and forced out, second rib from right bulged, angle-iron eracked; skin cracked and opened for length of $3^{\prime}$ below hole. Seventeen armour-plate holes, twenty-one rivets, and thirteen backing bolts broken. A great many splinters of iron, timber, boltheads, \&c., on raft. Shell on platform in rear of target broken up into four pieces.

\section{The 'Bellerophon' Target,}

The part of the ship which was tested by the target is that situated between the main and lower decks, and not in the line of ports, the object being to test the strength of the general side of the ship. Special arrangements are made to strengthon the side in the vicinity of the ports, which will be few in number, as the 'Bellerophon' carries a small number of very large guns. These fow ports are strengthened by the introduction of additional iron to an extent which would not be practicable if the number of ports were large.

Each frame of the target was made of angle-iron $10^{\prime \prime} \times 3 \frac{1}{2} \times \frac{1}{2}$, and two angle-irons $3 \frac{1}{2}^{\prime \prime} \times 3 \frac{x_{2}^{\prime \prime}}{2} \times \frac{5^{\prime \prime}}{8}$ riveted together; to the double angle-irons of this frame the skin, which is composed of two thicknesses of $\frac{3^{\prime \prime}}{4}$ plating, making together $1 \frac{11}{2}$, with a layer of painted canvas between, is riveted. On the outside of the skin-plating, four horizontal angle-jron stringers are attached, two under the upper armour-plato $9 \frac{1}{2} \times 3 \frac{1}{2}{ }^{\prime \prime} \times \frac{1}{2}$ ", the broad flange being square to the skin, and not reaching out to the armour by half an inch; the other two are placed behind the lower plate $10^{\prime \prime} \times 32^{\prime \prime} \times \frac{1}{2}$. The breadth of the broader flange being the same as the thickness of the backing, it reaches out to, and comes in contact with, the armour. Wood backing $10^{\prime \prime}$ thick, is worked longitudinally on the skin-plating and between the angle-stringers, bolted with nut and serew bolts through the skin-plating. The armour consists of two rolled plates, $6^{\prime \prime}$ thick, manufactured at the Millwall works, weighing upwards of 9 tons each. The upper armour-plate is bolted with bolts $2 \frac{1}{2}$ diameter, and the lower plate with bolts $24^{\prime \prime}$ diameter. In one half of the target, divided vertically, the armour-bolts have elastic washers, and are clenched on single nuts. In the other halt the bolts have common washers with double nuts, and bolts not elenched. In erecting the target, care was taken to support it behind with beam-ends, \&ce, so that the actual condition of the proposed ship's side might bo approximated to as elosely as possible. All the portions of the target were carefully weighed, and the weight, as reported by the Admiralty overseer, was $389 \mathrm{lbs}$. per square foot.

The range was 200 yards, and the shot named below struck the target. The most decided effects were, however, produced by the guns named in the Table on the next page :-

From $10^{\circ} 5^{\prime \prime}$ Armstrong rifled gun:Spherical cast-iron solid shot, one. Cylindrical cast-iron solid shot, one

From 7'1" Ordnance Select Copmmittee gun :Steel shell, one

From 7" Whitworth rifled gun:-

i. Stoel shell, one: 
From 5 s:" Whitworth rifled gun:-

1bs. woight

Steel shell, one

69

From 110-pounder Armstrong breech-loading rified gun:-

Solid cast-iron shot, four

From 68-pounder smooth-bore gun:-

Solid cast-iron shot, three

\begin{tabular}{|c|c|c|c|c|}
\hline $\begin{array}{l}\text { Nature of } \\
\text { Ord nance }\end{array}$ & $\begin{array}{l}\text { Weight in lbs. } \\
\text { of Projectlle }\end{array}$ & $\begin{array}{l}\text { Forms of } \\
\text { Projectile }\end{array}$ & $\begin{array}{l}\text { Charge } \\
\text { in } 1 \text { bs. }\end{array}$ & Remarks \\
\hline $\begin{array}{c}5 l^{\prime \prime} \\
\text { Whitworth } \\
\text { gun. }\end{array}$ & $\begin{array}{c}69 \\
\text { Steol shell }\end{array}$ & $\begin{array}{c}\text { Cylindrical. } \\
\text { Bursting } \\
\text { charge } \\
2 \text { lbs. } 6 \text { oz. }\end{array}$ & 12 & $\begin{array}{l}\text { Struck the lower plate } 8^{\prime \prime} \text { from top and } \\
5^{\prime \prime} \text { from right side, on a bolt ; nar- } \\
\text { row crack on face of indent; } \\
\text { armour-plate bolt driven in } \frac{1}{3} \text {-inch; } \\
\text { nut on bolt loose; one balk of } \\
\text { timber in backing split through. }\end{array}$ \\
\hline $\begin{array}{c}10 \cdot 5^{\prime \prime} \\
\text { Armstrong } \\
\text { gun. }\end{array}$ & 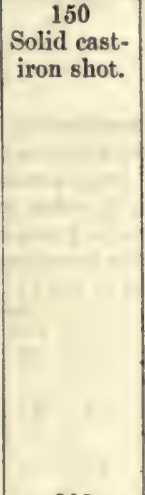 & $\begin{array}{l}\text { Spherical. } \\
10 \cdot 36 \text { diam. }\end{array}$ & 35 & $\begin{array}{l}\text { Struck the upper plate } 6^{\prime \prime} \text { from the } \\
\text { lower edge on a bolt; plate cracked } \\
\text { from bolt to bottom; crack } 9^{\prime \prime} \text { long } \\
\text { on face of indent; crack } 10^{\prime \prime} \text { long } \\
\text { and } 1^{\prime \prime} \text { wide at } 5^{\prime \prime} \text { from circum- } \\
\text { ferenee of indent on right side; } \\
\text { also two small cracks from bottom } \\
\text { of plate at } 5^{\prime \prime} \text { and } 10^{\prime \prime} \text { respectively } \\
\text { from left side of circumference; } \\
\text { plate driven in } 3 \frac{1}{2} \text { at lower edge in } \\
\text { length of } 3^{\prime} \text {; plate started out } 03^{\prime \prime} \\
\text { at top on left side, and } 0.2^{\prime \prime} \text { from } \\
\text { lower plate. At the back a throngh } \\
\text { armour-plate bolt driven out } 2^{\prime \prime} ; \\
\text { the heads of two backing-bolts and } \\
\text { one rivet broken off; one backing } \\
\text { bolt driven out } 14^{\prime \prime} \text {; skin bulged } \\
\text { slightly over area } 1_{4}^{1} \text { square. }\end{array}$ \\
\hline $\begin{array}{c}10 \cdot \tilde{\sigma}^{\prime \prime} \\
\text { Armstrong } \\
\text { gun. }\end{array}$ & $\begin{array}{l}308 \\
\text { Solid cast- } \\
\text { iron shot. }\end{array}$ & Cylindrical. & 35 & $\begin{array}{l}\text { Struck the armour-plate on the third } \\
\text { bolt from the right side, lower row; } \\
\text { plate driven in } 2 \cdot 1^{\prime \prime} \text { at bottom in } \\
\text { a length of } 5^{\prime} ; \text { a crack } 18^{\prime \prime} \text { long } \\
\text { through a bolt hole at } 2^{\prime} \text { from im- } \\
\text { pact plate, started at } 04^{\prime \prime} \text { from the } \\
\text { backing on the right side for a } \\
\text { length of } 2^{\prime} \text { at top. At the back one } \\
\text { through armour-plate bolt driven } \\
\text { out } 2^{\prime \prime} \text {, the heads of four rivets and } \\
\text { one backing-bolt broken off; vertical } \\
\text { frame-piece cracked through and } \\
\text { bent out slightly; beam-knee } \\
\text { crushed; skin slightly bulged. }\end{array}$ \\
\hline $\begin{array}{l}7^{\prime \prime} \\
\text { Whitworth } \\
\text { rifled-gun. }\end{array}$ & $\begin{array}{c}149 \cdot 5 \\
\text { Steel Shell }\end{array}$ & $\begin{array}{c}\text { Cylindrical. } \\
\text { Bursting } \\
\text { charge } \\
5 \text { lbs. } 8 \text { or. } \\
\text { in flannel } \\
\text { bag. }\end{array}$ & 27 & $\begin{array}{l}\text { Struck lower plate; head of shell } \\
\text { remained in hole; depth to nearest } \\
\text { point of shell } 91 \text { inches. At the } \\
\text { back of the skin bulged at junction } \\
\text { of skin plates, bottom plate } 2^{\prime \prime} \text {; } \\
\text { upper plate } 1^{\prime \prime} \text {; rib bulged out } 2^{\prime \prime} \text { at } \\
2^{\prime} \text { from ground; two deck-knees se- } \\
\text { parated from angle-iron } 0 \cdot 3 \text {; one } \\
\text { armour-plate bolt and three back- } \\
\text { ing-bolts broken; burst in backing. }\end{array}$ \\
\hline
\end{tabular}

\section{'Minotaur' Target,}

The armour is $5 \frac{1}{2}$ inches thick, the thickness of tho teak backing is reduced from 18 inches, as in the 'Warrior,' to $\theta$ inches, which is considered as equivalent to 1 inch of wrought-iren. The target used July 7, 1862, constructed on those principles, pres 
sented a frout of three armour-plates, one made by Messrs. Brown, of Sheffield, another by the Thames Iron Works, aud the third:by Messrs. Beale.

The guns used against this target wero the 12-ton. Armstrong muzzle-loading gun, throwing spherical $150-1 \mathrm{~b}$. cast-iron, and 162-lb. wronght-iron shot, with 50-1b. charges of powder, the former having an initial volocity of 1,750 feet, and the lattor of alout 1,700 foet per second; and a service 68-pounder throwing $67-1 \mathrm{~b}$. cast-, and 71-1b. wrought-iron shot, with 16 lbs. of powder; the cast shot having an initial velocity of 1,580 feet, the wrought-iron about 1,530 feet per second; all at 200 yards' range. The first $150-1 \mathrm{~b}$. cast shot struck the Thames Iron Company's plate, and made a hole about a foot square through the armour, and bedded itself deep in the teak. The plate was buckled considerably, several bolts were started, two ribs cracked, the skin much bulgel in, four bolts were broken, and a number of rivets.

The second $150-1 b$. shot struck the Sheffield plate, made a hole 13 inches by 12 inches in the armour, and sent pieces of the armour-plate, shot, and teak, through a large irregular hole in the skin, the armour-plate was buckled, three bolts broken, and other damage done. The third 150-1b. shot struck Messrs. Beale's plate, and did similar injury. These were the more important of the trials made upon this armour, proving thit the powers of resistance in the 'Minotaur' were inferior to those of the 'Warrior.'

\section{The 'Lord Warden' Target.}

The target, 20 feet by 9 inches, represented the ordinary construction of a wooden ship, armour-plated, with the addition of a thick iron skin worked outside of the frumetimbers of the ship. The following were the scantlings; frame-timbers moulded 121 inches; iron diagonal riders connecting the frame-timbers, 6 inches by $1 \frac{1}{4}$ inches: inner planking 8 inches thick; iron skin, $1 \frac{1}{2}$ inehes thick; outside planking $8 \frac{1}{2}$ inches thick; rolled armour plates 20 feet $\times 4$ feet 6 inches $\times 4 \cdot 5$ inches manufactured by the Millwall Company. The guns used were as follows (see Table, p. 247):

One 68-pounder smooth-bore muzzle-loading gun
One $9 \cdot 22$ inches' muzzle-loading rifle gun 11 feet long, $\dot{6}$ grooves . . , . , ,

One 9·22-inch muzzle-loading rifled gun 13 feet 3 inches long,

6 grooves . . . . . .

One 10.6-inch muzzle-loading rifle gun 11 feet 7 inches long,

10 grooves

One 7-inch muzile-loading rifled gun, 10 feet 9 inches long,

6 grooves

\begin{tabular}{cr|rrr} 
Tons & \multicolumn{3}{c}{ Weight } \\
0 & $95 t_{0}$ & qrs. & 1bs, \\
0 & 95 & 0 & 0 \\
6 & 11 & 2 & 11 \\
12 & 2 & 2 & 0 \\
11 & 15 & 2 & 0 \\
\hline 6 & 13 & 3 & 0
\end{tabular}

Some experiments were made to test the resistance of some rollod armour-plates made by Messirs. John Brown and Co

The plates were of the following dimensions :-

One 13 feet $4 \frac{1}{2}$ inches, by 3 feet 7 inches, and $5 \frac{1}{2}$ inches thick.

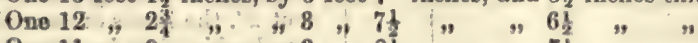

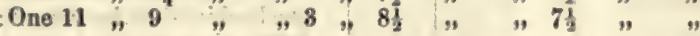

They were secured by $2 \frac{1}{2}$-in. screw-bolts to the skin and framo of Mr. Samuda's old targot; ono-half of each plate had a backing of from 7 inches to 9 inches of toak, and at the hack of the other half, it was left,hollow for an cqual interval between tho plates and the skin. India-rubber washers were used undor the nuts.

The guns in position for trial were:-

One 300-pounder Armstrong muzzle-loading.shunt gun.

One 9-inch Lynall Thomas gun.

One 7 inch whitworth rifle gun.

One 110-pounder. Armstrong breech-loader.

One 68-pounder service 9ó-cwt. gun.

All were fired at a range of 200 yards.

The first three shots (all cast-iron) were fired from the 68-pounder; one shot struck each plate and made indents $1 \frac{1}{2}$ inches deop in the $6 \frac{1}{2}$-inch and $7 \frac{1}{2}$-inch plates, and 2 inches deep in the 5 -inch plate. . ...............?

These were followed by three shots from the 110-pounder, also of cast-iron; the

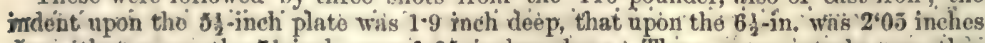
deep, sthat upon the $7 \frac{1}{2}$-inch was 1.65 inehes deep. "There was searcely any other

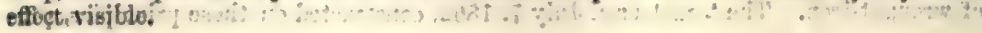


The most remarkable effects were as follows:-

\begin{tabular}{|c|c|c|c|c|c|}
\hline \multirow{2}{*}{$\begin{array}{l}\text { Nature of } \\
\text { Ordinance }\end{array}$} & \multicolumn{3}{|c|}{ Projectiles } & \multirow[b]{2}{*}{$\begin{array}{l}\text { Charge } \\
\text { in } 1 \mathrm{bs} .\end{array}$} & \multirow{2}{*}{ Remarks } \\
\hline & Nature & Weigh & & & \\
\hline $\begin{array}{l}10 \mathrm{ft} .5 \mathrm{in} . \\
\text { Armstrong } \\
\text { rifled gun. }\end{array}$ & $\begin{array}{l}\text { Steol } \\
\text { solid } \\
\text { shot. } \\
\ldots\end{array}$ & $168 \cdot 25$ & 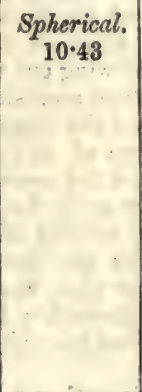 & ' & $\begin{array}{l}\text { Struck upper plate } 10 \mathrm{ft} \text {. from } \\
\text { bottom, and } 9^{\prime} 6^{\prime \prime} \text { from left } \\
\text { side on a bolt, penetrated the } \\
\text { armour-plate, making a hole } \\
\text { in plate } 11 \cdot 5^{\prime \prime} \times 11 \text {, depth to } \\
\text { surface of shot } 12^{\prime \prime} \text {, plate } \\
\text { driven in } 1^{\prime} 8^{\prime \prime} \text { at bottom } \\
\text { much damage was done at } \\
\text { the back; two inner timbers } \\
\text { were rent and splintered and } \\
\text { thrust out, fragments pro- } \\
\text { jecting about 1', Shot ro- } \\
\text { mained in hole apparently } \\
\text { whole. }\end{array}$ \\
\hline $\begin{array}{l}10 \mathrm{ft} .5 \text { in. } \\
\text { rifled guin. }\end{array}$ & $\begin{array}{l}\text { Steel } \\
\text { solid } \\
\text { shot. }\end{array}$ & $\begin{array}{r}301 \\
\\
\end{array}$ & $\begin{array}{c}\text { Cylindrical } \\
10.46\end{array}$ & . & $\begin{array}{l}\text { Struck at junction of plates } \\
5^{\prime} 10^{\prime \prime} \text { from left of target; } \\
\text { bolts at } 15^{\prime \prime}, 13^{\prime \prime} \text { and } 14^{\prime \prime} \\
\text { from hole started } 0 \cdot 8^{\prime \prime}, 0^{\prime \prime} \cdot 7^{\prime \prime} \text {. } \\
\text { and } 0 \cdot 4^{\prime \prime} \text { respectively; both } \\
\text { plates laminated round the } \\
\text { circumference of the hole, } \\
\text { and two small cracks on } \\
\text { front of lower plate from } \\
\text { edge of hole. At the back, } \\
\text { iron knee broken right off, } \\
\text { and lower limb ( } 4^{\prime} \text { long with } \\
\text { three bolts in it), driren } 50^{\prime} \\
\text { to the rear. Area of damage } \\
8^{\prime} \times 4^{\prime} \text {, five or six plank bolts } \\
\text { started and heads off; inner } \\
\text { timbers rent in fragments } \\
\text { and thrust out } 1^{\prime} 6^{\prime \prime} ; \text { large } \\
\text { splinters of wood scattered } \\
\text { all around, Shot struck a } \\
\text { large block of granite in } \\
\text { rear, and broke itself into. } \\
\text { four pieces. }\end{array}$ \\
\hline
\end{tabular}

The next shot was from the Armstrong 300-pounder, with a cylindrical stoel shot weighing $301 \mathrm{lbs}$, and fired with a $45-1 \mathrm{~b}$. charge of powder. This shot had a velocity of 1,295 feet per second at 30 yards in front of the target, and struck the 71 -inch plate where it had the teak backing. The indent made was 6.2 inches deop, and its diameter ahout 12 inches, or rather a circular piece of this diameter was driven in to a depth of .6.2 inches, and nearly, if not quite; separated from the plate, which.was of very good quality. There is, therefore, here, a well-defined measure of the full foree of this shot. Besides this local effect, the target had evidently received a serious shake; one rib was cracked through and bent out; a number of small rivets wero broken; the plate struck was buckled about $1 \frac{1}{2}$ inch and slightly cracked. The shot, which rebounded from the target, was set up about $2 \frac{1}{2}$ inches, and was of excollent material.

A cylindrical steol shell, with a cast-iron head, made on a principle designed by Sir William Armstrong for the purpose of penetrating iron plates by directing the force of the explosion of the bursting charge forward, was next fired from the same gun. It weighod $288 \mathrm{lbs}$, had a bursting charge of $11 \mathrm{lbs}$., and was fired with a charge of $45 \mathrm{lbs}$. of powder, which gave at 25 yards in from the target, a velocity of $.1,320$ foet per second. It struck the $5 \frac{1}{2}$-inch plate on the part supported by the tenk backing. It complotely penetrated the armour-plate, leaving a hole about 14 inches .in diameter, burst in the teak backing, tearing away the inner skin, and breaking a rib, and carried a shower of frngments and splinters in bonrd. The teak was sot' on 
fire by the explosion, but easily extinguished; one bolt was broken, and other injuries done.

Altogether, for completeness of penetration and for the destructive effects which would have been produced both upon the ship and crew, this experiment carries with it great significance.

After this a cylindrical flat-headed homogeneous metal shell, woighing 148 lhs., with a bursting charge of 5 lbs. $12 \mathrm{oz}$, was fired from the Whitworth 7 -inch gun, with a charge of $25 \mathrm{lbs}$. of powder, which gave velocity at 30 yards in front of the target of 1,265 feet per second. This shell struck the $5 \frac{1}{2}$-inch plate near the hole made by the last Armstrong shell, punched out a clean-cut hole about 9 inches in diameter, and burst in the toak backing; beyond blowing out some of the timber, it added very little indeod to the injury done by the Armstrong shell.

Lynall 'Thomas's 9-inch gun next missed the target with a round-headed solid steel shot weighing $327 \mathrm{lbs}$, fired with a charge of $50 \mathrm{lbs}$. of powder, which at 546 foet from the gun, gave a velocity of 1,220 feet per second.

The same gun next fired a wrought-iron solid flat-headed shot, weighing $302 \mathrm{lbs}$., with a charge of $50 \mathrm{lbs}$. of powder. The relocity of this shot was not obtained with cortainty, it struck partly on the $6 \frac{1}{2}$-inch and partly on the $7 \frac{1}{2}$-inch armour; the greatest depth of impression on the latter plate was 6 inches, and on the former 4 inches. The $7 \frac{1}{2}$-inch plate was cracked through a bolt-hole and round the indent. as was also the $6 \frac{1}{2}$-inch plate, but altogether the injury done was less than had been expected.

A hardened steel shot was next fired from the same gun; it weighed 330 lbs., was round-hended, was fired with a charge of $50 \mathrm{lbs}$. of powder, which gave a velocity of 1,220 feet per second at 25 yards in front of the target. It struck close to the lower edge of the $7 \frac{1}{2}$-inch plate, and made an irregular indentation, measuring about 1 foot by 1 foot 8 inches, and 7 inches deep; two bolts were broken, one rib broken through, two others much bent, and the skin bulged in. The shot itself broke in half lengthways.

After this the 300-pounder Armstrong shunt gun fired a spherical wrought-iron solid shot, weighing $163 \mathrm{lbs}$. with a charge of $45 \mathrm{lbs}$., which at 30 yards in front of the target, gave a velocity of 1,630 feet per second. It struck the $7 \frac{1}{2}$-inch plate where it had no teak backing, and made an indent $3 \frac{3}{4}$ inches deop and 13 inches in diameter, with a crack on the face of the indent; the plate was considerably bulged in; and at the back it showed a large starred crack. The shot was flattened out to a diameter of 13 inches.

The matorial of which these armour-plates was made proved itself to be of uniform and excellont quality.

The practical lessons to be learnt from such experiments seem to be these:-

18t. That guns are already in existence which can completely penetrate with shot tho best $7 \frac{1}{2}$-inch armour that can be made, and which can, with shell, pierce the side of a ship built, as to frame, much more strongly than our best ship, and protectod with our best $5 \frac{1}{2}$-inch armour.

2nd. That iron plates can now, with the improved manufacture of the country and the enorgy brought out by the occasion, be made of dimensions hitherto quite unattainable, and yet withont losing anything in quality.

With the exception of America, other nations have done little or nothing in the manufacture of guns throwing projectiles over $100 \mathrm{lbs}$. in woight. The United Statos also boast the possession of two large rifled guns.

$$
\begin{aligned}
& \text { 1st, an 8-inch gun - + . } 175 \mathrm{lb} \text {. projectile, } 16 \mathrm{lbs} \text {. charge. } \\
& \text { 2nd, a } 10, " \quad 250 \mathrm{lb} \text { " } " 25 \mathrm{lbs} .
\end{aligned}
$$

The Table (p. 249) gives the comparative values of the British muzzle-londing built-up ordnance and the American smooth-bore ordnance.

All cast hollow except the 10 -inch of 5.35 tons.

All shell-guns except 10-inch 125-pounder.

20-inch gun only, at present experimental.

Solid shot are only to be fired from the 15 -inch N. S. gun at iron-clad vessels, and thon with $50 \mathrm{lbs}$; 20 rounds may, however, be fired with $60 \mathrm{lbs}$.

The S. B. guns are formidable weapons, although they are merely cast-iron shellguns. Our guns being made of wrought-iron will not, on failure, break up like castiron or steel ordnance; and from their accuracy of fire, the capacity of their shells, and the power these latter have of maintaining a comparatively high velocity (in consequence of their elongated form) the British are in all probability greatly superior as weapons to the American guns.

Professor Major Owen, R.A., has ably dealt with tho following important question: 1 - One of the most important questions at the present time is this: are monster gurs 
British Muzzle-Loading Built-up Ordnance.

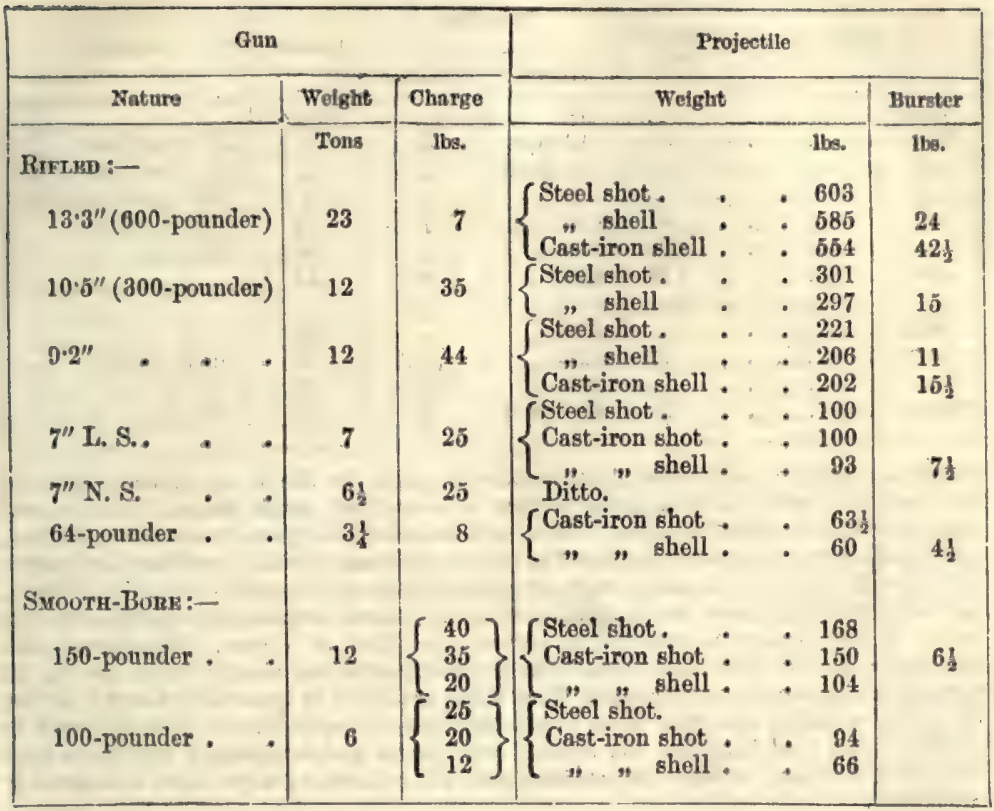

Smooth-Bore Cast-Iron American Ordnance.

\begin{tabular}{|c|c|c|c|c|c|c|c|}
\hline \multirow{2}{*}{\multicolumn{2}{|c|}{ Gun }} & \multirow{2}{*}{$\begin{array}{c}\text { Weight of } \\
\text { Gun }\end{array}$} & \multicolumn{2}{|c|}{ Charge } & \multirow{2}{*}{$\begin{array}{l}\text { Weight of } \\
\text { Shot }\end{array}$} & \multirow{2}{*}{$\begin{array}{l}\text { Weight of } \\
\text { Shell }\end{array}$} & \multirow{2}{*}{$\begin{array}{l}\text { Bursting } \\
\text { Charge of } \\
\text { Shell }\end{array}$} \\
\hline & & & Service. & Minimum & & & \\
\hline 20 -in. L.S. & & $\begin{array}{l}\text { Tons } \\
51 \cdot 42\end{array}$ & $\begin{array}{l}\text { lbs: } \\
100\end{array}$ & $\begin{array}{l}\mathrm{IbE}: \\
\ldots .\end{array}$ & 1,000 & 1be. & Hos. \\
\hline , N.S. & , & $44 \cdot 64$ & 100 & $\ldots$ & 1,000 & & \\
\hline 15 -in. L.S. & . & $21 \cdot 91$ & 50 & & 440 & 380 & 17 \\
\hline N.S. & . & $18 \cdot 75$ & 35 & 60 & 400 & & \\
\hline 13-in. L.S. & . & $14 \cdot 61$ & 30 & $\ldots$ & 300 & 224 & 7 \\
\hline$n$ N.S. & . & 16.07 & 40 & $\ldots$ & 280 & 224 & \\
\hline 11 -in. N.S. & - & $7 \cdot 14$ & 15 & 20 & 170 & 130 & \\
\hline 10-in. L.S. & - & $6 \cdot 72$ & $\left\{\begin{array}{l}15 \text { shell } \\
18 \text { shot }\end{array}\right\}$ & $\cdots$ & $172 \frac{1}{2}$ & 100 & 3 \\
\hline$"$ N.S. & & $5 \cdot 35$ & $12 \frac{1}{2}$ & 16 & 125 & 100 & \\
\hline (or 125-poun & ider) $\}$ & $7 \cdot 36$ & 40 & $\cdots$ & 125 & 100 & \\
\hline
\end{tabular}

required? Opinions are divided; but let us turn to facts, and see what has been done. I have endeavoured in the following Table (p. 250) to arrange some of the leading facts in order, so as to give an idea (necessarily a rough one) of the projectiles and charges necessary to actually penetrate cortain structures at different ranges. I have not chosen the targets in preference to any others, but simply believing that they represent the average resistance offered by sea-going vessels, and also for convenience of comparison.

- It appears then; from what has been already accomplished, that there in at present no occasion to employ monster ordnance for the destruction of ordinary plated ressels.

- The guns we are now making, which will throw projectiles of 200 or $800 \mathrm{lbs}$. weight with charges of $45 \mathrm{lbs}$, are, if properly used, which no doubt they will be, quito sufficient for the purpose. Further than this, however, it is probable that fow fron- 
ARTILLERY

Target Penetrated by Stecl Elongated. Projectiles.

\begin{tabular}{|c|c|c|c|c|c|c|c|c|}
\hline & & & & & & & & \\
\hline & Range & & & Weight of & Froj & ctules & & \\
\hline & & & & & Nature & Diameter & $\ldots$ & \\
\hline $\begin{array}{l}\text { Yarda } \\
200\end{array}$ & . . & & & $\begin{array}{c}\text { cwts, } \\
8\end{array}$ & $\frac{1 \mathrm{bs},}{12}$ shot & $\frac{\text { in }}{8}$ & $1 \mathrm{bs}$ & $2 \gamma^{\prime \prime}$ iron plate. \\
\hline$"$ & I in & & • & $"$ & 12 shell & " & $"$ & $\begin{array}{l}21^{\prime \prime} \text { iron plate and } \\
12^{\prime \prime} \text { wood breking. }\end{array}$ \\
\hline 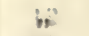 & • & 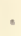 & • & 81 . & 110 shot & 77 & $\therefore \therefore 12$ & $5 \frac{1}{2}$ irón plate. \\
\hline$\ddot{-i}$ & . * . & - & - & 134 & 104 & $"$ & 25 & Warrior. \\
\hline 600 & , $\because \because$ & . & & $\begin{array}{r}148 \\
\text { tans }\end{array}$ & 130 & $\therefore 6.4$ & $\therefore 25$ & $=0$ \\
\hline 800 & : : : & . & & $\therefore$ & 130, & & 27 & \\
\hline $1,500^{?}$ &,$\therefore$ & . & & 12 & $221, y$ & $\therefore 9 \cdot 2$ & $\therefore 44$ & Small plate." \\
\hline,$^{3}$ & , . . & - & & ; & 301 & $10 \cdot 5$ & 45 & " \\
\hline 2,000 & , I" & • & & 22 & 610 shell & 13 & 70 & Warrior. \\
\hline
\end{tabular}

plated sea-going ressels (now afloat) could withstand the fire of our rifled $7^{\prime \prime}$ guns of $130 \mathrm{cwt}$, firecl with 25 -1bs. charges, at 800 or even 1,000 yards' rango ; ${ }^{4}$ for wo must remomber that $j$ actual warfare, vessels are constantly subjeoted to a continual firo, and not merely a few blows delivered at certain intervals of time; and that a structure may thus be kept in a constant state of vibration by the repeated impacts of shots, and will offer less resistance than. when time is allowed between the rounds, for the metal of the armour to resume its former condition of repose. We should also remember that the few plates upon which experimonts are usually made, are in all probability of sounder construction than those produced in quantities for the plating of several ressels; also that when a ship has been for some time at sea, and may in addition have been in action, the armour (bolt-plates and fastonings) will havo been subjected to many sliocks and strains, and will, therefore, have been considerably weakened.'

One of the most extraordinary of these large guns is the so-called 'Woolwicr IrFANT,' which has been recontly (1873) severely injured by the heary charges which hare been fired from it. The report of the Inspector of Ordnance upon the state of the interior of the first 35-ton muzzlerloading rifled gun built for the 'Devastation,' after 38 horizontal discharges from its 12 -inch bore, is illustrated by the accompanying

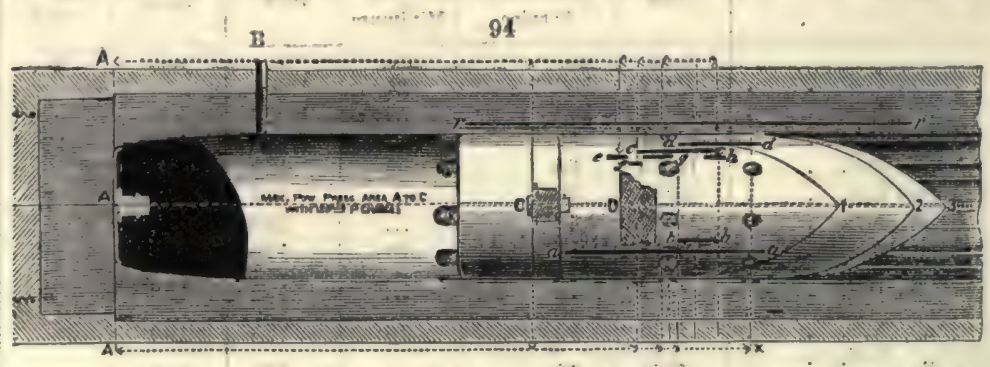

diagram, in which a section of the imner portion is shown to scale. $A$ is the inner ent of the bore, where the maximum pressure, varying from 20 to 66 tons per square inch from identical powder-charges, was registered by crushing gauge. $\mathrm{B}$ is the vent, where the lewest pressures in the chamber were generally registered. c shows the base of the shiot six inches in advanee of its seat; where it experienced the greatest pressure, varying from 18 to 53 tons per square inch from similar powder-charges, The positions of the 700.lbs." shot are shown with 120-1bs. powder-charge, lst, in its seat; 2nd, registering the greatest pressure; and; $3 \mathrm{rd}$, with the rear-studs coming into

i Ascertalned for this range by nsing a $30.1 \mathrm{bs}$. charge at 200 yards.

- $5 \frac{1}{2}$ inches of iron and 27 incheg of wood; the wood and iron nqt disposed in such an adrantageotis manner for resistance'as in the "Warrior' taitget."

4 Accertained for this range by using 235 .lbs. oharge at 200 yards.

- The result of a recent experlment shows " that a structure such ns the "Warrior" can be penetrated at 200 yards' rapge with elongated shot, and sbot cast in chill when the form of the latter is elliptical, .fired from a 7 -Inch rifled gun with 1 chirges': 'and the rebort adds, 'bit it is probiable that har the

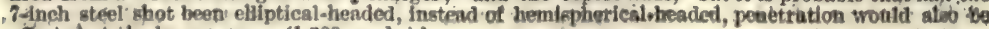
effected ot the longer range $(1,200$ gardan $)$ ? 
'driving' bearing eight inches in advance of their seat. 'This latter point corresponds nearly with that at which the front-studs hammer at starting. With shorter powdercharges these several positions of the shot would be nearor the chamber.

The longitudinal positions of the four cracks, four fissures, and the deop roughness or erosion caused by the escaping gases, are shown by dark lines; that of the greatest enlargement of the bore by dark shading, at $\mathbf{D}$; those of the burrs on the edges of the grooves are not stated in the official report. The nature of these injuries would be hardly visible on so small a scale, and the vertical positions could not be shown in a section. Two of the cracks were on the lower side of the bore, all the other injuries on the upper side, and their centres were $3 \frac{1}{2}$ to 4 feet from $\Delta$, where the greatest powder-pressure occurred, but coincided with the point where the front-studs hammer and the rear-studs come into 'driving' bearing. The gun is being rebuilt, at a cost of abont 700l. or $800 l$.

In detail, the drawing shows the following effects:-Four eracks, aa, $15 \frac{1}{3}$ inches long, centre $44+$ inches from the rear end of the bore at $A ; b b, 3$ inches long, centre 47 inches from $A ; c c, 5 \frac{1}{2}$ inches long, centre 45 inches from $A$; $d d, 6 \frac{1}{2}$ inches long, centre $48 \frac{5}{8}$ inches from $A$; four fissures, $e, 1 \frac{3}{4}$ inches long, centre 407 inches from $A$; $f, 1 \frac{1}{4}$ inches long, centre $42 \frac{3}{8}$ inches from $A ; g, 2 \frac{3}{4}$ inches long, centre $44 \frac{1}{4}$ inches from $\Lambda ; h, 2$ inches long, centre $48 \frac{1}{2}$ inches from $A ; r r$, a roughness extending from 1 inch in front of the seat of the shot ( $28 \frac{1}{2}$ inches from $A$ ), to 65 inches from $A ; \mathrm{x}$, burrs, of which the position is not specified, but approximately about 50 to 52 inches irom $\Delta$. The powder-pressure is relieved at $c, 33 \frac{1}{2}$ inches from $A$ ( 6 inches in front of seat of shot); the greatest enlargement of the bore is at $D, 42$ inches from $A$ (141 inches in front of seat of shot); the hammoring of front-stud is located $15 \frac{3}{4}$ inches away from seat of shot, at 434 inches from $A$; and the rear-studs come into driving contact $\frac{3}{4}$ of an inch further on.

In the 'Philosophical Magazine,' Captain Noble, of the Elswick Works, published an elaborate paper on the influence of the spiral in rifled ordnanee. The following abstract of that paper, freed from its mathematical formula, with the critical remarks, is from an experienced hand.

'Every one is aware that elongated rifled projectiles are the subjects of two principal motions during their exit from the bore, viz., a motion of translation and a motion of rotation. If the rotation be efficiently accomplished, no other considerable motion ought to take place. But if unmechanical derices be resorted to for the purpose of effecting the revolution of the elongated projectile, other undesirable movements are set up within the gun. However, under any sound mechanical arrangemont for supporting and rotating heavy elongated projectiles, it is an ascertained experimental fact, that the force necessary to impart rotation is only a small fraction of that required to give a high velocity of translation. Hence it follows, both in theory, and as an ascertained experimental fact, that the increment of gaseous 'pressure due to rifling is quite insignificant. It is with this small fraction of expulsivo force, and this insignificant increment of gaseous pressure, that Captain Noble deals in his very neat mathematical investigation. He takes two utterly unmechanical systems of rotating heary projectiles_one with an uniform angle of spiral, and the other with an ever-changing angle-both employing studs, and he yields the unmechanical precedence to the stud acting in an uniform spiral. Whether this be so or not, is a philosophical question which appears to us to have been decided by Captain Noble on erroneous data. Still, the formulas adduced will strike mathematicians as particularly neat; and, if they lenve out of sight the whole of the practical objections to tho increasing spiral, these formulas cannot fail to form most instructive beacons to all mathematical artillerists.

- Captain Noble assumes that the action of studs within their grooves is uniform in all the grooves. Now this cannot be, unless the major axis of the projectile coincides with that of the gun throughout the whole of its transit. He also assumes that, previous to starting, the studs are all in "driving" bearing, and at equal dopths in their several grooves; whereas the lower studs, on which the shot rests, and which are the only points in contact with the bore, are then touching the "loading" side of their groove, and all the other studs are more or less on the same, or "off" side of their grooves. Before thoy can come into "driving" bearing, the shot must move forward, slightly in the case of the uniform spiral, and about six or eight inches in the parabolic groove. In the former case, the so-called "pressure" is a succession of 'light blows all round the bore; and in the latter, the shot having attained about onethird of its velocity, it is a succession of very henvy blows. Now. these successive 'blows are concentrated on one-irich points of ench groove, and, constantly recurring upon the same spot, produce slight enlargements and roughnesses, which prevent the smooth stud slipping away easily. The tendency of the shot's momentum, acting on in circlo of points near the centro of gravity and of figure; is to exert an effort of rota. 
tion round those points, or, in other words, round the minor axis. Thus several os cillating motions aro set up, the force of which varies with the amount of the obstacle and the suddenness of the force applied; i.e., with the amount and nature of the powder-chargo. Moreover, in tho caso of the increasing spiral, further mechanical forces aro brought into operation at the muzzle, which have eseaped Captain Noble's investigation. An ever-changing angle of groove cannot be conformed to by a constant angle of shot, any more than a male screw of one pitch cain work into a female screw of a different pitch. To meet this obvious mechanical difficulty, only one stud, one inch long, bears in each groove all along the bore; but when within twelve inches of the muzzlo, an angle of twist is reached which coincides with the angle formed by the front and rear studs on tho shot. Now as this is the spot where most of the " increasing spiral "guns give way, a mathematical examination of the forces brought into play near the muzzle would be fraught with much interest. But these and other mechanical inquiries find no place in Captain Noblo's formula. He assumes that the original action on the stud is a pressure, not a blow, and that this pressure follows a uniform law throughout the gun. Granting this singular hypothesis. the conclusions are rather of a philosophical than practical character, dealing, as we have said, with but a small fraction of the force of translation, and a quite insignificant increment of gaseous pressure. Subject to those deductions, his conclusions are worthy of note.

- Captain Noble educes from his formulas what is, we doubt not, the fact, that in a nniform twist the pressure on the studs is a constant fraction of the pressure of the base of the shot, the value of the fraction depending on the angles of the rifling. The tension of the powder-gases at the muzzle being very small whos compared with their tension at the seat of the shot, the studs have, on the uniform system, scarcely any work to do at the muzzle, while they may be severely strained at the commencemont of motion. He estimates the pressure on the studs due to rifling as, in this case, about $2 \frac{1}{2}$ per cent. of that required to impart translation to a $400-1 \mathrm{~b}$. shot in the 10-inch 18-ton gun, or about $68 \frac{1}{2}$ tons' pressure when it has moved four inches, and 9 tons at the muzzle; whereas the substitution of the parabolic curves for the uniform angle of spiral, according to Captain Noble's formulas, reduces this pressure one-half, so that when the stud has moved four inches (i.e., before it tonches at all on the "driving" side), it sustains $31 \cdot 2$ tons' pressure, gradually rising to 36 tons at the muzzle!

"As Sir William Armstrong certifies that "the maximum pressure" (in the powderchamber) "causes the failure of the stud," the difference of powder-pressure arising from a change of spiral is noteworthy. Captain Noble tells us that, small as the inerement in gaseous pressure due to rifling is, it is still less in the parabolic than in the uniform spiral. Whereas the maximum bursting pressure is reduced from 19.7 tons per square inch to 19.5 tons per square inch, by suppressing the rifling altogether in the case of uniform spirals, the decrement of pressure due to the suppression of the parabolic rifling is a reduction from 19.7 tons to 19.62 tons per square inch. The gain then, to the powder-chamber, from the employment of the increasing spirnl, is .12 of a ton per square inch. We commend this philosophical fraction to our artillery philosophers, and would make them a present of this mathematical advantage. When, however, we turn to the Tables of Pressures registered by the Committee on Explosives in the 10-inch 15-ton, which is the subject of Captain Noble's learned investigation, we are rather puzzled to which of the pressures we are to apply the $\cdot 12$ of a ton. We find similar powder-charges fired under identical conditions producing most unlike results. We find these anomalous pressures with evory description of powder; and we observe that the gun has this parabolic system of rifling, with its consequent stud agency. Yet, with these great philosophical advantages, the powder-pressures registered in the 10 -inch gun, with $87 \frac{1}{2}$ lbs. P. charges and 400-lbs. shot, raried from 25 tons on the square inch to 63.4 tons, the latter expulsive force resulting in the least velocity and striking force in the projectile. Again 60 lbs. R.L.G. charges registered powder-pressures varying from 36.5 tons to 57.8 tons on the square inch, under identical conditions, the highest expulsive force imparting the lowest velocity to the projectile. Yet it is on the register of pressures within this gun that Captain Noble's calculations are based; true, he does not select any of the above figures for his formulas, but on the pressures registered with $85 \mathrm{lbs}$. P. charge, which happened to be $19 \cdot 7$ tons on the square inch. But, if a selection must be made betweon pressures varying from 19.7 tons to 63.4 tons on the squaro inch, it might be quite as well to close the Report of the Committeo on Explosives, and assume any number of tons at haphazard.

-Captain Noble has shown a mathematical gain of 12 of a ton pressure on the square inch by the adoption of the paraholic or increasing spiral. What if the greater part of this astounding variation of pressure from $19^{\prime} 7$ to 63.4 tons on the square inch was attributable to the parabolic curre, or, to speal more accurately, to the stud system 
which that curve necessitates? That gunpowder raries in explosive power in this way in mines, shells, or torpedoes, or anywhere, excopt in a stud-rifled gun, is stoutly denied. If the variation of powder-pressures be not due to the gunpowder it must be due to the shot; and if to the shot, then to the oscillations of the axis round the points of contact with the bore, which are exclusively the studs; but the studs are a necessity of the increasing spiral or parabolic groove. Hence it appears, that, while saving 12 of a ton pressure on the square inch, by employing this most unmechanical contrivance, we are adding at least 4 tons, and, probably, more, pressure occasionally to the gun. These are the deductions which we draw from Captain Noble's formulas, and from the Report of the Committoe on Explosives.'-Iron.

After the siege of Paris inquiries and experiments were made in all directions into the relative qualities of bronze and steel for cannon; amongst the metals tried was phosphorised bronze, of which MM. Montefiore, Léry and Kunzel claimed to be the inventors. This claim is now refuted, and it appears that sixteen years ago the very same alloy was introduced to the French artillery by two officers, MM. de Roulz and A. de Fontenay. In the years 1870 and 1871 a series of experiments were made at Liège, in presence of a commission of artillery officers of all nations, and the results were described in a pamphlet by M. Iséry, as highly favourable to the alloy in question. It is stated in that work, that an ordinary bronze gun founded at liège, having been fired forty-nine times with a charge of one kilogramme of powder and a shot, was so seriously injured that it wasimpossible to continue the experiments with it, while another gun of the same calibre (4)made of the new phosphorised bronze, and fired with the same eharge, and the same number of times, exhibited no sensible injury whatever. Furthor comparative trials with bursting eharges gave results equally favourable to the new metal. In this case, guns of each kind were fired under the same conditions, five times each, commeneing with one kilogramme of powder and a single shot, and carried

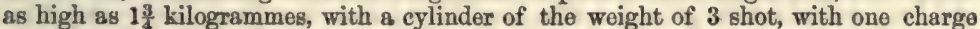
of $1 \frac{1}{4}$ kilogrammes of powder, and 2 shot, and still more so with the same amount of powder, and a cylinder equal to 3 shot. The interior of the chamber of both guns was visibly enlarged, but rather more so in the case of the old than of the new metal gun. Finally, the phosphorised bronze gun burst with a charge of $1 \frac{1}{2}$ kilogrammes of powder, and a cylinder of equal weight. These experiments seemed conclusive, but the French Government ordered further trials to be made at Bourges and other places by a commission of artillery officers, with bronze guns of various alloys, and of foreign as well as French make. The results as between ordinary and phosphorised bronze are very different from those obtained at Liège.

The projectiles used at Bourges were long solid shot, weighing from 10 kilogrammes to 20 kilogrammes for guns of 4 , with charges of powder ranging from the ordinary quantity to 1,700 grammes, the bursting charge. With a charge of 1,150 grammes, and a 10-kilogramme shot, the phosphorised bronze gun began to show cracks, while the ordinary gun exhibited no serious injury whatever. The experiments were afterwards continued with full charges of powder and 20-kilogrammes projectiles: at the seventeenth or eighteenth firing the phosphorised bronze gun burst, and produced a number of small fragments, without any appearance of enlargement of the circumference. At the nineteenth round, the ordinary bronze-metal gun also burst, nearly half the piece being blown off in one mass, which, as well as the rest of the gun, exhibited expansion of the metal with longitudinal fissures. The conclusion the Commission arrived at, after these absolutely conflicting results, was, that the ordinary bronze used for guns exhibits at least as much resisting power as phosphorised bronze, and is incomparably more malleable and less brittle.

SHrics. - The hollow explosive projectiles that we call shells, or bombs, are a very old invention. Under the name of ' coininges,' they consisted of rudely formed globes of plate-iron soldered together, filled with gunpowder and all sorts of miscellaneous 'mitraille.' These were thrown to short distances both from 'pierriers' (a sort of mortar) and from catapultæ, as early as 1495 at Naples, 1510 at Padua, 1520 at Heilsberg, 1522 at Rhodes, and 1542 at Boulogne, Liege. About the middle of the 15th century bombs of cast-iron seem to have come into use; an Englishman, named Malthus, learned the art of throwing them from the Dutch, and perfected the system for the French armies--being the first to throw shells in France, at the siege of $\mathrm{La}_{\mathrm{B}}$ Mothe, in 1643. The diameter of the bomb seems at that time to have become fixed at 13 inches - the old Paris foot; and at this it remains (with very fow exceptional cases) down to the present day.

A few attempts to inerease the size and power of these projectiles have been made at different periods, but never with the practical skill necessary to success; for example, 18-inch shells were thrown by the Fronch at the siege of Tournay, in 1745 ; whereas, just a century before, the Swedes threw shells of $462 \mathrm{lbs}$. weight, and 
holding $40 \mathrm{lls}$. of powder. The Froneh, when thoy occupied Algiers in 1830, found numbers of old shulls of nearly $900 \mathrm{lbs}$. in weight; and in almost every aryentil and furtress in Europe one or two old 16-inch and 18-ineh shells aro to bo found. No attompt was made in modern days to realise the vast accession of power that such large shells confer, until the year 1832, when the 'monster mortar,' as it was then ealled, of 24 inches calibre, designed by Colonel Paixhans (the author of the Paixhans gun), was constructod by order of Baron Evain, the Bolgian Minister of War, and attempted to be used by the Fronch at the siege of the citadel at Antwerp, but with the worst possible success. The mortar, a crude cylindrical mass of cast-iron; sunk in a bed of timber weighing about 8 tons, and provided neither with adequate means for 'laying' it, nor for eharging. it - the heavy shells weighing, when flled with $99 \mathrm{lbs}$. of powder, $1,015 \mathrm{lbs}$. each-could with difficulty be fired three rounds in two hours, while the shells themselves were very badty proportioned.

One of these shells fell nearly close to the powder-magazine, but did not, explode; had it fallon upon the presumed bomb-proof arch of the magazine, containing 300,000 1bs. of powder, it would have piereed it, aceording to the opinion of all the military ongineers present at the sioge, and so closed the enterprise at a blow. The ill: success of this mortar prevented for several years any attompt to develop bombs jnto their legitimate offico-as the means of suddenly transferring mines into the body of fartified places - of a power adequate to act with decisive effect upon their works; although some years aftorwards a 20 -inch mortar was made in England for the Pacha of Egypt, and proved at Woolwich.

But another cireumstance still more tended to the neglect of large shells thrown by vertical fire. After repeated trials and many failures, it was found practicable to throw 10-inch (and since that even 13-inch) shells from cannon, or 'shell-guns,' by projecting them nearly horizontally, or at such low angles that they should 'ricochet' and roll along the ground before they burst; and, thus fired, it was soon seen that their destructive power as against troops was greater than if fired at angles approaching $45^{\circ}$ of elevation from mortars. Paixhans and his school had pushed a good and useful invention beyond its proper limits, and had lost sight wholly of the gll-important fact that horizontal sholl-fire, powerful as it is against troops or shipping, is all but useless as an instrument of destruction to the works (the earthwork and masonry, \&ce.) of fortified places; for this end, weight and the penetrative power due to the velocity of descent in falling from a great height are indispensable,

A 13-inch shell, weighing about $180 \mathrm{lbs}$, is thrown, by a charge of $30 \mathrm{lbs}$. of powder, barely 4,700 yards. While, with not much more than double this amount of powder, the 36 -ineh shell, of more than 14 times its weight, can bethrown 2,650 yards, or much more than half the distance:

The explosive power, it. is obvious, is approximately proportionate to the weight of powder; but, by calculations, of which the result only can here be given, Mr. Mallet has shown that the total power of demolition-that is to say, the absolute amount of damage done in throwing down buildings, walls, \&ce. \&ce- - by one 39-inch shell, is 1,600 times that possible to be done by one 13-inch shell; and that an object which a 13-inch shell could just overturn at one yard from its centre, will be overthrown by the 36-inch shell at 40 yards' distance.

A 13-inch shell penetrates, on falling upon compact earth, about $2 \frac{1}{8}$ feet. The Antwerp shell penetrated 7 feet. The 36-inch shell penetrated 16 to 18 foet. The funnel-shaped eavity, or 'crater,' of earth blown out by the explosion of a buried sholl, is always a similar figure, called a 'paraboloid ; ' its diameter at the surface, produced by the 13 -inch shell, is about 7 feet, and by the 36 -inch shell about 40 feet.

No bomb-proof arch (so called) now exists in Europe capable of resisting the tremendous fall of such masses, and the terrible powers of their explosion when 480 lbs. of powder, fired to the very best advantage, puts in motion the fragments of more than a ton of iron. No precautions are possiblo in a fortress, no splinter-proof, no ordinary vaulting, porhaps no casemont, exists capable of resisting their fall and explosion. Such a shell would sink the largest ship or floating battery.

$A$ single 36-inch sholl in flight costs 25l., and a single 13-inch 21.28 ., yet the former is the cheaper projectile; for, according to Mr. Mallet's calculations, to transfer to the point of effect the same weight of bursting powder, we must give-

55 shells of 13 inches, at $2 l .2 s$.

Against 1 shell of 36 inches

Showing a saving in favour of the large shell of

\begin{tabular}{rrr}
115 & 10 & 0 \\
25 & 0 & 0 \\
\hline
\end{tabular}

$90 \quad 10 \quad 0$

In 1871 we have the following return of Cannon and Mortars exported :- 


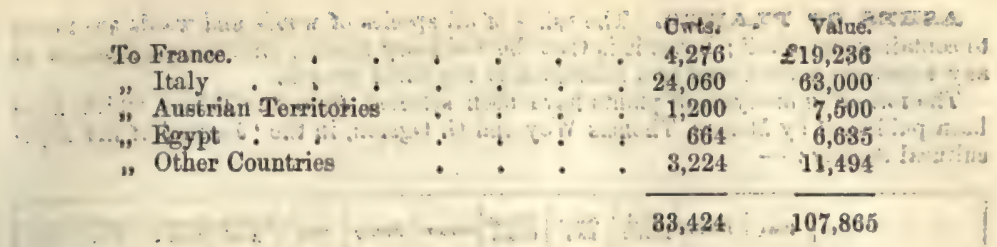

ArUar vUrGara. A. maculatum; The Wake-robin; Lords and Ladies. In tho island of Portland a kind of arrowroot was propared from this plant: See ArrowRoor.

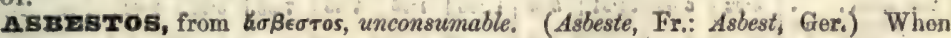
the fibres of the fibrous varieties of amphibolo aro so slender as to be flexible, it is called asbestos, or amianthus. It is found in Pjedmont, Savoy, Saliburg, the Tyrol, Dauphiné, Hungary, Silesia ; also in Corsica so abundantly as to have been mado use of by Dolomiou for packing minerals ; in the United States, St. Kovern' in Cornwall, in Aberdeenshire, in somo of the islands north of Scotland, and Greenland. Asbostos was manufactured into cloth by the ancients, who were well acquainted with its incombustibility. This cloth was used for napkins, which could bo cleansed by throwing them into the fire; it was also used as the wick for lamps in the ancient temples; and it is now used for the same purpose by the natives of Greenland. It has beon proposed to make paper of this fibrous substance, for the preservation of important matters. An Italian, Chevalier Aldini, constructed pieces of dress which are incombustible. Those for the body, arms, and legs were formed out of strong cloth steeped in a solution of alum; while those for the head, hands, and feet were made of eloth of asbestos. A piece of ancient asbestos eloth, preserved in the Vatican, appears to hare been formed by mixing asbestos with other fibrous substances; but M. Aldini has executed a piece of nearly the same size, which is superior to it, as it contains no foreign substance. The fibres were prevented from breaking by the detion of steam. The eloth is made loose in its fabric, and the threads'are about the fiftieth of an inch in diameter. The Society of Encouragement, of Paris, proposed a prize for the improvement of asbestos cloth. The use of it was publicly exhibited in London in 1858.

Common Asbeptos is found in fibres of a dull greenish colour, and of a somewhat pearly lustre.' In the Serpentine formations at the Lizard Point, in Cornwall, it is common. Dr. M'Culloch found it in the limestone of Glentilt in a pasty state, but it hardened upon exposure.

Mountain Leather, or Mountain Paper, is not in parallel fibres like the procoding, but the fibres are interwoven. The thinner pieces bear the latter name: Wanlock Head is the best known locality in this country.

Elastic Asbestos, or Mountain Cork, floats on water. It has, like the preceding, an interlaced fibrous texture. It varies in colour, being, white, grey, yellow, or brown, In appearance and feel it is not unlike comman codk, and it has a certain degreo of elasticity, hèce its namo.

Mountain Wood, or Lignfform Asbestos. This variety is usually massive, and of a brown colour, having much the appearance of wood. It is found in several localities in Scotland.

AsE. (Fraxinks excelsa.) Ash is superior to any other British wood for its toughness and elasticity. It is therefore used for the frames of machines, for agricultural implements, and the felloes of wheels. This wood is split into pieces for the springs of bleachers' rubbing-boards. Handspikes; hammer-haridles, rails for chairs, \&c., are made from the ash. All these and similar warks are much stronger when they follow the natural fibre of the wobd. Hoops are also frequently made of the young branches of the ash. Rankine gives its tenacity. as 17,000 , and its modulus of elasticity, or resistance to stretching, as 1,600,000.

Certain species of Fraxinus in the South of Europe yield a sweet exudation known as Manna.

Astres. In commerce, the word ashes is applied to the ashes of vegetable sub. stances from which the alkalis are obtained. Soe KrLP, BARiri, \&

It is the popular name of the vegetable alkali, potash, in an impure state, as procured from the ashes of plants by lixiviation and evaporation. The plants which vield the greatest quantity of potash aro wormwood and fumitory. Sea PorAsh, Prartasir, and, for the modo of determining tho value of àshes, Aizanimetrix.

Commercially ashes aredivided into soap-ashes, wood-ashes, and weed-ashes. . 
Asxzs Or PIAAxTs. The ashes of all species of woods and woods aro found to contain some alkali; hence it is that the residuary matter, after the combustion of any vegotablo mattor, acts as a stimulant to vegotable growth.

The following anulyses of plants havo beon selected from the Tables which have been publishod by Messrs, Thomas Way and G. Ogston, in tho 'Journal of the Agricultural Society:"-

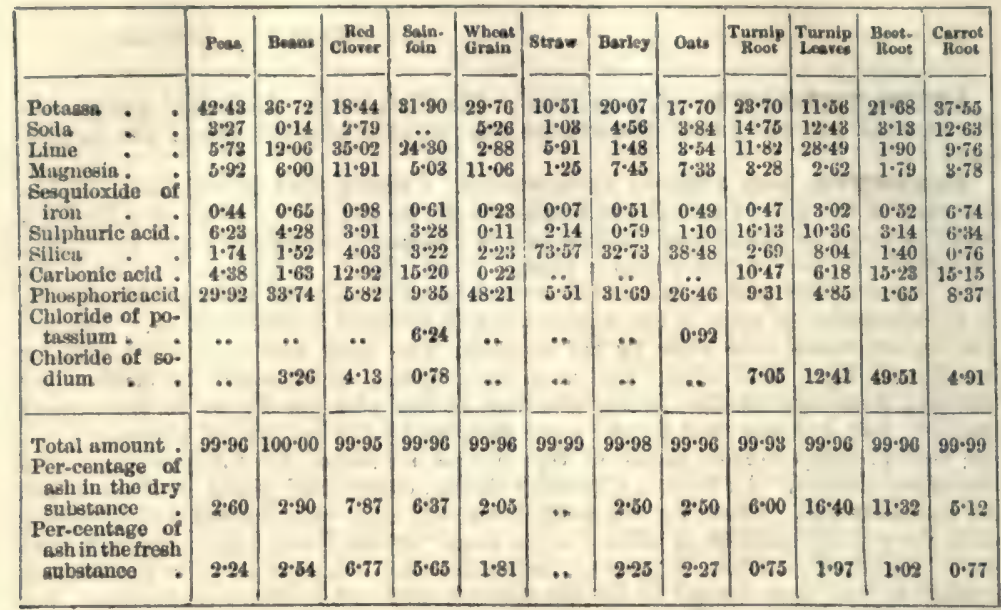

A few additional analyses, by Prof, Way and other chemists, are given for the purpose of showing the variations which exist in the constituents of plants as determined by the analysis of their ashes :-

\begin{tabular}{|c|c|c|c|c|c|c|}
\hline$\cdots, 5, \ldots$ & Potatoes ${ }^{2}$ & $\begin{array}{l}\text { Lettuce } \\
\text { Leaves and } \\
\text { Stalk }^{3}\end{array}$ & $\begin{array}{c}\text { Olive-tree } \\
\text { Wood }\end{array}$ & Hops * & Hay ${ }^{2}$ & $\begin{array}{l}\text { Spronts: } \\
\text { Ctupen } \\
\text { Sprotus. }\end{array}$ \\
\hline Potassa $\ldots$ & $25 \cdot 41$ & $22 \cdot 37$ & $20 \cdot 60$ & $24 \cdot 88$ & $11 \cdot 93$ & $17 \cdot 23$ \\
\hline Soda . . & $\ldots$ & $18 \cdot 50$ & $\ldots$ & $\ldots$ & $1 \cdot 07$ & $1 \cdot 19$ \\
\hline Lime : . . & $2 \cdot 84$ & $10 \cdot 43$ & $63 \cdot 02$ & $21 \cdot 59$ & $14 \cdot 76$ & $23 \cdot 57$ \\
\hline Magnesia $\quad . \quad$ & $4 \cdot 17$ & $5 \cdot 68$ & $2 \cdot 31$ & $4 \cdot 69$ & $5 \cdot 30$ & $3 \cdot 01$ \\
\hline Sesquioxide of iron . & 0.50 & $2 \cdot 82$ & $\cdots$ & $1 \cdot 75$ & $2 \cdot 75$ & $0 \cdot 28$ \\
\hline Sulphuric acid * & $4 \cdot 71$ & $3 \cdot 85$ & $3 \cdot 09$ & $7 \cdot 27$ & $0 \cdot 20$ & \\
\hline Silies : * & $3 \cdot 64$ & $11 \cdot 86$ & $3 \cdot 82$ & $19 \cdot 71$ & $53 \cdot 43$ & \\
\hline Carbonic acid & $\ldots$ & $\cdots$ & $\cdots$ & $2 \cdot 17$ & & \\
\hline Phosphorio acid & $10 \cdot 38$ & $9 \cdot 38$ & $4 \cdot 77$ & $14 \cdot 47$ & $6 \cdot 34$ & $43 \cdot 52$ \\
\hline Chloride of potassium & $12 \cdot 40$ & $\because$ & $1 \cdot 09$ & & & \\
\hline Chloride of sodium * & trace & $15 \cdot 09$ & $\cdots$ & $3 \cdot 42$ & $2 \cdot 27$ & $11 \cdot 19$ \\
\hline \multirow{4}{*}{$\begin{array}{l}\text { Total amount } \\
\text { Per-centage of ash in } \\
\text { the dry substance } \\
\text { Per-centage of ash in } \\
\text { the fresh substance }\end{array}$} & $100 \cdot 00$ & $99 \cdot 99$ & 100.00 & $99 \cdot 95$ & $100 \cdot 00$ & 100.00 \\
\hline & $4 \cdot 86$ & & 0.58 & $5 \cdot 95$ & 6.97 & \\
\hline & & & & & & \\
\hline & *ै & .. & .. & $\cdots$ & $6 \cdot 15$ & \\
\hline
\end{tabular}

The large amount of silica found in the grasses, constituting, as it does, much of their outer coating, cannot fail to be noticed. The variations in the quantities of phosphoric acid are instructive.

A vast number of analyses of the ashes of plants have been collected and systematically arranged by Dr. Emil Wolf in his 'Aschen-Analysen von landwirthschaftlichen Producton, Fabrik-Abfälen und wildwachsenden PAlanzen.' 4to., Berlin, 1871.

Griepenkerl. "Griepenkeri. " A, Muler. Way. "Hubert. - Way. 
AsmIAR or AsRIFR. When stones are worked in regular beds and joints, and aro dressed for facing work, thoy are called ashlar. The stone used as ashlar is called ashlaring, when in thin slabs, and made to serve merely as a case to tho regular body of the wall.

AsmIERING, in carpentry, are the short upright pieces of timbering or quartering fixed, in garrets, to the floor and rafters, to cut off the acute angle which they form.

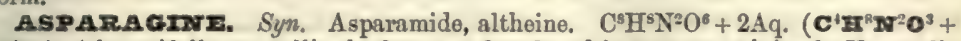
$\mathbf{H}^{2} \mathbf{O}$.) A beautifully crystallised substance, first found in asparagus juice, by Vauquelin and Robiquet, in 1805 . It not only exists in a great number of vegetables, but some which do not contain it naturally may be made to afford it by being grown in dark damp cellars. Many plants normally containing only small quantities of it may be made to yield more by being allowed to germinate in that manner. Among the vegetables from which it can be directly obtained may be mentioned the following:Althea officinalis, Asparagus acutifolins, A. off., Atropa belladonna, Convallaria majalis, C. mulliflora, Cynodon dactylon, Glycyrrhiza glabra, Lactuca sativa, Ornithogalum caudatum, Paris quadrifolia, Robinia pseudacacia, Solanum tuberosum, and Symphytum off. The following list contains the names of some plants normally containing no asparagine, but yielding it when allowed to germinate in darkness in damp cellars :Colutea arborescens, Cytisus laburnum, Ervum lens, Genista juncea, Hedysarum onobrychis, Lathyrus odoratus, L. latifolius, Phascolus vulgaris, Pisum sativum, Trifolium pratense, Vicia Faba, and V. sativa.

Preparation.- Perhaps the most convenient and economical mode of procuring asparagine is from the etiolated (blanched) shoots of vetches. When they have acquired a length of two inches-which, under favourable circumstances, will be in about three weeks - they are to be crushed, and the juice pressed out. The quantity yielded will be rather less than three-fourths of the weight of the plant. It is then to be boiled for a short time, to coagulate the regetable albumen, and strained. This clarified fluid is to be evaporated until almost syrupy, and put aside to crystallise. The product is at first brown, but by washing with cold water, afterwards dissolving in boiling water, and subsequent crystallisation, it may be obtained pure. If, provious to putting the hot fluid aside to crystallise, a little pure animal-charcoal be added, and the whole be digested a short time, and then filtered, the crystals will be obtained brilliantly white at one operation. Some chemists advise the germination to be allowed to go much further than was mentioned abore, so that the shoots may be as long as 15 inches. The crystals obtained by the process given have the formula $\mathrm{C}^{5} \mathrm{H}^{8} \mathrm{~N}^{2} \mathrm{O}^{6}+2 \mathrm{Aq}$., but the water is expelled at $212^{\circ}$. Asparagine possesses the peculiarity of behaving like a base towards strong oxides and like an acid towards bases. The erystals obtained by the method given contain, in the 100 parts, earbon $32 \cdot 00$, hydrogen $6 \cdot 67$, nitrogen 18.67 , oxygen $42 \cdot 66$. Dried at $212^{\circ}$, it has the folhowing composition: carbon $36 \cdot 36$, hydrogen $6 \cdot 06$, nitrogen $21 \cdot 21$, oxygen $36 \cdot 37 .-$ C. G. W.

ASPARAGOrTrE. A name given to a variety of apatite of the colour of asparagus.

Asparacts orricrasars. An esculent vegetable belonging to the natural order Liliacece. The young shoots sent up from the underground stem are the parts used,

AsPxis. The Populus tremula. A tree native of almost all parts of Europe as far north as Siberia. The wood is white, and is applied to many useful purposes. The chareoal prepared from the aspen is said to be well adapted for gunpowder. See Poptar Trez.

ASPEAIT, ASPEAITUM, or MrrszRAT PXTCE. (Asphalte, Fr.; Asphalt, Bergpech, Ger.) A name applied to the solid varieties of bitumen. In its purest form asphalt presents the appearance of a black or brownish-black solid substance, possessing a bright conchoidal fracture. It fuses at $212^{\circ} \mathrm{F}$., burning with a brilliant flame and emitting a bituminous odour. Specific grarity $=1$ to 1.2. Asphalt is insoluble in alcohol, but soluble in about five times its weight of naphtha.

The mineral substances included under the rather wide term of asphalt appear to differ considerably in chemical composition. They may generally be resolved proximately into certain oils, resins, and pitch-like solids; whilst ultimately they yield carbon, hydrogen, and oxygen, associated with a small proportion of nitrogen and mineral matter. The asphalts appear to have been formed by the evaporation, so'iditication, and consequent partial oxidation of certain liquid hydrocarbons, such as potroleum. Their ultimate origin may no doubt be traced to organic sources.

Asphalt is found in small quantity in the carboniferons limestone of Derbyshire, Vor. I. 
Staffordshire, and elsewhore. It is gonerally said that masses of asphalt flont on the surfuce of the Dead Soa, or Lako Asphaltites-whence tho name-but aceording to modorn observers, the quantity found at presont is but small. Tho rast deposit of asphalt in the Great Piteh Lake of 'Trinidad is deseribed under the hend of Butuares. A substance known as chapapote, or Mexican asphalt, is imported from Cuba.

Large masses of rock impregnated with asphalt are found in certain localitios on the Continent, and are important as furnishing most of the asphalt used commercially. Porhaps the best known of theso doposits is the great mass of Jurassic limestone in the Val do Travers, at Seyssel, on the Rhone. Similar rocks occur at Limmer, near Hanover; in the Isle of Brazza in Dalmatia ; at Hölle, near Heide, in Holstein ; in the Tyrol, and in Alsace.

Under the head of Brtumen will be found some historical notices of the ancient use of asphalt and kindred substances for building purposes. In modern times attention has frequently beon directed to the utilisation of the asphaltic rocks of Switzerland, espocially as a source of asphalt for paring. Without following tho vicissitudes of the Swiss workings since they were originally commenced by Erinus, in 1712 , it may suffice to say that within the last few years considerable attention has bren directed to their development, and that they are now activoly worked. Originally the asphalt obtained from the rocks of these quarries was employed in the form of a liquid, melted out from the limestone, and spread orer the pavement-durability being imparted to the matorial by admixture of coarse sand and by sprinkling sand or gravel over the surface. The following is the modern method of laying down asphaltic foot-paths, as practised in Paris :-A foundation is first formod by a layer of concrote, the surface of which is carefully flattened. On this even surface, when dry, the melted asphalt is spread. This asphalt is melted in a cauldron, and a proper proportion of sand added-the mixture being kept stirred to provent subsidence of the sand. The asphalt is spread with a woodon trowel over the concrete, and the surface is finally smoothed over.

Of late, a very great improvement in the construction of asplalt roads has been introduced, the merit of the new method being due to M. A. Merian, of Basle. The asphalt is laid down in the form of a hot powdor, and the powdor is then beaten into a compact mass. The process has been thus described by M. Léon Malo:- The asphalt stone is brought direct from the quarries, and broken up into small pieces about the size of those used for macadamized roads; it is then heated over a stove, in a drum-shaped iron vessel with feet, till it crumbles into powder; and in order that the powder may not lose its heat, the whole apparatus is conveyed on to the street where it is to be appliod. Then a foundation of beton (conerete) is laid, about 4 inches deep, which may, however, be thicker or thinner according to the nature of the soil.' The concrete having hardened, its surface is brought to the required curve, and a Inyer of powdered asphalt, 16 to 20 inches thick, laid down and compressed by stamping, the surface being finally smoothod by a heavy roller.

The Val de Travers asphalt quarries are now largely worked, and sereral experimental patches of paving with this asphalt have been laid down in some of the main thoroughfares of London, and have in general proved highly successful.

In addition to the use of asphalt for pavements and roadways, the material is of value as a waterproof lining for cisterns, and as a cement. It also finds application in the arts as a component of certain varnishes. Soo Bitumas and Pavemast, Aspratit,

ASPHALTENE. A product obtained from asphaltum by volatilising the oily and volatile product petrolene.

ASPHAITIC MAsTIC, used in Paris for largo works, is brought down tho Rhone from Pyrimont, near Seyssel. It is composed of nearly puro carbonate of lime, and about 9 or ten per cent. of bitumon.

When in a state of powdor it is mixed with about 7 per cent. of bitumen or mineral pitch, found near the same spot. The powdered asphalt is mixed with tho bitumen in a moltod state along with clean gravel, and consisteney is given to pour it into moulds. Sulphur added to about 1 per cont. makes it very brittlo. The asphalt is ductile, and has elasticity to enable it, with the small stones sifted upon it, to resist ordinary wear. Walls having cracked, and parts having fallen, the asphait his been soen to stretch and not crack. It has been rogarded as a sort of mineral leather. The sun and rain do not appear to affect it; and it answers for abattoirs and barracks, keops vermin down, and is uninjured by the kicking of horses.

AspmaIT orx. An oil obtained from asphaltum by dry distillation.

Aspiartiom. Seo Aspiait.

Asphastum, Gas-Tar, or Artificial. When, with the thick pitẹy residuo, 
obtained by evaporating off the more volatile portions of gas-tar, sand, chalk or lime are mixod, it is ealled artificial asphaltum. Tho minoral substances aro strongly heatod to expel all moisture, und then added to the pitch while in tho melted state. This is used for pavemonts, for lining tanks, \&c.

Aspriatron. An apparatus used for drawing a stream of air through a tube or other vessel. There are several varieties used by chemists in the analyses of air. One or two only noed be mentioned here.

Brumier's consists of two equal cylindrical vessels placed one above inother and communicating by tubes, which can be opened or elosed, so that when tho water has run from the upper to the lower vessel, the apparatus, turning for tho purpose on a horizontal axis, may be inverted, so as to repeat the process.

An aspirator devised some years ago by Mr. M. W. Johnson is of considerablo interest, as the principle of its action has of late received extensive application in the construction of certain forms of exhausting apparatus. The arrangement of Johnson's aspirator will be readily understood from the accompanying figure (fig. 95). A tap in connection with a supply of water is fitted by means of a piece of

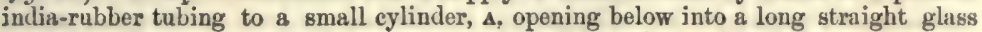
tube, $\mathrm{s}$, and communicating by means of a lateral branch, $\mathbf{c}$, with the ressol through which the stream of air is to bo drawn. On opening the tap, the water runs down the tubes A B, earrying with it air, which it sucks in througl the branch-tube, $\mathrm{c}$; in this way a stream of air continues to flow in at $c$ as long as water is allowed to run through A B. An aspirator of this form was fitted up by Mr. Jolnson at the Royal College of Chemistry, and was described before the Chemical Society in 1852. ('Journ. Chem. Soc.' vol. iv. p. 186.)

Johnson's aspirator is similar in principle to the water-blowing machine known as the trompe, used with the old Catalan iron-furnaces, still working in the Pyrenees and other parts of Southern Europe. In the trompe, a cistern of water is connected with wooden pipes having small openings called aspirateurs, communicating with the external atmosphere. As the stream of water descends from the eistorn

95

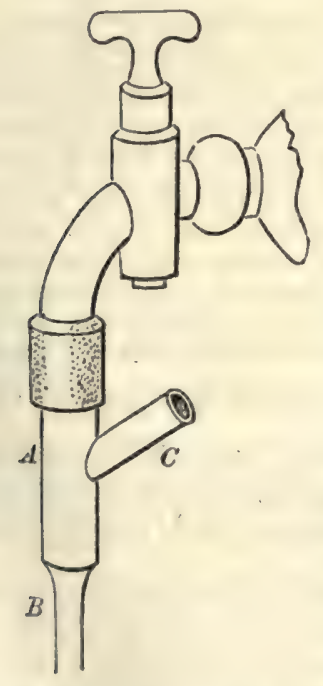

96

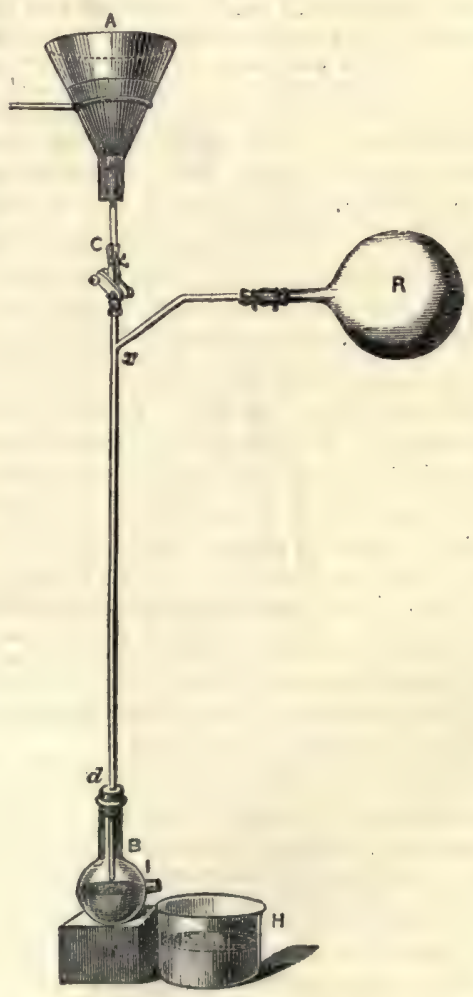

through the pipes, it draws down with it a current of air entering through these aspirateurs, and the mixod air and wator passes into a vessel below, whence the water flows out through a special aperture, whilst the air passes to the tuyere.

On roferring to the previous figuro of Johnson's aspirator, it is clear that if the 
latoral tube, c, instead of being connected with an open tubo through which air can bo drawn, be connected with a closed receivor, tho air will bo gradually sucked out of this vessel by the descending current of water, and a vacuum, more or less perfect, may be thus obtained. This is, indeed, the principlo of the admirable, though simple, exhausting apparatus introduced by Dr. Sprengel, in which a vacum is obtained by the descent of a column of mercury. The morcury is placed in a large funnel, A (fig. 96), which communicates with a glass tube, $c, d$, longer than the tube of the mercurial barometer, and open at both ends. The receiver, $\mathrm{R}$, to be exhaustod, is connected with a lateral tube, opening into the main tube at $x$. On pressing a spring-clamp attached to the india-rubber connection below $c$, the mercury flows down the tube, $c, d$. As the lower end of this tube opens a little below the lateral spout of the bulb, $\mathbf{B}$, the first portions of mercury hermetically seal this open end, and prevent any ingress of air. The descending column of mercury is broken up into detached cylinders separated by columns of air sucked in from the vessel $\mathbf{R}$. The mereury and air pass out at the lateral aperture of the bulb $\mathbf{B}$; and to economize the mercury, it is from time to time poured back from the vessel $\mathbf{H}$ to the funnel $\mathbf{\Delta}$. As rarefaction proceeds, the quantity of air enclosed with the mercury becomes less and less, and as the exhaustion approaches completion the mereurial column is almost uninterrupted by any air. At this stage of the operation, the falling morcury makes the characteristic sound of a liquid moving in a vacuum, familiar in the common water-hammer. When the exhaustion is complete, about 30 inches of mercury will bo supported in the tube, $c, d$, and the remaining part of the tube, together with the lateral branch $x$ and receiver $\mathbf{R}$, is in the condition of the Torricellian vacuum-that is to say, it is a space containing nothing but vapour of mercury at very low tension. By means of this morcurial pump a higher degree of rarefaction ean bo attained than with tho ordinary air-pump. Dr. Sprengel found no difficulty, even with common cold mercury, in rarefying the air to one-millionth of its original density. Tho-inventor recommends the use of an exhausting-syringe to remore the bulk of the air, employing the mercurial pump only as an auxiliary in completing the exhaustion. In practice, the Sprengel pump is not so simple in construction as that previously figured, but is furnished with certain accessory parts, which do not, however, affect the general principles of its action. Some improvements in the pump have been introduced by Prof. McLeod. (For Sprengel's original researches, see 'Journ. Chemical Soc.' 1865, p. 9 : for McLeod's improvements, seo same journal, 1867, p. 307).

Dr. Sprengel, in explaining the action of his mercurial pump, says: 'My instrument is merely the reverse of the trompe, with this addition, that the supply of air is limited, while that of the trompe is unlimited."

An important application of the principle of the Sprengel pump has been recently made by Professor Bunsen, in his new method of filtering. The operation of filtoring is usually very tedious, and in many branches of manufacturo a rapid method of filtration is highly desirable. Bunsen's great improvement consists in accelerating tho operation by filtering into a flask from which the air has been partially withdrawn: the pressure of the atmosphere on the surface of the liquid in the filter forces the liquid through the pores of the paper, and the partial vacuum on the other side offers but littlo resistanco to its passage. To effect the exhaustion, the vessel which receives the filtored solution is placed in connection with a water air-pump, that is, an apparatus on Sprengel's principle, but working with water instead of mercury. This water air-pump is recommended by Bunsen, not only for washing procipitates, but for crystallising substances from syrupy mother-liquors. Both the mercurial and water air-pumps may become of great use in the arts, where a ready and perfect means of exhaustion may be needed.

(For translation of Bunsen's paper on filtration with the filter-pump, seo. 'Philosophical Magazine,' Jan. 1869, p. 1.)

The following form of spirator, devised by Professor Guthrie, will be found useful in establishing a current of air for an indefinite length of time, by means of a continuous current of wator. It may be employed either as aspirator in drawing, or as expirator in forcing air through an apparatus. Its action will be readily understood by referring to the accompanying diagram (fig. 97.).

$\Delta$ is a wide-mouthed 12 -ounce bottle, whose bottom is covered with a few millimetres of mercury, $m$. Through its well-fitting cork, c, four holes are bored. Into the first of these, the wide tube open at both ends, $g$, is fitted, so that its lower ond is a little above the mercury, $m$. The upper end of $g$ is provided with a cork, through which the syphon-tube $f$ passes. In the second hole of $\mathrm{c}$ the bent tube $h$ is fixed, in such a manner that its lower end dips beneath the surface of the mercury, $m$. Through the third hole of the cork c, a straight tube, $c$, is passed, whose office is to convey water into the bottle, $\mathrm{A}$; the lower ond of $c$ is a littlo above the mercury, $m$. Through the fourth holo. in $\mathrm{c}$ a narrow tube, $e$, is fastoned, whose longor exterior 
limb dips under the surface of the mercury, $n$, in the tube $\mathbf{B} . \quad \mathbf{B}$ is a small test-tube, widened at the bottom in three diroctions, and containing a fow millimetres in depth of mercury, $n$. It is fastened with sealing-wax to $\mathbf{A}$, and is provided with a cork, the one orifice of which admits the tubo $c$; through tho other passes tho bent tube $d$.

The lower extremity of the tube $f$ being placod far down a sink, and a gradual stream of water being allowed to enter by $c$, as indicated by (1), the water in $\mathrm{A}$ risos (2), the air is driven out of $\mathbf{A}(3)$, bubbles througl the mercury, $n$, and passes by $d$. (4), through the apparatus employed. During this filling of $\Delta$ with water, the mercury, $m$, prevents the retrograde flux of water through $h$. $\Lambda$ fter $\mathbf{\Delta}$ is filled, the water

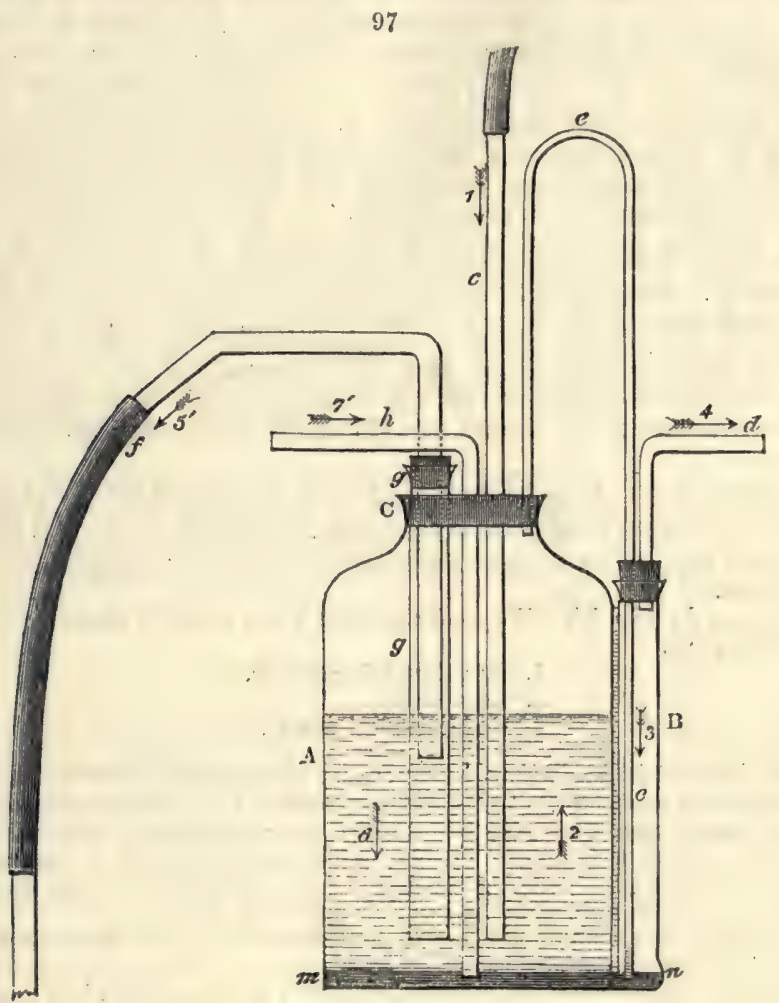

rises in the tubes $f$ and $e$ until it reaches the top of $f$, when this tube acts as a syphon $\left(5^{\prime}\right)$, and being wider than the ingress tube $c$, gradually empties the bottle $\mathrm{A}(6)$. To supply the place of this water, the air must enter by $h\left(7^{\prime}\right)$, and may thus be drawn through the apparatus in use, while the mercury $n$, in $k$, prevents the regression of the air through $e$.

Tho instrument has of course a simpler form when required to act only as an aspirator, for then the tube $\mathrm{B}$ and its appendages may bo dispensed with.

When an increased resistance has to be overcome(the instrument being used either as aspiritor or as expirator), the tube $f$ is drawn further out of the tube $g$.

This form of spirator has been found to be certain in its action; for. with a continuous stream of water, the volume of air which passes through is in definite proportion to the volume of water employed; and, indeed, the slower strenm of water tho more nearly equal are these volumes, for in this case the amount of water passing through $c$ during the action of the syphon $f$; is inconsiderable.

Assarcirida. (Assus, dried; fretidus, fetid.) A fetid gum-rosin obtained from the root of the Narthex Assafotida, and probably from some other species belonging to the Umbellifere.

AssAMAR. (Assare, to roast; Amarus, bitter.) A name given to the bitter 
substance producod whon gum, sugar, starch, or broad aro roastod until they turn brown. See Caravizi.

assay. (Essai, Fr., Probe, Ger.) assarivg. (Docimasie, Fr., Probirkunst, Ger.) Assaying is the art of estimating the proportion of metals in minorals, ores, metallurgical products, and various alloys used for monetary and other purposes. It is generally regarded as a branch of metallurgy, and should occupy an important place in metallurgical instruction. A knowledgo of chemistry is essential to a corroct understanding of the nature of the operations, and of the reactions which ocenr in the various assay-processes. To the practical motallurgist it is essential that the ores under treatment at smelting works should be submitted to assay, to afford a clear indication of the quantity of metal obtainable, and that the products of furnaces, \&c., obtained at various stages of the operations should also be tested, so as to exercise a salutary control over the working of the various processes. To the miner, also, it is indispensable to have the various parcels of ore submitted to assay, to ascertain their economical value. Assaying may likewise be advantageously employed to test the working capabilities of dressing machinery, or to control the dressing processes. It is also of the utmost importance that the alloys employed for monetary and other purposes should bo submitted to careful and exact assay, to ascertain that they aro of the correct standard, and to prevent fraud. To the colonist and explorer, a knowledge of assaying would often prove of great service, in enabling them to ascertain the nature and economical value of metalliferous minerals, or ores which they may discover. Assaying has beon practised for many generations; formerly the assays were made by the agency of fire, and were, more or less, but miniature smelting processes. Since tho study of chemistry has advanced, and within comparatively recent times, other methods by the use of liquid roagents have been introduced. The various methods of assaying, exclusive of those made by the blowpipe, are, therefore, conveniently divided into (1) assays by the dry way, (2) assays by the wet way.

1. Assays by the Dry Way.-These assays are made by the agency of fire, by fusion of the ores or other substances, with or without the addition of appropriate fluxes and reagents in crucibles or other suitable vessels of iron, clay, or black-lead; the requisite temperature being obtained in an air-furnace, muffe-furmace, or small blast-furnace.

2. Assays by the Wet Way.-These assnys are mado by the agency of water, or liquid reagents.
1. By volumotric methods.
2. By analysis.
3. By mechanical means.

1. The Volumetric Methods of assay are of comparatively recent introduction. These assays are made by the use of standard solutions of known strength, supplied from graduated vessels, as burettes, alkalimeters, or pipettes; unfortunatoly only a few of the numerous volumetric methods which are known can be appliod practically for the purposes of assaying, as thoy are not sufficiently reliable, consume too much time, or do not work with the requisite degree of aceuracy.

2. Analysis. - The methods of assay by analysis are only used when other methods are not available, or in special cases.

3. Mechanical Methods. - Theso assays consist in the soparation, by the agency of water, of the substances associated or occurring with metalliforous minerals and ores, and are performed by hand-washing on a vanning-shovel, or in a washing-bowl of wood ar metal. A known quantity of the pulverised ore is put on a shevel, or in a bowl with water, and by certain rotary and other movements, to be acquired only by practice, the lighter substances aro separated from thoso of greater specific gravity. The vanning-shovel is usually employed for tin ores, and the washing-bowl, or tin dish, for gold ores. Washing may also be practised to ascertain the nature and proportion of certain minerals, as galena, iron pyrites, \&c. present in samples of ores. It may likewise be had recourse to for ascertaining the proportion of motallie grains present in slags or other furnace-products, and which may be afterwards extractod from them by stamping and washing on the large scale.

\section{Explasation of Tenks tsed ix Assaying (by the Dry Mretrod).}

Fusion.-Rendering fluid by the aid of heat. Tho term fritting, or semi-fusion, is applied to materials which have softened sufficiently by heat to become remented together.

Reduction.-When a metal is separated from its compounds by another agent. it is said to be reduced, and the operation is called reduction. Thus the reduction of 
galena occurs whon it is heatod with iron, the products being metallic lead and sulphide of iron; tho iron in this instance is called tho reducing agent. When a metallic oxide passes from a higher to a lower state of oxidation, it is also said to be re. duced.

Calcination, Roasting.-When a powdered substance is exposed to the oxidising action of heat and air, in order to expel tho sulphur, arsenic, carbonic acid, and otlicr matters, the process is callod roasting, or calcination. If it is continued until practically complete, it is said to bo roasted sweet or dead. Accidental softening of the particles, so that they cohere, is malled clotting.

Scorification.-A roasting-fusion process, employed in assaying silver ores.

Cupellation.-An oxidising-fusion process conducted on a porous vessel called a cupel, used in silver assaying.

Distillation.-When a metal is rolatilisod by heat, and afterwards condensed in tho liquid form, the process is called distillation. The torm sublimation is applied when condensation occurs in the solid stato.

Liquation.- Whon an ore or other substance, part of which is fusible and the other infusible, or fusible at a higher temperature, is exposed to a tomperature sufficient to melt one portion and not the other, the fused product is said to be liquated, and the operation is named liquation. For example, when sulphide of antimony is liquated out from associated vein-stuff.

Flux.-The substance which is added to another to render it fusible by the application of heat, is callod a flux. Thus protoxide of lead or litharge is a flux for silica, the molted product being silicate of protoxide of lead.

Slag.-.The product resulting from the fusion of an ore or other substance, which floats on the top of the metal, regulus, or speiss. Those obtained in assay processes are very variable in composition, such as silicates, boro-silicates, metallic alkaline sulphides, metallic oxides, fluo-silicates, \&c.

Regulus. - A compound of one or more metals with sulphur.

Speiss.-A compound of one or more metals with arsenic.

Button of Metal.-The metal, or alloy, the result of assay, which is found at the bottom of the erucible or ingot mould, after cooling. The small more or less rounderl metallic particles are called shots, or globules, or pills.

For an explanation of the terms used in the wet way, such as filtration, precipitation, \&c., the reader is reforred to works on chemical analysis.

\section{Fursaces, Impleareants, Apparatus, \&c. used is Assayivg.}

Furnaces.-They aro of throe kinds. For a description of an air-furnace, see Coppre; of a cupellation furnace, seo SILvER; of a small blast-furnace, seo Iron.

Crucibles.-Open-monthed vessels of clay, iron, or black-lead. Clay crucibles should be well dried before use. When a erucible is charged with the assay mixture,

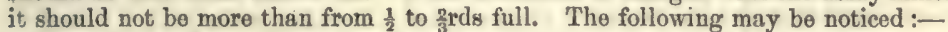

Cornish Crucibles. - These crucibles are tho best for general assay purposes, as they withstand great and sudden alternations of temperaturo without cracking. They also resist fairly the corrosive action of fluxes, and although they soften when exposed to extrome temperatures, yet iron assays can bo made in them, provided the precaution be taken of lowering the temperature somowhat, so that they become firm before remoral from the furnace. They are greyish-white, coarse in grain, and composed chicfly of silica. They are generally sold in nests of two, and sometimes three, each as used by copper assayers. The larger size are 3 inches diameter at top, and $3 \frac{1}{2}$ inches high, outside measure. They may now be obtained of various shapes and sizes.

Hessian Crucibles.-These crucibles, when genuine, have nearly the same qualities as Cornish pots, to which they approximate in composition. They are reddish-brown in colour, generally triangular at the top, and aro sold in nests of six crucibles, which fit successively into each other.

Iondon Crucibles.-These pots vary somewhat in shape and quality. Thoy are light reddish-brown in colour, close in grain, and havo a smooth surface. They resist the corrosive action of litharge and fluxes very woll, but require care in using to prevent eracking. They resist high temperatures well enough to bo employed in assaying iron ores. Crucibles known as 'skittle pots,' and mado of the samo materials as London crucibles, are still used by some assayers. They are deep, drawn in towards the top, to allow of plenty of room for effervescence during fusion, especially when undried materials are used.

French Crucibles.-These crucibles have a groyish-whito or cream colour, aro thin, firm, very perfect in form, and smooth externally. They withstand the highest temperature of ordinary furnaces, and resist well the action of fluxes. They 
are more expensive than other varietios of clay cmcibles. The so-called white fluxing pots made in London of Fronch fire-clay have the same shape, but are rather thicker than the true French crucibles. They also answer very woll for assay purposes.

Black-lead, or Plumbago Crucibles.-These pots are made of a mixture of plumbago and fire-clay. The best varieties withstand sudden alternations of temperatures; resist the highest temperatures without softening or cracking, but they slowly burn away externally by repented use. They are employed in iron and tin assaying.

Iron Crucibles.-Wrought-iron pots of various sizes and shapes are used in lead assaying. Those made out of one pieco of iron, without a weld, are preferable; but they cost more than those made from boiler-plate, or iron-tubing by hammering and welding.

Porcelain Crucibles.-These crucibles are made of various sizes and shapes. They aro chicfly nsed in assays by analysis.

Ronsting Dishes.-Flat, shallow, thin circular vessels of fire-clay of various sizes, used for the calcination of ores, \&ce, in muffles. The most useful sizes vary from 2 to 3 inches in diameter, and from $\frac{3}{4}$ to $\frac{7}{8}$ of an inch in depth, inside measure.

Scorifiers.-Cup-shaped vessels of fire-clay used in assaying silver ores; they should withstand sudden alternations of temperature, and resist the corrosive action of litharge and metallic oxides. Those commonly used are from $1 \frac{3}{4}$ to $2 \frac{1}{4}$ inches diameter at top, and from $\frac{B}{8}$ to $\frac{7}{8}$ of an inch in depth, inside measure.

Cupels. - Small circular vessels, having a shallow hemispherical cavity. They are generally made of bone-ash, or other material which is porous, and resists the corrosivo action of lithargo at the required temperature. 'They vary from $\frac{1}{2}$ inch to 2 inches in diameter, according to circumstances.

Tongs, Scoops, Stirring Rods, Ingot Moulds, \&c.-For a description of these implements, see the assays of. the various metals.

Balances. - For general assay purposes, three kinds of balances will suffice. (1.) To carry 500 grains, and turn with $\frac{1}{1000}$ th of a grain: this may be used for gold and silver assays, and for all purposes of exact weighing. (2.) To carry 1000 grains, and turn with from $\frac{1}{10}$ th to $\frac{1}{20}$ th of a grain. This will be found suitable for the dry assay of copper, tin, lead, \&ce. (3.) To carry 10,000 grains, and turn with $\frac{1}{10}$ th to $\frac{5}{10}$ th of a grain. Used for weighing out fluxes and other substances. In special branches of assaying, where a largo number of assays of the same kind have to be made, it is desirable to have balances constructed suitable for that specific work.

Weights.-For general assay purposes the grain weights divided on the decimal system are most convenient. Special weights are used by tin assayers, copper assayers, bullion assayers, and others, to facilitato calculation. Theso will be noticed under the respective metals when necessary.

Burettes, pipcttes, and any special apparatus employed in wet assays, or otherwise, will be described under the assays of the several metals, when necessary.

\section{Fluxes, Reagents, and othen Substayces vsed in Assaying.}

Thoy should be kept in covered earthenware jars, or in a covered long rectangular wooden box divided into compartments; all fluxes, when practicalule, should be used in the dry state. Those employed in the dry way may be classified as follows :-

Fivuxes. Carbonate of Soda, dricd.-Crystallised carbonate of soda contains about 63 per cent. of water, which it loses on heating. Bicarbonate of soda is a convenient substitute. Carbonate of potash may also bo used instead. The alkaline carbonates form fusible compounds with silica, \&c.

Borax or Bibortute of Soda, dried or calcined.-Crystallised borax contains about 47 per cent. of water; when heated it losos this water, and swells up to a very light bulky mass, which is dried or calcined borax. If the temperature is increased, it melts into a clear, colourless liquid, which on cooling constitutes glass of borax. It forms fusible compounds with carthy and metallic oxides, as lime, oxide of iron, \&ce.

- Glass. - White glass, free from oxide of lead, such as plate-glass or window-glass, is suitable for some purposes. Green bottle-glass may ulso be used where the presence of oxide of iron is not objectionable. It serves to increase the fusibility of earthy silicates, \&ce, and in some cases as a substitute for borax. \&c.

Silica. - White sand, or powdered quartz; it serres as a flux for oxides of iron, lime,

Eluor Spar, Fluor, or Fluoride of Calcium.-It should be selected free from galena, copper pyrites, and other minerals. It forms rery fusible compounds with sulphate of baryta, sulphate of lime, phosphate of lime, silica, \&c. 
China Clay, Kaolin, or Hydrated Silicate of Alumina.-Whèn pure it contains about 12 per cent, of water. It can bedeprived of this water by heating the powdered clay to a strong red heat. Fire-clays and shale are sometimes used as substitutes for china clay.

Lime.-Unslaked lime is preferable. It forms fusiblo compounds with silica, silicates of alumina, \&ce.

Litharge, or Protoxide of Lead.-It forms fusible compounds with silica, and earthy and metallic oxides, \&c. Red lead, or white load, or carbonate of lead may also be employed.

Oxides of Iron.-Hrmatite, or iron-scale, may be used as a flux, for silica, and difficultly fusible silicates, \&c.

Black Flux may be practically regardod as a mixture of carbonate of potash and charcoal. It is prepared as follows: 1 part of nitre, and from 2 to 3 parts of tartar, by weight, are mixed, and. deflagrated by stirring with a hot iron rod until action ceases, or by projecting portions of the mixture from time to time into a hot crucible. The product is reduced to powder and kept in a closely covered jar, as it is liable to absorb moisture rapidly. The quantity of carbon in the product varies with the proportion and purity of the tartar, or argol, employed. Very convenient substitutes are now used by mixing carbonate of soda with from 5 to 10 per cent. of charcoal powder, or with larger proportions of tartar, starch, or flour.

White Flux is essentially carbonate of potash. It is prepared in the same way as black flux, by deflagrating a mixture of equal parts by woight of nitre and tartar. The nitric acid of the nitre converts the carbon of the tartaric acid in the so-called bi-tartrate of potash into carbonic acid, which combines with the alkali, and carbonate of potash is formed.

Refining Flux is a variety of white flux, generally made in a similar manner, with salt intermixed. It is used by copper-assayers for refining impure copper.

Reductiva Agests. Charcoal Powder.-Coke dust, and culm or anthracite powder, are also used for some purposes, as they burn away less rapidly than charcoal powder.

Tartar. - Cream of tartar, known in the crude state as red argol and white argol, and when purified, as bitartrate of potash. Starch and flour form convenient substitutes.

Cyanide of Potassium:-Two varieties are used, one known as gold cyanide, and the other common, which contains a large amount of carbonate of potash. As cyanide of potassium has little fluxing power, for some purposes the latter variety is preferable, as the carbonate of potash forms fusible compounds with silica, \&c.

Iron.-Wrought-iron in the form of iron nails, iron rod, or hoop-iron.

Oxmising Agmats. Atmospleric Air.-This is the chief agent which acts in roasting processes, in removing sulphur, as sulphurous acid, \&c.

Nitre, or Nitrate of Potash. - It is an anhydrous salt, and acts as a powerful oxidising agent, on account of the large proportion of oxygen which it contains. Nitrate of soda may also be used.

Litharge. Red Lead.-These are also used for oxidising sulphur, \&c.

Salt, or Chloride of Sodium.-It acts as an oxidising agent upon metallic copper. By some assayers it is used in nearly erery dry assay process, to top-up with, or cover the other ingredients. On account of its easy fusibility and comparatively low specific gravity, it floats on the top of the products of fusion, and allays effervescence or ebullition when fused. It also acts as a lubricator to the interior of the crucibles; and enables the particles of matter to run down more freely. However, it can readily be dispensed with, and as in most cases its use injuriously affects the result of the assay mothods, it is: not advisable to employ it.

Sulphurtsivg Agents, used for forming metallic sulphides, or regulus. Sulphur.Flowers of sulphur or powdered brimstone may be used.

Iron Pyrites.-For some purposes it should be selected free from copper.

Sulphrde of Iron.-Made by heating iron and sulphur together, or by fusing iron pyxites with hoop-iron.

DesUlPHURIsisc Agrests, used for the removal of sulphur by forming other sulphides, or by oxidation into sulphurous acid. Iron.-Hoop-iron, thin bar-iron, ixon rod, or nails, may be used.

Cyanide of Potassium.

Carbonate of Soda, dried, or bicarbonate of soda.

Litharge or Red Lead.

Arsexicising Agents, used for forming motallic arsenides or spoiss. Arsenic, motallic.

Arsenious Aoid, in admixture with carbon.

Drarsenvicising Agenss, used for the removal of arsenic as a speiss, or by oxidation. Iron, hoop-iron, \&c. 
Atmospheric Air, in roasting.

Nitre, by conversion into arsenate of potash.

Confuctriva Agsants, used for collecting or dissolving gold and silver in an nssay. Lead, granulatod. Litharge, or galena, which yields lead when heated with earbon or iron respectively.
Mencury.

\section{RrAGeNTs, \&C., RMPLOYED IN THK WET WAY.}

Solvasrs, or agents used in dissolving various substances,-Nïric Acid. Hyllrochloric Acid. Sulphuric Acid.

REDUciso AGFNTs, or substances used to reduce a metallic compound in solution to " lower stato of combination. Zinc, granulated.-That in the form of bean shot, obtained by pouring the metal into hot water, is preferable.

Sulphite of Soda. Protochloride of Tin.

Mrtalic Precipitants. Zinc, in form of sheet. Iron.-Wrought-iron, in form of thin clean sheet, or round or flat bar. Protochloride of Tin.

Spectal Rfagests, as permanganate of potash, sulphide of sodium, iodide of potassium, \&c., will be noticed when necessary.

For the methods of assaying the various metals, sеe Copper, Lrad, Gotn, \&c.-R.S.

98

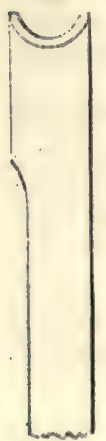

AstraxiA. (Starstone). The name first used by Pliny to denote those raricties of sapphire which display diverging rays of light.

Astracaz. An ornamental moulding, generally used to conceal a junction in either wood or stone.

AsTracaI PIANבS. Planes fitted with cutters for forming astrigal mouldings. They are commonly known as moulding-planes.

ASTRAGAI TOOT, for turning. By using a tool shaped as in fig. 98 , the process of forming a moulding or ring is greatly frcilitated, as one member of the moulding is comploted at one sweep, and we are enabled to repeat it any number of times with exact uniformity.

ASTrRAxT2. A glass resembling AVENTURINE, but containing crystals of a compound of coppor, which by reflected light exhibit a dichroic iridescence of dark red and greenish-blue. It is said to be made with 80 parts of flint, 120 parts of oxide of lead, 72 parts of carbonate of soda, and 18 parts of anhydrous borax. Te this aro added 24 parts of scalo oxide of copper, and 1 part of scale oxide of iron. These are melted in a Hessian crucible, at the hest of an ordinary air-furnace, and left to cool slowly without being moved.

ATACATrT2. A native oxychloride of coppor, originally found in the desert of Atacama in Peru. It forms small rhombic crystals, varying in colour from leek to omerald green. Splendid examples of this mineral have been found at Wallaroo, on Yorke Peninsula, and at Burra Burra, in South Australia.

ATHIRINA. See SARDINE,

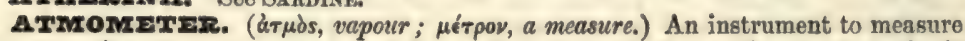
tho quantity of water eraporated in a giren time under ordinary atmospheric conditions.

ATruosprars. Tho gaseous onvelopo surrounding this globo. Tho term is also upplied to any gaseous body enveloping any mass of matter.

The extent of the earth's atmosphere has not been determined with any gront degree of accuracy. The height of the air above the surface of the earth should vary with the increase of the attractive force at the poles, and its diminution at the equator, and the variations of temperature also affect the mass, but, owing to the influence of centrifugal force, the spheroidal mass of air has a diameter shorter at the poles than at the equator. The volume which a given quantity of air occupies is directly dependent upon the pressure to which it is subjectod-and upon the temperature - so that the density diminishes as the distance from the earth's surface increases.

All the influences having been carcfully examined and allowed for, calculations have been made which appear to show that tho atmosphere reaches, in a state of density, which can be measured, to the height of about 45 miles. It may be assumed that in a state of continually increasing tenuity, it extends to a considerably greater distance from the earth's surface.

The composition of the air has been determined under a considerable number of conditions, the mean result being to show that it is a chemical mixture of about 20 , volumes of oxygen and 80 volumes of nitrogen (see Watts's 'Dictionary of Chomistry' for a full account of the chemical examinations of the air.) Carbonic acid exists in 


\section{ATMOSPHERE}

the atmosphere in variable proportions; the menn average being about 4 rolumes of carbonic acid in 10,000 of air. Ammonia and all the exhalations from tho earth exist as mixtures in the air, and on the presence or absence of these depends its healthfulness or otherwise.

The following remarks are from 'Travels in the Air,' by Mr. James Glaisher, F. R. S., and as giving the results of his practical experience and careful observations made during tho numerous balloon ascents by that gentleman, they have considerable value:-

'Every one knows that the pressure of the atmosphere is measured by means of the barometer. A column of air extending to its limit, of the same area as the barometer tube, is balaneed by the column of mercury in the tube; and if we weigh the mercury, we know the weight or pressure of the column of atmosphere upon that area. If the area of the barometor tube be one square inch, then this would tell us the pressure of the atmosphere on one square inch. The length of a column of mercury thus balanced by the atmosphere, near the level of the sea, is usually about 30 inches, and if this be weighed, it will bo found to be nearly $15 \mathrm{lbs}$; t therefore the atmospheric pressure on every square inch of surface is about $15 \mathrm{lbs}$, just one-half as many pounds as the number of inches which expresses the height of the column of mercury.

- Now, in ascending into the air, part of the atmosphere is below, and part above: the barometer, therefore, has to balance that which is above only, and will, therefore, read less.

'At the height of three miles and three-quarters, the barometer will read about 15 inches; there is, therefore, as much atmosphere above this point as there is below, and the pressure on a square inch is $7 \frac{1}{2}$ lbs.

'At a height of between five and six miles from the earth, the barometer-reading will be about ten inches; one-third of the whole atmosphere is then above and twothirds beneath, and the pressure of a square inch is reduced to $5 \mathrm{lbs}$.

'The reading of the barometer varies with the altitude at which it is observed, and indicates, by its increasing or docreasing readings, corresponding changes in the pressure of the atmosphere.

'At the hoight of 1 milo the baromotor roading is $24 \cdot 7$ inches.

- By the reading of the barometer in the balloon, the distance from the earth is known; and if the balloon be situated above clouds, or in a fog, the reading of the barometer indicates the near approach of the earth, and acts as a warning to the occupants of the car to prepare accordingly. In addition to this temporary use, the readings combined with those of temperature enable us to calculate the height of the balloon at every instant at which such readings have been taken.

- The temperature of the dew-point also deserves a few explanatory words.

- There is always mixed with the air a certain quantity of water, in the invisible shape of vapour, sometimes more, sometimes less, but there is a definite amount which saturates the air at every temperature, though this amount varies considerably with different temperatures.

- A cubie foot of air at the temperature of -

\begin{tabular}{|c|c|c|c|c|c|}
\hline $49^{\circ}$ & $"$ & " & 4 & ,' & 6 \\
\hline $70^{\circ}$ & $"$ & " & B & " & " \\
\hline $921^{\circ}$ & $\eta$ & g, & 16 & g & " \\
\hline
\end{tabular}

- The capacity of air for moisture, therefore, doubles for every increase of temperature of about 20 degrees. The temperature of the dew-point is the temperature to which air must be reduced in order to become saturated by the water then mixed with it; or it is that temperature to which any substance, such as the bright bulb of a hygrometer, must be reduced before any of the aqueous vapour present will he deposited as water, and become visible as dew. The tomperature at which this first bedewing or dulling of bright surfaces takes place is the temperature of the dew-point. 
For instance, I have already said that two grains of wator saturato n cubic foot of air at $30^{\circ}$; if, therofore, the temperature of the air be $40^{\circ}$, and there be two grains of moisture in a cubic foot of air, then if the bulb of the hygrometer be rediced to $30^{\circ}$, a ring of dew will appear on it, eaused by the deposition of the water in the air. The determination of the dew-point at once tells us, therefore, the amount of water present, and, combined with the tomperaturo, enables us to detormine the hygrometrieal state of the atmosphere.

'If the air be saturated with moisture, the temperature of the air and that of the dew-point are alike; if it be not saturated, the temperature of the dew-point is lower than that of the atmosphere; if there be a great difference between the two temperatures, the air is dry, and if this happen when the temperature is low, thero is very little witer present in the air. By the careful simultaneous readings of two thermometers, one with a moistened bulb, and the other dry, or by the use of a Daniell's or Regnault's hygrometer, the amount of water present in the air in the invisible shape of vapour can be determined, as well as the temperature of the dew-point and the degree of humidity.

- The degree of humidity of the air expresses the ratio between the amount of wator then mixed with it, and the greatest amount it could hold in solution at its then temperature, upon the supposition that the saturated air is representod by 100 , and the air deprived of all moisture by 0 . Thus: Suppose the water present to be one-half of the quantity that could be present, the degree of humidity in this case will be 50. If the air were at the temperature of $30^{\circ}$, and there were two grains of moisture in the air, it would be saturatod, and the degree of humidity would be 100 . If there were ono grain, that is, one-half of tho whole quantity that could bo present, the air would be one-half saturated, and the degree of humidity would be represented by 50 .

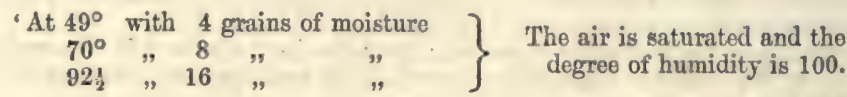

'But at $49^{\circ}$ with 2 grains of moisture $" 70^{\circ}, \begin{gathered}4 \\ " 72\end{gathered}$

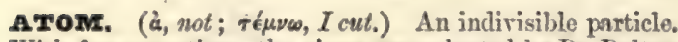

With few exceptions, the views promulgated by Dr. Dalton are received by chemists. They may be thus expressed: All elementary bodies are formed of individual atoms, the different species of which unite, generally by twos, in a small number of groups, constituting compound atoms of tho first order, always mechanically indivisible, but chemically divisible, and, in their turn, constituting all the other orders of composition by a series of analogous combinations.

We are not enabled by direct experiment to determine the condition of any ultimato atom of matter; but the results furnished by chemical science clearly point to the existence of elementary units, from which all tho infinite varioties of matter are formed. Sir Isaac Newton thus expresses himself :- All things considered, it seems probable that God, in the beginning, formel matter in solid, massy, hard, impenetrable, movablo particles, of such sizes, figures, and with such other properties, and in such proportions to space, as most conducerl to the end for which He formed them; and that these primitive particles, being solids, aro incomparably harder than any porous bodies compounded of them; eren so hard as never to wear or break to pieces; no ordinary power being able to divide what God Himself made one in the first creation. While the particles continue entire, they may compose bodies of one and the same naturo and texture in all ages; but should thoy wear away, or break in pieces, the nature of things depending on them would be changed. Water and earth composed of old worn particles would not be of the same nature and texture now with water and earth composed of entire particles at the beginning. And thereforc, that nature may be lasting, the changes of corporeal things are to be placed only in rarious separations, and new associations, and motions of these permanent particles; compound bodies being apt to break, not in the midst of solid particles, but where those particles are laid together and touch in a few points.'-Horsley's Newton.

With the metuphysical theories, which would lead us to regard all matter as mere accumulations of force, it would not be proper at present to deal.

Experimental philosophy has proved to us that the conditions of matter are determined by certain polar-attractive forces; and that these are opposed or balanced by heat, electricity, and the force which regulates chemical combination. Consequently, 
overy ultimate atom of matter may be regarded as the contre of such a set of physical forces surrounding it as an atmosphere.

In modern chemistry an atom is defined to be the smallest particle of any element which can exist in combination. The atom is thus distinguished from the molecule, the latter term being now applied to the smallest quantity of the substance capable of existing in a free state. The molecule and the atom may coincide, or the molecule may be made up of two or more atoms. Thus, an atom of chlorine, or 35.5 parts by weight, is represented by the symbol $\mathrm{Cl}$, since this denotes the smallest quantity of chlorine capable of existing in any of its compounds-hydrochloric acid ( $\mathrm{HCl}$ ) for example; while the moleeule of chlorine, or smallest quantity set free in any reaction, will be represented by two atoms, or $\mathrm{Cl}^{2}$.

ATOMrcIry, or Feurvarricr. Terms employed by modern chemical writers to denote the combining capacity of an element, or a radical, as determined by the number of atoms of hydrogen, or other monatomic element, with which it ean combine. Thus chlorine, oxygen, boron, carbon, and phosphorus (using the atomic weights, $\mathrm{Cl}=35 \cdot 5, \mathrm{O}=16, \mathrm{~B}=11, \mathrm{C}=12$, and $\mathrm{P}=31$ ) may be said to be respectively monatomic, diatomic, triatomic, tetratomic, and pentatomic elements ; or, to use equivalent expressions, they may be described as univalent, bivalent, tervalent, quadrivalent, and quinquivalent: in other words, the elements just citod may be termed respectively a monad, diad, triad, tetrad, and pentad. The atomicity, or equivalence, is often indicated by dashes or by Roman numerals, placed at the upper right-hand side of the symbol; thus, $B^{\prime \prime \prime}$ indicates the triad boron, $\mathrm{P}^{*}$ the pentad phosphorus, and so forth. It should be remembered, however, that mineralogists were formerly in the habit of employing dashes in this way to represent so many atoms of sulphur, just as they used, and still use, dots placed above a symbol to denote so many atoms of oxygen. Hence, to a mineralogical reader, such expressions hare now become ambiguous; $\mathrm{Sb}^{\prime \prime \prime}$, for instance, may either represent tersulphide of antimony, or merely jndicate that the metal antimony is a triad.

Elements of equal atomicity are termed artiads; and those of uneyual atomicity perissads. For a full discussion of the modern doctrine of atomicity, see Watts's 'Dictionary of Chomistry,' and the supplementary volume.

Aтомгс тrForх. The question as to whether matter be or be not infinitely divisible, has been debated from the earliest times, and is probably as far from a settlement as ever; we can, however, scarcely conceive of the existence of matter at all, if there be no limit to its divisibility. It is easy to demonstrate that a mathematical line is infinitely divisible, but a mathematical line is only an ideal thing; having only one dimensinn, it can have no physical existence. We have, therefore, no hesitation in admitting the existence of atoms of matter-of particles infinitely small, it is true, as regards our perceptions, far exceeding in minuteness the finest subdivision to which we can submit a body, but yet incapable of further subdivision. To such insectible molecules the term atom has been applied.

If we take any substance chemically complex, we may suppose the existence of atoms in this body, held together by the force of cohesion, which are themselves heterogeneous, being made up, in fact, of atoms of the elementary chemical constituents.

Dr. Dalton suggested the happy idea, which has been most fruitful in its results, of accounting for the constancy of chemical combinations by assuming that they were composed of one or more atoms of the several elements, the weight of which atoms is represented by the combining proportions; that carbonic oxide, for instance, contains singlo atoms of carbon and oxygon, whilst carbonic acid is composed of one atom of carbon and two of oxygen.

It must always be remembered that the combining proportions aro purely the results of experiment, and, therefore, incontestable, whatever may be the fate of this theory, which, however, has now stood its ground for many years, and done excellent service to science.

This theory offers a most satisfactory explanation of the different laws of chomical combination.

The fact of bodies uniting only in certain proportions, or multiples of those proportions, is a necessary consequence of the assumption that the weight of the elementary atoms is represented by the combining proportions ; for, if they united in any other ratio, it would involve the splitting up of these atoms, which are assumed to be indivisible.

And, of course, the combining proportion of a compound must be the sum of the combining proportions of the constituents, since it contrins within itself one or more atoms of the several constituents.

The term atom is, therefore, very often used instead of combining proportion, a body being said to conitain so many atoms of its eloments. 
All that is assumed in this theory is, that the atoms are of constnnt ralue by weight ; the $\operatorname{same}$ atoms may be arranged in a different way, and hence, although any particular compound contains always tho same elements in the atomic ratios, yet tho same atoms may, by difference in arrangement, give rise to bodies agreeing in composition by weight, but differing essentially in proporties. See Isomemsur.

The atomic theory is furthor confirmed by the obserration, that if the specific heat of the elements be compared, it is found that in a largo number of cases the specific heats of quantities of the bodies represonted by their atomic woights coincido with each other in a remarkable manner.

For a full examination of this subject, consult 'An Introduction to the Atomic Theory,' by Charles Daubeny, M.D.; and 'Memoirs of Joln Dalton and History of the Atomic Theory,' by Robert Angus Smith, $\mathrm{Ph}$. $\mathrm{D}^{\prime}$

ATomrc vorumrs. Of late years it has been assumed that the olements, when in the gaseous state, unite invariably in equal volumes, or, in other words, that the atoms of bodies have always the same volume. If this doctrine be maintained, it becomes necessary to alter the atomic weights or combining numbers of certain elements. For example, water contains two volumes of hydrogen to one of oxygon; but, according to the old idea, it consists of single atoms of each element; it is clear, therefore, that if. we are to assume that the atoms of hydrogen and oxygen have the same volume, we must either halve the atomic weight of hydrogen or double that of oxygen.

Berzelius suggested that all the atomic weights should remain the same, except those of hydrogen, nitrogen, phosphorus, chlorine, bromine, and iodine, which should halve their present values. Gerhardt, on the other hand, adopted the more convenient practice of allowing hydrogen and its congeners to retain thoir present atomic woights, doubling those of oxygen, sulphur, tellurium, and carbon. This practice has of late years been extended to many other elements. Seo Atoxre Wrigrrs, and Watts's 'Dictionary of Chemistry.'

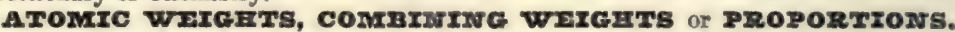

The atomic weights of the elements ropresent the proportions in which they severally combine with each other, referred to some standard element as unity. In accordance with Dalton's atomic theory, explained in a previous articlo, it is supposed that the numbers assigned to the elements as their respective combining proportions represent the relative weights of their atoms, and hence the adoption of the term atomic wight -a term extremely conveniont to retain whatever viows may be held as to the ultimate constitution of mattor. By Berzelius, oxygen was selected as the standard to which all atomic weights were referred, but it is now almost univorsal among chemists to take hydrogen as the standard of comparison, sinee it is found that of all the elements hydrogen has the smallest combining. number. It was believed by Prout that the atomic weights of all substances were multiples of the atomic weight of hydrogen, but it has beon shown by Stas - who has made the most refined experiments on this subject-that the theory is only approximately true.

In establishing the atomic weights, or proportional numbers, of the elements, it is not only necessary to determine exactly the ratios in which they severally combinowhich is merely a matter of accurate experiment-but also to interprot these ratios by the light derived from an extensive range of physical and chemical phenomenasuch as isomorphism, specific heat, combining volume of vapour, and tho like. Hence, as science has advanced, the necessity has boen recognised by most chemists, of altering the numbers assigned to certain of the elements as their atomic weights, and, in fact, within the last few years many of these numbers have been doubled. It has, however, been considered, in the preparation of the new edition of this Dictionary, that the convenience of manufacturers and others accustomed to the use of the old atomie weights would be best served by retaining these familiar figures; and hence in all cases throughout this work, unless otherwise stated, the furmulive are

'Dr. Angus Smith, in his 'Memolrs of Dalton,' thus sums up the labours of this deep thinker :- This Dalton did. He gave the first idea of atomic weights. Under this head cume Richter and Fischer's numbers. Richter, grappling with those numbers, never could obtain a rational theory from the phenomena. Dalton's plan explains these numbers with the greatest ease, and looks on such as a necessity of the fundamental law, instead of the beginning of the inquiry, as it wns to them. It seems to me, then, that what happened historically happened also intellectually. Dalton had included his predecessors in his more extensive system. He had gone to the summit of the hill, and when coming down found proofs that they had been making good progress upwards. Higgins had gone at once to the top, as it appears to me, but took no heed to make the needful observations when he was ap, or he found the prospect entirely obscured. We are compelled to put reciprocal proportions in a secondary position, as it seems to me it cannot be called a law, but one of the consequenoes of a lsw; and the evidence brought to support it, otherwise than empirically, presupposes some of the principles on which the general laws depend. It was by a careful mechanical juxtaposition of parts that Dalton arrived at the idea; it is eminently mechanical, and it is remarkable that all progressive views on the subject have been so. He introduced proportional eceights into the theory, and found it to agree with facts. His is, thercfore, the quantilative atomic theory." 
constructed with the old system of atomic weights. At the same time it appears desirablo also to introduce the use of the modern atomic weights; and, therefore, in almost erory chemical expression in this work both the old and the new formulæe are given - the latter being printed, for sake of distinction, in a thick black type.

In the following comparative Table the first column of figures gives (approximately) the old combining weights of the elements, whilst the second column gives the modern atomic weights, as employed by the most advaneed chemists of the present day. A glance at this Tablo is thereforo sufficient to show whieh of the elements have retained their old atomic weights, and which have had them doubled. Moreover, it is easy from these data to translate any of the formulpe on the old system into formulæe constructed with the recent values of the atomic weights. For example, in our article AxcoHoL, that compound is said to be composed of $\mathrm{C}^{4} \mathrm{H}^{6} \mathrm{O}^{2}$; but reference to the Table shows the atomic weights of carbon and of oxygen have been doubled, whilst that of hydrogen remains unnltered. It is, therefore, obviously necessary to halve the number of atoms of carbon and of oxygen in the old formula, and consequently the symbolic expression for alcohol, instoad of being $\mathrm{C}^{4} \mathrm{H}^{6} \mathrm{O}^{3}$, becomes on the modern system $\mathbf{C}^{2} \mathbf{Z}^{\mathbf{B}} \mathbf{0}$.

\begin{tabular}{|c|c|c|c|c|c|}
\hline & Elements & & Symbols & $\begin{array}{l}\text { Old } \\
\text { at. weights }\end{array}$ & $\begin{array}{l}\text { New } \\
\text { at. weights }\end{array}$ \\
\hline Aluminium & . & . & Al & $13 \cdot 75$ & $27 \cdot 5$ \\
\hline Antimony & . & . & $\mathrm{Sb}$ & 122 & 122 \\
\hline Arsenic . & . & . & As & 75 & 75 \\
\hline Barium. & & . & $\mathrm{Ba}$ & $68 \cdot 5$ & 137 \\
\hline Bismuth & . & ... & $\mathrm{Bi}$ & 210 & 210 \\
\hline Boron . & . & - & B & 11 & 11 \\
\hline Bromine & ; & . & $\mathrm{Br}$ & 80 & 80 \\
\hline Cadmium & . & . & $\mathrm{Cd}$ & 56 & 112 \\
\hline Cæesium . & . & , . & $\mathrm{Cs}$ & 133 & 133 \\
\hline Calcium. & . & . $\quad$. & $\mathrm{Ca}$ & 20 & 40 \\
\hline Carbon. & . & . . & c & 6 & 12 \\
\hline Chlorine & + & . . & $\mathrm{Cl}$ & $35 \cdot 5$ & $35 \cdot 5$ \\
\hline Chromium & . & .. & $\mathrm{Cr}$ & $26 \cdot 25$ & $52 \cdot 5$ \\
\hline Cobalt . & . & . & Co & $29 \cdot 5$ & 59 \\
\hline Copper . & . &.$\ldots$ & $\mathrm{Cu}$ & $31 \cdot 75$ & $63 \cdot 5$ \\
\hline Fluorine & . & . $\quad$. & $\mathrm{F}$ & 19 & 19 \\
\hline Gold & . & , . & $\mathrm{Au}$ & $98 \cdot 5$ & 197 \\
\hline Hydrogon & , & . & H & 1 & i \\
\hline Indium . & . & . $\quad$ & In & 76 & 76 \\
\hline Iodine . & . & . $\quad$ & I & 127 & 127 \\
\hline Iridium . & . & $\therefore \quad$. & $\mathrm{Ir}$ & $98 \cdot 5$ & 197 \\
\hline Iron & . & . & $\mathrm{Fe}$ & 28 & 56 \\
\hline Lead . & . & . & $\mathrm{Pb}$ & $103 \cdot 5$ & 207 \\
\hline Lithium & . & , , & $\mathrm{Li}$ & 7 & 7 \\
\hline Magnesium & . & . . . & $\mathrm{Mg}$ & 12 & 24 \\
\hline Manganeso & 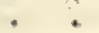 & . & $\mathrm{Mn}$ & $27 \cdot 5$ & 55 \\
\hline Mercury & . & . & $\mathrm{Hg}$ & 100 & 200 \\
\hline Molybdenum & . & . & Mo & 48 & 96 \\
\hline Nickel . & . & . & $\mathrm{Ni}$ & $29 \cdot 5$ & 59 \\
\hline Nitrogen & . & .. & $\mathrm{N}$ & 14 & 14 \\
\hline Osmium & . & , . & Os & $99 \cdot 5$ & 199 \\
\hline Oxygon. & 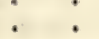 & $: \quad:$ & 0 & 8 & 16 \\
\hline Palladium & $\therefore$ & .. & $\mathrm{Pd}$ & 53 & 106 \\
\hline Phosphorus & . & .. & $\mathrm{P}$ & 31 & 31 \\
\hline Platinum & & . .... & Pt & $98 \cdot 5$ & 197 \\
\hline Potassium & . &.$\quad$. & $\mathrm{K}$ & 39 & 39 \\
\hline Rubidium & . & . . & $\mathrm{Rb}$ & 85 & 85 \\
\hline Selonium & . & . $\quad$ & Se & $39 \cdot 75$ & $79 \cdot 5$ \\
\hline Silicon . & . &.$\quad$. & $\mathrm{Si}$ & 21 & 28 \\
\hline Silver . & . & . $\quad$ & $\mathrm{Ag}$ & 108 & 108 \\
\hline Sodium . & . & $: \quad:$ & $\mathrm{Na}$ & 23 & 23 \\
\hline Strontium & . & . . . & $\mathrm{Sr}$ & $43 \cdot 75$ & $87 \cdot 5$ \\
\hline Sulphur & . & . & 8 & 16 & 32 \\
\hline Tellurium & . &.$\quad$. & To & $64 \cdot 5$ & 129 \\
\hline Thalliom & : & : & TI & 204 & 204 \\
\hline Tin . & . & , . & Sn & 59 & 118 \\
\hline
\end{tabular}




\begin{tabular}{|c|c|c|c|c|c|c|}
\hline \multicolumn{4}{|c|}{ Rlements } & \multirow{2}{*}{$\begin{array}{c}\text { Symbols } \\
\mathrm{Ti}\end{array}$} & \multirow{2}{*}{$\begin{array}{l}\begin{array}{c}\text { Old } \\
\text { at. weights }\end{array} \\
25\end{array}$} & \multirow{2}{*}{$\begin{array}{c}\begin{array}{l}\text { New } \\
\text { at. } \text { weights }\end{array} \\
50\end{array}$} \\
\hline Titaninm & . & • & .2 & & & \\
\hline Wolfram & . & & - $\quad \bullet$ & W & 92 & 184 \\
\hline Uranium & . & - & . . & U & 60 & 120 \\
\hline Vanadium & . & - & . & V & 51 & 51 \\
\hline Zine & . & 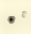 & $\therefore \quad$. & $\mathrm{Zn}$ & 32.5 & 65 \\
\hline Zirconium & + & . & - $\quad$. & $\mathrm{Zr}$ & $44 \cdot 5$ & 89 \\
\hline
\end{tabular}

The term 'atomic weight' was formerly employed as synonymous with 'chemical equivalent,' but the ideas invalved in the two terms, as applied in modern chomistry, are essentially distinet.

Every chemical manufacturer should be thoroughly aequainted with the conbining ratios, which are, for the same two substances, not only definite, but often multiple; two great truths, upon which are founded, not merely the rationale of his operations, but also the means of modifying them to useful purposes. The discussion of the doctrine of atomic weights belongs to pure chemistry; but several of its hap!niest applications are to bo found in the processes of art, as pursued upon tlie largest seale.

The following propositions may be regarded as the laws regulating atomic combination:-

1. The combining proportions of elementary bodies.represent the smallest proportions in which they enter into combination with each other.

2. The combining proportion of a compontnd body is the sum of the combining proportions of its elements.

3. Combination takes place, whether between elements or compounds, either in the proportions of their combining weights, or in multiples of these proportions, and never in sub-multiples.

4. The law of definite proportion teaches that individual compouncls always contain exactly the same proportions of their elements. Seo Equivalexts, Chemical. Soe also Watts's 'Dictionary of Chemistry.'

ATramenTum. An old name for iron-vitriol, or sulphato of iron. A product of tho partial oxidation of iron pyrites, which is sometimes. used in making ink.

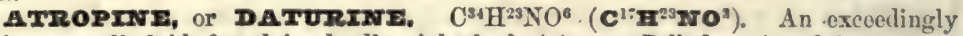
poisonous alkaloid, found in deadly nightshade (Atropa Belladonna) and in stramonium (Datura Stramonium), and probably in some other plants. One-sixth of a grain of atropine produces unconsciousness and delirium. To the freshly prepared extrict.t of belladonna add a strong solution of caustic potash, and well mix in a mortar. Digest the resulting mass at a temperature of $80^{\circ}$ with benzole; soparate the latter, and distil off the hydrocarbon in a retort on the water-bath. The residue in the retort is to be treated with water acidulated with sulphuric acid; the acid solution is to be precipitated by carbonate of soda, and the resulting atropine may then be obtained pure by crystallisation from alcohol. Atropine is used.in medicine, $\frac{1}{30}$ th of a grain being a full dose, and it is applied externally for producing dilatation of the pupil of the eye. The smallest portion of a very dilute solution rubbed on the eyelid suffices to produce the result.

AтTAг5a. A. funifera yields the coquilla nut mueh used in turnery. It was formerly supposed that this species of Attalea also yielded the Pinssaba fibre used in Brazil for ropemaking, and in this country for the manufacture of bast-brooms, but it is now known that the Piassaba fibre is the produce of another palm-the Liopuldina Piassaba. See Coqurus.

ATTAR or Rosss, more commonly, otTo or roszs. : An essential oil, obtained in India, Turkey, and Persia, from some of the finest varioties of roses. It is procured by distilling rose-leaves with water, at as low a temperature as pussihle. It is said that this perfume is prepared also by exposing the rose-leares in water to the sun; but, from the fact that under the circumstances fermentation would lo speedily established, it is not probablo that this is a method often resorted to. liy dry distillation from salt-water baths, no doubt the finest attar is obtained. This essential oil is only used as a perfume. Attar of roses is adulterated with spermaceti and with castor-oil dissolved in strong alcohol.

This adulteration may be detected by putting a small drop of the otto of roses on a piece of clean writing-paper; by agitation in the air, the volatile oil soon eraporates, leaving no stain if pure; if any fixed oil is present, a greasy spot is left on the paper. 


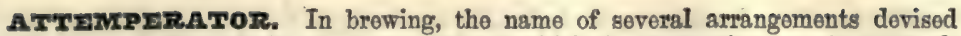
for tho purpose of regulating the temperature to which the fermenting wort is exposed. It is also employed to regulato the temperature of malting-rooms. Without them it is impracticablo to make malt in the summer equal to that made in winter. In all cases either air or water is the attemperating agent.

Axтrivuarrow. Brewers and distillers employ this term to signify the weakening of saccharine worts during fermentation, by the conversion of the sugar into alcohol and carbonic acid.

Aтrxx. A miner's term for the 'deads' or refuse-matter of a mine. The 'attleheap' is the mine-burrow or rubbish-heap.

AUGrr. The auger is a tool for boring either wood or stone. The single-lip auger is forged as a half-round bar; it is then coiled into an open spiral, with the flat side ontwards. The ordinary screw auger is forged; it is twisted red-hot; the end terminates in a worm, by which the auger is gradually drawn into the work as in the gimlet; and the two angles, or lips, are sharpened to cut at the extreme ends, and a little up the sides also. The American screw auger has a cylindrical shaft, around which is brazed a single fin or rib; the end is filed into a worm, as usual, and immediately behind the worm a small diametrical mortice is formed for the reception of a detached cutter, which exactly resembles the chisel-edge of the centre-bit. - Holtrapfel.

AUcIтr. (airit, brilliancy.) A sub-species of Pyroxene. The name is confined to the opaque and greenish-black varieties, common in basaltic, doleritic, and recent volcanic rocks, in which it forms an important constituent, but it is never found in granite. It has a base of magnesia, lime, protoxide of iron, and alumina. The term augite is often used by English geologists as synonymous with pyroxene. For the means of distinguishing between augite and hornblende-two minerals which often closely resemble each other-see HorNBurasdi.

AUratrs. Crystalline compounds of the peroxide of gold.

AURIC ACrD. (Aurum, gold.) A term sometimes used for the peroxide of gold. Aurrernous. Containing gold, as 'auriforous quartz,' 'auriforous pyrites,' \&c.

AUnrrva. A red colouring-matter obtained from phenol, or carbolic acid. It appears in commerce as a brittle resinous solid, having a beetle-green lustre, and yielding a red powder. See Carbortc Aom.

AURUM MUSTVUr or MOsarcum. Mosarc Gold,-For the preparation of Mosaic gold the following process is recommended by Woolfe. An amalgam of 2 parts of tin and 1 part of mercury is prepared in a hot crucible, and triturated with 1 part of sal-ammoniac, and 1 part of flowers of sulphur; the mixture is sublimed in a glass flask upon the sand-bath. In breaking the flask after the operation, the sublimate is found to consist, superficially, of sal-ammoniac, then of a layer of cinnabar, and then of a layer of mosaic gold.

Bergmann mentions a native aurum musivum from Siberia, containing tin, sulphur, and a small proportion of copper. Dr. John Davy gave the composition as-tin, 100; sulphur, $56 \cdot 25$; and Berzelius, as tin, 100 ; sulphur, 52.3.

Mosaic gold is employed as a bronzing powder for plaster figures, and it is said to enter sometimes into the composition of artificial aventurine.

AUSTRALFN binthine, obtained by neutralising English turpentine oil with an alkaline carbonato, and distilling the product.

AuTOGmivous sordmRIxG. A process of soldering by which metals are united, either by the ordinary solders or by lead, under the influence of a flame of hydrogen, or of a mixture of hydrogen and common air.

The process of using air and hydrogen was invented in France, by the Count de Richemont. Hydrogen gas is contained in a gasometer, to which a flexible tube is connected, and air is urged, from a bellows worked by the foot, through another tube, and on to the blowpipe, where the hydrogen is ignited. By means of the flexible tubes the flame can be moved up and down the line of any joint, and the connecting medium melted. Fig. 99.

99

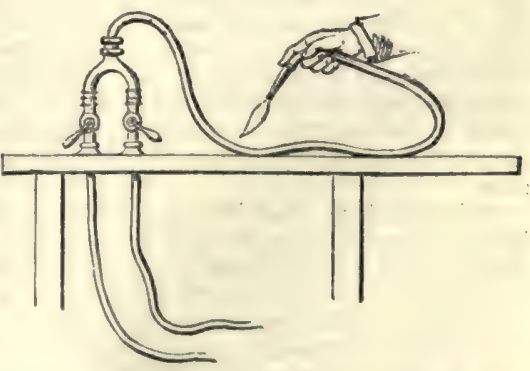

This process has been a good deal employed for plumbers' work, espocially in our Vor. I. 
naval arsenals. In Dovonport dockyard, the autogenic process has been laxgely used.

AUTOMaTrc. A term employed to designate such economic arts as are carried on by self-acting machinery. The word is employed by tho physiologist to express involuntary motions.

The torm automatic is now applied to self-acting machinery, or such as has within itself the power of regulating entirely its own movements, although the moving force is dorived from without; and to what pertains to such machinery; as automatic operations or improvements. -Webster.

The word 'manufacture,' in its etymological sense, means any system or objects of industry executed by the hands; but, in the vicissitude of language, it has now come to signify every extensive product of art which is made by machinery, with littlo or no aid of the human hand, so that the most perfect manufacture is that which dispenses entirely with manual labour. It is in our modern cotton and flax mills that automatic operations are displayed to most advantage; for there the elemontal power Hrat has been made to animate complex organs, imparting to forms of wood, iron, and brass, an agency of seeming intelligence. And as the philosophy of the fine arts, poetry, painting, and music, may be best studied in their individual master-pieces, so may the philosophy of manufactures in these its noblest creations.

The constant aim and effect of these automatic improvements in the arts are philanthropic, as they tond to relieve the workman either from niceties of adjustment, which exhaust his mind and fatigue his eyes, or from painful repetition of effort, which distort and wear out his frame. A well-arranged power-mill combines the operation of many work-people, adult and young, in tending with assidnous skill a system of productive machines continuously impelled by a central force. This great era in the useful arts is mainly due to the genius of Arkwright. Prior to the introduction of his system, manufactures were everywhere feeble and fluctuating in their development; shooting forth luxuriantly for a season, and again withering almost to the roots like annual plants. Their perennial growth then began, and attracted capital, in copious streams, to irrigate the rich domains of industry. When this new career commenced, about the year 1770, the annual consumption of cotton in British manufactures was under four millions of pounds' weight, and that of the whole of Christendom was probably not more than ten millions. In 1850 the consumption in Great Britain and Ireland had risen to five hundred and eighty-eight millions of pounds, and that of Europe and the United States together to one thousand and ninety-two millions. In our spacious factory apartments the benignant power of Steam summons around him his myriads of willing menials, and assigns to each the regulated task, substituting, for painful muscular effort upon their part, the energies of his own gigantic arm, and demanding in return only attention and dexterity to correct such little aberrations as casually occur in his workmanship. Under his auspices, and in obedience to Arkwright's policy, magnificent edifices, surpassing far in number, value, usefulness, and ingenuity of construction, the boasted monuments of Asiatic, Egyptian, and Roman despotism, have, within a short period, risen up in this kingdom, to show to what extent capital, industry and science may augment the resources of a State, while they ameliorate the condition of its citizens. Such is the automatic system, which promises, in its future growth, to become the great minister of civilisation to the. terraqueous globe, enabling this country to diffuse, along with its commerce, the life-blood of knowledge to myriads of people.

AUTOMATIC ARTs. Such arts or manufactures as are camiod on by selfacting machinery.

AUTOMATON. (airbuaros-automatos-8elf-moving.) In the etymological sense, this word (self-working) signifios every mechanical construction which, by virtue of a latent intrinsic force, not obvious to common eyes, can carry on, for some time, certain movements more or less resembling the rosults of animal exertion, without the aid of external impulse. But the term automaton is, in common language, appropriated to those mechanical artificos in which the purposely concealed power is made to imitate the arbitrary or voluntary motions of living beings. Human figures, of this kind, are sometimes styled Androides, from the Greek term, like a man.

Although, from what has been said, clockwork is not properly placed under the head Automaton, it cannot be doubted that the art of making clocks, in its progressive improvement and extension, has given rise to tho production of automata. The most of these, in their intorior structure, as well as in the mode of applying the moving power, have a distinct analogy with clocks; and these automata are frequently mounted in connection with watchwork. 'Towards the end of the 13 th century, several tower clocks, such as those at Strasburg, Lübeck, Pragne, and Olmütz, had curious mechanisms attachod to them. The most careful historical inquiry proves that automata, pro- 
perly speaking, are not older than wheel-clocks; and that the more perfect structures of this kind are subsequent to the general introduction of spring-clocks. Many accounts of ancient automata, such as the flying pigeon of Archytas of Tarentum, appear to have beon but poor mechanical contrivances. "The Pneumatics of Hero of Alexandria' have been rendered accessible to the English reader by the translation of $\mathrm{Mr}$. Bennett Wooderoft. In this work will be found descriptions and drawings of several curious contrivances which must be included amongst automata. The following, amongst othars, may be quoted:to it,

An automaton which drinks at certain times only, on a liquid being presented

'An automaton which may be made to drink at any time on a liquid boing presented to it.

'An automaton which will drink any quantity which may bo presented to it.

'An automaton, the head of which continues attached to the body after a knife has entered the head at one side, passed completely through it, and out at the other; which animal will drink immediately after the operation.'

Beckmann informs us, quoting from Plato, that Drdalns made statues which could not only walk, but which it was necessary to tie, in order that they might not move; and, on the authority of Aristotle, he speaks of a wooden Venus, and remarks, that the secret of its motion consisted in quicksilver having been poured into it.

The moving power of almost all automata is a wound-up steel spring; because, in comparison with other means of giving motion, it takes up the smallest room, is easiest concealed and set a-going. Weights are seldom employed, and only in a partial way. The employment of other moving powers is more limited; sometimes fine sand is made to fall on the circumference of a wheel, by which the rest of the mechanism is moved. For the same purpose water has been employed; and, when it is made to fall into an air-chamber, it causes sufficient wind to excito musical sounds in pipes. In particular cases quicksilver has been used, as, for example, in the Chinese tumblers, which is only a physical apparatus to illustrate the doctrine of the centre of gravity.

Fig. 100 exhibits the outlines of an automaton, representing a swan, with suitably combined movements. The mechanism may be described, for the sake of clearness of explanation, under distinct heads. The first relates to the motion of the whole flgure. By means of this pert it swims upon tho water, in directions changed from time to time without exterior agency. Another construction gives to the figure the faculty of bending its neck on several oceasions, and, to such an extent, that it can plunge the bill and a portion of the head nnder water. Lastly, it is made to move its head and neck slowly from side to side.

On the barrel of the spring exterior to the usual ratchet wheel, there is a main-

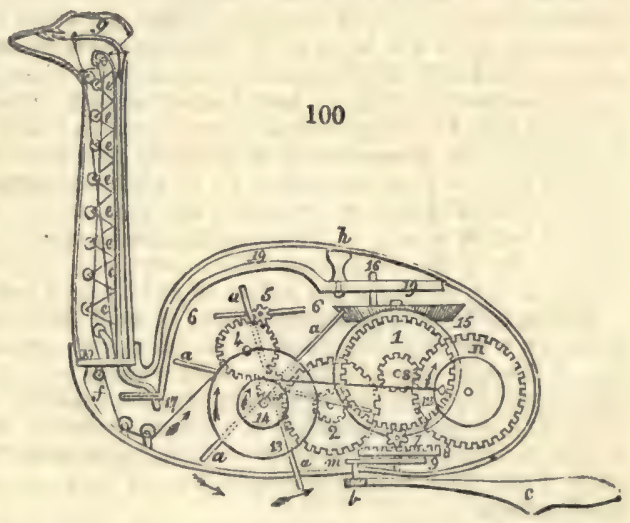
wheel, marked 1, which works into the pinion of the wheel 2. The wheel 2 moves a smaller one, shown merely in dotted lines, and on the long axis of the latter, at either end, there is a rudder, or water-wheel, the paddles of which are denoted by the letter a. Both of these rudder-wheels extend through an oblong opening in the bottom of the figure down into the water. They turn in the direction of the arrow, and impart a straightforward movement to the swan. The chamber in which these wheels revolve is made water-tight, to prevent moisture being thrown upon the rest of the machinery. By the wheol 4, motion is conveyed to the fly-pinion 5 ; the fly itself, 6 , serves to regulate the working of the wholo apparatus, and it is provided with a stop bar, not shown in the engraving, to bring it to rost, or set it a-going at pleasure. Here, as we may imagine, the path pursued is rectilinear, when the rudder-wheels are made to work in a squaro direction. An oblique bar, seen only in section at $b$, movable about its middle point, carries at each end a wob foot $c$, so that the direction 
of the bar b, and of both feet towards the rudder-wheels, determines the form of the path which the figure will describe. The change of direction of that oblique bar is effected without other agency. For this purpose the wheel 1 takes into the pinion 7 , and this carries round the erown-whoel 8, which is fixed, with an eccentric disc 9, npon a common axis. While the crown-wheol moves in the direction of the arrow, it turns the smaller eccentric portion of the olliptic dise towards the lever $m$, which, pressed upon incessantly by its spring, assumes, by degrees, the position corresponding with the middle line of the figure, and afterwards an oblique position; then it goes back again, and reaches its first situation; consequently, through the reciprocal turning of the bar $h$ and the swim-foot, is determined and varied the path which the swan must pursue. This construction is available with all automata which work by whoels; and it is obvious, that we may, by different forms of the dise 9 , modify, at pleasure, the direction and the velocity of the turnings. If the dise is a circle, for instance, then the changes will take place less suddenly; if the disc is an outward and inward curvature, upon whose odge the end of the lever presses with a roller, the movement will take place in a serpentine line.

The neck is the part which requires the most careful workmanship. Its outward case must be flexible, and the neck itself should therefore be made of a tube of spiral wire, covered with leather, or with a feathered bird-skin. The double line in the interior, where we see the triangles $e e e$, denotes a steel spring made fast to the plate 10, which forms the bottom of the neck; it stands loose, and needs to be meroly so strong as to keep the neck straight, or to bend it a little backwards. It should not be equally thick in all points, but it should be weaker where the first graceful bend is to be made; and, in general, its stiffness ought to correspond to the curvature of the neck of this bird. The triangles $e$ are made fast at their base to the front surface of the spring; in the points of each there is a slit, in the middle of which a movable roller is set, formed of a smoothly turned steel rod. A thin catgut string $f$, runs from the upper end of the spring, where it is fixed over all these rollers, and passes, through an aperture piereed in the middle of 10 , into the inside of the rump. If the catgut be drawn straight back towards $f$, the spring, and consequently the neck, must obviously be bent, and so much the more, the more tightly $f$ is pulled and is shortened in the hollow of the neck. How this is accomplished by the wheel-work will presently be shown. The wheel 11 receives its motion from the pinion $\varepsilon$, connected with the main wheel 1. Upon 11 there is, moreover, the dise 12, to whose circumference a slender chain is fastened. When the wheel 11 turns in the direction of the arrow, the chain will be so much pulled onwards through the corresponding advance at the point at 12, till this point has come to the place opposite to its present situation, and, consequently, 11 must have performed half a revolution. The other ond of the chain is hung in the groove of a very movable roller 14 ; and this will be turned immediately by the unwinding of the chain upon its axis. There turns, in connection with it, however, the large roller 13 , in which the catgut $f$ is fastened; and as this is pulled in the direction of the arrow, the neck will be bent until the wheel 11 has made a half revolution. Then the drag ceases again to act upon the chain and the catgut; the spring in the neck comes into play: it becomes straight, erects the neck of the animal, and turns the rollers 13 and 14 back into their first position.

The roller 13 is of considerable size, in order that through the slight motion of the roller 14, a sufficient length of the catgut may be wound off, and the requisite shortening of the neck may be effected; which results from the proportion of the diameters of the rollers 11, 13,14. This part of the mechanism is attached as near to the side of the hollow body as possible, to make room for the interior parts, but particularly for the paddle-wheels. Since the catgut $f$ must pass downwards on the middle from 10 , it is necessary to incline it sideways and outwards towards 13 , by means of some small rollers.

The head, constituting one piece with the neck, will be depressed by the complete flexure of this; and the bill, being turned downwards in front of the breast, will touch the surface of the water. The head will not be motionless; but it is joined on both sides, by a very movable hinge, with the light ring which forms the upper part of the clothing of the neck. A weak spring, $g$, also fastened to the end of the neck, tends to turn tho head backwards; but in the present position it cannot do so, because a chain at $g$, whose other end is attached to the plate 10 , keeps it on the stretch. On the bending of the neck, this chain becomes slack; the spring $h$ comes into operation, and throws the head so far back that, in its natural position, it will reach the water.

Finally, to render the turning of the head and neck practicable, the latter is not closely connected with the rump, while the plate 10 can turn in a cylindrical manner upon its axis, but cannot become loose outwardly. Moreover, there is upon the axis of the wheel 1, and behind it (shown merely as a circle in the engraving) a borel 


\section{AVENTURINE}

whoel, which works into a socond similar wheel, 15 , so as to turn it in a horizontal direction. The pin, 16, of the last wheel works upon a two-armed lever, 19, movable round the point $h$, and this lever moves the neck by means of the pin 17 . The shorter arm of the lever 19 has an oval aperture in which the pin 16 stands. As soon as this, in consequence of the movement of the bevel-wheel 15 , comes into the dotted position, it pushes the oval ring outwards on its smaller diameter, and thereby turns the lever upon the point $h$, into the oblique direction shown by the dotted lines. The pin 16, having come on its way right opposite to its present position, sets the lever again straight. Then the lever, by the further progress of the pin in its cireular path, is directed outwards to the opposite side; and, at last, when 15 has made an entire revolution, it is quite straight. The longer arm of the lever follows. of course, these alternating movements, so that it turns the neck upon its plate 10 , by means of the pin 17 : and, as 18 denotes the bill, this comes into the dotted position. It may be remarked, in conclusion, that the drawing of fig. 100 represents about half the size of which the automaton may be constructod, and that the body may be formed of thin sheet copper or brass,

In the former edition another example of an automaton was given, but it is thought unnecessary to retain it. In many of the machines now employed, we have examples of useful automata, superior in correctness of action to any of those which are at the best only scientific toys.

Autotypz. See Photography.

AVINA. A genus of corn-bearing grasses. The A. sativa is the common oat.

A VINTURINz. (Aventurine, Fr.) A variety of quartz, which is minutely spangled throughout with yellow scales of mica, is known as Aventurine quartz. It is usually translucent, and of a grey, brown, or reddish-brown colour. There is also an Aventurine felspar (Feldspath aventuriné, Fr.), frequently tormed sunstone (Pierre de soleil); some lapidaries, however, calling this stone by the name of Aventurine orientale. Aventurine quartz occurs at Capa de Gata, in Spain; and the aventurine felspar, or sunstone, at Tvedestrand, in Norway.

Aventurine, Artificiai, or Glass, called also Gold Flux, has been manufactured on a large scale, for a long period, at the glass-works of Murano, near Venice. According to Wöhler's examination, aventurine glass owes its golden iridescenee to a crystalline separation of metallic copper from the mass coloured brown by the peroxide of iron. C. Kursten analysed the artificial aventurine from the glass manufactory of Bigaglia, in Venice, and found it to contain-

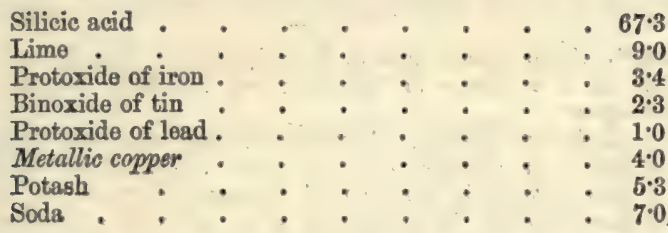

These numbers agree in a remarkable manner with the results formerly obtained by Péligot, and may therefore be regarded as truly representing the composition of the glass.

A VRRTURINe Glaze, for porcelain, exhibits a crystalline separation of green oxide of chromium from the brown ferruginous mass of the glaze producing a similar effect to the glass. This glaze is prepared as follows, according to A. Wächter:-

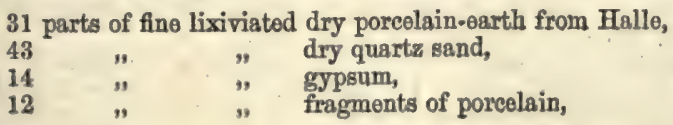

are stirred up with 300 parts of water, and by repeated straining through a linen sieve, uniformly suspended in it, and intimately mixod. To this paste are addod, under constant agitation, and one after the other, aqueous solutions of

19 parts bichromste of potash,

100 " protosulphate of iron,

47 , acetate of lead,

and then so much solution of ammonia that the iron is completely separatod. The salts of potash and ammonia are removed by frequent decantation with spring water.

The baked porcelain ressels are dippod into the pasty mixture obtained as above 
describod, in the same manner as with other glazes, and then fleod in the porcelain furnace. After this they aro covered with a brown glaze, which in reflected light appears to bo filled with a countless numbor of light gold spangles.

A thin fragment of the glazo appears, under tho mieroseopo, by transmittod light, as a clear brownish glass, in which numorous transparent green six-sided prisms of oxide of chromium, and some brownish crystals, probably of oxide of chromium and peroxide of iron, are suspended. The oxide of chromium, therefore, separates on the slow cooling of the glaze in the porcelain furnace, from the substance of the glazea silicate of potash, lime, and alumina, saturated with the peroxide of iron-and shines through the brownish mass with a golden colour. When the aventurine glaze is mixed with an equal amount of colourless porcelain glaze, the glassy mass no longer has a brown colour aftor the burning, but a light greonish-grey, and the eliminated crystalline spangles likewise exhibit in reflected light thoir natural groen colour.

AVIRRUNCATOR. A pair of pruning shears, which, on being mounted on a polo some ton foet long, and actuatod by a string of catgut, can be used for pruning at a considerable distance above the head.

AvOCADO-PrAR OII. An oil obtained from the oleaginous fruit, the Avocado pear-tree (Persea gratissima), a native of Trinidad. A portion of this oil having been submitted to Dr. Hofmann by the Governor of Trinidad, he roported on its character : - According to my present experience, the oil of the Arocado pear is less valuable as a lubricating material. To make it fit for the higher classes of machinery, its mucilaginous constituents must be removed by the same refining process requisite for its adaptation in illuminating purposes,

' On the other hand, the oil of the Avocado pear is very applicable for the production of good soap. I have the honour of transmitting to your Excellency specimens prepared with the oil : the smaller one, which possesses a yellow colour, is prepared with the oil in its original condition; the larger one is made with a portion of oil which had previously been bleached by chlorine. From this specimen it is obvious that the oil, although poor in stearine, nevertheless furnishes a soap which is tolerably hard and solid. I have even now no hesitation in stating that, for the purposes of the soap-maker, the oil of the Avocado pear will have, at least, the same value as palm oil.'

Axs. A tool much used by carpenters for cleaving and roughly fashioning blocks of wood. It is a thin iron wedge, with an oblong steol edge, parallel to which, in the short base, is a hole for receiving and holding fast the end of a strong wooden handle.

Axב-STOxz. A sub-species of jade, found in Corsica, Saxony, and on the banks of the Amazon. It is a silicate of magnesia and alumina, coloured by oxide of chromium. See JADE.

AxrNITrs, called also Thumite. A silicato of magnesia, alumina, and iron, containing boracic acid. It dorives its name from the axe-like bevelling of its lateral edges. This minerel is harder than felspar, and varies in colour from a violet-brown to a leek-green. It is found in many parts of the Continent, and at Botallack, St. Just, and at Trewellard, Cornwall, in fine brilliant clove-brown crystals,

Axrm-erzasz. Several kinds of unguents omployed to reduce the friction of wheels circulating on their axles, See ANTI-ATTRition.

AXImS, of carriages. See W HERT CARRIAGES.

Axuzrcez. Hog's lard. Seo Fat and Ors.

AYR STONE, called also Scotch stone and snake-stono, is much in request as a polishing stono for marble and for copper plates. These stones are always kept damp, or even wet, to prevent their becoming hard.

The harder varieties of Ayr stone are now employed as whotstones.

AzAIE (from Azala, Arabic for madder). A colouring-matter obtained from ' flowers of madder,' perhaps crude alizarine. It has been proposed to introduce azale in France as a dye-stuff.

АZAт̈̈гтr, A name for aniline-red.

AzIMUTE Compass. The arimuth compass is used chiefly to noto the actual magnetic azimuth, or that arch of the horizon intercepted between the azimuth, or vertical circle passing through the centre of any heavenly body, and the magnetic meridian.

The card of the arimuth compass is subdivided into exact degrees, minutes, and seconds. To the box aro fixed two 'sights,' through which the sun or a star may be viewed. The position into which the index of the sights must be turned to see it, will indicate on the card the azimuth of the star. When the observations are intended to be exact, telescopes take the place of the sights. By this instrument we note the 
actual magnetic aximuth : and as we know the azimuth calculated from the $\mathrm{N}$, and S. line, the variation of the needle is readily found.

AZOBENZINE, AZOBINZIDE, or AZOBENZOI, $\mathrm{C}^{2} \mathrm{H}^{10} \mathrm{~N}^{2}\left(\mathbf{C}^{12} \mathrm{Fr}^{10} \mathrm{~N}^{3}\right)$. A peculiar substance formed by acting with an alcoholic solution of potash upon nitrobenzole, or, as it is sometimes called, artificial oil of bitter almonds. If nitrobenzole, dissolved in alcohol, with the addition of solid potash, be distilled, a complex and by no means well understood reaction occurs. The azobenzide distils over mixed with aniline. The fluid treated with hydrochloric acid, to dissolve the aniline, is passed through a wet filter; the aniline salt passes through, leaving the azobenzide as a red oil, which in a few moments solidifies into a mass of rich golden-brown crystals of considerable size, even when working on a very small quantity. The alcohol enters into the reaction, and oxalic acid is formed, which unites with the potash. Four equivalents of nitrobenzole and two equivalents of alcohol appoar to yield one equivalent of azobenzide, two equivalents of aniline, four equivalents of oxalic acid, and eight equivalents of water. See Nrrrobenzor..

Azobenzene yields numerous derivatives. With fuming nitric acid it gives two nitro-compounds; viz, nitroazobenride and binitroazobenzide. Azobenzide, treated with sulphide of ammonium, yields an alkaline called benzidine, $\mathrm{C}^{24} \mathrm{H}^{12} \mathrm{~N}^{2}\left(\mathbf{C}^{12} \mathbf{z}^{12} \mathbf{T}^{2}\right)$. -C. G. W.

AzOBINZOIDE. When bitter almonds are distilled, per descensum, an oil is obtained; if the latter be treated with ammonia, and the substance thus formed bo treated with ether, a white powder remains, which is probably impuro hydrobonzamide.-C. G. W. See 'Watts's Dictionary of Chemistry.'

Azorsinzoyrs. A substance formed simultaneously with hydrobenzamido and benzydramide, when oil of bitter almonds is treated with ammonia.-C. G. W.

Azorz. An old name for Nitrogis.

AzOXISID, said of certain vegetable substances, which, as containing azote, were supposed at one time to partake, in some measure, of the animal nature. The vegotable products, indigo, caffeine, gluten, and many others, contain abundance of azote.

AzUz:. This term was applied by Pliny to the blues of the ancients. 'Cæruleum, or azure, is of three kinds: the Egyptian (artificial); the Scythian (natural), which is inferior; the Cyprian, the best.'-Theophrastus, also Pliny. Girardin, writing of the ancient colours, says, "This azure, which has thus endured above 1,700 years, may bo cheaply and easily mado thus : 15 parts, by weight, of carbonato of 6oda, 20 parts of opaque flints, and 3 parts of copper filings, are strongly heated for two hours, and the mixture will result in a fine deop sky-blue.' The Egyptian blue, or Alexandrian frit, is a pulverised blue glass; it was once thought to contain cobalt, but all analyses prove it to contain silicato of copper.

The term Azure has been applied to smalts. See Cobaut, Smalt, and UltraMARINE.

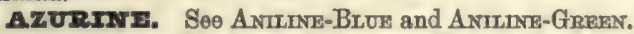

AzURIT2. This term is now usually restricted to the blue carbonate of coppor, otherwise known as Chessylite. It is a mineral of fine blue colour, crystallising in the oblique system. The old mines of Chessy, near Lyons, in France, were famous for yielding groups of magnificent crystals of this species. It is also found in the shallow workings of many other copper mines, often associated with malachite, or green carbonate of copper. Azurite contains, when pure, $55 \cdot 16$ per cent. of copper, and hence forms a valuable ore. It has also been used as a blue pigment, though too liable to turn green, the absorption of carbonic acid readily converting it into malachite.

It is right to remark that the torm Azurite has also been applied to certain other blue minerals, such as the phosphate of alumina and magnesia, usually known as Lazulite, and oven to the lapis lazuli.

The want of agreement between mineralogists-leading them to adopt names independent one of the other (names frequently taken from some locality in which the writer knows the mineral to be found)-produces great confusion, and retards the progress of knowledge.

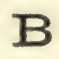

mazarx's mrrar. An alloy which, from its gmoothness of surface, is called an anti-attrition metal. It is composed of 25 parts of tin, 2 parts of antimony, and 11 a part of copper.

BABRSCTONTrE. An anhydrous silicate of iron and lime, found in small, greenish-black, doubly-oblique crystals, at Arendal, in Norway. A fibrous variety, 
much resembling hornblende, was discovered in a railway-cutting in Devonshire, in 1854, and was sufficiently abundant to be worked as an ore of iron. A spocimen roceived from the late Mr. S. Blackwell was analysed by Mr. David Forbes, with the following results :-Silica, 49.12 ; alumina, 1.60 ; peroxide of iron, $9 \cdot 78$; protoxide of iron, 12.87; protoxide of manganese, 1.25; lime, 20.87; magnesia, 3.67 ; loss on ignition, $0 \cdot 78$.

BAвIAf. The rind or shell of the fruit of the Mimosa cineraria, It is brought from the East Indies under the name of Neb-Neb. On account of the tamnin it contains, it has been used for dyeing cotton, and for producing various shades of drab.

BABUx cum. The gum of the Babul tree, a species of the Acacia, growing in Bengal. It is sometimes imported as Bengal gum.

Babul Bark is extensively used in India as a tanning material, and has occasionally been imported into this country.

BACI. In mining, that side of an inclined mineral lode which is nearest the surface of the ground. The back of a level is the ground between it and the level above it. In brewing, a brewer's utensil, a large vessel for receiving the wort. In building, that part of a stone opposite the face.

BACIr-MrII. A fulling mill.

BACUsus. A forked branch of hazel, used by the superstitious with a view to the discovery of springs and mineral lodes. See Divinuse Rod.

BADGER. (Blaireau, Fr.; Dachs, Ger.) A genus of carnivorous animals belonging to the family Mustelide. The common badger, Meles Taxus, inhabits the northern parts of Europe and Asia. The hide of the badger is employed for pistol furniture. The fine hair is used for making brushes for the use of the artist, and for the best class of shaving-brushes. The hind-quartors, salted and smoked, mako excellent hams.

BADICEOr. A mixture for stopping holes in wood or stone. The badigeon for stonework is composed of plaster of Paris and freestone ground together. That for wood is usually sawdust and glue, or sometimes putty and chalk.

BAG. A measure equal to a striked Winchester bushel. Twenty-five bags of lime make a ton. A bag of plaster of Paris is fourteen pounds. Eight bags are considered to equal a bushel.

BAGASSI. The sugar-cane, in its dry crushed state, much employed for fuel in the colonial sugar-houses.

BATN-MARIs. A vessel of water in which saucepans, \&c. aro placed to warm food, or to prepare it and some pharmaceutical preparations.

BATz:s. A coarse woollen stuff with a long nap, sometimes friezed on one side.

BAxERs' sAxT. The sesquicarbonate of ammonia, so called because it is often used as a substitute for yeast in bread and pastry.

BAтING. (Cuire, Fr.; Backen, Ger.) The exposure of any body to such a heat as will dry and consolidate its parts without wasting thom. Thus wood, pottery, and porcelain, are baked, as well as bread and meat. Seo Brscurt ; Bresd.

BAI. An ancient Cornish miner's term for a mine.

BAI-MAIDEN, BAI-BOY. A girl or boy working at a mine.

BAIACrowG. An articlo of food much used in the Eastern Archipelago, con. sisting of fish and shrimps pounded together.

BAтתמNA. A genus of cetacean mammals, including the Groenland, or Right Whale (Balena mysticetus), and the Southern Whale (B. australis). The former inhabits the Aretic Seas, and its capturo forms the object of the Northern whalefishery, while the latter is found in the Antaretic, South Pacific, and Indian Oceans. These species, in the adult state, are destitute of teeth, but the mouth is furnished with numerous plates of a horny substance, called baleen, or whalebone, which hang freely from each side of the palate, and thus form a sieve for straining off the water from the small prey taken into the mouth. Large quantities of oil are obtained from the blubber, or thick layer of fat which immediately underlies the naked skin, and serves to protect the warm-blooded whale from the cold of the surrounding medium. The Esquimaux not only eat the flesh of the whale, but use some of the internal membranes in the preparation of certain articles of clothing, and of a curious semi-transparent substance serving instead of glass for the windows of their huts. The species of Balena, or true Whalebone Whales, are to be distinguished from the Sperm Whales, which belong to a totally distinct genus, and though possessing teeth, and.therefore not yielding whalebone, are nevertheless valued for the salke of their spermaceti and ambergris. See AmBERgris; SPERMACETI; WHALRBONE.

BATANCE. To conduct arts and manufactures with judgment, recourse must be had to a balanco. Experience proves that all material bodies existing upon the 
surface of the earth are constantly solicited by a force which tends to bring them towards its centre, and that they fall to the earth when they are free to move. This force is called gravity. Though the bodies be not free, the effort of gravity is still sensible, and the resultant of all the actions which it exercises upon their material points constitutes what is called thein weight. Weights are, therefore, forces which may be compared together, and by means of machines may be made to correspond or be counterpoised.

To discover whether two weights be equal, we must oppose them to each othor in a machine where they act in a similar manner, and then see if they maintain an equilibrium; for example, we fulfil this condition if we suspend them at the two extremities of a lever supported at its centre, and whose arms are equal. Such is the general idea of a balance. The beam of a good balance ought to be a bar or double cone of metal, of such strength as to secure perfect inflexibility under any load which may be fitly applied to its extremities. Its arms should be quite equal in weight and length upon each side of its point of suspension; and this point should be placed in a vertical line over the centre of gravity; and the less distant it is from it, the more delicate will be the balance. Were it placed exactly in that contre, the beam would not spontaneously recover the horizontal position when it was once removed from it. To render its indications more readily commensurable, a slender rod or needlo is fixed to it, at right angles, in the line passing through its centres of gravity and suspension. The point, or rather edge, of suspension, should be made of perfectly hard steel, and turn upon a bed of the same. For common uses the arms of a balance can be made sufficiently equal to give satisfactory results; but, for the more refined purposes of science, that equality should never be presumed nor trusted to ; and, fortunately, exact weighing is quite independent of that equality. To weigh a body is to determine how many times the weight of that body contains another species of known weight, as of grains or pounds, for example. In order to find it out, let us place the substance, suppose a pioce of gold, in the left hand scale of the balance; counterpoise it with sand or shot in the other, till the index needle be truly vertical, or stand in the middle of its scale, proving the beam to be horizontal. Now remove gently the piece of gold, and substitute in its place standard multiple weights of any graduation, English or French, until the needle again resumes the vertical position, or until its oscillations upon either side of the zero-point are equal. These weights will represent precisely the weight of the gold, since they are placed in the same circumstances with it, and make the same equilibrium with the weight laid in the other scale.

This method of weighing is obviously independent of the unequal length as well as the unequal weight of the arms of the beam. For its perfection two requisites only are indispensable. The first is that the points of suspension should be rigorously the same in the two operations; for the power of a given weight to turn the beam being unequal, accordingly as we place it at different distances from the centre of suspension, did that point vary in the two consecutive weighings, we should require to employ, in the second, a different weight from that of the piece of gold, in order to form an equilibrium with the sand or shot originally put in the opposite scale; and as there is nothing to indicate such inequality in the states of the beam, great errors would result from it. The best mode of securing against such inequality is to suspend the cords of the scales from sharp-edged rings, upon knife-edges, at the ends of the beam, both made of steel so hard-tempered as to be incapable of indentation. The second condition is, that the balance should be very sensible-that is, when in equilibrium and loaded, it may be disturbed, and its needle may oscillate, by the smallest weight put into either of the scales. This sensibility depends wholly upon the centre of suspension; and it will be the more perfect the loss friction there is between that knife-edge surface and the plane which supports it. Both should therefore be as hard and highly polished as possible; and should not be suffered to press against each other, except at the time of weighing. Every delicate balance of moderate size, moreover, should be suspended within a glass case, to protect it from the agitations of the air, and the corroding influence of the weather. In some balances a ball is placed upon the index or needle (whether that index stand above or bolow the beam), which may be made to approach or recede from the beam by a fine-threaded screw, with the effeet of varying the centre of gravity relatively to the point of suspension, and thereby increasing, at will, either the sensibility or the stability of the balance. The greater the length of the arms, the less distant the centre of gravity is beneath the centre of suspension, the better polished its contral knife-edge of $30^{\circ}$, the lighter the whole balance, and the loss it is loaded, the greater will be its sensibility. In all cases the arms must be quito inflexible. A balance made by Ramsdon for the Royal Society is capablo of weighing ten pounds, and turns with one hundredth of a grain, which is the sevenmillionth part of the weight. See Wergunsa Machuse. 


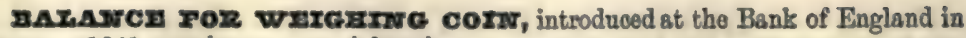

the year 1841, requires an especial notice.

Mr. William Cotton, then Deputy-Governor, and during the two succeeding years Governor of the Bank, had long regarded the mode of weighing by common handbalances with dissatisfaction, on account of its injurious effect upon the 'teller, or weigher, owing to the straining of the optio nerve by constant watching of the beamindicator, and tho necessity of reducing tho functions of the mind to the narrow offico of influoncing a fow constantly repeated actions. Such monotonous labour could vot be endured for hours together without moments of forgetfulness resulting in orrors. Errors more constant, although less in amount, were found to be due to tho rapid wearing of the knife-odges of the beam; currents of air also acting upon the pans produced undesired results; and even the breath of the 'teller' sometimes turned the scale; so that in hand-weighing the emors not unfrequently amounted to $\frac{1}{3} \mathrm{rd}$, and even $\frac{1}{2}$ grain. At the very best, the hand-scale working at the rate of 3,000 per six hours could not indicate nearer than $\frac{3}{\text { th th }}$ grain.

Upon taking into consideration the inconveniences and defects of the hand-weighing system, Mr. Cotton conceived the idea that it might be superseded by a machine defended from external influences, and contrived so as to weigh coins as fast as by hand, and within the fourth of a grain. He subsequently communicated his plan to $\mathrm{Mr}$. David Napier, of York Road, Lambeth, engineer, who undertook the construction of an experimental machine. Its capabilities were tested and reported upon by $\mathrm{Mr}$. William Miller, of the Bank. The result was most satisfactory : more 'automaton balances 'were ordered; and from time to time further additions have been made, so that at present there are ton in daily operation at the Bank of England. But it was not without a struggle that the time-hallowed institution of 'tellers' passed away. There were interests opposed to theintroduction of improved, more ready, and less expensive methods; and it required all Mr. Cotton's energy of character, the influence of his intelligence in mechanics, as well as that arising from his position in the Direction, to obtain the adoption of an invention by which a very large annual saving has boen effected.

The mechanical adaptation of the principles involved in the Automaton Balance, as contrived by Mr. Napier, may be shortly explained:-The weighing-beam, of steel, is forked at the ends, each extremity forming a knife-edge; and in the centre the fulcrum knife-edge extends on each side of the plate of the beam, and rests in hollows cut in a bowed cross-bar fixed to the under side of a rectangular brass plate, about 12 inches square, which is supported at the corners by columns fixed to a cast-iron table raised a convenient height on a stand of the same metal. To form a complete enclosing case, plates of metal or glass are slid into grooves down the columns. When the beam is resting with its centre knife-edge in the hollows of the cross-bar just referred to, its upper part is nearly on a level with the under-side of the brass plate, in which a long slot is made, so that the beam can be taken out when the feeding slide-box, and its plate, which covers this slot, are removed. On the top of the covering plate of the feoding slide a tabe-hopper is placed, and a holo in the plate communicates with the slide; another hole is pierced in the same plate exactly over one end of the beam, upon the knife-edges of which a long rod is suspended by hollows formed in a crossbar close to its upper end, where the weighing platform is fitted. A rod is also suspended at the other end of the beam in a similar manner; but instead of a woighingplate, it has a knob at top, which, when the beam is horizontal, comes into contact with an adjustable agate point. The lower end of this pendent rod is stirrup-shaped, for holding the counterpoise. Two displacing slides are provided, one on each side of the feeding-slide, and at right angles to each other; and a gripping apparatus is fixed to the under side of the brass top-plate, arranged so as to hold the pendant on which the scale-plate is fitted during the change of the coin. A dipping-finger is also attached to the frame of the gripping apparatus, its end passing into a small slot in the pendent rod, and acting upon a knife-edge at the lower end of the slot. There are four shafts crossing the machine; the one through which the power is applied is placed low and at the centro, and carrios a pinion which gears with a wheel of twice its diameter on a shaft above; this whoel gears with two similar wheels fixed to shafts on each side of the centre. Cams for acting upon the foeding slide, through the medium of a rocking frame, are carried by the shaft placed at the end of the machine where the counterpoise hangs, and the other two shafts on the same level bear cams for working the gripping apparatus, the dipping-finger, and the displacing slides.

Having describod, as clearly and as popularly as wo can, the general features of the mechanism, we will proceed to indicate its manner of action. Suppose, then, the hopper filled, and a hollow inclined plane about two feet long, which has been added to the hopper by the inventivo genius of one of the gentlemen in the woighing-room, also loaded its whole length with the pieces to be woighed, the machine is set in 
motion, and the feoding slide pushes the lowest piece forward on to the weighingplate, the grippers meantime holding fast by the neck of the pendant, so as to keep the plate perfectly steady; the dipping-finger is also at its lowest position, and resting upon the knife-edge at the bottom of the slot in the pendent rod, thus keeping the beam horizontal, and the knob on the counterpoise-pendant in contact with the agate point already mentioned. When the coin is fairly placed on the weighing-plate, the grippers let go their hold of the pendent rod, and the dipping-finger is raised by its cam; if then the coin is too light, the coin end of the beam will rise along with tho dipping-finger, and the counterpoise end will descend; if heavy, the beam will remain without motion, the agate point preventing it. As soon as the dipping-finger attains the proper height, and thus has allowed sufficient time for the weight of the coin to be decided, the grippers close and hold the pendant, and consequently the scale or weighing-plate, at the high lovel, if the coin has proved light, and been raised by the excess of weight in the counterpoise; and at the low or original level, if the coin has proved heavy. One of the displacing slides now comes forward and passes under the coin, if it is light, and therefore raisod to the high lovel; but knocks it off, if remaining on the low level, into the 'heavy box.' The other displacing slide then advances. This strikes higher than the first, and removes the light pieco which the other has missed, into the receptacle for the light coin. During these operations the fooding-slide has brought forward another coin, and the process just described is repeated. The attendant is only required to replenish the inclined plane at intervals, and remore the assorted coin from the boxes. The perfection of the workmanship, and the harmony of the various actions of the machine, will be best appreciated from the fact, that 25 pieces are weighed per minute to the fineness of $\frac{1}{100}$ th of a grain. This combination of great speed and accuracy would not have been possible with a beam made in the ordinary way, having the centre of gravity below the centre of action; and it was pronounced to be so by the late Mr. Clement, the constructor of Mr. Babbage's Calculating Machine. But Mr. Napier overcame the difficulty by raising the centre of gravity so as to coincide with the centre of action, which gave it much greater sensibility; and he provided the dipping-finger, to bring the beam to a horizontal position after each woighing, instead of an influencing weight in the beam itself.

The wear and tear of these machines is found to be very small indeed; those supplied in 1842 and 1843, and in daily use ever since, weigh with the same accuracy as at first, although they may be said to have cost nothing for repairs. The principal cause of this long-continued perfection is that the beam does not oscillate, unless the coin is light, and even then the space passed through does not exceed the thickness of the coin.

In 1851, when the Moneyers were no longer masters of the Royal Mint, and the now authorities began to regard the process of weighing the coin in detail by hand as a laborious, expensive, and inaccurate method, the firm of Napier and Son, at an interview with Sir John Herschel, the Master, and Captain Harness, the Deputy-Master, received an order for five machines, to be designed to suit the requirements of the Mint, which involved a complete change in the mechanical arrangement of the machine as used at the Bank, it being necessary to divide the 'blanks,' or pieces before they are struck, into three classes, 'too light,' 'too heavy,' and 'modium,' or those varying between certain given limits. It would occupy too much space to attempt a description of the mechanical disposition of this machine, and it could not be satisfactorily accomplished without the aid of drawings; let it suffice, then, to say that the displacing-slides are removed, and a long vibrating conducting-tube receives the blanks as they are in turn pushed off the weighing-plate by the on-coming blanks; but, according to the weight of tho blank, so the lower end of the tube is found to be opposite to one of throe openings leading into three boxes. The tube is sustained in its proper position, during the descent of the blank last weighed through it, by a stop-finger, the height of which is regulated by a dipping-finger, which comes down upon a knifo-odge at the lower end of a slot in the pendent rod just when the grippers have laid hold of the rod after the weighing is finished; this finger thus ascertains tho lovel which the knife-edge has attained, and as it brings down the stop-finger with it, the guide-tube, which is furnished with three rests, as steps in a stair, vibrates against the stop-finger, one of the three steps coming in contact with it, according to the level of the stop-finger; and the end of the guide-tube takes its place opposite the channel leading to the box in which the blank should be found. The counterpoise employed is less than the true standard weight, by the quantity which may be allowod as the limit in that direction; and in case a blank is too heary, not only is the countorpoise raised, but a small weight, equal to the range allowod betwoen the 'too light' and 'too heavy,' is raised also; this small woight comes to rest on supports provided for it when the beam is horizontal, and is only disturbod by a too heavy blank. 
These machines have proved even more accurate and rapid than those made for the Bank; and Professor Graham, the late Master, amongst the improvements introduced by him into the systom of the Mint, added to the number, and dispensed entirely with the hand-weighing. It is said that the saving accruing from this change alone amounts to nearly 2,000l. per annum. See Hydrostatic Balasce; Weigming Macrovs.

BAIAS, BAIIUS, or BAIAIS $\boldsymbol{g} U \mathbf{B X}$. The names applied to the rosered and reddish-white varieties of spinel. See RuBY.

BAII. A package of silk, linen, or woollen, is so called.

BAIIISTrRosITz. A varioty of iron pyritos found in Asturia.

BAIIISTIC PENDUIUR. An instrument for measuring the force of cannonballs. The ballista was an instrument used by the ancients to throw darts, \&c. The ballistic pendulum derives its name from this : it consists of an iron cylinder, closed at one end, suspended as a pendulum. A ball being fired into the open end, deflects the pendulum according to the force of the blow recoived from the ball, thus measuri.us its power.

BAIIOON. In France, a quantity of glass. Of white glass, 25 bundlos of six plates each; of coloured glass, $12 \frac{1}{2}$ bundles of three plates each, are called balloons. Chemists call receivers and flasks of a spherical form balloons.

\section{BaIxoONS. See Arirostation.}

BAII SODA, BIACK BAIIS, or BIACK ASE. Crude carbonato of soda, obtained in the manufacture of soda-ash. See Sops.

BaIm or GIIrad. Soo Batsam, Mreca.

zarsam. (Baume, Fr.; Balsam, Ger.) A native compound of ethereal or essential oils, with resin, and frequently benzoic acid. Most balsams have the consistence of honey; but a few are solid, or become so by keeping. They flow either spontaneously, or by incisions made in trees and shrubs in tropical climates. 'They have peculiar and sometimes powerful smells, aromatic hot tastes, but lose their odoriferous properties by long exposure to the air. They are insoluble in water; soluble to a considerable degree, in ether; and completely in alcohol. When distilled with water, ethereal oil comes over, and resin remains in the retort.

baisam, camada. Seo Canada Batsam.

batsam Coparva, or Capivi, or CAparba. (Baume de Copahu, Fr.; Kopaiva Balsam, Ger.) Capaiva balsam, balsam of copahu, or capivi, is obtained from incisions made in the trunk of the Copaifera officinalis, a tree which grows in Brazil and Cayenne. It is also very frequently obtained from the C. multijuga, C. Langsdorfi, and C. Coriacea. It is pale yellow, semi-liquid, clear and transparent, has a bitter, sharp, hot taste; a penetrating disagreeable smell; a specific gravity of from 0.950 to 0.996 . It dissolves in absolute alcohol, and partially in spirits of wine, and forms with alkalis crystalline compounds. It consists of 45.59 othereous oil, 52.75 of a yellow brittle resin, and 1.66 of a brown viscid resin. The oil contains no oxygen, has a composition like that of oil of turpentine; it dissolves caoutchoue (aceording to Durand), but becomes oxidised, in the air, into a peculiar species of resin.

This substance is extensively used in medicine. It was formerly often adulterated; some unctuous oil being mixed with it, but as this is easily discovered by its insolubility in alcohol, castor-oil has sinee been used. The presence of this cheaper oil may be detected, - 1 , by agitating the balsam with a solution of caustic soda, and setting the mixture aside to repose, when the balsam will come to float clear on the top, and leave a soapy thick magma of the oil below; 2 , when the balsam is boiled with water, in a thin film for some hours, it will become a brittle resin on cooling; but it will remain viscid if mixed with castor-oil ; 3 , if a drop of the oil on white paper bo held over a lamp, at a proper distance, its volatile oil will evaporate, and leave the brittle resin, without causing any stain around, which the presence of oil will produce; 4, when three drops of the balsam are poured into a watch-glass, alongside of one drop of sulphuric acid, it becomes yellow at the point of contact, and altogether of a saffion hue when stirred about with a glass rod; but if sophisticated with castor-oil, the mixture soon becomes nearly colourless, like white honey, though after some time the acid blackens the whole in either case; 5 , if three parts in bulk of the balsam be mixed with one of good water of ammonia (of 0.970 specific gravity) in a glass tube, it will form a transparent solution if it be pure, but will form a white liniment if it contain castor-oil ; 6 , if the balsam be triturated with a littlo of the common magnesia alba, it will form a clear solution, from which acids dissolve out the magnesia, and leave the oil transparent if it be pure, but opaque if it be adulterated. When turpentine is employed to falsify the balsam, the fraud is detected by the smell on heating the compound.

This balsam is used in the manufacture of some varieties of tracing paper; and many lacquers and varnishes have the balsam of copaiva as one of their constituents. 
It is no longer possible to ascertain the quantity of Balsam Copaiva imported; by, as it appears to us, a very mistaken regulation of the Custom-house, it, and a great many other articles, are entered under the head of 'Drugs unenumerated.'

BAISAMrTO and WHITr BATSAm. By digesting the fruit of the Balsam of Peru troe in rum, a liquid having a bitter taste, a light sherry colour, and the odour of the tonquin-bean, is produced, called Balsamito. It is taken internally, and usod as an application to sloughing sores-especially those of the chigoe. By subjecting this fruit to pressure, without heat, White Balsam is obtained. It resembles strained Bordeaux turpentine, and is sometimes confounded with balsam of Tolu.

Barsam, Mrcca. (Baume de la Mecque, Baume du Judée, Fr.) Mecoa balsam, or opobalsam, or Balm of Gilead, is obtained both by incisions in, and by boiling, the branches and leaves of the Balsamodendron opobalsamum, a shrub which grows in Arabia Felix and Egypt. When fresh it is turbid and whitish, but becomes by degrees transparent, yellow, thickish, and eventually solid. Its smell is peculiar, but agreeable; it tastes bitter and spicy; does not dissolve completely in hot spirit of wine, and contains 10 per cent. of ethereal oil of the specific gravity 0.876 . It is also obtained from $B$. Gileadense.

BaIsam or peru. (Baume du Pérou, Fr.; Peruvianischer Balsam, Ger.) Balsam of Peru is extracted from the Myroxylon Peruiferum, a tree which grows in Peru, Mexico, \&c. ; sometimes by incision, and sometimes by evaporating the decoction of the bark and branches of the tree. The former kind is very rare, and is imported in the husk of the cocoa-nut, whence it is called balsam en coque. It is brown, transparent only in thin layers of the consistence of thick turpentine, of an agreeable smell, an acrid and bitter taste; formed of two matters, the one liquid, the other granular, and somewhat crystalline. In 100 parts it contains 12 of benzoic acid, 88 of resin, with traces of a volatile oil.

The second sort, the black balsam of Peru, is much more common than the preceding; translucent, of the consistence of well-boiled syrup, very deep red-brown colour, an almost intolerably acrid and bitter taste, and a stronger smell than the other balsam. Stoltze regards it as formed of 69 parts of a peculiar oil, 20.7 of a resin little soluble in alcohol, of 6.4 of benzoic acid, of 0.6 of extractive matter, and 0.9 of water.

The colebrated Pomade Divine, which was a few years since very celebrated, contained a considerable quantity of the balasm of Peru. One of the best recipes for its preparation was the following :-

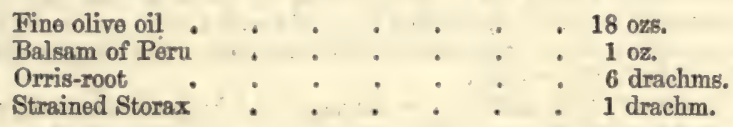

This, with some bruised nutmegs and cinnamon, was macerated in a water-bath for three hours, and then filtered.

A French authority states that, dissolved in four times its weight of alcohol, and spread upon sarsanet already covered with a layer of isinglass, it formed the taffetas d"Angleterre.

One thousand parts of good balsam should, by its benzoic acid, saturate 75 parts of crystallised carbonate of soda. It is employed as a perfume for pomatums, tinctures, lozenges, sealing-wax, and for chocolate and liqueurs, instead of vanilla, when this happons to be very dear.

M. Victor le Nouvel, who has been engaged in collecting this balsam since 1836, gives the following as the process used by the Indians to obtain it. An incision is made into the tree of about two or three inches broad, and three to four inches long. They raise the bark from the wood and apply cotton rags to it; a fire being lighted round the tree to liquefy the balsam. Fresh incisions are made higher and higher up the tree, till the cotton rags are quite saturated. It takes from ten to twelve days to effect this. The rags are next boiled, and when the liquor is cold, the balsam collects below.-Pereira's Materia Medica.

Balsam of Peru has been for some years exported from the State of Salvador On the coast of Chiquimulilla (Guatemala) there are many trees of the description that yield the balsam, but hitherto it has not attracted the attention of the people to collect it.

The Balsam of Peru of Salvador is procured within the department of Sonsonate. The British Consul thus describes its production:-

In the district of Cuisnagua there are 3,574 trees, which yield altogether only $600 \mathrm{lbs}$. of the gum annually. With proper care in the extraction, each tree would yield 2 lbs. to 3 lbs, making the total quantity capable of being produced in the 
before-mentioned district about 10,000 lbs. When the season has been more rainy than usual the product is much lower; but in order to meet this difficulty, the Indians heat the body of the tree by fire, by this means causing the gum to exude more freely; but this operation invariably causes the decay of the treo.

The Indians employed in collecting the gum say that such trees as are well shaded yiold a greater quantity; but that those which havo been planted by hand yield the most. This has been proved by experience, particularly in Calcutta, where a considerable quantity is yearly collected from trees which have been so planted. During the months of Decomber and January the gum oozes away spontaneously. This class of gum is called 'calcawzate.' It is orange-coloured, weighs less than the other, and omits a strong odour; is volatile and pungent.

BaIsam storax. See Storax.

BAIsAM ог тоIU. (Baume de Tolu, Fr.; Tolutanischer Balsam, Ger.) Balsam of Tolu flows from the trunk of the Myrospermum toluiferum, a tree which grows in South America, on the mountains of Tolu, Timbaceo, \&c. It is, when frosh, of the consistence of turpentine; is brownish-red, dries into a yellowish or roddish brittlo resinous mass, of a smell like benzoin; is soluble in alcohol and ether; affords, with water, benzoic acid. It appears probable that both the balsams of Peru and of Tolu are obtained from one tree. Balsam of Tolu is used to manufacture Tolu lozenges, and the Syrup of Tolu for irritating coughs. It is sometimes employed by confectioners to flavour sweetmeats, by perfumers, and in the formation of fimigating pastils,

BAIтrmorrmz. A variety of fibrous serpentine found at Baltimore.

Bamboo. (Bambou, Fr.; Indianisches Rohr, Ger.) A species of cane, the Bambusa arundinacea of botanists. A most important vegetable product in the East, where it is used in the construction of houses, boats, bridges, \&c. Its grain is used for bread; its fibre is manufactured into paper. Walking-sticks are said to be of bamboo; they are the ratan, a different plant. A siliceous secretion called tabasheor is frequently found in the joints of the bamboo. See RATAN and TABASHRTRR.

BAMrrTs. A silicate of alumina found at Bamle, in Norway.

BANANA. An herbaceons endogenous plant, Musa sapientum, growing in the West Indies, East Indies, and generally throughout the tropics. The Plantain has a fruit which is used for food to an immense extent by the inhabitants of hot climates, forming, indeed, a necessary article of diet.

BANDANTrA. A style of calico-printing, in which white or brightly-coloured spots are produced upon a red or dark ground. It seems to have been practised from time immemorial in India, by binding up firmly with thread those points of the cloth which were to remain white or yollow, while the rest of the surface was freely subjected to the dyeing operations.

The European imitations have now far surpassed, in the beeuty and precision of the design, the Oriental patterns, having called into action the refined resources of mechanical and chemical science. The white spots are produced by a solution of chlorine made to percolate down through the Turkey-red cotton eloth, in certain points defined and circumscribed by the pressure of hollow lead types in plates, in an hydraulic press. Fig. 101 is an elevation of one press ; A, the top of the entablature; B B, the cheoks or pillars; c, the upper block for fastening the upper lead perforated pattern to; $\mathbf{D}$, the lower block, to which the fellow pattern is affixed, and which moves up and down with the piston of the press; $\mathbf{E}$, the piston or ram; $\mathbf{F}$, the sole or base; $G$, the watertrough for the discharged or spotted calico to fall into; स, the small cistern for the aqueous chlorine or liquor-metre, with glass tubes for indicating the height of liquor inside the cistern; $e e$, glass stopcocks, for admitting the liquor into that cistern from the general reservoir; $f f$, stopcocks for admitting water to wash out the chlorine; $g g$, the pattern lead-plates, with screws for setting the patterns parallel to each other; $m m$, projecting angular pieces at each corner, perforated with a half-inch hole to receive the four guide-pins rising from the lower plate, which serve to secure accuracy of adjustment between the two faces of the lead pattern-plates; $h h$, two rollers, which seize and pull through the discharged pieces, and deliver them into the water-trough. To the left of $\mathbf{D}$ thero is a stopeock for filling the trough with water; $l$ is the waste tub for chlorine-liquor and water of washing. The contrivance for blowing a stream of air across the cloth through the pattorn holes is not repre. sented in the figure.

Sixteen engines, similar to the above, each possessing the power of pressing with sevoral hundred tons, are arranged in one line, in subdivisions of four, the spaces between each subdivision serving as passages to allow the workmen to go readily from. the front to the back of the presses. Each occupies 25 feet, so that the total length of the apartment is 100 foet.

To each press is attached a pair of patterns in lead (or plates as they are called), the 
manner of forming which will be described in the sequel. One of these plates is fixed to the upper block of the press. This block is so contrived that it rests upon a kind of universal joint, which enables this plate to be applied exactly to the under fellowplate. The latter sits on the movable part of the press, commonly called the sill. When this is forced up, the two patterns close on each other very nicely by means of the guide-pins at the corners, which are fitted with the ntmost care.

101

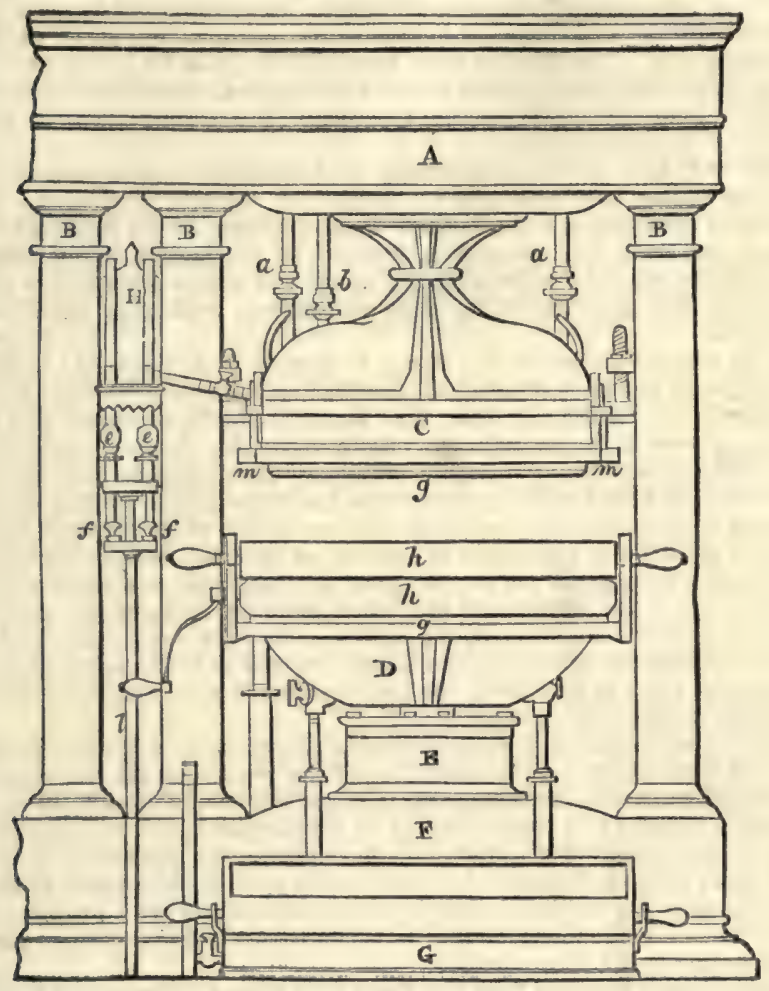

The power which impels this great hydrostatic range is placed in a separate apartment, called the machinery-room. This machinery consists of two press-cylinders of a peculiar construction, having solid rams accurately fitted to them. To erch of these cylinders three little force-pumps, worked by a steam-engine, are connected.

The piston of a large cylinder is eight inches in diameter, and is loaded with a top weight of five tons. This piston can be made to rise about two feet through a leather stuffing or collar. The other cylinder has a piston of only one inch in diameter, which is also loeded with a top woight of five tons. It is capable, like the other, of being raised two feet through its collar.

Supposing the pistons to be at their lowest point, four of the six small force-pumps are put in action by the steam-engine, two of them to raise the large piston, and two the little one. In a short time sa much water is injected into the eylinders that the loaded pistons have arrived at their highest points. They are now ready for working the hydrostatic discharge-presses, the water-pressure being conveyed from the one apartment to the other, under ground, through strong eopper tubes of small calibre.

Two valves are attached to each press, one opening a communication between the large driving cylinder and the cylinder of the press, the other between the small driving cylinder and the press. The function of the first is simply to lift the under block of the press into contact with the upper block; that of the second is to give the requisite compression to the cloth. A third valve is attached to the press for the purpose of discharging the water from its cylinder, when the press is to be relaxed in order to remove or draw through the cloth. 
From 12 to 14 pieces of cloth, previously dyed Turkey red, are stretched over each other as parallel as possible, by a particular machine. These parallel layers are then rolled round a wooden cylinder, called by the workmen a drum. This cylinder is now placed in its proper situation at the back of the press. A portion of the 14 layers of cloth, equal to the area of the plates, is next drawn through between them by hooks attached to the two corners of the webs. On opening the valve connected with the oight-inch driving cylinder, the water enters the cylinder of tho press, and instantly, lifts its lower block so as to apply the under plate with its cloth close to the upper one. This valve is then shut and the other is opened. The pressure of five tons in the oneinch prime cylinder is now brought to bear on the piston of the press, which is eight inches in diameter. The effective force here will therefore be 5 tons $\times 8^{2}=320$ tons, the areas of cylinders being to each other as the squares of their respective diametors, The cloth is thus condensed between the leaden pattern-plates with a pressure of 320 tons in a couple of seconds.

The next step is to admit the bleaching or discharging liquor (aqueous chlorine, obtained by adding sulphuric acid to solution of chloride of lime) to the eloth. This liquor is contained in a large cistern in an adjoining house, from which it is run at pleasure into small lead cisterns, $\mathbf{H}$, attached to the. presses, which cisterns have graduated index-tubes for regulating the quantity of liquor according to the pattern of discharge. The stopcocks on the pipes and cisterns containing this liquor are all made of glass.

From the measure-cistern, $\mathrm{k}$, the liquor is allowed to flow into the hollows in the upper lead plate, whence it descends on the cloth, and percolates through it, extracting in its passage the Turkey-red dye. The liquor is finally conveyed into the waste pipe from a groove in the under block. As soon as the chlorine liquor has passed through, water is admitted in a similar manner to wash away the chlorine, otherwise upon relaxing the pressure, the outline of the figure discharged would become ragged. The passage of the discharge liquor, as well as of the water through the cloth, is occasionally aided by a pneumatic apparatus, or blowing machine, consisting of a large gasometer from which the air, subjected to a moderate pressure, may be allowed to issue and act, in the direction of the liquid, upon the folds of the eloth. By an occasional twist of the air-stopcock, the workmen also can ensure the equal distribution of the discharging liquor over the whole excavations in the upper plate. When the demand for goods is very brisk, the air apparatus is much employed, as it enables the workman to double his product.

The time requisite for completing the discharging process in the first press is sufflcient to enable the other three workmen to put the remaining fiftoen presses in play. The discharger proceeds now from press to press, admits the liquor, the air, and the water; and is followed at a proper interval by the assistants, who relax the press, move forwards another square of the cloth, and then restore the pressure. Whenever the sixteenth press has been liquored, \&c., it is time to open the first press. In this routine about ten minutes are employed, that is, 224 handkerchiefs $(16 \times 14)$ are discharged every ten minutos. The whole cloth is drawn successively forward, to be successively treated according to the above method.

When the cloth is removed from the press it is passed between the two rollers in front, from which it falls into a trough of water placed below. It is finally carried off to the washing and bleaching department, where the lustre of both the white and the red is considerably brightenod.

By the above arrangement of presses, 1,600 pieces, consisting of 12 yards each $=$ 19,200 yards, are converted into bandannas in the space of ten hours, by the labour of four workmen.

The patterns, or plates, which are put into the presses to determine the white figures on the cloth, are made of lead in the following way:-A trellis frame of cast iron, one inch thick, with turned-up edges, forming a trough rather larger than the intended lead-pattern, is used as the solid groundwork. Into this trough a lead plate, about ono half-inch thick, is firmly fixed by screw-nails passing up from below. To the edges of this lead plate the borders of the pioce of sheet load aro soldered, which covers the whole outer surface of the iron frame. Thus a strong trough is formed, one inch deep. The upright border gives at once great strength to the plate and serves to confine the liquor. A thin sheet of lead is now laid on the thick lead plate, in the manner of a veneer on toilette tables, and is soldered to it round the edges. Both sheets must be made very smooth beforehand, by hammering them on a smooth stone table, and then finishing with a plane; the surface of the thin sheet (now attached) is to be covered with drawing-paper, pasted on, and upon this the pattern is drawn. It is now ready for the cutter. The first thing which he does is to fix down with brass pins all the parts of the pattern which are to be left solid. Ho now proceods with the little tools generally used by block-cutters, which are fitted to the 
different curvatures of the pattern, and he cuts perpendicularly quite through the thin sheet. The pieces thus detached aro easily lifted out, and thus the channels are formed which design the white flgures on the red eloth. At the bottom of tho channels a sufficient number of small perforations are made through the thicker sheet lead, so that the discharging liquor may have freo ingress and egress. Thus one plate is finished, from which an impression is taken in the hydrostatic press, by means of printers' ink, on paper pasted upon another plate. Each pair of plates constitutes a set which may be put into presses and removed at pleasure.

BANDOIIND, called also clysphitique and fixature, a mucilage of Carrageen moss; used for atiffening the hair and keeping it in order.

BANG or BHANG. When common hemp (Cannabis sativa) is grown in tropical countrios, its fibre becomes much less valuable, but its peculiar narcotic resin is much more abundantly secreted. The leaves and capsules of such hemp furnish tho substance known as bang, which is largely used in the East as an intoxicating drug.

BAIrTAM-work. Carved and painted work in imitation of Japan ware.

BAOBAB тRRт. Seo AdANSONu.

BAP or BAT. In Leicestershire a dark bituminous shale is so named.

BARBADOES TAR. A mineral pitch of a peculiarly odorous character. This bitumen was formerly obtained from Barbadoes; but several kinds now pass under the name.

BARBARX GUMr. Sometimes called Morocco gum. The product of the Acacia gummifera. Imported from Tripoli, Barbary, and Moroceo. Se日 Arabic, GUM.

BARBmRRY. (Berberis, Lat.; Epine-vinette, Fr.) It is probable that this name has been givon to this plant from its spines, or barbs. The name, Oxycanthus, also given to it, indicates a like origin.

The barberry is a shrubby plant, common in hedges in England; sometimes called the pipperidge bush. The berries are used in housewifery. The wood and bark of this plant contain a yellow colouring-matter which is soluble in water and alcohol, and is rendered brown by alkalis. The solution is employed in the manufacture of morocco leather. The yellow erystalline colouring-prineiple of the barberry is termed Beberine.

It is a common notion among farmers that barberry bushes cause the neighbouring wheat to become blighted. This was long regarded by botanists as nothing more than a popular projudice, but the recent researches of Oerstod and De Bary have shown that it really has foundation in fact. It is now proved that the two kinds of fungus, of which one infests the barberry and the other the wheat, though so different as to be placed in distinct genera, are really alternating forms of one and the same species. In one condition of its existence the fungus grows only on the barberry bush, but at a later period of its development it gives rise to an organism which produces the rust of wheat. Hence, a scientific relation is established between the appearance of the disease and the presence of the barberry.

Barrr.A. (Soude, Barille, Fr.; Barilla, Ger.) A crude soda, procured by the incinoration of the Salsola soda, a plant cultivated for this purpose in Spain, Sicily, Sardinia, and the Canary Islands. In Alieante the plants are raised from seed, which is sown at the close of the year, and they are usually fit to be gathered in September following. In October the plants are usually burned. For this purpose holes are made in the earth, capable of containing a ton or a ton and a half of soda. Iron bars are laid across these cavitios, and the dried plants, stratified with dry reeds, are placed upon them. The whole is set on firo. The alkali contained in the plants is fused, and it flows into tho cavity boneath, a red-hot fluid. By constantly heaping-on plants, the burning is continued until the pits are full of barilla; they are then covered up with earth and allowed to cool gradually. The spongy mass of alkali, when sufficiently cold, is broken out, and, without any further preparation, it is ready for shipment. Good barilla usually contains, according to Dr. Ure's analysis, 20 per cent. of real alkali associatod with muriates and sulphates, chiefly of soda, some lime, and alumina, with very little sulphur. . Caustic leys made from it wore formerly usod in the finishing process of the hard-soap manufacture.

The manufacture of barilla has greatly declined since the introduction of Le Blanc's process for artificially manufacturing soda from common salt.

BARIIIA DE COBRz. (Copper Barilla.) A commercial nnme for the nativo copper of Corocoro in Bolivia. The copper is obtained in the state of powder by crushing and washing the red Permian sandstone, which contains the metal.

marITz. $\Lambda$ form of spelling adoptod by Dana for the mineral usually called VOL. I. 
baryles or heavy spar. Professor Dann has proposod that a distinction should be establishod between the names of minerals and those of rocks. by torminating the former in -ite and the latter in -yte. Hence the change of spelling from baryte to barite.

BarIUax. (From Bapis, heavy.) The metallic basis of tho onrth baryta was obtained by Dary, in 1808 , by the voltaic decomposition of the moistenced curbonato of baryta in contact with mereury. It may likewise bo procured by jassing potassium in vapour over latryta hented to redness in an jron tube, and afterwards withdrawing the reduced barium which the residuum contains, by means of mereury. The latter motal is soparated by distillation in a glass retort, care being taken not to raise the temperature to redness, for the barium then decomposes glass. Mr. Crookes has preparod motallic barium by adding a saturatod solution of chloride of barium to his sodium-amalgam, and heating it to about $200^{\circ} \mathrm{F}$. Tho sodium is thus replacod by barium, and this barium-amalgam, when purified, is heated under naphtha, and tho mereury thus distilled off, leaving metallic barium.

Barium is a white metal, like silvor, fusible under a red heat, and densor than oil of vitriol, in which it sinks.-Graham.

BARIUM, OxTDEs Or. There aro two oxides of barium, a protoxido $\mathrm{BnO}$ (baryta) and a peroxido $\mathrm{BaO}^{2}$. The protoxide will be doseribed under BanYTA. Tho peroxide may be obtained by passing oxygen orer caustic baryta heated to dull rednoss; the oxygon thus absorbed may be expelled at $\Omega$ higher temperature, and the peroxide thus reduced to the state of protoxide. Availing himself of these reactions, Boussingault has proposed to prepare oxygen gas on a largo seale by first forming peroxide of barium by the passage of atmospheric air over baryta at a low red heat, and then decomposing this peroxide by ignition. The oxygen would thus be really derived from the air, and the baxium-oxide would meroly act as a medium for its alternate absorption and ovolution, and might thereforo be used over and over again indefinitely. 'Though economy seems to recommend this procoss, there are practical difficultios which have hitherto interfered with its working. Soo BARYTA.

\section{BARTUM, SAITS Or:-} acid.

Chloridr of Barrom. Mado from the native sulphate (henvy spar), by igniting it in a crucible with pounded coal, and then dissolving this sulphido in hydrochloric acid, or by fusing the native sulphate with chloride of calcium. The commercial chloride frequontly contains small quantities of the chlorides of strontium and calcium. Chloride of barium is especially used for the detection and estimation of sulphuric acid. acid.

Fruoridr of Barium. Prepared by neutralising baryta-water by hydrofluoric

Ionme of Bariug is formed when hydriodic acid is passed over baryta at a red heat.

BARx. The outer rind of plants. Many varieties of barks are known to commerce, but the torm is especially used to expross either Porurian or Jesuits' bark, a pharmacentical romedy, or Oak bark, which is very extensively used by tanners and dyer8. The varieties known in commerce are :-

Cork Bark. (Fr. Liége; Kork, Ger.). OAk Bark. (Tan brut, Fr.; Eichenrinde, Ger.). Perutian Bark. (Quinquina, Fr.; Chinarinde, Ger.). Quercitron Bark. WATtLi Bark, Seo these respectively.

BARJ RrEAD. A kind of bread propared in many parts of Norway by the poorer peasants from the inner bark of the Pinus sylvestris. See Pinus.

BARI.5Y. (Orge, Fr.; Gerste, Ger.) Hordeum, Linn. This term is supposed to be derived from hordus, heavy, because the bread made from it is very heary. Barley belongs to the class Endogens, or Monocotyledons; Glumel Alliance, of Lindley : natural order, Graminacece.

There are four species of barley cultivated in this country :-

1. Hordean hexastichon. Six-rowed barley, or Winter barley.

2. Hordeum vulgare. The Scotch bere or bigg; the four-rowed barley.

3. Hordeun zeocriton. Putney, fan, sprat, or battledore barley.

4. Hordeum distichon. Two-rowed long-eared barloy, or Summor barloy.

Barley and oats are the cerenls whose cultivation extends farthest north in Europe.

The specific gravity of English barley varies from 1.25 to 1.33; of bigg from 1.227 to 1.265 ; the weight of the husk of barley is $\frac{2}{3}$, that of bigg $\frac{2}{9}$. Specific gravity of barloy is $1 \cdot 235$, by Dr. Uro's trials. 1,000 parts of barley-flour contain, according to Finhof, 720 of sturch, 56 sugar, 50 mucilage, $36 \cdot 6$ gluten, $12 \cdot 3$ vegetable albumen, 100 wator, 2.5 pliosphate of lime, and 68 fibrous or ligneous matter.

From the examination instituted by the Royal Agricultural Society of England,and 
carried out under the directions of Messrs. Way and Ogston, the following results have beon arrived at:-

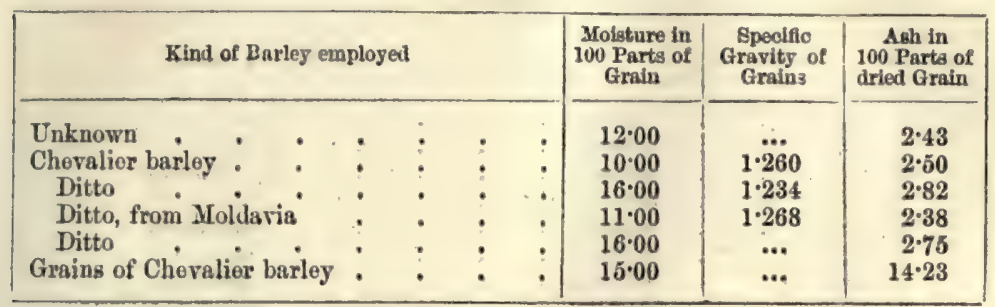

The annlyses of sereral varieties gave as the composition of the ashes of the grains of barley:-

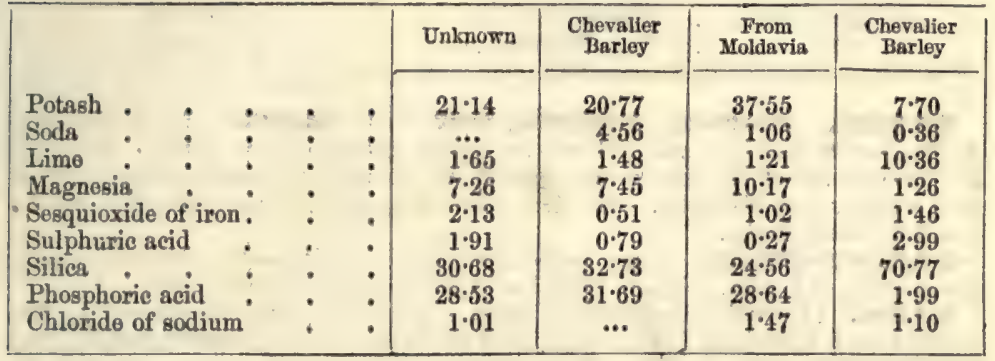

In the 'Synopsis of the Vegetable Products of Scotland,' by Peter Lawson and Son, will be found the best deseription of all the different varieties of barley; and, since the Lawsonian collection is in the museum of the Royal Botanic Gardens at Kow, the grains can be examined readily by all who take any interest in the subject. A few only of the varietios will be noticed.

The true six-rowed Barley, known also as Pomeranian and as six-rowed white winter barloy. - This is a coarse barloy, but hardy and prolific. It is oceasionally sown in Franeo, and also in this country, sometimes as a winter and sometimes as a spring barley, and is found to answer pretty well as either.

Naked two-rowed.-Ear long, containing twenty-eight or thirty very large grains, which soparate from the paleæ, or chaff, in the manner of wheat. This variety has been introduced to the notice of agriculturalists at various times, and under different names, but its cultivation has never been carried to any great extent.

Common Bere, Bigg, or rough Barley. - This variety is ehiefly eultivated in the Highlands of Scotland, and in the Lowlands on exposed inferior soils.

Victoria.-A superior variety of the old bigg, compared with which it produces longer straw, and is long-eared, often containing 70 or 100 grains in each. Instances lave beon known of its yielding 13 quarters per acre, and weighing as much as $96 \mathrm{lbs}$. per bushel.

Beyond these there are the winter black; the winter white; old Scottish four-rowed; naked, golden, or Italian; Suffolk or Norfolk, and Short-necked; cultivated in various districts, and with varying qualities. See Brek.

Total Acreage of Barley grown in Great Britain in each year from 1868 to 1872,

\begin{tabular}{|c|c|c|c|c|c|c|c|}
\hline \multicolumn{3}{|l|}{ Barley } & 1868 & 1869 & 1870 & 1871 & 1872 \\
\hline England . & . & - & $1,780,201$ & $1,864,088$ & $1,963,744$ & $1,964,210$ & $1,896,403$ \\
\hline Wales. & 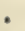 & - & 151,608 & 157,582 & 163,8503 & 169,751 & 168,014 \\
\hline Scotland. & - & - & 219,515 & 229,810 & 244,142 & 251,822 & 251,915 \\
\hline Great Britain & - & - & $2,151,324$ & $2,251,480$ & $2,371,739$ & $2,385,783$ & $2,316,332$ \\
\hline
\end{tabular}


Statement of Quantities of Barley produced in Foreign Countries in the following years:-

\begin{tabular}{|c|c|c|c|c|c|c|c|c|c|c|}
\hline \multicolumn{3}{|c|}{ Yearn } & \multicolumn{6}{|c|}{ Countrles } & \multicolumn{2}{|c|}{$\therefore \quad$ Quantity. } \\
\hline 1870 & & & & Sweden & & * & a & & & $\begin{array}{l}\text { Bushels } \\
12.377 .827\end{array}$ \\
\hline 1870 & . & . & & Norway & & : & : & : & & $3 ; 749,872$ \\
\hline 1871 & . & . & & Prussia & & : & : & $\vdots$ & & $113 ; 920,223$ \\
\hline 1871 & . & . & & Würtombe & & : & : & : & & $6,028,400$ \\
\hline 1863 & . & . & & Bavaria & & 4 & $\vdots$ & : & & $16,910,539$ \\
\hline 1870 & $\because$ & , & & Holland & : & ; & : & : & & $5,088,682$ \\
\hline 1866 & $\because$ & . & & Belgium & : & : & : & : & & $3,665,643$ \\
\hline 1869 & . & & & France & $\cdot$ & - & 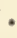 & • & & $56,495,697$ \\
\hline 1865 & & & & Portugal & & . & $\cdot$ & . & & $1,925,000$ \\
\hline 1857 & & & & Spain & & , & . & - & & $76,427,587$ \\
\hline 1871 & & & & Austria & & - & • & - & & $44,933,867$ \\
\hline 1865 & & & & Italy & & - & 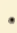 & - & & $20,534,907$ \\
\hline 1867 & & & & Greece & . & . & . & . & & $2,128,430$ \\
\hline 1871 & & & & United St & itates & $\therefore$ & . & . & & $26,718,500$ \\
\hline
\end{tabular}

BAtrEx, BCoTCE, ruxisD, Pox, and Prart. When barley is deprived of its husk by a mill, it forms the Scotch, Hulled, or Pót Barley. When all the integuments of the grains are removed, and the seeds are rounded and polishod, they constitute Pearl-Barley. The flour obtained by grinding pearl-barloy to powdor is called Patent Barley.

MARIBX-sUCAR. Sugar boiled, formerly in barley-water, until it is quite transparent and erisp. It is flavoured with either orange or lemon peel.

MARm. (Derived from the Saxon beorme; or from beer-rahm, beer-cream.) The yeasty top of fermenting beer. "It is used as leaven in bread, and to establish formentation in liquors. See BeER, Frrmertitiox.

BAR-MAstrar. In Derbyshire, the authority to whom all disputes in lead. mining are referred. He has charge of the standard 'dish' or measure used for measuring the ore. It is the same as Bargh-master.

BARmOT2 or BArGmorz. A court held for determining such questions as may arise in lead-mining. It is usually held in Derbyshire twice a year.

BAn OP GROUND. A course of rock dissimilar to the ordinary vein-stono-: which runs across a mineral lode.

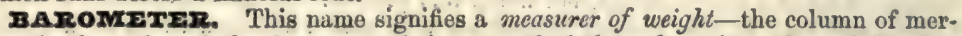
cury in the tube of the barometer being exactly balanced against the weight of a column of air of the same diameter, reaching from the surface of the earth to the extreme limits of the atmosphere. The length of this column of mercury is never more than thirty-one inches; below that point it may vary, according to conditions, through several inches. There have been many useful applications of the barometer, but the only one with which this Dictionary has to deal appears to be the use of the instrument in coal mines. It is now necessary, under the Coal Mines' Regulation Act, that a barometer and a thermometer should be found in every colliery. This has arisen from a prevailing idea that the explosions of fire-damp have, in numerous instances, arisen from the alteration of atmospheric pressure.

The relation between the state of tho barometer and the occurrence of colliery explosions has been investigated by Messrs. R. H. Scott, F. R. S., and W. Galloway, in a paper 'On the Connection between Explosions in Collieries and Weather.'-Proceodings of the Royal Society, April 18th, 1872.

Mr. T. Dobson read a paper 'On the relation between Fxplosions in Collieries and Rerolving Storms' at the meeting of the British Association at Glasgow in 1855, which is printed in the Reports for that year. Mr. Bunning has given in the 'Transactions of the North of England Institute of Mining Enginecrs' diagrams. showing the meteorologieal records from the observatories of $\mathrm{Kew}$ and Glasgow, and tho explosions in collieries reported in those years. It is not possible in this place to examine a question complicatod as this one is by the numerous conditions which surround the operations of working coals, and ventilating a colliery. The results of the examinations made from time to time before Committees of the House of Commons, and other Committees, and by individuals, many of which are carefully recorded in the paper already referred to, go to show that meteorological changes aro the proximate causes of a large majority of colliery explosions," and hence, thereforc, the necessity of carefully watching the changes of the barometer, and of regulating 
tho rentilation in obedience to its indications. So strongly was this folt by the Meteorological Committee who, in 1868, carefully examined the question, that they proposed to send telegraphic intelligence of storms-arising as they always do from disturbances of the atmospheric pressure-to colliery proprietors. Messrs. Scott and Galloway conclude their paper-above referred to-in the following words:-Whether, therefore, the barometer falls or the temperature rises, it is absolutely necessary to keep a most careful watch over the amount of air passing through the workings, in order to prevent the formation of dangerous accumulations of explosive. mixtures of air and fire-damp in all mines in which the margin between danger and safety is very small. . . . The one cry - whether we look to seeurity against explosion, or to afford to miners an atmosphere which is respirable without injury to health-is more air.' See Cost-Mistrg and Ventilation of Mines.

IARrzaz. A woollen fabric, in both warp and woof, which takes its name from the district in which it was first manufactured-the especidl locality being a little village named Arosons, in the beautiful valley of 'Barreges. " It was first employed as an ornament for the head, especially for sacred ceremonies, as baptism and marriage. Paris subsequently becamo celebrated for its bairrègés, but these were generally woven with a warp of silk. Enormous quantities of cheap barrèges are now made with a warp of cotton,

BARzI. (Baril, Fr.) A round vessel, or cask, of groater longth than breadth, made of staves, and hooped.

The English barrel-wine measure contains $31 \frac{1}{2}$ gallons.

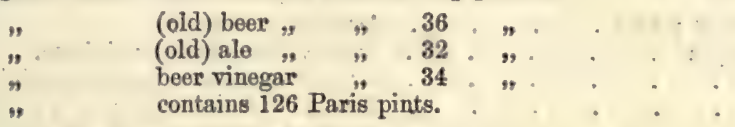

The ale and beer barrels wero equalised to 34 gallons by a statute of William and Mary. The wine gallon, by a statute of Anne, was declared to be 231 cubic inches; the beer gallon being usually reckoned as 282 cubic inches. The imperial gallon is. $277 \cdot 274$ cubic inches. The old barrels now in use are as follows :-

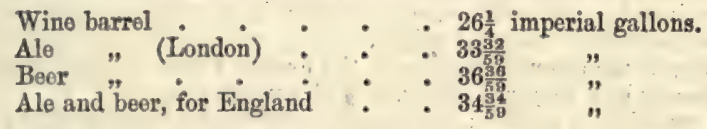

The baril de Florence is equivalont to 20 bottles.

The Connecticut barrel for liquors is $31 \frac{1}{2}$ gallons, each gallon to contain 231 cubic inches. The statuto barrel of America must be from 28 to 31 gallons. The barrel of flour, New York, must contain either $195 \mathrm{lbs}$. or $228 \mathrm{lbs}$. nett weight. The barrel of beef or pork in New York and Connecticut is $200 \mathrm{lbs}$. A barrel of Essex butter is 106 lbs. A barrel of Suffolk butter is $256 \mathrm{lbs}$. A barrel of herrings should hold 1,000 fish. A barrel of salmon should measure 42 gallons. In machinery, anything. hollow and cylindrical.

מarrows. In mining, heaps of waste stuff raised from the mine; rubbish, called in Cornwall ' deads.'-The conical baskets into which salt is put.

BArwooD. An African red lye-wood, the produco of the Baplita mitida, belonging to the Leguminose or pea-order-a tree which also yields Camwood. Al. though distinctions are made between sandal or saunders wood, camwood, and barwood, they appear to be very nearly allied to each other-at least, the colouringmatter is of the same composition. They come, however, from different places. See Carrwoon and SANDAL WoOD.

MM. Girardin and Preisser thus describe barwood:-

This wood, in the state of a coarse powder, is of a bright red colour, without any. odour or smell. It imparts scarcely any colour to tho saliva.

Cold water, in contact with this powder, only acquires a fawn tint after five days' maceration. 100 parts of water only dissolve $2 \cdot 21$ of substances consisting of 0.85 colouring-matter and of 1.36 saline compounds. Hoiling water becomes more strongly coloured of a reddish yellow; but, on cooling, it deposits a part of the colouringprinciple in the form of a red powder. 100 parts of water at $212^{\circ}$ dissolve 8.86 of substances consisting of $\mathbf{7 \cdot 2 4}$ colouring-principle, and 1.62 salts, especially sulphutes and chlorides. On macerating the powder in strong alcohol, the liquid almost immodiately acquires a very dark vinous red calour. To remove the whole of the colour from fifteen grains of this powder, it was pecessary to treat it soveral times with boiling alcohol. The alcoholic liquid contained 0.23 of colouring-principle and 0.004 of 
salt. Barwood contains, therefore, 23 per cent. of red colouring-matter; whilst saunders wood, according to Polletier, only contains $16 \cdot 75$.

The alcoholic solution behares in the following manner towards reagents :-

Distilled water added in great quantity. Produces a considerable yellow opalescence. The procipitate is re-dissolved by the fixed alkalis, and the liquor acquires a dark vinous colour.

Fixed alkalis
Lime-water
Sulphuric acid
Sulphuretted hydrogen
Salt of tin $:$
Chloride of tin $\quad \vdots$
Acetate of lead
Salts of the protoxide of iron
Copper salts,
Chloride of mereury :

- Turn it dark crimson, or dark violet.

- Ditto.

- Darkens the colour to a cochinoal red.

- Acts like water.

- Blood-red procipitate.

Brick-red precipitate.

- Dark violet gelatinous precipitato.

- Very abundant violet precipitates.

- Violet-brown gelatinous precipitates.

- An abundant precipitate of a brick-red colour.

Nitrate of bismuth . . . . Gires a light and brilliant crimson red.

Sulphate of zine . $\quad: \quad . \quad$ Bright red floceulent procipitato.

Tartar emetic $\quad: \quad: \quad:$ An abundant procipitato of a dark cherry colour.

Neutral salts of potash . . . . Act like pure water.

Water of baryta . . . . Dark violet-brown precipitate.

Golatine : : : Brownish-yellow ochreous precipitate.

Chlorine : ; : - Brings back the liquor to a light yellow, with a slight yellowish-brown precipitato, resembling hydrated peroxide of iron.

Pyroxylic spirit acts on barwood like alcohol, and the strongly coloured solution behaves similarly towards reagents. Hydrated ether almost immediatoly aequires an orange-red tint, rather paler than that with alcohol. It dissolres 19.47 per cont. of the colouring-principle. Ammonia, potash, and soda, in contact with powdered barwood, assume an extremely dark violet-red colour. These solutions, neutralised with hydrochloric acid, deposit the colouring-matter in the form of a dark reddish-brown powder. Acetic acid becomes of a dark-red colour, as with saunders wood.

Barwood is but slightly soluble; but the difficulty arising from its slight solubility is, according to Mr. Napier, overcome by the following very ingenious arrangement: -The colouring-matter while hot combines easily with the proto-compounds of tin, forming an insoluble rich red colour. The goods to be dyed are impregnated with proto-chloride of tin combined with sumach. The proper proportion of barwood for the colour wanted is put into a boiler with water and brought to boil. The goods thus impregnated are put into this boiling water containing the rasped wood, and the small portion of colouring-matter dissolved in the water is immediately taken up by the goods. Tho water, thus exhausted, dissolves a new portion of colouringmatter, which is again taken up by the goods, and so on till the tin upon the cloth has becomo (if we may so term it) saturated. The colour is then at its brightest and richest phase.

вапYTA. (Baryte, Fr.) One of the simple earths, protoxide of barium (BaO). It may be obtained most easily by dissolving the native carbonate of baryta in nitric acid, eraporating the neutral nitrate till crystals be formed, draining and then ealcining these, by successive portions, in a corered platina crucible, at a bright red heat. A less pure baryta may be obtained by igniting strongly a mixture of the carbonate and charcoal, both in fine powder and moistened. It is a greyish-white earthy looking substance, fusible only at the jet of the oxy-hyllogen blowpipe, has a sharp caustic taste, corrodes the tongue and all animal mattor, is poisonous even in small quantities, has a very powerful alkaline reaction; a specific gravity of 4.0 ; becomes hot, and slakes violently when sprinkled with water, falling into a fine white powder, called the hydrate of baryta, which contains $10 \frac{1}{2}$ per cent. of water, and dissolves in 10 parts of boiling water. This solution lets fall alundant columnar erystals of hydrate of baryta as it cools; but it still retains one-twentieth its weight of baryta, and is called baryta water. The above crystals contain 61 per cent. of water, of which, by drying, they lose 50 parts. This hydrate may be fused at a red heat without losing any more water. Of all the bases, baryta has the strongest affinity for sulphuric acid, and is hence employed-either in the state of the above water, or in that of one of its neutral salts, ตs the chloride-to detect the presonce and determine the quantity of that acid present in any soluble compound. 
2ARYTA, CARBorsATz or. The composition of tho native carbonato of barya, callod Witheritc, after Withering, who described it ('Phil. Transactions,' 1784), may bo regarded as baryta, $77 \cdot 59$, and carbonic acid $22 \cdot 41$. It is found in Shropshire, Cumberland, Westmoreland, and Northumberland. The carbonate of baryta is employed in our colour-manufactories as a base for some of the moro dolicate colours; it is also used in the manufacturo of plate-glass, and of Wodgwood-ware; and, in France, it is much used in the preparation of beet-root sugar.

A small quantity only of carbonate of baryta is now produced in this country; in 1870 about 2,613 tons, 19 cwt., were raised in Northumberland; a few tons were sold from Alston Moor, and from Snailbeach, in Shropshire.

BARXTA, NTTRAT2 Or. This salt is used in pyrotechny for making green fire. The best mixture is nitrate of baryta 77 parts, sulphur 13 parts, chlorate of potash o parts, metallic arsenic 2 parts, charcoul 3 parts. The nitrate is propared by dissolving carbonate of baryta in nitric acid.

BARYTA, SUIPHATE OF. This compound occurs native, forming the mineral-species known variously as barite, baryte, or barytes. Its comparatively high density for a non-metallic mineral (4.7) lod to its recognition by the older mineralogists as spathum ponderosum, or terra ponderosa, and it is still commonly known as heavy spar. The mineral frequently occurs in large crystals, usually tabular in form. Massivo varieties, common in many of the lead-mines worked in the mountain limestone of Derbyshire and Shropshire, are usually termed cawk; certain columnar varioties from Saxony are known to German mineralogists as Stangenspath; and a peculiar form occurring, near Bologna, in nodules which present a radiated structure, is called Bologna stone. This Bologna spar is notable for the phosphorescence which it exhibits when heated; the so-called 'Bologna Phosphorus' was made by powdering this stone, and cementing the powder into the form of sticks, by means of gum.

Sulphate of baryta is composed of baryta $65 \cdot 63$, sulphuric acid $34 \cdot 37$, with sometimes a little iron, lime, or silica.

This salt of baryta is rery extensively spread over various parts of the British islands. It might be obtained in very large quantities in Deronshire, Cornwall, and other places, if the demand for it sufficiently increased the price, so as to render the working of it profitable. It is worked in Derbyshire, Yorkshire, Shropshire, the Isle of Arran, and in Ireland.

Cawk, or massive sulphate of baryta, was introduced by Josiah Wedgwood as an important ingredient in his celobrated 'jasper warc,' and is still employed by the potter in producing a similar fine paste. But the mineral is chiefly used as a pigment, or rather for adulterating other pigments.

The white rarieties are ground after being heated and thrown into water, and the heavy white powder is employed in aduiterating whito lead. On this account it is very difficult to obtain correct returns. The production of 1871 was :-

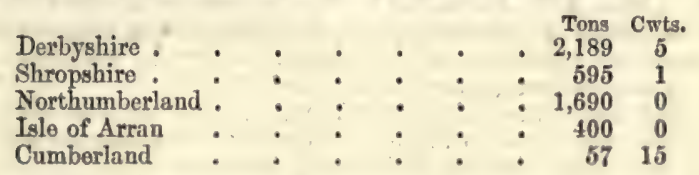

BAxXrzs. Heary spar, or native sulphate of baryta.

BASAIT. One of the most common varieties of trap-rock. It is a dark green or black stone, composed chicfly of augite and labradorite-felspar, rery compact in texture, and of considerable hardness, often found in regular pillars of three or more sides, called basaltic columns. Remarkable examples of this kind are seen at the Giant's Causeway in Ireland, and at Fingal's Cave, in Staffa, one of the Hebrides. Nessrs. Chance (brothers), of Birmingham, at one time adopted the process of melting the Rowley rag, a basaltic rock forming the plateau of the Rowley hills, near Dudley, South Staffordshire, and then casting it into moulds for architectural ornaments, tiles for paroments, \&e. Not only the Rowley rag, but basalt, greenstone, whinstone, or any similar mineral, might be used. The material was melted in a reverberatory furnace, and when in a sufficiently fluid stato poured into moulds of sand encased in iron boxes, these moulds having been previously raised to a rol heat in ovens suitable for the purpose. The object to be attained by henting the moulds previous to their reception of the liquid material is to retard the rate of cooling; as the result of slow cooling is a hard, strong, and stony substance, closely resembling the natural stone, while the result of rapid cooling is a dark brittle glass.

BAsIrICOx. Tho namo given by the old apotheenries to a mixture of oil, wax, and resin, which is representod by the Cerat resine of the present day. 
BAsxrTs. Weaving of rods into baskets is one of the most ancient of the arts amongst men; and it is practised in almost evory part of the globe, whether inhabited by civilised or sarago races. Basket-making roquires no description hore.

BAss, or $\mathbf{R A S T}$. The Russian mats usod by gardeners and upholstorers, made from the bark of the Lime or Linden treo, are called Bast mats. The name is also usod for the bark or tough fibres of the flax and hemp plants of which Bast brooms are made. The thick mat or hassock used by porsons to knool on at church is callod II Bass.

BAsszr. A miner's term for the outcrop of strata. When a seam of coal comes to the surface, it is said to 'basset.'

BAssora cUN. A gum obtained from the Acacia leucophlea, brought from Bassora. It has a specific gravity of $1 \cdot 3591$, and is yellowish whito in colour.

3Assorrave. A constituent part of gum Bassora, as also of gum tragacanth. It is semi-transparont, difficult to pulverise, swolls considerably in cold or boiling water, and forms a thick mucilage without dissolving.

BATra. A dish made slightly conical, which is employed for washing soil or gravel, in search of gold. It is usually about 20 inches in diameter, and $2 \frac{1}{2}$ inches deep. Before using the baten, to ascertain if thero bo any gold, and what amount, it is necossary to be very careful in the selection of the sample to be experimented upon. Tho usual manner of obtaining such a sample is as follows :- The produce of gold is so very irregular that it is necessary, in the first place, to break from the rock sevoral hundredweights to secure a good average sample; all of which should be broken in pieces about the size of a walnut, the whole well mixed together, and made into a round flat heap; this hoap is cut through the middle, and an equal quantity of stuff being taken from each side of the trench formed by the cutting, may, for a rough estimation, bo at onco pulverised and subjected to washing in tho batea or horn-spoon; but if greater accuracy be desired, it is well to tako about half a hundredweight from each side of the trench, and this be again redueed to pieces of about the size of a pea, then formed in a heap, and eut as before, and about two pounds should be taken from oach side the trench, and this after pulverisation, and being passed through a very fine wire sieve, is ready for a very accurate trial with the batea; or is quite fit for assay. This is, indeed, the process by which samples are taken of the poorer copper ores in Cornwall.

RATH aRrcz. A brick made of calcareous and siliceous earth, used in cleaning knives and for polishing purposes. They are made very extensively at and near Bridgewater from a deposit found in the estuary there; a similar deposit is also worked at Cumwick, at the entrance of the Parrot River, and at Highbridge. At Bridgewater the average make is about $3,000,000$ scouring bricks annually, which sell at about $2 l$. per thousand.

RATE MrTAI. An alloy in nearly equal quantities of coppor and zinc. Bath motal consists of $3 \mathrm{oz}$. of zine to $1 \mathrm{lb}$ of copper. See BrAss.

BATrs. (Bains, Fr.; Baden, Ger.) The importance attached by the Greeks and Romans to bathing is sufficiently attested by the remains of magnificent structures, which still excite the admiration' of the beholder, and by the beautiful specimens of fresco-painting and sculpture discovered in their baths:

It is computed that in the baths of Caracalla; as many as three thousand people could bathe at the same time, in 'water at various degrees of temperature, to suit their inclinations. The warm and hot baths were, however, almost exclusively in uso under the Emperors.

During the Republic the baths were cold. Mrecenns was the first to crect warm and hot ones for public use; they were called Thermæ, and wero placed under tho direction of rediles, who regulated the temperature, enforced cleanliness in tho establishment, and order and decorum among the visitors. Agrippa, during tho time ho was adile, increased the number of thermæ to 170 , and in the course of two centuries, there were no less than eight hundred in imperial Romo. The inhabitants resorted to the baths at particular hours, indicatod by striking a bell or gong. Adrian forbado their boing open before eight in the morning, except in cases of sickness; whereas Alexander Severus not only permitted them to be open during the wholo day, but also to be used through the night in the great heats of summer.

It was a common practice with the Romans to bathe towards evening, and particularly before supper: some of the more luxurious made use of the bath even after this meal. We are told of many citizens of distinction who wore in the habit of bathing four, or five, and even eight times a day. Bathing constitnted a part of public rejoicings, equally with the other spectacles, and like them was prohibited when the. country sufferod under any calamity. All classes resorted to the baths; the emperors themsdres, such as Titus, Adrian, and Alexander Severus, were occasionally seon 
among the bathers. The price of admission was very small, amounting to no more than a farthing, - E. Lee, on Mineral Waters and Baths.

Warm baths have come into very general use in England, and they are now considered as indispensably necessary in all modern houses of any magnitude, as also in club-houses, hotels, and hospitals; and the mode of constructing baths, and of obtaining the necessary supplies of hot and cold water, has undergone much improvement with the extension of their employment.

The several points in regard to warm baths are,

1. The materials of which they are constructed,

2. Their situation.

3. The supply of cold water.

4. The surpply of hot water.

5. Minor comforts and conveniences.

1. As to the materials of which they are constructed.-Of these the best are slabs of polished marble, properly bedded with good water-tight cement, in a seasoned wooden case, and neatly and carefully united at their respectivo edges. These, when originally well constructed, form a durable, pleasant, and agreeable-looking bath, but the expense is often objectionable, and, in upper chambers, the weight may prove inconvenient. If of white or veined marble, they are also apt to get yellow or discoloured by frequent use, and cannot easily be cleansed; so that large Dutch tiles, as they are called, or square pieces of white earthenware, are sometimes substituted. Welsh slate has now superseded marble to a great extent; and very superior baths are now manufactured of Stourbridge clay, at Stourbridge. Copper, tinned or galvanisod iron, are also employed; the first is most expensire in the outfit, but far more durable than the latter.

2. As to the situation of the bath, or the part of the house in which it is to be placed.-In hotels and club-houses, this is a question ensily determined; several baths are usually here required, and each should have annexed to it a properly warmod dressing-room. Whether they are upstairs or downstairs is a question of convenience, but the basement story, in which they are sometimes placed, should always be avoided : there is a coldness and dampness belonging to it, in almost all weathers, which is neither agreeable nor salubrious.

In hospitals, there are usually several baths on each side of the house (the men's and women's), and the supply of hot water is ready at a moment's notice.

In private houses, the fittest places for warm baths are dressing-rooms annexed to the principal bed-rooms; or, where such convenience cannot be obtained, a separate bath-room connected with the dressing-room, and always upon the bed-room floor.

3. The supply of water is a very important point, as connected with the present subject. The water should be soft, clean, and pure; and as free as possible from all substances mechanically suspended in it.

4 and 5.--In public bathing establishments, where numerous and constant baths are required, the most effective means of obtaining hot water for their supply are now employed. It is drawn directly into the baths from a large boiler, placed somewhere above their level. The hot water enters the bath by a pipe at least an inch and a half in diameter, and the cold water by one of the same dimension. The relative proportions of the hot and cold water, are of course to be adjusted by a thermometer; and every bath has a two-inch waste-pipe, opening about two inches from the top of the bath, and suffering the excess of water freely to run off; so that when a person is immersed in the bath, or when the supplies of water are accidentally left open, thero may be no danger of an overflow.

A contrivance of some ingenuity consists in suffering the water for the supply of the bath to flow from a cistern above it, through a leaden pipe of about one inch diameter, which is conducted into the kitchen or other convenient place, where a largo boiler for the supply of hot water is already fixed. The bath-pipe is immersed in this boiler, in which it makes many convolutions, and again, emerging, ascends to tho bath. The operation is simply this:- the cold water passing through the convolutions of that part of the pipe which is immersed in the boiling water, receives there sufficient heat for the purpose required, and ascending, in obedience to the law of fluid pressure, it is delivered in that stato by the ascending pipe into the bath, which is also supplied with cold water and waste-pipes as usual. The pipe may be of lead, as far as the descending and ascending parts are concerned, but the portion forming the worm or convolutions immersed in the boiler, should be copper, in order that the water within it may receive heat without impediment.

The facilities which are now afforded for the construction of baths in pritato housen, 
and for the use of them at a very cheap rate in public establishments, render it quite unnocessary to retain the remarks made by Dr. Uro.

Public baths and wash-houses have now become common amongst us, and with them an incroased eleanliness is apparent, and improved health throughout the population.

The steady increase of the revenue dorivol from the baths and wash-houses in Iondon, from the commencement of the undertaking in 18:6, shows the practical utility of these institutions, and their effect on the physical and social condition of the industrious classes; viz: :-

The aggregato receipts of nine establishments, during 1853 , amount to

1852. Eight establishments.

1851. Six establishments ..

1850. Four establishments .

1849. Three establishments.

1848. Two establishments . 1847. 1846. $\}$

A similar increase has continued to the present time, the receipts being now, $\mathbf{1 8 6 5}$, above 25,000l. Those conreniences-now, indeed, become absoluto necessities - are extending in every part of the country.

Baths, as curative agents, are of various kinds. Tunkish BATHs, for Rheumatism and other complaints - in which are adopted all the processes which have been so much extolled in the Oriental bath system - have been introduced with success during the last few years. Although much lauded by some, they have not been so satisfactory to others; and do not therefore appear to be extending. VAPOUR Batrs are stimulant and sudorific; they may be either to be breathed, or not to be breathed. Dr. Pereira has given the following Table as a comparative view of the heating powers of rapour and of water:-

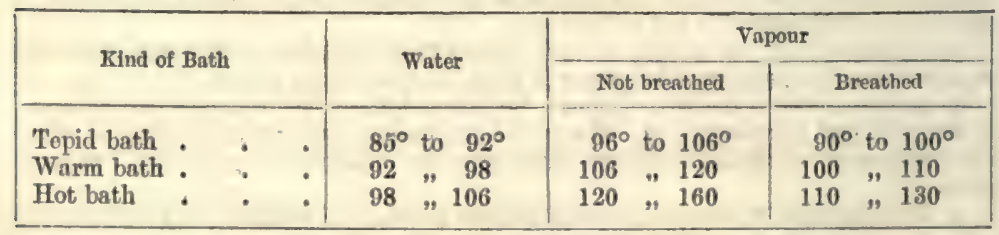

Local vapour baths are appliod in affections of the joints, and the like.

Vapour donche is a jet of aqueous vapour directed on somo part of the body.

Medicated vapour baths are prepared by impregnating vapour with the odours of medicinal plants.

Sulphur, chlorine, sulphurous acid, iodine and camphor, are occasionally employod in conjunction with aqueous vapour.

Warm, tepid, and hot baths aro sufficiently described above.

BATr STONE. A building stone raised in the ricinity of the city of Bath. Seo Oolite.

BAтrvirrтrz. Dr. C. Greville Williams, who has been largely employed in the distillation of bituminous minerals, and amongst others of tho Torbanite from Torbnne Hill, near Bathgate, in Scotland, has discovered a brown friablo substance occasionally filling the hollows of the above mineral. This is inflammable, and has been namod Bathvillite by its discoverer.

BATT. A name given to a highly bituminous shale, which is often found intorstratifiod with the coal. It oceurs in the South Staffordshiro coal-field, and has been woll described by Jukes in his 'Geology of the South Staffordshire Caal-Field.' In Lancashire this shale is called Black Bass, and in Flintshire, where thoy are distilling it in considerable quantities, it is known as Black Slag.

Batt or bat is ulso a term applied by the potter to a plate of gelatine, used in printing on to pottery, or porcelain over the glaze. In bat-printing, the impression is transforred from an engraved copper plate to a bat of gelatine or glue, whence it is printed on the glaze, in oil or tar. Enamel powder boing then dusted over the print, adheres to the oiled surface, and the porcelain is then fired at a low temperaturo.' Sie POTTRRY.

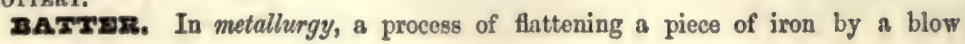


with a hammer, so as to compress it inwardly, and spread it outwardly on all sides around the place of impact. See Stamps.

BATтERx. In mining, a stamping mill. In clectricity, a combination of glass plates or jars, with both surfaces coated with tinfoil. A combination of zine and coppor, or of other dissimilar metal plates, which are placed in an acid solution, or some other exciting fluid. The galvanie battery. See Esccrricitr.

BAUIr. A piece of timber - the whole trunk of a tree. The torm is applied by Iondon timber-merchants to wood in lengths of from 20 to 25 feet and 10 inches square.

BAUxcrz. A mineral which was at one time regarded as an ore of iron. It is so called from Baux, near Arles, the name of one of the localities in France where it is found. Its composition varies, but the following analysis of a specimen from Revest, near Toulon, may be taken as typical .-

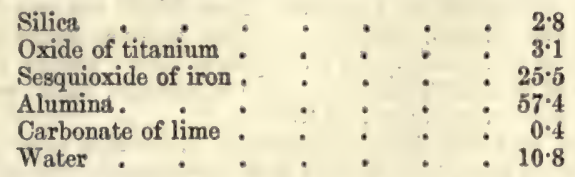

It is used as the source from which to obtain aluminium with the most facility and in the greatest purity. See Arumisiux.

BAY SAIT. The larger crystalline salt of commerce. See SALT.

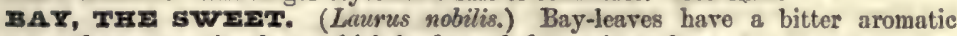
taste, and an aromatic odour, which leads to their use in cookery.

BATIDONIT2. A hydrated arsenate of lead and copper from Cornwall. It occurs in little concrotions of a grass-green colour; and was described by Prof. Chureh in the 'Journal of the Chemical Society' for 1865.

BArs, or工 or. This oil is imported in barrels from Trieste. It is obtained from the fresh and ripe berries of the bay-tree by bruising them in a mortar, boiling them for three hours in water, and then pressing them. When cold, the expressed oil is found floating on the top of the decoction. Its principal use is in the preparation of voterinary embrocations.

BDజrIrUar. Two gum-resins pass in commerce by this name. One is the false myrrh (the Bdellium of Seripture), the produce of the Amyris commiphora. The other is the African Bdcllium, obtained from Heudolatia Africana. Pelletier gives the composition of the African bdellium as-resin, $59{ }^{\circ} 0$; soluble gum, $9 \cdot 2$; bassorine, $30^{\circ} 6$; rolutilo oil and loss, 1:2.

zгaDs. (Grain, Fr.) Perforated balls of glass, porcelain, or gems, strung and worn for ornaments. Amongst some of the uncivilised races, beads are employed instead of money.

The use of beads is of the highest antiquity. They are found in the tombs of Thebes and in the ruined temples of Assyria. They are discovered buried with the mighty dead of Greece. The Roman lady had them placed with her in her grave; and even in the burjal-places of the ancient Britons we find beads, and these, too, of a similar pattern to such as we have every reason to believe aro as old as Moses. Indeed, the peculiar ornamented zigzag pattern of the most ancient beads has been nlways, and still is manufactured at Venice, and found over the entire continent of Africa.

Glass bends have long been made in very large quantities in the glass-houses of Murano, at Venice.

Glass-tubes, previously ornamented by colour and reticulation, are drawn out in proper sizes, from 100 to 200 feet in length, and of all possible colours. Not less than 200 shades are manufactured at Venice. These tubes are cut into lengths of about 2 feet, und then, with a knife, are cut into fragments, having about the same length as their diameter. The edges of these beads are, of course, sharp; and they are subjected to a process for removing this. Sand and wood-ashes are stirred with the beads, so that the perforations may be filled by the sand; this prevents the pieces of glass from adhering in the subsequent process, which consists in putting them into a revolving eylinder and heating them. The finished beads are sifted, sorted in various sizes, and strung by women for the market.

In the Jurors' Report of the Great Exhibition of 1851, are the following remarks on this manufacture:-

'The old Venetian manufactures of glass and glass-wares fully sustain their im. portance; and those of paper, jewellery, wax-lights, relvets, and laces, rather exceeded their ordinary production. The one nrticlo of beads employs upwards of $\delta, 000$ people at the principal fabric on the island of Murano; and the annual value is 
at least 200,000\%. They are exported to London, Marseilles, Hamburg, and thence to Africa and Asia, and the great Eastern Archipelago.' The perles a la lune are a finer, and, consequently, more expensive bead, which are prepared by twisting a small rod of glass, softened by a blow-pipe, about an iron wire. The preparation and cutting of gems into beads belong especially to the lapidary. The production of beads of Pastu, and of artificial Prancs, will be noticed under those heads respectively. In India beads of rock-crystal are often vory beautifully cut. In 1871 we importod 2'204,241 lbs. of glass beads, Soe Pastu ; Prarts.

BEAM Trmz. (Pyrus Aria.) Tho wood is used for axlo-trees, naves of wheels, and the cogs of machinery.

zва N. (Faba and Phaseolus.) See Legumnosas.

BгAw ors. (Bohnerz, Ger.) Brown iron ore occurring in ellipsoidal concretions.

\#DARrvas. The parts of a machine upon which the movable portions are supported. Upon the correct" adaptation of 'the rubbing surfaces to each other depends the value of a machine. If, for example, there should be much friction between the axles of a railway-carriage and its bearings, there would be a large amount of power lost in overcorhing that friction.

It has, therefore, been the study of ongineers to produce bearings which should offer great resistance to pressure, and from their smoothness produce as little friction as possible. Kingston's metal has been lately used in the large engines for our ironclad floot. Some of the railway companies aro using an alloy of oqual parts of tin and copper. Gun-metal is, however, commonly employed for the bearings of machines. Seo Bronze; Copper; Krngston's Mrtal; Unguents.

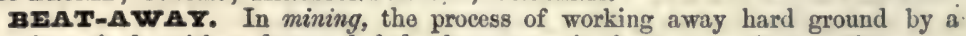
rough method-with wedges and sledge-hammers - in the process of excavation.

BaAUXrTz. See BaUxitr.

BEAVזr. (Castor Fiber.) This animal is captured for its skin, and for the castor (castoreum), which is employed medicinally. Seo Furs.

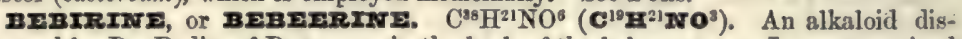
coverod by Dr. Rodie, of Demerara, in the bark of the bebeern tree. It was examinod more minutely by Madagan and Tilley, and still more recently by Von Planta, who has detormined its true formula. It is very bitter, and highly febrifugo.

APDE. In mining, a name given to a peculiar kind of pickaxe.

д²0E. (Hêtre commun, Fr.; Gemeine Buche, Ger.) The beech-treo (the. Fagus sylvatica of Linnreus) is one of the most magnificent of our English trees, attaining, in about sixty or seventy years, in favourable situations, a height of from 70 to 100 feet, and its trunk a diameter of 5 feet. The wood, when groen, is the hardest of British timbers, and its durability is increased by steeping in water; it is chicfly used by cabinet-makors, coopers, coach-builders, and turners.

REF wooD. An Australian wood, of red colour, the produce of certain specios of Casuarina. It is used for inlaying and marqueterie work.

BrFR. The fermontod infusion of malted barley, flavoured by hops, constitutes tho best species of beer; known also as ale, bitter ale, porter, or brown stout, according to its rariod flavour, colour, and strength. But there are many beverages of inferior quality to which tho name of beer is given; such as spruce-beer, ginger-beer, \&ce, all of which consist of a saccharino liquor, partially advanced into tho vinous fermentation, and flavoured with peculiar substances.

The ancients were acquainted with beer, and the Romans gave it the appropriate. name of Cerevisia (quasi Ceresia), as being the product of corn, the gift of Ceres. The most celebrated liquor of this kind in the old time was the Pelusian potation, so called from the town where it was prepared, at the mouth of the Nile. Aristotlo speaks of the intoxication caused by beer, and Theophrastus justly denominatod it the wine of barley. We may, indeed, infer, from the notices found in historians, that drinks annlogous to beer wore in use among the anciont Gauls, Germans, and, in fact, almost every people of our temperate zone; and they aro still the universal beverago in every land where the vine is not an object of rustic husbandry.

In the production of beer, the raw Barley, and Hops, which are the only materials necossary, have to undorgo various processes which will be more fully doscribed under the separate articles on Malting and Brewrive, but the changes which take place in those operations will now be considered.

\section{The Matmints.}

BArtay,-Barley, wheat, maize, and-several other kinds of grain, are capable of undergoing those changes which develop tho saccharine principle from which beer an bo made; but the first-named is by far the most fit, and in this country is almost 
exclusively used. There are two species of barley; tho 'Hordeum vulgare,' or common barley, having its corns arranged in two rows on its spikes; and the 'Hordeum hexastichon,' in which threo seeds spring from one point, so that its double row has apparently six corns. The former is the proper barley, and is much the larger sized grain. The latter is little known in England, but is much cultivated in Scotland under the name of bere, or bigg, being a hardy plant, adapted to a coldor climate. Bigg is a less compact grain than barley, the weight of an imperial bushol (2218.192 ins.) of the former being only $48 \mathrm{lbs}$, whilo that of the latter will be from 52 to 56 lbs. Their constituents are, however, similar.

By chemical analysis, 100 parts of barley-meal appear to consist of-

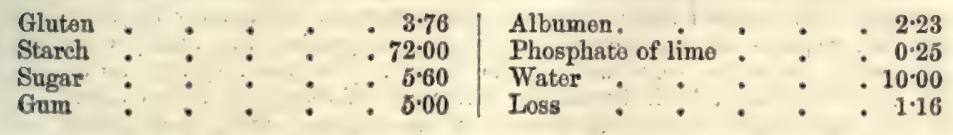

Another analysis gives:-

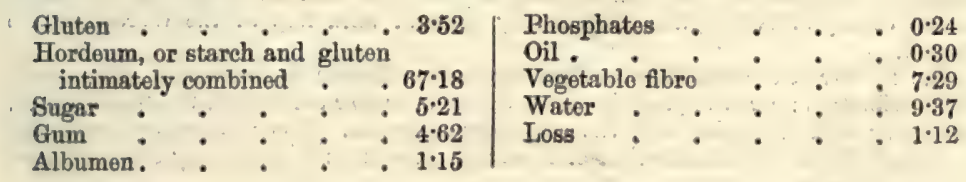

Hermstadt gives the mean of several analyses of barley to be:-

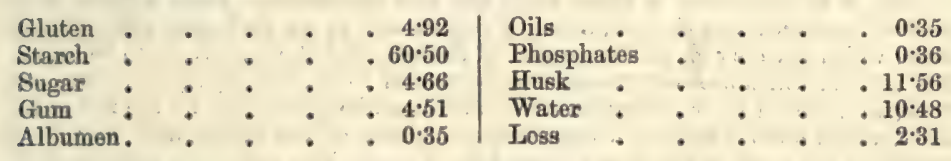

Proust thought ho had discovered in barley a peculiar principle, to which he gave the name of Hordeine, and which he separated from the starch by, the action of both cold and boiling water. He found that, by treating barley-meal successively with water, he obtained from 89 to 90 parts of a farinaceous substance, composed of from 32 to 33 of starch, and from 57 to 58 of hordeine. His analysis also gives, gluten, 3.0 ; sugar, 5.0 ; gum; 4.0 ; and resinous extract, $1 \cdot 0$.

Dr. S. Thomson gives no hordeine, but the starch as 88 per cent., sugar 4.

Einhof gives the constituents of barley as 70.05 flour; 18.75 husk; and 11.20 water.

The hordeine of Proust is a yellowish powder, contains no nitrogen, and is, therefore, dissimilar to gluten. In the process of malting the proportion of hordeine is greatly diminished by its conversion into starch and sugar, so that many chemists view hordeine as only an allotropic condition of sugar; but the subject will evidently bear yet more extended and careful research.

In giving the foregoing analyses, it may be remarked that they are not intended as a basis for any estimate of the value for brewing purposes of even the various samples from which they have been drawn, as the quality of the extract is affected by every variation of the soil on which the barley was grown, the quality of the seed, the climate, the season, and the care bestowed on its proper cultivation.

The quality of barley is much influenced by the soil on which it is grown; the -best being from a light calcareous soil, or that known by farmers as good turnipland; and crops of excellent quality are also grown on a rich land.

Much also depends on the seed, the climate, and the care of the husbandmen in the harvesting, stacking, and the threshing at the proper season.

Tho barley should have a thin, bright, clean, wrinkled husk, closely adhering to a plump, well-fed kernel, which, when broken, appears white and chalky, with a full uninjured germ of a pale yellow colour. If it breaks hard and flinty, it should bo avoided; and, although not in a proper condition for malting until it has sweated or seasoned in the stack or mow, care must be taken that it has not heated so as to destroy the vitality of the germ. Mixed or uneven samples should also be avoided, as it is important that all should grow simultaneously or evenly on the floors.

6. The Saalo district of Germany is generally considered to produce the finest quality 
of barley, although its weight per imperial bushel is less than the arorago of English barley, and much less than the Seotch.

The Saale woighs from 50 to 54 per bushel
The English " " 52 " 58 "
The Scotch " " 54,59

And as barleys, when equally woll malted, yield the valuable saccharine principlo nearly in proportion to their weight, tho heavier English or Seoteh barlcy, in a favourable season, is of the most value to the brewer of strong mild ales, where peculiar delicacy of flavour is not so much required.

Hops,-The female flowers, or eatkins, of a diœeious plant (Humulus lupulus) belonging to the natural order Urticacese, which grows wild in many English hodgerows, but requires the most careful cultivation to produce the highly odoriferous and cordial properties so valued by the brewer. The plant springs up annually from the old roots in April, flowers the latter end of June, and ripens towards the end of August and September, when they are gathered, dried, and packod very tightly in pockets or bags, for preservation and use. Hops are grown to the greatest extent in Kent and Sussox; but a strong hop is also grown in the north elay-district of the county of Nottingham, and a very grateful mild hop in the Worcestershire district.

The flavour of the Goldings, or Farnham hop, a district in Surrey, is rich, and in high estimation; but the plant is one of the most tender cultivated, the flowor small, but heavy with the farina, and the crop very uncertain. The Canterbury grape-hop is much cultivated in the districts of Kont and Sussex, and descrvodly esteomod as a good useful hop.

The Flemish plant produces a large flower, but of light weight and of inferior flavour ; it is considered a hardy kind, and very productive. Hops roquire a rich soil, well manured and cleaned, a sunny aspect, and to be sheltered from the east winds, which not only check the growth of the plants, but cause them to bo infested with vermin, which are sometimes 80 numerous as to destroy nearly the entire crop. The flower, during the ripening season, is also sometimes attacked by the red or blue mould, which often consumes a considerable portion of the farina, and may be discovered by the strig of the flower being bare of leaf. The catkins or strobils of the hop consist of the scales, or large and persistent bracts, which, in the early period of their growth, are of a light green colour (afterwards changing to a pale yollow), at the bottom of which are small round seeds, that, when ripe, have a hard shell of a brown or reddish colour. They are imbedded in the farina, or yellow powder, which is the most valuable part of the hop. No hop should be gathered till the seed is matured; not for the sake of the seed itself, but the nectarium, or farina, technically known as 'the condition,' will be in larger particles, and its essential aromatic and bitter qualities more perfectly developed when ripe. Good hops, when rubbed in the hand, leave an oily, or resinous, and rather clammy feeling, with a pungent and gratifying odour; the scales should also be even in colour, and without any green specks, or any appearance of mould on the sprig, or small stem of the flower.

The drying of the hop is an important part of its management, and requires great care; it is performed in kilns, in Sussex, termed oast-houses.

The heat should be moderate and regular, in no case exceeding $120^{\circ} \mathrm{F}$., as to overdry them would injure the flavour, and if not sufficiently dry they are liable to become mouldy.

The general practice is to try the strig or atalk of the flower, which, if it snaps from brittleness, is sufficiently dried, but if it bends without breaking, more drying is necessary.

In the process of drying every means should be used to aroid separating the farina from between the scales of the hop, and the practice of passing the hops, after drying, through what is tormed a mill, for the purpose of giving more evenness to the appearance of the sample, must be highly injurious, as it breaks up the hop and exposes to loss the most valuable part of the plant. The packing has also much influence in the preservation of the valuable but volatile aroma. The finer flavoured and pale hops are woll rammed into sacks of canvas, callod poekets, which weigh about $1 \frac{1}{\mathrm{~d}} \mathrm{ewt}$. each; the stronger and dark-coloured hops into sacks of a coarser texture, called hopbags, and weigh from $2 \frac{1}{2}$ ewts. to 3 ewts. each.

If intended for export, the bags are sometimes subjected to the action of the hydraulie press; and if not required for immediate use, the simple serew-press may be used with great advantage.

Dr. Ives first directed attention to the yellow pulverulent substance that has been 
alluded to as the farins or pollen of tho hop, which in good samples will amount to ono-sixth of their weight. This powder bears some resemblance to lycopodium; and its analysis by Dr. Ivos gives, tannin, 4.16 ; extractive, $8 \cdot 33$; bitter principle, $9 \cdot 16$; wax, 10.00 : resin, 30.00 ; lignin, 38.33 ; and loss, 0.02 . About 65 per cent. of the farina is soluble in alcohol, and the solution, distilled with water, leaves a resin amounting to 52.5 per cent., which has no bitter taste, and is soluble in alcohol or other. The distillate from which the resin has thus been separated contains the bitter prineiple, which has beon called lupuline (by Payen and Chevallier), mixed with a little tannin and malic acid.

To obtain this in a state of purity, the free acid must be saturated with lime, the solution evaporated to dryness, and tho residuum treated with ether, which removes a littlo resin; after which the lupuline is dissolved out by alcohol, leaving the malate of lime. On evaporating the alcohol, the lupuline romains, weighing from 8.3 to 12.5 por cent. It is sometimes white, or slightly yellowish, and opaque, sometimes orangeyellow and transparent.

At ordinary temperatures it is inodorous, but when heated omits the peculiar smell and possesses the characteristic taste and bitterness of the hop. Water dissolves it in the proportion of about 1 part to 20 , or 5 per cent., and acquires a yellow colour. It is quite soluble in alcohol and slightly so in ether.

Lupuline is neither acid nor alkaline, nor is it acted upon by solutions of the metallic salts ; it contains only a small quantity of nitrogen, and an essential oil.

The analysis by Payen and Chevallior gives the following:-Volatile oil, 2.00; lupuline, 10.30 ; resin, 55.00 ; lignin, 32.00 ; loss, 0.70 . There are also traces of futty, astringent, and gummy matters, malic and carbonic acids, and various salts.

The volatile oil was procured by $\mathrm{Dr}$. Wagner by distilling fresh hops with water. It constituted about 8 per cont. of the air-dried flowers, it possessed a clear brownishyellow colour, had an acrid taste and a strong odour of the hop. Its specific gravity is about 0.910 ; it is partially soluble in water, but moro so in alcohol and ether, and becomes resinified by keoping. The tannin of the hop is also important in brewing, as it serves to precipitate the nitrogenised or albuminous matter of the barley, and assist the clearing of the liquor. Iros thought the scales of the hop, when freod from the yellow powder, contained no principles analogous to it; but it is almost impossible to free them entirely from the lupulinic grains; and Payen and Chevallier found the same principles in the different parts of tho hop, but in difforont proportions.

\section{The Preparation of the Barley hy the Prodess of Malime.}

In this process (for the conduct of which we refer to the article Maltuve) the raw grain is steoped in cigterns of water until it has imbibed sufficient to cause it to germinate; it is then spread on the floor of the malt-house, and frequently turned, until the germination has advanced to the stage when the plumula is about to make its appearance, and its further germination is stopped by being rapidly dried on the malt-kiln.

During germination a remarkable change has taken place in the substance of the grain. Tho glutinous constituent has almost entirely disappeared, and is supposed to have passed into the matter of the radicles, or roots, which during the process will have grown rapidly to nearly one and a half the length of the grain, while a portion of the starch is converted into sugar and mucilage.

The change is similar to that which starch undergoes when dissolved in water and digested in a heat of about $160^{\circ} \mathrm{F}$. along with a little gluten. The thick paste becomes gradually liquid, transparent and sweet-tasted, and the solution contains now sugar and gum, with some unaltered starch. The gluten suffers a change at the same time, and becomes acescent, so that only a small quantity of starch ean thus be converted by a quantity of gluten.

By the artificial growth upon the malt-floor all the gluten and albumen presont in barloy are not decomposed, and only about one-half of the starch is converted into sugar, as a continuance of the gormination would exhaust the grain, and tho valuable products would bo taken up by the growth of the roots and stems of the plant. It is, therefore, the chief art of the maltster to regulate the germination and stop it at the point when the utmost conversion is attained with the least loss. This is generally considered to be done when the plumula, technically known as the acrospire, has advanced two-thirds the entire length of the grain, starting from the germ and proceoding under the skin toward the other end of the grain, beyond which it must never be suffered to protrude; the conversion of the hordeine into starch and sugar keeping pace with the growth of tho acrospirc, and being thus propared for its noarly complete conversion in the subsequent operations of the brower. 
Malt is genorully distinguishod by its colour-as pale, ambor, brown, or black malt -arising from the different degrees of hoat and management in the process of drying. The first is produced when the highest hent to which it has been subjected is from $90^{\circ}$ to $100^{\circ} \mathrm{F}$., the amber-coloured when tho heat has boen raised to $120^{\circ}$ or $125^{\circ}$, and the brown at a heat of from $150^{\circ}$ to $170^{\circ}$. The black malt, commonly called patent malt, is prepared by roasting in cylinders, like coffee, at a heat of from $360^{\circ}$ to $400^{\circ}$, and is the only legal colouring-mattor that may bo used in the brewing of porter.

The action of the kiln in drying is not confined to the mere expulsion of the moisture from the germinated seeds, but it serves to convert into sugar a portion of the starch which remained unchanged, not only by the action of the gluten upon the fecula at an elevated temperature, but also by the species of roasting which the starch undergoes, which renders it of a gummy nature. We have a proof of this if wo dry one portion of the malt in a naturally dry atmosphere, and another portion in a moderatoly warm kiln; wo shall find the former yield a less sacelarine extract than the latter. Moreover, kiln-dried malt has a peculiar, agreeable, and faintly-burned taste, probably from a small portion of empyreumatic oil formed in the husk, which not only imparts its flavour to the beer, but also contributes to its preservation.

As the quality of the malt depends much on that of the barley, so its skilful preparation has the greatest influence both on the quantity and quality of the worts made from it. If the germination has been imperfect or irregular, a portion of the malt will be raw, and too much of its substance remain unchanged and flinty; if it has been pushed too far, a part of the extractible matter is wasted.

If not thoroughly dried, the malt will not keep, but becomes soft and liable to mildew; and if too highly kiln-dried, a portion of its sugar will be caramelised and become bitter.

Good malt possesses the following characteristics:-The grain is round and full, breaks freely between the teeth, and has a sweetish taste, an agreeable smell, and is full of a soft flour from end to end. It affords no unpleasant flavour on being ehewed; is not hard, so that when drawn along an oaken board across the fibres it leaves a white streak like chalk. It swims upon water, while unmalted barley sinks in it.

The bulk of good malt exceeds that of the barley from which it is made by from 5 to 8 per cent., but at the same time it becomes lighter in weight, $100 \mathrm{lbs}$. of good barley, judiciously malted, weighing, after being dried and screened, no more than about $80 \mathrm{lbs}$., the loss being about 12 per cent. of water, 5 per cent. waste, and about 3 per cent. by the growth of the roots, which, in drying, have been rendered brittle, and are removed by passing the malt over a wire sereen.

The change which the barley has undergone by malting will be readily seen in the following comparative analysis by Proust:-

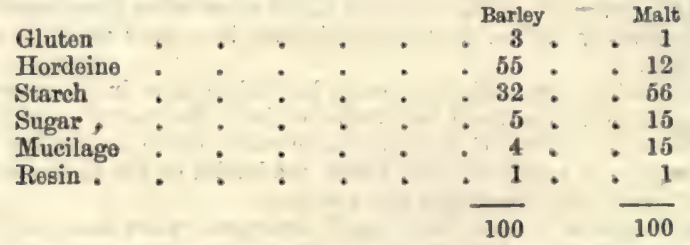

We thus see the amount of the convertible starch and sugar has been nearly doubled at the expense of the hordeine, a portion of which has also passed into the condition of mucilage, or a soluble gum, while the gluten is much diminished.

The researches of Payen and Persoz show there is also a new proximate principle formed during the malting, which may be considered as a residuum of the gluten or vegetable albumen, in the germinating grain.

If we moisten the malt-flour for a few minutes with cold water, press it out strongly, filter the solution, and heat the clear liquid in a water-bath to the temperature of $158^{\circ}$, the greater part of the albuminous azotised substance will bo coagulated, and should be separated by a fresh filtration, after which the cloar liquid is to be treated with alcohol, when a flocky precipitate appears, to which has been given the name of diastase. To purify it still further, especially from the nitrogenous matter, we should dissolve it in water, and precipitato again with alcohol. When dried at a low temperaturo it appears as a solid white substance, which contains no nitrogen, is insolublo in aleohol, but dissolves in water and proof spirit. Its solution is neutral and tasteloss; it changes with greater or less rapidity according to the temperature, and becomes 
sour at a tomperature from $149^{\circ}$ to $167^{\circ}$. It has the property of converting starch into gum, or dextrine (so called by the French chemists, from its polarising light to the right hand, whereas common gum does it to the left) and sugar; and, indeed, when sufficiently puro, the diastase operates with such energy that one part of it disposes 2,000 parts of dry starch to that change, but it operates the quicker tho greater its quantity.

Whenever the solution of diastase with starch is heated to the boiling point, it loses the converting property.

One hundred parts of the starch solution from good malt appear to contain about one part of this substance, which is of the greatest importance in effecting the further changes which take place in the process of brewing.

\section{The Formatiox of a SAccharme Liqum, on WorT,}

from the malt and hops, and production of the finished beer, is the province of the brewer; and the process will be found at length under the article BrEwLNG.

The peculiar properties contained in wort do not exist ready formed in malt, but are the result of the joint action of water and heat which is employed in the initiatory process of the brewer on that substance, and is termed the mashing.

The Mashing.-This operation requires the greatest care, as on it, almost as much as on the malt employed, depends the character of the liquor.

Payen and Persoz, already alluded to, show that the mueilage formed by the reaction of malt upon starch may be either convertod into sugar or be mado into a permanent gum, according to the temperature of the water in which the materials are digested. We take of pale barley-malt, ground fine, from 6 to 10 parts, and 100 parts of starch ; we heat, by means of a water-bath, 400 parts of water in a copper to about $80^{\circ} \mathrm{F}$.; we then stir in the malt, and increase the heat to $140^{\circ} \mathrm{F}$., when we add the starch, and stir well together. We next raise the temperature to $158^{\circ}$, and endeavour to maintain it constantly at that point, or, at least, to keep it within the limits of $167^{\circ}$ on the one side and $158^{\circ}$ on the other. At the end of twenty or thirty minutes the original milky and pasty solution becomes thinner, and soon after as fluid nearly as water. This is the moment when the starch is converted into gum or dextrine. If this merely mucilaginous solution, which seems to be a solution of gum with a little liquid starch and sugar, be suitably evaporated, it may serve for various purposes in the arts to which gum is applied; but, with this view, it must bo quickly raised to the boiling point, to prevent further change. If we wish, on the contrary, to produce a saccharine fluid, such as the wort for beer, we must maintain the tomperature at be tween $158^{\circ}$ and $167^{\circ}$ for three or four hours, when the greatest part of the starch will have passed into sugar, and by evaporation of the liquid at the same temperature, a starch syrup may be obtained like that procured by the action of sulphuric acid upon starch.

In the operation of mashing, the finished and mellowed malt, having been well cleansed from all extraneous matters by screening, is coarsely ground, or better if only crushed between iron rollers, as is now generally practised. It is then gradually mixed with water in the mash-tun, at the proper heat, and intimatoly blended by stirring with the mashing-rakes, so that it may be uniformly moistened and no lumps remain. After being allowed some time to stand and settle, the liquor is drawn off, and more water at a higher temperature is added, again intimately blended with the malt-now termed the 'goods '-again allowed to rest, and drawn off; the operation being ropeated until the complete erhaustion of the saccharine and amylaceous substances of the malt is effected.

We can now see, from Payen and Persoz's experiment just given, the temperature at which the liquor ought to be maintained in this operation; namely, the range between $158^{\circ}$ and $167^{\circ}$; and it has been ascertained, as a principle in mashing, that the best and soundest extract of the malt is to be obtained, first, by beginning to work with water at the lowest of these heats, and to conclude with water at the highest; secondly, not to operate the extraction at once with the whole of the water that is to bo employed, but with separate portions and by degrees.

The first portion has the task of penetrating equally the crushed malt, extracting the more soluble ingredients and subjecting the dissolved starch to the action of the diastase and free sugar; the second and further portions are for the purpose of converting the remaining starch and completing the extraction of all the available products. By this means also the starch is not allowed to run into a cohesive paste, or, as it is tormed, 'lock up the goods,' and the extract is more easily drained from the mass, and comes off a noarly limpid wort. The thicker, moreover, or the less diluted the mash is, so much the easier is the wort fined in the boiler or copper by the coagu-

Vor. I. 
lation of the albuminous matter. These principles indicate the true mode of conducting the mashing process, but different kinds of malt require a different treatmont; pale and slightly kilned malt requires a somowhat lower heat than malt highly kilned, because the former is more ready to become pasty, and, for the same reason, needs a more leisurely infusion than the latter; and this is still more applicable to the case of a mixture of raw grain with malt, for it requires still gentler heats and more cautious treatment.

It is quite practicable to obtain from 1 part of malt and 8 parts of barley, a wort precisely similar to that procured from 9 parts of pure malt alone. But, of course, this could not be done without modifying considerably the process of mashing; and it happens, unfortunately, that the practice of the present day, amongst brewers, is to maintain, as closely as possible, one uniform system of mashing, whatever may be the nature or quality of the malt employed. Thus a difference in the malt is made to produce a difference in the wort, and all the energy and skill of the practical brewer are sometimes insufficiont to compensate for the alterations which this difference induces in the subsequent working of the beer. With a regular and certain composition, as to the constituents of his wort, the operations of the brewer would assume a fixed and definite character, which, at present, they are very far indeed from possessing; and by which he not unfrequently suffers the most severe pecuniary loss and mental anxiety. With the excoption of a trifling quantity of vegetable albumen, the only solid ingredients of beer-wort are dextrine and sugar; the latter of which forments with great ease and rapidity, whilst the dextrine, though capable of fermentation, enters into the process only with difficulty, and requires, for its successful termination, not only much more yeast, but also a much higher temperature in the fermenting vat. At the same time, it is this very sluggishness in the fermentative quality of dextrine which is essential to the production of good beer; for, with sugar alone, the fermentation cannot be checked at ordinary temperatures, until the full measure of its decomposition has taken place, and it has become either a vapid admixture of alcohol and water, or, by the absorption of oxygen, is resolved into vinegar. It is indeed a notorious fact, that beer made with sugar will not keep so well as that made from malt; though, for rapid consumption, the use of sugar is, under some circumstances, to be eommended, more especially on the small scale and in cold weather. The peculiarity of dextrine is, however, as we have stated, to undergo fermentation only with difficulty and by slow degrees; hence its decomposition spreads over a long space of time, and, in very cold weather, amounts to nothing; so that for months, or even years, after all the sugar of the wort has been destroyed, the evolution of carbonic acid gas from the still fermenting dextrine, keeps up a briskness and vitality in the boer; and, by excluding oxygen, all chance of acidification is shut off. A perfect beer-wort should therefore have reference to the period of its consumption: if this be speedy and pressing, the proportion of sugar ought to be large; if remote, the dextrine should greatly predominate. Under the first condition, the attenuation would proceed quickly, and, provided the temperature of the fermenting vat was not allowed to exceed $78^{\circ}$, the beer would soon cleanse and become ripe and bright; under the secoud, the attenuation in the vat would be slow and trifling, and require, perhaps, several years for its completion in the cask. Nevertheless, if the attenuation in the vat had gone on to the complete destruction of all the sugar, this kind of beer would prove in the end both the better and more healthy beverage of the two; for by the mode of its formation the presence of cenanthic ether or fusel oil is avoided. The importance therefore of placing in the hands of the brewer a means of determining the relative amounts of sugar and dextrine in his wort is sufficiently obvious. Now, this may be done in two ways: either by ascertaining, in wort of a determinate strength, the proportion of the one or the other of these substances. The dextrine is easier of calculation than the sugar, in a rough or approximate way; but the sugar can be determined with much more minute accuracy than the dextrine. Yet, in practice, the former plan is preferable, from its simplicity, as we shall proceed to show. If, to a certain volume of strong wort (say of $30 \mathrm{lbs}$. per barrel), we add an equal amount of alcohol or spirits of wine, the whole of the dextrine will precipitate as a dense coagulum; and by examining the bulk of this deposit in the tube, its weight may be inferred pretty nearly if the tube has been previously graduated, so as to indicate, from actual experiment, the weight of the different measures of the coagulated dextrine. With weaker wort, more alcohol must be used, and with a denser wort, less alcohol, - the relations of which to each other may easily be kept recorded on a small card or scale affixed to the tube. This instrument is very easy of application, and has been found extremely useful to more than one practical brewer of the present day; and the accompanying record of brewing operations has reference to this mode of analysing wort. The determina- tion of sugar in wort is best effected by boiling 100 grains of it with about half a pint of the following solution, and collecting and weighing the red-coloured pre- 
cipitate which ensues,-every three grains of which indicato one grain of grape-sugar in the wort.

Grape-Sugar Test-Solution.

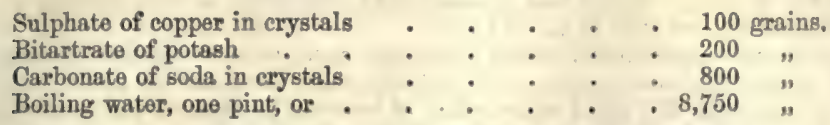

First dissolve the sulphate of copper, then the bitartrate of potash, after which add the carbonate of soda, and filter if necessary. This solution is not affected when boiled with cane-sugar, dextrine, gum, or starch.

We have retained from Dr. Ure's original article the result of two brewings, taken from one mash at two different periods, and analysed to determine their relative contents of dextrine and sugar, according to the tube or alcohol process :- March 28th, 1851 , proceeded to mash for experimental brewings; weather clear and open; thermometer outside at $51^{\circ}$, -in fermenting room $58^{\circ}$; difference botween wet and dry bulb, $5.750^{\circ}$; barometer, 39.4 inches. Composition of the malt :-Moisture, 6.1 ; insoluble matter, 27 ; extract, 66.9 . Quantity of malt employed, 70 bushels ; of water at $180^{\circ} \mathrm{F} ., 700$ gallons; made the mixture with a common mashing-oar, and finished in 15 minutes. One hour afterwards, drew off 200 gallons of wort; and three hours from commencing to mash, drew off 200 gallons more,-continuing the mash for table-beer wort. The first-drawn wort contained $7 \cdot 5$ parts of dextrine to 1 of sugar; the second, 6.3 parts of dextrine, $2 \cdot 2$ of sugar;--their dencities were, respectively, 30 and 36.5 lbs. per barrel. They were each boiled separately, with relative amount of hop, - the first having 30 and the second $36 \frac{1}{2} \mathrm{lbs}$. added; and the boiling in each case was kept up for three hours. At the ond of this time both were cooled and diluted with water to a gravity of $27 \frac{1}{2}$ lbs. per barrel, and 250 gallons of each let down into separate fermenting-rats placed side by side; after which, they both receired three quarts of good yeast,- the temperature being at $68^{\circ} \mathrm{F}$. Two hours afterwards, the following observations commenced:-No. 1 being the wort containing 7.5 parts of dextrine to 1 of sugar, and No. 2 the wort having 6.3 of dextrine to 2.2 of sugar.

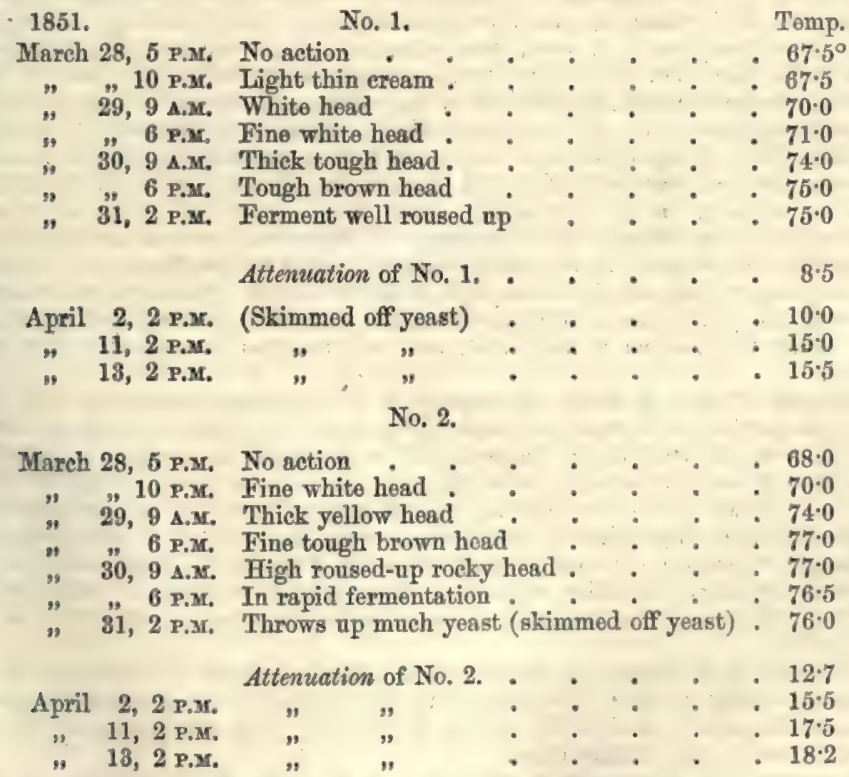

The temperature of both had now fallen to $69^{\circ} \mathrm{F}$, though each had been roused repeatedly; the yeast was therefore again skimmed off, and the beer run into barrels, and filled up with reserved wort three times a day as it worked over. On April the 18th the barrels were elosed, having thon lost, by attenuation-No. 1, 16.2 lbs., and No. $2,19.6 \mathrm{lbs}$. Six woeks afterwards these ales were oxamined:-No. 1 was found 
muddy and unpleasant; whilst No, 2 had a fine fragrant aroma, a brisk, lively appearance, and was perfoctly bright. On January 2nd, 1852, the casks wero again examinod;-No. 1 had now lost $17.9 \mathrm{lbs}$, and was bright, rich, and fine-flavoured; whilst No. 2, though bright and pleasant, had contracted a littlo acidity, and was bocoming flat: it had lost, in all, 21 t 1 lbs.

Two similar experiments, made about the same time in another quarter, gave almost exactly the same results; and, consequently, there can be little doubt that, where a quick sale and rapid consumption of beer can be ensured, the great object of the brewer should be to convert as much of the dextrine of his wort into sugar as is proportional to the rapidity of that consumption; whereas, for beer intended to keep, the opposite practice should be followed.

The conversion of any given amount of the dextrine-wort into sugar may be effected either by keeping up the temperature of the mash-tun, and prolonging the oporation of mashing; or, which is better and simpler, by merely preserving the wort for a fow hours at a heat of $165^{\circ} \mathrm{F}$., either in the underback or any other conveniont vessel. We have found from experiment that a wort which when run out from the mash-tun had only 3 parts of sugar to 16 of dextrine, became by 10 hours' exposure to a heat of $165^{\circ}$ converted almost altogether into sugar, - the proportions then being $17 \cdot 8$ of sugar to $1 \cdot 2$ of dextrine.

A very important part of the duty of a brewer should therefore be, first, the determination of the relative amounts of dextrine and sugar required to suit the tasto of his customers, or the circumstances of the market, and next, the continued careful examination of his wort, so as to ensure that these proportions are regularly maintained; for by no other plan is it possible to ensure that certainty of result and uniformity of quality which are essential to the proper conducting of an expensive business like browing. Far too little attontion has hitherto been given to the fluctuating qualities of beer-wort; in warm weather, this wort should probably contain at least twice as much dextrine as in winter; yet this is the very period when, from the increased temperature of the air and materials, the largest quantity of sugar must be formed by those who mash upon a fixed and unvarying principle. Hence the proneness of the wort to ferment violently in summer is still further inereased by the presence of an extra proportion of sugar;-whereas prudence would suggest, under such circumstances, a predominance of dextrine, and seek to effect this purpose by a low tomperature in the mash-tun, and by shortening the period of mashing. As a general rule, in the managoment of wort, more sugar is requisite where small quantities are brewed at a time, than where large operations are conducted, for the loss of heat is relatively larger in small masses than in large ones; and, from what has been stated, it must be apparent, that, as the fermentation of dextrine is more easily checked by cold than that of sugar, the beer brewed in trifling quantities could not preserro a fermentative temperature, but would become chilled and dead from the excessive radiation of heat, unless a principle existed in it capable of fermentation at the most ordinary temperatures of this country. If, therefore, beer-wort consisting chicfly of dextrine be fermented in very cold weather, or with an insuffeiency of yeast, or if the temperature happen to rise too high, so as to destroy or impair the fermentative power of the yeast, then a dull languid action will ensue, accompanied by what has been called the viscous fermentation, and beer becomes permanently ropy, and is spoiled.

Although, clearly, it would be impossible to lay down any specific rule for the proper proportion of dextrine and sugar in beer-wort, yet there could be no difficulty in each brewer determining for himself, and for the conditions of quantity, time of sale, time of year, and other contingencies, the requisite ratio to be established in his own case; and, as we have shown, nothing can be simpler than the means proposed for ascertaining the composition of wort, remembering that, though a dextrine-wort may bo thought to have a superior keeping property, it should be rather said that it is slower in arriving at maturity, whereas a full saccharine wort can be fermented more roadily, is more under control, and the beer sooner becomes a brilliant and maturod beveruge.

The quantity of extract per barrel weight, which a quarter of malt yields to wort, amounts to about $84 \mathrm{lbs}$. The wort of the first extract is the strongest; the second contains, commonly, one-half the extract of the first; and the third, one-half of the second, according to circumstances.

To measure the degrees of concentration of the worts drawn off from the tun, a particular form of hydrometer, called a saccharometer, is employed, which indicates the number of pounds' weight of liquid containod in a barrel of 36 gallons imporial measure. Now, as the barrel of water weighs $360 \mathrm{lbs}$, the indication of the instrument, when placed in any wort, shows by how many pounds a barrel of that wort is heavier than a barrel of water; thus, if the instrument sinks with its poige till the mark 10 is 
upon a line with the surface of the liquid, it indicates that a barrol of that wort weighs ton pounds more than a barrel of water. See SAccisarometre.

Or, supposing the barrel of wort weighs $396 \mathrm{lbs}$, to convert that number into specific gravity, we have the following simple rule:-

$$
360: 396:: 100: 1 \cdot 100 \text {; }
$$

at which density the wort contains about 25 per cent. of solid extract.

Now the ordinary German chemical thermometer, as supplied from all laboratorics, is by far the more reliable and useful instrument; some men have for years used them in the mashing, and are quite proud of the exactness and facility with which they are enabled thereby to manage the process. The hair stem is enclosed along with a paper scale (properly adjusted and accurately divided) within a glass tube annealed at the top, and the lower end on to the upper portion of the bulb; thus, with certainty, exposing the bulb alone to the influence of apparent heat. By fine copper wire twisted round the top and lower portions, this tube is attached to a long wood stick 1 inch $\times 1 \frac{1}{2}$, cheeked on either side with a lath of 3 or 4 feet long, and rising to the front nearly $\frac{3}{4}$ of an inch, to protect the thermometer from actual contact in the erent of an accidental knock; upon the lower end of the stick I attach a small tin box or cover about 4 inches long, just the width of the stick, and about an inch in depth. This covers the bulb, and is fitted at the lower end with a simplo tin trapdoor valve arrangement, which opens upward on the inside, whenever the slightest pressure is applied underneath it by contact with the mash, and the instrument is complete.

When this apparatus is thrust into the goods, their upward pressure pushes open the valve, and allows them to pass over the bulb, and immediately on attempting to withdraw the instrument the valve again closes. This of course can be repeated at pleasure, and changes the goods in immediate contact with the bulb, with every fresh motion of the hand, be it ever so slight, and these are distinctly marked by the click of the ralve as it closes being felt. By this simple and inexpensive arrangement, the brewer has a trusty, useful thermometer, with which he can easily obtain a sample of his mash from any part of his tub whatever. The thermometer can be read with the greatest deliboration; its indications are almost instantaneous and quite reliable, and a slight jerk is all that is required to free it entirely from every grain the box contains, when resding the stem.

We will here give a few moments' consideration to the amount of extract to be obtained from a certain quantity of malt, and for practical purposes let us say eight bushels, or one imperial quarter.

It is ascertained from experiment that, on an average, $60 \mathrm{lbs}$. of ordinary glucose (grape-sugar) are equal to 2 bushels of malt in producing a barrel of beer. A bushel of malt usually weighs about $40 \mathrm{lbs}$; ; if it weighs less, on account of being more thoroughly malted, it is all the better. This proves that $80 \mathrm{lbs}$. of dry malt, at least 60 lbs, , or 75 per cent., are taken up by the brewing liquor. It has been further ascertained by experiment that the amount of these substances just given, namely $60 \mathrm{lbs}$. of glucose, or $80 \mathrm{lbs}$. of malt, will produce a barrel of beer of $20 \mathrm{lbs}$. per barrel gravity.

From this the practical brewer may deduce that, to ascertain the amount of dry extract taken from the malt he has been brewing, he must multiply the lbs. per barrel gravity by 3 , and even this will but show the proportion of dry extract taken up by the brewing liquor alone. This multiplier is somewhat more than that allowed by the Excise, theirs being $2 \cdot 6$ instead of 3 ; but it must be borne in mind that their Tables were framed upon the basis of cane-sugar instead of malt, and that 90 parts canesugar are equal in atomic equivalent to 95 parts pure glucose. On this account, therefore, also, their multiplier ought to be 2.7368 instead of $2 \cdot 6$. The knowledge of this is of course extremely galling to the export brewer. It will at once be seen that, in order to determine by a rough and ready estimate what a certain amount of malt should produce, simply divide the total number of lbs. of malt by 4. This will give the total gravity of extract obtainable from good malt for common beers, and if it is desired to know the lbs. per barrel that should be obtained for a particular brewing, simply divide this total extract gravity by the number of barrels brewed; thus 20 quarters of malt at $40 \mathrm{lbs}$. per bushel $=6,400 \mathrm{lbs}$. malt :-

\section{4) 6400}

$\overline{1600}$ lbs. gravity of extract.

When cooled down and got into the fermenting-tun, if there were 66 barrels of it, $1600 \div 66=24 \cdot 2$ lbs. per barrel, would be tho weight of the beer. This is found practically correct, though it must not bo contended that you have hero an account 
of all that has beon obtained from the malt; there is no account of all the losses that aro inevitable in tho process of brewing, and these of course vary according to the peeuliarities of ench brewing. As to tho amount a quartor of malt ought to yiold, thero is much diversity of opinion, and of a necessity this must continue to be so according to the circumstances and class of trado of a brewer, and the kind of alo most suituble to him; but to ale adapted to tho prevailing taste of the present day, the experienced. brewer will find that the more he can afford to be below 80 lbs. per quarter when cooled down and got into the fermenting-tun, the quicker and more cortain will be his profits, and the less annoyances will he have; indeed, for ale that is not rattod it is most advisable, and for thoso that are, it is prudent, especially in warm weather.

The object of boiling the wort is not merely evaporation and concentration, but extraction, coagulation, and, finally, combination with the hops; purposes which may bo accomplished in a deep confined copper, by a moderate heat, or in an open shallow pan with a quick fire.

The copper, being encased above in brickwork, retains its digesting temperature much longer than the pan could do. The waste steam of the close kettle, moreover, can be economically employed in communicating heat to water or weak worts, whereas the exhalations from an open pan would prove a nuisanco, and would need to be carried off by a hood.

The boiling has a fourfold offect: first, during the earlier stages of henting, it converts the starch into sugar, dextrine, and gum, by means of the diastase ; secondly, it concentrates the wort; thirdly, it extracts the substance of the hops diffused through the wort; fourthly, it coagulates the albuminous matter present in the grain, or precipitates it by means of the tannin of the hops.

The degree of evaporation is regulated by the nature of the wort and the quality of the beer. Strong ale and stout, for keeping; require more boiling than ordinary porter or table-beer, brewed for immediate use. The proportion of the water carried uff by evaporation is usually from a seventh to a fourth of the.volume.

The hops are introduced at the commencement of the process. They serve to give the beer not only a bitter aromatic taste, but also a keeping quality, as they counteract its natural tendency to become sour-an effect partly due to the precipitation of the albumen and starch, by their resinous and tanning constituents, and partly to the antifermentablo properties of the lupuline, bitter principle, ethereous oil, and resin. In these respects, there is none of the bitter plants which can be substituted for hops with advantage.

For strong beer, powerful fresh hops should be selected; for weaker beer an older and weaker article will suffice.

The stronger the hops are, the longer time they require for the extraction of their virtues; for strong hops an hour and a half, or two hours' boiling may be proper; for a weaker sort, an hour may be sufficient; but it is never advisable to push this process too far, lest a disagreeable bitternoss, without aroma, be imparted to the beer. In somo breweries it is the practice to boil the lops with a part of the wort, and to filter tho decoction through a drainer, called the hop-back. The proportion of hops to malt is very various; but, in general, from $1 \frac{1}{1} \mathrm{lbs}$. to $1 \frac{1}{2} \mathrm{lbs}$. of the former are taken for $100 \mathrm{lbs}$. of the latter in making good table-beer.

For porter and strong ale, 2 lbs. of hops are used, or oven more: for instance, from 2 lbs. to $2 \frac{1}{2}$ lbs. of hops to a bushel of malt, if the beer be destined for consumption in India.

During the boiling of the two ingredients, much cosgulated albuminous matter, in various stages of combination, makes its appearance in the liquid, constituting what is called the breaking or curdling of the wort, when numerous minute flocks are seen floating in it. The resinous, bitter, and oily ethereous principles of the hops combine with the sugar and gum or dextrine of the wort; but for this effect they require time and heat; showing that the boil is not a process of mere evaporation, but one of chemical reaction. 1 yellowish green pellicle of hop-oil and resin appears upon the surface of the boiling wort, in a somewhat frothy form; when this disappears the boiling is presumed to be completed, and the beer is strained off into the cooler. The residuary hops may be pressed and used for an inferior quality of beer; or they may be boiled with fresh wort, and be added to the next brewing charge.

Many prefer adding the hops when the wort has just come to the boiling point. Their effect is to repress the passage into the acotous stage, which would otherwise inevitably ensue in a fow days. In this respect no other vegetable production hitherto discovered can be a substitute for the hop.

The odorant principlo is not so readily rolatilised as would at first be imagined; for when hop is mixod with strong beer-wort, and boiled for many hours, it can still impart a very considerable degreo of its fla vour to weaker beer. 
- By mere infusion in hot beer or water, without bolling, the hop loses very little of its soluble principles. The tannin of the hop combines, as wo have said, with the vegetable albumen of the barley, and helps to clarify the liquor.

If the hops be boiled in the wort for a longer period than five or six hours, they lose a portion of their fine flavour; but if their natural flavour be rank, a little extra boiling improves it. Many brewers throw the hops in upon the surface of the boiling wort, and allow them to swim there for some time, that the steam may penetrate them, and open their pores for a complete solution of their principles when they are pushed down into the liquor.

The quantity of hop to be added to the wort varies according to the strength of the beer, the length of time it is to be kept, or the heat of the climate where it is intended to be sent.

For weak beer $4 \frac{1}{2} \mathrm{lbs}$. of hops are required to a quarter of malt; but when it is intended to be highly aromatic and remarkably clear, and for the stronger kinds of ale and porter, the rule, in England, is to take $1 \mathrm{lb}$. of hops for every bushel of malt, or $8 \mathrm{lbs}$. to a quarter. Common beer has seldom more than $\frac{1}{4} \mathrm{lb}$. of hops to a bushel of malt.

The form, size, and setting, the extent of fire-bed and dimensions of the flues, as also the power of draught procurable of a wort copper, are each and all of such importance that none but men of experience should be entrusted with the work of placing it.

With respect to the first of these qualifications, the rapid evaporation required will suggest an open shallow basin, turned in at the top, so as to roll violent ebullition into the pan; the flue should not expose the lower part of the pan higher than can be covered by the first charge run off from the mash-tun, or the thin edge of its contents are liable to burn and thereby colour and perhaps flavour the brewing.

It should be set so that the whole bottom is freely exposed to the fire, and the bridge so placed as to direct the flame straight up against it, midway between the centre and edge in front. In size it should be capable of boiling away one-fourth of the brewing; a pan, say of 60 barrels contents, would turn out, after boiling, 45 barrels.

In estimating for a copper of any size, it is usual to reckon thus: suppose you desire a copper to boil for a 60 -barrol brewing, the contents of the copper should be 80 barrels, and this will only just allow for expansion of bulk and the violent ebullition.

The extent of fire-bed and power of draught should be such as would enable the boiling to be finished within two hours from the time the pan was fully charged. All beers should be boiled at least two hours, or much more of the value of the hops will be thrown away than is necessary: it will not endangor either the colour or the flavour of the palest ales, and it is time sufficient to ensure much of the benefit arising from the depuration of the flocks by the coagulation consequent on boiling.

The colour desired should also partially regulate the time required for boiling; for pale ales about two hours is sufficient, but not too much : for deep amber, two and a half to three hours; for rich brown ales from three to four hours will not be found too long; but in deciding this, the colour of the malt-extract, of the hops, and the gravity of the wort must each be considered.

The following considerations are submitted for the guidance of the brewer in this operation.

In the first place, except in steam-tight boilers, you cannot raise the heat above $212^{\circ}$, however hard you boil; by increasing the fire, therefore, you gain rapidity of concentration, and colour is obtained not by hard, but by long boiling.

Secondly, if wort, boiled either with or without hops, be examined by any of the simple tests for the albumenoids at different times in the process of boiling, it will be found that the longest boiled will be the most free from the albuminous constituents, and that it is almost impossible to boil wort long enough to free it entirely from them ; in this, again, a good boiling proves of value, for it enhances the keeping power of the beor by ejecting from it those constituents which are so troublesome to the brewer.

Again, the more concentration that can be permitted in the pan, the more of the valuablo properties that most deep-spring waters eontain are utilised, and these assist greatly the clarifying and soundness of the ultimate product.

Thirdly, with regard to the boiling with hops, the same, almost precisely, may be said, for the lupuline and tannin of the hop are very difficult to draw from their corert, but as this is being accomplished, it does its work of depuration, separating with its load of albuminous flocks, as tannato of albumen. 
In apportioning the hops that are to be boilod with the wort, quantity must be considered quite as much as quality, for it is on the amount of tannin utilised that the clarifying, and therefore the keeping power of the beer, deponds.

The hops used for this purpose may be mixed with yearlings and a small proportion of old hops, but these latter must be carefully selected, for in very old hops the tannin is apt to degenerate into the gallic form, and then it is useless as an agent to precipitate the albuminous flocks from the wort.

Refrigerators have now becomo so generally used, that the slow process of cooling is quite the exception in brewories, but the great benefit resulting from the quiet separation of the flocks as it rested on the cooler should not be lost sight of ; but it is very much foared that the great advantages derivable from using powerful refrigerators have led many away from the slow but prudent course, for by allowing the sediment to pass into the fermenting tun, the soundness and qualities of the beers concerned are very much impaired. But by the exercise of a little ingenuity and careful management, all the best benefits expected from using the most powerful refrigerators may bo obtained without any additional risk.

In the first place, let the hop-back be prorided with some arrangement which shall include a guard to keep tho lops, with ports to prevent a disturbance of the sediment settled under the plates, and a floating syphon provided with a flattened tin mouth-piece.

After the wort is discharged from the copper, allow it to rest in the hop-back until such time as the wholc of the flocks and hops shall have settled to the bottom, open the ports, and push the mouth of the syphon below the surface; it will then draw off all the wort clean, bright, and freed from every particle of flocks at any speed desired.

Now this is a simple and not expensive arrangement.

About an hour will be found long enough for a 90 or 100 barrel brewing to stand. It should then be passed through the refrigerator within two hours after, the machine of course being of such actual power as to be capable of cooling a whole brewing, no matter what the size, in that time.

It will then be found that the brewing has passed on to the refrigerator before the wort in the hop-back has fallen even to 150 degrees, and this precludes all probability of the wort attaining acetancy from exposure on account of cooling.

Another very excellent mode of fermentation, very general in the Northern Counties and Scotland, is that known as the stone-square system, and it has certainly a claim to the most serious consideration of the practical brewer on account of its many merits. It is very cleanly and simple in process, thorough in its work, most easily and perfectly under control, will prepare the beer for consumption in less time than any other process, and is worked at heats far removed from all dangerous tendencies, and these are all qualities which must greatly commend the system in a commercial point.

It is thus conducted:-

The stone square is a cistern made of hard blue stone or slate nearly six feet deop. This is for the reception of the wort to be fermented: it should hold from 20 to 25 barrels. Upon it is placed another stone cistern of sufficient dimensions to contain the whole of the yeast head that may be made. This is called the yeast-back. It is usually capable of holding about half the contents of the wort square; it has a man-hole in the centre, surrounded on the top by a circular stone ring of a section of about six inches square. Some twelve inches in front of this man-hole is another hole, about five or six inches diameter, fitted with a valve arrangement, to the underside of which is screwed a large tin pipe called the organ-pipe, conducting the wort to within about two inches from the bottom. There is also in the corner another hole, fitted with a plug at the top, and having a pipe attached to conduct the yeast (after the fermentation is finished) to the yeast waggon in the cellar below; the yeast-back usually projects some eighteen inches around the wort cistern, and is from thirty to thirty-six inches deep.

The wort cistern is contained within another stone cistern as deep as itself, within 3 or 4 inches from the top. This is called the shell; it allows of a free space all around the outside of the wort square of about 5 or 6 inches. This is to allow of the application of a suitable bath of warm or cold water, as may be required, to control the temperature of the fermenting wort; to assist in this operation a notch is cut at the back of the shell, at the top of the side slab: from this the water js allowed to overflow as required.

All the slabs composing these cisterns should be sawn plain and parallel on both sides. The bottom slab, of course, serves for both the wort cistern and shell : it has two taps let into it to drain each respectively, and in front, about $1 \frac{1}{2}$ or 2 inches up from the bottom, a racking pipe with tap attached is let in horizontally through the 
shell and wort-back slabs. To this a hose pipe is screwed, and the wort is thus drawn from the cistorn into the casks to be sent out to the consumer, bright, clean, and quite free from the sediment settled at the bottom of the square.

There are two ways of working the stone-square system : in the one, or what we may call the quiet process, as the wort is run into the square the yeast is added, and the whole remains undisturbed till the fermentation is finished (this is known by the falling of the yeast), when it is allowed to run off through the yeast-pipe in the corner, the wort remaining in the square till perfectly quiet, when it is also bright and ready for racking into the casks to be sent out to the consumer.

In the other process, after the wort and yeast are mixed in the square, it is allowed to rest and work of itself till there is an ovidence of the head just beginning to fall. This will usually occur about thirty-six hours after the square has been filled, sooner with light, later with heavy ones. Hand-pumps are then placed in the man-hole, and the wort is pumped on to the back above, well mixed with the new head, and let down into the bottom of the square again by the means of the valve and organ-pipe. This is repeated every $1 \frac{1}{2}$ or 2 hours, and the attenuation watched by the aid of the brewer's saccharometer. When it is desired to stop the progress of the fermentation, it is merely allowed to rest 6 hours; when the yeast is let off the back and the wort in the square allowed to rest till it is bright and ready for racking. The time occupied by the pumping varies with the character and gravity of the beer in the square from 12 to 30 hours; and the time for rest afterwards from 30 to 48 or 90 hours, according as it is required to be ready for the consumer, sooner or otherwise.

As to the amount of yeast to be apportioned to the setting on, the brewer must be principally guided by his experience, for in addition to the gravity of the wort and tho amount of attenuation required, many other points have to be considered, as the particular kind of ale, the state of briskness in which it is most acceptable to the consumer, the character of the malt, and the extent to which exhaustion has been carried, the amount of hops and the length of time it has boiled, the age and constitution of the yeast, the season and state of the atmosphero-all these considerations make it nearly impossible to construct a table of quantities, or even give a general rule as a guide that may be relied on by the brewer.

From circumstances peculiar to every brewer's trade, it will be seen at once how much difficulty there is in framing a table for the quantities of the yeast to be added to the wort at the setting on. In order to render this article as complete and practical as possible, it may be stated for wort mashed in the latter of the processes described, and fermented by the second of the processes of the stone-square systems, the extent of extract being about $83 \mathrm{lbs}$. per quarter when cooled down and got into the squares (this, of course, being less than the real amount taken from the malt), that for a gravity of about 24 lbs. per barrel, 2 l lis. of yeast per barrel has been found to be the quantity answering the requirements best, tho ale generally becoming bright in the squaro within six days, racking bright and in first-class condition for sending out within a weok afterwards.

As the gravity per barrel decruases, the proportion of yeast to be added must decrease in greater ratio, owing to the constitution of the wort being of a more albuminous nature, and for porters more so still, and vice vers $\hat{\imath}$, as the gravity is increased so must the quantity of yeast added be increased, but in a greater ratio, on account of the more saccharific quality of the wort.

The malt used in experimental trials was made from good Yorkshire barley, and weighed about 39 3 lbs. per bushel. It was dried a nice high amber, was three months old, and the time of the year was March, April, May.

For reasons very similar to those applied to yeast, it is equally difficult to make a rule as to the stopping-point in the process of fermentation, and the amount of attenuation necessary, other than this, the longer a beer has to remain on tap the further should attenuation be carried.

In porter it should be to nearly $\frac{2}{3}$ of the original gravity.

In common and mild ales at least $\frac{2}{3}$ of the original gravity.

In strong ales nearly $\frac{3}{4}$ of the original gravity.

In bitter ales quite $\frac{3}{4}$ or nearly $\frac{4}{5}$ of the original gravity.

It will be well here to consider some of the influences which act so adversely upon the reputation of the brewer from the quality of the materials he may have to use. Evory brewer should make malt and malting his anxious study, quite as much as he should any other portion of his operations, for on it mainly depends the quality of his productions, and he should not forget that it costs much less in time, care, and money, to make bad malt than good, and that it is oasy to mako bad malt yield overmeasure or overweight, just as it is desired by the local customs of the market: there- 
fore it is necessary for the brewer to be very careful in his selection and examination of all malt that comes to his hand, for he is very soon made to feel that any evil result from using it is not the fault of the maltster, but of his mismanagomont in brewing; therefore he must noglect nothing that will enable him to secure to his use a suitable and trustworthy article.

In an early paragraph of this article, under the head of Matrerars, is the description of good malt, and wo will here state the characteristic appearances of barley that has been improperly malted.

First, there is a deficiency in the growth of the acrospire; the cause of this is generally an insufficient steep in tho cistern; tho inducements to it are two-it will require less turning on the malting floor, and pay somewhat less duty. The results are a plumpnoss and weight in the malt, but with it a perceptible deficiency in the swectness and saccharine constituent in the product, though an apparently greator gravity of extract is obtainable from the excessive proportion of the albuminous constituent presont. It may be observed in brewing it, there is a large amount of sediment and flocks, and in the fermenting-tun abundance of yeast; the beer from it will bo fretful and soon turn off.

In the crushing of such malt in the rolls, it will be perceived there is an abundance of hard rice-like ends, and the more of these the more harm and loss to the brewer, for he is either deprived of the use of them if not crushed woll up, or disappointed by the fretfulness of his ale if he crushes them so as to gain a greater apparent gravity from his mash-tun. Another common fault is the tendency of the maltster to crowd his floors. This increases the evil begun with the inadequate steop, for the heat generated forces up the acrospire beyond what the real state of the grain would warrant, causing it to appear in an advanced state when really not so.

The maltster, taking advantage of this forward appearance, puts in on the kiln at seven or eight days old, which is utterly at variance with the interests of the brower, to whom the development of the saccharine principle is the true value of malting.

The treatment described above involving heavy sprinkling on the floor encourages acidity and mould, and it is not possible to eradicate this tendency when once fairly set in.

Perhaps it may be practically impossible to produce malt without a trace of acetic acid, but the trace should be all that should be there, and where mould has occurred, the consequences are much worse, for much of the sugar of the malt has become lactic, and of irreparable lactic tendency; a sound wort can never be made from such malt.

Malt of this description will have a close-fitting skin that good malt should not have, and many of the mouldy grains will have a dark spot where the mould has been attached before it was rubbed off by the sereening. Acidity is not always to be perceived by the taste, but mould is much easier of detection and should be most rigidly rejected.

We will next consider the results arising from injudicious drying. As in the former instance, the desire to tax to its utmost the working ability of the kiln is the incentive, and the kiln is therefore overloaded, sometimes to the depth of 15,16 , or 18 inches ; but it is not always the depth that is at fault-it is often the irregularity of density of different parts of the floor, an improper pack generally, unevenness in the distribution of the heat, want of draught power to penetrate the raw floor, or too much heat attained at first and insufficient at last. All these things have very baneful effects on the malt, varying according to the circumstances, and doing injury to the extent and degree of the irregularity-but the most common and serious among them are, overloading and insufficient fire at the finish. By the first of these, grain that has been in all other respects properly treated, and would have been turned off the kiln a first-rate article, will be disappointing in the mash-tun, and almost useless; the moisture that should pass quickly away is retained in the upper portion of the malt when it is permanently injured according to the extont of over-loading.

It is at all times difficult to detect this kind of malt, but it may be observed that there is a sort of biscuity crispness in breaking it with the teeth, quite unlike the soft friableness of good malt. Moreover, many of the grains will appear hard and glossy, as, from being subjected to an excess of heat at first, the outside has become hard before the moisture has been efficiently expelled from the interior.

In conclusion, let it be remembered that no art of the brewer can make a first-class quality of extract from inferior or unsound malt. Groat woights of extract may be obtained from the worst guite as easily as from the best of malts, but it is certain the less got the better, for with increase of extract out of indifforent samples of malt, there will be increase of trouble, anxiety, and loss, whilst by due care in the solection and use of good sound malt and healthy well-cured hops, no brewer need fail of procuring a sound and satisfactory ale. 
Beer in its perfect condition is an excellent and healthful beverage, combining, in some measure, the virtues of water, of wine, and of food, as it quenches thirst, stimulatos, cheers, and strengthons. The vinous portion of it is the alcohol, procoeding from the fermentation of the malt-sugar. Its amount, in common strong ale or beer, is about 4 per cent., or four measures of spirits, specific gravity 0.825 , in 100 measures of the liquor. The best brown stout porter contains 6 per cent., the strongest ale even 8 per cent., but common beer only one. The nutritive part of the beer is the undecompesed gum-sugar, and the starch-gum not changed into sugar. Its quantity is very variable, according to the original stareh of the wort, the length of the fermentation, and the age of the beer.

The main feature of good beer is fine colour and transparency; the production of which is an object of great interest to the brewer. Attempts to clarify it in the cask seldom fail to do it harm. The only thing that can be used with advantage for fining foul or muddy beer, is isinglass. For porter, as commonly brewed, it is frequently had recourse to. A pound of good isinglass will make about 12 gallons of finings. It is cut into slender shreds, and put into a tub with as much vinegar or hard beer as will cover it, in order that it may swell and dissolve. In proportion as the solution proceeds, more beer must be poured upon it, but it need not be so acidulous as the first, because, when once well softened by the vinegar, it readily dissolves. The mixture should be frequently agitated with a bundle of rods, till it acquires the uniform consistence of thin treacle, when it must be equalised still more by passing through a tammy-cloth, or a sieve. It may now be made up with beer to the proper measure of dilution. The quantity generally used is from a pint to a quart per barrel, more or less, according to the foulness of the beer. But before putting it into the butt, it should be diffused through a considerable volume of the beer with a whisk, till a frothy head be raised upon it. It is in this state to be poured into the cask, and briskly stirred about; after which the cask must be bunged down for at least 24 hours, when the liquor should be limpid. Sometimes the beer will not be improved by this treatment; but this should be ascertained beforehand, by drawing off some of the beer into a cylindric jar or phial, and adding to it a little of the finings. After shaking and setting down the glass, we shall observe whether the feculencies begin to collect in flocky parcels, which slowly subside; or whether the isinglass falls to the bottom without making any impression upon the beer. This is always the case when the fermentation is incomplete, or a secondary decomposition has begun. Mr. Jackson has accounted for this clarifying effect of isinglass in the following way.

The isinglass, he thinks, is first of all rather diffused mechanically, than chemically dissolved, in the sour beer or vinegar, so that when the finings are put into the foul beer, the gelatinous fibres, being set free in the liquor, attract and unite with the floating feculencies, which before this union were of the same specific gravity with the beer, and therefore could not subside alone; but having now acquired additional weight by the coating of fish-glue, precipitate as a flocculent magma. This is Mr. Jackson's explanation; to which we might add, that if thero be the slightest disengage* ment of carbonic acid gas, it will keep up an obscure locomotion in the particles, which will prevent the said light impurities, either alone or when coated with isinglass, from subsiding. The beer is then properly enough called stubborn by the coopers. The true theory probably of the action of isinglass is, that the tannin of the hops combines with the fluid gelatine, and forms a floceulent mass, which envelops the muddy particles of the beer, and carries them to the bottom as it falls, and forms a sediment. When, after the finings are poured in, no proper precipitate ensues, it may bo made to appear by the addition of a little decoction of hop.

Mr. Richardson, the author of the well-known brewer's saccharometer, gives the following as the densities of different kinds of beer:-

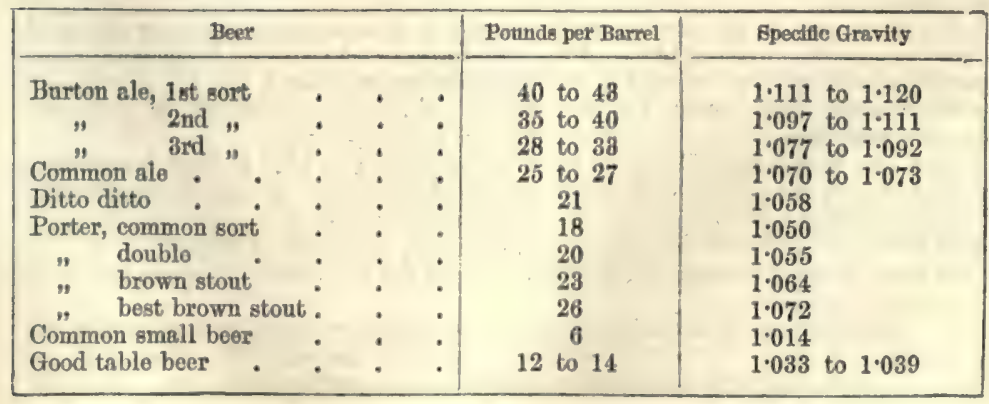


It may be remarked that Mr. Richardson somewhat underrates the gravity of porter, which is now soldom under $20 \mathrm{lbs}$. per barrel. Tho criterion for transferring from the gyle-tun to the cleansing butts is the nctenuation caused by the production of alcohol in the beer: when that has fallen to $10 \mathrm{lbs}$. or $11 \mathrm{lbs}$, which it usually does in 48 hours, the cleansing process is commenced. The heat is at this time generally $75^{\circ}$, if it was pitched at $65^{\circ}$; for the heat and the attenuation go hand in hand.

About forty years ago, it was customary for the London brewers of porter to keep immense stocks of their beer for eighteen months or two years, with the view of improving its quality. 'The beer was pumped from the eleansing butts into store-vats holding from twenty to twenty-five ' gyles' or browings of several hundred barrels each. The storovats had commonly a capacity of 5,000 or 6,000 barrels; and a fow were double, and one was treble, this size. The porter, during its long repose in these vats, became fine, and by obscure fermentation its saccharine mucilage was noarly all converted into vinous liquor, and partly dissipated in carbonic acid. Its hop-bitter was also in a great degree decomposed. Good hard beer was tho boast of the day. This was sometimes softened by the publican, by the addition of some mild new-brewed beer. Of late years, the taste of the metropolis has undergone such a complete revolution in this respect, that nothing but tho mildest porter will now go down. Hence, six woeks is a long period for beer to be kept in London; and much of it is drunk when only a fortnight old. Ale is for the same reason come greatly into vogue; and the two greatest porter houses, Messrs. Barclay, Perkins, and Co., and Truman, Hanbury, and Co., have become extensive and successful brewers of mild ale, to please the changed palate of their customers.

We shall add a fow observations upon the brewing of Scotch ale. This beverage is characterized by its pale amber colour and its mild balsamic flavour. The bittorness of the hop is so mellowed with the malt as not to predominate. The ale of Preston Pans is, in fact, the best substitute for wine which barley has hitherto produced. The low temperature at which the Scotch brewer pitches his fermenting tun restricts his labours to the colder months of the year. He does nothing during four of the summer months. He is extremely nice in selecting his malt and hops; the former being made from the best English barley, and the latter being the growth of Farnham or East Kent. The yeast is carefully looked after, and measured into the fermenting tun in the proportion of one gallon to 240 gallons of wort.

Only one mash is made by the Scotch ale brewer, and that pretty strong; but the malt is exhausted by eight or ten successive sprinklings of liquor (hot water) over the goods (malt), which are termed, in the vernacular tongue, sparges. These waterings percolate through the malt on the mash-tun bottom, and extract as much of the saccharine matter as may be sufficient for the brewing. By this simple method much higher specific gravities may be obtained than would be practicable by a second mash. With malt, the infusion or saccharine fermentation of the diastase is finished with the first mash; and nothing remains but to wash away from the goods the matter which that precess has rendered soluble. It will be found on trial that 20 barrels of wort drawn from a certain quantity of malt, by two successive mashings, will not be so rich in fermentable matter as 20 barrels extracted by ten successive sparges of two barrels each. The grains always remain soaked with wort like that just drawn off, and the total residual quantity is three-fourths of a barrel for every quarter of malt. The gravity of this residual wort will on the first plan be equal to that of the second mash ; but, on the second plan, it will be equal only to that of the tenth sparge, and will be more attenuated in a very high geomerrical ratio. The only serious objection to the sparging system is the loss of time by the successive drainages. A mash-tun with a steam-jacket promises to suit the sparging system well, as it would keep up an uniform temperature in the goods, without requiring them to be sparged with very hot liquor.

The first part of the Scotch process seems of doubtful economy; for the mash liquor is heated so high as $180^{\circ}$. After mashing for about half an hour, or till overy particle of the malt is thoroughly drenched, the tun is covered, and the mixture left to infuse about three hours; it is then drained off into the underback, or preforably into the wort-copper.

After this wort is run off, a quantity of liquor (water), at $180^{\circ}$ of hent, is sprinkled uniformly over the surface of the malt; being first dashed on a perforated circular board, suspended horizontally over the mash-tun, wherefrom it descends like a shower upon the whole of the goods. The percolating wort is allowed to flow off by three or moro small stopcocks round the circumference of the mash-tun, to insure the equal diffusion of the liquor.

The first sparge being run off in the course of twenty minutes, another similar one. is affused; and thus in succession till the wholo of the drainage, when mixed with the first mash-wort, constitutes the density adapted to the quality of the ale. Thus, the 
strong worts are prepared, and the malt is exhausted either for table beer, or for a return, as pointed out above. The last sparges are made $5^{\circ}$ or $6^{\circ}$ cooler than the first.

The quantity of hops seldom excoeds four pounds to the quarter of malt. The manner of boiling the worts is the same as that above described; but the conduct of the formentation is peculiar. The heat is pitched at $50^{\circ}$, and the fermentation continues from a fortnight to three weeks. Were three brewings made in the week, seven or eight working tuns would thus be in constant action; and, as they are usually in one room, and some of them at an elevation of temperature of $15^{\circ}$, the apartment must be propitious to fermentation, however low its heat may be at the commencement. No more yeast is used than is indispensable : if a little more be needed, it is made effective by rousing up the tuns twice a day from the bottom.

When the progress of the attenuation becomes so slack as not to exceed half a pound in the day, it is prudent to cleanse, otherwise the top barm might re-enter the body of the beer, and it would become yeast-bitten. When the ale is cleansed, the head, which has not been disturbed for some days, is allowed to float on the surface till the whole of the then pure ale is drawn off into the casks. This top is regarded as a sufficient preservative against the contact of the atmosphere. The Scotch do not skim their tuns, as the London ale browers commonly do. The Seoteh ale, when so cleansed, does not require to be set upon close stillions. It throws off little or no yeast, because the fermentation was nearly finished in the tun. The strength of the best Scotch ale ranges between 32 and 44 pounds to the barrel; or it has a specific gravity of from 1.088 to 1.122 , according to the price at which it is sold. In a good fermentation, seldom more than a fourth of the original gravity of the wort remains at the period of the cleansing. Between one-third and one-fourth is the usual degree of attenuation. Scotch ale soon becomes fine, and is seldom racked for the home market. The following Table will show the progress of fermentation in a brewing of good Scotch ale :-

20 barrels of mash-worts of $42 \frac{1}{2}$ pounds gravity $=860 \cdot 6$

20 returns $6 \frac{1}{10}, \quad, \quad=122$

$1 2 \longdiv { 9 8 2 \cdot \mathrm { C } }$

pounds woight of extract per quarter of malt $=81$

Fermentation :-

March 24, pitched the tun at $51^{\circ}$ : yeast 4 gallons.

\begin{tabular}{|c|c|c|c|c|}
\hline & 25. & $\begin{array}{c}\text { Temp } \\
52\end{array}$ & rrees. & $\begin{array}{l}\text { Gravity. } \\
41 \text { pounds. }\end{array}$ \\
\hline 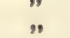 & 28. & 56 & $\Rightarrow$ & 39 " \\
\hline$"$ & 30 . & 60 & $"$ & 34 \\
\hline April & 1. & 62 & 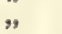 & 32 \\
\hline$\eta$ & 4. & 65 & , & 29 added $1 \mathrm{lb}$. of yeast. \\
\hline , & 5. & 66 & , & 25 pounds. \\
\hline 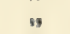 & 6. & 67 & $"$ & 23 \\
\hline$"$ & 7. & 67 & $"$ & 20 \\
\hline " & 8. & 66 & $"$ & 18 \\
\hline , & 9. & 66 & " & 15 \\
\hline y & 10. & 64 & $n$ & 14.5 eleansed. ${ }^{1}$ \\
\hline
\end{tabular}

Dr. Uro was employed to make experiments on the density of worts, and tho fermontative changes which they undergo, for the information of a Committeo of the Houso of Commons, which sat in July and August, 1830 : the following is a short abstract of that part of his evidence which bears upon the present subject:-

- My first object was to clear up the difficulties which, to common apprehension, hung over the matter, from the difference in the scales of the saccharometers in use among the brewers and distillers of England and Scotland. I found that one quarter of good malt would yield to the porter brewer a barrel imporial measure of wort, at the concentrated specific gravity of $1 \cdot 234$. Now, if the decimal part of this number be multiplied by 360 , being the number of pounds weight of water in the barrel, the product will denote the excess, in pounds, of the weight of a barrel of such concentrated wort over that of a barrel of water, and that product is, in the present case, 84.24 pounds.

-Mr. Martineau, jun., of the house of Messrs. Whitbread and Company, and a 
gentloman connected with another great London browory, had the kindnoss to inform me that their arerage product from a quarter of malt was a barrel of 84 lbs. gravity. It is obvious, therefore, that by taking the moan operation of two such great establishments, I must have arrived rery noarly at the truth.

- It ought to be remarked that such a high density of wort as 1.234 is not the result of any direct experiment in the brewory, for infusion of malt is never drawn off so strong; that density is deduced by computation from the quantity and quality of several successive infusions ; thus, supposing a first infusion of the quarter of malt to yiold a barrol of specific gravity $1 \cdot 112$, a second to yield a barrel at $1 \cdot 091$, and a third a barrel at 1.031 , we shall have three barrels at the mean of these threo numbers, or one barrel at their sum, equal to $1 \cdot 234$.

"I may here observe that the arithmetical mean or sum is not the true mean or sum of the two specific gravities; but this difference is either not known or disregarded by the brewers. At low densities this difference is inconsiderable, but at high densities it would lead to serious errors. At specifie gravity $1 \cdot 231$, wort or syrup contains one-half of its weight of solid pure saccharum, and at 1.1045 it contains one-fourth of its weight; but the brewer's rule, when here applied, gives for the mean specific gravity $1 \cdot 1155=\frac{1 \cdot 231+1 \cdot 000}{2}$. The contents in solid saccharine matter at that density are, however, $27 \frac{1}{4}$ per cont., showing the rule to be $2 \frac{1}{4} 1 \mathrm{bs}$. wrong in excess on 100 lbs., or 9 lbs, per barrel.

"The specific gravity of the solid dry extract of malt-wort is 1.264 ; it was taken in oil of turpentine, and the result reduced to distilled water as unity. Its specific volume is 0.7911 , that is, $10 \mathrm{lbs}$, of it will occupy the volume of $7.911 \mathrm{lbs}$. of water. The mean specific gravity, by computation of a solution of that extract in its own weight cf water, is $1 \cdot 1166$; but, by experiment, the specific gravity of that solution is $1 \cdot 216$, showing considerable condensation of volume in the act of combination with water.

'The following Table shows the relation between the specific gravitios of solutiuns of malt-extract and the percentago of solid extract they contain :-

\begin{tabular}{|c|c|c|c|c|c|}
\hline \multicolumn{2}{|c|}{ Extract of Malt } & Water & Malt-Extract in 100 & Sugar in 100 & Specific Gravity \\
\hline 600 & + & 600 & $50 \cdot 00$ & $47 \cdot 00$ & $1 \cdot 2160$ \\
\hline 600 & + & 900 & $40^{\circ} 0$ & $37 \cdot 00$ & $1 \cdot 1670$ \\
\hline 600 & + & 1,200 & $33 \cdot 3$ & $31 \cdot 50$ & $1 \cdot 1350$ \\
\hline 600 & + & 1,500 & $28 \cdot 57$ & $26 \cdot 75$ & $1 \cdot 1130$ \\
\hline 600 & + & 1,800 & $25 \cdot 00$ & $24 \cdot 00$ & $1 \cdot 1000$ \\
\hline
\end{tabular}

" The extract of malt was evaporated to dryness, at a temperature of about $250^{\circ} \mathrm{F}$., without the slightest injury to its quality or any empyreumatic smell. Bate's tables have been constructed on solutions of sugar, and not with solutions of extract of malt, as they agree sufficiently well with the former, but differ materially from the latter. Allen's tables give the account of a cortain form of solid saccharine matter extracted from malt, and dried at $175^{\circ} \mathrm{F}$., in correspondence to the specific gravity of the solution; but I have found it impossible to make a solid extract from infusions of malt, exeept at much higher temperatures than $175^{\circ} \mathrm{F}$. Indeed, tho numbers on Allen's saccharometer-seale clearly show that his extract was by no means dry : thus, at $1 \cdot 100$ of gravity he assigns 29.669 per cent. of solid saccharine matter; whereas thore is at that density of solid extract only 25 per cent. Again, at 1.135, Allen gives 40 parts per cent. of solid extract, whereas there are only $33 \frac{1}{3}$ present.'

The Table (p. 319) shows the origin and effect of fermentation in the reduction of geavity, in a number of practical experiments.

The second column here does not represent the solid extract, but the pasty extract obtained as the basis of Mr. Allen's saccharometer, and therefore each of its numbers is somewhat too high. The last column, also, must be in some measure erroneous, on account of thi quantity of alcohol dissipated during the process of fermentation. It must be likewise incorrect, because the density due to the saccharine matter will be partly counteracted by the effect of the alcohol present in the fermented liquor. In fact, the attenuation does not correspond to the strength of the wort; being greatest in the third browing and smallest in the first. The quantity of yeast for the ale brewings given in the Table was, upon an average, one gallon for 108 gallons; but it varied with its quality, and with the state of the woather, which, when warm, permits much less to be used with propriety.

The good quality of the malt, and the right management of the mashing, may be tested by the quantity of saccharine matter contained in the snccessively drawn worts. 


\begin{tabular}{|c|c|c|c|c|}
\hline $\begin{array}{l}\text { Original Gravity } \\
\text { of the Worta }\end{array}$ & $\begin{array}{l}\text { lbs, per Barrel } \\
\text { of Saccharine } \\
\text { Matter }\end{array}$ & $\begin{array}{c}\text { Speciflo Gravity } \\
\text { of the Ale }\end{array}$ & $\begin{array}{l}\text { 1bs. per Barrel } \\
\text { of Saccharine } \\
\text { Matter }\end{array}$ & $\begin{array}{l}\text { Attenuation, or } \\
\text { Saccharum decom- } \\
\text { posed. }\end{array}$ \\
\hline $1 \cdot 0950$ & $88 \cdot 75$ & 1.0500 & $40 \cdot 25$ & 0.478 \\
\hline $1 \cdot 0918$ & $85 \cdot 62$ & 1.0420 & $38 \cdot 42$ & $0.552^{\circ}$ \\
\hline 1.0829 & $78 \cdot 125$ & $1 \cdot 0205$ & $16 \cdot 87$ & $0.787^{\prime \prime}$ \\
\hline $1 \cdot 0862$ & $80 \cdot 625$ & $1 \cdot 0236$ & $20 \cdot 00$ & 0.757 \\
\hline I'0780 & $73 \cdot 75$ & $1 \cdot 0280$ & $24 \cdot 25$ & $0 \cdot 698$ \\
\hline 1.0700 & $65 \cdot 00$ & $\mathrm{I} \cdot 0285$ & $25 \cdot 00$ & 0.615 \\
\hline $1 \cdot 1002$ & $93 \cdot 75$ & $1 \cdot 0400$ & $36 \cdot 25$ & 0.613 \\
\hline $1 \cdot 1025$ & $95 \cdot 93$ & 1.0420 & $38 \cdot 42$ & 0.600 \\
\hline$I \cdot 0978$ & $91 \cdot 56$ & 1.0307 & $27 \cdot 00$ & 0.705 \\
\hline 1.0956 & $89 \cdot 37$ & $1 \cdot 0358$ & $32 \cdot 19$ & 0.640 \\
\hline $1 \cdot 1130$ & $105 \cdot 82$ & $1 \cdot 0352$ & $31 \cdot 87$ & 0.661 \\
\hline $1 \cdot 1092$ & $102 \cdot 187$ & $1 \cdot 0302$ & $26 \cdot 75$ & 0.605 \\
\hline $1 \cdot 1171$ & $110^{\circ} 00$ & $1 \cdot 0400$ & $36 \cdot 25$ & 0.669 \\
\hline $1 \cdot 1030$ & $96 \cdot 40$ & $1 \cdot 0271$ & $23 \cdot 42$ & 0.757 \\
\hline $1 \cdot 0660$ & $61 \cdot 25$ & 1.0214 & $17 \cdot 80$ & 0.709 \\
\hline
\end{tabular}

With this view, an aliquot portion of each of them should be evaporated by a safetybath heat to a nearly concrete consistence, and then mixed with twice its volume of strong spirit of wine. The truly saceharine substance will be dissolved, while the starch and other matters will be separated; after which the proportions of each may be determined by filtration and evaporation. Or an equally correct, and much more expeditious, method of arriving at the same result would be, after agitating the viscid extract with the alcohol in a tall glass cylinder, to allow the insoluble fecula to subside, and then to determine the specific gravity of the supernatant liquid by a hydrometer. The additional density which the alcohol has acquired will indicate the quantity of malt-sugar which it has received. The following Table, constructed by Dr. Ure, at the request of Henry Warburton, Esq. M.P., chairman of the Molasses Committeo of the House of Commons in 1830, will show the brewer the principle of this important inquiry. It exhibits the quantity in grains' weight of sugar requisite to raise the specific gravity of a gallon of spirit of different densities to the gravity of water $=1.000$.

$\begin{array}{cc}\begin{array}{c}\text { Specific Gravity of } \\ \text { Spirit, } \\ 0.995\end{array} & \begin{array}{c}\text { Grains' Weight of Sugar in the } \\ \text { Gallon Imperisl }\end{array} \\ 0.990 & \cdot 980 \\ 0.985 & 1 \cdot 890 \\ 0.980 & 2 \cdot 800 \\ 0.975 & 3 \cdot 710 \\ 0.970 & 4 \cdot 690 \\ 0.965 & 5 \cdot 600 \\ 0.960 & 6 \cdot 650 \\ 0.955 & 7 \cdot 070 \\ 0.950 & 8 \cdot 400 \\ & 9 \cdot 310\end{array}$

The immediate purpose of this Table was to show the effect of saccharine matter in disguising the presence or amount of alcohol in the weak feints of the distiller. But a similar Table might easily be constructed, in which, taking a uniform quantity of alcohol of 0.825 , for example, the quantity of sugar in any wort-extract would be shown by the increase of specific gravity which the alcohol received from agitation with a cortain weight of the wort, inspissated to a nearly solid consistence by a safetypan made on the principle of Dr. Ure's patent sugar-pan. (See Sugar). Thus, the normal quantities being 1,000 grains' measure of alcohol, and 100 grains by weight of inspissated mash-extract, the hydrometer would at once indicate, by help of the Table, first, the quantity per cent. of truly saccharine matter, and next, by subtraction, that of farinaceous matter present in it.

The advance of the arts is gradually assuming a charactor which will no longer permit any manufacturer to neglect the assistance of science ; and those who first take advantage of the power of knowledge will assuredly leave their fellow-labourers behind. From being an uncertain and hazardous operation, brewing must ere long become a fixed and definite principlo based upon facts well understood, and capable of perpetual repetition and reproduction at will. To sum up briefly the general details of ale-brewing, we may state, that, for most kinds of ale, the attenuation in the first instance should be finished in from six to twenty-one days, according to the strength 
of the wort; that this attonuation should approach to two-thirds of the whole woight; and that after tunning and cleansing, the ale itself should woigh about one-fourth of the original gravity of the wort. Thus, if the fermenting tun be set with wort of 27 lus., then the attenuation should bring it down to 9 or $10 \mathrm{lbs}$, and the subsequent operations produce an ale weighing from 6 to $7 \mathrm{lbs}$. When these conditions are fulfilled, without much extra troublo or attention, the alo is protty certain to turn out well, though, in some localitios, ale is never attenuated to more than one-half its original gravity: this kind of ale is, however, very apt to become sour in hot weather and ropy in cold.

Some additional remarks on the brewing of portor, which differs from that of ale both in the nature of the materials used and in the mode of finishing the fermentation, are required. Porter owes its peculiar colour and flavour to burnt saccharine or starchy matter; and this was formerly obtained by burning sugar until it exhaled the odour called by French writors caramel. At present, however, nothing but highly-torrefied malt is used; and of this thero are several kinds, as brown malt, imperial malt, and black malt; all of which are used by some brewers, whilst others employ only the brown and black, and a few the black alone, for giving colour and flavour. The fermentative quality or saccharine is, however, the same as that of ale, and is derived from palo or amber malt. As a general rule, the ratio of the colouring and flavouring malts are to the saccharine as about 1 to 5 or 1 to 4 ; but where black malt only is used, the proportion does not exceed 1 to 10.

The employment of these burnt malts permits a singular act of injustice on the part of the Excise, as regards the drawback on exportation. By the Excise regulations, it is assumed that a quarter of malt will produce four barrels of ale browed from wort of the sp. gr. 1.054, or $19.4 \mathrm{lbs}$. per barrel ; but, although this is hopeless even with pale malt, yet with an admixture of brown and black malt the assumption becomes absurd in the extreme. Admitting that, by good management, on the average, four barrels of wort, woighing $20 \mathrm{lbs}$, can be obtained from one quarter of fine pale malt, yet, in the operations of cooling, fermenting, tunning, skimming, and cleansing, a loss of fully 10 per cent. occurs under the most vigilant superintendence; and, taking the great bulk of our metropolitan breweries, it would be nearer the truth to estimate this loss at 12 per cent. In plain words, 100 gallons of wort will not, by any management, produce moro than about 88 gallons of saleable beer, though no allowance is made for this by the Excise; and the brewer who has paid duty upon 100 gallons gets a drawback upon but 88. This, however, is the most favourable view of the case; and we solicit attention to the force with which the argument returns in the instance of porter.

If a quarter of pale malt be assumed at $84 \mathrm{lbs}$. of saccharine strength, then such an edmixture of brown and black malt as is usually employed by brewers of porter will not give more than about $24 \mathrm{lbs}$; and as this constitutes at least one-fifth of the whole bulk used in porter-brewing, we see that a quarter of such mixed malt can never give more than 70 lbs. ; that is to say, 80 parts of pale malt, mixed with 20 of brown and black, instead of giving at the rate of $84 \mathrm{lbs}$., as pale malt alone does, would give but 70 lbs., or produce a difference between the actual return and that taken for granted by the Excise authorities, of no less than $16^{\cdot} \cdot \mathbf{p e r}$ cent.; to which, if we add the loss previously mentioned as arising from fermentation, yeast, \&ce, and which we have called 12 per cent., a total difference ensues of 28.6 per cent. between the duty paid by the brewer and the drawback allowed by Act of Parliament. But the grievance does not stop here; for the only return allowed by Act of Parliament is based upon the malt duty, and nothing whatever is said of the duty on hops. This, however, is at the rate of $19 s .7 d$. per cwt.; and since hops yield only about 35 per cent. of their weight of soluble matter, it would require 168 lbs. of hops to produce a barrel of fluid or wort weighing $19.4 \mathrm{lbs}$, or having the requisite parliamentary specific gravity of 1.054. Upon this barrel, when exported, the drawback is 5s.; but, as may easily be seen on calculation, the duty paid by the brewer has been 298 . $3 d$. In fact, upon every $168 \mathrm{lbs}$. of hops consumed by the export brewer, he suffers a dead loss of 248 . $3 d$. independently of the wasto incidental to his various processes. These things may seem startling; but the whole Board and Staff of the Excise are unable to provo that they are in the least over-estimatod. At the same time, the intelligent reader will gather that the profits of brewing are not by any means so large as a cursory glance at the subject might appear to warrant. No doubt the brewing business is at times very remunerative; but a continued high price of the raw materials sometimes proves ruinous to the large brewer, as it must not be forgotten that the capital required is large, and invested in very perishable materials, such as casks and other wooden utensils, the wear and tear upon which is a very large item; nor, again, as we have shown, must a speculator begin by assuming, with the Exeise authorities, that a quarter of malt will produce four barrels of beer, for be will be much nearer the truth if he estimates his 
saleable produce at throe barrels. As, however, it forms no part of our present task to onter into the financial statistics of brewing, we return to the object more immodiately in view, merely throwing out, en passant, the above hints for the bonefit of those whom they may concern.

If the analyses of malt and malt-wort are requisite to enable the brewer to perform. his operations with safety and success, the analysis of beer is not less indispensable to qualify him for the harassing labour of competition with his neighbours, and for the protection of his interest against Excise confiscation. Although beer may have been brewed of the requisite gravity for justifying a drawback on exportation, yet this is very far indeed from ensuring a return of the malt-duty, even to the limited extent awarded by law. The question is, How are the Excise officials to know the real weight of the wort from which the beer was brewed? This may be ascertained by the following method, which should take the place of tho present indefinite system :Having agitated a portion of the ale or beer so as to dissipate its carbonic acid gas, measure out exactly 3,600 grains' measure of it, and pour these into a retort; then distil with great care into a receiver surrounded by ice-cold water about one-third of the whole fluid, or rather more than this if the ale or beer is known to be highly alcoholic. Next weigh the distilled fluid, and then ascertain its specific gravity, from whence, by any, of the proper tables of alcohol, the total quantity of absolute alcohol in the distilled fluid may be known. This alcohol is to be converted by calculation into its equivalent of sugar, at the rate of 171 parts of sugar for every 92 of alcohol found; after which the sugar must be brought into pounds per barrel by the rule before given, which is $52 \frac{1}{2} \mathrm{lbs}$. of sugar for every $20 \mathrm{lbs}$. of gravity. The amount of vinegar is next to be determined by any of the known forms of acidimetry. (See ACDIMETRY.) This vinegar, or acetic acid, must, like the alcohol, be also converted into its representative of sugar, by assigning 171 of sugar to every 102 of anhydrous acotic acid present in the beer, this sugar being, as before, converted into pounds per barrel. To the beer remaining in the retort, sufficient distilled water is then to be added, that the entire bulk of fluid may once more be equal to 3,600 grains' measure; and the temperature of the mixture having fallen to $60^{\circ} \mathrm{F}$., its specific gravity must be determined in the usual way, and this reduced to pounds per barrel, by multiplying the excess above 1,000 by 360 , and dividing the product by 1,000 . The whole of these weights, added together, gives the original weight of the wort. Thus, for example, we will suppose that 3,600 grains of a particular beer have given 1,300 grains of a dilute alcohol, of specific gravity 0.9731 , and consequently containing about $17 \frac{1}{2}$ per cent. by weight of alcohol; again, that the same quantity of beer, when tested by ammonia, has indicated 30 grains of acetic acid; and lastly, that the spent wash, when filled up with distilled water to its primary bulk, has, at $60^{\circ}$, a specifie gravity of 1.016 ; then the total alcohol would be in 360 grains, or the representative of a barrel, $222_{4}^{3}$ grains, and the acetic acid in the same quantity, 3 grains : hence we have the following results :-

\begin{tabular}{|c|c|c|}
\hline Alcohol, $22 \frac{3}{3}$ grains, equnl to & Grs, of sugar. & Brewers' lbs \\
\hline Acetic acid, 3 grains & $\therefore \quad 5.4$ & $1 \cdot 9$ \\
\hline Spent wash, of specifie gravity 1.016 & ... & $5 \cdot 76$ \\
\hline
\end{tabular}

It might be thought that the proper kind of sugar to select in this instance as the representative of alcohol and acetic acid should be grape-sugar, whose atomic weight is 180 ; but it has been shown by Dr. Ure that the kind of sugar actually employed in the construction of our saccharometer tables must have been cane-sugar, the atom of which is 171 ; and hence the reason why it must be employed in this calculation.

Ane, Pale or Bitter; brewed chiefly for the Indian market and for other tropical countries.-It is a light beverage, with much aroma, and, in consequence of the regulations regarding the malt-duty, is commonly brewed from a wort of specific gravity 1.055 or upwards; for no drawback is allowed by the Excise on the exportation of beer brewed from worts of a lower gravity than 1.054. This impolitic interference with the operations of trade compels the manufacturer of bitter beer to employ wort of a much greater density than he otherwise would do; for beer made from wort of the specific gravity 1.012 is not only better calculated to resist secondary fermentation and the other effects of a hot climate, but is also moro pleasant and salubrious to the consumer. Under present circumstances the law expects the brewer of bitter beer to obtain four barrels of marketable beer from every quartor of malt he uses, which is just barely possible when the best malt of a good barloy year is employed. Witl

Vot. I. 
every quarter of such malt $16 \mathrm{lbs}$. of the best hops are used; so that, if we assume tho cost of malt at 60 s. por quartor, and the best hops at 28 . per $1 \mathrm{~b}$., we shall have, for the prime cost of each barrel of bitter beor-in malt, 15s. ; in hops, 88. ; together, 238. ; from which, on exportation, wo must deduct tho drawback of 58 . per barrol allowed by the Excise, which brings the prime cost down to 18s. per barrel, exclusive of the expense of manuficture, wear and toar of apparatus, capital investod in barrels, cooperage, \&ce, which constituto altogether a very formidablo outlay. As, however, this alo is sold as high as from 508 . to 65 s. por barrol, there can be no doubt that the bittcr-ale trado has long beon, and still continues, an exceedingly profitablo speculation, though somowhat hazardous, from the liability of the articlo to undergo decomposition ere it finds a market.

The East Indian pale ale, or bitter beer, is now brewed in large quantities for the home market at Burton-on-Tront, London, Glasgow, and Loeds, but differ's slightly from that exportod, as being less bitter and more spirituous. It is brewed sololy from the best and palost malts and the finest and most delicate hop, and much of its success depends on the care taken in selecting the best materials for its composition. It also requiros tho utmost care and attention at every stage of its progress to preserve the colour, taste, and other properties of this alo in thoir fulness and purity.

For further description of the brewhouse and its appliances, with the various modes of operations, see the article Brewiva.

The English ale-drinkers were a fow years since startled by a public report, apparently well authenticated, that the French chemists were largely engaged in preparing immense quantities of that most deadly poison stryclnine for the purpose of drugging the pale bitter ale, in such great vogue at present in Great Britain and its colonies. The following are a fow amongst many reasons which might be quoted, to show the absurdity of this report:-1. Strychnine is an exceedingly costly article. 2. It has a most unpleasant metallic bitter taste. 3. It is a notorious poison, and its use in any brewory being known would ruin the reputation of tho brewer. 4. It cannot be introduced into ordinary beer brewed with hops, because it is entirely precipitated by infusions of that wholesome and fragrant herb. In fact, the quercitannic acid of hops is incompatible with strychnia and all its kindred alkaloids. Hence hopped beer becomes in this respect a sanitary beverage, refusing to take up a particle of strychnia and other noxious drugs of like character. Were the nux vomica powder, from which strychnia is extracted, even stealthily thrown into the mash-tun, its dangerous principle would be all infallibly thrown down with the grounds in the subsequent boiling with the hops.

The varieties of beex depend either upon the difference of their materials, or upon a different management of the brewing processes. With regard to the materials, boers differ in the proportion of their malt, hops, and water, and in the different kinds of malt or othor grain. To the class of 'iable' or 'small beers,' all those sorts may be referred whose specific gravity does not exceed 1.025 , which contain about 5 per cent. of malt extract, or nearly $18 \mathrm{lbs}$. per barrel. Beers of middling strength may be reckoned those between the density of 1.025 and 1.040 , which contain, at the average, 7 per cent., or 25 lbs. per barrel. The latter may be made with 400 quarters of malt to 1,500 barrels of beer: stronger beers have a specific gravity of from $1 \cdot 050$ to $1 \cdot 080$, and take from 450 to 750 quarters of malt to the same quantity of beer. The strongest beer found in the market is somo of the English and Scotch ales, for which from 18 to 27 quarters of malt are taken for 1,500 gallons of beer: good porter requires from 16 to 18 quarters for that quantity. Beers are sometimes made with the addition of other farinacoous matter to the malt; but when the latter constitutes the main portion of the grain, the malting of the other kinds of corn becomes unnecessary, for the diastase of the barley-malt changes the starch into sugar during the mashing operation. Even with entiroly raw grain, beer is made in some parts of the Cantinent, the browers trusting tho conversion of the starch into sugar to the action of the gluten alone, at a low mashing temperature, on the principle of Saussure's and Kirchoff's researches.

The colour of the beer depends upon the colour of the malt and the duration of the boil in tho copper. The pale alo is made, as wo havo stated, from steam-or sun-dried malt and the young shoots of the hop; the deep yellow ale from a mixture of paleyellow and brown malt; and the dark-brown beer from well-kilned and partly car. bonised malt, mixed with a good deal of the pale to give body. The longer and more strongly heated the malt has been in the kiln, the less weight of extract, cateris paribus, does it afford. In making the fine mild ales, high temperatures ought to be avoided, and the yeast ought to be skimmed off, or allowed to flow very readily from its top, by means of the cleansing-butt systom, so that little ferment being left in it to decompose the rest of the sugar, the sweetness may remain unımpaired. With regard to porter, in certain breweries each of the three kinds of malt employed for it is 
separately mashed, after which the firstand the half of the second wort is boiled along with tho whole of the hops, and thence cooled, and set to ferment in tho gyle-tun. Tho third-drawn wort, with the remaining half of the second, is then boiled with the same hops, saved by the drainer, and, after cooling, added to the former in the Byle-tun, when the two must be well roused together.

It is obvious from tho preceding developmont of principles, that all amylaceous and saccharine materials, such as potatoes, beans, turnips, as well as cane- and starchsyrup, molasses, \&c. may bo used in brewing beer. When, however, a superior quality of brown beer is desired, malted barley is indispensable, and oven with these substitutes a mixture of it is most advantagoous. The washed roots of the common carrot, of the red and yellow beet, or of tho potato, must be first boiled in water, and then mashed into a pulp. This pulp must be mixed with water in the copper along with wheaten-or oat-meal and the proper quantity of hops, thon boiled during eight or nine hours. This wort is to be cooled in the usual way, and fermented with the addition of yeast. A much better process is that now practisod on a considerable scale at Strasbourg, in making the ale for which that city is celebrated. The mashed potatoes are mixed with from a twentieth to a tonth of their weight of finely-ground barley-malt and some water. The mixture is exposed in a water-bath to a heat of $160^{\circ} \mathrm{F}$. for four hours, whereby it passes into a saccharine state, and may then be boiled with hops, cooled, and properly fermented into good beer.

Maize, or Indian corn, has also been employed to make beer; but its malting is somowhat difficult, on account of the rapidity and vigour with which its radicles and plumula sprout forth. The proper mode of causing it to germinate is to cover it a fow inches deep with common soil, in a garden or field, and to leave it there till the bed is covered with green shoots of the plant. The corn must be then lifted, washed, and exposed to the kiln.

Tho Board of Excise, or Inland Revenue, having, a fow years ago, been permitted by the Legislature to grant leave to use sugar in the place of barley-malt in breweries, an extensire sugar-merchant in London, hoping, under this boon, to acquire a new and wealthy class of customers, employod Dr. Uro to ascertain by experiment the relative values of malt and sugar for the manufacture of beer. Ten samples of Muscovado sugar, of several qualities, were exnmined, and were found to vary very slightly in the proportions of alcohol they could furnish by fermentation in a brewer's tun, the avorage being 12 gallons of proof spirit for $112 \mathrm{lbs}$. of the sugar; whereas an equal quantity of proof spirit could be obtained from $4 \frac{8}{10}$ bushels of malt. One pound of malt yields $\frac{3}{4} \mathrm{lb}$. of extract capable of making as much beer as that weight of sugar. On comparing the actual price of sugar and malt, wo shall soe how ruinous a business it would bo to uso sugar instead of malt in a brewery, and hence the delusiveness of the Excise generosity towards the beer trade.

Although the object of the brewer is not the formation of a mere saccharine wort, a.s we have already shown (and malt contains other substances necessary to the formation of a sound beer), the amount of proof spirit producible from various sub. stances will be some index to their relative value and it has been found that, with proper management, a quarter of good malt, weigling $42 \mathrm{lbs}$. per bushel, or $336 \mathrm{lbs}$. per quarter, will yield 18 gallons of proof spirit; a quarter of barley, weighing $55 \mathrm{lbs}$. per bushel, or 440 lbs. per quarter, will yield from 18 to 20 gallons. An equal quantity of spirit, say 18 gallons at proof, can be obtained from $175 \mathrm{lbs}$. of best West India sugar; from $234 \mathrm{lbs}$. of inferior Jamaica raw sugar; from $275 \mathrm{lbs}$. of West India molasses; or from 295 lbs. of refined or sugar-house molasses. Bauerstock gavo the average of sugar 200 lbs., and of honey 226 lbs., as equivalent to a quarter of malt.

Ropiness is a morbid state of beer, which is best remedied, according to Mr. Black, by putting the beer into a vat with a false bottom, and adding, per barrel, 4 or 5 pounds of hops, taken away after the first boilings of the worts; and to them may be added about half a pound per barrel of mustard-seed. Rouse the boer as the hops are gradually introduced, and, in some months, the ropiness will be perfectly cured. The beer should be drawn off from below the false bottom.

For theoretical views, see Fermentation; and for wort-cooling apparatus, seo

\section{REFRIGERATOR.}

Bexr, Bavanzax. (Baierisches Bier, Ger.) The Germans from time immo. morial havo been habitually beer-drinkers, and havo exercised much of their technical and scientific skill in the production of beer of many different kinds, some of which nre little known to our nation, while one at least, called Bavarian, possesses excellent qualities, ontitling it to the attention of all brewers and consumors of this bevorage. The peculiarities in the manufacturo of Bavarian beor some time ago attracted the attontion of the most eminent chemists in Germany, especially of the late Professor 
Liebig, and much new light was thereby thrown upon this curious portion of vegetable chemistry.

The following is a list of the principal beors brewed in Germany :-

1. Brown beer of Merseburg; of pure barley malt.

2. " " , and beet-root sugar.

3. " barley malt, potatoes, and beet-root syrup.

4. " refined beet-root syrup alone.

5. Covent or thin beer.

6. Berlin white beer, or the champagne of the north.

7. Broyhan, a famous Hanoverian beer.

8. Double beer of Gruinthal.

9. Bavarian beer: 1. Summer beer; 2. Winter beer.

10. " Bock beer.

11. Wheat Lager-beer (slowly fermented).

12. White bitter beer of Erlangen.

Considerable interest among men of science, in farour of the Bavarian beer process, has been excited ever since the appearance of 'Liebig's Organic Chemistry.' In the introduction to this admirable work, he says, "The beers of England and France, and for the most part those of Germany, become gradually sour by contact of air. This defect does not belong to the beers of Bavaria, which may be preserved at pleasure in half-full casks, as well as full ones, without alteration in the air. This precious quality must be ascribed to a peculiar process employed for fermenting tho wort, called in German Untergährung, or fermentation from below ; which has solved one of the finest theoretical problems.

'Wort is proportionally richer in soluble gluten than in sugar. When it is set to ferment by the ordinary process, it evolves a large quantity of yeast, in the state of a thick froth, with bubbles of carbonic acid gas attached to it, whereby it is floated to the surface of the liquid. The phenomenon is easily explained. In the body of the wort, alongside of particles of sugar decomposing, there are particles of gluten being oxidised at the same time, and enveloping, as it were, the former particles, whence the carbonic acid of the sugar and the insoluble ferment from the gluten being simultaneously produced, should mutually adhere. When the metamorphosis of the sugar is completed, there remains still a large quantity of gluten dissolved in the fermented liquor, which gluten, in virtue of its tendency to appropriate oxygen, and to get decomposed, induces also the transformation of the alcohol into acetic acid (vinegar). But were all the matters susceptible of oxidisement as well as this vinegar ferment remored, the beer would thereby lose its faculty of becoming sour. These conditions are duly fulfilled in the process followed in Bavaria.

'In that country the malt-wort is set to ferment in open backs, with an extensive surface, and placed in cool cellars, having an atmospheric temperaturo not exceeding $8^{\circ}$ or $10^{\circ} \mathrm{C}$. $\left(46 \frac{1}{2}^{\circ}\right.$ or $50^{\circ} \mathrm{F}$.) The operation lasts from three to four weeks; the carbonic acid is disengaged; not in largo bubbles that burst on the surface of the liquid, but in very small vesicles, like those of a mineral water, or of a liquor saturated with carbonic acid, when the pressure is removed. The surface of the fermenting wort is always in contact with the oxygen of the atmosphere, as it is hardly covered with froth, and as all the yeast is deposited at the bottom of the back, under the form of a very viscid sediment, called in German Unterhefe.

'In order to form an exact idea of the difference between the processes of fermentation, it must be borne in mind that the metamorphosis of gluten, and of azotised bodies in general, is accomplished succossively in two principal periods, and that it is in the first that the gluten is transformed in the interior of the liquid into an insoluble ferment, and that it separates alongside of the carbonic acid proceeding from the sugar. This separation is the consequence of an absorption of oxygen. It is, however, hardly possible to decide if this oxygen comes from the sugar, from the water, or even from an intestine change of the gluten itself; or, in other words, whether the oxygen combines directly with the gluten, to give it a higher degree of oxidation, or whether it lays hold of its hydrogen to form water.

- This oxidation of the gluten, from whichever cause, and the transformation of the sugar into carbonic acid and alcohol, are two actions so correlated, that by an exclusion of the one, the other is immediately stopped,'

The superficial ferment (Oberhefe in German) which covers the surface of the fermenting works, is gluten oxidisod in a state of putrefaction; and the ferment of deposit is the gluten oxidised in a state of eremacausis, or slow combustion.

The surface yeast, or barm, excites in liquids containing sugar and gluten the same alteration which itself is undergoing, whereby the sugar and the gluten suffer a rapid and tumultuous metamorphosis. We may form an exact idea of the different 
states of these two kinds of yeast by comparing the superficial to vegetable matters putrefying at the bottom of a marsh, and the bottom yeast to the rotting of wood in a state of eremacausis. The peculiar condition of the elements of the sediment ferment causes them to act upon the elements of the sugar in an extremely slow manner, and excites the change into alcohol and carbonic acid, without affecting the dissolved gluten.

If to wort at a temperature of from $46 \frac{1}{3}^{\circ}$ to $50^{\circ} \mathrm{F}$. the top yeast be added, a quiet slow fermentation is produced, but one accompanied with a rising-up of the mass, while yeast collects both at the surface and bottom of the backs. If this deposit be removed to make use of it in other operations, it acquires by little and little the cha. racters of the Unterhefe, and becomes incapable of exciting the phonomena of the first fermenting period, causing only, at $59^{\circ} \mathrm{F}$., those of the second, namely, sedimentary fermentation. It must be carefully observed that the right Unterhefe is not the precipitate which falls to the bottom of backs in the ordinary fermentation of beer, but is a matter entirely different. Peculiar pains must be taken to get it genuine, and in a proper condition at the commencement. Hence the brewers of Hesse and Prussia, who wished to make Bavarian beer, found it more to their interest to send for the article to Würzburg, or Bamberg, in Bavaria, than to prepare it themselves. When once the due primary formentation has been established and well regulated in a brewery, abundance of the true Unterhefe may be obtained for all future operations.

In a wort made to ferment at a low temperature with deposit only, the presence of the Unterluefe is the first condition essential to the metamorphosis of the saccharum, but it is not competent to bring about the oxidation of the gluten dissolved in the wort, and its transformation into an insoluble state. This change must.be accomplished at the cost of the atmospherical oxygen.

In the tendency of soluble gluten to absorb oxygen, and in the free access of the air, all the conditions necessary for its eremacausis are to be found. It is known that the presence of oxygen and soluble gluten are also the conditions of acetification (vinegar-making), but they are not the only ones; for this process requires a temperature of a certain elevation for the alcohol to experience this slow combustion. Hence by excluding that temperature, the combustion (oxidation) of alcohol is obstructed, while the gluten alone combines with the oxygen of the air. This property does not belong to alcohol at a low temperature, so that during the oxidation in this case of the gluten, the alcohol exists alongside of it, in the same condition as the gluten alongside of sulphurous acid in the muted wines. In wines not impregnated with the fumes of burning sulphur, the oxygen which would have combined at the same time with the gluten and the alcohol does not seize either of them in wines which have been subjected to mutism, but it unites itself to the sulphurous acid to convert it into the sulphuric. The action called sedimentary fermentation is therefore merely a simultaneous metamorphosis of putrefaction and slow combustion; the sugar and the Unterhefe putrefy, and the soluble gluten gets oxidised, not at the expense of the oxygen of the water and the sugar, but of the oxygen of the air, and the gluten then falls in the insoluble state. The process of Appert for the preservation of provisions is founded upon the samo principle as the Bavarian process of fermentation, in which all the putrescible matters are separated by the intervention of the air at a temperature too low for the alcohol to become oxidised. By romoving them in this way, the tendency of the beer to grow sour, or to suffer a further change, is prevented. Appert's method consists in placing, in presence of vegetables or meat which we wish to preserve, the oxygen at a high temperature, so as to produce slow combustion, but without putrefaction or even fermentation. By removing the residuary oxygen after the combustion is finished, all the causes of an ulterior change are removed. In the sedimentary fermentation of beer, we remove the matter which experiences the combustion; whereas, on the contrary, in the mothod of Appert, we remove that which produces it.

The temperature at which fermentation is carried on has a very marked influence upon the quantity of alcohol produced. It is known that the juice of beets set to forment between $86^{\circ}$ and $95^{\circ} \mathrm{F}$., does not yield alcohol, and its sugar is replaced by a less oxygenated substance, mannite, and lactic acid, resulting from the mucilage. In proportion as the temperature is lowered the mannite fermentation diminishes. As to azotised juices, however, it is hardly possible to define the conditions under which the transformation of the sugar will take place, without boing accompanied with another decomposition which modifies its products. The fermentation of beer by deposit demonstrates that by the simultaneous action of the oxygen of the air and a low temperaturo, the metamorphosis of sugar is effected in a completo manner; for the vessels in which the operation is carriod on are so disposed that the oxygen of the air may act upon a surface great enough to transform all the gluten into insoluble yoasty and thus to present to the sugar a matter constantly undergoing do- 
composition. The oxidisement of the dissolved gluten goes on, but that of the alcohol requires a higher tomperature, whence it cannot suffer acetification, or conversion into vinegar.

In several States of Germany the favourable influence of a rational process of fermentation upon the quality of the beers has been fully recognised. In the Grand Duchy of Hesse considerablo premiums were proposed for the brewing of beer according to the process pursued in Bavaria, which were decroed to thoso brewers who were able to prove that their product (neither strong nor highly hopped) had kept six months in the casks without becoming at all sour. When the first trials were being made, several thousand barrels wero boing spoilod, till eventually experience led to the discovery of the true practical conditions which theory had foresoon and prescribed.

Neither the richness in alcohol, nor in hops, nor both combined, can hinder ordinary beer from getting tart. In England, says Liebig, an immense capital is sacrificed to preserve the better sorts of ale and porter from souring, by leaving them for sevoral years in enormous tuns quito full, and very well closed, while their tops aro coverod with sand. This treatment is identical with that appliod to wines to make them deposit the wine-stone. A slight transpiration of air goes on in this case through the pores of the wood; but the quantity of azotised matter contained in the beer is so great, relatively to the proportion of oxygen admitted, that this element cannot act upon the alcohol. And yot the beer thus managed will not keep sweet more than two months in smaller casks, to which air has access. The grand secret of the Munich brewers is to conduct the fermentation of the wort at too low a temperature to permit of the acetification of the alcohol, and to cause all the azotised matters to be completely separated by the intervention of the oxygen of the air, and not by the sacrifice of the sugar. It is only in March and October that the good store beer is began to be made in Bavaria.

The following Table exhibits the results of the chemical examination of the undermentioned kinds of Beer:-

\begin{tabular}{|c|c|c|c|c|c|}
\hline \multirow{2}{*}{ Name of the Beer } & \multicolumn{4}{|c|}{ Quantity in 100 parts by weight } & \multirow{2}{*}{ Analyst } \\
\hline & Water & Malt extr. & Aloohol & Carb. acid & \\
\hline Augustine double beer-Mu- & & & & & \\
\hline nich . $\cdot$. & $88 \cdot 36$ & $8 \cdot 0$ & $3 \cdot 6$ & $0 \cdot 14$ & Kaiser. \\
\hline $\begin{array}{l}\text { Salvator beer-do. } \\
\text { Bock boer from the Royal }\end{array}$ & $87 \cdot 62$ & $8: 0$ & $4 \cdot 2$ & 0.18 & Do. \\
\hline $\begin{array}{l}\text { Brewery-do. } \\
\text { Schenk (pot) beer, from a } \mathrm{Ba} \text { - }\end{array}$ & $88 \cdot 64$ & $7 \cdot 2$ & $4: 0$ & $0 \cdot 16$ & Do. \\
\hline $\begin{array}{l}\text { varian country brewery; a } \\
\text { kind of small beer }\end{array}$ & $92 \cdot 94$ & 4.0 & $2 \cdot 9$ & 0.16 & Do. \\
\hline Bock beer, of Brunswick, of & & & & & \\
\hline $\begin{array}{l}\text { the Bavarian kind } \\
\text { Lager (store) beer, of Bruns- }\end{array}$ & $88 \cdot 50$ & $6 \cdot 50$ & $5 \cdot 0$ & $\ldots$ & Balhorn. \\
\hline wick, of the Bavarian kind. & $91 \cdot 0$ & $5 \cdot 4$ & $3 \cdot 50$ & ... & Otto. \\
\hline Brunswick sweet small boer . & $84 \cdot 70$ & $14: 0$ & $1: 30$ & $\ldots$ & Do. \\
\hline Brunswick mum . & $59 \cdot 2$ & $39 \cdot 0$ & $1 \cdot 80$ & 0.1 & Kaiser. \\
\hline
\end{tabular}

Malting in Munich.-The barley is steoped till the acrospire, embryo, or seed-germ seems to be quickened, a circumstance denoted by a swelling at that end of the grain which was attached to the foot-stalk, as also when, on pressing a pile between two fingers against the thumb-nail, a slight projoction of the embryo is perceptible. As long, however, as the seed-germ sticks too firm to the husk, it has not been stoeped enough for exposure on the under-ground malt-floor. Nor can deficient stoeping be safely made up for afterwards by sprinkling the malt-couch with a watering-ean, which is apt to render the malting irregular. The steep-water should be changed repeatedly, according to the degree of foulness and hardness of the barley: first, six hours after immersion, having previously stirred the whole mass several times : afterwards, in winter every 24 hours, but in summer every 12 hours. It loses none of its substanee in this way, whatever vulgar prejudice may think to the contrary. After letting off the last water from the stone cistern, the Bavarians leave the barley to drain in it during 4 or 6 hours. It is now taken out, and laid on the couch floor in a square heap, 8 or 10 inches high, and it is turned over, morning and evening, with dexterity, so as to throw the middle portion upon the top and bottom of the newmade couch. When the acrospire has become as long as the grain itself, the malt is 
carried to the withering (Welkboden) or drying floor, in the open air, where it is exposed (in dry weather) during from 8 to 14 days, being daily turned over three times with a winnowing shovel. It is next dried in a well-constructed cylinder or flue-heated maltkiln, at a gontle clear heat, without being browned in tho slightest degree, while it turns into a fine friable white meal. Smoked malt is entirely rejected by the best Bavarian brewers. Their malt is dried on a series of wove wire horizontal shelves, placed over each other, up through whose interstices, or perforations, streams of air, heated to only $122^{\circ} \mathrm{F}$., rise, from the surfaces of rows of hot sheet-iron pipe-flues, arranged a little way below the shelves. Into these pipes the smoke and burned air of a little furnace on the ground are admitted. The whole is enclosed in a vaulted chamber, from whose top a largo wooden pipe issues for conveying away the steam from the drying malt. Each chargo of malt may bo completely dried on this kiln in the space of from 18 to 24 hours, by a gentle uniform heat, which does not injure the diastase or discolour the faring.

The malt for store beer should be kept three months at least before using it, and be freed by rubbing and sifting from the acrospires before being sent to the mill, where it should be crushed pretty fine. The barley employed is the best distichon or common kind, styled Hordeum vilgare.

The hops are of the best and freshest growth of Bavaria, called the fine spalter, or saatser Bohemian townhops, and are twice as dear as the best ordinary hops of the rest of Germany. They are in such esteem as to be exported even into France.

In Munich the malt is moistened slightly 12 or 16 hours before crushing it, with from two to three Maas' of water for every bushel, the malt being well dried, and several months old. The mash-tun into which the malt is immediately conveyed is, in middlo-sized breweries, a round oaken tub, about $4 \frac{1}{2}$ feet deep, 10 feet in diameter at bottom and 9 at top, outside measure, containing about 6,000 Berlin quarts. Into this tun cold water is admitted late in the evening, to the amount of 25 quarts for each scheffel, or 600 quarts for the 26 scheffels of the ground malt, which are then shot in and stirred about, and worked woll about with the oars and rakes, till a uniform paste is formed without lumps. It is left thus for three or four hours; 3,000 quarts of water being put into the copper and made to boil; and 1,800 quarts are gradually run down into the mash-tun and worked about in it, producing a mean temperature of $142 \cdot 5^{\circ} \mathrm{F}$. After an hour's interval, during which the copper has been kept full, 1,800 additional quarts of water are run into the tun, with suitable mashing. The copper being now emptied of water, the mash-mixture from the tun is transferred to it, and brought quickly to the boiling point, with careful stirring to prevent its settling on the bottom. and getting burned, and it is kept at that temperature for half an hour. When the mash rises by the ebullition, it needs no more stirring. This process is called, in $\mathrm{Ba}$ varia, boiling the thick mash, dickmeisch Kochen. The mash is next returned to the tun, and well worked about in it. A few barrels of a thin mash-wort are kept ready to be put into the copper the moment it is emptied of the thick mash. After a quarter of an hour's repose the portion of liquid filtered through the sieve part of the bottom of the tun into the wort-cistern is put into the copper, thrown back boiling hot into the mash in the tun, which is once more worked thoroughly.

The copper is next eleared out, flled up with water, which is made to boil for the after, or small-beer, brewing. After two hours' settling in the open tun, the worts are drawn off clear.

Into the copper, flled up one foot high with the wort, the hops aro introduced, and the mixture is made to boil during a quarter of an hour. This is called roasting the hops. The rest of the wort is now put into the copper, and boiled along with the hops during at least an hour or an hour and a half. The mixture is then laded out through the hop-filter into the cooling cistern, where it stands three or four inches deop, and is exposed upon an extensive surface to natural or artificial currents of cold air, so as to be quickly cooled. For every 20 barrels of Lagerbier there are allowed 10 of small beer; so that 30 barrels of wort are made in all.

For the winter or pot-beer the worts are brought down to about $59^{\circ} \mathrm{F}$. in the cooler, and the beer is to be transferred to the fermenting tuns at from $54.5^{\circ}$ to $59^{\circ} \mathrm{F}$.; for the summer or Lagerbier, the worts must be brought down in the cooler to from $43^{\circ}$ to $452^{\circ}$, and put into the fermenting tuns at from $41^{\circ}$ to $43^{\circ} \mathrm{F}$.

$\mathrm{A}$ few hours beforehand, while the wort is still at the temperature of $631^{\circ} \mathrm{F}$., a quantity of $l o b b$ must be made, called Vorstellen (fore-setting) in German, by mixing the proportion of Unterhefe (yeast) intended for tho whole brewing with a barrel or a barrel and a half of the worts, in a small tub called the Gähr-tiene, stirring them well together, so that they may immediately run into fermentation. This lobb is in this stage to be added to the worts. The lobb is known to be ready whon it is covered 
with a white froth from one quarter to ono half an inch thick, during which it must be well covered up. Tho large fermenting tun must in liko manner be kept covered even in the vault. The colder the worts, the more yeast must be used. For the above quantity, at

$$
\begin{gathered}
\text { From } 57^{\circ} \text { to } 59^{\circ} \mathrm{F} \text { : } \\
" 53^{\circ} \text { to } 55^{\circ} \text { : } \\
" 48^{\circ} \text { to } 50^{\circ} \\
" 41^{\circ} \text { to } 43^{\circ} \text { : }
\end{gathered}
$$

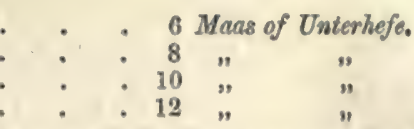

Some recommend that wort for this kind of fermentation (the Untergährung) should be set with the yeast at from $48^{\circ}$ to $57^{\circ}$; but the general practico at Munich is to set the summer or Lagerbier at from $41^{\circ}$ to $43^{\circ} \mathrm{F}$.

By following the preceding directions, the wort in the tun should, in the course of from 12 to 24 hours, exhibit a white froth round the rim, and even a slight whiteness in the middle. After another 12 to 24 hours, the froth should appear in curls; and, in a third like period, these curls should be changed into a still higher frothy brownish mass. In from 24 to 48 hours more, the barm should have fallen down in portions through the beer, so as to allow it to be seen in certain points. In this case it may be turned over into the smaller ripening tuns in the course of other five or six days. But when the worts have been set to ferment at from $41^{\circ}$ to $43^{\circ} \mathrm{F}$, they require from eight to nine days. The beer is transferred, after being freed from tho top yeast by a skimmer, by means of the stopcock near the bottom of the large tun. It is either first run into an intermediate vessel, in order that the top and bottom portions may be well mixed, or into each of the Lager casks, in a numbered series, like quantities of the top and bottom portions are introduced. In the ripening cellars the temperature cannot be too low. The best keeping beer can never be brewod unless the temperature of the worts at setting, and of course the fermenting vault, be as low as $50^{\circ} \mathrm{F}$. In Bavaria, where this manufacture is carried on under Government inspectors, a brewing period is prescribed by law, which is, for the under-fermenting Lagerbier, from Michaelmas (29th September) to St. George (23rd April). From tho latter to the former period the ordinary top-barm beer alone is to bo made. The ripening casks must not be quite full, and they are to be closed merely with a loose bung, in order to allow of the working over of the ferment. But should the fermentation appear too languid, after six or eight days, a little briskly fermenting Lagerbier may be introduced. The store Lagerbier tuns are not to be quite fillod, so as to prevent all the yeasty particles from being discharged in the ripening fermentation; but the pot Lagerbier tuns must be made quite full, as this beverage is intonded for speedy sale within a fow weeks of its being mado.

As soon as the summer-beer vaults are charged with their ripening casks and with ice-cold air, they are closed air-tight with triple doors, having small intervals between, so that one may be entered and shut again before the next is opened. These vaults are sometimes made in ranges radiating from a centre, and at others in rooms set off at right angles to a main gallery, so that in either case, when the external opening is well secured with triple air-tight doors, it may be entered at any time, in order to inspect the interior without the admission of warm air to the beer-barrels. The wooden bungs for loosely stopping them must be coated with the proper pitch, to prerent the possibility of their imparting any acetous fermont.

The Government has taken great pains to improve this national beverage, by encouraging the growth of the best qualities of hops and barley. The vaults in which the beer is fermented, ripened, and kept, are all under ground, and mostly in stony excavations called Felsenkeller, or rock-cellars. The beor is divided into two sorts, called summer and winter. The latter is light, and, being intended for immediato retail in tankards, is termed Schenkbier. The other, or the Lagerbier, very sensibly increases in vinous strength in proportion as it decreases in sweetness, by the judicious management of the Nachgährung, or fermentation in the casks. In several parts of Germany a keeping quality is communicated to beers by burning sulphur in the casks before filling them, or by the introduction of sulphite of lime; but the flavour thus imparted is disliked in Munich, Bayreuth, Regensburg, Nïrnberg, Hof, and the other chief towns of Bavaria, instead of which a preservativo virtue is sought for in an aromatic mineral, or Tyrol pitch, with which the inside of the casks are carefully coated, and in which the ripe beer is kept and exported. In December and January, after the casks are charged with the summer or store beer, the double doors of the cellars are closed, and lumps of ico are piled up against thom, to prevent all access of warm air. The cellar is not opened till next August, in order to take out the beer for consumption. In these circumstances the beer becomes transparent like champagne wine; and, since but little carbonic acid gas has been disengaged, littlo or none of the additionally generated alcohol is lost by evaporation. 
The winter or schenk (pot) beer is brewed in the months of October, November, March, and April; but the summer or store beer, in December, January, and February, or the period of the coldest weather. For the former beer, the hopped worts are cooled down only to from $51^{\circ}$ to $55^{\circ}$, but for the latter to from $41^{\circ}$ to $421^{\circ} \mathrm{F}$. The winter beer is also a little weaker than the summer beer, being intended to be sooner consumed; since four bushels ' (Berlin measure) of fine, dry, sifted malt, of large heavy Hordeum vulgare distichon, affords seven Eimers of winter beer, but not more than from five and a half to six of summer beer. ${ }^{2}$ At the second infusion of the worts small beer is obtained to the amount of 20 quarts for the above quantity of malt. For the above quantity of winter beer, $6 \mathrm{lbs}$. of middling hops are reckoned sufficient; but for the summer beer, from 7 to $8 \mathrm{lbs}$. of the finest hops. The winter beer may be sent out to the publicans in barrels five days after the fermentation has been completed in the tuns, and, though not quite clear, it will become so in the course of six days; yet they generally do not serve it out in pots for two or three weeks; but the summer beer must be perfectly bright and still before it is racked off into casks for sale.

Bock Beer of Bavaria.-This is a favourite double-strong beverage of the best lager description, which is so named from causing its consumers to prance and tumble about like a buck or a goat; - for the German word Bock has both these meanings. It is merely a beer having a specific gravity one-third greater, and is therefore made with a third greater proportion of malt, but with the same proportion of hops, and flavoured with a few coriander seeds. It has a somewhat darker colour than the general Lagerbier, cccasionally brownish, tastes less bitter on account of the predominating malt, and is somewhat aromatic. It is an eminently intoxicating beverage. It is brewed in December and January, and takes a long time to ferment and ripen; but still it contains too large a quantity of unchanged saceharine matter and dextrine for its hops, so that it tastes too luscious for habitual topers, and is drunk only from the beginning of May till the end of July, when the fashion and appetite for it are over for the year.

On the Clarifying or Clearing of Beers.-Clarifiers act either chemically,-by being soluble in the beer, and by forming an insoluble compound with a vegetable gluten, and other viscid vegetable extracts; gelatine and albumen, under one shape or other, have been most used: the former for beer, the latter, as whito of egg, for wine,-or mechanically, by being diffused in fine particles through the turbid liquor, and, in their precipitation, carrying down with them the floating vegetable matters. To this class belong sand, bone-black (in some measure, but not entirely), and other such articles. The latter means are very imperfect, and can take down only such matters as exist alroady in an insoluble state; of the former class, milk, blood, glue, calves'-feet jelly, hartshorn shavings, and isinglass, have been chiefly recommended. Calves'-feet jelly is much used in many parts of Germany, where veal forms so common a kind of butcher-meat; but in summer it is apt to acquire a putrid taint, and to impart the same to the beer. In these islands, isinglass, swollen and partly dissolved in vinegar, or sour beer, is almost the sole clarifier, called finings, employed. It is costly, when the best article is used; but an inferior kind of isinglass is imported for the brewers.

The solvent or medium through or with which it is administered is eminently injudicious, as it never fails to infect the beor with an acetous ferment. In Germany their tart wine has been used hitherto for dissolving the isinglass; and this has also the same bad property. Mr. Zimmermann professes to have discovered an unexceptionable solvent in tartaric acid, one pound of which dissolved in 24 quarts of water is capable of dissolving two pounds of ordinary isinglass; forming finings which may be afterwards diluted with pure water at pleasure. Such isinglass imported from Petersburg into Berlin costs there only $3 s$. per $1 b$. These finings are best added, as already mentioned, to the worts prior to fermentation, as soon as they are let into the setting-back, or tun, immediately after adding the yeast to it. They are best administered by mixing them in a small tub with thrice thoir volume of wort, raising the mixture into a froth with a whisk (twig-besom in German), and then stirring it into the worts. The clarification becomes manifest in the course of a fow hours, and when the fermentation is completed, the beer will be as brilliant as can be wished; the test of which with the German topers is when they can read a nowspapor while a tall glass beaker of beer is placed between the paper and the candle. One quart of finings of the above strength will be generally found adequate to the clearing of 100 gallons of well-brewed lager-beer, though it will be surer to uso double that proportion of finings. The Carrageen moss, as finings, is to be cut in fine shreds, thrown into the boiling

An. Fnglish quarter of grain is equal to 5 bushels (scheffel) and nearly one-third Prussian measure.

a 1 Eimer Prussian=15] English imperial gallons; I Munich scheffel is equnl to 4 Berlin scheffels; $1 \mathrm{Lib}$. Manich $=1 \cdot 285$ English poands aroird. ; $1 \mathrm{Lib}$. Berlin=1.031 ponnds avoird, 
thin wort, when the flocks begin to separato, and boforo adding the hops; after which the boiling is continued for an hour and a half or two hours, as neod be. The clarifying with this kind of finings takes place in tho cooler, so that a limpid wort may be drawa off into the fermenting back.

Zimmermann assumes the merit of having introduced Carrageen moss as a clarifier into the boor manufacturo. He says that 1 ounce of it is sufficiont for 25 gallons of beer; and that it operates, not only in the act of boiling with the hops, but in that of cooling, as also in the squares and backs before the fermontation has begun. Whenever this change, however, takes place, the commixture throws up the gluten and moss to the surface of the liquid in a black scum, which is to be skimmed off, so that the proper yeast may not be soiled with it. It accasions the separation of much of the vegetablo slime, or mueilage, callod by the German brewers Pech (pitch).

Berlin White or Pale Beer (Weiss-bier).-This is the truly national beverage of Prussia Proper. It is brewed from 1 part of barley malt and 5 parts of wheat malt, mingled, moistened, and coarsely crushed between rollors. This mixture is worked up first with water at $95^{\circ} \mathrm{F}$., in tho proportion of 30 quarts per scheffel of the malt, to which pasty mixture $\mathbf{7 0}$ quarts of boiling water are forthwith added, and the whole is mashod in the tun. After it has been left here a little to settle, a portion of the thin liquor is drawn off by the tap, transferred to the copper, and then for each bushel of malt there is added to it a decoction of half a pound of Altmark hops separately prepared. This hopped wort, after half an hour's boiling, is turned back with tho hops into the mash-tun, of which the temperature should now be $1622^{\circ} \mathrm{F}$.. but not more. In half an hour the wort is to be drawn off from the grains, and pumped into the cooler. The grains are afterwards mashed with from 40 to 50 quarts of boiling water per scheffel of malt, and this infusion is drawn off and added to the former worts. The whole mixture is set at $66^{\circ} \mathrm{F}$. with a due proportion of top yeast or ordinary barm, and very moderately fermented.

Potato Beer.-The potatoes being well washod are to be rubbed down to a pulp by such a grating cylinder machine as is represented in fig. 102, where $a$ is the

102

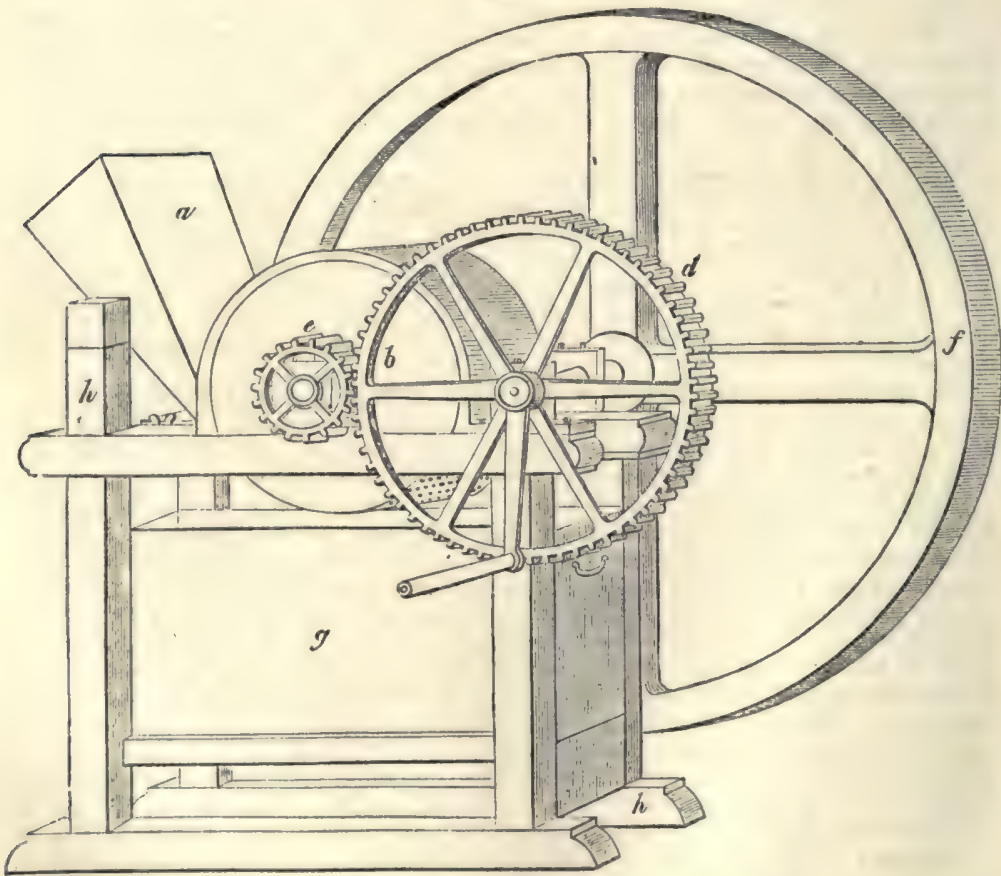

hopper for receiving the roots (whether potato or beot, as in the French sugar factories); $b$ is the crushing and grinding drum; $c$, the handle for turning the spurwheel $d$, which drives the pinion $e_{3}$ and the fly-wheel $f ; g, h$, is the frame. The 
dotted lines above o are the colander through which the pulp passes. For every scheffel of potatoes 80 quarts of water are to be put with them into the copper, and mado to boil.

Crushed malt, to the amount of 12 scheffels, is to bo well worked about in the mashtun with 360 quarts, or 90 gallons (English), of cold water, to a thick pap, and then 840 additional quarts, or about 6 barrels (English), of cold water are to be successively introduced, with constant stirring, and left to stand an hour at rest.

The potatoes having been meanwhile boiled to a fine starch paste, the whole maltmash, thin and thick, is to be speedily laded into the copper, and the mixture in it is to be well stirred for an hour, taking eare to keep tho temperature at from $144^{\circ}$ to $156^{\circ} \mathrm{F}$. all the time, in order that the diastase of the malt may convert the starch present in the two substances into sugar and dextrine. This transformation is made manifest by the white pasty liquid becoming transparent and thin. Whenever this happens the fire is to be raised, to make the mash boil, and to keop it at this heat for 10 minutes. The fire is then withdrawn, the contents of the copper are to be transferred into the mash, worked well there, and left to settle for half an hour; during which time the copper is to be washed out, and quickly charged once more with boiling water.

The clear wort is to be drawn off from the tun, as usual, and boiled as soon as possible with the due proportion of hops; and the boiling water may bo added in any desired quantity to the drained mash, for the second mashing. Wort made in this way is said to have no flavour whatever of the potato, and to clarify more easily than malt-wort, from its containing a smallor proportion of gluten relatively to that of saccharum.

A scheffel of good mealy potatoes affords from 26 to $2 \tau^{\frac{1}{2}} \mathrm{lbs}$. of thick well-boiled syrup, of the density of $36^{\circ}$ Baumé (see Areometer); and $26 \mathrm{lbs}$. of such syrup are equivalont to a scheffel of malt in saccharine strength. Zimmermann thinks beer so brewed from potatoes quite equal, at least, if not superior, to pure malt beer, both in appearance and quality.

Fig. 103 is the stopcock used in Bavaria for bottling beer.

The following analyses of German beers are by Loo:-

\begin{tabular}{|c|c|c|c|c|c|c|}
\hline \multirow{6}{*}{$\begin{array}{l}\text { Alcohol } \\
\text { Albumen } \\
\text { Extract } \\
\text { Water }\end{array}$} & \multirow{5}{*}{$\begin{array}{l}: \\
:\end{array}$} & Lichtenhain & $\begin{array}{l}\text { Upper } \\
\text { Weimar }\end{array}$ & Ilmenau & Jena & Double Jens \\
\hline & & $3 \cdot 168$ & 2.567 & $3 \cdot 096$ & $3 \cdot 018$ & $2 \cdot 080$ \\
\hline & & 0.048 & 0.020 & 0.079 & 0.045 & 0.028 \\
\hline & & $4 \cdot 485$ & $7 \cdot 316$ & $7 \cdot 072$ & $6 \cdot 144$ & $7 \cdot 153$ \\
\hline & & $92 \cdot 299$ & $90 \cdot 097$ & $89 \cdot 758$ & $90 \cdot 793$ & $90 \cdot 739$ \\
\hline & & $100 \cdot 000$ & 100.000 & 100.000 & 100.000 & $100 \cdot 000$ \\
\hline
\end{tabular}

Under the term 'extract,' in these analyses, is meant a mixture of starch, sugar, dextrine, lactic acid, various ealts, certain extractive and aromatic parts of the hop, gluten, and fatty matter. 
Hoffistedt's process for the detection of spurious bitters in beer is applicable to the detection of picrotoxin, absinthin, menyanthin, quassin, and colocynthin. The bitter principles likely to occur in beor may bo divided into two classes :-

\section{Preciptrable my acetate ox Iakad.}

Lupulin.-It is not precipitable by tannin. It is soluble in alcohol and ether, but not in water.

\section{II. - Not precrpitated aY Acetate of Limad.}

With tannin, after removal of lead by means of sulphuretted hydrogen.

a. Not precipitated by tannin:

Picrotoxin.-Soluble in water, alcohol, and other.

Absinthir.-Soluble in alcohol and ether, not in water.

b. Procipitated by tannin:

Menyanthin.-Sparingly soluble in ether and cold water; easily in hot water. Turns brown and then violet with strong sulphuric acid.

Quassin.-Sparingly soluble in ether; soluble in 222 parts of cold wator; not coloured by sulphuric acid.

Colocynthin.- Insoluble in ether; soluble in cold water. Turns first red and then brown with strong sulphuric acid.

The quantities needful for examination are six litres of Bavarian or bitter beer, or four of porter. This may seem excessivo, but it must be remembered that a vory small quantity of the above-mentioned drugs will impart a strong bitter taste to a large volume of liquid; and, again, that the hop is never entirely omitted, since its poculiar efficacy in preventing spurious or secondary fermentation appears to be possessed by no other bitter. The beer in question is to bo evaporated down, first over the naked fire, and afterwards on the water-bath. Great care must be taken that it does not dry or burn on the sides of the vessel, or bitter principles may be generated which mask the reactions to be sought for. The thick mass is well treated with alcohol in a tall beaker. At the bottom will be found a thick gummy mass, and a somewhat turbid stratum of liquor over it: this is set aside to become clear; it is then poured off and the alcohol distilled off; the residue is concentrated to a syrup and dissolved in alcohol. The solution is mixed with ton times its bulk of ether, which precipitates sugar; when clear, the liquid is decanted from the sediment and distilled. The residue is dissolved in warm water and a portion of it tested with tannin. A pure, well-hopped beer never gives a clear aqueous solution; a beer containing little of the hop may; if the solution does not elear up add a trace of alcohol. Besides lupulin, absinthin is insoluble in water. Filter off the resinous mattor which may have been doposited, then precipitate the warm filtrate with acetate of load, which must not be too acid; lupulin is then thrown down. Excess of lead must be carefully avoided, or menyanthin may fall down also: allow it to settle, filter, and wash the precipitate with hot water.

Filtrate.-Treat with sulphuretted hydrogen till all the lead is precipitated; filter and wash, first with warm water and then with alcohol; remove sulphuretted hydrogen and free acetic acid by evaporation almost to dryness. If the residuo is free from bitterness no adulteration is present; absinthin never gives a clear aqueous solution, and menyanthin never a clear cold one. A turbid solution may contain all the spurious bitters; add a little alcohol till the solution becomes clear, and then tannin.

1. The precipitate formed is dried up along with hydrated oxide of lead suspended in water, and extracted with boiling spirit. In the residue of this extract colocynthin, menyanthin, and quassin are separated by means of their behaviour with ether and water.

2. The precipitate is freed from tannin by means of acetate of lead, the precipitate filtored off, the lead removed by means of sulphuretted hydrogen and evaporated. Picrotoxin separates out in crystals; absinthin remains as a yellow mass.

Levin Ender recommends the following procedure:-

1. Procipitate with acetate of lead.

Lupulin. - It gives no mirror with ammoniacal solution of silver.

2. Not precipitated by acetato of lead, but by tannin.

A. Soluble in ether: Absinthin. Gives a mirror with the silver solution.

$B$. Sparingly soluble in ether. Menyanthin; Quassin. The former gives a mirror; the latter not.

Pierotoxin, absinthin, menyanthin, edocynthin, reduce solution of silver; lupulin and quassia do not. 
BEES-WAX

Beer and Ale-Exports.

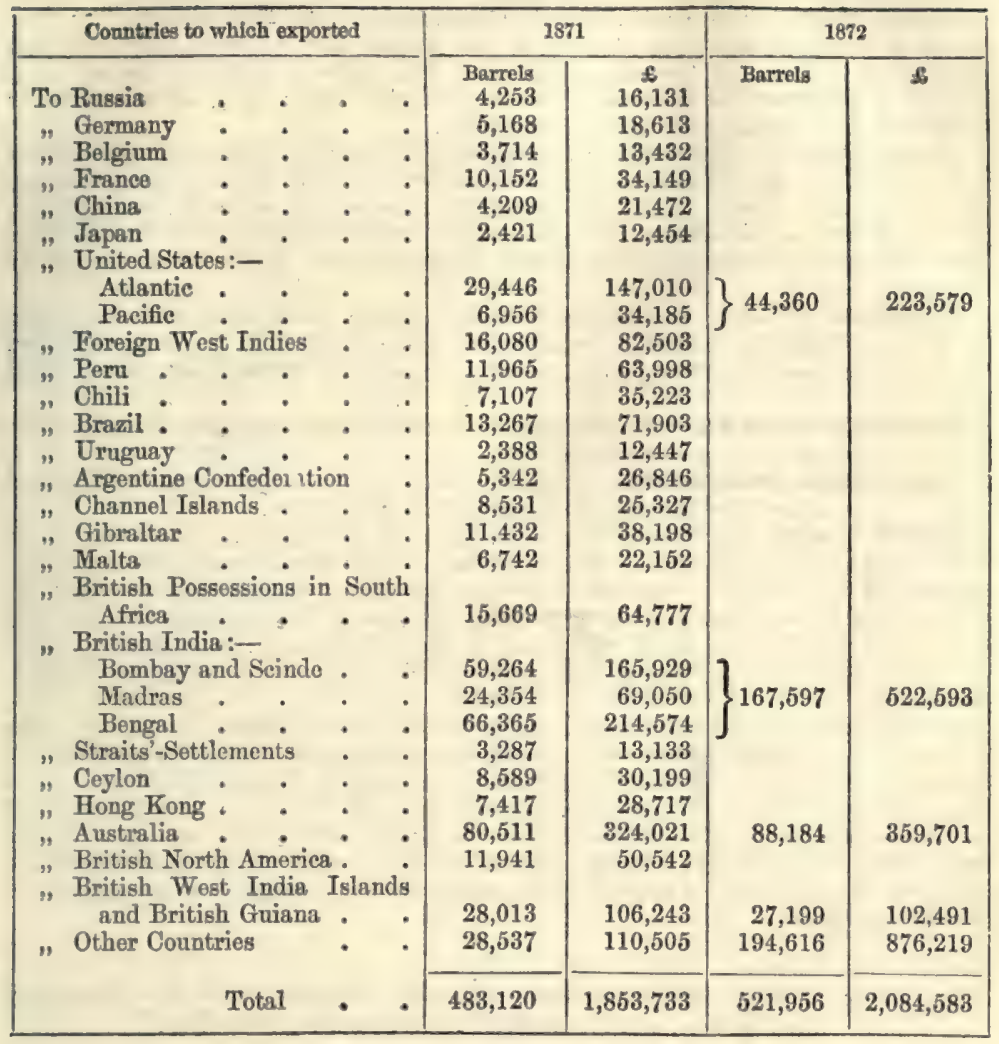

Beer and Ale.-Imports in 1872 .

\begin{tabular}{|c|c|c|c|c|}
\hline Countries whence imported & Quantities & Value & $\begin{array}{l}\text { Entered for } \\
\text { Home Con- } \\
\text { sumption }\end{array}$ & $\begin{array}{l}\text { Gross } \\
\text { Amonnt } \\
\text { received }\end{array}$ \\
\hline Mum - From all Countries & $\begin{array}{l}\text { Barrels } \\
2\end{array}$ & $\underset{f}{E}$ & $\begin{array}{l}\text { Barrels } \\
\quad 2\end{array}$ & $\frac{t}{2}$ \\
\hline $\begin{array}{r}\text { Spruce-From Germany } \\
\text { " Other Countries : }\end{array}$ & $\begin{array}{r}1,813 \\
7\end{array}$ & $\begin{array}{r}14,688 \\
\quad 30\end{array}$ & & \\
\hline Total & 1,820 & 14,718 & 1,720 & 2,062 \\
\hline $\begin{array}{r}\text { Other sorts-From Germany } \\
\text { "Belgium. } \\
\text { "Other Countries }\end{array}$ & $\begin{array}{r}326 \\
841 \\
1,277\end{array}$ & $\begin{array}{l}1,349 \\
2,858 \\
3,303\end{array}$ & & \\
\hline Total & 2,444 & 7,505 & 2,435 & 1,088 \\
\hline
\end{tabular}

BEתR-STONz. A peculiar stone, composed chiefly of carbonate of lime, which is quarried extensively at Beer, in Devonshire.

BzDs'-WAX. 'The solid matter forming the cells of the honeycomb, sccreted, according to Huber, by an organ situated in tho abdomen of the bee. Seo WAx. 
3DETzE. A name usually given to the insects of the Coleopterous order, especially to those of a dark colour. The specios of Coleoptorous insects known amount to nearly 40,000 . The cantharides, or blistering-flios, aro used medicinally. The larra of Cerambyx heros was regarded by tho Romans as a delicacy. The American Indians eat the larva of Calandra palmarum. Tho name is appliod to the annoying insects which infest the kitchens of houses in large towns; they are the blatta or cockroach, and belong to the Orthopterous order.

BEETLE's Wings. The elytra or wing cases of the more brilliantly coloured beetles (Coleoptera), are used in making head-dresses for ladies, and for decorating muslins, scarfs, and ball dresses. In Brazil especially, those wings are used for ornaments, and much art is bestowed in their production. Since the Exhibition of 1862, when Brazil exhibited a collection of the beetle's wing ornaments, they have been regularly imported.

BzFrxz, or Maul. A large mallet, with a handle about three feet in length, used for knocking the corners of framed work, and setting it in its proper position. Also a mallet used for driving piles, raised by ropes and pullies-sometimes called Boytle.

Bazriz sтonss. A name given in South Wales to septarian nodules of the clay ironstone from the coal-measures.

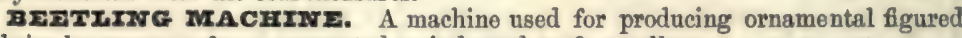
fabrics by pressure from corrugated or indented surface rollers.

В표-이. (Betterave, Fr.; rothe Rïbe, Ger.) The large fleshy root of the beet, a plant of the genus Beta. Thero are two distinct species cultivated, each containing several varieties. One called Hortensis, producing succulent leaves only; the other, the Vulgaris, distinguished by its long fleshy root. The variety of the Vulgaris, known as the red beet, is much cultivated in our gardens, and used as a vegetable. The white beet is in much repute in Belgium and France for the manufacture of sugar. Seo Sugar.

The common field beet, for cattle, which has been long known in Germany, was introduced into England at the latter end of the last contury; and its introduction is generally attributed to the late Dr. Lettsom, a physician of great repute, and one of the Society of Friends. The German name is mangold wurzel, or mangold root, but is commonly pronounced mangel wurzel, which means scarcity root; and, by a strange translation, it is called in Fronch racine d'abondance, or root of plenty, as well as racine de disette, or root of scarcity. The name feld beet is much more appropriate.Penny Cyclopedia.

The Analyses of Way and Ogston give the following Composition for two Varieties of the Beet-root, and the Analysis of Griepenkerl for another.

\begin{tabular}{|c|c|c|c|c|c|c|}
\hline \multirow{2}{*}{\multicolumn{2}{|c|}{ Substances contained }} & \multicolumn{2}{|c|}{$\begin{array}{l}\text { Yellow Globe } \\
\text { Mangold Wurzel }\end{array}$} & \multicolumn{2}{|c|}{$\begin{array}{l}\text { Long Red } \\
\text { Mangold Wurzel }\end{array}$} & \multirow{2}{*}{$\begin{array}{r}\text { Red Beet } \\
\text { Root }\end{array}$} \\
\hline & & Root & Leaves & Root & Leaves & \\
\hline Potassa & . & $25 \cdot 54$ & $8 \cdot 34$ & $21 \cdot 68$ & $27 \cdot 90$ & $51 \cdot 10$ \\
\hline Soda. & & $19 \cdot 08$ & $12 \cdot 21$ & $3 \cdot 13$ & 3.01. & \\
\hline Lime : & ? & 1.78 & $8 \cdot 72$ & $1 \cdot 90$ & $8 \cdot 17$ & $2 \cdot 45$ \\
\hline Magnesia. & . & 1.75 & $9 \cdot 84$ & $1 \cdot 79$ & $7 \cdot 03$ & $2 \cdot 94$ \\
\hline Sesquioxide of iron & . & 0.74 & $1 \cdot 46$ & 0.52 & 0.96 & 0.35 \\
\hline Sulphuric acid , & . & $3 \cdot 68$ & $6 \cdot 54$ & $3 \cdot 14$ & $4 \cdot 60$ & $3 \cdot 31$ \\
\hline Silica . & . & $2 \cdot 22$ & $2 \cdot 35$ & $1 \cdot 40$ & $2 \cdot 26$ & $0 \cdot 19$ \\
\hline Carbonic acid & . & $18 \cdot 14$ & $6 \cdot 92$ & $-15 \cdot 23$ & $6 \cdot 45$ & \\
\hline Phosphoric acid . & . & $4 \cdot 49$ & $5 \cdot 89$ & $1 \cdot 65$ & $6 \cdot 19$ & $10 \cdot 77$ \\
\hline Chloride of sodium & . & $24 \cdot 54$ & $37 \cdot 66$ & $49 \cdot 51$ & $34 \cdot 39$ & $17 \cdot 04$ \\
\hline Total amount & & $99 \cdot 96$ & $99 \cdot 95$ & 99.95 & $99 \cdot 96$ & $99 \cdot 99$ \\
\hline $\begin{array}{l}\text { Per-centage of ash } \\
\text { substance }\end{array}$ & the dry & $11 \cdot 32$ & $14 \cdot 00$ & $7 \cdot 10$ & $17 \cdot 90$ & $7 \cdot 8$ \\
\hline Per-centage of ash $i$ & 10 fresh & & & & & \\
\hline substance. & . & 1.02 & 1.40 & $0 \cdot 64$ & $1 \cdot 79$ & \\
\hline
\end{tabular}

The quantity of heet-root used in the Zollrerein States of Germany in the manufacture of sugar was as follows:- 


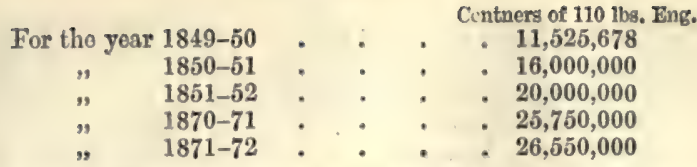

The centner varies, in different localities, from 100 to 112 avoirdupois pounds.

Tho cultivation of the sugar-beet has beon introduced into tho northern United States and Californis.

BrImadorva. (Belledame, Fr.) The Atropa Belladonna, or doadly nightshade, a poisonous plant belonging to the Solanacece or potato-order. It is omployed in modicine as an anodyne, and also for dilating the pupil of the eye in operation for cataract. It has been used in the preparation of an Italian cosmetic, whence the specific name-Belladonna ('fine lady'). The active principle of this plant is an alkaloid called atropine or atropia.

BrII-MIXAI. An alloy of copper and tin. The proportions of these constituonts vary within certain limits. The older bell-founders appear to have aimed at producing an alloy of 3 parts of copper to 1 of tin; these proportions yield a motal of high density - a good casting having a specific gravity of 8.9 , or equal to that of tho unalloyed copper-but rather too brittle to bo used for largo bells. An alloy of $3 \frac{1}{2}$ of copper to 1 of tin has been largely employed, and being easy to tune is a favourite metal with bell-founders. When 4 of copper to 1 of tin is used, a soft alloy is obtained, such as is ordinarily used for small house-bells. The large bells in the New Houses of Parliament at Westminster were cast in an alloy of 22 of copper to 7 of tin ; but Mr. Denison, who superintended the construction of these bells, has since suggested that the ratio of 13 to 4 , or $3 \frac{1}{4}$ parts of copper to 1 of tin, would probably form a better alloy, since these proportions exactly represent 6 equivalents of copper to 1 of tin. Such an alloy, being a definite compound, with its constituents in atomic proportions, would be more likely to remain homogeneous throughout-the component metals having less tendency to separate from each other during the cooling of the molten mass. 'I should now,' says Mr. Denison, speaking after his experience of tho Westminster bells, 'require large bells to be of this 76.5 copper to $23.5 \mathrm{tin}$, or $\mathrm{Cu}^{6} \mathrm{Sn}$; and they should then be rejected as unhomogonoous if any part of the bell is proved to be beyond the limits of either 77 per cent. of copper or 23 of tin.'

In addition to the two chief constituents of bell-metal, small quantities of other metals have occasionally been introduced into the alloy, but apparently without any decided advantage. Thus some celebrated old bells contain a small proportion of antimony-Old Tom of Lincoln containing about 03 per cent. The use of silver has also been recommended, but seems unwarranted.

An alloy of cast-iron and tin, callod 'Stirling's Union Metal,' has been employed for bells; but, though emitting a fair sound, is far inferior to ordinary bell-metal. The aame remark applies to cast-steel bells. See Bronze and Copper.

BבI-MrTAI ore, or Stannine; a sulphide of tin, copper, and iron. (Etain sulphuré, Haüy; Zinnkies, Hausmann.)

The composition of the ordinary varioty of this oro is-

\begin{tabular}{|c|c|c|c|c|c|c|c|}
\hline Copper & - & - & - & . & • & • & . \\
\hline Iron & • & 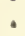 & • & . & . & . & • \\
\hline Tin & . & - & • & . & • & • & . \\
\hline Sulphur & & . & . & • & . & $\bullet$ & • \\
\hline
\end{tabular}

It was found in many of the Cornish mines, and especially at Carn Brea, but is now rare.

3aInows. See Metallungr.

B2x.s. Church bolls are said to have been originated in Italy; but bells wore certainly cast at a very early period in the East. They were evidently uscd by the ancient Egyptians, and at a very early date amongst the Chinese. All the more celebrated bells are manufuctured of bronze, or bell-metal (these alloys are described under their respective heads).

The following are the weights of a few of the largest bolls :-

The great bell of Moscow

The bell of St. Ivan

Another bell in the samo city.

Ditto ditto, cast in 1810 .
168.

443,772

127,836

39,827

112,000 


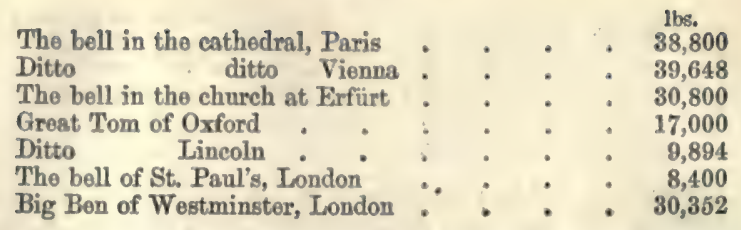

The Big Ben of the Now Houses of Parliament was designed by Mr. Denison, Q.C., who undertook all the responsibility of its construction, except the casting. The motal, as already stated, was an alloy of 22 parts of copper to 7 of tin. The original bell was cast on August 6, 1856, by Messrs. Warner, at Norton, near Stockton-onToes. It was thicker than the prescribed pattern, and exceeded the intended weight by $2 \mathrm{cwt}$. Moreover, the casting was defective, and when the bell was subsequently broken up, a natural erack, 18 inches long, was found in the sound-bow. In order to bring out the full sound, a clapper of 13 cwt. was required, and the bell was consequently cracked within a year after it had been cast.

On April 10, 1858, Big Ben was recast, with certain alterations of shape, by Mr. Mears. Soon after it had been placed in the clock-tower it was found that this second casting was much more defective than the first-the metal exhibiting a number of superficial cavities and still more internal blisters. Dr. Percy and Prof. Tyndall wero instructed to report officially on tho bell. It was found that instead of containing 22 of copper to 7 of tin, as required by contract, two fragments from the sound-bow contained respectively 19.4 of copper to 7 of tin, and 19.9 copper to 7 tin; whilst a samplo taken from the top yielded $22 \cdot 3$ of copper to 7 of tin. Thus, in addition to the porous structure of the casting, the alloy was far from being uniform throughout, and the lower part-which is, of course, the most important part of a bell-was harder and more brittle than the specified alloy. Both castings, therefore, were decided failures.

With regard to the general form of bells, Mr. Denison made the following remarks in a lecture at the Royal Institution:-

'Now, from these and other experiments, I have come to the conclusion that bells of the common and well-known shape, with a thick lip or sound-bow, are the most effective known instruments for producing a loud and musical sound, such as you want when you erect a large public clock, or put up a peal of church bells. And I confess, also, that after trying, at Messrs. Warners', a number of experiments with bells of the usual general form, but with various deviation in the details, I am equally satisfied that there is nothing to bo gained by deviating materially from the established proportions of the best old bells. And I think it is some confirmation of my views to tell you that Professor Wheatstone, having been commissioned by the Board of Works with Sir C. Barry, on his own suggestion, to collect information at the late Paris Exhibition respecting the most esteomed chimes in France and Belgium, and whether there are in those countries makers acquainted with the traditions of the art, or who have applied the discoveries of science to the improvement of bells, or to efficient substitutes for them, has come back with the conclusion that no such efficient substitutes have been discovered; nor is there any known improvement on the established mode and materials for casting them. Sir C. Barry and he, indeed, seem to have been rather impressod with the merits of the cast-steel bells, which you have seen noticed in the newspapers. I have not heard them myself, but I have heard such condemnation of their harshness of sound from other persons, of probably more experience in such matters, that I do not the least believe in their being rocoived generally as an efficient (though they may be a cheap) substitute for the more expensive compound of copper end tin; and, on the whole, that seems to be Professor Wheatstone's opinion also.'

BENGAI STRIPES. Ginghams; a kind of cotton cloth woven with coloured stripes, so called from the cottons which we formerly imported from Bengal.

BEx MUTS. (Noix de ben, Fr.; Salbnïsse, Ger.) The tree which furnishes these nuts is the Guilandina moringa of Linnæus, a native of India, Ceylon, Arabia, and Egypt.

BEN OII. The oil of ben, which may be obtained from the decorticated nuts, is said to be far less liable than other oils to become rancid, and hence it is much used by watchmakers. At a low temperature, the oil of ben separates into two parts - one solid and one fluid; the latter only is used for watch-work. At one time oil of ben was employed as a base for the enfleurage process of making perfumed oils from flowers in the south of France, brought thence from the Levant, but from some unknown reason it has long since ceased to be imported, and oil of ben is now 
a vory raro articlo of commerce. An attompt has been mado in Jamaica, by $\mathrm{Mr}$. Komblo, to raise tho moringa for the sako of this oil, but tho samples there produced remained a perfectly solid fat in England throughout tho whole year.-S.P.

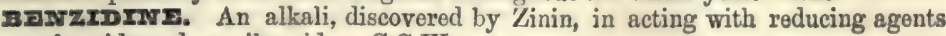
on arzobenride and azoxibenzide.-C.G.W.

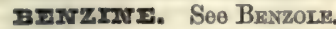

BENzorC ACID. $\mathrm{C}^{14} \mathrm{H}^{6} \mathrm{O}^{4}\left(\mathbf{C}^{7} \mathbf{I}^{6} \mathbf{O}^{2}\right)$. This acid may be obtained by placing bonzoin powdered, with sand, in an ovaporating basin, and above it a paper cap; on applying heat carefully to the sand, acid vapours ariso from the resin, and they are deposited in the form of fino light crystals within the paper cap. Stolze recommends the following process for extracting the acid:- The resin is to bo dissolved in three parts of alcohol, the solution is to be introduced into a retort, and a solution of carbonate of soda dissolved in dilute alcohol is to be gradually added to it, till the free acid be neutralised; and then a bulk of water equal to double the weight of the benzoin is to be poured in. The alcohol being drawn off by distillation, the remaining liquor contains the acid, and the resin floating upon it may be skimmed off and washed, when its weight will be found to amount to about 80 per cent. of the raw material.

Benzoic acid is also obtained by boiling hippuric acid, or the urine of cows or horses which contains this acid, with hydrochloric acid.

BrNzorr, or BrwJ Aarrw. (Benjoin, Fr.; Benzöe, Ger.) A species of resin, used chiefly in perfumery; improperly called a gum, since it is quite insoluble in water. It is extracted by incision from the trunk and branches of the Styras benzoin, or Lithocarpus benzoin, which grows in Java, Sumatra, Santa Fé, and in the kingdom of Siam. The plant belongs to the natural family of the Styracacer. The benzoin flows in small quantities swontaneously from the trees; but it is collected by making incisions in the stom, just below where the branches are given off, as soon as the tree has attained an ago of five or six years. These incisions are repeated each year for about twolve ycars, when the tree becomes exhausted. The resin flows out as a white fluid. It hardens readily in the air, and comes to us in brittle masses, whose fracture presents a mixture of red, brown, and white grains of various sizes, which, when white, and of a certain shape, have been called amygdaloid, from their resemblance to almonds. The benzoe in sortis is very impure, containing portions of wood and bark.

The fracture of benzoin is conchoidal, and its lustre greasy; its specific gravity varies from 1.063 to 1.092 . It has an agreeable smell, somewhat like ranilla, which is most manifest when it is ground. It enters into fusion at a gentle heat, and then exhales a white smoke, which may bo condensed into the acicular crystals of benzoic acid, of which it contains 18 parts in the hundred. Ether does not dissolve benzoin completely. The fat and volatile oils dissolve very little of it.

Unverdorben has found in benzoin, besides benzoic acid and a little volatile oil, no less than three different kinds of resin, none of which has, however, been turned, as yet, to any use in the arts.

Benzoin is principally used in perfumery; it enters into a number of preparations, among which may be mentioned fumigating pastilles, fumigating cloves (called also nails), poudre $\dot{a}$ la maréchale, \&ze. The alcoholic tincture, mixed with 20 parts of rose-water, forms the cosmetic virginal milk. Benzoin enters also into the composition of certain varnishes employed for snuff-boxes and walking-sticks, in order to give these objects an agreeable smell when they become heated in the hand. It is udded to the spirituous solution of isinglass, with which court-plaster is made.

ZExTOI.2. Syn. Benzine, benzene, benzol, hydruret of phenyl, $\mathrm{C}^{12} \mathbf{H}^{6}\left(\mathbf{C}^{6} \mathbf{H}^{6}\right)$. A compound of carbon and hydrogen discovered by Faraday in the products of the destructive distillation of whale-oil. The more volatile portion of coal-naphtha has been shown by Mansfiold to consist chiefly of this substance. It is produced in a great number of reactions in which organic bodies are exposed to high temperatures. It may at once be obtained in a state of purity by distilling benzoic acid with excess of quicklimo. The lime acts by removing two atoms of carbonic acid from the benzoic acid. The mothod of obtaining bonzole from coal-naphtha will bo found fully deseribed under the head of NapHtha, Coar. Benzole is also contained in considerable quantity in bone-oil; but it is accompanied by peculiar nitrogenised rolatile fluids, which are difficult of removal. The latter, owing to their powerful and fetid odour, greatly injuro the quality of the bone-oil benzole. Benzolo is an exceedingly volatile fluid, boiling at ordinary pressures at $187^{\circ} \mathrm{F}$. Its density is 0.850 . Owing to the levity of benzolo being regarded by manufacturers as a proof of its purity, it is not uncommon to find it adulterated with the naphtha from the Torbanehill mineral, or Boghead coal, which has a density as low as 0.750. Any benzole having a lower density than 0.850 is impure. Benzole is excessively inflammable, and its vapour mixed with air is explosivo. Numerous lives have been lost owing to these properties,

Vor. I. 
among thom that of Mr. Mansfeld, to whom wo aro indebtod for an excellent in. vestigation on coul-naphtha. Benzolo is greatly used in commerce, owing to its valuable solvont properties. It dissolvos caoutchoue and gutta-poreha readily, and, on evaporation, leaves them in a state well adapted for waterproofing and many other purposes. Its power of dissolving fatty, oily, and other greasy matters, has eaused it to become an article of commeree under tho nume of benzoline. It readily extracts grease even from the most delicato fabrics, and, as it soon, on exposure to the air, evaporates totally nway, no odour remains to betray the fact of its having been used. It dissolves rendily in very strong nitric acid, and, on the addition of water, it is precipitated as a heavy oil, having the composition $\mathrm{C}^{12} \mathrm{H}^{3} \mathrm{NO}^{4}\left(\mathbf{C}^{6} \mathbf{z}^{5} \mathbf{T} \mathbf{O}^{2}\right)$. The latter compound is nitrobenzole; it is regarded as benzole in which one atom of hydrogen is replaced by hyponitric acid. Nitrobenzole, in a stato of tolerable purity, is a paleyellow oil, having a sweetish taste, and an odour grently resembling bitter almonds. Owing to its conpurative cheapness, it is employed in perfumery. Nitrobenzolo ean be prepared with nitric acid of moderate strength, such as is ordinarily obtained in commerce; but it then becomes necessary to distil the acid and the hydrocarloon together several times. The product so obtained is darker in colour, and in other respects inferior to that obtained with highly concentrated acid. By treatment with acetate of protoxide of iron, nitrobenzolo becomes transformed into aniline. This change may be effected, but far less conveniently, by means of sulphide of ammonium. Benzole is extremely valuable in many operations of manufacturing chomistry. It dissolves several alkaloids, and, on evaporation, leaves them in a stato of purity. It dissolves quinine, but not einchonine, and may therefore be employed as a means of seperation. Morphia and strychnine aro also dissolved by it, but not in great quantity. To obtain many natural alkaloids existing in plants, it is merely necossary to digest the dry extract with caustic potash and then with bonzole. The latter is to be decanted, and then distilled off on a water-bath. The alkaloid will be left behind in a state well adapted for crystallisation or other means of purification. Benzole is becoming much used as a solvent in researches in organic ehemistry. Many substances, such as chrysene and bichloride of naphthaline, crystalliso better from benzole than from any other solvent.

Benzole may be employed in many ways for illuminating purposes. It is so easily inflamed that great, care is necessary in using it. It does not require a wick to enablo it to burn. If poured, even on an uninflammable surface and a light be applied, it takes fire like a train of gunpowder, and burns with a brilliant flame, emitting dense clouds of smoke, which, soon condensing into soot, presently fall in a shower of blacks. Even on the surface of water it burns as freely as anywhoro else. If a drachm or two be pourod on water contained in a pan, and a pollet of potassium be thrown in, the benzole inflames, and rises in a column of flame of considorablo height. A method of destroying enomies' shipping has been founded on this principle. In consequence of the smoky nature of the flame of benzole (caused by the comparatively larger percentage of carbon), it is often convenient to burn a mixture of ono volume of benzole and two volumes of alcohol. A stream of air driven through benzolo becomes so inflammable as to serve for the purposes of illumination. For this mode of using the hydrocarbon, it should be kept slightly warm to assist its raporisation. A machine on this principle, of American invention, has boen employed to illuminato houses. The air is driven through the benzolo by a very simplo contrivanec, tho motive power being a descending weight. See Gas, AIr.

When quite pure, benzolo freezes at $32^{\circ}$ to a beautiful snow-white substance, resembling eamphor. The mass retains the solid form until $n$ tempernture of $40^{\circ}$ or $41^{\circ}$ is reached. This property of solidifying under the influonce of cold may be made use of to produce pure benzole from the more volatile portion of coal-naphtha. To obtain it perfectly pure, it should be frozen at least three times, the portion not solidifying being removed by filtration through calico. The unfrozen portion con. tains hydrocarbons, homologous with olefiant gas.

Benzole dissolves free iodine and bromine, and has oven been used in analysis to separate them from kelp and other substances containing thom. They must of course be set free before acting with the hydrocarbon. The presence of benzole in mixtures may easily be demonstrated, even when present in rery small quantity, by converting it into aniline, and obtaining tho characteristic reaction with chloride of lime. For this purpose the mixture is to be dissolved in concentrated nitric acid and the nitrobenzole precipitated by water. The fluid is then agitated with ether, which dissolves the nitro-compound. The ethereal solution is mixed with an equal bulk of alcohol and hydrochloric acid: a little granulated zinc boing added, hydrogen is ovolved, and, by acting in a nascent state on the nitro-compound, reduces it to the stato of aniline. Tho base is thon to be separated by an excess of potash, and the alkaline fluid is shaken with other to dissol ve the base. The ethereal fluid being evaporated, leaves the aniline, 
On adding water and then a fow drops of solution of chloride of lime, the purplo colour indicative of aniline is immediately produced.-Hofmann. The writer of this article has by this process detectod minute traces of benzole in mixtures consisting almost entirely of homologues of olefiant gas.-C. G. W.

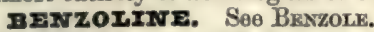

давсамот. (Bergamotte, Fr.) The Citrus bergama, a citron cultivated in the centre and south of Europe. By distillntion from the rind of the fruit is obtained the well-known essence of bergamot, See Ons, Fssratrat.

The ottoes, or essential oils, from fruits such as the bergamot, the lemon, and the orange, may all be procured by distillation, but these products thus procured are not valued by the manufacturing perfumer so much as when they are obtained by rasping the poel of tho fruit and collecting the essence thus disongaged, and finally filtering: The rasps employed for this purpose are hollow cones filled with spikes, in which the fruit is dexterously revolved by hand. All essences that are distilled are modified in their composition by the presence of the watery vapour and high temperature. -S. P.

Brigarot. A coarse tapestry, said to have been invented at Bergamo, in Italy, made of $o x$ and goats' hair, with cotton or hemp.

BEnIrN BzAcT. A black varnish, drying with almost a dead surface, used for coating the better kinds of iron-ware.

BrrInIN BIUE. A fine variety of the Prussian Blue.

BrRIIN CAsTrucs. Delicate ornamental objects cast in a rery fluid iron, smelted from bog-ores, and containing much phosphorus. These castings were formerly imported from Prussia, and used for personal decoration.

BERry. The term is commonly applied, not only to small fruit, but in some cases to soods. The following is Professor Lindley's definition of a berry:- 'A succulent or pulpy fruit containing naked seeds, or, in more technical language, a suceulent or pulpy pericarp, or soed-ressel without valves, containing several seeds, which aro uaked, that is, which have no covering but the pulp and rind. It is commonly round or cval. But in popular language, berry extends only to smaller fruits, as strawberry, gooseberry, \&c., containing seeds or granules. An indehiscent pulpy pericarp, many. celled and many-seeded; the attachment of the seeds lost at maturity, and the seeds remaining scattered in the pulp.'

Berries are used in some of the processes of manufacture, but they are not of much importance.

Bay Berries.-The fruit of the Laumes nobilis, or the sweet bay. Both the leaves and the fruit are employed as flavourings. A volatile oil, the oil of sweet bay, is obtained by distillation with water; and a fixed oil, by bruising the berries, and boiling thom for some hours in water; this oil, called also Laurel fat, is imported from Italy. See Bays, OrL or.

Turkey Yellow Berries.-The unripe fruit of the Rhamnus infectorious. They aro used in calico-printing, producing a lively but fugitive yellow colour.

Persian Yellow Berries.-These are said to be produced by the same species of plant; but the colour is considered more permanent, and they fetch higher prices.

Berries of Avignon.-Another name given to the Turkey and Persian berries.

Juniper Berries.-The fruit of the Juniperus communis. They are chiefly used for flavouring gin and some spirituous cordials, and in the preparation of some pharmacoutical articles, as the oil of juniper and the compound spirits of juniper.

Bear Berry.-The fruit of the Uva ursi. The leaves only are used medicinally.

Myrobolans. - The fruit of a tree which grows in India. It has a pale-yellow colour when new, but becomes darkor by age, and then resembles dried plums. It contains tannin, and has hence been used in dyeing. See Juniper Berries, \&c.

In 1871 we imported of Myrobolans 145,450 ewt., of the value of 100,695l.

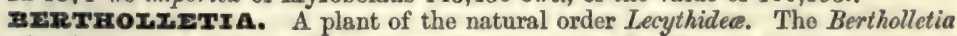
excelsa is a tree of large dimensions, forming extensive forests on the banks of the Orinoco. The Portuguese of Para have for a long time driven a great trade with the nuts of this tree, which the natives call Iuvia, and the Spaniards Almendron. They send cargoes to Fronch Guiana, whence they are shipped for England and Lisbon. These are the common BrazrL Nuxs. The kernols yield a large quantity of oil well suited for lamps.- Humboldt and Bonpland.

ฉבrx. (Béril, Fr.; Beryll, Ger.; Berillo, Ital.) A beautiful mineral or gom, usually of a green colour of various shades, passing into honey-yellow and sky-blue.

Boryl and emerald are varieties of the same species, the latter including the rich green transparent specimens which probably owe their colour to oxide of chrome; the former those of othor colours produced by oxide of iron. Gmelin gives the composition of a Swedish beryl as:- 


Silier
Alumina
Glucina
Peroxide of iron

'Beryls of gigantic size have beon found in the Unitod States, at Acworth and Grafton, Now Hampshire, and Royalston, Mass. One boryl from Grafton weighs 2,900 lbs; it is 32 inches through in one direction, and 22 in another transverse, and is 4 feet 3 inches long. Anothor crystal from this locality, according to Professor Hubbard, measures 45 inches by 24 in its diametors, and a single foot in length; by calculation, weighs $1,076 \mathrm{lbs}$, making it, in all, nearly $2 \frac{1}{2}$ tons. At Royalston, one crystal exceeded a foot in length.'-Dana. Seo Emmani.D.

Some of the natural crystals of phosphate of lime, or apatite, were formorly taken for beryls, and called the Saxony beryl.

Bzssemer proczss. A frocoss for making steel, invented by Mr. Henry Bessemer. See Strke.

BrTrz. A compound, in universal use in the East, consisting of the leaf of the betel-popper, with the betel-nut, a little catechu, and some chunam (lime obtained by calcining shells). This is almost universally used throughout central and tropical Asia; the people are unceasingly masticating the betel. The leaf of the pepper vino (Chavica betel) is extensively cultivated throughout tropical Asia, and forms a large article of Eastern traffic. See Preprar; Bertex.

בITDI-NVT, or Areca. The fruit of the Areca catechu, which is eaten both in its ripe and its unripe state. A tooth-powder used in this country is prepared by charring the aroca nut, and is sold as 'areca-nut charcoal.'

Bźrow. The French name for concrete. Self-slaked hydraulic lime is mixed with sand, and whon the mixture is complete it is beaten up with the ballast. See CorCRETk.

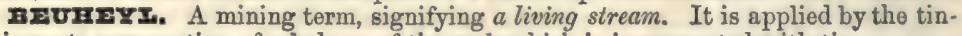
miners to any portion of a lode or of the rock which is impregnated with tin.

BzZOAR. (The most probable etymology of the word is from the Persian Padzahr, i. e. expelling poison, - Penny Cyclopadia.) A concretion found in the stomach of animals of the goat kind; it is said to bo especially produced by the Capra gazella. The finest bezoar is brought to India from Borneo and the shores of the Persian Gulf; the Capra Agagrus, or wild-goat of Persia, producing this concretion, which by way of eminence was called the Lapis bezoar orientalis. The bezoars, which were supposed to cure all diseases, have been found by the analysos of Fourcroy and Vauquelin, and of Proust, to be nothing more than some portions of the food of the animal agglutinated into a ball with phosphate of lime.

Fossil bezoars are found in Sicily, in sand and clay pits. They aro concretions of a purple colour around some, usually organic, body, and of the size of a walnut. Fossil bezoar is sometimes called Sivilian earth.

Bezoar Mineral. - An old preparation of the oxide of antimony.

BHANTG. SeO BaxG.

BrANCO-szCCo. A carefully slaked limo, mixed with powdered marble, em. ployed in fresco-painting.

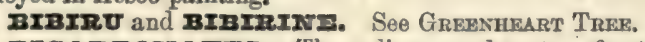

BICARB ONATES. The ordinary carbonates of potash and soda have a strong alkaline reaction and caustic taste, making them unfit for many purposes where a soluble carbonate is required. Moreover, there are many uses to which they aro applied, rendering it desirable that as large an amount of gas as possible should bo given off on the addition of a stronger acid.

Bicarbonate of Potash.-Thore are sevoral modes of converting the carbonato into bicarbonate. The most oconomical is by exposing the salt to a current of carbonic acid. For this purpose some manufacturers place it, slightly moistened, on stonewaro trays, and allow the vapours of burning coke to travel slowly over it. The sources of the gas used in this manufacture will vary according to the locality in which it is undertaken. It is not unusual to produce it by the action of sulphuric acid on limestone. The gas generated in fermentation has been employed, and ovon that which in somo places issues from the earth. The bicarbonate of potash is far less solublo than the carbonate, as it roquires four parts of cold water for solution, whereas the carbonate dissolves in 0.9 of its weight of water at $54^{\circ} \mathrm{F}$. Consequently, if a strong solution is saturated with carbonic acid, the bicarbonate crystallises out. When common pearl-ashes are dissolved in water, and the gas is passed in, a large quantity of a white precipitate is often thrown down; it consists chiefly of silica, but often contains alumina and other matters. Considerable hoat is developed when moistened carbonate of potash is exposed to a current of carbonic acid gas. When carbonato of potash is dissolred in water, and gradually treated with acetic acid, so as to form 
ncetate of potash, by no means tho whole of the carbonic acid is expelled, and a point is arrived at when a considerable quantity of crystals is deposited; they consist of very pure bicarbonate of potash. In making acetate of potash on the large scale, the quantity of crystalline precipitate obtained in this manner is sometimes very large. licarbonate of potash is usually tolerably pure. If well crystallised, all the impurities remain in the mother-liquor, and on heating to redness almost exactly the theoretical amount of residue is left, viz. 69.05 per cent. Crystallised bicarbonato of potash always contains one atom of wator, its formula being $\mathrm{KO}, 2 \mathrm{CO}^{2}+\mathrm{HO}$ (IxCO $\mathbf{C O}^{3}$.

Bicarbonate of Soda. - This salt is obtained by the same methods as the salt of potash. The crystals have a corresponding formula to the potash salt; namely, $\mathrm{NaO}$, $2 \mathrm{CO}^{2}+\mathrm{HO}\left(\mathbf{N a} \mathbf{M C O}^{3}\right)$. It requires about 13 parts of water at $60^{\circ}$ to dissolvo it. When pure, 100 parts leave $63 \cdot 18$ of $\mathrm{NaO}, \mathrm{CO}^{2}\left(\mathbf{M r a}^{2} \mathbf{C O}^{3}\right)$, on ignition.

The bicarbonates of potash and soda lose carbonic acid by the boiling of an aqueous solution.

Many chemists regard carbonic acid as being bibasic, the true formula being $\mathrm{C}^{2} \mathrm{O}^{4}$, instead of $\mathrm{CO}^{2}$. This view is probably the correct one, and it explains why the bicarbonates are neutral instead of acid salts. Moreover, $\mathrm{C}^{2} \mathrm{O}^{4}$ corresponds to 4 rolumes, like organic substances generally; whereas, if we assume $\mathrm{CO}^{2}$ as one atom of the gas, we are complled to admit a 2-volume formula. This view has, however, been considerably modified by our modern theoretical chemists.

BICE. A light blue colour prepared from smalt. There is a green bice prepared by mixing some yellow orpiment with smalt.

2rDERY. An Indian alloy of considerable interest, named Bidery, from Bider, a city N.E. of Hyderabad. Many articles are made, remarkablo for olegance of form and for gracefully-engraved patterns. Although the groundwork of this composition appears of a blackish colour, its natural tint is that of pewter or zinc.

Dr. Heyne says it is composed of, copper, 16 ; lead, 4 ; tin, 2 ; and to every 3 ounces of alloy 16 ounces of spelter (that is, of zinc) are added, when the alloy is melted for use. To give the esteemed black colour and to bring out the pattern, it is dipped in a solution of sal-ammoniac, saltpetre, common salt, and blue vitriol. Dr. Hamilton saw, zinc, 12,360 grains, copper 400, and lead 414, melted together under a mixture of resin and bees'-wax, introduced into the crucible to prevent calcination; it was then poured into moulds of baked clay, and the articles handed over to be turned in a lathe. Though called bidery and sometimes vidry, it is manufactured in other places. In some parts of the Nizam's dominions, specimens were obtained, for the Exhibition of 1851, of great beauty. Bidery does not rust, yields little to the hammer, and breaks only when violently beaten. According to Dr. Hamilton, bidery is not nearly so fusible as zinc or tin, but melts more easily than copper.-Dr. Royle, Lecture on the Great Exhibition of 1851 .

3rJourry. (Bijouterie, Fr.) Jowellery;-the manufacture of and dealing in jowellery. This work is not the place in which to describe the almost endless variety of articles which come under this denomination. The principal place for the manufacture, in England, is Birmingham, but formerly the trade was largely carried on in Derby, Edinburgh, and London. During the last twonty-five years the jewellery trade of Birmingham has made rapid progress. It has been estimated that the value of the gold annually consumed in the jewellery and gilt-toy trade of that town amounts to between $600,000 \mathrm{l}$ and $700,000 \mathrm{l}$, and of the silver to from $100,000 \mathrm{l}$. to $150,000 \mathrm{l}$, whilst the precious stones and their imitations have been ralued at a quarter of a million sterling. (See papers by Mr. J. S. Wright in "Tho Resources, Products, and Industrial History of Birmingham,' 1866; and by Mr. W. G. Larkins in 'Journal of tho Society of Arts,' March 1, 1872.)

The trade in jewellery forms one of the most important branches of French commerce; on which a French writer says: "La bijouterio est uno des branches les plus importantos du commerco Français, et c'est cllo que constate, de la manière la plus éridento, notre supériorité dans les arts du dessin et les progrès toujours croissans de l'industrio Parísienne. Dans cette partio essentielle, ello n'a pas de rivaux, et elle rend tributaire do notre pays presque toute l'Europo et uno grande partie de l'Asie et dle l'Amérique.' Of late years, however, the Parisian trade has been declining; but French jewellery has still so high a reputation that English manufacturers often find it to their interest to affix French labels to their goods. A quantity of cheap jewellery is also imported from Germany. See AGatre.

The ordinary practice has been to divido articles of this character into two principal kinds-fino jowellery and falso jewellery (bijoutier en fin and bijoutier en faux). Another division, among the French jewellers especially, has been to adopt four classes: 1 , flno jewellery, which is all gold; 2 , silver jewellery; 3 , false jewollery; and, 4 , jewellery of steel or iron. 
In tho article Alwors will be found the quantity of the baser metal which is pormitterl to be combined with gold; and also the proportions of the alloys forming tho brasses which are employed in the false jewellery.

Under their respective heads the true gems will bo described (soe Diaxosn, Exerald, \&c.); and under Gens, Artificiar, the imitations of them ; many of the false so nicely representing the peculiarities of the true gems as to deceive even tho practised eyo. Tho hardness is, however, an unfailing test; if, thoreforo, any gen is found to bo scratched with a stool file, wo may depend on its being artificial. Seo also Prarls, Artificial; Lapidary Worx; Giass, \&ce.

BIXE, BISE, or 2NABEg. An Indian poison prepared from the root of the Aconitum ferox, a native of Nepanl, and from other spocios of aconite. It is said to possess the concentrated power of all the European aconitos. This is doultfful; the poisonous properties of some of the monkshoods are little known. Rocently (1873), some childron from the Falmouth Workhouse, playing on the beach at Mainfortl, found some roots washed on shore, and four of them ate small portions-within two or three hours three of the boys died, and the life of the other was only saved by the greatest attention. See Aconitum.

BIrE. (Bile, Fr.; Galle, Ger.) The secreted liquor of the liver in animals.

Bile (ox's) is composerl, according to Berzelius, of-biline, fellinic acid, and fat of gall, 8.00 ; mueus, 0.30 ; alkali combined with biline, \&re., 0.41 ; muriato of soda. extractive matter, 0.74 ; phosphate of soda, do. of lime, \&ce, 0.11 ; water, 90.44 $=100.00$.

Thenard's analysis gives-resin of bile and picromel (acid gallenate of soda), 10.54; colouring-matter, 0.50 ; soda, 0.50 ; phosphate of soda, 0.25 ; muriate of soda, 0.40 ; sulphate of soda, 0.10 ; sulphate of lime, 0.15 ; traces of oxide of iron, water, 87.56 ; $=100.00$.

The analyses of Benach ('Ann. Ch. Phar.') give the following as the composition of the gall of several animals:-

\begin{tabular}{|c|c|c|c|c|c|c|c|}
\hline & Carbon & Hydrogen & Nitrogen & & Sulphur & & A \\
\hline Calve & $55^{\circ}$ & $7 \cdot 7$ & - $3 \cdot 3$ & & $4 \cdot 9$ & & $13 \cdot 15$ \\
\hline Shee & $57^{\circ}$ & $7 \cdot 8$ & $3 \cdot 9$ & . & $\therefore \quad 5 \cdot 7$ & & 11.86 \\
\hline Goat & 57 & $8 \cdot 2$ & $\ldots$ & • & $5 \cdot 2$ & * & 13.21 \\
\hline Ben & 5 & $8 \cdot 3$ & ... & . & . 5.8 & & 8.42 \\
\hline Fowls & 57 & $8 \cdot 3$ & $3: 5$ & . & - $5 \cdot 0$ & . & 10.99 \\
\hline & $56 \cdot 0$ & $8 \cdot 1$ & $2 \cdot 5$ & . & . 5.6 & & $14 \cdot 11$ \\
\hline
\end{tabular}

Strecker and Mulder have published two treatises on ox-gall. The riews adrocated by these chemists will be found in the 'Annual Report of the Progross of Chemistry of Liebig and Kopp,' translated by Hofmann and De la Rue.

It has been shewn by Stricker's researches that bile is largely composed of the soda-salts of two peculiar acids called glycocholic and taurocholic acids. Glycocholic acid may be prepared by extracting ox-bile with cold absolute alcohol, and treating the filtered extract with ether, when crystals of alkaline glycocholates are obtained; these were formerly termed crystallised bile. When the salts are decomposed by sulphuric acid, glycocholic acid is set free. This acid contains $\mathrm{C}^{32} \mathrm{H}^{43} \mathrm{NO}^{12}\left(\mathbf{C}^{26} \mathbf{H}^{13} \mathbf{N O}^{6}\right)$. Boiled with a solution of potash, it is resolved into cholic acid and glycocine. The second acid of the bile-taurocholic acid, formerly called bilin-has not hitherto been isolated in a state of purity. When boiled with alkalies the impure product is resolvod into cholic acid, and a substance called taurin, which contains $\mathrm{C}^{\prime} \mathrm{H}^{7} \mathrm{NS}^{2} \mathrm{O}^{6}$ $\left(\mathbf{C}^{2} \mathbf{F}^{\mathbf{2}} \mathbf{\mathbf { r }} \mathbf{5 O}^{\mathbf{3}}\right)$. A fatty matter, known as cholesterin is also present, to a small extent, in the bile, and forms the ehief ingredient in biliary calculi.

It is notable that the liver is capable of developing, in the blood which circulatcs through it, an amyloid substanco called glycogen, which is readily changed into liver-sugar, or glueose:

Heintz remarks (Poggendorff's 'Annalen'), that the change of colour sometimes produced-for it does not appear always-by nitric acid in liquids containing bile (first green, thon blue, violet, red, and lastly, yellow), is occasioned only by the colouring-matter, which Berzelius has named cholepyrrhin, and not by the essential constituents of the bile, and can therefore be regarded only as a test for the preseneo of this substance.

Pettenkofer's test for bile consists in adding a drop of sulphuric acid and a solution of sugar, whon a purple-violet colour is produced if bilo be present.

For the further chemical examination of bile, see 'Watts's Dictionary of Chemistry ;' for its uses in the arts, see Gart.

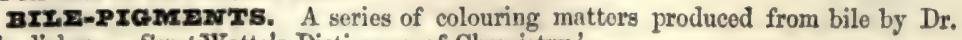
Thudichum, See 'Watts's Dictionary of Chemistry.' 
mrxv. Tho name by which many of the coal-measure shalos aro locally known in some districts. They aro generally more or less bituminous and calcareous. Since so much attention has been directed to the production of petroleum, many of those 'binds' have been subject to distillation, with variable results. See

\section{Perrolem.}

zINDIrTG coni. Seo Coat.

מrRpurme. (Glu, Fr.; Vogelleim, Ger.) The best birdlime may be made from tho middle bark of tho holly boiled seven or eight hours in water, till it is soft and tender, then laid by heaps in pits under ground, and covered with stones after the water is drained from it. There it must be loft during two or three woeks, to ferment, in the summer season, and watered, if necessary, till it passes into a mucilaginous state. It is then to be pounded in a mortar to a paste, washod in running water, and kneaded till it be freo from extraneous matters. It is noxt left for four or fivo days in carthen ressols to ferment and purify itself, when it is fit for use. Birdlime may be made by the samo process from the mistletoe (Viscum album), young shoots of elder, and the barks of other vegetables, as well as from most parasitical plants.

Good birdlimo is of a greenish colour and sour flavour, somewhat resembling that of linseed oil-gluey, stringy, and tenacious. By drying in the air it becomes brittle, and may be powdered; but its viscosity may be restored by moistening it. It contains resin, mucilage, a little free acid, colouring and extractive matter. The resin has boen called viscine.

Macaire has examined a substance which exudes from the receptacle and involucre of the Atractylis gummifera, and describes it as the pure matter of birdlime, which he calls viscine. Common birdlime may be regarded as a mixture of viscine, vegetable mucilage, and vinegar.

The mistletoe yields a peculiar viscid gluey substance, consisting of a green wax and birdlime.

BIscurTs. Biscuit-baking constitutes two separate branches of manufacture, —namely, that of ordinary biscuit, or, so to speak, biscuit 'proper,' for maritime purposes, and that of fancy biscuits. Ordinary, or sailors' biscuit consists of only flour and water kneaded into a paste, cut in the proper shape, docked, and baked in an oven ; fancy biscuits consist also of flour and wator, but with an addition of butter, sugar, eggs, spices, or 'flavourings,' all or either of them according to the kind.

Ships' biscuits are now made by machinery, and one of the reasons for this has been that the manual preparation of them was too slow and too costly. A landsman knows very little of the true value of a biscuit: with a seaman, biscuit is the only bread that he eats for months together. Thero are many reasons why common loaves of bread could not bo used during a long voyage: because, containing a fermenting principle, they would soon become musty and unfit for food if made previous to the royage, while the preparation of them on board ship is subject to insuperable objections. Biscuits contain no leaven, and, when woll baked throughout, they suffer little change during a long royage.

The allowance of biscuit to each seaman on board a Queen's ship is a pound per day (averaging six biscuits to the pound). The supply of a man-of-war for several months is, consequently, very large; and it often happened during the long war that the difficulty of making biscuits fast enough was so great, that at Portsmouth waggonloads, brought from a distance, were unpacked in the streets and conveyed to the ships.

We shall now describe the modo of making biscuits by hand, and afterwards speak of the improved method. The bakehouse at Gosport contained nine ovens, and to each was attachod a gang of five men,- the 'turner,' the 'mate, the 'driver,' the 'breakman,' and the 'idleman.' The requisite proportions of flour and water were put into a large trough, and the 'driver,' with his naked arms, mixed the whole up together into the form of dough $-\mathrm{a}$ very laborious operation. The dough was then taken from the trough, and put on a woodon platform called the break: on this platform worked a lever called the break-staff, five or six inchos in diameter, and seven feet long; one end of this was loosely attached by a kind of staple to the wall, and the breakman, riding or sitting on the other ond, worked this lever to and fro over tho dough by an uncouth jumping or shuffing movement. When the dough had become kneaded by this barbarous method into a thin sheet, it was removed to the moulding board and cut into slips by means of an enormous knifo; theso slips wero then brokon into pieces, each large enough for ono biscuit, and then workod into a circular form by tho hand. As each biscuit was shaped it was handed to a second workman, who stamped the King's mark, the number of the oven, \&c., on the biscuit. The biscuit was then docked, that is, pierced with holes by an instrument adapted to the purpose. The finishing part of the process was one in which remarkable dexterity was displayed. A man stood beforo the open door of the ovon, having in his 
hand the handle of a long shovel called a pool, the other ond of which was lying flat in the oven. Another man took the biseuits as fast as they woro formod and stamped, and jerked or threw thom into the oven with such undeviating accuracy that they should always fall on the peel. The man with the peel then arranged the biscuits side by side over the whole floor of the oven. Nothing could excoed (in manual labour alone) the regularity with which this was all done. Seventy biseuits were thrown into the oven and regularly arranged in one minute, the attention of each man being vigorously directed to his own department; for a delay of a single second on the part of any one man would have disturbed the wholo gang. The biscuits do not require many minutes' baking; and as the oven is kept opon during the time that it is being filled, the biscuits first thrown in would be overbaked were not some precaution taken to prevent it. The moulder therefore made those which were to be first thrown into the oven larger than the subsequent ones, and diminished the size by a nice gradation.

The mode in which, since about the year 1831, ships' biscuits have been mado by machinery inronted by T. T. Grant, Esq., of the Royal Clarence Yard, is this :-The meal or flour is conveyed into a hollow cylinder four or five feet long and about three feet in cliameter, and the water, the quantity of which is regulated by a gauge, admitted to it; a shaft, armed with long knives, works rapidly round in tho cylinder, with such astonishing effect, that in the short space of six minutes, $450 \mathrm{lbs}$. of dough are produced, infinitely better made than that mixed by the naked arms of a man. The dough is removed from the cylinder and placed under the breaking rollers; these latter, which perform the office of kneading, are two in number, and weigh $15 \mathrm{cwts}$. each; they are rolled to and fro over the surface of the dough by means of machinery, and in fire minutes the dough is perfectly kneaded. The sheet of dough, which is about two inches thick, is then cut into pioces half a yard square, which pass under a second set of rollers, by which each piece is extended to the size of six feet by three, and reduced to the proper thickness for biscuits. The sheet of dough is now to be cut up into biscuits; and no part of the operation is more beautiful than the mode by which this is accomplished. The dough is brought under a stamping or cutting-out press, similar in effect, but not in detail, to that by which circular pieces for coins are cut out of a sheet of metal. A series of sharp knives are so arranged that, by one movement, they cut out of a piece of dough a yard square about sixty hexagonal biscuits. The reason for an hexagonal (six-sided) shape is, that not a particle of wasto is thereby occasioned, as the sides of the hexagonals accurately fit into those of the adjoining biscuits, whereas circular pieces cut out of a large surface always leave racant spaces between. That a flat sheet can be divided into hexagonal pieces without any waste of material is obvious.

Fach biscuit is stamped with the Queen's mark, as well as punctured with holos, by the same movement which cuts it out of tho piece of dough. The hexagonal cutters do not sever the biscuits completely asunder, so that a whole sheet of them can be put into the oven at once on a large peel, or shovel, adapted for the purpose. About 15 minutes are sufficient to bake them; they are then withdrawn and broken asunder by the hand.

The corn for the biscuits is purchased at the markets, and cleaned, ground, and dressed at the Government mills; in quality it is a mixture of fine flour and middlings, the bran and pollard being removed. Tho ovens for baking are formed of fire-brick and tile, with an area of about 160 feet. About 112 lbs. weight of biscuits aro put into the ovens at once. This is called a suit, and is reduced to about $110 \mathrm{lbs}$. by the baking. From 12 to 16 suits can be baked in each oven every day, or after tho rato of $224 \mathrm{lbs}$. per hour. The men engaged are dressed in clean check shirts and white linen trousers, apron, and cap, and every endeavour is made to observe the most scrupulous cleanliness.

We may now make a few remarks on the comparative mexits of the hand and the machine processes. If the meal and the water with which the biscuits are made be not thoroughly mixed up, there will be some parts moister than othors. Now, it was formerly found that the dough was not well mixed by the arms of the workman; the consequence of which was, that the dry parts became burnt up, or else that the moist parts acquired a peculiar kind of hardness which the sailors called 'flint:' these defects are now removed by the thorough mixing and kneading which the ingredients receive by the machine.

We have seen that $450 \mathrm{lbs}$. of dough may be mixed by the machine in three minutes and kneaded in six minutes; we need hardly say how much quicker this is than men's hands could effect it. The biscuits are cut out and stamped 60 at a time, instead of singly: besides the time thus saved, the bisenits bocome more equally baked, by the oven being more speedily filled. The nine orons at Gosport used to employ 45 men to produce about $1,500 \mathrm{lbs}$. of liscuit per hour; 16 mon and 
boys will now produce, by the same number of orens, $2,240 \mathrm{lbs}$. of biscuits (ono ton) per hour.

The comparative expense is thus stated:-Under the old system, wages and woar and tear of utensils cost about 1s. $6 d$. per cwt, of biscuit; under the new system, the cost is $5 d$.

The bakehouses at Deptford, Gosport, and Plymouth could produce 7,000 or 8,000 tons of biscuits annually, at a saving of $12,000 l$. per annum from the cost under the old system. The advantages of machine-made over hand-made biscuits, therofore, aro many-quality, cleanliness, expedition, cheapness, and independence of Government contractors.

Fig. 104 represents the biscuit-machinery as executed beautifully by Messrs. Ronnie, engineers. $a$ is tho breaker roller, table, and toller; $b$, the finishing roller,

104

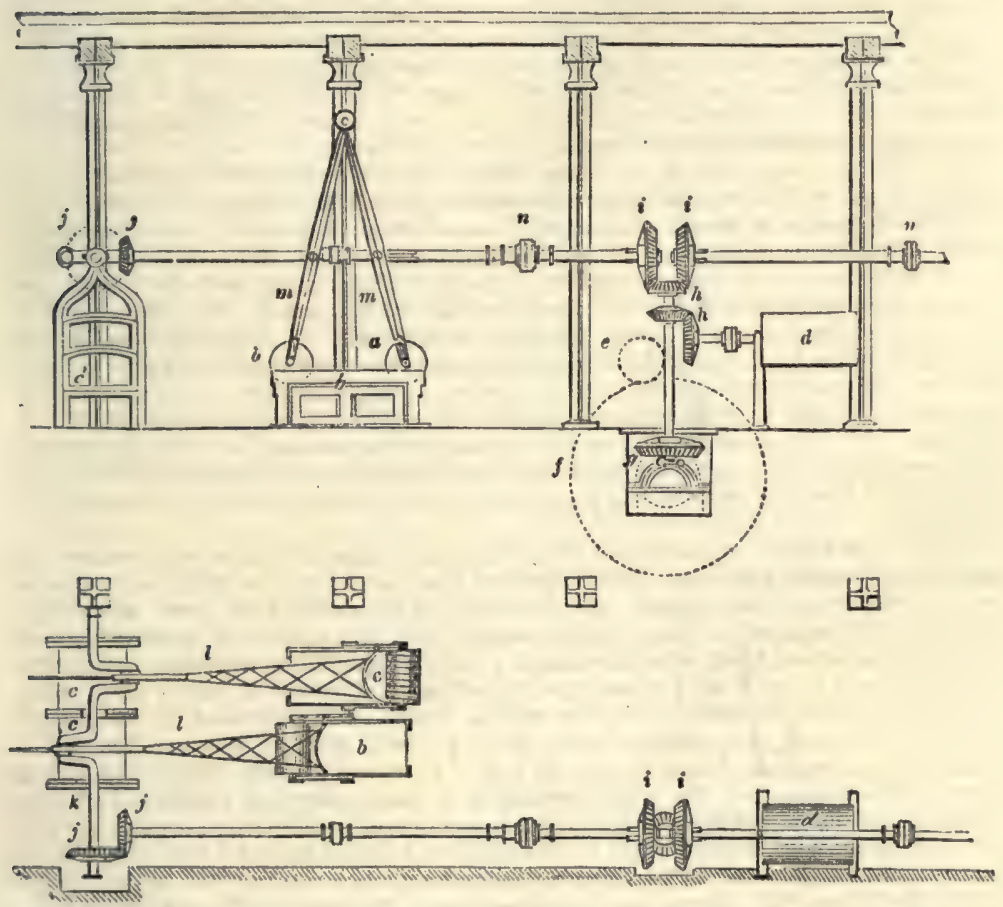

tablo, and toller; $c c$, docking machines, for stamping out the biscuits; $d$, mixing machine for making the dough; $e$, spur-pinion to engine-shaft; $f$, spur-wheel; $g g$, bevel mitre wheels, to give the upright motion; $h h$, bevel wheels for working the mixing machine; $i \boldsymbol{i} i$, ditto, for communicating motion to the rolling machines, $j j$; $k$, the crank shaft ; $l l$, connecting rods; $m m$, pendulums for giving motion to rollers; $n n$, clutches for connecting either half of the machinery to the other.

The manufacture of fancy biscuits, which in formor times was confined to the pastrycook and confectioner, has of late years assumed considerable importanco, and several firms are now exclusively engaged in this branch of industry, the products of which are sold under an extraordinary variety of names. Some of these, namely, the 'plain biscuit, arrowroot, captain, brown meal, cinnamon, caraway, vanilla biscuits,' \&c., are intelligible enough; but, if we except 'Abernethy biscuit, macaroons, and cracknels,' with the names of which the public, from long usage, are familiar, the rest of the products of the modern biscuit-baker, 'Africans, Jamaica, Queen's routs, ratafias, Bath and other sorts of olivers, exhibition, rings and fingers, pienies, cuddy,' \&c., \&c. form a list of upwards of eighty fanciful names, all expressive of articles of different form, appearanco, and taste, made of nearly the same materials, with but little variation in the proportion in which they are used,- the principal ingtedients in all boing flour and wator, butter, milk, eggs, and caraway, nutmeg, cinnamon, 
mace, ginger, essenco of lemon, neroli, or orange-flower water, called in technical language, 'flarourings.' The kneading of theso materials is always performed by a kueading or mixing machine. The dough or paste produced is passed several times between two rovolving cylinders adjusted at a proper distance, so as to obtain a flat, perfectly homogenoous mass, slab, or sheet. This is transferred to a stamping or cutting machine, consisting of two cylinders, through which the shoet of homogoneous pasto has to pass, and by which it is laminated to tho proper thickness, ind at the same timo pushed under a stamping and docking frame, which cuts it into dises, or into oval or otherwiso shaped pieces, as ocension may require. Tho stamps or cutters in the framo being intornally provided with prongs, push the eut piccos of dough, or raw cakes, out of the cutting frame, and at the same time dock the cakes, or cut piecos, with a series of holes, for the subsequent escapo of the moisture, which, but for these rents, would distort and spoil the cako or biscuit when put in the oven. The temperature of the ovon should be so regulated as to be perfectly uniform, noither too high nor too low, but just at such a heat as is sufficient to givo the biscuits a light brown colour. For such a purpose the hot-water oren of Mr. Perkins, or that of Mr. Roland, is the best that can possibly be used. (Seo Bread.) Roland's oren offers the peculiar adrantage that, by turning the screw, tho solo of the ovon can be brought nearer to the top, and a temperature is thus obtained suitable for baking thoroughly, without burning, the thinnest cakes.

One of the most curious branches of the bakor's eraft is the manufacture of gingerbread, which contains such a proportion of molasses that it cannot be fermented by means of yeast. Its ingredients aro flour, molasses or troacle, buttcr, common potashes, and alum. After the butter is melted, and tho potashes and alum aro dissolved in a little hot water, these three ingredients, along with the treacle, are poured among the flour which is to form the body of the bread. The whole is then incorporated by mixture and kneading into a stiff dough. Of theso five constituents the alum is the least essential, although it makes tho bread lighter and crisper, and renders the process more rapid; for gingerbread, dough requires to stand over for screral days, somo 8 or 10 , before it acquires the state of porosity which qualifies it for the oven; the action of the treacle and alum on the potashes, in evolving carbonic acid, seoms to be the gasifying principle of gingerbread; for if carbonate of potash is withheld from the mixture, the bread, when baked, resembles in hardness a pieco of wood.

Treacle is always acidulous. Carbonato of magnesia and soda may be used as substitutes for tho potashes. Dr. Colquhoun has found that carbonate of magnesia and tartaric acid may roplace the potashes and the alum with great advantage. affording a gingerbread fully as agreeable to the taste, and much moro wholesome than the common kind, which contains a notable quantity of potash. His proportions are $1 \mathrm{lb}$. of flour, $\frac{1}{4}$ of an ounce of carbonate of magnesia, and $\frac{1}{\alpha}$ of an ounco of tartaric acid, in addition to the treacle, butter, and aromatics, as at present used. The acid and alkaline carth must bo well diffused through the wholo dough; the magnesia should, in fact, be first of all mixed with the flour. The molted butter, the treacle, and the acid dissolved in a little water, are poured all at onco amongst the flour, and kneaded into a consistent dough, which being sct aside for half an hour or an hour, will be ready for the ovon, and should never be kept unbakod for moro than 2 or 3 hours. The following more completo recipe is givon by Dr. Colquhoun for making thin gingerbread cakes :-Flour $1 \mathrm{lb}$., treacle $\frac{1}{2} \mathrm{lb}$., raw sugar $\frac{1}{4} \mathrm{lb}$., butter 2 ounces, carbonato of magnesia $\frac{1}{4}$ ounce, tartaric acid $\frac{1}{8}$ ounce, ginger $\frac{1}{8}$ ounco, cinnamon $\frac{1}{8}$ ounce, nutmeg 1 ounce. This compound has rather moro butter than common thin gingerbread. In addition to these, yellow ochre is frequently added by cheap gingerlread makers, and altogether this preparation, more generally consumed by children, is very objectionable.

Within the last fow years there has been a very remarkable devolopment of the trade in biscuits. Biscuits of all sorts, and really many curious and agreeablo varieties, are now manufactured on a large scale, and machinery has been created to facilitate the process.

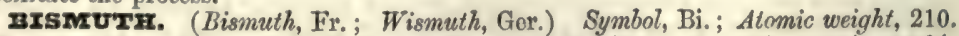
The motal bismuth occurs chiofly in a nativo state, but is also found is certain combirations, forming tho ores noticed in the following list of bismuth-bearing minerals:-

Native Bismeth is whitish, with a faint reddish tingo, and a metallic lustro which is liable to tarnish. Strcak, silver-white. Hardness, 2 to $2 \cdot$ é specific gravity, $9 \cdot 727$. It is lrittlo whon cold, but slightly malleablo when heatod. It generally occurs crystallised or in a dendritic form. It fuses readily at $476^{\circ} \mathrm{F}$. Beautiful crystals can be formed artificially by fusion and subsequent slow cooling. The native motal frequently contains silver, arsenic, or tellurium. It is the source of all the motal used in the 
Natiro bismuth has beon found, associated with other minerals, in Cornwall, at Dolcoath near Camborne; at Huel Sparnon, noar Rodruth, when that mine was worked; at Trugoo Mine, near St. Colomb (Grog), and at the Consolidated Mines, St. Ires; Caldbeck Fells, in Cumberland, with ores of cobalt. But the most abundant sources of native bismuth are the silver and cobalt mines of Saxony and Bohenial, especially those of Schneoborg, Aunaberg, Marienberg, Altenberg, Joachimsthal and Johanngeorgenstadt. It is also found at Sorato, in Bolivia.

Bismuthine, Bismuth glance, or sulphide of bismuth, occurs either in acicular crystals, or with a foliated, fibrous structure. It is isomorphous with stibnite, or sulphide of antimony. Hardness, 2 to $2 \cdot 0$ : specific gravity, 6.4 to 6.9 . It is composed of bismuth, $81 \cdot 25$; sulphur, 18.75 . It fuses in the flame of a candle.

Bismuthine occurs in Cornwall, at Botallack, and associated with tin at St. Just, and with copper at the mines near Redruth and Camborne. Large quantities aro now worked and smelted in South Australia.

Acicular Bismuth, or Aikinite, called also Needle Ore, is a plumbo-cupriforous sulphide of bismuth, composed of sulphur, 16 ; bismuth, 34.62 ; lead, 35.69 ; copper, $11 \cdot 79$.

Emplectite, or Tannenite, is a rare mineral, containing sulphur, 18.83 ; bismuth, $62 \cdot 16$; copper, $18 \cdot 72$.

Telluric Bismuth, or Tetradymite, oceurs in Cumberland, at Brandy Gill, Carrock Fells (Grog), and at Dolgelly in Merionethshire.

The purest varietios contain only bismuth and tellurium, in proportions ropresented by the formula, $\mathrm{Bi}^{2} \mathrm{Te}^{3}$, corresponding to tellurium, 48.1, and bismuth, 51.9 per cont. Other varioties contain sulphur and selenium. It is notable that tetradymite is often found associated with gold.

Bismuth Ochre.-A dull earthy mineral, found in the Royal Restormel Iron Mine, and in small quantities in the parish of Roach, in Cornwall. Its composition is stated by Lampadius to be :-Oxide of bismuth, 86.4 ; oxide of iron, $5 \cdot 1$; carbonic acid, $4 \cdot 1$; and water, $3 \cdot 1$.

Carlonate of Bismuth, or Bismutite. This ore is composod of a mechanical mixture of the carbonates of bismuth, of iron, and of copper.

Eulytine or bismuth-blende is a silicate of bismuth, occurring in small erystals in the mines of Schneeberg, in Saxony.

Bismuth, which was formerly known as Marcasite and as tin-glance, was shown to be a metal 'somewhat different from lead' by G. Agricola, in 1546. It was studied by Stahl and Dufay, and still more minutely by Pott and Geoffroy, about the middle of the last century.

This metal, the demand for which is limited, is chiefly procured in Saxony, from the mines of Schneeberg; where it occurs mixed with cobalt speiss, in the proportion

105

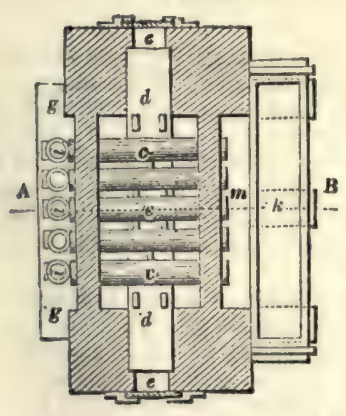

106
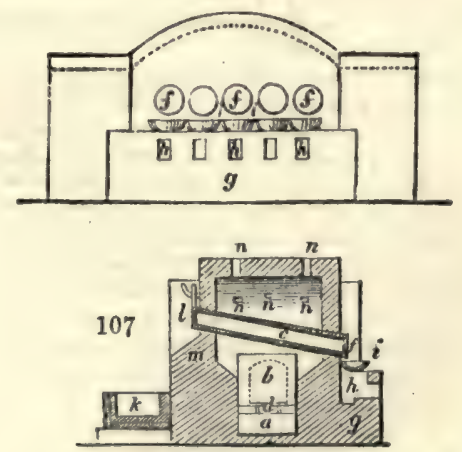

of about 7 per cent. In the metallurgical works at Schneoberg the motal is obtained by means of a peculiar furnace of liquation. This furnace is represented in figs. 105 and 106, of which the first is a view from above, the second a view in front; and fig. 107 is a transverse section on the dotted line A, B, of fig. 106. $a$ is the ash-pit; $b$, the fire-place; $c$, the eliquation pipes ; $d$, the grate, of masonry or brickwork, upon which the fuel is thrown through the fire-door, $e e$. The anterior deeper-lying orifice of the eliquation pipes is elosed with the clay-plate, $f$, which has beneath a small circular groore, through which the liquefied metal flows off; $g$ is a wall extending from the hearth-sole nearly to the anterior orifices of the liquation-pipes, in 
which wall there are as many fire-holes, $h$, as there are pipes in the furnace; $i$ are iron pans which recoive tho fluid nctal; $k$, a woodon wator-trough, in which the bismuth is granulated and coolod; $l$, tho postorior and higher-lying apertures of the eliquation pipes, shut moroly with a sheet-iron cover. The granulations of bismuth drained from the posterior openings fall upon the flat surfaces $m$, and then into the water-trough. $n n$ are draught-holes in the vault between the two pipes, which serve for increasing or diminishing the heat at pleasure.

The ores to be eliquated (sweated) are surted by hand from the gangue, brokon into pieces about the size of a hazol nut, and introduced into the ignited pipes; ono charge consisting of about $\frac{1}{2}$ ewt. ; so that the pipes are filled to half their diamoter, and three-fourths of their length. The shect-iron door is shut, and the fire strongly urged, whereby the bismuth begins to flow in ten minutes, and falls through the holes in the clay-plates into hot pans containing some coal-dust. Whenever it runs slowly, the ore is stirred round in the pipes, at intervals during half an hour, in which time the liquation is usually finishod. The residuum, ealled bismuth barley (Graupen), is scooped ont with iron rakes into a water-trough; and the pipes are charged afresh; the pans, when full, have their contents cast into moulds, forming bars of from 25 to 50 lbs. weight. About 20 cwt. of ore are smelted in 8 hours, with a consumption of 63 Loipsic cubic feot of wood. The bismuth thus procured by liquation upon the great scale contains no small admixture of arsenic, iron, and some other metals, from which it may be freed by solution in nitric acid, precipitation by water, and reduction of the sub-nitrate by black flux. By exposing tho crude bismuth for somo time to a dull red heat, under charcoal, arsenic is expelled.

Bismuth is also obtained as a by-product in treating cortain ores of cobalt and silver. A solution of the nitrate or chloride of bismuth is precipitated by addition of water, and the basic salt thus obtained is dried and reduced with carbonate of soda and charcoal.

Bismuth is white, and resembles antimony, but has a reddish tint; whereas the latter metal has a bluish cast. It is brilliant, and crystallises readily in small cubolike forms, often hollow, which are really rhombohedra, though long mistaken for true cubes. The beautiful iridescence often seen on specimens of crystallised bismuth is produced artificially. The metal is very brittle, and may be easily reduced to powder. Its specific gravity is 9.83 ; and it is said that by hammering it with care, the density may be increased to 9.8827 . It melts at $515^{\circ} \mathrm{F}$. $\left(268.3^{\circ} \mathrm{C}\right.$.. Riomsdijk), and may bo cooled $6^{\circ}$ or $7^{\circ}$ below this point without fixing; but the moment it begins to solidify, the temperature rises to $480^{\circ}$, and continues stationary till the whole mass is congealed. Bismuth, like cast-iron, expands during solidification.

When heated from $32^{\circ}$ to $212^{\circ}$, it expands $\frac{1}{710}$ in length. When pure it affords a very valuable means of adjusting the scale of high-ranged thermometers. At strong heats bismuth volatilises, may bo distilled in close ressels, and is thus obtained in erystalline laminæ.

Bismuth is readily soluble in nitric acid, but is almost unacted on by hydrochloric or by sulphuric acid.

Several alloys of bismuth are used in the arts. The alloy of bismuth and lead in equal parts has a density of $10 \cdot 709$, being greater than the mean of the constituents ; it has a foliated texture, is brittle, and of the same colour as bismuth. Bismuth, with tin, forms a compound more elastic and conorous than the tin itself, and is, therefore, frequently added to it by the pewterers. With 1 of bismuth and 24 of tir, the alloy is somewhat malleable: with more bismuth it is brittle. When much bismuth is present, it may be easily parted by strong muriatic acid, which dissolves the tin, and leaves the bismuth in a black powder. It has been said that an alloy of tin, bismuth, nickel, and silver hinders iron from rusting.

Tho alloy of bismuth with tin and lead was first examined by Sir I. Nowton, and has been called ever since fusible metal. The French give to this alloy the name of métal fusible de $D^{\prime}$ Arcet, and thus claim for him the merit of the discovery of it. 8 parts of bismuth, 5 of lead, and 3 of tin, molt at the moderate temperature of $202^{\circ} \mathrm{F}$; but 2 of bismuth, 1 of lead, and 1 of tin, melt at $200.75^{\circ} \mathrm{F}$., according to Rose. A small addition of mercury, or of cadmium, aids the fusibility. Such alloys serve to take casts of anatomical preparations. The value of these bismuth-alloys for taking casts is due in great measure to their expansion in cooling-a sharpimpression being thus secured. Indoed, the behaviour of fusible metal on exposure to heat is quite anomalous. It is said to dilate regularly from $32^{\circ}$ to $95^{\circ} \mathrm{F}$., then to contract to $131^{\circ}$, when it expands rapidly till it reaches $176^{\circ}$, and from that point again expands uniformly until it fuses.

An alloy of 1 bismuth, 2 tin, and 1 lead, is omployed as a soft solder by the pewterers; and the same has been proposed as a bath for tempering steel instruments. Cake-moulds for the manufacture of toilot soaps are made of the same metal; as alco 
excellent cliohes for sterootype, of 3 lead, 2 tin, and 5 bismuth-an alloy which melts at $199^{\circ} \mathrm{F}$. This compound should be allowed to cool upon a piece of pasteboard till it becomes of a doughy consistence, before it is appliod to the mould to receive the impross of the stamp. This alloy is also used for the metallic pencils for writing on the prepared paper of pocket-books.

The employment of plates of fusible metal as safoty rondelles to apertures in the tops of steam boilers was proposed in France, on the assumption that they would melt and give way at clevations of temperature under those which would ondanger tho bursting of the vessel, the fusibility of the alloy being proportioned to the quality of steam required for the engine. It has been found, however, that boilers, apparently secured in this way, burst, while the safety dises remained entire; the expansive forco of the steam causing explosion so suddenly, that the fusible alloy had not time to molt or give way.

Bismuth is interesting as being a highly diamagnetic metal. The distinction between magnetic and diamagnetic bodies was established by the late Dr. Faraday. This may be familiarly explained, by stating that one class of bodies is influenced by magnets, as iron is. being magnetic. That is, if a bar of iron was hung up between the poles of a horse-shoe magnet, it would arrange itself along the line which unites the two poles; which line has been called the axial line. But if another class of bodies be selected, bismuth being at the head of this class, and suspended in the same way between the poles of the magnet, they arrange themselves across the axial line, or, as Faraday has termed it, equatorially, these bodies being called in distinction diamagnetic bodies. See MAGNETrsar, for a further account of these phenomena.

The mines of Schneeberg produce annually about 4,000 kilogrammes of metallic bismuth; those of Johanngeorgenstadt and the cobalt mines of Saxony, about 600 kilogrammes-equal to about 10,500 avoirdupois pounds.

In 1844 bismuth was sold at from $10 d$. to $2 \delta$. the pound; in 1872 it had reached the high price of $30 \mathrm{~s}$. the pound; for some time this price was maintained. Up to 1844 a large quantity of bismuth was produced in this country from cobalt ores in the old way of refining, but a new mothod was then introduced which necessitated the loss of nearly all the bismuth. In 1845 there was a large demand for a composition to make rollers for calico-printers, which advanced the price. In 1858 the supply began to fall off, and in 1861-2 there was an increased demand for this metal for medicinal purposes, but there has been recently a considerable reduction. A company was formed in London, under the guidance of a German, who professed to have the secret of transmuting the baser metals into gold. This company had works in the Belvedere Road, Iambeth, and as bismuth was considered essential in the process, they bought all they could lay hands on. This, it is believed, more than anything led to the rapid increase in the price of the metal. The supply of bismuth is in but very few hands, and great care is taken to prevent any excess orer the demand from coming into the market; by this means the price is kept up.

Solid compounds of bismuth, when heated before the blowpipe with carbonate of soda and charcoal, yield a bead of metallic bismuth, surrounded by an incrustation of yellow oxide. Salts of bismuth in solution are recognised by becoming milky white when diluted with water, owing to the formation of sparingly soluble basic salts. These basic precipitates may be distinguished from similar sub-salts of antimony by being insoluble in tartaric acid, and by bocoming black when acted on by sulphuretted hydrogen.

In 'Watts's Dictionary of Chemistry' will be found various methods for the determination of bismuth. The following processes, howover, appear so useful as to warrant their insertion in this place:- To detect small quantitios of lead in bismuth, or in bismuth compounds, Chapman brings the somewhat flattened bead, reduced before tho blowpipe, in contact with some moist basic nitrate of teroxide of bismuth, when, in a short time, in consequenee of the reduction of the bismuth by tho lead, arborescent sprigs of bismuth are formed around the test specimen. Since zinc and iron interfere with this reaction, they must bo proviously removed, the former by fusion with soda, the latter with soda and borax, in the reducing flame.

Lead and bismuth can easily be quantitatively separated from each other by the following method, proposed by Ullgren:-The solution of the two metals is precipitated by carbonato of ammonia, and the carbonates are then dissolved by acetic acid, and a blado of pure lead, tho weight of which is ascertained beforehand, is plunged in the solution. This blade must be completely immersed in the liquor. The vessel is then corked up, and the experiment is left for several hours at rest. The lead precipitates the bismuth in the metallic form. When the whole of it is precipitated, tho blade of lead is withdrawn, washed, dried, and woighed. Tho bismuth is collected on a filter, washod with distilled water which hns boen pre- 
viously boilod, and cooled out of contact of the air; this metal is then treatod with. carbonate of ammonia, and the precipitato which is left, after washing and ignition, is thon woighed. The total loss of the motallie load employed indicates how much oxide of lead must be subtracted from the total woight of the protoxide of lead obtained.

Bisurutr, Oxmes of.-There are two well-dofined oxides of bismuth-the toroxide and the pentoxido, with an unimportant intermediate oxide. Of these compounds it is only necessary to notice the teroxide or bismuthous oxide $\mathrm{BiO}^{s}\left(\mathbf{m i}^{2} \mathbf{O}^{3}\right)$. This is found native as bismuth-ochre, and may bo readily formed by oxposing the metal to a redwhite heat in a muffle, when it takes fire, burns with a fuint blue flame, and emits fumes which condense into a yollow pulverulont oxido. But an easier process is to ignite the nitrate or earbonate. The oxide thus obtained has a straw-yellow colour, and fuses at a high heat into an opaque glass of a dark-brown or black colour; but which becomes luss opaque and yellow after it has cooled. Its speeific gravity is as high as 8.211 . It consists of 89.87 of metal and 10.13 of oxygen in 100 parts. The only salt of this oxide used in the arts is the nitrate, which is obtained by dissolving metallic bismuth in warm nitric acid. On largely diluting a solution of the nitrate with water, a sub-nitrate or basic-nitrate is procipitated. This precipitate was termed by the older ehemists, 'magestery of bismuth,' and is now sometimes called pearl-white, and is employed as a flux for cortain enamels, as it augments their fusibility, without imparting any colour to them. Henee it is used sometimes as a vehicle of the colours of other motallic oxides. When woll washed, it is employed in gilding poreelain; being added in the proportion of ono-fifteenth to the gold. But pearl-white is most used by ladies, as a cosmetic for giving a delicate whiteness to a fuded complexion. It is called blanc de fard by the Fronch. If it contains, as bismuth often does, a little silver, it becomes groy or dingy-coloured on exposure to light. Another sort of pearlpowder is prepared by adding a very dilute solution of common salt to the above nitric solution of bismuth, whereby a pulverulent sub-chloride of the metal is obtained in a light floceulent form. A similar powder of a mother-of-pearl aspect may be formed by dropping dilute muriatic acid into the solution of nitrate of bismuth. The arsonic always prosent in the bismuth of commerce is convertod by nitric acid into arsenic acid, which, forming an insoluble arsenate of bismuth, separates from the solution unless there be such an excess of nitric acid as to re-dissolve it. Hence tho medicinal oxide, proparod from a rightly-mado nitrato, can contain no arsenic. If wo write with a pen dipped in that solution, the dry invisiblo traces will become legible on plunging the paper in water.

The nitrato of bismuth, mixed with a solution of tin and tartar, has been employed as a mordant for dyoing lilac and violet in calico-printing.

When the oxide is propared, by dropping the nitric solution into an alkaline lye in excess, if this precipitate is well weshed and dried, it forms an excellent medicine; and is given, mixed with gum tragacanth, for the relief of cardialgin, or burning and spasmodic pains of the stomach.

This sub-nitrate of bismuth is now commonly employed as a romedial agent, under circumstances which are especially liable to attack the emigrant; it is, thereforo, thought advisable to give some account of its action. The following is extracted from Pereira's 'Elements of Materia Medica,' by Bentley and Redwood:-

'Physiolngical Effects.-In small doses it acts locally as an astringont, diminishing secretion. On account of the frequent relief given by it in painful affections of the stomach, it is supposed to act on the nerves of this viseus as a sedative. It has also been denominated tonic and antispasmodic. Vogt says, that when used as a cosmetic, it has produced a spasmodic trembling of the face, ending in paralysis.

- Large medicinal doses disorder the digestive organs, occasioning pain, vomiting, purging, \&c.; and sometimes affecting the nervous system, and producing giddiness, insensibility, with cramps of the extremities. On the other hand, M. Momeret states, after several years' trial of this medicine, that it may be givon in much larger doses than are usually administered, and that it is then of the greatest value in gastrointestinal affections, especially those attended with fluxes.

' Therapeutics. - It has been prineipally omployed in those chronic affections of the stomach which are unaccompanied by any organic disease, but which apparently depend on some disordered condition of the nerves of this viscus; and hence, the efficacy of the remedy is referred to its supposed action on these parts. It has been particularly used and recommonded to relieve gastrodynia and cramp of the stomach, to allay sickness and vomiting, and as a romedy for pyrosis or water-brash. In the latter disease I give it in the form of a powder, in doses of twenty grains thrice daily, in conjunction with hydrocyanic acid mixture, and the patient rarely fails to obtain marked benefit from its use. It is also used in ulcer of the stomach. Dr. Thoophilus Thompson recommends it in doses of fire grains, combined with gum arabic and 
magnesia, in the diarrhoea accompanying phthisis, and he thinks that both in efficacy and safety, it surpasses our most approved remedies for that complaint. I havo used it with advantage, in the form of ointment, applied to the septum nasi, in ulceration of this part, and as a loeal remedy in chronic skin diseases.'

Much of the sub-nitrate of bismuth of the shops has beon found to contain nitrate of silvor.

aISTRz. (Bistre; Fr.; Bister, Ger.) A brown colour which is used in watercolours, in the same way as China ink. It is prepared from wood-soot, that of beech being preforred. The most compact and best-burned parcels of soot aro collected from the chimney, pulverised, and passed through a silk sieve. This powder is infused in pure water, and stirred frequently with a glass rod, then allowed to settle, and the water decanted. If the salts are not all washed away, the process may be repeated with warm water. Tho paste is now to be poured into a long narrow vessel filled with water, stirred well, and left to settle for a fow minutes, in order to let the grosser parts subside. The supernatant part is then to be poured off into a similar vessel. This process may be repeated twico or thrico, to obtain a vory good bistre. At last the settled deposit is suffieiontly fine, and, when freo from its supernatant water, it is mixed with gum-water, moulded into proper eakes and dried. It is not used in oil-painting, but has the same effect in water-colours as brown pink has in oil.

Dr. MacCulloch objects to soot as a source of bistre, both from the carelessness used in collecting it, and the uncertainty of tone and colour. If the liquids resembling tar, obtained from the distillation of wood, be again carefully distilled, water, acetic acid, and hydrocarbonaceous substances, as naphtha, pass over, and leave a residuum-brown or black, pitch-like, or brittle-according to the time and temperature employed; by prolonging the heat with care, the brittle substance becomes a powder. Dr. MacCulloch states that, by care, bistre from wood-tar may be obtained, having the fine properties of sepia with great depth of colour.

The remarkable bronze-like varnish, with almost a metallie lustre, seen upon the interior of Highland cottages, are bistre-deposits from the smoke of peat.

BTTTER-AIMOND OIx. See ALMOND OIL.

MITTER-AIMOND WATER. Water containing the oil of bitter almonds in solution. It should be prepared by distillation from the bitter almonds. It is however frequently mado by first rubbing the essential oil of bitter almonds with magnesia and mixing this with water; or a solution of the oil in spirits of wine is simply added to the water and they are shaken together.

BITTER CUPS. Woodon eups in which water or other liquid is allowed to stand until it aequires a bitter taste, and thus serves medicinally as a tonic. These cups are turned in 'bitter wood,' or wood from a West Indian tree-the Picrena excelsa, Ldl.,- and from other plants belonging to the quassia order.

RITTERN. The mother-liquor of sea-water left after the crystallisation of the less soluble salts. The bittern is used as a source of bromine.

aITTER pRINCIPIE. (Amer, Fr.; Bitterstoff, Ger.) The 'bitter principles" consist of bodies which may be extracted from vegetable productions by the ageney of water, alcohol, or ether. These are not of much importance in the arts, with a few exceptions. Lupulin, the bitter principle of the hop, for example, is used for preserving beer.

Quassin is the bitter principle of quassia; Absinthin, that of wormwood; and Gentianin, that of Gentian. These are sometimes substituted for the hop.

For particulars of these, and numerous other bitter principles, see 'Watts's Dictionary of Chemistry.'

The following list gives the more important of the bitter substances which have boon used in the arts and in medicine. (See the articles respectivoly) -

\begin{tabular}{|c|c|c|c|c|c|}
\hline Name & & Part emy & ployed & Country & Obeervations \\
\hline Quassia . & & Wood & & Surinam, E. Indies & Powerfully bitter \\
\hline Wormwood & & Herb & & Great Britain & Ditto \\
\hline Aloe ... & & Inspissate & ed juico & South Africa & Ditto \\
\hline Angusture & - & Bark &.$\quad$. & South America & Ditto \\
\hline Orange : & . & $\ldots$ & • & South of Europe . & Aromatic bitter \\
\hline Acorus : & & Root & $*$ & Ditto . & Ditto \\
\hline Carduus bened & lictus & Herb & & Greek Archipelago & \\
\hline Cascarilla & . & Bark & . & Jamaica . & Ditto \\
\hline Centaury & - & Herb & $\therefore$ & Great Britain & \\
\hline Camomile & - & Flowers & - & Ditto : & Ditto \\
\hline Colocynth & . & Fruit & . & Iovant. & Intolerably bitter \\
\hline
\end{tabular}




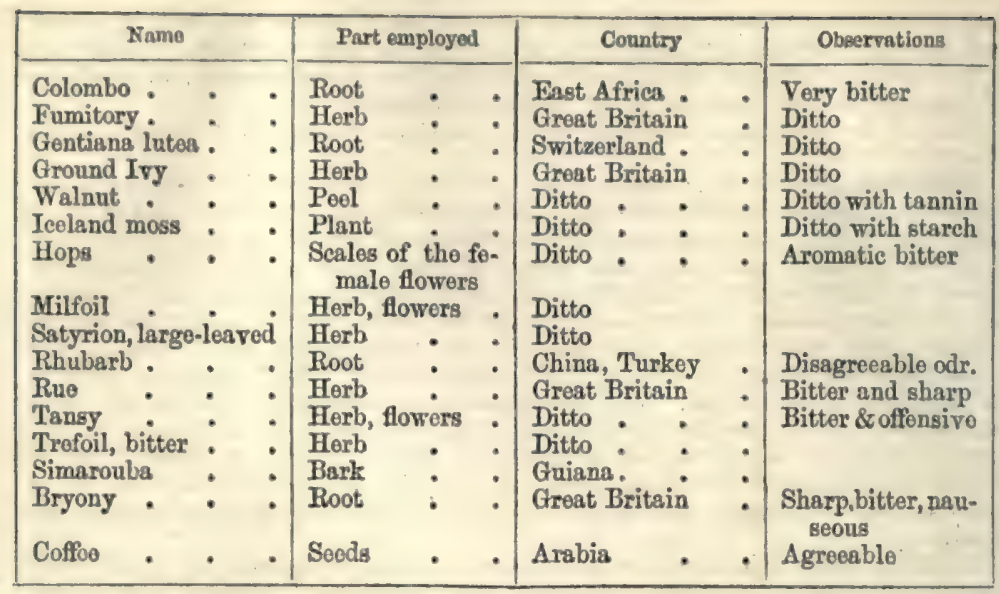

MITTER spare. A carbonate of lime and carbonate of magnesia. Soe DoloMrTE.

BITUMses. (Bitume, Fr.; Erdpech, Ger.)-This term, as commonly applied, comprises a number of solid viscid and liquid substances, resembling pitch, tar, naphtha and the like, and consisting mainly of native hydrocarbons, more or less oxygenated. Most of these appear to be too variable in composition to take rank as distinct mineralogical species, and our knowledge of the chemical constitution of many of them is still very imperfect. Professor Dana, in the last edition of his 'System of Mineralogy' (1868), has proposed a valuable scientific classification of the numerous hydrocarbons occurring in nature. It seems convenient, however, for practical purposes to adhere to the older and more popular, if less philosophical arrangement.

Bitumen comprises several distinct rarieties, of which the two most important are asphaltum and naphtha.

Asphaltum or mineral pitch is solid, and of a black, or brownish-black, colour, with a conchoidal brilliant fracture. It is sometimes called Bitumen of Judea, from its occurronce on the Dead Sea, or Lake Asphaltites. See Aspratr.

Naphtha.-Liquid and colourless when pure, with a bituminous odour. Seo Naphtra. The darker-coloured varieties are fully described under Petrolecrs.

Springs, of which the waters contain a mixture of petroleum, and the various minerals ullied to it-as bitumen, asphaltum, and pitch-are very numerous, and are, in many cases, undoubtedly connected with subterranean heat, by the agency of which organic remains undergo some of those romarkable changes which ultimately result in the formation of coal. Within a few years many discoveries have been made of sources of fluid bitumen and petroleum in both the Old and Now Worlds. The importance of these natural products renders it advisable to comprehend a description of them under one general head. In one locality there are said to be 520 wells, which yiold annually 400,000 hogsheads of potroleum. See Petroleur.

Fluid bitumen is seen to ooze from the bottom of the sea on both sides of the island of Trinidad, and to rise up to the surface of the water. It is stated that, about seventy years ago, a spot of land on the western side of Trinidad, nearly half-way between the capital and an Indian village, sank suddenly, and was immodiately replaced by a small lake of pitch. In this way, probably, was formed the celobrated Great Pitch Lake. Sir Charles Lyell romarks:- "The Orinoco has for ages been rolling down great quantities of woody and vegetable bodies into the surrounding sea, where, by the influence of currents and eddies, they may be arrested and accumulated in particular places. The frequent occurrence of earthquakes, and other indications of volcanic action in those parts, lend countonance to the opinion that these vogetable substances may have undergone, by the agency of subterranean fire, those transformations or chomical changes which produce petroleum; and this may, by the same causes, bo forced up to the surface, where, by exposuro to the air, it becomes inspissated, and forms the different varieties of pure and earthy pitch, or asphaltum, so abundant in the island.'

The Pitch Lake is one and a half miles in circumference; the bitumen is solid and cold near the shores, but gradually increases in temporature and softness towards the centre, where it is boiling. The solidified bitumen appears as if it had cooled, as the 
surface boiled, in large bubbles. The ascent to the lake from the sea, a distance of threequarters of a mile, is covered with a hardened pitch, on which trees and vegetables flourish; and about Point la Braye, tho masses of pitch look like black rocks among the foliage : the lake is underlaid by a bed of mineral coal.-(Manross, quoted by Dana.)

The Earl of Dundonald remarks, that vegetation contiguous to the lake of Trinidad is most luxuriant. The best pine-apples in the West Indies (called black pines) grow wild amid the pitch.

Asphaltum, or solid bitumen, is abundant on the shores of the Dead Sea. It occurs in the mountain-limestone of Derbyshire and Shropshire, and has been found in granite, with quartz and fluor spar, at Poldice, in Cornwall. There is a remarkable bituminous lime and sandstone of the region of Bechelbronn and Lobsann, in Alsace. From the observations of Daubrée, we learn that probably this bitumen has had its origin as an emanation from the interior of the earth; and indeed, in Alsace, with the great elevated fissure of the sandstone of the Vosges, a fissure which was certainly open before the deposit of the Trias, but was not yet closed during the tertiary epoch, affording during this latter, moreover, an opportunity for the deposition of spathic iron ore, imon pyrites, and heavy spar.-Annales des Mines.

Bituminous limestones are also found abundantly at Pyrimont, near Seyssel, in the Dép. de l'Ain, France, and in the Val de Travers, Neufchatel, in Switzerland. Both these rocks have been worked for the sake of their bitumen.

In addition to the bituminous substances already mentioned-asphalt, naphtha, and petroleum-there are a number of closely related minerals, such as pittasphalt and maltha, or mineral tar; elaterite or elastic bitumen; hatchettine, or mineral tallow, ozoccrite, \&c. The more important of these are described under their respectiva names.

Of ordinary bitumen, we give ultimate analyses of two specimens: one by Ebelmen, who obtained his sample from the Auvergne; and the other by Boussingault, of a Peruvian specimen :-

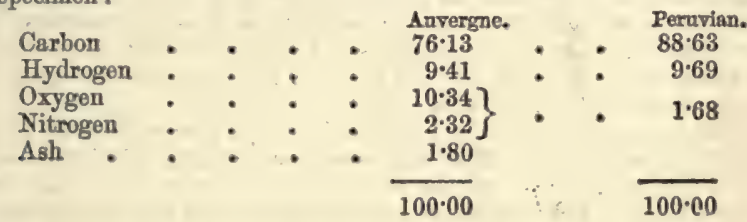

Bitumen in many of its varieties was known to the ancients. It was used by them combined with lime, in their buildings. Not only do we find the ruined walls of temples and palaces in the East, with the stones cemented with this material, but some of the old Roman castles in this country are found to hold bitumen in the cement by which their stones are secured. At Agrigentum it was burnt in lamps, and called 'Sicilian oil.' The Fgyptians used it for embalming.-Dana.

On the employment of bitumen for pavements, Dr. Ure has the following remarks :It is a very remarkable fact, in the history of the useful arts, that asphalt, which was so generally employed as a solid and durable cement in the earliest constructions upon record, as in the walls of Babylon, should for so many thousand years have fallen well nigh into disuse among civilised nations. For there is certainly no class of mineral substances so well fitted as the bituminous, by their plasticity, fusibility, tenacity, adhesireness to surfaces, impenetrability by water, and unchangeableness in the atmosphere, to enter into the composition of terraces, foot-pavements, roofs, and every kind of hydraulic work. Bitumen, combined with calcareous earth, forms a compact semi-elastic solid which is not liable to suffer injury by the greatest alternations of frost and thaw, which often disintegrate in a fow years the hardest stone, nor can it be gxound to dust and worn away by the attrition of the feet of men and animals, as sandstone, flags, and even blocks of granite are. An asphalt parement, rightly tempered in tenacity, solidity, and elasticity, seems to be incapable of suffering abrasion in the most crowded thoroughfares; a fact exemplified of late in a fow places in London, but much more extensively, and for a much longer time, in Paris.

The great Place de la Concorde (formerly Place Louis Quinze) is covered with a beautiful mosaic pavement of asphalt; many of the promenades on the Boulevards, formerly so filthy in wet weather, are now covered with a thin bed of bituminous mastic, free alike from dust and mud; the foot-paths of the Pont Royal and Pont Carousel, and the areas of the great public slaughter-houses, have been for several years paved in a similar manner with perfect success. It is much to be regretted that the asphalt companies of Iondon made the ill-judged, and nearly abortive, attompt Vor. I. 
to pave the carriage-way near the east end of Oxford Streot, and especially at a moist season, most unpropitious to the laying of bituminous mastic. Being formed of blocks not more than three or four inches thick, many of which contained much siliceous sand, such a pavement could not possibly resist the crash and vibration of many thousand heavy drays, waggons, and omnibuses daily rolling over it. This failure can afford, however, no argument against rightly-constructed foot-pavemonts and terraces of asphalt. Numerous experiments and observations have led mo to conclude that fossil bitumen possesses far more valuable propertios for making a durable mastic than the solid pitch obtained by boiling wood or coal tar. The latter, when inspissated to a proper degree of hardness, becomes brittle, and may be readily crushed into powder; while the former, in like circumstances, retains sufficient tenacity to resist abrasion. Factitious tar and pitch being generated by the force of fire, seem to have a propensity to decompose by the joint agency of water and air, whereas mineral pitch has been known to remain for ages without alteration.

Bitumen alone is not so well adapted for making a substantial mastic as the native compound of bitumen and calcareous earth, which has been properly called asphaltic rock, of which the richest and most extensive mine is unquestionably that of the Val de Travers, in the canton of Neufchatel. This interesting mineral deposit occurs in the Jurassic limestone formation, the equivalent of the English oolite. The mine is very accessible, and may be readily excavated by blasting with gunpowder. The stone is massive, of irregular fracture, of a liver-brown colour, and is interspersed with a few minute spangles of calcareous spar. Though it may be scratched with the nail, it is difficult to break by the hammer. When exposed to a very moderate heat, it exhales a fragrant ambrosial smell, a property which at once distinguishes it from all compounds of factitious bitumen. Its specific gravity is $2 \cdot 114$,-water boing $1 \cdot 000$, being nearly the density of bricks. It may be most conveniently analysed by digesting it in successive portions of hot oil of turpentine, whereby it affords 80 parts of a white pulverulent carbonate of lime, and 20 parts of bitumen in 100 . The asphalt rock of Val de Travers seems therefore to be far richer than that of Pyrimont, which, according to the statement in the specification of Claridge's patent of November, 1837, contains 'carbonate of lime and bitumen in about the proportion of 90 parts of carbonate of lime to about 10 parts of bitumen.'

The calcareous matter is so intimately cumbined and penetrated with the bitumen as to resist the action not only of air and water for any length of time, but even of muriatic acid; a circumstance partly due to the total absence of moisture in the mineral, but chiefly to the vast incumbent pressure under which the two materials have been incorporated in the bowels of the earth. It would indeed be a difficult matter to combine, by artificial methods, calcareous earth thus intimately with bitumen, and for this reason the mastics made in this way are found to be much more perishable. Many of the factitious asphalt cements contain a considerable quartity of siliceous sand, from which they deriva the property of cracking and crumbling down when trodden upon. In fact, there seems to be so little attraction between siliceous matter and bitumen, that their parts separate from each othor by a very small disruptive force.

Since the asphalt-rock of $\mathrm{Val}$ de Travers is naturally rich enough in concrete bitumen, it may be converted into a plastic workable mastic of excellent quality for footpavements and hydraulic works at very little expense, merely by the addition. of a very small quantity of mineral or coal tar, amounting to not more than 6 or 8 per cent. The union between these materials may be effected in an iron cauldron, by the application of a very moderate heat, as the asphalt-bitumen readily coalesces with the tar into a tenacious solid.

The mode adopted for making the asphalt pavement at the Place de la Concorde in Paris was as follows:-The ground was made uniformly smooth, either in a horizontal plane or with a gentle slope to carry off the water; the curb-stones were then laid round the margin by the mason, more than 4 inches above the level of the ground. This hollow space was filled to a depth of 3 inches with concrete, containing about a sixth part of hydraulic lime, well pressed upon its bed. The surface was next smoothed with a thin coat of mortar. When the whole mass had become perfectly dry, the mosaic pattern was set out on the surface, the moulds being formed of flat iron bars, rings, \&c., about half an inch thick, into which the fluid mastic was poured by ladles from a cauldron, and spread evenly orer.

The mastic was made in the following way:-The asphalt rock was first of all roasted in an oven, about 10 feet long and 3 broad, in order to render it friable. The bottom of the oven was sheet-iron, heated below by a brisk fire. A volatile matter exhaled, probably of the nature of naphtha, to the amount of one-fortieth the weight of asphalt ; after roasting, the asphalt became so friable as to be easily reduced 
to powder, and passed through a sieve having meshes of about one-fourth of an inch square.

The bitumen destined to render the asphalt fusible and plastic was melted; in small quantities at a time, in an iron cauldron, and then the asphalt in powder was gradually stirred in to the amount of 12 or 13 times the weight of bitumen. When the mixture became fluid, nearly a bucketful of very small, clean gravel, previously heated apart, was stirred into it; and, as soon as the whole began to simmer with a treacly consistence, it was fit for use. It was transported in buckets, and poured into the moulds.

For the reasons above assigned, I consider this addition of rounded, polished, siliceous stones to be very injudicious. If anything of the kind be wanted to give solidity to the parement, it should be a granitic or hard calcareous sand, whose angular form will secure the cohesion of the mass. I conceive also, that liquid bitumen in moderate quantity should be used to give toughness to the asphaltic combination, and prevent its being pulverised and abraded by friction.

In the able report of the Bastenne and Gaujac Bitumen Company, drawn up by Messrs. Goldsmid and Russel, these gentlemen have made an interesting comparison between the properties of mineral tar and vegetable tar: the bitumen composed of the latter substance, including various modifications extracted from coal and gas, has, so far as they were able to ascertain, entirely failed. This bitumen, owing to the qualities and effects of vegetable tar, becomes soft at $115^{\circ}$ of Fahrenheit's seale, and is brittle at the freezing point; while the bitumen into which mineral tar enters will sustain $170^{\circ}$ of heat without injury. In the course of the winter, 1837-38, when the cold was at $142_{2}^{\circ}$ below zero C., the bitumen of Bastenne and Gaujac, with which one side of the Pont Neuf at Paris is paved, was not at all impaired, and would, apparently, have resisted any degree of cold; while that in some part of the Boulevard, which was composed of vegetable tar, cracked and opened in white fissures. The French Government, instructed by these experiments, has required, when any of the vegetable bitumens are laid, that the pavement should be an unch and a quarter thick; whereas, where the bitumen composed of mineral tar is used, a thickness of three-quarters of an inch is deemed sufficient. The pavement of the bonding warehouses at Bordeaux has been laid upwards of 15 years by the Basterne Company, and is now in a condition as perfect as when first formed. The reservoirs constructed to contain the waters of the Seine, at Batignolles, near Paris, have been mounted six years, and notwithstanding the intense cold of the winter of 1837 , which froze the whole of their contents into one solid mass, and the perpetual water-pressure to which they are exposed, they have not betrayed the slightest imperfection in any point. The repairs done to the ancient fortifications at Bayonne have answered so well, that the Government many years ago entered into a very large contract with the company for additional works, while the whole of the arches of St. Germain and St. Cloud railways, and the parements and floorings necessary for these works, have been laid with Bastenne bitumen.

The mineral tar in the mines of Bastenne and Gaujac is easily separated from the earthy mattor with which it is naturally mixed, by the process of boiling, and is then transported in barrels to Paris or London, being laid down in the latter place to the company at $17 l$. per ton, in virtue of a monopoly of the article purchased by the Company at a sum, it is said, of $8,000 l$.

Mr. Harvey, the superintendent of the Bastenne Company, was good enough to supply me with various samples of mineral tar, bitumen, and asphaltic rock for analysis. The tar of Bastenne is an exceedingly viscid mass, without any earthy impurity. It has the consistence of baker's dough at $60^{\circ}$ of Fahrenheit; at $80^{\circ}$ it yields to the slightest pressure of the finger; at $150^{\circ}$ it resembles a soft extract; and at $212^{\circ}$ it has the fluidity of molasses. It is admirably adapted to give plasticity to the calcareous asphalts.

A specimen of Egyptian asphalt which he brought me gave, by analysis, the very same composition as the Val de Travers, namely 80 per cent. of pure carbonate of lime, and 20 of bitumen. A spocimen of mastic propared in France was found to consist, in 100 parts, of 29 of bitumen, 52 of carbonate of lime, and 19 of siliceous sand. A portion of stone called the natural Bastenne rock afforded me 80 parts of gritty siliceous matter and 20 of thick tar. The Trinidad bitumen contains a considerable portion of foreign earthy matter: one specimen yielded me 25 per cent. of siliceous sand; a second, 28 ; a third, 20 ; and a fourth, 30 ; the remainder was pure pitch. One specimen of Egyptian bitumen, specific gravity 1.2, was found to be perfectly pure, for it dissolved in oil of turpentine without leaving any appreciable residuum.

As the spocific gravity of properly made mastic is nearly double that of water, a cubic foot of it will woigh from 125 to $130 \mathrm{lbs}$; and a square foot, three quarters of an inch thick, will weigh very nearly 8 pounds.

It has been thought advisable to preserve these remarks on bitumen, although written several years ago, especially as the. recent attempts to introduce bituminous 
substances for paring soom likely to be successful. During tho last few years, several experimental patches of asphalt-paving havo been laid down in some of the most active thoroughfares of the motropolis. Such paving recommends itself by being much cleaner and quioter under heavy traffic than an ordinary granite pavement, but has the disadrantage of becoming extremely slippery when the surface is slightly moistened by rain. It is said, howorer, that this slipperiness only lasts while the parement is moderately damp, and that but fow horses fall on the asphalt during either dry or thoroughly wet weather. The liquid Val de Travers asphalt, the Limnor asphalt, and Barnett's liquid asphalt are all mixed with grit or sand, and thus present rougher surfaces than those pavings which consist of asphalt alone-such as the ordinary compressed asphalt of the Val de Travers Company. See PAveMcexr, Aspintt and MASTic.

Br TUMrrous coAx. Coal rich in bituminous matter. Pitch or caking coal, cherry cosl, splint coal, cannel coal, coking coal, and somo others, aro varieties of bituminous coal. See Cost.

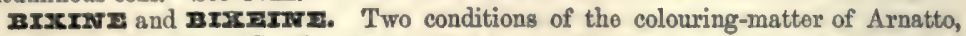
according to Preisser. See Arsatto.

BIACII AmBzir. Pitch coal is so called by the amber-diggers of Prussia, and it is manufactured by them into jet-like ornaments.

BIACK BAND. A varioty of the carbonate of iron, to which attention was first called by Mr. Mushet at the commencement of the present century. The iron manufacture of Scotland owes its present important position to the discovery of the ralue of the black-band ironstone. This ore of iron is also found in several parts of the coal-basin of South Wales, and in the north of Ireland. The chemical composition of the black-band iron ores will be given in connection with that of other minerals of the same class. See Iroy Ores.

BIACE CHAXr. A kind of clay containing a large amount of carbon. It is found in Carnarvonshire and in the Island of Islay.

BIACF COAI. Slate coal, cannel coal, and foliatod coal, were so called by Jameson and other mineralogists of his day.

BIACX-Cobaty ockrz. Seo Cobalt, Earthy.

BIACK COPPIR. An impure black oxide of copper. See Copper.

BIACK DYE. (Teinte noire, Fr.; Schwarze Farbe, Ger.) Textile fabrics are dyed by various processes, according to the quality of the black required, and the kind of stuff on which the dye is to be produced. Under A.NIIINE-BuAcK the process by which that black dye is prepared is already described; but the following process for using an anilino-black as a dye for cotton goods by $\mathbf{M}$. J. Persol properly finds its place here:-

'Chemists hare long tried to make use of the beautiful black precipitate produced by the action of bichromate of potassa for the solution of certain aniline salts as a dye for calicoes, but without success; if the solution was concentrated, the black was soon precipitated to the bottom of the bath; if, on the other hand, it was dilute, the black, owing to the absence of a sufficiently powerful oxidation, was not formed at all, or in insufficient quantity.

- This trouble it was attempted to remove by cooling down the solutions nearly to zero. But this produced another difficulty, the chromato of aniline crystallising out at that temperature whon the solutions were sufficiently concentrated to produce the desired dye. Wherever these spots existed in the cloth on subsequent drying, a mutual reaction took place between the constituents of the chromate of aniline, causing such a rise of temperature as not unfrequently to set fire to the cloth.

'To overcome these various difficulties the following expedients were adopted:By means of a horizontal brush, to which a reciprocating motion was given in a vertical direction, the solutions, either together or one after the other, were cast upon the cloth, while tightly stretched, in the form of a fine spray. By this means, however rapidly the reaction took place, it could not possibly do so until the solutions were intimately mixed together upon the cloth, the latter being at the same time thoroughly wetted with it.

"The salts found to be most suitable for this reaction are the sulphate, hydrochloride, and the nitrate. No black is obtainable with the acetate; and the tartrate, oxalate, and citrate are more or less unfitted for the production of a good colour.

'If a too nearly neutral solution is used there is great difficulty in producing the colour; if the solutions are too acid the black is formed so rapidly that the solutions have not time to mix sufficiently and to penetrate the cloth.

'As the result of numerous experiments with hydrochloric, sulphuric, and nitric acid salts, the author came to the conclusion that:-1. The employment of neutral aniline salts was ineffective. 2. The bi-acid aniline salts, especially the bi-sulphate, give good results. Of the tri-acid salts the hydrochloride is the best. 3. The sul- 
phates givo a reddish black; the hydrochloric and nitric acid salts yield a black with a blue lustro. 4. Equal volumes of bi-sulphato and bi-hydrochloride of aniline give excellont results. 5. The bi-chromate of potash solution must be concentrated, contrining not less than $\mathbf{8 0}$ grammes of salt to the litre.

'A dark green is first produced on passing the cloth into a hot-soap bath. After washing it thoroughly this passes into pure black.

'By printing the cloth with fatty matters or resins, previous to the application of the dyeing solutions, white patterns on a black ground can be obtained.'

The following processes for dyeing woollen stuff will be found to produce excellent results. For $1 \mathrm{cwt}$. of eloth previously dyed blue:-There is put into a boiler of middle size, $18 \mathrm{lbs}$. of logwood, with the same quantity of Aleppo galls, the whole being enclosed in a bag; this is boiled in a sufficient quantity of water for 12 hours : one-third of this decoction is transferred into another boiler with 2 pounds of verdigris; and the stuff is passed through this solution, stirring it continually during two hours, taking care to keep the bath very hot without boiling. The stuff is then lifted out, another third of the bath is added to the boiler, along with 8 pounds of sulphate of iron or green vitriol. The fire is to be lowered while the sulphate dissolves, and the bath is allowed to cool for half an hour, after which the stuff is introduced, and well moved about for an hour, and then it is taken out to air. Lastly, the remaining third of the bath is added to the other two, taking care to squeeze the bag well. 18 or $22 \mathrm{lbs}$. of sumach are thrown in; the whole is just brought to a boil, and then refreshed with a little cold water; 2 pounds more of sulphate of iron are added, after which the stuff is turned through for an hour. It is next washed, aired, and put again into the bath, stirring it continually for an hour. After this, it is carried to the river, washed well, and then fulled. Whenever the water runs off elear, a bath is prepared with weld, which is made to boil for an instant; and after refreshing the bath, the stuff is turned in to soften, and to render the black more fast. In this manner a very beautiful black is obtained, without rendering the cloth too harsh.

Commonly, more simple processes are employed. Thus the blue cloth is simply turned through a bath of gall-nuts, where it is boiled for two hours. It is next passed through a bath of logwood and sulphate of iron for two hours, without boiling, after which it is washed and fulled. But in all cases the cloth, after passing through the blue vat, should be thoroughly washed, because the least remains of its alkalinity would injure the tone to be given in tho black copper.

Hellot found that the dyeing might be performed in the following manner:-For 20 yards of dark blue cloth a bath is made of $2 \mathrm{lbs}$. of fustic (Machura tinctoria), $4 \frac{1}{4} \mathrm{lbs}$. of logwood, and 11 lbs. sumach. After boiling the cloth in it for three hours it is lifted out, 11 lbs. of sulphate of iron are thrown into the boiler, and the cloth is then passed through it during two hours. It is now aired, and put again in the bath for an hour. It is, lastly, washed and scoured. The black is less velrety than that by the preceding process. Experience convinced him that the maddering prescribed in the ancient regulations only gives a reddish cast to the black, which is obtained finer and more velvety without madder.

According to Lewis, the proportions which the English dyers most generally adopt are, for 112 lbs. of woollen cloth, previously dyed of a dark blue, about 5 lbs. of sulphate of iron, as much gall-nuts, and $30 \mathrm{lbs}$. of logwood. They begin by galling the eloth; they then pass it through the decoction of logwood to which the sulphate of iron has been added.

When the cloth is completely dyed, it is washed in the river, and passed through the fulling-mill till the water runs off clear and colourless. Some persons recommend, for fine cloths, to full them with soap-water. This operation requires an expert workman, who can free the cloth thoroughly from the soap. Several recommend, at its coming from the fulling, to pass the cloth through a bath of weld, with the view of giving softness and solidity to the black. Lewis says, that passing the cloth through weld, after it has been treated with soap, is absolutely useless, although it may be beneficial when this operation has been neglected.

The following German process is cheap and good. $100 \mathrm{lbs}$, of cloth or wool are put into tho copper with sufficient water and $15 \mathrm{lbs}$. of Salzburg vitriol (potash-sulphate of iron) and $5 \mathrm{lbs}$. of argol, heating the bath gradually to boiling, while the goods are well worked about for two hours, taking them out, and laying them in a cool place for twenty-four hours. They are then to be put in a lukewarm bath of from 25 to $30 \mathrm{lbs}$. of logwood, and $10 \mathrm{lbs}$. of fustic, and to be worked therein while it is made to boil during two hours. The goods are now removed, and there is put into the copper $1 \frac{1}{4} \mathrm{lbs}$. of rerdigris dissolved in vinegar; the goods are restored into tho improved bath, and turned in it for half an hour, after which they are rinsed and dried. 
The procoss for dyeing merinos black is, for 100 lbs. of them to put 10 lbs, of copperas into the bath of pure water, and to work therein for a quarter of an hour, as $800 \mathrm{n}$ as it is tepid, one-third of the goods; then to replaco that portion by the second, and aftor another quarter of an hour, to put in the last third. Each portion is to be laid aside to air in the cold. The bath being next heated to $140^{\circ} \mathrm{F}$., the merinos are to be treated as above piecemeal; but the third time it is to be passed through the bath at a boiling heat. Being now well mordanted, the goods are laid aside to air till the following day. The copper boing charged with water, 50 lbs. of ground log. wood, and $2 \mathrm{lbs}$. of argol, and heated, the goods are to be passed through, while boiling, for half an hour. They are then rinsed.

Different operations may be distinguished in dyeing silk black: the boiling of the silk,-its galling, - the preparation of the bath, - the operation of dyeing,-the softening of the black.

Silk naturally contains a gummy substance, which gives it the stiffness and elasticity peculiar to it in its nativo state; but this adds nothing to the strength of the silk, which is then styled raw; it rather renders it, indeed, more apt to wear ont by the stiffiness which it communicates; and although raw silk more readily takes a black colour, yet the black is not so perfect in intensity, nor does it so well resist the reagents capable of dissolving the colouring particles, as silk which is scoured or deprived of its gum.

To cleanse silk intended for black, it is usually boiled four or five hours with onefifth of its weight of white soap, after which it is carefully beetled and washed.

For the galling, nut-galls equal nearly to three-fourths of the weight of the silk are boiled during three or four hours; but on account of the price of Aleppo galls, more or less of tho white gall-nuts, or of evon an inferior kind called galon, berry or apple galls, are used. The proportion commonly employed at Paris is two parts of Aleppo galls to from eight to ten parts of galon. After the boiling, the galls are allowed to settle for about two hours. The silk is thon plunged into the bath, and left in it from twelve to thirty-six hours, after which it is taken out and washed in the river.

Silk is capable of combining with quantities, more or less considerable, of the astringent principle; whence results a considerable increase of weight, not only from the weight of the astringent principle, but also from that of the colouring particles, which subsequently fix themselves in proportion to the quantity of the astringent principle which had entered into combination. Consequently, the processes are varied according to the degree of weight which it is wished to communicate to the silk; a circumstance requiring some illustration.

The commerce of silk goods is carried on in two ways : they are sold either by the weight, or by the surface, that is, by measure. Thus the trade of Tours was formerly distinguished from that of Lyons; the silks of the formor being sold by weight, those of the latter by measure. It was therefore thoir interest to surcharge the weight at Tours, and, on the contrary, to be sparing of the dyeing ingredients at Lyons; whence camo the distinction of light black and heavy black. At present, both methods of dyeing are practised at Lyons, the two modes of sale having been adopted there.

Silk loses nearly a fourth of its weight by a thorough boiling, and it resumes, in the light black dye, one-half of this loss; but in the heavy black dye, it takes sometimes upwards of a fifth more than its primitive weight-a surcharge injurious to the beauty of the black and the durability of the stuff. The surcharged kind is denominated English black, because it is pretended that it was practised in England. Since silk dyed with a great surcharge has not a beautiful black, it is usually destined for weft, and is blended with a warp dyed of a fine black.

The peculiarity of the process for obtaining the heavy black consists in leaving the silk longer in the gall-liquor, in repeating the galling, in passing the silk a greater number of times through the dye, and even letting it lie in it for some time. The first galling is usually made with galls which have served for a preceding operation, and fresh gall-nuts are employed for the second. But these methods would not be sufficiont for giving a great surcharge, such as is found in what is called the English black. To give it this weight, the silk is galled without being ungummed; and, on coming out of the galls, it is rendered supple by being worked on the jack and pin.

The silk dyers keep a black vat, and its very complex composition varios in different dye-houses. These vats are commonly established for many years; and when their black dye is exhausted it is renovated by what is called in France a brevet. When the deposit which has accumulated in it is too great, it is taken out, so that at thoend of a certain time nothing remains of the several ingredients which composed the primitive bath, but which are not employed in the brevet.

For the dyeing of raw silk black, it is 'galled' cold, with the bath of galls 
which has already served for the black of boiled silk. For this purpose, silk, in its native yellow colour, is made choice of. It should be remarked, that when it is desired to preserve a portion of the gum of the silk, which is afterwards made flexible, the galling is given with the hot bath of gall-nuts in the ordinary manner. But here, where the whole gum of the silk, and its concomitant elasticity, are to be preserved, the galling is made cold. If the infusion of galls be weak, the silk is left in it for soveral days.

Silk thus prepared and washed takes very easily the black dye, and the rinsing in a little water, to which sulphate of iron may be added, is sufficient. The dye is made cold; but, according to the greater or less strength of the rinsings, it requires more or less time. Occasionally three or four days are necessary; after which it is washed, it is beetled once or twice, and it is then dried without wringing, to avoid softening.

Any of these processes will produce a black without the goods being previously dyed blue, but generally when such common blacks, as they are technically termed, are dyed, more of the dye drugs are required, and also a little modification in the operations. Sometimes they are 'bottomed' or 'rooted,' by first working them in a decoction of walnut-husks, and then dyed as above;-or, a good black may be dyed without any previous rooting, by working 1 cwt. of the stuff, for an hour, at a heat of $190^{\circ}$, in 6 lbs. of camwood: 6 lbs. of copperas are then added, and the stuff worked for another hour; the fire is then withdrawn from the boiler, and the stuff allowed to remain in the liquor for 10 or 12 hours. It is washed from this, and worked in a second bath with 60 lbs. of logwood for an hour and a half, then add 3 lbs. of copperas, and after another hour's working, it is washed.

Bichromate of potash is also used for dyeing blacks upon wool. A very good colour may be dyed direct by working, for 2 hours, 1 ewt. of the stuff in a solution of $5 \mathrm{lbs}$. of bichromate, $4 \mathrm{lbs}$. of alum, and $3 \mathrm{lbs}$. of fustic, then exposing it for an hour and washing well. It is again wrought for 2 hours, in a second bath, made up with $45 \mathrm{lbs}$. of logwood, 3 lbs. of barwood or camwood, and $3 \mathrm{lbs}$. of fustic; then adding $3 \mathrm{lbs}$. of copperas, and after half an hour's longer working, the dye is finished. A much cheaper blue black than that produced by previously dyeing the stuff in the indigo vat, is obtained by using a Prussian blue, and then proceeding as directed above.

Raw silk may be more quickly dyed by shaking it round the rods in the cold bath after the galling, airing it, and repeating these manipulations several times, after which it is washed and dried.

Macquer deseribes a more simple process for the black by which velvet is dyed at Genoa : and he says that this process, rendered still simpler, has had complete success at Tours. The following is his description.

For $1 \mathrm{cwt}$. (50 kilogrammes) silk, 22 lbs. (11 kilogrammes) of Aleppo galls, in powder, are boiled for an hour in a sufficient quantity of water. The bath is allowed to settle till the galls have fallen to the bottom of the boiler, from which they are withdrawn; after which $32 \mathrm{lbs}$. of copperas are introduced, and $22 \mathrm{lbs}$. of country gum, put into a kind of two-handled colander, pierced everywhere with holes. This kettle is suspended by two rods in the boiler, so as not to reach the bottom. The gum is left to dissolve for about an hour, stirring it from time to time. If, after this time, some gum remains in the kettle, it is a proof that the bath, which contains two hogsheads, has taken as much of it as is necessary. If, on the contrary, the whole gum is dissolved, from 1 to $4 \mathrm{lbs}$. more may be added. This colander is left constantly suspended in the boiler, from which it is removed only when the dyeing is going on; and afterwards it is replaced. During all these operations the boiler must be kept hot, but without boiling. The galling of the silk is performed with one-third of Aleppo galls. The silk is left in it for six hours the first time, then for twelve hours. The rest, secundum artem.

Lewis states that he has repeated this process in the small way; and that, by adding sulphate of irop progressively, and repeating the immersion of the silk a great number of times, he eventually obtained a fine black.

Astringents differ from one another as to the quantity of the principle which enters into combination with the oxide of iron. Hence, the proportion of the sulphate, or of any other salt of iron, and that of the astringents, should vary according to the astringents made use of, and according to their respective quantities. Gall-nut is the substance which contains most of the astringent principle; sumach, which seems second to it in this respect, throws down (decomposes), however, only half as much sulphate of iron.

The most suitable proportion of sulphate of iron appears to be that which corresponds to the quantity of the astringent matter, so that the whole iron precipitable by the astringent may be thrown down, and the whole astringent principle may be taken up in combination with the iron. As it is not possible, however, to arrivo at such 
precision, it is better that the sulphate of iron should predominate, because the astringent, when in excess, counteracts the precipitation of tho black colouring particles, and has the property of even dissolving them.

This action of the astringent is such that, if a pattern of black cloth be boiled with gall-nuts, it is reducible to grey. An observation of Lewis may thence be explained. If cloth be turned sevoral times through the colouring bath, after it has taken a good black colour, instead of obtaining more body, it is weakened, and becomes brownish. Too considerable a quantity of the ingredients produces the same effect; to which the sulphuric acid, set at liberty by the precipitation of the oxide of iron, contributes.

It is merely the highly oxidised sulphate which is decomposed by the astringent; whence it appears that the sulphate will produce a different effect according to its state of oxidation, and call for other proportions. Some advise, therefore, to follow the method of Proust, employing it in the oxidised state; but in this case it is only partially decomposed, and another part is brought, by the action of the astringent, into the lower degree of oxidation.

The particles precipitated by the mixture of an astringent and sulphate of iron have not at first a deep colour; but they pass to a black by contact of air while they are moist.

Black dye is only a very condensed colour, and it assumes more intensity from the mixture of different colours likewise deep. It is for this reason advantageous to unite several astringents, each combination of which produces a different shade. But blue appears the colour most conducive to this effect, and it corrects the tendency to dun, which is remarked in the black produced on stuffs by the other astringents.

On this property is founded the practice of giving a blue ground to black cloths, which acquire more beauty and solidity the deeper the blue. Another advantage of this practice is to diminish the quantity of sulphuric acid which is necessarily disengaged by the precipitation of the black particles, and which would not only counteract their fixation, but would further weaken the stuff, and give it harshness. For common stuffs, a portion of the effect of the blue ground is produced by the rooting.

The mixture of logwood with astringents contributes to the beauty of the black in a twofold way. It produces molecules of a hue different from what the astringents do, and particularly blue molecules, with the acetate of copper, commonly employed in the black dyes; which appears to be more useful the more acetate the verdigris made use of contains.

The boil of weld by which the dye of black cloth is frequently finished, may also contribute to its beauty, by the shade peculiar to its combination. It has, moreover, the advantage of giving softness to the stuffs.

The processes that are employed for wool yield, according to the observation of Lewis, only a rusty black to silk; and cotton is hardly dyed by the processes proper for wool and silk. Let us endeavour to ascertain the conditions which these three varieties of dyeing demand.

Wool has a great tendency to combine with colouring substances; but its physical nature requires its combinations to be made in general at a high temperature. The combination of the black molecules may therefore be directly effected in a bath, in proportion as they form; and, if the operation be prolonged by subdividing it, it is only with the view of changing the necessary oxidation of the sulphate and augmenting that of the colouring particles themselves.

Silk has not the same disposition to unite with the black particles. It seems to be assisted by the agency of the tannin, with which it is previously impregnated, especially after it has been scoured. A very deep black may be obtained upon 100 lbs. of silk, by working it for two hours in a solution of $20 \mathrm{lbs}$. of copperas and 3 pints of nitrate of iron. Wash from this thoroughly, and then wash for two hours more in a decoction of $100 \mathrm{lbs}$. of logwood and 20 lbs. of fustic. Lift up, and add to the bath a solution of 3 lbs. of copperas, and work half an hour longer, and wash. A beautiful rich blue-black is produced by dyeing the silk a deep royal blue, then working for an hour in a solution of copperas ( 2 ounces to the pound of silk), washing from this, and working in a bath of logwood, using half a pound to each pound of silk, and adding, after an hour's working, a few ounces of copperas; working half an hour longer, and finishing.

Cotton has no affinity for the black dye, and has always to be impregnated or combined with astringent substances, in order to produce the dye. A good deep black will be imparted to $100 \mathrm{lbs}$. of cotton by steeping it in a decoction of $30 \mathrm{lbs}$. of sumach, at a boiling heat, and allowing it to stand till perfectly cool; then passing it through lime-water, and, immediately after this, working for an hour in a solution of $20 \mathrm{lbs}$. of copperas. After this, expose for an hour to the air; then pass through lime- 
water again, and wash and work for an hour in a bath of $30 \mathrm{lbs}$ of logwood and $10 \mathrm{lbs}$. of fustic; lift, and add 2 lbs, of copperas, and work 30 minutes longer, and finish.

BIACE TIUX. Seo Assaring.

BIACR BRMATIT:. An ore of MANGANESE.

BLACRING roR sHozs. (Cirage des bottes, Fr.; Schuhschwärze, Ger.) Blacking consists of a black colouring-matter, generally bone-black, and substances that acquire a gloss by friction, such as sugar and oil. The usual method is to mix the bone-black with sperm-oil; sugar, or molasses, with a little vinegar, is then well stirred in, and strong sulphuric acid is added gradually. The acid produces sulphate of lime and acid phosphate of lime, which is soluble: a tenacious paste is formed by these ingredients, which can be smoothly spread; the oil serving to render the leather pliable. This forms a liquid blacking. Pasto blacking contains less vinegar. In Germany, according to Liebig, blacking is made by mixing bone-black with half its weight of molasses, and one-eighth of its weight of hydrochloric acid, and one-fourth of its weight of strong sulphuric acid, mixing with water, to form an unctuous paste. The following method for making liquid and paste blacking is given by William Bryant and Edward James : 18 ounces of caoutchouc are to be dissolved in about $9 \mathrm{lbs}$. of hot rape-oil. To this solution $60 \mathrm{lbs}$. of fine ivory-black and $45 \mathrm{lbs}$. of molasses are to be added, along with $1 \mathrm{lb}$. of finely ground gum arabic, proviously dissolved in 20 gallons of vinegar of strength No. 24. These mixed ingredients are to be finely triturated in a paint-mill till the mixture becomes perfectly smooth. To this varnish $12 \mathrm{lbs}$. of sulphuric acid are to be now added in small successive quantities, with powerful stirring for half an hour. The blacking thus compounded is allowed to stand for 14 days, it being stirred half an hour daily; at the end of which time $3 \mathrm{lbs}$. of finely ground gum arabic are added; after which the stirring is repeated half an hour every day for 14 days longer, when the liquid blacking is ready for use. In making the paste blacking, the patentees prescribe the above quantity of india-rubber oil, ivory-black, molasses, and gum arabic, the latter being dissolved in only $12 \mathrm{lbs}$. of vinegar. These ingredients are to be well mixed and then ground together in a mill till they form a perfectly smooth paste. To this paste 12 lbs. of sulphuric acid axe to be added in small quantities at a time, with powerful stirring, which is to be continued for half an hour after the last portion of the acid has been introduced. This paste will be found fit for use in about seven days.

BIACK-JACK. The miner's name for blende, or sulphide of zinc. See Zivc.

BIACI IEAD. The common name of Plumbago or GrapHite.

miack-xiad pencils. See Pench Manofacture.

BIACK Trs. The miner's name for tin ore ready for the smelter. See Tiv.

BLACK WADD. One of the ores of manganese. See Manganese.

BTADDER. (Vessie, Fr.; Blase, Ger.) A bag or sack, in animals, which serves as the receptacle of some secreted fluid. Bladders are chiefly employed for securing jars, bottles, \&zc.

BIANRIFT. (Blanchet. Fr.) A cover for a bed made of coarse wool loosely woven. Among printers the woollen eloth which is placed between the tympans is called a blanket.

Blankets are largely used in Californian gold-quartz mining, for catching the particles of metal as they eseape from the stamping mill. These blankets are generally of a coarse grey wool, and are woven expressly for the miner, at the woollen mills on the coast. They are made about 30 inches wide, and are spread over blanket-boards, or shallow wooden troughs, each about 16 inches wide, 3 inches deep, and from 9 to 12 feet long, and inclined in the direction of their length at an angle of from $3^{\circ}$ or $4^{\circ}$ to $15^{\circ}$ to the horizon. The finely pulverised ore, suspended in water, is carried in a current, from the battery-box, over the surface of a series of these blankets. In this way a larger proportion of the gold and auriferous pyrites, or of the gold-amalgam if mercury has been used in the battery, becomes entangled in the fibres of the fabric. Any particles that may escape are finally retained by a system of amalgamated copper riffles over which the current of water is generally caused to flow after leaving the blankets. The blankets, charged with finely-divided metal, are frequently washed (the upper ones, on which the heaviost sand is deposited, being in some cases washed every quarter of an hour), and the residue from the washings is then amalgamated. It has been attompted, but without success, to substituto ox-hides or sheepskins for the blankets. See Woomikn Manufacturess.

BIAST. The current of air driven into a furnace; it may be either cold or hot air. See Hот BLast.

mLAst-FurNACz. A furnace into which air is forcibly blown. See Iron, Metallungy.

BIAST-Hoxss. A mining term. The holes through which the water enters the bottom of a pump in the mines. 
BIASTrutc. The process of rending rocks by the use of some explosive com. pound, as gunpowder. Soe Murusa.

BLaAcerre (Blanchement, Fr.; Bleichen, Ger.) is the process by which the textile filaments, cotton, flax, hemp, wool, silk, and the cloths made of them, as well as various vegetable and animal substances, are deprived of their natural colour, and rendered nearly or altogether white. The term bleaching comes from the French verb blanchir, to whiten.

The principal bleaching agents, besides alkalis, are chlorine, sulphurous acid, and the combined action of air and light. These are destroyers of colour. The chief agents for removing colours which do not require to be previously decomposed, are alkalis. The principal amount of the colouring-materials are removed from the cloth by washing with alkalis: the last tint of whiteness is not removable by this means, and it is to this last tint that the word bleaching has been more definitely applied.

In ancient times bleaching, washing, and fulling were not distinctly separated; they were all practised. We read in the Seriptures of 'fine linen, white and clean," and in Greek authors, of 'raw linen,' of which towels were made, as well as of 'shining fine linen,' for the same purpose, thus at once making the distinction.' The pure white was apparently not so common as with us. A pure surface was, however, needful, in order to produce good colours, for which we are bound to give the ancients credit, as we know they were acquainted with them as pigments, and are not, therefore, to be suspected of being unable to distinguish good from bad, when transferred to textile fabrics. As their words for white and for colour are plain enough in general, we must conclude that they had the power of obtaining both fine whites and finely-dyed cloth; handkerchiefs were tied about the head in various ways, as now in Lancashire, white and coloured. The Babylonians wore white cloaks. ${ }^{2}$ By their method of washing, the discovery of bleaching was inevitable, the cloth boing washed and dried several times in the sun. But it was not left in the state of an accident only; the word insolation shows that the effects of the sun had been observed and classified, and this is stated to have been the chief method, as it is now, of bleaching wax. Egypt and the East seem to have been the teachers in bleaching. From Egypt were obtained alkalis, and soda mixed with lime. Both lime and alkalis were used in the process. Potashes, or the ashes of plants, were also used, and soap-plants, in all probability of various kinds, as it is not easy to decide on one. The Saponaria officinaiis, soap-wort, is still used, and the wako-robin or cuckowpint, Arum maculatum; the Gypsophila Struthium was considered by Linnæus to be the ancient one, and is still called Lanaria in Italy. Nor do we require to suppose that this plant was first incinerated, as has been supposed, in the case of Borith, the fuller's soap of the Bible. Vegetable decoctions are still used in China to bleach silk, and in France also; some have been patented even in England, although but little used. The Latin method of obtaining white cloth is very well preserved, and as they got their caustic soda from Egypt, it is probable that they got also their process thence; nor is it at all likely that Nicias of Megara invented fulling, as it was evidently well known before the existence of any well-founded Greek tradition. Pirtures exist in Pompeii of men dancing the fuller's dance, or stamping cloth with their feet, as women now practise in Scotland. Moderate-sized tubs were used: the clothes seem occasionally to have been taken up by the hand, in order that they might be well turned. They were then treated with ammoniacal liquors and soda. Urine was highly esteemed for the purpose. The fullers obtained it by placing vessels at the corners of the streets, which were removed when filled; this practice acting at the same time as a sanitary precaution. The same method of carefully collecting this fluid, or 'old lant,' as it is called, exists in the woollen districts of Lancashire and Yorkshire. A tax was laid on it by Vespasian, so that the fullers might not recoive it without payment. The cloth was then sulphured, if it was intended to be white: this process was performed under a conical frame like a small tent, the cloth being spread round the frame, and a vessel of sulphur burned under it. ${ }^{3}$ Potter's earth was then used according to circumstances. The fuller seems to have been a bleacher as well as domestic laundryman. He had, therefore, white as well as coloured dresses to deal with. For the first he used Sardinian potter's earth, which could not be employed for prints or such colours as easily changed (versicolores). For coloured cloth, sulphur was not used by the potters, but fine Cimolian earth. The potter's earth seems to have been used both before and after sulphuring, according to circumstances. This second process is allied partly to our mode of chalking white dresses, still somewhat in use: but more strongly allied to what is called dressing, stiffoning, and finishing. Pliny says that the Umbrian earth was only used for polishing vestments, also that it softened fine colours and gave lustre to those that were faded in sulphuring. This shows that

Thiloxenus in Athenms, ix. 77.

sompeif drawing; ;ee Smith's Dictionary, Lardner's Cyclopaedia. 
they used sulphur in washing, and not merely in preparing for the bleaching process.' They then gave a finish of very fine clay, gypsum being used instead of clay in Grooce, as amongst ourselves. If a nap was wanted, it was raised after sulphuring, by brushing, by carding, by the skin of a hedgehog, or by thistles and teasels. They seom to have got a fine nap on their woollen cloth, as garments of this kind once washed were considered less valuable, as would bo the case with our broadcloth for outer dresses. Wool for under dresses could not have been injured by one washing, especially as the fullones seom in old Italy to have been more attentive than our washerwomen, and to have formed a college, or at least a guild. The washing was seldom done at home, except in large establishments, especially in the country. Whiteness was very much esteemed, and great pains taken to obtain it. Coloured cloth seems a later invention. This love of whiteness was so great, that those who were too poor to have their cloths fulled, rubbed thom with a white fuller's earth, so as on holidays to appear clean and bright.

Clothes in ancient times required a good deal of washing, so much oil being used; alkalis alone could remove this, and people that used soft feather-beds, and pillows that sank under the weight of the head, would not be behind in having them also whitoned. In India the mode was different from that used in the western world. The preparation for printing was a series of washings, beatings, and exposure to the sun, as well as wearing next the skin, and steeping in goats' and sheeps' dung. Wearing next the skin was probably instead of the oiling process in Turkey red. Bleaching with boiled rice-water was practised in India. In Jamaica the aloe was used, and in China arbean is employed : this is smaller than the Turkey bean; five parts are used to five of salt, six of flour, and twenty-five of water: this is for raw silk. The exact action of the vegetable method on the colouring-matter is not well known; but it must not be ignored. The decompositions of fermentation and putrefaction have a great power of propagating themselves; we can, in fact, readily conceive the decomposition of gums by such means, provided they are not resinous matters, consisting chiefly of carbon and hydrogen. Mucilaginous plants are even now in some places used, and have been recommended also in the most modern times." It is, therefore, not easy to seo why so much difficulty has been raised amongst chemical historians as to the use of plants in washing and bleaching. Vegetable products, such as oatmeal, \&c., have powerful detergent qualities, and leave the skin exceedingly soft. In general we may conclude that these vegetable infusions and alkalis were the means of bleaching in ancient times, the influence of the sun being also employed. At present, alkalis are more generally used. Washing with alkalis is really the most important part of the process. The soaps of the ancients were also

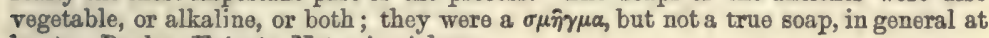
loast.-Paulus Egineta, Notes by Adams.

Until modern times no improvements of great importance took place affecting the principles of bleaching; and even now the only modern changes consist in the introduction of chlorine and machinery, to which may be added the greater abundance of soap. In the last century, Holland obtained the best name for bleaching. The process passed then to Ireland and Scotland, and thence into England. It was even customary to send goods from this country to be bleached in Holland. The first attempt to vio with Holland was made in Scotland in 1749.

We find in the patent lists many crude efforts made to improve the art. Alkalis and acids are recommended in various forms, and such a variety of substances as tartar, saltpetre, sal-ammoniac, marl, loam, clay, mud, chalk, fuller's earth, oystersholls, soot, turf, and ashes, with a great variety of washing machines.

The value of the plan in Holland was ascribed to the ashes of Museory (Russian potash) and the sea-water; but it is evident from the description, that it was not sea-, but very pure fresh water which was used. The Dutch process is thus described:-When a piece of linen is to be bleached, it is in the first place steeped in a lixivium, or lye, where other eloth has been trod; afterwards it is trod in a new lye of ashes poured upon it boiling hot. This is boiled in large copper cauldrons, and is never pourod upon the cloth till it is as clear as wine. The linen is left eight days in this lye, after which it is washed and pressed in this manner:- They empty some buckets of butter-milk into wooden vessels fixed in the ground; then they throw in a piece of linen, which three men tread with their feet as much as possible. Afterwards they pour in more butter-milk, and then another piece of cloth, proceeding thus alternately till the vessels are nearly filled, when thoy lay planks over the linen, upon which they raise a large round piece of wood, or great stake, touching the lower side of a beam, between which and the stake they drive wedges to press the cloth. Six or seven days after they take the cloth out of these vessels, and if it be not white enough, they steop it as we have described abovo. Afterwards it is washed and spread 
out upon the ground to bleach. It must be remarked that after overy dipping the cloth is washed first with black soap, thon with cloar wator, and aftor oach of the operations it is wrung by means of a machine that turns by means of a wheel. .... The whitening grounds are cut with canals in somo places, that there may be no trouble of fetching water from a distance. Tho cloth is watered with long narrow shovels made in shape of a scythe. The water of these canals comes from the dams, and it is that which contributes most to tho lustre of the Dutch eloth. To prevent the water from becoming thick and muddy, they are extromely careful in cloaning these canals. The washing tubs are built with bricks, with two trap-doors or sluices for admitting or excluding the water according as it is necessary.'- Select Essays, quoted by Parke.

The chief advantage here consists in the facility of obtaining soap, which in ancient times was either searce or badly made. This improvement began to be more and more used from the time of its earliest introduction. Modern times havo bogun to exclude it to a great extent again, finding it so much cheaper to work with the alkali alone without combining it with fatty matter.

Tho process of bleaching then became a series of operations, consisting of, 1st, stooping in water for about three or four days, or in weak alkali for forty-eight hours. 2nd, boiling in an alkaline lye, or, in other words, bucking or bowking : in this operation the hot lye was poured on the cloth; it then ran through it, was drawn off by a tap below, and then pumped up again. 3rd, crofting, or exposure to sun and air on the grass. 4th, souring : this was done by the butter-milk; it lasted several weeks. These operations were repeated four or five times, or until the goods were pure. The whole lasted from March to September. The best months for crofting were found to be March, April, and May. It was not known that it was the acid of the butter-milk which acted; but when sulphuric acid became cheaper, Dr. Home applied it instead of butter-milk, and caused a great revolution in bleaching, as the souring could now be done in a day which before had occupied weeks, exposing the cloth to much danger of decay by decomposition or putrefaction. Great fear was expressed in the country lest the vitriol should burn the cloth, when Dr. Home stated that he had kept linen in acid of the required dilution for some months without having it injured. Berthollet also said that the acid made a better white.

In 1784 Berthollet made known some investigations on chlorine, and in 1787 communicated them to the French Academy. By these investigations it was found. that chlorine had the power of destroying colouring-matters. The use of chlorine was brought to this country by the Duke of Gordon and Professor Copeland of Aberdeen, who then gave the process to be carried out by Messrs. Milnes, of the firm of Gordon, Barron, and Co., of that place. In this discovery the theoretical portion is due, first, to Scheole, who discovered the chlorine; and, secondly, to Berthollet, who discovered the peculiar property. The practical mode of effecting the object is the part which we claim; but it consists of such a long series of expensive trials and ingenions contrivances, that it will take a much longer time to describe them than to givo the first idea only. As the invention was at first applied only to cotton, which at that period was rising into importance, wo shall begin the description of modern bleaching with the mode adopted for that material.

James Watt at the date given was in intimate communication with Berthollet, and did not rest until he had made the process successful at the bleach-field of Macgregor, near Glasgow, requesting the results to be communicated to a meeting of manufacturers to be called together at Manchester; - so quick was Watt to see what would be for the permanent interest of a country, and so ready to act on it! Dr. Henry did much to make it known to the manufacturers about Manchester. This is one of the carly instances of scientific men being directly applied to by manufacturers for assistanco-an application seldom made unless under great difficultios.

In 1798, Charles Tennant, of Glasgow, introduced chloride of lime, which is proferred above all other compounds as a means of applying chlorine.

Tho true theory of bleaching has not been entirely agreed upon, but there can bo little doubt of the principal operations. It is known that oxygen deprives substances of colour; this may be performed by many high oxides; by nitric acid, manganic and chromic acids, chlorous acid, and ovon lower oxides which hold their oxygen lightly, as hypochlorous acid. The same offect may be produced by chlorine, bromine, and iodine. It has been said that chlorino unites with tho hydrogon of the water which is present, gives off oxygen, and so acts.just as oxygen would. Davy found that it would not act in dry air, so that water was noedful: but Dr. Wilson found that it would act, although slowly, in dry air, if exposed to the rays of tho sun. This might show that water is not necessary in order to supply oxygen, but only to allow the chlorine to be brought into thorough contact with the colouring-matter. It has also been supposed that the chlorine removes the hydrogen, or, rather, simply takes 
its place by an act of substitution. Now, whether the chlorine or the liberated oxygen removes the hydrogen, the result will be the same-the destruction of the compound. Chlorine so readily performs theso changes, that we should at once decide on calling it the active agent, wore it not for the fact that oxygen acts so readily, even when chlorine is not present : for example, peroxide of hydrogen, as well as the oxides just mentioned, and ozone also, which has no chlorine to help it. It is, then, certain that oxidation bleaches; and it is certain that dehydration bleaches, if performed by chlorine, and that the sun aids it by its active rays. We know also that water aids it: water aids bleaching or oxidation by air, partly because it contains air in solution. It aids also the bleaching performed by solutions in contact with porous bodies, bocause these bodies have a power of condensing gases in their pores and of compelling combinations. The next question is, Does it aid the bleaching by chlorine in the same way, by assisting the union mechanically, or by decomposing water? Chlorine acts slowly, unless water be present. The theory, therefore, does not demand the decomposition of water, and the known powerful affinities of chlorine do not require to be supplomented by oxygen. But, in order to see exactly the state of the case, let us look at the action of chlorine in bypochlorites or in chloride of lime, and we find that it is a direct oxidation. We obtain by it peroxides of metals, and not chlorides. Here we seem to be taught directly by experiment, that bleaching by hypochlorites is an oxidation of the colouring-matter. Bleaching by moist chlorine may therefore be looked on as the same; indeed, we oxidise by it; but in such cases we may obtain the base at the same time united to chlorine, giving another turn to the question, as Kane showed. The oxidation theory, therefore, seems to be sufficient when water is present. We are, however, finally to deal with dry chlorine in the sun; and in that case it is fair to conclude that it acts by direct combination with hydrogen or the colouring-matter or both. We have, then, two modes of bleaching; but the usual mode in the air becomes by that explanation an oxidation, and the direct action of chlorine obtainable only with difficulty. When sulphurous acid is used, another phenomenon may be looked for, as we find a substance whose chief quality is that of deoxidising. The removal of oxygen also decomposes bodies, and sulphuretted hydrogen can scarcely be supposed to act in any other way. Sulphurous acid, when it decomposes sulphuretted hydrogen, really acts as an oxidising agent, and we can therefore imagine it as such in the bleaching process. Intestigation has not told us if it enters into combination as $\mathrm{SO}^{2}$, and, like oxygen, destroys colour, altering tho compound by inserting itself.

We may fainly conclude that the processes by chlorine and sulphurous acid are performed in a manner as different as the mode in which a salt of ammonia acts on chlorine or an oxacid, or, in Dr. Wilson's general terms, 'specific differences may be expected to occur with all the gases named, as to their action on any one colouringmatter, and with different colouring-matters, as to their deportment with any one of the gases.'-Trans. R. S. E., 1848.

It has been attempted to introduce manganates, chromates, chlorates, chlorochromic acid, and sulphites, but without snccess, as bleaching agents.

\section{Buraching of Cotron.}

Substances dealt with in Bleaching. - The object of bleaching is to separate from the textile fibre all the substances which may mask its intrinsic whiteness, or, which, in the course of dyeing or printing, may produce injurious effects on the colours. The substances present in cotton goods, and to be treated in bleaching, are as follows :-

a. The resinous matter natural to the filaments.

b. The colouring-matter of the plant.

c. The paste of the weaver.

d. A fatty matter.

e. A cupreous soap.

$f$. A calcareous soap.

g. The filth of the hands.

h. Iron rust, earthy matters, and dust.

i. The cotton fibre itself.

$j$. The carbonaceous matter caused by singeing.

i. The seed-vessels.

a. Cotton is corered with a resinous matter, which obstructs its absorption of moisture. This alone would prevent it receiving colour, and it is known that if this could be removed, somo of the darker colours could bo dyed without any bleaching, providing also the impurities arising from manipulation wore absent, although the finest colours could not bo produced in this mannor on cotton in general. M. Bolly, 
however, has proposed the use of acetic acid, or of a sour bran liquor, as substances which are absorbed by the cotton and render it capable of absorbing colour or solutions. The matter which prevents the moistening has not been thoroughly examined. It is found to be soluble in alcohol or ether, and some of it in turpentine : it is there. fore callod a resinous, waxy, or fatty body. It is dissolved by alkalis, and thrown down by acids in strong solutions. The alcohol solution leaves thin yellowish scales, which may be dissolved in acid, or even in much water. But information concerning it is indistinct. For a long time the process commenced by removing this resin by means of alkali. It is called scouring.

b. The whole colouring-matter is not soluble in alkalis, but it becomes 80 after being altered by the action of chlorine, or by insulation or eroft-bleaching. It is not even capable of being bleached, or at least but slowly, unless it be previously acted on by alkalis. The amount of colour is much less with cotton than linen. The former is so white naturally, that washing and bleaching might be dispensed with, were it not for the substances which, during its manufacture, come in contact with it, if the gum were removed which prevents the moistening. The alkaline solution from the raw linen, when precipitated by acids, throws down a nearly black resinous mass, and the total loss of weight is very great.

$c$. The weaver's dressing is composed chiefly of farinaceous, glutinous, or gelatinous substances, starch, flour, or size. They are usually allowed to become sour before using. They are all dissolved by water or alkaline solutions, including lime. When the dressing gets dry, the hand-weaver occasionally renders his warp-threads more pliant by rubbing some cheap kind of grease upon them. Hence it happons that the cloth which has not been completely freed from this fatty mattor will not readily inbibe water in the different bleaching operations; and hence, in the subsequent processes, these greasy spots, under peculiar circumstances-somewhat like lithographic stones-strongly attract the aluminous and iron mordants, as well as the dyostuffs, and occasion stains which it is almost impossible to discharge. The acids act differently upon the fatty matters, and thence remarkable anomalies in bleaching take place. When oil is treated with the acetic or muriatic acid, or with aqueous chlorine, it evolves no gas, as it does with the sulphuric and nitric acids; but it puts theso substances into a condition in which they cannot be dissolved by a strong boiling lye of caustic soda. Carbonic acid is said to have a similar action with oil.

$d$. Both cotton and linen contain a little fatty matter, which is removed in the same manner as the resinous. Some of it comes from the mode of treating the warp, which is occasionally greased for weaving. This prevents, like resinous matter, the thorough saturation by solutions which are not alkaline, and soap, soda, or potash may be used to remove it by solution. Lime makes an insoluble soap, and is therefore not suited to the operation. If, however, lime has been used, the insoluble soap may be remored by treating with carbonate of soda, which forms a carbonate of lime, and leaves the fat in combination with the alkali. The carbonate of lime is then removed by an acid. 'This is, however, an indirect method; and the mode universally used is to decompose the lime-soap by an acid, and remove the lime, leaving the fat in the cloth; then to wash out the fat by an alkali, or by soap and alkali mixed, as is the custom almost everywhere. The soap used is in great measure a resinous one, for cheapness, and it is mixed with carbonate of soda.

$e$. When the hand-weavers' grease continues in contact for a night with the copper dents of his reed, a kind of cupreous soap is formed, which is sometimes very difficult to remove from the web. Lime-water does not dissolve it; but dilute sulphuric acid carries off the metallic oxide, and liberates the margaric acid, in a stato ready to be acted on by alkalis.

$f$. When eloth is boiled with milk of lime, the grease which is uncombined unites with that alkaline earth, and forms a calcareous soap, pretty soluble in a great excess of lime-water, and still more so in caustic soda. But all fats and oils, as well as the soaps of copper and lime, cease to be soluble in alkaline lyes when they have remained a considerable time upon the goods, and have been in contact with acetic, carbonic, or muriatic acids, or chlorine. These results have been verified by experiment.

g. Cotton goods are sometimes much soiled, from being sowed or tamboured with dirty hands; but they may easily be cleansed from this filth by hot water.

$h$. Any ferruginous or earthy matters which get attached to the goods in the course of bleaching are readily removable, if not allowed thoroughly to penetrate the cloth ; but the fine ferruginous clay found in suspension in water is very difficult to wash off, and it probably cannot, by any means, be romoved from printed goods without spoiling the colours.

$i$. In all these operations it is needful to consider the most important substance of all-the fibre. Each of the operations may weaken or destroy it, if managed unwisely. Caustic lime may be allowed to act for a long time on eloth without any injury, but 
if allowed to act on it with free access of air, it destroys it in a fow hours. Neither can cloth stand the action of alkalis of any kind very long: if very strong, they rapidly destroy it. The same thing may, in a still stronger sense, be said of acids; and chloride of lime or bleaching-powder acts in the same direction. Linen, although mechanically much stronger than cotton, has not an equal chemical resistance to decomposition. It has not, therefore, been possible to use chloride of lime so as entirely to complete the process of bleaching linen, but only to hasten it, the completion being still nearly in all cases made by crofting. The bleacher has found out these things by expensive experience, and every day shows the importance of guarding against the excessive action of any one of the bleaching agents. Goods are continually suffering from the desire of speed on the part of the trade, and especially of the buyer; nor is it easy to find them absolutely uninjured by the process of bleaching, although it seems possible to conduct the process so that no weakening will ensue. The precautions taken are such as cause the processes to appear very long and tedious. The boiling with lime is continued as long as it is safe; the cloth is then at once washed and scoured, so as to remove all the caustic earth from the fibre. The acid is not allowed to remain long, but is, within from two to four hours, washed out by machines which cause the cloth to be frequently and rapidly saturated with water; and when one of these processes is not enough, it is found better to return to it again than completely to finish it at once, to the danger of the fibre; in the same way as workmen, if they find it needful to put their hands into hot water, do it rapidly and for a short time, but bring them out to cool before they return to the charge. To dry the goods with even a very small amount of acid would infallibly render them rotten. When the chlorine has oxidised or otherwise acted on the colouring-matter, so as to render it soluble, it is washed out with alkalis, but the whole may not be acted on by the first process, and a second may be needful. Again, as to crofting: one exposure may not bo found enough; another washing and another crofting are then needed, and a third, and so on, according to the method employed and the nature of the material used.

The souring by vegetable substances or by fermentation may also injure the cloth, not by the amount of acid existing in the solution, but the decomposition which becomes communicated from the vegetable matter to the cloth, and so renders it weak and rotten. The same is peculiarly the case whon putrefactive action is allowed to commence. This was often the case when the gluten of the paste was removed by fermentation. It has been said that the action of carbonic and acetic acid on the fats is a great objection to the fermentation process, as they are thought to render the fat insoluble, and produce an indelible mark.

Experiments undertaken for the purpose have shown that the strength of the fibre is not impaired by being boiled in milk of lime for two hours, at the ordinary pressure, provided it is not exposed at the same time to air; but bleachers consider that, practically, the goods are not injured by boiling with lime for sixteen hours at the strength of $40 \mathrm{lbs}$. to 100 gallons. It has also been proved that caustic soda of the specific gravity of $1030^{\circ}$ does not hurt them, even boiled under the pressure of $140 \mathrm{lbs}$. to the square inch, or immersion for eight hours in chloride of lime solution containing $3 \mathrm{lbs}$. to 100 gallons, and afterwards in sulphuric acid of the specific gravity of $1067^{\circ}$, or eighteen hours at the specific gravity of 1035 .

$j$. The carbon left by the singeing is entirely removed, but it is not clear what becomes of it. It disappears in the alkaline solution, as no traces seem to exist after this action. Probably the blackness or darkness is not caused by any pure carbon, but by compounds soluble in alkalis. If any elementary carbon exist, it is carried away almost entirely, no doubt, by mechanical means.

$k$. The same method gets rid of the particles of pod which remain in the cotton, and after the first washing they seem to stand out very prominently, swelling up into large dark spots. The alkali probably renders them soft, and allows them to mix readily with liquids, if not altogether actually dissolved.

General Process of Bleaching.-The process of bleaching, from what we have seen, resolves itself into treatment with alkalis, and the action of chlorine or of light. In describing the operations they seem to be very numerous; but, as explained, some require to be repeated gently, instead of being finished by one decisive operation, so as not to injure the fibre; and some are intermediate operations, such as the frequent washings needed in passing from one process to the other. The alkaline solution in which the goods aro boiled does not contain above $250 \mathrm{lbs}$, of carbonate of soda to 600 gallons, but nearly always less. Limo is, however, used much more frequently than soda, which it will be seen is only employed in the second process, and the third if there be one. It is less hurtful to the cloth, and is much cheaper than the alkalis.

The chloride of lime is used at $\frac{1}{2}$ Twaddle, or 1002.5 . It is not considered so important now as formerly, and where $300 \mathrm{lbs}$. wero formerly employed, 30 to 40 are now used. The goods are made nearly white by the alkalis. The chlorine gires only 
the last finish, and is sometimes used to whiton the ground on coloured goods. The whole process may be expressed thus :-Wash out the soluble mattor; boil with lime to dissolvo still more, and to make a fatty compound with tho oily matter; wash out the limo by acids; wash out the fat with a soda-soap; clear tho white by chlorido of lime.

The impurities in the eloth have a certain power of retaining colour upon them. Mud and dirt, as well as grease, gluten, and albuminous mattors, have this property, and fatty soaps, such as lime-compounds of fatty acids. The puro fibro, however, has no power of taking up solutions of such colouring-matter as madder. When, thereforo, it is desired to try the extent to which cloth has been bleached, it is dyed or boiled up with madder exactly as in the process of dyeing. It is then treated with soap, as the madder-dyed goods are treated, and if it comes out without a stain, or nearly puro white, the goods are ready. Dyers or calico-printers who dye printod goods are exceedingly particular as to the bleaching, the dyeing and printing having now approached to such exactnoss, that shades invisible to any eyo not very much experionced are sufficiont to diminish in a material degree the value of the cloth. Any inequality from irregularity of blenching, which causes a similar irregularity of dyeing, is destructive to the character of the goods. Many patterns, too, have white grounds; these grounds it is the prido of a printer to have as white as snow. If delicate colours are to be printed, they will be deteriorated if the ground on which they are to be printed is not perfectly white.

The stains which come out upon maddered goods in consequence of defective bleaching are sometimes called spangs. Their origin is such as I have described above, as the following statements of facts will show. The weaver of calicoes receives frequently a fine warp so tender, from bad spinning, or bad staple in the cotton, that it will not bear the ordinary strain of the heddles, or friction of the shuttle and reed, and he is obliged to throw in as much weft as will compensate for the weakness or thinness of the warp, and make a good marketable cloth. He of course tries to gain his end at the least expense of time and labour. Hence, when his pasto dressing becomes dry and stiff, he has recourse to such greasy lubricants as he can most cheaply procure, which are commonly either tallow, or butter in a rancid state, but the former, being the lowest priced, is preferred. Accordingly, the weaver having heated a lump of iron, applies it to a piece of tallow held over the warp in the loom, and causes the melted fat to drop in patches upon the yarns, which he afterwards spreads more evenly by his brush. It is obvious, however, that the grease must be very irregularly appliod in this way, and be particularly thick on certain spots. This irregularity seldom fails to appear when the goods are bleached or dyed by the common routine of work. Printed calicoes, examined by a skilful eye, will be often seen to be stained with large blotches, evidently occasioned by this vile practice of the weaver. The ordinary workmen call these copper stains, believing them to be communicated in the dyeingcopper. Such stains on the cloth are extremely injurious in dyeing with the indigo-vat.

Old Methods still in use.-As a specimen of the older processes, we shall give the following, adding, afterwards, a minute account of some of the plans adopted by the most successful bleachers. When grease stains do not exist, as happens with the better kind of muslins, or when goods were not required to be finely finished, the following has been adopted:- After singeing, 1. Boiling in water. 2. Scouring by the stocks or dash-wheel. 3. Bucking with lime. 4. The bleaching properly so culled, viz., passing through chlorine or crofting. 5. Bucking or bowking with milk of limo. These two latter processes employed alternately several times, till the whole of the colouring-matter is removed. 6. Souring. 7. Washing.

Another routine has been, 1. Cleansing out the weavers' dressing, by steeping the cloth for twelve hours in cold water, and then washing it at the stocks or dash-wheel. 2. Boiling in milk of lime, of a strength suited to the quality of the goods, but for a shorter time than with the soda lye; two short operations with the lime, with intermediate washing, being preferable to one of greater duration. 3 and 4. Two consecutive lyes of ten or twelve hours' boiling, with about $2 \mathrm{lbs}$. of soda crystals for $1 \mathrm{cwt}$. of eloth. 5. Exposure to the air for six or eight days, or the application of chloride of lime and then sulphuric acid. 6. A lye of caustic soda. 7. Exposure to the air for six or eight days, or chlorine and acid as above. 8. Caustic soda lye as before. 9. Chlorine and the sour. 10. Rinsing in hot water, or scouring by the dash-wheel.

The Processes used in Bleaching. Singeing. -The singeing is performed by passing the cloth over a red-hot plate of iron or copper. The figure 108 shows this apparatus as improved by Mr. Thom. At $a$ there is a cylinder, with the eloth wound round it to be singed; it passes over the red-hot plate at $b$, becomes singed, passes over a small roller at $c$, which is partly immersed in water, and by this means has all the sparks extinguished; then is wound on to the roller $d$, when the process is finished. As the products of combustion from the singeing are sometimes very unpleasant, they 
are carried by this apparatus into the fire-place, where they are consumed. The arrows show the passage of these vapours from the surface of the eloth downwards into the hearth, and thence into the fire.

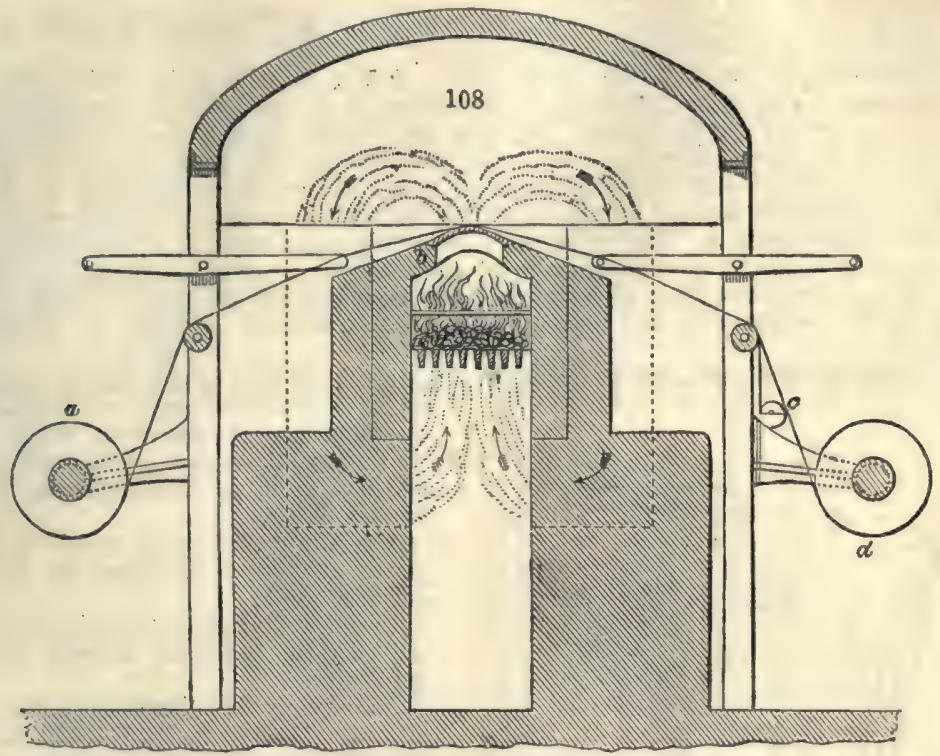

For goods to be finely printed both sides are singed; for market bleaching, one side. Sometimes, however, singeing is not at all desired.

The uso of a line of gas jets instead of a red-hot plate was introducod by Mr. Samuel Hall. It has not, however, found its way generally into bleach-works : the plate is preferred. Gas jets are used necessarily in singeing threads. Seo Singeive.

Shearing.-For fine printing, it is by some considered needful to shear the nap of the cloth instead of singeing it. The method is more expensive than singeing. Messrs. Mather and Platt have made a machine which will shear 60 to 80 yards per minute.

Bucking or Bowking.-This is the process of boiling goods. It is performed in alkaline liquids, generally lime, or soda, or both. The kier for bowking is a cylindrical iron vessel, the chief peculiarity of which is \& method of preventing the cloth from being burnt on the bottiom of the vessel, or allowed to dry on the vessel, or so to be pressed on the bottom as to prevent the boiling of the liquid in a uniform manner. This is done by simply having a false bottom to the kier, or a wooden perforated bottom, about eight or ten inches above the actual bottom.

The boiler, such as $\Delta$, fig. 109, has a stopecek, II $G$, at bottom, for running off the waste lye. Kiers are commonly made of cast-iron, and aro eapable of containing from 300 to 600 gallons of water, according to the extent of the business done. In order that the capacity of the beilers may be enlarged, they are formed so as to admit of a crib of wood, strongly hooped, or, what is preferable, of cast-iron, to be fixed to the upper

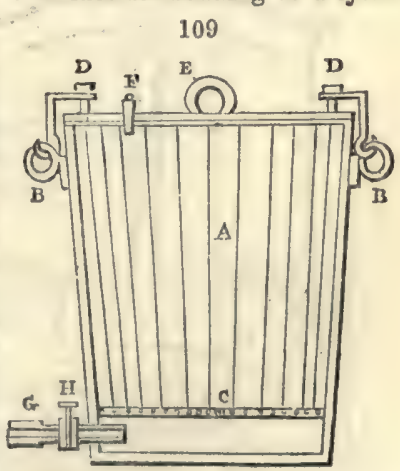
rim or edge of it. To keep the goods from the bottom, where the heat acts most forcibly, a strong iron ring, covered with netting made of stout rope, c, is allowed to rest six or eight inches above the bottom of the boiler. Four double ropes are attached to the ring E, for withdrawing the goods when sufficiently boiled, which have each an eye for admitting hooks from the running tacklo of a crane. Where more boilers than one are employed, the crane is so placed that, in the range of its sweep it may withdraw the goods from any of VoL. I. B B 
them. For this prtrnose, the crane turns on pivots at top and bottom; and the goods are raised or lowered at pleasure, with doublo pulleys and sheares, by means of a cylinder moved by cast-iron wheels. The lid is secured by the screw bolts $\mathbf{D} \mathbf{D}$, and rings $\mathrm{B}$ в. $\quad \boldsymbol{F}$ is a safety-valve.

To aroid the excessive heating neodful to drive the liquid through the goods, Mr. John Iaurie invented the kier shown at fig. 110.

In this figure, A B C D is the wooden kieve, or kier, containing the cloth ; C E F D represent the cast-iron boiler; $\mathrm{G} \mathrm{G}$, the pump; $g, \mathrm{~K}$, the pipe of communication betreen the kior and tho boiler. This pipe has a valve on each of its extromities: that on the upper extremity, whon shut, prevonts the lyo from xunning into the boiler, and is regulated by the attendant by means of the rod and handle $g$ B. The valve at $\mathrm{K}$ admits the lye : but, oponing inwards, it prevents the steam from escaping through the pipe $g$ K. The boiler has a steam-tight iron cover, $g \mathrm{~L}$; and at $\mathrm{C} \mathrm{D}$ in the kier is a wooden grating, a small distance above the cover of the boilor.

At $\mathrm{M} O$ is a broad plate of metal, in order to spread the lye over the cloth. It is hardly necessary to say that the boiler has a furnace, as usual, for similar purposes.

While the lye is at a low temperature, the pump is worked by the mill or steam-
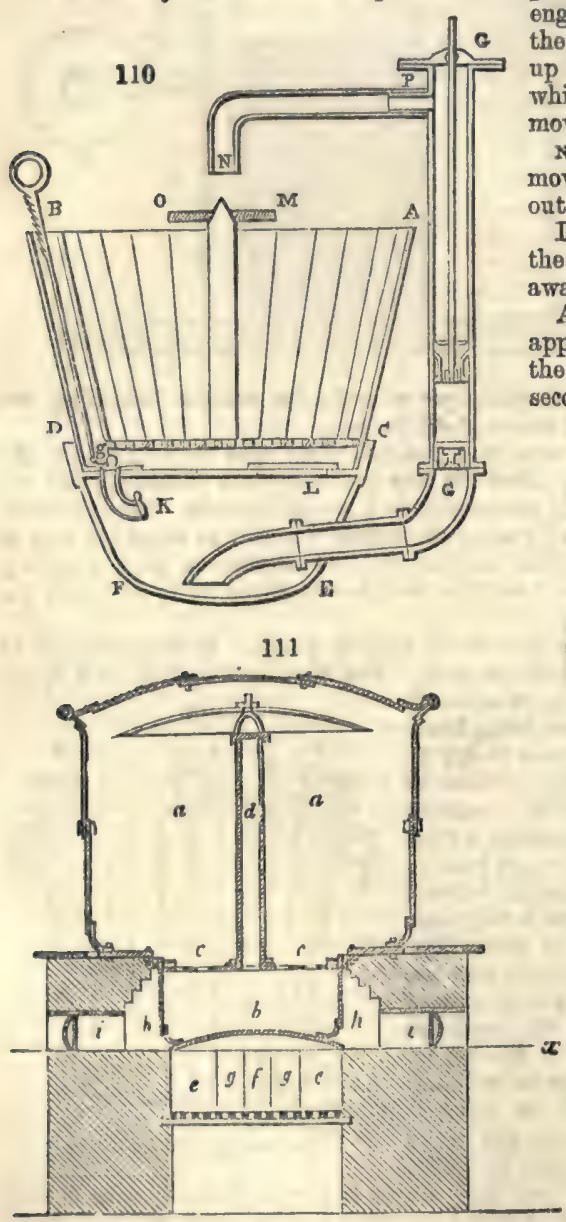
engine. When it is sufficiently heated, the elasticity of the steam forces it up through the valves of the pump, in which case it is disjoined from the moving power.

s $P$ is a copper spout, which is removed at the time of taking the eloth out of the kier.

In order still further to avoid labour, the pumping has been entirely done away with.

A simple modification of the bowking apparatus is shown in figs. 111, 112,113; the first being a vertical section, the second a horizontal section in the line $x$

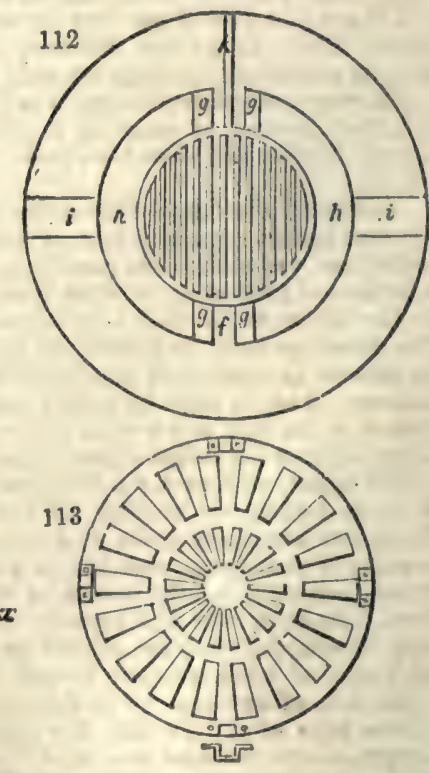

of the first. It consists of two parts: the upper wide part, $a$ a, serves for the reception of the goods, and the lower or pot, $b$, for holding the lye; $c c$ is an iron grating, shown apart in fig. 113. The grating has numerous square apertures in tho middle of the disc, to which the rising pipo $d$ is serewed fast. The npper cylinder is formed of 
cast-iron, or of sheet-iron well riveted at the edges; or sometimes of wood, this being secured at its under edge into a groove in the top edge of the lye-pot. The mouth of the cylinder is constructed usually of sheet-iron. $e e$ is tho fire-grate, whose upper surface is shown in fig. 111 : it is made of cast-iron in throo pieces. The flame is parted at $f$, and passes through the two apertures $g g$, into the flues $h h$, so as to play round the pot, as is visible in fig. 112, and escapes by two outlets into the chimney. The apertures $i i$ serve for occasionally cleaning out tho flues $h h$, and are, at other times, shut with an iron plate. In the partition $f$, which separates the two openings $g g$, and the flues $h h$, running round the pot, there is a circular space at the point marked with $k$, fig. 112, in which the large pipe for discharging the waste lye is lodged. The upper large cylinder should be encasod in wood, with an intermediate space filled with sawdust, to confine the heat. The action of this apparatus is exactly the same as that already explained.

Besides the boiling, bucking, and other apparatus above described, the machinery and utensils used in bleaching are various, according to the business done by the bleacher.

The kier of Messrs. Mather and Platt is very complete. The first figure (114) is the kier when shut or screwed down. The second $(115$, p. 372) is the section

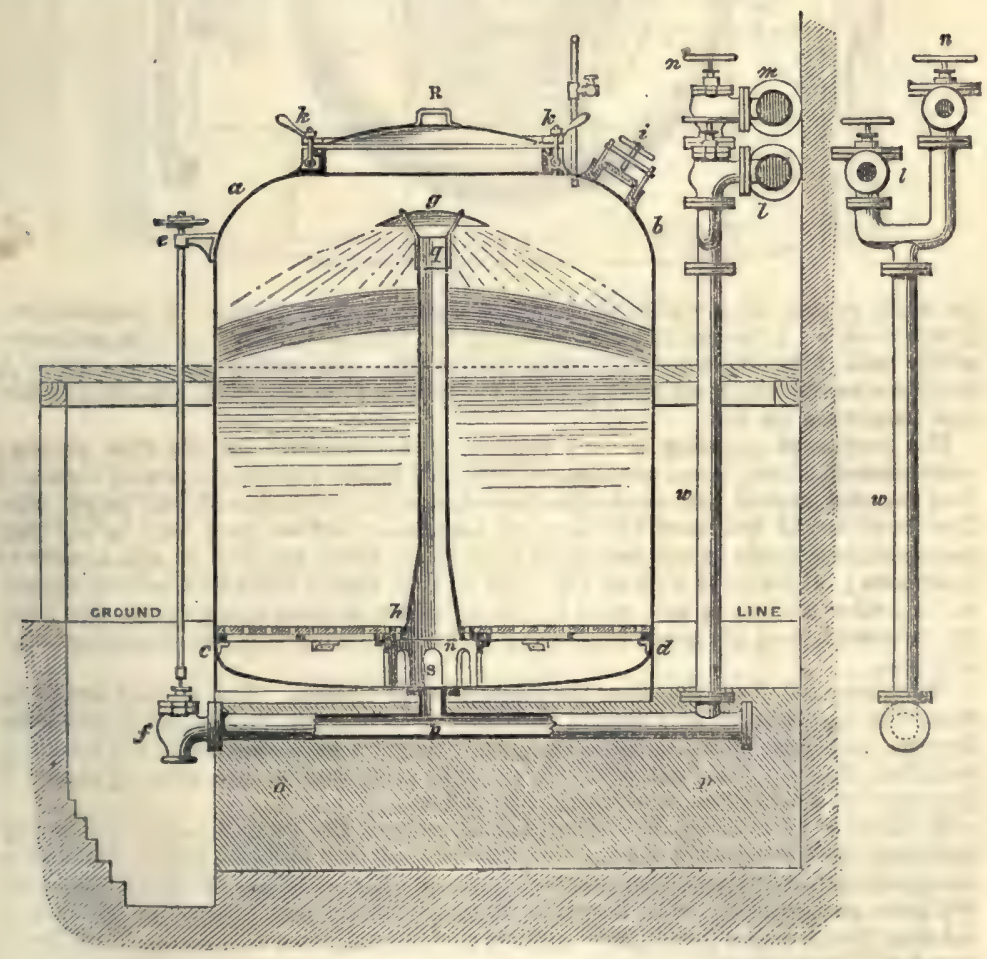

of the kier, which is very like that before given; but in this case it is steam-tight, and heated by steam which issues from a steam-pipe communicating beneath the false bottom. The dangers attending the kier before mentioned are by this means entirely averted, and all the inventions which give the washing liquid a separate and distinct place for heating are at once done awry with.

An exact description of these kiers is required. $a, b, c, d$, represent the body of the kier, which is a cylindrical vessel, generally mado of cast-iron, but sometimes of wood, or wrought iron. $h$ represents the false bottom; a cast-iron grating, sometimes covered with houlder stones, and sometimes with wood; $g$, a cylindrical disc, of wrought-iron, placed on the top of 'puffer-pipe' $q$, to spread the liquor over the cloth. $q$, 'pufferpiro, standing on falso bottom, $h, s$, cylindrical casting for supporting falso bottom 
and 'puffer-pipe,' whose periphery is 'slotted,' to admit of the liquor passing through. $\mathrm{R}$, cover for kier; the flanch on which this cover rests is groovod a littlo to admit of 'gasking' being inserted, so as to form a 'joint.' $k$, $k$, swivel-bolts, holding down the corer. $i$, a small aperture, covered with a lid capable of being remored easily, to enablo the attendant to soo that the cloth does not rise too high in the kier to endanger its working; if such happens, he checks the steam until

116

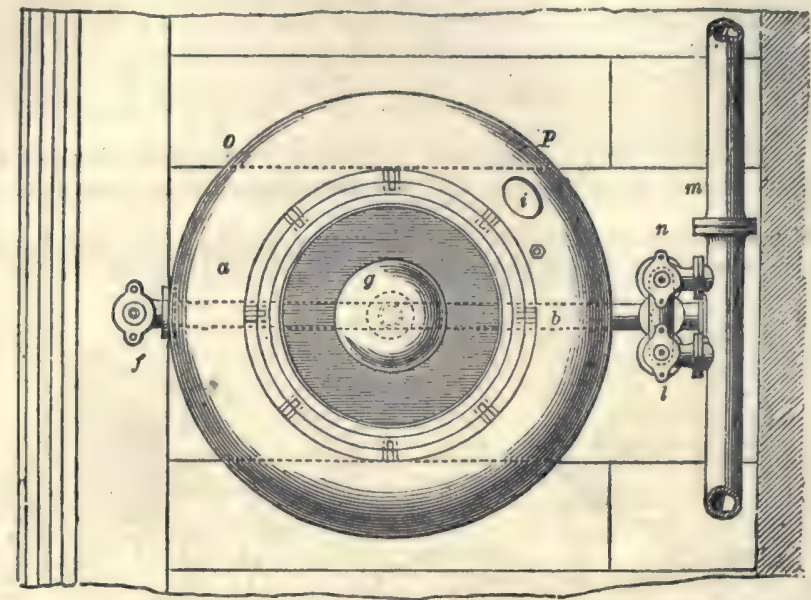

the cloth settles, after which it does not again attempt to rise. $n$, steam-valve; $l$, water-valvo; both communicate with pipo $w$, leading to kier. $p$, pipe communicating with kier for supplying steam and water-also serves as escape-pipe; $f$, escape-valve for letting off kier; $e$, wheel for opening ditto; $m$, steam-pipe from boiler. $o, p$, foundation for kier.

The process of cleansing is very various. Some use lime for the first process; some use soda alone; some use them mixed. Of course when carbonate of soda and lime are used, caustic soda is at once formed, and the carbonate of lime is left idle. The practices and fancies of bleachers aro numerous; and we have only to say that the principle consists in the use of alkaline lyes. Some use lime to the amount of 3 por cent. ; others go as high as 10 . The lime is slaked first and a portion thrown in ; a portion of cloth is laid upon it, and a portion of lime again covers that: but on no account must the goods be allowed to lie in contact with the atmosphere and the lime.

When remored from the kiers the goods must be washed. Now if they are to be washed in dash-wheels, it is needful that they be in separate pieces, and in this state they are sometimes boiled in the kiers; but if they aro to be washed in the washingmachines, they are lifted out of the kier in the same manner as a piece of string is drawn out of the canister in which the coil is kept.

M. Metz, of Heidelberg, has attempted to perform the work of boiling by merely extracting the air from the cloth. For this purpose the cloth is simply put into a strong upright cylinder, the top screwed down, and the air taken out by an air-pump. We have no knowledge as to the advantages gained by this process, or whether it has been found actually capable of putting cloth in a condition to be bleached for a very fastidious market.

High-Pressure Steam-Kiers. - These kiers greatly hasten the process of bleaching, and at the same time improve it. Fig. 116 (p. 373) is an elevation showing the original arrangement of these (which are necommended to be made of strong boiler-plate iron). Ono of these is shown in section. $a$ and $b$ are the kiers; $c$ is a perforated platform, on which the goods to be bowked are laid; $k k$ is the pipe connecting the bottom of the kier $b$ with the top of the adjoining kier $a$; and $l l$, the corresponding pipe connecting the opposite ends of the kiers $a$ and $b ; m m$ are draw-off cocks, connocted with the pipes $k$ and $l$, by which the kiers can be emptied of spent liquor, water, \&c. ; $n$ and $O$ are ordinary two-way taps, by which the stoam is admitted into the respective kiers from the main pipe, $p$, and the reversing of which shuts off the steam communication, and admits the bowking liquor as it becomes expelled from the 
adjoining kior; $q$ is a blowing-off valve or tap; $r$, the pipe through which the bowking liquor enters into the kier; 8 , manhole (closed by two cross-bars, socured by bolts and nuts) through which the goods are introduced and removed; $t$ t are gauges

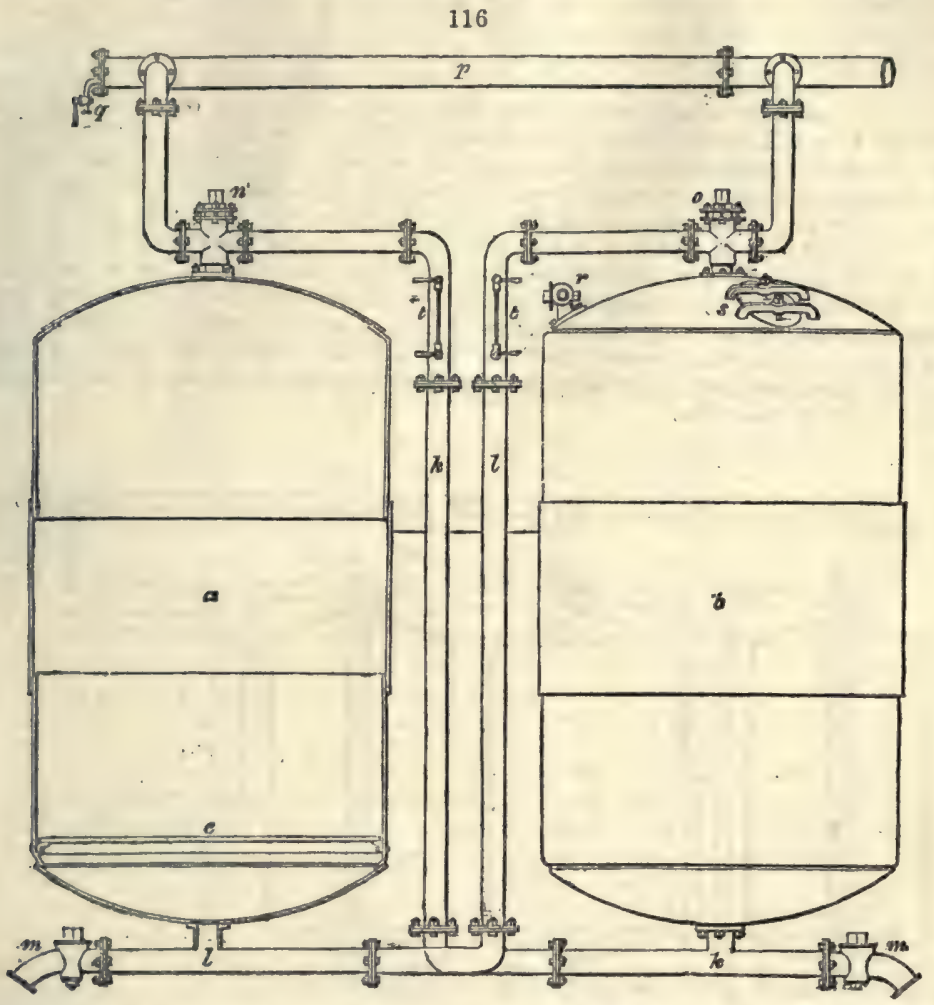

by which it is ascertained when the liquor has passed from one kier and has onterod the other.

The process adopted for bleaching is as follows:

1. The bos or water-trough of the washing-machine is then half filled with milk of lime of considerable consistence, and the goods are run through it, being carried forward by the winches and deposited in the kiers. The whole of the cloth in a kier is in one length, and a boy enters the vessel to lay it in regular folds until the kier is filled. All the cloth before entering the kier must pass through the lime.

2. When the kiers are filled, a grid of movable bars is laid on the top of the cloth, and the manhole of the kiers is closed. High-pressure steam is then admitted at the top; this presses down the goods and romoves the lime-water, which is drawn off at the bottom. At the same time the air is also removed from the goods and replaced by steam. When this is driven off, and nothing but steam issues from the tap at the bottom, $40 \mathrm{lbs}$. of lime, which have been previously mixed with 600 gallons of water, aro introduced into the first kier in a boiling state. High-pressure steam is again admitted, which forces the lime-liquor through the goods to the bottom of the vessel, then up the tube $l$, and on to the goods in the second kier. The tap is then closed which admits steam into the first kier, and the steam is now sent into the second. The same process occurs, only in this case the liquid is sent again on to the top of the goods in tho first kier. This process is continued about eight hours.

High-pressure and Distributing Kiers.-Mr Barlow has effected an important improvement in his original high-pressure kiers by the addition of distributors.

Fig. 117 (p. 374) is an elevation showing a pair of kiers, fitted with distributors, \&e. $A$ and $B$ are the kiers (which it is preferable to make of strong boiler-plate iron), the kier $\mathrm{A}$ being shown in section, and exhibiting the distributors, \&c.

At the bottom of the kier is a plate of an umbrella shape, $c$. This plate sproads 
over ebout three-fourths of tho bottom of the kier; it is perforated with holes all around its outer ridge, at $d d$, which rests upon the bottom of the kier; all the rest of the plate being solid. This plato is fastened in the contro to an iron block, $e$, which stands upon the bottom of the kior in the centro, over the outlet hole. The block $e$ is pigeonholed at tho bottom, so as to allow the liquor to pass from the kier. A socket is left in the upper part of the block $e$, to admit tho insertion of the distributor $f$. This distributor is made of dimensions corresponding to the sizo of the kier; it is solid for some distance from the bottom, and above that is hollow and perforated as full as possible with holes until within a few inches of the top of the kier, where it is connected with the tap $g$ (an ordinary two-way tap, which admits either steam or liquor, or shuts off both) by an inlet pipe, which dips for some distance inside the distributor. $O O$ is the pipe connecting the top of the kier $\Delta$ with the bottom of kier 13 , and $p p$ is the pipe which connects the top of kier $\mathrm{B}$ with the bottom of kier $\Delta ; q q$ are stoam-pipes from the main pipe $r ; m$ is the manway, through which the goods aro introduced and removed; $n$ the pipe and tap, through which the working liquor enters the kier; $s s$ are gauges, by which it is soen when the liquor has passed from one kier and has entered the other; $k k$ are draw-off taps connocted with the pipes 0 and $p$, by which the kiors can be emptied of spont liquor, water, \&c.

117

118

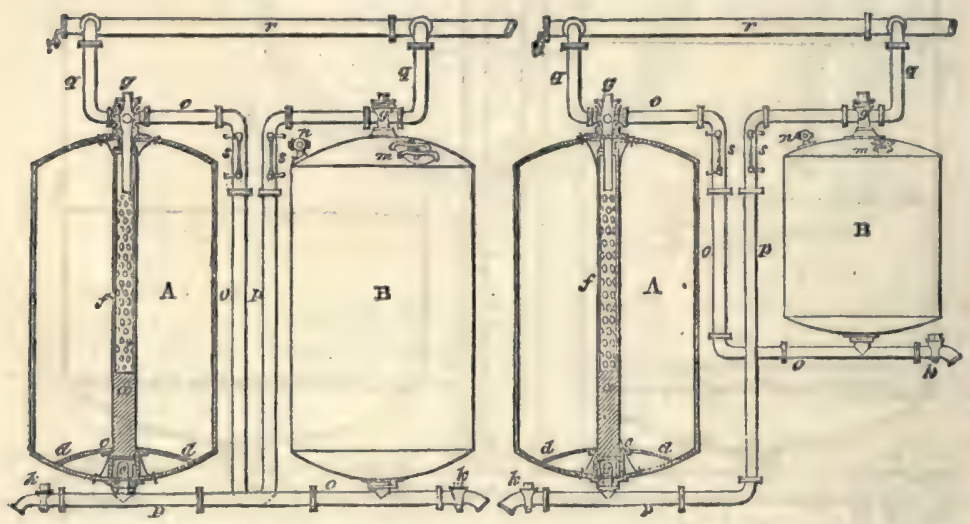

Fig. 118 is a modification of fig. 117. The various parts of the kiers correspond with the description of fig. 117 ; the principle and mode of working are precisely the same the only difference is, that kier $\mathrm{B}$ is reduced in size to about one-third that of kier $\Delta$; it is not charged with goods and is only intended to receive the liquor when forced through the goods in kier A. The liquor is boiled in kier B by a modified distributor; and this is repeated until the goods in kier $\Delta$ aro sufficiently worked.

This modification is only recommended where small quantities are done, and the kiers required would be too small for working in conveniently.

The distributors give a circulation of liquor and steam from the centre of the kiers to the goods all around, while by the action of the umbrella-plate the liquor is prevented escaping until all the goods are thoroughly saturated with it every time the liquor is forced through them; they also greatly aceelerate the circulation of the liquor through the goods, and there is no necessity for anything being laid upon the goods to keop them in their place. The kiors may be crammed full of goods, and either high or low pressure steam may be used in working them.

The process adopted for bleaching is as follows : it is the shortest and simplest in use.

1. After singeing, tho water-box or trough of the washing-machine is half filled with milk of lime of considerable consistence, and the goods are run through it, being carried forward by the winders and deposited in the kiers, a boy being in each kier, who lays the goods in regular folds until the kier is filled.

2. When the kiers are filled, the manholes aro closed. High-pressure steam is then admitted at the top; this presses down the goods and removes the lime-water, which is drawn off at the bottom; at the same time the air is also removed from the goods and roplaced by steam. When this is driven off and nothing but steam issues from the tap at the bottom, $40 \mathrm{lbs}$. lime, which have been previously mixed with 600 gallons of boiling water, are introduced into the first kier in a boiling stato. High- 
pressure steam is again admitted, which forces the lime-liquor through the goods to the bottom of the vessel, then up the pipe 0 , and on to the goods in the second kier. The tap is then closed, which admits steam into the first kier, and the steam is now sent into the second. The same process occurs, only in this case the liquid is sent again on to the top of the goods in the first kier. This process is continued about five hours.

In this method each 7,000 lbs. of cloth take into the kiers 2 cwts. of lime, which is equally distributed. The elear lime-water which is blown out of the steam at the commencement contains only 3 to $4 \mathrm{lbs}$. of lime in solution. At the closo of the operation the liquor has a specific gravity of $3 \frac{1}{2}$ to $4^{\circ}$ Twaddle (1017.5 to 1020$)$, instead of half that amount, or $1 \frac{1}{2}$ to $2^{\circ}$ Twaddle (1007.5 to 1010), as is usual.

3. When the liming is completed the steam-pressure in the kiers is removed, the manway opened, and the cloth in the kier attached to the washing-machine, which draws the goods out of the kiers and washes them.

4. The pieces are then passed by the winches through the souring machine, or soured by having muriatic acid of $2^{\circ}$ Twaddlo $(1010)$ pumped upon them. They must remain with the acid two to three hours, either steoped in it, or after having passed through it,

5. Again attach the cloth to the washing-machine, and wash it well, passing it on by winches, as before, into the kier.

6. Introduce steam and drive off the air and the cold water; these are let out by the tap at the bottom : add then 210 lbs. of soda-ash and 70 lbs. of resin, boiled in 600 gallons of water, for 7,000 lbs. of cloth. Work the kiers by driving the liquid from one to the other as before; about five hours is a sufficient time. These proportions of soda may be varied. If the cloth is very strong a little more may be used (or if the cloth has been printed upon in the grey state, from having been used to cover the blanket of the calico-printing machine).

7. After this the cloth is passed through the washing-machine, and then submitted to chloride of lime. This may be done either by the machine or by pumping. In either case it is an advantage to warm the bleaching-liquid up to $80^{\circ}$ or $90^{\circ} \mathrm{F}$. The strength of the solution when the machine is used may be aboit $\frac{1}{2}{ }^{\circ}$ Twaddle, or 1002.5 specific gravity ; but if the pump is used it must bo much weaker. When the bleaching is for finishing white, milk of lime is added to the chloride, in order to retard the operation; the goods are also washed from the bleaching-liquor before souring them. This causes a smaller escape of chlorine, and is a more careful method: it tends to preserve the headings, or the coloured threads, which are often put into the ends of pieces of cloth in order to see if tho bleaching has been performed roughly or not. The original use of this has almost been forgotten, but these headings are still carefully preserved. This method preserves also the cloth, which is also less apt to be attacked by the chlorine.

If the cloth has been well managed, it will be almost white when it leaves the second kier containing the resinate of soda; it will therefore require very little decolourising. If the goods have boen printed on, more chloride will be needed. The eloth should lie from two to eight hours in the liquor, or after saturation with it. The action is quickened if warmth is used. They are soured then, as before, in muriatic and sulphuric acid, at $2^{\circ}$ Twaddle, for three or four hours; then wash for drying.

The patenteo claims for these kiers considerable advantages over the old, amongst others - that they are not more costly-occupy less room, and whilst the goods are more thoroughly 'bottomed' or cleansed, cousiderable saving is effected in fuel, water, labour, chemicals, and time. 'As regards boiling of goods in bleaching, there is, as compared with the ordinary kier, a saving of three-fourths of the coal by using these kiers.' They are perfectly safe in their action and the fibre is not 'tendered.'

From what has been said, it will be seen that the operations of the bleacher are not so numerous as at first sight appears, when we call every washing a separate process ; and although it really is so, it is managed so rapidly that it can searcely bo said to occupy time, and as it is carried on at the same time as the other processes, it scarcely can be said to give trouble. The work may be divided into-

1. Singeing. 2. Bowking with lime. 3. Washing, souring, and washing. 4. Bowking with resinate of soda. 5. Washing and chlorinating. 6. Souring, washing, and drying.

Steeping. - Instead of boiling in the kier at first, the goods are sometimes, though now rarely, steeped from one to two days in water, from $100^{\circ}$ to $150^{\circ} \mathrm{F}$., for the purpose of loosening the gummy, glutinous, and pasty materials attached to the cloth. Fermentation ensues, and this process is dangerous, as the action of the ferment sometimes extonds to the goods, especially if they are piled up in a great heap without being previously washed. The spots of grease on the insoluble soaps become thereby capable of resisting the caustic alkalis, and are rendered in some measure indelible; an effect due, it is belioved, to the acetic and carbonic acids generated during fermentation. Some persons throw spent lyes into the fermenting vats to counteract the 
acids. The spots of grease are chietly to be found in hand-loom goods, and the diffculty concerning the fats is not therofore commonly felt whore power-loom goods are chiefly used, as in Lancashire.

Washing. - If the cloth is to be washed without having the pieces strung together, the following methods may be adopted. The stocks are still usod, but not in any large establishments in Lancashire.

Figs. 119, 120, represent a pair of wash-stocks. A A are called the stocks or feot. They are suspended on iron pivots at $B$, and receive their motion from wipers
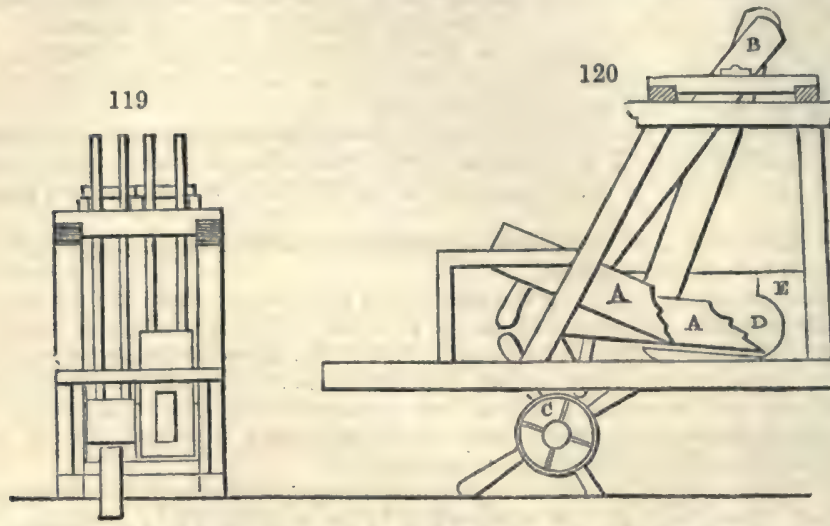

on the revolving-shaft $\mathrm{C}$. The cloth is laid in at $\mathrm{D}$, and, by the alternate strokes

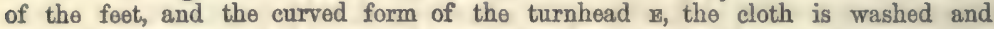
gradually turned. At the same time an abundant stream of water rushes on the cloth throughout holes in the upper part of the turnhead. Wash-stocks are much used in Scotland and in Ireland. In the latter country they are often made with double foet, suspended above and below two turnheads, and wrought with cranks instead of wipers. Wash-stocks, properly constructed, make from 24 to 30 strokes per minute.

This mode of washing is now entirely given up in Lancashire, where a preference is given to dash-wheels and washing-machines with squeozers. The dash are small water-wheels, the inside of which is divided into four compartments, and closed up, leaving only a hole in each compartment for putting in the cloth. There are, besides, small openings for the free admission and egress of the water employed in cleansing. The cloth, by the motion of the wheel, is raised up in one part of the revolution of the wheel; while, by its own weight, it fulls in another. This kund of motion is vory effectual in washing the cloth, while, at the same time, it does not injure its strength. The plan, however, where economy of wator is of any importance, is very objectionable; because the wheel must move at by far too great a velocity to act to advantage as a water-wheel.

121

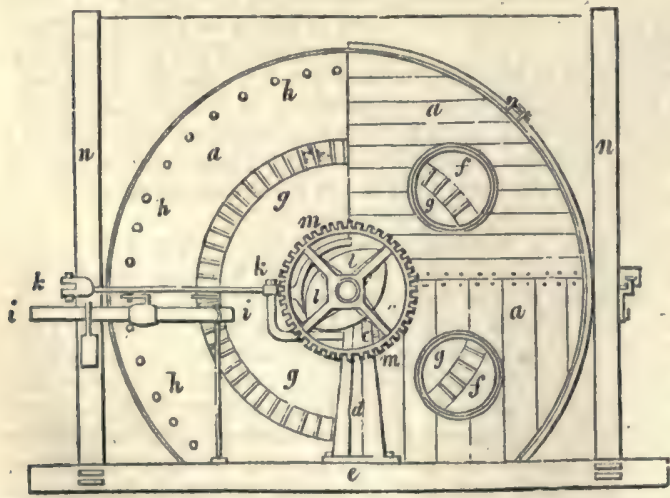

The wash or dash-wheel, now driven by steam-power in all good bleach- and print-works, is represented in fig. 121, upon the leit side in a back viow, and upon the right side in a front viow (the sketch boing halved). Fig. 122 is a ground plan.

$a a$ is the washing-wheel; $b b$ its shaft-ends; $c 0$ their brass bearings or plummerblocks, supported upon the iron pillars $d d$. The frame is made of strong beams of wood, $B$ \&, bound together by cross-bars with mortices. $f f$, two of the circular

apertures, each leading to a quadrantal compartment within the dash-wheel. In 
the back view (the left-hand half of the figure) the brass grating, $g g$, of a curvilinear form is seen, through which the jets of water are admitted into the cavity of the wheel; $h h$ are the round orifices, through which tho foul water runs off, as each quadrant passes the lower part of its revolution; $i$, a water-pipe, with a stopcock for regulating the washing-jets; $k k$, the lover for throwing the driving-erab $l$, or coupling-box, into or out of gear with the skaft of the whoel. This machine is so constructod, that the water-cock is opened or shut by the same loverage which throws the wheel into or out of gear. $m$, a whool, fixed upon the round extremity of

122

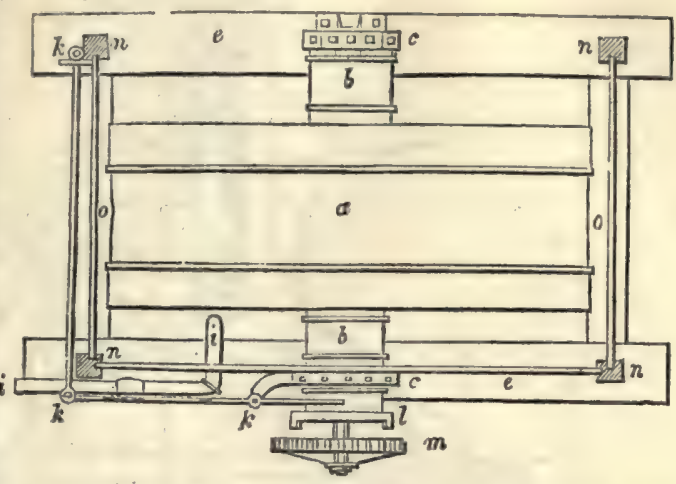

the shaft of the dash-wheel which works into the toothed pinion connected with the prime mover. When the end of the lever $k$, whose fork embraces the couplingbox upon the square part of the shaft, is pushed forwards or backwards, it shifts the clutch into or out of gear with the toothed wheel $m$. In the latter case, this wheel turns with its pinion without affecting the dash-wheol. $n n$, holdfasts fixed upon the wooden frame, to which the boards 00 are attached, for preventing the water from being thrown about by the centrifugal force.

The dash-wheel is generally from 6 to 7 feet in diameter, about 30 inches wide, and requires the power of about two horses to drive it.

A dash-wheel has one piece of cloth in each of the four compartments; these are washed in eight minutes, being 30 pieces an hour, or 300 pieces a day; sometimes two pieces are put in, when double the time is given. It generally requires 60 gallons of water per minute to feed it, 36,000 gallons a day, or 120 to a piece. Always after washing, the squeezers are applied, as they remove at once the superfluous water.

The machine made by Mr. Mather (figs. 123 and 124) washes 800 pieces per hour, or 8,000 pieces per day of ten hours, using 400 gallons per minute, or 120,000 gallons per day, or 20 gallons to a piece. This class of machine is now in its turn superseding the dash-wheel.

This washing-machine will be understood by the general plan ( $f g .124$, and corresponding section, fig. 123). $a$ and $b$ represent the squeezing-bowls, $a$, is 18 inches diameter and 8 feet 3 inches long; it is made of deal timber. (The lapping of strong canvas at $a^{\prime \prime}$ is for the purpose of giving the 'out-coming' pieces an extra squeeze, in order to prepare them for the kiers.) $b$ is 24 inches diameter and of the same lengtl as a, making 100 revolutions per minute; it is generally made of deal, sycamore, however, being better. $c, d$, a strong wooden rail, in which pegs are placed in order to guide the eloth in its spiral form from the edge to the centre of the machine. $h, h$, the water-trough, through which the pieco passes round the roller $\mathrm{R}$. $p$ (fig. 123), water-pipe. $t$, water-tap. $m, m$, pot-eyes, which may be adjusted to any angle, to gride and regulate the tension of the piece on entering the machine. $l$, side frame, for carrying bowls, \&c. $g$, engine (with cylinder, 8 inches diameter) and gearing for driving machine. $w$, weight and lever for regulating pressure on the bowl.

This machine washes 800 pieces per hour, and requires 400 gallons of water per minute. It will serve also to represent the chemick and souring machine, the only difference being that the bowls are 3 feet 6 inches, instead of 8 feet 3 inches, in length.

The chemick and sour are brought by turns into the trough, or into similar separate troughs, by a leaden pipe from the mixing cisterns, and are run in to 6 or 8 inches deep.

The washing-machine of Mr. Bridson ( fig. 125, p. 379) is worth attention. In its action the course of the cloth in the water is easily seen; it is chiefly horizontal. This motion had been givon by Hollowoll and Fearn in 1856; but they had a very complicated machine, and they did not attain the flapping motion which is given to the cloth when it becomes suddenly loose, and is driven violently against the board $a$ a 

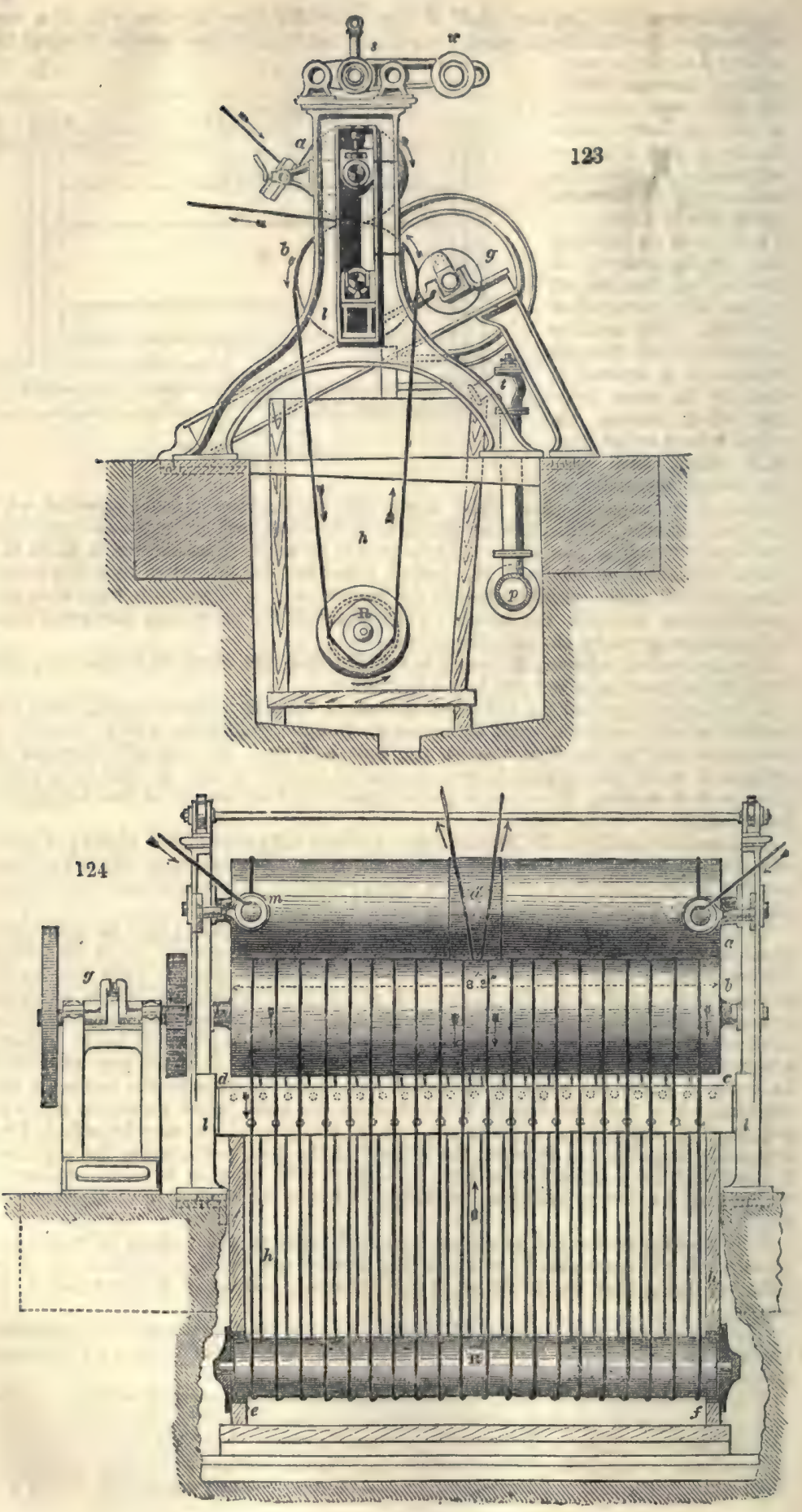
as often as $b c$ and $6 d$ are in one line. It is not shown by the drawing that the cloth passes eight times round these wheels. There is a constant stream of water from the pipe $f$, which is flattened at the mouth about one and a half inch in one diameter,

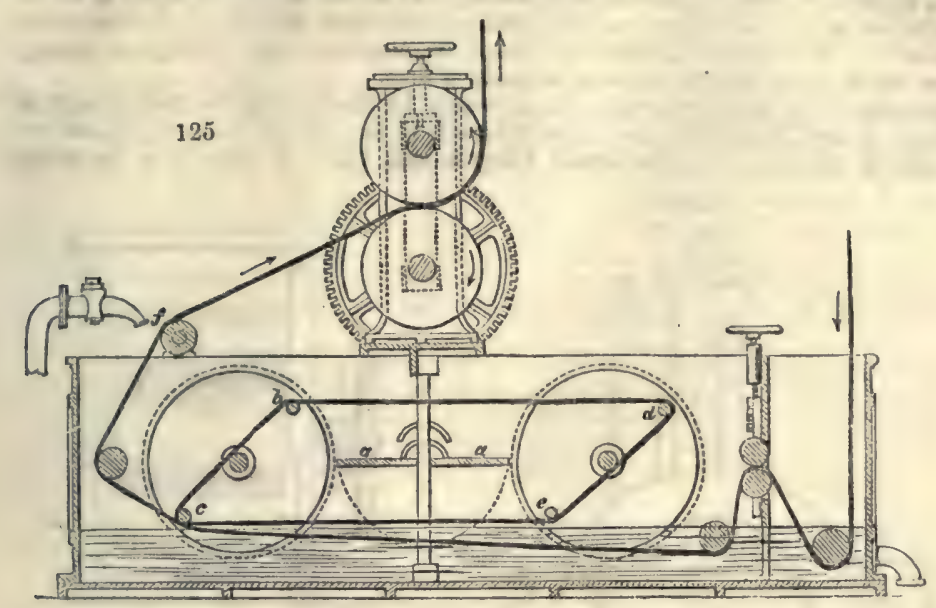

and about 10 inches in the other. This machine can wash 900 pieces in an hour. It requires about twice as much water as a dash-wheel, but washes seven and a half times more pieces. Its length is $\mathbf{9}$ feet.

Souring. - After boiling in the first kier and washing, the goods are soured in muriatic acid of $1010^{\circ}$ specific gravity, or $6 \frac{1}{2}$ gallons of the usual acid, which contains 33 per cent. of real acid, mixed with 100 gallons of water. This is equal to $2^{\circ}$ Twaddle. Muriatic acid may be replaced by sulphuric acid of $1024^{\circ}$ specific gravity, i.e. $3 \frac{1}{2}$ gallons liquid acid to 100 of water; - or the amount of the acid may be doubled in either case, and a shorter time allowed for the souring. The souring is performed in wooden or stone cisterns, where the oloth is laid regularly as it falls over one of the rollers of the calender; - or it is passed through the acid solution by the movement of the calender in the same manner as described in the process of washing. If this method is used, it is allowed to lie on the stillages from two to three hours to allow the acid to act. The acid decomposes any lime-soap formed, and washes out the lime. Hydrochloric or muriatic acid has been preferred in the process described, as the chloride of calcium is so much more soluble than the sulphate. After souring, of course the goods must be thoroughly washed as before.

The sixth operation with soda removes the remaining fatty materials. If lime be us ad, it may be allowed to settle; and it is better to allow it to do so, and thus to use pure caustic soda, which will with the resin remove the impurities in a more soluble form. If, instead of adding $170 \mathrm{lbs}$. of soda crystals to 600 gallons of water, 4.6 lbs. of liquid caustic soda of specific gravity 1320 . were added, the effect would be the same.

The solution of resin and carbonate of soda is a half-formed soap, which is considered to act beneficially in removing the soluble matter. It would not appear, from theory, to be capable of doing so well as the soda which has its carbonic acid removed; but tonder goods will not allow the action of caustic soda, and the carbonate is therefore safer.

Powder-bleaching.-Chloride of lime is added in stone vessels whore the goods are allowed to lie. It is universally called chemick in the manufactorios. The strength used at Brickacro is half a degree Twaddle, or 1002.5 . This is somotimes very much increased, so as to be evon $5^{\circ}$ in some establishmonts, according to the goods bleached; but it is not safe to allow the eloth to lio long in such strong solutions. In such cases it is needful to pass them rapidly through with the ealender, so as to soak them thoroughly, and then to pass them on to the acid, and forward to be washed. It may bo remarked that the use of the calender for these operations renders it possible to use strong solutions, eren for tender goods, as there is no time given for injurious action on the fibre.

Great care is to be taken to make the solution of the chloride of lime perfectly clear. Tho powder does not readily wet with water, and it must therefore bo pressed 
or agitated. This may be done by putting it in a revolving barrel with water, until complete saturation of the powder with moisture; the amount required is then thrown into the cistern, and the insoluble matter allowed to sink. This insoluble matter must not be allowed to come into contact with the cloth, as it will be equal of course to a concentrated solution of the liquor, and will produce rottenness, or burn the cloth, so as to leave holes. When removing from the trough, the cloth is drawn through squeezing rollers, which press out any excess of chloride of lime.

Squeezing. - The squeezing rollers or squeezers, for discharging the greater part of the water or any liquid from the yarns and goods in the process of bleaching, are represented in figs. 126, 127, the former boing a side view, to show how the roller

126

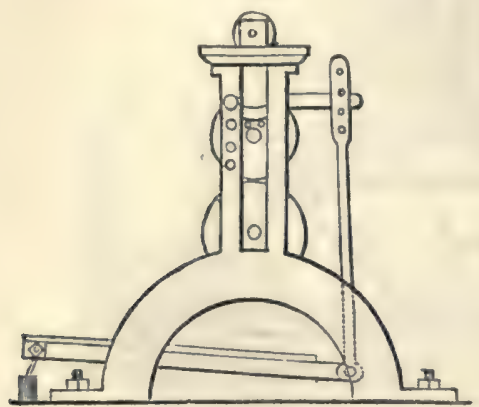

127

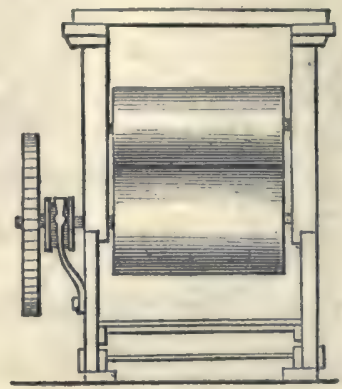

gudgeons lie in the slots of the frame, and how the shaft of the upper roller is pressed downward by a weighted lever, through a vertical junction rod, joined at the bottom to a nearly horizontal bar, on whose end the proper weight is hung. In fig. 128, these rollers, of birch-wood, are shown in face; the under one receiving motion through

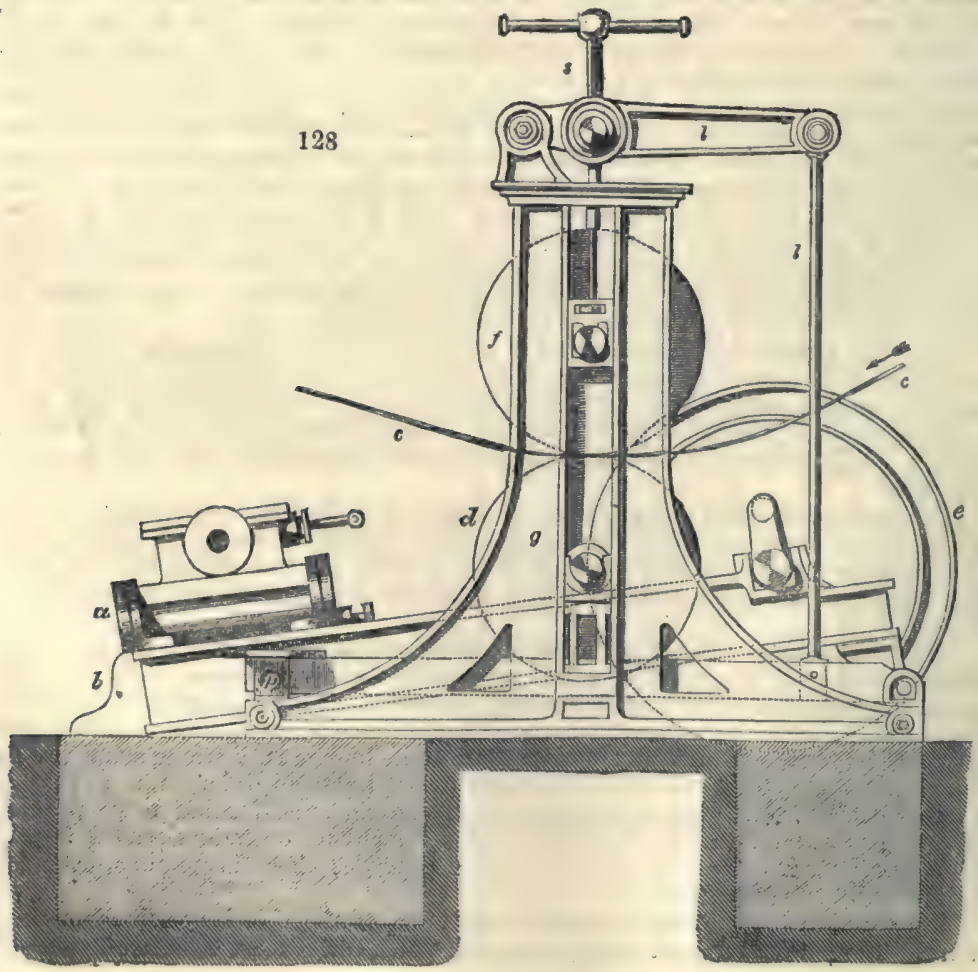


the toothed wheel on its shaft from any suitable power of water or steam. Upon the shaft of tho latter, between the toothed wheol and the roller, the lever and pulley for putting the machine into and out of gear is visible. The under roller makes about 25 rerolutions in the minute, by which time three pieces of goods, stitched endwise, measuring 28 yards each, may be run through the machine, from a water-trough on one side to a wooden grating upon the other.

A squeezing machine, with a small engine attached, is shown in fig. 128, for the drawing of which wo are again indebted to the makers, Messrs. Mather and Platt.

$d, f$, represent the squeezing-bowls. They are as large in diameter as possible, and are generally made of sycamore; but the bottom one is better made of highly compressed cotton. $a, b$, are the engine and frame for driving; $g$, frame for carrying bowls; $l, l$, compound levers for regulating the pressure; $s$ is a screw for the same purpose, and 0 is the cloth passing through the bowls.

Tho white-squeezers, or those used before drying, should have a box, supplied with hot water, fixed so that the piece may pass through it before going to the nip of the bowl.

When the goods are run through, they are carried off upon a grated wheolbarrow in a nearly dry state, and transferred to the spreading machine, called at Manchester a candroy. In many bleach-works, however, the creased pieces are pulled straight by the hands of women, and are thon strongly beat against a wooden stock to smooth out the edges. This being done, a number of pieces are stitched endwise together, preparatory to being mangled.

This squeezing machine is small, but, as will be seen, the rollers are introduced so as to act as long and as rapidly as cloth of whatever length is drawn through them.

The following figure (129) represents a pair of squeezers, for squeezing the cloth after several of the processes named, and are shown as being driven by a small

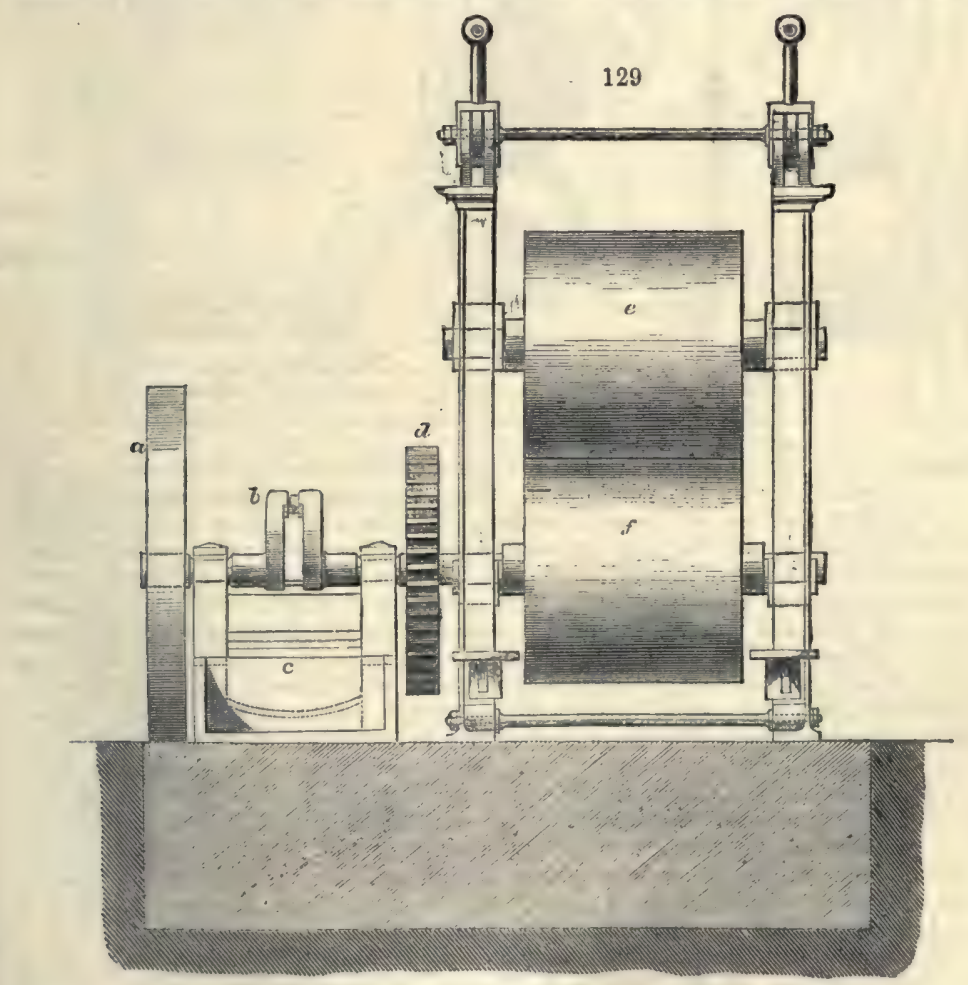

high-pressure engine. $a$ is the fly-wheel of engine; $b$, crank of ditto; $c$, frame of ongine; $d$, spur-wheols connecting the engino and squoezers; $e$ and $f$, sycamoro squeering bowls.

The cloth when passed over the steamed.rollers is not dry; but it is not smooth 
and roady for the market. If the cloth is wanted for printing, no further operation is needed; but if to bo sold as white calico, it is finished by being starched and calendered.

The starch at large works is prepared by the bleachers themselves. At Messrs. Bridson's it is made with the very greatest care from flour. Of course it would bo more expensive for them to buy it, as the mannfacturer would dry it, and they would require to dissolve it. They aro able also, in this manner, to obtain the purest starch. This is mixed with blue, according to the finish of the goods. A roller, which dips into the starch, lays it regularly and evenly on the cloth in the same manner as mordants are communicated in calico-printing, whilst other rollers expel the excess of the starch. The cloth is then dried over warm cylinders, or by passing into a heated apartment. It receives the final finish generally by the calender; but muslins recoive a peculiar treatment.

Calender.-Fig. 131 is a cross-section of this machine, and figs. 130, 132, are front views broken off. The goods are first rolled upon the wooden cylinder $a$, near the

130

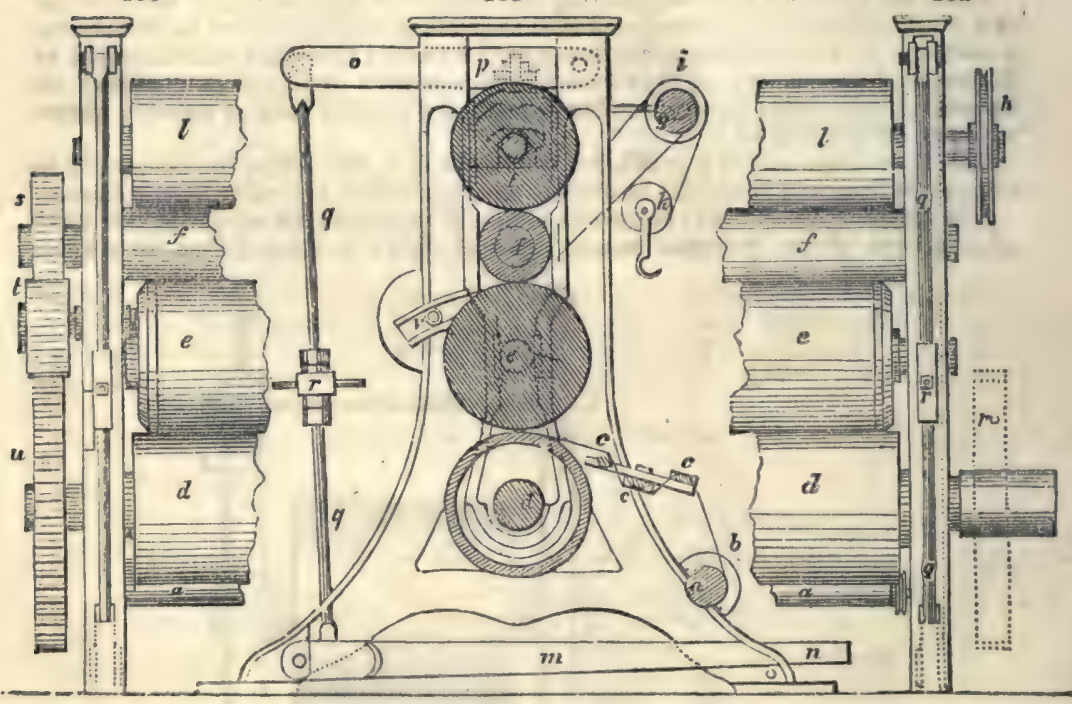

ground; by the tension-roller, $b$, upon the same cylinder, the goods receive a proper degree of stretching in the winding-off. They then pass over the spreading-bars, $c c c$, by which they are still more distended; next round the hollow iron cylinder, $d$, 16 inches diameter, and the paper cylinder, $e$, of like dimensions; thence they proceed under the second massive iron cylinder, $f$, of 8 inches diameter, to be finally wound sbout the projecting wooden roller, $g$. This is set in motion by the pulleys $h$ (fig. 132), and $i$ (fig. 131), and receives its proper tension from the hanging roller $k ; l$ is a pressing cylinder of 14 inches' diameter, mado of plane-tree wood. By its means wo can at all times secure an equal degree of pressure, which would be hardly possible did the weighted lever press immediately upon two points of the calender-rollers. The compression exercised by the cylinders may be increased at pleasure by the bent lever, $m$, weights being applied to it at $n$. The upper branch of the lever, 0 , is mado fast, by screws and bolts at $p$, to the upper press-cylinder. The junction-leg, $q$, is attached to the intermediate piece, $r$, by left-and right-handed screws, so that according as that piece is turned round to the right or the left, the pressure of the weighted roller will be either increased or diminished. By turning it still more, the piece will get detached, the whole pressure will be romoved, and the press-roller may be taken off, which is the main object of this mechanism.

The unequable movement of the cylinders is produced by the wheels $s, t, u$, of which the undermost has 69 , the uppermost 20 , and the carrier-wheel, $t$, either 33,32 , or 20 teeth, according to the difference of speed required. The carrier-wheel is bolted on at $v$, and adjusted in its proper place by means of a slot. To the undermost iron cylinder, the first motion is communicated by any power, for which purpose either a rigger (driving pulley) is applied to its shaft at $t_{h}$ or a crank motion. If it be desired 
to operate with a heated calender, the undermost hollow cylinder may be filled with hot steam, admitted through a stuffing-box at one end, and discharged through a stuffing-box at the other, or by a red-hot iron roller.

Before passing through the press they are slightly damped; this is done by a roller of brushes, which dips into the water, and throws it regularly on the cloth. They are then subjected to the powerful pressure of the calender rollers. The calendered piecos, by the powerful pressure of the rollers, are smooth and somewhat shining. There can be no doubt that cloth in this state looks to the best advantage. The pieces must, however, be put into a compact form. This is done by folding them into parcels, which are pressed by hydraulic power into firm and solid masses. Each parcel has the mark of the manufacturer, or any device that he may choose to have, stamped upon it, or bound round it.

Finishing. - Pure starch is not always used for the purpose of finishing. Fine clay, gypsum, or Spanish white is mixed with the cloth ; and if weight is desired to be given, sulphate of baryta is employed. Silicate of soda was patented for this purpose, but its uso has not been attended with success. Freedom from colour is of course a requisite property in whatever be added, or the excellency of the bleach is impaired. There can, however, be no doubt, that too much attention is given to this finish for home goods, or for all purposes which require the goods to be washed: they assume a solidity of appearance which they do not possess when the finishing material is removed from the pores, and the cloth appears without disguise. In some instances, however, this finish is a peculiarity of the goods, and is almost as important as the cloth itself. For example: in the case of muslins, when they are dried at perfect rest, they have a rigid inelastic feeling, somewhat allied to that of thin laths of wood, and feel very rough to the touch. They are therefore dried by stretching the cloth, and moving the lines of selvage backward and forward, so as to cause the threads of weft to rub against each other and so as to prevent them becoming united as one piece. Goods dried in this manner have a peculiar spring, and such thick muslins are for a time possessed of great elasticity. Several pieces folded up in a parcel spring up from pressure like caoutchouc.

Mr. Ridgeway Bridson invented an apparatus for giving this peculiar finish to muslins. Formerly it was done entirely by the hand, and in Scotland only. Since the invention of this machine, this trade has become a very important one in the Manchester district.

Sometimes goods are finished by the beotle, which acts by repeated hammering. This peculiar action has been transferred to a roller by T. R. Bridson, and called the 'Rotatory Beetle.' It consists of a cylinder having alternately raised and depressed surfaces, and two other cylinders which press upon it, and alternately press the cloth and give a freedom as it passes between the rollers. This is similar to the riso and fall of the hammers or mallets in the beetling process.

Sometimes a stiff finish is wanted; then muslins are dried in the nsual way.

Drying. - Figs. 133 and 134 represent a drying machine, with eleven cylinders, each 22 inches in diameter, capable of drying 1,000 pieces of bleached calico in a day. $a$, represents cylinders heated with steam ; $v$, vacuum-valves in ditto; $f$, frame for carrying cylinders; 0 , folding apparatus; $\varepsilon$, steam-pipe; $g$, gearing.

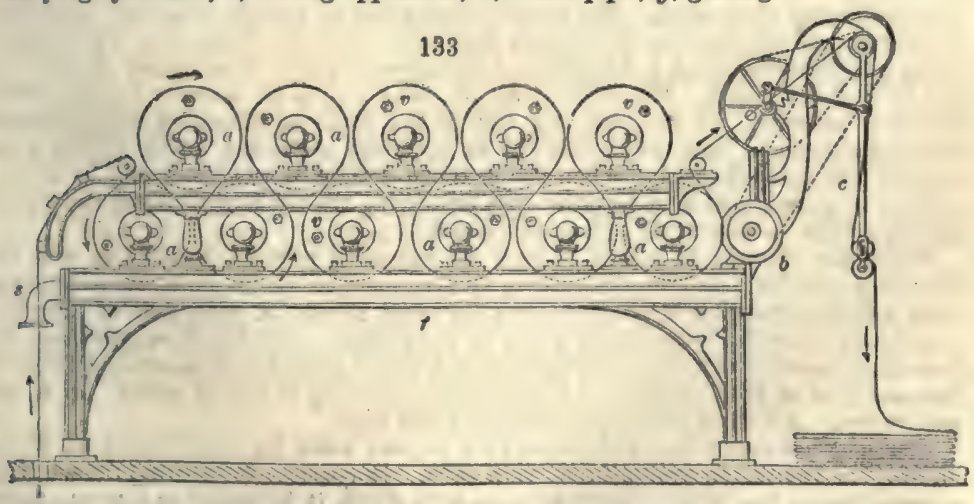

When goods are dried haring a raised pattern, such as brocades, or any other, such as striped white shirting, only ono side of the eloth is to be exposed; the pattern rises up from the heated surface on which the cloth is dried. For this reason, cylin- 
ders such as those just described cannot be used. Large wheels of cast-iron are employed, consisting of two concentric cylindors, between which is a closed space heatod by steam. The cloth is by this means heated on one side only, not passing from cylinder to cylindor, in which case the side next to the heating surface would be changed every time. The larger the cylinder or wheol, the more rapid is the drying,

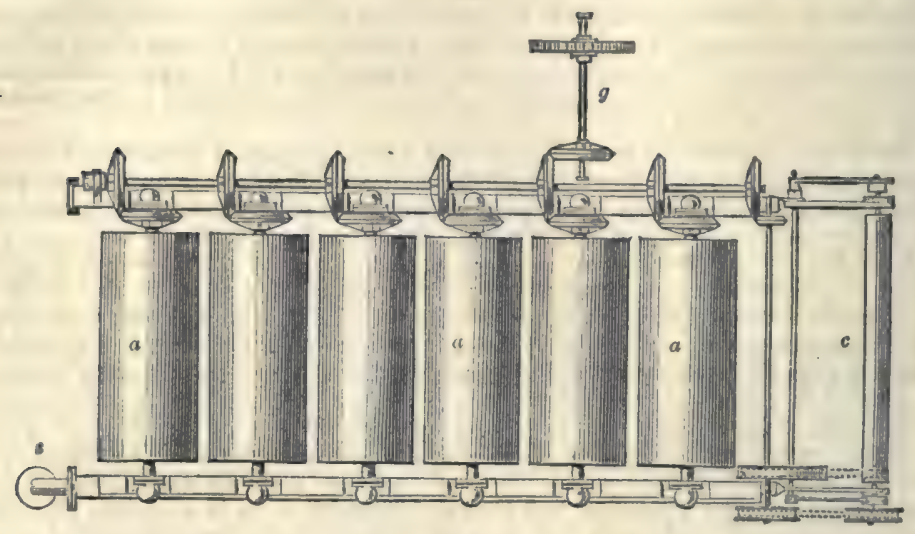

as there is more surface of cloth exposed to it at a time; it can, for the same reason, be turned more rapidly round. Well-finished goods will not rise when heated, except on the pattern. Messrs. Bridson have a large business in jacconets for artificial flowers on account of this peculiar finish. They are formed of a plain cotton cloth, but stand the pressure of hot irons without curling.

No essential difference is made in bleaching muslins, except that sometimes weaker solutions are employed for very tender goods. Mr. Barlow makes no difference as a rule in the strength given in describing his process; with very strong goods, he sometimes uses the liquids stronger.

It is desired occasionally to bleach goods which have coloured threads woven into them, or colours printed on them. In these cases great caution must be used. It is needful to use weak solutions, but more especially not to allow any one process to be continued very long, but xather to repeat it often than to lengthen it. This may be stated as a general rule in the bleaching of goods. It would indeed be possible to do the whole bloaching in one operation, but the cloth would be rotten. This arises from the fact that, at a cortain strength, bleaching.liquor or soda is able to destroy the fibre; but another and less strength does not act on the fibre, but only on such substances as colouring-matters. This care is needed when printed goods which have a white ground are treated. The white ground takes up colour enough to destroy its brilliancy, and soaping does not always remove it. The bleaching then is effected by using bleaching-liquor at $\frac{1}{2}$ Twaddle. Some persons put a Turkey-red thread into the ends of the pieces. The original use of this seems to be scarcely known among the manufacturors. It was used as a test of the modo of bleaching employed. If strong solutions be used, which are apt to spoil the cloth, the colour of the dyed threads will be discharged. When the separato system is employed, this is evaded easily; it is the practice to keep the ends containing the red threads out of the liquid, allowing them to rest on the side of the ressel.

Sometimes chlorate of potash is used for the same purpose, souring as with the bleaching-powder. The colours may, in this manner, be made much more brilliant than before, although a little excess will discharge them. A good deal of the effect may be owing to the better whito givon to the ground. Besides these processes for bleaching, another was at one time introduced, which consisted of immersing the cloth in a solution of caustic alkali, and afterwards steaming in a close vessel. It is not now in use. Alkali of $1020^{\circ}$ specific gravity was used.

The new or continuous Process. - This method owes its introduction to David Bentley, of Pendleton, who patented it in 1828. It consists in drawing the goods in one continuous line through every solution with which it is desired to saturate them. This is done by connecting the ends of all the pieces. The motion of rollers draws the chain of cloth thus formed in any desired direction, and through any number of solutions any given number of times. We shall allow him to use his own words. 
Fig. 135 is an ond view of two such calenders, each having two larger rollers, $B$ and $\mathrm{B} 1, \mathrm{a}$ smaller driving-roller $\mathrm{c}$, two racks $\mathrm{D}$ and $\mathrm{D} 1$, placed upon two cisterns $\mathrm{a}$

135

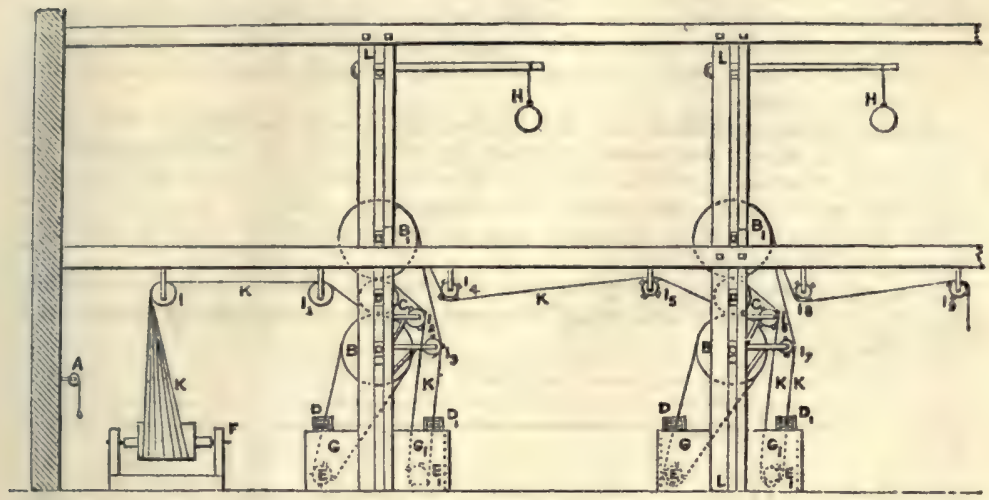

and $\mathrm{G} 1$, inside of which cisterns are two rollers $\mathrm{x}$ and $\mathrm{x} \mathbf{I}$, which rollers have four square ribs upon each, to shake the goods as they pass through the cisterns. At $F$ is a frame, upon which the batches of goods are placed upon rollers shown in fig. 136, where they are marked $\mathrm{x}, \mathrm{k}, \mathrm{x}, \mathrm{k}$. The calender-cheoks are made fast at the feet, at the middle, and to the top of the building, having levers and weights $\mathrm{x}$ to give pressure to the calender-bowls.

Near the end-walls of the building are two rollers, one of which is shown at $\mathrm{A}$; upon each of these is a soft cord used as a guido for conducting the goods through the machinery and cisterns. The operation is commenced by passing one end of the cord through the rollers B and c, down to cistern $\alpha$, under roller $\mathrm{B}$, through the furthermost division of rack $\mathrm{D}$, and again through calender-rollers at $B$ and $c$, repeating the same, but observing to keep the cord tight, and to approach one division nearer in rack $\mathbf{D}$ each revolution until each division is occupied, when the end must pass over $c$, under and round $B 1$, down to and over the guide-roller I 3 , through the nearest division of rack D 1 into cistern a 1 , under roller $x 1$, over guide-roller

136

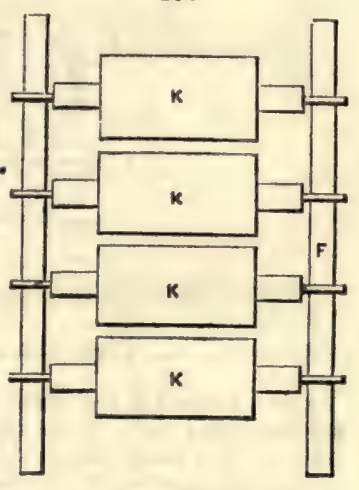
12 , and again over roller $\mathrm{c}$, under and round $\mathrm{B} 1$. This course must be repeated, observing as before to keep the cord tight, and to receive one division of rack $\mathrm{D} 1$ every revolution, until each division of rack $\mathbf{D} 1$ is occupied, when the ond must pass over from B 1 under I 4. The cord now forms a sort of spiral worm round and through the machinery and cisterns, beginning at $\mathrm{B}, \mathrm{C}$, and ending at the top of $\mathrm{B} 1$ to 14 , the number of revolutions being governed by the number of divisions in the racks $\mathrm{D}$ and D 1 , so that if there were fifteen divisions in each rack there would be fifteen revolutions under $\mathrm{C}$, round $\mathbf{B}$ through $\mathrm{G}$, under $\mathbf{E}$ through $\mathrm{D}$, and fifteen revolutions over C round B 1, over I 3 through $D 1$ and $a 1$, under $\mathrm{E} 1$ over I 2, and again over c, passing from the top of B 1 to $\mathbf{1 4}$; and by this means, if one end of the back of goods marked K, and placed upon the frame F (fig. 136), is fastened to the end of the guide-cord, tho goods will, when the calender is put in motion, be conducted and washed thirty times through the water in the cisterns, and squeezed thirty times through the calenders. As the operation proceeds and the guide-cord passes through the calonder, it is wound by hand upon roller $\mathrm{A}$ to prevent it from becoming entangled, and to keep it in readiness for the next operation. As soon as the first end of the goods has passed through fig. 136, and arrives at the guide-roller I 4, it is detached from the end of the guide-cord and attached to the guide-cord at the other end, or with the opposite set of calenders. After this, by putting these in motion, the goods are washed and squeezed through its cisterns, which eisterns are supplied with hot and strong lime-lye, and the goods passing orer guide-roller I 9, they are conreyed orer other guide-rollers to be placed for the purpose, and taken down

Vor. I. 
by some person or some proper machinery into one of the boiling rossels, whore, stoam or fire heat being addod, thoy are suffered to remain whilo the lime-boiling takes effect.

We need not follow the znventor into all the particulars. When the gools were sufficiently actod on by one solution, another solution was used, so that this mode of calendering not only was a method of moving the goods from place to place by means of rollors, but it was a method also of saturating goods thoroughly with a solution and of washing thom.

It was by a similar method that Mr. Bentley bleached skeins of yarn, of linen, or of cotton. The skoins are looped together by tying any soft material round the middle of the first skein, which will leave the loops from one end of the next skein to pass half way through, and which will always leave other two loops, and by repeating which any quantity of skeins may be looped together, tying the last loop with another soft material.

The mode of saturating the goods with solutions is effected by the arrangement shown in fig. 137. Rapid motion and frequent pressure are introdueed instead of a still soaking process.

137

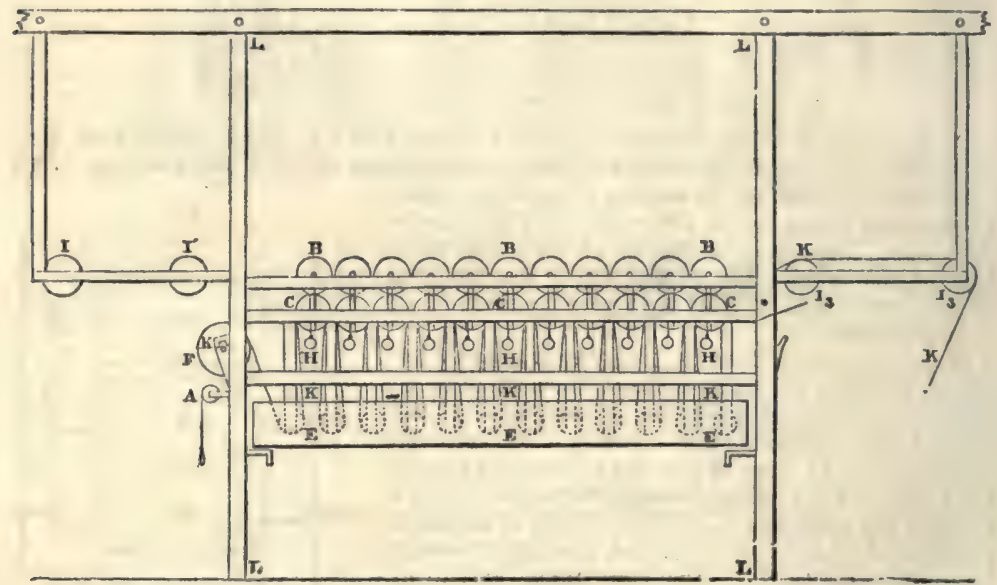

$\Delta$ is a roller for the guide-cords; $B, B, B$ are eleven washing-rollers; C, C, C are speed-rollers; $\mathrm{E}, \mathrm{E}, \mathrm{E}$ are twelve rollers immersed in twelve divisions of the cistern $G$. The eleven staple-formed irons which pass through the frame rails on each side of the centres of the eleven rollers $\mathrm{B}, \mathrm{B}, \mathrm{B}$, and the eleven rollers $\mathrm{C}, \mathrm{C}$, $\mathrm{C}$, serre to stay these rollers in their places, at the same time allowing the elevon washingrollers $\mathrm{B}, \mathrm{B}, \mathrm{B}$, to rise and fall according to the pressure by which they are held down by the eleven weights attached to these irons at placed such staves, brushes, or rollers, as may be found necessary for holding and brushing the goods in the best manner to keep them straight during the differont washings in water and bleaching-liquors. The goods are prepared by steoping, as before described, and placed in batches at $\mathbf{F}$, and passing under the immersing rollers $\mathbf{E}$ and the twelve divisions of cistern $a$, between the eloven speed-rollers $\mathbf{C}$ and the eleven washing-rollers $\mathrm{B}$, as seon at $\mathbf{x}$, are taken down straight and open into one of the vessels, and are then boiled by steam, which is succeoded by repeated washings alternately in water and bleaching-liquors, until they are sufficiently Wleached, as before described.

The elevation and ground-plan of a blerch-house and machinery capable of bleaching 800 pieces of $4 \mathrm{lbs}$. cloth por day (for best madder work), with tho labour of one man and three boys, working from 6 until 4 o'clock, exclusive of singeing and drying, are represented in figs. 138 and 139 (p. 387). The lettor $d$ represents two lengths of cloth of 400 piees each (end of pieces being stitched together by patont sewing-machine made by Mather and Platt), making together 800 pieces, passing through washing-machine $g$, and from thence delivered over winch $w$, into kier c,-this operation occupies one hour, -where they are boiled for twelve hours in lime. They are then withdrawn by the same washing-machine $g$, washed, and passed into second kier $b$ (operation occupying one hour), where they are boiled 


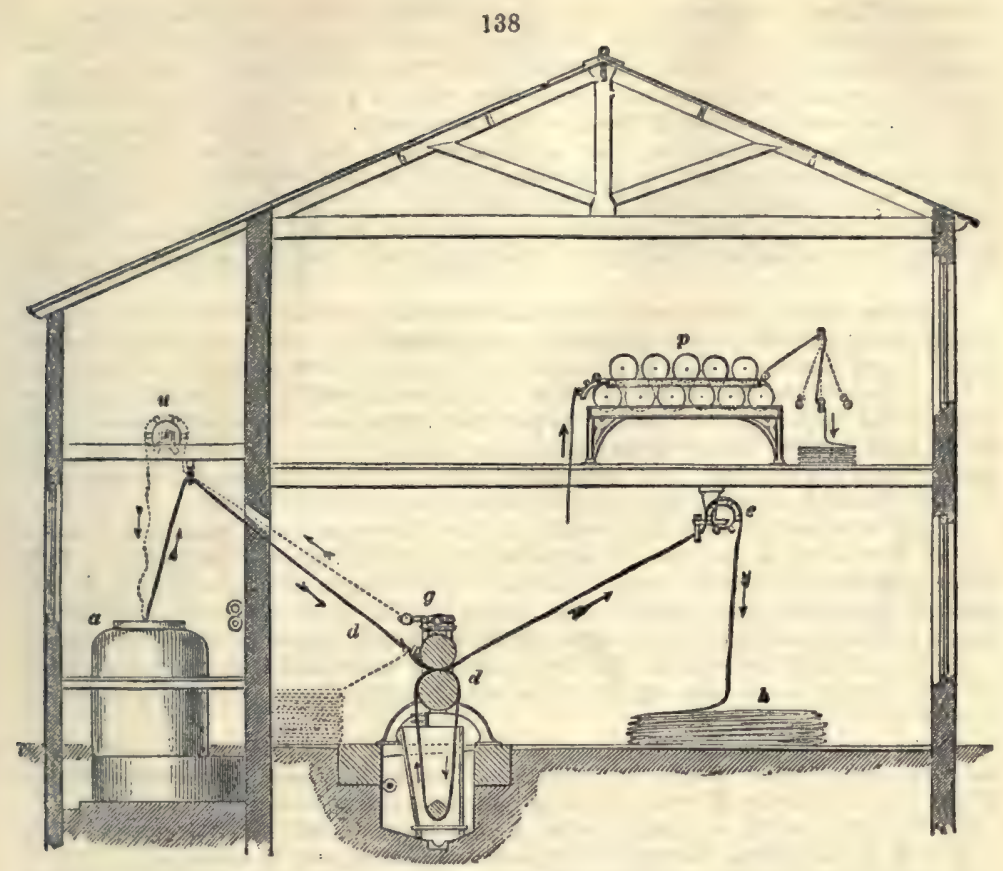

139

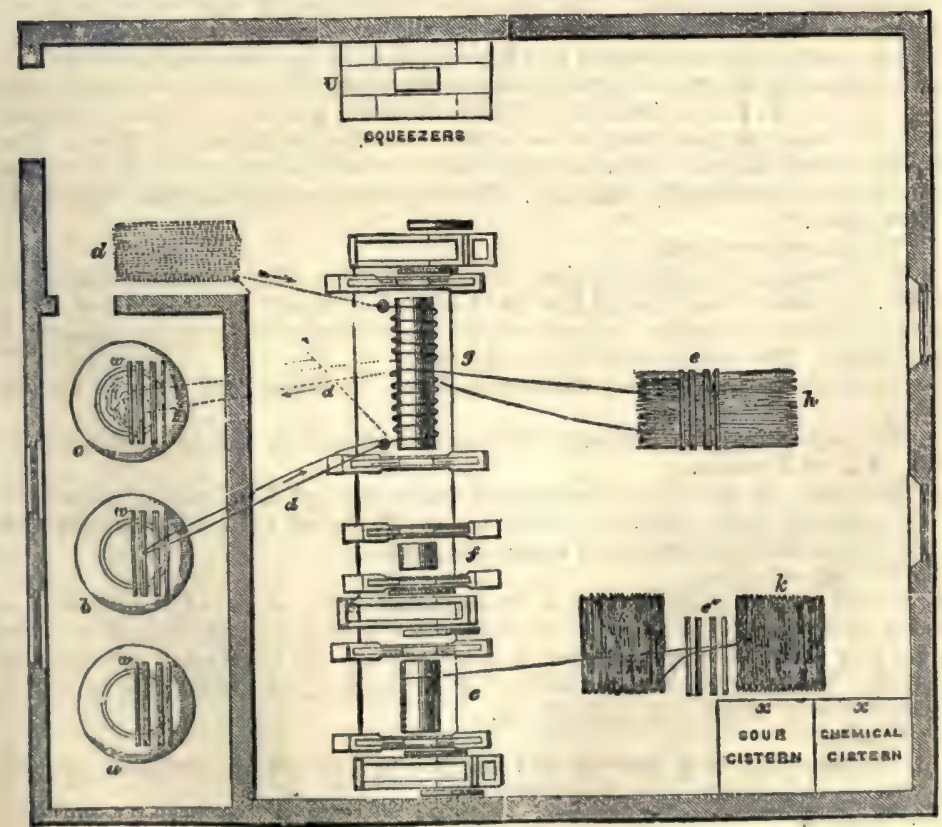

cc 2 
for twelre hours in ashes and resin; again withdrawn by the same machine $g$, washod, squeezed (seo plan at $v$ ), and passod over winch $e$, and pilod at $h$ (this oporation occupies ono hour). They are then taken from pile $h$, and throaded through sour-machine $e$, soured, passed over winch $e^{\prime \prime}$, and piled at $k$ (operation, one hour), where it remains in the pile for threo hours. It is then squeezed at $v$, and washed through machino $g$ (an hour's operation), delivered into third kier $a$, boiled for six hours, washed at $g$, squeezed at 0 (an hour's operation), and passod through chemick-machine (an hour's operation), and piled for one hour; after which it is sourod again (an hour's operation), squeezed, and washed at $g$ (an hour's oporntion), squeezed again at $f$ (an hour's operation), and dried by machine at $p$ (fig. 138).

There are several advantages in using the squeering process so often in the above arrangement :-Firstly, the bowels of the washing-machine are not so much damaged by the heavy pressure which is required to be applied, if no squeezers are used, in order to prepare the pieces for the sour and chemick machines. Secondly, a drior state of the cloth than can possibly be produced by the washing-machine alone, thus fitting it to become better saturated with the chemick or sour. Thirdly, the piece passing from the souring to the washing-machine, in this arrangement, carries with it less of the acid, and thus onsures a better washing, with less water.

It may be observed, that the velocity of the above-mentioned machines is much higher than usual, experience having shown that the various operations are thus better performed than when running slower. The reason of this appears to be, firstly, that the piece, running at such velocity, carries with it, by reason of capillary attraction, a greater quantity of liquid to the nip of the bowels ; secondly, the great velocity of the bowels, together with the grcater quantity of water carried up, produces a more powerful current at the nip and down the ascending piece, thus penotrating to every fibre of it.

It may alsa be remarked, that the abore-mentioned machines are not adapted to the bleaching of linen; for the latter cloth, not having the same elasticity as cotton, if it should become tight, would either be pulled narrow or torn.

In illustration of the continuous process as at present used, the plan of proceeding at Messrs. MeNaughten, Barton, and Thom's, at Chorley, mny be described.

1. In order that there may be no interruption in the process, the pieces are united in one continuous piece - each piece being about 30 yards, the whole varying with the weight of eloth-about 300 yards long. Each picee is marked with the name of the printer. This is sometimes done in marking-ink of silver, and sonetimes in coal-tar, at the extremity of the piece. The pieces are rapidly tacked together by girls, who use in some establishments a very simple sewing-machine. (Soe Sewing-Machine.) The whole amount to be bleached at a time is united in one piece, and is drawn from place to place like a rope. To give them this rope-form, the goods are drawn through an aperture whose surface is exceedingly smooth, being generally of glass or earthenware. Of these many are used in transferring the cloth from place to place. They serve instead of pulleys. The cloth when laid in a vessel is not thrown in at random, but laid down in a carefully made coil. The rope-form enables the water to penetrate it more easily.

2. The pieces are singed.

3. They are boiled in the first kier. In this, $3,500 \mathrm{lbs}$. of cloth have added to them $250 \mathrm{lbs}$. of caustic lime, $1 \mathrm{lb}$. of lime to 14 of cloth. The kier is cylindrical, 7 feet deep and 8 feet in diameter; as much water is added as will cover the cloth, aloout 600 gallons. This boiling lasts thirteen hours.

4. They are washed in the washing-machine. Robinson and Young's machino is used.

5. They are soured in a similar machine with hydrochloric acid of epecific gravity $1010^{\circ}$, of $2^{\circ}$ of Twaddle.

6. The same amount of cloth being supposed to be used, it is bucked in a solution of soda-ash and resin, $170 \mathrm{lbs}$. of sods-ash to $30 \mathrm{lbs}$. of resin. The boiling lasts sixteen hours, the same amount of water being used.

7. Washed as before.

8. Passed through chloride of lime, or chemicked. The cloth is laid in a stone or wooden cistern, and a solution of bleaching-powder is passed through it, by being poured over it and allowed to run into a vessel below; this is managed by continued pumping. This solution is about half a degreo Twaddle, or specific gravity $1002 \cdot 5$. The cloth lies in it from one to two hours.

9. Washed.

10. Boiled again in a kier for five hours with $100 \mathrm{lbs}$. of carbonate of soda crystals.

11. Washed. 
12. Put in chloride of lime as before.

13. Soured, in hydrochloric acid of 1012.5 specific gravity, or $21^{\circ}$ Twaddle.

14. Lies six hours on stillages.-A stillage is a kind of low stool used to protect the cloth from the floor.

15. Washed till clean.

16. Squeezed in rollers.

17. Dried orer tin cylinders heated by steam.

This is the process for calico generally; some light goods must be more carefully handled. The usual time occupied by all these processes is five days. Thoy are sometimes dried in a hydro-extractor; after singeing, laid twenty-four hours to steop, then washed before being put into the lime kier.

\section{Bleachisg of Lines.}

Linen contains much more colouring-matter than cotton. The former loses nearly a third of its weight, while the latter loses not more than a twentieth. The fibres of flax possess, in the natural condition, a light grey, yellow, or blond colour. By the operation of rotting, or, as it is commonly called, water-retting, which is omployed to enable the textile filaments to be separated from the boon, or woody matter, the colour becomes darker, and, in consequence probably of the putrefaction of the green mattor of the bark, the colouring-substance appears. Hence, flax prepared without rotting is much paler, and its colouring-matter may be in a great measure removed by washing with soap, leaving the filaments nearly whito. Mr. James Lee obtained a patent in 1812, as having discorered that the process of steeping and dew-retting is unnecessary, and that flax and hemp will not only dress, but will produce an equal if not greater quantity of more durable fibre, when eleanod in the dry way. Mr. Leo stated that, when hemp or flax-plants are ripe, the farmer has nothing more to do than to pull, spread, and dry them in the sun, and then to break thom by proper machinery. This promising improvement has apparently come to nought, having been many years abandoned by the patentee himself, though he was favoured with a special Act of Parliamont, which permitted the specification of his patent to remain soaled up for seren years, contrary to the general practice in such cases.

The substinco which gives steeped flax its peculiar tint is insoluble in boiling water, in acids, and in alkalis; but it possesses the property of dissolving in caustic or carbonated alkaline lyes, when it has by previous exposure been acted on only by chlorino. This process is effected in great mensure by the influence of air in combination with light and moisture acting on the linen cloth laid upon the grass : but chlorine hastens the operation. In no case, however, is it possible to dissolve the colour completely at once, but there must be many alternate exposures to oxygen or chlorine, and alkali, before the flax becomes white. It is this circumstance alone which renders the bleaching of linen an apparently complicated business.

Old Mcthod.-A parcel of goods consists of 360 pieces of those linens which are called Britannias. Each piece is 35 yards long, and weighs, on an average, $10 \mathrm{lbs}$; the weight of the pareel is, in consequence, about 3,600 lbs, avoirdupois weight. The linens are first washed, and then steeped in wasto alkaline lye, as formerly deseribed under these processes; they then undergo the following operations:-

\begin{tabular}{|c|c|c|c|c|c|}
\hline & & & & & \\
\hline to & 80 & ditto & ditto & ditto & ditto. \\
\hline 3. Ditto & 90 & potashes & ditto & ditto & ditto. \\
\hline 4. Ditto & 80 & ditto & ditto & ditto & 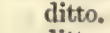 \\
\hline 5. Ditto & 80 & ditto & ditto & ditto & itto. \\
\hline 6. Ditto & 50 & ditto & ditto & ditto & its \\
\hline Ditto & 70 & ditto & ditto & ditto & ditto. \\
\hline Ditto & 70 & ditto & ditto & ditto & itt \\
\hline
\end{tabular}

9. Soured one night in dilute sulphuric acid; washed.

10. Bucked with $50 \mathrm{lbs}$. pearl-ashes, washed, exposed on tho field.

11. Immersed in the chloride of potash or lime 12 hours.

12. Boiled with $30 \mathrm{lbs}$. pearl-ashes, washed, exposed on the freld.

13. Ditto 30 ditto ditto ditto ditto.

14. Soured, washed.

The linons are then takon to the rubbing-board, and well rubbed with a strong lather of black soap, after which they aro woll washed in pure spring-water. At this period they are carefully examined, and those which are fully bleached are laid aside to be blued, and made up for the markot; while those which are not fully white are returned to be hoiled, and steeped in the chloride of lime or potash; then sourod, until they are fully white, 
By the above process, $690 \mathrm{lbs}$. weight of alkali is taken to bleach 360 preces of linon, each piece consisting of 35 yards in length; so that the expenditure of alkali would be somewhat less than $2 \mathrm{lbs}$. for each piece, were it not that some parts of the linens are not fully whitened, as above noted. Two pounds of alkali may therefore be statod as the averago quantity employed for bleaching each piece of goods.

What is called the old method, or that used from about the introduction of bleach. ing-powder, at the beginning of the century, till within ten or fifteen years, required bleaching on the grass; and the mode in which it was managed in Ireland and Scotland, where it held its ground longest, is as follows :

1. They were rot-steoped in a weak solution of potash, at about $130^{\circ} \mathrm{F}$, for two days, until the dressing used in manufacturing tho cloth was removed.

2. Washed.

3. Boiled or bowked in potash-lye at 10 Twaddle, for ten hours.

4. Washed, and the ends turned so that the whole might be equally exposed to the lye.

5. Boiled or bowked in a similar lye to the above for twelve hours.

6. Washed well.

7. Exposed on the grass for three days, and watered.

8. Taken up and soured with sulphuric acid, at $2^{\circ}$ Twaddle, for four hours.

9. Taken up and washed well.

10. Boiled again for eight hours in potash-lye, at $1^{\circ}$ Twaddle, to which had been added black or soft soap, about 20 lbs. to a kier of about 300 gallons.

11. Washed.

12. Crofted, or exposed on the grass, as before.

13. Treated with chloride of lime at $11^{\circ}$ Twaddlo, for four hours.

14. Washed.

15. Soured in sulphuric acid, at $2^{\circ}$ Twaddle, for four hours.

16. Washed.

17. Boiled for six or seven hours with soap and lye, using in this case more soap and one-third less lye than in the former bowkings.

18. Drawn out and put through rub-boards. This is a kind of washing-machine, made of blocks of wood, with hard-wood teeth. The goods are washed by it in a sorpy liquid. The teeth moving rapidly, drive tho soap into the clotls.

19. Boiled in the lye alone for six hours.

20. Washed.

21. Crofted, keoping them very clean, as this is the last oxposure.

22. Treated with chloride of lime.

23. They are then starched, blued, and beetled, to finish thom for the market. These operations last six weoks.

New System, as practised in Scotland and Ireland.-Directions given by an extensive

1. Wash.

2. Boil in lime-water ten or twelve hours.

3 Sour in muriatic acid, of $2^{\circ}$ Twaddlo, for three, four, or five hours.

4. Wash well.

5. Boil with resin and soda-ash twelve hours.

6. Turn the goods, so that those at the top shall be at the bottom, and boil again as at No. 5 .

7. Wash woll.

8. Chemick, at 30 Twaddle, or $1002 \cdot 5$, four hours.

9. Sour, at $2^{\circ}$ Twaddle, or $1010^{\circ}$ specific gravity.

10. Wash.

11. Boil in soda-ash ten bours.

12. Chemick again.

13. Wash and dry.

This is the system chiefly adopted when the goods are to be printed.

The following is the system practised in the neighbourhood of Porth, whore the chief trado is in plain sheetings :-

1. Before putting them into operation, they are put up into parcels of about 35 cwts.

2. They are then steeped in lye for twenty-four hours.

3. Then washed and spread on the grass for about two days.

4. Boiled in lime-water.

5. Turned, and boiled again in lime-water, those at the top being put at the bottom. $60 \mathrm{lbs}$. of lime are used at a time, and about 600 gallons of water.

6. Washed, then soured in sulphuric acid of $2^{\circ} \mathrm{T}$ waddle, or $1010^{\circ} \mathrm{sp} . \mathrm{gr}$. , for four hours, then washed again. 
7. Boiled with soda-ash for ton houts; 110 lbs. used.

8. Washed and spread out on the green, or crofted.

9. Boiled again in soda as before.

10. Crafted for three days.

11. They are then examined: the white ones are taken out; those that aro not finished are boiled and crofted again.

12. Next, they are scalded in water containing $80 \mathrm{lbs}$. of soda-ash, and washed.

13. The chloride of lime is then used at $1_{2}^{\circ}$ Twaddle, or 1002.5 specific gravity.

14. Washed and scalded.

15. Washed and treated with chloride of lime.

16. Soured, for four hours, with sulphuric acid, at $2^{\circ}$ Twaddle, or $1010^{\circ}$ specific gravity.

17. Washed.

If eloths lighter than sheetings are used, the washing liquids are used weaker. The great point is to observe them carefully during the process, in order to soe what treatment will suit them best.

It will be seen that the process of bleaching linen is still rery todious ; and although it may be managed in a fortnight, it is seldom that this occurs regularly for a great length of time. The action of the light introduces at once an uncertain element, as this varies so much in our climate. If, again, linen be long exposed to the air in a moist condition, it is apt to become injured in strength. To shorten the process, therefore, is important; and if no injurious agents are introduced, a shortening promises also to gire increased strength to the fibre. It has not been found possible to introduce chlorine into linen-bleaching at an early stage, as in the case of cotton; and the processes for purifying it without any chlorine, render it so whito that unskilled persons would call it as white as snow. The chlorine is introduced nearly at the end of the operation, after a series of boilings with alkalis, sourings, and expnsures on the grass. If introduced at an earlier stage, the colour of the raw cloth becomes fixed, and cannot be romoved. The technical term for this condition is ' set.' Mr. F. M. Jennings, of Cork, has patented a method which promises to obviato the difficulty. The peculiarity consists in using the alkali and the chloride of alkali at the same moment, thus giving the alkali opportunity to seize on the colouringmatter as soon as the chloride has acted, and thereby preventing the formation of an insoluble compound. He prefers the chlorides of potash or soda. His plan is as follows:-

1. Ho soaks the linen in water for about twelve hours, or boils it in lime or alkali, or alkali with lime, and then soaks it in acid, as he uses soaps of resin in other mixtures-the alkalis being from $3^{\circ}$ to $5^{\circ}$ Twaddle, $1015^{\circ}-1025^{\circ}$ specific gravity.

2. Boils in a similar alkaline solution.

3. Washes.

4. Puts it into a solution of soda, of $5^{\circ}$ Twaddle, $1025^{\circ}$ specific gravity, adding chloride of soda until it rises up to from $6^{\circ}-7^{\circ}$ Twaddle. It is allowed to romain in this solution for some hours, and it is better if subjected to heating or squeezing between rollers, as in the washing-machine.

5. He then soaks, sours, and washes.

6. Ho then puts it a second time into the solution of alkali and chloride.

7. Then washes, and boils again with soda. These operations, 6 and 7 , may be repeated until the eloth becomes almost white.

The amount of exposure on the grass by this process is said to be not more than from one-half to one-fourth that required by the usual method, or it may be managed so as entirely to supersede crofting.

Chevalior Claussen has opened up the filaments of flax by the evolution of gas from a carbonate in which the plant is steeped, and at the same time bleached by chloride of magnesia, but this has not been successful.

\section{Bueachtse of Silk.}

Silk in its raw state, as spun by the worm, is either white or yellow of various shades, and is covered with a varnish which gives it stiffness and a degree of elasticity. For the greater number of purposes to which silk is applied, it must be deprived of this native corering, which was long considered to bo a sort of gum. The operation by which this colouring-matter is removed is called scouring, cleansing, or boiling. A great many different processes have beon proposed for freeing the silk filres from all foreign impurities, and for giving it the utmost whiteness, lustre, and pliancy; but none of the now plans has superseded, with any advantage, the one practised of old, which consists essentially in steeping the silk in a warm solution. of soap; a circumstnnco placed beyond all doubt by the interesting experiments of 
M. Roard. The alkalis, or alkaline salts, act in a marked manner upon the varnish of silk, and effect its complete solution; the prolonged ngency even of boiling water or soap and water destroys the brillianey of silk. It would appear, however, that the Chineso do not employ this method, but something that is preferable. Probably tho superior beauty of their white silk may bo owing to the superiority of thoir raw material.

The most ancient method of scouring silk consists of three operations. For the first, or the ungumming, thirty per cont. of soap is dissolved in clean water at a boiling heat; then the temperature is lowered by the addition of a little cold water, by withdrawing the fire, or at least by damping it. The hanks of silk susponded upon horizontal poles over the boiler are now plunged into the soapy solution, kept at $n$ heat somowhat under ebullition, which is an essential point; for, if hotter, the soap would attack the substance of the silk, and not only dissolve a portion of it, but deprive the whole of its lustre. The portions of the hanks plunged in the bath get scoured by degrees: the varnish and the colouring-matter are removed, and the silk assumes its proper whiteness and pliancy. Whenever this point is attained, the hanks are turned round upon the poles, so that the portion formerly in the air may be also subjected to the bath. As soon as the whole is completely ungummed, they are taken out, wrung by the peg, and shaken out; after which the next step, called the boil, is commenced. About $25 \mathrm{lbs}$. or $35 \mathrm{lbs}$ of ungummed silk are enclosed in bags of coarso canvas, called pockets, and put into a similar bath with tho proceding, but with a smaller proportion of soap, which may therefore be raised to the boiling-point without any danger of destroying the silk. The ebullition is to be kept up for an hour and a half, during which time the bags must be frequently stirred, lest those near the bottom should suffer an undue degree of heat. The silk experiences in these two operations a loss of about 25 per cent. of its weight.

The third and last scouring operation is intended to give the silk a slight tinge, which renders the white more agreeable, and better adapted to its various uses in trade. In this way wo distinguish the China white, which has a faint cast of red, the silver white, the azure white, and the thread white. To produce these different shades, we begin by preparing a soap-water so strong as to lather by agitation; we then add to it for the China white a little arnotto, mixing it carefully in; and then, passing the silk properly through it, till it has acquired the wished-for tint. As to the other shades, we need only azure them more or less with a fine indigo, which has been previously washed several times in hot water, and reduced to powder in a mortar. It is then diffused through boiling water, allowed to settle for a few minutes, and the supernatant liquid, which contains only the finer particles, is added to the soap bath, in such proportion as may be requisite. The silk, on being taken out of this bath, must be wrung well, and stretched upon perches to dry; after which it is introduced into the sulphuring chamber, if it is to be made use of in the white state. At Lyons, however, no soap is employed at the third operation; after the boil, the silk is washed, sulphured, and azured, by passing through very clear river-water properly blued.

The present practice in the silk-works in Lancashire is as follows:-

The Italian silk arrives in this country with a little soap in it, put in by the throwsters there, amounting to one drachm to a pound of silk. It is received here in lanks, and bleached in that state. The hanks aro hung on sticks or small poles, about three pounds of silk being on each stick. The sticks being laid across a vessel, the silk hangs down, and in this way may be immersed in any liquid. The treatment of silk is then much more tender than that of cotton.

1. The hot lather is mado with $3 \mathrm{lbs}$. of soap in 50 gallons of water; to this is added $1 \mathrm{lb}$. of soda crystals. The silk is kept in this lather at a temperature of from $175^{\circ}$ in $190^{\circ} \mathrm{F}$. for three-quarters of an hour. It is then wrung or dried in tho hydroextractor (called hydro or whizzer in Lancashire works). 2. It is then, for tho purpose of straightening it, rolled on a cocoa-nut roll-pin 4 in. in diameter, a little turn being given it occasionally, by the finger and thumb, to prevent entangling. 3 . It is then put into bags of one yard square. The hanks are laid flat, and the bags stitelied down. In this state they are boiled for $3 \frac{1}{2}$ hours, using for the samo amount of water as before, $3 \mathrm{lbs}$. of soap to 20 lbs. of silk. 4 . The silk is then washed or moved about by the hand in a cistern one yard wide and one deop, retaining as much soap as will make a pretty permanent lather. To this there is generally addod a small quantity of archil, about $\frac{1}{4} \mathrm{oz}$. to $4 \mathrm{lbs}$. of silk. 5 . It is then dried in the hydro-extractor. 6. It is then straightened and sulphured. The sulphuring is dono in a small apartment, which should be very high. The size is froquently 10 feet square by 20 in height. The silk is hung up in it, and $4 \mathrm{lbs}$. of sulphur for each $40 \mathrm{lbs}$. of silk aro put on the floor and set fire to. Tho room is closed as well as possible, and the silk is allowed to remain 4 hours. This is the bleaching, and it requires now only to be 
washed by rinsing three to four times in cold water. A little indigo blue is usod to give it a pearly appearance. The use of archil, which has been mentioned, dopends upon the shado of white, so to speak, which is wanted. 7. The silk is now dried by the hydro-extractor first, and then by exposing to a temperature of $85^{\circ}-90^{\circ}$. If hearily laden with gums, silk must be dried at a still cooler tomperature. In this operation of bleaching, $1 \mathrm{lb}$. of good silk loses $4 \mathrm{oz}$; but as it seldom arrives very pure, the usual loss to the pound of silk is $5 \mathrm{oz}$.

The first, or simmering operation, mentioned here, is not necessary for the whito silk of Chins.

The silks intended for tho manufacture of blondes and gauzes are not subjected to the ordinary scouring process, because it is essential in these cases for them to presorve their natural stiffness. We must therefore select the raw silk of China, or the whitest raw silks of other countries; steep them, rinse them in a batl of pure water, or in one containing a little soap; wring them, expose them to the vapour of sulphur, and then pass them through the azure water. Sometimes this process is repeated.

Before the memoir of M. Roard appeared, extremely vague ideas were entertained about the composition of the native varnish of silk. He has shown that this substance, so far from being of a gummy nature, as had been believed, may be rather compared to bees'-wax, with a species of oil and a colouring-matter which exist only in raw silks. It is contained in them to the amount of from 23 to 24 per cent., and forms the portion of weight which is lost in the ungumming. It possesses, howerer, some of the proportios of vegetable gums, though it diffors essentially as to others. In a dry mass, it is friable and has a vitreous fracture; it is soluble in water, and affords a solution which lathers like soap; but when thrown upon burning coals, it does not soften like gum, but burns with the exhalation of a fetid odour. Its solution, when left exposed to the open air, is at first of a golden yellow, becomes soon greenish, and êre long putrefies, as a solution of animal matter would do in similar circumstances. M. Roard assures us that the city of Lyons alone could furnish several thousand quintals of this substance per annum, were it applicable to any useful purpose.

The yollow varnish is of a resinous nature, altogether insoluble in water, very soluble in alcohol, and contains a little volatile oil, which gires it a rank smell. The colour of this resin is easily dissipated, either by exposure to the sun or by the action of chlorine: it forms about one fifty-fifth of its weight.

Bees'-wax exists also in all the sorts of silk, even in that of China; but the whitor the filaments, the less wax do they contain.

II. Roard has obserred that, if the silk be exposed to the soap-baths for some time after it has been stripped of its foreign matters, it begins to lose body, and has its valuable qualities impaired. It becomes dull, stiff, and coloured in consequence of the solution, more or less considerable, of its substance; a solution which takes place in all liquids, and even in boiling water. It is for this reason that silks cannot be alumed with heat; and that they lose some of their lustre in being dyed brown, a colour which requires a boiling hot bath. The best mode, therefore, of avoiding these inconveniences, is to boil the silks in the soap-bath no longer than is absolutely necessary for the scouring process, and to expose them in the various dyeing operations to a temperature as moderate as may be sufficient to communicate the colour. When silks are to be dyed, much less soap should be used in the cleansing, and very little for the dark colours. According to M. Roard, raw silks, white or yellow, may be completely scoured in one hour, with 15 lbs. of water for one of silk, and a suitable preprortion of soap. The soap and the silk should be put into the bath half an hour before its ebullition, and the silks should be turned about frequently. The dull silks, in which the varnish has already undergone some alteration, never acquire a fine whito until they are exposed to sulphurous acid gas. Exposure to light has also a rory gond effect in whitening silks, and is had recourse to, it is said, with advantage, by the Chinese.

Baumé contrived a process which does not appear to have received the sanction of experience, but which may be a guide in the right way. He macerates the yellow raw silk in a mixture of alcohol at $36^{\circ}$ (sp. gr. ${ }^{\circ} 0837$ ) and one thirty-second part of pure muriatic acid. At the end of forty-eight hours, it is as white as possible, and the more so, the better the quality of silk. The loss which it suffers in the menstruum, is only one-fortieth; showing that nothing but the colouring-matter is abstracted. The expense of this menstruum is the grent obstacle to Baumé's process. The alcolinl, lowever, might be in a rery great measure recovered, by saturating the acid with chalk, and redistilling. 


\section{Bifschisa of Woor.}

Wool, like tho precolling fibrous matter, is covered with a peculiar varnish, which impairs its qualitios, and prevents it from being employod in the raw stato for the purposes to which it is well adapted when it is scoured. The English give tho name yolk, and the French suint, to that native coat: it is a fatty unctuous matter, of a strong smell, which apparently has its chiof origin in the cutaneous perspiration of the sheep; but which, by the agency of external bodies, may have undorgone some changes which modify its constitution. It results from the experiments of M. Vauquolin, that the yolk is composed of several substances; namoly, 1, a soap with basis of potash, which constitutes the greater part of it; 2 , of a notable quantity of acetate of potash; 3, of a small quantity of carbonato, and a trace of chloride of potassium ; 4, of a littlo lime in an unknown state of combination; 5 , of a specios of sebaceous matter, and an animal substance to which the odour is duo. There are seroral other accidental matters present on sheep's wool.

The proportion of yolk is variable in different kinds of wool, but in general it is more abundant the finer the staple; the loss by scouring being 45 por cont. for the finest wools, and 35 per cont. for the coarse.

The yolk, on account of its soapy nature, dissolves readily in water, with the exception of a littlo free fatty matter, which easily separates from the filaments, and remains floating in the liquor. It would then appear sufficient to expose the wools to simple washing in a stream of water; yet experience shows that this mothod nevor answers 80 well as that usually adopted, which consists in steoping the wool for some time in simple warm water, or in warm water mixed with a fourth of stale urine. From 15 to 20 minutes of contact are sufficient in this case, if wo heat the bath as warm as the hand can bear it, and stir it well with a rod. At the end of this time the wool may be taken out, set to drain, then placed in large baskets, in order to be completely rinsed in a stream of water.

It is generally supposed that putrid urino acts on the wool by the ammonia which it contains, and that this serves to saponify the remainder of the fatty matter not combined with the potash, although M. Vauquelin gave another opinion. Fresh urine contains a free acid, which, by decomposing the potash-soap of the yolk, counteracts the scouring operation.

If wools are better scoured in a small quantity of water than in a great stream, wo can conceive that this circumstance must depend upon the nature of the yolk, which, in a concontrated solution, acts like a saponaceous compound, and thus contributes to remove the free fatty particles which adhere to the filaments. It should also be observed that too long a continuance of the wool in the yolk water, hurts its quality very much, by weakening its cohesion, causing the filaments to swell, and eren to split. It is said then to have lost its nerve. Another circumstance in tho scouring of wool, that should always be attended to, is never to work the filaments together to such a degree as to occasion their felting; but in agitating we must merely push them slowly round in the ressel, or press them gently under the feet. Were it at all felted, it would neither eard nor spin well.

As the heat of boiling water is apt to decompose woollon fibres, we should be careful never to raise the temperature of the scouring bath to near this point, nor, in fact, to exceed $140^{\circ} \mathrm{F}$. Some authors recommend the uso of alkalino or soapy baths for scouring wool, but practical people do not deviate from the method ahove described.

When the washing is completed, all the wool which is to be sent whito into the market, must be exposod to the action of sulphurous acid, either in a liquid or a gaseous state. In the latter case, sulphur is burned in a close chamber, in which the wools are hung up or spread out; in the former, the wools are plunged into water moderately impregnated with the acid. (See SurpHuriva.) Exposure on the grass may also contribute to the bleaching of wool. Some fraudulent dealers are accused of dipping wools in buttormilk, or chalk-and-water, in order to whiten them and increase their weight.

Wool is sometimes whitened in the fleece, and sometimes in the state of yarn; the latter affording the best means of operating. It has been observed that the wool cut from certain parts of the sheop, especially from the groin, never bleaches woll.

After sulphuring, the wool has a harsh crispy foel, which may be removed by a weak soap-bath. To this also the wool-comber has rocourse when he wishes to cleanse and whiten his wools to the utmost. Ho generally uses a soft or potash soap, and after the wool is well soaked in the warm soap-bath, with gentle pressure ho wrings it well with the help of a hook, fixed at the end of his washing-tub, and hangs it up to dry.

The actual operations of purifying wool are so blended with the methods of weav. 
ing and working it, that, to show it fully, I shall givo here tho process of preparing flannels, out of which the parts relating to cleansing may be taken.

1. The wool is weighed out into parcels of $120 \mathrm{lbs}$. Add, on an average, 20 to $21 \mathrm{lbs}$. or 10 guarts of oil-rapo-oil or olive, or mixed, or, as is very common now, oleic acid, which may be so used as not to be hurtful to the machinery in this condition. This was introduced by Mr. M.Dougall.

2. It is then devilled or willowed, carded, slubbed, and spun.

The warp portion is made at this stage if wanted.

3. Scoured in the warp with urine and hot water, occasionally using a little ammonia.

4. Sized with a mineral sizing, and put into the looms.

5. If spun for weft, it is soaked, when on the bobbin, with cold water in a cistern, an air-pump being used to extract the air from the threads and to compel the water to enter.

6. The water is then removed by a revolving water-extractor. This process leaves the weft full and soft.

Skin-wool, so called, is taken from the skin by means of lime, which makes tho oil stiff, forming a compound.

7. The piece being now woven is grey. It is sent to the finishing or fulling mill, sprinkled over with urine and pigs' dung, and put under the fulling hammers until equally wet.

8. It is then washed out or scoured with cold water, raised with teasels, dricd out of doors or in a stove.

Treated a little differently, accordingly as Welsh or Lancashire is wanted.

9. It is then sprinkled again with soap-and-water, and milled one to two hours in the fulling-stock.

Three-quarters to $1 \mathrm{lb}$. of soep is given for each piece.

10. Cleared with cold water.

11. Hung up wet in a sulphur-stove; several pots of sulphur lighted. The door is shut till morning. Washed four to six hours in cold water, treated with finelyground indigo, dried, and a little further raised, pressed, and rolled up for sale.

If the flannel is Welsh. it is dried and sprinkled with fullers' earth (instead of the soap-and-water used for the Lancashire), well milled for some time, and then cleared in cold water. It is then put into a cistern fillod with water, having some soap thrown in as well as a few cakes of Prussian blue. This dipping is repeated threo or four times, and between each the flannel is milled in the fulling-stock. This levels the colour. When blue enough, the pieces are dried and made up for sale.

It appears that Welsh flannel is not sulphured; the cleaning is done entirely by nmmonia.

Sulphuring.-In the usual mode of sulphuring the cloth is hung on pegs or rails in rooms which are called the sulphur chambers or stoves. An iron pot containing sulphur is placed in each corner of the room, and the sulphur inflamed. The door is then shut and clayed. By the morning the process is finished, and the door is opened. This mode is objected to, because the sulphur, not being properly burnt, lodges in the cloth, and acts injuriously on it in the processes of dyeing or printing. Sparks also are apt to rise up and injure the pieces, the sulphur not being pure, and burning irregularly. Drops also of water impregnated strongly with sulphurous acid are apt to fall from the roof, doing injury to the eloth.

To aroid these inconveniences $\mathrm{Mr}$. Thom has invented a mothod by which the cloth is rapidly carried through the sulphuring chamber, and subjected to the influence of the vapour on the principle of the washing-machine. A great deal of time and space is of course saved; it is on the same principle as the washing apparatus, rapour being used instead of water. This has not yet been applied to thick woollen. See Catico-Pristira.

\section{Buzachise of Materials for Paper.}

The bleaching of prper is conducted on the same principle as the bleaching of cotton. Paper is made principally of two materials, cotton and flax, generally mixed. The cotton-waste of the mills, which is that inferior portion which has become too impure for spinning, or otherwise deteriorated, and cotton rags, are the principal, if not the only, sources of the cotton used by paper-makers. The waste is sorted by hand, the hard and soft boing separated, and all accidental mixtures which occur in it aro remored. This is done at first roughly on a large lattice, which is a frame of wire cloth, having squares of about three-quarters of an inch, through which impurities may fall. It is then put into a duster, which is a long rectangular box,--it may bo ten feet long,-lying horizontally, the inside diameter about two feet, and covered with wire gratings running horizontally, leaving openings of half ap 
inch in width. As this revolves, the waste is thrown from one angle to the other, and throws out whatever dust or other material falls into the holes or spaces. The fibrous matter has little tendency to separate from the mass, which is somewhat agglutinated by being damp, chiefly from tho oil obtained during the processes in the cotton-mill. A second duster, however, is used to retain whatever may bo of value; it is a kind of riddle. It is thon transferred to the lattices, which are a series of boxes corered with wire gauze, the meshes of which are about half an inch square, and so arranged as to form a series of sorting tables. The sorting generally is done by young women. Each table has a largo box or baskot beside it, into which the sorted material is thrown; this is removed whon filled, by being pushed along a railroad or tramway. Pieces of stone, clay, leather, wood, nails, and other articles, are taken out. The cotton is then put into a devil similar to that which is used in cotton machinery, but having larger, stronger teeth, which tear it up into small fragments.

The rags aro sorted according to quality, woollen carefully removed, and all the unarailable material sent back to the buyer. They are then chopped up by a knife, on the circumference of a heavy wheel, into pieces of an inch wide, devilled, and dusted.

The rags and the cotton wasto aro bleached in a similar manner. The cotton is put into kiers of about ten feet in diameter, of a kind similar to those described, and boiled with lime. The amount of lime used is about $6 \mathrm{lbs}$. to a cwt. of cotton or rags, but this varies according to the impurity. The lime remores a great amount of impure organic matter, and, as in bleaching cotton cloth, lays hold of the fatty matter, of which there is a great deal in the waste. When taken out, it is allowed to lie from two to three hours. The appearance is not much altered; it appears as impure as ever.

It is then put into the rag-engine and washed clean. This is a combined washingmachine and filter, the invention of Mr. Wrigley, near Bury. The washing may last an hour and a half, or more. See PAPpr.

The cotton has now a bright grey colour, and looks moderately clean. It is full of water, which is removed by an hydraulic press, the cotton being put into an iron cylindrical box with perforated sides. It is then boiled in kiers or puffing boilers, where soda-ash is usod, at the rate of 4 to $5 \mathrm{lbs}$. a cwt. Only as much water is used as will moisten the goods thoroughly. Much water would weaken the solution and render more soda necessary. It is then washed again in the rag-engine; afterwards put into chloride of lime, acidified as in cotton-bleaching, and washed again in the ring-engine.

The cotton rags are treated in a similar manner. The coloured rags are treated scparately, requiring a different treatment according to the amount of colour; this consists chiofly in a greater use of chloride of lime.

Some points relating to bleaching are necessarily trented of under CaticoPrintrate.

The following were the countries to which our bleaching materials woro oxported in 1871, and the quantities exported to each:-

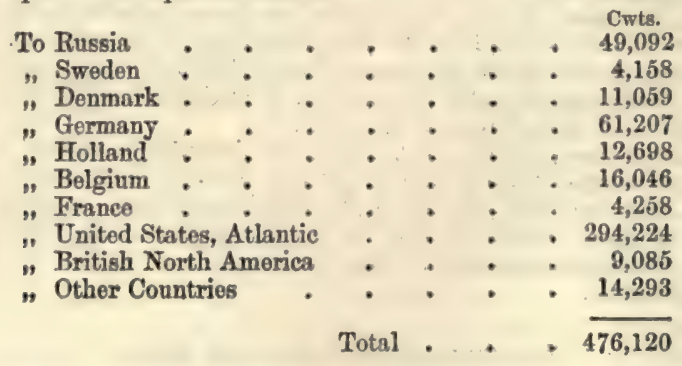

BIDAchrarc-rreurns. These liquids, manufuctured for the bleacher, contain metallic chlorides and hypochlorites. The most important are the liquid 'chlorides' of lime, magnesia, soda, and potash. See Chroridrs.

BIzACHING-POWDER. See Chronide of LiMe.

mIzax. (Cyprinus alburnus.) The scales of this fish are used for making the - essence of pearl,' or essence dorient, with which artificial pearls are manufactured. In the seales of the fish the optical offect is produced in the same manner as in the real pearl, the grooves of the latter being represented by the inequalities formed by the margins of the concentric laminze of which the scales are composed. These fish are caught in the Seine, the Ioire, the Saone, the Rhine, and several other rivers. They aro about four inches in length, and are sold rery cheap after the scales are washed 
off. It is said that 4,000 fish are necessary for the production of a pound of scales, for which the fishermen of the Chalonnois get from 18 to 25 livres.

The poarl essence is obtained merely by well washing the scales which have been seraped from the fish in water, so as to free them from the blood and mucilaginous matter of the fish. See Praris, Artificiat.

BIENDE, from Blenden, Ger., to dazzle. Sulphide or sulphuret of zine is a common ore of zine, composed of zine 67 , sulphur 33 ; but it usually contains a certain proportion of the sulphide of iron, which imparts to it a dark colour, whence the name of 'Black Jack,' applied to it by the Cornish miner. The ore of this country generally consists of ziue $61 \cdot 5$, iron $4 \cdot 0$, sulphur $33 \cdot 0$. Blende oceurs oither in a botryoidal form or in crystals (often of very complex forms), belonging to the tetruhedal division of the cubic or monometric system. $H=3.5$ to 4 . Specific gravity $=3.9$ to 4 . See Zixc.-H.W.B.

In some districts the presence of the sulphide of zine is regarded by the miners as a favourable indication, hence wo have the phrase, 'Black Jack rides a good horse." In other localities it is thought to be equally unfavourable, and the miners say, 'Black Jack cuts out the ore.' For many years the English zine ores were of little value, the immense quantity of zine manufactured by the Vieille Montagne Company, and sent into this country, being quite sufficient to meet the demand. Beyond this, there was some difficulty in obtaining xine which would roll into sheets, from English sulphides.

Much of the zine obtained from blende is used in the manufacture of brass.

Pure blende is a mineralogical rarity; the white and colourless variety (Cleiophane) of New Jersey, United States, was analysed by the late T. H. Henry, and found to be absolutely pure, with the exception of a trace of cadmium. Blende nearly always contains sulphide of iron. The following analyses of varieties of blende illustrate this :-

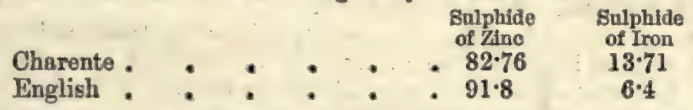

Production of Zine Ore in the United Kingdom in the years 1870 and 1871, showing the produce of each district.

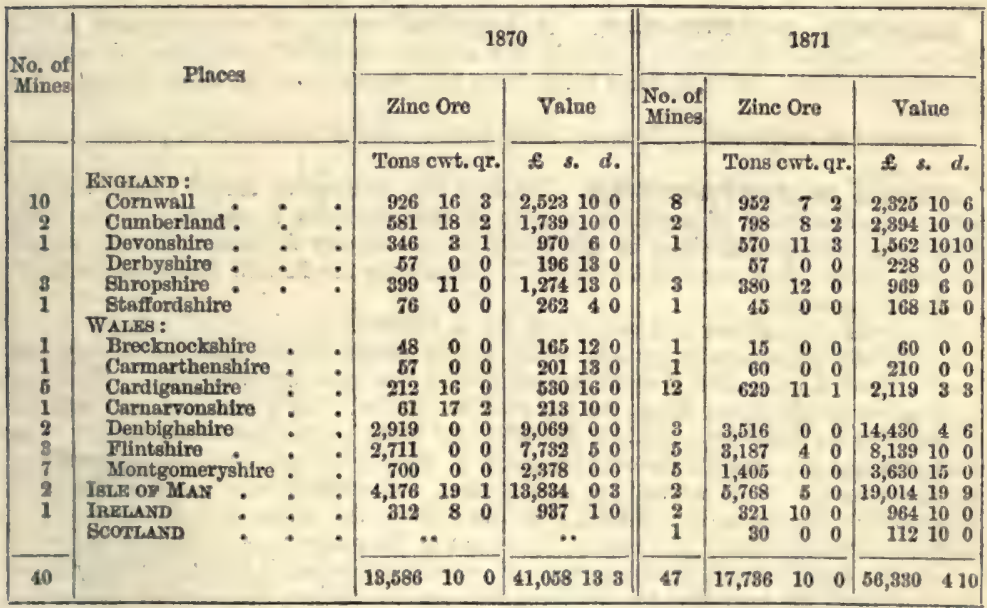

Metallic zine produced, $1870,3,936$ tons ; $1871,4,966$ tons.

\begin{tabular}{|c|c|c|c|c|c|c|}
\hline $\begin{array}{r}\text { Year } \\
1870\end{array}$ & Zine & $\begin{array}{c}\text { Tons } \\
28,775\end{array}$ & Zir & $\begin{array}{c}\text { Tons } \\
44,553\end{array}$ & of Zine & $\begin{array}{r}\text { Tons } \\
2,328\end{array}$ \\
\hline 1871 & Crude Zine & 20,929 & Ditto & 29,418 & Manufactured & 8,765 \\
\hline 187 & \multicolumn{6}{|c|}{ ExporTs, } \\
\hline 187 & Ditto & 6,452 & Zinc ore & 184 & & \\
\hline
\end{tabular}

In 13 out of 16 analyses of blende from different localities recorded by Rammols. berg, iron is present in proportions varying from $1 \cdot 18$ to $18 \cdot 1$ per cent. Copper oceasionally occurs in blende, but rarely above 1 per cent. Cadmium is a frequent, if not 
a general constituent of blende. The cadmiferous varieties are termed Praibranite. The blende from the King William mine at Clausthal contrins, according to Kulılemann, 0.63 per cent, of antimony, 0.79 of eadmium, and 0.13 of copper, besides 1.18 of iron. Blende is mot with in association with galena, iron-pyrites and copper-pyrites, from which it should be dressod as clean as possible, although in this there is some difficulty, owing to the very close approximation of the specific gravitios of these ores. Blende is occasionally argentiferous, and sometimes sufficiently so to allow of the profitable extraction of the silvor. In one of the mines in the Chiverton district, near Truro, Cornwall, some very fine examples of this argentiferous blende have been discovered. In one of the zinc ores of Silesia, the new metal Indur has been discovored by the aid of spectrum analysis. Plattner statos that blendo occasionally contains traces of tin and manganese.-(Percy's Metallurgy.) The red varieties of blende often contain from 2 to 3 per cent. of sulphido of cadmium, espocially that which is found at Marmato, near Popayan. Dana has quotod the following analyses of varietios of blende:-

\begin{tabular}{|c|c|c|c|c|c|}
\hline & & - Sulphux & Zine & Iron & Cadminm \\
\hline Carinthis & • & $32 \cdot 10$ & $64 \cdot 22$ & 1.32 & trace \\
\hline New Hampshiro . &.$\quad$. & $.32 \cdot 6$ & $52 \cdot 00$ & $10^{\circ} 0$ & $3 \cdot 2$ \\
\hline New Jersey & . & $32 \cdot 22$ & $67 \cdot 46$ & $\ldots$ & trace \\
\hline Tuscany . & . & $32 \cdot 12$ & $48 \cdot 11$ & $11 \cdot 44$ & $1 \cdot 23$ \\
\hline
\end{tabular}

\section{See Indiom; Marmatite.}

BIEU DE IYONT, See ANILINE-BLUR.

BIEU DE PARIS. See ANILINE-BLUR.

BIIGET. A disease in plants frequently produced by atmospheric, or by physical agencies. A peculiar blight has been referred to the action of the extra-spectral heat rays. Blight is sometimes of insect origin, but more frequently it arises from purasitic fungi. Seo Parathrmano Rays and Foxgi.

BIIND COAI, a namo given to anthracite in some parts of Scotland. Soo AxTHRACITR.

BXISTzR COPPER-ORF. A botryoidal variety of copper-pyrites, which has been found at the copper mines in the neighbourhood of Camborne, in remarkably fine masses. Of late years it has boen but rarely found in those mines. See Copper.

BIISTra-STrzx. Bars of steel which exhibit blister-like protuberances. See STrker.

BIOCK MATUEACIURI. Though the making of ships' blocks belongs rather to a dictionary of engineering than of manufactures, it may be expected that some account should be given of the automatic machinery for making blocks, so admirably devised and mounted by Sir M. I. Brunel, for the British Navy, in the dock-• yard of Portsmouth.

The series of machines and operations are as follows :-

1. The straight cross-cutting saw.- The log is placed. horizontally on a very low bench, which is continued through the window of the mill into the yard. The saw is exactly over the place where the log is to be divided. It. is lot down, and suffered to rest with its teoth upon the log, the back still being in the cleft of the guide. The crank boing set in motion, the saw reciprocates backwards and forwards with exactly the same motion as if worked by a carpenter, and quickly cuts through the tree. When it first begins to cut, its back is in the cleft in the guide, and this causes it to move in a straight line; but before it gets out of the gride, it is so doep in the wood as to guide itself; for, in cutting across the grain of the wood, it has no tendency to be diverted from its true line by the irregular grain. When the saw has descended through the troe, its handle is caught in a fixed stop, to prevent its cutting the bench. The machine is thrown out of gear, the attendant lifts up the saw by a rope, removes the block cut off, and advances the tree to receive a fresh cut.

2. The circular cross-cutting saw.-This saw possesses universal motion; but the axis is always parallel to itself, and the saw in the same plane. It can be readily raised or lowered, by inclining the upper frame on its axis; and to move it sidewise, the saw-frame must swing sidewise on its joints which copnect it with the upper frame. These movements are effected by two winches, each furnished with a pair of equal pinions, working a pair of racks fixed upon two long poles. The spindles of those winches are fixed in two vertical posts, which support the axis of the uppor frame. One of these pairs of poles is jointed to the extreme end of the upper frame; therefore, by turning the handle belonging to them, the frame and saw are elevated or do- 
pressed ; in like mannor, the other pair is attached to the lower part of the saw-frame, so that the saw can bo mored sidewise by means of their handles, which then swing the saw from its vertical position.

These two handles givo the attendant a complete command of the saw, which we suppose to be in rapid motion, the tree being brought forward and properly fixed. By one handle, he draws the saw against one side of the tree, which is thus cut into (perhaps half through); now, by the other handle, he raises the saw up, and by the firstmontionod handle he draws it across the top of the tree, and cuts it half through from the upper side; he then depresses the saw and cuts half through the next side; and lastly a trifling cut of the saw, at the lower side, completely divides the tree, which is then advanced to take another cut.

The great reciprocating saw is on the same principle as the saw-mill in common use in America.

3. The circular ripping saw is a thin circular plate of steel, with teeth similar to those of a pit saw, formed in its periphery. It is fixed to a spindle placed horizontally, at a small distance beneath the surface of a bench or table, so that the saw projects a few inches above the bench through a crovice. The spindle being supported in proper collars has a rapid rotatory motion communicated to it by a pulley on theopposite ond, round which an endless strap is passed from a drum placed overhead in the mill. The block cut by the preceding machine from the end of the tree is placed with one of the sides flat upon the bench, and thus slides forward against the revolving saw, which cuts the wood with a rapidity incredible to any one who has not seen these or similar machines.

4. Boring-machine.-The blocks prepared by the foregoing saws are placed in the machine represented in fig. 140. This machine has an iron frame, A $\mathbf{A}$, with three legs, beneath which the block is introduced, and the screw near $\mathrm{B}$ being forced down upon it, confines it precisely in the proper spot to receive the borers $\mathbf{D}$ and $\mathbf{E}$. This spot is determined by a piece of metal tixed perpendicularly just beneath the point of the borer $E$, shown separately on the ground at $x$; this piece of metal adjusts the position for the borer $\mathrm{D}$, and its hoight is regulated by resting on the head of the screw $x$, which fastens the piece $\mathrm{x}$ down to the frame. The sides of the block are

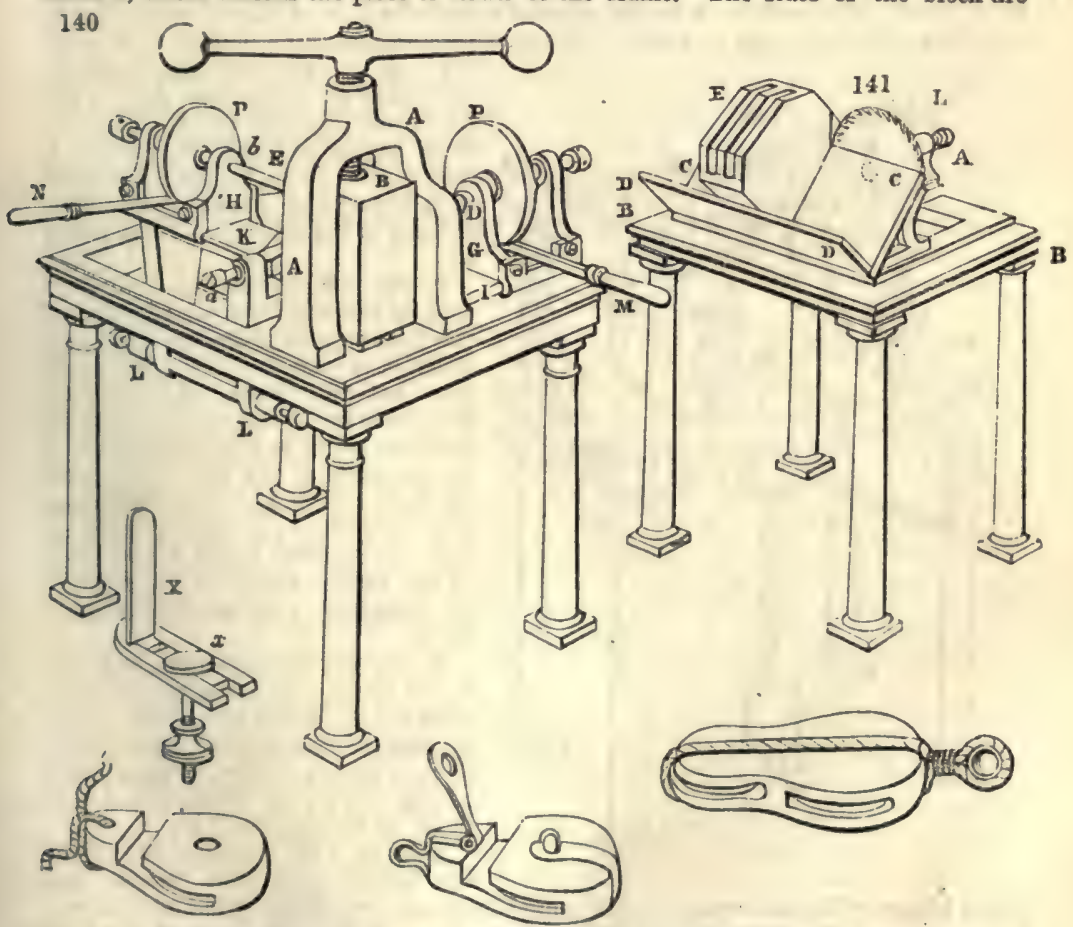

kopt in a parallel position, by being applied against the heads of three screws tapped into the double $\log$ of the frame A. The borcr $\mathrm{D}$ is adapted to bore the holo for the 
centre-pin in a direction exactly perpendicular to the surface resting against the three screws; the other, at $\mathbf{z}$, perforates the holes for the commencemont of the shedve holes. Both borers are constructed in nearly the same manner; they aro serewed upon the ends of small mandrols, mountod in frames similar to a lathe. Theso frames, $G$ and $\mathbf{~}$, are fitted with sliders upon the angular edges of the flat broad bars, 1 and $\mathbf{K}$. The formor of theso is screwed fast to the frame; tho lattor is fixed upon a frame of its own, moving on the centre-screws at L L, beneath the principal frame of the machine. By this means the borer $\mathrm{s}$ can be moved within cortain limits, so as to bore holes in difforent positions. These limits are determined by two serews, one of which is seen at $a$; the other being on the opposito side, is invisible. They are tapped through fixod pieces projecting up from tho frame. A projocting piece of metal from the underside of the slider $\mathbf{k}$ of the borer $\mathrm{k}$, stops agninst the ends of these screws, to limit the excursion of the borer. The frames for both borers aro brought up towards the block by means of levers $\mathrm{M}$ and $\mathrm{s}$. These are centred on a pin, at the opposite sides of the frame of the machine, and have oblong grooves through them, which reccive screw-pins, fixed into the frames $G$ and II, beneath tho pulleys $P$, which give motion to the spindles.

5. The mortising-nachine is a beautiful piece of mechanism, but too complicated for description within the limits prescribed to this article.

6. The corner saw, fig. 141, consists of a mandrel mounted in a frame A, and carrying a circular saw, $\mathbf{L}_{\text {, }}$ upon the extreme end of it. This mandrel and its frame being exactly similar to those at $\mathrm{\theta}$ and $\mathrm{H}, \mathrm{fig} .140$, do not require a separate view, although they are hidden behind the saw, except the end of the screw, marked A. This frame is screwed down upon the frame $\mathrm{B} \mathrm{B}$ of the muchine, which is supported upon four columns, c C, D D, is an inclined bench, or a kind of trough, in which a block is laid, as at $\mathrm{r}$, being supported on its edge by the plane $\mathrm{c} \mathrm{c}$ of this bench, and its end kept up to its position by the other part of the bench $\mathrm{D} D$.

By sliding the block along this bench, it is applied to the saw, which euts off its angles, as is evident from the figure, and prepares it for the shaping engine. All the four anglos are cut off in succession, by applying its different sides to the trough or bench. In the figure, two of them are drawn as being cut, and the third is just marked by the saw. This machine is readily adapted to different sizes of blocks, by the simple expodient of laying pieces of wood of different thiekness against the plane $D$ D, so as to

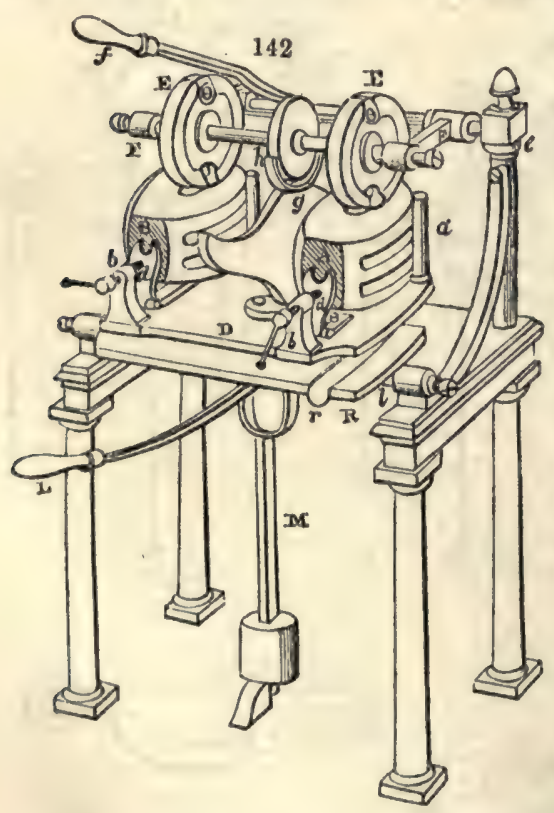
fill it up, and keep the block nearer to or farther from the saw; for all the blocks are required to be cut at the samo angle, though, of course, a larger piece is to be cut from largo than from small blocks. The block reduced to the state of $\mathrm{z}$ is now taken to

7. The shaping-machine-A great deal of the apparent complication of this figure arises from the iron cage which is provided to defend the workmen, lest the blocks, which are revolving in the circles or chuck with an immense velocity, should be loosened by the action of the tool, and fly out by their centrifugal force. Without this provision, the consequences of such an accident would be dreadful, as the blocks would be projected in all directions, with an inconcoivablo force.

8. The scoring-engine receives two blocks as they come from the shapingengine, and forms the groove round the longest diameters for the reception of their ropes or straps, as represented in the two snatch blocks and double block, under figs. 140, 141.

A, B, fig. 142, represent the above two blocks, each held between two small pillars $a$ (the other pillar is hid behind the block), fixed in a strong plate $\mathrm{D}$, and pressed against the pillars by a scrow $b$, which acts on a clamp $d$. Over the blocks a pair of circular planes or cutters, $\mathrm{z}$, are situated, both boing fixed on tho same 
spindle, which is turned by a pulley in the middle of it. The spindle is fitted in a frame F F, moving in centres at $e e$, so as to rise and fall when moved by a handlo $f$. This brings the cutters down upon the blocks; and the depth to which they can cut is regulated by a curved shape $g$, fixed by screws upon the plate $D$, between the blocks. Upon this rests a curved piece of metal $h$, fixed to the frame $F$, and inclosing, but not touching, the pulloy. To admit the cutters to traverse the wholo length of the blocks, the plate $\mathrm{D}$ (or rather a frame beneath it) is sustained between tho points of two centres. Screws are seen at $l$, on these centres. The frame inclines when the handle $x$ is depressed. At $\mathbf{M}$ is a lever with a weight at the end of it, counterbalancing the weight of the blocks, and plate $\mathrm{D}$, all which are above the centre on which they move. The frame $F$ is also provided with a counterpoise to balance the cutters, \&c. The cutters $\mathbf{E} \mathbf{E}$ are circular wheols of brass, with round edges. Each has two notches in its circumference, at opposito sides; and in these notches chisels are fixed by serews, to project beyond the rim of the wheel, in the manner of a planeiron before its face.

This machine is used as follows :-In order to fix the block, it is pressed between the two pins (only one of which, at $a$, can be seen in this view), and the clamp $d$, screwed up against it, so as just to hold the block, but no more. The clamp has two claws, as is seen in the figure, each furnished with a ring entering the double prints previously made in the ond of the block. These rings are partly cut away, leaving only such a segment of each as will just retain the block, and the metal between them is taken out to admit the cutter to operate between them, or nearly so. In putting the blocks into this machine, the workman applies the double prints to the ends of the claws of the clamps, but takes care that the blocks are higher between the pins $a$ than they should be; he then takes the handle $f$, and by it presses the cutters $\mathrm{E}$ it (which we suppose are standing still) down upon the blocks, depressing them between their pins at the same time, till the descent of the cutters is stopped by the piece $h$ rosting on the shape $g$. He now turns the screws $b \quad b$, to fix the blocks tight. The cutters being put in motion cut the scores, which will be plainly seen by the mode of adjustment just described, to be of no depth at the pin-hole; but by depressing the handle $\mathrm{L}$, so as to incline the blocks, and keeping the cutters down upon their shape $g$, by the handle $f$, they will eut any depth towards the ends of the blocks, which the shape $g$ admits.

By this means one quarter of the score is formed; the other is done by turning both blocks together half round in this manner. The centres $l$ are not fitted into the plate $\mathrm{D}$ itself, but into a frame seen at $\mathbf{R}$ beneath the plate, which is connected with it by a centre-pin, exactly midway between the two blocks $\mathbf{A}$ B. A spring-catch, the end of which is seen at $r$, confines them together; when this catch is pressed back, the plate D can be turned about upon its centre-pin, so as to change the blocks, end for end, and bring the unscored quarters (i. $e$. over the clamps) beneath the cutters; the workman taking the handles $f$ and $\mathbf{L}$, one in each hand, and, pressing them down, cuts out the second quarter. This might have been effected by simply lifting up the handle $x_{\text {; }}$ but in that case the cutter would have struck against the grain of the wood so as to cut rather roughly; but by this ingenious device of reversing the blocks, it always cuts clean and smooth, in the direction of the grain. The third and fourth quarters of the score are cut by turning the other sides of the blocks upwards, and repeating the above operation. The shape $g$ can be removed, and another put in its place, for different sizes and curves of blocks; but the pins $a$ and holding-clamps $d$ will suit many different sizes.

By these machines the shells of the blocks are completely formed, and they are next polished and finished by hand labour; but as this is performed by tools and methods which are well known, it is needless to enter into any explanation: tho finishing required being only a smoothing of the surfaces. The machines cut so perfectly true as to require no wood to be removed in the finishing; but as they cut without regard to the irregularity of the grain, knots, \&c., it happens that many parts are not so smooth as might be wished, and for this purpose manual labour alone can be employed.

The lignum vitx for the sheaves of the blocks is cut across the grain of the wood by two cross-cutting saws, a circular and straight saw, as before mentioned. These machines do not essentially diffor in their principle from the great cross-cutting saws wo have described, except that the wood revolves while cutting, so that a small saw will reach the centre of a large tree, and at the same time cut it truly flat. These machines cut off their plates from the end of a tree which are exactly the thickness for the intonded sheave. These pieces are of an irrogular figure, and must be rounded and centred in the crown saw,

9. The crown saw is represented in fig. 143 (p. 402), where $\Delta$ is a pulley revolving by means of an endless strap. It has the crown or tropan saw $a$ fixed to it, by a Vor. I. 
screw cut within the piece, upon which the saw is fixed, and which giros the ring or hook of the saw sufficient stability to perform its office. Both the pulleys and saw revolve together upon a truly cylindrical tube $b$, which is stationary, being attached by a flanch $c$ to a fixed puppet $B$, and on this tubs as an axis the saw and pulley turn,

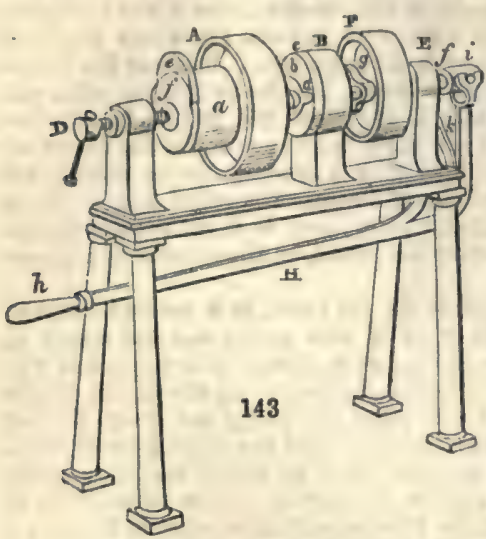
and may be slid endwise by a collar fitted round the centre-piece of the pulley, and having two iron rods (only one of which can be seen at $d$ in the figure), passing through holes made through the flaneh and puppet $\mathrm{B}$. When the saw is drawn back upon its central tube, the end of the latter projects beyond the teeth of the saw. It is by means of this fixed ring or tube within the saw, that the piece of wood $e$ is supported together during the operation of sawing, being pressed forcibly against it by $a$ screw $D$, acting through a puppet fixed to the frame of the machine. At the end of this screw is a cup or basin which applies itself to the piece of wood, so as to form a kind of vice, one side being the end of the fixed tube, the other the cup at the end of the screw $\mathbf{D}$. Within the tube $b$ is a collar for supporting a contral axis, which is perfectly cylindrical. The other end of this axis (seen at $f$ ) turns in a collar of the fixed puppet $\mathrm{x}$. The central axis has a pulley $\mathrm{F}$, fixed on it, and giving it motion by a strap similar to the other. Close to the latter pulley, a collar $g$ is fitted on the centre-piece of the pulley, so as to slip round freely, but at the same time confined to move endwise with the pulley and its collar. This collar receives the ends of the two iron rods $d$. The opposite ends of these rods are, as above mentioned, connected by a similar collar with the pulley $\Delta$ of the saw $a$. By this connection, both the centre-bit, which is screwed into the end of the central axis $f$, and the saw sliding upon the fixed tube $b$, are brought forward to the wood at the same time, both being in rapid motion, by their respective pulleys.

10. The coaking-engine. - This ingenious piece of machinery is used to cut the three semicircular holes which surround the hole bored by the crown saw, so as to produce a cavity in the centre of the disc.

11. Face-turning lathe.-The sheave is fixed against a flat chuck, similar to that in the coaking-engine, except that the centre-pin, instead of having a nut, is tapped into the flat chuck, and turned by a screw-driver.

A complete set of this block machinery has since been made, by Messrs. Maudslay and Field, for the Spanish Government, from the original drawings and models.

Iron blocks and sheaves have been introduced with great advantage by Messrg. Brown and Lenox, and are used extensively in the naval and merchant serrices. See Irov.

BIOCK Trvv. Metallic tin cast into a block, the weight of which is now about $3 \frac{1}{2}$ ewts. Formerly, when it was the custom to carry the blocks of tin on the backs of mules, the block was regulated by what was then considered to be a load for the mule, at $2 \frac{1}{2}$ ewts. Subsequently, the block of tin was increased in size, and made as much as two men could lift, or 3 cwts. It was the custom to order so many blocks of tin, and the smelter, being desirous of selling as much tin as possible, continued to increase the size of the block, so that, although $3 \frac{1}{2}$ ewts. is the usual weight, many blocks are sold weighing $3 \frac{3}{4}$ cwts. The term is also applied to articles made of tinned iron. See Tin.

mEOND Mrzrax. A Clayband ironstone found near Wednesbury. It is used, when smelted, for making tools.

BI00D. (Sang, Fr.; Blut, Ger.) The liquid which circulates in the arteries and veins of animals; bright red in the former and purple in the latter, among all the groups whose temperature is considerably higher than that of the atmosphere. Its specific gravity varies with the nature and health of the animal, being from 1.0527 to 1.0570 at $60^{\circ} \mathrm{F}$. It has a saline sub-nauseous taste, and a smell peculiar to each animal. It consists of a transparent, nearly colourless liquid, called the liquor sanguinis, or plasma, in which vast numbers of microscopic corpuscles are suspended. These corpuscles are of two kinds, some being white or colourless transparent colls, whilst others, far more numerous, are of a red tint, and impart the characteristic colour to the blood. The red corpuseles are present only in the blood of vertebrated 
animals, and vary considerably in size and shape in different groups of the vertebrata. They are always, however, flattened dise-like bodies, oval or circular in outline, and with or without a nucleus. These corpuscles consist apparently of an albuminoid substance, enclosing a red fluid. This fluid is now recognised as a definite crystallisable compound, containing iron, and tormed hamoglobin, homoglobulin or homatoglobulin. Hremoglobin may be resolved into two substances called globulin and hematin. The so-callod blood-crystals vary in shape, and are in many cases characteristic of the animal from which the blood was taken. The hremoglobin of the dog has been found to contain-

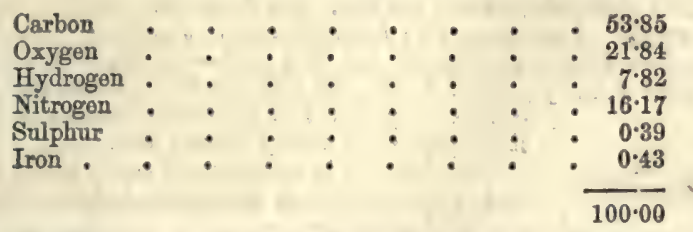

The liquor sanguinis, or colourless liquid in which the corpuseles freely float, contains fibrin (or rather two albuminous substances which readily form fibrin, and are called paraglobulin and fibrinogen), albumen, fatty matter, and certain saline substances. On the coagulation of blood, the fibrin separates in a gelatinous form, carrying with it the red corpuseles; this mixture of fibrin and corpuscles forms the dot, or crassamentum, whilst the remainder of the blood constitutes the liquid known as serum. If the warm blood be switehed with a bundle of twigs as it flows from the veins, the fibrin concretes and forms long fibres and knots, while it retains its usual appoarance in other respects. The clot contains fibrin and colouring-matter in various proportions. Berzelius found, in 100 parts of the dried clot of blood, 35 parts of fibrin, 58 of colouring-matter, 1.3 of carbonate of soda, 4 of an animal matter soluble in water, along with some salts and fat. The specific gravity of the serum varies from 1.027 to 1.029 . It forms about three-fourths of the weight of the blood, has an alkaline reaction, coagulates at $157^{\circ} \mathrm{F}$. into a gelatinous mass, and has for its leading constituent albumen to the amount of 8 por cent., besides fat, potash, soda, and salts of these bases. Blood does not seem to contain any gelatine. Fat and sugar are found in blood, the quantities varying with the health of the animal. For a very full account of the researches made by chemists on blood, see 'Watts's Dictionary of Chemistry.'

The red colouring-matter called hematosine may be obtained from the cruor, or hremoglobin, by washing with cold water and filtering. Professor Stokes, in a paper 'On the Reduction and Oxidation of the Colouring-Matter of the Blood,' has published some very curious results. By spectrum-analysis he has been led to infer that the colouring-matter of blood, like indigo, is capable of existing in two states of oxidation, distinguishable by a difference of colour and a fundamental difference in the action on the spectrum. It may be made to pass from the more to the less oxidised state, by the action of suitable reducing agents, and recovers its oxygen by absorption from the air. This colouring-mattor in its two states of oxidation, Professor Stokes proposes to call scarlet orworine and purple cruorine.-Proceedings of the Royal Society, vol. xuii. p. 355 (1864).

Mr. Sorby has also published in the 'Quarterly Journal of Serence.' No. VI., April 1865, a paper ' On the Detection of Blood Stains by Spectrum-Analysis,' in which he shows how it may be employed with great reliance in cases of the highest judicial importance. These two papers should be consulted.

Blood may be dried by evaporation at a heat of $130^{\circ}$ to $140^{\circ}$, and in this state has been transported to the colonies for purifying cane-juice. It is used for making animal charcoal in the Prussian-blue works, and, by an after-process, a decolouring carbon. It is employed in some Turkey-red dye-works. Blood is a powerful manure.

Mr. Pillans, in 1854, took out a patent for the separation of the colouring-matter of the blond, by which he obtained readily-1st, the clot, in a comparatively dry state, comprising hæmatosine, with a portion of serum and all the fibrin; 2 nd, a portion of serum, highly coloured with hæmatosine; 3rd, the clear serum.

The blood, in small fragments, is dried on wirework or trays, at a less temperature. than will coagulate the hæmatosiue, so that, when dry, it may be soluble in water; $110^{\circ}$ to $115^{\circ}$ is the temperature recommended.

The cloar serum is dried and ground and in a fit state to be used as albumen, and may be employed by the printers of textile fabries for fixing ultramarine blue and other colours, or as a substitute for egg albumen, both in printing colours and in refining liquids. 
BIoODgTowz. A very hard, compact variety of hæmatite iron ore, which, when reduced to a suitable form, fixed into a handle, and well polished, forms the best description of burnishor for producing a high lustro on gilt coat-buttons. The gold on china is burnished by the same means.

Bloodstone is a nane also applied to the jaspery variety of quartz known as the heliotrope, coloured deep green, with interspersod blood-red spots like drops of blood.

nxoom. Tho name given to a mass of iron after it leaves the puddling furnace.

\section{Seo Iron.}

מтoомary. The old iron-furnaces were so called.

nuow In Gaching and rans. Seo Iron, Metallurgy, Ventuation.

2I0wrrpz. (Chalumeau, Fr. ; Löthrohr, Ger.) Jowellers, mineralogists, chemists, enamellers, \&c., mako frequent use of a tube,-usually bont noar the end, terminated with a finely-pointed nozzle, for blowing through the flame of a lamp,

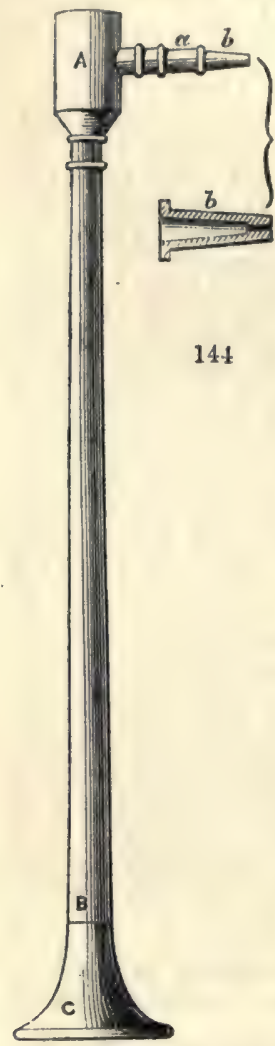
candle, or gas-jet, and producing thereby a small conical flame possessing a very intense heat.

The blowpipe is so extremely useful to the manufacturer and to the miner that a more exact description of the instrument is required.

When we propel a flame by means of a current of air blown into or upon it, the flame thus produced may be divided into two parts, as posseseing different properties - that of reducing under one condition, and of oxidising under another.

The reducing flame is produced by blowing the ordinary flame of a lamp or candle simply aside by a weak current of air impinging on its outer surface; it is therefore unchanged except in its direction. Unconsumed carbon, at a whito heat, giving the yellow colour to the flame, coming in contact with the substance aids in its reduction.

The oxidising flame is formed by pouring a strong blast of air into the interior of the flame; combustion is thus thoroughly established, and if a small fragment of an oxidisable body is held just beyond the point of the flame, it becomes intensely heated, and, boing exposed freely to the action of the surrounding air, it is rapidly oxidised.

The best form of blowpipe is the annexed (fig. 144), which, with the description, is copied from Blanford's excellent translation of Dr. Theodore Scheerer's 'Introduction to the Use of the Mouth Blowpipe.'

The tube and nozzle of the instrument are nsually made of German silver, or silver with a platinum point, and a trumpet-shaped mouth-piece of horn or ivory. Many blowpipes have no mouth-pieces of this form, but are simply tipped with ivory, or some similar material. The airchamber a serves in some degree to regulate the blast and receives the stem $B$, and the nozzle $a$, which are made separately, and accurately ground into it, so that they may be put together or taken apart at pleasure. The point $b$ is best made of platinum, to allow of its being readily cleaned, aud is of the form shown in the woodcut. When the instrument is used, the mouth-piece is pressed against the lips, or, if this is wanting, the end of the stem must be held between the lips of the operator. The former mode is far less wearying than the lattor; and whereas, with the trumpet mouth-piece, it is easy to maintain a continued blast for five or ten minutes, without it it is almost impossible to sustain an unbroken blast of more than two or three minutes' duration. While blowing, the operator breathes through his nostrils only, and, using the epiglottis as a valve, forces the air through the blowpipe by means of the cheek muscles.

Some years since, Mr. John Prideaux, of Plymouth, printed some valuable 'Suggestions ' for the use of the blowpipe by working miners. Some portions of this paper appear so useful, especially under circumstances which may proclude the use of superior instruments, \&c., that it is thought advisable to transfer them to these pages.

For ordinary metallurgic assays, the common blowpipe does very well. A mero tapering tube, 10 inches long, $\frac{1}{2}$ inch diameter at one end, and tho opening at the other scarcely oqual to admit a pin of the smallest kind, the smallor end curved off for $1 \frac{1}{2}$ inch to a right angle. A bulb at the bend, to contain the vapour condensed from 
the breath, is useful in long operations, but may generally be dispensed with. In selecting the blowpipe, the small aperture should be chosen perfectly round and smooth, otherwiso it will not command a good flame.

A common candle, such as the miner employs under ground, answers very well for the flame.

To support the subject of assay, or 'the assay,' as it has been happily denominated by Mr. Children, two different materials are requisite, according as we wish to calcine or reduce it. For the latter purpose, nothing is so good as charcoal ; but that from oak is less eligible, both from its inferior combustibility and from its containing iron, than that from alder, willow, or other light woods.

For calcination, a very convenient support, where platinum wire is difficult to procure, is white-baked pipe-clay or china-clay, selecting such as will not fuse nor become coloured by roasting with borax.

The supports are conveniently formed by a process of Mr. Tennant. The clay is to be beaten to a smooth stiff body; then a thin cake of it being placed between a fold of writing-paper, it is to be beaten out with a mallet to the thickness of a wafer, and cut, paper and all, into squares of $\frac{3}{8}$ ths inch diameter, or triangles about the same size. These are to be put in the bowl of a tobacco-pipe, and heated gently till dry, then baked till the paper, is burnt away, and the clay left perfectly white. They should be baked in a clear fire, to keep out coal dust and smoke as much as possible, as either of these adhering to the clay plates would colour the borax in roasting. A small fragment of the bowl of a new tobacco-pipe will serve instead in the absence of a more convenient material.

A simple pair of forceps ( $f i g .145$ ), to move and to take up the hot assay, may be made of a slip of stiff tin plate, 8 inches long, $\frac{1}{2}$ inch wide in the middle, and $\frac{1}{10}$ th inch at the ends. The tin being rubbed off the points on a rough whetstone, the slip is to be bent until they approach each other within $\frac{1}{2}$ an inch and the two sides are parallel; thus there will be spring enough in the forceps to open and let go the assay when not compressed by the finger and thumb.

145

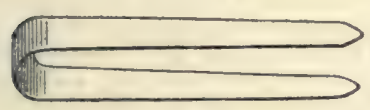

146

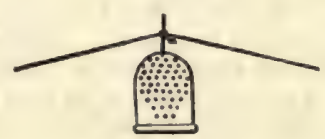

A magnetic needle, very desirable to ascertann the presence of iron, is easily made of the requisite delicacy where a magnet is accessible. A bit of thin steel wire, or a long fine stocking-needle, having $\frac{1}{4}$ inch cut of at the point, is to be heated in the middle that it may be slightly bent there (fig. 146). While hot, a bit of sealing-wax is to bo attached to the centre, and the point which had been cut off, being heated at the thick end, is to be fixed in the sealing-wax, so that the sharp end may serve as a pirot, descending about $\frac{1}{8}$ th inch below the centre, taking care that the ends of the needle fall enough below the pivot, to prevent it overturning. It must be magnetised, by sliding one end of a magnet half a dozen or more times from the centre to one end of the needle, and the other end a similar number of times from the centre of the needle to its other end. A small brass thimble (not capped with iron) will do for the support, the point of the pivot being placed in one of the indentations near the centre of the tap, when, if well balanced, it will turn until it settles north and south. If one side preponderate, it must be nipped until the balance be restored.

A black gun-flint is also occasionally used to rub the metallic globules (first attached, whilst warm, to a bit of sealing-wax), and ascertain the colour of the streak which they give. Thus minute particles of gold, copper, silver, \&c., are readily discriminated. A little refined borax and carbonate of soda, both in powder, will complete the requisites.

Having collected these materials, the next object for the operator is to acquire the faculty of keoping up an unintermittent blast through the pipe whilst breathing freely through the nose.

A very sensitive and, for most purposes, sufficiently delicato balance (fig. 147, p. 406) was also devised by Mr. Prideaux, of which the following is a description.

The common marsh-reed, growing generally in damp places throughout the kingdom, will yield straight joints, from 8 to 12 , or more, inches long; an 8 -inch joint will serve, but the longer the better. The joint is to be split down its whole length, 80 as to form a trough, say $\frac{1}{4}$ inch wido in the middle, narrowed away to $\frac{1}{2} r d$ inch at the onds. A narrow slip of writing-paper, the thinner the better (bank-post is very 
convenient for the purpose), and as long as the reed trough, is to be stuck with common pasto on the face of a carpenter's rule, or, in preference, that of an exciseman, - as the inches are divided into tenths instead of eighths; -in either caso observing that the dirisions of the inch on the rule be left uncovered by the paper. When it is dry, lines must be drawn the whole length of it, $\frac{1}{6}$ th inch apart, to mark out a stripe $\frac{1}{8}$ th inch wide. Upon this stripe the divisions of the inch are to be ruled off by means of a small square.

The centre divsion being marked 0 , it is to be numbered at every fourth line to the ends. Thus the fourth from the centre on each side will be 10 ; the eighth, 20 ; the twelfth, 30 ; the sixteenth, 40, \&c.; and a slip 10 inches long, graduated into tenths of an inch, will have on each arm 50 lines, or 125 degrees, divided by these lines into quarters. While the lines and numbers are drying, the exact centre of the reed-trough may be ascertained, and marked right across, by spots on the two edges. A line of gum-water, full $\frac{1}{6}$ th inch wide, is then laid with a camel-hair pencil along the hollow, and the paper being stripped from the rule (which it leaves easily), the graduated stripe is cut out with scissors, and laid in the trough, with the line 0 exactly in the centre. Being pressed to the gummed reed, by passing the round end of a quill along it, it graduates the trough from the centre to each end. This graduation is very true, if well managed, as the paper does not stretch with the gum-water after being laid on the rulo with the paste.

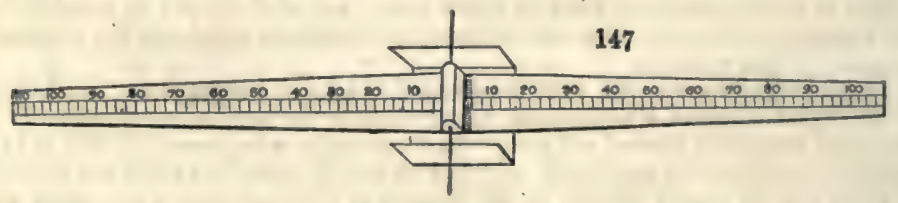

A very fine needle is next to be procured (those called bead-needles are the finest) and passed through a slip of cork the width of the centre of the trough, about $\frac{1}{4}$ th inch square, $\frac{1}{8}$ th thick. It should be passed through with care, so as to be quito straight. The cork should then be cut until one end of it fits into the trough, so that the needle shall bear on the edges exactly in the spots that mark the centre, as it is of importance that the needle and the trough be exactly at right angles with each other. The cork is now to be fixed in its place with gum-water, and, when fast dry, to be soldered down on each side with a small portion of any soft resinous cement, on the point of a wire or knitting-needle; a little cement being also applied in the same manner to the edges of the cork where the needle goes through, to give it firmness, the beam is finished. It may be balanced by paring the edges on the heaviest side: but accurate adjustment is needless, as it is subject to vary with the dampness or dryness of the air.

The support on which it plays is a bit of tin plate (or, in preference, brass plate), $1 \frac{3}{8}$ ths inch long, and 1 inch wide. The two ends are turned up square $\frac{3}{8}$ ths of an inch, giving a base of $\frac{5}{8}$ ths of an inch wide, and two upright sides $\frac{3}{8}$ ths high. The upper edges are then rubbed down smooth and square upon a Turkey stone, letting both edges bear on the stone together that they may exactly correspond. For use, tho beam is placed evenly in the support, with the needle resting across the edges. Being brought to an exact balance by a bit of writing-paper, or any other substance, placed on the lighter side, and moved toward the end until the equilibrium is produced, it will turn with extrome delicacy, a bit of horsehair, $\frac{1}{8}$ th inch long, being sufficient to bring it down freely.

It must not be supposed that any such instrument as this is recommended as in any way substituting the beautiful balances which are constructed for the chemist, and others requiring to weigh with great accuracy. The object is merely to show the miner a method by which he may construct for himself a balance which shall be sufficiently accurate for such blowpipe investigations as it may be important for him to learn to perform for himself. If the suggestions of the chemist who devised the above balance had been carried ont, much valuable mineral matter which has been lost might have been turned to profitable account.

The blowpipe is largely used in manufactures, as in soldering, in haxlening, and tempering small tools, in glass-blowing, and in enamelling. In many cases the blowpipes are used in the mouth, but frequently they are supplied with air from a bellows moved by the foot, by vessels in which air is condensed, or by means of pnoumatic apparatus.

A simple form of regulator for giving a perfectly constant blast has lately been devised by Messrs. Armin, Junge, and Mitzopulos, of Freiburg. The apparatus is thus 
described: "A common wide-mouthed bottle is carefully fitted with a caoutchone cork bored with two holes, into each of which passes a piece of glass tube bent at a right angle. On to one of these tubes is slipped the caoutchouc tube coming from an ordinary caoutchoue bellows, whilst the other is put in communication with the blowpipe nozzle by means of four pieces of caoutchouc tubing joined by three pieces of glass tube, drawn to a fine point at each end. This forms the main peculiarity of the arrangement. When air is forced into the bottle by the blower in jerks, it finds a difficulty in escaping as fast as it comes in, on account of the six fine openings in the glass tubes that it has to pass through on its way from the bottlo to the nozzle, and it thus acquires a certain pressure in the bottle, and flows out towards the nozzle as a regular blast. The bottle may be about 6 inches high by $3 \frac{1}{2}$ inches wide, with a neck $1 \frac{1}{2}$ inch in diameter; but the dimensions are of no great importance. On the whole a somewhat large bottle is better than a small one. The pieces of glass tube we employ are 2 inches long by $\frac{1}{3} r d$ inch in diameter. The apparatus will be stronger if instead of a glass bottle a tin cylinder is used, about 4 inches high by 2 inches in diameter, with two tin tubes opening into its top. Small metal cylinders with a fine hole at each end may be used instead of the little glass tubes.'

Many blowpipes have been invented for the employment of oxygen and hydrogen, by the combustion of which the most intense heat which we can produce is obtained. Professor Hare, of Philadelphia, was the first to employ this kind of blowpipe, when he was speedily followed by Clark, Gurney, Leeson, and others. The blowpipe, fed with hydrogen, is employed in many soldering processes with much advantage.

The general form of the 'workshop blowpipe' is that of a tube open at one end, and supported on trunnions in a wooden pedestal, so that it may be pointed vertically, horizontally, or at any angle as desired. Common street gas is supplied through one hollow trunnion, and it escapes through an annular opening, while common air is admitted through the other trunnion, which is also hollow, and is discharged in the centre of the hydrogen through a central conical tube; the magnitude and intensity of the flame being determined by the relative quantities of gas and air, and by the greater or less protrusion of the inner cone, by which the annular space for the hydrogen is contracted in any required degree. See Autogravous Soldering.

BXUanzr. The collular membrane of the whale, containing the oil. See OII.

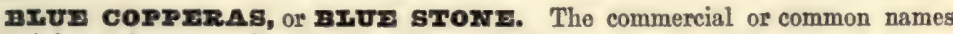
of sulphate of copper. See Coprer, Sulphate of.

Bxणr curm. The Eucalytus globulus (Lab.), a tree common in Tasmania and South-Fastorn Australia, and valuable for its timber and for the gum which it secretes.

BIUI IRON-ORF. See VIVLANITK.

BIUב JORN. A beautiful variety of fluor spar, found at Tray Cliff, near Castleton, Derbyshire, from which vases and other ornamental articles are wrought. It is now becoming scarce. See Fluor Spar.

BXU? IxAD. A name used sometimes by the miners to distinguish galena from the carbonate, or white lead. A variety of galena, to which this name has been applied, and which is pseudomorphous after pyromorphite, has been found at Herodsfoot mine, and Huel Hope in Cornwall, and at some mines in Saxony and France. The specimens from Huel Hope would burn in the flame of a candle like the supersulphide of lead.

Brua Premranss. The blues of vegetable origin, in common use, are indigo and litmus. The blue pigments of a metallic nature found in commerce are the following:-Prussian blue; sesqui-ferrocyanide of iron, called also Berlin blue; mountain blue, a carbonate of copper mixed with more or less earthy matter; Bremen blue, or verditer, a greenish-blue colour obtained from copper mixed with chalk or lime; iron blue, phosphate of iron, but little employed; cobalt blue, a colour obtained by calcining a salt of cobalt with alumina or oxide of tin; smalt, a glass coloured with cobalt and ground.

Molybdenum blue is a combination of this metal and oxide of tin, or phosphate of lime. A blue may also be obtained by putting into molybdic acid (mado by digesting sulphuret of molybdenum with nitric acid), some flings of tin, and a little muriatic acid. The tin deoxidises the molybdic acid to a certain degree, and converts it into the molybdous, which, when evaporated and beated with alumina recently precipitated, forms this blue pigment.

Ultramarine is a beautiful blue pigment.

Tumbull's and Chinese blues are both double cyanides of iron.

King's blue. $-\mathrm{A}$ carbonato of cobalt.

Saxon blue.-A solution of indigo in sulphuric acid.

BIUP VITrxor. Sulphate of copper. When found in nature, it is due entirely 
to the decomposition of the sulphides of copper, especially of the yellow copper pyrites, which are liable to this change when placed under the influence of moist air, or of water containing air. See Coppre.

BOG. The name given to accumulations of peat-earth. See Pgat.

BOG-BUTrxe. A hydrocarbon compound, like spermacoti, found in the bogs of Ireland. See ADIPOCKRE。

MOG-ZART2. A soil formed of vegetable fibre and sand.

goGrsad COAx, and other Brown Scotch Cannel Coals. The brown cannels are chiefly confined to Scotland, and have been wrought, with the exception of the celobrated Boghead, for the last forty years. They are found at Boghead, near Bathgate; Rocksoles, near Airdrie; Pirnie, or Methill ; Capeldrea, Kirkness, and Wemyss, in Fife. The first-named coal, about which there has been so much dispute as to its nature, has only been in the market about fourteen years. It is considered the most valuable coal hitherto discovered for gas- and oil-making purposes ; but, strange to say, the middlo portion of the Pirnie, or Methill, seam, which was unnoticed for a long period, is nearly as valuable for both puxposes.

Boghrad. Amorphous; fracture subconchoidal, compact, containing impressions of the stems of Sigillaria, and its roots (Stigmarie), with rootlets traversing the mass. Colour, clove-brown, streak yellow, without lustre; a non-electric; takes fire easily, splits, but does not fuse, and burns with an empyreumatic odour, giving out much smoke, and learing a considerable amount of white ash. H 2.5. Specific gravity, $1 \cdot 2$.

According to Dr. Stenhouse, F.R.S., its composition is :-

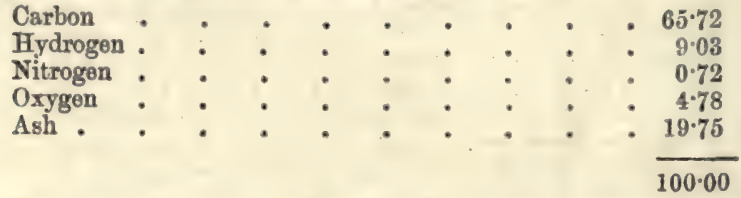

Dr. Stenhouse's analysis of the ash of Boghead coal (the mean of three analyses), was as follows :-

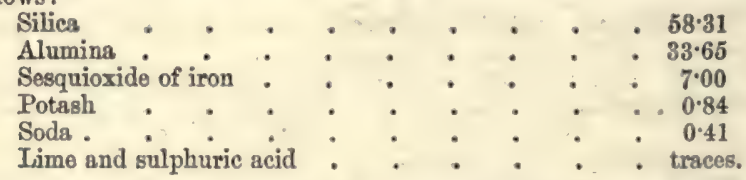

Dr. Andrew Fyfe, F.R.S.E., found on analysis that the coal yielded, from a picked specimen, 70 per cent. of volatile matter, and 30 per cent. of coke and ash. From a ton he obtained 14.880 cubic feet of gas, the illuminating power of which was determined by the use of the Bunsen photometer, the gas being consumed by argands burning from $2 \frac{1}{2}$ to $3 \frac{1}{2}$ feet per hour, according to circumstances. The candle referred to was a spermaceti candle, burning 140 grains por hour.

\begin{tabular}{|c|c|c|c|c|c|}
\hline $\begin{array}{l}\text { Cuble Feet of } \\
\text { Gas per Ton of } \\
\text { Coal }\end{array}$ & $\begin{array}{l}\text { Speoific } \\
\text { Gravity }\end{array}$ & $\begin{array}{l}\text { Condensation } \\
\text { by Chlorine } \\
\text { in } 100 \text { Parts }\end{array}$ & $\begin{array}{l}\text { Durability } 1 \\
\text { foot burns }\end{array}$ & $\begin{array}{l}\text { Illuminating } \\
\text { Power } 1 \text { foot } \\
=\text { Light of } \\
\text { Candles }\end{array}$ & $\begin{array}{l}\text { Pounds of } \\
\text { Coke per Ton } \\
\text { of Coal }\end{array}$ \\
\hline $14 \cdot 880$ & .802 & 27 & $\begin{array}{c}\text { Min. Sec. } \\
88 \quad 25\end{array}$ & $7 \cdot 72$ & 760 \\
\hline
\end{tabular}

The Pirnie or Methill brown cannel, on examination, gives the following results :-

Specific gravity .

Gas per ton

Illuminating power

Coke and ash

Hydro-carbons condensed by bromine

Sulphuretted hydrogen

Carbonic acid

Carbonic oxido

Volatile matter in coal

Specific gravity of gas
$1 \cdot 126$

13,500 feet

28 candles

36 per cent.

20

$\begin{array}{cc}20 \\ 4 \frac{1}{\frac{1}{2}} & \text { " } \\ 7 \frac{3}{4} & " \\ 700 & "\end{array}$


The Boghead coal occurs in the higher part of the Scotch coal-field, in about the position of the 'slaty band' of ironstono; its range is not more than 3 or 4 miles in the lands of Torbane, Incheross, Boghead, Capcer's, and Bathvale, near Bathgate, in the county of Linlithgow. In thickness it varies from 1 to 30 inches, and at the present rapid rate of consumption, it is feared it cannot last many years.

The following soction of a pit at Torbane shows that the cannel occurs in ordinary coal-measures, and under circumstances common to beds of coal :-

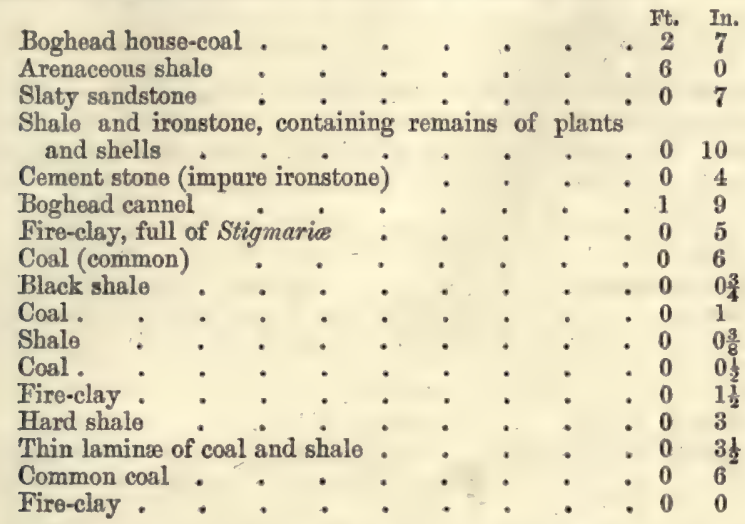

One of the chief characters of this cannel is its indestructibility under atmospheric agencies; for whether it is taken from the mine at a depth of fifty fathoms, or at the outcrop, its gas- and oil-yielding properties are the same. Even a piece of the mineral taken out of the drift-deposits, where it had most probably lain for thousands of years, appears to be just the same in quality as if it had been but lately raised from the mine.

In the earth the seam lies parallel to its roof and floor, like other beds of coal; and it is traversed by the usual vertical joints, dividing it into the irregular cubes which so generally characterize beds of cannel. The roof lying above the cement-stone contains remains of Calamites; and the ironstone nodules, fossil shells of the genus Unio. The floor of the mine contains Stigmarie; and the coal itself affords more upright stems of Sigillarie, and its roots (Stigmarice) and thoir radicles, running through the seam to a considerable distance, than the majority of coals show. In these respects it entirely resembles the Pirnio or Methill seam. Most cannels afford remains of fish; but in Boghead no traces of these fossils have yet been mot with, although they have been diligently sought after.

The roots in the floors, and the upright stems of trees in the seam itself, appear to show that the vegetable matter now forming the coal grew on the spot where it is found. If the mangroves and other aquatic plants, at the present day found growing in the black vegetable mud of the marine swamps of Brasstown, on the west coast of Africa, were quietly submerged and covered up with clay and silt, we should have a good illustration of the formation of a bed of carbonaceous matter showing no structure, mingled with stems and roots of trees showing structure, which is the case with Boghead coal, the structure being only detected in those parts showing evidence of stems and roots, and not in the matrix in which those fossils are contained.

The chemical changes by which vegetable matter has been converted into Boghead cannel will not be here dwelt on; but the chief peculiarity about the seam is its close and compact roof, composed of cement-stone, and shale. This is perfectly water-and air-tight, so much so that, although the mine is troubled with a great quantity of water, it all comes through the floor, and not the roof. This tight covering of the coal has doubtless exercised considerable influence on the decomposing vegetable matter after the latter had been submerged. It is worthy of remark that, above the Pirnie or Methill seam, - the coal nearest approaching Boghead, - a similar bed of impure ironstone occurs.

Away from whin dykes which traverse the coal-field, there are no appearances of the action of an elevated temperature, either upon the coal or its adjoining strata, to give any sanction to the hypothesis, that the cannel has resulted from the partial decomposition of a substratum of coal by the heat of underlying trap, the volatile matters having been retained in what has probably been a bed of shale. First, it must be understood that Boghead cannel, even whon treated with boiling naphtha, affords 
scarcely a trace of bitumen; and, secondly, when the seam of coal is examined in the neighbourhood of a whin dyke, whore heat has evidently actod on it, it is found nothing like cannel, but as a soft sticky substance, of a brown colour, resembling burnt india-rubber. Besides theso facts, tho seams of coal and their accompanying strata, both above and below the cannel, show no sigus of the action of hent, but, on the contrary, exhibit every appearance of having been deposited in the usual way, and of remaining without undergoing any particular alteration,-E. W. B. See CANNER Coat.

MOGmEAD INAPETEA (syn. Bathgate naphtha), naphthe from the Boghead coal. See Naphtha, Boghrad.

Moc-rRow oxa is an example of the recent formation of an ore of iron, arising from the decomposition of rocks containing iron, by the action of water charged with carbonic acid. The production of this ore of iron in the present epoch, explains to us many of the conditions under which some of the more ancient beds of iron ore have been produced.

Bog-iron ore is common in the peat bogs of Ireland and other places. See Iron Orres and Iror.

BoG mantGarrass. Wad, or earthy manganese. Seo Margarese.

nOGWOOD. The trunks and larger branches of trees dug up from peat bogs. The black oak of the bogs of Ireland, which is so largely employed in the manufacture of ornaments, is so called.

BOFFA. A kind of black tea, See Trs.

BOJIMIAX BOxE. A yellow variety of bole.

Bormmran CARNrms. Garnets belonging to the mineralogical species Pyrope. They occur embedded in serpentine at Zöblitz in Bohemia, and also loose in the sands of some of the rivers of Bohemia. These garnets are of a rich dark-red colour, and have been largely employed in cheap jewellery. Thoy are cut and polished in mills worked by water-power, and are mounted by working jewellers at Prague.

BOIr.m orx. Linseed oil boiled with litharge, which removes some of its oleaginous parts, and renders the oil more 'drying,' that is, it solidifies more readily.

BOTIFR. See Straur BomrR.

BOILFR PIAT2. Sheets of iron used for making boilers, and now largely employed for constructing railway bridges, ships, tanks, \&c. The average resistance of boiler plates is reckoned at 20 tons to the square inch, and the woight which they can carry safely is about 5 tons on the square inch. Riveting is calculated to reduce the strength to a degree corresponding to that of the area which the rivets occupy. Such are the principles by which the Railway Department of the Board of Trade are guided. See Iros.

Bors DuRCr. Finely powdered sawdust, and turnings of hard wood, such as rosewood, ebony, mahogany, and the like, are made into a paste with blood, which is pressed into moulds or formed by dies. It receires a beautiful polish, equal to jet, which it much resembles. This was first introduced to England in 1862 by $\mathbf{M}$. Latry, senior.

BOxz. A kind of clay, often highly coloured by iron. It usually consists of silica, alumina, iron, lime, and magnesia. It is not a well-defined mineral, and, consequently, many substances are described by mineralogists as bole. Amerian bole is of a bright red colour. This is frequently employed as a dentifrice, and in some cases it is administered medicinally. Bole of Blois is yellow, contains carbonate of lime, and effervesces with acids. Bohemian bole is yellowish red. French bole is of a pale red, with frequent streaks of yollow. Lemnian bole and Silesian bole are, in most respects, similar to the above-named varieties. The following analyses are by C. Von Hauer. Capo di Bove-Silica, 45.64 ; alumina, 29.33; peroxide of iron, 8.88 ; lime, 0.60 ; magnesia, a trace; water, $14 \cdot 27=98 \cdot 72$. New Holland-Silica, 38.22 ; alumina, 31.00 ; peroxide of iron, 11.00 ; lime, a trace; magnesia, a trace, water, $18 \cdot 81=99 \cdot 03$.

Borrrus. A genus of the mushroom order. See Amanot.

BOzOGNTAN STONe. A varioty of sulphate of baryta, found in roundish masses, which phosphoresces when, aftor calcination, it is exposed to the solar rays. Bolognian phosphorus was formerly made from this stone. Seo BarYTA, Sulprate of.

mommasrarz. A worsted stuff mixed with silk; it is a twilled fabric, of which the warp is silk and the weft worsted.

Bomaxx Morx. The moth to which the silkworm turns. The caterpillar (silkworm) is at first of a dark colour; but gradually, as with all other caterpillars, it becomes lighter coloured. This worm is about eight weoks in arriving at maturity, during which time it frequently changes its colour. When full grown, the silkworm commences spinning its web in some convenient place. The silkworm continues 
drawing its throad from various points, and attaching it to othors; it follows, therefore, that after a time the body becomes, in a great measure, enclosed in the thread. The work is then continued from one thread to another, the silkworm moving its head and spinning in a rigzag way, bending the forepart of the body back to spin in all directions within reach, and shifting the body only to cover with silk the part which was beneath it. As the silkworm spins its web by thus bending the forepart of the body back, and moves the hinder part of the body in such a way only as to enable it to reach the farther back with the forepart, it follows that it encloses itself in a cocoon much shorter than its own body; for soon after the beginning, the whole is continued with the body in a bent position. During the time of spinning the cocoon, the silkworm decreases in length very considerably; and after it is completed

- it is not half its original length: at this time it becomes quite torpid, soon changes its skin, and appears in the form of a chrysalis. The time required to complete the cocoon is five days. In the chrysalis state the animal remains from a fortnight to three weeks: it then bursts its case, and comes forth in the imago state, the moth having previously dissolved a portion of the cocoon by means of a fluid which it ejects.

The true silkworm (Bombyx mori) is a native of the North of China. Another specios, $B$. cynthia, oceurs in India.

The late Colonel Sir William Reid reported as follows on the Bombyx cynthia :-

'I made several reports on the Bombyx cynthia silkworm, which feeds on the castoroil plant, for the information of the Society of Arts. It had been introduced into Malta from India, and appenred both hardy and wonderfully prolific; yet it failed in Malta in 1855 .

'2. I had, however, previously distributed a great number of eggs, by sending them to Italy, France, and Algeria; and I contrived to watch the accounts of the trials made in those countries. I found that it had spread there, and had been carried to Spain and Portugal, and was creating considerable interest wherever it had been tried.

'3. I was, therefore, induced to reintroduce it into Malta. At the end of July last, I received a fow eggs by post, in a quill, from Paris, and these have multiplied in an extraordinary manner, so that I have not attempted to have them counted. The temperature of the winter season, now December, seems, however, to be affecting them, even in Maita, inasmuch as they grow more slowly than they did in the summer; but, nevertheless, they appear healthy.

'4. A very interesting paper, on the progress making in different countries in rearing the Bombyx cynthia, will be found in the last number of the papers of the French Societé d'Acclimation. This paper is by the able President of that Society, M. Geoffroy Saint Hilaire.

'5. I had, in 1854, successfully sent the insect to the West Indies. The French Society have sent it to Brazil, to the Southern United States, and into Egypt. It is beirig introduced into Germany, and we are now sending more eggs and worms from Malte to Sicily.

'6. Experiments are making in France on spinning the silk, which is found to be very fine, very strong, and to take dyes well. In France the cocoons are corded, and afterwards spun, as in Malta. It is said that the chrysalis, on extricating itself from the cocoon, and becoming a moth, does not, as was supposed, cut the thread; and the French have partially succeeded in unwinding the cocoons.

'7. The great interest I find taken in other countries in the attempts making to naturalise the Bombyx cynthic, has induced me to report to you its re-introduction into Malta, with the view of begging you to make this known to the Society of Arts. I enclose an extract from my despatch, dated 7th of July, 1855, which explains the manner in which I sucessfully sent the insect to the West Indies; and in the same manner it may lo easily conveyed from any one country to another. It may be found difficult to preserve the silkworm throughout the winter season, as well as difficult to grow the Ricinus, its proper food, in the climate of Europe. The proper climate for the Bu,nbyx cynthia is within, or on the borders of, the tropics. But the attempts now making ought not to be the less encouraged on that account, for they are producing a new raw material for thread and clothing within reach of mon of skill and science; and 127,000 cocoons have recently been sent from Algeria to be manufactured in Alsace.

' 8. The extraordinary manner in which the Bombyx cynthia multiplies, together with the abundance of food produced for it without culture in warm elimates, renders the study of the habits of this insect, and the nature of its cocoons, of considerable importance.

'9. I send herewith a small sample of the cloth made from the worms reared in Malta. 
Additional information on the Bombyx cynthia, or Eria Silkworm, will be found in the 'Society of Arts' Journal' of June 4, 1858.

Mr. Wells, writing from Grenada, in the West Indies, says of these silkworms forwarded to him by Sir William Reid:- 'I have the eighth generation of worms now hatching, having had seren crops of cocoons within tho year. These worms multiply one hundredfold in each generation; and there is no doubt of their being easily fed to any amount.' They are fed on tho castor-oil plant, Ricinus communis, which grows rapidly, can be cultivated without much expense, and yields a good return in its very abundant seeds. Sco Sirk.

3ON-BONs. Comfits and other sweetmeats of various descriptions pass under this name. They are manufactured largely in France, and a considerable quantity regularly importod into this country. The manufacture of swoetmeats, confectionery, \&c., does not enter so far into the plan of this work as to warrant our giving any special detail of the various processes employed; a general notice will, however, be found under the head of CoNfreriosery.

Liqueur bon-bons are made in the following manner. A syrup evaporated to the proper consistence is made, and some alcoholic liqueur is added to it. Plaster-ofParis models of the required form are made; and these are employed, several being fastened to a rod, for the purpose of making moulds in powdered starch, filling shallow trays. The syrup is then, by means of a funnel, poured into these moulds, and there being a powerful repulsion between the starch and the alcoholic syrup, the upper portion of the fluid assumes a spherical form; then some starch is sifted over the surface, and the mould is placed in a warm closet. Crystallisation commences on the outside of the bon-bon, forming a crust enclosing the syrup, which constantly gives up sugar to the crystallising crust until it becomes sufficiently firm to admit of being removed. A man and two boys will make three hundredweights of bon-bons in a day.

Crystallised bon-bons are prepared by putting them with a strong syrup contained in shallow dishes, placed on shelves in the drying chamber, pieces of linen being stretched over the surface, to prevent the formation of a crust upon the surface of the fluid. In two or three days the bon-bons are covered with erystals of sugar; the syrup is then drained off, and the comfits dried.

Painted bon-bons. - Bon-bons are painted by being first covered with a layer of glazing; they are then painted in body colours, mixed with mucilage and sugar.

The French have some excellent regulations, carried out under the 'Préfet de Police,' as to the colours which may be employed in confectionery. These are to the following effect:-

'Considering that the colouring-matter given to sweets, bon-bons, liqueurs, lozenges, \&ce, is generally imparted by mineral substances of a poisonous nature, which imprudence has been the cause of serious accidents; and that the same character of accidents have been produced by chewing or sucking the wrapping paper of such sweets, it being glazed and coloured with substanees which are poisonous; it is expressly forbidden to make use of any mineral substance for colouring liquours, bon-bons, sugar-plums, lozenges, or any kind of swoetmeats or pastry. No other colouringmatter than such as is of a vegetable character shall be employed for such a purpose. It is forbidden to wrap sweetmeats in paper glazed or coloured with mineral substances. It is ordered that all confectioners, grocers, dealers in liqueurs, bon-bons, sweetmeats, lozenges, \&c., shall have their name, address, and trade, printed upon the paper in which the above articles shall be enclosed. All manufacturers and dealers are personally responsible for the accidents which shall be traced to the liqueurs, bonbons, and other sweetmeats manufactured or sold by them.'

If similar provisions were in force in this country, it would prevont the uso, to an alarming extent, in our cheap confectionery, of such poisonous substances as
Arsenite of copper,
Acetate of copper,
Chromate of lead,
Sulphide of arsenic,
Oxide of lead,
Sulphide of mercury, \&c.

The colouring-matters allowed to be used in France aro indigo, Prussian blue, saffron, Turkey yellow, quercitron, cochineal, Brazil wood, madder, and the like.

zorv 25. (Os, Fr.; Knochen, Ger.) They form tho framework of animal bodies, commonly called the skeleton, upon which the soft parts are suspended, or in which they arg enclosod. Bones are invested with a membrane styled the periosteum, which is composed of a dense tissue affording glue; whence it is convertible into jelly, by ebullition with water. Bones are not equally compact throughout their whole substance: tho long ones have tubes in their contres, linod with a kind of periosteum of more importance to the life of the bones than oven their external coat; the flat, as 
well as the short and thick, bones exhibit upon their surface an osseous mass of a dense nature, while their interior presents a cavity divided into small cellules by their bony partitions.

In reference to the composition of bones, we have to consider two principal constituents : the living portion or the osseous cartilage, called osscin, and the inorganic or the earthy salts of the bones.

The ossoous cartilage is obtained by suspending bones in a large vessel full of dilute muriatic acid, and leaving it in a cool place at about $50^{\circ} \mathrm{F}$. The acid dissolves the earthy salts of the bones without perceptibly attacking the cartilage, which, at the end of a short time, becomes soft and translucid, retaining the shape of the bones; whenever the acid is saturated before it has dissolved all the earthy salts, it should be renewed. The cartilage is to be next suspended in cold water, which is to be frequently changed till it has removed all the acidity. By drying the cartilage shrinks a little, and assumes a darker hue, but without losing its translucency. It becomes, at the same time, hard and susceptible of breaking when bent, but it possesses great strength.

This cartilage is composed entirely of a tissue passing into gelatine. By boiling with water, it is very readily convertible into glue, which passes clear and colourless through the filter, leaving only a small portion of fibrous matter insoluble by further boiling. This matter is produced by the vessels which penetrate the cartilage, and earry nourishment to the bone. We may observe all these phenomena in a very instructive manner, by macerating a bone in dilute muriatic acid, till it has lost about the half of its salts; then washing it with cold water, next pouring boiling water upon it, leaving the whole in repose for 24 hours, at a temperature a few degrees below $212^{\circ} \mathrm{F}$.

The cartilage, which has been stripped of its earthy salts, dissolves, but the small ressels which issue from the undecomposed portion of the bone remain under the form of white plumes, if the water has received no movement capable of crushing or breaking them. We may then easily recognise them with a lens, but the slightest touch tears them, and makes them fall to the bottom of the vessel in the form of a precipitate; if we digest bones with strong hot muriatic acid, so as to accelerate their decomposition, a portion of the cartilage dissolves in the acid with a manifest disengagement of carbonic acid gas, which breaks the interior mass, and causes the half-softened bone to begin to split into fibrous plates, separable in the direction of their length. According to Marx, these plates, when sufficiently thin, possess, like scales of mica, the property of polarising light, a phenomenon which becomes more beautiful still whon we soak them with the essential oil of the bark of the Laurus Cassia. The osseous cartilage is formed before the earthy part. The long bones are then solid, and they become hollow only in proportion as the earthy salts appear. In the new-born infant, a large portion of the bones is but partially filled with these salts; their deposition in the cartilage takes place round certain invariable points of ossification, and begins at a certain period after conception, so that we may calculate the age of the foetus according to the progress which ossification has made.

Composition of Bones.

\begin{tabular}{|c|c|c|c|c|c|c|}
\hline & & & & Heintz & . & Berzelins \\
\hline & & & $\begin{array}{c}\text { Ox } \\
\text { Femur }\end{array}$ & Shoep & $\begin{array}{c}\text { Man } \\
\text { Forearm }\end{array}$ & $\underset{\text { Tooth }}{\text { Human }}$ \\
\hline Animal matter . & . & . & 30.58 & $26 \cdot 54$ & $31 \cdot 11$ & $28 \cdot 00$ \\
\hline Phosphate of lime & $\theta^{\circ}$ & . & $57 \cdot 67$ & $61 \cdot 99$ & $59 \cdot 14$ & 4.30 \\
\hline Fluoride of calcium & $\therefore$ & . & $2 \cdot 69$ & $2 \cdot 97$ & $2 \cdot 23$ & .6430 \\
\hline Carbonate of lime & . & . & $6 \cdot 99$ & 6.92 & 6.32 & $5 \cdot 30$ \\
\hline Phosphate of magnesia & • & . & 2.07 & $1 \cdot 58$ & $1 \cdot 20$ & $1 \cdot 40$ \\
\hline
\end{tabular}

Heintz found that the fixed bases in the bones were sufficient to saturate completely the acids contained in them, so that the phosphate of lime, as well as the phosphate of magnesia, which the bones contain, is composed according to the formula $3 \mathrm{RO}, \mathrm{PO}^{5}$. Bone phosphate of lime is, therefore, the tribasic or orthophosphate $3 \mathrm{CaO}, \mathrm{PO}^{5}$ $\left(\mathbf{C a}^{3} \mathbf{P}^{2} \mathbf{O}^{s}\right)$. True bony structure is perfectly free from chlorides and from sulphates, these salts being only found when the liquid pervading the bones has not completely removed. The bones in youth contain less earthy constituents to fose of adults; and, in advanced age, the proportion of mineral matters incr. Son Biria found more bone-earth in the bones of birds than in those of mamnals; he 
found also the ratio of the carbonate of lime to the phosphate to be generally greater. In the bones of amphibia, he found loss inorganic matter than in those of mammals and birds; and in the bones of fishes, the earthy matters rary from 21 to 57 por cont. The scales of fishes have a composition somewhat similar to that of bone, but they contain phosphate of lime in small quantity only.

In certain diseases (the craniotabes in children), the earthy salts fall in the spongy portion of the bone as low as $28 \cdot 16$ per cent. of the dry bone; and in several cases the proportion of earthy matter was found by Schlossberger as low as 50 per cent. At the age of 21 years, the weight of the skeleton is to that of the whole body in the ratio of $10.5: 100$ in man, and in that of $8.5: 100$ in woman, the weight of the body being about 125 or 130 lbs.

The quantity of organic matter in fossil bones varies very considerably: in somo cases it is found in as large a quantity as in fresh bones, while in others it is altogether wanting. Carbonate of lime generally oceurs in far larger quantity in fossil than in recent bones, which may arise from infiltration of that salt from without, or from a decompasition of a portion of the phosphate of lime by carbonic acid or carbonates. Magnesia often occurs in larger quantities in the fossil remains of vertebrated animals than in the fresh bones of the present animal world. Liebig found in the cranial bones excavated at Pompeii a larger proportion of fluoride of calcium than in recent bones; while, on the other hand, Girardin and Preisser found that this salt had greatly diminished in bones which had lain long in the earth, and, in some cases, had even wholly disappeared.

The gelatinous tissue of bones was found by Von Biria to consist of-

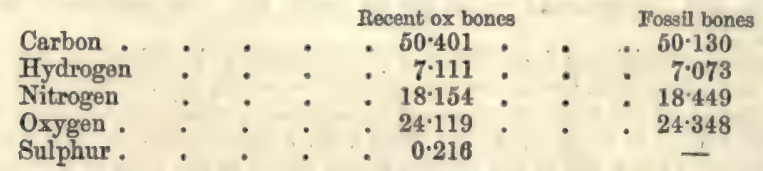

This is the same composition as that of the gelatinous tissues.

In the arts bones are employed by turners, cutlers, manufacturers of animal eharcoal, and, when calcined, by assayers, for making cupels. In agriculture, they are employed as a manure. Laid on in the form of dust, at the rate of 30 to 35 cwts. per acre, they have been known to increase the value of old pastures from 10s. or 15s. to 30 s. or 40 s. per acre; and after the lapse of 20 years, though sensibly becoming less valuable, land has remained still worth two or three times the rent it paid before the bones wore laid on. In the large dyeing establishments in Manchester, the bones are boiled in open pans for 24 hours, the fat skimmed off and sold to the candle-makers, and the size afterwards boiled down in another vessel till it is of sufficient strength for stiffening the thick goods for which it is intended. The size liquor, when exhausted or no longer of sufficient strength, is applied with much benefit as a manure to the adjacent pasture and artificial grass-lands, and the exhausted bones are readily bought up by the Lancashire and Cheshire farmers. When burned bones are digested in sulphuric acid, diluted with twice its weight of water, a mixture of gypsum and acid phosphate of lime is obtained, which, when largely diluted with water, forms a most valuable liquid manure for grass-land and for crops of rising corn; or, to the acid solution, pearl ashes may be added, and the whole then dried up, by the addition of charcoal powder or vegetable mould, till it is sufficiently dry to be scattered with the hand as a top-dressing, or buried in the land by means of a drill.

In France, soup is extensively made by dissolving bones in a steam heat of two or three days' continuance. Respecting the nutritive property of such soup, Liebig has expressed the following strong opinion:- 'Gelatine, even when accompanied by the savoury constituents of flesh, is not capable of supporting the vital process; on the contrary, it diminishes the nutritive value of food, which it renders insufficient in quantity and inferior in quality, and it overloads the blood with nitrogenous products, the presence of which disturbs and impedes the organic processes.' The erroneous notion that gelatine is the active principle of soup arose from the observation that soup made, by boiling, from meat, when concentrated to a certain point, gelatinises. The jelly was taken to be the true soup until it was found that the best meats did not yield the finest gelatine tablets. which wero obtained most beautiful and transparent from tendons, feet, cartilage, bones, \&ce. This led to an investigation on nutrition generally, the results of which proved that gelatine, which by itself is tasteless, and when eaten excites nausea, possesses no nutritive value whatever.

The following Table exhibits the relation between the combustible animal matter and the mineral substances of bones, as found by different observers:- 


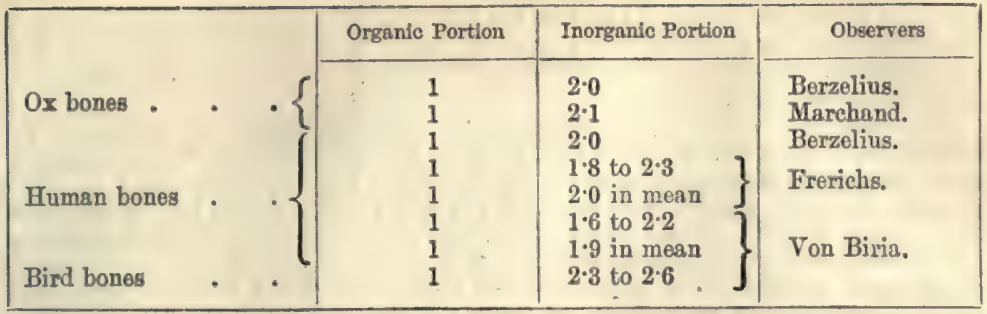

Pricr to the use of bones by the turner or carver, they require the oil, with which they are largely impregnated, to be extracted, by boiling them in water and bleaching them in the sun or otherwise. This process of boiling, in place of softening, robs them of part of their gelatine, and therefore of part of their elasticity and contractibility likewise, and they become more brittle.

The forms of the bones are altogether unfavourable to their extensive or ornamental employment: most of them are very thin and curved, contain large cellular cavities for marrow, and are interspersed with vessels that are visible after they are worked up into spoons, brushes, and articles of common turnery. The buttock and shin bones of the $0 x$ and calf are almost the only kinds used. To whiten the finished works, they are soaked in turpentine for a day, boiled in water for about an hour, and then polished with whitening and water.

Holtzapffel also informs us that after the turning tool, or scraper, has been used, bone is polishod, 1st, with glass-paper; 2nd, with Trent sand, or Flanders brick, with water on flannel; 3rd, with whiting and water on a woollen rag; 4 th, a small quantity of white wax is rubbed on the work with a quick motion; the wax fills the minute pores, but only a very minute portion should be allowed to remain on the work. Common bone articles, such as nail- and tooth-brushes, are frequently polished with slaked lime used wet on flannel or woollen cloth. See 'On Bone and its Uses,' by Arthur Aitkin, 'Trans. of Socioty of Arts,' 1832 and 1839.

Bones have recently been imported into this country, from Australia, in the form of bone-dust tiles, made by crushing the bones and compressing the powder into the form of cakes.

The importance of the trade in bones will be seen from the following statement of imports of the bones of animals and fish-not whalebone.

Bones of all kinds (except Whalefins) imported.

\begin{tabular}{|c|c|c|c|c|c|c|}
\hline & 1867 & 1868 & 1869 & 1870 & 1871 & 1872 \\
\hline & Tons & Tons & Tons & Tons & Tons & \\
\hline Quantity & - 246,767 & 245,120 & 229,223 & 215,748 & 302,079 & - \\
\hline Value & 437,436 & 430,442 & 600,019 & 629,619 & 659,416 & \\
\hline BoNE: & $\mathbf{r} 1$ & fon & Ire & $\begin{array}{c}\text { Tons } \\
92,032\end{array}$ & $\begin{array}{l}\text { Tons } \\
94,212\end{array}$ & $\begin{array}{l}\text { Tons } \\
\mathbf{7 , 7 7 8}\end{array}$ \\
\hline
\end{tabular}

BONE-BIACr (Noir d'os, Fr.; Knochenschwarz, Ger.), or Animal Charcoal, as it is less correctly called, is the black carbonaceous substance into which bones are converted by calcination in close vessels. This kind of charcoal has two principal applications-to deprive various solutions, particularly syrups, of their colouringmatters, and to furnish a black pigment. The latter subject will be treated of under IVORY BLACK.

The discovery of the antiputrescent and decolouring properties of animal chareoal in general is due to Lowitz, of Petersburg; but their modifications have occupied the attention of many chemists since his time. Kels published, in 1798, some essays on the decolouring of indigo, saffron, madder, syrup, \&c., by means of charcoal ; but he committed a mistake in supposing bone-black to have less power than the charcoal of wood. The first useful application of charcoal to the purification of raw colonial sugar was made by M. Guillon, who brought into the French markets considerable quantities of fine syrups, which he discoloured by ground wood-charcoal, and sold them to great advantage, as much superior to the cassonades (brown sugars) of that time. In 1811, M. Figuier, an apothecary at Montpellier, published a note about animal charcoal, showing that it blanched vinegars and wines with much more energy than vegetable charcoal; and lastly, in $1812, \mathrm{M}$. Derosnes proposed to employ animal charcoal, in the purification of syrups and sugar-rofining. The quantities of bone-black left in the retorts employed by MM, Payen, for producing crude carbonate of ammonia, 
furnishod abundunt materials for making the most satisfactory cxperiments, and onabled these gontlemen soon to obtain ten per cent. more of rofined sugar from the raw article than had been formerly extracted, and to improve, at the time, the characters of the lumps, bastards, treacle, \&c.

The calcination of bones is effected by two different systems of apparatus; by heating them in a retort similar to that in which coal is decomposed in the gas-works, or in small pots piled up in a kiln. On the second plan, which furnishes tho best charcoal, the bones, broken into pieces, are put into small cast-iron pots of the form shown in fig. 148, about three-eighths of an inch thick, two of which are dexterously placed with their mouths in contact, and then luted together with loam. The lip of the upper pot is made to slip inside the under one. These double vossels, containing together about fifty pounds of bones, are arranged alongside, and over each other, in an oven like a potter's kiln, till it is filled. The oven or kiln may be either oblong or upright. The latter is represented in figs. 149, 150, 151. $\Delta$ is the fireplace or grate for the fuel; $\mathrm{c}$ c are the openings in the dome of the furnace through which the flame flows; the divisions of these orifices are shown in fig. 151. B is the wall of brick-work. D the space in which the pots are distributed. $\mathbf{E}$ is the door by which the workman carries in the pots, which is afterwards built up with fircbricks, and plastered over with loam. This door is seen in fig. 149. $F$ F aro the lateral flues for conveying tho disengaged gases into the air.
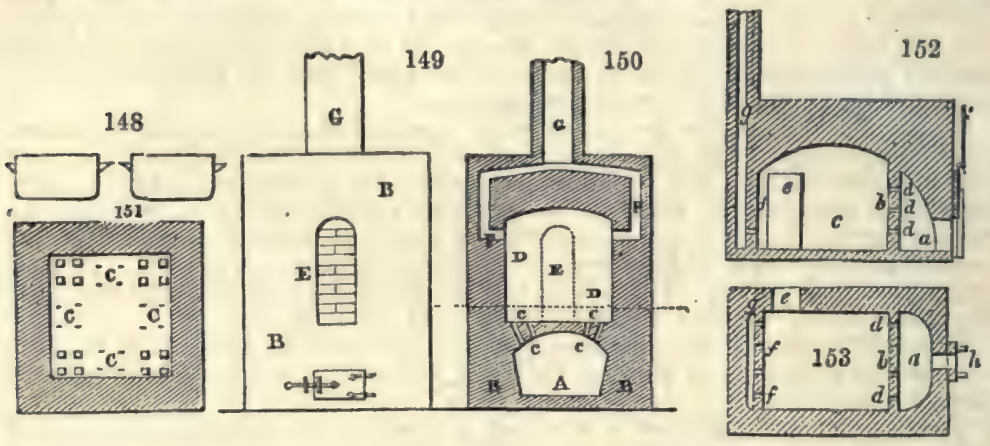

Fig. 152 is a longitudinal section, and fig. 153 a ground plan of a horizontal kiln for calcining bones. $a$ is the fire-chamber, lying upon a level with the sole of the kiln; it is separated by a pillar $b$, from the calcining hearth $c$. In the pillar or wall, several rows of holes, $d$, are left at different heights; $e$ is the entrance door; $f$, the outlet-vents for the gases, vapours, and smoke, into the chimney $g ; h$, a sliding damper-plate for regulating the admission of the air into the fire in the space $a$.

By this arrangement the offensive emanations aro partly consumed, and partly carried off with the smoke. To destroy the smell completely, the smoke should be made to pass through a second small furnace.

The number of pots that may be put into a kiln of this kind depends, of course, upon its dimensions; but, in general, from 100 to 150 are piled up over each other, in columns, at once; the greatest heat being nearest the roof of the kiln, which resembles, in many respects, that used for baking potteryware.

In both kilns the interior walls are built of fire-bricks. In the oblong one, the fiercest heat is near the vaulted roof; in the upright one, near the sole; and the pots, containing the larger lumps of bones, should be placed accordingly near the top of the former and the bottom of the latter. Such a kiln may receive about seventy double pots, containing in the whole thirty-flve ewts. of bones.

After the hearth is filled with the pots, and the entrance door is shut, the fire is applied, at first moderately, but afterwards it must be raised, and maintained at a brisk heat for eight or ten hours. The door of the ash-pit and the damper may now be nearly closed, to moderate the draught, and to keep up a steady ignition for six or eight hours longer, without additional firing; after which the doors must bo all opened to cool the furnace. When this is done, the brickwork of the entrance-door must be taken down, the kiln must be emptied, and immediately filled again with a set of pots previously filled with bones, and luted together: the pots which have been ignited may, in the course of a short time, bo opened, and the contents put into the magazine. But in operating with the large decomposing cylinder retort, the bones being raked out hot, must be instantly tossed into a receiver, which can be covered in air-tight till they are cool. 
The bones lose upon an average about one-half of their weight in the calcination. In reference to the quality of the black, experience has shown that it is so much more powerful as a decolouring agent, as the bones from which it was made have been freer from adhering fatty, fleshy, and tendinous matters.

The charcoal is ground in mills with grooved rollers, in order to prevent the formation of dust. The bones are thrown into a long quadrangular box, furnished at its lower aperture with moveable steel cheeks, between which the roller revolves; they are thus coarsely broken up, and the granulation is completed by another pair of bluntly grooved rollers, which can be placed nearer to, or further from, each other at pleasure. The crushed charcoal is collected on sieves, which separate the dust from the grains.

The composition of perfectly dry bone-black of average quality is as follows :Phosphate of lime, with carbonate of lime, and a little sulphuret of iron, or oxide of iron, 88 parts; iron in the state of silicated carburet, 2 parts; charcoal containing ahout $\frac{1}{15}$ th of nitrogen, 10 parts. None of the substances present, except the charcoal, possess separately any decolourising power.

It was formerly supposed that the peculiar absorbing and decolouring power of animal charcoal was only exerted towards bodies of organic origin; but it was found, by Graham, that inorganic substances are equally subject to this action; and later oxperiments hav'e demonstrated that there are few, if any, chemical compounds which altogether resist the absorbing power of charcoal. The action is of a mechanical nature, and in some cases it is sufficiently powerful to overcome chemical affinities of considerable power. It is not confined to charcoal, though pre-eminent in this substance, in consequence of the immense extent of surface which its porous structure presents. The action of charcoal in sugar-refining has been particularly studied by Lüdersdorf. When the defecated saccharine juice is allowed to flow upon a moist and firmly compressed charcoal filter, pure water is the first product that passes through; but a considerably larger quantity is ohtained than was employed for moistening the charcoal. Water is then obtained of a decidedly saline character, which increases in strength, and after this has passed through for some time, a sweet taste becomes perceptible, which gradually increases, and at last entirely masks the saline. This purely sweet fluid continues to flow for some time; after which, the liquid acquires an alkaline reaction from the presence of caustic lime; it then becomes coloured, the liquor getting gradually darker, till the action of the charcoal ceases. Limo is completely abstracted from lime-water by bone-charcoal; and, according to the experiments of Chevallier, lead-salts are likewise entirely absorbed, the acetate the most readily. It has also been shown by Graham, that iodine even is separated from iodide of potassium. The commercial value of animal charcoal has usually been estimated by its decolouring power on sulphate of indigo; its absorbent power, which is a property of equal, perhaps of greater importance, may, according to M. Corenwinder, be determined, approximately, by the quantity of lime which a given weight will absorb. For this purpose he employs a solution of saccharate of lime of known strength. An acid liquor is first prepared, composed of 20 grammes of pure oil of vitriol diluted with water to exactly 1 litre. A solution of saccharate of lime is then prepared, by dissolving 125 to 130 grammes of white sugar in water, adding thereto 15 to 20 grammes of quicklime, boiling the liquid, and then filtering to separate the undissolved lime. This solution is prepared of such a nature, that it will be exactly saturated by the same volume of the dilute sulphuric acid. By adding the latter to 50 cubic centimetres of the liquid filtered from the animal charcoal, it is easy to seo how many degrees of the burette are required to complete the saturation of the lime. Suppose 35 are required for this purpose, $100-35=65$, which represent the proportion of lime absorbed by the charcoal: this is, therefore, the number representing the standard, By operating with a burette graduated from the bottom, the degree of the charcoal experimented npon may be read directly.

This decolourising power does not belong alone to bone-black; different varieties of lignite, or even coal, when well carbonised in close vessels, afford a decolouring charcoal of considerable value. By reducing 100 parts of clay into a thin paste with water, kneading into it 20 parts of tar, and 500 of finely-ground pit-coal, drying the mixed mass, and calcining it out of contact of air, a charcoally matter may be obtained not much inferior to bore-black in whitening syrups.

The restoration of animal charcoal from burnt bones, for the purpose of sugarrefining, has been long practised in France. Mr. W. Parker has made the following process the subject of a patent. The chareoal, when taken from the vessels in which it has been omployed for the purposes of clarifying the sugar, is to be thoroughly washed with the purest water that can be obtrined, in order to remove all the saccharine matter adhering to it. When the washing process has been completed, the charconl is laid out to dry, either in the open air or in a suitable stove; and when per*

Vou. 1. 
fectly free from moisture, it is to be separated into small pieces, and sifted through of sieve, the wire or meshes of which are placed at distances of alout two and a half in overy inch. This sifting will not only divide the charcoal into small pieces, but will cause any bits of wood or other improper matters to be separated from it.

The charcosl thus prepared is then to be packed lightly in cylindrical vessels called crucibles, with some small quantity of bones, oil, or other animal matter, mixed with it. The crucibles are then closed by covers, and luted at the joints, leaving no other opening but one small hole in the centre of the cover, through which any gas generated within the ressel when placed in the oven or furnace may be allowed to escapo.

The crucibles are now to be ranged round the oven, and placed one upon another, in rertical positions; and when the oven is properly heated, gas will be generated within each crucible, and issue out from the central hole. The gas thus emitted, being of an inflammable quality, will take fire, and assist in heating the crucibles; and the operation being carried on until the crucibles become of a red heat, the oven is then to be closed, and allowed to cool ; after which the crucibles are to bo removed, when the charcoal will be found to have become perfectly renorated, and as fit for use as before.

A process for the restoration of bone-black, or animal charcoal, was made the subject of a patont by Messes. Bancroft and MacInnes of Liverpool, which consists in

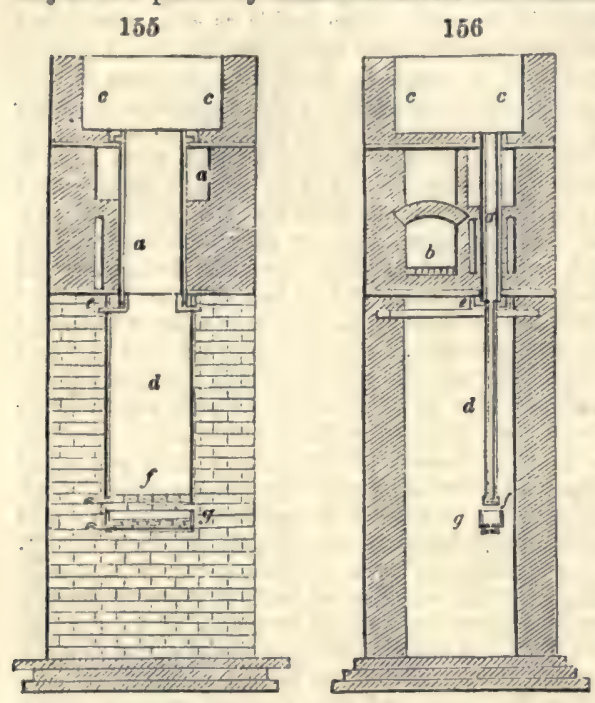
washing the granular charcoal, or digesting it, when finely ground, with a weak solution of potash or soda, of speeific gravity $1 \cdot 06$. The bone-black, which has been used in sugar-refining, may bo thus restored, but it should be first clearod from all the soluble filth by means of water.

Mr. F.Parker's method, patented in June, 1839, for effecting a like purpose, is by a fresh ealcination, as follows:-

Fig. 155 represents a front section of the furnace and retort; and fig. 156 a transworse vertical section of the same. $a$ is a retort, surrounded by the flues of the furnace $b$; $c$ is a hopper or chamber, to which a constant fresh supply of the black is furnished, as the preceding portion has been withdrawn from the lower part of $a$. $d$ is the cooling vessel, which is connected to the lower part of the retort $a$ by a sand-joint $e$. The cooler $d$ is made of thin sheet-iron, and is large; its bottom is closed with a slideplate, $f$. The black, after passing slowly through the retort $a$ into the vessel $d$, gets so much cooled by the time it reaches $f$, that a portion of it may be safely withdrawn, so as to allow more to fall progressively down; $g$ is the charcoal-meter, with a slide door.-H.M.N.

BONID-DARTH. The residue of bones after calcination; it is chiefly phosphate of lime. It finds many uses in the arts.

BONrs-IMUor. The liquor obtained by distilling bones. It is an impure solution of ammonia-a poor spirit of hartshorn.

BONE-oxx. See DrPPRI's Or.

BоOKBIX DIMT ' in all its branches' includos every process by which the sheets as received from the printer (from the pamphlet of a few pages to the folio of enormous size and thickness) are arranged in duo order, and the leares of which each sheet is composed secured and prepared, either simply or elaborately, for the use of the reader. It thus includes every gradation of style and finish, from the stitched and wrappered periodical to the costly and elaborate binding of the most magnificent library.

The sheots of paper on which books are printed are of various dimensions, beginning with the 'pott' and 'foolscap' (the smallest sizes that come from the papor-maker) up to the 'crown,' 'demy,' 'royal,' 'imperial,' ' atlas,' \&c. The printer arranges each of his pages 80 as to occupy, with the required margin, tho half, the fourth, the 
eighth, or the twelfth, and so on down to the forty-eighth, of each sheet, such divisional pages being called respectively folio, quarto, octaro, duodecimo, \&c., down to $48 \mathrm{mo}$; and it will be manifest that as the sheets themselves are larger or smaller from the atlas down to the pott as before stated, so their divisions will vary in proportion, thus, an $8 \mathrm{vo}$. rolume may be either a 'foolscap 8vo.,' a 'crown $8 \mathrm{ro.}$.' or a 'demy 8vo.,' \&c., and will be larger or smaller accordingly.

The 'gathering' of the sheets that form a volume-that is, the collecting together, in consecutive order into one or more 'gatherings,' of a copy of each of the sheots forming such volumes, is generally done by the printer before they are delivered to the binder. In such cases the first operation at the bookbinder's is that of folding (whether in folio, 4to., or $8 \mathrm{vo}$. , \&c.. as before said). In this operation the margin of the type, and not the margin of the paper, is the guide for the folder, who has to see that the head and sides of the printed matter of each page range or 'register' aceurately with those of the opposite page. The work of folding is done by women and girls, who use a bone or ivory folding stick to press the folds of ench sheet closely together, and by continued practice the process is so rapidly performed by them, that except in the case of newspapers and other publications where there are enormous numbers of the same sheet requiring no readjustment of machinery, little, if anything, is gained, either in speed, accuracy, or economy, by the use of folding machines, of which rarious kinds worked by steam-power have been introduced.

When the type of each leaf in a sheet is thus made to coincide it will be almost unirersally found that the outer edges of some of the leaves project more or less considerably beyond others, so that in order to give the book a neat and regular appearance, eren if the edges are not intended to be cut all round, the fore-edge and tail have to be 'trimmed,' that is, the rough and irregularly projecting edges are, after the book is sown, pared with a knife.

The folded sheets are then 'collated,' that is, examined and laid together in proper consecutive order, in which arrangement the letter or 'signature,' as it is termed, at the foot of the first page of each sheet, and not the general numerical paging of the book, is the collater's guide. If the volume about to be bound has belonging to it plates or maps, printed on distinct paper from that used for the letterpress, each such plate or map is secured by paste to the back edge of its appropriato pago af letterpress, or to a special strip of paper termed a 'guard.' Each rolume boing thus ' folded,' 'collated,' and (if needful) 'placed,' is subjected with others to hydraulic or other pressure to make the leaves lie smoothly and compactly together. The volume is then, if cords are to be used as the cross bands, slightly indented with saw cuts at regular intervals across its back, six such cuts being used for folios, five for quartos, and four or three for smaller sizes. If tape is to be used for the bands, pencil-marks are substituted for the saw ents.

The book is now ready to be fastened together. The simplest mode of doing this is by stabbing throngh each rolume three or four holes a short distance from its hinder edge, and passing a needle and thread alternately in and out through the holes, soeuring the ends of the thread together by a knot. . A book so fastened ('stitched,' not 'sewn') is of course prevented by the pressure of the thread from oponing freely to its back edge, and if the thread is cut or broken at any one part

157
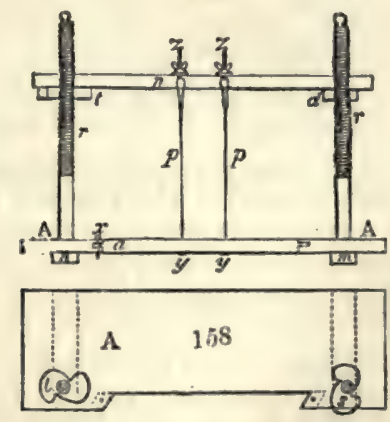

the whole falls to pioces. This rough and ready process is confined to pamphlets, periodical publications, or somo of the commoner sehool books.

The process of 'sowing' (as distinct from the beforo-named operation of stitch- 
ing) is that mostly employed for securing the folded shoots together. For this purpose the sewing press is employed.

Fig. 157 represents the sewing press as it stands upon the table before which the workwoman sits. Fig. 158 is a ground plan without tho parts $a$ and $n$ in the former figure, $\mathbf{\Lambda}$ is the base board supported upon the cross bars $m n$ marked with dotted lines in fig. 158.

Upon the serew rod $r r$, fig. 157 , the nuts $t d$ serve to fix the flat upper bar $n$ at any desired distance from tho base. That bar has a slit along its middle through which the hooks below $z z$ pass down for receiving the ends of the sewing cords or tapes (from two or three to six in number according to the size of the volumes under operation) $p p$ fixed at $y y$ and strotched by the thumbscrews $z z$. The bar $y y$ is let into an oblong space cut out of the front edge of the base board and fixed there by a moveable pin $a$ and a fixed pin at its other end round which it turns. The cords or bands are fixed at distances and in numbers corresponding to the saw or pencil-marks before named, made in the backs of the folded sheets, and the cross cords become embedded in the saw-marks by the pressure of the sewing thread. This is drawn through the middle of each sheet and turned round each band beginning at the first sheet and proceeding to the last. The first sheet being thus sewn and secured to the bands, the second sheet is laid upon it, the thread carried from the first sheet to the second, and the process is repeated, and so on to the concluding sheet of the volume, the whole being connected together by the cross bands (whether of cord or tape) round which the thread passes, first through one sheet, and then from each sheet to its successor.

A third method of securing the leaves of a book was some years ago introduced by Mr. W. Hancock, namely, by the adhesion of the back edges of each leaf of the book to a coating of caoutchouc and its superimposed lining eloth. In this method, instead of the fold of each sheet or section (consisting of $4,8,12,16$, or 24 pages) forming a back through which the needle of the sewer passes, as before described, the whole of the back of the book is cut through by a plough knife or guillotine so as to present a smooth level surface formed of the back edges of the book, which, if separated, would then consist of single detached leaves.

This smooth surface is slightly rasped so as to give greater facility for the adhesion to the edge of oach leaf of the caoutchouc, a solution of which is applied in two or three coats, at intervals sufficient to allow each coat to become firmly adhesive. Strips of cloth lining are laid over this, and fastened on with caoutchouc, the outermost strip being so much wider than the back of the book as to form a fly or projecting strip at each side for the purpose of securing the volume to the boards which form, with cloth or leather, the cover of the book. We thus see that, instead of leaves attached by thread at certain points or intervals, each single leaf is agglutinated continuously along the whole length of its back edge to the caoutchouc and cloth lining before described. Books bound in this way open so perfectly flat upon a table without strain or resilience, that they are equally comfortable to the student, the musician, and the merchant. And whore a book consists of paper at once thick, tough, and ausorbent, the adhesion of each single leaf by its back edgo to the caoutchouc coating and lining is generally perfect, and, with careful usage, moderately durable. On the other hand, whon the paper composing the book is thin or non-absorbent, the adhosion of each loaf is vory uncertain; and moreover, by frequent use, and especially under the chemical effect of atmospheric and climatic changes, the caoutchoue sooner or later loses its adhesive power over paper of any quality, hardens, cracks, and shrinks, and leaf after leaf becomes more or less insocuro and detached. The use of caoutchouc for bookbinding has therefore becomo far less general than was at one time anticipated, and the needle and thread of the workwoman is atill the general medium for connecting the leaves of a book together.

Hitherto we have dealt with the preliminary processes of securing the separate portions of a volume - processes which aro common to all kinds of binding, from the simple pamphlet with, or without its paper wrapper, to the most elaborately bound volume in russia or moroceo; except that in the more expensive styles of binding, additional care is taken in the process of sewing; the thread being more frequently passed through each section, round the cross bands, and from section to section, and more elaborately secured.

The next process is that of inserting the volume into its cover; and in this, two systems aro adopted: one, the original plan of attaching the boards of the cover by drawing the cross cords or bands through holes pierced through each board at its back edge, then fastoning the leather or other material used for covering the book over the board, and subsequently adding tho lettering and ornament xequired; the other that of the more modern 'case-binding.' This is now, from its comparative 


\section{BOOKBINDING}

cheapness and adaptation to the rapid execution of large numbers, the method universally adopted for the great mass of books, as thoy issuo from the publisher's warehouse. We will deal with this last-named process in the first instance. In 'case-binding' the case or covor (genorally formed of mill-boards covered with cotton cloth) is made, stamped and lettered with the desired amount of ornament, all complete before it is placed upon the volume. Thus (the exact size of the volume haring been first ascertained) the whole process of 'case-making' and 'blocking' can go on simultaneously with or in anticipation of the processes of folding, sewing, and backing up.

The boards used in bookbinding are formed of the pulp obtained from refuse brown paper, old rope, straw, or other vegetable material more or less fibrous; which pulp is pressed into sheets of varying size and thickness, to suit the requirements of the binder; from the sheet scarcely thicker than cartridge paper used for 'limp' bookcovers, to the thick substance now extensively used for books on which a cover with 'bevelled edges' (after the antique style of the old wooden book-covers used by early bookbinders) is prepared.

The size and style of the volume being ascertained, the board-cutter selects his mill-boards accordingly; and having with his shears 'squared,' i.e. cut of at right angles the rough outer edge of two adjoining sides of each board, adjusts the gauge of his cutting-machine to the exact requirement successively of the length and breadth of the volume, until he has completed the tale of 50 or it may be 5,000 pairs of boards for which his order is given. See Board Cutting Machine (fig. 159, as manufactured by F. Ullmer).

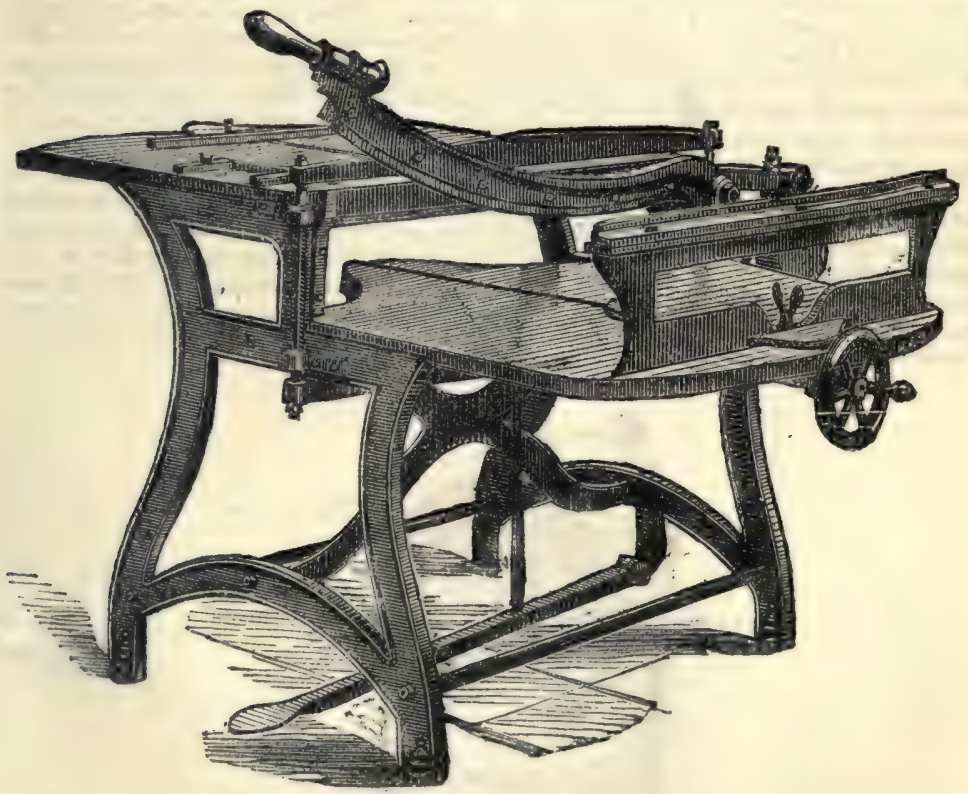

In the meantime, the cloth-cutter in like manner cuts up the corresponding numbers of covers of the dimensions proper for the book. The bookbinders' cloth now so extensively used, is a cotton fabric generally woven in Lancashire, and sometimes dyed and finished there; but these later processes are carried on to a considerable extent, also, in Iondon. The dyed cloth is passed through heated rollers, which being engraved with some grain or pattern, (sometimes in imitation of the grains of russia or morocco leather, sometimes with other patterns) impress the pattern or embossing upon the cloth. A third essential is a supply of a corresponding number of 'hollows.' These are strips of thick paper or of pasteboard, cut to the exact height and thickness required for the book for which the boards and cloth are intended, and which act as 
gauges for the guidance of the caso-makers, and as stiffners for the cloth at the back of the book, between the boards.

These three materials are then passed on to the case-makers: one of whom takes possession of the pile of cloth covers, laicl face downwards before him, rapidly passes the glueing brush from the pot of heated and diluted glue standing by his side upon the uppermost piece of the pile, lifts, and passes the glued piece of eloth to his righthand neighbour, who being provided with tho pile of pairs of boards and corresponding hollows, lays, in suceession, a board (1), a hollow (2), and a board (3) (fig. 160), on the

160

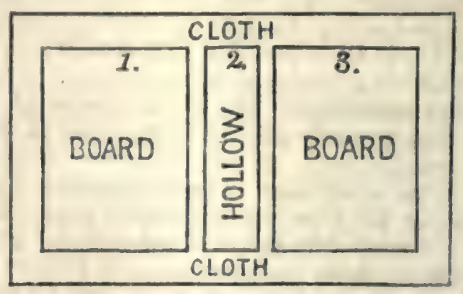
glued surfaco of the cloth, which is cut of a size sufficient to leavo a margin to turn over the edge of the boards and the requisite distance beyond. A narrow spaco, proportioned to the size and thickness of the rolume, is left between the inner enge of each board and the 'hollow,'so as to allow space for the hinge of the ease to turn round and be pressed into the ridge which is formed at each side of the back of a volume when prepared for the case.

This done, the second case-maker then rapidly and lightly (so rapidly as not to allow the hot glue to soak too much into the cloth and spoil its grain and gloss, and so lightly as not to produce the samo defect by too much pressure) passes the palm of his hand or a folding-stick over the cloth, to make it adhere smoothly to the boards and the intermediato hollow, and at once passes each to his right-hand neighbour. The third case-maker in his turn quickly snicks out, with a pair of seissors, the superfluous cloth at each of the four corners, folds the over-lapping margin of clnth round the edge of the boards and the top and bottom of the hollow, rubs the edges and inner margin smooth with a folding-stick, and each case is then taken by an attendant boy and hung up to dry. This process is soon accomplished, and the batch of cases is next passed on to the blockers.

The blocks or stamps used for lettering and ornamenting the cases of books aro of metal, generally of brass, cut in relief, and, besides the letterings, are of rarious kinds; border frames, bands for the back, corner or centre ornaments, \&ce.; adapted to the various characters of books, aud the tastes of publishers and purchasers. In many instances, special designs, pictorial, emblematical or otherwise, are cut for some particular books; and the number and variety of blocks that aceumulate in any large bookbinding establishment thus becomes very great, and absorbs a considerable capital.

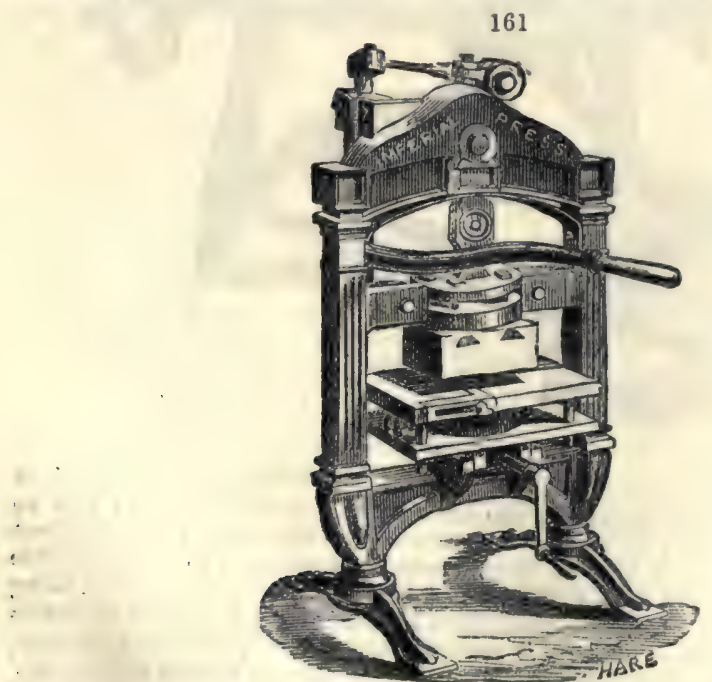

The requisite block, or group of blocks required for the cases in hand being prepared, they are accurately adjusted and secured (with the stamping surface downwards) to the upper bed of an arming press (fig. 161, as manufactured by F. Ullmer), which bed 


\section{BOOKBINDING}

is perforated by channels, for the admission of a jet of gas or steam, sufficient to give the required heat to the metal blocks susponded; heat being needful to give distinctness and permanence to the impression on the cloth case. Beneath the stamping block so propared and suspended, is a table on which has to be fixed a frame or gauge, adjusted to the exact proportions of the case about to be stamped; and the table is then brought by means of regulating serews, to the exact position under the stamping-block, to receive the required impression on the back and sides of each case. The application of a lever, moved either by hand or steam-power, brings the superincumbent and heated block forcibly upon the cloth case ; and when the pressure (which has to be carefully adjusted so as not to gire too faint an impression on the one hand, or on the other to burn too deeply and injure the fabric and colour of the eloth) the ease is withdrawn with the required impression stamped upon it; another is substituted, and the process is repeated throughout the required number of the sort. An impression given simply as above described, is technically tormed 'blind blocking,' and is a mere indentation in the cloth case of the pattern of the block applied. When gold or other metal for lettering or ornament is required, the cover of the book has to undergo the previous process of 'laying on' as follows:-

Gold-loaf is laid on a leather cushion and divided by the gentle pressure of a knifeedge into slips of the requisite size, which are then deftly and smoothly lifted by adhesion to a slightly greased pad and transferred from it to the part or parts of the cloth case about to be letterod or ornamented in gold. The case, with the gold-leaf thus laid on, is then subjected to the pressure of the arming-press as in blind blocking ; the gold-leaf is pressed by the heated block into the case of the book, which, on being withdrawn from the lower table of the press, is gently wiped with a rag or brushed; this removes the metal-leaf from every unpressed part, leaving the impression of the lettering or ornament clear and distinct.

The cases are then ready to be fixed upon the books to which they belong, and which having been, as before described 'folded, collated, placed and sewn,' and afterwards 'papered' (this last term being given to the pasting of the end papers with a fly-leaf to the beginning and end of each volume) have to undergo the further processes needful to fit them for insertion into the cases. In the first place the edges of each book are either cut at top, tail and fore-edge, or only 'trimmed.' This last operation merely pares down the rough and projecting leaves at the fore-edge and tail nearly to the level of those leaves which present a double or quadruple fold, tochnically tormed ' the bolt,' and leaving the top of the book entirely uncut, so that without reducing the general margin of tho book a neat and tolerably uniform edging is presented. The backs of the

\section{2}

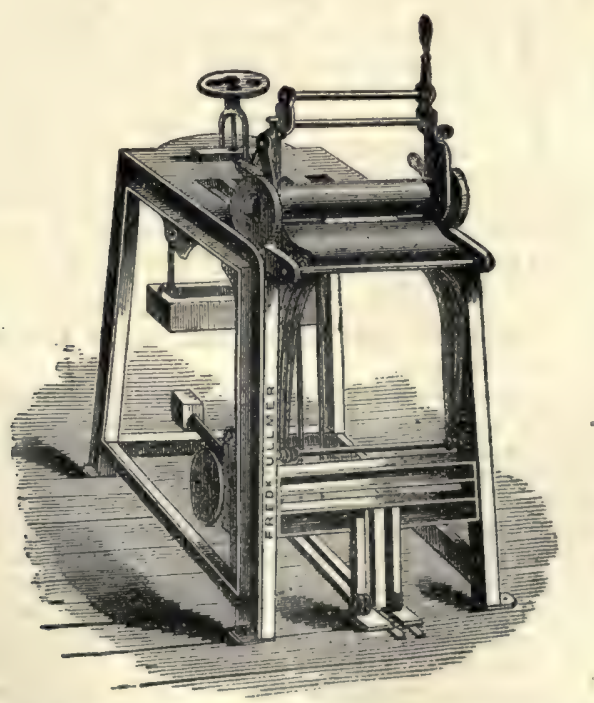

books are then coatod with gluo, on which is laid a strip of strong paper, and again over that a lining cloth or webbing of tough but loose and elastic texture, which 
projects beyond the width of the back so as to form two wings; these, with the projecting ends of the cross-cords or bands and the end papers, form the combined material for securing tho book in the case. Tho back of tho book, howover, before its insertion in the case, is rounded, either by a poculiar manipulation with the left hand and the use of the hammer in the right hand of the workman, or by the use of the rounding-machine. By this operation a concavo surfaco is presented at the foroedge of the book corresponding to the convex surface produced by rounding its back, and lastly each volume is tightly nipped (its back slightly projecting beyond the pressure) either between 'backing boards,' or by the jaws of a backing machine (fig. 162, as manufactured by F. Ullmer). The whole surface of the back is then hammerod and pressed till it spreads out beyond the thickness of the rest of the volume, and a projection is produced along each edgo of the back, forming two ridges, which fit into the space before described as being left by the case-makers between each board and the hollow of the ease, thus combining to form a hinge and give the needful play to the opening and shutting of the lid of the book.

As before indicated, the end papers, the flap of the lining cloth and the ends of the cords or bands are then pasted to the inside of the case first on one side and thon on the other, the projecting ridges of the back being at the same time pressed by the workman into the space left for them between the band and the hollow of the case - the volumes are carefully laid betwoen pressing-boards with their rounded backs put outside the edges of each pressing-board so as to escape the coming squeeze and the pile of boards and books is then subjected to pressure for a needful poriod, either in an hydraulic or screw-press. After a sufficient space of time and pressure to allow the paste to dry, the press is emptied of its contents, each volume opened and examined to see that it is correctly inserted, and the lot is then ready for delivery to the publisher.

Such, with some variations of detail in different establishments, are the processes by which the myriads of books issued by the large publishing houses throughout the year are bound with great rapidity, at a low price, and in ondless varieties of stylo to suit every taste.

We turn now to the more elaborate and complete style of binding, (technically termed 'extra binding,') which is substantially, with certain modifications, the system adopted ever since the book proper, with its rectangular figure, and its distinct leaves united together at their back edge, superseded the extended sheet or series of sheets which in ancient times were rolled round a cylinder and formed the 'volumen' or roll, from which our term 'volume,' no longer strictly applicable, is derived.

Instead of a case completely fitted and finished being pasted or glued upon the book prepared for it, as previously described, the cover of the book in extra binding is generally fitted on piecemeal, drawn over the boards, and the lettering or ornament added last when the cover has been fitted and attached to the book. The edges of a bound book after being cut round are generally either sprinkled, wholly coloured, marbled, or gilt. In the first, a brush slightly charged with colnuring fluid is struck smartly over the edges of the books so as sprinkle them with a uniformly distributed shower of small spots; in the second a sponge or brush dipped in colour is applied to the edges, which are tightly compressed so as not to allow the colour to penetrate the margin of the books ; in the third, the edges are applied to colours which by a peculiar process are made to float on water in patterns combined so as to produce a marbled effect, and which are transforred by contact from the surface of the water to that of the book-edge which is afterwards burnished; in the fourth, gold-leaf is laid upon the edges which have been previously coated with a solution of white-of-egg, \&c. termed 'glaire,' to which the gold-leaf adheres, and is then burnished with a polishing tool, tipped with agate.

The ends of the cords are then drawn by the 'forwarder' through holes pierced in the boards, near their back edge, unravelled, spread out and hammered down to the level of the board so as to present no unsightly lump under the leather. The leather for the cover is pared round its edges, softened by manipulation and the application of paste-water to make it pliable, and is then pasted evenly and smoothly over the boards and back of the book, worked well into the hinge and round the 'raised bands,' (if there are any), turned neatly over the edges and round the corners, and rubbed down with a folding-stick; 'head-bands' giving a neat and finished appearance and additional security to the turning in of the leather at the back are then added to the top and tail of the book. Sometimes lettering-pieces of a different colour to the rest of the book are used, being pared very thin so as to avoid any unsightly swelling, and pasted and rubbed down on that part of the book which is to receiro the lettering. If the book is ' half sbound,' instead of 'whole-bound,' the leather is limited to a strip at the back and a short distance from the back on each side, and to the corners; the sides of the book being covered with either marbled or coloured paper or cloth, 
ard tooled where the last material slightly overlaps the leather back and corners, so as to hide the join. 'Raised bands' are formed of strips of pasteboard or parchment placed at regular intervals across the back of the book, leaving a space termed 'panels' between them, and the pliable leather is stretched and worked over and round the edges of the bands, so as to give to the whole back a neat and uniform appearance.

'The 'forwarder' then passes the book on to the 'finisher,' whose duty it is to add the required lettering and ornament.

The tools used by him, whether single letters or figures, or 'pallets' (that is, the titlo of a book, \&ce, cut in a single metal block) are mounted on wooden handles, and applied before use to a gas burner, in order to obtain the requisite heat.

If the impression of the tool is intended to be gilt, the finisher lays on gold leaf in a manner similar to that described in case-blocking, and the tool moderately heated is applied, not by an arming press, but by the finisher, on whose steadiness of hand and accuracy of eye the proper and even application of the various tools successirely used in lettering or ornament is dependent. Additional pressure is given when needful for the larger tools, by the finisher's leaning his shoulder or chin against the end of the handle to assist the action of his hand. The superfluous gold-leaf is then cleaned off as in case blocking, by a greasy rag or a piece of India-rubber, after which the book is carefully examined, roughnesses smoothed down, and defective workmanship corrected, and a coating of polish or glaize given where required. Lastly, the end papers are pasted down, and after a final examination as to accuracy of lettering and other details, the book, after having a final squeeze to make it lie square and solid, is turned out complete.

The implement generally used by the extra binder for cutting the edges of single books is the plough (figs. 163 to 168).

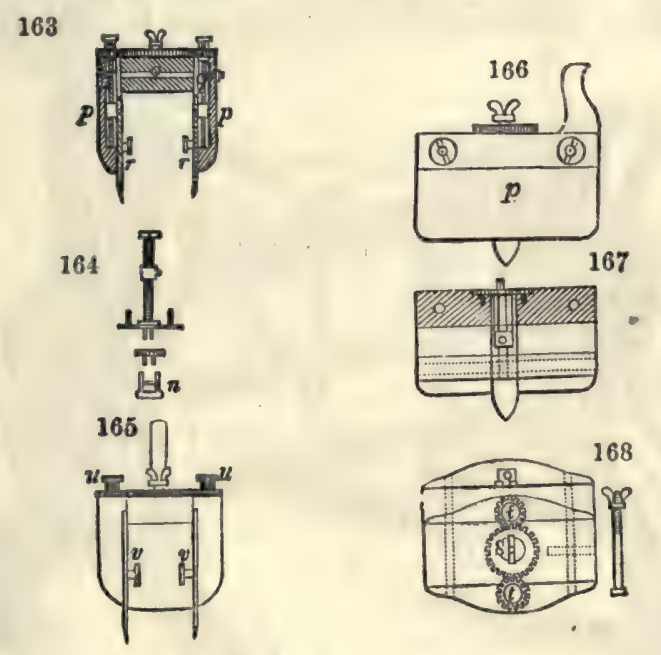

The plough (fig. 163) is made to receive two knives or cuttors, and which are situated in the plough in the following manner:-The plough is composed of three principal parts-namely, the top, and it two sides. The top, $o$, is made the breadth of the cross piece $a$, and with a handle made fast thereon. The sides, $p p$, are bolted thereto with bolts and nuts through corresponding holes in the top and sides. The figures give inside views and cross sections of the details of the manner in which the cuttors and adjustments are mounted. A groove is cut down each cheek or sido, in which are placed screws that are held at top and bottom from moving up and down, but, by turning, they cause the nuts upon them to do so; they are shown at $q q$. These nuts have each a pin, projecting inwards, that goes into plain holes mado in the top ends of eutters $r r$.

The cutters and the work for causing them to go up and down are sunk into the cheeks, so as to be quite level with their inner surfaces. Fig. 164 shows one of these screws apart, how fixed, and with moveable nut and projecting pin. The top of each screw terminates with a round split down; and above it a pinion-wheol and boss 
thereon, also similarly split. This pinion fits upon tho split pin. Alovo, thore is cross section of a hollow coupling cap, with stoel tongue across; that fits into both tho cuts of the serew pin and pinion boss, so that, when lowered upon each other, they must all turn togethor. In the middlo and on the top of the upper piece, $o$, the large wheel $s$, runs loose upon its centre, and works into tho two pinion whoels, $t t$ (fig. 168). The wheel $s$ has a fly nut with wings mounted upon it.

It will now be seen. when tho plough is in its place, as at fig. 165, that if it bo pushed to and fro by the right hand, and the nut occasionally turned by the left, the knives, or cutters, will be protruded downwards at the same time, and these either will or will not advance as the coupling caps, $u v$, are on or off.

169

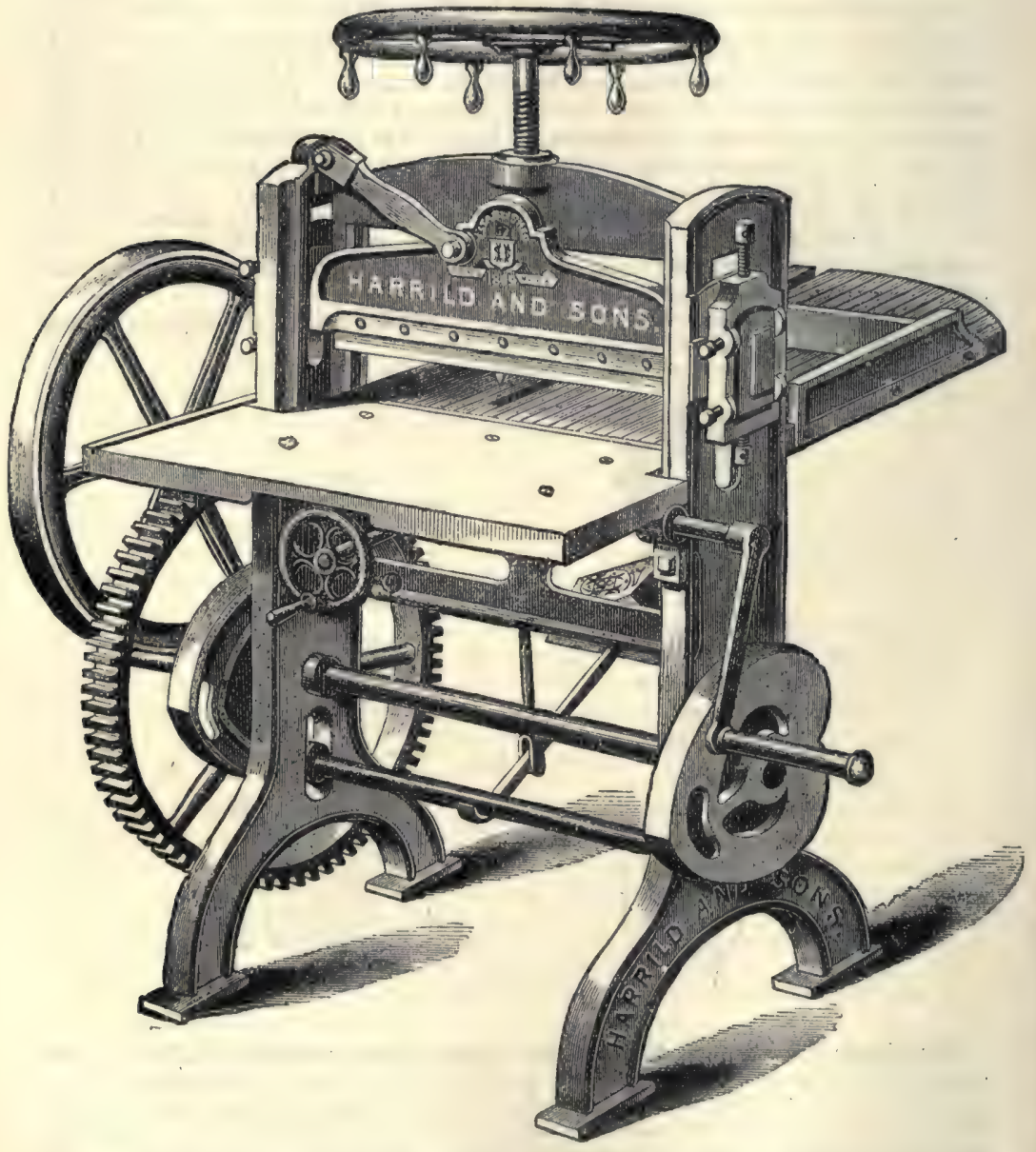

When the edges of a numbor of copies of the same book have to be cut, the operation is greatly aceelerated by the uso of Wilson's, or other cutting machines (fig. 169), which are adapted for working either by hand or by steam-power.

The cutting machines consist of an iron sliding table fitted with an upright plate at right angles to the surface of the table, against which the backs of the pile of books about to have their fore-edges cut are placed. By means of a turning wheel the foreedges of the pile of books are then brought in the exact position proper to receive the descending knife-edge. This knife, long enough to reach from side to side of the table, and therefore along the whole range of books placed upon it, is fitted into a. frame so as to act on the principle of the guillotine, and either by a diroctly downward or. 
by a diagonal movement. A fow turns of a fly-wheel, worked oither by hand or steam-power, bring the heary pressure of an iron bar upon the pile of books when thus brought into their proper position under the guillotine, by which the pile is squeezed into a firm compact mass, upon which, and simultaneously, the knife-edge is by the same power brought down, and the rough and superfluous foro-edges are at one stroke severed from the rest, leaving a smooth and polished surface on the foreedges of the books, instead of the rough and uneven one before presented. The position of the pile is then curefully shifted so as to bring in succession the tops and the tails of the pile of books to the like action of the guillotine, and in a very short space of time (varying according to the size and thickness of the rolumes operated on) the odges of many hundreds of books are cut smooth and in readiness for the sprinkler, the colourer, the marbler, or the gilder.

Booxum wooD. An Indian wood, used for dyeing red, the produce of the Sappan tree, Cesalpinia Sappan.

BORACIC ACID, or BORIC ACID. (Acide borique, Fr.; Borsäure, Ger.) Composition of the anhydride, $\mathrm{BO}^{3}\left(\mathbf{B}^{2} \mathbf{O}^{8}\right)$; of the erystallised acid, $\mathrm{BO}^{3}, 3 \mathrm{HO}$ $\left(\mathbf{n}^{2} \mathbf{O}^{3}, \mathbf{3} \mathbf{E}^{2} \mathbf{0}\right)$.

The chiof sources of this acid and its salts-the borates-are the hot vapours, or soffioni, which issue from the ground in certain parts of Tuseany, and are utilised by a process fully described below. In addition to its occurrence in these vapours, boracic acid is found native in a solid state, forming the mineral Sassoline or Sassolite, so called from Sasso, in Tuscany, the locality in which it was originally discovered by Mascagni. Sassoline is usually found in the form of small white pearly scales, with a soapy feel. Theoretically, the pure mineral should contain $56 \cdot 4$ per cent. of boracic acid (anhydride) and 43.6 of water. Klaproth, in examining the Sassoline of Sasso, found 80 per cent. of boracic hydrate, with 11 per cent. of protosulphate of manganese, and 3 per cent. of sulphate of lime, together with silica, carbonate of lime, and other mechanically-mixed impurities. Sassoline also occurs abundantly in the crater of Vulcano, one of the Lipari Islands, forming a layer on the sulphur and around tho fumaroles, or exits of the sulphurous exhalations.

Among the numerous compounds of boracic acid occurring ready formed in nature, the most important are native borax, or biborate of soda, and tincal, or crude borax: substances fully described under the head of Bonax. Of the other native borates, the following are the more interesting species :-

Boracite, a borate of magnesia with chloride of magnesium, containing when pure 62.5 per cent. of boracic acid (anhydride). It crystallises in the cubic system, often in hemihedral or tetrahedral forms, and is remarkable for being pyro-electric-that is, for exhibiting electrical polarity when exposed to a change of tomperature. The mineral is further notable for its anomalous optical properties; thus, a ray of light in passing through a crystal of boracite suffers double refraction, contrary to the general rule that crystals belonging to the cubic system are not capable of thus affecting light. The probable explanation of this anomalous behaviour on the part of boracite is beyond the scope of this article.

Boracite is usually found in association with deposits of rock-salt and gypsum. The mineral occurs crystallised at Lüneburg in Hanover, and at Stassfurt near Magdoburg; the latter locality also yields a massive boracite called Stassfurtite.

Ulexite or Boronatrocalcite is a hydrous borate of lime and soda, occurring in whito reniform masses, from the size of a hazel-nut to that of a potato, scattered over the dry plains of Iquique, in Southorn Peru, and in the Province of Tarapaca, where it is called tiza. It is also found in Nova Scotia and in Nevada. A specimen from Poru yielded-boracic acid, 45.46; lime 14.32; soda, 8.22 ; potash, 0.51 ; sulphuric acid, $1 \cdot 10$; chloride of sodium, $2 \cdot 65$; sand, $0 \cdot 32$. This analysis was made by Mr. A Dick, in the metallurgical laboratory of the Museum of Practical Geology. The torm Hayesine was formerly applied to this mineral, but some confusion has arisen in tho application of this name. According to Hayes the pure species contained no soda, and was simply a hydrous borate of lime. Mr. David Forbes discovered a borate of lime in the form of white silky flakes suspended in the waters of the hot springs called tho Baños del Toro, in the Cordilleras of Coquimbo. The formation of this substance was instructive, as throwing light upon the probable origin of the same compound elsewhere. When tho hot vapours, emanating from the neighbouring rolcanoes, passed into springs of water highly charged with carbonate of lime, the boracic acid of the rapours combined with the lime to form borate of lime, whilst carbonic acid gas was set free. The term Bechilite has been applied by Dana to a borate of lims from Tuscany.

Howlite or Silicoborocalcite is a hydrous boro-silicate of lime, containing about 43 per cent, of boracic acid, and occurring in nodular forms in gypsum and anbydrite in NoTa Scotia, 
Cryptomorphite is a hydrated borate of lime and soda, with 58.5 per cont. of boracio acid, closely related to Ulexite. It has boon found in Nevada.

Lardarellite is a borate of ammonia found in the lagoons of Tuscany, and named after the late Count Lardarel- - the founder of tho 'Iuscan boracic acid industry.

Lagonite is an earthy borato of poroxido of iron, ulso found in these lagoons.

In addition to the spocies noticed above, in which boracic acid forms a main constituent, there are several minerals which contain this acid in subordinate quantity. Thus, boracic acid is present in Danburite to the extent of about 28 por cent. ; in Datolite to about 22 per cent.; in T'ourmaline in variable proportions up to 12 per cont. ; and in Axinite it is present in quantity ranging from 2 to 5 per cent. But of all the boracic minerals it is only the boratos of soda, lime, and magnesia, which have hitherto been found in sufficient abundance to be oconomically omployed in the preparation of boracic acid and the alkaline borates.

The great supply of boracic acid, howevor, is derived from the boracic acid lagoons of Tuscany. Before the diseovery of this acid, in the time of the GrandDuke Leopold I., by the chemist Höffer, the fetid odour developed by the sulphuretted hydrogen gas and the disruptions of the ground occasioned by the appearance of new soffioni, or vents of vapour, had made the natives regard thom as a diabolical seisurge, which they sought to remove by priestly exorcisms; but since science has explained the phenomena, the fumaochi have become a great boon to the district, and a source of public prosperity.

The hot vapours of the soffioni consist of a mixture of permanent gases, condensible vapours, and mechanically-suspended solid particles. Among the usual constituents may be mentioned carbonic ncid gas, nitrogen, oxygen, sulphuretted hydrogen, watery vapour, ammonia, sulphate of ammonia, hydrochloric acid, organic matter, and boracio acid. To collect this boracic acid, which is nevor present in moro than very minute quantity, the soffioni aro enclosed by low walls of coarse masonry, or brick-work, glazed on the inside, and forming a series of circular basins, the diameter and depth of which vary greatly in different works. The larger basins may

170

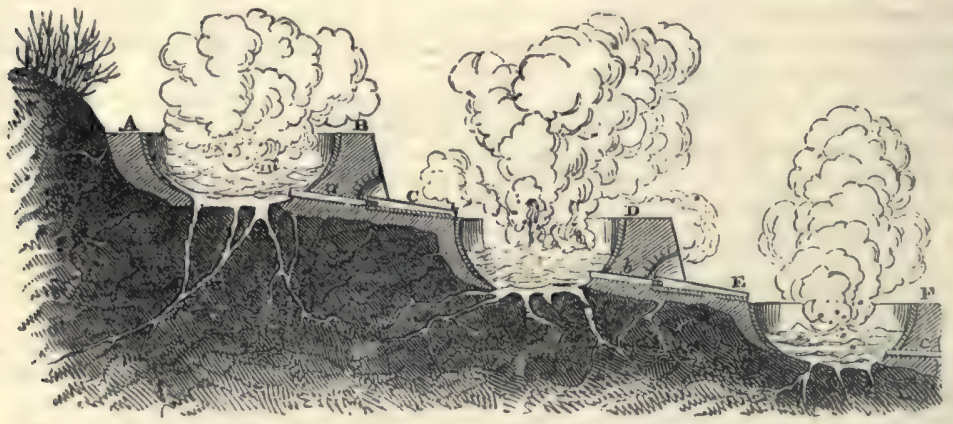

onclose several distinct vents. A series of these circular basins is arranged in terraces on the side of a hill, as represented in fig. 170. A small stream of water, from an adjacent spring, is introduced into the uppermost basin $\mathbf{A} B$, thus forming a small pool or artificial lagoon. By the escape of the hot vapours rising from bolow,

171

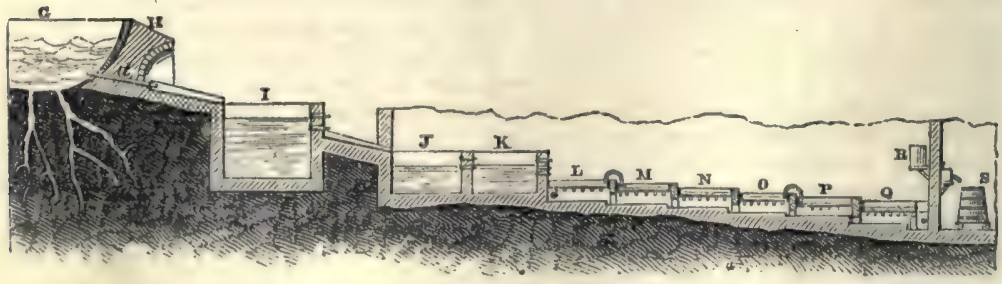

the wator of this lagoon bocomes more or less agitated and gradually heatod, and at the samo time impregnated with the boracic acid. After standing for 24 hours in tho 
upper basin $\mathrm{A} \mathrm{B}$, the weak solution is run out, through the channel $a$ into the socond basin C D. Here it takes up another dose of boracic acid, and after 24 hours is run off into the next lower basin E F. After having in this way traversed six or eight of the lagoons, the solution acquires a specific gravity of 1.007 , and contains about onohalf per cent. of boracic acid. This solution passes from the last basin a $\mathrm{H}$ (fig. 171) into a scries of clarifiers and evaporating vessels, represented in section in fig. 171,

172

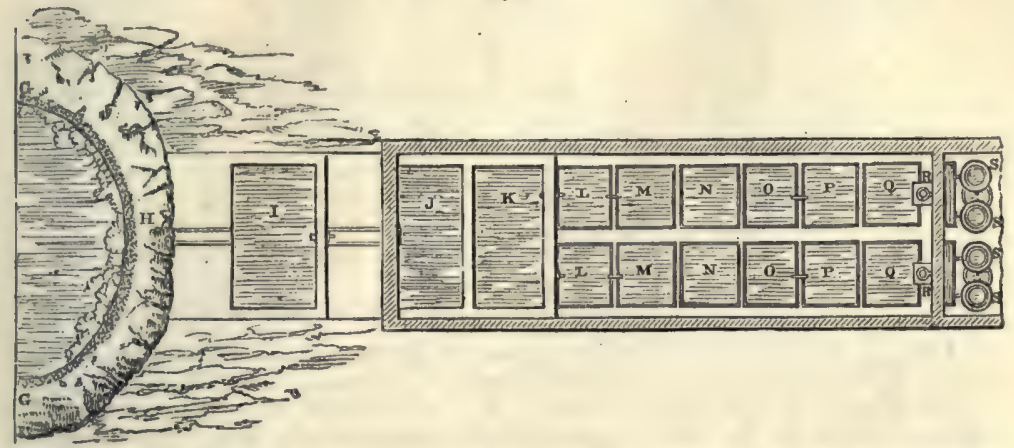

and in plan in fig. 172. In the large square brick vessel 1, called a vasco, the solution deposits much of the mud which it holds in suspension. After subsidence of a great part of its mechanical impurities, the comparatively clear liquor is drawn off into other settling tanks $\mathrm{J}, \mathrm{K}$, and thence into a long succession of squaro leaden ovaporating pans, of which half-a-dozen are represented by $\mathrm{L}, \mathrm{M}, \mathrm{N}, \mathrm{O}, \mathrm{P}, \mathrm{Q}$. The heat for effecting this evaporation was formerly obtained by the combustion of wood, but Count Larderel's capital improvement-an improvement without which the Tuscan soffioni could never have been profitably utilized-consisting in dispensing altogether with the use of artificial fuel, and conducting the evaporation by means of the natural leat of the volcanic emanations. Accordingly, jets of steam issuing from the ground are introduced through flues under the evaporating pans, and conducted successively from the lowest to the highest of the series. The solution during its passage through the system of evaporating vessels, which lasts about 62 hours, gradually becomes concentrated, and by the time it reaches the last vessel is sufficiontly strong to be passed to the crystallising tubs, s s. Fig. 173 represents an improved form of

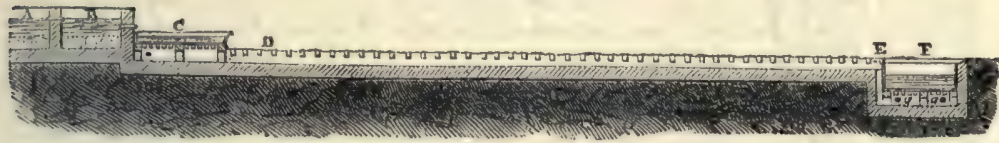

ovaporating apparatue, in which the liquid from the vasco $\mathrm{B}$ passes to a shallow boiler, c, whence it runs slowly over an inclined table of sheet lead, D s, about 150 feet long, and having its surface corrugated so as to form a series of channels through which the solution flows. During its passage, the solution slowly eveporates, by the heat of the soffioni introduced below, and finally reaches the vessel $\mathrm{s}$ in a sufficiently concentrated form.

The concentrated solution, obtained by oither of these methods of evaporation, is mixod with some of the mother-liquor of the pans, and set to crystallise in the round tubs -s s ( figs. 171, 172, 174), each having a capacity of about 8 cubic foet, and being mado of wood, lined with lead. The small crystals on remoral are drained in baskets J, at the top of the tub, and while still moist are sproad out in a layer on the floor $\mathrm{c}$ c of the drying chamber ( fig. 174), which is heated by stoam circulating in a spaco included botweon this floor and another floor below.

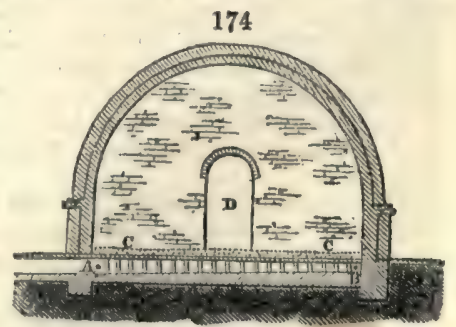


The boracic acid of the Tuscan lagoons is, lobtained from nine differont works :Snsso, Larderello, Lervazano, Monte Cerboli, Castel Nuoro, Monte Rotondo, San Frederigo, Lustignano, Lago ; producing 163,855 avoirdupois pounds per month.

The late Count Larderel furnished Mr. W. P. Jervis with the following statement of the production of boracic acid, from the commencement of the works to the year $.1859:-$

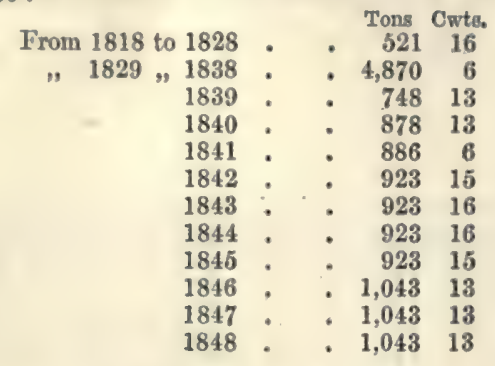

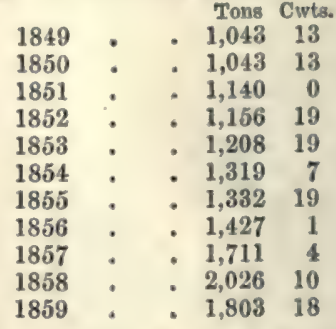

Although the boracic acid in the lagoons of Tuscany was discovered in 1777 by Peter Hoeffer, apothecary to the Grand-Duke of Tuscany, until 1818 all the efforts made to render it profitable to obtain it were of the most desultory character, and generally failures. In 1818 Count Larderel established the first works at Monte Cerboli, and used artificial heat to effect the evaporation. In 1828 ho began to use the natural heat of the soffioni to evaporate the boracic waters of the lagoons, effecting thus an enormous saving and greatly extending the works.

The late Professor Graham, in his 'Report on the Chemical Products of the Great Exhibition of 1851,' thus speaks of Larderel's discovery:-

'The preparation of boracic acid by Count F. de Larderel, of Tuscany, was rewarded by a Council medal. Although this well-known manufacture is not recent, having attained its full development at least ten years, still the bold originality of its first conception, the perseverance and extraordinary resources displayed in the successful establishment, and the value of the product which it supplies, will always placo the operations of Count de Larderel among the highest achiovements of the useful arts, and demand the most honourable mention at this epoch. The rapour issuing from a rolcanic soil is condensed, and the minute proportion of boracic acid which it contains (not exceeding 0.3 per cent.) is recovered by eraporation, in a district without fuel, by the application of volcanic vapour itself as a source of heat. The boracic acid thus obtained greatly exceeds in quantity the old and limited supply of borax from the upper districts of India, and has greatly extended the use of that salt in the glazes of porcelain, and recently in the making of the most brilliant crystal, when combined with the oxide of zinc instead of the oxide of lead.'-Reports of the Jurors of the Great Exhibition of 1851 .

Various hypotheses have been adranced to explain the origin of the boracic acid in the heated vapours of the Tuscan lagoons. Boracic acid is not known in an uncombined state as a constituent of any rock, but it seems likely that the deep-seated rocks whence the rapours issue may contain certain borates - such as boracite-and by the action of hot aqueous vapour on these compounds boracic acid may be eliminated.

The processes of chemical alteration taking place beneath the crater of Vuleano, already spoken of, may, according to the statement of Hoffmann, depend upon conditions very similar to those existing in Tuseany. There, likewise, sulphuretted lyydrogen is associated with the boracic acid, and, it would appear in much greater quantity, since the fissures through which the rapour issues are thickly lined with sulphur, which is in sufficient quantity to be collected for sale. A profitable factory is established at the place, which yields daily, besides boracic acid and chloride of ammonium, about 1,700 lbs. of refined sulphur and about 600 lbs. of pure alum.Bischof.

The boracic acid obtained from the waters of the Tusean lagoons by the process previously described is always more or less impure. M. Payen has given the following as the composition of this crude boracic acid for 100 kilogrammes:-

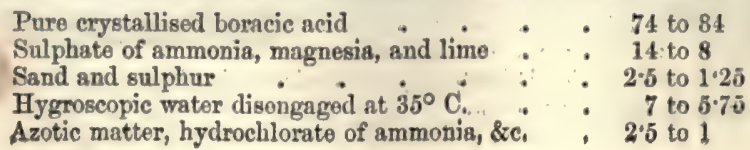


According to Wittstein, the commercial boracic acid is composod as follows :-

\begin{tabular}{|c|c|c|c|c|c|c|}
\hline Sulphat & nanganes & & & & & 80 t \\
\hline " & inon & : & & . & & 0.365 \\
\hline ", & alumina & . & & . & & . 0.320 \\
\hline$"$ & $\operatorname{lime}$ & . & : & . & & $1 \cdot 018$ \\
\hline " & magnesia & a. & & . & & $2 \cdot 632$ \\
\hline$"$ & ammonia & a. & & 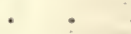 & & $8 \cdot 508$ \\
\hline$"$ & soda & . & & • & & 0.917 \\
\hline & potash & . & & • & & 0.369 \\
\hline al ar & iac & . & & & . & 0.298 \\
\hline lic & olution) & & & . & & $1 \cdot 200$ \\
\hline aly & acid (with & the boracic) & & . & & 1.322 \\
\hline Crystall & ble boracic & e acid. & & . & & . $76^{\circ} 494$ \\
\hline Water & . & . & . & - & . & . 6.557 \\
\hline
\end{tabular}

To obtain pure boracic acid, the crude commercial product should be converted into borax by saturating its solution with carbonate of soda, and a hot solution of this borax be then decomposed by addition of oil of vitriol, when sulphate of soda is formed, and boracic acid set free. 'Pure boracic acid crystallises in pearly, white, greasy scales, sparingly soluble in cold water; but dissolving in three times their weight of boiling water, and forming a solution possessing but feebly acid properties. It is more soluble in alcohol, and the solution burns with a characteristic green flame. This flamo oxamined by the spectroscope presents a peculiar system of green bands. At a moderate temperature boracic acid loses part of its water of erystallisation, and forms a compound known as metaboric acid. By further heating, the remainder of the water is expelled, and the anhydrous oxide $\left(\mathrm{BO}^{3}\right)$ left. This anhydride is readily fusible to a transparent glass of specific gravity $1 \cdot 83$. Many of the borates are also eminently fusiblo, and hence their value as fluxes. At a higher temperature, boracic anhydrido rolatilises.

Boracic acid was formerly called Homborg's sedative salt, but is not now officinal. It is sometimes used by the druggist to increase the solubility of cream of tartar.

BORACITE. See Boracic ACID.

Boraty or imms. See Boractc ACID.

Borats of SODA. See Borax.

Boratzs. Salts of boracic acid. See 'Watts's Dictionary of Chemistry.'

водаx. (Borax, Fr.; Borar, Ger.) Supposed to be the chrysocolla of Pliny. In the seventh century, Geber mentions borax; and it was described by Geoffroy and by Baron in the early part of the eighteenth century. Borax is a compound of boracic acid and soda (biborate of soda), found native in Thibet, in China, in Persia, the island of Ceylon, California, and in South America; it has also been found in small quantities in Saxony. The crude product from the former locality was imported into Europe under the name of tincal. Tincal was originally brought from a salt lake in Thibet; the borax was dug in masses from the edges and shallow parts of the lake; and in the course of a short time the holes thus made were again filled. The imported tincal was purified from some adhering fatty matter by a process kept a long time secret by the Venetians and the Dutch, and which consisted chiefly in boiling the substance in water with a little quicklime.

Attention has been directed within the last few years to some extraordinary deposits of borax in Borax Lake, California. This lake is a small body of wator, forming a narrow arm on the eastern side of Clear Lake, from which it is separated by a low ridge of loose voleanic materials. It is situated about 65 miles N. W. of Suisan Bay, and about 36 miles from the Pacific coast. Borax Lake was first described in 1856 , by Dr. Veatch, who detected the presence of borax in the water, and some months afterwards a large bed of crystals of borax was discovered at the bottom: Some of these crystals are microscopic, but others are unusually large, some of the faces measuring from 2 to 3 inches across. They form a layer of variable thickness, intermixed with blue mud at the bottom of the lake, from which they are collected during the dry season. A sample of water from the lake, collected in September. 1863 , and analysed by Mr. G. E. Moore, contained 2401.56 grains of solid matter per gallon, of which about one-half was chloride of sodium, one-fourth carbonate of soda, and the rest chiefly borax. Indeed, the water contained per gallon 281.48 grains of anhydrous biborate of soda, equivalent to 535.08 grains of crystallised borax. Samples collected from a coffer-dam sunk in the middle of the lake were even rịcher. The borate of soda has also been found at Potosi, in Peru; and it has boen discorered 
by Mr. T. Storry IIunt, of the Geological Survey, in Canada, from whose report the following extract is made:-

'In the township of Joly there occurs a very interesting spring on the banks of the Ruisseau Magnenat, a branch of the Rivière Souci, about five miles from the mills of Methot at Saint Croix. The spring furnishes three or four gallons a minute of a water which is sulphurous to the taste and smell, and deposits a white matter along its channel, which exhibits the purple vegetation generally met with in sulphur springs. The temperature of this spring in the evening of one 7 th of July was $46^{\circ} \mathrm{F}$., the air being $52^{\circ} \mathrm{F}$. The water is not strongly saline, but when concentrated is very alkaline and salt to the taste. It contains, besides chlorides, sulphates, and carbonates, a considerablo proportion of boracic acid, which is made evident by its power of reddening paper coloured by turmeric, after being supersaturated with hydrochloric acid. . . The analysis of 1,000 parts of the water gave as follows :-

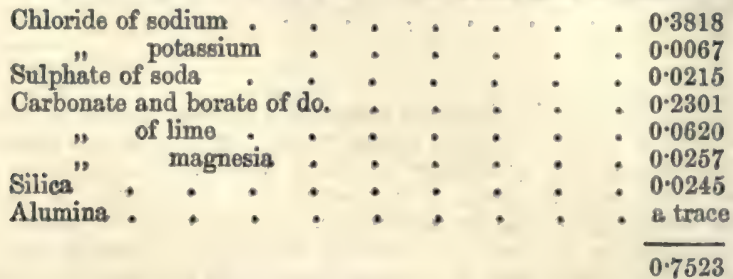

'The amount of boracic acid estimated was found to be equal to 0.0279.'

The following is the mode of purifying borax. The crude crystals are to be broken into small lumps, and spread upon a filter lined with a lead grating, under which a piece of cloth is spread upon a wooden frame. The lumps are piled up to the height of 12 inches, and washed with small quantities of caustic soda-lye of $5^{\circ} \mathrm{B}$. (specific gravity 1.033) until the liquor comes off nearly colourless; they are then drained, and put into a large copper of boiling water, in such quantities that the resulting solution stands at $20^{\circ} \mathrm{B}$. (specific gravity $1 \cdot 160$ ). Carbonate of soda equivalent to 12 per cent. of the borax must now be added; the mixed solution is allowed to settle, and the clear liquid is syphoned off into crystallising vessels. Whenever the motherwaters get foul, they must be evaporated to dryness in cast-iron pots, and roasted, to burn away the viscid colouring matter.

The following process for refining the native Indian borax, or tincal, has been published by MM. Robiquet and Marchand :-

It is put into large tubs, covered with water for 3 or 4 inches above its surface, and stirred through it several times during six hours. For $400 \mathrm{lbs}$. of the tincal there must now bo added $1 \mathrm{lb}$. of quieklime diffused through two quarts of water. Next day the whole is thrown upon a sieve, to drain off the water with the impurities, consisting, in some measure, of the fatty mattor combined with the lime, as an insoluble soap. The borax, so far purified, is to be dissolved in $2 \frac{1}{2}$ times its weight of boiling water, and $8 \mathrm{lbs}$. of muriate of lime are to be added for the above quantity of borax. The liquor is now filtered, evaporated to the density of $18^{\circ}$ or $20^{\circ} \mathrm{B}$. (1.14 to 1.16 specific gravity), and set to crystallise in vessels shaped like inverted pyramids, and lined with lead. At the end of a few days, the crystallisation being completed, the mother-waters are drawn off, the crystals are detached and dried. The loss of weight in this operation is about 20 per cent.

Borax is sometimes adulterated with alum and common salt: the former addition may be readily detected by a few drops of water of ammonia, which will throw down its alumina; and the latter by nitrate of silver, which will give with it a precipitate insoluble in nitric acid.

The native boracic acid obtained from tho lakes of Tuseany, being manufactured in France into borax, has greatly lowered the price of this article of commerce. When MM. Payen and Cartier first began the business, they sold the crystals at the same price

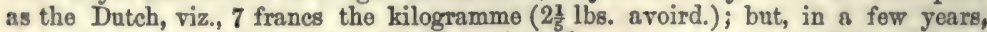
they could only obtain 2 franes and 60 centimes, in consequence of the market getting overstocked. The mode of making borax from the acid is as follows :-The lake water is evaporated in graduation houses, and then concentrated in boilers till it crystallises. In that state it is carried to Marseilles. About 1,100 lbs. of water are mado to boil in a copper, and 1,320 lbs. of crystallised carbonate of soda are dissolved in it by successive additions of about $40 \mathrm{lbs}$. The solution being maintained at nearly the boiling point, 1,100 lbs. of the crystallised boracic acid of Tuscany are introduced, in successive portions. At each addition of about $22 \mathrm{lbs}$. a lively effervescence ensue日, on which account the copper should be of much greater capacity than is sufficiont to 
contain the liquors. When the whole acid has been added, the fire must be damped by boing covered up with moist ashes, and the copper must be covered with a tight lid and blankets, to preserve the temperature uniform. The whole is left in this state during 30 hours; the clear liquor is then drawn off into shallow crystallising vessels of lead, in which it should stand no highor than 10 or 12 inches, to favour its rapid cooling. At the end of three days in winter, and four in summer, the crystallisation is usually finished. The mother-water is drawn off, and employed, instead of simple water, for the purpose of dissolving fresh crystals of soda. The crystals are carefully detached with chisols, re-dissolved in boiling water, adding for each $220 \mathrm{lbs}$. of borax, $22 \mathrm{lbs}$, of carbonato of soda. This solution marks $20^{\circ} \mathrm{B}$. (specific gravity $1 \cdot 160$ ); and, at least, one ton of borax should be dissolved at once, in order to obtain crystals of a marketable size. Whenever this solution has become boiling hot, it must be run off into large crystallising lead chests of the form of inverted truncated pyramids, furnished with lids, inclosed in wooden frames, and surrounded with mats to confine the heat. For a continuous business there should be at least 18 vessels of this kind, as the solution takes a long time to complete its crystallisation, by cooling to $30^{\circ} \mathrm{C}$. $\left(86^{\circ} \mathrm{F}\right.$.) The borax erystals are taken out with chisels, after the liquor has been drawn off and the whole has become cold.

One hundred parts of the purest acid, usually extracted from the lakes of Tuscany, contain only 50 parts of the real boracic acid, and yield no more, at the utmost, than 140 or 150 of good borax.

A considerable saving of expense in manufacturing borax, and a more ready application of the borax to use, has been proposed by Saulter, as follows:-Take about 38 parts of pure crystallised boracic acid, pounded and sifted; mix them well with 45 parts of crystals of carbonate of soda, in powder; expose the mixture upon wooden shelves to heat in a store-room; and rake it up from time to time. The boracic acid and the alkali thus get combined, while the carbonic acid and water are expelled; and a perfect dry borax is obtained.

Borax is an acid borate or biborate of soda, usually crystallised in oblique prisms, with 10 atoms of water. Under certain conditions it may also be obtained in octahedra, containing only half this proportion of water; and by application of heat the whole of the water may be expelled with intumescence. The composition of these three forms of the salt may be thus exhibited:-

Prismatic Borax (NaO, 2BO', 10HO).

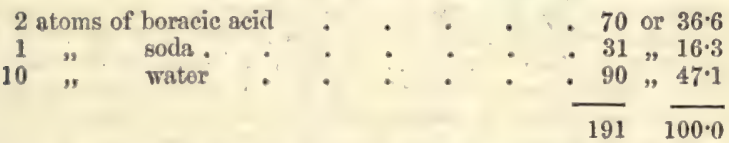

Octahedral Borax $\left(\mathrm{NaO}, 2 \mathrm{BO}^{3}, 5 \mathrm{HO}\right)$.

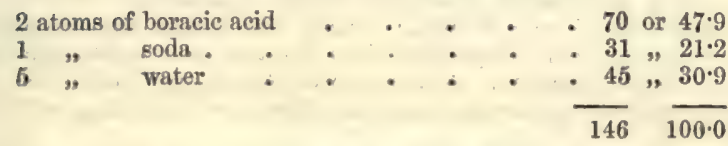

Anhydrous Borax $\left(\mathrm{NaO}, 2 \mathrm{BO}^{3}\right)$.

2 atoms of boracic acid

1 , soda.
- 70 or $69 \cdot 4$

- $31,30.6$

Borax has a sweetish, somewhat lixivial taste, and affects vegetable colours like an alkali ; it is soluble in 12 parts of cold, and in 2 parts of boiling water. It effloresces and becomes opaque in a dry atmosphere; it appears luminous, by friction, in the dark. It melts at a heat a little above that of boiling water, and gives out its water of crystallisation, after which it forms a spongy mass, called calcined borax. The octahedral borax, which is prepared by crystallisation, in a solution of 1.255 specific gravity, kept up at $145^{\circ} \mathrm{F}$., is not efflorescent. When borax is ignited, it fuses into a glassy-looking substance.

Dry borax acts on the metallic oxides, at a high temperature, in a very remarkable manner, melting and vitrifying thom into very beautiful coloured glasses. On this account it is a most usioful re-agent for the blowpipe. Oxide of chrome tinges it of an ememld green ; oxide of cobalt, an intense blue; oxide of copper, a pale green; oxide

Vor. I. 
of tin, opel ; oxide of iron, bottle green and yollow; oxide of manganese, violet; oxide of nickel, pale emerald groen. The white oxides impart no colour to it by themselves. In the fusion of metals, borax protects their surface from oxidisement, and eren dissolves awny any oxides formed upon them; by which twofold agency it becomes an excellent flux, invaluable to the goldsmith in soldering the precious metals, and to the brazier in soldering copper and iron.

Borax absorbs muriatic and sulphurous aeid gases, but no others, whereby it becomes, in this respect, a useful moans of analysis.

The strength or purity of borax may be tested by the quantity of sulphurie acid requisite to neutralise a given weight of it, as indicated by tineture of litmus.

When mixed witls shellac in the proportion of one part to five, borax renders that resinous body solublo in water, and forms with it a species of varnish.

The applications of borax in the manufacture of enamels, glazes, and of glass, will be noticed in the articles devoted to the consideration of those special industries.

BonING. Whether for the purpose of searching for coal or other minerals, or for obtaining water for the supply of towns or for land irrigation, the importance of boring as a branch of mining scionce is so important as to command careful consideration in a work of this description.

Under the head of Artesian WeLL, the various physical conditions under which water may be obtained by means of boreholes, have been described. It is now proposed to give an account of the different modes of prosecuting boroholes, and to refer to the purposes, other than the finding of water, for which the science of boring is resorted to.

It may be stated generally, that beyond the question of water supply, the boring of holes is chiefly carried out with the object of proving the existence, or otherwise, of rocks or minerals of more or less value. Whilst in putting down holes for the discovery of water, a simple hole, in a firm and durable condition, is all that is required, in the proving of minerals it is very important that the result of the borings should indicate very accurately the character and section of the strata passed through. The extent to which this end has been accomplished will be hereafter referred to.

Boring for water appears to have been in use from the earliest periods, in Egypt and in Asia. In many of the desert tracts there are remains of borings, which served, evidently at one period, to supply the wants of extensive populations which once inhabited those now deserted regions. In the 'Guide du Sondeur,' by M. J. Dégoussée, we find it stated, with reference to China: "There exists in the canton of Ou-TongKiao many thousand wells in a space of ten leagues long by five broad. These wells cost a thousand and some hundred taëls (the taël being of the value of $68.6 d$.), and are from 1,500 to 1,800 feet deep, and about 6 inches in diameter. To bore these wells, the Chinese commence by placing in the earth a wooden tube of 3 or 4 inches diameter, surmounted by a stone edge, pierced by an orifice of 5 or 6 inches ; in the tube a trepan is allowed to play, weighing 300 or $400 \mathrm{lbs}$. A man mounted on a scaffold, swings a block, which raises the trepan 2 feet high, and lets it fall by its own weight. The trepan is secured to the swing-lever by a cord made of reeds, to which is attached a triangle of wood; a man sits close to the cord, and at each rise of the swing seizes the triangle and gives it a half turn, so that the trepan may take in falling another direction. A change of workmen goes on day and night, and with this continuous labour they are sometimes three years in boring wells to the requisite depth.'

Hand boring. - The surface arrangements usually required for boring by hand, are shown by fig. 175. In ordinary practice, a well is first sunk of such a depth that the boring apparatus can be fixed in it; and thus a stage, raised from the surface of the ground, is dispensed with. A stout plank floor, well braced together by planks nailed transversely, and resting on putlocks, forms the stage. In the centre of the floor is a square hole, through which the boring rods pass. The plant required consists of a

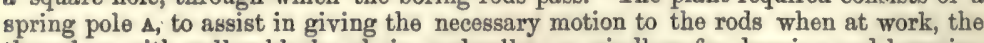
three legs with pulley blocks, chain, and roller, or windlass for drawing and lowering the rods, and the several lengths of rods required, with the various chisels, pumps, \&c.

The borehole is usually commenced by digging a small pit about 6 feet deep, and over this is set up the three legs, with pulley, \&c. A few feet of iron tubing aro also sometimes inserted at the commencement, to protect the sides of the borehole.

The boring rods are usually from 10 to 20 feet long. The chisel is first insorted, then rods added as the work progresses. At the top of the rods are attached two handles about $4^{\prime} 0^{\prime \prime}$ long, placed at right angles to ench other. By means of these the borers work the rods up and down, at the same time giving them a circular motion in order to alter the position of the chisel at each stroke.

As the depth increases, the men at the handles or cross-bar are assisted by means 
of a pole or lever at the surface; one end being firmly fixed in the ground between two posts, the other being allowed to pass over the hole. From the end of this pole the bore-rods are attached by means of a chain; thus every time the borers strike the chisel, the lever enables them to lift the rods high enough to give the necessary impetus to the rods for the next blow. The chisel is occasionally withdrawn, and a long bucket with a hinged valve at the bottom opening upwards, is attached to the rods, and lowered into the hole. The borers press this down upon the material broken up by the chisel, so that a quantity of débris becomes enclosed in the bucket, and is drawn up to the surface. This process is repeated until the hole is made clear, and ready again for the chisel.

The rods are drawn by means of a windlass attached to the three legs, and are unscrowed at every second joint, until the whole of them are drawn out. They have to lue again screwed together when they have to be lowered.

\section{5}

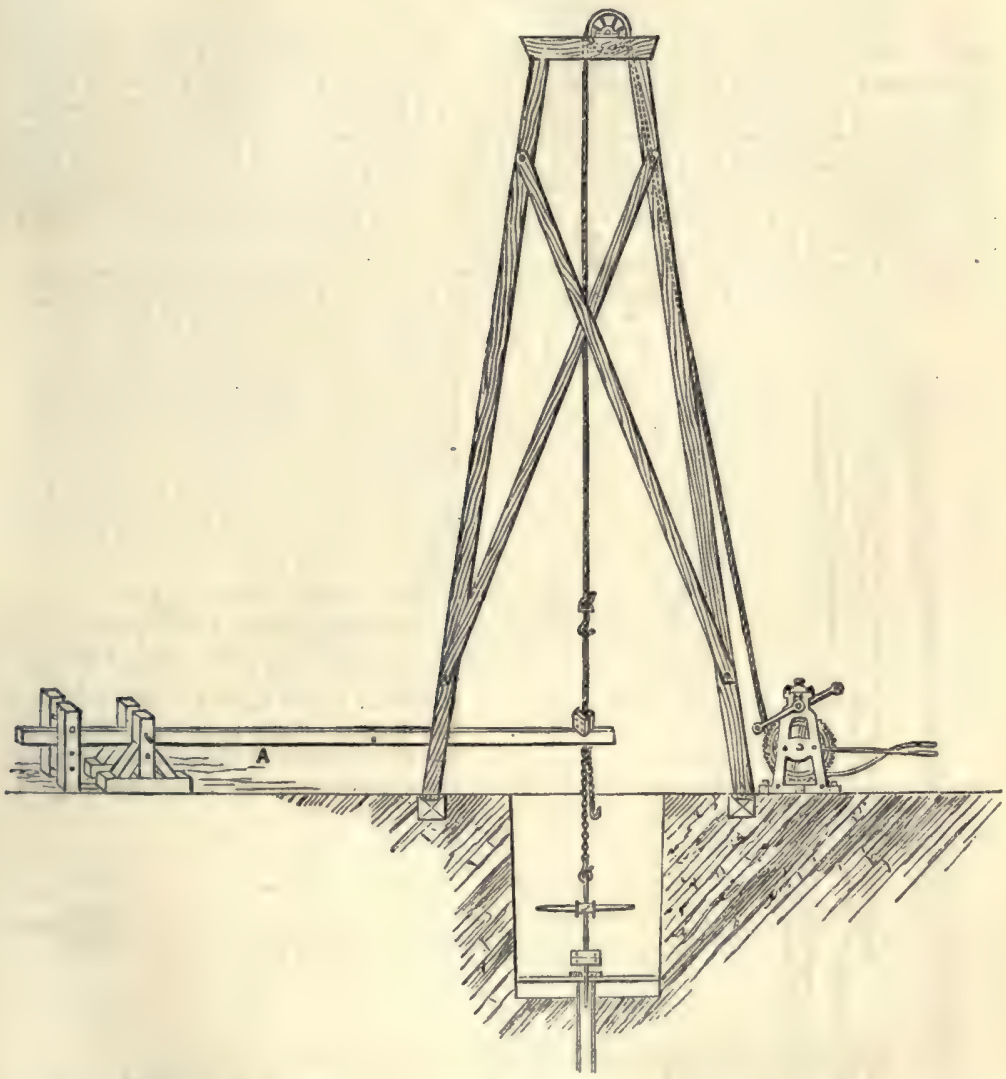

This style of boring is limited to a small depth; the weight of boring rods becomes so great with an increasing depth, that the borers, even when assisted by a lever, are unable to lift them. A depth of about 300 feet may be safely bored in this way.

The nature of strata bored through is ascertained from the material brought up by the bucket; and the borers having constantly hold of the cross-bar, any change of strata is at once indieated by the stroke of the chisel against the various beds met with, being imparted to the hands of the men, who aequire by experience great delicacy of touch. A now description of spring has lately been used with the handboring machine. This consists of several layers of india-rubber, about 1 inch square, and 2 feet 6 inches long, increasing the number of layers as the weight of rods becomes greater. Each end of the india-rubber is fixed in strong iron clamps, one 
attached by $n$ chain to the three legs, and the other coupled to the top of the rods; the elasticity of the india-rubber is thus brought to play upon the motion of the rods.

Horizontal Boring by Hand.-For boring in the sides of mountains, quarries, or other places where long horizontal holes are required, the machine illustrated by fig. 176, is sometimes used. The rods, chisels, pumps, \&c., are all of the same doseription as those used for vertical boring, except that they are of somewhat lighter

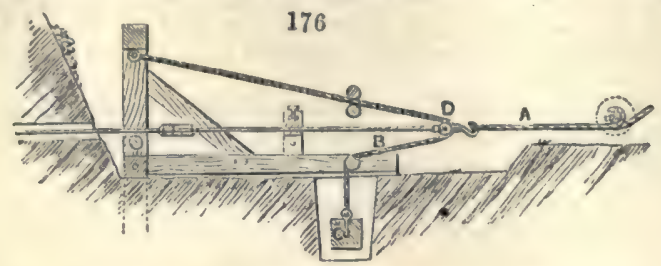

make. The rods are drawn out of the hole by means of the rope $\Delta$, the weight $\mathrm{c}$, which hangs in a small pit suspended by the rope $\mathbf{B}$, being raised at the same time. The rope $\Delta$ is then slipped, and the falling weight $c$ drives the rods into the hole again. The rods are kept steady and horizontal by being caused to run over a small rollor fixed on the frame, and also by moving in a slide block, adjusted by a screw and nut.

After using the chisel for a short time, the rods are withdrawn, and the pump or seourer introduced to clear away the débris, the other work boing carried on as with ordinary vertical hand-boring.

Boring by Steam Power.-Where boreholes are required of any considerable depth, and where speod is of importance, hand-labour has been superseded in recent years

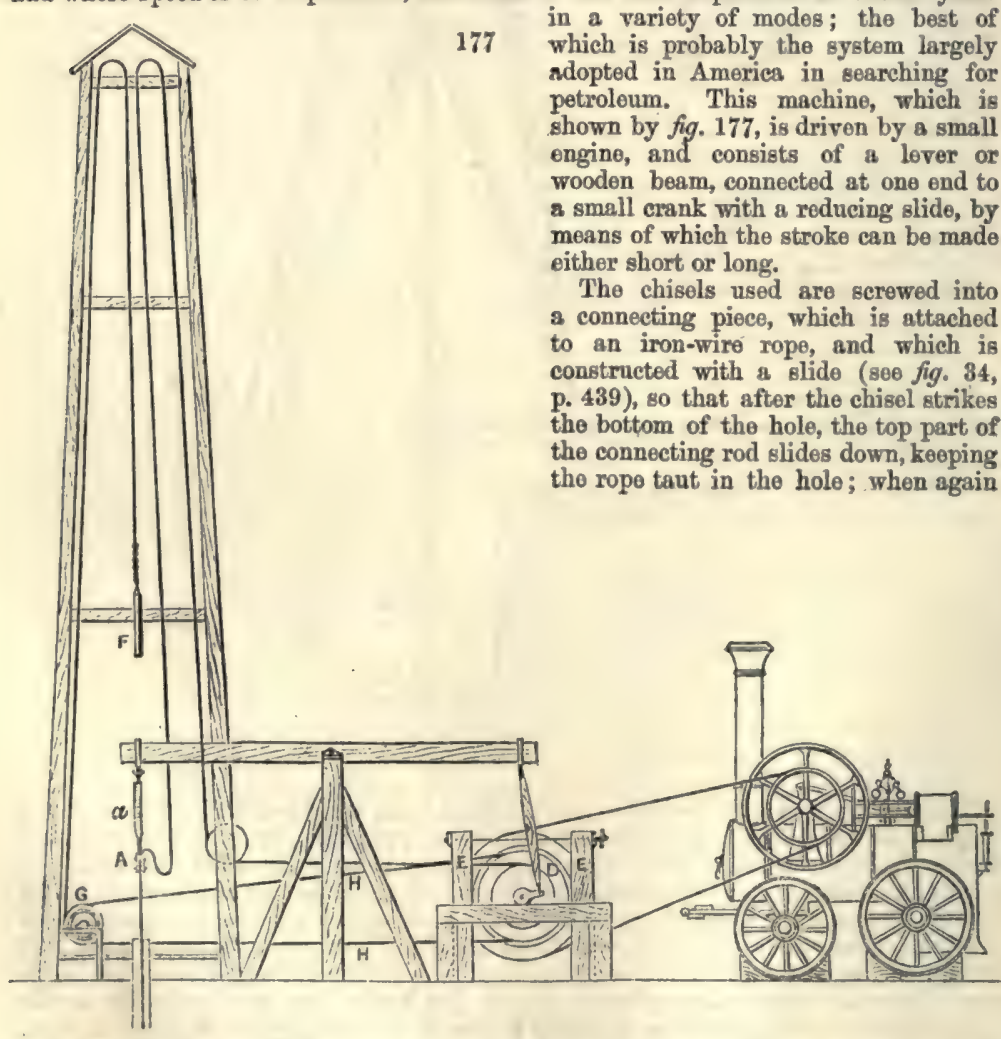

liftod np, it rises until it eatches the part to which the chisel is connected. This apparatus prevents the rope from becoming slack in the hole when the tool falls. 
The chisels omployed are of the ordinary description : whilst by hand boring, holes of not more than a fow inches diameter can be put down, with this apparatus holes of any diameter to 18 inches can be bored without any difficulty.

The rope is attached to the connecting piece by the clips, and is passed at the top of the hole through strong iron clamps, $\mathbf{A}$, over which it hangs loosely to allow for the upward and downward motion of the boring-tools. The iron clamps are also connected to a screw and slide, $a$, fig. 177, hanging from the end of the wood lever or beam, this screw being used to adjust the length of the rope after it has been attached securely into the iron clamp.

The rope is drawn up by a small drum, $D$, driven from the same shaft as the crank used for working the beam; a brake being attached to the drum for the purpose of lowering the chisel and connections into the bottom of the hole. This brake consists of two pieces of timber, $\mathbf{E} \mathrm{E}$, which are drawn together by means of a rod and screw.

After the chisel has been at work for a short time, it is drawn up by means of the drum $\mathrm{b}$, and the pump $\mathrm{F}$ is then introduced, being lowered by the small drum $\mathrm{G}$, which has also a brake attached. This pump is worked up and down a few times, then drawn up by the wire rope $\mathrm{H}$, driven by the wheel on the main driving shaft.

The engine used is an ordinary portable engine, with a cylinder 9 inches in diameter.

The boring rope is usually $\frac{3}{4}$ inch in diameter, and is covered with tarred hemp rope for protection.

The depth from the surface, and thickness of the various strata passed through is ascertained by observing the wire rope, and by carefully noting the nature of the samples drawn up by the steel pump.

In boring with this machine, a small hole is sometimes kept in advance a distance of about 4 feet, the sides of the hole being removed by the larger chisel, and the pieces thus broken off are brought up by the pump, and kept in sample boxes.

The average rate of boring varies from 10 to 20 feet per day of twenty-four hours. It is usual, whether in boring by hand or steam power, to line the hole from the surface down to the solid strata with tubes; and should any loose strata, as sand, be afterwards met with, tubes of smaller diameter than those first introduced are put down, this necessarily causing a contraction of the hole, and also of the tools.
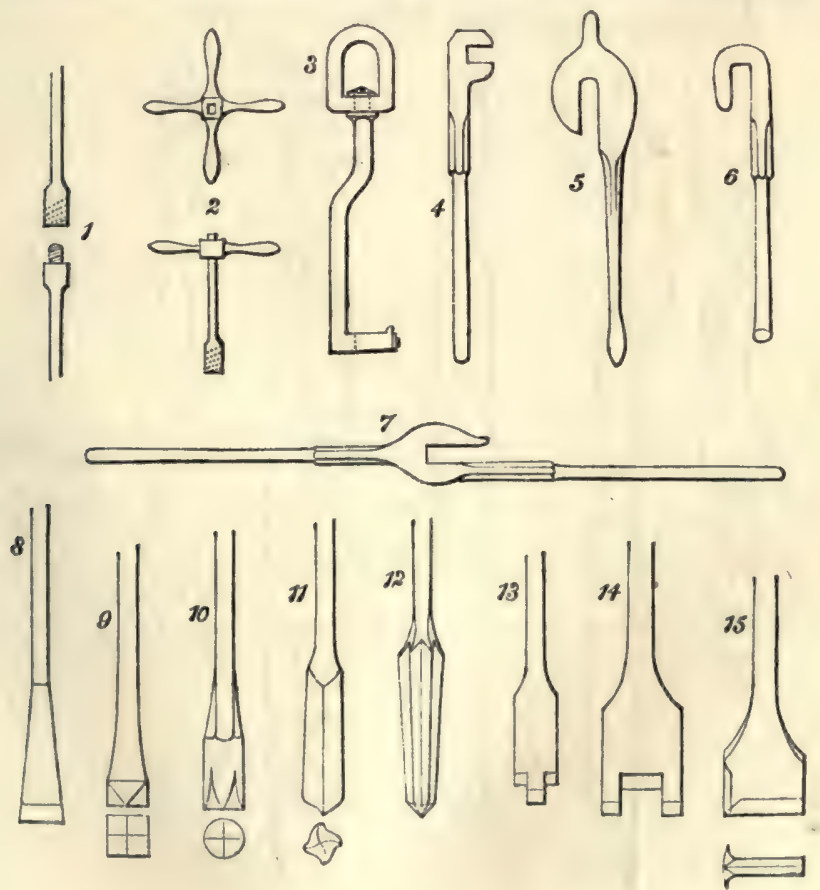

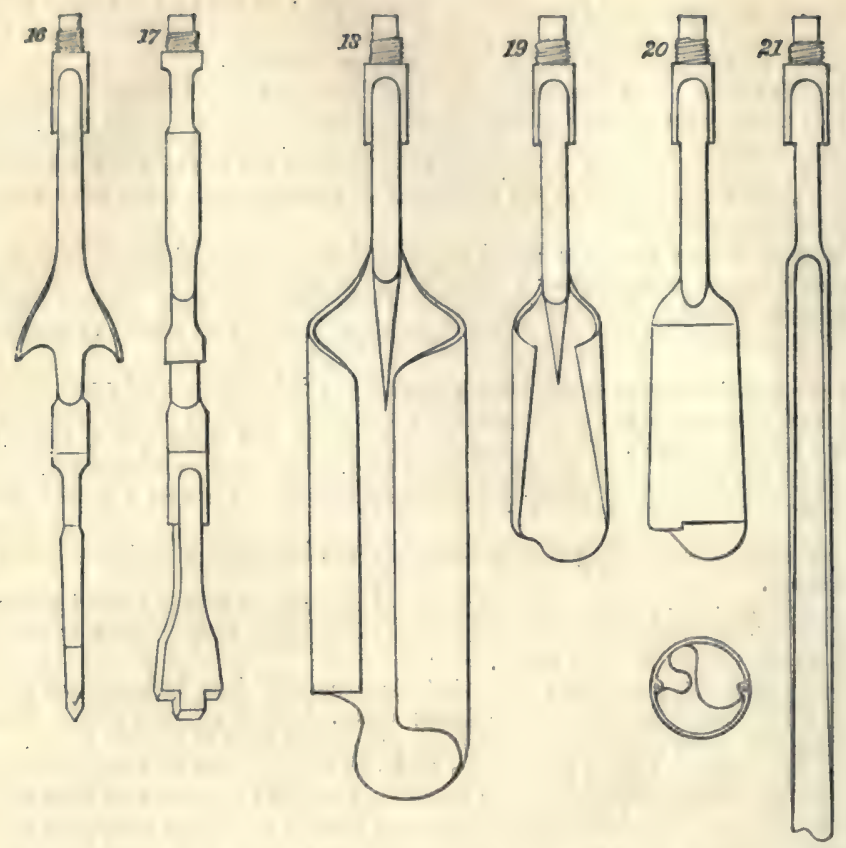

A description may now be given of the various tools used in the two systems of boring referred to:-

Fig. $\mathbf{I}$ is the joint used for attaching the boring rods together.

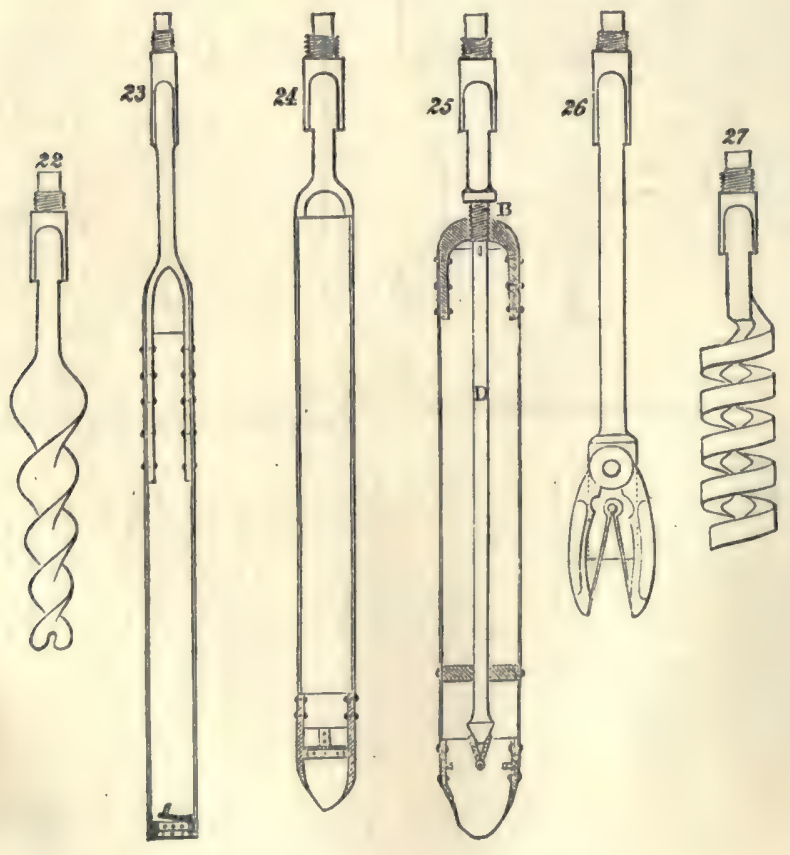


Fig. 2 is the brace-head, or cross-head, with the four handles held by tho borers. Fig. 3 is a catch for raising the rods from the borehole.

Figs. 4, 5, 6, and 7 , are spanners, used for screwing the rods together, and holding them steady at the top of the borehole. The shoulders of the rods rest between the claws.

Fig. 8 is the ordinary chisel used for boring.

Figs. 9, 10, 11, and 12, are various forms of chisels used for making the hole cylindrical, and for breaking up hard fragments of rock at the bottom of the hole.

Figs. 13 and 14 are also tools used for the latter purpose.

Figs. 15, 16, and 17, are chisels used for breaking off projections from the sides of the hole : figs. 16 and 17 showing two views of the same tool.
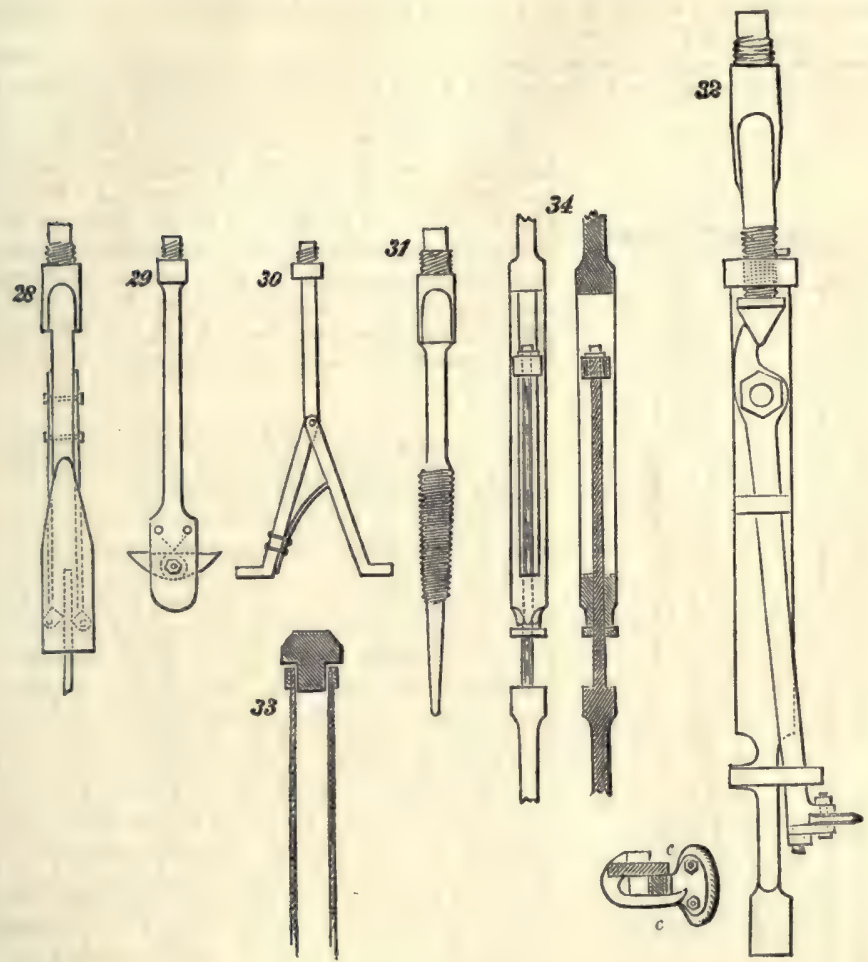

The implements used in extracting débris from boroholes are as follow:-

Figs. 18, I9, 20, and 21, are augers or wimbles, used for bringing up argillaceous strata.

Fig. 22 is an auger for boring through clay, generally used in commencing the borehole.

Figs. 23 and 24 are pumps or scourers, for bringing up the débris broken up by the chisels.

Fig. 25 is a scourer with valves, which are closed by turning the rods round, the action of the serew $\mathrm{B}$ causing the part $\mathbf{D}$ to descend.

Fig. 26 is an arrangement of pincers for extracting picces of rock which are too large to enter the pump.

The instruments employed for rectifying accidents are as follow :-

Fig. 27 is a special'screw for drawing out broken rods or tools.

Fig. 28 is a spring catch for extracting broken rods.

Figs. 29 and 30 are catches used for extracting broken lining tubes. One of the branches of the appliance shown by fig. 30 is fixed, and the other moveable.

Fig. $3 \mathbf{I}$ is a serew tap, also used for extracting tubes. 
Fig. 32 is a tonl used for cutting lining tubes when it is found that they cannot bo drawn out all at once. $c$ exhibits a plan of the cuttor.

Fig. 33 is a plug used for driving down lining tubes.

In some cases it is required after the tubes have been inserted, to recommence boring the hole, and make it of the same size as the outside diameter of the tubes. To recomplish this object several ingenious devices have beon designed.

In most of these the chisel intended to form the large diameter of the holo is passed down through the tube flush with the sides of the tool, and when reaching the bottom of the tube it is brought into position in several different ways. In one case, a serew whon turned, forces out the cutters. In another, the chisels are pushed out by springs.

Another mode is to attach to the moveable chisels a dry hemp cord, the contraction of which when soaked in water draws up the chisels.

In boreholes of a considerable diameter, a 'free falling ' arrangement, as shown by figs. 35 and 36 , is sometimes adopted. $\mathbf{A} \mathbf{A}$ are pins, holding in a fixed position two

35

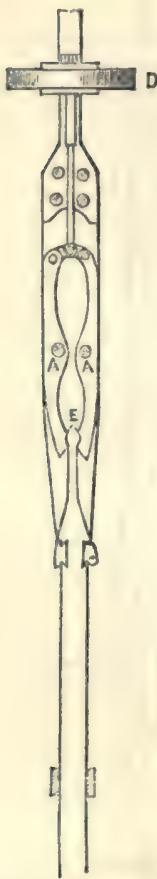

36

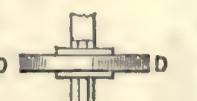
long clutches, which hold the end of the boring rods at 1 . The disc $D$ is of the same diameter as the hole being put down. When the sliding arms reach the bottom of the stroke, the arrow-head $\mathrm{E}$ is caught, and is drawn up to the top of the hole, as shown by fig. 35. Immediately the down stroke commences, the resistance against the disc, caused by the water standing in the borehole, causes it to open the clutches and disengage the tool. It will be seen that this apparatus is only applicable where a column of water is present.

Having now described the tools used in the ordinary modes of Boring, attention may be drawn to various modern improvements in this science.

A method of boring with hollow rods, combined with a force-pump, was introduced about the year 1846 by M. Fauvelle, in order to obviate the necessity of using the shell, the detritus produced by the tool being removed continuously by a downward current of water forced by the pump through the interior of the rod, and rising in the annular included spaee between the exterior of the rod and the lining tube. In spite of the apparent advantages of this method, namely, the maintenance of a clear face of rock for the cutting tool to work on, and the saring of time due to the abolition of the shell, and the adoption of the method of continuous discharge, it does not appear to have come much into use. Recently, however, a modification in which the discharge of the detrition is effected by an upward current of water through the rod, has been employed to advantage in borings for petroleum in Western Canada.

Trauvelle's system of boring is probably the most simple in existence, and has the power of boring at a quicker speed than other machines. A short description of its mode of working may therefore be desirable.

The machine is driven by engine-power, in the manner shown in fig. 178, p. 441.

The boring rods consist of ordinary steam tubing, 2 inches diameter, and jointed together by union boxes. The chisels are welded into short lengths of tubing, small holes being left at the bottom of the tube. Water is forced in at the top of the borerods by means of a small force-pump, worked by a crank on a pinion-wheel, which is connected to a larger pinion on the main shaft. The water from this pump is first driven into an air-cliamber, and is taken thence by india-rubber tubing to the top of the borerods, from which it escapes at the bottom of the rods a few inches above the chisel point. It is forced thence to the surface, carrying with it all the débris cut up by the chisel.

This débris is caught in pots or settling troughs, and from an examination of these the nature of the strata passed through is ascertained.

Independently of this test, however, the man in charge of the machine constantly has his hands upon the rods, and in this way can tell by the character of the stroke any change of strata, which is at once marked upon the rods. 
The boring rods aro allowed to have a percussive fall ; and are connected by iron clamps to the rope, which passing over a wheel fixed in the shear legs, is fastened to the lover $\mathrm{D}$. An up-and-down motion is imparted to this by the wheel $\mathrm{E}$, which has two blocks or cams fixed in its periphery.

The action of these cams gives two full strokes of the rods for every revolution of the wheel $\mathrm{k}$.

The stroke at commencing a borchole is about 1 foot 3 inches, but as the depth

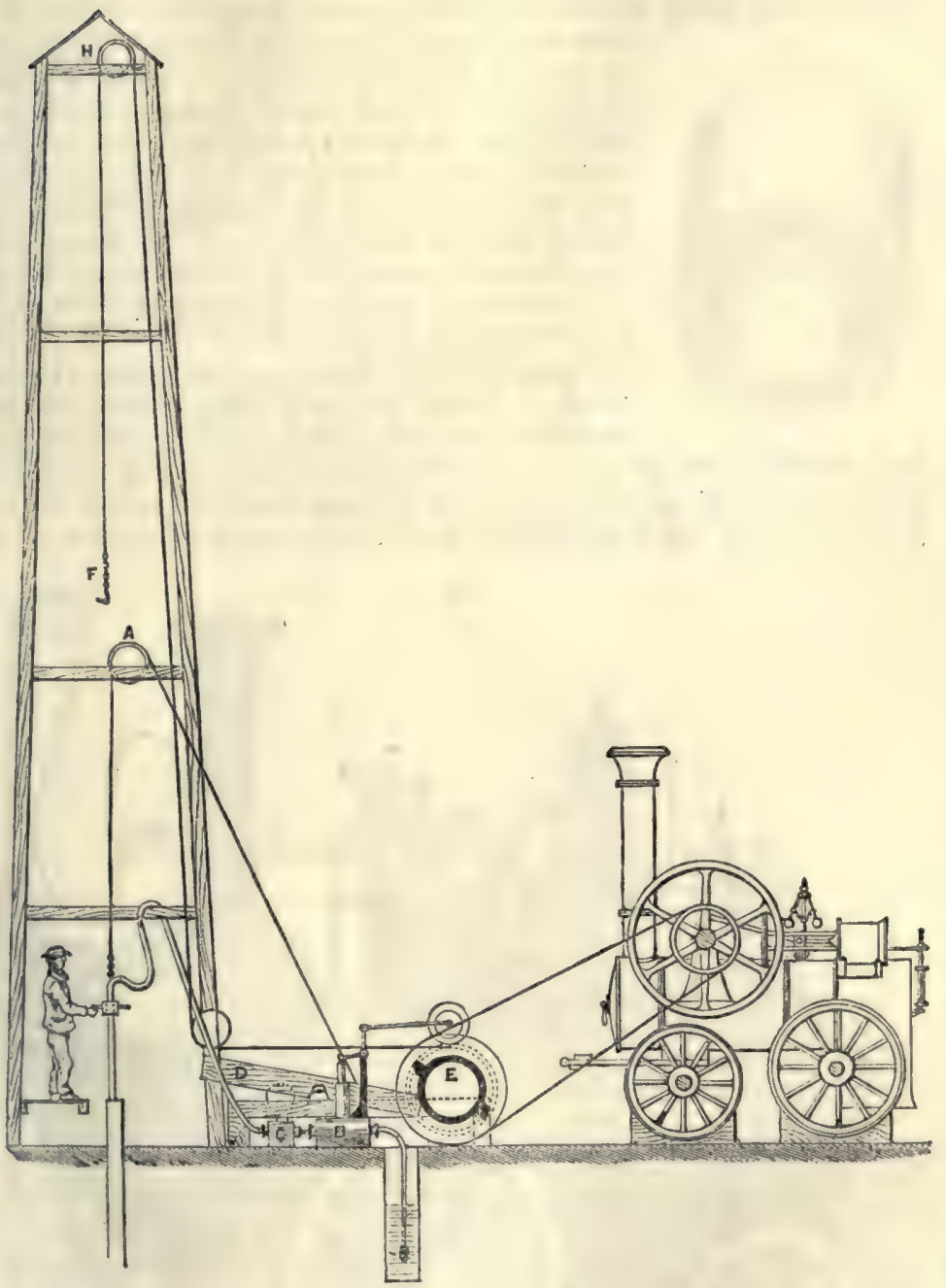

inereases, the stroke is shortened by attaching the rope nearer to the centre of the lover.

At a depth of 800 feet this stroke would be not more than a few inches, the weight of rods being sufficient to drive the chisel forward, with a very short stroke.

When the rods have to be drawn for changing the chisel they are attached to the hook $\mathrm{F}$, and are drawn up by means of a small drum on the main shaft to the height of the small wheel ir; the first length is then unscrewed and put on one side, the hook F 
being again lowered, connected to the rods and drawn as above until the whole of the rods are drawn out. There is a brake attached to the drum on the main shaft, which is used for lowering the rods into the hole.

In cases where the strata passed through are of a soft character, tubes are sometimes driven forward as the hole progresses, these at first being about $4 \frac{1}{2}$ inches diameter and faced with steel. They are driven forward by a large iron weight, and should the first pipe meet with anything that it cannot drive through, smaller sized tubes are introduced.

The chisel used is about $\frac{1}{8}$ th of an inch smaller than the lining tubes.

This machine has bored in ehalk a depth of 76 feet in 12 hours.

The Diamond Boring Machine.-In all the modes of boring described above, the

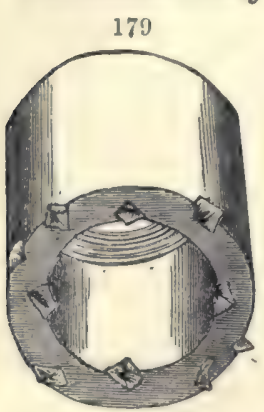
borer or drill acts by percussive impact against the face of the strata, producing a volume of powdered rock equal to the solid contents of the borehole. In the diamond machine, invented by M. Leschot, the cutting tool is of a tubular form and receives a uniform rotating motion; the result being the production of a cylindrical core from the rock of the same size as the inner periphery of the tube, showing to great depths the different stratification through which the boring tube passes. The general principles on which the machine is construeted are as follow:-

A continuously revolving and progressing boring head, having projecting diamond points, works in combination with a tubular boring bar.

The boring bit (fig. 179, is a steel thimble, about 4 inches in length, having two rows of Brazilian blaek diamonds (such as are used by lapidaries) in their natural rough state firmly imbedded therein, the edges projecting slightly from the outer and inner peripheries respectively. The diamonds in the centre row cut the path of the drill in its forward progress, while those npon the outer and inner periphery of the tool enlarge the cavity around the same and admit the free egress and ingress of the

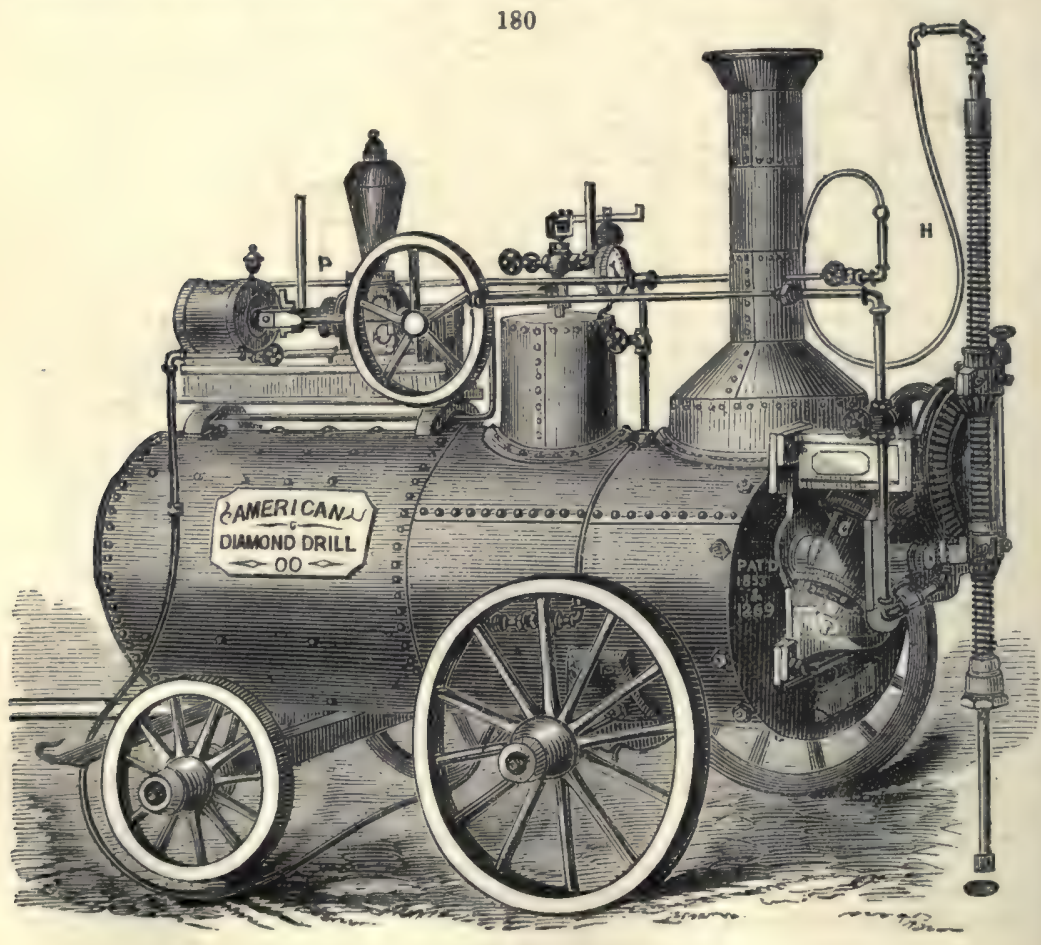


water as hereafter described. As the drill passes into the rock, cutting an annular channel, the portion of stone, encircled by this channel is, when of a compact nature, undisturbed, and the drill rod passing down over it preserves it intact until the solid cylindrical core thus formed is withdrawn with the rods.

The diamonds are placed at intervals of about $\frac{1}{3}$ rd of an inch apart both inside and outside the tube, the projection beyond the surface of the metal being not more than $\frac{1}{30}$ th of an inch.

The diamond teeth are the only parts of the tool which come in contact with the rock, and their hardness is such that more than 2,000 feet have been drilled by the same points with but little appreciable wear.

The drill rods are made hollow, in order that a volume of water may be conducted

181

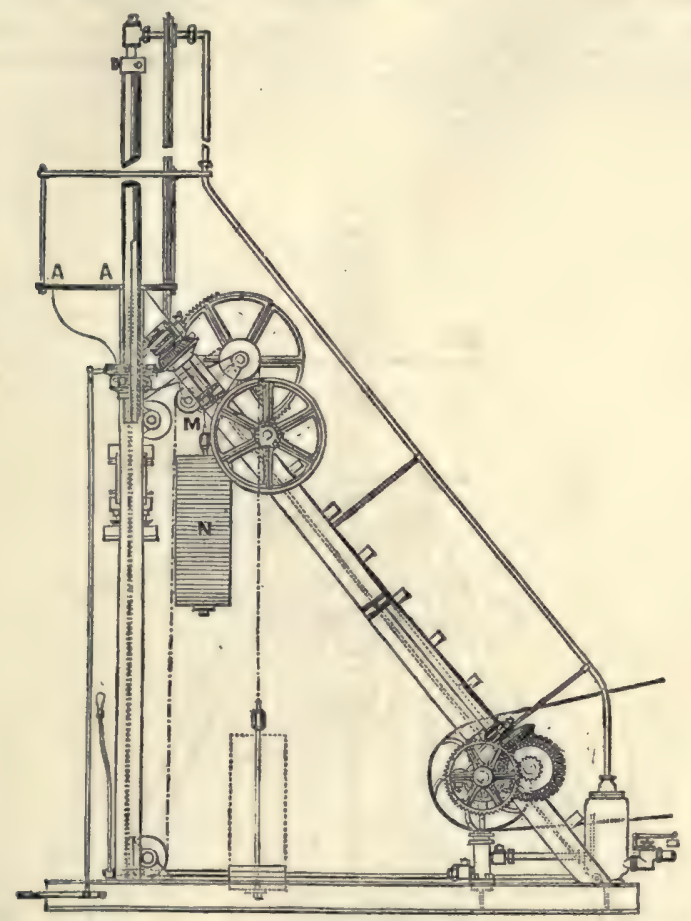

to the boring bit, at once moistening the rock, washing the cuttings away, and preventing the over heating of the diamonds.

The machinery for boring great depths is shown by fig. $180, \mathrm{p} .442$, and consists of a portable engine having two oscillating 6 -inch cylinders with a 6 -inch stroke. The boiler is tubular, $3 \frac{1}{2}$ feet $\times 7$ feet, and with flues 3 inches in diameter, the steam capacity being about 16 H. P.

The pump $\mathbf{P}$ and water hose $\mathrm{H}$ are connected by rubber hose with any convenient stream or reservoir of water, and also with the outer end of the drill pipe by similar hose having a swivel joint. Through this hose a stream of water is forced, by M. Fauvelle's system, into the hollow drill rod, from which it only escapes at the bottom of the diamond pointed bit.

The drill rods may be extended to any desirable length by simply adding fresh pieces of tubing. The successive lengths are quickly coupled togethor by an inside coupling 4 inches long, the drill being held firmly in its place by the chuck $\mathrm{a}$ at tho bottom of the screw shaft.

The speed of drilling depends chiefly of course upon the character of rock met with: in ordinary rock the drill being fed at the rate of 300 revolutions to the inchthe diamonds cutting the one three-hundredth part of an inch at each revolution, and in marble, hard sandstone, \&c., at the rate of an inch for every 200 revolutions.

The progress of the diamond bit or crown does not depend upon the pressure of the 
rods, which is arranged to be always the same. The diamonds work their way by steady and gentle attrition.

These machines will bore holes from one to three inches in diameter.

The advance per minute in pure Mount Cenis quartz equalled $2 \frac{1}{2}$ inches, in granite about 2 inches, and in very hard calcareous dolomito 3 inches.

The diamond machine shown by figs. 181 and 182 has lately been perfected by Messrs. Appleby and Beaumont.

The boring bit with diamonds set therein is exactly similar to that used in the machine described, but the boring machinery is of a different description. This machine is worked by an independent engine, and driven by leather or other belts, the circular motion given to the boring rods being conveyed by bevel gear. To this machine appliances are attached for the driving forward of the lining tubes. This is done by means of a series of weights $\mathbf{N}$ attached to a chain passing round the wheel $\mathbf{M}$, and connected to the sliding clutch $g$; this is made to press upon the tubes. At the same time an ordinary chisel is attached to the boring rods and worked from the table $\Delta \mathbf{A}$, the rods passing down the centre of the tubes.

182

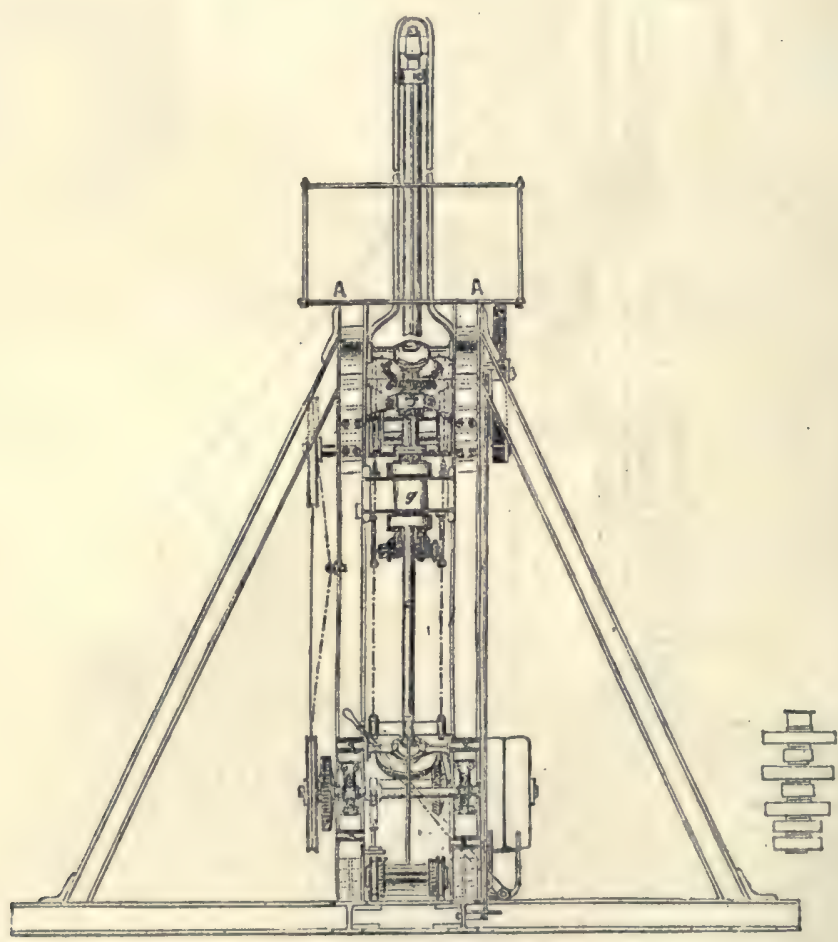

The debris is washed out at times by means of the pump fixed upon the machine.

After the lining tubes are made secure and water tight (it being an essential point that all water fored down the boring rods should come up on the inside of the lining tubes, so that all washings or indications of change of strata may be at once noticed), the diamond drill is introduced and boring proceeded with in the usual manner.

The rods aro drawn by means of a chain passing over shear legs about 45 feet high and wound upon a drum fixed on the machine. The rods aro unscrewed in lengths, and placed on one side of the machine.

The rods, by this system, are seldom drawn, except for the purpose of extracting the core made; the core tube being usually about $14^{\prime} 0^{\prime \prime}$ long, with a small spring at the bottom for the purpose of retaining the core while drawing the rods. 'The cores made in these machines are usually from 1 inch to $1 \frac{1}{2}$ inch in diamotor. 
The boring rods are of the best steel, and jointed together by unions or coupling boxes.

In soft strata it is somewhat difficult to obtain a core by the diamond borer, but in boring through hard rocks for which this machine is specially suitable a very perfect core is produced. The best average speed accomplished by this machine in hard rock equals abont 50 feet per day of 12 hours.

Mather and Platt's System of Boring.-The Chinese method of boring with ropes, instead of rigid rods, has been tried at different times in Europe, but without any great amount of success; the system of rigid suspension being preferred for working, not only the cutting tool, but also the shell. A very important modification of this principle has, however been introduced by Messrs. Mathor and Platt of Salford, who have succeeded in carrying bores of considerable size ( 8 or 10 inches in diameter) to depths of 1,200 feet and upwards.

In this system, instead of the implements being attached to rods, as in the previous systems, they are suspended by a flat hemp rope about $\frac{1}{2}$ inch thick and $4 \frac{1}{2}$ inches broad, such as is commonly used at collieries, and the boring tool and shell pump are raised and lowered as quickly in the borehole as the cages in a colliery shaft.

The flat rope $A \Delta, f i g .183$, from which the boring head B is suspended, is wound upon

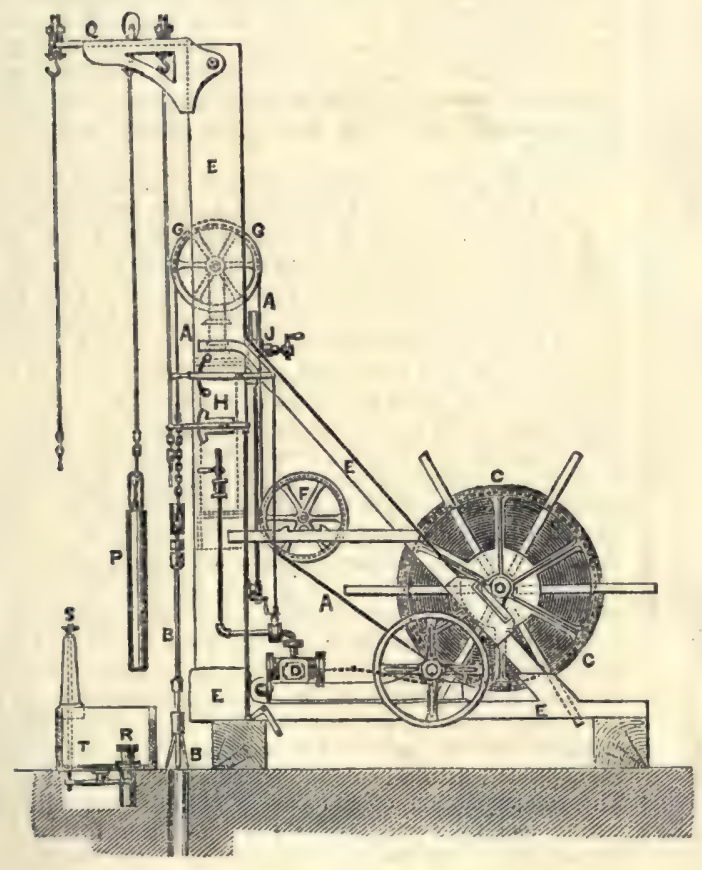

a large drum $\mathbf{C}$, driven by a steam-engine $\mathbf{D}$ with a reversing motion, so that one man can regulate the operation with the greatest ease. All the working parts are fitted into a wood or iron framing $\mathrm{E}$, rendering the whole a compact and complete machine. On leaving the drum $\mathbf{c}$, the rope passes under a guide pulley $\mathbf{F}$, and then over a large pulley $\mathrm{e}$, carried in a fork at the top of the piston-rod of a vertical single-acting steam-cylindor $\mathbf{H}$.

This cylinder, by which the percussive action of the boring head is produced, is fitted with a piston of 15 inches diameter, having a heavy east iron rod 7 inches square, which is made with a fork at the top carrying a flanged pulley.

The boring head having been lowered by the winding drum to the bottom of the borehole, the rope is fixed securely at that length by the clamp J; steam is then admitted underneath the piston in the cylinder $\mathbf{H}$ by the steam-valve, and the boring tool is lifted by the ascent of the piston-rod and pulley $\sigma$; and on arriving at the top 
of the stroke, the exhaust valve is opened for the steam to escape, allowing tho piston-rod and carrying pulley to fall frooly with the boring tool, which falls with its full weight to the bottom of the borehole. The exhaust port is 6 inches above the bottom of the cylinder, while the steam port is situated at the bottom; and there is thus ulways an elastic cushion of stenm retained in the cylinder of that thickness for the piston to fall upon, preventing tho piston from striking the bottom of the cylinder. The steam and exhaust valves are worked with a self-acting motion by tappets, which are actuated by the movement of the piston-rod; and a rapid succession of blows is thus given by the boring tool on the bottom of the borehole. As it is necessary that motion should be given to the piston before the valves can be acted upon, a small jet of steam is allowed to be constantly blowing into the bottom of the cylinder; this causes the piston to move slowly at first, so as to take up

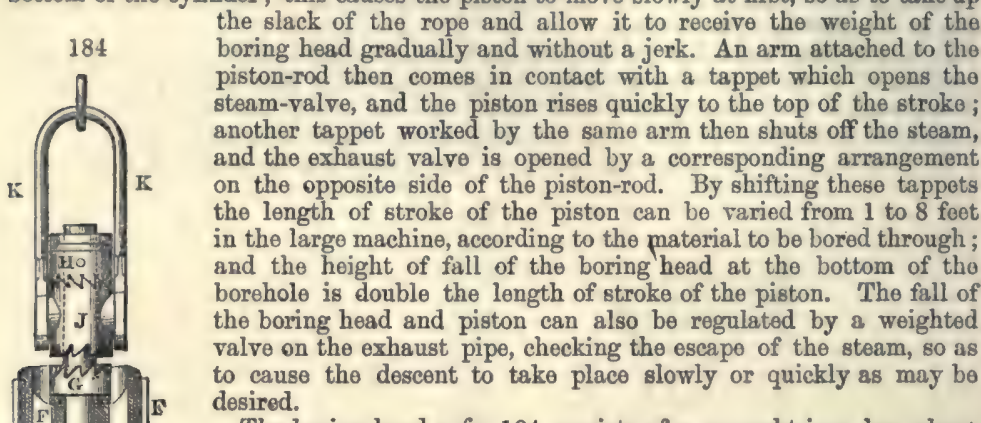

The boring head в fig. 184 consists of a wrought-iron bar about 4 inches diameter and 8 feet long, to the bottom of which a castiron cylindrical block $\mathbf{c}$ is secured. This block has numerous square holes through it, into which the chisels or cutters are inserted with taper shanks so as to be very firm when working. but to be readily taken out for repairing and sharpening. A little above the block $\mathrm{c}$ another cylindrical casting $\mathbf{E}$ is fixed upon the bar B, which acts simply as a guide to keep the bar perpendicular. Higher still is fixed a second guide $F$. To effect the rotation of the boring tool, two cast-iron collars $\mathrm{G}$ and $\mathrm{H}$ are cottered fast to the top of the bar $B$, and placed about 12 inches apart. The upper face of the lower collar $\theta$ is formed with deep ratchet-teeth of about two inches pitch, and the under face of the top collar $\mathrm{H}$ is formed with similar ratchet-teoth set exactly in line with those on the lower collar. Between these collars and sliding froely on the neck of the boring bar в is a deop bush J, which is also formed of corresponding ratchet-teeth on both its upper and lowor faces ; but the teeth on the upper face are set half a tooth in advance of those on the lower face, so that the perpendicular side of each tooth on the upper face of the bush is directly above the centre of the inclined side of a tooth on the lower face. To this bush is attached the wrought-iron bow $\mathrm{K}$ by which the whole boring bar is suspended with a hook and shackle from the end of the flat rope. The rotary motion of the bar is obtained as follows: when the boring tool falls and strikes the blow, the lifting bush J, which during the lifting has been engaged with the ratchet-teeth of the top collar $\mathrm{H}$, falls upon those of the bottom collar $\mathrm{G}$, and thereby receives a twist backwards through the space of half a tooth; and on commencing to lift again, the bush rising up against the ratchet-teeth of the top collar $\mathrm{z}$ receives a further twist backwards through half a tooth. The flat rope is thus twisted backwards to the extent of one tooth of the ratchet; and during the lifting of $\nu D=\sqrt{ }$ the tool it untwists itself again, thereby rotating the boring tool forwards through
that extent of twist between each successive blow of the tool. The amount of the rotation may be varied by making the ratchet-teeth of coarser or finer pitch. The motion is entirely self-acting, and the rotary movement of the boring tool is ensured with mechanical accuracy. This simple and most effective action taking place at every blow of the tool produces a constant change in the position of the cutters, thus inereasing their effect in breaking the rock.

The Shell-Pump, for raising the material broken up by the boring head, is shown 
in fig. 185, and consists of a cylindrical shell or barrel p of cast-iron, about 8 feet long and a little smallor in diameter than the size of the borehole. At the bottom is a elack A oponing upwards, somewhat similar to that in ordinary pumps; but its seating, instead of being fastened to the cylinder $P$, is in an annular frame $c$, which is held up against the bottom of the cylinder by a rod $\mathrm{D}$ passing up to a wrought-iron bridge $\mathrm{z}$ at the top, where it is secured by a cotter $F$. Inside the cylinder works a bucket $\mathrm{B}$, similar to that of a common lift-pump, having an india-rubber dise valve on the top side; and the rod $\mathbf{D}$ of the bottom elack passes freely through the bucket. The rod G of the bucket itself is formed like a long link in a chain, and by this link the pump is suspended. The bottom clack $\Delta$ is made with an india-rubber dise which opens sufficiently to allow the water and smaller particles of stone to enter the cylinder; and in order to enable the pieces of broken rock to be brought up as large as possible, the entire clack is free to rise bodily about 6 inches from the annular frame $c$, as shown in fig. 185, thereby affording ample space for large pieces of rock to enter the eylinder when drawn in by the upstroke of the bucket.

The general working of the boring machine is as follows:The boring head is hooked on to the end of the rope, and lowered to the bottom of the borehole, the rope is then secured by a clamp to prevent it from being drawn off the winding drum. Steam is admitted to the percussion cylinder, and the boring bar kept at work until it has broken up a certain quantity of material in the borehole. After this operation, the steam is shut off, the rope unclamped, and the boring head withdrawn. The shell-pump is next lowered down by the rope, and the débris pumped into it by lowering and raising the buckets in the pump about three times, by means of the winding engine, while the pump barrel rests upon the bottom of the borehole. These operations are repeated every fifteen or twenty minutes.

Three men are required to work the machine and sharpen the implements.

Cost of Boring.- It will be understood that the advantage of putting down boreholes, as compared with wells or shafts of large diameter, consists not only in the general convenience in the small size, but also in the relative cost, which may be said to vary according to the area of the perforation, but in an uneven ratio.

The cost of boring shallow holes is naturally much less per foot than the rate of charge for borings at a considerable depth. The general cost of boring by hand or by lever may bo taken as follows :-

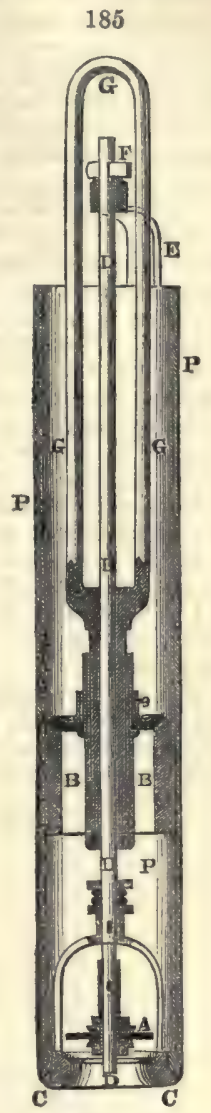

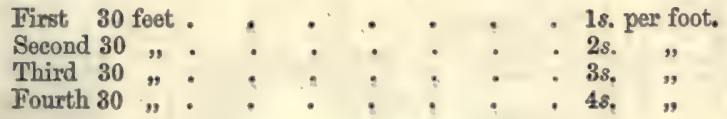

And so on, adding 18. per foot for each additional 30 feet.

In very hard strata the cost of boring may be taken at 50 per cent. more than the above prices.

The cost of boring by the 'Diamond' machinery amounts to no less than 13s. $6 d$. per font for the first 100 feet, and for each additional 100 feet 7s. $6 d$. per foot is added. The 'Diamond' machinery, however, is capable in hard strata in a day of twelve hours of boring a distance of 50 feet, whereas by hand boring, the average speed in similar rocks cannot be taken at more than 10 foet per day. This refers to a depth of, say 200 feet, but in the ordinary system of boring with rods, whether by hand or machinery, where the rods have to be disconnected and the debris has to be drawn from great depths, an important element has to be considered. Whilst the weight of the apparatus, consequent upon an increased depth, tends to augment the speed of boring, the drawing, disconnecting, and connecting of great lengths of rods for the purpose of clearing the hole, takes at a depth of about 250 feet really more time than is occupied in the actual boring, and at great depths these processes absorb no less than $\frac{7}{8}$ ths of the total working time. This serious difficulty is obviated in two systems 
of boring:-1st, the mode supposed to have been originated in China, of using ropes for working the boring tools, in place of rods. This system, which has been suecessfully carried out in America and elsewhere in the boring for potroleum, as previously described; but the principle has been most thoroughly adapted and re-arranged by Messrs. Mather and Platt. 2nd, by the invention of M. Fauvelle, by menns of which the beaten-up rock at the bottom of the borehole, instead of having to be drawn up by rods or ropos, is continuously removed by a current of water forced down the borehole by means of a pump placed at the top.

The foregoing briof reference to certain modes of boring has been made for the purpose of drawing attention to the chief conditions upon which economical boring depends.

On the Application of Boring to the Sinking of Shafts.-The science of boring has recently been applied in a manner, the practical economy of which is of great importance as bearing upon the investment of capital in the sinking of coal-mines through aqueous strata. M. Kind has within the past twelve years in Gornany and France put down a number of shafts, varying in diameter from 9 to 14 fect, a considerable distance. These pits have been sunk through strata yielding immense volumes of water, and the whole process has been conducted under water. To enable such shafts to be sunk down to their full depth by shutting off the feeders of waters met with, by means of tubing placed in the shaft, M. Chandron, a Belgian enginecr, came to M. Kind's aid, and by the application of several simple, but ingenious contrivances, hereafter to be described, has succeeded in fixing the necessary tubing under water, and in providing a perfectly efficient joint at the base of the tubing, between the water-bearing and the dry strata.

Of the future coal-fields of England, it is probable that nearly half of the remaining deposit of coal will have to be obtained by sinkings through newer formations than the coal-measures, where very large quantities of water will have to be encountered. This indicates the importance to this country of some economical system of piercing such strata.

The ordinary system of sinking through strata containing large quantities of water has been to provide a large pumping plant, and to pump each feeder of water out as met with, fixing on the rock forming the barrier for each feeder a firmly wedged irou curb, and making it the base of the tubing which is built in segments, such segments

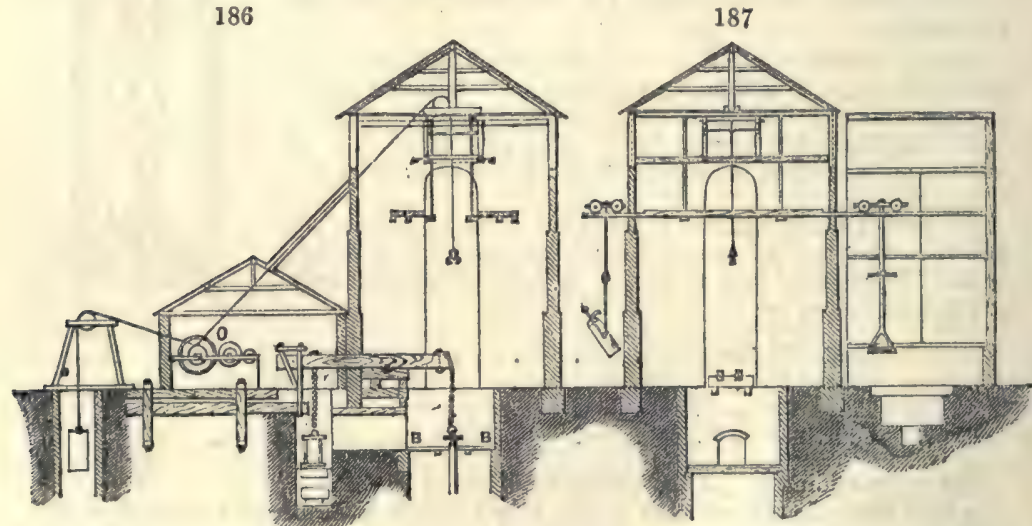

being generally about 4 feet long by 2 feet 9 inchos high. The joints between these segments are made secure by means of wooden sheeting wedged tightly. Where the feeders are large, several sets of pumps have sometimes to be introduced into the pit, and the workmen have almost continually to be working in water, and at a great disadvantage. This difficulty, combined with the heary cost of fucl, cost of buckets and powder, the great liability of accidents to the pumping plant, the cost of fixing the curbs and segmental tubing, and the risk entailed in the large number of joints in the tubing, constitute the chief disadvantages of this system of sinking pits through watery strata.

The Kind-Chaudron system may be briefly described by pointing out the various processes adopted:-

1st. The opening of the mine is commeneod by erecting the plant shown hy figs. 186 and 187, which exhibit two sectional elevations of the surface arrangements 
required. The general plant consists of the boring machine, this being a cylinder having steam applied to the upper side of the piston only. The piston-rod is attached to one end of a massive beam, to tho other end of which the boring tools are suspended. The capstan engine $\mathrm{c}$, is used for lowering and raising the boring tools, and for raising the débris made by the boring.

2nd. When the stage B B, on which the borers stand, has been erected, the boring of the pits is commenced. Supposing that the pit to be sunk has a diameter of 13 feet, the first process is to put down a borehole, with a diameter varying from 3 to 5 feet, and the tool used for this hole progresses in ordinary strata, at the rate of about 8 feet per day. When this holo has been put down, say a distance of 60 feet, the larger trepan used for the purpose of making the pit its full size is applied. This instrument is shown by figs. 188 and 189 ; it is made of wrought iron, is furnished with 28 teoth or chisels, and weighs about 16 tons. The smaller trepan is of similar design.

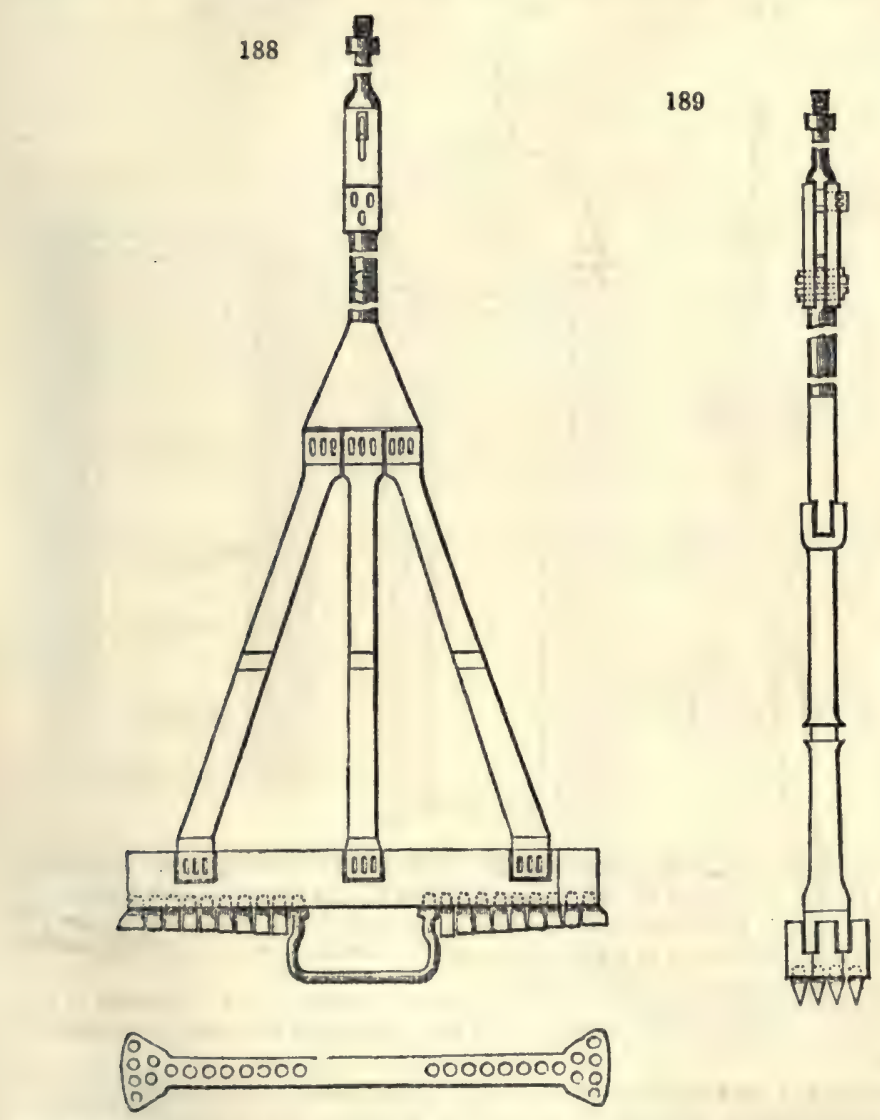

The ordinary speed of the boring when worked by the large trepan may be taken at about 3 feet per day. It will be observed that the faces of the chisels incline to the centre of the pit; this tends to assist the passing of the débris made in the large boring into the cavity formed by the small boring tool, whonce it is extracted by the ordinary scourer. This process is continued to the bottom of the aqueous strata, the small borohole being kept about 60 feet in advance of the larger hole. Should broken tools fall into the shaft, the instruments shown by figs. 190 and 191 are used, the former, by means of internal teeth, clutches with ease broken bars, \&cc., having no shoulder, and which cannot be taken hold of by means of the ordinary extractor. Fig. 191 is the 'grapin,' used for the purpose of raising broken teeth or other small objects which may have fallen to the bottom of the shaft. This tool has one part sliding into

VoI. I. G G 
the other, and is lowered with the elaws closed. An arrangement of light ropes handlod from the surface enables the ongincer to work this instrument.

3rd. Placing the Tubing. - Instoad of boing in small segments, the tubing is cast in complete cylinders, about 5 foet high, with inside flanges at both top and bottom, which are turned and faced. The tubing is suspended in the pit by rods, and is lowered down as each now cylinder is addod. Whon the bottom of the water-bearing strata is reachod, the arrangement shown by fig. 193 is adopted. A sliding moss-box, placed inside the base of the tubing, is allowed to rest on the rock floor made for the reception of the tubing, at the point below which no feeders of consequence are expected. The full weight of the tubing resting on this box, causes the moss to protrude at the back, and form a perfectly water-tight joint at the base of the tubing. To render this joint more secure, a few feet of tubing in segments resting upon two strong wedged curbs, aro placed in the pit below the moss-box.

190 191

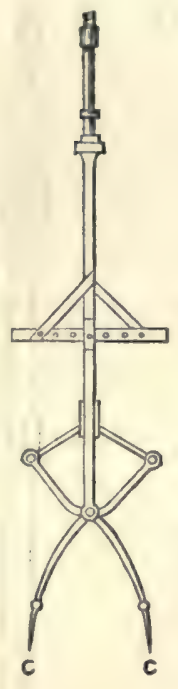

192

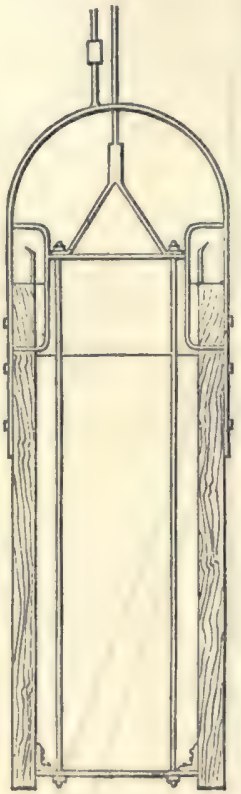

193

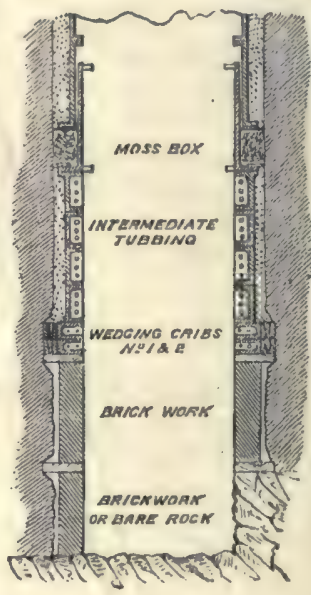

4th. The Securing of the Rigidity of the Tubing.-The tubing now, as it were, stands in the pit, resting on the foundation afforded by the moss-box, but without any lateral support. It is secured in its position by means of cement, which is dropped down behind the iron tubing by means of a coment-box with a moveable piston, worked by a rope from the surface. (See fig. 192).

5th. The shaft having now been made secure, the extraction of the water is pro. ceeded with, and the pit is then ready to be sunk either by the ordinary method or otherwiso.

'The labour and time taken in the changing of the rods becomes so great at considerable depths that the ordinary system of sinking has generally to be resorted to.

Boring in Coal.-An important operation in coal-mines, where explorations have to be made in the direction of old workings, or goofs, which may be holding large quantities of inflammable gas, or wator, is horizontal boring. In this work only very light rods are used, as the position of the hole makes the strength of the rods a question of secondary importance. The important points to consider are the driving of the hole exactly parallel with the strata, and arranging sufficient holos to render it impossible for the advancing ' heading' to come suddenly upon any old workings or narrow adits. In many cases water of immense pressure has to be borod against. The ordinary mode of boring is shown by fig. 194. Here the advancing head, $\mathrm{A}$, may be assumed to be 8 feet wide, and the minimum width of any old headings may be taken at 4 feet, Supposing the pressure anticipated is such that it is only necessary to ascertain 


\section{BORING}

the solidity of a thickness of 12 feet of coal on each side of the advancing rod, it is usual to bore three holes, one in the line of the heading, and two flank holes, which can either bo driven from the corners, or as shown in tho sketeh. The flank holes aro

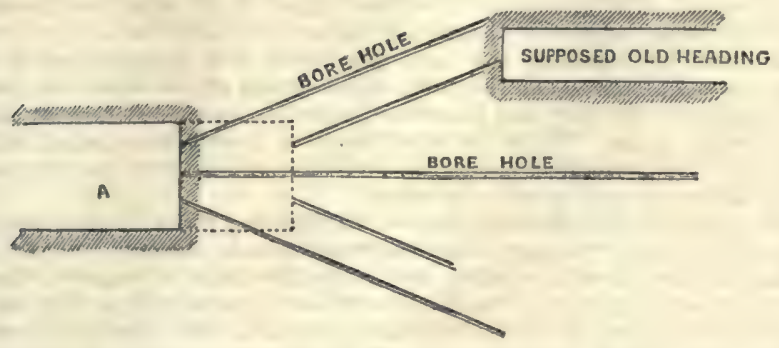

bored at such an angle as will enable them to detect, within a given distance, the prosence of any old works which may be in advance of them. Thus it will be seen on fig. 194, that the direction of the hole is such that no old head could exist without being discovered by the borers.

Where no extraordinary pressure exists, it is usual for the centre holes to be bored a distance of about 9 yards, after which the heading is advanced, say 3 yards farther, by ordinary mining; hence it will be seen that the use of the centre hole can be continued, while new flank or side holes have to be bored every few yards. The usual size of the holes driven for proving old works is about $1 \frac{1}{2}$ inch diameter, and the cost of boring varies from $9 d$. to $2 s$. per yard, according to the length of the hole.

In some cases, where a heavy pressure of water has to be encountered, the rush of water is so rapid, that it is impossible for the borers to stem its progress by means of plugs.

In an important boring conducted under the superintendence of the writer, the arrangement shown by fig. 195 was adopted. Here the bore-rod nearest to the handle

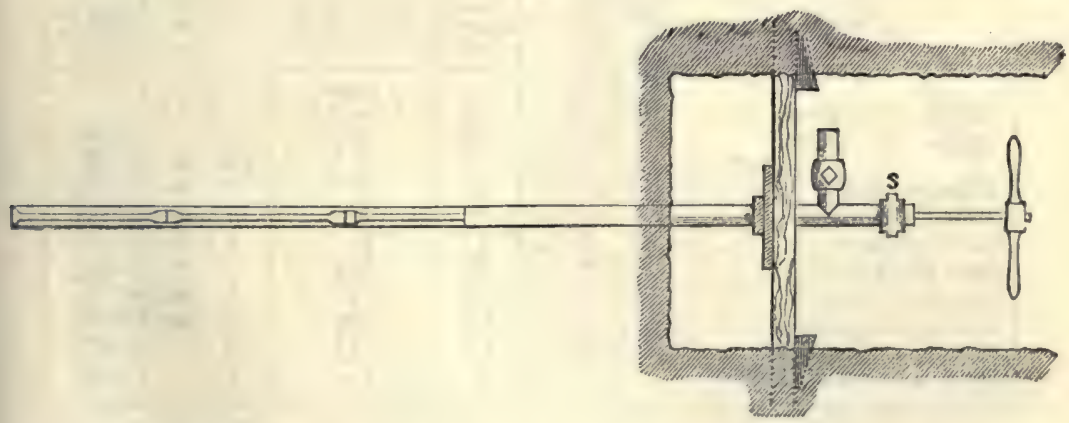

is of turnod stool, working through a stuffing-box, $\mathrm{s}$, which is attached to a tube, the end of the tube being wedged tightly into the borehole. Near the stuffing-box is a short elbow, in which is a valve or cock. When the old workings are pricked, and the wator is reached, the cock is closed to prevent the occurrence of any serious consequences. About the centre of the tube a disc-bracket is placed, bearing against two beams, one above, the other bolow the tube, the end of these beams being wedged tightly into the sides of the conl heading. The adoption of this mode not only ensures safety, but conduces to the accuracy with which the hole is driven.

Norton's Patent Well Tubes.-A method of well sinking, patented by Mr. J. L. Norton, of London, by which a supply of water can be obtained in a very short space of time, provided the geological conditions of the part selected for the sinking are favourable, deserves notice.

The arrangement consists of a wrought iron tube, about 11 feet long and $1 \frac{1}{4}$ to 3 
inches diameter, terminated at one ond by a sharp steel point, and perforated with numerous small holes for a distance of about 2 foet above the point. This tube is driven into the ground by repcated blows from a 'monkey,' or henvy weight, which slides freely on the tube, falling on a shoulder clamped to the lower end of the tube. The monkey is raised by ropos running over a pulley attached to a tripod, which at the same time preserves the upright position of the tube. When the clamp reaches the lovel of the earth, it is unscrewed and refixed higher up the tube, and the driving is continuod as before, additional lengths of piping being added as required. As soon as it is found, by plumbing the tube, that water has been reached, a cleansing pump is screwed to the top of the tube, which brings up the sand and loose earth from the bottom of the tube and immediately around the end of the same, forming a reservoir for tho water. Where the water has to be drawn from a sandy soil, a filter is attached to the perforated end of the well-tube, which prevents oven the finest sand from being pumped up with the water.

It will be seen at once that this method of obtaining water is not adapted for a rocky country ; but for purposes where the water is to be obtained within a comparatively short distance from the surface, and where the strata to be passed through are not of a very hard nature, these wells are very useful, especially as they can be sunk in light soils at the rate of 10 to 12 inches per minute. The quantity of water they can supply must depend upon the yield of the stratum to which they are sunk, but wells of this kind have beon known to gire a quantity of upwards of 800 gallons per minute.

The greatest depth at which these wells have been used is 120 feet.

Villepique Perforator. - This apparatus consists of two principal parts, namely, a standard or column, and a driving serew with its accessories. The column is formed of stout steel tube, fitted at its lower extremity with a twin claw working on a rocking joint, and at the upper end with a nut fixed inside, in which a screw works, which screw is terminated by a steol point. On this steel tube is a metal clip collar, free to slide from one end to the other, or to revolve around it. This collar can be clamped at any required point. To this collar is attached a malleable iron box, onclosing the mechanism for giving an automatic foed to the serew, and for carrying the driving or main screw. The weight of the whole apparatus is from 50 to $60 \mathrm{lbs}$. The time required for fixing the perforator in its place is about one minute. The steel augers are mechanically twisted, and have cutting ends to suit the material to be acted on, The following figures are taken from a valuable paper in the "Transactions of the North of England Institute of Mining and Mechanical Engineers,' vol. xx.

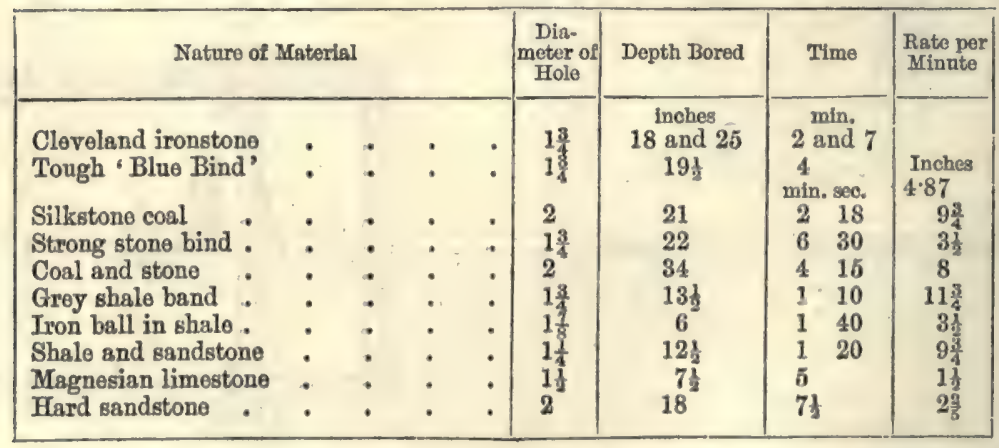

Ford's Borer.-A boring machine was invented by Mr. R. G. Ford, of Sandhurst, Victoria, about the year 1868, and patented in England on August 10, 1869. The motion of the tool is reciprocating, and the motive power compressed air, or steam, applied at a pressure of $60 \mathrm{lbs}$. per square inch. This pressure is constantly exerted on a small annular space in front of the piston, and intermittingly on the whole area of the back of the piston. The ports for the alternate admission of the pressure fluid, and for the exhaust, are opened and closed by a valve, worked by a small piston. The air ports and the movement of the valve are so arranged that the piston cannot strike the front and back of the cylinder.

The rotation of the boring tool is caused by the piston-rod, working a ratchet and paul round a cylinder attached to the front of the working cylinder; and as the piston reciprocates, it carries itself around the cylinder, and makes a complete revolution every tyenty-one blows, by which means the machine bores a round hole. The feed 
is self-advancing, and self-adjustablo, effected by the working cylinder being provided with an exterior cylinder, in which it can slide. The motive pressure is constantly tending to propel the working cylinder forward, but is retained by a screw, which is prevented from turning by a paul, which the piston strikes when it makes a full stroke, thus releasing the screw, and permitting tho working cylinder to advance forward as the hole increases in depth. The weight of blow which can be struck by this machine is $500 \mathrm{lbs}$; number of blows per minute 20 to 600 .

The inventor claimed the construction of rock boring machincs, in which the piston of the main cylinder distributes and exhausts the motive fluid to and from other cylinders at distinct portions of the stroke. For further particulars and illustration, see Specifleation No. 2,387, A.D. 1869.

Bergström's Borer. -This machine, used at the Persoberg Mines, near Philipstad, in Sweden, is a modification of that constructed by Schumann, of Freiberg. The machine consists of a cylinder $4 \frac{1}{2}$ inches diametor, and a balaneed slide valve. The movement of the piston and valve is automatic, the advance of the apparatus is effected by the hand. The length of stroke is 7 inches, eubic inches of air or steam required per stroke 200 , or $8 \frac{1}{2}$ strokes per cubic foot. Speed from 200 to 350 blows per minute. Prossure required to drive borer, 15 to 20 lbs. per square inch.

Weight of machine, $122 \mathrm{lbs}$. For additional particulars and illustrations, seo paper by C. Le Neve Foster, Miners' Association of Cornwall and Devonshire, 1867.

Sach's Borer.-The lightness, suitability, and economical working of this drill, have induced Prussian engineers to use it somewhat extensively, both in metalliferous mines and collieries. Fig. 196 illustrates an arrangement of the drill and stand, employed both in shaft and winze sinking. The cylinder is $2 \frac{1}{2}$ inches diameter, borer rod $1 \frac{7}{8}$ ths inch diameter, back rod 1 inch diameter, valve rod $\frac{s}{8}$ ths inch diameter. The valve is a simple plate; one face being on the portways leading to and from the cylinder, the other retained by a plate carrying the valve arbor. The top of the back rod carries a ring, to which is attached a small rod for rocking the valve-shaft. To this valvoshaft is also attached a horizontal rod, which carries two pauls, one for turning the borer, the other for advancing the cylinder through the medium of a nut travelling on the screw side bar. At a pressure of 25 lbs. per square inch, the borer makes 400 strokes per minute, the length of stroke being 5 inches. The blow pressure is 116 lbs., return pressure 72 lbs. About 45 cubic inches of air or steam are required per stroke, or 38 strokes por cubic foot. The area of steam portway is $\frac{6}{10}$ ths of a square inch. The rate of boring per minute in basalt is $\mathbf{3}$ inches, coal sandstone 3 inches, quartz grauwacke 2 inches, carbonate of iron $1 \frac{1}{2}$ inch. One borer requires about one horse-

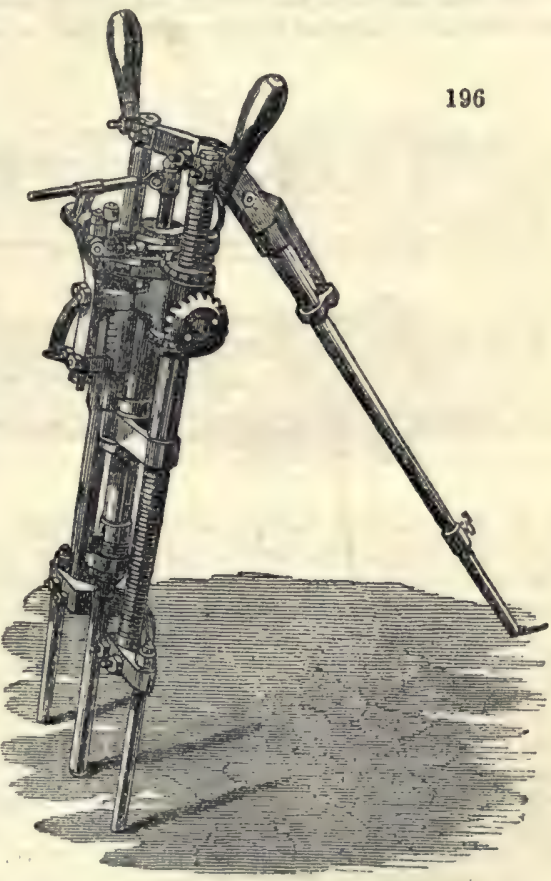
power to drive it.

Doering's Borer. - This machine is worked by compressed air or steam, at a pressure of about $35 \mathrm{lbs}$. per square inch. The pressure fluid acts alternately on the front and back of the main piston. The valre for the admission of compressed air, and for the exhaust, is worked by two chambers in the main piston, one of these chambers being always in communication with the main inlet of comprossed air, the other chamber in communication with the outer atmosphere. The valve has a separate piston to work it; on the smaller side of the piston a constant pressure of air is maintained, a passage connects the other end of the cylinder, in which tho valvo-piston works, with a certain point of the main cylinder. The chamber with compressed air 
passing over this port causes a pressuro on the larger nrea of the valve-piston, which overcomes the constant pressure on the smallor area, and reverses the valve so as to admit compressed air to the opposite end of the main piston. Rotation of the boror is effocted by means of a twist bar and ratchet-wheel, the feed or advanco is automatic. This machine was employed at Tineroft, and bored 277 holes from the 9th to the 30th of January 1868, or at the rate of 13 holes por 20 hours. In coal sandstono, at 270 strokes per minute, the rate of boring was 1 sths inch per minute. Seo Specifications of Patents, November 9, 1866, No. 2,922; January 7, 1867, No. 43; June 10, 1867, No. 1,704 .

Osterkamp's Borer.-Abont four years ago, the machine engineer, Osterkamp of Eschwoiler, near Aix-la-Chapelle, Prussia, contrived a light-boring machine, with a steam or air-moved valve. He also added a portable stand, with the object of dispensing with the columnar apparatus used in connection with Sach's Borer. The workmen held and directed the machine and also advanced the tool for deepening the hole.

The weight of a cylinder, having a piston-rod $2 \frac{6}{10}$ ths and piston 3 inches diameter, was $50 \mathrm{lbs}$. Weight of supporting stand, from 40 to $56 \mathrm{lbs}$.

This machine bored a hole in coal sandstone $1 \frac{1}{2}$ inch diameter, $\frac{8}{10}$ ths of an inch deep, in a minute; and in the same time a second hole $\frac{3}{4}$ inch diameter, $1 \frac{1}{4}$ to $1 \frac{1}{2}$ inch. The speed of the machine at $30 \mathrm{lbs}$. pressure is about 210 strokes per minute. Length of stroke, 5 inches. Cubic inches of steam or air required per stroke, 40 or 43 strokes por cubic foot. Pressure for blow 135 lbs., for returning piston 75 lbs. For particulars and illustration of Borer, see Specification, No. 1,466, A.D. 1870.

The McKean Rock-drill.-This rock-drill is worked oither by steam or compressed air, and can bo adjusted to any required position, so that holes may be drilled at any

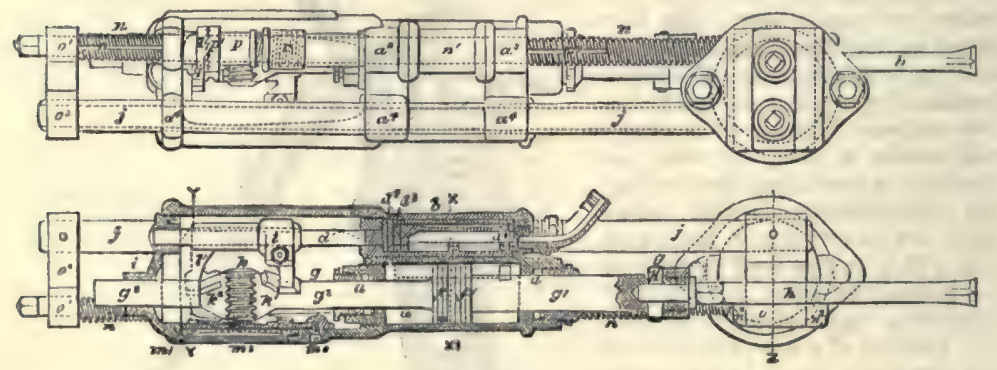

angle, the machine working with equal facility in erery direction. The ralvo is ulapted to deliver from 400 to 1,000 blows per minute, while the stroke of the piston and fall of the cutter bar is only $2 \frac{1}{2}$ inches or 3 inches; the shock to the cutter bar and piston when striking the rock is cushioned by the steam or air in the cylinder.

The cylinder, valve-chest, and frame for carrying the guides and bearings for the piston, valve-rods and other parts of the machine are cast in one piece. The percussion cylinder is marked $a$ and the valve-chest $b$; the valve seating is formed of a small cylinder $c$ placed within the valve-chest-situate on one sido of the cylinder $a$. The valve $d$ is cylindrical, its axis is parallel to that of the piston-rod, and on one side there is formed a projecting curved face $d^{\prime}$, forming the working part of the valre which alternately covers and uncovers the admission ports $e e$, that extend the whole length of the valve-chest $c$. The exhaust port is formed between the admission ports, and opens into the centre of the cylindrical valve.

The oscillating movement required for actuating the valve is given by the spindle through the key $d^{3}$, and the groove. At the bottom of the valve a shoulder $d^{4}$ is also formed, fitting tightly in the cylinder. By this means the escape of steam or air is provented, and sufficient friction is produced to avoid any excessive motion of the valve from the action of the tappets which actuate the spindle. These tappets are shown at $l l^{\prime}$, where it will be seen that they are attached to a sleeve fastened upon the valve spindle, and at such an angle on each side of the centre line passing through the valve spindle, and piston-rod, that they may be struck by the enlarged portions of the latter $k^{1} k^{2}$. The piston $f$ is formed of steel, in ono piece with the piston-rod $g^{2}$, and the tool holder $g^{1}$. Grooves are formed in the piston, as shown at $f^{\prime}$, to receive metallic piston-rings. The rod in advance of the piston is of a diumeter larger than that at the rear, in order that sufficient strongth may be obtained for 
carrying the cutting bar. The piston-rod $g^{2}$ passes through a stuffing box in tho cylinder, and takes a bearing at the end of the frame of the drill as shown at $i$; and as the rod on the front side of the piston also passes through a stuffing box, the chicf moving part of the machine is supported at three places in its length. Upon the piston-rod $g^{2}$ an cnlargement, $k^{1}, k^{2}, k^{3}$, is made with two conical faces; the valve tappets $l l^{\prime}$ are in contact with these faces, and the reciprocating motion or the pistonrod imparts to thom the necessary motion for actuating the valve. The angle of the tappots can be adjusted at will to regulate the distribution of the steam or air. Between the sloping or conical surfaces of the enlargement of the piston-rod there is a straight length $k^{s}$, in which are cut ratchet-teeth in an oblique direction around the surface; these teeth come in contact with a grooved bar $m$, the groores $m^{\prime}$ being cut to correspond with the teeth in $k^{3}$. In the frame of the drill is formed a recess, in which a spring $m^{2}$ is placod, the centre of the spring pressing against the back of the grooved plato $m$. At the forward stroke of the piston, the enlargement $k^{3}$ pressing against $m$ dopresses, it, forcing it into the rocess; but at the return stroke, the teeth of $k^{3}$ engaging in the grooves of $m$, give it a rotating morement to the piston-rod, piston, and cutter bar, so that a slow and constant turning of the cutter is effectod. Tho amount of rotary motion imparted during each stroke is about one-sixteenth of a revolution.

The automatic feed arrangement for keeping the end of the cutter in contact with the rock consists of a double threaded screw, with a key way in it, as shown, extending from end to end of the machine, the rear bearing being formed by a bracket. On the outside of the cylinder $a$, two stops $\theta^{5}$ are cast, and between these stops is placed a nut $n^{\prime}$, which is prevented from turning by being made with a flat side bearing against the outside of the cylinder $a$. Through this nut the screw $n$ passes ; fitting into the ratchet $p^{\prime}$ is a second ratchet $q$, with an arm $q^{\prime}$ that is attached to the tappets $l l^{\prime}$, which gives a reciprocating motion to the ratchet $q$, and a constant revolving motion to the sleeve $p$; the key in which drives the serew, which turns the nut $n^{\prime}$ and with it the whole drill, whilst the screw $n$ travels freely through the sleevo $p$. By this means a constant and regular feed is obtained. The end of the screw at $o^{\prime}$ is, however, made square for the reception of a lever, by which the automatic feed can be replaced by a hand movement. The cutter bar is made adjustable by means of a screw thread and nut, the face of the nut against the key being grooved, so that when it is screwed up tight and the key driven in, there is no possibility of the key shaking loose and the cutter becoming unfastened.

The machine of the size ordinarily used for quarry work or open cutting weighs about $150 \mathrm{lbs}$. A smaller, and for many purposes a still more convenient form is manufactured, which can be handled by one man.

With a steam pressure of $75 \mathrm{lbs}$, to the inch, it will drill as a maximum, a $2 \frac{1}{4}$-inch hole to a depth of 10 inches per minute in Aberdeen granite; but the average duty may be estimated at from 6 inches to 9 inches per minute, and the number of strokes from 500 to 1,000 .

For sinking shafts the machine is mounted on a column placed crosswise in the shaft, and fixed rigidly by the telescopic and serew adjustment above mentioned, from which column any required direction may be readily given to the boring tool, or the drill may be mounted upon adjustable stand or frame, as is designed for quarry and open work.

For driving tunnels where one or more machines may be worked against the face, the machines are mounted upon moveable and adjustable columns, supported upon a carriage moved upon rails.

In reference to this drill Sir George William Denys states as follows:-

(1) Weight of machine without stand, large size $1 \frac{1}{2}$ ewt., small do. 1 cwt. (2) Length of drill 38 inches. (3) Diameter of piston 5 inches, length of stroke 2 to 4 inches. (4) Strokes per minute, 500 to 1,000 ad libitum. (5) Pressure required 25 to 75, average $50 \mathrm{lbs}$. (6) Advance of drill, automatic or by hand; but where only one machine is working the hand is preferable, for it obliges the miner to pay attention and see that the drill advances regularly. (7) The nature of the stone varies; we have lime, chert, and grits of every degroe of hardness. (8) Diameter of water-wheel 38 feet or 28 feet would have done as well; bucket 4 feet wide; breadth of water trough 2 feet; depth of water varies with the supply from 3 to 8 inches. (9) Compression wet, a half-inch pipe carries the water up tho level, and squirts the water with grent force into the holes, and keeps them quite clean. (10) Dimensions of level 6 feet high clear of the rails, and about 5 feet wide. (11) Tho air-pipes are 2 inches in diameter, but I think 1 inch would suffice. (12) The level forehead is now nearly 400 fathoms from the compressor; the ventilation excellent, the air being as good inside the mine as out.

Some special measurements and particulars of this drill are as follow:-Diameter 
of eylinder 4 inches, piston-rod $2 \frac{1}{2}$ inches, diameter of back rod $1 \frac{8}{10}$ ths inch, aren for blow 10 square inches, for return $7 \frac{8}{10}$ ths square inches, pressure for blow at $50 \mathrm{lbs}$. per square inch $500 \mathrm{lbs}$, and for return of piston $380 \mathrm{lbs}$. Stroke of slide-valve $\frac{1}{4}$ to $\frac{1}{2}$ inch, opening of steam port $\frac{3}{10}$ th to $\frac{1}{4}$ inch, length of steam port 6 inches, area of steam port $1 \frac{1}{2}$ inch, diameter of stoam-pipe $1 \frac{1}{4}$ inch, stroke of piston-rod 2 to 4 inches. Turning motion one revolution to sixteen blows. The consumption of steam and air for a 4 -inch stroke including portway clearances is about 90 cubic inches, or 19 strokes per cubic foot. Soe Specifications of Patents, No. 1,104, A pril 14, 1870, and No. 3,131, Nor. 20, 1870.

The Burleigh Drill in its general arrangements does not differ essentially from some of those which have been described. It has been used in several mines and quarries in this country, and rather extensively in America. See Specification and Illustration of Drill, No. 3,065, A.D. 1866.

The main elements of this drill are the cage, the cylinder, and the piston. The cago is merely a trough, with ways on either side in which the cylinder, by means of a food-screw and an automatic feed-lever, is moved forward as the drill cuts away the rock.

The piston moves backwards and forwards in the cylinder, and is propelled and operated on substantially like the piston of an ordinary stoam-engine. The drill point is attached to the end of the piston-rod, which is a solid bar of steel.

The forward motion of the cylinder in the trough is regulated by an automatic feod, as the rock is cut away.

It is driven by steam or compressed air as a motive power, and with a pressure of 50 lbs. to the inch, strikes from 200 to 300 blows por minute, according to the sizo of the machine.

The valve used to control the entrance into and egress from the cylinder of tho impelling medium is the ordinary $D$ or locomotive slide-valve, which is operated by a ralve-rod nttached to a pivoted piece made sufficiently heavy to possess considerable momentum when put in motion.

The body of the machine is made in one casting, and constitutes the steam or air cylinder and covers and protects most of the feeding and turning mechanism. This body has two wings forming part of a support or carriage, so that the drilling machine may be presented to its work in any direction and at any angle.

The cylinder $a$ has its front end closed by a head $c$. into which a long stuffing box $d$ is serewed.

To prevent any injurious variation between the amount of the feed and the penetration of the drill, mechanism is introduced which automatically preserves a practical uniformity of feed and penetration. When the feed of the cylinder is in excess of the penetration of the drill, in which case the tappet $r$ does not in the forward stroke of the piston reach and displace the paul $\rightarrow$ and consequently no foed takes place until the penetration of the drill equals the previous feed or advance of the cylinder. As it may oceur when a feed of the cylinder takes place, that the piston on its back stroke might possibly come in contact with the back cylinder head $g$, a rubber cushion $c^{\prime}$ is placed against the rear-head $u$, to act as an elastic cushion to check the back stroke of the piston by impact of the tappet on the piston-rod with the protecting plate facing the cushion.

There are several forms of carriages for mounting drills for tunnel work. That used for the Burleigh drills consists of a trolly on four wheels, with a moveable support, which is actuated horizontally across the trolly and the face of the drift by means of a screw. The hollow bar or stretcher which fixes the carriage when it is in position for work, is held by a moveable support, and fixes itself by means of a screw at the bottom. To this hollow shaft or upright stretcher the drill is attached by a clamp; the drill is raised or lowered by means of a lever, having for its fulcrum a pin in either of the holes in the two bars of iron attached to the moveable support at their bottom ends, and to the hollow shaft at the top ends. When the drill is fixed in position, these bars are allowed to fall back by the removal of the pin at the top which connects them to the hollow shaft.

It is designed to be worked by one man, and may run on rails laid for the purpose.

The compressed air for those machines has been carried through $7 \cdot 150$ feet of an 8-inch iron pipe; and the average difference in pressure at the compressors, and the heading of the tunnel, with an average of six drills in operation, was but 2 lbs. to the inch.

Ingersol" Drill.--This drill, the invention of an American, is entirely automatic in its movements, that is, the borer is turned by a twist bar arrangement, as in Jordan and Darlington's Borer, patented in 1866, while the advance of the tool and movement 
of the valve are effected by tappet gear, struck by the head of the piston. In connection with this drill, the following particulars hare been obtained:-Diamcter of cylinder $3 \frac{1}{4}$ inches, diameter of piston-rod 2 inches, dimensions of valve portway $1 \frac{1}{8} \times \frac{3 \prime \prime}{8}$. Length of cylinder in the clear 22 inches, with attachments 39 inches. Pressure of steam required to drive tho drill, 45 lbs. per square inch. Length of stroko 6 inches, spoed of boring bar 280 strokes per minute, consumption of steam or air, forward stroke 81, backward 64, or together 145 cubicinches. Pressure for blow $373 \mathrm{lbs}$., for return 231 lbs. Time required to bore $1 \frac{1}{2}$ inch hole 1 inch deep in Cornish granite, 30 seconds. Strokes required to bore 1 inch deop, 140. Cubic feet of steam or air, consumed per inch of hole in granite, $11 \frac{7}{10}$ ths. Advance of machine on screw without change of borer, 18 inches.

Length of stroke necessary to advance borer 5 s ths inches. Number of stmkes required to make one revolution of tool 10. For illustration, see Specification No. 2,008, A. D. 1872.

Power Jumper.-This machine, the joint invention of Brydon, Davidson and Warrington, consists externally of a long cylinder and valve. The valre and turning gear are automatic; the advance of the tool is performed by hand. Tho pressure of steam required to work the drill is from 40 to $50 \mathrm{lbs}$. per square inch, the number of strokes from 300 to 400 per minute. Seo Specifications No. 3,507 and 3,921, A. D. 1872.

Darlington's Borer, Fig. 198. - The inventor, Mr. Darlington, obtains reciprocatory motion in rock-boring machinery without the intervention of a valve or valvular gear. In one modification a cylinder and motive piston are employed. The cylinder itself has an ordinary inlet portway, a long induction passage, and an outlet portway. The depth and length of the motive piston is proportioned to the position of the several portways, so that the induction and exhaust portways are alternately uncovered by the body of the piston which thus distributes the air or steam. The elastic fluid exerts its force continually against the smaller surface of the piston referred to. This piston, in moving in one direction, first covers the exhaust, then uncovers the induction portway and permits the air or steam to exert its force on the opposite side of the piston, which has a superior area to that on which the pressure is constant. In the return movement which follows, the induction portway is first covered, and immediately afterwards the exhaust is uncovered, releasing the suporior area of the piston from the pressure, and allowing the constant pressure on the small area to effect the opposite stroke.

In a second modification, which gives a like action, instead of keeping tho fluid prossure continually against the smaller surface of the motive distributing piston, the pressure is alternately admitted to and exhausted from both sides of the piston. To effect this, the piston is made of sufficient length to admit of a groove being turned in it of such a length as to always be in communication with the pressure portway formed about the centre of the length of the cylinder. Two induction passages and two exhaust portways are also formed in the cylinder. The motive piston, in its passage across these said portways, admitting the pressure to one induction portway, communicating with one end of the cylinder, at the same time as it uncovers one exhaust portway and releases the pressure from the opposite end of the cylinder.

Beyond these two modifications of one and the same invention, two other modes of distributing the steam or air have been devised. The objects gained by the foregoing method of obtaining reciprocatory motion for rock-boring and coal-cutting machines are--(1) There is only one working part used in obtaining the motion of the piston; with its rod attached, both being in one solid piece, without any loose or nttached part to be destroyed by the effect of rapid reciprocation and percussion. (2) Being enabled to uso large portways and passages, which are oponed and closed quickly, without having any reference to or being dependent on the movement of a valve, greater rapidity of motion and efficiency of blow are given. (3) Control of speed, weight of blow, and length of stroke given by the machine, and great limit of variation in the range of stroke, total freedom from stoppages due to displacement of valres, as by this arrangement while tho pressure is on the piston can never be in equilibrium, but must start at any part of the stroke. (4) Economy in working, as there is no clearance space to be filled with pressure and exhausted to wasto at erery stroke, the pressure being worked expansively to any degree required. The following are the dimensions of a machine designed to work in ordinary mine levels. Weight of cylinder and piston-rod without frame, $70 \mathrm{lbs}$; longth of cylinder over all 14 inches, diameter of cylinder $3 \frac{1}{2}$ inches, spoed 1,000 blows per minute, length of stroke from $1 \frac{1}{2}$ to 3 inches, cubic inches of air or stenm per stroke 34 , number of strokes per cubic fcot of steam or air 50, weight of blow cselusiro of effect dno to the weight and 
momentum of the tool at 30 lbs. pressure per square inch $150 \mathrm{lbs}$. In this machine the movements aro automatic, and complotely free from striking goar. For drawings, seo Specification No. 1,734, А. D. 1873.

In machine- or hand-boring it is of great importance to employ suitable stecl, properly sharpened.

For tough slate of variable hardness the cast steol should be of the very best quality. For hard brittle siliceous rock, steol of slightly inforior brand will afford good working results. The pointing of steel is a matter of great moment, overheating, or a white heat, must be aroided. The boror point should be formed with a hammer, well beaten from red to a black heat; a file, or grinding instrument, ought never to be used. The temper should be established at a dull red heat, the point, if dipped in oil or grease, is said to be toughened, as well as hardened. The hardest temper is indicated by a light straw colour, the softest by blue. The best rorking

\section{8}

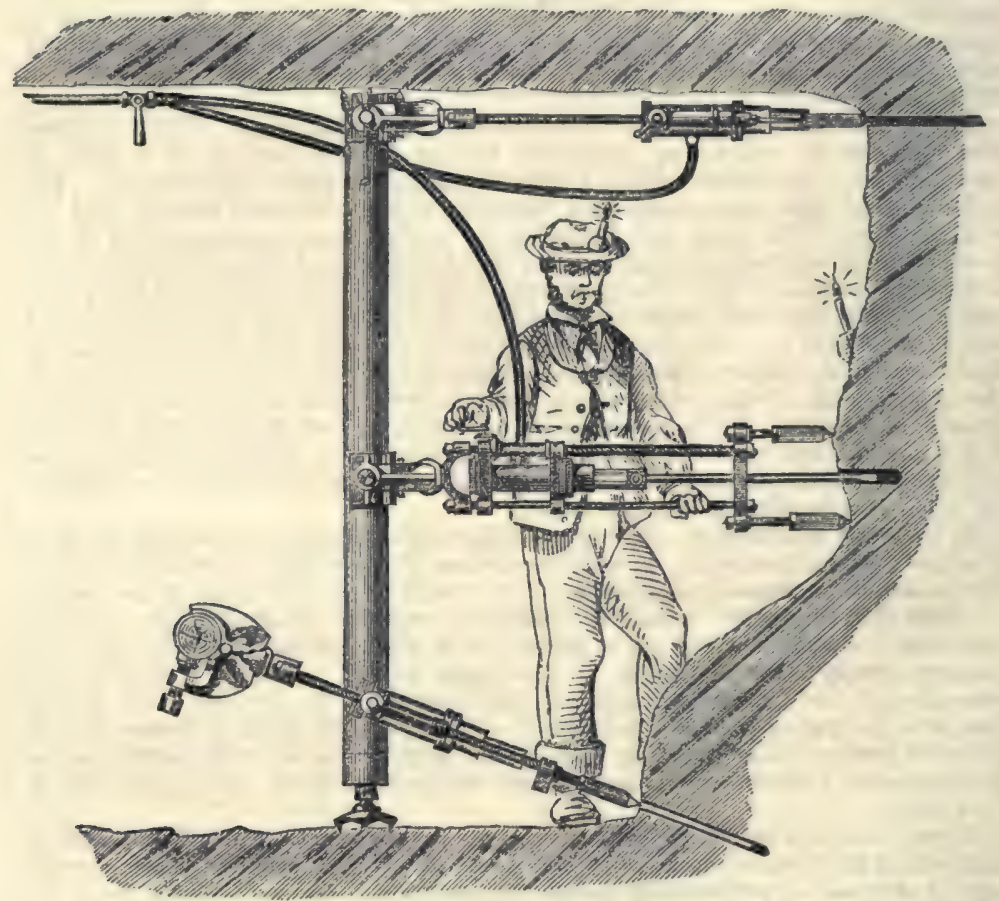

teraper is considered by some smiths to be just past straw, scarcely red purple; the tool at this colour to be instantly cooled in water.

The proper cutting angle of borers is a subject on which there is a great diversity of opinion, which probably arises from the circumstance that rocks differ even in ono and the same mine, and will differ more widely in mines far distant from each other, and also from the fact that smiths do not readily change their practice. But it is self-evident that for soft argillaceous stone tho point may be comparatively thin and flat, and that for flinty tough rock it should be well supported by lowness of angle. In machine-drilling, borer points do much more service than in liand-boring. In the former this is due to the uniform conditions of the blow. In the latter the blow is frequently an upset one, its effect being expended mostly on one side of the point. In hand-boring the chisel is almost invariably employed; but with boring machines the 'crown,' and $\mathrm{Z}$ or 'set' point is usod; the crown for starting a hole, and both the crown and $\mathrm{Z}$ for boring in cavernous ground. The Cornish minor is in favour of striking the borer both lightly and quickly. A single handed mallet or hammer weighs 4-5 lbs., a double handed one from 6-8 lbs. Each man givos about 20 blows per minute; the size of steel employed is from $\frac{3}{4}$ to $1 \frac{1}{8}$ th inch diameter, and whon sharponed will present points fully ono quarter of an inch wider: that is, a 


\section{BORNITE}

boring bar sainch diameter, when sharpened, will eut a hole 1 inch to 1 f th inch diametor.

Figs. 199, 200, 201 show chisel-point borers; fig. 202 a crown borer; and fig. 203 a $\mathrm{Z}$ borer.

The rate of hand-boring must necessarily depend on the workman, quality of steel, and hardness of rock, also on other minor circumstances. In a shaft $9 \times 5 \frac{1}{2}$ feet nino men sunk 15 feet in a period of 26 days of twenty-four hours per day. The average number of holes 11 inch diameter bored per shift of eight hours was 3 , arernge dopth of each hole 2 feet. During the run of 78 shifts the aggregate depth of the various holes was 468 feet; total cost of removing the ground, $77 l$. The average weight of rock removed per blast was $3 \frac{1}{2}$ ewts. In another shaft the rate of boring per minute, one man striking, was $\cdot 17$ inch, two men striking $\cdot 25$ inch. The number of blows required for a hole 30 inches deep was 4,500, number of borers blunted 7 ; depth bored per borer $4 \frac{1}{2}$ inches. In machine-boring the progress varies chiefly with the rapidity of the blow and toughness of the rock, or from $\frac{1}{2}$ to 6 inches per minute.

The boring machines employed in underground work are best driven by compressed

199
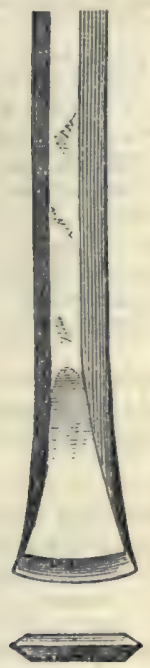

200
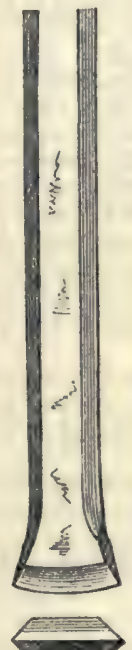

201
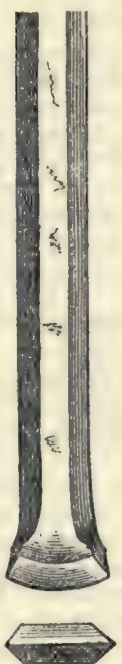

202

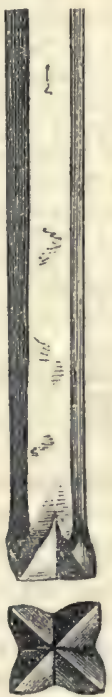

203

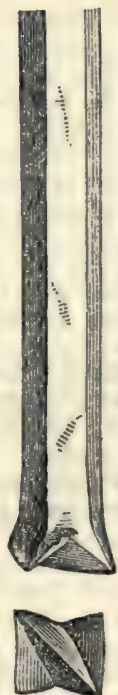

air. The air-pumps should be well constructed; and the pistons or rams worked in connection with water, with the double object of keeping down the temperature of the air, and expelling into the receiver a volume equal to the cubic contents of the airpump stroke. Jordan's compressor is a cheap and well-contrived apparatus. Low has also derised an efficient pump; while Ford of Melbourne, and Angström of Sweden, have produced simple and effective pumps worked in combination with ordinary pump rods.

In order to obtain the full effect of boring machines, the stuff must be removed rapidly after each blast. This object can only be accomplished by employing pneumatic tackles in connection with underground sinks, and having good forwarding ways. The advantages of using machine-borers in mining operations are-(I) Decided economy of time and money; (2) Diminution of the number of skilled miners, who can be advantageously employed in other parts of the mine; (3) Excellent venti. lation of shafts, sinks, and levels; (4) Possibility of making the roof, floor, and sides of a level more regular than can be effected by hand-boring; (5) Rate of driving levels or sinking shafts two to four times greater than is practicable by handboring.

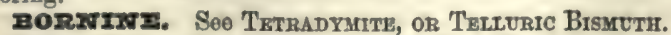

BornItr. Purple copper ore or Bunt Kupfererz. See Coppre.

It is to be regretted that names so similar as Bornine and Bornite, both complimontary to Von Born, should have been bestowed upon wiclely different minerals, 
zOzocAICXTz. A borate of lime. Seo Borscic Acid,

morox. Ono of the non-motallic olements; it oxists in nature in the form of boracic acid, and as borax, tincal, boracito, borocalcite, \&c.

Homberg is said to have obtained boron from borax in 1702; if so, his discorery appears to have been forgotton, since it was unknown, except hypothetically, to the more modern chemists until, in 1808, it was obtained by Gay-Lussac and Thénard, and by Dary in 1808, who decomposed boracic acid into boron and oxygen.

Boron is best obtained from the double fluoride of boron and potassium $\left(\mathrm{BF}^{3} \cdot \mathrm{KF}\right)$, which is prepared by saturating hydrofluoric acid with boracic acid, and then gradually adding fluoride of potassium. The difficultly soluble double compound thus produced is collected and dried at a temperature nearly approaching to rodness. This compound is then powdered and introduced into an iron tube closed at one end, together with an equal weight of potassium, whereupon heat is applied sufficient to melt tho latter, and the mixture of the two substances is effected by stirring with an iron wire. Upon the mass being exposed to a red heat, the potassium abstracts the fluorine. The fluoride of potassium may afterwards be removed by heating the mass with a solution of chloride of ammonium, which converts the free potassa into chloride of potassium, and thus prevents the oxidation of the boron, which takes place in the presence of potash; the chloride of ammonium adhering to the boron may be afterwards removed by treatment with alcohol. Boron thus prepared is a dark greenish-brown powder, tasteless, and inodorous; its atomic weight is $11 \cdot 0$.

MM. Wöhlor and Devillo have, by fusing boracic acid, or amorphous boron, with aluminium, succeeded in obtaining boron in the crystallised state. The form of the boron crystals thus obtained has been the subject of a remarkable enquiry by M. Quintino Sella. They are octahedra, belonging to the pyramidal or square prismatic system. They refract light powerfully, have a specific gravity of $2 \cdot 68$, and seem to be almost as hard as diamond. From the close resemblance of this form of boron to the diamond, it is generally known as Adamantine or Dianond Boron.

Accompanying this octahedral boron, as it crystallises from its solution in aluminium, aro certain copper-coloured six-sided scales, strongly resembling graphite, and hence called graphitoidal boron. It has been lately shown, however, that this substance, instead of being an allotropic form of boron, is really a definite compound of boron and aluminium. See BorAcic ACID.

gorowatrocazcrtr. A synonym of Ulexite. See Buracio Acid.

mosjzmasritz. A name given to Manganese Alum. See Alum, Nativi.

BOTAIIACKrTr. An oxychloride of copper found at Botallack Mine, in St. Just, Cornwall.

motrim ivavuracture. See Giass and Stone Ware.

воUсrs. A smooth, flexible, elastic, slender cylinder, introduced into the urethra, rectum, or cesophagus, for opening or dilating it, in cases of stricture and other diseases. The invention of this instrument is clained by Aldereto, a Portuguese physician; but its form and uses were first described by his pupil Amatus, in the year 1554. Some are solid and some hollow, some corrosive and some mollifying. They owed their elasticity, as formerly made, to linseed oil, inspissated by long boiling, and rendered dry by litharge. This viseid matter was spread upon a very fine cord or tubular wob of cotton, flax, or silk, which was rolled upon a slab, when it beceme nearly solid by drying, and was finally polished.

Pickel, a French professor of medicine, published the following recipe for tho composition of bougies :-Tako 3 parts of boiled linseed oil, 1 part of amber, and 1 of oil of turpentine; melt and mix these ingredients well together, and spread the compound at 3 successive intervals upon a silk cord or web. Placo the pieces so coated in a stove heated to $150^{\circ} \mathrm{F}$.; leave them in it for 12 hours, adding 15 or 16 fresh layers in succession, till the instruments havo acquired tho proper sizo. Polish them first with pumice-stone, and finally smooth with tripoli and oil. This process is the one still employed in Paris, with some slight modifications; the chiof of which is dissolving in the oil one-twontieth of its weight of caoutchouc, to render the substance more solid. For this purpose the caoutchouc must be cut into slender shreds, and added gradually to the hot oil. The silk tissue must be fine and open, to admit of the composition entoring freely among its filaments. Every successive layer ought to bo dried in a stove, and then in the open air, before another is applied. This process takes 2 months for its completion, in forming the best bougies called by distinction elastic bougies; which ought to bear twisting round the finger without cracking or scaling, and extension without giving way, but retracting when let go. When the bougies are to be hollow, a mandrel of iron wire, properly bent, with a ring at one end, is introduced into the axis of the silk tissue. Some bougies are mede with a hollow axis of tinfoil rolled into a slender tube. Bougies are now nsually made entirely of caoutchouc, by the intervention of a solution of this substance in salphuric ether, a 
menstruum sufficiently cheap in France, on account of the low duty upon alcohol, or of naphtha. Thero aro medicated bougies, the composition of which belongs to Surgical Pharmacy, The manufacture of these instruments of rarious kinds forms a separato and no inconsiderable branch of industry at Paris. Very superior bougies are now made by the surgical-instrument makors, and by tho workers in caoutchouc, in this country.

BOUxDan CrAx. The fine laminated clays of the Pleistocene epoch, which immediately overlie the true Boulder clay of geologists-so called from the bouldors and pebbles interspersed through their mass.

nourdFrive sxorrs. A name given by the Sheffield cutlers to the smooth flint pobbles with which they smooth down the faces of buff and wooden whoels.

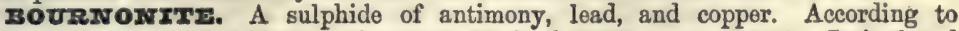
Rammelsberg, sulphur $19 \cdot 77$, antimony $24 \cdot 34$, lead $42 \cdot 88$, copper $13 \cdot 06$. It is found in several parts of Cornwall and Devonshire, and in many of the mining distriets of Europe.

Bovar coax. A lignite found in large deposits at Bovey-Tracey, in Dovonshire, whence its name. See Lrenite.

BOW PIN. A drawing pen. The parts which hold the ink is formed of two pieces, which are bowed out and adjustable by a screw.

Bowstrure rmine. A fibro prepared from the Sanscviera Zeylanica. Soo HEMc.

BOs wood. (Buis, Fr.; Buchsbaum, Ger.) Bumus sempervirens.-Two varieties of box wood are imported into this country. The European is brought from Leghorn, Portugal, \&c., and the Turkey box wood from Constantinople, Smyrna, and the Black Sea. English box wood grows plentifully at Bax Hill, in Surrey, and in Gloucestershire. The English box wood is used for common turnery, and is preferred by brass finishers for their lathe-chucks, as it is tougher than the foroign box, and bears rougher usage. It is of very slow growth, as in the space of 25 years it will only attain a diameter of $1 \frac{1}{t}$ to 2 inches. Box wood is used for making clarionets and flutes, carpenters' rules, and drawing scales, and is exclusively employed by the wood engraver. Its sawdust is used for cleaning jewellery. See Evaravisc ox Woon.

zRAcas. (Bretelles, Fr.; Hosenträger, Ger.) Narrow fillets or bands of leather or textile fabrics, which pass over the shoulders, and are attached behind and before to the waistbands of trousers, for supporting their weight. Braces are now made of an elastic material, into the structure of which Indian-rubber fibre enters.

BRAmMIN's BzADS. The seeds of a species of Elcocarpus, which are capped with silver and made into necklaces and bracelets.

IRAID. A plaitod, twisted or woven trimming.

BRAIDING MACrIrv. (Machine à lacets, Fr.; Bortenwerkerstuhl, Ger.) This being employed, not only to manufacture stay-laces, braid, and upholsterers' cord, but to cover the threads of caoutchoue for weaving brace-bands, deserves a description in this work. Three threads at least are required to make such a knitted lace, but 11, 13, or 17, and oven 29 threads are often employed, the first three numbers being

204

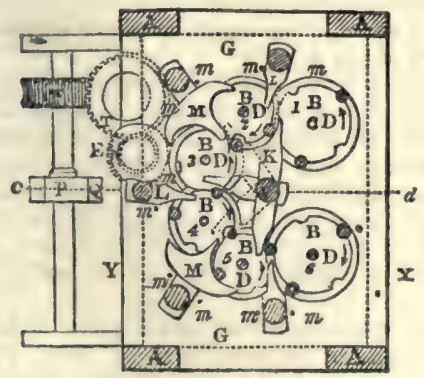

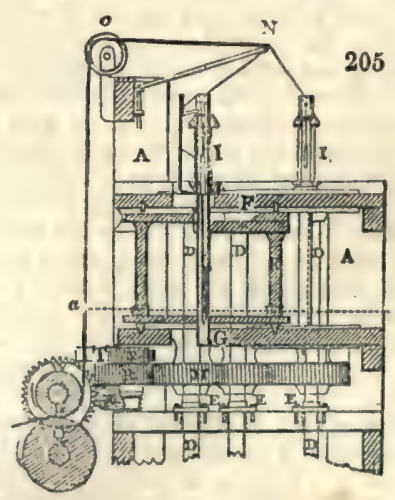

205

preferred. They are made by means of a framo of a very ingenious construction, which moves by a continuous rotation. Wo shall describe a frame with 13 threads, from which the structure of the others may bo readily conceived. The basis of the 
machino consists of four strong wooden uprights, s, figs. 204, 205, 206, occupying the four anglos of a rectangle, of which one side is 14 inches long, the other side 18 inches, and the hoight of the rectangle about 40 inchos. Fig. 204 is a section in a horizontal plane, passing through the line $a b$ of fig. 205, which is a vertical section in a plane passing through the centre of the machine $c$, according to the line $c d$, fig. 204. The side $\mathrm{X}$ is supposed to be the front of the frame; and the opposite side, $\mathrm{Y}$, the back. $B$, six spindles or skewers, numbered from 1 to 6 , placed in a vertical position upon the circumforence of a circle whose centre coincides with that of the machine at the

206 point c. These six spindles are composed-1, Of so many iron

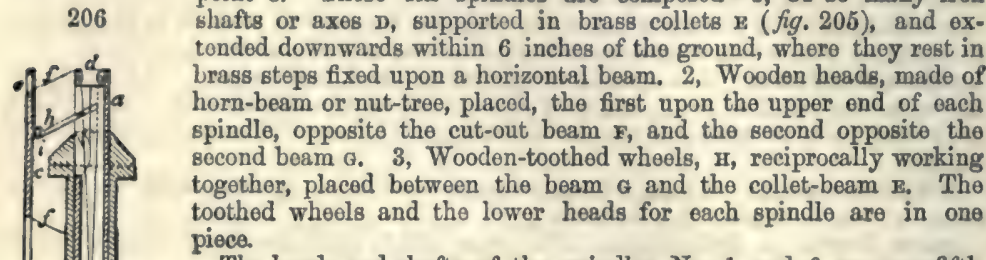

The heads and shafts of the spindles No. 1 and 6 are one-fifth stronger than those of the other spindles; their heads have five semi-circular grooves, and wheels of 60 teeth, while the heads of the others have only four grooves, and wheols of 48 toeth; so that the number of the grooves in the six spindles is 26 , one-half of which are occupied with the stems of the puppets 1 , which carry the 13 threads from No. 1 to 18.

The toothed wheels, which give all the spindles a simultaneous movement, but in different directions, are so disposed as to bring their grooves opposite to each other in the course of rotation.

$\mathrm{k}$, the middle winglet, triple at bottom and quintuple at top, which serves to guide the puppets in the direction they ought to pursue.

T, three winglets, single at top and bottom, placed exteriorly, which serve a like purpose.

$\mathbf{M}$, two winglets, triple at bottom and single at top, placed likewise exteriorly, and which serve the same purposes as the preceding; $m$, are iron pins inserted in the cut-out beam $G$, which serve as stops or limits to the oscillations of the exterior winglets.

Now, if by any moving power (a man can drive a pair) rotation be impressed upon the large spindle No. 1, in the direction of the arrow, all the other spindles will necessarily pursue the rotatory movement indicated by the respective arrows. In this case the 13 puppets working in the grooves of the heads of the spindles will be carried round simultaneously, and will proceed, each in its turn, from one extremity of the machine to the opposite point, erossing those which have a retrograde move. ment. The 13 threads united at the point $\mathrm{N}$, situated above the centre of the machine, will form at that point the braid, which after having passed over the pulley $o$, comes between the two rollers $\mathbf{P} \mathbf{Q}_{\text {, }}$ and is squeezed together, as in a flatting-mill, where the braid is calendered at the same time that it is delivered. It is obvious that the roller $\mathrm{P}$ receives its motion from the toothed wheel of the spindle No. 3 , and from tho intermediate wheols, $\mathrm{R}, \mathrm{S}, \mathrm{T}$, as well as from the endless screw $z$, which drives at proper speed the wheel w, fixed upon the shaft of the roller $P$.

The braid is denser in proportion as the point $\mathrm{N}$ is less elevated above the tops of the puppots, but in this case, the excontric motion of these puppets is much more sensible in reference to that point towards which all the threads converge than when it is elevatod. The threads, which must be always kopt equally stretched by means of a weight, as we shall presently see, are considerably strained by the traction occasioned by the constantly excentric movement of the puppots. From this cause, braiding machines must be worked at a moderate velocity. In general, for fine work, 30 turns of the large spindle per minute are the utmost that can safely be mado.

The puppet or spindle of this machine, being the most important piece, I have represented it in section, upon a seale one-fourth of its actual size, fig. 206. It is formed of a tube, $a$, of strong sheet iron well brazed; $b$ is a disc, likewise of sheet iron, from which a narrow fillet $c$, rises vertically as high as the tube, where both are pierced with holes, $d e$, through which the thread $f$ is passed, as it comes from the bobbin, $g$, which turns freely upon the tube $a$. The top of this bobbin is conical and toothed. A small catch or detent $h$, moveable in a vertical direction round $i$, falls by 
its own weight into the teeth of the crown of the bobbin, in which case this cannot revolve; but when the detent is raised so far as to disengago the teoth, and at the same time to pull the thread, tho bobbin turns, and lets out thread till the detent falls back into these same teeth.

A skewer of iron wire, $k$, is loaded with a small weight, $l$, melted upon it. The top of this skewer has an eyo in it, and the bottom is recurved, as is shown in fig. 206, so that supposing the thread comes to break, this skower falls into the actual position in the figure, where we see its lower end extending boyond the tube $a$, by about $\frac{1}{4}$ of an inch; but as long as the thread is unbroken, the skewer $k$, which serves to keop it always tense during the excentric movement of the puppet, does not pass out below the tube.

This disposition has naturally furnished the means of causing the machine to stop whenever one of the threads breaks. This inferior protrusion of the skewer pushes in its progress a detent, which instantly causes the band to slide from the driving pulley to the loose pulley. Thus the machine cannot operate unless all the threads be entire. It is the business of the operative, who has 3 or 4 under her charge, to mend the threads as they break, and to substitute full bobbins for empty ones, whenever the machine is stopped.

arax (Son, Fr.; Kleie, Ger.) The husky portion of ground wheat, separated by the boulter from the flour. It is advantageously employed by the calico printers, in the clearing process, in which, by boiling in bran-water, the colouring matters adhering to the non-mordanted parts of maddered goods, as well as the dun matters which cloud the mordanted portions, are removed. A valuable series of researches by M. Daniel Koechlin-Schouch, justified the following conclusions :-

1. The dose of two bushels of bran for 10 pieces of calico is the best, the ebullition being kept up for an hour. A boil for the same time in pure water had no effect in clearing either the grounds or the figures.

2. Fifteon minutes boiling are sufficient when the principal object is to clear white grounds, but in certain cases 30 minutes are requisite to brighten the dyed parts. If, by increasing the charge of bran, the time of the ebullition could be shortened, it would be, in some places, as Alsace, an economy; because in the passage of the 10 pieces through a copper or vat heated with steam, 1 cwt. of coal is consumed in fuel, which costs from $2 \frac{1}{2}$ to 3 franes, while 2 bushels of bran are to be bought for 1 franc.

3. By increasing the quantity of water from 12 to 24 hectolitres with 2 bushels of bran, the clearing effect upon the 10 pieces was impaired. It is therefore advantageous not to use too much water.

4. Many experiments concur to prove that flour is altogether useless for the clearing boil, and that finer bran is inferior for this purpose to the coarser.

5. The white ground of the calicoes boiled with wheat bran is distinguishable by its superior brightness from that of those boiled with rye bran, and especially with barley bran; the latter having hardly any effect.

6. There is no advantage in adding soap to the bran boil ; though a little potash or soda may be properly introduced when the water is calcareous.

7. The pellicle of the bran is the most powerful part; the flour and the starch are of no use in clearing goods, but the mucilage, which forms one-third of the weight of the bran, has considerable efficacy, and seems to act in the following way:- In proportion as the mucilaginous substance dissolves the colouring and tawny matters upon the cloth, the husky surface attracts and fxes upon itself the greater part of them. Accordingly, when used bran is digested in a weak alkaline bath, it gives up the colour which it had absorbed from the eloth.

From bran, Péligot obtained $\mathbf{8 . 0}$ per cent. of cellulose. Millon succeeded in extracting considerable quantities of glutinous substances from the bran with acetic acid and alcohol. He found in 100 parts, starch, dextrine, and sugar, 50.0 ; sugar, 1.0 ; gluten, 14.9; fat, 3.6 ; collulose, 9.7 ; salts, 5.7; water, 13.9 ; and of odorous and resinous matters, 1.2 per cont.

BRANCE COAI. A term applied in Yorkshire to cannel and other kinds of coal, which occurs in layers traversing the ordinary coal of the district. The branching coal of South Walos derives its name from the peculiar swelling which takes placo in the operation of coking, after which it becomes very light.

RRANDS. Imperfectly carbonised pioces of wood taken from a charcoal hoap.

Bravdy. (Eau de vie, Fr.; Branntwein, Ger.) The namo given in this country to ardent spirits distilled from wine, and possessing a peculiar taste and flavour, due to a minute portion of a volatile oil. Each variety of alcohol has an aroma characteristic of the fermented substance from which it is procured; whether it be the grape, cherries, sugar-cane, rice, corn, or potatoos; and it may be distinguished even as procured from different growths of the vine. The brandies of 
Languedoc, Bordeaux, Armagnac, Cognac, Aunis, Saintongo, Rochelle, Orleans, Bareelona, Naples, \&c., being each readily recognisable by an experieneed dealer. Aubergier showed by experiments, that the disagreeable taste of the spirits distilled from the marc of the grape is owing to an essontial oil contained in the skin of tho grapo; and found that the oil, when insulated, is so energetic that a fow drops aro sufficiont to taint a pipe of 600 litres of fine-flavoured spirit. Seo Fuskz Orr.

The most celobrated of the French brandies, those of Cognac and Armagnac, are slightly rectified to only from 0.935 to 1.922 : they contain more than half their weight of water, and come over therefore highly charged with the fragrant essential oil of the husk of the grape. When, to savo exponse of carriage, the spirit is rectified to a much higher degree, the doaler, on recoiving it at Paris, reduces it to the markot proof by tho addition of a little highly flavoured weak brandy-and-water; but ho cannot in this way produce so finely-flavoured a spirit as the weaker product of distillation of the Cognac wine. If the best Cognac brandy be carefully distilled at a low heat, and after distillation the strong spirit be diluted with water to restore it to its original strength, it will be found that the brandy has suffered much in its flavour.

Genuine French brandy evinces an acid reaction with litmus-paper, owing to a minute portion of vinegar; it contains, besides, some acetic ether, and, when long kept in oak casks, a little astringent matter.

The constituents of brandy are alcohol, water, volatile oil, acetic acid, acetic ether, colouring matter, and tannin.-Pereira.

Pale brandy acquires the slight colour which it possesses from the cask in which it is kept. Brown brandy is coloured by caramel. Brandy is sold of various strengths, but it is usually about 10 per cent. under proof. Tho quantities of brandy imported, and its computed value, have been as follow:-

\begin{tabular}{|c|c|c|c|c|c|c|}
\hline & & $\begin{array}{c}1867 \\
\text { Proof Galls. } \\
4,849,832\end{array}$ & $\begin{array}{c}1868 \\
\text { Proof Galls, } \\
4,062,885\end{array}$ & $\begin{array}{c}1869 \\
\text { Proof Galls } \\
3,937,266\end{array}$ & $\begin{array}{c}1870 \\
\text { Proof Galls, } \\
7,942,965\end{array}$ & $\begin{array}{c}1871 \\
\text { Proof Gallss } \\
5,228,568\end{array}$ \\
\hline & & $\underset{1,376,360}{\mathscr{E}}$ & $\stackrel{\mathscr{2}}{1,309,413}$ & $\stackrel{\stackrel{\varepsilon}{\boldsymbol{1}}}{1,249,579}$ & $\stackrel{\mathscr{L}}{2,153,699}$ & $1,895,378$ \\
\hline
\end{tabular}

For the last three years the returns are given, showing the quantity retained for home consumption :-

$\begin{array}{lrrr} & \text { Proof Galls. } & \text { Proof Galls. } & \text { Proof Galls. } \\ \text { Imports } & \text { - } 9,942,965 & 5,372,486 & 3,519,413 \\ \text { Home consumption : } & : \mathbf{3 , 5 2 6 , 1 3 2} & \mathbf{3 , 7 1 5 , 6 7 5} & \mathbf{3 , 9 4 4 , 7 2 5}\end{array}$

The duty on brandy was reduced in 1860 to $88,6 d$. per gallon.

Bravdy, BRIrrse. Dr. Ure gave the following formula for its proparation:-Dilute pure alcohol to the proof pitch; add to every hundred pounds weight of it from half a pound to a pound of argol, dissolved in water, a little acetic ether, and French wine vinegar, some bruised French plums, and flavour stuff from Cognac; then distil the spirit with a gentle fire in an alembic furnished with an agitator. British brandies are now sold as pure grain spirits, flavoured and coloured with caramel. See ArcoHor.

BRAss. (Laiton, cuivre jaune, Fr.; Messing, Ger.) An alloy of copper and zinc. The brass of the ancients appears, in very early times, to have chiefly consisted of a mixture of copper and tin, and to have consequently, been a species of bronze or bell motal. See Arrors.

Brass was formerly manufactured by cementing granulated coppor, ealled beanshot, or copper clippings, with calcined calamine (native carbonate of zine) and charcoal in a crucible, and exposing them to bright ignition. Three parts of copper were used for 3 of calamine and 2 of charcoal.

James Emerson obtainod a patent, in 1781, for making brass by the direct fision of its two metallic elements,. and it is now usually manufactured in this way.

It appears that the best proportion of the constituents to form fine brass is 2 equivalents of copper $=63 \frac{3}{2}+1$ of zinc $=32 \cdot 3$; or very nearly 2 parts of copper to 1 of zine.

In the procoss of alloying two metals of such different fusibilities as copper and zinc, a considerable waste of the latter metal by combustion might be expected; but in reality, their mutual affinities seem to prevent the loss, in a great measure, by the speedy absorption of the zine into the substance of the copper. Indeed, copper plates and rods are often brassed externally by exposure, at a high temperature, to the fumss of zinc, and afterwards laminated or drawn. The spurious gold wire of 
Iyons is made from such rods. Copper vessels may be superficially converted into brass by boiling them in dilute muriatic acid containing some tartar and zine amalgam.

The first step in making brass is to plunge slips of copper into melted zinc till an alloy of somewhat difficult fusion be formed, to raise the heat, and add the remaining proportion of the copper.

The brass of the first fusion is broken to pieces, and melted with a fresh quantity of zinc, to obtain tho finished brass. Each melting takes from 8 to 9 hours. The metal is now cast into plates, about 40 inches long by 26 broad, and from one-third to half an inch thick. The moulds were formerly slabs of granite mounted in an iron frame. Granite appears to have been preferred as a mould, because it preserves the heat, whilst, by the asperities of its surface, it keeps hold of the clay lute applied to secure the joinings.

The modern method of making brass, by the direct mixture of the component metals, is largely practised at Birmingham in small square furnaces, built of fire-brick, and measuring from 10 to 12 inches in the side, and about 2 feet in depth. Crucibles of Stourbridge fire-clay, or, rarely, of plumbago, are placed on the iron bars at the bottom of the furnace, and packed round with coke. The ingot copper is first introduced into the crucible, and when this is melted the proper proportion of zinc is cautiously added, the mixture being stirred with an iron poker to ensure union of the motals. As soon as the mixture is thoroughly effected, the erucible is withdrawn, and the molten brass is east, either into moulds of sand, or into iron ingot moulds, slightly oiled inside, and dusted over with charcoal powder. In the manufacture of the variety of brass called 'Muntz's metal,' an alloy extensively made for sheathing the bottoms of ships, the mixture of metals is generally effected in a reverberatory furnace, instead of in crucibles. See Muntz's Metax.

The east plates of brass are usually rolled into sheets. For this purpose they are cut into ribands of various breadths, commonly about $6 \frac{1}{2}$ inches. The cylinders of the brass rolling mill are generally 46 inches long and 18 inches in diameter. The ribands are first of all passed through the cylinders cold; but the brass soon becomes too hard to laminate. It is then annealed in a furnace, and, after cooling, is passed afresh through the rolls. After paring off the chipped edges, the sheets are laminated, two at a time; and if they are to be made very thin, even 8 plates are to be passed through together. The brass in these operations must be annealed 7 or 8 times before tho sheet arrives at the required thickness. These succossive heatings are expensive; and hence manufacturers have been led to try various plans of economy. The ancealing furnaces are of two forms, according to the size of the sheets of brass. The smaller are about 12 feet long, with a fire-place at each end, and about 13 inches wide. The arch of the furnace has a cylindrical shape, whose axis is parallel to its small side. The hearth is horizontal, and is made of bricks set on edge. In the front of the furnace there is a large door, which is raised by a lever, or chain and counterweight, and slides in a frame between two cheeks of cast-iron. This furnace has, in general, no chimney, except a vent slightly raised above the door, to prevent the workmen being incommoded by the smoke. Sometimes the arch is perforated with a number of holes. The sheets of brass aro placed above each other, but separated by parings, to allow the hot air to circulate among them, the lowest sheet resting upon bars of cast-iron placed lengthwise.

The larger furnaces are usually 32 feet long, by $6 \frac{1}{2}$ foet wide, in the body, and 3 fect at the hearth. A grate 13 inches broad extends along each side of the hearth, through its whole length, and is divided from it by a small wall, 2 or 3 inches high. The vault of the furnace has a curvature, and is pierced with 6 or 8 openings, which allow the smoke to pass off into a low bell-chimney above. At each end of the furnace is a cast-iron door, which slides up and down in an iron frame, and is poised by a counterweight. On the hearth is a kind of railway, composed of two iron bars, on which the carriage moves with its load of sheots of brass.

These sheets, being often 24 feet long, could not be easily moved in and out of the furnace; but as brass laminates well in the cold state, they are all introduced and moved out together. With this view an iron carriage is framed with bars, which rest on four wheels. Upon this carriage, of a length nearly equal to that of the furnace, are laid the shects, with brass parings between them. The carriage is then raised by a crane to a level with the furnace, and entered upon the grooved bars which lie upon the hearth. That no heat may be lost, two carriages are provided, the one being ready to put in as the other is taken out; the furnace is meanwhile uniformly kept hot. This method, however convenient for moving the sheets in and out, wastes a good deal of fuel in heating the iron carriage.

The principal places in which brass is manufactured on a large scale, in England, are Bristol and Birmingham, and at Holywell, in North Wales,

Vor. I. 
At tho brass manufactory of Hegormühl, upon the Finon Canal, noar Potsdam, the following are the materials of one charge: -41 pounds of old brass, 55 pounds refined copper (Garkupfor) granulated, and 24 pounds of zinc. This mixture, woighing 120 pounds, is distributed in four crucibles, and fused in a wind furnace with pitcoal fuel. The waste, upon the whole, varies from $2 \frac{1}{2}$ to 4 pounds.

Fig. 209 represents the furnace as it was formerly worked with charcoal; $a$, the laboratory, in which the crucibles wero placod. It was walled with fire-bricks. The

207

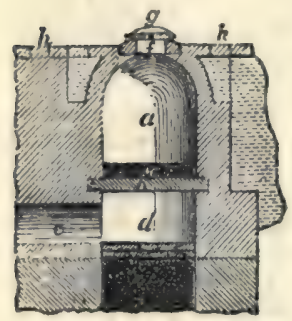

' 208

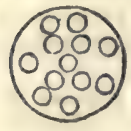

209

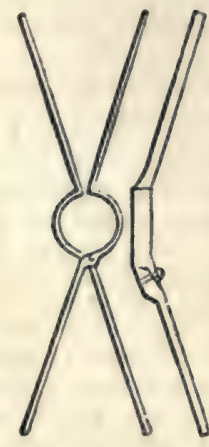
foundations and the filling-in walls were formed of stone rubbish, as being bad conductors of heat; sand and ashes may be also used; $b$, castiron circular grating plates, pierced with 12 holes (seo fig. 208), over them a sole of loam, $c$, is boaton down, and perforated with holes corresponding to those in the iron dises; $d$, the ash-pit; $\varepsilon$, the bock, a draught flue which conducts the air requisite to the combustion, from a sunk tunnel in communication with several melting furnaces. The terrace or crown of the furnace, $f$, lies on a level with the foundry floor, $h h$, and is shut with a tile of firo-clay, $g$, which may be moved in any direction by means of hooks and eyes in its binding iron ring. Fig. 209 the tongs for putting in and taking out the charges, as viewed from above and from the side.

Tho following description of a Continental brass manufactory, by Dr. Uro, it has been thought advisable to retain, with only a few rerbal alterations.

Figs. 210, 211, represent the furnaces more recently constructed for the use of pitcoal fuel; fig. 210 being an upright section, and fig. 211 the ground plan. In this furnace the crucibles are not surrounded with the fuel, but receive the requisite melting heat from the flame proceeding from the grate upon which it is burnt.

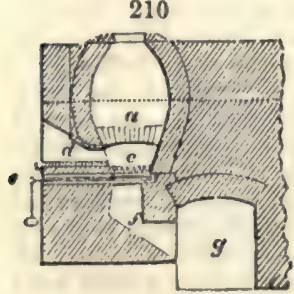

211

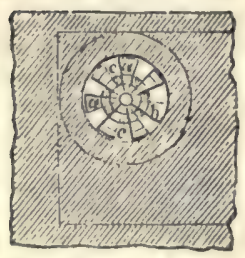
The crucibles stand upon soven arches $a$, which unite in the middle at the keystone, $b$, fig. 211; between the arches are spaces, through which the flame rises from the grate, $c$; $d$, is the fire door; $e$, a sliding tile or damper for regulating or shutting off the air-draught; $f$ an inclined plane, for carrying off the cinders that fall through the grate, along the draught tunnel $g$, so that the air in ontering below may not be heated by them.

The crucibles are 16 inches deep, $9 \frac{1}{2}$ wide at the mouth, $6 \frac{1}{2}$ at the bottom; with a thickness in the sides of 1 incl above and $1 \frac{1}{2}$ below; they stand from 40 to 50 meltings. The old brass, which flls their whole capacity, is first put in and melted down; the crucibles are now taken out and charged with the half of the zine in pieces of from 1 to 3 inches in size, covered over with coal-ashes; then onehalf of the copper is introduced; again dust; and thus the layers of zinc and copper are distributed alternately with coal-ashes botwixt them, till the whole charge becomes finally fused. Over all, a thicker layer of carbonaceous matter is laid, to prevent oxidation of the brass. Eight crucibles filled in this way are put into the furnace between the 12 holes of the grate; and over them. are laid two empty crucibles to be heated for the casting operation. In from $3 \frac{f}{2}$ to 4 hours the brass is ready to be poured. Fifteen English bushels of coals are consumed in one operation; of which six are used at the introduction of the crucibles, and four gradually afterwards.

When sheot brass is to be made, the following process is pursued :-

An empty crucible is taken out of the furnace through the crown with a pair of tongs, and kept rod hot by placing it in a hollow hearth surrounded with burning 
cosls; into this crucible the contents of four of the melting pots are poured; the dross is raked out with an iron scraper. As soon as the melting pot is emptiod, it is immediately re-charged in the manner above described, and placed in the furnace. The surface of the melted brass is swept with the stump of a broom, and then stirred about with the iron rake, to bring up any light foreign matter to the surface, which is then skimmed with a little scraper; the crucible is now seized with the casting tongs, and emptied in the following way:-

The mould or form for casting sheet brass consists of two slabs of granite, $a$ a, figs. 212, 213. These are $5 \frac{1}{2}$ feet long, 3 feet broad, 1 foot thick, and, for greater security,
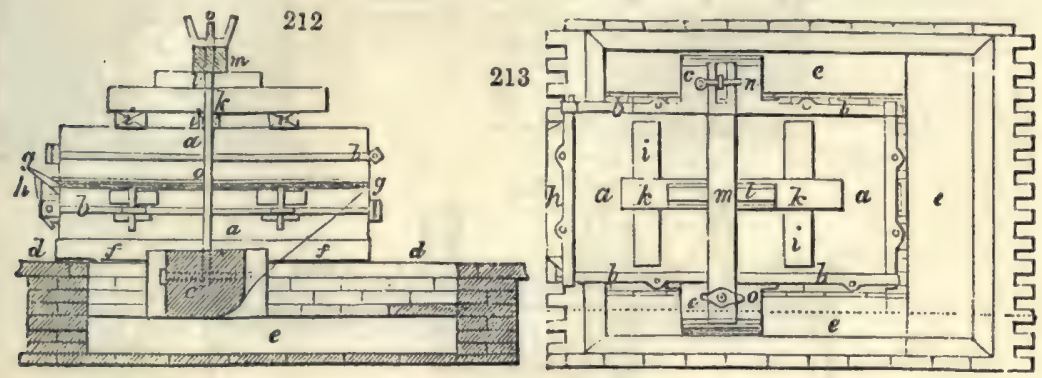

girt with iron bands, $b b, 2$ inches broad, $1 \frac{1}{2}$ thick, and joined at the four corners with bolts and nuts. The mould rests upon an oaken block, c, $3 \frac{1}{2}$ feet long, $2 \frac{1}{6}$ th broad, and $1 \frac{1}{4}$ thick, which is suspended at each end upon gudgeons, in bearing blocks, placed under the foundry floor, $d d$, in the casting pit, $e e$. This is lined with bricks; and is $6 \frac{3}{4}$ feet long, $5 \frac{1}{2}$ broad, and 2 deep; upon the two long side walls of the pit are laid the bearing blocks which support the gudgeons. The swing blocks are 10 inches long, 18 inches broad, 15 inches thick, and somewhat rounded upon their back edge, so that the casting frame may slope a little to the horizon. To these blocks two cross wooden arms, $f f$, are mortised, upon which the under slab rests freely, but so as to project about 5 inches over the block backwards, to securo an equipoise in the act of casting. $g g$ are bars, placed at both of the long sides, and one of the ends, between the slabs, to determine the thickness of the brass plate. Upon the other slab the gate $h$ is fastoned, a sheet of iron 6 inches broad, which has nearly the shape of a parallel trapezium (lozonge), and slopes a little towards the horizon. This serves for setting the casting pot upon in the act of pouring, and renders it more convenient to empty. The gate is coated with a mixture of loam and hair. The upper slab is secured to the under one in its slanting position by an armour or binding. This consists of tension bars of wood, $i, k, l, m$, of the iron bars $n$, ( 3 to $3 \frac{1}{2}$ inches broad, $1 \frac{1}{2}$ inch thick, see the top view, fig. 213) of a rod with holes and pins at its upper end, and of the iron screw spindle 0 . The mode in which these act may be understood from inspection of the figure. In order to lift the upper slab from the under one, which is offected by turning it round its edge, a chain is employed, suspending two others, connected with the slab. The former passes over a pulloy, and may be pulled up and down by moans of a wheel and axlo, or the aid of a counterweight. Upon each of the two long sides of the slab are two iron rings, to which the ends of the chains may be hooked. The casting faces of the slab must be casted with a layer of finoly ground loam; the thinner this is the better.

When calamine is employed, $\frac{1}{2} \mathrm{cwt}$. of copper, $\frac{3}{4} \mathrm{cwt}$. of calamine, and $\frac{1}{3} \mathrm{rd}$ the volume of both of charcoal mixod, are put into seven crueibles, and exposed to heat during 11 or 12 hours; the product being from 70 to $72 \mathrm{lbs}$. of brass.

Brass-Plate Rolling.-At Hegermühl there are two re-heating or annealing furnaces, one larger, 18 feet long, and another smaller, $8 \frac{1}{2}$; tho hot chamber is separated from the fireplace by iron beams, in such a way that the brass castings are played upon by the flames on both thoir sides. After oach passage through the laminating rolls, they are heated anew, then cooled and laminated, until they have reached the proper longth. The plates are smeared with grease before rolling.

Fig. 214 shows the ground plan of the furnace and its railway; fig. 215, the cross section; and fig. 216 the section longthwise; $a$ a, the iron way bars or rails upon the floor of the foundry for enabling the wheels of the waggon to movo rapidly back. wards and forwards; $b b$, the two grates; $c c$, the ash-pits; $d d$, the fire beams; 
B C e , vents in the roof of the hot chamber $f ; g, g$, two plates for shutting the hot chamber; $h$, the flue; $i$, the chimney. After rolling, the sheets, covered with black oxide of copper, are plunged for a few minutes into a mothor-water from the alum
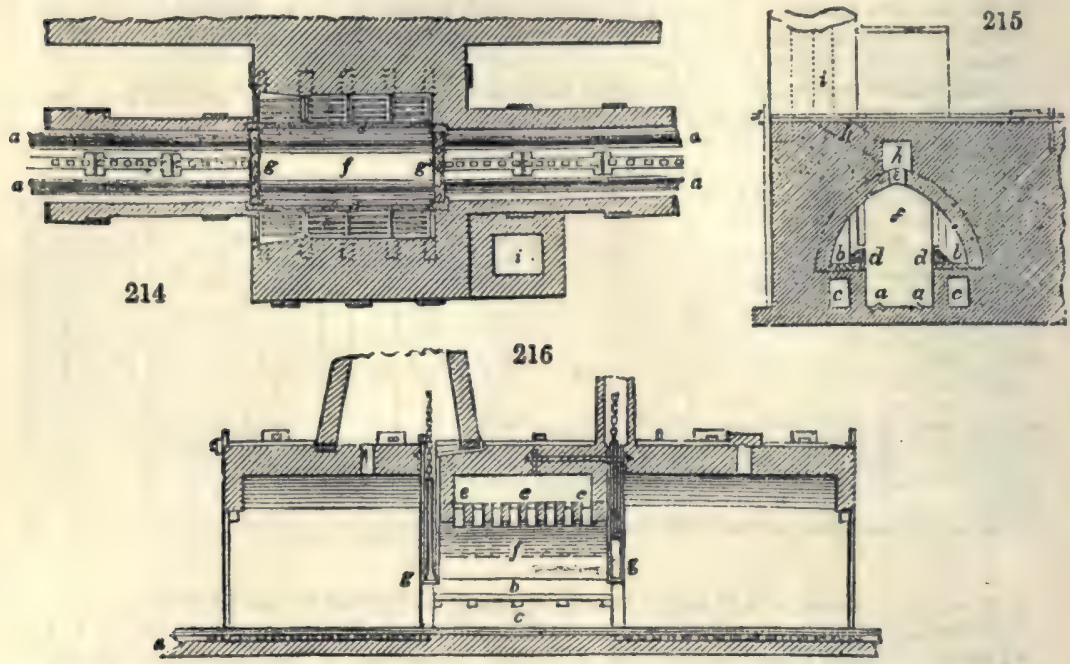

works, then washed in clean water, and lastly, smeared with oil, and scrapod with a blunt knife.

For musical purposes, the brass wire of Berlin had acquired great and merited colebrity; but that of Birmingham and of Cheadle is now preferred by foreigners.

The Table on the opposite page, for the compilation of which we are indebted to Mr. Robert Mallet, F.R.S., presente, in a very intolligible form, the chemical and physical conditions of the various kinds of brass.

It is known that common brass, containing from 27.4 to 31.8 per cent. of zinc and from 71.9 to 65.8 per cent. of copper, is not malleable while hot, but that articles of it must be made by casting. $\Lambda \mathrm{s}$ it would be of great adrantage in many branches of industry to have an alloy of this kind that could be worked while hot, like malleable iron, the information that such an alloy exists must be welcome to artists.

By melting together 33 parts of copper and 25 parts of zinc, there was a loss of 3 parts, thus making 60 per cent. copper and 40 per cent. zins. It differs from the English specimens by containing a larger proportion of zinc, and possesses, according to M. Machts, the proprietor of a brass foundry in Hanover, the precious property of malleability in a higher degreo than the English specimens.

A piece of 'yellow metal,' similar in colour to this alloy, was found on analysis to contain $60^{\circ} 16$ coppor and 39.71 zinc, which is the composition of malleable brass. It also showed great density or solidity.

An alloy was propared by molting together 60 parts copper and 40 parts zine, which had the following properties:-The colour was between that of brass and tombak, it had a strong metallic lustre, a fine close-grained fracture, and great solidity (density). Its specific gravity at the temperature of $10^{\circ} \mathrm{C}$. was 8.44 ; by calculation it ought only to have boen 8.08 ; thus showing that in the formation of the alloy a condensation must have taken place. Calculation shows that the alloy may be considered as a determinate chemical combination, for the results of the analysis very nearly accord with the assumption that it may be considered as composed of three atoms by weight of copper and 2 atoms by weight of zine $(3 \mathrm{Cu}+2 \mathrm{Zn})$. The hardness of the alloy is the same as that of fluor spar; it can be scratched by apatite (phosphate of lime), consequently its hardness is $=4$. The alloy is harder than copper, very tough, and is, in a properly managed fire, malleable; so much so that a key was forged out of a cast rod.

These important properties of this alloy warrant an expectation of its application to many purposes in the arts, and it would appear that they depend on its definite chemical proportions.

We learn some further particulars from the 'Gewerbeverein,' of Lower Austria, The commission obtained from an English specimen $65^{\circ} 03$ of copper and $34 \cdot 76$ zinc. 


\begin{tabular}{|c|c|}
\hline 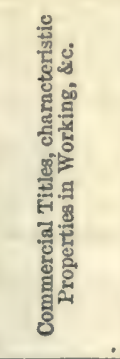 & 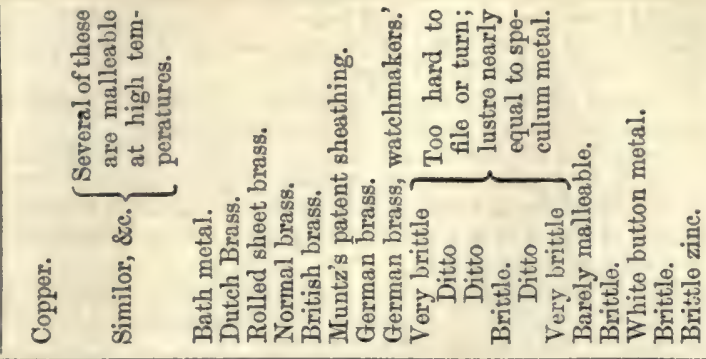 \\
\hline 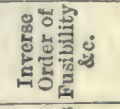 & 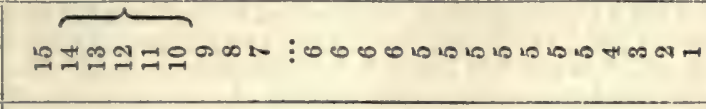 \\
\hline 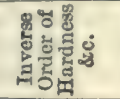 & 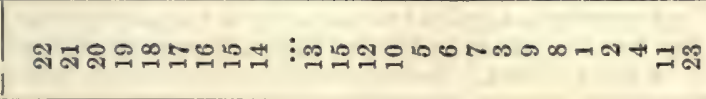 \\
\hline 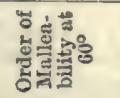 & 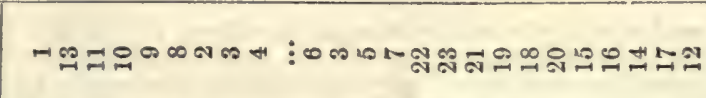 \\
\hline 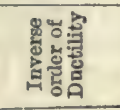 & $\infty 0$ H \\
\hline 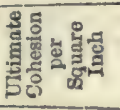 & 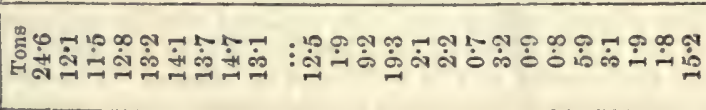 \\
\hline 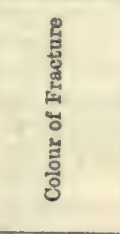 & 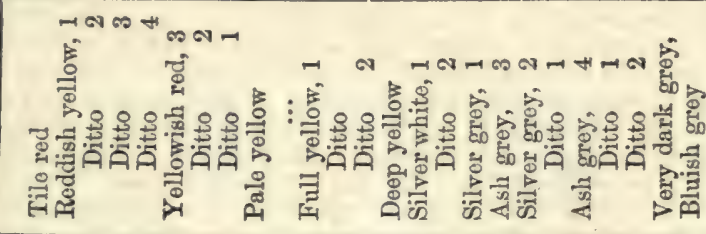 \\
\hline 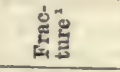 & 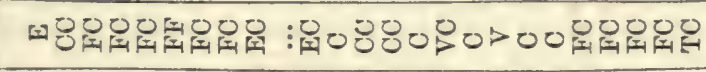 \\
\hline 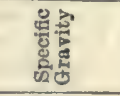 & 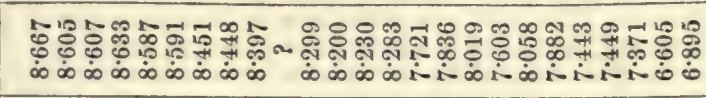 \\
\hline 일 & 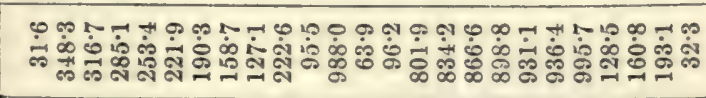 \\
\hline 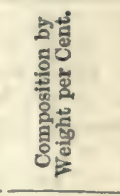 & 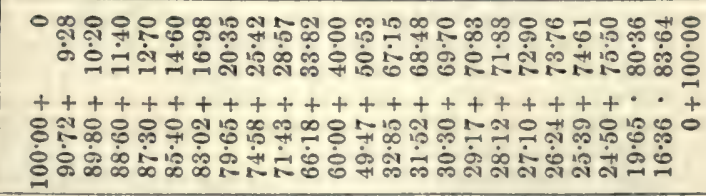 \\
\hline 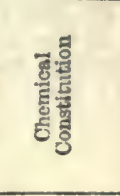 & 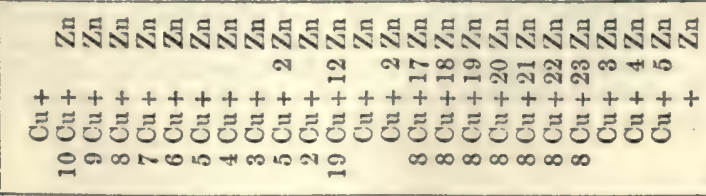 \\
\hline & 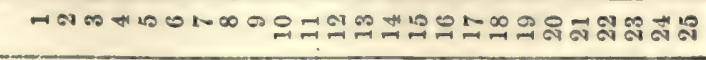 \\
\hline
\end{tabular}


Fisner analysed a malleablo brass, and found it to contain $61 \cdot 16$ coppor and $39 \cdot 71$ zinc. Theso numbers approximato to the composition $\mathrm{Cu}^{3} \mathrm{Zn}^{2}(59 \cdot 4$ per cent, copper and $40^{\circ} 6$ ainc). - Iiebig and Kopp's Report.

The alloy noticed in the preceding parngrapls is identical with that known as - Muntz's patent sheathing '(see table on proceding page).

The chief properties of valuo in brass, are its colour, hardness (which is superior to that of copper), and power of taking delicate impressions when cast in a mould.

The malleability of brass varies greatly with its chemical composition, and with the temperaturo at which it is workod. As a rule, the malleability diminishes with the increase in the quantity of zinc.

The effect of the admixture of foreign metals on brass is somewhat similar to that producod by them in copper. Antimony is most injurious, as it renders the motal brittle and liable to crack at the edges in rolling.

Lead also has a hardening effect on brass, and is useful for all work that requires to be turnod or filed; as the addition of about two per cent. causes the turnings to break short, and thus prevents the tool from becoming clogged. The same effect is produced in brass for engraving by the addition of a small quantity of tin.

Brass is apt to undergo a gradual molecular change, especially if subjected to vibration, and thus becomes extremely brittle. Hence, it happens that brass chains used for suspending heary objects, liko chandeliers, occasionally snap without any external violence.

Thin sheet brass may be readily stamped into shape, and in this way ornamental brass objects are now extensively made: the sheet brass being subjected to heavy blows delivered by steel dies. The metal requires, however, to be frequently annealed during the stamping, and the film of oxide which is formed on the surface by tho annealing, requires to be removed by dipping in aquafortis; the objocts are then washed with water, and lacquered. By varying the strength of the aquafortis, the colour of the brass may be considersbly modified.

The surface of brass work requires to be protected from the action of the air, by a coating of a less oxidisable metal, or a resinous varnish or lacquer. The metal is first brought up to a clean face by the process of 'dipping' or immersion into weak nitric acid, whereby the adherent scale or oxide formed during annealing is removed. By varying the strength of the acid employed, and repeating the dipping, the metal may be made to assume a 'matt,' dead, or frosted appearance. After dipping, the metal is washed in water, and dried by jmbedding it in hot sawdust. The lacquor, which is an alcoholic solution of shellac, more or less coloured with dragon's blood, according to the tint dosirod in the finishod work, is brushed over the articlo when in a heated state, and dried orer a stove. The colour in the lacquer helps to produce a higher or more golden tint than that due to the metal alone.

Brass work is bronzed in several difforent ways: the most usual are, immersion in a solution of arsenious acid, in hydrochloric acid, or in bichloride of platinum. The former is chiefly omployed for choap work, such as common ges fittings, while the latter is used for blacking or bronzing tolescopes, mathematical instrumonts, and similar fine work. The effect, in either case, is the production of a film of dark coloured metal on the surface of the brass, namely, arsenic in the former, and platinum in the latter case. This operation is performed after dipping, and before lacquering.

Another method now extensively used, is to apply a coating of a solution of corrosive sublimate (bichloride of mercury) in water, mixed with vinegar. A film of morcury is thus formed on the surface of the brass.

For the method of covering brass with a film of tin, see Pix Marufacturv.

Brass Fort. Dutch leaf, called Knitter or Rauschgold in Germany, is mado from a very thin sheet brass, beat out under a hammer worked by water-power, which gives from 300 to 400 strokes per minute, from 40 to 80 leaves being laid over each other. By this treatment it may be reduced to leaves not more than $\frac{1}{50000}$ th of an inch thick. The motal employed is one rich in copper.

Brass, Yelow. The following Table exhibits the composition of several varieties of this species of brass. No. 1 is a cast brass of uncertain origin; 2 , the brass of Jemappes; 3, the sheot brass of Stolberg, near Aix-la-Chapelle; 4 and 5 , the brass for gilding, according to D'Arcet; 6, the sheot brass of Romilly; 7, English brass wire; 8 , Augsberg brass wire; 9, Brass wire of Neustadt-Eberswald, in the noighbourhood of Borlin :- 


\begin{tabular}{|c|c|c|c|c|c|c|c|c|c|c|}
\hline \multirow{6}{*}{$\begin{array}{l}\text { Copper } \\
\text { Zine } \\
\text { Iead : } \\
\text { Tin : }\end{array}$} & & 1 & 2 & 3 & 4 & 5 & 6 & 7 & 8 & 9 \\
\hline & & $61 \cdot 6$ & $64 \cdot 6$ & $64 \cdot 8$ & $63 \cdot 70$ & $64 \cdot 45$ & $70 \cdot 1$ & $70 \cdot 29$ & $71 \cdot 89$ & $70 \cdot 16$ \\
\hline & . & 35.3 & $33 \cdot 7$ & $32 \cdot 8$ & 33.55 & $32 \cdot 44$ & $29 \cdot 9$ & $29 \cdot 26$ & $27 \cdot 63$ & $27 \cdot 45$ \\
\hline & . & $2 \cdot 9$ & $1 \cdot 4$ & $2 \cdot 0$ & 0.25 & $2 \cdot 86$ & ... & $0 \cdot 28$ & $\ldots$ & $0 \cdot 20$ \\
\hline & & 0.2 & 0.2 & 0.4 & $2 \cdot 50$ & $0 \cdot 25$ & ... & 0.17 & 0.85 & 0.79 \\
\hline & & $100 \cdot 0$ & $99 \cdot 9$ & 100.0 & 100.00 & 100.00 & $\ldots$ & 100.00 & $100 \cdot 37$ & 98.60 \\
\hline
\end{tabular}

Tombak, or Red Brass, in the cast state, is an alloy of copper and zinc, containing not more than 20 per cent. of the latter constituent. The following varieties are distinguished:-1, 2, 3, tombak for making gilt articles; 4, French tombak for swordhandles, \&c. ; 5, tombak of the Okar, near Goslar, in tho Hartz; 6, yellow tombak of Paris, for gilt ornaments ; 7 , tombak for the same purpose from a factory in Hanover ; 8 , chrysochalk; 9 , red tombak from Paris; 10 , red tombak of Vienna.

\begin{tabular}{|c|c|c|c|c|c|c|c|c|c|c|c|}
\hline \multirow{3}{*}{$\begin{array}{l}\text { Copper } \\
\text { Zine . } \\
\text { Lead . } \\
\text { Tin . }\end{array}$} & & 1 & 2 & 3 & 4 & 5 & 6 & 7 & 8 & 9 & 10 \\
\hline & • & $\begin{array}{r}82.0 \\
18.0 \\
1.5 \\
3.0\end{array}$ & $\begin{array}{r}82 \\
18 \\
3 \\
1\end{array}$ & $\begin{array}{c}82 \cdot 3 \\
1 \cdot 75 \\
\dddot{0} \cdot 2\end{array}$ & $\begin{array}{l}80 \\
17 \\
\cdots \\
3\end{array}$ & $\begin{array}{c}85 \\
15 \\
\ldots \\
\text { trace }\end{array}$ & $\begin{array}{c}85 \cdot 3 \\
14 \cdot 7 \\
\ldots\end{array}$ & $\begin{array}{l}86 \\
14 \\
\ldots .\end{array}$ & $\begin{array}{r}90.0 \\
7.9 \\
1.6\end{array}$ & $\begin{array}{r}92 \\
8\end{array}$ & $\begin{array}{r}97 \cdot 8 \\
2 \cdot 2\end{array}$ \\
\hline & & 4.5 & 104 & $100^{\circ} 0$ & 100 & 100 & $100 \cdot 0$ & 100 & 99.5 & 100 & 1000 \\
\hline
\end{tabular}

Mr. Holtzapffel, in his 'Mechanical Manipulation,' has given some rery important descriptions of alloys. From his long experience in manufacture, no one was moro capable than Mr. Holtzapffel to speak with authority on the alloys of copper and zine; and from his work, the following particulars have been obtained:-

The red colour of copper slides into that of yellow brass at about 4 or 5 ounces of zinc to the pound of copper, and remains littlo altered unto about 8 or 10 ounces; after this it becomes whiter, and when 32 ounces of zinc are added to 16 of copper, the mixture has the brilliant silvery colour of speculum metal, but with a bluish tint.

The alloys-from about 8 to 16 ounces to the pound of copper-are extensively used for dipping, a process adopted for giving a fino colour to an enormous variety of furniture work. The alloys with zine retain their malleability and ductility well unto about 8 or 10 ounces to the pound; after this the erystalline character slowly begins to prevail. The alloy of 2 zinc and 1 copper may be crumbled in a mortar when cold. In the following list, the quantity of zine employed to $1 \mathrm{lb}$. of copper 18 given:-

1 to 11 oz. gilding metal for common jewellery.

3 to 4 oz. Bath metal, pinchbeck, Mannhein gold, Similor; and alloys bearing various names, and resembling inferior jewellers' gold.

8 oz. Emerson's patent brass.

$10 \frac{2}{5} \mathrm{oz}$. Muntz's metal, or 40 zinc and 60 copper. 'Any proportions,' says the patentee, 'betwoen the extremes, 50 zine and 50 copper and 37 zinc and 63 copper, will roll and work well at a red heat.'

$16 \mathrm{oz}$. soft spelter solder, suitable for ordinary brass work.

$16 \frac{1}{2}$ oz. Hamilton and Parker's patent mosaic gold.

Brass is extensively employed for the bearings of machinery. Several patonts have been taken out for compositions varying but slightly. The following, for improvements in casting the bearings and brasses of machinery, appears important :- Mr. W. Howitson, of Leeds, directs, in his patent, that the proper mixture of alloy, copper, tin, and zinc, should be run into metal or 'chill' moulds, in place of the ordinary moulds. In largo castings, it is found more especially that the metals do not mix intimately in cooling, or, rather, they arrange themselves into groups when cast in sand, and the boarings are found to wear out more quickly; but if the bearings are cast so that the alloy comes in contact with metal, the mixture is more intimate, and tho bearings last longer than if cast in dry or green sand moulds.

Mr. Hewitson generally only applies these chill-metal surfaces of the moulds to those parts of a brass, or bearing, that are to receive the shaft or bear the axis of a machine. Tho chills are preferred of iron, perforated with holes ( $\frac{1}{10}$ th to $\frac{1}{6}$ th inch) 
for the passage of air or vapours ; the surface should bo thinly coated with loam, and hested to about $200^{\circ}$.

Fenton's patent metal consists of copper, spolter, and tin: it has less specific gravity than gun-metal, and is described as being ' of a more soapy nature,' by which, consequently, the consumption of oil or greaso is lessened.

Many of the patentees of bearing metals assure us, that the metals they now uso differ very considerably from the statemont in their specifications. Surely this requires a careful examination.

We exported of our Brass Manufactures, in 1864, as follows :-

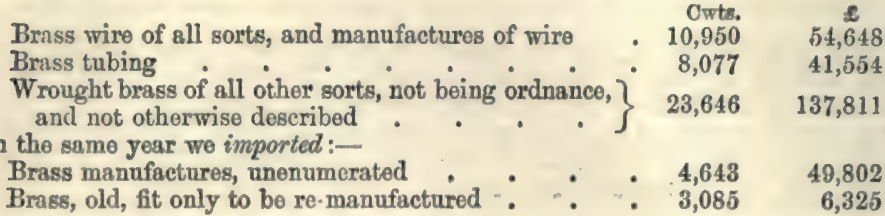

BRAss corour, for staining glass, is propared by exposing for several days thin platos of brass upon tiles in the leer, or annealing arch, of the glass house, till they are oxidised into a black powdor, aggrogated in lumps. This being pulvorisod and sifted, is to be again well calcined for several days more, till no particles remain in the metallic state, when it will form a fine powder of a russet-brown colour. A third calcination must now be given with a carefully regulated heat, its quality being tested from time to time by fusion with somo glass. If it makes the glass swell and intumesce, it is properly prepared; if not, it must be still further calcined. Such a powder communicates to glass greens of various tints, passing into turquoise.

When thin narrow strips of brass are stratified with sulphur in a cruciblo, and calcined at a red heat, they become friable and may be reduced to powder. This being sifted and exposed upon tiles in a reverberatory furnace for 10 or 12 days, becomes fit for use, and is capable of imparting a chalcedony-red or yellow-tinge to glass by fusion, according to the mode and proportion of using it.

The glassmakers' red colour may be prepnred by holding small plates of brass in a moderate heat in a reverberatory furnace till they are thoroughly calcined. When the substance becomes pulverulent, and assumes a red colour, it is ready for immodiate use.

Brass colour, as employed by the colourmen to amitate brass, is of two tints-the red or bronze, and the yellow, like gilt brass. Copper filings mixed with red ochre, or bole, constitute the former ; a powdered brass, imported from Germany, is used for the latter. Both must be worked up with varnish after being dried by heat, and then spread flat with a camel-hair brush evenly upon the surface of the object. The best varnish is composed of 20 ounces of spirits of wine, 2 ounces of shellac, and 2 ounces of sandarach, properly dissolved. (See VARsish.) Only so much of the brass powder and varnish should bo mixed at a time as is wanted for immediate use. See Bronze Powper.

BRASSIS, COAX, or BRASS or COAI, or BRAssmX COAI. Names given to iron pyrites found in the coal-measures. In 1872 it was estimated that the following quantities were produced and used:-

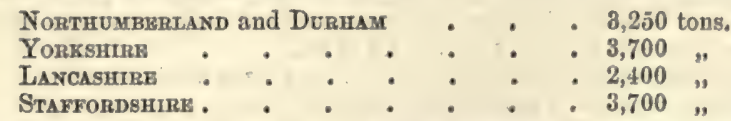

These sulphur ores are employed in Yorkshire, Lancashire, and on the Tyne, in the manufacture of the protosulphate of iron (copporas.) For this purpose they are extended in wide-spread heaps, and thus exposed to atmospheric influences. Tho result is the conversion of the sulphur into sulphuric acid, which ro-uniting with tho iron forms the sulphate of the protoxide of iron, which is dissolved out and crystallised. The presence of iron pyrites in coal (Brasses), often gives the tendency to spontaneons combustion. A large number of the ships which are sent from South Wales to Chili, with coals, which are used for smelting the copper ores of that country, take fire when they reach the tropies. This arises mainly from carelessness. In the first place, the coals are not carefully selected. They are bought cheaply, and being small, are put on board the ships, without being washed or picked, and frequently damp. During the voyage, docomposition goes on, and by the time they reach the warmer latitudes, a temperature sufficiently high to occasion actual coms. 
bustion is produced. By carefully washing the coal and drying it, these casualties might be almost entirely prevented. BRASS is a name given to an iron ore found in the coal-measures of South Wales, which is not a pyritic ore, although for a long period mistaken for it. See Cost Brass.

BRAssrI. A name given to iron pyrites in Derbyshire, 'a hard substance, and fiery, but yields no metal.'-Hodson, Complete Derbyshire Miner.

BRAssrug IRON. Iron ornaments are covered with copper or brass by proporly preparing the surface, so as to remove all organic matter, which would prevent adhosion, and then plunging them into melted brass. A thin coating is thus spread over the iron, and it admits of being polished or burnished. The electro-magnetic process is now employed for the purpose of precipitating brass on iron. This process was first mentioned in Shaw's 'Metallurgy,' in 1844, where he remarks: 'In depositing copper upon iron, a solution of the cyanide or acetate of copper should bo employed. The only value of these salts is, that the surface of iron may bo immersed in their solutions without receiving injury by the corrosion consequent upon tho deposition of a film of metal by chemical action. The following solutions are recommended by Dr. Woods, in the 'Scientific Amorican,' for coating iron with copper, zinc, or brass, by the electrotype process:-

To make a Solution of Copper or Zinc.-Dissolve 8 ounces (troy) cyanide of potassium and 3 ounces of cyanide of copper or zine in 1 gallon of rain or distilled water. These solutions to be used at about $160^{\circ} \mathrm{F}$. with a compound battery of from 3 to 12 cells.

To preparo a Snlution of Brass.-Dissolve $1 \mathrm{lb}$. (troy) cyanide of potassium, 2 ounces of cyanide of copper, and 1 ounce of cyanide of zinc, in ono gallon of rain or distilled water; then add two ounces of muriate of ammonia. This solution is to be used at $160^{\circ}$ F. for smooth work, and from $90^{\circ}$ to $120^{\circ}$, with a compound battery of from 3 to 12 cells. Seo Eiectro-Metaliurger.

BRATrICz. The division made in a shaft of a colliery is so called. It is used to ensure an up and a down-cast current of air. Brattices may be also used in any of the levels for the same purpose. They may be of metal, of wood, or tarred canvas. Mining engineers speak of a natuval brattice, i.e. one independent of any artificial arrangement-when the currents separate maturally in a shaft or a level-and thus produce a natural ventilation.

BRAUNITE. A sesquioxide of manganese, composed when pure of nearly $\mathbf{7 0}$ per cent. of manganese, and 30 por cent. of oxygen.

BRAZIIIAN ARROWROOT. SeO ARROWROOT.

BrazII murs. The hard-shelled fruit of the Bertholletia excelsa, which is roundish and about six inches in diameter, contains about two dozen of the elongated wrinkled triangular seeds - these are the 'nuts' of the shops. Seo Pars Nurs.

BrazrI Wood. (Bois de Pernamborc, Fr.; Brasilienholz, Ger.) This dyewood gires its name to the part of America, whence it was first imported. It has also the names of Pernambuca, Wood of St. Martha, and of Sapan, according to the places which produce it. Linnæus distinguishes the tree which furnishes the Brazil wood by the name of Cesalpinia crista. It commonly grows in dry places among rocks. Its trunk is very large, crooked, and full of knots. It is very hard, susceptible of a fine polish, and sinks in water. It is pale when newly cleft, but becomes rod on exposure to the air. The following is a very exact description of the tree producing this wood:-

The ibiripitanga, or Brazil wood, called, in Pernambuco, pao da rainha (Queen's wood), on account of its boing a Government monopoly, is now rarely to be soen within many leagues of the coast, owing to the improvident manner in which it has been cut down by the Government agents, without any regard being paid to the sizo of the treo or its cultivation. It is not a lofty troe. At a short distance from the ground, innumerable branches spring forth and extend in every direction in a straggling, irregular, and unpleasing manner. The leaves are small and not luxuriant; the wood is very hard and heavy, takes a high polish, and sinks in water; the only valuable portion of it is the heart, as the outward coat of wood has not any peculiarity. The name of this wood is derived from brasas, a glowing fire or coal ; its botanical name is Cesalpinia Brasileto. The leaves are pinnated, the flower white and papilionaceous, growing in a pyramidal spike: one species has flowers variegated with red. The branches are slender and full of small prickles. There are nine species. See Bell's ' Geography.'

The species Brasileto, which is inferior to the crista, grows in great abundance in the West Indies. The demand for the Brasileto, a few years ago, was so great, owing to its being a little cheaper than the crista, that nearly tho whole of the troes in the British possessions were cut down and sent home, which Mr. Bell rery justly terms improvidence. It is not now so much used, and is consoguently seareer in the English market. 
Tho wood known in commerce as Pernambuco is most estoemed, and has tho greatast quantity of colouring matter. It is hard, has a yellow colour whon newly cut, but turns rod by exposuro to the air. That kind tormod lima wood is tho samo in quality. Sapan wood grows in Japan, and in quality is next the two named above. It is not plontiful, but is much valued in the dyehouse for red of a certain tint; it gives a very clear and superior colour. The quantity of ash that these two qualities of wood contain is worthy of remark. Lima wood, as imported, gives the arerage of 2.7 per cent., while Sapan wood gives 1.5 per cent.; in both, the prevailing earth is lime. The quantity of moisture in the wood averages about 10 per cent. ; that in the ground wood in the market about 20 per cent.

Sapan wood is yielded by Casalpinia Sapan, and Lima wood by C. echinata.

Brazil wood has different shades of red and orange. Its goodness is determined particularly by its density. When chewed, a saccharine tasto is perceived. It may be distinguished from red saundors wood by its colouring water, which the lattcr loes not.

BRAzII wroOD DX25. Boiling water extracts tho wholo colouring matter of Brazil wood, and if the ebullition be long enough continuod, it assumes a fine red colour. The residuum appears black. In this case an alkali may still extract much colouring matter. The solution in alcohol or ammonia is still deeper than tho proceding.

The decoction of Brazil wood, called juice of Brazil, is observed to be less fit for dyeing when recent than when old, or even fermented. By age it takes a yellowishred colour. For making this decoction, Hellot recommends the use of the hardest water; but it should be remarked that this water doepens the colour in proportion to the earthy salts which it contains. After boiling this wood reduced to chips, or, what is preforable, to powder, for three lours, this first docoction is poured into a cask. Fresh water is poured on the wood, which is then mado to boil for throe hours, and mixed with the former. When Brazil wood is omployed in a dyeing bath, it is proper to enclose it in a thin linen bag.

Wool immersed in the juice of Brazil wood takes but a feeblo tint, which is speedily destroyed; it must therefore receivo somo preliminary preparations.

The wool is to be boiled in a solution of alum, to which a fourth or even less of tartar is added, for a larger proportion of tartar would make tho colour yellowish. The wool is kept impregnated with it, for at least eight days, in a cool place. After this, it is dyed with the Brazil juice with a slight boiling. But the first colouring particles that are deposited afford a less beautiful colour; hence it is proper to pass a coarser stuff previously through the bath. In this manner a lively rod is procured, which resists pretty well the action of the air.

Brazil wood is mado use of for dyeing silk the colour known as falso crimson, to distinguish it from tho crimson mado by means of cochincal, which is much more permanent.

The silk should be boiled at the rate of 20 parts of soap per cent., and thon alumed. The aluming need not be so strong as for the fino crimson. The silk is refreshed at the river, and passed through a bath more or less charged with Brazil juice, according to the shade to be given. When water free from earthy salts is omployed, the colour is too red to imitate crimson; this quality is givon it by passing the silk through a slight alkaline solution, or by adding a little alkali to the bath. It might, indeed, be washed in a hard wator till it had taken the desired shade. They thus become permanent colours. But what distinguishes them from madder and kormes, and approximates them to cochinoal, is their re-appoaring in their natural colour, when they are thrown down in a state of combination with alumina, or with oxide of tin. Theso two combinations seem to be the fittest for rondering them durable. It is requisite, therofore, to enquire what circumstances are best calculated to promote the formation of these combinations according to the naturo of the stuff.

The astringent principle, likewise, secms to contributo to the permanonce of the colouring matter of Brazil wood; but it deepens its hue, and can only be cmployed for light shades.

To make deeper false crimsons, a dark red juice of logwood is put into the Brazil bath aftor the silk has been impregnated with it. A little alkali may be added, according to the shade that is wanted.

To imitate poppy or flame colour, an arnotto ground is given to the silk, deeper even than when it is dyed with carthamus; it is then washed, alumod, and dyed with juice of Brazil, to which a littlo sonp water is usually addod.

The colouring particles of Brazil wood are casily affected and made yellow by the action of acids.

The colouring particles of Brazil wood are aleo very sonsible to the action of alkalis, 
which give them a purple hue; and there are several processes in which the alkalis, either fixod or volatile, aro used for forming violots and purples. But the colours obtained by these mothods, which may be easily varied according to the purpose, are porishable, and possess but a transiont bloom. The alkalis appear not to injure the colours derived from madder, but they accolerate the destruction of most other colours.

In England and Holland the wood-dyes are reducod to powder by means of mills erected for the purpose.

The bright fugitive red, called fancy red, is given to cotton of Nicaragua, or peach wood, a cheap kind of Brazil wood. See Prach Woop.

The cotton being scoured and bleached, is boiled with sumach. It is then impregnated with a solution of tin (at $5^{\circ} \mathrm{B}$., according to Vitalis). It should now bo washed slightly in a weak bath of the dyeing wood; and, lastly, worked in a somewhat stale infusion of the peach or Brazil wood. When the temperature of this is lukewarm, the dyo is said to take better. Sometimes two successive immorsions in the bath are given. It is now wrung out, aired, washed in water, and dried.

M. Vitalis says, that his solution of tin is prepared with two ounces of tin and a pound of aqua regia, made with two parts of nitric acid at $24^{\circ} \mathrm{B}$. and three parts of muriatic acid at $22^{\circ}$.

For a rose colour, the cotton is alumed as usual, and washed from the alum. It then gots the tin mordant, and is again washed. It is now turned through the dyebath, an operation which is repeatod if necessary.

For purple, a little alum is added to the Brazil bath.

1. For amaranth, the cotton is strongly galled, dried, and washod.

2. It is passed through tho black cask (tonneau noir), till it has taken a strong grey shade. See Buack Dys.

3. It receives a bath of lime water.

4. Mordant of tin.

5. Dyeing in the Brazil wood bath.

6. The last two operations are repeated.

Dingler has endeavoured to separate the colouring matter of the different sorts of Brazil wood, so as to obtain the same tint from the coarser as from the best Pernambuco. His process consists in treating the wood with hot water or steam, in concentrating the decoction so as to obtain 14 or 15 pounds of it from 4 pounds of wood, allowing it to cool, and pouring into it two pounds of skim milk; agitating, then boiling for a fow minutes, and filtering. The dun colouring matters are precipitated by the coagulation of the caseous substance. For dyeing, tho decoctions must be diluted with water; for printing, they must bo concentrated so that 4 pounds of wood shall furnish only 5 or 6 pounds of decoction, and the liquor may be thickened in the ordinary way. These decoctions may be employed immediately, as by this treatment they have acquired the same property as they otherwise could get only by being long kept. A slight fermentation is said to improve the colour of these decoctions; some ground wood is put into the decoction to favour this process.

Gall-nuts, however, sumach, the bark of birch or alder, renders the colour of Brazil wood more durable upon alumed linen and cotton goods, but the shade is a little darker.

In dyeing wool with Pernambuco Brazil wood, the temperature of the bath should never be above $150^{\circ} \mathrm{F}$., since higher heats impair the colour.

According to Dingler and Kurrer, bright and fast scarlet reds may be obtained upon wool, by proparing a decoction of 50 pounds of Brazil wood in threo successivo boils, and setting the decoction aside for 3 or 4 weeks in a cool place; 100 pounds of the wool are then alumed in a bath of 22 pounds of alum and 11 pounds of tartar, and afterwards rinsod in cold water. Meanwhile, we fill two-thirds with water a copper containing 30 pails, and heated to the temperature of $150^{\circ}$ or $160^{\circ} \mathrm{F}$. We pour in 3 pailfuls of the decoction, heat to the same point again, and introduce 30 pounds of wool, which does not take a scarlet, but rather a crimson tint. This boing removed, 2 pails of decoction are put in, and 30 pounds of wool, which becomes scarlet, but not so fine as at the third dip. If the dyer strongthens the colour a little at the first dip, a little more at the second, and adds at the third and fourth the quantity of docoction merely necessary, he will obtain an uniform scarlet tint. With 50 pounds of Pernambuco, 1,000 pounds of wool may be dyed scarlet in this way, and with the deposits another 100 may be dyed of a tile colour. An addition of weld renders the colour faster, but less brilliant. See WrID.

Karkutsch says the dyo may be improved by adding some ox-gall to the bath.

In dyeing cotton, tho tannin and gallic acid aro two necossary mordants; and the colour is particularly bright and durable when the cloth has been prepared with the oily process of Turkey red. 
It is said that stale urine heightens the colour of Brazil dye when the ground wood is moistened with it.

Cherreul obtained the colouring matter from Brazil wood in the following manner: Digest the raspings of the wood in water till all the colouring matter is dissolved, and ovaporate the infusion to dryness, to got rid of a little acctic acid which it contains. Dissolve the residue in water, and agitate the solution with lithargo, to get rid of a littlo fixed acid. Evaporato again to dryness; digest the residue in alcohol ; filter and evaporate, to drive off the alcohol. Diluto the residual mattor with water, and add to the liquid a solution of glue, till all the tannin which it contains is thrown down; filter again, and eraporate to dryness, and digest the residuo in alcohol, which will leave undissolved any excess of glue which may have been added. Tho last alcoholic solution, boing evaporated to dryness, leaves brazilin, the colouring matter of the wood, in a state of considerable purity.

BRAzIr. A torm given to a hard coal, approaching Anthracite in character, in South Staffordshire.

DRAZIIIN and BRAZIIIEN aro two colouring mattors which have been separated from Brazil wood, by Chevreul and Preisser. They are probably identical. See Watts's 'Dictionary of Chemistry.'

gRAZINGC. Soe Soldres and SoLDERTNG.

BREAD. One of the most important, if not altogether the most important, article of food, unquestionably, is bread; and although rye, barley, oats, and other cerenls, are sometimes used by the baker, wheat is the grain which is best fitted for the manufucture of that article, not only on account of the larger amount of gluten, or nitrogenous matter, which it contains, and than can be found in other ediblo grains, but also on account of the almost exact balance in which the nitrogenous and nonnitrogenous constituonts exist in that cereal, and owing to which it is capable of ministering to all the requirements of the human frame, and of being assimilated at once and without effort by our organs, whence the name of 'staff of life,' which is often given to it, wheat being, like milk, a perfect food.

Although gluten is one of the most important constituents of wheat, the nutritive powor of its flour, and its ralue as a bread-making material, should not be altogether considered as dependent upon the quantity of gluten it may contain, even though it be of the best quality. Doubtless a high per-centage of this material is desirable, but there aro other considerations which must be taken into account; for, in order to become availablo for making good bread, flour, in addition to being sound and genuine, must possess other qualities beyond containing merely a large amount of gluten. Thus, for examplo, tho blé rouge glucé d'Auvergne, which contains hardly 45 per cent. of starch, and as much as 36 per cont. of gluten, though admirably adapted for tho manufacture of macaroni, vermicelli, semolina, and other pattes d'Italie, is totally unfit for making good bread; the flour used for making best whito loaves containing only from 13 to 18 per cent. of gluten, and from 60 to 70 per cent. of starch.

Bread is obtained by baking a dough, previously fermented either by an admixture of yeast or learen, or it is artificially rendered spongy by enusing an acid, muriatic or tartaric, to react upon carbonate or bicarbonate of soda, or of ammonia, mixed in the doughy mass; or, is in Dr. Dauglish's process, which will bo described further on, by mixing the flour which has to be converted into dough, not with ordinary water, but with water strongly impregnated with carbonic acid.

Although a history of bread making cannot bo given in the present article, a few words on tho subject, reproduced from a former edition of this work, will not be deemed uninteresting.

Pliny informs us, that barley was the only species of corn at first used for food ; and even after the method of reducing it to flour had been discovered, it was long before mankind learned the art of converting it into cakes.

Ovens were first invented in the East. Their construction was understood by the Jows, the Greeks, and the Asiatics, among whom baking was practised as a distinct profossion. In this art, the Cappadocians, Lydians, and Phonicians, are said to have particularly excelled. It was not till about 580 years after the foundation of Rome that these artisans passed into Europe. The Roman armies, on their return from Macedonia, brought Grecian bakers with them into Italy. As these bakers had handmills besides their ovens, they still continued to be called pistores, from the ancient practice of bruising the corn in a mortar; and their bakehouses wore denominated pistorice. In the time of Augustus there were no fewer than 329 public bakehouses in Rome; almost tho whole of which were in the hands of Greeks, who long continued the only persons in that city acquainted with the art of baking good bread.

In nothing, perhaps, is the wise and cautious policy of the Roman government more remarkably displayod than in the regulations which it imposed on the bakers within the city. To the foreign bakers who camo to Romo with the army from Macedonia, 
a number of freodmen were associated, forming together an incorporation from which neither they nor their children could separate, and of which even those who married the daughters of bakers were obliged to become members. To this incorporation were entrusted all the mills, utensils, slaves, animals, everything, in short, which belonged to the former bakehouses. In addition to theso, they received considerable portions of land; and nothing was withheld which could assist thom in pursuing, to the best advantage, thoir highly prized labours and trade. The practico of condemning criminals and slaves, for petty offences, to work in the bakehouse, was still continued; and oven the judges of Africa were bound to sond thither, every five years, such persons as had incurred that kind of chastisement. The bakehouses were distributed throughout the fourteen divisions of the city, and no baker could rass from one into another without special permission. The public granaries were committed to thoir care; they paid nothing for the corn employed in baking bread that was to bo given in largess to the citizens; and the price of the rest was regulated by the magistrates. No corn was given out of these granaries except for tho bakehouses, and for the private use of the prince. The bakers had besides privato granaries, in which they deposited the grain which they had taken from the public granaries for immediate use; and if any of them happened to be convicted of having diverted any portion of the grain to another purpose, he was condemned to a ruinous fine of five hundred pounds' weight of gold.

Most of these regulations were soon introduced among the Gauls; but it was long before they found their way into the more northern countries of Europe. Borrichius informs us that in Sweden and Norway, the only bread known, so late as the middle of the 16th century, was unleaven cakes kneaded by the women. At what period in our own history the art of baking became a separate profession, we have not been able to ascertain ; but this profession is now common to all the countries in Europe, and the process of baking is also nearly the samo.

The French, who particularly excel in the art of baking, have a great many different kinds-of bread. Their pain bis, or brown bread, is the coarsest kind of all, and is mado of coarse groats mixed with a portion of whito flour. The pain de meteil is a bread mado with rye and barley flour, to which wheat flour is sometimes added also. The pain bis blanc, is a kind of bread between white and brown, made of white flour and fine groats. The pain blane, or white bread, is made of white flour, shaken through a sieve after the finest flour has been separated. The pain mollet, or soft bread, is made of the purest flour without any admixture. The pain chaland, or customers' bread, is a very white kind of bread, made of pounded paste. Pain chapelé, is a small kind of bread, with a well-beaten and very light paste, with butter or milk. This name is also given to a small bread, from which the thickest crust has been removed by a rasp. Pain comu is a name given by the Fronch bakers to a kind of bread made with four corners, and sometimes more. Of all the kinds of small bread this has the strongest and firmest paste. Pain $\dot{a}$ la reine, queon's bread, pain $\dot{a}$ la Ségovie, pain chapelé, and pain cornu, are all small kinds of bread, differing only in the lightness or thickness of the paste. Pain de gruau is a small very white bread made now in Paris, from the flour separated after a slight grinding from the best wheat. Such flour is in hard granular particles.

In England, however, we have but fow varieties of bread, the loaves known under the names of bricks, Coburg, cottage, and French rolls, being all made of the same dough; the only difference is in the shape given to them, their various flarours depending on the way in which they are affected by the heat of the oren in the baking. These loaves are crusted all over because thoy are deposited in the oven separate from each other, or baked in moulds made of tinned iron. The batch bread, the more usual variety, is crusted only at the top and bottom, because the loaves, which have a cubic form, touch each other in the oven; those, however, which lio round the oven have a crust on three of their sides. The cottage and French rolls are generally made of best flour,-known under the name of whites;-but batch bread is made of best flour and of households, or flour of second quality, and of seconds, which is flour of a third quality - that is, of flour containing more bran than the other kinds just enumerated.

We have also 'rye bread,' which is generally made of nothing else than ordinary wheat flour and bran.

Dr. Ure, in the former edition of this Dictionary, truly remarked, "The object of baking is to combine the gluten and starch of the flour into a homogeneous substance, and to excite such a vinous fermentative action, by means of its saccharine matter, as shall disengage abundance of carbonic acid gas in it for making an agreo. able, soft, succulent, spongy, and easily digestible bread. The two evils to be avoided in baking are, hardness on the one hand and pastiness on the other. Well-made bread is a chemical compound, in which the gluten and starch cannot be recognised 
or soparatod, as boforo, by a stronm of wator. Whon flour is knondod into a dough, and spread into a cake, this cake, whon baked, will bo horny if it bo thin, or if thick, will bo tough and clammy; whence we seo tho valuo of that fermontative process, which gonorates thousands of littlo colls in tho nuass or crumb, oach of thom dry yot tonder and succulent through tho intimato combination of tho moisture. By this con. stitution it becomes easily solublo in the juices of the stomach, or, in other words, light of digestion. It is, moreover, much less liablo to turn sour than cakes mado from unformentod dough.

' Rye, which also forms a true spongy bread, though inforior to that of wheat, consists of similar ingredients-namely, 61.07 of starch, 9.48 of gluten, 3.28 of vegrablo albumen, 3.23 of uncrystallisable sugar, 11.09 of gum, 6.38 of vegotable fibre; the loss upon the 100 parts amountod to 5.62, including an acid whoso naturo tho analyst M. Einhof, did not dotermine. Rye flour contains also sevoral salts, principally the phosphates of lime and magnesia. This kind of grain forms a dark coloured bread, rockoned very wholesome; comparativoly littlo used in this country, but very much in France, Germany, and Bolgium.

- Dough, fermonted with the aid eithor of leaven or yeast, contains little or none of the saccharine matter of the flour, but, in its stoad, a certain portion, nearly half its weight, of spirit, which imparts to it a vinous smell, and is volatilised in tho oven, whonco it might be condensed into a crude, weak alcolsol, on the plan of Mr. Hick's patont, were it worth while. But the increased complexity of the baking apparatus will probably prove an effectual obstaclo to the commercial success of this project, upon which a fow years ago upwards of 20,000l. sterling were foolishly squanderod.

- That the sugar of the flour is the true elomont of the fermentation which dough undergoes, and that the starch and gluten have nothing to do with it, may be proved by decisive experiments. The vinous fermentation continues till the wholo sugar is decomposed, and no longer; when, if the process be not checked by the heat of baking, the acetous fermentation will suporvene. Therefore, if a little sugar be added to a flour which contains little or none, its dough will become susceptible of fermenting, with extrication of gas, so as to make spongy succulent bread. But since this sponginess is produced solely by the extrication of gas and its expansion in the heat of the oven, any substance capable of emitting gas, or of boing converted into it under these circumstances, will answer the same purpose. Wore a solution of bicarbonato of ammonia obtained by exposing the common sesquicarbonate in powdor for a day to the air, incorporated with the dough, in the subsequent firing it will bo converted into vapour, and, in its extrication, render the bread very porous. Nay, if water highly impregnated with carbonic acid gas bo used for kneading the dough, tho rosulting bread will be somewhat spongy. Could a light article of food bo prepured in this way, then, as the sugar would remain undocomposed, the broad would be so much the sweeter and the more nourishing. How far a change propitious to digestion takes place in the constitution of the starch and gluten during tho formentative action of the dough has not been hitherto ascertained by precise experiments.

- Dr. Colquhoun, in his able essay upon tho art of making bread, has shown that its texture, when prepared by a sudden formation and disengagement of elastic fluid genorated within the oven, differs remarkably from that of a loaf which has boon made after the preparatory fermentation with yeast. Bread which has been raised with the common carbonate of ammonia, as used by the pastrycooks, is porous no doubt, but not spongy with vesicular spaces, like that made in the ordinary way. The former kind of bread never presents that air-coll stratification which is tho bonst of the Parisian bakor, but which is almost unknown in London. It is, moreover, very difficult to expol by the oven the last portion of the ammonia, which givos both a tinge and a tasto to the bread. The bicarbonate would probably be freo from this objection, which operates 80 much against the use of the sesquicarbonate.'-Ure.

The conversion of flour into bread includes two distinct operations: namely, the preparation of the dough and the baking. The preparation of the dough, however, though reckoned as one, consists, in fact, of threo operations: namely, hydrating, kneading, and fermenting.

When the baker intonds to make a batch of bread, his first care is, in technical language, to stir a ferment. This is done, in London, by boiling a fow potatoos, in the proportion of $5 \mathrm{lbs}$. or $6 \mathrm{lbs}$. of potatoes per sack of flour (which is the quantity we shall assume it is desired to convert into bread), peoling thom, mashing and straining thom through a cullonder, and adding thereto about throo-quarters of a pailful of water, 2 or 3 lbs. of flour, and one quart of yeast. The water employed need not be warmed beforehand, for the heat of tho potatoes is sufficient to impart a proper tomporature (from $70^{\circ}$ to $90^{\circ} \mathrm{F}$.) to the liquid mass, which should bo well stirred up with the hand into a smooth, thin, and homogeneous paste, and then left at rest.

In the course of an hour or two, the mass is seen to riso and fall, which swolling 
and heaving up is due to carbonic acid, generated by the fermentation induced in the mass, which may be thus left until wanted. In about three hours, this fermenting action will appear to be at an end, and when it has arrived at that stage, it is fit to bo used. The ferment, however, may be left for six or soven hours and be still very good at the end of that time, but the common practice is to use it within four or fivo hours after its preparation. After this the ferment rapidly becomes sour.

The noxt operation consists in ' setting the sponge.' This consists in stirring the ferment well, adding thereto about two gallons of lukewarm water, and as much flour as will make, with the forment, a rather stiff dough. This constitutes 'the sponge.' It is kept in a warm situation, and in the course of about an hour fermentation again begins to make its appearance, the mass becomes distended or is heaved up by the carbonic acid produced, the escape of which is impeded by the toughness of the mass. This carbonic acid is the result of the fermentation induced under the influence of water, by the action of the gluten upon the starch, a portion of which is converted thereby into sugrar, and then into alcohol. A time, however, soon comes whon the quantity of carbonic acid thus pent up becomes so great that it bursts through, and tho sponge collapses or drops down. This is called the first sponge.-But as the fermentation is still going on, the carbonic acid soon causes the sponge to rise again as bofore to nearly twice its volume, when the carbonic acid, bursting through the mass, causes it to fall a second time; and this constitutes what the bakers call the second sponge. The rising and falling might thon go on for twenty-four hours; but as the alcoholic would pass into the acetous fermentation soon after the second rising, the baker always interfores after the second, and rery frequently after the first sponge. The bread made from the first sponge is generally sweeter; but unless the best flour is used, and even then, the loaf that is made from it is smaller in size and more compact than that which is made with the second sponge. In hot weather, however, as there would bo much danger of the bread turning sour, if the sponge were allowed to 'take $a$ second fall,' the first sponge is frequently used. The next procoss consists in 'breaking the sponge,' which is done by adding to it the necossary quantity of water and of salt, - the quantity of the latter substance rarying from $\frac{1}{2} \mathrm{lb}$. to $\frac{3}{4}$ of a pound per bushel of flour; that is, from $2 \frac{1}{2} \mathrm{lbs}$. to $3 \frac{3}{4} \mathrm{lbs}$. per sack of flour (new flour, or flour of inferior quality, always requires, at the very least, $3 \frac{1}{2} \mathrm{lbs}$. per sack, to bind it, that is to say, to render the dough sufficiently firm to support itself while fermenting.) Salt acts, to a great extent, like alum, though not so powerfully. As to the quantity of water to be used, it depends also a groat deal on the quality of the flour, the best quality absorbing most ; though, as we shall have occasion to remark, the baker too often contrives to force and keep into bread made from inferior flour, by a process called under baking, tho same amount of water as is normally taken up by that of the best quality. Gonerally speaking, and with flour of good average quality, the amount of water is such, that the diluted sponge forms about 14 gallons of liquid. The wholo mass is then torn to pieces by the hand, so as to break any lumps that there may be, and mix it up thoroughly with the water. This being done, the rest of the sack of flour is gradually added and kneaded into a dough of the proper consistency. This kneading of the dough may be said to be one of the most important processes of the manufacture, since it not only produces a more complete hydration of the flour, but, by imprisoning a certain quantity of air within the dough, and forcibly bringing into closer contact the molecules of the yeast or leaven with the sugar of the flour, and also with a portion of the starch, the fermentation or rising of the whole mass, on which the sponginess of the loaf and its digestibility subsequently depend, is secured. When by forcing the hand into the dough, the baker sees that, on withdrawing it, none of the dough adheres to it, he knows that the kneading is completed. The dough is then allowed to remain in the trough for about an hour and a half or two hours, if either brewers' or German yeast havo been employed in making the sponge;-if, on the contrary, patent yeast or hop yeast have been used, three or even four hours may be required for the dough to rise up, or, as in tochnical language, to give proof. When the dough is sufficiontly 'proofed,' it is weighed of into lumps, shaped into the proper forms of $4 \mathrm{lbs}, 4 \mathrm{oz}$. each, and oxposed for about one hour in an oven to a temporature of about $570^{\circ} \mathrm{F}$, the heat gradually falling to 430 or $420^{\circ} \mathrm{F}$. The yield after baking is 94 quartern (not 4 -lb.) loavos, or from 90 to 92 really $4 \mathrm{lb}$. loaves, as largo again as they wore when put into the oven in the shape of dough.

The manner in which yeast acts upon tho flour-is, as yet, an unsolved mystory, or at any rate an, as yet, unsatisfactorily explained action; for the term 'catalysis,' which has sometimes been applied to it, explains absolutoly nothing.

A yeast, or formenting material, may bo prepared in various ways; but only three kinds of yeast are used by bakers: namely, brewors' yeast, or barm, - German yeast, -and patent, or hop yeast.

The most active of these ferments is the first, or browers' yeast; it is, as is well 
known, a frothy, thickish matorial, of a brownish or drab colour, which, when rocent, is in a stato of slight effervescenco, exhales a sour characteristic odour, and has an aeid reaction.

When viewed through the microscope, it is seen to consist of small globules of various size, generally egg-shaped. They wero first described by M. Desmayieres.

The best, and in fact the only, browers' yeast usod in broad-making is that from the alo breweries; portor yeast is unavailable for the purpose, becauso it imparts to the bread a disagreeable bitter taste.

German yeast is very extensivoly used by bakers. It is a pasty but easily crumbled mass, of an agreeable fruity odour, and of a dingy white colour. German yeast will remain good for a fow wooks, if kept in a cool place. When in good condition, it is an excellont articlo; but samples of it aro occasionally soized on bakers' premises, of a darker colour, viscid, and emitting an offensive choesy odour: such Gorman yeast, being in a putrefied state, is, of course, objectionable.

Tho so-called 'patent yeast' is the cheapest and at the same time the weakest of these ferments; very good bread, however, is mado with it, and it is most extensively used by bakers. It is made either with or without hops: when with hops, it is called hop yeast, and is nothing more than a decoction of hops to which malt is added while in a scalding hot state; when the liquor has fallon to a blood heat, a certain quantity of brewers' or German yeast is thoroughly mixod with it, and tho whole i: left at rest. The use of the hops is intended to diminish the tendency of this soluticis to become acid.

Potato yeast is a kind of 'patent yeast' in general use. See YEAST.

The theory of panification is not difficult of comprehension. 'The flour,' says Dr. Ure, 'owes this valuable quality to the gluten, which it contains in groater abundance than any of the other cerealia (kinds of corn). This substance does not constitute, as has been heretofore imagined, the mombranes of the tissuo of the perisperm of the wheat; but is enclosed in cells of that tissue under the epidermic conts, even to the centre of the grain. In this rospect the gluten lies in a situation analogous to that of the starch, and of most of the immediate principles of the vegetables. The other immediate principles which play a part in panification aro particularly the starch and the sugar; and they all operate as follows:-

'The diffusion of the flour through the water hydrates the starch, and dissolves the sugar, the albumen, and some other soluble matters. The kneading of the dough, by completing these reactions through a more intimate union, favours also the fermentation of the sugar, by bringing its particles into close contact with those of the leaven or yeast; and the drawing out and laminating the dough scftens and stratifies it, introducing at the same time oxygen to aid the formentation. The dough, when distributed and formed into loaves, is kept some time in a gentle warmth, in the folds of the cloth, pans, \&c., a circumstance propitious to the devolopment of their volume by fermentation. The dimensions of all the lumps of dough now gradually enlarge, from the disengagement of carbonic acid in the decomposition of the sugar, which gas is imprisoned by the glutinous paste. Were these phenomena to continue too long. the dough would become too vesicular; they must, therefore, be stopped at the proper point of sponginess, by placing the loaf lumps in the oven. Though this causes a sudden expansion of the enclosed gaseous globules, it puts an end to the fermentation, and to their growth; as also evaporates a portion of the water.

'The fermentation of a small dose of sugar is, therefore, essential to truo bread making: but the quantity actually fermented is so small as to be almost inappreciable. It seems probable that in well-made dough the whole carbonic acid that is generated romains in it, amounting to one-half the volume of the loaf itself at its baking temperature, or $212^{\circ} \mathrm{F}$. It thence results that less than one-hundredth part of the woight of tho flour is all the sugar requisito to produce well-raised bread.

'Although the rising of the dough is determined by the carbonic acid resulting from the decomposition of the sugar, produced by the reaction of the gluten on hydrated or moist flour, cousidering that the quantity of sugar necessary to produce fermentation does not amount, probably, to more than one-hundredth part of the weight of the flour employod, and perhaps to even considerably less than that, - the saving and economy which are said to accrue to the consumer from the use of unfermented bread (which is bread in which the action of yeast is replaced by an artificial ovolution of carbonic acid, by decomposing bicarbonato of soda with muriatic acid, as wo said before) is therefore much below what it has been estimated ( 25 per cent. !) by some writers; and is certainly very far from componsating for tho various and serious drawbacks which are poeuliar to that kind of bread, one of which-and it is not the least-is its indigestibility, notwithstanding all that may have beon said to the contrary.

'In a pamphlet, entitled, "Instructions for making Unfermented Bread, by a Phy- 
sician," published in 1846 , the formula recommended for bread made of wheat moal is as follows:-

Wheat meal
Bicarbonate of soda
Hydrochloric acid.
Water :
Salt

3 lbss avoirdupois.

$4 \frac{1}{3}$ drachms troy.

5 fluid drachms and 25 minims, or drops.

30 fluid ounces.

$\frac{2}{3}$ of an ounce troy.

'Bread made in this manner,' says the author, 'contains nothing but flour, common salt, and water. It has an agreeable, natural taste, keops much longer than common bread, is much more digestible, and much less disposed to turn acid,' \&c.

Liebig, in his 'Letters on Chemistry,' very judiciously remarks, 'that the intimate mixture of the saliva with the bread, whilst masticating it, is a condition which is favourable to the rapid digestion of the starch; wherefore the porous state of the flour in fermented bread accelerates its digestion.'

Now, it is a fact, which can be readily ascertained by anyone, that unfermented bread is permeated by fluids with difficulty. It will not absorb water, hence its heavy and clammy feel; nor saliva, hence its indigestibleness; nor milk, nor butter. Unfermented bread will neither make soup, nor toast, nor poultice. When a slice of ordinary bread is held before a bright fixe, a portion of the moisture of the bread, as the latter becomes scorched, is converted into steam, which penetrates the interior of the mass, and imparts to it the sponginess so well known in a toast properly made; but if a piece of unfermented bread be treated in the same manner, the steam produced by the moisture, not being able to penetrate the unabsorbent mass, evaporates, and the result is an uninviting slice, toasted, but hard inside and out, and into which butter penetrates about to the same extent as it would a wooden slab of the same dimensions.

'Fermentation,' says tiebig, 'is not only' the best and simplest, but likewise the most economical way of imparting porosity to bread; and besides, chemists, generally speaking, should never recommend the use of chemicals for culinary preparations, for chemicals are seldom met with in commerce in a state of purity. Thus, for example, the muriatic acid which it has been proposed to mix with carbonate of soda in bread is always very impure, and very often contains arsenic. Chemists never employ such an acid in operations which are certainly less important than the one just mentioned, without having first purified it.'

In order to remove this ground of objection, tartaric acid has been recommended instead of muriatic acid for the purpose of decomposing the carbonate of soda; but in that way, another unsafe compound is introduced, since the result of the reaction is tartrate of soda, a diuretic aperient, and consequently very objectionable salt, for it is impossible to say what mischief the continuous ingestion of such a substance may eventually produce; and whatever may be the divergence of opinion, - -if there be such a divergence, - as to whether or not the constant use of an aperient, however mild, may be detrimental to health, it surely must be admittod that, at any rate, it is better to eschew such, to say the least of it, suspicious materials ; and that, at any rate, if deprecating their use be an error, it is an error on the safe side;-after all, a bakehouse is not a chemical laboratory.

Before leaving this question of unfermented bread, we must not omit to speak of a remarkable process invented by Dr. Dauglish, and which has lately excited some attention. Without discussing the value of the idea which is said to have led Dr. Dauglish to invent the process in question, we shall simply describe Dr. Dauglish's method of making bread, and give his own version of its benefits :-

'Taking advantage of the well-known capacity of water for absorbing carbonic acid, whatever its density, in quantities equal to its own bulk, I first prepare the water which is to be used in forming the dough, by placing it in a strong vessel capable of bearing a high pressure, and forcing carbonic acid into it to the extent of say ten or twolve atmospheres ' (about 150 to $180 \mathrm{lbs}$. per square inch); 'this the water absorbs withont any appreciable increase in its bulk. The water so prepared will of course retain the carbonic acid in solution so long as it is retained in a close vessel under the same pressure. I therefore place the flour and salt, of which the dough is to be formed, also in a close vessel capable of bearing a high pressure. Within this vessel, which is of a spheroidal form, a simply-constructed kneading apparatus is fitted, worked from without through a closely-packed stuffing box. Into this vessel I force an equal pressure to that which is maintained on the aêrated watervessel; and then, by means of a pipe connecting the two vessels, I draw the water into the flour, and set the kneading apparatus to work at the same time. By this arrangement the water acts simply as limpid water among the flour, the flour and water are mixed and kneaded together into paste, and to such an extent as shall

Vor. I. 
give it the necessary tenacity. After this is accomplished the pressure is released, the gas escapes from the water, and in doing so raises the dough in the most beautiful and expeditious manner. It will be quite unnecessary for me to point out how perfect must be the mechanical structuro that results from this method of raising dough. In the first place, the mixing and kneading of the flour and water together, before any vesicular property is imparted to the mass, render the most complete incorporation of the flour and water a matter of very easy accomplishment; and this being secured, it is evident that the gas which forms the vesicle, or sponge, when it is released, must be dispersed through the mass in a manner which no other method-fermentation not excepted-could acoomplish. But besides the advantages of kneading the dough before the vesicle is formed, in the manner above mentioned, there is another, and perhaps a more important one, from what it is likely to effect by giving seope to the introduction of new materials into bread making, - and that is, I find that powerful machine-kneading, continued for several minutes, has the effect of imparting to the dough tenacity or toughness. In Messrs. Carr and Co.'s machine, at Carlisle, we have kneaded some wheaten dough for half an hour, and the result has been that the dough has been so tough, that it resembled birdlime, and it was with difficulty pulled to pieces with the hand. Other materials, such as rye, barley, \&c. are affected in the same manner. So that by thus kneading, I am able to impart to dough made from materials which otherwise would not make light bread, from their wanting that quality in their gluten which is capable of holding or retaining, the same degree of lightness which no other method is capable of effecting. And I am sanguine of being able to make from rye, barley, oatmeal, and other wholesome and nutritious substances, bread as light and sweet as the finest wheaten bread. One reason why my process makes a bread so different from all other processes where fermentation is not followed is, that I am enabled to knead the bread to any extent without spoiling its vesicular property; whilst all other unfermented breads are merely mixed, not kneaded. The property thus imparted to my bread by kneading, renders it less dependent on being placed immediately in the oven. It certainly cannot gain by being allowed to stand after the dough is formed, but it bears well the necessary standing and waiting required for preparing the loaves for baking.

' There is one point which requires care in my process, and that is,-the baking: as the dough is excessively cold ; first, because cold water is used in the process ; and next, because of its sudden expansion on rising. It is thus placed in the oven some $40^{\circ}$ Fahr. in temperature lower than the ordinary fermented bread. This, together with its slow springing until it reaches the boiling point, renders it essential that the top crust shall not be formed until the very last moment. Thus, I have been obliged to have ovens constructed which are heated through the bottom, and are furnished with the means of regulating the heat of the top, so that the bread is cooked through the bottom; and, just at the last, the top heat is put on and the top crust formed.

'With regard to the gain effected by saving the loss by fermentation, I may state what must be evident, that the weight of the dough is always exactly the sum of the weight of flour, water, and salt put into the mixing vessel: and that, in all our experiments at Carlisle, we invariably made 118 loaves from the same weight of flour which by fermentation made only 105 and 106. Our advantage in gain over fermentation can only be equal to the loss by fermentation. As there has been considerable difference of opinion among men of science with respect to the amount of this loss-some stating it to be as high as $17 \frac{1}{2}$ per cent., and others so low as 1 per cent.I will here say a few words on the subject. Those who have stated the loss to be as high as $17 \frac{1}{2}$ per cent. have, in support of thoir position, pointed to the extra yield from the same flour of bread when made by non-fermentation, compared with that mado by fermentation. Whilst those who have opposed this assertion, and stated the loss to be but 1 per cent. or little more, have declared the gain in weight to be simply a gain of extra water, and have based their calculations of loss on the destruction of material caused by the generation of the necessary quantity of carbonic acid to render the bread light. Starting then with the assumption that light bread contains in bulk half solid matter and half aëriform, they have calculated that this quantity of aëriform matter is obtained by a destruction of but one per cent. of solid material. In this calculation the loss of carbonic acid, by its escape through the mass of dough during the process of fermentation and manufacture, does not appear to have been taken into account. All who have been in any way practically connected with bakeries well know how large this loss is, and how important it is that it should be taken into account, that our calculations may be correct.

"One of the strongest proofs that the escape of gas through ordinary soft bread dough is very large arisos from the fact, that when biscuit dough, in which there is a mixture of fatty matter, is prepared by my process, about half the quantity of gas only is needed to obtain an equal amount of lightness with dough that is made of flour 
and water only, the fatty matter acting to prevent the escape of gas from the dough. Other matters will operate in a similar manner-boiled flour, for instance, added in small quantities. But the assumption that light bread is only half aëriform matter is altogether erroneous. Nover before has there been so complete a method of testing what proportion the aëriform bears to the solid in light bread as that which my process affords. The mixing vessel at Messrs. Carr and Co.'s works, Carlisle, has an internal capacity of ten bushels. When $3 \frac{1}{2}$ bushels of flour are put into this vessel, and formed into spongy bread dough, by my process, it is quite full. And when flour is mixed with water into paste, the paste measures rather less than half the bulk of the original dry flour. This will, therefore, represent about $1 \frac{3}{4}$ bushel of solid matter expanded into 10 bushels of spongy dough, showing in the dough nearly 5 parts aëriform to 1 solid; and in all instances, if the baking of this dough has not been accomplished so as to secure the loaves to 'spring' to at least double their size in the oven, they have always come out heavy bread when compared with the ordinary fermented loaves. This gives the relative proportion of aëriform to solid in light bread at least as 10 to 1 , and at once raises the loss by fermentation from 1 to 10 per cent., without taking into account the loss of gas by its passage through the mass of dough.

- Of the quality and properties of the bread manufactured by my process, there will shortly be amplo means of judging. I may be allowed, however, here to state, what will be evident to all, that the absence of everything but flour, water, and salt, must render it absolutely pure; - that its sweetness cannot be equalled, except by bread to which sweet materials are superadded;-that, unlike all other unfermented bread, it makes excellent toast; and, on account of its high absorbent power, it makes the most delicious sop puddings, \&c., and also excellent poultice. Sop pudding and poultice made from this bread, however, differ somewhat from those made from fermented bread, in being somewhat richer or more glutinous. This arises from the fact of the gluten not having been changed, or rendered soluble, in the manner caused by fermentation; but that this is a good quality rather than a bad one is evident from the fact, that the richer and purer fermented bread is, the more glutinous are the sop, \&c., made from it; and the poorer and more adulterated with alum it is, the freer the sop, \&ce, are of this quality.'

Such then is Dr. Dauglish's plan; and it is impossible to deny that it possesses great ingenuity.

From the fact that, in all his experiments at Carlisle, Dr. Dauglish invariably made 118 loaves from the same weight of flour which, by fermentation, made only 105 or 106 , to argue that the gain over fermentation can only be equal to the loss by fermentation, is to draw a somewhat hasty conclusion; for the gain may be, and is probably due, not to the preservation in the bread of what is generally lost by fermentation, but simply to a retontion of water.

It is of course certain that the production of the porosity required in bread produced by the carbonic acid and alcohol evolved by fermentation, entails the loss of a portion of the valuable constituents of the flour, but the amount of that loss should not be estimated, I think, from the proportions which the aëriform bear to the solid matter of the loaf after it is baked.

In effect, the fermentation induced in bread differs from that produced at the distillery, in as much as, instead of the fermenting material being sheltered from the air by an atmosphere of carbonic acid, the dough is on the contrary thoroughly permeated by, and retains a considerable quantity of atmospheric air introduced into it by the kneading process, and owing to the presence of which, in fact, the acetous fermentation is carried on to a certain extent, within the dough, simultaneously with the alcoholic fermentations, so that even the 10 parts of aëriform matter to 1 of solid matter in a quartern loaf, are not altogether carbonic acid resulting from the fermentation, but are carbonic acid from that source mixed with the atmospheric air with which the dough is permeated. On the other hand, the aëriform matter thus imprisoned in the dough, expands to at least twice its volume when exposed to the temperature of the oven, und accordingly the bread after breaking becomes as bulky again as the dough from which it was made, and this doubling of the volume being due to the expansion of the gases, and not to the fermentation, bears no proportion whatover to the amount of the sugar of the flour employed in the production of the alcohol and carbonic acid evolved. Moreover, as a quartern loaf, for example, measures about 9 inches by 6.5 inches by 5 inches, making a total of about 292 cubic inches, if we take nine-tenths of that to be aëriform matter, we have 262.8 inches as the aëriform cubic contents of the quartern loaf.

It is ascertained beyond doubt by numorous experiments, that genuine, properly manufactured now bread contains, on an avorage, 42.5 per cent. of water, and 57.5 of flour, and consequently a quartern loaf weighing really four pounds, would consist of 11,900 grains of water and 16,000 grains of solid matter, 422.5 grains of which aro 
salt and inorganic mattor, the rest, 15677.5 grains, being starch and gluton. Now a quartern loaf measuring about $9 \times 6.5 \times 5$ inches gives a total of 202 cubic inches. Assuming with Dr. Dauglish, nine-tonths of that to be ä̈riform mattor, we heve 262.8 inches of aëriform cubic contents of a quarten loaf, but as the gases expanded in the dough to double their volume during its boing baked into a loaf, we must divide by 2 the 262.8 inches above alluded to, which gives 131.4 as the number of cubic inches of aëriform matter contained in the dough before it went into the oren. Agrin, assuming with Dr. Dauglish that these 131.4 cubic inches consist altogether of carbonic acid resulting from the fermentation of the flour, they would represent in weight only 62 grains of that gas, and as 1 equivalent $=198$ of sugar produces 4 equivalents $=88$ of carbonic acid, it follows that, at most, about 140 grains of sugar or solid matter out of the 15677.5 of flour in the quartern loaf would have disappeared, which loss is less than 1 per cent., from which, however, it is necossary to make a considerable reduction, since a large quantity of air is mixed with that carbonic acid, and expanded with it in the oven. Unless, therefore, it can be satisfactorily proved that the unfermented bread manufactured by Dr. Danglish's process is more nutritious, weight for weight, or more digestible, or possesses qualities which unfermented broad has not, or is sold at a reduced price proportionate to the quantity of water thus locked up and passed off for bread, the benefits and advantages will be all on the manufacturer's side, but the purchasers of the unfermented bread will make but a poor bargain of it.

Of all the operations connected with the manufacture of bread, the most laborious, and that which calls most loudly for reform, is that of kneading. The process is usually carried on in some dark corner of a cellar, where the temperature is seldom less than $60^{\circ} \mathrm{F}$., and frequently more, by a man, stripped naked down to the waist, and painfully engaged in extricating his fingers from a gluey mass into which he furiously plunges alternately his clenched fists, heavily breathing as he, struggling, repeatedly lifts up the bulky and tenacious mass in his powerful arms, and with effort flings it down again with a groan fetched from the innermost recesses of his chest, and which almost sounds like an imprecation.

We know, on very good and unexceptionable authority, that a certain large bakery on the borders of a canal actually pumped the water necessary for making the dough directly and at once from the canal, and this from a point exactly contiguous to the dischargings of the cesspool of that bakery! And let us not imagine that this is a solitary instance of horrible filth. The following memoranda recorded by Dr. Wm. A. Guy, in his admirable lecture on "The Evils of Night-work and Long Hours of Iabour,' delivered on Thursday, July 6, 1848, at the Mechanics' Institution, Southampton Buildings, will serve to illustrate the condition of the bakehouses:-

1. Underground, two ovens, no daylight, no ventilation, very hot and sulphurous.

2. Underground, no daylight, two ovens, very hot and sulphurous, low ceiling, no ventilation but what comes from the doors. Very large business.

3. Underground, no daylight, often flooded, very bad smells, overrun with rats, no ventilation.

After mentioning several other establishments in the same, or even in a worse, condition then those just enumerated, Dr. Guy adds :

- The statements comprised in the foregoing memoranda are in conformity with my own observations. Many of the basements in which the business of baking are carried on are certainly in a state to require the assistance of the Commissioners of Sewers, and to invite the attention of the promoters of sanitary reform.'

If we reflect that bread, like all porous substances, readily absorbs the air that surrounds it, and that, even under the best conditions, it should never, on that account, be kept in confined places, what must be the state of the bread manufactured in such a villanous manner, and with a slovenliness greater than it is possible for our imagination to conceive? What can prove better the necessity of Government suporvision than such a fact? The heart sickens at the revolting thought, but after all there is really but little difference between the particular case of the bakery on the border of a canal above alluded to, and the mode of kneading generally pursued, and to which wo daily submit.

In the sitting of the Institute of France, on the 23rd of January, 1850, the late M. Arago presented and recommended to the Académie the kneading and baking apparatus of M. Rolland, then a humble baker of the 12th Arrondissement, which, it would appear, fulfils all the conditions of perfect kneading and baking.

'The kneading machine ( pétrin mécanique) of M. Rolland,' says Arago, 'is extremely simple, and can be easily worked, when under a full charge, by a young man from 15 to 20 years old : the necessity for horse-labour or steam-power may thus be obviated. The machine ( figs. 217 to 220 ) consists of a horizontal axis traversing a trough, containing all 
the dough requisite for one baking batch, and upon which axis a system of curvilinear blades, alternately long and short, are placed in such a manner that, while revolving, they describe two quarters of cylindrical surfaces with contrary curves, so that the convexity of one of those surfaces, and the concavity of the other, is turned towards the bottom of the trough. The axis has a fly-wheel, and is set in motion by two small cog-wheels connected with the handle, as xepresented in the following figures:-

217

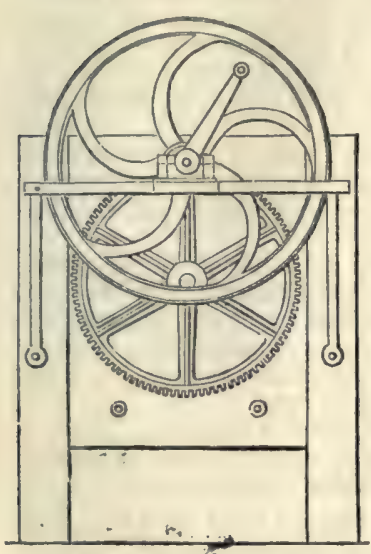

219
218

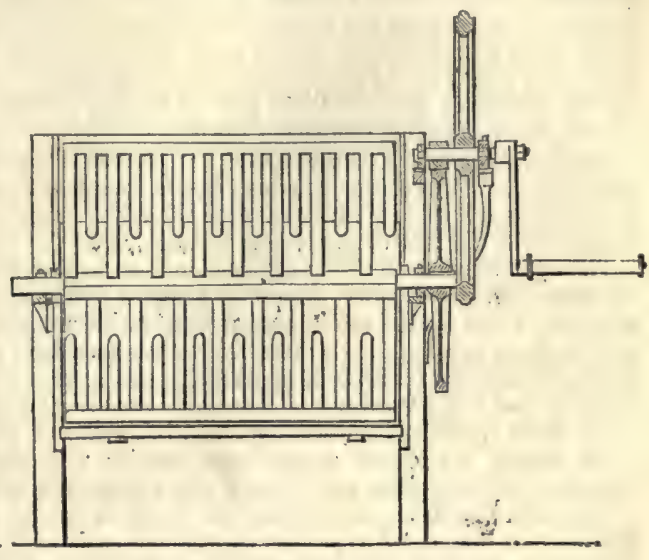

220

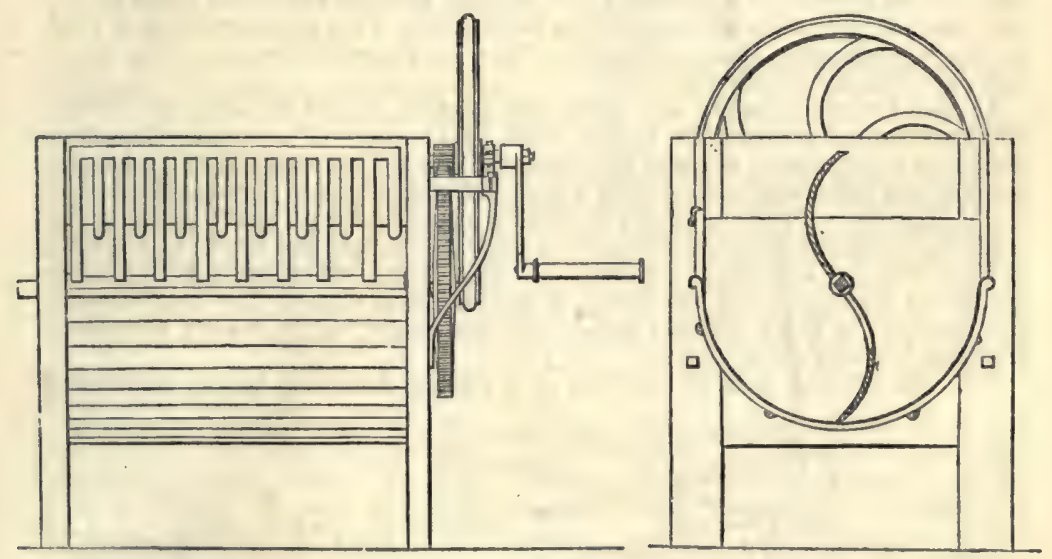

The action of the kneading machine is both easy andefficacious. In 20, and if necessary in 15, or even 10 minutes, a sack of flour may be converted into a perfectly homogeneous and aërated dough, without either lumps or clods, and altogether superior to any dough than could be obtained by manual kneading. The time requirod in kneading varies according to the greater or less density of dough required; and the quantity of dough manufactured in that space of time varies, of course, also with the dimensions of the kneading-trough; for instance, in the trough provided with 16 blades, one sack and a half of flour can be kneaded at once; in that of 14 blades one sack, and in that of 12 blades two-thirds of a sack.

M. Rolland gives the following instructions for the use of the machine, in order to impart to the dough the qualities produced by the operations known in France under the names of frasage, contrefrasage, and soufflage, which wo shall presently describo, and to which the bread manufactured in that country mainly owes, in the words of Dr. Ure, ' a flavour, colour, and texture, never yet equalled in London.'

The necessary quantity of leaven or yeast is first diluted with the proper quantity of water, as described before; and in order to effect the misture, the crank should be 
made to perform 50 revolutions altornately from right to left.-Frasage is the flrst mixture of the flour with the wator. The flour is simply poured into the kneadingtrough, or, better still, whon convenience permits it, it is let down from a room above through a linon hose, which may be shut by folding it up at the extremity.

Three-fourths only of the flour should at first be put into the trough; the first revolutions of the kneader should be rather rapid, but during the remainder of the operation the turning should be at the rate of two or three revolutions a minute, according to the density of the dough to bo propared. The dough thereby having time to be well drawn out between the blades, and to drop to the bottom of the trough. From 24 to 36 revolutions of the crank will generally be sufficient; but in order to obtain the dough in the condition which the frasage would give it in the usual way, it will be necessary to make about 250 revolutions of the crank alternately from right to left, about the same number of turns.

Contrefrasage is the completion of the process of mixing; and, in order to perform that operation, the last fourth part of the flour must now be added, the crank turned 150 revolutions, to wit, 75 turns rather slowly, alternately from right to left, and tho remsinder at the rate of speed above mentioned.

The operation of Soufflage consists in introducing and retaining air in the paste. To effect this, the kneader should be made to perform, during nearly the whole time occupied in the operation, an almost continuous motion backwards and forwards, by which means the dough is shifted from place to place; five revolutions being made to the right, and five to the left, alternately, taking care to accelerate the speed a littlo at the moment of reversing the direction of the revolving blades.

All these operations are accomplished in twenty or twenty-five minutes.

Of course, the reader should not imagine that these numbers must be strictly followed, they are given merely as a guide indicative of the modus operandi.

The kneading being completed, the dough is left to rest for some time, and then divided into lumps, of a proper weight, for each loaf. The workman takes one of these lumps in each hand, rolls them out, dusts them over with a little flour, and puts each of them separate in its panneton; he proceeds with the rest of the dough in the same manner, and leaves all the lumps to swell, which, if the flour have been of good quality, will take place at a uniform rate. They are then fit for baking, which operation will be described presently.

Another kneading trough, said to be very effectual, is that for which $\mathbf{M r}$. Edwin Clayton obtained a patent in August 1830. It consists of a rotatory kneading trough, or rather barrel, mounted in bearings with a hollow axle, and of an interior frame of cast iron made to revolve by a solid axle which passes through the hollow one; in the frame there are cutters diagonally placed for knoading the dough. The rovolving frame and its barrel are made to turn in contrary directions, 80 as greatly to save time and equalise the operation. This double action represents kneading by the two hands, in which the dough is inverted from time to time, torn asunder, and reunited in every different form. The mechanism will be readily understood from the following description:-

Fig. 221 exhibits a front elevation of a rotatory kneading trough, constructed

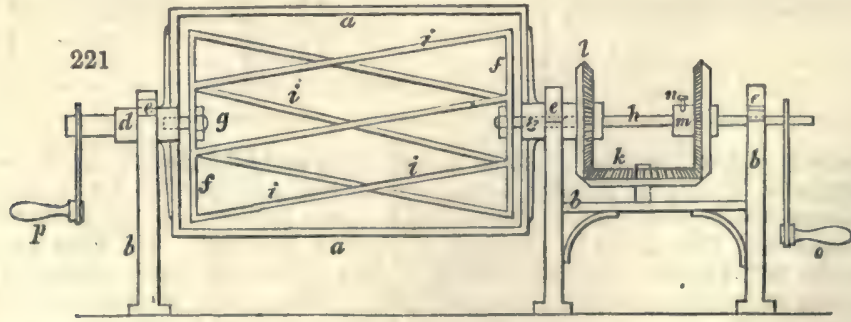

according to improvements specified by the patentee, the barrel being shown in section; $a$ is the barrel. into which the several ingredients, consisting of flour, water, and yeast, are put, which barrel is mounted in the frame-work $b$, with hollow axles $c$ and $d$, which hollow axles turn in suitable bearings at $e ; f$ is the revolving framo which is mounted in the interior of the barrel $a$, by axles $g$ and $h$. The ends of this revolving frame are fastened or braced together by means of the oblique cutters or braces $i$, which act upon the dough when the machine is put in motion, and thus cause the operation of kneading.

Either the barrel may be made to revolve without the rotatory frame, or the rota- 
tory frame without the barrel, or both may be made to revolve together, but in opposite ways. These several motions may be obtained by means of the gear-work, shown at $k, l$, and $m$, as will be presently described.

If it be desired to have the revolving motion of the barrel and rotatory frame together, but in contrary directions, that motion may be obtained by fastening the hollow axle of the wheel $m$, by means of a screw $n$, to the axle $h$ of the rotatory frame $f$ tight, so as they will revolve together, the other wheols $k$ and $l$ being used for the purpose of reversing the motion of the barrel. It will thon be found that by turning the handle $o$, the two motions will be obtained.

If it be desired to put the rotatory frame $f$, only into motion, that action will be obtained by loosening the serew $n$ upon the axle of the wheel $m$, when it will be found that the axlo $h$ will be mado to revolve freoly by means of the winch $o$, without giving motion to the wheels $k, l$, and $m$, and thus the barrel will remain stationary. If the rotatory action of the barrel be wanted, it will be obtained by turning the handle $p$, at the reverse end of the machine, which, although it puts the gear at the opposito end of the barrel into motion, yet as the hollow axle of the wheel $m$ is not fastened to the axle $h$ by the screw $n$, these whools will revolve without carrying round tho frame $f$.

Tho Hot-water Ovon Biscuit-baking Company possesses also a good machine with which 1 cwt. of biscuit dough, or 2 cwts. of bread dough, can be perfectly kneaded in
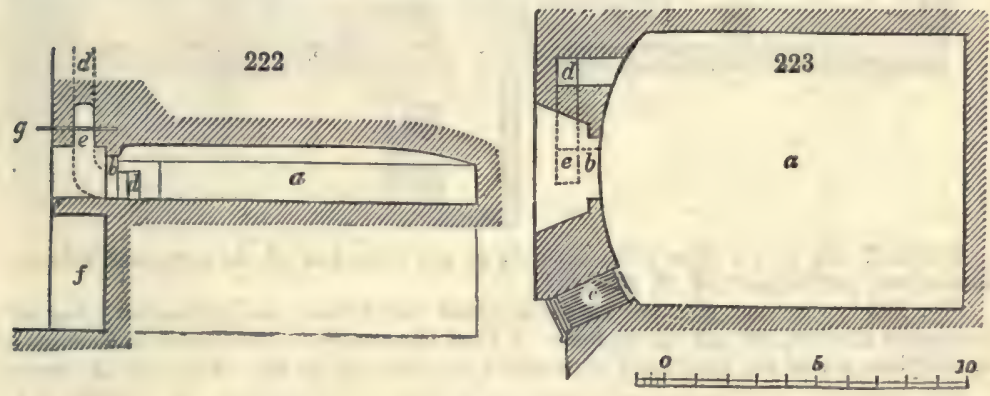

10 minutes. The machine is an American invention, and of extraordinary simplicity, for it is in reality nothing more than a large corkscrew, working in a cylinder, by means of which the dough is triturated, squeezed, pressed, torn, hacked, and finally agglomerated as it is pushed along. The dough as it issues from that machine can at once be shaped into loaves of suitable size and dimensions. A machine capable of doing the amount of work alluded to does not come to more than from $6 l$. to $7 l$.; the other forms of kneading machines are likewise inexpensive, so that, in addition to the economy of time which they realise, there does not seem to be any excuse for re. taining the abomination of manual knoading.

Among superior and very desirable apparatus for bread-making, there are at any rate three which fulfil the desiderata above alluded to, in the most complete and economical manner. One of them is M. Mouchot's aërothermal bakery; the second is A. M. Perkins' hot-water oven; the third is Rollana's hot-air oven, with revolving floors: all three are excellent.

Before proceeding to explain them, a plan and longitudinal section of an ordinary London baker's oven is given (figs. 222 and 223), that the reader may be the better able to judge of the rast improvement realised by the other ovens.

$a$, the body of the oven; $b$, the door; $c$, the fire-grate and furnace; $d$, the smoke flue; $e$, the flue abovo the door, to carry off the steam and hot air, when taking out the bread; $f$, recess. below the door, for receiving the dust; $g$, damper" plate to shut off the steam flue; $h$, damper plate to shut off smoke flue, after the oven has come to its propar heat; $i$ a small iron pan over the fire-place $c$, for heating water; $k$, ash-pit below the furnace.

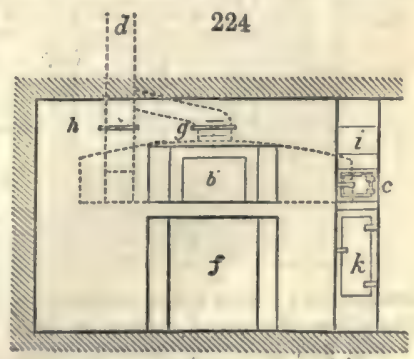

Fig. 224 is the front view; the same letters refer to the same object in all the figures. 
The flame and burnt air of the fire at $c$, sweeping along the bottom of the oven by the right-hand side, are reflectod from the back to the loft-hand side, and thence escrpe by the fluo $d$. Whenever the oven has acquired the proper degree of heat, the fire is withdrawn, the flues are closed by the damper plate, and the lumps of fermented dough are introduced.

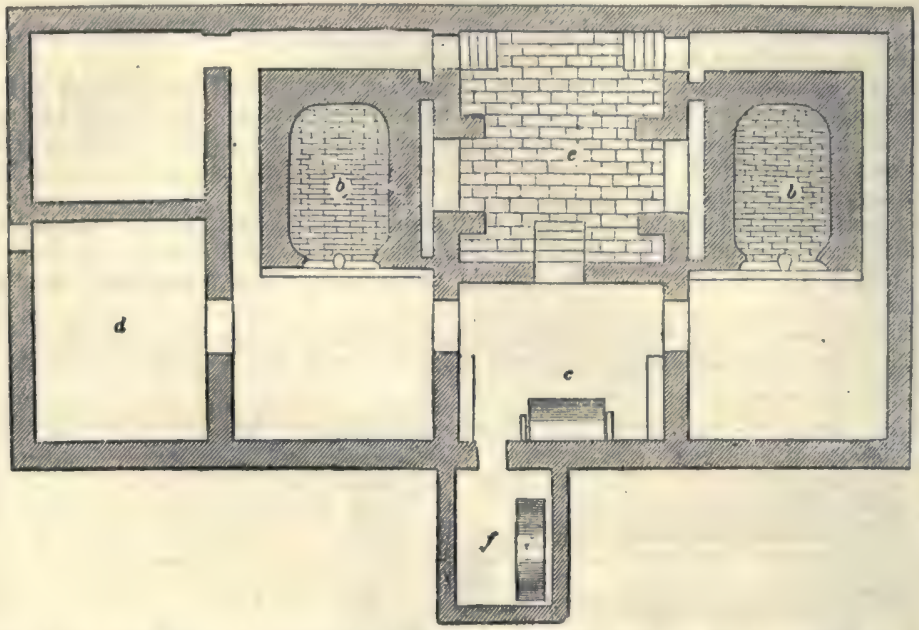

We shall now give a deseription not only of the oven, but of the improved bakery, boulangerie perfeotionie, of $\mathrm{M}$. Mouchot.

Fig. 225 is a ground plan of the ärothermal bakehouse: the granaries being in the upper storeys, are not shown here. $b b$ are the ovens; $c$, the kneading machine; $d$, the place where the machinery is mounted for hoisting up the bread into the store

226

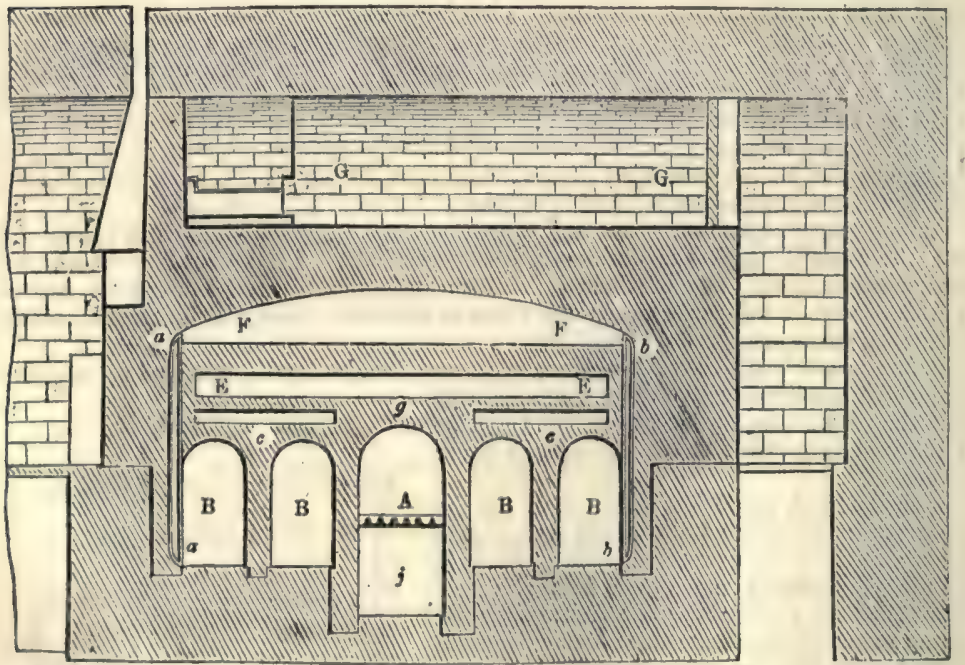

room above; $e$, va space common to the two ovens, into which the hot air passes; $f$, the place of a wheol driven by dogs, for giving motion to the kneading machine.

Fig. 226 is a longitudinal section of the oven; $\mathbf{A}$, the grate where coke or even pitcoals may be burned; B B, void spaces which, becoming heated, serve for warming small pieces of dough in; $c c$ are flues for conducting the smoke, \&c., from the fire- 
place; $D$, seen in fig. 227 (a transverse section through the middle of the oven), is the chimney for carrying off the smoke transmitted by the flues; $\mathbf{B E}$, void spaces immediately over the flues, and beneath the sole F F, of the oven. By this arrangement the air, previously heated, which arrives from the void spaces $\mathrm{B}$ through the flues $c c$,

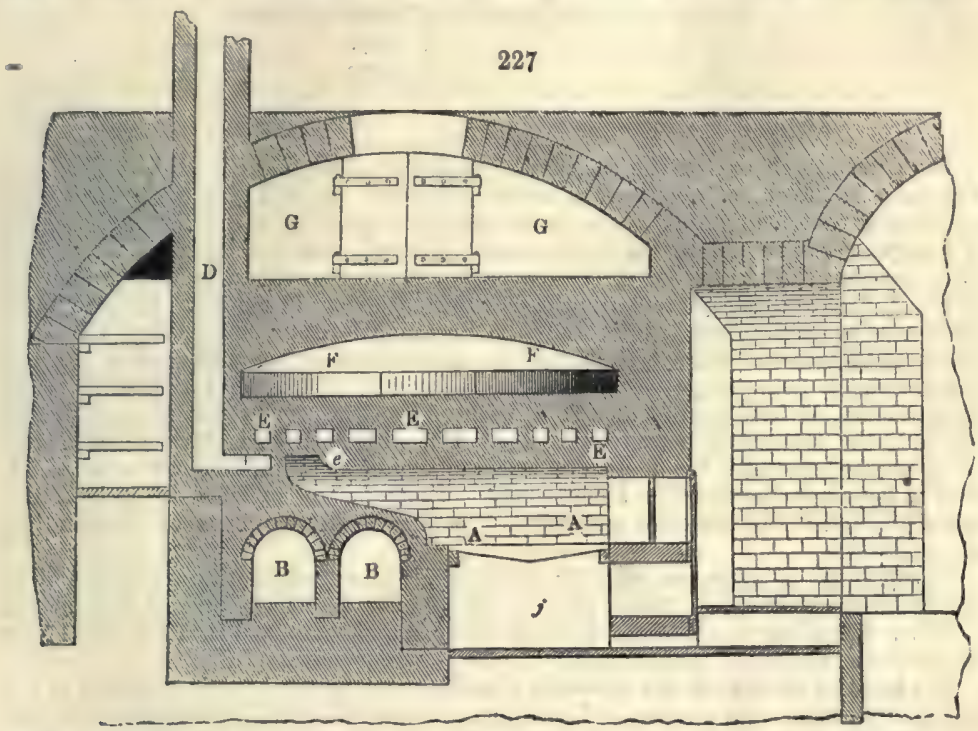

gets the benefit of the heat of the flame which circulates in these flues, and, after getting more heated in the spaces $\mathbf{E} \mathbf{E}$, asconds through channels into the oven $\mathbf{F} \mathbf{r}$, upon the sole of which the loaves are to be baked or laid. The hot air is admitted into it through the passages $a a$, being drawn from the reservoirs $\mathrm{B}$ В $\mathrm{B}$, and also by the passage $d \bar{d}$, drawn from the reserroirs $\mathbf{x}$. The sole is likewise heated by contact with the hot air contained in the space $\mathrm{E} \mathbf{\mathrm { E }}$, placed immediately below it. The hot air, loaded with moisture, issues by the passage $b b$, and returns directly into the reservoir B B. G $\mathrm{G}$, an enclosed space directly over the oven, to obstruct the dissipation of its heat; $g$, vault of the fire-place. Fig. 228, the kneading machine, a longitudinal section passing through its axis; P P, the contour of the machine, made of wood, and divided into three compartments for the reception of the dough. The wooden bars 00 are so placed in the interior of the compartments, as to divide the dough whenever the cylinder is made to revolve. One portion, $\mathrm{D}$, of the cylinder may be opened and laid over upon the other by means of a hinge joint, when the dough and flour are introduced. A, B, c, the three compartments of the machine, two for making the dough, and one for preparing the sponge, called levain, or leaven, by the French. $a$ a is the pulley which receives its motion from the engine, and transmits it to the cylinder through the pinion $b$, and the spur-wheel $e ; d d$, the fly-whe日l to regulate the motion; $g, a$ brake to act upon the fly $\bar{d}$, by means of a lever $h ; i$, the pillar of the fly-wheel.

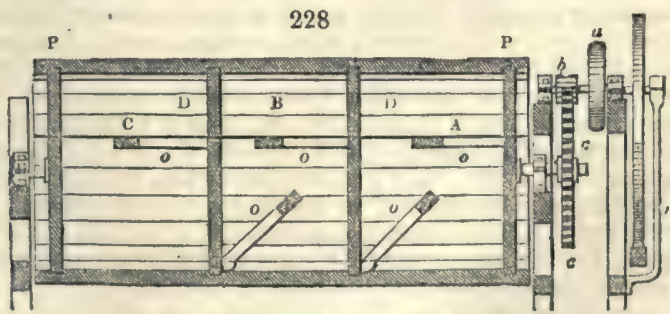

There is a ratchet-wheel counter for numbering the turns of the kneading machine, but it cannot be shown in this view ; $n$, cross-bars of wood, which are easily removed when the cylinder is opened; they divide the dough.

Fach of the three compartments of the kneader (fig. 228) is furnished at pleasure 
with two bars fxed crosswise, but which may be essily romoved, whenever the cylinder is oponed. These bars constitute the solo agents for drawing out the dough. In a continuous operation, the leavon is constantly preparod in the compartmont $A$; with which view thare is put into it-

125 kilogrammes of ordinary leaven or yeast.

$$
\text { ". . . flour, }
$$

\section{In all, 225 kilogrammes.}

The person in charge of the mechanical kneader shuts down its lid, and sets it a-going. At the end of about seven minutes he hears tho bell of tho counter sound, announcing that the number of rovolutions has beon sufficiont to call for an inspection of the sponge, in regard to its consistence. The eylinder is thorefore openod, and after verifying the right state of the leavon, and adding water to soften, or flour to stiffen it, ho closes the lid, and sets the machine once more in motion. In 10 minutes more the counter sounds again, and the kneading is completod. The 450 kilogrammes of leaven obtained from the two compartments are adequato to proparo dough enough to supply alternately each of the two ovens. For this purposo 75 kilogrammes of leaven are taken from each of the two compartments $\Lambda$ and $\Lambda^{\prime}$, and placed in the intermediate compartment $\mathrm{B}$. The wholo leaven is then $75+75=150$ kilogrammes; to which are added 100 kilogrammes of flour and 50 of water $=150$, so that the chest contains 300 kilogrammes. There is now replaced in each of the cavities $\mathbf{A}$ and $\mathbf{A}^{\prime}$ the primitive quantity, by adding 50 kilogrammes of flour and 25 of water $=75$.

The cylinder is again set a-going; and, from the nature of the apparatus, it is obvious that the kneading takes place at once on the leavens $\Delta$ and $\mathbf{\Delta}^{\prime}$, and on the paste $\mathrm{B}$; which last is examined after 7 minutes, and completed in 10 more $=17$, at the second sound of the counter-bell.

The kneader is opened, the paste on the sides and on the bars is gathered to the bottom by means of a scraper. The whole paste of the chest $\mathbf{B}$ being removed, 150 kilogrammes of the leaven are taken, to which 150 kilogrammes of flour and water are added to prepare the 300 kilogrammes of paste destined for the supply of the oven No. 2. These 75 kilogrammes of leaven from each compartment are placed as before, and so on in succession.

The water used in this operation is raised to the proper temperature, viz. $25^{\circ}$ or $30^{\circ} \mathrm{C}$. $\left(77^{\circ}\right.$ or $86^{\circ} \mathrm{F}$.) in cold weather, and to about $68^{\circ} \mathrm{F}$. in the hot season, by mixing common cold water with tho due proportion of water maintained at the temperature of about $160^{\circ} \mathrm{F}$., in the basis $\mathrm{F}$, placed above the ovens.

Through the water poured at each operation upon the flour in the compartment $B$, there is previously diffused from 200 to 250 grammes of fresh leaven, as obtainod from the brewery, after being drained and pressed (German yeast). This quantity is sufficient to raise properly 300 kilogrammes of dough. As soon as this dough is taken out of the kneader, as stated above, and while the machine goes on to work, the quantity requisite for each loaf is weighed, turned about on the table $\mathrm{D}$, to give it its round or oblong form, and there is impressed upon it with the forearm, or roller, the cavity which characterises cleft loaves. All the lots of dough of the size of one kilogramme, called cleft loaves (pains fendus), are placed upon a cloth, a fold of which is raised between two loaves, the cloth being first spread upon a board; which thus charged with 10 or 15 loaves is transferred to the wooden sholves $G G$, in front of the oven. The whole of them rise easily under the influence of the gentle temperatnre of this antechamber or foumil. Whenever the dough loaves are sufficiently raised hero, they are put into tho oven, a process called enfournement in France; which consists in setting each loaf on a wooden shovel dusted with coarse flour, and placing it thereby on the sole of the oven, close to its fellow, without touching it. This operation is made easy, in consequence of the introduction of a long-jointed gas-pipe and burner into the interior of the oven, by the light of which all parts of it may be minutely examined. The oven first is kept moderately hot, by shutting the dampers ; but whenever the thermometer attached to it indicates a tomperature of from $300^{\circ}$ to $290^{\circ} \mathrm{C}$. $\left(572^{\circ}\right.$ to $554^{\circ} \mathrm{F}$.), the damper or registers are opened, to restore the heat to its original degree, by allowing of the circulation of the hot air, which rises from the lower cavities around the fire-place into the interior of the oven. When the baking is completed, the gas-light, which had beon withdrawn, is again introduced into the oven, and the bread is taken out; called the process of defournement. If the temperature have been maintained at about $300^{\circ} \mathrm{C}$., the 300 kilogrammes of dough, divided into loaves of one kilogramme ( $2 \frac{1}{5} 1 \mathrm{bs}$. avoirdupois), will be baked in 27 minutes. The charging having lasted 10 minutes, and the discharging as long, the baking of each 
batch will take up 47 minuter. But on account of accidental interruptions, an hour may be assigned for each charge of 260 loaves of 1 kilogramme each; being at the rate of 6,240 kilogrammes (or 6.75 tons) of bread in 24 hours.

Although the outer parts of the loaves be exposed to the radiation of the walls, hented to $280^{\circ}$ or $300^{\circ} \mathrm{C}$, and undergo therefore that kind of caramelisation (charring) which produces the colour, the taste, and the other special characters of the crust, yet the inner substance of the loaves, or the crumb, never attains to nearly so high a temperature ; for $\mathbf{8}$ thermometer, whose bulb is inserted into the heart of a loaf, does not indicate more than $100^{\circ} \mathrm{C} .\left(213^{\circ} \mathrm{F}\right.$.)

Perkins' hot-water oven is an adaptation of that distinguished engineer's stove, which, as is well known, is a mode of heating by means of pipes full of water, and hermetically elosed; but with a sufficient space for the expansion of the water in the pipes. As a means of warning buildings, the invention has already produced the very benefical effects which have gained for it an extensive patronage. There is no doubt but that this novel application entitles the inventor to the warmest thanks of the public. The following figure (229), represents one of these ovens. A, stove; B, coil of iron pipe placed in the stove ; C C, flow-pipe; D, expansive tube ; $\mathrm{E}$, oven charged with loaves, and surrounded with the hot-water pipes; F, return hot-water pipe; $\boldsymbol{G}$, door of the oven; $\boldsymbol{H}$, flue for the escape of the vapours in the oven; $\mathbf{r}$, rigid bar of iron supporting the regulating box; J J, regulating box, containing three

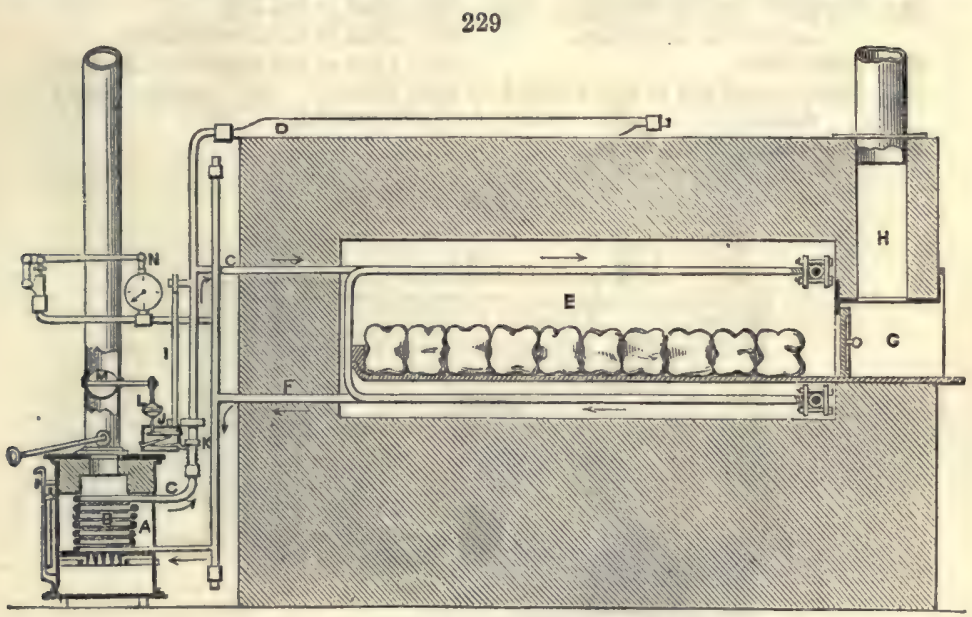

small levers ; K, nut adjusted so that if temperature of the hot-water pipe is increased beyond the adjusted point, its elongation causes the nut to bear upon the levers in the box $\mathrm{J}$, which levers, lifting the straight rod $\mathrm{I}$, shut the damper $\mathbf{M}$ of the stove ; $\mathrm{N}$ is an index indicating the temperature of the hot-water pipes.

The oven is first built in the ordinary manner of sound brickwork, made very thick in order to retain the heat. Then the top and bottom of the internal surfaces are lined with wrought-iron pipes of one inch external diameter, and five-eighths of an inch internal diameter, and their surface amounts in the aggregate, to the whole surface of the oven. These pipes are then connected to a coil in a furnace outside the oven. The coil having such a relative proportion of surface to that which is in the oven, that the pipes may be raised to a temperature of $550^{\circ} \mathrm{F}$., and no more. This fixed and uniform temperature is maintained by a self-regulating adjustment peculiar to this furnace, which works with great procision, and which cannot get out of order, since it depends upon the expansion of the upper ascending pipe close to the furnace acting upon three levers connected with the damper which regulates the draught. The movable nut at the bottom of that expanding pipe being adjusted to the requisite temperature, that precise temperature is uniformly retained. The smallest fluctuation in the heat of the water which circulates in the pipes instantly sets the levers in motion, and the expansion of one thirty-sixth part of an inch is sufficient to close the damper.

It will be observed that if the pipes be heated to $550^{\circ} \mathrm{F}$. the brickwork will soon attain the same temperature, or nearly so, and accordingly the oven will thus possess double the amount of the heating surface of ordinary ovens applicable to baking. 
The baking tomperature of the oven is from $420^{\circ}$ to $450^{\circ} \mathrm{F}$, which is ascortained by a thermometer with which the oven is provided.

With respect to Rolland's oven, Messiours Bonssingault, Payen, and Poncelet, in their report to the Institute of France; Gaultier de Glaubry, in a roport made in the name of the Committeo of Chemical Arts to the Societé d'Encouragement; and the late M. Arago, represented that oven as successfully mecting all the conditions of salubrity, cleanliness, and hygiène. Wood, coals, ashes are likewise banishod from it, and neither smoke nor the heated air of the furnace ean find access to it. As in Perkins', the furnace is placed at a distance from the mouth of the oven, but instead of conveying the heat by pipes, as in the hot-water oven, it is the smoke and hot air of the furnace which, circulating through fan-shaped flues, ramifying under the floor and spreading over the roof of the oven, impart to it the requisite temperature. The floor of the oven, on which the loaves are doposited, consists of glazed tiles, and it can thus be kept perfectly clean. The distinctive character of M. Rolland's ornia, howover, is that the glazed tiles just spoken of rest upon a revolving platform which the workman gradually, or from time to time, mores round by mesns of a small handle, and without effort.

Figures 230 to 239 represent the construction and appearance of M. Rolland's oven on a reduced scale.

230. Front elevation.

231. Vertical section through the

232. Ditto, ditto. axis of the fire-grate.

233. Elevation of one of the vertical flues.

234. Suspension of the floors.
235. Plan of the first floor.

236. Plan of the sole.

237. Plan of the second floor.

238. Plan of the fire-grate and flues.

239. Plan of the portion under ground.

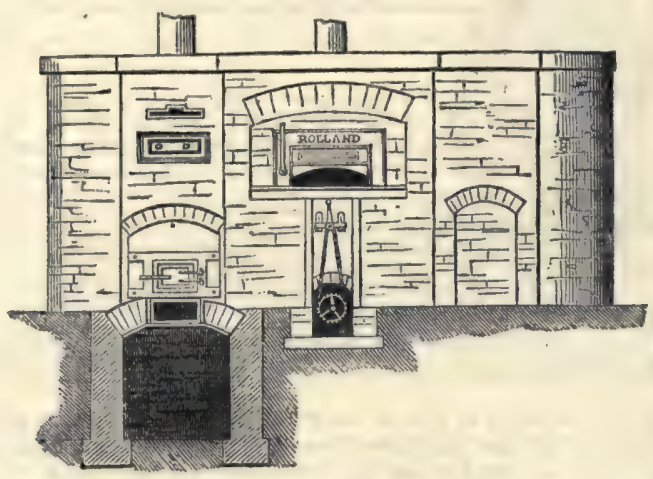

When the oven has to be charged, the workman deposits the first loaves, by means of a short peel, upon that part of the revolving platform which lies before the mouth of the oven, and when that portion is filled, he gives a turn with the handle, and proceeds to put the loaves in the fresh space thus presented before him, and so on until the whole is filled up. The door is then closed through an opening covered with glass, and reserved in the wall of the oven, which is lighted up with a jet of gas, or by opening the door from time to time the progress of the baking may be watched; if it appears too rapid on one point, or too slow on another, the journeyman can, by means of the handle, bring the loaves successively to the hottest part of the oven, and vice versâ, as occasion may require. The oven is provided with a thermometer, and, in an experiment witnessed, the temperature indicated $210^{\circ} \mathrm{C} .=420^{\circ} \mathrm{F}$, the baking of a full charge was completed in one hour and ten minutes, and the loaves of the same kind were so even in point of size and colour that they could not be distinguished from each other.

The top of the oven is provided with a pan for the purpose of heating the water necessary for the preparation of the dough, by means of the heat which in all other plans (Mouchot's excepted) is lost. The workman should take care to keep always some water in that pan, for otherwise the loaden pipe would melt and oceasion dangerous leaks. For this and other reasons, the safest plan, however, would be to 
replace this leaden pipe by an rron one. The sasd pan should be frequently scoured, for, if neglected, the wator will become rusty and spoil the colour of the bread. Bread baking may be considered as consisting of four operations: namely, heating
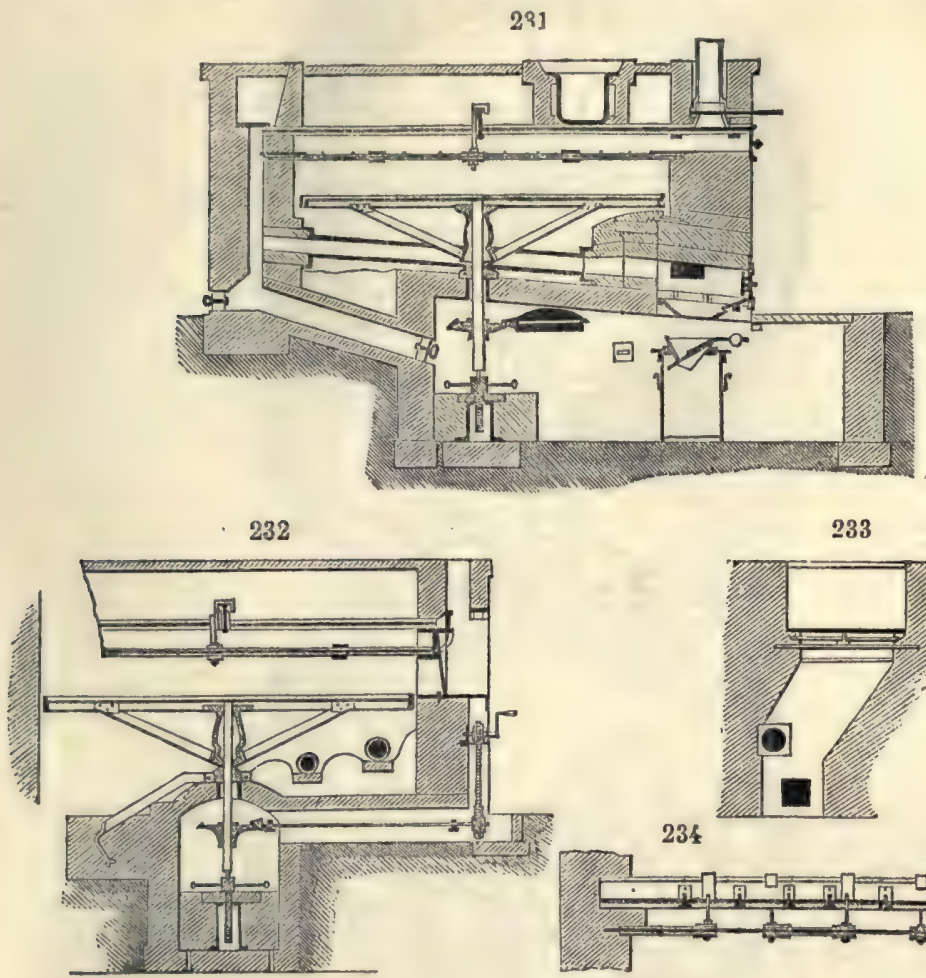

233

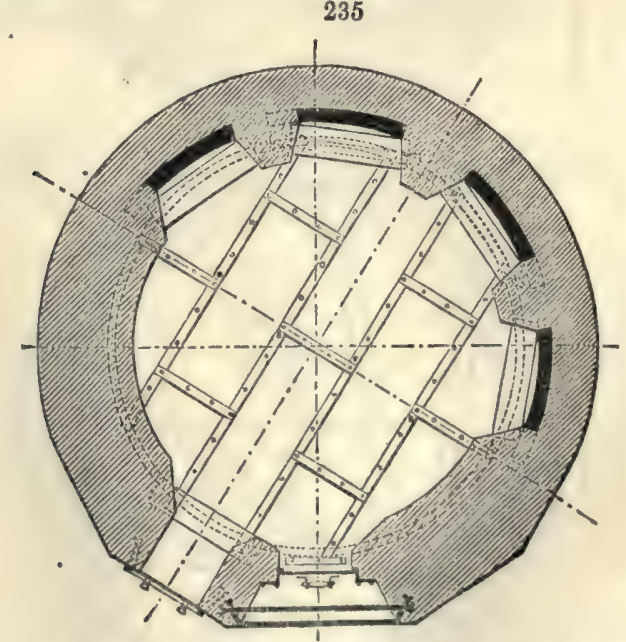

the oven, putting the dough into the oven, baking, and taking the loaves out of the oven. The general direction given by M. Rolland for each of these operations are as follows :- 

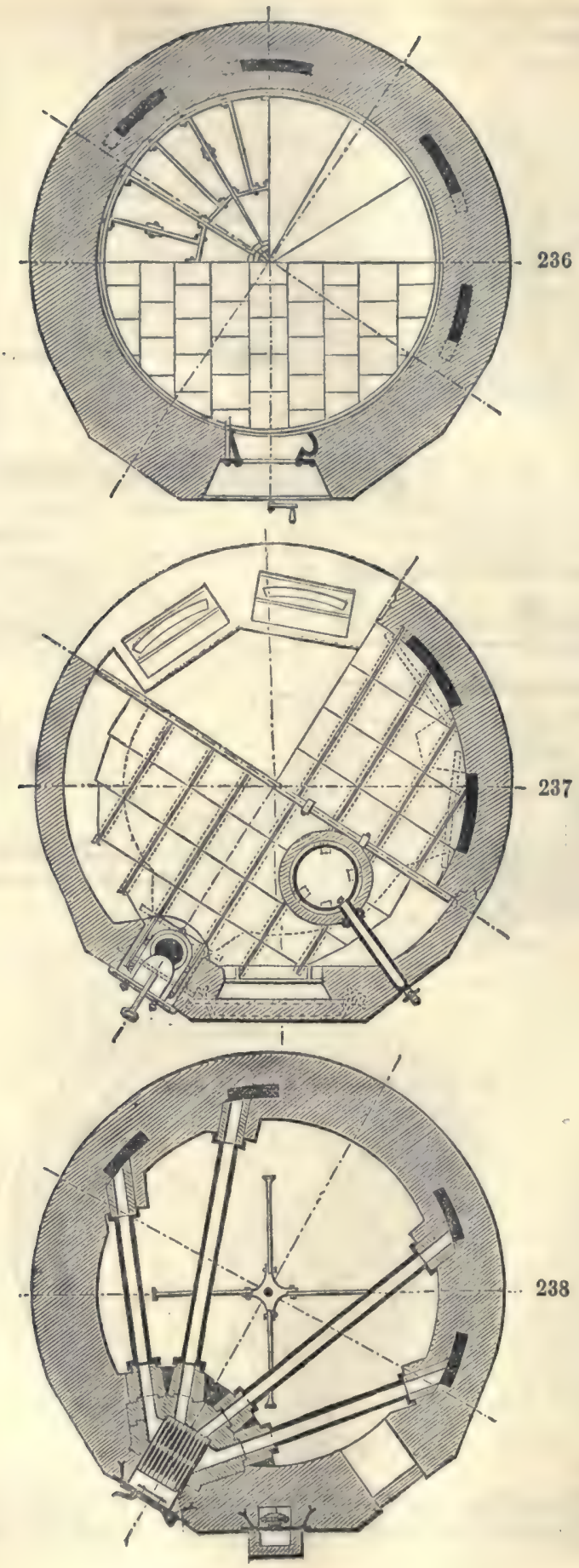
In order to obtain a proper hoat and one that may be easily managed, it is necessary to charge the furnace moderately and often, and to keep it in a uniform state.

239

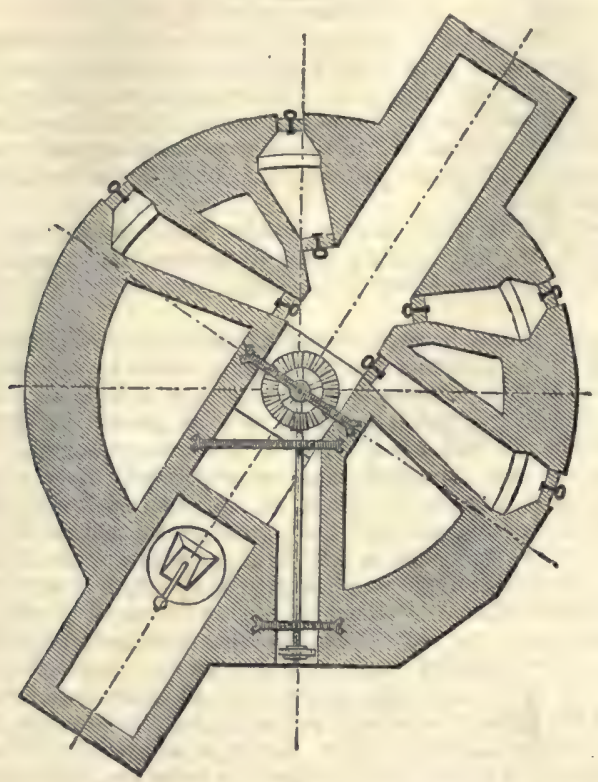

When the fire is kindled, the door should be kept perfectly closed, in order to compel the current of air necessary to the combustion to pass through the grate, and thence through the flues under and the dome over the oven. If, on the contrary, the furnace door were left ajar, the cold air from without would rapidly pass over the coals without becoming properly heated, and passing in that condition into the flues would fail in raising it to tho proper temperature. In order that the flame and heated products of the combustion may pass through all the flues, it is, of course, necessary to keop them clear by introducing into them once a month a brush made of wire, or

240

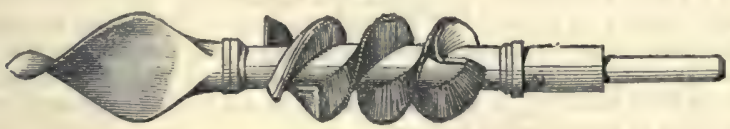

whalebone, or those which are now generally used for sweeping the tubes of marine tubular boilers, and the best of which are those patented and manufactured by Messrs. Moriarty of Greenwich, or How of London. The vertical flues which are built in the masonry are cleared from without or from the pit, according to the nature of the plan adopted in building the oven. These flues need not be cleaned more often than about once in three months.

Sweeping between the floors should be performed about every fortnight.

In case of accident or injury to the thermometers, the following directions, which indeed apply to all ovens, may enable the baker to judge of the temperature of his oven. If, on throwing a few pinches of tlour on the tiles of the oven, it remains white after the lapse of a few seconds, the temperaturo is too low; if, on the contrary, the flour assumes a deep brown colour, the temperature is too high; if the flour turns yellowish, or looks slightly scorched, the temperature is right.

The baking in Rolland's oven takes place at a temperature varying from $410^{\circ}$ to $432^{\circ} \mathrm{F}$. according to the nature and size of tho article intended to be baked. During the baking, the revolving floor is turned every ton or twelve minutes, so that the losves not remaining in the same place the baking becomes equal throughout.

As to the hot-water oven, a fow ostablishments only have as yet adopted it in England; 
one of them is the 'Hot-water Oven Biscuit-baking Company' on whose premises fancy biscuits only are baked; and another establishment is that of a baker of the namo of Noville, carrying on his business in London. With respect to M. Mouchot's system, it is not even known in this country, otherwise than by having been alluded to in one or two technological publications or dictionaries.

The quantity of bread which can be made from a sack of flour depends to a great extont upon tho quantity of gluten that tho flour of which it is mado contains, but the wheat which contains a large proportion of nitrogenous matter does not yield so light a flour as those which are poorer. From a great number of determinations it is found that the amount of gluten contained in tho flour to make best white bread ranges from 10 to 18 per cent., that of the starch being from 63 to 70 per cont., the ashes ranging from 0.5 to 1.9 per cent.

In tho ordinary plan of bread making, London bakers reckon that 1 sack of such a flour, weighing $280 \mathrm{lbs}$, will mako 94 roal 4-1b. loaves (not quartern) of pure genuine bread, although a sack of such flour may yiold him 94 or even 95 quartern (not 4-1b.) losves.'

From this account it may be easily imagined that if the baker could succeed in disposing at once of all the loaves of his day's baking either by sale at his shop, or, still better, by delivery at his customers' residences, such a business would indeed be a profitable one commercially speaking, for on that day he would sell from 28 to 34 lbs. of water at the price of bread, not to speak of the deficient weight.

As to those bakers who, by underbaking, or by the use of alum, or by the use of both alum and underbaking, manage to obtain $96,98,100$, or a still larger number of loaves from inferior flour, or material, their profit is so reduced by the much lower price at which they are compelled to soll their sophisticated bread, that their tamperings avail them but little; their emphatically hard labour yields them but a mere pittance, except their business be so extensive that the small profits swell up into a large sum, in which case they only jeopardise their name as fare and honest tradesmen.

Looking now at the improved ovens, of which we have been speaking merely in an economical point of viow, and abstractedly from all other considerations, the profits realised by their use appears to be well worth the baker's attention. But as with the improved ovens the economy bears upon the wages and the fuel, the advantages are much less considerable in a small concern than in a large one. Thus the economy which, upon 12 sacks of flour per week, would scarcely exceed 20 shillings upon the whole, would, on tho contrary, assume considerable proportions in establishments baking from 50 to 100 sacks per week.

The richness or nutritive powers of sound flour, and also of bread, are proportional to the quantity of gluten they contain. It is of great importance to determine this point, for both of these objects are of enormous value and consumption; and it may be accomplished most easily and exactly by digesting in a vater-bath, at the tomperature of $167^{\circ}$ F., 1,000 grains of bread (or flour) with 1,000 grains of bruised barley malt, in 5,000 grains, or in a little moro thon half a pint, of water. When this mixture ceases to take a blue colour from iodine (that is, when all the starch is convertod into a soluble dextrine), the gluten left unchanged may be collected on a filter cloth, washed, driod at a heat of $212^{\circ} \mathrm{F}$., and weighed. The colour, texture, and taste of the gluten ought also to be examined, in forming a judgment of good flour or bread.

The question of the relative value of white and brown bread, as nutritive agents, is one of very long standing, and the arguments on both sides may be thus resumed.

The advocates of brown bread hold:-

That the separation of tho whito from the brown parts of wheat grain, in making bread, is likely to be baneful to health;

That the general belief that bread made with the finest flour is the best, and that whiteness is a proof of its quality, is a popular error;

That whiteness may be, and generally is, communicated to bread by alum, to the injury of the consumer;

That the miller, in refining his flour, to please the public, removes some of the ingredients necossary to the composition and nourishment of tho various organs of our bodics; so that fine flour, instead of boing bettor than the meal, is, on the contrary, less nourishing, and, to make the case worse, is also more difficult of digestion, not to speak of the enormous loss to the population of at least 25 per cent. of branny flour,

2 It is absolutely necessary thus to establish a distinction between four pounds and quartern loaves, because the latter very seldom indeed have that weight, and this deficiency is, in fact, one of the profits calculated upon; for althongh the Act of Parliament (Will. IV. c. xxxvii.) is very strict, and directs (sect. vii.) that bakers delivering brend by cart or carriage shall be provided with scales, weights, \&c., for weighing bread, this requisition is seldom, if ever, complicd with.

There are of course a few bakers whose quartern loaves weigh exactly four pounds, but many are from four to sis ounces short. 
containing from 60 to 70 per cont. of the most nutritious part of the flour, a loss which, for London only, is equal to at least 7,500 sacks of flour annually;

That the unwiso preferenco given so universally to white bread, leads to the pernicious practice of mixing alum with the flour, and this again to all sorts of impositions and adulterations; for it enables the bakers who are so disposed, by adding alum, to make bread manufactured from the flour of inferior grain to look like the best and most costly, thus defrauding the purchaser and tampering with his health.

On the other side, the partisans of white bread contend, of course, that all these assertions are without foundation, and their reasons were summed up as follows in the Bakers' Gazette, in 1849 :-

"The preference of the public for white bread is not likely to be an absurd prejudice, seeing that it was not until after years of experience that it was adopted by them.

"The adoption of white bread, in preference to any other sort, by the great body of the community, as a general article of food, is of itself a proof of its being the best and most nutritious.

'The finer and better the flour, the more bread can be made from it. Fifty-six pounds of fine flour from good wheat will make seventy-two pounds of good, sound, well-baked bread, the bread, having retained sixteen pounds of water. But bran, either fine or coarse, absorbs little or no water, and adds no more to the bread than its weight.'

And, lastly, in conflumation of the opinion that white bread contains a greater quantity of nutriment than the same weight of brown bread, the writer of the article winds up the white bread defence with a portion of the Report of the Committee of tho Ilouse of Commons, appointed in 1800, 'to consider means for rendering more effectual the provisions of 13 Geo. III., intituled "An Act for the better regulating the Assize and making of Bread."'

In considering the propriety of recommending the adoption of further regulations and restrictions, they understood a prejudice existed in some parts of the country against any coarser sort of bread than that which is at present known by the name of 'fine household bread,' on the ground that the former was less wholesome and nutritious than the latter. The opinions of respectable physicians examined on this point are, - that the change of any sort of food which forms so great a part of the sustenance of man might for a time affect some constitutions; that as soon as persons were habituated to it, the standard wheaten bread, or even bread of a coarser sort, would be equally wholesome with the fine wheaten bread which is now generally used in the metropolis; but that, in their opinion, the fine wheaten bread would go farther with persons who have no other food than the same quantity of bread of a coarser sort.

It was suggested to them that if only one sort of flour was permitted to be made, and a different mode of dressing it adopted, so as to leave in it the fine pollards, $52 \mathrm{lbs}$. of flour might be extrącted from a bushel of wheat weighing $60 \mathrm{lbs}$., instead of $47 \mathrm{lbs}$., which would afford a wholesome and nutritious food, and add to the quantity $5 \mathrm{lbs}$. in every bushel, or somewhat more than $\frac{1}{8}$ th. On this they remarked, that there would be no saving in adopting this proposition; and they begged leave to observe, if the physicians are well founded in their opinions, that bread of coarser quality will not go equally far with fine wheaten bread, and increased consumption of wheaten bread would be the consequence of the measure.

From the baker's point of view, it is evident that all his sympathies must be in favour of the water-absorbing material, and therefore of the fine flour; for each pound of water added and retained in the bread which he sells represents this day so many twopences; but the purchaser's interest lies in just the opposite direction.

The question, however, is not, in the language of the Committee of the House of Commons of those days, or of the physicians whom they consulted, whether a given weight of wheaten bread will go farther than an equal weight of bread of a coarser sort; nor whether a given weight of pure flour is more nutritious than an equal weight of the meal from the same wheat used in making brown bread. The real question is, - Whether a given weight of wheat contains more nutriment than THE FLOUR obtained from that weight of wheat.

The inquiry of the Committee of the House of Commons, and the defence of white bread versus brown bread, resting as it does, in this respect, upon a false ground, is therefore perfectly valueless; for whatever may have been the opinion of respectable physicians and of Committees, either of those days or of the present times, one thing is certain-namely, that bran contains only 9 or 10 per cent. of woody fibre, that is, of matter devoid of nutritious property; and that the remainder consists of a larger proportion of gluten and starch, fatty, and other highly nutritive constituents, with a few salts, and water, as proved by the following analysis by Millon:-

Voz. I.

K K 


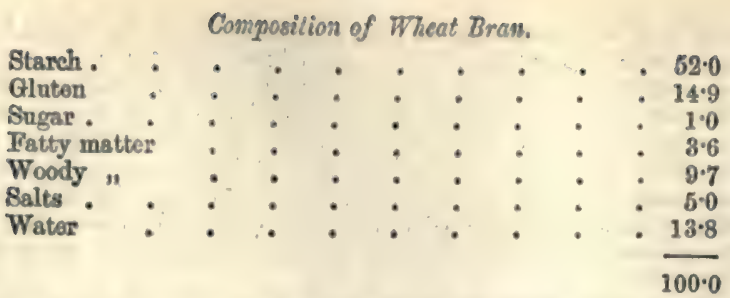

And it is equally certsin that wheat itsolf-the whole grain-does not contain more than 2 per cent. of unnutritious, or woody matter, the bran boing itself richer, weight for woight, in gluten than the fine flour; the whole meal contains, accordingly, more gluten than the fino flour obtainod therefrom. The relative proportions of gluten in the whole grain, in bran, and in flour of the same sample of wheat, were represented by the late Professor Johnston to be as follows:-

$$
\text { Gluten of Wheat. }
$$

Whole grain : $: \quad: \quad: \quad: \quad$ : 14 to 18 per cont,
Whole bran
Fine flour

Now, whereas a bushel of wheat weighing $60 \mathrm{lbs}$. produces, according to the mode of manufacturing flour for London, $47 \mathrm{lbs}$. - that is, 78 per cent. of flour, the rost boing bran and pollards; if we deduct 2 per cent. of woody matter, and $1 \frac{1}{2}$ per cont. for waste in grinding at the mill, the bushel of $60 \mathrm{lbs}$. of wheat would yield $58 \mathrm{lbs}$, or at least $96 \frac{2}{3}$ per cent., of nutritious matter.

It is, therefore, as clear as anything can possibly be, that by using the whole moal instead of only the fine flour of that wheat, there will be a difference of about $\frac{1}{6}$ th in the product obtained from equal weights of wheat.

In a communication mado to the Royal Institute nearly four years ago, M. Mégo Mouriess announced that he had found under the envolope of the grain, in tho internal part of the perisperm, a peculiar nitrogenous substanco capable of acting as a ferment, and to which he gave the name of 'céréaline.' This substance, which is found wholly, or almost so, in the bran, but not in the best whito flour, has the property of liquefying starch, very much in the same manner as diastase; and the decreased firmnoss of the crumb of brown bread is referred by him to this action. The coloration of bread made from meal containing bran is not, according to $\mathbf{M}$. Mège Mouriès, due, as has hitherto been thought, to the presence of bran; but to the peculiar action of corealin ; this new substance, like vegetable casein and gluten, being, by a slight modification, due perhaps to the contact of the air, transformed into a forment, under the influence of which the gluton undergoes a great alteration, yiolding, among othor products, ammonia, a brown-coloured matter analogous to ulmine, and a nitrogenous product capable of transforming sugar into lactic acid. M. Mège Mouriès having experimentally established, to the satisfaction of a committee consisting of MM. Chovreul, Dumas, Pelouze, and Peligot, that by paralysing or destroying tho action of cerealin, as described in the specification of his patent, bearing date June 14, 1856, white bread, having all the characters of first-quality bread, may be mado, in the language of the said specification, "with using either all the white or raw elements that constitute either corn or rye, or with such substances as could produce, to this day, but brown bread.'

Cerealin, according to M. Mège Mouriès, has two very distinet properties :- the first consists in converting the hydrated starch into glucose and dextrino; the second, which is much more important in its results, transforms the glucose into lactic, acetic, butyric, and formic acid, which penetrate, swoll up, and partly dissolve the gluton, rendering it pulpy and emulsive, like that of rye; producing, in fact, a series of docompositions, yielding eventually a loaf having all the charactoristics of bread mado from inferior flour.

In order to convert the whole of the farinaceous substance of wheat into whito bread, it is therefore necessary to destroy the cerealin; and the process, or series of processes, by which this is accomplished, is thus described by M. Mège Mouriès in his specification:-

- The following are the means I employ to obtain my new product:-

- 1st. The application of vinous formentation, produced by alcoholic ferment or yenst, to destroy the ferment that I call "cerereline" cxisting, together" with the frag- 
monts of bran, in the raw flour, and which in some measure produces the acidity of brown bread directly, whilst it destroys indirectly most part of the gluten.

'2ndly. The thorough purification of the said flour, either raw or mixed with bran, (after dilution and fermentation,) by the sifting and separating of the farinaceous liquid from the fragments of bran disseminated by the millstone into the inferior products of corn.

3rdly. The employing that part of corn producing brown bread in the rough state as issuing from the mill after a first grinding, in order to facilitate its purification by formentation and wot sifting.

' 4 thly. The employing an acidulated water (by any acid or acid salt) in order to prevent the lactic fermentation, preserving the vinous fermentation, preventing the yellow colour from turning into a brown colour (the ulmic acid), and the good taste of corn from assuming that of brown bread. However, instead of acidulated water, pure water may be employed with an addition of yeast, as the acid only serves to facilitate the vinous fermentation.

'5thly. The grinding of the corn by means of millstones that crush it thoroughly, increasing thereby the quantity of foul parts, a method which will prove very bad with the usual process, and vory advantageous with mine.

' 6 thly. The application of corn washed or stripped by any suitable means.

'7thly. The application of all these contrivances to wheat of every description, to rye, and other grain used in the manufacture of bread,

'8thly. The same means applied to the manufacture of biscuits.

'I will now describe the manner in which the said improvements are carried into effect.

'First Instance. When flour of inferior quality is made use of.-This description of flour, well known in trade, is bolted or sifted at 73, 74, 75, or 80 per cent. (a mark termed Scipion mark in the French War Department), and yields bread of middle quality. By applying to this sort of flour a liquid yeast, rather different from that which is applied to white flour, in order to quicken the work and remove the sour taste of bread, a very nice quality will be obtained, which result was quito unknown to everybody to this day, and which none ever attempted to know, as none before me was awaire of the true causes that produce brown bread, \&c.

'Now, to apply my process to the said flour (of inferior mark or quality) I take a part of the same - a fourth part, for instance-which I dilute with a suitable quantity of water, and add to the farinaceous liquid 1 portion of beer yeast for 200 portions of water, together with a small quantity of acid or acid salt, sufficient to impart to the said water the property of lightly staining or reddening the test-paper, known in France by the name of papier de tournesol. When the liquid is at full working, I mix the remaining portions of flour, which are kneaded, and then allowed to ferment in the usual way. The yoast applied, which is quite alcoholic, will yield perfectly white bread of a very nice taste; and I declare that if similar yeast were ever commended before, it was certainly not for the purpose of preventing the formation of brown bread, the character of which was believed to be inherent to the nature of the rery flour, as the following result will sufficiently prove it, thus divesting such an application of its industrial appropriation.

'Second Instance. When raw flour is made use of.-By raw flour, I mean the corn crushed only once, and from which 10 to 15 per cent. of rough bran have been separated. Such flour is still mixed with fragments of bran, and is employed in trade to the manufacture of so-called white flour and bran after a second and third grinding or crushing. Instoad of that, I only soparate, and without submitting it to a fresh crushing, the rough flour in two parts, about 70 parts of whito flour and 15 to 18 of rough or coarse flour, of which lattor the yeast is made; this I dilute with a suitable quantity of water, sufficient to reduce the whole flour into a dough, say, 50 por cont. of the whole weight of raw flour. To this mixture have been previously added the yoast and acid, (whenevor acid is applied, which is not indispensable, as before stated), and the whole is allowed to work for 6 hours at a temperature of $77^{\circ} \mathrm{F}$. for 12 hours at $68^{\circ}$, and for 20 hours at $59^{\circ}$, thus proportionally to the temperature. While this working or formentation is going on, the various elements (céréaline, \& c.) which by their peculiar action are productive of brown bread, have undergone a modification; the rough parts are separated, tho gluton stripped from its pellicles and disaggregated, and the same flour which, by the usual process, could have only produced deep brown bread, will actually yield first-rato bread, far superior to that sold by bakers, chiofly if the fragments of bran are separated by the following process, which consists in pouring on the sieve, described hereafter, the liquid contrining the rough parts of flour thus disaggregatod and modified by a well-regulated formentation,

' The sievo alluded to, which may be of any form, and consist of soveral tissues of x $\mathrm{k} \mathbf{2}$ 
different tightness, the closest being ever arranged underneath or the most forward, whon the siove is of cylindrical or vertical form, is intended to keep back the fragments of bran, which would by their interposition impair the whiteness of bread, and by their weight diminish its nutritive power. The sifted liquid is white, and constitutes the yeast with which the white flour is mixed after being separated, so as to make a dough at either a first or several workings, according to the baker's practice. This dough works or ferments very quickly, and the bread resulting therefrom is unexceptionable. In case the whiteness or neatness of bread should be looked upon as a thing of little consequence, a broader sieve might be employed, or even no sieve used at all, and yet a very nice bread be obtained.

- The saving secured by the application of my process is as follows:-By the common process, out of 100 parts of wheat, 70 or 75 parts of flour are extracted, which are fit to yield either white or middle bread ; whilst, by the improved process, out of 100 parts of wheat 85 to 88 parts will be obtained, yielding bread of superior quality, of the best taste, neatnoss, and nutritious richness.

'In case new yeast cannot be easily provided, the same shonld be dried at a tomperature of about $86^{\circ} \mathrm{F}$., after being suitably sepaxated by means of some inert dust, and previous to being made use of it should be dipped into 10 parts of water, lightly sweetened, for 8 to 10 hours, a fit time for the liquid being brought into a full fermentation, at which time the yeast has recovered its former power. The same process will hold good for manufacturing rye bread, only 25 per cent., about, of coarse bran are to be extracted. For manufacturing biscuits, I use also the same process, only the dough is made very hard and immediately taken into the oven, and the products thus obtained are far superior to the common biscuits, both for their good taste and preservation. Should, however, an old practice exclude all manner of fermentation, then I might dilute the rough parts of flour in either acidulated or non-acidulated water, there to be left to work for the same time as before, then sift the water and decant it, after a proper settling of the farinaceous matters of which the dough is to be made; thus the action of the acid, decantation, and sifting, would effectively remove all causes of alteration, which generally impair the biscuits made of inferior flour.

'The apparatus required for this process is very plain, and consists of a kneading trough, in which the foul parts are mixed mechanically, or by manual labour, with the liquid above mentioned. From this trough, and through an opening made therein, the liquid mixture drops into the fermenting tub, deeper than wide, which must be kept tightly closed during the fermenting work. At the lower part of this tub a cock is fitted, which lets the liquid mixture down upon an incline plane, on which the liquid spreads, so as to be equally distributed over the whole surface of the sieve. This sieve, of an oblong rectangular form, is laid just beneath, and its tissue ought to be so close as to prevent the least fragments of bran from passing through; it is actuated by the hand, or rather by a crank. In all cases that part of the sieve which is opposite to the cock must strike upon an unyielding body, for the purpose of shaking the pellicles remaining on the tissue, and driving thein down towards an outlet on the lower part of the sieve, and thence into a trough purposely contrived for receiving the waters issuing from the sieve, and discharging them into a tank.

'The next operation consists in diluting those pellicles, or rougher parts, which could not pass through the sieve, sifting them again, and using the white water resulting therefrom to dilute the foul parts intended for subsequent operations. The sieve or sieves may sometimes happen to be obstructed by some parts of gluten adhering thereto, which I wash off with acidulated water for silk tissues, and with an alkali for metallic ones. This washing method I deem very important, as its nonapplication may hinder a rather large operation, and therefore $I$ wish to secure it. This apparatus may be liable to some variations, and admit of several sieves superposed, and with different tissues, the broadest, however to be placed uppermost.

'Among the various descriptions and combinations of sieves that may be employed, the annexed figures show one that will give satifactory results.

- Fig. 241 is a longitudinal section, and fig. 242 an end view, of the machine from which the bran is ejected. The apparatus rests upon a cast-iron framing $a$, consisting of two chooks, kept suitably apart by tie pieces $b ; a$ strong cross-bar on the upper part admits a wood cylinder $c$, circled round with iron, and provided with a wooden cock $d$. The cylinder $c$ receives through its centre an arbor $f$, provided with four arms $e$, which arbor is supported by two cross-bars $g$ and $h$, secured by means of bolts to the uprights $i$. Motion is imparted to the arbor $f$ by the crank $j$, by pulleys driven by the ondloss straps $k$, and by the toothed wheel $l$, gearing into the wheel $m$, which is keyed on the upper ond of the arbor $f$. Beneath tho cylinder $c$, two sieves $n$ and $o$ are borne into a frame $p$, suspended on one end to two chains $q$, 
and on the other resting on two guides or bearings $r$, beneath which, and on the crank shaft, are cams 8 , by which that end of the frame that carries the sieves is alternately raised and lowered. A strong spring $u$ is set to a shaft borno by the framing $a$, whilst a ratchet wheel provided with a clink allows tho said spring,

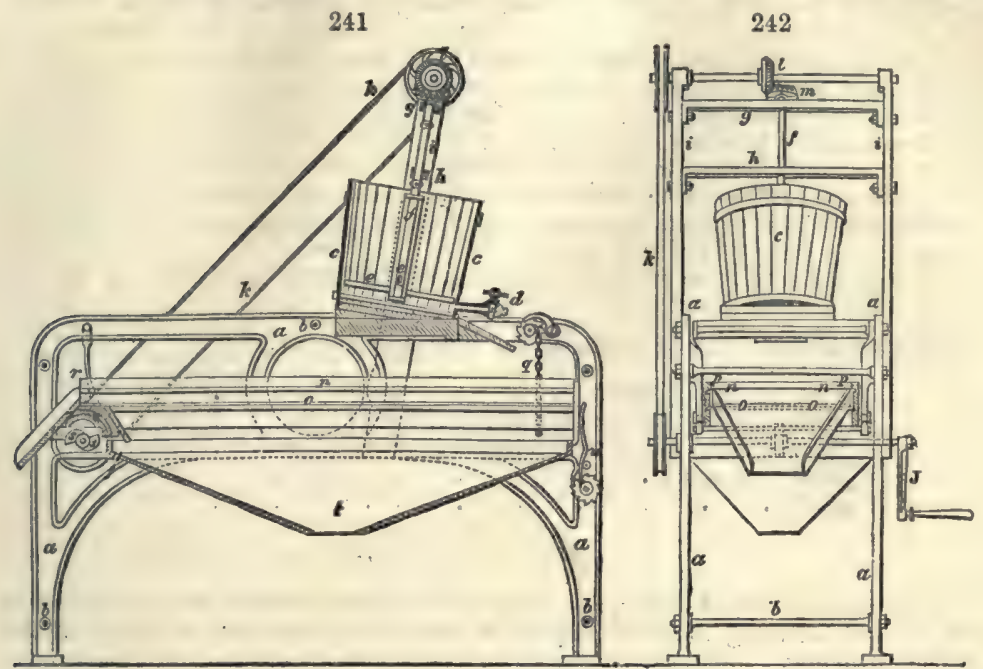

according to the requirements of the work, to give more or less impulse or shaking as the cams $s$ are acting upon the frame-sieve carrying the sieve. Beneath the said frame a large hopper $t$ is disposed, to receive and lead into a tank the liquid passing through the sieves. The filter sieve is worked as follows:-After withdrawing, by means of bolting hutches, 70 per cent., about, of fine flour, I take out of the remaining 30 per cont. about 20 per cont. of groats, noglecting the remaining 10 per cent., from which, however, I could separate the little flour still adhering thereto, but I deem it more available to sell it off in this state. I submit the 20 per cent. of groats to a suitable vinous fermentation, and have the whole taken into the cylinder $c$, there to be stirred by means of the arbor $f$ and the arms $e$; after a suitable stirring, the cock $d$ is opened and the liquid is let out, spread on the uppermost sieve $n$, which keeps back the coarsest bran. The liquid drops then into the second sieve or filter $o$, by which the least fragments are retained; the passage of the liquid through the filters is quickened by the quivering motion imparted by the cams $s$ to the frame carrying the sieve.'

The advantages resulting from such a process are obvious: first, it would appearand those experiments have been confirmed by the committee of the Acadermie des Sciences, who had to report upon them-that no less than from 16 to 17 per cent. of white bread of superior quality can be obtained from wheat, which increase is not due to water, as in other methods, but is a true and real one, the Commissioners having ascertained that the bread thus manufactured did not contain more water than that made in the usual way, their comparative examinations in this respect having given the following results:-

Loss by drying in Air.

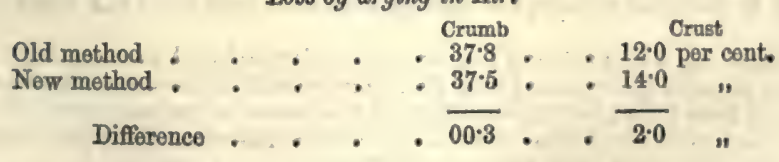

Another experiment by Poligot:-

Loss by drying in Air at $248^{\circ} \mathrm{F}$.

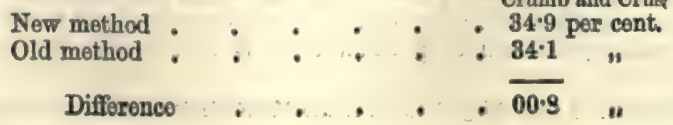


- Since the enrolment of his Specifleation, however, M. Mège Mouriès has mado an improvement, which simplifies considorably his original process, according to which the destruction of the cerealin, as we saw, was offectod by ordinary yeast; that is to say, by alcoholic fermentation. The last improvomont consists in preventing corealin from becoming a lactic or glucosic forment, by precipitating it with common salt, and not allowing it time to become a forment. In effect, in order that corealin may produce tho objectionablo effects alluded to, it must first pass into the stato of ferment, and, as all nitrogenous substances require a certain time of incubation to become so,' if, on the one hand, cerealin be precipitated by means of common salt, the glucosic action is neutralised; whilst, on the other hand, the levains boing mado with flour containing nocerealin-that is to say, with best white flour-if a short time before baking households or seconds are added thereto, it is clear that timo will be wanting for it (the ferment) to become doveloped or organised, and that, under this treatment, the bread will remain white.

The application of these scientific deductions will be better understand by the following description of the process :-

100 parts of clean wheat are ground and divided as follows:

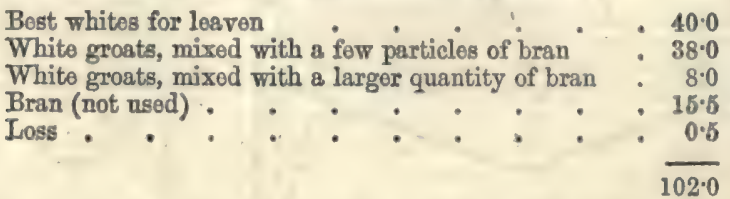

These figures vary, of course, according to the kind of wheat used, according to seasons, and according to the description of mill and the distance of the millstones used for grinding.

'In order to convert these products into bread,' says M. Mége Mouriès, 'a leaven is to be made by mixing the 40 parts of best flour above alluded to, with 20 parts of water, and proceeding with it according to the mode and custom adoptod in each locality. This leaven, no matter how prepared, being ready, the 8 parts of groats mixed with the larger quantity of bran above alluded to, are diluted in 45 parts of water in which 0.6 parts of common salt havo been previously dissolved, and the whole is passed through a sieve which allows the flour and water to pass through, but rotains and separates the particles of bran. The watery liquid so obtained has a whito colour, is flocculent, and loaded with corealin; it no longer possosses tho property of liquefying gelatinous starch, and weighs 38 parts (the romainder of tho water is retained in the bran, which has swelled up in consequonce, and romains on tho siove). The loaven is thon diluted with that water, which is loaded with best flour, and is nsed for converting into dough the 38 parts of white groats above alluded to; the dough is then divided into suitable portions, and aftor allowirg it to stand for one hour, it is finally put in tho ovon to be baked. As the operations just described take place at a temperature of $25^{\circ} \mathrm{C} .\left(=77^{\circ} \mathrm{F}\right.$. $)$, the one hour during which the dough is left to itself, is not sufficiont for the cerealin to pass into the state of ferment, and the consequence is the production of white bread. Should, howevor, the tomperature be higher than that, or were the dough allowed to be kept for a longer timo boforo baking, the bread produced, instead of boing white, would be so much darker, as the contact would have lasted longer. By this process, 100 parts in weight of wheat yield 136 parts of dough, and, finally, 115 parts in weight of bread' instead of 100, which the samo quantity of wheat would have yielded in tho usual way. This is supposing that the grinding of the wheat has been effected with close set millstones; if ground in the usual manner, the average yield does not exceed 112 parts in woight of bread.

The substances which are now almost-exclusively employed for adulterating bread are, water, alone or incorporated with rice, or water and alum : other substances, howover, are or havo been also oceasionally used for the same purpose.

This retention of water into broad is secured by underbaking, by the introduction of rice, of potatoes, and other feculæ, and of alum.

Underbaking is an operation which consists of keeping in the loaf the water which otherwise would escape while baking; it is, therefore, a process for selling wator at the prico of bread. It is done by introducing the dough into an oven unduly heatod, whereby the gases contained in the dough at once expand, and swell it up to the ordinary dimonsions, whilst a doep burnt crust is immediatoly afterwards formed; 
which, inasmuch as it is a bad conductor of heat, prevonts the interior of the loaf from being thoroughly baked, and at the same time opposes the free exit of the water contained in the dough, and which the heat of the oven partly converts into steam; while the crust becomes thicker and darker than it othorwise should be, a sensible loss of nutritive elements being sustained, at the same time, in the shape of pyrogenous products, which are dissipated.

The proportion of water retained in bread by underbaking is sometimes so large, that a baker may thus obtain as much as 106 loaves from a sack of flour.

The addition of boiled rice to the dough is also pretty frequently used to increase the yield of loaves; this substance, in fact, absorbs so much water that as many as 116 quartern loaves have thus been obtained from one sack of flour.

From a great number of experiments made with a view to determine the normal quantity of water contained in the crumb of genuine bread, it is ascertained that it amounts, in new bread, from 38 at least to at most 47 per cent.

The quantity of water contained in bread is easily determined, by cutting a slice of it, weighing 500 grains, for example, placing it in a small oven heated, by a gasburner or a lamp, to a temperature of about $220^{\circ} \mathrm{F}$, until it no longor loses weight, the difference between the first and last weighing (that is to say, the loss) indicating, of course, the amount of water.

Alum, however, is the principal adulterating substance used by bakers, almost without exception, in this metropolis; as was proved by Dr. Normanby in his evidence before the Select Committeo of the House of Commons, appointed in 1855, under the presidence of $\mathrm{Mr}$. W. Scholefield, to inquire into the adulteration of food, drinks, and drugs, which assertion was corroborated and established boyond doubt by the other chemists who were examined also on the subject.

The introduction of alum into bread not only enables the baker to give to bread made of flour of inferior quality the whiteness of the best bread, but to force and keop in it a larger quantity of water than could otherwise be done. We shall seo presently that this fact has been denied, and on what grounds.

Tho quantity of alum used varies exceedingly; but no appreciable effect is produced when the proportion of alum introduced is less than 1 in 900 or 1,000 , which is at the rate of 27 or 28 grains in a quartern loaf. The use of alum, however, has become so universal, and the Act of Parliament which regulates the matter has so long been considered as a dead letter, from tho trouble, and chance of pecuniary loss which it entails on the prosecutor should his accusation prove unsuccessful, that but few, and until quite lately none, of the public officers would undertake the discharge of a duty most disagreeable in itself and at the same time full of risk.

When alum is used in making bread, one of the two following things may happen: either the alum will be decomposed, as just said, in which caso the alumina will, of nocessity, be set free as soon as digestion will have decomposed the organic matter with which it was combined; and thus it is presumable that either alum will be ro-formed in the stomach, or that, according to Liebig, the phosphoric acid of the phosphates of the bread, uniting with the alumina of the alum, will form an insoluble phosphate of alumina, and the beneficial action of the phosphates will, consequently, bo lost to the system; and since phosphoric acid forms with alumina a compouud hardly decomposable by alkalis or acids, this may, perhaps, explain the indigestibility of the London bakers' bread, which strikes all foreigners. - Letters on Chemistry.

The last defence set up in bohalf of alumod bread to be noticed is, that, with cortain descriptions of flour, bread cannot be made without it; that by means of alum a large quantity of flour is made available for human food, which, without it, must be withdrawn, and turned to some other less important uses, to the great detriment of the population, and particularly of the poor, who would be the first to suffer from the increase of the price of bread which such a withdrawal must fatally produce.

The process usually adopted for the detection of alum is that known as Kuhlmann's process, which consists in incinerating about 3,000 grains of bread, porphyrising the ashos so obtained, treating them by nitric acid, evaporating the mixture to dryness, and diluting the residue with about 300 grains of water, with the help of a gontle heat; without flitering, a solution of caustic potash is thon added, the whole is boiled a little, filtered, the filtrate is testod with a solution of sal-ammoniac, and boiled for a fow minutes. If a precipitato is formed it is not alumina, as hitherto thought and stated by Kuhlmann and all other chemists, but phosphate of alumina, - a circumstance of great importance, not in testing for the presence of alumina, but for the determination of its amount, as will be shown further on, when entering into the details of the modifications which it is necessary to make to Kuhlmann's process.

In a paper read in April 1858, at the Socioty of Arts, Dr. Odling stated that out of 
46 examinations of ashes furnishod him by Dr. Gilbort, and treating them by the above process, he (Dr. Odling) obtained, to use his own words, 'in 21 instances, the celobrated whito procipitato said to bo indicative of alumina and alum, so that had these samples been in a manufacturod instoad of the natural state-had the wheat, for example, been made into flour-I should have boen justified, according to the authority quotod, in pronouncing it to bo adultorated with alum. But a subsequent examination of the precipitates I obtained, showed that in reality they were not due to alumina at all. M. Kuhlmann's process, as above described, is possessed of rare merits : it will never fail in detecting alumina when present, and will often succeed in detecting it when absent also. The idea of weighing this olla podrida of a precipitate, and from its weight calculating the amount of alum present, as is gravely rocommonded by great anti-adulteration adepts, is too preposterous to require a moment's refutation.'

In order, however, to render the process for the detection of alum in bread free from objections, the following method is recommended. It requires only ordinary care, and it is perfectly accurate:-

Cut the loaf in half; take a thick slice of crumb from the middle, carefully trimming the edges so as to remove the crust, or hardened outside, and weigh off 1,500 or 3,000 grains of it; crumble it to powder, or cut it into slices, and expose them, on a sheet of platinum foil turned up at the edges, to a low red heat, until fumes are no longer evolved, and the whole is reduced to charcoal, which will require from twenty to forty minutes, according to the quantity; transfer the charcoal to a mortar, and reduce it to fine powder; put now this finely-pulverised charcoal back again on the platinum foil tray, and leave it exposed thereon to a dark cherryred heat until reduced to grey ashes, for which purpose gas-furnace lamps will bo found very convenient. Only a cherry-red heat should be applied, because at a higher temperature the ashes might fuse, and the incineration be thus retarded. Remore the source of heat, drench the grey ashes with a concentrated solution of nitrate of ammonia, and carefully reapply the heat; the last portions of charcoal will thereby be burnt, and the ashes will then have a white or drab colour. Drench them on the tray with moderately strong and pure hydrochloric acid, and after one or two minutes' standing, wash the contents of the platinum foil tray, with distilled water, into a porcelain dish; evaporate to perfect dryness, in order to render the silica insoluble; drench the perfectly dry residue with strong and pure muriatic acid, and, after standing for five or six minutes, dilute the whole with water, and boil; while boiling, add carefully as much carbonate of soda as is necessary nearly, but not quite, to saturate the acid, so that the liquor may still be acid; add as much pure alcoholpotash as is necessary to render it strongly alkaline; boil the whole for about three or four minutes, and filter. If now, after slightly supersaturating the strongly alka.line filtrate with pure muriatic acid, the further addition of a solution of carbonate of ammonia produces, either at once or after heating it for a few minutes, a light, white, flocculent precipitate, it is a sign of the presence of alumina, the identity of which is confirmed by collecting it on a filter, putting a small portion of it on a platinum hook, or on charcoal, heating it thereon, moistening the littlo mass with nitrate of cobalt, and again strongly heating it before the blowpipe; when if, without fusing, it assumes a beautiful blue colour, the presence of alumina is corroborated. If the operator possesses a silver capsule, he will do well to use it instead of a porcelain one for boiling the mass with pure caustic alcohol-potash, in order to avoid all chance of any silica (from the glaze) becoming dissolved by the potash, and afterwards simulating the presence of alumina, though, if the boiling be not protracted, a porcelain capsule is quite available. It is, however, absolutely necessary that he should use potasse $\dot{a}$ l'alcohol, for ordinary caustic potash always contains some, and occasionally considerable quantities of alumina, and is totally unsuitod for such an investigation. Even potasse $\dot{a}$ l'alcohol retains traces of silica, either alone or combined with alumina; so that for this, and other reasons which will be explained presently, an extravagant quantity of it should not be used.

Lastly, carbonate of ammonia is preferable to caustic ammonia for precipitating the alumina, sinoe that earth is far from being insoluble in caustic ammonia.

The liquor from which the alumina has beon soparated should now be acidified with hydrochloric acid, and tested with chloride of barium, which should then yield a copious precipitate of sulphate of barytes.

The only precipitate which can, under the circumstances of the experiment, simulate alumina, is the phosphate of that earth, which behaves with all roagents as pure alumina. Such a precipitate, therefore, if taken account of as pure alumina, would altogether vitiate a quantitative analysis if the amount of alum were calculated from it; but the proof that a certain quantity of alum had been used in the bread from which it had been obtained would remain unshaken; since alumina, whether in that stato 
Or in that of its phosphate, could not have been found except a salt of alumina - to wit, alum-had been usod by the baker. When, therefore, the exact amount of alumina has to be determined, the precipitate in question should be submitted to further treatment in order to separate the alumina; and this can be done easily and rapidly by dissolving the precipitate in nitric acid, adding a little metallic tin to the liquor, and boiling. The tin becomes rapidly oxidised, and remains in the state of an insoluble white powder, which is a mixture of peroxide of tin and of phosphate of tin, at the expense of all the phosphoric acid of any earthy phosphate which may have been present. The whole mass is evaporated to dryness, and the dry residue is then treated by water and filtered, in order to separate the insoluble white powder, and the filtrate which contains the alumina should now be supersaturated with carbonate of ammonia. If a precipitate is formed, it is pure alumina. The white insoluble powder, after washing; may be dissolved in hydrochloric acid, and after diluting the solulion with water, the tin may be precipitated therefrom by passing through it a stream of sulphuretted hydrogen to supersaturation, leaving at rest for ten or twelve hours, filtering, boiling the filtrate until all odour of sulphuretted hydrogen has disappeared; an excess of nitrate of silver is then added, and the liquor filtered, to separate the chloride of silver produced, and exactly neutralising the filtrate with ammonia ; and if a lemon-yellow precipitate is produced, immediately soluble in the slightest excess of either ammonia or nitric acid, it is basic phosphate of silver (3AgO), $\mathrm{PhO}^{5}$, the precipitate obtained in the first instance being thus proved to be phosphate of alumina, The pure alumina obtained may now be collected on a filter, washed with boiling water, thoroughly dried, and then ignited and weighed. One grain of alumina represents 9.027 grains of crystallised alum.

In testing bread for alum, it should be borne in mind, however, that the water used for making the dough generally contains a certain quantity of sulphates, and that a precipitate of sulphate of barytes will therefore be very frequently obtained, though much less considerable than when alum has been used. Some waters called 'selenitous' contain so much sulphate of lime in solution, that if they were used in making the dough, chloride of barium would afford, of course, a considerable precipitate. For these reasons, therefore, the separation and identification of alumina are the only reliable proofs; because, as that earth does not exist normally in any shape in wheat or common salt otherwise than in traces, the proof that alum has been used becomes irresistible when we find, on the one hand, alumina, and, on the other, a more considerable amount of sulphate of barytes than, except under the most extraordinary circumstances, genuine bread would yield.

Sulphate of copper, like alum, possesses the property of hardening gluten, and thus, with a flour of inferior quality, bread can be mado of good appearance, as if a superior flour had been uset.

The use of sulphate of copper in bread is said to have originated about 25 or 30 years ago with the bakers of Belgium.

M. Kuhlmann, Professor of Chemistry at Lille, having been called upon several times by the courts of justice to examine, by chemical processes, bread suspected of containing substances injurious to health, collected some interesting facts upon the subject, which were published under the direction of the central council of salubrity of the department du Nord.

For some time public attention has been drawn to an odious frand committed by a great many bakers in the north of France and in Belgium,- the introduction of a cortain quantity of sulphate of copper into their bread. When the flour was mado from bad grain, this adulteration was very generally practised, as was proved by many convictions and confessions of the guilty persons. When the dough does not rise well in the fermentation (le pain pousse plat), this inconvenience was found to be obviated by the addition of blue vitriol, which was supposed also to cause the flour to retain more water. The quantity of blue vitriol added is extremely small, and it is never done in presence of strangers, because it is reckoned a valuable secret. It occesions no economy of yeast, but rather the reverse. In a litre (about a quart) of water, an ounce of sulphato of copper is dissolved; and of this solution a wine-glassful is mixed with the water necessary for 50 quartern or 4 -pound loaves.

Lime water has been recommended by Liebig as a means of improving the bread made from inferior flour, or of flour slightly damaged by keeping, by warehousing, or during transport in ships; and this method, at the meeting of the British Association at Glasgow, in 1855, was reported as having been tried to a somewhat considerable extent by the bakers of that town, and with success; the bread kneaded with lime. water, instead of pure water, being of good appearance, good taste, good texture, and free from the sour tasto which invariably belengs to alumed or even to genuine bread;admitting all this to be true, still wo should deprecate the use of lime-water in bread, because it cannot bo done with impunity; however small the dose of additional mattor 
may bo considered when takon sopnratoly, it is always large when considered as portion of an articlo of food like bread, consumed day aftor day, and at ench meal, without interruption. To allow articlos of food to bo tamporod with, undor any circumstancos, is a dangerous practico, oven if it were provod that it can be done without risk, which, however, is not tho case; and Liolig himself has said that chemists should never proposo tho use of chomical products for culinary preparations.

The quantity of ashes left after the incineration of genuine bread, varies from 1.5 at lesst to at most 3 per cent.; and if the latter quantity of ashes bo exceeded, the excess may safoly bo pronounced to bo due to an artificial introduction of some saline or aarthy matter.

As to the addition to broad of potatoos, beans, rice, turnips, maize, or Indian corn, which has occasionally beon practised to a considorablo extent, especially in yoars of scarcity, it is evident that they are actually permitted under the Act of Parliament, Will. IV. cap. 27. sect. 11.

In his 'Now Lotters on Chemistry,' Liobig makes the following remarks on the subject:-

'The proposals which have hitherto been made to use substitutes for flour, and thus diminish the price of bread in times of scarcity, prove how much the rational principles of hygiene are disregardod, and how unknown the laws of nutrition are still. It is with food as with fuel. If we compare tho price of the various kinds of coals, of wood, of turf, we shall find that the number of pence paid for a certain volume or weight of these materials is about proportionate to tho number of degrees of heat which they evolve in burning. . . . . The mean price of food in a large country is ordinarily the criterion of its nutritive valuo. . . . . Considered as a nutritive agont, ryo is quito as doar as wheat; such is tho case also with rice and potatoes; in fact, no other flour can replaco wheat in this respect. In times of scarcity, howover, theso ratios undergo modification, and potatoes and rice aequire then a higher value, bocause, in addition to their natural value as respiratory food, another value is superadded, which in times of abundance is not taken into account.

'The addition to wheat flour of potato starch, of dextrine, of the pulp of turnips, gives a mixture, tho nutritive value of which is equal to that of potatoos, or perhaps loss; and it is ovident that one cannot considor as an improvement this transformation of wheat flour into a food having only the same value as rice or potatoes.'

The detection of potato starch, of beans, peas, Indian corn, rico, and other feculæ, which is so easily effectod by means of the microscope in flour, is exceedingly difficult, if not impossible, in bread. Bread which has been mado with flour mixed with Indian corn is harsher to the touch, and has frequently a slight yellowish colour, and whon moistoned with solution of potash of ordinary strongth, a yellow or greenishyellow tinge is developed.-The late Dr. A. N. Seo Toast, Leaven.

Wo exported in 1871 the following quantities of Brfad and Biscuits :--

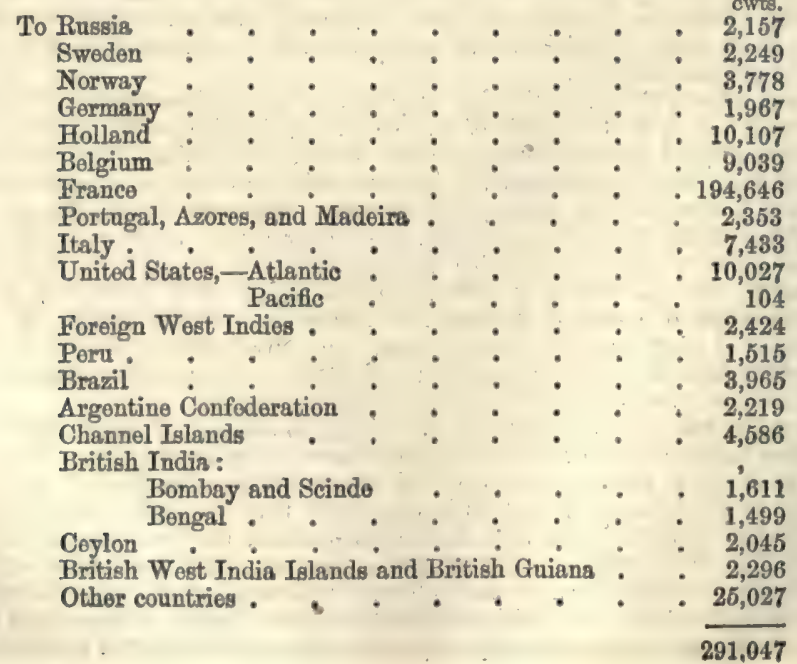




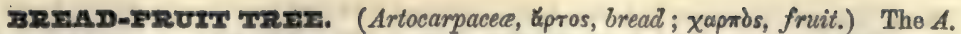
incisa, the true bread-fruit treo, is a native of the South Sea Islands, inhabiting such placos as aro hot and damp. The tree is about twolve inches diameter, and grows to tho height of forty feet. The fruit is about the size of a molon, and the seods are largo nut-like bodies. The fleshy receptacle is the valuable part of the fruit. It is very white, and of tho consistence of now bread. Whon washed it bocomes excellent food, tasting like wheaten bread, only a little sweeter.

A cloth is made of the flibres of the inner bark; the wood is used for building houses and boats. The leaves are used as towels and table-cloths, and to wrap provisions in. The male catkins serve as tinder, and the juice is employed as birdlime, and to mend the cracks in the water vessels of tho natives. Seo Cow Trre, JACK Trae, Upas TrRaz.

Braccra. An Italian term used for a rock composed of angular fragments of stone, comented together by an earthy or a mineral substance. It corresponds to the 'brockrans' of the Cumberland miners.

вRzCCIATID AGATr. An agate composed of fragments of jaspor, bloodstono, \&ce., comented by chalcedony.

Brirazzs. (Braise, Fr.) The dust of coke or charcoal. The coke burner applies this term to the small residual coke obtained in coke burning. The sifted ashes romorod from houses is called breeze, and sold under that name to brickmakers and others.

BrFrza OVIx. An oven for the manufacture of small coke. Mr. Joseph Davis, of Birmingham, has patented (Specification, A.D. 1856, No. 1,425) a breezo ovon, many of which are in use in South Staffordshire. The 'thick coal' of South Staffordshire is employed in this oven. The slack is screened, and the finer part is burnt on the grate adjoining the boiler, the remainder is converted into 'breezes.' Mr. Davis's specification will most completely illustrate and explain the character of this oven:-

This invention consists of a coke oven, constructed, arranged, and used as hereinafter described, and illustrated in the accompanying drawings, for the manufacture of the small coke called breezes, for oconomising of heat, and for the suppression or partial suppression of smoke.

243

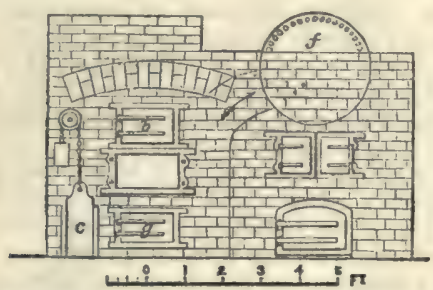

244

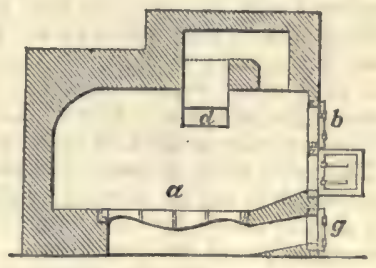

Fig. 243 of the accompanying drawing represents in elevation, fig. 244 in vertical soction, and fig. 245 in plan, a coke oven, combined with the furnace of a steam-

245

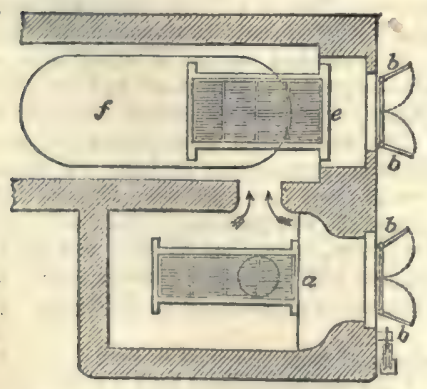
boiler, constructed according to this invention. The said coke oven consists of a chamber or furnace, $a$, provided with doors, $b, b$, by which said doors the coal to be coked is introduced and the admission of air regulated. The draught may bo further regulated by means of the damper, $c$. The oven, $a$, is also provided with flues, $d$, in which flues dampers may bo situated. The heated air and flame from the oven, $a$, may be conducted therefrom either into a stack or chimney, or into the furnace or fireplace, $e$, of a steam-boiler, $f$, or the said heated air and flame may be conducted elsewhere, where they can be applied to heating or other useful purpose. When the flame and heated air from the coke oven, $a$, are conducted into the furnace, $e$, of a steam-boiler, $f$, they may be deliverod at or near the bridge of the said furnace, when they will effect the suppression or partial suppression of the smoke from the said furnace. The heat from the coke over, $a$, also increases the production of steam in the boiler, $f$. 
When the coal in the oven, $a$, has been converted into the small coke, called breezes, the combustion is stopped by the closing of the doors, $b b$, demper, $c$, and the door, $g$, of the ash-pit; and the breezes are cooled by the introduction of a jet of water into the said oven, the said water being directed upon the breezes; or the said breozes may be withdrawn from the ovon in a heated state, and afterwards cooled by water of otherwise. Although I prefor to place the coke oven on the side of the boiler, as represented, yet it may be placed under the boiler, or in any other convenient situation.

Although I have only represented in the accompanying drawing the combination of a coke oven for manufacturing breezes with a stoam-boiler, yet the said oven may be combined with any other furnace for the purpose of increasing its heat and suppressing or partially suppressing its smoke; but I believe the nature of my said invention will be sufficiently understood by the description herein given, and illustratod in the accompanying drawing.

The size and form of the ovens may be varied to suit the quantity of breezes to be manufactured, and the particular purposes to which the flame and heated air from the said ovens are to be applied.

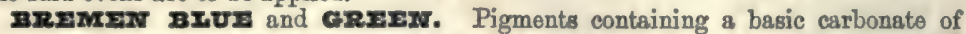
copper with alumina and carbonate of lime. According to one method, blue vitriol (sulphate of copper) is dissolved in 10 parts of water, and a little aquafortis added; the liquid is allowed to stand for a week, and is then filtered; after addition of limewater, it is precipitated by a solution of pearlash. By Gentele's method, these pigments are prepared from common salt and blue vitriol. A blue or a green colour is produced, according as the pigment is mixed with water or with linseed oil.

BREw Irre. (Brasser, Fr.; Brauen, Ger.) The art of making beer, or an alcoholic liquor, from a fermented infusion of some saccharine and amylacoous substance with water.

Figs. 246 and 247 represent the arrangement of the utensils and machinery in a porter brewery on the largest scale, in which it must be observed that the elevation fig. 247, is in a great degree imaginary as to the plane upon which it is taken, but the different vessels are arranged so as to explain their uses most readily, and at tho same time to preserve, as nearly as possible, the relative position which is usually assigned to each in works of this nature.

The malt for the supply of the brewery is stored in vast granaries or malt-lofts, usually situated in the upper part of the buildings. Of these, we have been able to represent only one, at A, fig. 246 : the others, which are supposed to be on each side of it, cannot be seen in this view. Immediately beneath the granary $\Delta$, on the groundfloor, is the mill; in the uppor storey above it, are two pairs of rollers (figs. 246, 248, and 249), under $a a$, for bruising or crushing the grains of the malt. In the floor beneath the rollers are the mill-stones $b b$, where the malt is sometimes ground, instead of being merely bruised by passing between the rollers, under $a a$.

The malt, when prepared, is conveyed by a trough into a chest $d$, to the left of $b$, from which it can be elevated by the action of a spiral screw, fig. 250, enclosed in the sloping tube $e$, into the large chest or bin $\mathrm{B}$, for holding ground malt, situated immediately over the mash-tun $\mathrm{D}$. The mash-tun is a largo circular tub with a double bottom; the uppermost of which is called a false bottom, and is pierced with many holes. There is a space of about 2 or 3 inches between the two, into which the stopcocks enter, for letting in the water and drawing off the wort. The holes of the false bottom, if of wood, should be burned, and not bored, to prevent the chance of their filling up by the swelling of the wood, which would obstruct the drainage: the holes should be conical, and largest below, being about $\frac{8}{8}$ ths of an inch there, and $\frac{1}{8}$ th at the upper surface. The perforated bottom must be fitted truly to the sides of the mash-tun, so that no grains may pass through. The mashed liquor is let off into a large back, from which it is pumped into the wort-coppers. The mash-tun is provided with a peculiar rotatory apparatus for agitating the crushed grains and water together, which we shall presently describe. The size of the wort-copper is proportional to the amount of the brewing, and it must, in general, be at least so large as to operate upon the whole quantity of wort made from one mashing; that is, for every quarter of malt mashed, the copper should contain 140 gallons. The mash-tun ought to be at least a third larger, and of a conical form, somewhat wider below than above. The malt is reserved in this bin till wanted, and is then let down into the mashing-tun, where the extract is obtained by hot water supplied from the copper $\alpha$, seen to the left of $B$.

The water for the service of the brewery is obtained from the well $\mathbf{k}$, seen beneath the mill to the right, by a lifting pump worked by the steam-engine; and the forcingpipe $f$, of this pump conveys the water up to the large reservoir or water-back $F$, placed at the top of the engine-house. From this cistern, iron pipes are laid to the copper a (on the left-hand side of the figure), as also to every part of the establishment where 
cold water can be wanted for cleaning and washing the vessels. The copper a can

be filled with cold water by moroly turning a cock; and the water when boiled therein,

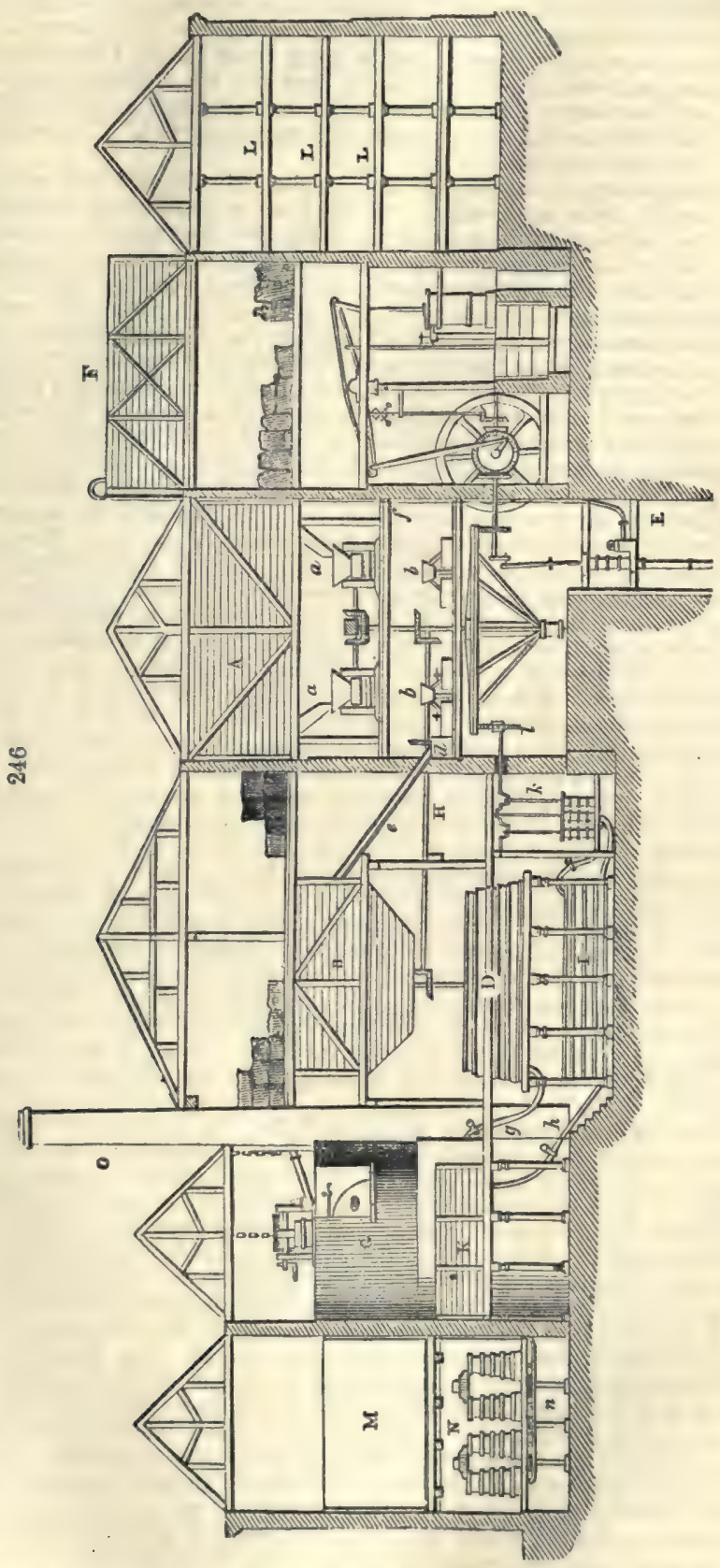

is conveyed by the pipe $g$ into the bottom of the mash-tun $\mathrm{D}$. The water is introduced beneath a false bottom, upon which the malt lies; and, rising up through the 
holes in the false bottom, it extracts the saccharine matter from the malt; a greater or less time being allowed for tho infusion, according to circumstances. The instant the water is drawn off from the copper, fresh water must be let into it, in order to be ready for boiling the second mashing, bocauso the copper must not be left ompty for a moment, otherwise the intense heat of the fire would destroy its bottom. For the convenience of thus letting down at once as much liquor as will fill the lower part of the copper, a pan or second boiler is placed over the top of the coppor, as seen in fig. 252; and the steam rising from the copper communicates a considerable degree of heat to the contents of the pan, without any expense of fuel. This will be more minutely explained hereafter.

During the process of mashing, the malt is agitated in the mash-tun, so as to expose every part to the action of the water. This is done by a mechanism contained within the mash-tun, which is put in motion by a horizontal shaft above it, $\boldsymbol{н}$, leading from the mill. The mash machine is shown separately in fig. 251. When the operation of mashing is finished, the wort or extract is drained down from the mait into tho vessel I, called the underback, immediately below the mash-tun, of like dimensions, and situated always on a lower level, for which reason it has received this name. Here the wort does not remain longer than is necessary to drain off the whole of it from the tun above. It is then pumped up by the three-barrelled pump $k$, into the pan upon the top of the copper, by a pipe which cannot be seen in this section. The wort remains in the pan until the water for the succeeding mashes is discharged from the copper. But this delay is no loss of time, because the heat of the copper, and the steam arising from it, prepare the wort, which had becomo cooler, for boiling. The instant the copper is emptied, the first wort is let down from the pan into the copper, and the second wort is pumped up from the under-back into the upper pan. The proper proportion of hops is thrown into the copper through the near hole, and then the door is. shut down and serewed fast, to keep in the steam, and cause it to rise up through pipes into the pan. It is thus forced to blow up through the wort in the pan, and communicates so much heat to it, or to water, called liquor by the browers, that either is brought near to the boiling point. The different worts succeed each other through all the different vessels with the greatest regularity, so that there is no loss of time, but every part of the apparatus is constantly employed. When the ebullition has continued a sufficient period to coagulate the grosser part of the extract, and to evaporate part of the water, the contents of the copper are run off through a large cock into tho jack-back $\mathrm{K}$, below $\mathrm{G}$, which is a ressel of sufficient dimensions to contain it, and provided with a bottom of cast-iron plates, perforated with small holes, through which the wort drains and leaves the hops. The hot wort is drawn off from the jack-back through the pipe $h$ by the three-barrelled pump, which throws it up to the coolers $I L L$; this pump being made with different pipes and cocks of communication, to serve all the purposes of the browery, except that of raising the cold water from the well. The coolers, I I x, aro very shallow vessels, built over one another in several stages; and that part of the building in which thoy are contained is built with latticowork or shutter flaps, on all sides to admit freo currents of air. When the wort is sufficiently cooled to be put to the first fermentation, it is conducted in pipes from all the different coolers to the large fermenting vessel or gyle-tun as, which, with another similar vessel behind it, is of sufficient capacity to contain all the beer of one day's brewing.

Whenever the first fermentation is concluded, the beer is drawn off from the great fermenting vessel $\mathrm{x}$, into the small fermenting casks or cleansing vessels $\mathrm{N}$, of which there are a great number in the brewery. They are placed four together, and to each four a common spout is provided to carry off the yeast, and conduct it into the troughs $u$, placed beneath. In these cleansing vessels the beer remains till the fermentation is completed; and it is then put into the store-rats, which are casks or tuns of an immense size, where it is kept till wanted, and is finally drawn off into barrels, and sent away from the brewery. The store-vats are not represented in the figure: they are of a conical shape, and of different dimensions, for fifteen to twenty feet diameter, and usually from fifteen to twenty foet in depth. The steam-engine, which puts all the machine in motion, is exhibited in its place on the right side of the figure. On the axis of the large fly-whoel is a bevelled spur-wheol, which turns another similar wheel upon the end of a horizontal shaft, which extends from the enginehouse to the great horse-wheel, set in motion by means of a spur-wheel. The horsewheol drives all the pinions for the mill-stones $b$, and also the horizontal axis which works the three-barrelled pump $k$. The rollers $a a$ are turned by a bevel wheel upon the upper end of the axis of the horse-wheel, which is prolonged for that purpose ; and the horizontal shaft $\mathrm{n}$, for the mashing engine, is driven by a pair of bevel wheols. There is likewise a sack-tackle, which is not represonted. It is a machine for drawing up the sacks of malt from the court-yard to the highest part of the building, whence 
the sacks are wheeled on a truck to the malt-loft $\mathbf{\Lambda}$, and the contents of the sack are discharged.

The horse-wheel is intonded to be driven by horses occasionally, if the steam-engine

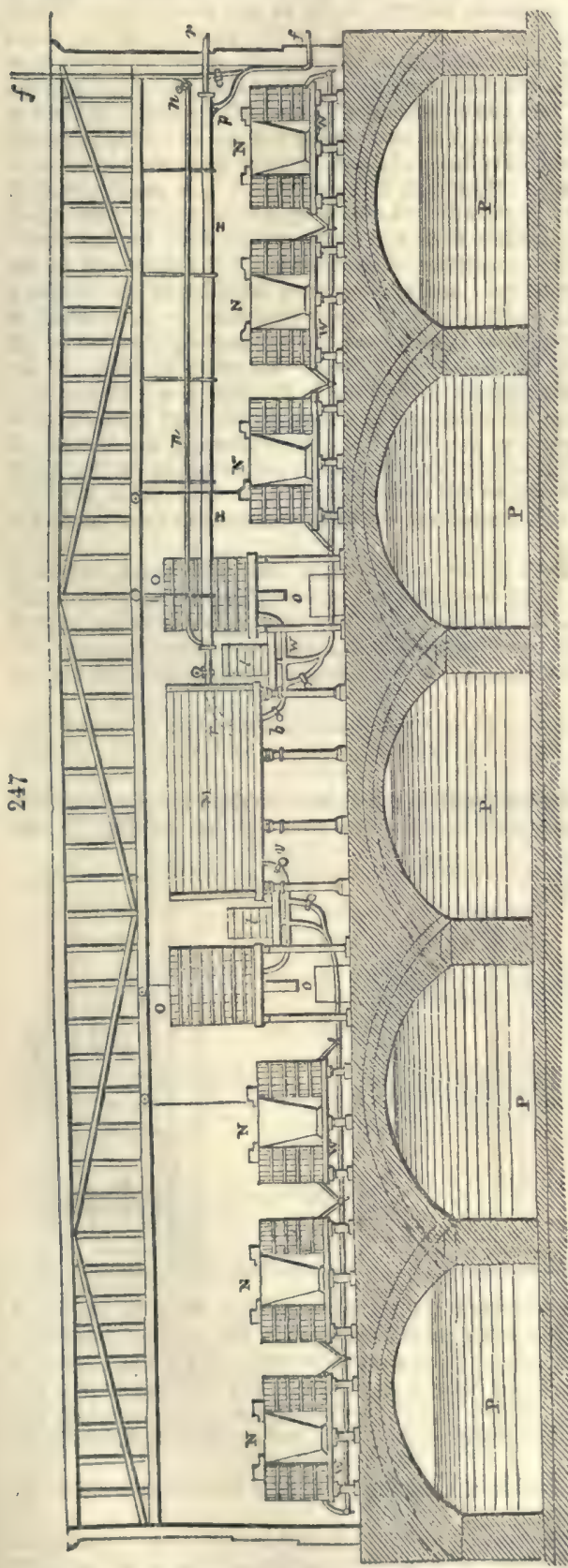
should fail; but these engines are now brought to such perfection that it is very seldom any resource of this kind is noeded.

Fig. 247 is a representation of the fermenting-house at the brewery of Messrs. Whitbread and Company, Chiswell Street, London, which is one of the most complete in its arrangement in the world : it was erected after the plan of $\mathrm{Mr}$. Richardson, who conducts the brewing at those works. Tho whole of fig. 247 is to be considered as devoted to the same object as the large vessel $\mathrm{M}$ and the casks $N$, fig. 246. In fig. $247, r r$ is the pipe which leads from the different coolers to convey the wort to the great fermenting vessels or squares $\mathrm{M}$, of which there are two, one behind the other; $f f$ represent a part of the great pipe which conveys all the water from the well $x$, fig. 246, up to the water cistern $\mathbf{r}$. . This pipe is conducted purposely up the wall of the fermenting-house, fig. 247, and has a cock in it, near $r$, to stop the passage. Just beneath this passage a branch-pipe $p$ proceeods, and enters a large pipe $x x$, which has the former pipe $r$ withinside of it. From the end of the pipe $x$, nearest to the squares M, "nother branch $n n$ procoeds, and returns to the original pipe $f$, with a cock to regulate it. The object of this arrangement is to make all, ox any part, of the cold water flow through the pipe $x x$, which surrounds the pipe $r$, formed only of thin copper, and thus cool the wort passing through the pipe $r$, until it is found by the thermomoter to have the exact temperature which is desirable before it is put to ferment in the great square $\mathbf{M}$. $\mathrm{By}$ means of the cocks at $n$ and $p$, the quantity of cold watar passing over the surface of the piper can be regulated at pleasure, whereby the heat of the wort, when it enters.into the square, may be adjustod within half a degreo.

When the first fermontation in the squares $\mathbf{M} \mathbf{M}$ is finished, the beer is drawn off from them by pipes marked $v$, and conducted by its branches $W W W$, to tho different rows of formenting-tung, marked $s w_{2}$, which occupy tho greator 
part of the building. In the hollow botween every two rows are placed large troughs, to contain the yeast which they throw off. The figure shows that the small tuns are all placed on a lower level than the bottom of the great vessels $\mathbf{M}$, so that the beer will flow into them, and, by hydrostatic equilibrium, will fill them to the same level. When they are filled, the communication-cock is shut; but, as the working off the yoast diminishes the quantity of beer in each vessel, it is necessary to replenish them from time to time. For this purpose, the two large vats 00 are filled from the great squares 3r $\mathrm{M}$, before any beer is drawn off into the small casks $\mathrm{N}$, and this quantity of beer is reserved at the higher level for filling up. The two vessels 00 are, in reality, situated between the two squares $\mathrm{M} M$; but $I$ have been obliged to place them thus in the section, in order that thoy may be seen. Near each filling-up tun o is a small cistern $t$, communicating with the tun o by a pipe, which is closed by a float-valve. The small cisterns $t$ are always in communication with the pipes which lead to the small fermenting vessels $\mathrm{N}$; and therefore the surface of the beer in all the tuns, and in the cisterns, will always be at the same level; and as this level subsides by the working off of the yeast from the tuns, the float sinks and opens the valve, so as to admit a sufficiency of beer from the filling-up tuns 0 , to restore the surfaces of the beer in all the tuns, and also in the cistern $t$, to the original level. In order to carry off the yeast which is produced by the fermentation of the beer in the tuns o 0 , a conical iron dish or funnel is made to float upon the surface of the beer which they contain; and from the centre of this funnel a pipe, o, descends, and passes through the bottom of the tun, being packed with a collar of leather, so as to be water-tight; at the same time that it is at liberty to slide down, as the surface of the beer descends in the tun. The yeast flows over the edge of this funnel-shaped dish, and is conveyed down the pipe into a trough beneath.

Beneath the fermenting-house are large arched vaults, $\mathrm{P}$, built with stone, and lined with stucco. Into these the beer is let down in casks when sufficiently formented, and is kept in store till wanted. These vaults are used at Mr. Whitbread's brewery, instead of the great store-vats of which we have before spoken, and are in some respects preferable, because they preserve a great equality of temperature, being beneath the surface of the earth.

The kiln-dried malt is sometimes ground between stones in a common corn-mill, like oatmeal; but it is more generally crushed between iron rollers, at least for the purpose of the London brewers.

The Crushing Mill. -The cylinder malt-mill is constructed as shown in figs. 248, 249. $\mathrm{I}$ is the sloping-trough, by which the malt is let down from its bin or floor to the hopper $\Delta$ of the mill, whence it is progressively shaken in between the rollers $\mathrm{B} D$. The rollers are of iron, truly cy. lindrical, and their ends rest in bearers of hard brass, fitted into the side frames of iron. A serow an goes through the upright, and gerves to force the bearer of the one roller towards that of the other, so as to bring them closer together when the crushing effect is to be increased. $G$ is the square end of the axis, by which one of the rollers may be turned either by the hand or by power; the other derives its

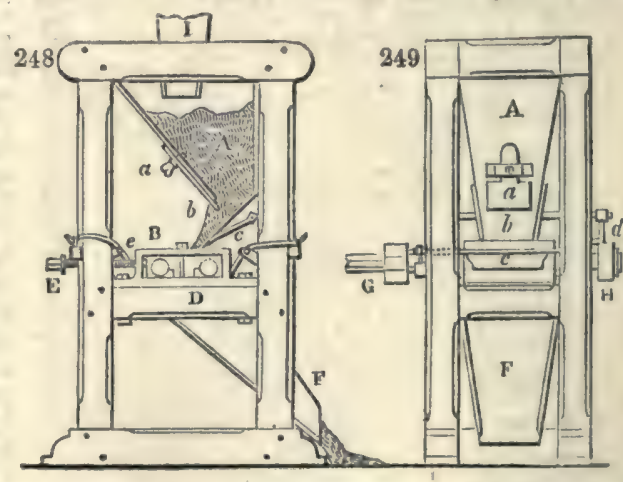
rotatory motion from a pair of equal-toothed wheels $\mathrm{H}$, which are fitted to the other end of the axes of the rollers. $d$ is a catch which works into the toeth of a ratchetwheel on the end of one of the rollers (not shown in this view). The lever $c$ strikes the trough $b$ at the bottom of the hopper, and gives it the shaking motion for discharging the malt between the rollers, from the side sluice a. $e e, f i g .248$, are scraper-plates of sheet iron, the edges of which press by a weight against the surfaces of the rollers, and keep them clean.

Instead of the cylinders, some employ a crushing mill of a conical-grooved form, like a coffee-mill upon a large scale.

Fig. 250 is the serew by which the ground or bruised malt is raised up, or conveyed from one part of the brewery to another. $K$ is an inclined box or trough, in the centre of which the axis of the screw $\mathrm{H}$ is placed; the spiral iron plate or worm, which is fixed projecting from the axis, and which forms the screw, is made very nearly to 
fill the inside of the box. By this means, when the screw is turnod round by the wheels $\mathrm{E} F$, or by any othor means, it raises up the malt from the box $d$, and delivers it at the spout $\mathrm{G}$.

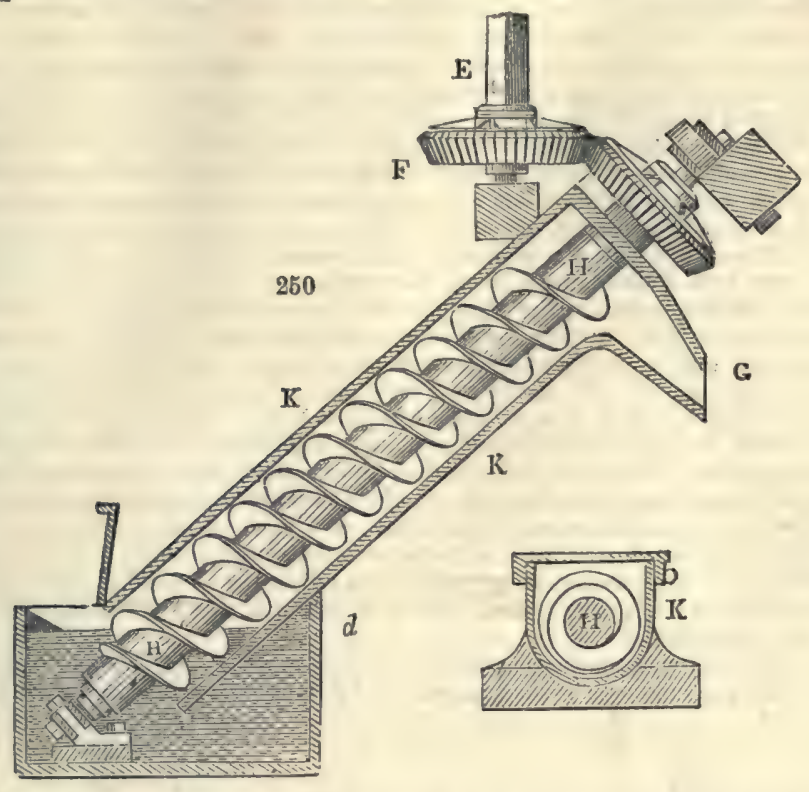

This screw is equally applicable for conveying the malt horizontally in the trough $\mathbf{x}$, as slantingly ; and similar machines are employed in various parts of breweries for conveying the malt wherever the situation of the works require.

Fig. 251 is the mashing-machine. $a a$ is the tun, made of wood staves hooped together. In the centre of it rises a perpendicular shaft $b$, which is turned slowly round

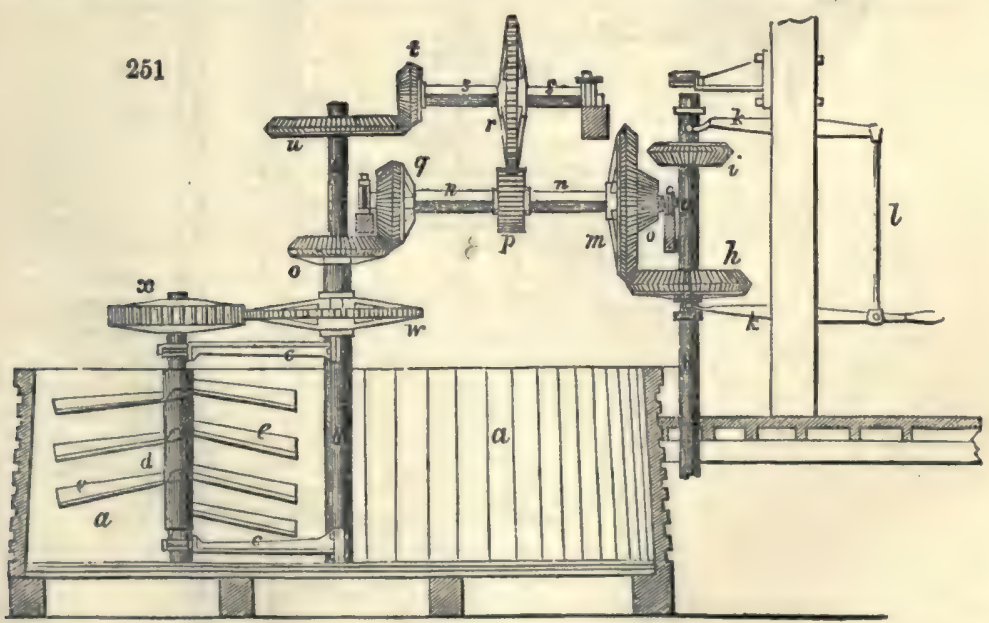

hy means of the bevelled wheels $t u$ at the top. $c$ o are two arms projecting from that axis, and supporting the short vertical axis $d$ of the spur-wheel $x$, which is turned by the spur-wheel $w$; so that, when the contral axis $b$ is made to revolve, it will carry the thick short axle $d$ round the tun in a circle. That axle $d$ is furnished with a number of arms, $\theta e$, which have blades placed obliquely to the plane of their Vor, I. 
motion. When the axis is turned round, these arms agitate the malt in the tun, and give it a constant tendency to rise upwards from the bottom.

The motion of the axlo $d$ is produced by a wheel, $x$, on the upper end of it, which is turned by a wheel, $w$, fastoned on the middle of the tube $b$, which turns freely round upon its central axis. Upon a higher point of the same tube $b$ is a bevel wheol 0 , receiving motion from a bevel wheel $q$, fixed upon the end of the horizontal axis $n n$, which gives motion to the whole machine. This same axis has a pinion $p$ upon it, which gives motion to the wheel $r$, fixed near the middle of a horizontal axle, which, at its left-hand end, has a bevel pinion $t$, working the wheel $u$, before mentioned. By these means, the rotation of the central axis $b$ will be very slow compared with the motion of the axle $d$; for the latter will make seventeen or eighteen revolutions on its own axis in the same space of time that it will be carried once round the tun by the motion of the shaft $b$. At the beginning of the operation of mashing, the machine is made to turn with a slow motion; but, after having wetted all the malt by one revolution, it is driven quicker. For this purpose, the ascending-shaft $f g$, which gives motion to the machine, has two bevel wheols $h i$, fixed upon a tubo $f g$, which is fitted upon a central shaft. These wheels actuate the wheels $m$ and $o$, upon the end of the horizontal shaft $n n$; but the distance between the two wheels $h$ and $i$ is such that they cannot be engaged both at once with the wheels $m$ and 0 ; but the tube $f g$, to which they are fixed, is capable of sliding up and down on its central axis sufficiently to bring either wheel $h$ or $i$ into gear with its corresponding wheel $o$ or $m$, upon the horizontal shaft; and as the diameters of $n o$ and $i m$ are of very different proportions, the velocity of the motion of the machine can be varied at pleasure, by using one or other. $k$ and $k$ are two levers, which are forked at their extremities, and embrace collars at the ends of the tube $f g$. These levers being united by a rod, $l$, the handle $k$ gives the means of moving the tube $f g$, and its wheels $h i$, up or down, to throw either the one or the other wheel into gear.

Figs. 252, 253 represent the copper of a London brewery. Fig. 252 is a vertical section; fig. 253, a ground plan of the fire-grate and flue, upon a smaller scale $: a$ is the close

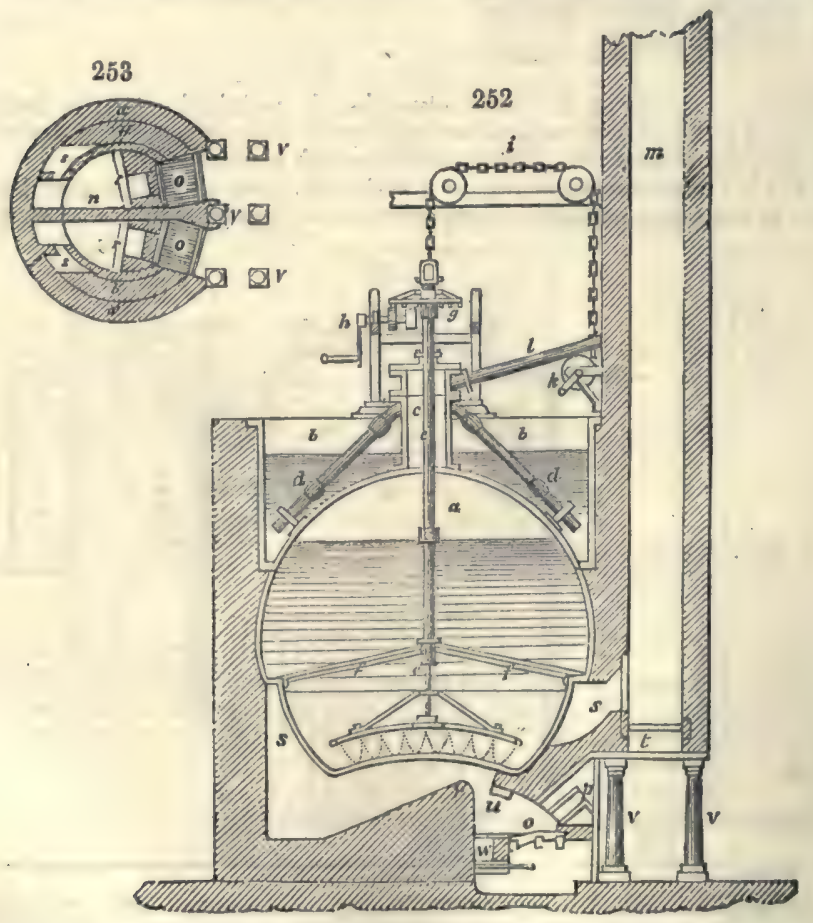

copper kettle, having its bottom convex within; $b$ is the open pan placed upon its top. From the upper part of the copper, a wide tube, $c$, asconds, to carry off the steam generated during the ebullition of the wort, which is conducted through four downwardse 
slanting tubes, $d d$ (two only are visible in this section), into the liquor of the pan $b$, in order to warm its contents. A vertical iron shaft or spindle, e, passes down through the tube $c$, nearly to the bottom of the copper, and is there mounted with an iron arm, called a rouser, which carries round a chain hung in loops, to prevent the hops from adhering to the bottom of the boilor. Three bent stays, $f$, are stretched across the interior, to support the shaft by a collet at their middle junction. The shaft carries at its upper end a bevel wheel $g$, working into a bevel pinion upon the axis $h$, which may be turned either by power or by hand. The rouser shaft may be lifted by means of the chain $i$, which, going over two pulleys, has its end passed round the wheel and axle $k$, and is turned by a winch : $l$ is a tube for conveying the waste steam into the chimney $m$.

The heat is applied as follows:-For heating the colossal coppers of the Irondon broweries, two separate fires are required, which are separated by a narrow wall of brickwork, $n$, figs. 252, 253. The dotted circle $a^{\prime} a^{\prime}$, indicates the largest circumference of the copper, and $b^{\prime} b^{\prime}$ its bottom; $o o$ are the grates upon which the coals are thrown, not through folding doors (as of old), but through a short slanting iron hopper, shown at $p$, fig. 252 , built in the wall, and kept constantly filled with the fuel, in order to exclude the air. Thus the low stratum of coals gets ignited before it reaches the grate. Above the hopper $p, a$ narrow channel is provided for the admission of atmospherical air, in such quantity merely as may be requisite to complete the combustion of the smoke of the corls. Behind each grate there is a fire bridge, $r$, which reflects the flame upwards, and causes it to play upon the bottom of the copper. The burnt air then passes round the copper in a semicircular flue, $s s$, from which it flows off into the chimney $m$, on whose under end a sliding damper-plate, $t$, is placed, for tempering the draught. When cold air is admitted at this orifice, the combustion of the fuel is immediately checked. There is, besides, another slide-plate at the entrance of the slanting flue into the vertical chimney, for regulating the play of the flame under and around the copper. If the plate $t$ be opened, and the other plate shut, the power of the fire is suspended, as it ought to be, at the time of emptying the copper. Immediately over the grate is a brick arch, $u$, to protect the front edge of the copper from the first impulsion of the flame. The chimney is supported upon iron pillars, $v v ; w$ is a cavity closed with a slide-plate, through which the ashes may be taken out from behind, by means of a long iron hook.

We have thus given the general plan and requisites for a brewery on a large scale. We need searcely say those arrangements will vary in every establishment, according to the requirements and facilities of the locality, and the various modes of operation. The few simple utensils required may be easily recapitulated:-

1. A mill for erushing the malt.

2. An iron pan for heating water.

3. A mash-tun or open tub fitted with a false bottom, a straincr, or with some other means of allowing the wort to run off freely, keeping back the grains.

4. An iron or copper pan for boiling the wort.

5. A shallow vessel or cooler, over which is placed the hop-jack or sieve for straining out the spent-hops.

6. A gyle tun or open tub for commencement of the fermentation.

7, A barrel or cask in which the cleansing is completed.

The first necessity is a plentiful supply of pure water, which it should be the chief aim in all arrangements to render available at the least labour and cost, as on its proper and judicious application greatly depends the regulation of the temperature in the various operations; and the most scrupulous cleanliness in every part is of the utmost importance. The fermenting rooms and store-cellars should be placed bolow the ground level, for the purpose of attaining a low and equable temperature; and for this purpose also the double stone fermenting square is highly esteemed. It consists of an inner cubical vessel, containing from fifteen to thirty barrels; each side formed by one slab of fine slate. This is placed in an exterior square or shell of inferior stone, leaving a space between the inner and outer squares, which can be filled with hot or cold water at pleasure. The inner or fermenting square has a manhole, with a raised rim, in the slab forming the top, on which also are raised four other fine slate slabs, which form a cistern for the expansion and overflow of the beer and yeast during the process of the formentation, and from which the yeast is readily removed at its close.

The process of brewing may be classed under three heads: the mashing, the boiling, and the formentation.

For the principle which should guide the brewer in the conduct of these operations, we refer to the article BEER, where it will bo seen that the ultimate success of the entire series depends greatly on the regulation of the temperature, the duration, and the proper management of the initial process of malting.

The Mashing,-Upon this very important process information, the result of Dr. A. 
Schwarzer's researches, is perhaps the most valuablo and precise of the present day, and it would bo well if every brewer wero familiar with them.

In the Brewers' Journal, January 15, 1871, this information is fully detailed, but as the number may not easily be obtainable, it is here reinserted:-

'As a general rule, the larger the quantity of diastaso and the greater the heat up to a certain point, the more rapid is the transformation of the gummy portion of the starch into sugar.

'At a temperature of about $167^{\circ} \mathrm{Fahr}$., and especially at $177^{\circ}$ or $178^{\circ}$, however, a diminution takes place in the action of the diastase, which incresses with the further increase of the temperature; and if the heat bo raised for a certain time to about $190^{\circ}$, the action of the diastase coases altogether.

' If also an extract of malt be heated to $190^{\circ}$ before the starch is added, a diminution in the action occurs, which is the more determined according to the length of time at which the hert has been maintained.

' The quantity of djastase thus rendered inactive may be ascertained by comparing the action of a cortain amount of the overheated extract, with that of a like quantity at the temperature of $145^{\circ}$. Observation must be taken of the time which elapses before all reaction ceases to be exhibited by the iodine test; and the same test must be applied to the action of the extract submitted to the lower temperature.

- Two per cent. of an extract of malt, not weakened by heating, effects the transformation more rapidly than four times that percentage kept at the heat of $178^{\circ}$ for one hour, or than twenty per cent. of the same extract maintained for the same period at a temperature of $190^{\circ}$. In the last case scarcely one-tenth of the diastase originally developed in the malt remains in the extract.

- With minute quantities of diastase the action proceeds more rapidly at first with a temperature of $190^{\circ}$, but afterward more slowly than at $145^{\circ}$.

- When the iodine test shows no discolouration, the saccharification is practically terminated, and the continuance of the action of the diastase yields an infinitesimal quantity of sugar.

'If, in one hour, and until the iodine shows no discolouration, fifty per cent. of sugar is formed, and if, during the next three hours, only two per cent. is produced, it is certain that the iodine test shows practically the action is finished.

'The cause of this feeble saccharification after the iodine test ceases to act, does not, however, arise from the exhaustion of the energy of the diastase, because if a new amount of starch be added, it will be rapidly transformed.

'At all temperatures from $167^{\circ}$ to $32^{\circ}$, even though employing very different proportions of diastase, from fifty to fifty-three per cent. of sugar is invariably produced from the starch in the extract.

'Admitting that the starch is transformed into an equivalent of sugar, and an equivalent of dextrine, analysis shows that the sugar to be obtained from the extract amounts to $52 \cdot 6 \mathrm{Ir}$ cent. of the extract.

- The amount of sugar obtained in practice differs so little from this rate that the small difference may fairly be supposed to arise from errors in the quantities and interruptions in the action of the diastase.

'At temperatures exceeding $167^{\circ}$ the quantity of sugar produced falls off in proportion.

'At $190^{\circ}$, after the iodine test ceases to act, the amount of sugar formed in the extract may fall off to twenty-seven per cent.; and even if the extract is heated to that temperature before the addition of the starch gum, and the greatesi care be taken to prevent cooling during the experiment, very little change indeed will be observed in the percentage.

'If all these facts be taken into account, it seems certain, that the action of the diastase at temperature below $167^{\circ}$ is very different from what it is under greater heats.

- The amount of sugar equal to twenty-seven per cent. which is the minimum value at which the iodine test ceases to exhibit any change, agrees exactly with the transformation of starch into an equivalent of sugar and an equivalent of dextrine.

A fact deserving of notice is, that between the temperatures of $167^{\circ}$ and $190^{\circ}$ the difference in the amount of sugar formed is moro than twenty per cent.

- In maintaining the extract of malt at $190^{\circ}$ for a long period, it is so greatiy altered that when the heat is afterward lowered, the amount of sugar is not, or scarcely at all, increased; but if, on the contrary, a solution of starch which contains about twenty-seven per cent. of sugar, be heated to $190^{\circ}$, and afterwards submitted to the action of diastase, not heatod and enfeebled at a lower temperature, the percentage may be carried to fifty-two per cent.

'During fermentation, the diastase continues to effect the transformation of starch ; and it is not improbable that jeast acts in the same manner as the former.' 


\section{BREWING}

With these very impurtant facts before us, it is comparatively easy for the brewer to determine his rule or mode of operation in the process of mashing.

As the heats approaching to $167^{\circ}$ tend to detract from the energy of the diastase, such must of course as much as possible bo avoided, it being known with equal certainty that when malt-wort is allowed to drop its temperature to a heat approaching $140^{\circ}$, it is liable to acetancy, and more readily so, as the temperature recedes below $140^{\circ}$; about $120^{\circ}$ being the most favourable to the change.

Therefore it is advisable that the wort should reach the copper before such a temperature is attained; still further it is ascertained that the value of the desired transformation in the malt-wort is best obtained with a temperature of about $151^{\circ}$, also that saccharification is promoted by agitation, and that the stronger the extract is, the less is the danger of acetification.

Therefore, in the first place, the brewer has to obtain a mash of $151^{\circ}$ temperature or thereabouts ; but he must do it with liquor as far removed below $167^{\circ}$ as possible, and when the goods are thoroughly mashed, $151^{\circ}$ of hoat should be maintained during their clarification; the wort should then be run from the mash-tun into a copper suitably warmed beforehand, and should then be slowly and carefully heated up to boiling, just by the time the subsequent exhaustion of the malt is completed by the 'sparging.'

In practice the following method will be found extremely easy, and safe in operation:-

Some time before commencing the mash, a sufficiency of boiling water is run into the tun to make it as hot as possible, and immediately before commencing the brewing this hot water is let off again into the underback, or the copper; this being done, tho mashing liquor having previously been very carefully prepared, is let into the tun under the false bottom, to the quantity of about one and a half barrels to the quarter of malt, where it arrives at about $164^{\circ}$ temperature; when a snfficiency has been let in, the grist is then added and thoroughly mashed, the infusion will then be about $150^{\circ}$, the temperature of reserve mashing liquor has been raised, and when the malt is effectually mashed, a further quantity of about half a barrel per quarter is run in at $166^{\circ}$, and thoroughly mixed up with the mash: the object of the additional liquor is to raise the heat of the mash to about $152^{\circ}$, after which settling and clarification are requisite; this usually occupies about two hours.

The tun having been thoroughly heated beforo the admission of the mash, it docs not abstract the heat from the infusion, either during the operation or when it is completed, but materially assists to maintain the heat for a very considerable time; with a well-protected and covered tun it will do it for four hours if required, without allowing the temperature to drop a single degree.

This is of the greatest importance, for the wort leaves the mash-tun at about $151^{\circ}$; and as it is passed from vessel to vessel is immediately preceded by the hot water from the vessel before it; thus, in turn, each vessel is thoroughly warmed, and we need hardly say most thoroughly cleansed, and being prepared in this way enables the operator to get the wort into the copper before it can fall even to $146^{\circ}$ or $147^{\circ}$, leaving the mash in the tun at such heat that all danger of acetification is entirely removed.

As the full value of the mash cannot be obtained by one maceration, recourse is now had to 'sparging,' and to do this properly requires a little careful thought and more careful management.

In the operation of mashing every possible care should be taken that the infused malt - called ' goods' - when drained should lay perfectly level, otherwise the sparging liquor, in running off, will form for itself courses through those portions of the mash that offer the least resistance, and as a consequence some parts of the 'goods' will have treatment in excess of what is desirable, while other portions will be kept with an imperfect sparge.

The liquor for sparging should be of such a heat that it will, on penetrating the ' goods,' raise their temperature gradually from, say $148^{\circ}$ or so, to $167^{\circ}$ or $168^{\circ}$ at the close, this will generally be found sufficient to dissolve out of them all the tractable portions of dextrine, \&ce. that are of any real value.

It is advisable during the operation to allow the infused malt to drain two or three times that the act of compression may express from them those portions of sweet wort detained in the texture of each individual grain; this will enable the brewer to obtain the whole of the value that is producible from his malt, which may on that account be treated with less pressure in the grinding; care in this latter particular will considerably facilitate all subsequent operations, and give a brilliancy and superior quality to the beer unattainable by other means.

In drawing the wort from the mash-tun, no mechanical means should be neglected that would aid in discharging it clear and bright into the copper, for all flocks and 
particles of malt that aro taken there, very materially interfere with the action and value of the hops upon the wort; but chemical agencies for this purpose should be avoided. where possible.

With regard to temperature, the brewer must not only regulate the heat of the water for the first mash by the colour, age, and quality of the malt, whether pale, amber, or brown, but he should also mark the temperature of the atmosphere, as influencing that of the malt, and the absorption of the heat by the utensils omployed; remarking that well-mellowed and brown malt will bear a higher mashing heat than pale or newly dried.

The following table of Mashing Heats is by Lovesque :-

Table of Masking Temperatures.

\begin{tabular}{|c|c|c|c|c|c|c|c|c|c|c|c|c|c|c|}
\hline \multirow{3}{*}{ 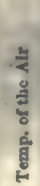 } & \multirow{2}{*}{$\begin{array}{c}\begin{array}{c}\text { Brown } \\
\text { Malt }\end{array} \\
\begin{array}{c}\text { Heat of } \\
\text { Mash, } \\
146^{\circ} \text { to } \\
1480\end{array}\end{array}$} & \multirow{3}{*}{ 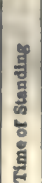 } & \multirow{3}{*}{ 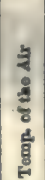 } & \multicolumn{2}{|c|}{ High-dried } & \multirow{3}{*}{ 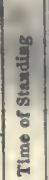 } & \multirow{3}{*}{ 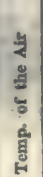 } & \multicolumn{2}{|c|}{ Amber } & \multirow{3}{*}{ 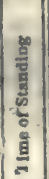 } & \multirow{3}{*}{ 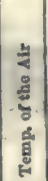 } & \multicolumn{2}{|c|}{ Pale Kalt } & \multirow{3}{*}{ 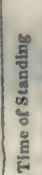 } \\
\hline & & & & $\begin{array}{l}\text { Beak of of } \\
1450 \text { to }\end{array}$ & $\begin{array}{l}\text { Igash, } \\
177^{\circ}\end{array}$ & & & \multicolumn{2}{|c|}{$\begin{array}{l}\text { Heat of } 348 \mathrm{sh}, \\
144^{\circ} \text { to } 14800\end{array}$} & & & \multicolumn{2}{|c|}{$\begin{array}{l}\text { Heat of Maub, } \\
\text { IH50 to } 145^{\circ}\end{array}$} & \\
\hline & $\begin{array}{l}6 \text { Firkins } \\
\text { per Qr. }\end{array}$ & & & $\begin{array}{c}7 \text { Firkins } \\
\text { per Qr. }\end{array}$ & $\begin{array}{l}8 \text { Firkin } \\
\text { per Qr. }\end{array}$ & & & $\begin{array}{l}\text { O Firkias } \\
\text { per Qr. }\end{array}$ & $\begin{array}{c}10 \text { Firking } \\
\text { per } Q \text {. }\end{array}$ & & & 11 Frings & $\begin{array}{c}12 \text { Firkins } \\
\text { per QR. }\end{array}$ & \\
\hline $\begin{array}{c}\text { Fah. } \\
10\end{array}$ & $197 \cdot 00$ & $\begin{array}{l}\text { I.x. } \\
4.00\end{array}$ & Fah. & 3900 & $184 \cdot 00$ & $\begin{array}{l}\mathbf{H}, \mathbf{M} \\
3.00\end{array}$ & $\begin{array}{r}\text { Fah. } \\
10^{\circ}\end{array}$ & 178.00 & $175 \cdot 00$ & $\begin{array}{l}\text { 8.u. } \\
2 \cdot 00\end{array}$ & & $172 \cdot 00$ & 170.00 & $\begin{array}{l}\text { H.M. } \\
1 \cdot 00\end{array}$ \\
\hline 15 & $195 \cdot 17$ & $4: 00$ & 15 & $187 \cdot 42$ & $182 \cdot 59$ & 3.00 & 15 & $176 \cdot 84$ & 173.92 & $2 \cdot 00$ & 15 & $171 \cdot 00$ & 19 & 1.00 \\
\hline 20 & $193 \cdot 34$ & $4 \cdot 00$ & 20 & $185 \cdot 84$ & $181 \cdot 18$ & 3.00 & 20 & $175 \cdot 68$ & $172 \cdot 84$ & $2 \cdot 00$ & 20 & $170 \cdot 00$ & .28 & $1 \cdot 00$ \\
\hline 25 & $191 \cdot 51$ & $4 \cdot 00$ & 25 & $184^{\cdot} \cdot 26$ & $179 \times 77$ & $3 \cdot 00$ & 25 & $174 \cdot 52$ & $171 \cdot 76$ & $2 \cdot 00$ & 25 & $169^{\circ} 00$ & $167 \cdot 37$ & 1.00 \\
\hline 80 & $189^{\circ} 68$ & $4 \cdot 00$ & 30 & $182 \cdot 68$ & $178 \cdot 36$ & 3.00 & 30 & $178 \cdot 36$ & $170 \cdot 68$ & $2 \cdot 00$ & 30 & $168 \cdot 00$ & .46 & $1 \cdot 00$ \\
\hline 35 & $187 \cdot 85$ & $4 \cdot 00$ & 85 & $180 \cdot 10$ & $176 \cdot 95$ & $3 \cdot 00$ & 35 & $172 \cdot 20$ & 369.60 & $2 \cdot 00]$ & 35 & $167 \cdot 00$ & .55 & $1 \cdot 00$ \\
\hline 40 & $186 \cdot 02$ & $4 \cdot 00$ & 40 & $179 \cdot 52$ & $175 \cdot 54$ & 3.00 & 40 & $171 \cdot 04$ & $168 \cdot 52$ & $2 \cdot 00$ & 40 & $166 \cdot 00$ & 64 & $1 \cdot 00$ \\
\hline 45 & $184 \cdot 19$ & $4 \cdot 00$ & 45 & $177 \cdot 94$ & 13 & 3.00 & 45 & 16 & $167 \cdot 44$ & $2 \cdot 00$ & 4 & $165 \cdot 00$ & 73 & $11 \cdot 00$ \\
\hline 50 & $182 \cdot 36$ & $4 \cdot 00$ & 60 & $176 \cdot 36$ & 72 & $3 \cdot 00$ & 50 & 72 & $166 \cdot 36$ & $2 \cdot 00$ & 50 & $164^{\circ} 00$ & 82 & $1 \cdot 00$ \\
\hline 55 & 180.53 & $4: 00$ & 56 & 78 & 31 & 3.00 & 55 & $167 \cdot 56$ & $165 \cdot 28$ & $2 \cdot 00$ & 5 & 163 & 16 & $1 \cdot 00$ \\
\hline 60 & $178 \cdot 70$ & $3 \cdot 40$ & 60 & 20 & 90 & 2.45 & 60 & $166 \cdot 40$ & $164 \cdot 20$ & 1.50 & 60 & $162 \cdot 00$ & 16 & 0.55 \\
\hline 65 & $176 \cdot 87$ & $3 \cdot 20$ & 65 & 62 & 49 & $2 * 30$ & 65 & $165 \cdot 24$ & $163 \cdot 12$ & $1 \cdot 40$ & 65 & 16 & & 0.50 \\
\hline 70 & $17.5^{\circ} 04$ & 3.00 & 70 & 4 & $167 \cdot 07$ & $2 \cdot 15$ & 70 & $164 \cdot 08$ & $162 \cdot 04$ & $1 \cdot 30$ & 70 & $160^{\circ} 00$ & $158 \cdot 28$ & $\{0.45\}$ \\
\hline $\begin{array}{r}\text { Hea } \\
18\end{array}$ & f the & p & & Iest o & 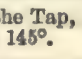 & & & Heat & 10 & & & 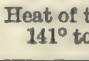 & 18 Tap, & \\
\hline
\end{tabular}

The first column gives the temperature of the air at the time of mashing.

The second column shows the heat of the water, the quantity used, and the resulting heat of the mash-noting, that if the water has been let into the mash-tun at the boiling point, and allowed to cool down, or the vessel has been thoroughly warmed before the commencement of the process, the heat may be taken several degrees lower.

The third column shows the time for the standing of the mash, but this will be modified, as before stated, by the quality of the extract requirod.

The bulk of the materials used must also enter into the consideration of the tem. perature, as a large body of malt will attain the required temporature with a mashing heat lower than a small quantity; the powers of chemical action and condensation of heat being increased with increase of volume.

Donovan, speaking of the temperature to be employed in mashing, lays down the following as a general rule:-For well dried pale malt the heat of the first mashing liquor may be, but should never exceed, $170^{\circ}$; the heat of the second may be, $180^{\circ}$; and, for a third, the heat may be, but need never exceed, $185^{\circ}$.

The quantity of wator, termed liquor, to be employed for mashing, depends upon the greater or less strength to be given to the beer; but in all cases, from one barrel and a half to one barrel and three firkins is sufficient for the first stiff mashing, but more liquor may be added after the malt is thoroughly wetted.

The grains of the crushed malt, after the wort is drawn off, retain from thirty-two to forty gallons of water for every quarter of malt. A further amount must be allowed for the lose by evaporation in the boiling and cooling, and the waste in fermentation, so that the amount of liquor required for mashing will, in some instances, be double that of the finished beer, but in general the total amount will be reduced about onethird during the various processes.

The following example has been given of the proportions for an ordinary quality of beer:-

Suppose thirteen imperial quarters of the best pale malt be taken to make 1,500 gallons of beer, the waste may be calculated at near 900 gallons, or 2,400 gallons of water will be requirod in mashing.

As soon as the water in the copper has attained the heat of $160^{\circ}$ in summer, or $187^{\circ}$ in winter, 600 gallons of it are to be run off into the mash-tun (which has pre- 
viously been well cleansed or scalded ont with boiling water), and the malt gradually but rapidly thrown in and well intermixed, so that it may bo uniformly moistened, and that no lumps remain. After continuing the agitation for about half an hour, more liquor, to the amount of 450 gallons, at a temperature of $180^{\circ}$, may be carefully and gradually introduced (it is an advantage if this can be done by a pipe inserted under the false bottom of the mash-tun), the agitation being continued till the whole assumes an equally fuid state, taking care also to allow as small a loss of temperature as possible during the operation, the resulting tomperature of the mass being not less than $148^{\circ}$, or more than $152^{\circ}$.

The mash is thon covered close, and allowed to remain at rest for an hour, or an hour and a half, after which the tap of the mash-tun is gradually opened, and if the wort that first flows is turbid, it should be carefully returned into the tun until it runs perfectly limpid and clear. The amount of this first wort will bo about 675 gallons.

Seven hundred and fifty gallons of water, at a temperature of from $180^{\circ}$ to $185^{\circ}$, may now be introduced, and the mashing operation repeated and continued until the mass becomes uniformly fluid as before, the temperature being from $160^{\circ}$ to $170^{\circ}$. It is then again quickly covered and allowed to rest for an hour, and the wort of the first mash having been quickly transferred from the underback to the eopper, and brought to a state of ebullition, the wort of the second mash is drawn off with similar precaution, and added to it. A third quantity of water, about 600 gallons, at a temperature of $185^{\circ}$ or $190^{\circ}$, should now be run through the goods into the mash-tun by the sparging process, or by any means that will allow the hot liquor to percolate through the grains, displacing and carrying down the heavier and more valuable products of the two first mashings. The wort is now boiled with the hops from one to two hours.

By the mashing process before described, the malt is so much exhausted that it can yield no further extract useful for strong beer or porter. A weaker wort might be, no doubt, still drawn off for small beer, or for contributing a little to the strength of the next mashing of fresh malt. But this, we believe, is seldom practised.

The wort is then transferred into tho copper, and made to boil as soon as possible, for if it remains long in the underback, it is apt to become acescent. The steam, moreover, raised from it in the act of boiling serves to screen it from the oxygenating or acidifying influence of the atmosphere.

Until it begins to boil, the air should be excluded by some kind of cover.

Dr. Piesse, in 1840, read a paper before the Chemical Society, shr wing that much extract was left in the malt after brewing, and that it was this matter which was convertiblo into sugar, which gave its feeding qualities to 'grains.

It was shown that the malt, after the first wort was drawn off still contained a portion of starch which was not converted into sugar, and that the presence of diastase was necessary to effect this conversion. As diastase is very soluble, there was none of course left in the infused malt.

It is, therefore, recommended by Dr. Piesse, that malt containing diastase should be added to the second wort.

In browing thirty quarters, I should take twenty-nine quarters for the first mash and add the remaining quarter to the second, by which all the starch, it was contended, would be converted into sugar. To prevent the access of air, which tends to induce acidity, to the wort, it is suggested that a board the size of the back in which it is contained should float on the surface of the fluid. (Transactions of the Chemical Society, 1841.)

BRICK. (Brique, Fr.; Backstein, Ziegelstein, Ger.) A solid rectangular mass of baked clay, omployed for building pnrposes. Brickmaking is exceedingly ancient: the tower of Babel was built with bricks, as we are told in Scripture, and also the city of Babylon. Over the ruins of Babylon, and the sites of the other great cities of the ancient monarchies, we still discover bricks of various kinds. Some are merely sun-dried masses of clay; others are well burnt; and others, again, are covered with a vitreous glaze. The Egyptians were great brick-makers; and the Romans were celebrated for their bricks and tiles, large quantities of them having been employed in the construction of their different military stations in England. Subsequently, the same bricks have at times been used on later structures, as for instance, in St. Alban's Abbey, which contains a large quantity of bricks from ruins of the Roman buildings of Verulamium. The Lollards' Tower of Lambeth Palace, built in 1454, and the older portions of Hampton Court Palace, built in 1514, are good examples of the English brick architecture in mediæval times.

The natural mixturo of clay and sand, called loam, as well as marl, which consista of lime and clay with little or no sand, are the matorials usually employed in the manufacture of bricks, 
There are fow places in this country which do not possess alumina in combination with silica and other earthy matters, forming a clay from which bricks can be manufactured. That most generally worked is found on or near the surface in a plastic state. Others are hard marls on the coal-measure, New Red Sandstone, and Blue Lias formations. It is from these marls that the blue bricks of Staffordshire and the fire bricks of Stourbridge are made. Marl has a greater resemblance to stone and rock, and varies much in colour; blue, red, yellow, \&c. From the greatly different and varying character of the raw material, there is an equal difference in the principle of proparation for making it into brick; while one morely requires to be turned over by hand, and to have sufficient water worked in to make it subservient to manual labour, tho fire-clays and marls must be ground down to dust, and worked by powerful machinery, before they can bo brought into even a plastic state. Now these various clays also shrink in drying and burning from 1 to 15 por cent, or more. This contraction varies in proportion to the excess of alumina over silica, but by adding sand, loam, or chalk, or (as is done by the London brick-makers) by using eshes or breeze-as it is technically called-this can be corrected. All clays burning red contain oxides of iron, and those having from 8 to 10 per cent. burn of a blue, or almost a black colour. The bricks are exposed in the kilns to great heat, and when the body is a fire-clay, the iron unites with a portion of the silica, forming a fusible silicate of protoxide of iron, which melts into an external glaze. Bricks of this description are common in Staffordshire, and, when made with good machinery (that is, the clay being very finely ground), are superior to any in the kingdon, particularly for docks, canal or river locks, railway-bridges, and viaducts. In Wolverhampton, Dudley, and many other towns, these blue bricks are commonly employed for paving purposes. Other clays contain lime and no iron; these burn white, and take less heat than any other to burn hard enough for the use of the builder, the lime acting as a flux on the silica. Many clays contain iron and lime, with the lime in excess, when the bricks are of a light dun colour, or white, in proportion to the quantity of that earth present; if magnesia, they have a brown colour. If iron is in excess, they burn from a pale red, to the colour of cast iron, in proportion to the quantity of that metal.

There are three classes of brick earths:-

1st. Plastic clay, composed of alumina and silica, in different proportions, and containing a small per-centage of other salts, as of iron, lime, soda, and magnesia.

2nd. Loams, or sandy clays.

3rd. Marls, of which there are also three kinds: clayey, sandy, and calcareous, according to the proportions of the earth of which they are composed, viz., alumina, silica, and lime.

Alumina is the oxide of the metal aluminium, and it is this substance which gives tenacity or plasticity to the clay-earth, having a strong affinity for water. It is owing to excess of alumina that many clays contract too much in drying, and often crack on exposure to wind or sun. By the addition of sand, this clay would make a better article than wo often see produced fromit. Clays contain magnesia and other earthy matters, but these vary with the stratum or rock from which thoy are composed. It would be impossible to give the composition of these earths correctly, for none are exactly similar; but the following will give an idea of the proportions of the ingredients of a good brick earth : silica, threo-fifths; alumina, one-fifth ; iron, lime, magnesia, manganese, soda, and potash forming the other one-fifth.

The clay, when first raised from the mine or bed, is, in very rare instances, in a state to allow of its being at once tempered and moulded. The material from which fire-bricks are manufactured has the appearance of ironstone and blue lias limestone, and some of it is remarkably hard, so that in this and many other instances in order to manufacture a good article, it is necessary to grind this material down into particles as fine as possible.

Large quantities of bricks are made from the surface marls of the New Red Sandstone and Blue Lias formations. These also require thorough grinding, but from their softer nature it can be effected by less powerful machinery.-Chamberlain.

Recently, some very valuable fire-bricks have beon made from the refuse of the China Clay Works of Devonshire. The quartz and mica left after the Kaolin has been washed out are united with a small portion of inforior clay, and made into bricks. These are found to resist heat well, and are largoly employed in the construction of metallurgical workes. See CLAT.

The general process of brick-making consists in digging up the clay in autumn; exposing it, during the whole winter, to the frost and the action of the air, turning it repeatedly, and working it with the spade; breaking down the clay lumps in spring, throwing them into shallow pits, to be watered and soaked for several days. The next step is to temper the clay, which is generally done by the treading of mon or oxen. In the neighbourhood of London, however, this process is performed in a 
horse-mill. The kneading of the clay is, in fact, the most laborious but indispensable part of the whole business; and that on which, in a great measure, the quality of the brick depends. All the stones, particularly the ferruginous, calcareous, and pyritous kinds, should be removed, and the clay worked into a homogeneous paste with as little water as possible.

Mr. F. W. Simms, C.E., communicated to the Institution of Civil Engineors, in April and May, 1843, an account of the process of brick-making for the Dover Railway. The plan adopted is called slop-moulding, because the mould is dipped into water before receiving the clay, instead of being sanded as in making sand-stock bricks. The workman throws the proper lump of clay with some force into the mould, presses it down with his hands to fill the cavities, and then strikes off the surplus clay with a stick. An attendant boy, who has previously placed another mould in a water trough by the side of the moulding table, takes the mould just filled, and carries it to the floor, where he carefully drops the brick from the mould, on its flat side, and leaves it to dry; by the time he has returned to the moulding table, and deposited the empty mould in the water trough, the brickmaker will have filled the other mould for the boy to convey to the floor, where they are allowed to dry, and are thon stacked in readiness for being burned in clamps or kilns. The average product is shown in the following Table:-

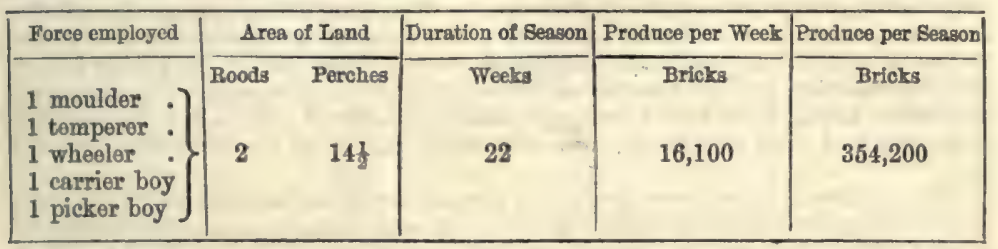

It appears that while the produce in sand-stock bricks is to that of slop-bricks, in the same time, as 30 to 16 , the amount of labour is as 7 to 4 ; while the quantity of land, and the cost of labour per thousand, are nearly the same in both processes. The quantity of coal consumed in the kiln was at the rate of 10 cwt. 8 lbs. per 1000 bricks. The cost of the bricks was 2l. 18.6d. per thousand. The slop-made bricks are fully 1 pound heavier than the sand-stock. Mr. Bennett states that at his brick-field at Cowley, the average number of sand-stock $₹$ :cks moulded per day was 32,000 ; but that frequently so many as 37,000 , or even 50,000 , were formed. The total amount in the shrinkage of his bricks was $\frac{13}{16}$ ths of an inch upon 10 inches in length; but this differed with the different clays. Mr. Simms objects to the use of machinery in brick-making, because it causes economy only in the moulding, which constitutes no more than about one-eighth of the total expense.

The principal machines which have been worked for this purpose are threo-1st, the pug-mill ; 2nd, the wash-mill ; $3 \mathrm{rd}$, the rolling-mill.

The pug-mill is a cylinder, sometimes conical, generally worked in a vertical position, with the large end up. Down the centre of this is a strong revolving vertical shaft, on which are hung horizontal knives, inclined at such an angle as to form portions of a screw, that is, the knives follow each other at an angle forming a series of coils round this shaft. The bottom knives are larger, and vary in form, to throw off the clay, in some mills vertically, in others horizontally. Some have on the bottom of the shaft one coil of a screw, which throws the clay off more powerfully where it is wished to give pressure.

The action of this mill is to cut the clay with the knives during their revolution, and so work and mix it, that on its escape it may be one homogeneous mass, without any lumps of hard untempered clay; the clay being thoroughly amalgamated, and in the toughest state in which it can be got by tempering. This mill is an excellent contrivance for the purpose of working the clay, in combination with rollers; but if only one mill is worked, it is not generally adopted, for, although it tempers, mixes, and toughens, it does not extract stones, crush up hard substances, or freo the clay from all matters injurious to the quality of the ware when ready for market. This mill can be worked by either steam, water, or horse-power; but it takes much power in proportion to the quantity of work which it performs. If a brick is made with clay that has passed the pug-mill, and contains stones, or marl not acted on by weather, or lime-shells, (a material very common in clays), or any other extraneous matter injurious to the brick, it is apparent from the action of this mill that it is not removed or reduced. The result is this, the bricks being when moulded in a very soft state of tempered material, or mud, considerably contract in drying, but the stones or hard substances not contracting, cause the clay 
to crack; and even if they should not be suffioiently large to do this in drying, during the firing of the bricks thero is a still further contraction of tho clay, and an expansion of the stone from the heat to which it is subjected, and the result is generally a faulty or broken brick, and on boing drawn from tho kilns, the bricks are found to be imperfoct.

The clay, being sufficiently kneaded, it is brought to the bench of the moulder, who works it into a mould mado of wood or iron, and strikes off the superfluous matter. The bricks are next delivered from the mould, and ranged on tho ground; and when they have acquired sufficient firmness to bear handling, they are dressed with a knife, and stacked or built up in long dwarf walls, thatched over, and left to dry. An able workman will make, by hand, 5,000 bricks in a day.

The different kinds of bricks made in England aro principally place bricks, grey and red stocks, marl facing bricks, and cutting bricks. The place bricks and stocks are used in common walling. The marls are made in the neighbourhood of London, and used in the outside of buildings, they are very beautiful bricks, of a fine yollow colour, hard, and well burnt, and, in every respect, superior to the stocks. The finest kind of marl and red bricks, called cutting bricks, are used in the arches over windows and doors, being rubbed to a centre, and gauged to a height.

Bricks, in this country, are generally baked either in a clamp or in a kiln. The latter is the preferable method, as less waste arises, less fuel is consumed, and the bricks are sooner burnt. The kiln is usually 13 feet long, by $10 \frac{1}{2}$ feet wide, and about 12 feet in height. The walls are one foot two inches thick, carried up a littlo out of the perpendicular, inclined towards each other at the top. The bricks are placed on flat arches, having holes left in them resembling lattice-work; the kiln is then covered with pieces of tiles and bricks, and some wood put in, to dry them with a gentle fire.

This continues two or three days before they are ready for burning, which is known by the smoke turning from a darkish colour to semi-transparency. The mouth or mouths of the kiln are now dammed up with a shinlog, which consists of pieces of bricks piled one upon another, and closed with wet brick earth, leaving above it just room sufficient to receive a fagot. The fagots are made of furze, heath, brake, fern, \&c., and the kiln is supplied with these until its arches look white, and the fire appears at the top; upon which the fire is slackened for an hour, and the kiln allowed gradually to cool. This heating and cooling is repeated until the bricks are thoroughly burnt, which is generally done in 48 hours. One of these kilns will hold about 20,000 bricks.

Clamps are also in common use. They are made of the bricks themselves, and generally of an oblong form. The foundation is laid with place brick, or the driest of those just made, and then the bricks to be burnt are built up, tier upon tier, as high as the clamp is meant to be, with two or three inches of breeze or cinders strewed between each layer of bricks, and the whole covered with a thick stratum of breeze. The fire-place is perpendicular, about three foet high, and generally placed at the west end; and the flues are formed by gathering or arching the bricks over, so as to lesve a space between each of nearly a brick wide. The flues run straight through the clamp, and are filled with wood, coals, and breoze, pressed closely together. If the bricks are to be burnt off quickly, which may be done in 20 or 30 days, according as the weather may suit, the flues should be only at about six feet distance; but if there be no immediate hurry, they may be placed nine feet asunder, and the clamp left to burn off slowly.

The following remarkx by Mr. H. Chamberlain, on the drying of bricks, have an espocial value from the great experience of that gentleman, and his careful observation of all the conditions upon which the preparation of a good brick depends.

- The drying of bricks ready for burning is a matter of groat importance, and roquires more attention than it generally receives. From hand-made bricks we have to ovaporato some 25 per cent. of water before it is safe to burn them. In a work requiring the make of 20,000 bricks per day, we have to evaporate more than 20 tons of water every 24 hours. Hand-made bricks lose in drying about one-fourth of their weight, and in drying and burning about one-third. The average of machine bricks - those mado of the stiff plastic clay - do not lose more than half the abovo amount from evaporation, and are, therefore, of much greater specific gravity than hand-made ones.

'The artificial drying of bricks is carried on throughout the year uninterruptedly in sheds having the floor heated by fires; but this can only be effected in districts whero coal is cheap. The floors of these sheds are a series of tunnels or flues running through the shed longitudinally. At the lower ond is a pit, in which are the furnaces she fire travels up the flues under the floor of the shed, giving off its heat by the way, and the smoke escapes at the upper end, through a series of (generally three or four) 
smaller chimneys or stacks. The furnace end of these flues would naturally be much more highly heated than the upper end near the chimneys. To remedy this, the floor is constructed of a greator thickness at the fire end, and gradually diminishes to within a short distance of the top. By this means, and by the assistance of dampers in the chimneys, it is kept at nearly an equal temperature throughout. Bricks that will bear rapid drying, such as are made from parly clays or very loamy or siliceous earths, will be fit for the kiln in from 12 to 24 hours. Before the duty was taken off bricks, much dishonesty was practised by unprincipled makers, where this drying could be carried on economically. Strong elays cannot be dried so rapidly. These sheds are generally walled round with loose bricks, stacked in between each post or pillar that supports the roof. The vapour given off from the wet bricks, rising to the roof, escapes. This system of drying is greatly in advance of that in the open air, for it produces the ware, as made, without any deterioration from bad weather; but the expense of fuel to heat these flues has restricted its use to the neighbourhood of collieries. In 1845 attention was turned to the drying of bricks, and experiments carried out in drying the ware with the waste heat of the burning kilns. The caloric, after having passed the ware in burning, was carried up a flue raised above the floor of the shed, and gave off its spent heat for drying the ware. Although this kiln was most useful in proving that the waste heat of a burning kiln is more than sufficient to dry ware enough to fill it again, it was abandoned on account of the construction of the kiln not being good.

'Another system of drying is in close chambers, by means of steam, hot water, or by flues heated by fire under the chambers. I will, therefore, briefly describe the steamchamber as used by Mr. Beart. This is a square construetion or series of tunnels or chambers, built on an incline of any desired length; and at some convenient spot near the lower end is fixed a large steam-boiler, at a lower level than the drying chamber. From the boiler the main steam pipe is taken along the bottom or lower end of the chamber, and from this main, at right angles, runs branch pipes of four inches diameter up the chamber, two feet apart, and at about three feet from the top or arch. From there being so close and shallow a chamber between the heating surface of the pipes and the top, and so large an amount of heating surface in the pipes, the temperature is soon considerably raised. At the top and bottom ends are shutters or lids, which open for the admission of the green ware at the upper end, and for the exit of the dry ware at the lower end of the chamber. Over the steam-pipes are fixed iron rollers, on which the trays of bricks, as brought from tít machine, are placed, the insertion of one tray forcing the tray previously put in further on, assisted in its descent by the inclination of the construction. The steam being raised in the boiler flows through the main into those branch pipes in the chamber, and from the large amount of exposed surface becomes condensed, giving of its latent heat. From the incline given to the pipes in the chamber, and from the main pipe also having a fall towards the boiler, the whole of the warm water from the condensed steam flows to the boiler to be again raised to steam, sent up the pipes, and condensed intermittently. The steam entering at the lower end of the chamber, it is of course warmer than the upper end. Along the top end or highest part of the chambers is a series of chimneys and windguards, through which the damp vapour escapes. The bricks from the machine enter at this cooler end charged with warm vapour, and as the make proceeds are forced down the chamber as each tray is put in. Thus, those which were first inserted reach a drier and warmer atmosphere, and, on their arrival at the lower end, come out dry bricks, in about 24 hours, with the strongest clays. In some cases the waste stoam of the working engines is sent through these pipes and condensed. Bricks will dry soundly without cracking, \&ce, in these close chambers, when exposed to much greater heat than they would bear on the open flue first described, or the open air, from tho circumstance of the atmosphere, although very hot, being so highly charged with vapour. In practice, these steam-chambers have proved many principles, but they are not likely to become universal, for they are very expensive in erection on account of the quantity of steam-pipes, and involve constant expense in fuel, and require attention in the management of the steam-boiler; but their greatost defect is the want of a current of hot air through the chamber to carry off the excess of vapour faster than is now done. The attaining a higher degree of temperature in these chambers is useless, unless there is a current to carry off the vapour. Why should this piping be used, or steam at all, when we have a large mass of heat being constantly wastod, night and day, during the time the kilns are burning? and after the process of burning the kiln is completed, we have pure hot air flowing, from 48 to 60 hours, from the mass of cooling bricks in the kilns, free from carbon or any impurities; this could be directed through the drying chambers, entering in one constant flow of hot, dry air, and escaping in warm vapour. The waste heat during the process of burning can be taken up flues under the chamber, and thereby all the heat of our burning 
kilns may be economised and a great outlay saved in steam-pipes, boilers, and attention. It must not be forgotten, also, that so large an atmospheric condenser as the stoam-chamber is not heated without a considerable expondituro in fuel. This drying by steam is a great stride in advance of the old flued shed; but practical men must see the immense loss incurred constantly from this source of the spent heat of the burning kilns, and that by economising it, an immense saving will be offected in the manufacture. The kilns are constructed as near the lower end of these chambers as convenient.'

A kiln for attaining the object of the one built in 1846 by Mr. Chamberlain is worked at Epson; but with this difference, that the smoke is consumed. The drying shed is kept quite close, that the hot flues may raise the temperature so high as to dry the ware. In this kiln the heated gases escape from tho top, after passing up through the ware, into flues, and are carried to the ground, and thence into the drying shed, which is a very large construction in proportion to the size of the kiln, and 'holds nearly sufficient ware to fill four kilns. In this shed the heat passes up a hollow wall, about six feet high, and after running through the length of the shed on one side, returns down similar flues on the opposite side of the shed, and is again carried to the kiln, through the bottom of which it passes in two close flues between the three kiln-furnaces, with the exception of smail apertures, through which the heat enters to consume the smoke. From these return flues the spent gases rise up a shaft at the end of the kiln. One result of carrying these return flues through the kiln, is the attaining a great draft or suction in the flues to carry off vapour.

The common brick kiln is a rectangular building, generally open, but sometimes arched over. In the side walls and opposite to each other, are built fireplaces, or holes for the insertion of the fuel. The furnaces are formed in the setting of the kiln with unburnt bricks, and above these the kiln is filled as above described. In these kilns, from the raw ware forming the furnace, the flash of the flame, from the fires of the walls, too often vitrefios and destroys the nearest bricks. In the open kiln, as the fire or heat reaches to the top, the fireman soils or earths it down, which throws the draft to another part more backward ; and, as it continues to rise, he proceeds with this operation until all the top is earthed in; he then continues the firing until the whole has sunk, by the contraction of the clay in the fire, to the desired depth. The fire-holes are then stopped up with mud, and the kiln is left to cool gradually. If the air were admitted too rapidly while the kiln was at this intenso heat, it would cause bricks, made with strong clays, to fly to pieces like glass; it is, in fact, the process of annealing, Cooling too quickly also affects, in many clays, the colour of the bricks.

Temporary kilns are constructed in the country, with unburnt bricks, and called clamps. In Staffordshire, the bricks are burnt in small round kilns, called ovens which hold from 7,000 to 8,000 bricks each; these are burnt from fire in the walls round the ovens, and the raw ware is set in, so as to form a flue from each fire, to direct the flamo to the centro. These ovens burn very quickly, and a most intonse heat can be obtained in them. Mr. Chamberlain must be again quoted on the burning of bricks:-

'I will now more fully describe a principle of burning which I have had in practice for the last six years, and which I can therefore recommend with great confidence. The great object in brick-making is to attain a sufficient heat to thoroughly burn the ware with as small a consumption of coal as possible; and with nearly an equal distribution of the heat over all parts, so that the whole of the ware, being subjected to the same temperature, may contract equally in bulk, and bo of one uniform colour throughout. The advantage is also gained of burning in much less time than in the old kilns, which, on an average, took a week; and the management is so simplified that any man, even though not at all conversant with the manufacture, after he has seen one kiln burnt, will be able to manage another; and the last, though not least, advantage is, that of delivering up to us the waste heat at the ground level, or under the floor of the kiln, to be used in drying the green ware, or in partially burning the next kiln.

' Hitherto the heat has been applied by a series of fireplaces, or flues and openings round the kiln, each exposed to the influence of the atmosphere; and in boisterous weather it is very difficult to keep the heat at all regular, the consequence of which is, the unequal burning we often see. The improvements sought by experimentalists have been the burning the goods equally, and, at the same time, more economically. These are obtained by the patent kilns, as improved by Mr. Robert Scrivener, of Shelton, in the Staffordshire Potteries. The plan is both simple and effective, and is as follows :-A furnace is constructed in the centre of the kiln, much below tho floor level, and so built that the heat can bo directed to any part of the kiln at the pleasure 
of the fireman. First, the heat is directed up a tube in the centre to the top of the oven or kiln, and, as there is no escape allowed to take place there, it is drawn down through the goods by the aid of flues in connection with a chimney. Thus, all the caloric generated in the furnace is made use of, and, being central, is equally diffused throughout the mass; but, towards the bottom, or over the exit flues, the ware would not be sufficionly burnt without reversing the order of firing. In order to meet this requirement there is a series of flues under the bottom, upon which the goods are placed, with small regulators at the end of each; these regulators, when drawn back, allow the fire to pass under the bottom, and to rise up among the goods which are not sufficiently fired, and thus the burning is completed. By means of these regulators the heat may be obtained exactly the same throughout; there is, therefore, a greater degree of certainty in firing, and a considerable saving of fuel, with the entire consumption of the smoke. From the fire or draught being under command, so as to be allowed either to ascend or descend through the ware during the time of burning or cooling, the waste caloric can be economised and directed through the adjoining kiln in order to partially burn it, or be used in drying of the raw wares on flues or in chambers. I have found the saving of fuel in these kilns, over the common kiln, 50 per cent.; and to give an indea of the facility with which they can be worked, it is common for my men to fill the kiln, burn, cool, and discharge it in six days.' Seo Krw.

In France attempts were long ago made to substitute animals and machines for the treading of men's feet in the clay kneading pit; but it was found that their schemes could not replace, with advantage, human labour where it is so cheap, particularly for separating the stones and heterogeneous matter, from the loam. The more it is worked, the denser, more uniform, and more durable, the bricks which are made of it. A good French workman, in a day's labour of 12 or 13 hours, it has been said, is able to mould from 9,000 to 10,000 bricks, 9 inches long, $\frac{1}{2}$ inches broad, and 21 thick; but he must have good assistants under him. In many brick-works near Paris, screw presses are now used for consolidating the bricks and paving tiles in their moulds. M. Molerat employed the hydraulic press for the purpose of condensing pulverised clay, which, after baking, formed beautiful bricks ; but the process was too tedious and costly. An ingenious contrivance for moulding bricks mechanically is said to be employed near Washington, in America. This machine moulds 30,000 in a day's work of 12 hours, with the help of one Lorse, yoked to a ginwheel, and the bricks are so dry when discharged from their moulds, as to be ready for immediate burning. The machine is described, with figures, in the 'Bulletin de la Société d'Encouragement,' for 1819.

Mechanical Brick Moulding.-Messrs. Lyne and Stainford obtained, in August 1825, a patent for a machine for making a considerable number of bricks at one operation. It consists, in the first place, of a cylindrical pug-mill of the kind usually employed for comminuting clay for bricks and tiles, furnished with rotatory knifes, or cutters, for breaking the lumps and mixing the clay with the other materials of which bricks are commonly made. Secondly, of two movable moulds, in each of which fifteen bricks are made at once; these moulds being made to travel to and fro in the machine for the purpose of being alternately brought under the pug-mill to be filled with the clay, and then removed to situations where plungers are enabled to act upon them. Thirdly, in a contrivance by which the plungers are made to descend, for the purpose of compressing the material and discharging it from the mould in the form of bricks. Fourthly, in the method of constructing and working trucks which carry the receiving boards, and conduct the bricks away as they are formed.

Fig. 254 exhibits the general construction of the apparatus; both ends of which being exactly similar, little more than half the machine is represented. $a$ is the cylindrical pug-mill, shown partly in section, which is supplied with the clay and othor materials from a hopper abore; $b b$ are the rotatory knives or cutters, which are attached to the vertical shaft, and, being placed obliquely, press the clay down towards the bottom of the cylinder, in the act of breaking and mixing it as the shaft revolves. The lower part or the cylinder is opened; and immediately under it the mould is placed in which the bricks are to be formed. These moulds run to and fro upon ledges in the side frames of the machine; one of the moulds only can be shown by dots in the figure, the side rail intervening: they are situated at $c c$, and are formed of bars of iron crossing each other, and encompassed with a frame. The mould resembles an ordinary sash window in its form, being divided into rectangular compartments (fifteen are proposed in each) of the dimensions of the intended bricks, but sufficiently deep to allow the material, after being considerably pressed in the mould, to leave it, when dischargod, of the usual thickness of a common brick. 
The mould being open at top and bottom, the material is allowed to pass into it, whon situated exactly under the cylinder; and the lower side of the mould, when so

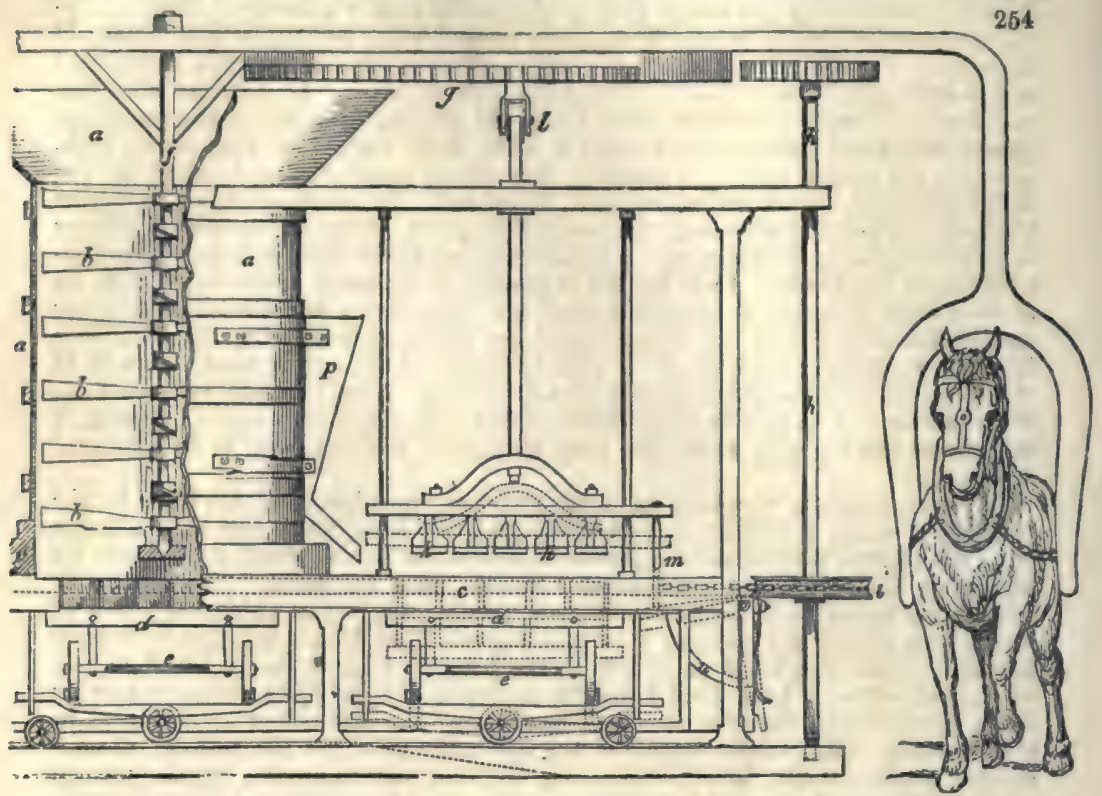

placed, it is to be closed by a flat board $d$, supported by the truck $e$, which is raised by a lever and roller beneath, running upon a plain rail with inclined ends.

The central shaft, $f$, is kept in continual rotatory motion, by the revolution of the upper horizontal wheel $g$, of which it is the axis; and this wheel may be turned by a horse yoked to a radiating arm, or by any other means. A part of the circumference of the wheel $g$, has teeth, which are intended at certain periods of its revolution to take into a toothed pinion, fixed upon the top of a vertical shaft $h h$. At the lower part of this vertical shaft there is a pulley $i$, over which a chain is passed that is connected to the two moulds $c$, and to the frame in which the trucks are supported; by the rotation of the vertical shaft, the pulley winds a chain, and draws the moulds and truck frames along.

The clay and other material having been forced down from the cylinder into the mould, the teeth of the horizontal wheel $g$, now come into gear with the pinion upon $h$, and turn it and the shaft and pulley $i$, by which the chain is wound, and the mould at the right hand of the machine brought into the situation shown in tho figure; a scraper or edge-bar under tho pug-mill having levelled the upper face of the clay in the mould, and the board $d$, supported by the truck $e$, formed the flat under-side.

The mould being brought into this position, it is now necessary to compress the materials, which is done by the descent of the plungers $k k$. A friction-rollor $l$, pendant from the under side of the horizontal wheel, as that wheel revolres, comes in contact with an inclined plane, at the top of the shaft of the plungers; and, as the friction-roller passes over this inclined plane, the plungers are made to descend into the mould, and to compress the material; the resistance of the board beneath causing the clay to be squeezed into a compact state. When this has been effectually accomplished, the further descent of the plungers brings a pin, $m$, against the upper end of a quadrant catch-level, $n$, and, by depressing this quadrant, causes the balance-lever upon which the truck is now supported to rise at that end, and to allow the truck with the board $d$ to descend, as shown by dots; the plungers at the same time forcing out the bricks from the moulds, whereby they are deposited upon the board $d$; when, by drawing the truck forward out of the machine, the board with the bricks may be removed, and replaced by another board. The truck may thon be again introduced into the machine, ready to receive the next parcel of bricks.

By the time that the discharge of the bricks from this mould has been effected, the other mould under the pug cylinder has become fillod with the clay, when the teeth of the horizontal wheel coming round, take into a pinion upon the top of a vertical 
shaft, exactly similar to that at $h$, but at the reverse end of the machine, and cause the moulds and the frame supporting the trucks to be slidden to the left end of the machine; the upper surface of the mould being scraped level in its progress, in the way already described. This movement brings the fretion-wheel, $o$, up the inclined plane, and thereby raises the truck, with the board to the under side of the mould, ready to receive another supply of clay; and the mould at the left-hand side of the machine being now in its proper situation under the plungers, the clay becomes compressed, and the bricks discharged from the mould in the way deseribed in the former instance; when this truck being drawn out, the bricks are remored to be dried and baked, and another board is placed in the same situation. There are boxes, $p$, upon each side of the pug cylinder containing sand, at the lower parts of which

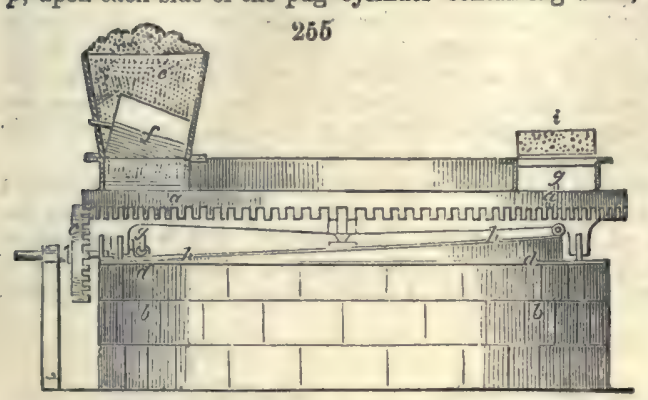
small sliders are to be opened (by contrivances not shown in the figure) as the mould passes under them, for the purpose of scattering sand upon the elay in the mould to prevent its adhering to the plungers. There is also n. rack and toothed sector, with a balance-weight connected to the inclined plane at the top of the plungerrods, for the purpose of raising the plunger after the friction-roller has passed over it; and there is a spring acting against the back of the quadrant-catch, for the purpose of throwing it into its former situation, after the pin of the plunger has risen.

An effective machine for brick-making is that patented by Mr. Edward Jones, of Birmingham, in August 1835. His improvements are deseribed under four heads: the first applies to a machine for moulding the earth into bricks in a circular frameplate horizontally, containing a series of moulds or rectaugular boxes, standing radially round the circumference of the circular frame, into which boxes successively the clay is expressod from a stationary hopper as the frame revolves, and after being

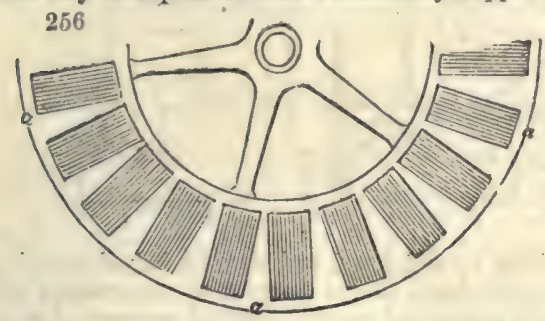
so formed, the bricks are succossively pushed out of their boxes, each by a piston acted upon by an jnclined plane below. The second head of the specification describes a rectangular horizontal frame. having a series of moulding boxes placed in a straight range, which are acted upon for pressing the clay by a corresponding range of pistons fixed in a horizontal frame, worked up and down by rods extending from a rotatory crank shaft, the moulding boxes being allowed to rise for the purpose of enabling the pistons to force out the bricks when moulded, and leave them upon the bed or board below. The third head applies particularly to the making of tiles for the flooring of kilns in which malt or grain is to be dried. There is in this contrivance a rectangular mould, with pointed pieces standing up for the purpose of producing air-holes through the tiles as they are moulded, which is done by pressing the clay into the moulds upon the points, and scraping off the superfuous matter at top by hand. The fourth or last head applies to moulding chimney-pots in double moulds, which take to pieces for the purpose of withdrawing the pot when the edges of the slabs or sides are sufficiently brought into contact.

Fig. 255 represents, in elevation, the first-mentioned machine for moulding bricks. Tho moulds are formed in the face of a circular plate or wheel, $a$ a, a portion of the upper surface of which is represented in the horizontal view, fig. 256. Any convenient number of these moulds are set readily in the wheel, which is mounted upon a central pivot, supported by the masonry $b$. There is a rim of teeth round the outer edge of the wheel $a a$, which take into a pinion, $c$, on a shaft connected to the first mover; and by these means the wheel $a$, with the moulding boxes, is made to revolve horizontally, guided by arms with anti-friction rollers, which run round a horizontal plate, $a$ a, fixed upon the masonry.

A hopper, $e$, filled with the brick earth, shown with one of the moulding boxes in section, is fired above the face of the wheel in such a way, that the earth may descond 
from the hopper into the several moulding boxes as the whoel passes round under it, the earth being pressed into the moulds, snd its surface scraped off smooth by a conical roller, $f$, in the bottom of the hopper.

Through the bottom of each moulding box there is a hole for the passage of a piston-rod, $g$, the upper end of which rod carries a piston with a wooden pallet upon it acting within the moulding box; and the lower end of this rod has a small antifriction roller, which, as the wheel $a$ revolves, runs round upon the face of an oblique ring or inclined way, $h h$, fixed upon the masonry.

The clay is introduced into the moulding boxes from the hopper fixed over the lowest part of the inclined way $h$; and it will be perceived that as the wheel revolves, the piston-rods, $g$, in passing up the inclined way, will cause the pistons to force the new-moulded bricks, with their pallet, or board, under them, severally up the mould, into the situation shown at $i$, in fig. 256, whence they are to be removed by hand. Fresh pallets being then placed upon the several pistons, they, with the moulds, will be ready for moulding fresh bricks, when, by the rotation of the wheel, $a$, they are severally brought under the hopper, the pistons having sunk to the bottoms of their boxes, as the piston-rods passed down the other side of the inclined way $h$.

The second head of the invention is another construction of apparatus for monlding bricks, in this instance in a rectangular frame. Fig. 257 is a front elevation of the machine; fig. 258, a section of the same taken transversely. $a a$ is the standard frame-work and bed on which the bricks are to be moulded. Near the corners of this standard frame-work, four vertical pillars, $b \quad b$, are erected, upon which pillars

257

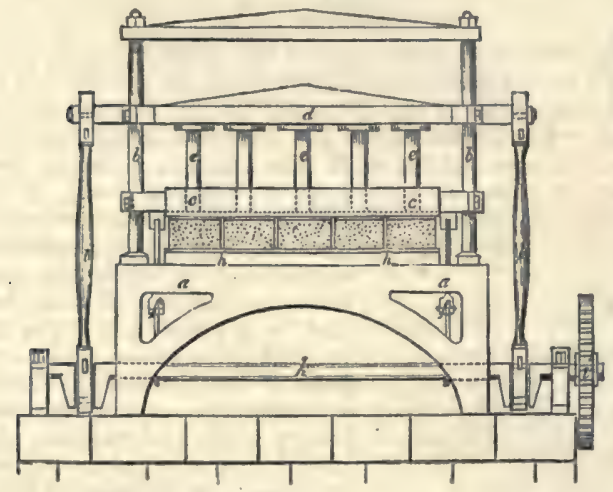

258

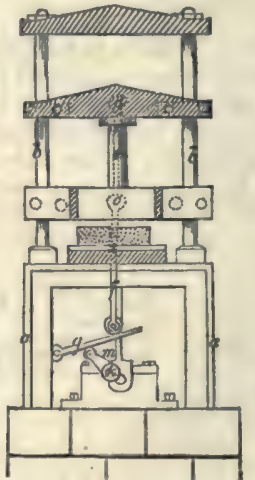

the frame of the moulding boxes, $c$, slides up and down, and also the bar, $d$, carrying the rods of the pistons, $e e e$. These pistons are for the purpose of compressing the clay in the moulding box, and therefore must stand exactly over and correspond with the respective moulds in the frame $c$, beneath.

The sliding frame, $c$, constituting the sides and ends of the moulding boxes, is supported at each end by an upright sliding rod, $f$, which rods pass through guides fixed to the sides of the standard frame, $a$, and at the lower end of each there is a roller, bearing upon the levers, $g$, on each side of the machine, but seen only in fig. 258, which levers, when depressed, allow the moulding boxes to descend and rest upon the bed or table of the machine $h h$.

In this position of the machine resting upon the bed or table, the brick-earth is to be placed upon, and spread over, the top of the frame $c$, by the hands of workmen, when the descent of the plunger or pistons $e e e$ will cause the earth to be forced into the moulds, and the bricks to be formed therein. To effect this, rotatory power is to be applied to the toothed wheel $i$, fixed on the end of the main driving crank-shaft $k k$, which on revolving will, by means of the crank-rods $l l$, bring down the bar $a$, with the pistons or plunger $e e e$, and compress the earth compactly into the moulds, and thereby form the bricks.

When this has been done, the bricks are to be released frum the moulds by the moulding frame, $c$, rising up from the bed, as shown in fig. 257, the pistons still remaining depressed, and bearing upon the upper surfaces of the bricks. The moulding frane is raised by means of cams, $m$, upon the crank-shaft, which at this part of the operation are brought under the levers $g$, for the purpose of raising the cams and the sliding rods $f$ into the position shown in fig. 258. 
The bricks having been thus formed and released from thoir moulds, thoy are to be removed from the bed of the machine by pushing forward, on the front side, fresh boards or pallets, which of course will drive the bricks out upon the other side, whence they are to be removed by hand.

There is to be a small hole in the centre of each pallet, and also in the bed, for the purpose of allowing any superfluous earth to be pressed through the moulding boxes when the pistons descend. And in order to cut off the projecting piece of clay which would be thus formed on the bottom of the brick, a knifo-edge is in some way connected to the bed of the machine, and as the brick slides over it, the knife separates the protubersnt lump; but the particular construction of this part of the apparatus is considered to be of little importance, and the manner of effecting the object is not clearly stated in the specification.

Fig. 259 represents Mr. Hunt's machine. The principal parts consist of two cylinders, each covered by an endless web, and so placed as to form the front and back of \& hopper, the two sides being iron plates, placed so that when the hopper is filled with tempered clay from the pug-mill, the lower part of the hopper, and consequently the mass of clay within it, has exactly the dimensions of a brick. Beneath the hopper an endless chain travels simultaneously with the movement of the cylinders. The pallet-boards are laid at given intervals upon the chain, and being thus placed under the hopper, while the clay is brought down with a slight pressure, a frame with a wire stretched across it is projected through the mass of clay, cutting off exactly the thickness of the brick, which is removed at the same moment by the forward movement of the endless chain. This operation is repeated each time that a pallet-board comes under the hopper.

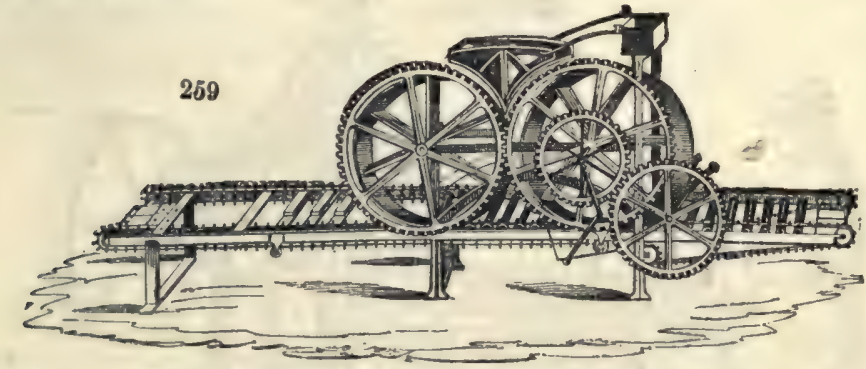

There are numerous machines in use for the manufacture of bricks. For the manufacture of perforated bricks, Mr. Beart's machine is the most generally employed. Mr. Chamberlain thus describes it:- The most universally used die-machine which has been extensively worked up to the present time is $\mathbf{M r}$. Beart's patent for perforated bricks. This gentleman, who is practically acquainted with these matters, in order to remedy the difficulties I have mentioned in expressing a mass of clay through a large aperture or die, hung a series of small tongues or cores, so as to form hollow or perforated bricks. By this means the clay was forced in its passago through the die into the corners, having the greater amount of friction now in the centre. Still, the bricks came out rough at the edge with many clays, or with what is tormod a jagged edge. The water-die was afterwards applied to this machine, and the perforated bricks, now so commonly used in London, are the result. In $\mathrm{Mr}$. Beart's machine, which is a pug-mill, tho clay is taken after passing through the rolling-mill, and being fed in at the top, is worked down by the knives. At the bottom are two horizontal clay-boxes, in which a plunger works backwards and for. wards. As soon as it has reached the extremity of its stroke, or forced the clay of ono box through the die, the other box receiving during this time its charge of clay from the pug-mill, the plunger returns and empties this box of clay through a die on the opposite side of the machine. The result is, that while a stream of clay is being forced out on one side of the machine the clay on the opposite side is stationary, and can, therefore, be divided into a series of five or six bricks with the greatest correctness by hand. Some of these machines have both boxes on one side and the plungers worked by cranks. This machine cannot make bricks unless the clay has previously passed through rollers, if coarse; for anything at all rough, as stone or other hard substance, would hang in the tongues of the die. But the clay being afterwards pugged in the machine is so thoroughly tempered and mixed, the bricks when made cannot be othorwise than good, provided they are sufficiontly fixed. As to the utility of hollow or perforated bricks, that is a matter moro for the consideration of the

Vor, I. M M 
architect or builder than for the brick-maker. Perforated bricks are a fifth less in weight than solid ones, which is a mattor of some importance in transit; but it takes considerably more power to force the clay through those dies than for solid brickmaking. In the manufacture of perforated bricks, there is also a royalty or patentright to be paid to Mr. Beart.'

Mr. Chamberlain's own machine is in principle as follows (fig. 260):-The clay is fed into a pug-mill, placed horizontally, which works and amalgamates it, and then forces it off through a mouth-piece or die of about 65 square inches, or about half an inch doeper and half and inch longer than is required for the brick, of a form similar to a brick on edge, but with corners well rounded off, each corner forming a quarter of a 3-inch circle, for clay will pass smoothly through an aperture thus formed, but not through a keon angle. After tho clay has escaped from the mill it is seized by four rollers, covered with a porous fabric (moleskin), driven at a like surface speed from connection with the pug-mill. These rollers are two horizontal and two vertical ones, haring a space of 45 inches between them; they take this larger stream of rough clay, and pross or roll it into a squared block, of the exact size and shape of a brick edgeways, with beautiful sharp edges, for the clay has no friction, being drawn through by the rollers instead of forcing itself through, and is delivered in one unbroken stream. The rollers in this machine, perform the functions of the die in one class of machinery, and of the mould in the other. They are, in fact, a die with rotating surfaces. By hanging a series of mandrels or cores between these rollers, or by

\section{0}

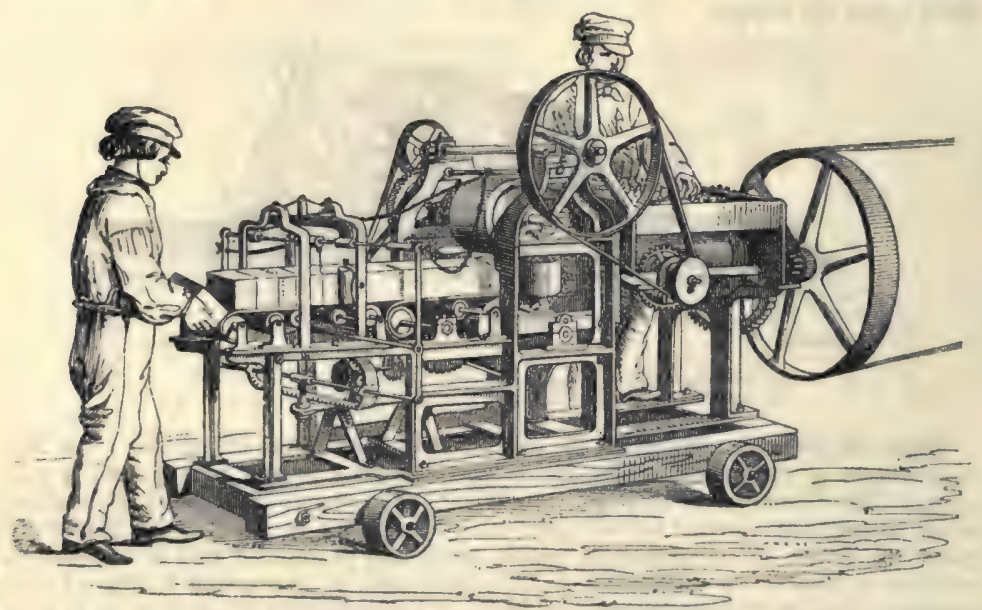

merely changing the mouth-piece, we make hollow and perforated bricks, without any alteration in the machine.

Messrs. Bradley and Craven, of Wakefleld, have invented a very ingenious brickmaking ( fig. 261) machine:-

It consists of a vertical pug-mill of a peculiar form, and greatly improved construction, into the upper part of which the clay is fed. In this part of the apparatus the clay undergoes the most perfect tempering and mixing, and on reaching the bottom of the mill, thoroughly amalgamated, is forcibly pressed into the moulds of the form and size of bricks required, which are arranged in the form of a circular revolving table.

As this table revolves, the piston-rods of the moulds ascend an incline plane, and gradually lift the bricks out of the moulds, whence they are taken from the machine by a boy, and placed on an endless band which carries the bricks direct to the waller, thus effecting the saving of the floor room.

The speed of the several parts of the machine is so judiciously arranged, that the operations of pugging, moulding, and delivering proceed simultaneously in due order, the whole being easily driven by a steam-engine of about six-horse power, which, at the ordinary rate of working, will make 12,000 bricks per day; or, with eight-horse power, from 15,000 to 18,000 .

In consequence of the perfect amalgamation of the clay, and the great pressure to which it is subjected in the moulds, the bricks produced by this machine are perfect; 
and from the stiffiness of the clay used, less water has to be evaporated in the drying, thus saving one half the time required for hand-made bricks, and avoiding the risk of loss from bad weather.

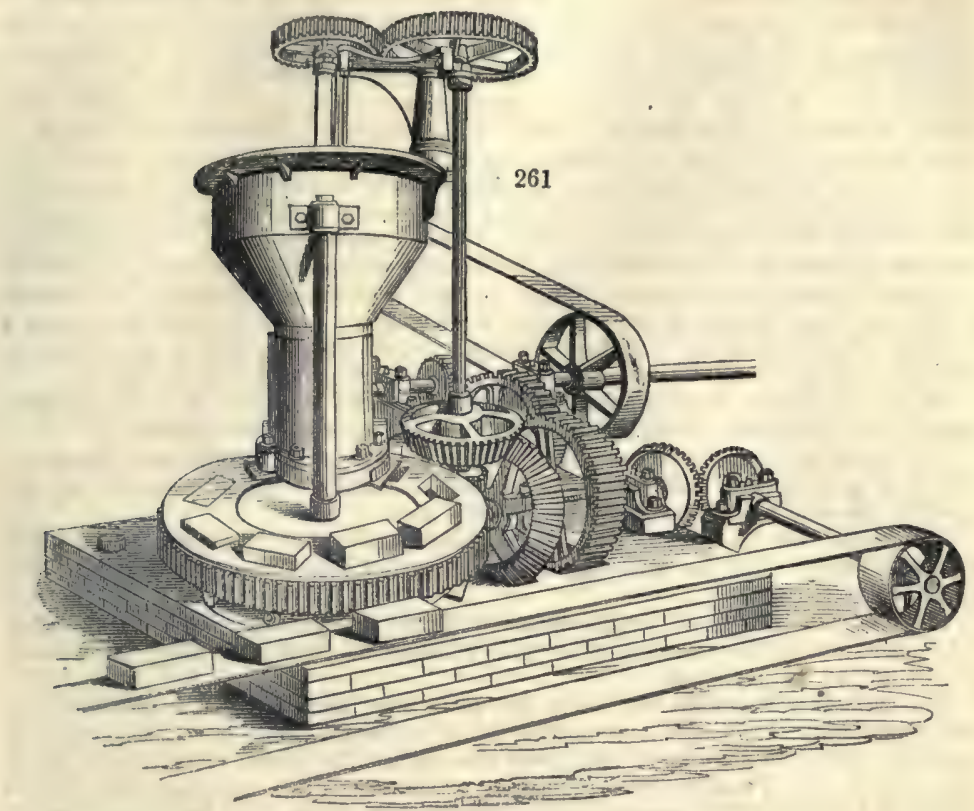

The following remarks by Dr. Ure are deserving of attention:-

'The brick kilns and clamps round London and other large cities, which are fired with the breeze rubbish collected from dust-holes that contain the refuse of kitchens, \&c., emit, in consequence, most unpleasant effluvia; but brick kilns fired with clean coke or coal give out no gases of a more noxious nature than common household fires. The consideration of this subject was closely pressed upon my attention on being consulted concerning an injunction issued by the Chancellor against a brick clamp in the Isle of Wight, fired with clean coke-cinders from the steam-engine furnace at Portsmouth Dockyard. The bricks, being of the description called sand stock, were of course made in moulds very slightly dusted with sand, to make them fall freely out. The sand was brought from Portsmouth Harbour, and, on being subjected . to a degree of heat more intense certainly than it could suffer in the clamp, was thought to give ont traces of hydrochloric acid.

'As it is well known to the chemist that common salt strongly ignited in contact with moist sand will emit hydrochloric acid, there was nothing remarkable in the above observation; but I ascertained that the sand with which the moulds wore strowed would give out no hydrochloric acid at a heat equal at least to what the bricks were exposed to in a clamp 10 or 12 feet high, and fired at its bottom only with a layer of cinders 3 or 4 inches thick. But 1 further demonstrated that the entire substance of the brick, with its scanty film of sand, on being exposed to ignition in a suitable apparatus, gave out-not hydrochloric or any other corrosive acid, but ammonia gas. Hence, the allegations that the clamp sent forth a host of acid gases to blight the neighbouring trees were shown to be utterly groundless; on the contrary, the ammonia evolved from the heated clay would act beneficially upon vegetation, whilo it was too small in quantity to annoy any human being. A fow yards to leeward of a similar clamp in full activity, I could percoive no offensive odour. All ferruginous clay, when exposed to the atmosphere, absorbs ammonia from it, and of course omits it again on being gently ignited.'

Floating bricks are a very ancient invention; they are so light as to swim in water; and Pliny tells us that they were made at Marseilles, at Colento, in Spain, and at Pittane, in Asia. This invention, however, was completely lost until M. Fabroni published a discovery of a method to imitate the floating bricks of the ancients. 
According to Posidoning, these bricks were made of a kind of argillacoous earth, which was employed to clean silver plate. But as it could not be our tripoli, which is too heavy to float in water, M. Fabroni triod several experiments with mineral agaric, guhr, lac-lunæ, and fossil meal, which last was found to be the very substance of which he was in search. This enrth is abundant in Tuscany, and is found near Casteldelpiano, in the territories of Sienna. According to the analysis of M. Fabroni, it consists of 55 parts of siliceous earth, 15 of magnesia, 14 of water, 12 of alumina, 3 of lime, and 1 of iron. It exhales an argillaceous odour, and, when sprinkled with water, throws out a light-whitish smoke. It is infusible in the fire, and, though it loses about an eighth part of its weight, its bulk is scarcely diminished. Bricks com. posed of this substance, either baked or unibaked, float in water; and $\frac{1}{20}$ th part of clay may bo added to their composition without taking away their property of swimming. These bricks resist water, unite perfectly with lime, are subject to no alteration from heat or cold, and the baked differ from the unbaked only in the sonorous quality which they have acquired from the fire. Their strength is little inferior to that of common bricks, but much greater in proportion to their weight; for M. Fabroni found that a floating brick, measuring 7 inches in length, $4 \frac{1}{2}$ in breadth, and 1 inch 8 lines in thickness, weighed only $14 \frac{1}{4}$ oz., whereas a common brick weighed 5 lbs. $6 \frac{3}{4}$ oz.

As an experiment, M. Fabroni constructed the powder magazine of a ship of these bricks; the vessel was set on fire, and sank without exploding the powder.

This earth has been found near Clermont, in the Auvergne. Ehrenberg has shown that it is entirely composed of microseopic siliceous shells. Bricks composed of this earth weigh only half as much as the ordinary ones.

Fire bricks are made extensively in the neighbourhood of Newcastle-on-Tyne and at Stourbridge. For the analyses of the clays of which these and others are constructed, see CI.AX.

Stone Bricks.-These are manufactured at Neath, in Glamorganshire, and are very much used in the construction of copper furnaces at Swansea. They are usually known as the 'Dinas bricks.'

The materialls of which the bricks are made are brought from a quarry in the neighbourhood. They are very coarse, being subjected to a very rude crushing operation under an edge stone, and, from the size of the pieces, it is impossible to mould by hand. There are three qualities, which are mixed together with a little water, so as to give the mass coherence, and in this state it is compressed by the machine into a mould. The brick which results is treated in the ordinary way, but it resists a much greater heat than the Stourbridge clay brick, expands more by heat, and does not contract to its original dimensions. The composition of the three materials is as follows :-

\begin{tabular}{|c|c|c|c|c|}
\hline \multirow{2}{*}{\multicolumn{2}{|c|}{ Silica }} & From Pendreyn & \multicolumn{2}{|c|}{ From Dinas } \\
\hline & & & $100^{\circ}$ & $91 \cdot 95$ \\
\hline $\begin{array}{l}\text { Alumina, with a trace } \\
\text { Lime and masnesia }\end{array}$ & $\theta$ of ox. iron & $4 \cdot 55$ & traces & $8 \cdot 05$ \\
\hline Lime and magnesia & - & - & traces & traces \\
\hline & & $98 \cdot 60$ & 100 . & $100 \cdot 00$ \\
\hline
\end{tabular}

\section{-Dr. Richardson: Knapp's Technology.}

Since the introduction of the Siemens gas-furnace, and the Bessemer process for cast-steel manufacture, into countries which, unlike our own, are not well supplied with fire-resisting materials, great difficulty has been exporionced in obtaining bricks of a sufficiently refractory character to withstand the extremely high temperature developed in the melting chamber, as well as the sudden and violent alternations in other parts of the furnace. In order to obviate this difficulty, Mr. Joseph Khern, a well-known Austrian metallurgist, has introduced a plan of manufacturing siliceous bricks, which he describes as being superior to any other refractory material obtained in Austria. The chief ingredient is quartz of the highest possible degree of purity, especial care being taken to reject all such samples as show any admixture of iron or eopper pyrites, carbonate of lime, or even mica, or felspar. The quartz so selected is heated in quantities of from 10 to 15 tons, in a Rumford lime-kiln for 10 or 12 hours, till it attains a full red heat, when it is quenched in water; the fragments are then cleaned by a simple jigging process, and subsequently are crushed under a tilt hammer, sufficiently fine to pass through a sieve having 60 holes to the square inch. The hammer weighs $2 \frac{1}{2}$ cwts., and is capable of erushing $3 \frac{1}{2}$ tons of quartz in 12 hours. Two varieties of clay aro used, differing slightly in plasticity; they are prepared by careful weathering, pulverisation under light stamp-heads, and grinding under edge rollers; a final sifting is performed through a sieve of 600 apertures to the inch. The purest quartz is reserved for the first quality of brick, which have to resist the greatest 
heat; while the second and third class, for less exposed positions, are made chiefly of the remains of bricks which have been already used, ground and sifted afresh. The following are the mixtures employed:-

First class : 16 parts of quartz to 1 plastic clay, or 14 parts of quartz to 1 of leaner elay. Second class: 16 parts of ground bricks of the 1st class, to 1 of clay. Third class : 8 parts of ground bricles of the 2 nd and 3rd classes, to 1 of clay.

The latter class are made more with a view to obtain mechanical strength than fireresisting power.

The materials are mixed together dry, and are thoroughly incorporated by kneading with water, and treading under men's feet; about 18 cubic feet are operated upon at one time. Sufficient water must be added to allow the mixture to be worked into a ball between the fingers without crumbling. The second and third class bricks are formed in open moulds, the stuff being beaten down by a metal rammer of about $4 \frac{1}{2} \mathrm{lbs}$. weight; che first class, however, are subjected to a pressure of about 3 tons to the square inch during a period of three quarters of an hour, before they are removed from the moulds. The drying takes place in chambers, through which a current of air passes, at the ordinary temperature in summer, but artificially warmed in winter; the bricks are fit for burning in from 4 to 6 days. The kilns are rectangular chambers, each having two step-grate fireplaces in one of the shorter sides, and a flue communicating with a high chimney at the opposite end. The capacity is about 2,300 or 2,500 bricks. As soon as the kiln is filled, the charging aperture is partly closed, and a gentle fire is kept in the grates, the flue damper being shut. After 36 hours, the charging hole is entirely closed, and the draught is urged by opening the damper inch by inch at intervals, until, at the end of 65 or 70 hours, the whole of the charge has attained a strong white heat. The fires are then removed, the damper is shut down, the grates are filled with sand, and any cracks that may have formed in the kiln are carefully luted up. After standing in this way for 24 hours, the charging place is gradually opened, and in from 36 to 48 hoprs more, the burnt bricks may be removed,

BRICE CIAX. The familiar torm for any clay used in the manufacture of bricks. A good clay for this purpose is a silicate of alumina, combined in various proportions with sand. Brick clay is used by geologists in contradistinction to Boulder clay.

BRICE IARTE. A maxly earth, containing much alumina, largely omployed in brick-making.

BRICX TrIN. See Krin.

Bercx orI. This is a relic of old pharmacy: it was propared by putting redhot roughly powdered brick into linseed oil. It is no longer used, except by old apothecaries and druggists in remote country towns.

BRICE RED COPPIR ORE. Seo TILE ORE.

BRIDGE. See IroN Bridar.

BrInstors. (Soufre, Fr.; Schwefel, Ger.) SuLPHUR.

Our Imports of Brimstone for the years ending 1871 were as follows:-

\begin{tabular}{|c|c|c|c|c|}
\hline \multicolumn{2}{|r|}{9} & \\
\hline $\begin{array}{l}\text { Quantity } \\
\text { Cwts. } \\
, 015,329\end{array}$ & $\begin{array}{c}\text { Value } \\
\& \\
388,723\end{array}$ & $\begin{array}{c}\text { Quantity } \\
\text { Cwsts, } \\
1,065,360\end{array}$ & $\begin{array}{c}\text { Value } \\
\& \\
386,660\end{array}$ & $\begin{array}{l}\text { Quantity } \\
\text { Cwwts. } \\
937,049\end{array}$ \\
\hline
\end{tabular}

MrIsxry. The stiff glossy hair of swine, which grows chiefly on the backs of those animals, both in the wild and the domesticated state. Bristles are used in the manufacture of brushes.

In 1864 our Imports of Bristles from Russia were 1,958,112 lbs., valued at 252,923l,; from Prussia 59,113 lbs., valued at 7,635l.; from Hamburg 207,274 lbs, valued at 26,772l.; from Belgium 34,880 lbs., valued at 4,505l.; from France 51,859 lbs., valued at $6,699 l$. ; from other parts $34,897 \mathrm{lbs}$., valued at $4,507 l_{\text {. : }}$ total amount, $2,346,135$ lbs., valued at 303,041 . In the same year we exported 12,395 lbs. valued at $2,264 l$. The importations for 1872 were:-From Russia, 1,800,933 lbs.; from Germany, 572,727 lbs.; from Holland, 492,921 lbs.; from Bolgium, 94,842 lbs.; from France, 60,030 lbs,; and from other countries, 46,642 lbs. The total value being $517,809 l$ :

BRISTOI Draxrow Ds. Brilliant crystals of quartz, found in the St. Vincent Rocks, near Bristol. They are often cut and polished for ornaments.

BRITANNTA MrTar. An alloy of tin with copper and antimony. In the best kinds a little nickel is used. See ALiors.

BRITxSE GUM. SoO DeXtrine.

BRITTIR SIIVIR-GIATCE. See SILVRR ORRs,

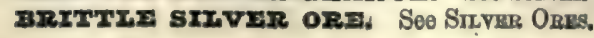




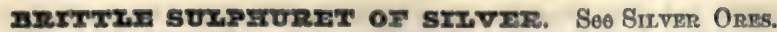

IROAD CIOTE. A fine kind of woollon cloth, which excoods twenty-nine inches in width. All of less width are known as narrow cloths.

mROAD CAUGE. Rails laid wide apart, as on the Great Western Railway and its branches, in contradistinction to tho narrow gauge, as on all the other railways of the Kingdom. The broad gauge rails are 6 feet apart; the narrow gaugo rails $4 \frac{1}{2}$ feet.

mRoAd mear. The Terminalia latifolia, a tree, native of Jamaica, the wood of which is used for boards, scantlings, shingles, and staves. It is sometimes mistaken for the almond tree, owing to the shape of its fruit. See Trirminalis.

BROADSIDE. A seaman's term - the full length or side of a ship. A printer's tern-a full printed page of any sized shoet.

及ROCADE. A rich stout silk, formerly much worn by ladies of rank. A namo commonly given to any variety of stuff upon which raised flowers aro embroidored. The name is also given to a cloth of silk and gold manufactured in Eastern countries. BRocatrrIs. Linsey-woolsey is so called in France. A silk material which is usod for lining carriages.

BROCATEIII MARBIE. An artificial marblo mado from fragments of natural marbles united by means of an artificial cement.

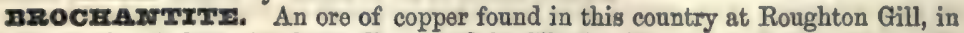
Cumberland. It has also been discovered in Siberia, Nassau, and elsewhere. Its composition is sulphuric acid, $17 \cdot 7$; protoxide of copper, $70 \cdot 3$; water, $12 \cdot 0$.

Bzockram. A Cumberland miner's term for a breccia.

mRoma. A preparation of chocolate.

BRomacerrc ACID.-Obtained by Messrs. Perkin and Duppa. They take a mixture of crystallisable acetic acid and bromine in the proportion of equal equivalents, introduce it into a sealed tube, which is placed in an oil bath and heated to $150^{\circ} \mathrm{C}$. The mixture which is nearly colourless, or of a light amber colour, is transferred to a retort, and the excess of acetic acid driven off by heating to $200^{\circ} \mathrm{C}$. On cooling, a beautiful white crystalline solid is obtained, which is bromacetic acid, together with hydrobromic acid and bibromacetic acid. The mixed acids are heated to $130^{\circ} \mathrm{C}$., carbonic acid passed until the reaction of hydrobromic acid, with nitrate of silver, is no longer evident. Carbonate of lead is then added, the whole heated to $100^{\circ} \mathrm{C}$., and allowed to stand for some hours; the liquid filtered off from the crystalline deposit.

The acid thus obtained crystallises in rhombohedra, is exceodingly deliquescent, and very soluble in water or alcohol. It fuses bolow $100^{\circ} \mathrm{C}$, and boils at $208^{\circ} \mathrm{C}$. When distilled with acetate of potassium, it gives off acetic acid; when heated with motallic zine, it yields acetato and bromide of zinc.

It attacks the epidermis powerfully, raising a blister like that produced by a burn. It forms crystallisable salts with most bases, many of which decompose rapidly.

Messrs. Perkin and Duppa have formed the salts of the alkalis and alkaline oarths. The lead salt is obtained by neutralising bromacetic acid with oxide of load, and recrystallising in water, washing the crystalline precipitate with cold water, and rocrystallising from water.

The silver salt is obtained by treating bromacetic acid with carbonate of silver, or by adding solution of bromacetic acid to solution of nitrate of silver. It is thrown down as a crystalline precipitato.

Bromacetate of methyl is a colourless mobile liquid, having an aromatic odour; it boils at $144^{\circ} \mathrm{C}$. The bromacetate of ethyl boils at $159^{\circ} \mathrm{C}$.; that of amyl at $207^{\circ} \mathrm{C}$.

By the action of ammonia on bromacotic acid, bromide of ammonium is formed, and glycocol, or a body isomeric with it.

Bibromacetic Acid. Formed when bromine and acetic acid are heated in presence of light, but it is difficult to obtain in large quantities. It is a liquid boiling at $240^{\circ} \mathrm{C}$., which is partially decomposed every time it is distilled, evolving hydrobromic acid. It does not solidify at $15^{\circ} \mathrm{C}$. It has a very high specific gravity. The silver salt is a crystalline precipitate which is, however, decomposed, by boiling with water, into bromide of silver and a soluble acid.

Bromatzs. Compounds of Bromic ACD with alkalis, alkaline earths, and motals.

BROMrC ACrD. A combination of bromine with oxygen, forming bromates.

BRomic sIrver. Seo SILVER Ores.

BROMIDEs. Compounds of Brourve with alkalis and metals.

aRomIDz or grIvas. A salt much used in photography. It is formed by adding a soluble bromide to nitrate of silver, whon a white precipitate is produced.

Soe Photographr.

nRomrry. Native bromide of silver.

arowze. (Bromes, Fr. and Ger.) A compound metal consisting of copper 
and tin, to which sometimes a little gine and lead are added. There is some confusion amongst Continental writers about this alloy; they translate their bronze into the English brass,

Seo, for an example of this, 'Dictionnaire des Arts et Manufactures.' This has arisen from the carelessness of our own writers. Dr. Watson, 'Chemical Essays,' remarks: "It has been said that Queen Elizabeth left more brass ordnance at her death than she found iron on her accession to the throne. This must, not be understood as if gun-metal was made in her time of brass, for the term brass was sometimes used to denote copper; and sometimes a composition of iron, copper, and calamine was called brass; and we, at this day, commonly speak of brass cannon, though brass does not enter into the composition used for casting cannon.'

Bronze is an alloy of copper and tin.

Brass is an alloy of copper and zine.

In many instances, we have zinc, lead, \&c., entering into the composition of alloys of copper and tin. However this may be, the alloy is called a bronze, if tin and copper are the chief constituents.

This alloy is much harder than copper, and was employed by the ancients to make swords, hatchets, \&ce, before the method of working iron was generally understood. Most modern archrologists, following the Danish antiquaries, recognise a bronze age; that is to say, an epoch of civilisation when bronze was the only metal in general use for cutting instruments, and other useful or ornamental objects. This period is generally held to have been subsequent to the so-called stone age and anterior to that of iron. Some authorities, however, arguing on metallurgical rather than on archreological grounds, have called this chronology in question, and have maintained that a knowledge of iron-must have preceded that of bronze. Be that as it may, it is certain that the use of bronze was general at a very early period in the history of Westorn eivilisation.

The art of casting bronze statues may be traced to the most remote antiquity, but it was first brought to a certain degree of refinement by Theodoros and Roeus of Samos, about 700 years before the Christian era, to whom the invention of modelling is ascribed by Pliny. The ancients were well aware that by alloying copper with tin, a more fusible metal was obtained, that the process of casting was therefore rendered easier, and that the statue was harder and more durable. It was during the reign of Alexander that bronze statuary received its greatest extension, when the celebrated artist Lysippus succeeded, by new processes of moulding and melting, in multiplying groups of statues to such a degree that Pliny called them the mob of Alexander. Soon afterwards enormous bronze colossuses were made, to the height of towers, of which the isle of Rhodes possessed no less than one hundred. The Roman consul Mutianus found 3,000 bronze statues at Athens, 3,000 at Rhodes, as many at Olympia and at Delphi, although a great number had been proviously carried off from the last town.

From the analyses of Mr. J. A. Phillips, we learn that most of the ancient coins were bronzes, the quantity of tin relatively to the copper varying slightly. The proportions of copper and tin in many of those coins are given below, the other ingedients being omitted :-

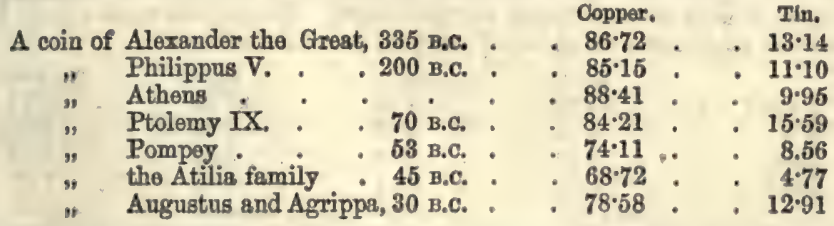

The arms and cutting instruments of the ancients were composed of similar bronzes, as the following proportions, also selected from Mr. J. A. Phillips' analyses, will show :-

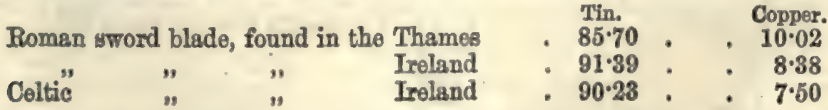

Layard brought from Assyria a considerable variety of bronze articles, many of them objects of ornament, but many evidently intended for use. Amongst others was a bronze foot, which was constructed for the purpose of support of some kind. This was submitted to the examination of Dr. Percy. It was then found that the bronze had been cast round a support of iron. By this means the appearance of cansiderable lightness was attained, whilo great strength was insurod. This discovery proves in a 
very satisfactory manner, that the metallurgists of Assyria were perfectly conversant with the use of iron, and that thoy employed it for the purpose of imparting strength to the less tenacious metals which they employed in thoir art-manufactures. This bronze, as analysed in the Metallurgical Laboratory of the Museum of Practical Geology, consists of copper $88 \cdot 37$, tin $11 \cdot 33$.

Examination has shown that all the bronze weapons of the Greeks and Romans were not only of the true composition for insuring the greatest density in the alloy itself, but that these, by a process of hammering the cutting edges, were brought up to the groatest degree of hardness and tenacity.

Before 1542, 'brass ordnance' (bronze) was founded by foroigners. Stow says that John Owen began to found brass ordnance, and that ho was the first Englishman who ever made that kind of artillery in England.

Bell founding followed. Bell-metal and other broken metal were allowed to be exported hitherto; but it being discovered that it was applied to found guns abroad, 'brass, copper, latten, bell metal, pan metal, gun metal, and shroff metal, are prohibited to be exported.'

Bronze has almost always been used for casting statues, bassi-rilievi, and works which are to be exposed to atmospheric influences. In forming such statues, the alloy should be capable of flowing readily into all the parts of the mould, however, minute; it should be hard, in order to resist accidental blows, be proof against the influence of the weather, and be of such a nature as to acquire that greenish oxidised coat upon the surface, which is so much admired in the antique bronzes, called patina antiqua. The chemical composition of the bronze alloy is a matter therefore of the first moment. The Brothers Keller, celebrated founders in the time of Louis XIV., whose chefs-d'œuvre are well known, directed their attention towards this point, to which too little importance is attached at the present day. The statue of Desaix, in the Place Dauphine, and the column in the Place Vendôme are noted specimens of most defective workmanship from mismanagement of the alloys of which they are composed. On analysing separately specimens taken from the bas-reliefs of the pedestal of this column, from the shaft, and from the capital, it was found that the first contained only 6 per cent; of tin, and 94 of copper, the second much less, and the third only 0.21 . It was therefore obvious that the founder, unskilful in the melting of bronze, had gone on progressively refining his alloy, by the oxidisement of the tin, till he had exhausted the copper, and that he had then worked up the refuse scoriæ in the upper part of the column. The cannon which the Government furnished him for casting the monument consisted of :-

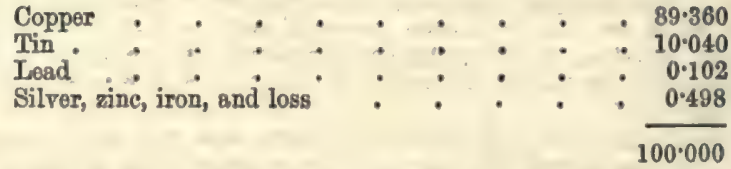

The moulding of the several bas-reliefs was so ill executed, that the chiselers employed to repair the faults removed no less than 70 tons of bronze, which was given them, besides 300,000 francs for their work. The statues made by the Kellers at Versailles were found, on chemical analysis, to consist of :-

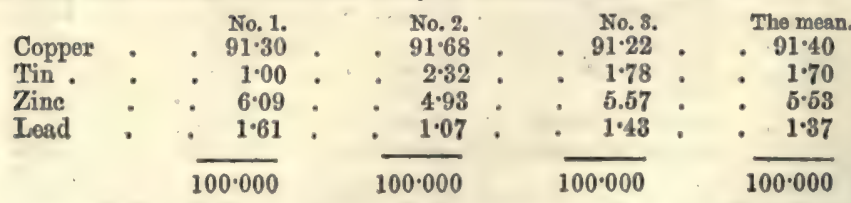

The analysis of the bronze of the statue of Louis XV. was as follows :-

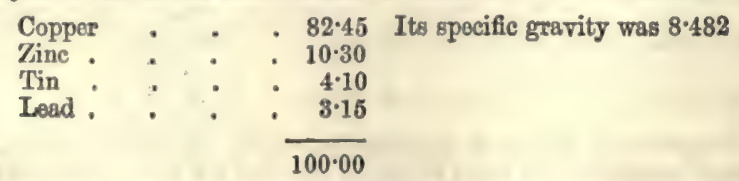

The bronzes of France, according to M. L. E. Rivot, contain nearly always four motals, copper, tin, lead, and zinc. They may contain also very small and variable quantitios of iron, nickel, arsenic, antimony, and sulphur. 
The alloys most proper for bronze to be afterwards struck for medals is composed of from 8 to 12 parts of tin and from 88 to 92 of copper; to which if 2 or 3 parts in the hundred of zine be added, they will make it assume a finer bronze tint. The alloy of the Kellers is famous for this effect. The metal should be subjected to three or four successive stamps of the press, and be softened between each blow by being heated and plunged into cold water.

The addition of a small proportion of phosphorus has recently been recommended to improve the quality of bronze.

The bronze of bells, or bell-metal, is composed, in 100 parts, of copper 78, tin 22. This alloy has a fine compact grain, is very fusible and sonorous. The other metals sometimes added are rather prejudicial, and meroly increase the profit of the founders. Some of the English bells consist of 80 copper, $10^{\cdot 1}$ tin, 5.6 zine, 4.3 lead; the latter metal, when in such large quantities, is apt to cause isolated drops, hurtful to the uniformity of the alloy.

The Tam-tams and Cymbals of Bronze.-The Chinese make use of bronze instruments forged by the hammer, which are very thin and raised up in the middle; they are called gongs, from the word tshoung, which signifies a bell. Klaproth has shown that they contain nothing but copper and tin, in the proportion of 78 of the former metal and 22 of the latter. Their specific gravity is 8.815 . This alloy, when newly cast, is as brittle as glass; but being plunged at a cherry-red heat into cold water, and confined between two dises of iron to keep it in shape, it becomes tough and malleable. The cymbals consist of 80 parts copper and 20 tin.

Bronze vessels, naturally brittle, may be made tenacious by the same ingenious process, for which the world is indebted to M. Darcet. Bronze mortars for pounding have their lips tempered in the same way.

Cannon Metal consists of about 90 or 91 copper, and 10 or 9 of tin. From the experiments of Papacino d'Antony, made at Turin, in 1770, it appears that the most proper alloy for great guns is from 12 to 14 parts of tin to 100 of copper; but the Comte Lamartilliere concluded, from his experiments made at Dousy, in 1786, that never less than 8 nor more than 11 of tin should be employed in 100 parts of bronze.

Gilt Ornaments of Bronze.-This kind of bronze should be easy of fusion, and take perfectly the impression of the mould. The alloy of copper and zine (brass) is, when fused, of a pasty consistence, does not make a sharp cast, is apt to absorb too much amalgam, is liable to crack in cooling, and is too tough or too soft for the chaser or turner; and if the quantity of zine was inereased, to make the metal harder, it would lose the yellow colour suitable to the gilder. A fourfold combination of copper, zine, tin, and lead is preferable for making such ornamental bronze articles; and the following proportions are probably the best, as they unite closeness of grain with the other good qualities. Copper 82 , zine 18 , tin 3 or 1 , lead $1 \frac{1}{2}$ or 3 . In the alloy which contains most lead, the tenacity is diminished and the density is increased, which is preferable for pieces of small dimensions. Another alloy, which is said to require for its gilding only two-thirds of the ordinary quantity of gold, has the following composition: copper $82 \cdot 247$, zine $17^{\circ} 481$, tin $0 \cdot 238$, lead $0 \cdot 024$.

The antique bronze colour is given to figures and other objects made from these alloys by the following process :-Two drachms of sal-ammoniac, and half a drachm of salt of sorrel, (binoxalate of potash,) are to be dissolved in fourteen ounce measures (English) of colourless vinegar. A hair pencil being dipped into this solution, and pressed gently between the fingers, is to be rubbed equally over the clean surface of the object slightly warmed in the sun or at a stove; and the operation is to be repeated till the wished-for shade is obtained.

The bronze founder ought to melt his metals rapidly, in order to prevent the loss of tin, zinc, and lead by their oxidisement. Reverberatory furnaces have been long used for this operation, the best being of an elliptical form. The furnaces with dome tops are employed by the bell founders, because, their alloy being more fusible, they do not require so intense a heat; but they also would find their advantage in using the most rapid mode of fusion. The surface of the melting metals should be covered with small charcoal or coke; and when the tin is added, it should be dexterously thrust to the bottom of the meltod copper. Immediately after stirring the melted mass so as to incorporate its ingredients, it should be poured out into the moulds. In general, the metals most easily altered by the fire, as the tin, should be put in last. The cooling should be as quickly as possible in the moulds, to prevent the risk of the metals separating from each other in the order of their density, as they are very apt to do. The addition of a little iron-in the form of tin plato-to bronze, is reckoned to be advantageous.

One part of tin, and two parts of copper (nearly one atom of tin and four of 
copper, or more exretly 100 parts of tin and 215 copper), form the ordinary speculum metal of reflecting telescopes, which is of all the alloys the whitest, the must brilliant, the hardest, and the most brittle, The alloy of 1 part of tin and 10 of copper (or nearly one atom of the former to eighteen of the lattor), is the strongest of the whole sories.

Ornamental objects of bronze, after boing cast, are commonly laid upon rod-hot coals till they take a dull red heat, and aro then exposed for some time to tho air. The surface is thereby freed from any greasy matter, some portion of the zinc is dissipated, and the alloy assumes more of a coppery hue, which propares for the subsequent gilding. The black tinge which it sometimes gets from the fire may be renoved by washing it with a weak acid. It may be made very clean by acting upon it with nitric acid, of spocifie gravity 1.324, to which a littlo common salt and soot have been added, the latter boing of doubtful utility; aftor which it must be well washed in water, and dried with rags or sawdust.

For the following Table we axe indebted to Mr. Robert Mallet, F.R.S., whoso investigations in this direction have been most extensive, and as accurate as they are extensive :-

\begin{tabular}{|c|c|c|c|c|c|c|c|c|c|c|c|c|}
\hline & $\begin{array}{c}\text { Chemienl } \\
\text { Constitusion }\end{array}$ & $\begin{array}{l}\text { Composition } \\
\text { by Weight } \\
\text { per Cent. }\end{array}$ & $\begin{array}{l}\text { है } \\
\text { है } \\
\text { है }\end{array}$ & 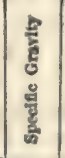 & है & Colour of Frseture & 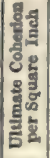 & 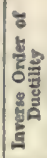 & 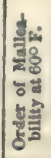 & 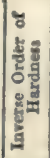 & 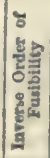 & $\begin{array}{c}\text { Commercial Titles, } \\
\text { ebsarketeristic } \\
\text { Froperties in } \\
\text { working, \&c. }\end{array}$ \\
\hline 1 & $\mathrm{Ca}$ & $100 \cdot 00+$ & $81 \cdot 6$ & $8 \cdot 667$ & $\mathbf{B}$ & Tile red & $24 \cdot 6$ & 1 & 2 & 10 & 16 & Copper. \\
\hline 2 & $10 \mathrm{Cu}+\mathrm{Sn}$ & $84 \cdot 29+15 \cdot 71$ & $374 \cdot 9$ & 8.561 & $\mathrm{FC}$ & $\begin{array}{l}\text { Reddish yel- } \\
\text { low, } 1\end{array}$ & $16 \cdot 1$ & 2 & 6 & 8 & 15 & Gun metal, \&cc. \\
\hline 3 & $9 \mathrm{Cu}+\mathrm{Sn}$ & $82 \cdot 81+17 \cdot 19$ & $848 \cdot 3$ & $8 \cdot 462$ & BO & $\begin{array}{c}\text { Reddish yel- } \\
\text { low, } 2\end{array}$ & $15 \cdot 2$ & 8 & 7 & 5 & 14 & Ditto. \\
\hline 4 & $8 \mathrm{Cu}+\mathrm{Sn}$ & $81 \cdot 10+18 \cdot 90$ & $311 \cdot 7$ & 8.459 & FC & Yellowish & $17 \cdot 7$ & 4 & 10 & 4 & 13 & Gun metal, tem- \\
\hline 5 & $7 \mathrm{Ca}+\mathrm{Sn}$ & $78 \cdot 97+21 \cdot 03$ & $280 \cdot 1$ & $8 \cdot 728$ & VC & $\begin{array}{l}\text { Yellowish } \\
\text { red, } 1\end{array}$ & $13 \cdot 6$ & 5 & 11 & 3 & 12 & $\begin{array}{l}\text { Fiard mill } \\
\text { brasses, \&c. }\end{array}$ \\
\hline 6 & $6 \mathrm{Cu}+\mathrm{Sn}$ & $76 \cdot 29+23 \cdot 71$ & $248 \cdot 5$ & $8 \cdot 750$ & $\mathbf{V}$ & Bluish red, 1 & $9 \cdot 7$ & 0 & 12 & 2 & 11 & Brittle. \\
\hline 7 & $5 \mathrm{C}$ & $72 \cdot 80+27 \cdot 20$ & 216.9 & $8 \cdot 575$ & C & Bluish red, 2 & $4 \cdot 9$ & 0 & 13 & 1 & 10 & Brittle. ${ }^{2}$ \\
\hline 8 & $4 \mathrm{Cu}+8 n$ & $68.21+81 \cdot 79$ & $185 \cdot 3$ & $8 \cdot 400$ & C & Ash grey & $0 \cdot 7$ & 0 & 14 & 6 & 9 & Orambles. \\
\hline 9 & $3 \mathrm{Ca}$ & $61 \cdot 69+38 \cdot 31$ & $153 \cdot 7$ & 8.539 & TC & Dark grey & 0.5 & 0 & 16 & 7 & 8 & Crumbles. ${ }^{2}$ \\
\hline 10 & $2 \mathrm{Cu}+\mathrm{Bn}$ & $51 \cdot 75+48 \cdot 25$ & $122 \cdot 1$ & $8 \cdot 416$ & $\nabla C$ & $\begin{array}{l}\text { Greyish } \\
\text { white, } 1\end{array}$ & $1 \cdot 7$ & 0 & 15 & 9 & 7 & Brittle. ${ }^{2}$ \\
\hline 11 & $\mathrm{Ca}$ & $34 \cdot 92+65 \cdot 08$ & 90.5 & $8 \cdot 056$ & TC & Whiter still, 2 & $1 \cdot 4$ & 0 & 9 & 1 & 6 & Small bells, \\
\hline 12 & $\mathrm{Ca}+2 \mathrm{Sn}$ & $21 \cdot 15+78 \cdot 85$ & $149 \cdot 4$ & $7 \cdot 387$ & $\mathrm{CO}$ & Ditto 8 & $3 \cdot 9$ & 0 & 8 & 1 & 5 & $\begin{array}{l}\text { brittle. } \\
\text { Speculum }\end{array}$ \\
\hline 13 & $\mathrm{Cu}+8 \mathrm{sn}$ & $16 \cdot 17+84: 83$ & $208 \cdot 3$ & $7 \cdot 447$ & $\mathrm{co}$ & Ditto & $3 \cdot 1$ & 0 & 5 & 1 & 4 & $\begin{array}{l}\text { Metal of au- } \\
\text { thors. }\end{array}$ \\
\hline 14 & $\mathrm{Ca}+4 \mathrm{Sn}$ & $11 \cdot 82+88 \cdot 18$ & $267 \cdot 2$ & $7 \cdot 472$ & $\mathrm{CO}$ & Ditto & $3 \cdot 1$ & 8 & 4 & 14 & 3 & Files, tough. \\
\hline 15 & $\mathrm{Cu}+5 \mathrm{Sn}$ & $9.68+90.32$ & $326 \cdot 1$ & $7 \cdot 442$ & E & Ditto & $2 \cdot 7$ & 6 & 8 & 15 & 2 & $\begin{array}{l}\text { Files, soft and } \\
\text { tough. }\end{array}$ \\
\hline 16 & Sn & $100 \cdot 00$ & $58 \cdot 9$ & $7 \cdot 291$ & $F$ & White, 7 & $2 \cdot 5$ & 7 & 1 & 16 & 1 & Tin. \\
\hline
\end{tabular}

Bronze, Phosphorised. By carefully dropping phosphorus on melted bronze or copper a product rich in phosphorus is produced. This alloy is claiming much attention, and will be fully treated of under the head of PHospror BroNar AND Coppren, which see. See also Alungrsiour Bronze.

Bronze Imports are now included under the heads of Brass, Bronze and Metal, Bronzed and Lacquered. Of these articles the following Imports are given in 1872:-

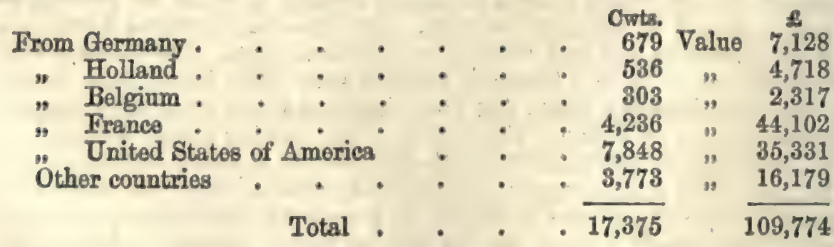

In the same year the Exportation of the same metals is given as-

To all countries . . $1,018 \mathrm{cwts}$, valued at $5,650 l$.

\footnotetext{
2. I signifies earthy ; c c, coarse crystalline; $\mathrm{v}$ C, fine crystalline ; 0 , concholdal; $\nabla$, vitreons ; $\nabla \mathrm{C}$, vitreous-concholdal ; T c, tabular crgstalline.

All these alloys are found occasionally in bells and opecula with mixtures of $\mathrm{Zn}$ and $\mathrm{Pb}$.
} 


\section{BRONZE POWDERS}

Browze pArvr. Bronzo paint, commonly called gold paint, is mado by mixing gold-coloured bronze powder with pure turpentine as a vehielo. The turpentine of commerce has at all times an acid reaction, which is detrimental to tho colour of the metal powder. The turpentino used for this purpose has, therefore, to bo mixod with lime to noutralise the acid bofore it is thus employed.

mRONZx POWDIRS have been much used of lato in tho decorativo painting of houses, \&c. They are prepared of every shade, from that of bright gold to orange, dark copper, emerald greon, \&c. Palo gold is produced from an alloy of $13 \frac{1}{4}$ of copper and $2 \frac{3}{4}$ of zine; crimson metallic lustre-from copper: ditto, paler, copper and a very little rinc; green bronze with a proportion of verdigris; another fine orange by 14.4 copper and $1 \frac{3}{4}$ zinc; another ditto, $13 \frac{3}{4}$ copper and 2.4 zinc : a beautiful pale gold from an alloy of the two metals in atomic proportions.

The alloy is laminated into very fine leaves with careful annealing, and these aro levigated into impalpable powders along with a film of fine oil to prevent oxidisement, and to favour the levigation.

Mr. Brandeis, in his account of his articles of manufacture furnished to the New York Exhibition, says :-

'Bronzes, or, more correctly, metallic powders resembling gold dust, were invented in 1648, by a monk, at Furth, in Bavaria, named Theophrastus Allis Bombergensis. Ho took the scraps or cuttings of the metallic leaves then known as 'Dutch leaf,' and ground them with honey. This roughly made bronze powder was used for ornamenting parchments, capital letters in Bibles, choral books, \&ce.'

At Furth bronze powders are largely made for Europe, and with little change or improvement. There are four sorts of Dutch leaf :-

Common leaf, soft, and of reddish cast, composed of 25 or 30 per cent, of zinc to 75 or 70 per cent. of copper.

French leaf contains more zinc, is harder, less ductile, and has a purer yellow colour.

Florenos leaf has a larger proportion of zinc, and is of a greenish gold colour; and lastly,

White leaf, composed of tin. The more zine these alloys contain, the harder, the more brittle, and more difficult are they to work into perfect leaves. The manner of beating is similar to the mode for producing gold leaves.

The scraps, cuttings, and fragments of these leaves are the materials for the German bronze powdors. First brushed through a sieve and ground with gum water on marble slabs for six hours, the gum washed out, the powders sorted, dried, and a coating of grease given to make them appear more brilliant, and to protect them from oxidation. Varioties of colour, such as orange, \&c., are produced by a film of suboxide upon the surface of the particles. The price of bronzo powders depends upon the demand, and the supply of the waste material of the metal leaves, and prices change accordingly.

Messrs. Brandeis patent their process, and in place of being dependent upon uncertain supplies of motal of unknown composition, they take the metals at once in a state of purity (say copper by voltaic precipitation): it is alloyed with zinc, cast into ingots, rolled into ribands, cut, annealed, and rolled until the metal is thin and leaflike; then it is taken to a sterm-mill, and ground. The bronze powder is washed out and dried, then introduced into an air-tight room, with an arrangement of boxes; the air of the chamber is set in violent motion by bellows, and the powder diffused throughout; the bronze powders are deposited, the finest in the upper boxes, and the coarser powders below. When settled, mineral varnish is introduced; the boxes fitted with tight lids are made to revolve, and the particles are thus rapidly coated, and the highest metallic brillianey imparted. Different shades of colour, pink, crim. son, \&c., are produced by submitting the powder to heat and oxidation before the rapid revolutions of the varnishing boxes.

The quantity thus produced by one firm, with three steam-engines at work, enables the finished bronze powders to be produced at a rate about equal to the price the German manufacturer has to pay for his materials-the cuttings and scraps of leaves. Hence, for the purposes of trade and art, a large exportation of bronze powders takes place from America to Europe, South America, and China.

The bronze powders are largely used in japanning, bronzing tin and iron goods, ornamental works of paper, wood, oil-cloth, loather, \&ce.; while sign-boards and the decoration of public buildings have effective metallic brilliant surfaces of boanty and durability. In fact, for ornamental decorations, the demand sterdily increases.

In Holland and Germany the subject has been examined, with the viow of ascertaining the effect of chemical composition.

Do Heer E. R. König has given a Table of the analyses of the best Europesn samples of bronze powders and leaves (Volksfight):- 


\begin{tabular}{|c|c|c|c|c|c|}
\hline & & Copper & Zine & Iron & Tin \\
\hline 1. Iight yellow & & $\begin{array}{c}\text { Per cent. } \\
82.38\end{array}$ & $\begin{array}{c}\text { Per cent. } \\
16 \cdot 69\end{array}$ & $\begin{array}{c}\text { Per cent. } \\
0 \cdot 16\end{array}$ & $\begin{array}{c}\text { Per cent. } \\
0\end{array}$ \\
\hline 2. Gold yellow . . . & & $84 \cdot 50$ & $16 \cdot 30$ & 0.07 & 0 \\
\hline $\begin{array}{l}\text { 3. Messing yellow, or brass } \\
\text { colour }\end{array}$ & yollow & 90. & $9 \cdot 61$ & 0.20 & 0 \\
\hline 4. Copper bronze, orange colou & . & $98 \cdot 93$ & 0.78 & 0.08 & 0 \\
\hline 5. Copper red, high shado of pur & e colour & $99 \cdot 90$ & 0.00 & trace & 0 \\
\hline 6. Purplo violet & - & $98 \cdot 22$ & 0.5 & $0 \div 30$ & traco \\
\hline 7. Light green & 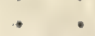 & $84 \cdot 32$ & $15 \cdot 02$ & 0.03 & traco \\
\hline 8. Tin white or leaden grey. & . & 0.00 & $2 \cdot 39$ & 0.56 & $97 \cdot 46$ \\
\hline
\end{tabular}

Vanadate of copper has recently been recommended as a new bronze. Dr. B. W. Gerland has shown that a solution of vanadate of copper in aqueous sulphurous acid doposits brilliant yellow crystals, after part of the sulphurous acid has been removed by boiling. Theso erystals are quite uniform in appearance, and contain cupric oxide, vanadic acid, and sulphurous acid. They change rapidly, under tho influence of air, their beautiful metallic lustre quickly disappearing, and the yellow colour changing to a dark groen. If, however, these crystals are removed from the mother-liquor, and treated with fresh sulphurous acid, although in the first instance formed in sulphurous acid solution, they now decompose, and two fresh kinds of crystals, the one brown the other orange yellow, are formed. Continuing to add sulphurous acjd, the brown crystals are redissolved, while the orange yellow are left intact.

After filtration, washing, and drying, a residue is left of microscopic scales of a deep orange-yellow colour, possessing a most beautiful lustre. They are free from coppor and sulphur, and are perfectly unalterable in the air.

The composition of this substance previously dried over vitriol is, according to analysis, as follows :-

$$
\begin{aligned}
& \text { Water (loss by heating) . . . . . } 8773 \\
& \text { Pentoxide of vanadium . . . . . 91.06 } \\
& \text { Impurities . . . . . . } 0.21 \\
& 10 \cdot 000
\end{aligned}
$$

These numbers correspond to the formula of metavanadic acid, which requires--

$$
\text { Wentoxide of vanadium }: \quad \vdots \quad \vdots \quad \frac{8.97}{91.03}
$$

In some instances, Dr. Gerland obtained the same bronze or gold-like substance by treating vanadate of copper suspendod in water with sulphurous acid gas; but in many others the effect of the gas was the formation of vanadic oxide in solution.

The vanadate of copper used in these experiments was prepared by precipitation from vanadate of ammonia by sulphate of copper. The mother-liquor contained both copper and vanadic acid. After evaporation the latter is found in the residue as metavanadic acid, with the same metallic appearance as that above described, and can be obtained by washing with water. The crystal, however, obtained in this manner most obstinately retains copper, sometimes to the extent of 12 per cent. Repeated treatment with sulphurous acid is the best method of purification. A sample of the bronze as thus prepared was aualysed by Professor Roscoe, who found-

$$
\begin{aligned}
& \text { Water . . . . . . . 9.12 } \\
& \text { Pentoxide of vanadium . . . } 90.88 \\
& 100.00
\end{aligned}
$$

Samples of vanadium bronze obtained by these three different methods have the same composition, the same appearance, and the same chemical properties. Vanadium bronze is essentially distinguished from the amorphous brick-red hydrated vanadic acid by its indifference to reagents. Sulphurous acid scarcely acts on it, neither does ammonia, and even a solution of carbonate of soda only dissolves it after longcontinued boiling. In the air it is perfectly permanent. It is probable that this metavanadic acid will become a favourite bronze, and be rated at even a higher value than gold bronze.

BnowzrarG. The process for giving to metals, plaster, wood, or any other body, a bronze-like surface. Various processes have beon adopted for producing this effect. 
When brass castings are to be bronzed, it is essential, in the first place, that they should be thoroughly cleansed from grease, and brightened either with the file or emery-paper, or by boiling in a strong ley and then scouring with fine sand and water. Vinegar alone is sometimes employed to produce the green bronze colour; sometimes dilute nitric acid, and often the muriate of ammonia (sal-ammoniac). This latter salt and vinegar are frequently combined, and often a little common table salt is added to the bronzing fluid.

Coins and medals may be bronzed with the following solution:-2 parts of verdigris and 1 part of sal-ammoniac are to be dissolved in vinegar; the solution is to be boiled, skimmed, and diluted with water till it has only a weak metallic taste, and upon further dilution lets fall no precipitate. This solution is made to boil briskly, and is poured upon the objects to be bronzed. These are well washed with clean water, and then lacquered.

To give fresh-made bronze objects an antique appearance, three quarters of an ounce of sal-ammoniac, and a drachm and a half of binoxalate of potash (salt of sorrel) are to be dissolved in a quart of vinegar, and a soft rag or brush moistened with this solution is to be rubbed over the clean bright metal till its surface becomes entirely dry by the friction. This process must be repeated several times to produce the full effect; and the object should be kept a little warm. Copper acquires very readily a brown colour by rubbing it with a solution of the common liver of sulphur, or sulphuret of potassium.

The Chinese are said to bronze their copper vessels by taking 2 ounces of verdigris, 2 ounces of cinnabar, 5 ounces of sal-ammoniac, and 5 ounces of alum, all in powder, making them into a paste with vinegar, and spreading this pretty thick like a pigment on the surfaces previously brightened. The piece is then to be held a little while over a fire, till it becomes uniformly heated. It is next cooled, washed, and dried; after which it is treated in the same way once, and again till the wished-for calour is obtained. An addition of sulphate of copper makes the colour incline more to chestnut brown, and of borax more to yellow. It is obvious that the cinnabar produces a thin coat of sulphuret of copper upon the surface of the vessel, and might probably be used with advantage by itself.

To give the appearance of antique bronze to modern articles, we should dissolve 1 part of sal-ammoniac, 3 parts of cream of tartar, and 6 parts of common salt in 12 parts of hot water, and mix with the solution 8 parts of a solution of nitrate of copper of specific gravity $1 \cdot 160$. This compound, when applied repeatedly in a moderately damp place to bronze, gives it in a short time a durable green coat, which becomes by degrees very beautiful. More salt gives it a yellowish tinge, less salt a bluish cast. A large addition of sal-ammoniac accelerates the operation of the mordant.

The best and most rapid bronzing liquid, which may be applied to copper, brass, iron, or to new bronze, with equal advantage, is a solution of the chloride of platinum (nitro-muriate of platinum), called chemical bronze; but it is expensive. With the chloride of platinum, almost any colour can be produced, according to the degree of dilution and the number of applications.

Some beautiful effects are produced upon bronze, and also upon iron castings, by treating them with dilute acids. The action here is scarcely to be described as bronzing; it is, in fact, merely developing the true colour of the metal or alloy.

With the view of rendering the action of the bronzing liquid as uniform as possible, small articles are dipped; for larger articles, the bronzing liquid is dabbed on plentifully with a linen rag. The dabbing process is to prevent the occurrence of streaks, which might arise if the liquid were applied in straight strokes. When properly bronzed and washed, the work is usually black-leaded, to give it a polished appearance.

Bronzing of Objects in Imitation of Metallic Bronze.-Plaster of Paris, paper, wood, and pasteboard, may be made to resemble pretty closely the appearance of articles of real bronze, modern or antique. The simplest way of giving a brilliant aspect of this kind is with a varnish made of the waste gold-leaf of the beater, ground up on a porphyry slab with honey or gum-water. A coat of drying linseed-oil should be first applied, and then the metallic powder is put on with a linen dossil. Mosaic gold ground up with six parts of bone-ashes has been used in the same way. When it is to be put on paper, it should be ground up alone with white of eggs or spirit varnish, applied with a brush, and burnished when dry. Whou a plate of iron is plunged into a hot solution of sulphate of copper, it throws down fine seales of copper, which being repeatedly washed with water, and ground along with six times its weight of bone-sshes, forms a tolerable brouxing.

Browning of Gun-Barrels and other Arms.-By this process the surface of several articles of irou acquire a shining brown colour. This preparation, which protects the 
iron from rust, and also improves its appearance, is chiefly employed for the barrels of fowling-pieces and soldiers' rifles, to conceal the fire-arms from the game and the enemy. The finest kind of browning is the Damascus, in which dark and bright lines run through the brown ground.

This operation consists in producing a vory thin uniform film of oxide or rust upon the iron, and giving a gloss to its surface by rubbing wax over it, or coating it with a shell-lac varnish.

Several means may be employed to produce this rust speodily and well. The effect may be obtained by inclosing the barrels in a space filled with the vapour of muriatic acid. Moistening their surface with diluted muriatic or nitric acid will answer the same purpose. But the most common material used for browning is the butter or chloride of antimony, which, on account of its being subservient to this purpose, has boen called bronzing salt. It is mixed uniformly with olive-oil, and rubbed upon the iron slightly heated, which is afterwards exposed to the air, till the wished-for dogree of browning is produced. A little aquafortis is rubbed on after the antimony, to quicken its operation. The brown barrel must be then carefully cleaned, washed with water, dried, and finally polished, either by the steel burnisher, or rubbed with white wax, or varnished with a solution of 2 ounces of shell-lac and 3 drachms of dragon's blood, in 2 quarts of spirits of wine.

The following process may also be recommended:-Make a solution with half an ounce of aquafortis, half an ounce of sweet spirits of nitre, 1 ounce of spirits of wine, 2 ounces of sulphate of copper, and 1 ounce of tincture of iron, in so much water as will fill together a quart measure. The gun-barrel to be browned must first of all be filed and polished bright, and then rubbed with unslaked lime and water to clear away all the grease. Its two ends must now be stopped with wooden rods, which may serve as handles, and the touch-hole must be fillod with wax. The barrel is then to be rubbed with the solution, applied to linen rags or a sponge, till the whole surface be equally moistened; it is allowed to stand 24 hours, and is then scrubbed off with a stiff brush. The application of the liquid and the brushing may be ro peated twice or oftener, till the iron acquires a brown colour. After the last brushing, the barrel must be washed with plenty of boiling water containing a little potash, then washed with elean water, dried, rubbed with polishing woods, and then coated with shell-lac varnish.

BROMIDEs. Compounds of bromine with electro-positive elements.

BRomrrs z. (Symb. Br.; Atomic weight, 80. Density in liquid state, 2:97. Density of vapour by experiment, $5 \cdot 39$; by calculation, $5 \cdot 536$, on supposition of the density of hydrogen being $0^{\circ} 0692$ ). - This element exists in very small quantity in sea-water, and to a larger extent in certain mineral springs, as those of Kreuznach and Kissingen. The water of the Dead Sea is comparatively rich in bromine-a sample taken from a depth of 300 mètres having yielded $\mathrm{M}$. Lartet 0.7 per cent. of this element. The mother-liquors of many brine springs, especially those of Schönebeck, near Magdeburg, are rich in bromides. The element s.130 occurs in carnallite and some of the other salts worked at Stassfurt, and in two silver ores found in the mines of Mexico and Chile, and known as bromyrite, or bromide of silver, and embolite or chloro-bromido of gilver. Megabromite and microbromite are names applied to ill-defined varieties of embolite, according as they contain more or less bromine.

Bromine was discovered in 1826, by Balard, of Montpellier, in the bittern produced from the water of the Mediterranean. Bromine is a very interesting substance, and its discovery has had great influence on the progress of theoretical and applied chomistry. It is the only eloment, save mercury, which exists in the liquid stato at ordinary temperatures. The liquid presents a deep brownish-red colour, and omits denso reddish vapours, which are extremely irritating when breathed. Its odour is sufficiontly charactoristic to have given the element its name (Bpw $\mu$ os, a stench). Bromine is slightly soluble in water, one part requiring 30 parts of water for its solu. tion. The aqueous solution is of a yellowish tint when freshly made, but is readily decomposed by exposure to light. It forms a definite crystalline hydrate with five atoms of water. Bromine is much more soluble in alcohol or in ether. Exposed to a temperature of about $145^{\circ} \mathrm{Fahr}$. bromine boils, and at $9.5^{\circ}$ it freezes to a red crystalline solid.

Preparation 1. From bittern.-Chlorine gas is passed in for some time; this has the effect of combining with the metallic base of the bromide present, the bromine being, in consequence, liberated. When the bittern no longer increases in colour, the operation is suspended or chloride of bromine would be formed, and spoil the operation. The coloured fluid is placed in a large globe, with a neck having a glass stopcock below like a tap funnel, the upper aperturo being closed with a stopper. Ether is then added, the stopper replaced, and the whole well agitated. After a short repose, the ether rises to the surface, retaining the bromine in solution. The stopper being 
removed to permit the entrance of air, the stopeock is opened, and the aqueous fluid is permitted to run out. As soon as the highly-coloured ethereal solution arrives at the aperture in the stopeock, the latter is shut; a quantity of solution of potash is then poured, by the upper aperture, into the globe, and the stopper is replaced. The whole is now to be agitated, by which means the bromine combines with the potash, forming a mixture of bromate of potash and bromide of potassium. The stopcock is again opened, and the aqueous fluid received into an evaporating vessel, boiled to dryness, and ignited. By this means the bromate of potash is all converted into bromide of potassium. The bromine may be procured from the bromide of potassium by distillation with peroxide of manganese and sulphuric acid. In this operation one equivalent of bromide, two equivalents of sulphuric acid, and one of peroxide of manganese, yield one equivalent of sulphate of manganese, one of sulphate of potash, and one of bromine; or, in symbols, $\mathrm{KBr}+2 \mathrm{SO}^{3}+\mathrm{MnO}^{2}=\mathrm{KO}, \mathrm{SO}^{9}+\mathrm{MnO}, \mathrm{SO}^{3}+\mathrm{Br}$. (2rerr +

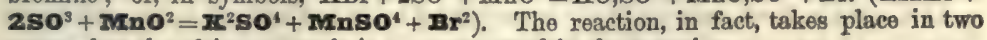
stages, but the ultimate result is as represented in the equation.

Preparation 2.-In some saline springs where bromine is present, accompanied by considerable quantities of salts of lime, \&c., the brine may be evaporated to one-fourth, and, after repose, decanted or strained from the deposit. The mother-liquid is to have sulphuric acid added in order to precipitate most of the lime. The filtered fluid is then evaporated to dryness, redissolved in water, and filtered; by this means more sulphate of lime is got rid of. The fluid is then distilled with peroxide of manganese and hydrochloric acid.

Preparation 3.-Bromine may be obtained by Leisler's method, patented in 1866, from the mother-liquors left in the treatment of the salts called carnallite and kainite, found in the mines of Stassfurt in Prussia, and Kaluez in Hungary. When these liquors are heated with bichromate of potash and dilute hydrochloric acid, the bromine is distilled off, and may be condensed in a receiver containing pieces of iron whereby a bromide of iron is formed. Stassfurt is now one of the chiof localities for the manufacture of bromine, and about $400 \mathrm{cwts}$. are produced there annually.

Bromine, like chlorine, may be employed as a disinfectant. In medicine and in photography it is used in the form of certain bromides, such as those of potassium, ammonium, and cadimium. Bromine has also been employed in the preparation of some of the aniline and anthracene colours.

With hydrogen, bromine forms hydrobromic acid; and with many of the metals it forms well-defined bromides. A chloride of bromine is known, as also a bromide of nitrogen-the latter being an explosive compound resembling chloride of nitrogen. The only well-developed oxide of bromine is bromic acid, $\mathrm{BrO}^{5} \mathrm{HO}\left(\mathbf{H B r O}^{3}\right)$.

Solutions of bromine in water may have their strength determined, even in presence of hydrochloric or hydrobromic acids, by means of a solution of turpentine in alcohol. One quarter of an equivalent of turpentine (34 parts) decolorises 80 parts or 1 equivalent of bromine.

BRONZITx. A silicate of magnesia and protoxide of iron, with a metalloid bronze-like lustre on the cleavage-planes. The species has been lately made to include cortain minerals formerly referrod to the species Diallago-such as the well-known lustrous mineral in the Cornish serpontine. A green variety of bronzite occurs with the diamonds of South Africa.

BRoom, DYrRs, or Greenweed. The Genista tinctoria, a dwarf shrub from the flowers of which a bright yellow colour is obtained, which for dyeing green with woad is said to be preferred by dyers to all other yellows. See WosD.

8rooms. Sweeping brushes. These are made of cocoa-nut fibre, of date-palm leaves, of broom-corn, and in this country of birch twigs, sedge, the common heath, and of the broom.

Broom Corrs. Sorghum dura. Cultivated in America to make brooms, the grains boing used as food for poultry. A closely allied grass, the Holcus saccharatus, a native of China, abounds in sugar. This grass is generally known by the name of Sorghs. It has been used from time immemorial by the inhabitants of China, who extract sugar from it. The Toulon Agricultural Association recommended its introduction into France, to take the place of beet-root. They state that it is richer in sugar than any known plant, except the vine. Beet-root contains from $\mathbf{8}$ to 10 por cent. of sugar; the sorgho from 16 to 20 per cent., from which about 8 per cent. of pure alcohol can be obtained. The refuse is said to be excellent food for cattle. The plant grows rapidly, and does not require irrigation.

Brora conx. An inferior coal lying in the Oolitic doposits of Brora, in Sutherland, Scotland, See Cost.

BRoussonITIA PAPYRIFraA. The Papor Mulberry. The fibrous bark is used in China and Japan for tho manufacture of a kind of paper, while the Polynesian islanders use it in the preparation of their Tapa cloth. 
mrows menrerss. A name occasionally given to the fruit of the Rubus fructicosus, or common bramble.

BnowN COAI is of a brownish-black colour, and presents, in some cases, the texture of wood, when it is called LigNiTs; but, in some varietios, all organic structure has disappeared, and it is then called Pitch coal, from its strong resemblance to true coal.

The beds of brown coal are usually of small extent, and are of later date than the true carboniferous strata, belonging generally to the Tertiary period.

Brown coal is worked in Saxony and in countries where there is an absence of true carboniforous deposits. It burns with an empyreumatic odour, and generally contains more pyrites than ordinary coal.

At Steieregg, in Southern Styria, brown coal occurs in the form of a basin; and has been opened out through a distance of more than two miles. The cosl, from 8 to 16 foet thick, is of good quality. It contains 9 to 14 per cent, of water, and leaves from 5 to 12 per cent. of ash after combustion.

The following is an analysis of a variety from Oregon:-volatile matter, 49.5 ; fixed carbon, $42 \cdot 9$; ash, $2 \cdot 7$; water, $4 \cdot 9=100 \cdot 00$.

A variety of brown coal, called the Paper-coal of Rott, near Bonn, and of Erpel on the Rhine, contains numerous remains of freshwater fishes, Leuciscus papyraceus; and of frogs, Palcophrygnos grandipes. The ashes of this coal are rich in infusorial remains.

For an account of the brown coals of this country, see Liemrte and Bogmrad Cost.

The following is a summary of the state of the brown coal-mines of Europe at the dates given, which are the latest returns obtainable, upon which reliance can be placed:-

HaNover.-Government mines near Münden, on the Steinberg, and near Wallensen, in the Wanzerbruche; and private mines near Drensfeld, produced in

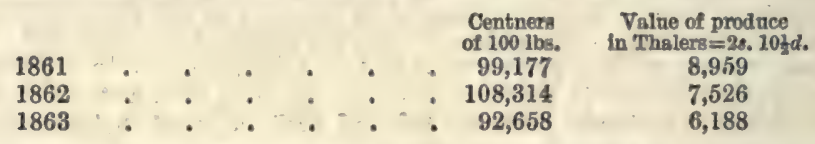

Nassau produces Iignit, a bituminous wood, preserving the ligneous structure; Pseudolignit, in which the woody structure is more or less obliterated; Meerkohl (literally sea-coal), a miry earthy coal resembling turf; Blätterkoh! (literally leaf-coal), a dark-coloured aggregate of decomposed leaves. The total produce in 1864 amounted to 1,032,000 centners (of 50 kilogrammes of $2 \cdot 204$ avoirdupois pounds).

Iтsir.-Leffe Gandino, in the Val Sterina, in Lombardy. This bed extends over 500 hectares. The lignite produced is of a kind resembling peat. It is sold at 15 franes per ton. Cadibona, near Savona,-in the Gulf of Genoa, produces about 1,000 tons annually. From Sarzanella, near Spezia, 25,240 to 31,300 tons of lignite are extracted yearly. At Monte Bamboli, between Grosseto and Piumbino, the production, which may amount to 30 tons daily, does not exceed 10,000 tons in the year, as owing to the unhealthiness of the climate, the works cannot be carried on more than seven or eight months. At the Monte Rufoli or Podemnovo mine, the field of lignite extends over 2,000,000 square mètres. At Monte Massi and Castel Tatti mines, in the year ending June 30,1865 , the amount of lignite produced was about 6,000 tons.

Prussia.-The annual production of brown coal from the beds in the vicinity of Stettin does not exceed 7,000 tons. The total production of brown coal in Prussia was as follows:-

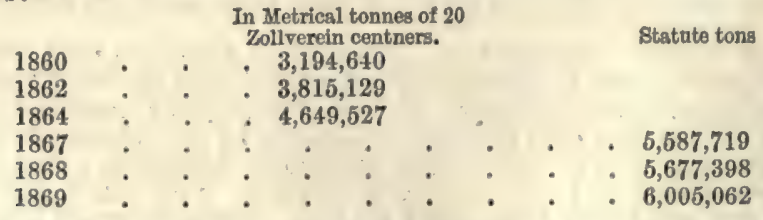

Brandenburg.-According to the best information, there were sent to Berlin 462,298 tonnen of Bohemian and Saxon brown coal. Kottbus.-The quantity taken from the pits Felix and Conrad, at Borsdorf, Julius at Friedrichshain, and Franz at Klein Kölzig, was about 400,000 tonnen. Silesia, Breslau.-One pit in the neighbourhood of Saaran produced 21,000 tonnen, of the walue of 2,000 thalers. Halle.-There were produced in the district of Meresburg, from the 3 government and 192 private works, the amount of $12,255,365$ tonnon; in the government district of Magdeburg,

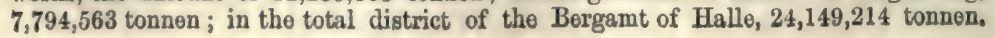


Rhine, Essen.-There were produced at the pit Nachtigall, near Höxter, 10,873 tonnen of brown coal, value 881 thalors. Of Brown coal, or Lignite, the produce in the Zollverein amounted in 1861 to $4,622,312$ tonnen; of the value of $16,631,122$ francs. Prussia alone produced 4,427,442 tonnen, of the value of 11,396,239 francs. The total produce in the Prussian States will be seen in the following table:-

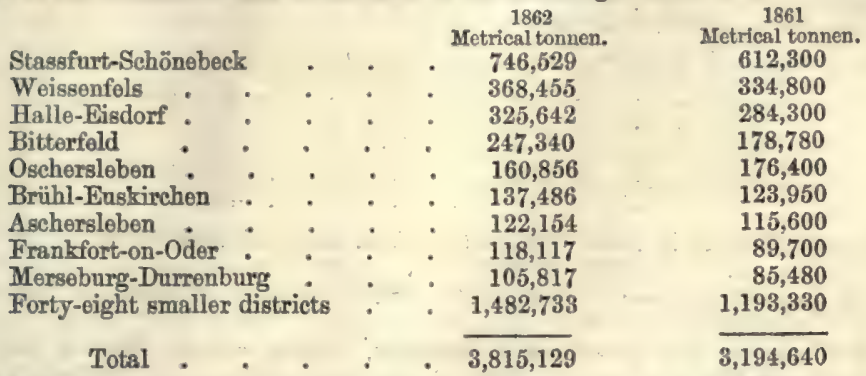

This shows an increase of 620,489 tonnen. Throughout the entire Zollverein, in the year 1863, the brown coal produced at 843 collieries yielded $109,189,899$ cwts., valued at 5,061,241 thalers.

AUsTria,-The production of Brown coal in Austria, in Vienna centners of $123 \frac{1}{2}$ pounds avoirdupois :-

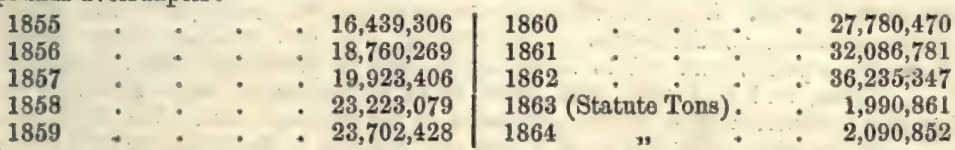

Saxory.-Here much of the brown coal is collected on or near the surface. This is much finer than that from the pits, and is made up like peat, in the shape of bricks. The bricks made at Margaret Hut amounted in 1862, to $3,000,000$, equal to 15,000 cwts., and in 1863 to $2,250,000$, equal to 12,000 cwts. In Upper Lusatia, and on the left bank of the Elbe, in 1863, there were only two pits which produced

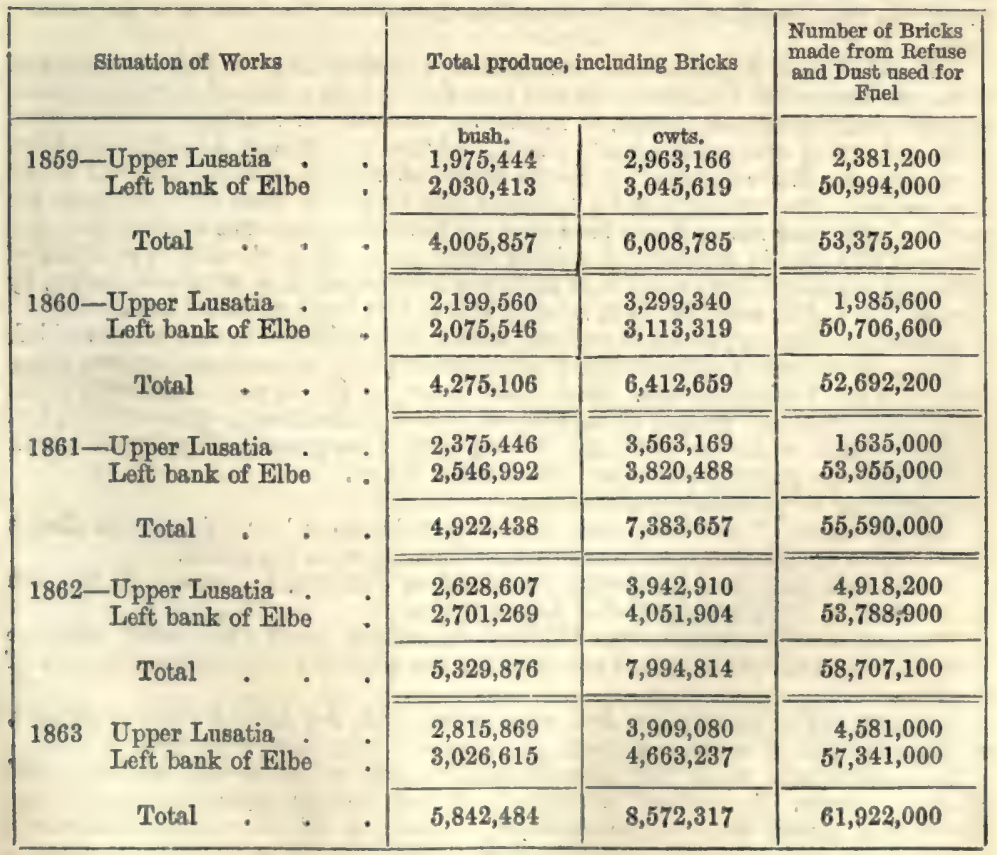

Vor. I.

$\mathrm{N} \mathrm{N}$


more than 400,000 bushels; 3 pits from 200,000 to 400,000 bushels; 4 pits from 100,000 to 200,000 bushels; 19 pits from 50,000 to 100,000 bushels; 36 pits from 20,000 to 50,000 bushels; 62 pits from 5,000 to 20,000 bushels; and 32 pits less than 5,000 . Besides these, there were- 1 pit belonging to the Crown, producing 424,712 bushols ; 4 pits belonging to sharoholders, producing 738,939 bushels ; and 159 privato pits, producing 4,681,833 bushels.

The Brown coal of Sixony was returned as follows in the years given :-

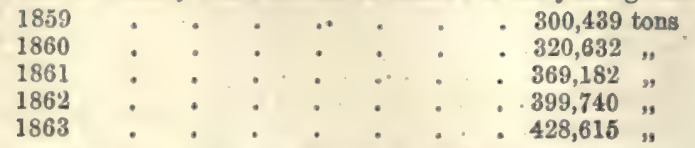

Bavaria produced in 186146,700 tons, from 56 Brown cosl works.

Bor"murs.-In the year 1864 , there were 306,345 tonnen of B"ohemian brown coal brought into the Berlin market.

Swrtzerland.-The coal-mines of Semsales; Bogens, and St. Martin, produce a lignite-coal of first-rate quality. From 31,300 to 37,560 quintals, or from 2,817 to 3,130 tons, are extractod yearly.

Gremcs. - The mines at Koumi produce a kind of coal known as lignite. According to the accounts furnished, 5,000 drachmas per annum is the maximum which has been obtained from these mines since 1849 , when the receipts amounted to 10,400 druchmas, and in 1847 to 12,000 drachmas.

BRowrv Dxa. Upon this subject some general views are given in the article Drenve, explanatory of the nature of this colour. The dye presents a vast variety of tints - from yellow and red to black-brown-and is produced either by mixtures of red, yellow, and blue with esch other, or of yellow or red with black, or by substantive colours, such as catechu or oxide of manganese alone. We shall here notice only the principal shades, leaving their modifications to the caprice or skill of the dyer.

Brown, from mixture of other colours,

Wool and woollen cloths must be boiled with $\frac{1}{8}$ th their woight of alum and sulphotartrate of iron, afterwards washed, and rinsed through the madder bath, which dyes the portion of the stuff imbued with the alum red, and that with the salt of iron black, the tint depending upon the proportion of each and the duration of the madder bath.

A similar brown is produced by boiling every pound of the stuff with two onnces of alum and one ounce of common salt, and then dyeing it in a bath of logwood containing either sulphotartrate, acetate, or sulphate of iron; or the stuff may be boiled with alum and tartar, dyed up in a madder bath, and then run through a black bath of iron mordant and galls or sumach. Here the black tint is added to the red till the proper hue be hit. The brown may be produced also by adding some iron liquor to the madder bath, after the stuff has beon dyed up in it with alum and tartar. A better brown of this kind is obtained by boiling every pound of wool with two ounces of alum, dyeing it up in cochineal, then changing the crimson thus given into brown, by turning the stuff through the bath after acetate of iron has been added to it.

Instead of cochineal, archil or cudbear, with a little galls or sumach, may be added.

A superior brown is produced apon cotton goods which have undergone the oiling process of the Turkey-red dye. Such goods must be galled, mordanted with alum, sulphate of iron, and acetate of lead (equal to $\frac{2}{3}$ rds of the alum); after washing and drying, dyed in a madder bath, and cleared with a soap boil. The tint of brown varies with the proportion of alum and sulphate of iron.

Brown may be produced by direct dyes.

The decoction of oak-bark dyes wool a fast brown colour, of different shades according to the concentration of the bath. Alum improves the colour.

The bastard marjorum (Origanum vulgare) dyes cotton and linen a reddish-brown with acotate of alumina, and wool of a dark brown.

The bark of the mangrove-tree (Rhizophora mangle) gives a red-brown colour to wool when boiled with alum and tartar, and a fast chocolate colour when sulphate of iron is added.

The pods of the East Indian Mimosa cineraria and the African Mimosa nilotica, called the bablat, give cotton a brown, with acetate or sulphate of copper.

The root of the water lily (Nymphea alba) gives to cotton and wool beautiful shades of brown. A mordant of sulphate of iron and zine is first given, and then the wool is turned through the decoction of the root till the wished-for shade is obtained. The cotton must be mordanted with a mixture of the acotates of iron and zinc. 
Walnut (Juglans regia) peelings, when ripe, contain a dark brown dyestuff, which communicatos a permanent colour to wool. The older the infusion or decoction of the peel, the botter dye does it make. The stuff is dyed in the lukewarm bath, and needs no mordant, though it becomes brighter with alum; or this dyo may bo combined with the madder or fustic bath, to give varieties of shade. For dyeing silk, this bath should be hardly lukewarm, for fear of causing inequality of colour.

The peelings of horse-chestnuts may be used for the same purpose: with muriate of tin they give a bronze colour, and with acetate of lead, a reddish brown.

Catechu gives cotton a permanent brown dye, as also a bronze and mordoré, when its solution in hot water is combined with acetate or sulphate of copper, or when the stuff is previously mordanted with the acetates of copper and alumina mixed, sometimes with a little iron liquor, rinsed, dried, and dyed up, the bath being at a boiling heat.

Ferrocyanide of copper gives a yellow-brown or a bronze to cotton and silk.

The brown colour called carmelite by the French is produced by $1 \mathrm{lb}$. of catechn to 4 ozs. of verdigris, with $\dot{5}$ ozs. of muriate of ammonia. The bronze (solitaire) is given by passing the stuff through a solution of muriato or sulphate of manganese, with a little tartaric acid, drying, passing through a potash-ley at $4^{\circ}$ Baumé, brightening and fixing with solution of chloride of lime.

These examples show in how many ways the browning of dyes may be modified, upon what principles they are founded, and how we have it in our power to turn the shade more or less towards red, black, yellow, blue, \&c.

Under the heads of different substances employed by the dyers will be found some description of the methods employed for the purpose of extracting their colours. For some good practical receipts, Love's ' Art of Cleaning, Dyeing, Scouring, and Finishing,' may be consulted.

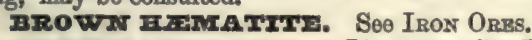

BRoWN Iron Orz, or Limonite. See IroN ORes.

BROWN OCHRE. The soft and decomposed varieties of brown iron ore.

Bzo wrs sucan. Strictly, the common dark Muscovado sugar ; ordinarily, the common varioties of West Indian sugar. See SUGAR.

BRowsz. A motallurgical term for a variety of slag.

BRUCIN2. ( $\mathrm{C}^{46} \mathrm{H}^{20} \mathrm{~N}^{2} \mathrm{O}^{8}\left[\mathbf{C}^{28} \mathbf{H}^{20} \mathbf{2}^{2} \mathbf{O}^{4}\right]$; syn. Canimarine, Vomicine.) A very bitter and poisonous alkaloid accompanying strychnine in nux vomica and in the false angustura bark (Brucia antidysenterica). It is somewhat less poisonous than strychnine, and, like that base, is used in the treatment of paralysis, but in rather larger doses. Brucine is prepared by the same process as strychnine.-C. G. W.

BRUCITI. Native hydrate of magnesia.-So called after Dr. Bruce, of Now York. This mineral accompanies other magnesia minerals in serpentine. It is broad foliated, folia several inches square being easily obtainable, and either opaque, silvery white, or translucent. Thomson gives its composition: magnesia, 66.67; protoxide of manganese, 1.57; protoxide of iron, 1.18; lime, 0.19 ; water, $30 \cdot 39=100$.

BRUNSwrces crman. An oxychloride of copper, used as a pigment. Copper filings or turnings are moistened with a solution of sal-ammoniac, and left in contact with the air. The oxychloride thus forms: it is washed off with water, and dried at a gentlo heat.

Brosers. (Brosses, Fr.; Bürsten, Ger.) Since Mr. Mason introduced his mode of securing the hairs in brushes, it has, in all the better class of brushes, been very generally adopted. His patent dates 1830. Modified contrivances, partaking more or less of the original conditions, have been introduced in the manufacture. The principles of Mr. Mason's invention and its subsequent modifications consist in a firmer mode of fixing the knots, or small bundles, of hair into the stock or the handle of the brush. This is done by forming grooves in the stocks of the brushes for the purpose of receiving the ends of the knots of hair, instead of the holes drilled into the wood, as in brushes of the common constructions. These grooves are to be formed like a dovetail, or wider at the bottom than the top; and when the ends of the knots of hair have been dipped into cement, they are to be placed in the grooves and compressed into an oval form, by which the

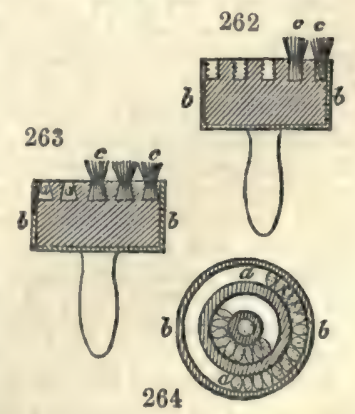

ends of the hair will be pressed outwards, into tho recess or wider part of the dovetailed groove; or the grooves may be formed with threads or teeth on the sides, instead of N 8 ? 
being dovetailed; and the cement and linirs, being pressed into the toeth or threads, will cause them to adhere firmly to the stock or liandle of the brush.

A metal ferrule may be placed on the outside of the stock of the brush, if necessary, and secured by pins or rivets, or in any other convoniont manner, which ferrule may also form one side of the outer grove.

Fig. 264 is a plan riew of the stock of the round brush; fig. 263 is a section of the same; $a a$ are the dovetailed grooves which are turned out of the wood; $b$ is the metal forrule; $c c$ are knots, or small buadles of hair, to form the brush. After a number of the knots of hair are prepared, the ends aro to be dipped into proper cement, and then placed into the grooves, when their ends are to be squeezed by a pair of pliers, or other means, which will compress them into the oval shape, as shown in fig. 264, and cause the ends of the hairs to extend outward under the dovetailed part of the recess.

The knots of hair are to be successively placed in the grooves, and forced up by a tool against the last knot putin, and so on, until the grooves are filled; fig. 262 is a brush with teeth or threads of a screw formed upon the sides of the groove; into these teeth or threads the cement and hairs will be forced by the compression, by which means they will be held firmly in the stock of the brush.

BRUSBrTz. A hydrated phosphate of lime occurring in the guano of Aves Island and Sombrero in the Caribbean Sea, and named after Professor Brush, of Yale Colloge, U.S.

mRUSH ORs or BIACK BRUsz. An iron ore found in the Forest of Doan.

BRUSH wrymss. In light machinery, wheels are sometimes made to turn each other by means of bristles fixed in their cireumference; these are called brush wheels. The term is sometimes applied to wheels which move by their friction only.

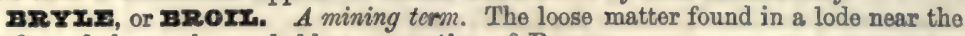
surface of the earth; probably a corruption of BeUHEYL.

BuCxrNG. A mining term. Bruising of the ore. A bucking iron is a flat iron fixed on a handle, with which the oro is crushed; and a bucking plate is an iron plate on which the ore is placed to be crushed.

BuCEING, or Bow bleaching. Ste Burachive.

BuCirram. A linen cloth of much strength, made very stiff with size.

ВUСхтиолж. (Rhanmus catharticus.) This plant is a native of England; it grows to the height of from 15 to 20 feet; its flowers are greenish coloured, and its berries four-seeded. It is the fruit of this plant which is sold under the name of French berries. The juice of these, when in an unripe state, has the colour of saffron; when ripe and mixed with alum, it forms the sap green of the painters; and in a very ripe state, the berries afford a purple colour. The bark also yields a fine yellow dye. See SAP GreKR.

The alder buekthorn (Rhamnus frangula) grows naturally, and is very abundant in woods and thickets in some parts of Britain. The berries of this species are often substituted for those of the above; but they are easily detected, since they contain only two seeds. In a green state, they dye wool green and yellow; when ripe, bluish grey, blue, and green. The bark also dyes yellow, and, with preparation of iron, black.-Lawson.

Rock buckthorn (Rhamnus saxatilis), the berries of which are used to dye moroceo leather yellow. These, in common with the narrow-leaved buckthorn berries $(R$. Clusii) and those of the yellow-berried buckthorn ( $R$. infectorius), are sold as Avignon berries. The wood of the Rhamnus erythroxylon (which is a native of Siberia, but grows freely in this climate), in a ground state, yields the bright red colour known to dyers under the name of red wood.

вUстwirzax. (Blé Sarrasin, Fr.; Bichweizen, Ger.) The common buckwheat (Polygonum Fagopyrum, from poly, many, and gonu, a knee, in reference to its numerous joints) is cultivated for feeding pheasants and other game; and is now being largely used in France and in this country in distilleries.

' In France, besides being used for feeding fowls, pigs, \&c., it is given to horses; and it is said that a bushel of its grains goes further than two bushels of oats, and, if mixed with four times its bulk of bran, will be full feeding for any horse for a wook. Its haulm, or straw, is said to be more nourishing than that of clover, and its beautiful pink or reddish blossoms form a rich repast for bees.'Lawson.

It has been stated that the leaves of the common buckwheat (Polygonum Fagopyrum), yield, by fermentation, Indigo-blue. On examining this plant, for the purpose of ascertaining whether this statement was correct, Schunck was unable to obtain a trace of that colouring matter; but he discovered that the plant contains a considerable 
quantity of a yellow colouring matter, which may very easily be obtainod from it. This colouring mattor crystallises is small primrose-yellow needles. It is very littlo soluble in cold water, but soluble in boiling water, and still more soluble in alcohol. Muriatic and sulphuric acid change its colour to a deop orange, the colour disappearing on the addition of a large quantity of water. It dissolves easily in caustic alkalis, forming solutions of a beautiful deop yellow colour, from which it is again deposited in crystallino needles on adding an excess of acid. It is, however, decomposed when its solution in alkali is exposed for some time to the air, being thereby converted into a yellowish-brown amorphous substance, resembling grm. Its compound with oxide of lead has a bright yellow colour, similar to that of chromate of lead. The compounds with the oxides of tin are of a pale but bright yellow colour. On adding protosulphate of iron to the watery solution, the latter becomes greenish, and, on exposure to the air, acquires a dark green colour, and appears almost opaque. The watery solution imparts to printed calico, colours, some of which exhibit considerable liveliness. Silk and wool do not, however, acquire any colour when immersed in the boiling watery solution, unless they have previously been prepared with some mordant. The composition of this substance in 100 parts is as follows:-carbon 50.00, hydrogen 5.55, oxygen 44.45 . Its formula is probably $\mathrm{C}^{30} \mathrm{H}^{20} \mathrm{O}^{20}\left[\mathbf{C}^{15} \mathbf{m}^{20} \mathbf{O}^{10}\right]$. It appears to be identical with Rutine, the yellow colouring matter contained in the Ruta graviolens, or common rue, and in capers; and with Mixanthim, a substance derived from the leaves of the common holly. From 1,000 parts of fresh buckwheat leaves, a littlo more than one part of the colouring matter may be obtained. As the seed of the plant is the only part at present employed, it might be of advantage to collect and dry the leaves, to be used as a dyeing material.-E. S.

The Tartarian Buckwheat (Polygonum Tartarium) differs from the former in having the edges of its seeds twisted. It is not considered so productive, but it is more hardy; and better adapted for growing in mountainous situations.

The Dyer's Buckwheat. (Polygonum tinctorium.) This plant was introduced to the Royal Gardens at Kew by Mr. John Blake, in 1776. Authentic information as to its properties as a dye-yielding plant was only received at a comparatively recent period, from missionaries resident in China, where it has always been cultivated for its colouring matter. In Europe, attention was first directed to its growth by M. Delille, of the Jardin du Roi at Montpellier, who in 1835 obtained seeds from the Baron Fischer, Director of the Imperial Gardens at St. Petersburg. It has since that time become sufficiently valuable to render its cultivation as a dye-drug of sufficient importance. The Japanese are said to extract blue dyes from Polygonum Chinensis, P. barbatum, and the common roadside weed, $P$. aviculare.-Lawson.

aUddze. See Dressing Oris.

BUDDIIirc. A mining term. The process of separating the metalliferous ores from the earthy matters with which they are associated, by means of an inclined hutch, called a buddle, over which water flows. It is indeed but an arrangement for availing ourselves of the action of flowing water to separate the lighter from the heavier particles of matter. See Dressing Orrs.

BUDE IIGrT. A highly illuminating flame invented by Sir Goldsworthy Gurney, of Bude, in Cornwall. Oxygen gas is driven through the centre of an argand flame of ordinary coal-gas, by which an intense combustion is established, and hence the high illuminating power is obtained.

BUFI IFATHFR. A leather propared with some albuminous substance and oil. See Tieather.

BUFr STrCrs. Pieces of wood upon which buff leather is fastened; they aro usod for polishing.

BUCx: A kind of bead used for ornamenting dresses.

BUEI. Buhl-work consists of inlaid veneers; and differs from marquetry in being confined to decorative scrollwork, frequently in metal, while the latter is more commonly used for the representation of flowers and foliage. Boule, or Buhl, was a celebrated cabinet-maker in France, who was born in 1642 and died in 1732. $\mathrm{He}$ was appointed 'Tapissier en titre du Rei,' and he gave his name to this peculiar procoss of enlaying wood with either wood or metal. Seo Marquetry, Parquetry.

BUMR-STON varieties of the quartz family. It forms a celebrated grit-stone, much used in France and other parts of the Continent for grist mills. Those of La Ferté-sous-Jouarre (Seine-et-Marne) are regarded as superior to all others. In consequence of the necessity for carefully piecing theso stones together, they are naturally expensive; yet the demand for buhr-stones continuos great. Written also Burr and BerRH.

BUxz or BUCK musIrr. A clear muslin woven for tambour working, and used principally for ladies' dresses. It is often called 'book' muslin.

מUIX-DOG. A name given to an irọn slag. See Iros. 


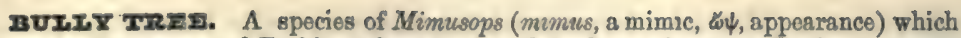
grows in Demerarn and Borbice, where it is used for house frames and other purposes in building, also for the spokes of wheels. It may be obtained nearly 30 feet long, and squares about 25 inches.

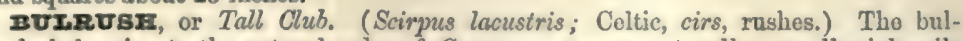
rush, belonging to the natural order of Cyperacece, grows naturally on alluvial soils which are occasionally covered with fresh water. A specios of Typha (Natural Order, Typhacee), is also known commonly as bulrush. It is much used by coopors for putting between the staves of barrels, and by chair-makers. Many other plants belonging to this order are employed for economical purposes, such as forming seats, ropes, mats, and fancy basket-work, also for thatching housos.

BUrv CH. A miner's term for an isolated mass of ore. A lode is seid to be bunchy when the metalliferous ore is found in irregular and sparsely distributed masses, the other portions boing made up of valueless earthy minerals.

Ruarcax. In mining. A lode in which the ore occurs in isolated masses scattered through it.

गU्यr C. A large cork for casks. The name in Persia for hemp, the Cannabis sativa.

Bunrary. In mining. A pipe of oro or a mass - not a vein or lode.

BUarTrarc. An open-made worsted stuff, used for making flags.

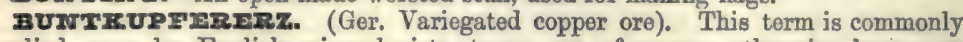
applied, even by English mineralogists, to an ore of copper, otherwise known us Erubescite, Phillipsite, Bornite, and Purple Copper Ore. By our Cornish miners it is generally known as Horse-Flesh Ore. See Copper, Ores of.

BURceurd PITCE. Burgundy pitch, whon genuine, is mado by melting frankinconse (Abietis resina) in water and straining it through a coarso cloth. Tho substance usually sold as Burgundy pitch is, however, common resin incorporated with water and coloured with palm-oil. In some cases American turpentine is employed. See PITcr and TAr.

BUrरmzs. Women are so called who are engaged in romoving from woollen cloths, with tweezers, all irregular threads or hair.

BURN FrT'S IIUID. A solution of ehloride of zine is commonly known as Sir William Burnett's disinfecting fluid. It is largely used as a powerful disinfectant.

2urvire I0usw. A miner's term. In Cornwall the kiln or oven in which the tin and other ores are placed to sublime the volatile constituents, sulphur and arsenic, is so called.

gurnt IEAvirgas. See Copper.

aURNT SUGar. Seo Caramet.

Burnov. A miner's term for a heap of rubbish.

zUsזs. The flat whalebones, or steel supports for the stays of women.

Bussorar cum. See Bassora.

BUTr. A large cask. The beer and wine butt should contain three barrels, or 108 imperial gallons. The wine butt formerly held 130 of the old wine gallons.

In the leather trade, a butt is a rounded crop, or a full hide.

zUrrsz. (Beurre, Fr.; Butter, Ger.) Butter is the fatty matter of mill, usually that of the cow. Milk is composed of butter, casoine, sugar of milk, soveral salts, and water. The butter exists in the form of vory small globules of nearly uniform sizo, quito transparent, and strongly refractire of light. Milk left in repose throws up the lighter particles of butter to the surface as cream. It was imagined that the butter was separated in the process of churning, in consequence of the milk becoming sour; but this is not the case, for milk rendered alkaline by bicarbonate of potash affords its butter fully more readily than aciduous mill. The best temperature for churning milk or cream is $53^{\circ} \mathrm{F}$.; that of $60^{\circ}$ is too high ; and under $50^{\circ}$ is too low. By the churning action the heat rises from $3^{\circ}$ to $4^{\circ} \mathrm{F}$. All the particles of butter are never separated by churning; many remain diffused through the butter-milk, and are easily discoverable by the microscope. These are more numerous in proportion to the bulk of the liquid; and hence it is more economical to churn cream than the whole milk which affords it. It is computed that a cow which gives 1,800 quarts (old English) of milk per annum eats in that timo 8,000 lbs. of hay, and produces $140 \mathrm{lbs}$. of butter. Analysis shows that this weight of hay contains $168 \mathrm{lbs}$. of fat. The finest flavoured butter is obtained from milk churned not long after it is drawn; but the largest proportion is derived from the cream thrown up by milk after standing 24 hours in a temperature of about $50^{\circ} \mathrm{F}$. Tho butter-milk, which contains the very fermentable substance, caseine, should be well soparated from the butter by washing with cold wator, and by beating with the hands, or preferably, without water, for the sake of fine flavour, by the action of a press.

The Tartars and French have long been in the habit of preserving butter, by 
melting it with a moderate heat, whereby are coagulated the albuminous and curdy matters remaining in it, which are very putrescible. This fusion should be made by the heat of a warm bath, about $176^{\circ} \mathrm{F}$, continued for some time, to effect the more complete purification of the butter. If in this settled liquefied state it be carefully decanted, strained through a tammy cloth, and slightly salted, it may be kept for a long time nearly fresh, without becoming in any degree rancid, more especially if it be put up in small jars closely covered.

In Cornwall and Western Devon, the first process in the manufacture of butter is the formation of the cream by heat. After the milk has stood for from twelve to twenty hours at rest, so that the cream globules may rise to the surface, it is exposed to a vory slow heat, until it aequires a temperature of about $200^{\circ} \mathbf{F}$.; care being taken that, if it exceeds this, the milk never boils. The pelliclo of cream heing formed, the vessel is removed from the fire, and allowed to cool; when cold, the butter is made from this cream in the usual way.

When subjected to mieroseopieal examination, milk is found to consist of infinitely minute globular particles floating in a serous fluid. Raspail says the largest of these globules are not above 1-2500th of an inch in diameter. These globules consist essentially of buttor. In the East, butter is preparod for keeping by carefully melting it over a very slow fire, and removing the scum as it rises. Thenard recommends this process, but directs that it should be carried out by the use of a water-bath. If good fresh butter is wrapped in a piece of linen and plunged into a strong brine, it will keep good, without saltness, for a long period.

Butter is preserved by salting in Ireland, in Holland, and in the Channel Islands. In 1872 we exported 56,322 cwts., of the value of 327,4316 . - of which 23,431 ewts. were sent to Brazil. See Mruk.

aUTTERs, MrNarAI. The old chemists called several of the metallic chlorides,--buttors; as butter of antimony, butter of tin, butter of bismuth, \&c.

RUTTER OI ANTrmony. An old name for the anhydrous chloride of antimony.

BUTTIR Or cacaO. Sec CaCaO.

ZUTTIR, VFerTABID. A greasy substance expressed from the kernels of the Bassia butyracea, a native of North India. This grease is said to make excellent soap. Shea butter is obtained from tho B. Parkii, of West Africa, and has been used in making candles and soap. The buttor-troe of Sierre Loone is the Pentadesma butyracea $(\mathrm{Br}$.), the fruit of which yields much grease, eaten by the negroes.

BUTYON MAATURACTURE. This art is divided into several branches, constituting so many distinct trades. Metal, horn, leather, bone, and wood, are the substances frequently employed for buttons, which are either plain, or covered with silk, mohair, thread, and other ornamental materials. The most durable and ornamental buttons are made of various metals, polished, or covered with an exceedingly thin wash, as it is termed, of silver or gold.

The buttons intended to be covered with silk, \&ce, are termed in general moulds. They are small circles, perforated in the centre, and made from those refuse chips of bone which are too small for other purposes. For the large and coarser buttons, pieces of hard wood, are sawn into thin flakes, of an equal thickness; from which, by a machine, the button moulds are cut out at two operations.

White metal and brass buttons, as well as plated buttons, are stamped by the flypress, out of copper-plate, covered on one side with silver at the flatting mill. The copper side is placed upwards in stamping, and the dye or hole through which they are stamped is rather chamfered at its edge, to make the silver turn over the edge of the button. The backs are stamped in the same manner as the gilt buttons. The shanks are soldered on with silver solder, and heated, one by one, in the flame of a lamp, with a blow pipe urged by bellows. The edges are now filed smooth in the lathe, care being taken not to remove any of the silver which is turned over the edge. They are next dipped in acid, to clean the backs, and boiled in cream of tartar and silver to whiton them; after which they are burnished, the backs being first brushed clean by a brush held against them as they revolve in the lathe. The mode of burnishing is the same as for gilt buttons. When the buttons are first cut they have exceed-

265

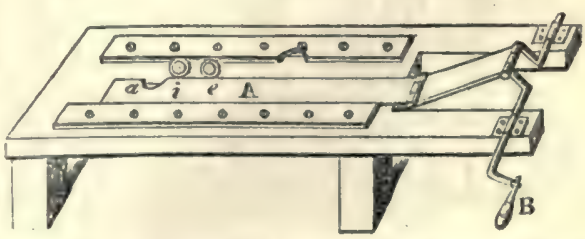
ingly sharp edges, to correct which and to produce a round, smooth, wire-like edge, they are rolled between two parallel pieces of stoel, one moving horizontally 
past the other, which is fixed, and both of them containing polished grooves on their corresponding faces. To the movable piece $\mathrm{A}$, fig. 265 , motion is given by means of the handle B. In both the grooved pieces, which are about eighteen inches in length, there are semicircular openings as at $a$, which, by corresponding once during each revolution of the hand, admit of the blank $e$ being dropped into the grooves, after which it is carried, revolving as it proceeds, between the pieces of steel, till coming to the hole $i$, it drops through into a basket. This operation is performed with amazing celerity by a boy, who drops the blanks into the cavity with his left hand, while he turns the handle with his right: they are now ready to receive the shanks.

Button shanks are made by hand from brass or iron wire, bent and cut by the following means :-

The wire is lapped spirally round a piece of steel bar. The steel is turned round by screwing it into the end of the spindle of a lathe, and the wire by this means lapped close round it till it is covered. The coil of wire thus formed is slipped off, and a wire fork or staple with parallel legs put into it. It is now laid upon an anvil, and by a punch the coil of wire is struck down between the two prongs of the fork, so as to form a figure 8 , a little open in the middle. The punch has an edge which marks the middle of the 8 , and the coil being cut open by a pair of shears along this mark, divides each turn of the coil into two perfect button shanks or eyes.

Buttons to be gilded are stamped out from copper (having sometimes a small alloy of zine), laminated in the flatting mill to the proper thickness. These circular pieces, called blanks, are annealed in a furnace to soften them; and the maker's name, \&c. is struck on the back by a monkey, which is a machine very similar to a pile engine. This stamp also renders the face very slightly convex, that the buttons may not stick together in the gilding process. The burnishing is performed by a piece of hrematite or blood-stone, fixed into a handle, and applied to the button as it revolves by the motion of the lathe.

A great number of the buttons, thus prepared for gilding, are put into an earthen pan, with the proper quantity of gold to cover them, amalgamated with mercury in the following manner:-The gold is put into an iron ladle, and a small quantity of mercury added to it; the ladle is held over the fire, till the gold and mercury are perfectly united. This amalgam being put into the pan with the buttons, as much aquafortis, diluted with water, as will wet them all over, is thrown in, and they are stirred up with a brush, till the acid, by its affinity to the copper, carries the amalgam to every part of its surface, covering it with the appearance of silver. When this is perfected, the acid is washed away with clean water. This process by the workman is called quicking.

The old process, in gilding buttons, called the drying-off, was exceedingly pernicious to the operator, as he inhaled the vapour of the mercury, which is well known to be a virulent poison. In order to obviate this, the following plan of apparatus has been employed with success:- The vapour, as it rises from the pan of buttons heated by a charcoal fire, is conducted into an oblong iron flue or gallery, gently sloped downwards, having at its end a small vertical tube dipping into a water cistern

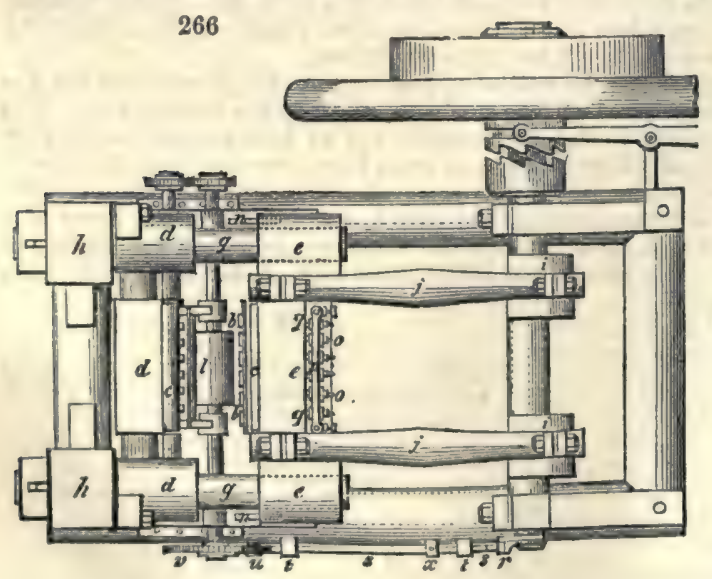
for condensing the mereury, and a large vertical pipo for promoting the draught of the products of the combustion. By act of parliament 5 grains of gold are allotted for the purpose of gilding 144 buttons, though they may be tolerable well gilt by half that quantity. In this last case, tho thicknesis would be about the 214,000th part of an inch.

Mr. Holmes of Birmingham patented a procoss which was fully described in the former edition of this work; a portion of the description is retained, especially such parts of his machinery as appear to be still in use. 
Having explained the peculiar form of his improved motallic shanks for buttons, and the tools employed in making tho same, he proceeds to describe the machinery or apparatus by which he carries his invention into effect. He takes a sheet of metal, say about 30 or 40 feet long, and of the proper width and thickness, which thin shoet is to be wound upon a roller, and placed above the machine, so that it can be easily drawn down into the machine as required for feeding the punches and dies. Fig. 266 is a planriow of a machine intended to work any convenient number of sots of punches and dies placed in rows. Fig. 267 is a side view, and fig. 268 a longitudinal section, taken through the $\mathrm{ma}$ chine ; figs. 269 and 270 are transverse sections taken through the machine between the pnnches and counter dies, fig. 269 representing its appearance at the face of the punches, and fig. 270 the opposito view of the counter dies. $a \boldsymbol{a}$ are the punches; $b \quad b$, the counter dies;

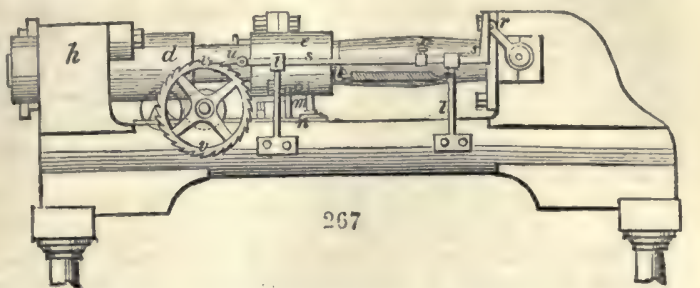
cach being mounted in rows in the steel plates $c c$, fixed upon two strong bars $d$ and $e$, by counter-sunk screws and nuts; the punches and dies being retained in their proper position by the plates, which are serewed $c$. to the front of the steel plates, and press against the collars of the punches and dies. The bars $d$ and $e$ are both
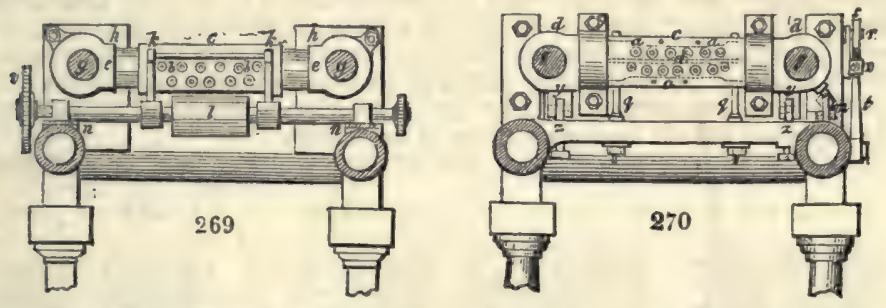

mounted on the guide-pins $g g$, fixed in the heads $h h$, of the frame, which guidepins pass through the bosses on the ends of the bars. The bar $d$ is stationary upon the guide-pins, being fixed to the heads $h h$, by nuts and screws passed through ears cast ou their bosses. The bar $e$ slides freely upon the guide-pins $g g$, as it is moved backwards and forwards by the crank $i$, and connecting-rods $j j$, as the crank-shaft revolves. The sheet of thin iron to be operated upon is placed, as before stated, above the machine; its end being brought down as at $a a$, and passed between the guide-rod and clearing plate $k$, and between the pair of feeding-rollers $l l$, which, by revolving, draw down a further portion of the sheet of metal between the punches and dies, after each operation of the punches.

As the counter dies advance towards the punches, they first come in contact with the sheet of metal to be operated upon; and after having produced the pressure which cuts out the discs, the perforations of the sheet are pushed on to the ends of the punches by the counter dies; and in order that the sheet may be allowed to advance, the carriage which supports the axles of the feeding-rollers, with the guide-rod and clearingplate, are made to slide by means of the pin $m$, which works in a slot in the slidingpiece $n$, bearing the axis of the feeding-roller $l l$, the slide $n$ being kept in its place on the framework by doretailed guides, shown in fig. 270.

When the counter dies have advanced near to the sheet of metal, the pin $m$ comes in contact with that ond of the slot in the pieco $n$ which is next to the punches, and forces the carriago with seed-rollers and clearing-plate, and also the sheet of metal, 
onwards, as the dies are advanced by the reaction of the cranks; and after they have cut out the dises, and raised the shanks, the shoot of metal will remain upon the punches; and when the bar $e$ returns, the finished backs and shanks are forced out of the countor dies, by the clearing-pins and rods 00 , which project through the bar $e$, and through the holes before mentioned in the counter dies; these clearing-pins being stationary between the bars $p p$, mounted upon the standard $q q$, on the cross bar of the frame, as shown in figs. 266, 268, 269. Immediately after this is done, the pins $m$ come in contact with tho othor ends of the slots in tho pioces $n$, and draw back the feeding-rollers $l l$, together with the clearing-plate $k$ and the sheet of metal, away from the punches, into the position represented in the figures.

At this time the feeding of the metal into the machine is effected by a crank-pin $r$, on the end of the crank-shafts coming in contact with the bent end of the sliding-bar s, supported in standards $t t$; and as the crank-shaft revolves, this pin $r$ forces the bar $s$ forward, and causes the tooth or pall $u$, on its reverse end, to drive the ratchet-wheel $v$, one or more teeth; and as the ratchet-wheel $v$ is fixed on to the end of the axle of one of the rollers $l$, it will cause that roller to revolve; and by means of the pair of spuxpinions on the other ends of the axles of the feeding rollers, they will both revolve simultaneously, and thereby draw down the sheet of metal into the machine. It will be perceived that the standards which support the clearing-plate and guide-bar are carried by the axles of tho feoding rollors, and partake of their sliding motion: also that the clearing-pins $o$, are made adjustable between the bars $p$, to correspond with the counter dies. There is an adjustable sliding-stop $x$ upon the bar $s$, which comes in contact with the back standard $t$, and provents the bar $s$ sliding back too far, and consequently regulates the quantity of sheet metal to be fed into the machine by the pall and ratchet-wheel, in order to suit different sizes of punches and dyes. In case the weight of the bar $c$, carrying the counter dies, should wear upon its bearings, the guidepins $g g$ have small friction rollers $y y$, shown under the bosses of this bar, which friction rollers run upon adjustable beds or planes, $z z$, by which means the guide-pins may be partially relieved from the weight of the bar $c$, and the friction consequently diminished.

Butross of Horn. - Mr. Thomas Harris obtained, in April 1841, a patent for improvements in the manufacture of horn buttons, and in their dies. His invention relates, first, to a mode of applying flexible shanks to horn buttons; secondly, to a mode of ornamenting horn buttons, by enlaying the front surface thereof; thirdly, to a mode of ornamenting what are called horn buttons, by gilding or silvering their surfaces; fourthly, to a mode of constructing dies, by applying separato boundary circles to each ongraved surface of a die, by which the process of engraving, as well as the forming of accurate dies, will be facilitated; fifthly, to a mode of constructing dies, used in the manufacture of horn buttons, whereby the horn or hoof employed will not be permitted to be expressed beyond the circumference of the button.

Fig. 271 represents, in section, a pair of dies, $\Delta$ and $B$, used in producing the

271
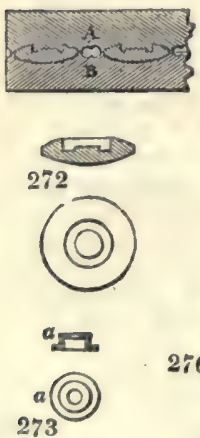

$c 0$

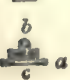

277
274
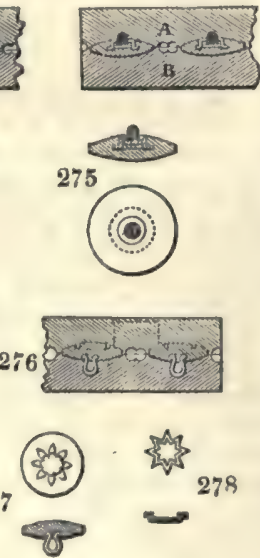

278
273

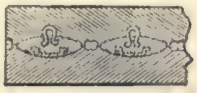

280

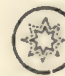

231

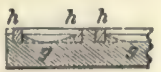

282

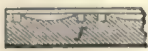

283

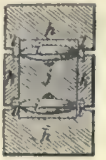

284
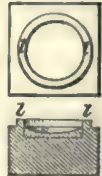

285
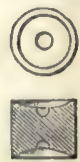

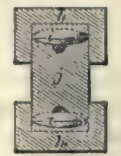

286

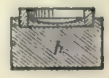

287

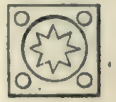

288

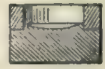

improved horn buttons, according to the first improvement; the upper die $\mathrm{A}$ is made to produce the back surfaces of the buttons, and the recess or groove for receiving the 
flexible shank. Fig. 272 shows, in section and back view, the form of a button produced by the dies.

Buttons thus formed are now ready to receive flexible shanks; and if the buttons are to have plain smooth front surfaces, then, in fixing the flexible shanks, the same kind of under die, B, may be used; but if the front surface of the button is to be embossed or ornamented, then, in place of that die, a similar one, having engraved or suitably ornamented surfaces, is to be used. When fixing the shanks to buttons, the lower or face die, containing the previously formed buttons, is to be heated till a drop of water will nearly boil upon it.

The shank is applied as follows :- a metal shell or collet $a$ (see fig. 273) is placed over the flexible shank $b$, and a plate of metal $c$ is laid under the shank; these are placed in tho groove or recess of the button, which had been previously heated in the lower die; the upper die $\Delta$ (fig. 274) is then to be placed on the lower die B, and the two submitted to pressure, until they become cool, when the shank will be firmly attachecl, as shown at fig. 275 , and the bottom may be finished in the usual way.

The second part of the invention, which relates to a mode of ornamenting horn buttons, by enlaying the front surface thereof, is performed in a manner similar to what has been above described, for fixing flexible shanks, and consists in first forming the front face or surface of a button, in suitable dies, for providing a recess ; and then, by a second-pressure in dies, to fix the ornamental surface; and, when desired, the surrounding front surface of the button may be embossed. Fig. 276 is a longitudinal section of a pair of dies, for forming a recess in the face of a button. Fig. 277 shows, in front view and section, a horn button produced by these dies. Fig. 278 shows a metal ornament, to be inlaid or fixed in the front surface of the button, but it should be stated that the ornamenting surface, to be fixed in the front surface of the button, may be of pearl or other material; and the size and device varied according to taste. Fig. 279 shows in section a pair of dies, for giving the second pressure for affixing the ornamental surface; and, if desired, the remaining front surface of the button may be ornamented, by having the lower die engraved, or otherwise suitably ornamented. Fig. 280 shows in front view and section a button made according to this part of the invention.

The third part of the invention relates to a mode of ornamenting horn buttons, by gilding or silvering their surfaces. This is effected by applying a suitable cementing or adhesive material with a soft brush tr the button, in order that gold- or silver-leaf may be attached to its surface. The cementing or adhesive materiai preferred to be used is dressing varnish rendered sufficiently liquid by essence of turpentine; and when the varnish is nearly dry, gold- or silver-leaf is applied thereto, and pressed in the same manner as practised when gilding and silvering other surfaces; by thus treating horn buttons a very novel manufacture of that description of buttons may be produced.

The fourth part of this invention relates to the construction of dies used in the manufacture of horn buttons. Fig. 281 is a section of a die, constructed according to this part of the invention; and fig. 282 is a section showing the die without the bounding circles, which confine the patterns ; $f$ is the die engraved at the parts $g g$; around each of which engraved surfaces are circular grooves or recesses to receive the bounding circles $h h$, which fit accurately. By the after-insertion of these circles, the workman is not confined to move his graver within the bounding line, as that line is not present when engraving the plate; and the graver may pass beyond, and the grooves and the bounding circles may readily be made with great accuracy to each of the engraved surfaces.

The fifth part of the invention also relates to a mode of constructing dies for the manufacture of horn buttons, and consists in forming the dies so that the bounding circle shall bo a sufficient depth for the counter die to slide within it, and fit accurately in order that the circumference of each button shall be smoothly and accurately formed. Fig. 283 represents in section two dies, and one counter die, made according to this part of the invention; fig. 284 shows one of the dies in plan and section; and fig. 285 a plan and section of a counter die suitable for flexible shank buttons. $h h$ are the dies, having the engraved surfaces $i i$ on separate circular dises of metal, such as have heretofore been used; $j$ is a counter die, and $k$ a tube within which the counter die is held, the object of this tube being to guide the projecting edges $l l$ of the dies as shown, and thus keep the dies and counter dyes correct to each other. Fig. 286 is a section of two dies $h$, and a counter die $j$; but in this case the tube $k$ is dispensed with, the dies being deeper sunk, and thus guiding the counter die correctly. By the use of theso dies, the edges of horn buttons will be more accurately formed, and consequently requiro less finishing. This description of dies may be made according to the mode described in the fourth part of this invention; that is, by forming the boundary circle separately, as will be understood by reforring to fig. 287 , which is a 
side-section of a die complete, with its boundary circle formed in a similar manner to that describod above. Fig. 288 represonts, in plan and soction, a variation in the means of affixing a separate bounding circle to each engraved surface; and it is suitable for working without the tubo. In using these dies they are to be hoated but slightly, whether for buttons with metal shanks, or to roceive flexible shanks, and are to bo pressed as heretofore. The patenteo claims, first, the mode of manufacturing horn buttons with flexible shanks, by first forming buttons by pressure and heat, and then by a second pressure in dies, to affix flexible shanks thereto, as above described. Secondly, the mode of ornamenting horn buttons, by causing suitable surfaces to be affixed in the front surfaces, by pressing the buttons with the ornaments in dies, as above deseribed. Thirdly, the mode of ornamenting horn buttons by gilding and silvering their surfaces as described. Fourthly, the mode of constructing dies used in the manufacture of horn buttons, by applying separate bounding circles to erch engraved surface, for a button; and fifthly, the mode of manufacturing horn buttons in dies, wherein the horn or hoof is preventod from being expressed at the circumferonce of the buttons as described.

Butrons, Covered.-Mr. Joseph Parkes obtained in 1840, a patent for improvements in the manufacture of covered buttons made by dies and pressure, by the application of horn as a covering material. The process resorted to by the patentees for carrying out this invention is very similar to that pursued in manufacturing Florentine buttons; such modifications being applied as are rendered necessary for adapting such process to the peculiar nature of the material employed for covering the face of each button. a (fig. 289) shows a plan of a dise of iron plato, with four projecting points,

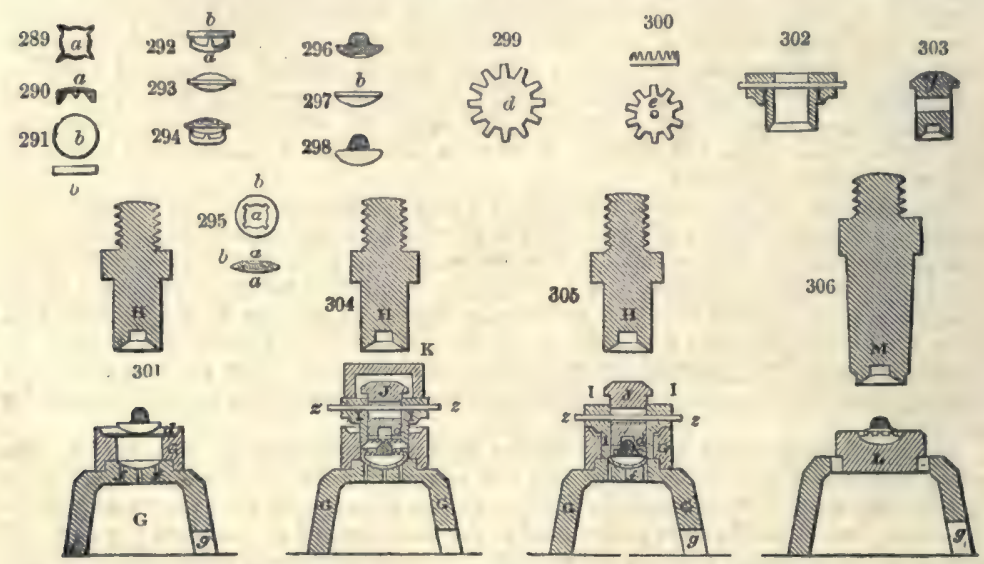

which is formed by suitable dies in a fly-press, as is well understood; the points aro then turned down, and the dise $a$ is sunk into the shape shown at fig. 290, and two such sunk discs are applied to the internal core of the button-board of each button: $b$ ( fig. 291) shows a plan and edge viow of a circular disc of button-board suitable for forming the internal core of a button.

The dies being placed in suitable presses, as is well understood in using similar dies in manufacturing Florentine and other covered buttons, one of the sunk dises $a$ is placed in the under die, with the points upwards, having a disc of button-board placed on the points, as shown at fig. 292; the upper die or punch is then caused to descend and press tho button-board $b$ into the shape shown at fig. 293 ; which, when thus formed, is to have a die $a$ applied on the other side, as shown at fig. 294. The disc $a$, to be next fixed to the button-board, is placed in a suitable die, the dise which has already been fixed being upwards; the die or punch is now to be pressed down, which will produce the button-board, with the discs $a a$, on either side, into the shape shown at fig. 295; and it will be seen, that one of the dises will by the shape of the die, be sunk concave, whilst the other disc $a$, on the other side, will be formed convex or according to the figure of the face of the intended button.

The core of button-board ( $f$ g. 295) is now ready to be inserted into the fabric which is to become the flexible shank of the button, and which flexible shank is formed by sinking a portion of fabric in suitable dies, as is well understood when making similar shanks in Florentine or other covered buttons; and the shank being 80 sunk, the button-board of core (fig. 295) is to be placed thereon, with the concave surface 
towards the protruding shank; and the edges of the fabric are then to be pressed over the core, as is well understood, which will produce the partly formed button ( fig. 296), which is a side view, and consists of the shank containing the core, which is next inserted into the metal shell $e$ ( fig. 297), and these parts being placed in a suitable die, are pressed together, and the partly manufactured button ( fig. 298) will be produced. consisting of the shank containing the core, corered on the front surface with the metal shell $c$, which, by the die, has its edges bent down on the fabric of the flexible shank. The button, thus far formed, is now in a condition to be covered with a thin plate of horn, which is performed in the following manner:- $d$ ( fig. 299) shows a dise of horn, cut out by suitable dies, the circumference being scolloped, in order that in folding over the mould (fig. 298) the horn may not be puckered. $e$ ( $f$ g. 300 ) shows a collet, for affixing the covering of horn to the button, the collet being similar to that used in what is called 'Sandar's plan of making Florentine and other covered. buttons.'

The method of covering the mould of the button with horn is described as follows :Fig. 301 represents, in section, a lower covering die, and also a proper punch for pressing the parts into the lower die; these dies being in a suitable press, as is well understood. The lower die is to be kept heated to such an extent that the workman can just bear his hand to rest, for a very short time, on the upper surface of the die; the heating is preferred to be accomplished by means of a flame of gas below the die; and it will be seen that there are holes $f f$, in the die, through which the heat of the flame may pass, and $g$ is an opening to allow of atmospheric air flowing under the lower die. The dise of horn $d$ is placed in the lower die $g$. The shape or mould (fig. 298) is then placed on the horn, and the punch or die $\mathbf{H}$, is caused to descend, and press the parts into the die $\mathrm{G}$; and the punch $H$ is then raised, in order to allow of the introduction of the parts shown at figs. 302 and 303, which consist of the tube $r$, and the punch or die $j$. The lower edge of the tube $r$ is made bell-mouthed, so as to cause the scolloped edges to be pressed on the back of the buttons, and the die or punch $j$ is to cause the collet to be forced through the horn in the button; and, in using these parts, the collet is placed in the tube $x$, which with its punch is inserted into the die $s$, as shown at fig. 304, which figure represents the die $\mathrm{G}$ and punch $\mathrm{H}$ in the condition just described, after having forced the parts into the die $\mathrm{G}$; and this figure also shows the tube $\mathrm{I}$, with a collet $d$ and the punch or die J placed in the tube I; and all things are in a condition to receive the pressure of the punch $\mathrm{H}$. In order to prevent the pressure coming on the punch or die $\mathrm{J}$ before the horn has been folded down by the tube $\mathbf{I}$, the hollow block $\mathbf{k}$ is $F_{\text {s }}^{2}$ ced over the die or punch $J$; consequently when the funch $H$ is caused to descend, it will force down the tuber, and cause it to gather the edges of the horn, and press them on the back of the mould of the button, when the punch $\boldsymbol{H}$ will be raised again, and the block removed, which will leave all things in the position shown at fig. 305 ; and then again, the bringing down of the punch $\mathrm{H}$ will cause the die or punch $\mathrm{J}$ to descend, and force the collet into the button, the die $\mathrm{s}$ being retained in the tube $\mathrm{I}$ by means of the pin $z$, passing through a slit formed therein, which allows of the die s rising and falling in the tube 1 , but prevents its coming out of that tube. The button, thus far formed, is now in a condition to be completed in the finishing dies ( $f g .306$ ), the lower dies being kept heated in a similar manner to the die c. The dies being fixed in a suitable press, the button to be finished is inserted into the die $\mathrm{I}$ (which may be ornamented or plain), with the shank upwards, and the punch or die $\mathbf{M}$ is caused to descend and press the button into shape.

When the front of the button is to be plain, the disc of horn should be polished before being used for covering; but when used to cover a button, and finished by an engraved or ornamented die, the polishing is not necessary. The button being thus made is to be finished by placing it in a lathe to be 'edged,' as is commonly practised in finishing horn buttons.

The patentee does not elaim the means of making the mould or shape shown at fig. 298, nor the dies employed when separately considered, very similar dies having been before used in the manufacture of other covered buttons; nor does he confine himself thereto, so long as the peculiar character and essence of the invention be retained; viz., that of manufacturing covered buttons, made by dies and pressure by the application of thin sheet horn as the covering material. He claims the mode herein described of manufacturing covered buttons by the application of horn as a covering material.

Porcelary Butrons. - These buttons were first manufactured by Messrs. Minton and Co. under Prosser's patent for the compression of dry porcelain clay in moulds (the process will bo fully described under the head Trsserse). Several houses have adopted the same process in this country. Mr. Bagaterasse has a largo ostablishment at Briare, where he manufactures these buttons on a large scale, and being in competition with 
Mr. Lobœuf of Breil, they aro sold very chenply. Bagatorasso has grontly improved the process by striking several hundred buttons at once, instead of doing them singly as by Prosser's original process. Seo Tilzs and Trssere:

In 1872 we imported of Buttons and Studs-not of metal-to the value of $134,015 l_{\text {. }}$; chiefly from Germany and Holland.

BUTTY. A miner who contracts to raise coal at a certain price per ton. He employs men to do his work, and they have usually an overlooker ongaged by the butty, and called tho 'Doggy.'

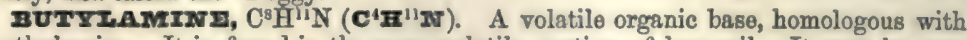
methylamine. It is found in the more volatile portion of bone-oil. It may be prepared artifically by processes analogous to those employed for methylamine, amylamine, \&c., substituting the butylic eyanate, urea, or iodine, for those of methyle and amyle. See Aarruansws- C. G. W.

מUTrתrC ACrD. A volatile fatty acid, discovered by Chevreul, who obtained it by saponifying butter with an alkali. Rancid butter owes its smell to the formation of this acid. See Watts's 'Dictionary of Chemistry.'

BUTYRIC ETHriss. Compounds formed by the direct action of butyric acid on alcohols. To the presence of small quantities of these others, the peculiar flavour of pine apples, melons, and some other fruits is due. Pine-apple rum owes its flavour to the presence of the butyrate of ethyl. 'A solution of butyric ether is very extensively used in perfumery, and in confectionery, under the name of pine-apple oil. It is prepared for this purpose by the following process :-Butter is saponified by a strong solution of potash-ley; the soap is dissolved in very little absolute alcohol, and to the solution is added a mixture of alcohol and sulphuric acid, until a strongly acid reaction is set up. The whole is then distilled; heat being applied ss long as anything comes over with a fruity odour.' See Watts's 'Dictionary of Chemistry.'

Buxưs. The box troe. The value of the box wood sent from Spain to Paris is given as 10,000 francs a year. The box-wood tree formerly grew abundantly at Boxhill, in Surrey. In 1815 the trees were cut down and produced upwards of 10,000l,-Baird. See Box Wood,

BUZzING, or BUSTLING. Terms sometimes applied to the process of working scrap iron in the charcoal hearth (Percy). See Iros.

CAmzacz вarz. The bark of the cabbage tree of the West Indies (Andira inermis), formerly used in medicine as a purgative and anthelmintic. It has been supposed that this tree furnishes the partridge-wood of the cabiret-maker.

CABBAGE PAIm. The young leaf-buds of this palm, the Areca oleracea, are boiled and eaton as a vegotable in India.

CABzIrNG. A term used amongst metallurgists. In Gloucestershire it is called Scabbling. Finery iron is smelted with charcoal, and when a soft mass of about two hundredweight is formed it is hammered out into a flat oval from two to four inches in thickness; this is allowed to cool, and is then broken up into small pieces, which is the process of cabbling or scabbling.

CAsnE. (Cable, Fr.; Ankertau, Ger.) A strong rope or chain, connecting the ship with the anchor for the purpose of mooring it to the ground. The sheet anchor cable is the strungest, and is used at gea after the bower, which is in constant use, goes, gives way, or requires help: the stream cable is smaller, being used chiefly in rivers. A cable's length is 100 to 140 fathoms in the merchant service; in the Royal Navy, 4 cables are employed each of 100 fathoms, 2 cables being attached end to end. The greatest improvement in mooring vessels has been the introduction of the chain cable, which, when duly let ont, affords in the weight of its long catenary curve, an elastic tension, and play to the ship under the pressure of the wind. The dead strain upon the anchor is thus greatly reduced, and the sudden pull, by which the flukes or arms are readily snapped, is in a great measure obviated. The best iron cables are chains made of links, whose sides are stayed by cross bars or stud, welded across the middle of the link. Experience has taught that the ends of these links wear ont much sooner than the sides. To remedy this evil, Mr. Hawkes, iron manufacturer, obtained a patent in July 1828, for constructing these anchor chains with links considerably stouter at the ends than in the middle. With this view he forms the short rods of iron, of which the links are to be made, with swells or protuberances about one-third of their length from each of their ends, so that when these are welded to- 
gether, the slenderer parts are at the sides, and the thicker at the ends of the elliptic links. Such rods as the above are formed at once by rolling, swaging, or any other means; but in practice, this plan has not been extensively carried out; the simple round iron soems best.

The first avowed proposal to substitute iron cables for cordage in the soa service is stated to have been made by Mr. Slater, surgeon of the Navy, who obtained a patent for the plan in 1808, though he does not seem to have had the means of carrying it into effect $\rightarrow$ s very general misfortune with ingenious projectors. It was Lieut. Samuel Brown, of the Royal Navy, who, in January 1808, had represented to the Naval Boards the policy of employing iron rigging and chain cable, and who, in February of that year, enrolled a patent for those articles, and in 1811 first employed chain cables in the vessel 'Penelope,' of which he was commander, for the purpose of experimental experience, this vessel of 400 tons having been fitted expressly for the trial by Captain Brown, Mr. Bruton, and other friends, at personal expense and risk, with iron rigging, chains, and cables, in place of hemp and rope.

He made a voyage in this ship from England to Martinique and Guadaloupe and home again, in the course of four months, having anchored many times in every variety of ground without any accident. $\mathrm{He}_{e}$ multiplied his trials, and acquired cortain proofs that iron might bo substituted for hemp in making cables, not only for mooring vessels, but for the standing rigging. Upon his return from the West Indies, Captain Brown strongly represented the advantages practically experienced of iron over hemp. A committee of naval officers reported upon the whole affair, and the Government ordered the 'Namur' of 74 guns, the 'Monmouth' of 64 , the 'Crescent' frigate, and the 'Alonzo' sloop, to be fitted with two chain cables of 100 fathoms each, and Lieut. Brown was promoted to the rank of captain. These chains were of various forms of links; those, for instance, supplied to the 'Crescent' were composed of very short links with parallel sides.

Since this period, chain cables have been universally introduced into all the ships of the Royal Navy; but the twisted links employed at first by Brown have been replaced by straight ones, stayed in the middle with a cross rod, the contrivance of Captain Brown, which was socured by patent in this country.

The twisting of the links was done in order to assimilate the chain to the form of rope, and for the purpose of making it run out with less concussion to the ship; but this in practice was found really to let the cable render out too easily, and was discontinued in practice.

Some of the cables supplied in 1811 to the ships of war were found to have defective links ; their general use was suspended until the beginning of 1812, when Captain Brown invented a modo of shutting the link with a long scarf, and introduced a machine to put upon the chain any amount of strain that ought to be brought to bear, and thus ascertain defects of workmanship and material. This proving machine lod to the introduction of stay pins in the links; about the middle of 1812, chain cables were thus brought to great perfection, and very generally introduced into the Royal Navy.

The first thing to be considered in the manufacture of iron cable is, to procure a material of the best quality, and, in using it, always to keep in view the direction of the strain, in order to oppose the maximum strength of the iron to it. The best form of the links may be deduced from the following investigation:-

Let A B ( fig. 307) be a circular link or ring, of one-inch rod iron, the outer circumference of the ring being 15 inches and the inner 9 . If equal opposite forces be applied to the two points of the link $\mathbf{c} \mathbf{D}$, pulling $\mathrm{C}$ towards $\mathrm{n}$, and $\mathrm{D}$ towards $\mathrm{F}$, the result will be, when the forces are sufficiently intense, that the circular form of the link will be changed into another form with two round ends and two parallel sides, as seen in fig. 308. The ratio of the exterior to the interior periphery, which was originally as 15 to 9 , or 5 to 3 , is no longer the same in fig. 308. Hence there will be a derangement in the relative position of the component particles, and consequently their cohesion will be progressively impairod, and eventually destroyed. In fig. 307 , the segment $\mathbf{m}$ s of the outside periphery being equal to $\mathbf{3}$ inches, the corresponding inside segment will be $\frac{4}{5}$ ths of it. If this portion of the link, in consequence of the stretching force, comes to be extended into a straight

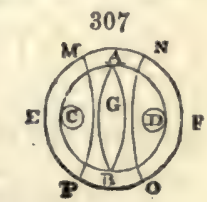

308

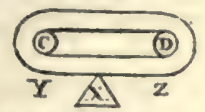
line, as shown in fig. 308, the corresponding segments, interior and exterior, must both he reduced to an equal length. The matter contained in the 3 inches of the outside periphery must therefore be compressed, that is, condensed into $1 \frac{4}{5}$ inch ; or the inside periphery, which is only $1 \frac{4}{5}$ inch already, must bo extended to 3 inches: that is to say, the exterior condensation and the interior expansion must take place in the 
reciprocal proportion. But, in every case, it is imposeiblo to effoct this contraction of one side of the rod and extension of the other, without disrupture of the link.

Let us imagine the outsido periphery divided into an infinity of points, upon each of which, equal opposite forces act to straighton the curvature; they must undoubtedly occasion the rupture of the corresponding part of the internal periphery. This is not the sole injury which must result; othors will occur, as we shall perceive in considering what passes in the portion of the link which surrounds CD (fig. 308), whose length is $4 \frac{1}{3}$ inches outside and $2 \frac{1}{10}$ th inside. The segments $\mathrm{M} P$ and $\mathrm{NO}$ ( fig. 307), are actually reduced to semi-circumferences, which aro inside no more than half an inch and outside as before. There is thus contraction in the interior with a quicker curvature or one of shorter radius than in the exterior. The derangement of the particles takes place here in an order inverse to that of the preceding case, but it no less tends to diminish the strength of that portion of the link; whence we may certainly conclude that the circular form of cable link is an extremely faulty one.

Leaving matters as we have supposed in fig. 307, but suppose that $\mathrm{G}$ is 8 rod introducod into the link, hindering its two opposite points a B from approximating.

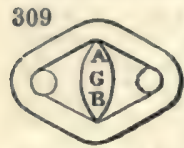
This circumstance makes a remarkable change in the results. The link, pulled as above described, must assume the quadrilateral form shown in fig. 309 . It offers more resistance to deformation than before; but as it may still suffer change of shape it will lose strength in so doing, and cannot therefore be recommended for the construction of cables which are to be exposed to very severe strains.

Supposing still the link to be circular, if the end of the stay comprehended a larger portion of the internal periphery, so as to leave merely the space necessary for the plan of the next link, there can be no doubt of its opposing more effectively the change of form, and thus rendering the chain stronger. But, notwithstanding, the circular portions which remain between the points of application of the strain and the stay would tend always to be straightened, and of consequence to be destroyed. Besides, though we could construct circular links of sufficient strength to bear all strains, we ought still to reject them, because they would consume more materials than links of a more suitable form, as we shall presently soe.

The effect of two opposite forces applied to the links of a chain is, as we have seen, to reduce to a straight line or a straight plane every curved part which is not stayed : whence it is obvious that twisted links, such as Brown first employed, even with a stay in their middle, must of necessity be straightened out, because there is no resistance in the direction opposed to the twist. A cable formed of twisted links, for a vessel of 400 tons, stretched 30 feet, when put to the trial strain, and drew back only 10 feet. This elongation of 20 feet proceeds evidently from the straightening of the twist in each link, which can take place only by impairing the strength of the cable.

Twisted cables are not now made, and but little of twisted chain. They were made to give the familiar form of rope to the chain, to please the sailors' prejudice.

From the preceding remarks, it appears that the strongest links are such as present in their original form, straight portions between the points of tension; whonce it is clear that links with parallel sides and round ends would be preforable to all others, did not a good cable require to be able to resist a lateral force, as well as one in the direction of its length.

Let us suppose that by some accident the link fig. 308 should have its two ex-

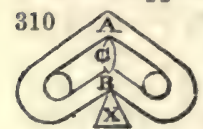
tremities pulled towards $\mathbf{Y}$ and $\mathrm{z}$, whilst an obstacle $\mathbf{x}$, placed right opposite to its middle, resisted the effort. The side of the link which touches $\mathrm{x}$ would be bent inwards; but if, as in fig. 310 , there is a stay, $\triangle \mathrm{G} \mathrm{B}$, the two sides would be bent at the same time; the link would notwithstanding assume a faulty shape.

In thus considering the vicious forms, we are naturally directed to that which has had at one time the preference, as is shown in fig. 311 ; but this form of link and

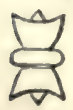

312

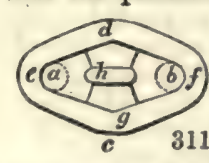

311 stay-pin is so faulty, as to give place now to the general use of the simple link of parallel sides (see fig. 312) and with a vory different stay-pin, as will bo shown hereafter. This old link has a cast-iron stay with large ends; it presents in all directions a great resistance to every change of form; for let it be pulled in the direction $a b$ against an obstacle $c$, it is evident that the portions $d e$ and $d f$, which are supported by the parts $g e$ and $g f$, cannot get doformed or be broken without the whole link giving way. As the matter composing ge and $g f$ cannot be shortened, or that which composes $d e$ and $d f$ be lengthened, these four sides will remain necessarily in their relative positions, by virtue of the large-ended stay $h$, whose profile is shown in fig. 314. Wo have examined the strength of a link in every direction, except that perpendicular to its plane. Fig. 313 represents the assemblage of three links in the 


\section{CABLE}

above predicament; but we ought to observe that the link c, placed between the links $\Delta \mathrm{B}$, could not renist the pressure or impact of the two lateral links.

Process of manufacturing Iron Cables.-The imploments and operations are arranged in the following order:-

1. The cutting, by a machine, of iron bars, in equal lengths, but with opposite bevels, to allow of the requisite crossing and splicing of the ends in the act of welding.

2. A reverberatory furnace, in which a number of

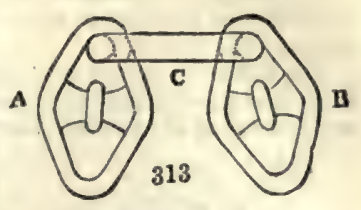
rods or round bars of the best possible wrought iron, and of proper dimensions, are heated to a red heat. The furnace is like those used in the sheet-iron works, but somewhat larger, and needs no particular deseription here.

3. The bending of each of these pieces by a machine, so as to form the links; the last operation is done rapidly while the iron is red hot.

4. The welding of the links at small forge fires, fitted with tools for this express purpose, and the immediate introduction of the stay, by a top tool and hammer.

5. Proving the cables by an hydraulic press, worked by a pump, with levers to ascertain the strain applied by working the pump.

Any ordinary shears will do to cut the iron, if furnished with a gauge or stop, to regulato the length of link.

The following forms of apparatus employed by the late Mr. Brunton and others, relate more to the history of the past manufactures, than to the present practice on a large scale.

Figs, 814 and 815 are a plan and elevation of the shears with which rods are cut

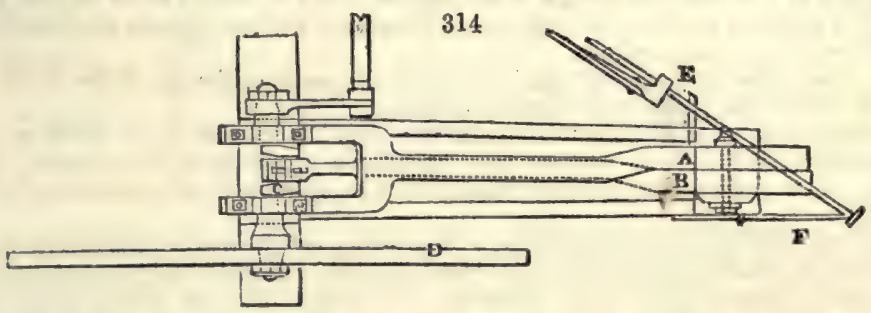

into equal pieces, for a link, moved by a steam-engine, or worked by four or more labourers. These must be relieved, however, frequently by others, for each shears machine is calculated to require nearly one horse in steam-power.

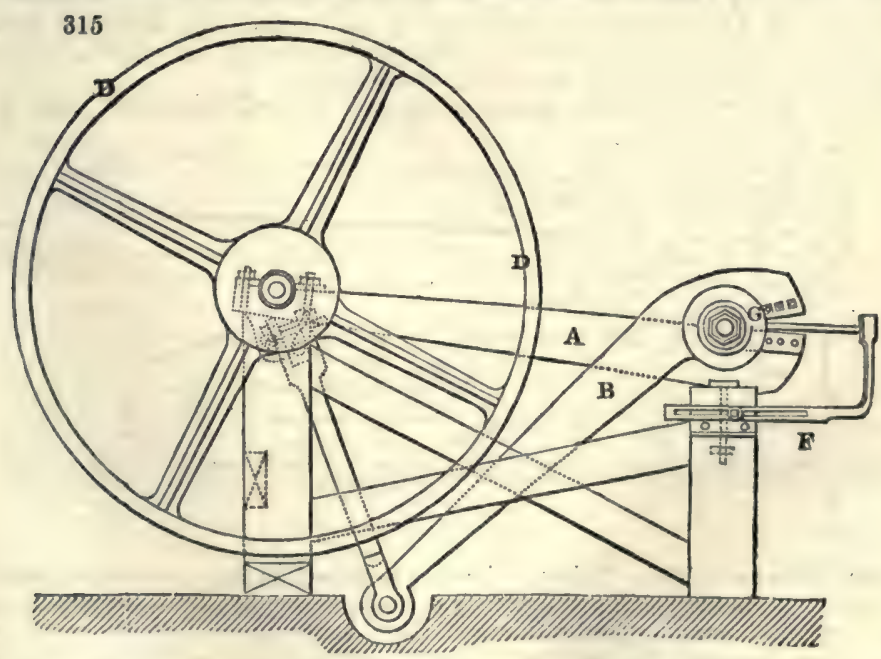

$\Delta$ and $\mathrm{B}$ are the two cast-iron limbs of the shears. The first is fixed and the second is movable by means of a crank-shaft $c$, driven by a heavy fly-wheel $\mathrm{D}$. The cutting VoL, I. 00 
jaws, a, aro of steel pieces made fast by bolts, and may be changed at pleasure. $F$ is a stop which dotermines the equal lengths of the pieces cut off, and can be shifted to suit different lengths: a piece of iron is shown as being cut off between the upright stop and the shears.

The following figures represent the plan and elevation of a machine for bending links into an elliptic form, superseded by tho machine hereafter to bedescribed. It is represented at the moment when a link is gotting bent npon it.
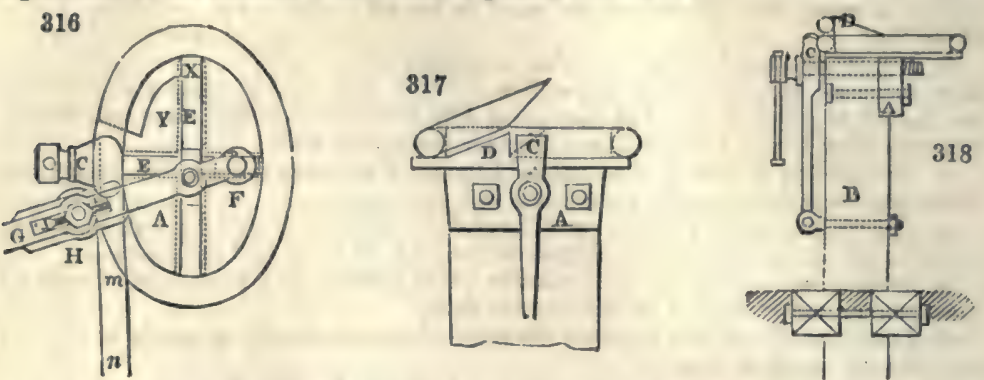

$\mathbf{A}$ is an elliptic mandrel of cast iron; it is fixed upon the top of a wooden pillar $\mathbf{B}$, solidly supported in the ground. $c$ is the jaw of the vice, pressed by a square-headed screw against the mandrel $\mathrm{A}$.

$\mathbf{D}$, part of the mandrel comprehonded between $\mathbf{x}$ and $\mathbf{y}$, formed as an inclined plane, so as to preserve an interval equal to the diameter of the rod between the two surfaces that are to be welded together.

x, rectangular slots (shears) passing through the centre of the nut of the mandrel, in which each of the pins $F$ may be freely slidden.

c, horizontal lever of wrought iron six feet long. It carries at $\mathrm{I}$ a pulley or friction-roller of steel, whose position may be altered according to the diameter of the links. It is obvious that as many mandrels are required as there are sizes and shapes of links.

The piece of iron intended to form a link being cut, is carried, while red hot, to the bending machine, where it is seized with the jaw of the vice $\mathbf{c}$, by one of its ends, the slant of the cut being turned upwards; this piece of iron has now the horizontal direction $m n$; on pushing the lever $\mathrm{G}$ in the line of the arrow, the roller $\mathrm{H}$ will force $m n$ to be applied succossively in the elliptic groove of the mandrel; thus, finally, tho two faces that are to be wivided together will be placed right opposite each other.

The length of the small diameter of the ellipse ought to exceed by a little the length of the stay-piece, to allow of this being readily introduced. The difference between the points $F \mathrm{E}$ is equal to the difference of the radii vectores of the ellipse. Hence it will be always easy to find the excentricity of the ellipse.

Fig. 319 is a lever press for squeezing the old form of links upon their stays

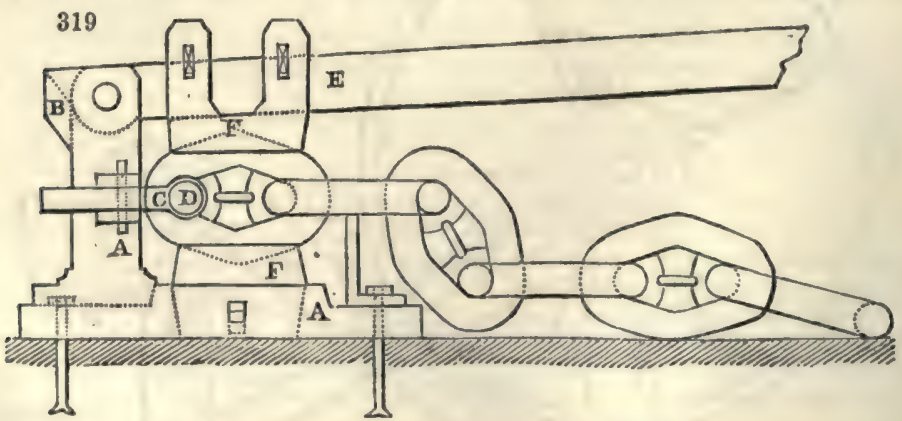

after the links are welded. This machine was contrived for the purpose of superseding manual labour, but the skill and dexterity of the workmen have quite superseded this machinery; however completely this and other machines may do the work, hand labour does tho work quicker and better, almost beyond comparison. The hand practice is as follows:-

The links bent are carried to the forge hearth to bo welded, and to receive their 
stay; two operations performod at one heating. Whonever the wolding is finished, while the iron is still red hot, the link is placed upright npon the stake, $i$. $e_{\text {. }}$, the shorter axis vertical and the longer axis of the link horizontal; then a workman introduces the stay with a pair of tongs or pincers; while another workman strikes down upon it. This mechanical compression first of all joins perfectly the sides of the link against the coneave onds of the stay, and afterwards the retraction of the iron on cooling increases still more this compression. If each link be made with the same care, the cable must be sound throughout. It is not delivered for use, however, till it be proved by the hydraulic press, at a draw-bench made on purpose, and examined link by link, on the side of the machine, or on a bench erected for the purpose, to detect any flaw the strain may have caused.

The following Table of compared materials and strains is given as a matter of historical eference. It is believed the dates of the experiments are 1815 and 1816 ; since then, alterations in the make of iron, and the introduction of new fibres, as well as hemp, render this Table of value, as the materials here employed were, no doubt, good exsmples, and subjected to eritical attention:-

Table of Iron Cables as substituted for Hemp, with the Strains applied at that Date.

\begin{tabular}{|c|c|c|}
\hline $\begin{array}{l}\text { Iron Cables } \\
\text { Diameter of Iron Rod }\end{array}$ & $\begin{array}{c}\text { Hemp Cableg } \\
\text { Circumference of Rope }\end{array}$ & $\begin{array}{l}\text { Old Proof, by } \\
\text { Mr. Branton }\end{array}$ \\
\hline $\begin{array}{l}\text { Inches } \\
0 \frac{7}{8} \\
1 \\
1 \frac{2}{9} \\
1 \frac{1}{4} \\
1 \frac{5}{16} \\
1 \frac{8}{6} \\
1 \frac{1}{1} \\
1 \frac{5}{6} \\
1 \frac{8}{4} \\
1 \frac{7}{8} \\
2\end{array}$ & $\begin{array}{l}\text { Inches } \\
9 \\
10 \\
11 \\
12 \\
13 \\
14 \text { to } 15 \\
16 \\
17 \\
18 \\
20 \\
22 \text { to } 24\end{array}$ & $\begin{array}{c}\text { Tons } \\
12 \\
18 \\
26 \\
32 \\
35 \\
38 \\
44 \\
52 \\
60 \\
70 \\
80\end{array}$ \\
\hline
\end{tabular}

It would be imprudent to put hemp cables to severer strains than those indicated in the Lloyd's Table, drawn up from experiments; but the iron cables of the above sizes will support a double strain without breaking. They ought never, in common cases, however, to be exposed to a greater stress. A cable destined for ships of a certain tonnage should not be employed in those of greater burden. Thus treated, it may be always trusted to do its duty, and will last longer than the ship to which it belongs. It has often been stated, that chain cables possess double the strength of the iron of which they are made, owing to the forms of the links employed : this, however, is an absurd error; for, suppose the two sides of a link to be of inch-iron, yet a part of the strength must be lost in the bending of the ends, for the straining force is at right angles, at the ends, to what it is at the side, or would be exerted upon portions of straight rods ; next, to make a link, the two ends have to be joined by welding, and wherever this join is made, there is every chance for less union, and no possible means of getting the fibre to be stronger than if they had never been separated; strength really must bo lost by heating the iron and shaping the link.

Mr. Lenox has found in practice, that an inch bolt will bear $21 \frac{1}{2}$ tons, while the inch cable will break at 34 tons, and not at the double strength, or 43 tons, of two lengths of straight iron.

One of the most valuable qualities of iron cables is their resisting lateral as well as longitudinal strains, as explained under figs. 311 and 313 .

Vessels furnished with chain cables have been saved by them from the most imm. nent peril. The 'Henry,' sent out with army stores during the Peninsular war, was caught on the zorthern coast of Spain in a furious storm. She ran for shelter into the Bay of Biscay among the rocks, where she was exposed for three days to the hurricane. She possessed fortunately 70 -fathoms chain cables, which held good all the time, but it was found afterwards to have had the links of its lower portion polished bright by attrition against the rocky bottom. A hemp cable would have been speedily worn to pieces in such a predicament.

In the contracts for the Admiralty of chain cables for the British Navy, it is stipulated that 'the iron shall have been manufactured in the best manner from pig iron smelted from ironstone only, and selected of the best quality for the purpose, and shall not have received in any process whatever, subsequent to the smelting, the admixture of either cinders or oxides produced in the manufacture of iron, and shall also have 
been puddled in the best manner upon iron buttons, and at least three times sufficiently drawn out at three distinct wolding heats, and at least twice properly fagotted.'

The following is a Table of the breaking proof of chain cables, and of the iron for the purpose of making them, and the proofs required by Her Majesty's Navy for chaiñts :-

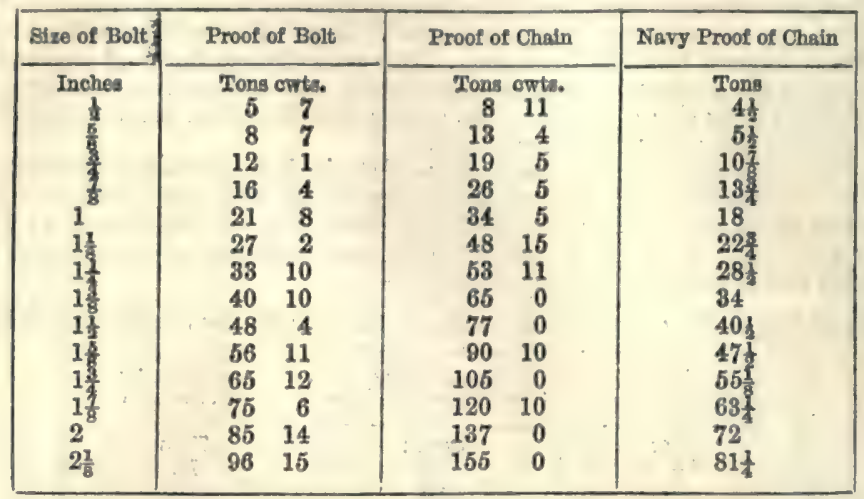

In these iron cables, the matter in the link is thrown very much into one plane; the link being of an oval form, and provided with a stay. As there are emergencies in which the cable must be severed, this is accomplished in those of iron by means of a bolt and sheckle (shackle), which is inserted in the Royal Navy cable at the end of every $12 \frac{1}{2}$ fathoms, and at the end of every 15 fathoms in the merchant service; so that by striking out this bolt or pin, this cable is parted with more ease than a hempen one can be cut. And the iron cable can be reconnected when the ship is clear, while with the hempen cable it would be necessary to cut it with an axe, and thus permanently injure the cable. Mr. Lenox's plan for securing these bolts is now made part of the Government contracts.

We have avoided all relating to the general history and application of chain cables, but in connection with the following particulars, obtained from Brown, Lenox, and Co.'s chain works at Millwall, we must admit the important part performed by this house in the improvement of this manufacture. The following remarks refer to chain cables for the Royal Navy, messenger and mooring chains for the Trinity Corporation, and ship cables for merchant service, showing the practice in 1858.

After selecting the best iron, cutting it off into required lengths, and heating it as before described, the links for chain cables may be bended at the rate of about 60 per minute, by machinery at Lenox's works, in Wales, worked by water-power, - the welding of the links in all cases being effected by hand labour.

In the practice with the bending machine at Newbridge works, Pont-y-Prid, Glamorganshire, it is as follows:- When the iron is cut to the requisite length for links, from 20 to 60 pieces, according to size, are put into the furnace, and when heated, are placed separately on the bending mandirel o (fig. 320) the machine is set in motion, and one revolution forms a link, which is pinched off the mandrel by a small crowbar, and another piece of iron applied, and so on, until from 40 to 60 links are formed in a minute.

The bending machine is connected with a water-wheel, or other power, by an ordinary coupling clutch, or box, which a lever throws into and out of gear at pleasure.

There is a stub or knob of iron on the mandrel under which the point of the piece of iron to be bent is fixed: the mandrel being oval, or of the inside shape of the link, when turned, is followed by the roller above, and this, pressing upon the piece of iron, forms it to the shape of the mandrel.

A B C ( fig. 320) are standards, D connecting-rod, x crank for lifting, F F the roller for pressing sides of links, $\theta$ mandrel, $\boldsymbol{H}$ mandrel spindle, $\mathrm{I}$ wheel for mandrel spindle, $\mathrm{J}$ pinion on main spindle, it crank spindle.

The form of the link, after being bended into shape (fig. 320) is shown with the two vilant-cut surfaces of the ends to be welded together and hammered into form.

For short lengths of chain the bending may be effected by hand; in this case the process is simple:-A sufficient length of the best iron is cut off, and, while hot, is partially bent by the workman over an iron ring, one end of the bar resting on the ground; the bend is finished upon the anvil; one entire end of the link is thus formed. 
The two slanting-cut ends are made to approach each other; heated up to a high temperature, the expert workman, by a peculiar blow, detaches the scales of oxide, and

320

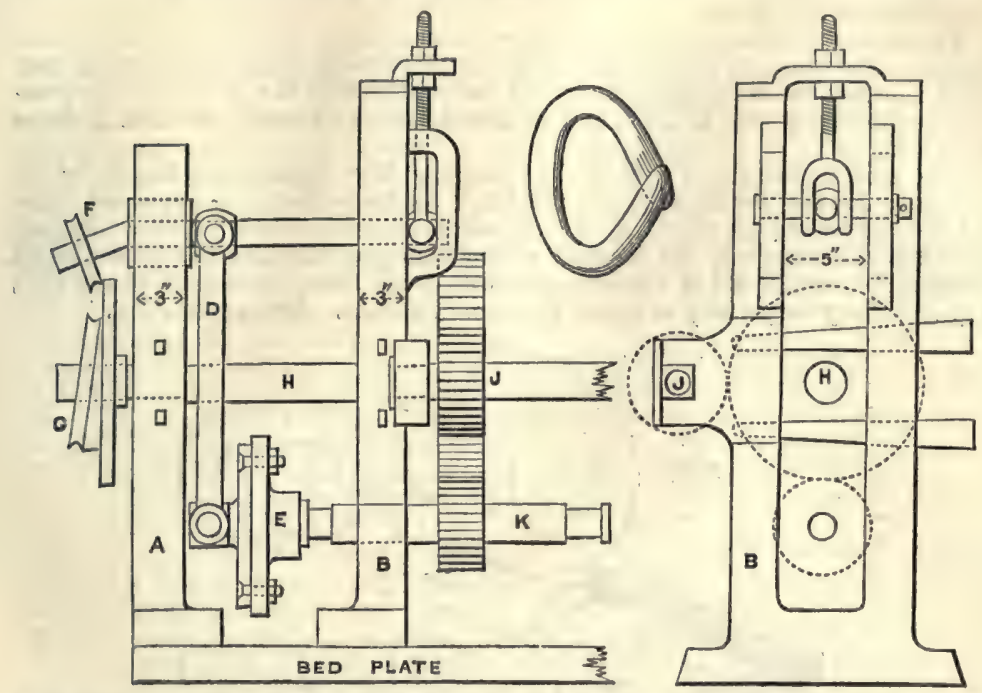

instantly presses both surfaces together; two men then by repeated blows effect the welding junction, and thus the link is formed.

The shape of the link, after due consideration of the advantages of particular patterns, seems to resolve itself into the decided preference for a link of parallel sides, unchanged in form from the round of the iron employed, while the ends may be reduced, somewhat flattened, and increased in breadth. The links thus in contact have the pressure sustained by a greater breadth of surface, and compression can scarcely alter the form.

The length of a good link may be of round iron 6 diameters in length of link A A (fig. 323), and from B to в 3.7 to 4 diameters of the iron rod employed, and 1.7 to 2 diameters inside.

The stub, staple, or cross-bit is of cast iron, and is placed across ; its use is to prévent the sides from collapsing by extension of the chain; in fact, to keep up a suecession of joints, and prevent the chain from becoming a rigid bar of metal.

The stud or cross-piece shown at $c$ is of cast iron, with dates and marks upon the surface. It is cast with a hollow bearing, having a curve to receive the round iron of the link; its shoulders, or feathering, enables the workman to insert it readily, and a few blows upon the yielding iron give the requisite grip; and all proper service only tends more firmly to keep it in position, very different indeed from the form fig. 312 which would positively injure the link.

In all cases this cross-piece has been of cast iron. Wrought iron was tried, but found to be too expensive. Malleable iron has been patented, but it is a question whether it can supersede common foundry iron, from the cheapness and facility of the latter.

The cables are proved and tested by regulated strains brought to bear continuously up to the proof strain, and then even up to the ultimate destruction of some of the links, if the final strength or opposition to resistance is required to be known. The proof of cable should be $600 \mathrm{lbs}$. for each circle of iron $\frac{1}{8}$ th of an inch in diameter.

The chain is attached at one end horizontally to a hydraulic press, the other end to the enormous heat of a bent iron lever, whose power is multiplied by second and third iron levers, all working upon knife-edges, and to the last lever a seale pan is attached; $1 \mathrm{lb}$. being here placed is equivalent to a strain of $2,240 \mathrm{lbs}$. upon the bar or chain that is being tested. This machine of Brown, Lenox, and Co., Millwall, is more powerful than that used in the Royal dockyard. The proving machine, invented by Captain Brown in 1813, was a great step towards the production of confidence.

In practice, length after length is tried up to the proof required; when the tension is to be exerted to the uttermost, a few links are taken: in such experiments it is usual for one link alone to give way, and the strength of the cable itself is uninjured by testing to find its ultimato strength. 
Perfection of practice is found when the link and the stay yield togother; in the largest chain cables ever produced, such were the due proportions and symmetry of form affording equality of resistance, that the cross-pieco split or broke at the time the link fractured and opened.

To measure these chains, or be near them when under such tension, is not without danger. The cable, on being struck, rings out with strange shrill sound, a link may suddenly snap, the chain lashes about, and the fragments fly to a great distance, penetrating the factory roof at times, and, at the moment of fracture, the link becomes very hot,

The cables are usually told-off into lengths. The Government length is $12 \frac{1}{2}$ fathoms; for the merchant service the length is 15 fathoms; as explained, these lengths are united by shackles. In the merchant service cable, larger links aro placed at each extromity for the anchor shackle to pass through, but in the Royal Navy cables, each length is alike provided with large links; thus, then, at any time, any end of any length may be placed to the anchor stock. See figs, 321, 322.

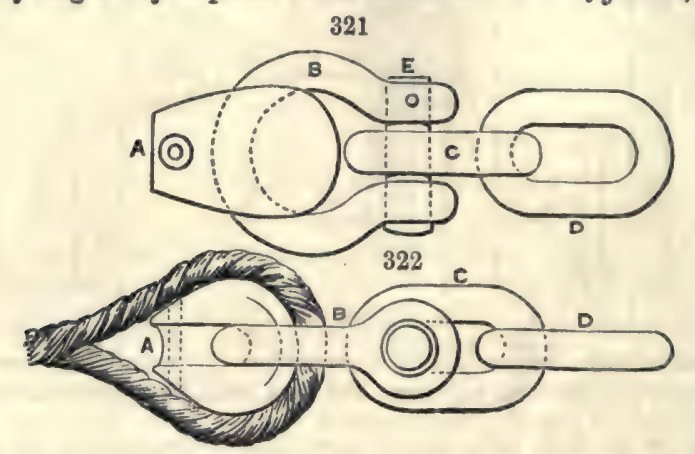

To obviate evils from the twisting of the chain cable, swivels are inserted: in the Government cables, a swivel is inserted in the middle of every other length; for the merchant service there does not appear to be any precise rule. Sometimes one, two, or more swivels may be in 100 fathoms; and in cheap chains, bought and judged by weight and figures, no swivel whatever exists in the cable.

The effect of such - twisting, or torsion, is to form a link, and give powerful lateral pressure upon the link; the stud or cross-piece is forced out, and the link itself may yield at the moment that any flaw from imperfection of welding occurs.

The mooring swivel is that by which a ship can ride with two anchors down at the same time and two bridles on board the ship. The mooring swivel, being equal in strength to the two cables, is over the bow, and enables the ship to swivel round her anchors without fouling hawse; in any direction the ship can swing round this swivel or point, leaving her anchors undisturbed, whereas by two cables out, without this, she would require great care to prevent them from fouling, and even being lost. This is an essential advantage of chain over hemp.

The splicing shackle is to unite or splice a hempen cable to be used on board ship, attached to the chain cable, which lies on the ground or bottom, so that the vessel rides lightly at her anchor, while the iron chain cable preserves the hempen cable from being destroyed by the rocky bottom, and the ship has the light hemp cable rendered buoyant by the water, which lifts portions of the chain cable by the motions of the vessel; and thus, the ship is relieved from weight and the anchor from jerks.

The splicing shackle, on the Hon. George Elliott's plan, is shown above (fig. 322). The rope is served round an iron thimble $\Delta$, on the shackle $B$, with end links, and onlarged links without stay-pins $\mathrm{C}, \mathrm{D}$, leading to the anchor, while the hempen cable A goes to the ship.

In the Royal Navy, 4 cables are employed to moor the ship, two being end to end.

When the ships lay long on certain shores, the pin or fastening often gets loose by constant tappings and vibrations of the chain cable on the rocky or shingly bottom. Men-of-war at some stations suffered severely in this way, and the commander at Malta had reason to represent it as a very serious matter. Mr. Lenox's plan for securing the bolts and pins is now made a point of contract to be adopted in all fastenings for the Royal Nary.

Simplo as it wonld seem to devise a plan, yet it was years before all the difficulties could be surmounted. This arrangement may be understood by reference to the 
figure of a shackle with links, ( fig. 323) : at $\mathrm{m}$ is seen the aperture at right angles to the bolt F, (of oval iron) through this channel, cut through the shackle and the bolt, a tapering but not quite cylindrical steol pin, fits exactly; but does not quite proceed through the iron; it is shown at gg. Various plans used to be resorted to before this final preference; for the stoel pins, of whatever form, got loose by repeated tapping on the rocky bottom, or the links upon each other. Mr. Lenox succoeded in cutting the cavity at $\mathrm{s}$ of the form of a hollow cone, and to complete the fastoning, a pollet or cylinder of lead that will just allow insertion at $\mathrm{E}$ is driven, and then by repeated blows the lead is made to fill up

323

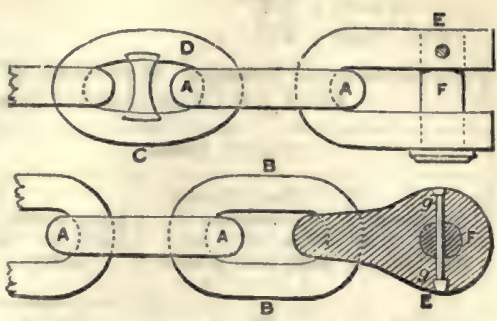
the cavity, the superfluous quantity of lead being cut off by the hammer at $\mathrm{B}$. To release the bolt it is only necessary to find the small space at the small end of the steel pin, to insert a punch, and then, with a fow blows, the steel pin $g g$ is driven out of its conical bearing, and its flat top and cutting edges enable it to emerge again at $\mathrm{k}$. Being forced out, the bolt $F$ is taken out, and the chain severed if required; the aperture at $\mathrm{z}$ can be cleared of its lead by a proper cutting-out tool, and the steel pin replaced to make all fast.

This operation can be effected on the darkest night; the sailor can sever the chain cable, and thus when one vessel is driving down upon another, more chain may be attached to the cable severed, and no harm done; while with hempen cable it might be found more than difficult, and even impossible, to cut them in time.

All the principles involved, and perfection of practice, in making chains and chain cables, have recently been deeply considered and fully verified by the firm of Brown, Lenox, and Co., Millwall, who, for the purpose of obtaining comparative results up to the greatest links required for the 'Leviathan'-now the 'Great Eastern,'-selected iron of the same identical quality and worked it into rods, links, and chains. The progression of resistance to increased strains, by increase of mass of iron, with all the influences of variation of make, flaws in the material, and other circumstances inseparable from practice, were thus mattors of critical experiment.

Commencing with $\frac{1}{2}$ inch chain, and trying four links of small chains up to $2 \frac{7}{8}$ ths, being the largest diameter of round iron for the greatest cable links ever hitherto made, being those for the sheet anchor of the 'Leviathan,' taking the breaking strains, and reducing all the links to the proportion borne upon a circle $\frac{1}{8}$ th of an inch in diameter, the minimum breaking force was $796.25 \mathrm{lbs}$, and the maximum $1052.8 \mathrm{lbs}$.

Sometimes the fracture was found to be dependent upon flaws, sometimes from over heating, or unequal heating, and other practical causes; but the whole series of experiments was important and interesting.

The iron lengthens to the intense strains employed, long before fracture. The comparison of actual extension, while under enormous force at ordinary temperatures was ascertained by the following impressive experiments :-

The 'Leviathan,' second size cable of $2 \frac{5}{8}$ diameters of iron employed in the links. Three links measured $35 \frac{1}{4}$ inches by strain of 10 tons (of course it requires power to extend them fairly).

And broko , 180,

\section{At 50 tons}

, 85, ,

, 110

(Proof)

" 124 "

, 140

, 150

, 160

" 170

A few links of the best bower anchor cable of the 'Leviathan' taken, proved, and destroyed.

Three links measured at 15 tons 39 inches.

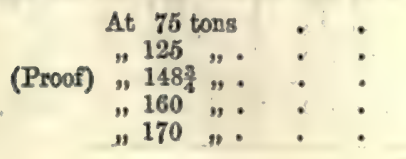

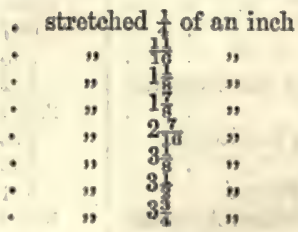


At 180 tons . . . . strotched $4 \frac{8}{8}$ of an inch.

,

" 190 , : : : : " $"$, 4 "

It bore ", 217 ,"

And broke " 218 "

Table showing the Prinoipal Dimensions of the Common Links, Weights, and Scale of Proof for Chain Cables to be supplied to Her Majesty's Navy.

\begin{tabular}{|c|c|c|c|c|c|c|}
\hline \multicolumn{3}{|c|}{ CoMnox IINTK } & \multirow{2}{*}{\multicolumn{3}{|c|}{$\begin{array}{l}\text { Weight of } 100 \text { Fathoms of } \\
\text { Cable in } 8 \text { Lengths, in. } \\
\text { oluding } 8 \text { joining Shackles } \\
\text { and } 4 \text { Swivels, not to be ex- } \\
\text { oeeded by more than to }\end{array}$}} & \multirow[b]{2}{*}{$\begin{array}{l}\text { Welghts by } \\
\text { which to be } \\
\text { proved equal to } \\
630 \text { lbs. per Cir- } \\
\text { cular t of Inoh }\end{array}$} \\
\hline \multirow{2}{*}{$\begin{array}{l}\text { Diameter of } \\
\text { Iron of Com- } \\
\text { mon Links } \\
\text { Inches }\end{array}$} & \multirow{2}{*}{ 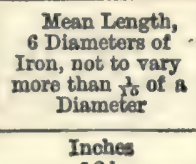 } & $\begin{array}{c}\text { Mean Wiath of } \\
\text { Links, 3.6 Dis- } \\
\text { meters }\end{array}$ & & & & \\
\hline & & Inches & Owts \& & & & Tons \\
\hline $2 t$ & $13 \frac{1}{3}$ & $8 \cdot 1$ & 243 & 0 & 0 & $91 \frac{1}{8}$ \\
\hline $2 \frac{1}{8}$ & $12 \frac{3}{8}$ & $7 \cdot 65$ & 216 & 3 & 0 & $81 \frac{y}{4}$ \\
\hline $2^{\circ}$ & $12^{\circ}$ & $7 \cdot 2$ & 192 & 0 & 0 & $72^{2}$ \\
\hline $1 \frac{7}{8}$ & $11 \frac{1}{4}$ & 6.75 & 168 & 3 & 0 & 681 \\
\hline $1 \frac{3}{4}$ & $10 \frac{1}{2}$ & $6 \cdot 8$ & 147 & 0 & 0 & $55 \frac{1}{8}$ \\
\hline $1_{\frac{h}{6}}^{b}$ & $9 \frac{3}{4}$ & 6.85 & 126 & 3 & 0 & $47 \frac{1}{2}$ \\
\hline $1 \frac{1}{2}$ & $9^{\circ}$ & $5 \cdot 4$ & 108 & 0 & 0 & $40 \frac{1}{2}$ \\
\hline $1 \frac{3}{3}$ & 84 & 4.95 & 90 & 3 & 0 & $34^{2}$ \\
\hline 1 & $7 \frac{1}{8}$ & $4 \cdot 5$ & 75 & 0 & 0 & $28 \frac{1}{2}$ \\
\hline $1 \frac{1}{a}$ & $6 \frac{5}{4}$ & 4.05 & 60 & 3 & 0 & $22 \frac{3}{4}$ \\
\hline 1 & 6 & $3 \cdot 60$ & 48 & 0 & 0 & 18 \\
\hline & 54 & $3 \cdot 15$ & 36 & 3 & 0 & $13 \frac{3}{4}$ \\
\hline & $4 \frac{12}{2}$ & $2 \cdot 7$ & 27 & 0 & 0 & $10 \frac{7}{8}$ \\
\hline & 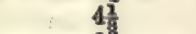 & $2 \cdot 467$ & 22 & 2 & 21 & $8 \frac{8}{2}$ \\
\hline & $8 \frac{3}{3}$ & $2 \cdot 25$ & 18 & 3 & 0 & \\
\hline$\frac{9}{16}$ & $3 \frac{3}{8}$ & $2 \cdot 025$ & 15 & 0 & 21 & $5 \frac{1}{2}$ \\
\hline$\frac{\pi}{2}$ & $3^{\circ}$ & 1.8 & 12 & 0 & 0 & $4 \frac{1}{2}$ \\
\hline$\frac{7}{10}$ & $2 \frac{5}{3}$ & $1 \cdot 575$ & 9 & 0 & 21 & $3 \frac{1}{8}$ \\
\hline
\end{tabular}

As the merchant marine may frequently be called upon for public service, it may be useful to know the particulars, which are promulgated from floyd's. A chain cable seldom breaks with the duty assigned, if proportioned to the tonnage, if the iron be oound and the workmanship good.

In 1871 and 1872 we exported of anchors, grapnels, chains, and cables as follows :-

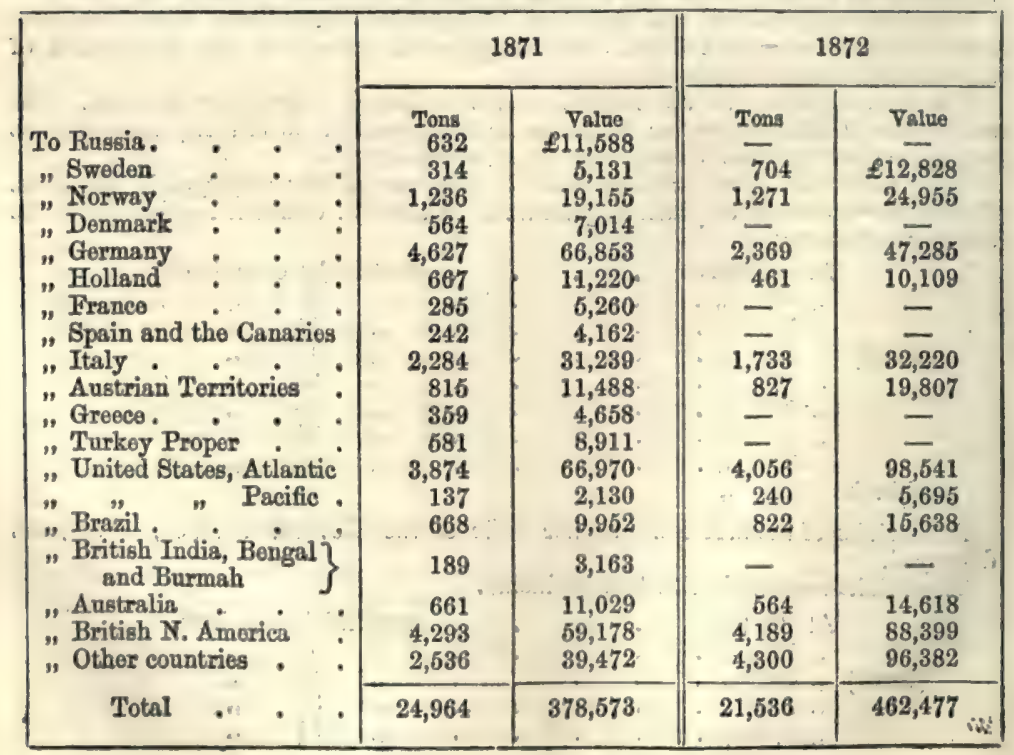


In the same years we exported of ' cordage, cables, and ropes of hemp or like material,' to nearly the same countries as above:-In 1871, 131,382 ewts., valued at $366,365 l_{\text {. }}$; and in $1872,139,083$ cwts., valued at $403,119 l$.

It is much to be regretted that we are unable to separate the anchors from the chains and cables.

Weights of Ordinary Anchors, Sizes and Lengths of Chain Cables, and Sizes and Lengths of Hawsers and Warps, to be recommended when the Surveyors are applied to by Ship Builders and Ship Owners.

\begin{tabular}{|c|c|c|c|c|c|c|c|c|c|c|c|c|c|c|}
\hline \multirow{3}{*}{ 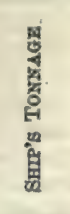 } & \multicolumn{8}{|c|}{ AxOHORS } & \multirow{2}{*}{\multicolumn{2}{|c|}{ CHATNS }} & \multirow{2}{*}{\multicolumn{4}{|c|}{ FAWAERS AND W $\triangle$ ARPS }} \\
\hline & \multicolumn{3}{|c|}{ Number } & \multicolumn{5}{|c|}{ Weight } & & & & & & \\
\hline & 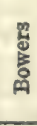 & 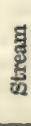 & 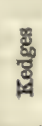 & 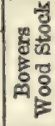 & 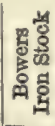 & $\begin{array}{l}\text { 竜 } \\
\text { 苟 }\end{array}$ & 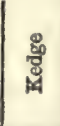 & 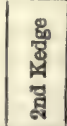 & 番 & 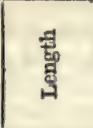 & 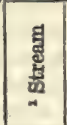 & 旡 & 参 & $\begin{array}{l}\text { 둉 } \\
\text { 莺 }\end{array}$ \\
\hline & & & & Owts & Wwts & Cwts. & Owts. & Owts. & In oh & Fathms. & Inch & Inch & Inch & \\
\hline 50 & 2 & 1 & 1 & 3 & 4 & $1 \frac{1}{2}$ & - & - & $\frac{11}{16}$ & 120 & 5 & 3 & - & \\
\hline 75 & 2 & 1 & 1 & 4 & 5 & $1 \frac{5}{4}$ & - & - & $\frac{10}{16}$ & 120 & 5 & 3 & - & \\
\hline 100 & 2 & 1 & 1 & 5 & .7 & $2 \frac{1}{2}$ & $1 \frac{1}{4}$ & - & $\frac{12}{36}$ & 150 & $5 \frac{1}{2}$ & 3 & - & है \\
\hline 150 & 2 & 1 & 1 & 8 & 10 & $3 \frac{1}{2}$ & $1 \frac{3}{4}$ & -1 & 10 & 180 & $6^{\circ}$ & 4 & - & $\&$ \\
\hline 200 & 3 & 1 & 1 & 10 & 12 & $4 \frac{1}{2}$ & 21 & - & i & 180 & $6 \frac{1}{2}$ & 4 & - & है \\
\hline 250 & 3 & 1 & 2 & 13 & 15 & $5^{2}$ & $2 \frac{7}{8}$ & - & $1 \frac{1}{8}$ & 200 & $7^{\circ}$ & 5 & - & ๕ \\
\hline 300 & 3 & 1 & 2 & 15 & 17 & 6 & $3^{2}$ & - & $1 \frac{7}{4}$ & 200 & $7 \frac{1}{2}$ & $5 \frac{1}{2}$ & - & 急 \\
\hline 350 & 3 & 1 & 2 & 17 & 20 & $6 \frac{1}{2}$ & 34 & - & $1 \frac{3}{8}$ & 240 & $7 \frac{1}{2}$ & $5 \frac{1}{2}$ & 一 & 4 \\
\hline 400 & 3 & 1 & 2 & 19 & 22 & $7 \frac{1}{2}$ & $3 \frac{3}{4}$ & - & $1 \frac{3}{8}$ & 240 & 8 & 6 & - & ద్ \\
\hline 500 & 3 & 1 & 2 & 23 & 26 & 9 & $4 \frac{7}{2}$ & - & $1 \frac{i}{2}$ & 270 & 9 & 7 & - & 18 \\
\hline 600 & 3 & 1 & $\overline{2}$ & 26 & 30 & 10 & $5^{2}$ & $2 \frac{i}{2}$ & $1 \frac{5}{8}$ & 270 & $9 \frac{1}{2}$ & 7 & 4 & 8 \\
\hline 700 & 3 & 1 & 2 & 29 & 34 & 11 & $5 \frac{1}{2}$ & $2 \frac{3}{4}$ & $1 \frac{3}{9}$ & 300 & $10^{\circ}$ & 8 & 5 & \\
\hline 800 & 3 & 1 & 2 & 31 & 36 & 12 & $6^{2}$ & 3 & $1 \frac{3}{4}$ & 300 & 10 & 8 & 5 & ㄱ. \\
\hline 900 & 3 & 1 & 2 & 33 & 39 & 12 & $6 \frac{1}{2}$ & $3 \frac{1}{4}$ & $1 \frac{3}{4}$ & 300 & 10 & 9 & $5 \frac{1}{2}$ & \\
\hline 1000 & 3 & 1 & 2 & 35 & 41 & 12 & $6 \frac{3}{4}$ & $3 \frac{7}{3}$ & $1 \frac{7}{8}$ & 300 & 10 & 9 & $5 \frac{1}{2}$ & \\
\hline 1100 & 3 & 1 & 2 & 37 & 44 & 12 & $7^{4}$ & $3 \frac{1}{2}$ & $1 \frac{9}{8}$ & 300 & 10 & $9 \frac{1}{2}$ & 6 & 营 \\
\hline 1200 & 3 & 1 & 2 & 39 & 46 & 12 & $7 \frac{1}{2}$ & $3 \frac{3}{4}$ & $1 \frac{7}{8}$ & 300 & 10 & $9 \frac{1}{2}$ & 6 & है \\
\hline 1400 & 3 & 1 & 2 & 41 & 48 & 12 & $7 \frac{3}{4}$ & $4^{4}$ & $2^{\circ}$ & 300 & 10 & 10 & 6 & 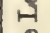 \\
\hline 1600 & 3 & 1 & 2 & 43 & 50 & 14 & $8 \frac{1}{4}$ & 4 & 2 & 300 & 11 & $10 \frac{1}{2}$ & $6 \frac{1}{3}$ & $\cong$ \\
\hline 1800 & 3 & 1 & 2 & 45 & 52 & 14 & $8 \frac{1}{2}$ & $4 \frac{1}{4}$ & $2 \frac{1}{8}$ & 300 & 11 & 11 & $7^{8}$ & $E$ \\
\hline 2000 & 4 & 1 & 2 & 47 & 54 & 14 & 9 & $4 \frac{1}{2}$ & $2 \frac{1}{8}$ & 300 & 11 & 11 & 7 & \\
\hline
\end{tabular}

The stream cables may be of iron, of proportionate sizes.

See Com Hramp, Wirs Rope.

CACAO (commonly, but improperly Cocos). The seeds of the Theobroma Cacao (or 'Food of the Gods,' as Linnæus named the tree). They are imported from the West Indies, the Caracas, from Brazil and some other parts of America. The seeds are oval, large as an olive, obtuse at each end. Simply torrified and bruised they constitute the Cocos of the shops, but frequently this is made from the fragments of the seed-coats, which impart to the preparation an astringent property. When the carefully prepared seeds are ground into a paste, sweetened, and flavoured with vanilla or cinnamon, it constitutes Crocosate (chocolata), which furnishes a nourishing beverage.

Cacao contains an active principle called theobromine, similar in its properties to theine and caffeine, the alkaloids in tea and coffee. A fatty or oily matter, called butter of cacao, is also present in the cocoa-seeds to the extent of one-half their weight.

In 1871 we imported of cocoa-seeds $17,368,302 \mathrm{lbs}$. of the value of $594,622 l$.; of the husks and shells, 10,533 cwts., valued at $11,030 l_{\text {. }}$; and of cocoa-paste or chocolate, $78,501 \mathrm{lbs}$., valued at 7,234l. And in $187215,044,134 \mathrm{lbs}$. of cocoa, valued at $467,144 l$. ; 8,725 cwts. of husks and shells, valued at 8,555l.; and of cocoa-paste or chocolate, $102,423 \mathrm{lbs}$. valued at $9,847 \mathrm{l}$.

From June 1853, the duty on the seeds has been $1 d$. per lb., on the husks and shells, 2s. per cwt, and on the paste or chocolate, $2 d$. per lb.

Cocoa or Cacao must not be confounded with Coca, a narcotic plant chewed by the Indians of South America, or Coco, one of the Aracece, the underground stems of which are used as food. Se Cocs and Coco.

CACHAIOT. A name for the sperm whale (Physeter macrocephalus). This whale yields sperm-oil, spermaceti, and ambergris.

CADmra. The Cadmiu Arabibus of old writers was the ancient name. of Calamine, the lapis calaminaris of the older chemists. Seo Watson's 'Chemical Essays.' 
cadmrum. (Symbol, Cd. ; Atomio weight, 56.) A motal discovered by Stromeyer about the beginning of the year 1818. It derives the name Cadmin from Cadmia fossilis - a denomination by which the common ores of zine were formerly distinguished. It occurs chiefly in Silesia in several ores of zine, and may be readily recognised by means of the blowpipe; for, at the first impression of the reducing or smoky part of the flame, the ores containing cadmium stain the charcoal all round them with a reddish-yellow circle of oxide of cadmium. The Silesian native oxide of gine contains from $1 \frac{1}{2}$ to 11 per cent. of cadmium. The only native compound of cadmium is the sulphide called Greenockite. Seo Grewarockrre.

Cadmium may be extracted by dissolving the ores containing it in sulphuric acid, leaving the solution acidulous, and diluting it with water, then transmitting through it a stream of sulphuretted hydrogen, till the yellow precipitate ceases to fall. The powder, which is sulphide of cadmium, is to be dissolved in concentrated muriatic acid, the excess of which is to be expelled by evaporation; and the salt being dissolved in water, carbonate of ammonia is added in excess; cadmium separates as a carbonate, while the adhering coppor or zine is retained in solution by the ammonia. Herapath has shown that, in distilling zine per descensum, the first portions of gaseous metal which are disengaged burn with a brown flame and deposit the brown oxide of cadmium. See Znvc.

Cadmium has the colour and lustre of tin, and is susceptible of a fine polish. Its fracture is fibrous; it erystallises readily in regular octahedrons: and when it suddenly solidifies, its surface gets covered with fine mossy vegetations. It is soft, easily bent, filed, and cut, soils like lead any surface rubbed with it. It is harder and more tenacious than tin, and emits a creaking sound when bent, like that metal. It is very ductile, and may be drawn out in fine wire, and hammered into thin leaves, without cracking at the edges. Its specific gravity, after being merely melted, is 8.604 ; and 8.6944 after it has been hammered. It is very fusible, melting at a heat much under redness; indeod, at a temperature little exceeding that of boiling mercury, it boils and distils over in drops. Its vapours have no smell. It is but slightly altered by exposure to air. When heated in the atmosphere it readily takes fire and burns with a brownish-yellow smoke, which is destitute of smell. In strong acids it dissolves with disengagement of hydrogen, and forms colourless solutions. Chromato of potash causes no precipitate in them, unless zine or lead be present.

There are two oxides of cadmium: a suboxide $\mathrm{Cd}^{2} \mathrm{O}$ and a protoxide CdO. The protoxide is a brown powder obtained by igniting the metal, or by heating its carbonate or nitrate. It is neither fusible nor volatile at a very high temperature. When in the state of a hydrate, it is white. This oxide of cadmium consists of 87.45 parts of metal and 12.55 oxygen, in 100 parts. The other, or suboxide of cadmium, is obtained by heating the oxalate of cadmium to about the melting point of lead. It is a green powder resembling oxide of chromium.

CADMrUM, AIIOYS Or. One hundred parts of copper at a red heat combine with eighty-two parts of cadmium. This alloy is of a yellowish-white colour. Platinum at a red heat will take up 117 parts of cadmium. This alloy is silver white, very brittle, and refractory in the fire. With mercury, cadmium forms a hard, brittle, white amalgam. These alloys have no commercial value or manufacturing interest.

Some of the alloys of cadmium are remarkable for their fusibility; thus, an alloy of 1 part cadmium, 6 parts of lead, and 7 of bismuth melts at $180^{\circ} \mathrm{F}$., or considerably below the temperature of boiling water. Alloys containing cadmium have been used for stopping teeth.

CADMrUa, sarTs or. Bromide, chloride, iodide, and sulphate of cadmium have been prepared and examined, but with the exception of the use of the bromide and iodide in photography, none of these salts are of any importance in the arts. The sulphate has been applied to the eyes by surgeons to remove specks on the cornea. -See Watts's 'Dictionary of Chemistry.'

CADMIUM YEIIOW or CADMIUN SUIPHIDa. The artificial sulphide of cadmium is prepared by fusing cadmium with sulphur, by igniting cadmic oxido (protoxide of cadmium) with sulphur, or it is precipitated in yellow flakes, as a hydrate, when sulphuretted hydrogen or an alkaline hydrosulphate is brought in contact with a cadmium salt. This forms the pigment cadmium yellow, or jaune brillant. The sulphide occurs native as Greenockite. See Gremenockite.

CAIn STOxrs. A variety of Oolitic limestone, which is largely quarried at Caen in Normandy, and which has been used for a long period in Gothic churches and other buildings. See OoIITIC Limmstosm.

CAzRUIFUM. A blue pigment, consisting of stannate of protoxide of cobalt, mixed with stannic acid and sulphate of limo.

CIFsrum. (Symbol, Cs.; Atomic weight, 133.) One of the new metals discovered by aid of the spectrum analysis in 1860 by Kirchoff and Bunsen. It has not boen 
found except in company with RuBrorox. Its name is derived from ocosius, 'skybluo,' which eolour it imparts to flame, and when its radiations are decomposed by a prism, it gives two blue lines, one rather faint in the middle of the blue space of the ordinary solar spectrum, and the other brighter, situated towards the violet end.

Cresium was first detected in the mineral water of Dürkheim, ten kilogrammes (about two pounds and a quarter) of which contained not quite two milligrammes ( 0.015 grain). It has sinee been detected in the waters of Kreuznach, and in those of Baden-Baden, Bourbonne-les-Bains, Haute-Marne; in the salines of Aussee; and in the lepidolite or lithium-mica of Zinnwald, of Rozena, and of Hebron in Maine, United States. But the most abundant source of crsium yet discovered is a rare mineral found in Flba, and known as Pollwx; Pisani has found as much as 32 per cont. of cosium in this mineral.

Puro metallic cæsium has not yet been obtained; but an emalgam of cresium can be procured by electrolysing a solution of chloride of cresium, using mereury as the negative pole. For the salts of cæsium, see Watts's 'Dictionary of Chemistry.'

CA is white, crystallisable in silky needles, fusible, volatile, and soluble in water, alcohol, and ether. It is identical with Theine and with Guaranin.

According to Robiquet, the proportion of caffeine in 1,000 of coffee is as follows :Martinique 6.4, Alexandrian 4.4, Java 4.4, Mocha 4, Cayenne 3.8, St. Domingo 3.2. It is probsble that 0.64 per cent. is an ordinary proportion. According to Liebig, the proportions are per lb.: Martinique 32 gr., Alexandrian 22, Java 22, Mocha 20, Cayenne 19, St. Domingo 16. To obtain eaffeine, H. J. Versman of Lubeck mixes 10 lbs. of bruised raw coffee with 2 of lime, made previously into hydrate; treats the mixture in a displacement apparatus with alcohol of $80^{\circ}$ till the fluid which passes through no longer furnishes evidence of the presence of caffeine. The coffee is then roughly ground and brought nearly to the state of a powder, and the refuse of the once digested mixture from the displacement apparatus, dried and ground again, and mixed with hydrate of lime, is once more macerated. The grinding is more easily. effected after the coffee has been subjected to the operation of alcohol, having lost its horny quality, and the caffeine is thus more certainly extracted. The bright alcoholic liquid thus obtained is then to be distilled, and the refuse in the retort to be washed with warm water to separate the oil. The fluid is now evaporated into a crystalline mass, filtered, and expressed. The impure caffeine is freed from oil by pressure between folds of blotting-paper, purified by solution in water with animal charcoal, and is thus obtained in shining white silky erystals. Seo Thense and Goaranin.

CArrzoxr. The aromatic principle of coffee is so called. It is a brown oil heavier than water, and slightly soluble in that fluid when boiling. It is prepared by distilling coffee with water, agitating the distillate with ether, and evaporating the other. Considerably less than a grain will flavour a pint or two of water.

CABOUN NOTS. The fruits of Attalea funifera, the A. cohune of Martius. The tree producing these nuts is a plume-like palm, a native of Honduras. The nuts grow in clusters like bunches of grapes. An oil is extracted from these nuts by $e x-$ pression equal to that of the cocoa-nut. See Cocon-Nut.

CAnrz. The Brazilian name for the American oil-palm, Elleis melanococca. Of its very long leaves the Brazilians make ropes, and its expressed oil is much used by them. See Fr.mis and Pary OrI.

CAIRIG ORM, or CAIruceorum, is the name generally applied to the more pellucid and paler coloured varieties of smoky quartz, with a tint resembling that of sherry or amber. It is so called from the district of Cairngorum, or the 'Blue Mountain,' in the south-west of Banff, where these crystals are frequently found. When of a good colour, this crystal is made into ornaments, and used for jewellery; indeed, so great a favourite is the Cairngorum with the people of Scotland, that brooches, pins, bracelets, and a variety of ornaments, are made with this stone, for use by all classes.

CAJUPUT orx is obtained from the leaves of the treo called Melalenca minor, which grows upon the mountains of Amboyna, and in other of the Molueca Islands. It is procured by distillation of the dried leaves with water, is prepared in great quantities in the island of Banda, and sent to Holland in copper flasks : hence, as it comes to us, it has a green colour.-Ure. M. Guibort appears to have detected copper in several samples of cajuput oil. Pereira says, "All the samples of the oil which I have examined, though green, were quite devoid of copper;' and Mr. Brande observes, that none of the samples which he has examined have contained even a trace of copper. It is very limpid, lighter than water, of a strong smell resembling camphor, and pungent taste like cardamoms. In 1831, oil of cajuput was greatly extolled as a remedy for cholera; and to meet the sudden and large demand, various adulterations and imitations were introduced. One of these consisted of oil of rosemary, flavoured with cardamoms or oil of cardamoms, and coloured. According to 
Blanchet, the composition of oil of cajuput is, carbon $77 \cdot 92$, hydrogen $11 \cdot 69$, oxygen 10.39. It is used in medicine as a stimulant. See Ors, Errmereous.

Carcrore COAI. A coal which fuses together when heated. All the caking coals are bituminous, and usually make good coke. See CoAt.

CAI or IRAI, sometimes CAIIIXX. A miner's torm for iron ochre, especially in St. Just in Cornwall. The term is sometimes applied to Wolfram.

CAIAna-TrEn, Calophyllum Calaba, so called from the beauty of its leaves. From the roots of several species an oil is expressed, which is used for burning in lamps, and from the incised trunk a resin is obtained, which is known in commerce by the name of Tacamahaca gum. The true calaba treo is a native of the Caribbee Islands; it grows to the height of sirty feet, and its wood is much valued for staves and for cask headings.

carabar meats. See Ordeat Beas.

CAIABAr-SEIN. The skin of the Siberian squirrel is sometimes socalled. It is imported and used for making muffs and trimmings.

CAIABAsmis. The pericarp of the fruit of the Calabash tree, one of the Bignoniacece (Crescentia cujete). It is a native of the tropical regions of America, and produces a melon-like fruit, containing a slightly acid pulp, which is sometimes eaton. After the removal of the pulp, the pericarp is used for cups, bottles, baskets, \&c.

catarts. See Turquors.

CAIAmarrco. A sort of woollen stuff of a shining appearance, chequered in the warp, so that the checks are seen only upon one side.

CAIAmanders. A wood, the produce of a species of Diospyros, from Ceylon, See Coromander.

CAIAMrro. Unfortunately, there is some confusion among mineralogists in the application of this term. It appears that the Lapis calaminaris of the older writers included two distinct ores of zinc-the carbonate and the hydrous silicate. In the early part of this century Smithson pointed out the differences between the two species. Brongniart soon afterwards gave the name of Calamine to the silicate, and several years later Beudant proposed the term Smithsonite for the carbonate. This usage of the names is still retained by Dana and by certain Continental writers, but English mineralogists have been accustomed to reverse this use of the terms. In this country, therefore, the carbonate of zine is invariably distinguished as calamine, whilst the silicate is known as Smithsonite or Flectric Calamine. See Zrsc.

CAIANINE, DIECTRIC.-Hydrous silicate of zinc, known otherwise as Smithsonite or Hemimorphite. The term 'electric,' applied to this mineral, refers to its pyroelectric properties, or its power of exhibiting electric polarity when heated, the two extromities of a heated crystal assuming opposite electrical conditions.

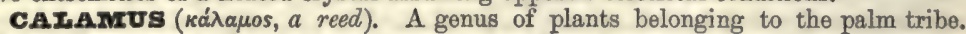
The C. Rotang and C. Scipionum are mentioned as two species which produce the Rattan canes of commerce. The Dragon's Blood of commerce is obtained from the C. Draco, a native of Sumatra and Borneo. See Dragon's Broon; Rattan.

CAIAmUs aromatrcus. The Acorus, or sweet Flag. The drug sold under this name in our shops is the produce of the Acorus calamus. The plant is a native of this country, of various parts of Europe, and of India. The Indian variety furnishes a famous medicine in the Levant, while the Turks candy it and regard it as a remedy against contagion. The rushes which were formerly strewn over the floors of rooms were the leaves of this plant. It has been said that the Acorus calamus is sometimes employed in rectifying gin. By distillation it yields a volatile oil used in scenting snuff, and in the production of aromatic vinegar.

calcantrhum. Seo Chatcanthum.

CAICAr. A name given by glass-makers to a small furnace-in which the first calcination is made of sand and potash-for the formation of a frit, from which glass is made.

CAICARzoUs EARTH (Terre calcaire, Fr.; Kalkerde, Ger.) commonly denotes lime, in any form; but, properly speaking, it is pure lime. This term is frequently applied to marl, and to earths containing a considerable quantity of lime.

CAICArzoUs SPAR. Crystallised native carbonate of lime, of which there are many varieties.

Carbonic acid $44^{\circ} 0$, lime $56^{\circ} 0$, may be regarded as the usual composition of calc spar; it often contains impurities upon which depend the colours assumed by the crystal. The carbonates of lime are extensively distributed in nature, as marbles, chalk, \&ce. See Icriand Spar, Marbir, \&ee.

CAXcAnzous TURA. This term is applied to varieties of carbonates of lime, formed by the evaporation of water containing that mineral in solution.

It is formed in fissures and caves in limestone rocks, about the borders of lakes, and , near springs, the waters of which are impregnated with lime. In the latter cases it is 
'frequently deposited upon shells, moss, and other plants, which it covers with a calcareous crust, producing frequently a perfect representation in stone of the substance :Bo enveloped.-H. W. B.

caIcrdowy. See Chatchont.

CaIcridonyx. See Chatcenonyx.

CAICINATION (from Calcine). The operation of expelling from a substance, by heat, either water or volatile water combined with it. Thus, the process of burning lime, to expel the carbonic acid, is one of calcination. The result of exposing the carbonate of magnesia to heat, and the removal of its carbonic acid, is the production of calcined magnesia. The term was, by the earlier chemists, applied only when the substance exposed to heat was reduced to a calx, or to a friable powder, this being frequently the oxide of a metal. It is now, however, used when any body is subjected oven to a process of roasting.

CAICIPHYRI. A name given by Brongniart to a compound of granular limestone with garnet or felspar, usually porphyritic.

CAICrTr. Crystallised carbonate of lime. See Ickind Spar.

CAxcrusx (Symbol, Ca.; Atomic weight, 20.) The metal contained in the oxide woll known as lime. It was first obtained by Davy, in 1808 , by the electrolysis of the hydrate, carbonate, chloride, or nitrate of lime. Matthiessen obtained it by heating, in a porcelain crucible, a mixture of two atoms of chloride of calcium with one of chloride of strontium, and a small quantity of ehloride of ammonium, until the latter is volatilised. The current from six cells of Bunsen's battery is then sent through the mixture by a charcoal pole of as large size as possible, and a piece of iron pianoforte wire (No.6), not more than two lines in length, which is united with the negative pole of the battery by means of a stronger line reaching close to the surface. A small crust is to be formed round the wire at the surface. To collect the small globules deposited on the wire, the latter must be taken out every two or three minutes, together with the crust. The globules are crushed in a mortar, and the flattened granules are then picked out. Calcium is a brilliant pale yellow metal, malleable and ductile. See Lnme.

CATCrUM, ChIORIDE OF. $\mathrm{Ca} \mathrm{Cl}\left(\mathrm{CaCl}^{2}\right)$. A deliquescent salt readily obtained by dissolving chalk in hydrochloric acid. A saturated solution boils at $355^{\circ} \mathrm{F}$., and is therefore sometimes employed as a bath where such a temperature is required. The hydrated chloride, obtained by evaporation of the solution, causes great depression of temperature when dissolved in water; hence its use in certain freezing mixtures. Mercury may be frozen by means of a mixture of crystallised chloride of calcium and snow. The anhydrous salt obtained by heating the hydrate, is extremely deliquescent, and is therefore used as a desiccating agent. After exposure to sunlight, the chloride appears luminous in the dark, and was formerly called Homberg's Phosphorus.

CAICIUM, FIUORIDr OF. CaF (Cari2). This salt occurs in the bones and teeth of animals, and in minute quantity in sea-water, and in the water of certain springs. It is found native as Fluor Spar.

\section{CAтCTUM, OXIDE OF. Seo LiMn.}

CAICrUM, PHOSPaIDI Or. By distilling phosphorus over lime at a low red heat, an impure phosphide of calcium is obtained. This salt, when thrown into water, evolves phosphuretted hydrogen, which is spontaneously imflammable. After tho phosphide has been slaked, by exposure to a moist atmosphere, it also yields phosphuretted hydrogen when brought in contact with water, but the gas thus obtained does not ignite spontaneously.

CAICIUM, SUIPHIDES OF. Several sulphides of calcium are known, but are of no importance in the arts. Canton's Phosphorus is an impure sulphide, obtained by calcining oyster-shells, and heating the product with one-third of its weight of flowers of sulphur, in a closed crucible.

CAIC-SINTIR. The incrustations of carbonate of lime upon the ground, or the pendulous conical pieces called stalactites, attached to the roofs of caverns, are so called.

CAIC-spar. Crystallised carbonate of lime or calcite.

CAICUIUS. The stony-looking morbid concretion oceasionally formed in the bladders and other parts of living animals. Its examination belongs to medical chemistry.

CAтrmbeg. An ornamental wood; it is often called aloes wood, and sometimes green sandal-wood, being of a greenish colour, and slightly scented.

CATFmrarax. A name sometimes given to coromandel wood. See CoromanDER WOOD.

CAImwdre (Calandre, Fr.; Kalender, Ger.), a word derived from the Greek

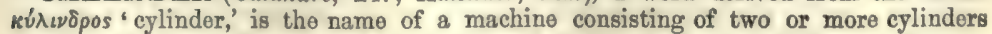
rovolving so nearly in contact with each other, that cloth passod through betwoen 
them is smoothed, and even glazed, by their powerful pressure. It is employed either to finish goods for the market, or to prepare cotton and woollen webs for the calico printer, by rendering their surfaces level, compact, and uniform. This condensation and polish, or satinage as the French call it, differ in degree according to the object in view, and may be arranged in three different series:-1. For goods which are to recoive the first impression by the block, a very strong pressure is required, for upon the uniformity of the polish the neatness and the regularity of the printing and the correspondence of its members depend. 2. The pieces already dyed up at the madderbath, or otherwise, and which remain to be fillod in with other colours, or grounded in, as it is technically called, must receive a much less considerable gloss. 3. The degree of glaxing given to finished goods depends upon the taste of purchasers, and the nature of the article; but it is, in general, much less than for the first course of block printing.

The calico printers of Alsace employ an improved form of calender to that usually employed in this country, which is the contrivance of M. Charles Dollfus. It is deseribed as possessing the following advantages:-1. It passes two pieces at once, and thus does double the work of any ordinary machine. 2. It supersedes the nocessity of having a workman to fold up the goods, as they emerge from the calender with the aid of a self-acting folder. 3. It receives, at pleasure, the finished pieces upon a roller, instead of laying them in folds; and, by a very simple arrangement, it hinders the hands of the workman from being eaught by the rollers.

The most remarkable feature of M. Dollfus's machine is its being managed by a single workman. Six or eight pieces are coiled upon the feed roller, and they are neither pasted nor stitched together, but the ends are merely ovierlapped half a yard or so. The workman is careful not to enter the second piece till one third or one half of the first one has passed through on the other side, to prevent his being engrossed with two ends at a time. He must, no doubt, go sometimes to the one side and sometimes to the other of the machine to see that no folds or creases occur, and to be ready for supplying a fresh piece as the preceding one has gone through. The mechanism of the folder in the Alsace machine is truly ingenious: it performs extremely well, and saves an extra workman. The lapping-roller works by friction, and does its duty better than similar machines guided by the hand.

The numerous accidents which have happened to the hands of workmen engaged in calenders should direct the attention towards an effective contrivance for preventing such misfortunes. These various improvements in the Alsace machine may be easily adapted to the ordinary calenders of almost every construction.

The folder is a kind of cage in the shape of an inverted pyramid, shut on the four sides, and open at top and bottom: the top orifice is about five inches, the bottom one an inch and a half: the front and the back, which are about four feet broad, are made of tin plate or smooth pasteboard, and the two sides are made of strong sheet-iron, the whole being bolted together by small bars of iron. Upon the sheet-iron of the sides, iron uprights are fixed, perforated with holes, through which the whole cage is supported freely by means of studs that enter into them. One of the uprights is longer than the other, and bears a slot with a small knob, which, by means of the iron piece, joins the guide to the crank of the eylinder, and thereby communicates to the cage a see-saw movement: at the bottom extremity of the great upright there is a piece of iron in the shape of an anchor, which may be raised, or lowered, or made fast by serews.

At the ends of this anchor are friction rollers, which may be drawn out or pushed back and fixed by screws: these rollers lift alternately two levers made of wood, and fixed to a wooden shaft.

The paws are also made of wood: they serve to lay down alternately the piles of the cloth which passes upon the cage, and is folded aigzag upon the floor, or upon a board set below the cage; a motion imparted by the see-saw motion of the cage itself.

To protect the fingers of the workmen, above the small plate of the spreading-board or bar, there is another bar, which forms with the former an angle of about $75^{\circ}$ : they come sufficiently near together for the opening at the summit of the angle to allow the cloth to pass through, but not the fingers. See Bulletin de la Sociéte Industrielle de Mulhausen, No. 18.

It is not thought necessary to give a drawing of the calender usually employed in this country; a few remarks may not, however, be out of place. The iron rollers are made hollow for the purpose of admitting either a hot roller of iron, or steam, when hot calendering is required. The other cylinders used formerly to be made of wood, but it was liable to many defects. The advantage of the paper roller consists of its being devoid of any tendency to split, crack, or warp, especially when exposed to a considerable heat from the contact and pressure of the hot iron rollers. The peper, moreover, takes a vastly finer polish, and being of an elastic 
nature, presses into every pore of the cloth, and smooths its surface more effectually than any wooden cylinder, however truly turned, could possibly do.

The paper cylinder is constructed as follows:-The axis of the cylinder is a strong square bar of tho best wrought iron, cut to the proper length. Upon this bar a strong round plate of cast iron is first put, somewhat less in diameter than the cylinder when finishod; a quantity of thick stout pastoboard is then procured, and cut into round pieces an inch larger in diameter than the iron plate. In the centre of the plates, and of every piece of the pasteboard, a square hole must be cut to receive the axis ; and the circle being divided into six equal parts, a hole must also be cut at each of the divisions an inch or two within the rim. These pieces of pasteboard being successively put upon the axis, a long bolt of malleable iron, with a head at one end, and screwed at the other, is also introduced through each of the holes near the rim; and this is continued until a sufficient number of pasteboards are thus placed to form a cylinder of the length required, proper allowance being made for the compression which the pasteboard is afterwards to undergo. Another round plate is then applied, and nuts being put upon the screws, the whole are screwed tight, and a cylinder formed. This cylinder is now to be placed in a stove, exposed to a strong heat, and must be kept there for at least several days; and, as the pasteboard shrinks by exposure to the heat, the screws must be frequently tightened until the whole mass has beon compressed as much as possible. When the cylinder is thus brought to a sufficient degree of density, it is removed from the stove; and, when allowed to cool, the pasteboard forms a substance almost inconceivably dense and hard. Nothing now remains but to turn the cylinder; and this is an operation of no slight labour and patience. The motion in turning must be slow, not exceeding about forty revolutions in a minute; the substance being now so hard and tough that tools of a very small size must be used to cut, or rather scrape it, until it is true. Three men are generally employed for the turning, even when the motion of the cylinder is effected by mechanical power, two being necessary to sharpen the tools for the third, who turns, as quickly as ho blunts them.

Let us suppose it to be a five-rollered machino: when a person stands in front of the calender, the cloth coming from behind above the uppermost cylinder 1, passes between 1 and 2 ; proceeding behind 2, it again comes to the front between 2 and 3 ; between 3 and 4 it is once more carried behind, and lastly, brought in front between 4 and 5 , where it is received and smoothly folded on a clean board, or in a box, by a person placed there for the purpose. In folding the eloth at this time, care must be taken that it may be loosely done, so that no mark may appear until it be again folded in the precise length and form into which the piece is to be made up. The folding may be done either by two persons or by one, with the aid of two sharp polished spikes placed at a proper distance, to ascertain the length of the fold, and to make the whole equal. When folded into lengths, it is again folded across upon a smooth clean table, according to the shape intended, which varies with different kinds of goods, or the particular market for which the goods are designed.

When the pieces have received the proper fold, the last operation previous to packing them is the pressing. This is commonly performed by placing a certain number of piecos, divided by thin smooth boards of wood, in a common screw press similar to those used by printers for taking out the impression left by the types in the printingpress. Besides the wooden boards, a piece of glazed pasteboard is placed above and below every piece of cloth, that the outer folds may be as smooth and glossy as possible. The operation of the common screw-press being found tedious and laborious, the hydraulic press is now had recourse to in all well mounted establishments. See Hydraunic Press.

For lawns and muslins of a light texture, the operation of smoothing requires a different process in some respects than close heavy fabrics. They only require to be slightly smoothed to remove any marks which they may have received at the bleaching; and, as their beauty depends rather on thoir transparency than their closeness, the more the cylindrical form of the yarn is preserved the better. They are therefore put through a small machine, consisting of three rollers or cylinders: and, as the power required to move this is small, the person who attends it generally drives it by a small winch; or the same effect may be produced by passing the muslins between only two or three rollers of the above calender, lightly loaded.

In the thick fabries of cloth, including those kinds which are used for many parts of household furniture, as also those for female dress, the operation of glazing is used both to add to the original beauty of the cloth, and to render it more impervious to dust or smoke. The glazing operation is performed entirely by the friction of any smooth substance upon tho cloth; and to ronder the gloss brighter, a small quantity of bleached wax is previously rubbed over the surface. The operation of glazing by the common plan is very laborious, but the apparatus is of the most simple kind. $\mathbf{A}$ 
table is mounted with a thick stout cover of level and woll-smoothed wood, forming an inclined plane; that side where the operator stands at work boing the lowest. The table is generally placed near a wall, both for convenience in suspending the glaxing apparatus, and for the sake of light. A long piece of wood is suspended in a groove formed between two longitudinal bosms, placed parallel to the wall, and fixed to it. The groove resembles exactly the aperture between the shears of a common turning lathe. The lever, of which the groove may be supposed to be the centre or fulcrum, is faced at the bottom with a semi-cylindrical piece of finely polished flint, which gives the friction to the cloth stretched upon the table below. Above the flint are two cross handles, of which the operator lays hold, and moves them backward and forward with his hands, keeping the flint pressing slightly upon the eloth. When he has glazed a portion equal to the breadth of the flint, he moves his lever between the shears sidewise, and glazes a fresh part: thus he proceeds from one side or selvage of the cloth to the other; and when all which is upon the table is sufficiently glazed, he draws it over, and exposes a new portion to the same operation. To preserve the cloth at a proper tension, it may be wound smoothly upon a roller or beam, which being set so as to revolve upon its own axis behind the table, another roller to receive the eloth may be placed before, both being secured by a catch, acting in a ratchet wheel. Of late years, however, a great part of the labour employed in glazing cloth has been saved, as the common four or five bowl calender has been altered to fit the purpose by direct pressure.

As a matter of accommodation, the different processes of packing, cording of boxes, sheeting of trunks, and, in general, all the arrangements preparatory to shipment, and also the intimations, and surveys necessary for obtaining drawbacks, debentures, or bounties, according to the Excise laws, are generally conducted at the calender houses where goods are finished.

CAImDONITE (from Caledonia). A cupreous sulphato-carbonate of lead found in minute bluish-green crystals associated with other ores of lead at the Lead Hills mines in Lanarkshiro. This minoral has also been found in Cumberland, at Tanné in the Hartz, at the Mine la Motte, Missouri.

CAIrara (Calibre, Fr.; Calibro, It.) The bore or diameter of a gun, or the diameter of a bullet; it is measured in inches, and in smooth-bore guns is larger than the diameter of the ball.

casrco (from Caliout, in India). A term for any white cotton cloth, which was first manufactured in, and introduced from, India. In this country we have shirting calicoes, unbleached calicoes, and the like. In the United States the term is re strictod to cotton cloths having patterns printed upon them.

CAIICO-PRINTIXse is the art of producing a pattern on cotton cloth, by printing in colours, or mordants, which become colours, when subsequently dyed. Calico derives its name from Calicut, a town in India, formerly celebrated for its manufactures of cotton cloth, and where calico was also extensively printed. Other fabrics than cotton are now printed by similar means, viz. linen, silk, wool, and mixtures of wool and cotton. Linen was formerly the principal fabric prinied, but since modern improvements have produced cotton cloth at a comparatively cheap rate, linen fabrics are now sparingly used for printing, and then principally for handkerchiefs, linen cloth not producing such beautiful colours, in consequence of the small affinity of flax for mordants, or colouring matters. Silk printing, also, is chiefly confined to handkerchiefs, but the printing of woollen fabrics or mousseline-de-laines is an important branch of the art. The earliest mode of ornamenting cloth with designs was, no doubt; by embroidery with the needle, and this mode was almost coeval with the art of dyeing, which is of very remote antiquity. Horodotus mentions that Amasis, king of the Egyptians, sent to the Lacedæmonians a pectoral of linen, adorned with many figures of animals, woven into the cloth, and enriched with gold and a variety of colours. A similar pectoral was taken among the spoils at the battle of Issus, and presented to Alexander the Great, who wore it afterwards as part of his military attire. Cloth was, however, stained in a rude manner by ancient tribes with fuices of plants. Herodotus mentions a Seythian tribe who stained their garments with figures of animals by means of the leaves of a tree bruised with water, which figures would not wash out, and lasted as long as the cloth. It is an interesting speculation as to what this dye was. The garments so stained were probably woollen, as in early times the outer garments were always woollen, and the particular dye might have been indigo in a soluble state, as produced by fermenting the leaves with water; according to Sir William Jones, the leaves of the shrub henna, when bruised in water, stain the skin or nails orange, and would doubtless do the same on woollen cloth. The first record of calico-printing as an art is that of Pliny, who describes the process followed by the Egyptians, who seemed to have attained a very considerable degree of refinement in the art. 'Robes snd white veils,' says he, 'are painted in 
Egypt in a wonderful way. They are first imbued, not with dyes, but with dyeabsorbing drugs, by which, though they seem to be unaltered, yet, when immersed for a litle while in a cauldron of the boiling dye-liquor, they are found to become painted. Yet, as there is only one colour in the cauldron, it is marvellous to see many colours imparted to the robe, in consequence of the influence of the excipient drug. Nor can the dye be washed out. A cauldron, which would of itself merely confuse the colours of cloths previously dyed, is thus made to impart several pigments from a single dye-stuff, painting as it boils.' The last expression, pingitque dum coquit, is perfeetly graphic and deseriptive of some processes in calico-printing.

Calico-printing is of very ancient date in India, and probably this country is the birthplace of the art, since, beyond doubt, cotton cloth originated in India, and the abundance of dye-stuffs, and the facility with which cotton receives dyes, rendered the staining it with figures a natural consequence, and there is good reason to suppose that the Egyptians learnt the art from India, since the Indians were highly civilised twenty-two centuries ago; and there was undoubtedly communication between India and Egypt before the time of Pliny. There is an account of Indian calico-printing by Father Ccurdoux, a missionary at Pondicherry, and in a manuscript account sent from thence by M. du Fay, and communicated to the Royal Academy of Sciences at Paris by the Abbé Mazeas, also from the report by M. Beaulien, of operations performed under his inspection at Pondicherry.-Bancroft.

These accounts describe the mode of producing the chintz calicoes, which were celebrated in Europe before the art had been introduced and simplified there. From these accounts of the cumbrous and tedious processes adopted by the natives, we have no difficulty in understanding the necessity that arose for the intervention of European skill and science, and can readily comprehend how it is that the European printer, to say nothing of superior artistic excellence, can compete successfully in India with the proverbially low-priced labour of Hindostan. After the eloths were partially bleached, they underwent several alternate stoeps in goats'-dung, beating, washing, and drying in the sun; they were then soaked in an astringent solution obtained from myrabolams, mixed with buffaloes' milk; squeezed out of this, they were dried in the sun, and, by pressure with wooden rollers, made smooth enough to have a pattern drawn on them with a pencil, applying various mordants: the general course was to paint on a mordant of iron liquor, similar in constitution to that at present used in calico-printing. This formed a black with the tannin substance proviously applied. The next step was to give the blue, and for this purpose the cloth required to be froed from the astringents by maceration in goats'-dung, well washing and drying in the sun; the parts intended to be white were then protected by a coat of melted wax; the cloth was then dipped in an indigo vat: when dyed, the wax had to be thoroughly removed by boiling in water, steeping in dung, washing with a sort of impure soda, renewed steeping in dung, washing and drying in the sun; after this the cloth was treated, as before, with the astringent milk mixture, dried and smoothed. It was then ready to receive the red and chocolate mordants, the red being simply alum mixed with a little soda to render it basic, and the chocolate, this red mixed with the iron mordant; (the use of acetate of alumina not being known, the albumen of the milk and the tannin combined with and fixed the alumina on the cloth). After careful sundrying, the cloth was well steoped and rinsed in water to remove the excess of mordants, \&ce, and then dyed with madder or chaya root. After this they were washed with dung and soap, exposed to the sun, and watered occasionally till the white parts were bleached. Yellow, made from alum and myrabolams was now pencilledin, and green formed by the yellow going over the blue. This process gave chintzes, the colours of which were generally very bright and lively, and most of them exceedingly durable. M. Koochlin Roder, of Mulhouse, brought home from India a rich collection of cloths in every state of preparation, which are in the cabinet of the Societe Industrielle of that interesting emporium of calico-printing. The native implements for applying the wax and colouring bases are placed alongside of the cloths, and form a curious picture of primeval art. There is among other ramples an ancient pallampoor, five French yards long and two and a half broad, said to be the labour of Hindoo princesses, which must have taken a lifetime to execute.

Calico-printing was not, however, in all oriental countries executed with the pencil. The shawl-printers of Cashmere use small wooden blocks for their complicated patterns. Mr. Buckingham states, that at Orfah, in Mesopotamia, the printers employ wooden blocks of 4 to 6 inches square, and use them nearly in the same manner as the block-printers in this country; and it is well known that the Chinese employed block-printing long before any species of printing was known in this country.

Calico-printing has been for several hundred years practised by the oriental methods in Asia Minor and the Levant; but it was unknown as an English art till

Vor. I. 
about the close of the seventeenth century. It is believed that the first attempts at imitating the printed calicoes of India were made in Holland, the Dutch Hast India Company having introduced the Indian chintzes there before their introduction into this country. It is uncertain where or whon these first attompts were made; but it appears the art soon spread to Germany, for about the close of the seventeenth century Augsburg lad obtainod a notoriety for printed linons and cottons. The art was most probably introduced into England about 1676, by Flemish emigrants. Mr. James Thompson, of Clitheroe, one of the most eminent English calico-printers, fixed the date at 1690, and supposed that a Fronchman, a refugee, at the time of the revocation of the edict of Nantes, was the first to print calicoes in this country, and that his works were at Richmond on the Thames; but there is evidence to show that prior to this date, calicoes were printed in this country, for Sir Joshua Child, a distinguished director of the East India Company, in a pamphlet published in 1677, mentions that calicoes were then brought over from India to be printed in this country, in imitation of the Indian printed chintzes. It appears, from a petition addressed to the House of Commons by the East India Company in 1627 , that Indian calicoes were at that timo imported, and in 1631, in a catalogue of legal imports from India, painted calicoes are montioned as to be allowed. In 1634, apparently, attempts were made to ornament fabrics with coloured patterns by mechanical means, for in that year Charles II. granted an exclusive patent for fourteen years, for the art or mystery of affixing wool, silk, and other materials of divers colours upon linen, silk, or cotton clotl, leather, and other substances, by means of oil, size, or other cements, to make them. useful for hangings, \&c., the patentee paying 10l. yearly to the Exchequer. Calicoprinting was commenced in 1689, at Neufchatel, by Jaques Deluze, a native of Saintonge, and this establishment rapidly became prosperous, and in time the parent of numerous offshoots in Germany, Portugal, and France.

Some time after the Richmond establishment, a considerable printing work was established at Bromley Hall, in Essex, and several others sprung up successively in Surrey, to supply the London shops with chintzes, their import from India having been prohibited in 1700 by Parliament. The art in its infancy had to struggle with many difficulties; an excise tax on all printed or dyed calicoes of $3 d$. per square yard was enacted in 1702 , and which was increased to $6 d$. per square yard in 1714 , only half these duties being laid on printed linens.

The silk and woollen weavers had all along manifested the greatest hostility to the use of printed calicoes, whether brought from the East or made at home. In the year 1680 they mobbed the India House in revenge for some large importations then made of the chintzes of Malabar. They next induced the Government, by incessant clamours, to exclude altogether the beautiful robes of Calicut from the British market. But the printed goods imported by the English and Dutch East India Companies found their way into this country, in spite of the excessive penalties annexed to smuggling, and raised a new alarm among the manufacturing population of Spitalfelds. The sapient legislators of that day, intimidated as would appear, by the East London mobs, enacted in 1720 an absurd sumptuary law, prohibiting the wearing of all printed calicoes whatsoever, either of foreign or domestic origin. This disgraceful enactment, worthy of the meridian of Cairo or Algiers, proved not only a death-blow to rising industry in this ingenious department of the arts, but prevented the British ladies from attiring themselves in the becoming drapery of Hindostan.

The effect of this law,' says Mr. Edmund Potter, in his lecture on CalicoPrinting, before the Society of Arts, as reporter on printed fabrics in the Exhibition of 1851 , 'was to put an end to the printing of calicoes in England, and to confine the printers to the printing of linens. In 1736, so much of this Act was repealed as iorbade the use or wear of printed goods of a mixed kind, containing cotton; and these fabrics were allowed to be printed, weighted with a duty of $6 d$. per square yard. In 17.50 , the entire production of Great Britain was estimated at 50,000 pieces por annum. In $\mathbf{1 7 6 4}$, printers established themselves in Lancashire, tempted, doubtless, by the cheapness of fuel, and by this being the locality in which the eloth was manufactured. In 1774, the printer was released from his fetters with regard to the kind of cloth he must use, by the repeal of this law, so as to leave him the choice of his material; but he was still saddled with a duty of $3 d$. per square yard, to which a halfpenny was added in 1806. On the accession of Lord Grey's government to office it was one of their first acts to repeal this duty. Thus, after a period of about 140 years from its first introduction, the print trade was allowed to enter into competition with other kindrod fabries on a fair footing.'

France pursued for some time a similar false policy with regard to calico-printing, but she emerged sooner from the mists of manufacturing monopoly than England. Her arowed motive was to cherish the manufacture of flax, a native product, instead of that of cotton, a raw material, for which prejudice urged that money had to bo 
exported. Her intelligent statesmen of that day repliod, that the money expended in the purchase of cotton was the product of French indusiry beneficially employed, and they therefore took immediate measures to put the cotton fabrics upon a footing of equality. Meanwhile the popular prejudices bocame irritated to such a degree, by the project of permitting the free manufacture and sale of printed cottons, that every French town possessed of a chamber of commerce made the strongest remonstrances against it. The Rouen deputies declared to the Government, 'that the intended measure would throw its inhabitants into despair, and make a desert of the surrounding country'; those of Lyons said, 'the news had spread torror through all its workshops:' Tours 'foresaw a commotion likely to convulse the body of the State:" Amiens said, "that the new law would be the grave of the manufacturing industry of France;' and Paris declared that 'her merchants came forward to bathe the throne with their tears upon that inauspicious occasion.'

The Government persisted in carrying its truly enlightened principles into effect," and with manifest advantage to the nation, for the despair of these manufacturing towns has been replaced by the most signal prosperity.

France probably produces at the present time nearly $5,000,000$ pieces of print per annum, which, considering the quality of many of them, may be considered a very important manufactare.

The great disadvantage under which the French printers labour is the higher price they pay for cotton fabrics and fuel above that paid by the English printers.

The repeal, in 1831 , of the consolidated duty of $3 \frac{1}{2} d$. per square yard upon printed calicoes in Great Britain is one of the most judicious acts of modern legislation. By the improvements in calico-printing, due to the modern discoveries and inventions in chemistry and mechanies, the trade had become so vast as to yield in 1830 a revenue of $2,280,000 \mathrm{l}$. levied upon $8,596,000$ of pieces, of which, however, about three-fourths were exported, with a drawback of $1,579,000 l$. $2,281,512$ pieces wero consumed in that year at home. When the expenses of collection were deducted, only $350,000 l$. found their way into the Exchequer, for which pitiful sum thousands of frands and obstructions were committed against the honest manufacturer. This reduction of duty enables the consumer to get this extensive article of clothing from 50 to 80 per cent. cheaper than before, and thus places a becoming dress within the reach of thousands of females in the humbler ranks of life. Printed goods, which in 1795 were sold for $2 s .3 d$. the yard, may be bought at present for $6 d$. The repeal of the tax has been no less beneficial to the fair dealers, by putting an end to the contraband trade, formerly pursued to an extent equally injurious to them and the revenue. It has, moreover, emancipated a manufacture eminently dependent upon taste, science, and dexterity from the venal curiosity of petty excisemen, by whom private improvements, of great value to the inventor, were in perpetual jeopardy of being piratod and sold to any sordid rival. The manufacturer has now become a free agent, a master of his time, his workmen and his apparatus; and can print at whatever hour he may receive an order; whereas he was formerly obliged to wait the convenience of the excise officer, whose province it was to measure and stamp the eloth before it could be packed-an operation fraught with no little annoyance and delay. Under the patronage of Parliament, it was easy for needy adventurers to buy printed calicoes, because they could raise such a sum by drawbacks upon the export of one lot as would go far to pay for another, and thus carry on a fraudulent system of credit, which sooner or later merged into a disastrous bankuruptcy. Meanwhile the goods thus obtained were pushed off to some foreign markets, for which they were, possibly not suited, or where they produced, by their forced sales, a depreciation of all similar merchandise, ruinous to the man who meant to pay for his wares.

Calico-printing was first practised in Scotland in 1738, twenty-six years previous to ite introduction into Lancashire. The following sketch of the early Lancashire printing is taken from Mr. Potter's pamphlet:- 'The trade was established in Lancashire in 1764, by Messrs. Clayton, of Bamber Bridge, near Preston; the cloth that was printed being made with linen warp and cotton weft, and produced principally at Blackburn; this was the reason of many printers settling near Blackburn, which was for a long time the great seat of the print trasle. The introduction of power-loom cloth caused the migration of a considerable print trade to Stockport, Hyde, Staleybridge, and North Derbyshire. The Claytons were followed by Mr. Robert Peel, who entered into the cotton business, and added to it the printing business. He carried on the business for some years at Brookside, near Blackburn, aided by his sons. The eldest son afterwards branched off from his father's concern, and established himself at Bury with his uncle, Mr. Haworth, and Mr. William Yates.

'During the period 1796 to 1821, the Forts, Hargreaves, and Thompsons fairly P $\mathbf{P} 2$ 
established themselves as extensive and wealthy printers, not more by their energy and business talent than by their scientific attainments, and by the unbounded and lavish support which they gave to overything which art and science could suggest to assist them. Mr. James Thompson of Primrose, near Clitheroe, was for forty years the recognised head of the print trade. The era of his commencement in the trado was the beginning of a series of discoveries and new applications in chemical science to the purposes of calico-printing. During forty years he devoted himself and the ample funds his business placed at his disposal to the advancement of tasto in connection with his trade. No sums, however large, were spared to draw into its servico the talent even of Royal Academicians, and of many other eminent men high in art.' Mr. John Mercer, of the house of Fort Brothers, and a contemporary of Mr. 'Thompson, for a long period worked enthusiastically and rendered valuable assistance to the trade by the introduction of chemical noveltios; and many styles founded by him are still popular. The house of Hargreaves Brothers and Co., during the same period, took a prominent position in the production of now and original colours and stylos.

In France M. Koechlin was looked up to as the leader of the trade, and was mainly instrumental in establishing sound scientific principles in the art. "During the progressive improvement, dating from 1831, one house may be named, of high standing, who introduced a colour superior in brilliancy, fastness, and utility for domestic wear, to any other previously known. This was the madder purple of Messrs. Thomas Hoyle and Sons, a colour which may be said to have superseded the old Navy blue print in English wear. Messrs. Hoyle and Sons maintained their well-deserved superiority for many years. The London printers, up to the repeal of the duty, still held their position for first-class goods. They made great use of the flat press printing-machine. Their plates were well engraved, and for a long time they succeoded in getting a smartness of impression, better than any at that time obtained from the cylinder. Some few of the Lancashire printers adopted the press, the better to compete with the town printor. The rapidly increasing trade in Lancashixe, and with it the power of so much cheaper production, gradually undermined the London printers, and brought about a complete change in their class of work.'-The London printers now print fine shawls, handkerchiefs, waistcoatings, and a superior class of cotton prints for furniture hangings. The present annual production of printed cloth of all kinds in Great Britain may be estimated at about $20,000,000$ pieces. In 1840 the quantity produced was about $16,000,000$. The quantity now, probably, rather exceeds $20,000,000$ of pieces; but, from the absence of any very authentic statistics, the quantity is very difficult to arrive at. The print trade, according to Mr. Bazley, consumes a weight of cotton about one-seventh the entire import into this country.

Owing to her natural advantages, England has by far the largest portion of the calico-printing trado, and especially of the export trade; and probably at the present time England produces as many printed pieces as all the rest of the world put together. The United States produces next to ourselves in quantity; France and Switzerland the next to America in quantity, but far superior to her in quality, and second only to ourselves in value of production. France is the only competitor we have to meet in the neutral markets of the world. The Zollverein, Austria, and Bohemia produce for their own markets, and by high protecting duties prevent any other supply, except of very fine French goods. Holland produces a small quantity of medium goods; Belgium also produces a fow; Naples has a few small print works; Russia produces for her own market, and the number of works has rapidly increased of late-her market is almost prohibited to us; Spain produces a limited quantity of inferior goods; Portugal has a slight production; Turkey produces a few printed goods, hardly worth notice; the Sultan Abdul Medjid has tried the experiment of organising print works on the English principle, with English artisans and foremen, but the experiment was a complete failure; Egypt also has revived the art, with very inferior rosults. The Chinese undoubtedly practised the art of calico-printing many centuries before ourselves. Mr. Potter was able to exhibit samples of Chineso work to the Society of Arts, which he described as of very primitive taste and rude execution. 'Mulhausen, it may not be uninteresting to mention,' says Mr. Potter, 'is certainly the seat of the finest printing in the world. Calico-printing was first established there in 1746, by the firm of Koechlin and $\mathrm{Co}$, and is still carried on by descendants of the original firm; and during the whole period, and not less so now, the house has had a high and justly deserved reputation for talent and taste; and to them the chemistry of the trado is most deeply indebted for many raluable processes and discoveries. Other houses of almost equal celebrity followed, and Mulhausen has justly maintained its reputation of being, for fine goods, the first calico-printing district in the world.'

The first step in calico-printing is to remove the fibrous down from the surface of 
the cloth, which is done by passing the piece rapidly through a flame of gas, or over a red hot semi-circular plate. The latter method will be found described under the head of Burachusg.

There are two methods in use of singeing with flame: one invented by Henry Gledhill, in which coke is burnt in a furnace provided with a cast-iron plate for a top, in which is left an aperture about 42 inches long and $\frac{5}{10}$ ths of an inch wide at the outside, but which is $\frac{3}{4}$ ths of an inch on the inside. The coke rests upon fire-bars in the usual manner, being fed from a close-fitting fire-door. The ash-pit, instead of being open as usual, is also provided with a closely-fitting door; a pipe from the blast fan is introduced into the side of the ash-pit. A fire being lighted, the furnace is filled with coke, and the fan being started, both the fire-door and the ash-pit door are closed and luted with clay; the flame now rises through the rectangular aperture to a height of about two feet, a hopper over the apparatus conducts the products of combustion into a chimney or flue, connected with the main chimney of the factory. The grey pieces are now passed rapidly over the flame, about 8 inches above the iron plate, being unwound from a roller, and after passing through a trough of water, boing rewound on another roller. The flame is depressed by the cloth, and extends about $\mathrm{a}$ foot in length. The effect of this singeing is to thoroughly remove all the

324

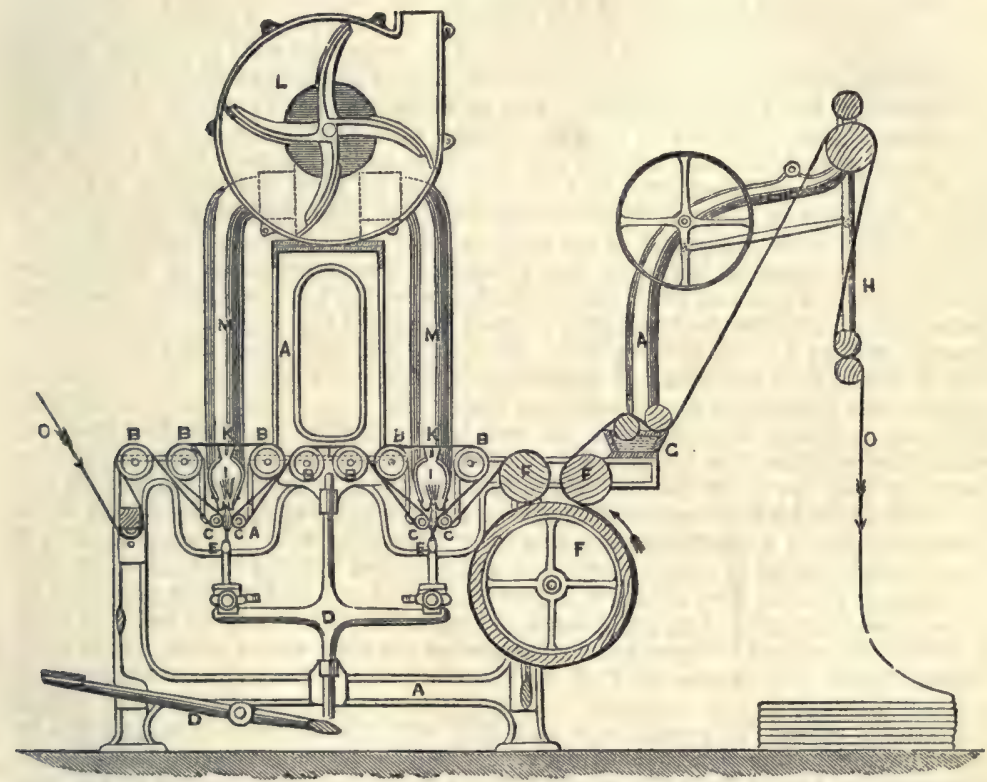

fibrous down on one side of the cloth without weakening it or making it appear thin, In some print works this singeing machine is supplemented by the copper semicircular hot plate (BufacHovg, fig. 109), which is arranged to receive the cloth on coming from the coke-gas flame. Singeing by coal-gas is a very cleanly and economical mode, but after its introduction it fell partly out of use on account of the thinning of the cloth by the gas flame being drawn through it. The gas singoing machine patented by Mather and Platt, overcomes this defect. The flame impinges against the surface of the cloth, which is thoroughly cleansed without being impoverished, and the gas is so perfectly consumed that there are no stains from imperfect combustion. There is also a considerable saving both of gas and time as compared with the old method. Fig. 324 is a vertical section of this machine, $\mathbf{\Delta}$, framing of the machine; $B$, binding rollers on rails; $c$, rollers where the flame impinges on the eloth; these are moveable to make a wide or narrow opening for the flame, for a mild or keen singe; D, arms and levers for supporting the burners, \&ce. across the machine, and bringing or taking away the flame from its work; $\mathrm{E}$, gasburners and flame; $F$, drawing drum and pressing rollers; $a$, water-box and rollers for killing sparks; $\mathrm{H}$, plaiter; $\mathrm{r}$, tubes for taking away the dust, heat, \&c., drawn through 
by the exhaust fan, which also canses a perfect combustion of gas, and produces a perfoctly clear flame. In the top of each tubo is fitted a steel doctor or scraper, $k$, for cloaning the singed side of the cloth. L, exhaust fan; $\mathbb{x}$, suction-pipe of fan; $\mathbb{N}$, delivery pipe of fan; 0 , eloth being singed.

The bleaching requisite for printing cloths is of much superior nature to that suffcient for calico intended to be sold in the whito state. It is sufficient for the latter to be white onough to please the oye: a result easily obtained by chlorine treatment after a comparatively mild alkaline boiling; but the former must be so well boiled with lime and alkali, as to remove every particle of resinous and glutinous matter previous to the chlorine steep. This, if not attondod to, becomes a source of great annoyance to the printer in his subsequent operations, from the difficulty of obtaining sufficiently good whites without injuring the colours. The high pressure kiers patented by Barlow, and which are fully deseribed in the article Brescunv, have been found to facilitate the thorough scouring of the cloth very much at a less cost than tite old kiers.

The pieces, on leaving the bleach-house drying machine are taken to the "white room,' a large room for storing the bleached goods, where they are unstitched, folded, sorted into the different qualities in use, and distributed to the proper compartments of the room, whence they are withdrawn as wanted for printing.

Till about the year 1760 , the printing of linens or calicoes was done by hand, wooden blocks being employed, on which the pattern is raised in relief. About this time a modification of the press used for printing engravings was adapted to printing with flat engraved copper plates on fabrics. This press was used to produce certain styles only, generally single colours, where delicacy of outline was required, shaded or stippled work being also introduced. The printing by blocks in several calours was the principal mode still, till in 1785 the cylinder printing-machine was invented by a Scotchman named Bell, and brought into suscessful use at Mossely, near Preston, by the house of Livesey, Hargreaves, and Co. The house of Oberkampf, of Jouy, in France, almost immediately adopted the invention, and have been frequently considered, in France at least, the originators of the machine; but it is now pretty certain that the honour of the invention is due to Great Britain. The introduction of the cylinder machine gradually caused the disuse of the flat press, the London printers continuing to use them long after the Lancashire printers had given them up; the first cylinder machine was used in Iondon in 1812. Blocks are still freely used for some description of prints, such as woollen or mousseline-de-laine goods, and also for introducing colours after printing by the cylinder and dyeing, \&c.--the cylinder not being capable of fitting in colours, after the piece has once left the machine. A blocking-machine, called the Perrotine, was introduced in France in 1834 by M. Perrot, and is still extensively used there, but though tried in this country, it never came into general use. It executes as much work as twenty hand printers, and for the special purposes for which it was invented is a satisfactory machine; the patterns capable of being printed by it are, however, limited in size, in consequence of the narrow width of the blocks. Surface printing, or printing from cylinders engraved in relief, was an invention preceding by a few years the engraved copper cylinder, but apparently not in general use. In 1800 , a Frenchman, named Ebingar, patented somewhat the same sort of thing, and in 1805, James Burton, of the house of Peel, at Church, invented the mule machine, which worked with one or two engraved copper cylinders, and one or two wooden rollers engraved in relief. This machine is very little used now, the impression produced by it not having the precision of that from copper rollers, and improvements in engraving copper rollers having given the printer many of the advantages possessed by the surface roller. Quite lately, however, Mr. James Chadwick has patented a species of surface roller which promises to becomo useful. The ordinary stereotyped pattorns described hereafter are adapted by screws to a brass or other metal roller, which in then fitted on the mandrel used with the ordinary engraved rollers, and a firmness and solidity thus given which was never possessed by the wonden surface roller.

Printing by block is thus performed:-The hand blocks are made of sycamore or pear-tree wood, or of deal faced with these woods, and are from 2 to 3 inches thick, 9 or 10 inches long, and 5 broad, with a strong box handle on the back for seizing them by. The face of the block is either carved in relief into the desired design, like an ordinary wood-cut, or the figure is formed by the insertion edgewise into the wood of narrow slips of flattened copper wire. These tiny fillets, being filed level on the one edge, are cut or bent into the proper snape, and forced into the wood by the taps of a hammer at the traced lines of the configuration. Their upper surfaces are now filed flat, and polished into one horizontal plane, for the sake of equality of impression. As the slips are of equal thickness in their whole depth, from having been made by running the wire through between the steel cylinders of in flatting mill, the lines of the figure, however much they get worn by use, are always 
equally broad as at first; an advantage which does not belong to wood-cutting. The interstices between the ridges thus formed are filled up with felt-stuff. Sometimes a delicate part of the design is made by the wood-cutter, and the rest by the insertion of copper slips.

The colouring matter or mordant, properly thickened, is spread with a flat brush, by a child, upon fine woollen cloth, stretched in a frame over the waxcloth head of a wooden drum or sieve, which floats inserted in a tubful of old paste, to give it elastic buoyancy. The inverted sieve drum should fit the paste-tub pretty closely. The printer presses the face of tho block on the drum-head, so as to take up the requisite quantity of colour, applies it to the surface of the calico, extended upon a flat table covered with a blanket, and then strikes the back of the block with a wooden mallet, in order to transfer the impression fully to the cloth. This is a delicate operatiun, requiring equal dexterity and diligence. To print a piece of cloth 28 yards long and 30 inches broad, no less than 672 applications of a block, 9 inches long and 5 inches broad, are requisite for each colour; so that if there are 3 colours, no less than 2016 applications will be necessary. The blocks have pin-points fixed into their corners, by means of which they are adjusted to their positions upon the cloth, so as to join the different parts of the design with precision. Each printer has a colour-tub placed within reach of his right hand; and for every different colour he must have a separate sieve. Many manufacturers cause their blocks to be made of three layers of wood, two of them being deal, with the grain crossed to prevent warping, and the third syeamore, for engraving.

The printing shop is an oblong compartment, lighted with numerous windows at each aide, and having a solid table opposito to each window. The table $\mathbf{B}($ fig. 325$)$ is

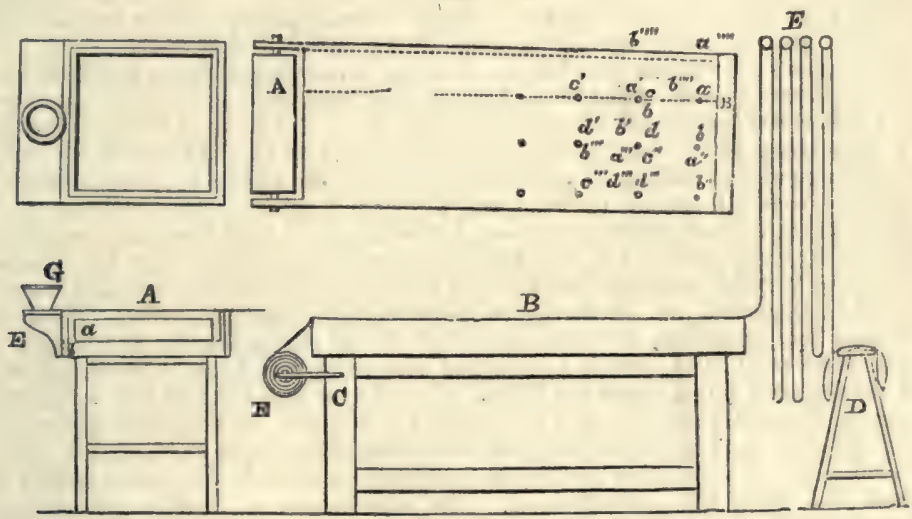

formed of a strong smooth flag, with a surface truly plane. Its length is about 6 feet, its breadth 2 feet, and its thickness 3,4 or 5 inches. It stands on strong feet, with its top about 36 inches above the floor. At one of its ends there are two brackets $\mathrm{c}$ for supporting the axles of the roller $\mathrm{k}$, which carries the white calico to be printed. The table is covered with blanket stretched tightly across and hooked at the side. The hanging rollers $\mathrm{E}$ are laid across joists fixed near the roof of the apartment above the printing shop, the coiling and floor between them being open bar work, at least in the middle of the room. Their use is to facilitate the exposure, and, consequently, the drying of the printed pieces, and to prevent one figure being daubed by another. Should they come to be all filled, the remainder of the goods must be folded lightly upon the stool $\mathrm{D}$.

The printer stretches a length of the piece upon his table $\mathrm{A} B$, taking care to place the selvage towards himself, and one inch from the edge. He presents the block towards the end to determine the width of its impression, and marks this line $\Delta \mathrm{X}$, by means of his square and tracing point. The spreador or teerer now besmears tho cloth with the colour, at the commencement, upon both sides of the sieve head; because, if not uniformly applied, the block will take it up unequally. The printer seizes the block in his right hand, and dips it twice in different directions upon the sieve cloth, then he transfers it to the calico in the line $\mathbf{A}$ B, as indicated by the four points $a b c d$, corresponding to the four pins in the cornors of the block. Having done so, he takes another dip of the colour, and makes the points $a b$ fall on $c d$, so as to have 
at the second stamp $a^{\prime} b^{\prime}$, covering $a b$, and $c^{\prime} d^{\prime}$; and so on, through the rest, as denoted by the accented letters. When one table length is finished, he draws the cloth along, so as to bring a new length in its place.

The grounding in, or re-entering (rentrage), of the other colours is the next process. The blocks used for this purpose aro furnished with pin-points, so adjusted that, when they are made to coincide with the pin-points of the former block, the design will be correct; that is to say, the new colour will be applied in its due place upon the flower or other figure. The points should not be allowed to touch the white cloth, but should be made to fall upon the stem of a leaf, or some other dark spot.

Every colour is printed separately, the printer going all through the piece with one block; the rest of the colours are next separately fitted into their places by the appropriate blocks, and the piece is then ready for the subsequent operations for raising the colours. Calico intended for printing by block is always smoothed by the calonder (s Caurnoer) the object being to leave the cloth stiff, so as to facilitate the printer joining the different block impressions. When pieces that have been printed by machine are required to have other colours inserted by block, as for instance, the grounding-in of blues, yellows, greens, \&c., after printing and dyeing in madder colours, the same sort of process is adopted, the pieces being dried and calendered, and then printed by blocks technically termed grounds; these grounds are cut from sketches or tracings, taken from the dyed piece when calendered, and, consequently, fit accurately those parts which are intended to be blocked. The grounding-in of colours, after the operations of dyeing, was formerly done by pencils, which were merely small thin pieces of wood, which were dipped in the colour, and the necessary portions of the patterns, such as leaves, \&c., painted in by hand. Of course, this method snon gave way to blocks; but the use of these pencils was continued down to a comparatively recent period for certain cslours, such as pencil-blue, which being a solution of reduced indigo, was too speedily oxidised when spread on the sieve, and required instantapplication of the pencil. Even this colour was eventually applied by block, by a peculiar kind of sieve.

Of late years the tedious hand labour of cutting or coppering blocks has been much reduced by stereotyping; when the pattern has several repeats on the block, a casting in type-metal being made of the pattern, and as many of these as requisite arranged on a plain block, and securely nailed down. It is obvious that the matrix once made, an infinite number of castings can be easily produced; the skilled labourer is therefore reduced to a small portion of what was formerly requisite. The ordinary way of making the mould is to draw or trace on a small block of pear tree (sawn across the grain, so that, the pattern is put on the end of the grain), the pattern to be typed. Slips of copper of varying thickness, but uniform width, are then driven down to a certain distance in the wood, just as in the orclinary way of coppering blocks. When the pattern is thus completed, the slips are pulled out, of course leaving the pattern indented in the wood; the block is now rubbed with chalk, and a border about $\frac{1}{16}$ th of an inch deep of card nailed round the block. Melted type-metal is now run in level with the top of the card, and when cold, a tap with a hammer on the under side of the block easily detaches the type, which requires very little trimming to bo ready for putting on the block; when a number of these are arranged on a block, the surface is filed and ground on a stone till perfectly level. The introduction of Burch's patent typing machine still further simplified the stercotyping process. In this beautiful invention the matrix is formed by steel punches of varying shapes, which are moved up and down by a stirrup and lever, and which are kept heated, by a gas flame ingeniously applied, to the temperature sufficient to char wood, and by moving the tlock about under these punches and depressing thom, the pattern is burnt into the wood to a uniform depth, and the labour of cutting and bending slips of coppor, \&c., dono away with.

There are some interesting modifications of block-printing apparatus which may be here described. In 1834, Mr. Hudson, of the Gale Print Works, near Rochdale, patented a mechanical teerer which was to dispense with the labour of children. The contrivance consists in a travelling endloss web, moved by power, which by passing progressively from the colour vat over the diaphragm, brings forward continuously an equable supply of the coloured paste for the workmen's block.

Fig. 326 represents the construction of this ingenious apparatus, shown partly in section. $a a$ is a ressel of iron, supported upon wooden standards, $b b$, over the upper surface of which vessel a sheet or diaphragm, $c c$, of oiled cloth, or other suitable elastic material, is distended, and made fast at its edges by being bent over a flange, and packed or cemented, to render the joints water-tight. A vertical pipe $d$ is intended to conduct water to the interior of the vessel $a$, and, by a small elevation of the column, to create such upward pressure as shall give to the diaphragm a slight bulge like the swimming tub.

An endless web, $e$, passing orer the surface of the diaphragm, is distended over 
three rollers, $f g h$, the lower of which, $f$, is in contact with the colour-roller $i$ in the colour-trough $x$. On the axle of the roller $i$ a palley wheel is fixed, which allows the roller to be turned by a band from any first mover; or the roller may receive rotatory motion by a winch fixed on its axle. On this said axle there is also a toothed wheel, taking into $d$ another toothed wheel on the axle of the roller $f$; hence, the rotation of the colour-roller $i$ in the one direction will cause the

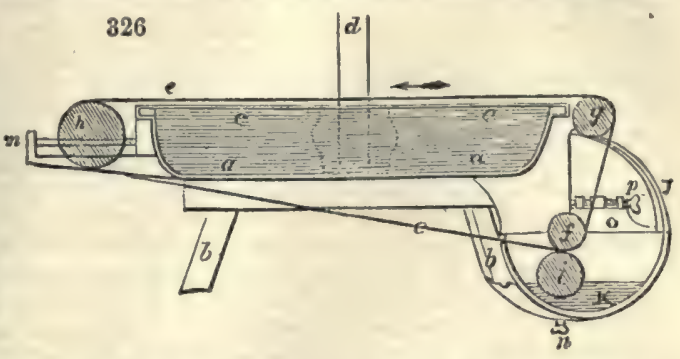
roller $f$ to revolve in the opposite, and to carry forward the endless web, $e$ e, over the elastic diaphragm, the wob taking with it a stratum of colour received from the roller $i$, equally distributed over its surface, and ready for the printer to dip his block into.

Tho axles of the rollers $f$ and $g$ turn in stationary bearings; but the axle of $h$ is mounted in sliding nuts, which may be moved by turning the screws $m$, for the purpose of tightening the endless web. The axle of the colour-roller $i$ turns in mortises, and

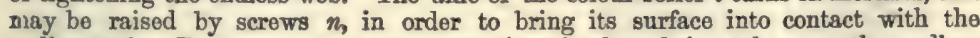
endless web. To prevent too great a quantity of colour being taken up, the endless web passes through a long slit, or parallel aperture in the frame 0 , which acts as a seraper or doctor, and is adjustable by a screw $p$, to regulate the quantity of colour carried up. The contents of the vessel $a$, and the colour-trough $\mathrm{k}$, may be discharged when required by a cock in the bottom of each. This contrivance did not come into general use, but is still employed at some print works in England. The Tobying sieve is a mode of applying with one block several colours at once, whereby the cost of several blocks is saved, and, what is of more consequence, the cost of labour is very much reduced, as one printer produces the same result as the combined efforts of several.

Whenever designs are composed of coloured parts, where each colour lies separate, and where the outlines of the coloured parts are not too close together, a sieve of the following construction is made use of (fig.327). A block of wood is scooped out in hollow compartments $x$, which vary in size and number according to the number and extent of the shades to be printed; these compartments communicate by tubes $\mathrm{B}$ at the bottom, with reservoirs A, at the sides of the sieve, over the compartments is then stretched tightly a woollen sieve; the surface of this cloth is cemented with melted resin string about $\frac{1}{6}$ th of an inch thick, following the configurations of the compartments; the use of this is to prevent the colours mixing and becoming blended at the edges. Colours are now put in the reservoirs, which are kept filled up above the height of the cloth, so that a gentle pressure is exerted against the under side of the sieve. The colours are made of such a thickness as to pass through the cloth, and keep the upper surface moist, but still not too thin, or they would spread when printed. The sieve being thus prepared, the block is furnished with guides, which, working against the sides of the sieve frame, constrain the block

327
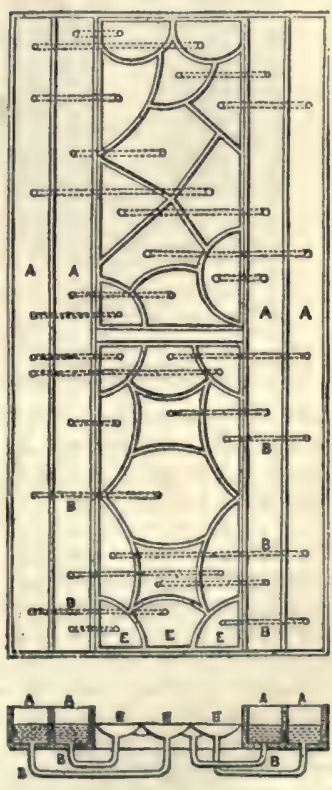
to be always dippod in one place, and thus each part of the pattern finds itself furnished with its proper colour. Sometimes the compartments for the colours are made of metal when required to be durable, so as to serve for a large number of pieces of the same pattern.

When colours are required to melt into one another, technically callod rainbowed (fondus, Fr.), the following apparatus is used:- $\mathbf{\Delta} \mathbf{\Delta}(\mathrm{fig} .328$ ) is a rectangular frame of wood, about 6 inches deep, 2 feet long, and about 1 foot broad. On this frame is stretched, by means of small hooks, a woollen cloth, and the frame then laid on the elastic surface of the usual swimming tub, the cloth downwards and pasted or gummed 
to the oilskin cover of the tub. At one end is now put the colour reservoir B B, which consists of a wooden or metal box, divided into water-tight compartments longitudinally by strips of thin metal; this box is of such a width as to fit easily into one end of the frame, and resting on a board of the same size, fixed across the frame; the depth of the box may be about 4 inches, and the width about 8 inches; but this is regulated by the number of colours to bo blended or rainbowed. A semi-circular pieco of wood, of nearly the same width as the frame, is corered with printers' blanket, and a handle formed on the top, so that the teerer can move backwards and forwards. The colour-lifter, c c, is a flat piece of wood just covering the colour-box; on the under sido of this are inserted wooden pogs, as $\mathrm{D}$, at cortain places determined by the width of the stripe of rainbowed colour and the number of shades composing it. These pegs are of turned wood, about $\frac{\lambda}{8}$ th of an inch thick at the small end, and about $\frac{3}{8}$ ths of an inch at the thick end, this end being also recessed so as to lift more colour; they are nearly as long as the coloux-box is deep. In the figure, suppose it is desired to produce on the sieve two stripes, say $\mathbf{x}$ of a dark green in centre, and two shades of green at each side, and $F$ of chocolato in centre, purple next, and drab next, at each
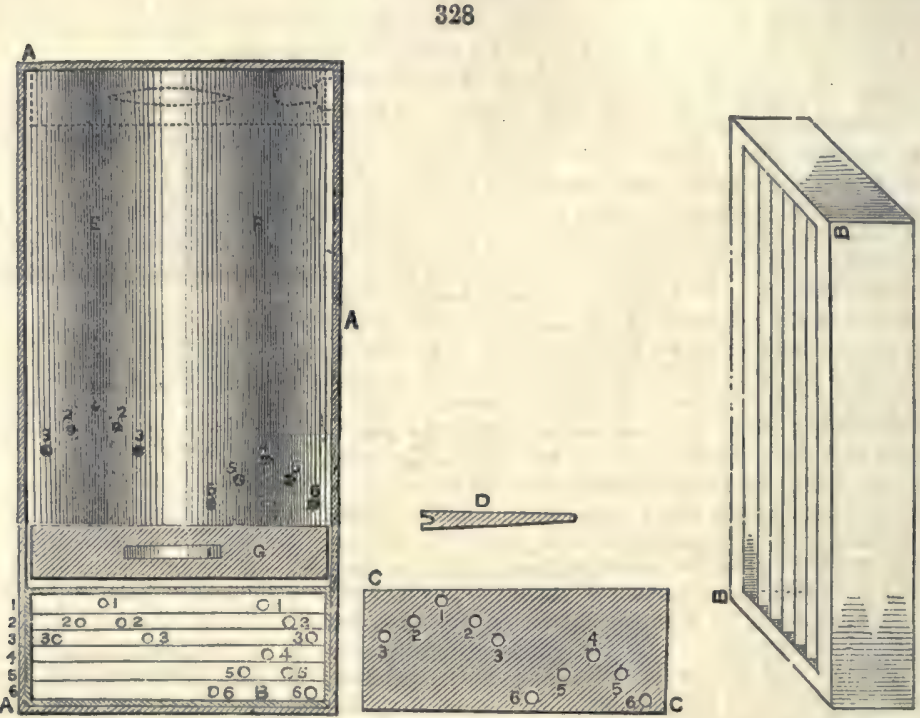

side, the colour-box is filled thus: In No. 1 compartment is put the darkest greon; in No. 2. the medium green ; in No. 3, the palest green; in No. 4, the chocolate; in No. 5, the purple; and in No. 6, the drab. The colour-lifter is so studded with pegs, that when put into the colour-box, tho pogs $1,2,3,4,5$, and 6 respectively dip into their appropriato colours. The brush, or semi-circular roller, $G$, is then moved up to the top, as shown in the dotted lines, the colour-lifter being then lifted up out of the colour-box is held a moment till the colour has ceased dropping from the pegs, and then lifted over, and the pegs allowed to deposit the colour on the sieve, as shown by the black spots $1,2,3,4,5$, and 6 . The lifter is then returned to the box, and a fresh portion of colour lifted, and deposited, as before, at a different part of the sieve, the spots of colour being of necessity all in straight lines; the brush $\mathrm{G}$ is then moved backwards and forwards by the tearer till the colours are sufficiently rubbed together or blended at the edges. It is necessary to observe, that the thickness of the colours must be pretty uniform, and sufficiently thin to allow thom to mix at the edges. By this means one colour is made to melt insensibly into snother, and a beautiful shaded effect produced on the sieve, and consequently on the piece, when printed from a block dipped on it.

The Perrotine is a machine for executing block-printing by mechanical power; and it porforms as much work, it is said, as 20 expert hands. It is in use in many factories in France and Belgium, in a very satisfactory manner; but there is reason to believe that there are none of them now working in this country. Three wooden blocks, from $2 \frac{1}{2}$ to 3 feet long, according to the breadth of the cloth, and from 2 to 5 inches broad, faced with pear-treo wood, engraved in relief, are mounted in a powerful 
east-iron framework, with their planes at right angles to each other, so that each of them may, in succession, bo brought to bear upon the face, top, and back of a square prism of iron coverod with cloth, and fitted to revolve upon an axis between the said blocks. The calico passes between the prism and the engraved blocks, and receires succossive impressions from them as it is successively drawn through by a winding cylinder. The blocks are pressed against the calico through the agency of springs, which imitate the elastic pressure of the workman's hand. Each block receives a coat of coloured paste from a woollen surface, smeared after every contact with a mechanical brush. One man, with one or two children for superintending the colourgiving surfaces, can turn off about 30 pieces English per day, in three colours, which is the work of fully 20 men and 20 children in block-printing by hand. It executes some styles of work to which the cylinder machine, without the surface roller, is inadequate.

The annexed cuts are taken from the 'Traite de l'Impression des Tissus,' of M. Persoz.

Fig. 329 is a vertical section, and fig. 330 an elevation.

A cast-iron framework. B B B cast-iron tables, planed smooth, over which circulate the blanket, the backeloth, and the piece that is printed; c c c sliding pieces, to which the block holders 3 , are screwed, and causing the engraved blocks, 2 , to move alternately

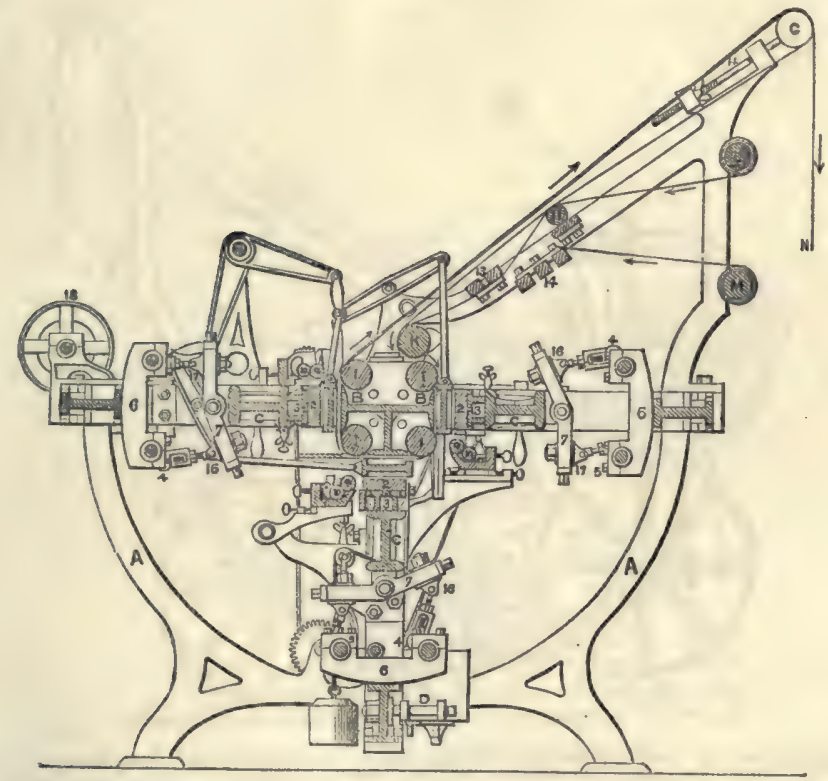

against the woollen surface, from which they receive the colours and the stuff to be printed, by the action of the arms 4 and 5 , the supports of which, 6 , rest on the frame $\mathrm{A}$, and which act, through the medium of connecting rods, on the beams, 7 , keyed to the slides c. The lower of these slides, being in a vertical position, takes by its own weight a retrograde movement, regulated by a counterweight. E E $\mathrm{E}$ are moveable colour-sieves, keyed to connecting rods, and receiving from the power applied to the machine the kind of movement which they require. These sieves, which are flat, and covered with cloth on the surface opposite to the blocks, slide in grooves on the sides of the tables, and receive from the furnished rollors the colours which they afterwards transmit to the blocks. F F F are the colour-troughs filled with colour, and furnished each with two rollers 8 and 10, the last of which, dipping into the tronghs, are charged with colour, which they communicate to the roller 8 , the latter being covered with woollen cloth; and these in their turn transmit their colour to the sieves $\mathrm{F}$, on which it is spread by the fixed brushes, 9. As it is important to be able to vary at pleasure the quantity of colour supplied to the sieves, and consequently to the blocks, the rollers, 10, are in connection with levers, 11, which, by means of 
adjusting screws, bring them into more or loss intimate contact with the rollers 8 , and consequently vary the charge of colour at pleasure.

The blanket, backcloth, and fabric are circulated as follows :-At the four angles formed by the three tables, B, are rollers, 1, armed on their surface with needle-points, which prevent the cloths from slipping as they pass round, and thus secure the regular movement of the stuff to be printed, a movement determined by the toothed wheels 21 (fig. 330) fixed at the extremities of the axes of these rollers. $a$ is a roller for stretching the endless web, resting with the two ends of its axes on two cushions forming the extremities of the screws 12 , by which the roller can be pushed further out when required, to give the cloth the necessary tension. $\mathrm{H}$ is another tension roller, supporting the blanket and backcloth. $\mathrm{K}$ is a roller which serves similar purposes for the blanket, the backcloth, and the fabric in course of being printed. $T$, the blanket, which in its course embraces the semicircumference of the roller $G$, passes over the roller $\mathrm{n}$, and behind $\mathrm{K}$, to circulate round the cylinders 1 , and over the surfaces of the tables B. $\mathbf{L}$ is a cylinder from which the backcloth is unwound, being first stretched by the roller $\mathbf{H}$, and then smoothed by the scrimping bars 13, from which it proceods to join the blanket on arriving at the roller K. I a roller, from which the fabric to be printed is unrolled by tho movement of the machine, first passing over the serimping bars 14, and joining at $\mathrm{x}$ the blanket and backeloth, which

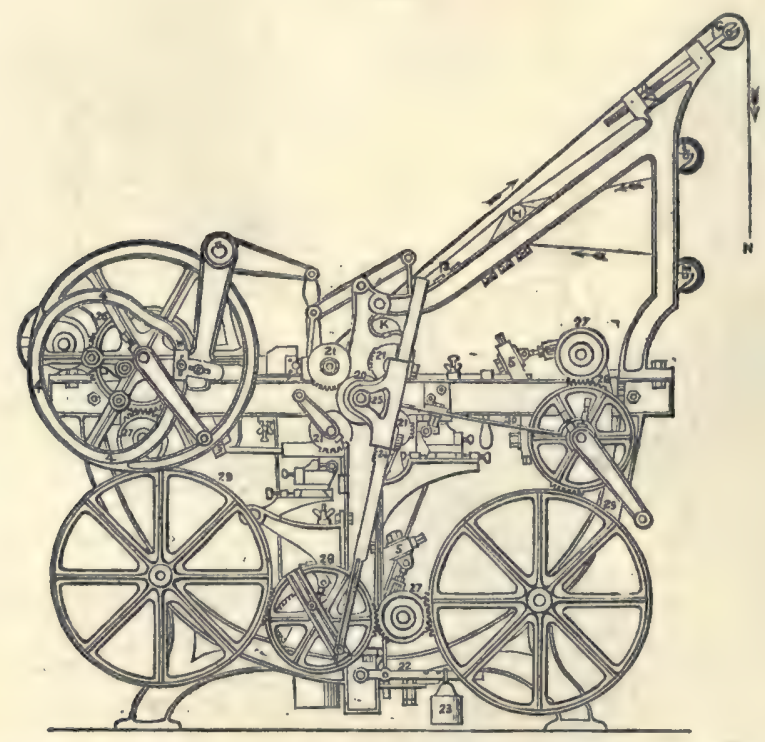

It accompanies in their course till it arrives at the roller $a$, when it separates and passes off in the direction of the line $\mathrm{s}$, to the hanging rollers, where it is dried.

The machine is put in movement, either by a man with a winch-handle, or by power communicated by a strap which passes over the pulley 18. This pulloy has several diameters, so as to give several speeds; it is loose on the driving shaft, and carries catches which lock into those of a sliding catch-box on the shaft, when the machine is to be put in movement. The movement of the machine is intermittent because the printing is intermittent; moreover, it must be so regulated that the fabric advances a distance exactly equal to the breadth of the blocks, and that it moves forward whilst the sieves are charged with colour from the rollers 88 . This result is obtained by means of a regulator, or dividing wheel 20. The wheels 21 , fixed at the extremities of the axis of the cylinders 1 , and having each the same number of teeth, receive their movement from a central wheel toothed in the same manner, and placed behind the wheel 20 . The last receives an alternating motion from a rack, 24 , fixed in a copper piece, 25 , and which rises and falls alternately, being keyed at its lower end to one of the spokes of the wheel 28. By varying the position of the point at which the end of the rack is connected with the spoke 26, the length or range of its movement is proportionally changed, and more or less of the teeth of 
the wheel 20 are made to pass, which renders proportionally, greater or less, the advance of the cloth at each movement; and this is further rogulated by a ratchet wheel placed at $\mathbf{D}$. At each half turn of this last, the lever 22 raises the catch or pallet, and throws out of gear the wheels 21 during the other half turn; but as in the working of these wheels there would be inevitably a backward movement, this is prevented by a break, consisting of a pulley, mounted on the shaft of the axis of the wheel 20, and a brass wire which after making a turn and a half, or two turns, on this shaft, is stretched by the weight 23 , which offers a sufficient resistance to any recoil. The slides or block-holders are put in motion by the wheels 27 and 28, gearing with tho larger wheel 29. And to vary their action at pleasure, both for causing the blocks to bear more or less strongly on the sieves, so as to be more or less charged with colour, and likewise for attaining the exact pressure, which suits best for the colour to be laid on, it is sufficient to move the points of junction, 16 and 17, to a greater or less distance from the point marked 15, which constitutes the centre of oscillation of the beams that work the slides. The movement of the sieves is controlled by that of the cam 11, 30, which works them all three by putting in motion a shaft with which they are respectively keyed. The furnishing rollers receive their movement from gearing with pinions on the axes of the rollers 88 . The general working of this complex machine remains to be described. When put into regular motion, and the three blocks have delivered their impression exactly at the same instant, three simultaneous morements then commence.

1st. The stuff advances a distance exactly equal to the breadth of the blocks, and with it the blanket and backeloth, so that the portion of the fabric which leaves the third block behind it, is fully printed; that which was under the second advances opposite the third; that which was under the first, moves along to the second; and a fresh breadth of white or imprinted fabric arrives opposite the first. 2nd. While the cloth is advancing as above stated, the sieves take the place which they occupy in the section, fig. 329 , that is to say, the first on the right hand rises, the second moves from left to right, the third descends, and in this movement all three press slightly on the furnishing rollers 8 , from which they receive the colour, which has been spread uniformly by the brushes 9 . 3rd. In the meantime, the slides, or blockholders, by a for ward movement, push the blocks against the sieves, to charge them with colour, and the blocks at the same time receive from the slides a gentle backward movement, during which the sieves deviate from their position; the blocks then return upon them, and are drawn back again after being applied to a new part of the colour surface. When these simultaneous movements have taken place, the action of the machine proceeding without intermission, the sieves move back from before the blocks, and these are pushed up against the latter, printing the position of the fabric that is stretched npon them. This brings the machine to that position at which the description commenced; and this succession of movements is renewed and repeated as long as the operation lasts; the printer having it always in his power to suspend the advance of the stuff whilst the working of the blocks and sieves continues, so that the colour may be re-applied to the same part of the fabric as often as may be required for a good impression.

There have been several attempts at block-printing by machinery in this country, amongst which the machines of $\mathrm{Mr}$. Joseph Burch have been most successful; but from one cause or another, none of them have ever come into general uso, and it is unnecessary therefore to particularise them.

The copper-plate printing of calico is almost exactly the same as that used for printing engravings on paper from flat plates, and being nearly superseded by the next machine, need not be described.

The cylinder printing-machine is one by which one or more colours are rapidly printed from engraved copper cylinders or rollers by the mere rotation of the machine, driven by the agency of steam or water. The productive powers of this printing automaton are very great, amounting for some styles to a piece of 30 yards per minute, or a mile of printed eloth per hour. Fig. 331 will give the reader a general idea of this elegant and expeditious plan of printing. The pattern is engraved upon the surface of a hollow cylinder of copper, and the cylinder is forced by pressure upon a strong iron mandrel, which serves as its turning shaft. To facilitate the transfer of the impression from the engraving to the cotton eloth, the latter is lapped

331

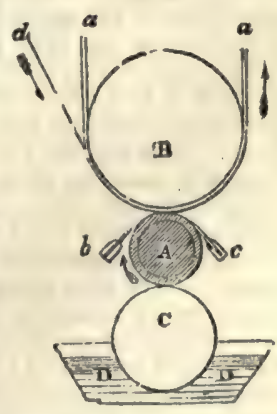
round another large cylinder, rendered elastic by rolls of woollen cloth, and the engraved cylinder presses the calico against this elastic cushion, and thereby prints it as it 
revolves. Let A be the engraved cylinder mounted upon its mandrel, which receives rotatory motion by wheels on its end, connected with the steam or water power of the factory. B is a large iron drum or voller, turning in bearings of the end frames of the machine. Against that drum the engraved cylinder $\mathbf{\Delta}$ is pressed by weights or screws; the weights acting stoadily, by levers, upon its brass bearings. Round the drum B the endless web of felt or blanket stuff $a a$, travels in the direction of the arrow, being carried round along with the drum B, which again is turned by tho friction of contact with the cylinder $\Delta$. c represents a clothed wooden roller, partly plunged into the thickened colour of the trough $\mathrm{D} D$. That roller is also made to bear, with a moderate force, against $\Lambda$, and thus receives, by friction, in some cases, a movement of rotation. But it is preferable to drive the roller $\mathbf{c}$ from the cylinder $\mathbf{A}$, by means of a system of toothed wheols attached to their ends, so that the surface speed of the wooden or paste roller shall be somewhat greater than that of the printing cylinder, whereby the colour will be rubbed, as it were, into the engraved parts of the latter.

$A s$ the cylinder $\mathbf{A}$ is pressed upwards against $\mathrm{B}$, it is obvious that the bearers of the trough and its roller must be attached to the bearings of the cylinder $\Delta$ in order to preserve its contact with the colour-roller c. $\quad b$ is a sharp-edged ruler of gun-metal or steel, called the colour doctor, screwed between two gun-metal stiffening bars; the edge of which wiper is slightly pressed at a tangent upon the engraved roller $\mathbf{A}$. This ruler vibrates with a slow motion from side to side, or right to left, so as to exercise a delicate shaving action upon the engraved surface, as this revolves in the direction of the arrow, $c$ is another similar sharp-edged ruler, called the lint doctor, whose office it is to remove any fibres which may have come off the calico in the act of printing, and which if left on the engraved cylinder, would be apt to occupy some of the lines, or at least to prevent the colour from flling them all. This lint doctor is pressed very slightly upon the cylinder A, and has no traverse motion.

What was stated with regard to the bearers of the colour-trough $\mathbf{D}$, namely, that they are connected, and moved up and down together, with the bearings of the cylinder A, may also be said of the bearers of the two doctors.

The working of this beautiful mechanism may now be easily comprehended. The web of calico, indicated in the figure by the letter $d$, is introduced or carried in along with the blanket stuff $a a$, in the direction of the arrow, and is moved onward by the pressure of the revolving cylinder $\Delta$, so as to receive the impression of the pattern engraved on that cylinder.

Before proceeding to describe the more complex machines which print upon eloth several colours at one operation, by the rotation of so many cylinders and rollers, it is advisable to give some insight into the modern method of engraving the copper cylinders. These were formerly engraved altogether by hand, in the same manner, and with similar tools, as the ordinary copper-plate engravings, till the happy invention of Mr. Jacob Perkins, of America, for transferring engravings from one surface to another by means of steel roller dies, was with great judgment applied by Mr. Joseph Lockett sen. to calico-printing, so long ago as the year 1808, before the first inventor came to Europe with the plan. The pattern is first reduced or increased in size to such a scale, that it will repeat evenly over the roller to be engraved; and as rollers are of varying diameters, owing to old patterns being turned off, \&c., this drawing to scale has to be adopted for every roller, the exact circumference of the roller being taken and the pattern arranged in accordance with this. This pattern is next engraved in intaglio on a roller of softened steel, which is of such a size that one repeat of the pattern exactly covers its surface; generally these rollers are about 3 inches long and from $\frac{1}{2}$ an inch to 2 or 3 inches in diameter. The engraver aids his eye with a lens when employed at this delicate work. This roller is hardened by heating it to a cherry-red in an iron case containing pounded bone-ssh, and then plunging it into cold water : its surface being protected from oxidisement by a chalky paste. This hardened roller is put into a press of a peculiar construction, called the clamming-machine, where by a rotatory pressure, it transfers its design to a similar roller in the soft state; and as the former was in intaglio, the latter must be in relievo. This second roller being hardened, and placed in the engraving-machine, is employed to engrave by indentation upon the full sized eopper cylinder the whole of its intended pattern. The first rollor engraved by hand is called the die; the second, obtained from it by a process like that of a milling tool, is called the mill. By this indentation and multiplication system not only has the cost of engraving been much diminished, but designs and styles have been brought into requisition which by no other means could have been obtained. The restoration of a worn-out cylinder becomes extremely easy in this way; the mill being preserved, need merely be properly rolled over the copper surface again. The die roller is made of such a size that its circumforence is exactly a fractional part of that of the mills, say one-half, one-third, one-fourth; then 
in the elamming-machine the die revolving in contact with the mill repeats its surface so many times on the surface of the mill. By this means as little skilled labour as possible is used. When a pattern having more than one colour is to be engraved, the drawing is reduced to scale as before, each roller being made of the samediameter; then a tracing is made of each colour, which is engraved on a separate die and milla mill being required for each colour-which engraves its separate copper roller; when these rollers come to be worked in the printing-machine, each roller fits its part of the pattorn into place, and the original pattern is reproduced. The annexed drawings of engraving machinery are from those made by Messrs. Gadd and Hill, of Manchester, to whose courtesy are due also the drawings of the printing-machines and their drying apparatus hereafter described. Fig. 332 is a front view of the clammingmachine, and fig. 333 is a side view of the same. A A cast-iron framework; $\mathrm{B}$ a headstock screwed on the frame work $\Delta ; 0$ s sliding piece, capable of movement from back to front on the headstock $B$; the position being determined, it is secured by the screw shown under 0 ; the roller $\mathrm{D}$ revolves in bearing attached to the sliding piece $\mathrm{c}$; the supporting pioce $\mathrm{x}$ has a motion backwards and forwards on the supporting piece $o$, which moves up or down; $e$ is a small steel roller, which again supports the die roller seen in the centre of the drawing. The roller $F$ is of softened steel called the mill, which revolves in bearings attached to the headstock, which has a sliding movement on the slide block $\mathrm{H}$, which is moved from right to left by the serew $\mathrm{x}$, worked by the lever $x . \quad x$ is a pinion gearing into the toothed wheel $x$, and turned

332

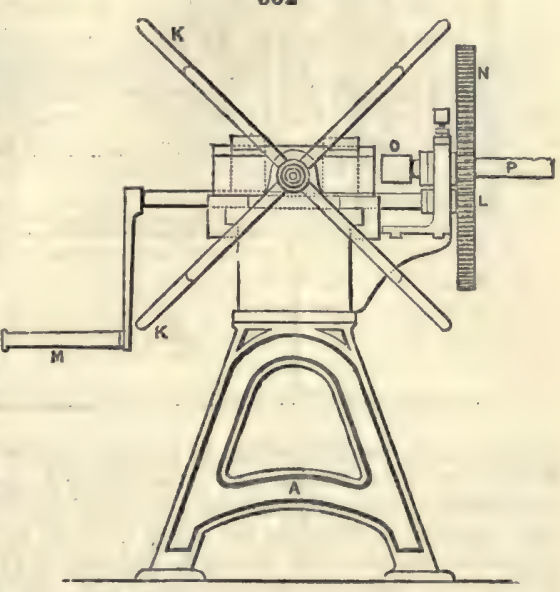
by the winch-handle $\mathrm{M}$; the shaft $\mathrm{P}$ has a sliding movement through the wheel $\mathrm{N}$, and carries the boss 0 , which has a square aperture to receive the centre of the mill, which is squared to fit into it. $q$ is a screw used to tighten and keep in the desired position the saddle pieces E 0 , which together are pushed up or down to meet the varying size of the die.

The die $d$ having been hardened, is inserted in the machine resting on the auxiliary hard steel roller e, which again rests on the supporting piece $\mathrm{E}$; the die being in contact with the hard steel roller D, the soft steel roller or mill $\mathrm{x}$ is next forcibly serewed up in contact with the die, rotatory motion being given to the roller $\mathrm{D}$ by the toothed wheols, those portions which are in intaglio in the die become in relievo on the mill. It is then ready for the machine engraver to transfer its pattern to the copper roller. Fig. 334 is an elevation of the engraving machine, $\Delta \mathrm{A}$ is a mandrel which carries the copper roller $B$; the mandrel is fitted in the universal joint $\mathrm{c}$, which is secured on the shaft of the wheels D D, which are a double pair of wheels for the purpose of altering the speed from fast to slow, and are moved by the winch-handle or

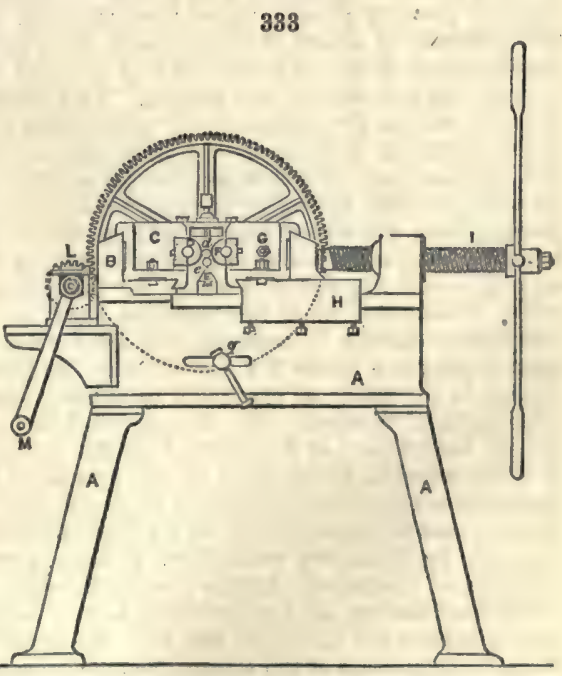
pulley. The lever $\mathrm{k}$ is fitted, works loosely on the shaft, on which is keyed the wheel 5 . By means of the serew $G$, the lover $\mathrm{E}$ can be secured to the wheel $F$, 
By this contrivance the motion termed rocking is effectod, that kind of motion being requirod when the pattorn repoats at great intorvals. The mill works in bearings attached to the pillar and carriage $\mathrm{m} \mathrm{H}$, which is moved from right to left by the serew I I; the mill is forcibly prossed against the copper roller by a weighted lever, which forces down the bearings of the mill in the pillar $\mathrm{H}$; this lever cannot be shown in the figure, but is at right angles to the roller. The mill being in contact with the

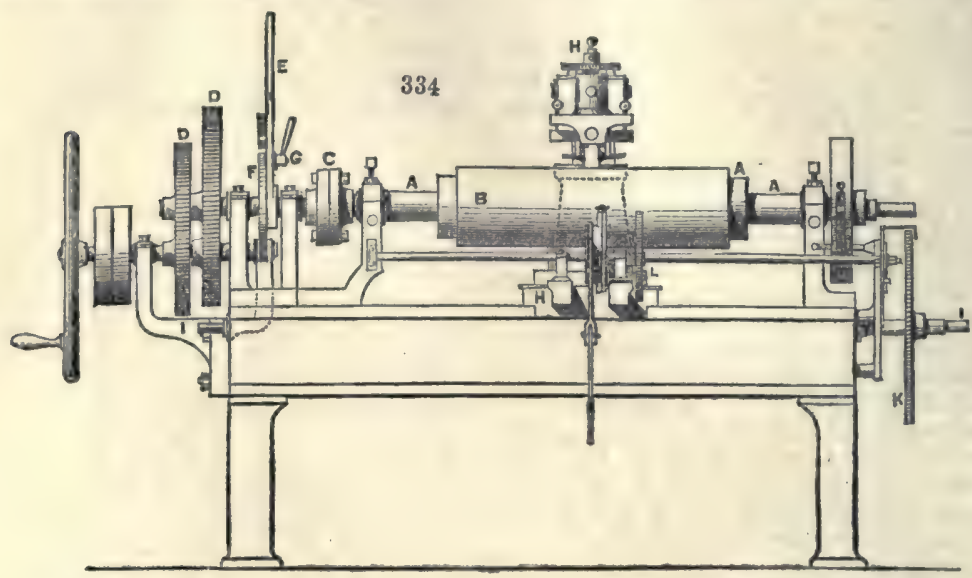

copper roller, revolves with it simultaneously on the roller being moved by the wheels $\mathrm{D}$ D or the lever $\mathrm{E}$, and consequently impresses or engraves its pattern on the copper roller; when the mill has traversed the circumference, it is then moved to its next relative position by the screw I, which moves the pillar and carriage $\mathbf{H}$; the exact distance the mill moves is determined by an index on the wheol $\mathrm{K}$, which is divided into segments, corresponding with the number of repeats laterally on the roller. The apparatus shown at $L$ is used occasionally when the machine is employed for turning off an engraved pattern, which, however, is generally performed in a slide lathe, and is unnecessary further to describe here,

Etching by nitric acid is largely employed in engraving for calico-printing. The roller is costed all over with a thin film of bituminous varnish. Lines of any required form or quantity are then traced upon it, which lay bare the copper surface beneath. On immersion in a bath of diluted acid, those parts of the roller only which are exposed become bitten or etched to any required depth, the remaining surface of the roller being protected by the varnish. This process is employed in combination with the mill process, for engraving all 'solids,' that is, those parts of an engraving which are intended to contain masses of colour, to be transmitted to the ealico in the printing-machine. For this purpose the outline of the solid to be engraved is first put on with the mill; the roller is then varnished, and a number of diagonal or angled parallel lines are traced upon it with a diamond-point. Those portions which are intended to be white on the calico, are 'stopped out,' or covered with varnish, by the operative, and the roller is ready for immersion in the bath of diluted acid. The roller is removed when the requisite depth is obtained, is well washed and the bituminous coating is removed, and after slight examination and addition by the hand engraver is ready for the printer. But the two most notable applications of etching in the trade of engravers to calico-printers have been the 'Eccentric' and 'Pontagraph' systems. The former owed its greatest development to the late Mr. Joseph Lockett, son of the founder of the eminent firm which still bears his name. It is not essential to the scope of this article, to give an elaborate description of the beautiful and ingenious machinery employed, nor would it be possible without a very elaborate set of drawings. It may suffice to record that by means of diamond-points, actuated by elaborato machinery, a most curious variety of configurations is produced on the roller. In this process the exact effect that will be produced by any given modification of the machine, cannot be determined beforehand, though an approximation can be made; but when a pattern is produced, and notes are taken of the relative position of the wheels, \&c., the same pattern can at any time be reproduced. This system is applicable principally to ground-works or, 28 they are termed, 'covers,' which form the bases of backgrounds of many classes of design much in vogue on 
account of their utility and variety. The Pentagraph system is of later development. The process is the following:-

The pattern is first enlarged to several times its size: this is conveniently done by the camera The paper pattern being put in the camera, an enlarged copy is thrown on a table in a darkened room, and is there easily traced on paper. It is then transferred to a thin zine plate, and this plate is then engraved with a graver, the lines of the engraving being adapted for the tracing-point to work easily in. The zine pattern, if of a 2- or more coloured pattern, is coloured for the guidance of the operative. It is then laid on the bed or table of the pentagraph machine, and a varnished roller being mounted in the machine, a number of tools, corresponding in number to the ropeats laterally, and carrying diamond points, are placed in contact with the roller. The operative then carries the tracer successively into all the lines of the pattern, a lever allowing the points to touch the roller only when necessary. The pattern is thus traced by the etching points on the roller a less size than that on the zine plate, or the same size as the paper drawing. The roller is then painted and etched with nitric acid, as before described. In 1834 Horton Deverill of Manchester patented the first application of the pentagraph principle to engraving cylindrical surfaces for calico-printing. It was the simplest form of the ordinary pentagraph, viz., a rhomboidal arrangement of levers, the relative adjustment of which, through appropriate connections, secured the transfer to the roller of the design from its enlarged copy. The time was not propitious for the adoption of this clever invention; a machine was tried by Mr. Lockett, and abandoned less on account of any inherent defect, than because of the very limited use which could be made of it in the then prevalent style of engraving. In 1848 Isaac Taylor patented the substitution for the single rhomboidal frame of Deverjll's pentagraph, several such frames in a continuous series. It was proposed to secure by this means a higher power of diminution in a compact machine, and as a result, a more perfect engraving. He also multiplied the number of bars supporting the tracing or etching tools. With this machine commenced the first commercial application of the pentagraph system of engraving in England, though on a very limited scale. In 1854 William Rigby introduced and patented an improved machine, based on the American invention of William Whipple. Whipple had constructed a machine with a curved bed for the zinc plate instead of the flat tables of Deverill and Taylor. The tracing point was attached to a swinging frame, and motion was communicated to the roller, and etching tools respectively in a similar manner to that shown in Rigby's machine, figs. 335 and 336. In a patent dated January 1, 1857, Rigby applied two rows of etching tools to his machine, whereby the time occupied in tracing the pattern was very considerably diminished. This method was soon very extensively adopted: a reference to the annexod figures will more clearly show the main features of this machine.

In figs. 335 and $336, a$ represents the cylinder to be operated upon; and $b$, the bed or table for the reception of the enlarged pattern or original device; $c$, the tracer, which is made to traverse in the direction of the are of the bed or table, and by means of its connection with the carriage $h$, the rail $d$, and the connecting arms $e e$, communicates part of a revolution to the bar or axis $f$, and thence to the cylinder through the dies $g g$, on which the cylinder rests. The cylinder being thus moved in a rotatory direction, will receive from the tools in contact with it diminished copies of the transverse lines which may have been gone over by the tracer on the enlarged pattern or device. The tracer $c$ being connected with the carriage $h$ which travels along the rail $d$, will, in passing over a line running longitudinally with the machine, communicate a partial revolution to the wheel $l$ by means of the bands of stoel $j j$, similar to watch-springs, which pass under and over the small wheels $k k$, and are passed round and secured to the large wheel $l$, which is mounted on the vertical shaft $m$, carrying at its upper end the small drum $m^{\prime}$, round which passes the steel band $n$, secured at each end to the pieces 00 . These pieces are secured by bolts or screws to the sliding frames $p$, to which the upper tool bar or bars $q$, which support the graving, drilling, or etching tools $r r r$, are fixed. Thus any motion of the large wheel $l$ will be imparted to the drum $m^{\prime}$, and by it through the steel band $n$ to the sliding frames $p$ and the tool bark $q$, and, consequently, to the tools $r$, thereby transferring to the cylinder diminished copies of any lines in a lateral direction that may be gone over by the tracer. It will be evident that the result of the simultaneous action or compounding of the two motions, by passing the tracer over any diagonal or curved line, will be the production of a diminished copy of such diagonal or curved line by each of the tools. 8 is a treadle with a vertical link and appropriate leverage, by which the tools may be brought in contact with the cylinder when required; $t t$ are counterbalance weights for the connecting arms $e e$, lower rail $d$, \&c.; $u$ and $v$ represent a worm and wheel for the purpose of giving the roller an extra partial revolution when it is required to engrave upon a different portion of the circumference of the cylinder; and to effect a

ฟoL. I. 

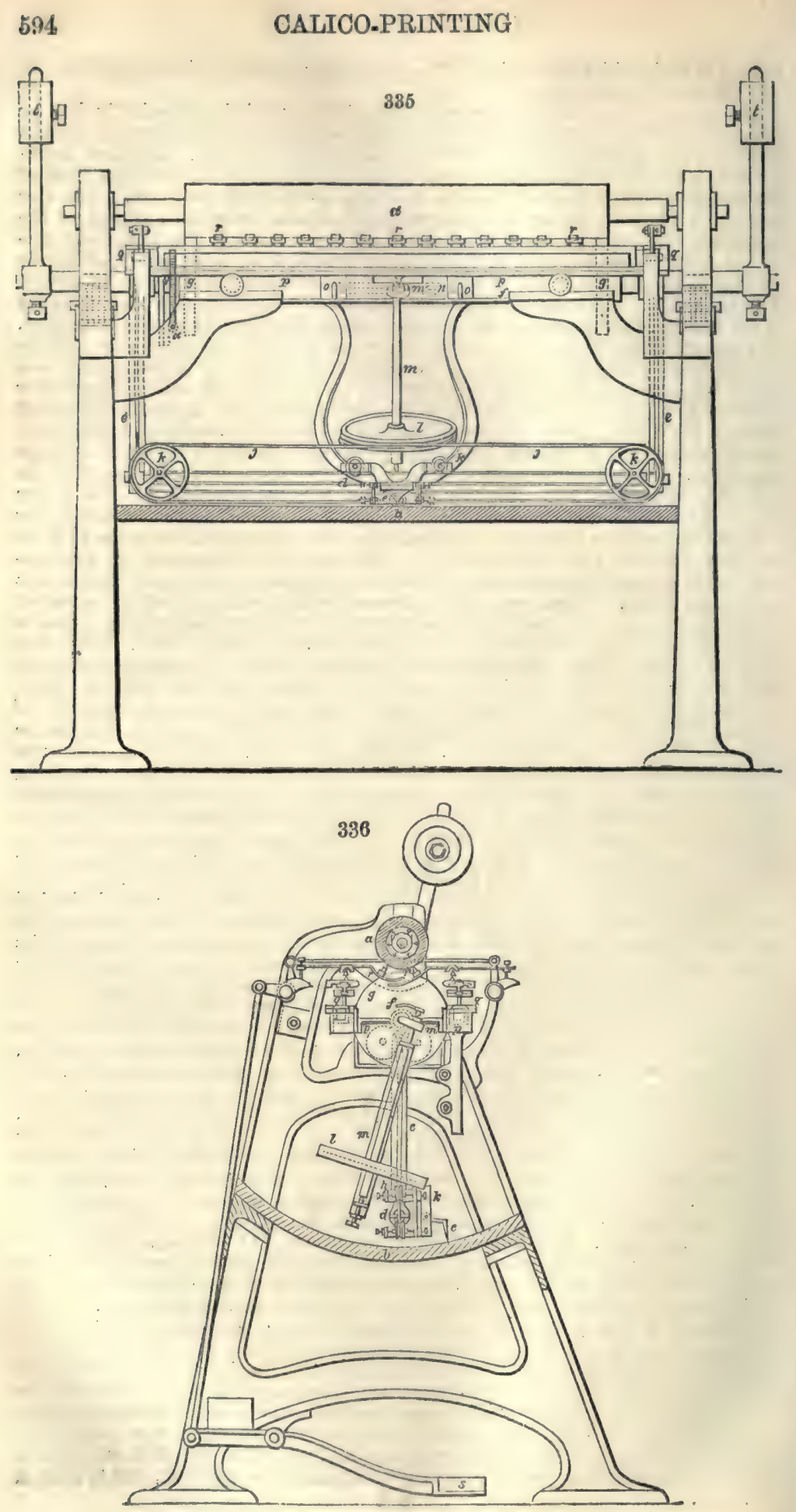
similar purpose in the longitudinal direction, the tool bar may be made to shift in its sliding frame with an adjusting screw attached to it, by means of which any degree of exrctitude in the setting of the tools may be obtained.

In the machine, as shown in the accompanying drawings, the design executed on the cylinder would bear the same proportion in size to the enlarged pattern on the bed or table that the small drum $m^{\prime}$ bears to the large wheel $l$, and the radius of the dises $g g$, to the radius of the circular bed; but by the adoption of wheels and dises of different diameters, any desired proportion between the pattern engraved and the enlarged pattern may bo adopted.

By minor improvements, an alternate reverse action is given to the tools and bars, thus adapting the machine for turnover patterns; also more than one row of points may be attached to each bar. In 1857 William Shields patented a machine in which he substituted a flat table for the curved table of Whipple and Rigby, and applied an ingenious and original method of varying the dimensions of the design on the zinc plate in transferring it to the roller. The tracing instrument communicates motion to the etching tools through the intervention of inclined planes, so that by varying the angle of these planes motion is transmitted in the required proportion; motion is communicated to the cylinder through similar apparatus. The remarkable facilities offered by this machine caused its immediate adoption by Messrs. Lockett, Leake, and Co., of Manchester, by whom it has been most extensively used in their own works, and manufactured for the trade in England and on the Continent. A reference to th. annexed diagram will more clearly show the peculiarities of this machine.

Fig. 337 is a longitudinal section of the machine; fig. 338 is a plan-view. The framework of the machine is shown at $a$, provided with a table $b$, upon which is placed

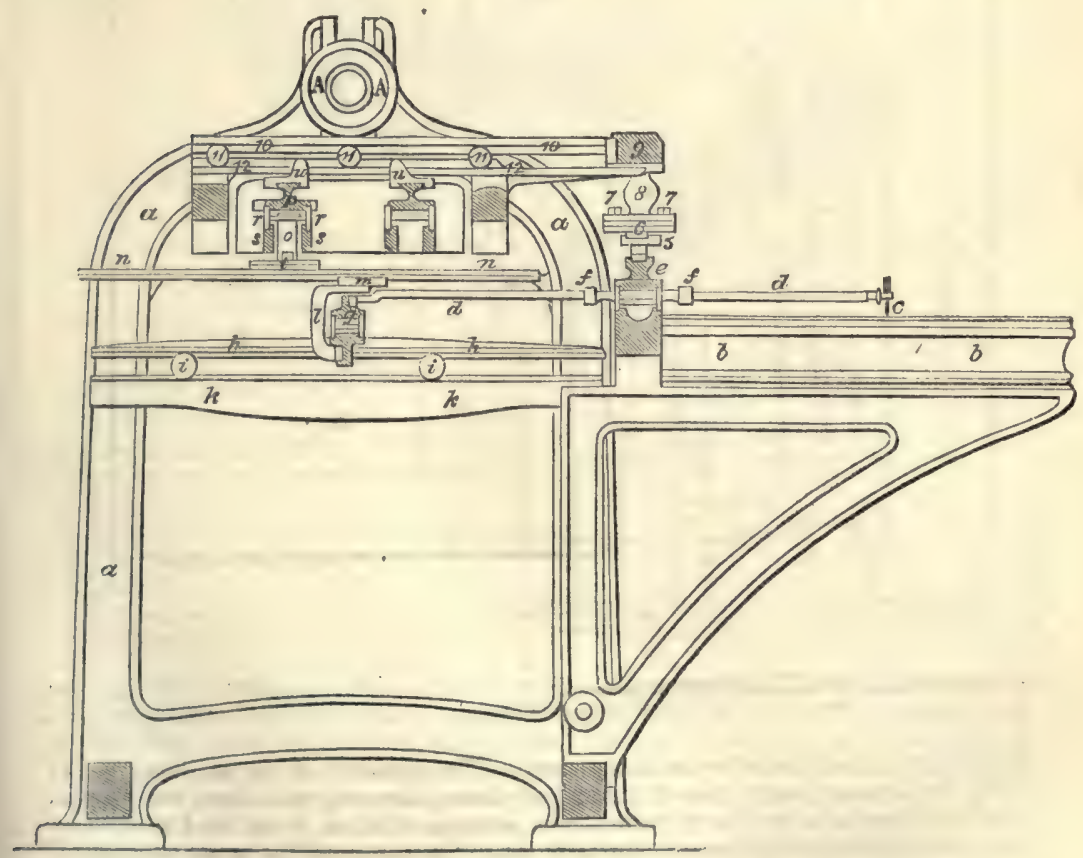

the design to be copied. The tracer $c$ is jointed upon a rod $d$, which rod $d$ passes freely through a bar $e$, and between slides $f$, mounted upon the said bar, which thereforo act as guides when the rod is moved to and fro in the direction of its length. This rod $d$ is attached at one end to a cross-bar $g$, carried by parallel bars $h$, which rest upon flanged rollors $i$, capable of rolling upon fixed rails $k$; as therefore the tracer $c$ and bar $d$ are moved longitudinally, the bar $g$ and its parallel supports are caused to partake of a like motion. To the bar $h$ is fixed a bracket $l$, which carries at its upper ond a stud $m$, projecting into a groove formed lengthwise in a lever $n$; this lover is 
attached by an arm 0 to a cross-bar $p$, and is capable of being turned upon its centre 80 as to oceupy any angle in referenco to the tracer rod $d$, being confined in any desired position by a tightening screw $q$, and thereby constituting an inclined plane for tho stud $m$ to act against; the cross-bar $p$ rests upon flanged rollers $r$, which roll upon a fixed bar $\delta$, provided with a groovo for the purpose of allowing the arm $o$ to travel along its length. To the bar $p$ are affixed the carriages $u$ of the tools. The upper part of the bar $e$ (through which the tracer rod passes) carries a stud 5 situato within a groove formed in a lever 6 , which lover, like that shown at $n$, is capable of being turned upon its centre to any angle, so as to constitute an inclined plane for the stud 5 to act against, and of being confined to any desired position by means of tightening screws 7. The boss of the lever 6 carries an arm 8, which extends upward, and is there connected with a cross-bar 9 , to which are attached parallel bars 10, running upon flanged rollers 11, situato upon fixed bars 12, upon which the cylinder

338

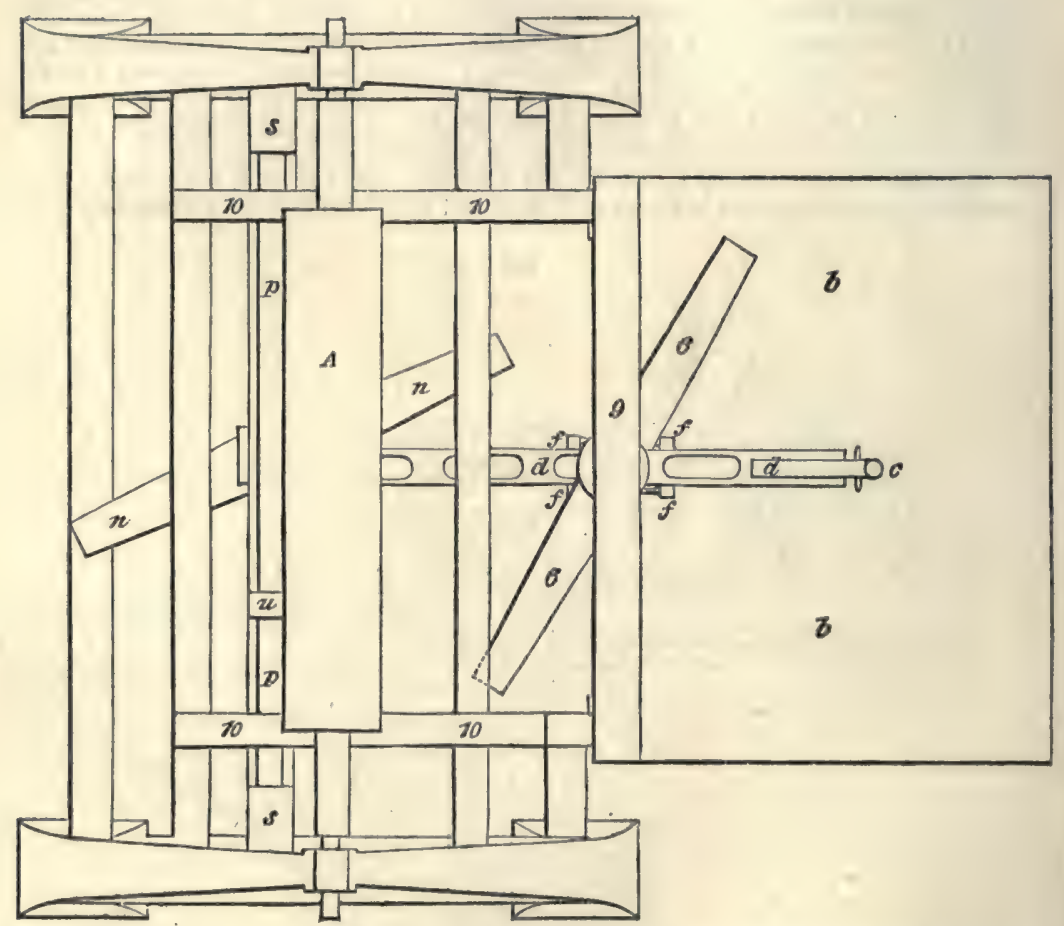

A bears so as to communicate rotatory motion thereto. The operation of the machine is as follows:- The tracer being pushed inward or drawn outward, will cause the parallel bars $h$ to move in a similar direction, thus carrying the bar $g$ and stud $m$ along the inclined groove in the lever $n$, by which means the bar $p$ will be caused to move transversely, carrying with it the tools along the surface to be operated upon, and thus the operation in one direction is accomplished. A crosswise movement of the tracer will cause the rod $d$ to move the bars $e g$ transversely, and the stud 5 will be forced against the inclined plane within the lever 6 , so as to effect a longitudinal motion of the bars 10, whereby the ribs 13 will act against a part upon the axis of the cylinder $\mathrm{A}, \mathrm{so}$ as to cause it to revolve, and thus the motion in the other direction is obtained. By subsequent improvements the pentagraph system has been greatly extended in its application to many styles of engraving hitherto unapproachable. Conspicuous amongst them may be noted the employment of the punch and a vibratory motion imparted to the roller, by means of which circumferential lines are engraved on the roller which have equal working qualities with those obtained by the mill.

With regard to the 2- and 3-coloured machines, we must observe, that as the calico 
in passing between the cylinders is stretched laterally from the central line of the web, the figures engraved upon the cylinders must be proportionately shortened, in their lateral dimensions, especially for the first and second cylinders.

Cylinder printing, although a Scotch invention, has received its wonderful development in England, and does the greatest honour to this country. The economy of labour introduced by these machines is truly marvellous; one of them, under the guidance of a man, to regulate the rollers, and the service of two boys, to supply the colour-troughs, \&ce, being capable of printing as many pieces as nearly 200 men and boys could do with blocks.

Pieces for printing by machine are stitched together end to end, in bundles containing from 20 to 40 pieces. This was formerly done by girls, but they are now more economically employed in tending stitching machines. Perhaps the original stitching machine employed in print-works is that shown in fig. 339. It is still used in dyehouses, where pieces are temporarily stitched together to enable them to undergo various dyehouse operations, and where rapid unstitching is of consequence, which is easily performed by unloosing the ends of the thread and pulling it straight out.

339

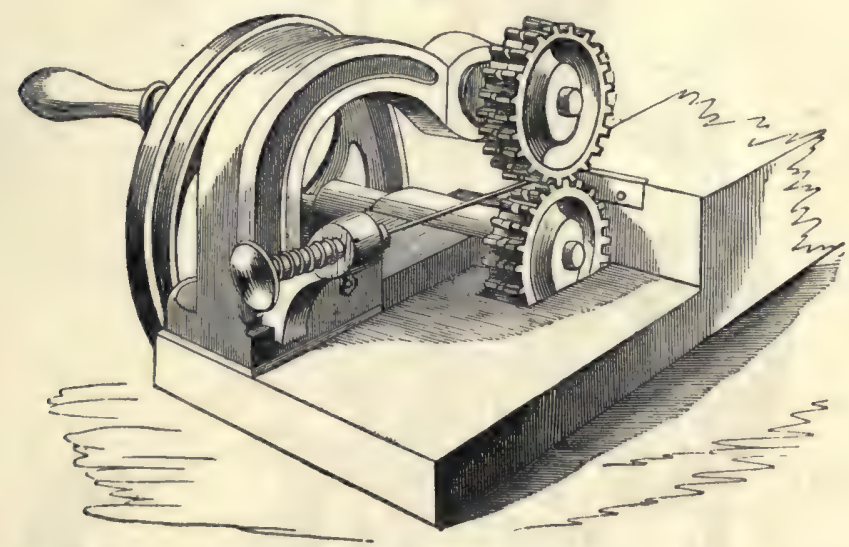

This machine was the invention of Charles Morey, in 1849. A pair of wheels are fitted with leaves on their peripheries, and gear into one another like cog-wheels. These wheols are mounted in suitable bearings fixed to a sole plate, and receive rotatory motion by means of a winch-handle. The centre of the teeth of both wheels is cut away, so as to form a circular groove between the two teeth which happen to be together. Opposite to this groove, and attached to the frame, there is a bracket which carries a sliding piece, with a spiral spring wrapped round it. In the end of the sliding piece, which passes through the bracket, there is a receptacle for the eye-end of a needle, the point of which rests in the groove formed by the wheel; the needle is threaded; and the fabric to be stitched placed behind the wheels, to which rotatory motion is communicated, whereby the fabric is successively folded into undulations, which, as the operation proceeds, are forced on the point of the needle; when the needle is full, and the piece at the other side of the wheels, the needle is pushed back on the spring, removed from the machine, and the thread drawn through the pieces, which are then basted or stitched together. This is a very rapid mode of stitching ends of pieces together; but where a number of pieces are stitched end to end for the purpose of being put through several operations without unstitching, a firmer description of stitching is required, and a machine of more elaborate construction is used. A machine invented in America, and hence called the American Machine, was for a long time used. In this machine the ends of the pieces were hooked round a circular table, three sets of pieces being put on at once; the needle now traversed the inside circumference, sewing the ends, which were then unhooked. This was found troublesome and not sufficiently expeditious, and afterwards a modification of the Wilcox and Gibbs sewing-machine was introduced. This is being rapidly superseded by a very beautiful machine invented by William Birch, of Manchester (fig. 340). It is not within the scope of this article to give a satisfactory description of the details of this machine; in general terms, we may say that it makes the common flat chain-stitch, which is easily drawn out again when required. It is simple in its working, and is so 
arranged that the attendant has nothing to do but to put the onds together and hook the right-hand side of the pioce ends upon the tenter hooks of the guide arm, and the left-hand side upon the hooks of the feod wheol; the machine will then start itself and guide the fabric across the machine. When the onds are sewn, the guido arm will leave the fabric, fall down, and stop the machine until another pair of piece ends is attachod. This arrangement onables one person to sew as many pieces per day as two or three can on othor machines. These machines sew wet or dry, thick or thin, cloth, with equal facility. They are driven by steam-power. Fig. 341 is the same machine, placed upon a portable stand to work by the foot; the stand is se

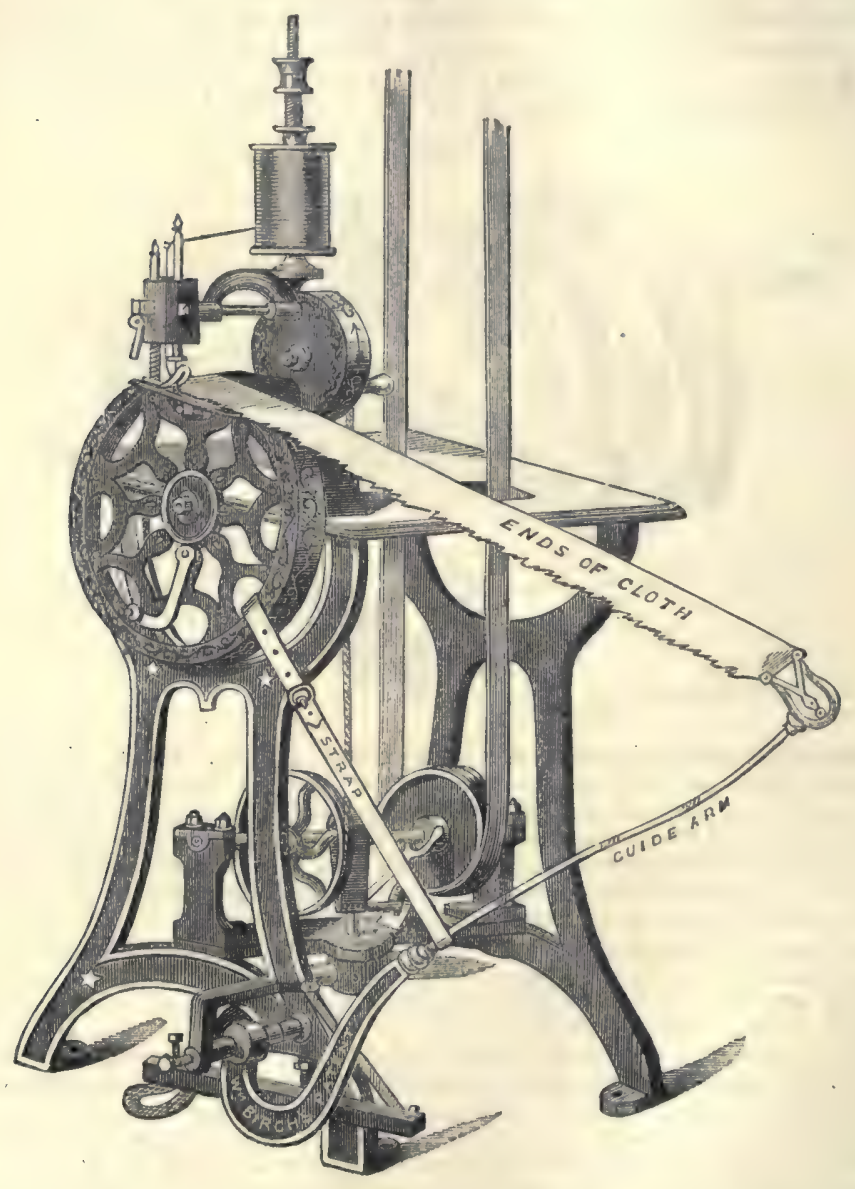

constructed that when stationary it rests upon two wheels and a log, and when being moved about, the act of pulling the handlo prosses a third wheel with a swivel upon the floor, and the machine is thus easily removed from place to place. There is no guide arm to this machine, which is used chiefly in the dyehouse.

Pieces are also frequently gummed together at the onds, which is done by pasting the ends for about $1 \frac{1}{2}$ inch with paste or gum, and, after laying one on the other, drying them immediately on a steam-pipe in front of the operator. This mode is advantageous for some purposes, as when the pieces come, in the subsequent operations, into hot water, they are easily detached one from the other.

By whichever of these modes the pieces are joined together, they are then wound 
in rolls of about 40 pieces by a machine called a candroy, which winds them on the wooden beam which fits in at the back of the printing-machine; the cloth during the operation of winding becomes stretched laterally quite smooth, by the aid of one or two grooved stretching bars, as described in fig. 347 , a due degree of strain being kept on the piece by its passing under and over several plain wooden bars, and to the axis of the

341

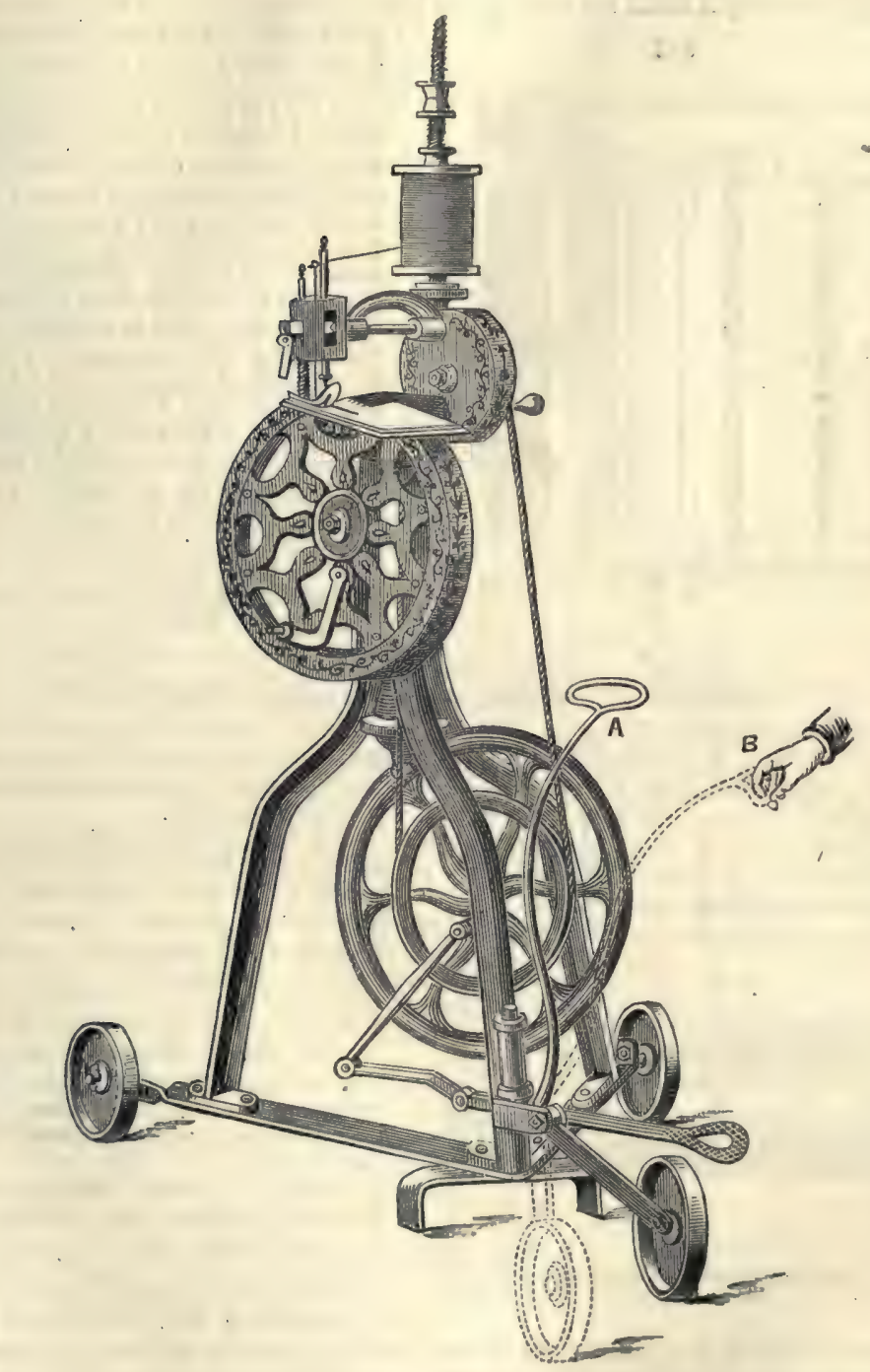

wooden beam which receives the pieces being suspended weights which keop it forcibly in contact with the wooden drum which turns it by friction. In this machine the ends of the axis of the beam pass through slots, which allow it to rise as the pieces become wound on, and the diametor consequently increases. If fewer pieces than 40 are to be printed in one pattern or colouring, it is usual to stitch a few yards of old cloth between two pieces where the change is intended to be made; by this means the 
printer, on coming to the wasto piece stops his machine, and fits another pattern or changes the colours without damaging good cloth.

A very good sort of candroy for this purpose is that made by Furnival, of Haslingden : plan, side and end elevation of which are annexed (fig. 342). A A are the castiron sides of the machine; B, a cast-iron roller turned by the pullies $\mathrm{c}$ c, keyed upon the same centre; $\mathrm{D}, \mathrm{a}$ roller or shell, upon which the eloth is wound, the roller $\mathrm{D}$ being pressed upon the roller $\mathrm{B}$ by the levers $\mathrm{E} \mathrm{E}$, which are keyed upon the shaft $F$, and on the end of the same shaft the brake pulley c is keyed; the friction of the wrought-iron strap a round the brake pulley holds the levers $\mathrm{E}$ tightly upon the onds

342
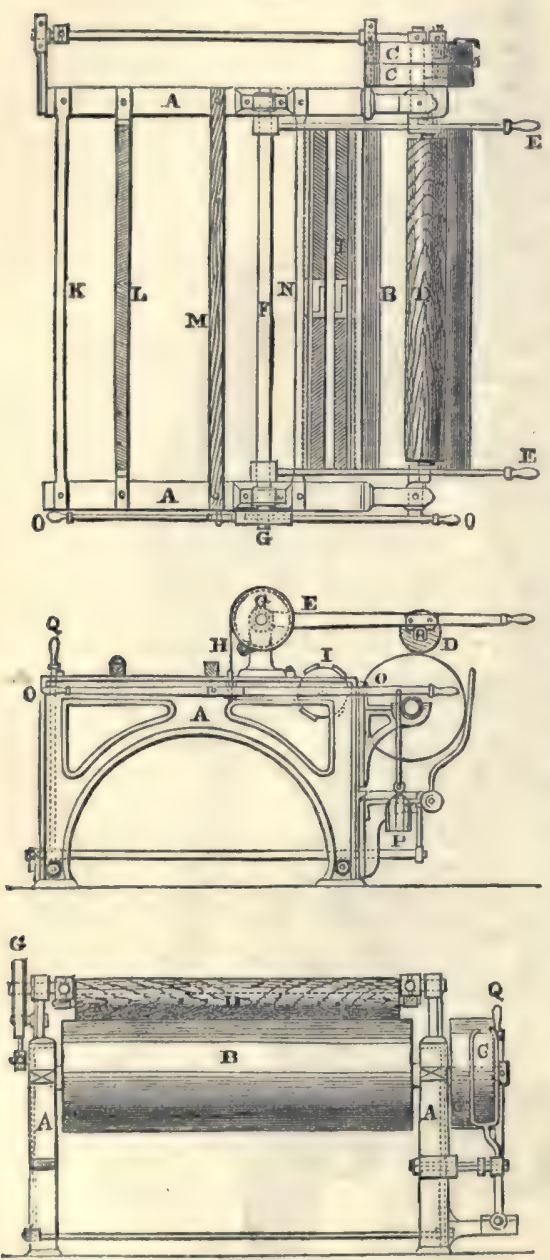
of the roller $\mathrm{D}$, but allows the levers to rise as the roller $\mathrm{D}$ increases in diameter by the cloth being wound upon it; $x$ is a brass spreading roller (further described where the finishing room is treated of) to stretch the cloth before the entering between the rollers $B$ and $D$; $\mathbb{K}$ and $\mathrm{N}$ are wroughtiron bars; $x$ and $x$ are scrimping bars. $O$ is a lever working on a pirot; to the longer end of this lever and near the pivot is fastened one end of the iron strap $\mathrm{H}$, and near the handle of the same end of the lever is a weight $\mathbf{P}$ depending from a chain ; \& a are handles for starting or stopping the machine by shifting the strap on or off the loose pulley. The eloth is passed under the iron bar $\mathrm{K}$ over the scrimping bar $\mathbf{L}$, under the scrimping bar $\mathbf{M}$, over the iron bar $s$, under the spreading roller $x$, and wound upon the shell $D$; to remove the roll of eloth when finished winding, the handle o with the woight is lifted, which relieves the pulley $\mathrm{G}$ from the pressure of the iron strap and allows the levers $\mathrm{E}$ to be lifted, when the shell with its load of eloth is detached.

The proper hygrometric state of calico when printing should be attonded to; very dry calico does not take colours or mordant nearly so well as when containing a certain amount of hygrometric moisture. Practically this is attained by the bleached pieces being stored in the "white room," generally severa! hundred pieces in advance, and they easily absorb sufficient moisturo from the air to be in a proper state for printing on.

Should, however, circumstances prevent this taking place, the pieces are conditioned by being passed through the spray of water. The machine for this purpose consists of a rectangular box, closed with a stratum of water at the bottom; one or more revolving brushes, set at a right angle to the piece, dip slightly into the water, ard being made to revolve very rapidly, a continuous fine spray is produced, and the pieces are drawn rapidly through the upper part of the machine, entering and leaving the machine through narrow apertures, a little wider than the pieces, and are wound on a batching roller. In its rapid passage through the spray the cloth becomes slightly damped. The 'white room' where the bleached calico is stored is generally placed in some cool part of the works, and sometimes built over a part of one of the reservoirs:

A shearing-machine is now generally employed to remove any knots, loose flbres, or 
duwn from the white cloth previous to printing by machine. The shearing-machine of Mather and Platt here shown, fig. 343, takes up the pieces from the stitched bundle, and winds them, after shearing, in a roll ready for the printing-machine. $a$ is the brush for the back of the cloth; $b$, spiral cutter; $c$, beds; $d$, brush for face

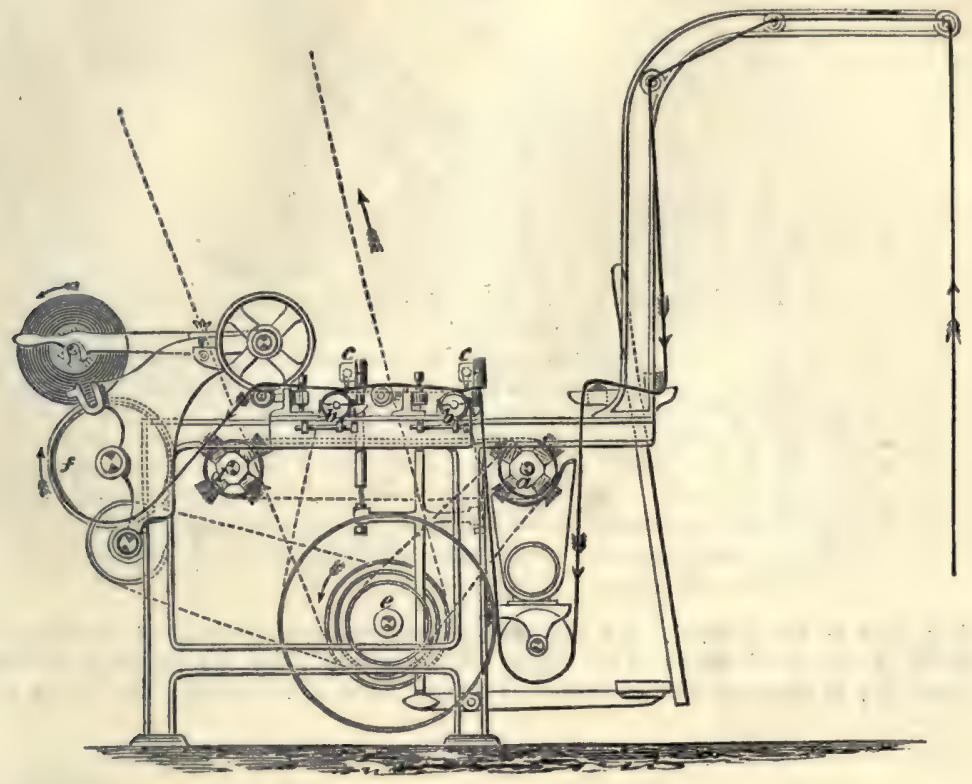

of cloth; $e$, main driving shaft; $f$, batching roller. The machine has fast and loose driving pullies 12 inches in diameter by $3 \frac{1}{2}$ inches broad, which make 250 revolutions per minute. The eloth passes rapidly over the spiral knives, which revolve also rapidly, and give a slicing effect from side to side of the cloth. In some cases this machine is used instead of singeing before bleaching, but is generally used as a final remover of loose matters before printing.

In mounting two or more cylinders in one frame, several adjustments become necessary. The first and most important is that which ensures the correspondence between the parts of the figures in the surcessive printing rollers, for unless those of the second and subsequent engraved cylinders be accurately inserted into their respective places, a confused pattern would be produced upon the cloth as it advances round the pressure cylinder.

Each cylinder must have a forward adjustment in the direction of rotation round its axis, so as to bring the patterns into correspondence with each other in the length of the piece; and also a lateral or traverse adjustment in the line of its axis, to effect the correspondence of the figures across the piece; and thus, by both together, each cylinder may be made to work symmetrically with its fellows.

Fig. 344 is an end-elevation of a 4-colour printing-machine, and fig. 345 is a section of same : the same letters of reference refer to both. $\Delta$ is the cast-iron framework, bolted to a corresponding framework by the bolts B, with a space of from 3 to 4 feet between; $\mathrm{c}$ is the pressure cylinder, about 2 feet diameter, of iron, but hollow, and between 3 and 4 feet long, according to the sort of cloth the machine is intended to print; $\mathbf{D}$ are the copper rollers, the width of a piece of cloth; $\mathbf{E}$ are wrought-iron mandrels on which the copper roller is forced by a screw press, the mandrel being about 4 inches in diameter where the roller fits on, but with journals of smaller diameter. The roller is made with a projecting piece inside, about $\frac{3}{2}$ an inch broad and $\frac{1}{4}$ of an inch deep, extending all the width of the roller; this tab, as it is called, fits in a slot cut in the mandrel, which causes it to turn without slipping on the mandrel; the pressure cylinder or bowl c, rests with its gudgeons in bearings or bushes, which can be shifted up and down in slots of the side cheeks $\Delta$; these bushes are suspended from powerful screws $F$, which turn in brass nuts mado fast to the 
frame A. These scrows counteract the pressure upwards of the two lowest rollors, and enable the bowl to be lifted out of the way of the rollers, \&c., whon

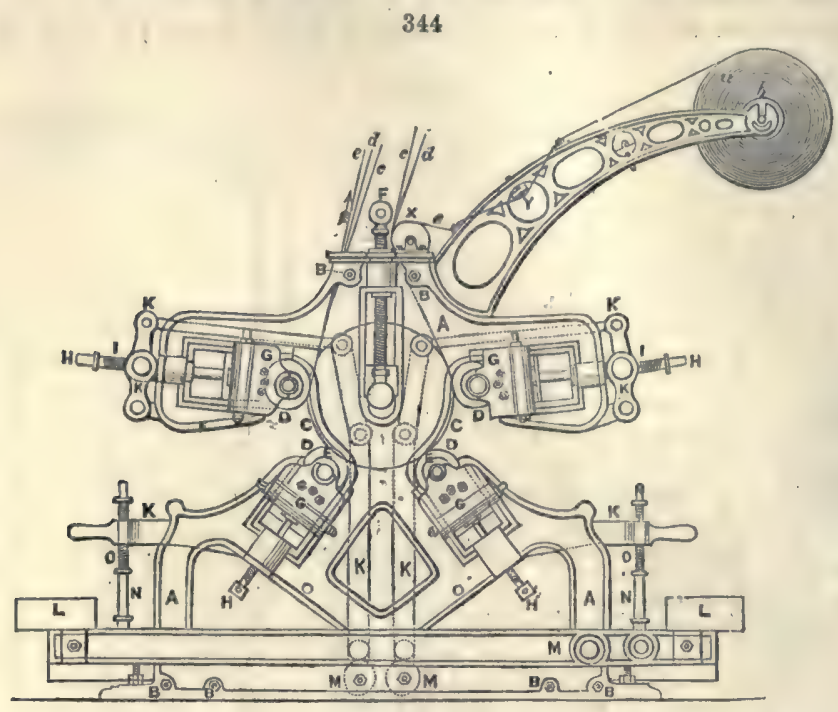

they have to be removed. a $\mathrm{G}$ are sliding pieces, moving in arms of the frame. work, by means of screws нн. These sliding pieces carry the bearings of the mandrels; to them are also attached the colour boxes and doctors. The screws in

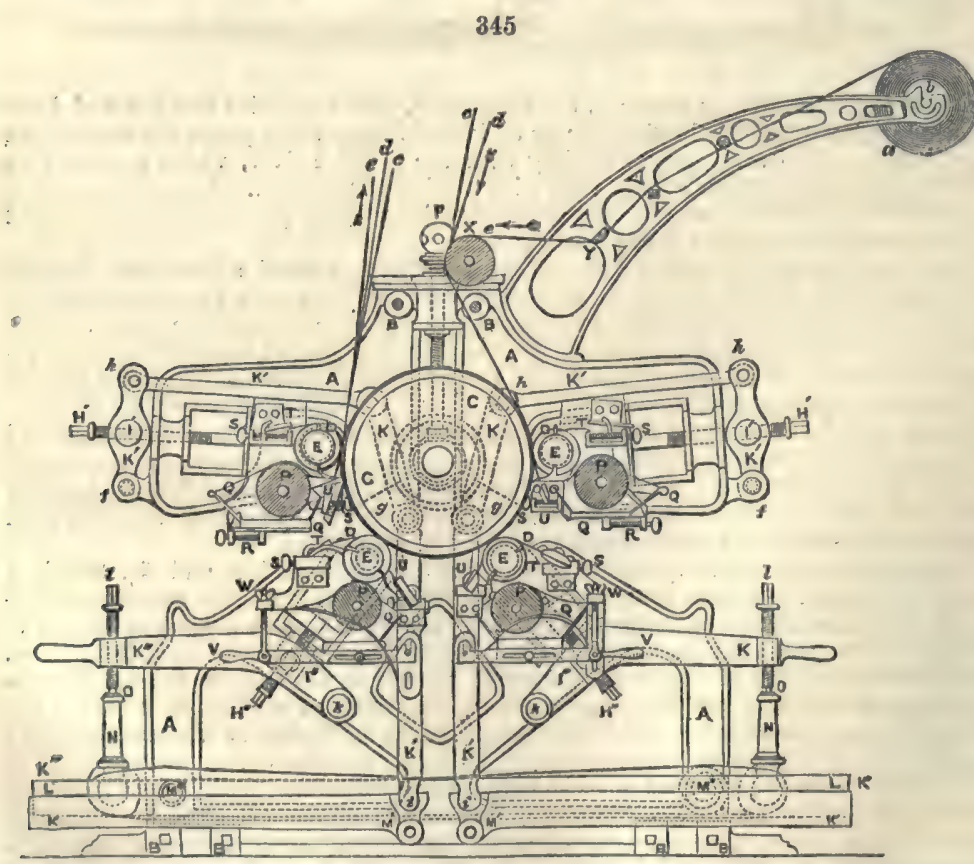

work in female serews $\mathrm{r}^{\prime}$, which form part of a system of jointed levers $\mathrm{k}$. These levers are for the purpose of giving an additional pressure or nip to the rollers $D$, the 
pressure boing also elastic. There are four pairs of levers, each pair bearing upor one mandrel. It will be sufficient to describe one side only, both sides being precisely alike. The two highost rollers are pressed against the cylinder by the compound levers $\mathrm{K}^{\prime}$, which have attachments to the arms of the framework at $f$, and to the inside of the main framework at $g$ and $\mathbf{m}^{\prime}$ as fulcrums, and are jointed together at $h h$, but the bent levers $h, g, i$, merely fit into sockets $i$, of the horizontal levers $\mathrm{M}^{\prime} \mathbf{x}^{\prime}$, which are weightod at the onds $\mathbf{x}^{\prime}$, by moveable weights made to fit expanded parts. The two lowest rollers are pressed against the cylinder by the system of compound levers $\mathrm{K}^{\prime \prime}$, which have attachments to the framework at $k$ and $\mathrm{M}^{\prime \prime}$ as fulcrums; the screws $\mathbf{H}^{\prime \prime} \mathrm{H}^{\prime \prime}$, working in female screws $\mathrm{x}^{\prime \prime} \mathrm{I}^{\prime \prime}$, as in the other set of levers. For cunvenience of removing the rollers, colour boxes, \&c., these levers are provided with a hinged piece 2 , in a socket 0 , on the top of which work the screws $l l$, which, by means of the female screw in the lever $k \mathrm{x}^{\prime \prime}$ serve still further to regulate the pressure; the lever $\mathbf{x}^{\prime \prime} k$ is shown as when the machine is printing, but when the rollers, \&c., are to be removed, the lever is lifted by the handle, and the hinged piece $\mathrm{x}$ pulled over, the lever with its burden being then lowered down; the weighting of these levers, which are partly outside the machine, is best seen in figs. 344 and 345 where $I$ are the weights, $Q$ are colour boxes, the sides and bottom of which are made of sheet copper, and the ends of gun-metal ; in each end is a slot which receives the brass journals of the wooden furnishing rollers $P$, which are wrapped with a few folds of coarse calico, and, by revolving in the colour and against the engraved rollers $\mathrm{D}$, supply it equally all over with the colour; the superfluous colour is next wiped off by the colour doctors T. These doctors are thin blades of steel or brass, which are mounted in doctorshears, or plates of metal serewed together with bolts; the shears have journals which rest in bearings moveable backwards and forwards by the serews $\mathrm{s}$; the doctors are kept in close contact with the engraved roller by levers and weights, for the way of arranging which, see fig. 346 , where $\mathrm{A}, \mathrm{B}, \mathrm{C}$, are the levers attached to the doctor

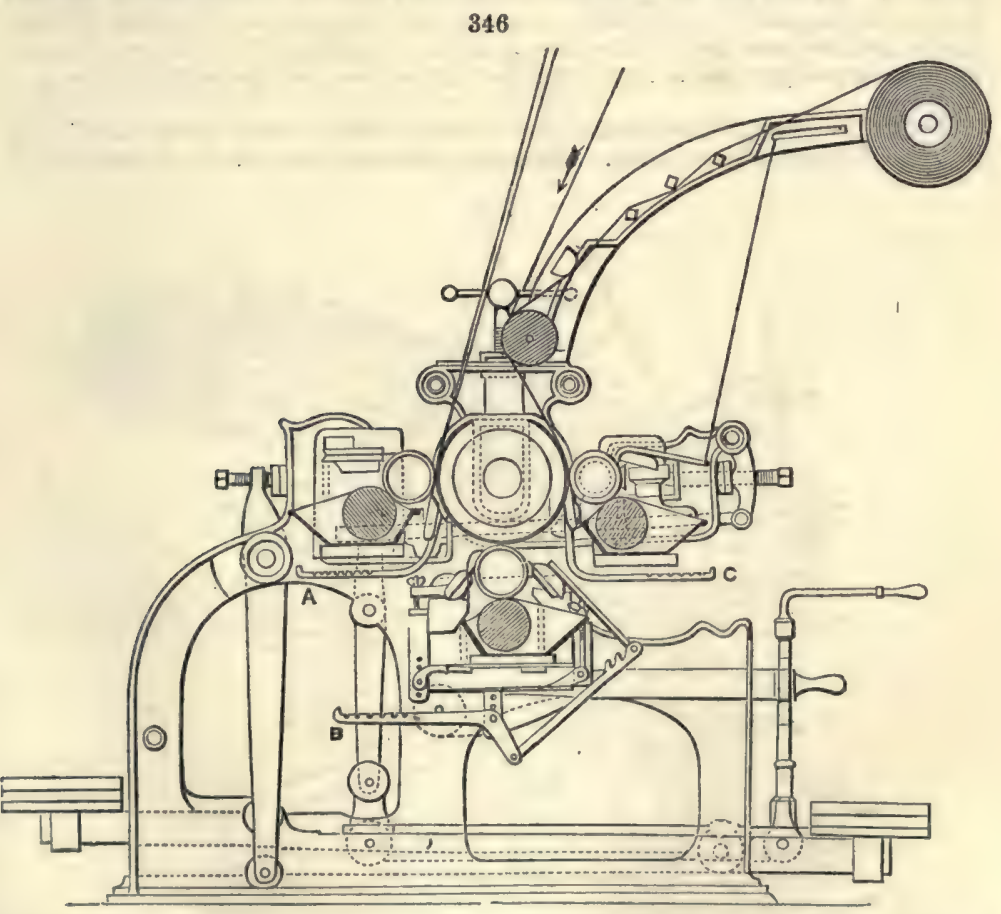

shears. On the ends of these levers weights are hung, and by this means the doctors are pressed forcibly against the roller.

After printing the pattern on the piece, the roller $\mathbf{D}$ is cleaned from threads or dust by the lint doctors v, pressed against the roller by the screws s, fig. 345 ; any loose threads from the piece are prevented by the lint doctors from going into the colour, and 
consequently under the cleaning doctors, where by preventing them from perfectly wiping the blank parts of the roller, smears on the piece would ensue. The colour boxes are mounted on wooden boards, to give them greater strongth, and are tightened up against the roller by the serews $\mathbf{R} \mathbf{R}$ and $\mathbf{W} \mathbf{W}$; the lower pair of colour boxes are removed from the copper roller when not in use by the handles $\mathrm{v}$, after detaching the screws $\mathrm{w}$ w. There is a toothed wheol slipped on to each mandrel, working into a toothed wheel on the axis of the furnishing roller, which ensures the copper roller and furnishing roller always turning together. By means of an excentric, fixed on the axis of the pressure bowl, and connectod with each cleaning doctor, a regular vibratory movement is given to them, which prevents the doctor being worn down unequally. Sometimes for the highest rollers, and especially in machines of more than four colours, the cumbrous colour box is dispensed with, and a doctor inserted in a curved frame is applied to the roller instead. In this arrangement the doctor forms the bottom of the colour reservoir, and is pressed strongly against the roller; the curved frame stopped off at the sides with a piece of copper curved to fit both roller and frame, and which is padded with a piece of folded cotton cloth, forms the colour box. This doctor box takes but little room, and wastes but little colour, but is only used for the uppermost rollers. Neither of these arrangements can be shown in fig. 345. The roll of pieces is shown at $a$, wound on the wooden roller $b$, the axis of which rests in bearings at the end of the arms. The piece is conducted under a

347 small wooden roller, next over a square iron bar, and next against the scrimping bax $\mathrm{Y}$, thence over the wooden roller $x$, round which also pass the grey piece $d$, and the woollen

DIDIDIDT blanket $e_{*}$. The scrimping bar is a bar of iron or brass, with curved surface, furrowed by grooves, cut right and left from the centre, as in fig. 347. In passing over this bar, the cloth is stretched equally from the centre, and any folds or creases removed. In order that the piece may be constantly stretched, the roller $b$ is provided with a wooden pulley, round which passes a leather strap, one end of which is made fast to the framework, and to the other is attached a weight; the friction of the strap against the pulley causes a retarding action of the piece, and consequently keeps it stretched.

Fig. 348 is an elevation of a 12-colour machine, which is inserted to show the way in which all machines are driven. The large spur-wheel is keyed on the axis of the

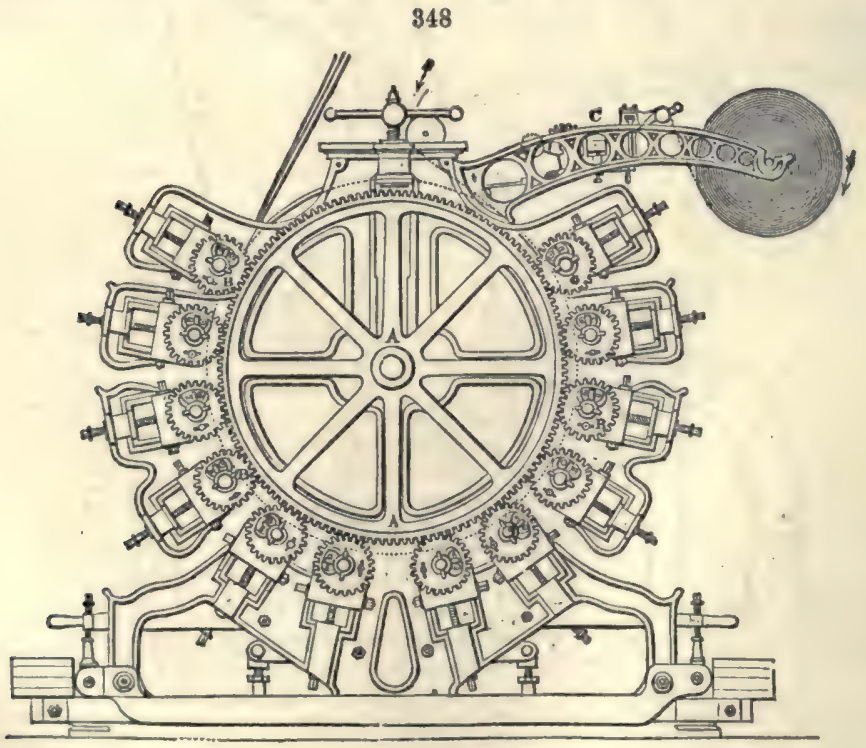

pressure bowl, and works into pinions staked on the mandrels; there is a peculiarity about these pinions, or box-wheels, as they are called, which may be observed in fig. 348 , but is shown on an enlarged scale in fig. 349 , which is a box-wheel detached. This wheel may be compared to the fine adjustment of a microscope, as by means of it 
the rollers receive the final and delicate adjustment so as to register accurately with one another. It consists essentially of two parts: the dise $\Delta$, carrying the cogs ; and the hollow axis $\mathrm{B}$, carrying a dise at one side, and the connecting piece and Berew $\mathbf{C} \mathbf{D}$ at the other. The part $\mathbf{A} \mathbf{A}$, or shell of the wheel, is about 10 inches in diameter and 3 inches broad across the oogs; one side of the shell is cut out to receive the plate shown by dotted lines. This plate is provided with the hollow axis $\mathbf{B}$, which comes through the shell, and projects about 3 inches, the part projecting being cut through at $\mathbf{F}$; fastened to it also is the connecting piece $\mathrm{c}$, in which works the screw $\mathbf{D}$; this screw just fits in two projecting lugs $a r$, cast on the shell $\mathbf{\Delta}$. The screw-nut $z$ forms part of the axlo piece, and works in the slide $\mathrm{r}$. When this wheel is used, it is slipped on the mandrel which carries the copper roller, and a cotter is driven through the cleft axle and through a corresponding cotter

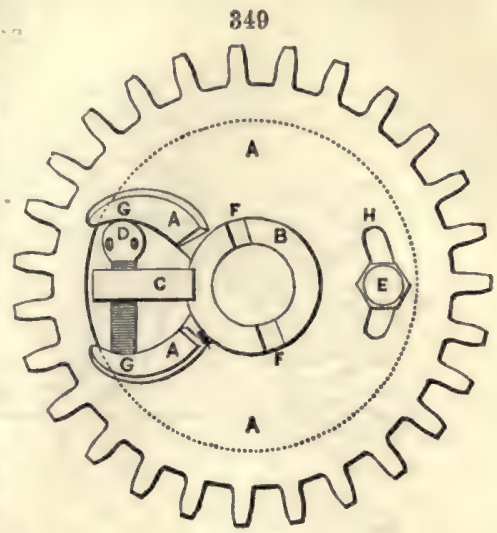
hole in the mandrel, thus firmly con-

necting the mandrel and wheel; the mandrel and roller being put in their place in the machine, the cogs of the mandrel wheel work into the main driving wheels, as shown in fig. 348. The coarse adjustment of the rollers being made when putting them in their places, the fine adjustment is made by turning the screw $\mathbf{D}$. It is obvious that the screw $D$, by pressing against the lugs of of the shell $\mathbf{A}$, which is geared into the driving wheel, will turn the mandrel and roller without moving the cogs. By this arrangement, any roller may be moved round about 2 inches at any time after being fixed in its place. All machines of more than one colour are fitted with these wheels, which indeed are indispensable.

Fig. 350 represents a 6-colour printing-machine as made by Mather and Platt. A is the bowl or cylinder; $B$, copper printing rollers upon iron mandrels; c, blocks, \&c.,

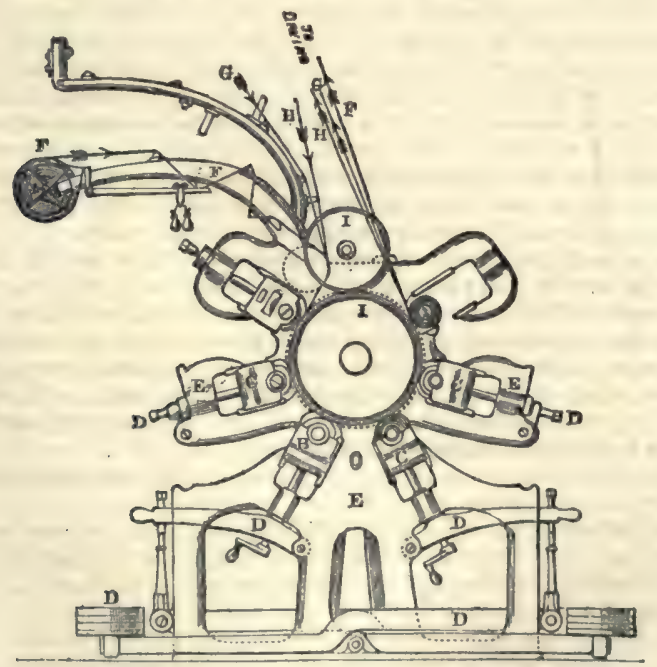

or journals for carrying the mandrels and copper rollers, the blocks being adjustablo by screws for fitting the pattern; $D$, setting up screws and levers for giving the pressure of copper rollers on to the cloth being printed; $E$, framing of machine, with nips or horns cast on, in which the blocks work; $F$, white cloth to be printed running from batch through the machine, and thence to the drying apparatus; $G$, grey or intermediate cloth; $\mathrm{k}$, blanket or back-cloth; $\mathrm{r}$, friction-wheel for drawing traverse 
motion. Fig. 351 represents a 12-colour printing-machine of Mather and Platt; A, the bowl or cylinder; $B$, copper printing rollers on mandrels; $c$, wooden furnishing-

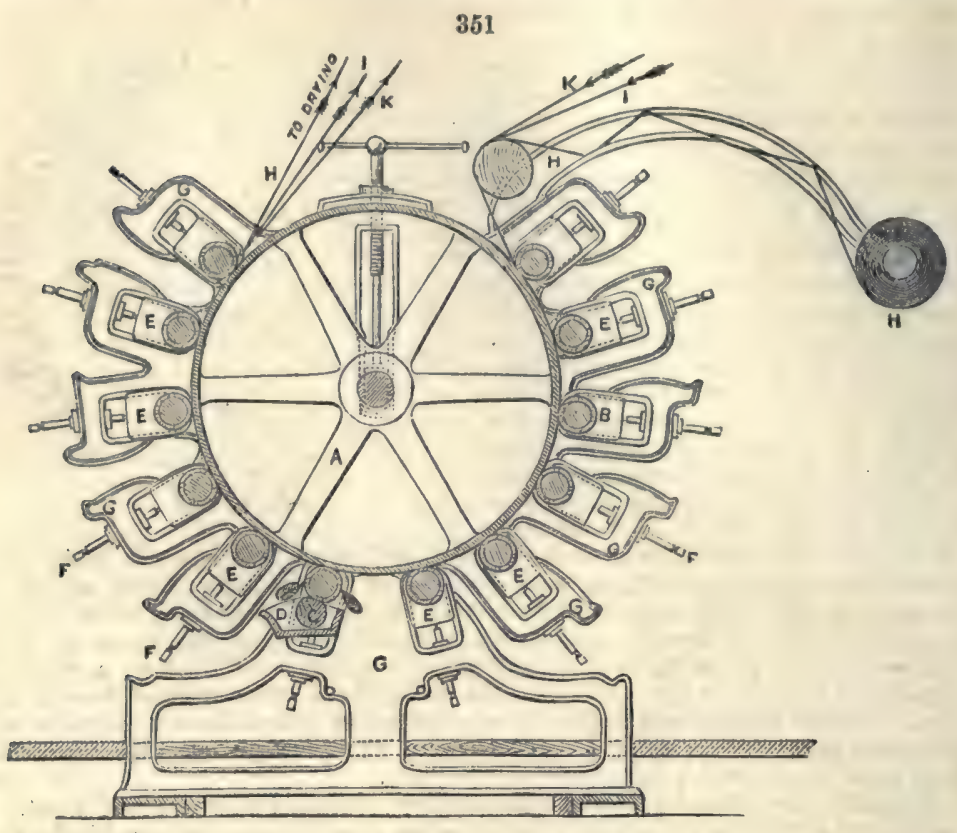

rollers, (one nip only shown in section); $\mathrm{D}$, copper colour box, (one nip only shown in section); $\mathbf{E}$, blocks or journals for carrying the mandrels and copper rollers, the blocks being adjustable by screws for fitting the pattern; $F$, setting-up screws for giving the pressure of copper rollers on to cloth being printed; $G$, framing of machine with nips or horns cast on in which the blocks work; $\mathrm{H}$, the white cloth to be printed running from batch through machine and thence to drying apparatus; 1 , grey or intermediate cloth; $\mathbf{x}$, blanket or back-cloth. The cuts here given with the exception of fig. 348 do not show any gearing or connection with the motive power, as to show this effectually several more drawings would be required, and the arrangement will naturally suggest itself to those conversant with mechanics.

The system of turning cylinder-machines, patented by Mr. Joseph Leese, possesses several advantages. In this plan a small high-pressure oscillating engine is attached directly to the axis of the large cylinder, thereby dispensing with the heavy gearing and shafting required when machines are turned by a large stationary engine; the machine printer also has perfect command over the speed of the machine, and can fit the pattern, when it is turning very slowly, with more convenience than on the usual system. On this system also machines can be put down in any portion of the works, and are independent of the stationary engne.

This system of turning is now employed in most modern print-works, or where new machines have been put down.

Four-, five-, and six-colour machines, similar to the above, are now at work in many establishments in Lancashire, which will turn off a pieco of 28 yards per minute, each of the three or four cylinders applying its peculiar part of the pattern to the cloth as it passes along, by ceaseless rotation of the unwearied wheels. At this rate, the astonishing length of one mile of many-coloured web is printed with elegant flowers and other figures in an hour. When we call to mind how much knowledge and skill are involved in this process, we may fairly consider it as the greatest achievement of chemical and mechanical science.

The printers of goods intended for hangings, which are generally of elaborate floral designs, employ machines capablo of printing from 10 to 20 colours at once. These machines are necessarily of very large dimensions. Fig. 352 is an ond-viow of a 20 -colour machine, made by Messrs. Gadd and Hill, of Manchester for Mr. Kay, of 
Castleton Print Works, and is employed in printing very beautiful floral patterns on woollen fabrics, in imitation of those produced by hand-labour in France.

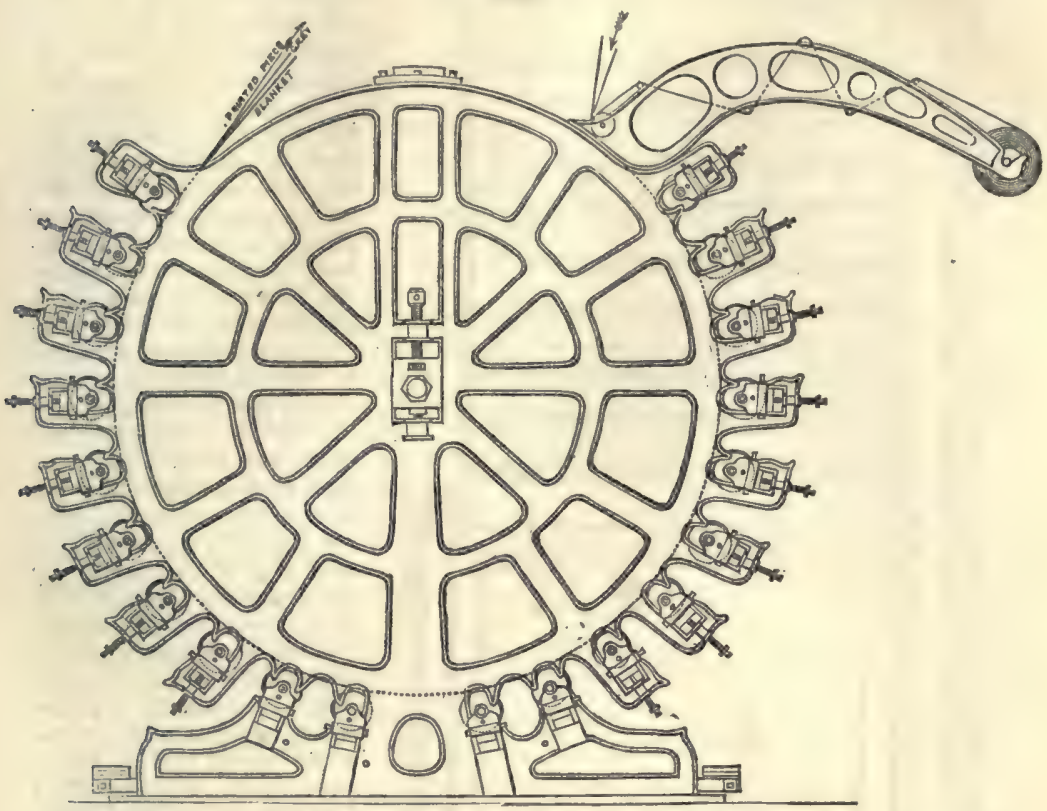

The general course of printing is thus performed:- The pieces to be printed are wound on a beam, and, last of all, a few yards of common coarse cotton or calico, kept for this purpose: this is for the printer to fit the pattern on, to save good cloth. The roll of cloth being put in its place behind the machine, the printer's assistant stations himself behind to guide the cloth evenly, and pluck off any loose threads he may see. The machine printer stands in front, and, after having fitted the pattern on the cloth, attends to supplying the colour boxes with colour, and regulating any misfitting or inequality in the printing. The machine then prints rapidly. After running through 30 or 40 pieces, the printer stops the machine, removes the doctors, and files them anew to a bevelled sharp edge.

To prevent the blanket being too soon soiled, it is usual to run grey or unbleached pieces between the blanket and the white pieces. The blanket, grey, and printed pieces are dried separately. There are several ways of drying after the machine. In the early days of machine-printing, the 'hot room' behind the machine was heated by a cast-iron furnace, termed the 'pot,' which was kept red-hot or nearly so, and large cast-iron pipes formed the flue, which after traversing the hot room, delivered the smoke, \&c. into a chimney. This arrangement gave way to cylinders, similar to those used in drying machines and were also employed in conjunction with the steam-chests hereinafter described for drying the printed cloth as well as the grey or intermediate cloth and the blanket,. but experience having shown that many colours and mordants are injured by the sudden drying given by the cylinders, these have been pretty generally abandoned in favour of steam-chests, in drying with which, the printed cloth does not absolutely touch the heated surface, but keeps moving on in very close proximity, the cylinders where they are retained being reserved for the 'grey' and the blanket. One of the most recent arrangements only, is shown in fig. 353, being that of Messrs. Mather und Platt; $\mathbf{A}$, the printing-machine with copper rollers, (an 8-colour machino here shown); B, place of steam-engine to drive the machine; c, white eloth to be printed, running from batch through machine, and thence to drying apparatus; $\mathrm{D}$, grey or intermediate eloth; $\mathbf{E}$ blanket or back-cloth; F, framing of apparatus for supporting and carrying the rollers and steamchests; $a$, hollow stoam-chests or chambors, the same width as the machine, about 1 foot broad and 3 or 4 inches deep, and connectod with one another by bent pipes a: the ond; $\mathrm{z}$, upright with tightoning trough apparatus for blanket; I, upright with 
353

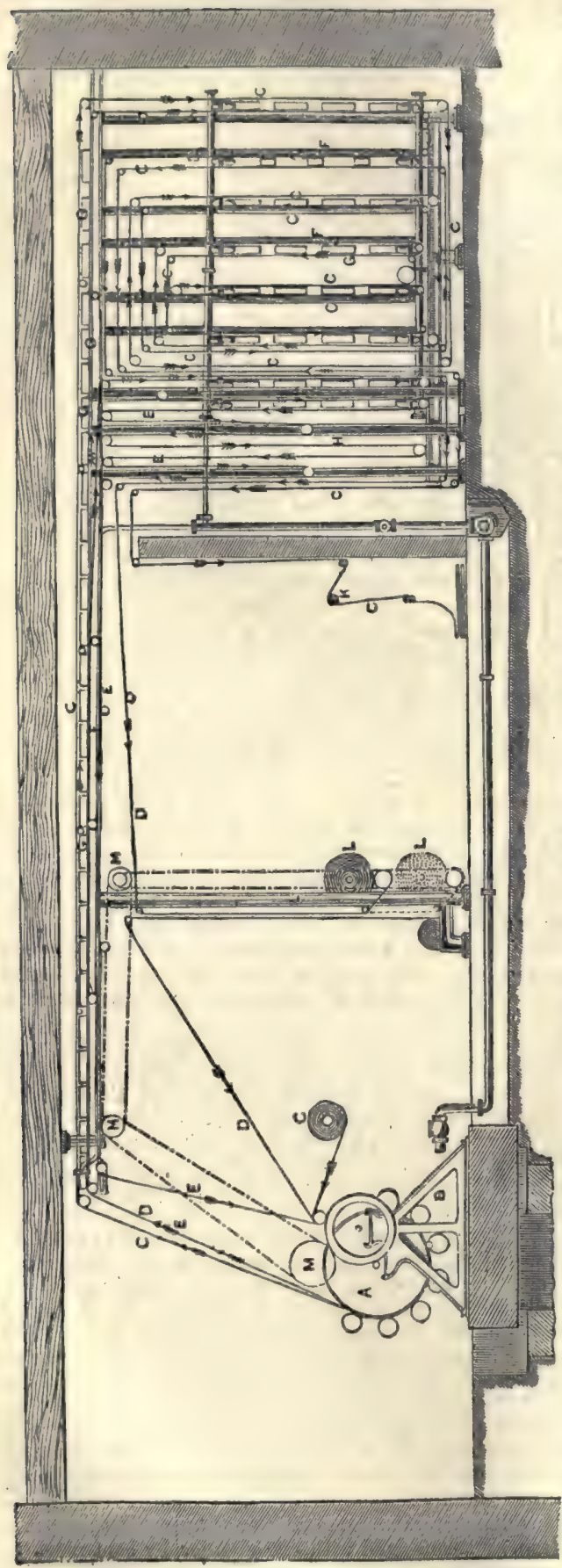

tightening rack apparatus fot blanket; $\mathbb{K}$, plaiting apparatus for printed cloth; $\mathbf{x}$, upright and batching apparatus for groy cloth; $\mathbf{x}$, driving pullies and straps for batching and plaiting apparatus.

The doctors used in cleaning off the superfluous colour from the rollers, are generally thin blades of steol, of a thickness varying from $\frac{1}{32}$ nd of an inch to $\frac{1}{16}$ th of an inch, according to the sort of engraving on the roller; but some colours, such as those containing salts of copper, would be too corrosive on a steel doctor, and in this case doctors of a composition like brass are used. They are filed to a bovelled edge, and require to be retouched with the file after printing from 10 to 30 pieces. The cylinder or drum, in contact with which revolve the copper rollers, is wrapped round with a cloth called 'lapping;' which is generally a coarso strong woollen eloth of peculiar make, and is folded tight on the cylinder about $\frac{1}{3}$ an inch thick. The blanket is next put on and drawn tight: this blanket is a very important part of the $\mathrm{ms}$ chine; it is a thick woollen web, about 40 yards long, and requires to be made with great care, so as to be uniform in texture, thickness, and elasticity. If the blanket is uneven, it has the effect of throwing the pattern into confusion at the uneven places.

A good blanket will serve to print 10,000 pioces, being washed whenever loaded with colour, and then is suitable for covering the tables of the block printer.

In the year 1835 Messre. Macintosh and Co. patented an India-rubber blanket, which consists of several thick cotton webs, cemented together with dissolved India-rubber. This blanket is very useful and economical for some purposes; the surface being very smooth, great delicacy of impression is obtained, and when soiled it is not nocegsary to remove it from the 
machine, as it is easily washed by being passed through. a special washing. machine; which will be found described subsequently. An India-rubber blanket will print 20,000 pieces, which is twice as much as a woollen one will do, the price per yard being also lower. Several descriptions of these blankets are made by Messrs. Macintosh, some of them having a coating of vuleanised Indiarubber on the face that is printed from, thereby giving a still more elastic surfaco. A great improvement has been recently made in these India-rubber blankets by shrinking or proparing the cotton previous to cemonting, according to. the patent process of Mr. John Mercer, viz. by soaking in strong alkali, and afterwards in dilute sulphuric acid; this process contracts the fibre to a certain extent, and the cloth is found to possess a great increase of strength. When made into blankets they are found to be more capable of resisting the severe strains of the printing process; and consequently many more pieces can be printed from them than from the old sort. They are made by $\mathrm{Kay}$ and $\mathrm{C}_{0}$. of Accrington, and others, and are now in general use. The woollen blanket, however, seems to be preferred for several styles. Several patents have been taken out for printing without blankets, but have never come into general use; but recently a mode of printing with grey or unbleached ealico has come into use, which is very favourably spoken of. In this method a roll of grey eloth is so disposed behind the machine that the fabric can be conducted five times through the machine before finally going away to be wound on a beam for removal. There are, therefore, five layers of cloth under the whito calico when printing, which gives a sufficiently elastic bed for printing from; and very delicate shapes can be got. Any given part of the grey eloth is five times uppermost on the pressure cylinder, and consequently one piece of grey cloth is used to print five pieces of white. Guttapercha pressure cylinders, or 'bowls,' have been suggested by Dalton, an Fnglish printer; but though theorotically preferable to iron, they do not appear to be much used.

In printing with aniline black (No. 240), the grey intermodiato cloth, unless removed from the machine without drying in the 'hot room,' and before this peculiar

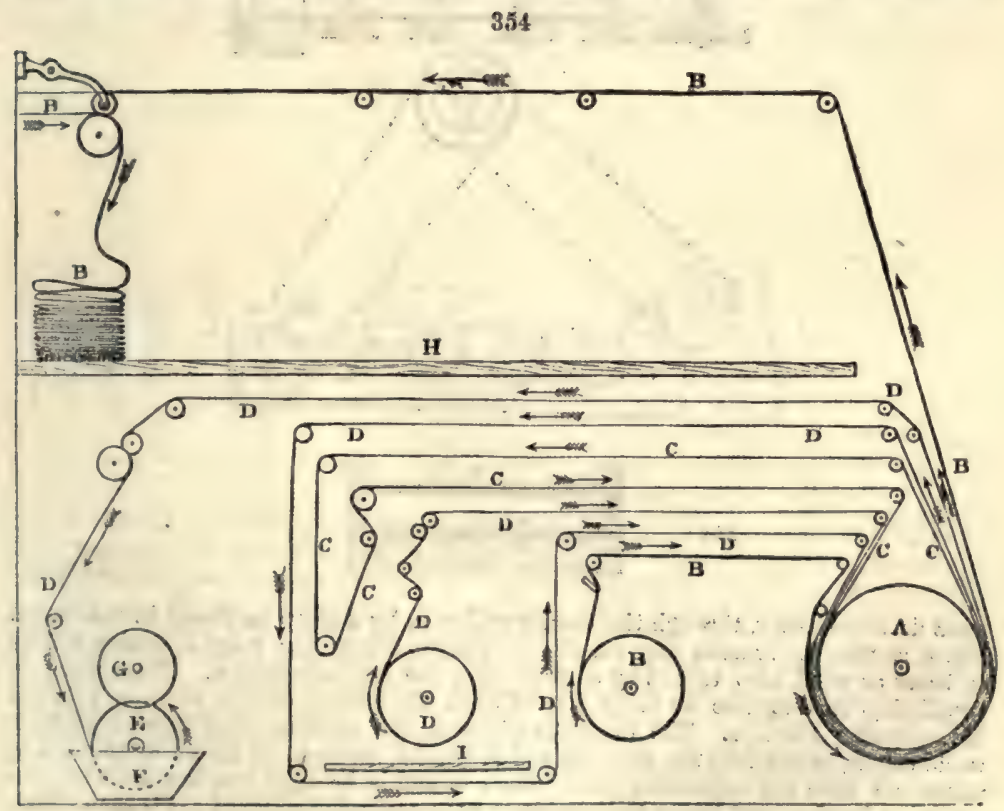

black has begun to form upon the eloth, will be stained and spoiled for future printing upon, in consequence of the extraordinary fastness of this colour, which resists the ordinary bleaching operations; and this is the place to describe a very simple arrangement for wetting the grey pieces, after they have served their purpose, and thus preventing the formation of the black. The arrungement (fig. 354 ) is that in use

at the Oriental Print Works at Apponaug, in the United States. A is the printing-
Vor. I. 
machine cylinder; $\mathrm{n}$, the roll of white cloth, and cloth passing through the machine, and hot room, and being plaited down; c, the blanket; D, the roll of grey cloth, and grey eloth passing twice through the printing-machine, and then through the wetting machine; $\mathrm{k}$, a wooden roller working in a water trough; $\boldsymbol{F}$, water trough, with a stream of water kept running through it; $G$, shell upon which the grey cloth is wound after passing through the water; $\mathbf{n}$, gallery above the machine where the printed eloth is plaited down ; 1, platform for putting up the grey rolls. Mr. Furnival of Haslingden has introduced a machine for washing India-rubber blankets: fig. 355 is a longitudinal section, 356 a plan, 357 is a sido elevation, and 358 a cross section. $\Delta$, an iron cistern containing water, and forming the framework of the machine; B, circular brushos, with a pulley keyed on the end of each; these brushes are turned by a strap from the

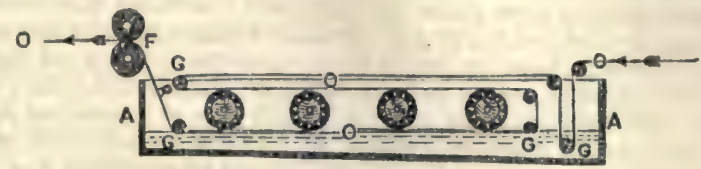

356

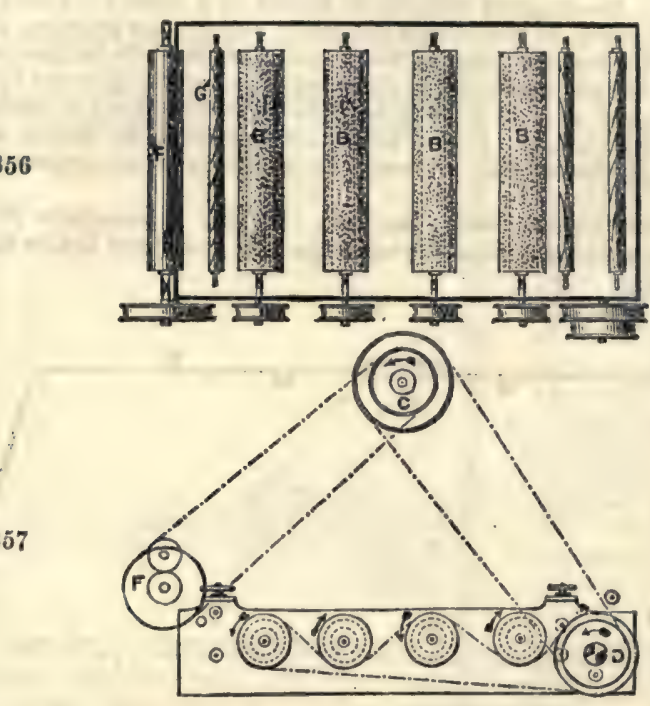

357

358

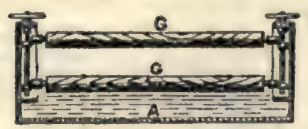

main driving-shaft $c$, through the carrier pulley $\mathbf{b}$, by'a strap as shown in the dotted line $\mathbf{B}$, so that the brushes may revolve in opposite directions; $\mathbf{F}$ are squeering rollers of brass or wood, also turned by a strap from the driving-shaft c. Clean water is supplied to the machine by being spirted upon the blanket before entering between the squeezing rollers $\mathbf{r}$. The blanket on leaving the printing-machine charged with waste colour, enters into the machine and is passed through, as shown by the line o, under and over the adjustable guide-rollers $\mathrm{G}$, and is brought into contact with the revolving brushes, and leaves the machine by the squeezing rollers $r$; it is then dried, and returns again to the printing-machine.

The thickening of mordants and colours is a subject of very great importance to the printer. It is obvious that a mere solution of salts or colouring matters, such as used in dyeing, cannot bo used in printing a pattern; capillary attraction speedily canses such a solution to spread beyond the limits of the pattern, and nothing but confusion is the result. A proper degree of inspissation is then essential. To the enpability of very thick colour being printed by engraved plates or rollers under 


\section{CALICO-PRINTING}

severe pressure is due the superior smartness of outline characteristic of goods produced by these means. Where colour can be laid on the outside of the eloth, so as to penetrate as little as possible to the other side, much brighter shades are produced. In order to obtain the most brilliant shades of colour, it is necossary that the eloth act as a sort of mirror behind the colour, which cannot be the case if the fibre is perfectly saturated with colour. Indepondent of this, a great economy of colouring material follows from the proper application of the colour or mordant to the face only. This is especially noticeable in madder goods, where the mordant, if printed in excess, is apt to give up a portion from the cloth in the dyebeck, thereby consuming a certain quantity of madder in pure loss.

The colour-house should be a spacious apartment on the ground floor, with the roof ventilated in such a manner that the steam produced finds a speedy exit; at one end, or down one side, is fixed a range of colour-pans, varying in size, and supplied with steam and cold water. Colour-pans are usually made to swing on pivots, whereby they are easily emptied and cleaned. A range of this sort, as manufactured by Messrs. Storey \& Co., of Manchester, is represented in fig. 359. This range con-
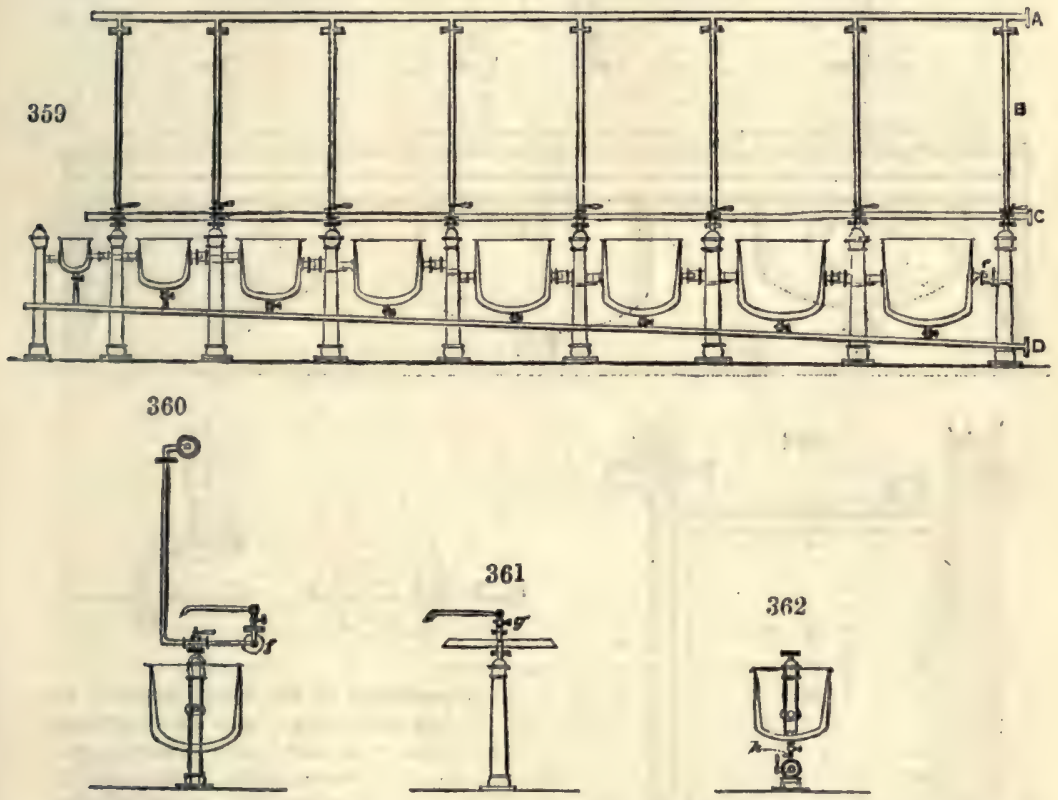

sists of 8 double-cased copper pans, containing from 1 to 28 gallons, riveted together at the top, wired at the edges, and made perfectly steam-tight; they are supported on cast-iron pillars, and are so arranged or fitted as to swivel or turn over when the colour is required to be emptied, by means of a brass stuffing-box attached to pan, and working in the corresponding part attached to pillar on the one side, and moving at the other on a plain brass nozzle, supported by a pedestal projecting from pillar, the nozale having a blank end, thereby cutting off the communication of steam, which is carried to the following pan. They are also supplied with a condense-tap to carry off the waste steam and water. Fach pillar in the range, except the last, is supplied with a brass tap on the top, with 3 flanges, to connect the steam and cold water pipes, . as more fully explained hereafter.

$\Delta, f i g .359$, is a copper pipe, with one blank end, and open at the other with flange for the admission of steam, which passes through the downward-bent pipe marked $B$, in connection with the brass tap on top of pillar, the plug of this tap being open at bottom to admit the steam down the pillar as far as the stuffing-box, marked $\mathbf{E}$, through which it rushes into the casing of pans, and out by the condense-pipe $\mathrm{D}$, when required. $c$ is a copper pipe, with one blank ond and open at the other, for the admission of cold water for cooling the colour after boiling, and is likewise connected with the tap on top of pillar, as shown in fig. 360 , marked $f$, the water passing through 

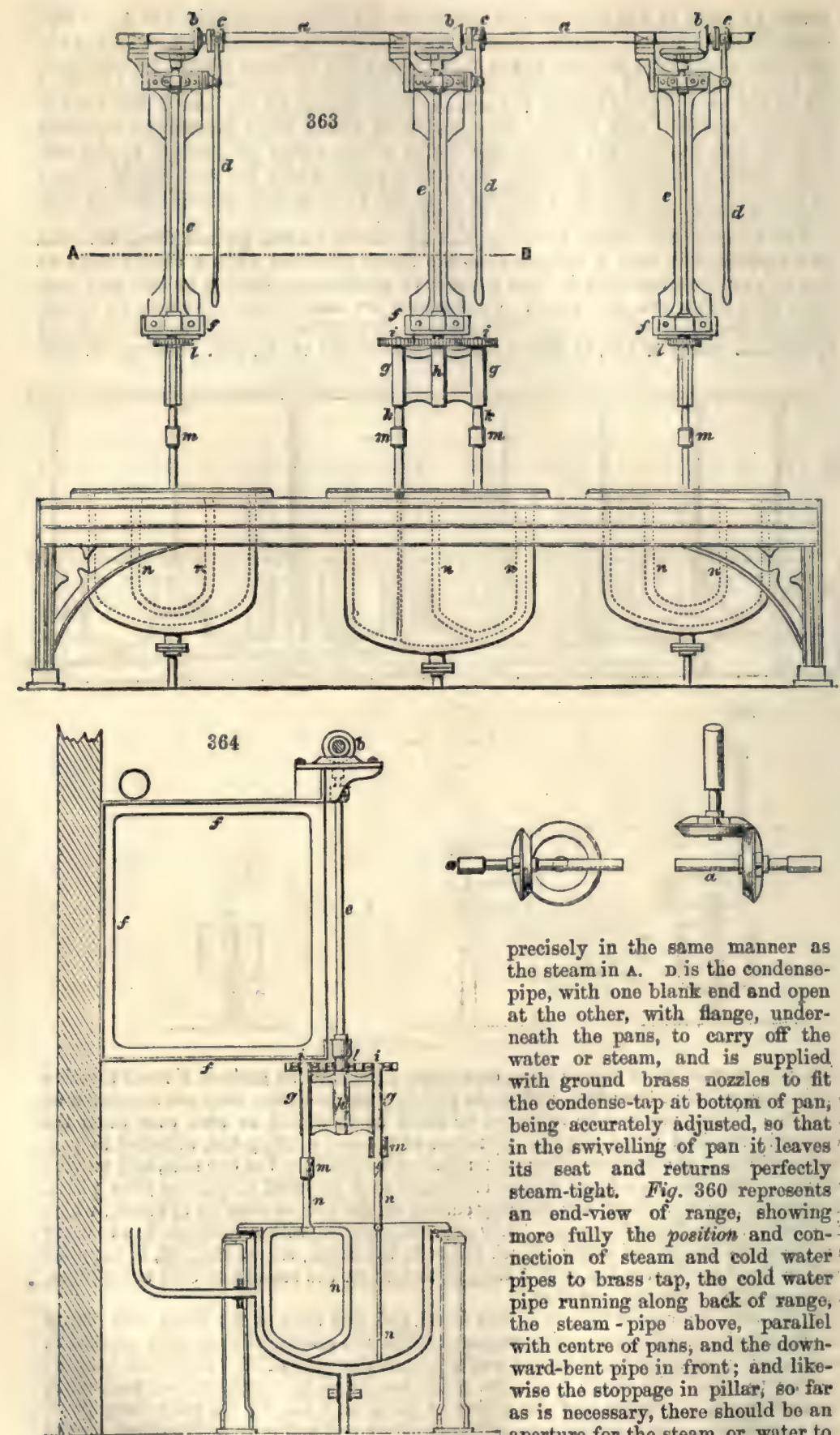

precisely in the same manner as the steam in $A$. $D$. is the condensepipe, with one blank end and open at the other, with flange, underneath the pans, to carry off the water or steam, and is supplied. with ground brass aozzles to fit the condense-tap at bottom of pan; being aceuratoly adjusted, so that in the swivelling of pan it leaves its seat and returns perfectly steam-tight. Fig. 360 reproserits an ond-view of range; showing more fully the position and connection of steam and cold water pipes to brass 'tap, the cold water pipe running along back of range, the steam-pipe above, parallel with centre of pans; and the dowhward-bent pipe in front; and likewise the stoppage in pillar, 80 far as is necessary, there should be an aperture for tho steam or water to meet the brass stuffing-box. In this fig. is also shown the copper pipe, with elbow swivel tap, for supplying pans with cold water (one pipo to supply two pans), and 


\section{CALICO-PRINTING}

fixed on top of cold water pipe exactly opposite pillar, as further shown in fig. 361 marked g. Fig. 362 is an end-view of range, with pillar cut, in order to show the position of condense-tap at bottom of pan, and its connection with condense-pipe, and where the point of separation takes place in swivelling, by the line marked $h$. It will be seen by the foregoing that the process of boiling and cooling is rapid and certain, everything being accurately adjusted and steam-tight throughout tho whole apparatus.

The colours are placed in these pans and stirred well all the time they aro being boiled; good stirring is very essential to produce smooth colourg. This was formerly done by hand with a flat stick, but lately the best print works have been fitted with machinery over the pans to stir mechanically. A very effective plan of this sort is represented in figs. 363 and 364. It is that of Messrs. Mather and Platt, of Manchester, the boilers in this drawing being not reversible, though the plan can be just as easily adapted to that description of pans. Fig. 363 is a front elevation; fig. 364 is a transverse section, and fig. 365 is a sectional plan, the same.letters referring to all. $a$ is a horizontal shaft above the pans, fitted with a pair of mitre wheels, $b b$, for ench pan. The vertical wheel $b$ is not keyed on the shaft $a$, but is brought into connection with it when required by the catch box $c$, which slides on a key on the shaft, and revolves with it (see small cuts); the catch box is worked by a lover-handle $d$, and thus motion is given to the vertical shaft $e$. The shafts $a$ and $e$ are both supported by the framework $f$, fastened to the wall ; the shaft $e$ is terminated by the frame $g h g$, the centre of which, $h$, is a continuation of the shaft $e$; and the wings $g$ are hollow to carry the shafts $k$, which are surmounted by the cog wheels $i$, which gear into a cog

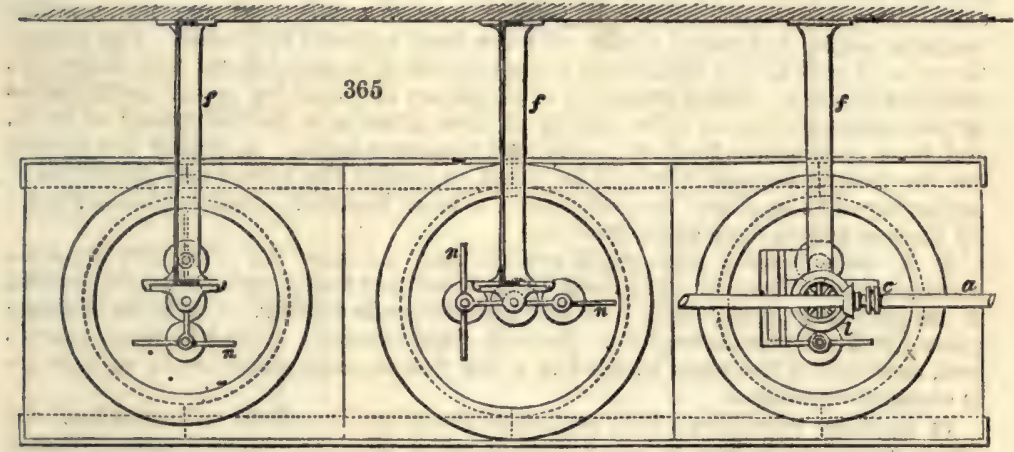

wheel $l$ on the shaft $e$. The agitators $n n$ are made of flat brass rod, and are curved to fit the bottom; they are connected with the shafts $k k$ by a hook joint, which is steadied by the conical sliding ring $m$; the agitators thus hang from the shaft $e$, and nearly touch the bottom of the boiler. When the shaft $e$ is put in motion, the agitators have two movements, one round each other, and also each on its own axis ; as they are set at right angles to each other, as shown in fig. 365 , it follows that no part of the pan can escape being stirred. When the colour is made, the piece $m$ is slid up on $k$, and the agitators unhooked and taken out, the waste of colour being very trifling, in consequence of the agitators being outlines only. The saving of labour cffected in a colour-house by this machinery is very great, as, after turning-on the steam, the pan may be left to itself till the colour is finished.

From the great variety of substances used in mordants and colours, of very different chemical properties, a varicty of thickening substances is required. Chemical combination between the mordants or colour and the thickening substance is to be avoided as much as possible, for such combination may be regarded as so much pure loss, the fibre of the fabric not being able to decompose and assimilate them. Several circumstances may require the consistence of the thickening to be varied; such as the nature of the mordant, its density, and its acidity. A strong acid mordant cannot be easily thickened with stareh; but it may be by roasted starch, vulgarly ealled British gum, and by gum arabic or Sonegal. Somo mordants which soem sufficiontly inspissated with starch, liquefy in the courso of a few days; and being apt to run in the printingon make blotted work. In France, this evil is readily obviated, by adding one ounce of spirits of wine to half a gallon of colour.

The very same mordant, when inspissated to different degrees, produces different tints in the dye-coppor; thus, the same mordant, thickened with starch, furnishes a darker shade than when thickened with gum, Yet there are circumstances in which 
the latter is preforred, because it communicates more transparency to the dyos, and because, in spite of the washing, more or less of the starch always sticks to the mordant. Gum has the inconvenience, however, of drying too speedily, and forming a hard crust on the cloth, which does not ensily allow the necessary capillary attraction to take place, and the tints obtained aro thin and meagre. The substances generally employed in thickening are:-

1. Wheat flour.

2. Wheat starch.

3. Torrefied wheat starch, or British gum.

4. Torrefied potato farina.

5. Gum substitutes or soluble gums.

6. Gum Senegal.

7. Gum tragacanth.

8. Salop. 9. Pipe-clay or china-clay mixed
with gum Senegal.

10. Sulphate of lead.

11. Molasses.

12. Dextrin.

13. Albumen of egge.

14. Lactarine.

15. Gluten.

16. Glue.

Thase most used are the first seven. The rest are only adapted for special styles or colours. The artificial gums produced by roasting starch or farina are very largely in use. The action of heat on starch causes a modification in it. According to the degree of heat and its duration a greater or less modification ensues; the higher the heat, the more soluble in water the gum, but also the browner and of less thickening properties. The addition of various acids and alkalis to starch or farina before caleination causes them to become soluble at lower temperatures than without: different acids also produce different results; those most generally used are nitric, acetic, muriatic, oxalic, and recently lactic acid has been proposed by Pochin. The proportion of acid used is very small, and, though the effect is produced, the acid disappears during calcination. Small quantities of alkalis are also used for special modifications of these gum-substitutes. The making of these gums is a distinct branch of trade, and finds employment for large capital and numerous hands. In giving the receipts for the various colours, care will be taken to specify the nature and proportion of thickening to be employed for each colour; a most important matter, often neglected by English writers upon calico-printing.

It is often observed that goods printed upon the same day, and with the same mordant, exhibit inequalities in their tints. Sometimes the colour is strong and decided in one part of the piece, while it is dull and meagre in another. The latter has been printed in too dry an atmosphere. In such circumstances a neutral mordant answers best, especially if the goods be dried in a hot flue, through which humid vapours are in constant circulation.

In padding, where the whole surface of the calico is imbued with mordant, the drying apartment or flue, in which a great many pieces are exposed at once, should be so constructed as to afford a ready outlet to the aqueous and acid exhalations. The cloth ought to be introduced into it in a distended state; because the acetic acid may accumulate in the foldings, and dissolve out the earthy or metallic base of the mordant, causing white and grey spots in such parts of the printed goods. Fans may be employed with greater advantage, combined with hot fiues. See Vmatinstion.

The mordant and thickening, or the dye decoction and thickening, being put in one of the copper pans, is stirred by hand or machinery and boiled till perfectly smooth; the steam being then shut off, cold water is admitted to the double casing, and the colour cooled. It is then emptied out of the pan into a straining cloth, stretched over a tub, and strained to remove all gritty particles, which would be very injurious to the copper rollers. A very useful straining machine was invented a fow years ago by Dollfus Mieg and Co. and patented in this country. This machine is shown in fig. 366. It consists of a case or cylinder, in which a piston is worked, either by hand or power, to press the colour through a cloth made of cotton, linen, hair, or other suitable material at the bottom of the case or cylinder; or, instead of the said eloth, a wire gauze may be used. The bottom of the piston may be made of wood, copper, brass, gutta percha, caoutchouc, or other suitable material. The manner of working the apparatus will be clearly understood by reference to the drawings, in which fig. 366 is a side elevation of the said machine or apparatus, and fig. 367 a front elevation of the same. A represents the case or cylinder, which is strengthened at its upper part by the iron band $\mathrm{B}$, and also at its lower part by the ring $a$. The skeleton plate $b$, which forms the bottom of the cylinder, is removable, and sustained by the four hooks $c$. To disengage the plate $b$, springs are fitted on the ring $d$, which act upon two of the hooks $c$, so as to throw them out from under the grid $b$. Upon the ring $a$ the second ring $d$ is laid, which supports the circular handle $e$. The upper parts of the four hooks $c$ lie upon four inclined planes fitted on the ring $d$. The 
modus operandi is as follows:-If the ring $d$ is tarned right or left, the skeleton plate $b$, on which one of the said cloths or wire gauze has previously been placed, will be brought firmly up to the extremity of the cylinder $A$; and if the said cylinder bo filled with colouring matter, the piston $\mathbf{M}$, being worked by the pulley $\mathbf{k}$, the wheels $\mathbf{r}, \mathbf{G}$, $\mathbf{H}, \mathbf{I}, \mathbf{K}$, and the rack $\mathbf{L}$, will force it through the cloth or sieve, to be recoived in a vessel under it for the purpose; and by a proper arrangement of the teeth of the said

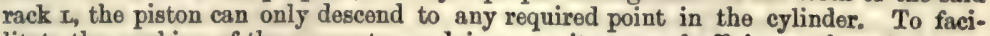
litate the working of the apparatus and increase its general efficiency, the cylinder is fixed on pivots at $\mathbb{N}$, so that it may be easily inclined or brought towards the operator for the purpose of introducing the colouring matter or cleaning the vessel. To the

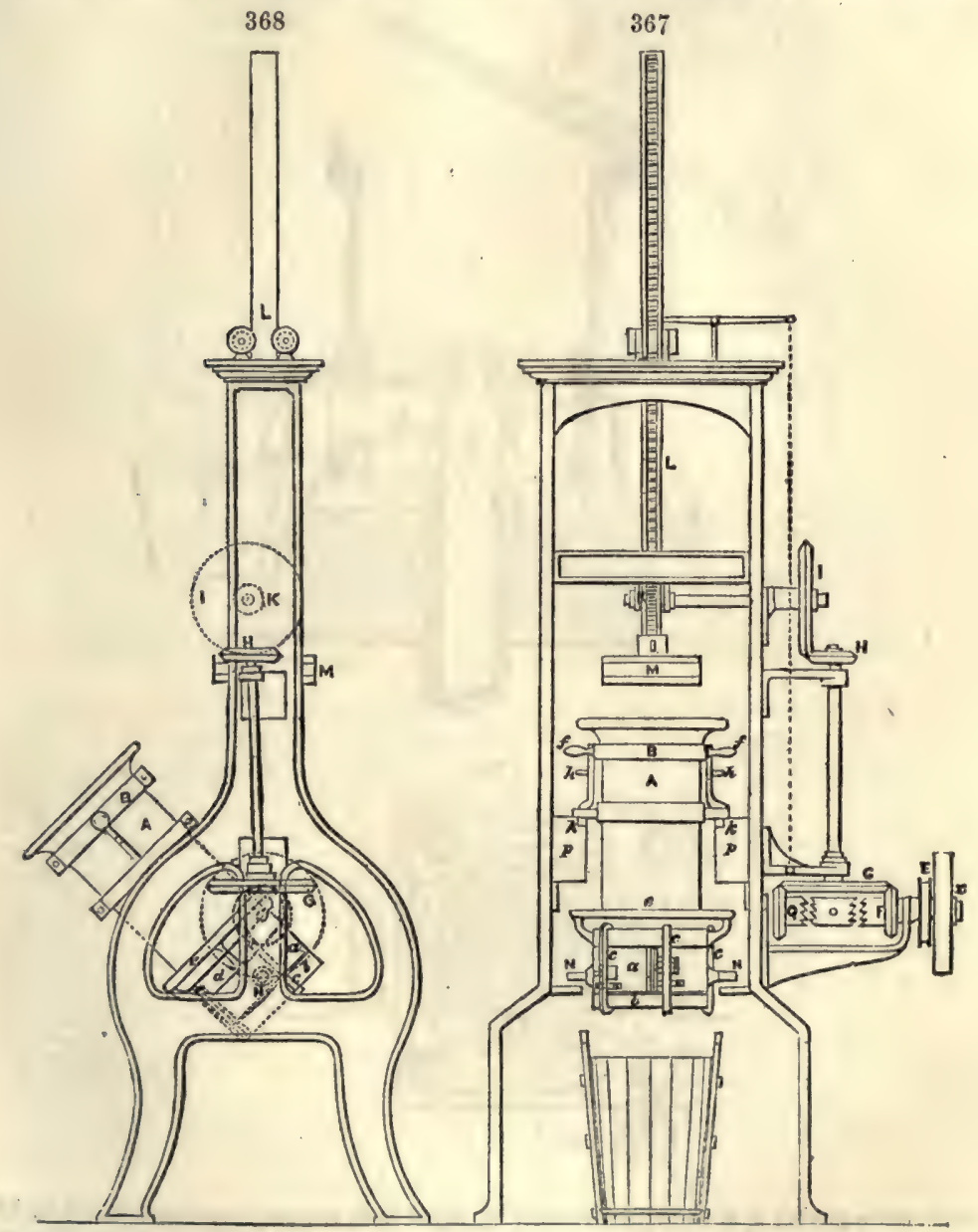

ring or band $B$ are fixed the two handles $f$ and the two catches $h$. The catches being raised from the notch $k$ on the frame $\mathbf{P}$, the cylinder may be pulled forward by meane of the handles $f$, till the hooks, being acted upon by a spring, re-engage themselves at $k$ on the lower part of the frame $\mathrm{P}$, and vice versa. On the shaft $x$ is placod a second wheel $Q$, by which a reverse motion is obtained, and the piston ar raised to its original position.

A straining machine patented by Samuel Ridge and Co. of Manchester has been introduced into some of the largest print works in Lancashire, and is very favourably spoken of (figs. 368, 369, 370, 371).

Fig. 368 , sectional viow of strainer ; fig. 369, ground plan, showing sieve, paddlo 
and colour-trib; fig. 370, ond elevation of pumps; fig. 371, side elevation of pumps; A, lid lever and balance weight; $\mathbf{B}$, cylinder for holding colour; $\mathbf{C}$ c, piston for supporting copper sieves, for the colour to pass through; $\mathbf{D}$, ram for raising piston; $\mathrm{E}$, - hydraulic cylinder; $\boldsymbol{F}$, floor-line; $\boldsymbol{a} \mathrm{G}$, trough for conducting colour to tub; $\boldsymbol{H} \mathrm{H}$, paddle for stopping colour whilst changing tubs ; 1 1, colour tubs ; 3, fixing for balance to rest on whilst filling with colour; $\mathfrak{k}$, for fastening down lid; $\mathbf{x}$, valve for raising and lowering piston; $\mathbf{M}$, end elevation of pumps, showing safety valve, cistern, and fly-wheel; s, eide elevation, showing cisterns, pumps, fly-wheel, fast and loose pullies,

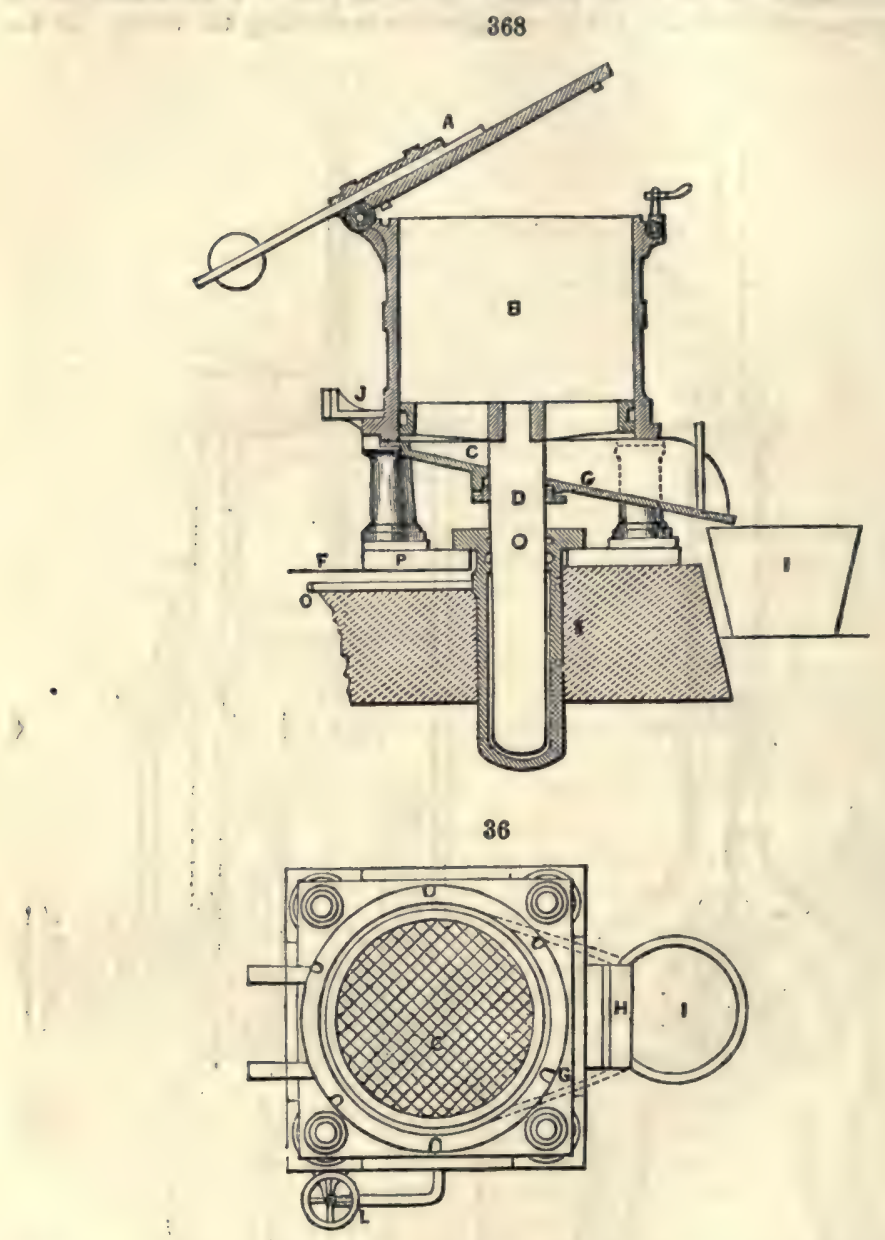

and strap gunde; 00 , showing the ends of pipes that require connecting together, let the distance be whatever it may between, the pumps and strainer; $\mathbf{P}$, foundation plate. Where two letters together are alike they refer to both sectional view and ground plan. This strainer will strain 80 gallons of thick colour in four minutes, and bo washed out ready for any other sort of colour in six minutes.

Colours for printing by block are for the most part thickened in the same manner as those for machine, but are much thinner, since very thick colour cannot be applied by hlock. Somo substances also can be used in block printing that are inapplicable to machine, such as pipe-clay and china-clay, which, howerer finely ground, still contain gritty particles which would speedily scratch and destroy the delicate engraving of the machine rollers.

A spacious drug-room is attichod to the colour-house where all the drugs used are 
aept away from the stenm of the colour-house. Near the colour-house should be a well appointed laboratory, where drugs can be tested and experiments made.

370

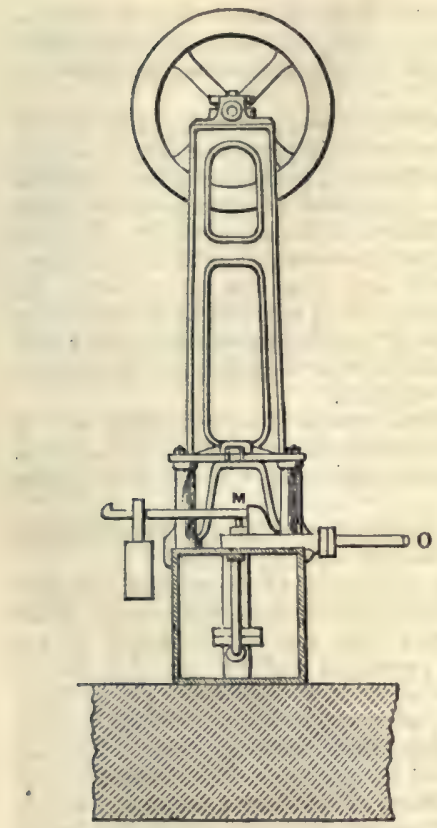

371

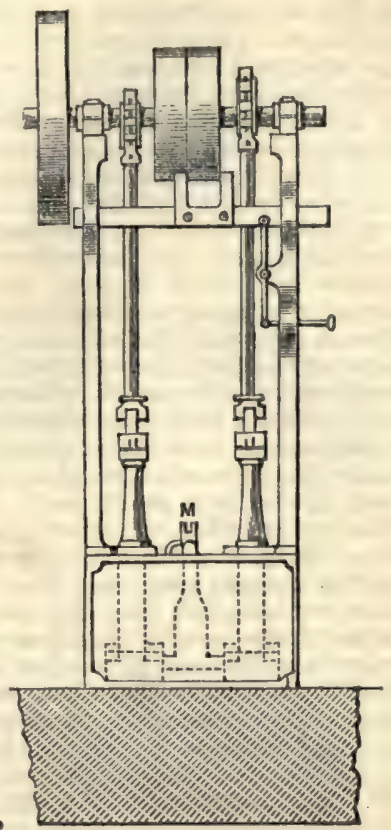

Formerly, all the decoctions and mordants used in print-works were made on the spot; but the trade having very much extended, the manufacture of the various mordants and decoctions of dyewood is now a separate business, and printers can be supplied with these articles at the same or in somo cases a lower rate than they could be produced for on the works, the quality also being uniform and good. The printer now only makes for himself a few unimportant articles. The province of the foreman colour-maker, who is generally a well-paid and responsible servant, is to combine these primary materials so as to form the different colours required for the different styles of work; as the taste of customers varies, he is required to be able to make any given variation of shade at will, and be able to judge of the quality of the various materials submittod to him. Tho ordinary decoctions that are kept in stock in the colour depurtment are:-

Logwood liquor.

Peachwood liquor.

Sapan liquor.

Quercitron-bark liquor.

Gall liquor.
Persian-berry liquor.

Cochineal liquor.

Fustic liquor.

Catechu liquor.
Ammoniacal cochineal liquor.

Extract of indigo.

Extracts of madder.

And the various mordants and solutions are:-

Red liquor, or acetate of alumina.

Iron liquor, or acetate of iron.

Buff liquor, or pyrolignite of iron.

Pernitrato of iron.

Permuriate of ixon.

Protomuriate of iron.
Protochloride of tin in solution.

Oxymuriate of tin in solution.

Nitrate of copper in solu. tion.

Acotate of copper in solution.

Lime juice.

Ammonia liquor.
Acetic acid.

Pyroligneous acid.

Nitric acid.

Muriatic acid.

Sulphuric acid.

Caustic-soda liquor.

Caustic-potash liquor.

Aniline hydrochlorate.

Aniline. 
Many other dry acids and salts are also kept in stock. For the constitution of the - various mordants and their preparation, see Mordasts.

It would be impossible to particulariso all the styles of calico-printing. The variety is infinite; but they may bo broadly classed as follows:-

I Madder styles, varieties of which are-

a. The simplest form is a pattern printed in mordants on white ground, such as black and red; black, red, and purplo; black and two reds, \&cc., chocolate being sometimes substituted for black, and brown from catechu being also introduced; these are dyed with madder, the ground remaining white.

$b$. Any or all of the above mordants, together with lime-juice, technically termed acid, printed, and a fine pattern printed all over or covered in purple or light chocolate, then dyed madder. In this style the red is a peculiar one, termed resist red; and the result when dyed is, that the acid and red have prevented the purple or chncolate fixing on those parts, the red remaining pure and the acid having formed a white, the rest of the ground being covered with the fine pattern or cover; of this style large quantities are printed in black, purple, and acid, and covered in paler purple, the cover roller being any small full pattern, and this not being required to fit to the other pattern, a great variety of effects may be produced by varying the cover: often a still weaker purple is padded or blotched in a plain shade all over the piece, and in this case the only white in the pattern is that reserved by the acid.

c. The French pink style, which is wholly various shades of reds or pinks, and is printed in one or more shades of red and acid, then covered or blotched in pale red, then dyed madder and subjected to a peculiar clearing with soap, whereby pink shades of very great delicacy are obtained.

All these are what are termed fast colours, and having, after dyeing, undergone severe soaping, cannot be altered by the usual domestic washing process.

In this category must now be classed goods, mordanted as for madder, but with weaker mordants, and dyed with alizarine artificially made from anthracene, which produces shades equal if not superior to those dyed with madder, and which do not require as much soaping or other brightening process as madder. At this date it cannot be absolutely affirmed that these colours are as fast to light and air as those of madder, but inasmuch as the alizarine of the purest sorts of the artificial product is identical with that of madder, it is almost certain that modes of dyeing, \&ce, will eventually be used which will give a result identical in every way with madder dyeing.

II. The same styles are dyed with garancin instead of madder; hesvier and darker colours being employed. These goods are not soaped, garancin producing bright colours at once; but the shades, though still classed as fast colours, do not possess the permanence of those dyed with madder.

III. The first style is frequently relieved by lively colours, such as green, blue, yellow, \&c., blocked in after dyeing and clearing; these colours are generally what are termed steam-colours, being fixed by steaming the cloth, and afterwards washing in water oniy, or the printed or dyed pattern is covered with a resist paste blocked on, and various shades of drab, slate, buff, \&c., printed with a small pattern all over; sometimes these colours are mordants, to be subsequently dyed with cochineal, quercitron bark, \&rc., or they may be colours composed of dyewood decoctions, mixed with mordants, and are fixed by passing through soda or other solutions; the result in either case being that tine original pattern, generally a group of flowers, being protected by the paste which prevented the subsequent colour fixing there, stand out pure, the rest of the ground being covered by the small pattern or cover. White may be also reserved by the paste, and frequently these white parts are blocked with blue, yellow, green, \&c., as before.

IV. Padded styles. - In these the cloth is first padded (as will be hereafter explained) all over with a liquid mordant, dried anà printed in spots or figures with strong acid, or discharge as it is called, then put through the dyeing operations necessary for the shade required; the printed spots remaining white, and the rest of the piece one plain shade. The white portions are frequently relieved by steam-colours blocked in.

V. Indigo-blue:-a style of considerable importance. In this, a resist paste, either alone or accompanied by resist yellow, or orange mordant, is printed on white calico, which is then dipped in the indigo vat, till the shade of blue wanted is obtained. If yellow or orange is present, these colours are raised with bichromate-ofpotash liquor. The peculiar colours printed in this style have the property of preventing the indigo fixing on the printed parts, and the result is dark blue ground, with white, orange, or yellow spots, steam-colours being sometimes blocked in the whites.

VI. China-blnes:-a modification of the indigo-blue style, but in this case the pattern is produced by indigo colours, printed on white cloth : the pieces are next put through a peculiar process, fixing the indigo in the cloth, the result being blue figures on white ground. All indigo styles are fast or permanent. 
VII. Turkey red and discharge.-On dyed Turkey-red cloth is printed an acid, or acid-solutions mixed with pigments or salt of lead; the printed pieces are passed through chloride-of-lime solution, when chlorine is eliminated by the acid colours, and discharges the red. The pigments or lead-salt being fixed in the cloth at the same time, sfter washing and chroming where yellow has to be obtained, the piece presents a pattern, bitten as it were in the Turkey-red ground. Black is also printed along with the other colours. A modification of this style is the well-known Bandanna style used for handkerchiefs. Turkey-red cloth is folded in a hydraulic press on a lead plate perforated with a pattern. When a sufficient number of folds are made on this plate, a precisely similar plate is put on the top, so as to register aceurately with the bottom one; pressure being now applied, the cloth is squeezed tightly between the two plates, a tap being opened above the upper plate, solution of chlorine is forced through the perforations, and in its passage through the cloth, discharges the dye; the chlorine liquor is followed by water, and the operation is finished: the pieces when removed from the press being discharged, according to the pattern of the lead plates.

VIII. Steam-colours. - In this style colours are formed from mixtures of dyewood extracts and mordants, together with various acids and salts, and being printed on calico which has been mordanted with peroxide of tin, the pieces are exposed to steam at $212^{\circ}$ in close vessels, which causes an intimate union of the calico with the dyewood extract and mordant, so that subsequent washing with water removes only the thickening substance, and leaves the cloth dyed according to the pattern in various colours. Woollen fabrics and de-laines are always printed in this manner, and also often silk; animal fabrics not being well adapted for mordanting and dyeing in the same manner as cotton fabrics, owing to the peculiar property of wool to absorb colouring matters, which renders the obtaining of whites, an impossibility where the wool is steeped in a dye decoction. These steam-colours are very brilliant and tolerably permanent to light, but do not withstand hot soap solution, which alters their shades.

The invention of the superb colours made from aniline, which only dates from 1856 has caused a considerable alteration in the composition of steam-colours; far more beautiful shades are now produced from aniline colours than formerly was possible from dyewood decoctions, and though not faster, in many cases not so fast to light as these, some of them will bear a slight soaping with improvement in their tone. Within the last five years the colouring matter of madder extracted in a tolerably pure form has been successfully applied as a steam-colour, various shades of red, pink, chocolate, and purple are produced from extracts of madder mixed with aluminous, ferruginous, or chromous salts of volatile acids and the colours thus composed are thickened, printed, and fixed by steaming. These colours, when properly made and fixed, are as fast to soap and light as those of maddered dyed goods. Still later the alizarine artificially made from anthracene has been applied to the same purposes as extracts of madder.

IX. Pigment-printing. - The colours in this class are the same pigments as used by painters, such as Scheele's green, ultramarine blue, chrome yellow, \&c., and being quite insoluble in water are, so to speak, cemented to the fibre. The vehicle used for fixing these, is generally albumen, which coagulates when the cloth is steamed, and imprisons both cloth and fibre with the coagulum; of course these colours, though not altered in shade by soap, are detached in part by severe treatment, such as rubbing, \&c.

Tho last two classes melt into each other in the very beautiful steam chintz styles, where aniline black (described hereafter), madder extract or alizarine reds, pinks, and purples, pigment green, blue, or other pigment shades, and aniline violets are simultaneously printed, fixed by steaming and soaped, thus producing delicate patterns which under no circumstances could be done by madder dyeing, aided by the subsequent introduction of steam-colours. When albumen is used in sufficient quantity for the pigments and aniline violets, these colours are quite as fast as those with madder extract or alizarine, and even compare favourably with madder-dyed shades.

X. Spirit-colours are made in somewhat the same manner as the steam-colours, but contain larger quantities of mordant and acid, and would not bear steaming, because the calico would be too much tendered by the acid, and are therefore only dried and hung up a day or two, and then washed in water. They are the most brilliant colours, but generally fugitive, and are not much used.

XI. Bronzes : formerly a style in large demand, but now almost obsolete; done by padding the cloth in solution of protochloride of manganese, precipitating the oxide by means of alkali, peroxidising this by chloride of lime, and then printing on colours composed of protochloride of tin and pigments or decoctions; the protochloride of tin immediately deoxidises, bleaching the brown oxide of manganese, and, where mixed with decoctions or pigment, leaving a dyed pattern cutting through the ground. 


\section{First Style: Madders.}

Madder styles being the most important, domand tho most detailed descriptions. The colours used are of the class termed mordants, which, not colouring mattors themselres, act by combining with both cloth and colouring matter. They are generally the acetates or pyrolignites of iron and alumina.

Red Liguor is the technical name of the pyrolignite of alumina used as mordant for red, \&c. See RkD Liquor.

Resist-red Liquor is a pyrolignite of alumina which containg little or no sulphateg, and has tho property of not being decomposed by protochloride of tin to so great an extent as the ondinary Red Liquor.

Iron Liquor is the pyrolignite of iron used as mordant for black, purple, \&cc. It is made by steoping iron borings, turnings, or thin pieces of scrap iron in crude pyroligneous acid (see ACETrC ACID, and Pyrulianeous AcID), occasionally allowing acess of air to the iron by drawing off the liquor and pouring it upon iron contained in another vat, the two vats being thus alternately full of liquor, the iron becoming oxidised by the air when uncovered is more easily dissolved. This iron liquor is not a mere solution of acetate of iron; such a salt would be of little use as a printer's mordant, being decomposed and peroxidised by the air with too great rapidity. It contains, in addition to the protoacetate of iron, the various substances found in the erude pyroligneous acid, such as pyrocatechin, pyroxanthogen, empyreumatic oil, resin, furfurol, creosote, \&c., which substances have a strong affinity for oxygen, and tond to prevent the acetate of iron passing into a peroxidised state. The preparation of these liquors on a, large scale forms a separate business.

Fixing Liquor. - For a long time it has been customary to add to black and purple colours, or mordants, some substance which has a tendency to prevent the oxide of iron from passing to the state of peroxide. The oxide of iron necessary to produce the best results with madder is a mixture of protoxide and peroxide of iron, probably the black or magnetic oxide, though this point is not precisely determined. If the oxide should pass to the red oxide state, inferior shades are produced; and the object of the printer introducing fixing liquor into his colour is to prevent this injurious tendency.

The earliest fixing liquor used was a solution of arsenious acid; and though other fixers have from time to time been introduced, the preparations of arsenic still hold their ground. A very good fixing liquor, that has been much used in France and England, is made as follows :-

No. 1. Purple Fixing Iiquor. $-7 \frac{1}{2}$ gallons water, 11 gallon acotic reid, 9lbs. salammoniac, 9lbs. arsenious acid; boil till the arsenic is dissolved, and let stand till quito clear.

In 1844, Mr. John Mercor patented an assistant mordant liquor for the same purpose, which was made as follows :-

No. 2. To 100 lbs. potato starch, add $37 \frac{1}{2}$ gallons water, 123 gallons nitric acid, specific gravity $1 \cdot 3$, and $4 \mathrm{oz}$. oxide of manganese. The chemical action which takes place amongst these ingrodients is allowed to proceed till the nitric acid is destroyed. To the residuum thus produced are added 50 gallons of pyroligneous acid, and the compound is the assistant mordant liquor in a fit stato to add to the various mordants used in printing and dyeing. The intention in making this liquor is to carry on the decomposition of the nitric acid and starch as far as possible without forming oxalic acid, and as little as possible of carbonic acid, which is greatly aided by the catalytic action of the oxide of manganese, preventing the formation of oxalic acid. Apparently there is formed by this process saccharic acid, or an acid in a low state of oxidation, which is the active agent in preventing the poroxidisement of the iron when added to purple mordauts. This liquor has been largely used, and is still preferred by some printers. Of late, various fixing liquors have boen made and sold by manufacturing chemists, pyroligneous acid and arsenious acid, or arsenite of soda, forming the staple of them; some of these have chlorate of potash added, the object being the formation of arseniate of iron when the eloth is dried, whereby the acetic acid is more speedily driven off; and since arseniate of iron does not pass beyond a certain degree of oxidisement in the air, the mordant is kept in a proper state for dyeing good colours. The following is also a good purple fixing colour:-

No. 3. Purple Fixing Liquor.-Boil together till dissolved 2 gallons water, $25 \mathrm{lbs}$. soda crystals, $22 \frac{1}{2}$ lbs. arsenious acid. When clissolved, add to 50 gallons wood acid, previously heated to $120^{\circ} \mathrm{F}$. ; let stand for a day or two till the tar of the acid is settled, and add 3 quarts muriatic acid.

The following madder colours are from some in practical use; and though almost every colour-maker has different receipts for his colours, they may be taken to represent the general principles on which these colours are composed. 
In all these colours the thickoning substance is first beaten up with a little of the liquid till quite fine and free from lumps, then the remainder of the liquid added, and the whole boiled and stirred in one of the double-cased steam-pans till quite smooth; cooled, and strained.

No. 4. Black for Machine (Madder).-4 gallons iron liquor at $24^{\circ}$ T., 4 gallons pyroligneous acid, 4 gallons water, 24 lbs. flour; boil, and add 1 pint oil.

No. う. Black for Garancin (Machine).- 7 gallons water, 3 gallons iron liquor at $24^{\circ}$ T., $1 \frac{1}{5}$ gallon purplo fixing liquor (No. 3), $24 \mathrm{lbs}$ flour, 1 pint oil.

No. 6. Dark red for Madder (Machine).-12 gallons red liquor at $18^{\circ}$ T., 24 lbs. flour.

No. 7. Pale reds for Madder (Machine) are made by reducing the standard liquor, No. 8, with gum-water to the shade wanted: for instance, No. 3 pale red is 1 of No. 8 and 3 of gum-water, No. 9 .

No. 8. Standard red Liquor.-10 gallons hot water, 40 lbs. alum, 25 lbs. white acetate of lead; rake up till dissolved, let settle, and decant the clear.

No. 9. 3 lbs.-Gum-substitute Water.-10 gallons water, 30 lbs. gum-substitute, No. 5 in the list of thickeners.

No. 10. Dark resist-red Madder (Machine),-12 gallons. resist-red liquor, $18^{\circ} \mathrm{T}$, $24 \mathrm{lbs}$. flour; boil, and when nearly cold add $12 \mathrm{lbs}$. of muriate-of-tin crystals.

No. 11. Dark resist-red Machine.-Same as No. 10, but 6 lbs. of tin crystals only.

Of these last two, No. 30 is used when it has to resist a chocolate cover, and No. 11 when it has to resist a purple cover.

No. 12. Pale resist-red Madder (Machine).-Mado by reducing resist-red liquor with water, and thickening it. For instance, No, 5 pale red: 12 gallons resist-red liquor at $5^{\circ}$ T., 9 lbs. flour; boil, and add, when cool, 2 lbs. tin crystals.

No. 13. Chocolates are made from iron liquor and red liquor mixed, and the red liquor is a multiple of the iron; as, for instance, 3 chocolate (madder) (machine):-3 gallons iron liquor at $24^{\circ} \mathrm{T} ., 9$ gallons red liquor at $18^{\circ} \mathrm{T} ., 24 \mathrm{lbs}$. flour, 1 pint oil. No. 6 Chocolate :-1 gallon iron liquor at $24^{\circ}$ T., 6 gallons red liquor at $18^{\circ}$ T., 14 lbs. flour, t pint oil.

No. 14. Strong red for Garaxcin (Machine).-10 gallons red liquor at $18^{\circ}$ T., 2 gallons water, 24 lbs. flour.

No. 15. Resist red for Garancin (Machine).-12 gallons resist-red liquor at $14^{\circ} \mathrm{T}, 24 \mathrm{lbs}$. flour; boil, cool, and add $9 \mathrm{lbs}$. tin crystals. This for resisting chocolate.

No. 16. Resist red for Garancin (Machine), -12 gallons resist-red liquor at $14^{\circ}$ T́. 24 lbs. flour; boil, cool, and add $4 \frac{1}{2} \mathrm{lbs}$. tin crystals. This for resisting purple.

No. 17. Brown Standard for Madder, -50 gallons water, 200 lbs. catechu ; boil 6 hours, then add $4 \frac{1}{2}$ gallons acetic acid, and add water to make up to 50 gallons; take out, and let stand 36 hours, and decant the clear; heat it to $130^{\circ} \mathrm{F}$., and add $96 \mathrm{lbs}$. sal-ammoniac, dissolve, and leave to settle 48 hours; decant the clear, and thicken it with 4 lbs. gum-Senegal per gallon.

No. 18. Brown Colour for Madder (Machine).-4 gallons No. 17, 1 gallon acetate of copper (No. 19), 2 quarts acetic acid, 2 quarts gum-Senegal water, 4 lbs. per gallon.

No. 19. Acetate of Copper.-1 gallon hot water, 4 lbs. sulphate of copper, 4 lbs. white acotate of lead; dissolve, lot settle, decant the clear, and set at $16^{\circ} \mathrm{T}$.

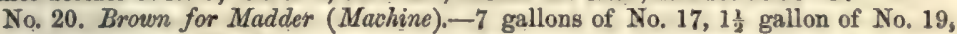
11 gallon gum red (No. 21).

No. 21. Gum red.-3 gallons red liquor at $18^{\circ} \mathrm{T} ., 12 \mathrm{lbs}$. gum-substitute; boil.

No. 22. Brown for Gurancin (Machine).-2 gallons of No. 18, 1 gallon 4 lbs.-gumsubstitute water.

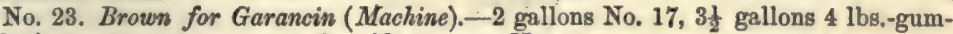
substitute water, 3 quarts acetic acid, 3 quarts No, 19.

No. 24. Drab for Madder (Machine). 4 gallons No. 17, I gallon protomuriate of iron at $9^{\circ}$ T., 3 gallons No. 19, 1 gallon 4 lbs.-gum-substitute water. For garancin, add 4 gallons gum-water instead of 1 gallon.

No. 25. Drab for Madder (Machine). -5 gallons No. 24, 1 quart muriate of iron at $9^{\circ} \mathrm{T}$., 5 gallons 4 lbs.-gum-substitute water, 3 quarts No. 19.

No. 26. Madder Fawns are made by adding to madder drab $\frac{1}{12}$ th, or so, of red liquor, according to the shade wanted.

No. 27. Madder Purples.-Iron liquor, mixed with purple fixing liquor, is diluted with gum-water according to the shade wanted. For instance, No. 4 purple for madder (machine):-1 gallon of iron liquor at $24^{\circ}$ T., 2 gallons No. 3,4 gallons farina gum-water No. 28. No. 12. purple:-1 gallon iron liquor at $24^{\circ}$ T., 2 gallons No. $3_{4}$ 12 gallons No. 28. boil.

No. 28. Dark Farina Gum Water.-10 gallons water, $60 \mathrm{lbs}$, dark calcined farina ; 
No. 29. Garawin Purples are reduced from iron liquor to the shade wanted with the following gum :-20 lbs. light British gum, 8 gallons water, 1 gallon purple fixing liquor No 3 ; boil well, then take out, and let stand 3 or 4 days before using. Colour: 1 messure iron liquor, 8, 10, 20, 30, \&ce., of the above gum, according to shade wanted.

No. 30. Padding Purples.-Reduce to shade with the following gum:- $6 \frac{3}{4}$ gallons water, 1 gallon No. 3, 1 quart logwood liquor at $8^{\circ}$ T., 9 lbs. flour; boil, and add 5 quarts farina gum, No. 28. For instance, 70-padding purple for machine:-1 gallon iron liquor at $24^{\circ} \mathrm{T}$., 70 gallons of the above gum.

Madder purples for some styles, such as the black, acid, and purple padded style, are thickened with $1 \frac{1}{2}$ to $2 \mathrm{lbs}$. of flour per gallon, the olject being to keep the colour on the surface of the fibre, thereby obtaining economy of dye-stuff, together with brilliancy of colour.

Colours, either red or purple, applied on the top of already printed colours are called 'covering' colours when a small pattern is applied, and ' padding' colours when a uniform shade without pattern is applied.

Block colours are made from any of the preceding receipts, by making them a little thinner; the strength of the mordants being also reduced. Colours for dyeing with extract of madder, artificial alizarine, and garancin require to be made much weaker in mordant, in consequence of the less severe treatment after dyeing which goods dyed with these substances require to bring out the full brilliancy of the colours.

No. 31. Alkaline red Mordant.-In a vessel capable of holding 12 gallons, put $10 \mathrm{lbs}$. alum, and dissolve with 5 gallons boiling water, then add gradually 3 quarts caustic soda at $70^{\circ} \mathrm{T}$., mixed with 1 gallon cold water, fill up with cold water; let settle, decant and repeat the washing till the clear liquor is tasteless; filter to a pulp, take off, and add to it 5 pints caustic acid at $70^{\circ} \mathrm{T}$.; boil down to 3 gallons, add 9 lbs. dark gum-substitute, and boil again a short time.

No. 32. Pale red Alkaline Mordant. -1 measure of the above colour and 2 or 3 measures of dark gum-substitute water.

No. 33. 10 Acid. - 1 gallon lime juice at $10^{\circ} \mathrm{T} ., 1 \mathrm{lb}$. starch; boil.

No. 34. 20 Acid. - 1 gallon lime juice at $20^{\circ}$ T., $1 \mathrm{lb}$. starch; boil.

No. 35. 30 Acid. -1 gallon lime juice at $30^{\circ} \mathrm{T}$., $1 \mathrm{lb}$. starch; boil.

No, 36. Acid Discharge. -1 gallon lime juice at $22^{\circ} \mathrm{T}, 1 \mathrm{lb}$. bisulphate of potash; filter, and thicken the eles $r$ with $1 \mathrm{lb}$. starch.

No. 37. Acid Discharge. -1 gallon lime juice at $28^{\circ}$ T., 2 lbs. bisulphate of potash; filter, and thicken the clear with $5 \mathrm{lbs}$, dark British gum.

In the last two colours, the bisulphate throws down a quantity of floceulent matter, which has to be filtered out.

No. 38. Reserve Paste. $-3 \frac{1}{2}$ gallons lime juice at $50^{\circ} \mathrm{T} ., 2 \frac{1}{4}$ gallons caustic soda at $70^{\circ} \mathrm{T}$.; beat to boil, then, in a separate vessel, beat up $56 \mathrm{lbs}$. pipeclay with $3 \frac{3}{4}$ gallons boiling water, and add $3 \frac{1}{4}$ gallons $6 \mathrm{lbs}$.gum-Senegal water; add to the other solution, and boil 20 minutes.

No 39. Reserve Paste. -4 gallons lime juice at $60^{\circ}$ T., 3 gallons caustic soda nt $70^{\circ} \mathrm{T}$.; boil, and add $48 \mathrm{lbs}$. pipeclay beat up with 2 quarts boiling water, and 4 gallons 6 lbss.-gum-Sonegal water; boil 20 minutes.

The above two pastes are used for blocking on madder work, to protect the pattern from the following covering shades, which are raised with quercitron bark, \&c. \&ce. No. 38 is a paste used where there are only black and reds to preserve, and No. 39 is used where there is also purple.

\section{Covering Shades.}

No. 40. 5 Drab. -1 quart iron liquor at $24^{\circ}$ T., 5 quarts water, $2 \frac{1}{4}$ lbs, light British gum.

No. 41.10 Drab. -1 quart iron liquor at $24^{\circ}$ T., 10 quarts water, $4 \frac{1}{2}$ lbs. light British gum.

No. 42.5 Drab. -1 quart iron liquor at $24^{\circ}$ T., 1 quart red liquor at $20^{\circ}$ T., 5 quarts water, $2 \frac{1}{2}$ lbs. light British gum.

No. 43. 10 Drab. - 1 quart iron liquor at $24^{\circ} \mathrm{T}, 1$ quart red liquor at $20^{\circ} \mathrm{T}, 10$ quarts water, 5 lbs, light British gum.

No. 44. Olive.-2 gallons red liquor at $12^{\circ} \mathrm{T}, 1$ gallon iron liquor at $14^{\circ} \mathrm{T}$., $6 \mathrm{lbs}$. light British gum.

No.45. Olive. -3 gallons red liquor at $18^{\circ} \mathrm{T} ., 2$ gallons iron liquor at $8^{\circ} \mathrm{T}$, $10 \mathrm{lbs}$. light British gum.

No. 46. Sage. -9 quarts red liquor at $9^{\circ} \mathrm{T}, 1$ quart iron liquor at $12^{\circ} \mathrm{T} ., 4 \mathrm{lbs}$. light British gum. 
No. 47. Suge, -14 quarts red liquor at $3^{\circ} \mathrm{T}$, 1 pint iron liquor at $12^{\circ} \mathrm{T}$., $5 \frac{1}{2} \mathrm{lbs}$. light British gum.

No. 48. Chocolate Brown. -6 gallons red liquor at $15^{\circ} \mathrm{T}, 1$ gallon iron liquor at $24^{\circ} \mathrm{T}$., $10 \frac{2}{2} \mathrm{lbs}$. light British gum, $3 \frac{1}{2} \mathrm{lbs}$. flour.

No, 49. Slate. -3 quarts logwood liquor at $8^{\circ} \mathrm{T}$., 2 quarts iron liquor at $24^{\circ} \mathrm{T}$., 1 quart red liquor at $18^{\circ} \mathrm{T}$, 1 quart No. 50, 7 gallons water, 18 lbs. light British gum; boil.

No. 50. Gall Liquor.-28 lbs, ground galls, 2 gallons acetic acid, 12 gallons water; stir occasionally for two days, and filter.

No. 51. Hazel. -4 quarts brown No. 18, 2 quarts bark liquor at $10^{\circ}$ T., 1 pint logwood liquor at $12^{\circ} \mathrm{T}$., 1 quart cochineal liquor at $8^{\circ} \mathrm{T}$., $16 \mathrm{oz}$. measure No. $52,4 \frac{1}{2}$ quarts $6 \mathrm{lbs}$. -gum. Senegal water.

No. 52.-1 quart nitrate of iron at $80^{\circ} \mathrm{T} ., 1$ pint nitrate of copper at $100^{\circ} \mathrm{T}$.

No. 53. Standard for Buffs.-10 gallons water, 40 lbs. copperas, 20 lbs. brown acetate of lead : stir till dissolved, settle, and use the clear ; reduced to shado wanted with gum-Senegal water.

No. 54. Chrome-oxide Standard.-3 gallons water, 12 lbs. bichromato potash; dissolve with heat, put in a mug of 12 gallons capacity, add $3 \frac{1}{4}$ pints oil of vitriol diluted with 6 quarts cold water, add gradually 3 lbs. sugar; when the effervescence has ceased, boil down to 3 gallons.

No. 55. Drab. -5 quarts gum-tragacanth water ( 8 oz. per gallon), 21 -quarts No. 55, pint cochineal liquor at $4^{\circ} \mathrm{T}$., $\frac{3}{4}$ pint bark liquor at $8^{\circ} \mathrm{T}$.

No. 56. Fawn.-1 gallon No. 55, 2 gallons 8 oz.-gum-tragacanth water, $\frac{1}{2}$ gallon brown No. 17.

No. 57. Slate, -1 gallon No. 55, 1 gallon 8 oz.-gum-tragacanth water.

No. 58. Gum-tragacanth Water,-10 gallons water, 5 lbs. gum tragacanth in powder; stir occasionally for 3 days.

No. 59. Fast Blue Standard.-150 gallons water, 18 lbs. indigo in pulp, 24 lbs. copperas, 28 lbs. lime previously slaked; stir oceasionally for 2 days, let settle, and draw off the clear liquor, and to every 10 gallons add 1 pint muriate-of-tin liquor at $120^{\circ} \mathrm{T}$.; filter on flannel to a thick paste.

No. 60. Fast Blue for Machine,-1 quart No. 59, 6 oz. muriate-of-tin crystals, 3 quarts of gum-water.

No. 61. Fast Blue Standard.-4 lbs. indigo ground to pulp, 3 quarts caustic soda at $70^{\circ} \mathrm{T}$., 3 quarts water, and granulated tin in excess ; boil in an iron pot till perfectly yellow, when tried on a piece of glass.

No. 62. Fast Blue (Block). - 1 quart No. 61, 12 oz. muriate-of-tin crystals, 12 oz. lime juice at $60^{\circ}$ T., 3 quarts 6 lbs.-gum-Senegal water.

No. 63. Fast Green.-1 $1 \frac{1}{4}$ quart No. 60,2 quarts lead gum No. $64, \frac{1}{2}$ lb. muriateof-tin crystale.

No. 64. Lead Gum. -1 gallon hot water, 8 lbs. white acetate lead, 4 lbs. nitrate lead; dissolve, and add 1 gallon 6 lbs.-gum-Senegal water.

The course of operation for the styles 1,2 , and 3 above, is to print in one or more of the madder colours; sfter dyeing, the goods are hung in the ageing room for a day or two, then brought to the dye-house. The 'ageing' room is a spacious airy chamber, with an arrangement of rails at the top of it, from which the pieces can be hung in long folds. These chambers are kept at an equable summer tomperature, and in proper hygroscopic conditions, due ventilation being also provided. These 'ageing' rooms, as they are called, are in several print-works of enormous dimensions, and are generally separate buildings. Those of Messrs. Edmund Potter and Co., and Messrs. Thomas Hoyle and $\mathrm{Co}_{0}$, in Lancashire, may be particularised as forming quite a feature in the works. The pieces stay in these chambers from 1 to 6 days, according to the style of work, during which time the colour which was deposited on the outside of the fibre gradually penetrates it, and becomes more firmly attached, a portion of the base being deposited, and acetic acid given off in vapours. Where colours are required to absorb a certain amount of oxygen, such as iron mordant, catechu browns, \&c., they find the necessary conditions here. On the proper ageing of printed goods. depends in a great measure the success of many styles; should the room be too hot, or too dry, imperfect fixation of the colour ensues, and meagre and uneven tints are obtained in the subsequent operations. In countries where in summer the atmosphere. is dry, great difficulty is found in ageing properly. In America catechu browns hare been known to require weeks before being of the proper shade. These are of course exceptional cases; the scientific printer knows how to combat these evils by the introduction of watery vapour, or even by erecting his ageing room over a reservoir of water, with rather open boarding for floor; many colours also may have deliquescent salts introducod. In England the process of ageing is of pretty uniform duration.

These ageing rooms have now almost lost their importance, owing to the introduc- 
tion of the 'ageing machine,' which lenves the printed pieces in such a condition, that a few hours is sufficient to perform tho work formorly oceupying days, and hence a notuble economy of space and timo. In a patent of $\mathrm{Mr}$. John Thom in 1849 , for sulphuring moussoline-de-laines, a claim is made for using tho samo apparatus, or a modification of it, for passing calico printod goods through a mixturo of air and acqueous vapour. Mr. Thom found that a printod piece as it came from the machine, absorbed nearly an ounce of moisture to the pound of eloth, without fueling damper; uud that an exposure to a moist atmosphere for one minute and a half was sufficient to produce this result. The ageing machino introduced by Mr. Thom into the Maytield Printworks, had a capacity of 30 cubic yards. The moisture was obtained by having a cistorn of water kept heated to $180^{\circ} \mathrm{F}$. in the machine. Mr. Crum was the next printer to take this idea and work it into more practical shape, by very much extending the size of the machine, and by other minor alterations. The use of this machine rayidly spread, and is now almost universal. Fig. 372 shows the machine as arranged by Mathor and Platt. It consists of a chamber about 36 feet long, 20 feet high, and 13 feet broad; generally it is partitioned off one of the disused ageing rooms. $\Delta$, is the framing forming the machine and for carrying rollers, \&c.; $B$, rollers 9 inches diameter for drawing the cloth, these are driven by gearing; c, rollers of tin plate, not driven; $\mathbf{D}$, entering rollers and rails; $\mathbf{z}$, plaiter for folding cloth on delivery from.

372

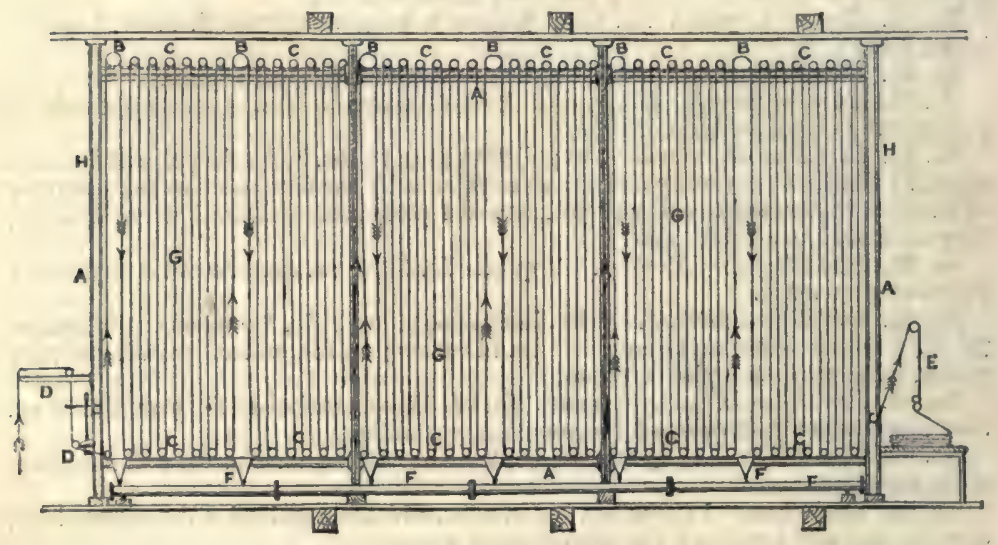

the machine; $\mathbf{r}$, steam or vapour pipes, with steam taps and funnels for spreading the vapour; $G$, the cloth passing through the machine. The machine is enclosed in a wooden room, the boards or walls fitting close at the ends $\boldsymbol{H}$ and $\mathrm{G}$; space being left on each side for passage and for getting to the rollers, taps, \&c.; one or two Daniell's hygrometers are put in different places of the machine. Two pieces, side by side, are run through at once, the temperature being $70^{\circ}$ to $75^{\circ} \mathrm{F}$., and sufficient steam being allowed to escape into the machine to cause a difference of $4^{\circ}$ on the hygrometers. The pieces occupy about 20 minutes during thoir passage through the machine; on emerging from which, they are plaited down into loose bundles of several pieees, which are then drawn away and left on the floor of the room all night. The temperature and hygrometic condition of this outside room should be as near as possible those of the ageing machine. What takes place in ageing in this manner has not been fully investigated; but it is probable that as the piece leaves the printing machine, the mordant is almost altogether on the surface of the fibre in an unaltered state, that the thorough permeation of the piece by the aqueous vapour dissolved in the air, restores to the eloth the normal water removed by the drying in the hotroom, and that under the influence of this, and the slightly elevated temperature, first, a separation of mordant from the thickening substance takes place, and the solution diffused through the fibre, and secondly, that the fibre now begins to play the part of an acid of superior affinity for the base of the mordant to the volatile acetic solvent, and gradually withdraws it from the mordant, acetic acid being freely given off, evident enough to the eyes and nose on going inside the machine. The change once begun is more slowly carricd on in the outside room, but still with much moro rapidity. than under the former conditions of agoing rooms. The chemical action of cellulose 
or cotton fibre in decomposing acetates of iron and alumina, has been denied by some chemists, especially by the late $\mathrm{Mr}$. Walter Cum, the eminent printer of Thornliebank, who, in a rery interesting paper printed in the Journal of the Chemical Society for 1863, propounded the theory, that capillary action of the fibre separates the mordant from the thickening substance, which is left at the outside of the fibre, that by desiccation the acetates are decomposed, and the bases left in the interior cavities. He also attributes to the cloth an attraction of surface, whereby bases are separated from their solvents, in the same manner as that in which charcoal decomposes certain salts. He points out also, that the fibre may act as a dialyser to retain within its - pores, or in the interior cavity of the cotton fibre tube, a colloid solution of alumina or iron; the crystalline portion of the mordant having passed through when the printed cloth is immersed in water (dunging operation). Mr. Crum gives some beautiful drawings of the appearance of dyed cotton fibres under the microscope, showing in many cases that a clot of mordant base has been formed in the interior cavity of cotton fibre and been subsequently dyed there. His drawings, however, for the most part show that the walls of the tubes are dyed throughout their substance; a fact not, as wo think, sufficiently explained by his theory. Other microscopists who have examined dyed cotton fibre, have failed to detect imprisoned particles of colour inside the tube, but have noticed that the wall of the tube throughout its substance is dyed; which, if correct, certainly points to a chemical action of the cell wall. That cotton fibre has a separating power, even when immersed in water, may be shown by putting bleached cotton cloth overhead in a solution of oxide of lead in lime (plumbite of calcium) for half an hour or so, removing, rinsing very well in water, and passing through a solution of bichromate of potash, whence the cloth will emerge dyed a bright yellow. This resction is noticed by Mr. Crum as an illustration of the surface attraction exercised by cotton fibre; the withdrawal of indigotine, or white indigo, from a solution of it, by cotton cloth immersed therein, being also given by Crum as an illustration of the same force; but the former, at all events, may equally serve as an illustration of the chemical power of cotton fibre to sieze and combine with bases, and this power is, we think, further illustrated by the rapidity with which acetates are decomposed under the influence of the ageing machine and torrents of acetic acid given off. There is room, apparently, for further investigation of this obscure point. That pigments such as prussian blue, chromate of lead, \&c., can be precipitated and imprisoned inside the cotton tubes, is not of course, denied; but the aetion here seems quite different from that of ageing. Probably even in well-aged goods, a precipitation of this latter sort takes place to some extent, when the arseniate phosphate or silicate of soda of the dunging solution reacts upon mordant separated from its thickening, and remaining inside the cotton tube. Thus, it is quite conceivable that two soparate actions have taken place in goods about to be dyed; viz., a chemical combination of the tube wall with the base of the mordant, and an imprisonment of an insoluble sait inside the tube, by double decomposition.

The next operation is that termed dunging, which is the same in principle for all varietios of madder or garancin goods: and as it is an operation the careful performance of which is of vital importance to the success of the subsequent operations, a somewhat detailed description of it will not be out of place. The process of dunging has for its object-

1. Precipitating on the fibre, by double decomposition, that portion of the mordant which has ercaped decomposition in the ageing room.

2. Rendering insoluble and inert those portions of the mordant which are not in direct contact with the fibre, and which, if allowed to diffuse in water only, would fix on and stain the white or unprinted parts of the cloth.

3. Softening and removal of the thickening substances.

4. Neutralising the acids which may have been added to the mordants, or used as resists or discharges, and which otherwise would dissolve in the water and weaken the colours.

5. The formation, in the case of iron mordants, of a compound of oxide of iron, and certain organic or inorganic acids which will not become peroxidised beyond a certain point. The use of cow-dung, derived from India, has been continued down to the present time, though for several years printers have largely introduced various substitutes.

No very exact analysis has boen made of cow-dung. Morin's is as follows:-

Water.

Water $\begin{aligned} & : \\ & \text { Vegetable fibre }\end{aligned}$
$\begin{aligned} & \text { Green resin and fat acids }: \\ & \text { Undecomposed biliary matter }\end{aligned}$
Peculiar extractive matter (bubuline)




$\begin{aligned} & \text { Albumen } \\ & \text { Biliary resin }\end{aligned}: \div \quad: \quad \div \quad \div \quad \div 8040$

Another analysis gives the total constituents of 100 parts of cow-dung as follows :Water, 69.58 ; bitter matter, 0.74 ; sweet substance, 0.93 ; chlorophyll, 0.28 ; albu$\min , 0.63$; muriate of soda, 0.08 ; sulphate of potash, 0.05 ; sulphate of limo, 0.25 ; carbonate of lime, 0.24 ; phosphate of lime, 0.46 ; carbonate of iron, 0.09 ; woody fibre, $26 \cdot 39$; silica, $0 \cdot 14$; loss, $0 \cdot 14$.

According to M. Koechlin's practical knowlodge on the great scale, it consists of a moist fibrous regetable substance, which is animalised, and forms about one-tenth of its weight; 2 , of albumen; 3 , of animal mucus ; 4 , of a substance similar to bile; 5 , of muriate of soda, muriate and acetato of ammonia, phosphate of lime, and other salts; 6 , of benzoïn or musk.

Fresh cow-dung is commonly neutral when tested by litmus-paper; but sometimes it is slightly alkaline, owing, probably, to some peculiarity in the food of the animal.

Probably the hot water in which the ealico-printer diffuses the dung exerts a powerful solvent action, and in proportion as the uncombined mordant floats in the bath it is precipitatod by the albumon, the animal mucus, and the ammoniacal salts; but there is reason to think that the fibrous matter in part animalised or covered with animal matter, plays here the principal part; for the great affinity of this substance for the aluminous and ferruginous salts is well known.

It would appear that the principal function of dunging is to hinder the uncombined mordant diffused in the dung-bath from attaching itself to the unmordanted portion of the cloth, as already observed; for if we merely wished to abstract the thickening stuffs, or to complete by the removal of acetic acid the combination of the mordanting base with the goods, dung would not be required, for hot water would suffice. In fact, we may observe, that in such cases the first pieces passed through the boiler are fit for dyeing; but when a certain number have been passed through, the mordant now dissolved in the water is attracted to the white portions of the cloth, while the free acid impoverishes the mordanted parts, so that they cannot afford good dyes, and the blank spaces are tarnished.

It seems to be ascertained that the mordant applied to the cloth does not combine entirely with it during the drying; that this combination is more or less perfect according to the strength of the mordants, and the circumstances of the ageing; that the operation of dunging, or passing through hot water, completes the combination of the cloth with the mordanting base now insoluble in water; that this base may still contain a very minute quantity of acetic acid or sulphate of alumina; that a long ebullition of water impoverishes the mordant but a little; and that even then the liquid does not contain any perceptible quantity of acetate or sulphate of alumina or iron.

A very able and learned memoir upon this subject, by M. Penot, Professor of Chemistry, appeared in the Bulletin of the Society of Mulhausen, in October 1834, and en ingenious commentary upon it, under the title of a Report by M. Camille Koechlin, in March 1835.

Experience has proved that dunging, is one of the most important steps in the process of calico-printing, and that if it be not well performed the dyeing is good for nothing.

In dunging calicoes the excess of uncombined mordant is in part attracted by the soluble matters of the cow-dung, and forms an insoluble precipitate, which has no affinity for the cloth, especially in presence of the insoluble part of the dung, which strongly attracts alumina. The most important part which that insoluble matter plays, is to seize the excess of the mordants, in proportion as they are dissolved by the water of the bath, and thus to render their reaction upon the cloth impossible. It is only in the deposit, therefore, that the matters carried off from the cloth by the dung are to be. found.

M. Camille Koechlin ascribes the action of cow-dung chiefly to its albuminous constituent combining with the alumina and iron, of the acetates of these bases dissolved by the hot water of the bath. The acids consequently set free soon become evident by the test of litmus-paper, after a few pieces are passed through, and require to be got rid of either by a fresh bath or by adding chalk to the old one. The dung thus serves also to fix the bases on the cloth, when used in moderation. It exercises, likewise, a deoxidating power on the iron mordant, and restores it to a state more fit to combine with colouring matter.

The use of cow-dung is open to some objections, amongst which are its giving a cortain amount of greenish colouring mattor to the white mordants, and its being apt 
to vary in its constituents from difference in the food of the animals, their health, \&c.; the method of using substitutes for it being now well known, and better colours and whites being more easily obtained from them than with dung, this substance has almost ceased to be used in calico-printing processes. The dunging operation ought to be a definite chemical decomposition, which cannot be the case with a variable substance like dung. The substitutes for dung in use are:-
1. Phosphate of soda and lime.
2. Arseniate of soda.
3. Arsenite of goda.
4. Silicate of soda.
5. Silicate of lime.

Each of these has its peculiar virtues, and the printer determines for himsolf which is best adapted for his styles. The first was patented by John Mercer, about 1842, and is made by calcining bones, then decomposing them with sulphuric acid, filtering out the sulphate of lime, and, to the clear superphosphate of lime, adding carbonate of soda till slightly alkaline; the resulting mixture of phosphate of soda and phosphate of lime is dried down to a powder; the use of arseniates formed part of the same patent. Arsenite of sode followed as a matter of course, though not so safe in use as phosphates and arseniates. Silicate of soda was suggested by Adolph Schlieper, of Elberfeld, and patented by Jäger in 1852. It is the ordinary soluble glass dissolved in water. It is open to the objection of being too alkaline, and requires care in the use. The silicate of lime was suggested by Higgin with a view to remove this objection. The silicate of lime is formed in the dung cistern, by mixing silicate of soda and muriate of lime, when sparingly soluble silicate of lime is formed; the quantity in solution at one time being never so much as to be dangerous, and fresh portions being dissolved as wanted. Dunging salts, or liquors, are now made by the manufacturing chemist, containing various mixtures, arseniates, phosphates, arsenites, \&c., which are adapted for every variety of dunging. Great economy of time and material result from the use of these dung-substitutes. In some of the largest print-works, instead of, as with dung, running off the spent-dung cistern after passing through from 100 to 200 pieces, and having to fill again, and heat to the proper temperature, it is found possible to run pieces through the same cistern charged with substitute, at the rate of two pieces per minute half a day, and with light goods a whole daybefore letting off; of course, occasionally adding some of the substitute, to make up for that saturated by the mordants. The dunging process is always performed twice: the first time in a cistern with rollers; and the second, in a beck similar to a dyebeck, washing well between. The first is called fly-dunging; the other, second dunging.

The manner of immersing the goods, or passing them through the dung-bath, is an important circumstance. They should be properly extended and free from folds, which is secured by a series of cylinders. The immersion should take place as fast as possible; for the moment the hot water penetrates the mordanted cloth, the acetic acid quits it, and, therefore, if the immersion was made slowly, or one ply after another, the acid, as well as the uncombined mordant, become free, would spread their influenee, and would have time to dissolve the aluminous subsalts now combined with the cloth, whence inequalities and impoverishment of the colours would ensue.

The fly-dung cistern should be set with about 30 gallons of dung to 1,000 gallons of water; or, to the same quantity, 3 to 4 gallons of dung-substitute liquor; a little chalk is added, to make the cistern slightly milky. The heat varies for different stylesfrom $150^{\circ} \mathrm{F}$. to boil. Where there is acid discharge or resist, and the colours are heavy, fly-dunging at boil is necessary, to enable the acid to cut properly through the colour; the nearer to $150^{\circ} \mathrm{F}$. that the bath will give good whites at, the better will be the subsequent dyed colour. With cow-dung, an excess of it is injurious, both to white ground and mordant.

Figs. 373 and 374 aro Furnival's arrangement of first and second dunging ressels. $\mathrm{A} 1$ to $\mathrm{A}$ is a smaller cistern provided with rollers, top and bottom, in which a stronger solution of dunging liquor than that in the main vessel is sometimes put; $\mathbf{A} \mathbf{A}$ is the main fly-dung vessel, divided into two compartments, mado of cast-iron plates boltod together. The whole fly-dung cistern is from 20 to 30 feet long, $4 \frac{1}{2}$ foet wide, and 5 to 6 feet deep. The pieces onter the machine at the end 1, passes over and under the rollers c c, being drawn through by the draw-rollers $\mathbf{D}$, which are placed over the divisions in the cistern; these rollers being connected and turned by the drivingeshaft I ; they then pass through the compartment $\mathbf{A ~ B}$, where they are spirted with water from perforated pipes fixed almost level with the top of the cistern, and beaten by the square beaters $\mathrm{H}$, which are also connected with and turned by the shaft $\mathrm{E} ; \mathrm{F}$ are the stcam-pipes for heating the liquid. The cistern $\mathbf{A} \mathbf{\Delta}$ is set with a solution of arseniate of soda, containing from 10 to 50 grains arsenic acid per gallon, according ta 
the strongth and nature of the mordants to be dunged; usually a little chalk is added sufficient to make the liquid slightly milky. The first compartment is either set with the same solution, or sometimes with a more concentrated one. The tomperature varies from $150^{\circ} \mathrm{F}$. to boil, according to the style under treatment. On leaving the cistern they pass successively through two or more becks, very similar to dyebecks (which see farther on) where they run spirally through a solution of arseniate of soda, much weaker than that of the fly-dung cistern, generally about 8 to 12 grains arsenic acid per gallon; to which some printers add a little cow-dung or bone-size solution. The time occupied in the transit of a piece through the second dung becks is from 20 to 30 minutes, and the temperature from $150^{\circ}$ to $170^{\circ} \mathrm{F}$. Fig. 374 shows a plan and sectional elevation of two second dung becks; the piece is brought from the
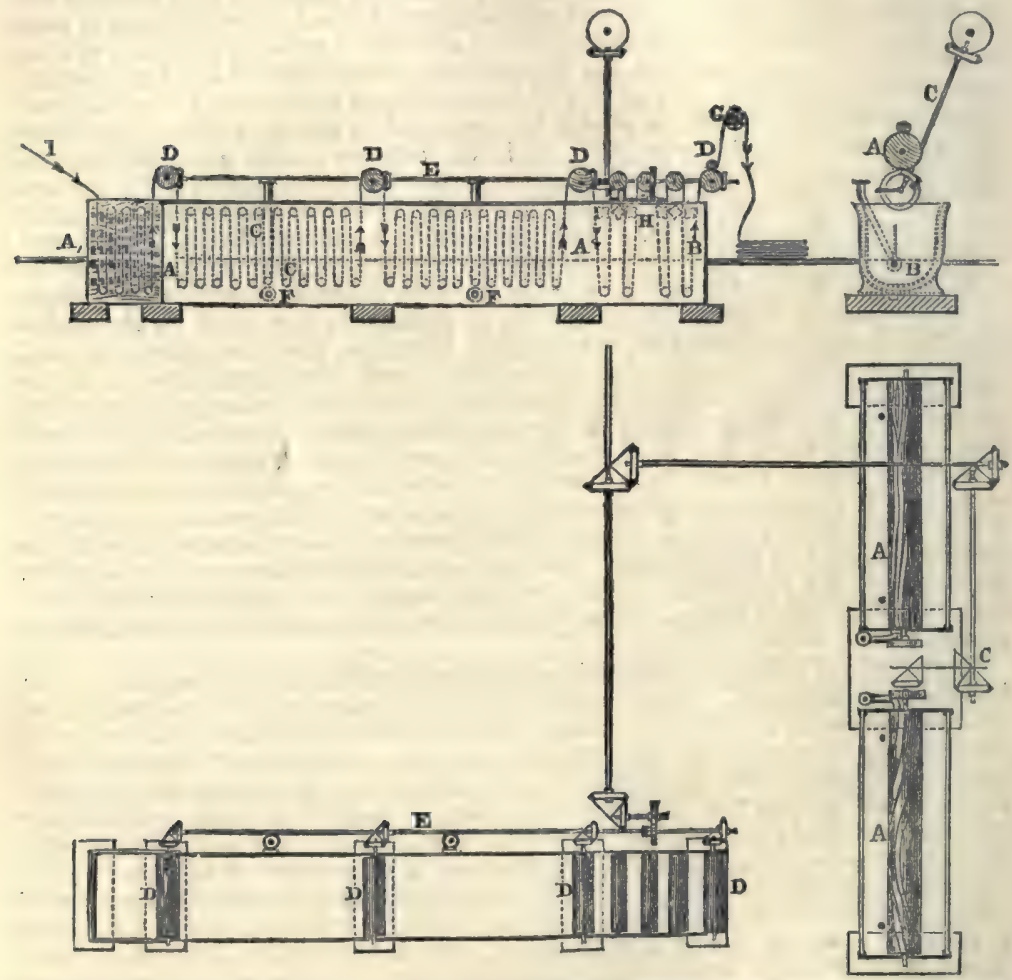

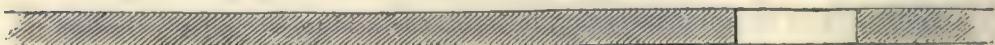

first dung cistern and entered into the end of one of the second dung becks, over the roller A, and under the steam-pipe B, being drawn through the entire length in a spiral and progressive manner; a few yards of cloth are left lying at the bottom of the cistern each time it passes over the roller A; B is the perforated steam-pipe for heating the

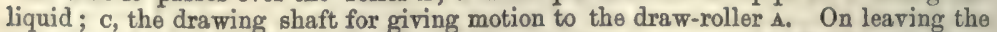
first second dung beck, the pieces pass in a similar manner through the second, or others, if more than two; they next pass through a washing machine. Fig. 375 is a section of the washing machine now most generally used for all purposes in printworks; $\Delta$, the framing and wooden trough of the machine; $\mathbf{B}$, large roller for drawing the cloth through machine; c, pressing roller above roller B; D, square beater rollor, running in a contrary direction to the cloth, at about 180 revolutions per minute; $\mathrm{E}$, wince rolier; F, perforated water-pipe; $\mathrm{G}$, pog rail; $\mathrm{H}$, plaiter wince; I, cloth passing through the machine; it enters at one end, and passes spirally through to the delivery end. The advantages of this machine are, great simplicity and efficiency. The square bowl causes the cloth to rise and fall upon the surface of the water, which, being allowed 


\section{CALICO-PRINTING}

to flow off at the overflow shown, is always kept at the right level. After this mnsing, the cloth passes between the squeezing rollers at the top of the machine, provious to

\section{5}

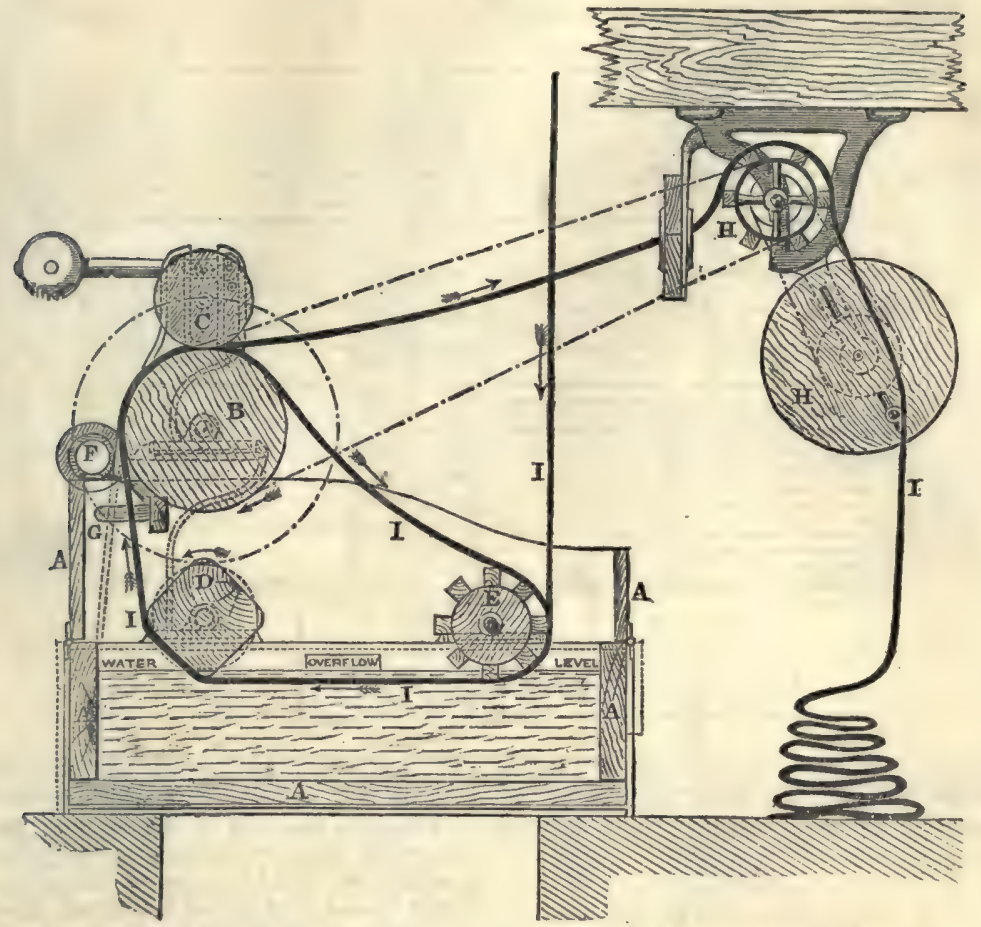

which it receives a spirt of clean water. It then passes into the trough over the lagged roller, as shown, and the operation is repeated until the cloth is sufficiently washed. This machine will wash about 90 yards of cloth per minute. Whenever a washing machine is mentioned in this article, it is to be understood that this sort of machine is meant. The drawing is of the machine as made by Mather and Platt.

Up to this point there is scarcely any difference in the operations on pioces destined for styles I $a, b$, \&c., and II. Those intended for dyeing with madder are printed in stronger colours than those for dyoing with garancin, since the soaping process reduces the strength of colour considerably, and garancin colours undergo no severe treatment after dyeing. The general proces of dyeing is thus performed:-

Fig. 376 represents a front elevation of a pair of dye-becks, with automatic winchreel, and fig. 377 is an end-elevation of one of them. The drawing is kindly supplied by Messrs. Mather and Platt, of Salford. $\Delta \Delta$ is a cast-iron cistern, 8 feet long by 4 feet deep by 3 feet wide, with curved bottom; brackets B B are cast on the ends to support the system on the stone foundation. This beck is fixed over a channel c, which communicates with the system of drains which carries away the waste liquors into the river. There are two holes in the curved bottom-one at each end-which, when the beck is in use, are stopped with moveable plugs; one of these holes communicates direct with the drain and the other with a trough $\mathrm{D}$, which communicates with a pit outside the dye-house, and where the spent madder can be run for the purpose of making into garanceux; $\mathbf{B}$ is a water-pipe, with a branch into each beck, with a screw tap attached ; $F$ is a main steam-pipe, which divides into branches $\mathrm{G}$, furnished with valves at $\mathrm{H}$; the pipes $\mathrm{G}$ subdivide in branches $\mathrm{I}$, one of which goes down each end of the dye-beck, the perforated pipe $\mathrm{K}$, which traverses the beck from end to end, connecting them; a perforated iron diaphragm is placed across the beck from end to end; above this is a strong rod $\mathbf{x}$, from end to end, carrying pieces s projecting at right angles from it. Bolted on the ends of the dye-beck is the framework 0 , which carries the bearings of the shaft $Q$ of the winch reel; keyed on the shaft are three sets of 
arms $\mathrm{R}$, which terminate in forks, in which fit the spars s; the reel is boarded betwoen the spars, as at $x$. The framework o of the two dye-becks is connected by the piece $\nabla$, which carries the bearings of the short shaft $\nabla$, on which is keyed one of a pair of

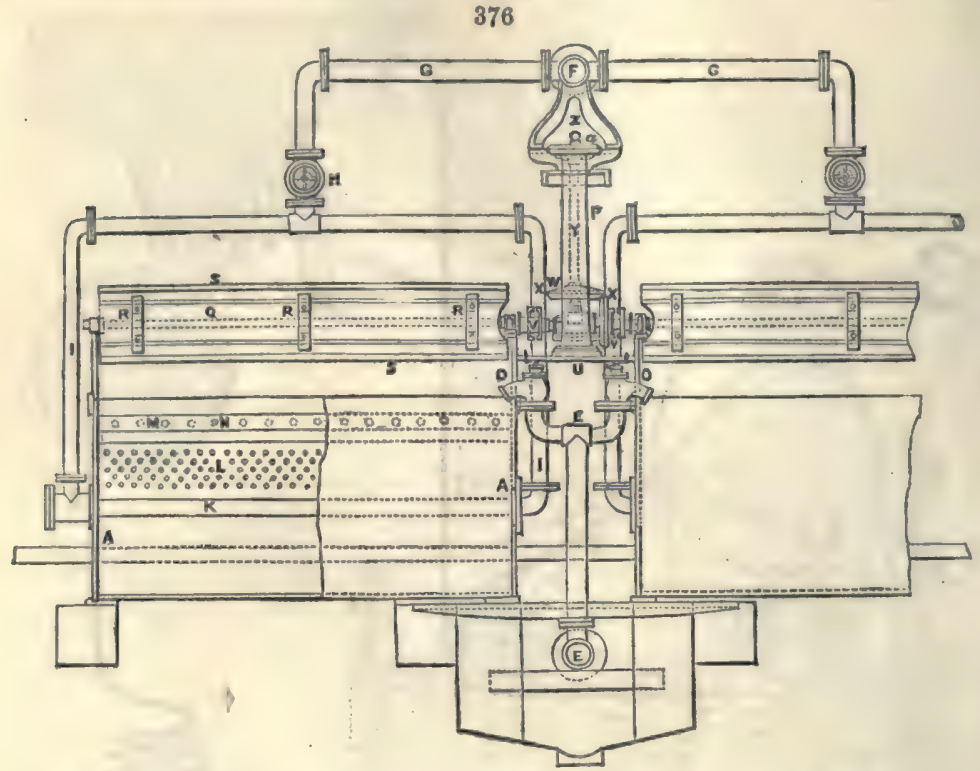

mitre wheols $\mathrm{W} W$; there are sliding catch boxes $\mathrm{x} \mathrm{x}$, on this shaft, which revolve with it; there are corresponding catch boxes keyed on the ends of the shaft $Q$; the

377

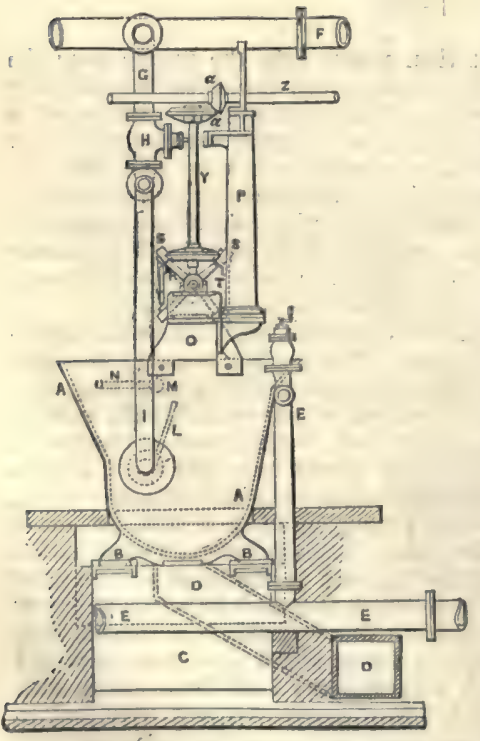
connecting piece $\mathrm{\sigma}$ carries also the pillar $P$, which carries the bearings of the vertical shafts $\mathbf{Y}$, and also of the horizontal shaft $\mathbf{z}$; keyed on the shafts $\mathbf{Y}$ and $\mathbf{z}$ are bevel wheel $a a$, and at the bottom of shaft $\mathbf{x}$; the mitre wheel w. Permanent motion being given to the shaft $\mathrm{v} v$, by this gearing, either of the reels can be put in motion or stopped by the catcli boxes $\mathrm{x} x$, worked by lever handles, in or out of the catch boxes on the ends of the reels. In working the becks, two pieces are knotted end to end, and each length passed over the reel down between two of the studs $x$, under the steam-pipe $\mathrm{x}$, up behind the diaphragm $\mathrm{L}$, being then knotted together so as to form an endless web, the bulk of which lies on the bottom of the beck. The drawing shows a beck adapted for 15 lengths of 2 pieces each, or 30 pieces. About 200 gallons of water are put in the beck before the pieces are put in; and, after the pieces, the dye-stuff is added, the reel set in motion, and the steam gently turned on; from the steam going in at each ond, the beck is uniformly heated; the heat is then gradually raised to boil, generally in about two hours, the pieces continually revolving with the reel so as to bring each portion successively into the air, agitating the dyeing materials at the same time.

- This gradual heating of the dyo-beck was a method arrived at by practice, and it 


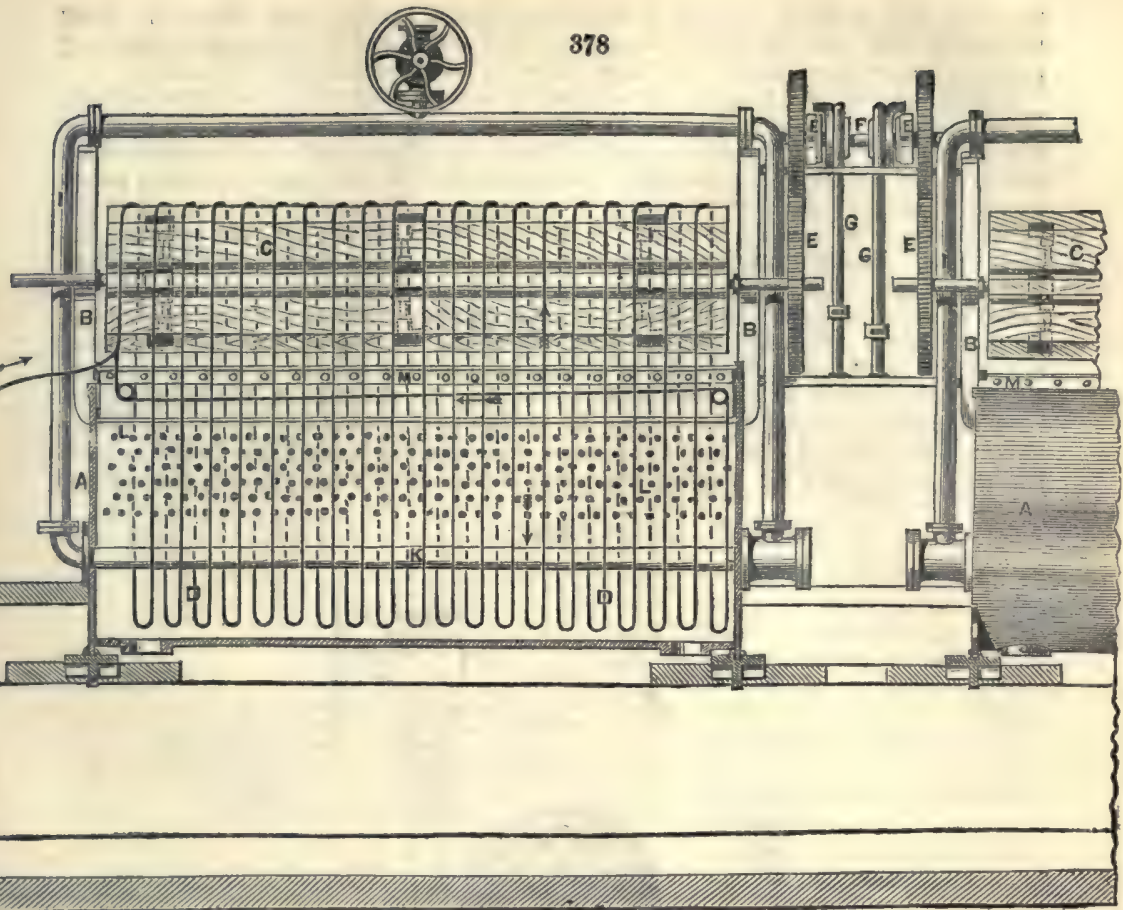

was only within a fow years that chemical seience has shown the wisdom, if not the necessity, of the system. Some dye-stuffs, and especially madder, do not contain their colouring matter ready formed in considerable quantity, but contain a colour-forming principle, which is acted on by nitrogenous ferment also present, whose activity is unlocked by the solution in water, and by the aid of which the colour-forming principle becomes decomposed into true colouring matter and some other substance. The newly-formed colouring matter is assimilated by the mordant nearly as fast as produced. As with most ferments, the temperature of boiling water destroys the ferments in dye-stuffs, and a very inferior result would in many cases be produced, were a boiling heat to be given during the first periods of dyeing. See MADDrR. With the colouring matters of dyestuffis properly formed and separated from woody fibre, these precautions are generally unnecessary, as is explained when treating of madder extract and alizarine. When the dyeing is finishod, the steam is shnt off, the knots untied, and the pieces pulled over into a pit of water

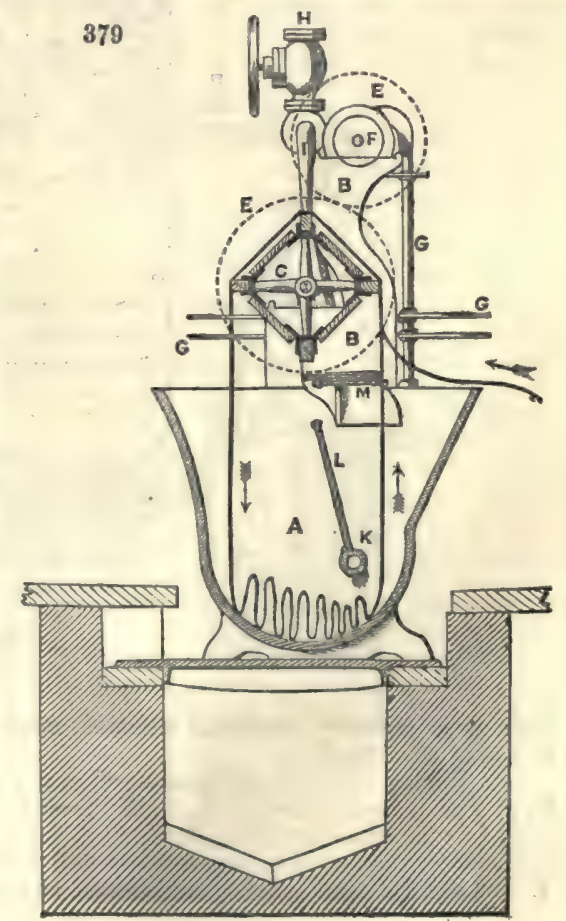


mounted with a winch rool, which is always plaood behind overy dye-beck. Aftor wincing in this, the pieces are fastened together again, and put through the washing machine two or three times; they then are ready for the subsequent operations.

Another method of dyeing is now adopted by several printers, termed spiral-dyeing. In this process 30 to 40 pieces are left stitched together, entered into the dye-beck at one end, and caused to revolve spirally through the liquor and over the wince from end to end of the beck; the end which was entered first is now passed through a porce. lain eye, brought back across the face of the beck, and knotted to the last end of the set of pieces, after passing through another eye which directs the cloth into the dye beck again. The process is now continuous, and the pieces constantly moving from end to end and back again, pass through all parts of tho dye-bock, and are all subjected to the same heat. It is said that much greater uniformity of dye is obtained by this method. Figs. 378 and 379 show the spiral or endless dye-beck of Mather and Platt. A, cast-iron dye-bock; $B$, fixings for carrying wince; $\mathrm{c}$, winces; $\mathrm{D}$, cloth being dyed; $\mathrm{s}$, spur whools, and catch boxes, \&ce., for driving; $\mathbf{F}$, driving shaft; $\mathrm{a}_{\text {, }}$ handles and tappet shafts for putting in motion or stopping the wince; $\mathrm{H}$, steamvalve; r, steam-pipes; $\mathrm{K}$, perforatod steam-pipe passing through the beck; I, midfeather; $\mathbb{x}$, peg-rail for keoping the pieces from entangling. Messrs. Mather and

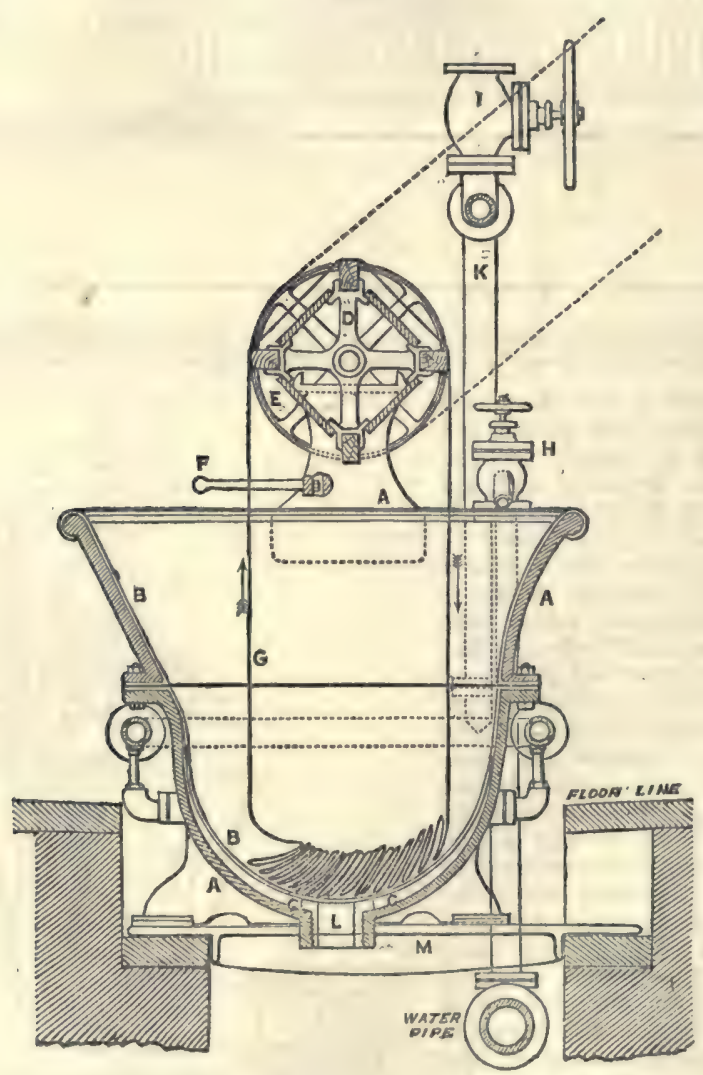

Platt have recently introduced a double-cased dye-beck, for which they claim the following advantages:-

1st. Having neither internal steam-pipe, nor midfeather, there is no possibility of tearing or damaging the goods in the process of dyeing. 2nd. As no steam is admitted to the dyeing solution, its strength is maintained uniform, and, in fact, rather increased to the end of the operation. 3rd. Perfoct freedom from stains is insured. 4. The work is more regular, and effected at less cost of material than with becks, to 
which steam is admitted in the ordinary way. It is double cased in the lowor part, the lining being made of copper, and the outer shell of cast-iron.

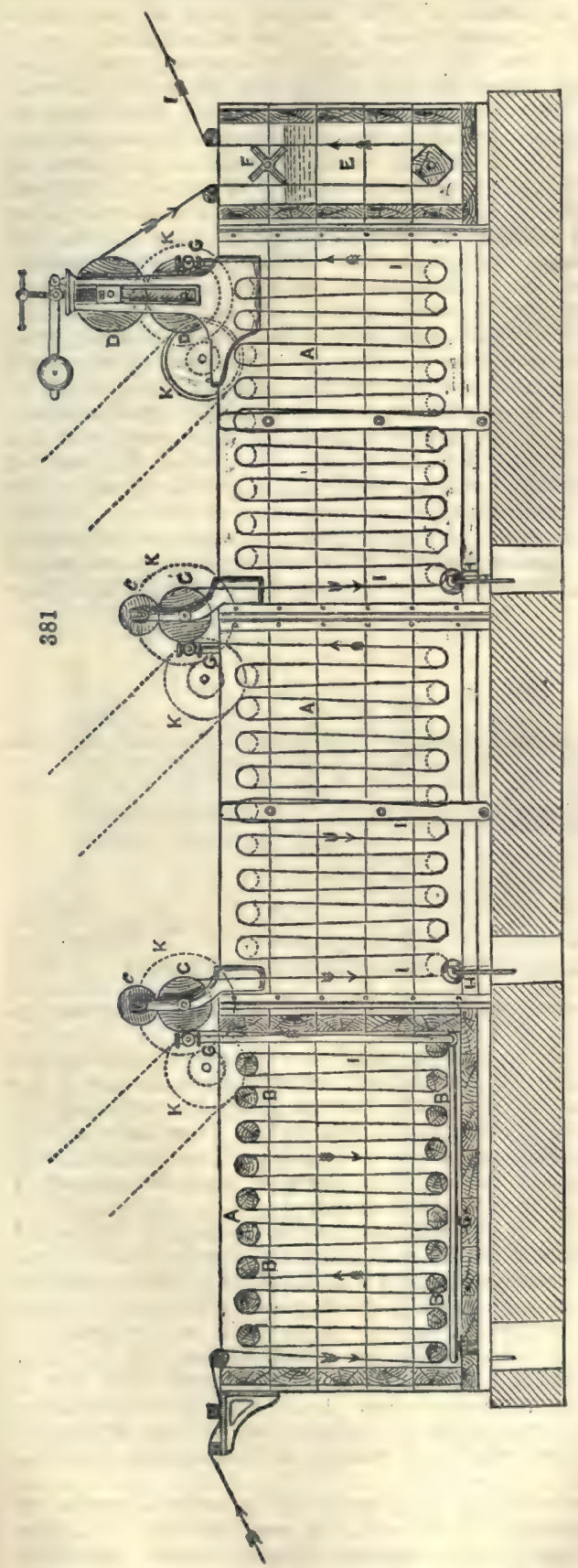

Fig. 380 shows a section of this bock. A, cast-iron outer casing of dye-beck, bolted together in two parts; $B$, copper lining of beck; $c$, steam space, in the bottom half of beck only; $\mathrm{D}$, wince, making 42 revolutions per minute; $\mathrm{s}$, driving pulley; $x$, peg rail; 6 , eloth being dyed; pipes ; r, steam-valve; $\mathbf{x}$, steam-pipes, supplying the stoam space at intervals along the body of beck; $x$, let-off plug and seating; $\mathbf{x}$, silk for carrying beck.

Chintz work is dyed with from $1 \mathrm{lb}$. to 5 lbs. madder per piece of 30 yards, according to the pattern; generally, a little chalk is added, and if there is no purple in the pattern, some sumac, which is found to oconomise madder, but will not do where there is purple, the shade of which it deadens.

The extensive use in topical printing of madder extract, which is the colouring matter of madder in a nearly puro state, lod Messrs. A. Duncan and Co., of Manchester, to experiment on dyeing pieces by running them stitched together, and extended to the full width through a series of cisterns similar to the ' $\mathrm{fly}$ dung' cisterns, charged with a pure extract of madder and boiling water. The theory was that if the madder extract were kept in large excess of the requirements of the pieces, and the cisterns kept boiling, the mordants would become saturated with colouring mattor in a very short time. Their experiments resulted in a patent taken out in 1871 ; and although it can scarcely at the present time be said that the trade is satisfled as to the economy of the process, very excellent results have been arrived at by some printers, and the process would appear one of great promise. Well-dyed pieces both of light and full shades have been obtained in from 3 to 5 minutes, being the tinie required for a given point in a piece to travel from the entering end to the issuing end of the apparatus. 
Tho cisterne ate kept constantly charged with extract diluted with water, in quentity corresponding to that requireck by the pieces if dyed in the ordinary beck; thus, the pisterns are kept up to the original strength, which, as above stated, is excessive compared with the actual wants of the mordants. This, however, is of no consequence If the arrangement be such that the issuing pieces bring away with thom only a very small percentage of the excess of dye. This is managed by squeezing thom undor bowls and passing them through water, which is employed to fill up the cisterns. Fig. 381 represents a section of the 'open dye-beck' of Mather and Platt, arranged specially for this process. A, wooden cisterns; B, wooden rollers with brass contres, fixtures, \&c. for immersing the cloth; c, drawing rollors, from cistern to cistern; $D$, strong squeering rollers; $\mathbf{B}$, washing-off cistern with beater $F$; $G$, steam-boiling pipes, \&c., which do not injoct steam into the liquor, but terminate in condensed wator-pipes I; I, cloth passing through tho machine; $\mathbb{K}$, drawing pullies and spur whoels. In reference to this open dyeing process, it is obvious that unless the extract of madder used is very puro and free from fine particles of woody fibre and chemical salts, the constant addition of it, without drawing anything off, would result in time in the cisterns being loaded with impurities which would weaken and oventually stop the dyeing power of the extract, no matter in what quantity added. The extract made by Tennants and Co., of Scotland, specially for this purpose, is claimed to be of the degree of purity required. It is probable, however, that the artificial alizarine now made in perfect purity, and consequently entirely free from woody fibre and resinous colouring matters, will be found most suitable for this process, if it becomes a well-established one in calico-printing works. Much will, of course, depend on the prices of the artificial and the natural alizarine as delivered to consumers. It may be here stated that the value of the open and quick dyeing process as an economiser of material and time has been somewhat reduced by the discovery that several sots of pieces may be dyed with artificial alizarine in the ordinary dye-beck without letting-

882

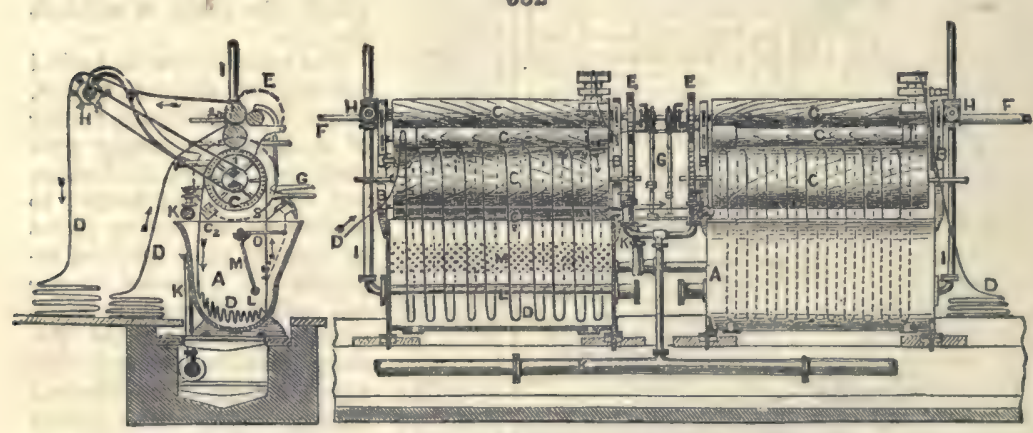

off between each set, but merely adding after the withdrawal of a set (of say 30 pieces) the quantity of alizarine necessary for the next set, entering these at once in the boiling hot liquor, which becomes cooled down by the wet pieces to about $130^{\circ} \mathrm{F}$., and recommencing the dyeing; the becks being brought to boil in an hour, and the pieces withdrawn. As many as 5 sets can be done in this way, and as the dyeing with artificial alizarine in an ordinary beck need not last above an hour, the economy in time of the open dyeing would appear somewhat doubtful.

Madderod goods, on issuing from the dye-beck, are far from possessing the beauty that they afterwards show, the colours are dull and heavy, and the white part stained with a reddish shade; various clearings are required, in which sosp plays a principal part. Garancined goods show pretty nearly the colour they are intended to be; but as the white is also stained, a poculiar clearing is given them, which will bo described further on. Madder goods are cleared with soap in a beck similar to a dye-beck. They receive generally two soapings at boil of about half-an-hour each, with from $\frac{1}{4}$ to $\frac{1}{2} \mathrm{lb}$. of soap per piece each time, washing between. If the white is not sufficiently good, the pieces are spread out on the grass for a day or two, and are afterwards winced in hot water to which a little solution of chloride of lime or soda is added. They are then washed and dried.

The success of the spiral system of dyeing led printers to adopt a similar systom for soaping, and at the principal print-works this operation is now porformed in a series of becks, generally 7 or 8 . The pieces enter at one end of the first beck of the series, and aftor passing spirally through this, traverse successively the remainder of the 
series. Clean soap liquor is kept flowing into the last beck of the series; and as each beck is connected by a pipe with the next, the soap solution is flowing all the time in a contrary direction to the pieces and leaves the first beck in a thoroughly exhausted condition; the pieces in their onward passage keep meeting with soap liquor less charged with colouring matter and impurities, until finally they pass through almost unsoiled soap solution. The passage of a given point of a piece through the series, occupies about half-an-hour. Fig. 382 shows two of these becks in action; they are those of Mather and Platt. A, cast-iron soap-beeks; $\mathbf{B}$, fixings for carrying rollers; c, wooden rollers; $C_{3}$, square wooden doffing roller; $D$, cloth being soaped on the con: tinuous system, any number of becks may be used for this purpose, they are generally worked in ranges of 7; 2 only here shown; $\mathrm{k}$, spur wheel and catch box for driving; $F$, driving shaft; $G$, handles and tappet shafts for putting in motion or stopping the rollers; $\mathbf{H}$, steam-valve; $\mathbf{x}$, steam-pipes ; $\mathbf{x}$, water-valves and pipes; $I$, perforated steam-pipes passing through becks; $\mathbf{M}$, midfeather; $s$, delivery wince on each bock, which can be used if necessary; 0 , peg rail.

The waste soap liquor at present allowed to run into the rivers, polluting them, and being the means of keeping in suspension for a long distance fine particles of dyestuffs, will probably be soon turned to advantage by the printer, for the experiments on the large scale of Mr. John Thom, of Birkacre, have conclusively shown that by a very simple and easy process, all the fatty matter with the coloured impurities dissolved in the soap can be precipitated and collected, and a patent taken out by Thom and Stenhouse in 1872 describes methods of separating useful and commercial articles. from these precipitates. After soaping, the pieces are well washed in the washing machine.

Pieces of any style, after undergoing the final process, are passed through a pair of squeezing rollers, or put in the hydroextractor, when the moisture is driven out by centrifugal force; they are then dryed on the cylinder drying machine. Seo HrdroEXTRACTOR.

Plate Purple is a style composed of black and one or more shades of purple only, and requires a little different treatment. Print in black No. 4, dark purple to shade No. 27 and acid, say No. 35 , cover pad in paie purple No. 30, age. Fly dung at $170^{\circ}$ F., second dung at $165^{\circ} \mathrm{F}$. half an hour; wash and ground with dry Turkey madder root giving $\frac{1}{20}$ th of its weight in chalk, and 3 quarts of bone size to the beck; bring to $175^{\circ} \mathrm{F}$. in two hours, and keep at $175^{\circ} \mathrm{F}$. half an hour; wash well and soap 15 pieces, $\frac{7}{8}$ ths, 30 yards, half an hour at boil with 5 lbs. soap to 15 pieces; wash well and wince 5 minutes at $140^{\circ} \mathrm{F}$. with 2 quarts chloride-of-lime liquor at $8^{\circ} \mathrm{F}$. to 300 gallons; winco and soap again at boil half an hour with $3 \mathrm{lbs}$. soap to 15 pieces; wash and wince 5 minutes in 4 quarts chloride of lime at $8^{\circ} \mathrm{F}$. and 2 lbs. carbonate-of-soda crystals to 200 gallons water at $160^{\circ}$; well wash and dry.

In this style, as in any where there is severe soaping, it is necessary to give a slight excess of madder in the dye, so as to ensure perfect saturation-if this is not done, the colour speedily degrades, and becomes impoverished. It may be observed here, that the style Plates are such as formerly were printed by the plate or flat press, and are generally small patterns, with padded or well covered grounds, the colours being fow, and frequently only different shades of one colour.

Plate Pinks or Swiss Pinks.-A style imported from Switzerland, consisting of various shades of red and delicate pinks, produced as follows. Print in No. 6 with second or third shades as No. 7-acid No. 34 may be also printed and a very palo shade of red covered, aged two or three days, dunged at $160^{\circ} \mathrm{F}$.- if dung-substitute is used, care must be taken to use one that is not caustic from free alkali : the dyeing must be done with the finest quality of French or Turkey madder. The pieces must have sufficient madder allowed to over-dye them, or dyo a heavy brownish red. For a full plate pink on $\frac{7}{8}$ ths cloth, from 4 to $6 \mathrm{lbs}$. of French madder will bo required. About 5 per cent. of chalk may be added to the dye where the water is soft. The heat should be raised to $150^{\circ} \mathrm{F}$. in 2 hours, and kept at that heat half an hour. It is necessary to keop the heat low in dyoing French pinks, to prevent the impurities from fixing on the mordants, as only the very finest portion of the colouring matter must be fixed-after dyeing, the pieces are well washed and soaped with about half a pound of soap per piece in a beck at $140^{\circ} \mathrm{F}$. for half an hour, they are then well washed and entered in a beck with cold water to which has been added sufficient oxymuriate of tin or sulphuric acid to make faintly sour, a little steam is turned on, and the heat raised to about $120^{\circ} \mathrm{F}$. in half an hour; the colours which on entering the beck wero full shades of red, gradually assume an orange tint, and when of a bright orange colour, the pieces are takgn out and winced in water. This operation, termed cutting, is the one that decides the depth of tint in the finished piecess The longer the pieces are kept in the beck and the greater the heat, the paler and moro delicato the shade of pink obtained. Aftor this treatment they are put in a beck 
with soap and boiled for an hour, takon out, washed woll, and put in a strong pan charged with sosp and water, the lid screwed down, and boiled at a pressure of two atmosphores, either by direct fire or high-pressure steam, for two or three hours, then taken out, washed, and put in a beck with water at $160^{\circ} \mathrm{F}$. charged with a little hypochlorite of soda; they stay in this about ten minutes, and are then washed and dried. In some print-works, after the high-pressure boil, the pieces are spread out on the grass for a night or two, and then cleared in hypochlorite, \&c. The use of the acid here is not very clear, it probably completely purifies the colour from iron which may have been in the mordant, but it also seems to render the combination of alumina, tin, lime, colouring matter and fat-acid a definite one by romoving a small quantity of the mordant. The French chemists assert, that after the final process, a definite atomic compound of lime and alumina, colouring matter, and fat-acid remains.

The quality of the soap used by printers is of great importance. It is made for them specially from palm-oil, and requires to be as neutral an oleo-stearate as possible; an alkaline soap like domestic soap would impoverish and degrade the shades.

The soaping process has a two-fold action:-

To clear the white while decomposing the compound of lime and colouring matter which forms the stain; this is done by double decomposition, forming oleo-stearate of lime, which dissolves or forms an emulsion wath the excess of soap; and a compound of sods and colouring matter, which dissolves. In its action on the dyed parts, it. probably first removes resinous and other impurities which are loosely held by the mordant, and secondly gives up a portion of its fat-acid to the dyed parts-the resinous acids or possibly phosphoric acid from the dyed parts, by combining with the soda, setting freo fat-acid for this purpose.

In reference to the above Swiss pink style, it may be observed that various shades of pinks are required for different markets, and where as in many cases a full rich inexpensive color $x$ is required, the method of dyeing at low heats as described above is unnecessary, and the becks are brought nearly to a boil. Where an orange-tinted shade is required the goods may be dyed with garancin of good quality or with a mixture of madder and garancin.

A few years ago Mr. Thomas Lightfoot, of Accrington, discovered that madder or garancin-dyed goods, if dried and steamed in the manner usual in fixing stoam colours, became much less degraded by soap in the clearing process for Swiss pinks, and that a considerable saving of madder or garancin might thus be effected, weaker mordants and consequently less dye-stuff being employed. Mr. Lightfoot patented the use of steam as an intormediate process between the dyeing and soaping of these colours, and the process is now generally used. Mr. John Graham had in 1855 patented the use of steam for 'fixing' the colours of dyed cloth, put his patent does not mention any subsequent process, and it is doubtful in what respects his so-called fixed colours were better than the ordinary dyed goods. Some printers pass the dyed goods through an oily emulsion previously to drying before steaming, with the object of eausing a combination between the base of the mordant, the colouring matter and the oily acid, and so dispense with some of the usual .oaping processes. Swiss pinks are sometimes ' covered ' with a pattern which harmonises with that of the pinks, in a discharge yellow made of salts of lead mixed with acid; and after ' covering,' the goods áre passed through strong chloride-of-lime solution which, being decomposed by the acid, the free chloride liberated destroys the pink-dyed part under the yellow, and the insoluble lead salt left in its place is dyed or raised with bichromate of potash. A style of madder work occasionally extensively printed is the madder orange style, where the orange is a salt of lead and called 'madder orange' because it has passed through the madder process along with the proper madder colours. The cloth is padded with solution of sulphate of soda containing 4 ounces of the crystallised salt per gallon, dried and printed in madder black, and purple, or black, red and purplo with orange, say of Nos. 4, 6, 27, the orange No. 64a, and cover, or cover and pad with weaker purples, an acid 33 to 35 may also be printed along with the other colours. After 'ageing,' instead of dunging, run through a cistern set with $2 \mathrm{lbs}$. crystallised sulphate of soda and $\frac{1}{4}$ ounce of erystallised phosphate of soda per gallon of water at $150^{\circ} \mathrm{F}$.; wash well and dye with madder as usual; wash and clear by running through a beck set with 300 gallons water at $140^{\circ} \mathrm{F}$., 2 quarts chloride of lime solution at $8^{\circ}$ Twaddell, and 2 quarts of solution of carbonate of soda at $8^{\circ}$ Twaddell, run through water, then through the spiral soaping becks at boil for half an hour, then run through water, next through a chloride of lime and soda cistern set as before but boiling, then through the spiral soaping becks at boil, again into chloride of lime and soda set as above but at boil; wash well and raise the load colour to yellow by passing for half a minute through a cistern containing 300 gallons of cold water, $30 \mathrm{lbs}$. of bichromate of potash and 3 quarts of sulphuric acid; run from this into water and wash well; to change the yellow into orange. run through 
a cistern containing 300 gallons of boiling water, 5 lbs. bichromate of potash, and $5 \mathrm{lbs}$. quicklime. After the passage of every 10 pieces add $1 \mathrm{oz}$. bichromate of potash and 1 oz. quicklime dissolved in 3 gallons of water; after this orangeing, wash well and dry.

No. 64a, Orange. $-7 \frac{1}{2}$ gallons of water; 60 lbs. white acetate of lead; $22 \frac{1}{2} 1 \mathrm{bs}$. litharge; boil 20 to 30 minutes till all dissolved, let settle and thicken the clear liquor with calcined wheat starch (British gum).

\section{Second Style: Garancin.}

Almost all the madder styles are imitated by dyeing with garancin, a concentrated proparation of madder (seO MADDER), which dyes fine brilliant colours at once, not requiring to be soaped to develope the shades, but not possessing the extreme solidity of madder colour. Garancin dyeing is the most economical way of using madder, since more colouring matter is obtained in this way than by using madder direct, and consequently garancin is principally used for full heavy colours, which, if dyed with madder and soaped, would be to a certain extent abraded and not stand so finely on the surface of the cloth. Chocolate grounds, black, red, and chocolate, with brown or drab, dark purple plates, black and scarlet ground, are thus dyed; in short, wherever the pattern is very full and cheapness essential, garancin is resorted to. The colours or mordants for garancin are usually about two-thirds of the strength of similar colours for madder (see the list of colours); the ageing and dunging, \&c., are the same as for madder; the dyeing is performed in the same manner, using from one-fourth to onethird the quantity that would be used of madder. A little chalk is also added, where the water is soft; and the dyeing is commenced at $110^{\circ} \mathrm{F}$., and carried to $185^{\circ} \mathrm{F}$, or

383
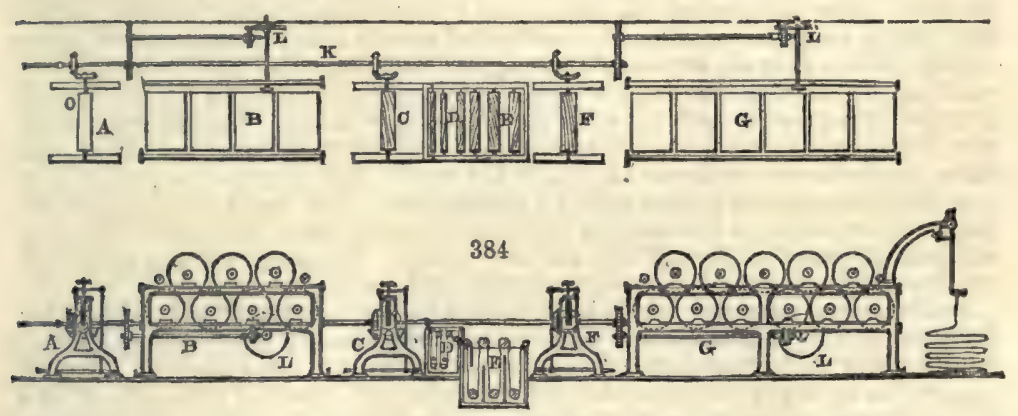

$190^{\circ} \mathrm{F}$. in two hours; then got out and well washed and rinsed in water at $140^{\circ} \mathrm{F}$, in a beck, for 10 minutes, then squeezed and dried. The white is always stained a little, though not to the same extent as in maddered goods, and this slight stain is removed by a process peculiar to garancin goods.

Fig. 383 is a plan, and fig. 384 an elevation of the chloring apparatus used for clearing the white grounds of garancin-dyed goods, for which wo are indebted to Mr. Furnival. A is a padding machine with two brass or one brass and one vulcanised India-rubber roller, and box to hold the solution of hypochlorite of lime; $B$ is a drying machine with copper cylinders, for partially drying the goods after the first chloring; $\mathrm{c}$ is a second padding machine similar to $\mathbf{A} ; \boldsymbol{D}$ is a steaming box, with rollers top and bottom, and perforated steam-pipe; $\mathrm{x}$ is a water cistern with divisions and rollers; $\mathbf{F}$ is a water mangle or padding machine, similar to $\mathrm{A}$ and $\mathrm{c}$, with water only in the trough; $\alpha$ is a drying machine large enough to thoroughly dry the cloth; these machines are connected with and turned by the shaft $\mathbf{x}$; to prevent pulling the cloth, and to adjust the speed of the machines with each other, the rollers o are made exactly the same size, and the speed of the drying machines is regulated by the friction plates c. The cloth is passed between the rollers in the first chloring machine $\Delta$, which is set with solution of hypochlorite of lime from $\frac{1}{2}{ }^{\circ}$ to $22^{\circ}$ Twaddell's hydrometer, aecording to the depth of stain on the white, over the first drying machine cylinders of B, into solution of hypochlorite of lime again in the troagh of, and through the rollers of $\mathrm{c}$, from this through the steaming-box $\mathrm{D}$, entering tho box by a narrow slit, which just admits the piece, and leaving by a similar opening, which is protected by a sort of hood which dips into the water in $\mathbf{E}$, then between the rollers of the water mangle $E$ and orer the cylinders of the drying machine $G$. The cloth passes through the various 
machines at the rate of 90 to 120 yards per minute, with the attendance of one man and two boys. By chloring in this manner the stained whites are blenched without degrading the dyed colours as would be the case if the goods were winced in a solution of the hypochlorite.

There are several varieties of garancin, each adapted to particular styles. For dark full black, ehocolate, and red, with brown or drab, and where there is no purple, a garancin termed 'chocolate garancin' made from the commonest descriptions of madder, answers very well, and this class of goods is usually dyed with chocolate garancin, assisted by small quantities of sumac, quercitron bark, and peachwood, which additions give full rich shades. Where there is purple, none of these adjuncts ean be used, and the garancin requires to be made from a superior description of madder.

Within the last few years great improvements in the manufacture of purple garancins have been made. Good purple garancin contains two colouring matters, alizarine and pupurine, one or two coloured resins and pectic acid. Of the two colouring matters, alizarine only dyes a pleasing purple, purpurine giving a reddisb and comparatively dull shade; the pectic acid and resins aro simply injurious, though they. can be to a considerable extent masked by the addition of a little chalk to the beck when dyeing. Mr. S. Pincoffs, experimenting on various ways of destroying the injurious parts of purple garancin without injuring the alizarine, discovered that sealing up the garancin in a strong tube with a little water, and exposing the tribe to a high temperature for a few hours, so that the contents would be under a pressure of two atmospheres, greatly improved the garancin. Further experiments in tho same direction by Dr. E. Schunck resulted in a joint patent taken out on Oct. 15,1852 , by Pineoffs and Schunck for the preparation of a substance called by them 'Commercial Alizarine.' The process consisted in first making garancin in the ordinary manner, but washing very carefully, so that no acid was left in the substance. The garancin was then filtered and pressed and packed into a strong iron cylinder, furnished with a false bottom perforated with holes, on which the pressed and broken-up cake of garancin was placed; near the bottom of the cylinder, and under the false bottom, was a small tap, for allowing the escape of condensed water, and both at the top and bottom, the ressel was provided with pipes for the admission of steam. The material having been placed on the false bottom, the man-hole lid in the upper part was closed, and high-pressure steam at about $30 \mathrm{lbs}$. to the square inch admitted and allowed to blow freely through the bottom tap for a few minutes. This was then closed, and the steam allowed to act on the material for about fourteen hours, the bottom tap being occasionally opened to blow off the condensed water. The steam was then turned off, and the contents of the cylinder removed, dried, and ground.

In this process the pectic acid is destroyod, and a large portion of the resins either destroyed or rendered insoluble in the solution of the alizarine when dyeing. It is a disputed point whether the purpurine is destroyed or converted into alizarine. A French chemist has asserted that he has changed purpurine into alizarine by beating it in a tube with water under a pressure of 4 atmospheres, but this statement has been negatived by other chemists. The garancin operated upon by Pincoffs and Schunck's patent has lost a considerable portion of its dyeing power, although it now dyes very pure shades, hence it would appear that the purpurine is really destroyed. Higgin followed soon after, patenting a mode of preparing a superior purple garancin, by boiling for several hours the ordinary purple garancin with water and small quantities of alkalies or alkaline salts. In this process the purpurine is not destroyed, but the resins and pectic acid are removed in solution in the menstruum omployed. This garancin, though not dyeing purple of such extreme purity as commercial alizarine, has the advantage over this latter of dyeing black and good reds with a vory good purple. Pernod, a French garancin maker, subsequently patented a method of boiling purple garancin in water in a tall wooden vat by high-pressure steam. In this process the pectic acid is changed into a soluble modification of it, and washed out with water afterwards. This preparation, as mado by the firm in which Mr. Pernod is a partner, is of great purity and strength, and compares very well with the commercial alizarine, both these preparations dyeing purples almost as pure as the finest soaped madder purples.

These improved garancins stain the white grounds very little, and produce con. siderably faster work than the ordinary garancins; the goods may even be soaped to a considerable extent. A garancin that will beas as severe soaping as madder, or a method of so dyeing with garancin as to produce the same effect, is still a desideratum. When this can be accomplished, there will bo an end of dyeing with madder, which will be considered a raw material, and be all manufactured into garancin.

Owing to the introduction of artificial alizarine there has been already a sensible falling off in the consumption of these fine purplo garancins, and it appears proballe 
that if the artificial product can be brought in at even a little higher relative price than these dye-stuffs, the superior colours producod by it and the comparative cleanliness of its uee, will drive garancins out of the market.

Garanceux.-In ordinary madder-dyeing, the madder can never be made to give up all its colouring matter; when all colouring matter soluble in water has been exhausted, there still remains about a quarter of the whole quantity combined with lime and mixed with the woody fibre.

Before the patent of Steiner in 1832 this spent madder was thrown away. He showed the printers that they were thus squandering thousands of pounds, and the collection of spent madder and its conversion into garanceux soon became universal; a second patent by Steiner in 1843 having shown a more practicable method of utilising waste madder.

The spent madder is run off into a pit outside the dye-house, where it is mixed with a small quantity of sulphuric acid, to precipitate any colouring matter in solution. It is then allowed to drain dry ; removed from the pit, it is boiled in a leaden vessel, with more sulphuric acid, for several hours, then washed on a filter till free from acid; and after draining is ready for use. It dyes to about one-third the strength of ordinary chocolate garancin, and is principally used for the commoner garancin styles: Mr. John Lightfoot, of Accrington, has patented an improvement in the ordinary process of making garanceux. He recommends large vats to be provided, two or more in number, each sufficiently large to contain all the waste dyeing liquor produced in the dye-house in one day, and so arranged that the liquor runs from the dye-becks into them; at a certain point in the trough that conducts the liquor to the vats is placed a lead cistern with a valve and perforated bottom: this cistern holds a regulated quantity of concentrated sulphuric acid, and whenever a dye-beck is let off and the liquor flowing down the trough, a quantity of acid, proportionate to the quantity of madder, is allowed to run down through the perforated bottom and mix with the hot liquor; the acidulated liquor then runs into the vat, a tightly fitting cover on which keops the liquor hot. When the day's dyeing is done, the vat is left covered up all night; next day the lid is raised, and by means of holes and pegs in the side of the rat, all the clear liquor is drained away, the vat filled anew with water, stirred up, and when settled, the clear drawn off again; this washing being repeated till all the acid is washed away, the garanceux is then run on a filter to drain for use. The advantages of this plan are, first, the saving of fuel, by economising the heat of the waste liquor, and, secondly, the production of one-fourth more colouring matter.

A beautiful modification of the garancin style was patented by Mr. John Lightfoot junior on Dec. 26, 1867. So long ago as $1846 \mathrm{Mr}$. Philippi of the firm of Mr. Benecke

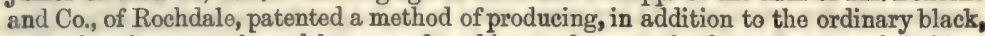
reds, chocolate, purple and browns of madder work, several other colours such as blue, greon, and yellow, all these colours being printed at once and undergoing the same operations ; the blue was an indigo colour, the yellow a chromate of lead, and the green a compound of these two. The patentee never got beyond the stage of printing a fow hundred pieces, for the style, though admitting of certain novelties in design, was not warmly received by the public on account of the inferiority of the blues, greens, and yellows. The style soon fell into disrepute and was almost forgotten. lightfoot revived it, making several important improvements and Messrs. F. W. Grafton and Co. of Accrington, in whose employment he was, became the ultimate owners of, and manufacturers under the patents of Mr. Lightfoot. We give here an outline of the process, using the words of the patentee:-

'I make a preparation of indigo, fulfilling the required conditions, by employing much less tin, whether as oxide or in the state of salt, in the process of dissolving the indigo than has hitherto been used, and also by dispensing with the addition of a sult of tin to the precipitated indigotine, whether this has been made by the aid of oxide or salt of tin, or, as is sometimes the case, by the aid of metallic tin, because I have ascertained that if the tin is in excess of a certain proportion to the indigo, oxide of tin is left in the fibre during the process of fixing the indigo colours and the mordants, and thus acting itself as a mordant becomes dyed by the dye-stuff, producing with the indigo compound colours which in the case of blue are more or less purple and dull, and in the case of green are more or less a sombre olive. This use of tin in excess has been one of the causes of the failures to which I have before allnded.

'I prepare a paste or pulp of indigotine and tin suitable for my improved process by any of the following methods:- I take of dry indigo in a ground or powdered state one and a quarter pounds, or when indigo pulp is used, using such a quantity as to be equal to one and a quarter pounds of dry indigo, of protochloride of tin in crystals one and a quarter pounds, and of caustic soda at thirty degrees on Twaddell's hydrometer, or of caustic potash at forty degrees on Twaddell's hydrometer, one gallon. I pat 
these materials in a pan and raise to boil in half an hour, and then add one gallon of boiling water. I prefer now to allow the mixture to become perfectly cold, and pour it into three gallons of cold water, in which I prefer to dissolve eight ounces of sugar or one pound of treacle (though this addition is not essential) I add to this solution two and a half pints of muriatic acid at thirty-two degrees of Twaddoll's hydrometer, or one pint of sulphuric acid of commerce, previously diluted with one pint of water, and allowed to stand till clear, or three quarts of acetic acid at eight degrees of Twaddell's hydrometer. The indigotine may also be precipitated by a mixture of protochloride of tin solution at one hundred and twenty degrees of Twaddell's hydro. meter, with any of the acids named, using one quarter of a pint of tin solution and only half the quantities of the acids given above: this quantity of tin solution being the maximum that I can use when precipitating the indigotine for producing a fine blue and green. Of all these precipitants I prefer to use "acetic acid alone." Instead of using protochloride of tin crystals in making the indigo solution, protoxide of tin made by precipitating a solution of protosalt of tin with an alkali may be used, the precipitate being washed with water and filtered to a thick paste, or anhydrous protoxide of tin made in any convenient way may be employed, in all cases taking such a proportion of oxide as shall contain an amount of tin about equal to but not greater than that in one and a quarter pounds of crystallised protochloride. In some cases the mixture of one and a quarter pounds of indigo and one gallon of alkali may be boiled with metallic tin in powder or granulated, the quantity of the latter being such that when the boiling is finished and the indigo all dissolved there shall bo metallic tin undiss ilved. In all these cases I precipitate the indigotine as proviously deseribed. I filter the indigotine precipitate through a deep conical filter so as leave as small a surface exposed to the air as possible; when filtered, the pulp should measure one gallon or thereabouts.

- To make a blue colour for printing I take four gallons of indigotine precipitate and fourteen pounds of gum-Senegal in powder, stirring until dissolved (other suitable thickening substances may be used), after straining, the colour is ready for printing.

'To make a green colour I take four and a quarter gallons of indigotine pulp and eighteen pounds of gum-Senegal in powder, stir till dissolved, and add eleven pounds of nitrate of lead in powder, and eleven pounds of white acetate of lead in powder, stir till dissolved and strain.

'Compound colours may be made by mixing the blue and green colours with each other or with the ordinary mordants for dyeing. With the blue and green above described and the ordinary ferruginous and aluminous mordants I print cotton and linen fabrics, and after cooling hang the pieces in a room known to printers as an ageing room for one night; I then cause them to undergo what is known as the fixing operation by passing them into a solution of silicate of soda or silicate of potash at eight degrees of Twaddell's hydrometer, or into a solution of carbonate of potash at twelve degrees of Twaddell's hydrometer, to which about one ounce of chalk in powder per gallon may be added, or into a mixture of silicate of soda or silicate of potash at eight degrees of Twaddell's hydrometer with carbonate of potash at twolve degrees of Twaddell's hydrometer. I heat the bath at ninety degrees Fahrenheit or thereabouts in a cistern fitted with rollers at the top and bottom, and the passago of the pieces may be at the rate of twenty-five yards per minute. On leaving this the piecos must be quickly winced in a pit of cold water fitted with a reel about four feet above the surface of the water. By this wincing the indigotine attached to the fibre becomes again indigo blue. If green has been printed, I next pass the pieces into a solution of bichromate of potash containing one ounce of bichromate of potash per gallon of water at one hundred degrees Fahrenheit for five minutes: the goods are then washed. If only blue has been printed along with the mordants this process may be omitted. I next subject the pieces to the operation known to calico printers as "second dunging," and which coasists in making the pieces circulate in a beck containing cow-dung and water at a temperature to about one hundred and sixty degrees Fahronheit for from fifteon to twenty minutes. They are then washed with water and dyed with madder, munjeet, flower of madder, garancin, extract of madder, cochineal, mixtures of garancin with sumac and bark, or with either of them, after which the ordinary operations of clearing the white grounds may be performed, preference being given to the chloride of lime clearing usually adopted with garancin colours.'

Another variety of garancin work is that where an orange is introduced along with black, red, and chocolate, The orange colour is made as follows:- -3 gallons of pyroligneous acid at 7 T., 1 gallon of water, 7 lbs. acetate of lime, dissolve and strain; then boil with 10lbs. of flour and 3 gallons of water, cool, and add 8 lbs. of muriate of tin crystals, dissolred in 1 gallon of water. The dyeing is with 3 ozs. garancin, $1 \frac{1}{2}$ ozs. of sumac, $1 \frac{1}{2}$ oz. of bark, 1 oz. of ground Persian berries per piece of $25 \frac{\mathrm{g}}{5}$ yards. The bark and berries are first put in the beck with the pieces, heat up to 
$120^{\circ} \mathrm{F}$. in half an hour, then add the garancin and sumac, and bring up to $150^{\circ} \mathrm{F}$, take out, hot water, and clear as usual.

\section{Third Style: Reserved.}

Maddered or garancined goods are often left with white spots, as leaves, \&c., and when dyed these spaces are filled with various bright colours, such as green, blue, yellow, \&c. These colours are the ordinary steam colours described hereafter, and are fixed in the same manner.

Another way of combining madder or garancin colours with steam colours is by blocking on the dyed object, generally groups of flowers, a reserve paste (No. 39), and when this is dry, covering by machine in small patterns with various shades of drab, olive, \&ce. (Nos. 5, 44, 46, \&re.), which then are dunged and dyed with quercitron bark, cochineal, madder and bark, \&c. \&c. When the paste has been applied, the colours underneath, or the white spots reserved, are unaffected by the covering colour, and stand out clear surrounded by the covering colour. In the white spaces reserved wre now blocked steam colours, which are raised by steam as described further on, This style admits of almost endless combinations of patterns as far as regards the 'covering,' for any small pattern roller of the 'covering' class can be printed over the reserved colours, and a great variety of shades can be produced by varying the dyeing of this ' cover.'

\section{Fourth Style: Padded.}

In this style the white cloth is mordanted all over by padding in red or iron liquor, or mixtures of them, and drying in the padded flue; then a pattern is printed on in acid, and the usual dunging and dyeing operations performed, the result being a dyed ground with a white pattern.

Fig. 385 represents a section of the padding flue used in mordanting for this style.

385
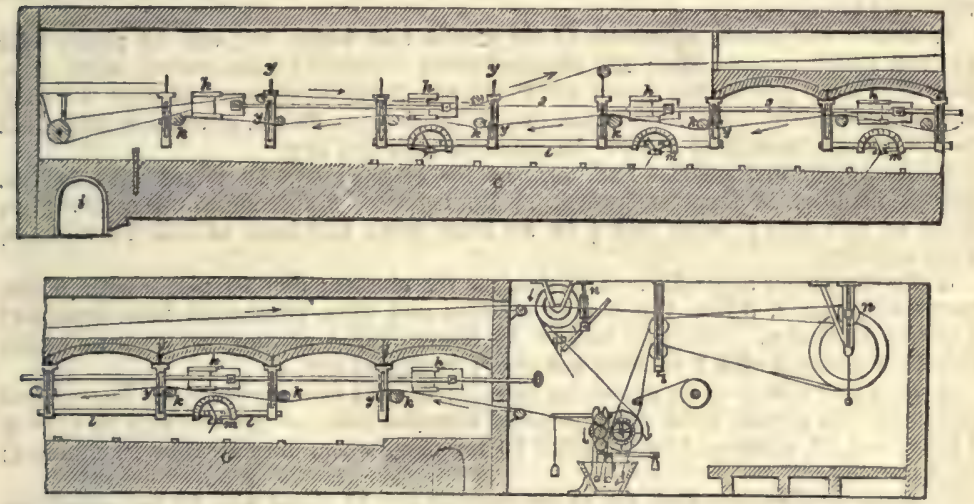

It consists of a long vaulted chsmber, about 35 yards long by 5 yards, and 4 yards high, cut in two at nearly half its length, by six small arches built in an opposite direction to that of the chamber, the object of which is to preserve the principal arch from the action of the heat, and to hinder the dried pieces from being exposed, on coming to the higher part, to moisture and acids, which are disengaged in great abundance and might condense there, $\mathrm{c} c$ is a long furnace, the flue of which forms the bottom of the chamber; the top of the flue is covered with plates of cast iron fitting one into another, and which can be heated to near red heat by the flame of the furnace. $F$ is an arched passage by which the interior of this stove can be reached. $h h$ are ventilating holes in the lateral wall which can be opened and closed at will, by means of the rod $j$, which is connected with sliding doors over the apertures. $k k$ are cast-iron supports for turned copper rollers, which are fixed to the cross pieces $y y$, and serve to conduct the piece. $l l$ are bars of iron which carry the fans $m m$, which aro covered by gratings, and make about 300 turns per minute.

In front of this hot flue is placed all the apparatus necessary for padding the pieces, and moving them through the drying chambers. This movement is caused by pullies R R driven from a prime mover.

The mordant liquor being put in the box of the padding machine, the pioces wound on a beam and placed above the machine are conducted through the box, then between the two lowest rollers above the box, from them through the liquor again, passing

Vox, I 
next through the highest sollere, and so on into the flue, the course being easily traced by the arrows; on leaving the flue dry, they are wound on a beam, or plated down on the wooden platform behind the machine. The 3 rollers of the paddingmachine are made of brass, and are wrapped with a few folds of calico; the iron journals of them work in slots, the lowest one being at the bottom of the slot working in brass bearings; a weighted lever presses the top roller in forcible contact with the others. Another method of padding, and one now bocoming very generally adopted, is, to dispense with the flues as above deseribed, and, after padding, to dry the pieces over an ordinary cylinder drying-machine, the cylinders of which are covered with woollen printing blanket.

Palded goods after printing in acid are passed through the ageing-machine, and after lying all night are dunged and dyed. A few of these shades are here given.

a. Claret and white. Pad in red liquor, at $10^{\circ} \mathrm{T}$., dry, cool, and pad again in same liquor, dry, cool, and print in acid No. 37, age three nights. Fly-dung at boil, wash, second dung at $160^{\circ} \mathrm{F}$., $\frac{1}{2}$ hour, wash, dry, and singe, wash and dye 12 pieces $7 \mathrm{ft} .8 \mathrm{in}$. 30 yards with $18 \mathrm{lbs}$. ground peachwood, $21 \mathrm{lbs}$. of French madder, 5 lbs. sumac, 5 lbs. prepared logwood, run the pieces in the beck cold for 20 minutes, and then bring to a boil in 1 hour and 10 minutes, boil 15 minutes, get out, rinse and wash, bran 10 minutes at boil in a bock with a fow pounds of bran, rinse in a pit, and bran again at boil, wash and dry.

Prepared logwood is thus made. - Ground logwood is spread out on a floor, damped with water, ind heaped up. It is then turned over once a day for a fortnight and occasionally wotted, during which time it changes from a dull red to a bright scarlet. It is then ready for use. Some change, probebly oxidation, has taken place, and the wood dyes further after this process.

b. Scarlet and white.-Padded and dunged as for clarets; then 10 pieces dyed with 15 lbs. French madder, 15 lbs. Dutch crop madder, 7 lbs. peach wood, 4 lbs. sumac with 3 quarts bone size : bring to a boil in $2 \frac{1}{4}$ hour, and boil a quarter of an hour; wash and bran, \&c.

c. Scarlet and yellow.-Proceed as for scarlet and white, but dye 10 pieces with $22 \frac{1}{1}$ lbs. Dutch crop madder; $22 \frac{1}{2}$ pounds French madder; $7 \frac{1}{2}$ lbs. sumac, wash, bran, and dry; then pad in red liquor at $10^{\circ} \mathrm{T}$., age 2 nights, fly-dung at $130^{\circ} \mathrm{F}$, wash and warm water at $120^{\circ} 10$ minutes, dye 10 pieces with $20 \mathrm{lbs}$. quercitron bark, heat to $120^{\circ}$ in one hour, keep at $120^{\circ} 15$ minutes, wash and dry.

d. Burgundy and white.-Pad, \&c., as for clarets; dye 10 pieces with 18 lbs. French madder, $18 \mathrm{lbs}$. peachwood, $1 \frac{1}{2} \mathrm{lb}$. logwood, 5 lbs. sumac, 4 quarts glue. Heat to boil in $1 \frac{3}{4}$ hour, boil $\frac{1}{4}$ of an hour, wash and bran at boil 10 minutos, wash and dry.

e. Tyrian purple and white.-Pad, \&c., as for clarets; dye 10 pieces with 5 lbs. prepared logwood, 5 lbs. Dutch crop madder, and 7lbs. peachwood 2 lbs. bran, and 3 quarts bone size. Bring to boil in $1 \frac{3}{4}$ hours, boil $\frac{1}{4}$ of an hour, wash and bran at $150^{\circ} 5$ minutes with $1 \mathrm{lb}$. bran per piece; wash and dry,

f. Puce and white.-Pad, \&c. as for clarets; dye 12 pieces with 3 lbs. fine ground cochineal, 1lb. ground galls, 4lbs. prepared logwood, 3lbs. peachwood, heat to $170^{\circ}$ in 1 hour, and 20 minutes, keop at $170^{\circ} 10$ minutes, wash, bran at $160^{\circ} 10$ minutes ; wash and dry.

g. Amber and white.-Pad, \&c. as for clarets; dye 10 pieces with $20 \mathrm{lbs}$. quercitron bark, $10 \mathrm{lbs}$. Dutch crop madder, 2 quarts bone size. Heat to $160^{\circ}$ in 1 hour and 15 minutes, keep at $160^{\circ} 15$ minutes, wash, bran 10 minutes at $150^{\circ}$; wesh and dry.

h. Peach and white.-Pad, \&c. as for clarets; dye 10 pieces with 2 lbs. ground cochineal, 2 lbs. peachwood, $6 \mathrm{or}$ logwood, heat to $140^{\circ}$ in $1 \frac{1}{4}$ hour, wash, bran at $140^{\circ} 10$ minutes ; wash and dry.

$i$. Black and white.-Pad in red liquor at $20^{\circ} \mathrm{T}$. once, print in No. 36, age 3 nights, fly-dung at boil, second dung at $140^{\circ}, 20$ minutes, wash, dry, and singe, wash and dye 10 pieces with $60 \mathrm{lbs}$. prepared logwood, 4 gallons of bone size, and 6 oz. carbonateof-soda crystals, heat to boil in 1 hour and 10 minutes; wash well and dry.

$k$. Olive, drabs, \&c. with white. - A great variety of shades may be obtained by varying the mordants. For drabs pad in iron liquor diluted about 10 times according to the shade wanted, and dye in bark, or bark and logwood. For olives, pad in mixtures of red liquor and iron liquor, diluted, and dye in bark, or bark and logwood. The acid used may be No. 33 .

l. Bark dyeing.-Dye 10 pieces with 25 lbs. bark, and 3 quarts bone size; heat to $190^{\circ}$ in $1 \frac{1}{2}$ hour, and keop at $190^{\circ} 10$ minutes, wash and bran at $160^{\circ} 10$ minutes, wash and dry.

m. Bark and Logwood dyeing.-Dye 10 pieces with $20 \mathrm{lbs}$, bark, and $30 \mathrm{oz}$, prepared logwood, with 3 quarts bone size; heat as in bark dyeing. 


\section{Fifth Style: Indigo.}

The indigo dye-house is always on the ground of a building, and is fitted up with a number of stone vats let into the ground. There are generally several rows of these vats, about 3 foet apart. They are about 8 feet long by 4 feet wide, and 8 to 10 foet doep. Some of them have steam-pipes inserted, which go to near the bottom, so that they can be heated whon necessary. There are about 10 vats in a row.

A. Blue and white. The simplest form of blue styles is blue and white; dark blue ground with white figures. The cloth is printed in one of the following reserve pastes :-

No. 65. Reserve paste for Block.-3 lbs. sulphate of copper dissolved in 1 gallon of water, $15 \mathrm{lbs}$. pipe-clay, heat up with some of the liquor: 1 gallon of thick gumSenegal solution, and 1 quart of nitrate of copper at $80^{\circ} \mathrm{T}$.

No. 66. Reserve paste for Machine, - 21 lbs. sulplate of copper, 1 gallon of water, thickened with 9 lbs. flour, and 2 pounds dark British gum.

No. 67. Rescrve paste for Machine. -5 lbs. sulphate of copper, 2 lbs. white acetate of lead, 2 gallons water, dissolve and thicken the clear with $3 \mathrm{lbs}$. flour and $2 \mathrm{lbs}$. pale British gum: when cold, add half a pint of nitrate of copper at $80^{\circ} \mathrm{T}$., to every 2 gallons of colour.

No 68. Reserve paste for Machine.-4 gallons boiling water, $16 \mathrm{lbs}$. of sulphate of copper, $8 \mathrm{lbs}$. white acetate of lead, let settle and pour off the clear liquor; thicken 3 gallons of this with $8 \mathrm{lbs}$. of flour, and 4 lbs. pale British gum. When boiled, add $4 \mathrm{lbs}$, sulphate of zinc, and dissolve. The foregoing are all to resist deep shades of blue. For light shades of blue dipping, any of the following:-

No. 69. Mild paste for Block. -25 lbs, dark British gum, 15 quarts of water, boil 10 minutes and add $7 \frac{1}{2} \mathrm{lbs}$. soft soap; stir well in, and when mixed, add $20 \mathrm{lbs}$, sulphate of ainc, stir well in, and add 10 lbs. pipe-clay beaten up into $7 \frac{1}{2}$ quarts of water, and $7 \frac{1}{2}$ gills of nitrate of copper at $80^{\circ} \mathrm{T}$. Mix all well together.

No. 70. Mild paste for Machine. -8 lbs. dark British gum; $3 \frac{3}{4}$ quarts water; boil, and add $2 \mathrm{lbs}$. soft soap, cool, and add $6 \mathrm{lbs}$. sulphate of zine dissolved in 2 quarts of boiling water, and one quart of nitrate of copper at $80^{\circ} \mathrm{T}$.

After printing in one of these reserves, hang in a rather humid atmosphere for 2 days, and then dip blue.

Indigo for use in the dye-hovise is ground with water to a fine pulp: a series of castiron mills with curved bottoms, are arranged in a line; one or two iron rollers are moved backwards and forwards on the curved bottom in each mill by an upright rod, which is furnished with a roller at the bottom, and is connected with a horizontal rod worked by an excentric. Indigo and a certain quantity of water are left in these mills several days, till the pulp is perfectly smooth. The method of blue dipping is as follows:-

In a line of ten vats, the first one is set with lime; as-

(No. 1.) 1,000 gallons water, $250 \mathrm{lbs}$, of hydrate of lime, or lime slaked to a dry powder; when used it is well raked up.

The indigo vats vary according to the style of work; for deep blue and white, or blue and yellow, or orange, the following is a good one:-

(No. 2.) 1,000 gallons water, 50 lbs. indigo previously pulped, 140 lbs. copperas, and 170 lbs. lime; dissolve the copperas in the water, then add the indigo, stir well up, and add the lime, previously riddled, to separate small stones. Rake up every two hours for two days, and let settle clear. The clear liquor, when taken up in a glass, must have a deep yellow colour, be perfectly transparent, and be immediately covered with a pellicle of regenerated indigo when exposed to the air. Eight or nine vats are all set alike.

The pieces to be dipped are hooked backwards and forwards on a rectangular frame which just fits the vats, so that the cloth can be immersed, but still not so deep as to touch the sediment of the vats. The process is thus performed:-The lime-vat No 1 being stirred up, the frame which contains two pieces, is lowered down into it, so as to completely immerse the pieces; a gentle up-and-down movement is given by hand. The frame is allowed to stay $\mathbf{1 0}$ minutes in, is then lifted out and supported over the vat by rods put across. After draining here a fow minutes, it is then removed and immersed in vat No. 2, or the first indigo vat. It stays here seven minutes, is lifted out, and drained as before over the vat 8 minutes, then removed to No. 3 vat, and so on till it has gone through the whole series, or till the shade of blue is considered strong enough. After the last dip the pieces are unhooked and winced in a pit of water, then wineod about 10 minutes in a pit containing sulphurie acid at $6^{\circ} \mathrm{T}$., washed well in the wheel, squoczed, and dried. In large dye-houses there is an arrangement for collecting all the waste indigo which is washed off the pieces, by running all the water used into a vaulted chamber under the dye-house, 
whore it passes from one compartment to another, gradually depositing the suspended indigo, which is periodically removed.

In heary bodies of colour, the paste sometimes slips or the shapes becomo irregular; this is counteracted by using the first indigo vat raked up instead of clear. The vats are used till nearly exhausted, and then the clear liquor pumped off to be used instead of water for setting fresh vats with.

B. Blue and Yellow, or Orange.-Print in one of the reserve pastes, and yollow or orange colour made as follows :-

No. 71. Chrome-yellow for Machine,-2 gallons water, 20 lbs. sulphate of copper, $20 \mathrm{lbs}$. nitrate of lead; dissolre, and boat up with $12 \mathrm{lbs}$. flour, and 2 gallons sulphateof-load bottoms; boil all together.

The sulphate of lead here is the bye-product in making red mordant No. 8 , and is drained to a thick paste.

No. 72. Orange.-Make a standard liquor by dissolving $24 \mathrm{lbs}$. white acotate of lead in 6 gallons water, and stirring 12 lbs. litharge in it till perfectly white, then let settle, and use the clear.

For the orange colour take 2 gallons of this standard liquor, instead of the gallons of water. in the above yellow coloux.

Follow the same routine in dipping, \&cc., as for blue and white. After wincing in sulphuric-reid sours, wash well, and wince 10 minutes in bichromate-of-potash golution; $2 \mathrm{oz}$. per gallon at $100^{\circ} \mathrm{F}$. Wash well, and wince in dilute muriatic acid at $3^{\circ}$ T., contaning $1 \mathrm{oz}$. oxalic acid per gallon, till the yellow is quite bright. The small quantity of chromic acid set free oxidises and destroys the indigo that may be attached to the yellow colour. After this souring, wash and dry.

If orange was printed instead of yellow, treat as for yellow; and after the muriooxalic sour, wash, and raise orange in the following:-10 lbs. bichromate of potash, 300 gallons water, and $3 \frac{1}{2}$ lbs. of lime previously slaked to a thin paste, heat to $180^{\circ}$ F., and wince the pieces in till the orange is full and bright; then take out and wash woll, and dry.

Other varieties of blue dyeing are:-

c. Two blues.

D. Two blues and white.

в. Two blues, white, and yellow or orange.

r. Dark blue and green.

G. Two blues and yellow.

For $\mathbf{C}$ and $\mathrm{s}$ a pale hade of blue is first given to the cloth. The light blue vat is thus composed:-

No. 3. (Light Blue Vat.) 1,000 gallons water, $40 \mathrm{lbs}$. indigo, 70 lbs. copperas, $80 \mathrm{lbs}$. limo. For c. Dip light blue by three immersions, draining well between; unhook, wince in water, then in sulphuric sours at $2^{\circ} \mathrm{T}$.; wash, squeeze, and dry; then print on a reserve paste, and proceed as for dark blue and white; when finished, the pale blue having been protocted by the reserve, has remained unaltered, all the rest being dark blue.

For F. Instead of reserve paste, print on yellow No. 71, and dip dark blue, sour and raise the yellow with bichromate of potash, omit the souring after chroming, and wash and dry. The yellow falling on the pale blue, makes a green.

For $\mathrm{v}$. On whito cloth print an object in muriate of manganese, thickened with dark British gum, raise this as described under the head Bronzes, dry and block in a reserve paste No. 65, then lime and dip in the dark blue vat, letting stay in half an hour, remore, oxidiso in the air, wash and sour with dilute muriatic acid to which some muriate-of-tin liquor. has been added, wash and dry; where the peroxide of manganese had boen is now dark blue, the ground pale blue with white object.

For \&. Print as $\mathrm{D}$, with yellow or orange in addition, and after the sulphuric sours, raise yellow or orange as before.

Dip light blue; print reserve paste and yellow; dip dark blue; wince; sour in sulphuric sours at $6^{\circ}$ T.; wince in water; chrome at $140^{\circ}$ F. 10 minutes at $2 \mathrm{oz}$. bichromate per gallon; wince, wash, and sour in the following:-7lbs. oxalic acid, $3 \mathrm{lbs}$. strong sulphuric acid; dissolve in water to stand $8^{\circ} \mathrm{T}$.; wince till the yellow is bright; then wash and dry.

A style formerly very mueh in vogue, but now scarcely ever used, is the neutral or lazulite style. It consists in combining mordants with reserves, and dipping blue; the colours throw off the blue, and are subsequently dyed with madder.

Neutrals are of two sorts:-

1. Where reds and chocolate, or black, with rosist white are printed, and dipped light blue, the resist white being only required to resist the blue.

2. Where the white is required to cut through the black, redṣ or chocolato in addition to the blue. 
The following are exnmples of lazulite colours for the first variety :-

No. 73. Black (Machine).-4 quarts logwood liquor at $12^{\circ}$ T., 1 quart ganl liquor at $9^{\circ}$ T., 1 quart red liquor at $20^{\circ}$ T., 1 quart iron liquor at $24^{\circ}$ T., 1 quart acetic acid, thicken with $3 \mathrm{lbs}$. flour, and $8 \mathrm{oz}$. starch: when boiled add 1 pint Gallipoli oil, and 1 pint turpentine.

No. 74. Chocolate (Machine). -5 quarts rod liquor at $12^{\circ}$ T., 1 quart iron liquor at $24^{\circ} \mathrm{T} ., 1 \frac{1}{\mathrm{l}} \mathrm{b}$. sulphate of coppor, $24 \mathrm{oz}$. measure of nitrate of copper at $100^{\mathrm{c}} \mathrm{T}$., thicken with $2 \frac{1}{2} \mathrm{lbs}$. flour, and $1 \mathrm{lb}$. dark British gum.

No. 75. Chocolate (Block). -5 quarts red liquor $12^{\circ}$ T., 1 quart iron liquor $24^{\circ}$ T., $2 \frac{3}{4} \mathrm{lbs}$. sulphate of copper, $36 \mathrm{oz}$. measure nitrate of copper at $100^{\circ} \mathrm{T} ., 9 \mathrm{lbs}$. pipoclay beat up well, and add 3 quarts of gum-Senegal solution at 5 lbs. per gallon.

No. 76. Dark resist Red (Block). -2 quarts red liquor $22^{\circ}$ T., $5 \frac{3}{4}$ oz. white acetato of lead, $4 \frac{1}{4} \mathrm{oz}$. sulphate of copper, dissolve, and beat up in $6 \frac{3}{4} \mathrm{lbs}$. pipe-clay. Thicken separately 2 quarts red liquor at $12^{\circ} \mathrm{T}$., with $12 \mathrm{oz}$. flour, and add when boiling hot $8 \mathrm{oz}$. of soft soap melted; mix well, add the pipe-clay mixture to this, and then 2 quarts red liquor at $2^{\circ} \mathrm{T}$., thickened by dissolving gum-Senegal in it. Stir the wholo .well together.

No. 77. Dark resist Red (Machine),-20 quarts nitrate of zine at 36 B., 10 quarts water coloured with a little peachwood, $121 \mathrm{lbs}$. alum, $10 \mathrm{lbs}$. acetate of lead; dissolve all togethor with heat, stir till cool, thickon all together with $8 \mathrm{lbs}$. flour, and $1 \frac{1}{2} \mathrm{lb}$. dark British gum.

No. 78. Any shade of pale red is made for block by substituting the red liquor in colour No. 76 by the mordant No: 8, reduced with water, according to the sliade wanted.

No. 79. Any shade of pale red for machine is mado by reducing the quantities of alum and acetate of lead in colour No. 77 .

The white reserve for this variety of neutrals is either of the mild pastes.

No. 80. Resist Brown.-2 gallons water, 24 lbs. catechu, 6 lbs. sal-ammoniac, 1 gallon acetic acid; boil 15 minutes, and add $7 \frac{1}{2}$ gallons gum-solution, 5 quarts nitrate of copper at $100^{\circ} \mathrm{T}$.

Process.- Tho colours after printing aro aged 3 days, then dipped light blue in the following blue vat:-

(No. 4.) Neutral vat.-1,000 gallons water, $120 \mathrm{lbs}$. indigo, $135 \mathrm{lbs}$. copperas, 150 lbs. lime; rake up for two days, and let settle.

A frame with rollers top and bottom is lowered into this, and the pieces are run through; after leaving the vat, they are made to travel over rollers in the air for a sufficient distance to turn them blue; then into a pit of water, from that into a beck with cow-dung and water, at $160^{\circ} \mathrm{F}$., where they run 15 minutes, then washed and dyed madder or garancin, \&c. \&c.

In the second variety of neutrals, the white is required to resist both mordants and blue, and is made thus :-

No. 81. Neutral White for Blocks.-7 quarts lime juice at $30^{\circ}$ T., 1 quart water, $4 \frac{1}{2}$ lbs. sulphate of copper, 24 lbs. pipe-clay, $5 \frac{1}{2}$ quarts lime juice at $30^{\circ} \mathrm{T}$., previously thickened with gum-Senegal.

No. 82. Neutral White for Machine.-1. gallon lime juice at $42^{\circ} \mathrm{T} ., 2 \mathrm{lbs}$. sulphate of copper, $32 \mathrm{oz}$. measure nitrate of copper at $100^{\circ} \mathrm{T}$., thickened with $1 \frac{1}{2} \mathrm{lb}$. starch.

The black is the ordinary madder or garancin black, Nos. 4 and 5 process.

The neutral white is first printed either by block or machine; if the latter, it cannot be in a pattern which should register accurntely with the subsequent colours, as it must be dried perfectly before the other colours are printed, to aroid obtaining jrregular shapes; the above reserve colours are then printed over the neutral white. Mild paste Nos, 69, 70 may also be printed along with the other colours, to reserve a white under the blue only. The subsequent process is the same as for the first variety.

After dyeing madder and garancin, and clearing with soap, \&c., steam or spirit colours are generally blocked in. Parts of the yellow being made to fall over the blue form green.

\section{Sixth Style: China Blues.}

China blues, so called from the shade of blue resembling that on porcelain. In this style indigo is printed on, and mado to penetrato and fix in the cloth by the subsequont process.

The colour is made thus :-

No. 83. Standard China Blue.-In an indigo mill aro put $45 \mathrm{lbs}$. indigo, 9 gallons iron liquor at $24^{\circ} \mathrm{T}$, and $18 \mathrm{lbs}$. copperas, the whole ground till quite fine; then add $7 \frac{1}{2}$ gallons gum-Senegal solution at $6 \mathrm{lbs}$. per gallon; grind an hour longer, take out and wash the mill with 6 quarts hot water, and add to the above. 
No. 84. China blue gum,-Gum.Senogal solntion at $3 \mathrm{lbs}$. por gallon, containing 4 or. copperas per gallor.

Colours are made by reducing the standard blue with the gum, according to the pattorn and strength required. For instance, for two blues of modium shades :-

No. 85. Strong Blue. -1 volume standard, 2 volumes gum.

No. 86. Pale Blue. -1 volume standard, 10 volumes gam.

After printing, age one night, and raise as follows:-Two rats similar to indigo vats aro set. No. 1. 1,000 gallons water, 500 lbs, slakod and dry lime.-No. 2. Solution of copperas at $5^{\circ} \mathrm{T}$. In each vat is lowered a frame, which is provided with rollers at top and bottom, and in addition, has a pair of bushes at each side of the frame, just above the surface of the liquor, in which are put beams, on which the pieces are wound alternately; the bearings of the beams being just above the surface of the liquor, allow the roll of pieces to be always half in and half out of the liquor. The course of proceeding is this:-A beam containing two or three pieces stitched end to end is placed on a small frame at one side of vat No. 1, and by means of a cord previously threaded through the rollers in the rat, the picees are slowly wound through the vat and on to a beam placed in the bearings at the opposite side of the vat, by means of a winch handle fitted on this beam; when the pieces havo thus passed through vat No. 1, which is kept in a milky state all the time, the beam is lifted out and transferred to one of the pair of bearings in vat No. 2; the pieces are wound through this vat in the same manner; after this vat, they are removed to No. 1 vat, and worked through; this alternate limeing and copperasing is continned till the pieces have been 4 times through each vat; then detach and wince in water; then put into sulphuric sours at $10^{\circ} \mathrm{T}$., immersing completely in the liquor till the whites appear quite clear; then wash well, soap in a beck at $120^{\circ} \mathrm{F}$. a quarter of an hour with $\frac{1}{4} \mathrm{lb}$. soap per pioce; wash again and sour in sulphuric sours at $1^{\circ} \mathrm{T}$. at $110^{\circ} \mathrm{F}$; ; wash well and dry.

The various phenomena which occur in the dipping of China blues are not difficult of explanation w:th the lights of modern chemistry. We have, on the one hand, indigo and sulphato of iron alternately applied to the cloth; by dipping it into the lime, the blue is deoxidised, because a film of the sulphate of iron is decomposed, and protoxide of iron comes forth to seize the oxygen of the indigo, to make it yellowgreen, and soluble at the same time in lime-water. Then, it penetrates into the hoart of the fibres, and, on exposure to air, absorbs oxygen, so as to become insoluble and fixed within their pores. On dipping the calico into the second vat of sulphate of iron, a layer of oxide is formed upon its whole surface, which oxide exercises an action only upon those parts that are covered with indigo, and deoxidises a portion of it; thus rendering a second dose soluble by the intervontion of the second dip in the lime-bath. Hence we see that while these alternate transitions go on, the same series of deoxidisement, solution, and re-oxidisement recurs : causing a progressively increasing fixation of indigo within the fibres of the cotton.

Other indigo styles are dipped greens, blue with white discharge.

Dipped Greens, - There are 4 vats similar to indigo vats in a row, set with-

First: (No. 5.) Light blue Vat for Greens.-1,000 gallons water, $25 \mathrm{lbs}$. indigo, $45 \mathrm{lbs}$. copperas, 65 los. lime, dry slaked, $17 \mathrm{lbs}$. caustic soda, $24^{\circ} \mathrm{T}$.; raked up 2 days, and settled clear.

Second: (No. 6.) Yellow Vat for Greens,-1,000 gallons water, $250 \mathrm{lbs}$, brown acetato of lead, 130 lbs. dry slaked lime; rake up till dissolved, and lot settle clear.

Third: (No. 7.) Filled with water only.

Fourth: (No. 8.) Set with bichromate of potash at $4^{\circ} \mathrm{T}$.

Each of these vats is mounted with a framo with rollers top and bottom; the pieces to be dipped are stretched end to end, and a length of cord being threader through all the rats and fastened to a drawing roller at the end of the fourth, the pieces are drawn slowly through between the first and second; the eloth is made to travel several yards so as to ensure oxidation of the indigo before going into the lead vat; after leaving the fourth, they are detached, winced, and washed well.

For dipped greens, either white cloth is printed in patterns, as spots, \&c., with mild paste, Nos. 69,70 ; or a pattern previously printed in madder colours and dyed, \&rc. is covered up with mild pasto by block; the cloth being now dipped green, tho pattern or spots are reserved or untouched by the green: a very good effect is produced by dipping the Burgundy and acid No. 4, green, when the Burgundy part comes out a beautiful chocolate, and the white part green.

Acid Discharge on Blue.-A bluo and whito style, but which permits the most delicate pattern to be printed, which is not the case with the ordinary blue and white style. The cloth is first dipped a medium shade of blue, washed and dried, then paddod in bichromate of potash at $6^{\circ} \mathrm{T}$. and carefully dried in the shade, without artificial heat, and printed in tho following colour :- 
No. 87. White Discharge for Blues,-1 gallon water, thieken with 2 lbs. flour, and 2 lbs. dark British gum; when partly cooled, add 2 lbs. oxalic acid, and when quite cold, $7 \frac{1}{2} \mathrm{oz}$. measure sulphuric acid. A few seconds after the colour is printed on the padded cloth the blue is discharged, and a dirty white left in the printed parts; after printing, tho pieces are dried so as to leave them slightly damp, and immocliately winced in chalk and water, then winced in sulphuric sours at $2^{\circ} \mathrm{T}$., winced and woll washod; the printed pattern is now a pure white, and if care has been taken not to dry the bichromate too hard, and not expose it to sunlight, the blue is bright and good.

This ingenious process was the invention of Mr. John Mereer. At the moment the block applies the preceding discharge to the bichromate dye, there is a sudden decoloration, and a production of a peculiar odour.

The pieces padded with the bichromate must be dried at a moderate temperature, and in the shade. Whenever watery solutions of chromate of potash and tartaric acid aro mixed an efferrescence takes place, during which the mixture possesses the power of destroying vegetable colours. This property lasts no longer than the effervescence.

In the 'Moniteur Scientifique' of Dr. Quesneville for July 1873 are described some now processes, invented by $P$. Schützenberger and F. Lalande for the fixation of indigo upon eloth. The active agent for the reduction of the indigo is the hydrosulphite of soda, discovered by the former, and which is made as follows:- 'Bisulphite of soda, standing at $30^{\circ}$ to $35^{\circ}$ Baumé, is brought in contact in a covered vessel with twisted sheet zine, or granulated zine, filling up to the top of the vessel without occupying more than one-fourth of its real volume. After the lapse of about one hour, the liquid is poured into an excess of milk of lime, which precipitates the salts of zine. The clear liquid is drawn off either by filtration and pressure, or by decantation after the addition of water. Air should be excluded as much as possible. By mixing the hydro-sulphite thus obtained with ground indigo, and the amounts of lime or soda necessary to dissolve the reduced indigo, wo obtain at once a yellowish liquid containing no insoluble matter except the earthy impurities of the indigo.' One pound of indigo may be thus reduced, so as to form a solution not exceeding 4 to 6 quarts in volume. This solution is diluted with water to form a blue vat or an alkaline solution of the reduced indigo, containing a large excess of hydro-sulphite of soda, is suitably thickened and printed by a copper roller in the ordinary printing-machine. The oxidation during printing is so slight that after an hour's work, the remnant of the colour is still yellow and soluble. The entire colour is utilised, 50 to 60 per cent. less indigo being consumed with the new process than with the old. The shades obtained are more beautiful and fast, and the impression is better defined. This new indigo blue, requiring no subsequent fixing process, can be printed along with a great number of other colours, such as aniline black, madder colours either for dyeing or as extracts, catechu shades, Guignet's groen and other colours fixed with albumen. Specimens of some of these colours printed along with the new blue, are given in the 'Moniteur,' and the new process would appear from these to be successful.

\section{Seventh Style: Discharge on Turkey-Red Ground.}

No. 88. White Discharge (Machine), -8 lbs. light British gum, 1 gallon tartaric-acid liquor $62^{\circ} \mathrm{T}$., 1 gallon acetic acid $6^{\circ} \mathrm{T}$.

No. 89. White Discharge (Block). - The above colour a little thinner.

No. 90. Black for Turkey Red. -7 gallons logwood liquor at $8^{\circ} \mathrm{T}$., 1 gallon pyroligneous acid, 10 lbs. starch; boil and add 2 lbs, $10 \mathrm{oz}$. copperas; boil again and cool, then add $3 \frac{1}{2}$ pints pernitrate of iron at $80^{\circ} \mathrm{T}$., and 1 gallon of blue paste.

No. 91. Blue Paste.-(a) 6 lbs. copperas, 2 quarts water; dissolve: (b) 4 lbs. prussiate of potash, 1 gallon of water ; dissolve. Mix $a$ and $b$ togethor, and add 1 quart standard red liquor No. 8, 1 quart nitric acid $60^{\circ} \mathrm{T}$.

No. 92. Yellow Discharge $(B l o c k),-1$ gallon lime juice at $50^{\circ}$ T., 4 lbs. tartaric acid, 4 lbs. nitrate of lead; dissolve, thicken with 6 lbs. pipe-clay, and 3 lbs. gumSenegal.

No. 93. Yellow Discharge (Machine).-Thicken the above with $1 \frac{1}{2} \mathrm{lb}$. starch, instend of the pipe-clay and gum.

No. 94. Yellow Discharge (Machine).-1 gallon lime juice at $40^{\circ} \mathrm{T} ., 4 \frac{1}{2}$ lbs. tartaric acid, $5 \mathrm{lbs}$. white acetate of lead, $1 \frac{1}{2} \mathrm{lb}$. stareh; boil and cool, then add $1 \mathrm{lb} .14 \mathrm{oz}$. nitric acid at $60^{\circ}$

No. 95. Blue Discharge (Machine).-(a) $1 \mathrm{lb}$. Prussian blue, $1 \mathrm{lb}$. oxalic acid, 1 quart hot water; grind well together, and leave to react on each other 24 hours; then $(b)$ 3 quarts of wator, $1 \frac{1}{4} \mathrm{lb}$. starch; boil, and add $2 \mathrm{lbs}$, tartaric acid, and mix $a$ and $b$ together.

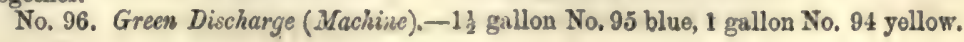


Process:-Print in any of the above colours, and as soon as dry from the machine, put through the decolouring vat.

(No. 9.) Decolouring Vat. $-1,000$ gallons of water, $1,000 \mathrm{lbs}$. chloride of lime; rake well up, till quite smooth and free from lumps, then immerse a frame with rollers top and bottom, as in dipping greons, \&c.; keep the vat stirred up so as to be milky, and run the pieces through at the rate of 1 piece of 28 yards in 3 minutes; on leaving the squeezing rollers, conduct into water and rinse, then wince 10 minutes in bichromate of potash at $4^{\circ} \mathrm{T}$.; wash and wince in very diluto muriatic acid; wash woll and dry.

In this style, such is the permanence of the Turkey-red dye, that it is not much altered by passing through chloride of lime, whilst in the parts printed in the discharge colours an instantaneous disengagement of chlorine takes place, which decolorises the dyed ground, and where a mineral colour or mordant formed part of the discharge, it is left in place of the red dye. This style was invented in 1811 by M. D. Kmelilin, and patented in England by Mr. James Thompson, of Primrose, who printed immonse quantities of it.

The Bandanna printing, being a business of itself, is more fitly described in another place, (See Bandarara.)

Eighth Style: Steam Colours.

The printing of steam colours may be considered as a mode of dyeing at one operation, for in most cases one or more mordants are mixed with dye-wood dococtions, and printed on the cloth, the subsequent steaming causing the mordant to combine with the colouring matter, and both with the cloth. Steam colours in some cases are mado so as to produce a fair colour when printed on ordinary white calico: but much superior colours are produced by mordanting the cloth first, so as to fix peroxide of tin in the fibre; and as this is the almost universal rule, it is this sort of steam printing alone that will be described. Woollen fabrics, indeed, require a good preparation by tin, \&c. before lively and substantial colours can be fixed on thom by steam.

The following is the mode of preparing calicoes for steam colours:-

Pad the pieces stitched together, in a padding machine with wooden bowls, through a solution of stannate of soda at $10^{\circ} \mathrm{T}$. twice over, letting them lie wet an hour between; immediately after padding the second time, run through a cistern with rollers, containing dilute sulphuric acid at $1 \frac{1}{2}^{\circ}$ to $3^{\circ}$ T., thence into a pit of water, wince well, and run through a washing-machine. It has been observed by Mr. James Chadwick, that if the cloth, with oxide of tin newly percipitated on it, is subjected to any severe washing, it loses a considerable quantity of oxide, so that no more washing must be given at this stage than will remove the free sulphuric acid. It appears that the cloth, once dried with the oxide in it, does not part with the oxide again by severe washing. After washing, the pieces are unstitched, and put into the hydro-extractor, then dried gently over the steam cylinders, and are then ready for printing.

The following list of steam colours comprises the usual rariety of shades printed on calico:-

No. 97. Steam Black (Machine), -1 gallon logwood liquor at $12^{\circ} \mathrm{T}$, 1 quart gall jiquor at $9^{\circ} \mathrm{T}$., 1 quart mordant, 2 lbs. flour, $6 \mathrm{oz}$. starch ; boil ton minutes, and add 1 pint nitrate of iron,

Stean Black Mordant.-I quart acetic acid, $1 \frac{1}{2}$ quart acetate of copper at $30 \mathrm{~T}$., $1 \frac{1}{2}$ quart iron liquor at $24^{\circ} \mathrm{T}$., 1 quart red liquor at $20^{\circ} \mathrm{T}$.

No. 98. Chocolate (Machine). - 3 gallons logwood liquor at $12^{\circ}$ T., 2 gallons Srpan liquor at $12^{\circ}$ T., 1 gallon nitrate of alumina, $\frac{1}{2}$ gallon bark liquor at $12^{\circ}$ T., 4 gallons water, $17 \mathrm{lbs}$. starch ; boil, and add $8 \mathrm{oz}$. chlorate of potash, $2 \frac{1}{2} \mathrm{lbs}$. red prussiate.

No. 99. Dark Blue (Machine). -7 gallons water, 14 lbs. starch, $2 \frac{3}{4}$ lbs. sal-ammonise; boil, and add whilst hot 12 lbs. yellow prussiate of potash in powder, $6 \mathrm{lbs}$. red prussiate of potash, 6 lbs. tartaric acid, and when nearly cold, 1 lb. sulphuric acid (specific gravity, 1.85), $1 \mathrm{lb}$. oxalic acid dissolved in 2 quarts hot water, and 6 gallons prussiate - of-tin pulp.

No. 100. Dark Blue. - 8 quarts water, 4 lbs. yellow prussiate of potash, 3 lbs. pale British gum; boil, and add $1 \mathrm{lb}$. bisulphate of potash, $2 \mathrm{lbs}$. muriato of ammonia, $8 \mathrm{oz}$. alum, 4 oz. oxalic acid, $4 \mathrm{oz}$. sulphuric acid at $170^{\circ}$ T., 4 quarts tin pulp No. 103.

No. 101. Cinnamon. - 1 quart cochineal liquor at $8^{\circ} \mathrm{T}$., 1 quart logwood liquor at $8^{\circ} \mathrm{T} ., 1$ quart berry liquor at $10^{\circ} \mathrm{T}$, $6 \mathrm{oz}$. alum, $4 \mathrm{oz}$. cream of tartar, $8 \mathrm{oz}$. starch ; boil, and whilst warm add $3 \mathrm{oz}$. muriate of-tin-crystals.

No. 102. Orange. -12 lbs. annatto, 1 gallon caustic soda at $70^{\circ} \mathrm{T}, 5$ gallons water; boil 20 minutes, strain, and add 3 quarts red mordant No. 198, 6 lbs. alum; heat till clear, and add 4 gallons thick gum-substituto water.

No. 103. Tin Pulp.-To protochloride-of-tin solution add as much yellow prussiate 
of potash in solution as will precipitate all the tin as ferroprussiate; this is washed by decantation, and filtered to a stiff paste.

No. 104. Light Blue for Machine,-1 gallon dark blue No. 99, 3 gallons 4-1b. gumsubstitute water.

No. 105. Green (Machine) -7 gallons Persian-berry liquor, at $12^{\circ} \mathrm{T} ., 15 \mathrm{lbs}$. yellow prussiate of potash, $8 \mathrm{lbs}$. alum, $28 \mathrm{lbs}$. gum-substitute; boil, and add $2 \mathrm{lbs}$. muriateof tin crystals, 2 lbs. oxalic acid.

No. 106. Pink (Machine), -4 gallons cochineal liquor at $6^{\circ}$ T., 2 lbs, alum, 2 lbs. bi-tartrate of potash, $8 \mathrm{oz}$. oxalic acid, 4 gallons thick gum-Senegal water.

No. 107. Purple (Machine). -2 gallons logwood liquor, at $12^{\circ}$ T., $12 \mathrm{oz}$. alum, $8 \mathrm{oz}$. red prussiate of potash, 4 oz. oxalic acid, 8 gallons gum-substitute water. If for block, add 12 gallons gum-water instead of 8 gallons.

No. 108. Dark Red (Machine.) -8 quarts Sapan liquor at $12^{\circ}$ T., 2 quarts bark liquor at $8^{\circ}$ T., 2 quarts nitrate of alumina No. 109, $6 \frac{1}{2} \mathrm{lbs}$. starch, $1 \mathrm{lb}$. gum-substitute, 4 quarts water, $4 \mathrm{oz}$. chlorate of potash, $12 \mathrm{oz}$. alum.

No. 109. Nitrate of Alumina. -8 gallons boiling water, 24 lbs. nitrate-of-lead crystals, 24 lbs. alum, 5 lbs. carbonate-of-soda crystals; let settle, and use the clear.

No. 110. Blue Standard.-1 gallon water, $12 \mathrm{oz}$, alum, $4 \frac{2}{2} \mathrm{oz}$. oxalic acid, $1 \frac{3}{4} \mathrm{lb}$. yellow prussiate of potash, 1 gallon gum-substitute water.

No. 111. Lavender Liquor. - 2 gallons red liquor at $18^{\circ}$ T., 6 lbs. ground logwood ; let steep for 48 hours, then strain off the liquor.

No. 112. Lavender. -4 gallons lavender liquor No. 111, 5 gallons blue standard No. 110 , from 24 to 48 gallons gum-water, according to shade wanted.

No. 113. Drab. 4 gallons lavender liquor, 4 gallons blue standard, 1 gallon bnrk liquor at $8^{\circ} \mathrm{T}$., from 40 to 70 gallons gum-water, according to shade wanted.

No. 114. Stone. -4 gallons lavender liquor No. 111, 6 gallons blue standard No. 110, 1 gallon bark liquor at $120^{\circ} \mathrm{T}$., reduced same as drab.

No. 115. Sage Greens for Blotch Grounds.-2 gallons yellow No. 118, 2 gallons blue standard No. 110, from 28 to 56 gallons gum-water, according to shade wanted.

No. 116. Yellow. -4 gallons berry liquor at $12^{\circ} \mathrm{T}, 1 \frac{1}{2} \mathrm{lb}$. alum.

No. 117. Brown Standard.-14 quarts bark liquor at $12^{\circ}$ T., 3. $\frac{1}{2}$ quarts Sapan liquor at $12^{\circ} \mathrm{T}$., $1 \frac{1}{2}$ quart logwood liquor at $12^{\circ} \mathrm{T}$., 12 quarts $8-1 \mathrm{~b}$. gum-substitute water, $3 \frac{1}{2}$ lbs. alum, $2 \mathrm{oz}$, chlorate of potash, $5 \mathrm{oz}$. red prussiate. All shades of light browns are made from this by reducing with gum-substitute water, according to shade wanted.

No. 118. Yellow -4 gallons bark at $8^{\circ}$ T., 2 quarts red liquor at $18^{\circ}$ T., 2 quarts nitrate of alumina No. 109, $12 \mathrm{oz}$. tin crystals, 5 lbs. starch.

No. 119. Green for Block,-28 lbs. yellow prussiate of potash, 6 gallons hot water; in a separate vessel 10 gallons 6 -1b. gum-Senegal water, 2 gallons water, 1 gallon muriate of tin at $120^{\circ} \mathrm{T}$.; mix the prussiate solution with the tin and gum by pouring one into the other, and violently agitating; when quite fine and free from floceulent matter, add 12 gallons berry liquor at $10^{\circ}$ T., then add $12 \mathrm{lbs}$. and $2 \frac{1}{2} \mathrm{lbs}$. oxalic acid dissolved in 5 gallons water, then 3 quarts acetic acid, and $2 \frac{1}{2}$ gills extract of indigo.

No. 120. Brown -6 quarts berry liquor at $20^{\circ}$ T., 6 quarts Brazil-wood liquor at $8^{\circ}$ T., 3 lbs. alum, 3 quarts lavender liquor, 6 quarts $6-1 \mathrm{~b}$. gum-Senegal water, $24 \mathrm{oz}$. nitrete of copper at $100^{\circ} \mathrm{T}$.

Printing with pigment colours, and fixing them by steaming was formerly a separate style; but owing to the introduction of aniline colours and madder extracts, goods are now printed so frequently with all these classes of colours at once, that it is useless to separate them. In printing pigments the ordinary pigments, such as used in oil painting, are mechanically attached to the eloth by a species of cementing. The first fixing vehicle used was a solution of caoutchouc in naphtha, which was mixed with the pigment so as to make colours of sufficient viscosity to print. The naphtha was then driven off by steaming, and the pigment was then cemented to the cloth by a film of caoutchouc. This method makes very fast colours, not affected by soaping and moderate frici.1on; but, unfortunately, the naphtha volatilising during the printing process, rendered the use of it too dangerous, and after it was found that explosions of the naphtha vapour frequently took place, calico printers turned their attontion to some other fixing vehicle. Animal substances, of which the white of eggs is the type, and which, solublo in water, are coagulated by heat, are now usually employed. 'Of these, fo it may be particularised :-Albumen of eggs; blood albumen, or dried sernm of blood ; lactarine, and gluten. The first is mado by simply drying gently the white of eggs into flakes. The second is the serum of blood dried in the same manner. The third is made by separating the solid part of buttermilk, purifying it from butter and free acid, and drying it. The fourth is the residue of starch-making from wheat-flour by the simple washing process, the gluten being gently dried. 
The lattor two thickonors require a small quantity of alkali to bring them in solution; they thon resemble albumen in thoir power of congulating by heat. There aro few colours of this style printed, chiefly ultramarine blue, carbon drab, and siennaand umber-browns; examples of these will bo found further on.

The invention of aniline colours, commencing with the discovery of maure in 1856 by W. H. Perkin, followed soon after by that of fuchsine or magenta, by Verguin, and subsequently by the production from aniline of a vast quantity of beautiful colours, has almost revolutionised steam-printing. These colours being of extraordinary purity and brilliancy, and in some cases being as fast to light as the colours above described, are now universally employed, to the almost extinetion of the old dyewood, \&ce, colours. In conjunction with the chrome green of Guignet and the various colours obtained from extracts of madder and artificial alizarine, most exquisite prints are now produced, where the genius of the designer, being no longer checked by the exigencies of the printer who, to make reds, purples, and chocolates fast to soap was compelled to dye them with madder, and thus make a break between the printing of these and their illumination by steam colours subsequently, has now full play, and a great rariety of colours being printed at one operation, and all raised or fixed together nfterwards : patterns of great delicacy of execution and beauty of colouring can be produced solid and fast to soap, which it was perfectly impossiblo to do before the introduction of these colours. It may therefore bo confidently affirmed that the introduction of madder extract has directly tended to make a marked improvement in the artistic taste applied to calicoprinting. The shades of colour produced from aniline colours, madder extracts, and pigments are infinite. It will only be within the scope of this work to give a selection which will illustrate the principles upon which they are made.

No. 121. Magenta Standard. - $1 \frac{1}{4} \mathrm{lb}$. magenta erystals, 4 gallons methylated spirit 2 gallons wator.

No. 122. Gum-tragacanth Water.--1 gallon water, $10 \mathrm{oz}$. gum-tragaeanth; boil 6 hours or till quito smooth, and make the bulk up to 1 gallon.

No. 123. Kigg-albumen-solution.-1 gallon of cold water, $8 \mathrm{lbs}$. of egg-albumen; stir till dissolved, and strain.

No. 124. Blood-albumen-solution.-1 gallon cold water, 8 lbs. blood-albumen; stir till dissolved, and strain.

No. 125. No. 45 Magenta Paste (for dark magenta),-2 quarts water, 2 lbs. fine wheat flour, $1 \mathrm{lb}$. of white British gum (slightly roasted whent starch), $45 \mathrm{oz}$. measure of No. 121 ; boil well, cool, and add 2 quarts of No. 123.

No. 126. No. 35 Magenta paste (for dark magenta).-The samo as No. 125, but $35 \mathrm{oz}$. measure of No. 121.

No. 127. No. 20 Magenta gum (for medium shado).-2 quarts of No. 122, 2 quarts of No. 123, $20 \mathrm{oz}$. measure of No. 121.

No. 128. No. 10 Magenta gum (for pale shade). -The same as No. 127, but $10 \mathrm{oz}$. measure of No. 121.

No. 129. No. 4 Magenta gum (for padding or 'blotching ').-The same as No. 127, but $4 \mathrm{oz}$. measure of No. 121.

No. 130. Dark Mauve paste.-2 lbs, whent flour, $1 \mathrm{lb}$. white British gum, 1 quart of water, $100 \mathrm{oz}$. measure of strong solution of mauve crystals in spirit; boil well, cool, and add 1 quart of No. 123.

No. 131. Medium shade Marve paste.-1 $1 \frac{1}{1 b}$. wheat flour, $3 \mathrm{lb}$. whito British gum, 1 quart of water, $60 \mathrm{oz}$. measuro mauve solution as No. 130 ; boil well, conl, and add 1 quart of No. 123.

No. 132. No. 20 Pale Mauve (for dark and palo mauvo),-2 quarts of No. 122, 2 quarts of No. $124,20 \mathrm{oz}$. measure maure solution as No. 130.

No. 133. No. 10 Pale Mauve (for a third shade).-2 quarts of No. 122, 2 quarts of No. 124, 10 or. measure mauve solution as No. 130 .

Mauves with glycerine, arsenions acid, and acetate-of-alumina fixing. In these colours there is no action until the goods are steamed, when arsenite of alunina is formed in an insoluble state in the eloth and the maure remains united with this mordant.

No. 134. Glycerine and arsenions-acid solution,-1 gallon of glycerine, 4 lbs. arsenious acid; boil well till dissolved.

No. 135. Acetate of ahmina. -1 gallon of water, $3 \mathrm{lbs}$. of alum, $2 \frac{1}{2} \mathrm{lbs}$. acetate-ofsoda crystals.

No. 136. Mauve-reducing paste. -2 gallons of water, 3 gallons of No. 135, 1 gallon of No. 122, 6 lbs. wheat stareh ; boil, cool, and add gradually 2 quarts of No. 134 .

No. 137. Dark Mauve. -5 lbs. starch, 5 quarts water, 3 quarts of No. 122, 1 quart of vinegar, $\frac{1}{4} \mathrm{lb}$. mauvo crystals; boil, cool, and add whon cooling 5 quarts of No. 135, and when cold $2 \frac{1}{2}$ pints No. 134 .

No. 138. - For pale or mediun shades, dilute No. 137 with No. 136 to the required shade. 
No. 139. Durk Cockineal Red. -1 gallon of cochineal liquor at $6^{\circ} \mathrm{T}$., $1 \frac{1}{2} \mathrm{lb}$. starch; boil, cool, and add $3 \mathrm{oz}$. muriato-of-tin erystals, $2 \mathrm{oz}$, oxalic acid.

No. 140. Scarlet. -5 lbs. starch, 3 gallons cochineal liquor at $7^{\circ}$ T., 2 quarts Persianberry liquor at $12^{\circ} \mathrm{T}$.; boil and add $\frac{3}{4} \mathrm{lb}$. binoxalate of potash, dissolve, and add when nearly cold $\frac{8}{4} \mathrm{lb}$. muriate-of-tin crystals, cool, and add 1 gallon of No. 141 .

No. 141. Peroxide-of-Tin Pulp. -6 gallons of water, $30 \mathrm{oz}$. measure strong ammonia liquor, $1 \frac{1}{4}$ quart oxychloride-of-tin solution at $120^{\circ} \mathrm{T}$., mix well, wash by decantation 3. times, and filtor to a stiff pulp.

No. 142. Drab.-1 gallon of water, $8 \mathrm{or}$. ultramarine, $4 \mathrm{oz}$. lamp-black, beat up fine, and add $1 \frac{1}{2}$ gallon of No. 124, $1 \frac{1}{2}$ gallon of No. 122 .

No. 143. Carbon Drab. $-8 \mathrm{lbs}$. lamp-black, 1 gallon of ncetic acid at $8^{\circ}$. T.; mix well together, and add 1 gallon of No. 124, and 1 gallon of No. 122.

No. 144. Ultramarine Blue with Lactarine, $-1 \frac{1}{3} \mathrm{lb}$. lactarine, $3 \frac{1}{6}$ pints water; $\mathrm{mix}$ well, and add $2 \frac{1}{5}$ oz. measure liquid ammonia specific gravity $\cdot 880,5 \mathrm{oz}$. measure caustic soda at $32^{\circ} \mathrm{T}$.; then having beaten up $3 \mathrm{lbs}$. ultramarine with $1 \frac{1}{4}$ pint water, mix with the lactarine solution.

No. 145. Ultramarine Blue with Gluten, -6 lbs. ultramarine, 5 quarts water; mix, and add gradually $3 \frac{1}{2}$ lbs. ground gluten; let it stand a few minutes, then add 1 quart caustic soda at $16^{\circ} \mathrm{T}$.; mix well, and let stand a few hours before using.

No. 146. (No. 4.) Ultramarine Blue with Albumen.-4 lbs. ultramarine, 1 quart of water, 1 quart of No. 122, 2 quarts of 123.

No. 147. (No. 3.) Ultramarine Blue. -As No. 146, but 3 lbs, ultramarine.

No. 148. (No. 2.) Ultramarine Blue.-As No. 146, but 2 lbs. ultramarine.

No. 149. Guignet's Green Standard,-13 lbs. Gujgnet's green in paste, 1 gallon of No. 150, grind in a mill till quite fine.

No. 150.3 lbs. Albumen-Solution.-1 gallon of water, $3 \mathrm{lbs}$, of blood-albumen, stir till dissolved.

No. 151. (No. 2.) Dark Green.-2 gallons of No. 149, 1 gallon No. 150.

No. 152. (No. 2.) Green. -1 gallon of No. 149, 2 gallons of No. 150.

No. 153. (No. 14.) Pale Green for Padding.-1 gallon of No. 149, 10 gallons of No. 150,4 gallons of water.

No. 154. Dark Brown.-26 oz. burnt sienna in pasto, $2 \frac{1}{2}$ quarts of No. 122, 1 pint of water, 2 quarts of No. 124.

No. 155. Medium Brown.-The same as with No. 154, but $12 \mathrm{oz}$. of burnt-sionna paste.

No. 156. Pale Brown for Padding. $-1 \frac{1}{4}$ oz. burnt-sienna paste, 3 pints of water, 1 pint of No. 22, 2 quarts of No. 124 .

No. 157. Orange. $-5 \mathrm{llss}$. pigment orange (dichromate of lead in paste), 2 quarts of No. 122,2 quarts of No. 124 .

No. 158. Drab. $-20 \mathrm{oz}$. of burnt-sienna paste, $14 \mathrm{oz}$. of ultramarine, 1 quart of water, 6 quarts of No. 122, 6 quarts of No. 124 .

- No. 159. Drab for Padding. -8 oz. of ultramarine, 8 oz. burnt-sienna paste, $16 \mathrm{oz}$. yellow ochre in paste, 7 gallons No. 124, 1 gallon of No. 122, 6 gallons of water.

No. 160. Black for Printing with Aniline Colours. -5 gallons of logwood liquor at $12^{\circ} \mathrm{T}$., 1 gallon of quercitron-bark liquor at $12^{\circ} \mathrm{T}$., $1+$ gallon of acetic acid at $8^{\circ} \mathrm{T}$. $14 \mathrm{lbs}$. starch, $3 \mathrm{lbs}$. delaine gum, boil, and add $1 \frac{1}{\mathrm{l}} \mathrm{lb}$. chlorate of potash dissolved in 5 quarts of hot water, cool, and add 4 quarts of No 161.

No. 161. Nitro-acetate of Chromium.-6 gallons of water, $12 \mathrm{lbs}$. of bichromato of potash, $16 \mathrm{lbs}$. sulphuric acid diluted with 3 quarts of water, add by degrees 2 lbs. hrown sugar, and when the effervescence has ceased, and $191 \mathrm{l}$ lbs. of nitrate of lead and $19 \frac{1}{2} \mathrm{lbs}$. of acetate of lead dissolved in 1 gallon of water, let settle, and use the clear liquor.

No. 162. Dark Red from Extract of Madder containing Acetate of Lime. -8 lbs. extract of madder, $4 \mathrm{lbs}$. acetic acid, $1 \frac{1}{4} \mathrm{lb}$. starch, boil in a pot immersed in a watorbath, when cold add th th of its bulk of No. 163.

No. 163. Acetate of Alumina.-1 gallon of water, 4 lbs. of alum, 4 lbs, acetate of lead, stir till dissolved, let settle, and use the clear liquor.

No. 164. Pale Reds from the above description of Extract of Mradder-4 1bs. of extract, 2 lbs. acetic acid, 6, 8, or 10 quarts of gum-gedda-solntion at 4 lbs. per gallon, 1 pint of No. 163.

No. 165. Dark Red from Extract of Madder not containing Acetate of Lime.-10 llss. extract of madder, 2 quarts of acetic acid at $12^{\circ} \mathrm{T}$., 6 quarts of starch paste at 2 lbs. per gallon, 1 pint of No. $122,1 \frac{1}{2}$ quart olive oil, $1 \frac{1}{4}$ quart of No. 163, 1 pint of acetate-of-lime solution at $28^{\circ} \mathrm{T}$.

No. 166. Reducing Paste for Pale Reds (Extract without Acetate of Lime).-7 pints of water, 1 pint of No 122, 1 pint of acetic acid, $2 \mathrm{lbs}$. stareh, boil and cool.

No. 167. Pale Reds from No. 165.-1 gallon of No. 165, 4 to 6 gallons of No. 166. 
No. 168. Dark Red with artificial Alizarine -1 lb. of No. 169, 7 lbs. wheat starch, 2 I lbs. acetic acid at $8^{\circ}$ T., $12 \mathrm{lbs}$ artificial slizarine of 10 per cent, strength, 5 quarts of acetate-of-lime solution at $18^{\circ} \mathrm{T}$., 2 gallons of water, 2 quarts olive oil, boil well, cool, and add $2 \frac{1}{\text { gallons of }}$ No. 163 reduced to $11^{\circ} \mathrm{T}$.

No 169. 1 gallon of wator, $1 \mathrm{lb}$. gum-tragacanth, boil till dissolved, and add water to make up to 1 gallon.

No. 170. Dark Red with artificial Alizarine,-1 quart of 10 per cent, alizarine, $15 \mathrm{oz}$. measure acetic acid $12^{\circ} \mathrm{T}$., $3 \frac{1}{2}$ pints of starch paste at 2 lbs. per gallon, $\frac{1}{2}$ pint of acetate-of-lime-solution at $20^{\circ} \mathrm{T}$., $12 \frac{1}{2} \mathrm{oz}$. measure of No. 163 , $\frac{1}{2}$ pint of olive oil.

No. 171. Pale Red from artificial Alizarine.-23 quarts of 10 per cent. alizarine of Meister, Lucius and Brünning, $1 \frac{1}{2}$ quart of acotic acid at $12^{\circ} \mathrm{T}$., $8 \frac{1}{2}$ quarts of starch paste at 2 lbs. per gallon, 1 quart of acetate-of-lime solution at $20^{\circ} \mathrm{T}$., $1 \frac{1}{4}$ quart of No. 168,11 quart of olive oil.

No. 172. Purple with artificial Alizarine. 2 lbs. of artificial alizarine as in No. 171, 1 pint acetic acid at $8^{\circ}$ T., $3 \frac{1}{2}$ quarts gum-gedda solution at 4 lbs. per gallon, $\frac{1}{2}$ pint of pyrolignite of iron liquor at $10^{\circ} \mathrm{T}$., $2 \frac{1}{2} \mathrm{oz}$. measure of acetate-of-lime solution at $20^{\circ} \mathrm{T}$.

Madder Extract Chocolate-M. Horace Koechlin discovered that oxide of chromium gave with madder extract a beautiful chocolate colour : an addition of solution of ncetate of chromium to any soiled madder-extract red colour left after printing, converts this latter into a very good chocolate, and is a good way of utilizing dirty colour, too valuable to throw away. A good chocolate may also be made by mixing madder-extract red with the chrome black No. 160, for instance 6 of old madderextract red and 1 of chrome black.

After printing, the pieces are hung up for some time to equalise their temperature, and are then steamed.

There are two methods of steaming now commonly employed-the column and the chest. The column is a hollow cylinder of copper, from 3 to 5 inches in diameter, and about 44 inches long, perforated over its whole surface with holes of about $\frac{1}{16}$ th of an inch, placed about $\frac{1}{4}$ of an inch asunder. A circular plate, about 9 inches diamoter, is soldered to the lower end of the column, destined to prevent the coil of cloth from sliding down off the cylinder. The lower end of the column terminates in a pipe, mounted with a stopeock for regulating the admission of steam from the main steam boiler of the factory. In some cases, the pipe fixed to the lower surface of the dise is made tapering, and fits into a conical socket, in a strong iron or copper box, fixed to a solid pedestal; the steam-pipe enters into one side of that box, and is provided, of course, with a stopcock. The condensed water of the column falls down into that chest, and may be let off by a descending tube and a stopcock. In other forms of the column, the conical junction pipe is at its top, and fits there into an inverted socket connected with a steam chest, while the bottom has a very small tubular outlet, so that the steam may be exposed to a certain pressure in the column when it is enceased with eloth.

The pieces are lapped round this column, but not in immediate contact with it; for the copper cylinder is first enveloped in a few coils of blanket stuff, then with several coils of white calico, next with the several pieces of the printed goods, stitched endwise, and lastly, with an outward mantle of white calico. In the course of the lapping and unlapping of such a length of webs, the cylinder is laid in a horizontal frame, in which it is made to revolre. In the act of steaming, however, it is fixed upright, by one of the methods above described. The stenming lasts for 20 or 30 minutes according to the nature of the dyes; those which contain much solution of tin admit of less steaming. Whenever the steam is shut off, the goods must be immediately uncoiled, to prevent the chance of any aqueous condensation. The unrolled pieces are free from damp, and require only to be exposed for a few minutes in the air to appear perfectly dry. Were water condensed during the process, it would be apt to make the colours run.

The other method of steaming, and the one now most generally employed, is that of the chest. This is an iron chamber generally now of cylindrical shape, as being the strongest form. This cylinder is about 10 feet long and 8 feet wide, fixed horizontally. It is closed at one end, the other is provided with strong closely-fitting folding doors, or with a strong door suspended from pullies, and balanced with a heavy weight. In either case the doors are fitted with iron screws, so that they can be screwed close to the cylinder and thus rendered steam-tight. Thero is a seeond roof insido the cylinder, which receives any drops of condonsed water, and carries them past the pieces at the sides. There is a perforated false bottom, underneath which is a perforated steam-pipe, laid from end to end of the chest; this pipe admits the steam, which is further diffused by the holes in the false bottom. On the false bottom is laid 
a pair of rails parallel with the sides of the chest; these rails are continued outside the chest into the room, the parts next the chest for about 3 feet being hinged so as to be moved on one side when the doors are opened or shut. Upon the rails moves a rectangular frame of iron which just fits inside the chest, and stands as high as the entrance to the chest will admit, generally about 6 feet high by 4 feet wide. This frame, when drawn out into the room, is filled with pieces in the following manner:They are first wound on an open reel, one by one, the selvages of each fold being kept as parallel as possible. The piece is then slid off the end of the reel, pulled flat, and a needle and thread passed through all the selvages of one side, and loops made, through which are passed wooden rods, which rest on the sides of the carriage, or they are wound on the reel and suspended on a thin wooden rod covered with calico, which rod is supported at the ends upon the sides of the wooden frame. Generally a grey piece is wound on the reel along with the printed piece on the face side, this is to prevent the colours marking off on the white parts. The piece being thus suspended with selvages downwards, the carriage being filled with the rods, is run into the chest,

\section{6}

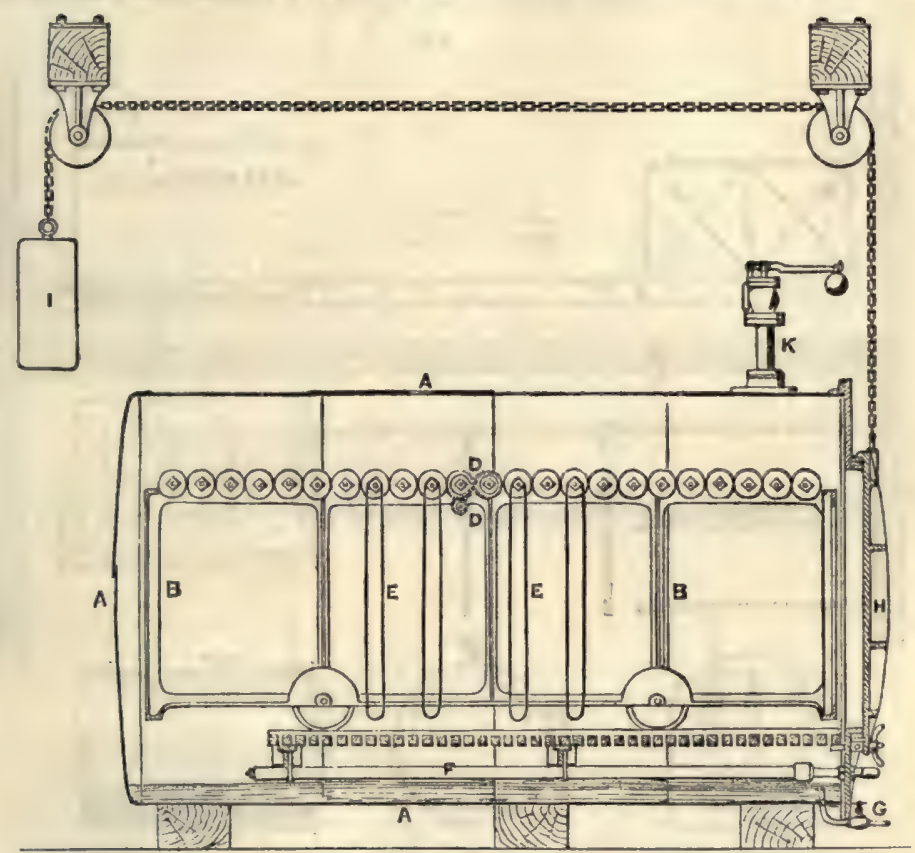

the doors firmly shut, and steam turned on, the steam escaping by a safety valve. They hang thus for $\mathbf{4 5}$ minutes, are taken out, unfolded, and loosely folded for washing off. They are next stitched end to end, and passed through a eistern with water, from that into a cistern containing a very weak solution of bichromate of potash; they are then put into a washing machine, hydro-extracted, starched, and dried.

Fig. 386 is a section of Mather and Platt's steaming chest: $\mathbf{\Lambda}$, the body; made cylindrical of wrought-iron plates; B, carriage fitted with square wooden rollers, all geared together by spur wheels, worked by a handle passing through a stuffing box on the outside of the cylinder; c, square wooden rollers; $\mathbf{D}$, handle and wheels; $\mathbf{E}$, cloth hanging on the rollers; $F$, perforated steam-pipe; $G$, tap and pipe for running off condensed water; $\mathbf{H}$, moveable door, arranged to be suspended by a chain, \&c. ; $\mathbf{T}$, balance weight for door; $\mathrm{K}$, safety valve. Fig. 387 , for which we are indebted to Mr. Furnival, shows a chest fitted with folding doors, tho arrangement of the interior roof, the stuffing-box and handle, and the railway arrangement for running the carriage in and out of the cylinder, and also the double line for allowing one carriage to be filled whilst another is in the steam-ehest, and thus avoid loss of time in flling and omptying. 
'The arrangement of moveable square wooden rollers is to allow of the roll of pieces plneed upon these rollers being ocensionally turned purt round, so that they may bo uniformly staamod, which would not bo the caso if the same part were always resting on the wooden roller.

Colours fixed by steaming are oither pigments insoluble in water, cemented to the cloth by the coagulation of the albumen or other nitrogenous vohicle with which they are mixed, or, as in the case of aniline colours, this cougulum becomes itself a mordant and at the time of coagulation and adherence to the fibre carrios with it the colour in chomical union; or, as in the case of dyewood and cochineal colours, these may be described as coloured lakes temporarily held in solution by acids, and during tho sterming, the cloth gradually withdraws these lakes from solution, the acid being either dissipated or 80 modified as to beincapable of holding the lakes dissolved. Tho state of the steam is an important matter. It is not the heat alone that produces tho effect; for it may easily bo demonstratod that heating cloth, when printed and dried, has no effect whatorer. The steam, to be effectire, must be as saturated with moisture as possible, and for this reason the stoaming apparatus should never be near the boiler: it is no disadvantage for the steam to travel a considerable distance before
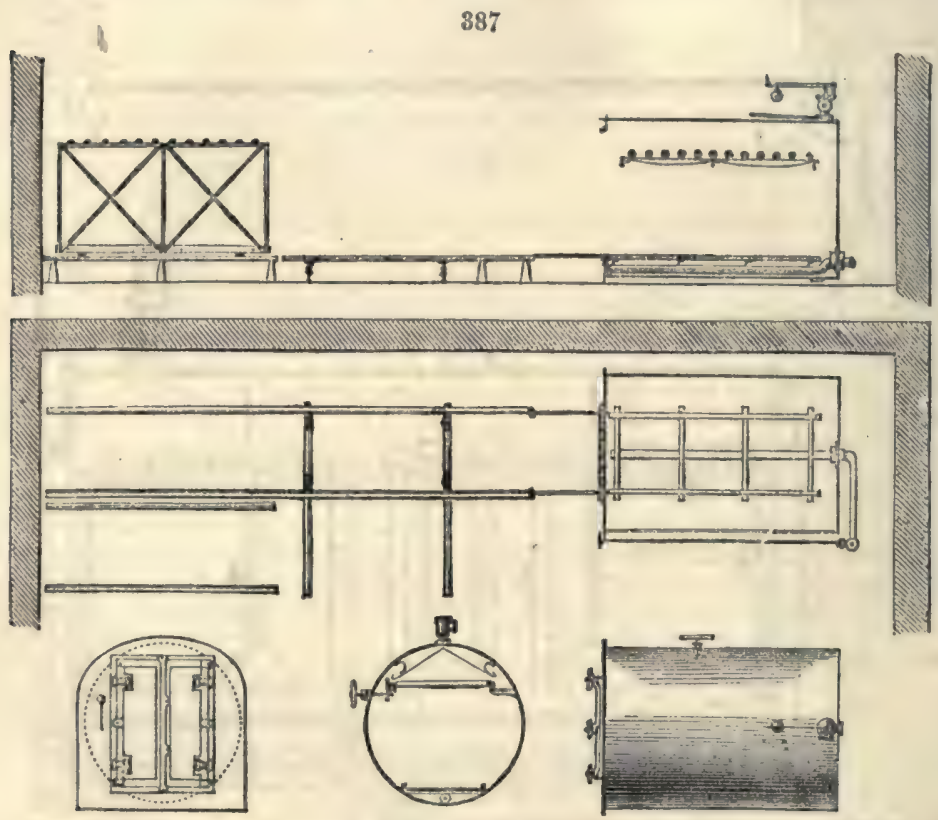

being applied. In some print-works the steam is made to pass through water in a vessel placed below the steam-chest, so that it arrives in the chest perfectly saturated with water. At the same time, the steam must not be of so low tension as to cause a deposit of moisture on the pieces, which would be very injurious, by causing the colours to run or mix. Steam-blue depends for its fixation on the decomposition of ferrocyanic acid by the high temperature and presence of vapour of water into white insoluble ferrocyanide of iron and potassium, which, by acquiring oxygen from the air or during the washing-off, becomes Prussian blue. The shade of it is much modified by the oxide of tin in the cloth and the prussiate of tin that forms part of the colour. It appears that tin substitutes iron, forming a compound ferrocyanide of tin and iron, or a ferro-stanno-cyanide of iron, which is of a deep violet-blue colour. Greens are mixtures of yellow lakes with the Prussian blue, formed by decomposition: In both these colours there is a large quantity of hydrocyanic acid disongaged during tho steaming; steam being decomposed, its hydrogen going to form hydrocyanic acid.

Madder-extract and alizarine colours require more care in steaming than ordinary dyewood colours. The steam-chest should be fitted with a manometor to indicate the pressure of the steam inside the chest. The steam at first should only be about 3 lbs. 


\section{CALICO-PRINTING}

pressure, and after ono hour the pressure increased, until at last a pressure of about 7 lbs. is given.

Mousseline-de-laines are treated somewhat in the same manner, the preparation of the cloth being different, and the colours are made in such a manner as to fix equally on both the wool and the cotton of the fabric. The steaming and washing-off are nearly the same as for calicoes. The following is the mothod in detail :-

The cloth is first well bleached and sulphured. This operation is usually performed by hanging the goods in a close stone or brick chamber. Trays of sulphur being lighted, the door is closed tight, and the pieces stay in the sulphurous gas for several hours, and are then removed and washed. An improvement on this method was patented by John Thom, and is here shown.
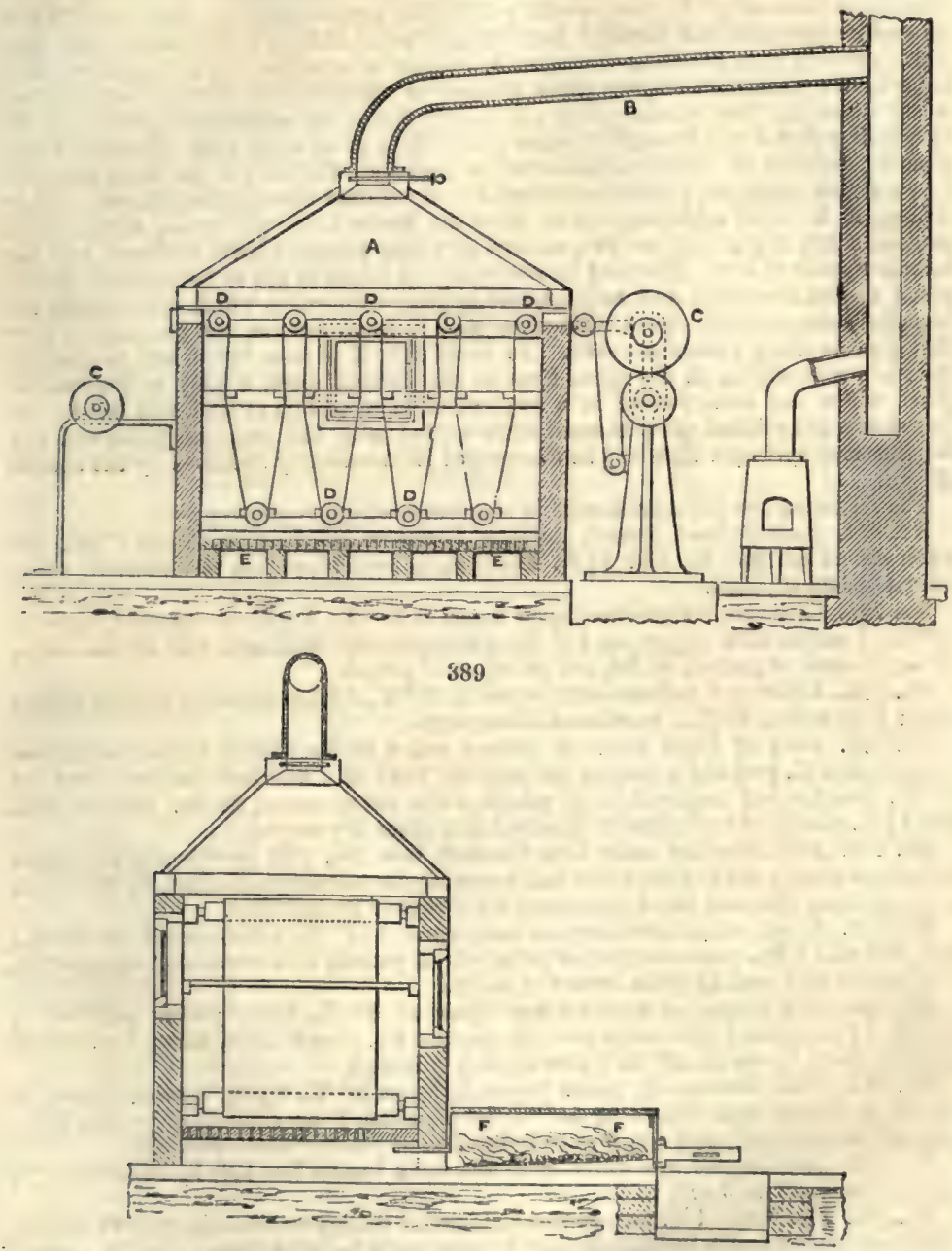

Thom's Sulphuring Apparatus.-Fig. 388. $\triangle$ is the roof, made of sheet lead, $4 \mathrm{lbs}$. to the foot. $B$ is a lead pipe, of one inch diameter, taking off the excess of sulphurous acid to the flue, $\mathrm{c}$ and $\mathrm{c}$ are rolls of pieces, going in.on one side and coming off at the other. $\mathrm{D}$ and $\mathrm{D}$, rollers of wood, three inches in diameter, with iron centres at the ends. $\mathbf{B}$ and $\mathbf{E}$, tiles, as in malt kilns, to let the gas pass up through to the cloth.

Fig. 389 shows the chamber: it is six feet in length by four foet in broadth, and about 
five feot high. There are two windowa; which are placed opposite each othor. $\mathbf{F}$ is a cast-iron tray for burning the sulphur. It is placed on a flag, inclining towards the chamber at about one inch to a foot. It is furnished with a slide, on which to put the sulphur to be pushed in, and to admit what air may be wanted. The space for air may be from half an inch to an inch wide. It costs $18 l$. to $20 l$.

Preparation.-Pad the pieces, previously well bleached in a wooden padding machine through stannate of soda at $10^{\circ}$ twice over, then pass through a cistorn with rollors, containing dilute sulphuric acid at $3^{\circ} \mathrm{T}$., wash gontly, and partially dry, then pad through sulphomuriate of tin at $4^{\circ} \mathrm{T}$. twice.

No 173. Sulphomuriate of Tin. -3 quarts muriate of tin at $120^{\circ} \mathrm{T}$., 1 quart sulphuric acid at $170^{\circ} \mathrm{T}$., mixed togethor gradually, and 4 quarts muriatic acid addod; reduce to $4^{\circ} \mathrm{T}$.

Run from this without washing into a large cistern with rollers, containing dilute chloride of lime at $\frac{1}{2}^{\circ} \mathrm{T}$, then wash, put in the hydro-extractor, and dry. When wanted for printing, pad through gum-Senegal water at $8 \mathrm{oz}$. to the gallon, and dry. After printing they are hung the same as calicoos to equalise the temperature, thon hung in the steam-chest in the same manner as calicoes, and steamed 45 minutes. After steaming, they are unrolled and loosely folded for washing-off, which is dono by wincing nvor a reel in a pit of water gently for $\frac{1}{4}$ of an hour, then transferred to a washing-machine or large automatic wince reel, and washed till no more coloured liquor comes away, then hydro-extracted, and dried over the steam cylinders. After drying, it is found advantageous to hang the pieces in a cool room, with covered shutter sides, for a day or two, so that they may imbibe a little moisture, and the colours appear richer. The wool in mousseline-de-laines is apt to be partially decomposed during steaming, and sulphuretted hydrogen liberated, which decomposes the metallic salts, such as nitrate of copper, used in some colours, and produces a very disagreeable effect, termed silvering. To avoid this, it is now customary to wind on the reel for steaming, at the same time as the printed piece, a grey or unbleached piece, which has been padded in a weak solution of acetate of lead, and dried. By this means the printed piece is steamed in contact with the prepared pieco, and any sulphuretted hydrogen that may be disengaged is immediately absorbed by the acotate of lead.

The following are the colours used in monsseline-de-laine printing :-

No. 174. Dark Red. -4 gallons cochineal liquor at $10^{\circ}$ T., 7 Ibs. starch; boil, and when cooled to $180^{\circ} \mathrm{F}$, add $1 \frac{1}{4} \mathrm{lb}$, oxalic acid, and when this is dissolved, $1 \frac{1}{4} \mathrm{lb}$. muriate-of-tin crystals.

No. 175. Chocolate. -6 gallons Sapan liquor at $12^{\circ} \mathrm{T}, 2$ gallons logwood liquor at $12^{\circ} \mathrm{T} ., 1$ gallon bark liquor at $12^{\circ} \mathrm{T}$., $16 \mathrm{lbs}$. starch; boil, and add $5 \frac{3}{4} \mathrm{lbs}$. alum, $12 \mathrm{oz}$. chlorate of potash, $4 \frac{1}{2} \mathrm{lbs}$. red prussiate of potash.

No. 176. Yellow. -4 gallons berry liquor at $10^{\circ} \mathrm{T}, 5_{4}^{\frac{1}{4}} \mathrm{lbs}$. starch, $1 \mathrm{lb}$, pele British gum; boil, and add $1 \frac{3}{4} \mathrm{lb}$. muriate-of-tin crystals.

No. 177. Dark or Royal Blue. -6 gallons water, $6 \frac{1}{2}$ lbs. starch, $2 \frac{1}{4}$ lbs. sal-ammoniac; boil well, and add 6 gallons tin pulp No. 103 ; mix well into the paste, and add $16 \mathrm{lbs}$. pounded yellow prussiate of potash, $8 \mathrm{lbs}$. red prussiate, $24 \mathrm{lbs}$, tartaric acid, and $1 \frac{1}{2}$ lb. oxalic acid previously dissolved in 4 pints hot water.

No. 178. Pale Blues are made from the dark blue No. 177, by reducing with gumsubstitute water; say 1 of dark blue and seven of gum-water for pale blue, for two blues, and 1 of dark blue and 14 of gum-water for blotch or ground blue.

No.179. Green. -4 gallons berry or bark liquor at $12^{\circ} \mathrm{T} ., 3 \mathrm{lbs}$. alum, 6 lbs. starch ; boil, and add $6 \mathrm{lbs}$. powdered yellow prussiate of potash, $1 \mathrm{lb}$. muriate-of-tin crystals, $1 \mathrm{lb}$. oxalic acid, and 23 pints extract of indigo.

No, 180. Pale Green.-3 quarts berry liquor at $6^{\circ} \mathrm{T}, 1 \frac{3}{4} 1 \mathrm{~b}$. yellow prussiate of potash, $9 \frac{1}{2}$ oz. alum, $\frac{3}{4}$ pint acetic acid, 16 quarts 4 lbs. gum-Senegal water, 8 oz. weight muriate-of-tin liquor at $12^{\circ} \mathrm{T} . \frac{3}{4}_{4}$ pint extract of indigo.

No. 181. Dark Brown.-21 quarts Sapan liquor at $8^{\circ} \mathrm{T}, 1$ pint logwood liquor at $12^{\circ}$ T., 5 quarts bark liquor at $10^{\circ}$ T., $12 \mathrm{oz}$. alum, 1 oz. chlorate of potash, $6 \mathrm{lbs}$. gum-substitute; boil, and add $4 \mathrm{oz}$. red prussiate of potash, $2 \mathrm{oz}$. oxalic acid.

No. 182. Pale Browns are made from the dark brown No. 181, by reducing with gum-water, say 1 to 3 or 1 to 5 .

No. 183. Pale Red.-1 lb. fine-ground cochineal, $1 \mathrm{lb}$. liquor ammonia, specific gravity 0.88 ; put in a jar with tight-fitting cover, which may be luted down; keop in a warm place for 48 hours, then mix with two gallons boiling water, and simmer in a mug down to 1 gallon, then strain off, wash the cochineal with a little water, and strain again; to the liquor made up to 1 gallon add $4 \mathrm{oz}$. alum, $4 \mathrm{oz}$. muriate-of-tin crystals, $4 \mathrm{oz}$. oxalic acid, and 1 gallon 6-1b. gum-Senegal wator.

No. 184. Scarlet. -2 gallons cochineal liquor at $12^{\circ}$ T., $4 \mathrm{lb}$. starch; boil, and add $4 \mathrm{oz}$. oxalic acid, $4 \mathrm{oz}$. binoxalate of potash, $8 \mathrm{oz}$, pink salts (double permuriate of tin and ammionis), and $8 \mathrm{oz}$. muriate-of-tin crystals. 
No. 185. Scarlet. -3 gallons standard No. 186, 1 quart berry liquor at $10^{\circ} \mathrm{T}$., $4 \frac{3}{4} \mathrm{lbs}$. starch ; boil, and add $8 \mathrm{oz}$. binoxalate of potash, $8 \mathrm{oz}$. oxalic acid, $1 \frac{1}{4} \mathrm{lb}$. pink valts, $\frac{1}{2}$ pint oxymuriate of tin at $120^{\circ} \mathrm{T}$.

No. 186. Standard.-2 lbs. fine-ground cochineal, 6 quarts water, 1 quart red liquor at $20^{\circ}$ T., $4 \mathrm{oz}$. nitric acid, $2 \mathrm{oz}$. oxalic acid ; boil 20 minutes, and strain off.

No. 187. Medium Blue. -6 gallons standard blue, No. 188, $1 \frac{1}{2}$ quart oxymuriate of tin at $120^{\circ} \mathrm{T}$., added gradually, and beaten fine, then $2 \frac{1}{4}$ quarts extract of indigo.

No. 188. Standard Blue.-10 lbs. yellow prussiate of potash, $3 \mathrm{lbs}$. alum, $2 \mathrm{lbs}$. oxalio acid, 4 gallons water, 4 gallons 6-lb. gum-water.

No. 189. Medium Green. -8 quarts berry liquor at $8^{\circ} \mathrm{T}$., 3 lbs. yellow prussiate of potash, $1 \frac{1}{2} \mathrm{lb}$. alum, 7 quarts 6 -lb. gum-water, 1 quart water, 1 quart acetic acid, 14 oz. weight muriate-of-tin liquor, 1 pint extract of indigo.

No. 190. Lilac. -8 quarts lavender liquor No. 191, 6 oz. oxalic acid, 2 oz. measure extract of indigo, 8 quarts $6 \mathrm{lbs}$. gum-Senegal water.

No. 191. Lavender Liquor. -2 gallons red liquor, $10 \mathrm{lbs}$. ground logwood; stoop 12 hours, and strain off.

No. 192. Dove. -6 quarts blue for doves, No. 193, 4 quarts lavender liquor No. 191, 8 quarts 6-lb. gum-Senegal water.

No. 193. Blue for Doves. -5 quarts water, 2 lbs. yellow prussiate of potash, 2 lbs. alum, 5 quarts 6 -lb. gum-water, 1 pint extract of indigo.

No. 194. Orange. -3 gallons berry liquor at $10^{\circ} \mathrm{T}$., $9 \mathrm{lbs}$. gum-Senegal, 3 pints red mordant No. 198, 12 oz. muriate-of-tin crystals ; boil 15 minutes.

No. 195. Drab Standard. - 6 quarts purple liquor No. 196, I quart bark liquor at $10^{\circ} \mathrm{T}$., $\frac{1}{2}$ pint red liquor at $20^{\circ} \mathrm{T}$., $\frac{1}{4}$ pint extract of indigo.

Drabs are made from this by reducing with gum-water, about 1 to 3 .

No. 196. Purple Iiguor. - 1 gallon lavender liquor No. 191, 3 oz. oxalic acid.

No. 197. Silver-drab Standard.-3 quarts gall liquor at $12^{\circ} \mathrm{T} ., 2$ quarts standard blue No. 188, 1 quart lavender liquor No. 191.

Colours reduced with gum-water from this, 1 to 2 or 3.

No. 198.-Red, Mordant.-1 gallon water, $6 \mathrm{lbs}$. alum, $3 \mathrm{lbs}$. white acetate of lead ; stir till dissolved, let settle and use the clear.

No. 199. Buff Standard.-1 quart cochineal liquor at $8^{\circ}$ T., 31 quarts berry liquor at $10^{\circ}$ T., 1 quart red mordant No. 198, $20 \mathrm{oz}$, oxalic acid.

Colours reduced from this with gum-water.

No. 200. Olive -1 quart lavender liquor No. 191,2 quarts berry liquor at $10^{\circ} \mathrm{T}$, 2 quarts 8-lb. gum-Senegal water.

\section{Aniline Colours for Mousseline-de-Laines.}

No. 201. Pale Magenta. -6 oz. mersure of solution of $4 \mathrm{oz}$. magenta crystals in 4 quarts methylated spirit, $3 \frac{2}{2}$ quarts of gum-gedda solution at $3 \mathrm{lbs}$. per gallon, 1 pint of 6-lbs, egg-albumen solution.

No. 202. Medium Magenta. - Same as No. 201, but 18 fluid ounces of magenta solution.

No. 203. Pale Violet. -6 oz. measure of Hofmann's Violet (spirituous solution), $3 \frac{1}{2}$ quarts of 3-lbs. gum-gedda solution, 1 pint of 6 -lb. blood-albumen solution.

No. 204. Medium Violet.-The same as No. 203, but $18 \mathrm{oz}$. fluid of Violot solution.

No. 205. Strong Solution. - 28 fluid oz. of Hofmann's Violet, $3 \frac{1}{2}$ quarts of water, 2 $1 \mathrm{lb}$. white British gum; boil, cool, add 1 pint of 6 -lb. blood-albumen solution.

No. 206. Strong Mauve. - $8 \mathrm{lbs}$. flour, 4 libs. white British gum, 10 quarts water, 5 quarts Mauve Standard; boil, cool, and add 6 quarts of 6-1b. blood-albumen solution.

No. 207. Mauve Standard.-6 oz. Brook, Simpson, and Spiller's R Violet, 2 quarts acetic acid, 2 quarts of water.

No. 208. Pale Mauve, - $1 \frac{1}{2}$ gallon of $6-1 \mathrm{~b}$. blood-albumen solution, $3 \frac{1}{2}$ gallons of gum-gedda solution, 30 fluid ounces of Mauve Standard.

No. 209. Iodine Green. -8 quarts of $6-1 \mathrm{lb}$. blood-albumen, 3 quarts of gum-gedda solution, $1 \frac{1}{2}$ pint of glycerine arsenicel solution No. 134, $1 \frac{1}{2}$ pint acetic acid, 1 quart

\section{- of iodine greon paste.}

In mousseline-de-laine printing success depends more on the bleaching and preparing of the cloth than in any other style. To Mr. John Mercer is due the merit of having effected an improvement in the preparation of woollen fabrics, the importance of which can hardly be overrated. Before his discovery of the power of prepared wool to absorb chlorine, mousseline-de-laines could only be effectively printed by block, which allows a large body of colour to bo laid on, and the fibre supersaturated with it. Machine colours were meagre and dull. But mousseline-de-laines prepared with tin, and then subjectod to the action of chlorine gas (as in the process given above, where the acid salt of tin remaining in the cloth disengages chlorine from the chloride Vor. I. 
of lime), have thoir power of absorbing and rotaining colour considerably enhaneed. Tho exact part tho chlorino plays is not well known, probably \& compound similar to the chloro-protein of Mulder is formed. The effect produced is not one, as might be supposed, of oxidation; but apparently a merely hoightened power of the wool to assinilate colouring matter. Wool subjected to chlorine without tin is much improved in its capacity for colour, but nothing liko the same when prepared with tin also. The whole of the chlorine may bo removed trom tho cloth by passing through an alkali, which renders it necessary to give the stannate-of-soda padding previously to the chlorinating. It may fairly be assumed that tho development of mousseline-de-laine printing by cylinder to the present perfection is due in a great measure to this chlorinating process. It ought also to be stated that, with rare liberality, Mr. Mercer gave the discovery to the trade, reserving for himself no right whatever.

\section{Ninth Style: Spirit Colours.}

Topical colours of great brilliancy, but possessed of rery little solidity, are made somewhat like steam colours, but with much larger proportions of 'spirits,' by which term is meant the metallic salts and acids, which, combining with the dye-stuff decoctions, give the peculiar tone and vivacity to these colours. These colours, from the largo adni"xture of these salts, are necessarily very acid, and cannot be steamed without the destruction of the cloth. They are merely gently dried after printing, nnd hung in the ageing room for several hours, then rinsed in water, washed, and dried.

The following are examples of spirit colours:-

No. 210. Black.-1 gallon logwood liquor at $8^{\circ} \mathrm{T}, 1$ gallon water, $10 \mathrm{oz}$. copperas, 3 lbs. starch; boil, and add $\frac{1}{2}$ pint pernitrate of iron at $80^{\circ} \cdot \mathrm{T}$.

No. 211. Pink. -1 gallon Sapan liquor at $8^{\circ}$ T., 1 gallon water, 2 lbs. common salt, $11 \frac{1}{\mathrm{lb}}$. starch; boil, cool, and add 1 pint oxymuriate of tin at $120^{\circ} \mathrm{T}$, $3 \mathrm{oz}$. measure nitrate of copper at $80^{\circ} \mathrm{T}$.

No. 212. Blue. -1 gallon water, $1 \mathrm{lb}$. yellow prussiate of potash, $6 \mathrm{oz}$ alum, $1 \frac{1}{\mathrm{a}} \mathrm{lb}$. starch; boil, and add $\frac{3}{4}$ pint nitrate of iron at $80^{\circ} \mathrm{T}$., $1 \frac{1}{2}$ gill oxymuriate of tin at $120^{\circ} \mathrm{T}$.

No. 213. Brown.-1 gallon berry liquor at $8^{\circ}$ T., 2 lbs. light British gum; boil, and add $1 \mathrm{lb}$. muriate-of-tin crystals, 2 quarts spirit pink No. 150, 2 quarts spirit purplo No. 214.

No. 214. Purple.-1 gallon logwood liquor at $8^{\circ} \mathrm{T}$., 1 gallon water, 10 oz. copperas, $2 \mathrm{lbs}$. starch; boil, and add 1 pint protomuriate of iron at $80^{\circ} \mathrm{T}$, I pint oxymuriato of tin at $120^{\circ} \mathrm{T}$.

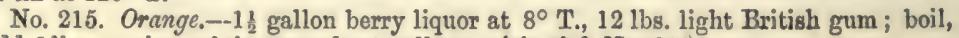
add 6 lbs. muriate-of-tin crystals, 4 gallons spirit pink No. 211 .

No. 216. Chocolate. $-2 \frac{1}{2}$ gallons spirit pink No. 211,1 gallon spirit blue No. 212.

No. 217. Red. -3 gallons Sapan liquor at $4^{\circ} \mathrm{T}$., $1 \mathrm{lb}$. sal-ammoniac, $1 \mathrm{lb}$. verdigris, $4 \frac{1}{2}$ lbs. starch ; boil, cool, and add 5 lbs. pink salts, $1 \mathrm{lb}$. oxalic acid.

No. 218. Yellow. - 1 gallon berry liquor at $10 \mathrm{~T}$., $\frac{1}{2} \mathrm{lb}$. alum, $1 \mathrm{lb}$. starch; boil, and add 1 pint of muriate-of-tin liquor at $120 \mathrm{~T}$.

No. 219. Green -1 gallon spirit blue No. 212, 1 gallon spirit yellow No. 218.

No. 220. Spirit Pink for Blocking Madder Work.-41 gallons Brazil-wood liquor at $108^{\circ}$ T., 9 lbs. pink salts, 3 lbs. sal-ammoniac, 2 lbs. sulphato of copper, $5 \frac{1}{4}$ oz. oxalic acid dissolved in 1 pint water, $4 \frac{1}{2}$ gallons of $6-1 \mathrm{~b}$. gum-Senegal water, $1 \frac{1}{8}$ quart $0 x y$ muriate of tin at $120^{\circ} \mathrm{T}$.

\section{Tenth Style: Bronzes.}

The cloth is padded in solution of sulphate of manganese, the strength of which determines the shade of brown produced; for a medium shade of brown, suitable for discharge colours, the liquor may be $80^{\circ} \mathrm{T}$.

After padding and drying, pad the pieces through caustic sode at $24^{\circ} \mathrm{T}$., and again through caustic soda at $12^{\circ} \mathrm{T}$, wince well in water, and then in solution of chlorido of lime at $2^{\circ} \mathrm{T}$. till perfectly brown; wash well in water, and dry.

The colours for printing on this dyed ground are so made as to dischargo the brown and substitute their own colour in place of it.

No. 221. Blue Discharge. - (a) 6 gallons water, $3 \frac{3}{3}$ lbs. yellow prussiate of potash, 10 lbs. starch, 6 lbs. light British gum; boil, and add 12 lbs. tartaric acid, 6 lbs. oxalic acid, $1 \frac{1}{4}$ quart pernitrate of iron : then take $(b) 5$ quarts of this standard, 3 quarts muriato of tin at $120^{\circ} \mathrm{T}$.

No. 222. Discharge Yellow for Chroming.-(a) 1 gallon water, 5 lbs, nitrate of load, 4 bs. light British gum; boil, and add 4 ibs. tartaric acid; then take $(b)$ s quarts this standard, 1 quart muriate of tin at $120^{\circ} \mathrm{T}$.

No. 223. Discharge Green. -2 quarts yellow standard No. 222 (a), 1 quart blue - ntandard No. 221 (a). 1 quart murinto of tin at $120^{\circ}$ 
No. 224. Discharge Pink.-(a) 2 gallons Brazil-wood liquor at $12^{\circ}$ T., 4 oz. sulphate of copper, 4 oz. sal-ammoniac, 4 lbs. starch ; boil, and add 8 oz. measur 3 oxy. muriate of tin at $120^{\circ} \mathrm{T}$. : then take (b) 2 quarts of this standard, 1 quart muriate of tin at $120^{\circ} \mathrm{T}$.

No. 225: White Discharge.-2 gallons water, 8 lbs. light British gum; boil, and add $8 \mathrm{lbs}$. tartaric acid, and 1 gallon muriate of tin at $120^{\circ} \mathrm{T}$.

Black.-Spirit black No. 210.

After printing, hang for a few hours, and wince in a pit with water freely flowing into it; then wince in chalky water, again in water, then wince in bichromate of potash at $4^{\circ} \mathrm{T}$., to raise the green and yellow; wash and dry.

The discharging agent in these colours is the protomuriate of tin, which, by its superior attraction for oxygen, robs the peroxide of manganese of a portion. "The protoxide of manganese formed by this change being then soluble in the acid, and subsequently washed away, the pigment Prussian blue and chromate of lead, also the Brazil lake, are left fixed in the discharged place.

Pencru Bloe.-Before the introduction of steam blues and the species of indigo blues tormed fast blues, the only blue that could be introduced into dyed prints was a solution of deoxidised indigo, dissolved in caustic alkali : this at flrst was applied by tho printer with a small flat bit of wood termed a pencil; he dipped this in the blue colour, and instantly applied it to the proper parts of the pattern, thus the colour arrived at the cloth before it had time to oxidise and the indigo to become insoluble. It is evident that this process was one of extreme clumsiness, as it requixed skill to apply the blne just to the proper places, and an apparatus for applying the blue, still called pencil blue, was at last devised. It consists of a copper case or box $A$, (fig. 390 ) in which is laid a frame $B$, filled with pretty stout canvas. The box communicates by a tube with the cistern C, mounted with a stopeock D. Fig. 391 represents the apparatus in plan: $\mathbf{A}$, the box; B the canvas; with its edges $a$ a $a$ a, fixed by pin-points to the sides. The coloured is teered, or spread even, with a wooden scraper as broad as the canvas. In working with this apparatus, the colour being contained in the vessel $\mathbf{c}$ is drawn off into the case $\mathrm{A}$, by opening the stopcock $\mathrm{D}$, till it rises to the level of the canvas. The instant before the printer daubs the block upon the canvas, the teerer, boy or girl, runs the scraper across it to renew its surface; and the printer immediately transfers the colour to the cloth. In this kind of printing great skill is required to give even impressions. As the blue is usually applied to somewhat large designs, it is very apt to run ; an inconvenience counteracted by dusting fine dry sand upon the cloth as soon as it is blocked. The goods must be washed within 24 hours after boing printed.

Pencil blue, before the introduction of China blues, was printed by cylinder with a doctor-box.

No. 226. Pencil Blue.-10 gallons of pulp of indigo, containing $40 \mathrm{lbs}$. indigo, $40 \mathrm{lbs}$. yellow orpiment, $11 \frac{1}{2}$ gallons of caustic soda at $70^{\circ} \mathrm{T}$., $18 \frac{1}{2}$ gallons of water, $4 \mathrm{lbs}$. lime; boil till quito yellow, when spread on glass; let settle and thicken the clear with 120 lbs. gru-Senegal.

Pieces printed in pencil blue are washed in water immediately after drying and sometimes soaped a little. Mr. Bennett Wooderoft, struck with the waste of indigo attending the printing of either China blue or pencil blue some few years ago invented and patented a method of printing pencil blue by the cylinder machine. His plan was to attach to an ordinary single-colour machine an India-rmbber apparatus which enveloped the colour-box and piece after printing: this apparatus was filled with coal-gas; a glass plate formed part of the long bag through which the piece travelled after printing, so as to enable tho printer to see the progress of his work. By this means tho deoxidised indigo was fairly applied to the eloth, and oxidation only ensued when the piece left the apparatus. The saving of indigo was said to be considerable, but the plan was not generally adopted.

Pencil blue is sometimes printed along with garancin mordants and dyed garancin. The blue withstands the dyeing and clearing. In America it is often thus printed.

SAFFLOWER DYeIse. - The beautiful but fugitivo colouring mattor of safflowor in 
applied in the printing for dyoing a self-colour, generally after the goods have boen printed in black and red mordant, or black alone, and dyed madder or garancin. It is commonly used for cotton velvets, the colour givon to velvot appearing very brilliant from the nature of the cloth. The process is as follows:- Safflower contains two distinct colouring matters; one yellow, being soluble in water; and the other pink, insoluble in water, the latter only being valuble. The yellow matter is therefore carefully washed sway. To effect this, the safflowor is put into canvas bags, $4 \mathrm{lbs}$ in a bag, and those bags put into running water and oceasionally trodden upon till the water runs off perioctly colourless from them. 12 of these bags are then emptied into a cask with 90 gallons of water and 10 quarts of pearlash liquor at $24^{\circ} \mathrm{T}$., stirred up for 2 hours after standing all night, drain off the liquor, add 90 gallons more water and 3 pints of pearlash liquor; stir up well, and after standing for three hours, drain off again; this weak liquor is saved for putting on fresh safflower: about 30 gallons of the safflower solution is put in a tub mounted with a wince over it, and a mixture of vinegar and lime-juice is added to it till it is feebly acid to test-paper. The carthamic acid, a red colouring matter of safflower, is thus precipitated, and romains as an exceedingly fine 7 wwder in suspension in the liquid; 2 pieces of 30 yards of velvet are put in and winced backwards and forwards five times, then wound upon the reel and allowed to stay there half an hour, then wince five times more, wind up again and let stay half an hour; wince again five times and wind up again; run off the liquor and put in 30 gallons of fresh liquor and acid as before; repeat the process wincing three times of fivo ends oach, and letting lie wound on the reel half an hour each time; then take out and wince in very dilute acetic acid, hydro-extract, and dry. The pieces when wound on the reel should be opened out flat or they might be uneven. Carthamic acid, being of a resinous nature, has the property of attaching itself to cloth, and dyoing it a beautiful pink like the petals of a rose : this dye is very fugitive, strong sunlight even being injurious to it. There has been no way yet discovered of making it permanent.

MURexide, - The purpurate of ammonia, or murexide, was discovered by Liebig and Wöhler in 1838, and in its pure state is one of the most beautiful products of chemistry. It is a crystalline substance of a beautiful metallic green, like the wings of the cantharides fly, and is produced when uric acid is dissolred in dilute nitric acid, the solution evaporated somewhat, and ammonia added; from the beautiful crimson liquid, murexide crystallises. This substance had, until a short time ago, no practical application. M. Albert Schlumberger discovered that metallic insoluble salts, possessing all the brilliancy of the original substance, could be made; and this fact was soon applied to a practical use by the French chemists, who succeeded in fixing a beautiful murexide crimson upon cotton cloth. The process was patented in this country for French interests in Fobruary 1857, and was for some time in extensive use. The process is as follows:-

Print in the colour.

No. 227. 1 gallon water, $4 \mathrm{lbs}$. nitrate of lead, $1 \mathrm{lb}$. murexide, $1 \frac{1}{2} \mathrm{lb}$. starch; boil. After printing, hang a few hours, then run through a cistern with rollers above and below, and provided with a cover, through apertures in which the pieces enter and leave. This cistern is kept supplied with ammoniacal gas; on leaving this cistern they pass into water, and from that into a cistern charged with $2 \mathrm{lbs}$. bichloride of mercury, $4 \mathrm{lbs}$. acetate of soda, $1 \mathrm{lb}$. acetic acid, 80 gallons water; run very slowly through this, wash and dry. In the first operation purpurate of load is formed on the cloth, and in the second, or changing bath, the lead is wholly or partly removed, and oxide of mercury left in its space; the resulting lake is a colour of great brilliancy and purity, so much so that few of the ordinary colours will bear to be looked at along with it. Though perfectly fast as to soap, it appears that strong sunlight is rather injurious to its pormanency.

This colour is now of historical interest only, aniline magenta having entirely rendered it obsolete.

A few outline illustrations of the varions maddor styles will render them more cloar.

1 a. Black, 2 reds, purple and browu on white ground. Print by machine in colours 4, 5, 6, 9, 27, (No. 12 shade) and 18 ; age 3 nights ; fly-dung at $160^{\circ} \mathrm{F}$., socond dung at $150^{\circ} \mathrm{F}$., wash and dye with French or Turkey madder, bringing to boil in $1 \frac{8}{4}$ hour, and boiling $\frac{1}{4}$ hour; wash and soap twice at $180^{\circ} \mathrm{F}$., wash; chloridoof-lime bath (see No. 1 plate purples), wash and dry.

1 b. Black, red, white and brown figures, covered in purple. Print in colours, $4,11,34$, and 18 ; when dry, cover with a fine pattorn in $27,(12$ shade); age 3 nights; fly dung at $170^{\circ} \mathrm{F}$., second dung at $160^{\circ} \mathrm{F}$.; wash, dyc, and clean as $1 \mathrm{a}$.

1 c. Print in colours 6,7 (No. 3 sbade), 34 ; dry and cover in $7,(6$ shade) and blotch (or pad with a rollor engraved with a pin, which has the effect of giving a uniform shade) in 7 (10 shade); age threo nights, and treat as described under the head Swiss Pinks.

1 d. Some printers prefer to mordant for Swiss pinks with alkaline mordants, con- 
sidering the composition of the colours to be a guarantes against their containing iron; in such case, they print in colours 31,32 , and 35 , covering in palor shades of 32 after dyeing; fly-dung with 3 cwts. cow-dung, 12 lbs. sal-ammoniac, 1,000 gallons waterat $110^{\circ} \mathrm{F}$.; second dung with $\frac{3}{4} \mathrm{cwt}$. cow-dung at $110^{\circ} 15$ minutes ; wash and dye as for $1 \mathrm{c}$. In this method of mordanting, the aluminate of soda that has escaped decomposition by the carbonic acid of the air is decomposed by the muriate of ammonia, and alumina precipitated on the cloth.

2 a. Black, chocolate, red, and brown on white ground. Print in colours 5, 13 (6 shade), 14, and 22 ; age 3 or 4 nights; fly-dung at $160^{\circ} \mathrm{F}$., second dung at $160^{\circ} \mathrm{F}$, and dye with chocolate garancin or garanceux (see p. 638).

$2 b$. Black, chocolate, red, and purple. Print as $2 b$, but dye with purple garancin (see p. 638).

3 a. For chintz work treat as $1 a$, then in the parts of the pattern meant for grounding-in, block the colours 118 yellow, 119 green, and 110. If the pattern is such as to admit of it, all these colours may be printed at once from one block, using the tobying sieve, p. 585; the colours, however, for this method must be thickened with gum; steam, \&c., as described for steam colours.

$3 b$. Black, 2 reds, blue, green, and yellow covered in drab, or other shades. Print in 4,6 , and 7 ; dye, \&ce, as $1 a$; block in colour 38 with a block which covers all the pattern, and also those portions which are intended for the steam colours: when this paste is dry, cover by machine in any of colours 40 to 47 , age 2 or 3 nights; fly-dung at $160^{\circ} \mathrm{F}$., second dung at $150^{\circ} \mathrm{F}$., and dye with bark, or bark and logwood or cover in colour 48, and dye madder and bark as dir: $g$ (p. 642) for chocolate; or cover in colour 49 or 51, and after drying and ageing, wincing in chalky water; or in any of colours 55,56 , or 57 , rinsing in carbonate of soda liquor at $5^{\circ} \mathrm{T}$. when dry. After obtaining the ground shade by any of these processes and drying, ground in by block colours 118, 119, and 110, steam, wash, and dry.

$3 \mathrm{c}$. For furniture hangings, which are generally printed in large groups of flowers, a very pretty pea-green ground is often blocked in as groundwork, which is mado and fixed as follows:-

No. 228. Pea-Green.-(a) Standard : 6 lbs. sulphate of copper, 1 gallon water, $4 \mathrm{lbs}$. brown acetate of lead; dissolve, let settle, and use the elear.-(b) Colour, 2 measures of standard, 1 measure of 7-1b. gum-Senegal solution.

After printing, age two nights, and pass through a cistern with roller, set with caustic potash liquor at $15^{\circ} \mathrm{T}$., which has $8 \mathrm{oz}$. per gallon of arsenious acid dissolved in it. The liquor should be heated to $110^{\circ} \mathrm{F}$; ; out of this wash and dry.

$3 d$. Instead of blocking-in steam blue and green, fast blue and green are introduced where the colours are required to be particularly permanent; colours 62 or 63 or both are blocked-in and raised as follows:-5 stone cisterns, each mounted with a hand reel, and containing about 200 gallons each, are set with carbonate-of-soda liquor, No. 1 at $7^{\circ}$ T., No. 2 at $6^{\circ}$ T., No. 3 at $5^{\circ}$ T., No. 4 at $4^{\circ}$ T., and No. 5 at $3^{\circ}$ T.; wince 10 times backwards and forwards in each pit, beginning with No. 1 , and ending with No. 5; wince in water and wash. The change that takes place here is similar to that in raising China blues. The indigo is maintained in a deoxidised state by the protoxide of tin formed, until it has fixed itself in the cloth by reoxidation in the air. Where fast green has been printed, the pieces are winced in bichromate-of-potash liquor at $4^{\circ} \mathrm{T}$. for 10 minutes, then washed and dried.

3 e. Black and purple and white with buff ground. Print in 4 and 27 (12 shade), age, dung, and dye, \&c., as directed for plate purples (p. 635); block over the pattern and portions of the unprinted part the paste No. 39 ; blotch with pad roller in No. 53 (6 shado), dry and raiso as follows :- Wince 14 minutes in caustic soda at $2^{\circ} \mathrm{T}$. at $110^{\circ} \mathrm{F}$., then wince the water till quite buff, then wince in 400 gallons water with 1 quart chloride of lime at $12^{\circ}$ T. 10 minutes; wash and dry.

\section{Silk-Printing.}

Silk, in its capacity for receiving colours, holds a medium place between cotton and wool. From its being an animal substance, it is difficult to obtain white grounds or objects after dyeing mordanted silk, the silk itself attracting colouring matter somewhat as a mordant. Proviously to printing silk, it is well scoured by boiling for 2 hours with $\frac{3}{4} \mathrm{lb}$. of soap to every pound of silk, then well washed and dried. For handkerchiofs, black, chocolate, and red mordants are printed, aged, and dunged off samo as for cottons, and dyod with madder or garancin, soapod, washed, and dried. Purples cannot be obtained on silk by mordanting and dyeing madder, the colour produced being a mixture of red and purple. All sorts of colours can be produced on silk by steam, the whites remaining brilliant. For steam colours, silk is mordanted with tin, by steeping 4 hours in a solution of sulphomusiate of tin at $2^{\circ} \mathrm{T}$., made by 
dissolving $1 \mathrm{lb}$. of muriate-of-tin erystals in water, and adding $1 \mathrm{lb}$. of sulphuric acid at $170^{\circ} \mathrm{T}$., and reducing to $2^{\circ} \mathrm{T}$. After stoeping, the silk is washed with water, and dried. The following aro specimens of steam colours for silk :-

No. 229. Black.-2 gallons logwood liquor at $8^{\circ} \mathrm{T} ., 1$ quart iron liquor at $10^{\circ} \mathrm{T}$., 1 lb. flour, 1lb. light British gum; boil, and add $6 \mathrm{oz}$. yollow prussiato of potash; cool, and add $2 \mathrm{oz}$. sulphate of copper, 1 pint muriate of iron at $80^{\circ} \mathrm{T}$., $\frac{1}{2}$ pint pernitrato of iron at $80^{\circ} \mathrm{T}$.

No. 280. Chocolate. -2 gallons of Sapan liquor at $12^{\circ} \mathrm{T}, 5$ quarts logwood liquor at $12^{\circ} \mathrm{T}, 1$ quart bark liquor at $16^{\circ} \mathrm{T} ., 2 \mathrm{lbs}$. alum, $1 \frac{1}{2} \mathrm{lb}$. sal-ammoniac, $14 \mathrm{lbs}$. gumSenegal.

No. 231. Red.-3 gallons of cochineal liquor at $4^{\circ} \mathrm{T} ., 1 \frac{1}{2}$ pint bark liquor at $12^{\circ} \mathrm{T}$., $3 \mathrm{lbs}$. starch; boil, then cool, and add $1 \mathrm{lb}$. oxalic acid, $1 \mathrm{lb}$. muriate-of-tin crystals.

No. 232. Yellow.-3 gallons of bark liquor at $16^{\circ} \mathrm{T}, 8 \mathrm{oz}$, alum, $3 \mathrm{oz}$. muriato-of-tin erystals, 8 oz. oxalic acid, 9 lbs. gum-Senegal.

No. 233. Green.-1 gallon of yellow, No. 232, $\frac{1}{2}$ pint extract of indigo, 2\% oz. measure of muriate of tin at $120^{\circ} \mathrm{T}$.

No. 234. Blue. -1 gallon of water, $1 \mathrm{lb}$. yellow prussiate of potash, $\frac{1}{4} \mathrm{lb}$. oxalie acid, $\frac{1}{2} \mathrm{lb}$. tartaric acid, $20 z$. sulphuric acid at $170^{\circ} \mathrm{T}$., 1 gallon of $6-1 \mathrm{~b}$. gum-Senegal water.

Aniline colours for silk do not require any fixing substance, such as albumen, the silk itsolf being capable of combining with such colours. A proliminary mordanting with muriate of tin, though not essential, improves the shades, and is given as follows :Wince the silk for one hour in muriate-of-tin solution at $11_{2}^{\circ} \mathrm{T}$, wince in water, and dry. The following colours will show the exceedingly simple nature of these preparations:-

No.235. Dark Magenta.-6 measures of 3-lb gum-Senegal solution, and 1 of Simpson's No. 2 Roseine.

No. 236. Pale Magenta. -5 measures of 3-1b. gum-Senegal solution, and 1 of Dark Magenta colour.

No. 237. Blotching Magenta,-8 measures of 3-1b. gum-Senegal solution, and 1 of No. 2 Roseine.

No. 238. Dark Primula.-20 measures of 3-1b. gum-Senegal solution, and one of Hofmann's Violot.

No. 239. Pale Primula.-4 measures of 3-1b. gum-Senegal solution, and one of Dark Primula colour.

Mauves are reduced the same way, but using Hofmann's Blue Violet Liquor.

Artificial gums will not do for silks, as thoy leave the silk stiff and harsh. Steam as usual for half an hour with rather a low pressure, wash and dry.

No. 240. Aniline Black.-This splendid and unique black was invented by the late Mr. John Lightfoot, of Accrington, in 1859, and patented in January 1863. This black, when developed on the cloth, is unaffected by light or soap. Strong acids merely turn it dark green, the black colour being restored in all its intensity by alkalis. Strong hypochlorite-of-lime solution turns the black into a chocolate or dark brown, but long washing with water restores the black, with scarcely any loss of strongth. It can be printed along with steam colours and madder colours, anci with lead oranges and yellows. There is no colour that can compare with it in fastness. Exposure to air containing sulphurous acid or sulphurotted hydrogen causes the black to turn dark green, but the black is immediately restored by a passage in soap solution. For the invention of this black Mr. Lightfoot received tho gold medal of the Société Industrielle of Mulhouse. The original colour was the following:-One gallon of starch paste at $1 \mathrm{lb}$. starch per gallon, $4 \mathrm{oz}$. chlorate of potash, $8 \mathrm{oz}$, aniline previously mixed with $8 \mathrm{oz}$. measure of muriatic acid of commerco; $4 \mathrm{oz}$, measure of chloride-of-copper solution at $88^{\circ} \mathrm{T}, 2 \mathrm{oz}$. of sal-ammoniac. It was soon found that from its great acidity and the action of the copper salt upon the stoel 'doctors' of the printing machine, this colour was too inconvenient for general use. H. Cordillot proposed in December 1863, a black made with ferricyanide of ammonium: which salt had, however, been previously mentioned in Lightfoot's specification; but the colour was dear, decomposed rapidly, required a high temperature for its development, and attacked the fibre of the cloth, inconvenionces which caused its speedy abandonment. Tho discovery of Charles Lauth, of Paris, that sulphide of copper used in placo of chloride of copper, whilst being nearly unacted upon by the other ingrodients of the colour until the printing had been performed, underwent speedy decomposition when on the cloth, and a soluble copper salt was formed by oxidation of tho sulphide by the chlorate of potash, gave the crowning impetus to this colour, and its uso became universal by printers. The action of the hydrochlorate of aniline upon the steel 'doctors' still proving an inconvenience, M. Camille Kochlin substituted tartrate of aniline for the hydrochlorate, adding chloride of ammonium, in order to decompose this tartrate when upon the cloth, and reproduce hydrochlorate of aniline, which salt is essential for the proper development of the black. The modified colour of C. Kochlin is still largely used in 
France, and the recipe for it will be found below. James Higgin patented subsequently the employment of salts of oxide of chromium, such as tungstate, arsoniate, \&c., mixed with an insoluble copper salt, such as arseniate or tungstate, hydrochlorate of aniline and chlorate of potash being used along with them. In this colour the chrome oxide acts as a carrier of oxygen, chromic acid being formed and instantly decomposed again by the aniline, some chloride of copper being also formed by the mutual reaction of the hydrochlorate of aniline, and the arseniate of copper further ussists in the reaction. This black has been and is largely used. There is still some action of this colour upon the 'doctor,' and there is a liability of tendering the cloth when printed in heavy masses. To prevent any action upon the steel 'doctors,' Higgin patented the use of the disulphocyanide of copper, a substitute for the sulphide of copper of Lauth, it having been found that under some conditions of printing the sulphide of copper was decomposed and a soluble copper salt formed in the furnishingbox of the printing machine, and even sometimes in the colour before being put in the furnishing-box. The disulphocyanide of copper is perfectly insoluble in hydrochloric acid, and, of course, equally so in the hydrochlorate of aniline; there is no soluble copper salt formed until the colour is printed and dried. This colour has been and is still very extensively used. The most recent improvement is that patented by Lightfoot in 1871. Chlorate of soda or ammonia is used instead of chlorate of potash and a much larger proportion of aniline is used than in former colours, this being not quite neutralised by hydrochloric acid, making, as the patentee says, a basic salt of. aniline; the chlorates of soda and ammonia being much more soluble than the potash salt, allows a larger quantity to be used. Sulphide of copper is the copper salt used in this black. The patenteo claims that no injurious action on the 'doctor' takes place in printing this colour, and that patterns of any size and body can be printed without tendering the fibre, also that the intensity of the black is so great owing to the large quantity of aniline used, that it resists the action of a sulphureous atmosphere like that of manufacturing towns longer than other blacks. Lightfoot proved by several experiments that the only metals which develope aniline black from a mixture of hydrochlorate of aniline and chlorate of potash are vanadium, copper, uranium, and iron. Their activity is in the order in which they are here placed. The black from iron salts is not nearly so good as that from copper, and practically a good black can only be made where a copper salt is employed. The exact reactions set up in the aniline-black colour have not been yet satisfactorily explained. M. Brandt, in a paper recently read before the Sociéte Industrielle of Mulhouse, asserts that there are two distinct blacks, one of them of a brownish shade and the other of a blue shade, and that a black of the best and most solid appearance is a mixture of the two blacks.

No. 241. C. Koechlin's Black. -20 lbs. of starch, 20 lbs. dark British gum, $2 \frac{1}{4}$ gallons of aniline, $2 \frac{1}{4}$ gallons of water, beat up well together, and add the following solution : 8 gallons of water, $11 \mathrm{lbs}$. chlorate of potash, $11 \mathrm{lbs}$. of sal-ammoniac; boil all well for $1 \frac{1}{2}$ hour and cool, then add 1 gallon of sulphide-of-copper paste. When about to print, add to every gallon of the above colour $22 \mathrm{oz}$. of tartaric acid, dissolved in 1 pint of hot water, it must be added gradually, and well stirred in. This is a very disagreeable colour to make, and a quantity of the aniline is boiled off. The sulphide of copper is made as follows:-

A. 2 quarts of caustic soda solution at $70^{\circ} \mathrm{T} ., 1 \mathrm{lb}$. of flour of sulphur, dissolve by constant stirring;

B. 9 gallons of water, $5 \frac{1}{2} \mathrm{lbs}$. sulphate of copper, heat to $170^{\circ} \mathrm{F}$.

Add A to B, stirring well, let sottle and wash by decantation 4 times, filter to 2 quarts of pasto.

No. 242. Higgin's Disulphocyanide-of-Copper Black.-1 gallon of water, 1 lb. wheat starch, $1 \mathrm{lb}$. dark British gum, 2 oz. of disulphocyanide paste, boil, and add $8 \mathrm{oz}$. chlorato of potash, cool, and add $1 \mathrm{lb}$. of hydrochlorate of aniline.

No. 243. Lightfoot's Chlorate-of-Soda Black.-1 gallon of chlorate paste, No. 244 $48 \mathrm{oz}$. measure basic hydrochlorate-of-aniline solution, No. 246,1 pint sulphide of copper

No. 244. Chlorate Paste. -10 gallons of water, 23 lbs. wheat stareh, $5 \frac{1}{2}$ lbs. salammoniac 4 lbs. chlorate of potash, 2 gallons chlorato-of-soda solution, No. 245, boil and cool.

No. 245. Chlorate-of-Soda Solution.-1 gallon of caustic soda solution at $70^{\circ} \mathrm{T} ., 13$ quarts of water in which has been dissolved $7 \frac{1}{4} \mathrm{lbs}$. tartaric acid, heat up to $170^{\circ} \mathrm{F}$., and add $12 \mathrm{lbs}$. chlorate of potash, dissolve, and add a solution of $7 \frac{1}{4} \mathrm{lbs}$. tartaric acid in $6 \frac{1}{2}$ quarts of water, stir till cold, filter and press the sediment with a weighted board, then take it off and mix with $1 \frac{1}{2}$ gallon of cold water, and filter and press again. Mix the filtrates, and set at $28^{\circ} \mathrm{T}$.

No. 246. Basio Bydrochlorate of Aniline, -8 measures of aniline and 6 of muriatic acid at $34^{\circ} \mathrm{T}$.

Aniline black unless basic should not be hard dried after printing, but only just enough 
to prevent the cosour from marking off on tho whito grounds. Tho piecos should not bo passed through the agoing machine, but hung up in a slightly moist atmosphero at about $70^{\circ} \mathrm{F}$., for about 24 hours; whon, if tho colour has assumod a dark myrtlogreen shado, almost black, tho goods aro ready for raising. If only black has boen printed, the goods are passed through milk of lime, or solution of carbonate of soda at $2 \mathrm{oz}$. per gallon, at $160^{\circ} \mathrm{F}$., washod and driod; or they may have a passage afterwards in weak hot solution of bichromato of potash, which gives a brownor shado of black; or they may be raised by a passage in boiling soap solution. If printod along with lead-orango (No. 64 a.), the process is as follows:-1st. Give a passage of half a minuto in a liquor composed of $2 \frac{1}{2}$ lbs. sulphate-of-soda crystals, and $3 \mathrm{oz}$. of bichromate of potash per gallon of wator at boil. 2nd. Wince in cold water. 3rd. Winco in a liquor composed of 100 gallons of boiling water, 1 gallon of bichromato of potash solution at $18^{\circ} \mathrm{T}, 24 \mathrm{oz}$. measure of caustic soda solution at $70^{\circ} \mathrm{T}$. Keop the goods in until the orange is well dyed, then wince in cold water and soap at $160^{\circ} \mathrm{F}$. for $\frac{1}{4}$ hour, in a soap solution containing $2 \mathrm{lbs}$. of soap to 100 gallons of water, thon wash and dry.

When aniline black is printed along with madder mordants, the ordinary dunging process raises the black at the same time as it prepares the mordants for dyeing.

Printed along with colours intended for steaming, a passago in ammonia vapour is given to the goods after ageing and before steaming. This is necessary in order to neutralize the acid in the black, which would tender the fibre, if not neutralised, This ammonia passage is given in a rectangular box, which is 7 feot long, 4 feet wide, and 6 feet deep; provided with rollers at the top and bottom; a lining of sheet lead for about 6 inches deep, is put in the bottom of tho box; a steam-pipe is placed along the bottom of the box, lengthwise, down the centre, a smallor pipo is placed along the steampipe, on the top of it; this pipe is perforated with smaller holes. A lid corers the whole of the box, except a slit through which the pieces enter, and another by which they leave after passing up and down over and undor tho rollors. Steam being turned on the pipe, liquid ammonia is allowed to run into tho small pipe from a reservoir outside, and to issue from the perforations, dropping on the steam-pipe. The pieces are now passed through, and are then ready for steaming.

The last operation in calico-printing is what is called the Finishing, which comprehends starching, drying, breadthening, calendering, measuring, and making-up. The starching is a very important operation, simple as it may appear, in consequence of the extremely varying requirements of the print buyors. A variety of starches are used, differing in the nature of the paste they give when boiled with water, some being gummy; and others firm and stiff, and at all degrees between the two. Compounded starches adapted to different styles, are made and sold by the manufacturing chemists.

Fig. 392 is an elevation and plan of a finishing room for calicoes, as arranged by Mather and Platt: A, circular boxes for receiving cloth; $\mathrm{B}$, wince for drawing cloth from the squeezers in the dye-house; c, opening wince for tho cloth from the boxes to the drying machine; D. drying machine; $\mathbf{E}$, chloring machine; $F$, steaming-box; $\theta$, water-mangle; machine; I, drying-machine; $\mathbf{M}$, engine and gearing for drying-machine; $\mathrm{N}$, engine and gearing for chloring arrangement $\mathrm{E}, \mathrm{F}, \mathrm{G}, \mathbf{H}$; $\mathrm{o}$, engine and gearing for starching arrangement $\mathbf{I}, \mathbf{x}, \mathbf{x}$;

Several details which cannot be shown in this plan, can also be but imperfectly described in words. The opening wince $\mathrm{c}$, is placed at a height of 30 to 40 feet above the cloth-box, the higher the better, the pieces arrive in the box $\Delta$ in the form of a rope; before any chloring or finishing can be done, this rope must be opened out, and the pieces presented to the drying-machine extended to the full breadth; this is done by a boy who sits on a platform near the wince, and the piece gradually untwisting during its passage from the box to the wince, is pulled flat out by the boy just before going over the wince c. Again the constant passago of the pieces over so many machines has pulled them lengthwise, and consequently diminished their breadth. To counteract this, and restore the pieces to somothing liko their usual breadth, a peculiar rollor, called a spreading roller, is fixed on every drying-machine. The 'spreading roller' is composed of brass or iron diagonal saw-tooth grooved plates or rails, each about 2 inches wide and $\frac{3}{8}$ ths inch thick, and of length according to necossity ; each of these rails has 2 small friction pullies at ono end, and 2 slide pieces in suitable places. The rails are mounted on the periphery of discs and cams supported in their centres by a fixed shaft; the discs are prepared with grooves to correspond with the 2 slides in each rail, and work on the shaft by the pull of the cloth. The cams are fixed at a variable angle, and give a throw causing a longitudinal motion of the rails, to open out the cloth whilst passing over. The cloth enters into contact with the spreader when the rails are closed and leaves it at the opposite side, when they 
CALICO-PRINTING

392
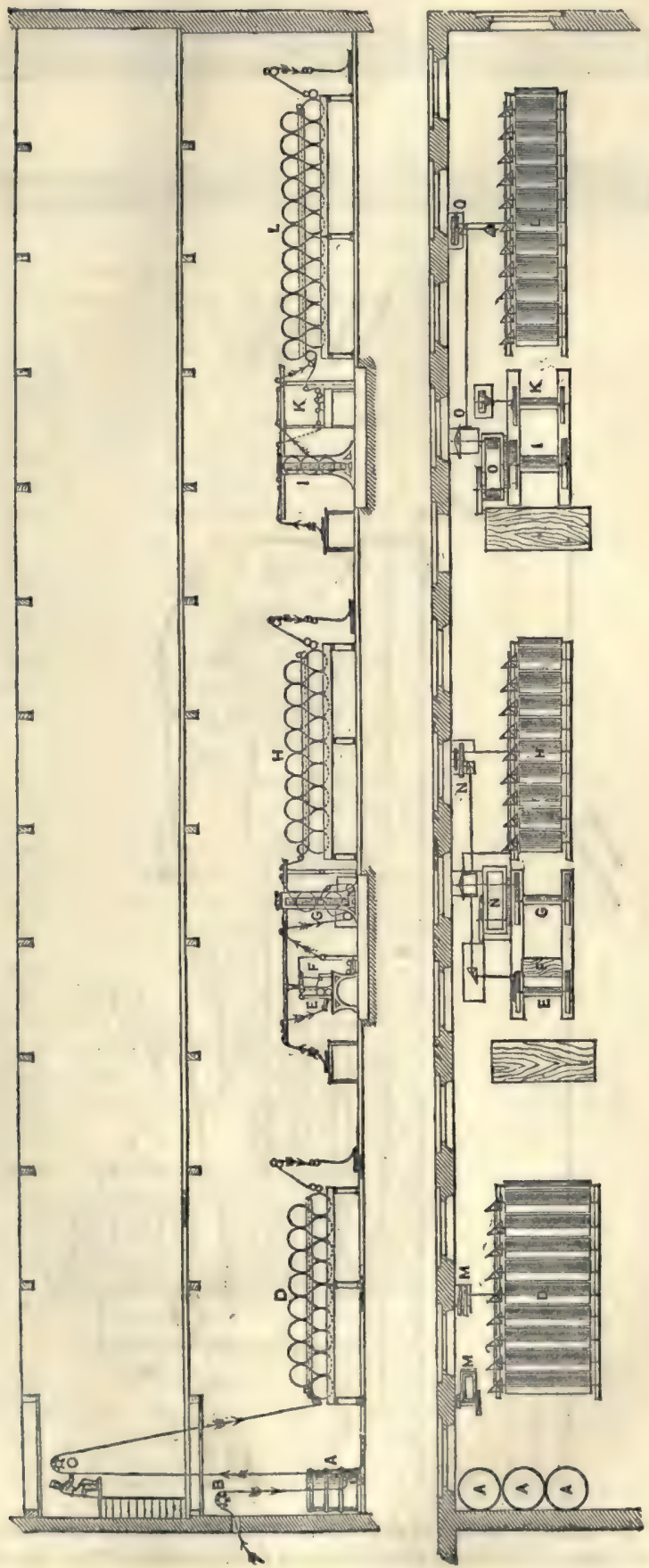
are widest apart or expanded longitudinally. Two rails compose one width as per figs. 393 and 394 , which aro shown closed and open, having beon moved right and left

393

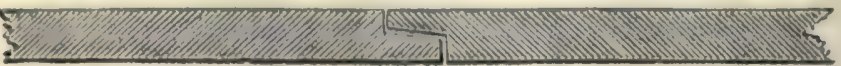

391
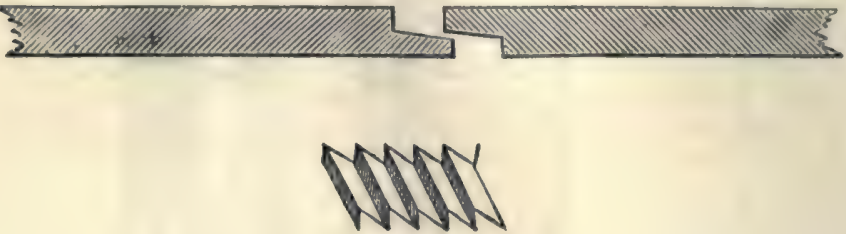

395

396

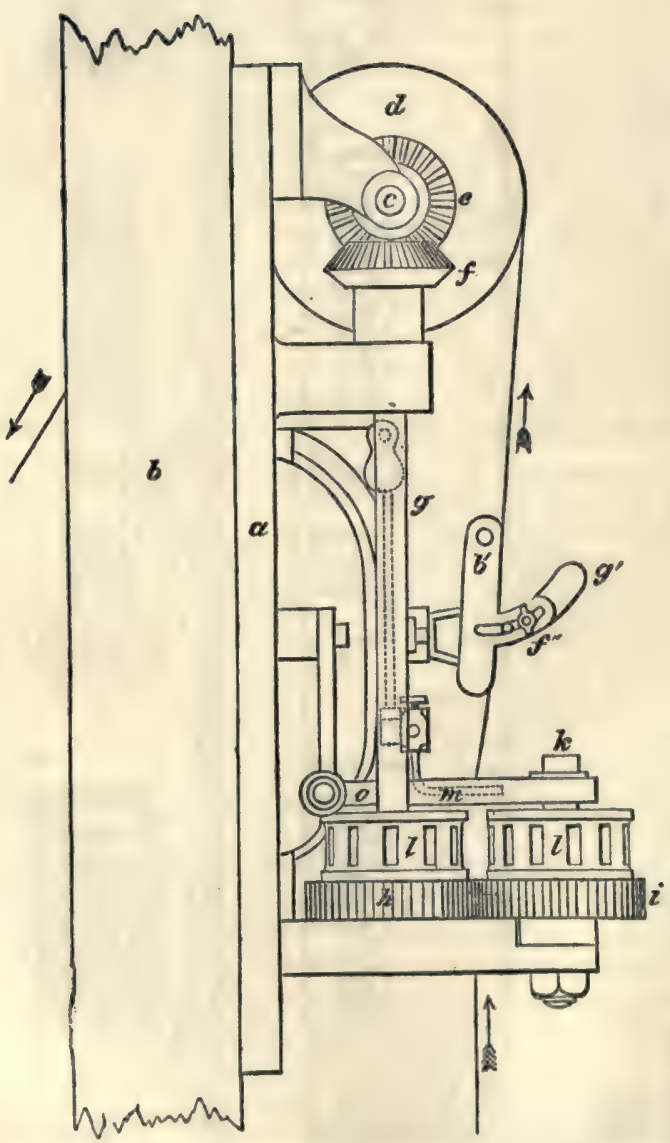

by means of the excentric motion of the cams. Fig. 395 shows the saw teeth, diagonal groores, of the bars. The bars move each about 1 inch outwards from the centre, and 
pull out the cloth to an extent of 2 inehes in the width. An opening machine, patented by William Birch of Salford in 1871, is now used by many calico-printers and bleachers. A detsiled description of this machine would involve too many drawings for the size and aim of this work: a general idea alone of it can be here given. Fig. 396 is an end elevation, and fig. $397 \mathrm{a}$ plan of part of the machine. The side frames $a$ of the machine are bolted to upright beams $b$, or other framework at the upper part of the building or other required place, and the fabric to be acted upon is drawn through the machine from vats, receptacles, or other places at the lower part of the building, by its being connected to the drying, folding, or other machine to which they require to go. In bearings at the top of the frames $a$ there is a shaft $c$ earrying a rollor $d$ and bevel wheels $e$, which gear into other bevel wheols $f$ on shafts $g$, to which are fixed spur-wheols $h$ goaring into spurwheels $i$ on stationary studs $k$. To the spur-wheels are cast or fixed chrin-wheels $l$, and on studs at the back there are chain-wheels $m$, and on cranked levers $n, 0$, chain-wheels $p$, the lovers working on the stationary studs $k$. On the several pairs of chainwheels there are two pairs of endless bands $q$, having external projections $r$ and internal projections e, the latter gearing with the chain-wheels, and between these ondloss bands the fabric to be acted upon is passed, and in order to obtain sufficient grip the external projections on one of each pair of bands passes between those on the other, and to prevent the bends from bulging inwards there are plates $t$, one plate at each side, being connected to the arm $n$, and the other plate at each side to a bracket $u$ connected to one of the cross frames.

When the fabric has to be opened, spread, and guided, it is passed between the endless bands and the single and double corrugated bars, and over the roller $d$ to the machine required, the central corrugated bar having been raised, to allow the passage of the fabric, the bolts being withdrawn from the slote for the purpose; but when the connections are complete the central bar is turned down and afterwards bolted. When the machine to which the fabric is connected is set to work, the fabric in its passage turns the roller $d$, which gives revolving motion to the gearing and horizontal travelling motions to the endless bands from the centre to the outside where they are in contact with the fabric, and these outward motions of the bands open and spread out the fabric.

The machine has also a very ingenious self-acting arrangement for keeping the cloth from swerving to either side during its passage through; but a description of it would be too elaborate for this work. Every machine saves the labour of a boy or girl. The drying-machine will be found described in the article BLEAchrsa. To extend the pieces to their utmost width a machine called a breadthening machine is employed. Fig. 398 is Mather and Platt's belt-stretching machine: $\mathrm{A}$ is the framing; B, moveable frame, supporting the breadthening pullies and belts, regulated for breadthening by вcrews; c, hollow bars, on which frame B slides, and through which the screws for regulating work; $D$, breadthening pullies; $\mathbf{x}$, tightening pullies for endless belts; $T$, endless belt, which works half round

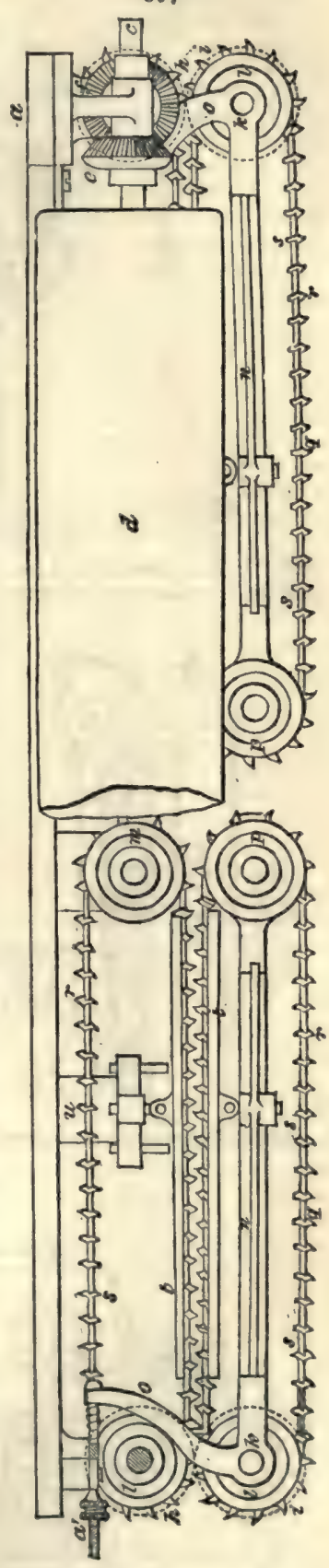
pulloy D and pullies $\mathrm{B}_{3}$ and between the pulley D and the belt, the cloth is hold 


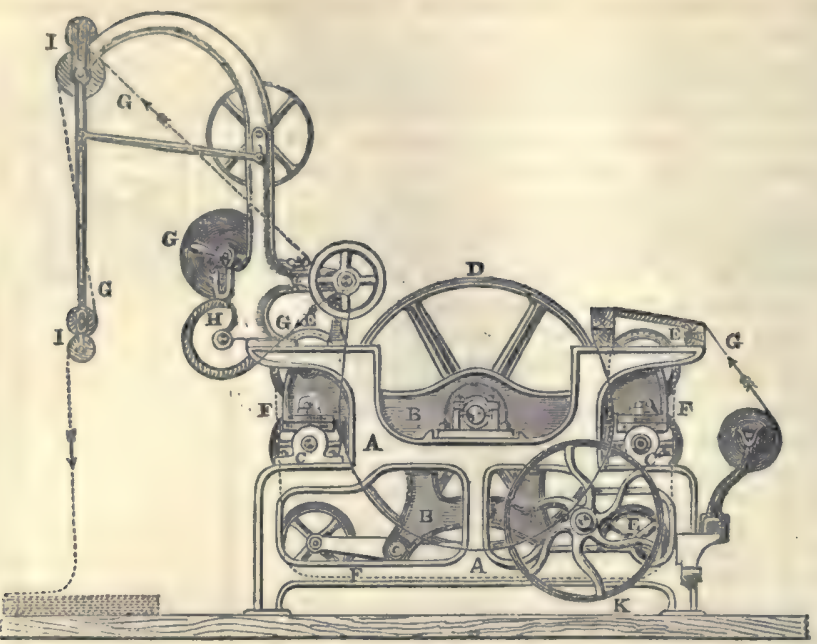

while being breadthened; $\mathrm{G}$, cloth passing through the machine; $\mathbf{~}$, batching apparatus; I, plaiting apparatus, the machine being prepared to either plait or batch;

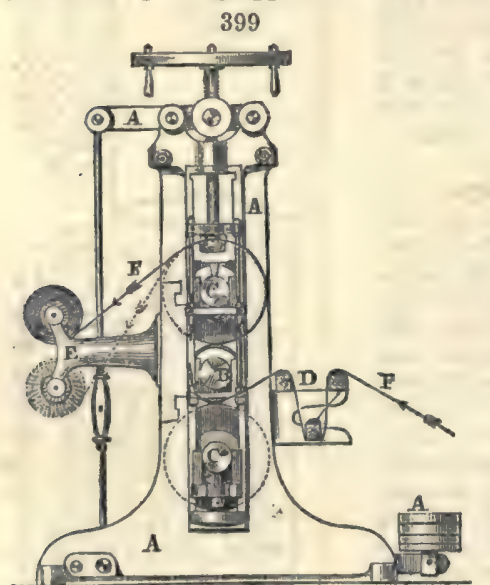
$\mathrm{x}$, driving pullies. For some deseriptions of goods such as furniture prints, a glazing calender is employed. Fig. 399 is an elevation of Mather and Platt's calender; $\Delta$, the framing with levers, scrows, \&ce; $B$, metal bowl heated by steam or gas; c, paper or cotton bowls ; $D$, entering rails, \&ce.; $\mathrm{x}$, batching apparatus; $\boldsymbol{r}$, cloth passing through the machine. The friction of the heatod motal bowl against the cloth, pressing against the largar bowls gives a glaze to the cloth. Pieces have now to be made up in folds of nniform length, generally about a yard. This operation is done by girls hooking one selvage of a piece backwards and forwards on long stoel hooks, fixed on a wooden frame, standing about 4 feet 6 inches from the ground; these hooks axe moveable, so as to give various lengths of fold as required. Plaiting and

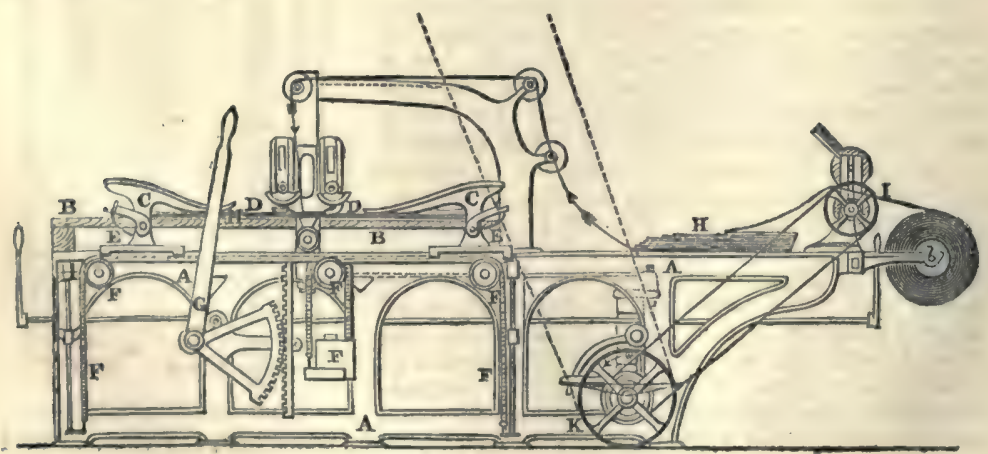


measuring machines have been introducod to economise labour. Fig. 400 is Knowles and Hayward's plaiting machine, of which Mather and Platt are the sole makers. It will plait goods of any quality or material, from the heaviest drill to the finest muslin, however highly glazed, laying them in folds of uniform length, which can be varied as required: $\mathrm{A}$ is the framing of the machine; $\mathrm{B}$, fixed table on which the cloth is plaited; c, cams for lifting the folding blade, to which are also attached the cards for holding cloth, rising as the thickness of the folds increases; $\mathrm{D}$, folding knives, moving longitudinally to make the fold, and vertically as the thickness of the folds increases on the table; $\mathbf{z}$, spring for relieving the pressure of the cards on the cloth whilst it is being passed under the cards which hold it on the return of the blade; $x$, rack motion and compensating chains and weights for cams, cards, and folding blades; $G$, levers and quadrant raising the cams, cards, \&c., when taking out cloth from machine; $\mathbf{H}$, cloth being folded; $\mathrm{I}$, draw rollers to give equal and regular delivery of cloth to folding blades; $\mathrm{x}$, driving-pulley, \&c.

The next step is to fold these lengths into three, and pile the folded pieces in an hydraulic press, where they are pressed, and are then ready for packing and delivery.

Fig. 401 is a plan of a modern print works, erected by Mather and Platt ; $\Lambda^{\prime}$, the singe house; $\mathbf{B}^{\prime}$, grey room; $c^{\prime}$, bleach house. The second story contains the dryingmachine after bleaching; $\mathbf{D}^{\prime}$, white room, the second story being the white store room; $\mathrm{s}^{\prime}$, printing room, above this is the ageing machine and ageing room; $\boldsymbol{x}^{\prime}$, colour house; $\mathbf{G}^{\prime}$, finishing room, above, the drying-machine after the dyeing, \&c.; $\mathrm{H}^{\prime}$, dye house, single story ; $\mathrm{I}^{\prime}$, boiler house; A, singe stove ; B, mixing cisterns for the kiers ; C, kiers ; D, mixing cisterns for chloride of lime and sours ; $\mathrm{E}$, washing machines and engine; $F$, squeezers and engine; $G$, chloring and souring machines and engine; $\mathrm{H}$, white squeezer and engine; 1 ,piling winces; $\mathbb{X}$, printing machines; $x$,colour pans; $\mathrm{x}$, chloring, water mangle, drying-machine, and engine; $\mathrm{s}$, starch mangle, and drying-machine; 0 , finishing calender and engine; $P$, friction calender and engine; $Q$, damping machine; $\mathbb{R}$, making-up tables ; $s$, first dunging ; $T$, second dunging; $v$, dyeing; $v$, soaping; $\mathrm{w}$, washing machines and engines; $\mathbf{x}$, squeezers and engines; $\mathbf{x}$, boilers.

Since 1840 there are no data as to the number of printers in Great Britain; but Mr. John Graham, in an unpublished 'History of the Lancashire Printers, gives a table, which he was at considerable care to compile from perfectly trustworthy sources, showing that in the Lancashire district, which includes also the contiguons counties, there were, in 1846, 128 firms employing-

540 cylinder machines.

39 perrotines.

7187 block tables.

The producing power of the Lancashire district having thus been doubled in 6 years.

Several printing firms, both in England and Scotland, have since that period much enlarged their powers of production. There aro 
many who manufacture 10,000 piecos of printod cloth por weok; and there are four coucerns of great magnitude whose united production at the present time probably does not fall short of four millions of pieces per annum, or nearly $\frac{1}{5}$ th of the total quantity printed.

Calico, and other printing has, since the repeal of the duty, risen steadily in importance, till it is now one of the most influontial manufactures of Great Britain. From a table compiled by the late Mr. Binyon, and communicated by Mr. John Graham, there were in 1840 :-

\begin{tabular}{|c|c|c|c|c|c|c|c|c|}
\hline 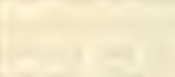 & & Firms & & $\begin{array}{l}\text { Cylinder } \\
\text { Machines }\end{array}$ & $\begin{array}{l}\text { Surface } \\
\text { Machiness }\end{array}$ & $\begin{array}{c}\text { Flnt } \\
\text { Presses }\end{array}$ & \begin{tabular}{|c} 
Discharg- \\
ing \\
Presseg
\end{tabular} & $\begin{array}{l}\text { Block } \\
\text { Tables }\end{array}$ \\
\hline $\begin{array}{l}\text { In Lancashire } \\
\text { "Scotland } \\
\text { "Ireland } \\
\text { " America }\end{array}$ & . & $\begin{array}{r}93 \\
70 \\
3 \\
47\end{array}$ & \} & $\begin{array}{r}247 \\
75 \\
13 \\
106\end{array}$ & $\begin{array}{l}\ldots \\
\cdots \\
5 \\
\cdots\end{array}$ & $\begin{array}{r}\ldots 2 \\
1 \\
\ldots\end{array}$ & $\begin{array}{l}\ldots \ddot{124} \\
\ldots \\
\ldots\end{array}$ & $\begin{array}{r}4355 \\
4997 \\
308 \\
834\end{array}$ \\
\hline
\end{tabular}

The following estimate of the exports of printed goods is from Mr. Potter's Lecture before the Society. of Arts, as reporter for printed fabrics exhibited in the Exhibition of 1851 :-

- In reference to the exports of printed goods, our information is rather obscure, owing to their being classed with dyed goods of all kinds. After considerable thought and calculation, I have ventured to estimate them for $185 \mathrm{I}$, at 15,544,000 pieces, or rather more than three-fourths of our entire production. These goods are, however, many of them of the cheap and more staple class of prints, or slight goods suited to warm climates, and for markets where cheapness is the great recommondation. In value, I should be disposed to estimate our export of printed goods at $5,775,000 l$.

'In reference to the entire export of manufactured cotton goods (exclusive of yarns), it may be assumed that out of $23,447,103 l$, given as the export of 1851 , about onefourth may be placed to the account for the print trade. I have endeavoured to estimate, from the Table of Exports for 1851, the probable quantity of low priced prints we export, and should be disposed to class them as follows :-

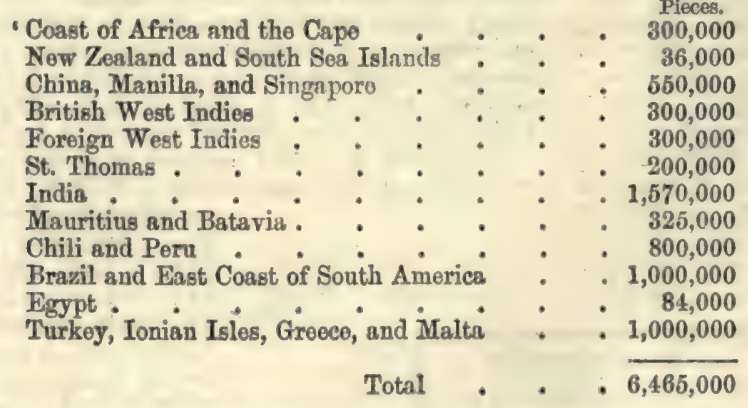

'I find those countries which take our lowest description of goods, and where the duties are chiefly very light-our Colonies, India and China-receive from us about 61 millions of pieces, or about 40 per cent. of our export in printed goods. A great proportion of the finer part of our exports, perhaps three-fourths, are very seriousily taxed, either for protection, as in the United States, the Zollverein, and Belgium, or for revenue, as in Brazil and the other South American markets. A part, however, of these better goods find their way into consumption in Canada, Turkey, tho Ionian Isles, Egypt, \&c., subject to very modorate dutios.'-(Potter.)

'The home-consumption,' says Mr. Potter, 'I estimate at 4,500,000; the excise returns for 1830 gave it as $2,281,512$ pieces. The repeal of the duty, and the decrease in the cost of production, giving the consumer goods in much better taste and ralue at one-half the price, have greatly tended to this increase.' 'The immense increase of production in lower goods has not decreased the taste in the higher in this country, though it may have caused it to mako loss apparont progress than when the larger part 
of the supply was of fine goods. We find specimens of good taste on the lowest material, printed at the lowest possible price for export, showing a taste superior to that in use for our best work twenty years ago, employing greater talent in design, greater skill in engraving - the cost of production cheap, because repaid by the quantity produced. This diffusion of art and of a better tasto cannot be otherwise than beneficial, even to the higher class of productions, as preparing a taste and demand for them in countries where high price would never have given prints any admission. The improvement of the lower cannot militate against that of the higher, either in the moral, intellectual, or artistic world. The productions of the highest class of French goods of to-day, whether furniture or dresses, are superior in taste and execution to those of any former period. The productions of the first-class printers of Great Britain maintain an equal advance, and are superior in taste and execution, in every respect, to those of former years. Great competition and rapidity of production are not immediately beneficial to high finish and execution in art; but this tendency to quickness of production, rather than perfection, rectifies itself; and machinery, which perhaps at first does not give the polish that excessive labour formerly supplied, ultimately exceeds it by its cheaper and more regular application. It is remarkable how taste or novelty in that class of demand, which would strike the casual observer as the one fitted for its greatest development, is limited in quantity. The limit or commencing point, in which taste or novelty enters freely into the composition of a print, is for the supply of the working and middle classes of society. They require it quict, modest, and useful; and any deviation, for the sake of novelty, which calls in the aid of the brighter and less permanent colour, quickly checks itself. The sober careful classes of society cling to an inoffensive taste, which will not look obsolete and extravagant after the lapse of such a time as would render a garment comparatively tasteless and unfashionable in a higher class. This trade is, to the printers, most extensive and valuable, and has its necessary and practical bearing on his taste; and hence it is in this branch of the business the English printer is most decidedly superior to his Erench competitors.'

The returns given in the 'Annual Statement of the Trade of the United Kingdom' for our Export of Piece Goods Printed in 1872 is as follows:-

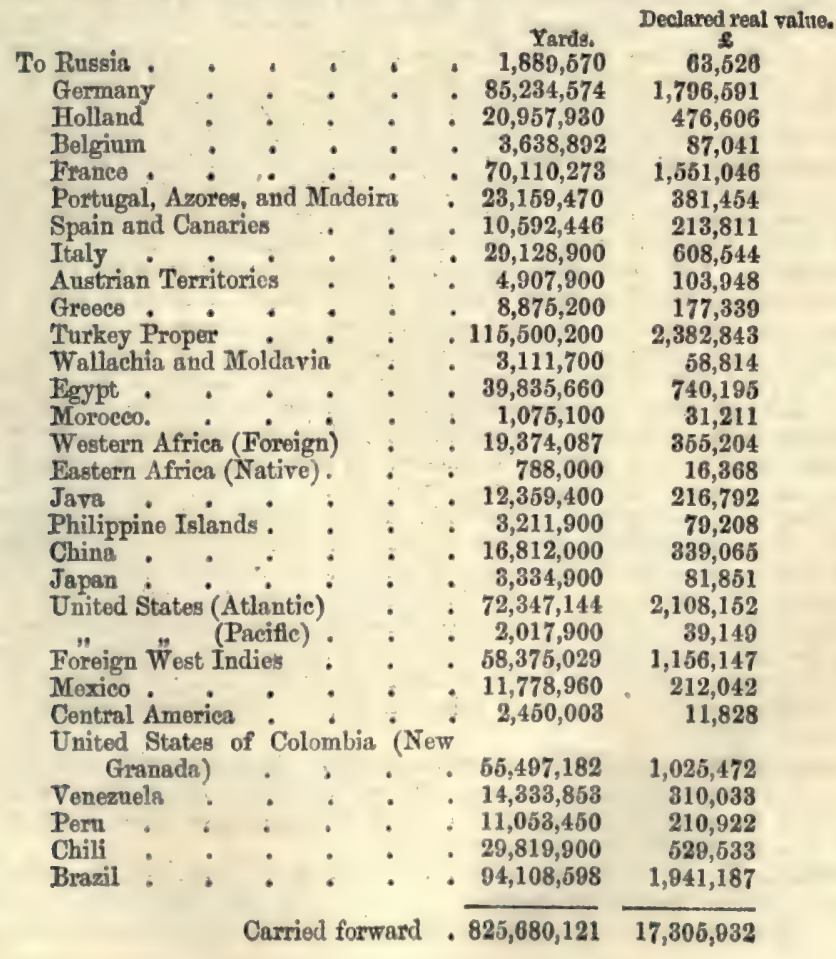




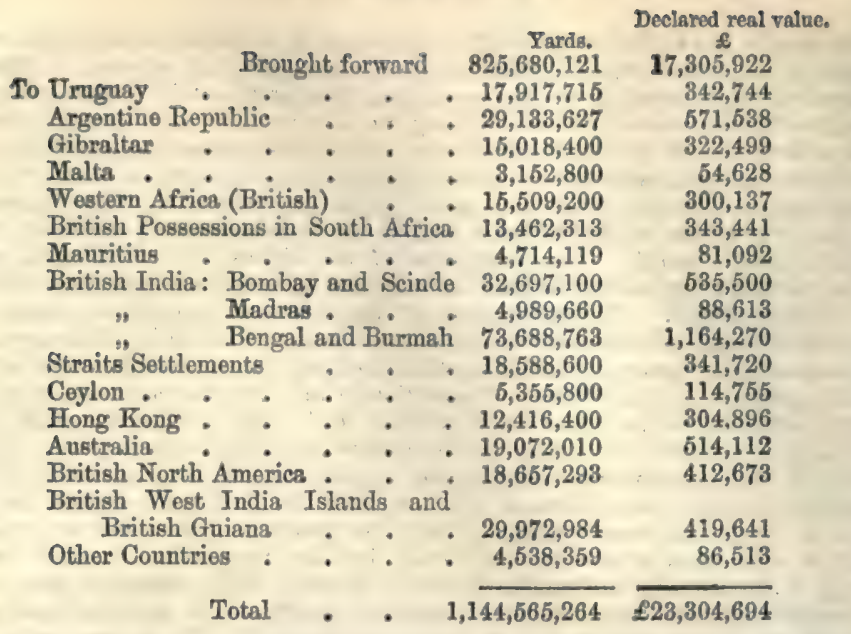

It would appear that occasionally attompts were made, during the early days of printing, to produce work possessing a high degree of artistic excellonce; and as the specimens that have been preserved to our time are very raro, it is fair to conclude that these experiments were not successful in a pecuniary point of viow. In the museum of the Peel Park, at Salford, there is a curious and interesting piece of printed linen, bearing the date $\mathbf{1 7 6 1}$ (at this period cloth of all cotton was prohibited), and which must have been printed from copper plates of very unusual size. Apparently, the pattern has been produced by two plates, each about 4 foet 6 inches by 3 feet. The design is printed in madder red, and is thus described by Mr. Plant, the curator of the museum. "The printed piece of linen measures, in the full length of the design, 6 feet 10 inches by 3 feet 2 inches in breadth. The composition in the design is very bold and free - in my opinion indicating very strongly the feelings of an artist who had been educated in the Flemish school. The grouping of the trees, figures, cattle, and fowls, is probably a direct copy from an engraving or sketch by Berghem, whose paintings and engravings of such subjects are well known for their truth to nature. His works bear date 1638 to 1680 . Perhaps, to fill up the design, and form a picturesque composition, the artist has borrowed from the French painters the classic ruins which form the sides of the design; it has had the effect of producing an anachronism. The upper group represents a peasant seated upon the wall of a well blowing a flute; near him stands a woman with a distaff; a gronp of sheep, cow, and a dog, in the foreground. The background shows a landscape, and on each side this group are ruins, columns, and trees, reflected in the stream below. On a broken bank, midway between the two groups, are two dogs chasing a stag. The lower group, although there is no defined line of separation between the groups, represents a peacock, fowls, and chickens, upon a bank and ruins; landscapo and river scenery beyond. Over, a hawk carrying a chicken, the sides occupied with a ruined portico, tomb, and pedestal and vase, trees, and broken ground; and bolow are ducks swimming, and water-plants on the bank. At the bottom of the piece are those parts of the pattern which would print or fit on the top part of the design. On the stonework of the well, in the upper group, is printed, ' $\mathrm{R}$. JONES, 1761 ;' on the broken stone-work, in the centre of the lower group, is printed, 'R. I. and Co., OLD FORD, 1761." "Old Ford is situated at Bow, where the East London Water Works now are, and where there was a print-works at the time specified. This design was no doubt printed for furniture hangings or tapestry, for which it is exceedingly well adapted, and the work being altogether a remarkable production for the period,

CaIrpyrs; carterr compasszs, Compasses with bowod logs, em. ployed to measure the size of any round or conical body. In use, the two points are opened to the required width, and so much wood, metal, or stone is turned off the piece of work, that the two points exactly fit.

CAIK. To drive oakum, or untwisted old tarred rope into the seams of any vessel to provent wator from entering it or to stop a leak. Aftor calking a ship, hot tar is applied to protect the oakum driven in. Calk is a term used amongst miners for lime. 
CAIro0 ㅍm. A fibre obtained from one of the Asiatic nettles-probably the Urtica tenacissima, which is a native of Sumatra and Rungpore. Its fibres are exceedingly strong, and are converted into cordage. China-grass cloth, and some other fabries are made from the fibres of the Nettle. See NETTLE.

CaII ow. The top or rubble bed of a quarry. This is obliged to be removed bofore the useful material is raised; it is the great source of expense in working a quarry.-(Brande). Frequently termed the 'overburthen.'

CAIIYS or KAIIYS. An old Cornish term, still used in the remote mining districts, and applied to hard ground-chiefly to a variety of the Killas or Clay Slate.

CAIOMrax. (Chlorure de Mercure, Fr.; Kalomel, Chlormercur, Versïsstes Quecksilber, Ger.) The sub-chloride of mercury, or mercurous chloride of modern chomists, $\mathrm{Hg}^{2} \mathrm{Cl}\left(\mathbf{H g}^{2} \mathbf{C l}^{2}\right)$. Calomel is found native in the Palatinate, in crystals often well defined, coating the cavities of a ferruginous gangue, associated with cinnabar; also at the quicksilver mines of Idria in Carniola, at Almaden in Spain, and Horzowitz in Bohemia. The native calomel is known as horn-quicksilver. The manufacture of this substance upon the large scale may be performed in various ways. Tho choapest, and most direct consists in mixing $1 \frac{1}{8}$ part of pure quicksilver with 1 part of pure nitric acid, of specific gravity from $1 \cdot 2$ to 1.25 ; and in digesting the mixture till no more metal can be dissolved, or till the liquid has assumed a yellow colour. At the same time, a solution is made of 1 part of common salt in 32 parts of distilled water, to which a little muriatic acid is added; and when heated to nearly tho boiling point, it is mixed with the mercurial solution. The two salts exchange bases, and a subchloride of mercury precipitates in a whito powder; which, after boing digested for some time in the acidulous supernatant liquor, is to be washed, with the greatest care, in boiling water. The circumstances which may injure the process aro the following:-1. When less mercury is employed than the acid can dissolve, there is formed a nitrate of mercury which gives rise to some corrosive sublimate and causes a proportional defalcation of calomel. 2. If the liquors are perfectly neutral at the moment of mixing them, some sub-nitrate of mercury is thrown down, which cannot be removed by washing, and which gives a noxious contamination to the bland calomel. The acid prescribed in the above formula obviates this danger.

The following are the directions given by the London College for the preparation of this salt:-2 lbs. of mercury are boiled to dryness in a suitable vessel with $3 \mathrm{lbs}$. of concentrated sulphuric acid : the sulphate of mercury thus obtained is rubbed when cold, first with $2 \mathrm{lbs}$. more mercury in an earthen vessel until perfectly mixed, and thon thoroughly incorporated with $1 \frac{1}{2} \mathrm{lb}$. of chloride of sodium; the whole is then sublimed in an appropriate vessel; the sublimate is rubbed to a fine powder, and then washed with boiling distilled water, until the washings are no longer tinged by sulphuretted hydrogen. This process is absolutely necessary in order to romove every trace of corrosive sublimate which is always formed in greater or less quantity.

At Apothecaries' Hall $50 \mathrm{lbs}$. of mercury are boiled with $70 \mathrm{lbs}$. of sulphuric acid' to dryness in a cast-iron vessel ; 62 lbs. of the dry salt are triturated with $40 \frac{1}{2} 1 \mathrm{lbs}$. of morcury until the globules disappear; and $34 \mathrm{lbs}$. of common salt are then added. The mixture is submitted to heat, and from $95 \mathrm{lbs}$. to $100 \mathrm{lbs}$. of sublimed cslomel are obtained. It is washed in large quantities of distilled water, after having been ground to a fine and impalpable powder.

According to the patent of Mr. Josiah Jewell, the vapour of calomel was to be transmitted into a vessel containing water, in order to condense it at once into an impalpable powder. But this process was beset with many difficulties. The vapour of the calomel was afterwards introduced into a large receiver, into which steam was simultaneously admitted; but this plan has also been found to be precarious in the execution. The best way is to sublime the calomel into a very large chamber from an iron pot, in the same way as the flowers of sulphur are formed. The great body of cool air serves to cause the precipitation of the calomel in a finely comminuted stato. It is afterwards washed with water, till this is no longer coloured by sulphuretted' hydrogen.

A patent was obtained in September 1841, by Anthony Todd Thomson, M.D., for an improved method of manufacturing calomel and corrosive sublimate, as follows:This invention consists in combining chlorine in the state of gas with the vapour of mercury or quicksilver, in order to produce calomel and corrosive sublimate. The apparatus employed consists of a glass, earthenware, or other suitable vessel, mounted in brickwork, and communicating at one end with a large air-tight chamber, and at tho other end by means of a bent tube, with an alembic, such as is generally used in generating chlorine gas, The alembic is charged with a mixturo of common salt, bin-

'Vor, I. 
oxide of manganese, and sulphur:c acid, or of binoxide of manganese and muriatic acid in order to produce chlorine gas.

The mode of operating vith this apparatus is as follows:-A quantity of mercury or quicksilver is placed in a glass vessel, and the temperature of the same is raised to between $350^{\circ}$ and $660^{\circ} \mathrm{F}$., by means of an open fire beneath. The chlorine gas, as it is genersted, passes from the alembic through the bont tube into the glass vessel, and there combining with the vapour of the mercury, forms either corrosive sublimate or calomel, according to the quantity of chlorine gas employed.

The product is found at the bottom of the air-tight chamber, and may be removed from the same through a door, when the operation is finished.

Prof. Wöhler proposes to preparo calomel in the humid way by decomposing a solution of corrosive sublimate by sulphurous acid. The commercial salt is dissolved in water at $122^{\circ}$ to saturation. Sulphurous acid gas, evolved by heating coarse charcoal powder with concentrated sulphuric acid, is passed into the hot solution: the separation of the calomel commences immediately. When the solution is saturated with the gas, it is digested for some time, then left to get cold, and filtered from the calomel, which is afterwards washed. The filtrate usually contains some unchangeable corrosive sublimate, which may be converted into calomel, either by heating to boiling, or by a fresh introduction of sulphurous acid and heating. Calomel obtained in this manner is a crystalline powder of dazzling whiteness, glittering in the sunlight.

The presence of corrosive sublimate in calomel is easily detected by digesting alcohol upon it, and testing the decanted alcohol with a drop of caustic potash, when the characteristic brick-coloured precipitate will fall, if any of that salt be present. To detect subnitrate of mercury in ealomel, digest dilute nitric acid on it, and test the acid with potash, when a precipitate will fall in case of that contamination.

117.75 parts of calomel contain 100 of quicksilver.

Great confusion has unfortunately arisen between the formulse of the two chlorides of mercury-calomel and corrosive sublimato.

If 100 be taken as the atomic weight of mercury, the formula of calomel is $\mathrm{Hg}^{2} \mathrm{Cl}$ (as given above), and that of corrosive sublimate is $\mathrm{HgCl}$. But if, as is often the case, 200 be assigned to mercury as its atomic weight, the formula of calomel becomes $\mathrm{HgCl}$ (or rather, for theoretical reasons, $\mathrm{Hg}^{2} \mathrm{Cl}^{2}$ ) and that of corrosive sublimate $\mathrm{HgCl}^{2}$. As fatal mistakes might occur by substituting one of these compounds for the other, the formulæ should never be used apart from the specific names.

CAIorrC. A term once extensively used in physical science, and perhaps somewhat too hastily abandoned. When employed there was a very general impression that heat was the effeot of some undeveloped canse, and to this cause the term caloric was applied. The modern hypothesis regarding heat as a mode of motion, the term has been dispensed with. Seo Hrat.

CALORIFInE OF WATER. In former editions, hot-water apparatus was described under this head: now see Hot-watzR Apparatus.

CAIORIMETER. An instrument intended to measure the quantity of heat producod by burning bodies, devised by Lavoisier and Laplace. The principle upon which these instruments depended may be easily understood. To convert a certain quantity of ice into water, a given amount of heat is necessary. Now suppose we construct a funnel-shaped ressel, and having placed in the middle of it a vessel containing boiling water, or hot mercury, or oil, or a red-hot piece of metal, it is packed round with ice, care being taken that no heat shall escape, except it is employed to thaw the ice. The water formed by the ice is collected in a vessel placed below the funnel, and the quantity collected during the cooling of the heated body will represent the heat given out and employed in liquefying the ice. For nice experiments certain exact determinations are required, for which, and the general rules to be observed, some good treatise on Physics should be consulted. See Watts's 'Dictionary of Chemistry:' article Hrat.

CaIotrpz (signifying beautiful picture). A name given by Mr. Henry For Talbot to a photographic process invented by him in 1840, and patented in 1841 .

Mr. Talbot's description of his process is as follows :-

Take a sheet of the best writing-paper, having a smooth surface, and a close and even texture. The water-mark, if any, should be cut off, lest it injure the appearance of the picture. Dissolve 100 grains of crystallised nitrate of silver in six ounces of distilled water. Wash the paper with this solution with a soft brush on one side, and put a mark on that side, whereby to know it again. Dry the paper cautiously at a distance from the fire, or else let it dry spontaneously in a dark room. When dry, or nearly so, dip it into a solution of iodide of potassium, containing 500 grains of that salt dissolved in one pint of water, and let it stay two or throe minutes in the solution. 
Then dip the paper into a vessel of water, dry it lightly with blotting-paper, and finish drying it at a fire, which will not injure it oven if held pretty near; or else it may be left to dry spontaneously. All this is best done in the evening by candle-light: the paper, so far prepared, is called iodised paper, because it has a uniform pale-yellow coating of iodide of silver. It is scarcely sensitive to light, but nevertheless it ought to be kept in a portfolio or drawer until wanted for use. It may be kept for some considerable time without undergoing change, if protected from sunshine. When the paper is required for use, take a sheet of it, and wash it with a liquid prepared in the following manner:-

Dissolve 100 grains of crystallised nitrato of silver in two ounces of distilled water; add to this solution one-sixth of its volume of strong acetic acid. Let this be called mixture A,

Make a saturated solution of crystallised gallic acid in cold distilled water. The quantity dissolved is very small. Call this solution B.

Mix together the liquids $\mathrm{A}$ and $\mathrm{B}$ in equal volumes, but only a small quantity of them at a time, because the mixture does not keep long without spoiling. This mixture Mr. Talbot calls the gallo-nitrate of silver. This solution must be washed over the iodised paper on the side marked, and being allowed to remain upon it for half a minute, it must be dipped into water, and then lightly dried with blotting-paper. This operation in particular requires the total exclusion of daylight; and although the paper thus prepared has been found to keep for two or three months, it is advisable to use it within $\mathrm{a}$ few hours, as it is often rendered useless by spontaneous change in the dark.

Paper thus prepared is exquisitely sensitive to light; an exposure of less than a second to diffused daylight being quite sufficient to set up the process of change. If a piece of this paper is partly covered, and the other portion exposed to daylight for the briefest possible period of time, a very decided impression will be made. This impression is latent and invisible. If, however, the paper be placed aside in the dark, it will gradually develop itself; or it may be brought out immediately by being washed over with the gallo-nitrate of silver, and held at a short distance from the fire, by which the exposed portions become brown, the covered parts remaining of their original colour. The pictures being thus procured, are to be fixed by washing in clean water, and lightly drying between blotting-paper, after which they are to be washed over with a solution of bromide of potassium, containing 100 grains of that salt, dissolved in eight or ten ounces of water; after a minute or two, it is again to be dipped into water, and then finally dried. The Collodion process has almost entirely superseded the Calotype. See Comodros and Photography.

catumba. See Coromba.

CAMBAY STOxr

camizocia. Seo Gambogr.

CAMrRIC. (Batiste, Fr.; Kammertuch, Ger.) A sort of very fine and rather thin linen fabric, first made at Cambray. An excellent imitation of it is made in Lancashire and in Scotland, woven from fine cotton yarn, hard twisted. Linen cambric of a good quality is also now manufactured in the United Kingdom from power-spun flax; this is frequently called Cambrio muslin.

\section{Camprimon mingrar. Seo Chamogran Mngrat.}

Campo. (Camée, Fr.; Cammeo, It.) Correctly a precious stone engraved in relief, as opposed to an intaglio, which is cut into the stone. The earliest cameos appear to have been cut upon the onyx, and subsequently, on the agate. The true cameo is formed upon a stone having two or more layers, differing in colour; and the art of the cameo-engraver consists in so cutting as to appropriate those differently coloured layers to distinct parts or elevations of the work.

Many of the varieties of chalcedony present in section transparent and opaque layers; and beautiful works have been cut upon such specimens of this material. The chalcedony and agate are, however, not unfrequently coloured artificially. The layers vary very much in their structure, some being absorbent and others not so. Such stones are taken, and if it is desired to have black and white layers, they are boiled in a solution of sugar or honey, and then in sulphuric acid. The sugar or honey is, in the first place, absorbed by the more porous layers, and then decomposed by the acid. Red or brownish-red layers are produced by occasioning the stone to absorb a solution of sulphate of iron, and then by exposure to heat effecting the peroxidation of the metal. This being done, layers very strongly contrasted in colour are the result; and very fine cameos have been cut upon stones so prepared. See Aante,

In Italy and in France, the art of producing the cammeo duro has been to some extent revived; but the immense labour which such hard materials require renders them so expensive, that these cameos have not come into general use.

The shells of several molluscous animals are now commonly used. Many of these $\mathbf{x} \times 2$ 
sholls afford the necessary variety of colour, and are soft enough to be worked with fucility, yet hard enough to wear for a considerable time without injury.

The natural history of the mollusea producing the shells, and the best account of the manufacture of cameos, was given by Dr. J. E. Gray, of the British Museum, in a paper read bofore the Society of Arts in 1847, to which, and to his paper in the Phil. Trans., we are indebted for much of the following information.

It was the custom in Holland to use tho pearly nautilus as a cameo shell, and several kinds of turbines or wreath-shells, which have an opaque white external coat over an internal pearly one. These are now rarely employed. The shells now used are those of the flosh-eating univalves (Gasteropoda ptenobranchiata zoophaga), which are peculiar for being all formed of three layers of calcareous matter, each layer being composed of three perpendicular laminæ placed side by side; the laminæ comprising the central layer, being placed at right angles with one of the inner and outer ones; the inner and outer being placed longitudinally with regard to the axis of the line of the shells, while the inner laminæ are placed across the axis, and concentrically with the edge of the mouth of the cone of the shell.-(Gray, Phil. Trans.)

This structure furnishes the cameo-cutter with the means of giving a particular surfaco to his work, a good workman always putting his work on the shell in such a manner, that the direction of the laminæ of the central coat is longitudinal to the axis of his figure. The central layer forms the body of the bas-relief, the inner laminæ being the ground, and the outer one, the third or superficial colour, which is some. times used to give a varied appearance to the surface of the figure. The cameo-cutter, therefore, selects for his purpose those shells which have three layers of different colours, as these afford him the means of relieving his work; and secondly, those which have the three layers strongly adherent together, for if they separated, his labour would be lost.

The following are the kinds of shells now employed:-1. The bull's mouth (Cassis rufum), which has a red inner coat, or what is called a sardonyx ground. 2. The black helmet (Cassis Madagascariensis), which has a blackish inner coat, or what is called an onyx ground. 3. The horned helmet (Cassis cornutum), with a yellow ground. 4. The queen's conch (Strombus gigas), with a pink ground.

The bull's mouth and the black helmet are the best shells. The horned helmet is apt to separate from the ground, or double; and the last, the queen's conch, has but seldom the two colours marked with sufficient distinctness, and the finish of the ground colour flies on exposure to light.

The red colour of the bull's mouth extends only a slight distance in the mouth of the shell, becoming paler as is proceeds backwards. The dark colour extends farther in the black and yellow varieties. Hence the bull's mouth only affords a single cameo large enough to make brooches of, and several small pieces for shirt-studs. The black helmet yields on an average about five brooches, and several pieces for studs, while the queen's conch affords only one good piece.

Forty years since, very fow cameos were made from any shells but the black helmet, and the number of shells then used amounted to about 300 annually, nearly all of which were sent from England, being all that were then imported. The black helmet is imported into England from Jamaica, Nassau, and New Providence. They are not found in Madagascar, though naturalists have for a long period called them Madagascar helmets.-(Gray).

Of the bull's mouth, half are received direct from the Island of Bourbon, to which place they are brought from Madagascar, and the other half are obtained from the Island of Ceylon, being received by the way of Calcutta ; hence they are often called 'Calcutta shells.'

So rapidly has the trade in these shells increased, that Dr. Gray informs us, that in Paris 100,500 shells are used for cameos annually. These are divided as follows :-

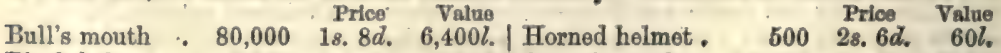

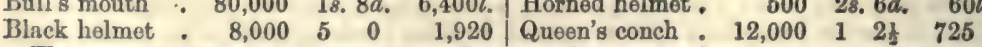

The manufacture of shell cameos was for some time confined to Italy; about twentyfiro years since, an Italian commenced making them in Paris, and now the trade belongs to the French capital, where not less than 300 persons are engaged in the manufacture. Nearly all the cameos made in France were sent to England, and in Birmingham mounted as brooches, and exported to America and the British Colonios.

CAmEra IUCida. An instrument invented by Dr. Wollaston, for the purpose of enabling anyone, without a knowledge of the rules of drawing or perspective, to delineate any external object with accuracy. It consists essentially of a quadrangular glass prism.

CAmzre onscura, literally dark chamber, An instrument inveatod by 
Baptiste Porta. It is employed for the production of photographic pictures, and will bo fully described in the article doroted to that art. See PHotograpry.

CAMEs. Slips of lead employed by glaziers in glazing church or cottage windows in the olden style. They were formerly called 'lattices,' and hence leaded windows were termed lattice windows.

CAMIIFT or CAMBrist. A light stuff, formerly much used for female apparol. It is made of long wool, hard spun, sometimes mixed in the loom with cotton or linen yarn. Several fabrics of the same kind are now introduced under other names.

Campracery wood (Hematoxylon Campechianum). Logwood brought from the bay of that name. See LoGwood.

CAMPHFN2. The radicle of camphor; various hydrocarbons isomeric or polymeric with oil of turpentine are so called. See Watts's 'Dictionary of Chemistry.

CAMPrInz. Rectified oil of turpentine is sold in the shops under this name for burning in lamps. Crude oil of turpentine is redistilled with potash, and then with water, and lastly, to secure its perfect purity, with chloride of calcium. The oil thus prepared forms a limpid colourless liquid; its specific gravity is about 0.870 , but it is subject to some slight veriations; $\mathrm{C}^{3} \mathrm{H}^{4}$ appears fairly to represent this, and several other similar oils. It is very inflammable, burning with a bright red flame, and without a proper supply of air it evolves much dense smoke, hence peculiax lamps (Camphine lamps) are required. Where it has, from exposure to air, absorbed oxygen, and become resinified, it is unfit for purposes of illumination. Such camphine very rapidly clogs the wick with a dense carbon, and is liable to the thick black smoke, which is so objectionable in the camphine lamps if they are not properly attended to.

To puxify old camphine, it must be redistilled from carbonate of potash, or some similarly active substance to deprive it of its resin. See ILMMPS.

CAMPEOIE. One of the oils obtained from coal-tar. Mansfield gave this name to the oils cumole and cymole, which boil at $284^{\circ}$ and $330^{\circ} \mathrm{F}$., when collected together. The specific gravity of crude camphole ranges from 0.88 to 0.98 , and the less volatile portions frequently contain naphthaline, which raises their specific gravity. This substance, either alone or mixed with pyroxylic spirit, is applicable for burning in lamps or for dissolving resins, as a substitute for oil of turpentine.

CAMPROR, or CAMPHIRE. (Camphre, Fr.; Kampher, Ger.) This immediate product of vegetation was known to the Arabs under the names of kamphur and kaphur, whence the name camphora. Camphor was not known to the ancients; it is first mentioned by Avicenna, and Serapion calls it cáfúr. Symeon Seth, who lived in the eleventh contury, describes it. It is found in a great many plants, and is secreted, in purity, by several laurels; it occurs combined with the essential oils of many of the Labiate; but it is extracted, for manufactnring purposes, only from the Laurus camphora, or Camphora officinarum, a member of the Laurel order, which abounds in China and Japan, as well as from a tree which grows in Sumatra and Borneo, called, in the country, Kapour barros, from the name of the place where it is most common. This Sumatra camphor is the produce of the Dryobalanops camphora. The camphor exists, ready formed, in these vegetables, between the wood and the bark; but it does not exude spontaneously. On cleaving the tree which produces the Sumatra camphor masses of pure camphor are found in the trunk. Sumatra esmphor is not imported into this country, as it is eagerly bought up by the Chinese, who prize it highly.

To prepare camphor the wood of the laurus is eut into small pieces, and put with plenty of water into large iron boilers, which are covered with an earthen eapital or dome, lined within with rice straw. As the water boils, the camphor rises with the steam, and attaches itself as a sublimate to the stalks, under the form of granulations of a grey colour. In this state it is picked off the straw, and packed up for exportation to Europe.

Formerly. Venice held the monopoly of refining camphor, but now France, England, Holland, and Germany refine it for their own markets. All the purifying processes proceed on the principle that camphor is volatile at the temperature of $400^{\circ} \mathrm{F}$. The substance is mixed, as intimately as possible, with 2 per cent. of quicklime, and the mixture is introduced into a large bottle made of thin uniform glass, sunk in ih sand-bath. The fire is slowly raised till tho wholo vessel becomes heated, and then its upper part is gradually laid bare in proportion as the sublimation goes on. Much attention and experionce are required to make this operation succeed. If the temperature be raised too.slowly, the neck of the bottle might be filled with camphor before the heat had acquired the proper subliming pitch; and, if too quickly, the whole contents might be exploded. If the operation be carried on languidly, and tho heat of the upper part of the bottle be somowhat under the melting-point of camphor, 
that is to say, a little under $350^{\circ} \mathrm{F}$., the condensed vapour would be snowy, and not sufficiontly compact and tmensparent to be saleable. Occasionally, sudden alterations of temporature cause little jets to be thrown up, out of the liquid camphor at the bottom, on the cake formed above, which soil it, and render its resublimation necessary.

If to the mixture of 100 parts of crude camphor and 2 of quicklime, 2 parts of bone-black, in fine powder, be added, the small quantity of colouring matter in the camphor will be retained at the bottom, and whiter cakes will be produced. A spiral slip of platina foil immersed in the liquid may tend to equalise its ebullition.

By exposing some volatile oils to spontaneous evaporation, at the heat of about $70^{\circ}$ F., Proust obtained a residuum of camphor:-from oil of lavender, 25 per cent. of its weight; from oil of sage, $12 \frac{1}{2}$; from oil of marjoram, 10 per cent.

Refined camphor is a white translucid solid, possessing a peculiar taste and smell. It may be obtained, from the slow cooling of its alcoholic solution, in octahedral erystals. It may be seratched by the nail, is very flexible, and can be reduced into powder readily by mixing it with a fow drops of alcohol and giving a few blows to the camphor. Its specific gravity varies from 0.985 to 0.996 . Mixed and distilled with six times its weight of clay, it is docomposed, and yields a golden yellow aromatic oil, which has a flavour analogous to that of a mixturo of thyme and rosemary; along with a small quantity of acidulous water tinged with that oil, charcoal remains in the retort. In the air, camphor takes fire on contact of an ignited body, and burns away with a bright fuliginous flame. Pieces of ignited camphor thrown into water execute peculiar rotatory movements.

Camphor is little soluble in water; one part being capable of communicating smell and taste to 1,000 of the fluid, this is the Misture Camphore of the apothecary. 100 parts of alcohol, specific gravity 0.806 , dissolve 120 parts of camphor, at ordinary temperatures. It is separated in a pulverulent state by water. Ether and oils, both expressed and volatile, also dissolve it.

When distilled with 8 parts of aquafortis, camphor is converted into camphoric acid. Camphor absorbs 144 times its volume of muriatic acid gas, and is transformed into a colourless transparent liquid, which becomes solid in the air, because the acid attracts humidity, which precipitates the camphor. One part of strong acetic acid dissolves 2 parts of camphor. By Dr. Ure's analysis, camphor consists of $77 \cdot 38$ carbon, $11 \cdot 14$ hydrogen, and $11 \cdot 48$ oxygen.

Dumas (1) and Blanchet and Sell (2) have given the following composition :-

\begin{tabular}{l} 
Carbon $: \quad: \quad: \begin{array}{l}78 \cdot 02 \\
\text { Hydrogen } \\
\text { Oxygen }\end{array}: \quad: \quad: 11 \cdot 39$ \\
\hline
\end{tabular}

There are two kinds of camphor imported :-

Japar CaMPHor, called DUTCH CAMPHOR, because it is always brought by the Dutch to England. It comes by the way of Batavia, and is imported in tubs (hence it is called tub camphor), covered with matting, and each surrounded by a socond tub, secured on the ontside by hoops of twisted cane.

Chira CaMphor, or Formosa CaMpHor, is imported from Singapore and Bombay, in chests lined with lead-foil, containing about $1 \frac{1}{2}$ cwt.

It has been suggested to introduce the camphor trees into other countries. South Georgia and Florida are named as suitable localities. The Laura camphora is commonly found in all the nurseries around Paris, and sold at 5 francs for a plant 30 inches high. At full growth the tree attains an altitude of from 40 to 50 feet. The wood of the camphor tree is in favour for carpenters' work; it is light, is easily worked, durable, and not liable to be attacked by insects. It is said that in Sumatra numbers of trees are cut down before one is found to repay. Not a tenth part of the trees attacked yield either camphor or camphor oil.

The camphor is distinguished by the names of head, belly, and foot, when in bulk. The head camphor is in large white flakes; the belly camphor, small brown flakes, transparent, like resin coarsely powdered; the foot, like dark-coloured resin. A native 'Catty' may be divided into:-1. Capallo, or large head, $=2 \cdot 2 ; 2$. Capallo cachell, or small head, $=3.5 ; 3$. Baddan, or belly, $=4.2 ; 4$. Cakee, or foot, $=6.1$; $=1$ Catty, 16 .

CAMrzioz, ArTrricrar. When hydrochloric (muriatic) acid is passed into oil of turpentine, surrounded by ice, two compounds are obtained, one solid and the other fluid. The first, solid artificial camphor $\mathrm{C}^{20} \mathrm{H}^{16} \mathrm{HCl}\left(\mathbf{C}^{10} \mathbf{H}^{10} \mathbf{m C l}\right)$ is white, transparent, lighter than water, and has a camphoraceous taste. The fluid is termed liquid artificial camphor, or terebine.

CAMPEOR JUxEP. Water in which camphor is dissolved. 
CAmpror, orr or IAURrs. When the branches of Camphora officinamu are distilled with water, a mixture of camphor and a liquid essential oil is obtained. This is the oil of camphor; it has a density of 0.910 , and its composition is $\mathrm{C}^{20} \mathrm{H}^{16} \mathrm{O}$. By exposure to oxygen gas, or to the action of nitric acid, it absorbs oxygen, and becomes solid camphor $\mathrm{C}^{20} \mathrm{H}^{16} \mathrm{O}^{2}\left(\mathbf{C}^{10} \mathbf{n}^{16} \mathbf{O}\right)$.

This is an esteemed article in the Eastern markets; it undergoes no preparation, and though named oil, it is rather a liquid and volatile resin. The natives of Sumstra make a transvorse incision in the tree to the depth of some inches, the cut sloping downwards so as to form a cavity of the capacity of a quart; a lighted reed is placed in it for about 10 minutes, and in the space of a night the eavity is flled with this fluid. The natives consider this oil of great use as a domestic remedy for strains, swellings, and inflammations.

Dr. Royle states the trees are of large dimensions, from $2 \frac{1}{2}$ to 7 feet in diameter. The same tree that produces the oil would have produced the camphor if unmolested, the oil being supposed to be the first stage of the camphor's forming, and is consequently found in younger trees.

CAMPIOR STORM GIAsszs. Glasses called usually storm glasses, and sold as indicators of atmospheric changes. 'Storm glasses' are made by dissolving camphor $2 \frac{1}{2}$ drachms, nitre 38 grains, sal-ammoniac 38 grains, water 9 fluid drachms, rectified spirit of wine 11 fluid drachms.

Plumose crystals form in the glass, and are said to condense and collect at the bottom of the bottle on the approach of a storm, and to rise up and diffuse themselves through the liquid on the approach of fine weather; but Dr. Parrion thinks that their weather-predicting qualities are false, and that light is the agent which, together with temperature, influences the condition.

CAM-wOOD. An African dye wood, shipped principally from Sierra Leone, in short logs. Mr. G. Loddiges, in his Botanical Cabinet, figures the plant producing it under the name of Baphia nitida; it is a leguminous plant, and has boen introduced into, and has flowered in this country.

CANADA BAISAM. A product of the Abies balsamea, or balm of Gilead fix. The finest variety of this balsam is used for mounting objects for the microscope. See Barsam.

Caxada pxatres. Tin plates, so called by having been made for the Canadian trade.

CATADA RICD. The Zizania aquatica, a Canadian grass.

CANADA SUGAR. A dark sugar mado from the sugar maple, Acer saccharinum. CANADA YDIIOW ROOT. The Hydrastis Canadensis (Xanthorhiza), a shrub growing in North America, so named from the colour of its root. It is intensely bitter, hence it is used as a tonic medicine, and a good yellow dye is extracted from its roots.

CANarium commurge. A small tree, growing in India, Ceylon, and various parts of the Eastern Archipelago. It is probably the source of the concrete resinous exudation commonly known as Gum Elemi. Seo Erxim.

CANARY WOOD. A wood is imported into this country under the name of Madeira mahogany, which appears to be this canary wood. It is the produce of the Royal Bay, Laurus indica, a native of the Canary Islands. It is rather a light wood, and of a jellow colour.

CANAsTrm. An American tobacco, so called from the peculiar rush basket in which it was imported.

CANAÜma. Tho leaves of the canaulba tree of Brazil (Copernicia cerifera) yield a wax which appears to be the same as that produced by the Carnaüba Palm.

CAND or ICAND. A name in some mining districts for Fluor spar.

CANDrz. (Chandelle, Fr.; Kerze, Licht, Ger.) Candles aro either dipped or moulded. As the quality of the candle depends on the material employed in its manufacture, the first part of the tallow-chandler's process is the sorting of the tallow. Mutton suet with a propurtion of ox tallow is selected for mould candles, because it gives them gloss and consistence. Coarser tallow is reserved for the dipped candles. After being sorted, it is cut into small pieces, preparatory to being melted or rendered; and the sooner this is done after the fat is taken from the carcase the better, because the fibrous and fleshy matters mixed with it promote its putrefaction. Tallow is too commonly molted by a naked fire appliod to the botton of the vessel, whereas it should be done either in a cold set-pan, where the flame plays only round the sides a little way above the bottom, or in a steam-cased pan. After being fused a considerable time, the membranous matters collect at the surface, constituting the cracklings used sometimes for feeding dogs, after the fat has been squeezed out of it by a press. The liquid tallow is strained through a sieve into another copper, where it is treated with water at a boiling temperature, in order to wash it. After a while, when the foul 
water has settled to the bottom, the purified tallow is lifted out, by means of tinned iron buckets, into tubes of moderate size, where it concretes, and is ready for use.

It is a remarkable circumstance, that the wicks for the best dipped candles are still cotton rovings imported from Turkey, notwithstanding the vast extension and perfection of cotton-spinning in this country. Four or more of these Turkey skeins, according to the intended thickness of the wick, are wound off at once into bottoms or clues, and afterwards cut by a simple machine into lengths corresponding to those of the candles to be made. The operations for cutting, twisting, and speading wicks, aro performed upon a series of threads at once. The apparatus is placed in a box, in front of which the operator sits. A reel extends across the box at the hinder part, upon which the cotton threads have been previously wound: from this reel they are drawn off into proper lengths, doubled, and cut by an ingenious mechanism. By dipping tho wicks into the melted tallow, rubbing them between the palms of the hands, and allowi.i. the tallow which adheres to harden, they may be arranged with facility upon the broaches for the purpose of dipping. The dipping room is furnished with a boiler for melting the tallow, the dipping monld or cistern, and a large wheel for supporting the broaches. From the ceiling of the workshop a long balanced-shaped beam is suspended, to one end of which a wooden frame is attached for holding the broaches with the wicks arranged at proper distances. The opposite arm is loaded with a weight to counterbalance the wooden frame, and to enable the workmen to ascertain the proper size of the candles. The end of the lever which supports the frame is placed immediately above the dipping cistern; and the whole machine is so balanced that, by a gentle pressure of the hand, the wicks are let down into the melted tallow as often as may be required.

The following is a convenient apparatus for dipping candles. In the centre of the dipping-room a strong upright post $\Delta \Delta,($ fig. 402$)$ is erected, with turning iron pirots

402

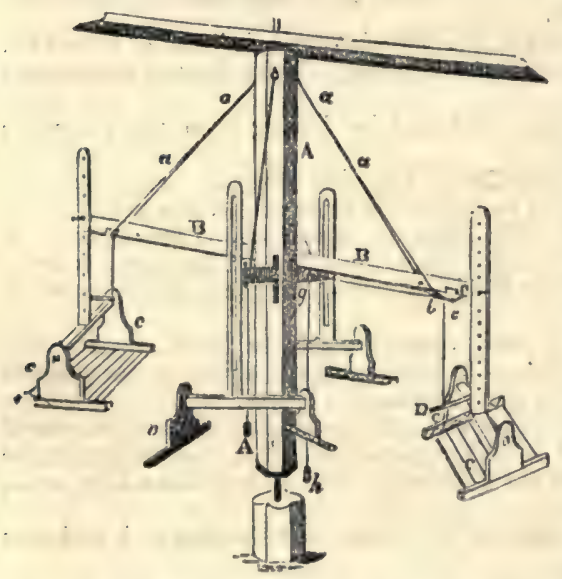
at its two onds. Near its middle, six mortices are cut at small distances from one another, into each of which is inserted a long bar of wood, $\mathrm{B} B$, which moves vertically upon an iron pin, also passing through the middle of the shaft. The whole presents the appearance of a large horizontal wheel with twelve arms. A complete view of two of them only is given in the figure. From the extremity of each arm is suspended a frame, or port, as the workmen call it, containing 6 rods, on each of which are hung 18 wicks, making the whole number of wicks upon the wheel 1,296. The machine, though apparently heavy, turns round by the smallest effort of the workman; and each port, as it comes in succession over the dipping-mould, is gently pressed downwards, by which means the wicks are regularly immersed in

melted tallow. As the arms of the lever are all of the same length, and as each is loaded with nearly the same weight, it is obvious that they will all naturally assume a horizontal position. In order, however, to prevent any oscillation of the machine in turning round, the levers are kept in a horizontal position by means of small chains, $a \boldsymbol{a}$, one end of which is fixed to the top of the upright shaft, and the other terminates in a small square piece of wood $b$, which exactly fills the notch $c$ in the lever. As one end of the levers must be depressed at each dip, the square piece of wood is thrown out of the notch by the workman pressing down the handle $\mathrm{D}$, which communicates with the small lever $e$, inserted into a groove in the bar $\mathrm{B}_{\text {. }}$ In order that the square piece of wood fixed in one extremity of the chain, may recover its position upon the workman's raising the port, a small cord is attached to it, which passes over a pulley inserted in a groove near $c$, and communicates with another pulley and weight, which draws it forward to the notch. In this way the operation of dipping may be conducted by a single workman with perfect ease and regularity, and even despatch. No time is lost, and no unnecessary labour expended, in removing the ports after each dip; and, besides, the process of cooling is much accelerated by the candles weing kept in constant motion tbrough the air. The number of revolutions which 
the whoel must make, in order to complete one operation, must obviously depend upon the state of the weather and the size of the candles; but it is said that, in moderately cold woather, not more than two hours are necessary for a single person to finish one wheel of candles of a common size. Upon the supposition, therefore, that six wheels are completed in one day, no less a number than 7,776 candles will bo manufactured in that space of time by one workman.

The process of moulding is even-less complicated in its details than that of dipping. The moulds are made of some metallic substance, usually pewter, and consist of two parts. The shaft or great body of the mould is a hollow cylinder, finely polished in the inside, and open at both extremities. The top of the mould is a small metallic cup, having a moulding within-side, and a hole to admit the wick. The two patts are soldered together, and when united, as will readily be imagined, have the shape of a moulded candle. A third piece, called the foot, is sometimes added : it is a kind of small funnel, through which the liquid tallow runs into the mould, and being serewed to the opposite extremity of the shaft, is removable at pleasure. This additional piece may certainly be useful in very mild weather; since, by removing it, the candles may be drawn more easily from the moulds; but, in general, it may be dispensed with.

Eight or twelve of these moulds, according to their size, are fixed in a frame, which bears a great resemblance to a wooden stool, the upper surface of which forms a kind of trough. The tops of the moulds point downwards, and the other extremity, which is open, is inserted into the trough or top of the stool, and made quite level with its upper surface. In order to introduce the wicks into the mould, the workman lays the frame upon its side on an adjoining table, and holding in his left hand a quantity of wicks, previously cut to the proper length, he introduces into the mould a long wire with a hooked point. As soon as the hook of the wire appears through the hole in the top of the mould, he attaches to it the looped end of the wick, and, immediately drawing back the wire, brings the wick along with it. In this manner each mould in succession is furnished with a wick. Another workman now follows, and passes a small wire through the loop of each wick. This wire is obviously intended to keep the wick stretched, and to prevent it from falling back into the mould upon the frame being placed in the proper position for filling. The frame is then handed to the person that fills the moulds, who previously arranges the small wires in such a manner that each wick may be exactly in the axis of the mould.

The moulds are filled by running tallow into each of them, or into the trough, from a cistern furnished with a cock, and which is regularly supplied with tallow of the proper temperature from an adjoining boiler. When the workman observes that the moulds are nearly half filled he turns the cock, and laying hold of that portion of the wick which hangs out of the mould, pulls it tight, and thus prevents any curling of the wick, which might injure the candles; he then opens the cock, and completes the process of filling. The frame is now set aside to cool; and when the tallow has acquired a proper consistence, which the workman eastily discovers by a snapping noise emitted by the candles upon pressing his thumb against the bottom of the moulds, he first withdraws the small wires which kept the wicks tense, and then, scraping off the loose tallow from the top of the frame with a small wooden spade, he introduces a bodkin into the loop of the wick, and thus draws each candle in succession from its mould. The candles are now laid upon a table for inspection, and afterwards removed to the storehouse. Previous to storing them up, some candlemakers bleach their candles, by exposing them to the air and dews for several days. This additional labour can be necessary only when the dealer is obliged to have early sales; for if the candles are kept for some months, as they ought to be, before they are brought to market, they become sufficiently whitened by age.

Wax Candles.-Next to tallow, the substance most employed in the manufacture of eandles is wax. Wax candles are made either by the hand or with a ladle. In the former case, the wax, being kept soft in hot water, is appliod bit by bit to the wick, which is hung from a hook in the wall: in the latter, the wicks are hung round an iron circle, placed immediately over a large copper-tinned basin full of meltel wax, which is poured upon their tops, one after another, by means of a large ladle. When the candles have by either process acquired the proper size, they are taken from the hooks, and rolled upon a table, usually of walnut-tree, with a long square instrument of box, smooth at the bottom.

Spermaceti Candles are moulded in the same manner as those composed of stearine, or stearic acid, to be described presently.

In June 1825, M. Gay-Lussac obtained a patent in England for making candles from margaric and stearic acids, called stearine, by converting tallow into the above fat atcids by the following process:-Tallow consists, by Chevreul's researches, of stearine, a solid fat, and oleine, a liquid fat; the former being in much the larger proportion. When tallow. is treated with an alkaline body, such as potash, soda, or lime, it is 
saponified; that is, its stearine and oleine become respectively stearic and oleic acids, and, as such, form compounds with these bases. When by the action of an acid, such as the sulphuric or muriatic, these combinations are docomposed, the fats reappear in the altered form of stearic and oleic acids; the former body being harder than tallow, and of a texture somewhat like spormaceti, the latter body being fluid like oil. "The docomposition of the soap should be made,' says the patentee, 'in a large quantity of water, kept well stirred during the operation, and warmed by steam introduced in any convenient way. When the mixture has been allowed to stand, the acid of the tallow or fat will rise to the surface, and the water being drawn off, will carfy the alkaline or saline matters with it; but, if the acids of the tallow should retain any portion of the salts, fresh water may be thrown upon it, and the whole well agitated, until the acids have become perfectly free from the alkaline matters; and when allowed to cool, the acids will be formed into a solid mass. The mass is now to be submitted to considerable pressure in such an apparatus as is employed in expressing oil from seeds; when the liquid acid will run off in the form of a substance resembling oil, leaving a solid matter, similar, in every respect, to spermaceti, which is fit for making candlos.'

The wick to be used in the manufacture of the stearine candles, and which forms one of the features of this invention, is to be made of cotton yren, twisted rather hard, and laid in the same manner as wiro is sometimes coiled round the bass strings of musical instruments. For this purpose, straight rods or wires are to be procured, of suitable lengths and diameters, according to the intended size of the candles about to be made; and these wires, having been covered with cotton coiled round them, as described, are to be inserted in the candle moulds as the common wicks are; and when the candle is made, and perfectly hard, the wire is to be withdrawn, leaving a hollow cylindrical aperture entirely through the middle of the candle. The first successful application of the fat acid, or stearic candles, appears to have been made by Messrs. Motard and Nilly. They made stearine candles, which they called 'bougies de l'etoile,' for which the 'Society of Encouragement' rotod them their silver medal. Seo Strarink.

Messrs. Hempbell and Blundell have given a very minute account of the process for making palm-oil, stearic and margaric acids, in their specification of their patent for this mode of manufacturing candles :-

1. Their first process is called crystallisation, which consists in pouring the melted palm-oil into iron pans, allowing it to cool slowly, whereby, at about $75^{\circ} \mathrm{F}$., the oleine separates from the crystalline stearine and margarine.

2. The concreted oil is subjected to the action of an hydraulic press, in order to separate the oleine from the solid fats.

3. This process is called oxidation. To $104 \mathrm{lbs}$. of the stearine and margarine, melted in an iron pan, about $12 \mathrm{lbs}$. of slaked and sifted quicklime are added, with diligont stirring, during which the temperature is to be slowly raised to $240^{\circ} \mathrm{F}$., and so maintained for about 3 hours, till a perfect chemical combination takes place. This is shown by the mass becoming thin, transparent, and assuming a glassy appearance when it cools. The fire being now withdrawn, cold water is added, very gradually at first, with brisk stirring till the whole mass falls into a state of powdery granulation, when it is passed through a wire sieve to break down any lumps that may remain.

4. Separation of the Stearic and Margaric Acids from the lime. For this purpose, as much muriate of lime (chloride of calcium) is taken as will, with its equivalent quantity of sulphuric acid (8lbs. of dry muriate of lime, require $7 \mathrm{lbs}$. of the strongest sulphuric acid), produce as much muriatic acid as will dissolve the lime combined with the fat acids: and therefore that quantity of muriate of lime dissolved in water must be treated with as much sulphuric acid as will saturate its lime and throw it down in the state of sulphate of lime. Add the supernatant solution of muriatic acid in such proportion to the stearate and margarate of lime as will rather more than saturate the lime. Three pounds of muriatic acid diluted with 9 lbs. of water are stated as enough for $1 \mathrm{lb}$. of lime. The mixture is to be let alone for 3 or 4 days, in order to insure the complete separation of the lime from the fat acids; and then the mixture is heated so as to melt and cause them to separate in a stratum on the top of the liquid. The resulting muriate of lime is drawn off into another tub, and decomposed by its dose of sulphuric acid, so as to liberate its muriatic acid for a fresh operation.

5. The fat acids, being well washed by agitation with hot water, are then set to cool and crystallise, in which state they are subjected to the action of the hydraulic press, at a temperature of $75^{\circ} \mathrm{F}$., whereat the margaric acid runs off from the solid stearic acid.

6. Bleaching. The stearic acid is taken from the press, and exposed upon water in large shallow vessels placed in the open air, where it is kept at the melting temperature from 1 to 12 hours, stirring meanwhile, in order to promote the blanching action of the atmosphere. The margaric acid is bleached in a similar manner in separate vessels.

7. Refining Process. The fat is warmed again, and poured in a liquid state into an 
agitating tub; where, for every $1,000 \mathrm{lbs}$. of the stearic acid, about $2 \frac{1}{2} \mathrm{lbs}$, of common black oxide of manganese, and $40 \mathrm{lbs}$. of concentrated sulphuric acid, diluted with $200 \mathrm{lbs}$. of pure water are to be used. This solution ('mixture'), while warm from the heat evolved in diluting the acid, is placed in a suitable vessel above the agitating tub. The stearic acid being at the melting point, in the vessel below, agitation is to be given with a revolving shaft, while the mixed manganese and acid are run slowly down into it, till the whole be well mixed, which generally requires about two hours. The mass is allowed to lie in this state for 48 hours; after which it may be boiled by steam for 2 or 3 hours, when it will be sufficiently refined. The sulphuric acid, which is at the bottom, is now run off, and the stearic acid which remains is well washed with pure water. It is then put into large conical ressels of stone-ware, inclosed in a box or jacket, kept warm by steam-heat, and lined with conical bags of a suitable strong filtering paper, through which, being warm, it finds its way: and when the stearic acid has been thus filtered, it is run into blocks, when it will be found to be a beautiful stearic acid or palm-wax, and is ready to be made into candles in the usual way.

The chief solid constituent of palm-oil is margaric acid. This they direct to be melted with tallow, in the proportion of from 10 to $20 \mathrm{lbs}$. of the former to $100 \mathrm{lbs}$. of the latter. See Newton's Journal, C. S., xi. 207.

Messrs. Price and Co. introduced, in 1840, on the occasion of Her Majesty's marriage (when, for the illuminations, a cheap self-snuffing candle was required), a new composite candle, which was a mixture of stearic acid and cocoa-nut stearine. Mr. George Gwynne, in 1840, patented a process for purifying the fatty acids by distillation; this was followed by a similar patent by Dubrunfant; and Mr. Wilson, of Belmont, Vauxhall, obtained in August 1842, a patent for improvements in treating fats for making candles. These advances led to many modifications in candle-manufacture.

If distilled fats are used in making composite candles, they are bleached and hardened in that operation. When palm-oil is the material, it is first saponified, then distilled, granulated by fusion and slow cooling, and cold-pressed; by which means stearic acid and a light-coloured oil are obtained, which may be mixed with the stearine of cocoa-nut oil, or other stearine. A cheaper article may be had by mixing the entire product of the above distillation with half its weight of distilled and cold-pressed stearic acid of tallow. Tallow is deprived of its oleine by pressure, accompanied by artificial cold if necessary; this being added to the other hard matter, the mixture is converted into fatty acids, and distilled, and the entire product of distillation is employed for making candles; or it may be pressed to make them harder. As distilled stearic acid is more crystalline than undistilled, 2 or 4 per cent. of wax may be added to assist the combination of the fatty acid with the stearine.

Candles consisting of alternate layers of tallow and stearine have been made by dipping their wicks alternately in these two fatty bodies in a fluid state. $\mathbf{M r}$. W. Sykes has gone to the expense of a patent on the contrivance. The wicks are impregnated with a solution of bismuth or borax.

In a lecture delivered before the Society of Arts by Mr. Wilson, and published in their Journal, he described the progress of the more recent improvements. In this he says:- 'Candles, beautiful in appearance, were made by distilling the cocos-nut acids; but, on putting them out, they gave off a choking vapour, which prodnced violent coughing.' 'This prevented those candles from being brought into the market. - By distilling cocoa-nut lime-soap, we made beautiful candles, resembling those made from paraffine, burning perfectly; but the loss of material in the process was so great, that the subsequent improvements superseded its use. Under one part of this patent, the distillation was carried on sometimes with the air partially excluded from the apparatus by means of the vapour of water, sometimes without, the low evaporating point of the cocoa-nut acids rendering the exclusion of air a matter of much less importance than when distilling other fat acids.' At this time, in conjunction with Mr. Jones, Mr. Wilson appears to have first tried using the vapour of water to exclude the air from the apparatus during distillation. This led, in 1842, Messrs. E. Price and Co. to patent, in the names of Wilson and Jones, a process which involved the treatment of fats, previously to distillation, with sulphuric acid, or nitrous gases. M. Frémy, in his valuable paper in the 'Annales de Chimie,' describes treating oils with half their weight of concentrated sulphuric acid, by which their melting point was greatly raised. Ho gave, however, particular directions that the matter under process should be kept cool. Instead of doing this, Mr. Wilson found it advantageous to expose the mixture of fat acid and fat to a high temperature, and this is still done at Price's works. -

- Our process of sulphuric acid saponification was as follows.-Six tons of the material employed-usually palm-oil, though occasionally we work cheap animal fat, vegetablo oils, and butter, and Japan wax-were exposed to the combined action of $6 \frac{3}{4} \mathrm{cwts}$. of concentratod sulphuric acid, at a temperature of $350^{\circ} \mathrm{F}$. In this process 
the glycerine is decomposed, large volumes of sulphurous acid are given off, and the fat is changed into a mixturo of fat acids, with a very high melting point, This is washod, to freo it from charred matter and adhering sulphuric acid, and is then transforred into a still, from which the sir is excluded by moans of steam. The sterm used by us is heated in a series of pipes similar to those used in the hot-blast apparatus in the manufacture of iron, the object of heating the steam being only to save the still, and reduce to a small extent gaseous loss in distillation.' 'Wo still,' says the patentee, 'employ this process, and in some cases reduce the quantity of acid employed to $4 \mathrm{lbs}$, and even $8 \mathrm{lbs}$., to a cwt. of the fat.'

Vegetable tallow melts at a degree of heat somewhat above that of animal tallow, but considerably below that of regetable wax. Mr. Wilson treats his tallow by putting 6 tons of it into an iron still capable of holding 9 tons, heats it gradually to $350^{\circ} \mathrm{F}$., and then adds gradually $1,440 \mathrm{lbs}$. of sulphuric acid of $1 \cdot 8$ specific gravity. At the expiration of about 2 hours, the tallow is pumped into a vessel, containing water slightly acidulated with sulphuric acid; and is therein agitated by freo steam passing through it for 2 hours. The materials are then left to repose for 6 hours; both these vessels, and the former, should be provided with a cover and a means of conveying the gases which may be evolved into a chimney. The vegetable tallow is next distilled in such a manner that the atmosphere is exciuded. This is best effected by the use of steam highly heated, which he introduces into the still, in numerous jets below the tallow. The distilled products are received into condensers, and they may be used alone, or they may be mixed with other matters for making the best class of candles. The patentee improves paraffine by a like process. He makes candles with 2 or 3 wicks, by mixing palm-oil pressed with tallow, or the above distilled fat, for burning in eandle lamps.

In 1854, Mr. Tighlman obtained a patent for the exposure of fats and oils to the action of water at a high temporature, and under great pressure in order to cause the combination of the water with the elements of the neutral fats; so as to produce at the same time free fat acid and solution of glycerine. See Glycerine.

He proposed to effect this by pumping a mixture of fat and water, by means of a force-pump, through a coil of pipe heated to about $612^{\circ} \mathrm{F}$., kept under a pressure of about $2,000 \mathrm{lbs}$. to the square inch; and he states that the vessel must be closed, so that the requisite amount of pressure may be applied to prevent the conversion of water into steam. Mr. Wilson improred upon this process, by passing steam into fat at a high temperature; and by this process hundreds of tons of palm-oil are now treated. The glycerine and fat distil over together, but no longer combined; and the former, being separated, is subjected to a redistillation, by which it is purified. This distillation is effected by transmitting through the fat contained in an iron still, steam at about $600^{\circ}$ or $700^{\circ} \mathrm{F}$., heated by passing through iron pipes laid in a fire. The steam is transmitted till the oily matter is heated to about $350^{\circ}$; the vapours produced being carried into a high shaft by a pipe from the cover of the iron vessel. The hot oily matter is then run into another vessel made of brick lined with lead, and sunk in the ground, for the purpose of supporting the brick-work under or against the internal pressure of the fluid. It has a wooden cover lined with lead, directly beneath which, and extending across the vessel, is a leaden pipe, 1 inch in diameter, having a small hole on each side, at every 6 inches of its length; and through this pipe is introduced a mixture of $1,000 \mathrm{lbs}$. of sulphuric acid, specific gravity $1 \cdot 8$, and the same weight of water. The introduction of the mixture, which falls in divided jets into the heated fat, produces violent ebullition; and by this means the acid and fat are perfectly incorporated before the action of the acid becomes apparent by any considerable discolouration of the fat. As the ebullition coases, the fat gradually blackens; and the matter is allowed to remain for 6 hours after the violent ebullition has ceased. The offensive fumes produced are carried off by a large pipe, which rises from the top of the ressel, then descends, and afterwards xises again into a high ehimney. At the downward part of this pipe a small jet of water is kept playing, to condense such parts of the vapours as are condensable. At the end of the six hours above mentioned, the operation is complete, and the product is then pumped into another close vessel and washed, by being boiled up (by means of free steam) with half its bulk of water. The water is drained off, and the washing repeated, except that in the second washing the water is acidulated with $100 \mathrm{lbs}$. of sulphuric acid. The vltimate product is allowed to settlo for 24 hours; after which it is distilled in an atmosphere of steam - once, or oftener-until well purified; and the product of distillation is again washed, and after being pressed in the solid state, is applied to the manufacture of candles.

The following definitions of terms applied to candles are by Mr. Wilson :-

Belmont Sperm.-Made of hot-pressed, distilled palm-acid.

Belmont Wax.-The same material, tinged with gamboge. 
Best Composite Candles, - Made of a mixture of the hard palm-acid, and stearine of cocoa-nut oil.

Composites, Nos. 1, 2, and 3, are made of palm-acids, and palm-acids and cocoanut stearine, the relative proportions varying according to the relative market prices of palm-oil and cocoa-nut oil at the particular time when the candles are manufactured.

Composite, No. 4.-A description of candle introduced at a price a very little above tho price of tallow dip candles. They are somewhat dark in colour, but give a good light.

The highest priced candles are usually made in the ordinary mould; but at Messrs. Price and Co.'s manufactory they have a machine for moulding the ordinary stearine candles, the others of a similar nature. When one set of candles is discharged from the mould, the moulds are re-wicked for the next process of filling. These moulds are arranged, side by side, 18 in number, on a frame; and for each mould there is a reel capable of holding 60 yards of wick, enclosed in a box. The moulded candle, being still attached to the cotton wick, when it is foreed out of the mould, brings the fresh wick into it. The moulded candles are, by a very ingenious contrivance, held firm in a horizontal position while a knife passes across and severs the wick. The wicks for the new set of candles are firmly secured, by forceps, to the conical caps of the moulds; these are carried into a vertical position, and slid upon a railway to a hot closet, where they become sufficiently warm to receive the fat, which, kept at the melting point by steam-pipes, is held in a cistern above the rails ; from this cistern the moulds are filled by as many cocks, which are turned by one impulse. If we imagine an extensive series of these sets of moulds travelling from the machine over a railway, in regular order, and that, when the fat has become solid, these return, the candles are discharged, and the process is renewed-the machine will be tolerably well understood. Each machine holds about 200 frames of moulds, and each contains 18 bobbins, starting each with 60 yards of cotton wick.

Night-Lights.-These are short thick cylinders of fat, with a very thin wick, so proportioned one to the other that they burn any required number of hours. The moulds in which these are made are metal frames, perforated with a number of cylindrical holes, and having a moveable bottom, with a thin wire projecting from it into every mould. These are filled with melted fat, and when cold, the bottoms are forced up, and all the cylinders of fat ejected, each having a small hole through which the wick, a cotton, previously impregnated with wax, is inserted. This being done, the night-light, being pressed on a warm porcelain slab, is melted sufficiently to cement the wick. These night-lights are burned in glass cylinders, into which thoy fit.

Child's Night-Lights are melted fat poured into card-board boxes, which hare a hole in the bottom, through which the wick and its metallic support are placed.

Dr. Ure made a set of experiments upon the relative intensities of light, and duration of different candles, the results of which are contained in the following Table:-

\begin{tabular}{|c|c|c|c|c|c|c|}
\hline Number in a Pound & $\begin{array}{l}\text { Duration of } \\
\text { a Candle }\end{array}$ & $\begin{array}{l}\text { Weight in } \\
\text { Grains }\end{array}$ & $\begin{array}{l}\text { Consumption } \\
\text { per Hour in } \\
\text { Grains }\end{array}$ & $\begin{array}{l}\text { Proportion } \\
\text { of Light }\end{array}$ & $\begin{array}{l}\text { Economy } \\
\text { of Light }\end{array}$ & $\begin{array}{c}\text { Candles } \\
\text { equal one } \\
\text { Argand }\end{array}$ \\
\hline $\begin{array}{l}10 \text { mould } \\
10 \text { dipped } \\
8 \text { mould } \\
6 \text { ditto } \\
4 \text { ditto } \\
\text { Argand oil flame : }\end{array}$ & $\begin{array}{lc}h . & m . \\
5 & 9 \\
4 & 36 \\
6 & 31 \\
7 & 21 \\
9 & 3 \cdot 6 \\
& \cdots\end{array}$ & $\begin{array}{r}682 \\
672 \\
856 \\
1160 \\
1707 \\
\cdots\end{array}$ & $\begin{array}{l}132 \\
150 \\
132 \\
163 \\
186 \\
512\end{array}$ & $\begin{array}{l}12 \frac{1}{4} \\
13 \\
10 \frac{1}{2} \\
14 \frac{2}{3} \\
20 \frac{1}{4} \\
69^{\circ}\end{array}$ & $\begin{array}{c}68 \\
65 \frac{1}{2} \\
59 \frac{1}{2} \\
66 \\
80 \\
100\end{array}$ & $\begin{array}{l}5 \cdot 7 \\
5.25 \\
6.6 \\
5 \cdot 0 \\
3.5\end{array}$ \\
\hline
\end{tabular}

A Scotch mutchkin, or $\frac{1}{8}$ th of a gallon, of good seal oil, weighs $6010 \mathrm{gr}$. , or $13 \frac{2}{10}$ or. avoirdupois, and lasts in a bright Argand lamp 11 hours 44 minutes. The weight of oil it consumes per hour is equal to 4 times the weight of tallow in candles 8 to the pound, and $\frac{1}{7}$ th the weight of tallow in candles 6 to the pound. But its light being equal to that of 5 of the latter candles, it appears from the above table that 2 pounds weight of oil, value 9d., in an Argand, are equivalent in illuminating power to 3 pounds of tallow candles, which cost about 2 shillings. The larger the flame in the above candles the greater the economy of light.

Minkrat Candlzs. Under this general head several varieties of candles have been manufactured at the works of Price's Candlo Company; and these candles manufactured by Price's Candle Company, at Belmont and Sherwood, are made according to processes patented by $\mathrm{Dr}$. Warren $\mathrm{De}$ La Rue. The novelty of these substances 
consists: -1 . In the material from which they are obtained. 2. In the mothod by which they are elaborated. 3. In their chemical constitution.

The raw material is a semifluid naphtha, drawn up from wells sunk in the neighbourhood of the river Irrawaddy, in the Burmese Empire. The geological characteristics of the locality are sandstone and blue clay. In its raw condition the substance is used by the natives as a lamp-fuel, as a proservative of timber against insects, and as a medicine. Being in part volatile at common temperatures, this naphtha is imported in hermetically-closed metallic tanks, to prevent the loss of any constituent. Reichenbach, Christison, Gregory, Reece, Young, Wiesman (of Bonn), and others have obtained from peat, coal, and other organic minerals, solids and liquids bearing some physical resemblance to those procured from the Burmese naphtha; but the first-named products have, in every instance, been formed by the decomposition of the raw material. The process of De La Rue, is, from first to last, a simple separation, without chemical change.

In the commercial processes, as carried out at the Sherwood and Belmont Works, the crude naphtha is first distilled with steam at a temperature of $212^{\circ} \mathrm{F}$; ; about one-fourth is separated by this operation. The distillate consists of a mixture of many volatile hydro-carbons; and it is extremely difficult to separate them from each other on account of their vapours being mutually very diffusible, however different may be their boiling points. In practice, recourse is had to a second or third distillation, the products of which are elassified according to their boiling points or their specific gravities, which range from 0.627 to 0.860 , the lightest coming over first. It is worthy of notice, that though all these volatile liquids were distilled from the original material with steam of the temperature of boiling water, their boiling points range from $80^{\circ} \mathrm{F}$. to upwards of $400^{\circ} \mathrm{F}$.

These liquids are all colourless, and do not solidify at any temperature, however low, to which they have been exposed. They are useful for many purposes. All are solvents of caoutchouc. The vapour of the more volatile, Dr. Snow has found to be highly anæsthetic. Those which are of lower specific gravity are called in commerce Sherwoodole and Belmontine; these have great detergent power, readily removing oily stains fron silk, without impairing even delicate colours. The distillate of the higher specific gravity is proposed to be used as lamp-fuel; it burns with a brilliant white flame; and, as it cannot be ignited without a wick, even when heated to the tomporature of boiling water, it is safe for domestic use.

A small percentage of hydro-carbons, of the benzole series, comes over with the distillates in this first operation. Messrs. De La Rue and Müller have shown that it may be advantageously eliminated by nitric acid. The resulting substances, nitrobenzole, \&ce., are commercially valuable in perfumery, \&ce.

After steam of $212^{\circ} \mathrm{F}$. has been used in the distillation just described, there is left a residue, amounting to about three-fourths of the original material. It is fused and purified from extraneous ingredients (which Warren De La Fue and H. Müller have found to consist partly of the colophene series) by sulphuric acid. The foreign substances are thus thrown down as a black precipitate, from which the supernatant liquor is decanted. The black precipitate, when freed from acid by copious washing, has all the characteristic properties of native asphaltum. The fluid is then transferred to a still, and, by means of a current of steam made to pass through heated iron tubes, is distilled to any required temperature. The distillates obtained by this process are classed according to their distilling points, ranging from $300^{\circ}$ to $600^{\circ} \mathrm{F}$. The distillations obtained, at $420^{\circ} \mathrm{F}$. and upwards, contain a solid substance, resembling in colour and in many physical and chemical properties, the paraffine of Reichenbach; like it, it is electric, and its chemical affinity is very feeble; but there are reasons for believing that a difference exists in the atomic constitution of the two substances. The commercial name of Belmontine is given to one of the fluids from the Burmese pitch. Candles manufactured from the solid material (Paraffine) possess great illuminating power. It is stated that such a candle weighing $\frac{1}{8}$ th $\mathrm{lb}$., will give as much light as a candle weighing $\frac{1}{6}$ th $\mathrm{lb}$. made of spermaceti or stearic acid. Its property of fusing at a very low temperature into a transparent liquid, and not decomposing below $600^{\circ} \mathrm{F}$, recommends this substance as the material of a bath for chemical purposes. As to the fluids obtained in the second distillation, already described, they all possess great lubricating properties; and, unlike the common fixed oils, not being decomposable into an acid, they do not corrode the metals, especially the alloys of copper, which are used as bearings of machinery. This aversion to chemical combination, which characterises all these substances, affords, not only a security against the brass-work of lamps being injured by the hydro-carbon burnt in them, but also renders these hydro-carbons the best detergents of common oil lamps. It is an interesting physical fact, that some of the non-volatile liquid hydro-carbons possess the fluorescent property which Stokes has found to reside in certain vegetable infusions. 
An important characteristic of the Burmese naphths is its being almost entirely destitute of the hydro-carbons belonging to the olefiant-gas series. See NAPHTHA.

The OzoxnRr candles, so called, have been recently introduced by the Messrs. Field and Co. These candles are made of ozokerit, that is to say mineral paraffine, also called mineral wax, which is found in beds in sandstone near Slanik in Moldavia, in the vicinity of coal-mines, and mineral salt. It is also found in the Carpathian Mountains, and it is from this source that the English merchants get their supply. This material has a rough appearance, is of a brown, greenish, or yellow colour; it is translucent at the edges, and its fracture is resinous. It is naturally fragile, but in softening it can be kneaded like wax. If exposed to the air it turns black. Friction electrifies it negatively, and it emits an aromatic hydro-carbonaceous odour. At the low temperature of $66^{\circ} \mathrm{C}$. it flows, and other substances, less fusible, necessary in the manufacture of candles can then be added. The power of the light of ozokerit candles can be deduced from the experiments of Dr. Letheby, who has shown that the light of 754 ozokerit candles equals that of 891 paraffine, or 1150 wax candles. Ozokerit candles can be coloured mauve or magenta, and then present a pleasing appearance. See Paraffine, and Mnnerat Candlas.

Our Exports of Candles of all sorts were as follow :-

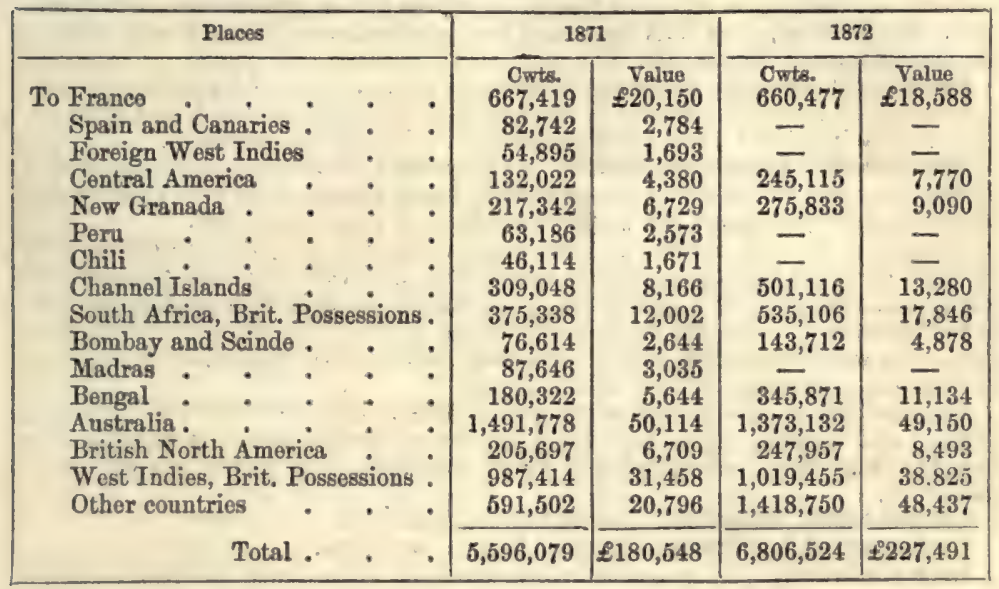

We Imported Candles from the following countries:-

\begin{tabular}{|c|c|c|c|c|c|c|c|c|}
\hline \multicolumn{5}{|c|}{ Places } & \multicolumn{2}{|c|}{1871} & \multicolumn{2}{|c|}{1872} \\
\hline \multicolumn{5}{|c|}{ STEARINE:- } & & Value & Cwts. & Value \\
\hline \multirow{4}{*}{\multicolumn{2}{|c|}{$\begin{array}{l}\text { From Russia . } \\
\text { Holland } \\
\text { Belgium } \\
\text { Other countries }\end{array}$}} & . & - & - & 11,968 & $\mathscr{E 4 7 , 4 8 5}$ & - & - \\
\hline & & . & . & * & 40,231 & 140,996 & 64,420 & $£ 230,605$ \\
\hline & & . & . & * & 30,265 & 96,329 & 41,214 & 151,536 \\
\hline & & . & , & - & 772 & 2,851 & 636 & 2,234 \\
\hline \multicolumn{2}{|c|}{ Total } & - & - & • & 83,236 & $£ 287,611$ & 106,270 & $£ 384,375$ \\
\hline \multicolumn{5}{|c|}{ OTHKR KINDs:- } & & & & \\
\hline From & Germany & + & & - & - & - & 2,423 & $£ 8,813$ \\
\hline & Holland & $\cdot$ & - & - & 932 & 3,084 & 1,129 & 8,007 \\
\hline & Belgium & * & - & • & 4,177 & 12,770 & 1,872 & 6,309 \\
\hline & Other countries & - & & - & 870 & 3,220 & 466 & $1 \cdot 729$ \\
\hline & Total & $\cdot$ & - & - & 5,979 & $£ 19,074$ & 5,890 & $£ 19,855$ \\
\hline
\end{tabular}

CAmsrxa, or Wild Cinnamon. The Canella alba, a tree growing in the West Indies. The bark is used as an aromatic and tonic. The negroes of the West Indies employ it as a condiment.

CANz-MIII. The mill employed for crushing the sugar-cane. See Sugar.

CAsras. The produce of rarious species of the genus Calamus. The Calamus Rotang and C. Scipionum are said to produce the rattan canes of commerce, but it is 
probable that several species are cut indiscriminately. The best rattans are obtained from Sumatra, Borneo, and Malaces ; from these places they aro exported to Europe, Bengal, and China ; in the latter country the consumption of rattans is immense. Tho average annual importation to this country amounts to about $11,000,000$. The stems of the rattan are coated with a flinty bark; they are easily split, and from them the bottoms of chairs and similar articles are manufactured. The resinous matters of the rattan is the dragon's blood of commerce.

Rattan anes are often counfounded with the bamboo. They are, however, as stated above, the produce of various species of the genus Calamus, whilst the bamboo is a grass belonging to the genus Bambrusa. Rattans are cylindrical, jointed, very tough, and strong, from the size of a goosequill to that of the human wrist, and from fifty to a hundred feet in length. They are used for wicker-work, seats of chairs, walking-sticks, \&cc.

The bamboo is a plant of the reed kind, growing in the East Indies, and other warm climates, and sometimes attaining the height of sixty feet. Old stalks grow to fire or six inches diameter, and are so hard and durable as to be used for building, and for all sorts of furniture, for water-pipes, and for poles to support palanquins. The smaller kinds are used for walking-sticks, flutes, \&c. Seө BАмвоо.

Canes of various kinds are employed in manufactures, as the Sugar-cane, \&ce. The arundo (the reed), a genus of plants belonging to the grasses, are also largely used. They are imported from Spain and Portugal for the use of the weaver, and for the manufacture of fishing rods.

CAsrarca wOOD, called also in England Angica. It is of a rose-wood colour; and is imported from the Brazils in trimmed logs, from eight to ten inches diameter. As a variety in cabinet work, small quantities of this wood are employed.

CANTrABIC Composirrow. This material for architectural decoration is deseribed by Mr. B. Albano to have a basis of homp, amalgamated with resinous substances, carefully prepared and worked into sheets of large dimensions.

Ornaments in high relief and with great sharpness of detail are obtained by pressure of metal dises, and they are of less than half the weight of papier-maché ornaments, sufficiently thin and elastic to be adapted to wall surfaces, bearing blows of the hammer, and resisting all ordinary actions of heat and cold without change of form. Its weather qualities had been severely tried on the Continent, as for coverings of roofs, \&ce, remaining exposed without injury.

This composition is of Italian origin, and in Italy it has been employed for panels, frames, and centres. It is well fitted to receive bronze, paint, or varnish; the material is so hard as to allow gold to be burnished, after gilding the ornaments made of it.

Camarasts Indrca. Indian hemp. See Hrirp.

cavrasis satrva. Common hemp. Seo Hemp.

CANranirs. A poisonous resin extracted from hemp (Cannabis sativa). The narcotic properties of chaschisch or bang depond on the presence of this resin.

CANTrA rouxIs. The rhizomes or tubers of this plant are supposed to yield the starch known in commerce as Tous-les-mois, but it is probably derived from C. glawca or C. Achiras. (Pereira; Bentley and Redwood, 1872). Seo Tous-LEs-Mors.

CANNEI COAI is generally considered to be a variety of bituminous coal. Its name Canwyl, a candle, is derived from the readiness with which it kindles and burns with flame. Cannel coal is obtained most abundantly in Lancashire, especially around Wigan, which district produces 650,000 tons per annum, while the Manchester district produces about $\mathbf{1 3 0 , 0 0 0}$ tons. It is found abundantly in Scotland, where it is known as gas-coal; the quantity produced in 1865 was 322,000 tons. In Flintshire a very fine cannel has been found, especially at Leeswood, near Mold; about 150,000 tons are raised, nearly all of which is used for the distillation of coaloil. Cannel coal is also found in Derbyshire, in Warwic's?.izo, and in Yorkshire.

\section{Detailed Statement of the Produotion of Cannel Coal in Great Britain.}

\section{ExaLAND.}

Staffordshire, North.-A Cannel coal of uncertain quality occurs in this district, especially between Hanley and Harecastle. It is usually termed in the locality 'pill' or 'peal,' and is sold at the pit's mouth at 108, the ton. The quantity of this Cannel which is raised annually is about
Nottinghamshirc. - The chief if not the only place in this county at which Nottinghamshire. - The chief if not the only place in this county at which
Cannel is worked is at Hucknall Torckard, near Nottingham. Nearly all that is raised is appropriated for oil-naking, the quantity produced being

Derbyshire.-The places produeing this variety of coal are the following:Tors

Ronishaw, near Chesterfield.-This is not strictly a Cannel.

Swanwick Colliery.-This is a Cannel of fair average quality.

Clay Cross.-But little is worked on this estate at present. 
The total production of Derbyshire is estimated at about

Yorkshire - The mines in Yorkshire producing Cannel a
1. Silkstone Fall, Barnsley.
5. College, Birstal.
2. Adwalton Moor, Leeds.
6. Oakwell, Lreds.
3. Bruntcliffe, Leeds.
4. Gilderstone, Leeds.
7. Ardsley Main, Ardsley.

Some of this is an inferior Cannel, known, locally, as 'drub' or 'stub' and a considerable quantity of the true Cannel of the West Riding will not coke. The quantity raised annually from the Yorkshire district may be taken at. $\mathbf{1 8 5 , 0 0 0}$

Lancashire. - The collieries in the East Lancashire (Manchester) district producing Cannel coal are the following:-
1. Bank, Little Hulton.
2. Blackrod, Chorley.
3. Blackrod, Blackrod.
4. Bridgewater, Worsley.
5. Hulton, Bolton.
6. Scot Lane, Blackrod.
7. Stonehill, Farnworth.
8. Rigby Pit, Anderton Hall.

The total estimated production being - . . . . . . 120,000

The collieries in the West Lancashire (Wigan) district producing Cannel are:-

1. Haigh, Haigh and Aspull. |3. Kirkless Hall, Kirkless.

2. Aspull, Haigh and Aspull. 4. Standish, Standish.

(These belong to the Wigan Coal and Iron Company.)
5. Rose Bridge, Ince.
6. Douglas Bank, Wigan.
9. Walthew House, Pemberton.
7. Norley Hall, Pemberton.
8. Ince Hall, Wigan.
10. Gidlow Lano, Wigan.
11. Rowenhead, St. Helen's.

The total production of these collieries was

Cheshire.-Duckinfield, Stockport, is the only colliory in this county pro-

ducing Cannel coal. The present yield being . . . . . . 12,000

\section{The Estimated Production of Cannel Coal in England.}

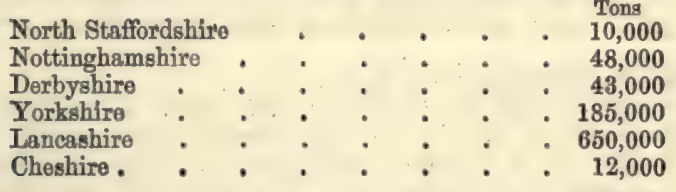

The total annual production in England being about . $\overline{948,000}$

At least 50,000 tons of this, possibly much more, is reserved for the manufacture of the mineral oils.

\section{WATIS.}

North Wales.-This is the only district at present producing Cannel. The collieries yielding it are:-
1. Leeswood Green, Mold.
2. Leeswood Hill, Mold.
8. Cood Talon, Mold.
4. Coppa, Mold.
5. Nertquis, Mold.
6. Wern, Bagilt.

From these works the quantity of Cannel coal raised was, as nearly as could be ascere tained, 150,000 tons, almost the whole of which was consumed in the oil works of Flintshire, which are now much reduced in extent.

\section{SCOTLAND,} field.

We have reliable information as to the output of Cannel coal from this important

At a meeting of coal-owners held in Glasgow, the estimated quantity raised annually was as follows :-

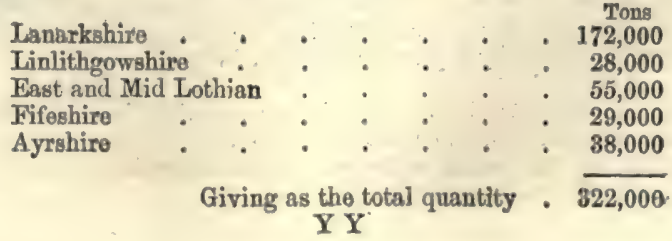


The stock of Cannel coal on hand when this estimate wns made was statod to have been 61,500 tons, making the total quantity raised as 383,500 tons. Mr. Binney informs us that nearly 100,000 tons of this is used in making oil.

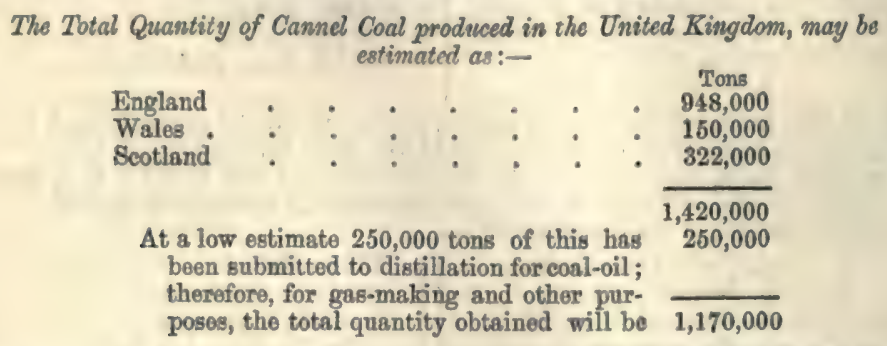

See Sramiss and Sham Orr.

This coal, when worked for ornament, is cut with a saw, and the pieces are roughshaped with a chopper. For making a snuff-box, whether plain, serewed, or excentric turned, the plank-way, or the surface parallel with the seam, is most suitable; it is also proper for vases, the caps and bases of columns, \&c. Cylindrical pieces, as for the shafts of columns, should be cut from either edge of the slab, as the lamine then run lengthways, and the objects are much stronger: cylindrical pieces thus prepared, say 3 inches long and $\frac{3}{8}$ ths of an inch diameter, are so strong they cannot be broken between the fingers. Similar pieces have been long since used for tho construction of flutes, and in the British Museum may be seen a snuff-box of Cannel coal, said to have been turned in the reign of Charles $I$., and also two busts of Henry VIII. and his daughter Lady Mary carved in the same material. The plank-way surfaces turn the most freely, and with shavings much like those of wood; the edges yield small chips, and at last a fine dust, but which does not stick to the hands in the manner of common coal. Flat objects, such as inkstands, are worked with the joiners' ordinary tools and planes. The edges of Cannel coal are harder and polish better than flat surfaces.-Holtrapffel. See CoAL and BogHEAD COAL.

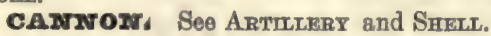

CANTON-BAII TREz. Couroupite Guianensis. A tree belonging to the Lecythidece, a suborder of the dicotyledonous plants belonging to the natural order Myrtacece. These trees are natives of South America, and are distinguished by their very large fleshy flowers and singular fruit. The cannon-ball tree obtains its name from the peculiar large heavy woody fruit, which is as big as a thirty-six-pound shot. The shells of this fruit are used for drinking vessels.

CANTHARIDzS. The blister beetle or Spanish fly, the Cantharis vesicatoria. Cantharides are chiefly collected in Hungary, Russia, and the south of France. They are imported from St. Petersburg in cases, each containing 160 to $170 \mathrm{lbs}$. and also - from Messina in barrels or cases holding each $100 \mathrm{lbs}$. See Pereira's 'Materia Medica,' Bentley and Redwood, 1872.

CANTOZN'S PEOSP HORUS. Sulphate of lime being heated with charcoal in a crucible is converted into sulphide or sulphuret of calcium. If this preparation is spread over a sheet of paper, and exposed to bright sunshine, it will, when taken into a dark room, emit a phosphoroscent light. This solar phosphorus was discovered by Canton, and is propared according to his directions by calcining for an hour at a red heat a mixture of 3 parts of ground oyster-shells and 1 part of sulphur. Seo Phospronts and Pxrophorus.

Carrvas. (Canevas, Fr.; Kanevas, Ger.) A coarse cloth made of hemp or flax, which is used for tho sails of ships and for tents. It has boen found that sails of ships made with the solvages and soams of the canvas running down parallel to their edges are vory apt to bag, and becomo torn in the middle, from the strain to which they are subjected by the pressure of the wind. To obviate this inconvenience a mode of making sails, with the seams and selvages running diagonally, was proposed by Admiral Brooking, and a patent granted to him for the same on Novomber 4, 1828. The invention of Messrs. Ramsay and Orr has a similar object, viz., that of giving additional strength to sails by a peculiar manner of weaving the canvas of which they aro mado.

The improrement consisted in weaving the canvas with diagonal throads; that is, placing the weft yarn, or shoot, in weaving, at an oblique angle to the warp yarns, instead of making the decussation of the wurp, or weft threads, or yarns, at right angles to each other, as in the ordinary mode of weaving. 
To accomplish this object the loom must be peculiarly constructed; its warp and work beams must stand at an oblique angle with the sides of the loom, and the batten and slay must be hung in a peculiar manner, in order to beat up the weft or shoot, in lines ranging diagonally with the warp.

Canvas paintod of various patterns is used for covering halls, \&c., and is generally callod floor-cloth. A finer kind of canvas, properly prepared, is employed by artists.

CAOUTCEOUC, GUM-ZIASTIC, or INDIA-RUBBER (Caoutchouc, Fr. ; Kautschuk, Federharz, Ger.) occurs as a milky juice in several plants, such as the Siphonia Cahuca, ealled also Hevea Guianensis, Cautschuc, Jatropha elastica, Castilleia elastica, Cecropia pelleta, Ficus religiosa and indica, Urceola elastica, \&ce. Dr. Lindley's account of the plants that yield the most important supplies of commercial caoutchouc, with plates of the leaves and flowers, will be found in Mr. Hancock's work on Caontchouc. These are stated to be Siphonia elastica, Hancornia speciosa, Ficus elastica, and Urceola elastica. It is, however, extractod chiefly from the first plant, which grows in South America and Java. The tree has incisions made into it through the bark in many places, and it discharges the milky juice, which is spread upon clay moulds, and dried in the sun, or with the smoke of a fire, which blackens it. Portions of this milky juice have been occasionally sent to Europe in bottles; some fow arrived with the milky emulsion, but generally they were found to be resolved into a coagulum floating in an aqueous solution. A small bottle from Cayenne or the Isle of France, in the possession of M. D'Arcet, passed some years without change, but the severe winter of 1788-9 caused it to pass to the solid state, and the bottle was broken.

Sir Joseph Banks is reported to have had a bottle of liquid unaltered, but which afterwards was decomposed. Fifty gold louis-d'ors, it is said, were offered by him at Lisbon in vain for a second supply.

Caoutchouc, Gum-Elastic, or India-Rubber, are the general names for a substance now so well known, familiar, and important, that it seems matter for surprise that the latter half of the preceding century should have passed away before it was made known to Europe by memoirs read to a learned body. For the remainder of the century, its extraordinary property of elasticity. and the grotesque objects made by the Indians, caused it to be met with in the cabinets of the curious; its use was still confined to erasing marks of black-lead pencil from paper, and in this country it received the common name of lead-eater.

Europe is indebted to the observations of M. de la Condamine, who, despatched from France nn a scientific mission, found the natives and residents of that part of South America which he visited constantly using syringes, bottles for non-corrosive liquids, boots, and many other articles; made of India-rubber. In 1736, he wrote to the Academy of Sciences at Paris an account of this substance; which during his te years' residence in Para, and journeys in the country, and along the banks of the Amazon, he had constant observation of the use of by the natives.

He describes the forms of bottles and articles moulded with clay, coated with the milky juice of trees, in successive layers, and when dried in the sun, the earth broken out, and says that the Indians, with a point of hard wood, impressed ornaments upon the soft mass.

He also states that the gum-resin spread upon eloth formed a waterproof covering of great use for bagging to keep biscuits, food, and clothing dry from rain, and as a substitute for tarpaulins; and ho especially notes the use of a great canvas prepared with liquid India-rubber, to cover the quadrant circle as it stood on its legs, which allowed it to be left in the rain end snow, and thus enabled them to make observations at intervals of weather, and avoid the great labour of removing the instruments to boxes and places of shelter. To obviate the adhesion of objects recently made to each other, especially if the sun is upon them, "Spanish white, and even dust is employed:' the inconvenience is thus prevented, and the articles immediately take the brown colour which is ultimately acquired by the exposure of the white juice to the sun and air, smoke and fire, methods employed by the Indians.

M. de la Condamine found in the provinee of Esmeraldes this substance, called by the natives Hhévé; it is obtained by a single incision from the plants, and is a white liquid like milk, which hardens and blackens in the air. The Indians made flambeaux 11 inch thick and 2 feet long, that burnt very well without a wick for 24 hours with a brilliant flame and without any disagreeable odour. This caoutchouc was wrapped in two leaves of bananier to form the flambeaux; he used these lights habitually, on his route along the river Esmeraldes, and especially in the wood of Sylanche, where he was detained for days.

It was in 1751, that M. de la Condamine brought the subject into notice, for M. Fresnoau had found and described trees in Cayenne, yielding elastic resin. Writing to hif

$$
\times \times 2
$$


friond, he records the localitios, trees, tools, and details for obtaining the sap, and forming articles.

M. do la Condamine concludes his remarks upon the memoir of M. Fresneau with words of suffieient import, and indeed with prophetic spirit, and says, 'it will be an exclusire object of commerce for that colony which possesses this species of treasure.'

MM. Herissent and Macquer in 1761, gave their chemical observations to the Académie Royale on caoutchouc, or elastic resin of Cayenne. They refer to the softening and solution by oils and heat, "but the caoutchouc does not again takes its solidity or elasticity.' By rectifying oil of turpentine upon lime and dissolving caoutchouc in it, they obtained a pasty mass which allowed the caoutchouc to regain its former elastic state. They point out that ether may be advantageously used, and they complain of the great expense of Dippel's animal oil as a solrent. While pointing out the surgical uses of caoutchouc, Macquer describes the necessity for carefully rectifying the ether, taking 8 or 10 pounds, and only receiving the first 2 pounds for the solution of caoutchouc. He used, instead of clay, moulds of wax, held by pincers, and plunged in the ethereal solution, and by repeated coatings, allowing the ether to evaporate, a thin covering was obtained; by the heat of boiling water the wax flowed out, and a tube of caoutchouc remained. He states that he found it difficult to get the tubes uniform. M. Grossart in 1768, published his experiments on preparing tubes of India-rubber by means of ether and boiling water: these memoirs seem to have remained without sufficient reference and study.

It has been received as an opinion in England and America, that one of the earliest notices of the useful properties of India-rubber is that given by Dr. Priestley, in a work evidently got up with gxeat care, called, 'A Familiar Introduction to the Theory and Practice of Perspective, by Joseph Priestley, LL.D., London, 1770.' At the end of the preface is the following addition: 'Since this work was printed off, I have seen a substance excellently adapted to the purpose of wiping from paper the marks of a black-lead pencil. It must, therefore, be of singular use to those who practise drawing. It is sold by Mr. Nairne, Mathematical Instrument-maker, opposite the Royal Exchange. He sells a cubical piece of about half an inch for three shillings, and he says it will last several years.'

It will be remarked that no name for the substance is mentioned; the preface is dedicated 'to Sir Joshua Reynolds, Knt., F.R.S., Leeds, March 20th, 1770;' and we may fairly conclude that the substance was at this time a novelty in art and trade.

It seems probable, that the experiments with balloons, and the application of airtight varnishes, especially those by Messrs. Charles and Robert, called more general attention to the properties of India-rubber in Europe.

Mr. Hancock says, "This substance came first into notice about the beginning of the last century, moulded into the shapes of bottles and animals. It was sold as high as a guinea the ounce, and used for rubbing out pencil-marks; but scarcely anything was known of its history, except that it came from America.'

The juice itself has been of late years imported. It is of a pale yellow colour, and has the consistence of cream. It becomes covered in the bottles containing it with a pellicle of concrete caoutchouc. Its specific gravity is 1.012. When it is dried it loses 55 per cent. of its weight; the residuary 45 is elastic-gum. When the juice is heated it immediately coagulates, in virtue of its albumen, and the elastic-gum rises to the surface. It mixes with water in any proportion; and when thus diluted, it coagulates with heat and alcohol as before.

Hitherto the greater part of the caoutchouc has been imported into Europe from South America, and the best from Para; but of late years a considerable quantity has been brought from Java, Penang, Singapore, Assam, and Africa. Many years ago, Mr. William Griffith published an interesting report upon the Ficus elastica, the caoutchouc tree of Assam, which he drew up at the request of Captain Jenkins, agent in that country to the Governor-General of India. This remarkable species of fig-tree is either solitary or in twofold or threefold groups. It is larger and more umbrageous than any of the other trees in the extensive forest where it abounds, and may be distinguished from the other trees at a distance of several miles, by the picturesque appearance produced by its dense, huge, and lofty crown. The main trunk of one was carefully measured, and was found to have a circumference of no less than 74 feet; while the girth of the main trunk along with the supports immediately round it, was 120 feet. The area covered by the expanded branches had a circumference of 610 feet. The height of the central tree was 100 feet.

It has been estimated, after an accurate survey, that there are 43,240 such noble trees within a length of 30 miles and a breadth of 8 miles of forest, near Ferozepore, in the district of Chardwar, in Assam.

Lieutenant Veitch has since discorered that the Ficus elastica is equally abundant in the district of Naudwar. Its geographical range in Assam seems to be between 
$25^{\circ} 10^{\prime}$ and $27^{\circ} 20^{\prime}$ of north latitude, and between $90^{\circ} 40^{\prime}$ and $95^{\circ} 30^{\prime}$ of east longitude. It occurs on the slopes of the hills up to an elevation of probably 22,500 feet. This tree is of the Banyan tribe, famed for its pillared shade, 'whose daughters grow about the mother tree,' which has furnished the motto, Tot rami, quot arbores, to the Royal Asiatic Society. Species of this genus afford grateful shade, however, in the tropical regions of America as well as Asia.

Many species of other trees yield a milky tenacious juice, of which bird-lime has been frequently made; as Artocarpus integrifolia and Lakoocha, Ficus indica and religiosa, also $F$. Tsiela, Roxburghii, glomerata, and oppositifolia. From some of these an inferior kind of caoutchouc has been obtained.

The juice of the Ficus elastica of Chardwar is better when drawn from the old than from the young trees, and richer in the cold season than in the hot. It is extracted by making incisions a foot apart, across the bark down to the wood, all round the trunk, and also the large branches, up to the very top of the tree: the quantity which exudes increasing with the height of the incision. The bleeding may be safely repeated once every fortnight. The fluid, as fresh drawn, is nearly of the consistence of eream, and pure white. Somewhat more than half a maund $(42 \mathrm{lbs}$.) is reckoned to be the average produce of each bleeding of one tree; or 20,000 trees will yield about 12,000 maunds of juice; which is composed in 10 parts, of from 4 to 6 parts of water, and, of course, from 6 to 4 parts of caoutehouc. The bleeding should be confined to the cold months, so as not to interfere with or obstruct the vigorous vegetation of the tree in the hot months.

Mr. Griffith says, that the richest juice is obtained from transverse ineisions made into the wood of the larger reflex roots, which are half exposed above ground, and that it proceeds from the bark alone. Beneath the line of incisions, the natives of Assam scoop out a hole in the earth, in which they place a leaf of the Phrynium capitatum (Linn.), rudely folded up into the shape of a cup. He observes that the various species of Tetranthera, upon which the Moonga silkworm feeds, as also the castor-oil plant, which is the chief food of the Eria silkworm, do not afford a milky caoutchoue juice. Hence it would appear that Dr. Royle's notion of caoutchoue forming a necessary ingredient in the food of silkworms, and being ' in some way employed in giving tenacity to their silk,' seems to be unfounded. If Botany discountenances this idea, Chemistry would seem to scout it altogether; for silk contains 11.33 per cent. of nitrogen, and caoutchouc contains none at all, being simply a solid hydro-carbide, and therefore widely dissimilar in constitution to silk, which consists of oxygen $34 \cdot 04$, nitrogen $11^{\cdot 33}$, carbon 50.69, and hydrogen 3.94 , in 100 parts.

This hydro-carbide emulsion is of common occurrence in the orders Euphorbiacece and Urticacee, which may be looked on as the main sources of caoutchouc. The American croutchouc is said to be furnished by the Siphonia elastica, or the Hevea Guianensis of Aublet, a tree which grows in Brazil, and also in Surinam.

Dr. Royle sent models of cylinders of $1 \frac{1}{2}$ to $2 \frac{1}{2}$ inches in diameter, and 4 or 5 inches in length, to both the Asiatic and Agricultural Societies of Bengal, to serve as patterns for the natives to mould their caoutchoue by. Mr. Griffith says that this plan of forming the esoutchouc into tumblers or bottles, as recommended by the committee of the London Joint-Stock Caoutchouc Company, is, in his opinion, the worst that can possibly be offered; being tedious, laborious, causing the caoutchouc to be blackened in the drying, and not obviating the viscidity of the juice when it is exposed to the sun. He recommends, as a far better mode of treating the juice, to work it up with the hands, to blanch it in water, and then subject it to pressure. Better methods have recently occurred while experimenting upon the caoutchoue juice. This fluid, with certain precautions, chiefly exclusion from air and much warmth, may be kept in the state of a creamy emulsion for a very long time. Mr. Hancock states, some barrels treated with ammonia arrived in England in a fluid state.

However plausible these observations may appear, the practical men wanted sheet rubber to cut into threads, \&c., and Mr. Hancock states he had a cylinder made of masticated rubber, of a convenient size, and sent it to Para as a pattern for the natives, and great numbers of cylinders were soon after in the market, well made, of the quality desired, and callod tubes. Such cylinders are still imported (1857).

Great interest was taken by Mr. Hancock to introduce the native juice into this country; and, after great expense, he had the disappointment of finding the barrels contained coagulated India-rubber and watery fluid. Some samples, by peculiar treatment, escapod; whatever might have been expected, there seemed but little confidence in these plans, and valuable as the native juice might be at one time, yet by solvents and by working the rubber with machinery, it is far more profitable to employ this state, than to import a large quantity of watery fluid, with all the expenses of casks and cooperage, while the solid article is excellently adapted to take care of itself. 
Sharp and clean casts wore taken with this liquid, and as it is susceptible of boing tinted with delicate colours, it might bo used for beautiful ornamental purposes; whon the solid rubber soparates, it is whito, but in small pieces or thin sheots, it is semitransparent.

The specific gravity of caoutchouc is 0.925 , and it is not permanently increased by any degree of pressure. By cold or long quiesconco it becomes hard and stiff. When the milky juice has become once coherent, no means hitherto known can restore it to the emulsive state. By long boiling in water it softens, swells, and becomes more readily soluble in its peculiar menstrua; but when exposed to the air it speedily resumes its former consistence and volume. It is quite insoluble in alcohol; but in ether, deprived of alcohol by washing with water, it readily dissolves, and affords a colourless solution. When the ether is evaporated, the caoutchoue becomes again solid, but is somewhat clammy for a while. When treated with hot naphtha, distilled from native petroleum, or from coal-tar, it swells to 30 times its former bulk; and if then triturated with a pestlo, and pressed through a sieve, it affords a homogeneous varnish, which being applied by a flat edge of metal or wood to cloth, prepares it for forming the patent water-proof cloth of Macintosh. Two surfaces of cloth, to which several coats of the above varnish have been applied, are, when partially dried, brought evenly in contact, and then passed between rollers, in order to condense and smoothe them together. This double cloth is afterwards suspended in a stove-room to dry, and to dispel the disagreeable odour of the naphtha,

Caoutchoue dissolves in the fixed oils, such as linseed oil, but the varnish has not the property of becoming concrote upon exposure to the air.

It has lately been asserted that caoutchouc is soluble in the oils of lavender and sassafras. Roxburgh found it perfectly soluble in the oil of cajeput.

It melts at $248^{\circ} \mathrm{F}$, and stands afterwards a much higher heat without undergoing any further change. When the melted caoutchouc is exposed to the air, it becomes hard on the surface in the course of a year. When kindled, it burns with a bright flame and a great deal of smoke.

Neither chlorine, sulphurous acid gas, muriatic acid gas, ammonia, nor fluosilicic acid gas, affect it, whence it forms very valuable flexible tubes for pneumatic chomistry. Cold sulphuric acid does not readily decompose it, nor does nitric acid, unloss it be somewhat strong. The strongest caustic potash-lye does not dissolve it even at a boiling heat.

According to Dr. Faraday, the pure caoutchouc, obtained from the sap, had a specific gravity of 0.925 , and no reduplication of it in a Bramah's press was found to offect permanent alteration.

The specifle gravity of the best compact Para caoutchouc, taken in dilute alcohol, is . . . . .

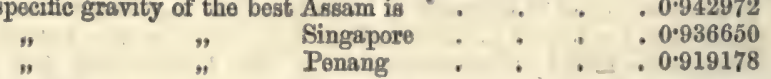

Having been favoured by Mr. Sievier, formerly managing director of the Joint-Stock Caoutchoue Company, and by $\mathrm{Mr}$. Beale, engineer, with two different samples of caoutchouc juice, Dr. Ure subjected each to chemical examination.

- That of Mr. Sievier, is greyish brown, that of Mr. Beale is of a milky grey colour ; the deviation from whiteness in each case being due to the presence of aloetic matter, which accompanies the caoutchoue in the secretion by the tree. The former juice is of the consistence of thin cream, has a specific gravity of 1.04125 , and yields, by exposure upon a porcelain capsule, in a thin layer, for a fow days, or by boiling for a fow minutes with a little water, 20 per cent. of solid caoutchouc. The latter, though it has the consistence of pretty rich cream, has a specific gravity of only 1.0175. It yields no less than 37 per cent. of white, solid, and very olastic caoutchouc.

' It is interesting to observe how readily and compactly the separate little clots or threads of caoutchouc coslesce into one spongy mass in the progress of the ebullition, particularly if the emulsive mixture bo stirred; but the addition of water is necessary to prevent the coagulated caoutchoue from sticking to the sides or bottom of the vessol and bocoming burnt. In order to convert tho spongy mass thus formed into good caoutchouc, nothing more is requisite than to expose it to a moderate pressure between the folds of a towel. By this process the whole of the aloetic extract, and other vegotable matters, which concrete into the substance of the balls and junks of caoutchouc prepared in Assam and Java, and contaminato it, aro ontirely separatod, and an article nearly white and inodorous is obtained. Some of the cakes of American cioutchouc when cut exhale the foetor of rotten cheese; a smell which adheres to the threads made of it after every process of purification.

'In the interior of many of the balls which come from both the Bravils and East 
Indies, spots are froquently found of a viscid tarry-looking matter, which, when exposed to the air, act in some manner as a ferment, and decompose the whole mass into a soft substance, which is good for nothing. Were the plan of boiling the fresh juice along with its own bulk of water, or a little more, adopted, a much purer article would be obtained, and with incomparably less trouble and delay, than has been hitherto brought into the market.

'I find that neither of the above two samples of caoutchouc juice affords any appearance of coagulum when mixed in any proportions with alcohol of 0.825 speciflc gravity; and, therefore, I infer that albumen is not a necessary constituent of the juice, as Dr. Faraday inferred from his experiments published in the 21st vol, of the Journal of the Roysl Institution.

- The odour of Mr. Sievier's sample is slightly acescent, that of Mr. Beales, which is by far the richer and purer, has no disagreeable smell whatever. The taste of the latter is at first bland and very slight, but eventually very bitter, from the aloetic impression upon the tongue. The taste of the former is bitter from the first, in consequence of the great excess of aloes which it contains. When the brown solution, which remains in the capsule after the caoutchouc has been separated in a spongy state by ebullition from 100 grains of the richer juice, is passed through a filter and evaporated, it leaves 4 grains of concrete aloes.

' Both of these emulsive juices mix readily with water, alcohol, and pyroxylic spirit, though they do not becomo at all clearer; they will not mix with caoutchoucine (the distilled spirit of caoutchouc), or with petroleum-naphtha, but remain at the bottom of these liquids as distinct as mercury does from water. Soda caustic lyo does not dissolve the juice; nitric acid (double aquafortis) converts it into a red curdy magma. The filtered aloetic liquid is not affected by the nitrates of baryta and silver; it affords with oxalate of ammonia minute traces of lime.'

The best solvent is a mixture of 100 parts of bisulphide of carbon with from 6 to 8 parts of anhydrous alcohol. If the alcohol be mixed with a little water a dough is obtained, from which the caoutchouc may be drawn out into threads and spun. By Gerard's process, gutta-percha is also soluble in the above mixtures of bisulphide of carbon and alcohol.

The sulphuration of caoutchouc, a valuable invention, is due to Mr. Charles Goodyear of Now York.

Caoutchouc, according to the experiments of Dr Ure, which have been confirmed by those of Dr. Faraday, contains no oxygen, as almost all other solid vegetable products do, but is a compound of merely carbon and hydrogen, in the proportion, by these results, of 90 carbon to 10 hydrogen, being three atoms of the former to two of the latter. Dr. Faraday obtained only $87 \cdot 2$ per cent. of carbon. Melted caoutchouc forms a very excellent chemical lute, as it adheres very readily to glass vessels, and withstands the corrosive action of acid vapours. Caoutchouc is much used for effacing the traces of plumbago pencils, whence it derived the name of India-rubber. It has been employed very extensively for making elastic bands or braces. The caoutchoue bottles aro skilfully cut into long spiral slips, which are stretched, and kept extended till nearly deprived of their elasticity, and till they form a thread of moderate fineness. This thread is put into a braid machine, and covered with a sheath of cotton, silk, linen, or worsted. The clothed caoutchouc is then laid as warp in a loom, and woren into an olegant riband. When woven, it is exposed upon a table to the action of a hot smoothing iron, which, restoring to the caoutchouc all its primitive elasticity, the riband retracts considerably in length, and the braiding corrugates equally upon the caoutchoue cores. Such bands possess a remarkable elasticity, combined with any desired degree of softness. Sometimes eloth is made of these braided strands of caoutchoue used both as warp and as weft, which is therefore elastic in all directions. When a light fabric is required, the strands of caoutchouc, either naked or braided, aro alternated with common warp yarns. For this mixed fabric a patent has been obtained. The original manufacturer of these elastic webs was a major in the Austrian service who erected a factory for them at St. Denys, near Paris.

\section{Caoutchodc Mavofactorres.}

But before entering upon these special divisions we may advert to some of the steps that have created this new employment for capital, commerce, and skill, especially as Mr. Hancock conceives it but just to the memory of the late Mr. Macintosh, to record the circumstances which led to his invention of the "Waterproof double textures,' that have been so long celebrated through the world by the name of 'Macintoshes.'

It will be recollected that on the introduction of coal-gas, the difficulties were very great to purify it from matters that gave a most disagreeable odour to the gas and 
gas-apparatus; the nuisance of these products led to many inconveniences. Mr. Macintosh, then employed in the manufacture of cudbear, in 1819 entered into arrangements with tho Glasgow Gas Works to receive the tar and ammoniacal products. After the separation of water, ammonia and pitch, the essential oil termed naphtha was produced, and it occurred to him that it might bo made use of as a solvont for India-rubber, and by the quality and quantities of the volatile naphtha, he could soften and dissolve the India-rubber. After repeated experiments to obtain the mixtures of due consistency, Mr. Macintosh, in 1823, obtained a patent for waterproof processes, and established a manufactory of articles at Glasgow, and eventually, with partners, entered upon the extended scale of business at Manchester, now so well known as the firm of Charles Macintosh and Co.

The action of many solvents of India-rubber is first to soften and then to form a sort of gelatinous compound with India-rubber, requiring mochanical action to break the bulk so as to get complete solution, when the original bulk is increased twenty or thirty times to form a mass: it may be imagined that in the early trials much time was occupied, and manual labour, to break up the soft coherent mass, \&ce., while hand-labour, sieves, the painters' slab and muller, and other simple means were resorted to.

Macintosh, Hancock, and Goodyear, alike record the simple manipulations they first employed, and the impression produced at the last, when they compared their small personal efforts with the gigantic machinery now used to effect the same results.

Mr. T. Hancoek's first patent was in April 1820: 'For an improvement in the application of a certain material to various articles of dress and other articles, that the same may be rendered elastic.' Thus, to wrists of gloves, to pockets to prevent their being picked, to waistcoats, riding-belts, boots and shoes without tying and lacing, the public had their attention directed. To get the proper turpentine to facilitate solution, and remedy defects of these small articles, and to meet the difficulties of practice and failures, Mr. Hancock gave constant zeal, and pursued the subject until, united with the firm of C. Macintosh and Co, he produced one of the most important manufactures known.

To get two clean pieces to unite together at their recently-cut surfaces, to obtain facile adhesion by the use of hot water, to cut the India-rubber by the use of a wet blade, to collect the refuse pieces, to make them up into blocks, and then cut the blocks into slices, were stages of the trade which required patience, years of time, and machinery to effect with satisfaction to the manufacturer.

To operate upon the impure rubber was a matter of absolute necessity for economic reasons : the bottles made by the natives were the purest form, but larger quantities of rubber could be cheaply obtained, full of dirt, stones, wood, leaves, and earth. To facilitate the labour of cutting or dividing, Mr. Hancock resorted to a tearing action,
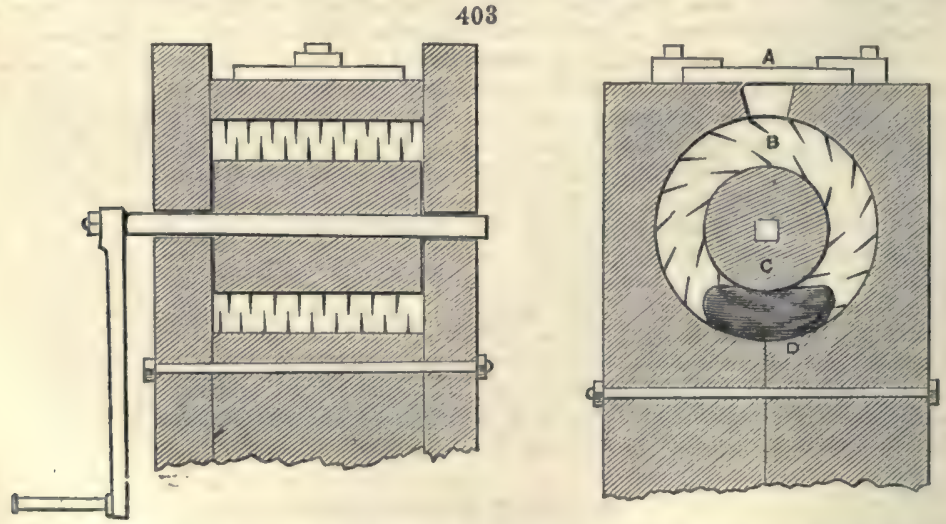

and constructed a simple machine for the purpose. (See fig. 403). \& shows the ontrance for pieces of rubber; $\mathbf{B}$, interior of fixed cylinder, with teeth; $\mathbf{c}$, cylinder to revolve with teoth or knives; $\mathrm{D}$, the resulting ball of rubber.

This machino had the effect of tearing the India-rubber into shrods and small fragments by the revolution of a toothed roller; the caoutchouc yielded, became hot, and ultimately a pasty mass or ball resultod; when cooled and cut it appearod homogeneous. Wasto cuttings put, in the first instance, on the roller, were dragged in, and there was evidence of action of some kind taking place; the machine was stopped, the 
pieces were found cohering together into a mass, this being cut showed a mottled grain, but being replaced and subjected to the revolving teeth of the rollers, it became very hot; and was found to be uniformly smooth in texture when cooled and cut open.

The first charge was about 2 ounces of rubber, and required the power of a man to work it. The next machine soon formed a soft solid, with speed and power, from all kinds of scraps of India-rubber, cuttings of bottles, lumps, shoes, \&c. : a charge of $1 \mathrm{lb}$. gave a smooth uniform eylindrical lump of about 7 inches in length and 1 inch in diameter. This process, including the use of heated iron rollers, was long kept secret; it is now known as the masticating process, and the machines are callod 'Masticators.' In the works at Manchester the charges are $180 \mathrm{lbs}$. to $200 \mathrm{lbs}$. of India-rubber each, and they produce, by steam-power, single blocks 6 feet long, 12 or 13 inches wide, and 7 inches thick. The Mammoth machine of $\mathrm{Mr}$. Chauffee, in the United States, weighs about 30 tons, and appears to have been invented about 1837. It is a valuable machine, differing in construction from Hancock's masticators, but it answers well in many respects; it may be considered as the foundation of the American trado.

In 1820 the blocks were cut into the form of square pieces and sold by the stationers to rub out pencil-marks, and then thin sheets were introduced for a variety of purposes. A cubical block cut by a keen sharp blade kept constantly wet, gave a sheet of India-rubber; the block raised by screws and the knife guided, enabled sheets of any thickness to be cut, sometimes so even and thin, as to be semi-transparent; when warm, the small sheets could be joined edge to edge, and thus large ones produced. From the blocks, rollers of solid rubber could be made, cylinders were covered for machinery, billiard tables had evenly cut pisces adjusted, tubes and vessels for chemical use were employed, and constantly increasing trials were made of the masticated rubber.

These remarks upon the early and successful manufacturers will better enable the outline of improvements to be followed. It can readily be imagined that when capital and interest combined to meet the changing requirements of the public, the trade applications became more numerous; many of these were secured by patents, but very many were worked as secret processes.

The department of operative industry which embraces caoutchouc manufactures has, within a few years, acquired an importance equal to that of some of the older arts, and promises, ere long, to rival even the textile fabrics in the variety of its designs and applications. The manufacture of caoutchone has, at present, these principal branches:-1. The condensation of the crude lumps or shreds of caoutchouc, as imported from South America, India, \&c., into compact homogeneous blocks, and the cutting of these blocks into cakes or sheets for the stationer, surgeon, shoemaker, \&c. 2. The filature of either the India-rubber bottles, or the artificial sheet caoutchouc, into tapes and threads of any requisite length and fineness, which, being clothed with silk, cotton, linen, or woollen yarns, form the basis of elastic tissues of every kind. 3. The conversion of the refuse cuttings and coarser qualities of caoutchouc into a viscid varnish, which, boing applied between two surfaces of cloth, constitutes the well-known double fabries, impervious to water and air ; and by special applications to one surface, constitute the single-texture fabrics. 4. The vulcanisation of India-rubber. 5. The mechanical applications resulting from the changed India-rubber. 6. The solarisation of caoutchouc.

The caoutchouc, as imported in skinny shreds, fibrous balls, twisted concretions, cheose-like cakes, and irregular masses, is, always more or less, impure, but sometimes it is fraudulently interstratified with earthy matter. It is first cleansed by being cut into small pieces, and then washed in warm water. It is next dried on iron trays, heated with steam, while being carefully stirred about to separate any remaining dirt, and is then passed through a pair of iron rolls, under a stream of water, whoreby it gets a second washing, and becomes at the same time equalised by the separate pieces being blended together. The shreds and cuttings thus laminated, if still foul or heterogeneous, are thrown back into a kind of hopper over the rolls, set one-sixteenth of an inch apart, and passed several times through between them.

In the establishment of William Warne and Co. at Tottenham, rinsing and lamination are superseded by a process of washing practised in Mr. Nickels's second operation, commonly called the grinding, or, as it should more properly be styled, the kneading. The mill employed for agglutinating or incorporating the separate fragments and shrods of caoutchouc into a homogeneous elastic ball, is a cylindrical box or drum of cast iron, 8 or 9 inches in diameter, set on its side, and traversed in the line of its horizontal axis (also 8 or 9 inches long) by a shaft of wrought iron, furnished with 3 rows of projecting bars, or kneading arms, placed at angles of $120 \mathrm{deg}$. to each other. These act by rotation against 5 chisel-shaped teeth, which stand obliquely up from the front 
part of the bottom of the drum. The drum itself coneists of 2 semi-cylinders; the under one of which is made fast to a strong iron framing, and the upper one is hinged to the undor one behind, but bolted to it before, so as to form a cover or lid-which may be opened or laid back at pleasuro-in order to examine the caoutchouc from timo to $\operatorname{time}$, and take it out when fully kneaded. In the centro of the lid a funnel is mado fast, by which the cuttings and shreds of the India-rubber aro introducod, and a stream of water is made to trickle in, for washing away the foul mattor often ombedded in it. The power required to turn the axis of one of these mills-as the drums or boxes are called-may bo judged of from the fact, that if it be only 2 inches in diameter, it is readily twisted asunder, and that it requires to be 3 inches to withstand every strain produced by the fixed teoth holding the caoutchouc against the revolving arms. Fivo pounds constitute a charge of the material. Mills vary in size, and charges range from 7 to 14 lbs.

One of the most remarkable phenomena of the kneading operation, is the prodigious heat disongaged in the alternate condensation and expansion of the caoutchouc. Though the water be cold as it trickles in, it soon becomes boiling hot, and emits copious vapours. When no water is admitted, the temperature rises much higher, so that the elastic lump, though a bad conductor of heat, cannot be safely touched with the hand. As we shall presently find that caoutchoue suffors no considerable or permanent diminution of its volume by the greatest pressure which can be applied, wo must ascribe the heat evolved in the kneading process to the violent intestine movements excited throughout all the particles of the elastic mass.

During the steaming much muddy water runs off through apertures in the bottom of the drum. In the course of half an hour's trituration the various pieces become agglutinated into a soft, elastic, ovoid ball, of a reddish-brown colour. This ball is now transferred into another similar iron drum, where it is exposed to the pricking and kneading action of 3 sets of chisel points, 5 in each set, that project from the revolving shaft at angles of $120 \mathrm{deg}$. to each other, and which encounter the resistance occasioned by 5 stationary chisel teoth, standing obliquely upwards from the bottom of the drum. Here the caoutchouc is kneaded dry along with a little quicklime. It soon gets very hot; discharges in steam through the punctures, the water and air which it had imbibed in the preceding washing operation; becomos in consequence more compact; and in about an hour assumes the dark brown colour of stationers' rubber. During all this time frequent explosions take place, from the expansion and sudden extrication of the imprisoned air and steam.

Instead of close boxes and tearing teeth or knives, rollers of iron are now employed (1858): their forms are corrugated, cut or indented, the piecos of India-rubber are thrown between, and by heat and pressure are cleansed and incorporated; streams of water, warm or cold, regulate these operations at will, of course with large rollers of motal exposed to air and streams of water. The temperature is now kept so low that the previous statement may appear exaggerated to those who work with more power but with less velocity in the modern arrangements. Mr. Hancock, however, says, 'the heat it acquires is very surprising; I have found in cutting a heavy charge open, and closing it upon the bulb of a thermometer, that the temperature reached $280^{\circ}$;' this heat was only due to motion of the machine and friction upon the rubber, as the materials and the machine were cold at the outset of the experiment.

From the second set of drums the ball is transforred into a third set, whose revolving shaft being furnished both with flat pressing bars, and parallel sharp chisels, perpendicular to it, exercises the twofold operation of pricking and kneading the mass, so as to condense the caoutchouc into a homogeneous solid. Seven of the finished balls, weighing, as above stated, 5 lbs, each, are then introduced into a much larger iron drum of similar construction, but of greater strength, whose shaft is studded all round with a formidable array of blunt chisels. Here the separate balls become perfectly incorporated into one mass, free from honeycomb cells or pores, and therefore fit for being squeezed into a rectangular or cylindrical form in a suitable cast-iron mould, by the action of a screw-press. When condensed to the utmost in this box, the lid is secured in its place by screw bolts, and the mould is set aside for several days. It is a curious fact, that Mr. Sievier tried to use this as a moulding force, by tho hydraulic press, without effect, as tho cake of caoutchouc after being so condensed, showed much more resiliancy than after the compressing action of the serew. The cake-form generally proforred for the recomposed, ground, or milled caoutchouc, is a rectangular mass, about 18 inches long, 9 inches broad, and 5 inches thick.

This is sliced into cakes for the stationer, and into sheets for making tapes and threads of caoutchouc, by an ingenious solf-acting machine, in which a straight steel blade, with its edge slanting downwards, is made to vibrate very rapidly to and fro in a horizontal plane; while the cake of caoutchouc clamped or embraced at each side between two strong iron bars, is slowly advanced against the blade by screw-work, 
like that of the slide-rest of a lathe. In cutting caoutchouc by knives of every form, it is essential that either the blade or the incision be constantly moistened with water; for otherwise the tool would immediately stick fast. As the above straight vibrating knife slants obliquely downwards, the sheet which it cuts off spontaneously turns up over the blade in proportion as it is detached from tho bottom niass of the cake. The thicker slices are afterwards cut by hand, with a wotted knife, into small parallelopipeds for the stationer, the sections being guided rectangularly by saw lines in a wooden frame. Slices may be cut off to almost any desired degree of thickness, by means of an adjusting serew-a mechanism that acts against a board which supports the bottom of the cake, and raises it by an aliquot part of an inch, the cutting blade boing caused to vibrate always in the same horizontal plane. These thin slices constitute what is called sheet caoutchouc, and they serve tolerably for making tubes for pneumatic apparatus, and sheaths of every kind; since, if their two edges bo cut obliquely with clean scissors, they may be made to coalesce, by gentle pressure, so intimately, that the line of junction cannot be discovered either by the eye, or by inflation of a bag or tube thus formed.

The inode of recomposing the cuttings, shreds, and coarse lumps of caoutchoue into a homogeneous elastic cake, specified by Mr. Nickels, for his patent, sealed October 24, 1836, is not essentially different from that above described. The cylinders of his mill are more capacious, are open at the sides like a cage, and do not require the washing apparatus, as the caoutchouc has been cleansed by previous lamination and rinsing. He completes the kneading operation, in this open cylinder, within the space of about two hours, and afterwards squeezes the large ball so formed into the cheese-form, in a mould subjected to the action of an hydraulic press. As ho succeeds perfectly in making compact cakes in this way, his eaoutchouc must differ somewhat in its physical constitution from that recomposed by Mr. Sievier's process. He uses a press of the power of 70 tons; such pressure, however, must not be applied suddenly, but progressively, at intervals of two or three minutes between each stroke; and when the pressing is complete, the caoutchoue is allowed to remain under pressure till it is cold, when ho thrusts it out of the mould entirely, or, placing his mould in the slide-rest mechanism, he gradually raises the caoutchouc out of it, while the vibrating knife cuts it into slices in the manner already described. The elegant machine by which these sheets are now so easily and accurately sliced, was originally contrived and constructed by Mr. Beale, engineer, Church-lane, Whitechapel,

\section{Finature of Caottchode for Matnse Finastio Fabrics}

The following particulars may be deemed as belonging to the history of the manufacture of threads of native rubber, - the cured, or vulcanised, or mineralised rubber having quite superseded the modes of preparing threads from native bottle caoutchouc.

Messrs. Rattier and Guibal mounted in their factory at St. Deny's, Dr. Ure says, in the year 1826 or 1827, a machine for cutting a disc of caoutchouc into a continuous fillet spirally, from its circumference towards its centre. This flat dise was made by pressing the bottom part of a bottle of India-rubber in an iron mould. A machine on the same principle was made the subject of a patent by Mr. Joshua Proctor Westhead, of Manchester, in February 1846; and, being constructed with the well-known precision of Manchester workmanship, it has been found to act perfectly well in cutting a disc of caoutchoue, from the circumference towards the centre spirally, into one continuous longth of tape. For the service of this machine, the bottom of a bottle of India-rubber of good quality being selected, is cut off and flattened by heat and pressuro into nearly a round cake of uniform thickness. The cake is made fast at its contre by a screw nut and washer to the end of a horizontal shaft, which may be made to revolve with any desired velocity by means of appropriate pullies and bands, at the same timo that the odge of the disc of caoutchouc is acted on by a circular knifo of cast steel, mado to revolve 3,000 times per minute, in a plane at right angles to that of the disc, and to advance upon its axis progressively, so as to paro off a continuous uniform tape or fillet from the circumference of the cake. During this cutting operation, the knife and caoutchoue are kept constantly moist with a slender stream of wator. A succession of threads of any desired fineness is afterwards cut out of this fillet, by drawing it in a moist state through a guide slit, against the sharp edge of a rovolving steel dise. This operation is dexterously performed by the hands of young girls. MM. Rattier and Guibal employed, at the above-mentioned period, a mechanism consisting of a series of circular steel knives, fixed perallel to each other at minute distances, regulated by interposed washors ucon a revolving shaft; which series of knives acted against another similar series, placed upon a parallel adjoining shaft, 
with the effect of cutting the tape throughout its length into eight or more thrends at once. An improved modification of that apparatus is described and figurod in the specification of Mr. Nickols's patont of Octobor 1836. He employed it for cutting into threads the tapes made from the recomposed csoutchone.

The body of the bottle of India-rubber, and in general any hollow eylinder of caoutchouc, is cut into tapes, by being first forced upon a mandril of soft wood of such dimensions as to keep it entirely distended. This mandril is then secured to the shaft of a lathe, which has one end formed into a fino-threaded serew, that works in a fixed nut, so as to traverse from right to left by its rotation. A circular disc of steel, kept moist, revolves upon a shaft parallel to the preceding, at such a distance from it as to cut through the caoutchouc, so that, by the traverse movement of the mandril shaft, the hollow cylinder is cut spirally into a continuous fillet of a breadth equal to the thickness of the side of the cylinder. Mr. Nickels has described two methods of forming hollow cylinders of recomposed caoutchouc, for the purpose of being cut into fillets by such a machine.

It is probable that the threads formed from the best India-rubber bottles, as imported from Para, are considerably stronger than those made from recomposed caoutchouc, and therefore much better adapted for making Mr. Sievier's patent elastic cordage. When, however, the kneading operation has been skilfully performed, it is found that threads of the ground eaoutchouc, as it is incorrectly called by the workmen, answer as well for any ordinary purpose of elastic fabrics, and are, of course, greatly more economical, from the much lower price of the material.

The following figs. 404, 405, 406, represent the machine for cutting the spiral riband. The dise $\mathbf{D}$, placed horizontally, turns round its vertical axis, so as to present ts periphery to the edge of a knife c, formed like a circular blade, whose plane is
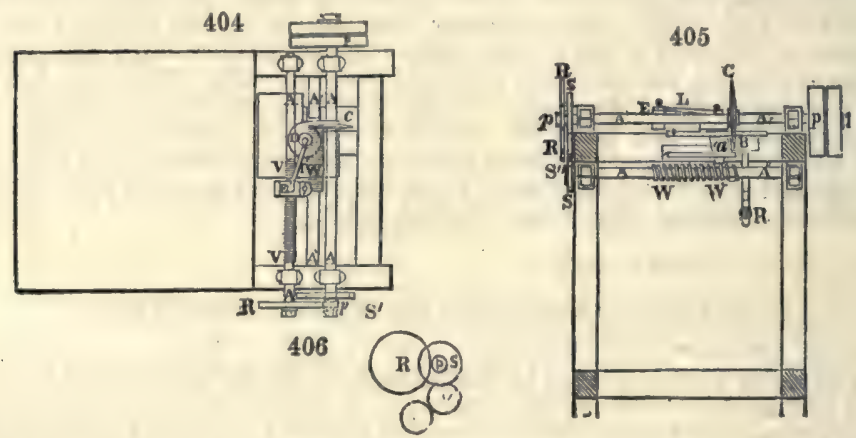

perpendicular to that of the bases of the dise. It is obvious, that if the dise alone revolved, the motionless knife could act only by pressure, and would meet with an enormous resistance. A third movement becomes necessary. In proportion as the dise is diminished by the removal of the spiral band, the centre of this disc must adrance upon the knife in order that the riband may have always the same breadth. The inspection of fig. 406 , will make the accordance of the three motions intelligible. The knife $\mathrm{c}$ is placed upon a shaft or axis $\mathrm{A}$, which carries a pulley, round which a belt or cord runs which drives the whole machine.

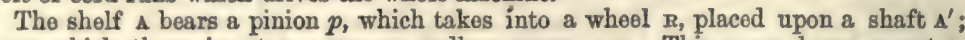
upon which there is cut a worm or endless screw, $\nabla \nabla$. This worm bears a nut $\mathbf{x}$, which advances as the serew turns, and carries with it a tie I, which in its turn pushes the dise D, carried upon a shoulder, constantly towards the knife. This shoulder is guided by two ears which slide in two grooves cut in the thickness of the table. The diameter of the pinion $p$ is about one fifth of that of the wheel $\mathbf{R}$; so that the arbour $\Delta$ turns five times less quickly than the arbour $\Delta^{\prime}$; and the fineness of the screw $\nabla$ contributes further to slacken the movement of translation of the disc.

The rotatory movement of the disc and its shoulder, is given by an endless screw $\mathrm{w}$ w, which governs a pinion $p$, provided with ton teeth, and carried by the shaft $\mathbf{A}$, upon which the shoulder is mounted. The arbour $\mathbf{A}^{\prime}$ of this endless screw receives its motion from the first shaft $\mathbf{A}$, by means of the wheels $\mathbf{s}$ and $\mathrm{s}^{\prime}$ mounted upon these shafts and of an intermediate wheel $\mathrm{s}^{\prime \prime}$. This wheel, of a diameter equal to that of the shaft $\mathbf{\Delta}^{\prime \prime}$, is intended merely to allow this shaft to recede from the shaft $\mathbf{A}$. The diameter of the wheel of this last shaft is to that of the two others in the ratio of 10 to 8. 
Second machine for subdividing the riband8. Figs. 407, 408.-The riband is engaged between the circular knives $\mathrm{c}$ C, which are mounted upon the rollers $\mathbf{R} \mathbf{R}$; thin brass washers keep these knives apart at a distance which may be varied, and two extreme washers mounted with screws on each roller maintain the whole system. The axes of these rollers traverse two uprights $\mathrm{M} M$, furnished with brasses, and with adjusting serews to approximate them at pleasure. The axis of the lower roller carries a wheel

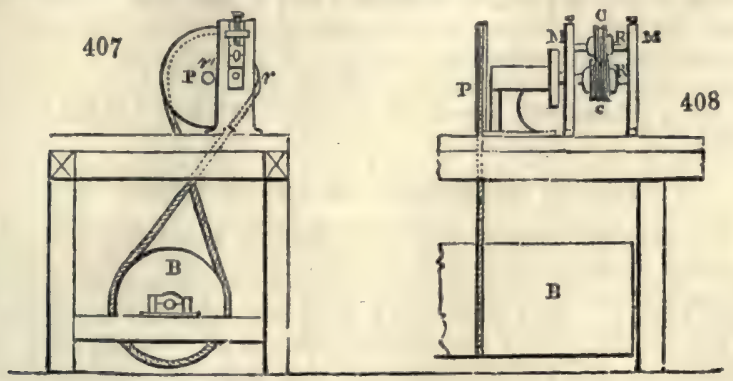

$r$, which takes into another smaller wheel $r^{\prime}$, placed upon the same shaft as the pulley $P$, which is driven by a cord. The diameter of the wheel $r$ is three times greater than the wheel $r^{\prime}$. The pulley $\mathrm{P}$ is twice the size of the wheel $r^{\prime}$; and its cord passes round a drum B, which drives the rest of the machine.

Threads of caoutchouc are readily pieced by paring the broken ends obliquely with scissors, and then pressing them together with clean fingers, taking care to admit no grease or moisture within the junction-line. These threads must be deprived of their elasticity before they can be made subservient to any torsile or textile manufacture. Each thread is inelasticated individually in the act of reeling, by the tenter boy or girl pressing it between the moist thumb and finger, so as to stretch it to at least eight times its natural length, while it is drawn rapidly through between them by the rotation of the power-driven wheel. This extension is accompanied with condensation of the caoutchouc, and with very considerable disengagement of heat, as pointed out in Nicholson's Journal upwards of 35 years ago, by Mr. Gough, the blind philosopher of Kendal. To stretch the thread, in the act of reeling, the sensation of heat is too painful for unseasoned fingers. The reels, after being completely filled with the thread, are laid aside for some days, more or fewer, according to the quality of the caoutchouc, the recomposed requiring a longer period than the bottle material. When thus rendered inelastic, it is wound off upon bobbins of various sizes, adapted to various sizes of braiding, or other machines, where it is to be clothed with cotton or other yarn.

In the process of making the ELASTIC TTSSUEs, the threads of caoutchouc being first of all deprived of their elasticity, are prepared for receiving a sheath upon the braiding machine. For this purpose they are stretched by hand, in the act of winding upon the reel, to 7 or 8 times their natural length, and left two or three weeks in that state of tension upon the reels. Thread thus inelasticated has a specific gravity of no less than 0.948732; but when it has its elasticity restored, and its length reduced to its pristine state, by rubbing between the warm palms of the hands, the specific gravity of the same piece of thread is reduced to 0.925939 . This phenomenon is akin to that exhibited in the process of wire-drawing, where the iron or brass gets condensed, hard, and brittle, while it disengages much heat; which the caoutchouc thread also does in a degree intolerable to unpractised fingers.

For the solution of India-rubber, for the manufacture of elastic tissues, the following has been strongly recommended:-

The raw India-rubber is soaked in clear water, and boiled for about an hour, to remove dirt. It is then taken out of the water and cut into round slices about one centim. in thickness. It is then rolled out into layers about 2 mètres long and 0.15 mètre broad. The rubber is then dried in a warm chamber. After the drying follows the solution. About 26 kilos of rolled caoutchouc are placed in a wooden vat, lined with zinc, and treated with a mixture of 50 kilos benzol, and 70 kilos oil of turpentine. Both these liquids must be perfectly free from fatty matters, or the solution of Indiarubber will be useless. The caoutchouc, before being brought into contact with the solvents, is torn up into small fragments. The mass is stirred occasionally, till it forms a thick, homogeneous liquid. To test the benzol and turpentine, small portions of each are evaporated to dryness in the water-bath. If any trace of fat remains, the sample is at once rejected. 
In the manufacture of elastic fabrics, the riband looms at Holloway displayed to great adrantage the mochanical genius of the patentee, Mr. Sievier. Their productive powers may be inferrod from the following statement:- ' 5,000 yards of 1 -inch braces are woven weokly in one 18-riband loom, whoreby the female operative, who has nothing to do but watch its automatic movements, earns 108. a-weok: 3,000 yards of 2-inch braces are woven upon a similar loom in the same time.' But one of Mr. Sievier's most curious patent inventions is that of producing, by the shrinking of the caoutchouc threads in the foundation or warp of the stuff, the appearance of raised figures, closely resembling coach-lace, in tho weft. Thus, by a simple physical operation, there is produced, at an expense of ono penny, an effect which could not be effected by mechanical means for less than one shilling. This manufacture is not now carried on in that locality.

\section{Of tho Watkr-proof Doumin Fabrics,}

In 1837, Mr. Hancock obtained a patent to produce waterproof cloth with greatly reduced quantities of dissolved caoutchouc, and in some cases without any solvent at all. The masticatod rubber, rolled into sheets, was moistened on both sides with a solvent and rolled up. The following day theso were submitted to rollers of different speeds, and the whole became a plastic mass. Instead of a wooden plank, as tho bed of the machine, a revolving iron cylinder was used, kept hot by steam or water, and the coated cloth passed over flat iron chambers, heated the same way, to evaporate the small quantity of solvent. Masticated rubber has been spread without any solvent by these machines; but the spreading is best effected by the rubber being in some degree softened by the addition of small quantities of the solvent.

Sheets of rubber have been prepared by saturating the cloth with gum, starch, glue, \&c., then rubber dough was placed on this smoothed surface; sufficient coatings of the rubber were spread to make up the desired thickness, the cloth was immersed in warm water to dissolve the gum, when the sheet of rubber cume off with ease, and the plastic, or dough state, was the precursor of vulcanisation experiments and success.

The clamminess of caoutchouc is removed by Mr. Hancock in the following manner: -10 lbs. of it are rolled out into thin sheets between iron cylinders, and at the same time $20 \mathrm{lbs}$. of French-chalk (silicate of magnesia) are sifted on and incorporated with it, by means of the usual kneading apparatus. When very thin films are required (like sheets of paper), the caoutchouc, made plastic with a little naphtha, is spread upon eloth previously saturated with size, and when dry is stripped off. Mixtures of caoutchouc so softened may be made with asphalt, with pigments of various kinds, and with plumbago, sulphur, \&c.

The first form of bags or pillows, or ordinary air-cushions, is woll known, and was manufactured by C. Macintosh and Co. as early as 1825 and 1826 ; when pressure is applied they yield for the instant to the compressing body, and then become rigid, and the whole strain is borne by the inelastic material of the bag. Mr. T. Hancock once tried an ordinary pillow between boards in a hydraulic press, and he records that it bore a pressure of 7 tons before it burst. To remedy the ovils of this form an ingenious arrangement was made of inserting slips of India-rubber into tho fabric, so that it expanded in every direction. This yielding of the case, and division into strengthened partitions, enabled seats, beds, and other applications to be made.

The gas-bags now so commonly used appear, by Mr. Hancock's statement, to have been made for experimental purposes in the year 1826; and in May 1826, at the suggestion and for the use of Lieut. Drummond, to be employed in the Trigonometrical Survey of these islands, with the oxy-hydrogen jets of gas on balls of lime.

They were made strong and of rough materials-fustian made air-proof with thin sheet rubber. Mr. Hancock, to try whether the rubber was absolutely impervious to water, had a bag made and weighed it from time to time during 30 years; the decrease of weight is shown:-

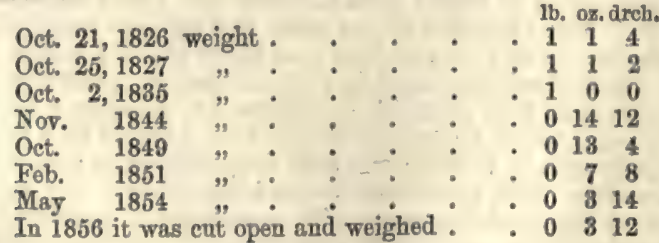

It was quite dry. Thus $12 \mathrm{oz}$. of water had evaporated or escaped in a quarter of a century, and $13 \mathrm{oz}, 8 \mathrm{dr}$. in 30 years of observation. 
He remarks that bags of such cloth made with a thin coating of rubber, soon evaporated sufficient water to cause mildew, when laid upon each other; but this slow evaporation does not interfere with their ordinary applications.

The porosity of caoutchouc would appear to explain the readiness with which it is permeated by different liquids which have no chemical action upon it. Thin sections of dry caoutchoue of the best kinds absorb from 18 to 26 per cent. of water in the course of a month, and become white from having been brown. It is, however, found that endosmose action is constantly active in India-rubber. In tubes through which gas flows there is always some escape of hydrogen deprived of much of its carbon.

To enumerate the applications of these double fabrics for cushions, life-preservers, \&ce, beds and boats, would be out of place here.

For single-texture fabrics, or eloth with one side only prepared, the process is the same as that described for double fabrics, only that one side only is proofed, or covered with India-rubber solution or paste; and this kind of waterproof has an advantage over the old, that the surface worn outside, being non-absorbent, imbibes no moisture and requires no drying after rain or wear. The objection to single-texture fabrics, of being liable to decomposition by the heat of the sun and from close packing, has been obviated by a discovery adopted by Messrs. Warne and Co., termed by them the Sincalor process (sine calore, without heat); by which the properties of the rubber are so changed that heat, grease, naphtha, and perspiration, which decompose the ordinary India-rubber waterproof, in no way affect the waterproof goods of the 'Sincalor' process. The singular changes effected by this process are especially shown by the application of a hot iron to the surface, which destroys it without the usual decompositions; the substance is burnt, but is not rendered sticky. The process is stated to be secret.

\section{Vutcantsamox.}

Of all the changes effected by chance, observation, or chemical experiments of late years, few cases have been so important as the change in India-rubber by the process ealled Vulcanisation. The union of sulphur with caoutchoue gives new properties so valuable, that it may be said the former well-known quality of elasticity is now rendered so variable that almost every range, from the most delicate tenuity to the hardness of metals, can be obtained at will by the manufacturer. These changes in the caoutchouc are produced with a degree of permanence to defy air, water, saline and acid solutions; the material is incapable of being corroded, and more permanent under harsh usage than any other set of bodies in the world. Such are the results of the processes that induce a 'change' in caoutchouc when sulphur and heat are employed; where metals and minerals are employed, 'metallised' and 'mineralised,' 'thionised,' and a number of other terms have been used.

When caoutchouc is mixed with from 2 to 10 per cent. of sulphur, and then heated to $270^{\circ}$ and $300^{\circ}$, it undergoes a change, it acquires new characters, its elasticity is greatly increased, and is more equable; it is not affected nor is the substance altered by cold, no climate effects a change, heat scarcely affects it, and when it does it does not become sticky and a viscid mass; if it yields to a high temperature it is to become harder, and will ultimately yield only at the advanced temperature to char and to decompose. All the ordinary solvents are ineffectual. The oils, grease, ether, turpentine, naphtha, and other solvents scarcely alter it, and the quantity of sulphur that will effect the change is known not to exceed 1 or 2 per cent. Further, if peculiar solvents, such as alkalies, remove all apparent sulphur from it, still the change remains; indeed, the analogy of steel to iron by the changes of condition effected by some small quantities of other bodies seems to be an analogous condition. Whatever the theory, which is exceedingly obscure, still the practice, by whatever name, is to obtain this changed state and exalted elastic properties.

'Vulcanisation' was discovered in America. Mr. Goodyear relates, that having made a contract for India-rubber mail bags, they softened and decomposed in service, and while ho thought a permanent article had been made, the colouring materials and the heat, united to soften and to destroy the bags; hence, by this failure, distress of all kinds arose, and the trade was at an end. During one of the calls at the place of abandoned manufacture, Mr. Goodyear tried a few simple experiments to ascertain the effect of heat upon the composition that had destroyed the mail-bags, and carelessly bringing a piece in contact with a hot stove, it charred like leather. He called the attention of his brother, as well as other individuals who were present, and who were acquainted with the manufacture of gum elastic, to the fact, as it was remarkable, and unlike any before known, since gum elastic always melted when exposed to a high degree of heat. The occurrence did not at the time appear to them 
to be worthy of much notice. He soon made other trials, the India-rubber always charring and hardening.

As ordinary India-rubber is always tending to adhere, many plans had been tried to prevent this. Chalk, magnesia, and sulphur had been patented in England and America, but no one seems to have supposed any other change would be produced by heat. Mr. Goodyear proceeded to try experiments, and produced remarkable results : samples of goods were shown about and sent to Europe.

The late Mr. Brockedon, so well known for his talents and love of scientific investigations, had long pursued means to obtain a substitute for corks, and, after much ingenuity, had devised India-rubber stoppers. As soon as all mechanical difficulties were over, objections were taken to the colour of the substance. Some samples of a changed rubber came into his possession, of which it was declared they would keep flexible in the cold, and were found not to have an adhesive surface. These caused numerous experiments, as it was recognised that a change had been effected, and although Mr. Brockedon failed, yet Mr. Hancock kept on working, combining sulphur, with every effect, but that of vulcanisation, as he was ignorant of the power of heat to effect this change. He used melted sulphur, and produced proof of absorption, for the pieces of caoutchouc were made yellow throughout; by elevating the temperature he found they became changed, the lower end of slips 'nearest the fire turning black, and becoming hard and horny' (the sulphur was melted in an iron pot). By these simple observations, as they now seem, Mr. Goodyear in America and Mr. Hancock in England were induced to take out patents, and commence that series of manufacturing applications to which there seemed no limit. The first English patent was by Mr. Hancock. The general method is to incorporate sulphur with caoutchouc, and submit it to heat; if any particular form is required, the mixture is placed in moulds, and takes off any delicate design that may be upon the metal mould, and if these are submitted to higher degrees of heat, the substance and evolved gases expand, and thus a very hard, horny, or light but very strong substance is produced, called hard India-rabber, or 'vulcanite.' Mouldings, gun-stocks, combs, cabinetwork, and hundreds of forms may be obtained by these curious means. The term 'vulcanisation' was given by Mr. Brockedon to this process, which seemed by the employment of heat and sulphur to partake of the attributes of the Vulcan of mythology. For the 'change' or 'vulcanising' to get a yielding but permanently elastic substance, steam beat is usually employed in England, but in America, ovens, with various plans for producing dry heat, are generally employed.

The articles thus made being more elastic, unaffected by heat, cold, or solvents, attracted much attention, and Mr. Parkes was engaged to find out a method of producing the same effects now secured by patent: all ordinary means were used and given up, but he finally succeeded. The process of cold sulphuring of Mr. Parkes consists in plunging the sheets or tubes of caoutchouc into a mixture of 100 parts of sulphuret of carbon and $2 \frac{1}{2}$ parts of protochloride of sulphur, for a minute or two, and then immersing them in cold water. Thus supersulphuration is prevented in consequence of decomposing the chloride of sulphur on the surface by this immersion, while the rest of the sulphur passes into the interior by absorption. Mr. Parkes prescribes another, and perhaps a preferable process, which consists in immersing the caoutchouc in a closed vessel for 3 hours, containing a solution of polysulphuret of potassium indicating a density of $25^{\circ}$ Beaumé, at the temperature of $248^{\circ}$ Fahr., then washing in an alkaline solution, and lastly in pure water. A uniform impregnation is thus obtained.

In the first instance sulphur, caoutchouc, and heat were alone employed; the temperature and the time to which the mixtures are subjected to heat being determined by practical experiments. Vulcanised rubber is not only the changed substance, as produced by sulphur, but it contains metallic oxides, \&c., metallic and mineral substances; and these compounds, are perhaps much better fitted for their respective uses than the pure sulphur and India-rubber. White lead, sulphide of antimony, black lead, and other substances enter into these combinations. After the early experiments with vulcanised rubber there seemed reason to believe that changes slowly took place. The rubber was found to become brittle, and bands strotched out broke immediately. To a great extent this has been remedied by the use of lead, which seems to combine with the sulphur, for changes are believed by practical men to take place with pure elastic vulcanised caoutchoue which do not occur when metallic matters are duly mixed. This is a trade statement, which may be true for some special uses. The brittleness may perhaps more fairly be admitted to be due to inexperience, and the difficulties to meet the demands of the public for 3 new article; but to those whom it may most concern, we have raised this question so far as to obtain the conscientious opinion of Mr. Thomas Hancock (now retired from business), who considers that by the peculiar plan of vuleanising by a bath of sulphtur, 
and employing high pressure steam (described in Patent of 1843), he obtains what he calls pure vulcanising, that is, the use of sulphur, rubber, and heat. He states 'That by this mode, the greatest amount of extensile elasticity is obtained, and that this quality is diminished in proportion as other matters are present in the compound.' It may, however, be useful to record some of the results of early trials mado by competent authorities, with the view of testing its ultimate employments. Mr. Brockedon stated at the Institution of Civil Engineers, that he had kept vulcanised Indiarubber in tranquil water for 14 years without visible change, and he summed up the then knowledge of trade production, that there was perhaps no manufacturing process of which the rationale was so little understood as that of vuleanising caoutchouc; all was conducted on the observation of facts, a given quantity of sulphur to a certain thickness of rubber, at a certain temperature; and certain results were reckoned upon with confidence, but more from practice than theory. Mr. Brockedon had placed vulcanised rubber for 10 years in damp earth, and it exbibited no change.

When articles were moulded, the metal of the mould was not a matter of indifference; if of tin, the article was usually delivered perfectly clean, but if of brass or copper, then the material adhered to it, probably from the greater affinity of the sulphur for the metal than for the caoutchouc: these surface effects may well be borne in mind, for it appears not to be an easy matter to vulcanise large masses of caoutchoue, while sheets and thin films are readily changed. The soft masses of materials are placed in moulds, strongly secured, if a high temperature is to be used, and the mass comes out with the form thus given to it, and more or less elastic; hence the surface of a mass is always likely to bo advanced in the vulcanising changes.

At present a very large proportion of the articles made have the forms given to them in the plastic state, and then subjected to heat; the change is effected, and they retain their form, although rendered permanently elastic.

Mr. Brockedon and Mr. Brunel tried this substance on the Great Western Railway, in place of felt, to be used between the under sides of bearing rails and sleepers of railways. It appeared, by constant trials of nearly a year, to be quite indestructible to any action to which it had been exposed; the slips were indented by the edge of the rail, but not permanently so, and the surface was glazed, as if by friction; the slips were 6 inches wide, and weighed $8 \mathrm{oz}$. to the yard in length : the transit of the carriages was easier over that part of the line.

To test the power of endurance to heavy blows, Mr. Brockedon subjected a piece of vulcanised India-rubber, $1 \frac{1}{2}$ inch thick and 2 inches in area, to one of Nasmyth's steam-hammers of 5 tons; this first rested on the rubber without effect, then was lifted 2 feet and dropped upon it without injury, then lifted 4 feet, and the rulcanised cake was torn, but its elasticity was not destroyed. Still more severe trials were made : a block of vulcanised caoutchouc was placed as between cannon balls, with the whole power of the heaviest steam-hammers employed, but the iron spheres split the block, and the elasticity of the vulcanised caoutchouc was not destroyed.

Sheets of enormous size - ship-sheets-have been made 50 yards long and 56 inches wide, others 10 feet square; these are intended to pass over a steam-vessel's side, to adapt a valve, fix a pipe, or repair, from the interior, the vessel itself, without going into dock. These stout sheets, $\frac{3}{8}$ ths inch thick, are let down by ropes over a ship's side, and brought over the hole or place for repair by the pressure of the water on the elastic sheet, when the leak may be stopped and the ship pumped dry, pipes renewed, shot-holes and leaks stopped. Indeed, an early application of compounds of native rubbers and other materials was applied directly as sheathing for ships with success; but litigation among the parties caused the business to cease. Since the various plans for getting a flexible material have been successful, there seems no doubt but many unexpected applications will be made.

Messrs. Macintosh had coated some logs of wood with rulcanised India-rubber, and caused them to be towed in the wake of a vessel all the way to Demerara and back, and it was found that the coated logs were quite intact, while the uncoated timber was riddled by marine borers. The same firm stated: "That the only effect they could trace upon long immersed vulcanised caoutchouc, was a slight change of colour, perhaps a hydrate produced by superficial absorption, but this change of colour disappeared on being dried. If they were called upon to select a situation for the substanee to retain its properties for the longest period, they would select immersion in water. After years of experience in the use of hose-pipes, pipe-joints, valves for pumps and steam-engines, they had never known an injury from the contact of any kind of water.'

Mr. Goodyear sums up the advantages of vulcanised rubber under the following heads, as being either properties new or superior to those possessed by the natural croutchone:-

Vot, I. 
1. Elasticity.

2. Pliability.

3. Durability.

4. Insolubility.

5. Unaltorability by climate or artificial hest or cold.

6. Inadhesiveness,

7. Impermeability to air, gases, and liquids.

$\mathrm{Mr}$. Burke, in describing his patented process, for the use of the golden sulphuret of antimony, says, that he avoids two principal defects of the usual article, viz. its efflorescence of sulphur with an offensive odour, and its consequent decomposition and becoming rotten. He employs crude antimony ore (the sulphuret of that metal, in fine powder), and converts it by boiling in water with soda or potash (carbonates) into the orange sulphuret of that metal (Kermes mineral) by the addition of hydrochloric acid to the fluid in slight excess. He combines this compound (after being well washed) with eaoutchouc or gutta-percha, either together or separately, according to the degree of elasticity which he wishes to obtain. This mixturo is afterwards subjected to a heat of from $250^{\circ}$ to $280^{\circ} \mathrm{F}$. He masticates the caoutchouc in the usual iron box, by means of the kneading fluted revolving rollers, subjecting the whole to heat. The antimonial compound is then added in quantities varying from 5 to $15 \mathrm{lbs}$, according to the strength and elasticity required in the compound. At the end of from one to two hours' trituration, the block is removed from the box, and while in a warm state it is strongly compressed in an iron mould; and after being under pressure for a day or two is subjected to a steam heat for a couple of hours. The block thus prepared may now be cut into sheets, and afterwards divided into threads, or formed into such other articles as are desired. This forms the elastic red rubber.

The chief improvements operated in caoutchouc by the process of vulcanisation are the properties of resisting and remaining unaffected by very high degrees of heat and cold, and increased compressibility and elasticity. In its natural state Indiarubber becomes rigid by exposure to cold, and soft and plastic by heat, or under the action of boiling water. Articles manufactured of this substance suffer and lose the qualities which constitute their value in cold and in hot countries. A piece of Indiarubber cloth, for instance, taken to Moscow in December or January, would assume all the qualities of a piece of thin sheet iron, or thick pasteboard; the same cloth would in India or Syria become uncomfortably pliable, and present, a moist and greasy appearance; and, indeed, after boing folded up some time, would be found to be glued together. Nothing but vulcanisation insures the equable condition of the articles in the most intense cold, and, in heat up to and above $300^{\circ}$, makes India-rubber fit for practical purposes. These advantages have conduced to its being very extensively used in connection with machinery of every description; and as steam power is still further employed, and as the numerous other advantages possessed by vulcanised India-rubber become known (for it is only of late that any idea of their extent has been realised) its application will be extended and its consumption proportionally increased.

The compressibility and the return to its former dimensions, when the pressure has ceased, in one word, the elasticity of the India-rubber, is increased to such a degree by vulcanisation, that comparing the improved with the original article, it may be said that the native India-rubber is almost devoid of elasticity. The high degree of elasticity which it obtains by vulcanisation is shown by the results of the following experiments, in which a block of the vulcanised India-rubber, of the kind used for the manufacture of railway carriage springs, measuring 6 inches outside disc, 1 inch inside disc, and 6 inches deep, was taken and exposed to pressure :-

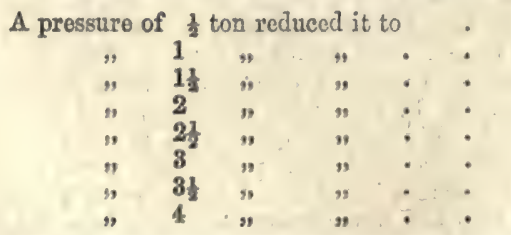

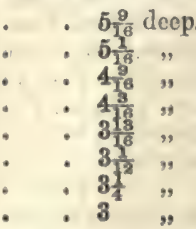

The block was left under pressure for 48 hours, and in each case returned to its original dimensions after a short period when the pressure was removed.

Among the most recent uses of India-rubber and canvas, are those of its manufacture into gas and ballast bags, the former are used for the transport of gas, and 


\section{CAOUTCHOUC}

applied to the various emergencies of gas engineering. India-rubber gas-tubing is now in general use, the groat advantage over metal tubes being, the ease with which gas can be conveyed to whatever part of the building it may be required in ; this, where any alterations are being effected, is a great desideratum. Ballast bags, large stout bags of India-rubber and canras, capable of holding from 1 to 5 or 10 tons of water, are coming into use as the most convenient form of ballast, thus saving valuable space which is made available for cargo. These bags may be emptied at any time, and when flattened down and rolled up, can be conveniently stowed away. India-rubber bags for inflation have also in a few cases been made use of for buoying up vessels, but hitherto the practice has been experimental only, and such floating machines are not as yet generally in use.

The vulcanising of India-rubber on silk or woollen goods was for a long time considered impracticable, because the process of vulcanisation destroyed the fibre and texture of the two substances; but it is stated that now this process is effected in a manner which deprives neither silk nor wool of their natural qualities and strength. By this improvement, combined with Silver's patent process of annihilating the unpleasant smell which all India-rubber goods used to acquire in the process of manufacture, the advantages of that substance for clothing purposes are extended to the lightest and the warmest of our textures. Silk and India-rubber garments are made without any deterioration of the strength and durability of the stuff, while they are perfectly free from odour of any kind.

\section{Mrghancat applications of Caodtchodc.}

Numerous important applications of caoutchouc have been made in the mechanical arts, among which we may mention, springs for railways and common road carriages, military carriages, lifting springs for mining ropes and chains, towing ropes and cables, rigging of ships, recoil of guns on ships, the tyres and naves of railway and other wheels, axles and axle bearings, windows of railway carriages, railway switches, bed of steam-hammer, couplings for locomotives and tenders, packing for steam and water joints, shields for axle boxes, soékets for water pipes, bands for driving machinery, valves for pumps, tubes for conveying acids, beer, water, and other fluids, packing for pistons, \&c.

The Exhibition of 1862 presented to us an enormous display of India-rubber manufacture. The applications of caoutchouc to driving belts for machinery, to washers and buffers, were numerous, and many of them excellent.

Messrs. Perreaux and Co. were exhibitors of a valve for pumps made of vuleanised caoutchouc. Its simplicity and efficiency were remarkable: the valve was simply a cylinder of India-rubber, with its two sides at the uttermost end pressed together like lips, and then vulcanised, which makes the material retain the form given to it. This valve, when in position, was open below, and closed above in the form of a wedge; the fluid easily passes upwards owing to the elasticity of the material, but the downward pressure firmly closes the lips and prevents return. Of ebonite and kamptulicon there were many examples, embracing a curious variety of articles from philosophical apparatus in the form of electrical machines, to pieces of furniture, and ornaments for ladies' wear. See EBonitr and KANTTULicos.

springs. - The first proposal to use caoutchoue for springs that we are aware of occurs in Lacey's patent, in 1825, when blocks of caoutchouc were proposed to be used, having dividing plates of iron between each series; but little seems to have been done towards any practical application at that time: later, in 1844, Melville proposed to use spheres of caoutchouc, enclosing air, and separated by dises of wood or metal, the whole being enclosed in iron cases, and used for buffers and bearing springs for railway carriages. In 1845 Walker and Mills proposed to use bags of croutchouc enclosing air, and contained in cases of iron, for use as buffer springs.

The next improvement is contained in Fuller's patent of 1845, which consists in the use of cylindrical rings of vulcanised India-rubber, in thicknesses varying from $\frac{1}{2}$ to 3 inches, and with diameter of ring suitable to the power of spring required; between each of these cylindrical rings he places a thin iron plate, through a hole in the centre of which passes a guide rod. Fig. 409 shows Fuller's spring in section and plan. These springs have been extensively used as buffer-, bearing-, and draw-springs for railway uses, alone and in combination with De Bergue's improvements : some defects have been found in practice in this form, to obviate which, the ingenuity of later inventors has been exercised; the defects alluded to are, the tendency to swell out at the central unsupported part of the ring, thus from the undue tension rendering it liable to break 
under sudden concussion, and occasioning complete disintegration of the material where not breaking.
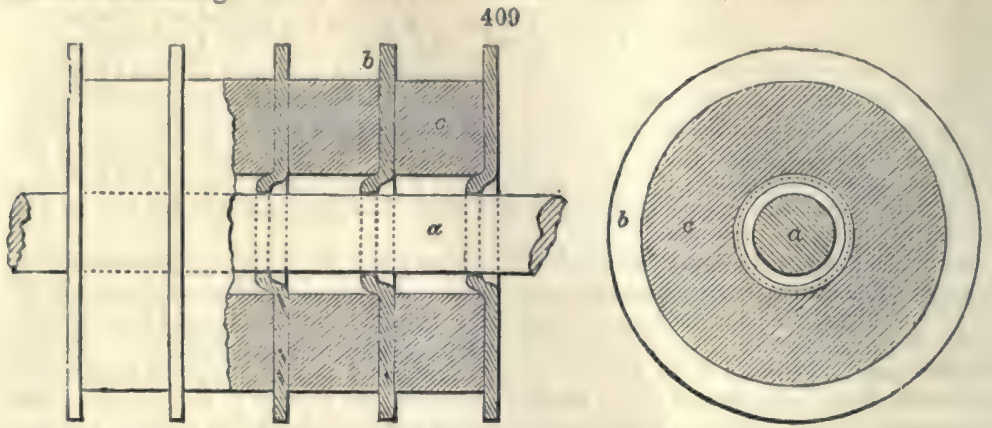

To obviate these defects, George Spencer (in 1852 and 1853) proposed to mould the caoutchouc at once in the form it assumes under pressure, and then to place a confining ring of iron on the larger diameter. (See fig. 410). By this ingenious plan, the caoutchouc loses its power of stretching laterally, being held by the ring $b$, secured in a groove moulded in the cone to receive it; when the pressure is applied to the ends, the rubber is squeezed into the cuplike spaces $c$, and thus the action of the spring is limited. By this plan, rubber of a cheaper and denser kind can be used than on the old cylindrical plan, and the patentee states that many thonsands of carriages and trucks are fitted with these springs which give entire satisfaction; among which, are those on the Brighton, South-Western, North London, South Wales, Vale of Neath, Bristol and Exeter, Taff Vale, Lancashire and Yorkshire, St. Helen's, Bombay and Baroda, Theiss Railways, and many others. These cones are used as buffer-, bearingand draw-springs for railway carriages, and are made in several sizes to suit various

410
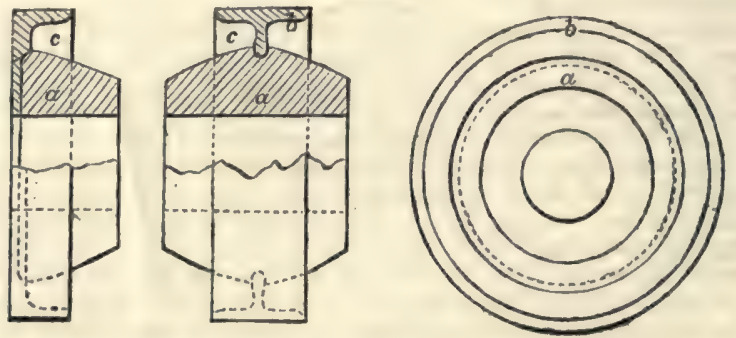

uses. To show the power that such springs are equal to, we append the result of an experiment on a No. 1 cone (for inside buffers), 3 inches in length, $3 \frac{3}{4}$ inches diameter at ring, 5 inches diameter of ring.

1 st Experiment, without the confining ring, weight of cone $1 \frac{1}{4} \mathrm{lb}$.

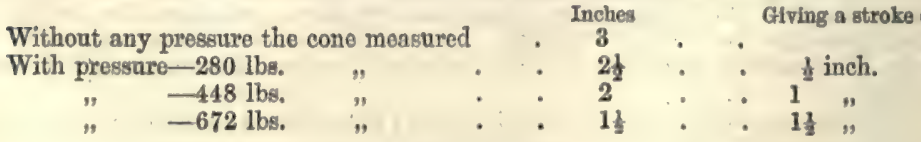

\section{2nd Experiment.}

With the confining ring $b$, on the same double cone; the following were the results:-

Without any pressure the cone measured .

With-448 lbs.

" $1,680 \mathrm{lbs}$.

, 2,912 lbs.

" $15,680 \mathrm{lbs}$.

$"$
$"$

3 inches, as before.

$2 \frac{1}{2}$

2 


\section{CAOUTCHOUC}

The adrantages are stated to be, less first cost than steel; less weight, $6 \mathrm{cwt}$. being saved in each carriage by their use ; and great durability.

Coleman's improvement consists in the use of iron rings to confine the lateral swellings of India-rubber cylinders. (See fig. 411). They are used as bearing-springs

411
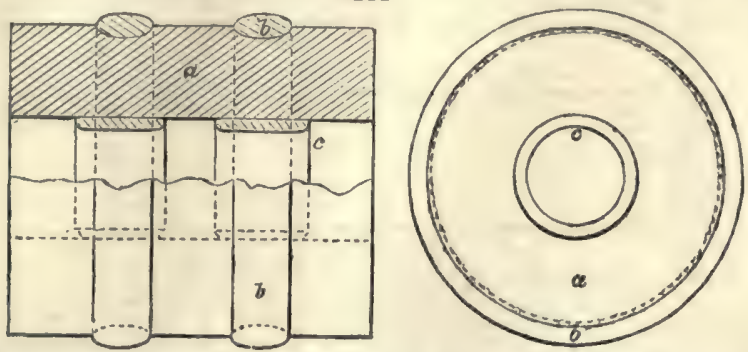

for engines and tenders on the North-Western Railway, by J. E. M'Connell, Esq., who prefers them to steel, as being easy in action, durable, safe, and easy of repair; they are used also as buffer- and draw-springs.

The next form of these springs is the invention of R. Eaton (see fig. 412). This spring seems to be peculiarly adapted to use where a powerful spring, acting through

412
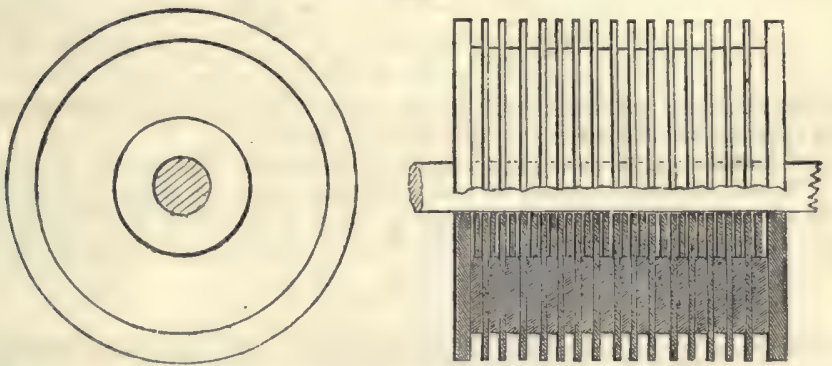

a small space, and taking little room, is required, as for use in mining ropes and chains, \&c. Faton's main idea is the use of laminæ of India-rubber, of a maximum thickness of $\frac{1}{2}$ an inch, with dividing plates, as in Lacey's and Fuller's, which avoids the objections stated above, by supporting the India-rubber at smaller intervals; for springs,

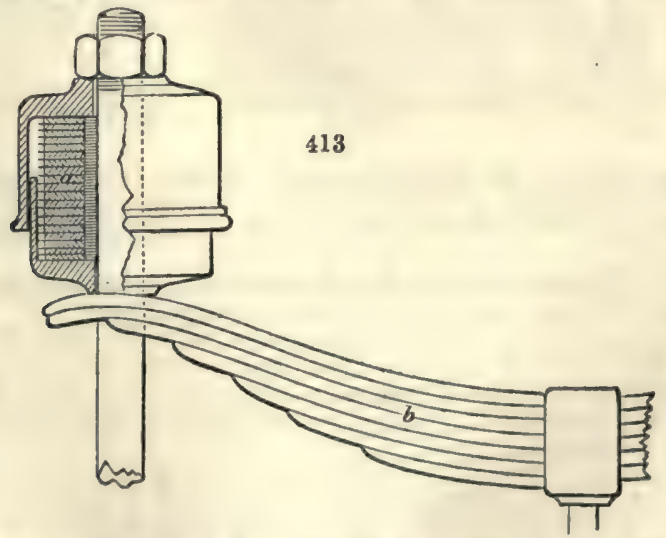

where great power is wanted in little compass, and to act through short distances, as in engine bearing-springs, lifting-springs, and somo kinds of draw-springs-this form proves to be well suited. 
Hoige's compound spring is designed to obviato the frequent breakage of the steol springs on locomotive engines. Fig. 413 shows one of these springs; a block of India-rubber is placed on each end of the steel spring, or is suspended under the engine frame; they are in use on several of the Euglish railways, and are said to answer tho purpose intended well.

Scott's patent (see fig. 414), dated May 1856, consists in the use of blocks of Indiarubber, or cones, placed over the centre of the spring; they are to obviate the danger of

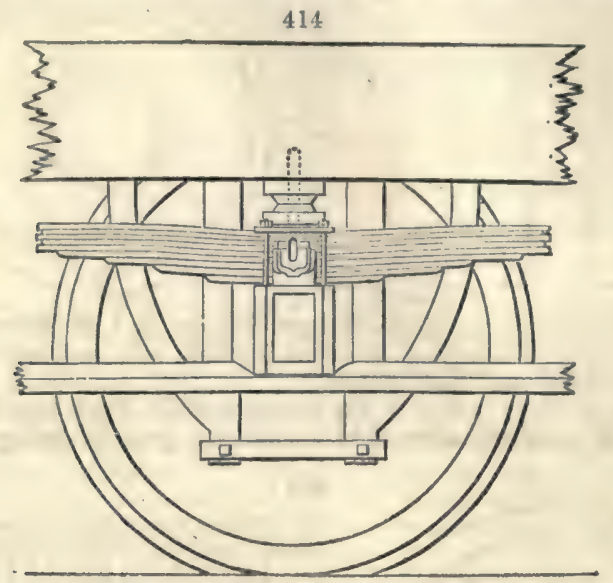

overloading carriages and trucks, \& frequent source of danger to the springs, and are made to take the whole load in case of a spring breaking.

Bridges' Patent (fig. 415). - This inventor ingeniously proposes to use Spencer's cones in blocks of wood, instead of iron confining rings. A series of them are enclosed in a case formed in the side timbers of the underframe of the railway truck or carriage; the cup space is formed in the block of wood, as our figure shows, and no

\section{5}

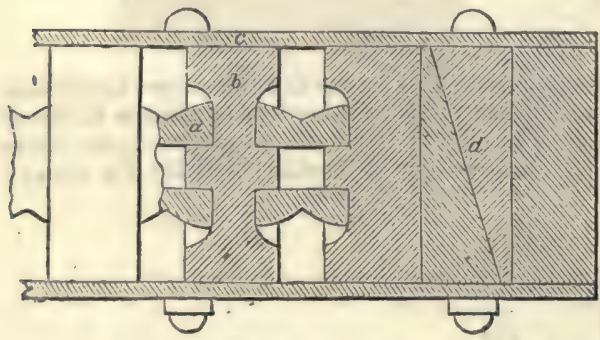

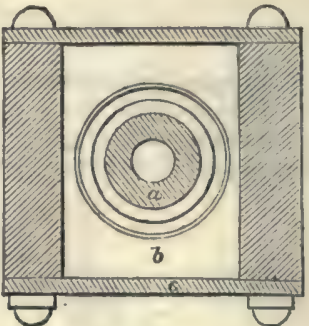

guide rods are required; the principle is applied to draw-and bearing-springs. The advantages proposed by this arrangement are, the dispensing with guide rods and the taking the ultimate blow on blocks of wood, which deadens its effect; they are said to answer very well.

In $1847, M r$. De Bergue patented some improvements in the application of Fuller's spring to buffer-, bearing-, and drawing-springs for railway uses.

The applications for common road carriages, patented by Mr. Fuller of Bucklersbury in 1852 and 1855, have been extonsively used, both in the form of cylindrical rings acting by compression and slso of suspension springs for lighter kinds of vehicles.

Respecting these springs, figs. 416, 417, we have been furnished by the patentee with the following particulars:-

The form generally used for heavy purposes, such as drays, vans, waggons, \&c.., consists of a series of rings of cylindrical or circular form, working in a perpendieular rod or spindle, on oach side the axle, with the usual separating plates or washers; the depth and diameter of the rings being regulated by the weight to be sustained and the speed required.

During the Cmmeen war, those eprings were introduced by Mr. Fuller to the notice 
of the Government authorities at the Royal Arsenal, Woolwich, and were in consequence adopted for all kinds of military carriages, store waggons, ammunition waggons, \&e. They were also applied in the suspensory form for the medical cars and ambulance waggons for the wounded, for which purposes the use of India-rubber on the principle of extension is found to produce the easiest and most satisfactory spring hitherto discovered.

416

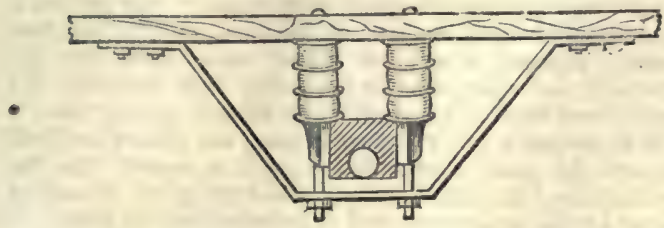

417

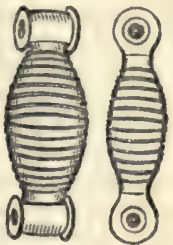

When the material is used as a suspension spring, the most advantageous form for the purpose is found to be that of round cord of the best and purest quality, prepared by solvents, and about $\frac{1}{4}$ th or $\frac{3}{8}$ the inch diameter.

A continuous length of such cord is wound at a considerable tension over the ends of two metal sockets or rollers, in shape something resembling a cotton reel, and whilst in a state of tension, bound at each end with strong tape or other suitable binding; the number of cords composing the spring, varying from 10 to 20,30, or 40, according to the strength required.

Another important adaptation of India-rubber by Mr. Fuller, is that of anchor springs, towing ropes, and springs for the recoil of guns and mortars.

418 Wheel tyres.-Fig. 418 shows an important application to the mympry tyres of whoels for railway purposes. A thin band of Indiarubber is inserted between the tyre and spoke ring, by first covering it with a thin plate of iron, to protect the India-rubber while the hot tyre is put on, when the wheel is instantly thrown into water and a cooled. This has been severely tested for some time, and found to answer very well; the advantage gained is the saving in the breaking and wear of the tyres.

For Windows. - Small ropes of India-rubber are inserted in grooves at each side of the window, and so stop out draught and prevent noise.

For steam-hammer beds. - A plate of India-rubber $\frac{5}{8}$ ths inch thick, is placed under the bed of the hammer; the effect is greatly to diminish the transmission of shocks to the building, and to cheapen the foundation: as an instance of useful application, we may state, that at Messrs. Ransome and May's works, at Ipswich, the working of the steamhammer shook the building and windows to an alarming extent; but the insertion of blocks of vulcanised rubber under the anvil, almost entirely obviated these effects.

Joints between engines and tenders,-Messrs. Lund, Spencer, and Fenton, have also introduced the use of rings of this material to form a joint between the locomotive and tender (fig. 419). They are extensively used, and ontirely prevent the leakage

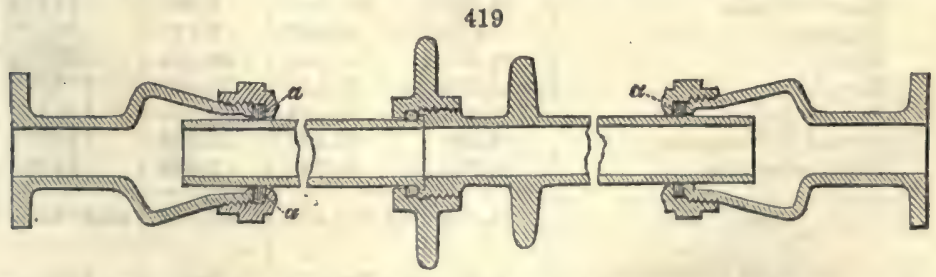

common to the old ball-and-socket joints, and are much cheaper in first cost. Rings of India-rubber were proposed by Mr. Wickstead, for closing the socket joint of water pipes, and they are used in a variety of forms for that purpose.

A large trade has been established in the supply of bands of India-rubber for driving machinery; for many purposes they answer better than leather, water having no effect on them and there being little or no slip and fewer joins; they are made in widths, and belts costing $150 \mathrm{l}$. each have been used in some cases. They are made with two or more layers of thread cloth between, and outside of which the rubber is placed. 
VI. Soluizisation of Caudtchodc.

Singular as caoutchouc is in its properties and its applications, it is probable that besides the meehanical and electrical qualities and general resistance to chemical action, it may yet be found to have other modifications peculiar and valuable. The practical mon most conversant with this substance, and deeply involved with patents and successful manufactures, record their conviction of the influence of solar light, and the marked distinctions supposed to exist between the influence of solar and terrestrial heat upon this substance.

Mr. Hancock says, 'In my early progress, I found that some of the rubber I employed was very quickly decomposed when exposed to the sun: as the heat was never more than $90^{\circ}$, and rubber exposed to a much higher temperature was not injured by it, I suspected that light had some effect in producing this mischief. To ascertain this, I cut two square pieces from a piece of white rubber, one of these $I$ coloured black, and exposed it to the sun's rays; in a short time, the piece which had been left white, wasted away, and the sharp angles disappeared, it seemed like the shape of a thin piece of soap after use; the blackened piece was not at all altered or affected. The lesson taught me by this experiment was of great value ever after.'

Speaking of the annoyances and failures in the early Macintosh goods by heat, grease, \&ce., Mr. Haneock says, 'The injurious effect of the sun's rays upon thin films of rubber we discovered and provided against before much damage accrued.'

Mr. Goodyear says, 'In anticipation of the future as relates to a mode of treatment in manufacture, which, though lightly esteemed and little thought of now, I believe will be extensively practised hereafter, I feel bound to make a strong though qualified claim to the process of Solarisation. This process consists in exposing caoutchouc when combined with sulphur, to the sun's rays.' Again, 'when exposed to the sun's rays for several hours, a change is produced which may becalled natural vulcanisation, in all thin fabrics or thin sheets of caqutchouc.' 'Solarisation is an effectual and cheap process of curing India-rubber.' He further says, 'it is well established that India-rubber melted at about $200^{\circ}$, and in the sun's rays at $100^{\circ}$ or less. Another effect yet more remarkable in the treatment of gum elastic, is that of the sun's rays upon it; when combined with sulphur and exposed to the sun, either in hot weather or cold, it becomes solarised, or divested of its adhesive quality; whereas, no other kind of light or heat has any similar effect, until the high degree of heat is applied to it, about $270^{\circ}$, which is used in vulcanising,'-Goodyear, p. 114, vol. i. Now Haven, U.S.

Our recent Imports of Caoutchoue have been as follow:-

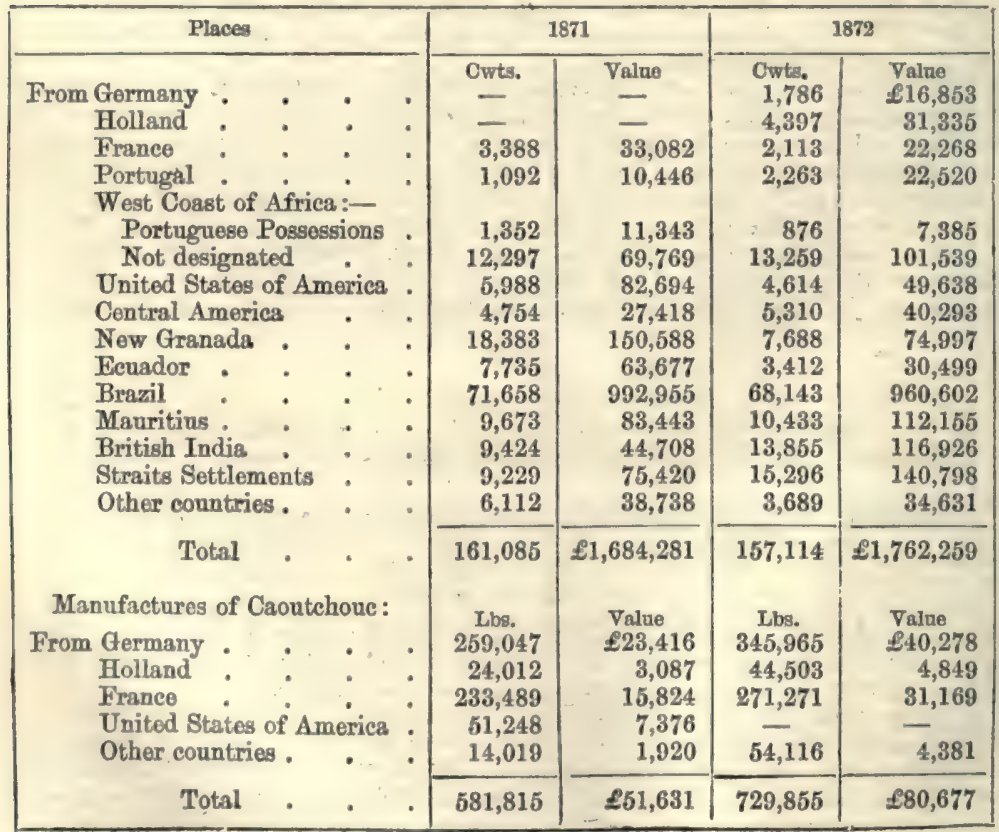


Our Exports of caoutchoue manufactures in the same year were valued at $854,539 l$.

In 1872 we imported 157,148 cwts., valued at $1,762,886 l$.

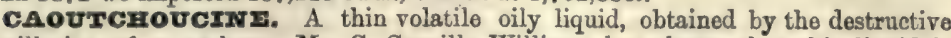
distillation of caoutchouc. Mr. C. Greville Williams has shown that this liquid is composed principally of two hydrocarbons, called caoutchin $\mathrm{C}^{20} \mathrm{H}^{16}\left(\mathbf{C}^{10} \mathbf{H}^{16}\right)$, and isoprene $\mathrm{C}^{10} \mathrm{H}^{8}\left(\mathbf{C}^{5} \mathbf{Z}^{8}\right)$.

Mr. William Barnard, in the course of some experiments upon the impregnation of ropes with caoutchouc, which he conducted at the factory of Messrs. Einderby at Greonwich, discovered that when this substance was exposed to a heat of about $600^{\circ} \mathrm{F}$. it resolved itself into a vapour, which, by proper refrigeratory methods, was condensable into a liquid possessing very remarkable properties, to which the name caoutchoucine has been given. For this invention ' of a solvent not hitherto used in the arts,' Mr. Barnard obtained a patent, in August 1833. His process for preparing it is described in his specification as follows:- 'I take a mass of the said caoutchouc, or Indiarubber, as imported, and having cut it into small lumps, containing about two cubic inches each (which I prefer), I throw these lumps into a cast-iron still (which I find adapted for the purpose, and a diagram of which is annexed to, and forms part of this my specification), with a worm attached (fig. 420): A is the still; B, the cover ground to a metallic fit, to admit of a thermometer to take the temperature; $\mathrm{c}$, the fire-place; $\mathrm{D}$, the ash-pit; $\mathrm{E}$, the worm-tub and worm; $\mathrm{F}$, the brickwork of the still; G, a roller and carriage, in connection with a crane, or other means, to raise the cover to take out the residue, and to charge the same; $\mathrm{H}$, the chain.

'I then apply heat to the still in the usual manner, which heat is increased until the thermometer ranges at about $600^{\circ} \mathrm{F}$, or thereabouts. And as the thermometer ranges upwards to $600^{\circ}$ F. a dark coloured oil or liquid is distilled over, which I claim as my said invention, such liquid being a solvent of caoutchouc, and other resinous and oleaginous substances. When the thermometer reaches $600^{\circ} \mathrm{F}$, or thereabouts, nothing is left in the still but dirt and charcoal.

'I have found the operation of distillation to be facilitated by the addition of a portion of this oil, either previous or subsequent to the rectification, as hereinafter mentioned, in the proportion of one-third of oil to two-thirds of caoutchoue.

- I afterwards subject the dark coloured liquid thus distilled, to the ordinary process of rectification, and thereby obtain fluids varying in specificgravity, of which the lightest hitherto

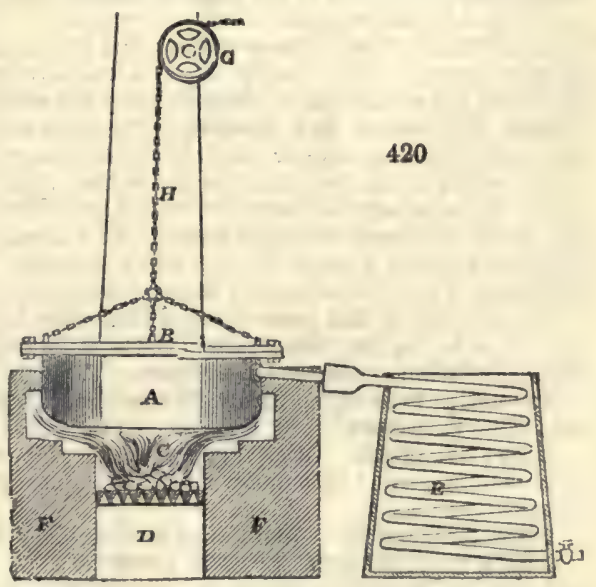
has not been under 670 , taking distilled water at 1,000 , which fluids I also claim as my said invention.

'At each rectification the colour of the liquid becomes more bright and transparent, until at the specific gravity of $0^{\circ} 680$, or thereabouts, it is colourless and highly volatile.

- In the process of rectification (for the purpose of obtaining a larger product of the oil colourless) I put about one-third of water into the still. In each and every state tho liquid is a solvent of caoutchouc, and several resinous and oleaginous substances, and also of other substances (such as copal), in combination with very strong alcohol.

'Having experienced much difficulty in removing the dirt which adheres to the bottom of the still, I throw into the still lead and tin in a state of alloy (commonly called solder), to the depth of about half an inch, and, as this becomes fused, the dirt which lies on the surface of it is more easily removed.

- Objections have been made to the smell of this liquid:-I have found such smell removed by mixing and shaking up the liquid with nitro-muriatic acid, or chlorine, in the proportion of a quarter of a pint of the acid (of the usual commercial strength) to a gallon of the liquid.'

The discovery of the chemical solvent, which forms the subject of the patent above described, has excited considerable interest in the philosophical world, not only from 
its probable usofulness as a new articlo of commerce, but also from two very extraordinary characteristics which it is found to possess, viz., that, in a liquid state, it has less specific gravity than any other liquid then known to the chemists, boing considerably lighter than sulphuric ether, and, in a state of rapour, is heavier than the most ponderous of the gases.

This matorial (when mixod with alcohol) is a solvont of all the resins, and particularly of copal, which it dissolves without artificial heat, at the ordinary temperature of the atmosphere; a proporty possessed by no other solvent known; and hence it is peculiarly useful for making varnishes in general. It also mixes readily with oils, and will be found to be a valuable and cheap menstruum for liquefying oilpaints; and without in the slightest degree affecting the most delicate colours, will, from its ready evaporation, cause the paint to dry almost instantly.

Cocoa-nut oil, at the common temperature of the atmosphere, always assumes a concrete form; but a portion of this caoutchoucine mixed with it will cause the oil to become fluid, and to retain sufficient fluidity to burn in a common lamp with extraordinary brilliancy.

Caoutchoucine is extremely volatile; and yet its vapour is so exceedingly hoavy, that it may bo poured, without the liquor, from one vessel into another, like water. One of the real practical objections to caoutchoucine seemed to be its easy decomposition. Messrs. Enderby and Barnard found that, if exposed to air, and especially if a small quantity of water was present, that it was speedily decomposed, changing colour to deep brown or black. Specimens, however, remain perfectly clear and without change, in bottles, after twonty years.

After caoutchouc has been subjected to destructive distillation, and the caoutchoucine or volatile oil of caoutchouc has distilled off, there remains in the retort a residual mass which, when dissolved in oil, forms a varnish impervious to moisture, and consequently much used by shipwrights.

CAPIrRs. The Capparis spinosa, the flower-buds of which constitute the capers of the shops. Their quality depends exclusively upon the age at which they are gathered, the smallest and youngest being the most delicate and the dearest; and the largest, the coarsest and cheapest. The buds are plucked before they open, and thrown into strong vinegar, slightly salted, where they are pickled. The erop of each day is added to the same vinegar tub, so that in the course of the six months during which the caper-shrub flowers, the vessel gets filled, and is sold to persons who sort the capers by means of copper sieves. This metal is attacked by the acid, wherofrom the fruit acquires a green colour, nuch admired by ignorant connoisseurs. About 60,000 lbs, a year are consumed in this country.

The capsules of the caper spurge, Euphorbia Lathyris, are sometimes pickled as a substitute for capers; but although the acid destroys some of the acrid properties, the free use of them is dangerons.-Percira.

The unripe fruit of the garden Nasturtium (Tropoohem majus) is also pickled as a substitute for capers.

CAPIIIAIRE. Originally a kind of syrup, extracted from maiden-hair. The term is now appliod to a finely clarifled simple syrup, which is made usually with orange-flower water.

CAPNOMOrz. A substance discovered by Reichenbach in wood-tar.

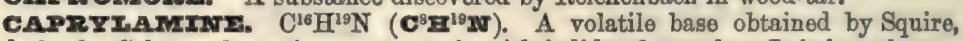
and also by Cahours, by acting on ammonia with iodide of capryle. It is homologous with mothylamine, \&ze-C. G. W.

CAPsicurs. The dried ripe fruit of Capsicum fastigiatum, imported from Zanzibar, known in commerce as Guinea Pepper and Pod Pepper. Seo PkPPER.

capstarr. (Cabestan, Fr.; Spille, Ger.) A machine whereon the cable is wound successively in weighing the anchor of a vessel. It is a species of wheel and axle; the axle being vertical, and piorced with holes near its top for the insertion of the ends of horizontal levers, called handspikes, which represent the wheel. These are turned by the force of men moving in a circle. The power applied to the lever is to the resistance to be overeome (the weight of the anchor, for example), when the forces are in equilibrio, as the radius of the cylinder round which the cable is coiled is to the circumference described by the power. It is manifest that the radius of the axle must be augmented in this computation by half the diameter of the cable, which is supposed to lie always one coil thick upon it. The force of a man thus applied, has been commonly estimated as equal to the traction of 27 pounds hanging over a pulley. Frietion being so variable a quantity in capstans, renders the exact calculation of its mechanical effect somewhat uncertain. A stout man, stationed near the bottom of the axle, holds fast the loose part of the cable, which has already made two or three turns; and, being aided by its friction upon the wood, he both prevents it from slipping backwards, and uncoils each turn as it is progressively made. 
Mr. Hindmarsh, of Newcastle, obtained a patent, in February 1827, for a contrivance to enable a capstan or windlass to be occasionally worked with inereased mechanical advantage. With this view, he placed toothed wheel-work, partly in the drum-head of the capstan, and partly in the upper part of the barrel, upon which the cable is coiled and uncoiled in successive portions.

The drum-head, and also the barrel, turn loosely upon a central spindle, independently of each other, and are connected together either by the toothed gear, or by bolts. On raising or withdrawing the connecting pinion from the toothed wheols, and then locking the drum-head and barrel together, the capstan works with a power equal only to that exerted by the men at the capstan-bars, as an ordinary capstan; but on lowering the pinion into gear with the wheel work, and withdrawing the bolts which locked the drum-head to the barrel, the power exerted by the men becomes increased in proportion to the diameter and number of teeth in the wheel and pinions.

Fig. 422 is the external appearance of this capstan. Fig. 421 a horizontal view of the toothed gear at the top of the barrel. The barrel, with the whelps $a a$, turn loosely upon a vertical spindle fixed into the deck of the vessel. The drum-head $b$ also turns loosely upon the same spindle. The circular frame $c c$, in fig. 421, in which the axes of the toothed wheels $d d d$ are mounted, is fixed to the central spindle. The rim eee, with internal teeth, is made fast to the top of the barrel, and the pinion $f$, which slides upon the spindle, is connected to the drum-head.

When it is intended to work the capstan with ordinary power, the pinion $f$ is raised up into the recess of the drum-head, by means of a screw $g$, fig. 422 , which throws it out of gear with the toothed wheels, and it is then locked up by a pin $z$ : the bolts $h h$ are now introduced, for the purpose of fastening the drum-head and barrel together, when it becomes an ordinary capstan.

But when it is required that the same number of men shall exert a greater power, the bolts $h$ are withdrawn, and the pinion $f$ lowered into gear with the toothed wheels. The rotation of the drum-head, then carrying the pinion round, causes it to drive the toothed wheels $d d d$; and these working into the toothed rim $e e$, attached to the barrel, cause the barrel to revolve with an increasing power.

Thus, under particular circumstances, a smaller number of men at the capstan or windlass (which is to be constructed upon the same principle) will be enabled to haul in the cable and

421

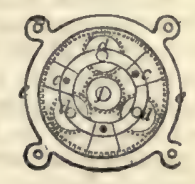

422

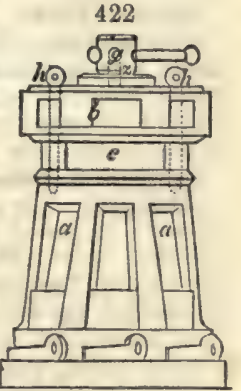
anchor, or warp off the ressel, which is an important object to be effected.

In 1819, Captain Philips obtained a patent for certain improvements in capstans, a part of which invention is precisely the same as this in principle, though slightly varied in its adaptation.

James Brown, ship-rigger, in his capstan, patented in 1833 , instead of applying the moving power by handspikes, having fixed two rims of teeth round the top of the capstan, acts upon them by a rotatory worm, or pinions turned by a winch.

Fig. 423 is an elevation of this capstan, and fig. 424 is a horizontal top view. $a$ is an upright shaft, fixed firmly to the deck, serving as an axle round which the body of the capstan revolves. A frame $c$, fixed to the top of a stationary shaft $a$, above the body of the capstan, carries the driving apparatus.

The upper part of the body of the eapstan has a ring of oblique teeth $d$, formed round its edge; and

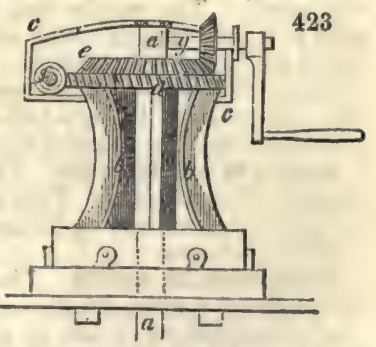
above this, on the top of the capstan, is a ring of bevel teeth $e$. A horizontal shaft $f$, mounted in the top frame $c$, has a worm or endless screw, which takes into the teoth of tho ring $d$; and a short axle $g$, having its bearings in the contral shaft $a$, and in the frame $c$, carries a bevel pinion, which takes into the bevel teeth of the ring $e$.

The bearings of the shaft $f$, in the top frame, are in long slots, with angular returns, something like the fastening of a bayonet, which is for the purposo of enabling the shaft to be readily lifted in and out of gear with the teeth of the ring $d$; the outer bearing of the axle $g$ of the bevel pinion is also supported in the frame $c$, in a similar way, in order to put it in and out of gear with the teeth of the 
bevel ring $\ell$. A mode of shifting these is essential; because the two toothed rings, and their driving worm and pinion, givo different speods, and, of course, cannot be both in operation at the same time.

The worm of the shaft $f$ being placed in gear with the teeth of the ring $g$, on

424

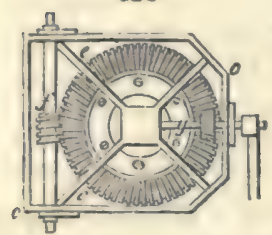
applying rotatory power thereto, by means of winches attached to the ends of the shaft, the barrel or body of the capstan will be made to revolve with a slow motion, but with great power; and thus two men at the winches will do the same work as many men with capstan bars in the ordinary way.

If a quicker movement than that of the endless serew is desired, then tho driving power may be applied by a winch to the axle $g$ of the bevel pinion, that pinion being put into goar with the bevel ring $e$, and the endless screw withdrawn. It should, however, be here remarked, that the patenteo proposes to employ two short axles $g$, placed opposite to each other, with borol pinions acting in the bevel-toothed ring, though only one is shown in the figure to. avoid confusion. He also contemplates a modification of the same contrivance, in which four short angles $g$, placed at right angles, with pinions taking into a bevel ring, may be employed, and made effective in giving rotatory motion to the barrel of a capstan by means of winches applied to the outer ends of the axlo, and turned by the labour of four men.

CAPUT MORTUUM. A term employed by the alchemists to express the residuum of distillation or sublimation, the volatile portions having been driven offo

carajura. A red dye stuff: see Chica, Rkd.

CARAMrI. Burnt or dried sugar, used for colouring spirits and gravies. It is a black, porous, shining substance, soluble in water, to which it imparts a fine dark brown colour. The French are in the habit of dissolving the sugar, after it has been exposed for some time to a temperature sufficiently high to produce the proper colour, in lime-water: this is sold under the name of 'colouring.'

\section{carapa oir. Seo Crab OrL.}

CARAT. The term carat is said to be derived from the name of a bean, the produce of a species of Erythrina, a native of the district of Shangallas in Africa, a famous gold-dust mart. The tree is called kuara, a word signifying 'sun' in the language of the country, because it bears flowers and fruit of a flame-colour. As the dry seeds of this pod are always of nearly uniform weight, the savages have used them from time immemorial to weigh gold. The beans were transported into India at an ancient period, and have been long employed there for weighing diamonds. The carat of the civilized world is, however, an imaginary weight, consisting of four nominal grains, a little lighter than four grains troy (poids de marc). It requires 74 carat grains and $\frac{1}{16}$ th to equipoise 72 of the other. The diamond carat, though containing 4 diamond grains, is equal to only $3 \frac{1}{5}$ grains Troy.

It is stated that the karat, a weight used in Mecca, was borrowed from the Greeks, and was equal to the 24th of a denarius or denier. The Encyciopedists thus explain the carat:- "The weight that expresses the fineness of gold. The whole mass of gold is divided into 24 parts, and as many 24th parts as it contains of pure gold it is called gold of so many carats. Thus gold of twenty-two parts of pure metal is gold of twenty-two carats. The carat of Great Britain is divided into four grains; among the Germans into 12 parts; and among the French into 32.' Among assayers, oven in this country, the German division of the carat is becoming common.

CARAWAY SFFDS. The fruits, commonly called seeds, of the Carum Carui, an umbelliferous plant. They are used for flavouring cordials. The oil of Caraway is employed to correct, by its aromatic properties, the nauseating and griping qualitios of some medicines.

CARBAMIC ACTD. The combination of gaseous ammonia with carbonic-acid gas produces a white substance formerly ealled anhydrous carbonate of ammonia, but now recognised as a salt of carbamic acid. This carbamate of ammonia readily combines with water, and passes into normal carbonate of ammonia.

CARBamidz. A white crystalline powder obtained by the action of gaseous ammonia on phosgene gas, or oxychloride of carbon.

carbazotrc acid. Seo Picric Acid.

CARBIDIs. A term synonymous with carburets. Thus, cast-iron being a combination of iron and carbon may be called a carbide or carburet of iron. 'In like manner the hydrocarbons are sometimes termed carbides of hydrogen.

CARBO-ZYPRATrs. Organic compounds containing carbon and the elements of water; such as starch and cellulose.

CARBO-HYDRIDE, commonly Hydrocarbon. A term used to denoto those 
bodies which consist of carbon and hydrogen only. The number of hydrocarbons now known is very great, and the list is increasing every day. They were formerly littlo understood, but so much has now been done that the anomalies and difficulties attending their history are rapidly disappearing. Although the number of individual bodies is very considerable, they are derived from a few great families. The principal are the following:-

Homologues of Olefiant gas.
Methyle.
$", \quad$ Marsh gas.
Benzole.
Isomers of Turpentine.

The other families which yield hydrocarbon derivatives are less important than the above, and will not be noticed here.

It is curious that the destructive distillation of organic matters is, of all operations, the most fruitful source of these bodies. Coal yields a great number, the nature varying with the temperature. When ordinary coals are distilled at very high temperatures, as in the production of gas, hydrocarbons belonging to the first four families are produced, and also a considerable quantity of naphthaline; but when, on the other hand, they are distilled at as low a heat as is compatible with their thorough decomposition, they yield fluid hydrocarbons, principally belonging to the first two classes, accompanied, however, by a considerable quantity of paraffine. The homologues of olefiant gas have acquired extreme interest, owing to the brilliant results obtained by MM. Berthelot, and De Luca, by Cahours, and Hofmann in the study of their derivatives. The homologues of methyle have attracted considerable attention, in consequence of the successful isolation, by MM. Frankland and Kolbe, of the singular group of hydrocarbons known as the organic radicals, and which, until then, were regarded as hypothetical bodies, existing only in cembination.

The hydrocarbons homologous with benzole not only exist in considerable quantity in ordinary coal naphtha, but are produced in a great variety of interesting reactions. Those contained in the following Table will show this :-

Table of the Physical Properties of the Benzole Series.

\begin{tabular}{|c|c|c|c|c|c|c|c|}
\hline \multicolumn{3}{|c|}{ Name } & Formulæ & \multirow{2}{*}{$\begin{array}{c}\begin{array}{c}\text { Boiling } \\
\text { point }\end{array} \\
176\end{array}$} & \multirow{2}{*}{$\begin{array}{l}\begin{array}{l}\text { Specific } \\
\text { gravity }\end{array} \\
0.850\end{array}$} & \multicolumn{2}{|c|}{$\begin{array}{l}\text { Specific gravity of } \\
\text { vapour }\end{array}$} \\
\hline Benzole & . & - & $\mathrm{C}^{12} \mathrm{H}^{6} \quad\left(\mathbf{C}^{6} \mathbf{H}^{6}\right)$ & & & $\begin{array}{c}\text { Experiment } \\
2 \cdot 77\end{array}$ & $\begin{array}{l}\text { Theory } \\
2 \cdot 699\end{array}$ \\
\hline Toluole & . & • & $\mathrm{C}^{14} \mathbf{H}^{8} \quad\left(\mathbf{C}^{7} \mathbf{x}^{8}\right)$ & 230 & 0.870 & $3 \cdot 26$ & $3 \cdot 183$ \\
\hline Xylole & - & • & $\mathbf{C}^{16} \mathbf{H}^{10}\left(\mathbf{C}^{8} \mathbf{E}^{10}\right)$ & 259 & $\ldots$ & $\therefore$ & $3 \cdot 668$ \\
\hline Cumole & • & • & $\mathrm{C}^{18} \mathrm{H}^{12}\left(\mathbf{C}^{9} \mathbf{I}^{12}\right)$ & 298 & ... & $3 \cdot 96$ & $4 \cdot 150$ \\
\hline Cymole & . & - & $\mathbf{C}^{20} \mathrm{H}^{14}\left(\mathbf{C}^{10} \mathbf{I}^{14}\right)$ & 347 & 0.861 & $4 \cdot 65$ & $4 \cdot 636$ \\
\hline
\end{tabular}

Benzole has already been sufficiently described, and will not, therefore, be further alluded to. All these hydrocarbons yield a great number of derivatives when treated with various reagents. By first treating them with strong nitric acid, so as to obtain nitro-compounds, that is to say, the original substance in which an equivalent of hydrogen is replaced by hyponitric acid $\mathrm{NO}^{4}\left(\mathbf{I N}^{2} \mathbf{O}^{4}\right)$, strongly odorous oils are produced. When treated with sulphide of ammonium or protacetate of iron, these oils become reduced, and yield a very interesting series of volatile organic bases or alkaloids; these are aniline, toluidine, xylidine, cumidine, and cymidine. Mr. Barlow has shown that special precautions are necessary in converting cymole into nitrocymole, preparatory to the formation of the alkaloid cymidine. Cymole is acted on too violently by nitric acid to allow of the nitro-compound being formed, unless the precaution is taken of cooling the acid and hydrocarbon, by means of a freezing mixture, before allowing them to react on each other. The nitro-compound, when well formed, may be reducod in the ordinary manner. These alkalis have of late years acquired special importance in consequence of the valuable dyes that Mr. Perkins has succeeded in producing from them. See Avilune.

Paraffine is a solid hydrocarbon of great interest; it is found both in wood and coal tar. When coal is distilled for the purpose of producing gas, tho temperature is so high as to be unfavourable for its production, and consequently mere traces only are found in ordinary coal-tar. But if any kind of coal be distilled at the lowest possible tomperature, not only is the resulting naphtha of much lower density than that produced in the ordinary manner, but considerable quantities of paraffine are found in the distillate. The last-mentioned substance is every day becoming more 
amportant, in consequence of the valuable illuminating propertios that have been found to belong to it. Colourloss, inodorous, hard at all modorate tomperatures, it forms the most elegant material for candlos yet discovered. See Parafrinv.

Modern researches have shown that the hydrocarbons generally are formod on one type-viz, hydrogen. Assuming hydrogen in the freo state to be a double molecule, HH, the hydrocarbons are formed by the substitution of one or two equivalents of a positive or negative radical for one or two of the equivalents of hydrogen; thus methyle, the formula of which (for four volumes) is $\mathrm{C}_{\mathrm{C}^{2} \mathrm{H}^{3}}^{3}$ or $\mathrm{C}^{4} \mathrm{H}^{6}$, is hydrogen in which both equivalents are replaced by methyle. Olefiant gas is hydrogen in which one equivalent is replaced by the negative radical acetyle, or vinyle, and so on.

There is one large class of hydrocarbons the rational formulæe for which are not known, and which will probably remain in this condition for some time. We allude to the numerous essential oils isomeric with oil of turpontine. Many of those havo almost the same boiling point and precisely the same vapour-donsity as their type; but in odour, fluidity, density in the liquid state, and various other minor points, are essentially different. The following Table exhibits some of their physical proporties:-

Table of the Physical Properties of some Isomers of Oil of Turpentine.

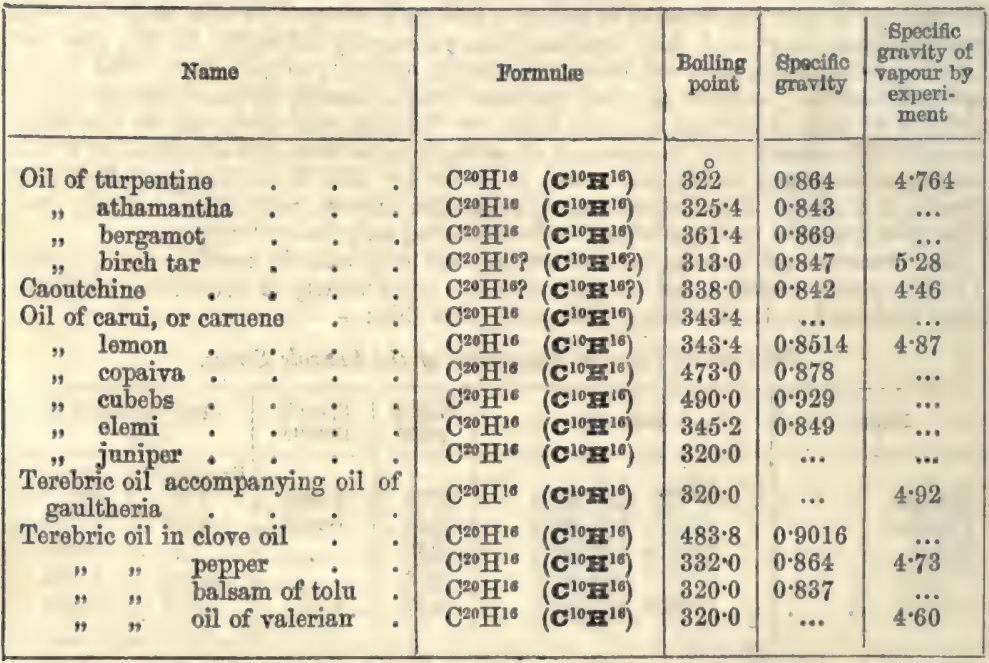

An inspection of the above Table will show that while, beyond doubt, a great number of essential oils are truly isomeric with turpentine, there are some the constitution of which is by no means well established.

The above account of some of the more prominent hydrocarbons is necessarily brief and imperfect; partly because the limits of this work preclude the possibility of entering minutely into the details of their history, and partly because many of them are described at greater longth in other articles, especially under NAPHTHA.-C.G.W.

CARBrmor. A synonym of methyl-alcohol or wood-spirit.

CARBoric ACrd, Phenic Acid, Phenol, Phenylic Alcohol, or Hydrate of Phenyl, $\mathrm{C}^{12} \mathrm{H}^{6} \mathrm{O}^{2}\left(\mathbf{C}^{6} \mathbf{H}^{6} \mathbf{O}\right)$. - The less volatile portion of the fluids produced by distillation of coal-tar contain considerable quantities of this substance. It may be extracted by agitation of the coal-oils (boiling between $300^{\circ}$ and $400^{\circ} \mathrm{F}$.) with an alkaline solution. The latter, separated from the undissolved portion, contains the carbolic acid in the state of carbolate of the alkali. On addition of a mineral acid, the phenol is liberated, and rises to the surface in the form of an oil. To obtain it dry, recourse must be had to digestion with chloride of calcium, followed by a new rectification. If required pure, only that portion must be received which boils at $370^{\circ}$. If, instead of extracting the carbolic acid from coal-products boiling between $300^{\circ}$ and $400^{\circ}$, a portion be selected distilling between $400^{\circ}$ and $428^{\circ}$, and the same treatment as before bo adopted, the acid which passes over between $347^{\circ}$ and $349^{\circ}$ will consist, not of carbolic acid, but of its homologue, cresylic acid $\mathrm{C}^{14} \mathrm{H}^{8} \mathrm{O}^{2}\left(\mathbf{C}^{7} \mathbf{H}^{8} \mathbf{O}\right)$. Commercial carbolic acid is generally very impure. Some specimons do not contain 
more than 50 per cont. of acids soluble in strong solution of potash. The insoluble portion contains naphthaline, fluid hydrocarbons, and small portions of chinoline and lepidine. Carbolic acid, when very pure and dry, is quite solid and colourless. The crystals often remain solid up to $95^{\circ}$, but a trace of water renders them fluid. Its specific gravity is 1.065. Carbolic acid, when mixed with lime and exposed to the air, yields rosolic acid. The lime acquires a rich red colour, during the formation of the acid. No means of dyeing reds permanently with this substance have yet been made known. Unfortunately, the red tint appears to require an excess of base to enable it to exist, consequently the carbonic acid of the air destroys the colour (Dr. Angus Smith). I find that homologues of carbolic acid exist which boil at a temperature beyond the range of the mercurial thermometer, and that all the acids above carbolic acid afford rosolic acid, or homologues of it, when treated with lime. Creosote of commerce appears to consist of a mixture of carbolic and cresylic acids. If only that portion be received which distils at the temperature given by Reichenbach as the boiling point of creosote, it will, if prepared from coal-oil, consist almost entirely of cresylic acid (Williamson and Fairlie). A splinter of deal wood, if dipped first in carbolic acid and then in moderately strong nitric acid, acquires a blue tint. For a comparison of the properties of Creosote and Carbolic Acid, see Creosotr.-C. G. W.

More than five-and-twenty years ago, Laurent extracted carbolic acid from coal-tar by fractional distillation, as described above; the products of distillation were treated with caustic potash and the alkaline solution then nentralised by an acid so as to liberate the carbolic acid. This process was modified in 1847 by Mansfield, and in 1856 by Baboeuf; these chemists substituted caustic soda for the potash, and worked upon all the light oils, instead of confining themselves to a portion only, as Laurent had previously done. Still the acid obtained by these methods was very impure, and it remained for the late Dr. F. Crace-Calvert to introduce successive ima provements into the manufacture until he finally obtained a pure product. In 1859 Calvert proposed to work upon the impure benzines or naphthas of commerce; these he treated with weak alkaline solutions, and thus obtained a product which was rich in carbolic acidcontaining as much as 60 per cent, - and capable of yielding a great part of this acid on distillation. By further modifications of his process, ho succeeded in obtaining a pure hydrate of carbolic acid (mono-hydrate of phenol) of the composition $\mathrm{C}^{12} \mathrm{H}^{6} \mathrm{O}^{2}$ HO [2 $\left.\left(\mathbf{C}^{6} \mathbf{E}^{6} \mathbf{O}\right) \mathbf{H}^{2} \mathbf{O}\right]$. The tarry and sulphurous odours, which clung to the earlier forms of carbolic acid, and were so prejudicial to its use for many purposes, have been entirely removed in some of Calvert's later preparations. In addition to the ordinary form of the acid, Messrs. Calvert and Co. now prepare carbolic-acid powder and carbolic-acid soap.

On an industrial scale, carbolic acid, or phenol, was first produced by the late Dr. Ernest Sell, a German chemical manufacturer, distinguished for his scientific attainments and predilections. Dr. Sell introduced the use of phenol as a substitute for the ever-varying creosote. The claims to chemical individuality of the latter substance, notwithstanding numerous investigations to which it has been submitted, are by no means finally established; many chemists, in fact, considering creosote as a mixture of phenol with larger or smaller quantities of its homologues, eresol and phlorol. The manufacture of coal-tar-derived creosote was extensively carried on by Dr. Sell in his factory at Offenbach-on-the-Main; and Dr. Hofmann saw, nearly twonty years ago, at that factory, hundredweights of phenol as white and beautifully crystallised as the specimens exhibited in London in $\mathbf{1 8 6 2 .}$

Of late years, carbolic acid has obtained extensive application as an antiseptic and disinfectant. Its rolatility gives it the advantage of being very rapidly diffused through an infected atmosphere. During the cattle-plague it was employed with great success by Mr. Crookes. Carbolic acid is also used in surgery for dressing offensive wounds, and as an application in certain forms of skin-disease. But the most interesting use of phenol is to be found in the manufacture of certain dye-stuffs from this acid and its derivatives. The following are the more important of these carbolic-acid dyes :-

YelLow Dyes: Picric acid, Carbazotic acid, or Trinitrophenylic acid. Though originally discovered by Welter, the composition of this body was not properly understood until Laurent examined it in 1841. It is obtained by the action of nitric acid on carbolic acid, or on phenyl-sulphato of soda. As far back as 1847, it was used for dyeing by Messrs. Guinon, of Lyons. It is employed for imparting a yellow colour to silk and wool; and also as a green dye when associated with indigo and blue colouring matters. Picrate of soda has been substituted for the pure acid, but this salt is dangerously explosive. See Picric Acm.

Aurin or Yellow Coralline. In 1861 Kolbe and Schmidt first obtained this compound by heating carbolic acid with oxalic acid and concentrated sulphuric acid. Commercial aurin is a brittle resinous substance, having the colour and lustre of cantharides, and 
fielding a red powder. The colouring principle has been isolated in a pure form, and carefully studied by Messrs. Dale and Schorlemmer.1 Aurin is used for giving a vory fine orange-yellow colour to silks and albuminized cotton.

RED Dyrs: Preonin, or Red Coralline. By heating aurin with aqueous ammonia to about $140^{\circ}$ or $150^{\circ} \mathrm{C}$. $\left(284^{\circ}\right.$ or $302^{\circ} \mathrm{F}$.), a now colouring agent is obtained, which dyes wool and silk with a redder colour than can bo obtained from aurin. This beautiful scarlet dye, known as pæonin, was discovered in 1860 by M. Persoz, jun., and its manufacture has been perfected by Messrs. Grunon, Marnas, and Bonnet, of Lyons.

It is believed that coralline is very closely related to, if not identical with, the rosolic acid originally obtained from coal-tar by Runge, and readily prepared by the oxidation of phenol.

Browy DYes: Picramic acid. This was obtained by Wöhler by acting on picric acid with proto-sulphate of iron, neutralising the product with caustic baryta, and finally removing the baryta by sulphuric acid. Picramic acid is a doep brown substance, used for dyeing silk.

Phenicienne or Phenyl brown. Roth discovered this substance in 1863, by acting on carbolic acid with nitro-sulphuric acid. The product is an amorphous brown powder, said to be a mixture of two distinct substances-the one yellow, and the other blackish-brown. A variety of shades, from a deep red to a golden buff, may be given to wool and silk by means of picramic acid.

Soluble Garnet (Grenat soluble). This is the isopurpurate of potash, obtained by acting with cyanide of potassium on a solution of picric acid. It has been introduced as a dye-stuff by M. J. Casthelaz. As it is explosive when dry, it should be kopt as a paste and moistened with glycerine.

Bưr Dye: Azuline, Azurine, Phenol-blue or phenyl-blue. By heating coralline, or rosolic acid, with aniline, and treating the product with sulphuric acid, a blue colouring-matter is obtained in the form of a reddish substance exhibiting golden tints. This is the dye known as Azuline.

Grees Dre: Viridine. A green dye has been obtained by Messrs. Grunon, Marnas, and Bonnet, by acting on rosolic acid with aniline and benzoic acid.

сArzor. (Symbol, C; Atomic weight, 6). Carbon exists in a considerable variety of forms, most of which are so unlike each other, that it is not surprising the older chemists should have believed them to be compounds.

Diamond.-The purest variety of carbon is the diamond. This gem crystallises in the regular octahedron and derived forms. The diamond does not owe its harclness and brilliancy solely to its purity, for many specimens of graphite consist of carbon as free from admixture as the best diamonds. The density of graphite and of diamond, however, is very different; for while the former seldom exceeds 2.45 , and is often much lower, the diamond is very constant, generally ranging between 3.51 and 3.55 . Diamonds, if they are perfectly transparent, leavo no residue when burnt in oxygen gas. If not clear, they yield from 0.05 to 0.20 of ash, consisting chiefly of peroxide of iron, but also containing traces of silica. The refractive power of diamonds is as high as $2 \cdot 439$. Sir Isaac Newton, observing that oily or intlammable bodies generally possessed the greatest refractive powers, inferred from the high index of refraction of the diamond, that it was 'an unctuous body congealed.' This idea will appear the more happy, when it is considered that the ashes of the diamond exhibit a structure resembling that of vegetable parenchyma.

In freedom from ashes, certain graphites nearly approach the diamond, some natural varieties not yielding more than $0 \cdot 33$ per cent. See Dramond.

Grophite.-This kind of carbon is found in many parts of the world, and in different degrees of purity: it is also formed artificially. Some native varieties are exceedingly soft, of a black or greyish tint, metallic lustre, and, in consequence of making a streak on paper, of various degrees of blackness, according to tho modo of preparation and other circumstances, are invaluable for the manufacture of artists' pencils, and are hence called 'Black Lead.'

A very hard graphite is found lining the retorts in which coal-gas is made: it is, when cut into plates or rods, used in galvanic arrangements, either for the poles or for the inactive elements of batteries. See Graphite and Plumbago.

A very dense form of carbon, having high electric conductivity, and sometimes used for the negative element in Bunsen's battery, may be obtained by heating to redness an intimate mixture of finely-divided coke and coal in the proportions of two parts of the former to one of the latter; this mixture having been ignited in an iron mould, is dipped several times in treacle, and again strongly heated. A hard compact substance is thus obtained, capable of being sawn to any required shape.

1 ' Journal of the Chemical Society;' [2] xd. 1873, p. 481. 
Coke.-This rariety of carbon is produced by the ignition of pit-coal. The largest quantities are produced in the menulacture of coal-gas. It of course raries greatly in quality with the coal from which it is procured. The density of coke varies not only with the quality of the coal, but also with the greater or less rapidity of the firing and the duration of the operation. See Cokr.

Anthracite is a very dense natural variety of carbon, its specific gravity varying from 1.390 to 1.7. It differs considerably in quality, some kinds being almost as frou from extraneous matters as graphite, while others approach nearer to the naturo of conls. Thus the hydrogen in anthracito oscillates between 1.0 and 4.0 per cent. Somo varictios of coal have only 4.5 to $5 \cdot 0$ per cent, of hydrogen, thus approximating to those anthracites which have high bydrogens. See Axturacres and Cost

Charcoal.- There are several varieties of charcoal : among them may be mentioned those from wood, bones, and the peculiar substance found between the layers of certain pit coals, and known as 'mineral charcoal.' Ordinary charcoal from wood contains many substances besides carbon, among which may be montioned oxygen, hydrogen, traces of nitrogen, and ashes.

Bone-charcoal contains a large quantity of earthy phosphates and carbonates, besides other matters. See CHarcons.

For $\mathrm{a}$ description of the method of preparing the variety of carbon known as Lamp-Black, see IuAmp-Brick,

The description of the charcoal best adapted for pyrotechnic purposes will be found under the head GunPowDrR.

Carbon combines with several elements, forming in genoral well-marked and highly important substances. Several of these compounds will bo found described undor the herds Carbonic Acid, Carbon Bisulphime, Hydrocarbon, \&c.

CARBON, BISUIPEIDE OF, or CARBON BISUIPAIDE (formerly Carburet of Sulphur or Sulphuret of Carbon, also called by the elder chemists tho Alcohol of Sulphur). A limpid, volatile, colourless, highly refractive liquid, possessing a penetrating foetid smell and an acrid burning taste. Its specific gravity is 1.27 ; its formula is CS?

Bisulphide of carbon may be prepared by distilling, in a porcelain retort, pyritos, the bisulphide (bisulphuret) of iron, with a fourth of its weight of well-dried charcoal, both in a state of fine powder and intimately mixed. The vapour from the retort is conducted to the bottom of a bottle filled with cold water to condense it.

The method now usually employed for preparing bisulphide of carbon consists in passing the vapour of sulphur over charcoal or coke heated to redness. In Peroucel's apparatus, a large cylindrical fire-clay retort, set vertically, is filled with pieces of coke, and strongly heated. Sulphur is then introduced through a special tubulure, and the vapour, formed by volatilisation of the sulphur, combines with the heated carbon. The vapours of bisulphide of carbon thus formed are conducted first into it receiver, where a portion condenses, and then through a worm in a condensing apparatus, where the remainder is liquefied.

The crude bisulphide of carbon thus obtained may be purified by re-distillation over either zinc or bichloride of mercury (corrosive sublimate).

The bisulphide of carbon is insoluble in water, but it is soluble in alcohol. It dissolves sulphur, phosphorus, and iodine. The solution of phosphorus in this liquid has been employed for electrotyping very delicate objects, such as grasses, flowers, feathers, \&c. Any of these are dipped into the solution: by a short exposure in the air the bisulphide of carbon evaporates and leaves a film of phosphorus on the surfaces; they are then dipped into nitrate of silver, by which silver is precipitated in an exceedingly minute film, upon which, by the electrotype process, any thickness of silver, gold, or copper can be deposited. If a fow drops of the bisulphide of carbon are put in a solution of the cyanide of silver, from which the metal is being doposited by the electroplating process, it covers the article quite brightly, whereas, without the bisulphide, the precipitated metal would be dull. See ErectrotYPE.

Bisulphide of carbon is an excellent solvent of caoutchouc, and is therefore largely used in the vulcanisation of India-rubber. It also dissolves fats and oils, and is consequently employed in extracting fat from bones, shoddy, and other greasy refuse, and in dissolving oils from seeds; the last traces of olive oil may thus be obtained from the compressed pulp of the olive after having yielded the greater portion of the oil by expression. Iiquid bisulphide of carbon is remarkable for its high dispersive power, and hollow glass prisms filled with the bisulphide are often used in optical researches. As the liquid has never been frozen, it has been occasionally employed in the construction of thermometers for measuring very low temperatures. Bisulphide of carbon is highly inflammable, and the vapour, if mixed with nitric oxide, burns with an intenso light possessing strong actinic properties; hence this light has beon employed for photographic purposes. The vapour of bisulphide of carbon is lighly Vor. I. 
poisonous, and adrantago has beon taken of this character to use it as a vermin. destroyer. A small quantity of the bisulphide, enclosed in an air-tight chamber with grain infested with weevils will destroy the insocts and their larvæ. It is curious that the vapour of bisulphide of carbon passed over wood undergoing destructive distillation, alters the character of the carbonaceous residue, and produces a material having high conducting powers for heat and electricity, and extromely sonorous when struck.

CARBONADO. A massive variety of diamond, found in small black pebbles in Brazil, and used for polishing hard stonos.

CARBOwAres. By this term is understood, in popular language, the salts formed by the union of carbonic acid with bases. In conformity with the language of modern chomistry, a carbonate may be definod as carbonic acid in which the hydrogen is replaced by a metal. Thus, earbonate of potash, according to the old definition, is a compound of carbonic acid gas (carbonic anhydride) with potash; whilst, according to the new definition it is merely carbonic acid (the true hydrate, not the anhydride), in which potassium takes the place of hydrogen. See Salrs.

The carbonates are among the most valuable of the salts, whether we regard their physical, geological, chemical, or technical interest. Were limestone and marble the only carbonates familiarly known, they would be sufficient to stamp this class of salts as among the most important. The carbonates of lime, potash, soda, ammonia, and lead, are articles of immense importance to the technologist, and are prepared on a vast scale for various purposes in the arts. The carbonates of iron and copper are among the most valued ores of those metals. Numerous processes of separation in analysis are founded on the various degrees of solubility in water and certain reagents of the different carbonates. By taking advantage of this fact, baryta, strontia, and lime, may be separated from magnesia and the alkalis. There are few analytical problems which have attracted more attention than the accurate determination of the carbonic acid in the carbonates. This has partly arisen from the frequency with which the potashes, soda-ashes, limestone, and other carbonates of commerce, are sent to chemists for analysis. The number of instruments contrived for the purpose is something extraordinary, especially when the simplicity and ease of the operation is considered. Among them all, there is none more convenient or easy to use than that of Parnell. 'It consists of a glass flask ( fig. 425) of about two ounces capacity, fitted with a sound cork, through which two tubes pass, one serving to connect a chloride-of-calcium tube, $a$, while the other, $b$, will be described presently.

425

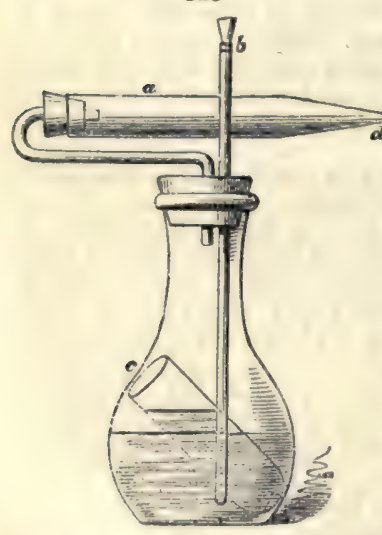
A small test-tube, $c$, is so placed in the flask, and is of such a size, that it cannot fall down, but its contents may be made to flow out by inelining the apparatus to one side. To perform the experiment, a weighed quantity of the carbonate is placed in the flask, and water added up to the level seen in the figure; the test-tube is then filled nearly to the top with concentrated sulphuric acid, and is carofully lowered into the flask; the cork with the tubes attached is then affixed, the aperture $b$ being closed with a small cork. The whole apparatus is now carefully weighed; the flask is then to be inclined so as to allow some of the acid to flow out, and, when the effervescence has subsided, a little more, and so on until no more carbonic acid is evolved. The flask is now to be so inclined as to cause the whole of the acid to mingle with the aqueous fluid, and thus cause a considerable rise of temperature; this expels the carbonic acid from the liquid; but as an atmosphere of the latter gas fills the flask, it must be removed and replaced by air, as the difference in density of the two is very considerable. For this purpose, the cork $b$ is remored and air is sucked out of $d$, until it no longer tastes of carbonic acid; the flask is then allowed to become perfectly cold, and, the little cork being replaced, it is then re-weighed: the difference in the two weighings is the amount of carbonic acid in the specimen. On drawing air for some time through the apparatus, it begins slowly to acquire weight, arising from the moisture in the atmosphere being absorbed by the chloride of calcium, and although the error introduced by this means is too minute to affect ordinary experiment, it must not be neglected where, from the quantity of material in the flask being limited, or other causes, a small difference has an important bearing on the result. In this latter case another chloride-of-calcium tube is to be attached to the aperture $b$, and the air must be drawn through by means of a suetion-tube applied at d.'-C. G. W.'s Chemical Manipulation. 
The commercial value of the carbonates of potash and soda may equally well be determined by ascertaining the quantity of dilute sulphuric acid required to neutralise them.

The following carbonates are such as find some use in the arts-or being found native, are brought under the notice of the miner :-

CARBONATE or AmMoña, commrrciar. The sesquicarbonate of chemists, sal volatile, or volatile or smelling salts of tho shops. Bicarbonate of ammonia has been found in considerable quantities, forming crystalline masses, in a bed of guano on the western coast of Patagonia.

CARBONAT⿱ OF BARYTA. This compound occurs in nature as the mineral Witherite, which is found in lead mines.

CARBONAT2 OI CAICIUIM, or of IIMrE. Carbonate of lime is found abundantly in the mineral kingdom. Calcareous spar, arragonite, Iceland spar, limestone, chalk, and marble, are carbonates of lime. Its uses are well known. Gay-Lrussite is a double carbonate of lime and soda.

carronatz oz cobazt. Seo Cobalt.

CARBONATP Or COPPIR. Malachite and azurite are the two carbonates of this metal found in nature. Seo Copper.

CArmovatres or rnon. Spathic ore is the most important native form which occurs. Soo Iros.

CARBONATE Or IFAD. White lead ore or comssite is found in the load mines of Cornwall, Devonshire, Cumberland, and Scotland. The pigment white lead is a carbonate of lead. See Lrand.

CARBONAT2 or MAGN pentine and other magnesian rocks. Magnesia alba of the pharmaceutists is a hydrocarbonate.

CARzorarg or maxGArrse. This salt is found in nature in rosecoloured rhombohedra known as manganese spar, or diallogite.

CARBONATz OF NrCzEI. A mineral called emerald nickel occurs at Texas, in Pennsylvania, and at Unst, Shetland. This is the only native carbonate of this metal.

Cazzonate or potasm. Vegetablealkali, salts of tartar, pearl-ashes, ashes, are several names given to this salt. See Potasi.

carbonate or strvir. Seo Silver Ores.

carbonatz or Soda. Commonly called Soda. Seo Soda.

CARBortatr or STromtra. Occurs native as Strontunite: it is found at Strontian in Argyleshire-hence its name; in the Hartz and other localities.

All these and the other rarer carbonates will be described under their respective heads.

CARBONIC ACID. (Symbol, $\mathrm{CO}^{2}$; Atomic weight, 22). The name commonly applied to a compound of carbon and oxygen. This gas was formerly termed faxed air, in consequence of its occurrence in a fixed form in chalk, marble, and other solid carbonates, from which the gas may be readily released by the action of stronger acids, conveniently by hydrochloric or muriatic acid.

In modern chemistry carbonic acid gas is usually termod carbonic anhydride, the name carbonic acid being restricted to the combination of the gas with water; indeed, the dry gas does not exhibit ucid reactions, Carbonic acid gas is also known in modern nomenclature as carbon dioxide.

Carbonic acid is destitute of colour, has a sour smell, and an acidulous pungent taste; it imparts to moist, but not dry, litmus-paper a transient reddish tint, and weighs, per 100 cubic inches, 47•19 grains, and per cubic foot, 815 44 grains, or little more than $3 \frac{3}{4}$ oz. avoirdupois. It may be condensed into the liquid state by a pressure of 40 atmospheres; and this liquid may then be solidified by its own sudden spontaneous evaporation. If air contains 4 per cent. in bulk of this gas, it is rendered unfit for respiration and combustion, animal life and burning bodies being speedily extinguished by it. See Dr. Angus Smith's 'Air and Rain.'

Curbonic acid is constantly given off by animals during respiration; and, ordinary combustion consisting mainly in the combination of carbon with oxygen, this acid is formed in enormous quantities in all our manufactories and by our ordinary fires.

Carbonic acid is, consequently, continually being poured into the air. The purity of the atmosphere is, however, maintained by the action of the vegetable world, all plants remoring carbonic acid from the air, and, under the influence of light, decomposing it again into carbon, which goes to the formation of wood and other vegetable products, and oxygen, which is given out to the atmosphere.

Notwithstanding this beautiful provision of Nature, we find carbonic acid accumulating in cavorns, deep wells, and long-closed cellars, rendering them dangerous. It is the 'bad air' and 'damp ' of the metal miner; the 'choke damp,' 'after damp,' and 'stythe' of the coal miner. The last two terms are especially employed to express the suffocating vapour which follows an explosion of 'fire-damp.' This gas, being 3 i 2 
much denser than common air, can be pumped out of any place containing it. Milk of lime-quicklime mired with water-may bo used with advantage to purify the air of a sunk apartment, by its affinity for, or power of absorbing this aërial acid.

In the actual condition of the atmosphere, from 4 to 6 rolumes of carbonic acid exist in every 10,000 volumes of air.

This acid gives the fresh and pleasant tasto to spring-water and to all fermented drinks. Champagne and bottled beer owe their sparkling properties to carbonic acid gas generated by fermentation after the liquid has been bottled.

Many springs are very highly charged with this acid, and form a sparkling beverage, as Soltzer water (Selterswasser) and the like. Large quantities of similar water are made artificially in this country, and sold under the names of Soda Water, Aërated Water, \&c. See Watts's 'Dictionary of Chemistry.'

Carzortc antroridr. See Carbonic Acid.

CARBowrC oxrdv. (Symbol, CO; Atomio weight, 14). This gas is known in modern chomistry as carbon monoxide or carbonous oxide. Carbonic acid is readily deprived of half its oxygen at a red heat by charcoal and other deoxidising substances, and so reduced to carbonic oxide.

This gas is presumed to contain two volumes of carbonic vapour and one volume of oxygen condensed into two volumes, so that its combining measure is two rolumes. Carbonic oxide is 14 times hearier than hydrogen. It is very fatal to animals, and whon inspired in a pure state almost immediately produces coma. It has nevor been liquefied. It is easily kindled, and burns with a pale blue flame like that of sulphur, combining with half its volume of oxygen, and forming carbonic acid, which retains the original volume of the carbonic oxide. This combustion is often witnessed in a coke or charcoal fire. The carbonic acid produced in the lower part of the fire is converted into carbonic oxide as it passes up through the hot embers, and afterwards burns with a pale-blue flame where it meets the air.-Graham.

Carbonic oxide plays an important part as a reducing agent in many metallurgical operations.

CARBONIPIROUS IIMESTONE, or MOUNTATN IIMESTONE. A sub-division of the Carboniferous System, which yields some of the finest British marbles, such as those of Derbyshire and Bristol. The same limestone in Ireland is also rich in marbles. Lead-ore is common in the Carboniferous Limestone of Derbyshire, Yorkshire and Cumberland, and red hæmatite in that of Ulverston and Whiteharen; whilst in the North of England and in Scotland the Carboniferous Limestone series contains interstratified beds of coal; indeed, a large part of the workable coal of Fast and Mid-Lothian is referable to this series.

CArzomrrzzous systrav. That geological formation which, in order of time, succeeds the OLD RED SAndstonk, and is succeeded by the Pramin Srstram, although in the absence of that system, they may be overlaid by rocks of still later geological age. The carboniferous systom comprehends :-

The Lower Limestone Shale: The Carboniferous or Mountain Limestone: Yoredale Rocles (Upper Limestone Shale): and the Coal-Measures, Lower, Middle, and Upper.

carzon privinug. Seo Photographr.

CAnzUracs. $\Lambda$ term applied to the garnet when cut with a convex face (en calsochon). This gem was much prized by the ancients, and in high reputo during the middle ages, from its supposed mysterious power of emitting light in the dark. Bonronuto Cellini affirms, in his treatise on jewellery, that he had seen the carbuncle glowing like a coal with its own light.

'The garnet was, in part, the carbunculus of the ancients, a term probably also applied to the spinel and oriental ruby. The Alabandic carbuncles of Pliny were so called because cut and polished at Alabanda. Hence the name Almandine now in use. Pliny describes vessels of the capacity of a pint formed from carbuncles, 'non claros ac plerumque sordidos ac semper fulgoris horridi,' devoid of lustre and beauty of colour, - which probably were large common garnets.'-Dana.

CArzurars. Compounds of carbon, now called Carbides.

CARBURTTras HYDRocrit. A compound of carbon and hydrogen. Marsh-gas or fire-damp is frequently called Light Carburetted Hydrogen. Soo GAsCos工, and consult Watts's ' Dictionary of 'Chemistry.'

CARD, CARDzOARD, called also Pasteboard, from the circumstance that several sheets of paper are pasted together to form the board or card, which is then subjected to a very great pressure between rollers.

A patent machine for cutting cards was invented by Mr. Dickinson. It consists of a pair of rollers with circular revolving cutters, the edges of which are intended to act against each other as circular shears; and the pasteboards, in passing between these rollers, are cut by the circular shears into cards of the required dimensions. These rollers are mounted in suitable standards with proper adjustments, and aro 
made to revolve by a band and pulley connected to the axle of a crank, or by any convenient means. Fig. 426 is a front view of this machine: $a a$ and $b b$ are the two rollers, the upper one turning upon an extended axle, bearing in the standards, the lower one upon pivots. These rollers are formed by a series of circular blocks, between a series of circular steel cutters, which are slidden on to iron shafts, and held together upon their axle by nuts screwed up at their ends. The accurate adjustment of the cutters is of the first importance to their correct performance; it is therefore found necessary to introduce spiral springs within the blocks, in order to press the cutters up to their proper bearings. A section of one of the blocks is shown at

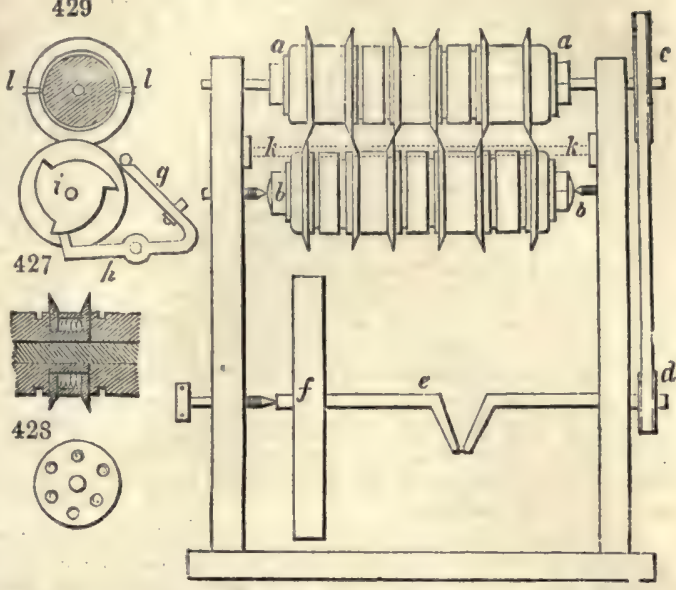

fig. 427 , and an end view of the same at fig. 428 , with the spiral springs inserted,

At the outer extremity of the axle of the roller $a$, a rigger $c$, is attached, whence a band passes to a pulley $d$, on the crank-shaft $e$, to which a fly-wheel $f$, is affixed, for the purpose of readering the action uniform. Rotatory motion being given to the crank-sheft, the upper roller is turned, the lower roller moving at the same time by the friction against the edges of the cutters.

Fig. 429 is an end view of the rollers, showing the manner in which tho pasteboards are guided and conducted between the cutters. In front of the machine a moveable frame $g$, is to be placed, for the purpose of receiving the pasteboards, preparatory to cutting them into cards, and a stop is screwed to this frame for the edge of the pasteboard to bear against, which stop is adjustable to suit different sizes. From the back part of this frame the arm $h$, extends, the extremity of which acts against the periphery of a ratchet wheel $i$, fixed at the end of the roller $b$, and hence, as the roller goes round, the frame is made to rise and fall upon its pivots, for the purpose of guiding the pasteboard up to the cutters; at the same time a rod $k$, hanging in arms from the sides of the standards (shown by dots in fig. 426), falling upon the pasteboard, confines it, while the cutters take hold, and racks, corresponding with the indentations of the rollers, are placed as at $l l$, by means of which the cards, when cut, are pushed out of the grooves.

As various widths of card will require to be cut by this machine, the patenteo proposed to have several pairs of rollers ready adjusted to act together, when mounted in tho standards, in preference to shifting tho circular cutters, and introducing blocks of greater or less width.

The second part of the invention is a machine for pasting the papers, and pressing the sheets together to make pasteboard. This machine consists of sereral reels on which the paper is to be wound, along with a paste trough, and rotatory brushes.

Damped paper is to be wound upon two reels, and conducted from thence over two other roels. Two fluted rollers revolving in the paste-trough supply pasto to two circular brushes; by these brushes the papers are to be pasted on one side, and then pressed together by rollers to make the pasteboard; after this, the pasteboard is drawn on to a table, and it remains there until sufficiently dry to be wound upon other rollers, by which it is subjected to the necessary pressure. Cards are glazed or enamelled by the use of porcelain-clay, white lead, and subnitrate of bismuth.

CarDs (Cardes, Fr.; Karden, Ger.) are instruments which serve to disentangle the fibres of wool, cotton, or other analogous bodies, to arrange them in an orderly lap of fleece, and thereby prepare them for being spun into uniform threads. The fineness and the lovelness of the yarn, as well as the beauty of the cloth into which it enters, depend as much on the regularity and perfection of the carding, as upon any subsequent operations of the factory. The quality of the carding depends more upon that of the cards than upon any attention or skill in the operative, since it is now nearly an automatic process, conducted by young women called card-tenters. 
Cands are formod of a sheot or fllet of leather pierced with a multitude of small holes, in which are implanted small staples of wiro with bent projecting ends called teetk. Thus every pioce of wiro is double toothed. The leather is afterwards applied to a flat or cylindrical surface of wood or metal, and the co-operation of two or more such surfaces constitute a card. The teoth of cards are made thicker or slenderer, according as the filaments to be carded are coarser or finer, stiffer or more pliant, more valuable or cheaper. It is obviously of grent importance that the teeth should be all alike, equably distributed, and equally inclined over tho surface of the leather, a degree of precision which is scarcely possible with handwork. To judge of the difficulty of this manipulation, we need only inspect the annexed figures, 430,431 . The wire must first be bent at right ang!es in $c$ and $d$, fig. 432 , then each branch must rocoive a second bend at $a$ and $b$ at a detorminate obtuse angle, invariable for each system of cards. It is indispensable that the two angles $c a e$ and $d b f$ be mathematically equal, not only as to the twin teeth of one staple, but through the wholo series: for it is easy to see that if one of the teeth be more or less sloped than its fellow, it will lay hold of more or less wool than it, and render the carding irregular. But though the perfect regularity of the teeth be important, it is not tho solo condition towards making a good card. It must be always kept in view that these teeth are to be implanted by pairs in a piece of leather, and kept in by the cross part $c d$. The leather must therefore be pierced with twin holes at the distance $c d$ : and piereed in such a manner that the slope of the holes, in reference to the plano of the leather, be invariably the same; for otberwise the length of the teeth would vary with this angle of inclination, and the card would be imegular.

A third condition essential towards producing perfect regularity, is that tho leather ought to be of the same thickness throughout its whole surface, othorwise tho teeth, though of the same length and fixed at the same angle, would be rendorod unequal by the different thickneşses of the leather, and the operation of carding would be in

430

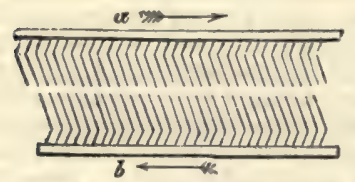

432

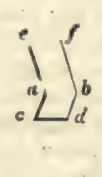

431

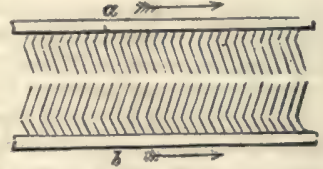

consequence extremely defective. Fig. 430 shows the card-teeth acting against each other, as indicated by the arrows in two opposite directions; in fig. 431 they work one way.

Of late years very complex but complete and well-acting machines have been constructod for splitting the leather or equalising it by shaving, for bending and cutting the vires, and implanting them in the leather, into holes pierced with perfect regularity. Card machines which fashion the teeth with great precision and rapidity, and pierce the leather, have been for a considerable time in use at Halifax, in Yorkshire, a town famous for the excellence of its card-cloth, as also Leeds, Glasgow, and several other places. The wires and the leather thus prepared are given out by the manufacturer to women and children, who put them together.

The simplest machine for equalising the leather which can bo employed, is that of MM. Scrive of Lille, where the leather is drawn forwards by a roller over a solid horizontal table, or bed, and passed under a nicely adjusted vertical blade, which shaves it by a seraping motion to a perfectly uniform thickness. About one-half the weight of the leather is lost in this process and in the subsequent squaring and trimming.

A machine for making cards, invented by a Mr. Filis of the United States, for which a first patent was obtained in this country by Joseph Cheoseborough Dyer, Esq., of Manchester, in 1811, and a second and third with further improvements in 1814 und 1824, is one of the most elegant automatons ever applied to productive industry. It is, however, necessarily so complicated with different mechanisms as to ronder its represontation impracticable in such engravings as are compatible with the scope of this Dietionary. The following general description of its constituont parts must therefore suffice :-

The first thing to be done after having, as above, prepared the long shcets or fillets of leather of suitable length, breadth, and thickness, for making the cards, is to stretch the leather, and hold it firmly; which is accomplished by winding the fillet of leather upon the roller or drum, like the warp-roller of a loom, and then conducting it upwards, between guide rollers, to a receiving or work roller at the top of the machine, where the fillet is held fast by a cramp, by which means the leather is kept stretched.

Secondly, the holes are pierced in the leather to receive the wire staples or teeth of 
the card, by means of a sliding fork, the points of which are presented to the face of the leather; while the fork is made to adrance and recede continually, by the agency of levers worked by rotatory cams upon a revolving main shaft.

The points of the fork being thus made to penetrate into the leather, the holes for receiving the staples are pierced, at regular distances and in correct order, by shifting the leather fillet so as to bring different parts of its surface opposite to the points of the sliding fork. This is done by cams, or indented wheels and gear, which shift the guide-rollers and confining drums laterally, as they revolve and consequently move the fillet of leather at intervals a short distance, so as to present to the points of the fork or piercers at every movement, a different part of the surface of the leathor.

Thirdly, the wire of which the teeth or points of the card are to be made, is supplied from a coil on the side of the machine, and is brought forward at intervals, by a pair of sliding pincers, which are slidden to and fro through the agency of levers actuated by rotatory cams upon the main shaft. The pincers having advanced a distance equal to the length of wire intended to form one staple, or two points, this length of wire is pressed upon exactly in the middle by a square piece of steel, and being there confined, a cutter is brought forward, which cuts it off from that part of the wire held in the pincers.

The length of wire thus separated and confined is now, by a movement of the machine, bent up along the sides of the square steel holder, and shaped to three edges of the square, that is, formed as a staple; and in the same way, by the continued movements of the machine, a succession of pieces of wire are cut off, and bent into staples for making the teeth of the card as long as the mechanism is kept in action.

Fourthly, the wire staple thus formed is held with its points or ends outwards, elosely contiguous to the forked piercer described above, and by another movement of the mechanism the staple is protruded forward, its end entering in the two holes made previously in the leather by sliding of the fork.

While the wire staple is being thus introduced into the leather, its legs or points are to be bent, that is, formed with a knee or angle, which is the fifth object to be effected. This is done by means of a small apparatus consisting of a bar or bed, which bears up against the under side of the wire staple when it has been passed half-way into the holes in the leather, and another bar above it, which being brought down behind the staple, bends it over the resisting bar to the angle required; that is, forms the knee in each leg. A pusher now acts behind the staple, and drives it home into the leather, which completes the operation.

The leather being thus conducted, and its position shifted before the piercer progressively, a succession of the above-described operations of eutting the wire, forming the staple, passing it into the leather, and bending its logs to the angular form, produces a sheet of card of the kind usually employed for curding or combing wool, cotton, and other fibrous materials. It may be necessary to add, that as these wire staples are required to be set in the leathers, sometimes in lines crossing the sheet, which is called ribbed, and at other times in oblique lines, called twilled, these variations are produced by the positions of the notches or steps upon the edge or periphery of the cam, or indented wheel, which shifts the guide rollers that hold the fillet or sheet of leather as already described.

CARDs, PIAyrwe. (Cartes a jouer, Fr.; Spielkarten, Ger.) Playing cards were probably invented in the East. In Italy cards originally bore the name of Naibi, and they are still, in Spain and Portugal, called Naipes, signifying, in the Oriental languages, divination. Cards were first painted by hand: the art of printing cards was discovered in Germany between 1350 and 1360. It has been stated that cards were in use in Spain in 1332: in 1387, John I., King of Castile, prohibited their use. In France, we find that card-playing was practised in 1361; and at the end of the 14th century, we find Charles VI. amusing himself with cards during his sickness. The figures on modern cards are of French origin, and are said to have been invented between 1430 and 1461 .

Mr. De La Rue obtained, in February 1832, a patent for certain improvements in the manufacture of playing cards, which he distributed under three heads : first, printing the pips, and also the picture or court-cards, in oil colours by means of types or blocks; secondly, effecting tho same in oil colours by means of lithography; and thirdly, gilding or silvering borders, and other parts of the characters, by the printing process, either by types, blocks, or lithography.

In the ordinary mode of manufacturing playing cards, their devices are partly procluced by copperplate printing, and they are filled up with water-colours by the means called stencilling.

The patentee does not purpose any material alteration in the devices or forms upon the cards, but only to produce them with oil colours; and, to effect this, he follows precisely the same mode as that practised by calico-printers. 
A set of blocks or types, properly dovised, are producod for printing the different pips of hearts, diamonds, spades, and clubs, or they are drawn, as other subjects, in the usual way, upon stone. The ink, or colour, whether black or red, is to be prepared from the best French lamp-black, or the best Chinese rermilion ground in oil, and laid on the types and blocks, or on the stone, in the same way as printers' ink, and the impressions taken on thick drawing-paper, by means of a suitable press, in the ordinary manner of printing.

The picture or court-cards are to be produced by a series of impressions in different colours, fitting into each other exactly in the same way as in printing paper hangings, or silks and calicoes, observing that all the colours are to be prepared with oil.

For this purpose a series of blocks or types are to be provided for each subject, and which, when put together, will form the whole device. These blocks aro to bo used separately, that is, all the yellow parts of the picture, for instance are to be printed at one impression, then all the red parts, next all the flesh colour, then the blue portions, and so on, finishing with the black outlines, which complete the picture.

If the same is to be done by lithography, there must be as many stones as there are to be colours, each to print its portion only; and the impression, or part of the picture, given by one stone must be exactly fitted into by the impression given from the next stone, and so on until the whole subject is complete.

A superior kind of card is supposed to be made, with gold or silver devices in parts of the pictures, or gold or silver borders round the pips. This is to be effected by printing the lines which are to appear as gold or silver, with gilder's size, in place of ink or colour; and immediately after the impression has been given, the face of the card is to be powdered over with gold-dust, silver, or bronze, by means of a soft cotton or wool dabber, by which the gold, silver, or bronze will be made to adhere to the picture, and the superfluous portions of the metal will wipe off by a very slight rubbing. When the prints are perfectly dry, the face of the card may be polished by means of a soft brush.

If it should be desirable to make these improved cards to resemble ivory, that may be done by preparing the face of the paper in the first instance with a composition of size and fine French white, and a drying oil, mixed together to about the consistence of cream; this is to be washed over the paper, and dried before printing, and when the cards are finished they will exactly resemble irory.

The only thing remaining to be described, is the means by which the successive impressions of the types, blocks, or stones forming the parts of the pictures, are to be brought exactly to join each other, so as to form a perfect whole design when complete; this is by printers called registering, and is to bo effected much in the usual way, by points in the tympan of the press, or by marks upon the stones.

The parts of the subjoct having been all accurately cut or drawn to fit, small holes are to be made with a fine awl through a quire or more of the paper at once, by placing upon the paper a gauge-plate, having marks or guide-holes, and by observing these, the same sheet laid on several times, and always made to correspond with the points or marks, the several parts of the picture must inevitably register, and produce a perfect subject.

In the year ended March 31,1873 , there were $1,043,384$ packs of cards manufactnred, on which the stamp duty of $3 d$. per pack was paid, producing $13,048 l .11 s$.

Playing cards imported at the duty fixed April 8, 1862, at 3s. 9d. per dozen packs, were as follows:-

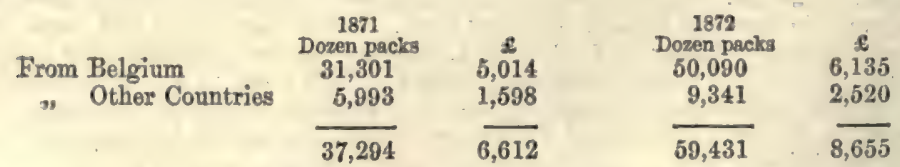

Cardanam. The fruit of the Elettaria Cardamomum, or the true officinal cardamom. Cardamoms are produced naturally and by cultivation in Malabar, at Travancore, and the western part of Soonda. The Malabar cardamoms are distinguished in commerce as shorts, short-longs, and long-longs. The three sorts are brought from Bombay in chests, the shorts fetching from $3 d$. to $6 d$. per lb. more than the longs.

CARII. A sort of hemp.

CARzock. Tho name given by the Russians to the isinglass obtained from the air-bladder of the sturgeon.

CARISBAD TWXIsS. Large felspar crystals which are porphyritically embodied in a regularly constituted rock, as in the granite of Carlsbad in Bohemia, and the granite of some parts of Cornwall.

CARMEI. A substance used by confectioner. for covering sweetmeats. 
Canmrirz. CARMruvic ACrD. (Carmin, Fr.; Karminstoff, Ger.) The colouring matter of the cochineal insect. See Cocmrnuar,

Thero are several methods of preparing carmine, the following being the most approved: Dr. Pereira speaks highly of this process. A decoction of the black cochineal is mado in water; the residue, called carmine grounds, is used by paperstainers. To the decoction is added a precipitant, usually bichloride of tin. The decoction to which the bichloride has been added is put into a shallow ressel and allowed to rest. Slowly a deposit takes place, which adheres to the sides of the vessel, and the liquid being poured off, it is dried: this precipitate is carmine. The liquid, when concentrated, is called Liquid rouge.

By the Old German Process, carmine is prepared by means of alum, without any other ruddition. As soon as the water boils, the powdered cochineal is thrown into it, stirred well, and then boiled for six minutes; a little ground alum is added, and the boiling is continued for three minutes more. The vessel is removed from the fire, the liquor is filtered, and left for three days in porcelain ressels, in the course of which time a red matter falls down, which must be separated and dried in the shade. This is carmine, which is sometimes previously purified by washing. The liquor, after three days more, lets fall an inferior kind of carmine; but the residuary colouring matter may also be separated by the muriate of tin.

The proportions for the abore processes are 580 parts of clear river water, 16 parts of cochineal, and 1 part of alum: there is obtained from $1 \frac{1}{2}$ to 2 parts of carmine.

Another Carmine with Tartar. - To the boiling water the cochineal is added, and after some time a little cream of tartar; in eight minutes more a little alum is added, and the boiling continued for a minute or two longer. Then take it from the fire and pour it into glass or porcelain vessels ; filter, and let it repose quietly till the carmine falls down. Then decant and dry in the shade. The proportions are $8 \mathrm{lbs}$. of water, $8 \mathrm{oz}$. of cochineal, $\frac{1}{2} \mathrm{Oz}$. of cream of tartar, and $\frac{3}{4} \mathrm{oz}$. of alum; and the product is an ounce of carmine.

The Process of Alxow or Langlois. - Boil two pails and a half of river wator (30 pints), throw into it, a little afterwards, a pound of cochineal, add a filtered solution of six drachms of carbonate of soda and a pound of water, and let the mixture boil for half an hour: remove the copper from the fire, and let it cool, inclining it to one side. Add six drachms of pulvorised alum, stir with a brush to quicken the solution of the salt, and let the whole rest 20 minutes. The liquor, which has a fine scarlet colour, is to be carefully decanted into another vessel, and there is to be put into it the whites of two eggs well beat up with half a pound of water. Stir again with a brush. The copper is replaced on the fire, the alumina becomes concrete, and carries down the colouring matter with it. The copper is to be taken from the fire, and left at rest for 25 or 30 minutes, to allow the carmine to fall down. When the supernatant liquor is drawn off, the deposit is placed upon filter-cloth stretched upon a frame to drain. When the carmine has the consistence of cream-cheese, it is taken from the filter with a silver or irory knife, and set to dry upon plates, covered with paper to screen it from dust. A pound of cochineal gives in this way an ounce and a half of carmine.

Process of Madanue Cenette of Amsterdam, with salt of sorrel.-Into six pails of river .water boiling hot, throw two pounds of the finest cochineal in powder; continue the cbullition for two hours, and then add $3 \mathrm{oz}$. of refined saltpetre, and, after a few minutes, $4 \mathrm{oz}$, of salt of sorrel. In ten minutes more take the copper from the fire, and let it settle for four hours; then draw off the liquor with a siphon into flat plates, and leare it there for three weeks. Afterwards there is formed upon the surface a pretty thick mouldiness, which is to be removed dexterously in one pellicle by a slip of whalebone. Should the film tear and fragments of it fall down, they must be removed with the utmost care. Decant the supernatant water with a siphon, the end of which may touch the bottom of the vessel, because the layer of carmine is very firm. Whatever water romains must be sucked away by a pipette. The carmine is dried in the shade and has an extraordinary lustre.

Carmine by the Salt of Tin, or the Carmine of China.-Boil the cochineal in river water, adding some Roman alum; then pass through a fine cloth to remove the cochineal, and set the liquor aside. It become brighter on keeping. After having heated this liquor, pour into it, drop by drop, solution of tin till the carmine be precipitated. 'The proportions are one pailful of water, $20 \mathrm{oz}$. of cochineal, and 60 grains of alum, with a solution of tin containing $4 \mathrm{oz}$. of the metal.

To make Ordinary Carmine. - Take 1 pound of cochineal in powder; 3 drachms and a half of carbonato of potash; 3 drachms of alum in powder; 3 drachms and a half of fish-glue.

The cochineal must be boiled along with the potash in a copper containing five pailfuls of water ( 60 pints); the obullition being allayed with cold water. After boiling a few minutes the copper must be taken from the fire, and placed on a table at such an angle as that the liquor may bo conveniently transvased. The pounded alum is then 
thrown in, and the decoction is stirred: it changes colour immediately and inclines to a more brilliant tint. At the end of fifteen minutes the cochineal is deposited at the bottom, and the bath becomes as elear as if it had been filtered. It contains the colouring matter, and probably a little alum in suspension. It is then decanted into a copper of equal capacity, and placed over the fire, adding the fish-glue dissolved in a great deal of water, and passed through a searce. At the moment of ebullition, the carmine is perceived to rise up to the surface of the bath, and a coagulum is formed, like what takes place in clarifications with whites of egg. The copper must be immediately taken from the fire, and its contents be stirred with a spatula. In the course of fifteen or tringty minutes the carmine is deposited. The supernatant liquor is decanted, and the deposit must be drained upon a filter of fine canvas or linen. If the operation has been well conducted, the carmine when dry crushes readily under the fingers. What remains after the precipitation of the carmine is still much loaded with colour, and may be employed very advantageously for carminated lakes. See LAKE.

There are sold at the shops different kinds of carmine, distinguished by numbers, and possessed of a corresponding value. The difference depends upon two causes, either upon the proportion of alumina added in the precipitation, or of a certain quantity of vermilion put in to dilute the colour. In the first case the shade is paler, in the second it has not the same lustre. It is always easy to discover the proportion of the adulteration. By availing ourselves of the property of pure carmine to dissolve in water of ammonia, the whole foreign matter remains untouched, and we may estimate its amount by drying the residuum.

Carmine is, according to Pelletier and Caventou, a triple compound of the colouring substance and an animal matter contained in cochineal, combined with an acid added to effect the precipitation. The most successful investigator into the colouring matter of the cochineal has been Mr. Warren De La Rue. This chemist had the opportunity of submitting the living insect to microscopical examination. He found it to be covered with a white dust, which was likewise observed on the adjacent parts of the cactus leaves on which the animal feeds. This dust, which he considered to be the excrement of the animal, has, under the microscope, the appearance of white curved cylinders of a very uniform-diameter. On removing the powder with ether, and piercing the side of the insect, a purplish-red fluid exudes, which contains red colouring matter, in minute granules assembled round a colourless nucleus. These groups seem to float in a colourless fluid, which appears to prove, that whatever may be the function of the colouring matter, it has a distinet and marked form, and does not pervade, as a mere tint, the fluid portion of the insect. To this colouring matter, Mr. De La Rue has given the name of CARMTINIC Acm.

It has been shown by Schützenberger that the carminic acid in carmine is combined with a nitrogenous base called tyrosine.

There are some remarkable peculiarities about the production of carmine. The shade and character of the colour are altered by slight, very slight, differences of the temperature at which it is prepared; and with every variation in the circumstance of illumination, a change is discovered in the colour. Sir $\mathrm{H}$. Dary relates the following anecdote in illustration of this:-

'A manufacturer of carmine, who was aware of the superiority of the French colour, went to Lyons for the purpose of improving his process, and bargained with a celebrated manufacturer in that eity for the aequisition of his secret, for which he was to pay $1,000 l$. He saw all the process, and a beautiful colour was produced, but he found not the least difference in the French method and that which had been adopted by himself. He appealed to his instructor, and insisted that he must have kept something concealed. The man assured him that he had not, and invited him to inspect the process a second time. He very minutely examined the water and the materials, which were in every respect similar to his own, and then, very much surprised, he said: "I have both lost my money and my labour; for the air of England does not admit of our making good carmine."- "Stay," said the Frenchman, "don't deceive yourself; what kind of weather is it now ?"-_ "A bright sunny day," replied the Englishman. "And such are the days," replied the Frenchman, "upon which I make my colour: were I to attempt to manufacture it on a dark and cloudy day, my results would be the same as yours. Let me advise you to make your carmine on sunny days." 'Experiments on this subject have proved that coloured precipitates which are brilliant and beautiful when they are precipitated in bright sunshine, are dull, and suffer in their general character, if precipitated in an obscure apartment, or in the dark.

To revive or brighten Carmine.-We may brighten ordinary carmine, and obtain a very fine and clear pigment, by dissolving it in water of ammonia. For this purpose we leave ammonia upon carmine in the heat of the sun, till its colour is extracted, 
and the liquor has got a fine red tinge. It must be then drawn off and precipitated by acetic acid and alcohol, next washed with alcohol, and dried. Carmine dissolved in ammonia has been long employed by painters under the name of Liquid carmine.

Carmine is the finest red colour which the painter possesses. It is principally employed in miniature painting, water colours, and to tint artificial flowers, because it is more transparent than the other colours. This valuable pigment is often adulterated with starch. Water of ammonia enables us to detect this fraud by dissolving the pure carmine, and leaving the starchy matter, as well as most other sophisticating substances. Such debased carmine is apt to spoil with damp.

CARTArIrmz. A hydrous chloride of potassium and magnesium, named after Von Carnall, of the salt-mines at Stassfurt, near Magdeburg, in Prussia. The carnallite occurs in the upper beds of these salt-deposits, known as the 'Carnallite region.' It is also found under similar conditions at Kalusz in Galicia, and is said to occur likewise at Maman in Persia. See Abratur Sarts.

Caruallite occurs massive or in coarsely-granular forms; when pure it is colourless, but in most cases it presents a reddish colour due to included six-sided plates of micaceous iron-ore (peroxide of iron). The specific gravity of the salt is 1.6. It deliquesces in the air, and dissolves freely in water. The formula of carnallite is $2 \mathrm{MgCl}+\mathrm{KCl}+12 \mathrm{HO}\left(\mathbf{M g C l}^{2}+\mathbf{K C l}+\mathbf{6} \mathbf{H O}^{2}\right)$. A specimen of pure carnallite would therefore contain $34 \cdot 2$ per cent. of chloride of magnesium, 26.8 of chloride of potassium, and 39 of water; as raised from the mine, however, it generally contains ulso chloride of sodium and sulphate of potash, sometimes with organic matter and microscopic crystals of anhydrite and quartz. The carnallite beds are so intermixed with the other minerals that the commercial potash-salts known as carnallite contain only 66 per cent of carnallite, representing $17 \frac{1}{2}$ per cent. of chloride of potassium. The mineral is associated with rock-salt, kieserite, sylvine, \&c. It is largely employed as a source of chloride of potassium, which is obtained from it by merely treating the salt with hot water, whereby the readily-soluble chloride of magnesium is dissolved out. The salt is also used in the preparation of chlorine gas, and in the manufacture of manures.

CArт A ÜBA. The name of a palm growing in Brazil, the Corypha cerifera. The fruit is edible. Candles are made from the wax which is obtained from the leaves by melting the coating. The wood is very strong, and used in building.

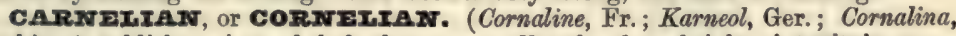
Ital.) A reddish variety of chalcedony, generally of a clear bright tint; it is sometimes of a yellow or brown colour, and it passes into common chalcedony through greyish red. Heintz, by his analysis, shows that the colour is due to peroxide of iron. He found per cent., Peroxide of Iron 0.050; Alumina 0.081; Magmesia 0.028; Potash 0.0043 ; Soda 0.075 ; the remainder being Silica.-Dana.

Carnelians are the stones usually employed, when engraved, for seals. The French give to those carnelians which have the utmost transparency and purity, the name of Cornaline d'ancienne roche. See AGATE.

The late J. Forbes, Esq., long a resident in India, and with ample means of reference to the province of Guzerat, thus describes the locality of the carnelian mines:-

'Carnelians, agates, and the beautifully variegated stones improperly called Mocha Stones, form a valuable part of the trade at Cambay. The best agates and carnelians are found in peculiar strata, thirty feet under the surface of the earth, in a small tract among the Rajepiplee hills on the banks of the Nerbudda; they are not to be met with in any other part of Guzerat, and are generally cut and polished in Cambay. On being taken from their native bed, they are exposed to the heat of the sun for two years: the longer they remain in that situation, the brighter and deeper will be the colour of the stone. Fire is sometimes substituted for the solar ray, but with less effect, as the stones frequently crack, and seldom acquire a brilliant lustre. After having undergone this process, they are boiled for two days, and sent to manufacturers at Cambay. The agates are of different hues; those generally called carnelians are dark, white, and red, in shades from the palest yellow to the deepest scarlet.

'The variegated stones with landscapes, trees, and water beautifully delineated are found at Copper-wange, or, more properly, Cubber-punge, 'The Five Tombs, a place sixty miles distant.'-Oriental Memoirs, 2nd ed. vol. i. p. 323.

'At Neemoudra, a village of the Rajepiplee district, and three miles east, are some celebrated carnelian mines. The country in the immediate vicinity of the mines is but little cultivated; and on account of the jungles, and their inhabitants the tigers, no human inhabitants are found nearer than Rattumpoor, which is seven miles off. The miners have huts at this place when stones are burned.

'The carnelian mines are situated in the widest parts of the jungle, and consist of numerous shafts worked down perpendicularly about 4 feet wide, the deepest about 50 foet. Some extend at the bottom in a horizontal direction, but usually not far, the 
nature of these pits being such as to prevent their boing worked a second year, on account of the heary rains eausing the sjdes to fall in; so that now ones must be opened at the conclusion of every rainy season. The soil is gravelly, and consists chiefly of quartz-sand, reddened with iron and a little clay. The nodules weigh from a few ounces to even two or three pounds, and lie close to each other, but for the most part distinct, not being in strata, but scattered through the masses in great abundance.

- On tho spot, the curnelians are mostly of a blackish-olive colour, like common dark flints, others somewhat lighter, others still lighter with a milky tinge; but it is quite uncertain what appearance they will assume after they have been burnt.

'From Neemoudra they are carried by the merchants to Cambay, where they are cut, polished, and formed into beautiful ornaments, for which that city is so justly celebrated.'-Copeland, Bombay Researches; Hamilton's Description of Hindostan, 4to. 1820 .

The stones from Cambay are offered in commerce, cut and uncut, as roundish pebbles from 1 to 3 inches in diameter. The colour of red carnelian of Cambay varies from the palest flesh-colour to the deepest blood-red; the latter being most in demand for seals and trinkets. The white are scarce, but when white and uniform they are valuable; the yellow and rariegated are of little estimation in the Bombay market.

The present trade in these stones cannot be correctly ascertained, as in the Board of Trade returns they are now included with precious stones 'of the inferior class, unset.' The value of the imports cannot be ascertained.

CARoB Trzx. Ceratonia Siliqua. This tree is often called St. John's Bread, from the idea that it furnished the 'locusts' and 'wild honey' to St. John when in the wilderness. It is an evergreen tree of large size, and is the only tree that grows in Malta. It grows abundantly in Palestine. The ripe fruit has a sweetish taste, and is eaten in times of scarcity by the country people. Large quantities of the fruit are sent annually from Palestine to Alexandria, and thence to Constantinople. The Turks pulp it with liquorice root and other fruits, to make their sherbet. The bark is sometimes, on account of its astringency, employed as a substitute for oak bark in tanning hides. M. Mennons, of Paris, has patented the application of the carob beans to the production of a species of glucose or fermentable sugar adapted to a variety of manufacturing purposes.

CARoTrws. The colouring principle of the carrot (Daucus Carota), which is associated in the root with a colourless substance called hydrocarotine.

CARPrr. (Tapis, Fr.; Teppich, Ger.) A thick woollen fabric of variegated colours, for covering the floors of the better sort of apartments. This luxurious manufacture took its origin in Persia and Turkey, whence the most beautiful patterns were wont to come into Europe; but they have been for some time surpassed by the workmanship of France, Great Britain, and Belgium. To form a just conception of tho elegant and ingenious processes by which carpets are made, we should visit the State establishment of thé Gobelins at Paris.

The warp, says Mr. Roland, being the foundation of the fabric, ought to be of fine wool, equally but firmly spun, and consists of three yarns twisted into one thread. The yarns that are to form the velvety surface of the carpet ought also to be of the best quality, but soft and downy in their texture, so that the dye may penetrate erery filament. Hemp or linen yarns are likewise employed in this manufacture, as a woof, to bind the warp firmly together after each shoot of the velvety threads. Thus we see that good carpeting consists essentially of two distinct webs woven at the same time, and firmly decussated together by the woof threads. Hence the form of the pattern is the same upon the two sides of the cloth, only the colours are reversed, so that what was green upon one side becomes red or black upon the other, and vice versi. The smaller the figures the more frequent the decussation of the two planes, and the firmer and more durable the fabric.

The carpet manufacture, as now generally practised, may be distributed into two systems - that of double fabrics, and that cut in imitation of velvet. Of late years the Jacquard loom has been much used in weaving carpets, the nature of which will be found fully explained under that title.

For the sake of illustration, if we suppose the double carpets to be composed of only two colours, the principle of weaving will be easily understood; for it is only necessary to raise the warp of each web alternately for the passage of the shuttle, the upper web being entirely above when the under web is being woven, or decussated, and vice versa. In the Brussels carpet the worsted yarn raised to form the pile, and make the figure, is not cut; in the Wilton the pile is cut to give it a velvety aspect and softness. In the imperial Brussels carpet the figare is raised above the ground, and its pile is cut, but the ground is uncut; and in the royal Wilton the pile is both raised higher than in the common Wilton, and it is cut, whereby it has a rich cushionlike appearance. The cloth of all these superior carpets consists of woollen and 
linen, or hemp; the latter being put upon a beam, and brought, of course, through heddles and a reed; but as its only purpose is to bind together tho worsted fabric, it should not be visible upon the upper face of the carpet. The worsted yarn is wound upon small bobbins or pirns, with a weight affixed to each, for giving proper tension to the threads. The number varies, for one web, from 1,300 to 1,800 , according as the carpet is to be 27 or 36 inches wide; and they are placed, in frames, bohind the loom, filled with differently-coloured yarn, to correspond with the figure. This worsted warp is then drawn through the harness, heddles, and reed, to be associated with the linen yarn in the compound fabric.

In Kidderminster carpeting, both warp and weft appear upon the face of the cloth, whereas, in the Brussels stylo, only the warp is seen, its binding weft being fine hempen or linen threads. The three-ply imperial carpet, called the Scotch, is coming very much into vogue, and is reckoned by many to be little inferior in texture, look, and wear to the Brussels. Kilmarnock has acquired merited distinetion by this ingenious industry. In this fabric, as well as in the two-ply Kidderminster, the weft predominates, and displays the design; but, in the French carpets, the worsted warp of the web shows the figure. Plain Venetian carpets, as used for stairs and passages, are woven in simple looms, provided merely with the common heddles and reed. The warp should be a substance of worsted yarn, so heavy as to cover in the weft completely from the view. Figured Venetian carpets are woven in the two-ply Kidderminster looms, and are provided with a mechanism to raise the pattern upon the worsted warp. The weft is an alternate shoot of worsted and linen yarn, and must be concealed.

The following figure and description will explain the construction of the three-ply imperial Scotch and two-ply Kidderminster earpet loom, which is merely a modification of the Jacquard metier. The Brussels carpet loom, on the contrary, is a draw-boy loom on the damask plan, and requires the weaver to have an assistant. Fig. 433, $\triangle \triangle \mathrm{A}$, is the frame of the loom, eonsisting of four upright posts, with caps and cross-rails to bind them together. The posts are about six feot high. $\mathrm{C}$, the cloth-beam, is a wooden cylinder, six inches or thereabouts in diameter, of sufficient length to traverse the loom, with iron gudgeons in the two ends, which work in bushes in the side frame. On one end of this beam is a ratchet wheel, with a tooth to keep it from turning round backwards by the tension of the web. D, the lay, with its reed, its under and upper shell,

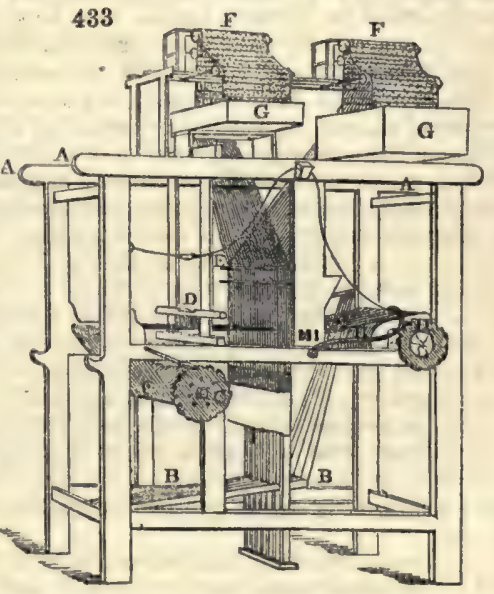

its two lateral rulers or swords, and rocking-tree above. There are grooves in the upper and under shell, into which the reed is fitted. $\mathbf{s}$, the heddles, or harness, with a double neck attached to each of the tower or card mechanisms, F F, of the Jacquard loom. The heddles are connected and work with the treddles в B, by means of cords, as shown in the figure. o o are wooden boxes for the. cards. H, the yarn, or warp beam.

In draw-looms of every kind, there is no sinking of any portion of the warp, as in plain cloth-weaving; but the plane of the cloth is placed low, and the threads under which the shuttle has to pass are raised, while all the rest remain stationary. The harness part of this carpet-loom is moved by an assistant boy or girl, who thus allows the weft to be properly decussated, while the weaver attends to working the front mounting or heddles. Fig. 434, a represents the frame of a carpet draw-loom; в is a box or frame of pullies, over which the cords of the harness pass, and are then made fast to a piece of wood, seen at $\mathrm{z}$, which the wearers call a table. From the tail of 
the harness the simples descend, and to the end of each is attached a small handle $a$, called a bob. These handles being clisposed in pairs, and thoir regularity preserved by means of a perforated board c, it is merely necessary to pull every handle in succession, the wearer, at the same time, working his treddles with his feet, as in any other loom. The treddles are four in number, the fabric being that of plain or alternate cloth, and two treddles allotted for each web. The harness part of the carpet draw-loom is furnished with mails, or metallic eyes, to save friction; two threads being drawn through each eye. The design or pattern of a corpet is drawn upon cross-rule paper, exactly in the same way as every other kind of fancy-loom work, and is transferrod from the paper to the mounting by the rules for damask weaving. Suppose that a double web is so mounted that every alternate thread of the one may be raised, so as to form a sufficient shed-way for the shuttle, without depressing the other in the least: then suppose another web placed upon the former, at such a distance that it will exactly touch the convexity of those threads of the former which are raised. Then, if the threads of the latter web are sunk while the others are raised, the two would be entirely incorporated; but if this be only partially done, that is, at particular places, only those parts immediately operated upon will be affected by the action of the apparatus. If the carpet is a two-coloured pattern, as black and red, and if upon the upper surface, as extended in the loom, red flowers are to be represented upon a black ground, then all those species of design paper which are coloured may be supposed to represent the red, and those which are vacant the black. Then counting the spaces upon the paper, omit those which are vacant, and cord those which are coloured, and the effect will be produced. But as the two webs are to be raised alternately, whatever is corded for the first handle must be passed by for the second, and vice vers $\hat{A}_{\text {, so }}$ that the one will form the flower, and the other the ground.

The board by which the simples are regulated appears at F. D shows the weights.

Mr. Simeox, of Kidderminster, has patented an invention for an improved manufacture of carpets, in which, by dispensing with the Jacquard loom, as well as the iron wires and tags usually employed to produce terry fabrics, such as Brussels carpets and coach-lace, he can work his machinery at greater speed and more economically. His second improvement relates to the manufacture of fabrics with cut pile, such as Wilton or Axminster carpets. He makes a ribbed fabric, greatly resembling the Brussels earpet, by a combination of woollen and linen warp and weft, arranged in such a manner that the woollen warp in the form of a ribbed surface may constitute the face of the fabric, while the linen warp forms the ground or back of the fabric. The plan he prefers, as most resembling the Brussels, consists in weaving the fabric as nearly as possible in the ordinary way, except that, instead of inserting a tag or wire to form the rib or terry, the patentee throws in a thick shoot or weft of woollen or cotton, over which the woollen warp is drawn, and forms a rib; the woollen warp being afterwards bound down with a linen shoot or weft in the ordinary way. The woollen warp employed being all of one colour, the fabric produced will be plain or unornamented, with a looped or terry pile; and upon this fabric any design may be printed from blocks.

The looms differ from the former chiefly in the employment of two separate shuttles, one for the woollen and one for the linen weft. These shuttles are both thrown by the same pickers and the same picking-sticks, and consequently the shuttle boxes must be moved up and down as may be required, in order to allow the picker to throw the proper shuttle. It will also be necessary to work the healds in a suitable manner to form the proper shreds, in order that the woollen face may be properly bound to the linen ground.

The second part of his invention relates to the production of fabrics with a cut pile, like the Axminster or Wilton rugs or carpets. The ordinary mode of making some of these fabrics is to weave the pattern in by means of a Jacquard apparatus, and pass the woollen warp over a rod or tag, which is afterwards cut by passing a suitable knife along it, thereby producing the cut pile. The patentee produces the design and surface of the fabric from the weft in place of the warp as heretofore. For this purpose the weft is made to consist of thick woollen shoots, which must be printed or stained with suitable colours, precisely as the woollen warps have been heretofore done; and the woollen shoot, when thrown in, is, by means of suitabiy formed hooks, pulled up and turned into loops, which, when they are properly secured to the foundation or ground of the fabric, are afterwards cut by means of knives or cutting instruments, with which the hooks are furnished, for the purpose of releasing them from the loops and producing the cut pile. The patentee observes, that cotton and other cheap materials may be employed with great advantage in the production of some of these fabries.

Another invention of improvements in manufacturing figured fabrics, principally. designed for the production of carpeting, patented by Mr. James Templeton, of 
Glasgow, consists in producing the pattern either on one or both sides of the fabric, by means of printed weft; also in the use of printed party-coloured fur or weft, in the manufacture of Axminster carpets and other similar fabries. This invention is also applicable to the production of figured chenille weft for the manufacture of chenille sfrawls.-Under the arrangements of the Patent Office the specifications of these patents are cheaply obtained.

Carpers, Printed. Mr. Wood has taken out a patent for weaving and printing carpets, using an ordinary Brussels carpet loom. After putting in the wire, or otherwise forming the loop, he throws in the usual linen shoot, on the face, to bind it; and then, for the back shoot, he throws in a thick soft weft. Or, to make a better edge and more elastic back, he employs the ordinary two linen shoots,- - one on the face, and the other in the back, - and then (or before throwing in the second linen shoot) he draws down only one-half of the lower portion of the linen warp (being one-quarter of the whole), and throws in the thick shoot which is driven up by the batten or lay, so as to cover the second linen shoot, which is then inside the fabric: from the thick shoot being bound only by each alternate yarn of the warp, it will be more elastic than if bound more closely by using every yarn; while the second linen shoot, having half the warp over it, holds down the face or first shoot; and any inequality in the taking up of the linen warp by one portion of it binding in a greater substance than the other, is remedied by drawing down the different portions in succession.

In printing Brussels and other pile carpets, the patentee first provides a table, long enough to receive the entire length or piece of the carpet to be printed: at each end of the table there is a frame of the some height or level, sufficiently long to receive the cylinder printing-machine when off the fabric; and on the surface of the table the printing blanket is laid between two rails or guides, which are fixed at exactly the same distance apart as the carpet is wide, so as to keep it in one position, and to form the guides for the printing eylinders. The carpet is fastened to one end of the table, and is then laid on the top of the same, and drawn tight at the other end by a roller, which is furnished with a ratchet wheel and click. The printing cylinders are mounted in a moveable frame, containing a corresponding number of colour cans and feeding rollers, to supply them with colour. The printing apparatus is passed over the table, and between the guide rails (the patterns on the cylinder being coloured, and , bearing upon the carpet), to the frame at the other end of the table, and then back again; and this process is repeated until the fabric is sufficiently coloured. In order to insure each part of the pattern or printing surface coming again and again on the same place, toothed wheels are affixed on the axis of the printing cylinders, which gear into racks fixed on the sides of the table; so that, however frequently the printing apparatus passes over the fabric, every part of the pattern will fall on the same place. Instead of the printing apparatus being passed back again over the same table, it may, by the application of moveable frames at the end of the table, be moved sideways on to another table, and so successively. See Rugs.

CArprorrs:. A silicate of manganese, alumina, and iron, found in the tinmines of Schlackenwald in Bohemia.

CARPrasis. The name of a coarse cloth which used to be manufactured in the North of England.

CARra Grav. Chondrus crispus: Irish moss. Seo Alax.

CArracrivin. The mucilaginous constituent of carrageen moss. It is called by some writers vegetable jelly or vegetable mucilage, by others pectin. "It appears to me (Pereira) to be a peculiar modification of mucilage.'

CAmRARA MARBur. A fine-grained white marblo largely quarried at Carrara in Tuscany, and valued as a statuary marble.

CArnorrme. A sulphide of cobalt and copper. Brush regards the mineral as cobaltic pyrites in which a portion of the cobalt is replaced by copper.

CAnTramos, or Safflower. (Carthame, Fr.; Färberdistel, Ger.) Car. thamus tinctorius, the flower of which alone is used in dyeing, is an annual plant cultivated in Spain, Egypt, and the Levant. There are two varieties of it-one which has large leaves, and the other smaller ones. It is the latter which is cultivated in Egypt, where it forms a considerable article of commerce.

Carthamus contains two colouring matters, one yellow and the other red. The first alone is soluble in water: its solution is always turbid; with reagents it exhibits the characters usually remarked in yellow colouring matters. The acids render it lighter, the alkalis deepen it, giving it more of an orange hue; both produce a small dun precipitate, in consequence of which it becomes clearer. Alum forms a precipitate of a deep yellow, in small quantity. Tho solution of tin and other metallic solutions cause precipitates which have nothing remarkable in them.

The yellow matter of carthamus is not employed; but in order to extract this portion, the carthamus is put into a bag, which is trodden under water, till no more 
colour can be pressed out. The flowers, which were yellow, become reddish, and lose in this operation nearly one-half of their woight. In this state they are used.

For extmeting the red part of carthamus, or carthamic acid, and afterwards applying it to stuff, the property which alkalis possess of dissolving it is had recourse to, and it is procipitated by an acid.

The process of dyeing consists, therefore, in extracting the colouring matter by means of an alksli, and precipitating it on the stuff by means of an acid.

This solution of carthamus is prepared with crystallised carbonate of soda, and it is procipitated by lemon-juico. It has been remarked that lemons beginning to spoil are fitter for this oporation than those which are less ripe, whose juice retains much mucilage. After squeezing out the lemon-juice, it is left to settle for some days. The precipitate of carthamus is dried at a gentle heat upon plates of stone-ware; from which it is detached and very carefully ground with tale which has been reduced to a very subtle powder by means of the leaves of shave-gxass (presle), and successively passed through sieves of increasing fineness. It is the fineness of the talc, aud the greater or less proportion which it bears to the carthamus precipitate, which constitute the difference between the high- and low-priced safflower rouges.

Carthamus is used for dyeing silk, poppy, nacarat (a bright orange-red), cherry, rose-colour, and flesh-calour. The process differs according to the intensity of the colour, and the greater or less tendency to flame-colour that is wanted. But the carthamus-bath, whose application should bo varied, is prepared as follows :-

The carthamus, from which the yellow matter has been extracted, and whose lumps have been broken down, is put into a trough. It is repeatedly sprinkled with crudo pearl-ashes or soda, well powdered and sifted, at the rate of 6 lbs. for $120 \mathrm{lbs}$. of carthamus; but soda is preferred, mixing carefully as the alkali is introduced. This operation is called amestrer. The amestred carthamus is put into a small trough with a grated bottom, first lining this trough with a closely-woven web. When it is about half filled, it is placod over a large trough, and cold water is poured into the upper one till the lower one becomes full. The carthamus is then set over another trough till the water comes from it almost colourless. A little more alkali is now mixed with it, and fresh water is passed through it. These operations are repeated till the carthamus be exhausted, when it turns yellow.

After distributing the silk in hanks upon the rods, lemon-juice, brought in casks from Provence, is poured into the bath till it becomes of a fine cherry-colour; this is called turning the bath. It is well stirred, and the silk is immersed and turned round the skein-sticks in the bath, as long as it is perceived to take up the colour. For ponceau (poppy-colour), it is withdrawn, the liquor is run out of it upon the peg, and it is turned through a new bath, where it is treated as in the first. After this it is dried and passed through fresh baths, continuing to wash and dry it between each operation, till it has acquired the depth of colour that is desired. Whon it has reached the proper point, a brightening is given it by turning it round the sticks seven or eight times in a bath of hot water, to which about half a pint of lemon-juice for each pailful of water has been added.

When silk is to be dyed ponceau, or poppy-colour it must be previously boiled. as for white; it must then receive a slight foundation of arnatto, as explained in treating of that substance. The silk should not be slumed.

The nacarats, and the deep cherry-colours, are given precisely like the ponceaux, only they receive no arnatto ground; and baths may be employed which have served for the ponceau, so as to complete their exhaustion. Fresh baths are not made for the latter colours, unless there be no occasion for the poppy.

With regard to the lighter chorry-reds, rose-colour of all shades, and flesh-colours, they are made with the second and last runnings of the carthamus, which are weaker. The deepest shades are passed through first.

The lightest of all these shades, which is an extremely delicate flesh-colour, requires a little soap to be put into the bath. This soap lightens the colour, and prevents it from taking too speedily, and becoming uneven. The silk is then washed, and a little brightening is given it in a bath which has served for the deeper colours.

All these baths are employed the moment they are made, or as speedily as possible, because they lose much of their colour upon keeping, by which thoy are even entirely destroyed at the end of a certain time. They are, moreover, used cold, to prevent the colour from being injured. It must have been remarked, in the exporiments just described, that the caustic alkalis attack the extremely delicate colour of carthamus, making it pass to yellow. This is the reason why crystals of soda are preferred to the other alkaline matters.

In order to diminish the expense of the carthamus, it is the practice in prepariug the deeper shados to mingle with the first and tho second bath about one-fifth of the bath of archil, 
Döbereiner regarded the red colouring matter of carthamus as an acid, and the yellow as a base. His carthamic acid forms, with the alkalis, colourless salts, decomposed by the tartaric and acetic acids, which precipitate the acid of a bright rose-red. Hoat has a remarkablo influenco upon carthamus, rendering its red colour yellow and dull. Honce, the colder the water is by which it is extracted, the finer is the colour. Light destroys the colour very rapidly; and hitherto no means have been found of counteracting this effect. For this reason this brilliant colour must be dried in the shade, its dye must be given in a shady place, and the silk stuffs dyed with it must be preserved as much as possible from the light. Age is nearly as injurious as light, especially upon the dye in a damp state. The colour is very dear: a thousand parts of carthamus contain only five parts of carthamine.

In preparing the finest rouge, the yellow colouring matter having been separated by washing with water, the red is dissolved by the aid of alkali, and is thrown down on linen or cotton rags, by saturating the solution with vegetable acid. The colour is rinsed out of these rags, dissolved anew in alkalis, and once more precipitated by lemon-juice. The best and freshest carthamus must be selected. It is put into linen bags, which are placed in a stream of water, and kneaded till the water runs off colourless. The bags are then put into water soured with a little vinegar, kneaded till the colour is all expelled, and finally rinsed in running water.

There is a considerable consumption of safflower in the manufacture of rouge, for which there is a far greater demand than is generally supposed, this colour being employed not only as a 'toilet appendage' of the monde, but for theatrical use.

Rouge consists of carthamine and very fine burnt talc made into a paste with water. It is made of five regular shades, known as Nos. 6, 8, 12, 18, 24, varying in depth of colour as the numbers increase; Nos. 6 and 8 are used by fair beauties, No. 12 is medium, while Nos, 18 and 24 are for dark complexions.

Pink saucers, formerly in great request for dyeing silk stockings at home, and even now not wholly unknown to trade, consist of a shallow porcelain saucer into which a film of carthamine has been allowed to precipitate, and then is dried. The Chinese make a 'Book Rouge ' in a somewhat similar way; a very strong solution of the carthamine is brushed over a cardboard, which when dry 'is fit for ladies' use.' The card is, however, no longer pink or red, but of a beautiful metallic green colour due to some special optical reason not yet explained; the pink saucers made in England exhibit in a very slight degree the same effect, which the surface of indigo also in some messure resembles.

Carthamus is the material used for dyeing ordinary red tape, and there is a curious prejudice against using other colours for this purpose, the trade objecting to any deviation from the conventional shade.

Salvétat has found it advantageous to mix the red of safflower with the pigments used in porcelain painting for purple, carmine, and violet, colours which, in consequence of the difference of their shade before and after firing, are very liable to mislead. To avoid this, he imparts to the pigment (consisting of flux, gold-purple, and chloride of silver) by means of the red of carthamus suspended in water, the same shade which he desires to obtain after firing.

The colouring matter of safflower has been examined by Salvétat, who has found much difference in carthamus of reputed good quality. The following Table gires some of his results :-

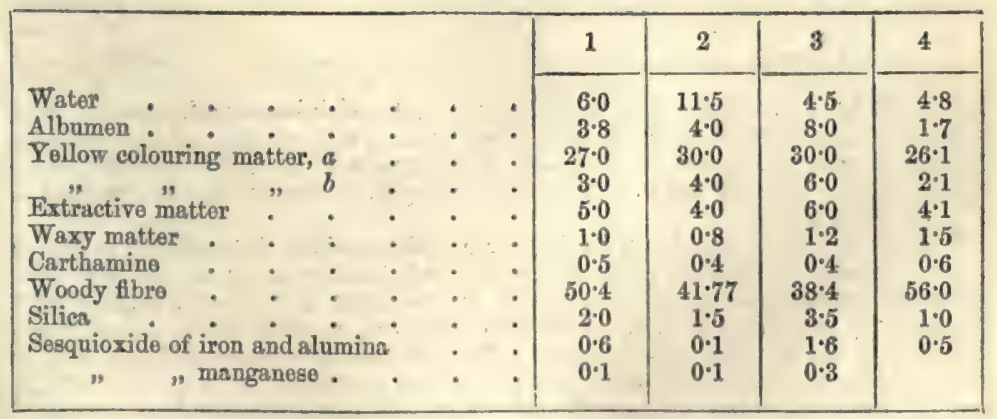

CArrox Prarra, A composition of paper mixed with whiting or glue for imitating stone or bronze. See PAPIKR MACHK.

CAnUro. The fruit of the Genipa Americana, a native plant of British Guiana, yields the dyo to which this name is given. It is of a boautiful bluish-black colour.

Vor, I. 


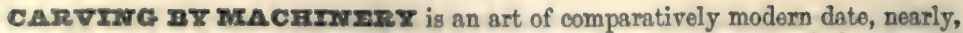
if not the whole of the originators and improvers of it, being men of tho prosont day. It is true that the Modallion Latho and many other appliances for ornamental turning and drilling can claim a much earlier origin, but these can scarcely bo called carving machines, and are altogether incapable of aiding the economy of producing architectural decorations of any kind. Wo aro not aware of any practical scheme for sccomplishing this object prior to the patont of $\mathrm{Mr}$. Joseph Gibbs, in 1829 , which wo beliero was used by Mr. Nash in ornamenting some of the floors of Buckingham Palace, and on many other works of inlaying and tracery. The cutting of ornamental forms in low relief seems to have been the principal object of the inrentor; and this he accomplished satisfactorily by a series of ingenious mechanical arrangements, which greatly reduced the cost, while securing unusual accuracy in this kind of work. Some modifications of machinery for copying busts, bosses, and other works in bold roliof are also described in Mr. Gibbs's patents, but these wore nevor carried into successful practice. The tracery and inlaying machine is illustrated by fig. 435 , which is a plan of the machine. $A$ is a shaft capable of vertical motion in its bearings, which are in the fixod framing of the machine; $\mathrm{B}$, $\mathrm{c}$, and $D, E$, are swing frames jointed together by a short vertical shaft $a$, and securely keyed to the shaft $\Delta$. The point $b$ is the axis of a revolving tool, which is driven

435

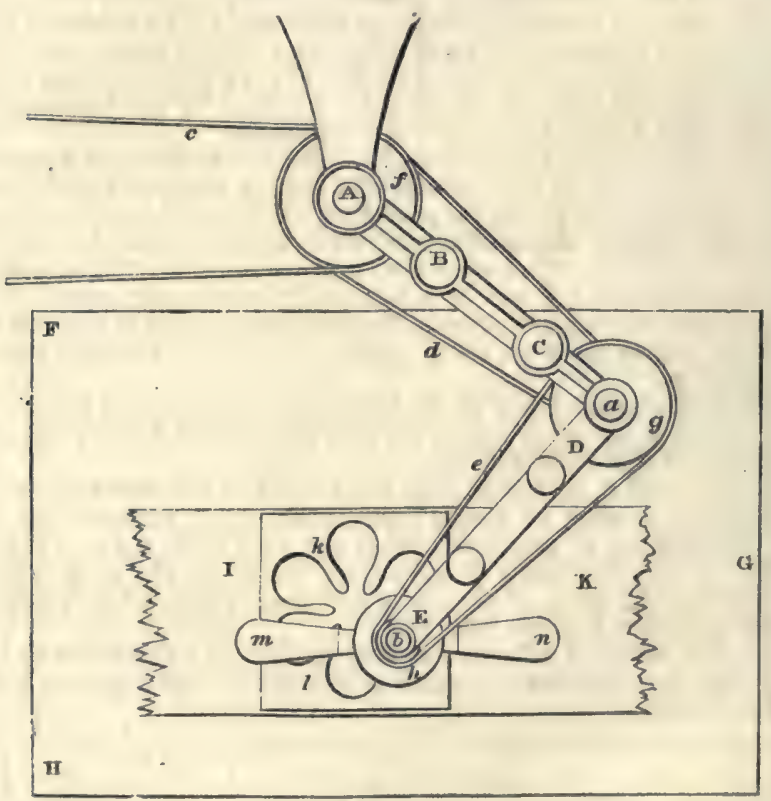

by the belts $c, d, e$, and the compound pullies $f, g, h$, which increase the speed at each step; $F, \mathrm{G}, \mathrm{E}$, is the table on which the work is fixed; $\mathrm{I}, \mathbf{x}$, the work; and $k, l$, a templet of brass pierced with the horizontal form of the pattern to be produced in the wood; this templet is securely fixed on the top of the work, or over it, and the machine is adjusted for action.

There is a treadle, not shown in the figure, which enables the workman to lift or depress the shaft $\Delta$, and the swing frames and tool attaehed to it; he can thus command the vertical position of the tool with his foot, and its horizontal position with his hand by the handles $m$, $n$, which turn freely on a collar of the swing frame surrounding the mandril or tool holder. The tool having been brought over one of the apertures of the templet when in rapid action, is allowed to sink to a proper depth in tho wood underneath, and the smooth part of its shaft is then kept in contact with the guiding edges of the templet and passed round and over the entire surface of the figure, until a recess of the exact size and form of that opening in the templet is produced; this process is repeated for every other opening, and thus a series of recosses are formod in the oak flooring-planks which correspond with the design of the tomplets used. To complote the work it is requisite to cut out of some darker 
or differently coloured material a number of thin pieces which will fit these recesses, and these are produced in the same way from templets which will fit the various aperturos of that first used; these pieces are next glued into the recesses, and the surface when planed and polishod exhibits the pattern in the various colours used. For inlaying it is important that the cutting edge of the tool should travel in the same radius as the cylindrical shaft, which is kept against the edge of the templet; but if the tool is a moulded one, a counterpart of its mouldings will be produced in the work, while the pattern, in planes parallel to that of the panel, will havo the form of the apertures in the templet used. In this way, by great care in the preparation of the templets and the tools, much of the gothic tracery used in church architecture may be produced, but the process is more applicable to Bath stone than to wood when moulded tools are requisite.

Mr. Irving's patents for cutting ornamental forms in wood and stone are identical in principles of action and in all important points of construction with the arrangements previously described. In that of 1843 ho particularly claims all combinations for accomplishing the purpose, 'provided the swing frame which carries the cutter, and also the table on which the article to be wrought is placed, have both the means of circular motion.' The pierced templet is the guiding power, and the work and templot are fixed on a circular iron table, which is at liberty to revolve on its axis. The swing frame which carries the cutter is single, as in Mr. Gibbs's curved moulding machine, and its radius so adjusted, that an are drawn by the tool would pass over the centre of the circular table. The mode of operating with this machine was to keop the shaft of the tool against the guiding edge of the templet, by the joint movements of the table on its centre, and of the swing frame about its shaft; and it will be obvious that by this means any point of the table could be reached by the tool, and therefore any pattern of moulded work within its range produced, in the way already described in speaking of Mr. Gibbs's machinery. But as these modifications of the original idea are not, strictly speaking, carving machines, seeing that they only produced curved mouldings, we need not further describe them.

Perhaps the most perfect carring machine which has been made for strictly artistic works is that used by Mr. Cheverton for obtaining his admirable miniature reductions of life-sized statuary; but we can only judge of the perfection of this machine by its work, seeing that the inventor has more faith in secrecy than patents, and has not made it public.

The carving machinery which is best known, and has been most extensively used, is that invented by Mr. Jordan and patented in 1845, and was used in producing the carved decorations of the interior of the Houses of Parliament.

Its principle of action and its construction are widely different from that above described, and it is capable of copying any carved design which can be produced, so far as that is possible by revolving tools; the smoothness of surface and sharpness of finish is neither possible nor desirable, because a keen edge guided by a practised hand will not only produce a better finish, but it will accomplish this part of the work at less cost; the only object of using machinery is to lessen the cost of production, or to save time; and in approaching towards the finish of a piece of carving, there is a time when further progress of the work on the machine would be more expensive than to finish it by hand. This arises from the necessity of using smaller tools towards the finish of the work to penetrate into its sharp recesses, and the necessarily slow rate at which these cut away the material; it is consequently a matter of commercial calculation, how far it is desirable to finish on the machine, and when to deliver it into the hands of the artist, so as to secure the greatest economy. This depends in a great measure on the hardness of the material; rosewood, ebony, box, irory, and statuary marble, should be wrought very nearly to a finish; but lime, deal, and other soft woods should only be roughly pointed.

Fig. 436 is a plan of the machine, fig. 437 a front elevation, and fig. 438 a side elevation. The same letters indicate the same parts in all the figures. The carving machine consists of two distinct parts, each having its own peculiar motions quite independent of the other, but each capable of acting simultaneously and in unison with the other. The first, or horizontal part, is the bed plate, 'floating-table,' \&c., on which the pattern and work are fixed; all the motions of this part are horizontal. The second, or rertical part, is that which carries the cutters and tracer, the only motion of which, except the revolution of the tools, is vertical.

The horizontal part consists of three castings: The bed plate $\mathbf{A}, \mathbf{B}, \mathbf{C}, \mathbf{D}$, which is a railway supported on piers from the floor and fixed strictly level. The carrying frame, I, J, K, I, mounted on wheols and travelling on the bed plate (the long sides of this frame are planed into ( $v$ ) rails), and the 'floating-table,' $\mathbf{M}, \mathbf{N}, \mathbf{0}, \mathbf{P}$, which is also mounted on wheels to travel on the rails of the carrying framo. It is called the 'floating-table' because it can be moved'in any horizontal direction with almout as 3 в 2 
much facility as if it were a floating body. Primarily this table has two stroightlined motions at right angles to each other, but by combination of these it may move over any figure in a horizontal plane; and because this is accomplished without angular motion about a centre, every point in the surface of the table moves through the same fgure at the same time; hence the power of producing many copies of a pattern simultaneously.

The second, or vertical part of the machine, is a cast-iron bridge supported on columns across the centre of the bed plate; on the centre of this bridge piece is a wide vertical slide, 5,6 , with a (T) slotted bar on its lower edge; to this bar the mandril heads or tool holders, $9,10,11$, are bolted, at such distances apart as suit the width of tho work in hand, and in such numbors as it is convenient to work at one time. If the framing of the machine is massive and well fixed, six or eight narrow pieces may be carved at once; but if the width of the work is equal to half that of the table,

436

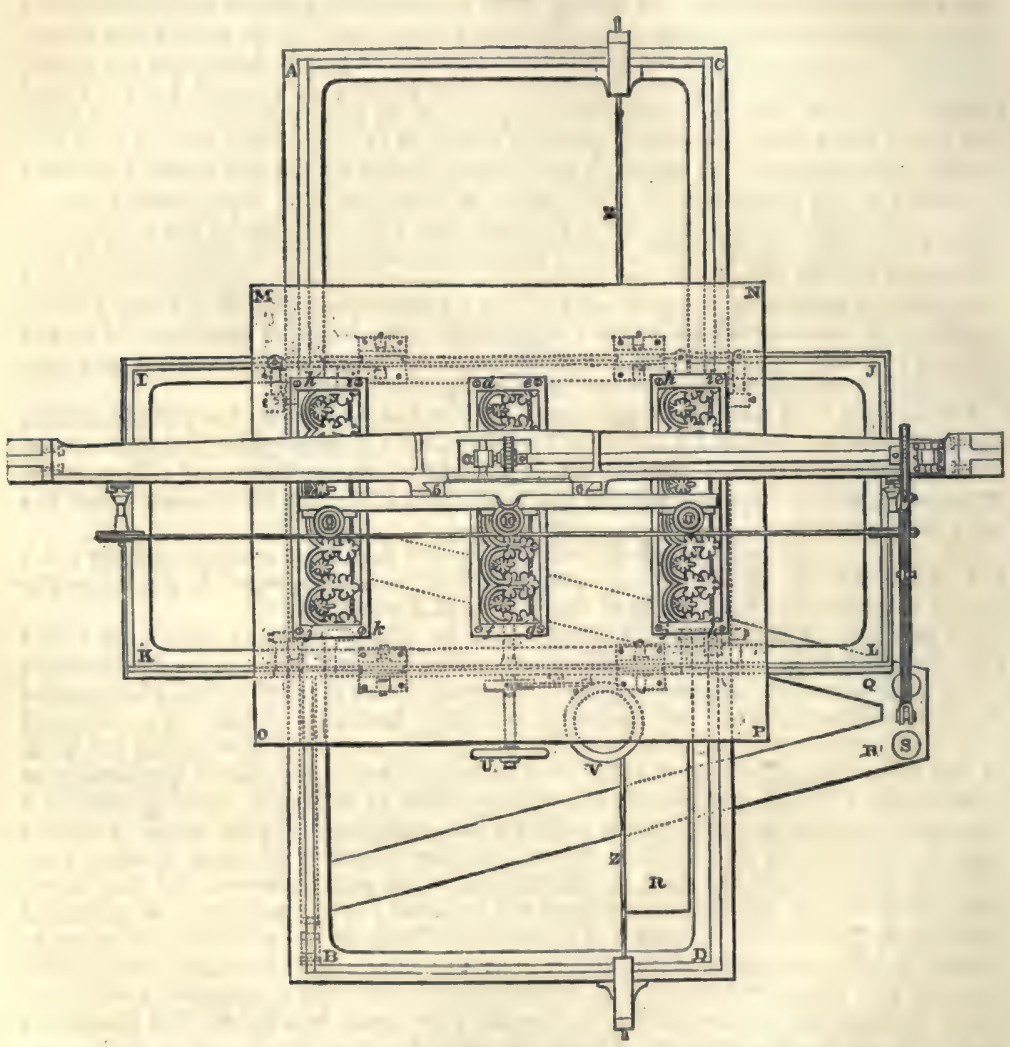

only one can be done, as in that case half the table is required for the pattern. The motion of the vertical slide is governed by the workman's foot on the treadle, $\mathrm{R}, \mathrm{Q}, \mathrm{s}$; at $s$ balance-weights are placed, so as to adjust the force with which the tools will descend on the work; any pressure on the foot-board $\mathrm{a}$ lifts the slide, and with it the tools and tracing point.

Returning to the horizontal part of the machine, $d, e, f, g$, is the pattern or original carving which is to be copied, and $h, i, j, k$, two copies in progress. The movements of the floating table are managed by the workman with the hand-wheels $\delta, \nabla$; the left hand, on $\mathrm{v}$, directs the lateral motion on the frame, and the right, on $\mathrm{v}$, directs the longitudinal motion on the bed plate; the left-hand movement is communicated by the cord $x, x$, which is fixed to brackets $w$, w, underneath the table, and makes one turn round a small pulley on the axis of the wheel $\tau$. The right-hand movement is communicated by the cord $\mathrm{z}$, which is fastened to each end of the bed plate, and makes one or two turns round the pulley 8 . When at work, the man stands inside 

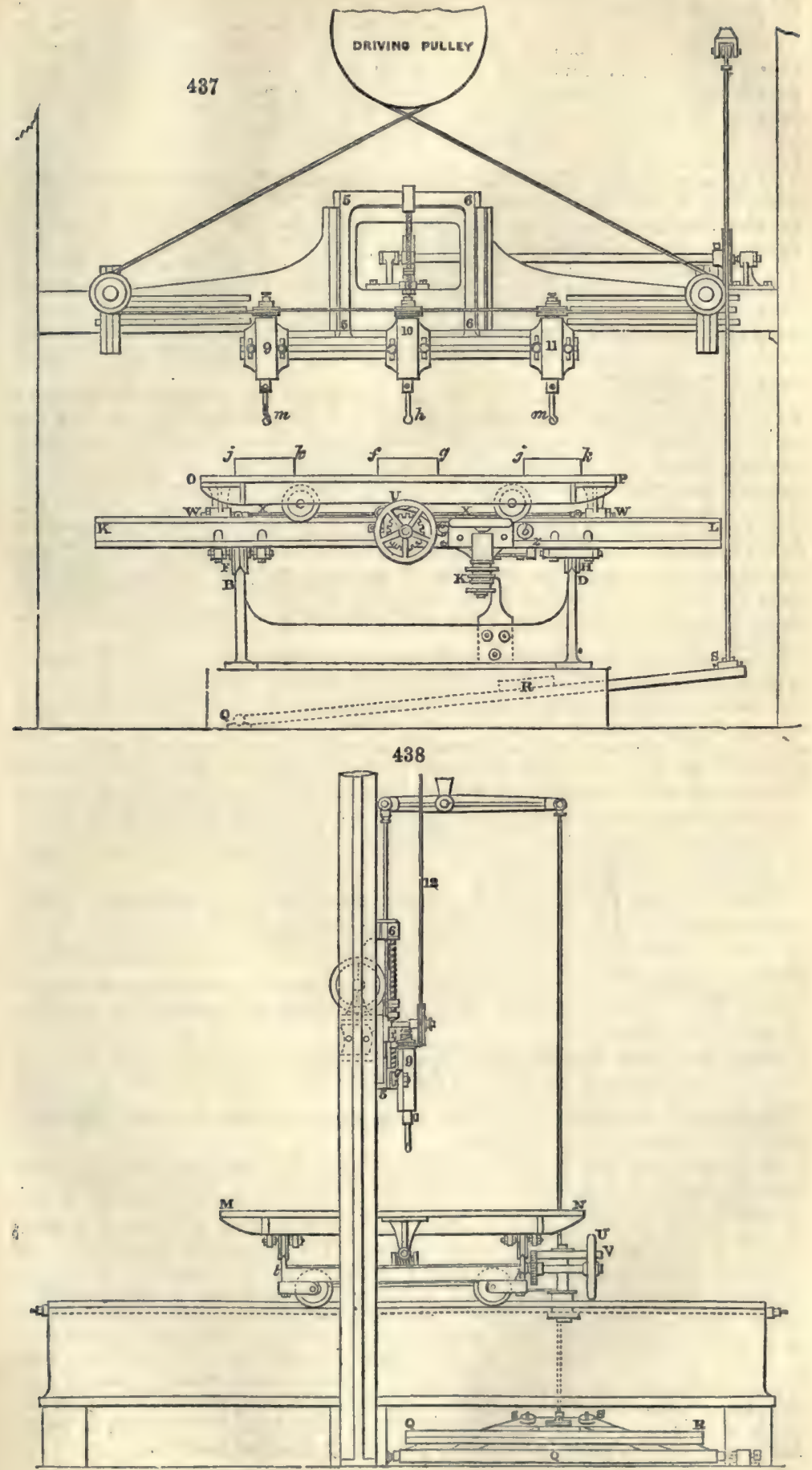
the frame of the bed plate, with his right foot on the board $\mathrm{R}$ and his hands on the stooring whoels; on releasing the pressure of the foot the vertical slide dosconds by its unbalanced woight until the tracer $l$ comes in contact with the pattern; the cutters $m, m$, are made to revolve by steam-power at the rate of seven thousand times per minute, and are so shaped as to cut like a revolving gouge, so that they instantly cut a way all tho superfluous material they como in contact with; and, by the time the tracer has been brought over every part of the pattern, the pieces $h, i, j, k$ will have become exact copies of it.

So far as panel carving is concerned the whole machine has been described; but it is requisite to elaborate its construction a littlo more for the purpose of carving on the round, and copying subjects which require the blocks to be cut into in all possible directions. Various modifications have boon used, but we shall only explain that which we think best adapted to ornamental carving. It is not requisite that we should go into the various applications of this machine, to tho manufacture of printing blocks, ships' blocks, gunstocks, letter cutting, tool handling, cabinot shaping, \&ce. \&c., all of which have been shown from time to time to be within its power; nor is it requisite to describe more recent inventions founded on it, as they will more properly come under other heads.

When the machine is intended to copy any form which can be carved by hand, the floating table is differently constructed, but all othor parts remain as before. In the floating table used for this purpose, there is an opening in the centre of the table, and a turning plate, which is mounted a few inches above the lovel of the table, to turn in bearings in standards. Underneath the turning plate, and forming a part of it, there 1s an are of rather more than half a circle, hâving its centre in the axis on which the plate turns, and this are is cogged on its edge to fit the threads of the tangent screw on the axis of the wheel, so that by turning this wheel, and dropping its detent into any cog, the workman can fix the plate at any angle with the horizon. There are three chucks fitted into sockets of the turn plate, and these are similarly divided on their edges by holes or eogs, into which detents fall, so as to secure them steadily in any required position.

In going through the process the workman will, of course, attack the work when it is placed in a favourable position for the tools to reach a large portion of its surface: and having completed as much as possible on that face, he would turn all the chucks through the same number of divisions; the pattern and work will still have the same relative position to each other as before, but an entirely new face of both will be presented to the tools; this will be carved in like manner, and then another similar change made, and so on until all has been completed which can be reached without changing the angular position of the turning plate. This can be done by the wheel, and when a sufficient number of these changes have been gone through, the work will be complete on every face, although the block may have required to be pierced through in fifty different directions.-T. B. J.

Notwithstanding the remarkable ingenuity displayed in the construction of this carving machine, it has not been so largely used as might have been expectod. In all cases where repetitions are required its advantages are obvious, yet prejudice appears to have stood in the way of its general adoption.

CAsCAIzB. After the first washing of sand for gold there is a deposit left, and this, in Brazil, is called Cascalho. The name is also given to the alluvial soil in which Brazil diamonds are found.

CAscarinta marz. (Spanish, Cascara, bark.) The bark of Crotons eleutheria and $C$. cascarilla, both of which shrubs grow in the West Indies. The bark possessing tonic properties is used medicinally.

CASE-ZARDIrNIre is the name of the process by which iron tools, keys, \&c., have their surfaces converted into steel.

Steel when very hard is brittle, and iron alone is for many purposes, as for fine keys, far too soft. It is therefore an important desidoratum to combine the hardness of a steely surface with the toughness of an iron body. These requisites are united by the process of ease-hardoning, which does not differ from the making of steel, except in the shorter duration of the process. The property of hardening is not possessed by pure malleable iron; but by a partial process of cementation the iron is converted externally into steel, and is subsequently hardened to that particular depth. Tools, utensils, or ornaments, intended to bo polished, are first manufactured in iron, and nearly finished, after which they are put into an iron box, together with vegetable or animal charcoal in powder, and cemented for a certain time. This treatment converts the external part into a coating of steol, which is usually rery thin, because the time allowed for the cementation is much shorter than when the whole mass is to be converted into steel. Immorsion of the heated pieces in wator hardens the surface, which is afterwards polished by the usual methods. 
When the case-hardening is required to terminate at any particular part, as a shoulder, the object is left with a band or projection; the work is allowed to cool without being immersed in water; the band is turned off, and the work, whon hardened in the opon fire, is only offected as far as the original cemented surface remains. This ingenious method was introduced by Mr. Roberts, of Manchester, who considers the success of the case-hardening process to depend on the gentle application of the heat; and that, by proper management not to overheat the work, it may be made to penetrate three-eighths of an inch in four or five hours.-Holtzapffel.

The application of prussiate (ferrocyanide) of potash to this purpose is a very interesting chemical problom. The picce of iron, after being polished, is to be mado brightly red-hot, and then rubbed or sprinkled over with the above salt in fine powder, upon the part intended to be hardenod. The prussiate being decomposed, and apparently dissipated, the iron is to be quenched in cold water. If the process has been well managed, the surface of the metal will have become so hard as to resist the file. It has been proposed to smear over the surface of the iron with loam made into a thin paste with a strong solution of the prussiate, to dry it slowly, then expose the whole to a nearly white heat, and finally plunge the wholo into cold water, when the heat has fallen to dull redness. Soe Strier.

CAsrrov (from Caseus, the Latin name for cheese). The principal part of the nitrogenised matters contained in the milk of mammiferous animals is casein. It forms the greatest part of cheese. It is coagulated by acids, but not by heat-in this it differs from either fibrine or albumen; it is also coagulated by rennet as in the curding of milk. See Watts's 'Dictionary of Chemistry.' See Renner.

Casoin is used by the calico-printer as a mordant. With lime it forms a good. cement, and with borax it has been used as a substitute for glue.

CAsErW NUTr. The fruit of the Anacardium occidentale of the West Indies. See Axacandiur Not.

CAsmm the kingdom of Cashmere (Kashmir), and now well imitated in France and Great Britain. The material of the Cashmere shawls is the downy wool found about the roots of the hair of the Thibet goat. The Oriental Cashmere shawls are woven by processes extremely slow and consequently costly, whence their prices are very high.

Prices of Cashmere shawls of various sorts kindly given by a gentleman who has resided at Cashmere for many years:-

1. Plain pashmina, ${ }^{2} 6 \mathrm{ft}$. square .

2. Pashmina, deeply embroidered

3. Doshala, or long and wide scarf of pashmina, with shawlwork at the edges and ends, as worn by the natives

4. Square shawl (loom-made), $6 \mathrm{ft}$. square

5. Long shawl (loom-made).

It is 4 and 5 that are the Cashmere shawls proper; the most nsual article in the European market is a long shawl costing 60l. or 70l. in Cashmere.

Finer sorts are made occasionally (as by order of the Maharaja for presents to tho Queen), whose value is as much as $300 l$.

The finest ever made was presented by the Maharaja to the Duke of Edinburgh at Lahore. The Duke would not receive it for himself, but for the Queen. The value of this was at least as much as $600 l$.

By the aid of the draw-loom, and still better of the Jacquard loom, M. Temaux flrst succeeded in weaving Cashmere shawls perfectly similar to the Oriental in external aspect, which became fashionable under the name of French Cashmere. But to construct shawls altogether identical on both sides with the Eastern was a more difficult task, which was accomplished only at a later period by M. Bauson, of Paris.

The year 1819 is remarkable in the history of French husbandry for the acquisition of the Cashmere goat, imported from the East under the auspices of their government, by the indefatigable courage and zeal of M. Jaubert, who encountered every fatigue and danger to enrich his country with these valuable animals, aided by the patriotism of M. Ternaux, who first planned this importation, and furnished funds for executing it, at his own expense and responsibility. He placed a portion of the flock brought by M. Jaubert at his villa of St. Ouen, near Paris, where the climato seemed to be very favourablo to them, since for several successive years after their introduction M. Ternaux was enabled to sell a great number of both malo and femalo goats. The quantity of fine fleece or down affordod by each animal annually is from a pound and a half to two pounds.

The wool imported into Europe comes by the way of Casan, the capital of a government of the Russian empire upon the oastern bank of the Volga ; it has naturally a greyish colour, but is easily bleached. Its price at Paris is about 6s. the pound

2 Pashmina is a general namo for the fabric made of pashm, or the ander-wool of the ahawl-goat. 
aroirdupois. The waste in picking, carding, and spinning, amounts to about one-third of its woight.

The mills for spinning Cashmero wools have multiplied very much of lato years in France, and the prices of the yarn have fallen by from 25 to 30 per cent., notwithstanding their improved fineness and quality.

In both modes of manufacture, the piece is mounted by 'reading-in' the warp for the different leaves of the heddles, as is commonly practised for warps in the Jacquard looms. The weaving of imitation shawIs is executed, as usual, by as many shuttles as there are colours in the design, and which are thrown across the warp in the order establishod by the 'reader.' The greatest number of these weft yarns being introduced only at intervals into the web, when the composition of the shawl requires it, they remain floating loose at the back of the piece and are cut afterwards, without affecting in the least the quality of the texture; but there is considerable waste of stuff in the weaving, which is worked up into carpets.

The weaving of the imitation of real Cashmere shawls is different from the above. The yarns intended to form the weft are not only equal in number to that of the colour of the pattern to be imitated, but, besides this, as many little shuttles or pirns (like thoso used by embroiderers) are filled with these yarns as there are to be colours repeated in the breadth of the piece; which renders their number considerable whon the pattern is somewhat complicated and loaded with colours. Each of these small bobbins or shuttles passes through only that portion of the flower in which the colour of its yarn is to appear, and stops at the one side and the other of the cloth exactly at its limit; it then returns upon itself after having crossed the thread of the adjoining shuttle. From this reciprocal intertexture of all the yarns of the shuttles, it results that although the weft is composed of a great many different threads, they no less constitute a continuous line in the whole breadth of the web,upon which the lay or batten acts in the ordinary way. We see, therefore, that the whole art of manufacturing this Cashmere cloth consists in avoiding the confusion of the shuttles, and in not strikiug up the lay till all have fulfilled their function.

In the Oriental process, all the figures in relief are made simply with a slender pirn without the shuttle used in European weaving. By the Indians the flower and its ground are made with the pirn, by means of an intertwisting, which renders them in some measure independent of the warp. In the Lyons imitation of this style, the leares of the heddles lift the yarns of the warp, the needles embroider as in lappet weaving, and the flower is united to the warp by the weft thrown across the pice.

Paris manufactures the French Cashmere, properly so ealled, of which both the warp and the weft are the yarn of pure Cashmere down. This web represents with fidelity the figures and the shades of colour of the Indian shawl, which it copies; the deception would be complete if the reverse of the piece did not show the cat ends. The Hindoo shawl, as woven at Paris, has its warp in spun silk, which reduces its prico withont much impairing its beauty.

Lyons, however, has made the greatest progress in the manufacture of shawls. It excels particularly in the texture of its Thibet shawls, the weft of which is yarn spun with a mixture of wool and spun silk.

Nîmes is remarkable for the low price of its shawls, in which spun silk, Thibet down, and cotton, are all worked up together.

It appears that M. J. Girard at Sèvres, near Paris, has succeeded best in producing Cashmere shawls equal in stuff and style of work to the Oriental, and at a lower price. They have this advantage over the Indian shawls, that they are woven without seams, in a single piece, and exhibit all the variety and the raised effect of the Eastern colours. Women and children alone are employed in his factory. See India SHawls.

casx. (Tonneau, Fr.; Fass, Ger.) Much ingenuity has beon displayed in cut. ting the curvilinear and bevelled edges of the staves of casks by circular saws. Sir John Robinson proposed many years back that the stave should be bent to its true curve against a curved bed, and that while thus restrained, its edges should be cut by two saws 88 , placed in radii to the circle, the true direction of the joint as shown by the 439 dotted circle fig. 439 , representing the head of the cask. Mr.

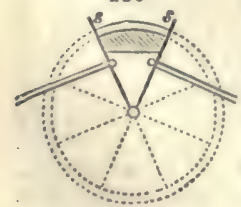
Samuel Brown obtained a patent in November 1825, for certain improvements in the machinery for making casks. His mechanism consists in the first place of a circular saw attached to a bench, with a sliding rest, upon which rest, each piece of wood intended to form a stave of a cask is fixed; and the rest being then slidden forward in a curved direction, by the assistance of an adjustable guide, brings the piece of wood against the edge of the rotatory saw, and causes it to be cut into the curved shape required for the edge of the stave. The second feature is an apparatus with cutters attached to a standard, and traversing round with their carrier upon a centre, by means of which 
the upper and lower edges of the cask are cut round and grooved, called chining, for the purpose of receiving the heads. Thirdly, an apparatus not very dissimilar to the last, by which the straight pieces of wood designed for the heads of the casks are held together, and cut to the circular figure required, and also the bevelled edges prcduced. And fourthly, a machine in which the cask is made to revolve upon an axis, and the cutting tool to traverse for the purpose of shaving the external part of the cask, and bringing it to a smooth surface.

The pieces of wood intended to form the staves of the cask having been cut to their required length and breadth, are placed upon the slide-rest of the first mentioned machine, and confined by cramps; and the guide, which is a flexible bar, having been previously bent to the intended curve of the stave and fixed in that form, the rest is slidden forward upon the bench by the hand of the workman, which, as it advances (moving in a curved direction) brings the piece of wood against the edge of the revolving circular saw, by which it is cut to the curved shapo desired.

The guide is a long bar held by a series of moveable blocks fitted to the bench by screws, and is bent to any desired curve by shifting the screws; the edge of the sliderests which hold the piece of wood about to be cut, runs against the long guide bar, and consequently is conducted in a corresponding curved course. The circular saw receives a rapid rotatory motion by means of a band of rigger from any first mover; and the piece of wood may be shifted laterally, by means of racks and pinions on the slide-rest, by the workmen turning a handle, which is occasionally necessary in order to bring the piece of wood up to, or away from, the saw.

The necessary number of staves being provided, they are then set round within a confined hoop at bottom, and brought into the form of a cask in the usual way, and braced by temporary hoops. The barrel part of the cask being thus prepared, in order to effect the chining, it is placed in a frame upon a platform, which is raised up by a treadle lever, so that the end of the barrel may meet the cutters in a sort of lathe above: the cutters are then made to traverse round within the head of the barrel, and, as they proceed, occasionally to expand, by which means the bevels and grooves are cut on the upper edgo of the barrel, which is called chining. The barrel being now reversed, the same apparatus is brought to act against the other end, which becomes chined in like manner.

The pieces of wood intended to form the heads of the cask are now to be cnt strajght by a circular saw in a machine similar to the first described; but, in the present instance, the slide-rest is to move forward in a straight course. After their straight edges are thus produced, they are to be placed side by side, and confined, when a seribing eutter is made to traverse round, and cut the pieces collectively into the circular form desired for heading the cask.

The cask having now been made up, and headed by hand as usual, is placed between centres, or upon an axle in a machine, and turned round by a rigger or band with a shaving cutter sliding along the bar above it, which cutter, being made to advance and recede as it slides along, shaves the outer part of the cask to a smooth surface.

$\mathrm{Mr}$. Smart cuts the edges of thin staves for small casks on the ordinary saw-bench, by fixing the thin wood by two staples or hooks to a curved block, the lower face of which is bevelled to give the proper chamfer to the edges, fig. 440. One edge having

440

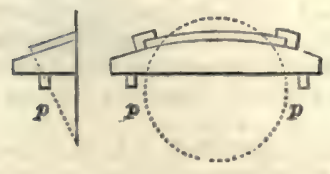

441

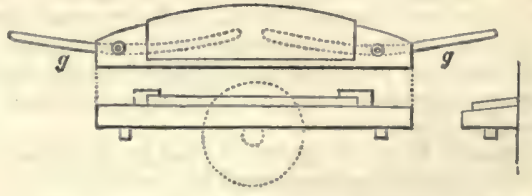

been cut, the stave is released, changed ond for end, and refixed against two pins, which determine the position for cutting the second edge, and make the staves of one common width. The curved and bevelled block is guided by two pins $p p$, which enter a straight groove in the bench parallel with the saws. This mode of bending is from various reasons found inapplicable to large staves, and these are cut, as shown in three views, fig. 441 , whilst attached to a straight bed, the bottom of which. is also bevelled to tilt the stave for chamfering the edge. To give the curve suitable to the edge, the two pins on the under side of the block run in two curved grooves $g g$ in the saw-bench, which cause the staves to sweep past the saw in the are of a very large circle, instead of in a right line, so that the ends are cut narrower than 
the middlo. Mr. Smart observes (Trans. Soo. of Arts, vol. xlvii.) that in staves cut whilst straight, the odges bocome chamfered at the same angle throughout, which although theoretically wrong is sufficiently near for practice; the error is avoidod when the staves are cut whilst bent to their true curvature.

The necessary flexibility which is required for bending the staves of casks is obtained by steaming them in suitable vessels in contact with rigid moulds. By Taylor's patent machinery for making casks, the blocks intended for the staves are cut out of white Canada oak, to the size of thirty inches by five, and smaller. They are well steamed and then sliced into pieces one-half or five-eighths of an inch thick, at the rate of 200 a minute, by a process far more rapid and economical than sawing, the instrument being a revolving iron plate, of 12 or 14 feet diameter, with two radial knives arranged somewhat like the irons of an ordinary plane or spokeshsve.

CAssarzep or CAssmzipz. The concentrated juice of the roots of the bitter cassava, flavoured by aromaties. It is used to flavour soups, and other dishes, and is the basis of the West-Indian dish pepper-pot. In French Guiana, the term cabion is applied to as similar condiment.-Pereira.

cassava. (Cassave, Fr.; Cassava, Ger.) Cassava, or Cassada Meal, are names given to the starch of the root of the Manihot utilissima, prepared, in the following manner, in the West Indies, the tropical regions of America, and upon the African coast. The tree belongs to the natural family of the Euphorbiacece.

The roots are washed, and reduced to a pulp by means of a rasp or grator. The pulp is put into coarse strong canvas bags, and thus submitted to the action of a powerful press, by which it parts with most of its noxious juice (used by the Indians for poisoning the barbs of their arrows). As the active principle of this juice is volatile, it is easily dissipated by baking the squeezed cakes of pulp upon a plate of hot iron. Fifty pounds of the fresh juice, when distilled, afford, at first, 3 ounces of a poisonous water, possessing an intolerable offensive smell; of which 35 drops being administered to a slave convieted of the crime of poisoning, caused his death in the course of 6 minutes, amid homible convulsions.'

The pulp, dried in the manner above described, conoretes into lumps, which become hard and friable as they cool. They are then broken into pieces, and laid out in the sun to dry. In this state they afford a wholesome nutriment, and are habitually used as such by the negroes, as also by many white people. These cakes constitute the only provisions laid in by the natives, in their voyages upon the Amazon. Boiled in water with a little beef or mutton, they form a kind of soup similar to that of rice.

The Cassava cakes sent to Europe are composed almost entirely of starch, along with a few fibres of the ligneous matter. It may be purified by diffusion through warm water, passing the milky mixture through a linen cloth, and evaporating the strained liquid over the fire, with constant agitation. The starch, dissolved by the heat, thickens as the water evaporates, but, on being stirred, it becomes granulated, and must be finally dried in a proper stove. Its specific gravity is 1.530 -that of the other species of starch.

The product obtained by this treatment is known in commerce under the name of tapioca; and, being starch very nearly pure, is often prescribed by physicians as an aliment of easy digestion. A tolerably good imitation of it is made by heating, stirring, and drying potato-starch in a similar way.

The expressed juice of the root of manioc contains in suspension a very fine fecula, which it deposits slowly upon the bottom of the vessels. When freed by decantation from the supernatant liquor, washed several times and dried, it forms a beautiful starch, which creaks on pressure with the fingers. It is called cipipa in French Guiana; it is employed for many delicate articles of cookery, especially pastry, as also for hairpowder, starching linen, \&cc. This is imported into England from Rio Janeiro as Brazilian arrowroot.

Cassava flour, as imported, may be distinguished from arrowroot and other kinds of starch by the appearance of its particles viewed in a microscope. They are spherical, all about $\frac{1}{1000}$ th of an inch in diameter, and associated in groups; those of potatostarch are irregular ellipsoids, varying in size from $\frac{x}{300}$ th to $\frac{1}{3000}$ th of an inch; those of arrowroot have the same shapo nearly, but vary in size from $\frac{1}{500}$ th to $\frac{1}{800}$ th of an inch; those of wheat are separate spheres, $\frac{1}{1000}$ th of an inch.

The empirical formula of Cassava starch is, $\mathrm{C}^{12} \mathrm{H}^{10} \mathrm{O}^{10}\left(\mathbf{C}^{6} \mathbf{m}^{10} \mathbf{O}^{5}\right)$, like the other starches.

Cassava has for some years been imported into France, from Martinique, as la noussache and la cipipa. Seo TAPTOCA.

'Memoir of Dr. Fermin, communicated to the Academy of Berlin, concerning experiments made at Cayenne, upon the juice of the Manioc. 
CAsszxI Trisnow. An oxychloride of lead. It seems to be the same as Patent Yellow and Turner's Yellow. Cassell yellow may be prepared by melting together one part of sal-ammoniac with ten parts of massicot, minium, or white lead.

CASSFIMANR'S GRIEN. A fine green pigment, destituto of arsenic. It is a lasic sulphate of copper, obtained by mixing together boiling solutions of sulphate of copper and an alkaline acetate.

CAssra. (Cinnamomum Cassia.) A bark employed for flavouring. The cinnamon cassia is a native of China, and is cultivatod in Java. It is imported from Singapore, Calcutta, Bombay, and Manilla. This bark is imported principally for the sake of its essential oil, which it yields by distillation. One hundred pounds of good cassia bark will thus yield $12 \mathrm{oz}$. of essential oil of cassia. This oil is principally consumed in scenting what is called Brown Windsor Soap.

CAssia BUDs. (Flores cassice immature.) The cassia buds and bark are both obtrined from the same tree (Reeves). "According to the latest observations which the older Nees has made known, cassia buds are the calyces (Fruchtkelche) of Cinnamomum aromaticum, about $\frac{1}{4}$ th of their normal size. It is also said that they are collectod from Cinnamomum dulce which is found in China.'-Martins, quoted by Pereira.

CAssia FISTUI.A. The pudding pipe tree, or purging cassia. The pods of this tree are imported from Madras and Ceylon, from Barbadoes, and from Carthagena and Savanilla.

CAssrn. The Acacia Farnesiana is grown extensively in the south of France for the sake of its fragrant flower balls : these are termed Cassie, and used in perfumery. The young plants are raised from seed in beds: in the third year they are planted out in fields and require for each tree about twelve square feet of land. When in maturity Acacia Farnesianc will yield flowers to the value of $30 \mathrm{l}$. an acre.

CAssIs, the black currant (Ribes nigra, Linn.), which was formorly celebrated for its medicinal properties, with very little reason.

The only technical use to which it is now applied is in preparing the agreeable liqueur called ratafia, by the following French recipe: Stone and crush 3 pounds of black currants, adding to the magma 1 drachm of cloves, 2 drachms of cinnamon, 4 quarts of spirits of wine, at $98^{\circ}$ Baumé, and $2 \frac{1}{2}$ pounds of sugar. Put the mixture into a bottle which is to be well corked; lot it digest for a fortnight, shaking the bottle once daily during the first 8 days : then strain through a linen cloth, and finally pass through filtering paper.

CAssrrmerTz. Oxide of Tin; Stream Tin. Stream tin is the alluvial débris of tin veins. Cassiterite is one of the objectionable names, of which a very great number have been introduced into the science of Mineralogy. The names which are derived from districts or towns give no indication of the character of a mineral, and they indicate one locality only, for example, REDRUTHITE (Copper glance) is found in many other places beyond Redruth, and CAssiterite, derived from the Cassiterides of the ancients, is used to designate a mineral which is found in all parts of the world. The confusion existing in mineralogical nomenclature is to be lamented. (See Trs ORRL)

CAssrus, Purple povder of. A preparation used in the arts as a colour, chiefly for stained glass and porcelain. It is also employed in medicine by some French physicians, and has been prepared in the following manner:-10 parts of acid chloride of gold are dissolved in 2,000 parts of water. In another vessel, 10 parts of pure tin are dissolved in 10 parts of nitric acid mixed with 20 parts of hydrochloric acid, and this solution is diluted with 1,000 parts of distilled wator. The solution of tin is added by degrees to that of the acid chloride of gold, so long as any precipitate results. This is allowed to subside; it is then washed, filtered, and dried at a very gentle heat. The tin salt above used contains both the protoxide and binoxide in cortain proportions. The doublo compound of chloride of tin with sal-ammoniac, called the pink salt of tin, is the preferable form ; as it is notaltered by the atmosphere, is of definite composition, and when boiled with metallic tin it takes up just so much as will form the protochloride: 100 parts of pink salt require for this purpose 10.7 parts of metallic tin.

Professor Graham, in his 'Elements of Chemistry,' gives the following account of the Purple of Cassius, and of its preparation:- 'When protochloride of tin is added to a dilute solution of gold, a purple powder falls. It is obtained of a finer tint when protochloride of tin is added to a solution of the sesquichloride of iron till the colour of the liquid takes a shade of green, and the liquid in that state added, drop by drop, to a solution of sesquichloride of gold, free from nitric acid, and very dilute. After 24 hours a brown powder is deposited, which is slightly transparent, and purple-rod, by transmitted light: when dried and rubbed to powder, it is of a dull bluo colour. Hoated to redness it loses a littlo water, but no oxygen, and retains its former 
appearance. If washed with ammonia, on the filter, while still moist, it dissolves, and a purple liquid passes, which rivals the hypermanganate of potash in beauty. . . . It may also be formed by fusing together 2 parts of gold, $3 \frac{1}{2}$ parts of tin, and 15 parts of silver, under borax, to prevent the oxidation of the tin, and treating the alloy with nitric acid, to dissolve out the silver; a purple residue is left, containing the tin and gold that were employed.'

'Berzelius proposed the theory that the powder of Cassius may contain the true protoxide of gold combined with sesquioxide of tin, $\mathrm{AuOSn}^{2} \mathrm{O}^{2}$, a kind of combination containing an association of three atoms of metal, which is exemplified in black oxide of iron, spinele, Franklinite, and other minerals. . . . A glanco at its formula shows how readily the powder of Cassius, as thus represented, may pass into gold and binoxide of tin, $\mathrm{AuOSn} \mathrm{O}^{3}-\mathrm{Au}+2 \mathrm{SnO}^{3}$.'-Graham and Watts.

CAsтIIr SOAP, or Spanish Soap, is prepared with olive oil and \& solution of caustic soda. There are two varieties, tho white and the marbled. Tho marbled appearance is produced in the soap, by adding, as soon as it is made and separated from the spent ley, a fresh quantity of ley, and immediately a solution of the protosulphate of iron. A precipitate of oxide of iron is at once formed, and this gives the dark coloured streaks to the soap. By exposure to the air these streaks become red, in consequence of the conversion of the black oxide of iron into the red or sesquioxide. See SoAp.

\section{CAst-Irox. See Iron.}

CAST-IRON SCOURING. Cast-iron surfaces are said to be easily scoured by adding a little of any kind of organic matter, such as glycerine, stearine, naphthaline, or creosote to dilute sulphuric acid; zinc and brass yield to the same method, with great economy of labour, time, and material.

CASTOR. A mineral occurring in Elba, associated with another species called Pollux. Castor is a silicato of alumina and lithia, closoly related to, if not identical with, petalite.

CASTOR. The Beaver. Seo Furs.

CAsto , or CAstorrum. This name is given to a secretion of the Beaver (Castor fiber), contained in pear-shaped cellular organic sacs, placed near the genital organs of both the male and female animals. It is a substance analogous to civet and musk, of a consistenco similar to that of thick honey. It has a bitter acrid taste; a powerful penetrating, foetid, and very volatile smell.

Several chemists, and in particular Bouillon, Lagrange, Laugier, and Hildebrandt have examined castor, and found it to be composed of a resin, a fatty substance, a volatile oil, an extractive matter, benzoic acid, and some salts.

The mode of preparing it is very simple. The sacs are cut off from the castors when they are killed, and are dried to prevent the skin being affected by the weather. In this state the interior substance is solid, of a dark colour, and a faint smell; it softens with heat, and becomes brittle by cold. Its fracture betrays fragmonts of membranes, indicating its organic structure. When chewed it adheres to the tooth somewhat like wax; it has a bitter, slightly acrid and nauseous taste.

The castor bags, as imported, are often joined in pairs by a kind of ligature. Sometimes the substance which constitutes their value is sophisticated; a portion of the castor being extracted, and replaced by lead, clay, gums, or some other foreign matters. This fraud may be easily detected, even when it exists in a small degree, by the absence of the membranous partitions in the interior of the bags, as woll as by the altered smell and taste.

Castor is used to a small extent in medicine and in perfumery manufacture, the latter particularly when ambergris is searce.

In English commerce, two varieties of American castor are made: one called the Hudson's Bay and the other the Canadian-though both are imported by the Hudson's Bay Company.

CASTORINE. A substance existing in castor. It is obtained by treating castor with hot aleohol, and filtering through a Platamour's ebullition funnel. On cooling, the alcohol deposits crystals of a fatty substance. The castorine is retained in the mother-liquor, and is procured by evaporation on the water-bath to a small bulk, and then setting aside to allow crystals to form. Castorine crystallises in needles possessing a slight odour of castoreum,-C.G.W.

CAsToz orI. The expressed oil of the seeds of the Palma Christi or Ricinus communis, a native of the West Indies and South America; but which has been cultivated in France, Italy, and Spain.

In England the castor oil is expressed from the seeds by means of powerful hydraulic presses fixed in rooms artificially heated. It is purified by repose, decantaticin, and filtration, being bleached in pale-coloured Winchester quart bottles, which are exposed to light on the tops of houses. 
CATAXYsIs. A term introduced to denoto the very peculiar phenomenon of one body establishing, by its mere presence, a like condition, in another body, to that which exists in itself. Thus a pieco of meat undergoing the putrefactive fermentation, almost immodiately sets up a similar action in fresh meat, or produces in a saccharine fluid that motion which is known as vinous fermentation. The action of the yeast plant, - a living organisation,-establishes an action throughout a large quantity of an infusion of malt, - fermentation, or that disturbance which leads to the conversion of sugar into alcohol. This catalytic power is ill understood, and wo are content to hido the imperfection of our knowledge under a sounding name.

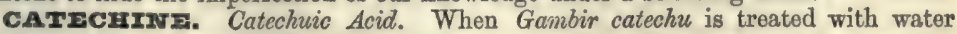
an insoluble residuo is left, which has boen termed by Nees resinous tannin. Its composition is $\mathrm{C}^{15} \mathrm{H}^{6} \mathrm{O}^{6}\left(\mathbf{C}^{13} \mathbf{H}^{12} \mathbf{O}^{6}\right)$.

CAтrcru. A Japanese term, from cate a tree, and chu juice. The term is applied to several astringent extracts made from the wood of several plants which grow in Bombay, Bengal, and other parts of India. The ordinary commercial catechu is prepared by boiling the chips of the interior of the trunk of the Acacia Catechu in water, evaporating the solution to, the consistence of syrup over the fire, and then exposing it in the sun to harden. It occurs in commerce in flat rough cakes, and under two forms. The first, or the Bombay, is of uniform texture, of a dark red colour, and of specific gravity 1.39. The second is more friable and less solid. It has a chocolate colour, and is marked inside with red streaks. Its specific gravity is $1 \cdot 28$.

According to Sir H. Davy, the Bombay variety is richer in tannin than that from Bengal, the former containing 54.5 , and the latter 41.5 in 100 parts.

Areca nuts are also found to yield catechu: and catechu is prepared from them in Ceylon, for which purpose they are cut into pieces, watered in an earthen pot with solution of nitre, and have a little of the bark of a species of Mimosa added to them. The liquor is then boiled with the nuts, and affords an inspissated decoction.

Good catechu is a brittle compact solid, of a dull fracture. It has no smell, but a very astringent taste. Water dissolves the whole of it, except the earthy matter, which is probably added during its preparation. Alcohol dissolves its tannin and extractive. The latter may be oxidised, and thus rendered insoluble in alcohol, by dissclving the catechu in water, exposing it for some time to a boiling heat, and ovaporating to dryness.

The tannin of catechu differs from that of galls, in being soluble in alcohol, and more soluble in water. It precipitates iron of an olive colour, and gelatine in a mass which gradually becomes brown.

It has been long employed in India for tanning skins, where it is said to effect this object in five days. Sole-leather has been completely tanned by it in this country in ten days, the ox-hide having been made into a bag, with the hair outside, and kept filled with the solution of catechu. In India it has also been used to give a brown dye to cotton goods, and of late years it has been extensively introduced into the calico-print works of Europe. The salts of copper with sal-ammoniac cause it to give a bronze colour which is very permanent; the proto-muriate of tin, a brownish yellow; the per-chloride of tin, with the addition of nitrate of copper, a deep bronze hue; acetate of alumina alone, $a$ reddish brown, and, with nitrate of copper, a reddish-olive grey; nitrate of iron, a dark-brown grey. For dyeing a golden coffeo-brown, catechu has entirely superseded madder; one pound of it being equivalent to six pounds of this root.

According to Pereira, the varieties of Catechu commonly met with are :-

1. Gambir Catecho (Catechu pallidum) or Pale Catechu, imported under the name of Gambir, from Singapore and some of the neighbouring islands, where the Uncaria Gambir is cultivated, and an extract prepared from it. The chief uso of this catechu is for tanning. In the trade it is distinguished from the black catechu and cutch by the name of Terra Japonica.

2. Beтr-Nut Catechu. See BrTht-Nut.

3. Curci.-Catechn of the Acacia Catechu described abovo.

Bombay Catechu, named above, and examined by Davy, is obtained from the Areca Catechu, and the Bengal Catechu, from the Acacia (Mimosa) Catechu.

Nubian Catecho, another variety occasionally met with, is obtained from the fruits of gummy Acaciss growing in Egypt and Nubia.

CATGUT. (Corde à boyau, Fr.; Darmsaite, Ger.) The name given to cords made of the twisted intestines of the sheep. The guts, being taken while warm out of the body of the animal, are to be cleared of feculent matter, freed from any adhering fit, and washed in a tub of water. The small ends of all the intestiues are noxt to be tied togother, and laid on tho odge of the tub, while the body of them is left to steep in some water, frequently changed, during two days, in order to loosen the peritoneal 
and mucous membranes. The bundle of intestines is then laid upon a sloping table which overhangs the tub, and their surfaco is scraped with the back of a knifo, to try if the external mombrane will come away freely in breadths of about half their circumforence. This substance is callod by the French manufacturers filandre, and the process filer. If we attempt to remove it by beginning at the large end of the intestine, wo shall not succeed. This filandre is employed as thread to sew intostinos, and to make the cords of raekets and battledores. The flayed guts are put again into fresh water, and after steeping a night, are taken out and scraped cloan next day, on the wooden bench with the rounded back of a knife. This is called curing the gut. The large ends are now cut off, and sold to the pork-butchers. The intestines are again steeped for a night in fresh water, and the following day in an alkaline lixivium mado by adding 4 ounces of potash, and as much pearlash, to a pail of water containing about 3 or 4 imperial gallons. This loy is poured in successive quantities npon the intestines, and poured off again, after 2 or 3 hours, till they are purified. They are now drawn several times through an open brass thimble, and pressed against it with the nail, in order to smooth and equalise thoir surface. They are lastly sorted, according to their sizes, to suit different purposes.

Whipcord is made from the abore intestines, which are sowed together endwise by the filandre, each junction being cut aslant, so as to make it strong and smooth. The cord is put into the frame, and each end is twisted separatoly; for whipcord is seldom made out of two guts twisted together. When twisted, it is to be sulphured once or twice. It may also be dyed black with common ink, pink with red ink, which sulphurous acid changes to pink, and green with a green dye which the colonr-dealors sell for the purpose. The guts take the dyes readily. After being well smoothed, the cord is to be dried, and coiled up for sale.

Hatters' Cord for Bowstrings. - The longest and largest intestines of sheop, after being properly treated with the potash, are to be twisted $4,6,8,10$, or 12 together, according to the intended size of the seams, which is usually made from 15 to 25 feet long. This cord must be free from cords and knots. When half dry, it must be exposed twice to the fumes of burning sulphur; and, after each operation, it is to be well stretched and smoothed: it should be finally dried in a state of tension.

Clockmakers' Cord.-This cord should be extremely thin, and be therefore made from very small intestines, or from intestines slit up in their length by a knifo fitted for the purpose, being a kind of lancet surmounted with a ball of lead or wood. The wet gut is strained over the ball which guides the knife, and the two sections fall down into a vessel placed beneath. Each hand pulls a section. Clockmakers also make uso of stronger cords made of two or more guts twisted together.

Fiddle and Harp Strings. - These require the greatest care and dexterity on the part of the workmen. The treble strings are peculiarly difficult to make, and are made at Naples, probably because the Neapolitan sheep, from their small size and leanness, afford the best raw material.

The first scraping of the guts intended for fiddle-strings must be very carefully porformed; and the alkaline leys being clarified with a little alum, are addod, in a progressively stronger state from day to day, during 4 or 5 days, till tho guts are well bleached and swollen. They must then be passed through the thimble, and again cleansed with the lixivium; after which they are washed, spun, or twisted and sulphured during two hours. They are finally polished by friction, and dried. Sometimes they are sulphured twice or thrice before being dried, and are polished betwoen horsehair cords.

It has been long a subject of complaint, as well as a serious inconvenience to musicians, that catgut strings cannot be made in England of the same goodness and strength as those imported from Italy. These are made of tho peritoneal covering of the intestines of the sheep; and, in this country, they are manufacturod-at Whitechapel, and probably elsewhere,-in considerable quantity; the consumption of thom for harps, as well as for the instruments of the violin family, being very great. Their chief fault is weakness; whence it is difficult to bring the smaller ones, required for the higher notes, to concert-pitch; maintaining at the same time, in their form and construction, that tenuity or smallness of diameter which is required to produce a brilliant and clear tone.

The inconvenience arising from their breaking when in use, and the expense in the case of harps, where so many are required, are such as to render it highly desirable to improre a manufacture which, to many individuals may, however, appear suffieiently contemptible.

It is well known to physiologists, that the membranes of lean aniraals are far more tough than those of animals which are fat or in high condition; and there is no reason to doubt that the superiority of the Italian strings arises from the state of the sheop in that country. In London, where no lean animals aro slaughtered, and where, indeod, 
an extravagant and useless degree of fattening, at least for the purpose of food, is given to sheep in particular, it is easy to comprehend why their membranes can never afford a material of the requisite tenacity. It is less easy to suggest an adequate remedy; but a knowledge of the general principle, should this notice meet the eyes of those interested in the subject, may at least serve the purpose of diminishing the evil and improving the manufacture, by inducing them to choose in the market the offal of such carcases as appear least overburthened with fat. It is probable that such a manufacture might be advantageously established in those parts of the country where the fashion has not, as in London, led to the use of meat so much overfed; and it is equally likely, that in the choice of sheep for this purpose, advantage would arise from using the Welsh, the Highland, or the Southdown breeds, in preference to those which, like the Lincoln, are prone to excessive accumulation of fat. It is equally probable that sheep dying of some of the diseases accompanied by emaciation, would be peculiarly adapted to this purpose.

That these suggestions are not merely speculative is proved by comparing the strength of the membranes in question, or that of the other membranous parts, in the unfattened Highland sheep, with that of those found in the London markets.

CATrARTrN3. A bitter, non-azotised, purgative substance found in senna. Its formula is not known. To prepare it, an alcoholic extract of senna leaves is to be evaporated to dryness, and then treated with water as long as anything is dissolved. The aqueous solution contains the eathartine mixed with several impurities. A considerable amount of the latter may be got rid of by adding a solution of acetate of lead as long as a precipitate is formed, and then filtering through a calico bag.

CArrisrra. A clay-stone so called after Catlin the American traveller. It is carved into tobacco pipes by the North American Indians.

CAT's гхт. A translucent quartz, presenting peculiar internal reflections. This effect is said to be owing to filaments of asbestos. When cut en cabochon, it is estecmed as an ornamental stone. This quartzose cat's-eye comes chiefly from Ceylon. A brown variety from South Africa, consists of fibrous quartz pseudomorphous after the mineral called crocidolite.

The Oriental Cat's-eye is a fibrous variety of Chrysoberyl. See Crmoprark.

CAT'S-2X2 RESTw. Seo DaMmar.

CArsup. A name given by Dr. Kitchiner to Ketchup. See KrTchop.

CATr2mustooo. A name given to an elastic gum or hydro-carbon obtained from some species of plants in India.

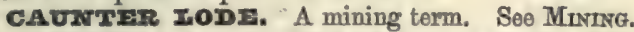

CAUsmic. Any chemical substance corrosive of the skin and flesh; as potash called common caustic, - and nitrate of silver, called lunar caustic, by surgeons.

Caviarz. The salted roo of certain species of fish, especially the sturgeon. This product forms a considerable article of trade, being exported annually from the town of Astrakhan alone, upon the shores of the Caspian Sea, to the amount of several hundred tons. The Italians first introduced it into Eastern Europo from Constantinople, under the name of caviale. Russia has now monopolised this branch of commerce. It is prepared in the following manner:-

The female sturgeon is gutted; the roo is separated from the other parts, and cleaned by passing it through a very fine searce, by rulbing it into a pulp between the hands: this is afterwards thrown into tubs, with the addition of a considerable quantity of salt; the whole is then well stirred, and set aside in a warm apartment. There is another sort of caviare,-the compressed - in which the roe, after having been cured in strong brine, is dried in the sun, then put into a cask, and subjected to strong pressure.

CAwri. The English miner's name for massive sulphate of baryta, or heavy spar,

CAx

CIDAR. (Cédre, Fr. ; Ceder, Ger.) The cedar of Lebanon, or great cedar (Pinus cedrus), is a cone-bearing tree. This tree has been famous since the days of Solomon, who used it in the construction of the Temple. The wood has been obtained from Crete and Africa.

Specimens have also been procured from Morocco, showing the probability that the range of the tree not only extends over the whole group of mountains which is situate between Damascus and Tripoli, and which includes the Libanus and Mounts Amanus and Taurus of antiquity, and various others, - but that its distribution on the mountainous regions of North Africa is extensive.

Indeed, if we are to suppose that the cedar and the cedar wood mentioned by many of the anciont writers referred exclusively to the Lobanon species, wo must believe that its distribution at one poriod extended over countries where no trace of its having existed now remains. Egypt, Crete, and Cyprus are mentioned by Pliny 
and Theophrastus as native habitats of the Cedrus; wo may thus fairly infor that the Cedrus of the ancionts as froquently had reference to the other Coniferce as to the Lobsnon species.

The pencil cedar is the Juniperus Virginiana. It is imported from America in pieces from 6 to 10 inches square. The grain of the wood is remarkably regular and soft, on which account principally it is used for the manufacture of pencils, and from its agreeable scent for the inside of small cabinets; it is also made into matches for the drawing-room.

The general use of the cedar wood dates from the highest antiquity. Pliny makes mention of cedar wood and the uses to which it was applied, and cites, as examples of its durability and imperishable nature, the timber of a temple of Apollo at Utica, in Africa, which, was nenrly 2,000 years old, was found to be perfectly sound,-and the famous statue of Diana in the temple of Saguntum in Spain. Cedria, an oil or resin extractod from a cedar, was also, according to Vitruvius, used to smear over the leaves of the papyrus to prevent the attacks of worms; and Pliny states that the Egyptians applied it with other drugs in the preparation of their mummies; but whether this extract was obtained from the Lebanon cedar or from trees belonging to the genus Cupressus or Juniperus, which also afford odoriferous resins, it is now impossible to ascertain.

In regard to the cedar and cedar wood mentioned in profane history, it is difficult, from what we have already stated, to determine what has reference to the true cedar, and what belongs to other coniferous species; all that we can know for certainty is that a wood called cedar, distinguished for its incorruptible nature, was frequently used for purposes most important in the eyes of the pagan, viz., in the building and decoration of their temples, and for the statues or images of their heroes and gods.

The peculiar balsamic odour of cedar has long been held as a means to preserve articles from the attacks of insects; chips and shavings of the wood have been in this way kept in collections of linen, papers, and objects of preservation. Cabinets have been recommended, or at least the drawers and fittings, to be made of cedar. It may be useful to call attention to some facts when cedar is employed as a means of preservation,

That the odoriferous substance when diffused may affect some forms of organic life, is not disputed, but it is as probable some of the effect may be due to covering the insect with a coating of varnish, alike irritating and interfering with the texture of the surfaces of the body; but the rule cannot be general; if the creatures have a sufficient hardihood they may, and indeed do, attack the wood itself.

The following cases will show that the substances emanating from cedar may produce unexpected interference. Mr. Vulliamy states that George III. had a cabinet in the observatory at Kew with drawers of cedar wood in them; watehes were placed with the intention of keeping them going. In a short time they all came to rest; the experiment, however, repeated had the same result: on examination, the oil used in different parts of the watches was found to be completely changed into a substance like gum. Mr. Farey's observations, also communicated to the Institution of Civil Engineers, still more show the extraordinary atmosphere produced in close cabinets of cedar wood, and of the effects upon delicate objects. The late Mr. Smith of Derby having shown him a small collection of minerals which have boen locked up in closely-fitted drawers of cedar wood; on opening the drawers for the first time after Bome months, the minerals were found to be covered with a gummy matter having the strong odour of cedar, and troublesome to remove; the bright surface of the crystals appeared ts if varnished in an unskilful manner. The cedar had given off a vapour that had condensed on all the minerals, and the same effect might be expected to be produced upon watches, metals, and other substances.

White cedar is a native of North America, China, and Cochin China ; in the United States it occupies large tracts, denominated cedar swamps. The wood is soft, smooth, and of the aromatic smell, and internally of a red colour, permanent in shape, vory durable, and is esteemed as a material for fences. Large quantities of shingles are made of it; it is a favourite material for wooden wares or the nicer kinds of coopers' work.

Colonel Lloyd, speaking of another species of cedar, the Juniperus Bermudiana, says, ' Up to this time there are great quantities of the finest cedar growing in the British island of Bermuda, and the best ships and schooners are always built of it; it is imperishable.'

The cedar known to cabinet makers as the Havannah cedar is the wood of the Cedrela odorata of Linnæus, and belongs to the same natural order as mahogany. All the eigar boxes from Havannah are made of this kind of cedar; it is importod from

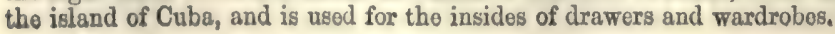


New South Wales produces a codar, Cedrela Toona, somewhat similar to the Havannah, but more red in colour.

A similar kind is found in the East Indies ; the Himalayan cedar, Juniperus excelsa, is harder and less odoriferous than the pencil cedar.

In the "Sketch of the Route and Progress of Lieutenant A. Burnes and Dr. Gerard, by a recent traveller' ('Journal of Asiatic Society of Bengal,' Calcutta, vol. i., 1832), in their adventurous journey to explore the Oxus, it is stated:-

'While on the banks of the Jelum they were much struck by the immense size of the firs floating down the river. The houses in all the towns along its banks are roofed therewith.

'Immense cedar trees were seen rolled down from the hills; it was these which supplied materials for Alexander's fleet. One tree measured 13 feet in girth, which may afford some idea of their applicability.'

There is much confusion in the application of the term Cedar, several trees which are not cedars being so called.

The cedar of Lebanon is usually called Pinus cedrus, but sometimes Cedrus Libanus. The lofty deodara, a native of the Himalayas, with fragrant and almost imperishable wood, and often called the Indian cedar, is sometimes referred to the genus Pinus, and sometimes to that of Cedrus or Larix, with the specific name of deodara.

The wood of several coniferee is, however, called cedar. The wood of Juniperus Virginiana is known as the red or pencil cedar, and that of $J$. Bermudiana is called Bermuda cedar; that of J. Barbadensis forms Barbadoes cedar, while the juniper of the north of Spain and south of France and of the Levant is called J. oxycedrus. The white cedar of North America, a less valuable wood than the red codar, is yielded by Cupressus thyoides, and the cedar wood of Japan, according to Thunberg, is a specios of cypress.

The name cedar is, however, applied to a number of woods in our different colonies which are in no way related to the coniferæ; thus, the cedar of Guiana is the wood of Icica altissima; the white wood or white cedar of Jamaica is Bignonia leucoxylon; and bastard cedar is Guazuma ulmifolia. In Now South Wales, again, the term white cedar is applied to Melia azedarach, and red codar, to the Flindersia australis, as well as to the wood toon tree, or Cedrela Toona.

Cedar wood yields a very fine essential oil by distillation, in the proportion of about $15 \mathrm{oz}$. to every hundred pounds weight of wood. The perfumery-makers find a considerable use for essential oil of cedar. It is now known that all the odoriferous woods, such as sandal wood, myall wood, rose wood, yield their perfumes at the ordinary temporature. If these woods in chips be put into a glass jar for a year the interior will be coated with a heavy dew of perfume.

CrDAR GUM. A gum used for many medicinal purposes, obtained from the branches and cones of the Widdingtonia jumiperoides.

CrDRA (Cédrat, Fr.) is the fruit of a species of orange, citron, or lemon, a tree which bears the same name. Its peel is very thick, and covered with an epidermis which encloses a very fragrant and highly-prized essential oil. The preserves flavoured with it are very agreeable. The citrons are cut into quarters for the dry comfits, but are put whole into the liquid ones. The liquorist-perfumer makes with the peel of the cedra an excellent liqueur; for which purpose, ho plucks them before they are quite ripe; grates down the peel into a little brandy, or cuts them into slices, and infuses these in the spirits. This infusion is distilled for making perfume; but the fiavour is better when the infusion itself is used. See PrRFumrer.

CrDiRow. Simaba Cedron. A tree which grows in the hottest parts of New Granada. It yiolds to alcohol a crystallisable substance - cedrin. It has an intensely and persistently bitter tasto.

Cerestrve or CaIssrrms. (Célestine, Fr.; Cölestin, Ger.) Native sulphate of strontia, $\mathrm{SrO}^{\mathrm{SO}} \mathrm{S}^{3}$ (SrSO ${ }^{4}$ ). This mineral occurs in prismatic or tabular erystals belong. ing to the rhombic system, and often resembling those of sulphate of baryta. In other physical characters, also, the two species are likely to be confounded. Density, if carefully determined, affords a fair means of discrimination, though the difference is not very striking; the specific weight of celestine never rises above 4, whilst that of barytes is about 4.5. Blowpipe reactions offer better-marked means of distinction; celestine imparting to the flame a crimson-red colour characteristic of all strontia-compounds, whilst barytes colours the flame yellowish-green.

The name Celestine (from colestis, 'sky-blue') has reference to the pale blue tint which the mineral frequently presents; in some cases this has been referred to the presence of a trace of phosphate of iron. It should be remembered, however, that this colour is neither a necessary characteristic nor a sufficient means of diagnosis; many of the finest specimens of celestine being colourless, whilst a blue colour, equally pronounced with that of the most typical celestine, is occasionally presented by erystals of barytes.

Vox. I. 
Calestine occurs in fine crystals, associated with sulphur and gypsum, at Girgenti in Sicily; and is also found under like circumstances at Conil in Spain. With rocksult and gypsum, it is found at Bex in Switzorland, and at Ischl in the Salakammergut. It occurs abundantly, also with gypsum, in the Now Red marls in the neighbourliood of Bristol ; it is found in nummulitic limestone (Eocene) at Mokattam, near Cairo ; and in Tronton limestone (Lower Silurian), at Strontian Island and elsewhere on Lake Huron. It is recorded from the eruptive rocks of Calton Hill, Edinburgh; and from the neighbourhood of Tantellan in the East Lothians. It is found in chalkflints at Meudon near Paris; and in the gypsum-quarries of Montmartre. Fine fibrous masses of blue celestine come from the neighbourhood of Jena.

Celestine is decomposed by ignition with charcoal into sulphide of strontium, which is converted into the nitrate by the action of nitric acid. The nitrate of strontia is employed for the production of the red light in theatrical fireworks.

CrIIUIAR BrAm. An application of wrought iron, in which wrought iron plates are rivetted with angle irons in the form of longitudinal cells with occasional cross struts. Fairbairn on Cast and Wrought Iron.

CrIrUzos 'Dictionary of Chemistry.'

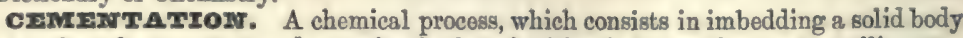
in a pulverulent matter, and exposing both to ignition in an earthen or metallic case. In this way, iron is cemonted with charcoal to form steel, and bottle glass with gypsumpowder, or with sand, to form Réaumur's porcelain.

CIMINT COPPER. The precipitate of metallic copper, formed by the action of iron on a solution of a salt of copper, is called Cement Copper. In like manner, the silver precipitated from a solution of a silver salt by metallic copper is known as Cement Silver.

Carrasrs. (Ciments, Fr.; Cämente, Kitte, Ger.) Substances which are capable of assuming the liquid form and of being applied between the surfaces of bodies so as to unite them firmly when solidifying. They are of very varied character.

Gum, glue, and paste are cements, the uses of which are well known.

Diamond cement is a preparation of isinglass and gum ammoniacum dissolved in alcohol (see Ammontacum GUM): it is employed to mend glass and china.

Sir John Robinson's coment he thus describes:-

- If it bo wished to dissolve good isinglass in spirits of wine, it should first bo allowed to soak for some time in cold water, when swelled it is put into the spirit, and the bottle containing it being set in a pan of cold water may be brought to the boiling point, when the isinglass will melt into a uniform jelly, without lumps or strings, which it is apt to have if not swelled in cold water previously to being put into spirits. A small addition of any essential oil diminishes its tendency to become mouldy.

'If gelatine, which has been swelled in cold water, be immersed in linseed oil and heated it dissolves, and forms a glue of remarkable tenacity, which, when once dry, perfectly resists damp, and two pieces of wood joined by it will separate anywhere else rather than at the joint. Ordinary glue may be thus dissolved, and sometimes a small quantity of red lead in powder is added.'

Shellac dissolvod in alcohol, or in a solution of borax, or still botter in naphtha, forms a good cement. White-of-egg alone, or mixed with finely sifted quicklimo, will answer for uniting objects which are not exposed to moisture. The latter combiuation is very strong, and is much employed for joining pieces of spar and marble ornaments. A similar composition is used by coppersmiths to secure the edges and rivots of boilers; only bullocks' blood is the albuminous matter used instead of white-of-egg. Another cement in which an analogous substance, the curd or caseum of milk is employed, is made by boiling slices of skim-milk eheeses into a gluoy consistence in a great quantity of wator, and then incorporating the mixture with quicklime on a slab with a muller, or in a marble mortar. When this compound is applied warm to broken edges of stoneware, it unites them very firmly after it is cold.

A cement which gradually indurates to a stony consistence may be made by mixing 20 parts of clean river sand, 2 of litharge, and 1 of quicklime, into a thin putty with linseed oil. When this cement is applied to mend broken pieces of stone, as steps of stails, it acquires after some time a stony hardness. A similar composition has been applied to coat over brick walls, under the name of 'Mastic.' Portland oolite powder with a little litharge and oil makes good mastic.

The iron-rust coment is mado of from 50 to 100 parts of iron borings, pounded and sifted, mixed with one part of sal-ammoniac, and whon it is to be appliod moistoned with as much water as will give it a pasty consistency. Formerly flowers of sulphur were used, and much moro sal-ammoniac, in making this cement, but with 
decided disadvantage, as the union is effected by the oxidisement, consequent expansion and solidification of the iron powder, and any heterogeneous matter obstructs the effect. The best proportion of sal-ammoniac is, one per cent. of the iron borings. Another composition of the same kind is made by mixing 4 parts of fine borings or filings of iron, 2 parts of potters' clay, and 1 part of pounded potsherds, and making them into a paste with salt and water. When this cement is allowed to concrete slowly on iron joints it becomes very hard.

For making architectural ornaments in relief, a moulding composition is formed of chalk, glue, and paper paste. Even statues have been made with it, the paper aiding the cohesion of the mass. Some French statuettes are so made.

Mastics of a resinous or bituminous nature which must be softened or fused by heat are the following:-

Mr. S. Varley's consists of 16 parts of whiting sifted and thoroughly dried by a red heat, adding when cold a melted mixture of 16 parts of black resin and 1 of bees'wax, and stirring well during the cooling.

Mr. Singer's electrical and chemical apparatus cement consists of $5 \mathrm{lbs}$. of resin, 1 of bees'-wax, 1 of red ochre, and 2 tablespoonfuls of Paris-plaster, all melted together. The ochre and the plaster-of-Paris should be calcined beforehand, and added to the other ingredients in their melted state. The thinner the stratum of cement that is interposed, the stronger, generally speaking, is the junction.

Boiled linseed oil and red lead mixed together into a putty are often used by coppersmiths and engineers to secure joints. The washers of leather or eloth are smeared with this mixture in a pasty state.

The resin mastic alone is sometimes used by jewellers to cement by heat cameos of white enamel or coloured glass to a real stone, as a ground to produce the appearance of an onyx. Mastic is likewise used to cement false backs or doublets to stones to alter their hue,

Melted brimstone, either alone or mixed with resin and brick-dust, forms a tolerably good and very cheap coment.

Plumbers' cement consists of black resin 1 part, and brick-dust 2 parts, well incorporated by a melting heat.

The cement for coating the fronts of buildings consists of linseed oil, rendered dry by boiling with litharge, and mixed with porcelain clay in fine powder, to give it the consistence of stiff mortar. Pipe-clay would answer equally well if well dried, and any eolour might be given with ground bricks or pottery. A little oil of turpentine to thin this cement aids its cohesion upon stone, brick, or wood. It has been applied to sheets of wire cloth, and in this state laid upon terraces, in order to make them water-tight; but it is little less expensive than lead.

The bituminous or black cement for bottle corks consists of pitch hardened by the addition of resin and brick-dust.

In certain localities where a limestone impregnated with bitumen occurs, it is dried, ground, sifted, and then mixed with about its own weight of melted pitch, either mineral, vegetable, or that of coal-tar. When this mixture is getting semifluid, itmay be moulded into large slabs or tiles in wooden frames lined with sheet iron, previously smeared over with common lime-mortar, in order to prevent adhesion to the moulds, which, being in moveable pieces, are easily dismounted so as to turn out the cake of artificial bituminous stone. This coment is manufactured upon a great scale in many places, and used for making Italian terraces, covering the floors of balconies, flat roofs, water reservoirs, water conduits, \&c. When laid down, the joints must be well run together with hot irons. The floor of the terrace should be previously covered with a layer of Paris-plaster, or common mortar, nearly an inch thick, with a regular slope of one inch to the yard. Such bituminous cement weighs 144 pounds the cubic foot; or a foot of square surface, one inch thick, weighs 12 pounds. Sometimes a second layer of these slabs or tiles is applied over the first, with the precaution of making the seams or joints of the upper correspond with the middle of the under ones. Occasionally a bottom bed, of coarse cloth or grey paper, is applied. The larger the slabs are made, as far as they can be conveniently transported and laid down, so much the bettor. For hydraulic cements, seo Hydraulic Cements and Mortar.

An excellent cement for resisting moisture is made by incorporating thoroughly eight parts of melted glue, of the consistence used by carpenters, with four parts of linseed oil, boiled into varnish with litharge. This cement hardens in about fortyeight hours, and renders the joints of wooden cisterns and casks air- and water-tight. A compound of glue with one-fourth its weight of Venice turpentine, made as above, serves to cement glass, metal, and wood to one another. The gluten of wheat, well prepared, is also a good cement. White-of-egg with flour and water well mixed, and smeared over linen cloth, forms a ready luto for steam joints in small apparatus.

White lead ground upon a slab with linseed-oil varnish, and kept out of contact of $3 \mathrm{c} \cdot 2$ 
air, affords a coment capable of repairing fractured bodies of all kinds. It roquires a few wooks to harden. When stone and iron are to be comented together, a compound of equal parts of sulphur and pitch answers very well.

Lapidaries' cement is made of resin, tempered with bees'-wax and a little tallow, and hardened with red ochre or Spanish brown and whiting.

Opticians' cement, for fixing glasses for grinding, is made by mixing sifted wood ashes with melted pitch, the essential oil of which is absorbed by the wood ashes, and the adhesiveness of the pitch is therefore reduced. The proportions are somewhat dependent on the temperature of the weather and the qualities of the pitch; but generally about $4 \mathrm{lbs}$. of wood ashes to $14 \mathrm{lbs}$. of pitch are employed, and the coment, if too hard and brittle, is softoned with hogs' lard and tallow.

Japanese cement is said to be prepared by mixing rice flour intimately with cold water, and then boiling the mixture; it is white, and dries nearly transparent. See MORTAR.

For Portland cement, Roman cement, Scott's cement, \&c. See Hrdratlic Crinevts.

CanTraury. Centaurea calcitrapa. The common star-thistle; an herb, the root and leaves of which are bitter. The term centaury is also applied to Erythrea Centaurium (the common centaury), one of the Gentian family. It is said to be sometimes used instead of hops.

crantraze. See Matric Srstem.

CDNTICRADE SCATT. See THRRMOMETHR.

centrigramirz. Seo Metric System.

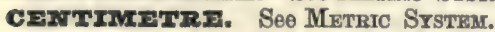

CENTraran. The Zollverein Centner contains 110.231 English 1bs, avoirdupois.

CENTrIFUGAI MACHINz. A machine in which advantage is taken of the foree produced by centrifugal motion. Several of the old mills were of this description. This term has been not very correctly applied to Barker's Mill as it is often called; but which was fully described by Desaguiliers (vol. ii. p. 468), in which water is made to act on a machine by its weight. Barker's mill consists of an npright pipe which communicates with two horizontal branches having holes near their ends opening in opposite directions. Desaguiliers affirms the pressure to be the weight of a column, which would produce a velocity of efflux equal to the difference of the velocity of the fluid and of the machine; and hence he deduces that its performance will be the greatest possible when its retrograde velocity is one-third of the velocity acquired by falling from the surface, in which case it will raise $\frac{8}{27}$ ths of the water expended to the same height, which is double of the performance of a mill acted on by the impulse of water.

But this is a very imperfect account of the operation. When the machine (constructed exactly as we have described) moves round, the water which issues descends in the vertical trunk, and then, moving along the horizontal arms, partakes of this circular motion. This excites a centrifugal force, which is exerted against the ends of the arms by the intervention of the fluid. The whole fluid is subjected to this pressure (increasing for every section across the arm in the proportion of its distance from the axis), and every particle is pressed with the accumulated centrifugal forces of all the sections that are nearer to the axis. Every section, therefore, sustains an actual pressure proportional to the square of its distance from the axis. This increases the velocity of effux, and this increases the velocity of revolution, and this mutual cooperation would seem to terminate in an infinite velocity of both motions. But, on the other hand, this circular motion must be given anew to every particle of water as it enters the horizontal arm. This can be done only by the motion already in the arm, and at its expense. Thus, there must be a velocity which cannot be overpassed even by an unloaded machine. But it is also plain, that by making the horizontal arm very capacious, the motion of the water from the axis to the jet may be made very slow, and much of this diminution of circular motion be prevented. 'System of Mechanical Philosophy, by John Robinson,' pp. 616, 617. Centrifugal pumps, correctly speaking, are such as have water admitted at the axis of a hollow wheel, traversed by vanes, which being made to revolve rapidly expels the water at the circumference. The pipe by which the water reaches the axis of the wheel or the reservoir which feeds it, becomes under these circumstances a suction-pipe, and if the reservoir into which the water is received from the periphery of the wheel be closed, and a pipe becarried from it upwards, the latter becomes a force pipe. Appold's Centrifugal Pump, and Gwynn's, are examples of this kind of machine in practico; they aro largely employed in raising large bodies of water to a small height. Centrifugal machines were used in America in 1830 ; Mr. M'Carty erected one in the navy yard, New York, in 1830. M. Ch. Combes describes a centrifugal machine in a papor entitled 'Sur les Roues de Réaction,' (Comptes Rendus, vol. vii. 2me. Semestre, 1838, p. 306). In this paper the theory of the centrifugal ventilator is discussed, and its obvious relations to the theory of the 
centrifugal pump pointed out. In August 1838, M. Combes took out a brevet d'invention, entitled 'Pour un machine universelle, à force centrale, propre à déplacer les liquides ot los fluides aëriformes' (Recherches théoriques et expérinentales sur les Rowes à Réaction ou à Tuyaux, par M. Ch. Combes, and 'Jurors' Report of Great Exhibition,'1851). Many ventilating machines for mines are constructed on this principle. (See Ventmatne Mines; also TUrBInk.) Whirling machines, which are used for drying cloth, sugar, and other things, are entirely dependent on the action of centrifugal force. These machines are called hydro-extractors.

Crrasin. The name given by Dr. John to those gums which swell but do not dissolvo in water; such as gam tragacanth. It is synonymous with Bassorine.

CrrAxr, from cera, wax. An unguent, of rather a stiff consistence, made of oil, or lard, and wax, thickened occasionally with pulverulent matters.

Crrisarm. (Céréaline, Fr.) A nitrogenous substance found by M. Mègo Mouries in bran. See Briad.

CrRpaIs. Grasses cultivated for sake of their grain, used as food-stuffs. They comprise wheat, barley, rye, oats, maize, and rice. The name is derived from the goddess Ceres.

CrRmSINz. A by-product obtained in purifying ozokerite for the manufacture of paraffin. By treating tho crude product with sulphuric and sulphurous acids, and then with alkalis, pure cerasine is obtained in the form of a white semi-transparent opaline substance, insoluble in water, but soluble in alcohol, fusing at about $143^{\circ} \mathrm{F}$., and having a specific gravity of 0.88 . In Vienna, ceresine has been used as a substitute for bees'-wax in certain pharmaceutical preparations. This article is sold in round, thin plates, a few inches in diameter. It is faultlessly white, scentless, harder than wax, and translucent at the edges. The fracture resembles somewhat that of wax, but it cannot be kneaded, either in warm water or between the fingers. It is not attacked by acids, either hot or cold, and is not in the slightest degree saponified by caustic alkalis. It is volatile at high temperatures and distils over unchanged. It is closely analogous to paraffin and stearine, but is rendered more waxy in appearance by being cooled slowly. See CANDIirs-OzoknRTte.

CrRIrs. A substance which forms from 70 to 80 per cent. of bees'-wax. It may be obtained by digesting wax for some time in spirit of wine at a boiling temperature. The myricine separates, while the cerine remains dissolved, and may be obtained from the decanted liquor by evaporation. Cerine is white, analogous to wax, fusible at $134^{\circ} \mathrm{F}$., hardly acted upon by hot nitric acid, but is readily carbonised by hot sul. phuric acid. When treated with caustic-alkali loy, it is converted into margaric acid and ceraine.

CaRrUam. A peculiar metal discovered in connection with lanthanum and didymium, in cerite, allanite, orthite, and a few other minerals of rare occurrence, found in Sweden. Cerium has also been detected by Prof. Church in a Cornish mineral, which he found to consist of a hydrous phosphate of cerium; this mineral has been named by Mr. Greville C. Williams, Churchite.

Cerium, extracted from its chloride by potassium, appears as a dark red or chocolate powder, which assumes a metallic lustre by friction. It does not conduct electricity well, like other motals; it is infusible; its specific gravity is unknown. It has been applied to no use in the arts. See Watts's 'Dictionary of Chemistry.'

CrRosing. A name applied to the wax of the sugar-cane.

Crroxrc ACID. An acid obtained by the action of boiling alcohol on bees" wax, or by the destructive distillation of Chinese wax.

crevse. A name of white lead. See Whutr Lias.

Creusirz or Crrussixz. Native carbonate of lead. See Lrad.

Cryion CATzcerv. A variety of the Bombay Catechu.

Crzion moss. (Plocaria candida.) See Ator.

CEAzAsItra. A hydrated silicato of alumina and lime. This zoolite is found in the fissures of some trap rocks.

Crararwores is a peculiar style of textile fabric, to which bosiery and tambouring belong. See Hosnar.

Craricara. A coarse sugar obtained from the cocoa-nut and other plants.

CrazcANTEITz. A namo occasionally applied to the nativo sulphato of copper. CrATCANTrum. The ancient name of the native sulphate of iron.

CratcrDoxy. A hard mineral of the quartz family, often cut into seals. Under it may be grouped common chalcedony, holiotropo, chrysoprase, plasma, agate, onyx, cat's-eye, sardonyx, carnelian, and sard.

CRAICrDONXX. The name of those agates in which opaque white chalcodony alternates with the translucent grey variety.

CEAxCrImUxтx. A green stone prized by the ancient Mexicans for ornamental purposes, It has been variously referred to jade, turquoise, emerald, \&ce. 
orAxcorrxy. A name applied to copper-uranite or torberite, a hydratod phosphato of uranium and copper.

C\&Axcor and copper-mica, the crystals being easily soparated into laminæ, like mica.

Craxcopxrixz. A mineralogical name for copper-pyrites. Seo Coppkr.

Craxcosiara. A synonym of copper-glance. See Copper.

Crajcostramrz. A sulphide of antimony and coppor, from Wolfsborg in the Harts.

Craxcorrercrrys. A name applied to the hair-like forms of red oxide of copper, known commonly as plash copper-ore.

CHAIx. (Craie, Fr.; Kreide, Ger.) An earthy carbonate of lime, white, opaque, soft, dull, or without any appearanco of polish in its fracture. Its specific gravity varies from $2 \cdot 4$ to $2 \cdot 6$.

The following analysis, by Mr. W. J. Ward, shows the composition of a sample of chalk from near Gravesend (after having been dried at $212^{\circ} \mathrm{F}$.): carbonate of lime, 98.52 ; carbonate of magnesia, 0.29 ; sulphato of $\lim \theta, 0.14$; peroxide of manganese, 0.04 ; phosphoric acid and organic matter, traces; ignited insoluble residue, chiefly silica, $0 \cdot 65$.

When purified, chalk is called whiting and Spanish white, in England; SchlemmKreide, in Germany; blanc de Troyes, and blanc de Meudon, in France. Pure chalk should dissolve readily in diluto muriatic acid, and the solution should afford no precipitate with water of ammonia. Chalk is burnt into lime in great quantities, in which state it is used as a manure, and for making mortar and whitewash. Some of the lower beds, which are argillacoous, afford a good hydraulic cement, equal in every respect to Roman cement.

Of late years, it has become the custom to manure land with unburnt chalk spread on the surface in the proportion of about 40 loads (tons) to an acre. The effect produced by chalk applied in its crude state is similar to that resulting from the application of quicklime, but more lasting, on some lands not requiring to be renewed for several years: it also has the advantage of rendering the soil mechanically lighter, from the larger quantity in which it is used.

In certain districts, chalk is sometimes used as a building matorial.

CEAxK, BIACx. An indurated clay, containing much carbon. The finer kinds are used for drawing on paper.

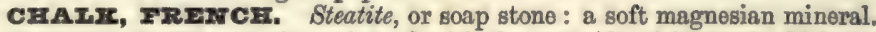

CrAx. RED. A clay coloured with the peroxide of iron, of which it contains about 17 per cent. Also a ferruginous carbonate of lime.

Craxrrs. About the year 1832 this article was introduced, and was cortainly an elegant silk and worsted fabric. It was made on a similar principle to the Norwich crape, only thinner and softer, composed of much finer materials; and instead of a glossy surface, as in Norwich crapes, the object was to produce it without gloss, and very pliable and clothy. The best quality of challis, when finished with designs and figures (either produced in the loom or printed), commanded the attention of the higher circles, and became a farourite article of apparel at their fashionable resorts and parties for several years. The worsted yarn for the weft of this article was spun at Bradford, from numbers 52's to 64's. The making of the challis fabric soon afterwards commenced in the North, but it has given way to the ever-varying ceprices of fashion.

CHATYBFAT2 is a name given in medicine to preparations of iron.

CHAIXBIrz. A synonym of carbonate of iron, or spathose iron-ore. See Irow.

CHAmrzisow, mrxarax. As this compound-so long known in chemistry as a mere curiosity, on account of the surprising changes of colour which it spontaneously assumed - has of late been largely employed for whitening tallow, palm-oil, and decolouring other organic matters, it merits description in this Dictionary. It exists in two states: one of which is called by chemists the manganate of potash, and the otber the permanganate; denoting that the first is a compound of manganic acid with potash, and that the second is a compound of permanganic acid with the same base. They are both prepared in nearly the same way: the former by calcining together, at a red-heat in a covered crucible, a mixture of one part of the black peroxide of manganese with three parts of the hydrate of potash (the fused potash of the apothecary). The mass is of a green colour when cold. It is to bo dissolved in cold water, and the solution allowed to settle, and become clear, but by no means filterod, for fear of the decomposition to which it is very prone. When the decanted liquid is evaporated under the exhausted receiver of an air-pump, over a surface of sulphuric acid, it affords crystals of a beautiful green colour, which should bo laid on a clean porous brick to drain and dry. They may be preserved in dry air, but should be 
kept in a well-corked bottle. They are decomposed by water, but dissolve in weak water of potash. On diluting this, decomposition of the salt ensues, with all the chameleon changes of tint; rod, blue, and violet. Sometimes a green solution of this salt becomes red on being heated, and preserves this colour evon whon cold, but resumes its green hue the moment it is shaken: it might, therefore, furnish the votaries of St. Januarius with an admirable means of mystifying the worshippers at his shrine. The original calcined mass, in being dissolved, always deposits a considerable quantity of a brown powder, which is a compound of the acid and peroxide of manganese combined with water. Much of the potash remains unchanged, which may be recovered.

The permanganate of potash is made by fusing with a strong heat a mixture of equal parts of peroxide of manganese and hydrate of potash, or one part of peroxide and two parts of nitre. The mass is to be dissolved in water, and if the solution bo green, it should be reddened by the cautious addition of a few drops of nitric acid. The elarified liquor is to be evaporated to the point of erystallisation. Even the smallest crystals of this salt have such an intense red colour, that they appear black with a green metallic reflection. In the air they gradually assume a steel-grey hue without undergoing any essential change of nature. A very little of the salt reddens a large body of water. The least portion of any organic matter added to the solution of this salt reduces the permanganic acid to the state of peroxide, which precipitates combined with water; the liquor becoming green or colourless, according to circumstances.

A more permanent permanganic salt may be mado as follows :- Melt chlorate of potash over a spirit lamp, and thwow into it a few pieces of hydrate of potash, which immediatoly dissolve and form a limpid liquid. When peroxide of manganese in fine powder is gradually introduced into that melted mixture, it immediately dissolves, with the production of a rich green colour. After adding the manganese in excess, the whole is to be exposed to a gentle red heat, in order to decompose the residuary chlorate of potash. It is now a mixture of manganate of potash, chloride of potassium, and peroxide of manganese. It forms with water a deep green coloured solution, which, when boiled, assumes a fine red colour, in consequence of its becoming a permanganate, and it ought to be decanted off the sediment while hot. By cooling, the permanganate of potash separates in crystals possessed of great lustre; but towards the end colourless erystals of chloride of potassium are deposited. See MLanganates.

CRAmorsirs. A hydrated silicate of alumina and protoxide of iron, occurring as a compact or oolitic iron-ore, at Chamoison, near Saint Maurice, in the Valais.

CEAmomin Fxowris. The Anthemis nobilis of Linnæus. The chamomile grows very abundantly in Cornwall, and some other parts of England. It is cultivated at Mitcham and in Derbyshire, for the London market. The chamomile is used medicinally, and is employed by some brewers to substitute hops in bitter beer. It would be well if no more objectionable bitter was employed.

The essential oil of chamomile is remarkable for its blue colour. In a paper read before the Chemical Society by Dr. Piesse, 'On the colouring matter of volatilo oils,' this blue body was shown in an isolated state, and was named by the author azulene.

\section{ceampagra. See Wriss.}

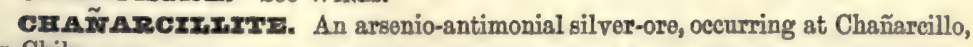
in Chile.

Crapapotz. A local name for Mexican asphalt.

Crarbon SUxFuriquz. This name is applied to the product obtained by treating pulverised madder, either at ordinary or at higher temperatures, with a considerable quantity of rather strong sulphurie acid. MM. Persoz and Gauthier de Claubry had shown that the true colouring principles of madder are soluble without alteration in strong sulphuric acid, and it was on this observation, turned to practical account by M. Robriquet, that the preparation of his charbon sulfurique was based. After the action of the acid has been continued for several hours, the mass is diluted with water, the liquid filtered, and the residue well washed and dried. Charbon sul. furique dyes very strongly, and produces fine colours.

CrancoAI. The fixed residuum of organic substances when they are exposed to ignition out of contact with air.

Wood Charcosi.-As wood charcoal may be regarded as, especially, the representative of the practically useful charred substances, a description of it naturally precedes that of the other varieties. The earliest plan of coaling wood, as the manufacture of charcoal was termed, and is still called, is carried on as follows :A piece of ground is levelled at some convenient spot in the forest, which is termed the 'hearth' or 'earth.' In the centre of this a thick pole or bundle of brushwood is placed, around which the wood is arranged, some of the pieces being laid horizontally and others set up at an inclination; or the wood may be placed 
altogether at any stoop angle, sloping outwards from the contre to form a flattoned cone, which when complete is usually called a heap; tho object, whichever way the wood is placed, is to obtain a freo cireulation of air undor tho hoap, to communicato with the chimney in the centre, which is formed by then withdrawing the central pole or bundle of brushwood. The large wood should if convenient bo at the bottom of the heap, and the outside packed as close as possible; the heap is then corered with small brushwood, and afterwards with turf, or the material most impervious to air which can be conveniently obtained. A fire is lighted in the centre chimney, and by leaving openings in the outside-covering at the bottom of the heap, the fire soon extends, and can be guided to any part by making temporary oponings to admit the air. When the heap is sufficiently fired all tho openings are closed, and lastly the chimney itself. The fire will always extond most rapidly-on tho side facing or towards the wind; and great care must be taken to watch and check this, by keeping the covering on that side in good order. The charcoal burner must always be careful to spread the fire as evenly as possible through the heap, and after it is coaled to stop it down carefully; he can always accelerate the process in any part of the heap if well built, by opening tho outside to admit air freely : but if he finds this does not act, from any fault in setting the wood, he had better open a hole with a bar at the place required, and light a fire in the hole; this will soon communicate with the main fire in the heap. As soon as the smoke and white flame cease to escape at the vents, the whole heap must be closed from the air as carefully as possible, until the charcoal is quite cooled and is ready to draw. The fire must never burn too fast; the slower the process, if the fire is steady and regular, the botter the yield of charcoal. Hard close-grained woods take a longer time to coal than soft open-grained woods, and should be placed in the heap accordingly. These technical instructions, handed down in the forests for ages as secrets from father to son amongst the 'coalliers' in every country in Europe, are the results of long practical experience, and strictly accord with the true principles on which the process is based.

To carbonise wood under a moveable covering, the plan of Meiler, or heaps, is employed very much in Germany. The wood is arranged either in horizontal layers, or in nearly vertical ones, with a slight slope, so as to form conical rounded heaps of difforent sizes. The former are called 'lying Meiler,' fig. 442; the latter 'standing Meiler' figs. 443 and 444. Both are distributed in much the same way.

442

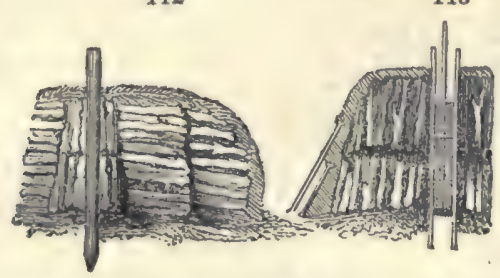

444

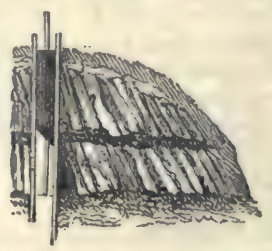

In districts where the wood can be transported into one place by means of rivers, or mountain slides, a dry flat space must be pitched upon, screened from storms and floods, which may be walled round, having a slight declivity mado in the ground, towards the centre. (See fig. 445.) Into this space the tarry acid will partially fall, and may be conducted outwards, through a covered gutter beneath, into a covered tank. The mouth of the tank must be shut, during the coking, with an iron or stone slab, luted with clay. A squaro iron plate is placed over the inner orifice or the gutter to prevent it being choked with cosl ashes. Fig. 445 represents a walled Meiler station: $a$, the station; $b$, the gutter; $c$, the tank, which is covered with the slab $d$; $e$, a slab which serves to keep the gutter clear of coals. The cover of the heaps is formed of earth, sand, ashes, or such other matter as may be most readily found in the woods. They should be kindled in the centre. From 6 days to 4 weeks may be required fot charring a heap, according to its size, hard wood requiring most time; and tho slowe the process, the better and greater is the product, generally speaking.

Charring of wood in mounds (Haufe or liegende Werke), figs. 446 and 447, differs from that in the Meiler, because the wood in the Haufe is successively charred, and the 
charcoal is raked out by little and little. The product is said to be greater in this way, and also better. Uncleft billets, 6 or 8 feet long, being laid over each other, are covered with ashes, and then carbonised. The station is sometimes horizontal, and sometimes made to slope. The length may be 24 feet, the breadth 8 feet; and the wood is laid crosswise. Piles are set perpendicularly to support a roof made of boughs and leaves covered with ashes. Pipes are occasionally laid within the upper part of the mounds, which serve to catch and carry off some of the liquid.

Fig. 448 is a vertical section, and fig. 449 a half bird's-eye view, and half cross-section at the height of the pit bottom, of Chabeaussiere's kiln for making wood charcosl. $a$ is the oven; $b$, vertical air-pipes; $c c$, horizontal flues for admitting air to the kiln; $d d$, small pits which communicato by short horizontal pipes $\theta \in$, with the vertical ones; $f$, the sole of the kiln, a circle of briekwork, upon which the cover or hood, $h$, reposes; $i$, a pipe which leads to the eistern $k ; l$, the pipe destined for earrying off the gaseons matter; $m m$, holes in the iron cover or lid.

The distribution of the wood is like that in the horizontal Meiler, or heaps; it is kindled in the central vertieal canal with burning fuel, and the lid is covered with a fow inches of earth. At the beginning of the operation all the draught flues are left open, but they are progressively closed,

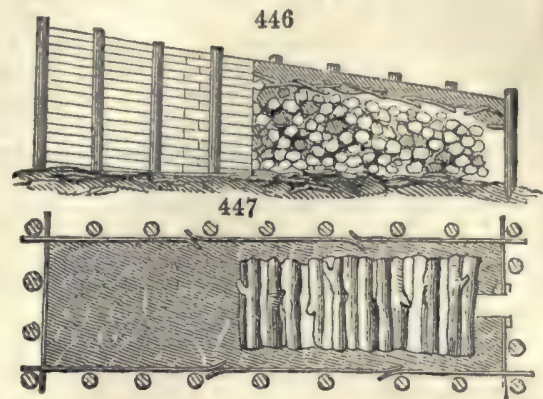
as occasion requires. In eight kilns of this kind, 500 decasters of oak wood are carbonised, from which 15,000 hectolitres of charcoal are obtained, equal to 64,000 pounds French, being about 25 per cent., besides tar, and 3,000 velts of wood vinegar, of from $2^{\circ}$ to $3^{\circ}$ Baumé.

449

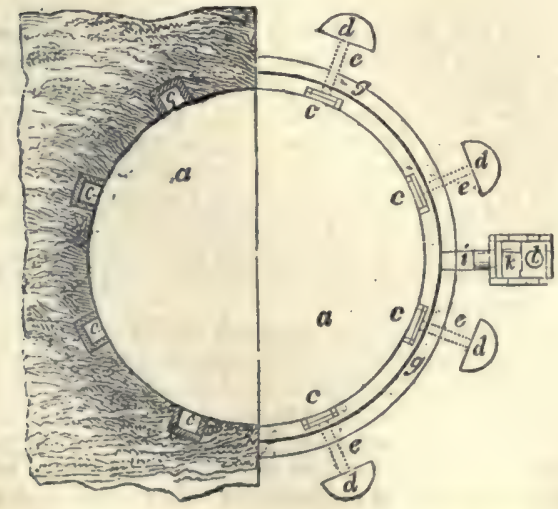

448
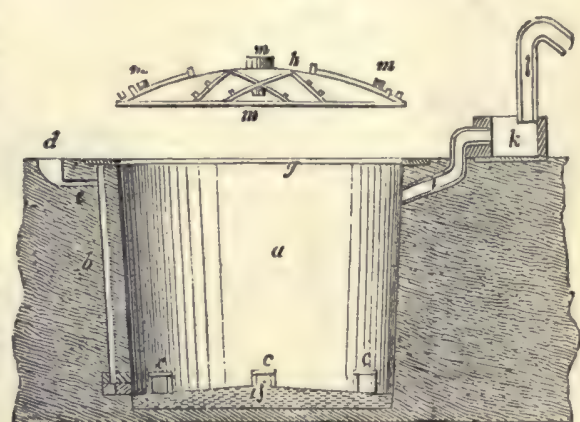
coking place, whose surrounding walls are heated by the flame which passer through the intermediate space $b$. The place itself is divided by partitions of fire tiles into three stages, through tho apertures in which the flames of the firo $c c$, rise, and heat the exterior of the coking apartment. In order to confine the heat, there is in the enclosing walls of the outer kiln, a cylindrical hollow space $d$, where the air is kept stagnant. Through the apertures left in the uppor end at $e$, the turf is introduced; they are then shut with an iron plate $f$, which is covered with ashes or sand. The fire-place opens above this aperture, and its outlet is provided with a moveable iron cover $g$, in which there is a small hole for the issue of the gases. The sole of the kiln consists of a cast-iron slab $h$, which may be raised by means of a hook, $i$, upon it. This is drawn back after the carbonisation is completed, whereby the charcoal falls from the coking space into a subjacent vault. The volatile products are carried off by the pipe $k$, and led into the condensing cistern; the gases escaping to the fire-place, where they are burned. The iron slab is protected from the corrosion of the acid vapours by a layer of coal ashes.

Charcoal obtained by the action of a rapid fire in close vessels is not so solid and so good a fuel as that which is made in the ancient way by the slow calcination of pyramidal piles covered with earth. One of the most economical ovens for making wood charcoal is that invented by M. Foucauld, which he calls a shroud, or abri. To construct one of these, 30 feet in diameter at the base, 10 feet at its summit, and from 8 to 9 feet high, he forms, with wood 2 inches square, a frame 12 foet long, 3 feet broad at one end, and 1 foot at the other. The figures 451, 452 will explain the construction.
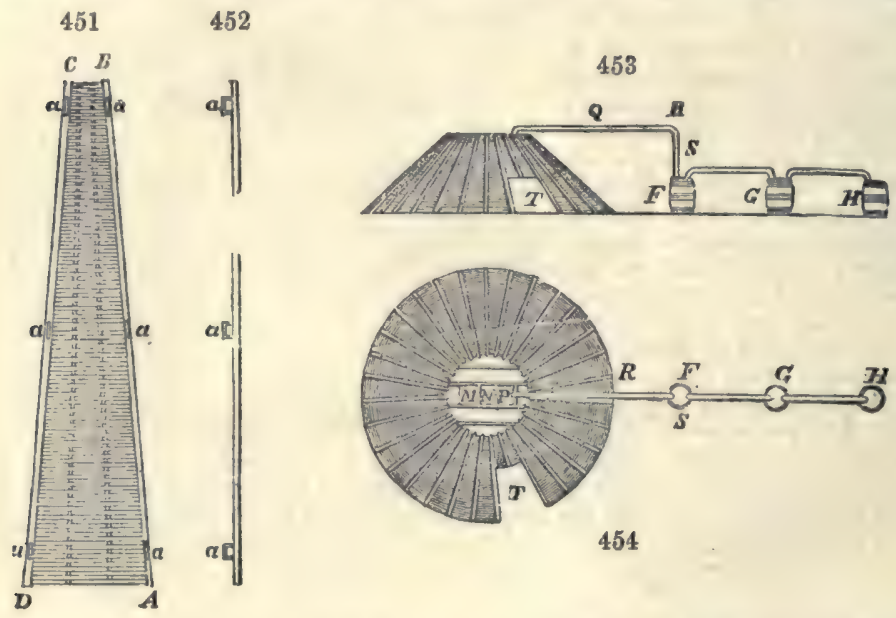

The uprights, $\mathbf{A}$ B and $\mathbf{C D}$, of this frame are furnished with three pairs of wooden handles $a a$, by means of which they can be joined together, by passing through two contiguous handles a wooden fork, the frame being previously provided with props, as shown in fig. 452, and covered with loam mixed with grass. A flat cover of 10 feet diameter, made of planks well joined, and secured by 4 cross bars, is mounted with 2 trap doors, M N, fig. 454, for giving egress to the smoke at the commencement of the operation; a triangular hole $\mathrm{P}$, cut out in the cover, receives the end of the conduit $Q$ R S, (figs. 453 and 454) of wood formed of three deals destined to convey the gases and condensed liquids into the casks $\mathrm{F} \& \mathrm{H}$. Lastly, a door $\mathrm{T}$, which may be opened and shut at pleasure, permits the operator to inspect the state of the fire. The charcoal calcined by this abri has been found of superior quality.

When it is wished to change the place where the abri is erected, and to transport it to a store of new-felled timber, the frame is taken down, after beating off the clay which covers it; the joints are then cut by a saw, as well as the ends of the fork which fixed the frames to one another. This process is economical in use, and simple and cheap in construction; since all the pieces of the apparatus are easily moved about, and may be readily mounted in the forests. For obtaining a compact charcoal, for the use of artisans, this mixed process of Foucauld is said to be preferable to either the close iron cylinder or the pile.

For making gunpowder-charcoal the lighter woods, such as the willow, dogwood, and alder answer best; and in their carbonisation care should be taken to let the 
vapours freely escape, especially towards the end of the operation, for when they are re-absorbed, they greatly impair the combustibility of the charcoal.

By the common process of the forests, about 18 per cent. of the weight of the wood is obtained; by the process of Foucauld about 24 per cent. is obtained, with 20 of crude pyroligneous acid of $10^{\circ}$ Baumé. By the process described under Acietic ACID, 27 of charcoal, 18 of acid at $6^{\circ}$, are procured from 100 parts of wood, besides the tar. These quantities were the results of careful experimenting, and are greater than can be reckoned upon in ordinary hands.

Charcoal for chemical purposes may be extemporaneously prepared by calcining pieces of wood covered with sand in a crucible, till no more volatile matter exhales.

The chareoal of some woods contains silica, and is therefore used for polishing metals. Being a bad conductor of heat, charcoal is employed sometimes in powder to encase small furnaces and steam-pipes. It is not affected by water; and hence, the extremities of stakes driven into moist ground are not liable to decomposition. In like manner casks when charred inside preserve water much better than common casks, because they furnish no soluble matter for fermentation or for food to animalcules.

Lowitz discovered that wood charcoal removes offensive smells from animal and vegetable substances, and counteracts their putrefaction. He found the odour of suceinic and benzoic acids, of bugs, of empyreumatic oils, of infusions of valerian, essence of wormwood, spirits distilled from bad grain, and sulphureous substances were all absorbable by freshly-calcined charcoal properly applied. A very ingenious filter has been constructed for purifying water, by passing it through strata of charcoal of different degrees of fineness.

When charcoal is burned, one-third of the heat is discharged by radiation, and twothirds by conduction.

The following Table of the quantity of charcoal yielded by different woods was published by Mr. Mushet, as the result of experiments carefully made upon the small seale. He says, the woods before being charred were thoroughly dried, and pieces of each kind were selected as nearly alike in every respect as possible. One hundred parts of each sort were taken, and they produced as under :-

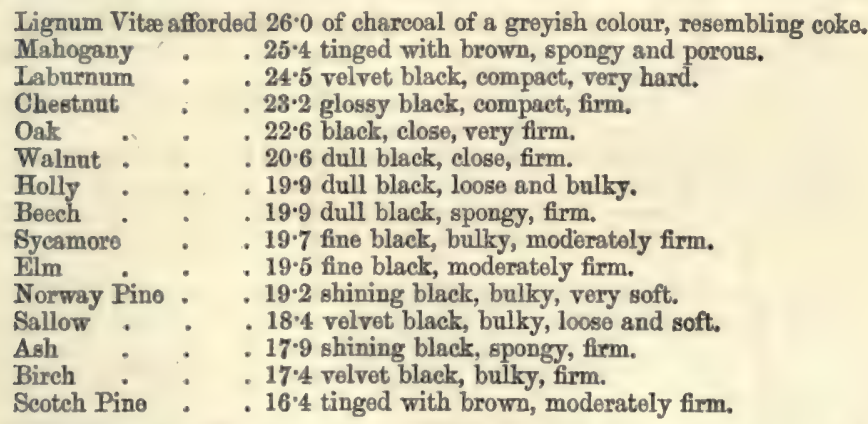

Messrs. Allen and Pepys, from 100 parts of the following woods, obtained the quantities of charcoal as under:-

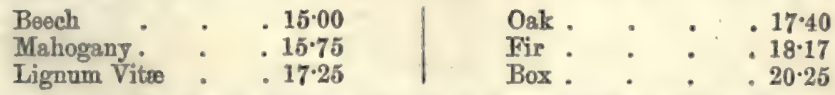

It is observable that the quantities obcained by Messrs. Allen and Pepys are in general less than those given by Mr. Mushet, which may be owing to Mr. Mushet not having applied sufficient heat, or operated long enough, to dissipate all the aqueous matter of the gaseous products.

To those persons who buy charcoal by weight, it is important to purchase it as soon after it is made as possible, as it quickly absorbs a considerable portion of water from the atmosphere. Different woods, however, differ in this respect. Messrs. Allen and Pepys found that by a week's exposure to the air, the charcoal of

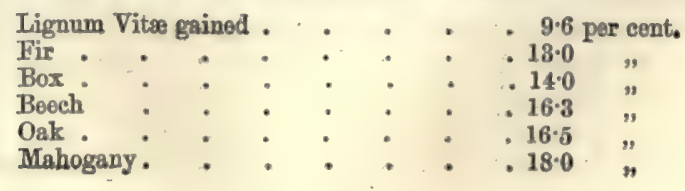


The following is a tabular viow of the volumes of the different gases which woro absorbed in the course of 24 hours, by one volume of charcoal, in the experiments of M. Théodoro de Saussure, which woro conducted in a way likely to produce correct results. Each portion of charcoal was heated afresh to a red heat, and allowed to cool under mercury. When taken from the mercury, it was instantly plunged into tho vessel of gas :-

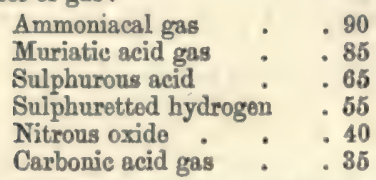

\begin{tabular}{|c|c|}
\hline$y \in n$ & \\
\hline Carbonic oxide & \\
\hline Oxygen gas . & \\
\hline cogen & \\
\hline hydrogen & \\
\hline Hydrogen gas : & \\
\hline
\end{tabular}

Neumann, who made many experiments on charcoal, informs us that for the reduction of the metallic oxides, the charcoal of the heavier woods, as that of the oak and the beech, is preferable, and that, for common fuel, such charcoal gives the greatest heat, and requires the most plentiful supply of air to keep it burning; while those of the lighter woods preserve a glowing heat with a much less draught of air ; and that for purposes where it is desirable to have a steady and a still fire, charcoal should be employed which has been made from wood previously divested of its bark, since it is the cortical part which crackles and flies off in sparks during combustion, which the coal or the wood itself seldom does.

For making crayons of charcoal, the willow is the best wood that can be employed, as the softness is uniform in all its parts. The durability of charcoal may be seen in several of our old churchyards, where the letters made with lampblack are still perfect, though the white lead with which the body of the stones was painted is entirely destroyed.

This property of carbon is shown, however, in a more striking manner by the writings that wers found in the ruins of Herculaneum, which have retained their original blackness for two thousand years. T'he ancients wrote with ink made from ground charcoal.

If it be required to purify any carbonaceous matter, to render it fitter for delicate pigments, this may be dono by first calcining it in a close ressel, and then lixiviating it in water slightly acidulated by nitric acid.

The incorruptibility of charcoal was well known to the ancients, and they availed themselves of this property upon all important occasions.

A bout sixty years ago a quantity of oak stakes were found in the bed of the Thames, in the very spot where Tacitus says that the Britons fixed a vast number of such stakes, to prevent the passage of Julius Cresar and his army. These stakes were charred to a considerable depth, had retained their form completely, and were firm at the heart.

Most of the houses in Venice stand upon piles of wood, which hare all been previously charred for their preservation. In this country, estates were formerly marked out by charred stakes driven to a considerable depth into the ground. These are oceasionally found, and usually the charred portions are quite perfect, although every other part has decayed.

Antmas Charcoar,-Animal charcoal especially, and other charcoal occasionally, has been much employed in the construction of filters for domestic use. The advantage of charcoal as a purifying agent-through which to pass water-has been long established. But it cannot be too generally known that the charcoal, in a fow weeks loses the property upon which its value depends. When first used, it separates not only the matters mochanically suspended in water, but much of the organic matter and salts held in solution. The property. appears to be entirely lost in about two months, after which time it is necessary either to renew or to restore the charcosl. See Fruter and Bore Buck.

Lignite Charcont.-Much of the lignite or brown coal of the Continent has been converted into charcoal, with a view to its use in motallurgical works. Mayer says that very firm charcoal is obtained by charring freshly-dug lignite without previously exposing it to the air. The complaint, however, amongst metallurgists is, that it is not generally sufficiently coherent for the furnace. The following are a few of the results of practical experiments :-

Lignito from Cologne, produced per cont. of charcoal
$"$ " Icoland


Prat Charcont, Numerous attempts have been made from time to time to utilise the peat of our bogs, and especially to bring peat charcoal into use for smolting iron, and other metallurgical processes. As far back as 1712, we are told by Carlowitz, that peat was charred in piles or stacks as wood is. Vogel informs us that in 1735 , peat charcoal was made in the Hartz. Numerous experiments have been made on the Continent to produce a charcoal from peat, which should answer the requirements of metallurgy. None of these appear to have been entirely successful. Within the last few years, experiments have been mado in Ireland to smelt the hrematite iron ore of Cumberland with peat charcosl. The exact results of the experiments have not been made known, but, certain it is, they did not promise to be commercially successful, and the idea has long been abandoned. We learn from the report of Sir Robert Kane, that the relative quantities of charcoal and other substances produced from a hundred parts of peat were found to be :-

\begin{tabular}{|c|}
\hline Chareoal \\
\hline Tarry matter \\
\hline Watery products \\
\hline Grases \\
\hline
\end{tabular}

From the same report we glean the following tables, giving the determinations of earbon, \&c., in different peats :-

\begin{tabular}{|c|c|c|c|c|c|c|}
\hline \multicolumn{4}{|c|}{$\begin{array}{c}\text { Rrogenautr } \\
\text { (Annal. der Pharm.) }\end{array}$} & \multicolumn{3}{|c|}{$\begin{array}{c}\text { MULDER } \\
\text { (Berz. Jahrb.) }\end{array}$} \\
\hline , & $\begin{array}{c}\text { Vulcatre } \\
\text { near } \\
\text { Abbeville }\end{array}$ & $\begin{array}{c}\text { Iong } \\
\text { near } \\
\text { Abbeville }\end{array}$ & $\begin{array}{l}\text { Champ de } \\
\text { Fer, near } \\
\text { Framont }\end{array}$ & $\begin{array}{l}\text { Friesland } \\
\text { comproct } \\
\text { turf }\end{array}$ & $\begin{array}{c}\text { Friesland } \\
\text { surface } \\
\text { turf }\end{array}$ & Holland \\
\hline Carbon & $60 \cdot 40$ & $60 \cdot 89$ & 61.05 & $50 \cdot 42$ & 60.41 & $59 \cdot 27$ \\
\hline Hydrogen & 5.90 & 6.21 & 6.45 & 5.87 & 5.57 & $5 \cdot 41$ \\
\hline Oxygen & $33 \cdot 64$ & $32 \cdot 90$ & $32 \cdot 50$ & $34 \cdot 71$ & $34 \cdot 02$ & $35 \cdot 32$ \\
\hline Ash, in 100 parts & 5.58 & $4 \cdot 61$ & $5 \cdot 33$ & 3.80 & 0.91 & $14 \cdot 25$ \\
\hline
\end{tabular}

The following results were obtained by Sir Robert Kane, from peats from the Irish bogs; and published in the Proceedings of the Royal Irish Academy:-

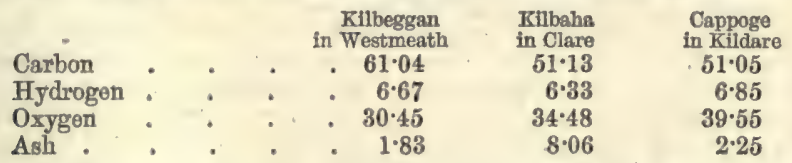

The determinations of charcoal and volatile matters found in peat in the following table, are: 1. from Rue, in Department of Somme; and 2. from Abbeville, by Berthier; 3. from Vaucluse, by Diday; 4. from Sécheral, by Sauvage; and 5. from Kildare; 6. from Kilbeggan; 7. from Kilbaha; and 8. Cappoge, by Sir Robert Kane :-

\begin{tabular}{|c|c|c|c|c|c|c|c|c|}
\hline & 1 & 2 & 8 & 1 & 5 & 6 & 7 & 8 \\
\hline & 21. & $23 \cdot 0$ & 17 & $22 \cdot 0$ & 23 & $22 \cdot 67$ & $72 \cdot 14$ & \\
\hline & 72 & 72 & 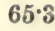 & 39 & $73 \cdot 63$ & 75 & 0 & $70^{\circ}$. \\
\hline & $7 \cdot 0$ & 488 & $17 \cdot 4$ & $8 \cdot 3$ & 2.55 & 1.83 & $8 \cdot 06$ & 6. \\
\hline
\end{tabular}

Peat is usually charred by methods similar to those employed for the carbonisation of wood. Kilns, variously constructed, have been used, but the success attending the production of peat charcoal by them, has not been sufficiently great to induce us to occupy space with any description of them.

In 1849, Mr. Vignoles, C.E., patented a process for charring peat by steam, superheated to the melting point of tin or lead. Pulpod peat is thrown in mass into a cylindrical drum-shaped vessel, divided, if necessary, into compartments, which is caused to revolve with great rapidity upon an axis-the velocity requisite being such as shall drive off the water or other fluid from the solid parts. of the peat or turf by centrifugal force. When all the water is removed from the peat by this process, and it is in a coherent mass, it is removed and moulded into blocks. These blocks are put into large cylindrical iron vessels, into which steam, at from $45 \mathrm{lbs}$. to $60 \mathrm{lbs}$. pressure per square inch, is admitted, after a process of superheating, by traversing iron tubes heated to bright redness. This process, although strongly recommended, has never been practically adopted.

Peat charcoal, as far as heating power is concerned, ranks among the best kinds of 
fuel, but it produces so much ash as to make its use very objectionable. 100 pounds of dry peat, say Messrs. Ronalds and Richardson, leave 21 lbs. of ash, and produce $47 \mathrm{lbs}$. of charcoal, therefore those $47 \mathrm{lbs}$. of peat charcoal will contain $21 \mathrm{lbs}$. or 46 per cont. of ash.

Kogers adopted in Ireland a system of drying peat and carbonising it, which was for some time carried forward with much promise of success. Green produced a draught of hot air through a drying house, by means of chimnoys and fires. $\mathrm{He}_{e}$ then distilled the dry peat in wrought-iron cylinders, similar to those employed in making illuminating gas, the gases obtained being used as part of the fuel bolow. Notwithstanding all the attempts which have been made to utilise peat charcoal, there is no process now carried out on a large scalo.

Platinised Cenarcoat.-This preparation, which appears destined to becomo excoedingly valuable, was introduced by Dr. Stenhouse, who thus describes the mode of preparation and some of its uses:-

-The lighter kinds of wood charcoal, owing to the nine volumes of oxygen gas contained in their pores, possess a considerable power of oxidising the greater number of easily alterable gases and vapours. The absorbent power of charcoal, however, is comparatively much greater than its capacity for inducing chemical combination. In this respect charcoal presents a remarkable contrast to spongy platinum, which, though inferior as an absorbent for some gaseous substances-such for instance, as ammonia, of which spongy platinum absorbs only 30 volumes, while charcoal absorbs ninety-is, nevertheless, immensely more effective, both as an oxidiser, and as a promoter of chemical combination generally. As it is desirable for some purposes, while retaining the absorbent power of charcoal unimpaired, to jncrease its oxidating influonces, it struck me that this important object might be easily effected by combining the chareoal with minutely-dividod platinum. In this way, a combination is produced, to which I have given the name of platinised charcoal, which possesses the good properties of both of its constituents. In order to platinise charcoal, nothing more is necessary than to boil the charcoal, either in coarse powder or in large pieces, in a solution of bichloride of platinum, and when the charcoal has become thoroughly impregnated with the platinum, which seldom requires more than ten minutes, or a quarter of an hour, to heat it to redness in a close vessel-a capacious platinum crucible being very well adapted for this purpose. When 150 grains of eharcoal were impregnated with nine grains of platinum, by the process just described, the charcoal was found to have undergone no change in its external appearance, though its properties have been very essentially altered. When a fow grains of this platinised charcoal wero passed up into a mixturo of dry oxygen and hydrogen in the proportions to form water, over mercury, the two gases combined in the course of a few minutes, precisely in the same way as when a clay ball of spongy platinum is employed. When, however, a fragment of charcoal containing a considerably larger proportion of platinum was passed up into a similar gaseous mixture, the gases instantly combined with explosive violence, just as if platinum-black had been used. If pieces of cold platinised charcoal are held in a jet of hydrogen, they speedily become incandescent, and inflamc the gas. Platinised charcoal, when slightly warmed, likewise rapidly becomes incandescent in a current of coal-gas, but the jet of gas is not inflamed, owing to the very high temperature, a white heat, which is required for this purpose.

'In the vapour of alcohol or wood spirit, platinised charcoal becomes red hot, and continues so till the supply of vapour is exhausted. In the course of a few hours, spirits of wine, in contact with platinised charcoal and air, is converted into vinegar. I find that two per cent. of platinum is sufficient to platiniso charcoal for most purposes. Charcoal containing this small amount of platinum, causes a mixturo of oxygen and hydrogen to combine perfectly in about a quartor of an hour, and this is tho strength of platinised charcoal that seems best adapted for charcoal disinfectant respirators. Charcoal containing one per cent. of platinum causes a mixture of oxygen and hydrogen to combine in about two hours; and chareoal containing the extremely minute quantity of $\frac{1}{4}$ per cent. platinum, produces the same effect in from 6 to 8 hours. Platinised charcoal soems likoly to admit of various useful applications; one of the most obvious of these is its excellent adaptability to air-filters and respirntors for disinfectant purposes. It is plain that no easily alterable organic vapours, such as effluvia or miasmata, can remain in contact, even for a fow minutes, with platinised charcoal without being destroyed, their carbon being converted into carbonic acid, and their hydrogen into water.

- Platinised charcoal also seems likoly to prove a highly useful application to malig. nant ulcers and similar sores, on which I confidently expect, from its powerful oxidating properties, that it will act as a mild, but effective, caustic. Porhaps, however, as an application to sores, platinised asbestos, either alono, or in combination with platinised charcoal, might be found more manageable. In thoso diseases also 
where the internal use of chareoal has beon found beneficial, I should think that platinised charcoal may bo advantageously substituted. In Bunsen's carbon battery also the employment of platinised charcoal may, I think, be advantageously tried.

- It is clear that the amount of platinum in the charcoal may be varied almost at pleasure, according to the strength of the platinum solution employed in its preparation, and the purposes to which the charcoal is intended to be applied. Almost any form, and even very considerable dimensions, may be given to the platinised charcoal - circumstances which greatly extend the range of its applications.'

Crancoars. The name by which the best tin plates are known; these are always made by charcoal fires.

CHAscriscE, or Hashash. Hadschy is not the correct term for this narcotic drug, for Hadschy means a pilgrim; the true name is, according to pronunciation, Chaschisch, the Arab word for hemp (Cannabis sativa). By this name, all intoxicating drugs whose chief constituent is this herb are well known over the whole of the East. The mode of preparing chaschisch is the following:-

The tops and all the tender parts of the hemp plant are collected after the period of inflorescence; dried and kept for use. It must be premised that the hemp plant is in the East distinguished by its narcotic properties, although botanists are unable to detect any difference between this and the European species. The dried hemp or chaschisch, is used-

1st. Boiled in fat, butter, or oil, with a little water; the filtered product is employed in all kinds of pastry.

2nd. Powdered for smoking: 5 or 10 grains of the powder are smoked from a common pipe (tsubuk) with ordinary tobacco (tiitïm), or from a water-pipe (nargiele) with another kind of tobacco (tombeki). The tombeki is probably the leaf of a specios of lobelia; it is smoked in a nargiele, and is uncommonly narcotic; so much so, that it is ordinarily steeped in water for a few hours before it is used, to weaken it, and the pipe is charged with it whilst it is yet wet.

3rd. Formed with tragacanth mucilage into pastiles, which are placed upon a pipe and smoked in similar doses. These last two preparations are termed esrar (esrar is the Arab word for secret); they are the most active of all the preparations of chaschisch, and the first pipe will cause cerebral congestion in beginners.

4th. Made into an electuary with dates or figs and honey. This preparation is of a dark brown, almost black colour, and tastes of dates and hemp; it is less active than the esrar.

5th. Lastly, another electuary is prepared of the same ingredients with the addition of spices, clove, cinnamon, pepper, amber, and musk. This preparation is used as an aphrodisiac.

Chaschisch is said not to produce stupor, but the most pleasant species of intoxication. The person under its infuence feels with perfect consciousness in the best of all humours ; all impressions from without produce the most grateful sensations; pleasant illusions pass before his eyes, and he feels comfortably happy; he thinks himself the happiest man on earth, and the world appears to him Paradise. From this imaginative state he passes into the every-day state, with a perfect recollection of all sensations, and of everything he has done and of every word he has spoken. The effects of a continual use of the nareotic are emaciation and nervous debility.

CrATHAN IICET. A flash-light usod for military purposes, obtained by blowing a mixture of powdered resin and magnesium through a spirit flame.

CRAX zOOT. The root of the Oldenlandia umbellata, a plant grown on the Coromandel Coast in India, and valued for the red dye-stuff which it yields.

CanCz. A cloth in which coloured lines or stripes cross each other rectangularly.

Blackburn in England, and Kirkaldy in Scotland, are $t^{\prime}$. 8 principal centres of the check manufacture. See Weaving.

CHבzsz. (Fromage, Fr.; Käse, Ger.) The curd of milk which has been allowed to undergo a peculiar change, by which it acquires the well-known flavour. The most celebratod English cheeses are manufactured in Cheshire, Gloucestershire, and Somersetshire. The milk of animals contains three principles, which are, by very slight changes, separated from each other. By a short repose, an oily portion rises to the surface of the milk-this consists of the cream globules, which are readily converted into butter. If any acid be added to the milk, a curd separates; this is the caseous matter, or casein, which being pressed in moulds, becomes cheese; the third portion being the serum or whey. The cheesy matter which exists in milk in a soluble state, is usually separatod by means of rennet (see RENNET) - that is, it is coagulated by it, or rendered insoluble. The action of rennet upon milk is still very imperfectly understood. In order to investigate tho subject, Berzelius washod and dried the stomach of a calf, and yet he found that one part of this, mixed with nearly 2,000 parts of skimmed milk, and heated to $120^{\circ} \mathrm{F}$., so completely coagulated the caseous matter that no trace of it remained in solution. All acids curdlo milk-muriatic (hydrochloric) 
acid is said to be used for this purpose in Holland. Somo regetables roadily coagulate milk, amongst others the juice of the fig-tree, and the flowers of the Galium ecrum, the yollow lady's bed-straw, or cheese rennet. The odour of the flowers of this plant is sweet, the stalks and flowers have been used in Cheshire to curdle milk, and they are said to give a peculiar flavour to that kind of cheese.

There are different kinds of cheeses according to the manner of preparing them.

Soft cheeses-as cream cheese, and Bath and Yorkshire cheese, which will not keep long, and are therefore sold as soon as made.

Hard cheeses - as Cheshire, Gloucester, Cheddar, Parmesan, and Dutch cheose.

Intermediate-we may regard the Gruyère and Stilton.

The following is the process used for cheese-making in general. The milk is first strained, then put into a large cauldron, which hangs from an iron crane over a wood fire, and heated to nearly blood-heat. It is then removed from the fire, the prepared rennet is added to it, and stirred till it is well mixed with the warm milk. Tho cauldron is then covered with a cloth, and loft for about half an hour or an hour, till the coagulum is formed. This done, the curd is cut horizontally, with a flat wooden skimming ladle, made for that purpose, into thin slices, which are poured against the side of the cauldron, so that every portion of the curd thus rises to the surface, and is thinly sliced. The cauldron is then replaced over the fire, and the whole is well stimed with a long staff with a knob made of hard wood at the end, and small sticks placed crosswise through holes bored in it; this breaks the curd into very small pieces; the stirring is continued until the heat is raised to $135^{\circ}$, whon the cauldron is again taken off the fire. It is then stirred for nearly an hour more with the staff, and when this is done, the curd is found in small pieces about the size of a pea, which feel elastic and rather tough. The curd is then collected in the bottom of the cauldron, and the whey floats at the top : a cloth is put under the curd, and lifted by the four corners on to an instrument, something like a small ladder which is placed across the top of the cauldron. The whey runs out through the cloth, then the curd in the cloth is put into a hoop, made of a thin piece of wood; and a board about two inches thick is placed over it; it is then put into the press. After remaining in the press about an hour, the curd is examined, and the edges which have pressed over the ring are pared off and put in the centre of the cheese. The cheese is then taken out of the hoop and turned, and a fresh cloth substituted. This is done two or three times during the day. A little finely-powdered salt is then rubbed on both sides of the cheese, and it is left in the press until the next morning; after that it is taken out and put on a shelf, where it is allowed to remain for six or eight weeks, having a little salt rubbed into it every day till it will not take any more. The weather has a great influence on the dairy. In Cheshire, cheeses are made in great perfection. In making them they are particularly careful to extract every particle of whey; to do this they press the curd very tightly in boxes with holes bored in them; they then put wooden skewers through these holes into the cheese, so that no whey can possibly remain in the curd. This process gives Cheshire cheeses the solid appearance which they possess. If there are any holes in them they are considered imperfect. In these cheeses the salt is mixed with the curd, instead of being rubbed on the outside of the cheese. This prevents fermentation, or tho formation of any elastic matter. Gloucester and Somersotshire cheeses aro made in just the same way as the Cheshire, except that the curd is not quite so much broken, or the skewers put through the cheese, and some portion of the cream is generally taken to make butter. Warm water is poured over the curd to wash from it any whey that may still be remaining in it, or to dissolve any of the oily part, or butter, that may have separated, before the milk had beon coagulated.

Stilton cheeses require great care in making them. They are mado by adding the preceding evening's cream to the morning's milk. The cream and milk must be well mixed together, and after the milk is coagulated, the curds must be strained through a sieve, only lightly pressed, and then put into a shape; when it is firm enough it is taken out, bound round with a cloth to keep it from breaking, and set on a shelf. It is sometimes powdered with flour and plunged into hot water to hardon the outside. Stilton cheese is generally preferred in a mouldy state, and to hasten this, pieces of another mouldy cheese are often inserted in the new Stilton, or wine or ale poured over it. But if the cheese is rich, it does not require this, but the inside will get soft liké butter, without getting mouldy.

Gruyere and Parmesan cheeses are made by the process first described; the only difference between them being, that a greater degree of heat is used in making Pur. mesan than in making Gruyere, and this renders the Parmesan cheose dry and grained, while the Gruyère is pressed and compact. A little saffron is also used to give Parmesan colour and flavour, but nothing is used for Grayère.

Dutch cheeses are mado in a very similar way to Gloucester cheoses; but instead of using rennet to curdle the milk, the Dutch use muriatic acid, or spirits of salt. 
They are very careful to prevent fermentation in this cheese, and in order to effoct this, the curd, after being well broken and pressed, is soaked in salt and water; salt is also rubbed on the outside of the cheeses, and it thus acquires the quality of keeping well, even in warm climates. These eheeses are generally known in Holland by the name of Edam cheeses, from the name of the place where they are made.

Another cheose much used in Holland is one made from skim milk; not having much flavour of its own, cummin seeds are mixed with the curd to supply this defect.

The Roquefort cheese is made in France from goats' or sheep's milk. The evening's and morning's milk are mixed, and rennet is used in the ordinary way to coagulate it. The curd is then subjected to great pressure, and when the whey is all extracted, the cheeses are laid on shelves, side by side, and frequently wiped earefully to dry thom without heat. They generally wrap the cheeses in coarse cloths, to prevent them from cracking in drying. When they are quite dried, they are taken to caves dug in the rocks, where the air is always cool, and placed on shelves. They are then salted, and after about two or three days, they are rubbed with a coarse cloth, and then scraped with a knife. They are then left for fifteen days, during which time they got hard, and begin to get mouldy on the outside: the mouldiness is removed by a knife, and this process is repeated every fifteen days, for two months, and sometimes oftener. During this time the crust formed on the cheeses becomes successively white, greenish and reddish; when it is this latter colour, they are fit for use.

The Brie cheese is another, the quality of which is nearly as good as the last, but it is only used in the places where it is manufactured, because it will not keep. It is very simply made; the milk curdled in the usual manner is put to strain on a wicker frame. When all the whey has been extracted, the cheese is salted and put in a very cool place; the salting is repeated every two days: when salted sufficiently it is removed to a cave or placed on a bed of hay, until it softens. This cheêse should be eaten when it is about the consistency of cream; it is then delicious, but if left longer, it becomes putrid.

The Neufchatel cheeses are merely cream thickened by heat, and then compressed in a small mould. They are estoemed a great delicacy.

There is a small cheese much relished in Germany, made from skimmed milk. The milk is curdled by being placed near the fire; the curds are then put into a linen bag, and all the whey pressed out of them. When this has become solid, the curd is again broken in a tub, and allowed to remain in this state for several days. Putrid fermentation then begins to take place; when in this state it is taken and rolled into little balls with the hand, and flavoured with carraway seed. After a fow days the inside of the ball becomes soft, and it is then esteemed a greatdainty by many. Sometimes they are placed in the smoke of a chimney over a wood fire; the smoke hardens the outside, and when they are eaten, it is taken off like the rind of an apple.

The Schabzieger, a Swiss cheese, is made in much the same manner; the curd is worked into a paste with a large quantity of an herb, called in the country dialect Ziegerkraut, finely powdered. It is then pressed into a mould, and becomes very solid, and capable of being kept a long time. When eaten it is scrsped and mixed with butter, and spread on bread. It possesses a very peculiar taste.

The Jura cheese is very much like the Gruyère, and is often sold for it.

There is a kind of cheese or hard curd made in Switzerland with the whey that is left after making other cheeses. The whey is heated over the fire until a thick scum arises; it is then put in a square box, all the whey muns off, and the curd, which is called serré, remains; " this is dried, and although nearly flavourless, is much eaten by the people on the mountains, who use it instead of bread. They cut it in slices, and spread butter on it, and it forms the principal food of some of the herdsmen.

Composition of Cheese.

(Payen, Journal Pharma.).

\begin{tabular}{|c|c|c|c|c|c|c|c|c|c|}
\hline \multirow{2}{*}{\multicolumn{2}{|c|}{ 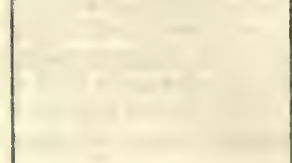 }} & \multirow{2}{*}{ Water } & \multicolumn{2}{|c|}{$\begin{array}{l}\text { Ash of the } \\
\text { substance }\end{array}$} & \multicolumn{3}{|c|}{ Nitrogen } & \multicolumn{2}{|c|}{ Fat } \\
\hline & & & Normal & Dry & Normal & Dry & $\begin{array}{c}\text { Free } \\
\text { trom ash }\end{array}$ & Normal & Dry \\
\hline Chees & from Chester & $\begin{array}{l}\text { Per ot. } \\
30.39\end{array}$ & $\begin{array}{l}\text { Per ct. } \\
4 \cdot 78\end{array}$ & $\begin{array}{r}\text { Per ct. } \\
6.88\end{array}$ & $\begin{array}{l}\text { Per ct. } \\
5.56\end{array}$ & $\begin{array}{l}\text { Per ct, } \\
8.00\end{array}$ & $\begin{array}{l}\text { Per ct. } \\
8.59\end{array}$ & $\begin{array}{l}\text { Per ct. } \\
25 \cdot 48\end{array}$ & $\begin{array}{l}\text { Per ct. } \\
36.61\end{array}$ \\
\hline & " Parmesan & $30 \cdot 31$ & $7 \cdot 09$ & $10 \cdot 18$ & $5 \cdot 48$ & $7 \cdot 87$ & 8.76 & 21.68 & $31 \cdot 12$ \\
\hline & "Neufchatel & $61 \cdot 87$ & $4 \cdot 25$ & $11 \cdot 17$ & $2 \cdot 28$ & $5 \cdot 99$ & $6 \cdot 07$ & $18 \cdot 74$ & $49 \cdot 15$ \\
\hline & " Brie & 53.99 & $6 \cdot 63$ & $12 \cdot 08$ & $2 \cdot 39$ & $5 \cdot 14$ & $5 \cdot 85$ & $24: 83$ & $53 \cdot 29$ \\
\hline$"$ & " Holland & $41 \cdot 41$ & 6.21 & $10 \cdot 61$ & $4 \cdot 10$ & $7 \cdot 01$ & 7.84 & $25 \cdot 06$ & $42 \cdot 78$ \\
\hline$"$ & " Gruyère & $32 \cdot 05$ & $4: 79$ & $7 \cdot 05$ & $5 \cdot 40$ & $7 \cdot 96$ & $8 \cdot 56$ & $28 \cdot 40$ & $41 \cdot 81$ \\
\hline
\end{tabular}

Vor, I. 
If a chocse is hard and dry it is much improved by washing several times in saft water, and then laying it in a cloth moistened with wine or vinegar. This method is ofton prictised in Switzerland, where cheoses aro often kept for many years.

In England, cheeses are frequently coloured : the substance most generally used for colouring is arnotto. Sometimes the juice of the orange or carrot, and the flower of the marigold, are used for this purpose. Foreign cheoses are seldom coloured.

Cheese of certain dairies and districts is apt to undergo a remarkable decomposition, whereby valerianic acid is formed. Messrs. Iljenko and Laskowsi distilled, along with water, a turbid ammoniacal liquor, which being redistilled along with some sulphuric acid, and the product neutralised by barytes, the resulting saline compound proved to be the valerianate of that base, mixed with compounds of butyric acid, caproic acid, caprylic acid, and capric acid; the cheese was from Limbourg. Valerianic acid was found by M. Balard in the choese of Roquefort.

CHEMTCAI Arrrmiry. The torm used to express the force, in obedience to which combinations of apparently dissimilar bodies are produced.

chzaricas rormuzer. Seo Formula, Chrmoar.

CHEMICK. See Br.rachina.

CHevevixise. A hydrous arsenate of peroxide of iron and protoxide of copper, from Cornwall.

CHIRRY COAI. A soft non-eaking coal. The coals of Staffordshire, of Derbyshire, and much of the Scotch coal appears to belong to this variety. See CoAr.

CERRRY TREs. The Tunbridge turners use the wood largely, considering the wood of the black-heart cherry the best. It is a hard, close-grained wood, of a palo red-brown colour.

CFIRT, a siliceous mineral nearly allied to chalcedony and flint. It generally occurs in limestone rocks, and beds of it are not uncommon in parts of the Mountain Limestone. A gradual passage from chert to limestone is not uncommon.-Lyell. Chert is a term often applied to hornstone, and to any impure flinty rock, including the jaspers.-Dana.

Chert is worked extensively out of the Carboniferous Limestone quarries of Flintshire, especially at Halkin and at Talacre. It is also produced in considerable quantities in the same formation in Derbyshire. It is used in the Potteries, for paving the mills in which flints are ground.

CEISSY COPPER or CHEssyrTrz. The blue carbonate of copper which is found in great beauty at Chessy near Lyons. See Azurite.

Cressivur. (Castanea vesca.) The wood of this, the sweet or Spanish chestnut, is sometimes used in house carpentry. The wood of an oak (Quercus sessilifloru) is often mistaken for it.

The wood of the horse chestnut (Fisculus hippocastanum) is one of the white woods much used by the turners of Tunbridge; it is also employed for brush backs. The white (inner) bark of the horse chestnut, when infused in boiling water, produces a yellow fluid, which possesses the remarkable power of fluorescence, that is, it throws back from its first surface a set of rays of high refrangibility, and of a blue colour, while the ordinary yellow rays are duly transmitted. The phenomena have been fully investigated by Professor Stokes, to whom the name is also due. See Fruorrscrance.

CHICA is a red colouring principle made use of in America by some Indian tribes to stain their skins. It is extracted from the Bignonia chica by boiling its leaves in water, decanting the decoction, and allowing it to settle and cool, when a red matter falls down, which is formed into cakes and dried. It is not much used in this country. The crajurv or carcuru, which is imported from Para in Brazil is more frequently employed. The dyes are probably identical, but one is purer than the other. See Carajura.

CEICORY. The root of the Chicorium intybus; Wild Succory or Chicory. This plant is cultivated in various parts of England, growing well in a gravelly and chalky soil; also in Belgium, Holland, Germany, and France. The roots of the wild suecory were formerly used medicinally ; it possesses properties in many respects resembling those of the dandelion, but it is rarely employed for curative purposes in the present day.

Chicory root roasted has been employed as a substitute for coffee for more than a century. (Constantini, Nachricht von d. Cichorianwurzel, 1771.) It is now employed extensively as a mixture with coffee, which, although allowed, cannot be regarded other than an adulteration.

Chicory root is heated in iron cylinders, which are kept revolving as in the roasting of coffee. In this country about two pounds of lard are added to every cwt. of chicory during the roasting process: in France butter is used; by this a lustre and colour resembling that of coffee is imparted to it. When roasted the chicory is ground to powder and mixed with the coffee. Chicory has been supposed by some persons to be wholesome and nutritive, while others contend that it is neither one nor the other; however, no obvious ill effects have been observed to arise from its mployment, if we except the occasional tendency to excite diarrhoea when it has 
been used to excess. The analysis of chicory root by John gave 25 parts watery bitter extractive, 3 parts resin, besides sugar, sal-ammoniac, and woody fibre. Waltl procured inulin from it, but the quantity varies greatly in different roots. The following remarks on the adulteration of chicory are by Dr. Pereira :-

'Roasted chicory is extensively adulterated. To colour it, Venetian red und, perhaps, reddle are used. The former is sometimes mixed with the lard before this is introduced into the roasting machine; at other times it is added to the chicory during the process of grinding. Roasted pulse (peas, beans, and lupines), corn (rye and damaged wheat), roots (parsnips, carrots, and mangold wurzel), bark (oak-bark tan), wood dust (logwood and mahogany dust), seeds (acorns and horse-chestnuts), the mare of coffee, coffee husks (called coffee-flights), burnt sugar, baked bread, dog biseuit, and baked livers of horses and bullocks (!), are substances which are said to have been used for adulterating chicory. A mixture of roasted pulse (peas usually) and Venetian red has been used, under the name of Hambro' powder, for the same purpose.

' The following are the chief modes of examining chicory with the viow to the detection of these adulterations:-

'1st. Careful examination of the odour, flavour, and appearance to the naked eye of the suspected powder. In this way foreign substances may sometimes be detected.

'2nd. A portion of the dried powder is to be thrown on water; the chicory rapidly imbibes the water and falls to the bottom, whereas some intermixed powders (as the mare of coffee) float.

' $3 r d$. The suspected powder is to be submitted to careful microscopical examination. Pulse and corn may be detected by the size, shape, and structure of the starch grains. The tissues of barks, woods, and other roots may also be frequently distinguished from those of the chicory.

' 4 th. A decoction of the suspected ehicory is then to be prepared, and, when cold, to be tested with solution of iodine and persulphate of iron.

'Iodine colours a decoction of pure chicory brownish; whereas it produces a purplish, bluish, or blackish colour with decoctions of roasted pulse, roasted corn, baked bread, roasted acorns, and other substances containing starch. Persulphate or perchloride or iron does not produce much effect on a decoction of pure chicory, but it communicates a bluish or blackish tint to a decoction of oak-bark, of roasted acorns, and other substances containing tannic or gallic acids.

' 5 th. By incineration, pure dried chicory yields from 4 to 5 per cent. of a grey or fawn-coloured ash. If Venetian red, or any earthy or mineral substances, be present, a larger amount of ash is obtained. Moreover, when Venetian red has been employed, the colour of the ash is more or less red.'

Our Importations of Chicory have been as follow:-

Raw or Kiln-dried at 1l, 63. 6d. per owt. duty, imposed April 17, 1863.

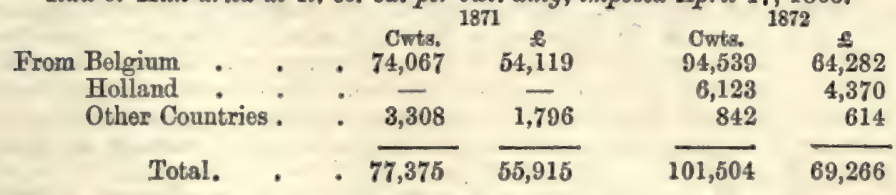

Roasted or Ground at 4d. per lb. duty, imposed April 17, 1863.

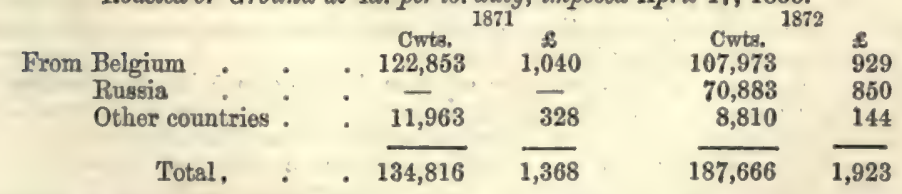

CHITDRzNrTz. This mineral may be regarded as a hydrous phosphate of alumina and iron. The composition of a specimen analysed by Rammelsberg was: phosphoric acid, 28.9 ; alumina, 14 ; protoxide of iron, 29.3 ; protoxide of manganese, 9.5 ; water, 18.3. At Crinnis'mine in Cornwall, childrenite is found on slate, and near Tavistock in Devonshire, with apatite.

CHIXI SAITPETRE. Native nitrato of soda, or cubic nitro.

Crrrerre A vanadate of copper and lead, occurring at the silver mine of Mina Granda, in Chile.

CrIIII, commonly Chillies. The fruit of certain species of capsicum, valued for their pungency.

CrImxry. (Cheminee, Fr.; Schornstein, Ger.) (The whole of this article is retained as written by Dr. Ure, his investigation on some of the points involved being 
of much value.) Chimnoy is a modern invention for promoting the draught of fres and carrying off the smoke, introduced into England so late as the age of Elizabeth, though it seems to have boen employod in Italy 100 years before. The Romans, with all their luxurious refinement, must havo had their epicurean cookery placed in perpetual jeopardy from their kitchen fires, which, having no vent by a vertical tunnel in the walls, discharged their smoke and froquently their flames at their windows, to the no small alarm of their neighbours, and annoyance of even the street passengers.

Chimneys in dwolling-houses serve also the valuable purpose of promoting a salubrious circulation of air in the apartments, when not foolishly sealed with anti-ventilating stove-chests.

Tho first person who sought to investigate the general principles of chimney draughts, in subserviency to manufacturing establishments, was the celebrated Montgolfier. As the ascent of heated air in a conduit depends upon the diminution of its specific gravity, or, in other words, upon the increase of its volume by the heat, the ascensional force may be deduced from the difference between the density of the elastic fluid in the interior of tho chimney, and of the external air; that is, between the different heights of the internal and external columns of elastic fluid supposed to be reducod to the same density. In the latter case, the velocity of the gaseous products of combustion in the interior of the chimney is equal to that of a heavy body let fall from a height equal to the difference in height of the two arrial columns.

To illustrate this position by an example, let us consider the simple case of a chimney of ventilation for carrying off foul air from a factory of any kind; and suppose that the tunnel of iron be incased throughout with steam at $212^{\circ} \mathrm{Fahr}$. Suppose this tunnel to be 100 yards high, then the weight of the column of air in it will be to that of a column of external air 100 yards high, assumed at $32^{\circ}$ Fahr., inversely as its expansion by $180^{\circ}$; that is, as 72.727 is to 100 . The column of external air at $32^{\circ}$ being 100 yards, the internal column will be represented by 72.727 ; and the difference $=27 \cdot 27$, will be the amount of unbalanced weight or pressure, which is the effective cause of the ventilation. Calculating the velocity of current due to this difference of weight by the well-known formula for the fall of heary bodies, that is to say, multiplying the above difference, which is $27 \cdot 27$, by the constant factor $19 \cdot 62$, and extracting the square root of the product; thus, $\sqrt{19 \cdot 62 \times 27.27}=23.13$ will be the velocity in yards per second, which, multiplied by 3 , gives 69.39 feet. The quantity of air which passes in a second is obtained of course by multiplying the area or cross section of the tunuol by this velocity. If that section is half a yard, that is $=\mathrm{a}$ quadrangle $2 \frac{1}{4}$ foet by 2 , wo shall have $23.13 \times 0.5=11: 56 j$ cubic yards, $=312 \frac{1}{4}$ cubic feet.

The problem becomes a little more complicated in ealeulating the velocity of air which has served for combustion, bocause it has changed its nature, a variable proportion of its oxygen gas of specific gravity 1.111 being converted into carbonic acid gas of specific gravity 1.534. The quantity of air passed through well-constructod furnaces may, in general, be regarded as double of what is rigorously necessary for combustion, and the proportion of carbonic acid generated, therefore, one half of what it would be were all the oxygen so combined. The increase of weight in such burned air of the temporature of $212^{\circ}$, over that of pure air equally heated, being taken into account in the preceding calculation, will give us about 19 yards or 57 feet per second for the velocity in a chimnoy 100 yards high incased in steam.

In comparing tho numbers resulting from the trials made on chimneys of different materials and of different forms, it has been coneluded that the obstruction to the draught of air is directly proportional to the length of the chimneys and to the square of the velocity, and inversely to their diameter.

With an ordinary wrought-iron pipe of from 4 inches to 5 inches diameter, attached to an ordinary stove, burning good charcoal, the difference is prodigious between the velocity calculated by the above theoretical rule and that observed by means of a stop watch, and the ascent of a puff of smoke from a little tow dipped in oil of turpentine thrust quickly into the fire. The chimney being 45 feet high, the temperature of the atmosphere $68^{\circ}$ Fahr, the velocity per second was:

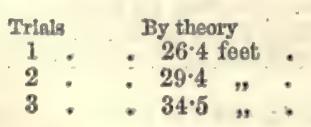

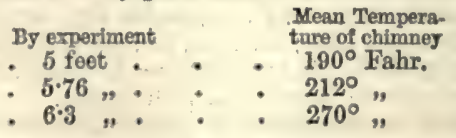

To obtain congruity between calculation and experiment, several circumstances must be introduced into our formulæ. In the first place, the theoretical velocity must be multiplied by a factor, which is differont according as the chimney is made of bricks, pottery, sheet iron, or cast iron. This factor must be multiplied by the square root of the dismoter of tho chimnoy (supposed to be round), divided by its 
length, increased by four times its diameter. Thus, for pottery, its expression is $2.06 \frac{\sqrt{D}}{\mathrm{~L}+4 \mathrm{D}} ; \mathrm{D}$ being the diameter, and $\mathrm{L}$ the length of the chimney.

A pottery chimney, 33 feet high, and 7 inches in diameter, when the excess of its mean temperature above that of the atmosphere was $205^{\circ} \mathrm{Fahr}$, had a pressure of hot air equal to 11.7 feet, and a velocity of $7 \cdot 2$ foet per second. By calculating from the last formula, the same number very nearly is obtained. In none of the experiments did the velocity exceed 12 feet per second, when the difference of temperature was more than $410^{\circ} \mathrm{Fahr}$.

Every different form of chimney would require a special set of experiments to bo made for determining the proper factor to be used.

This troublesome operation may be saved by the judicious application of a delicate differential barometer, such as that invented by Dr. Wollastnn; though this instrumont does not seem to have been applied by its very ingenious author in measuring tho dranghts or ventilating powers of furnaces.

If into one leg of this differential siphon, water be put, and fine spermaceti oil into the other, we shall have two liquids, which are to each other in density asthe numbers 8 and 7. If proof spirit be employed instead of water, we shall then have the relation of very nearly 20 to 19 . In experiments made on furnace draughts with tho instrument in each of these states, it is found that the water and oil siphon is sufficiently sensible: for the weaker draughts of common fire-places the spirits and oil will be preferable barometric fluids.

To the lateral projecting tube of the instrument, as described by Dr. Wollaston, it was found necessary to attach a stop-cock, in order to cut off the action of the chimney, while placing the siphon, to allow of its being fixed in a proper state of adjustment, with its junction-line of the oil and water at the zero of the scale. Since a slight deviation of the legs of the siphon from the perpendicular changes very considerably the line of the level, this adjustment should be made secure by fixing the horizontal pipe tightly into a round hole, bored into the chimney stalk, or drilled through the furnace door. On gently turning the stop-cock, the difference of atmospheric pressure corresponding to the chimney draught will be immediately indicated by the ascent of the junction-line of the liquids in the siphon. This modification of apparatus permits the experiment to be readily rectified by again shutting off the draught, when the air will slowly re-enter the siphon; because the projecting tube of the barometer is thrust into the stop-cock, but not hermetically joined; whereby its junction-line is allowed to return to the zero of the scale in the course of a few soconds.

Out of many experiments made with this instrument, it will be sufficient to describe a few, very carefully performed at the breweries of Messrs. Trueman, Hanbury, and Buxton, and of Sir H. Meux, Bart., and at the machine factory of Messrs. Braithwaite by Dr. Ure; in the latter of which he was assisted by Captain Eriessen. In the first trials at the breweries, the end of the stop-cock attached to the differential barometer was lapped round with hemp, and made fast into the circular peep-hole of the furnace door of a wort-copper, communicating with two upright parallel chimneys, each 18 inches square and 50 feet high. The fire was burning with fully its average intensity at the time. The adjustment of the level keing perfect, the stop-cock orifice was opened, and the junction level of the oil and water rose steadily, and stood at $1 \frac{1}{4}$ inch corresponding to $\frac{125}{8}=0^{\circ} 156$ of 1 inch of water, or a column of air $10^{\circ} 7$ feet high. This difference of pressure indicates a velocity of 26 feet per second. In a second set of $\theta \mathrm{x}$ periments, the extremity of the stop-cock, was inserted into a hole bored through the chimney stalk of the boiler of a Boulton and Wati steam-engine of twenty-horse power. The area of this chimney was exactly 18 inches square at the level of the bored hole, and its summit rose 50 feet above it. The fire-grate was about 10 feet below that level. On opening the stop-cock the junction-line rose $2 \frac{1}{4}$ inches. This experiment was verified by repetition upon different days, with fires burning at their average intensity, and consuming fully $12 \mathrm{lbs}$. of the best coals hourly for each horse's power, or nearly one ton and a third in twelvo hours. If wo divide the number $2 \frac{1}{4}$ by 8 the quotient 0.28 will represent the fractional part of 1 inch of water supported in the siphon by the unbalanced pressure of the atmosphere in the said chimney; which corresponds to $19 \frac{1}{4}$ feet of air, and indicates a velocity in the chimney current of 35 feet per second. The consumption of fuel was much more considerable in the immense grate under the wort-copper than it was under the steam-engine boiler.

In the exporiments at Messrs. Braithwaite's factory, the maximum displacement of the junction-line was 1 inch, when the differential oil and water barometer was placed in direct communication with a chimney 15 inches square, belonging to a steam boiler, and when the fire was made to burn so fiercely, that, on opening the safety-valve of the boiler, the excess of steam beyond the consumption of the engine rushed out with such violence as to fill the whole premises. The pressure of one-eighth of an inch of water denotes a velocity of druught of 23.4 feet per second. 
In bullding chimineys, we shonld be careful to make their area rather too largo than too small; because wo can readily roduco it to any desired size by means of a sliding register plate near its bottom, or a damper plate applied to its top, adjustable by wires or chains passing over pulleys. Wide chimneys are not so liable as narrow ones to have their draught affected by strong winds. In a factory, many furnace flues aro often conducted into one vertical chimney stalk, with great economy in the first orection, and increased power of draught in the several fires.

Vast improvements have been made in this country of late years in building stalks for steam boilers and chemical furnaces. Instead of constructing an expensive, lofty scaffolding of timber round the chimney, for the bricklayers to stand upon, and to place their materials, pigeon-holes, or recesses, are left at regular intervals, a few feet upart, within the chimney, for receiving the ends of stout wooden bars, which are laid across, so as to form a species of temporary ladder in the interior of the tunnel. By means of these bars, with the aid of ropes and pulleys, everything may be progressively hoisted for the building of the highest engine or other stalks. An expert bricklayer, with a handy labourer, can in this way raise, in a few weeks, a considerable chimney, 40 feet high, 5 feet 8 inches square outside, 2 feet 8 inches inside at the base, 28 inches outside, and 20 inches inside at the top. To facilitate the erection, and at the same time increase the solidity of an insulated stalk of this kind, it is built with three or more successive plinths, or recedures, as shown in fig. 455. It is necessary to make such chimneys thick and substantial near the base, in order that they may sustain the first violence of the fire, and prevent the sudden dissipation of the heat. When many flues are conducted into one chimney stalk, the area of the latter should be nearly equal to the sum of the areas of the former, or at least of as many of them as shall be going simultaneously. When the products of combustion from any furnace must be conducted downwards, in order to enter near the bottom of the main stalk, they will not flow off until the lowest part of the channel be heated by burning some wood shavings or straw in it, whereby the air siphon is set agoing. Immediately after kindling this transient fire at that spot, the orifice must be shut by which it was introduced; otherwise the draught of the furnace would be seriously impeded. But this precaution is seldom necessary in great factories, where a certain degree of heat is always maintained in the flues, or, at least, should be preserved, by shutting the damper plate of each separate flue whenever its own furnace ceases to act. Such chimneys are finished at top with a coping of stone-slabs, to secure their brick-work against the infiltration of rains, and they should be furnished with metallic conducting rods, to protect them from explosions of lightning.

When small domestic stoves are used, with very slow combustion, as has been proposed upon the score of a misjudged economy, there is great danger of the inmates being suffocated or asphyxied, by the regurgitation of the noxious burned air. The smoke doctors who recommend such a vicious plan, from their ignorance of chemical science, are not aware that the carbonic acid gas of coke or coal must be heated $250^{\circ} \mathrm{F}$. above the atmospheric air to acquire the same low specific gravity with it. In other words, unless so rarefied by heat, that gaseous poison will descend through the orifice of the ash-pit, and be replaced by the lighter air of the apartment. Drs. Priestley and Dalton have long ago shown the co-existence of these twofold crossing currents of air, even through the substance of stoneware tubes. True economy of heat and salubrity alike require vivid combustion of the fuel, with a somewhat brisk draught inside of the chimney, and a corresponding abstraction of air from the apartment. Wholesome continuous ventilation, under the ordinary circumstances of dwelling-houses, cannot be secured in any other way.

The figures upon the following page represent one of the two chimneys erected at the Camden Town station, for the London and North-Western Railway Company. The chimneys were designed by Robert Stephenson, Esq., engineer to the company, and executed by William Cubitt, Esq., of Gray's Inn Road. In the section. fig. 455,

A represents a bed of conerete, 6 feet thick and 24 feet square.

$B$, brick footings set in cement; the lower course 19 feet square.

c, Bramley-fall stone base, with a chain of wrought iron let into it.

D, a portion, 15 feet high, curved to a radius of 113 feet, built entirely of Malm paviours (a peculiarly good kind of bricks).

E, shaft built of Malm paviours in mortar.

$x$, ditto, built from the inside, without exterior scaffolding.

G, the cap, ornamented (as shown in the plan alongside) with Portland stone, the

dressings being tied together with copper cramps and an iron bond.

$\kappa$, the lightning conducting rod.

Fig. 456, represents the mouldings of the top, upon an enlarged scale.

Fig. 467, a plan of the foundation, upon an enlarged scale.

Fig. 458 , ditto, at the level of the entrance of the flue as seen in 
Fig. 459, the elevation of the chimney.

Fig. 460, plan at the ground level $\mathrm{x}$, in figs. 455 and 459.

Some modified arrangements in the mode of constructing the chimney and fire-place have been devised by Colonel Douglas Galton. These will be found fully described in the Report of the Committee 'On Waste of Coal in Combustion,' in the Report of the Royal Coal Commission.

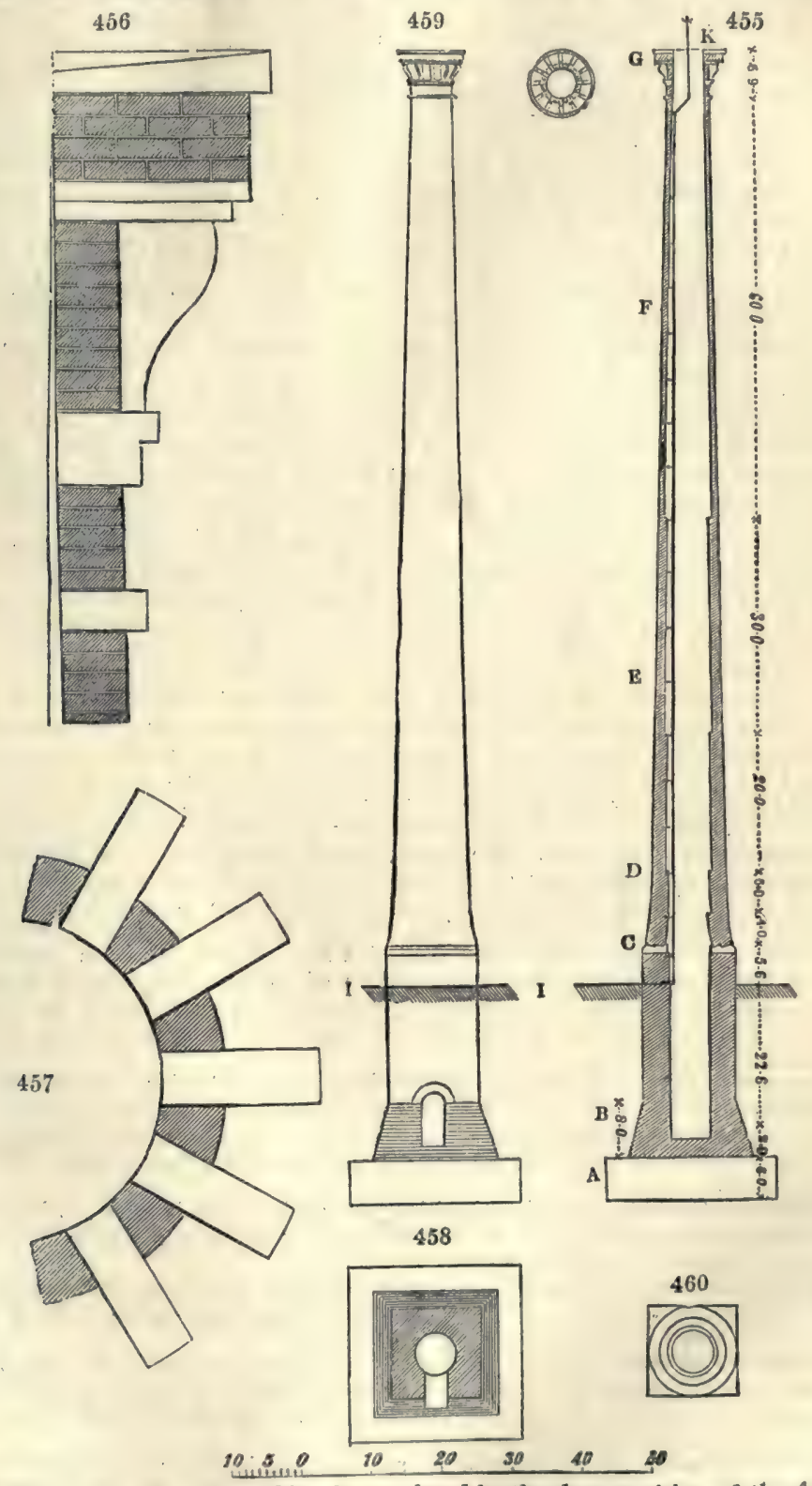

Crrva Crax. A fine white clay produced by the decomposition of the felspar of the granite rocks. It is found and prepared, in this country, in Cornwall and Devonshire. See Cray. 
Curva Grass. A fibre obtained from the stalk of the Bahmeria (Urtica) nivea, a plant belonging to the Urticaces, or Nettle order.

CBrava INI. (Encre de Chine, Fr.; Chinesischer Tusch, Ger.) It is said that the true China ink is made from the condensed smoke or soot of burned eamphor; and hence, when of the best quality, it has this odour.

Most of the China ink is mado from oil lampblack, disguised as to smell with musk, or with a littlo camphor-black. The binding substance is gelatine, commonly made from parchment; but isinglass answers equally well. A good imitation may bo made by dissolving isinglass in warm water, with the addition of a very littlo alkali to destroy the gelatinising power, and incorporating with that solution, by levigation on a porphyry slab, as much of the finest lampblack as to produce a mass of the proper consistence. Tho minute quantity of alkali serves also to saponify the oil which usually adheres to lampblack, and thoreby to make a pigment miscible with water.

Curna sTONz. This is usually regarded as a semi-decomposed granito (Petuntse) which has nearly the same composition as the China clay (see Cray). 'Indeed, the China clay can be considered as little moro than this granite in a more advanced state of decomposition.'-De la Beche. It is evident that we have adopted the word Pe-tun-tse somewhat hastily as indicating this peculiar granite. The following noto by M. A. Salvétat will explain its true moaning:-

- Nous forons observer une fois pour tout que l'expression Pe-tun-tse désigne les tablettes ou carreaux de matière blanche dont on va parler, et que la pate blanche s'appelle Pe-tun. Le mot Tse (vulgo fils) qui termine le mot Pe-tun-tse, sert à former des substantifs diminutifs. Ainsi Pe-tun signifie la matière blanche et Tse ajouté à Pe-tun indique des portions, des carreaux de pate, des briquettes de Pe-tun. Ily a des carreaux de pate de différentes couleurs: pour les distinguer, on fait précéder le mot Tun-tse du nom de la couleur. On dit, par exemple, Hoang-tun-tse, des carreaux de pate jaune.'-Histoire et Fabrication de la Porcelaine chinoise, Paris, 1856.

The China stone is a kind of granite, the felspar of which is in a peculiar state, as if it had undergone a partial decomposition. It is carefully selected so as to be entirely free of schorl, and requires no other preparation for the market than to be broken into a size convenient for carriage. This granite is of a peculiar nature; it does not contain any mica, but numerous glossy seales of greenish-yellow talc. It has been stated by some authors that ' this rock (Pegmatite or Graphic granite), after exposure to the decomposing action of the weather, is the chief source' of the China stone and clay. This represents but very imperfectly-indeed, incorrectly-the conditions. The decomposition of the granite, if it be decomposition, is not brought about by the action of the weather, but by some peculiar action proceeding to a considerable depth through the whole mass. In many places, from the very surface to the depth of more than 100 feet, this condition is equally apparent; and possibly it extends to much greater depths in some places. The same stone exposed to the air does not, in any ordinary time, exhibit any signs of disintegration. No satisfactory explanation has yet been offered of the conditions which operate on the granite to produce the Kaolin and the China stone. Indeed, in the minds of many, who have carefully observed all the conditions of the China clay and of the China stone in situ, there has originated the idea, that they rather represent the conditions of an imperfectly-formed granite, than of such as has suffered decomposition. China stone is much used in the manufacture of the finest varieties of porcelain, and especially for the production of tho most perfect glazes.

There was an agreement existing amongst the producers of China stone to send off annually only 12,000 tons; but when the demand is brisk, this has been extended to 18,000 tons, and sometimes even more. The value of the China stone at the works in Cornwall is annually about 1,800 . The whole that is raised is sent to the Staffordshire potteries.

CHIN nsE BIUz. Several compositions are sold under this name; it is sometimes a mixture of ultramarine with flako white, and sometimos a mixture of cobalt blue and white lead. See Blue Pigmants.

CHISESE GRAss CxOTH, or Ramec. A fabric woven from China grass.

CHIN ISE TAIIOW. A white regetable tallow covering the seeds of the Stillingia sebifera. It is used in making candles.

CHInEsE WAX. A white waxy secretion, resombling spermaceti, found on the branches of a species of ash (Fraxinus Chinensis), grown in China, and probably produced by the puncture of an insect (Coccus Pe-la). The wax is chiefly a cerotate of ceryl. It is used in China for making candles.

CHINOIDIJEz. A brownish resin-like substance obtained from the mother-liquor left in the preparation of quinine.

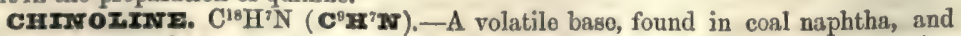
also, accompanied by several others, in the basic fluid obtained by distilling einchonine with potash.-C. G. W. 
Crrnoxirrs nxus, Cyanine or Lepidine Blue. A crystalline compound, prepared by heating chinoline oil, which consists chiefly of lepidine, with iodide of amyl, and treating the product with caustic soda. A black resinous precipitate is thus obtained, which dissolves in alcohol with production of a fine blue colour.

CrInTzz. (Zitz, Ger.) Probably derived from the East, the Hindoo name cheent, and the Persian chinz, signifying spotted or stained. The term is applied in this country to a fast-printed calico, in which several colours are imprinted upon a white or coloured ground, and usually glazed.

CrIos or CHIAN TURPENTIND. The Pistacia terebinthus, growing in Syria and the Greek Archipelago, yields this turpentine. The turpentine harrest of Scio, according to Tournefort, is made from the ond of July to October, by cutting crossways with a hatchet the trunks of the largest trees. The turpentine runs down on flat stones placed under the trees, where it hardens. The quantity produced from each tree does not exceed eight or ten ounces. Its consistency is that of honey; its colour greenish-yellow, with an agreeable turpentine odour. It is often called Cyprus turpentine, but is seldom seen pure in this country, other coniferons turpentines being sold for it.

CHLORAI. $\mathrm{C}^{4} \mathrm{H}^{3} \mathrm{O}^{2}\left(\mathbf{C}^{2} \mathbf{H C l}^{3} \mathrm{O}\right)$. This compound, discovered by Liebig in 1832 , is prepared by the long-continued action of chlorine on ethyl-alcohol. A current of dry chlorine gas is passed slowly but continuously through absolute alcohol, and the final product is a yellow liquid rich in chloral. Both the gas and the alcohol should be free from moisture, as otherwise the proportion of chloral is diminished, whilst that of aldehyde and acetic acid is increased; the reaction, towards the close, should be assisted by heat. It appears that an alcoholate of chloral is first formed by the action of chlorine on alcohol; but on agitating the product with about three times its volume of strong sulphuric acid, and warming the mixture, the free chloral soparates as an oily liquid floating on the acid. To purify this crude chloral it should be distilled first from oil of vitriol and then from quicklime, to remove hydrochloric acid. The production of chloral admits of being approximately represented by the following equation, but it should bo remembered that the reaction is really more complicated, and that other products accompany the chloral :-

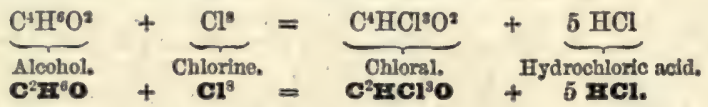

Chloral may also be obtained by other processes, such as the distlllation of stareh with hydrochloric acid and peroxide of manganese.

Pure chloral is a thin, oily, colourless, rolatile liquid, almost tasteless, but with a peculiar pungent odour. It is soluble in water, alcohol, ether, and benzole. Its specific gravity is 1.502 , and its boiling point about $200^{\circ} \mathrm{F} .\left(94^{\circ} \mathrm{C}\right.$.). Chloral may be regarded as acetic aldehyde, $\mathrm{C}^{4} \mathrm{H}^{4} \mathrm{O}^{2}\left(\mathbf{C}^{2} \mathbf{H}^{4} \mathbf{O}\right)$, in which three atoms of hydrogen are replaced by three of chlorine.

When chloral is long preserved in a hermetically-closed vessel it suffers a curious change, becoming converted into a solid white amorphous substance, called insoluble chloral or metachloral.

Chloral combines readily with water, forming a definite hydrate containing $\mathrm{C}^{4} \mathrm{HCl}^{3} \mathrm{O}^{2} .2 \mathrm{HO}\left(\mathbf{C}^{2} \mathbf{E C} \mathbf{C l}^{3} \mathbf{O} \cdot \mathrm{H}^{2} \mathrm{O}\right)$. This is a white, solid crystalline substance, slightly volatile on exposure to the air, and incapable of being distilled without decomposition. An aqueous solution of hydrate of chloral, treated with caustic alkali, is resolved into chloroform and an alkaline formiate. When hydrate of chloral is injected under the skin it undergoes a similar decomposition, reacting with the alkali naturally present in the blood, and generating chloroform. The physiological action of the chloroform thus liberated within the system has been studied by Dr. Liebreich, of Berlin, and by Dr. W. B. Richardson. In July 1869, Liebreich took out a patent for the use of chloral, hydrate of chloral, and trichloracetic acid for anæsthetic purposes. Since that time, the hydrate of chloral has been very largely employed as a powerful nareotic. According to Dr. Richardson, a dose of 90 grains taken internally will produce deep sleep, whilst 140 grains throw the patient into a sleep which is really dangerous. But the hydrate, like other narcotics, requires to be used with great caution. It unfortunately happens that persons who have been accustomed to take moderate doses to relieve pain and induce sleep, frequently acquire a tendency to use it habitually. This habit cannot be too strongly denounced; for, like opiumeating, it not only affects the physical organisation-leading eventually to confirmed disease - but it exercises a most baneful influence on the mental and moral constitution of the unhappy individual. 
[For the physiological action of chloral and its hydrate, see Dr. Richardson's Reports presented to the British Association in 1869, 1870 and 1871].-F. W. R.

crIoraIUM. Under this name a solution of chloride of uluminium has been introduced as an antiseptic and disinfectant. It is prepared in the form of a liquid of definite strength, and as a powder. Cotton-wool containing a certain per-centage of chloralum is made for the nse of the surgeon.

CHLORATIIINE. $\mathrm{C}^{12} \mathrm{H}^{\circ} \mathrm{ClN}\left(\mathbf{C}^{6} \mathbf{I}^{8} \mathbf{C l N}\right)$. An organic base obtained by distilling chlorisatin with a concentrated solution of caustic potash. It forms with acids a series of deflnite well-crystallised salts.

Chionate of potash, or ChIopate or potasstum, formerly called oxymuriste of potash. KO.ClO $\left(\mathbf{K C} \mathbf{C l O}^{3}\right)$. - This important salt has become the object of a pretty extensive manufacture, in consequence of its use in the preparation of lucifer matches, as a constituent of white gunpowder and certain mixtures used in pyrotechny, and as an ingredient in some of the detonating compounds for the cartridges of needle-guns; the chlorate is also used in calico-printing and in the preparation of certain aniline colours, while the chemist frequently employs it as a convenient oxidising agent and as a source of free oxygen gas. To supply these demands, about 750 tons of the salt, valued at nearly $80,000 l$, are annually produced in this country.

Chlorate of potash was formerly prepared by transmitting an excess of chlorine gas through a solution of potash, whereby a mixture of chlorate of potash and chloride of potassium was obtained, the former salt being readily separated from the latter by its sparing solubility in water.

Having made a strong solution of purified potash, or carbonate of potash, with from 2 to 3 parts of water, a current of chlorine gas is passed through it in a Woulfe's apparatus, till it ceases to absorb any more. 'Chloride of potash' and chloride of potassium alone are formed as long as there is an excess of alkali in the solution; but afterwards in the further reaction of the materials, the chloride passes into the state of a chlorate, and, as such, precipitates from the solution. During the first half of the operation (that is, till the potash is about one half saturated with chlorine, as indicated by litmus-paper ceasing to be darkened and beginning to be blanched), only the chloride of potassium or muriate of potash falls. The process should be interrupted at this point in order to remove the salt, to wash it, to add the washings to the liquor, and then to transmit the gas freely through the solution. As the operation advances, less muriate of potash is formed, and at length nothing but the pure chlorate is separated in crystals. When, finally, the bubbles of gas pass through without being sensibly absorbed, the process is known to be completed; the liquid may then be allowed to settle, and be poured off from the crystals of chlorate of potash, which are purified from the muriate by dissolving them in three times their weight of boiling water and filtering the solution while hot. On its cooling, the chlorate will separate in pearly-crystalline plates. It may be rendered quite puro by a second crystallisation, in which state it does not affect solution of nitrate of silver.

The above potash-ley usually gets a reddish tint in the course of the process, in consequence of a little manganesic acid coming over with the chlorine, but it gradually loses this colour as the saturation becomes complete, and then the solution turns yellow. The tubes for conveying the gas should be of large diameter, if they be plunged into the saline solution, because the crystallisation which takes place in it is apt to choke them up. This inconvenience may, however, be obviated by attaching to the end of the glass tube a tube of caoutchouc terminated in a small glass funnel, or simply the neck of a caoutchouc bottle with a part of its body, whose width will not be readily closed with a saline crust. The residuary lixivium may be used in another operation, or it may be evaporated down to half its bulk and set aside to crystallise, whereby some more chlorate will be obtained, mixed indeed with muriate and carbonate, from which, however, it may be separated by a seeond crystallisation. In general the pure chlorate obtained does not exceed $\frac{1}{10}$ th the weight of the potash employed; because in thus treating potash with chlorine, $\frac{5}{6}$ ths of it are converted into muriate of potash and only st th into chlorate, and a part of the lattor adheres to the muriate, or is lost in the mother-waters of the crystallisation.

In 1821 St. Romer patented at Vienna the following method for preparing chlorate of potash by the dry way:-Ten pounds of crystallised peroxide of manganese are to be finely pulverised, mixed with 10 pounds of plumbago, and 30 pounds of common salt, and put into the leaden retort represented in fig. 460, p. 783 . From the middle of tho holmet-shaped lid of this vessel, a lead tube, 2 feet long and 2 inches wide, conducts to the receiver, which is a square earthen pan, hard glazed both within and without, of the same capacity with the retort. The end of the tube must be made fast to a frame at the height of 6 inches above the bottom of the receiver. Upon its 
inner side, 4 inches apart, brackets are to be fixed for supporting a series of laths or shelves of whito wood on which a number of little paper or pasteboard boxes are to be laid. In these boxes 10 pounds of the purest carbonate of potash, prepared from tartar, are to be spread. The receiver must now be covered with a lid made tight by a water lute. Twenty pounds of concentrated sulphuric acid, previously diluted with 16 pounds of water, and then cooled, are to be poured upon the mixed materials in the retort, and the lid immediately secured, with the tube adjusted in the receiver. The wholo must be allowed to operate spontaneously without heat for 12 hours. At the end of this time the retort is to be surrounded with a water-bath and steadily heated during 12 hours, and then left to cool for 6 hours. The apparatus must now be opened, the cakes of chlorate of potash removed, and freed from muriate by solution and erystallisation.

Liebig proposed the following process for obtaining chlorate of potash :-

Heat chloride of lime in water till it ceases to destroy vegetable colours. In this case a mixture of chloride of calcium and chlorate of potash is obtained. This is to be dissolved in hot water, and to the solution concentrated by evaporation, chloride of potassium is to be added, and then suffered to cool. After cooling, a quantity of erystals of chlorate of potash is obtained, which are to be redissolved and crystallised again to purify them. Iiebig considered that this would be a cheap process for obtaining chlorate of potash. From $12 \mathrm{oz}$. of chloride of lime, of so bad a quality that is left 65 per cent. of insoluble matter, he obtained an ounce of chlorate of potash.

The only difficulty to overcome in this process arises from the chloride of lime not being so easily decomposed by heat as is generally supposed; a solution of it may be kept boiling for an hour without losing its bleaching power. The best method is to form a thin paste with chloride of lime and water, and then to evaporate it to dryness. If it be required to prepare it by passing chlorine into cream of lime, it is advantageous to keep it very hot. The chlorate of potash which separates from the solution by cystallisation has not the form of scales which it usually possesses, but is prismatic: whether this is oceasioned by some admixture has not been ascertained; but on recrystallising, it is obtained in the usual form. The solution ought not merely to be left to cool, in order to procure crystals, for the crystallisation is far from being terminated even after complete cooling; crystals continue to be deposited for 3 or 4 days.

The following modification of the process for making chlorate of potash is that of M. Vée :-A solution of chloride of lime marking $18^{\circ}$ or $20^{\circ}$ Baumé is to be set upon the fire in a lead or cast-iron pot, and when it begins to get hot, there is to be dissolved in it a quantity of chloride of potassium sufficient to raise the hydrometer $3^{\circ}$ or $4^{\circ}$. It must then be concentrated as quickly as possible till it marks $30^{\circ}$ or $31^{\circ}$, taking care that it does not boil over by the sudden extrication of oxygen. The concentrated liquor is set aside to crystallise in a cool place, when a deposit of chlorate of potash forms, mixed with chloride of potassium. The mother-waters being evaporated to the density of $36^{\circ}$, afford another crop of crystals, after which they may be thrown away. The salts, obtained at the first crystallisation are to be redissolved, and the solution being brought to $15^{\circ}$ or $16^{\circ}$ is to be filtered, when it will afford upon cooling pure chlorate of potash.

The following ingenious and easy way of making this valuable chlorate was suggested by the late Professor Graham:--Mix equal atomic weights of carbonste of potash and hydrate of lime (70 of the former, if pure, and 37 of slaked lime in powder), diffuse them through cold water, and transmit chlorine gas through the mixture. The gas is absorbed with great avidity, and the production of a boiling heat. When the saturation is complete, carbonate of lime remains, and a mixture of muriate and chlorate of potash, which latter salts are to be separated, as usual, by the difference of their solubity in water.

It has been remarked on the above process, that it effects no saving of potassa, and therefore is far inferior to the one long practised in several parts of Germany, especially at Giessen, and introduced into this country a good many years ago by Dr. Wagenmann, from Berlin. The chlorine is passed into a mixture of one equivalent of chloride of potassium (76), and 6 equivalents of hydrate of lime (222), previously stirred with water to the consistence of a thin paste. Thus the calcium of the lime unites with the chlorine to form chloride of calcium, while the chloride of potassium is converted into chlorate of potash, which salt is easily separated in crystals by its sparing solubility.

Chlorate of potash may also be made by saturating with chlorine a mixture of 74 parts of chloride of potassium (muriate of potash) and 168 parts of quicklime, brought to the consistency of a thin pap by the cantious addition of water. The mass being dissolved in warm water, and evaporated and cooled, yields crystals of chlorate of potash, while a mother-water of chloride of calcium (muriate of lime) remains. 
The following process has likewise been prescribed :-Nix 10 parts of good chloride of limo and water into a pap, and ovaporate to dryness, whereby it is comverted into a mixture of chlorido of calcium and chlorate of limo devoid of bleaching power; dissolve it in water, filter, concentrate the solution by eraporation, then add to it 1 part of chlorido of potasssum, and cool for crystallisation. The salt which may thereby be separated from the chloride of calcium will afford 0.83 of pure chlorate of potash. By this process of Professor Liebig $\frac{5}{6}$ ths of the potash are saved, but much oxygen is wasted in the evaporation to dryness of the cloride of lime; and consequently, much chloric acid is lost towards the production of the salt. Vée mixes the chloride of lime pap, before heating it, with the chloride of potassium, boils the mixture smartly, whereby much oxygon is undoubtedly thrown off, and then sets the liquor acid to crystallise. L. Gmelin suggests that saturation of the liquor with chlorine before boiling might be advantageons. Gay-Lussac proposed to make this valuable salt by precipitating a solution of chloride of lime with carbonate (or sulphate) of potash, saturating the liquor after filtration with chlorine gas, evaporating, and crystallising.

Mr. Calvert formed a mixture of $5 \frac{1}{2}$ equivalents of burnt lime for 1 equivalent of caustic potash, and passed a current of chlorine through the hot mixture. Under these conditions chloride of calcium and chlorate of potassium are produced, and the quantity of the latter is stated to be very nearly the theoretical amount.

Professor Juch's process is to pass chlorine gas into a mixture of 1 pound caustic lime and 1 pound carbonate of potash, with 8 pounds of water. The resulting chlorate of potash readily separates in the filtered liquid by crystallisation from the very soluble chloride of calcium. By this method potash is not wasted in the useless production of chloride of potassium.

The following process is now generally adopted for preparing chlorate of potash on a large scale :-An excess of chlorine gas is passed into a vessel containing milk of lime at a temperature of $120^{\circ}$ to $140^{\circ} \mathrm{F}$., the liquid being kept agitated by an iron stirred coated with lead. The reaction between the chlorine and the lime under these circumstances results in the production of chlorate of lime and chloride of calcium, with a small quantity of hypochlorite of lime; thus differing from the reaction which occurs between lime and chlorine at a lower temperature, as in the preparation of 'chloride of lime.' When the gas ceases to be absorbed by the lime, the liquid is run off into a tank lined with lead, where the suspended matter is allowed to subside, and whence the supernatant liquid is drawn off by a siphon into a leaden evaporating pan in which it is concentrated to $25^{\circ}$ to $30^{\circ} \mathrm{B}$. A hot solution of chloride of potassium is then added, by which the chlorate of lime is decomposed and chlorate of potash formed. Theoretically, every 168 parts by weight of caustic lime originally used require $74^{\circ} 5$ parts of chloride of potassium; but practically one part of the chloride is added for every three parts of lime. The chlorate of potash and chloride of calcium aro readily separated by taking advantage of the sparing solubility of the potash salt. The crude chlorate is purified by solution and re-crystallization.

Chlorate of potash, (the old exymuriate of potash), has a cooling, somewhat unpleasant and nitrous taste; it does not bleach. At $60^{\circ} \mathrm{F} .100$ parts of water dissolve 6 parts of the salt, and at its boiling point, or $220^{\circ}, 60$ parts. When heated to dull ignition in a glass retort, it gives out 39.21 per cent. of its weight of oxygen, and leaves a residue of chloride of potassium. The decomposition is effected at a much lower temperature if the salt be mixed with a small quantity of binoxide of manganese, sesquioxide of iron, or black oxide of copper. Chlorate of potash is an active oxidising agent: it deflagrates upon red hot cinders like nitre: when triturated along with sulphur or phosphorus, it detonates with great violence, not without danger to the hands of the operator. Similiar detonations may be produced with cinnabar or vermilion, sulphuret of potassium, rolatile oils, sugar, \&c ; but they can be effected only by the smart blow of a heated hammer on an anvil. See Lucrfer Matches and Prrcussion CAPS.

chioratras. Salts of chloric acid.

CHIOREYDric ACrd. The modern name for Hrdrochroric Acm. The gas was discovered by Priestley in 1772; dissolved in water, it has been long known as spirits of salts. See Mertatic Acm.

CBIORIC ACID. The acid constituent of the chlorates. This acid, which is only known in combination with one equivalent of water, is exceedingly unstable, being instantly decomposed by contact with organic matter, and undergoing gradual spontaneous decomposition in diffused daylight. It is prepared by decomposing chlorate of potash by the addition of hydro-fluosilicic acid, which forms with potash an insoluble compound.

CrIORIDEs. "The term chloride is applied to all compounds of chlorine, which may be derived from one or more atoms of hydrochloric acid, by the substitution of a metal or other radical (which may itself contain chlorine) for an equivalent quantity of 
hydrogen.'-Watts's ' Dietionary of Chemistry.' For descriptions of the chlorides, refer to their respective metals.

'The term ' chloride' is also applied in tho arts to a numbor of bleaching compounds, such as 'chloride of lime,' the exact chemical nature of which is still obscure, though it is certain that they aro not simple chlorides, or combinations of chlorino with metals. It is now generally believed, that these so-called chlorides of the alkalis and alkaline earths are either compounds or mixtures of true chlorides with hypochloritos.

CzIORIDz or Iraz. The compound so called was first employed in the liquid form as a bleaching agent in 1798; and in the following year the idea suggested jtself to Mr. Charles Macintosh, ait that time a partner of Messrs. Tenant and Knox, to impregnate quicklime in a dry state with chlorine, and a patent was taken out accordingly. The discovery of the bleaching property of chlorine is due to Berthollet, who announced the fact to the Academy of Sciences in 1785. In the following year, tho new method of bleaching was introduced into Great Britain by Mr. Watt. In 1788, Mr. Thomas Henry of Manchester exhibited ealico bleached by chlorine, without having any knowledge of the previous experiments of Watt; and in the following year a dotailed method of the process was published by Berthollet. To give some idea of the rapidity with which bleaching is conducted by the improved modern processes, the writer of the article on this subject in the Encyclopadia Britannica quotes the following illustration:-A bleacher in Lancashire received 1,400 pieces of grey muslin on a 'Tuesday, which on the Thursday immediately following were returned bleached to the manufacturers at the distance of 16 miles and they were packed up and sent off that very day to a foreign market.

It is ostimated that the present annual production of chloride of lime in Great Britain and Ireland amounts to 75,000 tons, of the value of three-quarters of a million sterling.

In the manufacture of chloride of lime, chlorine gas is transmitted at a proper temperature through milk of lime or over dry slaked lime, the product being thus obtained either as a liquid or as a powder. The different methods of generating the gas will be described under ChLorins.

A great variety of apparatus has been at different times contrived for favouring the combination of chlorine with the slaked lime for the purpose of commerce. The simplest construction for subjecting lime-powder to chlorine, is a large chamber 8 or 9 foet high, built of sandstone, having the joints of the masonry secured with a cement composed of pitch, resin, and dry gypsum in equal parts. A door is fitted into it at one end, which can be made air-tight by strips of cloth and clay lute. A window on cach side enables the operator to judge how the impregnation goes on by the colour of the air, and also gives light for making the arrangements within at the commencement of the process. As water lutes are incomparably superior to all others where the pneumatic pressure is small, a large valve or door on this principle might advantageously be made in the roof, and two tunnels of considerable width at the bottom of each side wall. The three covers should be simultaneously lifted off by cords passing over a pulley, without the necessity of the workman approaching the deleterious gas, when the apartment is to be opened. A great number of wooden shelves, or rather trays, 8 or 10 feet long, 2 feet broad, and 1 inch deep, are provided to receive the riddled slaked lime, containing generally about 2 atoms of lime to 3 of water. These shelves are piled one over another in the chamber, to the height of 5 or 6 feet, cross bars below each keeping them about an inch asunder, in order that the gas may have free room to circulate over the surfece of the calcareous hydrate.

The alembics for generating the chlorine, which are usually nearly spherical, are in some cases made entirely of lead, in others of 2 hemispheres joined together in the middle, the upper hemisphere being lead, the under one cast-iron. The first kind of alembic is enclosed for two-thirds from its bottom in a leaden or iron case, the intorval of two inches between the two being destined to receive steam from an adjoining boiler. Those which consist below of cast-iron have their bottom directly exposed to a very gentle fire; round the outer edge of the iron hemisphere a groove is cast, into which the under edge of the leaden hemisphere fits, the joint being rendered air-tight by Roman or patent cement. In this leaden dome there are four apertures, each secured by a water-Iute. The first opening is about 10 or 12 inches square, and is shut with a leaden valve, with incurvated edges, that fit into the water-channel at the margin of the hole. It is destined for the admission of a workman to rectify any derangement in the apparatus of rotation, or to detach hard concretions of salt from the bottom.

The second aperture is in the centre of the top. Here a tube of lead is fixed, which descends nearly to the bottom, and down through which the vertical axis passes. To its lower end the cross bars of iron or of wood, sheathed with lead, are attached, by whose revolution the materials receive the proper agitation for mixing the dense manganese with the sulphuric acid and salt. The motion is communicated eithor by the 
hand of a workman applied from time to time to a winch at top, or it is given by connecting the axis with wheol work, impellod by a stream of water or a steam-engine. The third opening admits the siphon-formed funnel, through which the sulphuric acid is introduced; and the fourth is the orifice of the eduction-pipe. The fourth aperture admits the eduction-pipe. This pipe is afterwards conveyed into a leaden chest or cylinder, in which all the other eduetion-pipes also terminate. They are connected with it simply by water-lutes, having hydrostatic pressure of 2 or 3 inches. In this general diversorium the chlorine is washed from adhering muriatic acid, by passing through a little water, in which each tube is immersed, and from this the gas is let off by a protty large leaden tube, into the combination-room. It usually enters in the top of the coiling, whence it diffuses its heavy gas equally round.

Four days are required, at the ordinary rate of working, for making good marketable bleaching-powder. A more rapid formation would merely endanger an elevation of temperature, productive of muriate of lime, at the expense of the bleaching quality. But skilful manufacturers use here an alternating process. They pile up, first of all, the wooden trays only in alternate shelves in each column. At the end of two days the distillation is intermitted, and the chamber is laid open. After two hours the workman enters, to introduce the alternate trays covered with fresh hydrate of lime, and at the same time rakes up thoroughly the half-formed chloride in the others. The door is then secured, and the chamber, after being filled for two days more with chlorine, is again opened, to allow the first set of trays to be removed, and to be replaced by others containing fresh hydrate, as before. Thus the process is conducted in regular alternacion; very superior bleaching-powder is manufactured, and the chlorine may be suffered to enter in a pretty uniform stream. But for this judicious plan, as the hydrate advances in impregnation, its faculty of absorption becoming diminished, it would be requisite to diminish proportionately the evolution of chlorine, or to allow the excess to escape, to the great loss of the proprietor, and, what is of more consequence, to the great detriment of the health of the workmen.

According to M. C. H. Mène ('Comptes Rendus,' Nov. 22, 1847), chloride of lime may be propared almost pure, and instantaneously, by pouring upon slaked lime, water saturated with chlorine. The chlorine is absorbed the moment the liquid comes into contact with the lime, and if the supernatant water is immediately decanted, and the lime remaining at the bottom of the vessel saturated by frequont repetition of the treatment with chlorine water, perfectly pure chloride of lime is obtained. Labarraque finds that the absorption of chlorine by moistened hydrate of lime is greatly facilitated by mixing it with $\frac{1}{20}$ th of its weight of common salt.

Manufacturers differ much from each other in the proportion of their materials for generating chlorine. In general, $10 \mathrm{ewt}$. of salt are mixed with from 10 to $14 \mathrm{cwt}$. of manganese, to which mixture, after its introduction into the alembic, from 12 to $14 \mathrm{cwt}$. of sulphuric acid are added in successive portions. That quantity of oil of vitriol must, however, be previously diluted with water, till its specific gravity becomes about 1'6. But, indeed, this dilution is seldom actually made, for the manufacturer of bleachingpowder almost always prepares his own sulphuric acid for the purpose, and therefore carries its concentration in the leaden boilers no higher than the density of 1.65 , which, from Dr. Ure's table of sulphuric acid, indicates $\frac{1}{4}$ th of its weight of water, and therefore trd more of such acid must be used.

The manufacturer generally reckons on obtaining from one ton of rock-salt, employed as above, a ton and a half of good bleaching-powder. But the following analysis of the operation will show that he ought to obtain two tons :-

When a mixture of sulphuric acid, common salt, and black oxide of manganese are the ingredients used by the manufacturer of bleaching-powder, the absolute proportions required by theory are:

1 atom chloride of sodium

1 atom binoxide of manganese : : : 43.5

2 atoms oil of vitriol

and the products ought to be:

$\overline{200 \cdot 0} \quad \overline{100 \cdot 00}$

1 atom chlorine disengaged

1 atom sulphate of soda

1 atom sulphate of manganese.

2 atoms water

$35 \cdot 5$

$71 \cdot 0$

$75 \cdot 5$

$18 \cdot 0$

$200 \cdot 0$
$17 \cdot 75$

$35 \cdot 50$

$37 \cdot 75$

$2 \cdot 00$

$100 \cdot 00$

These proportions are, however, very different from those employed by the manufacturers; and they ought to be so, on account of the impurity of their axide of man- 
ganese. Moreover, the oil of vitriol has a tendency to form the acid sulphate of soda or bisulphate, instead of the normal sulphate, and this salt is only decomposed at a high temperature.

From the preceding computation, it is evident that 1 ton of salt with 1 ton of the above native oxide of mangunese, properly treated, would yield 0.59 of a ton of chlorine, which would impreguate 1.41 ton of slaked lime, producing 2 tons of bleaching-powder, stronger than the average of the commercial specimens; or, allowing for a little loss, which is unaroidable, would afford 2 tons of ordinary powder, with a little more slaked lime.

Fig. 461 represents a retort of lead, well adapted to the evolution of chlorine from the mixture of salt, manganese, and sulphuric acid, or from manganese and muriatic acid.

The interior vessel is cast in lead, and it has round its bottom part a cast-iron steam case. The salt and manganese are introduced by the aperture $c$, and the sulphuric acid by the siphon-funnel $f$. The contact of these three substances is continually

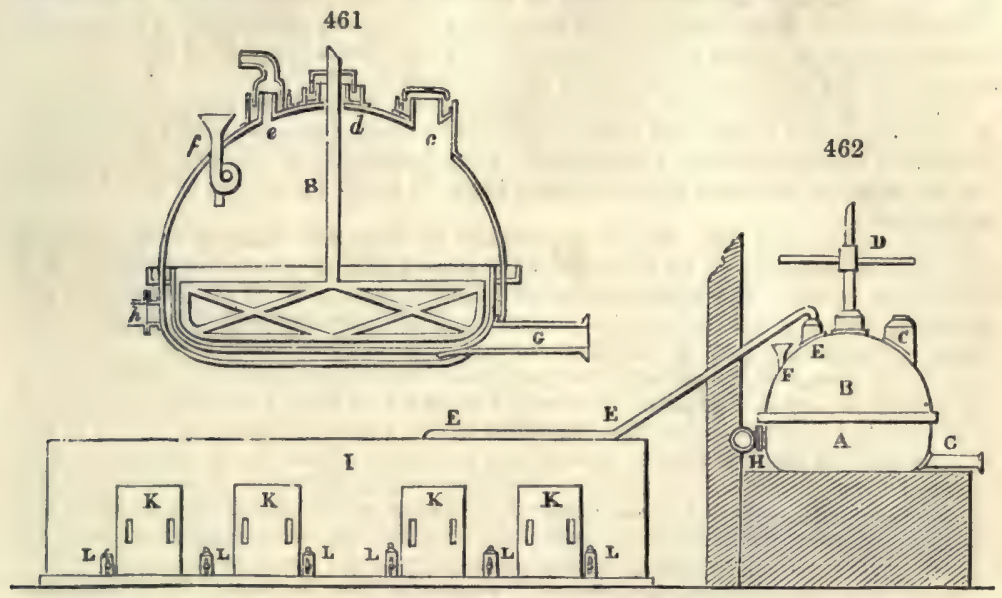

renewed by the agitator or stirrer $B$, which consists of wrought or east iron, sheathed with lead, $e$ is the gas-discharge pipe. The residuums are drawn off by the bottom discharge pipe $\mathrm{G}$. The heating case receives its steam by the pipe $h$.

The chlorine gas, is conveyed from the retort $\mathrm{B}$, fig. 462 , into the chamber I, by the tube $\mathbf{~ E ~} \mathbf{x}$. This chamber is divided into four compartments, to receive the gas disengaged from four retorts, like the above. The bottom of it is covered with a stratum. three or four inches thick of quicklime, newly slaked and sifted, which is stirred about from time to time, by the rakes I L LI. When the saturation is sufficient, the chloride of lime is taken out by the doors $\mathbf{K} \mathbf{K} \times \mathbf{r}$. The side of this apparatus allows 2 ewt. of manganese, and its equivalent quantity of salt and sulphuric acid, or of muriatic acid, to be introduced at once into the retort. $D$ is the handle of the agitator.

The same form of retort will suit perfectly well to prepare chlorine for making liquid chloride of $\lim \theta$, which is preferred by many bleachers and calico-printers who have conveniences for preparing it themselves. The most concentrated solutions of the dry chloride of lime do not mark more than $6^{\circ} \mathrm{B}$. (specific gravity 1.04), and discolour only 50 volumes of Gay-Lussac's solution of indigo, whilst the chloride made in the humid way marks from $8^{\circ}$ to $9^{\circ} \mathrm{B}$. (about $1 \cdot 060$ ), and discolours 80 volumes of the same solution.

In the chloride-of-lime apparatus, most generally used by the skilful calico-printers of Mülhausen, the mixture of muriatic acid and manganese is put into glass globes, with long necks, heated upon a sand-bath. The chlorine is conveyed by glass tubes into a cylindrical stone cistern, contrining milk of lime. The furnace of the sandbaths is made of cast-iron, and has brick partitions, to give each retort its own fire. The smoke of all these fires goes off by a flue into sheet-iron pipes. The cistern is made of siliceous sandstone. Its cover is of wood, coated with a resinous cement; and it fits at its edges into grooves cut in the stone. A wheel serves to agitate the liquid continually; its paddles being kept at 2 inches distance from the sides of the cistern. The milk of lime is introduced by a funnel, and the chloride is drawn off by a discharge pipe. The lead retort and agitator used in this country seem greatly preferable to 
the experimental laboratory plan described above. In all such apparatus we should avoid giving any pressure to the tubes or vessels, and should not therefore dip the extremitios of the gas-pipes beneath the surface of the liquid, but rather facilitate the combination of the chlorine and the lime, by enlarging the surfaces of contact and by agitating. Intermediate vessels containing water, or the chemical cascade of $\mathbf{M}$. Clement, are very useful for absorbing any muriatic acid which may be disengaged along with the chlorine, and thereby preventing the needless formation of muriate of lime in the chambers or cisterns of impregnation.

When the solution of the chloride of lime is mixed with hydrate of lime, it bears, without decomposing, a pretty high temperature, provided it be not too long continued; it may oven, in certain cases, be raised to near the boiling point without suffering a marked loss of its discolouring power; but when the chloride is deprived of that excess of lime, it is decomposed in a short time, even at a heat of $110^{\circ} \mathrm{F}$.

When chlorine is admitted to milk of lime, it infallibly produces some muriate of $\lim \theta$; but the quantity is kept at a minimum by constantly presenting an excess of lime to the gas with the agitator, and by keeping the temperature as low as possible. Hence the influx of gas should not be so rapid as to generate much heat. An automatic agitator, mored by steam or water power, is therefore much better than one driven hy the hand of the operator, who is apt to intermit his labours.

If the liquor becomes hot at the end of the process, it should be immediately drawn off into large stone bottles and cooled. The rose colour, which sometimes supervenes, is due to a minute quantity of manganese : the strongest liquid chloride of lime that can be prepared will not discolour more than 80 times its volume of Gay-Lussac's indigo test.

In the year 1846, Mr. Pattinson patented an improved mode of manufacturing chlorine. In this process he made use of a stone vessel or generator, enclosed in a double iron vessel. The hydrochloric acid, specifle gravity $1 \cdot 16$, is poured into the generator, and on a grating or false bottom is placed the binoxide of manganese in lumps. The temperature of the contents of the generating vessel is then raised to $180^{5} \mathrm{~F}$., by means of steam, made to circulate between the stone vessel and the iron casing. This heat is continued for about 18 hours ; and then, by means of a suitable pipe passing to the bottom of the generator, steam, under a pressure of $10 \mathrm{lbs}$, to the inch, is injected into the vessel for about two minutes, and this is repeated every half hour for about six hours. In this process no mechanical agitation is required, as the steam enters with sufficient force, under the pressure above mentioned, to effect the requisite agitation of the contents, and by clearing the lumps of manganese from all adhering matters, expose a fresh surface continually to the action of the acid.

In carrying this process into practical operation, Mr. Pattinson found that the apparatus is liable to be completely deranged, and the iron vessel destroyed by the action of the hydrochloric acid, if the stone generating vessel should happen to get broken; to obviate which inconvenience, and to enable the generator to be used though in a broken condition, the inner iron vessel is perforated; and the spaces between the two iron vessels, and between the inner iron vessel and the stone generator, are filled with coal-tar, or pitch, thickened by boiling to such a consistonce as to be tough, but not brittle, when cold. Steam, circulating through a coil of pipe passing between the iron vessels, serves to maintain the tar at the requisite degree of heat; and in the event of the breakage of the stone generator, the liquefied tar flows into the fissure, and prevents the escape of the hydrochloric acid into the steam vessel.

Chlorine-stills of metal or of earthenware, though used even now in manv continental works, have been generally displaced in this country by vessels of sandstone. In Lancashire they are usually constructed of Yorkshire flags, cemented together by a mixture of tar and clay, while on the Tyno they are cut out of solid blocks of stone, and rendered impervious on the surface by being boiled in coal-tar.

The receivers in which the chlorine acts on the lime are usually constructed either of stone slabs or of sheet lead supported on timber framework. The receiving chambers are fitted with shelves on which the slaked lime is conveniently exposed in layers for absorption of the chlorine. In other forms of apparatus the lime is placed in a series of pots or in rotating barrels. The absorption of chlorine is accompanied by evolution of heat, but as chlorate of lime is formed at a high temperature the gas is admitted but slowly and the temperature thus maintained sufficiently low to avoid formation of the chlorate.

Several proposals have been made for utilising the waste liquors from the chlorine stills, most of which aim at reproducing oxide of manganese which may be again available for the generation of chlorine.

A method of treating the residuum was patented in 1855 by Mr. C. Tennant Dunlop. 


\section{CHLORIDE OF LTME}

It consists in transforming the chloride of manganese, first into carbonate and then into oxide, by the action of heat. Whatever impurity the chloride of manganese may contain - as chloride of iron, for instance - is first separated, either by calcination or by the agency of a suitablo precipitant. Practical working has shown that the carbonate of manganese thus treated yields an oxide of a richness equivalent to that of 80 per cent. of peroxide. The carbonate of manganese may be obtained by precipitation from the chloride by carbonate of ammonia. The chloride of ammonium resulting from this treatment may either be employed as such, or it may be retransformed in the usual way into carbonate for the precipitation of fresh chloride of manganese. Hydrate of lime is also used as a precipitant, the resulting hydrated. oxide of manganese being subsequently converted into carbonate by the transmission through it of a stream of earbonic acid.

By another process, carbonate of manganese is obtained by passing carbonic acid through the solution of chloride of manganese which has been previously mixed with a quantity of carbonate of soda. The carbonate of soda, under the influence of carbonic acid, decomposes the chloride of manganese into carbonate, from which the oxide can be obtained. The essential feature of this invention is the production of artificial oxide of manganese, by first converting the chloride into carbonate, and afterwards this latter into oxide, by the joint agencies of heat and atmospheric air.

In a process suggested by Binks and Gatty, the chloride of manganese in the stillliquor is heated with nitrate of soda, whereby a mixture of nitrous acid and oxygen is erolred, while a residue of chloride of sodium and oxide of manganese is obtained.

But the most valuable process which has yet been proposed for regenerating the manganese from the spent liquors is that which has been lately introduced by Mr. Walter Weldon, and is now largely employed in this country. To understand the working of this process it should be remembered that the acid liquor left in the chlorine-stills, by the action of hydrochloric acid on peroxide of manganese, consists mainly of a solution of protochloride of manganese (manganous chloride), with the sesquichlorides of iron and aluminium, chloride of calcium, and such excess of hydrochloric acid as may have been employed. This liquid is run from the generators into a well, where it is neutralised by addition of finely-divided chalk, the neutralisation being facilitated by keeping the liquid stirred up by a mechanical agitator. Not only is the free acid thus neutralised, but the chlorides of iron and aluminium are decomposed, and the oxides precipitated. The neutral liquor is then pumped up into tanks, called 'chloride of manganese settlers,' where the sulphate of lime, the oxide of iron, and the alumina held in suspension are rapidly deposited, and a clear solution of manganous chloride, of a pale pink colour, is obtained. This clear liquor is run off into a tank below called the 'oxidiser,' in which it is treated with an excess of milk of lime to precipitate the manganese as hydrated protoxide, which is converted into a higher oxide by the injection of air. The oxidiser is generally a cylindrical iron vessel, about 12 feet in diameter and 22 feet deep. The reactions in this vessel are best effected at a temperature of from $130^{\circ}$ to $170^{\circ} \mathrm{F}$, and if the charge of manganous liquor is not sufficiently hot, its temperature is raised by injection of steam. When sufficiently heated, the liquor is subjected to a blast of atmospheric air introduced through a large pipe reaching nearly to the bottom of the oxidiser. The milk of lime is then rapidly run in from a reservoir above, and the blowing continued until peroxidation ceases-a point usually attained when from 80 to 85 per cent. of the manganese has been converted into peroxide. The thin black sludge resulting from this action contains chloride of calcium and peroxide of manganese associated with a variable proportion of protoxide of manganese and lime. Each cubic foot of mud contains about $2 \mathrm{lbs}$. of peroxide of manganese. The product is run off from the oxidiser to the 'mud settlers,' where a thick black deposit, containing about four lbs. of tho peroxide to the cubic foot, gradually subsides. This deposit, separated from the supernatant liquid, is transferred, while still moist, to the chlorine-stills, where it is treated with hydrochloric acid for production of chlorine, and the cycle or operations thus begins afresh, and may be continued indefinitely - the manganese merely playing the part of a carrier or medium of transference between the oxygen of the atmosphere and the hydrochloric acid. In an experiment continued for five hours, during which time 175,000 cubic feet of air was blown through the sludge in the oxidiser, it was found that more than $4 \mathrm{cwts}$. or 14.8 per cent. of the oxygen was absorbed, serving to reproduce $24 \mathrm{cwts}$. of peroxide of manganese. The cost of recovering the manganese by this process is said to be less than one-fourth the average cost of an equivalent amount of native manganese.

Nature of Bleaching Powder.-An elaborate series of experiments on the manufacture of chloride of lime was made in 1822 by Dr. Ure, the most important results of which are embodied in the subjoined Table:-

Vol. I. 


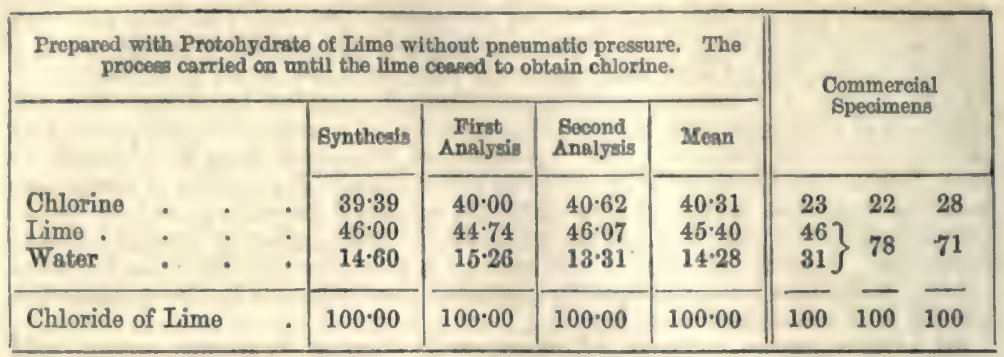

Mr. Graham found that hydrate of lime, dried at $212^{\circ}$, absorbed afterwards little or no chlorine; but that, when dried over sulphuric acid, it was in the most favourable condition for becoming chloride of lime. A dry, white, pulverulent compound is obtained by exposing the last hydrate to chlorine, which contains 41.2 to 41.4 chlorine in 100 parts, of which 39 parts are available for bleaching, the remainder going to form chloride of calcium and chlorate of lime. This appears to be the maximum absorption of chlorine by dry hydrate of lime; but the bleaching powder of commerce rarely, even when fresh prepared, contains more than 30 per cent. of chlorine, and after being kept for several months, the proportion often falls as low as 20 per cent. A compound containing one equivalent of chlorine and one equivalent of hydrate of lime, should contain 48.57 chlorine and 51.43 hydrate of lime; a compound of one equivalent of chlorine and two of hydrate of lime, should contain 32.42 chlorine and 67.58 hydrate of lime; and these are about the proportions in good commercial specimens. It would not be advisable to attempt to manufacture a more highly chlorinated product, as the stalility of the compound is increased by an excess of lime. Where a stream of chlorine is transmitted through water holding hydrate of lime in suspension, the lime is entirely dissolved, and the full equivalent of chlorine is absorbed. Different views have been held by chemists respecting the constitution of bleaching powder, but it is now generally admitted that the substance consists essentially of chloride of calcium and hypochlorite of lime, with excess of slaked lime. Water poured upon bleaching powder dissolves out the bleaching combination, learing a large residue of lime. Ten parts of water are required for one part of dry chloride. The solution emits the peculiar odour of hypochlorous acid. The reaction which occurs in the formation of 'chloride of lime' may be thus represented:-

$$
\begin{aligned}
& 2(\mathrm{CaO} \cdot \mathrm{HO})+2 \mathrm{Cl}=\mathrm{CaCl}+\mathrm{CaO} \cdot \mathrm{ClO}+2 \mathrm{HO} \text {. } \\
& 2 \mathrm{CaH}^{2} \mathrm{O}^{2}+\mathrm{Cl}^{4}=\mathrm{CaCl}^{2}+\mathrm{CaCl}^{2} \mathrm{O}^{2}+2 \mathrm{Ir}^{2} \mathrm{O} \text {. }
\end{aligned}
$$

Slaked lime and chlorine thus yield chloride of calcium, hypochlorite of lime and water. Whether the chloride and hypochlorite are united as a combination or merely mixed together, may be open to question. But good bleaching powder is not deliquescent, neither does alcohol dissolve anything from it, both which should occur if the compound contained free chloride of calcium. It is possible, however, that the two salts may exist in bleaching powder in the form of a double salt, or that the chlorine is in direct combination with the oxide. If the compound be supposed to be pure chloride of lime, the reaction is simply an absorption of chlorine; and the same should be the case with the other bleaching compounds-chloride of soda, for instance. But when carbonate of soda, saturated with chlorine (Labarraque's Liquor), is evaporated, no chlorine is evolved, and the residue still possesses bleaching properties. The true nature of bleaching powder is open, therefore, to speculation.

The bleaching action of solution of chloride of lime is very slow unless an acid be added to it. When dilute sulphuric acid in insufficient quantity is employed, no chlorine is evolved, but hypochlorous acid, which may be distilled off and condensed in a suitable receiver; but with excess of acid, chlorine only is liberated. When calicoes and other woven goods are to be bleached, they are first thoroughly cleansed by boiling successively with lime-water and a weak solution of canstic soda; they are then digested in a solution of bleaching powder, specific gravity 1.02, containing about 21 per cent. of chloride of lime; after which they are immersed in very dilute sulphuric acid, which, by liberating the chlorine within the filres of the cloth, rapidly remores the colour. The goods are then washed, a second timo steeped in alkali, and again passed through a weaker solution of chloride, and then through dilute acid ; after which they are thoroughly washed in water. The quantity of liquor necessary for $700 \mathrm{llbs}$. of cloth is 971 gallons, containing $388 \frac{1}{2}$ lbs. of chlorido. When white figures are required on a coloured ground, the pattern is printed on the cloth with tartaric acid, thickened 
with gum. The colour is discharged in those places where the acid was present, but elsewhere untouched. Sulphate of zinc or chloride of zine may be employed, instead of acids, for liberating chlorine from bleaching powder.

On acting upon cotton-cloth with a concentrated solution of chloride of lime, at from $110^{\circ}$ to $120^{\circ} \mathrm{F}$., pure carbonic acid is disengaged, and the texture of the cloth is injured. Here the hydrogen of the water, and of the cotton, being seized by the chlorine, the liberated oxygen combines with the carbon to form carbonic acid. In the dischargetroughs, where printed calicoes are passed through strong solutions of chloride of lime, stalactitic crusts of carbonate of lime come to be formed in this way.

When chloride of lime is heated, it evolves oxygen gas, and sometimes chlorine, and it becomes converted into a mixture of chlorate of lime and chloride of calcium, which has no bleaching properties. Half an ounce of chloride of lime boiled in two ounces of wates yields, according to Keller, 165 cubic inches of oxygen contaminated with chlorine.

The property of chlorine, to destroy offensive odours and to prevent putrefaction, gives to the chlorides of lime and soda a high value. On this important subject Pexeira, has the following remarks (Mat. Med. vol. i.) with reference to medical police:- If air bo blown through putrid blood, and then through a solution of chloride of lime, caxbonate of lime is precipitated, and the air is disinfected; but if the air be first passed through putrid blood, then through caustic potash, or milk of lime, to abstract the carbonic acid, and afterwards through the solution of chloride of lime, it retains its stinking quality. Chloride of lime may be employed to prevont the putrefaction of corpses previous to interment;- to destroy the odour of exhumed bodies during medico-legal investigations;-to destroy bad smells and prevent putrefaction in dissecting-rooms and workshops in which animals substances are employed (as catgut manufactories); - to destroy unpleasant odours from privies, sewers, drains, wells, docks, \&c. ;- to disinfect ships, hospitals, prisons, stables, \&cc. The various modes of applying it will readily suggest themselves. For disinfecting corpses, a sheet should be soaked in a pailful of water containing a pound of chloride, and then wrapped round the body. For destroying the smell of dissecting rooms, \&c., a solution of the chloride may be applied by means of a gardening pot.' Of equal importance is this substance to the medical practitioner. The poisonous exhalations from foul sewers may be counteracted by a slight inhalation of chlorine gas, as obtained from a little chloride of lime placed in the folds of a towel wetted with acetic acid. The importance of chlorine as a disinfecting agent appears to have been fully proved during the period of the cattle plague. Both Dr. Angus Smith and. Mr. Crookes, in their reports, give several very striking examples of the power of this agent in cleansing sheds and houses which had been occupied by cattle suffering under and dying from the disease. Carbolic acid, however, appears to be preferred by one of these chemists. Both these preparations may, it is elear, be used with satisfaction when required.

For estimating the amount of chlorine in bleaching powder, and thus determining the commercial value of a given sample, see Chooromerny.

\section{CAIORIDE OT potasstum. See Potassium.}

CHIORIDFS OF POTASR AND SODA. When a weak solution of caustic potash or soda is saturated with chlorine, it affords a bleaching liquor, still used by somo bleachers and calico-printers for delicate processes; but the price of the alkalis has led to the disuse of these chlorides as a general means, and has occasioned an extensive employment of chloride of lime. The chloride of potash is known as Water of Jarelle, and the chloride of soda as Labarraque's Liquor. They may bo prepared by transmitting chlorine gas through a solution of caustic alkali or alkaline carbonate, or by decomposing chloride of lime with an alkaline sulphate or carbonate. These so-called chlorides are now generally considered to be mixtures or compounds of chlorides and hypochlorites. See CHLORmz of Lring.

CEIORINATION. The method of extracting gold and silver from certain ores by means of chlorine. This process is especially employed for separating gold from sulphides and arsonides which cannot be treated by the ordinary methods of amalgamation. By the aid of chlorine gas, the gold is converted into a chloride, and from a solution of this salt the metal may bo precipitated as a sulphide by sulphuretted hydrogen, or reduced at once to the metallic state by means of sulphate of iron. The process enables a very small percentage of gold to be extracted with advantage from an ore; indeed, according to Mr. Allain, it is possible to extract gold from pyrites by means of chlorine, when the metal is present to the extent of only one part in 10,000 parts of roasted ore.

The chlorination-process was originally proposed by Professor Plattner, and was applied, as far back as 1851, to the extraction of gold from the arsenical pyrites of Reichenstein in Silesia-an ore containing about 200 grains of gold to the ton. In 
consequence of the introduction of this process, the mines, after haring been abandoned for more than five centuries, wero re-opened and worked with profit. The ore is first roasted in a reverberatory furnace, and the arsenic thus becomes converted into arsenious acid, which sublimes, and is condensed in a large chamber. The residue, consisting chiefly of iron, is subjected to the action of chlorine, and the iron, with the gold which it contains, then passes into the state of chloride; the chlorides are dissolved out by water, and the gold precipitated by sulphuretted hydrogen,- - the precipitation of the iron being prevented by previously acidifying the solution with hydrochloric acid. The sulphide of gold is readily decomposed by heat, yielding metallie gold on simple ignition.

Since the introduction of the chlorination-process at Reichenstein, it has been applied elsewhere with considerable success. At Schemnitz, in Hungary, the tailings left after extraction of silver by Ziervogel's method, are treated with chlorine, and the gold, though amounting to only about 0.012 per cont. of the tailings, is thus profitably separated. In California the chlorination-process was first introduced, in 1858, by Mr. Deetken; and several chlorination-establishments have since been erected in the neighbourhood of Grass Valley. The process, as conducted in California, has been deseribed by Mr. Knstel.'

If the ore to be chlorinated contains finely-divided gold in quartz, it needs no preparatory treatment beyond that of pulverization. But if sulphides and arsenides are to be treated, they require to be previously roasted. The concentrated ores should be roasted while damp, but pan-tailings should be first dried and then finely pulverized. The roasting is most advantageously effected in a reverberatory furnace in which the flame of the fuel is allowed to come into direct contact with the ores. A low temperature should be employed at first, and when much of the sulphur and arsenic is expelled the heat should be gradually raised until any sulphates or arsenites which are formed may be decomposed, and all the metals converted into oxides, excepting the gold, which is set free in a metallic state. The loss of gold during roasting, though stated by some to be considerable, seems really to be insignificant.

In some cases it is desirable to add common salt (chloride of sodium) to the ore during the roasting. If lead and antimony are present, no salt should be used, but if the ore be associated with minorals containing lime, magnesia, or baryta, the addition of salt is desirable. About 5 per cent., or $100 \mathrm{lbs}$. of salt to the ton of sulphides, will be found sufficient. The salt should be thoroughly incorporated with the ore, so as to favour separation of the gold. It appears that the gold first forms a terchloride $\left(\mathrm{AuCl}^{3}\right)$, which is converted at a higher temperature into protochloride $(\mathrm{AuCl})$, and this at a red heat, is reduced to metallic gold.

The roasted ore, before being treated with chlorine, must be slightly moistened; this is effected by spreading the charge over the floor of an iron chamber, and sprinkling it with water by means of a hose; the ore being well stirred so that the wet stuff is well mixed with the dry. After being sifted, to remove lumps, the ore is transferred to the chlorination-vats. These are large circular wooden vessels, each capable of holding two or three tons of roasted ore. The inside of the rat is coated with a mixture of pitch and tar, or with some similar substance, to prevent absorption of the gold-solution by the wood. At a height of about one inch from the bottom of the vessel, is fixed a false bottom, formed of boards laid together, with spaces between, and perforated with holes for the passage of the chlorine gas. A layer of fragments of quartz, or other rock, is first spread over the false bottom, and the coarser fragments covered by finer and finer layers till a stratum of fine sand is placed on the top. The charge is laid on this prepared bed, which serves as a filter, and chlorine gas is introduced through the false bottom. The gas is generated in a leaden vessel. Sufficient chlorine for treating a charge of three tons of roasted sul. phides may be obtained, according to Kustel, from the following mixture:--Pulverized manganese (peroxide) $30 \mathrm{lbs}$., common salt, 30 or $40 \mathrm{lbs}$, oil of vitriol of 66 degrees, $75 \mathrm{lbs}$., and water, $45 \mathrm{lbs}$. Having introduced the water, salt, and manganese into the generator, the vessel should be covered air tight; the acid is then gradually poured in through a funnel, and the chlorine thus produced is washed by passing through a ressel containing water to absorb any hydrochloric acid, the presence of which is prejudicial. The evolution of chlorine is assisted by heat applied under the gasgenerator. When the chlorine, which passes from the wash-bottle to the chlorinevat, has permeated through the charge, the cover of the rat is luted on. The operator should carefully avoid inhaling the chlorine, as this is a highly poisonous gas, and extremely irritating when inhaled even in small quantity.

The ehlorine is allowed to act upon the ore for from 12 to 18 hours; the covor is then removed from the vat, and a stream of water introduced. The solution of

2 'A 'Treatise on Concentration of all kinds of Ores, including the Chlorination Process.' By Guido Kustel. Ban Francísco : 1868. 
chloride of gold is then conveyed through a trough lined with sheet-lead into the precipitating-vat, which is a large wooden vessel lined with lead or with asphalt. To precipitate the gold, a solution of sulphate of iron (green copperas) is employed; this is best prepared a few days before use, by pouring dilute oil of vitriol on old pieces of wrought iron. A bucket or two of this solution is poured into the precipitating-vat before the gold-solution enters, and a sufficient quantity is afterwards employed to throw down the whole of the gold. This precipitated gold gradually subsidos as a dark brown powder, and the supernatant liquor when clear is drawn off. The gold is then carefully scooped out from the vat into a clear porcelain dish, and the last traces washed out by water. This finely divided gold is dried over a fire, and then melted in clay crucibles; a little borax, salt, or saltpetre being added as a flux. The use of plumbago crucibles is not advisable. The metal obtained in this way generally contains about 995 -thousandths of pure gold.-F.W.B.

CHIorisrz. (Chlorine, Fr., Chlor, Ger.) Symbol, Cl. ; Atomic wèight, 35.5.This element was discovered by the Swedish chemist, Scheele, in 1774, and its true elementary character demonstrated by Sir H. Dary in 1810. It exists, under ordinary circumstances, as a greenish-yellow gas; but, when exposed to a pressure of 4 atmospheres, at $60^{\circ} \mathrm{F}$., it condenses to a transparent liquid, which remains unfrozen even at the cold of $-220^{\circ} \mathrm{F}$. In the first state, its density, compared to air (reckoned. $1.00)$, is 2.47 ; in the second, its density, compared to water $(1.00)$, is 1.33 . It is generally obtained either by the action of sulphuric acid on a mixture of common salt and binoxide of manganese, or by the action of moderately strong hydrochloric acid on binoxide of manganese alone. In the first case, the proportions are 7 parts by weight of oil of vitriol, previously diluted with 7 parts of water and 4 parts of common salt, intimately mixed with 3 parts of binoxide of manganese; in the latter, which is the most convenient method, hydrochloric acid, specific gravity $1 \cdot 15$, is gently heated with the finely-powdered binoxide, in the proportions of about three oz. of oxide to half a pint of acid. The hydrochloric acid should not be more diluted than above indicated, otherwise an explosion may occur, probably in consequence of the formation of one of the explosive oxides of chlorine. The gas must be collected either over brine or over warm water.

In the preparation of chlorine from binoxide (peroxide) of manganese and hydrochloric acid, 43.5 parts by weight of manganese and 73 of acid yield 35.5 parts of free chlorine. If the peroxide of manganese be acted on by a mixture of hydrochloric and sulphuric acids, 43.5 parts of the manganese, 36.5 of hydrochloric and 49 of sulphuric acid, will produce 35.5 of chlorine gas. When the generating materials are common salt, sulphuric acid and peroxide of manganese, 58.5 parts of salt, 147 of acid, and 43.6 of manganese yield 35.5 of chlorine. In this case a bisulphate or acid sulphate of soda remains in the retort, but if the supply of acid should only be sufficient to form a neutral sulphate the complete decomposition will not be effected until a much higher temperature has been reached than was required with the larger proportion of acid. With respect to the calculations given above, it should be remarked that they refer only to theoretical reactions with pure materials, and that in practice the manufacturer will find it necessary to use more than the specified quantities of acid in order to allow for combination with lime, oxide of iron, and whatever other bases may be present as impurities in the raw materials.

In addition to the ordinary methods of generating chlorine already described, several others havo been proposed for obtaining this element from its combinations, chiefly from hydrochloric acid. Some of these methods work with the hydrochloric acid in the state of gas. For example, the gas may be caused to act directly upon peroxido of manganese; or it may be mixed with carbonic acid gas, and the mixture transmitted through red-hot earthenware tubes; or again a mixture of hydrochloric acid and the gases evolved by heating nitrate of soda may be similarly treated. In all these cases an oxidising agent is employed to remove the hydrogen from the hydrochloric acid and thus liberate the chlorine. The simplest method of effecting this oxidation is to pass hydrochloric acid gas and atmospheric air over red-hot pumice-stone or asbestos or spongy platinum. A somewhat similar process has lately been ingeniously devised by Mr. Henry Deacon, of the Widnes Alkali Works, and has been successfully worked on a manufacturing scale. A mixture of hydrochloric acid gas and atmospheric air, heated to from $700^{\circ}$ to $750^{\circ} \mathrm{F}$, is passed over a mass of brick-work which has been soaked in a solution of sulphate of copper (blue vitriol) and dried. Under these circumstances the oxygen of the air and the hydrogen of the acid unite to form water, while the chlorine is liberated, mixed of course with the nitrogen of the atmospheric air, but this dilution of the chlorine does not prevent its employment in the manufacture of chloride of lime. The action of the copper salt in this process is rather obscure; many other salts are capable of playing a similar part, and their action is supposed to bo merely catalytic, that is to say, they induce the reaction botween the oxygen and 
hydrogen merely by their presence or by contact, without themselves taking any direct part in the process : the chomist is familiar with many similar examples of catalytic action. For an elaborato discussion of the principles involved in Deacon's new process we may refer to tho 'Journal of the Chemical Society,' Sept. 1872.

On the Industrial Manufacture of Chlorine, M. F. Lalande and M. Prud'homme have the following remarks:-

The reaction of anhydrous sulphuric acid, mixed with air or oxygen, upon heated alkaline chlorides, which has given riso to Deacon's patont for tho preparation of chlorine and of sulphate of soda, has led us to generaliso this process. We have chiofly experimentod with silicic, boric, stannic, and phosphoric acids, and also with alumina. When any of these bodies, silica, for instance, is mixed with an alkaline chloride, or with an alkaline earth or earthy chloride, and over this mixture, while being heated to red heat, a current of dry oxygen or air is passed, chlorino is evolved, and a silicate formed of the base of the chloride employed:

$$
\mathrm{SiO}^{2}+\mathrm{NaCl}+\mathrm{O}=\mathrm{SiO}^{2} \mathrm{NaO}+\mathrm{Cl}\left(\mathbf{S i O}^{2}+\mathbf{2 N a C l}+\mathbf{O}=\mathbf{2 N a}^{2} \mathrm{SIO}^{3}+\mathbf{C l}^{2}\right)
$$

At a red heat, therefore, oxygen expels chlorine, when there is simultaneously present an acid capable of uniting with the base which is formed. Chlorides heated by themselves in a current of oxygen (chloride of calcium, for instance) do not give off chlorine. When a current of hydrochloric acid gas is passed, along with oxygen, over the mixture of silics and chloride, the acid regenerates the chloride and decomposes the silicate :

$$
\mathrm{SiO}^{2} \cdot \mathrm{NaO}+\mathrm{HCl}=\mathrm{SiO}^{2}+\mathrm{NaCl}+\mathrm{HO}\left(\mathrm{Na}^{2} \mathrm{SiO}^{3}+2 \mathrm{RCl}=\mathbf{S i O}^{2}+2 \mathrm{NaCl}+\mathrm{Fr}^{2} \mathrm{O}\right) \text {. }
$$

In this way a continuous evolution of chlorine is obtained. Instead of taking the mixture of chlorine and silica, the silicate of the metal in which the chlorine is contained, or a mixture of silica and the oxide of that metal, is taken. Since analogous reactions occur with the acids above mentioned, we may state that it is general. The steam formed during the phase of reaction :

$$
\mathrm{SiO}^{2} \cdot \mathrm{NaO}+\mathrm{HCl}=\mathrm{SiO}^{2}+\mathrm{NaCl}+\mathrm{HO}
$$

gives rise to two secondary actions :-

(1). The chlorine decomposes the water at red heat:

$$
\mathrm{HO}+\mathrm{Ol}=\mathrm{HCl}+\mathrm{O}\left(\mathbf{Z}^{2} \mathbf{O}+\mathrm{Cl}^{2}=\mathbf{2} \mathrm{ECl}+\mathbf{0}\right) \text {. }
$$

(2). The stesm decomposes the chlorides at red heat:

$$
\mathrm{NaCl}+\mathrm{HO}=\mathrm{NaO}+\mathrm{HCl}\left(2 \mathrm{NaCl}+\mathbf{x}^{2} \mathbf{O}=\mathbf{2} \mathbf{a}^{2} \mathbf{O}+\mathbf{2 r C l}\right) \text {. }
$$

It is probable that under these conditions an equilibrium exists between the quantities of chlorine, aqueous vapour, and hydrochloric issuing from the apparatus, or a limit of maximum between the quantity of chlorine produced and the hydrochloric acid evolved and issuing from the apparatus. This limit of maximum will bo the greater according as the temperature required for the reaction be less high. Some of our experiments having been made by causing the substances operated with to be imbibed with pumice-stone, we have been induced to try whether pumice-stone alone would yield any result, that material being a complex silicate and a substance belonging to the category of those indicated by us. We have found that with pumice-stone an evolution of chlorine is obtained comparable to that obtained from the other substances, moro particularly silica and lime, boric acid and lime, stannic acid and lime, alumina and chloride of sodium; since the same result is obtained with lumps of brick, it might be ascribed to the porosity of these substances; but this opinion is gainsaid by the fact, that under the same conditions pipeclay, a silicate of alumina and a very porous body, does not give riso to any appreciable reaction; it therefore appears possible that hydrochloric acid converts the surface of pumicestone into a mixture of silica and chlorides. We have compared the production of chlorine in our experiments with that obtained by using bricks impregnated with sulphate of copper (Deacon's patent); the quantity of chlorine obtained in both cases is the same; but with bricks impregnated with sulphate of copper the reaction takes place at a lower temperature. The imperfection of our apparatus has hitherto prevented us from estimating the respective quantities of chlorine, hydrochloric acid, aqueous vapour, contained in the gases issuing from the apparatus; neither have wo been able to estimate exactly the degree of temperature at which the reaction takes place.

Many other processes have been used with more or less success for the generation of chlorine. Thus, peroxide of manganese may be acted on by a mixture of hydrochloric and nitric acids, when chlorine, water, and nitrate of manganese will be obtained; this nitrate, on calcination, reproduces peroxide of manganese and some of the lower oxides of nitrogen, which, by the action of air and water, may again be oxidised to nitric acid. When a concentrated solution of chloride of magnesium (specific gravity 1.435) is mixed with peroxide of manganese, a product is obtained 
which on exposure to heat yields chlorine, magnesia, and chloride of manganese. The chloride of magnesium used in this process may be obtained from carnallite, a mineral largoly worked in the salt mines of Stassfurt and Kalucz. According to Longmaid's mothods, chlorine is prepared by passing dry air over a mixture of common salt and iron-pyrites, roasted together; or a mixture of salt and some metallic sulphate, such as sulphate of iron. In Mac Dougal and Rawson's process any chromate or bichromate is acted on by hydrochloric acid. Shank's method is very similiar, chromato of lime being decomposed by hydrochloric acid; but the mode of reproducing the chromate is different. By Lauren's method protochloride of copper (cupric chloride), mixed with sand, is heated, and free chlorine evolved, while the residue of subchloride (cuprous chloride) is reconverted into the higher chloride by exposure to air in contact with hydrochloric acid. Mallet directs a current of air over subchloride of copper, thus forming an oxychloride, from which chlorine may be eliminated by hydrochloric acid.

Mr. Dunlop has also obtained a patent for the production of chlorine by a very elegant method, which dispenses with the use of manganese altogether. It consists in mixing common salt with nitrate of soda, and submitting the mixture to the action of sulphuric acid. Chlorine and nitrous gas are evolved, and are caused to traverse a vessel containing strong sulphuric acid, by which the nitrous gas is readily absorbed, and the chlorine passes off. A current of atmospheric air is now passed through the nitrous sulphuric acid, until the nitrous is converted into nitric acid. These mixed acids are then made to act upon common salt without any addition of nitrate of soda, and the same gaseous products are obtained as before.

A description of $\mathrm{Mr}$. Weldon's process by which the refuse liquors left in the ordinary mode of preparing chlorine are utilized and the spent manganese re-oxidised, will be found under ChLORme of Lmes (p. 785).

Chlorine has a peculiar smell and irritates the nostrils and other air-passages most violently when inhaled. It is eminently noxious to animal life, and if breathed in its undiluted state, would prove instantly fatal. It supports the combustion of many bodies, and, indeed, several substances, such as antimony and phosphorus, spontaneously burn in chlorine without being previously kindled. The resulting combinations are called chlorides, and act most important parts in many manufacturing processes.

Water absorbs, at the ordinary temperature of the atmosphere, about $2 \frac{1}{2}$ times its volume of chlorine, and acquires the colour, taste, and smell of the gas, as well as its power of destroying or bleaching vegetable colours. When this aqueous solution of chlorine is cooled down to $36^{\circ} \mathrm{F}$., dark yellow crystalline plates appear in it of the hydrate of chlorine, which are composed in 100 parts of 27.7 chlorine and 72.3 water. If these crystals be heated to about $45^{\circ}$, they liquefy, and the gas is evolved.

Chlorine has a powerful affinity for hydrogen, not only combining with it rapidly in the gaseous state, but seizing it in many of its liquid and solid combinations : as in certain volatile hydrocarbons, which it inflames. The compound of chlorine and hydrogen gases is hydrochloric or muriatic acid gas. Binoxide of manganese, whon mixed with liquid hydrochloric acid, as in the above process, abstracts the hydrogen and eliminates the chlorine. When chlorine is passed into water, it decomposes some of it, seizes its hydrogen to form a little hydrochloric acid, and enables its oxygen to unite, either with the chlorine into chlorous acid, or with the remaining water, and to constitute oxygenated water. Hence an aqueous solution of chlorine, exposed to the sunbeam, continually evolves oxygen, and ere long becomes hydrochloric acid.

In the presence of moisture, chlorine acts powerfully upon vegetable colouring matters; water is decomposed, its hydrogen combining with the chloride to form hydrochloric acid, whilst its oxygen is liberated, and in its nascent state, or at the moment of its liberation when most active, oxidises the colouring principles, and thus bleaches them. Bleaching by means of chlorine is therefore a true process of oxidation; in some cases, however, it appears that colourless substitution-products containing chlorine are produced. The value of chlorine as a deodorizer and disinfectant consists also in its power of decomposing water, and setting free oxygen, which thus oxidises the poisonous miasmatic exhalations, and converts them into more or less innocuous products. For such purposes, chlorine is usually best employed in the form of bleaching powder, or chloride of lime; but in some cases the pure gas may be conveniently employed : it requires, however, from its poisonous nature to be used with the greatest care. In fumigating the Millbank Penitentiary, Dr. Faraday found that a mixture of 1 part of common salt and 1 part of binoxide of manganese, when acted upon by two parts of oil of vitriol previously mixed with one part of water (all by weight), and left till cold, produce the best results. Such a mixture, at $60^{\circ}$, in shallow pans of red earthenware, liberatod its chlorine gradually, but perfectly, for four days. The salt and manganese were well mixed, and used in charges of $3 \frac{1}{2}$ pounds 
of the mixture. The acids and water were mixed in a wooden tub, the water being put in first, and then about half the acid; after cooling, the other half was addod. The proportions of water and acid were 9 measures of tho former to 10 of the latter. Soo Watts's 'Dictionary of Chemistry.'

Many of the compounds of chlorine are of great value in the arts and manufactures. Its hydrogen acid is fully described under HYDrochLoric Acm, while its compounds with the metals, forming important substances called chlorides, are noticed under the names of their respective metals. With oxygen, chlorine forms some unimportant anhydrides; but with oxygen and hydrogen it gives rise to a series of ox-acids, known respectively as hypochlorous, chlorous, chloric, and perchloric acids. Some of tho salts of hypochlorous acid are important as constituents of the bleaching compounds popularly called 'chlorides,' as chloride of lime; aud some of the salts of chlorous acid, as chlorate of potash, are also of great value in the arts. Seo CHoratre of Potasn; Cmonmen of Ltare.

CrIORITE. A term appliod to a family of minerals of a dark green colour and lamellar structure. They are hydrated silicates of alumina, magnesia, protoxide of iron, \&c. The chlorite occurring in the Cornish tin-veins is known as Peach.

Crroromrmz. The name given to the process or processes by which the amount of available chlorine is estimated in substances containing it, which are employed in bleaching, or as disinfectants. The chlorides (hypochlorites) of lime, of potash, or of sode are the most important of these compounds. All these decolourising chlorides are decomposed by the action of acids, even by that of the carbonic acid of the atmosphere, chlorine being set free. The amount of chlorine capable of being thus evolved is required to be known for two reasons; to ascertain its commercial value, and to learn whether the strength of the solution employed is within the limits which enable it to be effectively and yet safely used in bleaching. A number of processes have been devised for this purpose. The method proposed by Gay-Lussac has been extensively used in manufactories; it depends upon the fact that arsenious acid in solution is oxidised by free chlorine and converted into arsenic acid with the simultaneous formation of hydrochloric acid. A solution of arsenious acid in hydrochloric acid was first propared, of a known strength, and also a solution of the chloride of lime to be tested, by triturating a weighed quantity with water and making the mixture up to a certain bulk. A measured quantity of the arsenious acid liquor was placed in a beaker and coloured with a solution of sulphate of indigo, and the chloride of lime solution run into it from a graduated burette till the blue colour was destroyed. This process, though capable of giving accurate results when due caro was taken, was liable to some objections. It was difficult to hit the exact point when the whole of the arsenious acid was converted into arsenic acid, and a little chlorino was apt to be evolved if the chloride solution was run in too quickly; moreover, it necessitated the measurement of a turbid liquid which required frequent agitation to keep the suspended matter equally diffused through it, and consequently of uniform strength throughout. To obviate these defects, the following modification of this process has been proposed by Penot. It is based upon the same reaction as Gay-Lussac's, but the change is effected in an alkaline solution, and a test-paper moistened with starch paste containing iodide of potassium is used to show when the reaction is completed. Whatever process may be employed, the mode of preparing the solution of the chloride of lime is the same. Weigh out 100 grains of the chloride to be examined, after it has been well mixed so as to obtain a fair average sample, put them into a mortar with a little water and triturate together so as to break up all lumps and produce a smooth paste, continue to rub whilst gradually adding more water, allow the heavier particles to subside, pour off the supernatant liquid into a measuring flask or cylinder capable of holding 10,000 grains, treat the residue in the mortar as before, and finally rinse the mortax out into the flask and make up the solution to exactly 10,000 grains measure and mix thoroughly.

Preparation of the Solution of Arsenite of Soda. -139.44 grains of the purest arsenious acid prepared either by crystallisation from hot dilute hydrochloric acid or by re-subliming a quantity of the arsenious acid of commerce free from sulphide, are dissolved in a flask in a few ounces of water with the addition of about 700 grains of crystallised carbonate of soda free from sulphide or hyposulphite. To facilitate the solution, the arsenions acid should be in fine powder and the mixture should be kept near the boiling point end frequently shaken. The contents of the flask with the water required to rinse it out are now transferred to 810,000 grains measure, and when quite cold filled up to the mark and the whole then carefully mixed. The starch test may be prepared by boiling 3 parts of starch with 500 of water, and afterwards adding 1 part of iodide of potassium. The starch should be first mixed smoothly with a portion of cold water, then the remainder of the water may be added boiling, and the whole boiled for a short time. Slips of white unsized paper (filtering paper) are 
dippod into the mixture and used whilst still damp, in which state they are far more sensitive than when dried as Penot proposed. To perform the operation, 1,000 grains of the solution of chloride of lime are measured out with a pipette immediately after thorough mixing, and placed in a beaker or other suitable vessel; the graduated burette having been filled up to the proper height with the arsenite-of-soda solution, its contents aro gradually run into the chloride of lime till a drop of the latter taken out with a glass rod ceases to produce any colouration upon a piece of the starch testpaper above described. The operation is then completed. If the burette used contains 1,000 grains divided into 100 parts, each division corresponds to 1 per cent. of chlorine, when the solutions used are of the strength just described. The reason of this will be evident from the following considerations : one molecule of arsenious acid requires four atoms of chlorine with two atoms of water to form one molecule of arsenic acid and four molecules of hydrochloric acid. Taking the molecule of arsenious acid as 198 and the four molecules of chlorine as 132 , that is $35.5 \times 4$, a simple calculation will show that 139.436 grains of arsenious acid are equivalent to 100 grains of chlorine, and the 1,000 grains measure of the arsenious test-liquor in the burette contain one-tenth of this quantity, and are consequently equal to 10 grains of chlorine. Hence the number of divisions read off upon the burette indicates at onee the percentage of chlorine in the sample examined. As it may sometimes be preferable to operate upon a larger quantity than 10 grains of chloride of lime, 2,000 grains of its solution may be taken, or it may be made of double strength; of course, in this case the number of divisions observed must be divided by two to obtain the correct result. It is as well to mention that the solution of arsenite of soda is liable to change slightly by the action of the air converting a portion into arsenate of soda, and it is recommended to keep it in small stoppered bottles entirely filled. When the storo bottle is provided with the means of drawing off the solution from the bottom, a layer of petroleum oil floating upon the top of the liquid will defend it from the action of the air.

Bunsen's Method. - When free chlorine is brought in contact with an excess of solution of iodide of potassium, iodine is set free and hydrochloric acid is formed. Each atom of chlorine sets free one atom of iodine, which by means of the exceedingly delicate reaction with starch may be estimated with the greatest accuracy. 100 or 200 grains of the solution of chloride of lime prepared as before described are measured, placed in a beaker or mixing jar, and about 60 or 120 gxains measure of solution of iodide of potassium containing 1 part iodide in 10 parts of watex added, diluted with about 1,000 grains of water, acidulated with hydrochloric acid, and a standard solution of arsenite or hyposulphite of soda run in from a burotte, till only a yellow tint remains. A little starch-paste is now added and the arsenite again added cautiously drop by drop till the blue colour just disappears. When an occasional test only is required, the method subjoined may be followed with advantage, as it obviates the necessity of preparing a standard solution.

This process is founded upon the following reaction:-One atom of chlorine, in presence of free sulphuric acid and water, converts two atoms of protosulphate of iron into one atom of persulphate of iron, with the production of one atom of hydrochloric acid:

$$
\begin{aligned}
& 2 \mathrm{FeO} \cdot \mathrm{SO}^{3}+\mathrm{SO}^{2}+\mathrm{HO}+\mathrm{Cl}=\mathrm{Fe}^{2} \mathrm{O}^{3} \cdot 3 \mathrm{SO}^{3}+\mathrm{HCl} \text {. } \\
& 2 \mathrm{FeSO}^{4}+\mathrm{I}^{2} \mathrm{SO}^{4}+\mathrm{Cl}^{2}=\mathrm{Pe}^{2}\left(\mathrm{SO}^{4}\right)^{2}+\mathbf{2 m C l}
\end{aligned}
$$

For the experiment either pure sulphate of iron, free from peroxide, or the double sulphate of iron and ammonia, or finally a weighed quantity of thin annealed iron wire may be used, dissolved in hydrochloric or preferably in dilute sulphuric acid. Of the crystallised sulphate of iron, as 278 parts correspond to 35.5 parts of chlorine, it is easily calculated that 7.831 grains will be peroxidised by one grain of chlorine. 31.324 grains $(7 \cdot 831 \times 4)$ of sulphate of iron are, therefore, weighed out, dissolved in water, with the addition of a few drops of sulphuric acid, and the volume made up to 2,000 grains measure. One fourth of this, or 500 grains, is taken out with a pipette, diluted with 1,500 to 2,000 grains of water, acidulated with hydrochloric acid, and the chloride-of-lime solution, made as previously described, is run into it from a 1,000 . grain burette, with constant agitation, till the whole of the iron is brought into the state of peroxide. To ascertain when the reaction is finished, a dilute solution of ferricyanide of potassium is used. A drop being placed upon a white plate, a stirring rod, dipped into the mixture of the iron salt and chloride of lime, is brought into contact with it. As long as a blue colour is produced it shows that the whole of the protosalt of iron is not yet oxidised, and the addition of the chloride-of-lime solution is, therefore, cautiously continued, until, on mixing the drops, no further shade of blue is porceptible. The reading of the burette is then taken; and, as the volume used contains one grain of chlorine, the percentage of that element is easily obtained by dividing 10,000 (the number of grains of solution in which 100 grains of chlorido 
of lime have been dissolved) by the number of grains of solution deliverod from the burette. Thus, suppose 437 grains have been used, 43.7 divisions:as $437: 1:: 10,000: 22-89$ per cent. of chlorino in the samplo in question. If iron wiro be takon, 6.308 grains, or, allowing 0.3 per cent. for impuritios, 6.327 grains may be weighod, dissolved in dilute sulphuric acid, made up to 2,000 grains measure, and the solution thus obtained employed instead of that of the crystallized protosulphato.-W.J.W.

CHIOrororm. When a mixture of chlorine and gaseous chloride of methyl is exposed to the sun's rays, ehloroform is produced. It is prepared usually by distilling alcohol with chloride of lime. It is used to produce insensibility. See Watts's 'Dictionary of Chemistry.'

CEIorepzasrz. A name given to some of the varieties of fluor epar, which omit a phosphorescent light when heated. See Fruor Spar.

Crioropayrx. The green colouring matter of leaves and other herbaceous parts of plants.

Cxocorarr. (Eng. and Ger.; Chocolat, Fr.) This is an alimentary preparstion of very ancient use in Mexico, from which country it was introducod into Europe by the Spaniards in the year 1520, and by them long kept a secrot from the rest of the world. Linnæus was so fond of it, that he gave the specific name, Theobroma, 'food of the gods,' to the cacao tree which produced it. The cacao-beans lie in a fruit somewhat like a cucumber, about 5 inches long and $3 \frac{1}{2}$ thick, which contains from 20 to 30 beans, arranged in 5 rogular rows with partitions betwoon, and which are surrounded with a rose-coloured spongy substance, like that of water melons. There are fruits, however, so large as to contain from 40 to 50 beans. Those grown in the West India islands, Berbice, and Demerara, are much smaller, and have only from 6 to 15 beans; their development being less perfect than in South America. After the maturation of the fruit, when their green colour has changed to a dark yellow, they are plucked, opened, their beans cleared of the marrowy substance, and spread out to dry in the air. Like almonds, they are covered with a thin skin or husk. In the West Indies they are immediately packed up for the market when they are dried; but in the Caraccas they are subjected to a species of slight fermentation, by putting them into tubs or chests, covering them with boards or stones, and turning them over every morning, to equalise the operation. They emit a good deal of moisture, lose the natural bitterness and acrimony of their taste by this process, as well as some of their weight. Instead of wooden tubs, pits or trenches dug in the ground are sometimes had recourse to for curing the beans; an operation called earthing (terrer). They are lastly exposed to the sun and dried. The latter kind are reckoned the best, being larger, rougher, of a darker brown colour, and when roasted, throw off their husk readily, and split into several irregular fragments; they have an agreeable mild bitterish taste, without acrimony. The Guiana and West India sorts are smaller, flatter, smoother skinned, lighter coloured, more sharp and bitter to the taste. They answer best for the extraction of the butter of cacao, but afford a less aromatic and agreeable chocolate. According to Lampadius, the kernels of the West India cacao-heans contain, in 100 parts, besides water, 53.1 of fat or oil, 16.7 of an albuminous brown matter, which contains all the aroma of the bean, 10.91 of starch, 7.75 of gum or mucilage, 0.9 of lignine, and 2.01 of a roddish dye stuff, somewhat akin to the pigment of cochineal. The husks form 12 per cent. of the weight of the beans; they contain no fat, but, besides lignine, or woody fibre, which constitutes half their weight, they yield a light brown mucilaginous extract by boiling in water. The fatty matter is of the consistence of tallow, white, of a mild agreeable taste, called butter of cacao, and not apt to turn rancid by keeping. It melts only at $122^{\circ} \mathrm{F}$, and should, therefore, make tolerable candles. It is soluble in boiling alcohol, but precipitates in the cold. It is obtained by exposing the beans to strong pressure in canvas bags, after they have been steamed or soaked in boiling water for some time. From 5 to 6 ounces of butter may be thus obtained from a pound of cacao. It has a reddish tinge when first expressed, but it becomes white by boiling with water.

The beans, being freed from all spoiled and mouldy portions, are to be gently roasted over a fire in an iron cylinder, with holes in its ends for allowing the vapours to escape; the apparatus being similar to a coffee-roaster. When the aroma begins to be well developed, the roasting is known to bo finished; and the beans must be turned out, cooled, and freed by fanning and sifting from their husks. The kernels are then to be converted into a paste by trituration in a mortar heated to $130^{\circ} \mathrm{F}$., or by the following ingenious and powerful machine. The chocolate paste has usually in France a little vanilla incorporated with it, and a considerable quantity of sugar, which varies from one-third of its woight to equal parts. For $1 \frac{1}{2} \mathrm{Ib}$. of cacao one pod of vanilla is sufficient. The roasted beans soon lose their flavour by exposure to the air. 
Fig. 462 represents the chocolate mill. Upon the sole $\mathrm{A}$, made of marble, six conical rollers B B are made to run by the revolution of the upright axis or shaft $q$, driven by the agency of the fly-wheel $\mathbf{z}$ and bevel wheels I K. The sole A rests upon a strong iron plate, which is heated by a small stove, introduced at the door $\mathrm{K}$. The wooden framework $\mathrm{F}$ forms a ledge, a few inches high, round the marble slab, to confine the cocoa in the act of trituration. $\mathrm{c}$ is the hopper of the mill through which the roasted beans are introduced to the action of the rollers, passing first into the flat vessel $\mathrm{D}$, to be thence evenly distributed. After the cacao has received the first trituration, the paste is returned upon the slab, in order to be mixed with the proper

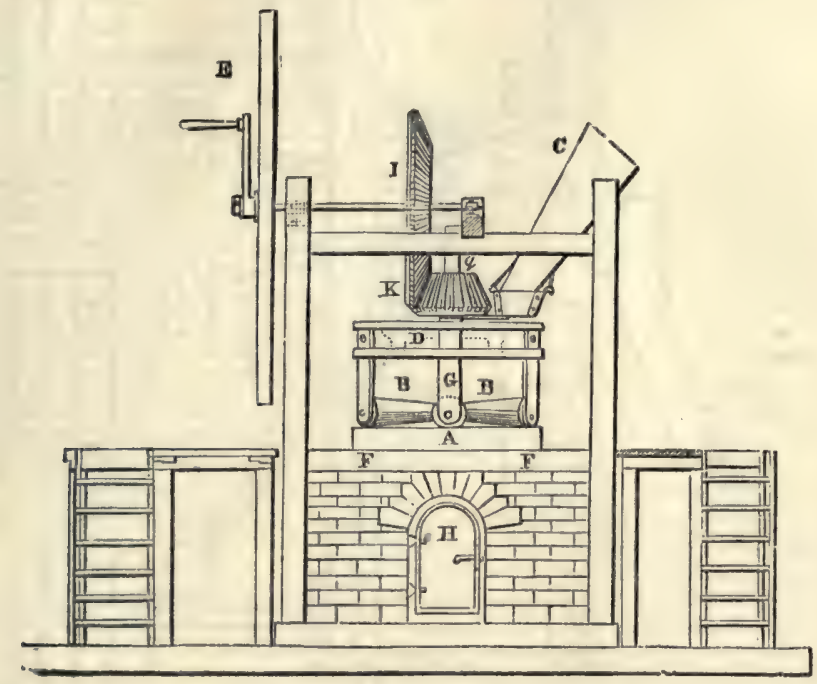

quantity of sugar and vanilla, previously sliced and ground up with a little hard sugar. When the chocolate is sufficiently worked, and while it is thin with the heat and trituration, it must be put carefully into the proper moulds. If introduced too warm, it will be apt to become damp and dull on the surface; and, if too cold, it will not take the proper form. It must be previously well kneaded with the hands to ensure the expulsion of every air bubble.

In Barcelona, chocolate mills on this construction are very common, but they are turned by a horse-gin set to work in the under story, corresponding to $\mathbf{H}$ in the above figure. The shaft $\mathrm{a}$ is, in this case, extended down through the marble slab, and is surrounded at its centre with a hoop to prevent the paste coming into contact with it, Each of these horse-mills turns out about ten pounds of fine chocolate in the hour, from a slab two feet seven inches in diameter.

Chocolate is flavoured with cinnamon and cloves in several countries, instead of the more expensive vanilla. In roasting the beans the heat should be at first very slow, to give time for the humidity to escape; a quick fire hardens the surface, and injures the process. In putting the pasto into the tin plate, or other moulds, it must be well shaken down, to insure its filling up all the cavities, and giving the sharp and polished impression so much admired by connoisseurs. Chocolate is sometimes adulterated with starch; in which case it will form a mass pasty of consistence when treated with boiling water. The harder the slab upon which the beans are triturated the better; and thence porphyry is far preferable to marble. The grinding rollers of the mill should be made of iron, and kept very clean.

Fig. 463 represents the chocolate mills at the victualling-yard, Deptford, as mounted by the celebrated engineers, Messrs. Rennie. There are four double mill-stones, A, B, C, D, each three feet in diameter, of which the nether rests upon a bed of castiron, like a drum-head, kept at the temperature of about $220^{\circ}$ by the admission of steam to the case below. Over each mill there is a feeding hopper, $1,2,3,4$, in communication by the pipes $5,6,7,8$, with the general reservoir $\mathrm{E}$, charged upon the floor above with the cocoa through the funnel placed over it. The vertical shafts which turn these mills are marked, $E, G, H, L$; they are moved by the train of bevel-wheels 

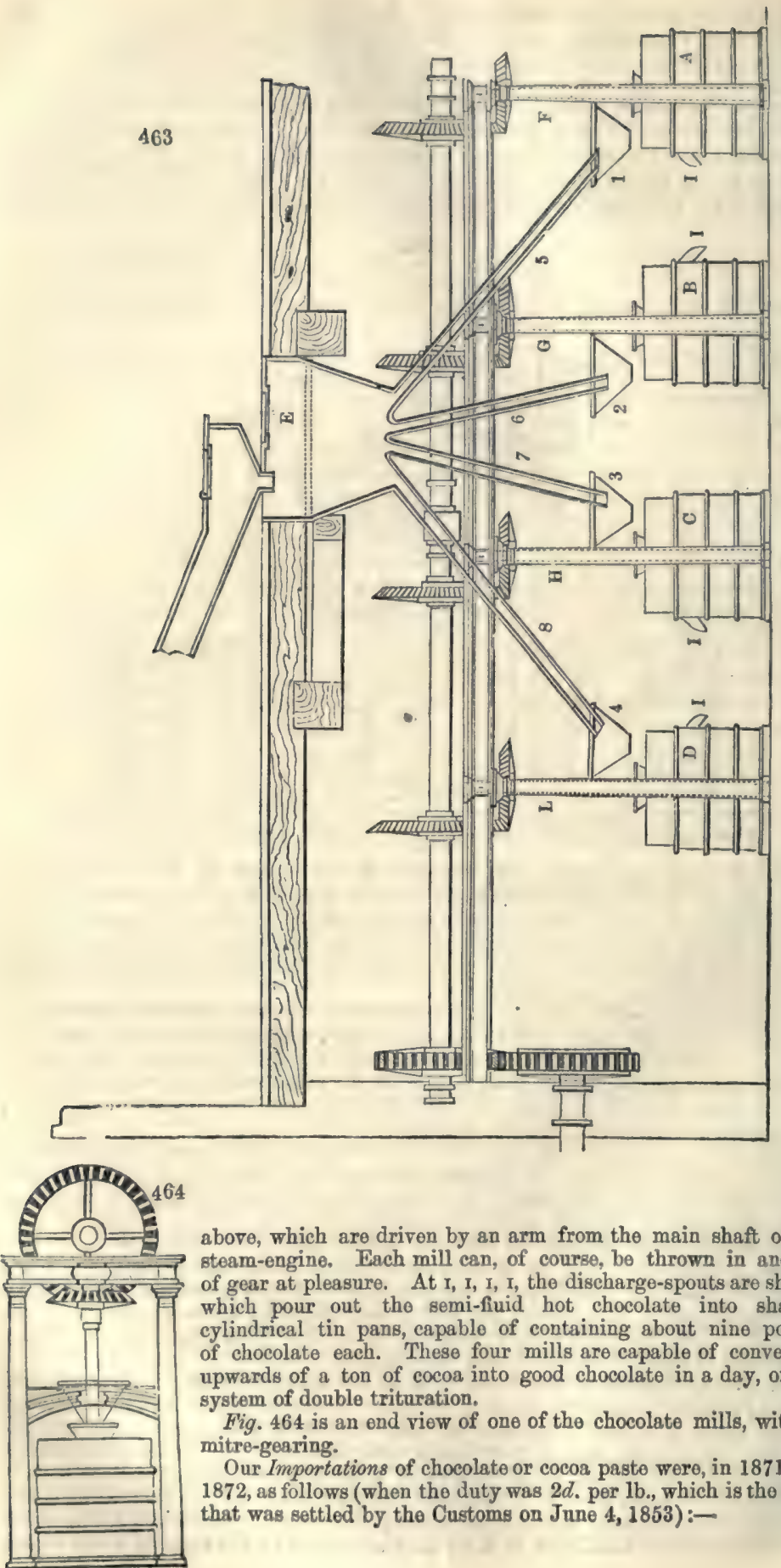

above, which are driven by an arm from the main shaft of the steam-engine. Each mill can, of course, be thrown in and out of gear at pleasure. At I, I, I, I, the discharge-spouts are shown, which pour out the semi-fiuid hot chocolate into shallow cylindrical tin pans, capable of containing about nine pounds of chocolate each. These four mills are capable of converting upwards of a ton of cocoa into good chocolate in a day, on the system of double trituration.

Fig. 464 is an end view of one of the chocolate mills, with its mitre-gearing.

Our Importations of chocolate or cocoa paste were, in 1871 and 1872 , as follows (when the duty was $2 d$. per $1 b$., which is the duty that was settled by the Customs on June 4, 1853):- 


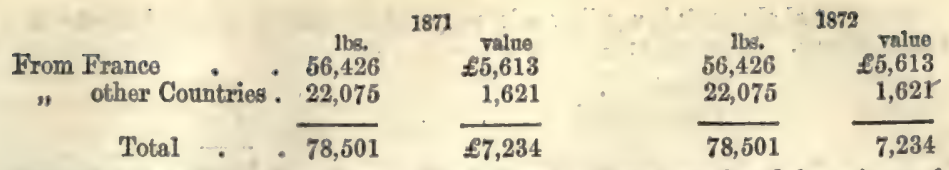

CHоxу DAMr, After Damp, Black Damp. Terms employed by miners for the atmosphere of coal-mines, after an explosion of fire-damp, which is usually air deprived of its oxygen and largely mixed with carbonic acid. Choke damp is also used to express the atmosphere of carbonic acid, which accumulates in deserted shafts, in abandoned workings, in wells, or in brewers' vats. It is removed by throwing limewater into the space it occupies, and agitating the air.

Chromo-IIthograpry. Printing in colours from lithographic stones. Seo Lithography.

CHROMASCOPE. An instrument for showing colours.

Chromatrs. Saline compounds of chromic acid with bases ; or, chromic acid in which the hydrogen is replaced by a metal. See Chromrum and Crromic Acid.

Crgomates or mad. Some of these compounds occur native, forming the minerals known as Crocoisite and Melanochroite. The chromates of lead are also prepared artificially, and largely used as pigments, the neutral chromate forming Chrome Yellow, and the basic chromate Chrome Red. The chrome yellow of the painter is a rich pigment of various shades, from deep orange to the palest canary yellow. It is made by adding a limpid solution of the neutral chromate of potash, to a solution equally limpid of acetate or nitrate of lead. A precipitate falls, which must be well washed and carefully dried out of the reach of any sulphuretted vapours. A lighter shade of yellow is obtained by mixing some solution of alum or sulphuric acid with the chromate before pouring it into the solution of lead; and an orange tint is to be procured by the addition of subacetate of lead in any desired proportions.

It was ascertained by MM. Riot and Delisse, that the proportion of chromic acid in chromate of lead may be much diminished without any injury to the colour, and that the same colour is produced with 25 parts of neutral chromate for 100 of chrome yellow, as when 54 parts are used. They give the following formula for the preparation of this pigment:-Acetate of lead is dissolved in water, and sulphuric acid in quantity necessary to convert the oxide of lead into sulphate is added. The clear liquid contains acetic acid and may be drawn off, and preserved for the preparation of fresh acetate of lead. The sulphate of lead is washed and treated with a hot solution of neutral ehromate of potash, 25 parts being used for every 75 parts of sulphate of lead. The liquid then contains sulphate of potash which may be made available, and the precipitate consists of chromate of lead.

Chrome yellow may be prepared according to Liebig, by digesting sulphate of lead in a warm solution of chromate of potash. It may also be obtained by digesting 100 parts of freshly-precipitated chloride of lead with 47 parts of bichromate of potash. This is Anthon's process.

To prepare chrome red, Rungé directs an intimate mixture to be made of $448 \mathrm{lbs}$. of litharge, $60 \mathrm{lbs}$. of common salt, and $500 \mathrm{lbs}$. of water. As soon as the mass becomes white and swells up considerably, more water is added to prevent it from becoming too hard. After four or five days, the mass becomes a compound of chlorido and hydrated oxide of lead. Without separating the mother-liquor, which contains undecomposed chloride of sodium and soda, 150 lbs. of powdered bichromate of potash are to be added, and the whole well stirred together, and finally washed.

Liebig and Wöhler contrived a process for producing a subchromate of lead of a beautiful vermilion hue. Into saltpetre, brought to fusion in a crucible at a gentle heat, pure chrome yellow is to be thrown by small portions at a time. A strong ebullition takes place at each addition, and the mass becomes black, and continues so while it is hot. The chrome yellow is to be added till little of the saltpetre remains undecomposed, care being taken not to overheat the crucible, lest the colour of the mixture should become brown. Having allowed it to settle for a few minutes, during which the dense basic salt falls to the bottom, the fluid part, consisting of chromate of potash and saltpetre, is to be poured off, and it can be employed again in preparing chrome yellow. The mass remaining in the crucible is to be washed with water, and the chrome red being separated from the other matters, it is to be dried after proper edulcoration. It is essential for the beauty of the colour, that the saline solution should not stand long over the red powder, because the colour is thus apt to become of a dull orange hue. The fine crystalline powder subsides so quickly to the bottom after every ablution, that the above precaution may be easily observed.

CHRomatrs or potash. Three of these salts are known, but only two of them are used in the arts, namely, the neutral ehromate of potash KO.CrO $\left(\mathbf{x}^{2} \mathbf{C r O}^{4}\right)$, and the acid or bichromate of potash, $\mathrm{KO} .2 \mathrm{CrO}^{3}\left(\mathbf{x}^{2} \mathbf{C r}^{2} \mathbf{O}^{7}\right)$. 
The neutral chromate is a yellow salt, prepared by heating chromo iron-ore with a salt of potakh. It contains :-

Potash
Chromic acid : $: \quad: \quad: \frac{48.0}{52.0}$
$\frac{100 \cdot 0}{4}$

The value of a solution of chromate of potash, if it be tolerably pure, may be inferred from its specifle gravity by the following table :-

At specific gravity 1.28 it contains about 50 per cent. of the salt.

\begin{tabular}{|c|c|c|c|c|c|c|c|}
\hline " & $n$ & $1 \cdot 21$ & " & $n$ & 83 & " & " \\
\hline " & $y$ & $1 \cdot 18$ & $n$ & ") & 25 & $n$ & $y$ \\
\hline " & "3 & $1 \cdot 15$ & "s & $n$ & 20 & " & I1 \\
\hline " & $"$ & 1.11 & $"$ & $y$ & 10 & $n$ & $n$ \\
\hline 3 & 39 & $1 \cdot 10$ & $"$ & $n$ & $\begin{array}{l}14 \\
19\end{array}$ & ") & 'ง \\
\hline 99 & s" & & "y & $"$ & & "s & 3 \\
\hline
\end{tabular}

The acid chromate or bichromate (callod by modern chemists the dichromate or anhydrochromate of potassium) is a red salt, which by slow cooling, may bo obtained in the form of square tables, with bevelled edges, or flat, four-sided prisms. They are permanent in the air, have a metallic and bitter taste, and dissolve in about one-tenth of their weight of water at $60^{\circ} \mathrm{F}$, but in one half of their weight of boiling water. The composition of bichromate of potash is : potash, 31.6 ; chromic geid, 68.4 .

In making the red bichromate of potash from solutions of the yellow salt, nitric acid was at first chiefly used; but, in consequence of its relatively high price, sulphuric, muriatic, or acetic acid has been frequently substituted upon the large scalo.

(For the preparation of both these salts refer to CHrome Iron.)

These salts are much employed in Calico PrINInvg and in Dyenve. The bichromate of potash is also used in the photogxaphic process known as Carbon-printing; a sensitive surface being obtained by bichromate of potash and gelatine. Another modern use of the bichromate is in a convenient form of galvanic cell, where it serves to oxidise the bydrogen set free by the action of an acid upon zinc.

Chromate of Potash, Adulteration of, to detect.-The chromate of potash has the power of combining with other salts up to a certain extent without any very sensible change in its form and appearance; and hence it has been sent into the market falsified by very considerable quantities of sulphate and muriate of potash, the presence of which has often escaped observation, to the great loss of the dyers who use it so extensively. The following test process has been devised by M. Zuber, of Mülhouse :-Add a large excess of tartaric acid to the chromate in question, which will decompose it, and produce in a few minutes a deep amethyst colour. The supernatant liquor will, if the chromate be pure, afford now no precipitate with the nitrates of baryta or silver; whence the absence of the sulphates and muriates may be inferred. We must, however, use dilute solutions of the chromate and acid, lest kitartrate of potash be precipitated, which will take place if less than 60 parts of water be employed. Nor must we test the liquid till the decomposition be complete, and until the colour verge rather towards the green than the yellow. Eight parts of tartaric acid should be added to one of chromate to obtain a sure and rapid result. If nitrate of potash (saltpetre) is the adulterating ingredient, it may be detected by throwing it on burning coals, when deflagration will ensue. The green colour is a certain mark of the transformation of the chromic acid partially into the chrome oxide; which is effected equally by the sulphurous acid and sulphuretted hydrogen. Here this metallic acid is deoxygenated by the tartaric, as has been long known. The tests which I should prefer are the nitrates of silver and baryta, having previously added so much nitric acid to the solution of the suspected chromate as to prevent the precipitation of the chromate of silver or baryta. The smallest adulteration by sulphates or muriates will thus be detected.

A mixture of sulphate of soda and chloride of sodium tinged with strong solution of chromium is sometimes sold for pure bichromate of potash.-H. M. N.

Cminomatyre. A photographic process. Seo Photographr.

CHRomatypograrzy. A Fronch process for printing letter-press in colours.

Carnoms Arvar. A double sulphate of sesquioxide of chromium and potash. It is obtained as a by-product in the preparation of the aniline colours, and is sometimes used in dyeing. See Alum.

CHnoms GR2rm. This term is applied both to the green oxide (sesquioxide) of chromium; and to a green pigment prepared by mixing chrome yellow with Prussian blue. 
Crnoma Inox, or CrRomriz. The only ore of chromium which occurs in sufficient abundance for the purposes of art is the octahedral chrome ore, commonly called chromate of iron, though it is rather a compound of the oxides of chromium and of iron. It is formed on the type of magnetic iron-ore, being a combination of a protoxide with a sesquioxide - the protoxide metal in chrome iron-ore being chiefly iron, and the

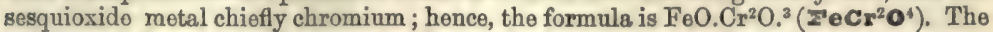
fracture of this mineral is imperfect conchoidal, or uneven. Hardness $=5.5$; specific gravity 4.4 to 4.5 ; but the usual chrome ore found in the market varies from 3 to 4 . It lustre is semi-metallic or resinous ; colour, iron or brownish black; streak, yollowish to reddish brown. It is sometimes magnetic. Before the blowpipe it is infusible alone, but in borax it is slowly soluble, forming a beautiful emerald-green bead; fused with nitre it forms a yellow solution in water.

Chrome ore was first discovered in the Var department in France; it is also found, chiefly in serpentine, in Saxony, Silesia, Bohomia, Transylvania, the Banat, and Sty ria; in Norway at Röraas, in the Ural near Katherinenberg, in the United States at the Barehills near Baltimore, Chester in Massachusetts, and Hoboken in New Jersey. In Scotland it is found in the parishes of Kildrum and Towie in Aberdeenshire, in the limestone near Portsoy in Banffshire, near Ben Lawes in Perthshire, and at Buchanan in Stirlingshire. It occurs massive and in considerable quantity in serpentine-rocks at Swinaness, and Haroldswick in Unst, one of the Shetlands; in Fetlar and in other of the smaller Shetland Islands.

Composition of Chrome Iron Ores.

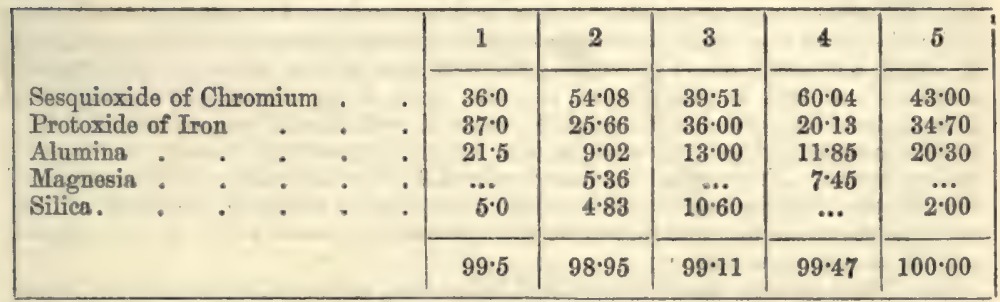

(1) from St. Domingo, analysed by Berthier; (2) from Rörass, in Norway, analysed by Von Kobell ; (3) from Baltimore, analysed by Seybert; (4) crystallised, from Baltimore, analysed by Abich ; (5) analysed by Klaproth.

The chief application of this ore is to the production of Chromate of Potash, from which salt the various other preparations of this metal used in the arts are obtained.

Treatment of the Ore.-According to the old method it is reduced to a fine powder, by being ground in a mill under ponderous edge-wheels, and sifted. It is then mixed with one third or one half its weight of coarsely-bruised nitre, and exposed to a powerful heat for several hours, on a reverberatory hearth, where it is stirred about occasionally. In the large manufactories of this country, the ignition of the above mixture in pots is laid aside as too operose and expensivc. The calcined matter is raked out and lixiviated with water. The bright yellow solution is then evaporated briskly and the chromate of potash falls down in the form of a granular salt, which is lifted out from time to time from the bottom with a large ladle, perforated with small holes, and thrown into a draining box. The saline powder may be formed into regular crystals of neutral Chromate of Potash, by solution in water and slow evaporation; or it may be converted into a more beautiful crystalline body, the Bichromate of Potash, by treating its concentrated solution with nitric, muriatic, sulphuric, or acetic acid, or indeed any acid exercising a stronger affinity for the second atom of the potash than the chromic aoid does.

The first great improvement in this manfacture was the dispensing with nitre, and oxidising entirely by means of air admitted into the reverberatory furnace, in which the ore mixed with carbonate of potash is calcined. Stromeyer afterwards suggested the addition of limo, by which the oxidation was much quickened, and Mr. Cliarles Watt substituted the sulphates of potash and soda for the nitrates of those alkalis. The sulphate was first intimately mixed with the ground ore, and then the lime well incorporated with the mixture, which was heated to bright redness, for four hours with frequent stirring.

In $1847 \mathrm{Mr}$. 'Tilgmann obtained a patent for the use of felspar in the manufacture of certain alkaline salts, and amongst them of chromate of potash: he directs 4 parts by woight of felspar, 4 parts of lime, or an equivalent quantity of carbonate of lime, and one part of chromic ore, all in fine powder, to be intimately mixed together, and 
kept at a bright red heat for from 18 to 20 hours in a reverberatory furnace, the mixture being turned over frequently, so that all parts may be exposed equally to heat and air ; the temperature is not to rise high enough to cause even incipient fusion, and the charge should be kept in a porous state; when, on being examined, the charge is found to contain the proper quantity of alkaline chromate, it is withdrawn from the furnace and lixiviated with water.

In treating chromium (chromate of iron), the ore is pulverised and mixed with common salt, muriate of potash, or hydrate of lime, and exposed in a reverberatory furnace to a red or even a white heat, the mixture being stirred every ten or fifteen minutes, and steam at a very elevated temperature introduced during the operation, until the desired effect is obtained, which may be ascertained by withdrawing a portion from the furnace and testing it, as customary. The products of this operation are finally treated in the manner usual for the chromic and bichromic salts.

The mixture of chromium and common salt produces chromate of soda, the greater portion, or perhaps all of the iron contained in the chromium being absorbed by the hydrochloric acid evolved from the salt, and carried off in the form of sesquichloride of iron. From the first mixture is manufactured pure bichromate of soda, which, by the addition of hydrochloric acid, may be converted into chlorochromate; and from the last, or lime mixture, is produced a chromate of that earth, from which, by the addition of soda or potash, there may be obtained a compound salt, which, with those previously mentioned, may be advantageously employed.

M. Jacquelin first prepares chromate of lime by calcining at a bright red heat in a reverberatory furnace, for $\mathbf{9}$ or $\mathbf{1 0}$ hours, an intimate mixture of chalk and chromo ore. The friable and porous mass is then crushed, suspended in water, and sulphuric acid added until the liquid slightly reddens blue litmus-paper; the chromate of lime is hereby converted into bichromate; chalk is now added, until the whole of the sesquioxide of iron is precipitated, and the clear liquid, which now contains only bichromate of lime and a little sulphate, may be used for the preparation of the insoluble chromates of lead, zinc, baryta, \&ce., by mixing it with the acetates or chlorides of these metals. To prepare bichromate of potash, the bichromate of lime is mixed with solution of carbonate of potash, which gires rise to insoluble carbonate of lime, which is easily washed, and a solution of bichromate of potash which is concentrated and set aside to crystallise.

Mr. Booth (patent sealed Nov. 9th, 1852) mixes powdered chrome ore with onofifth of its weight of powdered charcoal, and heats it on the hearth of a reverberatory furnace, protecting it carefully from the air. The ore is by this means decomposed, and the iron reduced to the metallic state, and is dissolved out by dilute snlphuric acid ; the residue is washed and dried, and afterwards mixed with carbonate of potash and salpetre, and heated in the same manner that the chrome ore itseif is heated in the process usually employed. The solution of sulphate of iron is evaporated to crystallisation so as to produce copperas in a state adapted for commerce.

Analysis of Chrome Iron Ore.-Various methods have been proposed. The following, suggested by Mr. T. S. Hunt, gives accurate results:-The ore, finely levigated in an agate mortar, is mixed with 10 or 12 times its weight of fused bisulphate of potash, and preserved at a gentle heat for about half an hour. The fused mass is extractod with hot water, and boiled for a few minutes with excess of carbonate of soda; the precipitate is dried and fused with five times its weight of a mixture of equal parts of nitre and carbonate of soda, in a platinum or silver crucible. The mixture is kept in fusion for 10 or 15 minutes, and when cold is extracted with water. The alkaline chromate thus obtained may be precipitated by a salt of lead, or it may be supersaturated by hydrochloric acid, and boiled with alcohol, by which it is convertod into chloride of chromium, from which the oxide is to be precipitated by adding anmonia in excess and boiling for a few minutes. Chrome iron ore is so difficult of decomposition, that the method of fusing it at once with nitre and an alkaline carbonate frequently fails in oxidising the whole of the chromium into chromic acid.

Mr. Calvert mixes the well-pulverised ore with three or four times its weight of a mixture made by slaking quicklime with caustic soda, and then dries and calcines the mass. He then adds one-fourth part of nitrate of soda, and calcines for two hours more, by which time he finds the whole of the chromium is converted into chromic acid. Another process, which Mr. Calvert finds to produce good results, consists in calcining the pulverised chrome ore with nitrate of baryta, adding a littlo caustic potash from time to time towards the end of the process.-H.M.N.

Crromz RED and CHROME YrIIOW. Seo Chromates of Lead.

Crraoms sTows. The name given to Chrome Ochre when it is so intimately mixed with the rock in which it is contained as only to be separated from it by chemical means. Such mixtures are met with at Creuzot in France, Waldenberg in Silesia, Mortenberg in Sweden, and elsewhere. See Bristow's 'Glossary of Mineralogy.' 
Crromac ACrD. There aro several methods of proparing this acid; the simplest consists in decomposing bichromate of potash by oil of vitriol :-1. An excess of oil of vitriol is mixed with a warm solution of bichromate of potash; the liquid is poured off from the chromic acid, which separates in small red crystals; the crystals are drained in a funnel having its stem partly filled with coarsely-pounded glass, and are afterwards dried on a porous tile under a bell glass: 2. Mr. Warrington mixes 10 measures of a cold saturated solution of bichromate of potash with from 12 to 15 measures of oil of vitriol free from lead, and presses the red acicular crystals which separate as the liquid cools, between porous stones. If it be desired to remove the last traces of sulphuric acid, the crystals should be redissolved in water, and a solution of bichromate of baryta should be added in quantity just sufficient to throw down tho whole of the sulphuric acid as sulphate of baryta; the solution may be recrystallised by evaporation in vacuo: 3. Meissner prepares the acid direct from chromate of baryta by digesting that salt with a quantity of diluto sulphuric acid, not sufficient for complete saturation : the solution which contains chromic acid and acid chromate of baryta is precipitated by the exact amount of sulphuric acid required, so that the solution is affected neither by sulphuric acid, nor by a salt of baryta: it is then evaporated to dryness.

Mr. Charles Watt obtains the acid by treating chromate of baryta with considerable excess of pure strong nitric acid. The chromic acid is separated from the insoluble nitrate of baryta by filtration through asbestos, and the solution is then evaporated to drymess, while nitric acid is expellod.

Chromic acid is obtained in quadrangular crystals, of a deep red colour; it has a very acrid and styptic taste. It reddens powerfully litmus-paper. It is deliquescent in the air. When heated to redness, it emits oxygen and passes into the sesquioxide. When a little of it is fused along with vitreous borax, the compound assumes an emerald green colour.

As chromic acid parts with its last dose of oxygen very easily, it is capable, in cortain styles of calico-printing, of becoming a valuable substitute for chlorine where this more powerful substance would not, from peculiar circumstances, be admissible. For this ingenious application, the arts are indebted to that truly scientific manufacturer, M. Daniel Kochlin, of Mülhouse. He discovered that whenever chromate of potash has its acid set free by its being mixed with tartaric or oxalic acid, or a neutral vegetable substance (starch or sugar, for example), and a mineral acid, a very lively action is produced, with disengagement of heat, and of several gases. The result of this decomposition is the active reagent chromic acid, possessing valuable properties to the printer. Water-solutions of chromate of potash and tartaric acid being mixed, an effervescence is produced which has the power of destroying vegetable colours. But this power lasts no longer than the effervescence. The mineral acids react upon the chromate of potash only when vegetable colouring matter, gum, starch, or a vegetable acid, are present to determine the disengagement of gas. During this curious change, carbonic acid is evolved; and when it takes place in a retort, there is condensed in the receiver a colourless liquid, slightly acid, exhaling somewhat of the smell of vinegar, and containing a little empyreumatic oil. This liquid, heated with the nitrates of mercury or silver, reduces these metals. On these principles M. Koechlin discharged indigo-blue by passing the cloth through a solution of chromate of potash, and printing nitric acid thickened with gum upon certain spots. It is probable that the employment of chromic acid would supersede the necessity of having recourse in many cases to the more corrosive chlorine.-H.M.N.

Chromic acid is now used in microscopy for hardening the preparations of soft tissues.

\section{crroomrty. See Chrom Iron-ore.}

CEromrura. (Symbol, Cr; Atomic weight, 26.27.) The metallic base of the oxide of chromium. It may be obtained by exposing to a very high temperature, in a crucible lined with charcoal, an intimate mixture of sesquioxide of chromium and chareoal. The spongy mass obtained is powdered in an iron mortar, and mixed with a little more sesquioxide of chromium (to oxidise as much as possible of the carbon); it is then again exposed in a porcelain crucible to a very high temperature, when a coherent metal is obtained. This metal is greyish in colour, hard and brittle, and is magnetic at low temperatures. It has received no practical applications.

CrRomrum, OxIDz OF. There are several oxides of chromium, known to chemists, but the only one used in the arts is the sesquioxide $\left(\mathrm{Cr}^{2} \mathrm{O}^{3}\right)$, known commonly as green oxide of chromium. This has come so extensively into use as an enamel colour for porcolain, that a fuller account of the best modes of manufacturing it muat prove acceptable to many of our readers.

That oxide, in combination with water, called the hydrate, may be economically

Vot. I. 
prepared by boiling chromate of potash, dissolved in water, with half its weight of fluwers of sulphur, till the resulting greon precipitate ceases to increase, which may be easily ascertained by filtering a little of tho mixture. The addition of some potash accelerates the operation. This consists in combining the sulphur with the oxygen of the chromic acid, so as to form sulphuric acid, which unites with the potash of the chromate into sulphate of potash, while the chrome oxide becomes a hydrate. An extra quantity of potash facilitates the deoxidation of the chromic acid by the formation of hyposulphite and sulphide of potash, both of which have a strong attraction for oxygen. For this purpose the clear lixirium of the chromate of potash is sufficiontly pure, though it should hold some alumina and silica in solution, as it generally does. The hydrate may be freed from particles of sulphur by heating dilute sulphuric acid upon it, which dissolves it; after which it may be precipitated, in the state of a carbonate, by carbonate of potash, not addod in excess.

By calcining a mixture of bichromate of potash and sulphur in a crucible, chromic acid is also decomposed, and a hydrated oxide may thus be obtained; the sulphur being partly converted into sulphuret of potassium, and partly into sulphuric acid (at the expense of the chromic acid), which combines with the rest of the potash into a sulphate. By careful lixiviation, these two new compounds may be washed away, and the chrome green may be freed from the remaining sulphur, by a slight heat.

Another method of preparing green oxide of ehromium, is to mix intimately 45 parts of gunpowder with 240 parts of perfectly dry chromate of potash, and 35 parts of chloride of ammonium (sal-ammoniac), reduce to powder, and pass through a fine sieve; fill a conical glass or other mould with this powder, gently pressed, and invert so as to leave the powder on a porcelain slab of any kind. When set on fire at its apex with a lighted match, it will burn down to the bottom with brilliant coruscations. The black residuum, being elutriated with warm water, affords a fine brightgreen oxide of chromium.

Preparation of Green Oxide of Chromium for Calica-Printing.-The following directions are given by De Kerrur. At the commencement of the process the green hydrate of the oxide of chromium is first prepared by dissolving 4 kilogrammes of bichromate of potash in 22 litres ( 39 pints) of boiling water. Then into a boiler or vessel containing 108 litres (24 gallons) of boiling water, 4 or 5 kilogrammes ( 8 or 10 lbs.) of pulverised white arsenic are thrown, and boiled for 10 minutes : a precipitate will be formed, and must be allowed to settle: the clear liquor is then run off, and immediately mixed with the solution of bichromate of potash, stirring all the time: in a short time the mixture acquires a green tint, and the hydrated oxide of chromium will be formed and precipitated. After being several times well stirred, and allowed to cool, the whole is thrown upon a filter of white wool, and the hydrate of chromium remaining on the filter is carefully washed with boiling water. It is then dried, and ready to be employed for the preparation of the chloride. In order to obtain that salt, lyydrochloric acid of $22^{\circ}$ Baumé is diluted with water, until the acid no longer gives off vapour. It is then heated, and whilst hot, as much of the hydrated oxide of chromium, prepared as abore, is added as will saturate the acid and leave a slight excess of the oxide undissolved. The whole is then left to settle, and the clear liquor is decanted from the dissolved matter. In this state the solution of chloride of chromium still presents some traces of free acid, which would act injuriously upon the fibres of the cotton. To remove this, and to obtain the product in a neutral state, potash-ley (marking $36^{\circ}$ Baumé) is poured in very gradually, until the oxide of chromium begins to be precipitated. The solution of choride of chromium thus prepared, and which is of a dark green colour, is evaporated until it marks $46^{\circ}$ Baumé; nfter cooling, oxide of chromium of the finest green colour is obtained. This preparation is sold under the name of Sea-green.

This oxide may also be prepared by decomposing, with heat, the chromate of protoxide of mercury, a salt made by adding to nitrate of protoxide of mercury (mercurous nitrate), chromate of potash, in equivalent proportions. This chromate has a fine cinnabar red, when pure; and, at a dull red heat, parts with a portion of its oxygen and its mercurial oxide. From M. Dulong's experiments it would appear that the purest chromate of mereury is not the best adapted for preparing the oxide of chrome to be used in porcelain painting. Ho thinks it ought to contain a little oxide of mangrnese and chromate of potash to afford a green colour of a fine tint, especially for jieces that are to receive a powerful heat. Pure oxide of chrome preserves its colour well enough in a muffle furnace; but, under a stronger fire, it takes a dead-leaf colonr.-H.M.N.

A hydrated sesquioxide of chromium is used as a green pigment, under the name of Guignet's Grums.

CHnomrum, BIUב OxIDz or. The following directions have been given for the preparation of a blue oxide of chrominm :-The concentrated alkaline. solution 
of chromate of potash is to be saturated with weak sulphuric acid, and then to crery $8 \mathrm{lbs}$. are to be added $1 \mathrm{lb}$. of common salt, and half a pound of concentrated sulphuric acid; the liquid will now acquire a green colour. To be certain that the yellow colour is totally destroyed, a small quantity of the liquor is to have potash added to it, and filtered; if the fluid is still yellow, a fresh portion of salt and of sulphuric acid is to be added: the fluid is then to be evaporated to dryness, redissolved, and filtered; the oxide of chrome is finally to be precipitated by caustic potash. It will be of a greenish-blue colour, and being washed, must be collected upon a filter.H.M.N.

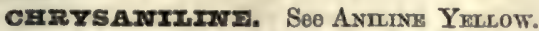

CERYsozmexI, or Golden Teryl, is composed of alumina 80.2 and glucina $19 \cdot 8=100$. It is of various shades of yellowish and light green, sometimes with a bluish opalescence internally. It has a vitreous lustre, and varies from translucent to transparent. Fracture, conchoidal, or uneven. Specific gravity $=\mathbf{3 . 5}$ to $3 \cdot 8$. It belongs to the trimetric system.

This stone, when transparent, furnishes a beautiful gem of a yellowish-green colour, which is cut with facets, unless it be opalescent, in which case it is cut en cabochon. It occurs in the Brazils and Ceylon, in rolled pebbles in the alluvial depasits of rivers; in the Ural, in mica-slate; and at Haddam, Connecticut, U.S., in granite, traversing gneiss,-H.W.B.

For varieties of Chrysoberyl, see Cat's Ere, and Crmophank.

Crm rocorra. The Greeks gave this name to Borax. It is now applied to the hydrous silicate of copper, a mineral with the composition of silica $34 \cdot 3$, protoxide of copper 45.2 , water 20.5 . It is often mixed with carbonate and oxide of copper. It is found in Chili, in the Lake Superior district, in the copper mines of South Australia, and the West Indian Islands; also in Cornwall, Saxony, and indeed in most localities where copper-mines occur. Bischof observes, that silicate of copper may be formed through the action of an alkaline, lime or magnesia silicate, on sulphate or nitrate of copper in solution. He also shows that this silicate is decomposed by carbonated waters, producing carbonate of copper. In nature the alkaline silicates are often furnished by the decomposing granite, and the sulphate of copper by the changes which constantly oecur in copper pyrites.

CrRzsorxwr, or Peridot. The name given to the paler and more transparent crystals of olivine, the latter name being restricted to imbedded masses or grains of inferior colour and clearness. It is usually found in angular or rolled pieces, rarely crystallised. The crystals (generally, 8, 10, or 12-sided prisms), belonging to the rhombic system, are variously terminated, and often so compressed as to become almost tabular. They are generally very fragile, and therefore unfit for ornamental purposes. The chrysolite is a silicate of magnesia, with more or less protoxide of iron. An analysis of an oriental chrysolite by Stromeyer gave: silica $39 \cdot 73$, magnesia $50 \cdot 13$, protoxide of iron $9 \cdot 19$, alumina $0 \cdot 22$, protoxide of manganese 0.09 , oxido of nickel $0.32=99 \cdot 68$.

As a gem, chrysolite is deficient in hardness and play of colour; but when the stones are large and of good colour, and well cut and polished, it is made into necklaces, \&c., with good effect. From its softness, which is little less than that of glass, it requires to be worn with care, or it will lose its polish. The best mode of displaying the colours to the greatest advantage is to cut it in small steps. To give it the highest polish, a copper wheel is used, on which a little sulphuric acid is dropped. During the process a highly suffocating smell is given out, produced, probably, by the oxidation of the copper and the decomposition of the acid. Chrysolite is supposed to have been the topaz of the ancients. It is found near Constantinople; at Vesuvius; and the Isle of Bourbon; at Real del Monte, in Mexico; in Egypt; and at Expailly, in Auvergne,-H.W.H.

CrRzsorrasp. An apple-green or leek-green variety of chalcedony, the colour of which is caused by the presence of nickel. It occurs at Kosemütr, in Silesia, and Belmont's lead-mine, St. Lawrence County, New York.

This stone was probably the chrysoberyl of the ancients.-H.W.B.

CERYSOrruz. A fibrous or asbestiform variety of serpentine.

Crur Am. A cement made of shell-lime and sea-sand, commonly used in India.

CHuncarrs. A hydrated phosphate of protoside of cerium, found in minute crystals, forming a thin coating on quartz and clay-slate, in a Cornish tin-lode. Mr. (:. Greville Williams has detected didymium in churchite.

crckorrua rerzats. See Chicony.

CIDIR. See CxDre.

CImorrary zarme. Cimolite (Cimolia, Pliny). This earth is found in the island of Argentiers, the ancient Cimolus, in Bohemia, and some parts of Russia. It has been frequently confounded with fullers' earth, because it has been used for 
the same purposes-romoving grease from silk or woollen fabrics. It is an aluminous silicate, consisting of silica 63 , alumina 23 , oxide of iron $1 \cdot 25$, water 12 . It absorbs water rapidly, and splits into thin laminæ. Powdered and mixed with water it forms a creamy mass, which absorbs oil from any fabric over which it may be spread.

Crarcenowa marx. A Parliamentary paper on the progress of India in 1872 gives information respecting the cultivation of the cinchona plant, from which quinine is obtained. It was introduced into the Hill districts of India, from South America, in 1860. The total expenditure of the experiment was $61,719 l$. The return represents a value which is simply incalculable. There are now $2,639,285$ plants in the Government plantations on the Neilgherry Hills alone, without counting those of private planters in this and in other districts. The largest trees are $30 \frac{1}{2}$ feet high, and over 3 feet in girth round the trunk. The area covered by the plantations amounts to 950 acres, and is being largely added to every year. The bark under cultivation is much richer in quinine and the other febrifuge alkaloids than the wild bark of South America. During last year 7,295 lbs. of excellent bark were sold in the London market, while $65,688 \mathrm{lbs}$. were supplied to the local manufactory. This year 20,000 lbs. will be sent home. The alkaloid is manufactured on the spot in an exceedingly cheap form for the use of local medical stores, and hundreds of fever patients are thus annually cured. The object of providing an abundant supply of the febrifuge at a price within the means of the population at large is rapidly being realised.

From the report on the Government cinchona plantations at Ootacamund for 1870-71 we learn that the growth of the plants has been very satisfactory. The older plants of the various medicinal kinds have grown from shrubs into trees 22 feet or 23 feet high, and 18 inches to 21 inches in girth. Of the Cinchona succirubra the finest samples reach a height of 30 feet, with a girth of three feet. Three thousand five hundred plants of the Calisaya kind have been permanently planted out on two acres of new land. With this exception, the work of the yoar has been confined to flling up failures and planting along the roadside. Among the new species of plants lately introduced is the Pitayo bark, which appears to be hardy and well suited to the climate. A lanceolate variety of Calisaya introduced from Java makes but slow growth at Ootacamund. During the year 51,353 lbs. of fresh bark were supplied to Mr. Broughton, the Government Quinologist, for the manufacture of amorphous quinine. From 1,000 eight-year-old plants of $C$. succirubra as much as 2,560 lbs. have been or will be extracted during this year. This average of more than $2 \frac{1}{2} \mathrm{lbs}$. to each tree will yield, at the present rates of $2 s .8 d$. to $3 s$. per lb., a clear profit of at least a rupee a pound. After the tenth year an increasing profit may be yearly expected, with a steady improvement in the quality of the bark. In the cheaper kinds of bark it is unlikely that India will ever compete successfully in the home market with America, where the plant grows wild, and its culture, which is yearly spreading, costs nothing. In the finer kinds of bark, however, a sucessful competition is far from unlikely, if the mossing process is steadily applied. Under this process each successive renewal of bark will become more valuable than the last, until red bark yields from 10 to 12 per cent. of crystallisable alkaloids, containing from 6 to 8 per cent. of pure quinine, while 8 or 10 per cent. of the latter may be expected from the crown barks. In that case India would defy competition, for no such bark, it appears, could be obtained from America. See Preruviar Bark.

Crncriona RaD. An amorphous dark reddish-brown substance, obtained from cinchona, or Peruvian bark.

Crmchorrermz. $\mathrm{C}^{40} \mathrm{H}^{2} \mathrm{~N}^{2} \mathrm{O}^{2}\left(\mathbf{C}^{20} \mathbf{B}^{2 !} \mathbf{N}^{2} \mathbf{O}\right.$.) An alkaloid isomeric with cinchonine and cinchonidine. It is produced by the action of heat on any of the saline combinations of cinchonine,-Pasteur.

CrNCEONIDINI. $\mathrm{C}^{40} \mathrm{H}^{24} \mathrm{~N}^{2} \mathrm{O}^{2}\left(\mathbf{C}^{20} \mathbf{H}^{21} \mathbf{N}^{2} \mathbf{O}\right.$.) This alkaloid, the quinidine of Leers, is one of the isomers of einchonine.

CrNChONINI. $\mathrm{C}^{40} \mathrm{H}^{24} \mathrm{~N}^{2} \mathrm{O}^{2}\left(\mathbf{C}^{20} \mathbf{H}^{21} \mathbf{N}^{2} \mathbf{O}\right.$.) An alkaloid or organic base acmmpanying quinine. Some of the differences of properties on which processes for their separation may be founded are the following:-Cinchonine crystallises more readily than quinine from an alcoholic solution, in consequence of its being less soluble in that fluid. Sulphate of quinine, on the other hand, is less soluble than sulphate of cinchonine. Cinchonine is insoluble, while quinine is freely soluble in ether. See Watts's 'Dietionary of Chemistry.'

When cinchonine is distilled with a hydrated alkali it yields a product known as chinoline oil, containing three homologous bases-chinoline, lepidine, and krytidine. From this oil, a brilliant blue pigment may be obtained.

CrN DEr. The slags produced in the processes of iron manufacture.

CINDER RzD. A bed of oyster-shells; found in the Middle Purbeck series.

Cimdar rivz. See Irox. 
CINDIfr mon. See Iron. CINDER TAP. See IroN.

CINTABAR is the principal and only valuable ore of mercury which is prepared from it by sublimation.

It is a sulphide (sulphuret) of mercury, composed, when pure, of quicksilver 86.2 , sulphur 13.8, in which case it is a natural vermilion, and identical with the vermilion of commerce; but it is sometimes rendered impure by an admixture of clay, bitumen, oxide of iron, \&c. Cinnabar is of a cochineal-red colour, often inclining to brownishred, and lead-grey, with an adamantine lustre, approaching to metallic in dark varieties, and to dull in friable ones. It varies from sub-transparent to opaque, has a scarlet streak, and breaks with a sub-conchoidal uneven fracture. $\mathrm{H}=2$ to $2 \cdot 5$, specific gravity $=8.99$. In a matrass it entirely sublimes, and with soda yields mercury with the evolution of sulphurous fumes. When crystallised, it belongs to the rhombohedral system,

Cinnabar occurs in beds in slate-rocks. The chief European beds are at Almaden, near Cordova, in Spain, and at Idria in Carniola, where it usually occurs in a massive form, and is worked on a thick vein belonging to the carboniferous series. It also occurs abundantly in China, Japan, Huanca Vilica in South Peru, and at New Almaden in Santa Clara Co., and in other parts of California, where extensive mines of cinnabar are worked. It is also abundant in Idaho. The chief source of the mercury used in England, is Spain. Our recent imports of native Cinnabar eannot now be ascertained, as it is all entered at the Custom House under the head of 'Ores unenumerated.'

Cinnabar in the arts is used as a pigment, in the state of a fine powder, which is known by the name of rermilion. See Mrroury and Vermuros.

Crmstamox. (Cannelle, Fr.; Zimmt, Ger.) The inner bark of the Laurus cinnamomum, used chiefly for flavouring cordials. Cinnamon yields an exquisite essential oil by distillation which has considerable use in medicine and perfumery. It is largely used for incense, but its principal consumption is in Spain, for the fabrication of chocolate, where it is said not less than $50,000,000 \mathrm{lbs}$. are used annually, nearly the whole of which is brought from Ceylon.

Of cinnamon we imported as follows:-

\begin{tabular}{|c|c|c|c|c|c|}
\hline 918 & & & & & lbs. \\
\hline 1871 . & * & & & . & $1,574,946$ \\
\hline 1872 . & . & & & . & . $1,082,134$ \\
\hline
\end{tabular}

Value

$£ 252,375$

143,520

111,496

Crmpamow stowz. A name given to Essonite, one of the varieties of the lime garnets. Many so-called hyacinths are really nothing more than finecoloured cinnamon stones.

crporrvo. An Italian marble, of white colour, with pale green markings.

CITrIC ACID. (Acide citrique, Fr.; Citronensaure, Ger.) This acid exists in the juice of fruits, especially the lemon, orange, currant, and quince. It was first procured from lemon-juice in a pure state by Scheele, who adopted the following process:-Lemon-juice was put into a large tub, and saturated with dry chalk in fine powder, noting carefully the quantity employed. The citrate of lime which precipitates being freed from the supernatant liquor is to be well washed, with repeated affusion and decantation of water. For every ten pounds of chalk employed, nine and a half pounds of sulphuric acid diluted with six times its weight of water are to be poured while warm upon the citrate of lime, and well mixed with it. At the end of twelve hours, or even sooner, the citrate will be decomposed, dilute citric acid will float above, and sulphate of lime will be found at the bottom. The acid being drawn off, the calcareous sulphate must be thrown on a canvas fliter, drained, and then washed with water to abstract all the acid.

The citric acid thus obtained may be evaporated in leaden pans, over a naked fire, till it acquires the specific gravity 1.13 ; after which it must be transferred into another vessel, evaporated by a steam or water bath till it assumes a syrupy aspect, when a pellicle appears first in patches, and then over the whole surface. This point must be watched with great circumspection, for if it be passed, the whole acid runs a risk of being spoiled by carbonisation. The steam or hot water must be instantly withdrawn, and the concentrated acid put into a crystallising ressel in a dry, but not very cold apartment. At the end of four days the crystallisation will be complete. The crystals must be drained, re-dissolved in a small portion of water, the solution set aside to settle its impurities, then decanted, re-evaporated, and re-crystallised. A third or fourth crystallisation may be necessary to obtain a colourless acid.

If any citrate of lime be left undecomposed by the sulphuric acid, it will dissolve in the citric acid, and obstruet its crystallisation, and hence it will be safer to use the 
slightest excess of sulphuric acid, than to leave any citrate undecomposed. There should not, however, be any great excess of sulphuric acid. If there bo, it is easily detected by nitrate of baryta, but not by the acetate of lead as prescribed by some chemical authors; because the citrate of lead is not very soluble in the nitric $\Omega$ id, and might thus be confounded with the sulphate, whereas citrate of baryta is perfectly soluble in that test acid. Sometimes a little nitric acid is added with advantage to the solution of the coloured crystals, with the effect of whitening them.

Twenty gallons of good lemon-juice will afford fully ten pounds of white crystals of citric acid.

Citric acid erystallises from a cold saturated solution in prisms belonging to the rhombic system, and containing $\mathrm{C}^{12} \mathrm{H}^{8} \mathrm{O}^{14}, 2 \mathrm{HO}\left(\mathbf{C}^{6} \mathbf{F}^{8} \mathbf{0}^{7} \cdot \mathbf{x}^{2} \mathbf{O}\right)$. If however, the crystals be deposited from a hot solution, they present different forms, and contain only one-half the normal proportion of water.

The specific gravity of the crystals of citric acid is 1.617 . They are unalterablo in the air. When heated, they melt in their water of crystallisation; and at a higher heat, they are decomposed. They contain 18 per cent. of water, of which one-half may be separated in a dry atmosphere, at about $100^{\circ} \mathrm{F}$., when the crystals fall into a white powder. Citric acid is soluble in 0.75 parts of cold, and in 0.5 parts of boiling water.

Citric acid in crystals is composed, by Dr. Ure's analysis, - of carbon, $33 \cdot 0$, oxygen, 62.37, and hydrogen, 4.63; results which differ very little from those of Dr. Prout, subsequently obtained. Dr. Ure found its atomic weight to be 8.375 , compared to oxygen 1,000. The composition of crystallised citric acid has been thus represented :-

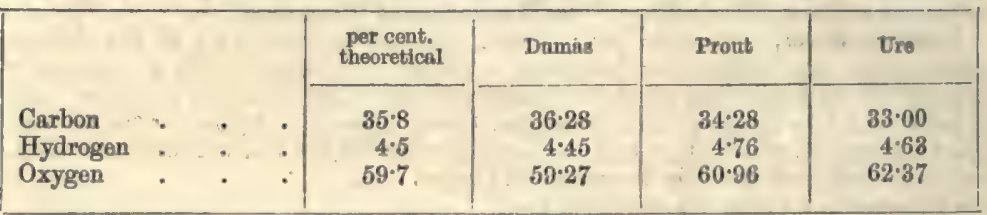

Attempts were made, both in the West Indies and Sicily, to convert the lime and lemon-juice into citrate of lime, but they seem to have failed through the difficulty of drying the citrate for shipment.

Citric acid in somewhat crude crystals is employed with much advantage in calico-printing; for many of the finer colours it cannot be replaced by any other. If adulterated with tartaric acid, the fraud may be detected by adding potash to the solution of the acid, which will cause a precipitate of cream of tartar.

The manufacture of eitric acid so closely resembles that of tartaric acid, that tho makers of one commonly fabricate the other. The raw material in this case is pretty generally a black fluid, like thin treacle, which comes from Sicily, and is obtained by inspissating the expressed juice of the lemon,- the rind having previously beon removed from the lemon for the sake of its essential oil. This black juice is impure citric acid, and requires to be treated with chalk, as practised with respect to the first operation on tartar; by which means an insoluble citrate of lime is formed; and this after being well washed with cold water, is decomposed by sulphuxic acid; and the solution, after undergoing the action of animal charcoal and proper evaporation, yields lrownish crystals on cooling. These are re-dissolved, decoloured, and crystallised three or four times ere they can be sent into the market, for citric acid is more tenacious of colouring matter than most of the other vegetable acids. At Nice, and in the South of France, a portion of chloride of lime is digested upon the citrate of lime, to bleach it prior to decomposition by sulphuric acid. For this purpose, the washed citrate is exposed in shallow vessels to the action of the sun's rays covered by at weak solution of chloride of lime. In a fow hours decolouration ensues; and it is moreover stated that the mucilage which hangs about the citrate of lime, and impedes the subsequent crystallisation of the acid, is in this way destroyed, and the number of re-crystallisations requisite to give a saleable aspect to the citric acid thereby diminished.

According to Perret's process, tho lemon-jujce is clarified with excess of magnesia, and the salt thus obtained after having been washed with cold water, in which it is insoluble, is dissolved in hot lemun-juice. The solution, on evaporation, yields a bibasic citrate of magnesia in a form convenient for exportation.

The use of a vacuum-pan for the final evaporation of a solution of citric acid has been introluced by Mr. E. A. Pontifex.

CrTratras. Combinations of citric acid with alkalis, earths and metals forming salts, See Watts's 'Dictionary of Chemistry.' 
Crrzon. (Citradier, Fr.; Cedrato, Ital.) The fruit of the Citme medica, belonging to the family of dicotyledonous plants, the Aurantiacee or Hesperidec. The rind of this fruit caudied is well known as a delicate sweetmeat. The tree producing the citron is the most beautiful of the genus. A curious variety of the citron is cultivated in China, and called the fingered citron, from its lobes all separating into fingers in different shapes and sizes. The fruits of several other genera belonging to the fumily are greatly esteemed in the countries which produce them. See HessPRRIDE.

CITrowrax oxI. An oil from the Lemon-grass (Andropogon citratum).

Crrzos. A genus of the family Aurantiacece, producing the orange, the lemon, \&c.

Crtreg Aurastium, The common or sweet orange.

Citrus Bergamis. The Bergamot orange.

Crmaus Bigaradra. The bitter or Seville orange, or bigarade.

Crrads Decomana. The shaddock. See SHadoock.

Citrus Limetra. The lime. See Lime.

Citros Lrmoniux. The lemon. See Lexos.

Crtros Mrdica. The citron.

Crvғr. (Civette, Fr.; Zibeth; Ger:) The odour of this substance is peculiar and very characteristic, and is ever more persistent than musk. It forms the base of the famous 'Jockey Club' perfume. It is the product of two small quadrupeds of the genus Viverra ( $V$. zibetha and $V$. civetta), of which the one inhabits Africa, and the other Asia. They are reared with tenderness, especially in Abyssinia. The civet is contained in a sac situated between the anus and the parts of generation in either sex. The animal frees itself from an excess of this secretion by a contractile movement which it exercises upon the sac, when the civet issues in a rermicular form, and is carefully collected. The negroes are accustomed to increase the sêeretion by irritating the animal; and likewise introduce a little butter, or rather grease, by the natural slit in the bag, which mixes with tho odoriferous substance, and increases its weight. It is employed only in perfumery.

According to M. Boutron-Chalard, it contains a volatile oil, to which it owes its smell, some freo ammonia, resin, fat, and extractiform matter, and mucus. It affords, by calcination, an ash, in which there are some carbonate and sulphate of potash, phosphate of lime and oxide of iron.

CXACr. The valve of a pump. See PuMP.

CIAUSTrAIITr. A selenide of lead which occurs in the veins of hrematite at Clausthal and other mines in the Hartz, and at Rio Tinto in Spain.

CIAY. (Argile, Fr.; Thon, Ger.). The term clay is applied to certain hydrous silicates of alumina, derived for the most part, from the decomposition of felspathic rocks, and which are generally rendered impure by the admixturo of other substances. Economically, the term is applied to any finely-divided mineral matter, which becomes plastic on being wetted, and retains its shapo when moulded or pressed into any particular form. Lime, magnesia, oxide of iron, with some other colouring metallic oxides, are occasionally present in small quantities in certain natural clays: when iron is present, the clay burns red.

1. They are readily diffusible through water, and are capable of forming with it a plastic ductile mass, which may be kneaded by hand into any shape. This plasticity exists, however, in very different degrees in the different clays.

2. They concrete into a hard mass upon being dried, and assume, upon exposure to the heat of ignition, a degree of hardness sometimes so great as to give sparks by collision with hardened steel. In this state they are no longer plastic with water, even when pulverised. Tolerably pure clays, though infusible in the furnace, becomo readily so by the admixture of lime, iron, manganese, \&c.

3. All clays, even when previously freed from moisture, shrink in the fire by virtue of the reciprocal affinity of their particles; they are very absorbent of water in their dry gtate, and adhere strongly to the tongue.

4. Ochrey, impure clays, emit a disagreeable earthy smell when breathed upon; the odour is indecd observable to a greater or less extent in all clays.

Brongniart distributes Clays into:-

(1.) Fire-clays (argiles apyres, Fr ; Feuerfeste, Ger.).

(2.) Fusible (schmelzbare, Ger.).

(3.) Effervescing (brausende, Ger.), from the presence of chalk.

(4.) Ochrey (ocreuses, Fr. ; ochrige, Ger.).

The following are the chief varieties of clay usually recognized :-

1. Sratr-clar. (Schiefer-thon, Ger). Its colour is grey or greyish-yellow; massive, dull, or glimmering from admixture of particles of mica. Fracture slaty, approaching sometimes to earthy. Fragments tabular; soft, sectile, and easily 
broken; sp. gr. $=2.6$; adheres to the tongue, and breaks down in water. Slate-clay is ground and reduced into a paste with water for making fire-bricks ; for which purpose it should be as free as possible from lime and iron.

2. Fire-CLAY. In this country the geological position of the fire-clay, which is largely employed in the manufacture of fire-bricks, glass-house pots, \&ce., is immediately beneath the coal, each bed of which rests upon a stratum of greater or less thickness of a clay possessing the peculiar qualities of fire-clay, and distinguished in the mining districts, from the position it occupies with referenee to the coal, by the name of under-clay. The Stourbridge clay is of this character. The following analysis of Stourbridge clay was made by Mr. C. Tookey in Dr. Percy's laboratory :-

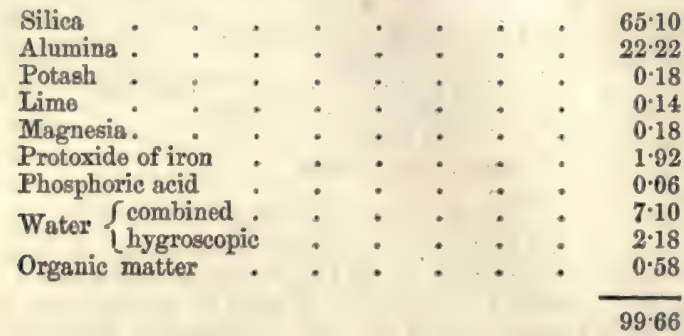

3. Cosmox Crax on Loas. - This is an impure coarse pottery-clay, mixed with ironochre, and occasionally with mica. It has many of the external characters of plastic clay. It is soft to the touch, and forms, with water, a somewhat tenacious paste; but is in general less compact, more friable than the plastic clays, which are more readily diffusible in water. It does not possess the property of acquiring in water that commencement of translucency which the purer clays exhibit. Although soft to the touch, the common clay wants unctuosity, properly so called. The best example of this argillaceous substance, is afforded in the London-clay formation, which consists chiefly of bluish or blackish clay, mostly very tough. Those of its strata which effervesce with acids partake of the nature of marl. This clay is fusible at a strong heat in consequence of the iron and lime which it contains. It is employed in the manufacture of bricks, tiles, and coarse pottery ware.

4. Potters' Clay, or Pipe-Clat.-This species is compact, soft, or even unctuous to the touch, and polishes with the pressure of the finger; it forms, with water, a tenacious, very ductile, and somewhat translucent paste. It is infusible in a porcelain kiln, but assumes in it a degree of hardness. Werner called it pipe-clay. Good plastic clay remaine white, or if grey before, becomes white in the porcelain-kiln. The elay from Poole in Dorsetshire is a celebrated potters' clay, and the clay from the neighbourhood of Newton Abbot in Devonshire is a well-known pipe-clay. The following is an analysis of Poole clay, made in Dr. Percy's laboratory, by Mr. W. Weston :-

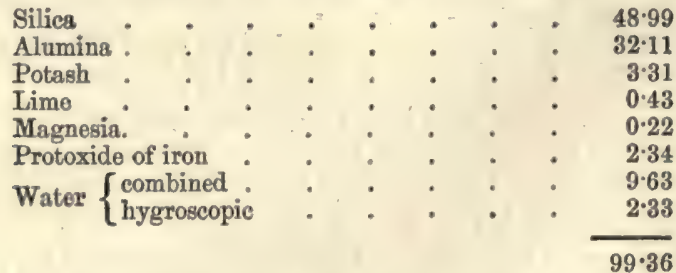

Plastic Clay,-The geological position of the 'Plastic clay,' of geologists, (the old name of the 'Woolwich and Reading beds'), is beneath the London-clay, and above the sand (Thanet sand) which covers the chalk-formation. The Plastic clay of the Paris basin is described as consisting of two beds separated by a bed of sand. The lower bed is the proper plastic elay. The plastic clay of Abondant, near the Forest of Dreux, analysed by Vanquelin, gave-Silica, 43.5 ; alumina, 33.2; lime, 0.35 ; iron, 1; water, 18.

This clay is employed as a fire-clay for making the bungs or seggars, or coarse earthenware cases, in which china-ware is fired.

The plastic clay of Dorsetshire which supplies the great Staffordshire potteries, occurs near the base of the Bagshot beds (Middle Eocene). It is grey coloured, less unctuous than that of Dreux, and consequently more friable. It becomes white in the 
pottery kiln, and is infusible at that heat. It causes no offervescence with nitric acid, but falls down quickly in it, and becomes more highly coloured. Its refractoriness allows of a harder glaze being applied to the ware formed from it without risk of the heat requisite for making the glaze flow affecting the biscuit either in shape or colour. 'Most of the plastic clays of France,' says M. Brongniart, 'employed for the same ware, have the disadvantage of reddening a little in a somewhat strong heat; and hence it becomes necessary to coat them with a soft glaze, fusible by means of excess of lead at a low heat, in order to preserve the white appearance of the biscuit. Such a glaze has a dull aspect and cracks readily into innumerable fissures by alternations of hot and cold water.' Hence one reason of the vast inferiority of the French stoneware to the English. See Dorsershure Crur.

5. Porckinar Cray or Kaomin Earth. (Terre à porcelaine, Fr.; Porzellanerde, Ger.). Kaolin is the name given by the Chinese to the fine white clay with which they fabricate the biscuit of their porcelains. This is the purest known form of clay, its chemical composition being generally reducible to the formula $\mathrm{Al}^{2} \mathrm{O}^{3} .2 \mathrm{SiO}^{2}+2 \mathrm{HO}$ $\left(\mathrm{AI}^{2} \mathbf{O}^{3} . \mathbf{2 5 1 0 ^ { 2 }}+\mathbf{2 H}^{2} \mathrm{O}\right)$, corresponding to silica, 46.3 ; alumina, 39.8 ; water, 13.9 per cent. Ordinary massive kaolin, examined under the microscope, always exhibits certain six-sided scales characteristic of the mineral species called Kaolinite. Kaolin and some allied substances are included by Messrs. Johnson and Blake under this species.

The term Kaolin appears to have been derived from the name of a mountain Kao-ling (Lofty ridge), from which this argillaceous earth was extracted. The kaolins possess very characteristic properties. They are friable in the hand, meagre to the touch, and difficultly form a paste with water. When freed from the coarse and evidently foreign particles interspersed through them, they are absolutely infusible in the porcelain kiln, and retain their white colour unaltered. They harden with heat like other clays, and perhaps in a greater degree; but they do not acquire an equal condensation or solidity, at least when they are perfectly pure. Most of the kaolinclays contain some spangles of mica, which betray their origin from granite.

This origin may be regarded as one of their most distinctive features. Almost all the porcelain clays are evidently derived from the felspars contained in granite, principally in those rocks of felspar and quartz called 'graphic granite.' Hence they are to be found only in primitive mountain districts, among banks or blocks of granite, forming seams often of very considerable thickness. In the same bed quartz and mica occur, while some portions of the kaolin retain the external form of felspar.

The most valuable kaolins have been found-

In China and Japan. The specimens imported from these countries appear pretty white; but are more unctuous to the touch, and more micaceous than the porcelain clays of France.

In Saxony. The kaolin employed in the porcelain manufactories of that conntry has a slight yellow or flesh colour, which disappears in the kiln, proving, as Wallerius observed, that this tint is not owing to any metallic matter.

In France, at Saint-Yrieix-la-Perche, about 10 leagues from Limoges. The kaolin occurs there in a bed, or perhaps a vein, in beds of granite, or rather of that felspathic rock called Pe-tun-tse, which exists there abundantly. This kaolin is generally white, but sometimes a little yellowish, with hardly any mica. It is meagre to the touch, and some beds include large grains of quartz, called pebbly by the China manufacturers. This variety, when ground, affords, without the addition of any fusible ingredient, a very transparent porcelain.

Near Bayonne. A kaolin possessing the lamellated structure of felspar in many places. The rock containing it is a graphic granite in every stage of decomposition.

In England, in the counties of Devonshire and Cornwall. The kaolin or Chinsclay is very white, and more unctuous to the touch than those upon the continent of Europe mentioned above. Like them it is supposed to result from the decomposition of the felspars entering into the composition of granite.

Nature has, up to a certain point, provided the article which man requires for the elaboration of the most perfect production of the potters' art. The clay-China clay, as it is commonly called, or kaolin, as the Chinese have it-is quarried from amidst the granitic masses of Dartmoor and of Cornwall. We are not at all satisfied with any of the theories which have been put forward to account for the formation of porcelain clay. It is commonly stated to be a docomposed graxite; this rock, as is well known, consisting of mica, quartz, and felspar, with sometimes schorl and hornblende. The felspar is supposed to have decomposed; and, as this forms the largest portion of the mass, the granite is disintegrated by this process. We have, therefore, the mica, quartz, and the clay, forming together a soft mass, lying but a short distance below the surface, but extending to a considerable depth. It is quite evident that this stratum is not deposited; had it been so, the particles constituting the mass would have arranged themselves in obedience to the law of gravity, towards which 
there is not the slightest attempt. But we do not know by what process the decomposition of the solid granite could have been effected to a depth from the surface of upwards of one hundred feet, and then, as it often does, suddenly to coase. This, however, is a question into which we cannot at present enter. The largest quantity of porcolain or China-clay is manufactured in Cornwall, espocially about St. Austell and St. Stephen's.

A spot being discovered where this substance sbounds, the operation is commenced by removing the vegetable soil and substratum, called by the workmen the overburden, which varies in depth from about three to ten feet. The lowest part of the ground is thus selected, in order to secure an outlet for the water used in washing the clay. The overburden being removed, the clay is dug up in stopes: that is, in successive layers or courses, and each one being excavated to a greater extent than the one immediately below it, the stopes resemble a flight of irregular stairs. The depth of the China-clay pits is various, extending from twenty feet to fifty feet.

The clay when first raised has the appearance and consistenco of mortar; it contains numerous grains of quartz, which are disseminated throughout in the same manner as in granite. In some parts the clay is stained of a rusty colour, from the presence of veins and imbedded portions of shorl and quartz; these are called by the workmen weed, caple, and shell, which are carefully separated. The clay is next conveyed to the floor of the washing place, and is then ready for the first operation of the process.

A heap of the clay being placed on an inclined platform, on which a little stream of water falls from the height of about six feet, the workman constantly moves it and turns it over with a piggle and shovel, by which means the whole is gradually carried down into an oblong trench beneath, which is also inclined, and which onds in a covered channel that leads to the catch-pits about to be described. In the trench the grains of quartz are deposited, but the other parts of the clay, in consequenco of their greater levity, are carried away in a state of suspension.

This water is conductod into a series of pits, each of which is about eight feet long, four in breadth and in depth, and is lined on the sides and bottom with cut moorstone, laid in a waterproof cement. In these pits the porcelain earth is gradually deposited. In the first pit the grosser particles collect; and being of a mixed nature, are always rejected at the end of each day's work by an opening provided for that purpose at the bottom of the pit. When the water has filled the first pit, it overflows into the second, and in like manner into the third; and in these pits, particularly in the second, a deposit also takes place, which is often preserved, and is called by the workmen mica. The water, still holding in suspension the finer and purer particles of porcelain clay, next overflows into larger pits, called ponds, which are of the same depth as the first pits, but about three times as long and wide. Here the clay is gradually deposited, and the clear supernatant water is from time to time discharged by plugholes on one side of the pond. This process is continued until, by successive accumulations, the ponds are filled. At this stage the clay is in the state of a thick paste, and to complete the process, it remsins to be consolidated by drying. Formerly, the elay was dried by exposure to atmospheric influences only; but the demand for clay has been so large, that artificial heat is now applied to tho briek tanks, so as to quicken the evaporation of the water. When sufficiently dry, the clay is cut into oblong cakes, and is ready for the market.

The following remarks on the clays and plastic strata of Great Britain are from the pen of Mr. George Maw, who has brought to bear upon the subject much geological and chemical knowledge, coupled with great experience as a practical potter. ${ }^{1}$

Plastic strata may be defined as beds of mechanical origin, containing alumina as an essential constituent, which have undergone little or no consolidation, or been subject to metamorphic action.

Although common to various geological formations from the palseozoie to the most recent deposits, a very large proportion of plastic strata applicable to coramic manufactures occurs in the recent and tertiary beds.

'Plastic strata diminish in frequency as the older deposits are approached: in the earlier palrozoic formations, the beds which were at the time of decomposition soft clays and marls, occur for the most part as shales and slates, or have undergone further metamorphism into hard porcelainites and other altered rocks unavailable for the potters' use. Indeed, the very changes which the potter effects by artificial heat have, as regards the earlier rocks, been anticipated in the laboratory of nature, pressure in combination with heat having altered their original soft and plastic condition, changing them into the hardest rocks.

'It must not, however, be supposed that all clays of economic applicability occur in a soft and plastic state, as every gradation exists between hard metamorphic rocks

- These remarks are taken, with slight alterations, from Mr. Maw's Appendix to the 'Catalogue of Iritish Pottery in the Kuscum of Practical Geology.' 2nd edition, 1871. 
and the softest clays, and many of the most valuable clays occur in a semi-indurated condition, are mined by the process of blasting, and brought to the surface in hard rock-like masses. These, by exposure to atmospheric changes and aiternations of wet and drought, frost and thaw, are speedily, by the process known as weathering, disintegrated and reduced to the plastic condition.

- The state of mechanical subdivision is of no little importance in the applicability of clays to the various purposes of ceramic manufacture. Every gradation exists between an almost impalpable condition and a mixture of coarse and fine matter, the coarse residue of which sometimes forms as much as 10 or 20 per cent. of the entire weight.

- Bearing in mind that most clay-strata result from tho mechanical disintegration of older rocks, it will be easily understood that their state of subdivision has been dependent for the most part on the transporting and sorting agencies of water, carrying away and separating the finer particles held longer in suspension than the coarse matter.

'The origin of some of the finer white clays must, however, be attributed to the chemical dissolution of calcareous rocks by the agency of carbonated water, the insoluble silica, alumina, magnesia, \&c., associated with carbonate of lime in chalk and limestone in the finest state of subdivision, being left behind as an impalpable residuum. The writer, in a paper on 'The sources of the materials composing the white clays of the Iower Tertiaries,' published in the 'Quarterly Journal of the Geological Society' (vol, xxiii. p. 387), suggested such a derivation from the chalk, of the smooth clays of Bovey Tracey and Newton Abbot, and of similar clays from the Lower Bagshot beds of Wareham, and it seems scarcely open to question that the white clays resting on the carboniferous limestone of North Wales, Derbyshire, and Tipperary, are the remnants of the subaërial dissolution of the limestone.'

The chemical composition of plastic strata varies as much as their mechanical condition. Thoy may be generally described as an association of silicate of alumina, alumina, free silica, and magnesia, with more or less water of combination. Clays and marls scarcely over occur entirely free from iron, to which their colour is mainly due; it exists in various states of combination, further referred to below. Carbonaceous matter, especially in the tertiary and carboniferous clays, is frequently associated in a fino siate of subdivision, and the alkalis are generally present in variable proportions both as silicates and carbonates.

Contraction in Burning. - This character is of so much importance in all branches of ceramic manufacture that it may be of interest to notice one or two features that the experiments exhibit.

The amount of contraction in burning, due partly to the loss of water of combination and of the carbonic acid in the carbonates, when present, and to the ignition of any carbonaceous matter contained in the clays, but more especially to the drawing togethor of the particles in the production of vitreous silicates, is very variable, and depends both on the chemical and mechanical composition of the clays. The presence of the alkalis and iron tends to complete vitrification, which is always accompanied by a great amount of contraction, and the production of a glass-like body with a bright conchoidal fracture. On the other hand, in clays containing much free silica or even silicate of alumina without the accompaniment of the fluxing alkalis, a small amount of contraction takes place and an open porous 'body' is the result.

The amount of contraction is not less due to the state of mechanical subdivision of the constituent particles. Clays in a coarse state of subdivision and contrining a large proportion of gritty matter, especially of silicious sand, invariably contract less in burning than those of smooth and fine texture, in which the constituents are in an impalpable state of subdivision; this will be at once seen by a comparison of the amount of contraction of slabs composed of the coarse clay in its natural state with those moulded from the clays from which the cosrse particles have been removed; and the larger the proportion of coarse matter in the native clay the greater is the difference between the amount of contraction of the clay in its natural and refined states. The average contraction observed in a considerable number (122) of burnt slabs composed of the native unrefined clay amounted to $6^{\circ} 01$ per cent., and of the burnt slabs of refined clay to 7.53 per cent, of the original moulded size. This appears clue to two causes: first, that a mixture of large and small particles is, to begin with, ectually more dense than a mass of particles of equal size, ${ }^{2}$ and therefore admits of less contmetion in the drawing together of the particles in vitrification; and, secondly, that the coarser subdivision and less intimate contact seem to hinder the recombination of the constituents as vitreous silicates in the process of burning.

1 See papers on this snbject by the writer at pp. 241 and 299 , vol. Iv., of the 'Ceological Magazine.' Illustrated by the fact that a busnel of shot weighs the same as a bushel of bullets, but is exceeded in weight by a bushel of mixed shet and bullets, the small shot filling the vacancies bebween without displecing the lnrger spheres. 
Fow clays produce a perfectly vitreous and unabsorbent body. Some burnt slabs approach to a glassy toxture, while others are so highly silicious and coarse in grain as to be held together by a very slight cohesion. These are in the burnt state open and spongy, and have undergone but little contraction in the kiln. Such clays, as for example those from the North Worcestershire coalfield, of which the celebrated Stourbridge fire-bricks are made, are from their refractory character eminently suitable for the manufacture.

The great majority of clays are intermediate in character between these two extremes, and after the process of burning, form a compact but slightly porous body, subject to a moderate amount of contraction, and are available for general pottery purposes.

The Colouring Matter of Clays.-No native clay is entirely free from the presence of iron which occurs in aluminous earths in various proportions and states of combination. Those most free from iron are the white lower tertiary clays of Devon and Dorset, largely exported from Teignmouth and Poole for the manufacture of white earthenware; for this purpose the absence of iron is a matter of great importance, as it imparts to the ware a yellowish tint, to obviate which it is found necessary to cloak and neutralise the natural cream colour of the burnt clay by the admixture of very small proportions of cobalt blue.

Iron in the white and grey tertiary clays occurs principally in the form of grey carbonate of protoxide, generally in association with finely-divided carbonaceous matter, in proportions varying from a mere trace up to 4 or 5 per cent.

Iron, which is so prejudicial in clays employed for white pottery, is an essential colouring matter in those used in the manufacture of torra cotta, encaustic tiles, bricks, and all common pottery.

These may be considered separately as (a) Grey clays. (b) Yollow clays. (c) Red clays.

(a) Grey clays, so largely developed as 'clunches' and fire-clays in the carbonaceous beds of the coal-measures, owe their colour, in addition to the presence of carbonaceous matter, to carbonate of protoxide of iron in a fine state of subdivision, and oceasionally to the presence of finely-divided pyrites, or bisulphide of iron, which also occurs in the London clay, gault, \&c.

A pale grey clay, almost white, from the base of the Ashdown Sands (Wealden), near Hastings, contains a much larger proportion of iron than its colour would seem to indicate, from its occurring in the form of the comparatively colourless basic sulphate, of which there is 1.68 per cent. present.

(b) Yellow clays axe coloured by hydrous sesquioxide of iron, and generally occur as surface deposits, or where red and gray clays have been subject to weathering, as on exposed surfaces or along lines of jointing. They occasionally occur interstratified with red and grey beds, but appear moro generally to be the result of a kind of rusting process. Grey carbonate of iron on exposure to watery infiltrations, accompanied by atmospheric oxidation, becomes converted into the yellow hydrous sesquioxide; and bisulphide of iron, which readily decomposes under similar circumstances, becomes converted partly into sulphate of protoxide, and partly into hydrous sesquioxide, to the presence of which the yellow joint-surfaces of the London clay and gault are due.

Yellow clays have also been derived from red beds by the red anhydrous sesquioxide and the lower hydrates receiving water of combination.

(c) Red clays and marls, e.g. those of the Keuper, Old Red beds, permian, coalmeasures, the middle wealden, the Neocomian strata of France, the plastic clay of the London and Paris basins, and other tertiary strata, derive their colour from the presence of anhydrous sesquioxide and the lower hydrous oxides of iron which oceur in variable proportions, and are generally associated with small quantities of iron in other states of combination, the colour of which the red oxide obscures. Red hæmatite may be cited as a familiar example of almost pure anhydrous sesquioxide of iron, which, when finely divided, has a strong colouring power. The red Keuper marls receive their colour from about 3 per cent. of this anhydrous sesquioxide, whilst the red clays of the argile plastique of Paris, and of the Neocomian beds of Beauvais, used in the manufacture of the celebrated Beauvais pottery, contain as much as 20 per cent., equivalent to 15 or 16 per cent. of metallic iron. Nearly all such red clays are variegated by lighter patches from which the oxide of iron has been abstracted; partly by a segregational process, drawing together the iron into ferruginous nodules of hydrous sesquioxide, and also from its dissolution by the acids of organic 
decomposition derived from imbedded organic remains. Details of the analysis of red and other elays will be found in a paper by the writer 'On the Disposition of Iron in Variegated Strata,' Quarterly Journal of the Geological Society, vol. xxix. p. 351 .

The Colouring of Burnt Clays. - The colour of burnt ferruginous clays is entirely due to the amount of iron present, irrespective of its previous state of combination, but subject to certain conditions in the general composition of the clay. The action of the kiln, with some exceptions referred to below, is uniform on nearly every state of combination in which the iron occurs ; viz., to reduce it to anhydrous sesquioxide associated as silicates in a more or less intimate state of combination with the other silicates developed in the process of burning.

Yellow clays coloured with hydrous sesquioxide (e.g. yellow oehre), and red clays coloured with anhydrous sesquioxide, and the lower hydrates merely lose their water of combination and become bright brick reds (e.g. red ochre and venetian red).

Grey clays containing finely-divided pyrites or bisulphide of iron are also converted by the kiln into bright reds, the sulphur being driven off, leaving the terra cotta charged with the red anhydrous sesquioxide.

In clays charged with grey carbonates of iron the following reaction takes place: The carbonic acid $\left(\mathrm{CO}^{2}\right)$ is driven off as carbonic oxide $(\mathrm{CO})$, part of its oxygen peroxidising the iron.

Grey clays containing less than 1 or $1 \frac{1}{2}$ per cent. of iron change in the kiln to various shades of cream colour and buff, whilst those containing from 2 to 10 or 12 per cent. range in colour from yellowish fawn to dark reds; from 3 to 4 per cent. of iron produces in the kiln the bright red bodies used in the manufacture of red terra cotta, encaustic tiles, red building bricks, \&c. There seems to be no essential difference (with the exception noticed below) in the colouring matter of the clays that burn buff and those that burn red in the kiln, the depth of colour depending merely on the amount of iron present, the buff shades regularly graduating into the deeper shades of red.

The brightest shades of red and buff are, however, produced with but a partial vitrification of the body. At a heat sufficient to insure its complete vitrification a further change of colour takes place. The bright buff shades are changed to neutral greys, and the reds to a slaty-greyish-black, which probably results from a partial reduction of the metallic colouring matter and its more intimate combination with the other vitreous silicates produced at the higher temperature. In clays containing a large proportion of carbonaceous matter the complete peroxidation and consequent colouring power of the iron seems to be arrested. In a black carbonaceous clay from Bovey Tracey, containing 13 per cent. of organic matter, the combustion of the carbon in contact with the ferruginous oxides seems wholly or partially to have reduced them to a metallic state, or lower oxide having less colouring power than the sesquioxide, and a remarkable bleaching of the burnt elay has been the result. The presence of the alkaline earths in ferruginous clays, especially of lime and magnesia, has also a singular bleaching power in the kiln, arresting the development of the bright red colour. A Permian marl, containing 6 per cent. of sesquioxide of iron and 35 per cent. of carbonate of lime, burned of a greyish buff instead of the rich red such a proportion of iron would otherwise have produced. From some experiments made by the writer, it has been ascertained that as small a proportion as 5 per cent, of caustic magnesia mixed with a red clay entirely destroys its red colour in the kiln, probably from the production of a pale-coloured double silicate of iron and the alkaline earth. A familiar example of this reaction occurs in the process of manufacturing yellow bricks in the neighbourhood of London, the colour of which is dependent on the admixture of ground chalk with brick earth, the brick earth by itself burning of a red colour.'

The composition of ordinary clay will be seen from the following analyses, by Mr. T. H. Henry :-

1. Fire-clay (Stourbridge, Brierly Hill):

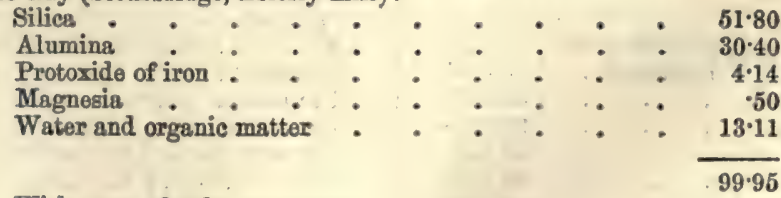

With trace of soda.

2. Three samples of Fire-clay from Wales:-No. I. inferior; the other two good; No. III. the best. 


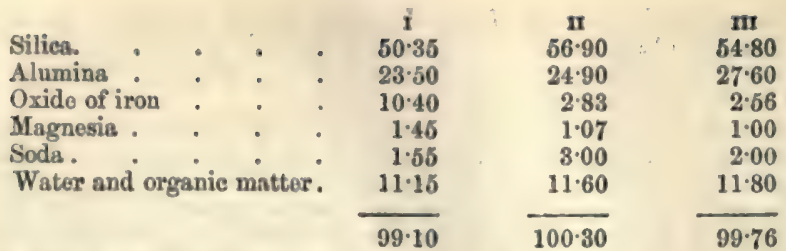

3. Clay making good red bricks :-

Silica . . . . , . 50.40

Alumins and oxide of iron . . . . . . 24.00

Carbonate of lime . $\quad . \quad \div \quad: \quad: \quad 270$

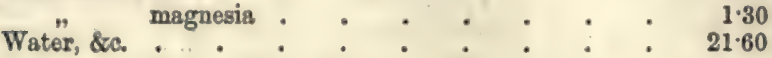

$100 \cdot 00$

4. Clay inferior, on account of excess of carbonate of lime; effervesced strongly with acids :-

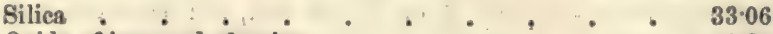

Oxide of iron and alumins : : : : : . 1120

Carbonate of lime. $\quad: \quad \vdots \quad \vdots \quad \vdots \quad 3980$

" magnesia . • : * . : 6.00

Water, \&e. . . . . . . . . 1000

6. Black shale from coal-measures, Dudley :-

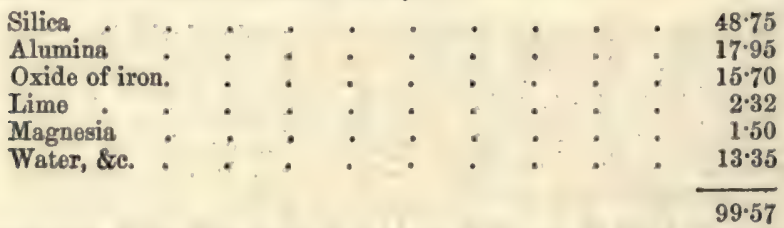

The production of the finer kinds of clay was as follows, in the years given :-

Corawali.

Ksous, commonly called China Clay.

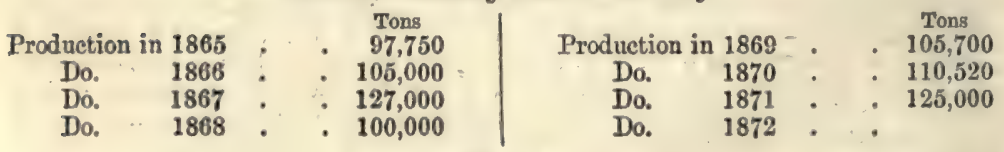

China Stone.

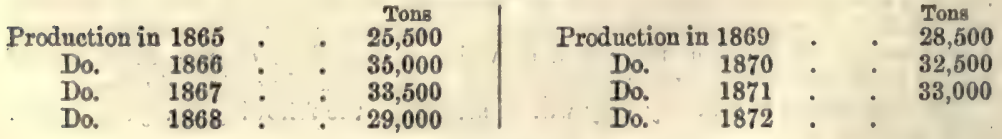

Shipments in 1872 of China Clay and China Stone.

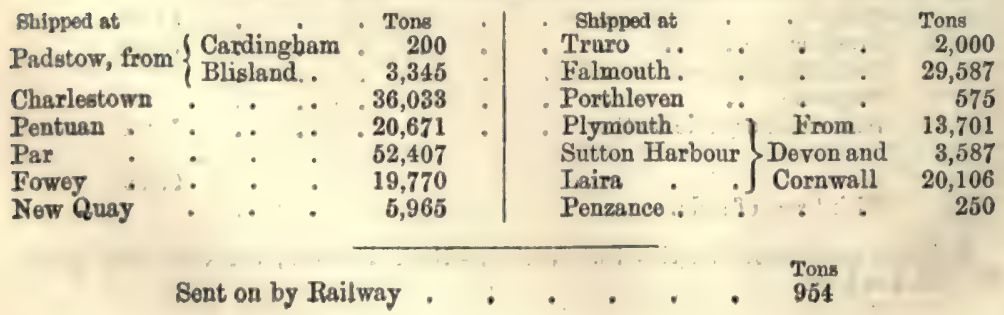




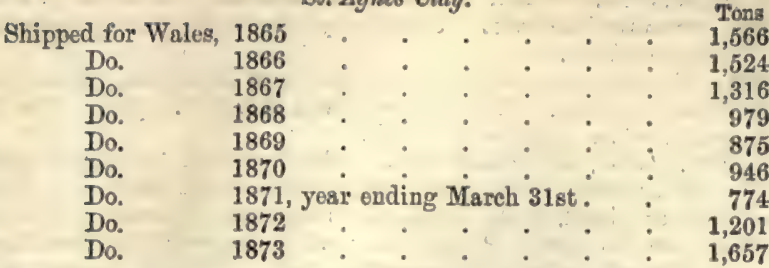

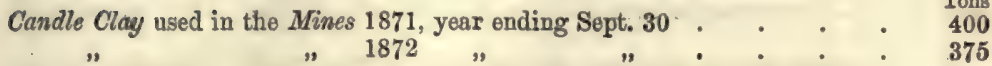

\section{DevonsHire,}

\section{China Clay.}

Lee Moor and other China Clay Works, on Dartmoor, 1872 .

Pipe and Potters' Clay shipped at Teignmouth, the production of Newton and Neighbourhood

\section{Total of Devonshire}

Of Potters' Material there were imported into the Potteries by Trent and

Mersey Navigation, (Clay, \&ce.) ......... . . And by North Staffordshire Railway, (Clay, \&c.) . . . . . . .

Otay IRON STONE. See IroN OrRs.

CIAYITE. A variety of Galena. Seo Lian.

Cisay-siatz. Argillaceous Schist. Seo Sutte.

CIAXSTONE. An earthy felspathic rock, occurring in veins and in mountain masses. It becomes porphyritic by the intermixture of felspar crystals, and is then known as Claystone porphyry.

CIsaT. It is well known that most coals have a tendency to split not only along the plane of bedding, but also along two sets of divisional planes, nearly at right angles to the bedding and to one another. The coal may thus be split into rough. cuboidal fragments. One of these two sets of vertical planes is much more marked than the other, and the smooth clean surface of the coal coinciding with this welldefined set of joints is known as the cleat, face, or slyne; whilst the other vertical surface is callod the end or back of the coal. The direction of the cleat is often constant over a large area, and advantage is taken of this constancy in laying out the workings of a colliery.

CIFAvACE. The tendeney of a crystal, or erystalline substance, to split more readily in cortain directions than in others. The property was originally observed in calc-spas (carbonate of lime), a mineral which crystallises in a great varioty of external forms, but may always be readily cleaved along the planes of a definite rhombohedron. The natural cleavage of a mineral is frequently taken advantage of by the lapidary, in cutting hard stones ; thus, a crystal of diamond, though excessively difficult to cut, can be very easily split or cleaved parallel to the faces of a regular octahedron.

By geologists, the term cteavage is used in a different sense. Cortain rocks, such as slate, exhibit a tendency to split into very thin parallel plates, and the direction of this cleavage is often constant throughout great masses of rock. This slaty cleavage is not to be confounded with the 'lamination' of a rock, or the facility with which it divides into parallel laminz along the planes of stratification. The cleavage, though it may accidentally coincide with the bedding, usually cuts across it, at various angles, and is evidently a structure superinduced in the rock after its original deposition. Physical researches on this subject have shown that slaty cleavage may be developed by lateral compression, the direction of the planes of cleavage being at right angles to the direction in which the pressure has been applied. The ordinary clay-slates used for roofing are prepared by splitting the mass along its planes of cleavage.

CrIcry waxim. A white lead manufaetured at Clichy in France. See Whire Lead. 


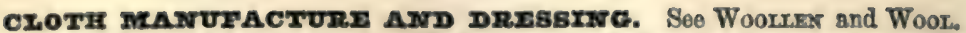
CIOUDY CraxCEDOrX. Chalcedony displaying eloudy spots in a palo groy, semi-transparent base.

Cxovar. Fodder plants belonging to the genus Trifolium. The common red clover is Trifolium pratense. The white or Dutch clover T. repens. The shamrock is generally considered as a species of Trefoil.

Crova, Tres. (Clou, Fr.; Clavo, Span.) The clove tree, Carophyllus aromaticus, is a small tree, from fifteen to thirty feet high, a native of the Moluccas. The flowers are odorous, and the bark, fruit, leaves, and roots are all more or less aromatic. The flower buds are the cloves of commerce; when prepared they have the appearance of a nail, hence their name from clavo, a nail. The buds as soon as they are gathered are dried in smoke over a fire, in the sun, or in a kiln. They are exported in small boxes.

The clove trade was at one time the monopoly of the Dutch. The clove tree is, however, now cultivated both in the East and West Indies, but the finest are still brought from Amboyna in the Moluccas.

Cxovs orx. Eugenic acid, or Carophyllic acid. $\mathrm{C}^{20} \mathrm{H}^{12} \mathrm{O}^{4}\left(\mathbf{C}^{10} \mathbf{I}^{12} \mathbf{O}^{2}\right)$. When cloves are distilled with water, a large quantity of oil passes over. It has been examined by Dumss, Ettling, Böckmann, Stenhouse, Calvi, and, more recently, by Greville Williams. Treated with solution of potash, the greater portion dissolves, leaving a small quantity of a hydrocarbon isomeric with oil of turpentine. See Carborkttre Hrdrogks. The potash solution, on being supersaturated with a mineral acid, allows the eugenic acid to rise to the surface in the form of an oil. When freshly distilled it is colourless, and boils at $438^{\circ} \mathrm{S}$. Its density at $57^{\circ} .2 \mathrm{~F}$. is 1.0684. On analysis it gave:-

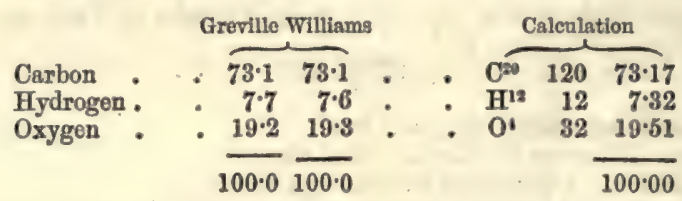

The density of its vapour was found to be $5 \cdot 86$; theory requires 5.67 . The above results were confirmed by a determination of the percentage of baryta in the eugenate.-C. G. W.

Croves. The new bulbs produced from the subterranean axis of the hyacinth, lily, onion, and the like.

CIUTHAIrTz. A mineral which occurs in the amygdaloid rocks of Kilpatrick Hills. It is found in flesh-red vitreous crystals, and consists of silica, alumina, iron, and soda. It takes its name from Clutha, the name sometimes given to the valloy of the Clyde.

conx. (Houille, Fr.; Steinkohle, Ger.) Coal is a mineral of vegetable origin. There is abundant evidence to prove this, but there still exists considerable uncertainty as to the mode of conversion and as to the conditions under which our coalbeds were formed. This is not the place for the discussion of these questions; but it appears necessary that the hypotheses of the best authorities on the subject should be briefly given.

'The occurrence of vegetable remains in all kinds of cosl is such convincing evidence of its formation from vegetable substances, that all further proof is superfluous.'-Gustav Bischof.

'There are fow varieties of coal in which their vegetable origin can be detected by anatomical examination.'-Göppert.

'It appears from the researches of Liebig, and other eminent chemists, that when wood and vegetable matter are buried in the earth, exposed to moisture, and partially or entirely excluded from the air, they decompose slowly, and evolve carbonic acid gas, thus parting with a portion of their original oxygen. By this means they become gradually converted into lignite or wood-coal, which contains a larger portion of hydrogen than wood does. A continuation of decomposition changes this lignite into common or bituminous coal, chiefly by the discharge of carburetted hydrogen or the gas by which we illuminate our streets and houses, According to Bischof, the inflammable gases which are always escaping from mineral coal, and are so often the cause of fatal accidents in mines, always contain carbonic acid, carburetted hydrogen, nitrogen, and olefiant gas. The disengagement of all these gradually transforms ordinary or bituminous coal into anthracite, to which the names of splint coal, glance coal, hard coal, culm, and many others have been' given.'-Lyell. 
- These drifts of plants now forming streaks of conlly mattor in the sandstones or shales including them, aro sufficiont to show that though numerous conl-beds may be tho result of tho growth of a peculiar vegetation in places, the roots of which required and penetrated a suitable soil boneath, it might so happen that extonsive and doep accumulations of drifted plants may wholly form coal-beds under favourable circumstances.'-De la Beche.

True coal is so altered from its original vegetable condition as to have left scarcely any trace of its history. It is generally, however, associated with sands and clays, exhibiting numerous fragments of the ancient vegetation that obtained at the time of its formation; but these fragments are so far removed in every respect from the existing form of vegetation as to afford little clue to the condition of the Earth in this respect. In coal all trace of true woody fibre has disappeared; the water originally present, and so injurious in the less altered forms of vegetable fuel, is entirely absent, or if present at all, is so rather mechanically than chemically, while the water originally in the plant appears to have undergone decomposition, the hydrogen uniting with some part of the carbon, to form carburetted hydrogen gas, often existing in the cells, and between the plates of the coal under considerable pressure, and the oxygen being almost entirely removed. The former vegetable has now become a mineral substance, and lies in vast beds of variable thickness, and overlaying each other to the extent sometimes of more than a hundred in a single district; such beds being regularly interstratified with deposits of sand and clay, and occupying a distinct geological position, being, with only a few exceptions, confined to rocks belonging to the newer part of the Palaozoic series.

The changes undergone by vegetable matter when buried in the earth, and accumulated in large quantities, and the length of time needed to produce any marked alteration, are subjects rather more interesting, it may seem, to the chemist than to the practical man, who looks only for fuel that he may employ economically. But inasmuch as the real condition of coal varies considerably, it is desirable that the whole history of coal and lignite beds should be generally understood by any one using these substances.

Vegetable matter consists of carbon in combination with oxygen and hydrogen, as its principal constituents; nitrogen forming but a small, although an important, part in its economy. A large quantity of water is also present; and so long as the vegetable lives, there is a constant change and circulation of material particles kept up, replacing and renewing the different portions. When death takes place, there is a tendency to decomposition, or the separation of the whole into minute atoms having no further relation to each other. But this is frequently checked by various conditions, such as the presence of some substances derived from plants themselves, or the absence of sufficient oxygen gas to allow the change to take place by combining with the carbon to form carbonic acid gas, the first step in the process of destruction. These causes act constantly but partially, and thus a large quantity of regetable matter is always in the course of decomposition, while in particular spots a large quantity is constantly being accumulated. The latter condition is seen in our climate in the gradual but steady increase of peat bogs.

That coal is derived from the vegetable kingdom no longer admits of a doubt, but the class of plants to which more especially we are to look for the origin of coal, is still a matter of much uncertainty. The idea generally entertained is that which supposes a natural basin in which vegetable matter is deposited, the layers varying in thickness, becoming covered with mud or sand.

Some microscopic observers assure us that they are enabled to detect ligneous structure in bituminous coal. Mr. Quekett has given a great number of drawings in proof of this, and he refers the coal to the woody matter of an extinct class of the Conifera. Botanists of eminence, however, assure us that there is no evidence of ligneous structure in any of the examples brought forward in proof of that hypothesis. Others maintain that such structure, though observable in the charcosl-like layers called ' mother of coal,' cannot be detected in the bituminous parts, and that by far the greater portion of the coal is composed of the macrospores and microspores of Lepidodendra, and other lycopodiaceous plants. The spores of these, or of allied plants, are found more or less abundantly in all true coal; indeed, in some cases they appear to make up the mass of the mineral, whilst in others they seem to have been crushed together, thus forming the brown streaks commonly seen in microscopic sections of coal.

Sir Charles Lyell, in his excellent 'Manual of Elementary Geology,' enters largely and with his usual lucid manner, into the consideration of the carboniforous plants. There can be no doubt of the existence of the remarkable flora described by him during the period when our beds of fossil fuel were forming. Referring to Sir William Iogan as his authority, Sir Charles says, 'It was observed that while in the overlying Vor. I. $8 \mathrm{G}$ 
shales or "roof" of the coal, ferns and trunks of trees abound, without any stigmaria, and are flattoned and compressed, those singular plants of the underclay (the stigmario) very ofton retain their natural forms of branching freely, sending out their slender leaf-like rootlets, formorly thought to be leaves, through the mud in all directions.' This plant is singularly indicativo of the class of plants from which coal has been derived.

M. Adolph Brongniart states that the number of species of carboniferous plants amounts to about 500. Lindley informs us that no less than 250 ferns havo been obtrinod from the coul-strata. Forty specios of fossil plants of the coal period have been referred to the Lepidodendrons. These with Equisetacea, Calamites, Asterophyllites, Sigillaria, of which about thirty-five species are known with their roots, Stigmarie, and Conifera make up the remarkable flora which have been preserved to us in our coal series.

Trees and humbler plants in grcat variety are found in the carboniferous sandstones and shales, and in the coal itself; but it does not appear that we have any one evidence of tho actual conversion of the woody fibre of these plants into coal ; that is, there is no evidence of the direct conversion of wood into bituminous coal. The trees are almost invariably silicified, or converted into columns of sandstone; the carbon which constituted the original woody fibro being substituted by silica, or sometimes by carbonate of lime, and sometimes by iron. Sir Charles Lyell has carefully examined the phenomena, now in progress, of the great Delta of the Mississippi, and he perceives in them many facts which fully explain, to his mind, the progress of coal deposit. It cannot, however, be disguised that even while he refers the coal to the supposed submerged forests, he does not venture to explain any of those changes, which he evidently believes depend upon some peculiar conditions of climate.

Professor John Phillips, who has devoted much study to this subject, says, "There is no necessity to enlarge upon the proofs of the origin of coal from vegetables, drawn from an examination of its chemical constitution, as compared with the vegetable products, and the composition of the ligneous parts of the plants, and from the unanswerable identity of the carbonaceous substance, into which a vast multitude of fossil plants have been converted. The chemical constitution of this carbonaceous product of the individual vegetables, is exactly analogous to the chemical constitution of coal; and it is quite probable that hereafter the reason of the variations to which both are subject, whether dependent on the original nature of the plant or produced by unequal exposure to decay after inhumation, or metamorphic subsequent operations, will be as apparent as that of the general argument arising from a common vegetable origin.'Manual of Geology.

Mr. Jukes says, 'If therefore, we suppose wood (or vegetable matter) buried under accumulations of more or less porous rock, such as sandstone and shale, so that it might rot and docompose, and some of its elements enter into new combinations, always using up a greater quantity of oxygen and nitrogen than of carbon and hydrogen, or of oxygen and hydrogen than of carbon, we should have the exact conditions for the transformation of vegetable matter into coal.'-The Student's Manual of Geology.

Much stress has been laid upon the fact that we have brown coal still retaining all the unmistakable charactors of wood, and the apparent passage of this into true coal.

'Göppert states that the timber in the coal-mines of Charlottenbrunn is sometimes converted into brown coal. The same conversion was many years ago found in an old gallery of an iron-mine at Turrach in Styria. A. Schröttor explains, according to the analysis made by him, this conversion, by the separation of marsh gas and carbonic acid from the ligneous fibre of oak wood.'-Bischof.

The same authority says, "This conversion of wood into coal may take place in four different ways, namely:-

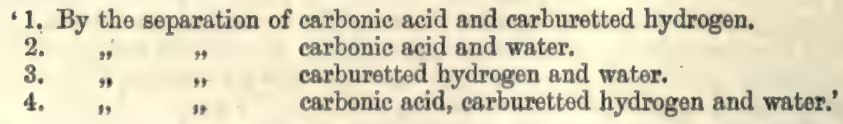

Quoting the information accumulated by Bischof for the purpose of showing the chomical changes which take place, the following analyses (see Table at top of next page) are given.

Such is, in the main, the evidence brought forward in support of the view that coal is the result of the decomposition, upon the place where it is found, of woody fibre. The following remarks by Professor Henry Rogers on the structure of the Appalachian coal exhibits some of the difficulties which surround this view:-

- Each bed is made up of innumerable very thin laminæ of glossy coal, alternating 


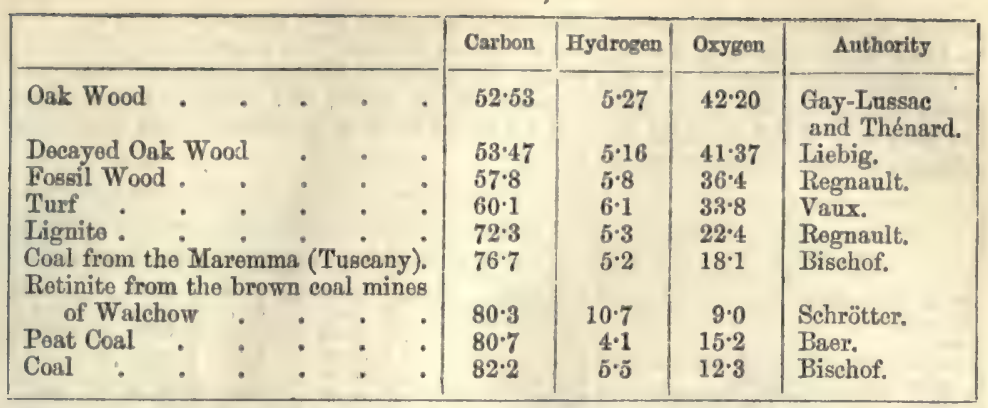

with equally minute plates of impure coal, containing a small admixture of finelydivided earthy matter. These subdivisions, differing in their lustre and fracture, are frequently of excessive thinness, the less brilliant leaves sometimes not exceeding the thickness of a sheet of paper, In many of the purer coal-beds these thin partings between more lustrous layers consist of little laminæ of pure fibrous charcoal, in which we may discover the peculiar texture of the leaves, fronds, and even the bark of the plants which supplied a part of the vegetable matter of the bed. All these ulti. mate divisions of a mass of coal will be found to extend over a surprisingly large surface, when we consider their minute thickness. Pursuing any given brilliant layer, whose thickness may not exceed the fourth part of an inch, we may observe it to extend over a superficial space which is wholly incompatible with the idea that it can have been derived from the flattened trunk or limb of any arborescent plant, lowever compressible. When a large block of coal is thus minutely and carefully dissected it very seldom, if ever, gives the slightest evidence of having been produced from the more solid parts of trees, though it may abound in fragments of their fronds and deciduous extremities.'

It is not possible within the space which can be afforded to this article in the present work, to examine further the various views which have been entertained by geologists and chemists of the formation of coal. A brief summary must now suffice :-

1. Coal is admitted upon all hands to be of vegetable origin.

2. Many refer coal to some peculiar changes which have taken place in wood; others to the formation and gradual subsidence of peat bogs (Unger). Fuci have also been thought by some to supply the materials for coal-beds.

3. By some the coal is thought to be formed upon the spots on which the trees grew and decayed. By others it is supposed that vast masses of vegotablo matter wero drifted into lakes or deltas, to be there decomposed.

4. Whether the plants grew on the soil-the under clay-upon which the coal is found, or were drifted to it; there must have been long periods during which nothing but vegetable matter was deposited, and then a submergence of this land, and vast accumulations of mud and sand. The number of coal-seams in some of our coal-fields and the thicknesses of the strata will be given.

Professor Henry Rogers and others suppose, that the whole period of the coal-measures was characterised by a general slow subsidence of the coasts on which we conceive that the vegetation of the coal grew; that this vertical depression was, however, interrupted by pauses and gradual upward movements of less frequency and duration, and that these nearly statical conditions of the land, alternated with great paroxysmal displacements of the level, caused by the mighty pulsations of earthquakes. (Seo Faults, Heaves.)

The difficulties are mainly the facts-

1. That the evidence is not clear that anything like ligneous structure can bo detected in coal, while microspores and macrospores are sometimes found in abundance.

2. That the woody matter found in coal is never converted into coal, although sometimes it appears as if the bark was so changed.

3. That the coal arranges itself always in exact obedience to the underlying surface, as though a semi-fluid mass had been spread out on a previously-formed solid bed.

4. The thinning out of true coal to extreme tenuity, as mentioned by Professor Henry Rogers; numerous examples of which appear in this country.

5. The extreme difficulty connected with the subsidence of the surface of the earth to such a depth as that to which the lowest seams of coal extend.

We do not intend to answer any of these difficulties, but to leave the question open for further examination; merely remarking, in conclusion, that there can be no doubt of the vegetable origin of coal. The only question is, the conditions of 
change by which bituminous coal has been produced from vegetable fiore, and that wo have not completed all the links in the chain between brown coal and true coal.

The following Table shows at a glance the chemical difference between wood and brown coal on the one hand, and anthracite on the other; and serves to explain what has been said of the chemical changes by which wood is supposed to pass into bituminons and, oventually anthracite coal:-

\begin{tabular}{|c|c|c|c|c|c|c|c|c|c|c|}
\hline \multirow[b]{2}{*}{ Pure wroody flbre } & \multicolumn{3}{|c|}{ Locality } & \multicolumn{3}{|c|}{ Authority } & \multirow{2}{*}{$\frac{\text { Carbon }}{52 \cdot 65}$} & \multirow{2}{*}{$\frac{\text { Hydrogen }}{\cdot 5 \cdot 25}$} & \multirow{2}{*}{$\frac{\text { Oxygen }}{42 \cdot 10}$} & \multirow[t]{2}{*}{ Nitrogen } \\
\hline & & .. & & Schodler & . & & & & & \\
\hline Beech. & 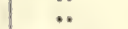 & $\because$ & & Chevandier & . & & $48 \cdot 89$ & $6 \cdot 07$ & $43 \cdot 11$ & 0.93 \\
\hline Oak , & & .. & & Ditto. & : & & $50 \cdot 64$ & $6 \cdot 08$ & $42 \cdot 05$ & 1.28 \\
\hline Peat . & Holland & & & Milder . & . & & $59 \cdot 27$ & $5 \cdot 41$ & $35 \cdot 82$ & \\
\hline Ditto & Long . & & & Regnault & : & : & 60.06 & 6.21 & $83.73^{\circ}$ & \\
\hline Ditto & Bog of All & len & . & Kane & . & - & $61 \cdot 02$ & $5 \cdot 77$ & $82 \cdot 40$ & 0.80 \\
\hline Ditto & Upper Sha & annon & & Ditto & : & : & $61 \cdot 21$ & $5 \cdot 61$ & $31 \cdot 44$ & $1 \cdot 62$ \\
\hline Lignite & Cologne & . & 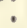 & Regnault & : & 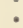 & $63 \cdot 42$ & 4.98 & $27 \cdot 11$ & \\
\hline Ditto & Patagonia & & & J. A. Phillip & & & $62 \cdot 13$ & 5.08 & $10 \cdot 44$ & \\
\hline Brown cosl & Neider All & pen & & Regnault & & & 60.05 & $5+20$ & $22 \cdot 74$ & \\
\hline Ditto & Wigan & . & & J. A. Phillip & & & $80 \cdot 21$ & $6 \cdot 30$ & $8 \cdot 54$ & \\
\hline Ditto & Bog Head & 1. & & Hofmann & 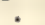 & & $65 \cdot 66$ & $8 \cdot 90$ & & \\
\hline Ditto & Ditto & & . & Anderson & : & - & $64 \cdot 02$ & $8 \cdot 09$ & $5 \cdot 66$ & $0 \cdot 55$ \\
\hline Cannel coal & Wigan. & $\because$ & & Richarison & : & 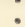 & 88.75 & 5.66 & 8.03 & 0.00 \\
\hline Cherry coal & Newcastie & & & Ditto & : & & $81 \cdot 20$ & $5 \cdot 04$ & $8 \cdot 43$ & \\
\hline Carr's Hartley & Ditto & , & & Admiralty In & nqui: & & $79 \cdot 83$ & $5 \cdot 11$ & 7.26 & $1 \cdot 17$ \\
\hline Steam Wallsend & Ditto & & & Ditto : & . & & $83 \cdot 71$ & $5 \cdot 30$ & $2 \cdot 79$ & 1.06 \\
\hline Resolven . & South Wal & les & & Ditto . & . & . & $79 \cdot 38$ & $4 \cdot 75$ & & $1 \cdot 38$ \\
\hline Neath Abbey & Ditto & . & & Ditto : & : & . & $89 \cdot 04$ & 5.05 & & 1.07 \\
\hline Graigola & Ditto & . & & Ditto : & $\therefore$ & & $84 \cdot 87$ & $3 \cdot 84$ & $7 \cdot 19$ & \\
\hline Aberaman: & Ditto & & & Ditto: & ? & . & $90 \cdot 94$ & $4 \cdot 28$ & 0.94 & $1 \cdot 21$ \\
\hline Anthracite. & Ditto & & & Ditto - & : & & $91 \cdot 44$ & $8 \cdot 46$ & 0.79 & 0.21 \\
\hline Ditto & Slievardag & $\mathrm{gh}$, Ire & & Ditto & & & $80 \cdot 03$ & $2 \cdot 30$ & & \\
\hline Ditto & Vizille & $\dot{0}$ & & Jacqueline & : & . & $94 \cdot 09$ & 185 & ** & $0 \cdot 28$ \\
\hline Ditto & Swansea & - & - & Regnault & . & . & $91 \cdot 29$ & $9 \cdot 98$ & 0.82 & 0.45 \\
\hline
\end{tabular}

Brooke and Miller divide coal into three varieties:-1. Anthracite; 2. Black cosl ; 3. Brown coal. The chemical differences between those being:

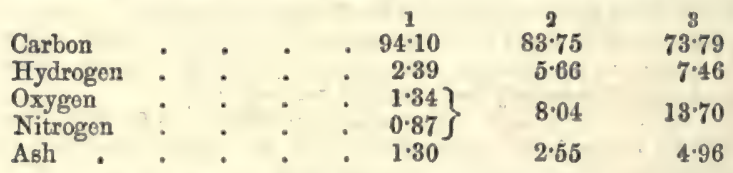

Other mineralogists, as Dana, divide coal into bituminous and non-bituminous; and under these divisions they group the numerous varieties of coal which occur, as -

Bitumnous Varietres. 1. Pitch or caking coal (Dana), which burns roadily with a yellow flame, and which on receiving the heat, unites into a solid mass; thus requiring poking to prevent a too complete consolidation of the mass. 2. Cherry coal somewhat resembles caking coal, but in burning it does not soften or cake. It burns more rapidly than caking coal, with a clear yellow flame. 3. Splint coal or splent coal is in Scotland a term for a hard laminated variety of bituminous coal, intermediate in texture, between caking and cannel coal. The name is derived from its splitting (or splenting) up in large flaggy or board-like laminæ (Page). It is a coarse kind of cannel coal (Dana). A variety of bituminous (cannel) coal, with a slaty structure, and of a harder and tougher nature than cherry coal. Splint coal is used by Lyell as the equivalent to anthracite. 4. Cannel coal. A coal with a fine compact texture, a large conchoidal fracture; it receives a good polish; is sonorous when struck. It is coal more perfectly bituminised than No. 1. In Scotland this coal is called parrot coal from the crackling, chattering noise which it makes when first thrown into the fire.

Jet resembles cannel coal, but is blacker, and has a much more brilliant lustre.

Flint Coal. A kind of coal resombling anthracito in appearanco; but containing bitumen.

Flew Coal. A coal resembling flint coal. It must be regarded as a local name for the coal found at Wedgebury in Staffordshire.

Crow Coal. A coal found near Alston, containing but a small quantity of bitumen.

Albert Coal or Albertite. A bituminous coal found in Nova Scotia; it has the appearance of asphaltum, and is partially soluble (about 20 per cent.), but it has not the fusibility of asphaltum. 
Now-Bitumanous Varueties. Anthracite. A coal with a sharp-edged, shining, conchoidal fracture, not easily ignited, but when burning it gives out an intense heat, unaccompanied by smoke, and produces but little flame. Often called stone coal.

Culm. An impure shaly kind of coal, or anthracite shale, as the culmniferous or anthracite shales of Devon. The torm is used in Parliamentary returns to signify anthracito.

Fossil Coke. An American variety, more compact than artificial coke, supposed to be produced by the action of trap rocks on anthracite.

Recest Cont. The true coal era is a well-marked one. Geologically it lies between the Old Red Sandstone and the New Red Sandstone rocks. A newer coal is found in the lias at Richmond in Virginia, United States; and a coal belonging to the Oolites, at Brora in Sutherland, Scotland, and other places. These newer coals are very insignificant as compared with the true old coals.

Brown CoAt. This comparatively recent coal sometimes resembles bituminous coal. Other varieties have a brownish-black colour, with a coal-like lustre. It is called, when it approaches ordinary coal in hardness and appearance, stone coal.

Lignite. Wood in a process of change; when it still retains its woody structure, it is called wood or board coal; when it consists of thin layers, it is termed paper coal, and when soft and earthy it is known as peat coal.

The relative importance of mineral fuel in various countries, as indicated by the actual coal area and the real production of some of the principal coal-fields, may be understood by a reference to the subjoined Table:-

\begin{tabular}{|c|c|c|c|}
\hline Countries & $\begin{array}{c}\text { Doal Area in Squaro } \\
\text { Miles }\end{array}$ & $\begin{array}{l}\text { Proportion of whole } \\
\text { Area of the Country }\end{array}$ & $\begin{array}{l}\text { Annual Production } \\
\text { according to the } \\
\text { latest Returns }\end{array}$ \\
\hline British Islands . & 12,800 & $1-10$ & $123,573,600$ \\
\hline France., & 2,000 & $1-100$ & $12,148,223$ \\
\hline Belgium & 520 & $1-22$ & $12,544,038$ \\
\hline Spain . & 4,000 & $1-52$ & 500,000 \\
\hline Prugsia. & 1,200 & $1-90$ & $14,751,729$ \\
\hline United States of America & 220,166 & $3=0$ & $21,558,329$ \\
\hline British North America . & 13,000 & $1-10$ & $2,750,000$ \\
\hline India . & uncertain & $\ldots$ & 401,279 \\
\hline
\end{tabular}

\section{CoAL-FIRLDS OF THE UATTED Kingdom,}

It will thus be seen how extremely important the coal-fields of the British Islands really are when compared with any others. This is the case, not merely in the total anmual production and the proportionate extent of the deposits, but also from the great number of points at which the coal can be advantageously worked. This will be best seen by reference to the Table given at the end of the article.

The distribution of coal in the United Kingdom is of vast importance to the country. It is spread over large areas, commencing with Devonshire in the south, and extending to the northern divisions of the great Scotch coal-fields. A careful examination of all these deposits cannot but prove useful.

DevonshrRe. Iignite of Bovey-Heathfield. - Lysons, 'Magna Britannia,' informs us that this so-called Bovey coal was worked for use early in the last century; and Dr. Maton described those beds in 1797 as being from 4 to 16 feet in thickness, alternating with clay, and he stated that the pits were about 80 feet deep, and worked for the supply of a neighbouring pottery. A pottery was established at Ideo in 1772, and one at Bovey Tracey in 1812, both of which were supplied with fuel from thoso lignite beds.-De la Beche.

Mr. William Pengelly carefully investigated the Bovey Tracey lignites. The result of his enquiry was "that the Bovey lignites are the contemporaries of the "Hempstead Bods" in the Isle of Wight, first discovered by the late lamented Edward Forbes in 1852, and described by him in the following year. Though their discoverer always regarderl them as Upper Eocene, they have recently been grouped amongst the Lower Miocene.' Professor Heer, of Zürich, determined forty-fivo species of plants from this lignite, forty-one of them being decidedly of the Lower Miocene age.

Bideford Anthracite. - The beds of anthracite stretch across the country from Barnstaple Bay. by Bideford and Averdiscot, towards Chittlehampton, a distance of about twelve miles and a half. The anthracite is mixed with the black shales of the carbonaceous deposits.

'The anthracite is mixed with those shales in the manner represented beneath 
fig. 465 ; $a$, sandstones; $b$, shales; $c$, culm or anthracite ; so that the culm jtsolf soems the result of irregular accumulations of regotiblo mattor intermingled with mud and

465

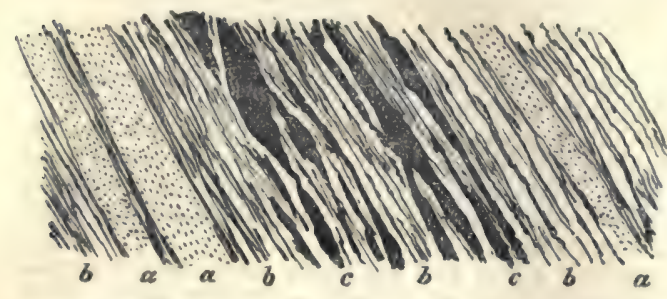

sand. As so frequently happens with carbonaceous deposits of this kind, nodules of argillaceous iron-stone are often found in the same localities with the shales and anthracite, rominding us of the intermixture of iron ores and regetablo matters in the bags and morasses of the present day.'-De la Beche.

Sommesetshirn and Grodcestershirr, - The Dean Forest coal-field, and tho coalmoasures extending further south forming the Bristol coal-field, are included in this division. The workable seams of coal in the Forest are the following:-

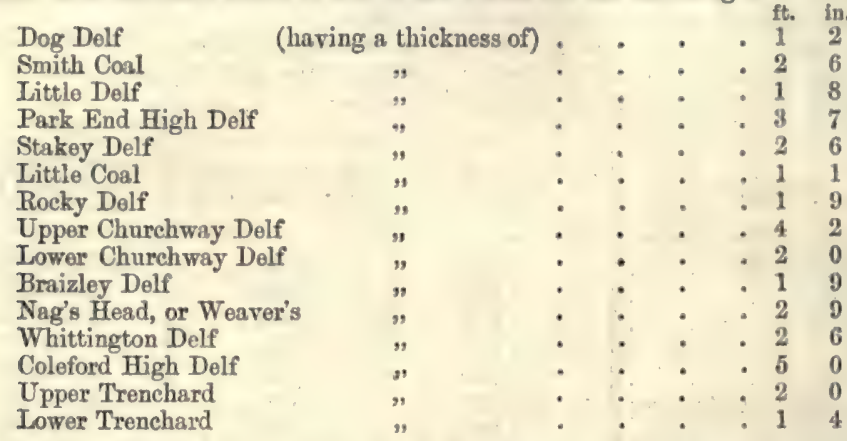

There is a small coal-field north of the Forest of Dean, which is a long narrow strip, containing two and a half square miles or 1,600 acres.-Maclauchlan, Geological Transactions, vol. $\nabla$.

About nine miles and a half to the south of Dean Forest a considerable mass of coal-measures has been preserved from destruction, by the denuding causes which have carried off the connecting portion between it and Dean Forest, leaving at least two outlying patches on the north of Chepstow.

The Bristol coal-fiold oceupies about fifty square miles, or 32,000 acres. The seams of coal are very thin in comparison with those which are worked in other districts. Buckland and Coneybeare ('Geological Transactions,' vol. i.) have well described this coal-field.

The total thickness of the whole series of strata in this Bristol coal fleld has been shown by De la Beche to be as follows:-

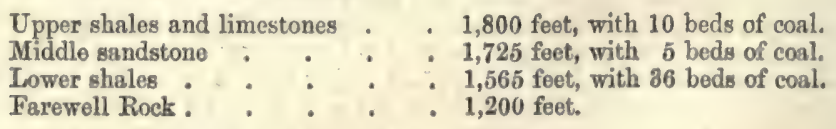

Total : . $\overline{6,290}$ feet.

Soutr Wates COAL-FIELD. - The total thickness of the coal-strata in this important district is very great. Logan and $D e$ la Beche havo accumulated evidence which appears to justify the admission of 11,000 , or even 12,000 feet thickness from the carbrniforous limestone to the highest part of tho coal series about Llanelly; in other parts of the ficld tho series is found to be on proportions only less gigantic. The most general view which can be afforded scems thus, giving the true coal-measuro about 8,000 feet :-

Llanelly series, with several beds of coal. . . . . $\quad$ Feet

Penllergare series of shales, sandstones, and beds of coal, 110 beds; 26 beds of cosl

Central series (Townhill sandstones of Swansea, Pennant grit of the

Bristol field); 62 beds, and 16 beds of coal . 3,000 
Lower shalos, coals, and iron-stones (Mertliyr); 266 beds, 34 beds of coal.

Abundance of iron-stone beds and Unionidee occur.

Farewell-Rock and Gower shales above; the carboniferous limestone below.

The coal on the north-eastern side of the basin is of a coking quality, excellent for the iron manufacture; on the north-western it contains little or no bitumen, being what is called stone-coal or anthracite; on the south side, from Pontypool to Caermarthen Bay, it is of a bituminous or binding quality.-Phillips.

Shropsure.-This district includes the small coal-field of Coalbrook Dale and that of the plain of Shrewsbury. The Coalbrook-Dale field, according to Mr. Prestwich, has some remarkable features. (Geological Transactions.) Perhaps there is no coal tract known, which in so small a compass, about twelve miles long, and, at most, three and a half miles wide, exhibits so many curratures in the outcrops, crossed by so many continuous faults, some varying north by east, others east-northeast; these crossed by many of shorter length, and directed west-north-west, and in several other lines. The total thickness is supposed to be 1,000 or 1,100 feet, divided. into 80 distinct strata. The coal varies in total thickness from 16 feet to 55, and in the number of its beds from 7 to 22 , the increase being to the north. The 'cleat' or system of joints runs from west-north-west to east-south-east. The coal is, for the most part, of the variety called slate-coal in Scotland, and hard coal in Derbyshire. Cannel coal is rare-sulphureons coal (pyritous) very common. Petroleum abounds in the central and upper part of the field. The beds are mostly thin; the ten uppermost are too sulphureous for other uses than lime burning, and are called stinkers; twelve beds of good coal, in all 25 feet thick, the thickest being five feet, succeed, and the lowest bed of the whole formation, eight inches thick, is sulphureous.-Phillips, Prestwich.

STAFFORDSHIRE. - The Coal-field of South Staffordshire, which has been described by Mr. J. Beete Jukes, who states, its boundary would be roughly described as the space included within a boundary line drawn from Rugeley through Wolverhampton to Stourbridge; hence to the southern end of the Bromsgrove Lickey, and returning through Harborne (near Birmingham) and Great Barr back to Rugeley. This geologist classes these coal-strata in three divisions, by the well traced band of thick coal. The total thickness of coal near Dudley being about 57 feet, and between Bilston and Wolverhampton upwards of 70 feet. The thick coal is formed of eight, ten, or thirteen distinguishable parts, the whole seam varying in thickness from three feet to thirtynine feet five inches; it is very irregular in parts, divided by sandstones, splitting with wide-shaped offshoots, and cut into 'swiles' or 'horse-backs,' which rise up from the floor. Below the thick coal, are numerous beds of sandstone-shales, coal, and ironstone, having on the average a thickness of 320 foet; and above the thick coal the thickness is 280 feet on the average.-Records of the School of Mines.

North Staffordshire Coal-field. - This field is comprised in the space between Congleton, Newcastle-under-Lyne, and Lane End. About 32 beds of coal have been determined, rising eastward between Burslem in the centre of the field and its eastern limit near Norton Church.

DrRBtshiRe and NotTINGHasshirk.-The Derbyshire and Nottinghamshire coals are classed as to structure in two varieties, as 'hard' coal, in which the divisional structures are chiefly derived from the planes of stratification, crossed by one set of 'cleat' or natural joints (called 'slines,' 'backs,' \&c.) so that large prismatic masses result; ' soft' coal where the cleat fissures are numerous, and broken by cross cleat. In respect of the quality, some of the coal is of a 'crozling' or coking nature, easily fusible and changing its figure by 'coking'; the rest (and this is specially the case with the 'hard 'variety) makes both good furnace coal and excellent coke, which, however, is hardly melted at all, and the masses are not changed in figure by the process,-Phillips's Manual of Geology.

The names by which the more important beds of coal worked within this district are known, are as follow: Tupton coal, hard coal, soft coal, black shalo or elod coal, low hard coal and low soft, windmill coal, Dansil coal, Ganister coal, Parkgate coal, Aston coal, Kilburn coal, furnace coal, Hazel coal, Euroka coal, main and deep coal.

LieicestershiRe AND WARWICKSHIRE,-The Leicester coal-field is best developed about Ashby-de-la-Zouch (seo Mammatt on 'The coal-field of Ashby-de-la-Zouch'), where the coal is much like the hard coal of Derbyshire. Amongst the seams of coal is one variety called cannel; and another, formed by the concurrence of more than one band, from seventeen to twenty-one feet in thickness. The beds near Ashby-de-laZouch are as follow:- 
In the Moira districtEuroka coal

Stocking coal

Woodfield coal

Slate coal

Nether main conl

Fourfoot coal

The Earl coal

In the Coleorton district-

Heath End coal.

Lount coal

Main coal

Thickness of beda

- 4 to 6 feet.

- 6 to 7 ,

- 5

- 3 t to 4 "

- 14 to 15 ,"

- 4 to 5 ,

- $4 \mathrm{ft}$. 6 in.

- 9 feet.

- (3 beds).

- 10 to 12 feet.

The Warwickshire Coal-field extends from a point east of Tamworth to a point east of Coventry, about twenty miles from N.W. to S.E. parallel to the Ashby coal tracts. The strata are most productive of coal near the southern extremity, where, by the coming together of two seams,-worked separately at Griff,-the fire-yard seam is worked. The beds are known as the seven-fect coal and rider, slate coal, two yards, lower seam, cannel, and Ell coal.

Yorkshine.-Professor John Phillips gives the following mode of classification as the most natural and convenient for the Yorkshire coal :-

Magnesian limestone unconformably covers the coal seams.

$$
\text { Upper coals . }\left\{\begin{array}{l}
\text { Shales and Badsworth coal. } \\
\text { Ackworth rock. } \\
\text { Wragby and Sharlston coals. }
\end{array}\right.
$$

Red rock of Woolley, Hooton-Roberts, \&c.

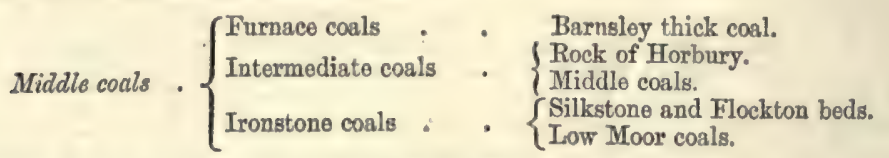

Flagstone rock of Woodhouse, Bradford, Elland, Peniston, \&c.

$$
\text { Lower coals : }\left\{\begin{array}{l}
\text { Shales and ganister stone. } \\
\text { Coals. } \\
\text { Shales and ganister stone. } \\
\text { Coals. } \\
\text { Shales, \&c. }
\end{array}\right.
$$

Millstone grit lies below the 'coal series.'

The important middle-coal series are again divided by Professor Phillips as follows :-

Red rock of Woolley Edge.

Furnace coals of Barnsley, \&c. including the eight or ten feot seam.

Rock of Horbury and Wentworth House. Iron-stone coals $\left\{\begin{array}{l}\text { Swift burning coals of Middleton, Dewsbury, \&c., with } \\ \text { bands of 'mussels,' } \\ \text { Bituminous coals of Silkstone and Low Moor. } \\ \text { Flagstone rocks beneath. }\end{array}\right.$

The small coal-field of Ingleton and Black Burton in Lonsdale is thrown down on the south side of the great Craven fault.

LANCASHIRE.-The coal-field of Lancashiro occupies an area extending from Macelesfield to Colne, 46 miles, and from Torboch, near Liverpool, to Todmorden, abont 40 miles. Excluding the millstone grit, its area is about 250 square miles.Heywood.

In a line through Worsley, Bury, and Burnley to the limestone shales of Pendle Hill, we have 36 seams of coal, 10 of them not exceeding 1 foot in thickness, making in all 03 feet of coal.

The series is divisible into three parts above the millstone grit:-

Upper part, containing a bed of limestone at Ardwick, near Manchestor.

Middle part, containing the greater part of the thick and raluable soams, especially

cannel coal of Wigan.

Lower part, corresponding to the ganister series of Yorkshire. 
Creshirs:-The coal-field of Cheshire is not of great importance.

Nortu Wales.-Flintshire and Denbighshire. -The Flintshire coal-basin extends from north to south, somewhat more than 30 miles from Llanassa to near Oswestry in Shropshire. The coal-strata dip generally eastward, and form in the northern part a trough beneath the estuary of the Dee. This coal-basin in Flintshire commences with beds of shale and sandstone. The coal is of various thickness, from $\frac{3}{4}$ to 5 yards, and consists of the common, cannel, and peacock varieties.-Phillips and Conybeare.

CUMBERTAND. - This coal-field extends as a narrow crescent from Whitehaven to near Hesket Newmarket: around Whitehaven and at Workington the coal is worked extensively. At the latter place, a few years since, a very valuable colliery was destroyed by the bursting in of the sea.

There are three workable seams in the Cumberland coal-field in the neighbourhood of the three under-mentioned towns, and these are known in each place by the names given :-

\begin{tabular}{|l|l|l|}
\hline \multicolumn{1}{|c|}{ Whitehaven } & \multicolumn{1}{|c|}{ Workington } & \multicolumn{1}{|c|}{ Naryport } \\
\hline $\begin{array}{l}\text { Bannock band. } \\
\text { Main band. }\end{array}$ & $\begin{array}{l}\text { Moorbanks. } \\
\text { Main seam. } \\
\text { Six-quarter coal or } \\
\text { Low-bottom seam. }\end{array}$ & $\begin{array}{l}\text { Hamilton seam. } \\
\text { Ten quarters. } \\
\text { Cannel and metal seams (di- } \\
\text { vided with shale from 2 } \\
\text { feet to 5 fathoms thick). }\end{array}$ \\
\hline
\end{tabular}

NoRThUMBERTAND AND DURHAM. - The total thickness of the coal-measures of this district is about 1,600 feet. The number of distinct layers or beds, as usually noted by the miners, is about 600 . The total thickness of the beds of coal rarely exceeds - does not on the average equal-60 feet. No bed of coal is of greater thickness, even for a short distance, than 6 or $\mathbf{7}$ feet; several are so thin as to be of no value at present; but many of these will be worked with profit. The total thickness of 'workable coal,' supposing all the beds to be found in a given tract, is not to be estimated at above 20 or 30 feet. The most part of the coal in this great district is of the coking quality, but, in this respect, there is much variation; advantage has been taken of this, in rendering available very large quantities of the dust of coal, and the small coal which formerly was burnt to waste on the pit bank. The best coke for locomotive engines is now made from the lower coals in the Auckland district of Durham, and the Shotley Bridge district of Northumberland. The best 'steam coal' is obtained from the north side of the Tyne and the Blyth district. The best 'house coal' still comes from the remains of the 'High chain' on the Tyne, and from the 'Hutton seam' on the Wear; but the collieries north of the Tees have acquired a high reputation.

As a general view of the groups of strata the following summaries may suffice:-

Upper groups of coal-measures, including chiefly thin seams of small value (8 or more) in a vast mass of sandstone in shales, with some iron-stone. At the base is a mussel band; estimated at 900 feet.

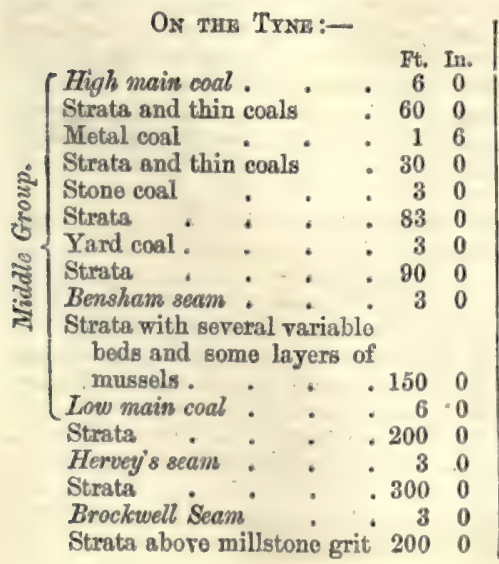

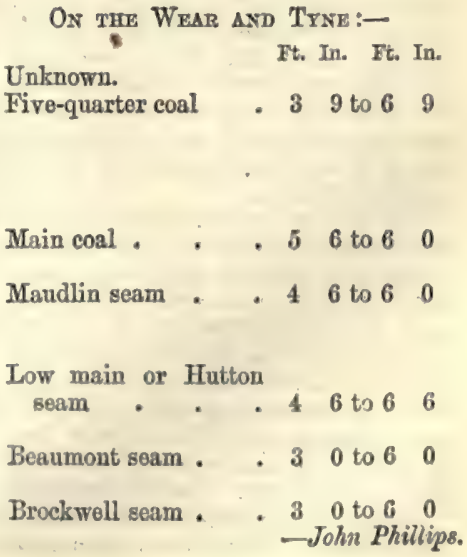


Tho sonms which are principally worked in this district are the high main, fire. quarter main, Bensham seam, Hutton senm, Beaumont seam, low five-quarter, threequarter seam, Brockwell, and stone coals. These seams are known by other names, euch district usually adopting its own peculiar term to designate the workablo seams. Thus the Bensham seam of the Tyno is known as the Maudlin seam of the Wear. The Beaumont or Harvey seam is the Townley seam of the Townley colliery and the main coal of Wylam colliery. At Hetton the high main seam of the Cramlington district separates into two, and is called tho three-quarter seam at Pontop; where it unites again it is known as the Shieldrow seam. The Cramlington grey seam is the metal-coal seam and stone coal-seam of Sherriff Hill, where it is divided; while it unites at Hetton and forms the five-quarter seam of that and the Auckland district. The Cramlington yard seam becomes the main coal-seam at Hetton, Haswell, and some other localities, the Brass Thill at Pontop, and the main coal in Auckland. Again, the Cramlington five-quarter seam divides and forms the six-quarter, and the fivequarter at Sherriff Hill the Brass Thill seam at Pittington; they again unite and form the Hutton seam at Pontop colliery, and so with regard to a few others.-Mineral Statistics.

Scomand.- 'A memoir on the Mid-Iothian and East-Lothian Coal-fields,' by Darid Milne, gives the most exact account of the carboniferous system of Scotland. From this description it will be seen that the Scotch conl-field extends from the eastern unto the western shore.

There are three principal coal-basins in Scotland: 1, that of Ayrshire; 2, that of Clydesdale; and 3 , that of the valley of the Forth, which runs into the second in the line of the Union Canal. If two lines be drawn, one from Saint Andrews on the north-east coast, to Kilpatrick on the Clyde, and another from Aberlady, in Haddingtonshire, to a point a few miles south of Kirkoswald in Ayrshire, they will include between them the whole space where pitcoal has been discovered and worked in Seotland.

According to Mr. Farey there are 337 principal alternations of strata between the surface in the town of Fisherow, on the banks of the Frith of Forth (where the highest of these strata occur) and the commencement of the basaltic rocks, forming the general floor and border of this important coal-field. These strata lie internally in the form of a lengthened basin or trough, and consist of sandstone, shale, coal, limestone, ironstone, \&c. Sixty-two seams of coal, counting the double seams as one; 7 limestones; 72 assemblages of stone and other strata: in all 5,000 feet in thickness.

Professor Phillips remarks of this district, ' On the whole, allowing for waste, un. attainable portions, and other circumstances, this one district may be admitted as likely to yield to the miner for actual use 2,250 millions of tons of coal.' The coal is partly 'splint,' partly 'rough,' or 'cherry,' partly of the 'cannel ' or ' parrot 'variety. The first containing most oxygen; the last, most hydrogen and nitrogen, and the least carbon.

Irruard.-The coal-mensures of Ireland, if we include in this term the millstone grit, occupy large tracts of land in that country, and are upon the whole analogous, in general mineral characters and organic contents, to those of England. The same absence of limestone, the same kind of successions of sandstones and shales is remarked in them. Anthracite or stone-coal like that of South Wales is found in the Leinster and Munster districts; bituminous coal occurs in Connaught and Ulster. In Ulster the principal collieries are at Coal Island and Dungannon. The Munster coal district is stated by Mr. Griffith to be of greater extent than any English coal-fiold, but it is much less productive. At Ballycastle the coal is found in connection with basalt.-Phillips.

Mr. Hull's remark, in the 'Coal-fields of Great Britain,' 'that Ireland was once covered over two-thirds of its extent by coal-fields,' is a proposition which we may confidently affirm on geological grounds; but the misfortunes of the sister isle began long before the landing of Stronglow, for old father Neptune had swept the coal and coal-strata clean into his lap, and left little but a baro floor of limestone belind. In plain words, if we examine a geological map of Ireland, we shall find that the carboniforous limestone overspreads its greater part; and as we always find in England that this formation is ultimately surmounted by coal-measures, so wo may infer that was the same order of succession here, before the sea, which more than once orerwhelmed the count $\%$ after the carboniferous epoch romorselessly swept away the more valuable portion of this system of rocks.

The following remarks on the coal-fields of Ireland are translated, hy Mr. W. H. Baily, from a description of them by Dx. H. B. Geinitz:- 
'Althongh the carboniferous limestone extends over the greater part of Ireland, and wo may assume that the coal-bearing strata of the carboniforous formation may also, at one time, have exhibited a considerable extension in that country, thore is, howevor, very little of it remaining, in consequence of the subsequent denudation observable thore. In the south of Ireland the carboniferous limestone is accompanied by a sories of black shales or grey sandstone, and aronaceous or sandy shales, which contain thin beds of anthracite coal in the uppor strata. Professor J. Beete Jukes, who is at the hoad of the Geological Survey of Ireland (sinco deceased), distinguishes in this district (Castlecomer, Queen's County) the following groups of the carboniferous formation, from the lower to the upper:-1. Carboniferous limestone, 3,000 feet thick. 2. Black shale, with oceasional thin beds of sandstone, about 8,000 feet thick. In these occur the fossil shells, Aviculopecten papyraceus, Posidonomya Becheri, Goniatites sphericus, Orthoceras Stcinhaueri, and other marino animals, indicativo of a lower horizon. 3. Greenish-grey sandy shale, and black shale (flagstone series) characterised by vormicular impressions or markings, which have been referred to wormtracks, as well as crustacean and molluscous tracks, 500 feet thick. 4. Black shales and grey sandstone, with thin beds of coal, 1,800 feet thick; making a total maximum thickness of coal-measure strata above the carboniferous limestone of 3,100 feet.

- An extensive working is carried on in the Castlecomer coal-field, which lies on the borders of Kilkenny and Queen's Counties. Accurate sections referring to this field, for which we are indebted to Professor Jukes, exhibit in this district five beds of coal, of which, however, the upper only are observable, and that over a very limited area, in consequence of denudation (probably from sea action upon what was once the coastline). The richer deposits of coal in the lower beds are found north-east of Castlecomer, where, at the Garrow Colliery, at the time of my visit, a coal-bearing seam, $3 \mathrm{ft} .10 \mathrm{ins}$. thick, was working, which yielded excellent anthracite coal, the normal Kilkenny coal of Werner. The circumstances just described are perfectly analogous to those in the neighbouring Geneva Colliery. Opinions still vary as to whether this Garrow bed is the second or third from the bottom. At all events, it is the most important in the district. In the small Leinster coal-field, somewhat further to the north-east, in the direction from Castlecomer to Athy, a seam is also worked from 18 to 20 inches thick only. The numerous remains of plants collected in this coal-district, compared with those of other coal-basins, show that the anthracite coal does not belong to the zone (horizon) of the culm, or our first zone, but rather to the commencement of the second, or Sigillaria zone. Marine shells occur over the Garrow seam, among which the characteristic and widely-distributed coal-measure fossils before mentioned, Aviculopecten papyraceus and Posidonomya Becheri are most frequent. Numerous well-preserved bivalve shells occur, Anthracosia, Myalina, \&c., locally called " beams" by the miners, together with some remarkable crustacea of the genus Belinurus, from the black shales of Bilboa Colliery, situated about six miles S. W. of Carlow, the coal of which is believed to correspond with the second seam of the Castlecomer district. At this colliery, also, the well-known fossil plants Alethopteris lonchitidis and Sagenaria elegans, were easily recognisable, two forms, which, in the productive coal-formation of England especially, have the widest distribution. In the north of Ireland, where coal-bearing beds occur, in the counties of Leitrim, Fermanagh, as well as at Dungan. non, in Tyrone, and Ballycastle, in Antrim, they rest upon a thick sandstone formation, apparently representing the millstone grit which, separates them from the carboniferous limestone group. Sir Richard Griffiths, Bart., in his geological map of Ireland, divides the latter into an upper and lower limestone, between which the "calp" is deposited, consisting of shales and sandstones, and often more than 1,000 feet thick, It is remarkable that while in the whole of the south of Ireland the typical anthracite or Kilkenny coal predominates, in the north the prevailing deposits are bituminous, or good gas-coal. According to the investigations of Sir R. Griffiths, the coal-field of Dungannon, in Tyrone, is divided into two districts, which are distinguished as the Coal Island district and the Annaghone district. The first is six miles long, with a mean breacth of about two miles; the latter is only one mile long and half a mile broad. In this important coal-field there are eight beds of coal, of variable thickness, from $2 \mathrm{ft}, 2 \mathrm{in}$. to $1 \mathrm{ft}, 9 \mathrm{in}$. viz., the Annagher coal. Under these thore are also two or three other seams known. These coals are, for the most part, of good quality. The occurrence of the productive coal-formation at Ballycastle, on the northern coast of Ireland, in tho county of Antrim, is very interesting, where it extends, from Fair Head, in a westerly and southerly direction, to a distance of about four miles, with a mean breadth of one mile and a half. It contains, at Murlough Bay, six coal seams, from one to eight feet thick, of which four yiold good gas-coal, while the two deepest are anthracite. The first four occur botwoon two columnar isolated basaltic masses, while the two lower anthracite scams aro almost in direct contact with the lower 
basaltic mass, which is about 70 feet thick. Thoso coal-boaring beds appear to rest directly on the mica-schist.

'As an additional characteristic of the Irish coal-fields, there follows a summary of the fossil plants which we had the opportunity of examining. I do not think that we should be justified, from a consideration of these plants, to refer the coal-bearing beds of Iroland to an older zone than to the lower, generally poor, étage of the Westphalian coal-formation, which represents very well the relations of their deposition. They indicate here, as there also, the commencement of the Sigillaria zone, whose later and richer beds were, in Ireland, destroyed, and of which only a very small area may still be preserved in some northern localities, as at Dungannon.'

The following general statement of the geological conditions of the coal-measures of these islands is continued from the pen of the late Dr. Ure:-

The great Carboniferous Formations of these islands may be subdivided into three orders of rocks : 1. the coal-measures, including their manifold alternations of coalbeds, sandstones, and shales; 2 . the millstone grit and shale towards the bottom of the coal-measures; 3 . the carboniferous limestone, which, projecting to considerable heights above the outcrop of the coal and grit, acquires the title of mountain limestone; resting on the Old Red Sandstone, which may be regarded as the connecting link with the transition and primary rock basin in which the coal system lies.

The coal series usually, but not invariably, consists of a regular alternation of mineral strata deposited in a great concavity or basin, the sides and bottom of which are composed of transition rocks. This arrangement will be clearly understood by inspecting fig. 466, which represents a section of the coal-field south of Malmesbury.

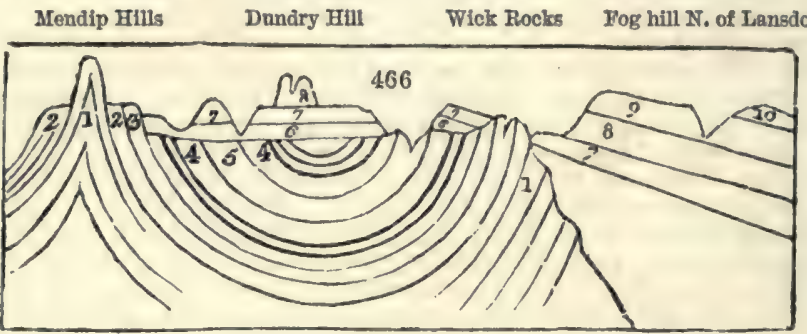

1, 1, Old Red Bandstone. 4, 4, coal-seams. 7,7 , Las.
2, mountain limestone.

6, New Red Sandstone, or red mar

No. 1, or the Old Red Sandstone, may therefore be regarded as the characteristic lining of the coal-basins; but this sandstone rests on transition limestone, and this limestone on highly-inclined beds of slaty micaceous sandstone which, on the one hand, alternates with, and passes into a coarse breccia, having grains as large as peas; on the other, into a soft argillaceous slate. The micaceous sandstone stands bare on the north-eastern border of the Forest of Dean, near the southern extremity of the chain of transition limestone which extends from Stoke Edith, near Hereford, to Flaxley on the Severn. It is traversed by a defile, through which the road from Gloucester to Ross winds. The abruptness of this pass gives it a wild and mountainous character, and affords the best opportunity of examining the varieties of the rock.

The limestone consists in its lower beds of fine-grained, tender, extremely argillaceous slate, known in the district by the name of water-stone, in consequence of the wet soil that is found wherever it appears at the surface. Calcareous matter is interspersed in it but sparingly. Its upper beds consist of shale alternating with extensive beds of stratified limestone. The lowest of the calcareous strata are thin, and alternate with shale. On these repose thicker strata of more compact limestone, often of a dull blue colour. The beds are often dolomitic, which is indicated by straw-yellow colour, or dark pink colour, and by the sandy or glimmering aspect of the rock.

The Old Red Sandstone, whose limits are so restrictod in other parts of England, here occupies an extensive area. The space which it covers, its great thickness, its high inclination, the abrupt character of the surface over which it prevails, and the consequent display of its strata in many natural sections, present, in this district, advantages for studying the formation, which are not be met with elsewhere in South Britain. In the neighbourhood of Mitchel Dean, the total thicknoss of this formation, intorposed conformably between the transition and mountain limestone, is from 600 to 800 fathoms.

The mountain or carboniferous limestone is distinguished from transition limestone 
rather by its position than by any very wide difference in its general character or organic remains. According to the measurements of Mr. Mushet, the total thickness of the mountain limestone in the Gloucestershire coal-fiold is about 120 fathoms. The zone of limestone belonging to this coal-basin is from a furlong to a mile in breadth on the surface of the ground, according as the dip of the strata is more or less rapid. The angle of dip on the northen and western border is often no more than $10^{\circ}$, but on the eastern it frequently amounts to $80^{\circ}$.

The aggregate thickness of the coal-measures amounts to about 500 fathoms. 1. The lowest beds, which repose on the mountain limestone, are about 40 fathoms thick, and consist here, as in the Bristol coal-basin, of a red siliceous grit, alternating with conglomerate, used for millstones; and with clay, occasionally used for ochre. 2. These beds are succeeded by a series about 120 fathoms thick, in which a grey gritstone predominates, alternating in the lower part with shale, and containing 6 seams of coal. The grits are of a fissile character, and are quarried extensively for flag-stone, ashlers, and fire-stone. 3. A bed of grit, 25 fathoms thick, quarried for hearth-stono, separates the preceding series from the following, or the $4 \mathrm{th}$, which is about 115 fathoms thick, and consists of from 12 to 14 seams of coal alternating with shalo. 5. To this succeeds a straw-coloured sandstone, nearly 100 fathoms thick, forming a high ridge in the interior of the basin. It contains several thin seams of coal, from 6 to 16 inches in thickness. 6. On this reposes a series of about 12 fathoms thick, consisting of 3 seams of cosl alternating with shale. 7. This is covered with alternate beds of grit and shale, whose aggregate thickness is about 100 fathoms, occupying a tract in the centre of the basin about 4 miles long and 2 miles broad. The sandstone No. 5 is probably the equivalent of the Pennant in the preceding figure. The floor, or pavement, immediately under the coal-beds is, almost without exception, a greyish-slate clay, which, when made into bricks, strongly resists the fire. This firoclay varies in thickness from a fraction of an inch to several fathoms. Clay-ironstone is often disseminated through the shale.

The above description holds generally correct for the great coal-fields of southwestern England, where we have coal-measures, millstone grit, and mountain limestone in regular order, the latter being at the base of the system. As we proceed northward to Yorkshire and Northumberland, however, the limestone begins to alternate with the true coal-measures, the two deposits forming together a series of strata about 1,000 feet in thickness. To this mixed formation succeeds the great mass of genuine mountain limestone. In Fifeshire, in Scotland, we observe a still great departure from the type of the south of England, or a more complete intercalation of dense masses of marine limestone, with sandstone and shales containing coal.

At Brora, in Sutherlandshire, we have a coal-formation belonging to the lower divisions of the Oolite period; and in the nort-east of Yorkshire, we have a similar formation.

The Brora coal-field, to which of late much attention has been directed, is a remarkable example of a coal-basin among the deeper secondary strata, but above the Now Red Sandstone formation. The Rev. Dr. Buckland and Sir C. Lyell, after visiting it in 1824, had expressed an opinion that the strata there were wholly unconnected with the proper coal-formation below the New Red Sandstone, and were in fact the equivalent of the oolitic series; an opinion fully confirmed by the subsequent researches of Sir R. Murchison. ('Geol. Trans.' for 1827, p. 293.) The Brora coalfield forms a part of those secondary deposits which range along the south reast coast of Sutherlandshire, occupying a narrow tract of about 20 miles in length and 3 in its greatest breadth.

Ono stratum of the Brora coal-pit is a coal-shale, composed of a reed-like striated plant of the natural order Equisetacece, which seems to have contributed largely towards the formation of that variety of coal. From this coal-shale the next transition upwards is into a purer bituminous substance approaching to jet, which constitutes the great bed of coal. This is from 3 feet 3 inches to 3 feet 8 inches thick, and is divided nearly in the middle by a thin layer of impure indurated shale charged with pyrites, which, if not carefully excluded from the mass, sometimes occasions spontaneous combustion upon exposure to the atmosphere; and so much indeed is that mineral disseminated throughout the district, that the shales might be generally termed 'pyritiferous.' Inattention on the part of the workmon, in 1817, in leaving a large quantity of this pyritous matter to accumulate in the pit, occasioned a spontaneous combustion, which was extinguished only by excluding the air; indeed, the coal-pit was closed in and remained unworked for four years. The fires broke out again in the pit in $\mathbf{1 8 2 7}$.

The purer part of the Brora coal resembles common pitcoal; but its powder has the red ferruginous tinge of pulverised lignites. It may be considered one of the last links between lignite and true coal, approaching very nearly in character to jet, 
though less tenacious than that minoral; and, when burnt, exhnling but slightly tho vegetable odour so peculiar to all imperfectly-bituminised substances. The fossil remains of shells and plants prove the Brorn coal to be analogous to that of the oastorn moorlands of Yorkshire, although the extraordinary thickness of the former, compared with any similar duposit of the latter (which never exceeds from 12 to 17 juches), might have formerly led to tho belief that it was a dotached and anomalous deposit of true coal, rathor than a lignite of any of the formations above the Now Red Sandstone: such misconcoption might more easily arise in the infancy of geology, when the strata were not identified by their fossil organic remains.

The beds of coal at Brorn havo been recently (1873) opened up more thoroughly for the Duke of Sutherland, with a view to supplying the district with fuel. Tho latest report, August 1873, says:- 'The output of coal at the Duke of Sutherland's colliery at Brors is as yet small, but it is rery satisfactory as regards quality and in the promise of quantity. In tho seam which is at present being worked there is a narrow band about three inchos thick, which is somewhat sulphurous; but this can easily bo chipped off and dotached. From various causes, it is at present inconvenient to supply the public with coals at the pit mouth, and due notice will be given when the workings are in such a condition as to admit of the public being freely supplied. A few weeks ago a 6-ton truck of Brora coal was sent south to Invernoss; and coal has also been supplied from the mine to the inhabitants of Laing and the eastern districts of Sutherlandshire at alout $1 l$. and in some cases rather less, per ton. This is only about half the price paid for coal from the south. The Duke of Sutherland's limo-kilns at Laing are now almost constantly supplied from the Brora mine. Whon the new seam is opened up and worked, it is expected that the supply will be both regular and plentiful.' This expectation does not appear to have been realised (1874).

For description of the oolitic coals of Scotland, see Mr. J. Judd's paper on 'The Secondary Rocks of Scotland,' in 'Quart. Journ. Geol. Soc.', Lond. xxix. 1873, p. 97.

On the coast of Yorkshire the strata of a similar formation appear in the following descending order, from Filey Bay to Whitby:-1. Coral-rag. 2. Calcareous grit. 3. Shale, with fossils of the Oxford clay. 4. Kelloway rock (swelling out into an important arenaceous formation). 5. Cornbrash. 6. Coaly grit of Smith. 7. Pierstone (according to Mr. Smith, the equivalent of the great oolite). 8. Sandstone and shale, with peculiar plants and various seams of coal. 9. A bed with fossils of the inferior oolite. 10. Marl-stone? 11. Alum-shale or lias. All the above strata are identified by abundant organic remains.

The most complete and simplest form of a coal-field is the entire basin-shape, which we find in some instances without a dislocation. An example of this is to be seen at Blairengone, in the county of Porth, immediately adjoining the western boundary of Clackmannanshire, as represented in fig. 467 , where the outer elliptical

467
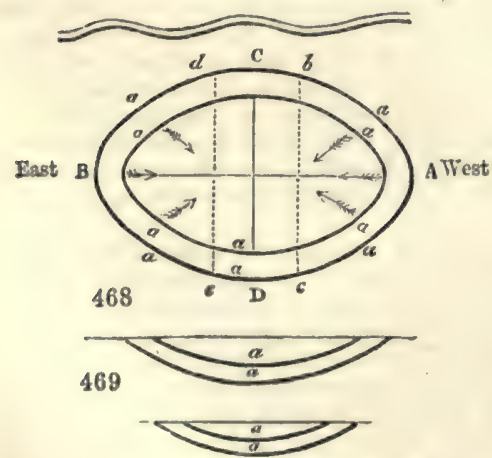

470.

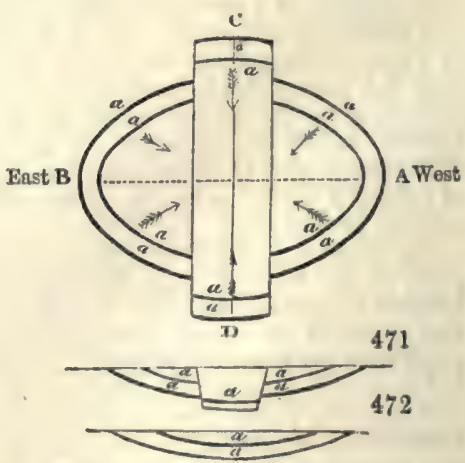

line, marked $\Delta, B, C, D$, represents the crop, outburst, or basset edge of the lower conl, and the inner elliptical line represents the crop or basset edge of the superior cosl. Fig. 468 is the longitudinal section of the line $\triangle \mathrm{B}$; and fig. 469 , the transverse section of the line $C$ D. All the accompanying coal-strata partake of the same form and parallelism. These basins are generally elliptical, sometimes nearly circular, but are often very eccentric, being much greater in length than in breadth; and frequently one side of the basin on the short diameter has a much greater dip than the other, which circumstance throws the trough or lower part of the basin concavity much nearer to the one side than to the other. From this viow of one entire basin, it 
is evident that the dip of the coal-strata belonging to it runs in opposite directions, on the opposite sides, and that all the strata regularly crop out, and meet the alluvial cover in every point of the circumferential space, like the edges of a nest of common basins. The waving line marks the river Devon.

It is from this basin shape that all the other coal-fields are formed, which are segments of a basin produced by slips, dikes, or dislocations of the strata. If the coal (fig. 467) were dislocated by two slips $b c$ and $d e$. the slip $b c$ throwing the strata down to the east, and the slip $d e$ throwing them as much up in the same direction, the outerops of the coals would be found in the form represented in fig. 470 , of which fig. 471 is the section in the line $\mathbf{A ~ B}$, and fig. 472 the section in the line $\mathbf{C ~ D .}$

The absolute shape of the coal-fields in Great Britain has been ascertained with surprising precision. To whatever depth a coal-mine is drained of its water, from that depth it is worked, up to the rise of the water-level line, and each miner continues to advance his room or working-place, till his seam of coal meets the alluvial cover of the outcrop, or is eut off by a dislocation of the strata. In this way the miner travels in suceession over every point of his field, and can portray its businshape most minutely.

Fig. 473 represents a horizontal plan of the Clackmannanshire coal-field, as if the strata at the outerop all around were denuded of the alluvial cover. Only two of the concentric beds, or of their edges $a, a$, are represented, to avoid perplexity. It is to be remembered, however, that all the series of attendant strata lie parallel to the above line. This plan shows the Oehill mountains, with the north coal-fields, of an oblong elliptical shape, the side of the basin next the mountains being precipitous, as if upheaved by the eruptive trap-rocks; while the south, the east, and the west edges of the basin shelve out at a great distanee from the lower part of the coneavity or trough, as miners call it. Thus the alternate beds of coal, shale, and sandstone, all nearly concentric in the north coal-field, dip inwards from all sides towards the central area of the trough. The middle cosl-field of this district, however, which is formed by the great north slip, is merely the segment of an elliptical basin, where the strata dip in every direction

473

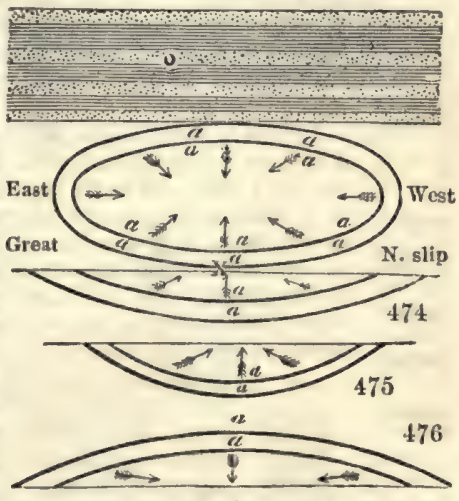
to the middle or the axis marked with the letter $\mathrm{x}$; being the deepest part of the segment. The south coal-field, formed by the great south slip, is likewise the segment of another elliptical basin, similar in all respects to the middle coal-field. Beyond the outcrop of the coals and subordinate strata of the south coal-fields, the counter dip of the strata takes place, producing the mantle-shaped form; whence the coal-strata in the Dunmore field, in Stirlingshire, lie in a direction contrary to those of the south cosl-field of Clackmannanshire. o, are the Ochill mountains.

Fig. 477 is a very interesting section of the main coal-basin of Clackmannanshire, as given by Mr. Bald in the Wernerian Society's Memoirs, vol. iii. Here we see it broken into three subordinate coal-fields, formed by two great faults or dislocations of

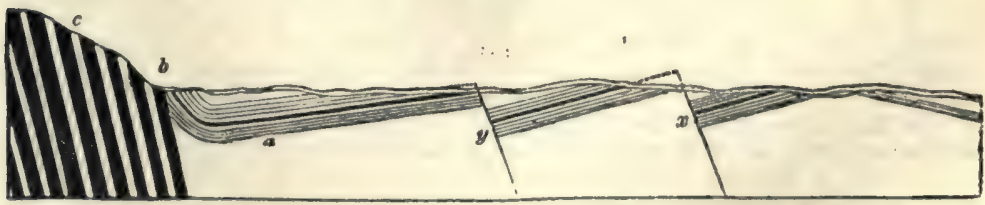

the strata; but independently of these fractures across the whole series, the strata continue quite regular in their respective alternations, and preserve nearly unchanged their angle of inclination to the horizon. The section shows the south coal-field dipping northerly, till it is cut across by the great south slip $x$, which dislocates the coal and the parallel strata to the enormous extent of 1,230 feet, by which all the coals have been thrown up, not simply to the day, but are not found again till we advance nearly a mile northward, on the line of the dip, where the identical seams of coal, shale, \&c., are observed once more with their regular inclination. These coals 
of the middle area dip regularly northward till interrupted by the great north slip $y$, which dislocates the strata, and throws them up 700 feet; that is to say, a line prolonged in the direction of any one well-known seam, will run 700 feet above the line of the same seam as it emerges after the middle slip. Immediately adjoining the north slip, the coals and coal-field resume their course, and dip xegularly northwarl, running through a longer range than either of the other two members of the basin, till they arrive at the valley of the Devon, at the foot of the Ochill mountains, where they form a concave curvature, or trough $a$, and thence rise rapidly in an almost vertical direction at $b$. Here the coals, with all their associated strata, assume conformity and parallelism with the face of the syenitic-greenstone strata of the Ochill mountains $c$, being raised to the high angle of 73 degreas with the horizon. The coal-seams thus upheaved, are called edge-metals by the miners.

In this remarkable coal-field, which has been accurately explored by pitting and boring to the depth of 703 feet, there are no fewer than 142 beds, or distinet strata of 478 coal, shale, and sandstone, \&ce, variously altornating, an idea of which may 478 be had by inspecting fig. 478 . Among these are 24 bods of coal, which

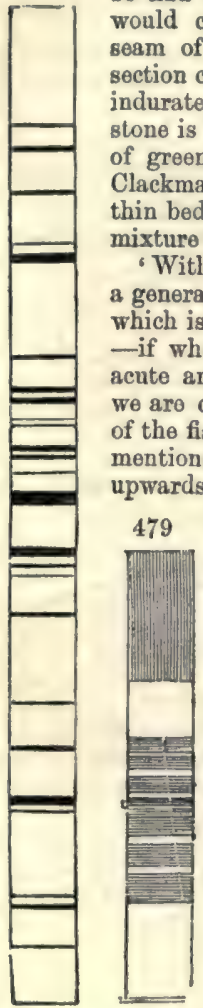
(thite an aggregate thickness of 59 feet 4 inches: the thinnest on contain numerous varieties of sandstone, slate-clay, bituminous shale, indurated clay, or fire-clay, and clay ironstone. Neither trap-rock nor limestone is found in connection with the workable coals; but an immense bed of greenstone, named Abbey Craig, occurs in the western boundary of Clackmannanshire, under which lie regular strata of slate-clay, sandstone, thin beds of limestone, and large spheroidal masses of clay ironstone, with a ixture of lime.

'With regard to slips in coal-fields,' says Mr. Bald, 'we find that there is a general law connected with them as to the position of the dislocated strata, which is this:- When a slip is met with in the course of working the mines -if when looking to it, the vertical line of the slip or fissure, it forms an cute angle with the line of the pavement upon which the observer stands, we are certain that the strata are dislocated downwards upon the other side of the fissure. On the contrary, if the angle formed by the two lines above mentioned is obtuse, we are certain that the strata are dislocated or thrown upwards upon the other side of the fissure. When the angle is $90^{\circ}$, or a right 479 angle, it is altogether uncertain whether the dislocation throws up or down on the opposite side of the slip. When dikes intercept the strata, they generally only separate the strata the width of the dike, without any dislocation, either up or down; so that if a coal is intercepted by a dike, it is found again by running a mine directly forward, corresponding to the angle or inclination of the coal with the horizon.'-Wernerian Society's Memoirs, vol. iii. p. 133.

The Johnstone coal-field, in Renfrewshire, is both singular and interesting. The upper stratum of rock is a mass of conpact greenstone or trap, above 100 feet in thickness, not at all in a conformable position with the coal strata, but overlying; next there are a fow fathoms of soft sandstone and slate-clay, alternating, and uncommonly soft. Beneath these beds, there are no fewer than 10 seams of coal, lying on each other, with a fow divisions of dark indurated clay. These coal-seams have an aggregate thickness of no less than 100 feet; a mass of combustible matter, in the form of coal, unparalleled for its accumulation in so narrow a space. The greater part of this field contains only 5 beds of coal; but at the place where the section shown in fig. 479 is taken, these 5 coals seem to have been overlapped or made to slide over each other by violence. This structure is represented in fig, 480 , which is a section of the Quarrelton coil in the Johnstone field, showing the overlapped coal and the double coal, with the thick bed of greenstone, overlying the coal-field.

Fig. 481 is intended to represent an extensive district of country, containing a great coal-busin, divided into numerous subordinate coal-fields by dislocations. The lines marked $b$ are slips, or faults; the broad lines marked $c$ denote dikes: the former dislocate the strata, and change their level, while dikes disjoin the strata with a wall, but do not in general affect their elevation. The two parallel lines marked $a$, represent two seams of coal, variously heaved up and down by the faults; whereas the dikes are seen to pass through the strata without altering their relative position. In this manner partial coal-fields are distributed over a wide area of country in overy direction.

Fig. 482 is an instance of a convex coal-field exhibited in Staffordshire, at the Castle-hill, close to the town of Dudley. 1, 1, are limestone strata ; 2, 2, are coal. 
Through this hill canals have been ent, for working the immense beds of carboniferous limestone. These occur in the lower series of the strata of the coal-field, and therefore at a distance of many miles from the Castle-hill, beyond the outcrop of all the workable

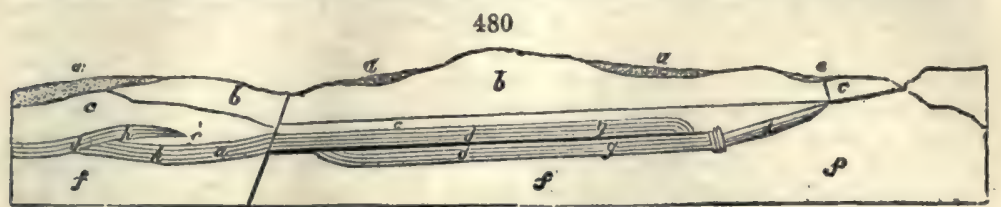

a. Alluvial cover.

b. Bed of trap or greenstone.

c. Alternating coal strata,

d. Coal-seams. e. Position of greenstone, not ascertained.

f. Strata in which no coals have been found.

g. The overlapped coal.

$h$. The double coal.

coals in the proper basin-shaped part of the field; but by this apparently inverted. basin-form, these limestone beds are elevated far above the level of the general surface of the country, and consequently above the level of all the coals. We must regard this seeming inversion as resulting from the approximation of two coal-basins, separated by the basset edges of their mountain limestone repository.

Fig. 483 is a vertical section of the Dudley coal-basin, the upper coal-bed of which has the astonishing thickness of 30 feot; and this mass extends 7 miles in length, and 4 in breadth. Coal-seams 5 or 6 feot thick are called thin in that district.

For a satisfactory description of the coal-field of South Staffordshire, the reader is referred to a memoir, "On the Geology of the South Staffordshire Coalfleld,' by J. Beete Jukes, published in the 'Records of the School of. Mines.'

It is not possible in the present work to enter into any further description of the coal-fields of this country. In the selections which have been made, striking types have been chosen, which are sufficiently characteristic to serve the purposes of general illustration. There are many variations from the conditions which have been described, but these are due to disturbances which have taken place either since the formation of the coal, or during the period of the actual deposition of the coss.

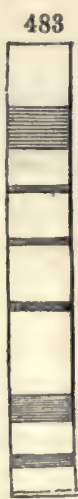

481

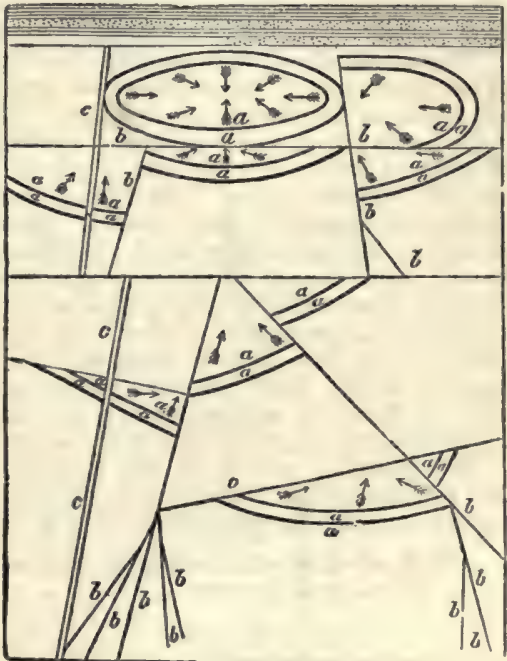

482

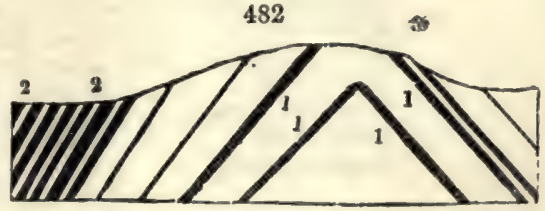

The probability of finding coal in the South of England has, of late, been invested with especial interest; and we are from time to time startled with the announcement that coal has been discovered.

Not long since a paragraph appeared announcing a remarkable discovery of cosl in the Isle of Wight. The late gales had denuded the shore of Whitecliff Bay of its usual mass of sand and shingle, laying bare 'a seam of coal' extending from the front of the cliff down to low-watermark, and how fax beyond could not, of course, be said. The seam was alleged to be from six to seven feet wide, and it had been dug out by the fishermen and others to a depth of six feet without any signs of exhaustion, the seam mather widening as it deepened. The coal was described as resembling the ordinary kind, and burning well. The existence of coal in the Isle of Wight was a fact by no means unknown, though it has been in a great measure overlooked. During Vor, I. 
a visit made to the island in 1860 Professor Ramsay and Mr. H. W. Bristow observed certain beds of a coal on the wost side of the island, in Alum Bay.

These cosl-seams at Alum Bay aro found in the Bracklesham beds of the Middle Bagshot series, apportaining therefore to the Middle Eocene Tertiaries. The formation reappears at the opposite extremity of the Isle of Wight, namoly, at Whitecliff Bay near Undercliff, the spot already montioned. But the existence of the coal-seams at the latter spot was not detected by the Geological Surrey until very lately, when the scams in question were observed by Mr. Penning, in company with a miner from the Forest of Dean, named Richard Gibbs. The seams were apparently of greater width than those at Alum Bay, although a thickness of seven feet could not be made out unless it were by adding several contiguous seams together. The discovery was communicated to Mr. Bristow, who thus describes them :-

- The strata comprised between the well-known glasshouse sands at the bnse of Headon Hill and the pipe-clay-bearing sands and clays overlying the London Clay, and grouped together by Mr. Joshua Trimmer as the Middle Bagshot series, wcre subdivided by him into Barton Clay and Bracklesham Beds.

- The latter are represented in Alum Bay by clays and marls in the lower part, and by white, yellow, and crimson sands above. The lower beds are remarkable for the quantity of vegetable matter contained in them, not, however, in the shape of leaves, as is the case in some of the Lower Bagshot Beds, but in the form of lignite, constituting solid beds from fifteen inches to two feet three inches thick. Four of these beds, when fully displaced, constitute conspicuous objects in the cliff, where they project out of the softer strata, and on the shore, from their black and coal-like appearance.

'These beds of coal were recently more than usually well displayed in consequence of the prevalence of long-continued wet weather having worn away the soft intervening strata in which they are imbedded. On examining them during a brief visit made to the Island, in company with Professor Ramsay, during the autumn of 1860 , it appeared evident that the beds in question occur more in the manner of ordinary cosl than of mere lignite. Like true coal, each bed was based upon a stratum of clay, containing, apparently, the rootlets of plants, as in the underclay of the true coalmeasures. The underclays, which oceur beneath each bed of coal of carboniferous date, having been the soil which supported the regetation which, by certain chemical changes, became subsequently mineralised and converted into coal, it is reasonable to infer from the presence of similar underclays beneath the coal in the Bracklesham Beds at Alum Bay, that the plants out of which it was formed grew on the spot, and were not drifted from elsewhere and deposited afterwards in the places where they are now found, as was undoubtedly the case with regard to the vegetable remains contained in the pipe-clay beds of the Lower Bagshot series.'

The following section of the Bracklesham beds in Alum Bay, as given by Mr. Bristow, in his 'Memoir on the Geology of the Isle of Wight,' will show the true position occupied by those lignite beds in the series (in ascending order):-

Dark chocolate-coloured marls and carbonaceous clay, with much ft. in. Lignite and Gypsum . . . . . . ... .

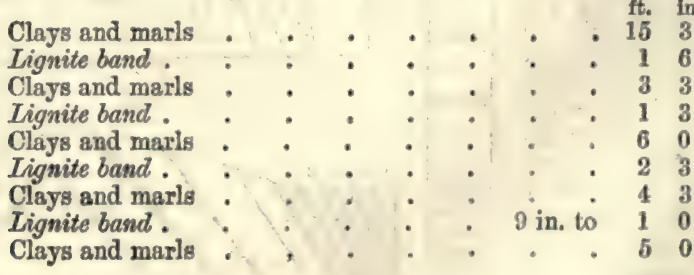

Whitish marl clay (principally white), light tawny yellow in the upper part; the

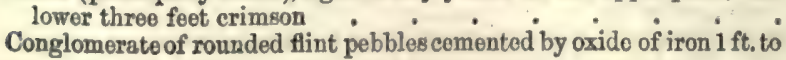

$45 \quad 0$

16

$$
\text { Total . . . . . . . } \overline{1110}
$$

The pebbles are of various sizes, the largest a foot in diameter.

The Lignite of Bovey already referred to, may be regarded as of a similar character to this of the Isle of Wight, notwithstanding the differences which have been pointed out, and the coal of Brora is not very dissimilar in its general character.

The coal of the Wealden strata is generally termed 'lignite,' though some approaches very closely to the character of a true coal. Whether we call it lignite or 
coal, it appoars to belong to the lowest stratum of the Hastings sands, known as the Ashburnham beds, which have never yet been fully explored. They rarely crop out to the surface, and would be less known than they are were it not that they are occasionally laid bare by water-courses, and are at times exposed at the base of eliffs when the sea makes deeper inroads than usual. It was this same kind of coal which gave so great a zest, at the beginning of the present century, to the costly speculation at Bexhill, near Hastings. In a boring near the seashore 'smut coal,' three feet thick, is reported to have been found at the depth of 30 yards ; 'strong coal,' two feet thick, at a depth of rather more than 50 yards; and sulphureous coal of bad quality, more than four feet thick, at a further depth. Being very near the sea the salt water percolated into the boring, and although a powerful steam-engine was employed, the exploration at that spot had to be abandoned. Another attempt was made further inland, on Bexhill Down; but coal does not seem to have been found in this second effort, and after a large outlay-ruinous to the principal adventurer-the attempt was abandoned. The Bexhill coal, there is reason to believe, belonged to an upthrow of the Ashburnham beds.

The question of the existence of coal in our Southern Counties was carefully examined by the Royal Coal Commission. Several witnesses, more especially Mr. Godwin Austen and Mr. Prestwich, were examined, and the reports of their evidence are full of interest.

The following is the general purport of the report made to the Royal Coal Commission by Mr. Prestwich on the probabilities of finding coal in the South of England.

'About two centuries ago the Belgian coal-field was found to extend, beneath the newer formation on the frontiers, into France as far as Valenciennes. An uninterrupted chalk district extended northward, and the coal-measures were supposed to be lost. But at a later period valuable coal was found to exist at Anjin. This lod to further search, and the coal-measures have been gradually followed in a western direction under the chalk to within thirty miles of Calais. Looking at these facts, and reasoning on theoretical considerations connected with the formation of coal in the west of Europe, Mr. Godwin Austen concluded that cosl-measures might possibly extend beneath the south-eastern part of England. He showed that the coal-measures which thin out under the chalk near Therouanne probably set in again near Calais, and are prolonged in the line of the Thames Valley, parallel with the North Downs, and continuing thence under the Valley of the Kennet extend to the Bath and Bristol coal area. He showed, upon theoretical grounds, that the coal-measures of a large portion of England, France, and Belgium were once continuous, and that the present coal-fields were merely fragments of the great original deposit preserved in hollows. These views are supported by many eminent geologists who gave evidence before the Commission; but they have been controverted by Sir Roderick Murchison, who contends that in consequence of the extension of Silurian and Cambrian rocks beneath the secondary strata of the south-east of England, and of the great amount of denudation which the carboniferous rocks had undergone over the area of the South of England previous to the deposition of the secondary formations, little coal could be expected to remain under the Cretaceous rocks. Upon a general review of the whole subject, Mr. Prestwich adopts, with slight variations, the views of Mr. Godwin Austen, and is led to the conclusion that there is the highest probability of a large area of productive coal-measures existing under the Secondary rocks of the South of England. He shows that the thickness of these overlying rocks is not likely to exceed 1,000 to 1,200 feet, and considers that there is reason to infer that the underground coal-basins may have a length of 150 miles, with a breadth of two to eight miles,limits within which are confined the rich and valuable coal-measures of Belgium.'

Mr. Prestwich shows that there are grounds for believing in the existence of coal on the south side of the Mendips, and under adjacent parts of the Bristol Channel, but at a dopth of not less than 1,500 to 2,000 feet, and mentions also a small new coal basin in the Severn valley, near New Passage.

As the existence of coal under the unexplored area of the South of England is still a question of theory, an attempt is now (1873) being made to settle this important question.

The boring now going on in the parish of Netherfield, near Battle, is designed to accomplish something more than a mere exploration of the Wealden strata. In this case there is the hope of finding the Primary rocks, an expectation which was deemed chimerical by the early school of geologists, but which is now viewed in a very different light. Experience has shown than all the strata which probably intervene between one formation and another do not invariably occur. Several members of the series may be absent, and we may experience a kind of plunge from upper secondary rocks into those of the primary order. This is the probability which encourages the sub-Wealden explorers. The Ashburnham beds have been 'pierced,' and it appears that the boring tool is in some lower stratum. This may be so; but the geology of this Netherfield boring is not very clear, the depth of the boring is now above 300 
foet. A bed of gypsum has been found, which is altoguther about thirty feet in thickness, sometimes in thick massos, at other times nodular and irregular. This is an important addition to the Purbeck series of Sussex. Nearer the surface, limestono has been found, said to be available both for burning and building purposes. It is desirable to know whether the Palrozoic rocks are really as near the surface in the south-east of England as some geologists have supposed. The sub-Wealden explorers do not now profess to be actually in search of coal, but, without doubt, they would bo glad if their enterprise should load the way to a discovery of this kind.

From the Report of the Royal Coal Commission the following Table is taken: showing, according to their careful computation, the-

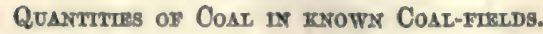

-Adopting 4,000 feet as the limit of practicable depth in working, and accepting the estimate of each Commissioner for the waste and loss incident to working the coal in the district assigned to him, we now present the following estimate of the quantities of available coal contained in the several districts which together comprise the coalfields above enumerated:-

Summary of Results of Reports as to Quantities of Coal worked and unworked in certain districts.

\begin{tabular}{|c|c|c|c|c|c|}
\hline $\begin{array}{l}\text { Conmisuloner and } \\
\text { Number on his } \\
\text { Report }\end{array}$ & No. & Nume of Coal-field & $\mid \begin{array}{c}\text { Amount of Coal in } \\
\text { Statute Tons to } \\
\text { depths not exceod. } \\
\text { ing } 4,000 \text { fo, and } \\
\text { afier the neees. } \\
\text { aary deduetions }\end{array}$ & $\begin{array}{l}\text { Amount of Coal in } \\
\text { Statute Tons in } \\
\text { depth exceding } \\
4,000 \text { ft., and afier } \\
\text { the nocesaury } \\
\text { deduetions }\end{array}$ & $\begin{array}{l}\text { Totals of Cosl in } \\
\text { Statur Tons in } \\
\text { each Cosl-fleld } \\
\text { after the } \\
\text { necessary } \\
\text { deductions }\end{array}$ \\
\hline $\begin{array}{l}\text { 1. Mr. Vivian } \\
\text { 2. Mr. Clark }\end{array}$ & 1 & South Wales & - $32,456,208,913$ & $4,109,987,004$ & $36,566,195,917$ \\
\hline \%. Mr. Dickinson & 2 & Forest of Dean. & - $265,000,000$ & Nil. & $265,000,000$ \\
\hline 10. Mr. Prestwich & 3 & Bristol & $4,218,970,762$ & $1,885,340,220$ & $6,104,310,982$ \\
\hline 9. Mr. Woodhouse & 4 & Warwickshire & $458,652,714$ & Nil. & $458,652,714$ \\
\hline $\begin{array}{l}\text { 8. Mr. Hartley : } \\
\text { Do. }\end{array}$ & $\begin{array}{l}5 \\
6\end{array}$ & $\begin{array}{l}\text { South Staffordshire } \\
\text { Coalbrook Dale and Fo- }\end{array}$ & & & \\
\hline & & rest of Wyre & $1,906,119,768$ & .. & $1,906,119,768$ \\
\hline 9. Mr. Woodhouse & $\begin{array}{l}7 \\
8\end{array}$ & $\begin{array}{l}\text { Clee Hills } \\
\text { Leicestershire : }\end{array}$ & $836,799,734$ & .. & $836,799,734$ \\
\hline 11. Mr. Dickinson & 9 & North Wales & - $2,005,000,000$ & $\therefore$ & $2,005,000,000$ \\
\hline Do. & 10 & Anglesey & - $\quad 5,000,000$ & 1000.785 .489 & $\begin{array}{r}5,000,000 \\
4.826,273,593\end{array}$ \\
\hline $\begin{array}{l}\text { 7. Mr. Buliot } \\
\text { 6. Mr. Dickinson }\end{array}$ & $\begin{array}{l}11 \\
12\end{array}$ & $\begin{array}{l}\text { North Staffordshire } \\
\text { Lancashire and Cheshire }\end{array}$ & $\begin{array}{l}3,825,488,105 \\
5,546,000,000\end{array}$ & $\begin{array}{r}1,000,785,488 \\
90,000,000\end{array}$ & $\begin{array}{l}4,826,278,793 \\
5,636,000,000\end{array}$ \\
\hline 9. Mr. Woodhouse & 13 & Midland * * & - $18,172,071,433$ & $234,728,010$ & $18,406,799,443$ \\
\hline Do. & 14 & Black Burton & $70,964,011$ & Nil. & $70,964,011$ \\
\hline $\begin{array}{l}\text { 4. } \mathrm{Mr} \text {. Forster } \\
\text { 5. Mr. Elliot }\end{array}$ & 15 & $\begin{array}{l}\text { Northumberiand } \\
\text { Durham }\end{array}$ & $10,036,660,236$ & •" & $10,036,660,236$ \\
\hline 4. Mr. Forster & 16 & Cumberland : & $405,203,792$ & *. & $405,203,792$ \\
\hline & & SCOTLAND. & & & \\
\hline 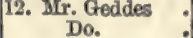 & 17 & Edinburgh & - 2,153,703,360 & $\because$ & $\begin{array}{l}2,153,708,360 \\
2,044,090,216\end{array}$ \\
\hline $\begin{array}{l}\text { Do. } \\
\text { Do. }\end{array}$ & $\begin{array}{l}18 \\
19\end{array}$ & $\begin{array}{l}\text { Lanarkshire : } \\
\text { Tifeshire }\end{array}$ & - 2,044 & $\because$ & $1,098,402,895$ \\
\hline Do. & 20 & Ayrshire: & $\begin{array}{l}: 1,098,402,895 \\
\\
1,785,397,089\end{array}$ & 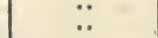 & $\mathbf{1}, 785,397,089$ \\
\hline Do. & 21 & East Iothian : & $86,849,880$ & .. & $86,849,880$ \\
\hline Do. & 22 & Frith of Forth & 1,800, & .. & 1,800 \\
\hline Do. & 28 & Dumfriesshire & 3,995 & . & $358,173,995$ \\
\hline Do. & 24 & West Lothian & 800 & . & $127,621,800$ \\
\hline Do. & 25 & Perthshire. & 109 & .. & 5,040 \\
\hline Do. & 26 & Stirlingshire & 106,475 & .. & $106,475,436$ \\
\hline Do. & 27 & Clackmannanshire. & 87,56 & *. & $87,563,494$ \\
\hline Do. & 28 & Dumbartonshire & $48,618,820$ & & $48,618,320$ \\
\hline Do. & 29 & Renfrewshire . & 25,8 & .. & $25,881,285$ \\
\hline Do. & 80 & Argyleshire & $7,228,120$ & • & $7,223,120$ \\
\hline Do. & 81 & Sutherlandshire & $8,500,000$ & • & $3,500,000$ \\
\hline Do. & 32 & Roxburghshire. & 70,000 & * & 70,000 \\
\hline 13. ProfessorJnkes, & 38 & $\begin{array}{l}\text { Irrm.AND. } \\
\text { Ballycastle (Antrim Co.) }\end{array}$ & $16,000,000$ & .• & $16,000,000$ \\
\hline $\begin{array}{l}\text { Commissioner } \\
\text { (deceased), and } \\
\text { Mr. Hull }\end{array}$ & & & & $\because$ & \\
\hline Do. & 34 & Tyrone & $6,800,000$ & ". & $6,300,000$ \\
\hline Do. & 35 & Leinster (Queen's Co, ) & $77,580,000$ & .. & $77,580,000$ \\
\hline Do。 & 36 & Tipperary & 0,000 & * & $25,000,000$ \\
\hline Do. & 37 & Mnnster (Clare, \&c. & $20,000,000$ & * & $20,000,000$ \\
\hline \multirow[t]{2}{*}{ Do. } & 38 & Connanght & $10,800,000$ & $\cdots$ & $10,800,000$ \\
\hline & & Total & $90,207,285,398$ & $7,820,840,722$ & $97,528,126,210$ \\
\hline
\end{tabular}

Nom, - It was an instruction to the Commissioners to whom the districts were assigned to exclude from their returns all beds of coal of less than 1 foot in thickness. 
From the same Report the following remarks on the duration of coal in these islands is abstracted :-

'The Commissioners, before proceeding to investigate the question of the duration of this quantity, based upon increasing consumption, think it useful to state the relation which $146,480,000,000$ of tons bear to our present consumption, estimated at $115,000,000$ per annum, in order that the vast magnitude of our stores of coal may be better appreciated. Thus we find that $146,480,000,000$ of tons will support our present production for 1,273 years; the same quantity would support an annual production of $146,000,000$ for 1,000 years; of $175,000,000$ for 837 years; and of $230,000,000$, being double our present production, for 636 years.

'The question of the duration of the total available quantity turns, therefore, chiefly upon the statistics of consumption contained in the Report of Committee E (which was the sole labour of the Editor of this Dictionary), from which the following facts are collectod:-

'In the year 1660, the coal produce of the United Kingdom appears to have been only about $2,250,000$ tons, and 40 years later the increase was only 364,000 tons. Fifty years after this, or in 1750 , the quantity raised in the kingdom had increased to nearly $5,000,000$ tons. In 1800 , the quantity exceeded $10,000,000$ tons.

'A bout this period the system of canal navigation was rapidly extending, and the result was that coals were gradually finding their way into new districts, by which means the consumption of coal was greatly increased. In 1816, the production reached $16,000,000$ tons according to one statement, and $27,000,000$ as given with considerable probability by another.

- Advancing to a later period, when coal statisties were more carefully collected, it appears that in 1854 the production of coal was $64,500,000$ tons.

' From that period up to, and including, 1869, the progressive increase was exhibited in a Table which has been completed up to the present time.

- From the following Table (p. 838) it will be seen that the total quantity of coal raised in this country had reached upwards of $123,000,000$ in 1872 .

'In attompting to form an opinion as to what the future consumption of coal is likely to be, it is necessary to consider the question which was referred to Committee B ; namely, "Whether there is reason to believe that coal is wasted by carelessness or neglect of proper appliances for its economical consumption?" The conclusion arrived at by this Committee was, that "for some time past in our manufactures there have been constant and persevering efforts to economise coal, by the application of improved appliances for its consumption." The Committee had reason to believe that "in some branches of manufacture, the limits of a beneficial economy appeared to have been nearly reached, and that in other cases a gradual effort would continue to be made for saving fuel." It may be assumed, therefore, that the progress of economy in using cosl is not likely to operate in future with greater effect in keeping down the increase of consumption than it has hitherto done.

'The present consumption of coal for domestic use is generally estimated at one ton per head of the whole population, and may be assumed to absorb nearly one third of the entire production. It is probable that this rate per head will continue pretty constant; because, although more economical methods of using coal in dwellings may probably be introduced, yet the increasing wealth of the nation will cause coal to be more liberally used for domestic purposes. The future increase of consumption under this head may, therefore, be expected to coincide with the increase of the population.

"As regards the future exportation of coal, although a very large increase has taken place within the period embraced by the preceding Table, yet there is reason to doubt whether much further increase will take place in this direction. Upon this point Committee $\mathrm{E}$ have reported that the probable development of the enormous coal-fields of North America, and those of India, China, Japan, and other countries, and the more effective working of the known coal-fields of Europe, will probably prevent any considerable increase in the future exportation of British cosl.

- The large increase which, in recent years, has taken place in the consumption of coal, has an intimate connection with the introduction and extension of the railway system, but for several years past the progress of railways has not been exceptionally great, and yet the consumption of cosl has continued to increase with unabated rapidity.'

"Professor Jevons, in a work " On the Coal Question," which first appeared in 1865, and which was in a great measure founded upon a previous work by Mr. Hull on the Coal-fields of Great Britain, estimated that the rato of growth at that period in the aggregate annual consumption of coal amounted to about three and a half per cent. per annum, reckoning each annual percentage on the previous year's consumption. He contended that coal, being the source of power, and required for every great 
COAL

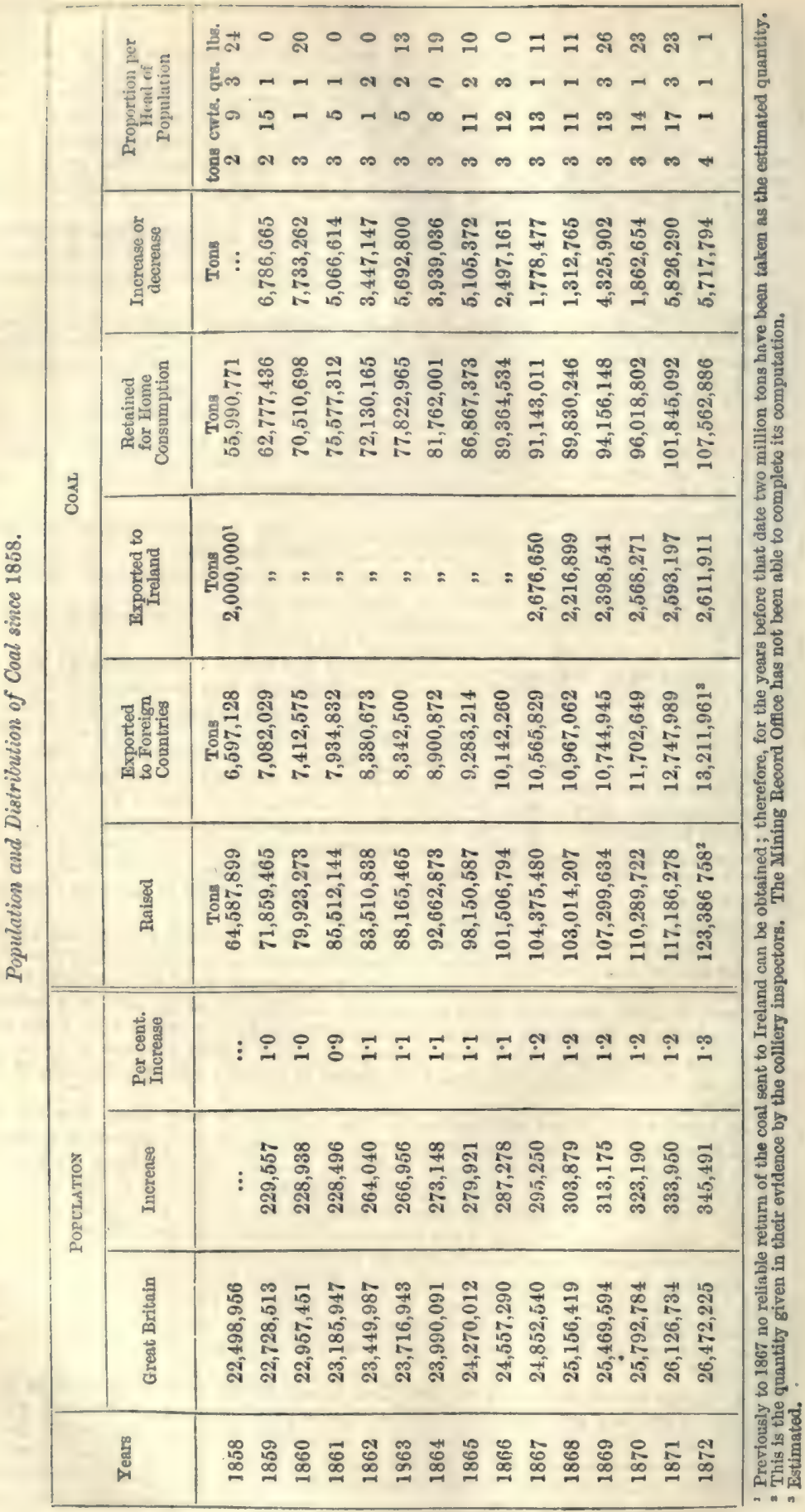


extension of industry, the consumption of it would keep pace with the progress of population and industry.

'He further showed that a continuance of the same progress in population and productive industry would lead to an annual consumption of no less than 2,607,000,000 tons of coal at the end of a century. Upon this assumed rate of increase, the whole quantity of coal which we have reported as available in this country would be exhausted in 110 years.

'But although Mr. Jevons's theory has, up to this time, nearly aecorded with observation, yet the results involved in the supposition that the annual percentage of increase in the consumption of coal will continue unabated for a very lengthened period are so prodigious that very few persons will bo found to accopt his theory without considerable qualifications.

'It had been suggested by Mr. Price Williams, who gave great attention to questions of this kind, that Mr. Jevons's conclusions at all events require modification to meet the fact, that although the population of this kingdom has long been and still is rapidly increasing, yet the rate of its annual growth is diminishing.

'From the year 1811 to 1821 , the increase was 16 per cent., while in the last decade, from 1861 to 1871 , it was 118 per cent.

Mr. Price Williams constructed a Table, of which the following is an abstract, showing how the Average Diminution of the Decennial Rate of Increase of the Population is obtained:-

\begin{tabular}{|c|c|c|c|}
\hline Year & $\begin{array}{c}\text { Population of } \\
\text { Great Britain }\end{array}$ & $\begin{array}{c}\text { Rate of Increase } \\
\text { per 10 years }\end{array}$ & $\begin{array}{c}\text { Diminution of Rate } \\
\text { per cent. }\end{array}$ \\
\hline 1811 & $12,338,573$ & $15 \cdot 977$ & \\
1821 & $14,309,989$ & $15 \cdot 008$ & $-6 \cdot 065$ \\
1831 & $16,457,596$ & $13 \cdot 549$ & $-9 \cdot 721$ \\
1841 & $18,687,537$ & $12 \cdot 249$ & $-9 \cdot 595$ \\
1851 & $20,976,532$ & $11 \cdot 197$ & $-8 \cdot 588$ \\
1861 & $23,325,305$ & $11 \cdot 736$ & $+4 \cdot 814$ \\
1871 & $26,062,721$ & $\ldots$ & $-33 \cdot 969$ \\
& & & $+4 \cdot 814$ \\
\hline
\end{tabular}

Computing, from this and another Table constructed by Mr. Price Williams, the Commissioners arrived at the following conclusions :-

The total available coal in the United Kingdom was estimated at $146,480,000,000$ of tons. Then, according to the views entertained, the annual consumption of coal at the end of a hundred years would be $274,000,000$ of tons. A further conclusion was that, the now estimated quantity of coal available for use would upon this view represent a consumption of 360 years.

It will of course be observed that, assuming the rate of increased consumption as above, there would be a nearly corresponding increase in the products of manufacturing industry, and the figures representing such an increase would raise questions ns difficult and problematical as those raised by the assumption of a population of $131,000,000360$ years hence.

The Commission then give a calculation founded upon arithmetical instead of geometrical increase in the rate of consumption of coal, that is to say:-they discard the principle of an increase in the nature of a per-centage on the previous quantity, and simply add a constant quantity equal to the average annual increase of the last fourteen years, which may be taken at $3,000,000$ of tons.

Upon this basis we arrive at the following results, namely, at the end of a hundred years the consumption would be $415,000,000$ of tons per annum, and the now estimated quantity of coal available for use would represent a consumption of 276 years.

There was yet another view considered. It was that the population of the whole country and the consumption of coal per head remained constant or merely oscillated without advancing. Then our available coal would represent a consumption of upwards of 1,273 years at the rate of $115,000,000$ of tons per annum.

The Commissioners proceed to advert to the large amount of coal excluded from our previous estimates on the ground of excessive depth. The quantity of coal lying 
beneath the Permian and other newer strata at depths exceeding 4,000 feet is computed at upwards of $41,144,000,000$, as appears by the following Table:-

Estimate of Quantities of Coal at Depths over 4,000 feot beneath the Permian. New Red, and other Strata.

\begin{tabular}{|c|c|c|c|c|}
\hline Dtatricts & $\begin{array}{l}\text { Square } \\
\text { Miles }\end{array}$ & $\begin{array}{c}\text { From } 4,000 \text { to } \\
6,000 \text { feet }\end{array}$ & $\begin{array}{l}\text { From } 6,000 \text { to } \\
10,000 \text { feet }\end{array}$ & Total in tons \\
\hline $\begin{array}{l}\text { Between Cannock Chrse, Coalbrook Dale, } \\
\text { and the North Staffordshire Coal-field, } \\
\text { under the Now Rod Marl, Eco, of Ire- } \\
\text { cleshall, Stafford, Breewood, and High }\end{array}$ & & tons & tons & tons \\
\hline $\begin{array}{l}\text { Offley } \\
\text { Sonthern borders of the North Stafford: }\end{array}$ & 112 & $8,846,022,400$ & $\cdots$ & $3,846,022,400$ \\
\hline $\begin{array}{l}\text { shire Coal-field } \\
\text { Plains of Cheshire, between the Denbigh: }\end{array}$ & 75 & $2,240,640,000$ & $\cdots$ & $2,240,640,000$ \\
\hline $\begin{array}{l}\text { shire and North Staffordshire Coal-fields } \\
\text { Southern borders of the Lancashire Coal- } \\
\text { fleld, and thecountry around Manchester }\end{array}$ & 340 & $11,850,496,000$ & $11,850,496,000$ & $28,700,992,000$ \\
\hline $\begin{array}{l}\text { and Btockport } \\
\text { The Wirrell, Mersey, and country to the }\end{array}$ & 208 & $6,904,490,667$ & $8,452,245,393$ & $10,356,786,000$ \\
\hline north,.,+ & $108 ?$ & $1,500,000,000$ & *. & $1,500,000,000$ \\
\hline Total & 848 & $29,341,649,007$ & $15,802,741,333$ & $41,144,800,400$ \\
\hline
\end{tabular}

Of this quantity it will be seen that more than $29,000,000,000$ of tons are assumed to lie at depths of between 4,000 and 6,000 feet, at which latter depth the temperature of the earth, it is supposed, would be $150^{\circ} \mathrm{F}$. The remainder, amounting to more than $15,000,000,000$ of tons, is assumed to lie at depths varying between 6,000 and 10,000 feet, at which maximum depth the temperature of the earth, according to the prevailing views, would be $215^{\circ} \mathrm{F}$., or three degrees above the temperature of boiling water at the sea level. To these quantities are to be added $7,320,000,000$ of tons returned as being at greater depths than 4,000 feet within the area of the known coal-fields. Of this quantity probably 5,922,000,000 of tons lie between the limits of 4,000 and 6,000 feet in depth, and the remaining $1,397,000,000$ of tons between 6,000 and 10,000 feet.

With these additions the total quantity of coal lying at depths exceeding 4,000 feet will be a little more than $48,465,000,000$ of tons.

It is entirely a matter of conjecture whether any or what portion of this coal can ever be worked; but if we were to suppose the whole to become available, we should have to make the following corrections in the number of years' duration given above as the result of the different modes of viewing the question :-

1st. The 360 years would be altered to 433 years.

2 nd. The 276 years based on an increasing consumption in arithmetical ratio would be altered to 324 years.

$3 \mathrm{rd}$. The 1,273 years computed on the supposition of non-increasing consumption would be altered to 1,695 years.

Whatever view may be taken of the question of duration of coal, the results will be subject to contingencies, which cannot in any degree be foreseen. On the one hand, the rate of consumption may be thrown back to any extent by adrerse causes affecting our national prosperity; and on the other hand, new discoveries and developments in new directions may arise to produce a contrary effect upon the consumption of coal. Every hypothesis must be speculative, but it is certain that if the present rate of increase in the consumption of coal be indefinitely continued, even in an approximate degree, the progress towards the exhaustion of our coal will be very rapid.

The numbers given in the preceding Table (p. 838) are sufficiently consistent with each other to represent the changes which have taken place over a period of years.

We find the following differences in the number of the collieries in 1858 and 1868 :-

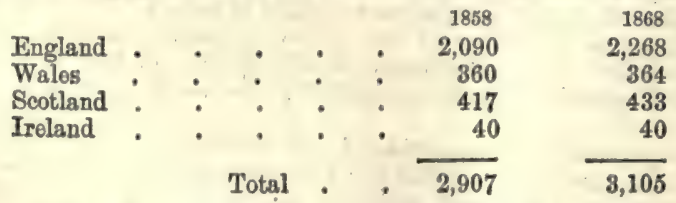

The increase being 198 collieries in the ten years. In 1872 the number of collieries returned were 3,001 .

With the viow of showing at a glance the condition of our coal trade, the produc. 
The following List of Collieries of the United Kingdom is corrected from the Returns of the Inspeotors of Coal Mines, up to February 1873.

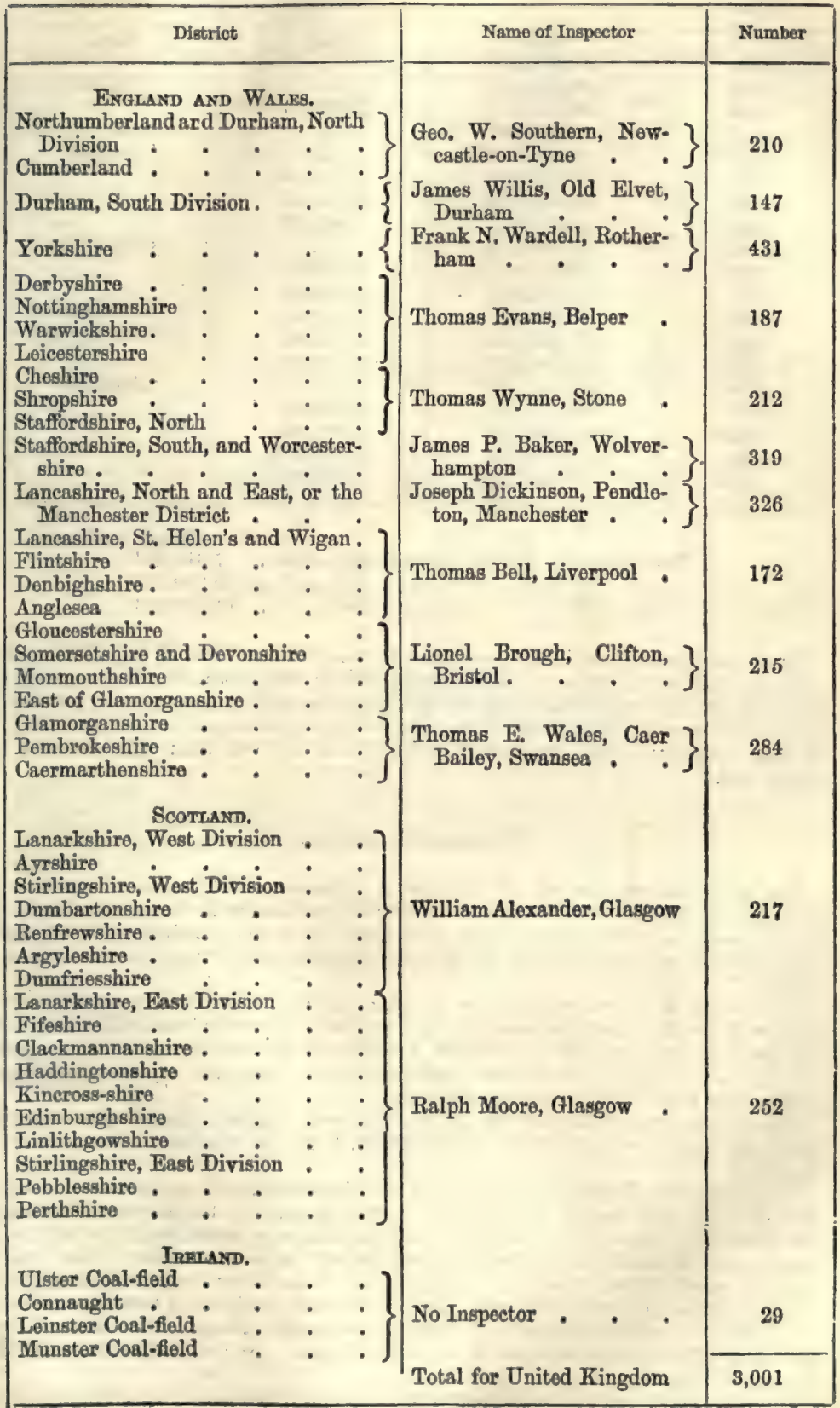


tion in the United Kingdom, since $\mathbf{1 8 5 4}$ is given in tho following Table, with the rates of incroase and decrease in each year:-

\begin{tabular}{|c|c|c|c|}
\hline Yeare & & Increaso & Deorease \\
\hline 1854 & $\begin{array}{c}\text { Tons } \\
64,661,401\end{array}$ & Tons & Tons \\
\hline 1855 & $64,453,070$ & & 208,322 \\
\hline 1856 & $66,645,450$ & $2,192,371$ & \\
\hline 1857 & $65,394,707$ & $\ldots$ & $1,250,743$ \\
\hline 1858 & $65,008,649$ & $\ldots$ & 386,058 \\
\hline 1859 & $71,979,765$ & $6,971,116$ & \\
\hline 1860 & $84,042,698$ & $6,062,933$ & \\
\hline 1861 & $86,039,214$ & $1,996,516$ & \\
\hline 1862 & $81,638,338$ & $\ldots$ & $4,400,876$ \\
\hline 1863 & $86,292,215$ & $4,653,877$ & \\
\hline 1864 & $92,787,873$ & $6,495,658$ & \\
\hline 1865 & $98,150,587$ & $5,362,714$ & \\
\hline 1866 & $101,630,544$ & $3,479,957$ & \\
\hline 1867 & $104,500,480$ & $2,869,936$ & \\
\hline 1868 & $103,141,157$ & & $1,359,323$ \\
\hline 1869 & $107,427,557$ & $4,286,400$ & \\
\hline 1870 & $110,289,722$ & $2,862,165$ & \\
\hline 1871 & $117,352,028$ & $7,062,306$ & \\
\hline 1872 & $123,386,735$ & $6,034,707$ & \\
\hline
\end{tabular}

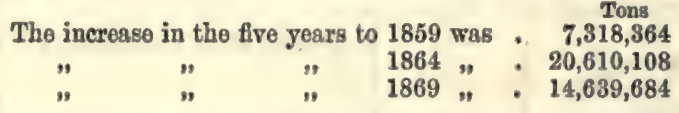

Or we have an increase in ten years, that is, from 1859 to 1869 , of $35,447,792$ tons. The quantity of coal produced in the years 1854 to 1869 , both inclusive, amounts to $1,343,793,705$ tons, which added to the quantity estimated as having been produced before 1859, namely, 2,850,000,000 tons, shows that up to the end of last year, (1872) we shall have drawn from our original stores of fuel not less than $4,300,000,000$ of tons of coal.

\section{Forkigr Cost-Fikids.}

We have not yet arrived at the poriod when we could pronounce with any approach to certainty on the actual number of caal-basins in the world; the total number must, however, amount to at least from 250 to 300 principal coal-fields, and many of these are subdivided by the disturbed position of the strata into subordinate basins.

The basins or coal areas may be, however, grouped into a comparatively small number of districts, and even many of these are little known and not at all measured. The greater number occur in Western Europe and Eastern North America, while Central and Southern Africa, South America, and a large part of Asia, are almost without any trace of true carboniferous rocks.

The principal coal-fields of Europo, are those of Belgium, France, Spain (in the Asturias), Germany (on the Rhur and the Saar), Bohemia, Silesia, and Russia (on the Donetz).

Bronum.-The Belgian coal-field is the most important, and occupies two districts : that of Liége and that of Hainault, the former containing 100,000 and the latter 200,000 acres. In each the number of coal-seams is very considerable, but the beds are thin and so much disturbed as to require special modes of working. The quality of cosl is very various, including one peculiar kind, the Flenu coal, unlike any found in Great Britain, except at Swansea. It burns rapidly with much flame and smoke, not giving out an intense heat, and having a somewhat disagreeable smell. There are nearly fifty soams of this coal in the Mons district. No iron has been found with the coal of Belgium.

Belgium is traversed, in a direction from nearly west-south-west to east-north-east, by a large zone of bituminous coal-formation. The entire region is generally described under two principal divisions :- 
1. The western or Hainault division, comprising

$a$. The two basins known as Lovant and Couchant of Mone.

That of Charleroi.

b. The basin of Namur.

2. The eastern or Liége division.

The Quantities of Coal of different Qualities raised in Belgium ${ }^{2}$ from 1869 to 1866.

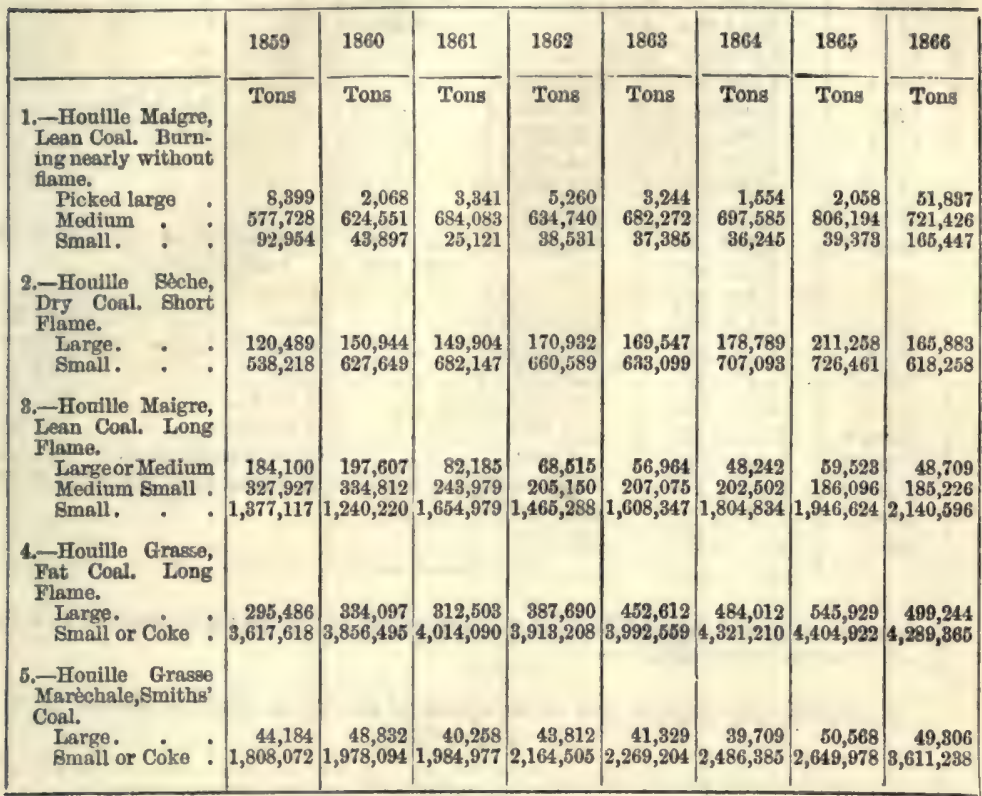

3Te Coal of Belgium is divided commercially into the following qualities :-Maigre, Lean Coal Seche, Dry Cosl; and Grasse, Fat Coal. These kinds are again divided into Gros, picked large cosl; Tout Venant, the remainder after the selection of the Gros, equivalent to 'through and through' coal ; Gaillette, smaller than the Gros, but not less than six inches square; Menu, coal which pagses throngh a sieve with 1 -inoh meshes; Gailletterie, the coal which remains after the separation of the Gaillette and Menu.

Netherrands,- There is only one coal-mine in Holland. It is situated in the province of Limburg.

FrANCE, - The most important coal-fields of France are those of the basin of Loire and those of St.-Etienne, which are the best known and largest, comprising about 50,000 acres. In this basin are eighteen beds of bituminous coal, and in the immediate neighbourhood several smaller basins containing anthracite. Other valuable localities are in Alsace, several in Burgundy worked by very deep pits, and of considerable extent; some in Auvergne with coal of various qualities; some in Languedoc and Provence with good coal; others at Aveyron; others at Limosin; and some in Normandy. Besides these, there are several others of smaller dimensions and less extent, whose resources have not yet been developed. The total area of coal in France has not been ascertained, but is probably not less than 2,000 square miles. The annual production in 1865, exceeded $11,000,000$ tons. But the conl of France is of an inferior description; and, therefore, when good and strong coals are required, the supply is obtained from the English conl-fields. The mineral combustibles of France were estimated in 1864 by the Government engineers as follow :-

$$
\begin{aligned}
& \text { Anthracites } \\
& \text {. . . . . . } 800,000 \\
& \text { Hand coal-short flame }: \quad: \quad \cdot \quad \cdot \quad \cdot 1,400,000 \\
& \text { Forging cosl . . . . } 600,000 \\
& \text { Bituminous caal - long flame : : : : : } 44,000,000 \\
& \text { Soft cosl-long flamo. . . . . . . . } 3,000,000 \\
& \text { Lignites . . . * . . , . . , 200,000 } \\
& \text { Total . . . . 10,000,000 }
\end{aligned}
$$


The Rate of Increase in the Production of Coal from the French Coal-fields has been as follows, to the end of 1867 :-

\begin{tabular}{|c|c|c|c|c|}
\hline Years & & Increave & Decrease & Another Return gives \\
\hline & Tons & Tons & Tons & Tons \\
\hline 1847 & $5,065,109$ &... & $1,047,234$ & $5,061,183$ \\
\hline 1848 & $4,017,875$ & $\ldots$ & 40,531 & $3,928,996$ \\
\hline 1849 & $3,977,344$ & $\ldots$ & ... & $3,976,910$ \\
\hline 1850 & $4,354,840$ & 377,496 & ... & $4,354,396$ \\
\hline 1851 & $4,404,941$ & 50,101 & $\ldots$ & $4,404,943$ \\
\hline 1852 & $4,816,306$ & 411,365 & $\ldots$ & $4,816,355$ \\
\hline 1853 & $5,831,939$ & $1,015,633$ & $\ldots$ & \\
\hline 1854 & $6,709,535$ & 877,596 & $\ldots$ & \\
\hline 1855 & $7,317,226$ & 607,691 & ... & \\
\hline 1856 & $7,784,165$ & 466,939 & 28,178 & The details of the return for \\
\hline 1857 & $7,755,987$ & $\ldots$ & 634,720 & 1852 being - \\
\hline 1858 & $7,221,267$ & $\ldots$ & $\ldots$ & Loire. \\
\hline 1859 & $7,337,326$ & 116,059 & $\ldots$ & $1,053,687$ \\
\hline 1860 & $8,155,394$ & 818,068 & $\ldots$ & SoAne-et-Loire \\
\hline 1861 & $9,125,040$ & 969,646 & ... & 379,114 \\
\hline 1862 & $10,102,116$ & 977,076 & $\ldots$ & $\quad 251,892$ \\
\hline 1863 & $10,518,406$ & 416,290 & $\ldots$ & - 179,560 \\
\hline 1864 & $11,046,794$ & 528,388 & $\ldots$ & Sarthe-et-Mayenne 111,44 ? \\
\hline 1865 & $11,098,258$ & 51,464 & $\ldots$ & Bouches du Rhóne 103,616 \\
\hline 1866 & $11,785,714$ & 687,456 & $\ldots$ & \\
\hline 1867 & $12,148,223$ & 372,509 & $\ldots$ & \\
\hline
\end{tabular}

The following Table will place the whole question of the coal consumption in France in a more satisfactory light:-

Produotion, Importation, and Consumption of Coal of all kinds in France from 1787 to 1866.

\begin{tabular}{|c|c|c|c|c|c|c|c|}
\hline \multirow[b]{2}{*}{ Years } & \multirow[b]{2}{*}{$\begin{array}{c}\text { Production } \\
\text { of Coal } \\
\text { in France } \\
\text { Bince } 1787\end{array}$} & \multicolumn{6}{|c|}{ Quantities of Coal imported into France from } \\
\hline & & Belgium & $\begin{array}{c}\text { Prussia } \\
\text { and } \\
\text { Bavaris }^{3}\end{array}$ & $\begin{array}{l}\text { Great } \\
\text { Britain }\end{array}$ & $\begin{array}{l}\text { Total } \\
\text { Imports }\end{array}$ & $\begin{array}{l}\text { Differenoe } \\
\text { between } \\
\text { Imports } \\
\text { and } \\
\text { Exports }\end{array}$ & $\begin{array}{c}\text { Total } \\
\text { Consump- } \\
\text { tion in } \\
\text { France }\end{array}$ \\
\hline $\begin{array}{l}1787 \\
1788 \\
1789 \\
1802 \\
1811 \\
1816 \\
1821 \\
1826 \\
1881 \\
1836 \\
1841 \\
1846 \\
1851 \\
1856 \\
1861 \\
1862 \\
1868 \\
1864 \\
1865 \\
1866\end{array}$ & $\begin{array}{c}\text { tons } \\
211,160 \\
220,982 \\
235,714 \\
829,105 \\
759,878 \\
924,828 \\
1,114,448 \\
1,513,482 \\
1,728,950 \\
2,789,858 \\
8,349,303 \\
4,389,532 \\
4,404,940 \\
7,337,741 \\
9,227,710 \\
10,133,148 \\
10,516,752 \\
11,001,249 \\
11,785,714 \\
11,807,142\end{array}$ & $\begin{array}{r}\text { tons } \\
49,107 \\
50,893 \\
49,107 \\
86,428 \\
99,303 \\
267,206 \\
247,305 \\
403,278 \\
435,628 \\
703,088 \\
974,507 \\
1,326,095 \\
1,989,811 \\
2,995,619 \\
3,503,028 \\
3,619,687 \\
3,648,945 \\
\mathbf{3}, 945,916 \\
4,189,144 \\
4,070,557\end{array}$ & $\begin{array}{r}\text { tons } \\
9,821 \\
11,785 \\
9,821 \\
17,678 \\
24,558 \\
28,973 \\
41,823 \\
56,428 \\
67,694 \\
111,858 \\
192,993 \\
224,326 \\
292,875 \\
937,400 \\
1,259,116 \\
1,106,069 \\
1,136,496 \\
1,264,666 \\
1,261,867 \\
1,985,622\end{array}$ & $\begin{array}{r}\text { tons } \\
154,568 \\
181,474 \\
176,785 \\
9,821 \\
18 \\
18,719 \\
26,041 \\
36,237 \\
35,270 \\
166,482 \\
422,272 \\
600,384 \\
591,377 \\
1,038,605 \\
1,413,323 \\
1,379,832 \\
1,273,446 \\
1,258,051 \\
1,443,040 \\
1,674,164\end{array}$ & $\begin{array}{c}\text { tons } \\
213,496 \\
244,152 \\
235,713 \\
113,927 \\
117,856 \\
814,898 \\
315,169 \\
495,943 \\
588,592 \\
981,428 \\
1,589,772 \\
2,150,805 \\
2,874,063 \\
4971,624 \\
6,175,467 \\
6,105,588 \\
6,058,887 \\
6,468,638 \\
6,844,050 \\
7,730,348\end{array}$ & $\begin{array}{r}\text { tons } \\
186,117 \\
212,724 \\
206,250 \\
89,375 \\
88,392 \\
285,135 \\
242,715 \\
492,310 \\
581,674 \\
955,634 \\
1,550,590 \\
2,101,336 \\
2,840,160 \\
4,881,743 \\
5,900,007 \\
5,850,831 \\
5,701,487 \\
6,177,865 \\
6,623,650 \\
7,482,811\end{array}$ & $\begin{array}{r}\text { tons } \\
847,277 \\
4483,706 \\
441,954 \\
918,480 \\
848,270 \\
1,209,960 \\
1,357,163 \\
2,005,792 \\
2,260,624 \\
3,745,492 \\
4,899,893 \\
6,490,868 \\
7,245,100 \\
12,219,484 \\
15,127,717 \\
15,983,970 \\
16,218,189 \\
17,179,114 \\
18,409,364 \\
19,269,453\end{array}$ \\
\hline
\end{tabular}

Irom Mons, Charlerol, Namur, Iége, szc.

- From Saarbrtick and St.-Imbert, Prussia, and Rhenish Bavaria.

- This Table has been compiled from the 'Statistique de l'Indnstrie Minerale, which is isgned in France about every five years by the Minister of Agriculture, Commerce, and Publio Works, but has not appeared since 1867, and then the returns were to the end of $1864 \mathrm{only}$.

Tho motrical quintal of the original tables is taken at $220 \mathrm{lbs}$. avoirdupois, and the Finglish ton at 2,240 lbs. 
Coal Production in chrtatn Departments of Frayce for Five Yuans.

L'Aveyron (en quintaux métriques).

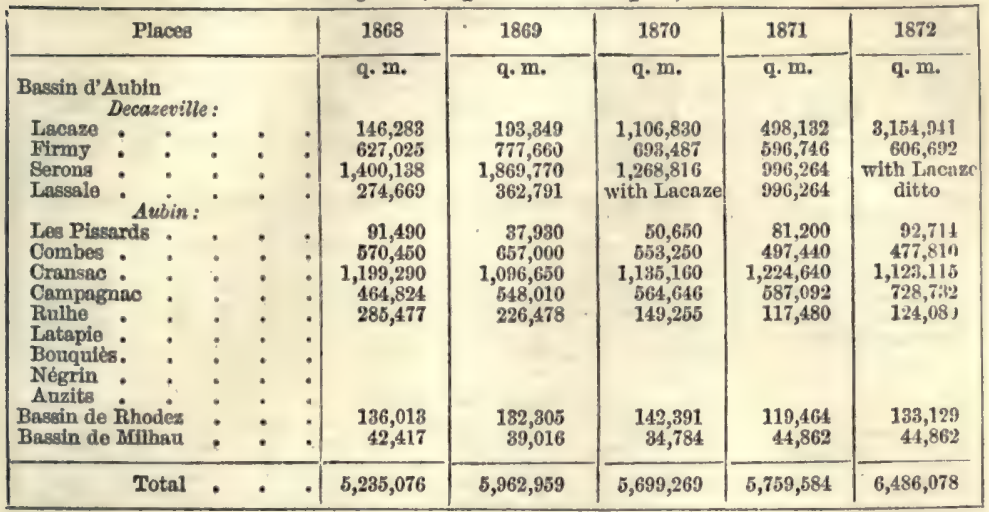

Saône-et-Loire.

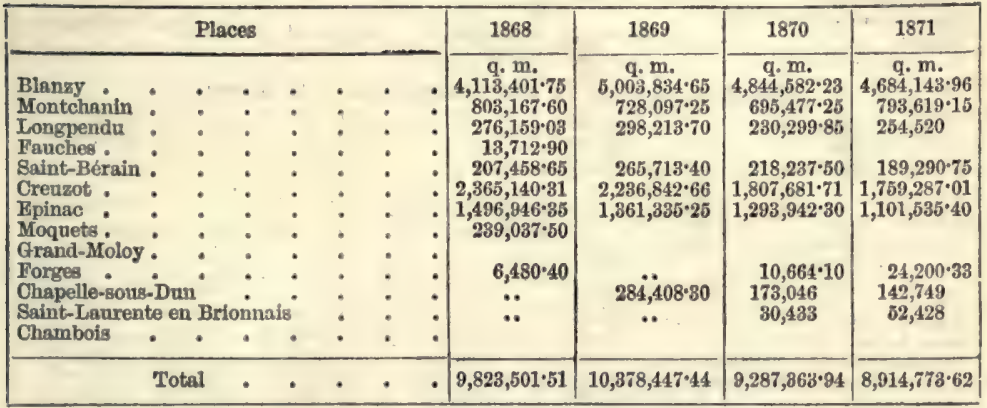

Les quantités sont exprimées en quintaux métriques à raison de 85 kil. par hectolitres.

Département de l'Allier.

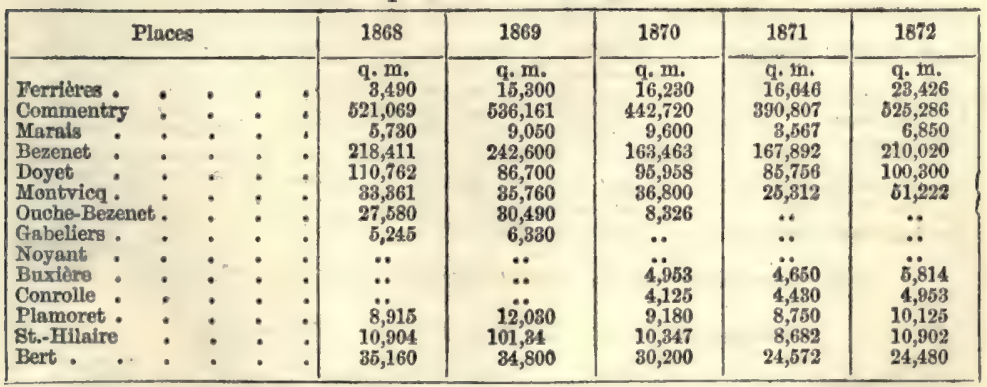

De la Production de la Houille dans les Trois Départements du Puy-de-Dôme, de la Haute-Loire, et du Cantal.

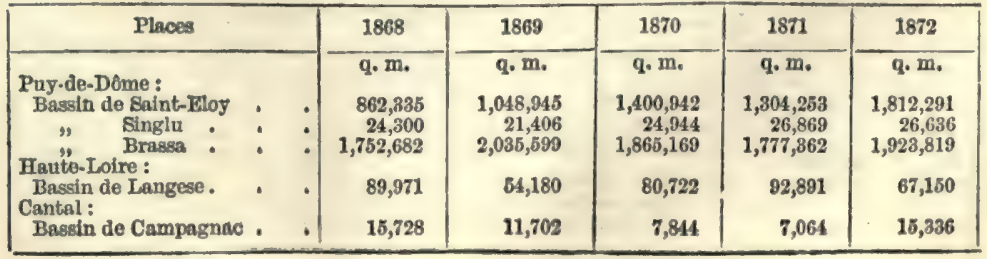




\section{Creuse.}

$\begin{array}{rrrrr}1868 & 1869 & 1870 & 1871 & 1872 \\ \text { kilog. } & \text { Kilog. } & \text { Kilog. } & \text { kllog. } & \text { Kilog. }\end{array}$

Aluan. * . . . 167,079,372 167,666,740 189,170,295 198,870,765 275,786,593

Departement de la Mayenne.

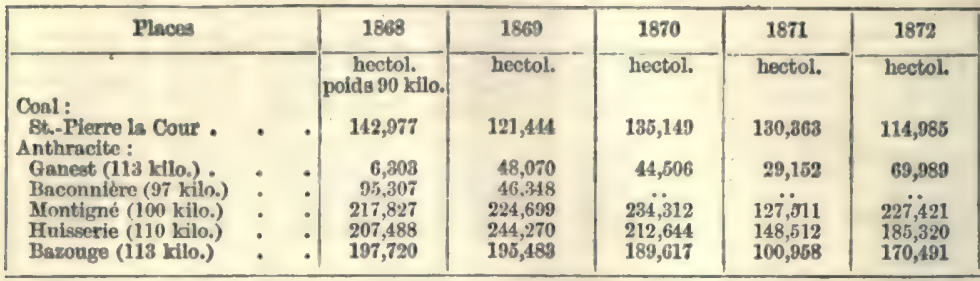

Département du Gard.

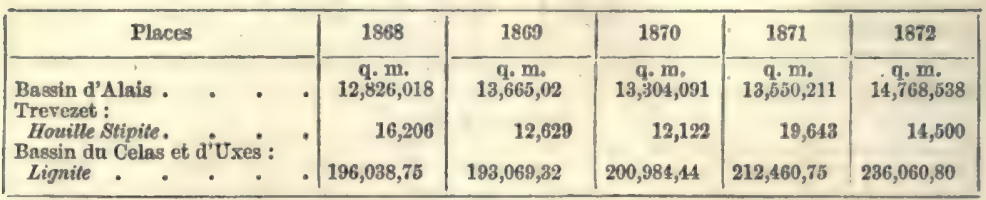

Concessions diverses du sous-arrondissement de Marseille.
1868 197,000
1869
1870

Bassin de la Loire.

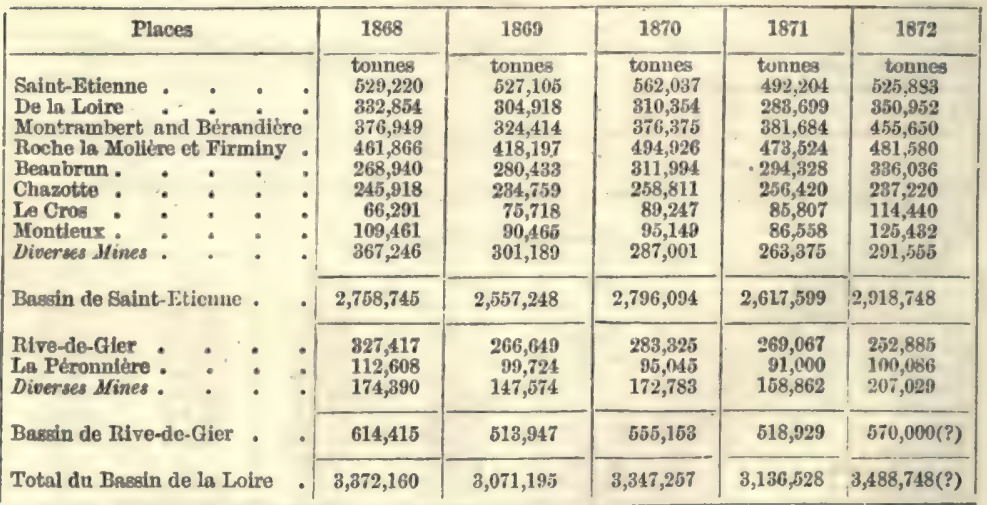

Under divers mines those are Incinded which produce less than 100,000 tonnes.

Concession des Touches (anite de la Loire-Inférieure).

1868

1869

1870

$$
\begin{array}{r}
q . m . \\
26,478 \\
5,687 \\
8,514
\end{array}
$$

1871
1872 q. $\mathrm{m}$.

94,223

Mines of Anthracite, Département de la Sarthe.

\begin{tabular}{|c|c|c|c|c|c|c|c|}
\hline & Places & & 1808 & 1869 & 1870 & 1871 & 1872 \\
\hline \multirow[t]{2}{*}{$\begin{array}{l}\text { Mine de } \\
n\end{array}$} & $\begin{array}{l}\text { Manpertais } \\
\text { Saulneries . }\end{array}$ & $: \quad:$ & $\begin{array}{l}\text { bectol. } \\
179,733 \\
14,926\end{array}$ & $\begin{array}{r}\text { hectol. } \\
\mathbf{1 8 7 , 3 4 0} \\
83,998\end{array}$ & $\begin{array}{r}\text { hectofl. } \\
146,829 \\
22,503\end{array}$ & $\begin{array}{r}\text { hectol. } \\
146,175 \\
1,002\end{array}$ & $\begin{array}{r}\text { hectol. } \\
170,445 \\
40,735\end{array}$ \\
\hline & Total : . & . & 194,659 & 251,323 & 169,802 & 147,177 & 211,180 \\
\hline
\end{tabular}

Hectolitres polds moyen $=100 \mathrm{kilo}$. 
Bassins du Nord et du Pas-de-Calais.

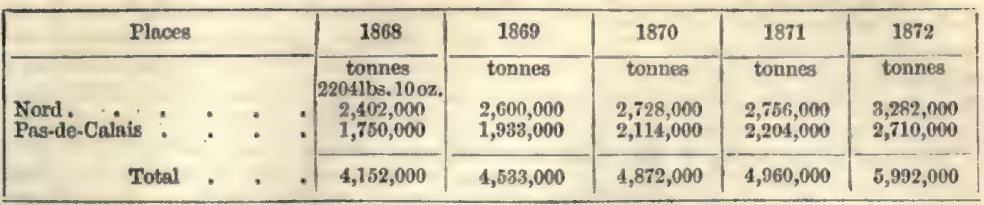

Lignite du sous-arrondissement minéralogique d'Avignon.

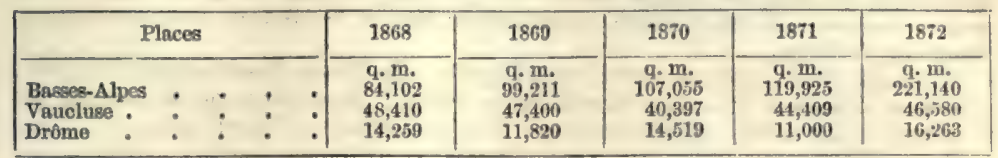

Coal and Lignite, $l$ Hérault et $\bar{C}$ Aude.

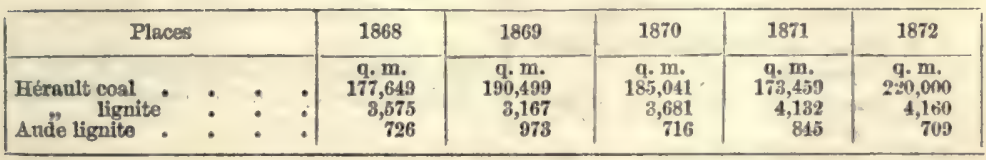

Spars.-Spain contains a large quantity of coal, both bituminous and anthracite. The richest beds are in Asturias, and the measures are so broken and altered as to bo worked by almost vertical shafts through the beds thomselves. In one place upwards of 11 distinct seams have been worked, the thickest of which is only 14 feet. The exact ares is not known, but it has been estimated by a French engineer that about $12,000,000$ of tons might be readily extracted from one property, without touching the portion existing at great depths. In several parts of the province the conl is now worked, and the measures seem to resemble those of the coal districts generally. The whole coal area is said to be the largest in Europe, presenting upwards of 100 workable seams, varying from 3 to 12 feet in thickness.

The Asturias Mining Company are working many mines in this region, and they are said to produce 400,000 tons annually, or to be capable of doing so. In Catalonia and in the Basque Provinces of Biscay there are found anthracite and bituminous coals.

In the Balearic Islands also coal exists.

Portugas. - Beds of lignite and some anthracite are known to exist, but the production of either is small.

Itary. - The principal coal-mines of Italy are in Saroy and near Genoa. In the Apennines some coal is found, and in the valley of the Po are largo deposits of good lignite; and a small quantity of good coal is worked in Sardinia.

SwITzerLaND.-Coal has only been found in the cantons of Berne, Fribourg, and the Valais. Beds of anthracite coal are developed in the Central and Western Alps.

Geraravy. - The Germanic Union-the Zollverein-embraces the following principml coal-beds :-

$$
\begin{aligned}
& \text { Gorman States }\left\{\begin{array}{l}
\text { Saxony. } \\
\text { Bavaria, } \\
\text { Duchy of Hesso. }
\end{array}\right. \\
& \text { Prussian States }\left\{\begin{array}{l}
\text { The Ruhr, in Westphalia. } \\
\text { Silesia. } \\
\text { Saarbrick, and provinces of the Bas Rhin. }
\end{array}\right.
\end{aligned}
$$

The true coal of Prussian Stlesia stretches for a distance of serenteon leagnes. The most recent information we have been able to obtain as to its production would appear to give above 850,000 English tons. The coal-fields of Westphalia were described by Sedgwick and Murchison in 1840. The productive coal-beds are on the right bank of the Rhine, and possess many features in common with the English coal-fields. Bituminous coal, and lignite or brown coal occur extensively in some districts. The coal-basin of Saarbruick, a Rhenish province belonging to Prussia, has thus been described by Humboldt, chiefly from a communication received from M. Von Dechen:- 
- The depth of the coal-measures nt. Mont St.-Gilles, Liége, I have estimated at 3,650 feet below the surface, and 3,250 feet below the sea level. The coal-basin at Mons lies fully 1,750 feet deeper. These depressions, however, are trifling when compared with that of the caal-strata of the Saar rivers (Saarbrück). After repeated trials, I have found that the lowest strata known in the county of Duttweiler, near Bettingen, north-eastward from Saarlouis, dip 19,406 feet, and 20,656 under the level of the sea.'

The cosl of the valley of the Glane is bituminous, and of good quality; it is procurable at a depth of 112 feet, and the seam is about two feet in thickness: about 50,000 tons annually are produced from this valley. Coal is found in Würtemberg, but not much worked. In Saxony are extensive mines of bituminous coal ; at Schonfield, near Zwickau, the coal alternates with porphyry. Near Dresden a bituminous coal is also worked, and the coke manufactured from it is used in the metallurgical works at Freiberg.

Electoral Hesse produces little beyond lignite. In Hesse Cassel some bituminous coal is worked, but to a very inconsiderable extent. Real stone coal is only as yet produced in the county of Schaumburg. It is rich in beds of brown coal.

In the Thuringerwald or Thuringian forest some coal is produced.

The following is a list of the coal-basins of Germany and Austria, and their situa. tions :-

1. Bassin de la Worm, or de Kohl-

schneider. weilex.

3. Bassin de Saarbrück.

4. Bassin de la Forêt-Noire. In the Grand-Duchy of Baden.

5. Bassin de la Westphalie or de la Ruhr.

6. Lo Bassin d'Tbbenbüren.

7. Bassin de Piesberg. Near Osnabrïck.

8. Bassin d'Illefeld. To the south of the Hartz.

10. Les Bassins do Wettin, Löbejun, and Plotz.

11. Thuringerwald. Near Manebach.

12. Gelberg. Near Ilmenau.

13. Mordflech. Near Goldlauter.

14. De Crock.

15. Stockeim et Neuhaus. In the Upper Pfalz of Bavaria.

16. Erbendorf.

17. Bassin de Zwickau-Chomnitz. In Saxony.

18. Bassin de Flohaer.

19. Bassin de Plauen. Near Dresden.

20. Schonfield. Near Altenburg.

21. Brandau. In Bohemia.

22. Bassin de Radmitz. In Bohemia,

23. Bassin de Pilsen.

24. Bassin de Schlau-Rakonitz.

25. Bassin de la Basse Silésie. In Bohemia.

26. Bassin de lo Haute Silésie. Partly in Prussia and partly in Austria.

27. Bassin de Mahr-Ostrau.

28. Bassin de Rossitz. In Moravia.

The coal-pits of the most importance are in the Rhenish provinces and Westphalia, in the western parts of Prussia, as well as in the province of Silesia. Among the coalbasins there are three of importance: these are the basins of Upper Silesia, of the Ruhr, and the Saar. Two other basins, near Waldenburg, in Lower Silesia, and near Aix-la-Chapelle, in the Rhenish province, are less considerable. The basins of Wellin and Löbejun in the prorince of Saxony, and of Ibbenbüren in the northern part of Westphalia, are of less extent. There is also some coal mining, but inconsiderable, near Minden, on the Weser (in Weald clay), and near Löwenburg in Silesia.

The brown coal of most importance is found in the eastern part of the country; that is, in the provinces of Saxony and Brandenburg. There this lignite is exceedingly useful, as coals are excessively dear, owing to the cost of transporting them so great a distance.

The coal-pits of the river Ruhr extend over ten miles in length on the Lower Rhine. This country supplies almost half of the coal prodnce, and it sends supplies to Alsatia and Switzerland, to Bavaria and Thuringia, to Berlin and the seaports of the Hanseatic Towns, as well as to Holland. 
The following, from an article on 'Westphalia and the Ruhr Basin,' by Mr. T. E. Cliffe Leslie, contains most valuable information:-'

'Twenty years ago the Ruhr Basin was nowhere in the industrial race; now it produces nearly half as much coal as the great northern coal-field of England. Twenty years ago it had only just completed a single line of railway; now the basin is a network of branches, connecting not only the towns, but the principal manufactories and collieries with the three main lines which traverse it. The following figures show the rate at which the production of coal has advanced:-

\begin{tabular}{|c|c||c|c|}
\hline Date & Finglish tons" & Date & Inglish tons \\
\hline 1851 & $1,771,454$ & 1860 & $4,276,254$ \\
1852 & $1,921,962$ & 1861 & $4,964,621$ \\
1853 & $2,146,275$ & 1862 & $5,701,201$ \\
1854 & $2,670,099$ & 1863 & $6,300,981$ \\
1855 & $3,252,323$ & 1864 & $8,146,433$ \\
1856 & $3,510,502$ & 1865 & $9,276,685$ \\
1857 & $3,635,256$ & 1866 & $9,329,503$ \\
1858 & $3,898,502$ & 1867 & $10,526,015$ \\
1859 & $3,793,356$ & & \\
\hline
\end{tabular}

- The immense increase of production shown in these figures is mainly attributable to the introduction of railways and the low charge for the carriage of coal. Down to 1851 the Ruhr and the Rhine were the only means of transport in districts beyond the immediate neighbourhood of the collieries, and the greater part of the coal was of an inferior kind, raised where it came to the surface by small collieries along the Ruhr. In 1851 the Cologne-Minden Railway came into use for the transport of coal, and led not only to deep-pit-sinking, and the discovery of seams of superior coal in other parts of the basin, but also to the establishment of ironworks and other manufuctures, affording a local market for the coal. To this local market, down to 1859 , it was in a great measure confined. In that year the charge for railway carriage of coal for long distances was reduced to one pfennig per centner (a fifth of a farthing per $100 \mathrm{lbs}$.) per German mile, ${ }^{3}$ and the above figures show the subsequent inerease of production. The railways and coal-mines render each other reciprocal service; the carriage of Westphalian coal is now one of the most important branches of traffic on several of the chief Prussian lines, and the low rates at which it is carried enable it to find a distant market. The projected reduction of the rate for the transport of iron ore to the same tariff as that for coal, when carried into effect, will greatly augment the market for coal as well as for manufacture of iron. Until the last few years the Ruhr Basin excelled only in the manufacture of steel; but its iron manufactures are now of the highest quality.'

The Westphalian coal-field is stated to be at least 400 square miles in extent. The number of workable seams are from 60 to 80 , and the average thickness of coal is about 200 feet. The coal is of good quality, as the analysis of Essen cosl shows :-

\section{Carbon $88 \cdot 08$, hydrogen $5 \cdot 00$, nitrogen $1 \cdot 39$, oxygen $3 \cdot 12$, sulphur $1 \cdot 06$, ashes $1 \cdot 35=100$.}

Mr. Consul-General J. A. Crowe, in his 'Report on the Trade of the Rhenish Provinces and Westphalia,' gives the following:-

The coal-fields of the Ruhr extend over a surface of 115 square miles, and are supposed to contain $40,000,000,000$ tons of fuel. They are comprised chiefly in the Government districts of Duisburg, Essen, Bochum, Dortmund, and Hamm. The rapid increase of their production may be seen by a glance at the following Table:-

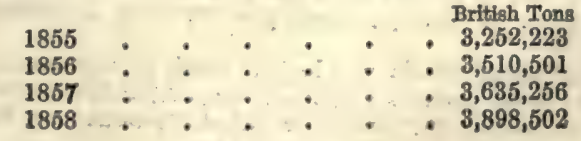

Fortnightiy Review, No. XXVII. March 1, 1869.

2. The Prussian Tonne is a measure of capacity, and varies therefore in weight ns applied to different articles-cosl and iron, for example. The quantity of cosl in a Tonne is about one-fifth of an English ton. In nome of the reports in Finglish blue-books the Tonne is translated 'ton,' which may mislead readers.

The German mile is abont 4 Inglish miles.

Vorn I. 


\begin{tabular}{|c|c|c|c|c|}
\hline 1859 & & • & $\begin{array}{r}\text { British tons } \\
.3,793,355\end{array}$ & \\
\hline & . & . & $\quad 4,276,253$ & Increase since $18551,024,030$ tons. \\
\hline & $\cdot$ & : & $\therefore \quad 4,964,621$ & \\
\hline & . & - & - $\quad 5,701$ & \\
\hline & $\therefore \quad \therefore$ & $\therefore$ & $\begin{array}{r}\quad 6,300,318 \\
7,474,935\end{array}$ & \\
\hline & & 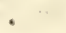 & . $8,535,614$. & Increase since $18604,259,361$ tons. \\
\hline & 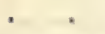 & . & $8,583,362$ & \\
\hline
\end{tabular}

The Working of the Royal Mines of the Coal-Basin of Saarbrick.

\begin{tabular}{|c|c|c|c|c|c|c|}
\hline \multirow{2}{*}{ Years } & \multirow{2}{*}{$\begin{array}{l}\text { Total Quantity } \\
\text { produced }\end{array}$} & \multirow{2}{*}{$\begin{array}{c}\text { Quantity } \\
\text { sold }\end{array}$} & \multicolumn{4}{|c|}{ Value of Bales in Francs. } \\
\hline & & & Prussia & Zollverein & Switzerland & France \\
\hline 1863 & $\begin{array}{c}\text { tons } \\
2,157,879\end{array}$ & $\begin{array}{c}\text { tons } \\
1,944,884\end{array}$ & 494.421 & 445,270 & 78.966 & 961.589 \\
\hline 1864 & $2,551,129$ & $2,307,257$ & 576,078 & 574,596 & 116,278 & $1,082,256$ \\
\hline 1865 & $2,821,695$ & $2,490,418$ & 703,776 & 574,371 & 125,709 & $1,131,843$ \\
\hline 1866 & $2,951,034$ & $2,612,328$ & 682,185 & 537,361 & 119,030 & $1,321,254$ \\
\hline
\end{tabular}

M. Vuillemin gives the following as the result of the workings for four years :-

Bassin de Saarbriick.

\begin{tabular}{|c|c|c|c|}
\hline Years & $\begin{array}{c}\text { Metrical Tons, } \\
2,200 \mathrm{lbs}\end{array}$ & Value in Franes & $\begin{array}{c}\text { Value per Tonno } \\
\text { in Francs }\end{array}$ \\
\hline 1864 & $2,597,516$ & $24,011,048$ & $9 \cdot 24$ \\
1865 & $2,872,999$ & $27,638,250$ & $9 \cdot 62$ \\
1866 & $3,004,690$ & $32,341,262$ & $10 \cdot 93$ \\
1867 & $3,171,125$ & $33,994,460$ & $10 \cdot 72$ \\
Mean & $2,911,582$ & $29,621,256$ & $10 \cdot 17$ \\
\hline
\end{tabular}

Bassin de la Ruhr (District de Dormund).

\begin{tabular}{|c|c|c|c|}
\hline Years & $\begin{array}{c}\text { Metrical Tons, } \\
2,200 \text { lbs. }\end{array}$ & Value in Francs & $\begin{array}{c}\text { Value per Tonne } \\
\text { in Franas }\end{array}$ \\
\cline { 1 - 3 } 1864 & $8,146,483$ & $49,856,170$ & $6 \cdot 12$ \\
1865 & $9,165,675$ & $62,693,217$ & 6.84 \\
1866 & $9,291,250$ & $\ldots$ & $\ldots$ \\
1867 & $10,366,035$ & $\ldots$ & $\ldots$ \\
Mean & $9,242,348$ & $\ldots$ & $\ldots$ \\
\hline
\end{tabular}

Mr. Lowther in his Report remarks, "The western parts of Prussia are so richly furnished with mineral fuels, particularly coals, that they do not depend for their supply on foreign countries, but rather give up a great part of their coal produce to the latter. The eastern provinces of the state have to look to the import of foreign coal, partly from Westphalia, partly from foreign countries.

- This is particularly the case with countries on the Baltic, which receive by sea cosl cheaper from British harbours than from distant inland mining districts. The provinces of Saxony and Brandenburg, particularly whon near the watercourses of the Elbe, recoivo important quantities of British coal and Bavarian brown coal, as well as great imports of coal by railway from the pits of Zwickau and Chemnitz in the neighbouring kingdom of Saxony; these pits are daily improving in extent and value.'

The coal production of Silesia was in 1871 much greater than anybody expected. It reached a height never previously attained, viz., $32,723,824$ tonnen (about $5 \frac{1}{2}$ to an English ton), which is $4,403,767$ tonnen, or $16,154,479 \mathrm{cwts}$. more than last year.

The importation of coal into Berlin amounted to-

1870. $2,697,043$ tonnen Silesian cosl. 1871. 3,228,391 tonnen.

1870. 600,000 " English coal. 1871. 1,160,919 " .

This is an increase in one year of 531,348 tonnen Silesian coal, and 560,240 tonnen English coal, while during the five preceding years the importation amounted to- 


\begin{tabular}{|c|c|c|c|c|c|}
\hline & Years & & Silesian Coal & Finglish Coal & Finglish Cokc \\
\hline 1869 & - & . tonnen & $1,965,927$ & 689,952 & 77,386 \\
\hline 1868 & . & - tonnen & $2,002,807$ & 706,406 & 111,608 \\
\hline 1867 & . & . tonnen & $1,888,353$ & 525,464 & 103,742 \\
\hline 1866 & . & . tonnen & $1,466,400$ & 917,056 & 96,358 \\
\hline 1865 & . & . tonnen & $1,962,000$ & 686,096 & 104,362 \\
\hline
\end{tabular}

Coal in Pmissia, as returned to January 1871 :

Centners

of 100 metrical pounds $=$

$110 \cdot 231$ lbs, avoirdupois

Upper Silesia

. $181,144,049$

Lower Silesia : $: \quad: 39,400,740$

Wettin ... . . . $\quad 820,958$

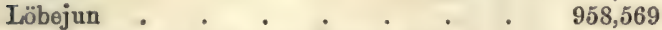

The Province of Hanover (Weald coal) ， 5,972,845

The County of Hohnstoin . . . . . 469,356

Schaumburg $\left(\frac{1}{2}\right)$. . . . . . . . $1,978,429$

Minden . . . . . . 109,347

Ibbenbuiren . . . . . . 4,066,314

The district of the Ruhr _ . . . . $249,235,184$

Aachen . . . . . 19,923,919

The district of the Saar . . . 65,261,165

Total . . . : $\overline{519,340,875}$

Quantity raised to January $1870^{\circ} \quad \cdots \quad 466,324,753$

$$
\text { Increase - . . } \overline{58,016,122}
$$

\section{Brown Coal in Prussia to January 1871:-}

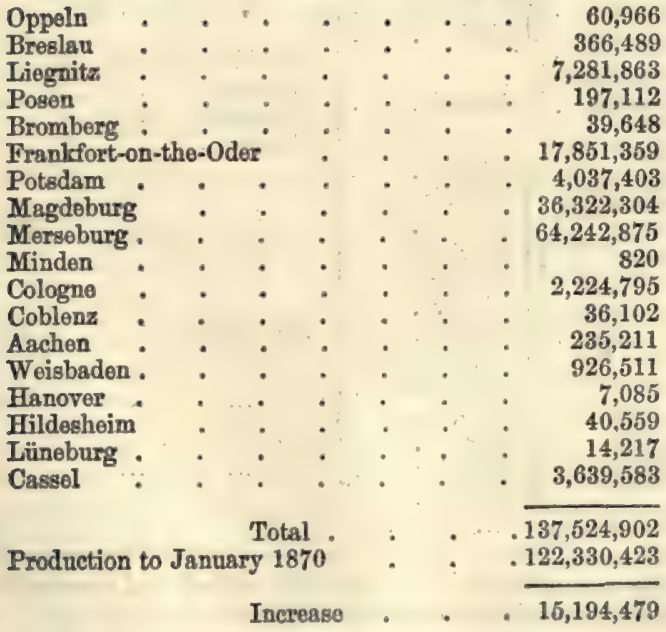

The quantity of cosl raised in Prussia in 1872 was as follows:-

\begin{tabular}{|c|c|c|c|c|}
\hline & & & Quan & \\
\hline as & 428 & 140,544 & $590,473,512$ & $85,118,828$ \\
\hline $\mathrm{Can}$ & : 532 & 17,447 & $148,992,730$ & $7,957,125$ \\
\hline
\end{tabular}

Poras, - There are several coal-mines in Poland. The crown coal-mines produce about $1,000,000$ sacks of $3 \frac{1}{2}$ bushels. The coal-mines of the western district produce $8,000,000^{\circ}$ lbs. per annum.

HUNGARY and other countries in the east of Europe contain true coal-measures of the carboniferous period; but the resources of these districts are not at present developed. The coal worked at Fünflkirchen, in Hungary, is of Liassic age. On the banks of the Donetz, in Russia, cosl is worked to some extent, and is of excellent quality. 
Avgrnu.-Coal oceurs in Styria, Carinthia, Dalmatia, the Tyrol, Moravia, Lom. bardy, and Venice; but 700,000 tons appears to be the maximum annual produce of the empire. The basin of Vienna, in Luwer Austria, produces meveral varfeties of coal, which belong to the brown coal of the Tertiary period.

The following Table gires the geographical position of coal now worked (1872), and the estimated production :-

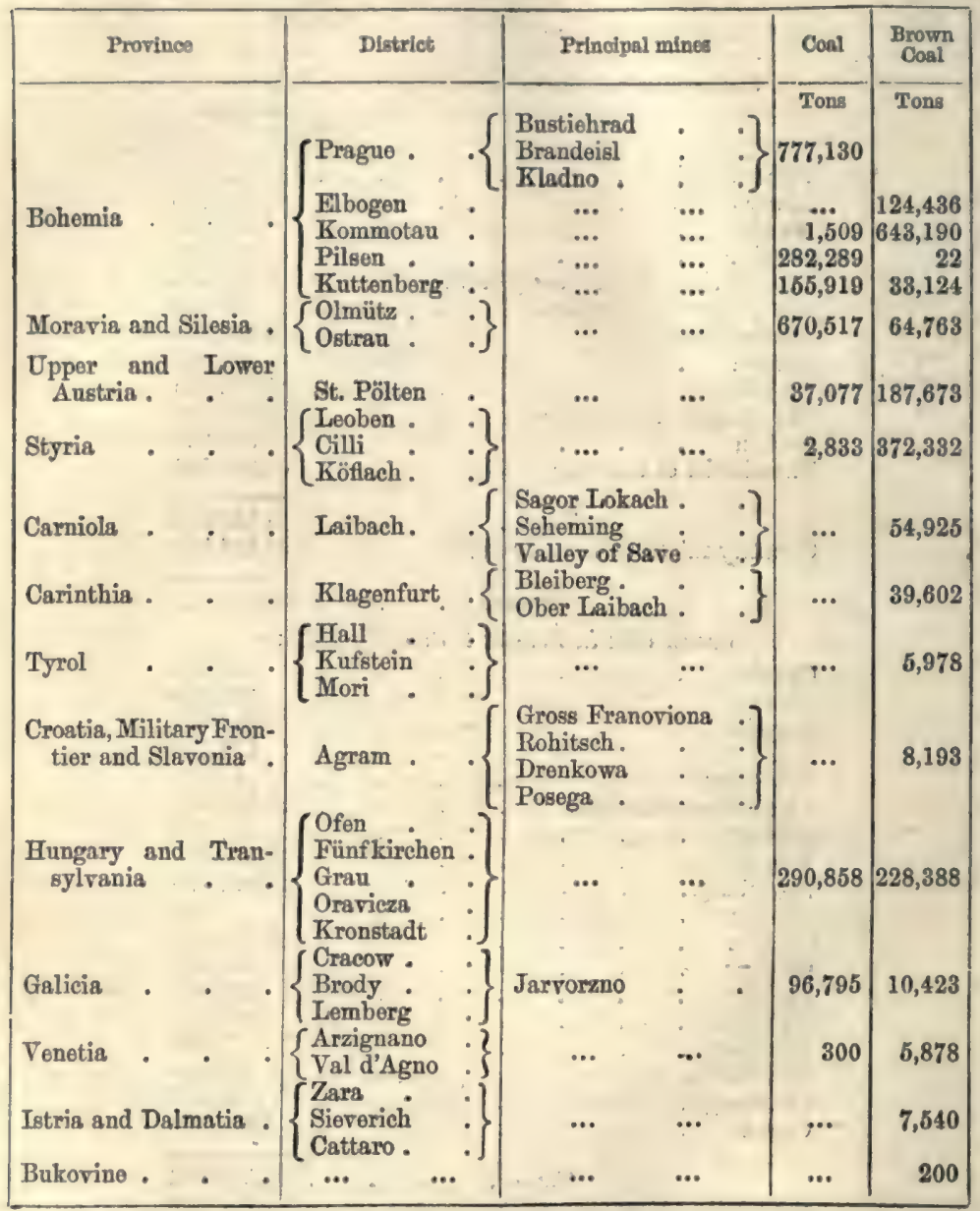

The following statement of the productions and of the consumption of coal in Austria has recently been issued upon anthority :-

\begin{tabular}{|c|c|c|}
\hline Years & Conl produced & Coal consumod \\
\hline 1848 to 1851 & Tons & Tons \\
1852 "1855 & $14,973,121,328$ & $8,087,567$ \\
1856 "1859 & $22,114,874$ & $14,239,041$ \\
1860 "1863 & $33,390,964$ & $22,182,280$ \\
1864 "1867 & $41,466,292$ & $33,288,233$ \\
1868 " 1871 & $60,018,071$ & $40,253,099$ \\
\hline
\end{tabular}

A considerable quantity of that reported as coal is lignite or brown coal, of which there are very large deposits in Austria, 
Swkips. - Anthracito is found in small quantities at Dannemora ; and bituminous coal, probably of Liassic or of oolitic age, is worked at Helsingborg, at the entrance of the Baltic.

Dewsark. - The island of Bornholm and some other islands belonging to Denmark. produce coal, but it would appear to belong to the brown coal variety.

Russis.- The Donetz coal-field is the most important. In that extensive district many good seams, according to Sir R. I. Murchison, of both bituminous and anthracitic coal exist.

TORKEY, - Coal is found bordering on the Carpathian mountains, in Servia, Roumelia, and Bulgaria.

The coal of Heraclia, on the south coast of the Black Sea, in Anatolia, has been, since the Crimean war, exciting much attention.

Syrrana. - Moderately good coal is found at Nazli near Olidin. There are other coal-mines of very inferior quality.

Graccs.-There is no coal, properly so called, known to exist in Greece, but lignite has been found in several places.

North America. - The coal area of the United States of America, ${ }^{1}$ is thus described by Professor C. H. Hitchcock, Hanover, N.H., U.S.A.

There are eight distinct areas of the Coal-measures in the United States. They are-(1.) The New England Basin. (2.) The Pennsylvania Anthracite. (3.) The Appalachian Basin. (4.) The Michigan Basin. (5.) The Illinois Basin. (6.) The Missouri Basin. (7.) The Texas Basin. (8.) Areas of unknown extent, probably inconsiderable, in the Rocky Mountain Region.

1. The New England Basin.-This is in Massachusetts and Rhode Island estimated to cover 750 square miles. The eoal is a plumbaginous anthracite, used to advantage in some smelting furnaces. Perhaps eleven beds exist, best seen in Portsmouth, R.I. Their maximum thickness is $23 \mathrm{ft}$. The coal-measures are about $2,500 \mathrm{ft}$. thick. ${ }^{2}$

2. The Pennsylvania Anthracite. - This is the most important coal district in the United .States. Including the Broad Top semi-anthracite, of 24 square miles, the five separate basins amount to 434 square miles. The measures are from $2,000 \mathrm{ft}$. to 3,000 ft. thick. The number of distinet beds varies from two to twenty-five, according to the depth of the basin. The maximum thickness at Pottsville is $207 \mathrm{ft}$, while the average cannot be far from $70 \mathrm{ft}^{3}$

3. The Appalachian Basin.-This occupies an area of 63,475 square miles, extending from Pennsylvania to Alabama, all of bituminous coal.

In Pennsylvania the area amounts to 12,222 square miles, with an average thickness of $40 \mathrm{ft}$. of coal. The aggregate thickness of the measures varies from $825 \mathrm{ft}$. to $2,535 \mathrm{ft.}^{3}$

In Maryland the area is 550 square miles, in three separate baeins. The strata are 1,500 ft. thick. There are thirty-two beds of coal, one of $14 \mathrm{ft}$, three of $6 \mathrm{ft}$. each, and the others from $1 \mathrm{ft}$. to $5 \mathrm{ft}^{4}$

In West Virginia (with a little in Virginia) the coal area oceupies 16,000 square miles. On the Kanawha River the strata are 1,250 ft. thick, with twenty-four beds of coal, of which eleven have an aggregate thickness of $51 \mathrm{ft}$. The coals are best developed along this river. ${ }^{\circ}$

In Ohio Dr. J. S. Newberry mentions that the ares is greater than 10,000 square miles, with a thickness of $1,500 \mathrm{ft}$. of sediment, and ten workable beds of coal.

In Eastern Kentucky the ares is stated to be 10,000 square miles. ${ }^{3}$

In Tennessee the area of the measures is 5,100 square miles. A characteristic section gives a thickness of $578 \mathrm{ft}$. There are seven beds of coal, with a total thickness of $14 \mathrm{ft}$. The beds vary locally in their dimensions, more than has been reported elsewhere; perhaps more carefully explored. ${ }^{6}$ The conviction is increasing among American geologists that coal-beds are not evenly persistent on large areas, and constantly vary in thickness.

In Georgia the area cannot be more than 170 square miles.

In Alabama, a hasty measurement of a map furnished in manuscript by Professor Safford indicates an area of 9,000 square miles. The general character of the measures must be like those of Tennessee.

4. The Michigan Basin.-The area is about 6,700 square miles, with $123 \mathrm{ft}$. thickness of measures, and $11 \mathrm{ft}$. (maximum) of coal. In the centre the coal is thickest, thinning out to a mere line around the edges.?

2 'Geology of Island of Aquidneck,' by C. H. Hitcheock. Proc. Amer, Ass, $\Delta d$ d. Sci. 1860.

" 'Geology of Panngylvania,' by H. P. Rogers.

- 'First Report upon the Geology of Maryland, by P. T. Tysson.

' 'Report to Chesapeake and Ohio R.R.' by T.'s. Ridgway.

- 'Geology of Tennessee,' by James M. Safford.

"Geology of Michigan, 1861, by $\mathbf{A}$. Winchell.

" 'Final Report on the Geology of Illinois;' bj A. H. Worthen. 
5. The Tlinois Basin.-This occupies an ares of 51,700 square miles, including IIlinois, Indians, and Western Kentucky.

In Illinois the measures occupy an area of 41,500 square miles, are from $600 \mathrm{ft}$. to $2,500 \mathrm{ft}$. thick, and contain ten beds of cosl, with an aggregate thickness of $35 \mathrm{ft} .{ }^{1}$

In Indiana the measures occupy an area of 6,500 square miles, are $650 \mathrm{ft}$. thick, and contain thirteen beds of coal, with an aggregate thickness of $31 \mathrm{fto}^{1}$

In Western Kentucky ' the measures are $612 \mathrm{ft}$. thick, including the Millstone grit. They contain eleven beds of coal.

6. The Missouri Basin.-This is the largest of all the areas, comprising more than 100,000 square miles. It is also reached by several navigable rivers. It extends from Iowa to Texas. It is separated from the Texas field only by cretaceous beds, which probably overlie coal-beds continuous from one basin to the other.

In Iowa, ${ }^{2}$ Professor White's late map shows an area of about 25,000 square milis of coal-measures. He has divided the group into three divisions, each about $200 \mathrm{ft}$. The two lower ones contain the workable coal, which amounts to $8 \mathrm{ft}$. in the second, and only $20 \mathrm{in}$. in the upper division. As the lower divisions everywhere pass under the higher, the whole area may be regarded as productive.

Nebraska contains 3,600 square miles of the Upper Coal-Measures, according to map in 'Final Report upon the Geology of Nebraska,' by F. V. Hayden.

In Missouri (private communication) Professor G. C. Swallow estimates the coal area at 27,000 square miles, and in Kansas at 17,000 square miles. The measures are $2,000 \mathrm{ft}$. thick, carrying twenty coal-beds, from a few inches to $6 \mathrm{ft}$. thick.

In Arkansas, Dr. D. D. Owen ${ }^{3}$ describes two coal-beds, the thickest $5 \mathrm{ft}$. thick, and very valuable. The area is stated to be 12,000 square miles. The cosl, however, underlies the conglomerate, and does not belong to the true coal-measures.

Scarcely anything is known of the coal in Indian territory. From the map, the area must be as great as that of Arkansas. I am assured by the officers of the Missouri, Kansas, and Texas Railway that they find good beds of coal all through the territory near their line of travel.

7. The Texas Basin.-This is barely separable from the preceding. Dr. B. F. Shumard estimated the coal area at 5,000 square miles. A. B. Roessler ${ }^{5}$ estimates the same at 6,000 square miles. Beds of good conl are reported at Fort Bilknop, $4 \mathrm{ft}$. in thickness.

8. Mr. G. K. Gilbert, ${ }^{6}$ of the expedition under the immediate clirection of Lient. George M. Wheeler, reports coal near Camp A pache, in Axizona. Carboniferous strata are known in many places in the mountainous territories, and valuable discoveries of coal may be looked for among them at no distant day.

The total coal area as thus described amounts to 230,659 square miles. No notice is to be taken of any coals which do not belong to the Carboniferous system. There are many others of commercial importance, as the Triassic of Virginia, the Cretaceous of the territories west of the Missouri River, an immense amount in California, Alaska, \&ce.

These facts will afford data for those who are interested in estimating the amount of coal in different countries by the number of cubic miles or tons. The statements are too brief to permit any notice of the best or of the inferior cosl.

The anthracite coal-fields of Pennsylvania are divided into three great distriets, viz.: 1st, Southern or Schuylkill district, embracing the Lehigh and Lykens valley coal ; 2nd, the Middle district, embracing the Beaver Meadow, Shamokin and Trerorton coal; the 3rd, Northern or Wyoming and Lackawanna, embracing the Seranton, Pittston and Lackawanna coals.

Produce of these districts from 1858 to 1863.

\begin{tabular}{|c|c|c|c|c|}
\hline Years & Schuylkdl District & Middle District & Wyoming District & Total \\
\cline { 5 - 5 } & tons & tons & tons & tons \\
\cline { 5 - 5 } 1858 & $3,212,879$ & 909,000 & $2,186,094$ & $6,524,838$ \\
1859 & $3,598,531$ & $1,050,659$ & $2,731,236$ & $7,517,516$ \\
1860 & $3,815,822$ & $1,091,032$ & $2,856,896$ & $8,059,017$ \\
1861 & $3,114,254$ & 994,705 & $2,918,458$ & $7,487,672$ \\
1862 & $3,549,844$ & 396,227 & $3,13,480$ & $7,640,905$ \\
1863 & $4,151,882$ & 609,548 & $3,760,374$ & $9,420,135$ \\
\hline
\end{tabular}

: 'Second Report on the Geology of Indiana,' by E. T. Cox,

" 'Geology of Iowa, by C. A. White.

' 'Second Report, Geology of Arkansas,' by D. D. Owen.

- 'Teras Almanack for 1861."

s 'Texas Almanack for 1872,

- Oflicial Report. 
Total produce of Pennsylvania in 1864 :-

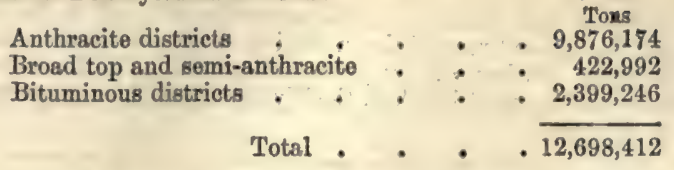

Coal product of the Loyal United States, year ending June 1864, from the returns made to the Internal Review Department :-

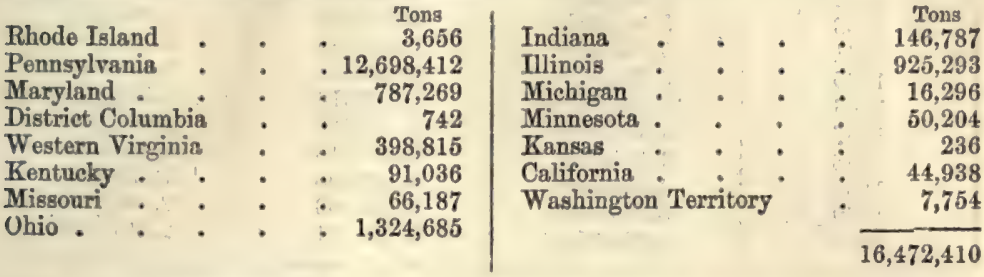

The following summary of the condition of the American coal trade in 1871 is of interest:-

The quantity of anthracite coal sent to market during the year 1871 is returned officially at $14,965,501$ tons, and the quantity consumed in the coal regions is estimated at 2,720,000 tons. The quantity of bituminous coal sent towards the seaboard, including 443,955 tons imported, is returned officially at $4,894,914$ tons, and the quantity of bituminous mined and consumed in the United States, but not at present returned in the official tables, is estimated unofficially at $11,500,000$ tons. The aggregate production of the year thus comes out at $34,081,415$ tons, of which all but 269,751 tons exported, was retained as the supply of the Republic for the year. At this rate the average coal production of each American amounted last year to about 17 cwts. per head, while in 1869 the average coal production of each inhabitant of tho United Kingdom was as nearly as possible $3 \frac{1}{2}$ tons per head. It is clear from this comparison, that, great as the coal wealth of the United States is supposed to be, A merican coal-mining is still in its infancy; while the corresponding branch of industry has been carried on in Great Britain to an extraordinary development. Tho openings for human effort are so numerous in the United States, that no very large amount of the population can be induced to apply themselves to mining industry - at any rate at present; and hence the average coal extraction of the United States per head of the population is only about one-fourth of the corresponding extraction in Great Britain. Far-seeing and thoughtful minds may come to the conclusion that, after all, there is no very great ground for national complacency, at the results brought out by the comparison, sinee it may be argued that we are rapidly working up our coal supplies, while the Americans are husbanding theirs. It should, perhaps, be xemarked that we have accepted the American statisties as to the American coal trade as they have come upon our hands. At the same time it cannot be denied that they are characterised by great ragueness, one-third of the whole total of $34,081,415$ tons being derived from unofficial estimates.'

Although the returns are fairly open to the imputation of vagueness, and uncertainty as a whole, they are tolerably conclusive and satisfactory, however, as respects the anthracite coal production of the United States. The total of $14,965,501$ tons already given as representing this production in 1871, compares with $15,274,029$ tons, the production of 1870 ; so that the extraction fell off in 1871 to the extent of 308,528 tons - a result attributable, we presume, to the prolonged and troublesome strikes which prevailed last year in the Pennsylvania coal regions. The two totals of $14,965,501$ tons and $15,274,029$ tons include the semi-anthracite production of tho Lykens Valley in each year, and they were mado up as follow :-

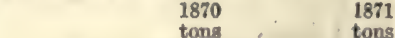

Schuylkill, Columbia, \&c. ․ . $3,720,403 \quad 5,124,780$

Northumberland . . . . $\quad 486,174 \quad 628,866$

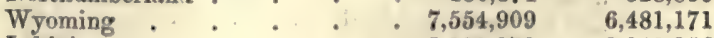

Lehigh $\quad . \quad 2,2,990,878 \quad 2,249,356$

Lykens Valley (semi-anthracite) $\ldots \quad 521,665 \quad 481,328$

Total . . . 15,274,029 14,965,501 
It will be observed that there was a considerable increase in the production of the Schuylkill region last year, while the extraction fell off in the Wyoming and Lehigh regions : the general result being a decline of 308,528 tons in the last year, as compared with 1870 . During the ten years ending with 1871 , inclusive, the production of anthracite coal in the Schuylkill, the Wyoming and the Lehigh regions appears to have been as follows:-

\begin{tabular}{|c|c|c|c|}
\hline Year & Schuylk11 & Wyoming & Lohigh \\
\hline 1862 & $2,890,598$ & $3,145,770$ & $1,351,054$ \\
1863 & $3,443,265$ & $3,759,610$ & $1,984,713$ \\
1864 & $3,642,218$ & $3,960,836$ & $2,054,669$ \\
1865 & $3,735,802$ & $3,256,658$ & $1,822,535$ \\
1866 & $4,633,487$ & $3,736,616$ & $2,128,867$ \\
1867 & $4,334,820$ & $5,328,329$ & $2,062,446$ \\
1868 & $4,414,356$ & $5,990,813$ & $2,507,582$ \\
1869 & $4,748,960$ & $6,068,395$ & $1,929,583$ \\
1870 & $3,720,403$ & $7,554,909$ & $2,990,878$ \\
1871 & $5,124,780$ & $6,481,171$ & $2,249,356$ \\
\hline
\end{tabular}

The aggregate extraction of each year will, however, illustrate more conclusively the progress which has been made. The figures come out as follow :-

\begin{tabular}{|c|c||c|c|}
\hline Years & Tons & Years & Tons \\
\hline 1862 & $7,387,422$ & 1867 & $11,725,588$ \\
1863 & $9,187,588$ & 1868 & $12,912,751$ \\
1864 & $9,657,723$ & 1869 & $12,746,988$ \\
1865 & $8,814,995$ & 1870 & 14,$266 ; 190$ \\
1866 & $10,498,970$ & 1871 & $13,855,307$ \\
\hline
\end{tabular}

It will be observed that the production very nearly doubled itself during ten years. It would, probably, have completely doubled itself but for the strike troubles of last year. In this current 1872 it is expected that the ground lost last year will be more than recovered; and that the progress realised will be considerable, as the aspect of the labour market has become more settled. Provision has also been made for an arbitration and conciliation tribunal to adjust any difficulties which may present themselves.

British America contains coal in the provinces of New Brunswick and Nova Seotia. The former presents three coal-fields, occupying in all, no less than 8,000 square miles; the latter exhibits several very distinct localities where coal abounds. The Now Brunswick coal-measures include not only shales and sandstones, as is usual with such deposits, but bands of lignite impregnated.with various copper ores, and coated by green carbonate of copper. The coal is generally in thin seams lying horizontally. It is chiefly or entirely bituminous.

Nova Scotra possesses three coal regions, of which the Northern presents a total thickness of no less than 14,570 feet of measures, having 70 seams whose aggregate magnitude is only $\mathbf{4 4}$ feet, the thickest beds being less than four feet. The Pietou or central district has a thickness of 7,590 feet of strata, but the coal is far more abundant, one seam measuring nearly 30 feet; and part of the coal being of excellent quality and adapted for steam purposes. The southern area is of less importance. Besides the Nova Scotia coal-fields, there are three others at Cape Breton, yielding different kinds of coal, of which one, the Sydney coal, is admirably adapted for domestic purposes. There are here 14 seams about 3 feet thick, one being 11, and one 9 feet.

The areas of these coal-fields are respectively:-

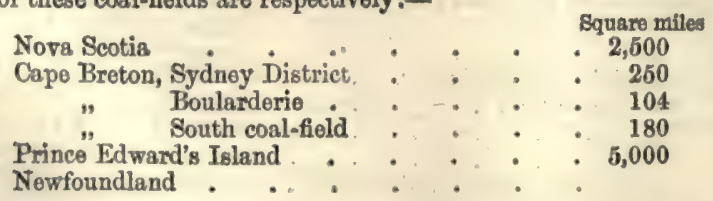

The production of coal in Nova Scotia in the year 1865 to 1870 was as given in the following Table:- 


\begin{tabular}{|c|c|c|c|c|c|c|c|}
\hline \multirow[t]{2}{*}{ Plnoes } & & \multicolumn{2}{|c|}{$\begin{array}{c}\text { 1866, } \\
\text { Year ending } \\
\text { September } 30\end{array}$} & \multicolumn{2}{|c|}{$\begin{array}{l}\text { 1868, } \\
\text { Year ending } \\
\text { September } 80\end{array}$} & \multicolumn{2}{|c|}{$\begin{array}{l}\text { 1869, } \\
\text { Year ending } \\
\text { September } 30\end{array}$} \\
\hline & & Round & Slack & Round & Slack & Round & slack \\
\hline $\begin{array}{l}\text { Cumberland } \\
\text { Pictou. } \\
\text { Cape Breton } \\
\text { Inverness } \\
\text { Richmond } \\
\text { Victoria }\end{array}$ & \begin{tabular}{ll|} 
& $\vdots$ \\
$\vdots$ & $\vdots$ \\
$\vdots$ & $\vdots$ \\
$\vdots$ & $:$
\end{tabular} & $\begin{array}{r}\text { tons } \\
15,008 \\
183,244 \\
325,515 \\
2,093 \\
739 \\
7,828\end{array}$ & $\begin{array}{r}\text { tons } \\
1,441 \\
22,485 \\
14,065 \\
1,206 \\
223 \\
453\end{array}$ & $\begin{array}{r}\text { tons } \\
9,093 \\
126,642 \\
276,045 \\
131 \\
12 \\
1,633\end{array}$ & $\begin{array}{r}\text { tong } \\
1,010 \\
18,209 \\
20,696 \\
\ldots . \\
8 \\
141\end{array}$ & $\begin{array}{c}\text { tons } \\
7,648 \\
175,286 \\
287,027 \\
292 \\
\ldots . . \\
372\end{array}$ & $\begin{array}{c}\text { tons } \\
867 \\
22,925 \\
17,292 \\
40 \\
\ldots . \\
45\end{array}$ \\
\hline Total . & . & 561,428 & 39,873 & 413,557 & 40,067 & 470,625 & 41,169 \\
\hline \multirow{2}{*}{\multicolumn{4}{|c|}{ Places }} & \multicolumn{2}{|c|}{$\begin{array}{l}\text { 1870, } \\
\text { Year ending } \\
\text { September } 30\end{array}$} & \multicolumn{2}{|c|}{$\begin{array}{l}\text { 1872, } \\
\text { Year ending } \\
\text { Decomber } 31\end{array}$} \\
\hline & & & & Round & Slack & Round & Slack \\
\hline $\begin{array}{l}\text { Cumberland } \\
\text { Pictou } \\
\text { Cape Breton } \\
\text { Inverness . } \\
\text { Richmond . } \\
\text { Victoria . }\end{array}$ & : & $: \quad:$ & $\begin{array}{l}\dot{ } \\
\dot{0} \\
\dot{.}\end{array}$ & $\begin{array}{c}\text { tons } \\
6,906 \\
191,465 \\
306,097 \\
15 \\
\ldots . \\
271 \\
\end{array}$ & $\begin{array}{c}\text { tons } \\
978 \\
35,059 \\
27,480 \\
\quad 3 \\
\ldots . \\
\cdots \\
\end{array}$ & $\begin{array}{c}\text { tons } \\
13,272 \\
340,142 \\
360,036 \\
2,879 \\
\cdots \\
\cdots \\
\end{array}$ & $\begin{array}{c}\text { tons } \\
881 \\
48,275 \\
20,237 \\
191 \\
\ldots \\
\ldots \\
\end{array}$ \\
\hline \multicolumn{2}{|c|}{ Total } & • & . & 504,756 & 63,520 & 616,329 & 69,584 \\
\hline
\end{tabular}

Britisu Columbia. - The following is the report of Mr. Hilary Bauerman in 1860 :

Two seams of coal, averaging six to eight feet each in thickness, occur and are extensively worked for the supply of steamers plying between Victoria and Frazer's River.

The coal is a soft black lignite, of a dull earthy fracture, interspersed with small lenticular bands of a bright crystalline coal, and resembles some of the duller varieties of coal produced in the South Derbyshire and other central coal-fields in England.

In some places it exhibits the peculiar jointed structure, cansing it to split into long prisms, observable in the brown coal of Bohemia.

For economic purposes these beds are very valuable. The coal burns very freely, and yields a light pulverulent ash, giving a very small amount of slag and clinker.

NEWFOUNDIAND COAL-FIBID.-This field is estimated at about 5,000 square miles. According to Mr. Jukes, formerly Director of the Geological Survey in Ireland, the entire western side of the island, along a space of 356 miles in breadth, is occupied by secondary and carboniferous rocks. The coal on the south-western point of the island has been traced at intervals along a space of 150 to 200 miles to the northeast.

Greencard. - Captain Scoresby discovered a regular coal-formation here. At Hasen Island, lignite or brown coal has been found, and also at Disco Island on the western coast.

Arctic Ocnas.--At Ryam Martin's Island coal-formations exist, and at Melville Island several varieties of coal have been discovered, much of it being of an anthracitic or of a semi-anthracitic character. We learn that at Prince Regent's Inlet indications of coal have been observed.

Russian America, - Beyond the Icy Cape and at Point Barrow coal was observed on the beach; and it has been found by digging but a few feet below the surface at Point Franklin.

Oregon TrRritony.-Coal has been discovered and worked in Wallamette valley, nearly 100 miles above Oregon City; and anthracite has been observed by Sir George Simpson about 30 miles up one of the tributaries of the Columbia river.

U MTTED STATES of Colombia.-Immense deposits of cannel coal have been digcovered in the Province Rio Hacha.

Caciforyia, - Colonel Fremont states that a coal-formation exists in Upper California, north lat. $41 \frac{1}{2}$, and west long. $107 \frac{1}{2}{ }^{\circ}$ : - ' The position of this coal-formation is in the centre of the Rocky Mountain chain, and its elevation is 820 feet above the 
level of the sea. In some of the coal-seams the coal did not appear to be perfectly mineralised, and in others it was compact and remarkably lustrous.'- Fremont's Report, 1843.

In 1847 a coal-mine was discovered near San Luis Obisco, North lat, $35^{\circ}$. There are three coal-mines within $\mathbf{3 0 0}$ miles of Monterey.

Mexico. - On Salado river coal is worked by an American company. A coal. formation 50 miles in breadth crosses the Rio Grande from Texas into Mexico at Loredo; and on the Mexican shore, within 200 yards of the Rio Grande, a remarkably fine voin of cosl 8 feet thick occurs.

TExAs.-Coal is known to exist in Texss, though the country has not been geologically examined. The 'Trinity Coal and Mining Company' was incorporated by the Texan Congress in 1840, who worked both anthracite and a semi-bituminous coal. Kennedy in his work 'Texas, its Geography, \&c.,' says 'Coal, both anthracite and bituminous, abounds from the Trinity River to the Rio Grande.'

Sovtr Amertca, - In the Republic of Now Granada, especially at Santa Fé do Bogotá, coal occurs: also in the island of Santa Clara, and brown coal in the province of Panama.

Vexezukra is said to contain coal, but whether brown or bituminous does not appear certain.

Peru appears to possess some coal, but a fossil charcoal of considerable value is more abundant.

Chmi.-The coal of this district has been examined by many American engineors, and by Captains Fitzroy and Beechy and Mr. Darwin. In 1844 upwards of 20 coalminos were open in the neighbourhood of Conception. At Tulcahnano a new seam of $4 \frac{1}{2}$ feet was proved. The coal is deseribed by W. R. Johnson as, 'in external appearance nearly related to many of the richest bituminous coals of America and Furope;' and Mr. Wheelwright, in his report on the mines and coal of Chili, says, 'in fact, the whole southern country is nothing but a mine of colal.'

BRAzIL does not possess much cosl of value.

The following communications from John Miers, Esq., F.R.S. and from N. Plant, Esq., give all that is known respecting the two coal-fields of Brazil :-

- The coal-mine for which the Visconde de Barbacena obtained a privilege is in the province of Sta. Catherina, where extensive deposits are found in a district near the junction of two small rivers flowing from the Serragerul de Sta. Catherina, and which unite to form the Rio Tuberao, which runs eastward for about 70 miles, when it enters the Lake of Laguna, in lat. $28^{\circ} 23^{\prime} \mathrm{S}$. The lake or bay is sufficiently deop and capacious, and is available for sea-gaing ressels with a draft of 11 feet, which is the depth of water over the bar in its mouth. The Rio Tuberao is navigable by ressels of that capacity as far as Piedade, which is 27 miles from the point of its entrance into the bay; but above that distance the river is only navigable by smaller craft for a few miles; so that Piedade will be the shipping port for the coal, which will have to be conveyed by a tramway from the mine, 45 miles long, along a course nearly due east and west, upon a gentle gradient. At that distance no less than 20 different sites have been seen on both sides of the Tuberao where the coal depusits erop out, the river running over several beds. This district is situated in a beautiful and well-wooded country, about 880 English feet above the level of the sea, and upon the northern flanks of the Morro de Sta. Martha or de Congouhas, a spur of the main serra that extends for a long distance parallel with the coast. This spur runs eastward, and terminates at a point 4 leagues south of Laguna. This coal-formation is evidently of considerable extent, for similar deposits are found on the southern flanks of the same spur, near the sources of the Rio Ararangua, which runs eastward, and falls into the ocean in lat. $28^{\circ} 48^{\circ} \mathrm{S}$., and which is navigable for a distance ef 20 miles to Tresportes, where it is broad and deep. The Visconde de Barbacena's mining ground is on the Rio Bonito, an affluent of the Tuberao, where the coal shows itself wherever the surface of the hills has become denuded by gullies formed by springs, presenting cliffs, 40 feet high. Here the coal-deposits are visible to a depth of from 10 to 14 feet, and these are covered by a roofing of hard sandstone 20 feet deep. Immediately below the upper bed is another layer of coal 6 inches deop; then a stratum of greyish schist with fossil regetable remains, succeeded by a bed of good solid coal 8 feet thick; then a layer of black sehist, and another bed of coal 1 foot thick. How much deeper these beds may be is unknown, as the ground has not been bored; but it is probable that doep beds of more valuable conl exist. There is, however, a sufficient store above ground to admit of extensire workings without the necessity of sinking shafts, or of the ordinary expense of raising the coal to the surface by means of steam machinery. The existence of a thick sandstone roofing, containing vegetable impressions, offors good promise as regards this coal-formation. The samples exhibited in the Paris Fxhibition brought from this mine were much broken by long carriage, but they showed a tolerably bright coal, 
of a very laminated structure, with indications of pyrites scarcely visible; but it is not unlikely that seams yet undiscorered may elicit a coal of a still better quality.

- The second sample of Brazilian coal exhibited in Paris was scarcely equal in quality to the former, and seemingly was more slaty and more charged with pyrites. This was brought from a mine in the provinee of Rio Grande do Sul, which has been scantily worked for soveral years, but hitherto with little encouragement. It is situated on the Arroyo dos Ratos, a small stream that runs into the Rio Jacuahy, about half a league distant from Saō Jeronymo, which is near 16 leagues to the westward of Porto Alegre, the capital of the province, and which has an excellent water conveyanco between it and the seaport town of Rio Grande; but with all the advantages of easy transport, the coal has not been able to obtain a good reputation among the owners of the numerous steam vessels that are constantly plying between the places above mentioned, for which traffic English coal, notwithstanding its high price, is also preferred.

- There is another extensive deposit of coal in the same province, and which is said to be a coal of very good quality; but its distance, too far inland, and the want of water conveyance, are said to be great obstacles against the probable success of any attempt to work it in the present state of the country. Mr. N. Plant thus describes them :-

'The only localitios on the eastern coast of South America, between the river Amazon and the river Plate, where the existence of coal has actually been determined are the two southern Brazilian provinces of Saō Pedro do Rio Grande do Sul and Santa Catherina, and the neighbouring Republic of Banda Oriental, or Uruguay.

- Lignite, brown coal, and bituminous schists, to which the name of "carvão de pedra," stone coal, has been applied in Brazil occur in thin beds along the coast and in the interior; but it is only in the two extreme southern provinces and the adjoining Republic of Uruguay that beds of bituminous coal containing palæozoic fossils have yet been discovered. Nor should I think it likely from the observations I have made during many years in nearly every province of the Brazilian. Empire and the Republics of the River Plate, that coal of so early an age as even the lower oolitic rocks will ever be found in Brazil north of the province of Sta. Catherina, unless it be in the adjoining one of Paraná.

"In the province of Sao Pedro do Rio Grande do Sul, three distinet coal-basins have been discovered, and the extent and thickness of the beds of coal to some degree determined. The district in which these coal-deposits are found is contained within the limits of latitude $30^{\circ}$ and $32^{\circ}$ and long. $51^{\circ}$ and $54^{\circ}$. The basins are separated from each other by rolling hills of granite, syenite, and mica schist.

- The largest of these coal-basins is perhaps that occupying the valleys of the rivers Jaguarao and Candiota, between lat. $31^{\circ}$ and $32^{\circ}$ and longitude $53^{\circ}$ and $54^{\circ}$, ealled the Candiota coal-field. A detailed report of this appeared in the "Reports from Her Majesty's Secretary of Embassy and Legation respecting Coal, 1867." Along a line ranging from N.E. to S.W. the upper coal-beds of this deposit can be seen at intervals for about 50 miles, where the superincumbent sandstone has been denuded, or the strata worn through by streamlets, and the same may be observed from N.W. to S.E. for about 30 miles.

'The falling away of one side of a hill some years ago on the banks of the River Candiota, near the basset-edge of the basin, laid bare five beds of coal, varying in thickness from 9 feet to 25 feet, giving 65 feet as the total thickness of coal exposed. The coal from the lower beds, which are also the thickest, is highly bituminous; but that from the upper is shaly and poor.

"The second basin lies in the valley of the Sta. Sepe, one of the tributaries of the River Jacuahy, in about lat. $30^{\circ} 20^{\prime}$ and long. $53^{\circ} 30^{\prime}$. Two beds appear here, one of 7 feet and the other of 14 feet, which have been traced over an area of some 15 miles, and along the margins of other neighbouring streams. The coal in this deposit underlies sandstone, like that of Candiota.

'The third basin is near Saö Jeronymo, a town on the banks of the River Jacuahy, in about lat. $30^{\circ}$ and long. $51^{\circ} 30^{\prime}$. This is the only coal-field being worked at the present moment on the eastern side of the Andes, on the South American continent. Although the beds of coal in this deposit are of less thickness than those of Candiota and Sta. Sepé, the nearness of the basin to the port of embarkation at the town of Saó Jeronymo, which is only eight miles distant from the coal, ronders the cost of land carriage very trifling, compared to what it would be from the other two fields, to a navigable river.

- Two shafts have been sunk in this basin to a bed of coal 6 feet thick, and a horseway made into it, along which the coal is drawn to the surface. Borings have been made in different parts, by which it has been ascertained that the wholo basin spreads over an area of about 20 miles, and that other bods of coal exist under the 6-feot soam. 
- Owing to the want of miners and machinery to work this mine on a large scale, the arerage amount of coal being taken out at present does not exceed 200 tons per month, which is barely sufficient to supply the stuamers navigating the River Jacuahy. This river falls into the Lago dos Patos, which has its outlet in the Atlantic Ocean at the seaport of Rio Grande do Sul. The coal is said to be as good for steam purposes as Newcastle coal, with the exception of leaving more ash.

- The coal-beds in the Republic of Banda Oriental are situated on the head waters of the river Negro, between lat. $31^{\circ}$ and $32^{\circ}$, long. $54^{\circ}$ and $55^{\circ}$, where they can be traced for several miles along the margins of the river and some of its tributaries; the beds vary in thickness from 3 to 6 foet.

'The cosl-deposits actually determined and surveyed in the province of Sta. Catherina are at Boa Vista and at the river Tubarao, at "Passa-dous," between lat. $28^{\circ}$ and $29^{\circ}$, long. $49^{\circ}$ and $50^{\circ}$. Here beds of cosl of considerable thickness can be seen along the margin of the river, and appear to extend over an area of several leagues, though the limits of the deposits have not yet been ascertained. The fossil plants found in this coal are of the same character as those found in the ironstone shales interstratifying the coal-beds on the River Candiota in the province of Kio Grande do Sul.

'Beds of bituminous schist extend over considerable areas at Missão da Corda, on the river Mearim, and in the valley of the river Itapicuru in the province of Maranhão, and, associated with lignite, it is found along the margin of the river Camaragibe in the province of Alagoas, also at the mouth of the river Camamú in Bahia, and in various parts of the province of Pernambuco. From experiments I have had made in England, these schists yielded from 15 to 20 per cent. of crude oil, though some specimens I saw in Pernambuso were so rich in oil as to readily ignite when applied to the flame of a candle.

- Lignite and brown coal are found in thin isolated beds in many places along the coast of Brazil, and in the provinces of Minas Geraes, Goyaz, and in the valleys of the Ribeira de Iguape and Tieté in Saō Paulo.'

The West INDIAN Iscaxds. Cuba, in the vicinity of Havannah, produces a kind of asphaltum much resembling coal, the analysis of which gives, carbon $34 \cdot 97$, volatile matter 63.00, ashes 2.03. At Now Havannah a similar combustible is found; but it contains 71.84 of carbon. True coal does not appear to have been found in Jamaica. Sir H. de la Beche ('Trans. Geological Society of London'), describes three or four thin seams of coal imbedded in shale near the north-eastern extremity of the island.

BarBaDors. - Bitumen is found plentifully: and, on Grove Plantation estate, a good coal is atated to have been found.

TrinIDaD. - The pitch lake of this island is well known. Near it, and it is believed, extending under it, a true coal of superior quality is worked.

India.- From the 'Memoirs of the Geological Survey of India,' we extract the following authentic information as to the coal resources of India :-

\section{Coal Resources of India.}

1. Rajmahal Hills.-In this district coal-deposits occur in many places. In the basin of the Brahmini, four or five seams are known, varying in thickness from 3 to 12 feet. In the valley of the Bansloi stream further north, several valuable beds of coal also occur, and in the Goomani Valley, and towards the north-western end of the range near Rorah. While the East Indian Railway was being constructed, many of these beds were worked; but since the opening of the line, very little has been done. This railway should have opened a trade from this coal-field to Calcutta.

2. Raneegunge. - This coal-field is at a distance of 120 to 160 miles N.W. of Calcutta; its greatest length being nearly E. and W. about 30 miles, and the greatest breadth nearly $\mathrm{N}$. and $\mathrm{S}$. about 18 miles. The area of coal-bearing rocks is about 500 square miles.

The coal of this field is a non-coking bituminous coal, composed of distinct laminæ of a bright jetty coal, and of a dull more earthy rock. The average amount of ash is some 14 or 15 per cent., varying from 8 to 25 per cent. The western end of the coalfield produces coking coal at Sanktoria.

The Raneegunge coal-field has the advantage of two branches of the Indian Railway, which traverse its richer portion. Professor Oldham has estimated that 14,000,000,000 tons are fairly obtainable from this coal-field.

3. Kurhurbali. - Situated north of the river Barakur, about 80 miles south of the Luckieserai station of the East Indian Railway. Its greatest length is 61 miles; its breadth nowhere being more than 23 miles. There are several beds of good coal from 8 to 14 feet thick. The coal of Kurhurbali is of better quality than most of the 
Indian coal, and is used for locomotives by the East Indian Railway Company, who have purchased the greater part of the field. After carefully excluding one-fourth for loss, waste, \&c., it is estimated that $168,000,000$ of tons of coal remain in this field.

4. Jherria.-This coal-field extends along the valley of the Damudah river. Its greatest length is 21 miles, in an $\mathrm{E}$. and $\mathrm{W}$. direction; its maximum breadth is about 9 miles $\mathrm{N}$. and S. Total area 200 square miles. It is roughly calculated that this field would yield $465,000,000$ tons.

5. Bokaro.-This coal-field is but a mile west of the Jherria coal-field. It extends along the valley of the Damudah, forming a long narrow band of coal-bearing rocks of more than 40 miles from $\mathrm{E}$. to W.; its breadth $\mathrm{N}$. and $\mathrm{S}$. never exceeding $7 \frac{1}{2}$ miles. It is a poor coal, used only for brick and lime burning; but much has been raised and carted to Hazareebagh, and even to Gya. It is estimated that $1,500,000,000$ tons of cosl are in this field.

6. Ramgurh.-This small field occupies a triangular space. Near Ramgurh the breadth in a N. and S. direction is only a few hundred yards. It extends eastward about 12 miles, and at the eastern end expands to a breadth of 6 miles. Available cosl $2,000,000$ or $3,000,000$ tons.

7. Hoharo and Kamimpoora.-The field is of large extent, covering a surface of at least 450 square miles. The South Karunpoora field is thought to occupy 120 square miles. These fields are poor in coal, and what does occur is of inferior quality.

8. Eetcoora.-Near Eetcoora, to the N.W. of Hazareobagh, coal occurs of poor quality, but usable for brick and lime burning.

9. Palamow.-The principal fields of coal occur along the valley of the Damudah and its tributaries. The actual limits of the field have not been determined, but the district is poor in coal, and if worked it will be only to supply local demand.

10. Singrowlie.-Not far to the west of the village of Singhpur the colliery of Kotah has for years been known, and a fair amount of good coal has been produced: Coal exists in eight or nine localities, spreading over an area of about 40 miles in length from E. to $W_{\text {, }}$, and 20 in breadth from N. to S. No details can be obtained.

11. Upper Sone.-The prospects of any large amount of coal being available from this district are not good. Some thin seams, apparently unworkable, were noted by the Geological Survey in the Mahanuddy Valley, near the district of Bijiragoogurh.

12. Hutsoo or Chotteesgurh. - Coal occurs in the Hutsoo near Koorba; in the Beoja Kurra, 25 miles N.W. of Koorba; and in the Labed, a few miles further in the same direction. It is said to extend 200 yards along the bed of the former, and about a mile in the latter. Coal was also found in the Chornai stream, a tributary of the Hutsoo.

13. Talcher.-There is but little coal in this field, and that little poor in quality. The total area of the coal-bearing rocks in the field is not more than 10 square miles. 'These coal-fields, in conjunction with the reported coal-fields near Chanda, on the Wurda, and also the reported coal-fields in the vicinity of Sumbhulpor, will in a very few years become of far higher value than they are now.' Oldham.

This opinion appears to be founded on the probability of the formation of a line of railway from Calcutta to Bombay. The Nerbudda Coal and Iron Company have their works near Mopani, which are connected by a branch, fourteen miles in length, with the main line of the Great Indian Peninsular Railway. The coal does not appear to be of a high quality, but still it is of considerable local importance. Many small thin seams exist in several localities within Tawa Valley of the Nerbudda district, but they are unimportant.

14. Pench River.-Coal occurs at Sirgori village: One seam was more than 4 feet thick, and another seam 2 feet thick. (2.) Chenda or Digawani.-A sedm with more than 12 feet of coal. (3.) Hurrye--A seam; thickness unknown. (4.) Rawunwarra. -One seam with 5 feet 4 inches of coal. A second with more than three feet of coal. (5.) Parassia.-Above five feet of coal. (6.) Bhundaria-Seam of 15 feet 2 inches, with more than 7 foet of good coal: (7.) Pootaria.-More than 5 feet of good coal. (8.) Burkoi,-About 6 foet of good coal. (9.) Gogri.-Sis feet seam, with 5 feet of coal.

This coal-feld is nearly 100 miles from Nagpoor; and this distance prevents any profitable working. Coal has only been raised from one seam, that at Burkoi. Since 1860 one colliery has been held on lease; but little has been done.

15. Chanda. - The existence of coal has been proved by borng, in the Wurdah Valley, and on the opposite side of the river. One or two sinkings have been commenced.

16. Kota.-Near Kota, on the Godavery, coal has been more than once reported.

17. Cutch.-The coal occurring at Cutch affords no hopes of proving valuable.

18. Scinde.-There is coal ; but 'as a source of fuel it was utterly worthless.'

19. Salt Range.-The Salt Range may, therefore, in my opinion be rejected from 
all calculation ' of the arailable supplies of good coal in India, excepting for immediately local demands, and for such common purposes as this inferior coal may suit.'

20. Murree, \&c.-Patches of coal and lignite only.

21. Darjeeling.--Coal-bearing rocks near Punkabaree, at the foot of the Darjeeling Hills, and nests of cosl and liguite.

22. Assam.-At Terap a minimum thickness of 5 feet of clean bright coal was seen. At Namchik, a tributary of the Deehing, within 200 feet in length, 3 thick beds of good sound coal were seen; one of them 8 feet in thickness. At Jaipur a seam of 17 feet occurs, of which 10 feet is a good coal. Several other seams exist; but from the inaccessible nature of the country it is not possible to obtain an approximate idea of the amount of fuel svailable. 'It can, however, be unhesitatingly asserted that this amount is very large and most valuable.'

23. Khasi Hills.-Good coal occurs, especially well adapted for making gas. Professor Oldham estimates the available coal of the Khasi Hills at $3,000,000$ tons. 'It is not improbable that double this quantity will prove to be near the true amount.'

24. Coal-measures exist in the Garo Hills; but the Geological Survey failed to find any coal. At (25) Cachar small pockets of coal occur. Similar samples occur at (26) Chittagong. In the island of (27) Cheduba a lignite is met with.

28. Burmah. - In British Burmah no workable coal is known to oceur. In Upper Burmah some irregular beds of inferior coal were examined by Dr. Oldham. "They hold out no prospect of supplying anything more than a very local and limited demand, and even this with inferior fuel.'

29. Tenasserim. - Beds of coal near. Mergui, in the Tenasserim Provinces, were examined by Prof. Oldham in 1854. They hold out little prospect of affording a large supply, while the coal is of inferior quality.'

Coal Distriots of India, with their Areas and estimated Coal Contents.

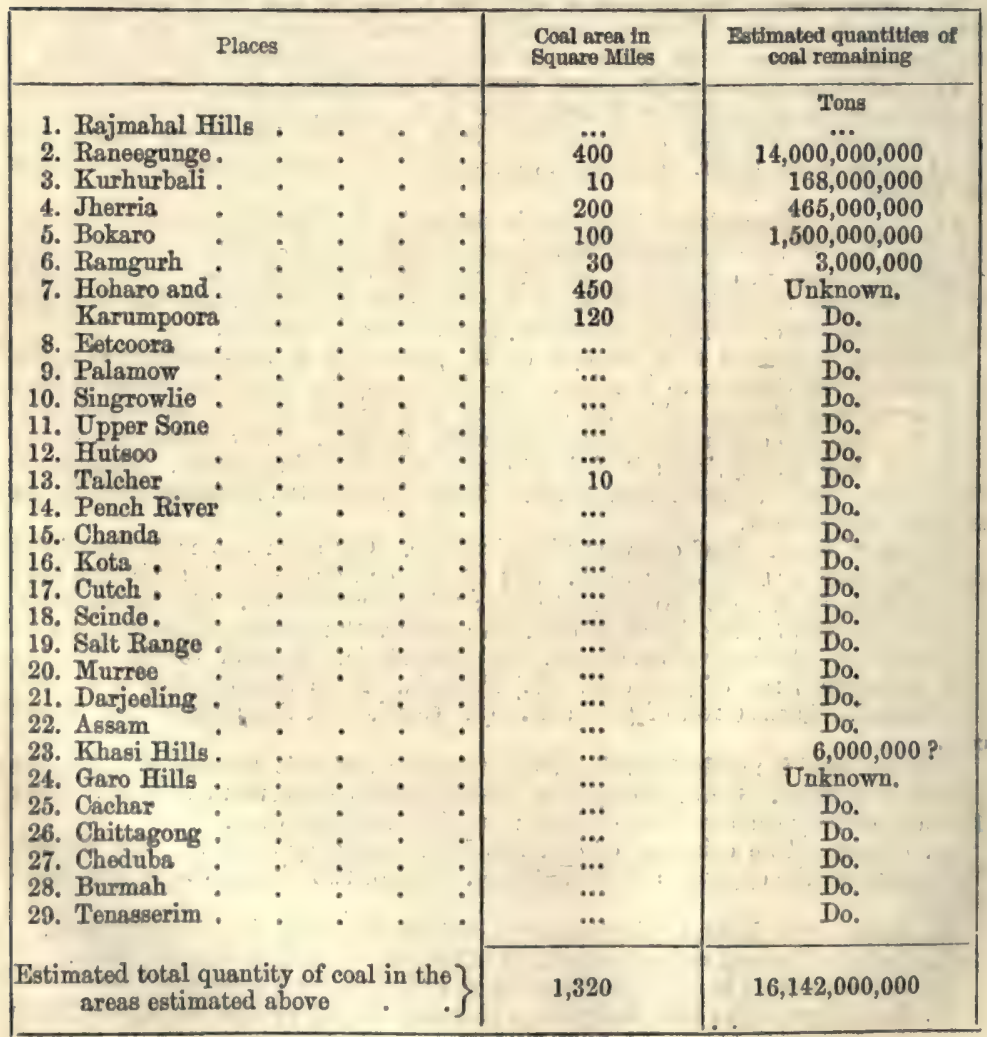


According to the Report by Dr. Oldham, the Assam coal-field must be regarded as the most important amongst those of which the coal areas cannot be satisfactorily ascertained. The inaccessible nature of the country renders it impossible to obtain an approximation to the ralue of its coal-formations; and from its situation, supposing some local industry leads eventually to the development of its native resources, tho coal of Assam can never come into competition with the fuel of other countries.

The Production of Coal in India from 1858 to 1866.

Compiled from Dr. Oldham's Report.

\begin{tabular}{|c|c|c|c|c|c|c|c|c|c|c|c|}
\hline Name of Coal-tield. & $\mid \begin{array}{l}\text { No. of } \\
\text { Col- } \\
\text { Heries }\end{array}$ & $\begin{array}{l}\text { Na. of } \\
\text { Pith or } \\
\text { Quarries }\end{array}$ & 1858 & 1859 & 1860 & 1861 & 1862 & 1803 & 1864 & 1865 & 1806 \\
\hline BANEEGUNCE, & & & Tons & Tons & Tons & Tons & Tons & Tons & Tons & Tons & Tons \\
\hline Valley & 22 & 43 & 32,220 & 86,994 & 80,792 & 83,060 & 105,191 & 104,222 & 114,561 & 124,162 & 169,313 \\
\hline Raniganj . & 11 & 31 & $\mid 181,119$ & 172,697 & 171,261 & {$[153,882$} & 166,727 & 184,778 & $\mid 163,838$ & 162,521 & 190,766 \\
\hline Nunia Valley $\mathbf{E}$ & 13 & 26 & 17,064 & 21,284 & 17,348 & 467 & 3,736 & 7,106 & 11,649 & 14,217 & 17,345 \\
\hline W" of the field and & 5 & 16 & 9,908 & 11,748 & 10,642 & * & $\cdots$ & 18,353 & 20,417 & 9,546 & 11,501 \\
\hline others . & 9 & 16 & 26,825 & 32,036 & 34,051 & 30,148 & 30,532 & 41,649 & 24,807 & 9,859 & 10,704 \\
\hline IL Hiris. & & & & & & & & & & & \\
\hline ai Nuddi & 7 & 11 & 8,000 & 31,222 & 45,217 & & & & $\cdots$ & \% & $\cdots$ \\
\hline Banslol Nuddi . & 7 & 7 & 1,000 & 8,583 & 8,000 & 2,367 & 1,641 & 11 & .. & .. & .. \\
\hline Goomani Nuddi . & 3 & 4 & 8,708 & 5,550 & 9,665 & .. & $\cdots$ & $\bullet$ & .. & . & *. \\
\hline N.W. of Hills & 3 & 4 & 3,703 & 1,500 & 29,111 & 878 & 10909 & $\ddot{g} 0$ & $\cdot \cdot$ & .. & .. \\
\hline Kurhurbali & $\frac{1}{1}$ & 7 & 148 & 4,006 & 10,193 & 1.234 & $\begin{array}{l}10,2093 \\
16921\end{array}$ & 300 & "* & 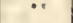 & .. \\
\hline $\begin{array}{l}\text { Palamow } \\
\text { Khasia and Jyn- }\end{array}$ & 1 & . & 148 & 1,061 & 1,144 & 1,254 & 1,621 & .. & ". & .. & .. \\
\hline $\begin{array}{l}\text { teah. } \\
\text { Singrowlie }{ }^{\circ} \text { and }\end{array}$ & 2 & - & 868 & 1,023 & 1,144 & 598 & .. & $\ddot{*}$ & "* & *. & * \\
\hline Rewah \& & 2 & ". & 868 & 1,028 & 1,444 & 185 & 481 & 259 & 148 & .. & 1,108 \\
\hline $\begin{array}{c}\text { Scinde, } \\
\text { Valley }\end{array}$ & 1 & 4 & & 1,680 & .. & & & & & & \\
\hline Salt Range. & $\ldots$ & $\ldots$ & .. &.. & .. & . & 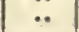 & & 360 & 1,019 & ${ }^{8} 40$ \\
\hline
\end{tabular}

Coal raised in India. The original returns are given in Bengal mannds $=40$ seers each $=2,057 \mathrm{lbs}$. These have been brought into the English statute tons :-

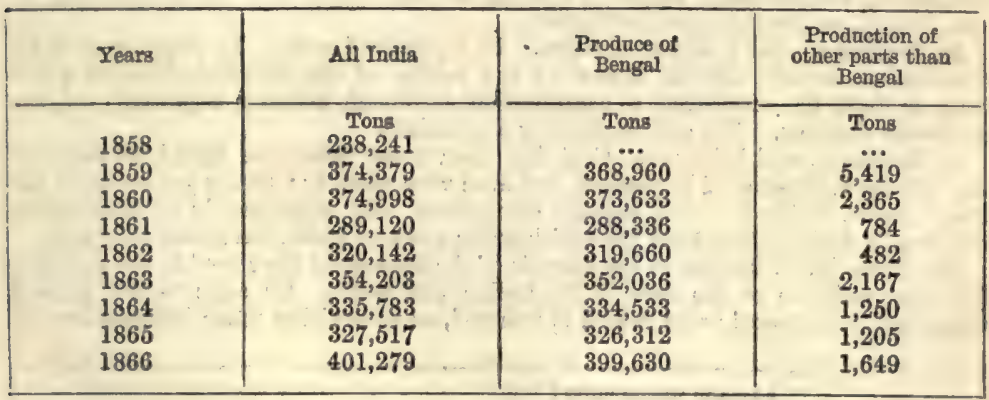

The above figures show that within the last nine years but little progress has been made in the development of the coal-fields of India. The coal-fields of Bengal are the only ones which have been worked in anything approaching to a systematic plan, and even in these there appears to have been a want of any well regulated method. There can be no doubt that, if there should spring up a large demand for native coal, there would speedily be an increased production of it. Still, since 1861, we have given us by Dr. Oldham, from the East Indian Railway's Deputy Agent, a return of the coal used on that line, which is as follows:-

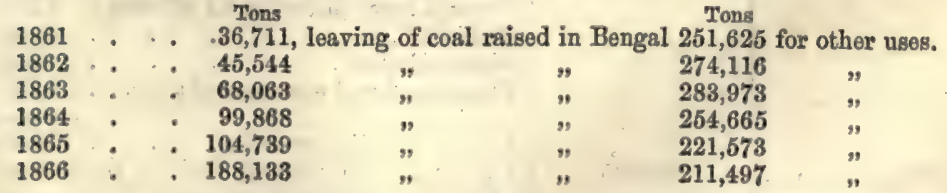

From this we see that as the locomotives of the railway have been burning more Indian coal, the increased supply from the Bengal collieries has not kept pace with 
the demand, and in each year since 1863 there has been a steady docline in the quantity of native coal left to meet the requirements of the country.

Dr. Oldham tells us, "That during the last eight years there has been an aggregate consumption of coal supplied for Bengal alone and the port of Calcutta, of more than $3,000,000$ tons, of which about eight-ninths were raised in India, and about one-ninth imported; that there has on the whole been but little increase in the demand for coal during that period.'

Coal of India.

\begin{tabular}{|c|c|c|c|c|}
\hline Years & $\begin{array}{l}\text { Coal raised in } \\
\text { all India }\end{array}$ & $\begin{array}{l}\text { Coal raised } \\
\text { in Bengal }\end{array}$ & $\begin{array}{l}\text { Coal imported } \\
\text { into Calcutta }\end{array}$ & $\begin{array}{l}\text { Total consumption } \\
\text { for Bengal }\end{array}$ \\
\hline 1858 & $\begin{array}{l}\text { Maunds } \\
61,62,319\end{array}$ & $\begin{array}{l}\text { Maunds } \\
61,62,928\end{array}$ & Maunds & Maunds \\
\hline 1859 & $99,61,928$ & $99,61,928$ & $12,29,160$ & $1,11,91,088$ \\
\hline 1860 & $1,00,88,113$ & $1,00,88,113$ & $4,96,585$ & $1,05,84,698$ \\
\hline 1861 & $78,06,252$ & $77,85,085$ & $12,85,203$ & $90,70,288$ \\
\hline 1862 & $86,43,843$ & $86,30,843$ & $6,76,687$ & $93,07,530$ \\
\hline 1863 & $95,12,174$ & $95,04,975$ & $10,36,408$ & $1,05,41,382$ \\
\hline 1864 & $90,46,147$. & $90,32,405$ & $18,18,132$ & $1,08,50,537$ \\
\hline 1865 & $88,37,953$ & $88,10,425$ & $16,16,143$ & $1,04,26,568$ \\
\hline 1866 & $1,08,34,551$ & $1,07,90,035$ & $9,14,427$ & $1,17,04,462$ \\
\hline 1867 & $1,18,61,031$ & $1,18,47,178$ & $11,46,734$ & $1,29,93,912$ \\
\hline 1868 & $1,35,62,274$. & $1,34,65,829^{1}$ & $19,28,591$ & $1,63,94,420$ \\
\hline
\end{tabular}

Tho produce of Assam is incladed in this.

In Oude no coal is known to occur. In the Punjaub no coal is known to ocenr, if we except as above, in the North-western Provinces, the patches of lignite which have been found in several localities along the base of the outer Himalaya, as well as in the Salt Range.

In Scinde the only coal raised was that of Synah Valley, as given above, but the irregularity and the small extent of this deposit has caused it to be absndoned. It was, in fact, an irregular patch of lignite.

In Bombay no coal is known to oecur. In Hyderabad none. In Nagpore a small cosl-field is known near to Muret, on the border of the Nerbudda District, which may, in fact, be considered a continuation (although actually separated) of the Nerbudda deposits. The coal is not now economised.

In Madras no coal is known. Coal has been more than once stated to occur on the Godavery, or some of its feeders, and even very recently; but as yet nothing but black shales, which will not support combustion, and which are, in all probability, of a totally different age from the coal-bearing rocks of India, have been met with.

Mr. Theo. W. H. Hughes in the Records of the Geological Survey of India, gives the following estimate of the area of the Indian coal-fields. He adds to it a general statement of other coal areas, which is retained, as it furnishes much information :-

\begin{tabular}{|c|c|c|}
\hline Places & $\begin{array}{l}\text { Area in square miles } \\
\text { over which coal rocks } \\
\text { may te presumed to } \\
\text { extend. }\end{array}$ & Ilemarks \\
\hline India $\cdot$ & 35,000 & $\begin{array}{l}\text { This mileage is made up as follows : } \\
\text { Godavari area (including its affluents) } 11,000 \\
\text { Son } \\
\text { Sirgujjảh and Gangpur area } \\
\text { Assam } \\
\text { Narbadá area (including its affluents) } \\
\text { Damúdá } \\
\text { Rájmahál area } \\
\text { Unsurveyed and uncomputed areas }\end{array}$ \\
\hline United Statos & 600,000 & $\begin{array}{l}\text { Square miles. } \\
\text { The productive area of coal is much less. } \\
\text { Professor Hitcheock estimates the ares of } \\
\text { the true carboniferous system at } 230,659 \\
\text { square miles. }\end{array}$ \\
\hline
\end{tabular}




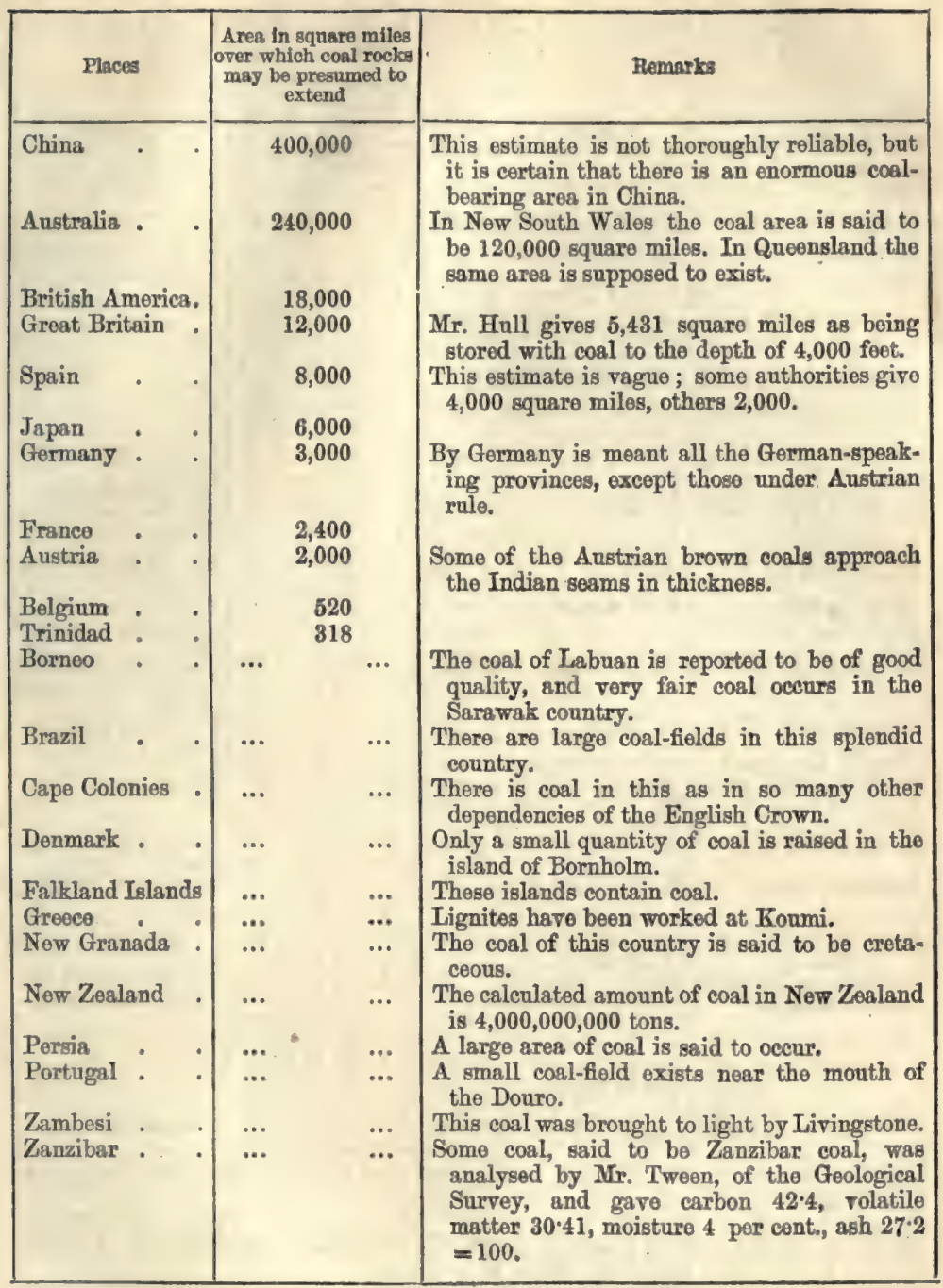

New Sovth WALEs.-Running down the rivers Bremer and Brisbane to the town of Brisbane, numerous outerops of coal may be seen in the banks. Several works are situated about half way between Ipswich and Brisbane. This coal is of much importance to the steam navigation of Moreton Bay. In the parish of Maggil several collieries are established. "The coal varies from five to six feet in thickness, dipping south forty degrees east, angle from seven to eight degrees, although not worked to its whole thickness, excepting where they wanted head room for the "horse way." The main coal separates from the top coal at about four feet; the two feet of the top coal is good, but mixed with a small quantity of earth.'Stuchbury.

The following Table (p. 866) will show the number and condition of the collieries of New South Wales. The Neweastle district produces a good bituminous coal, useful for steam and household purposes, and also splint and cannel. The Southern district yields a semi-bituminous variety of good quality; and from the American creek in that district an abundance of petroleum is obtained. The Western district gives a stiperior splint coal, and oil shales in abundance.

VoI. I. 
list of Collicries and Kerosene and Shale Mines in New South Wales, with the Number of Persons employed, and Quantity of Mineral raised, in 1869.

\begin{tabular}{|c|c|c|c|c|c|}
\hline Mines & Proprietors & $\begin{array}{c}\text { Tons of } \\
\text { Cosl } \\
\text { railsed }\end{array}$ & $\begin{array}{c}\text { Tons of } \\
\text { Otl-coal } \\
\text { and Shale } \\
\text { raised }\end{array}$ & $\begin{array}{c}\text { No. of } \\
\text { people } \\
\text { employed }\end{array}$ & Remarlos \\
\hline 2. Borehole. . & Australia Agricul- & 168,108 & .. & 876 & \\
\hline 2. Burwood . . & $\begin{array}{l}\text { Trustees of Dr. Mit- } \\
\text { chell. }\end{array}$ & 2,980 & .. & 12 & $\begin{array}{l}\text { Worked only about } \\
\text { three months. }\end{array}$ \\
\hline 8. Nonmt i : & J. and A. Brown & $\begin{array}{r}18,523 \\
109,850\end{array}$ & . & 827 & \\
\hline 4. Wallsend & Walto ${ }^{\text {Ditsend Coal Co. : }}$ & $\begin{array}{l}109,850 \\
154,000\end{array}$ & . & 320 & \\
\hline B. Lambton . & $\begin{array}{l}\text { Soottish and Austra- } \\
\text { lian Mining Co. }\end{array}$ & 158,368 & 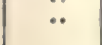 & 257 & \\
\hline 7. Waratah & $\begin{array}{l}\text { Waratah Coal Co. } \\
\text { Laidley, Ireland, } \\
\text { and Company. }\end{array}$ & $\begin{array}{r}127,184 \\
4,000\end{array}$ & $\because$. & $\begin{array}{l}285 \\
180\end{array}$ & \\
\hline 9. Dark Creek . & Wm. Steel : & 800 & .. & 1 & $\begin{array}{l}\text { Oniy supplying saw- } \\
\text { mill. }\end{array}$ \\
\hline $\begin{array}{l}\text { 10. Sunderland } \\
\text { 11. Notts' Inganee seam }\end{array}$ & Mr. Tolip : : : & $\begin{array}{r}500 \\
8,266\end{array}$ & $\because$. & $\begin{array}{r}3 \\
18\end{array}$ & $\begin{array}{l}\text { Local consumption. } \\
\text { Supplied to steamer at }\end{array}$ \\
\hline $\begin{array}{l}\text { 12. Mitchell's Boam : } \\
\text { 13. Rathinba Pit . }\end{array}$ & $\begin{array}{l}\text { John Mitchell. : } \\
\text { Mr. Tunks : }\end{array}$ & $\begin{array}{r}9,426 \\
500\end{array}$ & $\ddot{\prime}$ & $\begin{array}{r}18 \\
2\end{array}$ & $\begin{array}{l}\text { Ditto, } \\
\text { For Maitland gas- } \\
\text { works. }\end{array}$ \\
\hline 14. Stoney Creak . & Frank Rassell. & 900 & .. & 4 & $\begin{array}{l}\text { Local consumption, } \\
\text { Maitlend and envi- } \\
\text { rons, }\end{array}$ \\
\hline 15. Anvil Creels & Wm. Farthing & 14,400 & . & 30 & $\begin{array}{l}\text { Good household and } \\
\text { steam conl, Bplinty } \\
\text { cannel. }\end{array}$ \\
\hline 16. Rix's Creek - . & Jas, Flliott . & 1,900 & .. & 6 & For supply of Single- \\
\hline $\begin{array}{l}\text { 17. Bull Wollongong } \\
\text { 18. Osborne, Wallisend : }\end{array}$ & $\begin{array}{l}\text { Bulli Coal Company } \\
\text { Robson and Co. }\end{array}$ & $\begin{array}{l}64,000 \\
16,677\end{array}$ & $\ddot{m}$ & 90 & \\
\hline 18. Mount Pleasant : & $\begin{array}{l}\text { The Hon. James } \\
\text { Byrnes. }\end{array}$ & 17,014 & $\because$ & 25 & \\
\hline 20. Americs Creek & John Graham. & 1,800 & $\cdots$ & 1 & $\begin{array}{l}\text { For the oil-shale fur. } \\
\text { nace. }\end{array}$ \\
\hline 21. Sutton Forest . & M. Larkin and Co. . & 100 & .. & 4 & $\begin{array}{l}\text { Scarcely in working } \\
\text { order. }\end{array}$ \\
\hline $\begin{array}{l}\text { 22. America Creek } \\
\text { 23. West Kerosene Coal } \\
\text { Mine. }\end{array}$ & $\begin{array}{l}\text { John Graham. } \\
\text { Western Kerosene } \\
\text { Oil Company. }\end{array}$ & $\because$. & $\begin{array}{l}2,076 \\
3,000\end{array}$ & $\begin{array}{l}10 \\
12\end{array}$ & $\begin{array}{l}\text { For kerosene ofl. } \\
\text { Ditto. }\end{array}$ \\
\hline \multirow[t]{3}{*}{$\begin{array}{l}\text { 24. Hartley Kerosene } \\
\text { Cosl Mine. }\end{array}$} & $\begin{array}{l}\text { Hartley Kerosene } \\
\text { Oil and Petroleum } \\
\text { Coal Company. }\end{array}$ & .. & 1,200 & 7 & $\begin{array}{l}\text { For kerosene oil and } \\
\text { supply of gasworks. }\end{array}$ \\
\hline & Oil-coal and shale. & $\begin{array}{r}913,246 \\
6,276\end{array}$ & 6,276 & 2,012 & \\
\hline & $\begin{array}{c}\text { Total cosl and oil- } \\
\text { shale. }\end{array}$ & 910,522 & & 2,012 & Persons employed. \\
\hline
\end{tabular}

In 1872 there were 20 collieries raising coal, and 3 raising petroloum oil, cannel oil, and shale.

In the Newcastle District 7 collieries .

Anvil Creek, Stony and Four Mile Creek

District 4 collieries

Tons

producing 858,716

Valne

Murrurundi District 1 colliery .

Illawarra District 5 collieries :

New South Wales Shale Oil Co.

\begin{tabular}{rrr} 
& 24,032 & 4,449 \\
$"$ & 300 & 600 \\
$"$ & 123,681 & 48,780 \\
$"$ & 5,221 & 1,656 \\
$"$ & 8,000 & 24,000 \\
\cline { 2 - 3 } & $1,019,950$ & 153,046
\end{tabular}

$$
\text { Total Coal and Oil Shale ... . . } \overline{1,019,950} \overline{153,046}
$$

Qukanstasd in 1863 exported 4,228 tons. The produce of the collieries being in $1861,14,212$ tons ; in $1862,24,067$ tons; and in $1863,2,400$ tons.

Mr. R. Daintree, in his 'Notes on the Geology of the Colony of Queensland,' has the following remarks on the coal of that colony:-

- Up to the present time, within all tho area occupied by Carbonaceons Mosozoic Strata, only the Maryborough beds have yielded fossil remains other than plants. Or the tributaries of the Condamine, Brisbane, and Mary rivers, where numerous coalseams aro known to exist, sevoral of which have been and are worked, the plant remains are of the same character.' Mr. Daintree then gives a list of the fossils from the Tiroli cosl-mine, and then proceeds:- 
' The Burrum coal-seams, worked for some time on a branch of the Mary river, lie below the Maryborough Cyprina-Sandstones. . . Bars of coal are a marked feature in these Mesozoic lacustrine beds. . . . The appearance of these lacustrine coal-measures diffors in a very marked manner from their supposod marine representatives to the westward. Coarse grits and thick-bedded sandstones form the majority of the strata, though shales, sandstones, and limestones are interstratified throughout the system.

'Whilst the affinities of the sonthern coal-field of Queensland are Mesozoic, a northern field of even larger extent has a distinct fauna more resembling the Palrozoic carboniferous of Europe. . . . The Dawson, Comet, McKenzie, and Bowen rivers drain this Carboniferous area, and numerous outcrops of coal have been observed on these streams. No commercial use, however, has yet been made of any of them, as the coal-measures, generally, are too far inland to be made available until the railwaysystem of the country is extended in that direction.'

New ZraraNd. - The hydrous coal-measures (lignite) lie on the eastern slopes of the axial rocks in both of the islands of Now Zealand. This coal is of a brown colour, but hard and glossy, and frequently contains a quantity of fossil resin, which greatly facilitates its combustion. It is in the locality a valuable fuel, and is largely used both for domestic purposes and for raising steam. It is also applied to the manufacture of bricks and pottery, and for other economic purposes. The best known deposits of this coal in the North Island are those mined at Drury, twenty miles to the south of the city of Auckland. Seams of this coal, varying from 6 to 15 foet in thickness, occur at intervals over a large area of country. In the South Island the total quantity of this coal has been estimated at $100,000,000$ tons. Two mines have been opened on this field. The Clutha mine is on the sea coast, about three miles from the mouth of Clutha river. The other mine is in the vicinity of Tokomairo. The seam is 9 feet thick, and worked by a level in the side of a hill.

In 1864 the quantity of coal produced from the mines in this district was about 4,000 tons.

Anhydrous coal. -The most important development of the coal-seams of this class is on the west coast of the South Island. At Preservation Inlet, in the province of Otago, and at varions points on the coast, the same formation occurs, but without any valuable seams of coal, as far as we yet know, until the Grey river is reached.

At Grey river the coal extends over an area which Dr. Haast has estimated at 15 miles in length by 6 in width. 'At present (1866) the output of the coal is from 250 to 300 tons per week, and is taken down the river in canoes and in 16-ton barges, and delivered alongside the vessels at $40 \mathrm{~s}$. per ton. It has been used to a large extent on board the steam-ressels that frequent the coast, and gives great satisfaction. A small quantity of this coal is also reported to have been tried in the Dunedin gasworks, and to have yielded a large quantity of illuminating gas.'

In the immediate neighbourhood of the Buller river the existence of workable coal-seams is considered certain over an area of at least 15 square miles. Reckoning none of the seams having a less thickness than five feet, there is a total of 38 feet of pure coal, and the quantity available in this one locality has been estimated at $200,000,000$ tons. The following summary of the anhydrous coal is given in the report by Mr. Stafford to Lord Carnarvon:-

\begin{tabular}{|c|c|c|c|c|}
\hline Locality & & Quality of Coal & Thickness of Seam & Worked by \\
\hline 1. Grey River & & Superior & 25 foet & Level free \\
\hline 2. Buller River & & Do. & 38, & Do. \\
\hline 3. West Wanganu & & Medium & $8 "$. & Uncertain \\
\hline 4. Pakawau. & & Do. & Uncertain & Do. \\
\hline 5. Aorere : & & Superior & Do. & Level freo \\
\hline 6. Batten River & . & Medium & Do. & Do. \\
\hline 7. Wangapeka & . & Do. & Do. & Do. \\
\hline 8. Wangarei & . $\quad$. & Inferior & 6 feet & Do. \\
\hline 9. Kawa-kawa & & Superior & $13 n$ & Shaft \\
\hline
\end{tabular}

JAPAs:-The following are the only available notes on the coal-fields of Japan :-

Iwanai. Tomazi. $-\mathbf{A}$ bed of coal from four to six feet in thickness, consisting of very good bituminons coal, in places very pure, in others intermixed with slate, which is easily separated from it. The coal-mines lie two miles inland from a village called Kaianoma, some seven miles across the bay. Four seams of coal have been discovered : 2 are from 4 feet to 6 feet thick, 1 is about 1 foot thick, 1 not yet examined.

Serenty-nine pounds of coal burnt in the galley fire of Her Majesty's ship 'Salamis,' $3 \mathrm{~K} 2$ 
under the superintendence of the chief engineer, yielded 17.27 per cent. of $a$ sh, 1.5 per cent. of elinker, an average volume of smoke, and a strong durable flame. The probable cost of the coal delivered would be four dollars the ton on the spot; freight to Lakodate would be three dollars the ton, and to Yokohama or Nagasaki six dollars the ton.

Coal-field near the Port of Hiogo.-Sir Harry S. Parkes communicates (1867) to the Foreign Office particulars of 'another coal-field in the immediate vicinity of the port of Hiogo,' from Mr. Frederick W. Sutton, Chief Engineer of H.M.S. 'Serpent.'

The coal is of tertiary formation, and it crops out in a number of places on the gides of a low range of hills, about six miles to the westward of Hiogo. These beds have been occasionally worked by the natives. In February 1867, 30 men were employed, and up to May they had obtained more than 170 tons of cosl. Mr. Sutton says, " the best coal brought out in my presence was quite equal to Takasima (Nagasaka) coal. It is not of exactly the same deseription, but more of an anthracite kind.'

There is no evidence of the value of this coal, and evidently the statement of the coal being of the tertiary age and of 'anthracite kind ' wants consistency.

At a meeting of the Geological Society of London, April 14,1869, a paper was read ' On the Coal-mines at Kaianoma, in the island of Yezo,' by F. O. Adams, Esq., Hon. Secretary of Legation in Japan: communicated by the Secretary of State for Foreign Affairs.

The writer states that the works at Kaianoma have made considerable progress since they were reported upon by Mr. Mitford last year. There are four seams of cosl, each about seven feet thick, from 50 to 100 feet apart. A tunnel has been driven through one of the seams for a distance of between 150 and 250 feet, and at an elevation of $\mathbf{4 3 0}$ feet above the sea. From this the coal obtained is carried down to the shore on the backs of men, mules, and ponies. The writer adds, that there is abundance of coel ' of the cannel description.'

At the end of 1870, according to the 'Nagasaki Gazette,' the coal from Takasima, which has long been known to be a good coal, was ordered to be used by all steamers in the Japanese waters. The export of coal from Nagasaki has not increased of late, but this is referable to the want of ships to take it away. 1,174 tons have been shipped for, and several vessels were engaged in taking coal to China.

The Netherlands Trading Company are using the utmost energy in their efforts to develope this coal. These mines are managed by an English mining engineer, Mr. Frederick Potter. A large quantity of machinery, tools, \&c. have been sent out from England, and a large steamer has been purchased for the entire use of the collieries.

The following information has recently (1874) been received from the colliery of Takasima :- 'Our coal fossils here are oolitic. We have none of the coal-measure flora, but great stumps of trees, which the Japanese call " matzii," the name for Scotch fir, and which are no doubt coniferous woods. These hard stumps of trees give us great trouble in our working, it being impossible to cut through them with the pick. Only the islands are coal-measures, the main land being clay-slate with syenite ridges; and above the clay-slate, greenstone of the same age probably as the slate. We are now (December 1873) getting 350 tons a day, but are very short of men.'

Borseo.- In the province of Labuan, on the north-west coast, there is abundance of coal of good quality. The seam is generally 9 feet in thickness. The coal found here burns fast, and emits a bright flame; it soon acquires a red heat, and continues in that state until it smoulders into a white ash, like that of wood; there is not much smoke from it. For several years past the coal-mines of Moara have ceased to be worked.

Crinsa.-There appears to be much good coal in China. It is procured from a mine on the river Yang-tse-kiang, about 400 miles from its mouth; of the other coalproducing districts we know little.

Consul Braune in his report to Sir F. Bruce, says, writing from Tamsuy, January 21, 1864: 'It is to the coal-mines of Kelung that we must look to the future prosperity of this place as a port of trade; a depôt has been already established there by a large firm to supply their own steamer, running on the coast.'

In 1863 there were exported from the port of Tamsuy, in British vessels, 10,801 piculs of $133 \mathrm{lbs}$. avoirdupois ; in foreign vessels, $1,259,152$ cotties of $1 \frac{3}{4} \mathrm{lb}$. avoirdupois ; also from the port of Tien-tsin, 180 tons, value 1,800 taels, of $68.4 \frac{1}{2} d$. each.

Mr. Consul Mongan's report for 1864 on the trade of Tien-tsin, says: 'I allude to coal, extensive mines of which exist in the mountains to the north and west of Peking. It costs about 168. per ton at the pit's mouth, and more than double this amount per ton is paid for transport to the coast; but the mines are worked in the rudest way, and the little coal which finds its way from the western range of Tientsin is conreyed on mules and camels from the mountains to Tang-chow on the Peiho, 
and thence down the river in boats to this port. From the mines in the northern range, there is water communication of an indifferent kind to Tien-tsin, but the quality of their coal is much inferior to that which comes from the western mountains.

At the meeting of the British Association at Bradford (1873) Baron von Richthofon read a paper ' On the Distribution of Coal in China.' The Baron has devoted several years to the investigation of the geology, products, and resources of the interior proyinces of China, and has travorsed the whole country, with the exception of tho southwestern portions. He stated, as the result of his researches, that China exhibits the great peculiarity of containing no geological formation later than the Triassic; all the groat secondary deposits, from the lias to the chalk, and all the Tertiary series being absent. It, has, therefore, been dry land throughout the whole period of these later formations; and to this peculiarity are owing the stupendous results of sub-aërial denudation which it furnishes; among which are the deop narrow gorges which its rivers have eroded nearly up to their sources, the rarity of cataracts and rapids, and the removal of rocks overlying the great coal-beds. The coal strata belonged to various geological epochs, from the Silurian upwards; but by far the greater portion belonged to the same formation as the coal of Europe and North America, viz., the Carboniforous. The coal-beds were deposited around mountains of metamorphic and primary rocks which then constituted the land, and have lain horizontally, with little disturbance, ever since that remote epoch. The deep ravines worn by the rivers cut through these coal-bearing strata, and lay them bare on the precipitous sides; so that the coal is easily accessible on the banks of the great streams. The author proceeded to describe the extent of the coal in each province, beginning with Southern Manchuria and terminating with Honan. In Manchuria the coal is confined to valleys in the hilly parts, and is not readily accessible to foreign commerce; it is accompanied, however, by an abundance of rich iron ore, which at some future date may be worked with immense advantage to the country. Coal exists further west all along the Great Wall; and there are beds 95 feet thick near Peking, in which city it is the fuel in universal use; but it is an error to suppose, as some have done, that the high hills round Peking consist of coal-measures; coal is found only in limited valleys at a great elevation. The coal of Shantung, although not situated near good harbours, is the most accessible of all Chinese coal from the sea. It exists also in the other maritime provinces, but in districts offering much fewer facilities. The greatest of all the coal districts is in the west and north-west; at the southern foot of the great mountain range (the eastern continuation of the Kuen-Lun), which here stretches across Western China. In Sze-chuen coal occupies an area of 100,000 square miles. At the centre of this vast basin the coal is bad and inaccessible, but round its borders it is of excellent quality, and near means of communication by water, although too distant from the sea to be available to foreigners. The whole surface of Northern China is covered by rich yellow earth, or loess, to a depth often of 1,000 and 2,000 feet, which overlies all the coal-fields. The great plain of China is bordered on the west by a vast limestone wall, 2,000 to 3,000 feet high, on the top of which extends a plateau of coal in a state of excellent preservation, owing to its capping of hard limestone which has resisted denudation. There are here 30,000 square miles of coal-bearing ground of the very best quality, in which the coal-beds lie perfectly horizontal, 30 foet thick, for a length of 200 miles. They extend westward into Shensi and Kansu, and are reported by all travellers to continue beyond the frontiex of China fax into Mongolia. Coal costs here, at the pit-mouth, $7 d$. per ton, and the wages of miners are $6 d$. per day. The Baron believed that the readiest way of getting at this vast coal-field from Europe was by a railway from Ili and Kulja, in Russian territory, to the north-western corner of Kansu.

South Africar Coat-Field.-For the following account we are indebted to the Geographical Report on the Stormberg coal-field, made by Mr. E. J. Dunn, to the Governor of Cape Town:-

'In proceeding northward from Port Elizabeth to Dassyklip on Bushman's River the beds crossed appoar to be of secondary age (Sunday River Beds) covered by tertiary and post-tertiary deposits, comprising calcareous beds, bods of red clay, and beds of clay conglomerate. At Dassyklip hard sandstone and quartz rock crop out. This series is passed at a distance of siz miles north of Graham's Town. Between Katberg and Winchelsea beds of shalo occur, and extensivo dykes of dolerite traverse the country: most of the surrounding hills have a capping of igneous rock. These series of beds continue as far as the Stormberg Mountains and from the base of that range 4,900 feet above the sea level. Above this, and forming the coal-measures, are the sandstones, shales, \&c. of which the Stormberg Range consists. The coal-measures are composed of beds of sandstone, usually very thick, brown, grey, or white, in colour, frequently showing no traces of lamination through many feet in thickness.

'Sheets and dykes of igneous rock are of frequent occurrence over the whole area 
occupied by the cosl-measures. The general dip of the cosl-measures is slightly to tho north-east, but modified by the action of intrusive rocks.'

From tho localities opened, the Government Reporter is onabled to give the description of the condition of the coal-field from which the following abstract has beon made. At Vice's workings a shaft is sunk to a depth of 70 feet. It cuts the coal at 41 feet. The seam consists of alternate layers of coal and shale to a thickness of 3 feet 7 inches. Of coal there are 25 inches. The lower seam 13 inches thick is of poor quality. The middle one 9 inches thick is much superior, while the top $3 \frac{1}{2}$ inches is very inferior.

The coal is firm and hard, breaking out in good-sized blocks that do not readily break up in transport. It is laminated, splitting into thin layers; the faces of the layers are of a dull, earthy black colour, while the edges are lustrous. When burnt a good heat is given out, and the fire burns for a long time. The cinder is, howevor, very considerable, and retains the form of the fuel when first ignitod. It does not fall into an ash. When burnt in a steam-engine or where a strong heat is induced, the result forms a pasty cinder.

Hattingh's workings are about one mile to the south-east of Vice's shaft, situate on the same seam. Of coal there are $24 \frac{1}{2}$ inches, with 3 partings of shale ( 15 inches) between the 4 layers. The third seam from the bottom, $6 \frac{1}{2}$ inches thick, is the best in quality.

At Kapok Krasl, about seven miles to the south-east of Burghersdorp there are 28 inches of coal in 4 seams, distributed through about 16 feet of shale, on the opposito side of the valley close to a neck where a trial has been made by means of a small shaft, there are two seams, one 16 inches, then 2 feet of shalo and 9 inches of coal. Buekley's drive is opposite and about 300 feet above the hotel at the base of Bushman's Hoek. The outcrop of coal and shale is of very inferior quality, and about $3 \frac{1}{2}$ feet thick. About nine miles eastward from Dordrecht a seam of coal occurs 8 inches thick at November's Kraal, Tambookie Location. All the appearances are favourable to the presence of coal in this neighbourhood and throughout the district. Besides the above localities, the seam worked by Vice and Hattingh can be traced for miles along the hill sides. Numerous other outcrops are known, but they are not oponed out.

Mr. Dunn says :- - There can be little doubt that more extensive deposits, and probably superior quality of coal, will bo found further north and north-eastward (in Basutoland and Kafirland Proper, extending towards Natal).

For the Heating Power of British coal, see Furt. For Coal Mining, see Mrnive.

COAI Brass. This name is given to the iron pyrites (bisulphide of iron), found in the coal-measures, and which are employed in Yorkshire, and on the Tyne in the manufacture of copperas-the protosulphate of iron-and also in the alkali works, for the sulphur they contain. See Sulpror Orrs.

The iron ores called Brass, occurring in the coal-measures of South Walos, were particularly described by E. Chambers Nicholson and David S. Prico, Ph. D., F.C.S., at the meeting of the British Association at Glasgow. Their remarks and analyses wore as follow :-

- There are three kinds of ores to which the name brass is applied; they are considered to be an inferior class of ore, and are even rejected by some iron-masters. One is compact, heavy, and black, from the admixture of coaly matter, and exhibits, when broken, a coarsely pisiform fracture. A second is compact and crystalline, not unlike the darkest-coloured mountain limestone of South Wales in appearance. The third is similar in structure to the first-named variety; the granules, consisting of iron pyrites, are mixed with coaly matter, and cemented together by a mineral substance, similar in composition to the foregoing ores. It is from the yellow colour of this variety that the name brass has been assigned to the ores by the miners.

'The ores have respectively the following composition:-

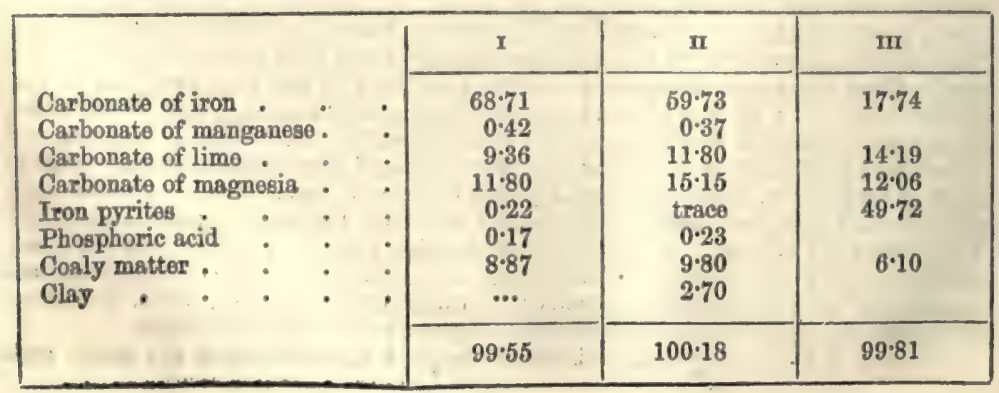


' It is unnecessary to allude to the third variety as an iron-making material; its colour admits of its being at all times separated from the others. The pyrites which it contains, we may remark, is bisulphuret of iron.

'It is to the ores I. and II. that we would direct attention. The reason of their having hitherto been comparatively disregarded may bo attributed either to their having been mistaken for the so-callod brass of coal, or to their being difficult to work in the blast-furnace in the ordinary manner, through the belief that they were similar in constitution to the argillaceous ores of the district. It will be seen from the above analyses that they are varieties of spathic iron ore, in which the manganese has been replaced by other bases. If treated judiciously, they would smelt with facility and afford an iron equal to that produced from the argillaceous ores. From the large amount of lime and magnesia which they contain, their employment must be advantageous in an economic point of view.

'An interesting feature in these ores is their fusibility during calcination on the large scale. When this process is conducted in heaps, the centre portions are invariably melted. This, considering the almost entire absence of silica, is apparently an unexpected result. The fused mass is entirely magnetic and crystalline. Treated with acids, it dissolves with great evolution of heat.

The following is its composition:-

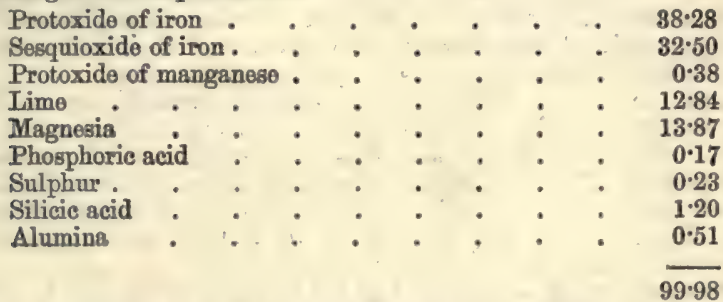

'From the above analysis, it is probable that the fusibility of the compouna 18 owing to the magnetic oxide of iron acting the part of an acid. When thoroughly calcined and unfused, the ores retain their original form; and if exposed to the air for any length of time crumble to powder from the absorption of water by the alkaline earths.'

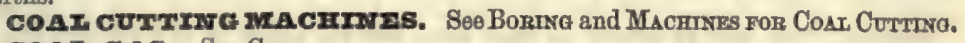
COAI GAS. SOO GAS.

CовAIт. (Symbol, Co; Atomic weight, 29.5.) Little is known of the properties of this substance in the metallic state, as it has not hitherto been employed as such in the arts, and has only been obtained in a state of purity in small quantities by delicate operations in the laboratory. Like the allied metals, nickel, iron, manganese, and chromium, it may be obtained in a dark coloured pulverulent state by heating its oxide to redness, in a current of hydrogen, or in a compact button, by effecting the reduction with carbon at the highest temperature obtainable in an air furnace. Prepared in the last-mentioned way it is a brilliant white, or slightly reddish metal, less fusible than nickel or iron, but more so than chromium. It is decidedly magnetic, coming next in the scale to iron. If carbon be present in excess, a portion combines with the reduced metal, forming a compound analogous to cast-iron. It may be obtained melted, in a perfectly pure state, by heating oxalate of cobalt in a closely-covered crucible, to a strong white heat, when the oxide of cobalt is reduced by the carbon of the oxalic acid, thus: $\mathrm{CoO} \cdot \mathrm{C}^{2} \mathrm{O}^{3}=\mathrm{Co}+2 \mathrm{CO}^{2}$. According to the older observers, it is very brittle, but Deville states that pure cobalt is extromely tenacious, even to a higher degree than iron. The specific gravity is 8.5 . It forms a brittle alloy with iron. Quite recently metallic cobalt has been prepared on a commercial scale in Germany, and is said to be used as such in certain new alloys.

Ores of CoBatt.- The number of minerals containing cobalt is but small, and they are restricted to a very few localitios. The following are the most important spocies :-

1. Smaltine, Tin-white Cobalt, Speiss Cobalt (Co, Fe, Ni) As. (Co, Te, IN1) As2. This is of a steol or lead-grey colour, and metallic lustro, crystallising in the cubical system. The hardness is about that of felspar. Sp. gr. 6.5 to 7.2. According as one or other of the three bases prevails, three simple types of composition may bo deduced, as follow:-
I. CoAs $\left(\right.$ CoAs $\left.^{2}\right)=$ Cobalt, 28.2 ; Arsenic, 71.8 per cont. Speiss cobalt, Cubical,
III. NiAs $\left(\operatorname{MrAs}^{2}\right)=$ Nickel $28 \cdot 3$;
III. $F \odot A s\left(r e \Delta s^{2}\right)=$ Iron, $27 \cdot 2$; $71 \cdot 7, \quad "\left\{\begin{array}{l}\text { Chloanthite } \\ \text { Rammelsberg }\end{array}\right.$ Trismatic. ". $72 \cdot 8, "$ Löllingite 
The varieties I. and II. occur in considerable quantities at Schneeberg, in Saxony, associated with silver, copper, and other pyritic ores, quartz and hornstone replacing sulphate of baryta, fluor, and spathic carbonates; also at Riecholsdorf, in Hesse; in Bohemia, Sweden, and in a fow localities in the United States. They have also been found in Cornwall; among other places, at Wheal Sparnon, and Saint Austell Consols. The nearest natural mineral in approach to typo No. I. contains about 23 per cont. of cobalt; as a rule, however, the nickeliferous varieties are the more common. The predominance of one or other metal may be determined by exposing the specimens to damp air, when a green crust of arsenate of nickel forms on the varieties in which nickel is most abundant, while a pink incrustation is producod on those richest in cobalt. Type No. III. is the mineral known as arsenical pyrites, which, in reality, often contains a little cobalt, and is treated as a cobalt ore.

2. Cobaltine, Grey Cobalt, Bright White Cobalt, or Cobalt Glance $\left(\mathrm{CoS}^{\S}+\mathrm{CoAs}\right)$ $\left(\mathbf{C o s}^{2}+\mathbf{C o A s}^{2}\right)$ differs from Speiss cobalt chiefly by having part of the arsenic replaced by sulphur. It crystallises in the cubical systom in forms similar to those of iron pyrites, is of a whito colour, with a slightly bronze-red tinge, and a brilliant motallic lustro. Its hardness is less than that of felspar, and specific gravity about 6 . The typical composition is : cobalt, 35.5 per cont. ; arsenic, 45.2 per cont. ; sulphur, $19 \cdot 3$ per cent. The purest natural varieties contain about 33 por cont. of cobalt; these occur in Sweden. At Siegen, in Rhenish Prussia, a variety is found containing only 8 or 9 per cent. of iron, forming three quarters of the whole amount of bases present. Unlike the preceding species, it does not contain nickel.

3. Linncite or Cobalt Pyrites, -This, although crystallising in the cubical system, is analogous in composition to copper pyrites, being represented by the formula $\mathrm{CoS}+\mathrm{Co}^{2} \mathrm{~S}^{3}\left(\mathbf{C o s}+\mathbf{C o}^{2} \mathbf{S}^{3}\right)$, with 58 per cent. cobalt and 42 per cent. sulphur: part of the base in either term may be replaced by copper, nickel, or iron. It is the

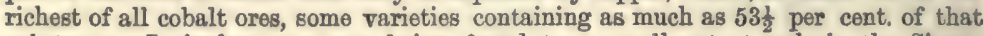
substance. It is, however, rare, being found to a small extent only in the Siegen district, and in Sweden.

A mongathe products of the alteration of the above minerals, aro-4. Cobalt Bloom, of the composition $3 \mathrm{CoO} \cdot \mathrm{AsO}^{5}+8 \mathrm{HO}\left(\mathbf{C o}^{3} \mathbf{A s}^{2} \mathbf{O}^{8}+\mathbf{8} \mathbf{z} \mathbf{2}^{2} \mathbf{O}\right)$ with $37 \frac{1}{2}$ per cent. of oxido of cobalt: and 5. Earthy Cobalt Ore, a variety of the substance known as Wad or Bog Manganese, which at times contains as much as 15 or 20 per cent. of the same oxide, together with the oxides of iron, manganese; and copper. 6. Cobaltic Bismuth Ore, is a finely crystalline mixture of Speiss cobalt and bismuth-glance, found occasionally in the Schneoberg district, containing 10 per cont cobalt and 4 per cent. bismuth. The ores of cobalt, as they come into the hands of the smelter, are, as a rule, so much mixed up with those of nickel, that they are worked for both metals.

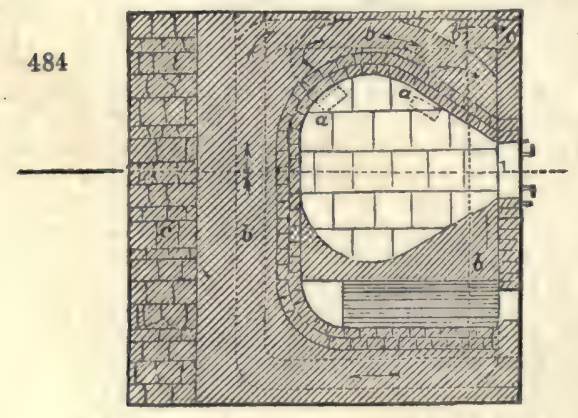

See NICKFI.

The use of cobalt in the arts is mainly confined to the production of glasses coloured blue by oxide of cobalt, which, when finoly pulverised and levigated, are used as pigments under the name of smalts. Oxide of cobalt, in a pure state, is also prepared, to a certain extent, for the use of porcelain manufacturers. The first point to be considered, therefore, is the production of the oxide, and next the fusion with a sufficient quantity of siliceous matter. Earthy cobalt ore may be used in the fusion process, without any further

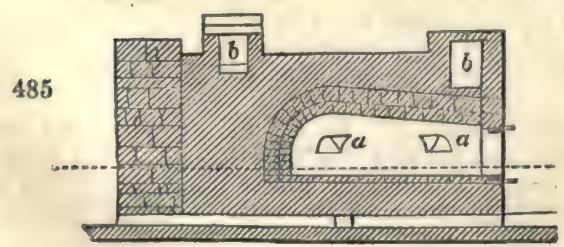
preparation; but those that contain sulphur aud arsenic must be subjected to a preliminary calcination. Fig. 484 is a horizontal, and fig. 485 a vertical section of the roasting furnace employed for this purpose in the Saxon smalt works. The firo-grate is placed on one side of the hearth, which is of irregular form, being about six feet in breadth at the further end of the furnace, diminishing to 27 inches at the working door. Wood is the fuel employed. The arsenical vapours given off during roasting, pass out through the openings, $a, a$, by a 
eystom of flues, $b, b$, in the direction shown by the arrows, into a chamber where they are deposited, and may be subsequently used in the mannfacture of arsenical products. The charge is from 3 to 5 cwts.; the degreo to which the roasting is carried varies with the composition of the ores. When many foreign metals are present, they must not be roasted 'dead ' or ' sweet,' but sufficient arsenic or sulphur must be left to form with the less oxidisable metals, more especially copper and nickel, a speiss or regulus which separates from the blue glass produced in the fusion process, and subsides to the bottom of the melting pot. The oxides of those metals that are more easily oxidisable than cobalt, such as iron and bismuth, may be partially removed by an addition of metallic arsenic in the fusion, whereby the latter oxide is roduced and passes into the speiss with the production of arsenious acid, which tends to heighten the colours produced. Earthy cobalt ores containing much iron, require an addition of arsenious acid, in order to convert the protoxide of iron, which has a strong colouring power, into peroxide, which is of less consequence. In some works, the roasting is performed in muffle furnaces, the hearth being heated from below, so that the ore does not come into contact with the flame. Ores containing bismuth are subjected to the process of liquation previously to roasting.

When the ores are very impure, or when it is wished to produce very deep blue colours, they are subjected to the process of concentration. Common ores, and those that are rich in copper, are first slightly roasted, and then fused in crucibles in the smalt furnace described below, with broken glass fragmonts, charcoal dust, and carbonate of potash. When the mixture is in a state of complete fusion, the slag is partly removed at intervals, and fresh quantities of ore are added, until a sufficient quantity of speiss is produced. In this process, the oxides of iron and copper in the ore form an easily fusible elag with the glass, while the cobalt is reduced by the charcoal to the metallic state, and unites with the arsenic to form a speiss. If the roasting is carried too far, or if there is an insufficiency of arsenic or of reducing matters in the mixture, a certain amount of oxide of cobalt will be taken up by the slag. The concentrated speiss produced is then pulverised and roasted 'sweet,' leaving a nearly pure basic arsenate of oxido of cobalt.

The smalt furnace, figs, 486 and 487 , is similar in construction to that used for melting flint glass. The firo-place, in which wood is burnt, is a long narrow rectangular chamber, with a vaulted floor perforated by draught holes, $d, d$; $c$, the lower chamber, is the ash-pit; the flame passes through the hole, $a$, into the melting chamber above, which is a low domed structure, containing six melting pots; $f, f, f$, are the working doors, - they are faced with cast-iron frames in the ordinary way. The waste flame, after heating the pots, passes through the small flues, $i, i$, into a system of chambers, where it is employed in the accossory operations of calcining quartz, or drying wood for fuel. $h, h$, are arches through which the pots are inserted or removed,-they are built up when the furnace is at work. At $e, e, e$, are small holes, corresponding with similar ones in the pots, for removing the speiss formed during the fusion. The charge consists of roasted ore, with from 1 to 16 times its weight of quartz previously calcined and reduced to a fine powder, and carbonate of potash to the extent of about $\frac{3}{4}$ ths or $\frac{4}{5}$ ths of the amount of the quartz. Each pot contains about 3 cwts.; the fusion takes place at a low temperature, the glass 
being frequently stirred with an iron rod in order to render it perfectly homogeneous ; when this point is attained, the stirring is discontinued, and the speiss is allowed to settle. In 6 or 8 hours, the glass is sufficiently fluid, and it is then ladled out with iron ladles, and thrown into water. After the contents of two pots have been romoved, the working doors are clased, and the fire is urged for some time, in ordor to bring the contents of the remaining pots which have somewhat cooled down to a propar degree of fluidity.

Grinding the smalts. - The blue glass, rendered brittle by sudden cooling, is stamped or crushed, sifted, and ground wet, under hard stones. The finely-divided glass from the mill is then passed through a system of washing-vats; the first three givo a coarse sand which is usually returned to the mill for further grinding, the finer slime is allowed only a fow minutes to deposit in No. 4, about a quarter of an hour in No. 5 , then it filtars more slowly through No. 6, and finally into a slime-pit, where the whole of the suspended matter is deposited. The darkest coloured smalts, known as King's blue or azure, are found in vats Nos. 4 and 5 . No. 6 gives the lighter coloured tub smalts. The pale blue powder from the slime-pit is mostly returned to the melting furnace.

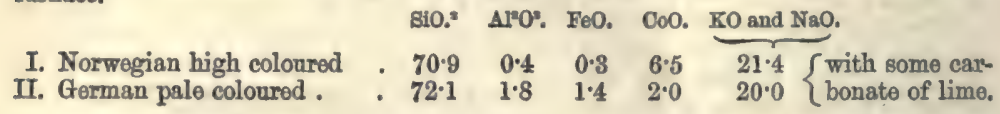

Smalt is therefore a kind of glass coloured by oxide of cobalt. One part of oxide of cobalt communicates a distinctly bluish tinge to 240 parts of glass; with more than 18 per cent. the glass becomes black. In practice a potash-glass is always employed, and hence smalt may be regarded as a double silicate of potash, and protoxide of cobalt. Soda cannot be substituted for the potash, as it produces an inferior colour, and the same remark applies to some other oxides. The following are the principal facts observed with regard to the influence of the oxides of other motals on the colour of smalts. Baryta heightens the tone, giving an indigo tinge; soda, lime, and magnesia, have a decidedly lowering effect, and produce a reddish shade; alumina has no effect on the purity of the colour, but lowers its intensity; iron produces a blackish-green dull tinge, which is very prejudicial to the higher coloured varieties; manganese communicates a violet shade, as does also nickel, but in a much higher degree. Copper, zinc, bismuth, and antimony, all give a dull dirty shade, especially to the higher colours. Oxide of lead has no effect on the colour, but as it increases the specific gravity of the glass, and causes the ground smalts to subside too rapidly in the washing vats, producing an incomplete separation, its presence in the mixture is not desirable.

In commerce, smalts are classified both according to their contents in cobait, and the size of the grain, the following being the chief marks :-

F.C. Fine colour. F.C.B. Fine Bohemian colour. F.E. Fine Eschel.

M.C. Middling colour. M.C.B. Middling Bohemian colour. M.E. Middling Eschel.

O.C. Ordinary colour, O.C.B. Ordinary Bohemian colour. O.E. Ordinary Eschel.

In the above scheme, the words fine, middling, and ordinary, indicate the relative quantities in cobalt, while colour, Bohemian colour, and Eschel, express the state of division. The two former are composed of angular fragments, while tho latter are the finest-rounded grains deposited in the lowest washing vats and the slime-pits. Smalts that are darker in colour than F. are indicated by multiples of that letter, the highest being FFFFC; while, on the other hand, those that are lower than $\mathrm{O}$ are distinguished by exponents following the second letter, thas $\mathrm{OC}^{2}, \mathrm{OC}^{4}$ contain respectively only $\frac{1}{2}$ and $t$ as much cobalt as 0.0 .

Zaffre, or Safflor, is the name given to a mixture of roasted ore and quartz, similar, in fact, to that employed in smalt-making, but without potash. It is used either for pottery purposes, or for bringing up the colour in smalts made from low-classed ores.

Formerly smalts were used to a great extent in colouring paper, but at present artificial ultramarine is almost exclusively used for this purpose (see Ultramarane).

For an account of the method of assaying cobalt ores, see NickEI.

The imports of cobalt can no longer be given; the Custom-house authorities now returning the cobalt ores as 'Ores unenumerated.'

COAAIT IIOOM. Hydrous arsenate of Cobalt commonly occurring as a peach-blossom coloured incrustation on arsenical ores of cobalt. It is also found in crimson or peach-red crystals, and is known mineralogically as Erythrine. Whon abundant it is used in the preparation of cobalt colours. See CoBarr.

COzAIT BIU工z, or Thénard's Blue, is prepared by precipitating a solution of sulphate or nitrato of cobalt by phosphato of potash, and adding to the resulting 
gelatinous deposit from three to four times its volume of freshly-deposited alumina, obtained by the addition of carbonate of soda to a solution of common alum. This mixture, aftor being well dried, calcined, and ground, affords a bluo pigment.

COBAIT BRONRz. A violet-coloured substance, with strong metallic lustre. It consists of phosphate of protoxide of cobalt, and phosphate of ammonia.

COBATT, FARTHY. A protoxide of cobalt with oxide of manganese, from Saalfeld, in Thuringia.

CoBaIt erastce. A synonym for cobaltine. Seo Coвatr.

СовАIT GRIIN, or Rinmann's Green. This pigment is a compound of oxide of cobalt and oxide of zinc. It is prepared by mixing a solution of sulphate of zine with a salt of protoxide of cobalt, and precipitating with carbonate of soda; the precipitate is then washed, dried, and heated.

COBAIT SPIrSs. A substance obtained in the preparation of smalts, and consisting chiefly of arsenide of nickel, derived from the nickel associated with the cobalt ore. The speiss is used in the manufacture of nickel.

cobart urtrramarinz. Thénard's Blue. See Cobatt Bluk.

COBAIT YIIIOW. An orange-yellow pigment precipitated from an acidified solution of nitrate of protoxide of cobalt by means of nitrate of potash.

COCA. The leaves of Erythroxylon coca; mixed with burnt lime they are chewed by the natives in parts of South America. See Erryruoxyuor Coca.

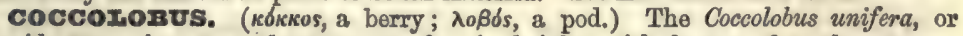
seaside grape, is a tree about twenty feet in height, with leares of a glossy green colour-the nerves being a deep red. The fruit is in bunches, and is eaten in the West Indies. The wood yiolds a red colouring matter, which is used for a dye; and the wood itself, which is hard, is employed in cabinet work. A decoction of this plant is evaporated to form a substance known as Jamaica Kino.

COCCUIUS INDICUS, or Indian berry, is the fruit of the Anamirta panioulata, a large tree, which grows upon the coasts of Malabar, Ceylon, \&c., and belongs to the Menispermacece or Moon-seed order. The fruit is blackish, and of the size of a large pea. It owes its narcotic and poisonous qualities to the vegeto-alkaline chemical principle called picrotoxia, of which it contains about one-fiftieth part of its weight. It is sometimes thrown into waters to intoxicate or kill fishes; and it is said to have been employed to increase the inebriating qualitites of ale or beer. Its use for this purpose is prohibited by Act of Parliament, under a penalty of $200 l$. upon the brewer, and $500 l$. upon the seller of the drug.

However, Dr. Pereira states, 'I am not acquainted with any official roturns of the quantity annually brought over. From a druggist's private books I find that in 1834 above 2,500 bags entered-and this probably is much below the quantity imported. The greater part is consumed for illegal practices-principally for adulterating 'beer and ale.' Morrice, in his treatise on brewing, directs that in the manufacture of porter, three pounds of Cocculus indicus should be added to every ten quarters of malt. 'It gives,' says he, 'an inebriating quality, which passes for strength of liquor ;' and he adds, "that it prevents the second fermentation in bottled beer, and conseguently the bursting of the bottles in warm climates.'

The Editor of this work had a fluid extract, the name of which was unknown to the Custom-house officers, submitted to him some years since. This was an extract of this deleterious drug, of which a very large quantity was then in the London docks.

The powder of the berries mixed with lard is used to destroy pediculi : hence the Germans call those grains Läusekörner, or lousegrains.

cockrrisas. (Cochenille, Fr.; Kochenille, Ger.) Cochineal was first taken for a soed, but was proved by Leeuwenhoock to be an insect, the female of that species of shield-louse, or coccus, discovered in Mexico so long ago as 1518. It is brought to us from Mexico, where the animal lives upon the Cactus opuntia or Nopal. Two sorts of cochineal are gathered : the wild from the woods, called by the Spanish name Grana silvestra; and the cultivated, or the Grana fina, termed also Mesteque, from the name of a Mexican province. The first is smaller, and covered with a cottony down, which increases its bulk with a matter useless in dyeing ; it yields, therefore, in equal weight, much less colour, and is of inferior price to that of the fine cochineal. But these disadvantages are compensated in some measure to the growers by its being reared more easily and less expensively; partly by the effect of its down, which enables it better to resist rains and stormg.

The wild cochineal, when it is bred upon the field nopal, loses in part the tenacity and quality of its cotton, and acquires a size double of what it has on the wild opuntias. It may, therefore, be hoped that it will be improved by persevering care in the rearing of it, when it will approach more and more to fine cochineal.

The fine cochineal, whon well dried and well preserved, should have a grey colour 
bondaring on purple. The grey is owing to the powder which naturally covers the insects, of which a little adheres, and to a waxy fat. The purple shade arises from the colour extracted by the wator in which they were killed. The grain is wrinkled with parallel furrows across its back, which are intersected in the middle by a longitudinal ono: hence, when viowed by a magnifier, or evon a sharp naked eye, especially after being swollen by soaking for a short time in water, it is easily distinguished from the factitious, smooth, glistening, black grains, of no value, called East India cochinoal, with which it is often shamefully adulterated by certain London merchants. The genuine cochineal has the shape of an egg, bisected through its long axis, or of a tortoise, being rounded like a shield upon the back, flat upon the belly, and without wings.

These female insects are gathered off the leaves of the nopal plant after it has ripened its fruit, a few only being left for brood : they are killed either by a momentary immersion in boiling water, by drying upon heated plates or in ovens: the last become of an ash-grey colour, constituting the silver cochineal, or jaspeada; the second are blackish, called negra, and are most esteemed, boing probably driest: the first aro reddish brown, and reckoned inferior to tho other two. The dry cochineal being sifted, the dust, with the imperfect insects and fragments which pass through, are sold under the name of Granillo. Cochineal keeps for a long time in a dry place. Hellot says that he has tried some 130 years old, which produces the same effect as new cochineal.

We are indebted to MIM. Pelletier and Caventou for a chemical investigation of cochineal, in which its colouring matter was skilfully eliminatod.

Purified sulphuric ether acquired by digestion with it a golden-yellow colour, amounting, according to Dr. John, to one-tenth of the weight of the insect. This infusion left, on evaporation, a fatty wax of the same colour.

Cochineal, exhausted by ether, was treated with alcohol at $40^{\circ} \mathrm{B}$. After 30 infusions in the digester of M. Chevreul, the cochineal continued to retain colour although the alcohol had ceased to have any effect on it. The first alcoholic liquors were of a red verging on yellow. On cooling, they let fall a granular matter. By spontaneous evaporation, this matter, of a fine red colour, separated, assuming more of the crystalline appearance. These species of crystals dissolved entirely in water, which they tinged of yellowish red.

This matter has a very brilliant purple-red colour; it adheres strongly to the sides of the vessels; it has a granular and somewhat crystalline aspect, very different, however, to those compound crystals alluded to above; it is not altered by tho air, nor does it sensibly attract moisture. Exposed to the action of heat, it melts at about $50^{\circ}\left(122^{\circ} \mathrm{F}\right.$.). At a higher temperature it swells up, and is decomposed with the production of carburetted hydrogen, much oil, and a small quantity of water, very slightly acidulous. No trace of ammonia was found in these products.

The colouring principle of cochineal is very soluble in water. By evaporation, the liquid assumes the appearance of syrup, but never yields crystals. It requires of this matter a proportion almost imponderable to give a perceptible tinge of bright purplish red to a large body of water. Alcohol dissolves this colouring substance, but, as we have already stated, the more highly it is rectified, the less of it does it dissolvo. Sulphuric other does not dissolve the colouring principle of cochineal; but weak acids do, possibly owing to their water of dilution. No acid precipitates it in its pure state. This colouring principle, however, appears to be precipitable by all the acids when it is accompanied by the animal matter of the cochineal.

The affinity of alumina for the colouring matter is very remarkable. When that earth, newly precipitated, is put into a watery solution of the colouring principlo, this is immediately seized by the alumina. The water becomes colourless, and a fine red lake is obtained, if we operate at the temperature of the atmosphere; but if the liquor has been hot, the colour passes to crimson, and the shade becomes more and more violet, according to the elevation of the temperature, and the continuance of the ebullition.

The salts of tin exercise upon the colouring matter of cochineal a remarkable action. The proto-chloride of tin forms a very abundant violet precipitate in the liquid. This precipitate verges on crimson, if the salt contains an excess of acid. The bi-chloride of tin produces no precipitate, but changes the colour to scarlet-red. If gelatinous alumina be now added, we obtain a fine red precipitate, which does not pass to crimson by boiling.

To this colouring principle the name carminic acid has been given. It forms the basis of the beautiful pigment called carmine. A very complete examination of the colouring matter of the cochineal insect has been made by Dr. Warren De La Rue. See Carmine.

The carmines found in the shops of Paris have been analysed, and all yielded the same 
products. They were decomposed by the action of heat, with the diffusion at first of a very strong smell of burning animal matter, and then of sulphur. A white powder remained, amounting to about one-tenth of the matter employed, and which was found to be alumina. Other quantities of carmine were treatod with a solution of caustic potash which completely dissolved them; with the exception of a fine red powder, not acted on by potash and concentrated acid, and which was recognised to be red sulphurot of mercury or vermilion. This matter, evidently foreign to the carmine, appears to have boen added, in order to increase its weight.

The preceding observations and experiments seem calculated to throw some light on the art of dyeing scarlet and crimson. The former is effected by employing a cochineal bath, to which there have been added, in determinate proportions, acidulous tartrate of potash and nitro-muriatic deutoxide of tin. The effect of these two salts is now well known. The former, in consequence of its excess of acid, tends to redden the colour, and to precipitate it along with the animal matter; the latter acts in the same manner, at first by its excess of acid, then by the oxide of tin which falls down also with the carmine and animal matter and is fixed on the wool, with which it has of itself a strong tendency to combino. MM. Pelletior and Caventou remark, that "to obtain a beautiful shade, the muriate of tin ought to be entirely at the maximum of oxidisement; and it is in reality in this state that it must exist in the solution of tin prepared according to the proportions prescribed in M. Berthollet's treatise on dyeing.'

We hence see why, in dyeing scarlet, the employment of alum is carefully aroided, as this salt tends to convert the shade to a crimson. The presence of an alkali would seem less to be feared. The alkali would occasion, no doubt, a crimson-coloured bath; but it would be easy in this case to restore the colour, by using a large quantity of tartar. We should, therefore, procure the advantage of having a bath better charged with colouring matter and animal substance. It is for experience on the large scale to determine this point. As to the earthy salts, they must be carefully avoided.

To obtain crimson, it is sufficient, as we know, to add alum to the cochineal bath, or to boil the scarlet cloth in alum water. It is also proper to diminish the dose of the salt of tin, since it is found to counteract the action of the alum.

The alkalis ought to be rejected as a means of changing scarlet to crimson. In fact, crimsons by this process cannot be permanent colours, as they pass into red by the action of acids.

According to M. Von Grotthuss, carmine may be deprived of its golden shade by ammonia, and subsequent treatment with acetic acid and alcohol. Since this fact was made known, M. Herschel, colour-maker at Halle, has prepared a most beautiful carmine.

The officers of Her Majesty's Customs detected some time since a system of adulterating cochineal, which had been practised for many years upon a prodigious scale by a mercantile house in London. Dr. Ure stated that he had analysed about 100 samples of such cochineal, from which it appears that the genuine article is moistened with gum-wator, agitated in a box or leather bag, first, with sulphate of baryta in fine powder, afterwards with bone or ivory black, to give it the appearance of negra cochineal, and then dried. By this means about 12 per cent. of worthless heavy spar is sold at the price of cochineal, to the enrichment of the sophisticators, and the disgrace and injury of British trade and manufactures.

The specific gravity of genuine cochineal is 1.25 ; that of the cochineal loaded with the barytic sulphate, 1.35. This was taken in oil of turpentine, and reduced to water as unity, because the waxy fat of the insects prevents the intimate contact of the lattor liquid with them, and the ready expulsion of air from their wrinkled surface. They are not all acted upon by the oil, but are rapidly altered by water, especially when they have been gummed and barytified.

Humboldt states that so long ago as the year 1736, there was imported into Europe from South America, cochineal to the value of 15 millions of francs. Its high price had for a long time induced dyers to look out for cheaper substitutes in dyeing red; and since science has introduced so many improvements in tinctorial processes, both madder and lac have been mado to supersede cochineal to a very great extent.

In order to ascertain the value of cochineal for dyeing we must have recourse to comparative experiments. We are indebted to MM. Robiquet and Anthon for two methods of determining the quality of cochineals, according to the quantity of carmine they contain. The process of $\mathbf{M}$. Robiquet consists in decolourising equal volumes of decoction in different cochineals by chlorine. By using a graduated tube, the quality of the cochineal is judged of by the quantity of chlorine employed for decolourising the decoction. The process of M. Anthon is founded on the property which the bydrate of alumina possesses of precipitating the carmine from the decoction so as to 
decolourise it entirely. The first process, which is very good in the hands of a skilful chomist, does not appear to us to be a convenient method for the consumer; in the first place, it is difficult to procure perfectly identical solutions ; in the next place, it is impossible to keep them a long time without alteration. Wo know that chlorino dissolvod in water roacts, oven in diffused light, on this liquid; decomposes it, appropriates its olements, and gives rise to some compounds which possess an action quito different from that of the chlorine solution in its primitive state. The second process seems to us to be preferable, as the proof liquor may be kept a long while without alteration. A graduated tube is also used; each division represents one hundredth of the colouring matter. Thus the quantity of proof liquor added exactly represents the quantity in hundredths of colouring matter contained in the decoction of cochineal which has been submitted to examination. The following remarks from a practical dyer are valuable:-

- The colouring matter of cochineal being soluble in water, I have used this solvent for exhausting the different kinds which I have submitted to examination in the colourimeter. I operated in the following manner: I took a grain of each of the cochineals to be tried, dried at $120 \mathrm{Fahr}$.; I submitted them five consecutive times to the action of 200 grains of distilled water at water-bath heat, each time for an hour; for every 200 grains of distilled water I added two drops of a concentrated solution of acid sulphate of alumina and of potash. This addition is necessary to obtain the decoctions of the different cochineals exactly of the same tint, in order to be able to compare the intensity of the tints in the colourimeter. ${ }^{1}$

'In order to estimate a cochineal in the colourimeter, two solutions, obtained as described above, are taken; some of these solutions are introduced into the colourimetric tubes as far as zero of the scale, which is equivalent to 100 parts of the superior scale ; these tubes are placed in the box, and the tint of the liquids enclosed is compared by looking at the two tubes through the eye-hole; the box being placed so that the light falls exactly on the extremity where the tubes are. If a difference of tint is observed between the two liquors, water is added to the darkest (which is always that of the cochineal taken as type) until the tubes appear of the same tint. ${ }^{2}$

- The number of parts of liquor which are contained in the tube to which water has been added is then read off; this number, compared with the volume of the liquor contained in the other tube, a volume which has not been changed, and is equal to 100 , indicates the relation between the colouring power and the relative quality of the two cochineals. And if, for example, 60 parts of water must be added to the liquor of good cochineal, to bring it to the same tint as the other, the relation of volume of the liquids contained in the tubes will be in the case as 160 is to 100 , and the relative quality of the cochineals will be represented by the same relation, since the quality of the samples tried is in proportion to their colouring power.'-Napier.

The exports from Guatemala consist principally of cochineal, the staple and almost the only article of exportation for a number of years past. It is chiofly produced in Old Guatemala, nine leagues distant from Guatemala, and also in Amatellan about six leagues distant. The rearing of this insect is subject to so many accidents and contingencies that it is excessively precarious, and, above all, the weather has a great effect upon it. Taking all this into consideration it is surprising that attention has not been directed to the cultivation and production of other articles suited to the alimate and soil of Guatemala, and less liable to destruction by unseasonable rains and atmospheric changes than cochineal. It is reasonably to be feared that, if a longer time be suffered to pass, the cochineal of this country cannot compete with that of Teneriffe, and other parts of the world, where it is now cultivated.

Our Imports of cochineal have been as follow:-

In 1870 we imported 47,790 cwts., valued at $£ 581,956$.

$\begin{array}{llll}1871 & \quad 64,542, " & & 706,713 . \\ 1872 \quad & 38,160, & \prime & 494,641 .\end{array}$

Cock-Mrrar. An inferior metal; a mixture of copper and lead used for making cocks, See Arroy.

Coco. A species of Aracere, to which the genus Arum gives the name. The rhizomes or underground stems of the cocos (Colocasea) are sometimes substituted for potatoes and yams; they abound in starchy matter.

- Care must be taken not to add to the water, which serves to extract the colouring matter from the different cochineals, more than the requisite quantity of acid sulphate of alumina and solution of potash, becanse a stronger dose would precipitate a part of the colouring matter in the state of lake.

a For diluting the liquors the same water must always be nsed which has served to extrnct the colouring matter of the cochineals under cxamiuation, otherwise the darkest decoction would pasa into violet, us water was added to it, to bring back the tint to the same degree of intensity as that of the decoction to which it is compared. 
cocos. A well-known preparation from the seeds of the Theobroma cacao. It is stated to be made from the fragments of the seed-coats mixed with portions of the kernels. See Cacao.

COcoA-2NUTS. (Noix de Coco, Fr.; Cocosnuss, Ger.) The cocoa-nut tree (Cocos nucifera) is a native of tropical climates. It is one of the most important and valuable of the palms.

CocoA-2rux orr. Cocoa-nut oil is obtained by two processes: one is by pressure, the other by boiling the bruised nut and skimming off the oil as it forms on the surface.

It is a white solid, having a peculiar odour. It fuses a little above $70^{\circ} \mathrm{F}$.; becomes readily rancid, and dissolves easily in alcohol. It consists of a solid fat called cocin or cocinine (a combination of glycerine and cocinic, or coco-stearic acid), and of a liquid fat or oloine. Cocos-nut oil is used in the manufacture of soap and candles.

Mr. Spames obtained a patent in September, 1829, for making stearine and elaine by the following process :-

Ho takes the substance called cocos-nut oil, in the state of lard, in which it is imported into this country, and submits it to a strong hydraulic pressure, having made it up in small packages, 3 or 4 inches wide, 2 feet long, and 1 or $1 \frac{1}{2}$ inch thick. These packages are formed by first wrapping up the said substance in a strong linen cloth, of close texture, and then in an outward wrapper of strong sail-cloth. The packages are to be placed side by side, in single rows, between the plates of the press, allowing a small space between the packages for the escape of the elaine.

The temperature at which the pressure is begun, should be from about $50^{\circ}$ to $55^{\circ}$, or in summer as nearly at this pitch as can be obtained, and the packages of the said substance intended for pressure should be exposed for several hours previously to about the same temperature. When the packages will no longer yield their oil or elaine freely at this temperature, it is to be gradually raised; but it must at no time exceed $65^{\circ}$, and the lower the temperature at which the separation ean be effected, the better will be the quality of the oil expressed.

When the packages are sufficiently pressed, that is, when they will give out no more oil, or yield it only in drops at long intervals, the residuum is then to be taken out and cleansed and purified, which is done by melting it in a well-tinned copper vessel, which is fixed in an outer vessel, having a vacant space between, closed at the top, into which steam is admitted, and the heat is kept up moderately for a sufficient time to allow the impurities to subside; but if a still higher degree of purity is required, it is necessary to pass it through filters of thick flannel lined with blotting paper.

Having been thus cleansed or purified, it is fit for the manufacture of candles, which are made by the ordinary process usod in making mould tallow eandles. Having thus disposed of the stearine, or what is called the first product, he proceeds with the elaine or oil expressed from it, and which he calls the second product, as follows: that is to say, he purifies it by an admixture, according to the degree of its apparent foulness, of from 1 to 2 per cent. by weight of the sulphuric acid of commeree, of about 1.80 specific gravity, diluted with six times its weight of water. The whole is then to be violently agitated by mechanical means, and he prefers for this purpose the use of a vessel constructed on the principle of a common barrel churn. When sufficiently agitated, it will have a dirty whitish appearance, and is then to be drawn off into another vessel, in which it is to be allowed to settle, and any scum that rises is to be carefully taken off. In a day or two the impurities will be deposited at the bottom of the oil, which will then become clear, or nearly so, and it is to be filtered through a thick woollen eloth, after which it will be fit for burning in ordinary lamps and for other uses.

The process of separating the elaine from the stearine, by pressure, in manner aforesaid, had never before been applied to the substance called cocoa-nut oil, and consequently no product had heretofore been obtained thereby from that substance fit for being manufactured into candles in the ordinary way, or for being refined by any of the usual modes, so as to burn in ordinary lamps, both which objects are attained by, the method of preparing or manufacturing the said substance.

Candles well made from the above material are a very superior article. The light produced is more brilliant than from the same sized candle made of tallow; the flame is perfectly colourless, and the wick remains free from cinder, or any degree of foulness during combustion. See Castdres; Rianne; Stratrine.

COD. The Morrhua vulgaris, a fish belonging to the family of Gadide.

It is calculated that tho take in Scotland of cod and ling amounts to about $4,000,000$ individuals annually, of which upwards of $1,000,000$ aro from the Shetland Islands.

COD-IIVan ors. The oil obtained from the livers of several varieties of the 
Gadide family; especially from the torsk, Brosimus brosme. It is administored modicinally : it acts mainly as a nutritivo body, and the old idoa that its modicinal value depended on the iodine it contained is now proved to be false, since it holds no iodino in composition. Since the demand for cod-liver oil has been large, it has been extensively adulterated with other fish-oils.

CODErws. $\mathrm{C}^{38} \mathrm{H}^{21} \mathrm{NO}^{6}+2 \mathrm{HO}\left(\mathbf{C}^{18} \mathbf{E}^{2 i} \mathbf{N O O}^{3}+\mathbf{H O}^{2}\right)$. An alkaloïd obtainod from opium, whore it occurs associated with morphine and several othor bases. Codoine was discovered by Robiquet, and has been studied by Dr. Anderson and other chemists. The substitution-compounds formed from codeine by the action of hydrochloric acid were investigated by Drs. Matthiessen and Wright, and those produced by the action of hydrobromic acid have recently been studied by Dr. Wright alone.

CODIIIA OF Fruax. The coarsest parts of the fibre sorted out by itself. See Fina.

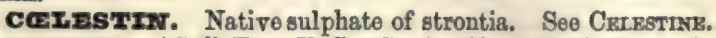

Corrza. (Cafe, Fr. ; Kaffee, Ger.) The coffee is the seed of the Coffea Arabica, a treo of the order Rubiacea, and belongs to the Pentandria monogynia of Linnæus. There are several species of the genus, but the only one cultivated is the Coffea Arabica, a native of Upper Ethiopia and Arabia Felix. It rises to the height of 15 or 20 foot; its trunk sends forth opposite branches in pairs above and at right angles to each other; the leaves resemble those of the common laurel, although not so dry and thick. From the angle of the leaf-stalks small groups of white flowers issue, which are like those of the Spanish jasmine. These flowers fade very soon, and are replaced by a kind of fruit not unlike a cherry, which contains a yellow glairy fluid, onveloping two small seeds or berries convex upon one side, flat and furrowed upon the other in the direction of the long axis. These seeds are of a horny or cartilaginous nature; they are glued together, each being surrounded with a peculiar coriacoous membrane. They constitute the coffee of commerce.

It was not till towards the end of the 15 th century that the coffee-tree began to be cultivated in Arabia. Historians usually ascribe the discovery of the use of coffee as a beverage to the superior of a monastery there, who, desirous of preventing the monks from sleeping at their nocturnal services, made them drink the infusion of coffee upon the report of shepherds, who pretended that their flocks were more lively after browsing on the fruit of that plant. The use of coffee was soon rapidly spread, but it encountered much opposition on the part of the Turkish governmont, and became the occasion of public assemblies, Under the reign of Amurath III. the mufti procured a law to shut all the coffee-houses, and this act of suppression was renewed under the minority of Mahomet IV. It was not till 1554, under Solyman the Great, that the drinking of coffee was accredited in Constantinople; and a century elapsed before it was known in London and Paris. Solyman Aga introduced its use into the latter city in 1669, and in 1672 an Armenian established the first cafe at the fair of Saint-Germain.

When coffee became somewhat of a necessary of life from the influence of habit among the people, all the European Powers who had colonies between the tropics projected to form plantations of coffee-trees in them. The Dutch were the first who transported the coffee plant from Mocha to Batavia, and from Batavia to Amsterdam. In 1714 the magistrates of that city sent a root to Louis XIV., which he caused to be planted in the Jardin du Roi. This became the parent stock of all the French coffee plantations in the Martinique.

The most extensive culture of coffee is still in Arabia Felix, and principally in the kingdom of Yemen, towards the cantons of Aden and Mocha. Although these countries are very hot in the plains, they possess mountains where the air is mild. The coffee is generally grown half-way up on their slopes. When cultivated on the lower ground it is always surrounded by large trees, which shelter it from the torrid sun, and prevent its fruit from withering before their maturity. The harvest is gathered at three periods: the most considerable oceurs in May, when the reapers begin by spreading cloths under the trees, then shaking tho branches strongly, so as to make the fruit drop, which they collect, and expose upon mats to dry. Thoy then piss over tho dried berries a very heavy roller, to break the envelopes, which aro aftorwards winnowed away with a fan. The interior boan is again dried before being laid up in store.

In Demerara, Berbice, and some of our West India islands, where much good coffee is now raised, a different mode of treating the pulpy fruit and curing the beans is adopted. When the cherry-looking berry has assumed a deep-red colour, it is gathered, and immediately subjected to the operations of a mill composed of two wooden rollers, furnished with iron plates, which revolve near a third fixed roller called tho chops. The berries are fed into a hopper abovo the rollers, and falling down between them and the chops, they are stripped of their outer skins and pulp, 


\section{COFFEE}

while the twin beans are separated from each other. These beans then fall upon a sieve, which allows the skin and the pulp to pass through, while the hard beans accnmulate and are progressively slid over the edge into baskets. They are next steeped for a night in water, thoroughly washed in the morning, and afterwards dried in the sun. They are now ready for the peeling mill, a wooden edge-wheel turned vertically by a horse yoked to the extremity of its horizontal axis. In travelling over the coffee, it bursts and detaches the coriaceous or parchment-like skin which surrounds each hemispherical bean. It is then freed from the membranes by a winnowing machine, in which four pieces of tin made fast to an axle are caused to revolve with great velocity. Corn fanners would answer better than this rude instrument of negro invention. The coffee is finally spread upon mats or tables, picked clean and packed up for shipment.

The most highly-esteemed coffee is that of Mocha. It has a smaller and a rounder bean; a more agreeable taste and smell than any other. Its colour is yellow. Next to it in European reputation is the Martinique and Bourbon coffeo; its colour is greenish, and it preserves almost always a silver grey pellicle, which comes off in the roasting.

The Bourbon coffee approaches nearest to the Mocha, from which it originally sprung. The Saint Domingo coffee has its two extromities pointed, and is much less esteemed then the preceding.

The coffee-tree flourishes in hilly districts where its root can be kept dry, while its leaves are refreshed with frequent showers. Rocky ground, with rich docomposed mould in the fissures, agrees best with it. Though it would grow, as we have said, to the height of 15 or 20 feet, yet it is usually kept down by pruning to that of 5 feet for increasing the production of the fruit, as well as for the convenience of cropping. It begins to yield fruit the third year, but is not in full bearing till the fifth, does not thrive beyond the twenty-fifth, and is useless in general at the thirtieth. In the coffee husbandry the plants should be placed 8 feet apart, as the trees throw out extensive horizontal brenches, and in holes 10 or 12 feet deep to secure a constant supply of moisture.

Coffee has been analysed by a great many chemists, with considerable diversity of results. The best analysis perhaps is that of Schräder. He found that the raw beans distilled with water in a retort communicated to it their flavour and rendered it turbid, whence they seem to contain some volatile oil. On reboiling the beans, filtering, and evaporating the liquor to a syrup, adding a little alcohol till no more matter was precipitated, and then evaporating to dryness, he obtained 17.58 per cent. of a yellowish. brown transparent extract, which constitutes the characteristic part of coffee, though it is not in that state the pure proximate principle, called caffeine. Its more remaris. able reaction is its producing, with both the protoxide and the peroxide salts of iron, a fine grass-green colour, while a dark green precipitate falls, which re-dissolves when an acid is poured into the liquor. It produces on the solution of the salts of coppers scarcely any effect, till an alkali be added, when a very beautiful green colour is produced which may be employed in painting. Coffee beans contain also a resin, and a fatty substance somewhat like suet. According to Robiquet, ether extracts from coffee beans nearly 10 per cent. of resin and fat, but he probably exaggerates the amount. The peculiar substance caffeine contained in the above extract is crystallisable. It is remarkable in regard to composition, that after urea and the uric acid, it is among organic products the richest in nitrogen. It was dissolved and described in 1820 by Rungé. It does not possess alkaline properties. Pfaff obtained only 90 grains of caffeine from six pounds of coffee beans. There is also an acid in raw coffee to which the name of caffeic acid has been given. When distilled to dryness and decomposed, it has the smell of roasted coffee. See CaFreins.

Coffee undergoes important changes in the process of roasting. When it is roasted to a yellowish brown it loses, according to Cadet, $12 \frac{1}{2}$ per cent. of its weight, and is in this state difficult to grind. When roasted to a chestnut brown it loses 18 per cent. i and when it becomes entirely black, though not all carbonised, it has lost 23 per cent. Schräder has analysed roasted coffee comparatively with raw coffee, aud ho found in the first $12 \frac{1}{2}$ per cent. of an extract of coffee soluble in water and aleohol, which possesses nearly the properties of the extract of the raw coffee, although it has a deeper brown colour, and softens more readily in the air. He found also 10.4 of a blackish brown gum; 5.7 of an oxygenated extract, or rather apardeme soluble in alcohol, insoluble in water; 2 of a fatty substance and resin; 69 of burnt vegetable fibre, insoluble. On distilling roasted coffee with water, Schräder obtained a product which contained the aromatic principle of coffee; it reddened litmus paper, and exhaled a strong and agreeable odour of roasted coffee. If we roast coffee in a retort, the first portions of the aromatic principle of coffee condense into a yellow liquid in the receiver; and these may be added to the coffee roasted in the common way, from which this matter has boen expelled and dissipatod in the air.

Vox. I. 
Chenevix affirmed that by the roasting of coffee a certain quantity of tannin posressing the property of precipitating gelatine is generated. Cadet made the same observation, and found moreover, that the tannin was most abundant in the lightly roasted coffee, and that there was nearly none of it in coffee highly roasted. Passe and Schräder, on the contrary, state that solution of gelatine does not precipitate either the decoction of roasted coffee or the alcoholic extract of this coffee. Rungé likewise asserts that he could obtain no precipitate with gelatine; but he says that albumen precipitates from the decoction of roasted coffee the same kind of tannin as is precipitated from raw coffee by the acetate of lead, and set free from the lead by sulphuretted hydrogen. With these results Dr. Ure's experiments agreed. Gelatine certainly does not disturb clear infusion of roasted coffee, but the salts of iron blacken it.

Schräder endeavoured to rosst separately the different principles of coffee, but none of them exhaled the aromatic odour of roasted coffee, except the horny fibrous matter. He therefore concluded that this substance contributes mainly to the characteristic taste of roasted coffee, which cannot be imitated by any other vegetable matter, and which, as we have seen, should be ascribed chiefly to the altered caffeic acid. According to Garot, we may extract the caffeine without alteration from roasted coffee by precipitating its decoction by subacetate of lead, treating the washed precipitate with sulphuretted hydrogen, and evaporating the liquid product to dryness.

To roast coffee rightly we should keep in view the proper objects of this process, which are to develop its aroma, and destroy its toughness, so that it may be readily ground to powder. Too much heat destroys those principles which we should wish to preserve, and substitutes new ones which have nothing in common with the first, but add a disagreeable empyreumatic taste and smell. If, on the other hand, the rawness or greenness is not removed by an adequate heat, it masks the flavour of the bean, and injures the beverage made with it. When well roasted in the sheet-iron cylinder set to revolve over a fire, it should have a uniform chocolate colour, a point readily hit by experienced roasters, who now manage the business for the principal coffee dealers both of London and Paris. The development of the proper aroma is a criterion by which coffee roasters frequently regulate their operations. When it loses more than 20 per cent. of its weight, coffee is sure to be injured. It should never be ground till immediately before infusion, since the fine essential oil rapidly escapes from the finely-divided coffee.

Liebig's views of the process of nutrition have given fresh interest to every analysis of articles of food. A watery infusion of coffee is used in almost every country as a beverage, and yet it is uncertain whether it is an article of nutrition or merely a condiment. A minute examination of the raw seed, or coffee bean as it is called, must precede the determination of that disputed point. Caffeine is the principle best known, being most easily separated from the other substances, resisting most powerfully chemical reagents, and by assuming a crystalline state is discoverable in very small quantitios.

Roasted coffee affords a much richer infusion to hot water containing a minute quantity of carbonate of soda, and improves the quality of the coffte on the stomach, by neutralising the caffeic acids.

Coffee, as sold in the shops in its roasted and ground state, is often adulterated with a variety of substances, but chiefly with chicory. See CHICory.

If tannin exists in roasted coffee, as maintained long ago by Chenevix, and generally admitted since, it must be very different from the tannin present in tea, catechu, kino, oak-bark, willow-bark, and other astringent vegetables ; for it is not, like them, precipitated by either gelatine, albumen, or sulphate of quinine. With regard to the action upon the animal economy of coffee, tea, and cocoa, which contain one common chemical principle called caffeine or thoine, Liebig advanced some ingenious views, and, in particular, endeavoured to show that, to persons of sedentary habits in the present refined state of socioty they afford eminently useful beverages, which contribute to the formation of the characteristic principle of bile. This important secreted fluid, deemed by Liebig to be subservient to the function of respiration, requires for its formation much azotised matter, and that in a state of combination analogous to what exists in caffeine. The quantity of this principle in tea and coffee being only from 2 to 5 per cont., might lead one to suppose that it could have little effect upon the system even of regular drinkers of these infusions; but if the bile contains only one-tenth of solid matter, called choleic acid, which contains less than 4 per cont. of azote, then it may be shown that 3 grains of caffeine would impart to 500 grains of bile the azoto which occurs in that crystalline precipitate of bile called taurine, which is thrown down from it by mineral acids.

One atom of caffoine, 9 atoms of oxygen, and 9 of water, being added together, produce the composition of 2 atoms of taurine. Now this is a very simple combi- 
nation for the living organism to effect; one already paralleled in the generation of hippuric acid in urine, by the introduction of benzoic acid into the stomach : a physiological discovery which promised to lead to a more successful treatment of some of the most formidable diseases of man, particularly gout and gravel.

Cofress Roasting aND GrINDing. The gratefulness of the beverage afforded by this seed depends upon many circumstances, which are soldom all combined. It may be ruined in the roasting; for if some berries be under and some over done, the whole when ground will yield an unpalatable infusion. The due point to which the torrefaction should be earried may be determined partly by the colour and partly by the loss of weight, which points, however, are different for each sort of coffee. But perfect equality of ustulation is difficult of attainment with the ordinary cylindrical machines. Messrs. Law, of London and Edinburgh, had long been dissatisfied with the partial mannex in which the cylinder performed its duty, as it generally left some part of its contents black, some dark brown, and others paler; results which greatly injure the flavour of the beverage made with the coffee. Mr. William Law overcame these difficulties by his invention of the globular roaster, actuated by a compound motion like that of our earth. This rosster, with

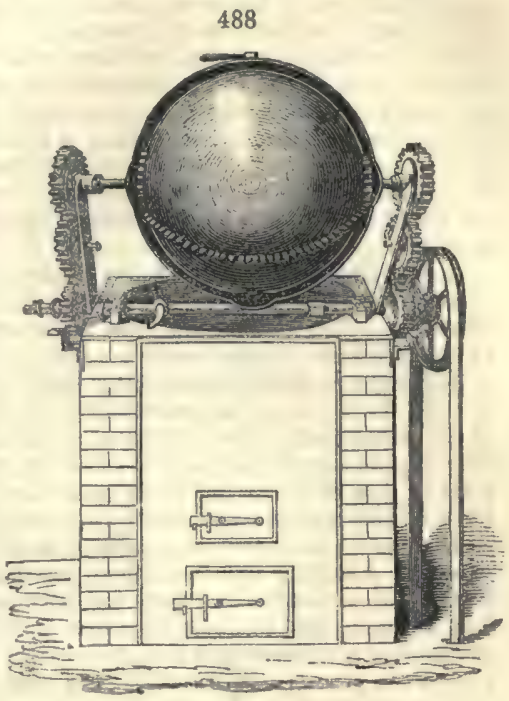
its double rotatory motion, is heated not over an open fire, but in an atmosphere of hot air, through a cast-metal casing. The globe is so mounted as to revolve horizontally, and also from time to time vertically, whereby the included beans are tossed about and intermingled in all directions, and inequality of torrefaction is scarcely possible. The position of the globe in fig. 488 shows it as turned up by a powerful levorage out of the cast-iron heater, preparatory to its being emptied and re-charged.

Messrs. Dakin and Co. have patented, and have now for some years used, another kind of apparatus, which consists mainly of steam-chests upon the upper surface of which the coffee is roasted. The advantages of this arrangement appear to be that the coffee berries are spread over the heated surface in a thin layer, and thus every berry is equally exposed to the regulated action of the steam heat. The whole being exposed to view, it can be seen when the roasting has been carried to its proper point.

Our Imports of coffee have been as follow:-

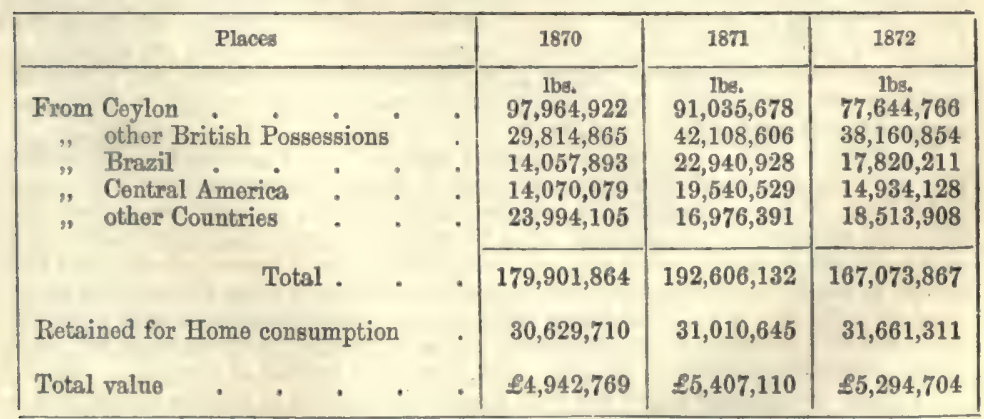

coavsc. The finest kind of French brandy, distilled from wine-so called from the town of Cognac, in the department of Charente. See Brandy.

corr. The outer coating of the cocoa-nut, often weighing one or two pounds, when stripped off longitudinally, furnishes the fibres called by the native name of Coir, and used for small cables and rigging.

In England these fibres are used in matting and for coarse brush work. In Price

$$
3 \text { I. } 2
$$


and Co.'s works they are advantageously employed, placed betweon iron trays and on the surface of the cocos-nut and other concrete oils and fats, and subjected to great pressure; the liquid oil flows out, leaving solid fats behind. From the abundance, cheapness, and durability of this substance it is likely to come into more general use, and it has even been seriously proposed as a material for constructing ocean tolegraphs, from its lightness and power of resisting sea-water. The qualities of coir for many purposes have been established for ages in the East Indies. Dr. Gilchrist thus describes the properties of coir ropes:- "They are particularly elastic and buoyant, floating on the surface of the sea; therefore when, owing to the strength of the current, a boat misses a ship, it is usual to veer out a quantity of coir, having previously fastened an oar, or small cask, \&c., to its end. Thus the boat may be easily enabled to haul up to the ship's stern. Were a coir hawser,' he adds, 'kept on board every ship in the British marine, how many lives would probably be saved!'

It is stated that fresh water rots coir in a very short time, corroding it in a surprising degree, whereas salt water absolutely strengthens it, seeming to increase the elasticity. Coir is therefore unfit for running rigging, especially for vessels subject to low latitudes, it being easily snapped in frosty weather.

Nothing can equal the ease with which a ship rides at anchor, when her cables are of coir. As the surges approach the bows, the vessel gradually recedes in consequence of the cables yielding to their force; but as soon as they have passed, it contracts again, drawing the vessel gradually back to her first position : the lightness of the material adds to this effect, for the eable would float if the anchor did not keep it down. At the present time the forces exerted upon cables and the angles assumed under different circumstances, in paying out submarine telegraphic. cables, have been the subject of practical attention and theoretical investigation. Some of the greatest authorities have assumed that the forces exerted, between the bottom of the sea and the ship's stern, had reference only to forms or waves of the cables, representing some curve between the vertical and horizontal line, but always concave to the water surface. For a curre to exist, in the opposite direction, was named only as a condition, without evidence of Bny practical kind to show that it really existed, or called for any attention to investigate it. So long since, however, as 1825 , Dr. Gilchrist, among others, had described this very opposite curve of the coir ; viz., of being, when in action as a cable, curved with a concave surface toward the bottom of the sea; a fact well known to the experienced sailors of England, as well as to the natives who employ these coir cables so extensively on the East Indian coast.

-A hempen cable always makes a curve downwards, between the vessel and the anchor, but a coir cable makes the curve upwards. Therefore, if a right line were drawn from the hawse-hole to the ring of the anchor, it would be something like the axis of a parabolic spindle, of which the cables would form, or nearly so, the two elliptic segments."

In the employment of materials for ocean telegraphs, especially for deop-sea purposes, the use of iron and the proposal for using coir and other light substances, have caused the telegraphic means to be spoken of as 'heavy' or 'light' cables. Dr. Allan, of Edinburgh, proposed the abundant use of coir to make a light cable, say, half the weight of the lightest hitherto made, the Atlantic cable. He states that a deep sea cable may be compounded to weigh not more than $10 \mathrm{cwt}$. per mile: while the cheapness, durability in salt water, lightness, and abundant supply, will give it advantages over gutta percha and other substances used to form the bulk of the lightest cables hitherto employed.

When cocoa-nuts are sawed into two equal parts across the grain of the coir coating, they form excellent table brushes, causing wood planks to assume a very high polish by friction. If the shell should be left, the edges should be perfectly smooth, and then they will not scratch. It is a good mode to strip off the coir, and, after soaking it in water, to beat it with a heavy wooden mall until the pieces become pliant, when they should be firmly bound together with an iron ring; the ends being levelled, the implement is fit for use; a little bees'-wax, rubbed occasionally upon them, adds greatly to the lustre of the furniture; of course the polish is mainly due to strength and repid action producing the friction upon the wood, and other articles of furniture.

In India, the coarse bark of the nuts is extensively used to cleanse houses, and in washing the decks of vessels. Coarse stuff, matting, and bagging, are made of the fibres, as well as ropes, sails, and cables.

The general preparation is simple:-The fibrous husks or coats which envelope the cocoa-nuts, after being for some time soaked in water, become soft; they are then beaten, to separate other substances with which they are mixed, which fall away like saw-dust, the strings or fibres being left; this is spun into long yarns, woven into sailcloth, and twisted into cables, even for large ressels. Cordage thus mado is con. 
sidered preferable, in many respects, to that brought from Europe, especially the advantage of floating in water.

coxr. (Eng. and Fr.; Coaks, Ger.) It is necessary to distinguish between what is called gas-coke and oven-coke. The word 'coke' applies, properly, to the latter' alone; for in a manufacturing sense, the former is merely cinder. The production of good coke requires a combination of qualities in coal not very frequently met with; and hence first-rate coking coals can be procured only from certain districts. The essential requisites are, first, the presence of very little earthy or incombustible ash; and, secondly, the more or less infusibility of that ash. The presence of any of the salts of lime is above all objectionable; after which may be classed silica and alumina; for the whole of these have a strong tendency to produce a vitrification, or slag, upon the bars of the furnace in which the coke is burnt; and in this way the bars are speedily corroded or burnt out; whilst the resulting clinker impedes or destroys the draught, by fusing over the interstices of the bars or air passages. Iron pyrites is a common obstacle to the coke-maker; but it is found in practice, that a protracted application of heat in the oven dissipates the whole of the sulphur from the iron, with the production of bisulphuret of carbon and metallic carburet of iron, the latter of which alone remains in the coke, and, unless silica be present, has no great disposition to vitrify after oxidation. Where the iron pyrites exists in large quantities it is separated by the coal-washing machines, some of which will be described in a general article.-See Wasmive Mucrunes. One object, therefore, gained by the oven-coke manufacturer over the gas-maker is the expulsion of the sulphuret of carbon, and consequent purification of the residuary coke. Another, and a still more important consequence of a long sustained and high heat is, the condensation and contraction of the coke into a smaller volume, which, therefore, permits the introduction of a much greater weight into the same space; an advantage of vast importance in blast furnaces, and above all, in locomotive engines, as the repeated introduction of fresh charges of coal fuel is thus prevented. Part of this condensation is due to the weight of the superineumbent mass of coal thrown into the coke oven, by which (when the coal first begins to cake or fuse together) the particles are forced towards each other, and the cavernous character of cinder got rid of : but the chief contraction arises, as we have said, from the natural quality of carbon, which, like alumina, goes on contracting, the longer and higher the heat to which it is exposed. Hence, good coke cannot be made in a short time, and that used in locomotive engines is commonly from 48 to 96 , or even 120 hours in the process of manufacture.

The prospects of improvement in coke-making point rather to alterations in the oven than in the process. Formerly it was not thought possible to utilise the heat evolved by the gaseous constituents of the coal; but now, as an example of the incorrectness of this idea, it may be stated that at the Felling Chemical Works, 200 tons of salt per woek are made by the waste heat alone, and it is also employed in partially heating the blast for one of the furnaces. There appears no valid reason why sets of coke-ovens might not be so arranged as mutually to compensate for each other, and produce upon one particular flue a constant and uniform effect. Contrivances of this kind have been projected; but hitherto, wo may suppose, without uniform success, as many of our large cokemakers still continue the old mode of working.

The following figure, 489, represents a Shachtofen, or pit-kiln, for coking coals in Germany, $a$ is the lining (chemise), made of fire-bricks; the enclosing walls are built of the same material ; $b, b$, is a cast-iron ring covered with a cast-iron plate $o$. The floor of the kiln is massive. The coals are introduced, and the coke taken out, through a hole in the side $d$; during the process it is bricked up, and closed with an iron door. In the surrounding walls are four horizontal rows or flues $e, e, e, e$, which are usually iron pipes; the lowest row is upon a level with the floor of the kiln; and the others are each respectively one foot and a half higher than the preceding. Near the top of the shaft there is an iron pipe $f$, of from 8 to 10 inches in diameter, which allows the incoercible vapours generated in the coking to escape into the condenser, which consists either of wood or brick chambers. For kindling the coal, a layer of wood is first placed on the bottom of the kiln.

The coking of small coal is performed upon vanited hearths, somewhat like bakers? 
ovens, but with still flatter roofs. Of such kilns, several are placod alongside one another, each being an ollipse deviating little from a circle, so that the mouth may project but a small spaco. The dimensions are such, that from 10 to 12 cubic foot of coal culm may be spread in a layer 6 inches deep upon the sole of the furnace.
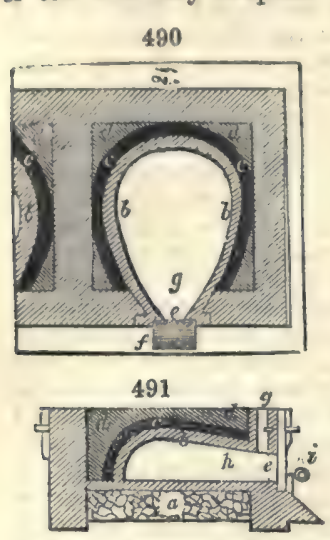

The top of the flat arch of fire brick should be covered with a stratum of loam and sand.

Figs. 490 and 491 represent such a kiln as is mounted at Zabrze, in Upper Silesia, for coking small coal. Fig. 490 is the ground plan; fig. 491 the vertical section in the line of the long axis of fig. 490 . $a$ is the sand-bed of the hearth, under the brick sole; $b$ is the roof of large fire-bricks; $c$, the covering of loam; $d$, the top surface of sand; $e$ the orifice in the front wall, for admission of the culm, and removal of the coke over the sloping stone $f$. The flame and vapours pass off above the orifice, through the chimney marked $g$, or through the aperture $h$, into a lateral chimney. $i$ is a bar of iron laid across the front of the door, as a fulerum to work the inon rake upon. A layer of coals is first kindled upon the hearth, and when this is in brisk ignition, it is covered with the culm in successive sprinklings. When the coal is sufficiently coked, it is raked out and quenched with water.

Fig. 492 represents a simple coking Meiler or mound, constructed in a circular form round a central chimney of loose bricks, towards which small horizontal flues are laid among the lumps of coals. The sides and top are covered with culm or slack, and the heap is kindled from certrin openings towards the circumforence. Fig. 493 represents an oblong Meiler, sometimes made 100 or 150 feet in length, and from 10 to

492

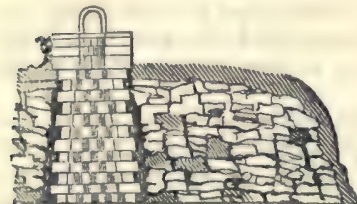

493

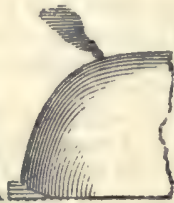

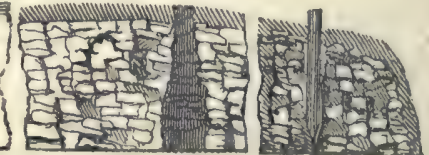

12 in breadth. The section in the middle of the figure shows how the lumps are piled up, ; the wooden stakes are lifted out when the heap is finished, in order to introduce kindlings at various points; and the rest of the Meiler is then covered with slack and clay, to protect it from the rains. A jet of smoke and flame is seon issuing from its loft end.

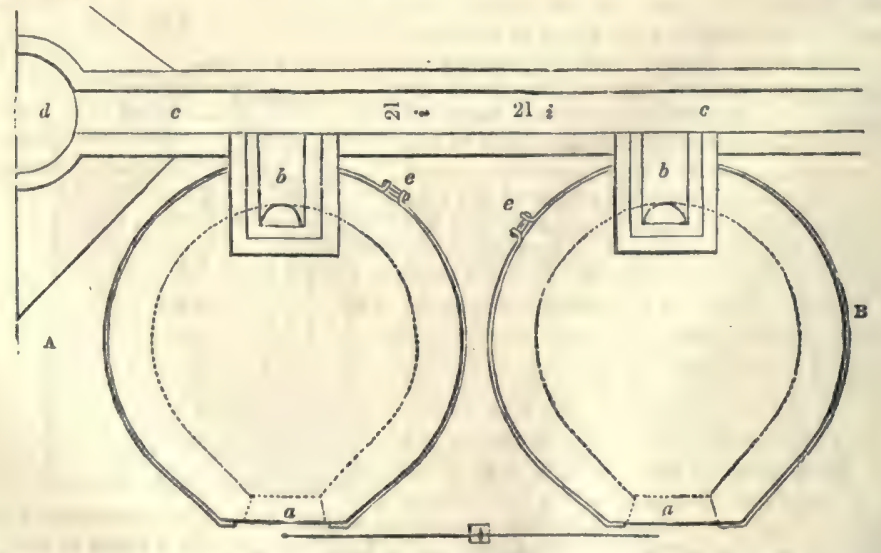

An excellent range of furnaces for making a superior article of coke, for the service of locomotive engines of the London and Birmingham Railway Company, has been 
erected at the Camden Town station, consisting of 18 ovens in two lines, the whole discharging their products of combustion into a horizontal fluo, which terminates in a chimney-stack 115 feot high. Fig. 494 is a ground plan of the elliptical ovens, each being 12 feet by 11 internally, and having 3 feet thickness of walls. $a, a$ is the mouth, $3 \frac{1}{2}$ feet wide outside, and about $2 \frac{3}{4}$ feet within. $b, b$ are the entrances into the

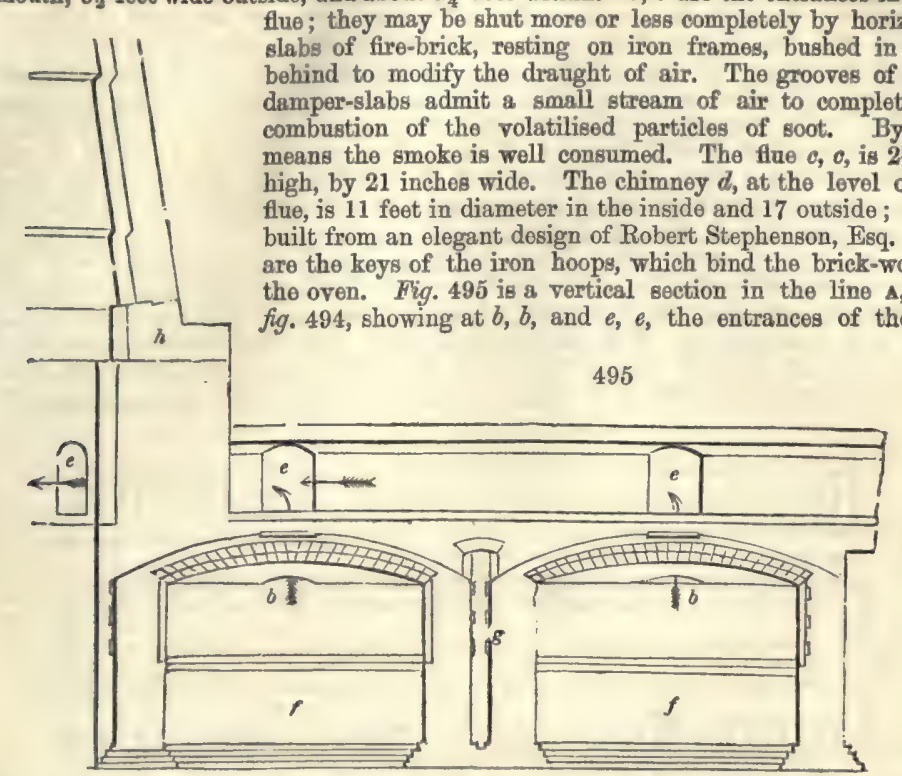

ferent ovens into the horizontal flue; the direction of the draught being indicated by the arrows. $f, f$, is a bed of concrete, upon which the whole furnace range is built, the level of the ground being in the middle of that bed. $g$ is a stanchion on which the crane is mounted; $h$ is a section of the chimney wall, with part of the interior to the left of the strong line. Fig. 496 is a front elevation of two of these elegant coke ovens; in which the bracing hoops $i, i, i$, are shown; $k, k$ are the cast-iron doors, strengthened outside with diagonal ridges; each door being $5 \frac{1}{4}$ foet high, by 4 feet wide, and lined internally with fire-bricks. They are raised and lowered by means of chains and counterweights, moved by the crane $l$.

496

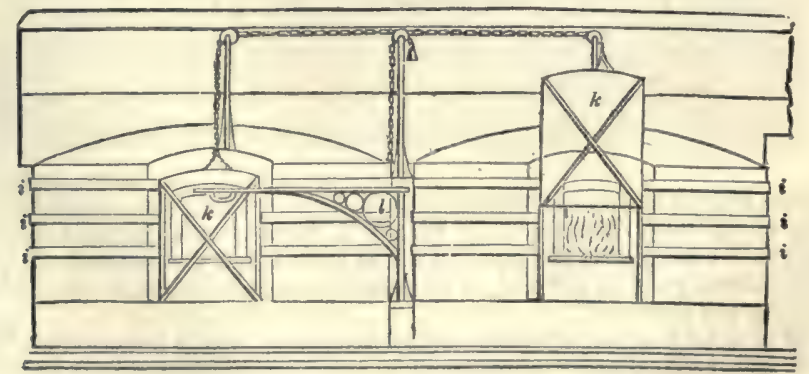

Each alternate oven is charged, between 8 and 10 o'clock every morning, with $3 \frac{1}{2}$ tons of good coals. A wisp of straw is thrown in on the top of the hesp, which takes fire by the radiation from the dome (which is in a state of dull ignition from the preceding operation), and inflames the smoke then rising from the surface, by the reaction of the hot sides and bottom upon the body of the fuel. In this way the smoke is consumed at the very commencement of the process, when it would otherwise be more abundant. The coking process is in no respect a species of distillation, 
but a complete combustion of the volatile principles of the caal. The mass of coals is first kindled at the surface, where it is supplied with abundance of atmospherio oxygon; because the doors of the ovens in front, and the throat-vents behind, are then left open. The consequence is, that no smoke is discharged from the top of the chimney, at this, the most sooty period of the process, than is produced by an ordinary kitchen fire. In these circumstances, the coal gas, or other gas, supposed to be generated in the slightly heated mass beneath, cannot escape combustion in passing up through the bright open flame of the oven. As the coking of the coal advances more slowly and regularly from the top of the heap to the bottom, only one layer is affected at a time, and in succession downwards, while the surface is always covered with a stratum of red-hot cinders, ready to consume every particle of carburetted or sulphuretted hydrogen gases which may escape from below. The greatest mass, when calcined in this downward order, cannot emit into the atmosphere any more of the above-mentioned gases than the smallest heap.

The coke being perfectly freed from all fuliginous and volatile matters by a calcination of upwards of 40 hours, is cooled down to a moderate ignition by sliding-in the dampers, and sliding-up the doors, which had been partially closed during the latter part of the process. It is now observed to form prismatic concretions, somewhat like a columnar mass of basalt. These are loosened by iron bars, lifted out upon shovels furnished with long iron shanks, which are poised upon swing chains with

497

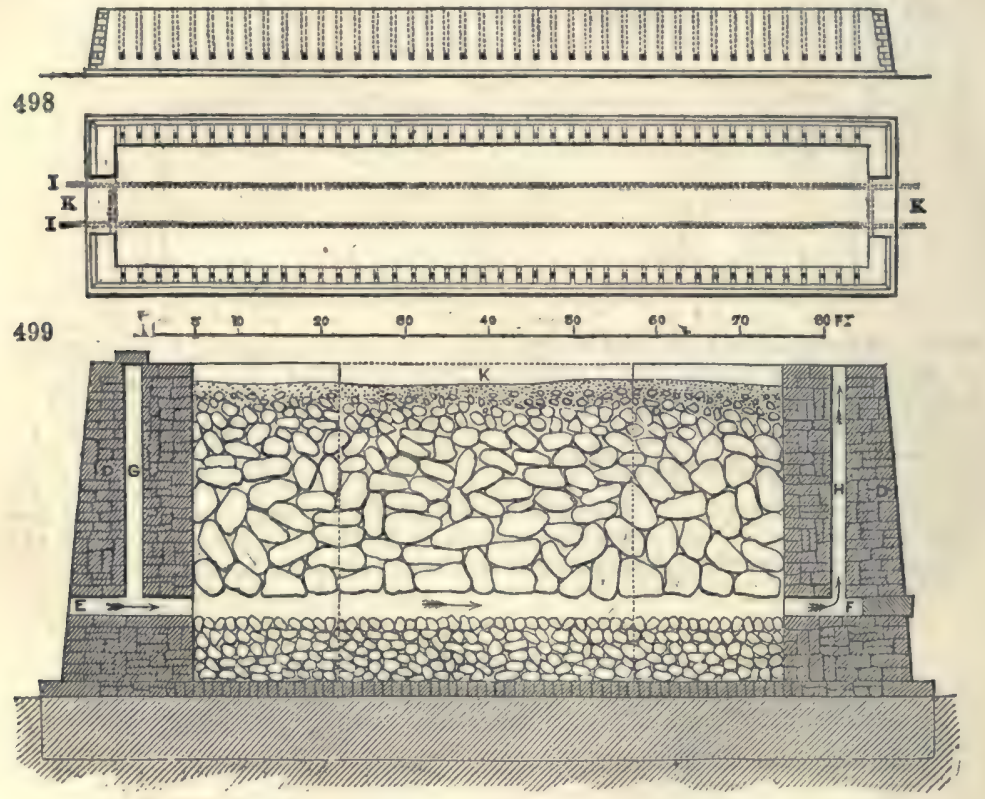

hooked ends, and the lumps are thrown upon the pavement, to be extinguished by sprinkling water upon them from the rose of a watering can; or, they might be transferred into a large chest of sheet-iron set on whoels, and then covered up. Good cosls, thus treated, yield 80 per cent, of an excellent compact glistening coke, weighing abont 14 cwts. per chaldron.

The loss of weight in coking in the ordinary ovens is usually reckoned at 25 per cont.; and coal, which thus loses one-fourth in weight, gains one-fourth in bulk.

Labourers who have been long employed at rightly-constructed coke ovens seent to onjoy remarkably good health.

Mr. Ebenezer Rogers, of Abercarn, in Monmouthshire, introduced a method of coking, which ho thus described:-

-A short time ago a plan was mentioned to the writer as having been used in Westphalia, by which wood was charred in small kilns : as the form of kiln described was quite new to him, it led him to some reflection as to the principles on which it actod, which were found to bo so simple and effective, that ho determined to apply 
them on a large scale for coking coal. The result has been that in the course of a fow months the original idea has been so satisfactorily matured and developed, that instead of coking 6 tons of coal in an oven costing $80 l .150$ tons of coal are now being coked at once in a kiln costing less than the former single oven.

' Figs, 497 and 498 are a side elevation and plan of one of the new coking kilns to a small scale; fig. 499 is an enlarged transverse section.

'D D are the walls of the kiln, which are provided with horizontal flues, $\mathrm{B}, \mathrm{F}$, which open into the side or bottom of the mass of coal. Connected with each of these flues are the vertical chimneys $\mathrm{G}$, H. The dotted lines I I, fig. 498, represent a moveable railway, by which the coal may be brought into the kiln and the coke removed from it. In filling the kiln with coal, care is taken to preserve transverse passages or flues for the air and gases between the corresponding flues $\mathrm{E} F$ in the opposite walls. This is effected by bailding or constructing the passages at the time with the larger pieces of coal, or else by means of channels or flues permanently formed in the bed of the kiln. When the coal is of different sizes, it is also advantageous to let the size of the pieces diminish towards the top of the mass. The surface of the coal when filled in is covered with small coal, ashos, and other suitable material.

'When the kiln is filled the openings $\mathbf{K}$ at the ends are built up with bricks, as shown dotted; the kiln is not covered by an arch, but left entirely open at the top. The apertures of the flues $F$ and the chimneys $\mathrm{G}$ are then closed, as shown in fig. 499, and the coal is ignited through the flues $\mathbf{x}$; the air then enters the flues $\mathrm{E}$, and passes through the coal, and then ascends the chimneys $\mathrm{H}$, as shown by the arrows. When the current of air has proceeded in this direction for some hours, the flues $\mathrm{x}$ and chimneys $\mathrm{H}$ are closed, and $\boldsymbol{F}$ and $\mathrm{G}$ are opened, which reverses the direction of the current of air through the mass. This alteration of the current is repeated as often as may be required. At the same time air descends through the upper surface of the mass of coal. When the mass is well ignited, which takes place in from 24 to 36 hours, the external apertures of the flues $\mathrm{E}$ and $\mathrm{F}$ are closed, and the chimneys $\mathrm{G}$ and F opened : the air now enters through the upper surface of the coal only, and descends through the mass of the coal, the products of combustion passing up the chimneys.

'The coking gradually ascends from the bottom of the mass to the top, and can be easily regulated or equalised by opening or closing wholly or partially the apertures of the flues or chimneys. The top surface of the coal being kept cool by the descending current of air, the workman is enabled to walk over it during the operation; he inserts from time to time at different parts of the surface an iron bar, which is easily pushed down until it reaches the mass of coke, by which its further descent is prevented. In this way the workman gauges the depth at which the coking process is taking place, and if he finds it to have progressed higher at one part than at another, he closes the chimneys communicating with that part, and thus retards the progress there. This gauging of the surface is carried on without difficulty until the coking process has arrived close to the top. The gases and tarry vapours produced by the distillation or combustion descend through the interstices of the incandescent mass below, and there deposit a portion of the carbon contained in them, the residual gases passing up the chimneys. The coke at the lower part of the kiln is effectually protected from the action of the air, by being surrounded and enveloped in the gases and rapours which descend through it, and an non-supporters of combustion.

'When the mass of coal has been coked up to the top, which takes place in about seven days, it is quenched with water, the walls closing the end openings, $\mathbf{x}$, are taken down, and the coke is removed. When a portion has been removed, a moveable railway is laid in the kiln, so as to facilitate the removal of the remainder of the coke.

'The flues $\mathrm{s}$ and $\mathrm{F}$ may enter at the bottom of the kiln, or at the sides above the bottom, as in fig. 499; in the latter case the space below, up to the level of the bottom of the flues, may be filled with small coal, which becomes coked by the radiated heat from the incandescent mass above. The transverse passages through the mass are then constructed upon this bed of small coal with the larger lumps of coal, as before mentioned. The flues and chimneys need not necessarily be horizontal and vertical; and instead of connecting a separate chimney with each transverse flue, flues may be constructed longitudinally in the walls of the kiln, so as to connect two or more of the transverse flues, which are then regulated by dampers, conveying the gaseous products from them into chimneys of any convenient height; the arrangement first described, however, and shown in the drawings is preferred. The gaseous products may bo collected, and tar and ammonia and other chemical compounds manufactured from them by the usual modes. The coking or charring of peat and wood may be effected in a similar manner to that already described with regard to cosl.

'The new kilns have proved entirely successful; they are already in use at some of 
the largest iron-works in the kingdom, and are being erected at a number of other works. The great saving in first cost of oren, economy in working and maintonance, increased yield, and improved quality of coke, will probably soon cause this mode of cuking to supersede the others now in sse. The kilns are most advantageously made about 14 foet in width, and 90 feet in length, and 7 feet 6 inches in height; this sizo of kiln contains about 150 tons of cosl.'

From the long experience of this gentleman we are induced to quote yet further from his memoir :-

'The process of coking converts the coal into a porous mass; but this is done during the melting of the coal, at which moment the gases in liberating themselves form very minute bubbles; but the practical result is the same as in wood-coal, allowing a large surface of carbon in a small space to be acted upon by the blast. As a general rule, coke made rapidly has larger pores and is lighter than coke mado slowly; it accordingly bears less blast, and crumbles too easily in the furnace.

- The process of coking in the ordinary ovens may be thus explained:- When the oven is filled with a proper charge, the coal is fired at the surface by the radiated heat from the roof; enough air is admitted to consume the gases given off by the coal, and thus a high temperature is maintained in the roof of the oven. The coal is by this means melted; and those portions of it which, under the influence of a high tomperature, can of themselves form gaseous compounds, are now given off, forming at the moment of their liberation small bubbles or cells; the coke now left is quite safe from waste, unless a further supply of air is allowed to have access to it. At this stage of the process, the coke assumes a pentagonal or five-sided shape and columnar structure. When the coke is left exposed to heat for some time after it is formed, it becomes harder and works better, from being less liable to crush in the furnace and decrepitate on exposure to the blast.

'It has been often remarked as a strange fact, that the hotter the oven, the better the yield of coke; hence all the arrangements of flues to keep up the temperature of the ovens. This fact is, however, the result of laws well known to chemists. When the coal is melted as above mentioned, the hydrogen in the coal takes up two atoms of carbon for each two atoms of hydrogen, forming bicarburetted hydrogen gas $\left(\mathrm{C}^{2} \mathrm{H}^{2}\right)$; this at once escapes, but it has to pass upwards through the red-hot coke above, which is at a higher temperature than the melted coal below. Now when bicarburettod hydrogen gas is exposed to a bright red heat it is decomposed, forming carburetted hydrogen gas $\left(\mathrm{CH}^{2}\right)$, and depositing one atom, or one half of its carbon, in a solid form. Consequently in the process of coking, if the oven is in good working order, snd the coke hot enough, the liberated carbon is detained in its passage upwards, and either absorbed by the coke, or crystallised per se upon it. This is simply illustrated by passing ordinary illuminating gas through a tube heated to a bright rod heat; the tube will soon become coated internally, and ultimately filled with a carbonaceous deposit produced by the decomposition of the bicarburetted hydrogen contained in the gas.

'It is found that some coal which is too dry or not sufficiently bituminous to coke when put into the oven by itself in lumps, will coke perfectly if crushed small and well wotted with water and charged in this state. This fact, if followed out, would lead to an examination of the chemical nature of the effect produced by the water, and would point the way to further improvements.'

Charred Coal, as it is called, must be regarded as a spocies of coke. It has been largely employed in lieu of charcoal in the manufacture of tin plates. This preparation was also a discovery of Mr. Ebenezer Rogers, who thus described its manufacture :-

The preparation of the 'charred coal' is simple. The coal is first reduced very small, and washed by any of the ordinary means; it is then spread over the bottom of a reverberatory furnace to a depth of about four inches; the bottom of the furnace is first raised to a red heat. When the small coal is thrown over the bottom, a great rolume of gases is given off, and much ebullition takes place; this ends in the production of a light spongy mass, which is turned over in the furnace and drawn in one hour and a half. To completely clear off the sulphur, water is now freely sprinklod over the mass until all smell of the sulphuretted hydrogen producod ceases. Charred coal has been hitherto produced on the floor of a coke oven, whilst red hot after drawing the charge of coke. See Thw-Plata Manufacture.

A process has for some time been gaining ground in France known as the 'Systeme Appolt,' from its being introduced by two brothers of that name. The coking furnaces employed are vertical, and they are in compartments. The authors published a deseription of their process and a statement of its results, 'Carbonisation de la Houille Systeme Appolt, décrit par les Auteurs, MM. Appolt Frères :' Paris, 1858; to which we must refor our readers.

M. Coppée has a coke oven which has been largely used in Belgium, and it has 
been introduced at the Thorncliffe collieries. The following objects are aimed at in the construction of the Coppese ovens :-

1. The retention (as coke) of as large a proportion of the carbon contained in the coal as possible.

2. The utilisation of the heat of the gases given off, by the use of flues arranged to maintain a high temperature, and applied in such contiguity to the oven itself as to hasten, by imparting an intense heat to the inside of the oven, the expulsion of the gases.

3. As a secondary, but consequent consideration, the application of the heat retained by the gases when they leave the stack of ovens, to the production of steam.

The Coppese ovens have each the form of a parallelogram, the usual dimensions being: longth, 29 feet 6 inches; breadth, 18 inches; height, 4 feet. These are charged with coals every 24 hours. When built to be drawn every 48 hours, the ovens have the same length, but are built 5 feet 7 inches high and 24 inches broad. The thickness of brickwork between the ovens is $13 \cdot 2$ inches.

The coals, whether washed or otherwise, are crushed or disintegrated before being placed in the oven to the consistency of very coarse meal. At each end of the oven are two metal doors moving on hinges, and fixed securely in metal frames, the lower being 3 feet, and the upper 1 foot in height. Between each two ovens are about 28

500

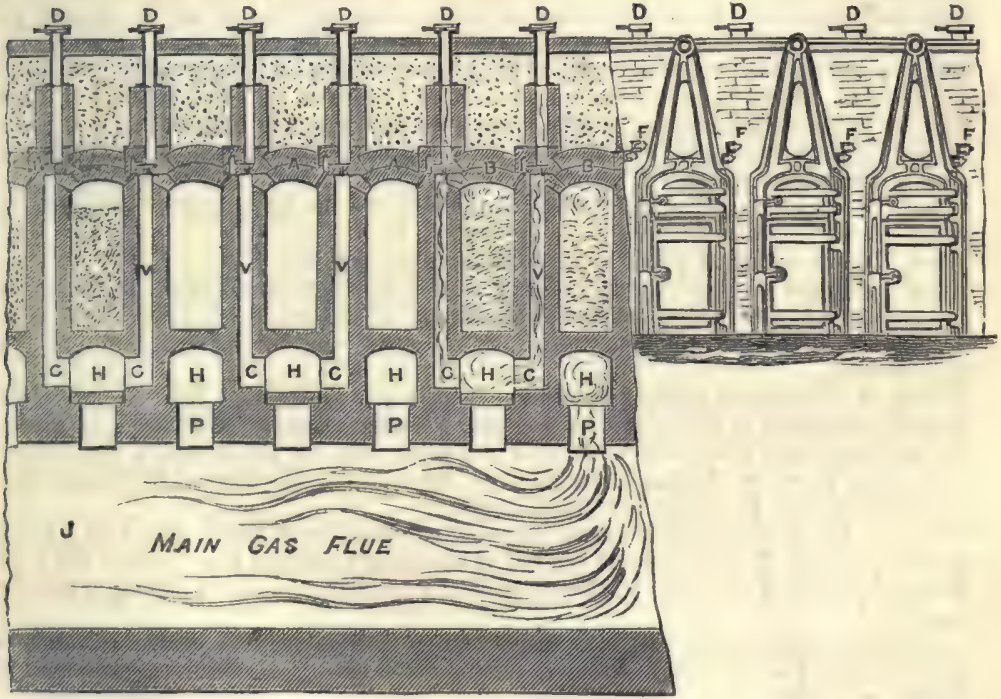

vertical channels, $\mathrm{v} v$ (fig. 500), which, leading from one side of each oven, convey the gases down to the horizontal flues, $\mathbf{H}$ H, one of which runs under each oven. The ovens are arranged in groups of two. The gases from each two ovens, $\mathbf{A} \mathbf{A}$ or B B, take their course down vertical channels to the horizontal flue under one of the ovens, entering such flue by the apertures $\mathrm{c} c$. The combined gases, after passing along this flue to the end of the oven, return by the flue under the other oven, and enter, at the point $P$, into a large channel running at right angles to the ovens, J. They pass from this channel, either directly into a chimney, or are carried under one or several boilers.

At the commencement of the burning the admittance of air is regulated by three small channels, by means of each of which air can be conveyed to the top, either of the oven, or of the vertical flues. One of these air-passages is in the centre of the oven, and is worked by the doors, $\mathbf{D} \mathbf{D}$, and the others are fixed at each end of the oven, at the side of the doorway, as shown by F F, a very simple arrangement of sliding doors allowing the air to be applied or shut off, with great facility and promptness.

The coke is removed from the ovens by means of a ram, propelled by a cogged driving-wheel, which is worked by a small portable engine. 
When the coke is ready to be taken from the oven, the engine and ram are placod opposite to the end of the oven, and three wagons of coal are placed over the three openings at the top. The coke is then pushed out by the ram, this operation occupying about two minutes. A jet of water is at once applied to the coke whilst it is being spread out on the floor. At the same time the lower doors are closed, and the coal dropped into the oven, the apertures through which the coal passes being immediately covered up by sliding doors. The coal is levelled in the ovens by means of rakes passed through the opening of the upper doors. The upper doors are then closed. The time occupied from the moment the doors are opened to their being sealed up again is eight minutes. These ovens have been found, by a large number of experiments to yield only 2 per cont. short of the actual quantity of fixed carbon contained in the coal used.

The workings of the ovens at Thorncliffe, Chapeltown, gave the following comparative rosults :-

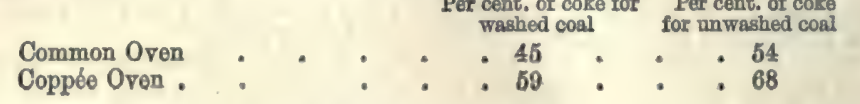

The high per-centage of carbon yielded by the Coppée oven is probably chiefly due: 18t. To the delicate manner in which the admittance of air to the ovens can be regulated. The temperature of the common oven is comparatively low when the combustion of the coal commences, and some time is occupied before the coal is in a state of combustion. During this time a considerable quantity of air is allowed to enter the oven, and carbonic acid is formed. This naturally takes up a large proportion of the carbon in the coal, which is thus removed almost before the coking process begins. When, however, the temperature of the oven in being refilled is very high, as in the Coppee system, it is not unlikely that the free carbon, being thrown off quickly, will unite with the air and form the oxide, taking up no more carbon than if, by the introduction of a larger quantity of air, carbonic acid were formed.

2nd. To the very small space allowed in the oven for the movement of the burning gases.

3rd. To the care with which leakages are provided against.

The quickness with which coke is manufactured by the Coppée system is doubtless due to the rapid combustion caused by the action of the constantly-maintained high temperature of the side, top, and bottom of the ovens, up the enclosed thin upright layer of coal.

Such is a sufficient general description of this new method of coking cosl. For further particulars as to the cost of erection, working expenses, utilisation of gases, and other matters, the reader is referred to a paper read by Mr. Emerson Bainbridge before the North of England Institute of Mining and Mechanical Engineers, and published in their 'Transactions' 1873, p. 81 .

Messrs. Breckon and Dixon have patented a coke-oven in which the heat of the ignited products of combustion is utilised by circulating through anes beneath the floor of the ovens. It is said that by thus heating the floor, the process of coking is greatly facilitated.

In the so-called anchor-ovens the entire charge of coke may be drawn out in one mass, by means of an iron instrument termed an anchor, thus saving a considerable amount of labonr.

corrss. A name given to tin plates which have been prepared with coke.

Corcotran or virriox (Rouge d' Angleterre, Fr.; Rothes Eisenoxyd, Ger.), the antiquated name of oxide of iron. It is the brownish-red peroxide of iron, produced by calcining sulphate of iron at a strong heat, levigating the resulting mass, and elutriating it into an impalpable powder. A better way of making it, so as to complete the separation of the acid, is to mix 100 parts of the green sulphate of iron with 42 of common salt, to calcine the mixture, wash away the resulting sulphate of soda, and levigate the residuum. The best sort of polishing powder, called jewellers' rouge, or plate-powder, is the precipitated oxide of iron prepared by adding solution of soda to solution of copperas, washing, drying, and calcining the powder in shallow ressels with a gentle heat, till it assumes a deep brown-red colour.

Cord miast. Soo Iron and Hot Buast.

CoxIrdrve. A volatile base discovered by Anderson in bone oil, and subsequently found in shale naphtha.

Coxxodrox. M. Malgaigne communicated to the French Medical Journals some romarks on the preparation of gun-cotton for surgical purposes. Several French chemists, at the suggestion of M. Malgaigne, attempted to make an ethereal solution of this compound by pursuing the process recommended by Mr. Maynard in the American 
Journal of Medical Science, but they failed in procuring the cotton in a state in which it could be dissolved in ether. It appears that these experimentalists had employed a mixture of nitric and sulphuric acids; but M. Miallio ascertained, after many trials, that the collodion in a state fitted for solution was much more easily procured by using a mixture of nitrate of potash and sulphuric acid.

For the information of our readers, we give here a description of M. Miallie's process for its preparation. It appears from the rosults obtained from this chemist, that cotton in its most explosive form is not the best fitted for making the ethereal solution :-

Finely-powdered nitrate of potash $: \quad: \quad \begin{array}{r}40 \\ \text { Concentrated sulphuric acid }:\end{array}$
Carded cotton

Mix the nitrate with the sulphuric acid in a porcelain vessel, then add the cotton, and agitate the mass for three minutes by the aid of two glass rods. Wash the cotton, without first pressing it, in a large quantity of water, and when all acidity is removed (indicated by litmus paper), press it firmly in a cloth. Pull it out into a loose mass, and $\mathrm{dry}$ it in a stove at a moderate heat.

The compound thus obtained is not pure fulminating cotton; it always retains a small quantity of sulphuric acid, is less inflammable than gun-cotton, and it leaves a carbonaceous residue after explosion. It has, however, in a remarkable degree the property of solubility in ether, especially when mixed with a little alcohol, and it forms therewith a very adhesive solution, to which the name of collodion has been applied.

Preparation of Collodion.-Prepared cotton, 8 parts; rectified sulphuric ether, 125 parts; rectified alcohol, 8 parts: all by weight.

Put the cotton with the ether into a well-stopped bottle, and shake the mixture for some minutes. Then add the alcohol by degrees, and continue to shake until the whole of the liquid acquires a syrupy consistency. It may be then passed through a cloth, the residue strongly pressed, and the liquid kept in a well-secured bottle.

Collodion thus prepared possesses remarkable adhesive properties. A piece of linen or cotton cloth covered with it, and made to adhere by evaporation to the palm of the hand, will support a weight of twenty or thirty pounds. Its adhesive power is so great that the cloth will commonly be torn before it gives way. The collodion cannot be regarded as a perfect solution of the cotton. It contains suspended and floating in it a quantity of vegetable fibre, which has escaped the solvent action of the ether. The liquid portion may be separated from these fibres by a filter, but it is doubtful whether this is an advantage. In the evaporation of the liquid these undissolved fibres, by felting with each other, appear to give a greater degree of tenacity and resistance to the dried mass.

Small balloons for holding gas are also made of collodion by pouring it into a glass globe, and turning it about, so that every part is covered with the fluid. As the ether evaporates, a thin film is left on the surface of the glass. To remove this, a glass tube is inserted into the neck of the flask, so that the collodion film may adhere; the air is sucked out, and the collapsed film is easily withdrawn.

For the application of collodion to photography, see PнotograpHy.

coxocystr. Citrullus Colocynthis. The Coloquintida, bitter cucumber or bitter apple, a drastic medicine.

COIOGRT EARTH. A brown pigment, prepared from lignite or brown coal.

COIOGNa YaIIOW. This pigment consists of a mixture of chromate and sulphate of lead with sulphate of lime. It is prepared by precipitating a mixture of nitrate of lead and nitrate of lime, with sulphate of soda and chromate of potash.

Cогомва. Cocculus palmatus. A climbing shrub, a native of the intertropical regions of the earth. It is now cultivated in the Mauritius and the Isle of Bourbon. The root is of a large, fleshy, doop yellow colour, and divided into many irregular forks. These are eut off, sliced, strung on cords, and hung to dry in the shade.

CoropHowy. Black resin, the solid residuum of the distillation of turpentine when all the oil has been worked off.

cororms. An alcoholic extract of garancine, when evaporated, is known in commerce as colorine. It contains the colouring principles of madder (alizarine and purpurine), together with fatty matter and other components of the garancine, which are capable of extraction by alcohol.

Cozovs. In Physics, a property of light, producing peculiar impressions through the eye. Every surface, differing in its mechanical structure or chemical character, acts differently towards the light falling on it, and according to tho amount of absorption, or reflection, or refruction, so is the colour of the ray. White is an entire reflection, and black a total absorption of all the rays, and consequently are not colours. The following Table is by Mr. William Linton:- 
1 Table of Oil Paintors' Colours, with notices of their Chemical and Artistical Properties.

\begin{tabular}{|c|c|c|c|c|}
\hline \multirow[b]{2}{*}{ 茪 } & Colours & $\begin{array}{c}\text { Chemleal Denignation } \\
\text { and Proparation }\end{array}$ & $\begin{array}{l}\text { Artiatical Properties and } \\
\text { Durablifty }\end{array}$ & Additional Colours, - with Remarks \\
\hline & Flake White. & $\begin{array}{l}\text { Carbonate of } \\
\text { Lead, with an } \\
\text { excess of Oxide. } \\
\text { Plates of lead, } \\
\text { exposed to the } \\
\text { action of vine- } \\
\text { gar-steam in } \\
\text { beds of ferment- } \\
\text { ing tan. }\end{array}$ & $\begin{array}{l}\text { The best White extant } \\
\text { for Oil or Resin vehicles } \\
\text { when pure, which is } \\
\text { generally ascertained } \\
\text { by its exceeding white- } \\
\text { ness and opacity. Its } \\
\text { usual adulterations are } \\
\text { Sulphate of Barytea, } \\
\text { Chaik, Pipe-clay, \&so., } \\
\text { all of which are par- } \\
\text { tially transparent, and } \\
\text { consequently appear } \\
\text { darker in unctuous or } \\
\text { resinous vehicles. It } \\
\text { has no injurious action } \\
\text { upon Vegetable and } \\
\text { other colours, as some } \\
\text { have conjectured. It } \\
\text { is perfectly soluble in } \\
\text { diluted Nitric or Acetic } \\
\text { Acid when free from } \\
\text { Pipe-clay or Sulphate } \\
\text { of Barytes. It is black- } \\
\text { ened by the foul gases } \\
\text { common to most do- } \\
\text { mestio atmospheres, } \\
\text { and is moresecure in a } \\
\text { rapidly drying and pro- } \\
\text { tective vehicle. }\end{array}$ & $\begin{array}{l}\text { There are other Whites of Lesd, } \\
\text { varying in body and brilliancy, } \\
\text { and equally obnoxious to the } \\
\text { action of mephitic vapours; as } \\
\text { Krems, Roman, and Venetian } \\
\text { Whites, and Swiphate of Lead. } \\
\text { The Whites of Bismudh, Pearl, } \\
\text { and Antimony are injured by } \\
\text { light as well as by mephic } \\
\text { vaponrs. Those of Zinc, Tin, } \\
\text { and Barytes, although they are } \\
\text { comparatively secure against } \\
\text { the foul gases, are too feeble } \\
\text { in body to be satisfactory in } \\
\text { unctuous or resinous vohicles. } \\
\text { Pattinson's Oxichlorideof Lead, } \\
\text { a denser preparation, forms } \\
\text { an admirable substratum for } \\
\text { works in which a powerful } \\
\text { lmpasto is desired. }\end{array}$ \\
\hline \multirow{3}{*}{$\frac{m}{3}$} & $\begin{array}{l}\text { Strontian } \\
\text { Yellow. }\end{array}$ & $\begin{array}{l}\text { Ohromate of } \\
\text { Strontia. A } \\
\text { Solution of Chlo- } \\
\text { ride of Stron- } \\
\text { tium added to } \\
\text { one of Chromate } \\
\text { of Potash. }\end{array}$ & $\begin{array}{l}\text { Of a pale Canary Colour. } \\
\text { Resists the action of } \\
\text { the fonl gases andight, } \\
\text { and is perfectly dur- } \\
\text { able. }\end{array}$ & \multirow{3}{*}{ 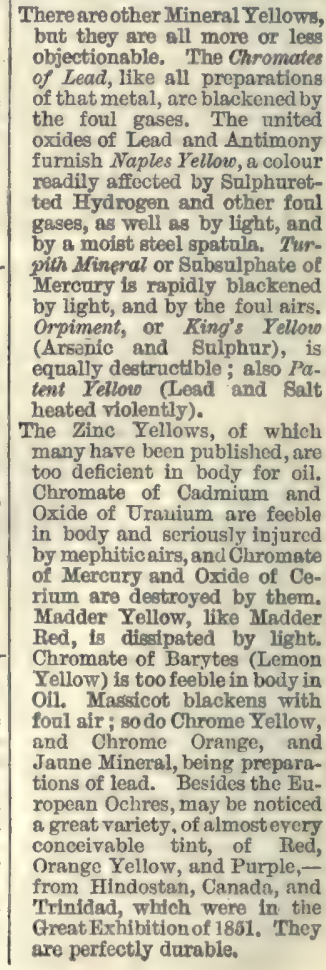 } \\
\hline & $\begin{array}{l}\text { Cadmium } \\
\text { Yellow. }\end{array}$ & $\begin{array}{l}\text { Salphide of Cad- } \\
\text { mium. A com- } \\
\text { bination of Cad- } \\
\text { minm and Sul- } \\
\text { phur. }\end{array}$ & $\begin{array}{l}\text { A rich and brilliant } \\
\text { Orange. Resists the } \\
\text { action of the fonl gases, } \\
\text { light, \&c.. and is per- } \\
\text { fectly durable. A good } \\
\text { sabstitute, like Stron- } \\
\text { tian Yellow, for Naples, } \\
\text { Chrome, and other } \\
\text { changeable Mineral } \\
\text { Yellows. }\end{array}$ & \\
\hline & $\begin{array}{l}\text { Yellow, Oxford, } \\
\text { Roman, Slome, } \\
\text { Brown, Sienna, } \\
\text { with Orange, } \\
\text { Puce, Purple, } \\
\text { and other In- } \\
\text { dian and Ame- } \\
\text { rican Ochres. }\end{array}$ & $\begin{array}{l}\text { Native Tarths, } \\
\text { consisting of Si- } \\
\text { lica and Alu- } \\
\text { minn, coloured } \\
\text { by Oxide of Iron. }\end{array}$ & $\begin{array}{l}\text { All permanent Coloars, } \\
\text { whether Native, Arth- } \\
\text { ficial, or Burnt. The } \\
\text { Oxides of Iron are } \\
\text { among the mast staple } \\
\text { colours of the palette. } \\
\text { When properly washed } \\
\text { and prepared for oll } \\
\text { painting, they are in- } \\
\text { capable of injuring } \\
\text { other colours, and may } \\
\text { be said to constitute } \\
\text { the soundlest materials } \\
\text { with whloh the che- }\end{array}$ & \\
\hline
\end{tabular}




\begin{tabular}{|c|c|c|c|c|}
\hline \multirow[b]{2}{*}{ 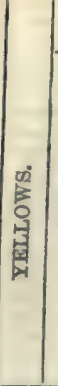 } & Colours & $\begin{array}{l}\text { Chemical Deaignation } \\
\text { and Preparation }\end{array}$ & $\begin{array}{l}\text { Artistical Properties and } \\
\text { Durability }\end{array}$ & Additlonal Coloura, - with Remarka \\
\hline & Jaune de Mars. & $\begin{array}{l}\text { A Chemical Pre- } \\
\text { paration with si- } \\
\text { milar properties. }\end{array}$ & $\begin{array}{l}\text { mistry of Nature has } \\
\text { furnished the painter } \\
\text { for the imitation of her } \\
\text { works. }\end{array}$ & $\begin{array}{l}\text { Palladinm Yellow is better } \\
\text { avoided, so 1s Golden Sul- } \\
\text { phuret of Antimony. Chro- } \\
\text { mate of Tin darkens in oil, and } \\
\text { Platinum Yellow is wortbless. } \\
\text { Gamboge (vegetable) bleaches } \\
\text { in the light. Brown-pink, and } \\
\text { others of the same class are } \\
\text { also evanescent in their layers. } \\
\text { The Vegetable Yellows are not } \\
\text { to be depended npon. They } \\
\text { soon disappear when applied in } \\
\text { delicate tints or thin glazings, } \\
\text { especially if subjected to the } \\
\text { action of the solar rays. }\end{array}$ \\
\hline \multirow{5}{*}{ 菓 } & Light Red. & $\begin{array}{l}\text { Yellow Ochre, } \\
\text { Burnt. }\end{array}$ & \multirow{2}{*}{$\begin{array}{l}\text { Sound, useful, and } \\
\text { durable colours. }\end{array}$} & \multirow{5}{*}{$\begin{array}{l}\text { There are other Mineral Reds } \\
\text { which are durable, but they are } \\
\text { of inferior quality, and are not } \\
\text { needed. Native Cinnabar is in- } \\
\text { ferior is every respect to Ver- } \\
\text { milion, Venetian Red is an } \\
\text { inferior representative of In- } \\
\text { dian Red, and Colcothar a still } \\
\text { coarser one. Red Lead blackens } \\
\text { in oil; and Iodide of Mercury } \\
\text { has no claim to durability. Red } \\
\text { Precipitate is worthless. Phos- } \\
\text { phate of Cobalt (a Pink Sili- } \\
\text { cate) wants body, and darkens } \\
\text { in oil, Mineral Lake (Tin and } \\
\text { Chromium) is too feeble in } \\
\text { body. Lilac and Crimson Sili- } \\
\text { cates, from Gold, have not body } \\
\text { enoagh for oil painting. } \\
\text { Among Vegetable Reds, the } \\
\text { Madders have the best repu- } \\
\text { tation for standing. All vege- } \\
\text { table colours, howerver, should } \\
\text { be looked upon with suspicion } \\
\text { when used in thin glazings. }\end{array}$} \\
\hline & Indian Red. & $\begin{array}{l}\text { A Native Iron } \\
\text { Oxide. }\end{array}$ & & \\
\hline & Vermition. & $\begin{array}{l}\text { Bisulphide of Mer- } \\
\text { cary. Mercury } \\
\text { and Sulphur } \\
\text { combined. }\end{array}$ & $\begin{array}{l}\text { A beantiful colour, and } \\
\text { of an excellent body. } \\
\text { Quite permanent, and } \\
\text { not affected by acids or } \\
\text { caustic alkalis. V\&- } \\
\text { porised by a red heat, } \\
\text { if pure. }\end{array}$ & \\
\hline & Madder Lakes. & $\begin{array}{l}\text { Vegetable dyes on } \\
\text { earthy bases. }\end{array}$ & $\begin{array}{l}\text { Rich and beantiful } \\
\text { colours. }\end{array}$ & \\
\hline & Palladium Red. & $\begin{array}{l}\text { Ammonio-perchlo- } \\
\text { ride of Palladium. }\end{array}$ & $\begin{array}{l}\text { A rich, deep, and } \\
\text { beautifal colcur. }\end{array}$ & \\
\hline \multirow{2}{*}{ 总 } & $\begin{array}{l}\text { Uttramarine, } \\
\text { Native. }\end{array}$ & \multirow{2}{*}{$\begin{array}{l}\text { A compound of } \\
\text { Silicate of Alu- } \\
\text { mina and sili- } \\
\text { cate of soda, } \\
\text { with Salphide of } \\
\text { Sodium: the co- } \\
\text { lour is owing to } \\
\text { the reaction of } \\
\text { the latter on the } \\
\text { two former con- } \\
\text { stituents. } \\
\text { The Native is pre- } \\
\text { pared from Lapis } \\
\text { laznli. }\end{array}$} & \multirow{2}{*}{$\begin{array}{l}\text { A brilliant purplish- } \\
\text { blue. None of the me- } \\
\text { phitic gases or light do } \\
\text { it any injury. Acids } \\
\text { will remove its colour. } \\
\text { It is perfectly durable } \\
\text { for the painter. The } \\
\text { artificial preparation } \\
\text { by Zuber, of Alsace (in } \\
\text { the Great Frhibition } \\
\text { of 1851), is of a less } \\
\text { purple hue, and better } \\
\text { fitted for the aërial } \\
\text { tints of landscape. }\end{array}$} & \multirow{2}{*}{$\begin{array}{l}\text { There are other Mineral Blues, } \\
\text { but they are better avoided. } \\
\text { The Sllicate of Cobalt and } \\
\text { PotassamakesCobalt Blue; and } \\
\text { the Oxide of Cobalt and Po- } \\
\text { tassa makes Smalt: both feeble } \\
\text { pigments. Verditer and all the } \\
\text { Copper Bines tarn Green, and } \\
\text { darken in oil. A Blue Frit, } \\
\text { Bilicate of Copper(Alexandrian } \\
\text { Blue), brought by Mr. Layard } \\
\text { from Nineveh, which has re- } \\
\text { tained its colour for probably } \\
\text { 4000 years, turned black when } \\
\text { mixed with oil. The perma- } \\
\text { nence of Vitreous (called } \\
\text { silica) colours, when levigated } \\
\text { for pigments, is a delasion. } \\
\text { They are subject to all the } \\
\text { changes and anfinities of the } \\
\text { substances which compose } \\
\text { them. Prussian Blue is liable } \\
\text { to change, Indigo fades in the } \\
\text { light. }\end{array}$} \\
\hline & $\begin{array}{l}\text { Utramarine, } \\
\text { Artificial. }\end{array}$ & & & \\
\hline 窗 & $\begin{array}{l}\text { Chromium } \\
\text { Green. }\end{array}$ & $\begin{array}{l}\text { Sesquioxide of } \\
\text { Chrominm. } \\
\text { When Chromate } \\
\text { of Mercury (the } \\
\text { Orange precipl- } \\
\text { tate on mixing } \\
\text { Nitrate of Mer- } \\
\text { cury and Chro- } \\
\text { mate of Potash) } \\
\text { is strongly igni- } \\
\text { ted, Oxdde of } \\
\text { Chromium re- } \\
\text { mains in a pow- } \\
\text { der. }\end{array}$ & $\begin{array}{l}\text { An opaque light Green, } \\
\text { of a full body, and per- } \\
\text { manent in all respents. } \\
\text { It is the colouring mat- } \\
\text { ter of emeralds. }\end{array}$ & $\begin{array}{l}\text { There are other Mineral Greens, } \\
\text { Thosefrom theArtiflcial Ultra- } \\
\text { marine process, though dura- } \\
\text { ble, are wanting in body and } \\
\text { richnees. The Phosphates, } \\
\text { Carbonates (Mrlachite), Disul- } \\
\text { phates, and Acetates of Copper, } \\
\text { with Mineral, Verditer, and } \\
\text { Verdigris Greens,are blackened } \\
\text { by foul gases, and deoxidised } \\
\text { or darkened by oil. Nickel } \\
\text { Green also darkens and black- } \\
\text { ens in oll. Scheele's Green } \\
\text { (Vert Metis), Arsenate of Cop- }\end{array}$ \\
\hline
\end{tabular}




\begin{tabular}{|c|c|c|c|c|}
\hline \multirow[b]{2}{*}{ 央 } & Colourn & $\begin{array}{c}\text { Chemical Designation } \\
\text { wad Preparation }\end{array}$ & $\begin{array}{l}\text { Artededeal Properties and } \\
\text { Durabllity }\end{array}$ & Additlonal Coloars, 一with Remerks \\
\hline & Terre Verte. & $\begin{array}{l}\text { Bilicate of Prot- } \\
\text { oxide of Irou, } \\
\text { with Water, Po- } \\
\text { tassa, and Mag- } \\
\text { nesia. A Native } \\
\text { Mineral. }\end{array}$ & $\begin{array}{l}\text { A deliente neatral } \\
\text { Green. Quite perma- } \\
\text { nent. Like Uitrama- } \\
\text { rine, its colour is de- } \\
\text { stroyed by adids. }\end{array}$ & $\begin{array}{l}\text { per, darkens in ofl and foul } \\
\text { air. Zinc or Cobalt Green } \\
\text { (Rinmann's Green) is perma- } \\
\text { nent, but inferior in body and } \\
\text { colour to the Green of Chro- } \\
\text { mium. }\end{array}$ \\
\hline \multirow{5}{*}{ 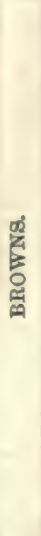 } & $\begin{array}{l}\text { Vandyke, Cap- } \\
\text { pah, kubens, } \\
\text { Cassel, and Co- } \\
\text { logne Browns. }\end{array}$ & $\begin{array}{l}\text { Decomposed Ve- } \\
\text { getable, with Bi- } \\
\text { tuminous Mat- } \\
\text { ter. }\end{array}$ & $\begin{array}{l}\text { The best when deep, } \\
\text { rioh, and transparent. }\end{array}$ & \multirow{5}{*}{$\begin{array}{l}\text { There are other Browns, but } \\
\text { many of them are less durable, } \\
\text { Madder Brown, like all the } \\
\text { other Madders, is liable to fade. } \\
\text { Ivory and Bone Browns are not } \\
\text { so permanent as the blacks } \\
\text { of those substances. Oatechu } \\
\text { Brown, rich and transparent, } \\
\text { is subject to fly when laid on } \\
\text { thiniy. Manganese Brown is } \\
\text { semi-opaque, but \& good drier. } \\
\text { Prussian Brown (Ferrocyanide } \\
\text { of Copper) is affected by foul } \\
\text { gas, and destroyed by alkalis. } \\
\text { Calcined Prusedan Blue is a fine } \\
\text { Brown, but troublesome to } \\
\text { prepare. The peaty Brown, } \\
\text { probably from their vegetable } \\
\text { colouring matter, have a slight } \\
\text { tendency to become paler when } \\
\text { used very thinly, and exposed } \\
\text { to strong light. }\end{array}$} \\
\hline & $\begin{array}{l}\text { Terre Verle } \\
\text { Brovon. }\end{array}$ & $\begin{array}{l}\text { Terre Verte. } \\
\text { Burnt. }\end{array}$ & A quiet beautiful colour. & \\
\hline & Umber. & $\begin{array}{c}\text { An Iron Ore with } \\
\text { Manganese. }\end{array}$ & $\begin{array}{l}\text { An useful colour both } \\
\text { Raw and Burnt. }\end{array}$ & \\
\hline & Mrummy. & $\begin{array}{l}\text { White Pitch and } \\
\text { Myrrh, com- } \\
\text { bined with Ani- } \\
\text { mal Matter. }\end{array}$ & Rioh transparent Brown. & \\
\hline & Asphaltum. & $\begin{array}{l}\text { Bitumen, A MI- } \\
\text { neral Pitch or } \\
\text { Resin found } \\
\text { flosting on the } \\
\text { Dead Bea ; also } \\
\text { after the distil- } \\
\text { lation of Natural } \\
\text { Naphtha. }\end{array}$ & $\begin{array}{l}\text { Asphaltum is liable to } \\
\text { crack, except when in } \\
\text { an unctuous or waxy } \\
\text { vehicle. }\end{array}$ & \\
\hline \multirow[b]{2}{*}{ 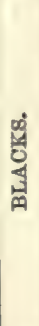 } & Ivory Black. & Burnt Ivory. & $\begin{array}{l}\text { Of a Brownish-black } \\
\text { tint, quito permanent. }\end{array}$ & \multirow[b]{2}{*}{$\begin{array}{l}\text { There are other perwanent } \\
\text { Blacks, as Bone-black (Burnt } \\
\text { Bones), Lamp-black (pureCar- } \\
\text { bon), the Soot of Burnt Resin, } \\
\text { and Turpentine. Manganese- } \\
\text { black, a native product, and a } \\
\text { good drier (Peroxide of Man- } \\
\text { ganese). There are also Black } \\
\text { Oohres, native earths, but they } \\
\text { are not required. }\end{array}$} \\
\hline & Blue-black. & $\begin{array}{l}\text { Burnt Vine } \\
\text { Twigs, \&c. Com- } \\
\text { mon Blacks } \\
\text { mixed with in- } \\
\text { secure Blues are } \\
\text { sometimes sub- } \\
\text { stituted by em- } \\
\text { pirics. The Blue- } \\
\text { black of the an- } \\
\text { cients was made } \\
\text { with the lees of } \\
\text { wine. }\end{array}$ & $\begin{array}{l}\text { Of a Bluish-black tint, } \\
\text { perfectly durable. }\end{array}$ & \\
\hline
\end{tabular}

COzouRING MaTrERs. The colour of any object, either natural or artificial, owes its origin to the effect produced on it by the rays of light. This effect is either due to the mass or substance of the body itself, as may be seen in the colours of metals and many shells, or it arises from the presence of some foreign substance or substances not absolutely essential to it, and which may in many cases be separated and removed from it. It is in speaking of these foreign substances which are often found colouring natural objects, or which are employed in the arts for the purpose of imparting colours to various materials, that we generally make use of the term colourive MaTTRR. By chemists, however, the term is only applied to organic bodies, and not to mineral substances, such as oxide of iron, cinnabar, ultramarine, \&c., which, though they aro employed as pigments in the arts, differ very widely in their properties from one another and from colouring matters in the narrower sense of the word. Colouring matters may be defined to be substances produced in animal or vegetable organisms, or easily formed there by processes occurring in nature (such as oxidation or fermentation), and which are either themselves coloured or give coloured compounds with bases or with animal or vegetable fibre. According to this definition, bodies like carbazotic acid and murexide, which are formed by complicated processes such as never occur in nature, are excluded, though they resemble true colouring matters in many of their properties, such as that of giving intensely-coloured compound bases. Whether, however, even after accepting the above definition, colouring matters can be considered as constituting a natural class of organic bodies, such as the fats, resins, \&cc., must still remain doubtful, though modern research tends to prove that these substances are related to one another by other properties besides the accidental one of colour, and will probably be found eventually to belong in reality to one natural class. 


\section{COLOURING MATTERS}

Colouring matters occur in all the organs of plants, in the root, wood, bark, leaves, flowers and fruit ; in the skin, hair, feathers, blood, and various secretions of animals ; in insects, for example, in various species of coccus; and in mollusca, such as the murex. Indeed there are very fow plants or animals whose organs do not produce somo kind of colouring matter. It is remarkable, however, that the colours which are most frequently presented to our view, such as those of the leaves and flowers of plants and the blood of animals, are produced by colouring matters with which we are but very little acquainted, the colouring matters used in the arts, and which have been oxamined with most care, being derived chiefly from less conspicuous organs, such as the roots and stems of plants. In almost all cases the preparation of colouring mattors in a state of purity presents great difficulties, so that it may even be said that very fow are known in that state.

Some colouring matters bear a great resemblance to the so-called extractive matters, others to resins. Hence they have been divided into extractive and resinous colouring matters. These resemblances are, however, of no great importance. The principal colouring matters possess such peculiar properties that they must be considered as bodies altogether sui generis.

As regards their most prominent physical characteristic, colouring matters are divided into three principal classes: viz, the red, yellow, and blue, the last class comprising the smallest number. Only one true green colouring matter occurs in nature, viz., chlorophyll, the substance to which the green colour of leaves is owing. ${ }^{1}$ Black and brown colouring matters are also uncommon, the black and brown colours obtained in the arts from animals or vegetables being (with the exception of sepia and a few others) compounds of colouring matters with bases. The colours of natural objects are often due to the presence of more than one colouring matter. This may easily be seen in the petals of some flowers. If, for instance, the petals of the orangecoloured variety of the Tropcoolum majus be treated with boiling water, a colouring matter is extracted which imparts to the water a purple colour. The petals now appear yellow, and if they be treated with boiling spirits of wine, a yellow colouring matter is extracted, and they then become white. When the purple colouring matter is absent the flowers are yellow; when, on the contrary, it is present in greater abundance, they assume different shades of brown. Precisely the same phenomena are observed in treating the petals of the brown Calceolaria successively with boiling water and spirits of wine. In many cases colouring matters exhibit, when in an uncombined state, an entirely different colour from what they do when they enter into a state of combination. The colouring matter of litmus, for instance, is, when uncombined, red, but its compounds with alkalis are blue. The alkaline compounds of alizarine are of a rich violet colour, while the substance itself is reddish-yellow. Many yellow colouring matters become brown by the action of alkali, and the blue colouring matters of flowers generally turn green when exposed to the same influence. The classification of colouring matters, according to colour, is therefore purely artificial. The terms red, yellow, and blue colouring matter, merely signify that the substance itself possesses one of these colours, or that most of its compounds are respectively red, yellow, or blue. In almost all cases, even when the colour is not entirely changed by combination with other bodies, its intensity is much increased thereby, substances of a pale yellow colour becoming of a deep yellow, and so on.

Colouring matters consist, like most other organic substances, either of carbon, hydrogen, and oxygen, or of those elements in addition to nitrogen. The exact relative proportion of these constituents, however, is known in very few cases, and in still fewer instances have the chemical formulæe of the compounds been established with any approach to certainty. This proceeds on the one hand from the small quantities of these substances usually present in the organs of plants and animals, and the difficulty of obtaining sufficient quantities for examination in a state of purity, and on the other hand from the circumstance of their possessing a very complex chemical constitution and high atomic weight.

Only a small number of colouring matters are capable of assuming a crystalline form ; the greater number, especially the so-called resinous ones, being perfectly amorphous. Among those which have been obtained in a crystalline form, may be mentioned alizarine, indigo-blue, quercitrine, morine, luteoline, chrysophan, and rutine. It is probable, however, that when improved methods have been discovered of preparing colouring matters, and of separating them from the impurities with which they are so often associated, many which are now supposed to be amorphous will be found to be capable of crystallising.

Very little is known concerning the action of light on colouring matters and their

- Another green colouring matter, derived from different species of Rhamnu, has been described under the name of 'Chinese Green.' It is stated to be a peculiar sabstance, not as might be snp. posed, a mixture of a blue and a yellow colouring matter.

Vor. I. 
compounds. It is woll known that these bodies when exposed to the rays of the sun, especially when deposited in thin layers on or in fabrics made of animal or vegetablo materials, lose mueh of the intensity of their colour, and sometimes even disappoar entirely, that is, they are converted into colourless bodies. But whether this process depends on a physical action induced by the light, or whether, as is more probable, it consists in promoting the decomposing action of oxygen and moisture on them, is uncertain. The most stable colouring matters, such as indigo-blue and alizarine in its compounds, are not insensible to the action of light. Others, such as carthamine from safflower, disappear rapidly when exposed to its influence. Colours produced by a mixture of two colouring matters are often found to resist the action of light better than those obtained from one alone. In one case, viz., that of Tyrian purple, the action of light seems to be absolutely essential to the formation of the colouring matter. The leaves of plants also remain colourless if the plants are grown in darkness, though in this case the formation of the green colouring matter is probably not due to the direct chemical action of the light.

The action of heat on colouring matters varies very much according to the nature of the latter and the method of applying the heat. A moderate degree of heat often changes the hue of a colouring matter and its compounds, the original colour being restored on cooling, an effect which is probably due to physical causes. Sometimes this effect is, without doubt, owing to the loss of water. Alizarine, for instance, crystallised from alcohol, when heated to $212^{\circ} \mathrm{F}$. loses its water of crystallisation, its colour changing at the same time from reddish-yellow to red. At a still higher tomperature most colouring matters are entirely decomposed, the products of decomposition being those usually afforded by organic matters, such as water, carbonic acid, carburetted hydrogen, empyreumatic oils, and, if the substance contains nitrogen, ammonia, or organic bases such as aniline. A few colouring matters, as for example alizarine, rubiacine, indigo-blue, and indigo-red, if carefully heated, may be volatilised without change, and yield beautiful crystallised sublimates, though a portion of the substance is sometimes decomposed, giving carbon and empyreumatic products.

Colouring matters, like most other organie substances, undergo decomposition with more or less facility when exposed to the action of oxygen; and the process may, indeed be more easily traced, in their case, as it is always accompanied by a change of hue. Its effects may be daily observed in the colours of natural objects belonging to the organic world. Flowers, in many cases, lose a portion of their colour before fading. The leaves of plants, before they fall, lose their green colour and become red or yellow. The colour of venous blood changes, when exposed to the air, from dark red to light red. When exposed to the action of oxygen, blue and red colouring matters generally become yellow or brown; but the process seldom ends here: it continues until the colour is quite destroyed ; that is, until the substance is converted into a colourless compound. This may be easily seen when a fabric, dyed of some fugitive colour, is exposed to the air. The intensity of the colour diminishes, in the first instance; it then changes in hue, and, finally, disappears entirely. Indeed, the whole process of bleaching in the air depends on the concurrent action of oxygen, light, and moisture. The precise nature of the chemical changes which colouring matters undergo during this process of oxidation is unknown. No doubt, it consists, generally speaking, in the removal of a portion of their carbon and hydrogen, in the shape of carbonic acid and water, and the conversion of the chief mass of the substance into a more stable compound, capable of resisting the further action of oxygen. But this statement conveys very little information to the chemist, who, in order to ascertain the nature of a process of decomposition, requires to know exactly all its products, and to compare their composition with that of the substances from which they are derived. The indeterminate and uninteresting nature of the bodies into which most colouring matters are converted by oxidation has probably deterred chemists from their examination. The action of oxygen on colouring matters varies according to their nature and the manner in which the oxygen is applied, and it is the degree of resistance which they are capable of opposing to its action that chiefly determines the stability of the colours produced by their means in the arts. Indigo-blue shows no tendency to be decomposed by gaseous oxygen at ordinary temperatures; it is only when the latter is presented in a concentrated form, as in nitric or chromic acid, or in a nascent state, as in a solution of ferrideyanide of potassium containing caustic potash, that it undergoes decomposition. When, however, indigo-blue enters into combination with sulphuric acid, it is decomposed by means of oxygen with as much facility as some of the least stable of this class of bodies. Some colouring matters are capalle of resisting the action of oxygen even in its most concentrated form. Of this kind are rubianine and rubiacine, which, when treated with boiling nitric acid, merely dissolve in the liquid and crystallise out again, when the latter is allowed to cool. The action of atmospheric oxygen on colouring matters is generally promoted by alkalis, and retarded in the presence of 
acids. A watery solution of hematine, when mixed with an excess of caustic alkali, becomes of a beautiful purple; but the colour when exposed to the air, almost im. mediately turns brown, the hematino being then completely changed. It is almost needless to observe, that the bodies into which colouring matters are converted by oxidation are incapable, under any circumstanees, of returning to their original state.

The action of reducing agents, that is of bodies having a great affinity for oxygen, on some colouring matters, is very peculiar. If indigo-blue, suspended in water, be placed in contact with protoxide of iron, protoxide of tin, or an alkaline sulphuret, sulphite, or phosphite, or grape sugar, or, in short, any easily oxidisable body, an excess of some alkali or alkaline earth being present at the same time, it dissolves, forming a pale yellow solution without a trace of blue. This solution contains, in combination with the alkali or alkaline earth, a perfectly white substance, to which the name of reduced indigo has been applied. When an excess of acid is added to the solution, it is precipitated in white flocks. By exposure to the air, either by itself or in a state of solution, reduced indigo rapidly attracts oxygen, and is reconverted into indigo-blue. Hence the surface of the solution, if left to stand in uncovered vessels, becomes covered with a blue film of regenerated indigo-blue. It was for a long time supposed that reduced indigo was simply deoxidised indigo-blue, and that the process consisted merely in the indigo-blue parting with a portion of its oxygen, which was taken up again on exposure to the air. It has, however, been discovered that in every case water is decomposed during the process of reduction which indigo-blue undergoes, the oxygen of the water combining with the reducing agent, and the hydrogen uniting with the indigo-blue, water being again formed when reduced indigo comes in contact with oxygen. Roduced indigo is therefore not a body containing less oxygen than indigo-blue, but is a compound of the latter with hydrogen. There are several red colouring matters which possess the same property, that of being converted into colourless compounds by the simultaneous action of reducing agents and alkalis, and of returning to their original state when exposed to the action of oxygen. There can be little doubt that the process consists, in all cases, in the colouring matter combining with hydrogen and parting with it again when the hyduret comes in contact with oxygen.

The action of chlorine on colouring matters is very similar to that of oxygen, though, in general, chlorine acts more energetically. The first effect produced by chlorine, whether it be applied as free chlorine, or in a state of combination with an alkali, or alkaline earth as an hypochlorite, usually consists in a change of colour. Blue and red colouring matters generally become yellow. By the continued action of chlorine all trace of colour disappears, and the final result is the formation of a perfectly white substance, which is usually more easily soluble in water and other menstrua than that from which it was formed. Since it is most commonly by means of chlorine or its compounds that colouring matters are destroyed or got rid of in the arts, as in bleaching fabries and discharging colours, the process of decomposition which they undergo by means of chlorine has attracted a good deal of attention, and the nature of the chemical changes, which take place in the course of it, has often been made a subject of dispute, though the matter is one possessing more of a theoretical than a practical interest. It is a well-known fact that many organic bodies are decomposed when they are brought into contact, in a dry state, with dry chlorine gas. The decomposition consists in the elimination of a portion of the hydrogen of the substance and its substitution by chlorine. When water is present at the same time, the decomposition is, however, not so simple. It is well known that chlorine decomposes water, combining with the hydrogen of the latter and setting its oxygen at liberty, and it has been asserted, that in the bleaching of colouring matters by means of chlorine when moisture is usually present, this always takes place in the first instance, and that it is in fact the oxygen which effects their destruction, not the chlorine. This appears, indeed, to be the case occasionally. Rubian, for instance, the body from which alizarine is derived, gives, when decomposed with chloride of lime, phthalic acid, a beautifully-crystallised substance, containing no chlorine, which is also produced by the action of nitric acid on rubian, and is, therefore, truly a product of oxidation. In many cases, however, it is certain that the chlorine itself also enters into the composition of the new bodies produced by its action on colouring matters. When, for instance, the chlorine acts on indigo-blue, chlorisatine is formed, which is indigo-blue, in which one atom of hydrogen is replaced by one of chlorine, plus two atoms of oxygen, the lattor being derived from the decomposition of water.

The behaviour of colouring matters towards water and other solvents is very various. Some colouring matters, such as those of logwood and brazilwood, are very easily soluble in water. Others, such as the colouring matters of madder and quercitron bark, are only sparingly soluble in water. Many, especially the so-called resinous ones, are insoluble in water, but more or less soluble in alcohol and other, or alkaline liquids. $\mathbf{A}$ 
fow, such as indigo-blue, are almost insoluble in all menstrua, and can only be made to dissolve by converting thom, by means of reducing agents, into other bodies soluble un alkalis. Those which are soluble in water, are, generally speaking, of the greatost importance in the arts, since they admit of more ready application, when they possoss this property.

The behaviour of colouring matters towards acids is often very characteristic. Most colouring matters are complotely decomposed by nitric, chloric, manganic, and chromic acids, in consequence of the large proportion of oxygen which these acids contain. With many colouring matters the decomposition takes place even at the ordinary temperature: with others, it only commences when the acid is warmed, especially if the latter be applied in a state of considerable dilution. Concentrated sulphuric acid also destroys most colouring matters, especially if the acid be heated. It seems to act by depriving them of the olements of water, and thereby converting them into substances containing more carbon than before, as may be inferred from the dark, almost black, colour which they acquire. At the same time the acid generally loses a portion of its oxygen, since sulphurous acid is almost always evolved on heating. Some colouring matters, such as alizarine, are not decomposed by concentrated sulphuric acid eren when the latter is raised to the boiling point; they merely dissolve, forming solutions of various colours, from which they are precipitated unchanged, on the addition of water, when they are insoluble or not easily soluble in the latter. Others, again, like indigo-blue, dissolve in concentrated or fuming sulphuric acid, without being decomposed, and at the same time enter into combination with the acid, forming true double acids, which are easily soluble in water and combine as such with basos. Many colouring matters undergo a change of colour when exposed to the action of acids, the original colour being restored by the addition of an excess of alkali, and this property is made use of for the detection of acids and alkalis. The colour of an infusion of litmus, for instance, is changed by acids from blue to red, and the blue colour is restored by alkalis. An infusion of the petals of the purple dahlia or of the violet becomes red on the addition of acids, and this red colour changes again to purple or blue with alkalis, an excess of alkali making it green. The yellow colour of rutine becomes deeper with strong acids. In most cases, this alteration of colour depends on a very simple chemical change. Litmus, for example, in the state in which it oecurs in commerce, consists of a red colouring matter in combination with ammonia, the compound being blue. By the addition of an acid, the ammonia is removed, and the uncombined red colouring matter makes its appearance. Ammonia and most alkalis remove the excess of acid, and, by combining with the red colouring matter, restore the blue colour. When a colouring matter, like alizarine, is only sparingly soluble in water, its solubility is generally diminished in the presence of a strong acid. Hence, by adding acid to the watery solution, a portion of the colouring matter is usually precipitated. It is very seldom that colouring matters are really found to enter into combination with acids. Indeed, only one, viz. berberine, is capable of acting the part of a true base, and forming definite compounds with acids. Some acids, such as sulphurous and hydrosulphuric acids, do certainly seem to combine with some colouring matters and form with them compounds, in which the colour is completely disguised, and apparently destroyed. If a red rose be suspended in an atmosphere of sulphurous acid it becomes white, but the red colour may be restored by neutralising the acid with some alkali. On this property of sulphurous acid depends the process of bleaching woollen fabrics by means of burning sulphur. In this case the colouring matter is not destroyed, but only disguised by its combination with the acid.

Most colouring matters are capable of combining with bases. Indeed, their affinity for the latter is generally so marked that they may be considered as belonging to the class of weak acids. Like all other weak acids, they form, with bases, compounds of a very indefinite composition, so much so, that the same compound, prepared on two different occasions, is often found to be differently constituted. Hence the great difficulty experienced by chemists in determining the atomic weight of colouring matters. There are very fow of the latter for which several formulæ, all equally probable, may not be given, if the compounds with bases be employed for their determination. The compounds of colouring matters with bases hardly ever crystallise. Those with alkalis are mostly soluble in water and amorphous; those with the alkaline earths, lime, and baryta, are sometimes soluble, sometimes insoluble; those with the earths and metallic oxides are almost always insoluble in water. The compounds with alkalis are obtained by dissolving the colouring matter in water to which a little alkali is added, and evaporating to dryness: an operation which must be carefully conducted if the colouring matter is one easily affected by oxygen. The insoluble compounds, with earths and metallic oxides, are obtained either by double decomposition of a soluble compound with a soluble salt of the respective base, or by adding to a solution of the colouring matter, in water or any other menstruum, a salt of the base containing some 
weak acid, such as acetic acid. It is remarkable that of all bases none show so much affinity for colouring matters as alumina, peroxide of iron, and peroxide of tin, bodies which occupy an intermediato position between acids and bases. If a solution of any colouring matter be agitated with a sufficient quantity of the hydrates of any of these bases, the solution becomes decolourised, the whole of the colouring matter combining with the base and forming a coloured compound. It is accordingly these bases that are chiefly employed in dyeing, for the purpose of fixing colouring matters on particular portions of the fabric to be dyed. When used for this purpose they are called mordants. Their compounds with colouring matters are denominated lakes, and are employed as pigments by painters. The colours of the compounds usually differ, either in kind or degree, from those of the colouring matters themselves. Red colouring matters often form blue compounds, yellow ones sometimes give red or purple compounds. The compounds with peroxide of iron are usually distinguished by the intensity of their colour. When a colouring matter gives with alumina and oxide of tin red compounds, its compound with peroxide of iron is usually purple or black; and when the former are yellow, the latter is commonly olive or brown. Almost all the compounds of colouring matters with bases are decomposed by strong acids, such as sulphuric, muriatic, nitric, oxalic, and tartaric acids, and even acetic acid is not without effect on some of these compounds. The compounds with earths and metallic oxides are also decomposed, sometimes by alkalis. A solution of soap is sufficient to produce this effect in many cases, and dyes are therefore often tested by means of a solution of soap in order to ascertain the degree of permanence which they possess.

No property is so characteristic of colouring matters, as a class, as their behaviour towards bodies of a porous nature, such as charcoal. If a watery solution of a colouring matter be agitated with charcoal, animal charcoal being best adapted for the purpose, the colouring matter is generally entirely removed from the solution and absorbed by the charcoal. The combination which takes place under these circumstances is probably not due to any chemical affinity, but is rather an effect of the so-called attraction of surface, which we often see exerted by bodies of a porous nature, such as charcoal and spongy platinum, and which enable the latter to absorb such large quantities of gases of various kinds. That the compound is indeed more of a physical than a chemical nature seems to be proved by the circumstances that sometimes the colouring matter is separated from its combination with the charcoal by means of boiling alcohol, an agent which can hardly be supposed to exert a stronger chemical affinity than water. It is this property of colouring matters which is made use of by chemists to decolourise solutions, and by sugar manufacturers to purify their sugar. The attraction manifested by colouring matters for animal or vegetable fibre is probably also a phenomenon of the same nature, caused by the porous condition of the latter, and the powerful affinity of the so-called mordants for colouring matters, may, perhaps, be in part accounted for by their state of mechanical division being different from that of other bases. Colouring matters, however, vary much from one another in their behaviour towards animal or vegetable fibre. Some, such as indigo-blue, and the colouring matters of safflower and turmeric, are capable of combining directly with the latter, and imparting to them colours of great intensity. Others are only slightly attracted by them and consequently impart only feeble tints; they therefore require, when they are employed in the arts for the purpose of dyeing, the interposition of an earthy or metallic base. To the first class Bancroft applied the term substantive colouring matters, to the second that of adjective colouring matters.

One of the most interesting questions connected with the history of colouring matters is that in regard to the original state in which these substances exist in the animal and vegetable organisms from which they are derived. It has been known for a long time that many dye-stuffs, such as indigo and archil, do not exist ready formed in the plants from which they are obtained, and that a long and often difficult process of preparation is required in order to eliminate them. The plants which yield indigo exhibit, while they aro growing, no trace of blue colour. The colouring matter only makes its appearance after the juice of the plant has undergone a process of fermentation. The lichens employed in the proparation of archil and litmus are colourless, or at most light brown, but by steeping them in liquids containing ammonia and lime a colouring matter of an intense red is gradually generated, which remains dissolved in the alkaline liquid. Other phenomena of a similax nature might be mentioned, as for instance the formation of the so-called Tyrian purple from the juice of a sholl-fish, and new ones are from time to time being discovered. In order to explain these phenomena various hypotheses have been resorted to. It was supposed, for instance, that the indigoferce contained whito or reduced indigo, and hence were devoid of colour, and that the process of preparing indigo-blue consisted simply in oxidising the white indigo, which was for this reason denominated indigogene, by some chemists. The same 
assumption was made in regard to other colouring matters, all of which wore supposed to exist originally in a deoxidised and colourless state. In regard to indigo, however, the hypothesis is disprored at onee by tho fact that reduced indigo is only soluble in alkaline liquids, and that the juice of the indigo-bearing plants is always acid. In regard to the other colouring matters it seems also to be quite untenable. The first person to throw some light on this obscure department of organic chemistry was Robiquet. This distinguished chomist succeeded in obtaining from lichens in their colourless state a beautifully crystallised, colourless substance, soluble in water, having a sweet taste, and consisting of carbon, hydrogen, and oxygen. This substance he denominated orcine. By the combined action of ammonia and oxygen, he found it to be converted into a red colouring matter, containing nitrogen, and insolublo in water, which was in fact identical with the colouring matter of archil. This beautiful discovery furnished chemists with a simple explanation for the curious phenomena observed in the formation of this and other colouring matters, and it was soon followed by others. Heeren and Kane obtained from various lichens other colourless substances of similar properties, and it was shown by Schunck that orcine is not even the first link in the chain, but is itself formed from another body, lecanorine, which, by the action of alkalis, yields orcine and carbonic acid, just as sugar by fermentation gives alcohol and carbonic acid. In like manner it was discorered by Erdmann that the colouring matter of logwood is formed by the simultaneous action of oxygen and alkalis from a crystallised colourless substance, hematoxyline, which is the original substance existing in the wood of the tree, and is like the others, not itself strictly speaking, a colouring matter, but a substance which gives rise to the formation of one.

There is, however, another class of phenomena connected with the formation of coiouring matters, entirely different from that just referred to. It was discovered by Robiquet that if madder be treated for some time with sulphuric acid, and the acid be afterwards completely removed, the madder is found to have acquired a much greater tinctorial power than before. This fact was explained by some chemists by supposing that the sulphuric acid had combined with or destroyed some substance or substances, contained in the madder which had previously hindered the colouring matter from exerting its full power in dyeing, such as lime, sugar, woody fibre, \&c. By others it was suspected that an actual formation of colouring matter took place during tho process, and this suspicion has been verified by recent researches. Schunck succeeded in preparing from madder a substance resembling gum, very soluble in water, amorphous, and having a very bitter taste, like madder itself, and to which he gave the name of rubian. This substance, though not colourless, is incapable of combining with mordants, like most colouring matters. When, however, it is acted on by strong acids, such as sulphuric or muriatic acid, it is completely decomposed, and gires rise to a number of products, the most important of which is alizarine, one of the colouring matters of madder itself. Among the other products are a yellow crystallised colouring matter, mbianine, two amorphous red colouring matters resembling resins, rubiretine and verantine, and lastly, grape-sugar. When subjected to fermentation, the same products are formed, with the exception of rubianine, which is replaced by a yellow colouring matter of similar properties. This process of decomposition evidently belongs to that numerous class called by some chemists 'catalytic,' in which the decomposing agent does not act, as far as we know, in virtue of its chemical affinities. It is erident, then, that when madder is acted on by sulphuric acid an actual formation of colouring matter takes place, and it is even probable that the whole of the colouring matter found in madder in its usual state was originally formed from rubian, by a process of slow fermentation, the portion of the latter still remaining undecomposed being that which is acted on when acids are applied to madder. From the Isatis tinctoria or common woad plant, Schunck, in like manner, extracted an amorphous substance, easily soluble in water, called by him indican, and which, by the retion of strong acids, is decomposed into indigo-blue, indigo-red, sugar, and other products, among which are also several resinous colouring matters. Looking at them from this point of view, colouring matters may bo divided into two classes, viz., first, such as are formed from other substances, not themselves colouring matters, by the action of oxygen and alkalis; and, secondly, such as are formed from other substances by means either of ferments or strong acids, without the interrention of oxygen. To the first class belong the colouring matters of archil, litmus, and logwood; they yield comparatively fugitive dyes, and are usually decomposed ky allowing the very process to which they owe their formation to continue. To the second class belong indigoblue, indigo-red, and alizarine, bodies which show a remarkable degree of stability for organic compounds. More extended research will probably show that many other colouring matters are formed either in one manner or the other, which will probably afford us the means of arriving at a rational mode of classifying these bodies, and of distinguishing them as a class from others. $-\mathrm{E}$. S.

corT's-roor. See Tussinago. 
COzUMreren or Niobium. A peculiar metal originally extracted from a rare minoral brought from Haddam in Connecticut, and known as Columbite. This is a columbate and tantalate of iron and manganese. Columbium also occurs, with tantalium, in several raro Swedish minerals. The metal received its name from Columbia (America). See Nromuar.

COIUMTAn COAI. A name given to ANTHRACTTE.

Comma is a variety of cabbage,--the Brassica oleracea,-whose seeds afford by pressure an oil much employed in France and Belgium for burning in lamps, and for many other purposes. This plant requires a rich but light soil ; it does not succeed upon either sandy or clay lands. The ground for it must be deeply ploughed and well dunged. It should be sown in July, and be afterwards replanted in a richly manured field. In October it is to be planted out in beds, 15 or 18 inches apart. Colza may also be sowed in furrows 8 or 10 inches asunder.

Land which has been just cropped for wheat is that usually destined to colza ; it may be fresh dunged with advantage. The harvest takes place in July, with a sickle, a little before the seeds are completely ripe, lest they should drop off. As the seed is productive of oil, however, only in proportion to its ripeness, the cut plants are allowed to complete their maturation, by laying them in heaps under airy sheds, or placing them in a stack, and thatching it with straw.

The cabbage stalks are thrashed with flails; the seeds are winnowed, sifted, and spread out in the air to dry; then packed away in sacks, in order to be subjected to the oil mill at the beginning of winter. The oil-cake is a very agreeable food to cattle; it serves to fatten them, and is reckoned to defray the cost of the mill.

When proper manure was not applied, it was reported that colza impoverished the soil very much, as do indeed, all the plants cultivated for the sake of their oleaginous seeds. The same soil must not, therefore, be come back upon again for six years, if fine crope be desired. The double ploughing which it requires effectually eleans the ground.

The colza or wild cabbage itself is a plant of sufficient interest to call special attention to its properties. Besides yielding an oil which gives a brilliant light for the lamps of lighthouses, its seed has other properties that should induce the plant to be in favour with agriculturists, emigrants, and colonists. The recent accounts, according to $\mathrm{Du}$ Bow, state 'it to be admirably adapted for cattle as food; that the seeds after the oil is expressed yield a cake highly prized for fattening cattle, and as manure.' 'There is a spring variety which will succeed in almost any part of the United States, and will ultimately become an article of great importance.'

The real history of this valuable plant seems to be this:-The Abbe de Commerell, in a letter to Dr. Lettsom, dated from Paris at the Abbey of St. Victor, 1789, calls especial attention to the colza which he had cultivated for some time in the neighbourhood of Paris, 'and last year under the inspection of the Royal Society of Agriculture. The severe winter just experienced, which had destroyed great abundance of turnips and cold, had not done the least injury to my plants, which is a proof of resisting the sererest cold.' The following description of the plant may lead to its adoption as sources of oil and food. To induce this we may refer to the original communication, now of course sufficiently rare: it is entitled: 'Mémoire sur la Culture, $l$ usage et les avantages du Choux à faucher, par M. l' Abbé de Commerell, à Paris,' 1789. He states he found the plant in Germany, where it was only used for seed; that there are three distinct rarieties, known by the colours of the 'nerves' of the leaves: violet, yellow, and green. He gives preference to the violet, 'il est plus abondant, plus sapide, et resiste mieux à l'impression du froid et à la rigueur des hivers.' Ho adds, that he presents to the Royal Society (Agric. de Paris) the plants which had resisted the cold of the preceding winter, 'the most rigorous of which mention is made in our annals.'

Again Commerell says: "This plant is a kind of wild cabbage, that may be cut four or six times in the year it is sown; each cut is as plentiful as trefoll or lucerne; we leave it afterwards for the winter. About the month of February it shoots up, and the leaves of it may be cut; but in the month of April it begins to grow up, send off stalks, and bears its seed, which may be gathered in June. The first year this wild cabbage does not send stalks; its leaves appear to rise out of the ground, and thus it may be cut like grass; it may also be dried for hay. Its leaves extend to 10, 12, and 15 inches in length and 6 to 8 broad, which have not the bitter and herbaceous taste of other cabbages. It is a pulse very agreeable for man during the whole year, and a fodder equally as good as plentiful for all kinds of cattle. The milk of cows does not acquire a bad tasto, nor do the cows get tired of it.

This plant bears more and larger sized seed than turnips or cole, and the oil which I have extracted from it cold is very superior as food for man to that from poppy or cole, and is equal to the common oil of olives, in the opinion of good judges. I give the name of the mowing cahbage to this plant. If you will make a trial of it,' he adds to Dr. Lettsom, 'you will have every reason to be satisfied, for this cabbage yields one 
third more oil than turnips in proportion to the equal quantity of ground, and we may sow it in spring or in entumn.'

When Commerell wrote the trials were limited for want of seed; but it now appears to be well worthy the attention of agriculturists, as a plant whose rapid growth and general favour, may remedy the scarcity of other erops more in use. The production of the oil alone forms a considerable article of the trade of France, Belgium, England, and America.

To mining districts, to manufacturers, and othors in remote localities, the valuablo properties of this plant and seed, as sources of oil, food, and manure, are commended. Even six crops a year are said to have been taken. Thus the bitterness of famine by the failure of other crops might be mitigated; and as the oils of seeds are now confusedly mixed together in commercial transactions, we have thought the usefulness of this plant should be more generally known by references to those qualities recorded on its original cultivation.

Colza oil is now extensively used for burning in lamps and for lubricating machinery. The Carcel, Moderator, and other lamps are contrived to give a continuous supply of oil to the wick, and by a rapid draught of air brilliant combustion of the oil is maintained without smoke.

In the lighthouses of France and England it has been employed with satisfaction, so as to replace the use of sperm-oil; the preference has been given on the grounds of greater brilliancy, a steądier flame, the wick being loss charred, and the advantage of economy in price.

The Corporation of the Trinity House and the late Mr. Hume took great in. terest in the question of the relative merits of colza, rape, and seed oils, as compared with sperm-oil, and in $\mathbf{1 8 4 5}$ referred the investigation of the power and qualities of the light from this description of oil, to Professor Faraday. He reported "that he was much struck with the steadiness of the flame, burning 12 or 14 hours without being touched;' 'taking above 100 experiments, the light came out as one and a half for the seed-oil to one of the sperm; the quantity of oil being used in the same proportion;' and he concludes by stating his ' confidence in the results.'

The advantages then were, less trouble, for the lamps with sperm had to be retrimmed, and the same lamp with seed-oil gave more light, and the cost then was as 3s. $9 d$. per gallon seed-oil, against $6 s .4 d$. imperial gallon of sperm.

Those interested should consult returns, ordered by the House of Commons, "Lichrnouses: on the motion of Mr. Hume, "On the substitution of Rape-seed oil for Sperm-oil, and the saving accruing therefrom." 17th Feb. 1855 ; No. 75; 18th March, 1857,196 and 196 '.'

In the Supplementary Returns laid before the House of Commons by the Commis. sioners of the Northern Lights, there is the following report of Alan Sterenson, Esq., their engineer :-

'In the last annual report on the state of the lighthouses, I directed the attention of the Board to the propriety of making trial, at several stations, of the patent culzà or rape-seed oil, as prepared by Messrs. Briggs and Garford, of Bishopsgate Strect. These trials have now been made during the months of January and February, at three catoptric and three dioptric lights, and the results have from time to time been made known to me by the light-keepers, according to instructions issued to them, as occasion seemed to require. The substantial agreement of all the reports as to the qualities of the oil renders it needless to enter into any details as to the slight varying circumstances of each case; and I have therofore great satisfaction in briefly stating, as follows, the very favourable conclusion at which I have arrived:-

-1. The culzà oil possesses the advantage of remaining fluid at temperatures which thicken the spermaceti oil.

-2. The culzà oil burns both in the Fresnel lamp and the single argand burner, with a thick wick, during seventeen hours, without requiring any coaling of the wick, or any adjustment of the damper; and the flame seems to be more steady and free from flickering than that from spermaceti oil.

3. There seems (most probably owing to the greater steadiness of the flamo) to be less breakage of glass chimneys with the culza than with the spermaceti oil.'

The above firm, who from thirty years' experience in the trade, were enabled to induce the Trinity Corporation to give this oil a fair and extended trial, state that ' $\mathrm{A}$ )r manufacturing purposes it is equally useful; it is admitted by practical men to be the best known oil for machinery; equal to Gallipoli ; and technically it possesses moro " body," though perfectly free from gummy matter.'

Whilo sperm, Gallipoli, nut, or lard oils are rendered useless by the slightest oxposure to frost, the colza oil will, with ordinary care, retain its fluidity: this has been acknowledged as a very important quality to railway and steam-boat companies.

It should be here stated that tho terms rape-oils, seed-oils, colza or culza, are all 
now blended together, and, however much this may be regretted, yet it does not seem easy to keep distinctness in the general usages of oil, for the Customs returns class all under one head,-Rape Oil.

A number of British and colonial seed-bearing plants appear to be now employed for the sake of their oils, although on account of the mucilaginous matter contained in many of the oils, they are far inferior to the colza which they are employed to adulterate.-T. J. P.

Of the importance of the trade some estimate may be formed by the following entry.-The Importations of seed-oils were as follow :-

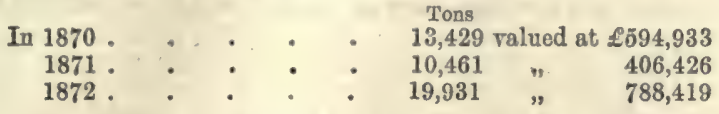

comrz. The name of an instrument which is employed to disentangle, and lay parullel and smooth the hairs of man, horses, and other animals. They are made of thin plates, either plane or curved, of wood, horn, tortoise-shell, ivory, bone, or metal, cut upon one or both sides or edges with a series of somewhat long teeth, not far apart.

Two saws mounted on the same spindle are used in cutting the teeth of combs, which may be considered as a species of grooving process; one saw is in this case larger in diameter than the other, and cuts one tooth to its full depth, whilst the smaller saw, separated by a washer as thick as the required teeth, cuts the succeeding tooth part of the way down.

A few years back Messrs. Pow and Lyne invented an ingenious machine for sawing boxwood or ivory combs. The plate of ivory or boxwood is fixed in a clamp suspended on two pivots parallel with the saw spindle, which has only one saw. By the revolution of the handle a cam first depresses the ivory on the revolving saw, cuts on $\theta$ notch, and quickly raises it again; the handle, in completing its circuit, shifts the slide that carries the suspended clamp to the right, by means of a screw and ratchet movement. The teeth are cut with great exactness, and as quickly as the handle can be turned; they vary from about thirty to eighty teeth in one inch, and such is the delicacy of some of the saws, that even 100 teeth may be cut in one inch of ivory. The saw runs through a cleft in a small piece of ivory, fixed vertically or radially to the saw, to act as the ordinary stops, and prevent its flexure or displacement sideways. Two combs are usually laid one over the other and cut at once; occasionally the machine has two saws, and cuts four combs at once.

In the manufacture of tortoise-shell combs, very much ingenuity is displayed in soldering the back of a large comb to that piece which is formed into teeth. The two parts are filed to correspond; they are surrounded by pieces of linen, and inserted between metal moulds, connected at their extremities by metal screws and nuts; the interval between the halves of the moulds being occasionally curved to the sweep required in the comb; sometimes also the outer faces of the mould are curred to the particular form of those combs in which the back is curled round, so as to form an angle with the teeth. Thus arranged it is placed in boiling water. The joints when properly made cannot be detected, either by the want of transparency or polish. Much skill is employed in turning to economical account the flexibility of tortoise-shell in its heated state : for example, the teeth of the larger descriptions of comb are parted, or cut one out of the other with a thin frame saw; then the sholl, equal in size to two combs with their teeth interlaced, is bent like an arch in the direction of the length of the teeth. The shell is then flattened, the points are separated with a narrow chisel or pricker, and the two combs are finished whilst flat, with coarse single cut files, and triangular scrapers; and lastly, they are warmed, and bent on the knee over a wooden mould by means of a strap passed round the foot, in the manner a shoemaker fixes a shoo last. Smaller combs of horn and tortoise-shell are parted whilst flat, by an ingenious machine with two chisel-formed cutters, placed obliquoly, so that every cut produces one tooth, the repetition of which completes the formation of the comb.

Mr. Rogers's comb-cutting machine is described in the "Transactions of the Society of Arts, vol. xlix., part 2, page 150. It has been since remodelled and improred by Mr. Kelly. This is an example of slender chisel-like punches. The punch or chisel is in twro parts, slightly inclined and curved at tho ends to agree in form with tho outline of one tooth of the comb, the cutter is attacherl to the end of a jointed arm, mored up and down by a crank, so as to penetrato almost through the mat rial, and the uncut portion is so very thin that it splits through at each stroke and liaves the two combs detached.

The comb-makers' double saw is called a 'stadda,' and has two blades contrived so as to give with great facility and exactness the intervals between the teeth of combs, 
from the coarsest to those having from forty to forty-five teeth to the inch. The gage-saw or gage-vid is used to make the toeth square and of one depth. The saw is frequently made with a loose back, like that of ordinary back-saws, but much wider, so that for teeth $\frac{1}{2}, \frac{5}{8}, \frac{3}{4}$ inch long, it may shield all the blade except $\frac{1}{2}, \frac{6}{8}, \frac{3}{4}$ inch of its width respectively, and the saw is applied until the back prevents its further progress. Sometimes the blade has teeth on both edges, and is fixed between two parallel slips of steel connected beyond the ends of the saw blade by two small thumb-screws. After the teeth of combs are cut they are smoothed and polished with flles, and by rubbing them with pumice-stone and tripoli.-Holtzapffel.

COMRINING NUMBERS AND CERMICAI COMBINATION.Constancy of composition is one of the most essential characters of chemical compounds; by which is meant that any particular body, under whatever circumstances it may have been produced, consists invariably of the same elements in identically the same proportion; the converse of this is not, however, necessarily true, that the same elements in the same proportion always produce the same body. See Isomersisur.

But not only is there a fixity in the proportion of the constituents of a compound; but also, if any one of the elements be taken, it is found to unite with the other elements in a proportion which is either invariable, or changes only by some very simple multiple.

The numbers expressing the combining proportions of the elements are only relative. In England it is usual to take hydrogen as the starting-point, and to call that number the combining number of any other element which expresses the proportion in which it unites with one part by weight of hydrogen : and these numbers are now likewise adopted on the Continent, although in France the combining numbers are sometimes referred to oxygen, which is taken as 100 . It is obvious that, whichever system is used, the numbers have the same value relatively to each other.

These combining numbers would have but little value if they expressed nothing more than the proportion in which the elements combine with that body arbitrarily fixed as the standard; but they also represent the proportions in which they unite among themselves, in the event of union taking place. Thus, not only do 8 parts of oxygen unite with one of hydrogen, but also 8 parts of oxygen unite with 39 of potassium, 23 of sodium, 100 of mereury, 108 of silver, \&c. It is in virtue of this law that the combining proportions of many of the elements have been ascertained. Some of them do not combine with hydrogen at all, and in such cases the quantity which unites with 8 parts of oxygen, or 16 of sulphur, \&c., has to be ascertained. See A томпо Wetghts; EquTVatzents.-H.M.W.

ComnUsтrzxs. (Eng. and Fr.; Brennstoff, Ger.) Any substance which, exposed in the air to a certain temperature, consumes with the emission of heat and light. All such combustibles as are cheap enough for common use go under the name of Fuel. Every combustible requires a peculiar pitch of temperature to be kindled, called its accendible point. Thus phosphorus, sulphur, hydrogen, carburetted hydrogen, carbon, each takes fire at successively higher heats. See Fuave; Furd.

Comaustrow. (Eng. and Fr.; Verbrennung, Ger.) The phenomena of the development of light and heat from any body, as from charcoal combining with the oxygen of the air, from phosphorus combining with iodine, and from some of the metals combining with chlorine. Combustion may be exerted with rery various degrees of energy. We have a low combustion often established in masses of vegetable matter; as in haystacks, or in heaps of damp sawdust. In these cases, the changes going on are the same in character, only varying in degree, as those presented by an ordinary fire, or by a burning taper-oxygen is combining with carbon to form carbonic acid. The heat thus produced (the process has been termed by Liebig Eremacausis), increasing in force, gives rise eventually to visible combustion.

Cases of spontaneous combustion are by no means uncommon. Some years since, the editor investigated the conditions under which H. M. ships the 'Imogen' and 'Talavera' were burnt in Devonport Dockyard, and he was enabled to trace the fire to a large bin, in which there had been allowed to accumulate a mass of oiled oakum, pieces of old flannel covered with anti-attrition, sawdust, shavings, and the sweepings of the painters', wheelwrights', and some other shops.

Tho subject of combustion belongs to Watts's 'Dictionary of Chemistry,' where it is fully treated. See Spontanious Cominestros.

Concrers. The name given by architects to a compact mass of pebbles, sand, and lime, cemented together in order to form the foundation of buildings. Semple says that the best proportions are 80 parts of pebbles, each about 7 or 8 ozs. in weight, 40 parts sharp river sand, and 10 of lime; the last is to be mixed with water to a thinnish consistonce, and grouted in. It has been found that Thames ballast, as taken from the bed of the river, consists of nearly 2 parts of pebbles to 1 of 
sand, and therefore answers exceedingly well for making concrete, with from onoseventh to one-eighth part of lime. The best modo of making concrete, according to Mr. Godwin, is to mix limo, previously ground, with tho ballast in a dry state; sufficient water is now thrown over it to effect a perfect mixturo; after which it should be turned over at least twice with shovels, or oftener; then put into barrows, and wheeled away for use instantly. It is generally found advisable to employ two sets of men to perforin this operation, with three sets in each set, and thoy repeating the process, turn over the concrete to the barrow-men. After being put into the barrows, it should be at once wheeled up planks so raised as to give it a fall of some yards, and thrown into the foundation, by which means the particles are driven closer together, and groater solidity is given to the whole mass. Soon after being thrown in, the mixture is observed usually to be in commotion, and much heat is evolved, with a copious emission of rapour. The barrow-load of concrete in the fall spreading over the ground will form generally a stratum of from 7 to 9 inches thick, which should be allowed to set before throwing in a second.

Another method of making concrete, is first to cover the foundation with a certain quantity of water, and then to throw in the dry mixture of ballast and lime. It is next turned and levelled with shovels; after which more water is pumped in and the operation is repeated. The former method is undoubtedly preferable. In some cases it has been found necessary to mix the ingredients in a pug-mill, as in mixing clay, \&c., for bricks. For the preparation of a concrete foundation, as the hardening should be rapid, no more water should be used than is absolutely necessary to effect a perfect mixture of the ingredients. Hot water accelerates the induration. There is about one-fifth contraction in volume in the concrete, in reference to the bulk of its ingredients. To form a cubical yard of concrete, about $30 \mathrm{ft}$. cube of ballast and $3 \mathrm{zt}$. cube of ground lime must be employed, with a sufficient quantity of water.

Several other methods have been adopted by builders and engineers: these, however, involve the same principles and general condition; a detail of them is, therefore, unnecessary in this work. The reader desiring information is referred to works especially devoted to engineering and building. See Hydrautic LTME.

CONDY'S DISINryCrruc SOIUTroN. A solution of an alkaline manganate, or permanganate. See Manganates and Disireectarrs.

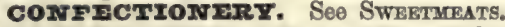

Concristrox. (Eng. and Fr. ; Gefrierung, Ger.) The act of freezing liquids. The processes employed are chiefly chemical, but some are mechanical. These will have further attention under the heads Frmanvg Mrxtures, Ice, and Ine Mavufacrure.

CONICRAICITr. An arsenate and phosphate of copper found in Andalusia.

ConIfraze. The natural order of cone-bearing plants, including some of the most important trees, such as all kinds of fir, cedar, juniper, pine, cypress, \&c.

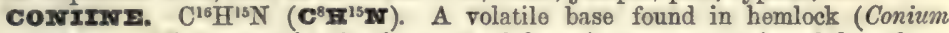
maculatum). It is supposed to be the cause of the poisonous properties of that plant.

covrma. A fragrant resin used for making pastiles. It is extracted from the Hyawa, or incense tree, which grows in British Guiana.

conrum. Conium maculatum. Spotted Hemlock. The celebrated Athenian state poison by which Socrates and Phocion died.

coorosterrs. Under this name a peculiar substance has recently been imported from South Australia. It resembles caoutchouc in many of its physical properties, and has been called by some authorities mineral caoutchouc or Elaterite; it seems, however, to be essentially different from that substance, and is probably a product of regetable origin. It is found in thin layers spread over the surface of the ground in a sandy plain in the district known as the Coorong, in South Australia.

Coorongite is a dark brown elastic substance, which exhibits under the microscope a cellular structure interwoven with fibres. It does not vuleanize with sulphur. By destructivo distillation it evolves gaseous products; and oils and acetic acid may be obtained by condensation. According to an examination in tho Colony, by $\mathbf{M r}$. G. Francis, it is capable of being resolved into two substances: (1) a soft, semi-fluid balsam, of an olive-green colour, insoluble in water, mineral acids, absolute alcohol, and wood-naphtha, but soluble in ether, chloroform, benzole, turpentine, and essential oils; (2) a tough, pulverulent, olastic substance, combustible, but insoluble in any of the abore-named menstrua. The specific gravity of Coorongite is 0.98 or 0.99 . An analysis by Dr. Bernays yielded:

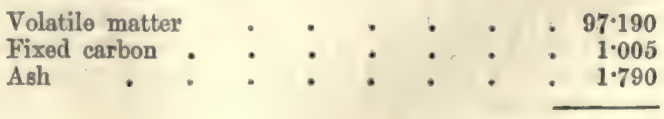


The amount of moisture was only 0.4682 per cent.; but separating this from the volatile matter, the complete analysis may bo thus ropresented:

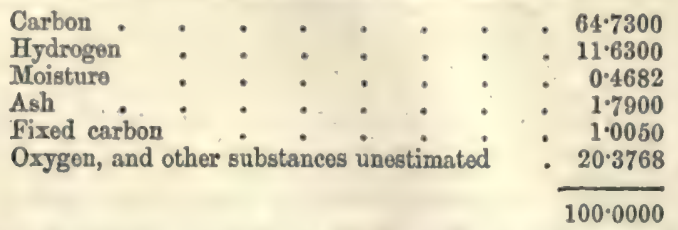

coorms, watran. Vessels of porous unglazed earthenware, in which a liquid can be kept cool by constantly exuding through the substance of the ware and evaporating from the outer surface of the vessel. Such coolers, of great use in hot climates, are often known by their Spanish name Alcarrazas.

coortzra FuUIDs. See Refrigeratory.

Coparba, BAIsAm or. This oleo-resin is imported from Peru, and is the produce of several species of Copaifera.

COPAI. A resin which exudes spontaneously from two trees, the Rhus copallinum, and the Eloocarpus copaliferus, the first of which grows in America, and the second in the East Indies. A third species is said to grow on the banks of some rivers in, and near the coasts of, Quinea.

Much information has been received of late years from various sources concerning this somewhat ill-understood product. It is now known that there are three different kinds of copal in commerce, but little is known of their distinguishing characteristics. Wo have East Indian and West Indian copal, and, under the latter name, two very different substances. The East Indian, called also African, is more colourless, soft, and transparent, than the others; it forms a fine surface, and when heated emits an agreeable odour. It furnishes the finest varnish; fresh essence of turpentine dissolves it completely, but not old. Essence digested upon sulphur will dissolve double its own weight, without letting any fall. Fresh-rectified oil of rosemary will dissolve it in any proportion, but if the oil is thickened by age it serves only to swell the copal.

When cautiously melted, it may be then dissolved in good essence of turpentine in any proportion, producing a fine varnish of little colour.

A good varnish may be made by dissolving 1 part of copal, 1 of essence of rosemary, with from 2 to 3 of pure alcohol. This varnish should be applied hot, and when cold, becomes very hard and durable.

The West Indian species, or American, comes to us not in lumps of a globular form, but in small flat fragments, which are hard, rough, and without taste or smell. It is usually yellow, and never colourless like the other. Insects are very rarely found in it. It comes from the Antilles, Mexico, and North America. It wili not dissolve in essence of rosemary.

The third kind of copal, known also as West Indian, was formerly sold as a product of the East Indies. It is found in fragments of a concavo-convex form, the outer corering of which appears to have been removed. It contains many insects. When rubbed it emits an aromatic odour. It gives out much ethereous and empyreumatic oil when melted. It forms a soft varnish, which dries slowly.

Fusel-oil, or amyl spirit, has been lately used as a solvent of the hard copal; but it does not dry into a very solid varnish.

It is now believed that the East Indian copal is the produce of Hymencea Courbaril, and perhaps also of $H$. verrucosa. It is probable that the Brazilian copal is yielded by soveral species of the same genus, and by Trachylobium martianum. The African copals are supposed to be the produce of certain species of Hymencea and of the Guibourtia copallifera.

COPEx. The wood of the Clusia rosea used for dyeing.

COPPrR. (Symbol, Cu. ; Atomic weight, 31\%.) One of the metals most anciently known. It was named from the island of Cyprus, where it was extensively mined and smelted by the Greeks. It has a roddish-brown colour inclining to yellow; a faint but nauseous and disagreeable taste; and when rubbed between the fingers imparts a smell somewhat analogous to its taste. Its specific gravity is from 8.8 to 8.9 . It is much more malleable than it is ductile; so that far finer leaves may be obtained from it than wire. It is said to melt at $1,996^{\circ} \mathrm{F}$, and at a higher temperature it evaporates in fumes which tinge flame of a bluish green. By exposure to heat with access of air, it is rapidly converted into black seales of protoxide. In tenacity it yields to iron; but considerably surpasses gold, silver, and platinum, in this respect. 
Pure copper may be obtained in the solid state either by the reduction of the pure oxide by a stream of hydrogen gas passed over it in an ignited tube, or by the electrotype process. See FíLctro-Mestatiuror.

ORES OF COPPER.-Copper ores do not possess any one general physical character by which they may be recognised; but they are readily distinguished by chemical reagents. Ammonia digested upon any of the cupreous ores in a pulverised state, after they have been calcined either alone or with nitre, assumes an intense blue colour, indicative of copper. Few copper ores are to be met with which do not betray the presence of this metal by more or less of a greenish film.

The following are the principal minerals which contain copper:-

Native or Virgin Copper (Cuivre natif, Fr.; Gediegen Krupfer, Ger.) This metal commonly occurs in dendritic and arborescent masses, or is indistinctly erystallised in complex and distorted forms of the cubic system. It is a frequent associate of the ordinary ores of copper, and appears to have been formed in mest cases by reduction from some of these ores. Native copper is found not only in copper-bearing veins. but in the fissures and cavities of certain trappean rocks, in deposits in serpentine, and occasionally in sandstones and conglomerate. The native metal is not uncommon in some of the Cornish copper-mines, and is found abundantly in certain copperbearing deposits in Siberia, Chili, Bolivia, and South Australia. But the most important occurrence of native copper yet recorded is that at Keewenaw Point, Lake Superior, where certain amygdaloidal trap-rocks hold large quantities of copper, in association with calcite and zeolitic minerals. The native copper of Lake Superior also occurs as the cementing materials of a conglomerate, and in a finely-divided form disseminated through sandstone. Some of this copper is remarkable for containing silver, which appears in distinct masses sharply separated from the copper in which it is embedded, the two metals not having formed an alloy. Masses of native copper have been found at Lake Superior, weighing upwards of 400 tons.

Copper Glance, Vitreous Copper Ore, Chalcocite, Redruthite, or Disulphide of Copper, (Cuive vitreux, Fr.; Kupferglanz, Ger.). This mineral, which forms a most valuable ore, occurs both crystallised and massive. Some of the composite crystals from St. Just, in Cornwall, are known, from their form, as nail-head copper ore. The fracture of copper-glance is conchoidal; surface sometimes dull ; colour, iron-black or leadgrey, often bluish, iridescent, or reddish from a mixture of oxide. It is easily melted even by the heat of a candle; but is more difficult of reduction than protoxide. This ore yields to the knife, assuming a metallic lustre when eut. Its density varies from $4 \cdot 8$ to $5 \cdot 34$. Copper-glance is a disulphide (cuprous sulphide), containing when pure $\mathrm{Cn}^{2} \mathrm{~S}$, corresponding to 79.8 per cent. of copper and 20.2 of sulphur; but as found in nature it generally contains more or less iron. This is, therefore, one of the richest ores, and forms very important veins. It is to be found in all considerable copper districts; in Siberia, Saxony, Sweden, and especially Corawall, where the finest crystals occur.

Copper Pyrites, Chalcopyrite, Towanite, or Yellow Copper Ore, (Cuivre pyriteux, Fr.; Kupferkies, Ger.). This is the common ore of copper in Cornwall and many other copper-mining districts. It resembles in its metallic yellow hue bisulphide of iron (iron pyrites); but the latter is paler, harder, and strikes fire easily with steel. Copper pyrites sometimes presents the most lively rainbow colours, and is then known, from this iridescence, as Peacock ore. Its specific gravity is $4 \cdot 3$.

Copper pyrites is a double sulphide of copper and iron, containing theoretically 34.5 per cent. of copper, 30.5 of iron, and 35 of sulphur, corresponding to the formula $\mathrm{Cu}^{2} \mathrm{~S}, \mathrm{Fe}^{2} \mathrm{~S}^{3}$, or, as sometimes expressed, CuS,FeS. Copper pyrites occurs in vast masses and extended veins, in primitive and transition districts; and is commonly accompanied with grey copper, iron pyrites, sparry iron, galena, and zinc blende.

Bornite, Erubeseite, Phillipsite, Purple Copper Ore, Variegated Ore, or Horse-flesh Ore, (Cuivre panaché, Fr.; Buntkupfererz, Ger.). A double sulphide of copper and iron. A Cornish specimen, analysed by Plattner, yielded-copper, 56.76 ; iron, 14.84 ; sulphur, 28.24. The composition of the crystallised mineral appears to be reducible to the formula $3 \mathrm{Cu}^{2} \mathrm{~S}, \mathrm{Fe}^{2} \mathrm{~S}^{3}$. The ore usually occurs massive, presenting a colour between copper-red and pinchbeck-brown, but often obscured by a purple tarnish. Its specific gravity varies from $4 \cdot 4$ to $5 \cdot 5$. Horse-flesh ore is abundant in some of the copper-mines of Chili and Canada. It is also found in Cornwall and in Ireland. It occurs with serpentine at Monte Catini in Tuseany, and forms thin seams in the copper-slates of Mansfeld in Prussia.

Fahl Ore, Tetrahedrite, or Grey Copper Ore, (Fahlerz, Ger.). An ore of variable composition, generally containing between 30 and 40 per cent. of copper. It is essentially an antimonio-sulphide or arsenio-sulphide of copper, but the copper may be partially replaced by iron, zinc, silver, or mereury. Fahlerz is a steel-grey mineral, with a metallic lustre, and frequently assumes well-defined tetrahedral forms. The 
ore is generully ralued for the silvor which it contaius rather than for its copper. It is very difficult to convert into pure copper by smelting, on account of the presence of antimony and arsonic. Seo Faruzez.

Temuantite. An arsenio-sulphide of copper and iron, closely alliod to Fahlerz. It was formerly obtained crystallised in rhumbic dodecahedra, from Wheal Jewoll, in Gwonnap, and from somo other Cornish mines. This species is raro.

Cuprite, Red Oxide of Copper, Ruby Copper Ore, or Sub-oxide of Copper, (Cuiure axidule, Fr.; Rotnkupfererz, Ger.). This mineral frequently occurs in fine octahodral and cubic crystals, associated with other ores in the shallow workings of coppor-mines. Its colour is a deep red, sometimes very bright, especially when bruised. It is friable, difficult of fusion at the blowpipe, reducible on burning charcoal, soluble with efforvesconce in nitric acid, forming a green liquid, and also soluble in ammonia. Cuprito is a sub-oxide of copper (cuprous oxide), $\mathrm{Cu}^{2} \mathrm{O}$, containing, when pure, copper 88.8 , and oxygen 11.2. The impure compact varieties, associated with much iron ochre, have been called tile-ore, whilst certain delicato capillary varioties are known as plush copper ore or chalootrichite.

Melaconite, or Black Oxide of Copper, (Cuivre oxidé, Fr.; Kupferschuiarzc, Ger.). The native protoxide of copper (cupric oxide), CuO, generally occurs as a black earthy or pulverulent incrustation on other copper ores, from which it has been producod by decomposition.

Malachite or Green Carbonate of Copper. This mineral is found in most deposits of copper ore, especially in the upper parts whore ntmospheric influences have been active. It is a hydrous carbonate of copper, corresponding, when pure, with the formula, $2 \mathrm{CuO}, \mathrm{CO}^{2}+\mathrm{HO}\left(\mathbf{2} \mathbf{C u} \mathbf{C O}^{3}+\mathbf{H}^{2} \mathbf{O}\right)$ : this requires 71.9 per cent. of protoxide of copper, 19.9 of carbonic acid, and 8.2 of water. Malachite is found earthy, compact, stalactitic, or mammillated. The finest examples, sometimes polished as ornamental stones, come from Siberia, and from Burra Burra in South Australia.

Azurite, Chessylite, Blue Malachite, or Blue Carbonate of Copper. A hydrous carbonate of copper containing in its purest forms $2\left(\mathrm{CuO}^{\left.\mathrm{C} \mathrm{CO}^{2}\right)}+\mathrm{CuO}^{\mathrm{HO}}\left(\mathbf{2 C u C O}^{3}\right.\right.$ $+\mathbf{C u}^{3} \mathbf{O}^{2}$ ); which corresponds to protoxide of copper $69 \cdot 2$, carbonic acid $25^{\circ} 6$, and water 5.2. Azurite thus diffors from malachite in chemical composition and in colour; it is also more prone to crystallise, and magnificent specimens were formerly obtained from the copper-mines of Chessy, near Lyons.

Chrysocolla. This mineral, which is a hydrous silicate of copper of variable composition, somewhat resembles carbonate of copper, and commonly occurs as a greell, bluish-green, or turquoise-blue incrustation on other copper ores. A black resinous variety, impure by the presence of much iron, is generally known as pitch copper cre.

Dioptase or Emerald Copper. A beautiful but rare silicate of copper occurring in emerald-green crystals, composed of $\mathrm{CuO}_{\mathrm{SiO}}{ }^{2}+\mathrm{HO}\left(\mathbf{C u S i O}^{3}+\mathbf{m}^{2} \mathbf{O}\right)$. It is found in the Kirghese Steppes and in Chili, but cannot be called an ore of copper.

Atacamite. An oxychloride of copper containing $\mathrm{CuCl}+3$ ( $\mathrm{CuO} . \mathrm{HO}$ ) or $\mathbf{C u C l}^{2}$ $+\mathbf{3 u r r}^{2} \mathbf{O}^{2}$. This corresponds to copper 15, chlorine 16, protoxide of copper 56 , and water 12. It occurs in fine rhombic crystals of a greenish black colour, and is worked as an ore at the Wallaroo and other copper-mines on Yorke Peninsula, South Australis. It is also found in tho form of a green sand in the Desert of Atacama.

Phosphates of Copper. Several copper-bearing minerals containing phosphoric acid, with or without its isomorph arsenic acid, are known to the mineralogist, but none of them occur in sufficient abundance to be used as ores. Libethenite is a hydrous phosphate, occurring in rhombic crystals at Libethen in Hungary; and Ehlite is a similar mineral from Ehl in Nassau.

Arsenates of Copper. A large numker of these compounds are known, but are only of mineralogical interest. Among these are the species named olivenite, euchroite, tagilite, tyrolite, liroconite, cornwallite, and chalcophyllite or copper-mica.

Chalcanthite, Cyanosite, Blue Vitriol, or Sulphate of Copper. A mineral similar to the artificial salt of the laboratory. The blue water which flows from certain coppermines is a solution of this salt. The copper is easily procured in the metallic state by plunging into it pieces of iron.

It should be remarked that many of the minerals noticed above are not available as ores of copper. Dr. Scherer, of Freiberg, has arranged the principal ores of copper as follow:-
1. Copper Glance, $\mathrm{Cu}^{2} \mathrm{~S}$
2. Copper pyrites, $\mathrm{Cu}^{2} \mathrm{~S}, \mathrm{Fe}^{2} \mathrm{~S}^{3}$
3. Buntkupfererz, $3 \mathrm{Cu}^{2} \mathrm{~S}, \mathrm{Fe}^{2} \mathrm{~S}^{3}$.
4. Fahlerz, $4\left(\mathrm{Cu}^{2} \mathrm{~S}, \mathrm{FeS}, \mathrm{ZnS}, \mathrm{AgS}\right)\left(\dot{\mathrm{SbS}}^{3}, \mathrm{AsS}^{3}\right) *$

\begin{tabular}{|c|c|c|}
\hline & & Copper in 100 \\
\hline & & - $\quad 79 \cdot 7$ \\
\hline & & $34: 8$ \\
\hline & & $55 \cdot 7$ \\
\hline & & $14-41$ \\
\hline
\end{tabular}


5. Red oxide of copper, $\mathrm{Cu}^{2} \mathrm{O}$.

6. Malachite, $2 \mathrm{CuO}, \mathrm{CO}^{2}+\mathrm{HO}$

7. Azurite, $2\left(\mathrm{CuO}, \mathrm{CO}^{2}\right)+\mathrm{CuO}, \mathrm{HO}$
Copper in 100

. $88 \cdot 5$

- $67 \cdot 4$

- $55 \cdot 3$

We shall here give a brief account of the copper slates of Mansfeld, in Prussia, and of the copper veins of Cornwall.

Copper-slate (Kupfersoliefer) of Mansfeld, in Prussian Saxony. The Kupferschiefer is a dark coloured bituminous sehist, impregnated with copper ore, occurring with singular persistence over a large area on the south-east of the Hartz. Its geological position is in the Permian formation, below the Zechstein, or Magnesian limestone, and above the red sandstones and conglomerates known as the Rothtodtliegende; indeed, the latter name (' red dead strata') has reference to the fact that the ores in the overlying schist die away on approaching these beds. The actual floor of the cupreous schist is formed by a thin bed of white sandstone, known as the Weissliegendes, tho upper part of which is slightly cupriferous, and is smelted under the name of Sanderz. Upon this sandstone rest the true copper-bearing schists, forming three or four thin beds, with an aggregate thickness rarely exceeding two feet, and sometimes not attaining more than half this thickness. The schists are highly fossiliferous, and are espocially rich in remains of ganoid fish belonging to the genera Palcomiscus and Platysomus. Towards their upper part, the schists become calcareous, and are sometimes known as the Noberge. The beds of cupreous schists, or copper-slate, are thrown into a synclinal form, and are chiefly worked near their basset; the intrusion of masses of melaphyre has divided the deposit into three separate areas: those of Eisleben, Sangerhausen, and Frankenhausen. The schist is richest in the neighbourhood of faults, and the thinner beds are said to hold more copper than the thicker deposits. Only a fow inches-not generally more than five-are worth working. The ore is disseminated in the form of eopper pyrites, copper-glance, and purple-ore. The schist contains from 1.8 to 3.7 per cent. of copper, and this copper always contains traces of silver, 1 cwt. of copper holding from 0.53 to $0.58 \mathrm{lb}$. of silver. Although the German copper-slate is thus a very poor ore, yet by an admirable organisation of the mines and smelting-works both the copper and silver are profitably extracted, and a large population entirely supported by this industry. The coppermines of Mansfeld have been worked since the close of the twolfth century; Martin Luther's father was a miner in this district. The metallurgical treatment of the copper-slate will be describod bolow.

Cornish Copper Veins.-The deposits of copper in Cornwall occur as veins in granite, or in the schistose rocks which surround and cover it; and hence, the Cornish miners work mostly in the granite and clay-slate; the former of which, when metalliferous, is usually in a coarse and often a disintegrated state; this they call growan, the latter killas.

Copper veins are abundant in killas and more rare in granite; but most numerous near the line of junction of the two rocks. The different kinds of copper veins in Cornwall may be classed as follow :-

1. Copper veins, gonerally running east and west.

2. Second system of copper veins, or contra lodes.

3. Crossing veins; cross-courses.

4. Olay veins, called Cross-Flookans or Slides.

The width of these veins does not often exceed 6 feet, though occasional enlargements to the extent of 12 or more feet sometimes take place. Their length is unknown, but one explored in the United Mines has been traced over an extent of seven miles. The gangue of these veins is generally quartz, either pure, or mixed with green particles analogous to chlorite. They contain iron pyrites, blende, sulphide, and several other compounds of copper, such as the carbonate, phosphate, arsenate, chloride, \&c. The most part of the copper lodes are accompanied by small argillaceous veins, called by the miners flookans of the lode. These are often found on both sides of the vein, so as to form cheeks or walls.

When two veins intersect each other, the direction of the one thrown out becomes an object of interest to the miner. In Saxony it is regarded as a general fact, that the rejected portion is always on the side of the obtuse angle; this also holds generally in Cornwall, and the more obtuse the angle of incidence, the more considerable the heave. The great copper vein of Carharack, in the parish of Gwennap, is an instructive example of intersection. The width of this vein is 8 feet; it runs nearly from east to west, and dips towards the north at an inelination of 2 feet in a fathom. Its upper part is in the killas, its lower in granite. This vein has suffered two intersections: the first results from encountering the vein called Steven's flookan, which runs from north-east to south-west, throwing it out several fathoms. The second has been caused by another vein, almost at right angles to the first, and which has heared it 20 fathoms 
to the right. The throw of the vein oceurs, thereforo, in one caso to the right, and in the other to tho left; but in both instancos, it is to tho side of tho obtuse angle. This disposition is very singular; for one portion of the vein appears to have asconded, whilo another has sunk. See FavLts.

The copper-mines of the isle of Anglesea, those of North Walos, of Westmoreland, the adjacent parts of Lancashiro and Cumberland, of the south-west of Scotland, of the Islo of Man, and of the south-east of Ireland, also occur in primitive or transition rocks. The ores lie sometimes in masses, but more frequently in veins. The mine of Ecton, in Staffordshire, and that of Cross-gill-burn, near Alston-moor, in Cumberland, occur in carboniferous or mountain limestone.

The copper ores extracted both from the granitic and schistose localities, as woll as from the calcareous, are uniformly copper pyrites more or less mixed with mundic; the red oxide, carbonate, aresenate, phosphate, and chloride of copper, are very rare in these districts.

The working of copper-mines in the isle of Anglesea may be traced to a rory remote era. It appears that the Romans were acquainted with the Amlwch mine near Holyhead. Mr. Thomas F. Evans has been so fortunate as to obtain and preserve three copper cakes found at Bryndu, Amlwch, which are evidently of Roman origin. The copper-mines in Parys Mountain were not worked with activity until the year 1779. In 1784 these mines produced 3,000 tons of copper annually. Ihis deposit lies in a greenish clay slate, passing into talc slate; a rock associated with serpentine and euphotide. The veins of copper are from one to two yards thick; and converge towards a point where their union forms a considerable mass of ore. On this the mine was first commenced by an open excavation, which is now upwards of 300 feet deep, and appears from above like a vast funnel. Galleries are formed at different levels upon the flanks of the excavation to follow the several smaller veing, which run in all directions, and diverge from a common centre like so many radii. The ore receives in these galleries a kind of sorting, and is raised by means of hand windlasses to the summit of a hill, where it is cleaned by breaking and jigging.

The water is so scanty in this mine that it is pumped up by a small steamengine. A great proportion of it is charged with sulphate of copper. It is conveyed into reservoirs containing pieces of old iron; the sulphate is thus decomposed and metallic cupper obtained by cementation. The Anglesea ore is poor, yielding only from 2 to 3 per cent. of copper; a portion of its sulphur is collected in roasting the ore.

The copper-mices of these islands, now so important, were so little worked until a recent period that in 1799 we are told in a Report on the Cornish mines, 'it was not until the beginning of the last century that copper was discovered in Britain.' This is not correct, for in 1250 a copper-mine was worked near Keswick, in Cumberland. Edward III. granted an indenture to John Ballanter and Walter Bolbolter, for working all 'mines of gold, silver, and copper;' but that the quantity found was very small is proved from the fact that Acts of Parliament were passed in the reigns of Henry VIII. and Edward VI. to prevent the exportation of brass and copper, 'lest there should not be metal enough left in the kingdom, fit for making guns and othor engines of war, and for household utensils :' and in 1665 the calamine works were encouraged by the Government, as 'the continuing these works in England will occasion plenty of rough copper to be brought in."

At the end of the seventeenth century, some 'gentlemen from Bristol made it their business to inspect the Cornish mines, and bought the copper ore for $2 l$. $10 \mathrm{~s}$. per ton, and scarce ever more than $4 l$. a ton.'

In 1700, one Mr. John Costor introduced an hydraulic engine into Cornwall, by which he succeeded in draining the mines, and 'he taught the people of Cornwall also a better way of assaying and dressing the ore.'

The value and importance of copper-mines since that period regularly increased, until within the last fow years. There has been of late a steady decline in the produce of our copper-mines, and a falling off in the per-centage of metal in the ore.

Mechanical Preparation of the Copper Ores in Cornwall.- The ore receives a first sorting, the object of which is to separate all the pieces larger than a walnut; after which the whole is sorted into lots, according to their relative richness. The fragments of poor ore are sometimes pounded in stamps, so that the metallic portions may be separated by washing.

The rich ore is broken into small bits, either with a flat beater, or by means of a crushing-mill. The ore to be broken by the bucking iron is placed upon plates of cast iron; each about 16 inches square and $1 \frac{1}{2}$ inch thick. These plates are set towards the edge of a small mound about a yard high, constructed with dry stones rammed with earth. The upper surface of this mound is a little inclined from behind forwards. The work is performed by women, each furnished with a buckingiron: the ore is placed in front of them beyond the plates; they break it, and strew 
it at their feet, whence it is remored and disposed of as may be subsequently required.

The crushing-mill has of late years been brought to a great degree of perfection, and is almost universally made use of for pulverising certain deseriptions of ore. For an account of this apparatus, see Grinding and Crushisg Apparatevs.

Stamping-mills are less frequently employed than crushers for the reduction of copper ores. At the Devon Great Consols Mines, the concentration of the erushed copper ores is effected in the following manner:-From the crushing-mill the stuff js carried by a stream of water into a series of revolving soparating sieves, where it is divided into fragments of $\frac{1}{20}$ th inch, $\frac{1}{10}$ th inch, and $\frac{1}{10}$ th inch diameter, besides the coarser particles which eseape at the lower end of the sieves. The slimes flow over a small water-wheel called a separator, in the buckets of which the coarser portions settle, and are from thence washed out by means of jets of water into a round buddle, whilst the finer particles are retained in suspension, and are carried off into a series of slime-pits, where they are allowed to settle.

The work produced by the round buddle is of three sorts; that nearest the circumference is the least charged with iron pyrites, or any other heavy material, but still contains a certain portion of ore; this is again buddled, when a portion of its tail is thrown away, and after submitting the remainder to a buddling oporation, and separating the waste, it is jigged in a fine sieve, and rendered morchantable.

The other portions of the first buddle are rebuddled, and after separating the waste, the orey matters are introduced into sizing cisterns, from which the finer particles aro made to flow over into a buddle, from whence a considerable portion goes directly to market. That which requires further manipulation is again buddled until thoroughly cleansed. The coarser portions of the stuff introduced into the sizing cisterns pass downward with a current of water into the tye, and after repeated projections against the strenm, the orey matter is separated, leaving a residue of mundic in a nearly pure state.

The stuff falling from the lower extremities of the separating sieves is received into bins and subsequently cleansed; each of the three sizes is jigged, and in proportion as the worthless matters are separated, they are seraped off and removed. Those portions of the stuff that require further treatment are taken from the sieres, washed down from behind the hutches, and treated by tyes, until all the valuable portions have been extracted.

In this way rein-stuff that originally contained but $1 \frac{1}{2}$ per cont. of copper is so concentrated as to afford a metallic yield of 10 per cent., whilst by means of sizing-sieves, dressing-wheels, jigging-machines, and round-buddles, \&c., from 40 to 50 tons of stuff are elaborated per day of 9 hours, at a cost of $12 s$. per ton of dressed ore.

Captain Richards, the agent of these mines, has also introduced considerable improvements in the slime-dressing department. The proper sizing of slime is as necessary as in the case of rougher work, and in order to effect this, he has arranged a slime-pit, which answers this purpose exceedingly well. This pit has the form of an inverted cone, and receives the slimes from the slime-separator, in an equally-divided stream. The surface of this apparatus being perfectly level, and the water passing through it at a very slow rate, all the valuable matters are deposited at the bottom. If slime be valuable in the mass, it can evidently be more economically treated by a direct subdivision into fine and coarser work; since a stream of water, acting on a mixture of this kind, will necessarily carry off an undue proportion of the former in freeing the latter from the waste with which it is contaminated.

The ordinary slime-pit is of a rectangular form, with vertical sides, and flat bottom. The water enters it at one of the ends by a narrow channel, and leaves it at the other. A strong central current is thus produced through the pit, which not only carries with it a portion of valuable slime, but also produces eddies and creates currents towards the edges of the pit, and thus retains matters which should have been rejected. The slime-pits at Devon Consols are connected with sets of Brunton's machines, which are thus kept regularly supplied by means of a launder from the apex of the inverted cone, through which the flow is regulated by means of a plugvalve and screw.

A waggon cistern is placed under each frame for receiving the work, which is removed when necessary, and placed in a packing-kieve. This is packed by machinory, sot in motion by a small water-wheel. The waste resulting from this operation is either entirely rejected, or partially reworked on Brunton's machines, whilst the orey matters contained in the kieve are removed by a waggon to the orehouse where they are discharged.

Cornwall being destitute of coal, the whole of the copper ore which it produces is sent for smelting to South Wales.

Welsh Copper-Smelting: Furnaces.-The furnaces employed are of the reverberatory

Vó.. I, 
construction; they rary in their dimensions and in the number of their openings according to the operations for which they are intended. There are five of them:-1. The calcining furnace, or calciner; 2 . The melting furnace; 3 . The roasting furnace, or roaster; 4 . The refining furnace; 5 . The heating or igniting furnace.

1. The Calcining Furnace rests upon a vault, c, into which the ore is raked down after being calcined; it is built with bricks, and bound by iron bars, as shown in the elevation, fig. 501. The hearth, B B, fig8. 502 and 503, is placed upon a level with the lower horizontal binding bar, and has nearly the form of an ellipse, truncated at the two extremities of its greater axis. It is horizontal, bedded with fire-bricks set on edge, so that it may be romoved and repaired without disturbing the arch upon which it reposes. Holes, not visible in the figure, are left in the sole before each door $e c$, through which the roasted ore is let fall into the subjacent vault. The dimensions of the hearth B B, vary from 17 to 19 feet in length, and from 14 to 16 in breadth. The fire-place A, fig. 503, is from $4 \frac{1}{2}$ to 5 feet long and 3 feet wide. The bridge or low wall b, fig 504, which separates the fire-place from the hearth, is 2 feet thick, and in Messrs. Vivians' smelting-works is hollow, as shown in the figure, and communicates at its two ends with the atmosphere, in order to conduct a supply of fresh air to the hearth of the furnace. This judicious contrivance will be described in explaining the roasting operation. The arched roof of the furnace slopes down from the bridge to the beginning of the chimney $f$, figs. 501, 503, its height above the hearth being at the first point about 26 inches, and from 8 to 12 at the second.

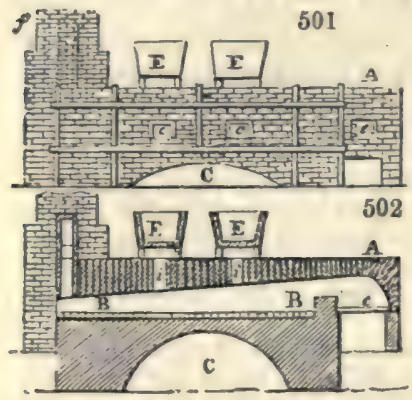

503

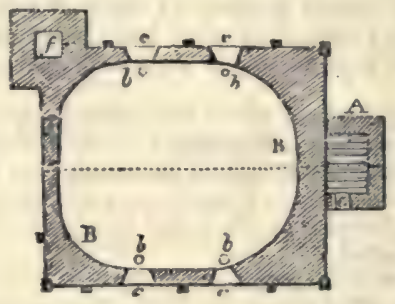

504

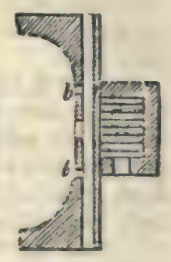

Such calcining furnaces have five doors, cccc, fig. 503, and one for the fire-place, as shown at the right hand in fig. 501 ; four are for working the ore upon the reverberatory hearth. These openings are 12 inches square, and are bound with iron frames. The chimney is about 22 feet high, and is placed at one angle of the hearth, as at $f$, fig. 503, being joined by an inclined flue to the furnace.

For charging it with ore, two hoppers $\mathrm{B} \mathrm{E}$, are usually placed above the upper part of the vault, in a line with the doors; they are formed of four plates of iron, supported in an iron frame. Beneath each is an orifice for letting the ore down into the hearth.

These furnaces serve for calcining the ore and matts : for the latter purpose, indeed, furnaces of two stories are sometimes employed, as represented in fig. 507. 'The dimensions of each floor in this case are a littlo less than the preceding. Two doors, $c c$, fig. 501, correspond to each hearth, and the workmen, while employed at the upper story, stand upon a raised moveable platform.

2. Melting Purnace, figs. 505 and 506.-The form of the hesrth is, in this case, also elliptical, but the dimensions are smaller than in the calcining furnace. The length does not exceed 11 or 11 , feet, and the breadth raries from 7 to 8 . The fire-place is, however, larger in proportion, its length being from $3 \frac{1}{2}$ to 4 feet, and its breadth from 3 to $3 \frac{1}{2}$; this size being requisite to produce the high temperature of the furnace. It has fewer openings, there being commonly three; one to the fire-place at $D$, a second one, $o$, in the side, kept generally shut, and used only when inerustations need to be seraped off the hearth, or when the furnace is to be entered for repairs; and the third or working door, $a$, placed on the front of the furnace beneath the chimney. Through it, the scoriæe are raked out, and the melted matters stirred and puddled, \&ce.

The hearth is bedded with infusible sand, and slopes slightly towards the side door, to facilitate the discharge of the metal. Above this door is a hole in the wall of the chimnoy (fig. 506) for letting the metal escape. An iron gutter, o, leads it into a pit, $\mathrm{k}$, bottomed with an iron receiving-pot, which may be lifted out by a crane. The pit, $M$, is filled with water, and the metal becomos granulated as it falls into the receiver. Tbese melting furnaces are surmounted by a hopper $\mathrm{x}$, as shown fig. 605 . 
Melting furnaces are sometimes also used for calcination. Some of those near Swansea serve this double purpose; they are composed of 3 floors (fig. 507). The floor $\Delta_{1}$ is destined for melting the calcined ore; the other two, B, c, serve for calcination. The heat being less powerful upon the upper sole c, the ore gets dried upon it, and begins to be calcined: a process completed on the next floor. Square holes, $d$, left in the hearths B and $\mathrm{c}$, put them in communication with each other, and with the lower one $\mathbf{A}$; these perforations are shut during the operation by a sheet of iron, removable at pleasure.

The hearths $\mathrm{B}$ and c, are made of bricks ; these are horizontal at the top and slightly vaulted beneath; they are two bricks thick, and their dimensions larger than those of the inferior hearth, as they extend above the fire-place. On the floors destined for calcination the furnace has two doors on one of its sides: on the lower story there are also two; but they are differently placed. The first, being in the front of the furnace, serves for drawing off the scorise, for working the metal, \&ce; and the second, upon the side, admits the workmen to make necessary repairs. Below this door is placed the discharge or tap-hole, which communicates by a cast-iron gutter with a pit filled with water. The dimensions of this furnace in length and breadth are nearly the same as those of the melting furnace above described; the total height is nearly 12 feet. It is charged by means of either one or two hoppers.

3. Roasting Furnace. - The furnaces employed for this purpose are in general similar to the calciners; but in the smelting-works of Messrs. Vivian, the furnaces above alluded to present a peculiar construction; this is for the purpose of introducing a continuous current of air
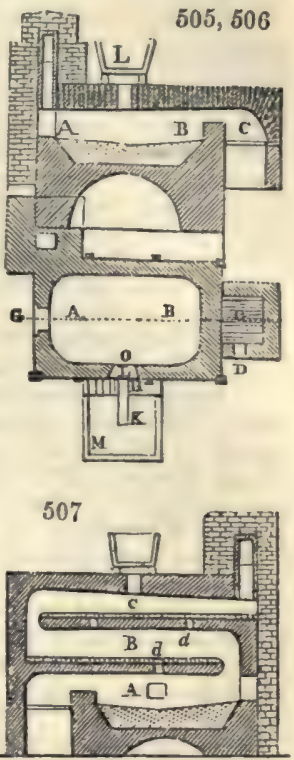
upon the metal, in order to facilitate its oxidation. This process was originally invented by Mr. Sheffield, who disposed of his patent right to Messrs. Vivian.

The air is admitted by a channel through the middle of the fire-bridge, which extends all its length; it communicates with the atmosphere at its two extremities, whilst square holes, left at right angles to this channel, conduct the air into the furnace. This very simple construction produces a powerful effect in the roasting. It not only promotes the oxidation of the metals, but burns the smoke, and assists in the vaporisation of the sulphur; while by keeping the bridge cool it preserves it from wasting, and secures uniformity of temperature to the hearth.

4. Refining Furnace.-In this, as in the melting furnace, the side slopes towards the front door instead of the side doors, because in the refining furnace the copper collects in a cavity formed in the hearth near the front door, from which it is lifted out by ladles; whereas, in the melting furnaces, the metal is run out by a tap-hole in the side. The sole is laid with sand; but the roof is higher than in the melting furnace, being from 32 to 36 inches in height. If the top arch were too much depressed, there might be produced upon the surface of the metal a layer of oxide very prejudicial to the quality of the copper. In that case, when the metal is run out, its surface solidifies and cracks, while the melted copper beneath breaks through and spreads irregularly over the cake. This accident, called the rising of the copper, prevents it from being laminated, and requires it to be exposed to a fresh refining process, when lead must be added to remove the oxide of copper. This is the only occasion upon which the addition of lead is proper in refining copper. When the metal to be refined is mixed with others, particularly with tin, as in extracting copper from old bells, then very wide furnaces must be employed, to expose the metallic bath on a great surface, and in a thin stratum, to the oxidising action of the air.

The door, on the side of the refining furnace, is very large, and shuts with a framed brick door, balanced by a counter-weight.

5. Heating Furnaces, being destined to heat the pigs or bars of copper to be laminated, as well as the copper sheets themselves, are made much longer in proportion to their breadth. Their hearth is horizontal, the vault not much depressed; they have only one door, placed upon the side, but which extends nearly the whole length of the furnace; this door may be raised by means of a counter-weight, in the same way as in the furnaces for the fabrication of sheet-iron and brass.

Series of Operations to which the Ore is subjected. -The ores which are smelted in the Swansea works are chiefly copper pyrites, more or less mingled with gangue (vein-stone). 
This pyrites is composed of nearly equal proportions of sulphide of copper and sulphide of irun. Richer ores, consisting of oxides and earbonates of copper, \&c., are also worked in.

The enthy matters which acconpany the pyrites are usually siliceous, though in some mines the minornl is mixed with either clay or fluor-spar. Along with these sub. stances, tin ore and arsenical pyrites oecusionally oceur with the copper; and though these two minerals are not chemically combined, yet they cannot be ontirely separated by mechanical preparation. The constituent parts of the ore prepared for smelting are, therefore, copper, iron, and sulphur, with earthy matters, and, in some cases, tin and arsenic. The different ores wro mixed in such proportions that the average motallic contents may amount to 8 per cont. The smelting procoss consists in alternato ronstings and fusions.

In the roasting operation the rolatile substances are mostly disengaged in tho gaseous state, while the metals that possess a strong affinity for oxygen become oxidised. In the fusion the earthy substances combine with these oxides, and form glassy scorix or slags, which float upon the surface of the melted metal.

The calcinations and fusions take place in the following order:-

1. Calcination of the ore. 2. Fission of the calcined ore. 3. Calcination of coarsemetal. 4. Melting the calcined cuirse-metal. 5. Calcination of fine-metal (second matt). 6. Melting calcined fine-metal. 7. Roasting coarse-copper. In some smeltingworks, this roasting is ropeated four times; in which case a calcination and a melting aro omitted. In other works, however, a saving is made without increasing the number of roastings. 8. Refining or toughening the copper.

Besides these operations which constitute the treatment of copper properly speaking, two others are sometimes performed, in which only the scoriæ are smelted. These may be designated by the letters $a$ and $b: a$ is the re-melting of a portion of the scorix of the second process, which contain some metallic granulations; $b$ is a particular melting of the scoriæ of the fourth operation. This fusion is intended to concentrate the particles of copper in the scoria, and is not practised in all smeltingwork8.

First Operation: Calcination of the ore.-The different ores on arriving from Cornwall and other localities where they are mined, are discharged in continuous cargoes at the smelting works, in such a way, that by taking out a portion from several heaps at a time, a tolerably uniform mixture is obtained; which is very essential, since the ores, being different in quality and contents, act as fluxes for each other. The mixed ore is transported to the works in wooden moasures each holding a hundred-weight. The workmen entrusted with the calcination convey the oro into the hoppers of the ealcining furnace, whence it falls into the hearth; other workmen spread it uniformly on the surface with iron rakes. The charge of a furnace is from 3 tons to $3 \frac{1}{2}$ tons. Fire is applied and gradually increased, till towards the end of the operation, the temperature is as high as the ore can support without melting or agglutinating. To prevent this running together, and to aid the extrication of the sulphur, the surfaces are renewed, by stirring up the ore at the end of every hour. The calcination is usually eompleted at the end of 12 hours, when the ore is raked into the areh under the sole of the furnace, and when cold enough to be moved, is taken out of the arch, and conveyed to the calcined heap.

The ore in this process scarcely changes weight, having gained by oxidation nearly as much as it has lost in sulphur and arsenic; and if the roasting has been rightly managed, the ore is in a black powder, owing to the oxides present.

The utilisation of the sulphurous vapours evolved during calcination is explained, p. 928.

Second Operation: Frusion of the calcined ore.-The calcined ore is likewise given to the smolters in measures containing a hundred-weight. They throw it into hoppers, and, after it has fallen on the hearth, spread it uniformly. They then let down tho door, and lute it tightly. In this fusion there are added about 2 cwt. of scoriæ (' metal slag') proceeding from the melting of the calcined matt, to be afterwards described. The object of this addition is not only to extract the copper that those scoriæ may contain, but also to increase the fusibility of tho mixture. Sometimes, when the composition of the ore requires it, lime, sand, or fluor-spar is added, more particularly the latter.

The furnace being charged, fire is applied, and the sole care of the founder is to keep up the heat so as to have a perfect fusion; the workman then opens the door, and stirs about the liquid mass to complete the separation of the 'metal' (regulus, or matt) from the scorixe, as well as to hinder the melted matter from sticking to the sole. The furnace being ready, that is, the fusion being perfect, the founder takes out the seorix by the front door, by means of a rake. When the mass is thus freed from the scoriæ, a second charge of calcined ore is introduced to increase the metallic bath; which second fusion is executod like the first. New charges of roasted ore are put 
in till the matt collected on the hearth rises to a lerel with the door-way, which happens commonly after the third charge. The tap-hole is now opened, and the matt flows out into a pit filled with water, where it is granulated and collects in the pan placed at the bottom. The granulated matt is next conveyed into the matt warehouse. The oxidation with which the grains get covered by the action of water does not allow the proper shade of the matt or coarse-metal to be distinguished; but in the bits which stick in the gutter it is seen to be of a steel-grey colour. Its fracture is compact, and its lustre metallic. The scorix, known as ore-furnace slag, often contain metallic grains ; they are broken and picked with care. All the portions which include metallic particles are re-melted in an accessory process.

In this operation the copper is concentrated by the separation of a great part of the matters with which it was combined. The granulated matt produced contains in general 33 per cent. of copper; it is therefore four times richer than the ore; and its mass is consequently diminished in that proportion. Its constituents are principally copper, iron, and sulphur. It is termed coarse-metal.

The most important point in the fusion just described, is to make a fusible mixture of the earths and oxides, so that the matt of copper may, on account of its greater specific gravity, sink below and separate exactly from the slag. This is attained by means of metallic oxides contained in the scoriæ of the fourth operation, of which $2 \mathrm{cwt}$. were added to the charge. These consist almost entirely of oxide of iron. When the ores are very difficult to melt, about half a hundred-weight of fluor-spar is added; but this must be done with precaution, for fear of too much increasing the scoriæ.

The work proceeds day and night. Five charges are commonly put through in the courso of 24 hours; but when all circumstances are favourable, that is to say, whon the ore is fusible, when the fuel is of the first quality, and the furnace in good conditinn, even six charges a day have been despatched.

The charge is a ton and a half of calcined ore, so that a smelting furnace nearly corresponds to a calcining furnace ; the latter turning out 7 tons of calcined ore in 24 hours. The workmen are paid by the ton.

Third Operation: Calcination of coarse-metal. - The object of this operation is principally to oxidise the iron, which is more easily accomplished than in the first calcining, because the metal is now disengaged from the earthy. substances, which screened it from the action of the air.

This calcination is executed in the furnace already represented in figs. 505, 506, 507, page 915, exactly in the same way as the ore was calcined. The metal must be perpetually stirred, to expose all its surfaces to the action of the hot air, and to hinder clotting together. The operation lasts 24 hours; during the first six, the fire should be very moderate, and gradually increased to the end of the calcination. The charge is, like that of the first, $3 \frac{1}{2}$ tons.

Fourth Operation: Melting the calcined coarse-metal.-In the fusion of this first calcined matt, some scoriæ of the latter operations must be added, which are very rich in oxide of copper, and some crusts from the hearth, which are likewise impregnated with it. Addition may also be made of richer crude ores, such as oxides and carbonates. The proportion of these substances raries according to the quality of the calcined matt.

In this second fusion, the oxide of copper contained in the scoriæ is reduced by the affinity of the sulphur, one portion of which passes to the state of sulphurous acid, while the other forms a subsulphide with the free copper. The matt commonly contains a sufficient quantity of sulphur to reduce the oxide of copper completely; but if not, which may happen if the calcination of the matt has been pushed too far, a small quantity of uncalcined matt must be introduced, which, by furnishing sulphur, diminishes the richness of the scoriæ, and facilitates the fusion.

The scorix are taken out by the front door by means of a rake. They have a great specific gxavity; are brilliant with a metallic lustre, very crystalline, and present, in the cavities, crystals like those of pyroxene; they break easily into very sharp-edged fragments, and contain no granulated metal in the interior; but it sometimes occurs, on account of the small thickness of the stratum of scorix, that these carry off with them, when withdrawn, some metallic particles.

These scorix, as we have already stated (under Fusion of the rocsted Ore), are in general melted with it. In some cases, however, a special melting is assigned to them.

The matt obtained in this second fusion is either run out into water like the first, or moulded into pigs (ingots), according to the mode of treatment which it is to undergo. This matt, termed by the smelters fine-metal when it is granulated, and blue-metal when it is in pigs, is of a light grey colour, compact, and bluish at the surface, and contains about 60 per cent. of copper.

(b.) Particular Fusion of the Scorice of the fourth Operation.-In re-melting these scoriæ, the object is to procure the copper which they contain. To effect this fusion, 
the scorixe are mixed with pulrerised coal, or other carbonaceous matters. The coppes and several other metals are deoxidised, and furnish a white and brittle alloy. The scorise resulting from this melting are in part employed in the first melting, and in part thrown away. They are crystalline, and often present erystals in the cavities, which appear to be those of bisilicate of iron. They have a metallic lustre, and break into very sharp-edged fragments. The white metal is melted again, and then added to the product of the second fusion.

Fifth Operation: Calcination of the second Matt, or fine-metal.- This is executed in precisely the same way as that of the first matt. It lasts 24 hours; and the charge is usually 3 tons.

Sixth Operation: Melting of the calcined fine-metal. -This fusion is conducted like that of the first matt. The black, or coarse copper, which it produces, contains from 70 to 80 per cent. of pure metal; it is run into ingots, in order to undergo the operation of roasting.

The scoriz are rich in copper; they are added to the fusion of the calcined coarsemetal of the fourth operation.

In many smelting-houses, the fifth and sixth operations have of late years been omitted. The second matt is run into pigs, under the name of blue-metal, to be immediately exposed to roasting.

The disposition of the canal $c c$, fig. 501, which introduces a continuous current of air to the hearth of the furnace, accelerates and facilitates the calcination of the matt; an advantage which has simplified the treatment, by diminishing the number of calcinations.

Seventh Operation: Roasting the coarse-copper, the product of the sixth Operation. - The chief object of this operation is oxidation; it is performed either in an ordinary roasting furnace, or in one similar to fig. 507, which admits a constant current of air. The pigs of metal derived from the preceding melting are exposed, on the hearth of the furnace, to the action of the air, which oxidises the iron and other foreign metals with which the copper is still contaminated. The duration of the roasting varies from 12 to 24 hours, according to the degree of purity of the crude copper. The temperature should be graduated in order that the oxidation may be complete, and that the volatile substances which the copper still retains may escape in the gaseous form. The fusion must take place only towards the end of the operation.

The charge varies from a ton and a quarter to a ton and a half. The metal obtained is run out into moulds of sand. It is covered with black blisters, like cementation steel; whence it has got the name of blistered copper. In the interior of these pigs the copper presents a porous texture, occasioned by the ebullition produced by the escape of gases during the moulding. The copper being now almost entirely freed from sulphur, iron, and the other substances with which it was combined, is in a fit state to be refined. This operation affords scoriæ, which are very heavy, and contain a great deal of oxide of copper, and sometimes even metallic copper. They are known as roaster-slag.

These scorix, as well as those of the third melting and the refining, are added to the second fusion, as we have already stated, in describing the fuurth operation.

In some works, the roasting is several times repeated upon the blue-metal, in order to bring it to a state fit for refining.

Eighth Operation: Refining or Toughening.-The pigs of copper intended for refining are placed on the sole of the refining furnace through the door in the side. A slight heat is first given, to finish the roasting or oxidation, in case this operation has not already been pushed sufficiently far. The fire is to be increased by slow degrees, so that, by the end of six hours, the copper may begin to flow. When all the metal is melted, and the heat is very considerable, the workman lifts the door in the front, and withdraws with a rake the few scoriæ which may cover the copperbath. These are red, lamellated, rery heary, and closely resomble protoxide of copper.

The refiner then takes an assay with a small ladle, and when it cools, breaks it in a rice, to ascertain the state of the copper. From the appearance of the assay, the aspect of the bath, the state of the fire, \&tc., he judges if he may proceed to the toughening, and what quantity of wooden spars and wood-charcoal he must add to render the metal malleable, or, in the language of the smelters, bring it to the proper pitch. When the operation of refining begins, the copper is dry or brittle, and of a deep red colour approaching to purple. Its grain is coarse, open, and somewhat crystalline.

To execute the refining, the surface of the metal is covered with wood-chareoal, and stirred with a spar or rod of birch or other wood. The gases which escape from the wood oceasion a brisk effervescence. More wood-charcoal is from time to time added, so that the surface of the metal may be always corered with it, and the stirring is continued until the operation of refining is finished; a circumstance indicated 
by assays taken in succession. The grain of the copper becomes finer by degrees, and its colour gradually brightens. Whew the grain is extremely fine, or close, whon the trial-pieces, half cut through and then broken, present a silky fracture, and the copper is of a fine light red, the refiner considers the operation to be completed; but he verifies still further the purity of the copper, by trying its malleability. For this purpose, he takes out a sample in a small ladle, and pours it into a mould. When the copper is solidified, but still red-hot, he forges it. If it is soft under the hammer, and does not crack on the edges, the refiner is satisfied with its ductility, and pronounces it to be in its proper state. He then orders the workmen to mould it: they lift the copper out of the furnace in large iron ladles lined with clay, and pour it into moulds of a size suitable to the demands of commerce. The ordinary dimensions of the ingots or pigs are 12 inches broad, 18 long, and from 2 to $2 \frac{1}{2}$ thick.

The period of the refining process is $\mathbf{2 0}$ hours. In the first six, the metal heats and suffers a kind of roasting; at the end of this time it melts. It takes four hours to reach the point at which the refining, properly speaking, begins; and this last part of the process lasts about four hours. Finally, six hours are required to arrange the moulds, east the ingots, and allow the furnace to cool.

The charge of copper in the refining process depends upon the dimensions of the furnace. In different works the charge varies from 3 to 5 tons.

When the copper offers difficulties in refining, a fow pounds of lead are added to it. This metal, by the facility with which it seorifies, acts as a purifier, aiding the oxidation of the iron and other metals that may be present. The lead ought to be added immediately after removing the door to skim the surface. The copper should be constantly stirred to expose the greatest possible surface to the action of the air, and to produce the complete oxidation of the lead; since the smallest quantity of this metal in copper causes a difficulty in the lamination; $i, e$, the scale of oxide does not come clean from the surface of the sheets.

The operation of refining copper is delicate, and requires, upon the part of the workmen, great skill and attention to give the metal its proper ductility. Its surface ought to be entirely covered with wood-charcoal; without this precaution, the refining of the metal would go back, as the workmen say, during the long interval which elapses in moulding; whenever this accident happens, it must be stirred anew with the wooden pole.

Too long employment of the pole causes the copper to become more brittle than it was prior to the commencement of the refining; that is, when it was dry. Its colour is now of a very brilliant yellowish red, and its fracture fibrous. When this occurs, the refining, as the workmen say, has gone too far, and the refiner removes the charcoal from the top of the melted metal; he opens the side door, to expose the copper to the action of the air, and it then resumes its malleable condition.

The theory of refining may be thus explained:-We may conclude that the copper in the dry state before refining, is combined with a small portion of oxygen, or, in other words, that a small portion of sub-oxide of copper is diffused through the mass, combined with it; and that this proportion of oxygen is expelled by the deoxidising action of the wood and charcoal, whereby the metal becomes malleable. 2. That when the refining process is carried too far, the copper gets combined with a little carbon. Thus copper, like iron, is brittle when combined with oxygen or carbon; and becomes malleable only when freed entirely from these substances.

It is remarkable, that copper, in the dry state, has a strong action upon iron; and that the tools employed in stirring the liquid metal become glistening, like those nsed in a faxrier's forge. The iron of the tools consumes more rapidly at this time than when the copper has acquired its malleable state. The metal requires also, when $d r y$, more time to become solid, or cool, than when it is refined; a circumstance depending, probably, upon the difference in fusibility of the copper in the two states, and which seems to indicate the presence of oxygen.

When the proper refining point has been passed, another very remarkable cireum. stance has been observed; namely, that the surface of the copper oxidises less easily, and that it is uncommonly brilliant, reflecting clearly the bricks of the furnace vault. This fact is favourable to the idea suggested above, that the metal is in that case combined with a small quantity of carbon; which absorbs the oxygen of the air, and thus protects the metal from its action.

Copper is brought into the market in different forms, according to the purposes which it is to serve. That which is to be employed in the manufacture of brass is granulated. In this condition it presents more surface to the action of zinc, and combines with it more readily. To produce this granulation, the metal is poured into a large ladle, pierced with holes and placed above a cistern filled with water, which must be hot or cold, according to the form of the grains required. When it is hot round grains are obtained analogous to lead shot; and the copper in this state is called 
bean shot. When the melted copper falls into cold water perpetually renewed, the granulations are irregular, thin, and ramified; constituting feathered shot. The bean shot is the form employed in brass making.

Copper is also made into small ingots, about six ounces in weight. These are intonded for exportation to the East Indies, and are known in commerce by the name of Japan copper. Whenever these little pieces are solidified, they are thrown, while hot, into cold water. This immersion slightly oxidises the surface of the copper, and gives it a fine red colour.

Lastly, copper is often reduced into sheets, for the sheathing of ships, and many other purposes.

The eylinders for rolling copper into sheets are usually 3 feet long and 15 inches in diameter. They are uniform. The upper roller may be approached to the undor one by a screw, so that the cylinders are brought closer in proportion as the sheet is made thinner.

The ingots of copper are laid upon the sole of a reverberatory furnace to be heated; they are placed alongside each other, and are formed into piles in a cross-like arrangemont, so that the hot air may pass freely round them all. The door of the furnace is shut, and the workman looks in through a peep-hole from time to time, to see if they lave taken the requisite temperature; namely, a dull red. The copper is now passed between the cylinders; but although this metal is very malleable, the ingots cannot be reduced to sheets without being several times heated; because the copper cools, and acquires, by compression, a texture which stops the further progress of lamination. Seo AnNeading.

These successive heatings are given in the furnace above indicated; though, when the sheets are to have a very great size, furnaces somewhat different are had recourse to. They are from 12 to 15 feet long and 5 feet wide. See Brass.

The copper, by successive heating and lamination, gets covered with a coating of oxide, which is removed by stoeping the sheets for a few days in a pit filled with urine; they are then put upon the sole of the heating furnee. Ammonia is formed, which acts on the copper oxide, and lays bare the metallic surface. The sheets are next rubbed with a piece of wood, then plunged, while still hot, into water, to make the oxide scale off; and are lastly passed cold through the rolling press to smooth them. They are now cut square, and packed up for home sale or exportation.

The following estimate was given by MM. Dufrénoy and Elie de Beaumont of the expense of manufacturing a ton of copper at the time of their visit to South Wales in 1822 :-

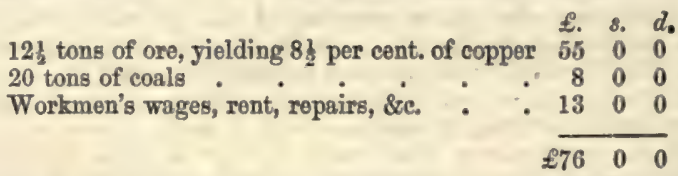

The exhalations from the copper smelting-works are exceedingly detrimental to both vegetable and animal life. They consist of sulphurous acid, sulphuric acid, arsenical

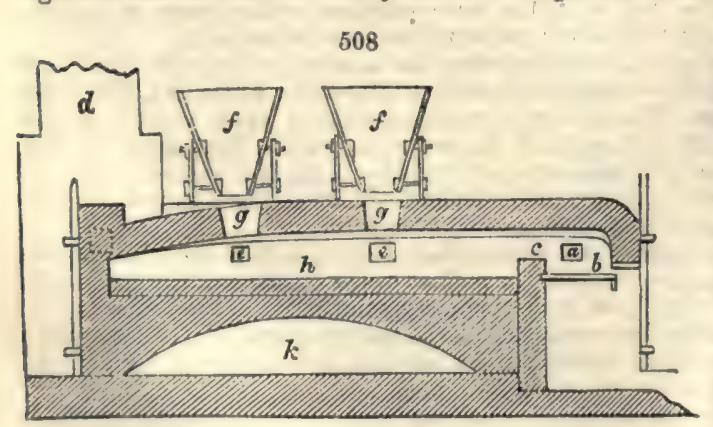
and arsenious acids, various acid and other vapours, with solid particles mechanically swept away into the air. See p. 928.

The following figures represent certain modifications of the copper calcining and smelting copper furnaces of Swansea.

Fig. 508 is the section of the roasting furnace lengthwise; fig. 509, the ground plan; in which $a$ is the fire-door; $b$, the grate; $c$, the firebridge; $d$, the chimney; $e e$, apertures on each side of the long sides of the furnace, through which the ore is spread, and turned over; $f f$, iron hoppers; $g g$, openings in the vaulted roof; $h$ the hearth-sole; $i i$, holes in this; $k$, a vaulted space under the hearth. The hearth has a suitable oval shape, and is covered with a flat arch. Its length is 16 foet, breadth 181 foet, mean height 2 feet. 
Fig. 510 is a longitudinal section of the melting furnace; fig. 511 the ground plan, in which $a$ is the fire-dnor; $b$, the grate; $c$, the fire-bridge; $d$, the chimney; $e$, the

509

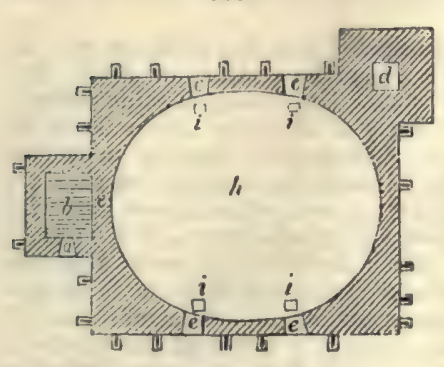

510

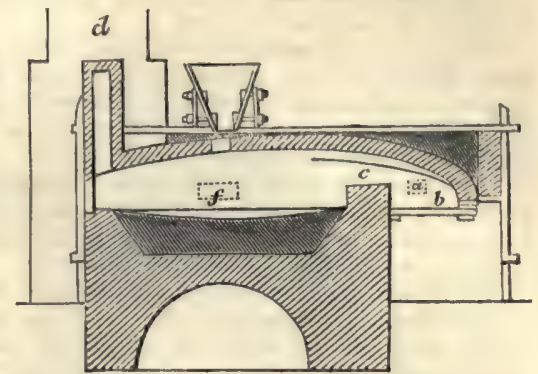

side openings; $f$, the working door; $g$, the raking-out hole; $h$, iron spouts, which conduct the melted metal into pits filled with water.

The melting furnace is altogether smaller, but its firing hearth is considerably larger than in the roasting furnace. The long axis of the oval hearth is 14 feet; its short axis 10 feet; its mean height 2 feet.

Napier's Process for smelting Copper Ores. - As the copper ores of this country often contain small portions of other metals, such as tin, antimony, arsenic, \&c. which are found to deteriorate the copper, Mr. Napier's process had in view to remove these metals, and at the same time to shorten the operations of the smelting process.

The first two operations, that of calcining and fusing the ore, are the

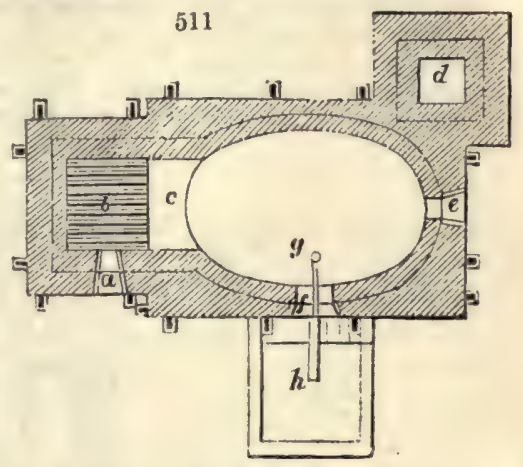
same as the ordinary processes; but the product of this last fusion-viz., the coarse-metal-is again fused with a little sulphate of soda and conl mixed; and whenever this becomes solid, after tapping the furnace, it is thrown into a pit of water, where it immediately falls into an impalpable powder; the water boils, and then contains caustic soda and sulphide of sodium, dissolving from the powder those metals that deteriorate the copper; the ley is let off, and the powder washed by allowing water to run through it. The powder is then put into a calcining furnace, and calcined until all sulphur is driven off, which is easily effected from the finoly-divided state of the mass. This calcined powder is now removed to a fusing furnace, and mixed with ores containing no sulphur, such as carbonates and oxides, and a little ground coal, and the whole fused ; the result of this fusion is metallic copper and sharp-slag-that is, a scoria containing much protosilicate of iron, which is used as a flux in the first fusion of the calcined ore, so that any small trace of copper which the slag may contain is thus recovered.

The copper got from this fusion is refined in the ordinary way, and is very pure.

This process, which was patented in 1846, and carried on for some few years at Loughor, near Swansea, has been found in practice to be incapable of completely separating antimony, arsenic, and tin from copper, and has in consequence been abandoned.

When the copper ores contain tin to the extent of from $\frac{1}{2}$ per cent. to 2 per cent., which many of them are found to do, $\mathrm{Mr}$. Napier proposed to extract this tin, and make it raluable by a process which has also been the subject of a patent. The ore is first ground and calcined, till the amount of sulphur is a little under one-fourth of the copper present ; the ore is then fused with a little coal. The result of this fusion, besides the seoria, is a regulus composed of sulphur, copper, and iron, and under this is a conrse alloy of copper, tin, and iron. This alloy is ground fine, and calcined to oxidise the metals, which are then fused in an iron pot with caustic soda, which combines with the tin and leaves the copper. The oxide of copper is now fused with the regulus. The stannate of soda is dissolved in water, and the tin precipitated by 
slaked lime, the precipitate is dried and fused with carbonaceous matters and a little sund, and metallic tin obtained; the caustic soda-solution is eraporated to dryness and used over again. This process was proposed for the treatment of very poor copper ores that are mixed with tin, or poor tin ores mixed with copper, but does not appear to have been practically applied.

Process formerly employed at Chessy.-The prineipal ore smelted at Chessy was the azure copper, or blue carbonate, which was discovered by accident in 1812. Red copper ore, also, came into operation there aftor 1825. The average metallic contents of the richest azure ore was from 33 to 36 per cent.; of the poorer, from 20 to 24 . The red ore contained from 40 to 67 parts in 100. The ore was sorted to an average of 27 per cent. of metal, to which 20 per cent. of limestone was added; whence the cinder will amount to 50 per cent. of the ore. A few per cents. of red copper slag, with some quicklime and Gahrslag, was added to each charge, which consisted of 200 pounds of the above mixture, and 150 pounds of coke. When the furnace (fourneau à manche, see the Scotch smelting hearth, under LFAD) was in good action, from 10 to 14 such charges could be worked in 12 hours. When the crucible was full of metal at the end of this period, during which the cinder had been frequently raked off, the blast was stopped; the matt floating over and the metal being sprinkled with water and taken off, left the black copper to bo treated in a similar way, and converted into rosettes. The refining of this black copper was performed in a kind of reverberatory furnace.

The cinders produced in this reduction process were either vitreous and light blue, which were most abundant; cellular, black, imperfectly fused from excess of lime; or, lastly, red, dense, blistery, from defect of lime, from too much heat, and the passage of oxide of copper into the cinders. They consisted of silicate of alumina, of lime, and of protoxide of iron; the red contained some silicate of copper.

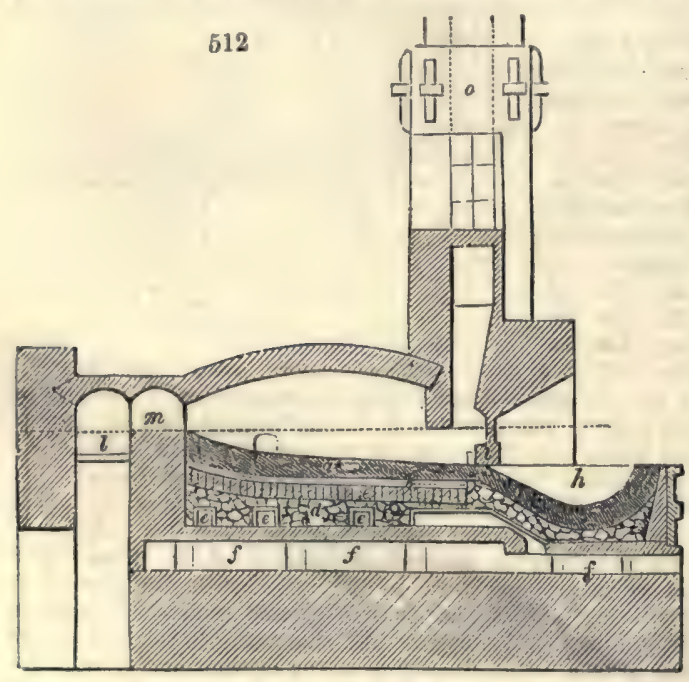

The copper - refining furnace at Chessy was of the kind called Spleissofen (split-hearth) by the Germans. Fig. 512 is a section lengthwise on the dotted line $\Delta \mathrm{B}$ of fig. 513, which is the ground plan.

The foundation-walls were made of gneiss ; the arch, the fire-bridge, and the chimney, of firebricks. The hearth a was formed of a dense mixture of coal-dust, upon a bottom of wellbeat clay $b$, which reposed upon a bed of brick-work o. Beneath this there was a slag bottom $d ; e$ is the upper, and $f$ the under discharge-hole. The hearth was egg-shaped; the longer axis being 8 feet, the shorter $6 \frac{1}{2}$ feet; in the middle it was 10 inches deop, and furnished with the nutlets $g \mathrm{~g}$, which lead to each of the split-hearths, $h h$, fig. 513. These outlets were - contracted with fire-bricks $i$, till the proper period of the discharge. The two hearths were placed in communication by a canal $k$; they were each $3 \frac{1}{2}$ feet in diameter, 16 inches deep; floored with well-beat coal-ashes, and received about 27 cwts. for a charge.

$l$ is the grate; $m$, the fire-bridge; $n$, the boshes in which the tuyeres lie; 0 , the chimney; $p$, the working door, through which the slags may be drawn off. Above this was a small chimney, to carry off the flame and smoke whenever the door was opened.

The smelting post or charge to be purified at once, consisted of $60 \mathrm{cwts}$. of black copper, to which a little granular copper and copper of cementation were added; the consumption of pit-coal amounted to $36 \mathrm{cwts}$. As soon as the copper was melted, the bellows were set a-going, and the surface of the metal soon became covered with a moderately thick layer of cinder, which was drawn off. This was the first skimming or décrassage. By-and-by, a second layer of cinder formed, which was in like manner removed; and this skimming was repeated, to allow the blast to act upon fresh 


\section{COPPER}

metallic surfaces. After 4 or 5 hours, no more slag appeared, and then the fire was increased. The melted mass now began to boil or work (travailler), and continued so to do for about $\frac{3}{4}$ of an hour, or an hour, after which the motion ceased, though the fire was kept up. The Gahrproof was now taken ; but the metal was seldom fine in less than

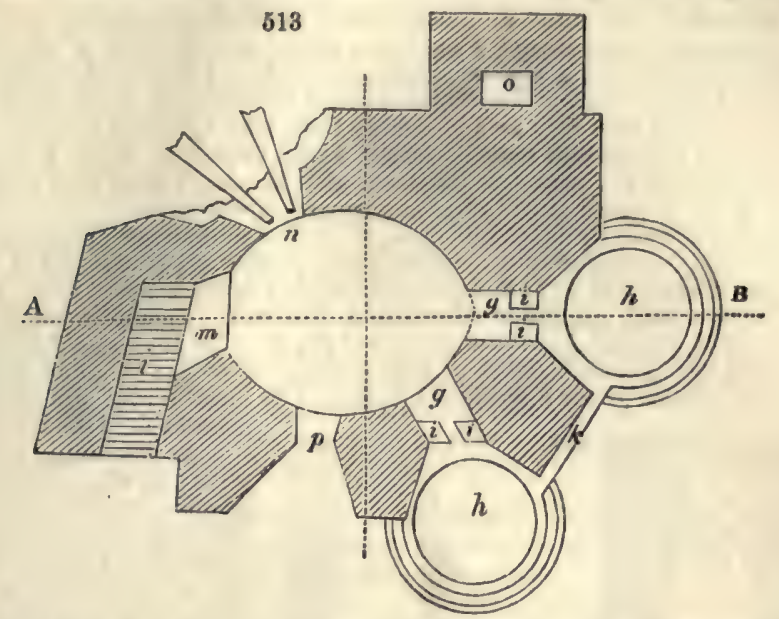

a of an hour after the boil was over. Whenever the metal was run off by the tap-hole into the two basins, $h h$, called split-hearths, a reddish vapour or mist arose from its surface, composed of an infinite number of minute globules, which revolved with astonishing velocity upon their axes, constituting what the Germans called Spratzen (crackling) of the copper. They were composed of a nucleus of copper covered with a film of oxide, and were used as sand for strewing upon manuseript. The copper was separated by sprinkling water upon the surface of the melted metal, in the state of rosettes, which were immediately immersed in a stream of water. This refining process lasted about 16 or 17 hours; the skimmings weighed about $50 \mathrm{cwts.}$; the refuse was from 15 to 17 per cent.; the loss from 2 to 3 per cent. The Gahrslag amounted to 11 cwts.

Smelting of the Mansfeld Copper-Schist, (Kupferschiefer). -The cupreous ore is first roasted in large heaps of 2,000 cwts., interstratified with brush-wood, and with some schists rich in bituminous matter mixed with the others. These heaps are 3 ells high, and go on burning 15 weeks in fair and 20 in rainy weather. The bituminous matter is decomposed; the sulphur is dissipated chiefly in the form of sulphurous acid; the metal gets partially oxidised, particularly the iron, which is a very desirable circumstance for the production of a good smelting slag. The calcined ore is diminished $\frac{1}{10}$ th in bulk and $\frac{1}{8}$ th in weight; becoming of a friable texture and a dirty yellow colour. The smelting furnaces are cupolas (Schachtöfen), 14 to 18 feet high ; the fuel is partly coal and partly coke from the Berlin gas-works and from Silesia. The blast is now given by a cylinder, but formerly with the old barbarous Blasebälgen, or wooden bellows of the household form. The smelting was formerly conducted as follows :-

The copper-slate is sorted, according to its composition, into slate of lime, clay, iron, \&c. ; by a mixture of which the smelting is facilitated. For example, 1 post or charge may consist of $20 \mathrm{cwts}$. of the ferruginous slate, 14 of the calcareous, 6 of the argillaceous, with 3 of fluor-spar, 3 of rich copper slags, and other refuse matters. The nozzle at the tuyere is lengthened 6 or 8 inches, to place the melting heat near the centre of the furnace. In 15 hours 1 Fuder of $48 \mathrm{cwts}$. of the above mixture may be smelted, whereby 4 to 5 cwts. of matt (Kupferstein) and a large body of slags are obtained. The matt contains from 30 to 40 per cent. of copper, and from 2 to 4 Loths ( 1 to $2 \mathrm{oz}$.) of silver. The slags contain at times $\frac{1}{10}$ th their weight of copper.

The matt is composed of the sulphides of copper, iron, silver, zinc, along with some arsenical cobalt and nickel. The slaty slag is raked off the surface of the melted matt from time to time. The former is either, after being roasted six successive times, smelted into black copper, or it is subjected to the following concentration process:It is broken to pieces, roasted by brushwood and coals three several times in brickwalled kilns, containing 60 cwts., and turned over after each calcination; a process of 4 weeks' duration. The thrice-roasted mass, called Spurröst, being melted in the 
cupola, fig. 514, with ore-cinder, yields the Spurstein, or concentrated matt. From 30 to 40 cwts. of Spurröst aro smelted in 24 hours; and from 48 to 60 per cent. of Spurstein are obtained, the slag from the slate-smelting being employed as a flux. The Spurstein contains from 50 to 60 por cent, of copper, combined with the sulphides of copper, of iron, and silver.

The Spurstein is now mixed with Dïnnstein (a sulphide of copper and iron produced in the original smelting), roasted 6 successive times, in quantities of 60 cwts., with brushwood and charcoal ; a process which requires from 7 to 8 wooks. The product of this six-fold calcination is the Gakrröst of the Gormans; it has a colour like red copper ore, varying from blue grey to cochineal-rod, with a granular fracture; and may be immediately roduced into metallic coppor, which procoss is called Kupfermachen. But before smelting the mass, it is lixiviated with water, to extract from it the soluble sulphate, which is concentrated in leaden pans and crystallised.

The lixiviated Gahrröst mixed with from $\frac{1}{4}$ th to $\frac{1}{5}$ th of the lixiviated Dinnsteinrost and $\frac{1}{6}$ th to $\frac{1}{10}$ th of tho copper-slate slag are smelted with charcoal or coke fuel in the course of 24 hours, in a mass of 60 or 80 ewts. The product is black copper, to the amount of about $f$ th the weight, and $\frac{1}{6}$ th of Dïnstein or thin matt. This black copper contains in the ewt. from 12 to 20 Loths (6 to $10 \mathrm{oz}$.) of silver. The Diinnstein consists of from 60 to 70 per cent. of copper combined with sulphur, sulphide of iron, and arsenic ; and when thrice roasted, yields a portion of metal. The black copper lies undermost in

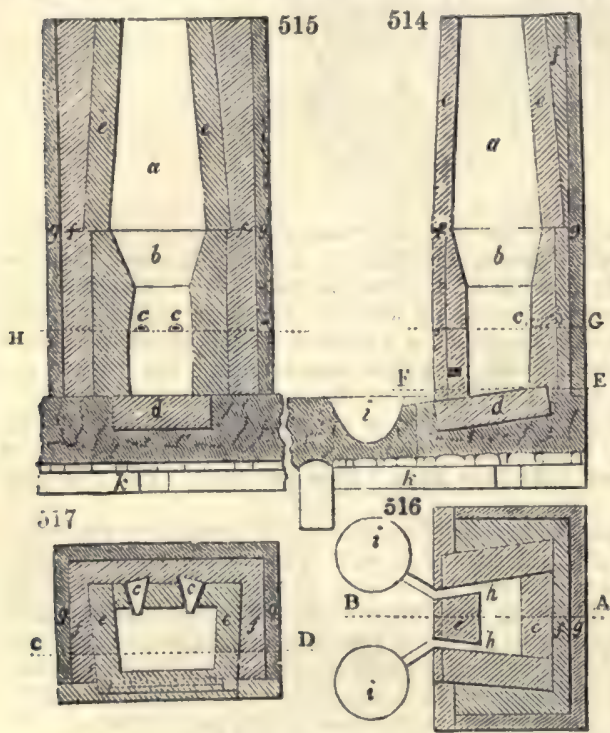
the crucible of the furnace; above it is the Diinnstein, covered with the strong slag, or copper cinder, resulting from the slate-smelting. The slags boing raked off, and the crucible sufficiently full, the eye or nozzle hole is shut, the Dinnstein removed by cooling the surface and breaking the crust, which is about $a+$ to $\frac{1}{2}$ inch thick. The same method is adopted for taking out the black copper in successive layers. For the desilvering of this, and similar black coppers, see Smvar.

Fig. 514 is a vertical section through the tuydre in the dotted line A B of fig. 516. Fig. 515 is a vertical section through the dotted line c D of fig. 517. $a$ is the shaft of the furnace; $b$, the rest, $c c$, the tuyires; $d$, A the sole or hearth-stone, which has a slope of 3 inches towards the front wall ; $c e$, \&ce, casing walls of fire-bricks; $f f$, \&c., filling-np walls, built of rubbish stones; 9.9 , a mass through which the heat is slowly conducted; $h h$, the two holes through one or other of which nlternately the product of the smelting process is run off into the fore-hearth. Beneath the hearth sole there is a solid body of loam; and the fore-hearth is formed with a mixture of coal-dust and clay; $k$ is the discharge outlet for moisture. Fig. 516 is a horizontal section of the furnace through the hole or eye, on the dotted line $\mathbf{F}$ of fig. 514 ; fig. 517 , a horizontal section of the shaft of the furnace through the form along the dotted line $\mathrm{A} H$ of figs. 514 and 515. The height of the shaft, from tho line $\mathrm{E}$ F to the top, is 14 feet; from $\mathrm{E}$ to $\mathrm{G}, 25$ inches; from $c$ to the line below $b$, 2 feet; from that line to the line opposite $g g, 2$ feet. The width at the line $g g$ is 3 feet 3 inches, and at $c, 26$ inches. The basins, $i, f i g .516$, are each 3 foet in diameter and 20 inches deep.

During the last twenty years few processes have undergone more extensive modifications than that used for the extraction of copper and silver from the copper-schists of Mansfeld; and it is therefore necessary to present a short account of tho series of operations at present employed.

The cupola furnaco now used diffors greatly, both in construction and dimensions, from that formerly employed in the district; boing in almost all respects, excepting size, similar to the closed blast-furnaces usod in this country for the manufacture of cast iron. This furnace is circular, and 30 feot in height by about 7 feet in diameter 
at the top, which is the widest part; the interior dimension decreasing gradually towards the hearth, where the width is 6 feet.

The blast is supplied by six water-tuyers placed radially, or nearly so, at a height of from 4 to 5 feet above the hearth. On one side is a dam plate over which slag flows continuously whilst on the opposite side is a tapping hole through which the matt is drawn off from time to time.

The top of the furnace is closed by a cup-and-cone, and the waste-gases are conveyed to the stoves employed for heating the blast, the temperature of which is raised to $370^{\circ} \mathrm{F}$.

The reverberatory furnace employed in the calcination processes is in all essential respects similar to that used in Wales for the rcasting of the various matts produced in the English method of smelting.

The following is an outline of the routine generally employed:-

1. Roasting the schists in the open air, in large heaps, in order to drive off water, bitumen, and other volatile matters and at the same time to induce such a state of division between the particles of the ore as shall be most suitable to subsequent melting operations.

2. The first melting in a cupola-furnace, the object being the separation of siliea and earthy matters in the form of slag and the concentration of the copper in a matt containing 36 per cent. of that metal, together with some silver.

3. The matt formed in the last operation is roasted in the open arr in rectangular 'stalls, the object being the elimination of a portion of the sulphur and the oxidation of some of the iron.

4. The roasted matt or coarse-metal is concentrated in a reverberatory furnace in order to fit it for the next process for extracting the silver by Ziervogel's method. During the roasting, lead, arsenic, and zine are to some extent eliminated and a regulus produced which is rich in copper and silver; at the conclusion of the operation this is granulated by being run into a vat of water.

5. For the extraction of the silver, the granulated matt is ground to a fine powder, and roasted in a reverberatory furnace at such a temperature that as much as possible of the copper shall be converted into oxide, whilst the silver remains in the form of sulphate; this is washed from the roasted material by means of hot water and the silver subsequently precipitated from solution by metallic copper.

6. Fusion for blister-copper. The residue from the lixiviation vats is made inito balls with about 8 per cent. of clay, which serves the double purpose of supplying materials for slag and at the same time forming the powder into lumps and preventing its elogging the furnace.

The fusion is conducted in a cupola-furnace and fluid products flow continuously from it into a basin, and arrange themselves according to their specific gravities; namely,
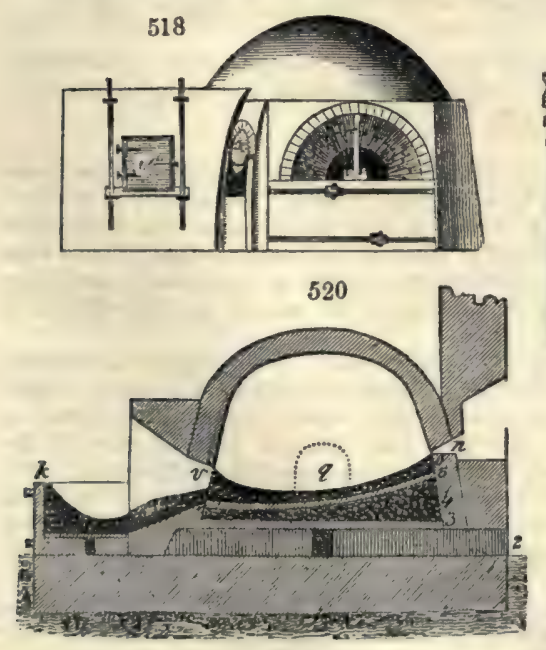

519

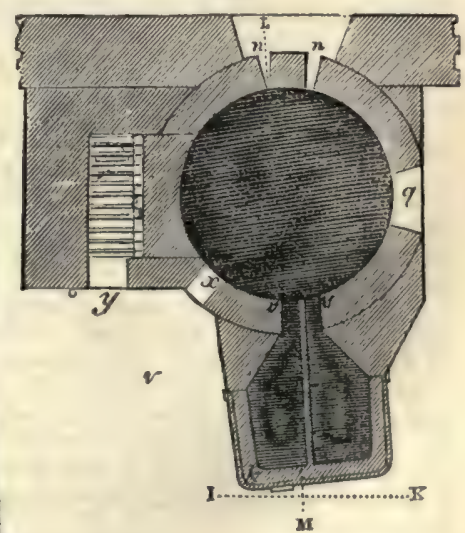

first, metal ; second, a thin layer of rich matt; and thirdly, slag. The latter is broken and sorted, and any containing copper is, together with the matt, worked over in one of the earlier operations. 
7. Refining the blister-copper. This is effected either in the German hearth by the action of glowing charcoal and a highly-inclined blast, and the fine-copper obtained in the form of rosettes; or the Welsh reverberatory furnace is employed, and the copper cast into ingots. For extraction of silver from the Mansfeld copper regulus, see Silvikr.

Figs. 518, 519, 520, represent the reverberatory furnace generally omployed in the Harz, in the district of Mansfeld, Saxony, Hungary, \& c., for the treatment of black copper, and for refining rosette copper upon the great scale. An analogous furnace is used at Andreasberg for the liquefaction or purification of the matts, and for workable lead when it is much loaded with arsenic.

Fig. 518 presents the elevation of the furnace parallel to the line I $\mathbf{x}$, of the plan fig. 519, which plan is taken at the level of the tuyere $n$ of fig. $520 ;$ fig. 520 is a vertical section in the line $\mathbf{I} \mathbf{M}$, fig. 519. $k$ represents one of two basins of reception, brasqued with clay and chareaal ; $n n$, two tuyeres through which enters the blast of two pairs of bellows; $q$, door by which the matter to be melted is laid upon the sole of the furnace; $v, v$, two points where the sole is perforated, when necessary to run off the melted matter into either of the basins $k ; x$, door through which the slags or cinders floating upon the surface of the melted metal are raked out; $y$, door of the fire-place. The fuel is laid upon a grate above an ash-pit, and below the arch of a reverberatory, which is contiguous to the dome or cap of the furnace properly so called. In the section, fig. 520, the following parts may be noted: 1, 2, 3, mason-work of the foundation; 4 , vapour-channels or conduits, for the escape of the humidity; 5 , bed of clay; 6 , brasque, composed of clay and chareoal, which forms the concavity of the hearth.

Figs. 521, 522, 523, show the furnace formerly employed for liquation in one of

521

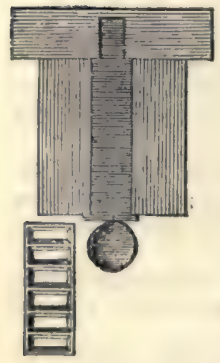

622

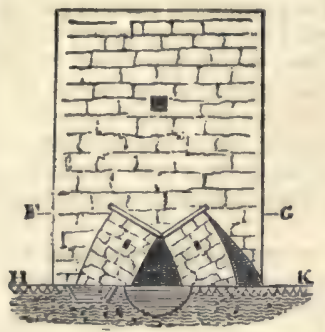

523

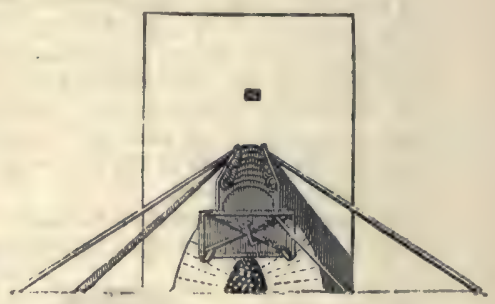

the principal smelting works of the Harz. Fig. 523 exhibits the workng area charged with the liquation cakes and charcoal, supported by sheets of wrought-iron; Fig. 521 is the plan, in the line $x \mathrm{G}$, of fig. 522 .

A liquation cake is composed of-

Black copper holding at least 5 or 6 Loths $\left(2 \frac{1}{2}\right.$ or 3 oz.) of silver per cwt., and weighing 90 to $96 \mathrm{lbs}$. Iread obtained from litharge, 2 cwts. Litharge, $\frac{1}{2}$ ewt.

From 30 to 32 cakes are successively worked in one operation, which lasts about 4 hours; the furnace is brought into action as usual, with the aid of slags; then a little litharge is added; when the lead begins to flow, the copper is introduced, and when the copper flows, lead is added, so that the mixture of the metals may be effected in the best possible way.

From 8 to 16 of these cakes (pains) are usually placed in the liquation furnace, figs. 521, 522, 523. The operation lasts 3 or 4 hours, in which time about $1 \frac{1}{2}$ quintal of charcoal is consumed. The cakes are covered with burning charcoal supported, as before stated, by the iron plates. The workable lead obtained flows off towards the basin in front of the furnace; whence it is laded out into moulds set alongside. (See fig. 518). If the lead thus obtained be not sufficiently rich in silver to be worth cupellation, it is employed to form new liquation cakes. When it contains from 5 to 6 Loths of silver per cwt., it is submitted to cupellation in the said smelting works. See SILVER.

The refining of the eliquated copper (called Darringe) from which the silver has been sweated out by means of lead, can be performed only in small hearths. The following is the representation of such a furnace, called in German Kupfergahrheerd. Fig. 524 is the section lengthwise; fig. 525 is the section across; and fig. 526 is the ground plan: in these figures $a$ is the hearth-hollow; $b$, a massive wall; $c$, the mass out of which the hearth is formed; $d$, cast-iron plates covering the hearth; 
$e$, opening for running off the liquid slag; $f, a$ small wall; $g$, iron curb for keeping the coals together.
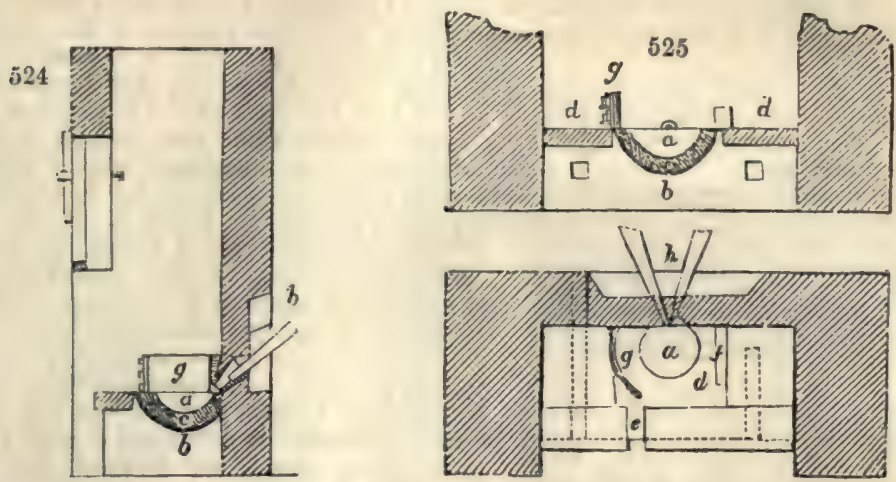

The hearth being heated with a bed of charcoal, $\frac{3}{4} \mathrm{cwt}$. of Darrlinge are laid over it, and corered with more fuel: whenever this charge is melted, another layer of the coal and Darrlinge is introduced, and thus in succession till the hearth becomes full or contains from $2 \frac{1}{4}$ to $2 \frac{1}{2} \mathrm{cwts}$. In Neustadt $7 \frac{1}{2}$ cwts. of Darrlinge have been refined in one furnace, from which 5 cwts. of Gahrkupfer have been obtained. The blast oxidises the foreign metals, namely, the lead, nickel, cobalt, and iron, with a little copper, forming the Gahrslag; which is, at first, rich in lead oxide and poor in copper oxide; but at the end, this is reversed. The slag, at first blackish, assumes progressively a copperred tint. This slag flows off spontaneously along the channel $e$, from the surface of the hearth. The Gahre is tested by means of a proof-rod of iron, called Gahreisen, thrust through the tuyere into the melted copper, then drawn out and plunged in cold water. As soon as the Gahrspan (scale of copper) appears brownish-red on the outside, and copper-red within, so thin that it seems like a net-work, and possessing sufficient tenacity to admit of its being bent backwards and forwards several times without breaking, the refining is finished. The blast is then stopped; the coals covering the surface, as also the cinders, must be raked off the copper, after being left to cool. The surface is now further cooled by sprinkling water uron it, and the thick cake of congealed metal (rondelle) is lifted off with tongs, a process called Schleissen (slicing), or Scheibenreissen (shaving), which is continued till the last convex cake at the bottom of the furnace, styled the kingspiece, is withdrawn. These rondelles are immediately immersed in cold water, to prevent the oxidation of the copper: whereupon the metal becomes of a cochineal-red colour, and gets covered with a thin film of red oxide. Its under surface is studded over with points and hooks, the result of tearing the congealed dise from the liquid metal. Such cases are called rosette copper. When the metal is pure and free from oxide, these cakes may be obtained very thin, $\frac{1}{24}$ th of an inch, for example. The refining of $2 \frac{1}{2}$ cwts. of Darrlinge takes $\frac{3}{4}$ of an hour, and yields $1 \frac{1}{2} \mathrm{cwt}$. of Gahr copper in 36 rosettes, as also some Gahrslag. Gahr-copper generally contains from $1 \frac{1}{2}$ to $2 \frac{1}{2}$ per cent. of lead, along with a littlo nickel silver, iron, and aluminium. Most of the Mansfield copper is now refined in a reverberatory furnace, and sold as Raffwadkupfer, and not as Gahrkupfer.

Utilisation of the Copper Smoke.-The application of the sulphur disengaged in the calcination or roasting of copper ores to the production of liquid sulphuric acid, has long been an important object of copper-smelters. In the case of a small proportion of copper ores, containing a comparatively large proportion of sulphur, and in the state of hard lumps, free from earthy matter, this is effected in the ordinary 'pyrites burner' of the sulphuric acid works. But copper ores, for the most part, are small, poor in sulphur, with much earthy matter; and are therefore calcined in reverberatory furnaces with fire-grates. The gases from these calciners do not consist of more than 0.5 rol. of sulphurous acid in 100 vols., and are moreover largely mixed with the products of combustion from the fire-place : they were therefore unfit for the vitriol chamber, and were allowed to escape.

An important invention, by which this loss is avoided, is the calcining furnace of M. Gerstenhöfer; in which the ore, in a finely-divided state, is allowed to fall on a number of fire-brick bars or bearers, placed in rows, at different elevations. The ore, which is supplied in a continuous stream at the top, meets in its descent with a current of air in the reverse direction. The furnsce being moderately heated on starting, 
the ore ignites, and afterwnrds maintains its own combustion, without the aid of fuel, for any length of time; while the ascending current of air becomes charged with sulphurous acid of sufficient strength and purity to be conveyed to the ritriol chambers.

The annexed figures represent perpendicular transverse sections of M. Gersten-

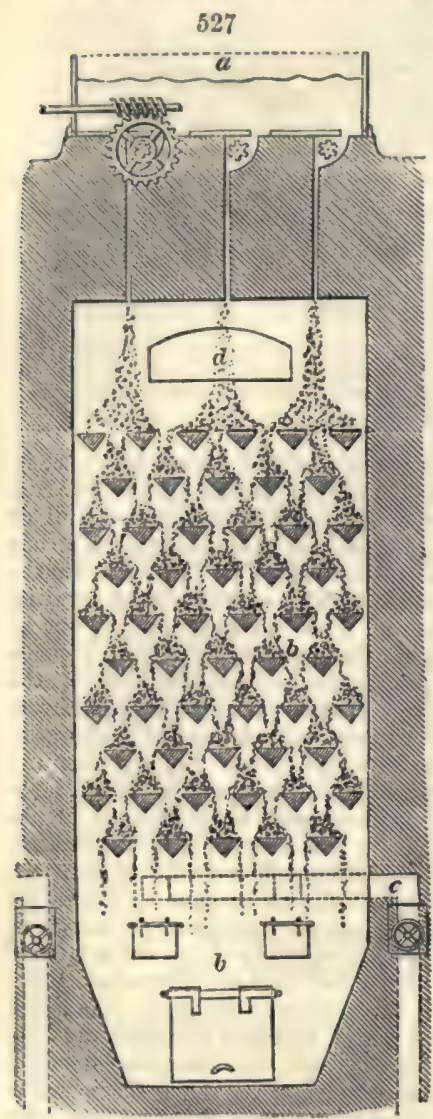

Gerstenhofer's Furnace for calcining Copper Ores. höfer's furnace. Fig. 527 is a cross-section parallel with the front; fig. 528 represents a section from front to back. The length of the earthen bars is about two feet six inches: twenty rows oceupy about twelve feet in height; $a$ is an iron box or hopper for containing a supply of ground ore, having at bottom two or more cast-iron grooved rollers, which are worked without interruption, at a speed adapted to supply ore equal to a discharge of about 10 ewts. of sulphur per twenty-four hours. As the ore sometimes has a tendency to form a cake on the bars, it is necessary to screpe these occasionally, by means of an iron rod, with curved ond, which is introduced through plugholes in cast-iron boxes, shown in fig. 528 . The calcined ore collects in the cavity $b$ at the bottom, and is raked out when convenient. The necessary amount of air is admitted partly at the back through the flue $c$, regulated by means of a screw-Valve, and partly through the plug-holes in front. After passing upwards through the calciner, the air, now charged with sulphurous acid to the extent of 6 or $8 \mathrm{pex}$ cont, in volume, passes over the bridge, and through the flue $c$ in the direction of the $8 \mathrm{r}$ rows, fig. 528. Provision is mado at the bottom of these flues for collecting and removing par. ticles of roasted ore carried over by the current ; $f$ is a brick-built chamber for a similar purpose, from the back of which the gas passes through the flue $g$ to the vitriol chambers. The latter are supplied with nitric acid in the usual way; either from a nitrate-pot or oven worked independently, or with liquid nitric acid allowed to percolate a tower and mix with the gas from the calciners before entering the leaden chambers.

The Gerstenhöfor calciner is applicable to all varieties of sulphuretted copper ores, although varying in proportion of sulphur from 16 to 40 per cent.; it is also adapted to the 'coarsemetal' or regulus obtained as the result of the first reducing operation.

The calcination is conducted with ease, so that no more sulphur remains in the ore than is necessary for the smelting processes.

As the superiority of this calciner over every other, having a similar object, has been proved, in the Government copper smelting establishments of Freiberg and Mansfeld, and in this country, in the works of Messrs. Vivian and Sons, near Swansea (who are the propriotors of the English patent), it is expected that their general introduction into copper works will be the means of greatly reducing the smoke arising therefrom and turning it to profitable account. This was written in 1867. Although still used advantageously, its use has not, in 1874, been extended.

Mr. Peter Spence, of Manchester, has also a calciner which has been four years in operation, and has calcined 20,000 tons of copper ore. Mr. Spence, in his patent of the 8rd July 1861, thus describes his arrangements:-

- The essential feature of invention consists in submitting such ores to the action of a roasting heat as they are passed from one end of a furnace to the other, during which transference a current of air is caused to travel over them in an opposite direction; to accomplish this, a furnace of considerable length, having several doors for the purpose of introducing apparatus by which the transference may be effected, is used. It will be obvious that by this arrangement the ores may be submitted to heat in a thin stratum, that the amount of roasting may bo modified by a quicker or slower 
transference, and that the heat communicated may be greater towards the tormination of the operation than at the commencement thereof.

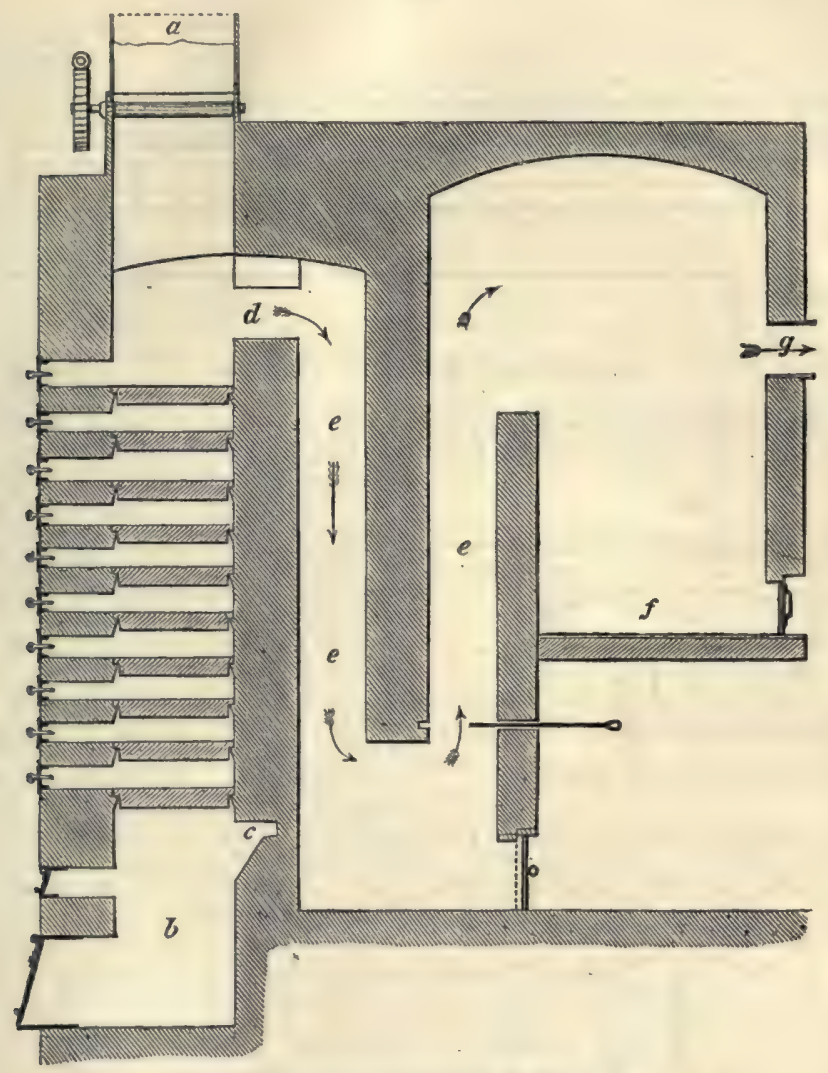

Gerstenhöfer's Furnace for calcining Copper Ores.

This inrention will be fully understood by the accompanying drawings of apparatus.

Fig. 529 is a side view of the furnace, and fig. 530 a longitudinal section, in both of which the two ends only are shown, the whole being of considerable length. One

529

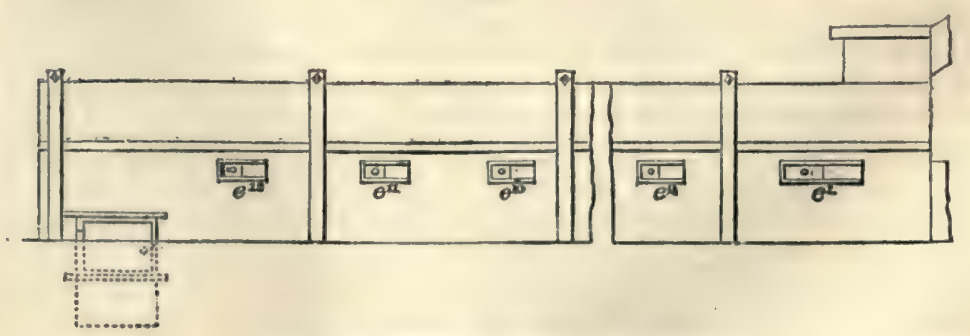

Spence's Furnace for calcining Copper Ores.

now in operation is fifty feet long, with twelve doors for the transference of the ores; but these dimensions may be varied, and aro only montioned for the purpose of Vox. I. 
explaining that the furnace should be of considerable length. Fig. 581 represents a cross-section; and in describing the apparatus in the first instance we suppose that the manufacture of sulphuric acid is the object in riew.

530
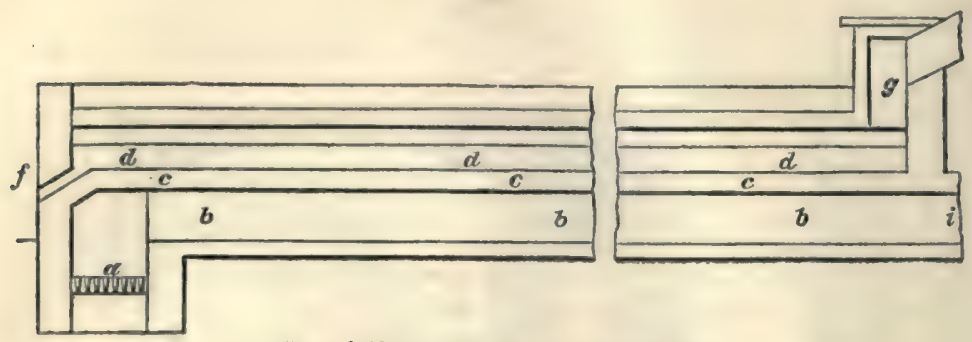

Spence's Furnace for Calcining Copper Ores.

At $a$ are the fire-bars of the furnace, and at $b$ the fire-chamber, formed by channels, as seen in fig. 531, extending under a partition $c$, of fire brick, which partition forms

581

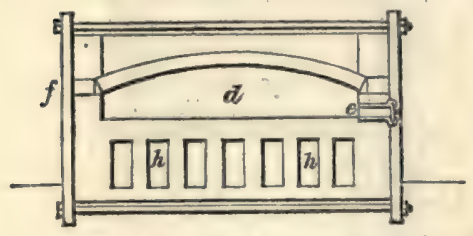

Spence's Furnace for calcining Copper Ores.

the bottom of another and distinct chamber $d$, furnished at one or both sides with a number of doors $\epsilon^{1}, \epsilon^{2}$, \&ce. At $f$ is an aperture formed through the brickwork, and constituting a communication from the external atmosphere to the chamber $d$. The ores from which sulphuric acid is to be obtained are passed through the door $e^{1}$ into the chamber $d$ in sufficient quantity to lio about two or three inches in thickness upon the bed $c$, and to extend, say, halfway towards the door $e^{2}$. This bateh having beon submitted to heat, passing from the firechamber $b$ for the required time, is pushed forward by any convenient instrument, so as to be brought opposite the door $e^{2}$, and another batch is introduced through the door $e^{1}$; then the first charge is removed from $e^{2}$ to $e^{3}$, and the second from $e^{1}$ to $e^{2}$, and so on until the first-deposited batch shall have arrived at the other end of the furnace at $e^{12}$, and it is then pushed through the aperture $f$ into any receptacle placed to receive it. During this transference of the material, it has gradually become heated, and a current of air entering at $f$ has been passing over it, and the operation has caused the sulphur to be driven off from the ore and conveyed through the channel $g$ to the ordinary sulphurie acid apparatus. The end of the fire-chamber $d$ leads to a flue at $i$. The degree of roasting to be effected, and so as to secure the best results, can be ascertained only by experience; but working with a furnaco fifty feet long and with twelve doors, the first charge is allowed to remain for one hour, then transferred to the second position, and a fresh charge put into the first, and so on, waiting an hour between each charge.

An advantage arising from this invention is its capability of being applied to the manufacture of sulphuric acid from any description of ores. It is well known to those engaged in the extraction of copper from ores that the sulphur thereof is frequently wasted from the impracticability of roasting certain mixed ores so as to render them into a fit condition for reduction, and also for the manufacture of sulphuric acid; but by this improved method, ores of any description, large or small, may be roasted so as to produce sulphuric acid with economical results. In calcining the mixed copper ores preparatory to smelting, as practised at the Swansea and other copper works, and with the riew of economising the manufacture of sulphuric acid, the process of calcination by this method is effected with equal advantage as by the modes now practised, as the heat is made to act on a large body of ore without an exhaustive, but with an accumulative effect, so that the heat which has calcined or driven off nearly the last portion of sulphur from the ore which lies at the exit of the furnace then passes on, heating every successive charge, and gaining strength from the combustion of the sulphur until it comes into contact with the cold charge, which it rapidly heats to ignition, partly from the under heat of the bed derived from the fire, which, after leaving the furnace, is passed to a chimney, and partly from all the hot gases passing over it on their way out of the furnace to the vitriol chamber, into which they are conveyed after being mixed with nitrous gases, as is well known. 
As another advantage arising from the use of this invention, it is found in the practical carrying out thereof, that the production of a given amount of sulphuric acid is effected by the expenditure of far less nitre than is eommonly used, a certain amount of sulphuric acid being formed in the chamber of the furnace and passing forward in a state of vapour. Thus far we have spoken of this invention as applicable to the manufacture of sulphurie acid, but it is also applicable to roasting ores of copper preparatory to reduction. In this application the operation is precisely that above duscribed, and will not, therefore, noed a repetition of description, it being of course understood that if the ores have been previously used for the manufacture of sulphuric acid, and contain, therefore, but a small amount of sulphur, the vapours from the chamber $d$ may pass into the atmosphere instead of into the vitriol chamber, if desired.

On the 14th June 1864, Mr. Peter Spence and Mr. J. B. Spence effected another patent for 'calcining and smelting copper ores.' This will be fully understood by the following description :-

' Our invention consists in applying the heat used in smelting copper ores to the purpose of calcining such materials, and in transforring the calcined ores direct to the smelting furnace. To accomplish these objects, we place the two furnaces in connection with each other, and cause a suitable flue to convey the heat used in smelting to the material to be calcined; and when this operation is complete, and the smelted ore is removed, we rake the calcined ore direct on to the bed of the smelting furnace. The calcining furnace we at present prefer to uso is of that construction for which Letters Patent were granted to Peter Spence, bearing date July 3rd, 1861, No. 1695 .'

In addition to these processes, it may be stated that a company has been established at Oldbury, in Staffordshire, for working the process know as 'Henderson's salt process.' We have been favoured with the following description:-

'The works are erected for the purpose of extracting the copper, and utilising the residue from the Spanish pyrites, imported mostly by the alkali makers, and the sulphur extracted by them for tho purpose of making sulphuric acid. We then calcine the ore with salt in such a way as to decompose every trace of copper, and peroxidise the whole of the iron that is not already oxidised during the burning out of the sulphur. We then put the calcined ore into vats or tanks; and as the chloride of copper is roadily soluble, that metal is washed out perfectly clean with hot water, and the residue of peroxide of iron is sold at the iron works in the immediate neighbourhood. This is used in the puddling furnaces, and answers well for two purposes : first, to keep the pig iron from cutting the bottom of the furnaces, and, secondly, to improve the quality of the wrought iron produced. We sell of this residue about 600 tons per month. The copper is precipitated from the solution by scrap iron, the precipitate run down and refined; and the whole of the copper is sold as B. S. ingot. We produce 15 to 18 tons of copper per month.' See PrrrTrs.

Spanish Process of refining Copper. -The refining of copper is well executed at Seville, in Spain ; therefore some account of the mode of operating is required.

The first object is to expel in a reverberatory furnace all the volatile substances, such as sulphur, arsenic, antimony, \&c., which may be associated with the copper; and the second, to oxidise and convert into scorix the fixed substances, such as iron, lead, \&c., with the least possible expense and waste. The minute quantities of gold and silver which resist oxidation cannot be in any way injurious to the copper. The hearth is usually made of refractory sand and clay with ground chareoal, ench mixed in equal volumes, and worked up into a doughy consistence with water. This composition is beat firmly into the furnace bottom. But a quartzose hearth, such as a bed of fire-sandstone, is found to answer better, and to be far more durable.

Before kindling the furnace, its inner surface is smeared with a mixture of fire-clay and water.

The cast pigs, or blocks of crude copper, are piled upon the hearth, each successive layer crossing at right angles that which is beneath it, in order that the flame may have access to play upon the surface of the hearth, and to heat it to a proper pitch for making the metal flow.

The weight of the charge should be proportional to the capacity of the furnace, and such that the level of the metallic bath may be about an inch above the nozzle of the bellows ; for, were it higher, it would obstruct its operation, and, if too low, the stream of air would strike but imperfectly the surface of the metal, and fail to effect, or would at least retard, the refining process, by leaving the oxidation and volatilisation of the foreign metals incomplete.

As the scorix form upon the surface, they are drawn off with an iron rabble fixed to the end of a wooden rod.

Soon after the copper is melted, charcosl is kindled in three iron basins lined with 
losm, placed alongside the furnace, to prepare them for receiving their charge of copper, which is to be converted in them, into rosettes.

The bellows are not long in action before tho bath assumes a boiling appearanee; some drops rise up to the roof, others escape by the door, and fall in a shower of minute spherical globules. This phenomenon proves that the process is going on well; and, when it ceases, the operation is nenrly completed. A small proof of copper, of the form of a watch-case and therefore called montre, is taken out from time to time upon the round end of a polished iron rod, previously heated. This rod is dipped 2 or 3 inches into the bath, then withdrawn and immersed in cold water. The copper cap is detached from the iron rod by a few blows of a hammer, and judgment is formed, from its thickness, colour, and polish, as to the degree of purity which the copper has acquired. These watches need not be drawn till the small rain, above spoken of, has ceased to fall. At the ond of about 11 hours of firing, the numerous small holes observable in the first watch samples begin to disappear; the outer surface passes from a bright red to a darker hue, the inner one becomes of a more uniform colour, and always less and less marked with yellowish spots. It has acquired the greatest pitch of purity that the process can bestow when the watches become of a dark crimson colour.

Care must be taken to stop this refining process at the proper time; for, by unduly prolonging it, a small quantity of cuprous oxide would be formed, which, finding nothing to reduce it, would render the whole body of the copper hard, brittle, and incapable of lamination.

The tuyère being closed, the basins must be emptied of their burning charcoal, and the melted copper allowed to flow into them through the tap-hole, which is then stopped with loam. Whenever the surface is covered with a solid crust, it is sprinkled with water; and as soon as the crust is about $1 \frac{1}{2}$ inch thick it is raised upon hooks above the basin, to drain off any drops, and then carried from the furnace. If these cakes, or rosettes, be suddenly cooled by plunging them immediately into water, they assume a fine red colour, from the formation of a film of oxide.

Each refining operation produces, in about 12 hours, $1 \frac{7}{10}$ ths ton of copper, with the consumption of about $\frac{4}{5}$ ths of a ton of dry wood.

Care should be taken that the copper cake, or rosette, be perfectly solidified before plunging it into water, otherwise a very dangerous explosion might ensue. On the other hand, the cake should not be allowed to cool too long, lest it get oxidised upon the surface, and lose those fine red, purple, and yellow shades, due to a film of the suboxide, which many dealers admire.

When antimony or oxide of copper are combined with copper, they occasion the appearance of micaceous scales in the fractured faces. Such metal is hard, brittle, yellowish within, and can be neither laminated nor wire-drawn. These defects are not owing to arsenic, as was formerly imagined; but, most probably, to antimony in the lead, which is sometimes used in refining copper. They are more easily prevented than remedied.

According to Mr. Frèrejean, proprietor of the great copper works of Vienne, in Dauphiny, too low a temperature, or too much charcoal, gives to the metal a cubical structure, or that of divergent rays; in either of which states it wants tonacity. Too high a temperature, or too rapid a supply of oxygen, gives it a brick-red colour, it radiated crystallisation without lustre, or a very fine grain of indeterminate form; the last structure being unsuitable for copper that is to be worked under the hammer or in the rolling-mill. Tho form which indicates most tenacity is radiated with minute fibres glistening in mass. Melted copper will sometimes pass successively through these three states in the space of ten minutes.

Fig. 532 represents a roasting mound of copper pyrites in the Lower Harz, near

532

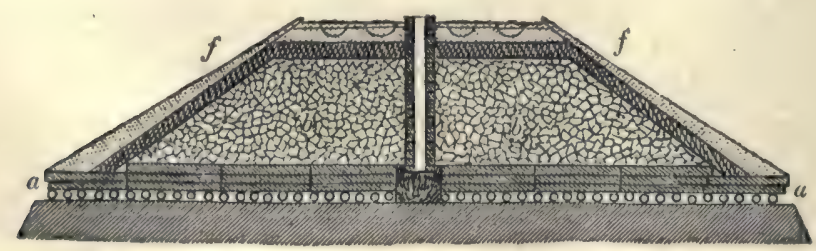

Goslar, where a portion of the sulphur is collected. It is a vertical section of a truneated quadrangular pyramid. $\Lambda$ layer of wooden billets is arranged at the base of the pyramid in the line $a$ a. 
c, a wooden chimuey, which stands in the centre of the mound, with a small pile of charccal at its bottom; $b b$ are large lumps of ore surrounded by smaller pieces; $f^{\prime} f$, are rubbish and earth to form a covering. A curront of air is admitted under the billets by an opening in the middle of each of the four sides of the base, $a$, so that two principal currents of air cross under the vertical axis, c, of the truncated pyrnmid, as indicated in the figure.

The fire is applied, through the chimney, c, to the charcoal at its bottom; the pile, $b \quad b$, is kindled, and the sulphurous ores are raised to such a high temperature as to expel the sulphur in the state of vapour.

In the Lower Harz a roasting mound continues burning during four months. Some days after it is kindled the sulphur begins to exhale, and is condensed by the air at the upper surface of the pyramid. When this seems impregnated with it, small basins are excavated, in which some liquid sulphur collocts; it is removed from time to time with iron ladles, and thrown into water, where it solidifies, It is then refined and cast into roll brimstone.

A similar roasting mound contains, in the Lower Harz, from 100 to 110 tons of ore and 730 cubic feet of wood. It yields in four months about one ton and a half of sulphur from copper pyrites. Lead ore is treated in the same way, but it furnishes less sulphur.

There are usually from 4 to 5 roasting heaps in action at once for each smelting works of the Lower Harz. After the first roasting two heaps are united to form a third, which is calcined anew, under a shed; the ores are then stirred up and roasted for the third time, whence a crude mixture is procured for the smelting-house.

The most favourable seasons for roasting in the open air are spring and autumn; the best weather is a light wind accompanied with gentle rain. When the wind or rain obstruct the operation, this inconvenience is remedied by planks distributed round the upper surface of the truncated pyramid over the sulphur basins.

\section{Wet Methods of Extracting Copper.}

The Process of extracting Copper from Ores, at the Mines in the Rio Tinto District, Province of Huelva, Spain, by what is termed 'Artificial Cementation.'This method, which was first applied here by Don Felipe Prieto, a mine proprietor of Seville, in the year 1845, is the only one employed in the present day in the copper-mines of that district.

The operation begins with the calcination of the ores, proviously reduced to small pieces; piles or heaps of these ores (sometimes in the form of cones) are made on beds of stubble fire-wood of about a yard thick; ench pile is made up with from 400 to 500 tons of mineral, and allowed to burn for six months; the smoke destroying all vegetation within its reach.

The ores, after being thus burnt or calcined, are thrown into wooden troughs let into the ground, about 6 yards long, 4 wide, and $1 \frac{1}{2}$ deep, called 'dissolvers.' In each of these troughs, or cisterns, aro placed about twelve tons of calcined ore, and the trough is then filled with water; which water is, after remaining in contact with the ores for forty-eight hours, drained off into a similar trough placed at a lower level, and called a 'depositor.' The ores remaining in the dissolver are covered by a second quantity of water, left on, this time, for three days; and the process repeated four times successively, the water being always drained off into the same depositor.

From the depositors the water flows on to another set of troughs called 'pilones,' into which is placed a quantity of pig iron, troken inta pieces of about the size of bricks, and piled loosely together so that the vitriol in the water may better act on its whole surface. Each of these troughs (pilones) will hold from 12 to 18 tons of pig iron (wrought iron answers the purpose as well, but it is much more expensive); and, as experience has demonstrated that a slow continuous movement in the water hastens the process, a man is employed for the purpose of agitating it, until all the copper suspended in the vitriol-water is deposited, which, in summer, is effected in about 2 days, and in from 3 to 5 days in winter. After the water has been renewed four or five times, and the agitation process repeated, the scales of copper deposited on the iron, as well as that in the form of coarse grains of sand found in the bottom of the trough, are collected together, washed, and melted, when it is found to produce from 65 to 70 per cent. of pure copper.

From the remains of the first washings of the above copper-scales, \&c., another quality is obtained, worth about 50 per cent. for copper, which is mixed with the after-washings, yielding about 10 per cent. of copper, and passed on to the smelting furnace.

The method is very defective. Minerals containing 5 per cent. of copper, treated by this system of reduction, will scarcely give a produce of 2 per cent. of that metal, 
It is, however, the only method that has hithorto boen profitably omployed in the Rio Tinto district.

The produce of some of the mines of this district is under 1 per cent, About 2,500 tons of the richest of the ores produced by the mines in tho Rio Tinto district, are shipped from Huelva, a port near Seville, for England; and the result has beon that a very large industry has been created. See Prrures.

The Process of extracting Copper from the Water that drains out of the Mine, at Rio Tinto, called the 'System of Natural Cementation' (Precipitation).-The mine worked by the Spanish Government at Rio Tinto is formed in a mass of iron pyrites containing copper; and its immense labyrinth of excavations is known to extend over a length of 500 yards and a width of 100 yards (and prcbably to a much greater extent); the earliest of which workings must date back to rery remote times; for in the different excarations are still to be found the impressions of hands, evidently guided by the science of the ancients, middle ages, and of more modern times.

The sixth, or lowest level in the mine, where all the operations of the present day are carried on, is 80 yards deep (from the top of the hill in which the lode is found), and it is from this level that the mine is (naturally) drained by an adit. From the roof, at the extreme end of a gallery at this level, flows, from an unknown source, a stream of water rich in copper, which, together with the drainage from other points of the mine, is directed through a channel to the adit 'San Roque,' that empties its waters at the foot of the hill, where the copper is extracted.

An able engineer has thus explained the phenomena of 'natural cementation:''The natural rentilation through the open excarations of this mine, combined with the humidity of the ground, produces a natural decomposition of the materials composing the lode or vein, and thereby forming sulphates of iron and copper, which the water is continually dissolving and carrying off, thus forming the substance of this " natural cementation."

This said adit 'San Roque,' which empties its waters on the south sido of the hill, has placed in it two wooden launders, or channels, about 12 inches wide and 15 inches deep, and (in the year 1853) 400 yards long; in the bottom of these launders are placed pieces of pig iron, and to this iron adhere the particles of copper which the slowly-flowing water contained in solution. In ten days the iron becomes coated with copper, so pure as to be worth 80 per cent. for fine-copper, and so strongly formed in scales as to resist to a certain extent the action of a filo, and give a strong metallic sound on being struck with a hammer. At the expiration of ten days, or earlier, the scales of copper so formed on the iron are removed, that the surface of the iron may be again exposed to the action of the mineral water; and the process repeated to the entire extinction of the iron. The copper thus obtained passes at once to the refining furnace.

Since 1853 it has been discovered that the water escaping from the launders in the adit, 400 yards long, still contained copper, and they have been lengthened to nearly 1,000 yards with good effect.

The production of copper by cementation is carried on not only at Rio Tinto, but also at the Tharsis Mines in Spain, and at San Domingos in Portugal.

Linz Copper Process.-At Linz on the Rhine, and some other localities in Germany, the poorer sulphides of copper, containing from 2 to 5 per cent. of that metal, are treated by the following process:-

The ores coming directly from the mine, and without any preliminary dressing, are first roasted in a double-soled furnace, and then taken to a series of tanks sunk in the ground, and lined with basalt. These tanks are also provided with a double bottom, likewise formed of basalt, so arranged as to make a sort of permeable diaphragm, and on this is placed the roasted ore, taking care that the coarser fragments are charged first, whilst the finer particles are laid upon them.

The eavity thus formed between the bottom of the tank and the diaphragm, or false bottom, is connected, by means of proper flues, with a series of oblong retorts, through each of which a current of air is made to pass from a ventilator, or a pair of large bellows, set in motion by steam or water power.

In order to use this apparatus, a quantity of ore is roasted in the reverberatory furnace, and subsequently placed in the tanks, taking care that the first layer shall be in a coarser state of division than those which succeed it.

The retorts--which are formed of fire tiles, and are alvout 6 inches in height by 1 foot in width and 6 feet in length-are now brought to a red heat, charged with blende, and the blast applied.

The sulphurous acid thus formed is forced by the draught through the flues, where it becomes mixed with nitrous fumes, obtained from a mixture of nitrate of soda and sulphuric acid, and ultimately passes into the chambers benoath the diaphragms on which are laid the roasted ores, which must be previously damped by the addition of a 
little water, of which a small quantity is also placed in the bottoms of the tanks. The sulphuric acid thus generated attacks the oxide of copper formed during the preliminary roasting, giving rise to the production of sulphate of copper, which percolates through the basaltic diaphragm into the reservoir beneath.

The liquors which thus accumulate are from time to time distributed over the surface of the ore, and the operation repeated until the greater portion of the copper has been extracted, when, by shifting the damper, the gases are conducted into another tank similarly arranged. The liquors from the first basin are now pumped into the second, and the operation continued until the ores which it contains have ceased to be acted on by the acid. When sufficiently saturated, the liquors are drawn off into convenient troughs, and the copper precipitated by means of scrap iron. The sulphate of iron thus formed is subsequently crystallised out, and packed into casks for sale.

On removing the attacked ores from the tank, the finer or upper portions are thrown away as entirely exhausted, nearly the whole of the copper having been removed from them, whilst the coarser fragments are crushed and re-roasted, and finally form the upper stratum in a subsequent operation.

It has been found that, by operating in this way, ores yielding only 1 per cent, of copper may bo treated with considerable advantage, since the sulphate of iron produced, and the increased ralue of the roasted blende, are alone sufficient to cover the expenses of the operation.

By this process, 3 cwts. of coal are said to be required to roast one ton of ore, whilst the same quantity of blende is roasted by an expenditure of 4 cwts. of fuel.

Treatment of Copper Ores by Hydrochloric Acid.-At a short distance from the village of Twiste, in the Waldeck, several considerable bands of sandstone, more or less impregnated with green carbonate of copper, have been long known to exist. Although varying considerably in its produce, this ore, on the average, yields 2 per cent. of copper, and was formerly raised and smelted in large quantities; but this method of treatment not having apparently produced satisfactory results, the operations were ultimately abandoned.

The insoluble nature of the granular quartzitic gangne with which the copper is associated, suggested, some years since, to Mr. Rhodius, of the Linz Metallurgic Works, the possibility of treating these ores by means of hydrochloric acid, and a large establishment for this purpose was ultimately the result. The following is a description of this process, as witnessed by Mr. J. A. Phillips, in 1856 :-

These works consist of a crushing mill, for the reduction of the cupreous sandstone to a small size, 16 dissolving tubs, and a considerable number of tanks and reserroirs for the reception of the copper-liquors and the precipitation of the metal by means of scrap-iron. Each of the 16 dissolving tubs is 13 feet in diameter, and 4 feet in depth, and furnished with al arge woodon revolving agitator, set in motion by a run of overhead shafting in connection with a powerful water-wheel. This arrangement admits of the daily treatment of 20 tons of ore, and the consequent production of from 7 to 8 cwts. of copper. Each operation is completed in 24 hours, the liquor being removed from the tanks to the precipitating trough by the aid of wooden pumps. The ore is stoped and brought into the works at 48 . per ton.

The acid employed at Twiste is obtained from the alkali works in the neighbourhood of Frankfort, contains 16 per cent. of real acid, and costs, delivered at the works, 2s. per $100 \mathrm{lbs}$. Each ton of sandstone treated requires $400 \mathrm{lbs}$. of acid, which is diluted with water down to 10 per cent. before being added to the ore. Every ton of copper precipitated requires $1 \frac{1}{4}$ ton of scrap iron, at $4 l .58$. per ton.

These works yielded in 1857,120 tons of metallic copper, and afforded a net profit of nearly 50 per cent. The residues from the washing vats, run off after the operation, contained but $\frac{1}{10}$ th per cent. of copper.

The works at Twiste were ultimately abandoned on account of a falling off in the yield of the ores.

Humid Process of extmating Copper from Burnt Cupreous Pyrites.-Very large quantities of cupreous pyrites, amounting in the aggregate to about 400,000 tons per annum, aro imported into this country from Spain and Portugal. The sulphur is utilised in the manufacture of sulphuric acid, and from the buxnt residue the large quantity of 1,200 tons of copper is annually extracted.

The burnt pyrites contains on the average $2 \frac{1}{3}$ to 3 pex cent. of sulphur, and 3 to $3 \frac{1}{2}$ per cent. of copper. The extraction of the latter is effected as follows:-The ore is ground between rolls or under edge runners, and a sufficient quantity of unburnt sulphur added to it to raise the per-centage of sulphur to an amount slightly in excess of the copper, at the same time a sufficient quantity of salt, to convert the copper into' chloride, is introduced. This mixture passes through a sieve of about 36 holes to the square inch, and is then ready for ronsting, which is commonly offected in reverbera- 
tory furnaces having flues beneath the led through which the flame passes before coming into contact with the ore. The furnaces are sometimes supplied with independent fireplaces, but more frequently a series of them is worked by gas generated in Siemens's producers. A form of furnace is also employed, in some cases, in which the oro is placed in a sort of muffle, betweon which and the outer walls of the furnaces the flames are allowed to play; in this arrangement the flame and products of combustion do not come into contact with the ore.

The mixture is charged into the furnaces through hoppers in the arch, and spread evenly over the bed to a depth of 4 to 5 inches, a charge usually consisting of about 3 tons; this is stirred and rabbled from time to time in order to expose fresh surfaces to oxidation, the temperature not being allowed to exceed a dull redness. Hydrochloric acid is evolved during the roasting, and is conveyed to a condenser, from which a weak acid solution is obtained, which is subsequently employed in the lixiriation of the roasted ore.

After the expiration of about 6 to $6 \frac{1}{2}$ hours the whole of the copper in the ore should have been converted into a soluble chloride: a sample is taken from each furnace and washed twe or three times with hot water and a little dilute hydrochloric acid, the residue is then treatod with nitric acid, and the solution obtained rendered alkaline by the addition of ammonia ; should more than a trace of blue colour be discernible in the solution, the sulphide of copper in the mixture has not been decomposed, and the roasting must be prolonged until a satisfactory test can bo obtained. The charge is then drawn out on to the floor of the furnace-house and allowed to cool considerably, after which it is transferred in iron tram-waggons to wooden lixiviating vats capable of holding about 16 tons, and provided with false bottoms, over which a layer of cinders is spread to serve as a filter.

The hot roasted ore is then treated with hot water, and finally with a little hydrochloric acid; the stronger solutions are ready for precipitation by means of metallic iron, whilst the weaker are run into a reservoir, whence they are pumped back and serve as washing-water to a fresh lot of furnaced ore, and so in turn become strong solutions.

In many works the silver present in these solutions is, previously to the precipitation of the copper, extracted by Claudet's process, which consists in mixing with them a quantity of a soluble iodide, usually that of potassium, sufficient to combine with the silver present, which amounts to from 3 to 4 grains per gallon. The insoluble iodide of silver is allowed to subside, and the copper solution drawn off from it into the precipitating vats.

When a considerable quantity of iodide of silver has been collected, it is well washed to free it from copper, and whilst suspended in water, metallic zine in thin fragments is added, and the whole kept boiling by a jet of steam. The iodide of silver is thus decomposed, and metallic silver and solution of iodide of zine produced; the latter serves to precipitate a fresh quantity of silver, whilst the former after being dried is ready for the smelter.

The copper present in the strong solutions is precipitated in large wooden vats, by immersing in them scrap wrought iron; if the solution be kept boiling by a steam jet, the precipitation is completed in about 12 hours. The spent liquid, which is valueless, is drawn off by a siphon from the precipitated copper, and the latter freed from iron by washing through perforated plates. After being allowed to drain, the precipitate is dried on a bed of fire-tiles heated by flues passing beneath, and is then ready for smelting; it contains, on the average, 70 to 75 per cent. of fine-metal.

The material from which the copper has been washed should contain about $\frac{1}{10}$ th per cent. of that metal, and when good pyrites have been used, consists almost entirely of sesquioxide of iron: it is largely employed under the name of 'Purple ore' or 'Blue Billy' for the fettling of puddling furnaces. See Pxrites.

Alderley Edge in Cheshire.-The hydrochloric acid process is, at the present time, carried on to a considerable extent at Alderley Edge in Cheshire, where the ore is in many respects similar to that at Twiste, with the exception that in the last-mentioned locality, mechanical agitation is dispensed with, and the acid is pumped from beneath the false bottom of one tank to the top of the next, in order that the whole solvent power of the acid may be utilised. The hydrochloric acid process was originally introduced at Alderley Edge by Mr. W. Henderson, who protected his method by a provisional Specification, dated September 30, 1857. Another specification was filed by Mr. Henderson in 1859, which included the extraction of copper from pyrites by converting the copper into chloride by calcination with common salt-a process similar to that originally suggested by Mr. Longmaid, and now extensively applied to the treatment of burnt pyrites. The sandstone of the curious elevation abore the plain of Cheshire, known as Alderley Elge, is impregnated with copper existing in the state of blue and green carbonates. The bods containing this copper lie at a short distance below the surface, and are therefore easily worked. 
The quantities of ore raised and copper obtained are given as follow in the 'Mineral Etatistics' for the last few years :-

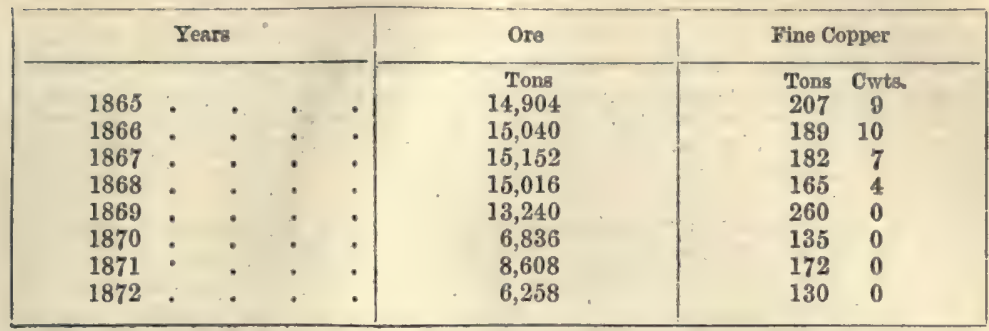

Mona and Pary's Mines, Anglesea.-The waters draining from the onormous heaps accumulated from the old and remarkable workings of these mines are collected in large ponds and precipitated by iron. In 1872, the Mona Mine produced 2,400 tons of copper are, but there was no precipitate; but Pary's Mine produced 155 tons of precipitate and 2,813 tons of copper ore.

Wicklow Mines. - Similar precipitating processes were carried on in these mines. The process is thus described by Mr. W. W. Smyth, in his 'Mines of Wicklow and Wexford':-

From a letter of the Rev. William Henry, D.D., inserted in the 'Philosophical Transactions' for 1752, we learn that the existence of copper in solution in the water flowing from Ballymurtagh has only lately been discovered by accident, but had given rise to extensive apparatus where 500 tons of iron were at the same time employed to effect the precipitation of the costlier metal. The mode of operating was very rude; pits were dug, 10 feet long, 4 feet wide, and 8 feet deep, floored with flags and lined with stone, and the iron bars were laid on rough wooden beams fixed across from wall to wall. Dr. Henry's statement of the everlasting efficiency of the water, shows that, by him at least, the rationale of its action was little understood. 'Chains of these pits are continued along the stream, as far as the directors please, for the water never abates of its quality, if it were conveyed from pit to pit through a thousand.'

By this mode a ton of the precipitant obtained from the pits yielded 16 cwts. of the finest copper. Dr. Rutty states, that in seven years previous to 1765 the Cronebane water had yielded $17,260 l$., from precipitate-copper; the precipitate affording abovo half of pure metal.

Towards the close of last century, copper was obtained by the same means at Cronebane and Tigroney. According to the 'Journal des Mines,' vol. jii., it was usual to add to the strength of the solution, by placing in the water a quantity of poor pyritous ore which has undergone a process of roasting. The form of the pits is not described, but the ratio of pure copper to the precipitated powder was only 0.328 .

The quantity of precipitate or cementation-copper exported to England was, in 1788, $11 \frac{1}{2}$ tons; in 1789,37 tons; in $1790,59 \frac{3}{4}$ tons.

During Mr. Weaver's management, the water was run into tanks in which the muddy particles were allowed to subside, and then passed into pits filled with plate and scrap iron; the quantity precipitated during that series of years was $442 \frac{1}{2}$ tons, which sold on an average at $27 l$. $8 s$. $9 d$. per ton, being in aggregate value $12,126 l$.; whilst the consumption of iron was rather less than one ton to the ton of the precipitate.

At the present date the water is economised at all the Oroca Mines, but by a somewhat different method it is led through a series of narrow troughs or launders, inclined at angles of $10^{\circ}$ or $12^{\circ}$, and interrupted at intervals by a deop chest or 'hutch.' At Tigroney the precipitate is swept down with brooms every night and morning into the hutches, the contents of which are afterwards mixed, and realise 50 to 60 per cent. of copper. The expenses of obtaining it are about $3 l$. per ton for attendance, sifting, \&ce. At Connarie people are kept sweeping down the launders throughout the day, by which means the precipitate, although more rapidly collected, is more impure, yielding but 43 to 54 per cant. of copper.

M. Bischoff junr. has patented a process for obtaining copper by precipitation which certainly effects the separation of the copper with great readiness, and, as he assures us, with great economy. His process differs from any other in the circumstance of his obtaining the iron in a peculiar condition. He takes the ordinary iron pyrites (Mundic), and reduces this to a stato of coarse powder. This is then mixod with coke and exposed to heat in close furnaces. The sulphur is volatilised, and the incan- 
descont coke absorbing all the oxygen, the iron is prevented from oxidising, and it remains in a motallic state combined with some carbon. When this procoss is completed, the powder is drawn off into close vessels and kept until required for use, carefully guarded from the air. If this preparation is thrown into a solution of sulphato of copper, there is a violent action, and the copper in a metallic state is at once thrown down. In practice, M. Bischoff prefers spreading out this preparation in thin layors, and passing the copper water through it. The process has been in use at some of the works on the banks of the Dee, and, as we understand, considerable success has attended its use. The only difficulty appears to be in preserving the finolydirided metallic iron from oxidation, which is essentially necessary to the success of the process.

Mr. S. Higgs junr. has greatly accelerated tho process of precipitation by forcing steam through the solution of copper in which pieces of iron have been placed. This process has been successfully applied by him at Huel Margery copper-mine, St. Ives, Cornwall.

Aluoxs of Coppre.-Copper forms the basis of a greater number of important alloys than any other motal. With zinc it forms brass in all its varieties. See Brass.

Bronze and bell-metal are alloys of copper and tin. This compound is prepared in crucibles when only small quantities are required; but in reverberatory hearths, when statues, bells, or cannons are to be cast. The metals must be protected as much as possible during their combiuation from contact of air by a layer of pounded charcoal, otherwise two evils would result; waste of the copper by combustion, and a rapid oxidation of the tin, so as to change the proportions and alter the properties of tho alloy. The fused materials ought to be well mixed by stirring, to give uniformity to the compound. See Brit-Mrtal; Browzis.

By an unalysis of $M$. Berthier, the bells of the pendules, or ornamental clocks, made in Paris, are found to be composed of copper $72 \cdot 00$, tin 26.56 , iron 1.44 per cent.

An alloy of 100 of copper and 14 of tin is said by M. Dussaussy to furnish tools, which, hardened and sharpened in the mannor of the ancients, afford an edge nearly equal to that of steel (?).

Cymbals, gongs, and the tam-tams of the Chinese are made of an alloy of 100 of copper with about 25 of tin. To give this compound the sonorous property in the highest degree, it must be subjected to sudden refrigeration. M. D'A rcet, to whom this discovery is due, recommends to ignite the piece after it is cast, and to plunge it immediately into cold water. The sudilen cooling gives the particles of the alloy such a disposition that, with a regulated pressure by skilful hammering, they may bo mado to slide over each other, and remain permanently in their new position. When by this means the instrument has received its intended form, it is to be heated and allowed to cool slowly in the air. The particles now take a different arrangement from what they would have done by sudden refrigeration; for, instead of being ductile, they possess such an elasticity, that, on being displaced by a slight compression, they return to their primary position after a series of extremely rapid vibrations; whence a very powerful sound is emitted. Bronze, bell-metal, and probably all other alloys of tin with copper, present the same peculiarities.

Sume valuable researches on alloys of copper and tin have recently been made by M. Alfred Riche (Annales de Chimie et de Physique, xxx. [4], 1873, p. 351). It has been asserted that the Chinese work their bronze at a red heat, but M. Riche has succeeded in making tam-tams by manipulating his bronze at much lower temperatures.

The bronze-founder should study to obtain a rapid fusion, in order to avoid the causes of waste indicated above. Reverberatory furnaces have been long adopted for this operation; and among these, the elliptical are the best. The furnaces with spheroidal domes are used by the bell-founders, because, their alloy being more fusible, a more moderate heat is required; however, as the rapidity of the process is always a matter of consequence, they also would find advantage in employing the elliptical hearths. Coal is now universally preferred for fuel.

The process of coating copper with tin exemplifies the strong affinity between the two metals. The copper surfice to be tinned is first cleaned with a smooth sandstono; it is then heated and rubbed over with a little sal-ammoniac, till it be perfectly clean and bright; the tin, along with some pounded resin, is now placed ou the copper, which is made so hot as to melt the tin, and allow of its being spread over the surfice with a dossil or pad of tow. The layer thus fixed on the copper is exceedingly thin. Bayen found that a copper pan, 9 inches in diameter and $3 \frac{1}{4}$ inches deep, being weighed immediately before and after tinning, became only 21 grains heavier. Now as the area tinned, including the bottom, amounted to 155 square inches, 1 grain of tin had been spread over nearly $7 \frac{1}{2}$ square inches; or obly 20 grains over ercry sq. foot.

Copper and Arsenic form a white-coloured alloy, sometimes used for the scales of 
thermometers and barometers, for dials, candlesticks, \&c. To form this compound, successive layers of copper clippings and white arsenic are put into an earthen crucible; which is then covered with sea-salt, closed with a lid, and gradually heated to redness. If 2 parts of arsenic have been used with 5 of copper, the resulting compound commonly contrins onc-tenth of its weight of metallic arsenic. It is white, slightly ductile, donser, and more fusiblo than copper, and without action on oxygen at ordinary temperatures; but, at higher heats, it is decomposed with the exhalation of arsenious acid. The white copper of the Chinese consists of 40.4 copper; 31.6 nickel ; 25.4 zinc; and 2.6 iron. This alloy is nearly silver white; it is very sonorous, well polished, malleable at common temperatures, and even at a cherry red, but very brittle at a red-white heat. When heated with contact of air, it oxidises, burning with a white flame. Its specific gravity is $\mathbf{8 . 4 3 2}$. When worked with great care, it may be reduced to thin leaves and wires as small as a needlo. See Germar Silver.

Tutenag, formerly confounded with white copper, is a different composition from the above. Keir says it is composed of copper, zinc, and iron; and Dick describes it as a short metal, of a greyish colour, and scarcely sonorous. The Chinese export it, in large quantities, to India.

M. Pélouze states that an alloy of equal parts of copper and nickel is greatly preferable to an alloy which contains also zinc. Even 2 of copper and 1 of nickel form a valuable alloy. See Prrites (Iros).

Copper, Statistics of.-During a term of about 30 years, the copper-mines have sold their ores at the public sales. The following Table, from ' Records of Mining and Metallurgy,' by Messrs. Phillips and Darlington, represents the progress of copper mining from 1726 to 1855 :-

Copper Ore raised and sold in Cormwall and Devon in Decenvial Periods for 126 Years, from 1725 to 1855.

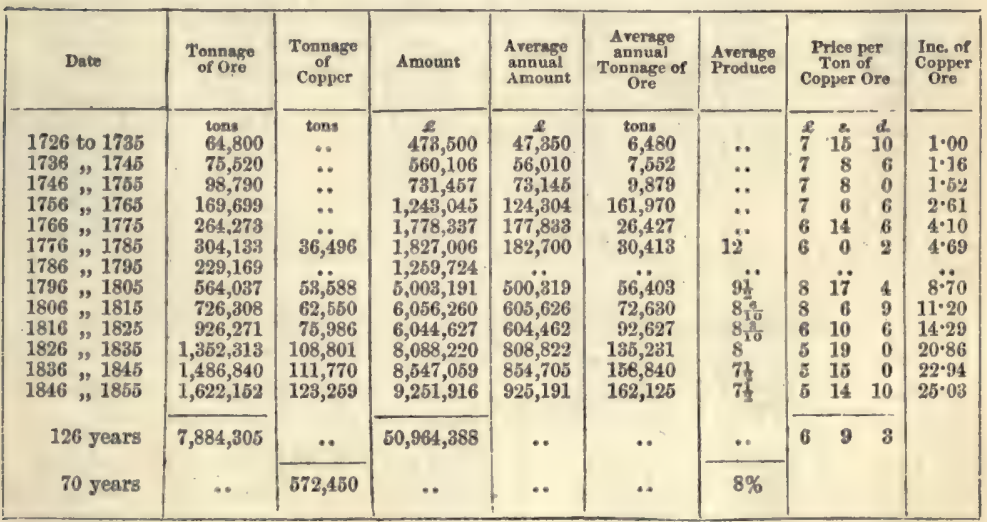

From 1855 to 1866 the following have been the quantities of copper produced in Cornwall and Deyonshire:-

\begin{tabular}{|c|c|c|c|c|c|c|c|}
\hline Date & $\begin{array}{l}\text { Copper } \\
\text { Ore }\end{array}$ & $\begin{array}{c}\text { Value of } \\
\text { Ore }\end{array}$ & $\begin{array}{l}\text { Fine Copper } \\
\text { produced }\end{array}$ & $\begin{array}{l}\text { Mean AT } \\
\text { Price of C }\end{array}$ & $\begin{array}{l}\text { verage } \\
\text { Copper }\end{array}$ & $\begin{array}{l}\text { Average } \\
\text { Standard }\end{array}$ & $\begin{array}{l}\text { Average } \\
\text { per cent. } \\
\text { produce }\end{array}$ \\
\hline 18506 & $\begin{array}{c}\text { tons } \\
206,177\end{array}$ & $\stackrel{\text { f }}{1,241,835}$ & $\begin{array}{c}\text { tons } \\
13,583\end{array}$ & $\begin{array}{c}6 \\
123\end{array}$ & $s_{0}$ & $\begin{array}{cc}2 & 8 \\
103 & 13\end{array}$ & $6 \frac{1}{2} \frac{1}{10}$ \\
\hline 1857 & 191,798 & $1,201,270$ & 12,179 & 124 & 0 & 1407 & $6 \frac{3}{8}$ \\
\hline 1858 & 182,391 & $1,057,534$ & 11,831 & 108 & 2 & 13114 & $6 \frac{1}{2}$ \\
\hline 1859 & 181,848 & $1,096,207$ & 11,827 & 110 & 7 & 1386 & $6 \frac{1}{2}$ \\
\hline 1860 & 180,883 & $1,065,376$ & 11,797 & 107 & 13 & $\begin{array}{ll}133 & 18\end{array}$ & $6 \frac{1}{2}$ \\
\hline 1861 & 180,778 & $1,004,915$ & 11,486 & 102 & 12 & 1301 & $6 \frac{3}{8}$ \\
\hline 1862 & 183,313 & 928,213 & 11,562 & 100 & 12 & $127 \quad 13$ & $6 \frac{1}{16}$ \\
\hline 1863 & 169,971 & 832,152 & 10,896 & 98 & 17 & 1209 & $6 \frac{3}{8}$ \\
\hline 1864 & 165,611 & 854,116 & 10,373 & 101 & 8 & $124 \quad 17$ & $6 \frac{8}{8}$ \\
\hline 1865 & 159,409 & 753,427 & 9,850 & 94 & 7 & 1253 & $6 ?$ \\
\hline
\end{tabular}


Summary of Produce of Copper Ore and Copper for ench of the Ten Iears ending 18:2, with the respective Values and the Number of Mines worked.

Constwat.r.

\begin{tabular}{|c|c|c|c|c|c|}
\hline Year & $\begin{array}{l}\text { No. of } \\
\text { Mines }\end{array}$ & Oopper Ore & Value & Copper & Value \\
\hline 1863 & 166 & $\begin{array}{c}\text { tons } \\
129,229\end{array}$ & $\begin{array}{c}\mathscr{2} \\
642,944\end{array}$ & $\begin{array}{c}\text { tons } \\
8,411\end{array}$ & 831,417 \\
\hline 1864 & 173 & 127,633 & 659,919 & 7,963 & 807,532 \\
\hline 1865 & 148 & 121,253 & 572,618 & 7,413 & 699,432 \\
\hline 1866 & 130 & 103,670 & 431,083 & 6,551 & 600,726 \\
\hline 1867 & 109 & 88,603 & 413,533 & 5,990 & 493,875 \\
\hline 1868 & 100 & 86,722 & 373,005 & 5,725 。 & 450,986 \\
\hline 1869 & 84 & 71,790 & 316,364 & 5,144 & 398,698 \\
\hline 1870 & 77 & 56,526 & 242,227 & 4,147 & 331,337 \\
\hline 1871 & 79 & 46,766 & 205,025 & 3,340 & 258,850 \\
\hline 1872 & 63 & 41,414 & 225,402 & 2,926 & 306,757 \\
\hline
\end{tabular}

DefosshiRe.

\begin{tabular}{|c|c|c|c|c|c|}
\hline Year & $\begin{array}{l}\text { No. of } \\
\text { Mines }\end{array}$ & Copper Oro & Value & Copper & Value \\
\hline 1863 & 24 & $\begin{array}{l}\text { tons } \\
40,742\end{array}$ & $\stackrel{\mathscr{L}}{189}$ & $\begin{array}{c}\text { tons } \\
2,485\end{array}$ & $\stackrel{2}{24.701}$ \\
\hline 1864 & 19 & 37,978 & 194,197 & 2,309 & 234,162 \\
\hline 1865 & 21 & 38,156 & 184,776 & 2,337 & 223,497 \\
\hline 1866 & 21 & 34,471 & 151,481 & 2,248 & 206,141 \\
\hline 1867 & 23 & 31,163 & 143,898 & 2,036 & 167,867 \\
\hline 1868 & 20 & 30,540 & 128,748 & 1,941 & 152,900 \\
\hline 1869 & 18 & 22,723 & 86,056 & 1,394 & 108,040 \\
\hline 1870 & 15 & 24,752 & 84,096 & 1,458 & 105,970 \\
\hline 1871 & 14 & 24,352 & 79,409 & 1,342 & 104,005 \\
\hline 1872 & 12 & 23,972 & 89,841 & 1,203 & 124,641 \\
\hline
\end{tabular}

Summary for the United Kingdom during the last Ten Years.

\begin{tabular}{|c|c|c|c|c|c|}
\hline Yenr & $\begin{array}{l}\text { No. of } \\
\text { Mines }\end{array}$ & Copper Ore & Value & Copper & Value \\
\hline & & tons & $\ell$ & tons & \\
\hline 1863 & 222 & 210,947 & $1,100,554$ & 14,247 & $1,409,608$ \\
\hline 1864 & 201 & 214,604 & $-1,155,471$ & 13,302 & $1,350,699$ \\
\hline 1865 & 203 & 198,298 & 927,938 & 11,888 & $1,134,664$ \\
\hline 1866 & 173 & 180,378 & 759,118 & 11,153 & $1,019,168$ \\
\hline 1867 & 164 & 158,544 & 699,693 & 10,233 & 831,761 \\
\hline 1868 & 152 & 157,335 & 642,103 & 9,817 & 761,602 \\
\hline 1869 & 136 & 129,953 & 519,912 & 8,291 & 654,060 \\
\hline 1870 & 124 & 106,698 & 437,851 & 7,175 & 551,309 \\
\hline 1871 & 122 & 97,129 & 387,118 & 6,280 & 475,143 \\
\hline 1872 & 117 & 91,893 & 443,738 & 5,703 & 583,232 \\
\hline
\end{tabular}

Copprir Assaying.-The substances which are submitted to assay for copper are various ores of copper, slags, and other metallurgical products, commercial varieties of copper and its alloys. The assays are made by the dry and wet methods.

Dry Assay. 1. English or Cornish method.-This process is very extensively employed in England; all the copper ores being bought and sold by this method. The mode of working by this method varies somewhat as to the manipulation, nature, and proportion of fluxes, form of apparatus, and other minute details; but it may be regarded as consisting essentially of the four following processes :-

1. Fusion for regulus, or sulphide of copper and iron.

2. Roasting or Calcination of the regulus into oxides of copper and iron. 


\section{COPPER ASSAYING}

3. Reduction or Fusion for coarse-copper; the oxide of copper being reduced to metal, and the oxide of iron retained in the slag.

4. Refining of the coarse-copper.

All the ores of copper, even rich carbonates, are submitted to the whole of the abore processes by some assayers; part of the processes are dispensed with by others, and the rich oxidised ores fused direct for coarse-copper, regulus and calcination.

Furnace and Implements. A wind-furnace or air-furnace - Fig. 533 represents a vertical section of a furnace suitable for assaying copper ores: $a$, the fire-place, lined with firebrick, 9 inches square by 12 inches deep; $b$, the ash-pit; $c$, flue or stack; $d$, damper, $e$, firebrick covers of two sizes, each clamped with a piece of flat bar-iron; $f$, register or iron ashpit door, provided with a revolving disc of sheet-iron, about 5 inches diameter, having a semicircular opening; $g$, the fire-bars of castor wrought-iron. The draught is regulated by means of the damper, the register, or by partly removing the fire-brick cover. The fuel used is coke.

Cornish Crucibles of three sizes, (see Assax). Copper scoop, fig. 535, 12 inches in length, 4 inches in width at the widest part, and $1 \frac{1}{4}$ inch at the mouth, provided with a handle about $7 \frac{1}{2}$ inches in length, for projecting the fluxes into the crucible. Iron mould, fig. 534, having two hemispherical cavities, each $1 \frac{5}{8}$ inch in width and $\frac{7}{8}$ ths of an inch in depth. Calcining rod, fig. 537,28 inches in length, inclusive of the blade 4 inches in length. Crucible tongs, fig. 536, represents those used for general assay purposes, those employed by copper assayers are of a different shape and larger dimensions. Flux spoon or ladle, sieves, pestle and mortar,

533

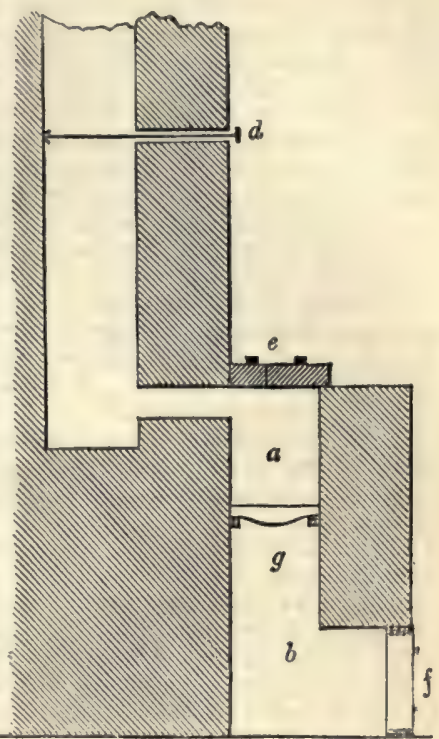
anvil, hammer, chisel, forceps. Balance; it should carry 500 grains, and turn with from $\frac{1}{20}$ th to $\frac{1}{50}$ th of a grain. Weights; grain weights are convenient, but special weights are used by copper assayers: the heaviest is

534

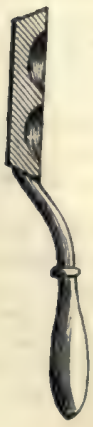

535

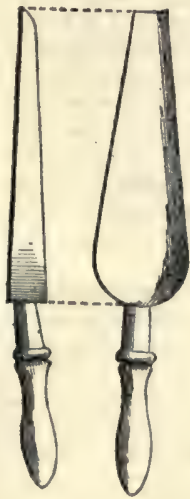

536

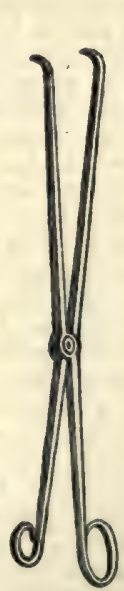

557

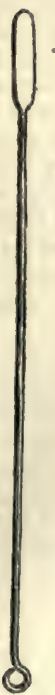

marked 100 , and weighs 400 grains, the unit being subdivided into $\frac{1}{2}, \frac{1}{4}, \frac{1}{8}$, and $\frac{1}{16}$.

Fluxes. Those omployed are, borcts caleined; glass, green or white, freo from oxide 
of lead; tartar or argol; lime, dry ; Aur-spar, free from copper or lead; nitre ; charcoal. powder; carbonate of soda, dried; salt; sulphur; iron pyrites, free from coppor, or sulphide of iron. Refining flux; the product obtainod by doflagrating a mixture of from 2 to $2 t$ parts by measure of tartar, 3 of nitre, and 1 of salt.-(Soo Assax). The fluxers aro usually measured, but the better plan is to woigh them, espocially until experience is gained.

For purposes of assay by this method, the ores, \&c. may be conveniently divided into: 1. Ores contrining over 30 per cent. of copper : $a$, oxides, carbonater, silicates, \&c., which require fusion for coarse-copper and refining; $b$, various sulphides comparatively pure or containing arsenic or antimony, which require calcining 'sweet,' fusion for' coarse-copper and refining; 2. ores containing less than about 30 per cent. of copper, which roquire tho fusion for regulus; $a$, yellow copper ores, with or without iron pyrites, blende, galona, \&c., which require partial calcination or warming, or the addition of nitre, or both, to remove the excess of sulphur; $b$, grey copper ores, poor oxides, carbonates, silicates, and poor antimonial and arsenical copper ores, which have been rossted sweet, which require the addition of sulphur or of sulphur and iron or iron pyrites to yield a proper regulus; $c$, purple copper ore, mixed oxides and sulphides, which yield a proper regulus by direct fusion.

To assist in forming an opinion as to the character of the ore, nature of the associated roin-stuff, and approximate per-centage of copper, a portion of tho samplo may be vanned or washed, calcined, or tested by the mouth blowpipe, or by a preliminary fusion for regulus. This will enable the assayer to determine boforehand how the ore is to be treated.

For the formation of a proper regulus, containing about 50 per cent of copper, the following data may serve as a guide:-

100 grains of copper pyrites will require sbout 75 grains of nitre to yield a good regulus.

100 grains of iron pyrites will require about 180 grains of nitre to oxide it, and cause it to pass into the slag.

100 grains of grey copper ore or disulphide of copper will require about 60 grains of sulphide of iron, about 82 grains of iron pyrites, or about 55 grains of hrmatite and excess of sulphur to yield a proper regulus.

The quantity of ore operated on will vary from 100 to 400 grains according to amount of copper present.

Process : Fusion for regulus, - The largest size Cornish crucible is employed. I'he raw or calcined ore is mixed with the appropriate fluxes; about from 150 to 200 grains each of lime, glass, borax, and fluor-spar, will form a suitable mixture for many ores. The nitre, or iron pyrites or sulphur, is added according to circumstances. The crucible and its contents are submitted to a high temperature for about 15 minutes, and when tranquil the fused products are poured out into the iron mould, fig. 534. Whon the slag has set, the whole is dipped into water, removed, and when cold the regulus afterwards carefully detached from the slag. A good regulus is reddish-brown in colour, very tender, and easily reduced to powder; it should contain about 50 per cent. of copper, but may vary from 40 to 60 per cent. If the regulus is too coarse it resombles iron pyrites or copper pyrites in appearance, and contains too much iron and sulphur, and the ore requires more calcining, or the addition of more nitre during fusion. If the regulus is too fine, it is black in colour, hard, and the external surface is very smooth and bright: it indicates that the ore requires more sulphur or iron pyrites, or a substitute for them in the fusion. The slag should be uniform in colour, but it may be white, green, or black, according to the nature of the ore. It should be free from all trace of red colour, which would indicate the presence of copper. The slag should be examined for any shots of regulus.

Roasting or calcination. - The smallest or intermediate-sized crucible is used according to the weight of the ore or regulus. A dull fire is required to commence with. The finely-powdered substance is transferred to the crucible; this is placed on the top of the fuel, mouth frontwards, and in an inclined position to facilitate oxidation. The substance is constantly stirred with the calcining rod, fig. 537, especially at the first part of the operation, and the heat is afterwards increased to a bright red. The calcination should be completed in about 30 minutcs. If any sign of clotting or softening of the particles occur, the crucible must be romoved, and, if necessary, the product repowdered, mixed with some anthracite-powder or coke-dust, and recalcined. Calcinations may also be made in scorifiers or roasting dishes in a muffe furnace.

Fusion for course-copper.--This is effected in the crucible used for calcination. The calcined ore or regulus is mixed with about from 50 to 150 grains of carbonate of soda, from 50 to 150 grains of tartar, and from 20 to 30 grains of borax, and exposed to a high temperature for about 15 minutes. The temperature should be sufficient to reduce the oxide of copper and melt the metal, and leave the oxide of iron in the slag. The 
products are poured out into the ingot mould, quenched in water, and when coid the slag detached from the button of coarse or impure copper. The character of this will vary according to the impuritios present. If any regulus is attached to the upper surface it indicates that the previous calcination has been imperfect, and the assay of the ore must be repeated. The slag should be black, and free from all trace of red colour. If the fusion has been properly made, the slag will not require to be cleaned or to be re-fused with reducing agents; although it is customary for some assayers to clean all such slags. When raw or calcined ores are fused for course-copper the addition of more carbonate of soda and borax are generally necessary. A little lime or oxide of iron may also be added with ores comparatively free from vein-stuff, to keep the slag free from oxide of copper : nitre and tartar are also used for reduction, instead of the carbonate of soda and tartar.

Refining. - This may be conducted in the crucible which has been used for the previous process. If $\mathrm{a}$ new one is employed a little borax should be previously fused in it. A hot fire is necessary. The crucible is fixed in the fuel so that the process can be easily observed through the space of the fire-brick covers, and when hot the copper button is thrown in. When the surface of the metal appears bright and clean about from 100 to 150 grains of the refining flux are quickly projected from the copper scoop, fig. 535, upon it, and the furnace closed. After the lapse of about 2 minutes the crucible is removed and the contents poured out into the mould. The button should be flat and have a pit or depression on the upper surface. The fracture is generally dull, granular, and of a red colour, or finely fibrous or silky, and of a salmon-red colour. The slag varies somewhat in colour; it should be grey, flesh-colour, pink, or pale red. It is powdered, re-fused with tartar or other reducing agents for about 10 minutes, the product poured out, and carefully examined for any shots of metal it may contain. With some assayers it is customary to submit impure copper buttons to one or more washings previous to refining. In washing, the metal and refining fluxes are projected together into the crucible and the product poured out when fused.

The following is another Method of conducting the Cornish Process.-A portion of the pounded and sifted ore is first burnt on a shovel, and examined as to its supposed richness and the amount of sulphur, arsenic, and other impurities it may contain. A little practice in this operation will enable the operator to judge with considerable accuracy of the quantity of nitre necessary in order to obtain a good regulus.

Fusion for regulus. - Two hundred grains of the mixed ore are now weighed out and carefully mixed with a flux consisting of nitre, borax, lime, and fluor-spar, and the fusion for matt or regulus is begun. The quantity of nitre used will of course vary with the amount of sulphur and arsenic present ; but the other ingredients are commonly employed in the following proportions:-Borax, 5 dwts.; lime, $1 \frac{1}{2}$ ladleful; fluor-spar, 1 ladleful." After being placed in the crucible, the whole is generally covered by a thin stratum of common salt. After remaining in the fire for about a quarter of an hour, the fusion will be found complete, and the contents of the pot may be poured into a suitable iron mould. The button of regulus is now examined, in order to determine whether a suitable proportion of nitre has been used. If the right quantity has been employed, the button, when broken, should present a granular fracture, and yield from ' 8 to 10 for 20 ' of copper, i.e. from 40 to 50 per cent. However rank a sample may be, it should never be mixed with above 9 or $9 \frac{1}{2}$ dwts. of nitre; and if the amount of sulphur be small, 3 dwts. are often sufficient. The grey sulphides, the red and black oxides, and carbondtes, have sulphur added to them for the purpose of obtaining a regulus. Highly-sulphurised samples, requiring above $9 \frac{1}{2}$ drits. of nitre, are sometimes treated in a different way. In this case the ores are first carefully roasted, and afterwards fused with about 5 dwts. of nitre, 9 dwts. of tartar, and 3 dwts. of borax.

Roasting.-The roasting of the regulus thus obtained is performed in a smaller. crucible than that used in the fusion for matt. During the first quarter of an hour, a very low temperature is sufficient. The heat is then increased to full redness, and the assay allowed to remain on the fire for a further period of about 20 minutes. During the first 15 minutes it should be kept constantly stirred with a slender iron rod; but afterwards, an occasional stirring will be found sufficient. When nearly the whole of the sulphur and arsenic has been expelled, the temperature must be raised nearly to whiteness during a few minutes, and the assay thon withdrawn and allowed to cool.

Fusion for coarse-copper. -The fusion for copper is effected in the same crucible in which the roasting has been carried on. The quantity of flux to be used for this purpose varies in accordance with the weight of the button of regulus obtained. A mixture of 2 dwts. of nitre, $7 \frac{1}{2}$ dwts. of tartar, and $1 \frac{1}{2} \mathrm{dwt}$. of borax, is sufficient for

- The ladle used for this purpose is three-quarters of an inch in diameter and hals-an-inch in depth. 
the roduction of a calcined regulus that, previous to roasting, weighed from 45 to 50 grains. In the case of a button weighing from 90 to 100 grains, $3 \frac{1}{2}$ dwts. of nitre, 9 dwts. of tartar, and 2 dwts. of borax, should be employed. These quantities are, howover, seldom weighed, since a little practice renders it easy to guess, with a sufficient degree of accuracy, the necessary amounts.

Refining. - The prill of copper thus obtained is seldom fine, and consequently requires purification. A crucible is heated to redness in the furnace, the metallic button is taken from the mould and thrown into it, and some refining flux and salt are placed in a scoop for immediate use. ${ }^{1}$ In a few minutes the fusion of the prill is effected. The crucible is now taken from the fire by a pair of tongs, the contents of the scoop introduced, and a gentle agitation given to it ; an appearance similar to the brightening of silver on the cupel now takes place, and the crucible is returned to the fire for about four minutes. The crucible is now removed, and its contents rapidly poured into a mould. The button thus obtained will consist of refined copper, and present a slight depression on its upper surface. The slags from the reducing and refining operations are subsequently fused with a couple of spoonfuls of crude tartar, and the prill thus obtained weighed with the larger button.

2. German Method.-This mothod is used in Germany and other parts of Europe, for the estimation of copper in ores and other substances. It consists essentially of the following processos:- 1. Roasting or calcination; 2. Reduction or fusion for black copper; 3. Refining.

Furnace, \&c.-A muffle furnace is employed. The form and dimensions of a muffle furnace vary somewhat; the construction depending on the nature of the fuel employed and other circumstances. If bituminous coal is used, the muffle is heated by the flame of the fuel, and the fire-grate is placed at some distance below. When anthracite coal or charcoal is employed the muffle is surrounded with hot fuel. Scorifiers or roasting dishes; flat shallow vessels made of fire-clay about $2 \frac{1}{2}$ inches diameter and 1 inch deep inside measure. Crucibles; thin oval shaped vessels of fire-clay, provided with a flat foot about $4 \frac{1}{2}$ inches high and $1 \frac{3}{4}$ inch intornal diameter at the widest part. Tongs, scoop, pestle and mortar, and other implements are required. For a description of a muffle furnace, see SiLvkR.

Weights. - Special assay weights are used in Germany. 1. Assay centner $=100$ assay pounds; 1 assay pound $=100$ assay part pounds. The assay centner weighs 3.75 grammes, or about 58 grains. 50 grains of the substance may be taken to operate on when grain weights are used.

Fluxes, $\& c$ - Black flux, the product obtained by deflagrating 1 part of nitre and $2 \frac{1}{2}$ of tartar, or a mixture of alkaline carbonate and charcoal, or substitute for them borax, glass, salt, graphite, charcoal-powder, lead, arsenic, antimony, iron pyrites. For the purpose of assay by this method the ores and other substances may be divided into: 1 , Oxidized ores, \&c. which require reduction and refining; 2. Sulphurous ores, \&ce. which require calcination, reduction, and refining; 3 . Ores, \&cc. poor in copper, which require fusion with iron pyrites to obtain a regulus: the regulus is afterwards treated according to $2 ; 4$. Varieties of impure copper, which require refining with lead.

Process: Roasting. - 50 grains of the ore mixed with about $\frac{1}{4}$ of its weight of graphite powder or other substitute and spread over the bottom of a scorifier or roasting dish previously coated inside with red oxide of iron. The scorifier is now placed in a muffle at a low red heat, and the calcination continued until the graphite ceases to glow and the odour of sulphurous and other gases is no longer perceptible. This is then removed, and the product powdered, remixed with about 5 per cent. or more of charcoalpowder, and recalcined. This operation is repeated a third time if necessary. The calcination is completed in about 36 minutes. Carbonate of ammonia is sometimes added to complete the calcination.

Fusion for Black Copper. - The powdered calcined product is transferred to the ovalshaped crucible, and covered with about twice its weight of black flux, and borax or glass or both added as required. The whole is covered with a layer of salt, and over this is placed a piece of charcoal about $\frac{1}{2}$ an inch square ; a cover is then inserted. Lead, arsenic, or antimony or their oxides are also added when necessary as collecting agents to alloy with the copper and ronder it more fusible. The fusion is conducted in a muffle; the temperature is gradually increased from a rod heat and the operation completed in from 30 to 40 minutes. The crucible is removed, and when cold, broken open. The button of copper should be perfectly fused and free from regulus. The slag should be well fused, glassy, and free from a red colour.

Refining. - This is conducted in a muffle upon a flat shallow piecn of broken crucible about from 1 to $1 \frac{1}{2}$ inch square. This is placed between hot charcoal, as, a high tem-

The refining flux consists of two parts of aitre and one of white tartar fused together, and sub. sequently pounded. 
porature is roquired. The button is then wrapped in paper with about an equal weight of borax, and placed upon the hot clay vessel. When the button is melted, the door of the mufflo is partly opened to allow the air to enter, and the operations continued until the copper brightens, appears of a green colour, and finally becomes solid. The clay ressel is then removed from the muffle, and cooled by dipping it slowly in water. The slag is then detached from the button, which should have the characteristic properties of fine copper. The refining is also conducted on a cupel. Two cupels are treated in a muffle, and upon one is placed the button of black copper with an equal weight or more of lead, and upon the other the same weight of pure copper and lead. When meltod, air is admitted by partly removing the muffle-door, and the process continued until the copper brightens. The buttons are then covered with charcoal-powder, remored, and cooled in water, and weighed. The loss upon the pure copper is added on to the assay button, and the result calculated.

Wet Assay: 1. By Cyanide of Potassium.-This is the best adapted and most direct method for estimating copper in copper ores by means of standard solutions. It was first proposed by Mr. H. Parkes. The process depends on the following reaction: when a solution of cyanide of potassium is added to a blue ammoniacal solution of copper it becomes colourless by the formation of a double cyanide of ammonia and copper. By ascertaining by direct experiment the amount of cyanide of potassium required to dischargo the colour in an ammoniacal solution containing a given weight of copper, it is easy by a comparatire experiment to determine the amount of copper in a given weight of ore. The standard solution of cyanide of potassium required, is prepared by dissolving about from 2,000 to 2,500 grains of cyanide of potassium (gold cyanide) in 4 pints ( 35,000 grains) of water. One thousand grains of this solution should be equal to about 10 grains of copper. The solution is standardised as follows:-A piece of clean, electrotype copper, of from 4 to 7 grains, is accurately weighed, dissolved in a small quantity of dilute nitric acid in a small flask, boiled to expel nitrous fumes, and diluted with water to about half a pint. Ammonia is added in excess, when it will becomo blue. A burette (for a description of this instrument, seo AlKaLIMETRT) is filled to the top line with the solution of cyanide of potassium, and run in very slowly to the cold blue solution of copper until it becomes nearly colourless, or of a faint lilac tint. The number of divisions which are read off will be equal to the amount of copper operated on. The experiment is repeated on a second or third piece of copper, and the mean result taken as standard. The decolorisation will require from $\frac{1}{2}$ to $\frac{3}{4}$ of nn hour, according to the amount of copper present. A piece of white paper should be placod under and behind the flask, to aid in observing the tint of the solution during decolorisation. As the standard solution undergoes slow decomposition, it will require correction about once a week.

Process.-From 10 to 50 grains of the ore are placed in a small flask or beaker glass, and moistoned with strong sulphuric acid; nitric acid is then added from time to time, and the whole digested at a gentle heat until nitrous acid fumes cease to be given off. Water is cautiously added, and the solution reheated, to dissolve the basic sulphates of copper and iron. When the ore is completely decomposed, the whole is transferred into a pint flask and diluted to about $\frac{1}{2}$ a pint; ammonia is added in excess, and when the solution is cold, the standard solution is gradually run in until there remains only a faint lilac tint. The number of divisions used are read off, and the per-centage of copper calculated. Example:-1,000 grains of the burette are equal to 10 grains of copper, and 20 grains of the coppor ore require 500 grains of solution; therefore,

$$
\begin{aligned}
& 1,000: 500:: 10: 5 \text { and } \\
& 5 \times 5=25 \text { per cent, of copper. }
\end{aligned}
$$

When peroxide of iron is present it should not be filtered off during the assay, as it would retain copper. The copper thus retained passes into solution during decolorisation. The alteration in the tint of the peroxide of iron will also afford a rough indication of the completion of the assay. The addition of sulphuric acid is only requirod for the decomposition when sulphides are present in the ore. The presence of silver, zine, nickel, and cobalt, interfere with the estimation of copper by this method. Silver is ensily removed by adding hydrochloric acid or chloride of sodium, and filtering before adding the ammonia. When zinc, nickel, and cobalt are present, the copper is separated with the usual precautions by means of sulphuretted hydrogen, hyposulphite of soda, or metallic iron, and the precipitates afterwards redissolved in acids, and the solution assayed as before described.

2. Precipitation Method.-This is one of the oldest wet methods of estimating copper, and when the requisite care is taken the results are accurato. It is also usoful when burettes are not available. In some localities, and particularly in South America and the United States of America, the assay of copper ores is performed by this pro.

Vox. I. 
cess. The whole of the ores belonging to the different classes may be estimatod in this way.

A weighed quantity of the pulverised ore is introduced into a long-necked flask of hard German glass. Nitric acid is now added, and tho flask exposed to the hent of a sand-bath. A little hydrochloric acid is subsequently introduced, and the attack continued until the residue, if any remains, appears to be free from all metallic stains. The contents of the flask are transferred to a porcelain evaporating dish, and evaporated to dryness, taking care, by means of frequent stirring, to prevent the mass from epirting. The whole must now be removed from the sand-bath and allowed to cool, a littlo hydrochloric acid subsequently added, and afterwards, some distilled wator. The eontents of the basin must then be made to boil, and, whilst still hot, filtered into a beaker. A piece of bright wrought iron, about two inches in length, three-quarters of an inch in width, and a quarter of an inch in thickness, is now introduced, and tho liquor gently heated on the sand-bath until the whole of the copper has been thrown down. The liquor is now removed by means of a glass siphon, and the metallic copper freed from all adhering chlorides, by means of repeated washings with hot water, and then dried in a water-bath, and weighed.

In case the mineral operated on should contain tin or antimony, very minute traces only of these metals will be found with the precipitated copper. When lead is present, it is best to add a few drops of sulphuric acid during the process of the attack; by this means the lead will be precipitated as sulphate of load, and be removed by filtration.

\section{Assay of Commercial Varieties of Copper, Brass, Bronze, \&c.}

By Hyposulphite of Soda. This is a very accurate method of estimating copper: it was first made known by Mr. E. O. Brown. The reagents required are, 1. A standard solution of hyposulphite of soda. This is made by dissolving 1,300 grains of the erystals in 4 pints of water; the solution is standardised by means of weighed pieces of electrotype copper by the process afterwards described, and the mean result takon as standard. 2. Iodide of potassium. The crystals should be free from iodate. About six times the weight of the copper present is required for each assay. 3. Solution of Starch. Prepared by boiling from 1 to 2 grains of starch in about half a pint of water. When cold the clear solution is decanted off for use. The estimation of the copper by this method depends on the following reactions. When iodide of potassium is added to acetate of protoxide of copper, subiodide of copper is formed, and freo iodine is set free, which dissolves in the excess of iodide of potassium. By means of a standard solution of hyposulphite of soda, the free iodine is converted into iodide of sodium with the formation of tetrathionate of soda. The solution of starch serves to indicate the completion of the reaction, by the bleaching of the blue iodide of starch at first formed. The reactions may be expressed by the following equations :-

$$
\begin{aligned}
& 2(\mathrm{CuO}, \overline{\mathrm{A}})+2 \mathrm{KI}=\mathrm{Cu}^{2} \mathrm{I}+\mathrm{I}+2(\mathrm{KO}, \mathrm{A}) \\
& \mathrm{I}+2\left(\mathrm{NaO}, \mathrm{S}^{2} \mathrm{O}^{2}\right)=\mathrm{NaI}+\mathrm{NaO}, \mathrm{S}^{4} \mathrm{O}^{3} .
\end{aligned}
$$

Process. - From 8 to 10 grains of the copper or alloy are dissolved in dilute nitric acid, and the nitrous acid expellod. The solution diluted with watcr, and carbonate of soda added until carbonate of protoxide of copper remains precipitated. Acotic acid is added in excess, and afterwards about 60 grains or less of iodido of potassium. The solution of hyposulphite of soda is now run in from a burette, until the solution acquires a yellow colour. A little of the starch-solution is now addled, and the addition of the hyposulphite of soda cautiously continued until the solution becomes colourless. The number of divisions on the buretto are read off, and the amount of copper calculated therefrom. Example:-If 1,000 grains of the standard solution are equal to 10 grains of copper, and 10 grains of the alloy require 600 grains of the same solution, then

$$
\begin{aligned}
1,000: 600:: 10: 6 \text { and } \\
6 \times 10=80 \text { per cent. of copper. }
\end{aligned}
$$

When lead is present, a little sulphurie acid should be added during the solution of the metal in nitrie acid, to convert it into insoluble sulphate of protoxide of lead. To render it applicable to copper ores, after the solution of the ore and dilution, the copper must first be separated by means of sulphuretted hydrogen, hyposulphite of soda, or by iron or zinc, and the precipitate redissolved in nitric acid, taking care to expel all trace of nitrous acid before proceeding with the assay.

\section{Assay of Slags and other Substances containing Small Quantities of Copper.}

Coloration Test.--This mothod depends on the fact that the intensity of the blue colour of an ammoniacal solution of copper is proportionate to the quantity of metal present. There are two plans which may bo followod: $-a$. A standard coloration 
test solution is proparod by dissolving a known woight of copper ( 0.5 or 1 grain) in dilute nitric acid, adding oxcess of ammonia and diluting to 10,000 grains. This solution is kept in a woll-stoppered, long, glass graduated rossel. Tho ammoniacal solution of copper obtained by the decomposition of a given weight of the substance is transferrod to a graduated measure of the same shape and capacity, and water added until tho tint corresponds to that of the standard coloration test. The percontage of copper is calculated as follows:-10,000 grains of the standard coloration test contain 0.5 grain of copper; the solution of 100 grains of the substance required dilution to 8,000 grains-

\section{$10,000: 8,000:: 0.5: 0.4$ per cent. of copper.}

b. A series of standard coloration test solutions of copper are prepared and kept in a number of colourless square or round glass bottles. The bottles should be of the same shape and capacity. If the series comprise ten bottles, the first bottle may contain $\frac{1}{10}$ th of a grain of copper, the second $\frac{2}{10}$ ths, and so on to the last of 1 grain. From 50 to 100 grains of the powdered slag or other substance are treated with nitric or with nitro-hydrochloric acid, until decomposition is effected. In some cases previous fusion with carbonate of soda may be found necessary. The solution is diluted with water, excess of ammonia added, and filtered, or the precipitate allowed to settle and the solution decanted. If necessary, the precipitate obtained by ammonia is redissolved in acid, and reprecipitated by ammonia ; and if the filtrate has a blue tint it nust be added to that proviously obtained. The whole of the solution is then diluted with water until there is the same volume of solution as in one of the test bottles. The colour is then compared with the coloration test bottles, and the one it corresponds to represents the amount of copper present in the quantity of substance opcrated on. From this datum the per-centage of copper is calculated. If nickel or cobalt bo present the copper must first be separated by means of sulphuretted hydrogen or otherwise, and the precipitate redissolved in acid.

COPPER Orzs. See Copprr.

COPP rr, OXIDrs or. Two oxides are known : the red or suboxide (cuprous oxicle) $\mathrm{Cu}^{2} \mathrm{O}$, and the black or protoxide (cupric oxide), CuO. Both occur native, and are valuable ores. The suboxide may be prepared artificially by adding grape-sugar to a solution of the blue sulphate of copper, treating the product with an excess of caustic potash, and then boiling the solution. The suboxido communicates a fine ruby-rod colour to glass; this oxide is soluble to some extent in metallic copper, the metal becoming thereby 'dry' or brittle. Protoxide of copper may be prepared by calcining copper, or by igniting the nitrate or tho sulphate. It communicates a green colour to glass. The protoxide is employed in organic analysis for yielding oxygen during combustions.

CopprR PхRтт5. Seo Copper.

COPPIR, SAITS OF. Copper, ACETATE of. A basic acotate of copper (cupric ncetate) is largely used as a pigment under the name of Verdigris. The neutral ncetate is also prepared commercially, and termed Distilled Verdigris. It appoars that the well-known pigment, Schweinfurth or Emerald Green, is a combination of acetato and arsenite of copper. See Verdigris; Schwernfurtu Green.

Copprr, Arsenitu of. The fine green pigment called Scheele's Green is an arsenite, and Schweinfurth Green is an aceto-arsenito of copper. See ScherLe's Graren; SchweINeUR'TH Green.

Copper, Carbonates of. Two of these occur native, forming the minerals known ns Malachite and Azurite. An artificial basic carbonate is prepared as a pigment under the names of Brunswick and mineral-green. See Azurite; CoPper; Matachitw; VARDITER.

COPPER, Chlormes of. Two chlorides are known; the subchloride (cuprous chloride) $\mathrm{Cu}^{2} \mathrm{Cl}\left(\mathbf{C u}^{2} \mathbf{C l}^{2}\right)$, and the protochloride (cupric chloride) $\mathrm{CuCl}\left(\mathbf{C u C l}^{2}\right)$. The latter is readily oltained by dissolving either oxido or carbonate of copper in hydrochloric acid; it is a deliquescont salt soluble in alcohol, the alcoholic solution burning with a fine green flame. The subchloride may he reduced from the protochloride by boiling its solution with sugar. A solution of the subchloride in hydrochloric acid is used in gas-analysis for the absorption of carbonic oxide. There are also sereral oxychlorides of copper; one of these occurs native, forming an ore known as Atacamito. See Coppre.

Copper, Nitratr of. This salt is prepared by dissolving copper in moderately strong nitric acid, and evaporating to crystallisation. Its formula when anhydrous is $\mathrm{CuO}, \mathrm{NO}^{s}\left(\mathbf{C u 2 r r o} \mathrm{O}^{3}\right)$. This salt is used to produce a fine green in fireworks.

Copprer, Selpirate of, called in commerce Blue Vitriol, Blue Stone, or Blue Copperas.-This salt is frequently preparod by roasting copper pyrites with freo access of air, but sulphato of iron is formed at the samo time, and the two salts are not roadily separated. It is also oltained by heating old coppor with sulphur, by which $~$ 
subsulphide of copper is producod; this is converted into sulphate. by roasting in contact with air. Iarge quantities of sulphato of copper are obtaned in the process of silver refining, and as a by-product in Ziervogel's method of silver-extraction. Crystallised sulphate of copper contrins $\mathrm{CuO} \cdot \mathrm{SO}^{3}+\mathbf{5 H O}\left(\mathbf{C u s O}^{4}+\mathbf{5 H}^{2} \mathbf{O}\right)$. It is largely employed in calico-printing, and as a source of other salts of copper. The salt is also used in certain forms of constant batteries, as Daniell's.

Several basic sulphates are known, some of which occur native. Casselman's green is an artificial basic sulphate. Seo Copprr; Pxrites; Suvrer.

Copper, Sulpuriss of. At loast two distinet sulphides of copper may be prepared. Copper and sulphur when melted together combine with rivid incandescence, and produce the subsulphide or disulphide (cuprous sulphide), $\mathrm{Cu}^{2} \mathrm{~S}$. This compound also occurs native as copper glance; and is produced as a regulus in the ordinary process of copper-smelting, when it is called by the smelter fine metal. The higher sulphide (cupric sulphide), $\mathrm{CuS}$, is found native as Covelline, and may be artificially prepared, as a hydrate, by precipitating $\pi$ salt of copper with sulphuretted hydrogen. The sulphides are decomposed when heated with free access of air; at high temperatures sulphurous acid is evolved, and oxide of copper formed, whilst at low temperatures a sulphate of copper is produced.

copprisas. A common name for green vitriol or sulphate of protoxide of iron (ferrous sulphate). Iron pyrites is also sometimes called copperas. See Prritrs.

CoprozrTms. ( $66 \pi \rho \circ$, dung; $\lambda i \theta 0 s$, stone). A term properly restricted to fossilized fecal matter. True coprolites usually present the form of ovoid nodules, resembling kidney potatoes, marked on the surface with corrugations, and exhibiting internally a spiral structure due to the form of the intestinal canal through which they have passed. Some of these coprolites were formerly described, from their shape, as fir-cones; but their true origin was clearly pointed out many years ago by Dr. Buckland. They often contain scales, teeth, and bones, of fish, which represent the undigested portions of food voided in the excrements; and they are occasionally found in such connection with the skeletons of fossil saurians as to show their original position in the intestines. The coprolites of reptiles and fish are found in many of our Secondary and Tertiary rocks, and are especially abundant in the Lias. These true coprolites consist mainly of phosphate and carbonate of lime. The analyses below by Mr. Thornton J. Herapath represent the composition of a coprolite from the lias strata of Lyme Regis. It was rather large, being above $9 \mathrm{oz}$. in weight, was of a greyish colour, and when broken exhibited some traces of a crystalline structure. It furnished a greyish-white powder. Many scales of different extinct fishes, and other organic remains, were to be perceived on the external surface; the greater proportion of them appeared to belong to a species of fish which is known by the name of Pholidophorus limbatus. Its specific gravity was about $2 \cdot 644$ or $2 \cdot 700$, and the composition per cent. was as follows:-

\begin{tabular}{|c|c|c|c|}
\hline & I & II & Mean \\
\hline $\begin{array}{l}\text { Water driven off at from } 300^{\circ} \text { to } \\
350^{\circ} \mathrm{F} \text {. }\end{array}$ & $2 \cdot 560$ & $2 \cdot 668$ & $2 \cdot 6140$ \\
\hline Water and orgenic mattors expelled & & & \\
\hline $\begin{array}{l}\text { at a red heat } \\
\text { Chloride of sodium, with some sulphate }\end{array}$ & $3 \cdot 680$ & $3 \cdot 456$ & 3.5680 \\
\hline of soda $. \quad . \quad . \quad$. & traces & traces & traces \\
\hline Carbonate of lime $\quad \therefore \quad$. & 23.640 & $23 \cdot 708$ & 23.6740 \\
\hline $\begin{array}{l}\text { do. of magnesia } \\
\text { Sulphate of lime. }\end{array}$ & none & $\begin{array}{c}\text { none } \\
1.801\end{array}$ & ${ }_{1 \cdot 770 \tilde{n}^{\text {none }}}$ \\
\hline Phosphate of do. (tribasic) : & $60 \cdot 726$ & $60 \cdot 813$ & $60 \cdot 7695=\mathrm{PO}^{5} 28 \cdot 047$ \\
\hline do. magnesia & a little & a little & a little \\
\hline Perphosphate of iron . & $3 \cdot 980$ & $4 \cdot 135$ & $4.0575=\mathrm{PO}^{3} \quad 1.992$ \\
\hline Phosphate of alumina . & a little & a little & a little \\
\hline Peroxide of iron . & $2 \cdot 094$ & 1.894 & $1 \cdot 9940$ \\
\hline 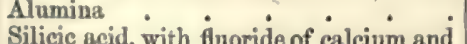 & none & none & none \\
\hline loss . . . . . . & 15580 & 1.525 & 1.5525 \\
\hline & $100 \cdot 000$ & $100 \cdot 000$ & $100 \cdot 0000=\mathrm{PO}^{5} 29 \cdot 969^{*}$ \\
\hline
\end{tabular}

* In the first of these analyses, the phosphoric acid was estimated by M. Schulze's method, as perphosphate of iron ; in the second, as phosphate of lead. 
From the high per-centage of phosphate of lime recorded in these analyses, it must be evident that coprolites are of grent value to the ngriculturist as fertilising agents. Indeed, large quantities of so-called 'coprolites' are thus employed. It should, however, be distinctly understood that the term has been considerably extended in its meaning, and is now applied to all phosphatic nodules used in the formation of artificial manures, whatever may have been their origin. These nodules, or pseudo-coprolites, are especially abundant in the Red Crag of Suffolk. This Crag is a marine deposit of Plioceno (Upper Tertiary) age, rich in shells and other organic remains; and at the base of the Crag there is a bed of rolled bones, teeth, and various animal exuviæ, derived from the London Clay, and associated with rolled pebbles rich in phosphate of lime. These pebbles or nodules were first described by the late Prof. Henslow as true coprolites, but it is certain, from their physical characters, that they have not a coprolitic origin. Nevertheless, these pseudo-coprolites are equally rich in phosphate of lime, as will be seon by the following analyses of two samples from the coast of Suffolk by Mr. Herapath :-

\begin{tabular}{|c|c|c|}
\hline & I & II \\
\hline $\begin{array}{l}\text { Water with a little organic matter } \\
\text { Salts soluble in water (chloride of } \\
\text { sodium and sulphate of soda) }\end{array}$ & $4: 00$ & $3 \cdot 560$ \\
\hline Carbonate of lime & $10 \cdot 280$ & $8 \cdot 959$ \\
\hline Sulphate of lime $\quad: \quad: \quad$ : & $\begin{array}{l}\text { a trace } \\
\text { distinet traces }\end{array}$ & $0 \cdot 611$ \\
\hline Phosphate of lime $\left(3 \mathrm{CaO}, \mathrm{PO}^{5}\right)$. . & $\begin{aligned} 70 \cdot 920= & =\mathrm{PO}^{5} 32 \cdot 765 \\
& \text { traces only }\end{aligned}$ & $69 \cdot 099=\underset{\text { traces }}{\mathrm{PO}^{5} 31 \cdot 924}$ \\
\hline Perphosphate of iron $\left(2 \mathrm{Fe}^{2} \mathrm{O}^{3}, 3 \mathrm{PO}^{5}\right)$ & $6 \cdot 850=\mathrm{PO}^{5} \quad 3 \cdot 244$ & $8 \cdot 616=\mathrm{PO}^{5} \quad 4.081$ \\
\hline Phosphate of alumina $\left(2 \mathrm{Al}^{2} \mathrm{O}^{3}, 3 \mathrm{PO}^{3}\right)$ & $1.550=\mathrm{PO}^{5} \quad 0.870$ & $2 \cdot 026=\mathrm{PO}^{5} \quad 1 \cdot 158$ \\
\hline Oxide of manganere & traces & traces \\
\hline Silicic acid, coloured red by a little & & \\
\hline undecomposed silicato of iron & $5 \cdot 702$ & $6 \cdot 309$ \\
\hline & $100 \cdot 000=\mathrm{PO}^{3} 36 \cdot 889$ & $100 \cdot 000=\mathrm{PO}^{5} \quad 37 \cdot 16$ \\
\hline
\end{tabular}

Phosphatic nodules, passing under the name of coprolites, are found at the junction of the Lower Greensand and Gault, at the junction of the Gault and Upper Greensand, and at the base of the Chalk.

In an excellent paper ' On the Phosphoric Strnta of the Chalk Formations,' published in the first number of Vol. IX. of the 'Journal of the Royal Agrieultural Society of England,' Mr. Way observes, that he has found the coprolites from Farnham in Surrey to contain from 52 to 54 per cent. of bone-earth phosphate; and that Dr. Gilbert had informed him, that in soveral analyses which he had made of samples taken from. several tons of the ground coprolites, he had found the proportion of phosphate of lime to vary between 55 and 57 per cont. Mr. Nesbit ('Quart. Journ. of Chem. Soc.' III. p. 235) found from $22 \cdot 30$ to $28 \cdot 74$ per cent. of phosphoric acid, which is equivalent to from 48.31 to 59.07 of tribasic phosphate, in those from the tertiary deposits of this county.

Phosphatic nodules, or coprolites, are obtained from the base of the Gault at Folkestone, and from the base of the Chalk at Cambridge. "The Cambridgeshire phosphatic nodules,' says the Rev. $\mathrm{O}$. Fisher, 'are extracted by washing from a stratum (seldom exceeding a foot in thickness) lying at the base of the Lower Chalk, and resting immediately, without any passage-bed, upon the Gault. There is, however, a gradual passage upwards from the nodule-bed into the Lower Chalk or clunch. The average yield is about 300 tons per acre; and the nodules are worth about $50 \mathrm{~s}$. a ton. The diggers usually pay about 140l. an acre for the privilege of digging, and return the land at the end of two years properly levelled and re-soiled. They follow the nodules to a depth of about 20 feot; but it scarcely pays to extract them from that depth. - 'Quart. Joum. Geolog. Soc.' Feb. 1873.

The following analyses of Cambridge coprolites are published by Dr. Voelcker in the Journ. of the Roy. Agric. Soc. for 1860:-

\footnotetext{
- Journal Roy. Agric. Soc, Ing. 1851,
} 


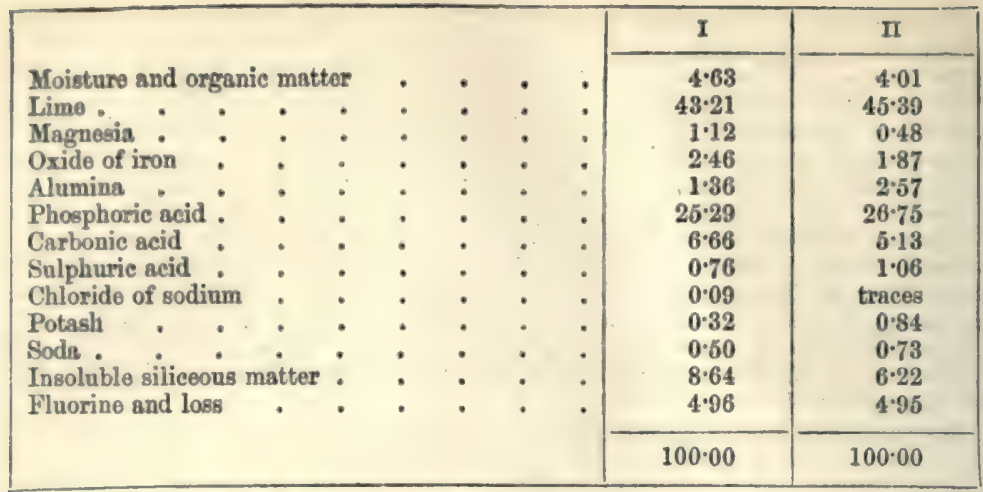

In the first analysis the proportion of tribasic phosphate of lime (bono-earth) reaches 54.89 , and in the second analysis $57 \cdot 12$ per cent.

Mineral deposits rich in phosphate of lime oceur in the Bala Limestone at Cwmgwymnen, about 16 miles from Oswestry; but they do not appear to be of coprolitic origin. They have been worked for agricultural purposes, and were described by Dr. A. Voelcker before the British Association in 1865.

Attention has recently been called to a remarkable phosphatic deposit in South Carolina, which is now extensively worked. This deposit oceurs in beds of post-pliocene age, but is largely composed of nodules of altered marl derived from an Eocene rock known as the great Carolinian Marl Bed. These nodular fragments from the motherrock are rich in organic remains, and are associated with bones and toeth of land animals of more recent age. For a description of the deposit, see 'The Phosphato Rocks of South Carolina,' by Francis S. Holmes, A.M. Charleston, 1870. Seo Apatrte;

\section{Manure.}

CoPyrre. A new and important quality of writing-inks was introduced by the indefatigable James Watt, in 1780 , who in that year took out a patent for copying letters and other written documents by pressure. The modus operandi was to have mixed with the ink some saccharine or gummy matter, which should prevent its entire absorption into the paper, and thus render the writing capable of having a copy taken from it when pressed against a damp sheet of common tissue paper. But although this process was very imperfect, the writing generally being much besmeared by the damping, and the copies, in many cases, only capable of being read with great difficulty, it was not for serenty-seven years after the invention of Watt that any improvements in such inks were attempted. The firm of Underwood and Burt patented a method of taking copies by the action of a chemically-prepared paper, in a chemical ink, by which, not only are far superior copies taken, and the original not at all damaged, but many copies may be taken at one time from a single document. Printed matter may be also copied at the same time, on the same beautiful principle. Wo give the specification of Mr. Underwood:-

- But while the means employed for producing the desired effects may bo varied, I prefer the following for general use :-I damp the paper, parchment, or other material which I desire to copy upon, with a solution of 200 grains of the yellow or neutral chromate of potash dissolved in 1 gallon of distilled water, and either use it immediately, or dry it and subsequently damp it with water as it is required for use. I then prepare the material which I use for producing the characters or marks, and which may be called copying ink, by simply dissolving (in a wator-bath) pure extract of logwood in distilled water; or, for printing, I use a varnish or other similar material soluble in water, and dust, or throw over it powdered extract of logwood. If I desire to take twenty copies from an original, I use about six pounds of the pure extract of logwood to a gallon of distilled water; but a larger number of copies may be taken by dusting or throwing over the original, before the ink has thoroughly dried, a powder composed of five parts of powdered extract of logwood, one part of powdered gum arabic, and one part of powdered tragacanth. When I desire to print from an original, in producing which I have used ink preparod as beforo described, I proceed by damping six shoets of paper, prepared as before described, and having taken off all superfluous moisture with good blotting paper, I place the original upon the upper sheet and press the whole for about half a minute in a copying press; I then romove the original, and in its place put six other shects of the prepared paper in a damp state, and subject 
the whole to pressure for about a quarter of an hour. I then take five other prepared sheots in a damp state, and having laid the original upon them, press them together for about two minutes, then roplace the original by three other proparod and damped shoets, and press the whole together for about a quartor of an hour. Tho extract of logwood so acts upon the neutral chromate of potash that I thus obtain twenty good clear fac-similes of the original matter or design.'

They have also produced an Indian ink on the same principle, which, when used in the preparation of architectural plans, maps, \&c., will give one or more clear copies of even the finest lines. The only point to be observed in the taking of such copies, is that as they are taken to a seale, they must be kept pressed in close contact with the original, till they are perfectly dry, because if not, they will shrink in drying, and the scale will be spoilt.

The most complete information on this subject, and that of inks generally, is to be found in a memoir published in the 'Journal of the Society of Arts' by Mr. J. Underwood.

COQUIIIA NUTs. These nuts are produced in the Brazils by the Attalea funifera. They are suitable for a great rariety of small ornamental works, and are manufactured into the knobs of umbrellas and parasols. The same palm which yields coquilla nuts also furnishes the Piassaba fibre, or monkey-grass.

CORAf. An Indian pattern silk handkerchief.

Corna (Corail, Fr.; Koralle, Ger.) is a calcareous substance, formed a species of marine polype, which constructs in concert immense ramified habitations, consisting of an assemblage of small cells, each the abode of an animal. The coral is, therefore, a real polypary, which resembles a tree stripped of its leaves. It has no roots, but a foot not unlike a hemispherical skull-cap, which applies closely to every point of the surface upon which it stands, and is therefore difficult to detach. It merely serves as a basis or support to the coral, but contributes in no manner to its growth, like the root of an ordinary tree, for detached pieces have often been found at the bottom of the sea in a state of increase and reproduction. From the above base a stem, usually single, proceeds, which seldom surpasses an inch in diameter, and from it a small number of branches ramify in very irregular directions, which are studded over with cells, each containing a polype. These polypes when they extend their arms, feelers, or tentacula, resemble flowers, whence, as well as from the form of the coral, they wero formerly classed among vegetable productions. They are now termed zoophytes by many writers upon Natural History.

The polype which yields the ordinary red coral of commeree is known to naturalists as the Corallium rubrum, and is placed in the family of the Gorgonide, or Sea shrubs, a group of the order Alcyonaria. This red coral, therefore, occupies a different systematic position from that of the common corals which, by the accumulation of their solid parts, form the rocky masses known as coral islands, and which are referred to the order Zoantharia. As much misconception prevails with respect to the true nature of the corallium or hard parts, in these two kinds of polype, it may be useful to point out their essential difference. In the common corals, the solid calcareous structure is formed within the body of the polype, whilst in the red coral it is secreted by the outer surface of the organism, notwithstanding its position as a branched axis supporting the soft parts of the body. Both types of coral belong to the Actinoza: a class of the great division of the Animal Kingdom termed the Ceelenterata. It is the red coral which is described in this article.

The finest red coral is found in the Mediterranean. It is fished for on the coasts of Provence, and constitutes a considerable branch of the trade at Marseilles. The coral is attached to the submarine rocks, as a tree is by the roots; but the branches, instead of growing upwards, shoot downwards towards the bottom of the sea: a conformation farourable to bieaking them off and brining them up. For this kind of fishing, eight men, who are excellent divers, equip a felucea or small boat called commonly a coralline. They carry with them a large wooden cross, with strong, equal, and long arms, each bearing a stout bag-net. They attach a strong rope to the middle of the cross, and let it down horizontally into the sea, having loaded its centre with a weight sufficient to sink it. The diver follows the cross, pushes one arm of it after another into the hollows of the rocks, so as to entangle the coral in the nets; then his comrades in the boat pull up the cross and its accompaniments.

Coral fishing is nearly as dangerous as pearl fishing, on account of the number of sharks which frequent the seas where it is carried on. One would think the divingbell in its now very practicable state might be employed with great advantage for both purposes.

Coral is mostly of a fine red colour, but occasionally it is flesh-coloured, yellow, or white. The red is preferred for making necklaces, crosses, and other female ornaments. It is worked up like precious stones. Seo Lapidary.

Coral beads have over boen fashionable ornaments. Dr, Gilchrist states :-- Coral 
bends aro in high ostimation throughout Hindostan for necklaces and bracelets for women. These beads are manufactured from the red coral fished up in various parts of Asia; thoy are vory costly, especially whon they run to any size; and thoy aro generally sold by their weight of silver.'

Coral heads wero always favourite articles for ornament even in this country; and in the 'Illustmations of Manners and Expences of Antient Times in England,' by Nichols, 1798, we find the following entries from 'the churchwardens' accompts of St. Mary's Hill, London,' containing 'the inventory of John Port, layt the king's servant, as after followeth:

'Item of other old gear found in the house:-

'Item one oz, and t of corall

- Jewels for her body.

- Itom, a pair of coral beds, gaudyed with gaudys of silvor and gilt, $10 \mathrm{oz}$. at $38.4 d$.

(John Port died in 1524.)

2. $\% d$.

$\begin{array}{lll}0 & 2 & 6\end{array}$

$1134^{\prime}$

conaxzIxz. A scarlet dye, prepared by heating a mixture of carbolic, oxalic and sulphuric acids, Seo Carbourc Acm.

Cordacs. (Cordage, Fr.; Tauwerk, Ger.) Cordage may be, and is, made of a great variety of materials. In Europe, however, it is mostly formed of hemp, although now much cordage is made of coir. See Corn.

Profossor Robinson proposed the following rule for determining the strength of cordage:- Square the circumference of a rope in inches ; one fifth of the product will be the number of tons' weight which it will bear : this is, however, uncertain.

condovaxr. A leather made at Cordova in Spain from goat-skin, Leather made from horso-hide is called cordovan in this country.

corpurox. A kind of ribbed cloth. See Fustias.

corr or corve. A basket for carrying coals ; a frame of wood to losd coals on; a sledge to transport minerals on.

CORIATDIR SIPD. The fruit of the Coriandum satioum, used as a seasoning, and also for the preparation of an essential oil.

Cork. (Liege, Fr.; Kork, Ger.) is the bark of the Quercus suber, Linn., a species of oak-tree which grows abundantly in the southern provinces of France, Italy, and Spain. The bark is taken off by making coronal incisions above and below the portions to be removed; vertical incisions are then made from one of these circles to another whereby the bark may be easily detached. It is steeped in water to soften it, in order to be flattened by pressure under heavy stones, and is then dried at a fire, which blackens its surface. The corks are bound up in bales and sent into the market.

There are two sorts of cork, the white and the black; the former grows in France and the latter in Spain. The cakes of the white are usually more beautiful, more smooth, lighter, freer from knots and cracks, of a finer grey, and of a yellowish-grey colour on both sides, and cut more smoothly than the black. When this cork is burnt in close vessels it forms the pigment called Spanish black.

Cork is employed to fabricate not only bottle corks, but small architectural and geognostic models, which are very convenient from their lightuess and solidity.

The cork-cutters divide the boards of cork first into narrow fillets, which they afterwards subdivide into short parallelopipeds, and then round these into the proper conical or cylindrical shape. The bench bofore which they work is a square table, where four workmen are seated, one at every side, the table being furnished with a ledge to prevent the corks from falling over. The cork-cutter's knife has a broad blade, very thin, and fine edged. It is whetted from time to time upon a fine-grained dry whetstone. The workman ought not to draw his knife-edge over the cork, for ho would thus make misses, and might cut himself, but rather the cork over tho knife-edgo. He should seize the knife with his left hand, rest the back of it upon the edge of the table, into one of the notches, made to prevent it from slipping, and merely turn its edge sometimes upright and somotimes to one side. Then holding the squared piece of cork by its two ends, between his finger and his thumb, he presents it in the direction of its length to the edge; the cork is now smoothly cut into a rounded form by being dexterously turned in the hand. He next cuts off the two ends, when the cork is finished and thrown into the proper basket alongside, to be afterwards sorted by women or boys,

Of late years much thicker kinds of cork boards have been imported from Catalonia, from which longer and better corks may be made. In the art of corkcutting the Fronch surpass the English, as any one may convince himself by coraparing the corks of thoir champagne bottles with thoso made in this country.

Cork, on account of its buoyancy in water, is extensively employed for making 
floats to fishermon's nets, and in the construction of life-boats. Its impermeability to water has led to its employmont for inner soles to shoes.

When cork is rasped into powder, and subjectod to chomical solvents, such as alcohol, \&c., it leaves 70 per cent. of an insoluble substance, called suberine. When it is treated with nitric acid, it yields the following remarkable products:- White fibrous matter $0 \cdot 18$, resin 14.72 , oxalio acid 16.00 , suboric acid (peculiar acid of cork) $14 \cdot 2$, in 100 parts.

A patent was obtained some years ago for machine cork-cutting. The cutting of the cork into slips is effeeted by fixing it upon the sliding bed of an engine, and bringing it, by a progressive motion, under the action of a circular knife, by which it is cut into slips of equal widths. The nature or construction of a machine to be used for this purpose may be easily conceived, as it possesses no new mechanical feature, except in its application to cutting cork. The motion communicated to the knife by hand, steam, horse, or other power, moves at the same time the bed also which carries the cork to be cut.

The second part of the invention, viz, that for separating the cork into square pieces, after it has been cut into slips as above, is effected by a moving bed as before, upon which the slips are to be placed and submitted to the action of a cutting lever, which may be regulated to chop the cork into pieces of any given length.

The third part of the invention, viz., that for rounding or finishing the corks, consists of an engine to which is attached a circular knife that turns vertically, and a carriage or frame upon its side that revolves on its axle horizontally.

The carriage or frame contains several pairs of clamps intended respectively to hold a piece of the square cut cork by pressing it at the ends, and carrying it lengthways perpendicularly. The wood of the Anona palustris, growing in the West Indies, is so soft that corks are made of it. It is hence called CorI-Wood.

Corvzo. An ore of quicksilver found in Spain, is so called locally.

COROMANDmI WOOD. The wood of the Diospyros hirsuta.

corozo, corosso. Vegetable Ivory.-The commercial names for the fruit of the Phytelephas macrocarpa, a species of Brazilian palm. It is called the Tagua Nut in South America. It grows on the borders of the river Magdalena in great abundance, and the nuts are largely imported. The natives of the districts where the tree grows have been in the habit from time immemorial of using this vegetable ivory for making buttons, heads of walking-sticks, \&c. It is used largely in this country in the place of ivory, but it does not keep its colour well. See Ivory, Vegretabiz.

comrosive gubirmatr, Chloride, or Protochloride, of Mercury, (Deutochlorure de mercure, Fr.; Aetzendes Quecksilber Sublimat, Ger.), is mado by subliming a mixture of $2 \frac{1}{2}$ parts of sulphate of oxide of morcury, and 1 part of sea-salt, in a stone-ware cucurbit. The sublimate rises in vapour, and encrusts the globular glass capital with a white mass of small prismatic needles. Its specific gravity is $6 \cdot 225$. Its taste is acrid, stypto-metallic, and exceedingly unpleasant. It is soluble in 16 parts of water, at the ordinary temperature, and in less than three times its weight. It dissolves in $2 \frac{1}{4}$ times its weight of cold alcohol. It is a very deadly poison. Raw white-of-egg swallowed in profusion is the best antidote. A solution of corrosive sublimate has been long employed for preserving soft anatomical preparations. By this means the corpse of Colonel Morland was embalmed, in order to be brought from the seat of war to Paris. His features remained unaltered, only his skin was brown, and his body was so hard as to sound like a piece of wood when struck with a hammer.

In the work upon the dry rot, published by Mr. Knowles, Secretary of the Committeo of Inspectors of the Navy, in 1821, corrosive sublimate is enumerated among the chomical substances which have been prescribod for preventing the dry rot in timber; and it is well known that Sir H. Davy had, several years before that date, used and recommended to the Admiralty and Nary Board corrosive sublimate as an anti-dry rot application. It has been since extensively employed by a joint-stock company for the same purpose, under the title of Kyan's Patent.

The preservative liquid known as Goadby's Solution, which is employed for preserving wood and anatomical preparations, is composed as follows:-Bay salt 4 oz., alum 2 oz., corrosive sublimate 2 grains, water 2 pints.

CORRUGATED IrON. See IroN.

Corurdur. This mineral species contains sapphire, corundum stone, and emery. It consists of alumina, more or less coloured by metallic oxides.

The perfectly white crystals of sapphire are pure alumina.

There are two varieties of the perfect corundum : the sarphire so called, and the oriental ruby; of which the latter has a rather less specific gravity, being 3.9 against 3.97. Their form is a slightly acute rhombohedron, which possesses double refraction, and is inferior in hardness only to the diamond. The sapphire occurs also in 6-sided prisms, See EMrERY; RUBY; SAPPIIRK, 


\begin{tabular}{|c|c|c|c|c|}
\hline & \multicolumn{2}{|c|}{$\begin{array}{l}\text { Blue Sapphire, } \\
\text { China }\end{array}$} & $\begin{array}{l}\text { Corundura, } \\
\text { Bengal }\end{array}$ & $\begin{array}{l}\text { Immery, } \\
\text { Naxos }\end{array}$ \\
\hline \multirow{5}{*}{$\begin{array}{l}\text { Alumina : } \\
\text { Lime : } \\
\text { Silica } \\
\text { Oxide of iron } \\
\text { Water : }\end{array}$} & $98 \cdot 5$ & $84 \cdot 0$ & $89 \cdot 5$ & $68 \cdot 53$ \\
\hline & 0.5 & $\cdot 0$ & 0.0 & 0.86 \\
\hline & 0.0 & $\cdot 5$ & 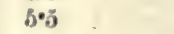 & $3 \cdot 10$ \\
\hline & $1 \cdot 0$ & $7 \cdot 0$ & $1 \cdot 25$ & $24 \cdot 10$ \\
\hline & $\ldots$ & $\ldots$ & ... & $4 \cdot 72$ \\
\hline 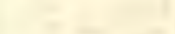 & 100.0 Klapr. & 98.0 Chen. & 98.2 Tennant. & 101.31 J.L.Smith. \\
\hline
\end{tabular}

Corundum, or Corone, is the Indian name for the mineral called also Adamantine spar. The Chinese specimens have a grey colour, but the varioty from India is whiter. The extreme hardness of this substance, scratching everything excopt diamond, renders it romarkably valuable to lapidaries and seal-cutters. It is but littlo softor than the ruby, sapphire, or oriental topaz. It is far superior to emery for grinding, and is used throughout India and China for polishing stones, \&cc. Large deposits of crystalline corundum have recently been discovered in North Carolina, U.S., and have been worked commercially, espocially at Corundum Hill, in Macon Co. The corundum occurs in veins running through sorpentine, and is associatod with ripidolite, jefferesite, \&cc.

costrantrarG, a mining term, from the old Cornish Cothas stean, fallen or dropped tin. It signifies the practice of sinking pits in search of lodes across the line of direction which the tin lodes usually traverse in Cornwall.

COTTON is a vogetable fibre covering the entire surface of the seeds of species of Gossypium-plants which belong to the natural order Malvacea. Cotton is unlike any other textile fibre used in commerce in the fact that it is a hair formed of a single cell. In the vegetable world there is an almost endless series of cell-forms, marked by the two extremes of a perfectly spherical cell, and of a cell so attenuated as to be nearly cylindrical. The latter form is characteristic of cotton. In its early stage, the cotton hair consists of a hollow tube, a transverse section of which would present an elliptical outline; but in the course of growth, partly from the pressure of contiguous hairs, and partly from a loss of moisture, the cylinder collapses and becomes tape-like in form. The collapse is not uniform, however; all down the centre of the hair the sides are parallel, but at each edge a ridge is formed by the folding of the cell-walls. The hair thus comes to have the form of an ordinary railway rail, and its transverse section presents the general appearance of a dumb-bell. The two rounded edges of the hair have, however, a tendency to twist upon themselves, and give to it a certain curly roughness. It is this characteristic of cotton which makes it especially valuable for spinning purposes; the corrugations of the surface helping to bind the fibres together, and to give strength and elasticity to the thread.

Many other vegetable hairs have been tried as substitutes for cotton, such as the ' cotton grass,' (Eriophorum) often seen upon bogs and moors, but they have all failed, because they are not formed of a single hair, but of a linear series of hairs united by their extremities. In all such cases the points of union of the several cells form knots in the fibre which render the hairs liable to entanglement and severance when acted upon by machinery. Other substitutes for cotton, which have resembled it in being uni-cellular, have nevertheless failed from being too short.

The fibre of cotton is the longest uni-cellular hair known in the vegetable kingdom, and its length varies with the species, or according to the locality in which it is grown. It is this variability in the length of the fibre-in commercial language its ' staple'which is the most important factor in determining the price; the longer the staple the more it is worth. The description of cotton which has the longest staple. is known as Sea Island, the average length being $\mathbf{1 . 6 1}$ inch; Egyptian stands next, with a fibre of 1.41 inch; then Peruvian, 1.30 inch; Brazilian, $1 \cdot 17$ inch; New Orleans, 1.02 inch; and finally Indigenous East Indian, 0.89 inch of average length.

Comparatively littlo attention has yot been directed (and that only within recent years), to the improvement of the essential qualities of cotton fibre. And it may be asserted that a wide field lies yet before the cultivator for the adoption of such methods of irrigation, manuring, hybridisation, and goneral treatment as will lead to future developments of these qualities, and to an enlarged production of the plant. The euormous changes which modern agriculturists have brought about by the treatment, on sciontific principles, of ceroals and root crops are probably not more marvellous than those which another genoration may see, with due care and method, in the culture of cotton. Cultivators have got to discover, from careful analysis, what materials they can add to the soil to increase the strength, length, whiteness, and lustre of the hair; 
what modifications they can make in the management of the plant for the purpose of making its growth possiblo in districts hithorto found unsuitable. There was a time when not even the cherry - much less the grape-would scarcely ripen upon the Rhine, a district which the fostering care of man has at length transformed into a fertile wine-producing region. Much progress is also possible in the way of hybridisation and careful selection of seed, by bringing into existence fresh varieties, which shall perpetuate the best characteristics of their progenitors. The practice of hybridisation is a department of cotton-culture which has not met with the attention it deserves. The experiments in this direction of Major Trevor Clurke, whose persevering efforts to improve the native Indian varieties are well known, have shown the great value of this class of research.

The number of species of Gossypium producing the cotton of commerce has been very variously estimated; but Parlatore, the writer of one of the most recent monographs upon this genus (Le Specie dei Cotoni, Firenze, 1866), describes and figures seven good species. So great, however, is the multitude of forms at present in cultivation, that authoritios are by no means agreed as to which of these forms are to be reckoned as specific. But the opinion of competent botanists is gaining ground that all these forms have descended from the three following species:-Gossypium arboreum, the true cotton of the Indian peninsula, and of the island of Celebes; $G$. barbadense, the Barbadoes or Bourbon cotton, from which has probably originated the celebrated Sea Island cotton; and $G$. herbaceum, or the common cotton of the East Indies, China, and the Malay Archipelago.

\section{Coxtox Dy risc. See Drang.}

Corrox rACTORX, or CoTrow mrus. These terms are omployed indis. criminately for the purpose of describing any building or set of buildings, in which the processes of spinning or of weaving cotton by the aid of machinery are carried on. Establishments of this kind are often of great magnitude, giving employment to large numbers of work-people, and producing many varieties of fabric. But whether it be large or small, whether its productions be of one kind or of many kinds, a cotton factory needs to be arranged in a certain order, and its successive processes must be suitably organised. The hands and heads of those who labour must be specially trained and habituated to their several parts, and the whole operation conducted with the greatest attainable regularity, and with the least possible interruption at the successive stages of the work. In this manner is secured that harmonious co-operation which is scarcely less essential to economy of manufacture than is the use of steam or water power.

The successive operations carried on in the manufacture of cotton will be more fully described elsewhere; meanwhile the following synthetic view may furnish the reader with a general notion of their nature and their order:-

1. The mixing and opening up or loosening the cotton wool, as imported in the bales, so as to separate at once the coarser and heavier impurities as well as those of a lighter and finer kind.

2. The willowing, seratching, or blowing, an operation which removes the seeds and dirt, and prepares the material in the form of a continuous lap or rolled sheet for the next process.

3. The carding, which is intended to disentangle every tuft or knot, to remove every romaining impurity which might have eluded the provious operation, and finally to prepare for arranging the fibres in parallel lines, by laying the cotton first in a fleecy web, and then in a riband form.

4. The doubling and drawing out of the card-ends or ribands, in order to complete the parallelism of the filaments, and to equalise their quality and texture.

5. Tho slubbing operation, whereby tho drawings made in the preceding process are greatly attenuated, with no more twist than is indispensable to preserve the uniform continuity of the spongy cords.

6. The intermediate slubbing, another doubling and further attenuation, which is however, omitted in the spinning of coarse yarns.

7. The roving, which is simply a repotition of the doubling and drawing accomplished in the intermediate process, but leaving the cotton in a still more attenuated condition ready for the next process.

8. The spinning process, which complotes the extension and twisting of the yarn. This is accomplished either with the throstle or the mule. By means of the former machine tho thread is spun with a larger proportion of turns or twists, and is thus made tough and strong for purposes requiring yarn that will not readily break - as in the warps of stout fabries. By means of the latter, yarns of less strength aro produced, such as the warps of lighter fabries, and wefts of all kinds. The latter process, being the less costly of the two, is preferrod in cases where its results are otherwise suitallo to the particular end in viow, 
9. The ynom thus completed is now converted into the special fabric for which it has been prepared-by one or other of tho following processes:-

a. Weaving for which, however, cortain preparatory operations are needed. Tho warps must be formed, first, by winding the yarn on large bolbins, one continuous thread on each. From a given number of bobbins the requisito number of throids are thon laid side by side upon beams. These again are passod through the sixing machine, which adds strength and elasticity to the threads of the warp, enubling thom more successfully to resist the necessary strain of wearing. The weft needs no preparation after leaving the mule, but is placed at once in the form of a 'cop' into the shuttle.

b. Doubling.-In this process two or more threads are twisted together; the doubled yarn being then either converted into warps for woven goods of special strength, such as double warp calicoes, fustians, velveteens, and cotton velvets; or is carried forward through one or other of the following operations.

c. Reeling and Binding.--Here the yarn is wound into reels or skeins and packed in bundles for the purpose of exportation, or for dyeing or bleaching, or for the manufacture of hosiery.

d. Singeing and Polishing.-By the first of these processes the thread passes through jets of gas flame, and thus loses its superfluous fibres; by the latter, having first been dyed, it acquires a high degree of glossiness, and is then used for admixture with silk in the mixed goods which of ląte years have become popular, under the designation of Japanese silks, \&c.

There are few instances in which the whole of these operations are carried on in the same factory. To a large extent even the two main divisions-spinning and weaving -are the work of separate firms and establishments. Usually, however, these two are combined. In order the better to illustrate these main features of the factory system, wo now proceed to describe a concrete example - that of a cotton mill at Stockport, containing 61,400 throstle and mule spindles, and 1,320 looms.

The mill consists of a main body with two lateral wings, projecting forwards, the latter being appropriated to store-rooms, a counting-house, rooms for winding the yarn on bobbins, and other miscellaneous purposes. The building has six floors, besides the attic story. The ground-plan comprehends a plot of ground 280 feet long by 200 broad, exclusive of the boiler sheds.

The right-hand end, $\mathbf{A}$ (fig. 538) of the principal building, is separated from the main body by a strong wall, and serves in the three lower stories for accommodating two ninety-horse steam-engines, which are supplied with steam from a range of boilors contained in a low shed exterior to the mill.

The three upper stories over the steam-engine gallery are used for unpacking, sorting, picking, cleaning, willowing, and lapping the cotton wool. Here are the willow, the blowing, and the lap machines, in a descending order, so that the lap machine occupies the lowest of the three floors, being thus most judiciously placed on the same level with the preparation room of the building. On the fourth main floor of the factory there are, in the first place, a line of carding engines arranged near and parallel to the windows, as shown at $\mathbf{B}$ B, in the ground plan (fig. 538), and, in the second place, two rows of drawing frames, and two of bobbin and fly frames, in alternate lines, parallel to each other, as indicated by $D, C, D, C$, for the drawing frames, and $\mathbf{E}, \mathbf{E}, \mathbf{E}, \mathbf{E}$, for the bobbin and fly frames in the ground plan. The latter machines are close to the centre of the apartment.

The two stories next under the preparation room are occupied with throstle frames, distributed as shown at F F, in the ground plan. They stand in pairs alongside of each other, whereby two may be tended by one person. These principal rooms are 280 feet long and nearly 50 feet wide. The two stories, over the preparation room, viz., the fifth and sixth floors from the ground, are appropriated to the mule jennies, which are placed in pairs fronting each other, so that each pair may be worked by one man. Their mode of distribution is shown at $G G$, in the ground plan. The last single mule is seen standing against tho end wall, with its head-stock projecting in the middle.

The ground floor of the main building, as well as the extensive shed abutting behind it, marked by $\mathrm{N}, \mathrm{H}, \mathrm{H}$, in the plan, is devoted to the power looms, the mode of placing which is plainly seen at $\mathbf{H}, \mathbf{H}, \mathbf{H}$.

The attic story accommodates the winding frames, and warping mills, and the warp sizing machines, subservient to power weaving.

Some extre mules (self-actors), are placed in the wings.

We shall briefly sum up the references in the ground plan as follows:-

A, the ground apartment for the steam-engines.

$\mathrm{B}_{3}$, the distribution of the carding engines, the moving shaft or axis running in a straight line through them, with its pulleys, for receiving the driving bands, 
C c, the drawing frames.

D D, the jack, or coarse bobbin and fly frames.

538

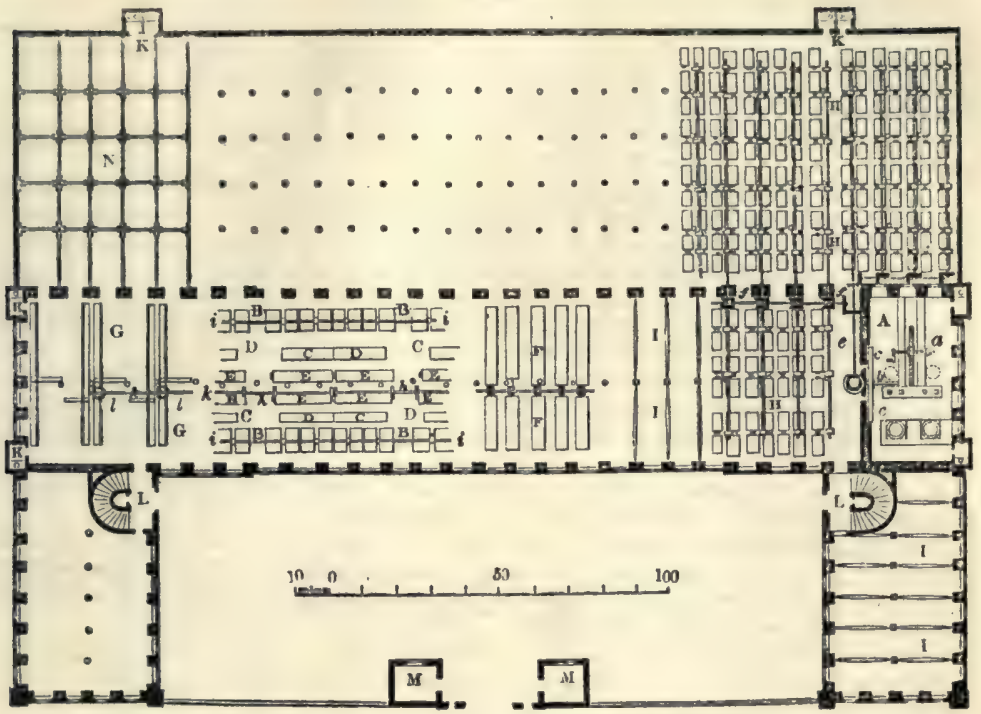

538
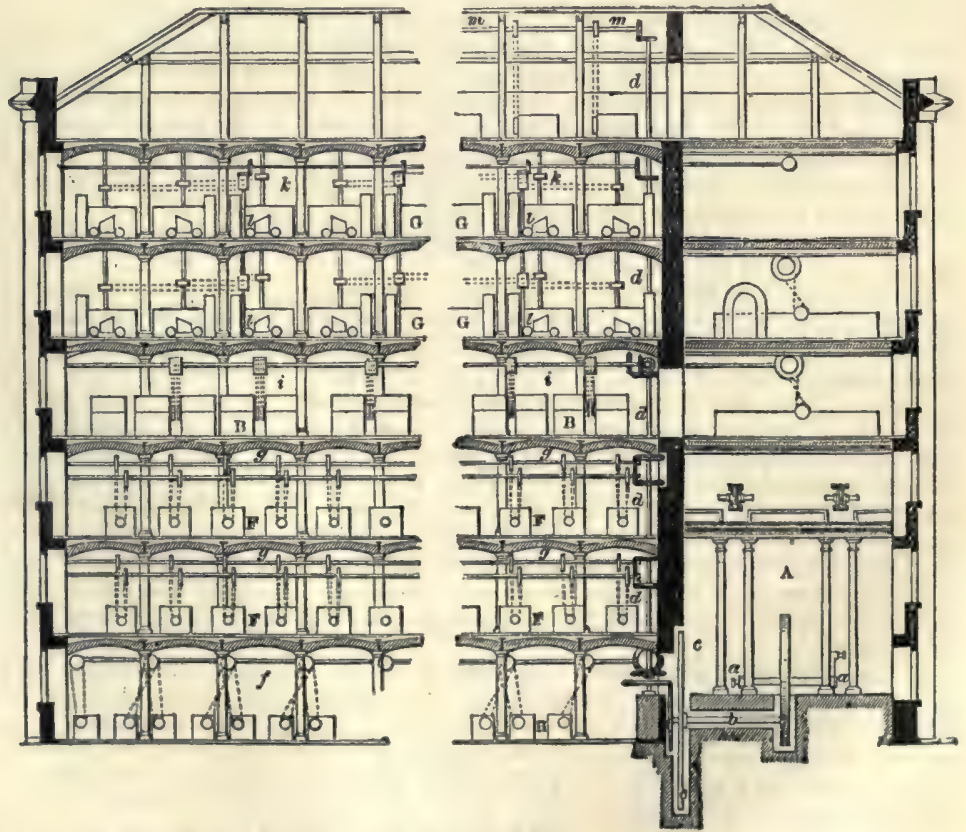

s $\mathrm{n}$, the fine roving, of bobbin and fly frames.

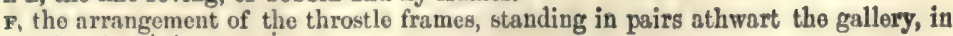
the 2nd and 3rd flats. 
G, the mules aro horo represented by thoir roller beams, and tho outlines of their head-stocks, as placed in the 5th and 6th stories.

$\mathbf{n}$, the looms, with their driving pulleys projecting from tho ends cf their main axos. Sometimes the looms are placed in parellol struight lines, with the rigger pulleys of tho one altarnately projected more than the other, to permit the free play of the driving-belts ; sometimes the looms are placed, as generally in this engraving, alternately to the right and left, by a small space, when the pulleys may all project equally. The former plan is the one adopted in Mrr. Orrell's mill.

I, represents the cast-iron girders which support the floors of this fire-proof building.

$\mathbf{r}, \mathbf{K}$, are closets placed in each floor, in the recesses of a kind of pilasters built against the outside of the edifice. These hollow shafts are joined at top by horizontal pipes, which all torminate in a chest connected with the suction axes of a fan, whoreby a constant draught of air circulates up the shafts, ventilates the apartments, and prevents the reflux of offonsive effluvia from the water-closets, however careless the workpeople may bo. The closets towards the one end of the building are destined for the men; towards the other for the women.

$\mathbf{L}, \mathbf{L}$, are the staircases, of a horse-shoe form, the interior space or shaft in the middle being used for the teagle or hoist. In the posterior part of the shaft a niche or groove is left for the counter-weight to slide in, out of the way of the ascending and descending platform.

$\mathrm{M}, \mathbf{M}$, are the two porters' lodges, connected to the corner of each wing by a handsome iron balustrade. They are joined by an iron gate.

It will be observed that the back loom-shed has only one story, as shown in section (fig. 540). In the ground plan of the shed, $s$ represents the roofing, of wood-work. Tho rafters of the floors rest at their ends upon an iron plate, or shoe with edges (as it is called), for the girders to bear upon.

The power for driving the machinery is conveyed from the engine-rooms by shafting in the usual manner. To the horizontal ramifications from the upright shaft any desired velocity of rotation may bo given by duly proportioning the diameters of the bevelled wheels of communication between them; thus, if the wheel on the end of the horizontal shaft have one-half or one-third the diameter of the other, it will give it a double or a triple speed.

In the lowest floor, the second bevel wheel above the stone block drives the horizontal shaft $e$, seen in the ground plan; and thereby the horizontal shaft $f$, at right ungles to the former, which runs throughout the length of the building, as the other dic through its breadth, backwards. The shaft $f$ lies alongside of the back window wall, near the ceiling; and from it the transverse slender shafts proceed to the right and left in the main building, and to the shed behind it, each of them serving to drive two lines of looms. These slender or branch shafts aro mounted with pulleys, each of which drives four looms by four separate bands.

In the second and third floors, where the throstlos are placed, the shaft $d$ is seen in the section to drive the following shafts :-

Upon the main upright shaft $d$ (fig. 540), there are in each of these stories two horizontal bevol wheels, with their faces fronting vach other (shown plainly over $d d$ ), by which are moved two smaller vertical bevel wheels, on whose respective axes are two parallel shafts, one over each other, $g g$, which traverse the whole length of the building. These two shafts move therefore with equal relocities, and in opposito directions. They run along the middle space of each apartment; and wherever they pass the rectangular line of two throstle frames (as shown at $F$ in the ground plan) they are each provided with a pulley; whilo tho steam pulloys on the axes of two contiguous throstles in one line are placel as far apart as the two diameters of the said shaft-pulleys. An ondless strap goes from the pulley of the uppermost horizontal shaft round the steam or driving-pulley of one throstle frame; then up over the pulley $g$, the second or lower shaft, $g$; next up over the steam pulley of a second throstle; and, lastly, up to the pulley of the top shaft, $g$. Seo $g g$ in the throstle floors of the eross section.

In tho preparntion room, three horizontal shafts are lod pretty close to the ceiling through the wholo length of the building. The middle one, $h$ (see the plan, fig. 538) is driven immediately by bevel wheels from the main upright shaft $d$ (fig. 539). The two side ones $i$, $i$, which run near tho window walls, are driren by two horizontal shafts, which lead to these side shafts. The latter are mounted with pulleys, in correspondence with the sterm pulleys of the two lines of carding engines, as seen between the cards in the plan. The middle shaft $h$, drires the two lines of bobbin and fly frames, $x, x, x, x$ (see cross section), and short shafts $i$; $i$, seen in the cross section of this floor, moret from the middle shaft $h$, turning the gallows fixed to the 
ceiling, over the drawing and jack frames, give motion to the latter two sets of machines. See $\mathbf{c} \mathbf{b}$ in the cross section.

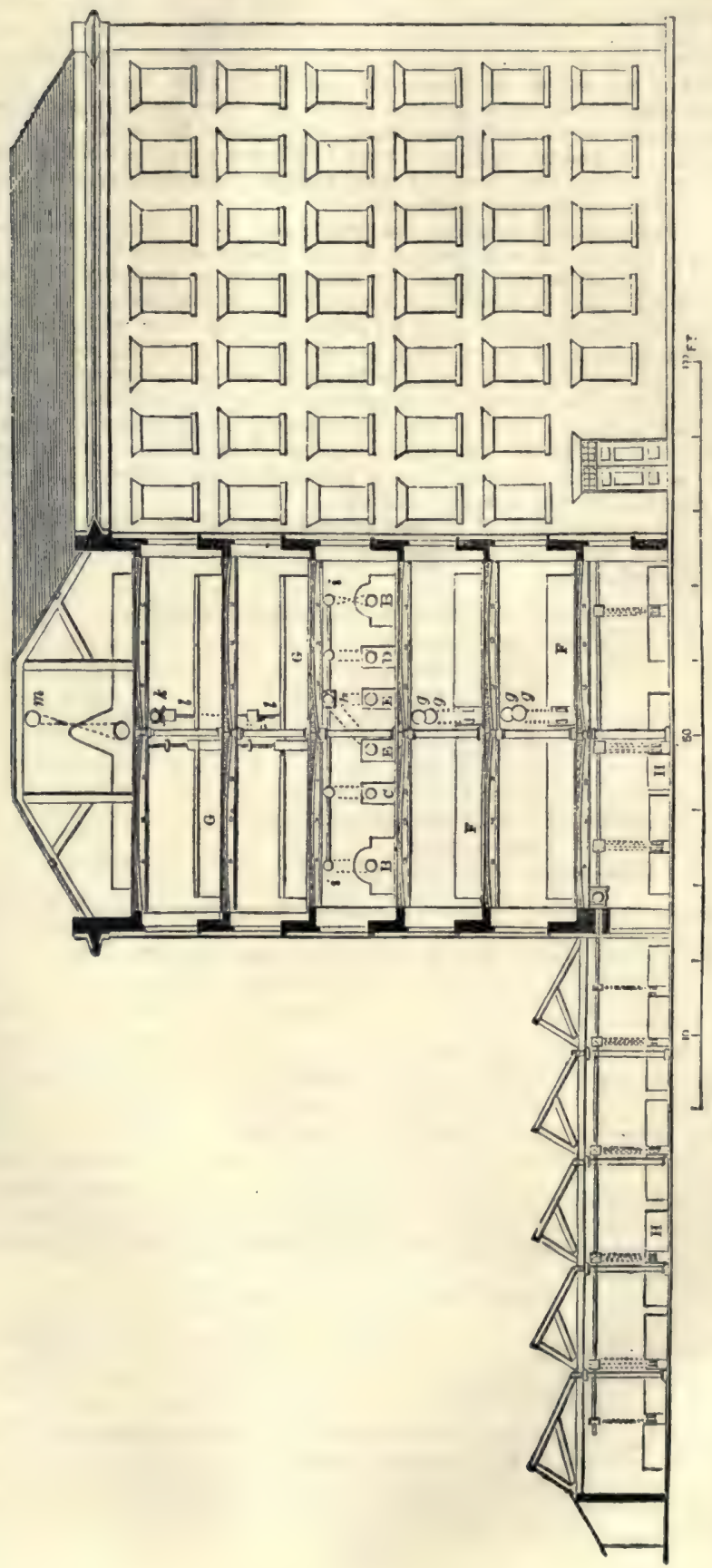


To drive the mules in the uppermost story, a horizontal shaft $k$ (seelongitudinal and eross sections, as well as ground plan) runs through the middle line of the building, and receires motion from bevel wheels placed on the main upright shaft, $d$, immediately beneath the ceiling of the uppermost story. From that horizontal shaft, $k$, at every second mule, a slender upright shaft, $l$, passing through both stories, is driven (see both sections). Upon these upright branch shafts are pulleys in each story, one of which serves for two mules, standing back to back against each other. To the single mules at the ends of the rooms, the motions are given by still slenderer upright shafts, which stand upon the head stocks, and drive them by wheel-work, the stops (top bearings) of the shafts being fixed to brackets in the ceiling.

In the attic, a horizontal shaft $m m$, runs lengthwise near the middle of the roof, and is driven by wheel-work from the upright shaft. This shaft, $m$, gives motion to the warping mills and dressing machines.

In Great Britain and throughout the greater part of the European continent the motive power used in cotton factories is derived almost entirely from steam; but in the United States and in Switzerland the large amount of available river fall is so great that water-power is extensirely employed in the manufacture of cotton : the proportion in the United States being 2.1 horse-power of water to 1 horse-power of steam, and in Switzerland 3.4 of water to 1 of steam. A further peculiarity in the construction of American cotton mills is that the motion is communicated from the steam-engine or turbine or water-wheel, as the case may be, not through the medium of wheels and shafting as shown in our illustrations, but by means of huge leather belts and drums or pulleys. This plan, originating, no doubt, in the formerly very high price of iron in the United States, is stated to be more effective and economical than the European method already described.

The latest complete roturn of the number and extent of the cotton factories of the United Kingdom was presented to Parliament in 1871. From this return we give the following particulars:-

\section{Cotton Factories in Great Britain and Ireland.}

\begin{tabular}{|c|c|c|c|}
\hline Number & otton factories & & 2,483 \\
\hline " & carding ongi & • & 65 \\
\hline " & g machines. & & 1, \\
\hline & & & 34,695 \\
\hline " & indles & & 3,5 \\
\hline & powes & • & \\
\hline & power loom weaver & & 165 \\
\hline count & (hor & $w$ & 00 \\
\hline tal & 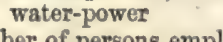 & & \\
\hline
\end{tabular}

The growth during recent years of the cotton manufacture in the United Kingdom may be pretty accurately gauged by the comparison of a few particulars from the last four Parliamentary returns, those of $1856,1861,1868$, and 1871 . Thus :-

\begin{tabular}{|c|c|c|c|c|c|}
\hline Number of & & 1856 & 1861 & 1868 & 1871 \\
\hline Spinning spindles & . & $28,010,217$ & $30,387,467$ & $32,000,014$ & $34,695,221$ \\
\hline Power looms & . & 298,847 & 399,992 & 379,329 & 440,676 \\
\hline Persons employed & . & 379,213 & 451,569 & 401,064 & 449,087 \\
\hline
\end{tabular}

In the United States the progress of the cotton manufacture has been since 1860 relatively much more rapid than in this country. From the Census returns of 1860 and 1870 , the only complete and official statistics bearing upon this subject, we take the annexed figures:-

\section{Cotton Factories in the United States.}

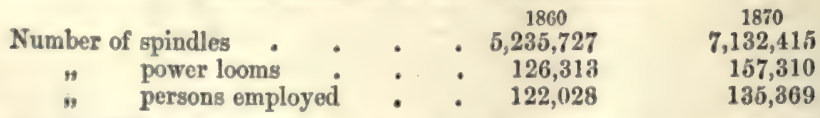

The following estimate, based upon official returns or upon a comparison of the best accessible information, of the number of cotton spinning spiudles and the weight of cotton consumed in each of the manufacturing countries in Europe and America will furnish un approximately correct idea of the rolative importance of the cotton industry in each at the present time (1874):- 


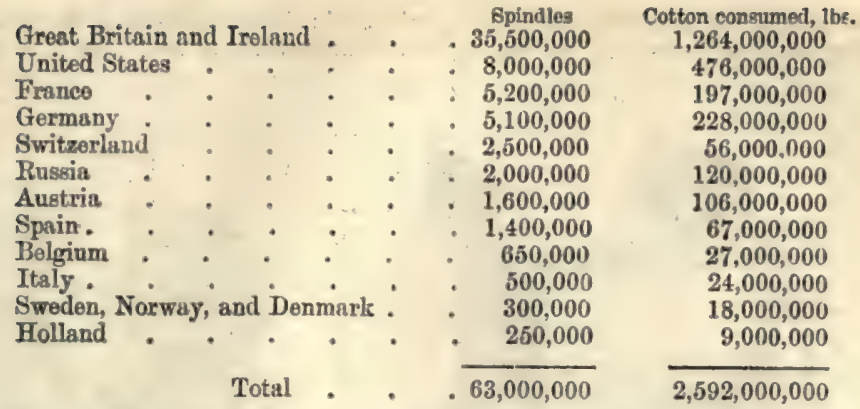

Corrow-GIr. The great mass of seeds with which the woolly fibres of cotton aro accompanied, and to which they adhere most tenaciously, must of necessity be removed before the cotton can be turned to any useful purpose. This operation, called ginning, is always performed as near as possible to the place of growth. Formerly the object was effected by the rudest handicraft methods, such as are indeed still followel in some parts of India. These methods were, howerer, very slow and costly. It was, therefore, an important step forward in the history of cotton culture when Eli Whitney, an American, in the year 1793, made known his invention of the saw-gin. The working of this machine will be readily understood on reference to fig. 541 , where $A B$ is a sectional viow of a roller, about 9 inches in diameter, revolving in the direction of the arrow. Thronghout the length of the roller a series of circular saws, at intervals of about an inch and \& half, projects beyond the wooden portion of it. Above the cylinder, a hopper $\mathrm{B} F$ contains the seed cotton, which falls upon a grating, so placed as to allow the teeth of the saws when revolving to lay hold of the fibres of cotton and pull them through the grating. The seeds being thus left behind roll down the slope of the grating, and are discharged at the spout $\mathbf{I} \mathbf{K}$. The brush $\mathbf{m}$ revolves against the teeth of the saws for the purpose of clearing them of the adherent fibres.

The saw-gin has in several respects been modified since its invention, with the effect. of considerably increasing the out-turn, but the principle remains essentially the same as we hare described. Tho quality of its work, however, leaves room for improrement. The rough teeth of the saws do not use the fibre gently enough, but cut and 'nep ' or knot it, especially when the machine is allowed to get out of order or is carclessly employed. Quite recently, an American inventor has hit upon an expedient which is said to go far towards remedying these defects. This modification consists in substituting, for the circular saws, rows of pointed smooth steel wire teeth, which, whilst effectual for the purpose of drawing away the cotton from the seeds, present no rough corners or surfaces to the passing fibre.

Calvert's toothed roller gin, illustrated by fig. 542 , may be considered as a modification of the saw-gin. $a$ is a perspective and $b$ a sectional view. $\Delta$ is a box to hold the seed cotton, $\mathbf{B}$ is the hopper, and $\mathbf{D}$ is the toothed roller which draws away the cotton, whilst the disengrgement of the seeds is aided by the fluted roller c.

The Macarthy gin differs from the saw-gin mainly in substituting for the saws \& roller, covered longitudinally, at intervals, with strips of leather set on spirally. A blunted knife, working alternately up and down at right angles to the axis of the roller und along its entire length, serves to strike off the adherent seeds at the point where the fibre is being drawn through. This machine is not capable of doing as much work as the saw-gin; but, on the other hand, it delivers the cotton in very good condition, and is on this account preferred for the longer stapled and more valuable varieties. Successful efforts have been made to remove the objection to the Macarthy gin on the ground of its comparatively small out-turn, but the inability of the native labourers in most cotton-producing countries to work and keep in order any but the simplost mechanical combinations has hitherto prevented the extensive adoption of the improred michines.

Another variety of cotton-gin is known as the Lock Jaw or Cowper's Gin. This machine is intermittent in its action. It nips the fibre firmly, whilst an instrument pushes off the seed; then it releases its hold, allowing the clean fibre to pass awny. A fresh quantity is thon brought in, hold in liko mauner, and so detached from Vor. I. 
the sood. Thero is no sliding and rubbing action, as in the Macarthy Gin; or sawing of the fibre, as in the saw-gin. The closing of the nipping blade upon the flbre is so

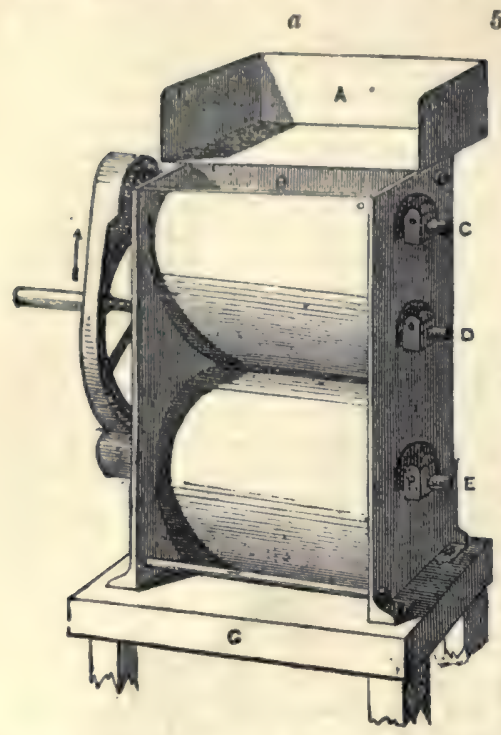

542

$b$

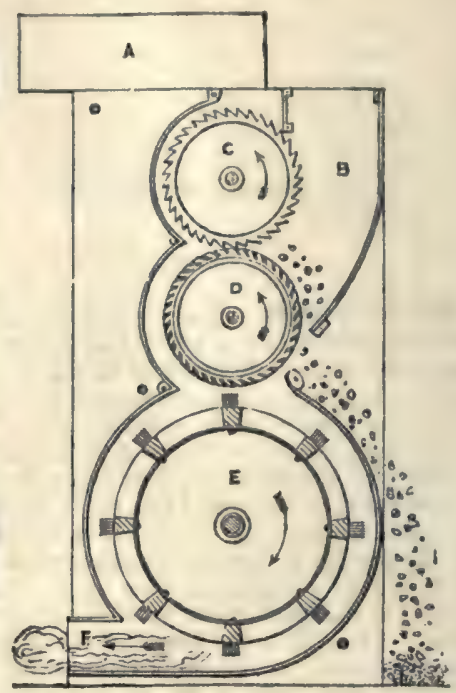

like the action of a jaw, that the resemblance has given to this machine its best known name.

Fig. 543 shows a perspective view, and fig. 544 a section, of it: $N$ is the nipping
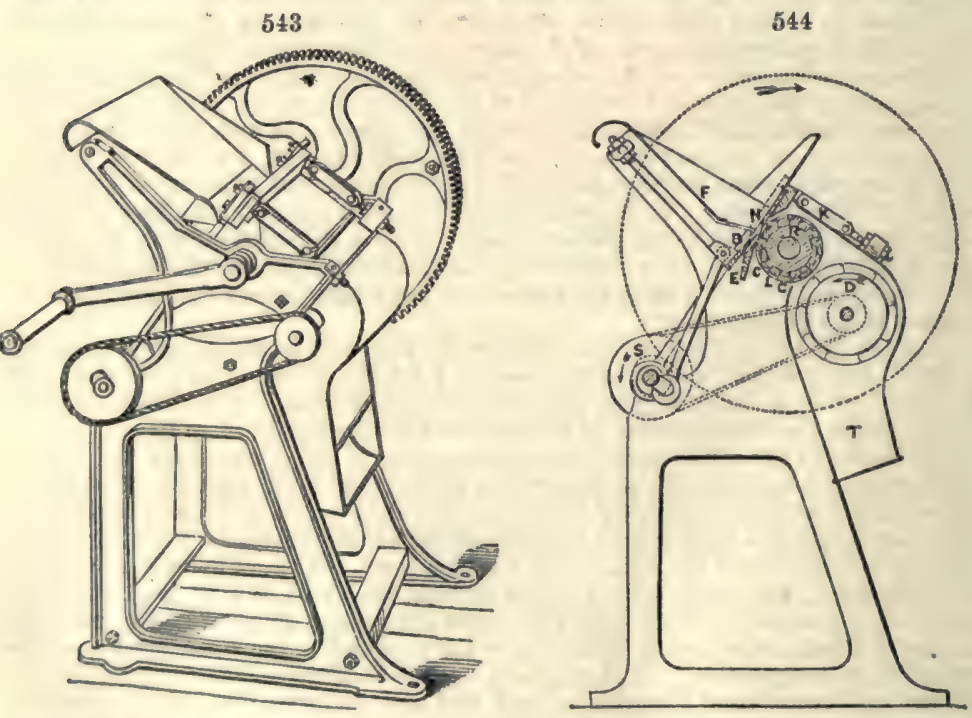

blade, $x$ the roller on which are arranged the strips of leather, r. These come into action one after the other, in order to form one side of the pair of jaws to act with the nipping blade; fine combs, c, are also attached to the roller between the strips of leather (when short-stapled cotton is being combed). $\mathrm{s}$ is a shaft, with cranks, to move the beater, $B$, and excentrics to move the nipping blade, $N$, which latter is confined by links, $\mathrm{x}$, causing it to movo in a curved elliptical path. $F$ is a bar to fill up the 
space between the roller and beater, and $\mathrm{D}$ is a doffer to take the clean cotton off tho rollor. At the time when the nipping blado $\mathrm{N}$ approaches the roller $\mathrm{R}$, it is moving in the same direction, and at the samo surface speed; and as it approaches it nips upon the fibre, or locks it fust between its edge and the strip of leather on the rollor; the beater I then comes up quickly, and pushes off the seed a short distance, thus separating the fibre when it is held close up to the seed, and obtaining it, necessarily, of its full natural length.

It will be inferred from the description of this invention that it answers one of the essentials of a good cotton-gin in that it inflicts little damage upon the fibre. There is, however, in this case as in others the practical difficulty that those who haro to use the machine have too little skill and intelligence to use it profitably. $\Lambda$. further obstacle to the adoption of the best inventions when they happen, as is usually the ease, to be somowhat complex, is the great distance of the cotton-fields from ongineering establishments, and the consequent impossibility of promptly executing tho needful repairs.

Enough has been adduced to show that the operation of cotton-ginning, simple as it may uppear to be, occupies a prominent place in the chain of processes by which this natural product is at last made useful for the service of mankind. It is also evident that the various means at present within reach for effecting the separation of the fibre from the seed are all more or less objectionable. The great losses arising from bad ginning can scarcely be appreciated by those who are not practically acquainted with the various qualities as they come to market. We may observe, however, that the Report of the Commissioners appointed to examine and value the samples of cotton exhibited at the International Exhibition of 1862 estimated the average loss from this source at $2 \cdot 67 d$. per $1 \mathrm{~b}$.

cotrow Gur. See Gus Corros.

COTTON-s25. The practical utility of cotton-seed for any other purpose than the limited one of reproduction or for manure has until quite recently been almost unknown. In the United States it was occasionally used for fodder, and cattle were very fond of it. It was found, however, that unless given in very small quantitios and only occasionally, the shorter fibres still left on the seed after the ginning process proved very unwholesome. Means have been found, however, for removing this difficulty, and the cotton-seed is now taking high rank, amongst agricultural products, as the source of no less than three valuable commodities, viz.: Oil, cattle-food, and paper material. The method of treatment as practised in the United States is as follows:-first, Re-ginning, by a special apparatus which entirely clears off the short fibres from the seed, making them available for paper manufacture; secondly, Decorticating, by which the external husk is removed, and this again, after decomposition, is utilised as manure; thirdly, the kernel is subjected to the process of Crushing. The products of this last operation are a valuable oil and a cattle-food cake, both of which have now taken their place as important articles of commerce. After re-ginning, the seed consists on an average of one half husk and one half kernel. The kernel yields when crushed about one third crude oil and two thirds cake.

Some idea of the wide field opened up by this new branch of manufacture may be formed from a rough statement of the quantity of seed available for its purposes. Taking the American cotton crop at $4,000,000$ bales or 800,000 tons annually, the production of seed, on an average of estimates, may be put down at three times as much by weight, or 2,400,000 tons. Allowing one half this quantity for waste and resowing, there remain 1,200,000 tons of the American crop for manufacturing purposes. The available yield of the crop of India, Egypt, Brazil, and other countries can scarcely be less than two thirds of this amount. We thus arrive at an estimate of $2,000,000$ tons as the annual supply of cotton-seed capable of being turned to valuable uses, most of which is now being wasted. The quantity of cotton-seed imported into the United Kingdom almost entirely for manufacturing purposes was in $1871,174,392$ tons, valued at $1,526,652 l$; in $1872,167,936$ tons, valued at $1,404,724 l$; and during $1873,207,755$ tons, valued at $1,608,975 l$.

COTTON-SPINANIre. The many varieties of cotton with which the tropical world abounds, and the widely-differing purposes for which each, according to its peculiar fitness, is destined, have led to considerable divergence in the methods of manufacture. The treatment suitable for the shorter and rougher stapled cottons, or for their conversion into bagging, candle-wick, or the coarser kinds of elothing, is obviously very far removed from the processes proper to the finest New Orleans, whose clear and silky fibres fit it for the manufacture of velvets and cambrics. Still further removed is the treatment necessary in spinning Sea Island, which is used in the pro. duction of the most delicate muslins and laces. To describe with minuteness orery part of the many kinds of operations comprised within these extremes lies obviously beyond the scope of this article. There are, nevertheless, certain well-marked strges 
or processes common to all kinds of cotton-spinning. These we shall follow in due oriler, pointing out by the way such modifications of treatment as the chief rarieties of raw matorial and of finished product require.

The long distances trarorsed by the cotton in its passage from the field to the factory require that orery available expedient be adopted to abridge the cost of transmission. A gront part of the cost consists in the charge for sea freight, which is reckoned according to bulk and not according to woight. Hence nearly all kinds of cutton, and especially East Indian descriptions, aro compressed by means eithor of a scrow or a hydraulic press into an exceedingly small space. The effect of this process is to cause the fibres to bo almost welded together in solid masses; and when the bales aro opened the cotton is seen to lie in hard layers, very unlike the fleecy and cloud-like condition in which it leares the gin. The first purpose, therefore, to which the use of machinery is directed in cotton-spinning is the loosening or opening of the fibres. With this process is necessarily associated the cleansing of the cotton, the separation from it of as much as possible of the sand and other impurities with which it is often too freely associated, as well as of the seeds, which in a whole or a broken condition have escapod through the gin, and adhere very tenaciously to the fibros. The various kinds of machines in use for these purposes are known as Willows, Openers, or Scutchers.

Before proceeding to describe this class of machinery, however, it is desirable to say something with reference to the mixing of cotton. Under the head of Cotronwe have alluded to the most distinctive characteristics of the principal varieties. But there are many minuter differences in every description, differences of length, strength, colour, texture, and general working qualities, with which only the practical cottonspinner is acquainted. This is a soction of technical knowledge which nothing but experience can give; and it is a mark of skill to be able so to combine or to set off these qualities against each other as to turn them to the best account. Hence the selection and the proper admixture of varions growths and deseriptions of cotton is no small part of the work of the spinner. Moreover, it is found in practice that even in the same 'grade,' or quality, there is considerable variation between one bale and another, and not unfrequently even in the same bale. The period of the harvest-time at which the cotton is picked, the kind of weather at the time, the part of the plant on which it is grown, and other circumstances, contribute to diversify greatly the produce of the same plantation. And however carefully it may be classified, important differences will remain. But there is reason to believe that care of this kind is not common. The best mothod of overcoming these last-namod differences is to mix together as large a number of bales and to blend them as intimately as possible by spreading out the contents of each balo on a large stack in parallel layers, in such manner as that when it is raked down from top to bottom a small portion of each bale will come away. By this means the irregularities in the subsequent processes and in the thickness, strength, and evenness of the yarn, will be minimised.

The freoing of cotton from its grosser impurities is not an easy undertaking, and during the American Civil War when coarse, dirty, and badly-gained growths were forced into consumption, much difficulty was experienced for wani of effective cleaning machinery. During this period the Cotton Opener of Messrs. Crighton and Co. was

545

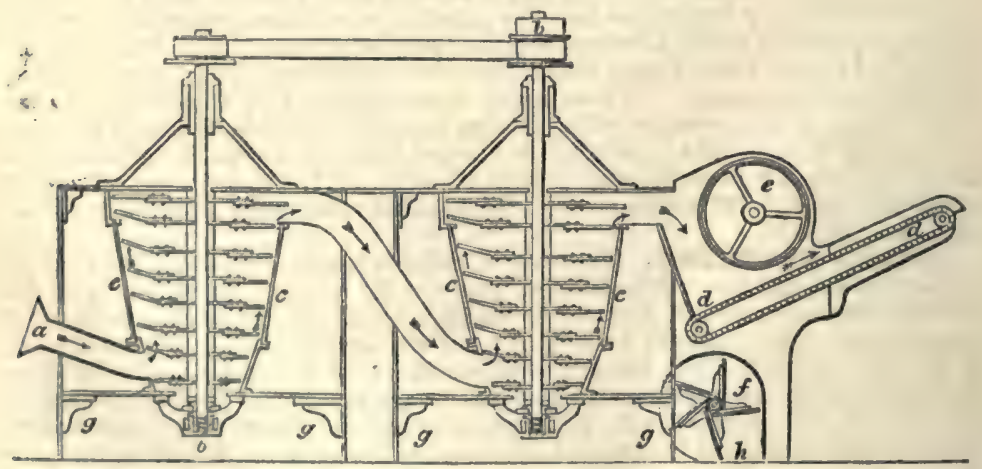

introduced, and extensively adopted for the special purpose just named. This machipe is illustrated ip vertical section by fig. 545. The cotton is fed down the 
open pipe $a$, to tho bottom of the vertical beater $b$. This beater revolves rapidly, its arms striking the cotton against the grids $c c$, by which it is surrounded, and separating the dirt and leaf from the cotton. These fall into the dirt grid $g$, below, whilst the draught from the fan, aided by the shape of the beater, causes the cotton gradually to ascend, till it arrives at the discharge pipe, whence it descends to the bottom of the next beater, and is again similarly operated upon. The cotton, after passing the second beater, is discharged through a pipe to a cage $e$, and creeper $d$, and without any pressure leaves the machine in an open, woll cleaned, fleecy state.

Various other forms of Opener are in use. All, however, rest upon the application either of centrifugal force, or of toothed rollers or cylinders for the loosening of the cotton, and upon the pneumatic force derived from the fan for the purpose of drawing Iway dust and other impurities. One recent improvement, however, we may mention (that of Messrs. Lord, of Todmorden) which is not so much a separate machine as a new method of feeding the opener. It consists in passing the cotton through a long horizontal tube, the lower portion of which is grated. The cotton is forced through the tube by pneumatic pressure, created by the revolution of a fan in the ordinary way. The distance traversed by the cotton serves to loosen it to a considerable extent, and to disengage much of the dirt from it, whilst at the same time there is no violent tearing or strain upon the fibre.

Perhaps the most common description of Opener in use is known as the Scutcher, a name which is associated with a particular form of construction easily recognised in fig. 546. The cotton is placed upon the travelling creeper marked $a$, which is made

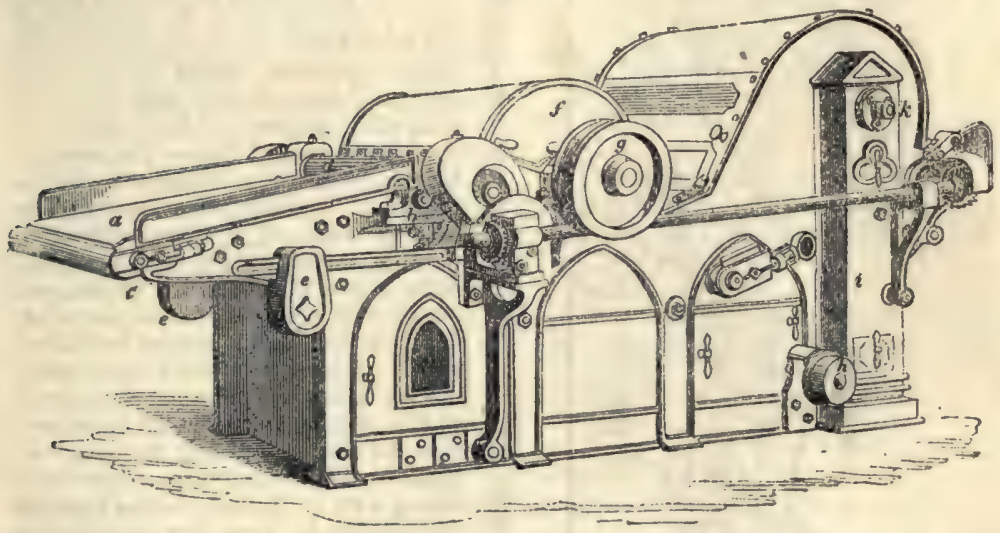

of a number of narrow slips, or laths, of wood, screwed to three endless bands of leather, the pivots of which are marked $b$ and $c$. Motion is given to the roller $e$, by a wheel on the end of the feed roller, thus causing the creeper to advance, carrying with it the cotton to the feeding rollers $d$; these revolving slowly pass the cotton to the second smaller pair of fluted rollers, which serve it to the beater. The top feeding rollers are weighted by levers and weights $e e$, and hold the cotton sufficiently tight fur the beater to act upon it. The beater is placed inside the machine at $f$, and extends quite across its breadth, its shaft or axis being shown with the pulley upon it at $g$. The form of the beater varies, but we give the following as an example - On a shaft are placed four or five spiders, each having three or four arms ( $\mathrm{fg} .547$ ); to the ends of these arms are attached steel blades, which pass along the whole length of the beater; two of the arms being shorter than the others, allow the blades attached to them to contain a double row of spikes in each, the points of the spikes being at the same distance from the axis as the other two blades. As the beator revolves about 800 turns per minute, tho blades and spikes strike the cotton with considerable force as it is passed from the feeding rollers, and thus loosen

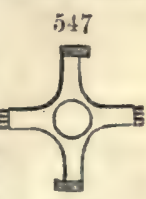
and disengage it from much of its impurity. Immediately under the feed rollers and beater aro placed a number of wedgo-shaped bars, which form a semicircular grid, through tho narrrow openings of which the dirt and seeds fall to the floor, their removal being effected through the doors in the framing. To prevent the cotton passing with the dirt through the grid, a current of air, to draw the cotton from the beater to the cage, is produced by an exhuust fan (its axis being shown at $h, f i g .546$ ) 
receiving its motion from a pulley on the beater shaft. The projection $i$ on the framing forms a pine, through which the fan draws the air from the beater, passing on its wny through a large revolving cage or cylinder, the periphery of which is formed of sheets of porforatod motal, or wire gauze. Its axis is shown at $k$.

From the cage the cotton is delivered by a secund travelling creoper, and falls into a receptacle, from which it is taken and passed through the second Seutcher or Lap machine, to which we shall refer presently.

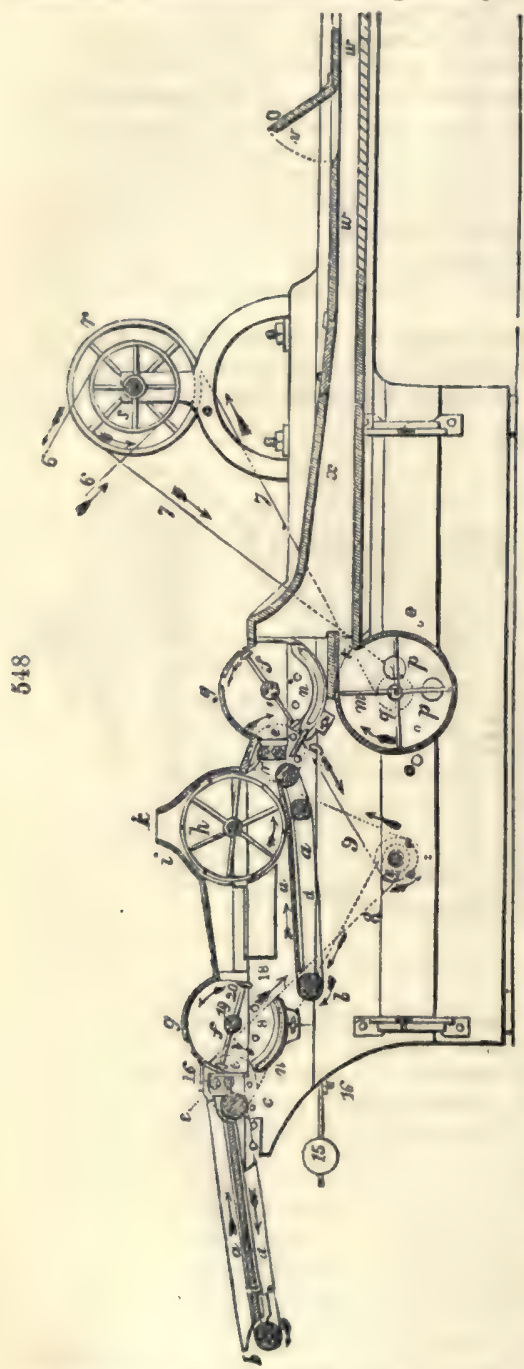

Fig. 548 exhibits a longitudinal section of another kind of Opener, or first Scutcher. The machine is about 18 or 19 feet long, and three feet across within the case. The whole frame is made of cast iron, forming a close bor, which has merely openings for introducing the raw cotton wool, for taking out the cleansed wool, and removing the dust as it collects at the bottom. These doors are shut during the operation of the machine, but may be opened at pleasure to allow the interior to be inspected and repaired. The introduction of the cotton is effected by means of an endless cloth or a wooden lath creeper similar to the one in fig. 543, which moves in the direction of the arrows $a$, at the left end of the figure, by passing round the continually rovolving rollers $b$ and $c$. It is then delivered to the feed rollers $e_{\text {. }}$ The double-armed beater $f_{c}$ turns in the direction of the arrow, and strikes the cotton violently as it enters, so as to throw down any heavy particles upon the iron grating or grid at $n$, while the light cotton filaments are wafted onwards with the wind, in the direction of arrow $a^{\prime}$ slong the second travelling apron. $h$ is a cylindrical cage made of wire gauze or perforated zinc, from which the air is exhausted by means of a fan formerly placed at some digtance from the machine, and communicating with it through a pipo fixed at the opening $k$. Now, however, the fan is placed below the machine, and communicates with the cylindrical cage, as deseribed in fig. 546. The cage $h$, by its rotation, presses down the halfcleaned cotton upon the cloth $a^{\prime}$, which carries it forward to the second beater $f^{\prime}$, by the second set of feed rollers $d$. The second beater throws down the heavy dust upon the recond grid $n^{\prime}$, through which it falls upon the bottom of the case. The cotton is wafted by the second beater into the space $x w w$, provided with a fine grid bottom. In fig. 548 an additional ventilator is in. troduced beneath at $m o$, to aid the action of the scutchers in blowing the cotton onwards into the oblong trough $a$. The outlet of that fan is at $t$; and it draws in the air at its axis $q$. $u$ and $v$, are two doors or lids for removing the cleaned cotton wool. This last fan is suppressed in many scutchers, as the scutching arms supply a sufficient stream of air. The dotted lines show how the motion is transmittod from the first mover at 8 , to the rarious parts of the machine. $6^{\prime} 6^{\prime}$ represent the bands leading to the main shafting of the mill.

The second Seutcher or Lap machino differs from the first Scutcher only in two respects. The cotton as it passes out of the machine is coiled in a continuous broad sheet 
or coil upon a roller. This coil, called a lap, it is necessary to make as even in thickness as possible, hence a further distinction of the second scutcher consists in its being provided with the means of approximately securing the desired evenness. Formerly this was accomplished by making the feed-cloth or apron into equidistant spaces and spreading equally a given weight of eleaned cloth upon each space. This plan is still followed in many factories. It has, however, been largely displaced, on account of the costliness of the manual labour involved, by a mechanical contrivance invented by Messrs. Lord, of Todmorden, and known as Lord's regulator. This apparatus is connected with the feed rollers, and is made to increase or diminsh their speed inversely as the thick. ness of cotton passing between them, thus approximately securing a uniform rate of delivery to the beater. This invention has been further improved by Messrs. Lord, who have added what is known as the 'Piano Motion' the purpose of which is to obtain greater delicacy in the action of the regulator, and to counteract the minuter variations in the thickness of the cotton as it enters the machine. In the improved regulator, the trough or plate under the roller, or rollers, which supply cotton to the beater, instead of boing in one piece as heretofore, is divided into any convenient number of parts, each part being acted upon by a weight or spring, their object being to press the fibres between the trough, or plate, and the feed roller, so as to prevent them being drawn forward unevenly by the beater, and to prevent 'snatching.' The variations in the quantity of cotton fed to the machine produce corresponding variations in the distance between the divided trough or plate and the feed roller, and thus increase or diminish the quan. tity of cotton supplied to the machine.

In cases where Lord's regulator is applied the opener must be made to lap the cotton, instead of delivering it into a loose mass. Three or four laps are then made to

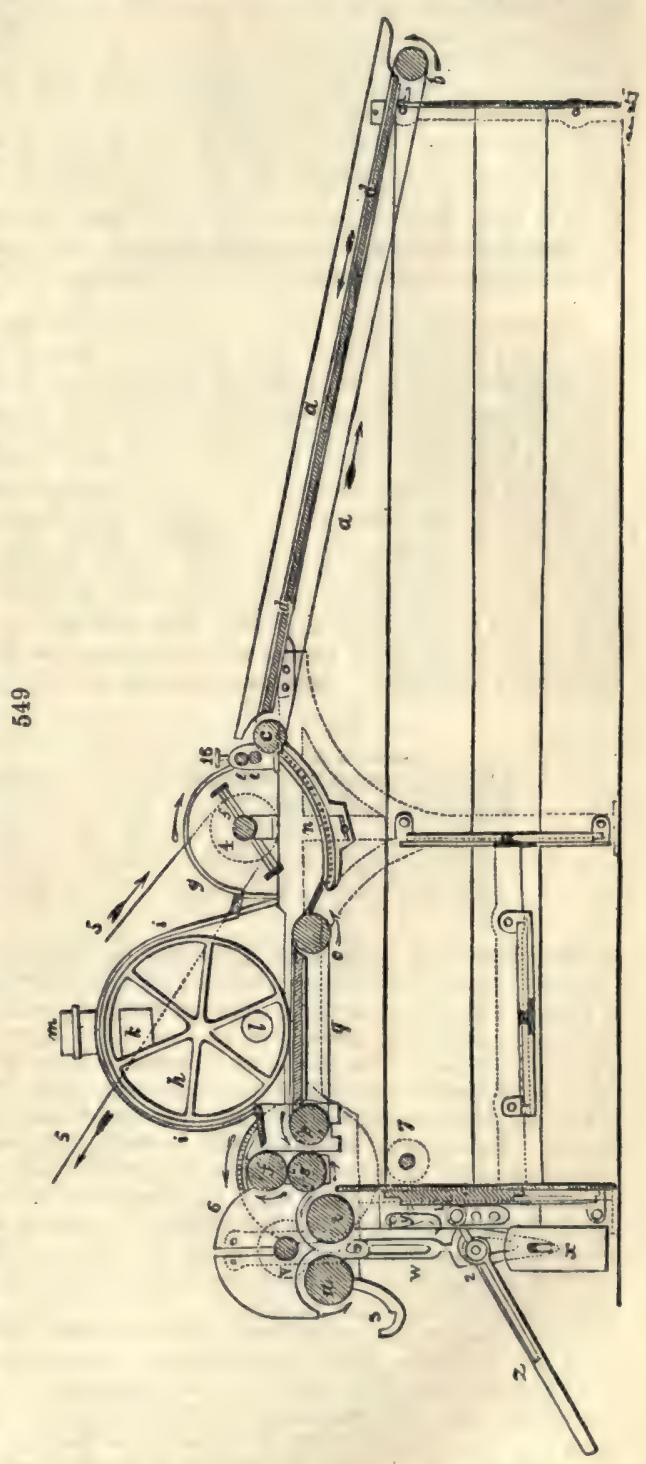
anfold together upon tho feeding lattice or apron of the seutcher, with the object of making the inevitable unevenesses of the first lap machine correct each other as far as possible, and thus aid the work of the regulator.

A section of a Lap machine is shown in fig. 549, where we see the feed-cloth, the scutching beater, tho cylindrical cage, and tho rollers for coiling up the lap. The lever shown below is for removing the pressure weight from the axis of the lap rollers, when 
a full lap is to be removed, and roplaced by an empty ono. $m$, at tho top, is the commencement of the pipe which leads to the suction fan, or ventilator.

It is to be observed that different kinds of cotton require different kinds of treatment in the opening and scutching operations. Some scutchers have only one bentor, others two or even threo. Somotimos the opener las two beaters, so that altogethor the cotton is mado to pass through as many as firo beaters. As a rulo, however, it is well to beat or tear it as littlo as is possiblo, having in view the main purpose of this operation, viz. to loosen and to cloanse it from the more palpablo impurities. For not only does the staplo suffer from repeated strain, but the longer stapled cottons aro liablo occasionally to get knotted or 'nopped,' and thus to introduco almost irroparablo mischief into the subsequent operations.

The operations of oponing and scutching are exposed to frequent risks of firc. Pieces of stone. of flint, of metal, and even boxes of lucifor matches aro not unfroquently found in cotton. Whenerer these pass into the machines and are struck by the beaters, there is imminent danger of fire. Hence the rooms in which these operations are carried on are almost invariably fireproof, and are built quito separate from the rest of the factory, communicating with it only by a covered fireproof gangway.

The cotton thus opened and to a certain extent cleaned is yet, however, in a very unfit state for being spun into yarn. The fibres, on examination, will be found mingled in a confused mass, and whether straight, or curled, or folded, lying in every possible direction. Moreover, it will be seen that there are amongst the longer fibres many 80 short as to be unfit for combination with them, some also will be knotted, or 'neppy,' and added to these imperfections many ' moats,' small pieces broken off the pod or the seed of the cotton, besides other forms of finer impurity. For the purpose of separating the useful fibres from the forcign accompaniments and laying them in parallel order, we have the operation of Carding. 'The essential part of this operation may be described as the mutual action of two opposite surfaces, which are studded thick with oblique-angled hooks. The wires of which these hooks are made must be very hard drawn in order to render them stiff and elastic. The middle part of the figures shows one of the staples or double teoth, the structure of which has been partly explained under CazD. Suppose $a, f i g .550$, to be a piece of a card fillet, and $b$ to be

550

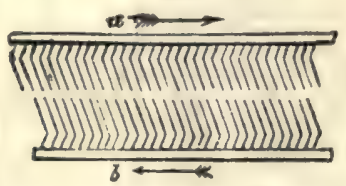

551

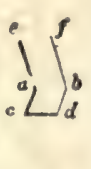

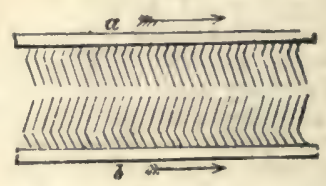

another piece, ench being mado fast with pins to a board; the toeth of these two cards are set in opposite directions, but are rery near together, and parallel. Now suppose a flock or tuft of cotton placed between two such bristling surfaces. Let $a$ be moved in the direction of its arrow, and let $b$ be moved in the opposite direction, or even let it remain at rest. The filaments of cotton will be laid hold of by each set of teeth, when their surfaces are thus drawn over each other; the teeth of $a$ will pull them in a forward direction, while those of $b$ will tend to retain them, or to pull them backwards. The loops or doublings will, by both movements, be opened or drawn out, so that the tangled fibres will be converted into rows of parallel filaments, lying alongside or bofore each other. Each tooth will secure to itself one or more of them, and by tho friction of its sides as well as the hooks of its points, will draw them to their utmost olongation. Though one stroke of the opposite cards be inadequate to produce this equable arrangement, yet many repeated strokes must infallibly accomplish the end in riew, of laying the fibres parallel.

Let us suppose this end effected, and that all the fibres have been transferred to the carl $a$, a trinsverse stroke of $b$ will draw over to it a cortain number of them, and in. doed at cach stroke there will be a new partition between the two eards, with increased parallclism, but still each card will retain a great deal of the cotton. To make one card strip another, the teeth of one of them must be placed in a reverse position, as shown in fig. 551. If $a$ be now drawn in the direction of its arrow along the face of $b$, it will inevitably comb out all, or almost all, the flaments from it, since the hooks of $h$ have, in this position, no power of retaining thom. Even the doubled fibres or loops will slip orer the sloping point of $b$, in obedience to the traction of $a$. By consilering these two relative positions of the cards, which tako place in hand cards simply ly reversing one of them, uny person will be able to understand the play of a cylinder card against its flat top, or against another cylinder card, the respective toeth 
being in what we may call the tensing position of fig. 550 ; and also the play of a cylinder card against the doffer cylinder, in what may be called the stripping position of fig. 551.

Cylinder cards, so essential to the continuity and despatch of cotton-factory labour, were the ingenious invention of Lewis Paul, of Northampton, but were greatly improved and brought into nearly their present operative state by Sir Richard Arkwright. A carding engine consists of one or more cylinders, covered with eardleather (sometimes card-cloth), and a set of plain surfaces similarly covered, made to work against each other, but so that their points do not come into absolute contact. Some cards consist entirely of cylinders, the central main cylinder being surrounded by a series of smaller ones called rollers. These are generally used for preparing the coarser stapled cotton.

Fig. 552 may be taken as a typical illustration of the carding engine. $a$ is one

552

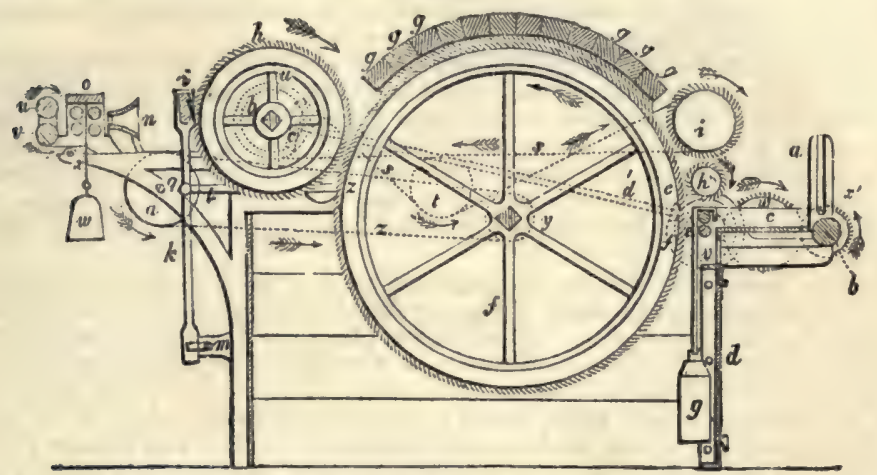

of the two upright slots, which are fixed at each side of the engine for receiving the iron gudgeons of the wooden rollers round which the fleece of the lapping machine is rolled. The circumference of this coil rests upon a roller $b$, which is made to turn slowly in such a direction as to aid the unfolding of the lap by the fluted cylinders $e$. The lap proceeds along the table seen beneath the letter $c$, in its progress to the fluted rollers; $g$ is a weight which hangs upon the axis of the upper roller, and eauses it to press upon the under one: $f$ is the main card cylinder; $g g g$, the arch formed by the flat top cards ; $h$, the small card cylinder for stripping off the cotton, and therefore called the doffer; $i$, the doffer-knife or comb for stripping the fleecy web from the doffer; $k l q m$, the lever mechanism for moving these parts. At $d$ there is a door giving

553

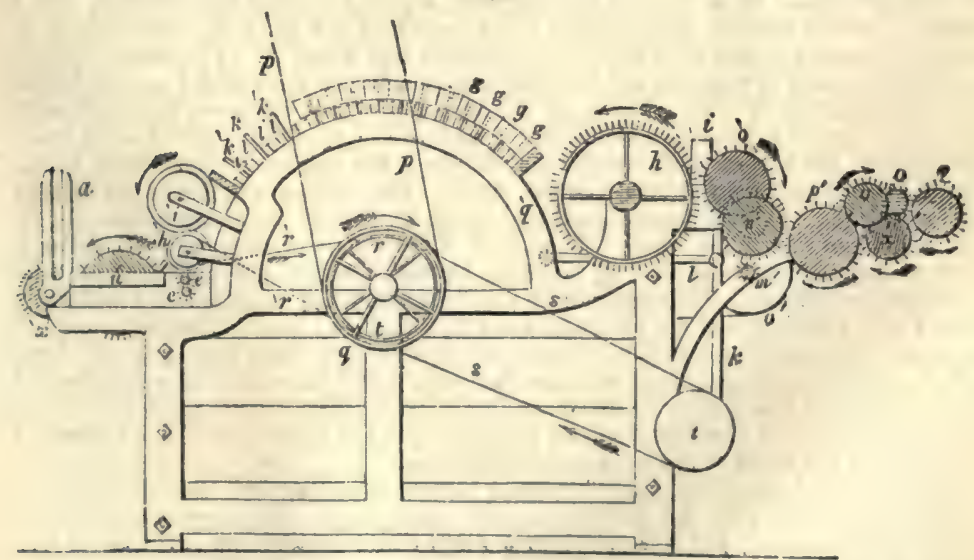

aecess to the interior of the engine, for the purpose of removing whatever dirt, \&c. may happen to fall into it. In fig. 553 we see the manner of fixing the flat tops $g g$ 
over the cylinder; and to make the matter clearer, three of the tope are removed. Upon the archod cast-iron side of the frame, a row of strong iron pins $k$ is made fast in the middle line; and each top piece has, at each of its ends, a hole, which fits down upon two such opposite pins. Il are screws whose heads serve as supports to the tops, by coming into contact with the bottom of the holes, which are not of course bored through the woods of the tops. By turning the heads of these serews a little the one way or the other, the pins may be lengthened or shortened in any degree, so as to set the tops very truly in adjustment with the cylinder teeth revolving beneath them. $h^{\prime}$ is the small runner or urehin, and $i^{\prime}$ the large runner; both of which are spirally corered from end to end with narrow card fillets, in the same manner as the doffer. The main cylinder is, on the contrary, covered with card-cloth, in strips laid on parallel to its axis, with interjacent parallel smooth leather borders. The teeth of these several cards are set as represented in the figure, and their cylinders revolve as the arrows indicate. The runners as well as the doffer cylinder may be set nearer to or further from the cylinder $f$; but the screws intended for this adjustment are omitted in the drawings, to avoid confusion of the lines.

The card-end or fleece taken off the doffer $k$ by the crank and comb mechanism $i k m$ passes through the tin-plate or brass funnel $n, f i g .552$, whereby it is hemmed in and contracted into a riband, which is then passed through between a pair of drawing rollers $o$. It is next received by the rollers $u v$, which carry it off with equal velocity, and let it fall into the tin cans placed below, when it is not to be re-carded, or conduct it over a friction pulley, to be wound along with many other card-ends upon a lap roller or large bobbin. In the latter case it is then conveyed to the 'finisher' carding engine.

The two pairs of rollers at 0 effect the extension of the card-end, and reduce its size. The under rollers are made of iron and fluted; the upper ones are also made of iron, but they are covered with a coat of leather, nicely glued on over a coat of flnnnel, which two coats render them both smooth and elastic. Two weights, $w$, press the upper cylinder steadily down upon the under ones. Between the first and second pair there is a cortain interval, which should be proportioned to tho length of the cotton staple. The second, or that furthest from the funnel, revolves with greater velocity than tho first, and therefore turns out a greater length of riband than it receives from its fellow ; the consequence is, a corresponding extension of the riband in the interval between the two pairs of rollers.

The motions of the several parts of the engine are effected in the following way:The band, $p p$, fig. 553, which comes down from the pulley upon the main shaft near the ceiling of the work-room, drives, by means of the pulley $q$, the cylinder $f$, fig. 552 . From another pulley, $r$, on the axis of the cylinder, the axis of $t$ is driven by the band $s$ working round the pulley $t$ on its end. This shaft drives the crank and lover mechanism of the stripper knife i. A third pulley of the same side as $r$ is fixed just within the frame to the other end of the cylinder, and from it a crossed or close band $r^{\prime}$ goes to a pulley upon the small runner $h^{\prime}$, to give this its rapid rotation. Upon the opposite end of the engine in fig. 552, these wheels and pulleys are marked with dotted lines. Here we may observe, first, a pulley $y$ upon the cylinder, and a pulley $a^{\prime}$, which receives motion from it by means of the band $z$. The axis of $a^{\prime}$, carries in front a pinion $m^{\prime}$, which sets in motion the wheel $n^{\prime}$. The latter imparts motion, by means of a pinion and intermediate wheel $o^{\prime}$, to the wheel $h$ on the doffer, and consequently to that cylinder on the one hand; and it turns, by the carrier wheel $p^{\prime}$, a wheel $x$, whose axis is marked also with $x$ in fig. 553, upon the other hand. The axis $x^{\prime}$, fig. 552, carries, towards the middle of the engine, a very broad wheel, which is represented by a small dotted circle. The toothed wheel $v$ of the smooth roller $v^{\prime}$, fig. 552, and the two toothed wheels 00 , fig. 553, of the under rollers 00 , fig. 552 , work into that broad wheel. The wheel of the second or delivery fluted roller is seen to be smaller than that of the first, by which means the difference of their volocities is obtained. The large runner $i$ is driven from the main cylinder pulley, by means of the band $s^{\prime}$, and the pulley $u^{\prime}$, fig. 552. The said band is crossed twice, and is kept in tension hy the pulloy $t^{\prime}$, round which it passes. The motion of the fluted rollers $e$, which feod in the cotton floce, is accomplished by moans of a berel wheel $b^{\prime}$ on the end of the doffer, which works in a similar whel $c^{\prime}$ on the oblique axis $d^{\prime}$ (dotted lines across the drum) of the pinion $e^{\prime}$ upon the lower end of the same axis which turns the wheel $f^{\prime}$, upon the under feed roller.

Each of the foed rollors, fig. 553, bears a pinion $e e$ at one end, so that the apper roller turns round with the under one. The roller $b$, fig. 552, is set in motion by means of its wheel $x^{\prime}$; which is driven by a wheel $v^{\prime}$ on the other end of the under feed roller, through the intervention of the large carrier-wheel $w^{\prime}$. The original or first motion of $b$ must be as quick as that of the fluted feed rollers $e$, in order that the former may uncoil as much lap as the latter can pass on. 


\section{COTTON-SPINNING}

The annexed Table exhibits the relative velocities of the different cylinders and rollers of the carding engine, which, however, are not invariable, but may be modified

\begin{tabular}{|c|c|c|c|c|c|}
\hline Names of the Parts & & $\begin{array}{c}\text { Diameter in } \\
\text { inches }\end{array}$ & $\begin{array}{l}\text { Circumference } \\
\text { in inches }\end{array}$ & $\mid \begin{array}{c}\text { Revolutions } \\
\text { in one minute }\end{array}$ & Velocity \\
\hline Main cylinder $f$ & . & 35 & $109 \cdot 9$ & $130^{\circ}$ & $142 \cdot 87$ \\
\hline Doffer $h$. & - & 14 & $43 \cdot 96$ & $4 \cdot 38$ & $192 \cdot 5$ \\
\hline Runner or urchin $\varepsilon^{\prime}$ & . & $6 \cdot 25$ & $19 \cdot 62$ & 5 & $98 \cdot 1$ \\
\hline Ditto $h^{\prime}$. & 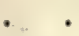 & $3 \cdot 5$ & 11. & $470^{\circ}$ & $5170^{\circ}$ \\
\hline Fluted feed roller $\epsilon$. & & $1 \cdot 167$ & $3 \cdot 664$ & $0 \cdot 696$ & $2 \cdot 55$ \\
\hline First drawing roller o & & 1. & $3 \cdot 14$ & $68 \cdot 71$ & $215 \cdot 75$ \\
\hline Second ditto & . & $1 \cdot 167$ & $3 \cdot 664$ & $114 \cdot 52$ & $419^{\circ} 6$ \\
\hline Smooth delivery roller $v$. & . & $2 \cdot 5$ & $7 \cdot 85$ & $54 \cdot 66$ & $429 \cdot 08$ \\
\hline
\end{tabular}

according to circumstances, by changing the pinions $e^{\prime}$, fig. 552, and $w^{\prime}$ according to the quality or length of the cotton staple.

Fig. 554 is a plan of the card and the fleece, where $h$ is the cylinder, $n$ is the funnel, $u$ the pressing rollers, and $h^{\prime}$ the card-ends in the can.

Figs. 555, 556 may bo studied in order to facilitate the comprehension of these complex machines. Fig. 555 is a plan: $\mathrm{F}$ is the main cylinder; 3 ar is the doffer knife or comb; $G$, the carded fleece hemmed in by the funnel $a$, pressed between the rollers $b$, and then falling in narrow fillets into its can. Fig. 556, $\mathrm{x} \mathrm{x}$ are the feed rollers; $\Delta \mathrm{B}$, the main cylinder; $\mathrm{C} \mathrm{D}$, the tops; $\mathrm{EF}$, the doffer card; ir $\mathrm{N}$, the doffer knife; $d, b, c$, the card-end passing between compressing rollers into the can $a$.

Fig. 557 is a carding-engine without top-flats, being entirely covered with rollers and clearers. This kind of engine is very well suited to the preparation for the lower

555

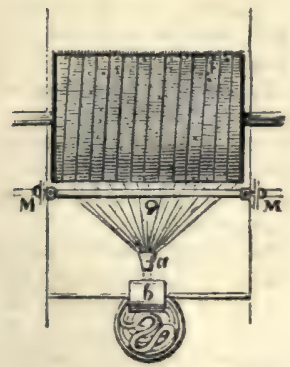

556

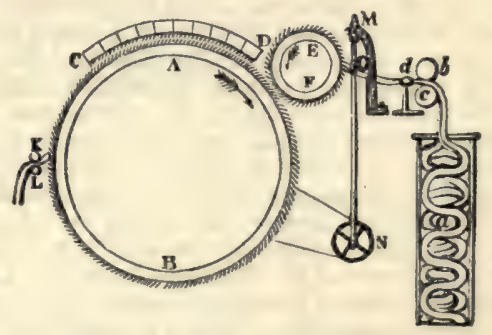

and lower medium 'counts' of yarn. For finer descriptions, the use of 'flats' or ' tops ' is almost universal.

The same fig. (557) will serve to illustrate one of the most modern forms of carding engine, if the reader will suppose two additional rollers placed under the licker-in; all three rollers being of the same size, clothed alike and revolving in the same direction, in close proximity to each other and to the main cylinder. This engine known as the Patent Automatic Carding Engine, is made by the patentees, Messrs. W. Higgins \& Sons, and Messrs. Hetherington \& Sons. Its action is as follows: -The cotton is delivered by the feed rollers to the licker-in as usual; but before it reaches the main cylinder it is brought into contact with and is carded by the intermediate roller, which allows only the carded portions of cotton to pass through to the cylinder, the larger masses being taken hold of by the intermediate roller, and carried forward until they are brought into contact with the bottom roller, which still further cards and separates the fibres of cotton, allowing an additional portion to be delivered to the cylinder, the remainder being taken on by the bittom roller and likewise deposited on the cylinder. Thus, instead of the eotton being delivered in flaky 
masses upon the cylinder by the licker-in, as in the ordinary mode, it is first well opened sod earled, separited from dirt and seeds at the most convenient point, and then distributed lightly and evenly over the main cylinder at three separate points.

Fig. 557 also exhibits a view of a 'coiler' immediately in front of the carding

557

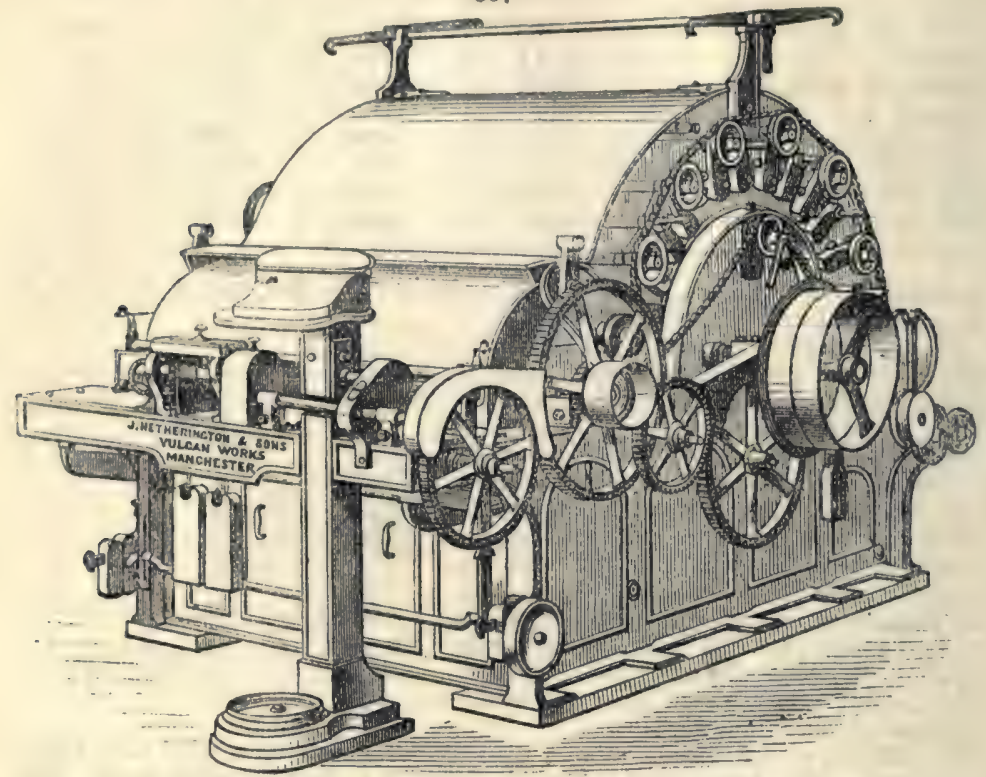

engine. By means of this ingenious contrivance the sliver or riband of cotton instead of coming directly out of the rollers and falling into the can, is made to pass through the upper portion of the coiler, which folds itself with a circular coil into the can placed upon the flat dise-stand at the foot of the coiler. The dise, and with it the can, is made to rerolve very slowly in a contrary direction to that of the coiler, and thus every part of the can is equally filled, and the appearance of the sliver when takon out of the can is that of a serios of neat and closely-packed spiral convolutions.

In some districts where the yarns spun are of medium 'counts' or thickness 'double carding engines' are used, engines in which there is a second cylinder fed directly by the doffer of the first. These machines turn out a large quantity of work, and in the preparation of coarse cottons are efficient and economical.

The efforts to improve the carding engine have of late years been directed rather to clenning and maintaining the efficiency of the engine than to any changes in its methorl of working. 'The delicate points of the wires both on the cylinder doffer" rollers and flats of the machine need to be kept constantly sharp. Frequent 'grinding' is therefore necessary. This is accomplished by friction against prepared surfaces of emery. Formerly manual labour was necessary to effect this purpose as regards the cards on the cylinders. Now, however, it is accomplished entirely by mechanical means. The most recent improvement of this kind is the grinding roller of $\mathrm{Mr}$. Horsfall, which is made to traverse the width of the cylinder in contact with the wires whilst they are revolving rapidly in a backward direction. It is obvious that the impurities remaining after seutching must tend to clog up the wires of the carcling engine. And, as a matter of fact, some parts of the eards need to be cleansed many times in a day. Especially is this the caso with flats. This operation called 'stripping' was until late years a very laborious one; now, bowever, the labour is much lighter, in consequence of the various contrivances for machine stripping. The most important inventions of this kind are the self-stripping flats of Mr. Evan Leigh, of Mr. Buchanan, and Mr. Wellman. Of the last two there are many modifications, and indeed the mechanical stripping of carding engines is still in a state of progress.

It has already been stated that in preparing the longer stapled cottons for the spinuing of fino yarns, a single carding operation is not sufficient. After passing through the first or 'breuker card,' the cotton is put through the 'finisher.' Even the 
finisher card, however, has now been superseded where it has been desired to spin the best fine yarns, by the Combing Machine. This important invention, the discovery of M. Heilmann, an Alsacian, though patented in England in 1846, was first brought into general notice at tho London Exhibition of 1851. The putent right 80 far as it relatod to the preparation of cotton was purchased by a company of five Manchester spinners for $30,000 l$, and for a time these firms enjoyed the exclusive use of the

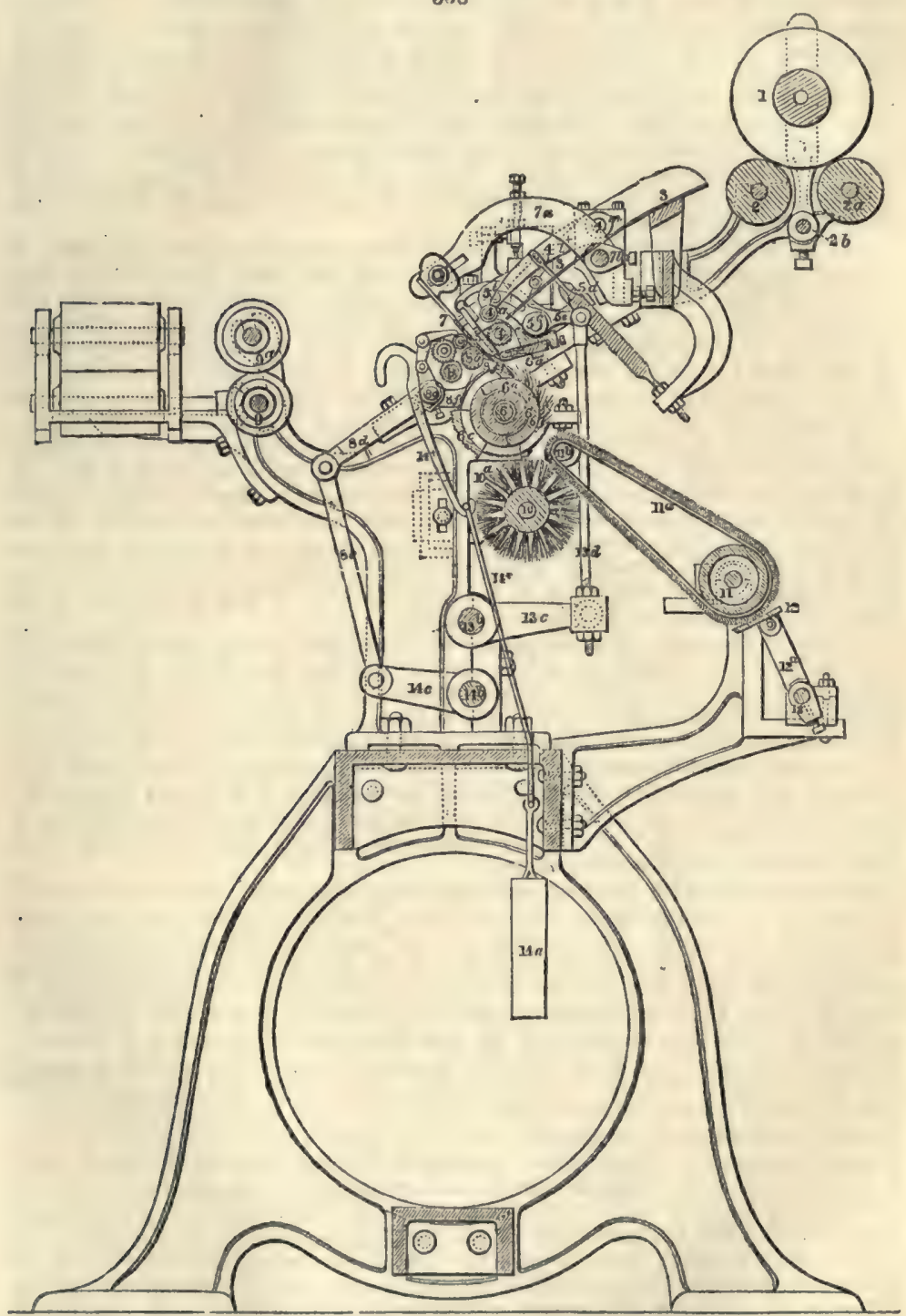

machine. Several improvements have been effected in M. Heilmann's invention by Messrs. John Hetherington \& Sons of Manchester. The great advantages derived from the use of the combing machine consist in its power entirely to remove the mosts and dirt from the cotton, and to separate with far greater precision than is possible with the carding engine the long from the short fibres. The spinning of the finest yarns for the manufacture of muslins, laces, and sewing cotton has thus been 
wonderfully improved. The construction and operation of the combing machine will be readily understood upon a study of the accompanying illustration of a cross-section of the machine (fig. 558).

1 is the lap of cotton resting upon the two wooden rollers $2,2 a$. When motion is given to these rollers, they cause the lap to unwind and deliver the sheet of cotton duwn the inclined conductor 3 , and between the fluted steel feeding roller 4, and the leather-covered pressure roller $4 a$; to these rollers an intermittent motion is given by means of a star wheel; they make $\frac{1}{10}$ th of a revolution to one revolution of the cylinder 6 , this motion being effected during the time the cushion plate $5 a$ is forward, and the nipping plate 5 is lifted from it. The cushion plate $5 a$ is hung upon the centre $5 b$, and the nipping plate upon the shaft $5 c$, and this shaft receives motion from a cam at the end of the machine through the lever $5 e$, the connecting rod $13 d$, lever $13 c$, and shaft $13 b$,- the parts being so arranged that the cushion plate $5 a$ is pressed backward by the nipping plate 5 , but as soon as the pressure is removed it is drawn forward by a spring until it arrives at the strap. Besides this movement, the nipping plate is caused to move on its own axis, which enables it to quit contact with the cushion, while the cotton is being fed in between them.

The cushion $5 a$ is represented as thrown back by the nipping plate 5 , and while in this position the cotton is held between them, until the combs on the cylinder pass between the fibres of cotton which protrude, and remove from them all impurities and the fibres which are too short to be held by the nipper. The combing cylinder $6 a$ is attached to the shaft, or axis 6 , by which it is made to revolve. The periphery of this crlinder is divided into four unequal parts, by the combs $6 b$ on one side, and the fluted segment $6 c$ on the other side; the spaces between them being plain to allow time for the nipper and leather detaching roller $8 a$ to change their positions.

The combs on the cylinder are made with teeth at various distances, the coarser ones taking the lead, and finer teeth following, the last combs having more than 80 teeth in a lineal inch. All impurity or waste mixed with the fibres held by the nipper is carried away by these combs, which at every revolution are cleaned by the cylindrical brush $10 a$, stripping the waste from them, and depositing upon the travelling creeper 11 $a$, formed of wired cloth, which carries it down until the doffing knife, or stoel blade 12 removes it in the usual manner; it then drops into a wasto box, and is afterwards used for inferior purposes.

As soon as the combs have all passed the fibres held by the nipper, the cushion plate $5 a$ is drawn forward, the nipper plate 5 is lifted from it, and the fibres are released. The fluted segment $6 c$ on the cylinder is at the same time passing immediately under the cushion plate $5 a$, the ends of the combed fibres lying upon it, and as the leather detaching roller $8 a$ has been lowered into contact with the fluted segment, they are then drawn forward; but as it is necessary to prevent any fibres passing that have not been properly cleaned or combed, the top comb 7 is placed between the nipper and the roller and as this comb falls and penetrates the fibre, just in front of the part uncombed by the cylindrical combs it prevents any waste from being drawn forward with the combed cotton.

The leather detaching roller $8 a$, in addition to its oceasional contact with the fluted segment $6 c$, is always in contact with the fluted steel detaching roller 8 , and participates in its movements. These rollers are stationary while the cylinder combs are cleaning the fibres projecting from the nipper, but as soon as that operation is completed they are put into motion, and make part of a revolution backward, taking back with them the fibres previously combed, but taken out of the way to allow the cylinder combs to pass, in order for the next fibres coming forward to be joined or pieced to them, so as to form a continuous sliver or riband. As soon as the backward movement is completed, the leather-detaching roller $8 a$ is made to approach the cylinder by the lever $8 f$, which receives motion from a cam at the end of the machine, through the lever $8 d$, connecting rod $8 e$, lever $14 c$, and shaft $14 b$. Before, however, it comes in contact with the fluted segment $6 c$, the movement of the fluted roller is reversed, and it is caused to turn forward, producing a corresponding movement of the detaching roller $8 a$, the speed being so arranged that, before they are allowed to touch each other, the peripheries of the fluted segment $6 c$ and the roller $8 a$ travol with an equal relocity. At this stage, the ends of the fibres cleaned by the cylinder combs and projecting from the nipper are resting upon the fluted segment; and the roller $8 a$, in coming in contact with it, presses upon those fibres, and immediately draws them forward; the front ends are then lifted by the leather roller and placed on the top of those fibres previonsly cleaned, and brought back to receive them. The pressure of the rollers 8 and $8 a$ completes the piecing of the fibres; the motion of the rollers being continued until the hindermost ends of the fibres are drawn through the top comb, and a length of cotton is delivered to the callender rollers, - - sufficient slack being left between to allow for the next baekward movement. The roller $8 a$ is then. 
zaised from the fluted segment and ceases to revolve. From the callender rollers, the combed cotton passes along the front plate or conductor, where it joins the slivers from the other five heads of the machine, and with them passes through the drawing hoad, and is then deposited in a can ready to be removed to the drawing frame.

Such is a minute analysis of the movements of this complicated piece of mechaniøm. Perhaps a cloarer and more complete viow of its operation may be gathered from a summary statement.

The lap of cotton having been placed on a pair of revolving lap rollers, the fleece, or sheet of cotton, is conducted down an inclined guide to a fluted steel feeding roller, which places the cotton between the open jaws of an iron nipper. The nipper is then closed and made to approach the comb cylinder, where it holds the fibres in such a position that the combs of the revolving cylinder pass between and remove from the fibres all impurities and short or broken cotton, which are afterwards worked up into yarns of a coarser quality. As soon as the combs have all passed through the first portion of the fleece under treatment, the nipper recedes from the cylinder, and when it has reached the proper distance, opens its jaws, and allows the partially combed fibres to be drawn out of the fleece, by means of a leather-covered roller. The drawing out of these fibres brings their uneombed ends, which were before held in the nipper, under the teeth of a fine top comb, thus completing the combing of each separate fibre. Previous to the movement for drawing out fresh fibres from the uncombed fleoce, the detaching roller has made a partial revolution backwards, and taken with it the combed cotton previously delivered, in order to piece to it the fibres just combed. The machine is so arranged that the forward movement of the detaching roller brings out the cotton in a continuous sliver to the front of the machine.

Both the detailed and the summary statement just presented describe only one 'head' or section of the machine. There are, however, working simultaneously side by side and identical in construction, six heads on one machine. Each of these delivers a continuous sliver of combed cotton, and the whole six slivers are finally combined into one, which is then ready for the next operation, viz, Drawing.

When the cotton leaves the carding engine (or in the case of very fine yarn the combing machine) it ought to be quite clean, having regard to the particular degree of purity and brightness required in the kind of yarn it is proposed to spin. If the dirt and other impurities have not been sufficiently got rid of at this stage, there is no hope of their being extracted afterwards. What remains now to be done before the actual spinning can take place is simply to equalise the sliver and complete the straightening of the fibres. To a great extent this last operation has been already accomplished by the carding engine; but a slight examination of the fleece as it comes from the doffer of the engine will show that the parallelism of the fibres is as yet in a very imperfect state. This double object of equalising the sliver and making the fibres quite parallel and straight, is effected by means of the Drawing Frame. The former end is reached by frequent combination of the sliver, by doubling as it is termed -whilst the latter is accomplished by drawing out or attenuating the combined slivers. The drawing operation, or draught, is indeed repeated in all the subsequent processes also, and it will therefore be convenient to deseribe it once for all.

Let $a$ and $b$, fig. 559, represent the section of two rollers lying over each other, which touch with a regulated pressure, and turn in contact upon their axes in the direction shown by the arrows. These rollers will lay hold of the fleecy riband presented to them at the point of contact, draw it through between them, and deliver it quite unchanged. The length of the piece passed through in a given time will be equal to the circumference of one of the rollers multiplied by the number of its entire revolutions in the same time. Now if the speed and circumference of the three sets of rollers shown in the figure be the same, that is to say, if their surfaces travel at the same rate, the riband will

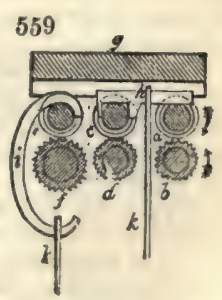
be delivered from the last pair of rollers of exactly the same thickness or 'counts' as at its entrance at the first pair. But if the surface speed of $c$ and $d$ be greater than that of $a$ and $b$, then the former pair will deliver a greater length of riband than the latter receives and transmits to it. The consequence can be nothing else in these circumstances than a regulated drawing or elongation of the riband in the interval betwixt $a, b$, and $c, d$, and a tendency of the filaments as they glide over each other, to assume a straight parallel direction. In like manner the drawing may be repeated by giving the rollers $e, f$, a greater surface speed than that of the rollers $c$ and $d$. This increase of velocity may be produced, either by enlarging the diameter, or by increasing the number of turns in the same time, or finally by both methods conjoined. In general the rollers are so adjusted, 
that the chief elongation takes place between the second and third pairs, while that between the first and socond is but slight and preparatory. It is obvious, besides, that the speed of the middle pair of rollers can have no influence upon the amount of the extension, provided the speed of the first and third pairs remains unchanged. The rollers $a, b$, and $c, d$, maintain towards erch other continually the the same position, but they may be remored with their frame-work, more or less, from the third pair, e, $f$, according as the length of the cotton staple may require. The distance between the points of contact of $a, b$, and $c, d$, is, once for all, so calculated, that it shall exceod the length of the cotton filaments, in order that these filaments may wot be torn asunder, by the second pair pulling them while the first holds them fust. Between $d$ and $f$, where the greatest extension takes place, the distance must bo as small as it can be without risk of tearing. If the distance between $d$ and $f$ be very great, a riband passing through will be found to be much less erenly drawn than when the two sets of rollers are set so close to each other as to act upon the shortest possible longth of riband at once.

The under rollers $b, d, f$, are made of iron, and to enable them to lay firmer hold of the filaments their surfaces are fluted with triangular channels parallel to their axes. The upper rollers, $a, c, e$, are also made of iron, but they are smooth, and covered with a double coating, which gires them a certain degree of softness and elasticity. A cant of flannel is first applied by sewing or glueing the ends, and then a coat of leather in the same way. The junction edges of the leather are cut slanting, so that when joined by the glue, the surface of the roller may be smoothly cylindrical. The top rollers are sometimes called the pressers, because they press by means of weights upon the under ones. 'These weights are suspended to the slight rods $k^{\prime} k$; of which the former operates on the roller $e$ alone, the latter on the two rollers $a$ and $c$ together. For this purpose the former is hung to a c-shaped curve $i$, whose upper hook embraces the roller, not the curved portion of it used in the drawing operation and called the boss, but the central portion between the bosses, which is turned smooth for the purpose. The latter is suspended upon a brass saddle $h$, which rests upon $a$ and $c$. A bar of hard wood, $g$, called a clearer, whose under surface is covered with flannel, rests, with merely its own weight, upon the top rollers, and strips off all the loose hanging filaments, which gather upon the roller when at work. Similar bars with the same riew are made to bear up under the futed rollers $b, d, f$, and press against them by a weight acting through a cord passing over a pulley. Instead of the upper clearers, light wooden rollers covored with flannel are occasionally applied.

Were the drawing of a riband continued till all its fibres acquired the desired degree of parallelism, it would be apt, from excessive attenuation, to tear across, and thereby to defeat the purpose of the spinner. The difficulty is got rid of in a very simple way, namely, by laying several ribands together at every repetition of the process, and incorporating them by the pressure of the rollers. The practice is called doubling. It is an exact imitation of what takes place when we draw a tuft of cotton wool between our fingers and thumb in order to ascertain the length of the staple, and replace the drawn filaments over each other, and thus draw them forth again and again. till they are all parallel and of nearly equal length. The doubling has another advantage, that of causing the inequalities of thickness in the ribands to disappear, by applying their thicker to their thinner portions, and thereby producing uniformity of substance.

The drawing frame, as shown in section in figs. 559, 561, and in a back view in fig. 560, will require, after the above details, little further explanation. $l l$ are the weights which press down the top rollers upon the under ones, by means of the rods $k k^{\prime}$ and hook $i$. Each fluted roller, is, as shown at $f$, fig. 561, provided in the middle of its length with a thinner smooth part ealled the neck, whereby it is really divided into two fluted portions, represented by $e e$ in the figure. Upon this middle neck in the pressure rollers, the hook $i$, and the saddle $h$ immediately bear, as shown in the former fig. 559. The card-ends or slivers to the number probably of six, are introduced to the drawing frame either from cans, placed at e e, fig. 561, and $\mathrm{A}$, fig. 560, or from lap-bobbins; and, after passing through it, the ribands or slivers aro received either into similar cans as $g$, or upon other lap-bobbins upon the other side. These appendages may be readily conceived, and are therefore not exhibited in all the drawings. Three of the slivers being laid together, are again introduced to the one fluted portion $a b$, fig. 559, and three other slivers to the other portion. The sloping curved tin or brass plate $s$, fig. 560 , with its guide pins $t$, serves to conduct the slivers to the rollers. When the two threefold slivers have passed through between the three pairs of rollers, they are gathered together behind the last roller pair e f, fig. 559, and issue from the conical funnel $m$, fig. 560 , into a single riband, which is immediately carried off with equable volocity by two smooth cast-iron rollers, $n$ o, figs. 560 and 561, and either dropped into \& can or wound upon a large bobbin. 
Four fluted drawing portions are usually mounted in one drawing frame, which are ret a-going or at rest togethor.

560

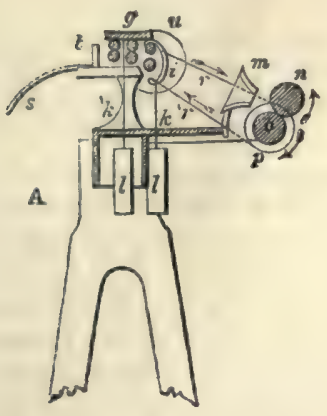

561

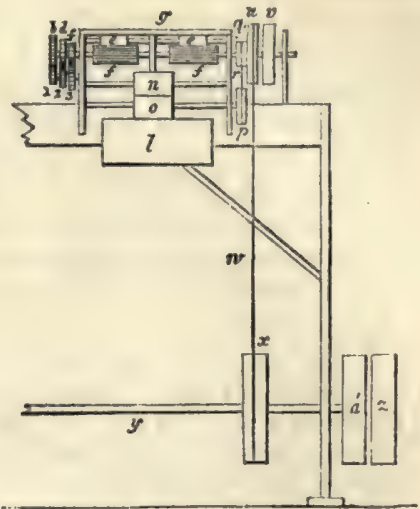

A drawing frame usually consists of three heads or portions, each similar to the other. The cotton passes once through each head, and in order to save the labour of carrying the cans from one side to another of the frame, the centre head is set in a direction contrary to the other two. By this alternate arrangement, the work goes on without interruption.

The fast pulley $u$, fig. 561, by which the whole machine is driven, derives its motion from the main shaft of the mill by means of the band $w$. The similar pulley $z$, which sits loose upon the axis, and turns independentiy of it, is called the loose pulley. When the operative desires to stop the machine, he transfers the band from the fast to tho loose pulley by means of a lever bearing a fork at its end, which embraces the band. This arrangement of fast and loose pulley is common to all machines used in cotton manufacture. Upon $y$, four pulleys such as $x$ are fixed, each of which sets in motion a drawing head, by means of a band like $w$ going round the pulleys $x$ and $u$. The toothed wheel-work, by which the motions are communicated from the backmost fluted roller to the middle and front ones, is seen in fig. 561.

The more frequently the drawing process is repeated, the more perfectly will its object be accomplished. The fineness of the appearance of the sliver after the last draught depends upon the number of doublings conjointly with the original fineness and number of drawings. By changing the wheels which drive the back roller, for others with a different number of teeth, the speed of the roller may be increased or

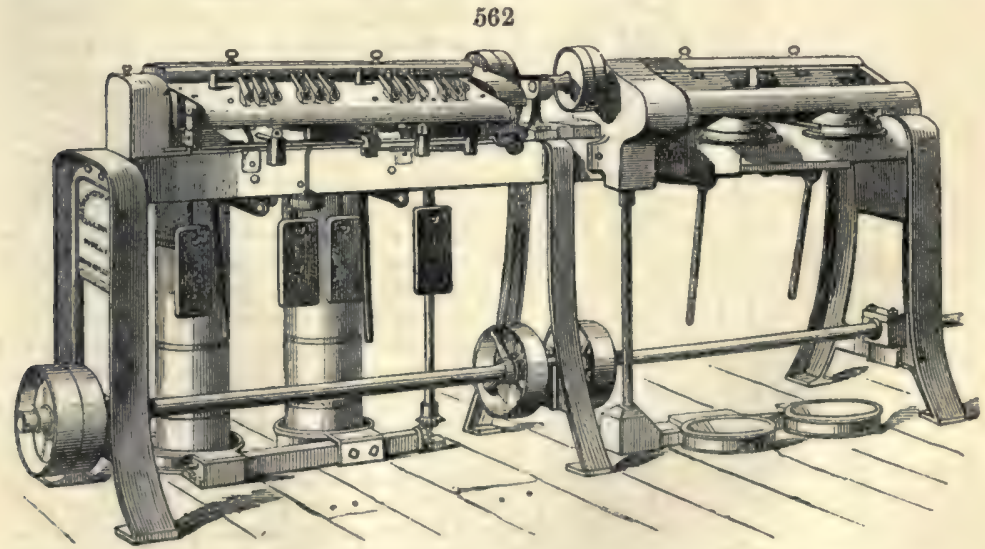

diminished, and thus the fineness of the sliver may be modified in any desired degree; for, when the subsequent processes of the mill remain the same, the finer the drawings Vor.. 1. 
the finer will be the yarn. For spinuing caarse numbers or low counts, for example, six card-ends are usually transmitted through the first drawing head, and converted into one riband. Sis such ribands again form one in the second draught; six of these again go together into the third sliver; and this sliver passes five-fold through the last draught. By this combination 1,080 of tho original card-ends are united in the finished drawn sliver $=6 \times 6 \times 6 \times 5$. The fineness of the sliver is, however, in consequence of these doublings, not increased but rather diminished. For, by the drawing, the card-end has been made 625 times longer, and so much smaller; by the doubling alone it would have become 1,080 times thicker; therefore the original thickness is to the present as 1 , to the fraction $\frac{838}{2080}$; that is, supposing 1,072 feet of the riband delivered by the card to weigh one pound, 625 feet, the sliver of the last drawing, will also weigh a pound.

The rearmost or last drawing roller has a circumference of nearly 4 inches, and makes about 150 revolutions per minute; hence each of these drawing heads may turn off 35,000 foet of sliver in 12 hours.

Fig. 562 is a complete view of a drawing frame as manufactured by Hetherington and Sons, having the usual modern contrivances for increasing the quality and the amount of work done, viz., a stop motion behind the frame to stop the frame when a sliver breaks; a roller plate to prevent roller laps. The coiler motion, by means of which the sliver is placed in the can in circles overlapping each other on the principle described in fig. 563, the can roving frame; 4 rows of draught rollers instead of 3 ; and lastly, an apparatus for lifting all the roller weights from off the rollers at any time when the frame may be stopped.

A further improvement is now added to drawing frames, viz., a front stopping motion. The object of the back stopping motion is to prevent inequalities arising in the sliver from the occasional breakage of one of the slivers which are being combined together. The front one has a different purpose. It is obvious that whenever the rollers which carry forward the sliver from the front drawing rollers to the can fail to take up the cotton as fast as it is delivered, there arises an accumulation, which, from the great speed at which the sliver passes through the machine, rapidly increases. All this accumulation was formerly so far wasted that it was necessarily sent back into the mixing room to be worked over again. By the use of a front stopping motion the drawing frame is now stopped instantly, and all accumulation of this kind prevented.

Hitherto the riband or sliver has received no twist, but the fibres have lain in a linear, parallel manner, holding together in a loose mass. But in the further preparatory processes, some reduction of the thickness of the riband is necessary, so that it may gradually approach the thickness finally required in the yarn. The natural cohesion of the fibres when placed side by side will not now be sufficient to carry the sliver forward. At the next stage, therefore, a slight convolution or twist is given to it simply for this purpose. This operation takes place in the Slubbing Frame. The twisting should go no further than to fulfil the purpose of giving cohesion, otherwise

568

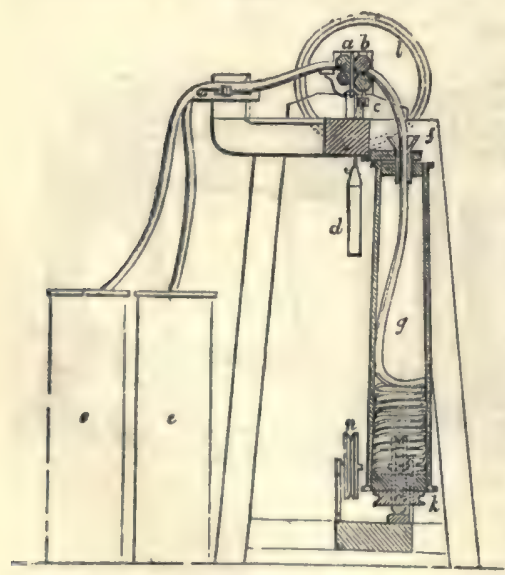
it would place an obstacle in the way of the future attenuation into level thread. The combination of drawing and twisting is what mainly characterises the spinning processes, and with this fifth operation therefore, commences the formation of yarn. As, however, a sudden extension to the wished-for fineness is not practicable, the draught is thrice repeated.

Fig. 563 is a section of the can, or lantern slubbing frame, the ingenious invention of Arkwright, which, until within the last 50 years, was the principal machine for communicating the incipient torsion to the spongy cord furnished by the drawing frame. It differs from that frame in nothing but the twisting mechanism; and consists of two pairs of drawing rollers, $a$ and $b$, between which the sliver is extendud in the usual way; at $e$ there are brushis for cleaning the rollers; and $d$ is the weight which presses the upper set upon the lower. The wiping covers (not shown here) rest upon $a b$. The surface speed of the posterior or second pair of rollers is 3,4 , or 5 times greater than that of the front or receiving pair, ac- 
cording to the desired degree of attenuation. Two drawn slivers were generally united into one by this machine, as is shown in the figure, where they are seen coming from the two cans $e e$, to be brought together by the prossure rollers, before they reach the drawing rollers $a b$. The sliver as it escapes from these rollers, is conducted into the revolving conical lantern $g$, through the funnel $f$ at its top. This lantern-can receives its motion by means of a cord passing over a pulley $k$, placed a little way above the step on which it turns. The motion is steadied by the collet of the funnel $f$, being embraced by a brass busk. Such a machine generally contained four drawing heads, each mounted with two lanterns; in whose side there was a door for taking out the conical coil of roving. The motion imparted to the back roller by the band pulley or rigger $n$, was conveyed to the front one by toothed wheel-work.

The can slubbing frame has long been superseded by the Bobbin and Fly frame, a kind of machine which, with some modifications, is still used not only for the Slubbing process, but also for the two following preparatory ones, viz, Intermediate and Roving. Figs. 564 and 565 exhibit a perspective and a sectional view of this frame. It is to be

564

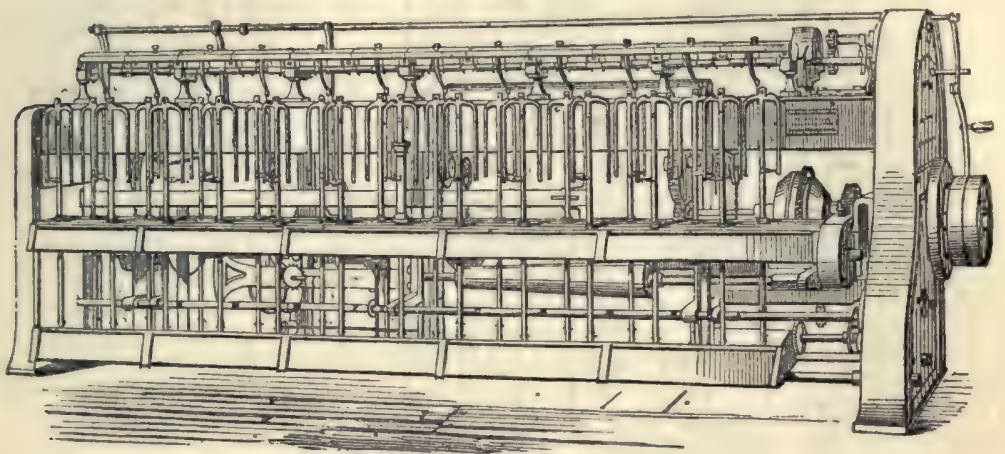

observed, however, that these illustrations, though serving well enough to explain the general construction and operation of the Bobbin and Fly frame, do not show the recent improvements, which will be described further on.

Fig. 565 exhibits a back view of this machine; and fig. 566 a section of some of the parts not very visible in the former figure. The cotton is introduced between the drawing rollers. The cans, filled with slivers from the drawing frame, are placed in the situation marked at the back of the machine $\mathrm{B}, \mathrm{fig} .565$, in rows parallel with the length of the machine. The sliver of each can is conducted upwards along the surface of a sloping board $f$, and through an iron staple or guide $e$, between the usual triple pair of drawing rollers, the first of which is indicated by $a b$. In fig. 565, for the purpose of simplifying the figure, the greater part of these rollers and their subordinate parts are omitted. After the slivers have been sufficiently extended and attenuated between the rollers, they proceed forwards, towards the spindles $i i i$, where they receive the twist, and are wound upon the bobbins $h$. The machine delineated contains thirty spindles, but many bobbin and fly frames contain double or even four times that number. Only a few of the spindles are shown in fig. 565.

The drawing rollers in this machine do not differ essentially from those described under the section devoted to the drawing frame. This part of the machine, called the roller beam, is a cast-iron bench, upon which the bearers $c$, are mounted for carrying the rollers. The fluted portions of the rollers $a a a$, fig. 567, alternate with smooth 'necks' $z z z$. The whole length of roller is subdivided into sections. The coupling of these roller sections or subdivisions into one cylinder is secured by the square holes $x$, and square pins $y$, fig. 567, which fit into the holes of the adjoining subdivision. The top or pressure rollers $b$, are two-fold orer the whole set; and the weighted saddle presses upon the neck $w$, which connects every pair, as was already explained under fig. 560.

The structure and operation of the spindles $i$ may be best understood by examining the section fig. 568. They are made of steel, are cylindrical from the top down to $a^{2}$, but from this part down to the steel tipped rounded points they.are conical. The wooden bobbin $h$, slides upon the cylindrical part, which must move freely upon it, as will be presently explained. The upper end of the spindle bears a fork $s t$, which, though rerolving with it, may be taken off at pleasure when the machine is standing still.

$$
3 \text { R } 2
$$


This fork or flyur, has a funnel-formed hole at $v$. One arm of the fork is a tube $s, u$, upen at top and bottom; the $\log t$, is added merely as a counterpoise to the other.

665

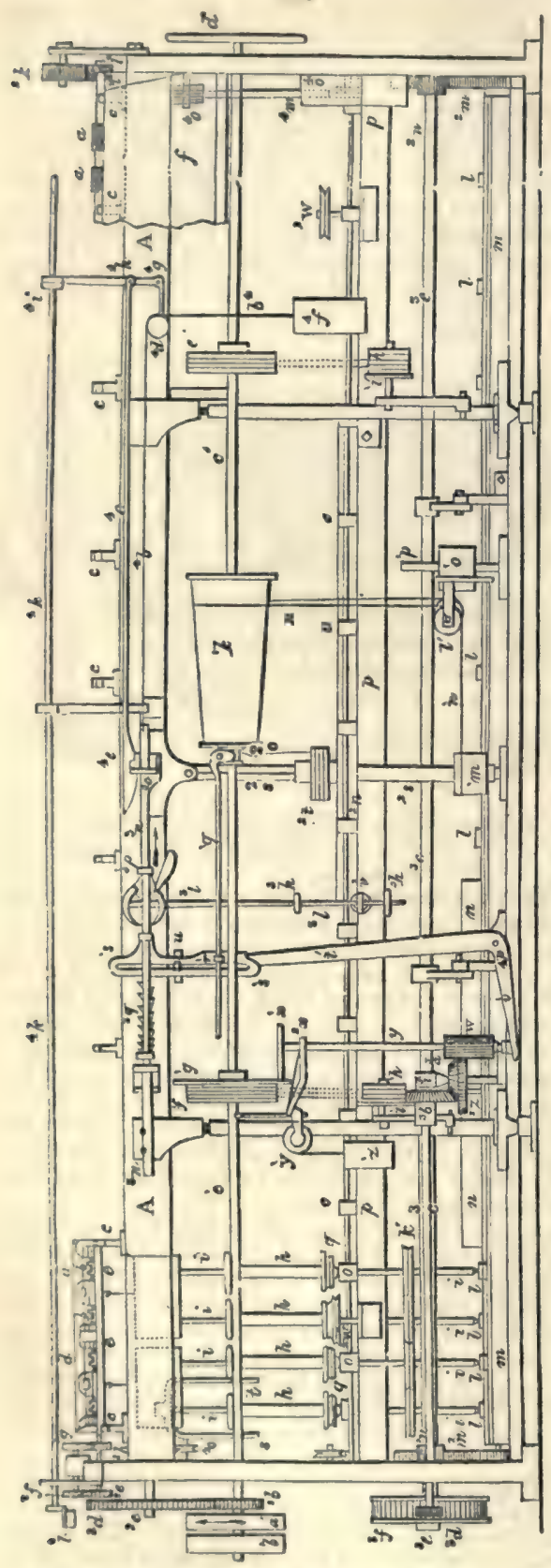

566

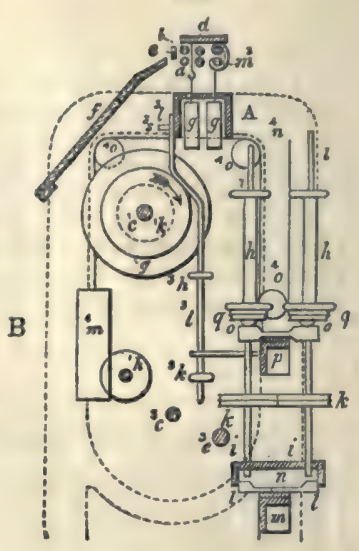

567

568
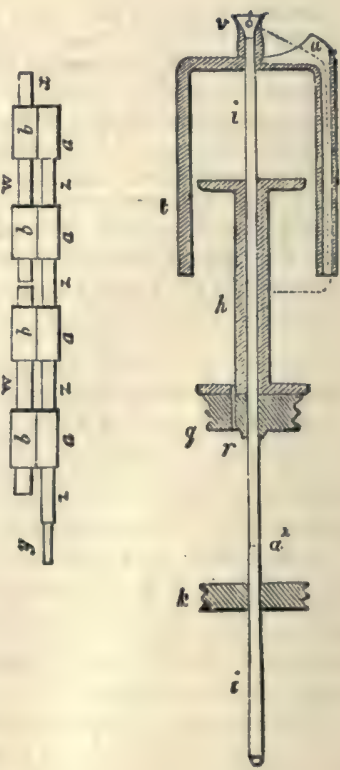

In fig. 566, for the sake of clearness, the forks or flyers of the two spindles here represented are left out; and in fig. 565 only one is portrayed for the same reason. If 
we suppose the spindles and bobbins (both of which have independent motions) to revolve simultaneously and in the same direction, their operation will be as follows: The sliver properly drawn by the fluted rollers, enters the opening of the funnelv, proceeds thence downwards through the hole in the arm of the fork, runs along its tube $u, s$, and then winds round the bobbin. This path is marked in fig. 568 by a dotted line.

The revolution of the spindles in the above circumstances twists the sliver into a soft cord; and the flyer $8, t$, or particularly its tubular arm 8 , lays this cord upon the bobbin. Were the speed of the bobbins equal to that of the spindles, did the bobbin and spindle make the same number of turns in the same time, the process would be limited to mere twisting. But the bobbin makes in a given time a somewhat greater number of revolutions than the spindle, and thus the cord is wound upon it. Suppose the bobbin to make 40 revolutions, while the spindles make only $30 ; 30$ of these rerolutions of the bobbin will be inoperative towards the winding-on, because the flyers follow at that rate, so that the cord or twisted sliver will only be coiled 10 times round the bobbin, and the result as to the winding-on will be the same as if the spindle had stood still, and the bobbin had made $40-30=10$ turns. The 30 turns of the spindles serve, therefore, merely the purpose of communicating twist.

Simple as the winding-on process may seem, a little reflection will show that there are complications involved in it which do not at first sight appear. It must be observed, in the first place, that as the cord is wound on the bobbin, the diameter of the latter increases with every succeeding layer of cord put upon it, and the length taken up by each turn becomes continually greater. It is, therefore, necessary so to reduce the number of revolutions of the bobbin as that they may not stretch nor break the cord by taking it up faster than it is delivered from the fluted rollers. A second point to be noticed is, that in order to secure a proper distribution of the cord upon the bobbin, and the regular increase of its diameter, two of its successive convolutiong should not be applied over each other, but that they should be laid close side by side. This object is attained by the slowly ascending and descending motion of the bobbin upon the spindle. This up-and-down motion must become progressively slower, since it increases the diameter of the bobbin at each range by a quantity equal to the diameter of the sliver. What has now been stated generally will become more intelligible by an example.

Let it be assumed that the drawing rollers deliver, in 10 seconds, 45 inches of roving, and that this longth receives 30 twists. The spindles must, in consequence, make 30 revolutions in 10 seconds, and the bobbins must turn with such speed, that they wind up the 45 inches in 10 seconds. The diameter of the bobbin barrels being $1 \frac{1}{2}$ inch, their circumference of course $4 \frac{1}{2}$ inches, they must make 10 revolutions more in the same time than the spindles. The effective speed of the bobbins will be thus $30+10=40$ turns in 10 seconds. Should the bobbins increase to 3 inches diameter, by the winding-on of the sliver, they will take up 9 inches at each turn, and consequently 45 inches in 5 turns. Their speed should therefore be reduced to $30+5=35$ turns in 10 seconds. In general, the excess in number of revolutions which the bobbins must make over the spindles is inversely as the diameter of the bobbins. The speed of the bobbins must remain uniform during the period of one ascent or descent upon their spindle, and must diminish at the instant of changing the direction of the up-and-down motion; because a fresh range of convolutions then begins with a greater diameter.

The motions of the drawing rollers, the spindles, and bobbins are produced in the following manner:-A shaft $c^{\prime}$, figs. 565 and 566, extending the whole length of the machine, and mounted with a fly-wheel $d^{\prime}$, is set in motion by a band from the running pulley upon the shaft of the mill, which actuates the pulley $a^{\prime}$. $b^{\prime}$ is the loose pulley upon which the band is shifted when the machine is set at rest. Within the pulley $a^{\prime}$, but on the outside of the frame, the shaft $c^{\prime}$ carries a toothed wheel $b^{2}$, which by means of the intermediate wheel $c^{2}$ turns the wheel $d^{2}$ upon the prolonged shaft of the backmost fluted roller $\left(m^{2}, f i g .566\right)$. This wheel, $d^{2}$, may be changed for one with a greater or less number of teeth when the cotton is to receive more or less twist; for as the spindles revolve with uniform velocity, they communicate the more torsion the less length of sliver is delivered by the rollers in a given time. Upon the same shaft with $d^{2}$, a pinion, $e^{2}$, is fixed, which works in a wheel, $f^{2}$. Within the frame another changeable toothed wheel, or 'change pinion' $g^{2}$, is made fast to the shaft of $f^{2}$. This pinion regulates the drawing, and thereby the fineness or number of the roving. It works in a wheel, $h^{2}$, upon the end of the backmost fluted roller a, fig. 566. The other extremity of the same roller, or, properly speaking, line of holder and the pulley is sufficient to distend the endless band $n^{\prime}$, which runs from the cone $k^{\prime}$, through under the pulley $l^{\prime}$, and round the small drum $m^{\prime}$ on the shaft $s^{2}$. A pulley or whorl $t^{2}$ with four grooves, is made fast by means of a tube to this shaft, and slides along it backwards and forwards, without ever ceasing to follow its revolutions. The shaft pos- 
sesses for this purpose a long fork, and the interior of the tube a corresponding tongue or catch. There is besicles upon the tube benenth the pulley, at $u^{2}$, a groove that goes round it, in which the staple or forked end of an arm like $v^{2}, f i g .565$, made fast to the copping beam $p$, catches. By the up-and-down movement of that beam, the pulley $t^{2}$ takes along with it the arm that embraces the tube, which therefore rises and falls equally with the bobbins $h^{\prime}$, and their pulleys or whorls $q$. This is requisite, since the bobbins are made to revolve by the pulleys $t^{2}$ by means of two endless cords or bands.

Such was the bobbin and fly frame prior to the improvements introduced by Mr. H. Houldsworth in the 'equation box' mechanism and the 'spring fingers.' By means of the former the differential movement of the bobbins already referred to was accomplished with much greater precision and neatness than previously. The object of the latter was, by laying the soft cord or sliver upon the bobbin with a certain degree of pressure, to put an increased weight of cotton upon it, and thus to save some portion of the time and labour lost in the then frequent operations of 'doffing' or removing the full bobbins and placing empty ones in their places.

Fig. 569 represents a portion of a fly frame with Mr. Houldsworth's invention.

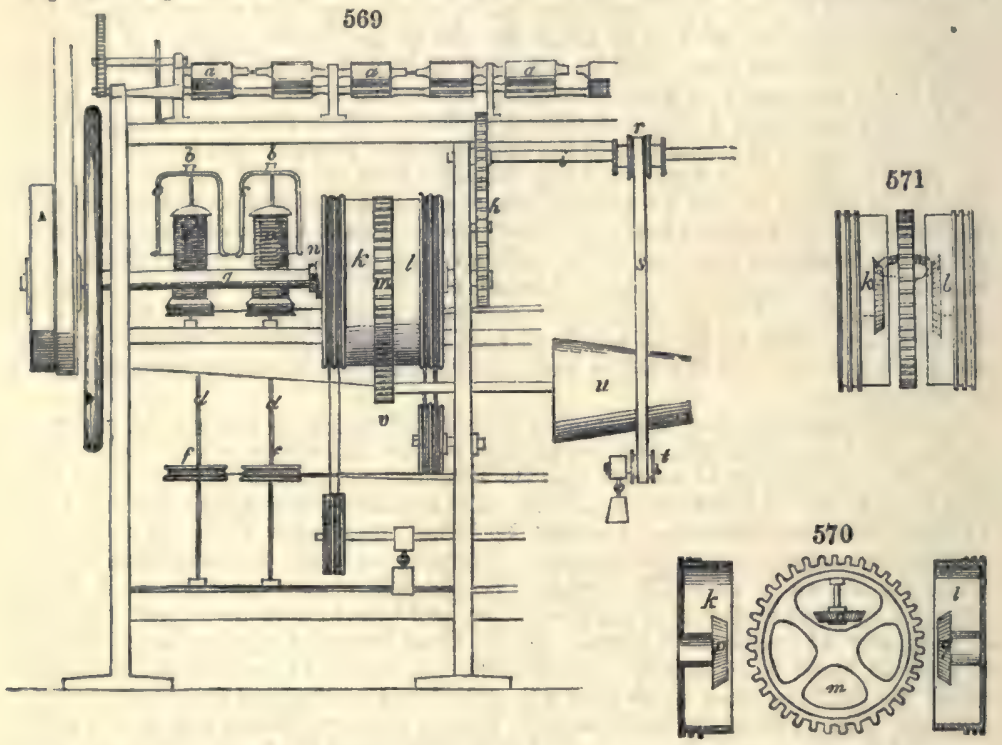

a $a$ are the front drawing rollers, turning upon bearings in the top of the machine, and worked by a train of toothed wheels, in the way that drawing rollers are usually actuated. From the drawing rollers, the flaments of cotton are brought down to $b$, and passed through the arms of the flyers $c c$, mounted or the tops of the spindles $d d$, which spindles also carry the loose bobbins $e e$. According to the former mode of constructing such machines, the spindles were turned by cords or bands passing from a rotatory drum round their respective pulleys or whorls $f$, and the loose bobbins $e$, curned with them by the friction of their slight contact to the spindle, as before said; in the improved machine, however, the movements of the soindles and the bobbins are independent and distinct from each other, being actuated from different sources.

The main shaft $g$, turned by a band and rigger $\mathbf{A}$ as usual, communicates motion by a train of wheels $h$, through the shaft $i$, to the drawing rollers at the reverse end of the machine, and causes them to deliver the filaments to be twisted. Upon the main shaft $g$, is mounted a cylindrical hollow box or drum pulley, whence one cord passes to drive the whorls and spindles $f$ and $d$, and another to drive the bobbins $e$. This cylindrical box pulley is made in two parts, $k$ and $l$, and slipped upon the axle with a toothed wheel $m$, intervening between them. The box and wheel are shown detached in fig. 570, and partly in section at fig. 571. That portion of the box with its pulley marked $l$, is fixed to the shaft $g$; but the other part of the box and its pulley $k$, and the toothed wheel $m$, slide loosely round upon the shaft $g$, and when brought in contact and confined by a fixed collar $n$, as in the machine shown at fig. 569 , they constitute two distinct pulleys, one being intonded to actuate the spindles, and the 
other the bobbins. In the web of the wheel $m$, a small bevel pinion 0 , is mounted upon an axle standing at right angles to the shaft $g$, which pinion is intended to take into the two bevel pinions $p$ and $q$, respectively fixed upon bosses, embracing the shaft in the interior of the boxes $k$ and $l$. Now it being remembered that the pinion $q$, and its box $l$, are fixed to the shaft $g$, and turn with it, if the loose wheel $m$ be independently turned upon the shaft with a different velocity, its pinion $o$, taking into $q$, will be made to revolve upon its axle, and to drive the pinion $p$ and pulloy box $k$, in the same direction as the wheel $m$; and this rotatory movement of the box $k$ and wheel $m$, may bo faster or slower than the shaft $g$, and box $l$, according to the relocity with which the wheel $m$ is turned.

The main shaft $g$, being turned by the band and rigger $\mathbf{\Delta}$, as above said, the train of wheels $h$, connected with it, drives the shaft $i$, which at its reverse end has a pinion (not seen in the figures) that actuates the whole series of drawing rollers $a$. Upon the shaft $i$ there is a sliding pulley $r$, carrying a band $s$, which passes down to a tension pulley $t$, and is kept distended by a weight. This band $s$, in its descent, comes in contact with the surface on the cone $r$, and causes the cone to revolve by the friction of the band running against it. The pulley $r$ is progressively slidden along the shaft $i$, by means of a rack and weight not shown, but well understood as common in this kind of machines, and which movement of the pulley is for the purpose of progressively shifting the band $s$ from the smaller to the larger diameter of the cone, in order that the speed of its rotation may gradually diminish as the bobbins fill by the winding-on of the yarns.

At the end of the axle of the cone $u$ a small pinion $v$ is fixed, which takes into the teeth of the loose wheel $m$, and as the cone turns, drives the wheel $m$ round upon the shaft $g$, with a speed dependent always upon the rapidity of the rotation of the cone. Now the box pulley $l$, being fixed to the main shaft $g$, turns with one uniform speed. and by cords passing from it over guides to the whorls $f$, drives all the spindles and flyers, which twist the yarns with one continued uniform velocity; but the box pulley $k$, being loose upon the shaft, and actuated by the bevel pinions within, as described, is made to revolve by the rotation of the wheel $m$, independent of the shaft, and with a different speed from the pulley box $l$; cords passing from this pulley box $k$, over guides to small pulleys under the bobbins, communicate the motion, whatever it may be, of the pulley box $k$, to the bobbins, and cause them to turn, and to take up or

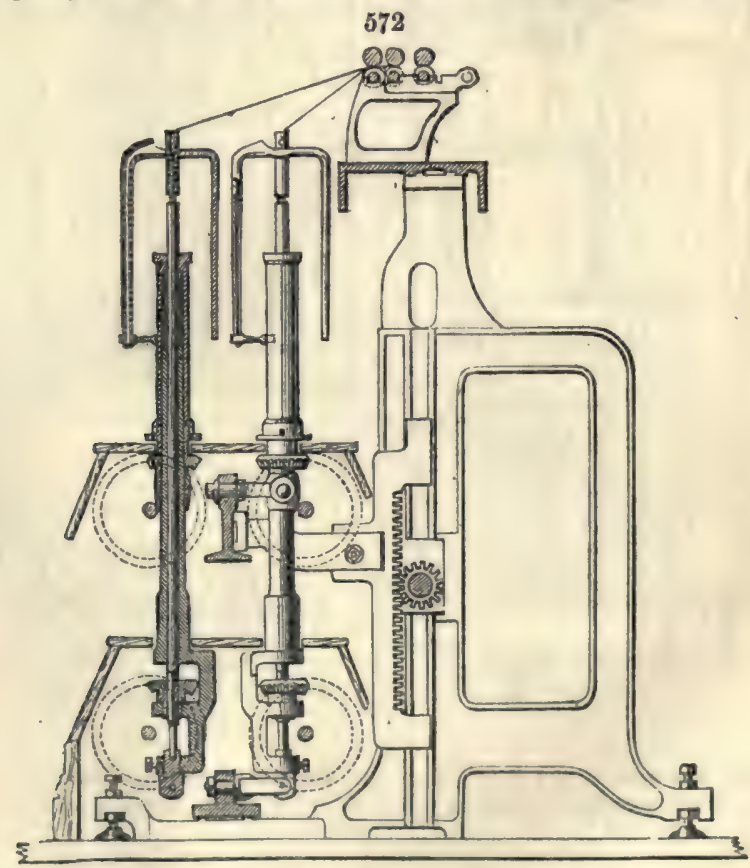

wind the yarn with a speel derived from this source, independent of, and different from, the speed of the spindle and flyer which twist the yarn. 
It will now be perceired, that these parts being all adjusted to accommodate the anking up movements to the twisting or spinning of any particular quality of yarn intended to be produced, any variations between the velocities of the spinning and taking up, which another quality of yarn may require, can easily be effected by merely changing the pinion $v$ for one with a different number of teeth, which will cause the

573

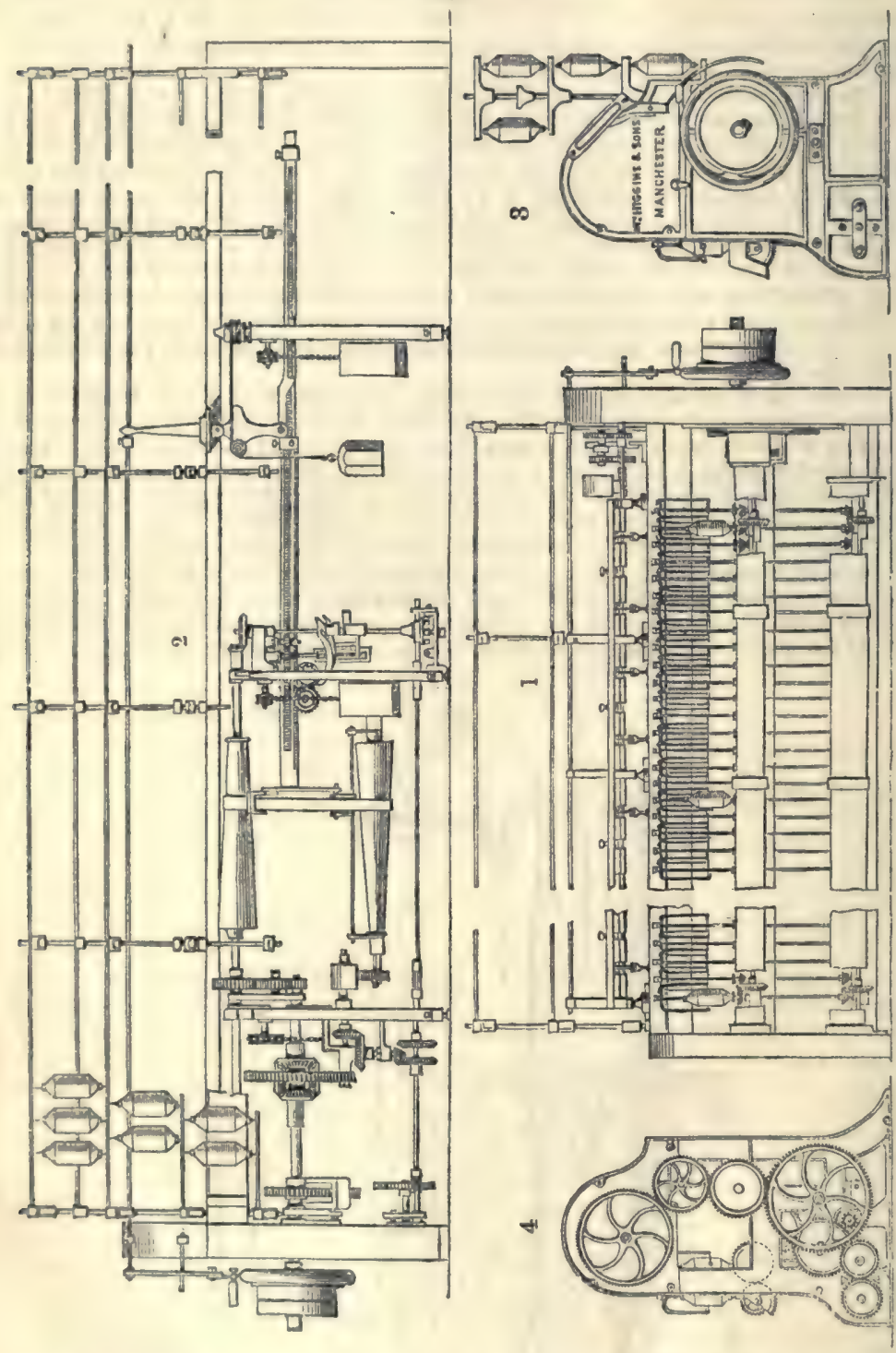

wheel $m$, and the pulley box $k$, to drive the bobbins faster or slower, as would be required in winding-on fine or conrse yarn, the speed of the twisting or spinning being the rame.

The bobbin and fly frame as thus detailed is now no longer constructed; but it seemed desirable, if not necessary, to describe with some minuteness a machine which 
has formed the basis of the more modern slubbing, intermediate, and roving frames. These three operations, which follow each other in the order named, come next after that of drawing. They have one common object, to gradually attenuate the sliver until it is at last sufficiently reduced in thickness to be advantageously spun into yarn. But the intermediate and roving processes have one other purpose, analogous to that fulfilled by the drawing frame, viz. to secure uniformity of thickness throughout by the operation of doubling. Thus two slubbings are united together, and are drawn out into one intermediate slubbing, and two intermediate slubbings into one roving.

The slubbing frame usually consists of from 40 to 80 spindles. Its main features will be easily comprehended after the preceding descriptions, by a glance at fig. 572 , which presents the principal details seen in a tranverse section. The sliver is brought from the drawing frame in deep cylindrical cans and placed behind the slubbing frame (on the right hand of the figure), after being drawn through the rollers in the manner already pointed out. It will be observed, however, that instead of bobbins, as in the old bobbin and fly frame, simple wooden tubes are now used. By means of the presser attached to one end of each flyer the slubbing, as the cotton sliver is now called, is built up in a solid hard mass upon the tube, not in a cylindrical form, as in the case of the bobbin, but with the ends tapering conically as shown in fig. 573 .

The construction of the intermediate and roving frames is identical in plan, and so far as the principal working parts are concerned it is similar to that of the slubbing frame. In fig. 573, No. 1 illustrates a front sectional view of an intermediate frame, No. 2 a back view, and Nos. 3 and 4 the two ends. Here we see in the back, and in one of the end views, the spools of cotton produced by the slubbing frame, and hence called slubbings, placed in the 'creel' behind the frame. The 'ends' of these slubbings are now 'drawn' or attenuated in pairs through the rollers, after the fashion previously described, and are once more slightly twisted and wound upon smaller tubes than before. These when full are transferred to the creel of the roving frame, and there receive treatment precisely similar to that of the intermediate frame. The 'roving', as the cord is called, after passing through the roving frame is, however, much more attenuated than the 'intermediate,' and is now ready for the final spinning processexcept in mills where very fine yarns are spun. In these cases, a second or fine roving or ' jack' frame is used for the purpose of again doubling and equalising the cord and further reducing its thickness. The intermediate frame usually contains from 80 to 120 spindles, and the roving frame from 120 to 200 spindles. In fig. 574 the front view of a roving frame is given with greater completeness than is presented in fig. 573.

574

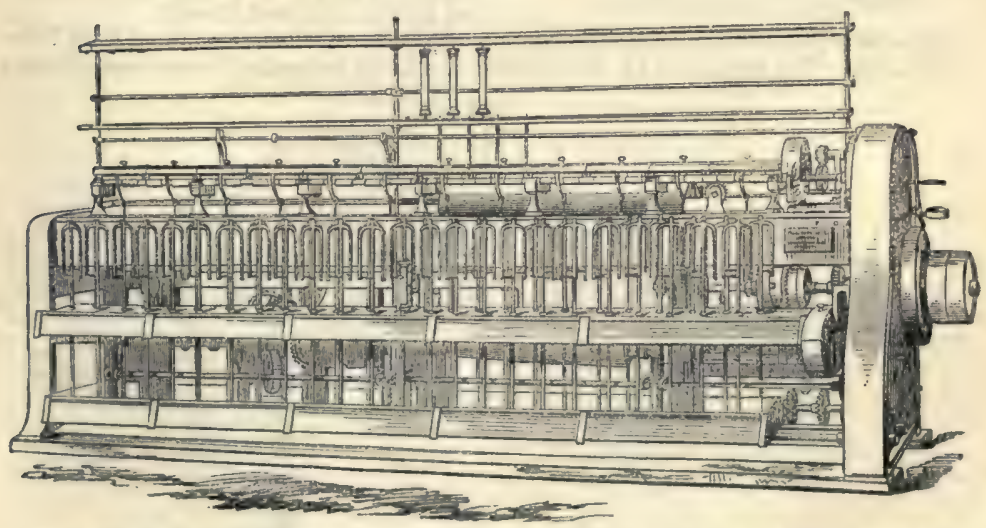

The earlier processes of the cotton spinning industry which we have thus traced are usually comprehended under the technical term, the 'preparation;' and it will seadily bo understood from even the cursory view we have endearoured to present, that the quality of the yarn when spun will greatly depend upon the manner in which these processes are conducted. So widely is this truth recognised amongst practical spinners, that it has become almost an axiom that a bad roving can never make \& good yarn. Since the 'cotton famine' which arose out of the American civil war of 1861-65, much progress has been mado in the practical management of tho preparatory processes. The searcity and high price of raw cotton during the period in question, the necossity of resorting to new and inferior descriptions, their varying 
characteristica, and the difficulty of adapting them to the kind of production for which American cotton had proved eminently suitable, laid upon persons engaged in cottonspinning, at that time, a task which required much ingenuity and patienco. The result has been, however, to improve greatly the technical methods used in the art, and especially as we have stated, in the details of the proparation. This end has not been reached by any fundamental alteration, but rather by a number of minor improvements, which are the result of a closer study and a better knowledge of the working qualities of the fibre in its many varioties than had previously been thought necessary, and the consequence has been a more careful and successful treatment in every part of the chain of operations under consideration.

The great object to be kept in view throughout the 'preparation' is to produce a roving as free from impurity as possible, and as uniform in weight and texture as it ean be made, consistently with due economy. For the former end, the efficiency and right management of the scutching and carding machinery are requisite; for the latter a due adjustment of the draughts and doublings, regard being always had towards the fineness and quality of the yarn required.

Without attempting anything in the way of a history of cotton-spinning, it may be well to refer briefly to some of the principal stages through which this, one of the

575

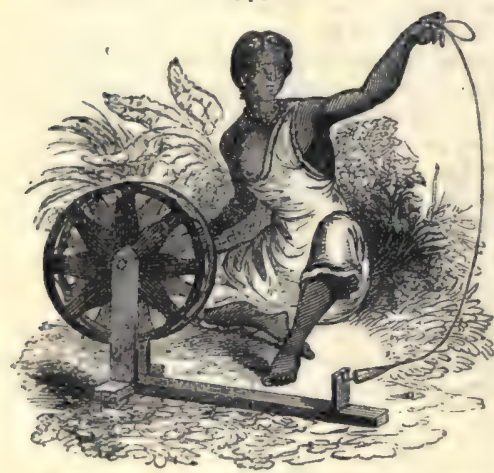

earliest of the industrial occupations of civilised life, has at length reached its present advanced position. Except the spindle and distaff, the use of which was purely handicraft, the earliest contrivance for the production of yarn was the spin. ning wheel of ancient India (fig. 575), an instrument very like the common ' wheel,' which, until the dawn of this mechanical age extinguished it, was the familiar companion of every English spinster.

The first-successful effort to improve upon this primitive piece of mechanism was that of James Hargreaves, who about the year 1764 invented his 'spinning jenny,' which differed little from the spinning wheel, except in enabling one person to spin simultaneously upon several spindles, instead of one.

Fig. 576 represents the original jenny of Hargreaves, which contained from 16 to 40 spindles. The soft cords of rovings wound in double conical cops upon skewers

\section{6}

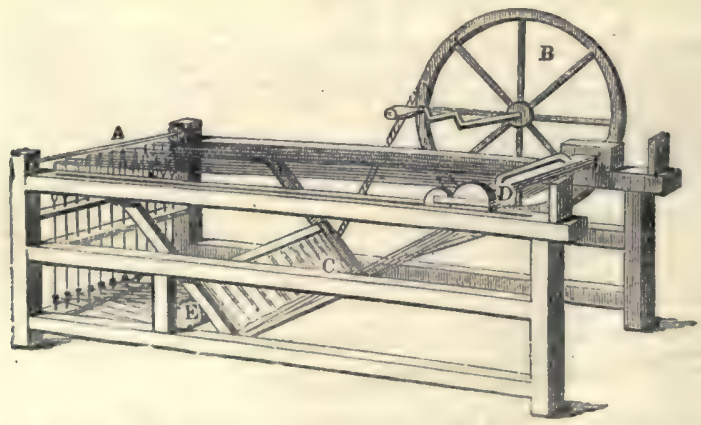

were placed in the inclined frame at $\mathrm{c}$; the spindles for first twisting and then windingon the spun yarn were set upright in steps and bushes at $\mathbf{A}$, being furnished near their lower ends with whorls, and endless cords, which were driven by passing round the long-revolving drum of tin-plate, $\mathbf{E} . \quad \mathrm{D}$ is the clasp or clove, having a handle for lifting its upper jaw a little way, in order to allow a few inches of the soft roving to be introduced. "The compound clove $D$ being now pushed forward upon its friction wheels to $\Delta$, was next gradually drawn backward, while the spindles were made to revclve with proper speed by the right hand of the operative turning the flywheel $\mathrm{B}$. Whenever one stretch was thereby spun, the clove frame was slid home towards $\mathbf{A}$; 
the spindles being simultaneously whirled slowly to take up the yarn, which was laid on in a conieal cop by the due depression of the faller wire at $\mathbf{A}$, with the spinner's left hand.

It will be observed that no attempt was made in Hargreaves' machine to 'draw' the cotton through rollers, which is the chiof distinction between ancient and modern spinning. The discovery of this principle really preceded that of Hargreaves, and was the work of Lewis Paul of Birmingham, who, in the year 1738, took out a patent for 'spinning wool and cotton by rollers.' The patentee appears to have received important aid in the working out of the idea from John Wyatt, also of Birmingham, if indeed, the original conception be not attributable to him. It does not appear, however, that the invention attributed to Paul was ever adopted upon any large or useful scalo.

We come now to the spinning machine of Arkwright, the invention of which was almost contemporaneous with that of Hargreaves' jenny.

Fig. 577 is a diagram of Arkwright's original water-frame spinning machine, called afterwards the water-twist frume. The rovings mounted upon bobbins in the creel $\mathbf{A} \mathbf{A}$,

577

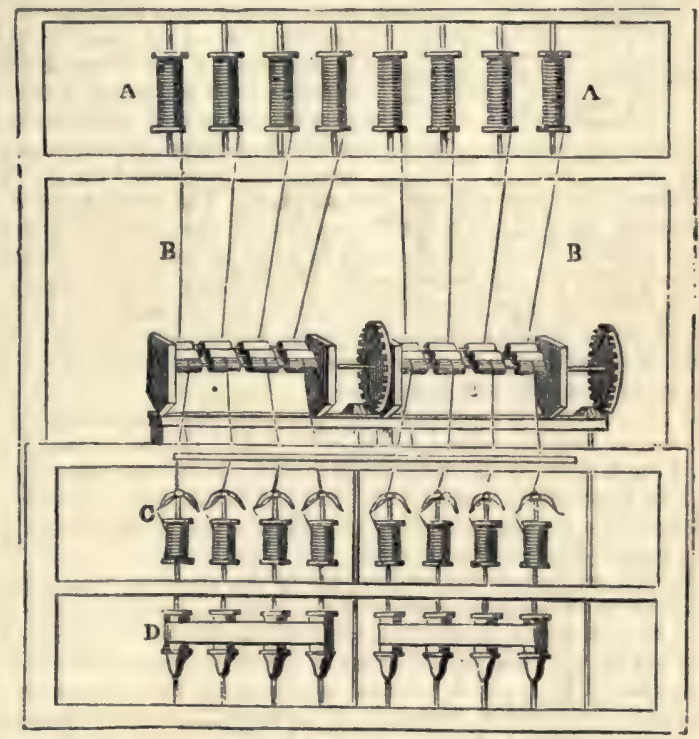

have their ends lod through between the three sets of twin rollers below B B, thence down through the eyelet-hooks upon the end of the flyers of the spindles c, and finally attached to their bobbins. The spindles being driven by the band $\mathbf{D D}$ upon their lower part, continuously twist and wind the finished yarn upon the bobbins; constituting the first unremitting automatic machine for spinning which the world ever saw.

The water-twist frame, so called by its inventor because it was first driven by water, is now superseded by the throstle frame, of which the name has no assignable origin. Fig. 578 exhibits a vertical section of this machine, which has upon each side of its frame, a row of spindles with all their subsidiary parts. The bobbins filled with rovings from the bobbin and fly, or the tube frame, are set up in the creel $a a$, in two ranges. $b, c, d$, are the three pairs of drawing rollers through which the yarn is attenuated to the proper degree of fineness, upon the principles already explained. At ity escape from the front rollers, every thread runs through a guide eyelet $e$ of wire, which gives it the vertical direction down towards the spindles $f, g$. The spindles, which perform at once and uninterruptedly the twisting and winding-on of the thread delivered by the rollers, are usually made of steel, and tempered at their lower ends. They stand at $g$ in steps, pass at $v$ through a brass bush or collar which keeps them upright, and revolve very rapidly upon their axes. The bobbins $h$, destined to take up the yarn as it is spun, are placed loosely upon the spindles, and rest, independently of the rotation of the spindles, ucon the copping-beam $l$, but with the intervention of a strip of woollen cloth, the use of which in acting as a 'drag ' upon the motion of the 
hobbin will be understood by-and-bye. Upon the top of the spindles, $a$ wire fork. callod a fy or flyer, $i, k$, is mado fast by a loft-hand serew, and has one of its forks

\section{8}

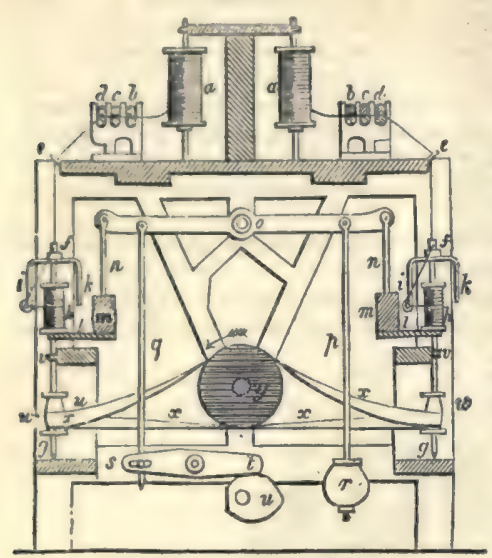
turned round at the ond into a little ring. The branch of the flyer at $f$ is tubular, to allow the thread to pess through, and to escape by a little hole at its side, in order to reach the eyelet at the end of that fork. From this eyelet $i$, it proceeds directly to the bobbin. By the revolution of the spindle, the twisting of the portion of thread between the front roller $d$, and the nozzlo $f$, is effected. The winding-on takes place in the following way:- Since the bobbin has no other connection with the spindle than that of the thread, it would but for it remain entirely motionless, relatively to the spindle. But the bobbin is pulled after it by the thread, so that it must follow the rotation of the spindle and fly. When we consider that the thread is pinched by the front roller $d$, and is thereby kept fully upon the strotch, we perceive that the rotation of the bobbin must be the result. Suppose now the tension to be suspended for an instant, while the rollers $d$ deliver, for example, one inch of yarn. The weight of the bobbin, and its friction upon the copping beam $l$, will, under this circumstance, cause the bobbin to hang back in a state of rest, till the inch of varn be wound on by the whirling of the fly $i$, and the former tension be restored. The delivery of the yarn by the drawing rollers, however, does not take place, inch after inch, by starts, but at a certain continuous rate; whence results a continuous retardation or loitering, so to speak, of the bobbins behind the spindles, just to such an extent as to secure the winding of the yarn upon the bobbin as fast as it is spun.

This process in spinning is essentially the same as what occurs in the fine bobbin and fly frame, but is here simplified, as the retardation regulates itself according to the diameter of the bobbin by the drag of the thread. In the fly frame the employment of this tension is impossible, because the roving has too little cohesion to bear the strain; and hence it is necessary to give the bobbins that independent movement of rotation which so complicates this machine.

The up-and-down motion of the bobbins along the spindles, which is required for the equal distribution of the yarn, and must have the same range as the length of the bobbin barrels, is performed by the following mechanism:-Every copping rail $l$, is made fast to a bar $m$, and this, which slides in a vertical groove or slot at the end of the frame, is connected by a rod $n$, with an equal-armed, moveable lever $o$. The rod $p$ carries a weight $r$, suspended from this lever; another rod $q$, connects the great lever $o$, with a smaller one $s, t$, upon which a heart-shaped dise or pulley $u$, works from below at $t$. By the rotation of the disc $u$, the arm $t$, being pressed constantly down upon it by the reaction, the weight $r$ must alternately rise and fall; and thus the copping rail $l$ must obviously move with the bobbins $h$ up and down; the bobbins upon one side of the frame rising, as those upon the other sink. Strictly considered, this copping motion should become slower as the winding-on proceeds, as in the flyroving frame; but, on account of the smallness of the finishod thread, this construction, which would render the machine complicated, is without inconvenience neglected, with the result merely that the coils of the yarn are successively more sparsely laid on, as the diameter of the bobbin increases. The movement of the whole machine proceeds from the shaft of a horizontal drum, which drives the spindles by means of the endless bands $x x$. Each spindle is mounted with a small pulley or wharf $w$, at its lower part, and a particular band, which goes round that wharf or whorl, and the drum $y$. The bands are not drawn tense, but hang down in a somewhat slanting direction, being kept distended only by their own weight. Thus every spindle, when its thread breaks, can readily be stopped alone, by applying a slight pressure with the hand or knee, the band meanwhile gliding loosely round the whorl. A more complete view of the throstle frame is presented by fig. 579.

Several modifications of the throstle have been attempted, but very fow of any importance have stood the test of experience, at least, in England. The Danforth throstle, inventod in 1829 , had for its main object to increase the speed of the spindles, and so to enlarge the productive capacity of the machine. It failed, however, amongst 
other reasons, for the very sufficient ones that it required much more power than the ordinary throstle, and that it could not be stopped without causing ' snarls' or twisted trngles in the yarn. The Montgomery throstle, introduced in 1832, was more successful. In this machine the spindle does not revolve, but is stationary, the flyer revolving

579

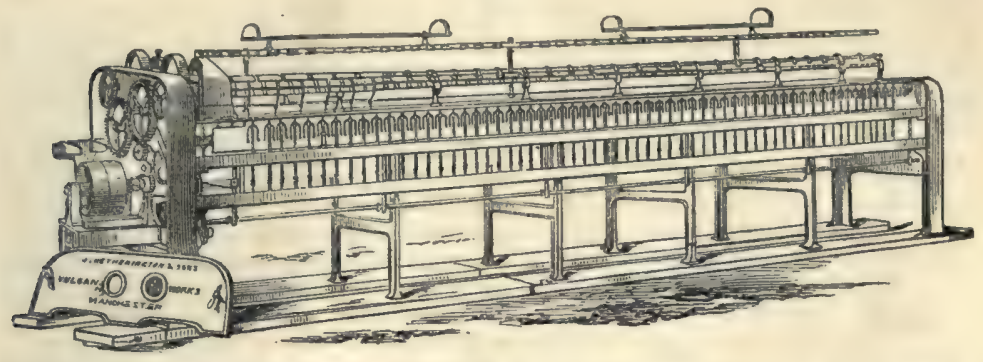

round it and winding the yarn upon the bobbins in the usual manner. It is said to produce good yarn, and although it has been discarded in this country, it still holds its ground in the United States. Perhaps the most remarkable, as well as one of the most recent improvements in the throstle frame is that of Bernhardt's 'doffing-motion.' The labour of 'doffing' or taking off the bobbins when filled with yarn, as well as the substituting of empty ones in their places, is usually accomplished by boys, who, notwithstanding the agility with which they perform the operation, necessarily keep the machine for a considerable time standing still. This loss of time, as well as the labour of the 'doffers,' is abolished by Bernhardt's invention. For a full description of this ingenious contrivance, we refer the reader to Mr. Evan Leigh's 'Science of Modern Cotton Spinning' (Manchester, 1873). The same work contains also a very full account of the ring throstle spinning frame, which in its various forms has taken deep root in the United States, and is beginning to find appreciation even in English mills, where there was formerly a strong prejudice against it.

It may be as well here to explain what is meant by the 'counts' or ' numbers of yarn. It is obvious that in all the textile industries some plan of indicating the varying thickness of the yarns is absolutely necessary. At present the methods employed are different in various countries, and, even in the same country, the systems used in the principal textiles are not identical, cotton, linen, silk, and jute having as a rule each a separate system. But all these methods are found to rest at bottom in one of two principles: First, the adoption of a fixed length as a standard, the counts being expressed by the weight of this fixed length in any given examplo. This plan is rarely followed, except in the case of silk, in which therefore the higher the counts the coarser is the thread. The other principle is founded upon a fixed unit of weight, and determines the counts by the length required to reach that weight. On this plan the numbering of all other kinds of yarns proceeds in nearly every country. In cotton yarns the English system has the pound (Avoirdupois) for the unit of weight and the 'hank' of 840 yards for the unit of length, and the counts is determined by the number of hanks which in any given case is needed to make up one pound in weight. The French system adopts the half kilogramme (equal to $1 \mathrm{lb} .1 \frac{1}{2} \mathrm{oz}$.) as the unit of weight and the hank of 1,000 mètres (1,093 yards 22 inches) as the unit of length. The English system is exclusively used in this country, in America, in India, and in a portion of the Continent. The French system prevails in France, in Alsace, and in some portions of Germany, Austria, and Italy. An effort, originating at the Vienna Exhibition of 1873 , has been made to secure a uniform method in the numbering of yarns. No definite agreement has yet been arrived at, but in France, and in Alsace there is a strong desire that the French or 'metric' system should be generally adopted. The method of practically testing the counts of yarn is this:-A short length of the sample to be tested is wound upon a carefully-adjusted measuring apparatus called a 'wrap-reel,' and then weighed by means of small delicately balanced scales. The fineness is then easily determined by calculation or by reference to a previously-compiled table of equivalents.

Yarn spun upon the throstle frame needs to be made very strong in order that it may resist the strain produced by the 'drag' of the bobbin whilst being spun. On this account the throstle can selclom be omployed with advantage in spinning the finer and more delicate yarns. As a matter of fact, it is scarcely over used in the produc- 
tion of counts finer than No. $36^{\prime} \mathrm{s}$. Moreover, it is necessary that throstle yarn, for the renson just stated, should be highly twisted, should receive a comparatively large number of turns or twsts per inch, and hence its principal characteristic is its hard, wiry and closely compact appearance. On this account it is especially suitable for tho manufacturo of goods which cannot be woven without much strain upon the warp, or which when finished need to be very durable.

Besides the special characteristics of throstle yarn to which we have alluded, and which greatly limit its applicability, one other must be noticed, viz. the smallness of the rate of production. This, it is obvious, increases the cost of spinning, and the throstle is therefore rarely employed, except where yarn is required possessing the characteristics already indicated.

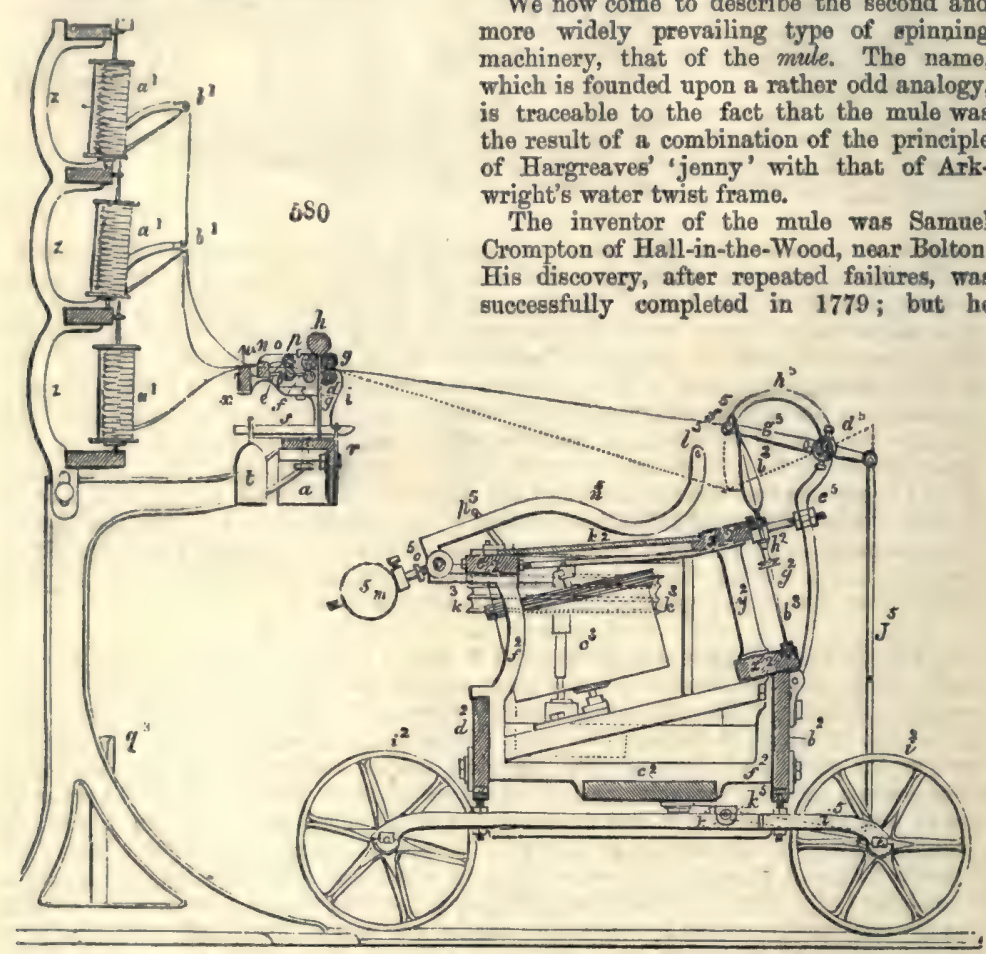

We now come to describe the second and more widely prevailing type of apinning machinery, that of the mule. The name, which is founded upon a rather odd analogy, is traceable to the fact that the mule was the result of a combination of the principle of Hargreaves' 'jenny' with that of Ark's water twist frame. successfully completed in 1779 ; but ho

reaped only a slender pecuniary reward from it, not being able to keep his secret long, nor to pay the cost of securing a patent-right in it. The beneficial results of Crompton's labours upon the economy of manufactures have, however, placed his name in the front rank of inventors. He received a parliamentary grant of $5,000 z$. a sum which, when compared with what he might have obtained under the protection of a patent, was insignificant. A statue to his memory was erected in Bolton in the year 1857 , thirty years after his death.

It will have been already observed that the spinning operation, as carried on by means of the throstle is continuous, that is to say, the motion of the rollers, the spindles, and the bobbin goes on simultaneously, and the cotton is drawn, twisted and wound upon the bobbin without intermission during the whole of the time that the machine is at work. In the mule, on the contrary, the action is intermittent or rather alternate. A given length is first drawn and spun, then the motion of the rollers ceases, the spindles stop spinning, and proceed to wind up the length that has been spun. Spinning is then resumed and the alternate movements are repeated ad libitum. The details of this process will be readily understood on reference to fig. 580, which is a transverse section of the mule, and in which its principal parts are shown. The machine consists of two main sections: a fixed one, corresponding in some measure to the water frame or throstle, and a moveable one, corresponding to the jenny. The furst contains in a suitable frame the drawing roller-beam and the chief moving 
machinery ; the second is called the carriage, in which the remainder of the moving mechanism and the spindles are mounted.

The frame of the fixed part consists of two upright sides, and two or more intermediate parallel bearings, upon which the horizontal roller beam $a$, the basis of the drawing rollers is supported. $b, c, d$ are the three ranges of fluted iron rollers; $e, f, g$, are the upper iron rollers covered with leather; $h$, the wooden wiper-rollers, covered with flannel, which being occasionally rubbed with chalk, imparts some of it to the pressure rollers beneath, so as to prevent the cotton flaments adhering to them.

The skewers upon which the bobbins containing the rovings from the roving frame are set up are seen at $a^{1}, a^{1}, a^{1}$, arranged in three rows in the creel z. The soft threads unwound from these bobbins, in their way to the drawing rollers, pass first through eyelets in the ends of the wire arms $b^{1}$, then through the rings or eyes of the guide bar $w$, and enter between the back pair of rollers. The number of these bobbins is equal to the number of spindles in the mule, and twice as great as the number of fluted portions of the rollers; for two threads are assigned to each portion.

The carriage consists of two cast-iron side pieces, and several cast-iron intermediate similar pieces, such as $f^{2}$, which altogether are made fast to the planks $b^{2}, c^{2}, d^{2}$. The top is corered in with the plank $k^{2}$. The carriage runs by means of its cast-iron groored wheels, upon the cast-iron railway $l$, which is fixed level on the floor.

The spindles stand upon the carriage in a frame, which consists of two slant rails $x^{2}, x^{2}$, connected by two slender rods $y^{2}$, and which frame may be set more or less obliquely. The lower rail carries the brass steps for the points of the spindles $b^{2}$; upon the upper rail brass slips are fixed pierced with holes through which the tops of the spindles play. The spindles are as usual made of steel, perfectly straight, turned truly round, and are all arranged in one plane. To each of them a small wooden or cast-iron whorl $g^{2}$ is made fast. They are distributed into groups of 24 , and the whorls are arranged at such different heights, that only two of them in each group are upon a level with each other. A small brass head $h^{2}$, which every spindle has beneath the upper slant rail of the frame $x^{2}$, prevents their sitting down into the step during their rotation, or sliding off their cop of yarn.

$c^{3}$ are drums, mounted in the carriage in a plane at right angles to the plane in which the spindles are placed. At top they have a double groove for a cord to run in, and the motion which they receive from the great fly wheel, or nm of the mule (not visible in this view) they impart to the spindles. Such a drum is assigned to every 24 spindles; and therefore a mule of 480 spindles contains 20 drums. In the middle of the carriage is seen the horizontal pulley $k^{3}$, furnished with three grooves, which stands in a line with the drums $c^{2}$.

The motion is given to the drums $c^{3}$, upon the right hand half of the carriage by a single endless band or cord which proceeds from the middle groove of the pulley $k^{3}$. The rotation of the spindles is produced by a slender cord, of which there are 12 upon each drum $c^{3}$; because every such cord goes round the drum, and also every two wharves which stand at the same level upon the spindles. It is obvious that the drums, and consequently the spindles, must continue to revolve as long as the main rim of the mule is turned, whether the carriage be at rest or in motion upon its railway.

If we suppose the carriage to be run in to its standing point, or to be pushed home to the spot from which it starts in spinning, its back plant $d^{2}$ will strike the post $q^{8}$ upon the fixed frame, and the points of the spindles will be close in front of the roller beam. The rollers now begin to turn and to deliver threads, which receive immediately a portion of their twist from the spindles; the carriage retires from the roller beam with somewhat greater speed than the surface speed of the front rollors, whereby the threads receive a certain degree of stretching, which affects most their thicker and less twisted portions and thereby contributes greatly to the levelness of the yarn. When the carriage has run out to the end of its course, or has completed a stretch, the fluted rollers suddenly cease to revolve (and sometimes even earlier, when a second stretch is to be made), but the spindles continue to whirl till the fully extended threads have received the proper second or after twist. Then the carriage must be put up, or run back towards the rollers, and the threads must be wound upon the spindles. This is the order of movements which belong to the mule. It has been shown how the rotation of the spindles is produced.

For winding-on the yarn the carriage has a peculiar apparatus, which we shall now describe. In front of it, through the whole extent to the right hand as well as the left, a slender iron rod, $d^{b}$, runs horizontally along, in a line somewhat higher than the middle of the coping portion of the spindles, and is supported by several props, such as $e^{s}$. Upon each end of the two rods, $d^{3}$, there is an arm, $g^{5}$; and betwixt these arms an iron wire, called the copping wire, $f^{s}$, is stretched, parallel with the rod $d^{3}$. For the support of this wire, there are several slender bent arms $h^{3}$ extended from the 
rod $d^{b}$ at several points betwixt the straight arms $g^{5}$. The rod $d^{5}$ has, besides, a wooden handle at the place opposite to where the spinner stands, by which it can be readily grasped. This movement is applied at the left division of the machine, and it is communiested to the right by an apparatus which resembles a crane's bill. The two arms, $g^{5}$, in the middle of the machine, project over the rods $d^{3}$, and are connected by hinges with two vertical rods $j^{3}$, which hang together downwards in like manner with two arms $i^{3}$, proceoding from a horizontal axis $k^{3}$.

By means of that apparatus the yarn is wound upon the spindles in the following manner:-As long as the stretching and twisting go on, the threads form an obtuse angle with the spindles, and thereby slide continually over their smooth rounded tips during their revolution, without the possibility of coiling upon them. When, however, the spinning process is completed, the spinner seizes the carriage with his left hand and pushes it back towards the roller beam, while with his right hand he turns round the handle of the rim or fly wheel, and consequently the spindles. At the same time, by means of the handle upon the rod $d^{5}$, he moves the copping-wire $f^{4}$, so that it presses down all the threads at once, and places them in a direction nearly perpendicular to the spindles; as shown by the dotted line $y^{5}$. That this movement of the copping wire, however, may take place without injury to the yarn, it is necessary to turn the rim beforehand a little in the opposite direction, so that the threads may get uncoiled from the upper parts of the spindles, and become slack; an operation called in technical language the backing-off. The range upon which the threads should be wound, in order to form a conical cop upon the spindle, is hit by depressing the copping wire to various angles, nicely graduated by an experienced eye. This faller wire alone is not, however, sufficient for the purpose of winding-on a seemly cop, as there are always some loose threads which it cannot reach without breaking others.

Another wire called the counter-faller, $l^{5}$, must be applied under the threads. It may be raised to an elevation limited by the angular piece $p^{5}$; and is counterpoised by a very light weight $m^{5}$, applied through the bent lever $n^{5}$, which turns upon the fulcrum $b^{5}$. This wire, which applies but a gentle pressure, gives tension to all the threads, and brings them regularly into the height and range of the faller $f^{3}$. This wire, must be raised once more, whenever the carriage approaches the roller beam. At this instant a new stretch commences; the rollers begin again to revolve, and the carriage resumes its former course. These motions are performed by the automatic machinery.

There is a little excentric pulley mechanism for moving the guide beam to and fro with the soft yarns, as they enter between the back rollers. On the right hand end of the back roller shaft, a worm screw is formed which works into the oblique teeth of a pinion attached to the end of the guide beam, in which there is a series of holes for the passage of the threads, two threads being assigned to each fluted roller. In the flat dise of the pinion, an excentric pin stands up which takes into the jointed lever upon the end of the guide beam, and as it revolves, pushes that beam alternately to the loft and the right by a space equal to its excentricity. This motion is exceedingly slow, since for each revolution of the back roller, the pinion advances only by one tooth out of the 33 , which are cut in its circumference.

After counting the number of teeth in the different wheels and pinions of the mule, or measuring their relative diameters, it is easy to compute the extension and twist of the yarns; and when the last fineness is given to ascertain their marketable value. Let the ratio of speed between the three drawing rollers be $1: 1 \frac{3}{22}: 7 \frac{1}{2}$; and the diameter of the back and middle roller three quarters of an inch : that of the front roller one inch; in which case the drawing is thereby increased $1 \frac{1}{3}$ time, and $7 \frac{1}{3} \times 1 \frac{1}{3}=10$. If the rovings in the creel bobbins have been No. 4 , the yarn, after passing through the rollers, will be No. 40. By altering the change pinion (not visible in this view) the fineness may be changed within certain limits, by altering the relative speed of the rollers. For one revolution of the great rim or fly wheel of the mule, the front roller makes about 6 -tenths of a turn, and delivers therefore $22 \cdot 6$ lines or 12ths of an inch of yarn, which, in consequence of the tenfold draught through the rollers, corresponds to $2 \cdot 26$ lines of roving fed in at the back rollers. The spindles or their whorls make about 66 revolutions for one turn of the rim. The pulleys or grooved wheels on which the carriage runs, perform 0.107 part of a turn while the rim makes one revolution, and move the carriage $24^{\circ} 1$ lines upon its rails, the wheels being six inches in diameter.

The 22.6 lines of soft yarn delivered by the front rollers will be stretched $1 \frac{1}{2}$ line by the carriage advancing $24^{\prime} 1$ lines in the same time. Let the length of the railway, or of each stretch be 5 feet, the carriage will complete its course after 30 revolutions of the rim wheel, and the 5 -feet length of yarn (of which $56 \frac{1}{2}$ inches issue from the drawing rollers, and 3 inches proceed from the stretching) is, by the simultaneous whirling of the spindles, twisted 1,980 times, being at the rate of 33 twists for every inch. The second twist, which the threads receive after the carriago has come to 
repose, is regulated according to the quality of the cotton wool, and the purpose for which the yarn is spun. For warp yarn of No. 40 or 50 , for example, 6 or 8 turns of the rim wheel, that is, from 396 to 528 whirls of the spindles for the whole stretch, therefore from 7 to 9 twists per inch, will be sufficient. The finished yarn thus receives from 40 to 42 twists per inch.

One spinner attonds to two mules, which face each other, so that he need merely turn round in the spot where he stands, to find himself in the proper position for the other mule. For this reason the rim wheel and handle, by which he operates, are not placed in the middle of the length of the machine, but about two-fifths of the spindles are to the right hand and three-fifths to the left; the rim wheel being towards his right hand. The carriage of the one mule is in the act of going out and spinning, while that of the other is finishing its twist, and being put up by the spinner.

The quantity of yarn manufactured by a mule in a given time, depends directly upon the number of the spindles, and upon the time taken to complete every stretch of the carriage. Many circumstances have an indirect influence upon that quantity, and particularly the degree of skill possessed by the spinner. The better the machine, the steadier and softer all its parts revolve, the better and more abundant is its production. When the toothed wheels do not work truly into their pinions, when the spindles shake in their bushes, or are not accurately made, many threads break, and the work is much injured and retarded. The bettor the staple of the cotton wool, and the more careful has been its preparation in the carding, drawing, and roving processes, the more easy and excellent the spinning will become: warmth, dryness, cold, and moisture have great influence on the ductility, so to speak, of cotton. A temperature of $65^{\circ} \mathrm{F}$.; with an atmosphere not too arid, is found most suitable to the oporations of a spinning-mill. The finer the yarn, the slower is the spinning. For numbers from 20 to 36 , from 2 to 3 stretches of warp may be made in a minute, and nearly 3 stretches of weft; for numbers above 50 up to 100 , about 2 stretches; and for numbers from 100 to 150 one stretch in the minute. Still finer yarns are spun more slowly, which is not wonderful, since in the fine spinning-mills of England, the mules usually contain upwards of 500 spindles each, in order that one operative may manage a great number of them, and thereby earn such high wages as shall fully remunerate his assiduity and skill.

In spinning fine numbers, the second speed is given before the carriage is run out to the end of its railway; during which course of about 6 inches, it is made to move very slowly. This is called the second stretch, and is of use in making the yarn level by drawing down the thicker parts of it, which take on the twist less readily than the thinner, and therefore remain soft and more extensible. The stretch may therefore be divided into three stages. The carriage first moves steadily out for about 4 feet, while the drawing rollers and spindles are in full play; now the rollers stop, but the spindles go on whirling with accelerated speed, and the carriage advances slowly, about 6 inches more; then it also comes to rest, while the spindles continue to revolve for a little longer, to give the final degree of twist. The acceloration of the spindles in the second and third stages, which has no other object but to save time, is effected by the mechanism called the counter, which shifts the driving band, at the proper time, upon the loose pulley, and, moreover, a second band, which had, till now, lain upon its loose pulley, upon a small driving pulley of the rim shaft. At length, both bands are shifted upon their loose pulleys, and the mule comes to a state of quiescence.

In the machine as thus described the winding-in motion is performed by means of the necessary mechanism, by manual labour. This method is, howerer, now used only in the spinning of the very finest yarns, and the winding-on is performed by an automatic arrangement which gave to the machines to which it was first applied tho name of self-acting mules. Mules of this kind were first constructed, we believe, by Messrs. Eaton, formerly of Manchester, who mounted ton or twelve of them in that town, four at Wiln, in Derbyshire, and a few in France. From their great complexity and small productiveness, the whole were soon relinquished, except those at Wiln. M. de Jong obtained two patents for self-acting mules, and put twelve of them in operation in a mill at Warrington, of which he was part proprietor; but with an unsuccessful result.

The first approximation to a successful accomplishment of the objects in view, was an invention of a self-acting mule, by Mr. Roberts, of Manchester : one of the principal points of which was the mode of governing the winding-on of the yarn into the form. of a cop; tho entire novelty and great ingenuity of which invention were universally admitted, and proved the main step to the final accomplishment of what had so long been a desideratum. For this inrention a patent was obtained in 1825. In 1830, Mr. Roberts obtained $\AA$ patent for other improvements ; and by a combination of both his inrentions, he produced a solf-acting mulo, which is generally admitted to havo Vox. I. 
excecled the most sanguine expectations, and which has been extensirely adopted. The advantages gained by Mr. Roberts's improrements are the saving of a spinner's wiges to each pair of mules, piecers only being required, as one overlooker is sufficient to manage six or eight pairs of mules; and the production of a greater quantity of yarn, in the ratio from 15 to 20 per cont. The yarn possesses a more uniform degreo of twist, and is not liable to be strained during the spinning, or in winding-on, to form the cop ; consequently fewer threads are broken in these processes, and the yarn, from having fower piecings, is more regular.

The cops are made firmer, of better shape, and with underiating uniformity; and, from being more regularly and firmly wound, contain from one-third to one-half moro yarn than cops of oqual bulk wound by hand; they are consequently less liable to injury in packing or in carriage, and the expense of packages and freight (when chnrged by measurement) is considerably reduced. From the cops being more regularly and firmly wound, combined with their superior formation, the yarn intonded for warps less frequently breaks in winding or reeling, consequently there is a considerable saving of waste in those processer.

Several improvements have beon made in the construction of self-acting mules since the date of Roberts's patent, but none of them have marked so decided a step forward. The more prominent of these may be briefly noticed.

In 1834 Mr. James Smith obtained a patent for a method of dispensing with the 'backing-off motion' of Roberts. Instead of reversing the moroment of the spindles, in order to clear the spindle point and arrange the thread conreniently for the winding-on process, he elevated the counter-faller and thus, whilst the spindle was stationary, 'stripped off' the spiral coil of thread from the spindle in an upward direction. This method is still in use; but as it subjects the thread to considerable strain, it is generally used only for the coarser and stouter yarns. More recently patents have been taken out for improvements in mules, by Mr. Potter, of Manchester. Messrs. Higgins and Whitworth of Salford, Mr. Montgomery of Johnstone, Messrs. Craig and Sharp of Glasgow, and many others.

Mr. Roberts's self-acting mule, which was practically the first introduced, and with several modifications in its construction, is still the mule which is most extensively used and approved in the cotton trade. Some of the more prominent of these modifications have been effected by Messrs. Parr, Curtis, and Madeley of Manchester. Of these the fowing summary gives the principal features, viz, the substitution of a catch-box with an excentric box, in lieu of a cam shaft, to produce the required changes; an improved arrangement of the faller motion, which causes the fallers to act more easily upon the yarn, and not producing a recoil in them when the 'backingoff' takes place, thus preventing 'snarls' and injury to the yarn; in applying a spiral spring for the purpose of bringing the backing-off cones into contact, by which the operation of 'backing-off' can be performed with the greatest precision. The backingoff movement is also made to stop itself, and to cause the change to be made which affects the putting-up of the carriage, which it does in less time than if an independent motion was employed. They have also an arrangement for driving the back, or drawing-out, shaft by gearing in such a manner that in the event of an obstruction coming in the way of the carriage going out, the motion ceases and prevents the mule being injured.

By means of a friction motion, the object of which is to take the carringe in to the rollers, the carriage will at once stop in the erent of any obstruction presenting itself. For the want of an arrangement of this nature, lives have been lost and limbs injured, when careless boys have been cleaning the carriage whilst in motion, and have been caught between it and the roller beam, and thus killed or injured.

Another improvement consists in connecting the drawing-out shaft and the quadrant pinion shaft by gearing, instead of by hands, thereby producing a more perfect winding-on, as the quadrant is moved the same distance at each stretch of the carriage. They have also made a different arrangement of the headstock-or selfneting portion of the mule-causing its height to be much reduced, which makes it more steady, offers less obstruction to the light, enables the spinuer to see all the spindles from any part of the mule, and allows a larger driving strap, or belt, to be used, which in low rooms is of considerable importance. The result of these various improvements is the production of one of the most perfect 'spinning machines now in the trade.

For spinding very coarse numbers, say 6 's, they have patented an arrangement, by which the rotation of the spindles can be stopped, and the operation of backingoff performed, during the going-out of the carriage, thus effecting a considerable saving of timo.

The following is a description of one of these mules:-

Fig. 581 is a plan view; fig. 582 a tranverse section; and fig. 583 an end view, of so much of a mule as is requisite for its illustration here. 
581

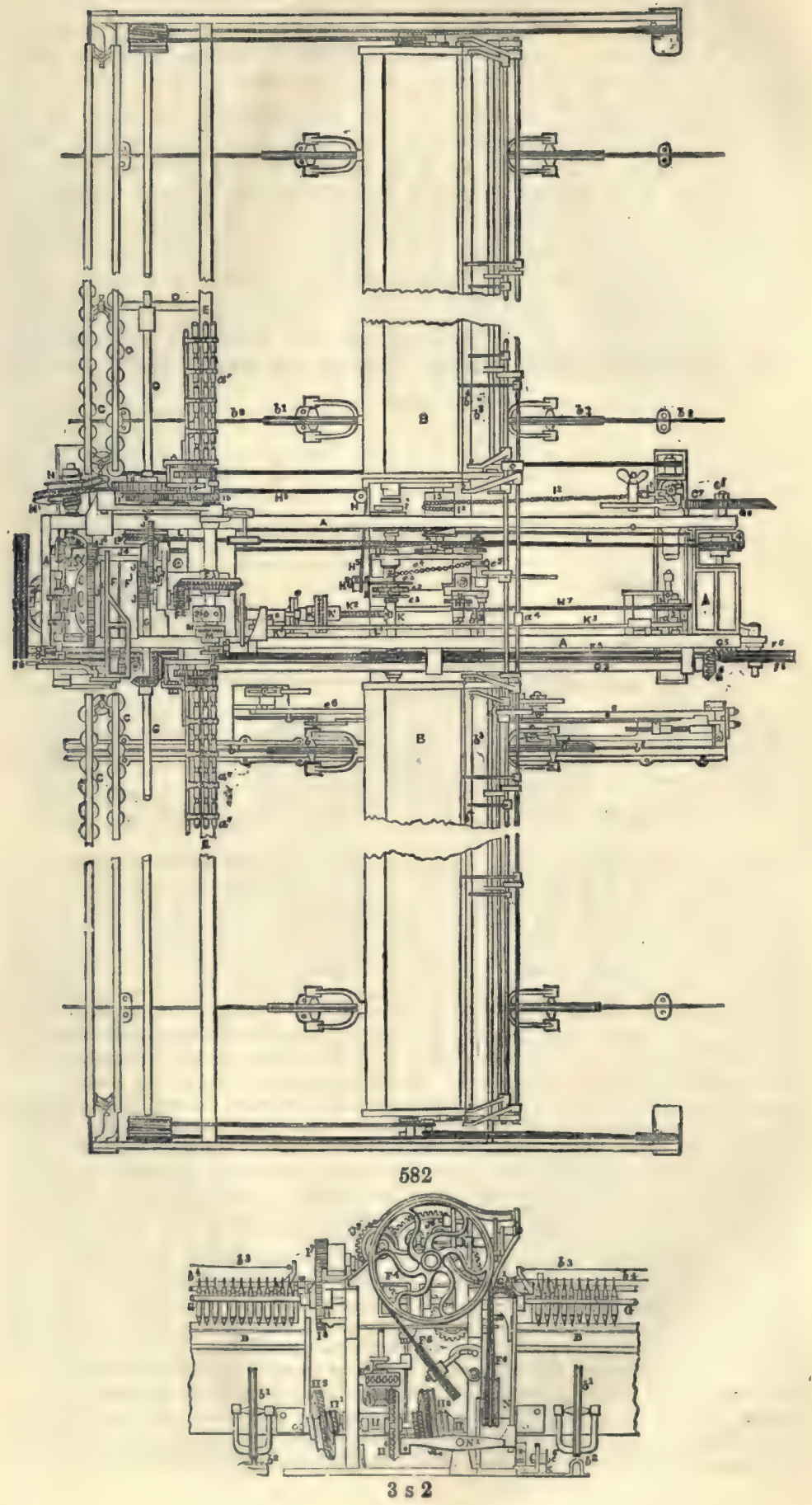


As there are many parts which are common to all mules, most of which have been previously described in the notice of the hand mule, we shall therefore only notice the more prominent portions of the self-acting part of the mule. Among such parts nre, the framing of the headstock $\mathbf{A}$; the carriage $\mathbf{B}$; the rovings $\mathbf{C}$; the supports $\mathbf{D}$, of the roller beam $\mathrm{E}$; the fluted rollers $a$; the top rollers $a^{1}$; the spindles $b$; the carriage wheols $b^{1}$; the slips, or rails, $b^{2}$, on which they move; the faller wire $b^{3}$; the counter-faller wire $b^{4}$. The following are the parts chiefly connected with the self-acting portion of the mule:-The fast pulley $r$, the loose pulley $\boldsymbol{F}^{\prime}$, the bevels $\mathbf{F}^{2}$ and $\mathrm{F}^{3}$, which give motion to the fluted rollers; the back, or drawing-out shaft $\mathrm{G}$; wheels $a^{1}$ and $a^{2}$, by which, through the shaft $G^{3}$ and wheels $a^{4}$ and $a^{5}$, motion is communicated to the pinion $G^{6}$ on the shaft $G^{8}$, and thence to the quadrant $G^{7}$. The scroll shaft $\mathrm{H}$, the scrolls $\boldsymbol{H}^{1}$ and $\mathbf{H}^{2}$, the catch-box $\mathbf{H}^{6}$, for giving motion through the bevel wheels $\mathbf{H}^{3}$ and $\mathbf{H}^{4}$ to the scroll shaft. Drawing-in cord $\mathbf{H}^{5}$. Screw in radial nrm $\mathrm{I}$, nut on same $\mathbf{1}^{1}$, winding-on chain $\mathrm{I}^{2}$, winding-on band $\mathbf{1}^{3}$, drawing-out cord $\mathbf{1}^{4}$. Pinion $\mathrm{I}^{5}$ on front roller shaft, to give motion through the wheels $1^{6}, x^{7}$, and $1^{8}$, to drawing-out shaft $\mathrm{G}$. Pinion $\mathrm{J}$, and wheels $\mathrm{J}^{\mathbf{2}}, \mathrm{J}^{2}$, and $\mathrm{J}^{\mathbf{3}}$ for giving motion to sluaft $\mathrm{J}^{4}$; pinjon $\mathrm{J}^{5}$, giving motion to backing-off wheel $\mathrm{J}^{6}$. On the change shaft $\mathrm{K}$ is keyed a pinion which gears with the wheel $\mathrm{J}^{5}$, and receives motion therofrom.

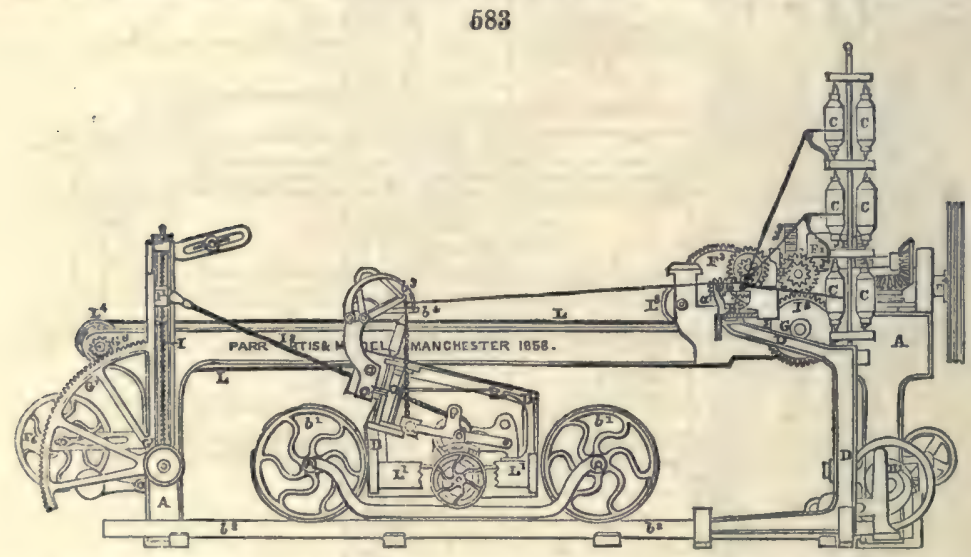

One lalf of the catch-box $\mathbf{x}^{1}$ is fast to one end of a long hollow shaft on which sre two cams, one of which is used to put the front drawing roller catch-box in into and out of contact, the other is used for the purpose of traversing the driving strap on or off the fast-pulley $\mathrm{F}$ as required. The other half of the catch-box $\mathrm{K}^{1}$ is placed on the Elhaft $\mathbf{K}$, a key fast on which passes through the boss of the catch-box, and causes it to be carried round by the shaft as it rotates. Though carried round with the shaft, it is at liberty to move lengthwise, so as to allow it to be put into and out of enntact with the other half when required. The spiral spring $\mathbf{K}^{2}$ is also placed on the shaft $K$, and continually bears against the end of the catch-box next to it, and endearours to put it in contact with the other, which it does when permitted and the changes are required. The change lever $\mathrm{x}^{3}$ moves on a stud which passes through its boss $a^{2}$ : near which end of this lever are the adjustable pieces $a$. When the machine is put in motion, supposing the carriage to be coming out, the eriving strap is for the most part on the fast pulley $\mathrm{F}$ when motion is given through the leerel wheels $\mathrm{F}^{2}$ and $\mathrm{F}^{3}$ to the drawing rollers $a$, which will then draw the rovings c off the bobbins, and deliver the slivers so drawn at the front of the rollers, and the same loing fast to the spindles; as the carriage is drawn out the slivers are taken out also, and as the spindles at this time are turned round at a quick rate (say (6,000 revolutions per minute), they gire twist to the slivers, and convert thom $i$ to yarn or twisted threads. Motion is communicated to the spindles from the rim pulley $\mathbf{F}^{\prime}$, through the rim band $\mathbf{F}^{3}$, which passes from the rim pulley to a grooved pulley on the tin roller shaft, round which it passes and thence round the grooved pulley $\mathbf{F}^{\prime}$ back to the rim pulley, thus forming an endless band. It will be seen that the rim band pulley and the other pulleys, over or round which the rim band passes, are formed with double grooves, and the band being passed round each, it forms a r.ouble band, which is found of great advantage, as it will work with a slacker band than if only one groove was used; thore is, consequently, less strain on the band, and it is luarger, $\triangle$ string or corł passes round the tin roller to a wharve on each spindle, 
round which it passes, and thence back to the tin roller, and thus, when the tin roller receives motion from the rim band, it gives motion to the spindles. The carriage is caused to move outwards by means of the cord $L$, one end of which is attached to a ratchet pulley fixed on the carriage cross, or square $\mathrm{L}^{\prime}$, and is then passed over the spiral grooved pulley $\mathrm{L}^{2}$ fast on the drawing out shaft $\mathrm{G}$, and passes thence under the guide pulley $I^{3}$ round the pulley $I^{4}$ to another ratchet pulley, also on the carriage square where the other end is then fastened. The cord receives motion from the pulley $\mathrm{L}^{2}$, round which it passes and communicates the motion it receives to the carriage, the carriage-wheels $b^{2}$ moving freely on the slips $b^{2}$.

When the carriage has completed its outward run, the bowl $a^{4}$ on the counter faller shaft comes against the piece $a^{3}$, depresses it and the end of the lever $\mathrm{K}^{3}$ to which it is attached, and zaises the other end, and with it the slide $c$, on which aro two inclines. A round pin (not seen) passes through the boss of the catch-box next to the slide, and bears against the sliding half of the catch-box, and holds it out of contact.

When the slide $c$ is raised, the part of the incline which bore against the pin and kept the catch-box from being in contact is withdrawn, nn which the spring puts them in contact, and motion is given to the hollow shaft, and the cams thereon; one of which causes the catch-box $\mathbf{x}$ to be taken out of contact when motion ceases to be given to the drawing rollers and to the going-out of the carriage; and the other causes the driving strap to be traversed off from the fast pulley on to the loose one, when motion ceases to be given to the rim pulley and thence to the spindles. The inclines on the slide are so formed that, by the time the shaft has made half a revolution, they act on the pin and cause it to put the catch-box out of contact. The next operation is the backing-off or uncoiling the threads coiled on the spindle above the cop, which is effected by causing the backing-off cones attached to the wheel $\mathrm{J}^{\mathrm{B}}$ to be put into contact with one formed in the interior of the fast pulley $F$, when a reverse motion will be given to the rim pulley and thence to the tin roller and the spindles.

The backing-off cones are put into contact by means of a spiral spring, which, when the strap fork is moved to traverse the strap on to the loose pulley, it is allowed to do. Simultaneously with the backing-off the putting-down of the faller wire takes place, which is effected through the reverse motion of the tin roller shaft, which cruses the catch $c^{1}$ to take into a tooth of the ratchet wheel $c^{2}$, when they will movo together, and with them the plate $c^{3}$, to a stud in which one end of the chain $c^{4}$ is fastened, the other end of which is attached to the outer end of the finger $c^{5}$, fast on the faller shaft. When this chain is drawn forward by the plate, it draws down the end of the figure $c^{5}$ to which it is attached, and thereby partially turns the faller shaft and depresses the faller wire $b^{3}$, and, at the same time, raises the lever $c^{3}$, the lower part of which bears against a bowl attached to a lever which rests on the builder rail $c^{8}$. As soon as the lever $c^{5}$ is raised sufficiently high to allow the lower end to pass over, instead of bearing against the bowl, it is drawn forward by a spiral spring, which causes the backing-off cones to be taken out of contact, when the backing-off ceases, and the operations of running the carriage in and winding the yarn on to the spindles must take place. When the cones are taken out of contact the lower end of the lever $\mathrm{N}$ is withdrawn from being over the top of the lever $\mathrm{N}^{1}$, leaving that lerer at liberty to turn, and the catch-box $\mathrm{H}^{6}$ thereupon drops into gear, and motion is communicated to the serolls $\mathrm{x}^{2}$ and $\mathrm{H}^{2}$, and to the cords $\mathbf{H}^{5}$ and $\mathbf{H}^{7}$. The cord $\mathrm{H}^{5}$ is at one end attached to the scroll $\mathbf{H}^{1}$, and passes thence, round the pulley $\mathbf{H}^{8}$, to the ratchot pulley $\mathbf{H}^{9}$ fixed to the back of the carriage square. The cord $\mathbf{H}^{7}$ is at one end attached to the scroll $\mathrm{H}^{2}$, and passes thence round the pulley $\mathrm{H}^{10}$ to the ratchet pulley $\mathrm{H}^{\prime \prime}$ fixod to the front of the carriage square. It will thus be seen that the carriage is held in ono direction by one band, and in another by the other band, and that it can only be moved in either direction by the one scroll giving off as much cord as the other winds on. When the catch-box $\mathbf{H}^{6}$ drops in gear, the scroll $\mathbf{H}^{1}$ winds the cord $\mathrm{H}^{3}$ on and draws the carriage in. It will thus be seen that the carriage is drawn out by means of the back or drawing-out shaft $\mathrm{G}$, and is drawn in by the scroll $\mathrm{H}^{3}$. The windingon of the thread in the form of a cop is effected by means of Mr. Roberts's ingenious application of the quadrant or radial arm $\mathrm{A}^{7}$, screw $\mathrm{I}$ and winding-on chain $\mathrm{I}^{2}$ and $\mathrm{I}^{3}$. The chain $\mathrm{I}^{2}$ is at one ond attached to the nut $\mathrm{I}^{1}$ and at the other to the band $\mathrm{I}^{2}$. During the coming out of the carriage the drawing-out shaft through the means of the wheels $G^{4}, G^{2}, a^{4}$, and $G^{5}$, shafts $G^{3}$ and $G^{8}$, and pinion $G^{6}$, moves tho quadrant which, by the time the carriage is quite out, will have been moved outwards a little past the perpendicular. The chain is moved on to the barrel by means of the cord $\mathrm{o}$, which being fixed and lapped round the barrel as the carriage moves outward causes it to turn. On the barrel is a spur wheel which gears into a spur pinion on the tin roller shaft (these wheels being under the frame side are not seen in the drawing). The spur pinion is loose on the tin roller shaft, and as the carriage comes out it turns loosely thereon, but as the carriage goes in the chain $1^{3}$ turns the barrel round, and 
with it the spur pinion. A catch on a stud fixed in the side of the pinion, at that time taking into a tooth of tho ratchet wheel $i$ fast on the tin roller shaft, the motion of the spur pinion is communicated to the tin roller shaft, and thence to the spindles, causing the thread or yarn spun during the coming-out of the carriage to be wound on the spindles, in the form of the cop, while the carriage goes in. At the commencemont of the formation of a set of cops, when the yarn is being wound on the bare spindles, tho spindles require to have a groater number of turns given to them than they do when the cop bottom is formed; to produce this variation the following means are employed. At the commencement of each set, the screw in the radial arm is turned so as to turn the nut $I^{1}$ to the bottom of the serew, where it is near to the shaft on which the quadrant moves ; consequently, little or no motion is given to the chain, and the carriage, as it goes in, causes the chain to be drawn off the band. As the formation of the cop bottom proceeds, the screw is turned and the nut is raised; by which means a less quantity of chain is drawn off the barrel; the chain, at the point of attachment, gradually following the carriage as it goes in.

During the going-in of the carriage the quadrant is drawn down or made to follow the carriage by the chain pulling it, the speed at which it is allowed to descend is regulated by the motion of the carriage; the quadrant, during the going-in of the carriage, through the pinion $G^{6}$, shafts $G^{8}$ and $G^{8}$, and wheels, $G^{1}, G^{2}, G^{4}$, and $G^{5}$ driving the drawing-ont shaft.

When the carriage has completed its inward run, the bowl $\Lambda^{\prime}$ comes in contact with the piece $\mathbf{A}^{6}$, and depresses it and the end of the lever $\mathrm{K}^{3}$ to which it is attached, and also the slide $\mathrm{c}$, which then allows the catch-box $\mathrm{K}^{1}$ to be put in contact, and causes the cam shaft to make another half revolution. During this half revolution of the cam shaft, the cams cause the catch-box $\mathrm{m}$ to be put in contact, and the driving strap to be traversed on to the fast pulley, and, by the latter movement, the catch-box $\mathbf{r}^{8}$ is taken out of gear and the winding-in motion of the serolls ceases, and the carriage will again commence its outward run, and with it the spinning of the thread.

Other improvements have been effected by Messrs. Dolsen and Barlow, and by Messrs. P. and J. Me Gregor, of Manchester. The latter firm have combined some of the advantages of Roberts's and Smith's mule. The chief alterations made by Messrs. Me Gregor are: first, the regulation of the amount of 'twist' givon to the yarn from the tin roller or dium shaft of the carriage; and second, the completion of the automatic action of Roberts's mule, which left to the spinner the duty of oceasionally regulating the copping apparatus so that as the cop was gradually built upward upon the spindle, its speed might be slightly increased to meet the lessening diameter due to its slightly spiral form. Neglect of this duty on the part of the spinner was followed by a defect in the building of the upper part of the cop, which was rendered soft. The necessity of this attention has been abolished by the improrement in question.

The mule is used for the production of both 'twist' or warp, and weft. The twist cop is usually of a larger size than the weft cop. The latter undergoes no further operation until it is placed in the shuttlo of the loom, except when intended for bleaching or dyeing, or for export to distant markets. In the latter case it is wound into skeins, and it may then with advantage be spun in cops of larger size. But when taken directly to the loom, its bulk is necessarily limited by the fact, that the 'shuttle space' in weaving is very narrow, and the weight of the shuttle with its contained cop cannot with profit be indefinitely increased, because of the force absorbed in its transmission across the web.

Of the diverse purposes to which yarn is applied after the spinning process is com. pleted, some account will be found under the head of CotTON FActoRY.

COTTON Trams. It is impossible to call to mind the fact, that nearly the whole of the human race is elothed ontirely, or in part, with cotton fabric, without realising in some measure the important place amongst the activities of mankind which is filled by the growth, manufacture, and distribution of this matorial. This is the ground which is covered by the term 'Cotton Trade,' when used in its widost sense. And it is obvious that the chain of operations included in the term is much enlarged by the manifold diversity of the fabrics produced, as well as by the great distances which lie between the countries where the raw material is grown, the region of its manufacture, and the world-wide fields of consumption to which it is at last distributod. Cotton is exclusively a tropical or semi-tropical product. Its conversion into clothing by means of machinery is an industry belonging, as yet, almost exclusively to temperate zones. In seeking to present a general view of the nature and extent of the Cotton Trade it will be nocessary therefore to indicate the relative importance of the sources from which the raw material is supplied, as well as of the countries where it is manufuctured, and the proportions in which it is distributed to the regions of consumption. A considerable quantity of cotton is still spun by purely handieraft methods, especially in China and in India. But of this we do not here take 
any account. The manufucture of cotton in India by means of machinery has also of late years reached important dimensions. With this exception, there is no part of the world where cotton is converted into clothing by mechanical means, on any seale worth notice, except in Europe and in the United States. Under the head of Oorros FActorT we have indicated the relative importance of the various manufacturing countries as shown in the estimated number of spinning spindles possessed by each. Now, the supply of raw material consumed in the manufacture is drawn from many fielde; and the order in which these stand may be readily inferred from the following table, which shows the average annual quantities contributed by each source of supply for the use of the factories of Europe and the United States, during the three years 1871-73. The statistics referring to the production of Central Asia, which is entirely consumed in Russia, refer to the year 1867 , none of a later date being accessible.

Quantities and Sources of Supply of the Cotton consumed in Europe and the United States.

Average of the

three years 1871-73.

United States .

East India

Egypt, Asia Minor and Greece

Brasil Indies and Peru : .

Contral Asia

Total

This enormous aggregate which, reckoning 300 working days in the year, is equal to $8,592,000 \mathrm{lbs}$, or upwards of 3,835 tons per day, was distributed to the manufacturing countries in the following proportions:-

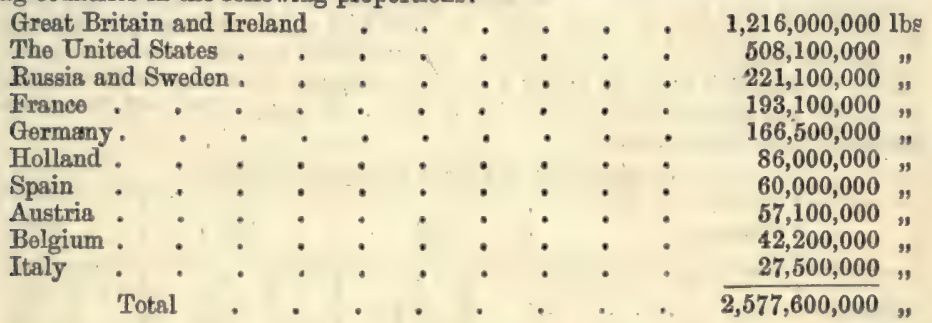

It thus appears that of all the raw cotton consumed in the manufacturing countries of Europe and America very nearly one half is used in the United Kingdom.

With regard to the distribution of the manufactured product, it is to be observed that most of the countries named in the preceding table retain for home consumption nearly all that they produce, and are also, in varying degrees, dependent for their supply of clothing upon the manufactures of other countries. The only ones which, besides supplying the wants of their own home market, have any considerable surplus for exportation, are Great Britain, France, Switzerland, Holland and Germany. Prior to 1862 the United States also exported a large proportion of their cotton manufactures. But, owing mainly to the high degree of protection with which the legislature surrounded American manufactures during and since the civil war, the exports of cotton goods from the United States have greatly declined. The well-known effects of a protective system in increasing the cost of production have not prevented a very rapid growth of the home consumption of native manufactures in the United States, thanks to their growing wealth, to their increasing population, and to the large exclusion of foreign productions by heavy import duties. But this increase in the cost of production has effectually weakened the power of American manufacturers to compete successfully with their foreign rivals in neutral markets abroad. The exports of cotton goods from France, Switzerland, and Germany go principally to South America and Africa, those of Holland to the Dutch East Indies and China. But by far the largest proportion of the population of the non-manufacturing countries derive their supplies of machine-made cotton goods from the United Kingdom. Of the proportions in which the exports of British manufactures are distributed to the several consuming countries, a very clear idea may be formed from a perusal of the statistics given at the close of this article.

Of the trables given in tho following pages not the least interesting is that which furnishes an account of the quantities of yarns and piece goods, and the aggregate ralue of these and other cotton products exported to the various countries of the world in 1872. From statistics for 1871, compiled by Messrs Ellison and Co., of Liverpool, 
wo find the consumption of British cotton manufactures in Russia increased from $\frac{3}{9} d$. per head in 1851 to $1 \frac{1}{8} d$. in 1861, and $1 \frac{8}{8} d$. in 1871. In Sweden and Norway the consumption increased from $4 \frac{6}{8} d$. in 1851 to over $13 \frac{1}{2} d$. in 1871 . Germany advanced from 28. 9 t d. to 58. $2 f d$. per head. Belgium stood at rather less than 11 fd. per head in 1851, and about the same in 1861 , but in 1871 the figures rose to $28.5 \frac{3}{4} d$., owing in a great measure to the diversion of trade occasioned by the war. In 1851 France did not use more English cotton manufactures than was equal to about $1 d$. per head of her entire population; in 1861 the figures rose to $4 \frac{1}{2} d$., but in 1871, thanks to the Commercial Treaty, the consumption reached 18. $2 d$. per head. Spain, including Gibraltar, consumed $7 \frac{1}{4} d$. per capita in 1851, $9 \frac{8}{6} d$. in 1861, and $13 \frac{3}{8} d$. in 1871. Portugal took $38.7 \frac{5}{8} d$. per head in $1851,48.9 \frac{1}{2} d$. in 1861 , but only $48.2 \frac{1}{d}$. in 1871 . In Italy the consumption increased from about 1s. $8 d$. per head in 1851 to $2 s .3 d$. in 1861 , but fell to $1 s .10 d$. in 1871 . Turkey in Europe took 2s. $2 \frac{5}{8} d$. per head in 1851, and 4s. $3 \frac{4}{4} d$. in 1871; Turkey in Asia figures for about $5 d$. in 1851, but $203 d$. in 1871, owing to extended commerce with the interior. The trade with China is still very small per capita, but the increase during the past 20 years has been from $1 d$. per head to nearly $4 d$. With India the increaso has been from $7 \frac{1}{2} d$. to $16 \frac{3}{4} d$. Japan took nothing in 1851 , but in 1871 her consumption reached more than $7 d$. per head. It is with these Eastern countries that the greatest expansion of trade is to be expected in the future. The whole of Africa consumed about $2 \frac{3}{4} d$. per head in 1851 , and $7 d$. in 1871 . Turning to America, there is an apparent decrease in the consumption of Canada, the figures being $58.8 \frac{3}{4} d$. in 1851 , but only $4 s .10 \frac{5}{8} d$. in 1871 , but part of the increased shipments to the United States passed through Canada ; and it is this transit trade that partly accounts for the United States' figures rising from 1s. $8 \frac{8}{8} d$. per head in 1851 , to $28.8 \frac{1}{8} d$. in 1871 . The West Indies do not show much increase; in some instances there is a decrease. Mexico advanced from $7 \frac{5}{8} d$. per head in 1851 to $16 \frac{7}{8} d$. in 1871 . In South America the consumption of Brazil only rose from 5s. $9 d$. to $6 s .2 \frac{3}{4} d$. per head, but Paraguay, \&c., increased from 1s. $5 \frac{1}{3} d$. to 6s. $10 \frac{7}{8} d$. Chili, Peru, \&c., remained almost stationary. Part of these discrepancies, however, are owing to the changes which have occurred in the course of trade. Taking the whole of South America the figures show an average consumption of 48. $5 \frac{1}{2} d$. per head in 1851, and 58. $9 d$. in 1871. A recapitulation of the figures for the world is as follows :-

Consumption of Cotton Products per Head.

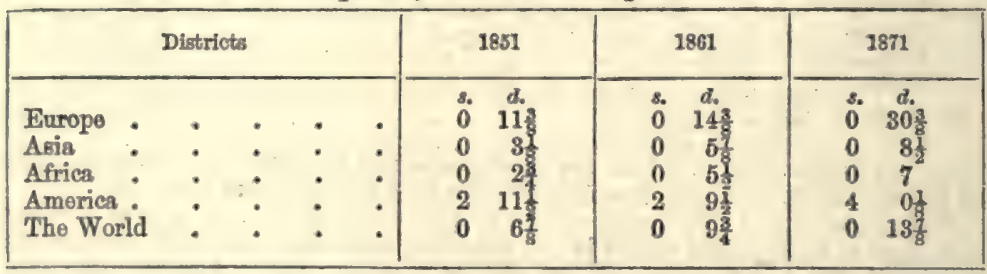

Respecting the business results of the year 1872, Messrs. Ellison and Co. say that ' from every point of view regarding the interests of the cotton trade the past year has formed quite a contrast to its immediate predecessor. 1871 was a year of unexampled prosperity to everyone in the industry, whether as importers, exporters, or consumers ; but 1872 has been a year of constant anxiety, disappointment, and general unprofitablenessi.' The table given on the last page of Messrs. Ellison and Co.'s report shows how unprofitable the business of the year was to consumers:- 'The balance left for wages, other expenses, interest of capital, and profits was $54,216,000 l$. or $12 \frac{1}{2} d$. per $\mathrm{lb}$. on the cotton consumed, against $61,147,000 l$. or $13 \frac{3}{4} d$, in 1871. This loss of $1 \frac{1}{4} d$. per lb. (nearly $7,000,000 l$.), was entirely borne by the millowners, for there was no reduction in the rate of wages. Moreover, there was an important additional item of expense in the shape of a serious advance in the price of coal. Altogether the year has been the most unsatisfactory one since 1869, during which some scores of failures occurred in the manufacturing districts, but the profits realised in 1871 saved Lancashire from bankruptcy during the past 12 months.'

'Importers of the raw material,' say Messrs. Ellison and Co., 'have also had a harassing time of it. They made some money in the early part of the year, but they have lost since, and the net result has been the reverse of gratifying. To importers of East-Indian cotton the year has been one of unmixed disappointment, owing to the tenacity with which consumers, notwithstanding the high range of prices, have clung to American and long-stapled cotton. The explanation is, first, that the demand has been chiefly for the better grades and finer numbers of yarn; secondly, that the "hands" have persistently refused to work Surats; and thirdly, that the quality of the Enst-Indian crop was exceptionally poor, 
- The total direct import of cotton into Europe in 1872, amounted to $5,488,000$ bales, of which $3,880,000$ bales were received into British and $1,608,000$ bales into Continental ports. Of the $3,880,000$ bales received into Great Britain 743,000 were ro-exported to the Continent, making the total supply to foreign Europe 2,351,000 bales, and leaving $3,137,000$ bales for British consumption. The stocks at the close of the year showed an increase of 70,000 bales as compared with those of twelve months previously, so that the deliveries were $5,418,000$ bales, of which $3,215,100$ bales were to English, and 2,203,000 to Continental spinners. The average weekly deliveries were: to English spinners, 61,820 bales, against 62,820 in 1871 ; and to Continental spinners, 42,365 , against 45,270 bales. We believe, however, that the stocks at the mills are fully 50,000 bales in Great Britain and 100,000 on the Continent less than they were at the close of 1871 , so that the actual consumption has been at the rate of 62,800 bales per week in Great Britain, against 59,900 in 1871 , and 44,5000 , against 43,560 bales.'

Messrs. Ellison and Co. estimate the number of cotton spindles in Europe at $39,500,000$ in Great Britain, and $18,580,000$ on the Continent. In the United States there are about $8,350,000$. The deliveries of each description of cotton in each of the past two years were as follow:-

\begin{tabular}{|c|c|c|c|c|c|}
\hline \multirow{2}{*}{ 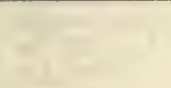 } & \multirow[b]{2}{*}{ 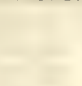 } & \multicolumn{2}{|c|}{ Deliveries $1,000^{\prime}$ 's of Bales } & \multirow{2}{*}{ Average } & \multirow{2}{*}{ Total } \\
\hline & & 1871 & 1872 & & \\
\hline $\begin{array}{l}\text { American . } \\
\text { Brazilian : } \\
\text { Egyptian, \&c. } \\
\text { West Indian } \\
\text { East Indian }\end{array}$ & 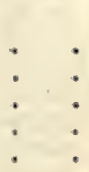 & $\begin{array}{r}3,004 \\
628 \\
435 \\
245 \\
1,257\end{array}$ & $\begin{array}{r}2,142 \\
1,043 \\
507 \\
233 \\
1,493\end{array}$ & $\begin{array}{r}2,573 \\
835 \\
471 \\
239 \\
1,375\end{array}$ & $\begin{array}{c}\text { lbs, } \\
1,126,974,000 \\
133,600,000 \\
219,015,000 \\
50,190,000 \\
493,625,000\end{array}$ \\
\hline Total & $\therefore$. & 5,569 & 5,418 & 5,493 & $2,023,404,000$ \\
\hline
\end{tabular}

For a full consumption a supply of $2,064,000,000$ lbs. is requisite, or about $40,000,000$ 1bs. more than the average deliveries of the past two years. Messrs. Ellison and Co. estimated the supply in 1873 as compared with the actual imports in 1872 and 1871 as follows :-

\begin{tabular}{|c|c|c|c|c|c|c|}
\hline \multirow[b]{2}{*}{ American } & & \multirow{2}{*}{$\begin{array}{c}1871 \\
\text { Balea } \\
\mathbf{3 , 1 1 4 , 0 0 0}\end{array}$} & \multirow{2}{*}{ 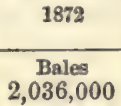 } & \multicolumn{2}{|c|}{ Weight of Import, 1878} & \multirow{2}{*}{$\frac{\text { Total }}{\text { Ibes. }_{1,082,574,000}}$} \\
\hline & & & & $\begin{array}{c}\text { Bales } \\
2,466,000\end{array}$ & $\begin{array}{c}\text { Average } \\
439\end{array}$ & \\
\hline Brazilian & - & 680,000 & $1,006,000$ & 760,000 & 160 & $121,600,000$ \\
\hline Moditerranean & $\mathbf{n}$ & 445,000 & 513,000 & 560,000 & 480 & $268,800,000$ \\
\hline West Indian & - & 240,000 & 237,000 & 240,000 & 210 & $50,400,000$ \\
\hline East Indian & - & $1,538,000$ & $1,696,000$ & $1,650,000$ & 360 & $558,400,000$ \\
\hline Total & - $\quad$. & $6,017,000$ & $5,488,000$ & $5,576,000$ & 273 & $2,081,774,000$ \\
\hline
\end{tabular}

On these figures Messrs. Ellison and Co. remark that, allowing for no increase in spindles during the past twelve months, the above supply shows an excess of only $17,774,000$ lbs., or 47,600 bales of $373 \mathrm{lbs}$. over the requirements of consumers, and, bearing in mind the fact that the year commences with a stock in all Europe 80,000 bales less than at the close of 1871 (that is 150,000 bales less than in the hands of spinners, but 70,000 more in the ports), there does not appear to be very much room for an expansion of spindle power during the ensuing twelve months. With a prospective supply very little, if at all, greater than the present consuming power of Europe, and only about $2 \frac{7}{8}$ per cent. greater than the actual average deliveries of the past owt years, preies on the average, for the year round will not, perhaps, greatly vary from the mean of $1871-72$, say $9 \frac{1}{2} d$. for Middling Uplands, and $7 \frac{1}{8} d$. for fair Dhollerah. Any important average advance upon these rates would lead to economy and reduced consumption, as in 1872. Any material decline would stimulate demand, as in 1871. For the immediate future the course of the market will be ruled by the amount of the weekly receipts at the American ports. Continued free arrivals would weaken the hands of holders; but as the supply from the East will be much smaller during the first half of this year than last, owing to the lateness of the Bombay crop, prices would not give way very much. On the other hand, a material reduction in the receipts at the American ports pointing to smallor figures than those we have adopted, would bring the smaller crop estimates into favour, and lead to some speculative excitement, and a sharp advance in values, 
The preceding remarks, when read in connection with the Tables of Imports and Exports given, eannot but be of high value in any considerations of the state of our cotion manufactures.

\section{CotToN IMPORTKD IX 1872.}

Cotton Raw, and Waste of:

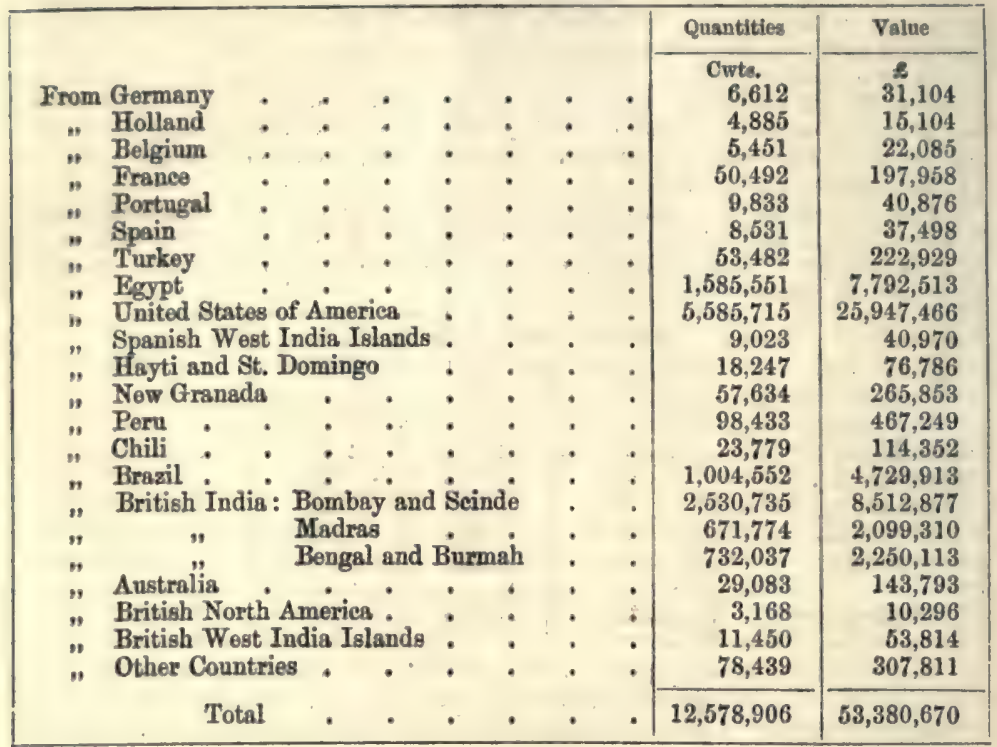

Cotton Yarn, and Waste of:

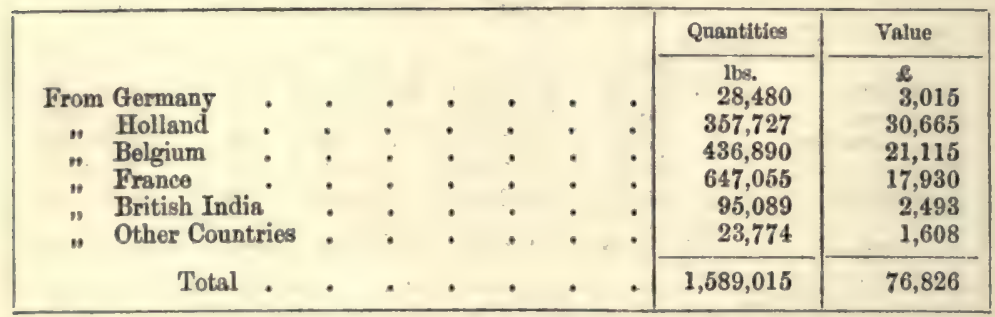

Cotton Manufactures.

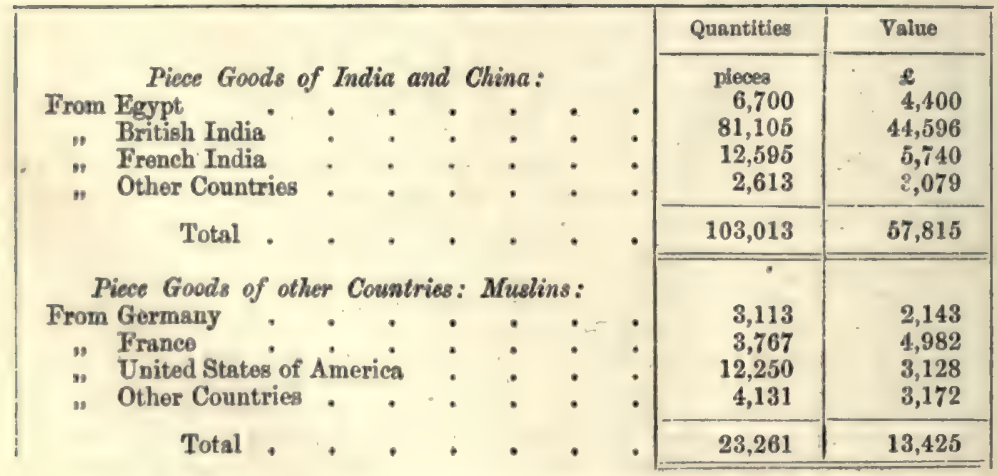




\begin{tabular}{|c|c|c|c|c|c|c|c|c|c|}
\hline \multirow{9}{*}{$\begin{array}{c}\text { From } \\
", " \\
" \\
"\end{array}$} & \multirow[b]{2}{*}{ Piece Goods } & \multirow{2}{*}{\multicolumn{2}{|c|}{ other than }} & \multirow{2}{*}{\multicolumn{2}{|c|}{ Muslins: }} & \multirow[b]{3}{*}{. } & \multirow[b]{3}{*}{. } & \multirow[t]{2}{*}{ Pieces } & \multirow{2}{*}{$\begin{array}{c}\text { Falue } \\
\end{array}$} \\
\hline & & & & & & & & & \\
\hline & Germany & . & . & $\cdots$ & $\ldots$ & & & 28,603 & 18,082 \\
\hline & Holland & - & . & . & $\therefore$ & . & . & . 138,580 & 154,473 \\
\hline & Belgium & . & - & . & $\cdots$ & . & $\cdots$ & 43,562 & 48,172 \\
\hline & France & - & ? & . & $\therefore$ & . & . & 19,947 & 34,410 \\
\hline & Other Countries & - & - & - & • & . & • & 3,559 & 2,691 \\
\hline & Total , & • & - & - & $\cdot$ & $\therefore$ & • & 229,251 & 257,828 \\
\hline & Hosiery & of & all So & rts: & & & & Quantities & \\
\hline From & Germany : & . & · & . & . & • & $\therefore$ & - & 115,559 \\
\hline " & Other Countries & • & 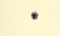 & - & . & - & • & & 2,148 \\
\hline & Total . & - & - & . & - & . & $\therefore$ & - & 117,707 \\
\hline & Une: & num & viated & & & & & . & \\
\hline From & Germany & . & . & . & . & - & . & - & 90,281 \\
\hline " & Holland & . & . & . & • & . & . & - & 343,587 \\
\hline 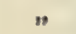 & Belgium & - & - & . & . & . & . & - & 210,763 \\
\hline " & France & . & - & . & - & . & . & - & 360,767 \\
\hline$"$ & Other Countries & - & - & - & - & • & - & - & 36,724 \\
\hline & Total & - & . & . & . & 4 & • & - & $1,042,122$ \\
\hline
\end{tabular}

\section{Corros Exponts nx 1872.}

Cotton Yarn and Twist.

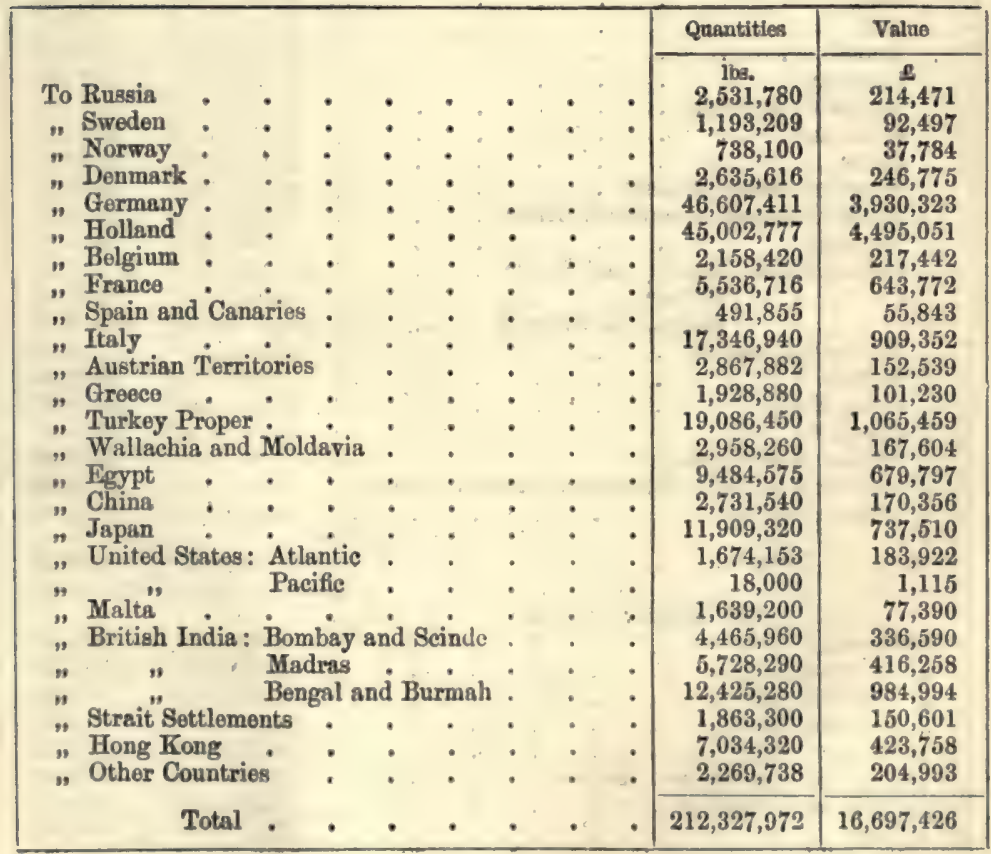


Cotton Manufactures.

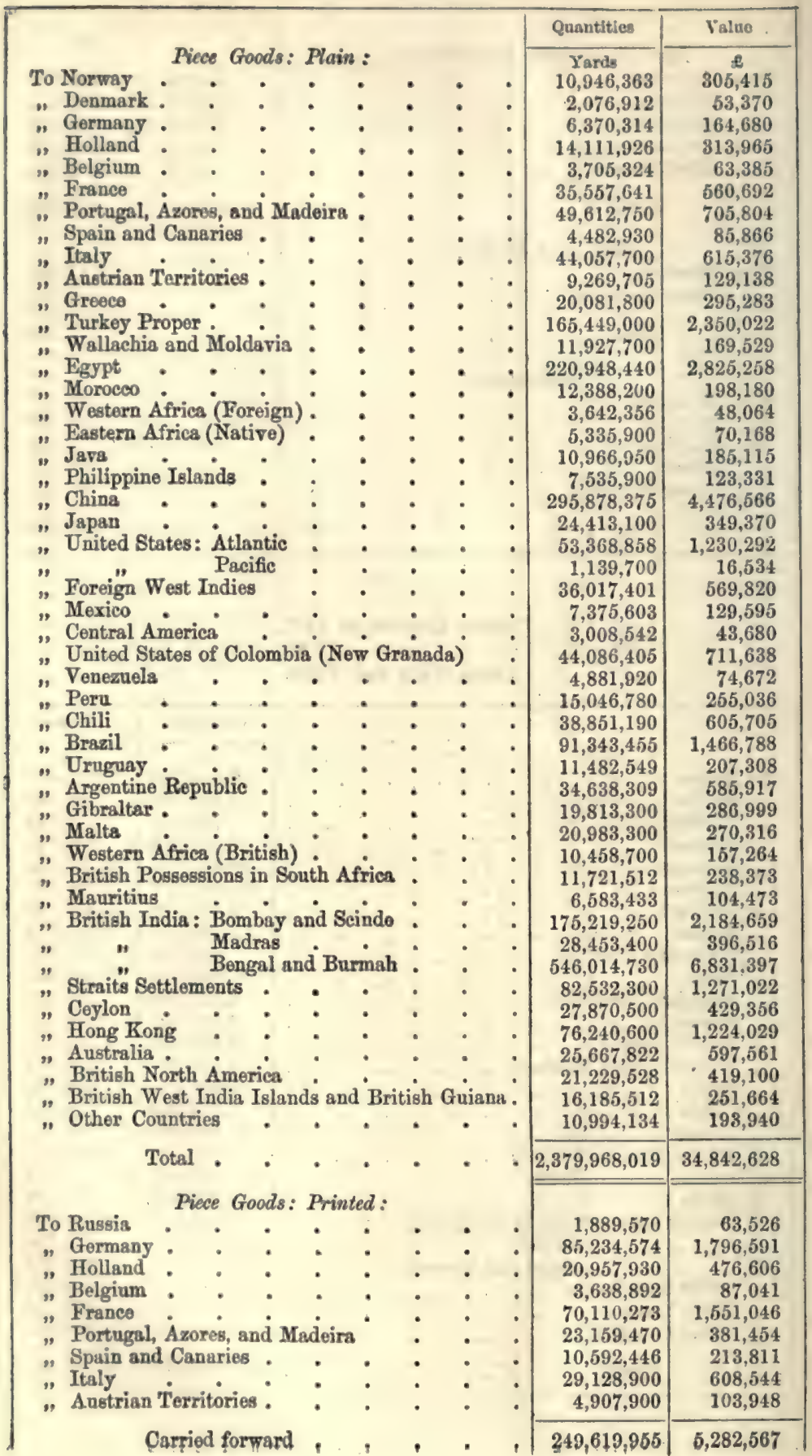




\begin{tabular}{|c|c|c|c|c|c|c|}
\hline \multirow{2}{*}{\multicolumn{2}{|c|}{ Brought forwand }} & & & \multirow[b]{2}{*}{ • } & Quantities & Value \\
\hline & & & & & $\begin{array}{c}\text { Yards } \\
249,619,955\end{array}$ & {$\left[\begin{array}{c}\stackrel{\mathcal{E}}{\mathcal{E}}, 28,567 \\
\end{array}\right.$} \\
\hline To & Greece. & - & . & . & $8,875,200$ & 177,339 \\
\hline & Turkey Proper & - & - & - & $115,500,200$ & $2,382,843$ \\
\hline & Wallachia and Moldavia & & - & - & $3,111,700$ & 58,814 \\
\hline & Egypt • • • & - & - & - & $39,835,660$ & 740,195 \\
\hline & Moroceo & - & . & - & $1,075,100$ & 31,211 \\
\hline & Western Africa (Foreign) & - & - & - & $19,374,087$ & 355,204 \\
\hline$"$ & Eastern Africa (Native) & . & . & - & 788,000 & 16,368 \\
\hline & Jam & , & - & & $12,359,400$ & 216,792 \\
\hline & Philippine Islands . & . & . & - & $3,211,900$ & 79,208 \\
\hline & China . . & . & - & : & $16,812,500$ & 339,065 \\
\hline & Japan $\cdot$ & . & - & ; & $3,334,900$ & 81,851 \\
\hline & Unitod States: Atlantic & . & - & - & $72,347,144$ & $2,108,152$ \\
\hline$"$ & Pacific & . & . & . & $2,017,900$ & 39,149 \\
\hline & Foreign West Indies & . & - & - & $58,375,029$ & $1,156,147$ \\
\hline$"$ & Mexico & - & . & $\bullet$ & $11,778,960$ & 212,042 \\
\hline & Central America & & & - & $2,450,003$ & 41,828 \\
\hline & United States of Colombia & (New Gra & anada) & - & $55,497,182$ & $1,025,472$ \\
\hline$"$ & Venezuela : . & - & - & . & $14,333,853$ & 310,033 \\
\hline & - $\cdot$ & - & . & . & $11,053,450$ & 210,922 \\
\hline & Chili & - & . & . & $29,819,900$ & 529,533 \\
\hline & Brazil . & - & - & - & $94,108,598$ & $1,941,187$ \\
\hline & Uruguay * $\cdot$ & . & - & - & $17,917,715$ & 342,744 \\
\hline & Argentine Republic . & * & . & , & $29,133,627$ & 571,538 \\
\hline & Gibraltar . . & . & . & . & $15,018,400$ & 322,499 \\
\hline$"$ & Malta & . & . & . & $3,152,800$ & 54,628 \\
\hline & Western Africa (British) & & - & . & $15,569,200$ & 300,137 \\
\hline & British Possessions in Sor & uth Africa & . & - & 13,4 & 343,441 \\
\hline & Mauritius _ • & & . & - & $4,714,119$ & 87,092 \\
\hline & British India : Bombay ar & nd Scinde & - & - & 32,6 & 555,500 \\
\hline & " Madras & . & , & . & $4,989,660$ & 88,613 \\
\hline & Bengal an & id Burmah & . & - & $73,688,765$ & $1,164,270$ \\
\hline & Straits Settlements . & - & - & - & $18,588,600$ & 341,720 \\
\hline & Ceylon . . & . & . & . & 5, & 114,755 \\
\hline & Hong Kong & . & . & . & $12,146,400$ & 304,896 \\
\hline & Australia. & . & . & & 19 & 12 \\
\hline & British North America & & & . & 7,293 & 412,673 \\
\hline & British West Indiis Island & Is and IBrit: & tish Guiang & & 22,2 & 419,641 \\
\hline$"$ & Other Countries & $\cdot$ & $\cdot \quad \cdot$ & - & $4,538,359$ & 86,513 \\
\hline & Total. & , & - & - & $1,137,625,766$ & $23,360,694$ \\
\hline & $\begin{array}{l}\text { Picce Goods of Afixe } \\
\text { Cotton predomi }\end{array}$ & $\begin{array}{l}\text { ed Mater } \\
\text { inating: }\end{array}$ & & & & \\
\hline & Germany . & . & . & - & $2,626,702$ & 75,117 \\
\hline & Holland . & . & . & - & 972 & 41, \\
\hline & Belgium . & . & . & . & 4,706 & 183,874 \\
\hline & France & . & . & . & $1,063,200$ & 35,757 \\
\hline & Egypt $*$. & . & - & . & 327,610 & 13,588 \\
\hline & Western Africa (Foreign) & & - & & 568,960 & 7,983 \\
\hline & China (exclusire of Hong & Kong) & . & . & 729 & 28,383 \\
\hline & Japan &.$\quad$ & . & - & 310,100 & 14,825 \\
\hline & United States : Atlantic & & . & & $2,734,234$ & 97,423 \\
\hline & Pacific & $\cdot$ & . & & 9,500 & 588 \\
\hline & Foreign West Indies & . & : & . & 591,750 & 20,423 \\
\hline & States of Colomb & a (New C & auadu) & & 471,527 & 20,8 \\
\hline & Brazil : & & & & 366,239 & 18,419 \\
\hline & Argentine Republic . & : & . & - & 75,300 & 3,574 \\
\hline & British India: Bengal and & d Burmah & & 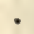 & 121,900 & 5,413 \\
\hline & Australia . & v & $\bullet$ & & $2,005,800$ & 66,616 \\
\hline & British North America & : & : & & $1,442,742$ & 46,998 \\
\hline & Other Countries & . & . & 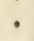 & $1,267,917$ & 46,487 \\
\hline & Total : & $\because$ & 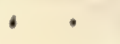 & & $20,391,526$ & 727,891 \\
\hline
\end{tabular}




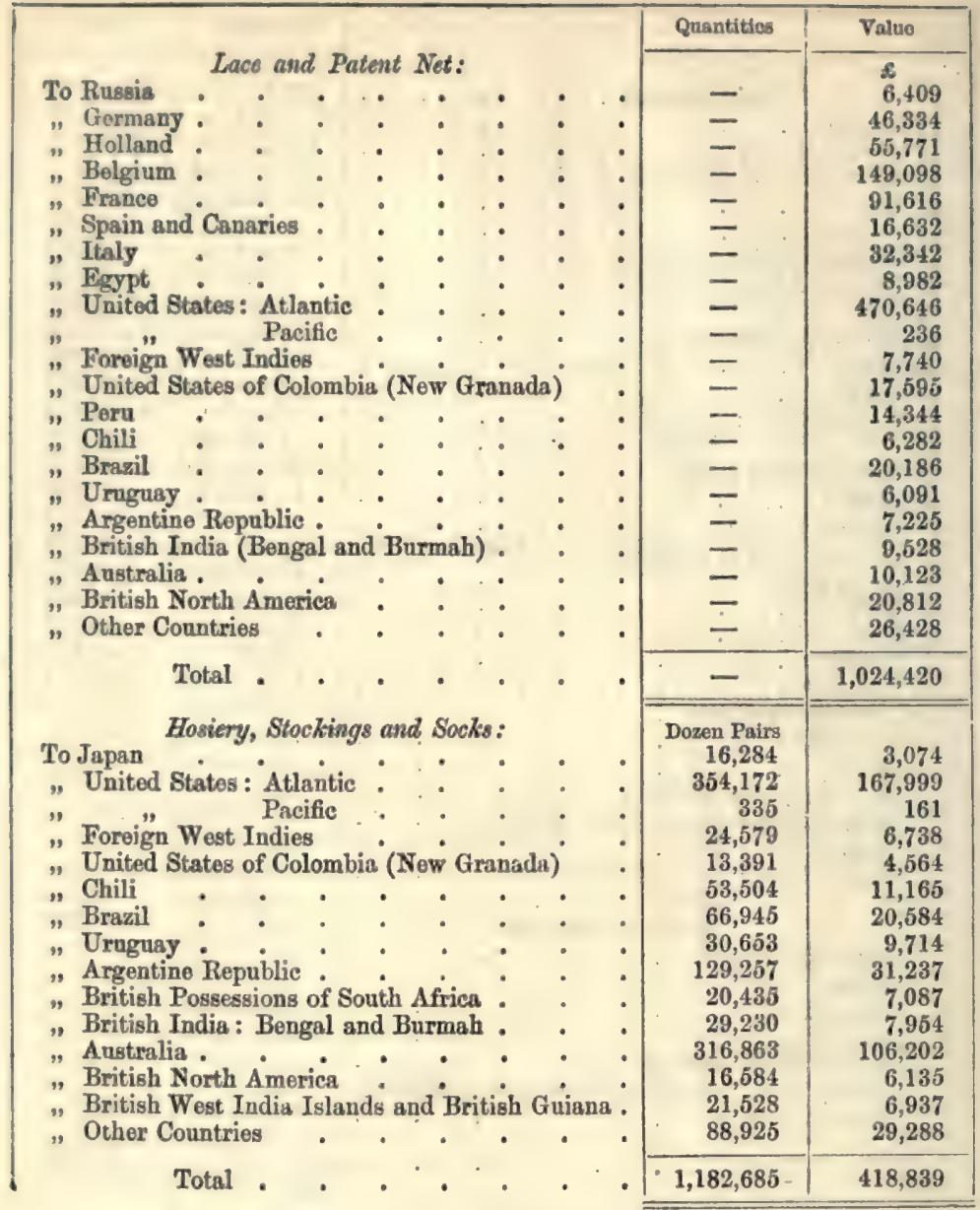

Hosiery of other Sorts (quantities not given).

To Germeny Value

" Folland : • • • • : $: 6,863$

"Italy $\therefore$ • • • • : . 7,831

"Turkey Proper . . . . . . 9,351

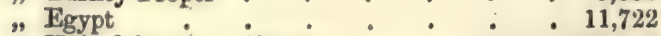

"United States: Atlantic : . . . 359,037

" $"$ Pacific : : 203

"Foreign West Indies . $\quad . \quad \vdots \quad$\begin{tabular}{l}
13,817 \\
\hline
\end{tabular}

". United States of Colombia (New Granada) . 17,183

" Peru. : . • . : . . 12,358

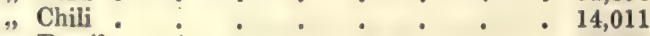

"Brazil : . . . . . . 19,231

"Uruguay $: \quad: \quad \vdots \quad \vdots \quad \vdots 16,869$

" Argentine Republic : . : . . 36,878

"British India : Bombay and Scinde : $\quad 6,698$

"Australia : . : : : : $\quad 20,109$

"British North America $\quad . \quad$. 46,951

"British West India Islands and British Guiana 10,694

"Other Countries . . . . . . 66,547

Total . . . . . $\overline{687,624}$ 


\begin{tabular}{|c|c|c|c|c|c|c|}
\hline \multirow{2}{*}{\multicolumn{5}{|c|}{ Thread for Sewing: }} & Quantities & Value \\
\hline & & & & & lbs. & \\
\hline \multirow{2}{*}{ "Sweden } & . & ${ }^{\circ}$ & . & - & 404,498 & 62,084 \\
\hline & - & - & - & . & 105,096 & 16,621 \\
\hline "Norway & . & . & - & - & 21,780 & 3,916 \\
\hline \multirow{2}{*}{ " Germany } & . & . & . & . & 950,005 & 190,339 \\
\hline & - & - & . & . & 189,223 & 30,680 \\
\hline $\begin{array}{l}\text { Holland } \\
\text { "Belgium }\end{array}$ & - & - & . & . & 96,621 & 23,379 \\
\hline "France & & . & . & . & 213,225 & 89,672 \\
\hline \multirow{2}{*}{$\begin{array}{l}\text { " Portugal, } \\
\text { „. Spain and }\end{array}$} & Azores, and $\mathrm{M}_{\mathrm{a}}$ & adeirı & & . & 109,308 & 13,845 \\
\hline & 1 Canaries . & . & . & . & 142,818 & 26,922 \\
\hline "Italy. & $\dot{0} \cdot$ & - & - & - & 202,521 & 20,178 \\
\hline "Austrian " & Territories . & . & . & - & 31,621 & 3,168 \\
\hline "Turkey P & roper . & . & . & . & 192,403 & 20,524 \\
\hline "Java & . & . & . & . & 111,390 & 13,201 \\
\hline "United St & tates: Atlantic & . & - & . & $2,106,996$ & 473,341 \\
\hline & Pacific & . & . & - & 26,600 & 4,551 \\
\hline "Foreign V & West Indies & - & . & . & 282,945 & 40,528 \\
\hline "Mexico & . & . & . & . & 154,532 & 21,238 \\
\hline " United St & of Colombi & ia (Now & Grannd: & & 153,175 & 19,874 \\
\hline "Venezuels & . $-\infty$ & - & - & - & 84,905 & 8,261 \\
\hline " Peru & - $\quad$. & . & - & . & 136,757 & $15, \tilde{0} 06$ \\
\hline "Chili & . & . & . & . & 150,893 & 18,138 \\
\hline \multirow{2}{*}{$\begin{array}{l}\text { " Brazil } \\
\text { Uruguay }\end{array}$} & . & . & . & . & 444,159 & 77,340 \\
\hline & . & - & . & . & 64,772 & 13,101 \\
\hline "Argentir & O Republic & & . & . & 145,500 & 38,182 \\
\hline " British In & ndia : Bombay a & and Scin & & . & 226,233 & 24,573 \\
\hline & Bengal an & ind Burm & ah. & - & 313,411 & 35,421 \\
\hline "Straits S & ettlements . & . & - & . & 80,790 & 7,757 \\
\hline \multirow{3}{*}{ " Australi } & & . & . & . & 137,266 & 21,006 \\
\hline & iorth America & & & $\therefore$ & 282,737 & 61,468 \\
\hline & Vest India Island & dds and $\mathrm{B}$ & British G & tuiana . & 68,186 & 11,166 \\
\hline "Other Cor & untries & - & - & - & 413,490 & 44,368 \\
\hline & Cotal , & - & - & - & $8,043,856$ & $1,400,243$ \\
\hline
\end{tabular}

Other Manufactures Unenumerated:

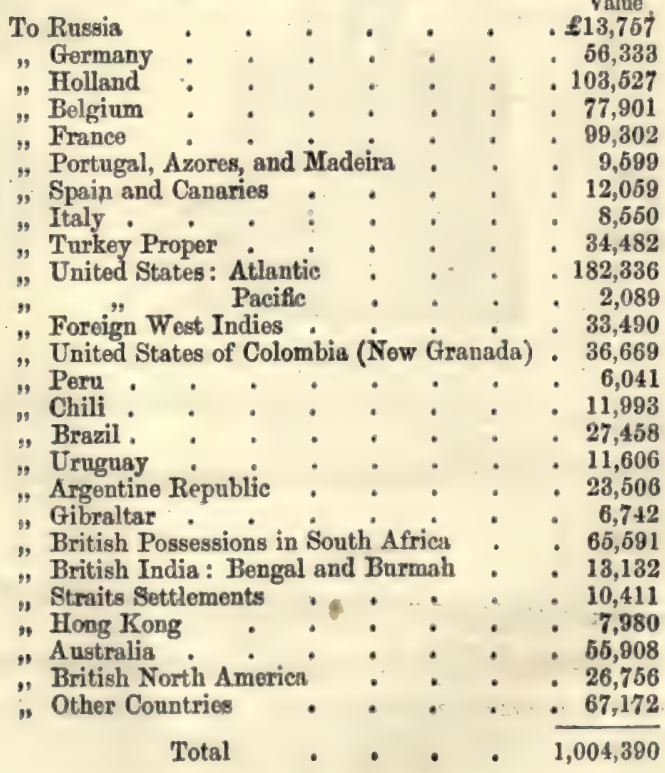


COURT PIASTER. Silk or some similar fabric covered on one side with isinglass in spirits of wine, with some Bonzoin or gum Benjamin.

COW-DUNG is employed in the processes of dunging in Calico-printing.

Cow-DUNG SUBsTrTUx and soda. See Cantco-Printing.

COWDIE PIXs. Cowrie or Kaurie Pine. The Dammara australis, a native of Now Zoaland, producing timber which is much valued for making masts and long spars of great strength. This pine also yields the gum-resin known as Cowrie gum, used in the preparation of certain varnishes.

CRATrBmRRY. The red acid fruit of the Oxycoccus palustris. The O. macrocarpus is the American variety. The fruit is preserved in water, and largely used.

CRAsras, Tubular. Among the many applications of the hollow-girder system, this is one of the most ingenious.

Fig. 584 is a vertical section of a crane, constructed according to a recent invention, and calculated for lifting or hoisting weights up to about 8 tons. Fig. 585, is an elevation of the same; figs. 586, 587, 588, and 589, are cross-sections, on the lines $a b$, c $d, e f, g h$; and $f g$. 590, a transverse vertical section on the line $i k$. $\Delta \Delta$ is the jib, which in its general outline, is of a crane-neck form, but rectangular in its crosssection, as particularly shown in figs. 587, 588, and 589. The four sides are formed of metal plates, firmly riveted together. Along the edges the connection of the

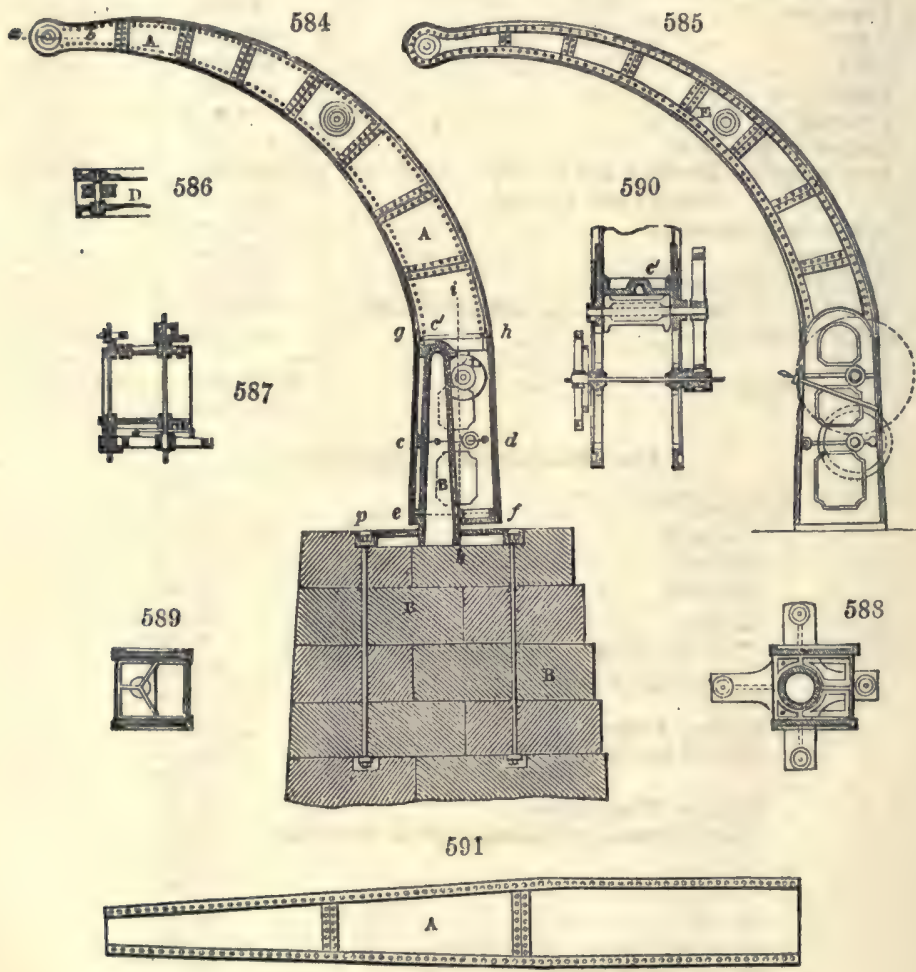

592

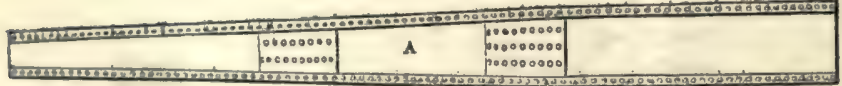

plates is effected by thearis of pleces of angle iron. The connection of the plates at the cross-joints on the convox or upper sido of the jib are made by the riveting on of a plate, which covers or orerlaps the cnds of the two plates to be joined; the rivets at 
this part are disposed as ropresented in fig. 592 (a plan of the top plates), and known as 'chain riveting'; $\mathrm{B}$ B is the pillar, which is firmly secured by a base plate $p$, to a stone foundation в; and fits at top into a cup-shaped besring $c^{\prime}$, which is so firmly gecured to the side plates of the jib, at or near to the point where the currature commences, and on which bearing the jib is free to revolve. Fig. 590 is a transverse vertical section of the lower part of the jib, showing the manner of fitting the bearings for the chain-barrel (which is placed in the interior), and the spindles and shafts of the wheel gearing, by which the power is applied there to $\mathrm{D}$, the chain-pulley, which is inserted in an aperturo formed in the top of the jib. The chain passing over this pulley enters the interior of the crane and is continued down to the chainbarrel. E is a pulley or roller, which is interposed about half-way between the chainpulley and the chain-barrel, for the purpose of preventing the chain rubbing against the plates. Fig. 591 is a plan of the lower plates.

Fig. 593 is a vertical section of another crane constructed upon the same principle as that just describod, but calculated for lifting much greater woights (say 20 tons); it differs in having the lower or concave side $\Delta \mathbf{\Delta}$, of the jib strengthened by means of three additional plates $\mathrm{B} \mathrm{B} \mathrm{B}$, whereby the interior is divided into one large and three

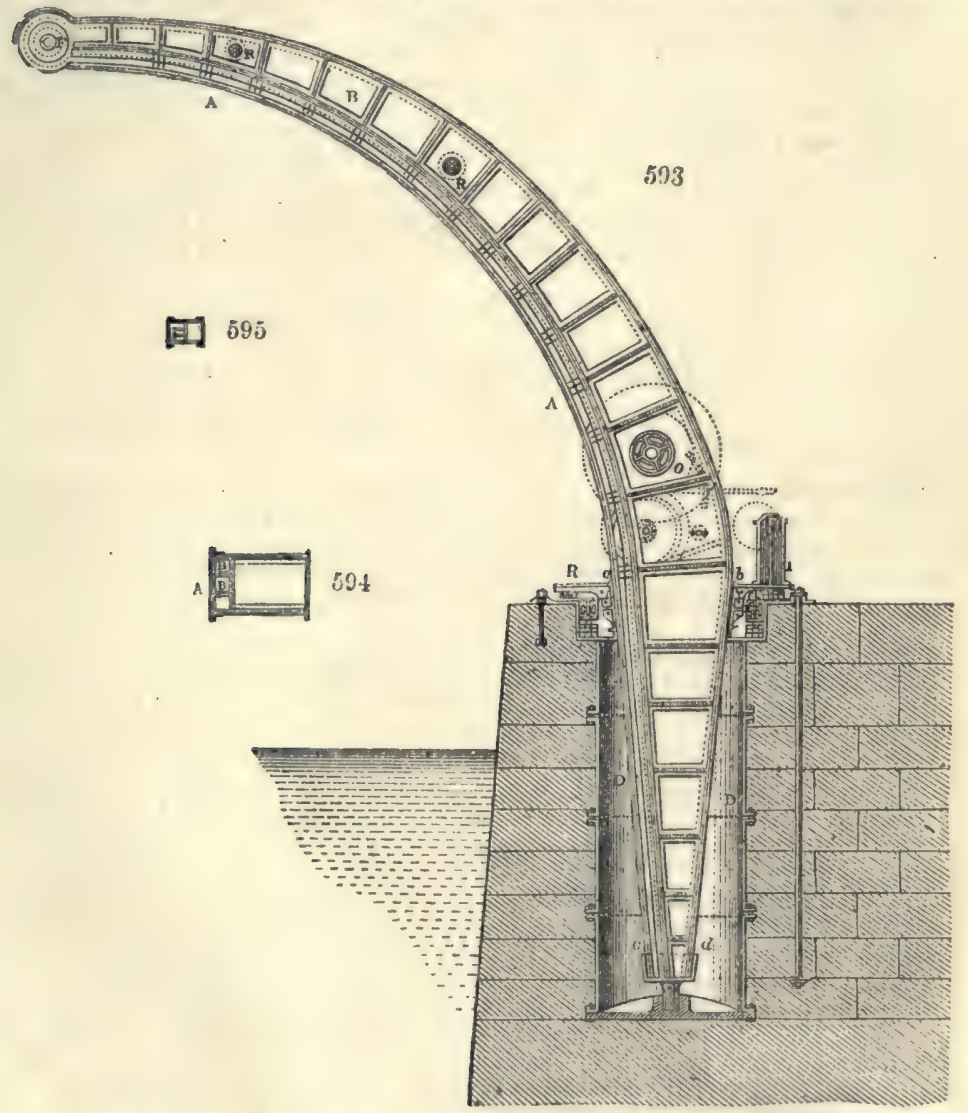

smaller cells, as shown in figs. 594 and 595, which are cross-sections upon the lines $a b$, and $c d$ of fig. 593. This arrangement of the cells to strengthen the lower or concave side is advisable, in order to obtain sufficient rosistance to the compression exerted by the load lifted, without unnecessarily increasing the weight of the other parts. The tension exerted upon the upper or convex plates does not require so much materials to withstand it; $\mathrm{c}$, is the toe of the jib, which rests in a stop formed in the bottom of the cylindrical castings $D$, which is built into the masonry forming the basis of the machine. E E are two of a set of pulleys, which are mounted between two rings $F$.

Vor. I. 
and serve as anti-friction rollers for the upper bearing of the jib. The lowermost of the riugs $F r$, rests upon a set of rollors $G G$, which are fitted into the top of the easting $D$, so that as the jib is turned round, the rings $F \mathbf{F}$, and the anti-friction rollers which they carry, have perfect freedom to movo along with it; which the persons working the machine may stand, and which supports tho column I, within which there is mounted a spindle $\mathbf{K}$, the lower end of which has keyed to it a pinion $\mathrm{L}$, which gears into a eircular rack $\mathbf{M} \mathbf{M}$, bolted to the top of the cylindrical casing $\mathrm{D}$. $\mathrm{N}$ is a worm-wheel keyed to the top of tho spindle $\mathrm{k}$, into which an endless serew worked by $a$ hand-wheel, is geared, so that, by turning the hand-wheol, the jib of the crane is made to moro round in any required direction. 0 is the chain-barrel; $\mathbb{P}$ the chain-wheel; $\mathbf{R} \mathbb{R}$ pulleys or rollers which support the chain, and prerent its rubbing against the plates of the jib.

In the cranes and hoisting machines described, the chain barrels are inclosed within the jib, and the spindles of the wheel-gearing are also inside; and this is the disposition of thoso parts preforred; but it will be obvious that they may be also placed outside of the jib, in a manner similarly to that generally followed in the construction of ordinary cranes.

Crapz. (Crîpe, Fr.; Krepp, Ger.) A transparent textile fabric, somewhat like ganze, made of raw silk, gummed, and twisted at the mill. It is woven with any crossiug or tweel When dyed black, it is worn by ladies as a mourning dress, Crapes are crisped (cripes) or smooth; the former being double, are used.in close mourning; the latter is less deep. The silk destined for the first is spun harder thun for the second; since the degree of twist, particularly for the warp, determines the degree of crisping which it assumes after being taken from the loom. It is for this purpose steeped in clear water, and rubbed with prepared wax. Crapes are all woven uncl dyed with the silk in the raw state. They are finishod with a stiffening of gumwater. White crape is appropriated to young unmarried females, and to virgins on taking the veil in nunneries.

Crape is a Bolognese invention, but has been long manufactured with superior excellence at Lyons in France, and Norwich in England. There is now a large manufactory of it at Yarmouth, by power-loom machinery.

There is another kind of stuff, ealled crépon, made either of fine wool, or of wool and silk, of which the warp is twisted much harder than the weft. The crepons of Naples consist altogether of silk. Airophanes, crape-lesse, and gauze are either white or coloured crapes.

Crayows. (Eng. and Fr.; Pastellstifte, Ger.) Slender, soft, and somewhat friable cylinders, variously coloured for delineating figures upon paper, usually called chalk drawings. Red, green, brown, and other coloured crayons, are made with fine pipe or china clay paste, intimately mixed with earthy or metallic pigments, or in general with body or surface colour, then moulded and dried. See Drawing

\section{Снаткя.}

Crian of tartar. The Bitartrate of Potash.

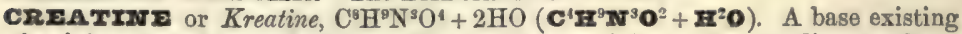
in the juice of flesh and in urine along with creatinine. It was discorered by Chevreul, but chiefly investigated by Liébig.

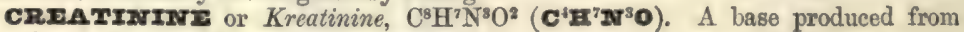
creatine by the loss of two atoms of water.

CrmxP. In working a colliery on the pillar-and-stall principle it occasionally happens that the pillars are forced into the floor by the weight of the superincumbent strata, and the pavement is gradually heaved up until it may finally settle against the roof; this rise of the floor is called a creep. Soo Mintra for Cont.

Crzosorz or Kreosote. One of the many singular bodies discovered by

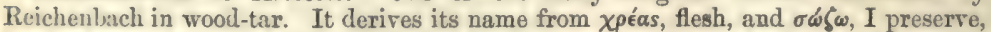
in allusion to its remarkable antiseptic properties. A great deal of confusion exists in the published accounts of wood creosote, owing to the variable nature of the results ohtrined by the chemists who have examined it. This confusion is not found with that from coal, which undoubtedly contains two homologous bodies, $\mathrm{C}^{12} \mathrm{H}^{6} \mathrm{O}^{2}\left(\mathbf{C}^{6} \mathbf{H}^{6} \mathbf{O}\right)$ and $\mathrm{C}^{14} \mathrm{H}^{8} \mathrm{O}^{2}\left(\mathbf{C}^{7} \mathbf{x}^{8} \mathbf{0}\right)$; the first being carbolic, and the second cresylic acid. Tho composition of carbolic acid has long been known, owing to the researches of Laurent: cresylic acid was afterwards discovered by Williamson and Fairlie. Commercial coul crensote sometimes consists almost entirely of cresylic acid. Coal oils, of very high loiling point, contain acids apparently homologues of carbolic acid, higher up in the series than even cresylic acid, and yet perfectly soluble in potash.-(Greville Williams.) There is little doubt that wood creosote consists essentially of the same substances as that from coal. The great difference in the odour arises chiefly from the fact of the product from coal retaining with obstinacy traces of naphthaline, parroline, and chinoline, 
all of which are extremely odorous. No creosote found in commerce is ever perfectly homogeneous, nor, in fact, is it necessary that it should be so. If perfectly soluble in potish and acetic acid of the density 1.070 , and if it does not become coloured by exposure to the air, it may be considered pure enough for all medicinal purposes. The oils from wood-and coal-tar may be made to yicld creosoto by the following process. 'The oils are to bo rectified until the moro volatile portions (which are lighter than water) havo passed over. As soon as tho product runuing from the still sinks in water the receiver is to le chauged, and the oils may bo received until the temperature required to sund over the oil is as high as $480^{\circ} \mathrm{F}$. Tho oil so obtained is to be dissolred in caustic soda, all insoluble in it being rejected. The alkaline solution, after being mechanically separated, as far as possible, from the insoluble oil, is to be boiled for a very short time. Two advantages are gained by this operation : any volatile bases become expelled, and a substance which has a tendency to become brown on keeping, is destroyed. Sometimes the oil on treatment with potash yields a quantity of a crystalline paste. This is naphthaline, and should be removed by filtration through coarse calico or canvas. The alkaline liquid is then to be supersaturated with dilute sulphuric acid, on which the creosote separates and rises in the form of an oil to the surface. This creosote is already free from the greater number of impurities, and, if rectified, may bo used for many purposes. To obtain a purer article the operations commencing with solution in caustic soda are to be repeated. If the alkaline solution on boiling again becomes coloured, the purification must be gone through a third time. It is essential not to boil the alkaline solution long, or a serious loss of creosote would take place. According to Reichenbach the boiling point of creosote is $397^{\circ}$. Carbolic acid boils between $369^{\circ}$ and $370^{\circ}$. Cresylic acid boils at $397^{\circ}$. From this it would appear that Reichenbach's creosote consisted of eresylic acid. The specific gravity of ereosoto according to Reichenbach is 1.037 at $68^{\circ}$, that of carbolic acid is 1.065 at $64^{\circ}$. Carbolic acid and its homologues, when mixed with quicklime and exposed to the air, yield a beautiful red colour, owing to the formation of rosolic acid. Seo Disinfectants.

crassor. $\mathrm{C}^{14} \mathrm{H}^{8} \mathrm{O}^{2}\left(\mathbf{C}^{7} \mathbf{m}^{8} \mathbf{O}\right)$. A compound found with carbolic acid in coal-tar, creosote, and the tar of pine-wood.

CrasyIIC ACID. $\mathrm{C}^{14} \mathrm{H}^{8} \mathrm{O}^{2}\left(\mathbf{C}^{7} \mathbf{H}^{8} \mathbf{O}\right)$. A homologue of carbolic acid. See Creosotr ; Disinfectants.

CRITrF. ( $(\rho|\theta\rangle)$, a barleycorn). A unit of weight, introduced into modern chemistry by Dr. Hofmann. A crith is the weight of a litro of hydrogen at $0^{\circ} \mathrm{C}$. and $760 \mathrm{~mm}$. bar. pressure; this amounts to 0.089636 gramme. The use of the crith is convenient in calculating the weights of given volumes of different gases on the metric system.

CROCIDOIrTz. A fibrous asbestiform mineral occurring in the Griqua Country, Soutl Africa. White, brown, red, and blue varieties are known; and some of these, when cut en cabochon, resemble coloured cat's-eye, and are used in jewellery. The truo crocidolite is a hydrous silicate of protoxide of iron, magnesia, and soda; but much of the so-called crocidolite from South Africa has been proved to be pseudomorphous fibrous quartz coloured with oxide of iron and other foreign matter.

Crocorsrmz. Native chromate of lead, $\mathrm{PbO}, \mathrm{CrO}^{3}$ ( $\mathbf{P b} \mathbf{C r O}^{4}$ ) occurring in small hyacinth-red erystals. in some of the Siberian mines, in Hungary, in Brazil, and in the Philippine Islands. It was in this mineral that the metal chromium was discovered in 1794, by Vauquelin. The name of the species is sometimes written, Crocoite.

Crocus. The commercial name for a polishing powder made with oxide of iron. The older chemists gave this name to many of the metallic compounds, which had something of the nature of rust of iron. Crocus Martis, sesquioxide of iron; Crocus Metallomim, liver of antimony, glass of antimony, oxysulphide of antimony; Crocus Veneris, oxide of copper.

Crocus. A genus of plants belonging to the natural order of Iridacece. The Crocus sativus is the species from which the saffron of commerce is produced. The stigniata of the flowers are of a deep orange colour, and these, when dried in the loose state, form hay saffron; when compressed it forms the cake saffron. Saffron is cultivated in Cambridgeshire; it is also imported from Sicily, France, and Spain; but English saffion is always preferred.

Cross coursz. A mining term. See Mrwrk.

cross cur. A mining term. See Mrara.

CROSS-FIUCKANS or FIoorass. The name given by the Cornish miners to clay veins crossing the mineral lodes. See Frookas.

Crotataria JUrrcra. The plant which yiolds Sumn or Bengal hemp.

Croxow oxx. A poworful purgative oil, obtained from seeds of the Croton Tiglirem, a shrub grown in India and the neighbouring isles.

$$
3 \text { T } 2
$$


czorrzns. A name givon to certain lichons in Scotland which aro usod for dyeing woollen stuffis brown.

CrownzrRy. The berries of Empotrum nigmu, eaten in the north of Europe, and used in Groenland in the preparation of a fermented boverage.

crowre GIass. See Grass.

CrucrBzms (Creusets, Fr.; Schmelatiegel, Ger.) aro small conical ressels, nnrrower at tho bottom than the mouth, for reducing ores in assaying by the dry way, for fusing mixtures of earthy and other substances, for melting metals, and compounding metallic alloys. They ought to be refractory in the strongest heats, not readily acted upon by the substances ignitod in them, not porous to liquids, and capalle of beuring considerable altornations of temperature without cracking; on which account they should not be made too thick. Soe Assay; Mective Pots.

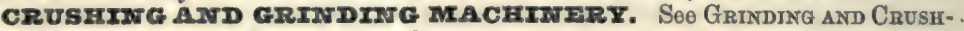
INe MachnNery, under Dressinct of Ores.

Cryorrm2. (Kryolith, Eisstein, Ger.) This mineral derives its name from rpúos, ice,-from the circumstance of its being fusible in the flame of a candlo. It is $\Omega$ double fluoride of aluminium and sodium, represented by the formula $A^{2} \mathrm{~F}^{3}+3 \mathrm{NaF}$ $\left(\mathbf{A l}^{2} \mathbf{I}^{6} .62 \mathrm{Nar}\right)$, which corresponds to aluminium 13.00 ; sodium 32.8 ; fluorino $54 \cdot 2$ per cont. Tho minoral usually occurs in cleavable masses of snow-whito colour, having a specific grarity of about 3. Cryolite is found only at Evigtok, in Arksutford in West Greenland, where it forms a large deposit in gneiss, associated with galena, pyrites, and spathic iron ore. It is now obtained in large quantitios, and is used in the preparation of sulphate of alumina and alum; it has also been used in Germany in the manufacture of soda and soap, and of late has found a new application in America for the production of eryolite glass. The mineral is also employed in the manufacture of aluminium, and was at one time the chief source of the metal. Sce ALOMINTOM.

CryorrTz cruss. At Pittsburg in Pennsylvania an opaque milk-white glass resembling porcelain is made with cryolite. This glass may be obtained by melting together the following ingrodients: silica, $67 \cdot 19$; cryolite, 23.84 ; and oxide of zine, 8.97 per cent.

CRXPTIDINI, $\mathrm{C}^{22} \mathrm{H}^{11} \mathrm{~N}\left(\mathbf{C}^{11 \mathbf{I X}^{11}} \mathbf{N}\right)$. A volatile base homologous with chinoline, found by Greville Williams in the less volatile portion of coal-tar.

CRYsTAI. A crystal is a body which has assumed a certain geometric form. It is produced by nature, and may be obtained by art.

The ancients beliered quartz to be water converted into a solid by intense cold, and

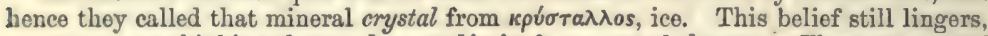
many persons thinking that rock crystal is, in fact, congealed water. The term orystal is now applied to all solid bodies which assume certain regular forms. A crystal is any solid bounded by plane surfaces symmetrically arranged. Each mineral has its own mode of crystallisation, by which it may be distinguished, and also its own peculiarity of internal structure.

We may have a mineral in a considerable variety of external forms, as pyrites, in cubes, octahedrons, dodecahedrons, \&c.; but these are all related to a single typethe cube. Thus galena, whatever external form it may assume, has an internal cubical structure. Fluor-spar, usually occurring in cubical forms, may be cleaved into a regular octahedron. A little reflection will enable the student to see that nature in her simple arrangements maintains an unvarying internal type, upon which sho builds up her varying and beautiful geometric forms. There are certain imaginary lines which are called the axes of the crystal : these may be

Rectangular and equal, as in the cube.

Rectangular and one unequal, as in the right square prism.

Rectangular and three unequal, as in the right rectangular prism.

The three axcs anequal, vertical inctined to one of the lateral, at right angles to the other, two lateral at right angles with one another, as in the oblique rhombic prism. prism.

The three axes unequal and all the intersections ollique, as in the doubly oblique

Three equal lateral axes intersecting at angles of $60^{\circ}$ and $a$ vertical axis of varying length at right angles with the lateral, as in tho lioxagonal prism.

Upon these simplo arrangements of tho axial lines all the crystalline forms depend. the particles of matter arranging themselves around these axes according to some law of polarity which has not yet been developed. For an outline of Crystallography, see Watts's "Dictionary of Chomistry."

Crystax. A name given to flint glass. See Funt Guass.

CUBz Orz. (Würfelerz, Ger.) A common name for Pharmacosiderite : a hydrous arsenate of sesquioxide of iron, occurring in some of the Cornish copper-mines, and in Saxony, in the form of small cubes, generally of a green colour. 
Corrc rxrre. Native nitrate of soda, or Chili saltpetro. The name is supposed to rofor to the form of tho crystals, which, however, are rhombohedra and not cubes. The mineral is used in the preparation of nitric acid and of nitre.

CUDBzAr. This colouring mattor was first mado an articlo of trade by Dr. Cuthbert Gordon, from whom it derived its name; and was originally manufactured on a great seale by Mr. G. Mackintosh, at Glasgow, nearly 80 years ago.

It is prepared in the samo manner as archil, from the samo species of lichen; only, towards the end of the process, the substance is dried in the air, and is then ground to a very fine powder. See ArCHL; Limus.

Cưruzr. Broken refuse glass.

CUImx, a term applied to anthracito or coal-stone in somo districts. It is still used in Parliamentary returns. See ANтнracitx.

CUIMIDIN2, $\mathrm{C}^{18} \mathrm{H}^{13} \mathrm{~N}\left(\mathbf{C}^{9} \mathbf{z n}^{13} \mathbf{M N}\right)$. An alkali homologous with aniline, formed by Nicholson by the action of reducing agents on nitrocumole. Its density is 0.9526 , and its boiling point $437^{\circ}$.

CUMrN SmpD. The fruit of the Cuminum Cyminum. This plant is much cultivated in Sicily and Malta for the sake of the seeds. These have a warm aromatic tuste, and a strong and rather agreeable smell. The Dutch sometimes flavour their cheese with cumin seed. In Germany they are put into bread; and in this country they are employed to flavour curries.

comors, or Cumène, Hydruret of Cumenyle. $\mathrm{C}^{18} \mathrm{H}^{12}\left(\mathbf{C}^{9} \mathbf{E}^{12}\right)$ A hydrocarbon found in coal naphtha ; it is also produced by the destructive distillation of cuminic acid with caustic baryta. See Naphtha, Coat, and Carberetted Hydrogen.

CUPzI. A shallow cup made of bone-ashes. See Assar.

cupriteatrow. Seo Assaying and Silver Refining.

CUprzous mangatrisz. Seo Mavganese.

CUrAcos. A Dutch liquour flavourod with Sevillo orange peel, cinnamon, and mace. Seo Liouners.

CURCUMA Arg GUSxrrozxA. The narrow-leaved Turmeric. (East Indian Arrow Root.) This plant is found in the forests, extending from the banks of the Lona to Nagpore. At Bhagulpore the root is dug up and rubbed on a stone bed or in a mortar, and afterwards rubbed in water with the hand and strained through a cloth; the fecula having subsided, the water is poured off, and the tikor (fecula) dried for use. The East Indian arrow-root is a fine white power, readily distinguishable, both to the eye and the touch, from West Indian arrow-root. To the oyo it somewhat resembles a finely-powdered salt (as bicarbonate of soda or Rochelle salt). When pinched or pressed by the fingers, it wants the firmness so characteristic of West Indian arrow-root, and it does not crepitate to the same extent when rubbed between the fingers.-Pereira.

At Travancore this starch forms a large portion of the diet of the inhabitants.

Currinc smorva. A stone used in Scotland in playing the national game of curling, which is practised upon the ice during the winter. The stone is made of some hard primary rock. That of Ailsa Craig, in the Firth of Clyde, is very celebrated. Ailsa Craig consists of a single rock of greyish compact felspar, with small grains of quartz, and. very minute particles of hornblende.-Bristow.

CUrRasrs. The fruit of the Ribes rubrum and $R$. nigrum. The name currant is derived from the similarity of the fruit to that of the Corinth raisins, or small grape of Zante, which are commonly called corinths or currants.

Curersos. A resin obtained from the hemp-plant (Camabis sativa) grown in India.

Curaz. A seasoning originally prepared in the East Indies. The following aro excellent recoipts for curry :-

Coriander seeds powdered

Black popper

Oayenne pepper

Fenegric seeds

Cumin seeds

To be thoroughly well mixed together.

Coriander seeds powdered

Fonegric seeds

Cumin seeds

Black pepper

Turmeric root

Cayenne pepper

Well mix. Ginger root

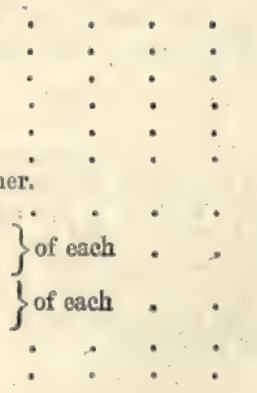

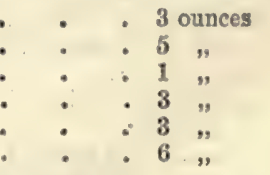

$$
\begin{aligned}
& \text { - } \quad \frac{1}{4} \text { a pound } \\
& \text { - } 2 \text { ounces } \\
& \text { - } 4 \text { ounces } \\
& \text { - } 6 \text { drachms } \\
& \text { - } 1 \text { ounco }
\end{aligned}
$$




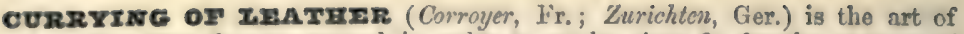
dressing skins after they are tanued, in order to render them fit for the purpose of the shoo-maker, coach-maker, harness-maker, \&e. Seo Leathær, Cokryixg of.

cuscus ז00т. A grass which yields a fragrant ossential oil, called Vitever.

CUsparia marz. The bark of a South American tree, the Galipen Cusparia, much esteemed for its medicinal properties.

CUrCr or Kutch. The catechu of the Acacia Catechu. Seo Acacia Catrocho.

CUтI-RY. (Contelleric, Fr. ; Messerschmicdwaare, Ger.) Three kinds of steel are made use of in the manufacture of different articles of cutlery, viz., common steel, shear steel, and cast steel. Shear steel is exceedingly plastic and tough. All the edge tools which require great tenacity without great hardness are made of it, such as table knives, scythes, plane-irons, \&c.

Cast steel is formed by melting blistered steel in covered crucibles, with bottle glass, and pouring it into cast-iron moulds, so as to form it into ingots : these ingots are then taken to the tilt, and drawn into rods of suitable dimensions. No other than cast steel can assume a very fine polish, and hence all the finer artieles of eutlery are made of it, such as the best scissors, penknives, razors, \&c.

Formerly cast steel could be worked only at a very low heat; it can now be made so as to be welded to iron with the greatest case. Its use is consequently extended to making rery superior kinds of chisels, plane-irons, table knives, \&c.

Forging of table knives. - Two men are generally employed in the forging of table knives; one called the foreman or maker, and the other the striker.

The steel called common steel is employed in making the very common articles; but for the greatest part of table knires which require a surface free from flaws, shear steel and cast steel are generally preferred. That part of the knife termed the blade is first rudely formed and cut off. It is next welded to a rod of iron about $\frac{1}{2}$ inch equare, in such a manner as to leave as little of the iron part of the blade exposed as possible. A sufficient quantity of the iron now attached to the blade is taken off from the rod to form the bolster or shoulder, and the tang.

In order to make the bolster of a given size, and to give it at the same time shape and neatness, it is introduced into a die, and a swago placed over it; the swage has a few smart blows given it by the striker. This dio and swage are, by the workmen, called prints.

After the tangs and bolster are finished, the blade is heated a second time, and the foreman gives it its proper anvil finish : this uperation is termed smithing. The blade is now heated red hot, and plunged perpendicularly into cold water. By this means it becomes hardened. It requires to be tempered regularly down to a blue colour : in which state it is ready for the grinder.

Mr. Brownill's method of securing the handles upon table knives and forks, is, by lengthening the tangs, so as to pass them completely through the handle, the ends of which are to be tinned after the ordinary mode of tinning iron; and, when passed through the handle, the end of the tang is to be spread by beating, or a small holo drilled through it, and a pin passed to hold it upon the handle. After this, caps of metal, either copper plated, or silver, are to be soldered on the projecting end of the tang, and while the solder is in a fluid state, the cap is to be pressed upon the end of the handle and held there until the solder is fixed, when the whole is to be cooled by being immersed in cold water.

Mr. Thomason's patent improvements consist in the adaptation of steel edges to the blades of gold and silver knives. These steel edges are to be attached to the other metal, of whaterer quality it may be, of which the knife, \&c., is made, by means of solder, in the ordinary mode of effecting that process. After the edge of steel is thus attached to the gold, silver, \&c., it is to be ground, polished, and tempered by immersion in cold water, or oil, after being heated. This process being finished, the other parts of the knife are then wrought and ornamented by the engraver or chaser, as usual.

A patent was obtained in 1827, by Mr. Smith of Sheffield, for rolling out knifes at one operation.

In the ordinary mode of making knives, a sheet of steel being provided, the blades are cut out of the sheet, and the backs, shoulders, and tangs, of wrought iron, aro attached to the steel blades, by welding at the forge. The knife is then ground to the proper shape, and the blade polished and hardened.

Instead of this welding process, the patentee proposes to make the knives entirely of steel, and to form them by rolling in a heated state between massive rollers; tho shoulders or bolsters, and the tangs for the handles being produced by suitable reesses in the peripheries of the rollers, just as railway rails are formed. When the kuife is to be mado with what is called a scale tang. that is, a broad flat tang, to which the handle is to be attached in two pieces, rireted on the sides of the tang, the 
rollers are then only to have recesses cut in them, in a direction parallel to the axis for forming the bolster.

The plate of steel having been heated, is to be pressed between the two rollers, by which the blades and the parts for the scale tangs will bo pressed out flat and thin, and those parts which pass between the grooves or recess will be left thick or protuberant, forming the bolster for the shoulder of the blade. But if the tangs are to be round in order to be fixed into single handles, then it will be necessary also to form transverse grooves in the rollers, that is, at right angles to those which give shape to the bolsters, the transverse grooves corresponding in length to the length of the intended tang. When the plates of steel have been thus rolled, forming three or more kinves in a breadth, the several knives are to be cut out by the ordinary mode of what is callod slitting, and the blades and shoulders ground, hardened, and polished in the usual way.

Forks are generally a distinct branch of manufacture from that of knives, and are purchased of the fork makers by the manufacturers of tables knives, in a state fit for receiving the handles.

The rods of steel from which the forks are made, are about sths of an inch square. The tang and shank of the fork are first roughly formed. The fork is then cut off, leaving at one end about 1 inch of the square part of the steel. This part is afterwards drawn out flat to about the length of the prongs. The shank and tang are now heated, and a proper form given to them by means of a die and swage. The prongs are afterwards formed at one blow by means of the stamp; this machine is very similar to that used in driving piles, but it is worked by one man. It consists of a large anvil fixed in a block of stone nearly on a level with the ground. To this anvil are attached two rods of iron of considerable thickness, fixed 12 inches asunder, perpendicularly to the anvil, and diagonally to each other. These are fastened to the ceiling. The hammer or stamp, about $100 \mathrm{lbs}$. in weight, having a groove upon either side corresponding to the angles of the upright rods, is made to slide freely through its limited range, boing conducted by its two iron supporters. A rope is attached to the hammer, which goes over a pulley on the floor of the room above, and comes down to the person who works the stamp: two corresponding dies are attached, one to the hammer, and the other to the anvil. That part of the fork intended to form the prongs, is heated to a pretty white heat and placed in the lower die, and the hammer containing the other die is made to fall upon it from a height of about 7 or 8 feet. This forms the prongs and the middle part of the fork, leaving a very thin substance of steel between each prong, which is afterwards cut out with an appropriate instrument called a fly-press. The forks are now annealed by surrounding a large mass of them with hot coals, so that the whole shall become red hot The fire is suffered gradually to die out, and the forks to cool without being disturbed. This process is intended to soften, and by that means to prepare them for filing. The inside of the prongs are then filed, after which they are bent into their proper form and hardened. When hardened, which is effected by heating them red-hot and plunging them into cold water, they are tempered by exposing them to the degree of heat at which grease inflames.

Ponknives are generally forged by a single hand, with the hammer and the anvil simply.

The hammer in this trade is generally light, not exceeding $3 \frac{1}{2}$ lbs. The breadth of the face, or the striking part, is about one inch; if broader, it would not be convenient for striking so small an object. The principal anvil is about 5 inches, and 10 upon the face, and is provided with a groove into which a smaller anvil is wedged. The smaller anvil is about 2 inches square upon the face. The blade of the knife is first drawn out at the end of the rod of steel, and as much more is cut off along with it as is thought necessary to form the joint. The blade is then taken in a pair of tongs, and heated a second time to finish the joint part, and at the same time to form a temporary tang for the purpose of driving into a small haft used by the grincier. Another heat is taken to give the blade a proper finish. The small recess called the nail-hole, used in opening the knife, is made while it is still hot by means of a chisel, which is round on one side and flat upon the other.

Penknives are hardened by heating the blade red hot, and dipping them into water up to the shoulder. They are tempered by setting them side by side, with the back downwards, upon a flat iron plate laid upon the fire, where they are allowed to remain till they are of a brown or purple colour.

The blades of pocket knives, and all that come nnder the denomination of spring knives, are made in the same way.

The forging of razors is performed by a foreman and striker, as in making table knires. 
They are genorrily hade of cast stecl. The rods, as they como from the tilt, are about $\frac{1}{2}$ inch brond, and of a thickness sufficient for the back of a razor.

There is nothing peculiar in the tools made use of in forging razors; the auril is a little rounded at tho sides, which affords the opportunity of miking the edge thinner, and saves an immense labour to the grinder.

Razors are hardened and tempered in a similar manner to penknives. They are, however, left harder, being only let down to yellow or brown eolour.

The forging of scissors is wholly performed by the hammer, and all the sizes are made by a single hand. The anvil of the scissor-maker weighs about $1 \frac{1}{2} \mathrm{cwt}$; it mensures, on the face, about 4 by 11 inches. It is provided with two gates or grooves for the reception of various little indented tools termed by the workmen bosses: one of these bosses is employed to give proper figure to the shank of the scissors; auother for forming that part which has to mako the joint; and a third is made use of for giving a proper figure to the upper side of the blade. There is also another anvil placed on the same block, containing two or three tools called beak-irons, each consisting of an upright stem about 6 inches high, at the top of which a horizontal beak projects; one of these beaks is conical, and is used for extending the bow of the scissors; the other is a segment of a cylinder with the round side upwarls, containing a recess for giving a proper shape and smoothness to the inside of the bow.

The shank of the seissors is first formed by means of one of the bosses, above clescribed, leaving as much steel at the end as will form the blade. A hole is then punched about $\frac{1}{3}$ inch in width, a little above the shank. The blade is drawn out and tinished, and the scissors separated from the rod a little above the hole. It is heated a third time, and the small hole above mentioned is extended upon the beak-irons so as to form the bow. This finishes the forging of scissors. They aro promiscuously made in this way, without any other guide than the eye, having no regard to their being in pairs. They are next annealed for the purpose of filling such parts of them as cannot be ground, and afterwards paired.

The very large scissors are made partly of iron, the blades being of steel.

After the forging, the bow and joints, and such shanks as caunot be ground, are filecl. The rivet hole is then bored. through which they are to be screwed or rivetod together. This common kind of seissors is only hardened up to the joint. They are tempered down to a purple or blue colour. In this state they are taken to the grinder.

Grinding and polishing of cutlery.-The various processes which come under this denomination are performed by machinery, moving in general by the power of the steam-engine or water-wheel.

Grinding wheels or grinding mills are divided into a number of separate rooms; each room contains six places ealled troughs; each trough eonsists of a convenience for running a grindstone and a polisher at the same time, which is generally occupied by a man and a boy.

The business of the grinder is generally divided into throe stages, viz., grinding, glazing, and polishing.

The grinding is performed upon stones of various qualities and sizes, depending on the articles to be ground. Those exposing much flat surface, such as saws, fenders, \&cc., require stones of great diameter, while raisors, whose surface is concave, require to be ground upon stones of very small dimensions. Those articles which require a certain temper, which is the case with most cutting instruments, are mostly ground on a wet stone; for which purpose the stone hangs within the iron trough, filled with water to such a height that its surfuce may just touch the face of the stone.

Glazing is a process following that of grinding: it consists in giving that degree of lustre aud smoothness to an article which can be effected by means of emery of various degrees of fineness. The tool on which the glazing is performed, is termed a glazer. It consists of a circular piece of wood, formed of a number of pieces in such a manner that its edge or face may always present the endway of the wood. Were it made otherwise, the contraction of the parts would destroy its circular figure. It is fixed upon an iron axis similar to that of the stone. Some glazers are covered on the fuce with leather, others with metal, consisting of an alloy of lead and tin; the lattor are termed caps. In others, the wooden surface above is made use of. Some of the leather-faced glazers, such as are used for forks, table knives, edge tools, and all the canrser polished articles, are first coated with a solution of glue, and then covered with emery. The surfaces of the others are prepared for use by first turning the face very true, then filling it with small notehes by means of a sharp-ended hammer, and lastly filling up the interstices with a compound of tallow and emery.

The pulley of the glazier is so much less than that of the stone, that its velocity is more than double, having in general a surface speed of 1,500 feet in a second.

The process of polishing consists in giving the most perfect polish to the different 
articles. Nothing is subjected to this operation but what is made of cast steel, and has been previously hardened and tempered.

The polisher consists of a circular piece of wood covered with buff leather, the surface of which is covered from time to time, while in use, with the crocus of iron, called also colcothar of vitriol.

The polisher requires to run at a speed much short of that of the stone, or the glazer. Whatever may be its diameter, the surface must not move at a rate exceeding 70 or 80 feet in a second.

СUттхх гтSн. Mollusea belonging to the class Cephalopoda. The internal calcareous sholl of one species (Sepia officinalis) is used as pounce. See SEPIA.

CYANANIIINz. A crystalline body formed by the action of cyanogen upon anilize. See Cravogran.

CYAXATES. The combinations of the various bases with cyanic acid, C'HNO" (Crro). The cyanate of potash, $\mathrm{C}^{2} \mathrm{NKO}^{2}(\mathbf{C r \pi m})$, is employed for the preparation of artificial urea. There are two modes of preparing cyanate of potash, both of which yield a good product: the first is that of Clemm, the second of Liebig. (1.) 8 parts of ferrocyanide of potassium and 3 parts of carbonate of potash are intimately mixed and fused, care being taken not to urge the heat too much. The fluid mass is allowed to fall somewhat in temperature, but not to such an extent as to solidify; 15 parts of red lead are then added by small portions. The crucible is now to be reheated with stirring, then removed, and the contents poured on to a clean iron plate. (2.) The cyanide of potassium of commerce (prepared by the method described in the article under that head) is to be melted in an iron crucible or ladle, and $3 \frac{1}{4}$ parts of dry litharge in fine powder are to be added with constant stirring. When the lead has all collected at the bottom, the whole is poured on to an iron plate. The mass obtained by either of the above processes is to be reduced to powder, and boiled with repeated quantities of alcohol, until no more cyanate is extracted. This may be known when the alcohol filtered from the residue no longer yields crystals of cyanate in cooling.-C. G. W.

\section{CYaNhydrrc aCrd. See Hydrocyanic Acid.}

CYArIDEs. The combinations of cyanogen with metals or other bodies. It is remarked in the article HYDrocy ANIC AcID that cyanogen, $C^{2} \mathrm{~N}(\mathbf{C N})$ is a compound salt-radical, analogous to the halogens chlorine, iodine, and bromine. Like the latter, it unites with metals without the intervention of oxygen, and with hydrogen to form a hydracid corresponding to the hydrochloric, hydriodic, and hydrobromic acids. The cyanides are both an important and interesting class of salts. The most important is the cyanide of potassium. The latter is formed under a great variety of circumstances, especially where carbonate of potash is heated in contact with carbonaceous matters. The nitrogen to form the cyanide in the greater number of instances is principally, and in a few entirely, derived from the atmosphere. Many chemists have experimented on this subject, and their results are by no means in harmony; but thus much is certain, that success or failure depends solely upon the circumstances under which the experiments are conducted. It has been shown that, when carbonate of potash mixed with charcoal prepared from sugar (seo CARBON) is exposed to a very high temperature in a current of uitrogen gas, the potash in the carbonate is, at times, absolutely converted into cyanide, not a trace of carbonic acid remaining. Experiments of this class, when made with animal charcoal or coal, are less conclusive because those matters contain nitrogen. But even then the amount of cyanogen found is out of proportion to the quantity of nitrogen in the coal or other carbonaceous matters. In fact, it would seem that the presence of a certain quantity of nitrogen in the coal, \&c., exercises a predisposing tendency on the nitrogen of the air so as to induce its combination with carbon with greater facility than would be the case if pure carbon were employed. Cyanide of potassium has been found on more than one occasion oozing from apertures in iron-smelting furnaces. In fact, it has been produced in such abundance at one furnace in Styria as to be sent into the market for sale to electro-platers.

Cyanide of potassium is largely prepared for the use of electro-platers and gilders. The proportions of the materials used are those of Liebig, who first made known the process. The modes of manipulation, however, differ in the details in all laboratories. The following method can be recommended from the experience of the author of this article as giving a white and good product. It can, moreover, be worked on a very Inrge scale. The ferrocyanide of potassium and salt of tartar are to be separately dried, pulverised, and sifted through eane sieres. The salt of tartar must be free from sulphates. To 8 parts of dry ferrocyanide of potassium 3 of dry salt of tartar are to be added, and the two are to be incorporated by sifting. A large and strong iron pot is then to be suspended by a chain from a crane in such a position that it can be lowered into the furnace and raised with ease; there must also bo an arrange- 
ment to enable the pot to be urrested at any desired height. The pot being heated to redness, the mixture is to bo thrown in by small portions until tho vessel is half full; the heat being allowed to rise gradually until tho whole flows pretty quiotly. During the fusion the contents are to be stirred with a clean iron rod to promote the aggregation of the spongy sediment. As soon as the rod, on being dipped into the fused mass and remored, brings with it a pure white porcelain-like product, the operation may he regarded as terminated, and tho pot is to bo raised from the fire by means of the crane and sling in a slightly inclined position. One of the operators now holds a large clean iron ladle under the edge of the pot, while another elevates the latter with the aid of tongs, so that the ladle becomes filled. The contents of the first ladle are then poured off into another held by the assistant who tilted the pot. The latter then pours the contents of his ladle into a large, shallow, and brilliantly clean brass basin standing in another containing a little water so as to cool the fused cyanide rapidly. Extreme care must be taken to prevent even the smallest drop of water from finding its way into the brass vessel, because on the hot cyanide coming in contact with it an explosion would occur, seattering it in erery direction to the great danger of the persons in the vicinity. The two ladles are to be kept very hot, by being held orer the firo until wanted, in order to prevent the cyanide from chilling until it is poured into the brass basin. The latter should be about 18 inches in diameter and $1 \frac{1}{2}$ deep. It should be quite flat-bottomed. The object of so many pourings off is to prevent any of the sediment from finding its way into the product, and thus causing black specks in it. The pot, on being emptied as far as convenient, is to have the sediment remored and a fresh charge inserted. As soon as the coke of cyanide is cool, it is to be broken up into moderate-sized pieces and placed in dry and well closed jars.

The cyanide of potassium possesses great points of interest for the technical and theoretical chemist. It is the salt from which an immense number of compounds of importance may be obtained. Very large quantities are made for the purpose of preparing the auro- and argento-cyanides of potassium for the electro-platers and gilders.

Auro-cyanide of potassium is capable of being formed in several ways. The following are convenient processes. The selection of a mode of preparing it will depend upon the circumstances under which the operation is situated. 1. By the battery. This process is perhaps the most generally convenient and economical for the electro-gilder. A bath is prepared by dissolving the best commercial cyanide of potassium in good filtered or distilled water. The best salt is that sold under the name of 'gold cyanide.' A Daniell's battery of moderate size being charged, two plates of gold are attached to wires and connected with it. The larger, which is to be dissolved, is attached to the positive, and the smaller, which need be but the size of a flattened wire, to the negative pole. The action of the battery is kept up until the desired amount is dissolved. It is easy to remove the plate used, dry and weigh it at intervals so as to know the proper time to stop the operation. 2. Teroxide of gold (prepared with magnesia) is to be dissolved in a solution of cyanide of potassium.

Argento-cyanide of potassium. This solution is easily prepared for the electroplater by the following process. Metallic silver is dissolved in nitric acid and the solution evaporated to dryness. The residue is dissolved in distilled water and filtered. To the solution cyanide of potassium, dissolved in distilled water, is added as long as precipitation takes place, but no longer, The precipitate is filtered off on calico strainers, and well washed with distilled water. It is then to be dissolved in solution of cyanide of potassium and diluted to the desired strength. The solution is frequently dark coloured at first, but it becomes colourless in a few hours, and should then be filtered from a small black precipitate which will be obtained. Many operators neglect the filtration and washing of tho precipitated cyanide of silver, and merely continue the addition of the solution of eyanide of potassium to the nitrate of silver until the precipitate at first formed is re-dissolved. The first method is however to be preferred. Some, instead of precipitating with cyanide of potassium, clo so with solution of common salt, and then, after washing off the precipitated chloride of silver, dissolve it in cyanide of potassium. Argento-cyanide of potassium can also be prepared with the battery by the process mentioned under auro-cyanide of potassium; this method is so convenient where the proper apparatus is at hand, that few professional electro-platers would use any other method.

CyATIDIs rerro. See Ferrocyantors.

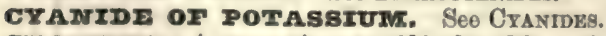

Cxarrarz. A name given to Chinoline Bluc. See Chroone Bude.

CXAsrrxz. Kyanite or Disthene. A native silicato of alumina, occurring generally in flattened prisms of a bluo colour. Transparent, finely-coloured specimens have occasionally been cut and polished as gem-stones, and bear some resem- 
blance to sepphires; the hardness, however, is considerably less, It is notable that the hardness of cyanite varies in different faces of the same crystal-the old name Disthene ( $\delta$ is, twice; $\sigma \theta$ évos, strength), having reference to this unequal resistance which the crystal offers in two different directions.

CXanocrar. C'N (CX). A compound salt-radical, analogous in its character to chlorine and the other halogens. It was the first body discovered possessing the characters of a compound radical, and the investigations made upon it and its derivations have thrown more light upon the constitution and proper mode of classifying organic substances than any other researches whatever. In consequence of its acting in all its compounds as if it were a simple body or element, chemists generally have acquired the habit of designating it by the symbol Cy. Liko the haloids, it combines with hydrogen to form an acid, and with metals, without the necessity for the presence of oxygen. For a fow illustrations of its analogies with chlorine, \&c., see HxDrocranic Acm. In the article Cyanides several of the conditions under which it is formed have also been pointed out. The modern French chemists of the school of Gerhardt very justly regard eyanogen in the light of a double molecule, thus Cy Cy, or $\mathrm{C}^{4} \mathrm{~N}^{2}$. The reason of this is because most of the phenomena of organic chemistry are more easily explained by the use of four-volume formulæe than any others. This latter mode of condensation has been shown by M. Wurtz, in his admirable work on the compound radicals, to undoubtedly exist in the case of radicals belonging to the strict hydrogen type, not as ethyle and its homologues; and numerous theoretical and experimental results are in favour of the supposition that all radicals in the free state are binary groups.

If we assume the truth of the above hypothesis, we shall regard cyanogen in the free state as a cyanide of cyanogen, analogous to hydrocyanic acid, which is a cyanide of hydrogen.

Cyanogen may very conveniently bo prepared by heating cyanide of mercury in a retort of hard glass. A considerable quantity of the gas is given off, but a portion remains behind in the state of paracyanogen. The latter substance is a black matter, the constitution of which is by no means understood. It has, however, the same composition in the hundred parts as cyanogen itself, and is therefore isomeric with it.

Cyanogen is a colourless combustible gas, with a sharp odour. Its density is 1.81 . theory requires for two volumes $1 \cdot 80$. If cooled to a temperature of between $-13^{\circ}$ aud $-22^{\circ} \mathrm{F}$., it liquefies into a transparent, colourless, and very mobile fluid, having a specific gravity of 0.866 . A little below $22^{\circ}$ the fluid congeals to a mass resembling ice. The flame of cyanogen is of a pale purple or peach-blossom colour.

Some of the properties of cyanogen are very remarkable, and quite distinct from those of the true halogens. For instance, it combines directly with aniline to produce it body having basic properties. The latter is calred Cyaniline or Cyananiline, and is formed by the coalescence of two molecules of cyanogen with two of aniline, the resulting formula being consequently $\mathrm{C}^{28} \mathbf{H}^{14} \mathrm{~N}^{4}\left(\mathbf{C}^{14} \mathbf{H}^{14} \mathbf{I S}^{4}\right)$. There are a variety of singular compounds produced by the action of cyanogen and its halogen compounds upon aniline; they have been studied with remarkable skill by Hofmann,--C.G.W.

\section{Cyarrosz or Cyanosite. Native sulphate of copper.}

CYAIrURIC ACID. When cyanate of ammonia is heated it passes into urea; and urea by further heating may be converted into ammonia and cyanuric acid. This acid may also be prepared by the action of sulphuric acid on melam. Cyanuric acid contains $\mathrm{C}^{6} \mathrm{H}^{3} \mathrm{~N}^{3} \mathrm{O}^{8}\left(\mathbf{C}^{3} \mathbf{H}^{3} \mathbf{N}^{3} \mathbf{O}^{3}\right)$, and at a very high temperature may te resolved into three molecules of cyanic acid.

CrDma (Cidre, Fr.; Apfelwein, Ger.): the vinous fermented juice of the apple. The ancients were acquainted with cyder and perry, as we learn from the following passage of Pliny the naturalist: 'Wine is made from the Syrian pod, from pears and apples of every kind.' (Book xiv. chap. 19.) The texm cyder or cidre in French, at first written sidre, is derived from the Latin word sicera, which donoted all other fermented liquors, except grape-wine. Cyder seems to have been brought into Normandy by the Moors of Biscay, who had preserved the use of it after coming into that country from Africa. It was afterwards spread through some other provinces of France, whence it was introduced into England, Germany, and Russia. It is supposed that the first growths of Normandy afford still the best specimens of cyder. Devonshire and Herefordshire are the counties of England most famous for this beverage.

Strong and somewhat elevated ground, rather dry, and not exposed to the air of the sea, or to high winds, are the best situations for the growth of the cyder apple. The fruit should be gathered in dxy weather. The juice of apples is composed of a great deal of water; a little sugar analogous to that of the grape; a matter capable of causing fermentation with rontact of air ; a pretty large proportion of mucilage, with malic acid, acetic acid, and an azotised matter in a very small quantity. The seeds contain a bitter substance and a little essential oil; the pure parenchyma or cellular 
membrane constitutes not more than two per cent. of the whole. After the apples are gathered, they are left in the barn-loft for fifteen dsys or upwards to mellow; some of them in this case, however, become soft and brown. This degree of maturution diminishes their mucilage, and developes alcohol and carbonic acid; in consequence of which the cyder suffers no injury. There is always, however, a little loss; and if this ripening goes a little further it is very apt to do harm, notwithstanding the vulgar prejudice of the country people to the coutrary. Too much care, indeed, cannot bo taken to separate the sound from the spoiled apples; for the latter merely furnish an acid leaven, giring a disagreeable taste to the juice, and hinder the cyder from fining, by leaving in it a certain portion of the parenchyma, which the gelatinous matter or the fermentation has diffised through it. Unripe apples should be separated from the ripe also, for they possess too little saccharum to be properly susceptible of the vinous fermentation,

Where cyder-making is scientifically practised, it is prepared by crushing the apples in a mill with revolving edge-stones, turned in a circular stone cistern by one or two horses. When the fruit is half mashed, about one fifth of its weight of river water is added.

In some places a mill composed of two cast-iron fluted cylinders placed parallel to each other under the bottom of a hopper, is employed for crushing the apples. One of the cylinders is turned by a winch, and communicates its motion in the opposite direction by means of the flutings working into each other. Each portion of the fruit must he passed thrice through this rude mill in order to be sufficiently mashed; and the same quantity of water must be addecl as in the edge-stone mill.

After the apples are crushed they are usually put into a large tub or tun for 12 or 24 hours. This steeping aids the separation of the juice, because the fermentative motion which takes place in the mass breaks down the cellular membranes; but there is always a loss of alcohol carried off by the carbonic acid disengaged, while the skins and seeds develope a disagreeable taste in the liquid. 'Ihe vatting might be suppressed if the apples were so comminuted as to give out their juice more readily.

After the vatting, the mashed fruit is carried to the press and put upon a square wicker frame or into a hair bag, sometimes between layers of straw, and exposed stratum above stratum to strong pressure till what is called a cheese or cake is formed. The mass is to be allowed to drain for sorne time before applying pressure, which ought to be very gradually increased. The juice which exudes with the lenst pressure affords the best cyder; that which flows towards the end acquires a disugreeable taste from the seeds and the skins. The must is put into casks with large bungholes, where it soon begins to exhibit a tumultuous fermentation. The cask must be completely filled, in order that all the light bodies suspended in the liquid when floated to tho top by the carbonic acid may flow over with the froth; this means of clearing cyder is particularly necessary with the weak kinds, because it cannot be expected that these matters in suspension will fall to the bottom of the casks after the motion has ceased. In almost every circumstance besides, when no saccharine matter has been added to the must, that kind of yeast which rises to the top must be separated, lest by precipitation it may excite an acid fermentation in the cyder. The casks are raised upon gauntrees or stillions, in order to place flat tubs below them to receive the liquor which flows over with the froth. At the end of 2 or 3 days for weak cyders, which are to be drunk somewhat sweet, of 6 or 10 days or more for stronger cyders, with variations for the state of the weather, the fermentation will be sufficiently adranced, and the cyder may be racked off into other casks. Spirit puncheons preserve cyder better than any other, but in all cases the casks should be well seasoned and washed. Sometimes a sulphur match is burned in them before introducing the cyder, a precaution to be generally recoinmended, as it suspends the activity of the fermentation, and prevents the formation of vinegar.

The cyder procured by the first expression is called cyder without water. The cake remaining in the press is taken out, divided into small pieces, and mashed anew, adding about half the weight of water, when the whole is carried back to the press, and treated as above described. The liquor thus obtained furnishes a weaker cyder which will not keep, and therefore must be drunk soon.

The cake is once more mashed up with water, and squeezed, when it yields a liquor which may be used instead of water for moistening fresh ground apples.

Tho processes above described, although they have been long practised, and have therefore the stamp of ancestral wisdom, are extremely defective. Were the apples ground with a proper rotatory rasp which would tear all their cells asunder, and the mash put through the bydraulic press in bags between hurdles of wicker-work, the juice would be obtained in a state of perfection fit to make a cyder superior to many wines. An experimental process of this kivd has been actually exeented in Franco upon a considerable scale, with the best results. The juice had the fine flarour of the 
npple, was fermented by itself without any previous fermentation in the mash, and afforded an excellent strong cyder, which kept well.

When the must of the apples is weak or sour, good cyder cannot be made from it without the addition of some saccharine matter. The syrup into which potato farina is convertible by diastase (saccharine ferment, see Starci and Sugar), would answer well for enriching poor apple juice.

The value of apples to produce this beverage of good quality is proportionate to the specific gravity of their juice. M. Couverchel has given the following table, illustrative of that proposition: water being 1000 :-

Juice of the green renette, queen apple (reinette verte) has a spec. graw. 1094

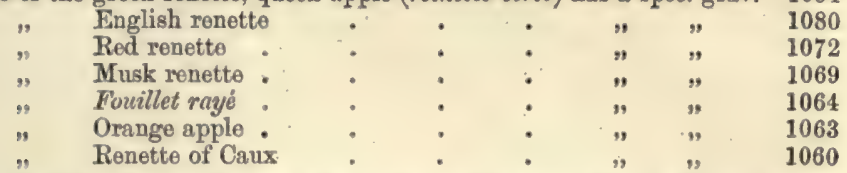

Cyder apples may be distributed into three classes: the sweet, the bitter, and the sour. The second are the best; they afford a denser juice, richer in sugar, which clarifies well, and when fermented keeps a long time; the juice of sweet apples is diffienlt to clarify; but that of the sour ones makes bad cyder. Late apples are in general to be preferred. With regard to the proper soil for raising apple-trees, the reader may consult with advantage an able essay upon "The Cultiration of Orchards and the Mraking of Cyder and Perry, by Frederick Falkner, Esq., in the fourth rolume of the 'Royal Agricultural Journal.' He adverts judiciously to the necessity of the presence of alkaline and earthy bases in the soils of all deciduous trees, and especially of such as produce acid fruits.

CXarrser. $\mathrm{C}^{20} \mathrm{H}^{14}\left(\mathbf{C}^{10} \mathbf{Z}^{14}\right)$. A hydrocarbon obtained from the essential oil of cumin (Cuminum cyminum), and from other sources. Thus it may be prepared by the action of either chloride of zine or pentachloride of phosphorus on camphor or on myristicol. The varieties of cymene have been lately studied by Dr. Wright (Journ. Chem. Soc., July 1873, p. 686).

CYMOIz. $\mathrm{C}^{21} \mathrm{H}^{14}\left(\mathbf{C}^{21} \mathbf{I n}^{28}\right)$. Syn. Camphogen. A hydrocarbon found in oil of cumin and in coal naphtha. See Carburetted Hrdrogras.

CXMOPRANז. A variety of Chrysoberyl, which exhibits a peculiar milky or opalescent appearance. When cut en cabochon, it shows a white floating band of light, and is much prized as a ring stone. Seo Chrysorrry.

CyPrivis. A pale blue mineral coloured by copper, now considered to be a rariety of idocrase. It occurs at Tellemark in Norway.

CXSTrws. An alkaloïd obtained from the laburnum (Cytisus laburnum).

END OF THE FIRST VOLUMR,

$\times$ 



\section{ENCYCLOPADIAS AND DICTIONARIES.}

CATES AND WOODWARD'S ENCYCLOP ÆDIA OF CHRONOLOGY, HISTORICAL AND BIOGRAPHICAL: Comprising the DATEs of all the Great Events of History, including Treaties, Alliances, Wars, Battles, \&tc.; Incidents in the lires of Eminent Men, and their Works, Scientific and Geographical Discoveries, Mechanical Inventions, and Social, Domestic, and Economical Improvements. Medium 8ro. pp. 1,496 (double columns, brevier), price 42s, cloth.

BRANDE'S DICTIONARY OF SCIENCE, LITERATURE, AND ART; Comprising the Definitions and Derivations of the Scientific Terms in general use, together with the History and Description of the Scientific Principles of nearly every Branch of Human Knowledge. Fourth Edition, reconstructed by the late Author and the Rev. G. W. COX, M.A. assisted by Contributors of eminent Scientific and Literary Acquirements. In Three Volnmes, modium $8 \mathrm{vo}$, price 638 .

\section{KEITH JOHNSTON'S GENERAL DICTIONARY OF GEOGRAPHY}

Descriptive, Physical, Statistical, and Historical. Forming a Complete Gazetteer of the World. New Edition, revised and corrected to the Present Date by the Author's Son, Kerth Johsston, F.R.G.S. In One Volume, medium 8ro.

[In the Spring.

\section{M'CULLOCH'S DICTIONARY OF COMMERCE AND COMMERCIAL}

NAVIGATION. New Edition, revised and corrected throughout; with Supplements containing Notices or Abstracts of the New Tariffs for the United States and Spain, and of our New Bankruptcy, Naturalisation, and Neutrality Laws. Edited by HचGH G. Rem. With Eleven Maps and Thirty Charts. In One Volume, medium 8vo, price $63 s$.

\section{URE'S DICTIONARY OF ARTS, MANUFACTURES, AND MINES.}

Serenth Edition, rewritten and enlarged by RoBert Hunt, F.R.S. Keeper of Mining Records; assisted by numerous Contributors eminent in Science and familiar with Manufactures. With about 2,600 Engravings on Wood. In Three Volumes, medium 8vo. price $£ 5.58$.

\section{GWILT'S ENCYCLOP IDDIA OF ARCHITECTURE.}

Illustrated with more than 1,100 Engravings on Wood, revised, with Alterations and considerable Additions, by WyatT PAPWorth, Fellow of the Royal Institute of British Architects. Additionally Illustrated with nearly 400 Engravings on Wood by 0 . Jewitt; and more than 100 other Woodeuts. In One Volume, medium 8vo. price 52s. 6d.

\section{CATES'S NEW DICTIONARY OF GENERAL BIOGRAPHY;}

Containing Concise Memoirs and Notices of the most Eminent Persons of all Countries, from the Earliest Ages to the Present Time. With a Classified and Chronological Index of the Principal Names. In One Volume, medium 8vo. price $21 s$.

\section{COPLAND'S DICTIONARY OF PRACTICAL MEDICINE,}

Abridged from the larger work by the Author, assisted by J. C. CopJ.AND, M.R.C.S. and throughout brought down to the present state of Medical Science. In One Volume, medium 8vo, price 368 .

A DICTIONARY OF CHEMISTRY AND THE ALLIED BRANCHES OF OTHER SCIENCES, founded on that of the late Dr. Ure. By HENRY Watts, B.A. F.R.S. F.C.S. assisted by Eminent Scientific and Practical Chemists. In Srx Voloures, medium 8vo. price $28.148 .6 d$.

SUPPLEMENTARY VOLUME of Recent Chemical Discoveries, price $42 s$. 


\section{POPULAR TREASURIES.}

CONTAINING UNIVERSALIY REQUIRED INFORMATION, IN A PORTABLE SHAPE, $\triangle N D$ CONVENIENTLY ARRANGED.

\section{THE TREASURY OF GEOGRAPHY:}

Physical, Historical, Descriptive, and Political. Comprising an Account of every Country in the World. Completed by W. Hughes, F.R.G.S. Revised and Corrected throughout; with 7 Maps and 16 Plates. Price 6s. cloth lettered; or 10s. calf.

\section{THE BIOGRAPHICAL TREASURY:}

An Alphabetical Dictionary of the Lires of all Eminent Men. Reconstructed and partly Rewritten, with about 1,000 Additional Memoirs and Notices, by W. I. R. Catris. Price 6s. cloth lettered; or 108. calf.

\section{THE TREASURY OF NATURAL HISTORY:}

Or, Popular Dictionary of Beasts, Birds, Fishes, Reptiles, Insects, and Creeping Things. Revised and supplemented by E. W. H. HoLDsworth, F.L.S. F.Z.S. With above 900 Woodeuts. Price 68. cloth lettered; or 108 . calf.

\section{THE HISTORICAL TREASURY:}

Comprising a General Introductory Outline of Universal History, Ancient and Modern, and a Series of Separate Histories of every Nation. Carefully Revised and brought down to the Present Date, by the Rev, G. W. Cox, M.A. Price 6s. cloth lettered; or 10s. calf.

\section{THE SCIENTIFIC AND LITERARY TREASURY:}

Or, Popular Cyclopædia of Science, Literature, and Art. Revised and in great part Rewritten, with upwards of 1,000 Now Articles, by JAMrs Y YTE Jomsson, Corr. M.Z.S. Price 6s. cloth lettered ; or 10s, calf.

\section{THE TREASURY OF KNOWLEDGE AND LIBRARY OF REFERENCE:}

A Copious Popular Encyclopædia of General Knowledge. Revised Edition, Corrected and Enlarged, Price 6s, cloth lettered; or 10s. calf.

\section{THE TREASURY OF BIBLE KNOWLEDGE:}

Or, Dictionary of the Books, Persons, Places, Events, and other Matters of which mention is made in Holy Seripture. By the Rev. JoHn A Yre, M.A. With about 300 Woodeuts, 15 Plates, and 5 Maps. Price 6s. cloth lettered; or 10s. calf.

\section{THE TREASURY. OF BOTANY:}

Or, Popular Dictionary of Trees, Shrubs, Plants, Flowers, and all Vegetable Growths, with which is incorporated a Glossary of Botanical Terms. Edited by J. LINDLEX, F.R.S. and T. Moore, F.L S. With 300 Woodeuts and 20 Steel Plates, In Two Parts, price 12s. cloth lettered; or 20s. calf. 

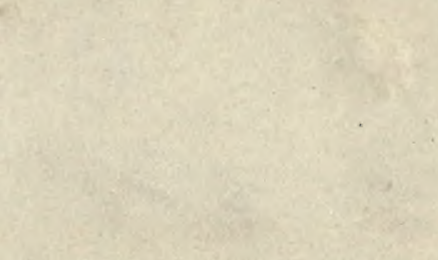

an $=$

30

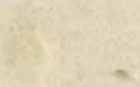

ac

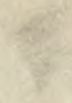

II 



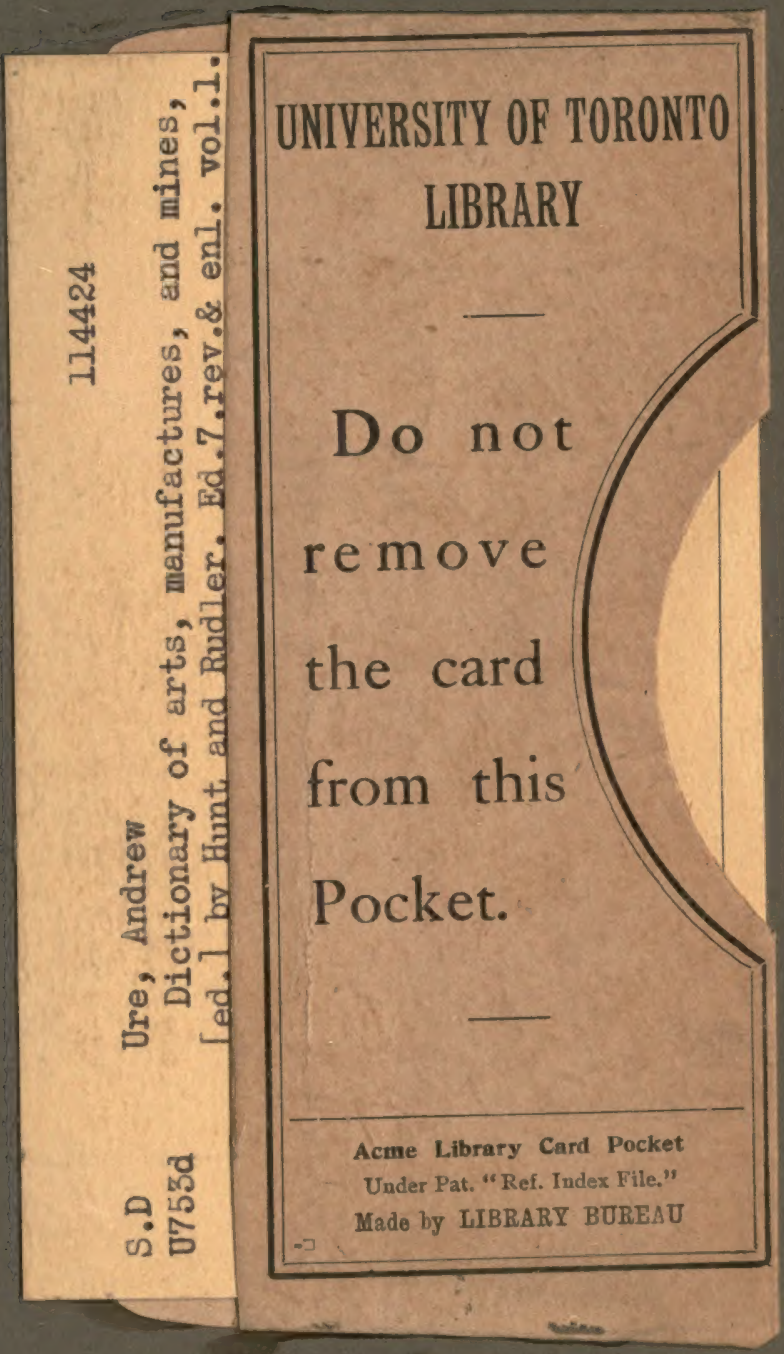




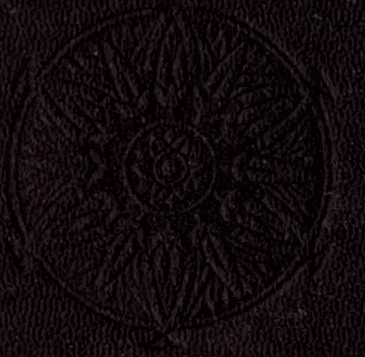

